\title{
Abstracts of the 27th Annual Conference of APASL, March 14-18, 2018, New Delhi, India
}

(C) Asian Pacific Association for the Study of the Liver 2018

\section{Plenary Clinical}

16 March 2018

$P O-C-01$

Post-treatment wisteria floribunda agglutinin-positive Mac-2binding protein combined with platelet predict hepatocellular carcinoma development in chronic hepatitis $\mathrm{C}$ patients

Ming Lun Yeh ${ }^{1}$, Chung Feng Huang ${ }^{1}$, Jee Fu Huang', Chia Yen $\overline{D a i}^{1}$, Ming Lung $\mathbf{Y u}^{1}$, Wan Long Chuang ${ }^{1}$

${ }^{1}$ Kaohsiung Medical University Hospital, Taiwan

Background: Wisteria floribunda agglutinin-positive Mac-2-binding protein (WFA+-M2BP) is a novel marker for liver fibrosis assessment. We aimed to predict the hepatocellular carcinoma (HCC) occurrence after antiviral therapy in Taiwanese patients with chronic hepatitis $\mathrm{C}(\mathrm{CHC})$ using WFA+-M2BP.

Method: Seventy patients with HCC and another 140 age, gender, sustained virological response (SVR) propensity matched, non-HCC patients were recruited. Pre- and 24 weeks post-treatment (SVR24) serum WFA+-M2BP levels were measured. The primary endpoint of the study was whether serum WFA+-M2BP level predicts HCC development.

Result: There was no difference of WFA+-M2BP level at pretreatment, but a significantly higher WFA+-M2BP level at SVR24 in patients with HCC. The accuracy of WFA+-M2BP at SVR24 in the prediction of HCC was $0.628(0.543-0.712)$ in all, 0.621 (0.512-0.730) in patients with SVR, and $0.651(0.522-0.780)$ in patients without SVR. Cox-regression hazard analysis demonstrated Platelet (HR 0.99, 95\% CI 0.982-0.997, p = 0.005) and WFA+M2BP at SVR24 (HR 1.11, 95\% CI 1.022-1.194, p = 0.012) as independent factors associated with HCC. Combined Platelet and WFA+-M2BP at SVR24, a better predictive ability for HCC development was found in all, and patients with/without $\mathrm{HCV}$ SVR24.

Conclusion: Post-treatment WFA+-M2BP, especially combined with platelet, predict $\mathrm{HCC}$ development in Taiwanese CHC patients after antiviral therapy.

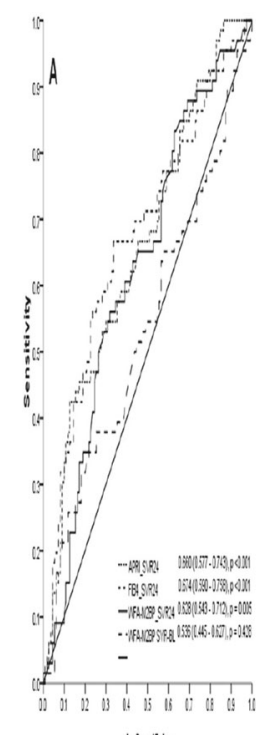

1.5pesfitit
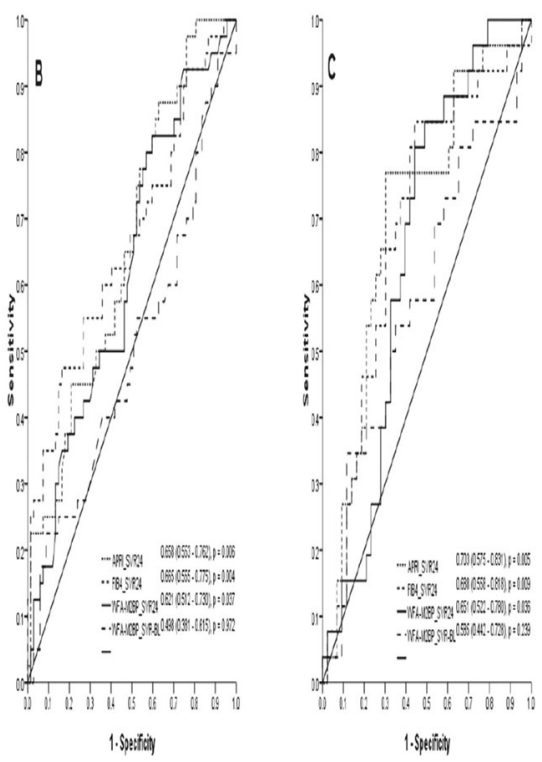
Cox-regression hazard analysis to identify factors associated with HCC

\begin{tabular}{|c|c|c|c|c|c|c|}
\hline & \multicolumn{3}{|c|}{ Crude } & \multicolumn{3}{|c|}{ Adjust } \\
\hline & HR & $95 \% \mathrm{CI}$ & $\mathrm{p}$ & $\mathrm{HR}$ & $95 \%$ CI & $\mathrm{p}$ \\
\hline Age, per 1 year increase & 1.00 & $0.975-1.034$ & 0.755 & & & \\
\hline Male gender & 1.26 & $0.767-2.064$ & 0.363 & & & \\
\hline Baseline APRI, per 1 increase & 1.11 & $0.994-1.249$ & 0.064 & & & \\
\hline Baseline FIB-4, per 1 increase & 1.05 & $0.989-1.115$ & 0.110 & & & \\
\hline Baseline platelet, per $1000 / \mathrm{mm}^{3}$ increase & 0.99 & $0.988-0.998$ & 0.004 & & & \\
\hline $\mathrm{HCV}$ genotype 1 & 0.88 & $0.545-1.404$ & 0.579 & & & \\
\hline HCV RNA, per $1 \log _{10} \mathrm{IU} / \mathrm{mL}$ increase & 0.93 & $0.742-1.159$ & 0.507 & & & \\
\hline HCV SVR & 0.68 & $0.417-1.112$ & 0.125 & & & \\
\hline APRI at $S_{V R} R_{24}$ per 1 increase & 1.28 & $1.099-1.485$ & 0.001 & 1.16 & $0.801-1.686$ & 0.428 \\
\hline FIB-4 at SVR 24 per 1 increasc & 1.09 & $1.027-1.155$ & 0.004 & 0.89 & $0.746-1.058$ & 0.185 \\
\hline Platelet at $S R_{24}$ per $1000 / \mathrm{mm}^{3}$ increase & 0.99 & $0.986-0.996$ & $<0.001$ & 0.99 & $0.982-0.997$ & 0.005 \\
\hline Baseline $\mathrm{WFA}^{+}$-M2BP, per $1 \mathrm{COI}$ increase & 1.07 & $1.006-1.148$ & 0.034 & & & \\
\hline $\mathrm{WFA}^{+} \cdot \mathrm{M} 2 \mathrm{BP}$ at $\mathrm{SVR}_{24}$, per $1 \mathrm{COI}$ increase $\mathrm{s}^{\hat{5}}$ & 1.14 & $1.063-1.214$ & $<0.001$ & 1.11 & $1.022-1.194$ & 0.012 \\
\hline$\triangle W_{F A} A^{+}-\mathrm{M} 2 B P$, per 1 COI increase ${ }^{5}$ & 1.05 & $0.958 \cdot 1.156$ & 0.284 & & & \\
\hline
\end{tabular}

\section{$\mathrm{PO}-\mathrm{C}-\mathrm{O} 2$}

The effect of Modified Alternate Day Calorie Restriction (MACR) on Non-Alcoholic Fatty Liver Disease (NAFLD)

\section{Muhammad Izzad Johari $^{1}$, Yeong Yeh Lee ${ }^{1}$, Muhammad Ilham Abdul Hafidz $^{1}$}

${ }^{1}$ Malaysian Society of Gastroenterology and Hepatology, Malaysia

Background: Non-alcoholic fatty liver diseases are an important cause of morbidity and mortality due to adverse cardiovascular outcomes and also liver-related complications. Currently, there is no approved therapy for non-alcoholic fatty liver diseases; weight reduction is typically recommended. Modified alternate day calorie restriction is more feasible to the patient compared to daily calorie restriction; however, no trials have been done in non-alcoholic fatty liver diseases patients focusing on changes in liver fibrosis and steatosis

Method: The aim of this study is to evaluate the effect of modified alternate day calorie restriction on the changes in liver steatosis and fibrosis, anthropometry as well as biochemical parameters in nonalcoholic fatty liver diseases patients after 8 weeks of modified alternate day calorie restriction. We performed a prospective study involving adult gastroenterology patients with non-alcoholic fatty liver disease from August 2015 to July 2016 in Hospital Universiti Sains Malaysia.

Result: A total of 105 patients were screened, 41 consented but 11 withdrew and 30 participants (mean age 43.9 years, BMI $31.5 \mathrm{~kg} / \mathrm{m} 2$, males $70 \%$, diabetes $53 \%$ ) completed the study. With 8 week MACR, significant reductions were observed of grading of liver steatosis
(40\% reduction in those with moderate steatosis, $\mathrm{P}=0.001)$, SWE (mean difference 0.9, $\mathrm{P}=0.001$ ), BMI (mean difference $0.6 \mathrm{~kg} / \mathrm{m} 2, \mathrm{P}$ $=0.003)$, glucose (median difference $0.3 \mathrm{mmol} / \mathrm{L}, \mathrm{P}=0.01$ ), ALT (median difference $20.5 \mathrm{U} / \mathrm{L}, \mathrm{P}=0.001$ ) and AST (median difference $9 \mathrm{U} / \mathrm{L}, \mathrm{P}=0.002$ )

Conclusion: In summary, the results indicate that modified alternate day calorie restriction appear to be an effective diet strategy to help reducing the risk of progression for non-alcoholic fatty liver diseases especially in patients with moderate steatosis and mild fibrosis.

\section{PO-C-03}

Pharmacogenetic testing for warfarin use in patients with BuddChiari syndrome-Does it make a clinical difference?

Akash Shukla ${ }^{1}$, Akash Shukla ${ }^{2}$, Vinit Kahalekar ${ }^{3}$, Abhinav Jain ${ }^{3}$, Shobna Bhatia $^{3}$, Shital Bendkhale ${ }^{3}$, Nithya Gogtay ${ }^{3}$, Urmila Thatte $^{3}$

${ }^{1}$ LTMMC LTMGH, India; ${ }^{2}$ LTMMC \& LTMGH, India; ${ }^{3}$ Seth GSMC \& KEMH, India

Background: Medical management using anticoagulation is recommended in all patients with Budd-Chiari syndrome [BCS]. Most commonly used anticoagulant is warfarin and the patient's genotype (VKORC and Cyp2C9) is known to be a major determinant of warfarin dose requirements.

Method: Patients diagnosed with BCS underwent genotyping for three single nucleotide polymorphisms [SNPs]-two for the liver enzyme CYP2C9 and one for the VKORC1 haplotype. Based on genotyping results, the patients were divided into two groups- those with mutations and those without. Between group analysis was carried out for warfarin dose, INR values and bleeding complications. A crude odds ratio [crude OR] was also derived for the association between bleeding and presence or absence of mutant alleles.

Result: Eighty patients (35 males [43.8\%]) with BCS underwent genetic testing. The mean[SD] age of the patients was 27.47[8.93] years. The median [range] duration of disease was 5 months [0.5150]. 37 [46.2\%] had at least one mutation. No significant difference was seen both in dose requirements and INR between those with and without the SNPs. Those with mutant SNPs had thrice the odds of bleeding relative to those without $\{14 / 37 v s .7 / 43, \mathrm{p}=0.04$, crude $\mathrm{OR}$ $[95 \% \mathrm{CI}] 3.13[1.09,8.9]$

Table 1 Genotyping analysis [Single Nucleotide Polymorphisms of CYP2C9 and VKORC1] of $n$ : 80 BCS patients

\begin{tabular}{l|l|l|l|l|}
\hline Polymorphisms & CYP2C9*1/*1 & CYP2C9*1/*2 & CYP2C9*1/*3 & Total \\
\hline VKORC GG & 43 & 4 & 11 & 58 \\
\hline VKORC AG & 15 & 3 & 4 & 22 \\
\hline VKORC AA & 0 & 0 & 0 & 0 \\
\hline Total & 58 & 7 & 15 & 80 \\
\hline
\end{tabular}


Table 2 Characteristics of patients with hemorrhagic complications

\begin{tabular}{|c|c|c|c|c|c|c|}
\hline & $\begin{array}{l}\text { Sr. } \\
\text { No. }\end{array}$ & Сур2С9 & VKORC & $\begin{array}{l}\text { Dose of } \\
\text { warfarin }\end{array}$ & $\begin{array}{l}\text { Hemorrhagic } \\
\text { complication }\end{array}$ & Treatment given \\
\hline \multirow{6}{*}{$\begin{array}{l}\text { Patients } \\
\text { having } \\
\text { major bleed }\end{array}$} & 1 & ${ }^{*} 1 / * 2(\mathrm{~m})$ & $A G(m)$ & 4 & $\begin{array}{l}\text { Intracerebral } \\
\text { bleed }\end{array}$ & FFP \\
\hline & 2 & $* 1 / * 3(\mathrm{~m})$ & $\mathrm{GG}(\mathrm{w})$ & 5 & $\begin{array}{l}\text { Intracerebral } \\
\text { bleed }\end{array}$ & FFP \\
\hline & 3 & ${ }^{*} 1 / *^{*} 1(w)$ & $A G(m)$ & 2 & Hematemesis & FFP and EVL \\
\hline & 4 & ${ }^{*} 1 /{ }^{*} 1(w)$ & $\mathrm{GG}(\mathrm{w})$ & 2 & Menorrhagia & FFP \\
\hline & 5 & $* 1 / * 1(w)$ & $\mathrm{GG}(w)$ & 3 & $\begin{array}{l}\text { Muscular } \\
\text { hematomas }\end{array}$ & FFP \\
\hline & 6 & $* 1 / * 3(\mathrm{~m})$ & $A G(m)$ & 7.5 & Malena & FFP and EVL \\
\hline \multirow{14}{*}{$\begin{array}{l}\text { Patients } \\
\text { having } \\
\text { minor bleed }\end{array}$} & 7 & $* 1 / * 1(w)$ & $\mathrm{GG}(\mathrm{w})$ & 5 & Menorrhagia & $\begin{array}{l}\text { Antifibrinolytic } \\
\text { drug }\end{array}$ \\
\hline & 8 & ${ }^{*} 1 / * 3(\mathrm{~m})$ & $G G(w)$ & 1.5 & $\begin{array}{l}\text { Posttraumatic } \\
\text { ecchymoses }\end{array}$ & $\begin{array}{l}\text { Temporary } \\
\text { stoppage of Warf }\end{array}$ \\
\hline & 9 & $*_{1} 1 /{ }^{*} 1(w)$ & $G G(w)$ & 5 & Menorrhagia & $\begin{array}{l}\text { Antifibrinolytic } \\
\text { drug }\end{array}$ \\
\hline & 10 & $* 1 / * 1(w)$ & $G G(w)$ & 4 & Menorrhagia & Shifted to Aspirin \\
\hline & 11 & ${ }^{*} 1 /{ }^{*} 1(w)$ & $A G(m)$ & 5 & Bleeding gums & $\begin{array}{l}\text { Temporary } \\
\text { stoppage of Warf }\end{array}$ \\
\hline & 12 & $*_{1} 1 / *_{1}(w)$ & $\mathrm{GG}(\mathrm{w})$ & 7.5 & Bleeding gums & $\begin{array}{l}\text { Temporary } \\
\text { stoppage of Warf }\end{array}$ \\
\hline & 13 & $* 1 / * 1(w)$ & $A G(m)$ & 1 & Epistaxis & $\begin{array}{l}\text { Temporary } \\
\text { stoppage of Warf }\end{array}$ \\
\hline & 14 & $*^{*} 1 /{ }^{*} 1(w)$ & $A G(m)$ & 8 & Bleeding gums & $\begin{array}{l}\text { Temporary } \\
\text { stoppage of Warf }\end{array}$ \\
\hline & 15 & $* 1 / * 1(w)$ & $\mathrm{GG}(\mathrm{w})$ & 2.5 & Bleeding gums & $\begin{array}{l}\text { Temporary } \\
\text { stoppage of Warf }\end{array}$ \\
\hline & 16 & $* 1 / * 3(\mathrm{~m})$ & $G G(w)$ & 4 & $\begin{array}{l}\text { Pulmonary } \\
\text { hemorrhage }\end{array}$ & $\begin{array}{l}\text { Temporary } \\
\text { stoppage of Warf }\end{array}$ \\
\hline & 17 & ${ }^{*} 1 / * 2(\mathrm{~m})$ & $\mathrm{GG}(w)$ & 4 & Hematemesis & $\begin{array}{l}\text { Temporary } \\
\text { stoppage of Warf }\end{array}$ \\
\hline & 18 & $* 1 / * 1(w)$ & $A G(m)$ & 2.5 & Bleeding gums & $\begin{array}{l}\text { Temporary } \\
\text { stoppage of Warf }\end{array}$ \\
\hline & 19 & ${ }^{*} 1 / * 3(\mathrm{~m})$ & $\mathrm{GG}(\mathrm{w})$ & 4 & Bleeding gums & $\begin{array}{l}\text { Temporary } \\
\text { stoppage of Warf }\end{array}$ \\
\hline & 20 & ${ }^{*} 1 / * 3(\mathrm{~m})$ & $\mathrm{GG}(\mathrm{w})$ & 2.5 & $\begin{array}{l}\text { Hematoma in } \\
\text { muscle }\end{array}$ & FFP \\
\hline
\end{tabular}

Conclusion: Presence of mutants in VKORC or CYP2C9 is associated with increased risk of bleeding. Genotyping for two SNPs of CY2C9 and one for the VKORC1 haplotype prior to initiating warfarin therapy in patients with BCS may be useful.

\section{$\mathrm{PO}-\mathrm{C}-\mathrm{O} 4$}

Early HCC occurrence in $\mathrm{HCV}$ patients treated with direct acting antivirals: all that glitters is not gold

Luca Rinaldi ${ }^{1}$, Alessandro Parrella ${ }^{3}$, Massimo De Luca ${ }^{2}$, Antonio Ascione $^{4}$, Guido Piai ${ }^{6}$, Nicola Coppola ${ }^{1}$, Antonio Izzi $^{3}$, Filomena Morisco $^{5}$, Giovanna Valente ${ }^{6}$, Maria Guarino ${ }^{5}$, Luca Rinaldi ${ }^{1}$, Luigi Elio Adinolfi ${ }^{1}$

${ }^{1}$ Université della Campania Luigi Vanvitelli, Italy; ${ }^{2}$ Cardarelli Hospital, Italy; ${ }^{3}$ Cotugno Hospital, Italy; ${ }^{4}$ Fatebenefratelli Hospital, Italy; ${ }^{5}$ Federico II University, Italy; ${ }^{6}$ S.Anna e s. Sebastiano Hospital, Italy

Background: This study aimed to evaluate rate and risk factors of early $\mathrm{HCC}$ occurrence in a $\mathrm{HCV}$ population regularly followed during and after antiviral treatment with DAAs.

Method: According to Italian ministerial guidelines for DAAs treatment, 1022 consecutive HCV patients without HCC history and treated with DAAs were enrolled. Abdominal ultrasound was performed before starting antiviral therapy and thereafter repeated at the end of therapy, 12 weeks and six months later. The HCC diagnosis was performed according to international guidelines.

Result: 985 patients completed the 36 weeks follow-up after the end of treatment. In that cohort, a sofosbuvir-based regimen was administered in $74.9 \%$, while ribavirin was used in $71.6 \%$ (of those patients $71.6 \%$ had ribavrin in their schedule). A successful treatment outcome with SVR-12 achievement was documented in 966 patients $(98.2 \%)$. During the post treatment follow-up, HCC was detected in 35 patients with a cumulative incidence rate of $3.55 \%$. At multivariate analysis, five variables resulted independently associated with HCC development: cirrhosis (p 0.034, OR: 8.83, 95\% CI: 1.18-66.31), therapeutic schedule including sofosbuvir without ribavirin ( $\mathrm{p} 0.0001$, OR: 6.675, 95\% CI: 3.25-13.70), Child Pugh B class (p 0.04, OR: 3.32, 95\% CI: 1.47-7.53), male gender(p 0.014, OR: 2.69 , 95\% CI: 1.23-5.91) and presence of diabetes (p 0.05, OR: 2.23, 95\% CI: $1.00-$ 5.00).

Conclusion: Consistent with our data, early HCC occurrence seems to be more frequently correlated to sofosbuvir-based therapy without ribavirin; conversely, the use of this latter antiviral seems to be protective. Therefore a careful follow-up should be done in DAAs, particularly in patients without ribavirin in their treatment schedule.

\section{$\mathrm{PO}-\mathrm{C}-05$}

Early HCC occurrence in $\mathrm{HCV}$ patients treated with direct acting antivirals: all that glitters is not gold

Luca Rinaldi ${ }^{1}$, Alessandro Parrella ${ }^{3}$, Massimo De Luca ${ }^{2}$, Antonio Ascione $^{4}$, Guido Piai ${ }^{6}$, Nicola Coppola ${ }^{1}$, Antonio Izzi $^{3}$, Filomena Morisco $^{5}$, Giovanna Valente ${ }^{6}$, Maria Guarino ${ }^{5}$, Luca Rinaldi ${ }^{1}$, Luigi Elio Adinolfi ${ }^{1}$

${ }^{1}$ Université della Campania Luigi Vanvitelli, Italy; ${ }^{2}$ Cardarelli Hospital, Italy; ${ }^{3}$ Cotugno Hospital, Italy; ${ }^{4}$ Fatebenefratelli Hospital, Italy; ${ }^{5}$ Federico II University, Italy; ${ }^{6}$ S.Anna e s. Sebastiano Hospital, Italy

Background: This study aimed to evaluate rate and risk factors of early $\mathrm{HCC}$ occurrence in a $\mathrm{HCV}$ population regularly followed during and after antiviral treatment with DAAs

Method: According to Italian ministerial guidelines for DAAs treatment, 1022 consecutive HCV patients without HCC history and treated with DAAs were enrolled. Abdominal ultrasound was performed before starting antiviral therapy and thereafter repeated at the end of therapy, 12 weeks and six months later. The HCC diagnosis was performed according to international guidelines.

Result: 985 patients completed the 36 weeks follow-up after the end of treatment. In that cohort, a sofosbuvir-based regimen was administered in $74.9 \%$, while ribavirin was used in $71.6 \%$ (of those patients $71.6 \%$ had ribavrin in their schedule). A successful treatment outcome with SVR-12 achievement was documented in 966 patients $(98.2 \%)$. During the post treatment follow-up, HCC was detected in 35 patients with a cumulative incidence rate of $3.55 \%$. At multivariate analysis, five variables resulted independently associated with HCC development: cirrhosis (p 0.034, OR:8.83, 95\% CI: 1.18 66.31), therapeutic schedule including sofosbuvir without ribavirin ( $\mathrm{p}$ 0.0001, OR:6.675, 95\% CI: 3.25-13.70), Child Pugh B class (p 0.04, OR:3.32, 95\% CI: 1.47-7.53), male gender(p 0.014, OR: $2.69,95 \%$ CI: $1.23-5.91)$ and presence of diabetes (p 0.05, OR: $2.23,95 \% \mathrm{CI}$ : 1.00-5.00)

Conclusion: Consistent with our data, early HCC occurrence seems to be more frequently correlated to sofosbuvir-based therapy without ribavirin; conversely, the use of this latter antiviral seems to be protective. Therefore a careful follow-up should be done in DAAs, particularly in patients without ribavirin in their treatment schedule 
PO-C-O6

Usefulness of lactate-free Asian Pacific Association for the Study of Liver acute-on-chronic liver failure (ACLF) Research Consortium (AARC) "ACLF score for predicting short-term mortality in patients with alcoholic liver disease

Dong Joon Kim ${ }^{1}$, Tae Yeob Kim ${ }^{12}$, Ashok Kumar Choudhury ${ }^{11}$, Ji Dong Jia $^{5}$, Shiv K Sarin ${ }^{11}$, Priyanka Jain ${ }^{11}$, Irene Paulson ${ }^{11}$, Mamum Al Mahtab ${ }^{4}$, Salimur Rahman ${ }^{4}$, Harshad Devarbhavi ${ }^{14}$, Zhongping Duan', Chen Yu' ${ }^{6}$, CE Eapen', Ashish Goel ${ }^{9}$, Q Ning ${ }^{15}$, George K Lau ${ }^{10}, \mathrm{P} \mathrm{N} \mathrm{Rao}^{3}$, Diana Alcantarapayawal ${ }^{7}$, A. Kadir Dokmeci $^{2}$, Man Fung Yuen ${ }^{13}$, V G Mohan Prasad ${ }^{16}$, Osamu Yokosuka ${ }^{8}$

${ }^{1}$ Hallym University College of Medicine, Korea, Republic of; ${ }^{10}$ Humanity and Health Medical Group, China; ${ }^{11}$ Institute of Liver and Biliary sciences, India; ${ }^{12}$ Leeheart Clinic, Korea, Republic of; ${ }^{13}$ Queen Mary Hospital, China; ${ }^{14}$ St John Medical College, India; ${ }^{15}$ Tongji Hospital, Tongji Medical College, China; ${ }^{16}$ VGM Hospital, India; ${ }^{2}$ Ankara University School of Medicine, Turkey; ${ }^{3}$ Asian Institute of Gastroenterology, India; ${ }^{4}$ Bangabandhu Sheikh Mujib Medical Univerity, Bangladesh; ${ }^{5}$ Beijing Friendship Hospital, Capital University, China; ${ }^{6}$ Beijing Youan Hospital, Capital Medical University, China; ${ }^{7}$ Cardinal Santos Medical Center, Philippines; ${ }^{8}$ Chiba University, Japa; ${ }^{9} \mathrm{CMC}$, India

Background: Asian Pacific Association for the Study of Liver (APASL) acute-on-chronic liver failure (ACLF) Research Consortium (AARC) proposed new prognostic scoring system of ACLF including hepatic encephalopathy, bilirubin, international normalized ratio (INR), creatinine, and lactate. However, lactate is not routinely checked in clinical practice of patient with ACLF in some countries. Therefore, we aimed to investigate the predictive accuracy of lactatefree AARC-ACLF score for predicting short-term mortality in patients with alcoholic liver disease (ALD).

Method: A total of 749 ALD patients who had liver failure (bilirubin $=5 \mathrm{mg} / \mathrm{dL}$ and INR $=1.5$ ) in AARC database were investigated. Diagnostic performances for short-term mortality were compared according to the area under receiver operating characteristic (AUROC) curve. Predictive accuracy of AARC-ACLF score and lactate-free AARC-ACLF score were compared with Child-TurcottPugh (CTP) score, model for end-stage liver disease (MELD), and MELD-Sodium (Na), chronic liver failure-sequential organ failure assessment (CLIF-SOFA) scores. The findings were validated in Korean ACLF cohort (212 ALD patients with liver failure and 108 ALD patients with ACLF according to AARC definition).

Result: Of 708 patients, $286(40.4 \%)$ and $363(51.3 \%)$ patients died within 30 days and 90 days, respectively. The area under receiver operating characteristics curve (AUC) of AARC-ACLF, DF, ABIC, GAHS, CTP, MELD, and MELD-Na was 0.752 (0.705-0.799), 0.630 (0.575-0.685), $0.658 \quad(0.604-0.711), 0.577 \quad(0.523-0.631), \quad 0.641$ (0.589-0.694), 0.705 (0.653-0.756), 0.703 (0.651-0.755), respectively, for 30-day mortality. The AUC of various prognostic scores for the prediction of 90-day mortality is similar. The performance of AARCACLF was superior to that of DF, ABIC, GAHS, CTP, while comparable to that of MELD and MELD-Na in predicting short-term mortality. According to AARC-ACLF grades, short-term cumulative survivals was statistically different (30-day, 82.4, 70.4, and $35.3 \%$, p $<0.001$; 90-day, 76.9, 56.0, and 26.4\%, p <0.001).

Conclusion: Compared to the previous AH prognostic scores, AARC-ACLF score and grades are simple and useful for predicting the short-term mortality in patients with $\mathrm{AH}$. Further studies are needed to confirm these implications.
Plenary Basic

\section{March 2018}

$P O-B-01$

Mucosal-Associated invariant $\mathbf{T}$ cells (MAIT) are functionally impaired during chronic liver diseases and promote fibrosis

Pushpa Hegde ${ }^{1}$, Emmanuel Weiss', Valrie Paradis ${ }^{1}$, Jinghong Wan $^{1}$, Morgane Mabire ${ }^{1}$, Sukriti Bajewa ${ }^{1}$, Pierre Emmanuel Rautou $^{2}$, Miguel Albuquerque ${ }^{1}$, Olivia Picq ${ }^{1}$, Abhishak Gupta ${ }^{1}$, Gladys Ferrerre ${ }^{1}$, Helene Gilgenkrantz ${ }^{1}$, Badr Kiaf ${ }^{3}$, Amine Toubal $^{3}$, Lucie Beaudoin ${ }^{3}$, Philippe Leterron ${ }^{1}$, Richard Moreau ${ }^{1}$, Agnes Lehuen ${ }^{3}$, Sophie Lotersztajn ${ }^{1}$

${ }^{1}$ Inserm UMR-1149, Centre de Recherche sur 1' Inflammation, 75018 Paris, France, France; ${ }^{2}$ Département Hospitalo-Universitaire (DHU) UNָITY, Service d'patologie, HÃpital Beaujon, Assistance PubliqueHÃ́pitaux de Paris, Clichy, France, France; ${ }^{3}$ Inserm U-1016, CNRS UMR 8104, Institut Cochin, université Paris-Descartes, Paris, France, France

Background: Chronic inflammation and immune dysfunction are major driving forces of chronic liver disease progression to fibrosis. Mucosal-Associated Invariant T (MAIT) cells are unconventional $\mathrm{T}$ cells characterized by the invariant TCR-chain, Va7.2 in humans (V?19 in mice) and restricted by MR1. Owing to their involvement in the pathogenesis of various pathologies, we hypothesize that MAIT cells may be involved in immune-dysfunction associated with liver fibrosis.

Method: MAIT (CD161hi V?7.2+) cells were analyzed in the PBMC of cirrhotic patients (decompensated $(n=59)$, compensated $(n=15)$ ) of alcoholic and non-alcoholic origin, in comparison with healthy donors $(n=47)$ by FACS. Liver MAIT cells were analyzed by FACS and immunofluorescence labeling. Functional relevance of MAIT cells was studied in mice deficient (MR1-/-) or enriched (Va19TCRTg) in MAIT cells, following chronic challenge with CCl4 or bile duct ligation. Interactions between human MAIT cells and human hepatic myofibroblasts (HMF) were studied in in-vitro coculture experiments

Result: MAIT cell frequency was reduced in PBMC from cirrhotic patients and was independent of cirrhosis etiology and severity. Peripheral MAIT cells from cirrhotic patients displayed an activated phenotype with increased CD25 and CD69, and produced increased levels of intracellular IL-17 and granzyme B. Despite their reduced frequency, MAIT cells in cirrhotic livers preferentially accumulated along the fibrotic septa. However cirrhotic liver MAIT cells showed an activated phenotype with increased intracellular IL-17 production. Moreover, cirrhotic MAIT cells displayed exhaustion features, as revealed by increased expression of TIM- 3 and PD- 1 compared to MAIT cells from control livers. In vivo, following chronic administration of $\mathrm{CCl} 4$ or bile duct ligation, Va19TCRTg mice showed exacerbated fibrosis and accumulation of fibrogenic cells, while MR1-/- mice were resistMAIT cell frequency was significantly reduced in PBMC from cirrhotic patients and was independent of cirrhosis etiology and decompensation. Moreover, peripheral MAIT cells from cirrhotic patients displayed an activated phenotype with up-regulated surface expression of CD25 and CD69, and produced higher levels of intracellular IL-17 and granzyme B. MAIT cell frequency was significantly reduced in cirrhotic livers compared to that of controls. Of note, despite their reduced frequency, MAIT cells preferentially accumulated along the fibrotic septa with a discrete incidence in the sinusoids. However cirrhotic liver MAIT cells displayed an activated phenotype compared to those from healthy livers and displayed exhaustion features as compared to MAIT cells from 
control livers. In vivo, following chronic administration of $\mathrm{CCl} 4$, or bile duct ligation, V $\alpha 19 T C R T g$ mice showed exacerbated fibrosis and accumulation of fibrogenic cells, while MR1-/- mice were resistant. Profibrogenic properties of MAIT cells were related to mitogenic effects on HMF in vitro, as demonstrated in co-culture experiments. ant. Profibrogenic properties of MAIT cells were related to mitogenic effects on HMF, as demonstrated in co-culture experiments. HMF expressed MR1, and MAIT cell-induced proliferation of HMF was reduced by an anti MR1 antibody.

Conclusion: Our results reveal a novel pro-fibrogenic role for MAIT cells during chronic liver injury, via direct effects on the interaction with hepatic myofibroblasts and thus identify MAIT cells as a novel target for anti-fibrogenic therapy.

PO-B-O2

Pregnane $X$ receptor activation by Ginkgolide-A attenuates inflammation and improves tight junction integrity in experimental cirrhosis

\section{$\underline{\text { Sundhar Mohandas }}^{1}$, Balasubramaniyan Vairappan ${ }^{1}$}

${ }^{1}$ Jawaharlal Institute of Postgraduate Medical Education and Research (JIPMER), India

Background: Pregnane X receptor (PXR) is a ligand-activated transcription factor and nuclear receptor that is expressed ubiquitously along the gut-liver-axis. Previous studies in inflammatory bowel disorders have implicated PXR in maintaining tight junction protein integrity and countering inflammation. However, the role of PXR activation in liver cirrhosis has not been explored. Ginkgolide-A (GA), a terpene trilactone present in Gingko Biloba extract, is a natural ligand of both rodent and human PXR. The aim of this study was to investigate the effect of GA on inducing PXR pathway and preserving tight junction integrity in a mouse model of $\mathrm{CCl} 4$ induced liver cirrhosis.

Method: Male Swiss albino mice were administered with CCl4 (0.5 $\mathrm{ml} / \mathrm{kg}$ body weight, i.p.) in corn oil twice a week for over the period of 12 weeks. Following the induction of ascites, animals were randomized and administered $100 \mathrm{mg} / \mathrm{kg}$ bodyweight of Ginkgolide-A to control and cirrhotic animals through oral gavage daily for next 2 weeks. At termination, serum and liver tissue were collected for biochemical and molecular studies

Result: Compared to naïve mice, liver function parameters such as AST, ALT and bilirubin were significantly elevated whilst albumin was significantly reduced in serum of CCL4 induced cirrhotic mice. Following treatment with GA to cirrhotic mice show significantly decreased serum AST, ALT and bilirubin and significantly increased albumin levels. Protein expression of PXR, CYP3A and occludin were found to be significantly downregulated in CCL4 induced cirrhotic mice when compared to naïve mice. Treatment with GA significantly induced the expression of PXR, CYP3A and Occludin. Moreover, when compared to naïve mice, overt expression of NF-kB was observed in CCL4 induced cirrhotic mice. Following GA treatment to CCL4 mice, significant downregulation in the expression of NF-kB was observed.

Conclusion: In conclusion, our results demonstrate that GA might have a therapeutic effect in CCL4 induced liver cirrhosis by countering inflammation and protecting the expression of $\mathrm{TJ}$ proteins, through the activation of PXR.
PO-B-03

A systematic comparison reveals dynamic differences in early adaptive immune responses of acute-resolving versus chronic HBV replication

$\underline{\text { Qin Wang }}^{1}$, Yanan Liu ${ }^{1}$, Jinzhuo Luo ${ }^{1}$, Qing Yu ${ }^{1}$, Shangqing $\overline{\text { Yang }}^{1}$, Lu Wang ${ }^{1}$, Yinping Lu ${ }^{1}$, Xuemei Feng ${ }^{1}$, Xuecheng Yang ${ }^{1}$, Ulf Dittmer', Mengji Lü ${ }^{2}$, Dongliang Yang', Jia Liu ${ }^{1}$

${ }^{1}$ Union Hospital, Tongji Medical College, Huazhong University of Science and Technology, China; ${ }^{2}$ University Hospital of Essen, University of Duisburg-Essen, Germany

Background: Chronic hepatitis B virus (HBV) infection has been characterized by lack of effective adaptive immune responses which are vital for the viral clearance. However, very little is known about the dynamics of adaptive immune responses during the early phase of chronic HBV infection especially in the spleen and the liver.

Method: We kinetically characterized the feature differences of adaptive immunity, including the frequencies, phenotypes and function of antigen presenting cells and T cells in the spleen, the PBMCs and the liver, of chronic versus acute-resolving HBV replication by using hydrodynamic injection (HI) mouse model.

Result: We found that, both mice with acute-resolving $\mathrm{HBV}$ replication (AR mice) and mice with chronic HBV replication ( $\mathrm{CH}$ mice) showed early splenomegaly accompanied by $\mathrm{T}$ cell expansion in spleen but not in liver after $\mathrm{HI}$. Interestingly, $\mathrm{CH}$ mice showed early and continuous increase of HBV specific CD8 + T cells in spleen in a comparable extent to that in the AR mice. However, the splenic $\mathrm{T}$ cells of $\mathrm{CH}$ mice showed no activation phenotype compared with those in AR mice. Besides, increases of activated effector CD8 + T cells in PBMCs and liver at later time points were only observed in $\mathrm{AR}$ mice but not $\mathrm{CH}$ mice. $\mathrm{CH}$ mice also showed insufficient dendritic cells (DCs) expansion in spleen and increased DCs PD-L1 expression in liver compared to AR mice. Adoptive transferring total splenocytes or splenic CD8+ T cells of AR mice to $\mathrm{CH}$ mice revealed that their ability in breaking $\mathrm{HBV}$ tolerance varies at different stages of HBV clearance. Moreover, inducing functional activation of endogenous $\mathrm{HBV}$ specific $\mathrm{CD} 8+\mathrm{T}$ cells of $\mathrm{CH}$ mice was achieved through adoptive transfer of splenocytes from AR mice. Our results suggest that early $\mathrm{T}$ cell priming and expansion firstly happen in periphery after HBV antigen exposure in both acute-resolving and chronic replication.

Conclusion: The paucity of T cell activation, and following migration and liver infiltration is a key feature of the adaptive immune responses during the early phase of chronic HBV replication, which is probably caused by the dysfunction of DCs. Fully viral clearance and activation of endogenous CD8+ T cells are achievable during this phase through adoptive transfer of splenocytes from AR mice.

\section{$P O-B-04$}

Circulating dendritic cell subsets and serum interleukin-12 levels in schistosomal hepatic fibrosis: relation to severity of liver disease and renal injury

\section{Hayam El Aggan ${ }^{1}$, Mona Salem ${ }^{1}$, Wessam El Gendy ${ }^{1}$, Wageh El $\overline{\text { Gebaly }}^{1}$, Eman El Gohary ${ }^{1}$ \\ ${ }^{1}$ Faculty of Medicine, University of Alexandria, Egypt}

Background: Chronic infection with schistosomiasis is associated with downregulation of $\mathrm{T}$ cell immune responses that require activation by innate immune cells like dendritic cells (DCs). The present work was designed to study DC subsets (myeloid and plasmacytoid) and serum interleukin (IL)-12 levels in peripheral blood in patients with schistosomal hepatic fibrosis (SHF) in relation to severity of liver disease and renal injury. 
Method: Forty five patients with SHF and 15 healthy subjects were included in the study. The percentages of $\mathrm{CD} 11 \mathrm{c}+$ myeloid and CD123 + plasmacytoid DC subsets in peripheral blood were quantified using 3-color flow cytometry. The serum levels of IL-12p70 heterodimer were measured using enzyme-linked immunosorbent assay. Renal injury was assessed by urinary albumin excretory rate (UAER). Renal biopsies from patients with macroalbuminuria were examined by immunohistochemical staining for DCs and angiogenesis using antibodies against OX62 and factor VIII-related antigen respectively. Result: The percentages of circulating CD11c+ and CD123+ DC cells, CD11c+DC/CD123+DC ratio and serum IL-12 levels showed significant decreases in patients with SHF compared with healthy subjects and in patients with micro- and macro-albuminuria compared with patients with normo-albuminuria $(P<0.01)$. Renal tissues from patients with macroalbuminuria showed significant increases in the number of DCs and angiogenesis compared with normal renal tissues $(P<0.01)$. The percentages of circulating DC subsets and serum IL12 levels were inversely correlated with Child-Pugh score, Model of End Stage Liver Disease score, number of renal DCs, renal angiogenesis and UAER and were positively correlated with creatinine clearance. The number of renal DCs showed positive correlations with UAER and renal angiogenesis and negative correlation with creatinine clearance $(P<0.05)$.

Conclusion: Dendritic cells, particularly myeloid DC subset, and IL12 production seem to play a role in the progression of liver disease and renal injury in SHF. DC-based vaccines may provide a potential new goal for immunotherapy in SHF.

\section{$P O-B-05$}

Disruption of myeloid PTEN induces indoleamine 2,3-dioxygenase activation and alleviates acetaminophen-induced liver injury in mice

\section{Longfeng Jiang ${ }^{1}$, Youde $\mathrm{Yan}^{1}$, Michael $\mathrm{Ke}^{3}$, Ping Shi ${ }^{1}$, Bibo $\mathrm{Ke}^{2}$, Jun Li ${ }^{1}$}

${ }^{1}$ The first affiliated hospital with Nanjing Medical University, China; ${ }^{2}$ David Geffen School of Medicine at UCLA, China; ${ }^{3}$ David Geffen School of Medicine at UCLA, United States

Background: Acetaminophen (APAP)-induced hepatotoxicity is initially triggered by a P450-generated toxic metabolite and subsequently mediated by activated liver Kupffer cells and neutrophils. This study was designed to investigate the role of myeloid PTEN in the regulation of innate immune responses in APAP-triggered liver inflammation.

Method: Using a mouse model of APAP-induced liver injury, myeloid specific PTEN knockout (PTENM-KO) and floxed PTEN (PTENFL/FL) mice were injected intraperitoneally with APAP or PBS. Some animals were injected with a mannose-mediated indoleamine 2,3-dioxygenase (IDO) siRNA or non-specific (NS) siRNA. For the in vitro study, bone marrow-derived macrophages (BMMs) from these conditional knockout mice were transfected with IDO siRNA and NS siRNA and stimulated with LPS.

Result: PTENM-KO mice were resistant to APAP-induced liver damage, with significantly decreased ALT levels, increased phosphorylation of Akt (p-Akt) and Stat3 (p-Stat3), and IDO expression but diminished HMGB1, TLR4, and NF-kB after APAP challenge. Myeloid PTEN deficiency reduced macrophage and neutrophil accumulation and hepatocellular apoptosis in APAP-challenged livers. However, knockdown of IDO with siRNA treatment in PTENMKO mice reversed myeloid PTEN deficiency-mediated protection in APAP-triggered liver injury, as evidenced by increased APAP-induced liver damage and ALT levels. Unlike in PTENFL/FL controls, PTENM-KO BMMs augmented p-Akt, p-Stat3, IDO and reduced
HMGB1 and TLR4, with diminished mRNA levels of TNF- $\alpha$, IL-1 $\beta$, and IL-6 following LPS stimulation. However, disruption of IDO with siRNA treatment in PTENM-KO BMMs increased HMGB1, TLR4, and NF-kB activity. Moreover, IDO siRNA knockdown increased M1 (Nos2+) while reducing M2 (Mrc-1/Arg-1+) phenotype in LPSstimulated PTENM-KO BMMs.

Conclusion: These results demonstrate that myeloid PTEN deficiency inhibits HMGB1/TLR4 activation and alleviates hepatocellular damage through regulation of IDO signaling pathway and reprogramming macrophages toward the M2 phenotype. Our findings demonstrate that the myeloid PTEN-IDO axis is a novel regulator of innate immunity in APAP-induced liver injury and imply the therapeutic potential for the management of sterile liver inflammation.

\section{PO-B-06}

Morphological and elemental mapping of human gallstones using novel synchrotron XRF and microtomography

Mayank Jain ${ }^{1}$, Jayanthi Venkataraman ${ }^{1}$, Joy Varghese ${ }^{2}$, Balwant Singh $^{5}$, Manoj Kumar Tiwari ${ }^{5}$, Ramana Ramya $\mathbf{J}^{4}$, Narayana Kalkura $\mathbf{S}^{3}$

${ }^{1}$ Gleneagles Global Health City, India; ${ }^{2}$ apollo Hospitals, India; ${ }^{3}$ Crystal Growth Centre, Anna University, Chennai, India; ${ }^{4} \mathrm{~d}$ Science and Humanities, India; ${ }^{5}$ Indus 2, Raja Ramanna Centre for Advance Technology, India

Background: Pathogenesis of gallstones (GS) remains unclear. Nuclear Instruments and Advanced Methods in Physics Research have made break throughs in understanding the bio-mineral concrements of human pathogenic stones. There are few studies on GS using sophisticated techniques of synchrotron XRF and Microtomography. Aim: To perform morphological and multi-elemental mapping of GS using various techniques and to determine their composition of elements and minerals.

Method: 15 random samples were selected based on morphology and biochemistry to identify cholesterol, mixed and pigment gallstones. The selected GS were subjected to optical microscopy (gross morphology.i.e. raw image), scanning electron microscopy (SEM) (surface morphology at micro and nano level), synchrotron microtomography (SMT) (3-D image), powder X-ray diffraction (XRD) (amorphous or crystalline and phase identification). Representative samples were obtained for SR-XRF (elemental constituents) and FTIR (functional groups) from the 3-D cross sectional imaging (SMT).

Result: Table 1 and 2 shows the major elemental components (in $\mu \mathrm{g} / \mathrm{g}$ ) and functional groups respectively of the 3 different types of gallstones.

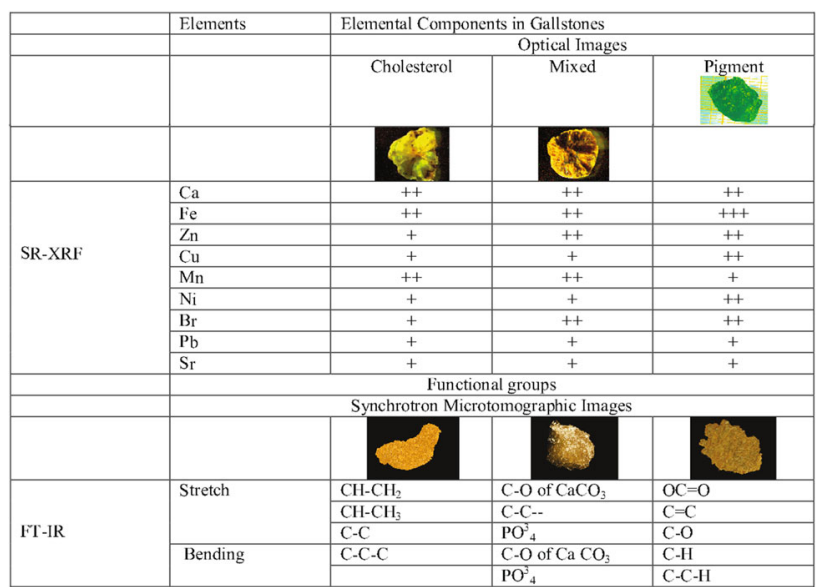


Conclusion: The study for the first time has shown the intelligible morphology and elemental mappingof gallstones using 3D images from synchrotron microtomography and SRXRF. The functional group characteristics was possible using FT-IR

\section{Yia Clinical}

\section{March 2018}

YIA-C-01

Risk factors in cirrhotic variceal bleeders that determine urgency of liver transplantation and mortality

\section{Moinak Sen Sarma ${ }^{1}$, Surender Kumar Yachha ${ }^{1}$, Anshu Srivastava $^{1}$, Ujjal Poddar ${ }^{1}$ \\ ${ }^{1}$ Sanjay Gandhi Postgraduate Institute of Medical Sciences, Lucknow, India}

Background: Prognostic scoring systems triage liver transplantation (LT) solely based on synthetic functions and organ damage, not accounting for portal hypertension. Variceal bleeding, an important complication is an independent predictor of mortality, more so in children. We aimed to study the outcome of variceal cirrhotic bleeders and whether scoring systems adequately predict the urgency for LT.

Method: Cirrhotics with variceal bleeding $>1$ year age were recruited. All patients underwent 3-4 weekly endoscopic sessions till eradication following by 3-6 monthly endoscopic surveillance. After resuscitation and stabilization, liver synthetic functions, Pediatric end-stage liver score (PELD) and Child score at 3 weeks (baseline), 6 weeks and 12 weeks were analysed. Risk factors of mortality were assessed at 12 weeks and 48 weeks. Outcome at longest follow-up were noted. Patients lost to follow-up $>3$ months after initial visit were considered as under non-survivors in intention-to-treat analysis. Result: 48 cirrhotics with variceal bleeding had an overall follow-up period of 36(3-120) mo. Mortality was $27 \% 35 \%$ and $44 \%$ at 12 weeks, 48 weeks and longest follow-up. Table 1 shows the baseline characteristics and outcome of survivors vs. non-survivors at 12 weeks. PELD and Child score were non-significant in the two groups at 12 and 48 weeks. Significant risk factors of mortality at 12 weeks were pre-bleed ascites, severe ascites, large gastric varices, gastric variceal bleeders, failure to eradicate esophageal varices (EV), variceal re-bleed, persistent hypoalbuminemia, hyponatremia and PELD score rise $>5$ by 6 weeks from baseline. Risk factors of mortality at 48 weeks were EV recurrence after eradication, EV re-bleed, hypersplenism, recurrent ascites, $>3$ hospital admissions, baseline height $\mathrm{z}$-score $<-2 \mathrm{SD}$. On regression analysis, 12 week (failure to eradicate EV, hyponatremia, PELD score rise $>5$ ) and 48 week (EV recurrence after eradication, EV re-bleed and recurrent ascites) mortality risk factors were significant. In outcome at last follow-up among survivors $(n=26), 36 \%$ were referred for liver transplantation, $15 \%$ developed hepato-pulmonary syndrome and $10 \%$ developed hepato-renal syndrome.

Conclusion: Variceal bleeding in cirrhotics is a potential cause of mortality and is not considered by the existing prognostic scoring systems. Failure to eradicate EV, hyponatremia and PELD score rise $>5$ are risk factors for 12 week mortality.

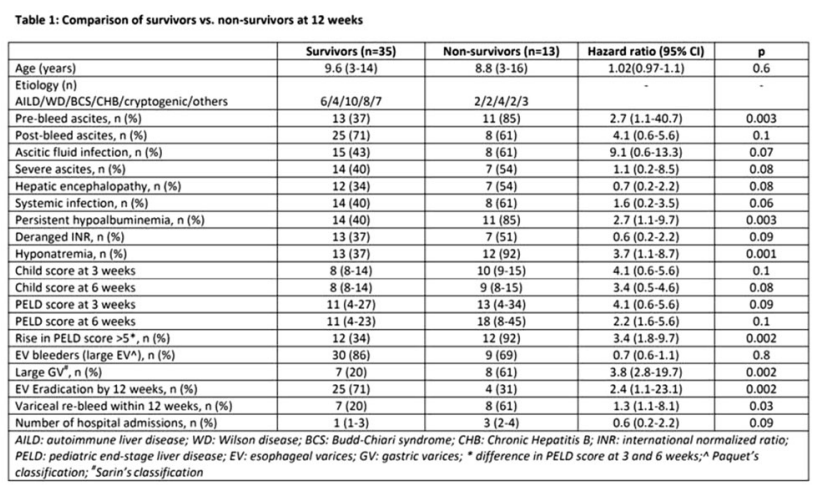

YIA-C-02

Reactivation of overt and occult hepatitis $B$ in patients with chronic hepatitis $C$ undergoing treatment with DAAs

$\underline{\text { Sevda Aghayeva }}{ }^{1}$

${ }^{1} \mathrm{MD}$, Azerbaijan

Background: In Azerbaijan the prevalence of viral hepatitis B and C is $3.2 \%$ and $4.3 \%$ respectively.

In our previous studies it was estimated that $9.3 \%$ of hepatitis C patients have $\mathrm{HCV} / \mathrm{HBV}$ co-infection and $25 \%$ of patients have occult hepatitis B. The aim of the study is to evaluate the risk of HBV reactivation during the DAA therapy in both groups.

Method: From 2015 until 2017, 194 patients with chronic hepatitis C received peroral DAA therapy. The patients were monitored at the week 4, 8, 12 during the treatment and at week 12 and 24 after the end of the treatment. The patients were divided in 2 groups:

Group I included 170 (85.4\%) HBsAg- negative, anti-HBc- positive patients (118 men and 52 women; mean age \pm SD $48.7 \pm 9.8$ years) Group II included 24 (14.6\%) HBsAg positive patients (19 men and 5 women; mean age \pm SD $43.6 \pm 9.8$ years). All patients received treatment with DAA regimens and evidence of virological/clinical reactivation was monitored.

Result: Out of 194 patients, 171 (88.1\%) had negative HCV RNA at week 4 and $182(93.8 \%)$ patients had SVR at week 12. Group I: Out of $170 \mathrm{HBsAg}$-negative, anti-HBc-positive patients, pretreatment detectable HBV DNA was found in $46(27 \%)$ and HBV DNA range was $34-2356 \mathrm{IU} / \mathrm{mL}$. During the treatment with DAAs, 4 patients (2.35\%) had $1 \log$ HBV DNA increase but normal ALT level. Group II: Out of 24 patients with positive HBsAg (HBV DNA range 147-12.580 IU/mL), in 7 patients HBV DNA was increased by $1-2 \mathrm{log}$ with parallel increase in ALT level (median $45 \mathrm{IU} / \mathrm{L}$ ), especially in patients with pretreatment viral load $>2000 \mathrm{IU} / \mathrm{mL}$. The rest 7 patients of group II had no increase in HBV DNA and ALT.

Conclusion: DAAs provoked clinical/virological reactivation in $50 \%$ of $\mathrm{HBV} / \mathrm{HCV}$ patients, and virological in $2.35 \%$ of occult $\mathrm{HBV}$. NA treatment is recommended in patients with dual infection, especially in those with HBV viral load $>2000 \mathrm{IU} / \mathrm{mL}$.

\section{YIA-C-03}

Clinical curative effect of transcatheter iodine-125 implantation combined with TIPS in treating tumor emboli of portal vein trunk and their complications

${ }_{\text {Fuliang He}}{ }^{1}$, Fuquan Liu ${ }^{1}$, Lei Wang ${ }^{1}$, Zhendong Yue ${ }^{1}$, Yifan $\mathrm{Wu}^{1}$ 
${ }^{1}$ Beijing Shijitan Hospital, Capital Medical University, China

Background: This study aimed to analyze the clinical curative effect of transcatheter iodine-125 implantation combined with transjugular intrahepatic portosystemic shunt (TIPS) in treating tumor emboli of portal vein trunk and their complications.

Method: A total of 40 patients with tumor emboli of portal vein trunk treated successfully using catheter iodine-125 implantation combined with TIPS were selected from January 2011 to March 2015 (the ITT group). From January 2007 to January 2011, 45 patients with tumor emboli of portal vein trunk treated with only TIPS were selected (the TT group). The survival rate, median survival time, stent stenosis rate, and rate of symptom recurrence of the two groups were recorded.

Result: The cumulative 6-, 12-, and 24-month survival rates for the two groups were $80.0 \%$ and $80.0 \%, 42.5 \%$ and $17.5 \%$, and $71.1 \%$ and $26.7 \%$, respectively. The median survival time of the groups was 11.8 and 8.5 months, respectively. The symptom recurrence rates were $12.5 \%$ and $85.0 \%, 35.6 \%$ and $71.1 \%$, and $57.5 \%$ and $85.0 \%$, respectively. The stent restenosis rates were $17.5 \%$ and $54.5 \%, 66.7 \%$ and $62.2 \%$, and $70.6 \%$ and $66.7 \%$, respectively.

Conclusion: TIPS was effective in complications caused by portal hypertension and had a curative effect on the hemorrhage of the digestive tract. Iodine-125 could obviously reduce the volume of tumor emboli. Iodine-125 combined with TIPS helped in treating tumor emboli of portal vein trunk and their complications, improving the quality of life of patients and reducing the mortality.

\section{YIA-C-04}

Randomized controlled trial of methocarbamol in the treatment of muscle cramps in cirrhotic patients

\section{Sherief Abd Elsalam ${ }^{1}$, Mona Arafa ${ }^{1}$, Rehab Badawi ${ }^{1}$, Walaa Elkhalawany $^{1}$, Asem Elfert ${ }^{1}$, Shaimaa Soliman ${ }^{2}$ \\ ${ }^{1}$ Tanta University Faculty of medicine, Egypt; ${ }^{2}$ Menofia University, Egypt}

Background: The prevalence of muscle cramps in cirrhotic patients ranges from $29 \%$ to $88 \%$. They adversely affect the quality of life in cirrhotic patients. The aim of this study was to assess the safety and efficacy of methocarbamol in the treatment of muscle cramps in cirrhotic patients.

Method: 100 patients with post-hepatitis $\mathrm{C}$ liver cirrhosis with an average CTP score of $7+2$ suffering from frequent muscle cramps ( $\geq 3$ cramps per week) were enrolled in the study. They were randomized to receive either methocarbamol (50 patients) or placebo (50 patients) for one month. Thirty one patients in the methocarbamol group and 33 patients in the placebo group were on diuretic therapy. Muscle cramp questionnaire was fulfilled. Severity, duration, and frequency of muscle cramps were assessed before, after one month of treatment and two weeks after washout of treatment. Liver functions, renal functions, and electrolytes have been checked every visit to determine safety and side effects were recorded.

Result: After one month of treatment with methocarbamol, the frequency of muscle cramps decreased significantly from $11 \pm 4$ at baseline to $0.5 \pm 1$ per week ( $<0.001$ ). The pain score improved significantly from a score of $6.52 \pm 1.29$ to $0.66 \pm 1.18(\mathrm{p}<0.001)$. The duration of muscle cramps decreased from $4.22 \pm 1.1 .55 \mathrm{~min}$ to $0.71 \pm 1.06$ min after treatment $(p<0.001)$. No significant changes in the frequency, severity and duration of muscle cramps were noted in the placebo group. Few side effects in the form of dry mouth and drowsiness were noted in the methocarbamol group when compared to placebo group.
Conclusion: Methocarbamol was effective, safe, and well tolerated in the treatment of muscle cramps in patients with liver cirrhosis.

\section{YIA-C-05}

Does early on-treatment alanine aminotransferase (ALT) normalization reduce the risk of hepatocellular carcinoma (HCC) in patients with chronic hepatitis B?-A territory-wide study of 21,182 subjects

Cheuk Fung Yip ${ }^{1}$, Grace Lai Hung Wong ${ }^{1}$, Yee Kit Tse ${ }^{1}$, Henry Lik Yuen Chan $^{1}$, Vincent Wai Sun Wong ${ }^{1}$

${ }^{1}$ The Chinese University of Hong Kong, Hong Kong

Background: Alanine aminotransferase (ALT) normalization is considered to be the biochemical response to antiviral treatment. We aimed to evaluate the impact of ALT normalization achieved at different time points after the start of entecavir and/or tenofovir disoproxil fumarate (TDF) treatment, on the risk of hepatocellular carcinoma (HCC) in patients with chronic hepatitis B (CHB).

Method: We identified a territory-wide cohort of CHB patients who received entecavir and/or TDF for at least 1 year from January 2005 to December 2016 in Hong Kong. Serial on-treatment ALT levels were collected and analyzed. ALT normalization (ALT-N) was defined as the ALT level lower than the upper limit of normal (ULN), which is $30 \mathrm{U} / \mathrm{L}$ in males and $19 \mathrm{U} / \mathrm{L}$ in females. Early on-treatment ALT-N was defined as ALT-N within 12 months. The primary outcome was HCC based on ICD-9-CM diagnosis codes. Patients with cancers previously or during the first year of treatment were excluded. Result: 21,182 CHB patients (10,437 with and 10,745 without ALT$\mathrm{N}$ at 12 months after antiviral treatment) were identified and followed for a median (interquartile range) of 4.1 (2.4-6.0) years. Patients with or without ALT-N at 12 months differed in gender distribution (76.9\% vs. 58.4\% male), baseline ALT (58 vs. $61 \mathrm{U} / \mathrm{L}$ ), baseline serum HBV DNA (4.9 vs. $5.1 \log 10 \mathrm{IU} / \mathrm{mL})$, proportion of positive hepatitis $\mathrm{B}$ e antigen $(31.5 \%$ vs. $37.1 \%)$, proportion of having cirrhosis $(8.8 \%$ vs. $10.5 \%)$ and prevalence of diabetes mellitus $(8.1 \%$ vs. $9.1 \%) ; 509$ (2.4\%) patients developed HCC. Compared to no ALT-N, ALT-N at 3, 6, 9 and 12 months were associated with a reduced risk of HCC (Figures 1A-D), with adjusted hazard ratios (95\% confidence interval [CI]) of 0.55 (0.42-0.71), 0.52 (0.41-0.64), 0.47 (0.38-0.58) and $0.46(0.37-0.56)$, respectively (all $P<0.001)$. In contrast, patients with ALT 1 to 2 times the ULN and ALT greater than 2 times the ULN at 12 months were associated with a higher risk of HCC as compared to patients with ALT-N at 12 months, with adjusted hazard ratios (95\% CI) of 2.07 (1.67-2.56) and 3.21 (2.32-4.44), respectively, after adjustment for baseline ALT and other important covariates. The cumulative incidence $(95 \% \mathrm{CI})$ of $\mathrm{HCC}$ at 6 years was $2.7 \%(2.3 \%$ $3.2 \%$ ) in ALT-N at 12 months, $4.6 \%(4.1 \%-5.2 \%)$ in ALT 1 to 2 times the ULN at 12 months, and $6.0 \%(4.7 \%-7.7 \%)$ in ALT greater than 2 times the ULN at 12 months (log-rank test, $P<0.001$ ) (Figure 2).

Conclusion: Early on-treatment ALT-N can be translated into reduced risk of $\mathrm{HCC}$ development in $\mathrm{CHB}$ patients having nucleos $(\mathrm{t})$ ide anaolgue treatment. On-treatment ALT above 1 and 2 times the ULN at 12 months were associated with higher risk of HCC. 

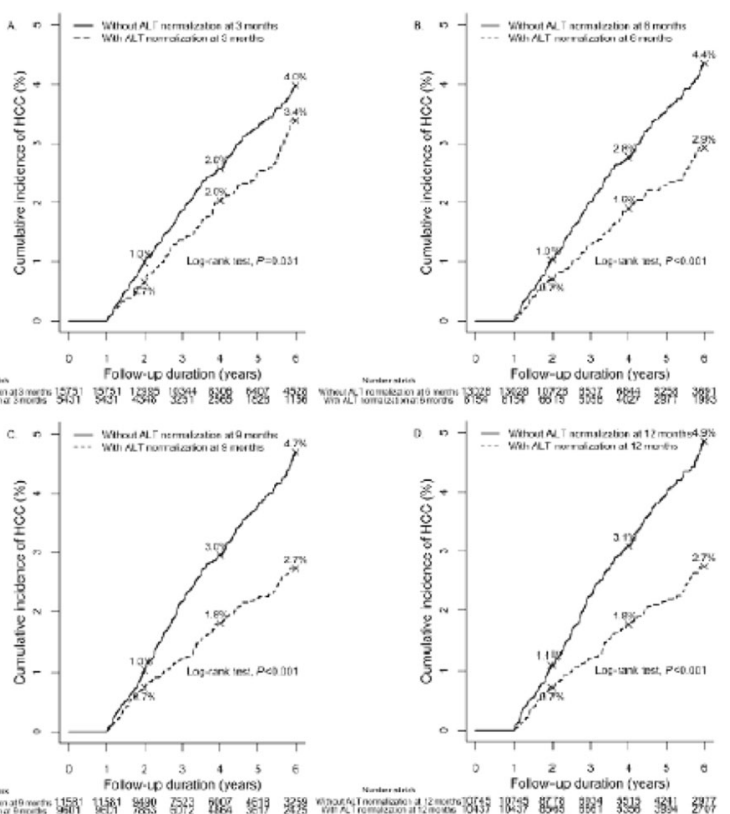

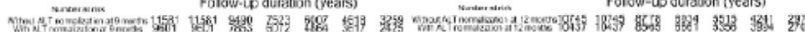

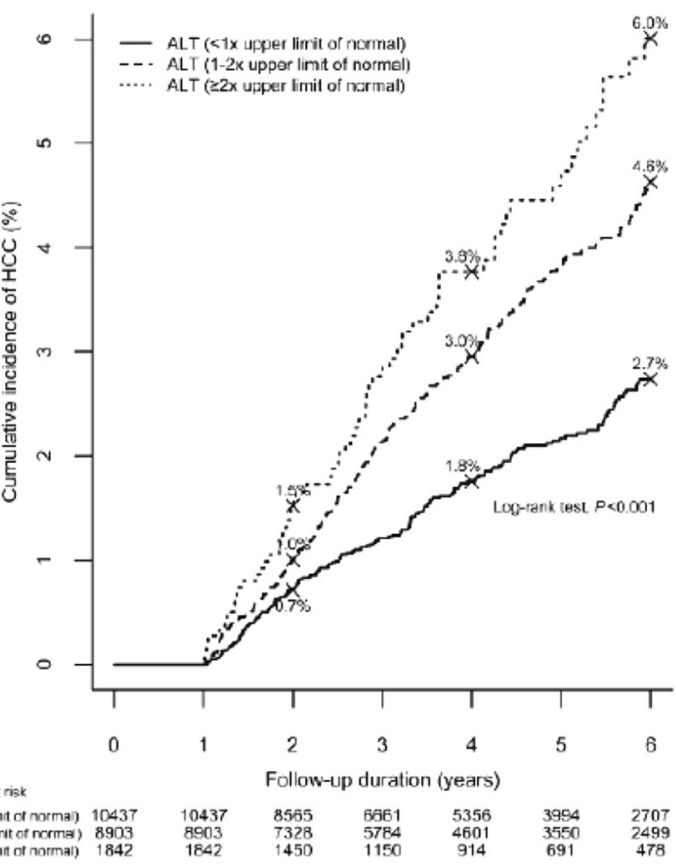

YIA-C-06

Clinical significance of measuring serum M2BPGi level in patients with acute liver injury

Takuya Seike ${ }^{1}$, Takuya Komura', Yoshiaki Shimizu' ${ }^{1}$, Hitoshi Omura $^{1}$, Tatsuo Kumai ${ }^{1}$, Takashi Kagaya ${ }^{1}$, Hajime Ohta ${ }^{1}$, Atsuhiro Kawashima', Kenichi Harada ${ }^{3}$, Shuichi Kaneko ${ }^{2}$, Masashi Unoura ${ }^{1}$
${ }^{1}$ National Hospital Organization Kanazawa Medical Center, Japa; ${ }^{2}$ Graduate School of Advanced Preventive Medical Science, Japa; ${ }^{3}$ Kanazawa University Graduate School of Medical Science, Japan

Background: Serum Wisteria floribunda agglutinin positive human MAC-2-binding protein glycosylation isomer (M2BPGi) level has been recently recognized as a marker of liver fibrosis in patients with chronic liver injury. However, its clinical significance is not well known in patients with acute liver injury. In this study, we evaluated the clinical significance of measuring serum M2BPGi levels in patients with acute liver injury.

Method: Serum M2BPGi levels were measured at diagnosis (78 patients) and at the time that alanine aminotransferase (ALT) levels were normalized (26 patients) in patients with acute liver injury. Forty-six patients were observed until normalization of ALT level by conservative medical treatment, such as supplemental liquids and bed rest. We evaluated the differences in serum M2BPGi level for each cause of acute liver injury, and the correlation between serum M2BPGi level at diagnosis and the duration which ALT was normalized.

Result: The causes of acute liver injury in the cases were: Autoimmune liver injury, five patients; infectious mononucleosis, 17 patients; drugs, 21 patients; acute hepatitis A, nine patients; acute hepatitis B, nine patients; and unknown etiology, 17 patients. Serum M2BPGi level at diagnosis increased significantly (2.3 COI; range 0.31-14.9 COI); the M2BPGi level increased significantly in autoimmune, as compared to that in non-autoimmune liver injury (autoimmune, $8.4 \mathrm{COI}$; non-autoimmune, $2 \mathrm{COI}$; $\mathrm{p}=0.02$ ), although ALT level was not significantly different between the two groups. Furthermore, the ratios of M2BPGi to serum parameters of liver injury were significantly different in each cause. Especially M2BGPi/ aspartate aminotransferase and M2BPGi/ALT in patients with infectious mononucleosis $(\mathrm{p}<0.0001)$ and M2BPGi/lactate dehydrogenase in patients with autoimmune liver disease $(p=0.0075)$ were significantly higher than the other causes. Serum M2BPGi levels decreased significantly at the time of normalization of ALT levels in patients treated conservatively $(0.55 \mathrm{COI}, \mathrm{p}<0.0001)$. Furthermore, serum M2BPGi level at diagnosis was positively correlated with duration of achieving ALT normalization (Spearman rho $=0.53, \mathrm{p}=$ $0.0001)$, compared with other parameters.

Conclusion: Serum M2BPGi levels increased in patients with acute liver injury, but its mechanism might differ with each cause. Serum M2BPGi levels could be useful for predicting the duration of subsided liver injury.

\section{Yia Basic}

\section{March 2018}

YIA-B-01

Placental expression of asialoglyco protein receptor showed its role and association in Hepatitis $B$ virus transmission from mother to child

Ashish Kumar Vyas ${ }^{1}$, Sharda Patra ${ }^{3}$, R Usha ${ }^{2}$, Bijoya Sen ${ }^{1}$, Karan Singh $^{2}$, Gayatri Ramakrishna ${ }^{1}$, Archana Rastogi ${ }^{1}$, Shiv Kumar Sarin $^{1}$, Nirupma Trehanpati ${ }^{1}$

${ }^{1}$ Institute of liver and Biliary Sciences, India; ${ }^{2}$ ESI Hospital, Noida, Sector 24, India; ${ }^{3}$ Lady Hardinge Medical College, India

Background: Vertical transmission of Hepatitis B virus (HBV) from infected mother to the newborn is the major cause of HBV chronicity. Asialoglycoprotein receptor (ASGPR) expression on hepatocytes has 
been associated with HBV entry and endocytosis. However, there is a big lacuna regarding expression of ASGPR expression on placental cells and its role in HBV vertical transmission.

Method: $34 \mathrm{HBsAg}+\mathrm{ve}$ and 13 healthy pregnant mothers were enrolled along with their newborns. QHBsAg, HBV DNA and liverfunction-test were performed among the groups. HBsAg+ve women were grouped into transmitting and non-transmitting mothers on the basis of newborns HBsAg and HBVDNA. Expression of ASGPR and HBsAg were analyzed in placental tissue using Immunohistochemistry and immunofluorescence staining. Peripheral and cord bloodmononuclear cell together with dendritic cells (mDCs and pDCs) were analyzed for the expression of DC-ASGPR, using flow cytometry and QPCR in all subjects.

Result: The incidence of HBV vertical transmission to the newborn was $18 \%$ among the $\mathrm{HBsAg}$ positive pregnant females. HBV transmitting mothers showed increased expression of ASGPR in trophoblasts of placenta. Immunofluorescence microscopy revealed co-localization of HBsAg and ASGPR in placenta as well as in DCs of HBV transmitting mothers. HBV transmitting mothers and their $\mathrm{HBsAg}+\mathrm{ve}$ newborns showed increased mRNA levels of DC-ASGPR in PBMCS. However, flowcytometry revealed no significant difference in the expression of ASGPR on PBMCs or CBMCs cells between the 2 groups. The HBV transmitting mothers and their HBsAg+ve newborns also showed an increased expression of DCASGPR on both myeloid and plasmocytoid dendritic cells compared to HBV non-transmitting mothers and their HBsAg negative newborns.

Conclusion: The present work highlights for the first time a role of ASGPR in the intrauterine mother to baby HBV transmission. Blocking of ASGPR appears to be novel therapeutic strategy for prevention of HBV mother to baby vertical transmission.

\section{YIA-B-02}

\section{Pristine C60 fullerenes affect the hepatic and extrahepatic manifestations of experimental cholangitis}

\section{Halyna Kuznietsova ${ }^{1}$, Natalia Dziubenko ${ }^{1}$, Oksana Lynchak ${ }^{1}$, Olexandr Ogloblya ${ }^{1}$, Yuriy Prylutskyy ${ }^{1}$, Volodymyr Rybalchenko $^{1}$, Uwe Ritter ${ }^{2}$, Peter Scharff ${ }^{2}$}

${ }^{1}$ Taras Shevchenko National University of Kyiv, Ukraine;

${ }^{2}$ Technische Universität Ilmenau, Germany

Background: Sclerosing cholangitis is the liver disease of uncertain etiology, unfavorable prognosis and lack of effective medication therapy. A significant role at all the stages of the disease is attributed to reactive oxygen species, therefore the evaluation of possible protective properties of water-soluble pristine C60 fullerenes (effective free radical scavengers) using the simulation of acute and chronic cholangitis models in rats was aimed to be discovered.

Method: Acute cholangitis (AC) was simulated by single $\alpha$-naphthyl isothiocyanate (ANIT, $100 \mathrm{mg} / \mathrm{kg}$ ) per os administration. The pristine C60 fullerene aqueous colloid solution (C60FAS; initial concentration $0.15 \mathrm{mg} / \mathrm{ml}$ ) was administered daily either per os or intraperitoneally (i.p.) at dose of $0.5 \mathrm{mg} / \mathrm{kg} \mathrm{C} 60$ for 2 times after $\mathrm{AC}$ induction. Chronic cholangitis (CC) was simulated by 4 weekly ANIT ingestions, C60FAS was administered at the same dose and manner every other day. Reference drug prednisolone $(0.7 \mathrm{mg} / \mathrm{kg}$ daily $)$ was used in both models. The states of the liver, kidney and pancreas were assessed by histological (hematoxylin-eosin staining) and biochemical (serum alanine aminotransferase (ALT), aspartate aminotransferase (AST), alkaline phosphatase (ALP), lactate dehydrogenase (LDH), $\alpha$-amylase activities, serum total and direct bilirubin, total protein, triglycerides, urea and creatinine levels) methods.
Result: AC rats demonstrated increase of total and direct bilirubin, ALT, AST, ALP and LDH (1.5 to 4 times), indicating the cholestasis and hepatocyte cytolysis, and raise of urea, creatinine and total protein (1.3 to 2 times), suggesting the kidney failure. Under CC only direct bilirubin, ALP, LDH and $\alpha$-amylase were increased (1.3 to 3 times), suggesting the hepatic compensatory process. Hepatic portal tracts fibrotic alterations with surrounding inflammatory cell infiltrate were also detected, confirming the sclerosing cholangitis development. Prednisolone normalized ALT, AST, direct bilirubin and ALP and diminished fibrotic alterations when applied under both models. However, growth of creatinine, urea, total protein and triglycerides (2 to 4 times) in $\mathrm{AC}$ rats could indicate pancreas dysfunction and kidney failure aggravation. I.p. C60FAS normalized direct and total bilirubin, ALT, ALP, decreased liver parenchyma fibrotic alterations in AC rats, but only improved liver morphology in CC animals. Per os C60FAS was less effective under AC compared to i.p. one, but under $\mathrm{CC}$ the situation was opposite. However, $\boldsymbol{\alpha}$-amylase remained elevated and triglycerides even increased, suggesting the pancreas failure, probably through C60 fullerenes accumulation in this organ.

Conclusion: Thus, C60FAS partially improved liver morphological and functional state under AC and CC when applied by both ways. However, pancreas suffered after C60FAS impact, especially after chronic one. I addition, the same and sometimes better C60FAS ability to treat liver failure and cause much less side effects on kidney compared to reference was concluded.

\section{YIA-B-03}

Study to assess chitinase 3- like 1 as a non-invasive bio-marker of hepatic fibrosis in patients with HBV related compensated chronic liver disease

Amit Das ${ }^{1}$, Mamun Al Mahtab ${ }^{3}$, Sheikh Mohammad Noor E Alam Dew $^{2}$, Salimur Rahman ${ }^{4}$

${ }^{1}$ Indoor Medical Officer, Bangladesh; ${ }^{2}$ Assistant professor, bangabandhu sheikh mujib medical university., Bangladesh; 3 associate Professor, Bangabandhu Sheikh Mujib Medical University., Bangladesh; ${ }^{4}$ Professor, Bangabandhu Sheikh Mujib Medical University., Bangladesh

Background: The disease spectrum of CHB infection ranging from an inactive carrier state to progressive $\mathrm{CHB}$, which may evolve to cirrhosis and HCC.The assessment of the severity depends on necroinflammatory and fibrosis status. The aim of this study is to find out the correlation between CHI3L1 and histological severity and thereby to measure serum CHI3L1 level to assess the grade of fibrosis with METAVIR and to assess the correlation of serum CHI3L1 level with hepatic fibrosis.

Method: This was a prospective observational cross sectional study. Result: Total 60 patients were divided in five groups. F0 includes patients with no fibrosis $(\mathrm{n}=1), \mathrm{F} 1$ includes patients with mild fibrosis $(n=9)$ and F2-F3 includes patients with moderate fibrosis (n $=49)$ and F4 is cirrhosis $(\mathrm{n}=1)$ at liver biopsy.

In this study, most of the patients were young with 32 patients $(53.4 \%)$ below the age of 30 years. Mean age of the patients found $27.27 \pm 6.77$ with the highest frequency at 21-30 years age group.

Among $60 \mathrm{CHB}$ patients a male predominance was observed in this study. Male $48(80 \%)$ and female $12(20 \%)$. Among the 60 patients, 19 patients were $\mathrm{HBeAg}$ positive and 41 patients were $\mathrm{HBeAg}$ negative.

In this study, $16.7 \%$ patients did not have significant fibrosis (F0, F1) and 83.3\% patients have significant fibrosis (F2, F3, F4). Since F0 and $\mathrm{F} 4$ group have total 2 patients only (1 in each group), we exclude them from further statistical evaluation as because these are 
statistically insignificant. Serum CHI3L1 level were significantly increased in a stepwise fashion from F1 to F3.

Correlation between CHI3L1 and Fibrosis METAVIR score on pearson's correlation test reveals, Serum CHI3L1 level had weakly positive correlation with stages of fibrosis (F1 to F3) ( $\mathrm{r}=0.08, \mathrm{p}=$ $0.550)$.

The area under the receiver-operator characteristic (ROC) curves for prediction of serum CHI3L1 level reveals - area under curve(AUC) 0.741 , which gave a cut off value of $159 \mathrm{ng} / \mathrm{ml}$ with $98.3 \%$ sensitivity and $50 \%$ specificity for prediction of significant fibrosis (F2 and F3).

Conclusion: Further study with large number of patients is required to determine whether serum CHI3L1 level significantly correlate with the histological severity in patients with HBV related compensated CLD or not.

Track ID : YIA-C-06

Table 1 Baseline characteristics of the study population :

\begin{tabular}{|c|c|c|c|c|}
\hline \multirow[t]{2}{*}{ Laboratory parameters } & \multicolumn{4}{|c|}{ Total number $\mathrm{N}=60$} \\
\hline & Minimum & Maximum & Mean & Std. Deviation \\
\hline Hemoglobin \% & 9.80 & 16.30 & 14.28 & 1.43572 \\
\hline Total WBC count $\left(/ \mathrm{mm}^{3}\right)$ & 4.00 & 95.00 & 8.9 & 11.40851 \\
\hline Neutrophil\% & 43.00 & 76.00 & 60.20 & 7.88218 \\
\hline Lymphocyte\% & 19.00 & 46.00 & 31.27 & 6.65841 \\
\hline Monocyte\% & .00 & 12.00 & 4.63 & 2.37905 \\
\hline Eosinophil\% & .00 & 13.00 & 4.07 & 2.95082 \\
\hline Basophil\% & 0.00 & 0.00 & 00.00 & 0.00 \\
\hline ESR & 5.00 & 75.00 & 22.33 & 14.44 \\
\hline Platelet count $\left({ }^{\times} 10^{9} / \mathrm{L}\right)$ & 100.00 & 390.00 & 233.83 & 56.89 \\
\hline Fasting blood sugar & 4.30 & 51.00 & 5.96 & 5.93 \\
\hline $\begin{array}{l}\text { Two hours after blood } \\
\text { sugar }\end{array}$ & 3.60 & 61.00 & 7.13 & 7.12 \\
\hline Total cholesterol & 76.00 & 295.00 & 144.93 & 43.68 \\
\hline LDL & .00 & 198.00 & 90.13 & 31.20 \\
\hline HDL & 26.00 & 61.00 & 40.23 & 6.05 \\
\hline TG & 39.00 & 438.00 & 134.45 & 61.83 \\
\hline AST & 17.00 & 118.00 & 35.05 & 17.58 \\
\hline ALT & 11,00 & 241.00 & 46.85 & 32.16 \\
\hline Prothrombin Time & 10.50 & 13.60 & 12.17 & 0.48 \\
\hline INR & 0.88 & 1.13 & 1.02 & 0.04 \\
\hline GGT & 9.00 & 54.00 & 34.50 & 11.26 \\
\hline Serum Creatinine & 0.54 & 1.35 & .8235 & 0.17 \\
\hline HBV DNA PCR (IU/ml) & 65.10 & 2.38 & 13.54 & 16.63 \\
\hline
\end{tabular}

All values are expressed as mean \pm SD or number (\%) and with standard unit *

HBV DNA PCR values were transformed into logarithmic scale.
Table 2 Distribution of the study patients by age $(n=60)$

\begin{tabular}{|l|c|c|}
\hline \multicolumn{1}{|c|}{ Age groups } & Number of patients & Percentage \\
\hline$\leq 20$ & 12 & 20.0 \\
\hline $21-30$ & 32 & 53.4 \\
\hline $31-40$ & 14 & 23.3 \\
\hline 40 & 2 & 3.3 \\
\hline Range (min, max, ) & 18,43 & \\
\hline Mean $=$ SD & $27.27 \pm 6.77$ & \\
\hline
\end{tabular}

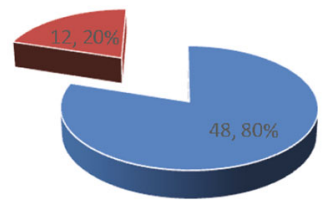

- Male - Female

Figure 1 Pie chart shows sex distribution of the study patients

Table 3 Distribution of study patients on monthly income

\begin{tabular}{|l|c|c|}
\hline Income & Frequency (n) & Percent (\%) \\
\hline$\leq 5000$ & 15 & 25 \\
\hline $5001-10000$ & 12 & 20 \\
\hline $10001-20000$ & 27 & 45 \\
\hline$>20000$ & 6 & 10 \\
\hline
\end{tabular}




\section{Chart Title}

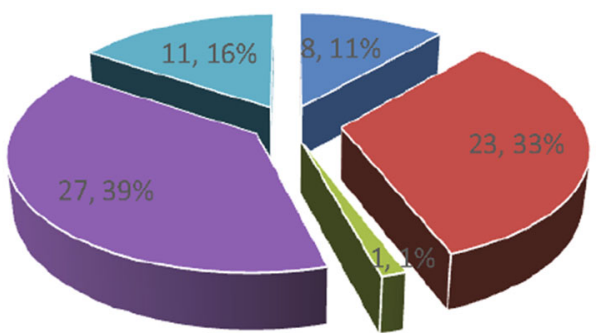

\begin{tabular}{|l|r|r|r|r|r|}
\hline & \multicolumn{1}{|c|}{$\begin{array}{c}\text { Total } \\
\text { Number }\end{array}$} & \multicolumn{1}{|c|}{ Minimum } & Maximum & \multicolumn{1}{|c|}{ Mean } & Std. Deviation \\
\hline $\begin{array}{l}\text { Chitinase } \\
\text { 3- like 1 } \\
\text { (CHISL1) }\end{array}$ & 58 & 130.50 & 1877.00 & 716.64 & 507.81 \\
\hline
\end{tabular}

Table 6 : Association of stages of fibrosis with CHI3L1.
All values expressed as mean $\pm \mathrm{SD}$ or number $(\%)$

Table-5 Distribution of the study patients by serum CHI3L1 level

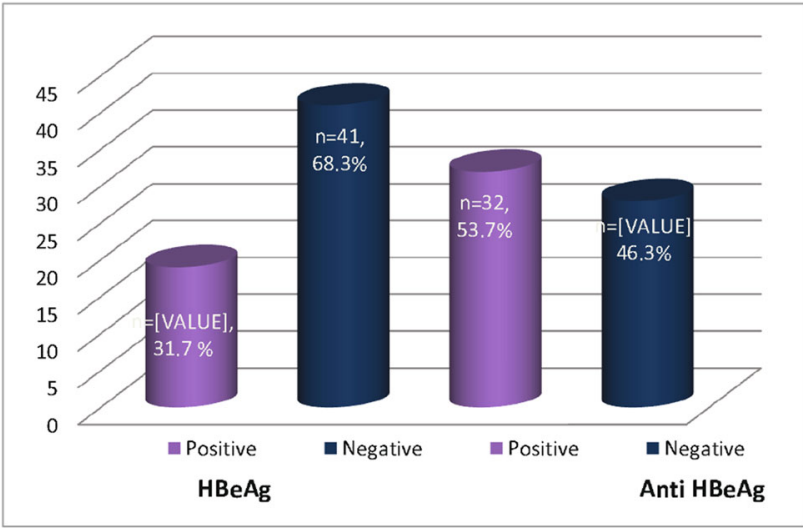

Figure 3: Bar diagram shows HBeAg status of the study patients

Table 4: Distribution of study patients according to fibrosis Metavir score

\begin{tabular}{|c|c|c|}
\hline Liver biopsy METAVIR score & Frequency & Percent \\
\hline F0 & 1 & 1.7 \\
\hline F1 & 9 & 15.0 \\
\hline F2 & 39 & 65.0 \\
\hline F3 & 10 & 16.6 \\
\hline F4 & 1 & 1.7 \\
\hline Total & 60 & 100.0 \\
\hline
\end{tabular}

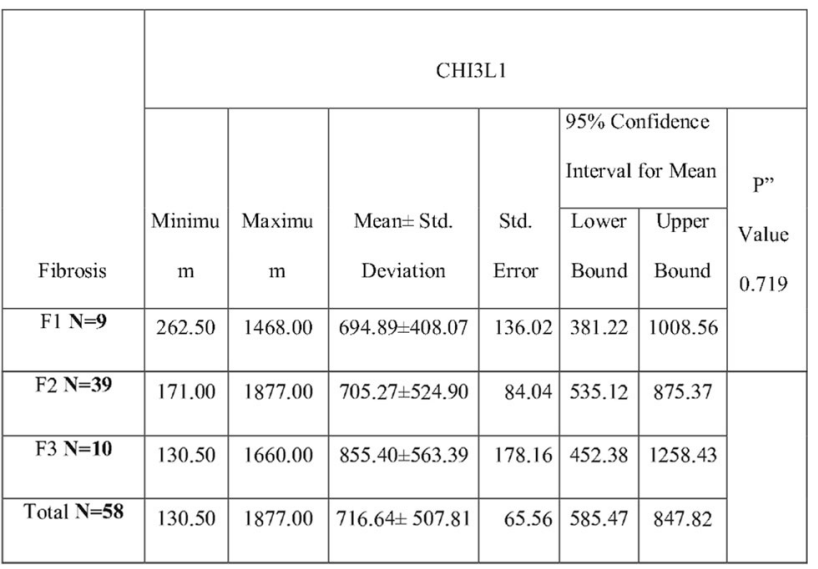

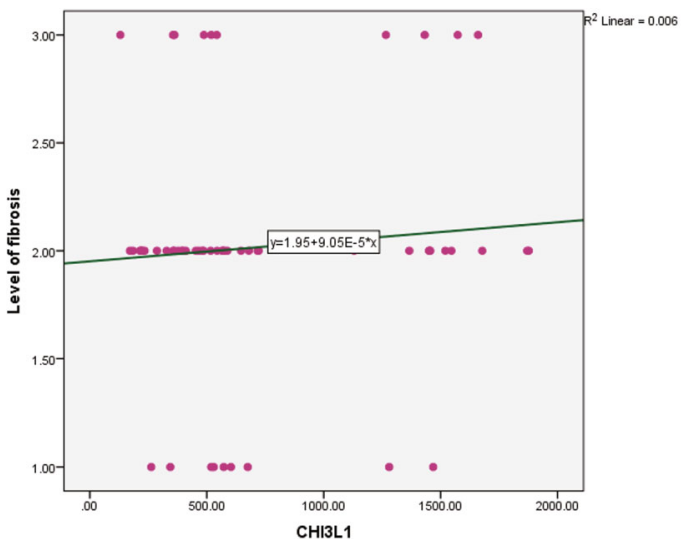

Figure 4: Scatterd diagram shows weak positive correlation $(r=0.08, p=0.550)$ between fibrosis score and serum CHI3L1 level. Pearson's correlation test done. $p$ value $<0.05$ considered significant Serum CHI3L1 expressed as $\mathrm{ng} / \mathrm{ml}$. Hepatic fibrosis expressed as F1, $\mathrm{F} 2$ and $\mathrm{F} 3$ 


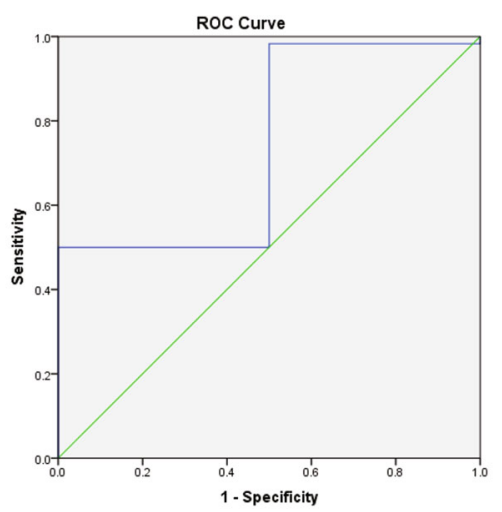

Figure 5: Receiver-operator characteristic (ROC) curve of serum CHI3L1 level for prediction of significant fibrosis (F2 and F3) patients.

Table 7 : Receiver-operator characteristic (ROC) curve of serum CHI3L1 for prediction of significant fibrosis

Area Under the Curve

Test Result Variable(s): CHI3L1

\begin{tabular}{|c|c|c|c|c|}
\hline \multirow[b]{2}{*}{ AUC } & \multirow[b]{2}{*}{ Std. Error ${ }^{a}$} & \multirow[b]{2}{*}{$P$ value } & \multicolumn{2}{|c|}{ Asymptotic $95 \%$ Confidence Interval } \\
\hline & & & Lower Bound & Upper Bound \\
\hline 0.741 & .176 & .249 & 0.397 & 1.000 \\
\hline
\end{tabular}

a. Under the nonparametric assumption

b. Null hypothesis: true area $=0.5$

Cut off value $=159.0 \mathrm{ng} / \mathrm{ml} \quad$ Sensitivity $=98.3 \% \quad$ Specificity $=50.0 \%$

\section{YIA-B-04}

Blocking CLEC-2 mediated liver damage enhances liver recovery after a toxic liver injury

\section{Abhishek Chauhan ${ }^{1}$ \\ ${ }^{1}$ University of Birmingham, United Kingdom}

Background: Acute liver failure remains a significant cause of mortality and indication for liver transplantation. Causes include toxic injury from drugs including paracetamol (APAP), the main cause of acute liver failure in the west. The mechanisms that lead to irreversible liver injury are poorly understood but the innate immune response is important. We hypothesised that platelets could be important players in this process given their roles beyond haemostasis which include liver regeneration. Little is known about the role platelets play in driving toxic liver injuries such as paracetamol.

The platelet receptor CLEC-2 mediates powerful platelet activation independently of haemostasis through its ligand podoplanin (PDPN) which is ubiquitously expressed and upregulated on a variety of cells during inflammation. We sought to investigate whether blocking
CLEC-2 dependent platelet activation reduces liver damage during toxic liver injury.

Method: APAP or carbon tetrachloride $(\mathrm{CCl} 4)$ were used to induce acute liver damage in mice and the role of CLEC-2 mediated platelet activation investigated using mice with conditional deletions for either the platelet CLEC-2 receptor (PF4creCleclbfl/fl), or its activating ligand podoplanin (Vav1-iCre+PDPNfl/fl) or using specific function blocking antibodies. Liver damage was assessed by measuring serum ALT levels and histological analyses. The innate/ inflammatory response was measured by the hepatic infiltrate and cytokine production. Liver damage and the subsequent inflammatory response was measured by assessing hepatic leukocyte infiltration, histological and serum markers.

Result: Initial liver injury was comparable in both WT and CLEC2 deficient mice. The absence of CLEC2 receptor accelerated liver healing from both APAP and CCl4 injury (ALT:WT $1264 \pm 296.5$ $\mathrm{IU} / \mathrm{L}$ vs CLEC1bfl/flPf4Cre $52.00 \pm 5.000 \mathrm{IU} / \mathrm{L} \mathrm{P}=0.0078, \mathrm{n}=5-8$ ). Targeting this pathway therapeutically using a specific PDPN function blocking antibody in WT mice enhanced healing (isotype $\mathrm{AB}$ treated ALT- $6768 \pm 2048 \mathrm{IU} / \mathrm{L}$ vs AB treated $238.0 \pm 86.67 \mathrm{IU} / \mathrm{L}, \mathrm{P}$ $=0.0007, \mathrm{~N}=6-8)$. In vitro experiments reveal that CLEC2 deficient platelets interact with Kupffer cells (KC) to enhance TNF-a production and increase hepatic neutrophil accumulation. Healing was prevented by either blocking TNF-a or depleting neutrophils in $\mathrm{PF} 4 \mathrm{creClec} 1 \mathrm{bfl} / \mathrm{fl}$ mice. Upregulation of PDPN on KCs in human ALF suggests this pathway is also activated in human disease.

Conclusion: Platelets are involved in determining the outcome of the sterile inflammatory response to toxic liver injury. Platelet activation via CLEC-2 in the context of an acute liver injury inhibits TNF-a driven reparative inflammation mediated by neutrophils. The fact that blocking CLEC-2 mediated platelet activation enhances neutrophil driven restorative hepatic inflammation without causing bleeding suggests this may represent a completely novel treatment for human acute liver failure.

\section{YIA-B-05}

\section{Hepatitis $\mathbf{E}$ induces mitochondrial fusion to facilitate viral replication}

$\underline{\text { Jingmin Zhao }}^{1}$, Yijin Wang ${ }^{1}$

${ }^{1}$ Beijing 302 Hospital, China

Background: Although hepatitis E virus (HEV) infection is generally self-limiting, severe complications and chronic hepatitis have been reported in special populations. Mitochondrial dysfunctions in hepatitis patients have been reported in many clinical studies. Liver cells contain abundant copies of mitochondria, and mitochondrial morphological dynamics, including the processes of fusion and fission, play important roles in physiology and pathogenesis. This study aims to understand mitochondrial morphological alteration in response to HEV infection and its implication in HEV replication.

Method: Liver biopsies of 17 patients with positive anti-HEV immunoglobulin (Ig) $\mathrm{M}$ and 5 control patients with hepatic hemangioma were reviewed for mitochondrial morphology by confocal immunofluorescent imaging. Human liver Huh-7 cells were used to model HEV infection in vitro and mitochondrial dynamics were observed by electron microscope and confocal immunofluorescent imaging. Lentiviral mediated RNAi was applied to silence genes that regulate mitochondrial dynamics. Viral replication was analyzed by quantitative real-time polymerase chain in reaction (qPCR).

Result: Immunofluorescent staining of liver biopsies showed that 11 out of $17(65 \%) \mathrm{HEV}$ infected patients presented aggregated and fused mitochondria; whereas mitochondria in all 5 uninfected control patients displayed uniform and spotty distribution. Ultrastructural 
analysis of HEV-infected Huh7 cells by transmission electron microscopy displayed elongated mitochondria (mitochondrial fusion) with obscure cristae. In contrast, mitochondria in uninfected cells displayed short and rod-like mitochondria with clear cristae. Consistently, confocal observation of HEV-infected cells by immunofluorescence substantiated tubular mitochondria, in contrast to dispersive and fragmented mitochondria in uninfected cells. Optic Atrophy 1 (OPA1) and mitofusion 1 (Mfn1) are the well-known positive regulators of mitochondrial fusion. In OPA1 and Mfn1 silencing cells, both the uninfected and HEV-infected cells presented fragmented mitochondria because of the impairment of the fusion machinery. Importantly, silencing of OPA1 or Mfn1 resulted in significant decrease of HEV RNA, suggesting that HEV-induced mitochondrial fusion facilitates viral infection. Further, exogenous mitochondrial dynamic regulator, ginsenoside $\operatorname{Rg} 3$ (G-Rg3), which had been reported to abrogate hepatitis $\mathrm{C}$ induced mitochondrial fission, was used to treat stable HEV infected cells. Importantly, G-Rg3 was able to promote HEV replication significantly.

Conclusion: HEV infection results in mitochondrial fusion which facilitates viral replication. Targeting mitochondrial dynamics represents a viable option for antiviral drug development against HEV.

\section{YIA-B-06}

Prometheus in the XXI century: analysis of the crosstalk between cell division and lipid metabolism in the liver

\section{Matias Jose Caldez ${ }^{1}$, Helena Salaberria Tellechea ${ }^{1}$, Philipp Kaldis $^{1}$}

${ }^{1}$ Institute of Molecular and Cell Biology, Singapore

Background: Cell division and metabolism are essential for tissue homeostasis and renewal. However, little is known if is there any crosstalk between these pathways. Early studies have suggested that both processes are changed during development of liver diseases; moreover it has been indicated that steatosis impairs hepatocyte division. Therefore, we hypothesized that there is an overlap between pathways that control cellular division with lipid metabolism in hepatocytes.

Method: To test our hypothesis we used a new strategy, by which we delete Cdk1, a master regulator of mitotic cell division, using the hepatocyte specific Albumin-cre. 2/3 partial hepatectomy was used and transcriptomic and lipidomic analysis was performed. Bioinformatic analysis was used to integrate both data sets to common pathways. Western blots and RT-qPCR, in combination with histopathology analysis of liver samples were used to validate the results.

Result: Initially, livers were collected at 8 weeks of age; mutant mice displayed changes in hepatocyte size, with enlarged hepatocytes composing up to $60 \%$ of the hepatic parenchyma, in addition to anisokaryosis. To understand the function of enlarged cells, we allowed mice to age for 18 months. Histopathology analysis indicated that mutant mice developed micro and macro vesicular steatosis, fibrosis, and inflammation; correlated with increased in expression of Fasn, Acaca, and Acly, central enzymes of fatty acid metabolism. These initial results suggested that changes in cell and nuclear size correlate with development of liver disease. In order to understand the relationship between cell division and lipid metabolism, we used a protocol of $2 / 3$ partial hepatectomy, which is useful to study cell division in vivo. During regeneration, wild type livers regenerated by cell division, while mutant livers regenerated by increases in cellular size, or cellular hypertrophy. We initially measured total lipids from blood at several time points after resection. Total triglycerides were increased in total blood from mutant mice, suggesting changes in lipid metabolism. Then, liver samples were subjected to transcriptomic analysis. Bioinformatic analysis indicated that both groups displayed decreased fatty acid synthesis, while lipid peroxidation was increased. We also found a significant increase in fatty acid elongation in wild type livers only; while inositol metabolism was decreased in mutant livers only. This suggested that blocking cell division in hepatocytes changes specific pathways of lipid metabolism during liver regeneration. To further validate the changes observed, we subjected the samples to lipidomic analysis. We observed that before liver resection, triglyceride metabolism was significantly decreased in mutant livers. However, ceramides and phosphatidyl-inositol metabolism were increased. On the contrary, during liver regeneration, total triglycerides were significantly increased in mutant livers, while phosphatidylinositol was significantly reduced. We further validated these results using qPCR, western blotting and immunohistochemistry.

Conclusion: These results validate that reduced cell division in hepatocytes induces changes in liver lipid metabolism. Moreover, we found that inositol metabolism may play a central role in this interaction, a new pathway useful for therapy.

\section{Oral Presentation}

Session: Alcoholic Liver Disease. Chairperson: Alaaeldin Ibrahim; Pankaj Tyagi; V Rajan

$O-A L-01$

Iron accumulation in alternatively activated macrophages modulates matrix metalloproteinase ADAM17 and mediates inflammation in severe alcoholic hepatitis

\section{Jaswinder Singh Maras ${ }^{1}$}

\section{${ }^{1}$ Institute of liver and biliary sciences, India}

Background: Macrophages are the secondary site for iron accumulation. Enhanced phagocytic activity in alternatively activated macrophages $(\mathrm{CD} 163+)$ results in iron accumulation and could correlate with inflammation in severe alcoholic hepatitis (SAH). Molecular mechanisms linking iron accumulation and inflammation in $\mathrm{SAH}$ are not clear.

Method: Frequency of CD163+ cells and Labile iron pool (LIP) was determined in SAH patients $(n=40)$, alcoholic cirrhosis (AC $\mathrm{n}=20)$ and Healthy control $(\mathrm{HC} ; \mathrm{n}=20)$. Gene expression of sorted $\mathrm{CD} 163+$ macrophages was compared to healthy monocyte derived macrophages (MDM) and Tohoku Hospital Pediatrics-1(THP1) macrophages treated with iron/Fenton-reagent in presence/absence of deferiprone. TAPI1 mediated ADAM17 inhibition was performed on THP1-macrophage under Iron/Fenton stress to establish the causality between iron-mediated activation of ADAM17, sCD163 and inflammation. Differentially regulated genes/pathways were confirmed using ELISA, Immunohistochemistry and Western blot in $101 \mathrm{SAH}$ and correlated with outcomes.

Result: The frequency of CD163+ macrophages, CD163+ TNF alpha + macrophages and LIP in CD163+ macrophages were higher in SAH than AC and HC ( $\mathrm{p}<0.005)$. Genes linked to iron-loading, oxidative-stress, inflammation together with CD163 and ADAM-17 were upregulated $(\mathrm{p}<0.05)$ in CD163+ macrophages than negative phenotypes. Expression of CD163, ADAM17 and CD68 was increased in SAH patients $(\mathrm{p}<0.01)$. Serum TNF- $\alpha$, iron, ferritin and sCD163 levels were higher $(\mathrm{p}<0.01)$ and haemoglobin-hepatoglobin (He-Hp) complex was lower $(\mathrm{p}<0.05)$ in SAH particularly nonsurvivors. Levels of sCD163 and TNF- $\alpha$ correlated with poor outcome $(\mathrm{HR}>3, \mathrm{C}$-index $=0.70, \mathrm{p}<0.01)$. CD163+ vemacrophages of non-survivors and MDM/THP1 macrophages under iron/Fenton stress showed markedly increased expression of iron-processing, metalloprotease activation, oxidative-stress and inflammation genes 
$(\mathrm{p}<0.01)$ which got neutralized by iron-chelation $(\mathrm{p}<0.05)$. Macrophage expression of CD163 and TNF- $\alpha$, IFN $\gamma$ were increased, while their secretory (cell supernatant) levels decreased $(p<0.05)$ under ADAM17 inhibition irrespective of iron/Fenton stress. Expression of inflammatory genes significantly decreased and iron regulatory genes were increased when ADAM17 was inhibited this suggests that iron regulates inflammation and CD163 expression via ADAM17 activation in macrophages. Further iron-chelation decreased ADAM17 $(\mathrm{p}<0.01)$ thereby preventing TNF-alpha maturation, CD163 shedding and inflammation.

Conclusion: Macrophage iron accumulation triggers inflammation and oxidative-stress via ADAM17 induction and lipid peroxidation. These events were reduced under iron-chelation or ADAM17 blocking and this could be used as new therapeutic options in treating SAH with iron-overload.

\section{$O-A L-02$}

\section{Bile acids and intestinal dysbiosis in alcoholic hepatitis}

\section{Dragos Ciocan ${ }^{1}$}

${ }^{1}$ INSERM, France

Background: The intestinal microbiota (IM) plays an important role in bile acid (BA) homeostasis, influences the gut barrier, and dysbiosis promotes inflammation. We aimed to study the structure of the $\mathrm{IM}$ and its function in BA homeostasis in alcoholic patients based on the severity of alcoholic liver disease (ALD).

Method: In this prospective study, we included four groups of active alcoholic patients $(\mathrm{N}=109)$ : two non-cirrhotic, with (noAC_AH, $\mathrm{n}=14$ ) or without alcoholic hepatitis (noAC_noAH, $\mathrm{n}=61$ ), and two cirrhotic, with (AC_sAH, $\mathrm{n}=17$ ) or without severe alcoholic hepatitis (AC_noAH, $\mathrm{n}=17$ ). Plasma and fecal BA profiles, as well as IM composition, were assessed.

Result: Plasma levels of total BAs, primary BAs, conjugated BAs, and total ursodeoxycholic acid (UDCA) were higher in AC_sAH than AC_noAH whereas total BA and secondary BA levels were lower in feces. AC_sAH patients had a different IM than AC_noAH patients: at the phyla level, the abundance of Actinobacteria was higher and that of Bacteroidetes was lower, and at the genus level, 11 taxa were modified. Moreover, the IM of AC sAH patients showed changes in the abundance of genes involved in 15 metabolic pathways, including upregulation of glutathione metabolism, and downregulation of biotin metabolism.

Conclusion: Patients with AC_sAH show specific changes of the BA pool with a shift towards more hydrophobic and toxic species that may be responsible for the specific IM changes. Conversely, the IM may also alter the BA pool by transforming primary BAs to secondary BAs, leading to a vicious cycle between modifications of IM and BAs.

\section{$O-A L-03$}

Effect of candesartan cilexetil on striatin-cGMP-nitric oxide axis in regulating endothelial dysfunction in alcoholic liver disease

\section{Balasubramaniyan Vairappan $^{1}$, Anjana Chandrasekar ${ }^{1}$ \\ ${ }^{1}$ JIPMER, India}

Background: Our recent clinical study in decompensated cirrhotic patients show decreased systemic striatin concentration, which was negatively correlated with systemic cGMP concentration; indeed the underlined molecular mechanism remains obscure. Our aims were to identify the striatin/NO pathway in a mouse model of alcoholic liver disease (ALD) and further to determine the effect of candesartan cilexetil (CC) in regulating endothelial dysfunction through modulation of striatin/NO pathway in ALD.

Method: Male Swiss mice ( $\mathrm{n}=10$ /group) were studied for 60 days. Four groups were studied. 1) naive, 2) na\&amp;iuml;ve + CC (8 mg/ $\mathrm{kg}$ for two weeks from day 45), 3) alcohol (6.32 $\mathrm{g} / \mathrm{kg}$ daily by gastric lavage, for 60 days) and 4) alcohol + CC (as prior dosing).

Result: Compared to naive mice, alcohol supplementation significantly $(\mathrm{p}<0.05)$ increased plasma levels of ALT, TNF- $\alpha$, Ang II, and von Willebrand factor. CC treatment to alcohol-fed mice corrected these indices significantly. Moreover, alcohol administration had markedly increased eNOS and caveolin-1 protein expression ( $p<0.01$, for both); but iNOS expression was insignificantly increased $(\mathrm{p}=0.1)$. CC treatment to alcohol-fed mice showed significantly $(\mathrm{p}<0.05)$ decreased eNOS and caveolin-1 expression, but iNOS expression was unchanged. Hepatic striatin expression was significantly $(\mathrm{p}<0.01)$ lowered in the alcohol-fed group when compared to na\&amp;iuml;ve. CC treatment to alcohol-fed mice showed significantly $(\mathrm{p}<0.05)$ increased striatin protein expression. Furthermore, compared with alcohol alone; hepatic cGMP was increased to naive levels following CC treatment whilst remaining significantly lower in the alcohol group. Liver histology showed that mice given alcohol had ballooning and macrovesicular type of \&lt;/ p\&gt; \&lt;p\&gt; fatty changes in the hepatocytes. CC treatment to alcohol-fed mice showed reduced these pathological changes.

Conclusion: These data provide the first in vivo evidence in rodents that lowered hepatic striatin correlated with decreased hepatic cGMP levels and increased caveolin-1 and thus endothelial dysfunction in ALD. CC treatment to alcohol-fed mice resulted in the increase of hepatic striatin and NO synthesis and improved endothelial dysfunction in ALD. Our further study will pinpoint the effect of $\mathrm{CC}$ on nitric oxide pathway and portal pressure reduction in alcoholic hepatitis patients.

\section{$O-A L-04$}

The prognostic stratification using acute-on-chronic liver failure scoring system for predicting short-term mortality in patients with alcoholic hepatitis

Dong Joon Kim ${ }^{1}$, Tae Yeob Kim ${ }^{13}$, Ashok Kumar Choudhury ${ }^{12}$, Ji Dong Jia $^{5}$, Priyanka Jain ${ }^{12}$, Irene Paulson ${ }^{12}$, Mamum Al Mahtab $^{4}$, Salimur Rahman ${ }^{4}$, Harshad Devarbhavi ${ }^{15}$, Zhongping Duan $^{6}$, Chen Yu ${ }^{6}$, CE Eapen ${ }^{9}$, Ashish Goel ${ }^{9}$, Q Ning ${ }^{16}$, George K Lau $^{11}$, P N Rao ${ }^{3}$, Diana Alcantarapayawal ${ }^{7}$, A. Kadir Dokmeci ${ }^{2}$, Man Fung Yuen ${ }^{14}$, V G Mohan Prasad ${ }^{17}$, Osamu Yokosuka ${ }^{8}$, Ananta Shrestha Prasad ${ }^{10}$

${ }^{1}$ Hallym University College of Medicine, Korea, Republic of; ${ }^{10}$ Foundation Nepal Sitapaila Height, Nepal; ${ }^{11}$ Humanity and Health Medical Group, China; ${ }^{12}$ Institute of Liver and Biliary Sciences, India; ${ }^{13}$ Leeheart Clinic, Korea, Republic of; ${ }^{14}$ Queen Mary Hospital, China; ${ }^{15}$ St John Medical College, India; ${ }^{16}$ Tongji Hospital, Tongji Medical College, China; ${ }^{17}$ VGM Hospital, India; ${ }^{2}$ Ankara University School of Medicine, Turkey; ${ }^{3}$ Asian Institute of Gastroenterology, India; ${ }^{4}$ Bangabandhu Sheikh Mujib Medical University, Bangladesh; ${ }^{5}$ Beijing Friendship Hospital, Capital University, China; ${ }^{6}$ Beijing Youan Hospital, Capital Medical University, China; ${ }^{7}$ Cardinal Santos Medical Center, Philippines; ${ }^{8}$ Chiba University, Japan; ${ }^{9} \mathrm{CMC}$, India

Background: To compare the existing various prognostic scoring models and newly proposed scores for acute-on-chronic liver failure (ACLF) and evaluate usefulness of stratification for the prediction of short-term morality in patients with alcoholic hepatitis (AH).

Method: A total of 708 clinical AH patients, enrolled in the APASLACLF Research Consortium (AARC) with 90-day follow-up, were analyzed. AARC-ACLF score, Maddrey discrimination function (DF) 
score, age, bilirubin, international normalized ratio and creatinine score (ABIC), Glasgow Alcoholic Hepatitis Score (GAHS), ChildTurcott-Pugh (CTP) score, model for end-stage liver disease (MELD), and MELD-Sodium (Na) scores were used to compare the performance for predicting 30-day and 90-day mortality. AARC-ACLF scores were categorized into three grades (Gr I: 5-7; II: 8-10; and III: 11-15 points) and survival curves by the Kaplan-Meier method were created and compared using log-rank test.

Result: Of 708 patients, $286(40.4 \%)$ and $363(51.3 \%)$ patients died within 30 days and 90 days, respectively. The area under receiver operating characteristics curve (AUC) of AARC-ACLF, DF, ABIC, GAHS, CTP, MELD, and MELD-Na was 0.752 (0.705-0.799), 0.630 (0.575-0.685), 0.658 (0.604-0.711), $0.577 \quad(0.523-0.631), \quad 0.641$ (0.589-0.694), 0.705 (0.653-0.756), 0.703 (0.651-0.755), respectively, for 30-day mortality. The AUC of various prognostic scores for the prediction of 90-day mortality is similar. The performance of AARCACLF was superior to that of DF, ABIC, GAHS, CTP, while comparable to that of MELD and MELD-Na in predicting short-term mortality. According to AARC-ACLF grades, short-term cumulative survivals was statistically different (30-day, 82.4, 70.4, and 35.3\%, $\mathrm{p}<0.001$; 90-day, 76.9, 56.0, and 26.4\%, $\mathrm{p}<0.001$ ).

Conclusion: Compared to the previous $\mathrm{AH}$ prognostic scores, AARC-ACLF score and grades are simple and useful for predicting the short-term mortality in patients with AH. Further studies are needed to confirm these implications.

\section{$O-A L-05$}

Extracellular vesicles from mice with alcoholic liver disease carry a distinct protein cargo and induce macrophage activation via Hsp90

\section{Banishree Saha ${ }^{1}$, Scott A Shaffer ${ }^{4}$, Gyongyi Szabo ${ }^{4}$, Fatemeh} Momen Heravi ${ }^{2}$, Istvan Furi ${ }^{4}$, Karen Kodys ${ }^{4}$, Donna Catalano ${ }^{4}$, Anwesha Gangopadhyay ${ }^{4}$, Reka Haraszti ${ }^{4}$, Abhishek Satishchandran ${ }^{4}$, Arvin Iracheta Vellve ${ }^{4}$, Adeyinka Adejumo ${ }^{3}$

${ }^{1}$ University of Massachusetts, United States; ${ }^{2}$ Columbia University, United States; ${ }^{3}$ North Shore Medical Center, United States;

${ }^{4}$ University of Massachusetts Medical School, United States

Background: A salient feature of alcoholic liver disease (ALD) is Kupffer cell (KC) activation and recruitment of inflammatory monocytes/macrophages (Mo/MØ). These key cellular events of ALD pathogenesis may be mediated by extracellular vesicles (EVs). EVs transfer biomaterials, including proteins and miRNAs, and have recently emerged as important effectors of intercellular communication.

Method: We hypothesized that circulating EVs from mice with ALD have a protein cargo characteristic of the disease and mediate biological effects by activating immune cells. We performed mass spectrometric analysis to determine the molecular composition (proteomic signature) of circulating ALD EVs and control EVs. To test the functional significance of ALD-EVs, in vitro and in vivo experiments were performed. Circulating ALD EVs were injected intravenously into naive mice, and liver mononuclear cells (LMNCs) and hepatocytes were isolated from the recipient mice for further analysis.

Result: The total number of circulating EVs was increased in mice with ALD (ALD-EV) compared to pair-fed controls (control-EV). Mass spectrometry analysis of circulating EVs revealed a distinct signature for proteins involved in inflammatory responses, cellular development and cellular movement between ALD-EVs and controlEVs. We also identified uniquely important proteins in ALD-EVs that were not present in control-EVs. When ALD-EVs were injected intravenously into alcohol-naïve mice, we found evidence of uptake of ALD-EVs in recipient livers in hepatocytes and MØs. Hepatocytes isolated from mice after transfer of ALD-EVs, but not control-EVs, showed increased MCP-1 mRNA and protein expression suggesting a biological effect of ALD-EVs. Compared to control-EV recipient mice, ALD-EV recipient mice had increased numbers of F4/80hi CD11blo KCs and increased percentages of TNF $\alpha+$ IL-12/23+ (inflammatory/M1) KCs and infiltrating monocytes (F4/80int CD11bhi) while the percentage of CD206+ CD163+ (anti-inflammatory/M2) KCs was decreased. In vitro, ALD-EVs increased TNF $\alpha$ and IL-1 $\beta$ production in MØ and reduced CD163 and CD206 expression. We identified Heat shock protein 90 (Hsp90) in ALD-EVs as the mediator of ALD-EV-induced MØ activation.

Conclusion: Our study indicates a specific protein signature of ALDEVs and demonstrates a functional role of circulating EVs containing $\mathrm{Hsp} 90$ in mediating $\mathrm{KC} / \mathrm{M} \varnothing$ activation in the liver.

\section{$O-A L-06$}

The TIM3-Gal9 immune checkpoint axis is inter-linked with severity of Alcoholic Liver Disease

Shilpa Chokshi ${ }^{1}$, Antonio Riva ${ }^{1}$, Huyen Adams ${ }^{1}$, Vish Patel ${ }^{1}$, Sarah Azarian $^{1}$, Gavin Wright ${ }^{2}$, Sarah Tarff ${ }^{2}$, Krum Katzarov ${ }^{3}$, Marieta Simonova $^{3}$, Tanya Hadzhiolova ${ }^{3}$, Slava Pavlova ${ }^{3}$, Roger Williams ${ }^{1}$

${ }^{1}$ Foundation for Liver Research, United Kingdom; ${ }^{2}$ Basildon Hospital, United Kingdom; ${ }^{3}$ Military Medical Academy, Bulgaria

Background: Susceptibility to overwhelming bacterial infection represents a significant cause of mortality in alcoholic liver disease (ALD). T-cell immunoglobulin and mucin domain (TIM)3 is a well characterised immune checkpoint receptor that modulates the equilibrium between protective anti-pathogen immunity and host-induced immunopathology. We have previously shown (Markwick et al, Gastroenterology, 2015) that hyper-expression of TIM3 on adaptive T-cells suppresses antibacterial immunity in ALD and in vitro blockade can rescue impaired innate and adaptive immunity. However, the role of soluble (s)TIM3, its ligand Galectin(sGal)9 and other checkpoint receptors in antibacterial immunity and in ALD is unknown and is the aim of this study.

Method: We measured levels of soluble checkpoint receptors in plasma samples from patients with alcohol-related cirrhosis (ARC, Child-Pugh A/B/C, $n=6 / 8 / 8$ ), severe alcoholic hepatitis (SAH, Maddrey's DF $>32, n=13$ ), alcohol-related acute-on-chronic liver failure (ACLF, $n=6$, longitudinal) and healthy controls $(n=13)$ and correlated these findings with demographic and clinical characteristics. Receiver operating characteristic area under the curve analysis (AUROC) was used to investigate the discriminatory power of STIM3 as a disease biomarker.

Result: Plasma sTIM3 was significantly different in patients and controls $(p<0.0001)$, with greater amounts in ARC $(p=0.0004)$, SAH $(\mathrm{p}<0.0001)$ and ACLF $(\mathrm{p}=0.0065)$ patients. sGal9 was also significantly higher in SAH vs. controls $(\mathrm{p}=0.0116)$. Stratification of ARC patients according to Child-Pugh score (CP) indicated greater levels of sTIM3 in CP-C compared to CP-A or CP-B patients. Levels of STIM3 in CP-C, SAH and ACLF patients were higher than controls $(\mathrm{p}=0.0004, \mathrm{p}=0.0003, \mathrm{p}=0.0156$ respectively). AUROC analysis for sTIM3 produced a value of 0.936 (95\% CI 0.868-1.000, $\mathrm{p}<0.001$ ) and at a cut-off of $2427.053 \mathrm{pg} / \mathrm{mL}$ the discriminatory sensitivity and specificity between ALD patients and controls were $90.2 \%$ and $88.5 \%$ respectively.

Conclusion: Our results show that the TIM3-Gal9 axis plays an important role in the pathogenesis of ALD and that sTIM3 represents a novel biomarker of disease severity. 
Session: Acute Liver Failure and Acute on chronic Liver failure; Chairperson: Osamu Yokosuka; Piyush Ranjan; Chetan Kalal

\section{$O-A C L F-01$}

Significant increase in intestinal permeability in acute-on-chronic liver failure (ACLF) is reversed after slow albumin furosemide infusion with or without terlipressin

Gaurav Pandey ${ }^{1}$, Arun Singh Badoria ${ }^{1}$, Shreya Bhutala ${ }^{1}$, Prabhat Narayan Sharma ${ }^{1}$

${ }^{1}$ SGPGI, India

Background: Increased intestinal permeability (IP), increased bacterial translocation (BT), small intestinal bacterial overgrowth (SIBO) and gut dysbiosis are well known in liver cirrhosis (LC). In acute-onchronic liver failure (ACLF), marked increase in IP and BT may occur due to a surge in portal hypertension

Method: Measuring lactulose/mannitol ratio (LMR) in the urine using $1 \mathrm{H}-\mathrm{NMR}$ spectroscopy is a simple, efficient way to assess intestinal permeability (IP). Urinary LMR was measured in 20 healthy controls, 20 patients with decompensated (CTP-B,C) LC, 20 patients of ACLF before therapy out of which 20 patients after ascites mobilisation of large ascites which was treated with continuous slow albumin and furosemide infusion with or without terlipressin were included in study. Among this last group average duration of therapy was 14 days. Result: IP was highest in ACLF patients before treatment with SAFI \pm TMedian value urinary LMR 1.4, intermediate in decompensated cirrhotics 0.55 and lowest in healthy volunteers 0.11 . After control of ascites with SAFI \pm T in ACLF, urinary LMR was similar to normal controls 0.19 ( $\mathrm{P}$ value in comparison to pre-treatment value was 0.03 ) suggesting that control of ascites in ACLF normalized the increased IP. Conclusion: ACLF patients have higher intestinal permeability compared with decompensated cirrhotics and controls. Intestinal permeability decreases after decongestive therapy, suggesting that intestinal decongestion may reverse increased IP in ACLF.

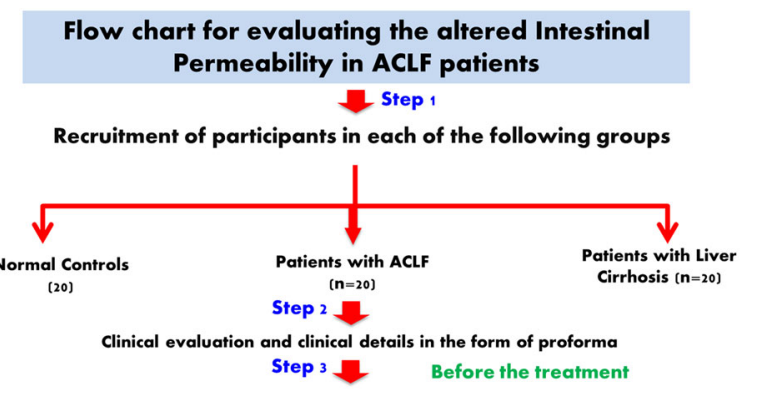

Collection of early morning urine samples and then oral administration of (lactulose/ mannitol solution to the participants and collection of urine samples after 30, 60, 90, 150 and 210 minutes Step 4

All the ACLF patients will be managed as per the standard clinical protocol of management Step 5 After the treatment

After clinical improvement, the urine samples of ACLF patients will be collected following the step 3 (for follow up investigation] Step o 1

The collected urine samples will be cooled and stored at $-80 \mathrm{C}$ as per the standard protocol before the start of NMR experiments Step 7

NMR sample preparation and data collection Step 8

To process and analyze all the NMR data using multivariate/Univariate statistical analysis tools Step 9

Biological and clinical interpretation Step $10-1$

Report the results to the scientificibiomedical community

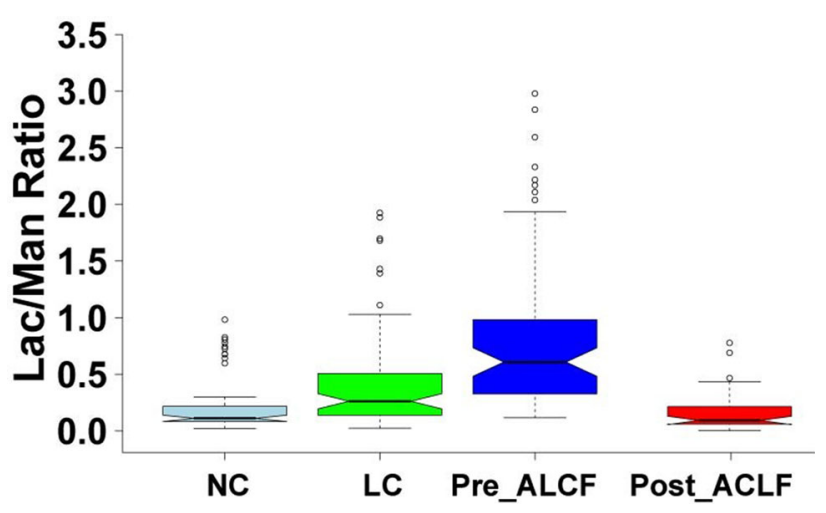

$O-A C L F-02$

\section{PROFILE OF ACLF CASES IN ASIA: A MULTICENTRE STUDY}

APASL ACLF Working party ${ }^{1}$, Mamun Al Mahtab ${ }^{6}$, Salimur Rehman $^{6}$, Harshad Devarbhavi ${ }^{27}$, Zhongping Duan ${ }^{8}$, Chen Yu ${ }^{8}$, Dong Joon Kim ${ }^{16}$, Ji Dong Jia ${ }^{7}$, C E Eapen ${ }^{13}$, Ashish Goel ${ }^{13}$, Qin Ning $^{28}$, Yogesh Kumar Chawla ${ }^{24}$, R K Dhiman ${ }^{24}$, Ajay Duseja ${ }^{24}$, Sunil Taneja ${ }^{24}$, Saeed Sadiq Hamid ${ }^{3}$, Amna Subhan Butt ${ }^{3}$, Wasim Jafri $^{3}$, Soek Siam Tan ${ }^{17}$, Hasmik Ghazinian ${ }^{23}$, Deepak N

Amarapurkar', Sombat Treeprasertsuk ${ }^{12}$, Guan Huei Lee ${ }^{31}$, Seng GEE $\mathrm{Lim}^{31}$, Jin Hua $\mathrm{Hu}^{2}$, Laurentius A. Lesmana ${ }^{22}$, Cosmas Rinaldi Lesmana ${ }^{22}$, Akash Shukla ${ }^{21}$, Samir Shah ${ }^{15}$, Chetan Kalal $^{15}$, Zaigham Abbas ${ }^{32}$, Jose D Sollano ${ }^{29}$, Gian Carpio ${ }^{29}$, Mohammed Fazal Karim ${ }^{26}$, George K Lau ${ }^{18}$, P N Rao ${ }^{5}$, Diana

Alcantra Payawal ${ }^{10}$, A.Kadir Dokmeci ${ }^{4}$, Manfung Yuen ${ }^{25}$, V G

Mohan Prasad $^{30}$, Osamu Yokosuka ${ }^{11}$, Ananta Prasad ${ }^{14}$, Priyanka Jain $^{19}$, Irene Paulson ${ }^{19}$, Shiv Kumar Sarin ${ }^{19}$, Manoj Kumar Sahu $^{20}$, Ayaskant Singh ${ }^{20}$, Ashok R Choudhury ${ }^{19}$

${ }^{1}$ APASL, India; ${ }^{10}$ Cardinal Santos Medical Center 10 Wilson St. Greenhills West San Juan City, Philippines; ${ }^{11}$ Chiba University Japan, Japan; ${ }^{12}$ Chulalongkorn University, Bangkok., Thailand;

${ }^{13} \mathrm{CMC}$ Vellore, India; ${ }^{14}$ Foundation Nepal Sitapaila Height, Kathmandu, Nepal; ${ }^{15}$ Global Hospital, Mumbai, India, India;

${ }^{16}$ Hallym University College of Medicine, Korea, Democratic People's Republic of; ${ }^{17}$ Hospital Selayang, Bata Caves, Selangor, Malaysia; ${ }^{18}$ Humanity and Health Medical Group, China, China;

${ }^{19}$ ILBS, India; ${ }^{2} 302$ Millitary Hospital Beijing, China, India; ${ }^{20}$ IMS \&SUM hospital, Odisa, India; ${ }^{21}$ KEM Hospital and Seth GSMC, India; ${ }^{22}$ Medistra Hospital, Jakarta, Indonesia, Indonesia; ${ }^{23}$ Nork Clinical Hospital of Infectious Disease Armenia, Armenia;

${ }^{24}$ PGIMER, Chandigarh India, India; ${ }^{25}$ Queen Mary Hospital Hong Kong, China, China; ${ }^{26}$ Sir Salimullah Medical College, Mitford Hospital Bangladesh, Bangladesh; ${ }^{27}$ St Johns Medical college, India; ${ }^{28}$ Tongji Hospital, Tongji Medical College, China; ${ }^{29}$ University of Santo Tomas, Manila, Philippines, Philippines; ${ }^{3}$ Aga Khan University Hospital, Karachi, Pakistan; ${ }^{30}$ VGM Hospital Coimbatore, India, India; ${ }^{31}$ Yong Loo Lin School of Medicine, National University of Singapore, Singapore; ${ }^{32}$ Ziauddin University Hospital, Karachi, Pakistan; ${ }^{4}$ Ankara University School of Medicine, Turkey; ${ }^{5}$ Asian Institute of Gastroenterology Hyderabad, India, India; ${ }^{6}$ Bangabandhu Sheikh Mujib Medical university, Bangladesh; ${ }^{7}$ Beijing Friendship Hospital, Capital University, Beijing, China, China; ${ }^{8}$ Beijing You'an 
Hospital, China; ${ }^{9}$ Bombay Hospital \& Medical Research Center, Mumbai, India, India

Background: Acute on Chronic Liver Failure (ACLF) is an emerging entity. There is scarce data on profile of ACLF cases in the literature. The present study was undertaken to study the profile of ACLF cases in Asia.

Method: ACLF concept was defined as per Asian Pacific Association for the Study of Liver (APASL) consensus criteria 2014. Consecutive cases attending different centers in India and other Asian countries satisfying the above criteria were included and prospectively evaluated for three months.

Result: Out of total 2000 patients with ACLF (mean age: $45.61 \pm 12.87$ years), male outnumbered the female (Male: Female5:1). Most common underlying chronic liver disease (CLD) and acute insult were alcoholic liver disease $(46.8 \%)$ and drinking of alcohol $(43.6 \%)$ respectively. Ascites and hepatic encephalopathy were present in $74.2 \%$ and $35.55 \%$ cases respectively. Mean serum bilirubin was $18.85 \pm 10.08 \mathrm{mg} / \mathrm{dl}$. $44.25 \%$ patients died within 3 months (early mortality). Early mortality was most common in alcoholics (49.15\%). The prognostic scorings at base line were CLIF SOFA: $11.7 \pm 2.91$; APASL ACLF Research Consortium (AARC) SCORE: $9.75 \pm 2.11$; APACHAE: $15.63 \pm 6.68$; SOFA: $8.71 \pm 3.11$; CTP: $11.7 \pm 1.59$; MELD: $27.92 \pm 7.49$; MELD Na: $30.95 \pm 7.15$. All these prognostic scorings were significantly higher in died patients compared to survived patients (p: 0.0001). Multivariate logistic regression analysis showed that AARC scoring is the single most important predictor for early mortality.

Conclusion: ACLF patients in Asian countries had high early mortality with majority of death occurring in alcoholics. AARC scoring is the single most important prognostic determinant in prediction of early mortality in ACLF cases.

\section{$O-A C L F-03$}

The prognostic determinant in acute on chronic liver failure: a multicentre prospective cohort study

APASL ACLF Working party ${ }^{1}$, Manoj Kumar Sahu ${ }^{21}$, Preetam Nath $^{20}$, Ashok Kumar Choudhury ${ }^{19}$, Mamun Al Mahtab ${ }^{6}$, Salimur Rehman ${ }^{6}$, Harshad Devarbhavi ${ }^{28}$, Zhingping Duan ${ }^{8}$, Chen $\mathrm{Yu}^{8}$, Dong Joon Kim ${ }^{16}$, Ji Dong Jia ${ }^{7}$, C E Eapen ${ }^{13}$, Ashish Goel $^{13}$, Qin Ning ${ }^{29}$, Yogesh Kumar Chawla ${ }^{25}$, R K Dhiman ${ }^{25}$, Ajay Duseja ${ }^{25}$, Sunil Taneja ${ }^{25}$, Saeed Sadiq Hamid ${ }^{3}$, Amna Subhan Butt ${ }^{3}$, Wasim Jafri ${ }^{3}$, Soek Siam Tan ${ }^{17}$, Hasmik Ghazinian $^{24}$, Deepak N Amarapurkar', Sombat

Treeprasertsuk $^{12}$, Guan Huei Lee ${ }^{32}$, S G Lim ${ }^{32}$, Jin Hua $\mathrm{Hu}^{2}$, Laurentius A. Lesmana ${ }^{23}$, Cosmas Rinaldi Lesmana ${ }^{23}$, Akash Shukla $^{22}$, Samir Shah ${ }^{15}$, Chetan Kalal ${ }^{15}$, Zaigham Abbas ${ }^{33}$, Jose D Sollano $^{30}$, Gian Carpio ${ }^{30}$, Mohammed Fazal Karim ${ }^{27}$, George K Lau ${ }^{18}$, P N Rao ${ }^{5}$, Diana Alcantra Payawal ${ }^{10}$, A.Kadir Dokmeci $^{4}$, Manfung Yuen ${ }^{26}$, V G Mohan Prasad ${ }^{31}$, Osamu Yokosuka $^{11}$, Ananta Prasad ${ }^{14}$, Priyanka Jain ${ }^{19}$, Irene Paulson ${ }^{19}$, Shiv Kumar Sarin ${ }^{19}$

${ }^{1}$ APASL, India; ${ }^{10}$ Cardinal Santos Medical Center 10 Wilson St. Greenhills West San Juan City, Philippines; ${ }^{11}$ Chiba University Japan, Japan; ${ }^{12}$ Chulalongkorn University, Bangkok., Thailand; ${ }^{13} \mathrm{CMC}$ Vellore, India; ${ }^{14}$ Foundation Nepal Sitapaila Height, Kathmandu, Nepal; ${ }^{15}$ Global Hospital, Mumbai, India, India;

${ }^{16}$ Hallym University College of Medicine, Korea, Republic of;

${ }^{17}$ Hospital Selayang, Bata Caves, Malaysia; ${ }^{18}$ Humanity and Health Medical Group, China, China; ${ }^{19}$ ILBS, India; ${ }^{2} 302$ Millitary Hospital Beijing, China, China; ${ }^{20}$ IMS \&SUM hospital, India; ${ }^{21}$ IMS \&SUM hospital, Odisa, India; ${ }^{22}$ KEM Hospital and Seth GSMC, India;
${ }^{23}$ Medistra Hospital, Jakarta, Indonesia, Indonesia; ${ }^{24}$ Nork Clinical Hospital of Infectious Disease Armenia, Armenia; ${ }^{25}$ PGIMER, Chandigarh India, India; ${ }^{26}$ Queen Mary Hospital Hong Kong, China, China; ${ }^{27}$ Sir Salimullah Medical College, Mitford Hospital Bangladesh, Bangladesh; ${ }^{28}$ St Johns Medical college, India; ${ }^{29}$ Tongji Hospital, Tongji Medical College, China; ${ }^{3}$ Aga Khan University Hospital, Karachi, Pakistan; ${ }^{30}$ University of Santo Tomas, Manila, Philippines, Philippines; ${ }^{31}$ VGM Hospital Coimbatore, India, India; ${ }^{32}$ Yong Loo Lin School of Medicine, National University of Singapore, Singapore; ${ }^{33}$ Ziauddin University Hospital, Karachi, Pakistan; ${ }^{4}$ Ankara University School of Medicine, Turkey; ${ }^{5}$ Asian Institute of Gastroenterology Hyderabad, India, India; ${ }^{6}$ Bangabandhu Sheikh Mujib Medical university, Bangladesh; ${ }^{7}$ Beijing Friendship Hospital, Capital University, Beijing, China, China; ${ }^{8}$ Beijing You'an Hospital, China; ${ }^{9}$ Bombay Hospital \& Medical Research Center, Mumbai, India, India

Background: Acute on Chronic Liver Failure (ACLF) is an evolving concept. There is scarce data on prognostication of ACLF cases mentioned in the literature. The present study was undertaken to find out the prognostic determinant in ACLF patients.

Method: ACLF concept was defined as per Asian Pacific Association for the Study of Liver (APASL) consensus criteria 2014. Consecutive cases attending different centers in India and other Asian countries satisfying the above criteria were included and prospectively evaluated for three months

Result: Out of total 2000 patients with ACLF (mean age: 36 years; M/F-1669:331), 30\% and 44.25\% patients died within 1 and 3 months respectively. Most of the deaths occurred in cases having acidosis [Adjusted odds ratio (AOR):30.04]; fever (AOR: 3.08); multiple organ failure (AOR: 1.95); cerebral failure (AOR: 1.44); renal failure (AOR: 1.4); prolonged ICU stay (AOR: 1.36); higher serum lactate (AOR: 1.17); higher serum bilirubin(AOR: 1.06); higher INR(PT) with AOR: 1.02; higher leukocyte count (AOR: 1.01); higher heart rate (AOR: 1.01). APASL ACLF Research Consortium (AARC score with AOR: 1.29) correlated better with early mortality compared to other prognostic scorings [SOFA (AOR: 1.11); MELD (AOR: 1.05); APACHAE (AOR: 1.04); CTP (AOR: 1.01); Glasgow coma scale (AOR: 0.95); CLIF SOFA (AOR: 0.92)].

Conclusion: ACLF patients in Asian countries had high early (3 months) mortality with majority of death occurring in cases having acidosis, fever, and multiple organ failure. AARC scoring is the single most important prognostic determinant in prediction of early mortality in ACLF cases.

\section{$O-A C L F-04$}

Role and regulation of mitophagy by ROS in hepatic stellate cells during acute liver failure

\section{Zhen Tian', Yingli $\mathrm{He}^{2}$, Tianyan $\mathrm{Chen}^{2}$, Yingren $\mathrm{Zhao}^{2}$}

${ }^{1}$ Department of Infectious Diseases, the First Affiliated Teaching Hospital, School of Medicine, Xian JiaoTong University, China; ${ }^{2}$ Department of Infectious Disease, the First Affiliated Hospital, Xi'an Jiaotong University, Xi' an 710061, China, China

Background: We previously found that acute liver failure (ALF) is accompanied by hepatic stellate cells (HSCs) activation. HSCs also integrate cytokine-mediated inflammation responses in the sinusoids and relay them to the liver parenchyma in immune-mediated hepatitis. Oxidative stress has been shown to promote inflammation. Whether and how oxidative stress is involved in ALF and HSCs inflammation remains unclear.

Method: ALF patients were recruited to investigate the correlation between plasma superoxide dismutase (SOD) levels and clinical 
features. Liver tissues were collected from chronic hepatitis patients by biopsy and from ALF patients who had undergone liver transplantation. The expression of SOD2, hepatic stellate cell activation $(\alpha-$ SMA) and mitophagy activity (Tom40) were investigated by immunohistochemistry. Inflammation, mitophagy and apoptosis were investigated by immunoblot analysis, confocal microscopy and flow cytometry in HSCs treated with LPS and reactive oxygen species (ROS) donors.

Result: Plasm SOD level was significantly increased in patients with ALF compared to those with cirrhosis $(444.4 \pm 23.58 \mathrm{U} / \mathrm{mL}$ vs $170.07 \pm 3.52 \mathrm{U} / \mathrm{mL}, P<0.01)$ and positively correlated with MELD-score $(\mathrm{r}=0.5053, P<0.01)$. In vivo observation revealed that mitophagy was inhibited in HSCs based on increased Tom40 immunostaining, and in vitro experiments demonstrated that ROS can promote inflammation via inhibition of mitophagy. Moreover, regulation of inflammation in HSCs was apoptosis independent.

Conclusion: Increased oxidative stress induced by LPS participants in promoting inflammation through mitophagy inhibition in HSCs during the process of ALF, providing a novel strategy for the treatment of patients with ALF.

Table 1 Demographic data and clinical characteristics of controls* and ALF patients

\begin{tabular}{|c|c|c|c|}
\hline Parameter & $\begin{array}{l}\text { Healthy Control } \\
(n=30)\end{array}$ & $\begin{array}{l}\text { Cirrhosis } \\
(\mathrm{n}=30)\end{array}$ & $\begin{array}{l}\text { Acute Liver Failure } \\
(\mathrm{n}=30)\end{array}$ \\
\hline Age(yrs) & $31.13 \pm 6.8$ & $38.58 \pm 5.19$ & $37 \pm 8.38$ \\
\hline Gender(M/F) & $20 / 10$ & $20 / 10$ & $20 / 10$ \\
\hline PTA(\%) & $86.17 \pm 11.62$ & $76.15 \pm 17.2$ & $42.79 \pm 17.98$ \\
\hline $\mathrm{FIB}(\mathrm{g} / \mathrm{L})$ & $2.53 \pm 0.67$ & $2.14 \pm 0.5$ & $1.66 \pm 0.67$ \\
\hline INR & $1.06 \pm 0.07$ & $1.16 \pm 0.15$ & $1.78 \pm 0.46$ \\
\hline WB(109/L) & $5.54 \pm 1.44$ & $4 \pm 1.53$ & $7.69 \pm 3.61$ \\
\hline $\operatorname{PLT}\left(10^{9} / \mathrm{L}\right)$ & $211.97 \pm 38.88$ & $113.29 \pm 74.23$ & $135.93 \pm 61.83$ \\
\hline $\operatorname{ALT}(\mathrm{U} / \mathrm{L})$ & $22.2 \pm 15.33$ & $33.81 \pm 21.28$ & $405.76 \pm 519.76$ \\
\hline $\mathrm{GLU}(\mathrm{mM})$ & $4.19 \pm 0.36$ & 4.29 0.79 & $4.97 \pm 2.01$ \\
\hline TBIL $(\mu \mathrm{M})$ & $10.13 \pm 3.81$ & $19.26 \pm 9.88$ & $339.33 \pm 160.1$ \\
\hline $\mathrm{CHOL}(\mathrm{mM})$ & $3.72 \pm 0.67$ & $3.41 \pm 0.8$ & $2.8 \pm 0.92$ \\
\hline $\operatorname{CREA}(\mu \mathrm{M})$ & $54.67 \pm 12.64$ & $53.81 \pm 12.86$ & $60.07 \pm 25.64$ \\
\hline MELD & & & $23.7 \pm 4.43$ \\
\hline
\end{tabular}
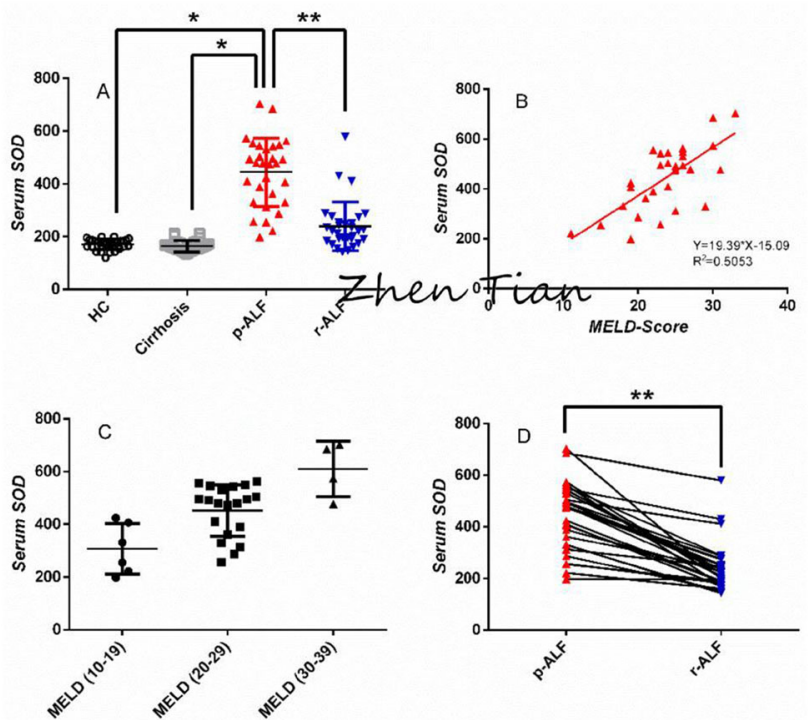

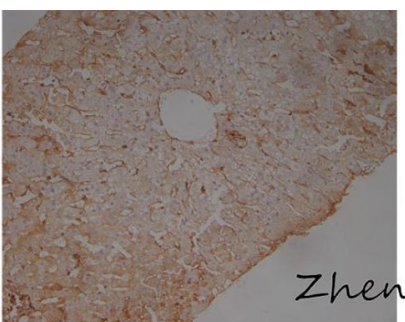

ALF SOD2 100X

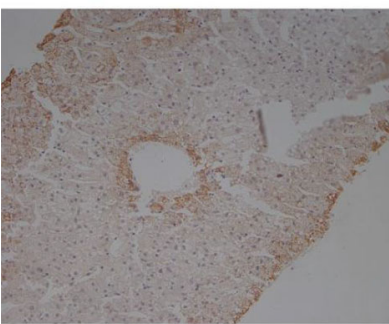

CHB SOD2 100X

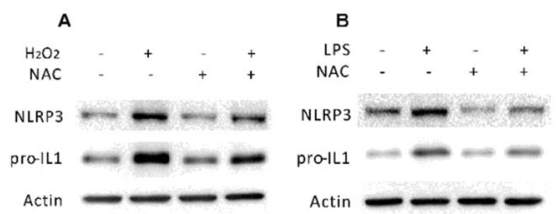

$$
\text { D }
$$

E

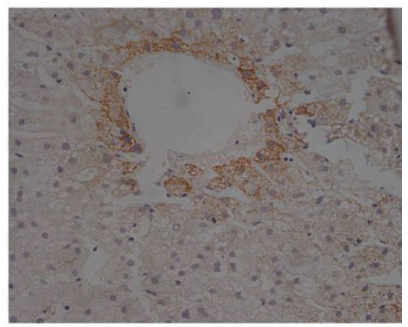

CHB SOD2 200X
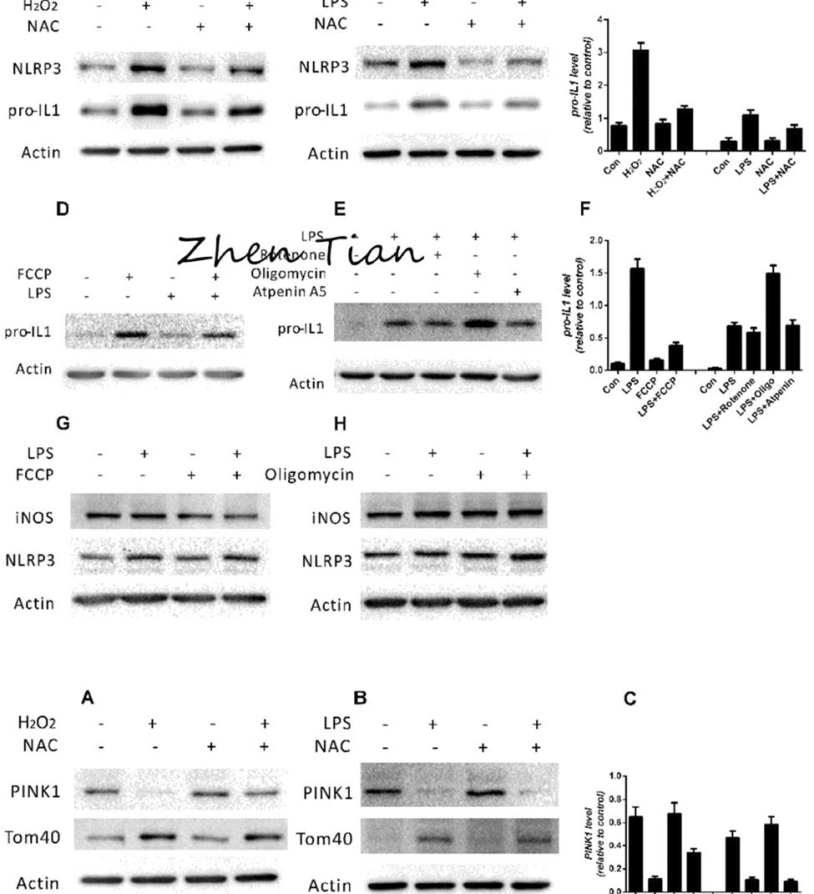

c

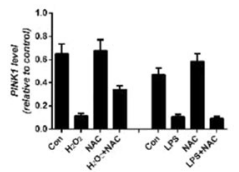

$\mathrm{F}$

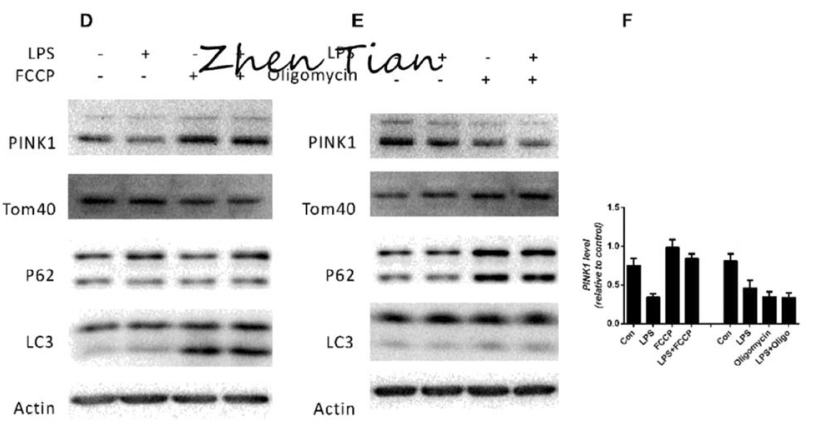



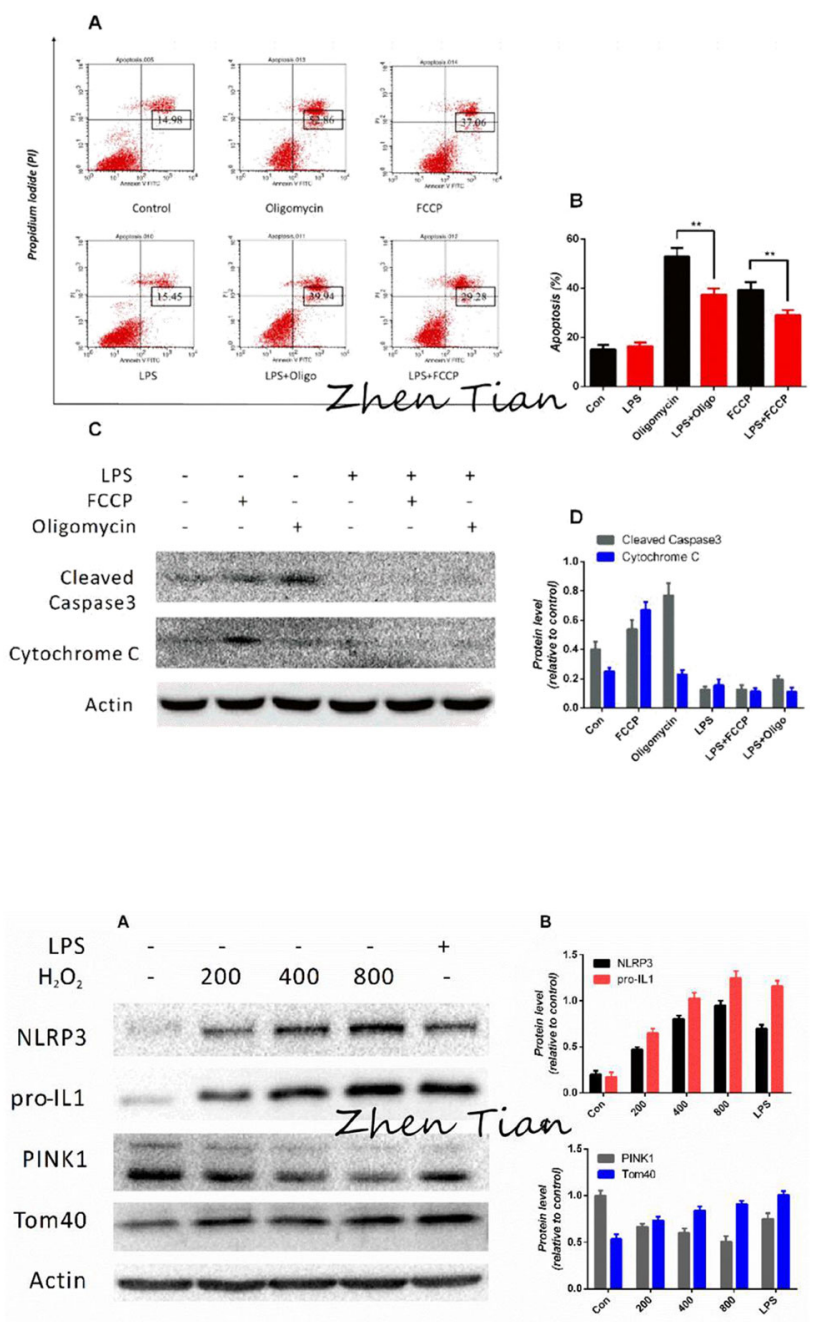

O-ACLF-05

Validation of CLIF-C AD score in prediction of post-admission development of ACLF in patients with $\mathrm{HBV}$ associated decompensated cirrhosis

\section{$\underline{\text { Yuhan Liu }}^{1}$, Zhujun Cao ${ }^{1}$, Qing Xie ${ }^{1}$}

${ }^{1}$ Ruijin Hospital, China

Background: Acute-on-chronic liver failure (ACLF) is associated with high short-term mortality. Early prediction of ACLF development in patients admitted without ACLF is essential for risk stratification and individualized management. Recently, a specific score, CLIF-C AD for these patients has been developed using the CANONIC study database. The aim of this study was to validate this score in patients with HBV associated decompensated cirrhosis (DC) and further compare it to other available scores.

Method: A retrospective cohort with HBV-DC without ACLF at admission were included. ROC analysis was performed to determine predictive accuracy of different scores. Outcome was post-admission development of ACLF by within 28-day. Proportional hazards models using competing risk approach with the Fine and Gray method were further used to identify risk factors (parameters for scores). Liver transplantation before ACLF onset was considered as a competing event for ACLF development.

Result: A total of 436 patient with HBV associated decompensated cirrhosis was included in this analysis. Seventy-four patients (16.9\%) developed ACLF within 28-day of hospitalization. Six scores (CLIF-C AD, CLIF-OF, CLIF-SOFA, CTP, MELD, MELD-NA) were calculated for comparisons. Among them the most accurate score was MELD-NA score (AUROC, 0.89, [95\% CI: 0.86-0.92]) followed by MELD (AUROC, 0.89), CLIF-SOFA (AUROC, 0.873), CLIF-OF (AUROC, 0.867), CTP (AUROC, 0.830) and CLIF-C-AD (AUROC, 0.803). The CLIF-C AD was the poorest scores for the prediction of ACLF in our HBV-DC cohort. Further multivariate analysis demonstrated that the presence of hepatic encephalopathy (sHR, 2.2), serum total bilirubin (sHR, 1.1), serum creatinine (sHR, 2.9), INR (sHR, 1.96), albumin (sHR, 0.99), sodium (sHR, 0.96) and blood leukocytes (sHR, 1.004) were independent predictors for the development of ACLF. Conclusion: In patients with HBV associated DC patients, CLIF-C AD score was inferior to MELD or MELD-NA score. The distinct pathophysiology between alcoholic and HBV aetiology may be the reason of disadvantage of CLIF-C AD in HBV patients.

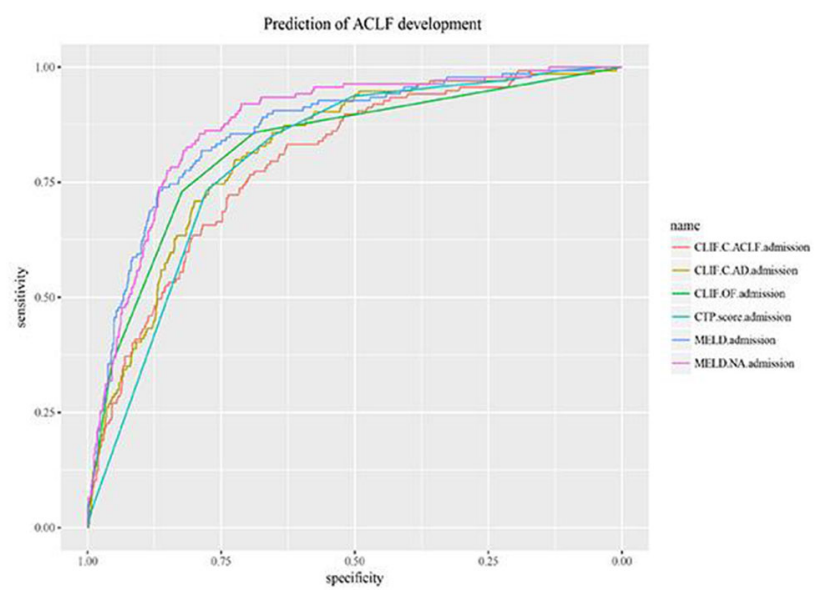

$O-A C L F-06$

Characteristics and outcome of acute-on-chronic liver failure patients with hepatic encephalopathy

Guan Huei Lee ${ }^{1}$, Wah Wah Phyo', Ashok Choudhury ${ }^{15}$, Jindal Ankur $^{15}$, Rakhi Maiwall ${ }^{15}$, Manoj Kumar Sharma ${ }^{15}$, B.C Sharma ${ }^{15}$, Viniyendra Pamecha ${ }^{15}$, Mamun Mahtab ${ }^{5}$, S Rahman ${ }^{5}$, Yogesh Kumar Chawla ${ }^{17}$, Sunil Taneja ${ }^{17}$, Soek Siam Tan ${ }^{18}$, Harshad Devarbhavi ${ }^{21}$, Zhongping Duan ${ }^{7}$, Chen $\mathrm{Yu}^{7}$, Qin Ning ${ }^{23}$, Ji Dong Jia ${ }^{6}$, Deepak Amarapurkar ${ }^{8}$, C.E. Eapen ${ }^{11}$, Ashish Goel $^{11}$, Saeed Hamid ${ }^{2}$, Amna Subhan Butt ${ }^{2}$, Wasim Jafri' ${ }^{2}$, D.J. Kim $^{10}$, Hasmik Ghazinian ${ }^{16}$, Ajit Sood ${ }^{12}$, L.A. Lesmana ${ }^{24}$, Abbas Zaigham $^{19}$, Gamal Shiha ${ }^{13}$, Diana Payawal ${ }^{9}$, A.Kadir Dokmeci ${ }^{3}$, J.D. Sollano" ${ }^{9}$, Gian Carpio ${ }^{9}$, G.K. Lau ${ }^{22}$, Fazal Karim ${ }^{20}$, P.N. Rao $^{4}$, Richard Moreau ${ }^{14}$, P. Jain ${ }^{15}$, P. Bhatia ${ }^{15}$, G. Kumar ${ }^{15}$, Shiv Kumar Sarin ${ }^{15}$

${ }^{1}$ Yong Loo Lin School of Medicine, National University of Singapore, Singapore; ${ }^{10}$ Center for Liver and Digestive Diseases, Chuncheon, Korea, Democratic People's Republic of; ${ }^{11}$ Christian Medical College, India; ${ }^{12}$ Dayanand Medical College, India; ${ }^{13}$ Egyptian Liver Research Institute and Hospital, Egypt; ${ }^{14}$ Inserm, U1149, Centre de recherche sur l'Inflammation (CRI), UMR_S 1149, Labex INFLAMEX, Université Paris Diderot Paris 7, France; ${ }^{15}$ Institute of Liver and Biliary Sciences (ILBS), India; ${ }^{16}$ Nork Clinical Hospital of Infectious 
Diseases, Armenia; ${ }^{17}$ Post Graduate Institute of Medical Education and Research, India; ${ }^{18}$ Selayang Hospital, Malaysia; ${ }^{19}$ Sindh Institute of Urology and Transplantation, Pakistan; ${ }^{2}$ Aga Khan University Hospital, Pakistan; ${ }^{20}$ Sir Salimur Rehman Medical College, Mitford Hospital, Bangladesh; ${ }^{21}$ St John Medical College, India; ${ }^{22}$ The Institute of Translational Hepatology, China; ${ }^{23}$ Tongji Medical College, Tongji Hospital, Huazhong University of Science and Technology, China; ${ }^{24}$ University of Indonesia, Indonesia; ${ }^{3}$ Ankara University School of Medicine, Turkey; ${ }^{4}$ Asian Institute of Gastroenterology, India; ${ }^{5}$ Bangabandhu Sheikh Mujib Medical University, Bangladesh; ${ }^{6}$ Beijing Friendship Hospital, Capital Medical University, China; ${ }^{7}$ Beijing Youan Hospital, Capital Medical University, China; ${ }^{8}$ Bombay Hospital and Medical Research Centre, India; ${ }^{9}$ Cardinal Santos Medical Center, Philippines

Background: Acute-on-chronic liver failure (ACLF) is associated with high mortality. Hepatic encephalopathy (HE) has been identified as a negative prognostic factor. We examine the impact of HE on the outcome of ACLF patients in an Asian cohort.

Method: A total of 2,611 ACLF patients enrolled in the APASLACLF Research Consortium (AARC) database were analyzed. Clinical, laboratory and survival data of the patients were compared according to the degree of $\mathrm{HE}$ at the time of presentation.

Result: There are marked differences between ACLF subjects with $\mathrm{HE}(\mathrm{n}=1153)$ and without HE $(\mathrm{n}=1458)$. 90-day mortality worsens significantly in relation to the presence $(59.4 \%$ vs $31.3 \%$, $\mathrm{p}<0.001$ ) and grade of HE (Figure 1). HE is associated with sepsis, gastrointestinal bleed, and hepatitis $\mathrm{C}$ infection. Subjects with HE have lower hemoglobin and platelet levels, higher white cell count, urea/creatinine, bilirubin, INR, and transaminases. Prognostic scores, including MELD, SOFA, APACHE II and CLIF-SOFA scores, are consistently higher in ACLF patients with HE.

Conclusion: The presence and grade of HE identify a subgroup of ACLF patients with more severe disease and poorer prognosis.

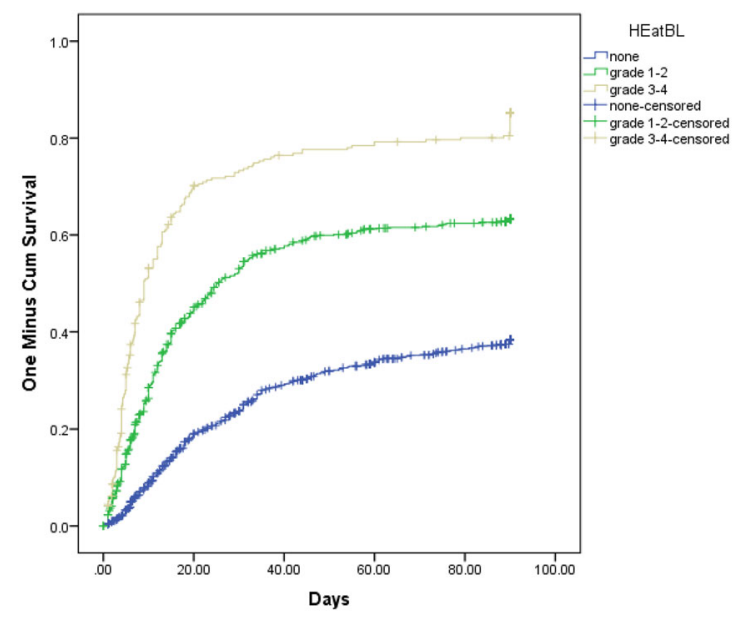

Figure 1. Mortality of ACLF patients in relation to the severity of HE at inclusion (competing risk assessment).
Session: Autoimmune Liver Diseases. Chairperson: Khin Maung Win; Sandeep Nijhawan; Dev Raja Rangegowda

$O-A I H-01$

Evaluation of half yearly spectrum of Autoimmune Liver Diseases (AILDs) and their prevalence from a single tertiary health care referral center of North India

Kumari Priyanka $N^{1}$, Kumari Priyanka $\mathbf{N}^{\mathbf{2}}$, Gude Geethanjali ${ }^{2}$, Suvradeep Mitra $^{2}$, Yogesh Kumar Chawla ${ }^{2}$, Biman Saikia ${ }^{2}$, Yashwant Kumar ${ }^{2}$, Shashi Anand ${ }^{2}$, Ashim Das ${ }^{2}$, Sunil Taneja ${ }^{2}$

${ }^{1}$ Researcher, India; ${ }^{2}$ PGIMER, Chandigarh, India

Background: World over an increase in prevalence of autoimmune liver related diseases has been noted with very few epidemiological studies from India only. Therefore current study was planned to characterize the spectrum and prevalence of autoimmune liver diseases in a tertiary referral health care set up to estimate prevalence of these in Northern India.

Method: A hospital based descriptive retrospective study was conducted to evaluate the prevalence of AILDs among adults patients attending the Hepatology clinic at PGIMER, Chandigarh. Age, sex, clinical presentation, findings from USG, firoscan, Biochemical parameters, Auto-antibody profile, viral markers etc. were screened. Autoimmune screening for antibody positivity was done for ANA, SMA, AMA, or LKM using a composite tissue i.e on rat liver, kidney and stomach and was analysed in the Department of Immunopathology, PGIMER. Statistical analysis was performed using mean \pm S.D.

Result: With the advent of viral markers (PCR based detection techniques and extensive screening for autoantibody profile) the diagnosis of AILD cases has increased at our centre in North India. Out of 3116 screened files, over a period of study (between 1st Jan 2015 to 30th Jun 2015) 42 patients with AILD presented AIH as the most common form of all AILDs constituting $71.4 \%$ of all AILD (93.33\%, \% type I AIH, 6.66\% type II AIH) and rest $28.57 \%$ with PBC and PBC/AIH overlap patients respectively. Based on IAHIG criteria, Hospital based prevalence was calculated by dividing the total number of cases of AIH over the period of six months and the total number of patients attending the Liver Clinic. The overall prevalence of AIH was $0.96 \%$ of all patients visiting hospital with hepatic diseases with age distribution over a wide range of $39.30 \pm 15.41$ years (range between 19-76 years) with female preponderance for AILDs. SMA was the most prevalent antibody $(30.26 \%, \mathrm{n}=42)$ found in younger AIH patients. Overall results of the study points towards AIH which is the predominant AILD affects younger age groups with cirrhosis at presentation as a hall mark in many patients. The next important group comprises $\mathrm{PBC}$ and $\mathrm{PBC} /$ $\mathrm{AIH}$, the latter having a more aggressive course.

Conclusion: Current study covers information on burden of disease in Northern India, emphasises strength of the AILD diagnostics in rest of the country to identify it at early stages to prevent its cirrhotic progression by institution of therapies. 


\section{$O-A I H-02$}

\section{Spectrum of liver involvement in celiac disease}

\section{Snehavardhan Radheyshyam Pandey ${ }^{1}$, Rajeev Khanna ${ }^{1}$, Vikrant Sood $^{1}$, Archana Rastogi \\ ${ }^{1}$ I.L.B.S., India}

Background: Liver involvement in children with Celiac disease (CD) may vary from asymptomatic celiac hepatitis to chronic liver disease in the setting of autoimmunity. The present study was aimed to study the spectrum of hepatic involvement in children with $\mathrm{CD}$ and to correlate the severity of hepatic involvement with intestinal disease. Method: All children under-18 years of CD diagnosed as per ESPGHAN guidelines were enrolled. Liver involvement was studied in patients in terms of elevation of transaminases ( $>25.8 \mathrm{U} / \mathrm{L}$ for boys, and $>22.1 \mathrm{U} / \mathrm{L}$ for girls), and/or clinical, sonographic or histological evidence of chronic liver disease and/or portal hypertension.

Result: 115 (72 males) children with CD were identified out of which 37 were potential CD. Elevation of transaminases was seen in 64 $(55.6 \%)$ children - of these 46 had celiac hepatitis, 16 had autoimmune liver disease (autoimmune hepatitis in 13, autoimmune sclerosing cholangitis in 2 and primary sclerosing cholangitis in 1) and 2 patients had hepatic venous outflow tract obstruction (HVOTO) based on liver biopsy findings. Liver biopsy available in 22 children showed features compatible with autoimmune liver disease in 11, steatohepatitis in 06, and chronic hepatitis in 04. One of the child had hepatocellular carcinoma in the setting of HVOTO. There was no significant correlation seen between the levels of transaminases with that of tTG or with the degree of villous atrophy. Out of the 20 patients with Celiac Hepatitis who were on regular follow up, 14 showed resolution of transaminases over a period of 4 months.

Conclusion: More than half of the patients with Celiac Disease have liver involvement. The spectrum of liver involvement in these patients is wide, however there is no correlation with the severity of intestinal disease.

\section{$O-A I H-03$}

Clinical spectrum and treatment outcomes of severe autoimmune hepatitis

Nikhil Sonthalia ${ }^{1}$, Samit Jain ${ }^{1}$, Sunil Pawar ${ }^{1}$, Suhas Udgirkar ${ }^{1}$, Sanjay Chandnani ${ }^{1}$, Ravindra Surude ${ }^{1}$, Pravin M Rathi ${ }^{1}$, Qais Contractor ${ }^{1}$

${ }^{1}$ Topiwala National Medical College and BYL Nair Ch Hospital, India

Background: Previously thought to be an uncommon disease, Autoimmune hepatitis (AIH) is on the rise in the Indian population. Severe acute presentation is an uncommon manifestation of AIH and it remains poorly characterized. The aim of this study was to study natural history and treatment outcomes of AIH variants presenting with Severe-AIH.

Method: Out of 1153 patients with acute or chronic liver disease who were screened from January 2011 till June 2017, 125 (10.84\%) were diagnosed as AIH. Of them 101 patients where finally recruited. Patients were classified as Seropositive and Seronegative AIH based on pre-specified diagnostic criteria. Patients with acute liver failure (ALF), acute on chronic liver failure (ACLF) and severe acute hepatitis were defined as Severe-AIH. Clinical, biochemical, serological, histological parameters and treatment outcomes with follow up till 12 months were analyzed between different groups.
Result: Out of 101, 24 (23.76\%) had Severe-AIH. Of them 9 (37.5\%) were severe acute hepatitis, 3(12.5\%) ALF and 12 (50\%) ACLF. Seronegative-AIH patients presented as Severe-AIH, significantly more than seropositive-AIH ( $50 \%$ vs. $20.27 \%, \mathrm{P}=0.022)$. However, response to treatment were not significantly different between the two groups $(\mathrm{P}>0.05)$. Severe-AIH had 50\% complete responders, $25 \%$ partial responders, and $25 \%$ treatment failures. Jaundice $(88.88 \%$ vs. $68.7 \%, \mathrm{P}=0.048)$, encephalopathy $(55.55 \%$ vs. $6.66 \%, \mathrm{P}=0.014)$, and higher international normalized ratio values $(2.17 \pm 0.60$ vs. $1.82 \pm 0.14, \mathrm{P}=0.038$ ) were factors associated with nonresponse rather than the presence or absence of autoantibodies in severe-AIH. The hazard ratio for predicting remission in the non-severe AIH group as compared with the severe-AIH group was 1.502 , which was statistically not significant $(95 \%$ CI, 0.799-2.827; $\mathrm{P}=0.205)$ (Figure 1A, B, C).

Conclusion: Approximately $24 \%$ of patients with AIH have severeAIH. Conventional autoantibodies are often absent in severe-AIH; however it does not alter the outcome. Immunosuppressants should be given expediently in patients with severe-AIH.

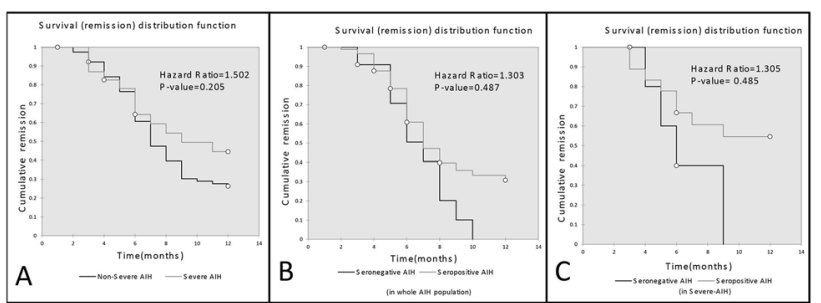

O-AIH-04

Intrahepatic recruitment of cytotoxic NK cells contributes to autoimmune hepatitis progression

Jinshang $\mathrm{Hu}^{1}$, Guo $\mathrm{Ai}^{1}$, Weiming Yan ${ }^{1}$, Xiaoping Luo ${ }^{1}$, Xiaoyang Wan $^{1}$, Qin Ning ${ }^{1}$

${ }^{1}$ Tongji Hospital, Tongji Medical College, Huazhong University of Science and Technology, China

Background: It is widely accepted that multiple subsets of immune cells are involved in the pathogenesis of autoimmune hepatitis AIH during which the autoimmune response is initiated by self-antigen recognition by the uncommitted CD4 helper T-cells (Th0) leading to Th1/Th2/Th17 differentiation in response to different cytokine stimulation. This is followed by the differentiation and activation of monocyte/Macrophage, natural killer (NK) cells, cytotoxic CD8 T cells, B cells leading to the destruction of liver parenchyma. However, exact contribution of $\mathrm{NK}$ cells to $\mathrm{AIH}$ remains poorly understood. In this study, we aim to dissect the role of peripheral and liver resident NK cells in AIH progression.

Method: Peripheral blood mononuclear cells were isolated from $\mathrm{AIH}$ at patients at active phase and remission phase and subjected to flow cytometry for NK cells detection. Liver NK cells was isolated from mice of experimental autoimmune hepatitis and analyzed by flow cytometry. CD56dim/ CXCR3- and CD56bright/ CXCR3+ NK cells in $\mathrm{AIH}$ patients/mice were drafted as cytotoxic and regulatory NK cells, respectively.

Result: Frequency of peripheral NK cells, more accurately CD56dimNK subset, was reduced in AIH patients. The reduction of CD56dimNK was negatively correlated with disease progression. In experimental autoimmune hepatitis (EAH), hepatic accumulation of NK cells was observed along with a decrease of NK cells in the periphery. In addition, infiltrated NK cells are almost conventional CXCR3 - NK cells, the counterpart of CD56dimNK cells in the 
human. Both conventional CXCR3 - NK cells of infiltration and liver resident NK cells are activated progressively at active phase as demonstrated by expression of granzyme B, Perforin, and IFN- $\gamma$

Conclusion: Recruitment of peripheral cytotoxic NK cells into the liver, but not liver resident NK cells expansion, accounts for AIH progression.

\section{O-AIH-05}

\section{Pattern of autoimmune liver disease in Southern Pakistan}

Shoukat Ali Samejo ${ }^{1}$, Zaigham Abbas', Muhammad Asim ${ }^{1}$, Javed Yakoob $^{2}$, Wasim Jafri' ${ }^{2}$, Saeed Hamid ${ }^{2}$

${ }^{1}$ Dr Ziauddin University Hospital Clifton, Pakistan; ${ }^{2}$ Agha Khan University Hospital, Pakistan

Background: Autoimmune liver disease is an uncommon cause of chronic liver disease in Pakistan. Not much is known about the spectrum of autoimmune liver disease in our country. The aim of this study was to describe the pattern of autoimmune related liver disorders in patients presenting to a tertiary care hospital.

Method: Patients presenting to the gastroenterology clinics of two university hospitals having the autoimmune liver problems in the last three years were identified through the ICD coding. Patients of autoimmune hepatitis (AIH) were evaluated by AIH criteria (MAYO clinic criteria). Autoimmune liver disorders other than autoimmune hepatitis were identified and included in the study.

Result: Total number of patients identified were 57. Female 37 $(64.9 \%)$. Age range was $14-84$ years with a mean of 49.7. Definite AIH was found in $8 / 57$ (14\%), probable AIH in 21/57 (36.8\%), possible AIH in 20/57 (35.1\%). Out of 49 cases of AIH, Type II AIH was identified in 6 patients due to presence of LKM antibody. IgG4 related cholangiopathy was present in $3 / 57$ (5.3\%). Primary biliary cholangitis (PBC) with overlap syndrome was seen in $2 / 57(3.5 \%)$ cases and PBC alone in 1/57 (1.8\%). Other autoimmune disorders included hepatic sarcoidosis $1 / 57(1.8 \%)$ and celiac hepatitis $1 / 57$ $(1.8 \%)$.

Conclusion: Wide spectrum of autoimmune liver disease exist in Pakistan. Autoimmune liver disease though rare but is an important cause of liver related morbidity and mortality.

\section{$O-A I H-06$}

Prioritization of histomorphological features in autoimmune hepatitis: are emperipolesis and rosetting losing their significance?

Kaushik Majumdar ${ }^{1}$, Puja Sakhuja ${ }^{1}$, Sunayana Misra ${ }^{1}$, Kavita Gaur $^{1}$, Sanjeev Sachdeva ${ }^{1}$, Siddharth Srivastava ${ }^{1}$, Amarender Singh Puri ${ }^{1}$

${ }^{1}$ G B Pant Institute of Postgraduate Medical Education and Research (GIPMER), India

Background: The simplified criteria for the diagnosis of autoimmune hepatitis (AIH) involve the constellation of serological markers, serum immunoglobulin $\mathrm{G}$, liver histology and negative workup for viral etiology. In contrast to the histological triad of interface hepatitis with lymphoplasmacytic infiltrate, rosettes, and emperipolesis described earlier, a revised histological criteria has been recently proposed. Rosettes and emperipolesis through characteristic, have been shown to lack sensitivity in the diagnosis of AIH.

Method: The liver biopsies with the clinico-serological diagnosis of AIH submitted to the department were reviewed retrospectively, and age, gender, drug history, autoantibody titers (smooth muscle antibodies, anti-actin antibodies, antinuclear antibodies, antimitochondrial antibodies), immunoglobulin levels, viral hepatitis markers, and liver function test results were tabulated. All the selected cases lacked serological evidence of viral hepatitis or alcohol abuse. The results for CK7 stain were recorded as positive if at least three periportal hepatocytes were positive in at least two foci (Balitzer et al, 2017)

Result: Of the 38 cases of AIH evaluated in this study, 21 cases $(55.3 \%)$ revealed the classically described histomorphological triad for AIH. While autoimmune serology and histological activity contributed consistently as reliable positive indicators, rosetting and emperipolesis were not seen in rest of the 17 cases. Cases with high activity index (6) and those with bridging fibrosis (8) showed positivity for intermediate hepatocytes and extraportal ductules as a consequence of hepatocyte regeneration. Accentuated Orcein staining for copper associated protein was appreciated in cases with associated chronic cholangiopathy and overlap (11). In addition, steatohepatitis was also noted in 6 cases.

Conclusion: Emphasizing the histological evidence of rosetting and emperipolesis led to poor performance of the simplified scoring and thereby missing otherwise characteristic cases of AIH. Hence, it seems rational to revise the histological parameters and correlate histological activity, autoimmune serology and negative viral serology for the diagnosis of AIH. Negative staining for Orcein and CK7 favor hepatitic over biliary pattern of injury. In addition, steatohepatitis is an interesting association in this study, and its presence should not rule out $\mathrm{AIH}$.

\section{Session: Cholestatic Liver Diseases; Chairperson: Laurentius A Lesmana; Praveen Sharma; Sumeet Kainth}

\section{O-CHOL-O1}

Quantification of biliary tree volume in patients with PSC using three dimensional (3D) reconstruction of magnetic resonance cholangiopancreatography (MRCP): Clinical associations and prognostic significance

Jina Lee ${ }^{1}$, Mehdi Taghipour ${ }^{2}$, Peter Behringer ${ }^{2}$, Elmira Hassanzadeh $^{2}$, Anik Sahni ${ }^{2}$, Nisha Sainani ${ }^{2}$, Dora Ding ${ }^{1}$, Jie Zhang $^{1}$, Bryan Mccolgan ${ }^{1}$, Macky Natha ${ }^{1}$, Chuhan Chung ${ }^{1}$, Robert P Myers', Mani Subramanian', John G Mchutchison', Zachary Goodman ${ }^{5}$, Bertus Eksteen ${ }^{7}$, Michael Manns ${ }^{4}$, Roger Chapman ${ }^{6}$, Christopher L Bowlus ${ }^{8}$, Cynthia Levy9, Andrew J Muir $^{3}$

${ }^{1}$ Gilead Sciences, Inc., United States; ${ }^{2}$ Brigham and Women's Hospital, Department of Radiology, Harvard Medical School, United States; ${ }^{3}$ Duke Clinical Research Institute, United States; ${ }^{4}$ Hannover Medical School, Germany; ${ }^{5}$ Inova Fairfax Hospital, United States; ${ }^{6}$ Oxford University, United Kingdom; ${ }^{7}$ University of Calgary, Canada; ${ }^{8}$ University of California at Davis, United States; ${ }^{9}$ University of Miami, United States

Background: MRCP assessment of the biliary tree in PSC lacks quantification, which limits evaluation of treatment response and disease progression. The goals of this study were to develop a quantitative method to measure biliary tree volume (BTV) using MRCP, determine associations between BTV and qualitative parameters, and describe changes in BTV and their prognostic significance. Method: MRCP was performed at baseline (BL), Week 48 (W48), and W96 in 234 PSC patients enrolled in a trial of simtuzumab (SIM). Since SIM was ineffective in this study, treatment groups were 
combined. Consensus reading of BL MRCP images by two radiologists characterized $18 \mathrm{MR}$ features including stricturing, dilatation, stones, and hepatic dysmorphy. For quantitative assessment, using the coronal thin slice MRCP, hepatic borders were manually segmented in 3D-Slicer using coronal T2W as a reference guide and MeVisLab was used to create a 3D biliary tree image. After processing, a signal threshold was applied to a multi-scale tube-like output image to exclude non-biliary fluid and the connected regions of voxels were extracted to calculate BTV. Associations between BL BTV and qualitative parameters were evaluated, and Cox regression assessed the association between BL BTV and its change with PSC-related clinical events (e.g. decompensation, ascending cholangitis, cholangiocarcinoma, and transplantation).

Result: The median age was 45 years, $64 \%$ were male, $48 \%$ had ulcerative colitis, $51 \%$ had advanced cirrhosis, and the median ALP was $260 \mathrm{U} / \mathrm{L}$ (IQR 129-401). The quantitative MRCP technique was feasible in $96 \%$ of cases. At BL, the median (IQR) BTV for the whole liver, and left and right lobes were 24.3 (17.3-36.0), 10.2 (5.5-16.1), and $10.7 \mathrm{~mL}$ (5.5-17.3), respectively. At W96, BTV decreased by a median of 2.9, 1.3, and $2.1 \mathrm{~mL}$, respectively (all $\mathrm{p}<0.005$ ). BL BTV did not differ according to the presence of strictures in the CBD, left or right hepatic ducts; however, subjects with $>50 \%$ involvement of the left or right lobes had greater BTV $(\mathrm{p}=0.078)$. BTV was also greater in subjects with stones $(p=0.042)$, caudate/right lobe ratio $>0.6(\mathrm{p}=0.011), \mathrm{T} 2 \mathrm{~W}$ sequence heterogeneity $(\mathrm{p}=0.017)$, and suspicion for cholangiocarcinoma $(p=0.001)$. Over a median follow-up of 23.0 months, 47 patients $(20 \%)$ developed a PSC-related clinical event. BL BTV and its change over 96 weeks were not associated with the risk of events (both $\mathrm{p}>0.05$ ).

Conclusion: This method of 3D MRCP reconstruction is the only noninvasive technique to measure BTV. In patients with PSC, BTV is associated with qualitatively-assessed parameters and declines over 96 weeks of follow-up, but does not have prognostic significance.

\section{$\mathrm{O}-\mathrm{CHOL}-\mathrm{O2}$}

The increasing risk of clinical events of primary biliary cholangitis patients presenting with early biochemical disease stage - the experience of the Global PBC Study Group

Bettina Elisabeth Hansen ${ }^{1}$, Jorn Goet ${ }^{3}$, Nikolaos K Gatselis ${ }^{9}$, Kalliopi Zachou', Willem J Lammers ${ }^{3}$, Harry L A Janssen', Gideon Hirschfield $^{15}$, Christophe Corpechot ${ }^{5}$, Keith D Lindor ${ }^{7}$, Pietro Invernizzi ${ }^{17}$, Marlyn J Mayo ${ }^{19}$, Pier M Battezzati ${ }^{12}$, Annarosa Floreani ${ }^{18}$, Albert Pares ${ }^{14}$, Frederik Nevens ${ }^{6}$, Andrew L Mason $^{13}$, Kris V Kowdley ${ }^{10}$, Cyriel Y Ponsioen ${ }^{2}$, Tony Bruns ${ }^{16}$, Douglas Thorburn $^{11}$, Xavier Verhelst ${ }^{4}$, Maren H Harms ${ }^{3}$, Henk R Van Buuren ${ }^{3}$, George N Dalekos ${ }^{9}$

${ }^{1}$ Toronto General Hospital, Canada; ${ }^{10}$ Swedish Medical Center, United States; ${ }^{11}$ The Royal Free Hospital, United Kingdom;

${ }^{12}$ Universita“ degli Studi di Milano, Italy; ${ }^{13}$ University of Alberta, Canada; ${ }^{14}$ University of Barcelona, Spai; ${ }^{15}$ University of Birmingham, United Kingdom; ${ }^{16}$ University of Jena, Germany; ${ }^{17}$ University of Milan-Bicocca, Italy; ${ }^{18}$ University of Padua, Italy;

${ }^{19}$ UT Southwestern Medical Center, United States; ${ }^{2}$ Academic Medical Center, Netherlands; ${ }^{3}$ Erasmus University Medical Center, Netherlands; ${ }^{4}$ Ghent University Hospital, Belgium; ${ }^{5} \mathrm{Ho}^{\wedge}$ pital SaintAntoine, France; ${ }^{6} \mathrm{KU}$ Leuven, Belgium; ${ }^{7}$ Mayo Clinic, United States; ${ }^{8}$ School of Medicine Thessaly University, Greece; ${ }^{9}$ School of Medicine, Thessaly University, Greece

Background: The majority of PBC patients are more and more frequent diagnosed at an early biochemical disease stage. We aimed to investigate the time to progression of disease into biochemical moderate or advanced disease stages and the following impact on event-free survival.

Method: Patients data were obtained from the Global PBC Study Group database. Patients with an early biochemical stage (normal albumin and bilirubin) at diagnose were included. At every follow-up visit patients who transited from biochemical early- to moderate(abnormal bilirubin or albumin) or advanced stage (abnormal bilirubin and albumin) were identified. We used a composite endpoint of cirrhotic decompensation, development of hepatocellular carcinoma, liver transplantation or death.

Result: The Global PBC Study Group is an international and multicenter collaboration between 19 liver centers from 12 countries, which combined individual patient data from major long-term followup cohorts. All patients had an established diagnosis of PBC. From the database of more than 6000 PBC patients we included for this study 2097 PBC patients with mild disease stage at diagnosis [92\% female, 84\% UDCA treated, median follow-up 7.7 years (IQR 4.011.9)]. During follow-up 1084 transited to moderate disease and sequentially 238 to advanced disease. Transition rates to moderate disease at $1,3,5$ year(s) were $14 \%, 33.8 \%$, and $46.1 \%$, and $4.8 \%$, $16.9 \%$ and $20.7 \%$ for transition from moderate to advanced disease. During follow-up 301 patients experienced a clinical event. Patients with normal bilirubin and normal albumin throughout follow-up had an excellent event-free survival (figure). Once patients reached a moderate disease stage however, the event-free survival rate at 1,3 and 5 year(s) were $98 \%, 94.3 \%$ and $90.1 \%$. For transition to advanced disease stage these rates were $91.2 \%, 72.6 \%$ and $59.1 \%$. Cox-proportional hazard analyses were used to assess the impact of transition on event-free survival with time until the first transition as a timedependent covariate adjusting for age, sex, UDCA use, diagnose year, ALP and platelets. The risk of a clinical event from a moderate disease stage was HR 3.3 (2.4-4.6) compared to early disease stage and increased to HR 4.7 (3.7-6.1) from an advanced disease stage compared to moderate disease stage.

Conclusion: One out of two patients presenting with a mild $\mathrm{PBC}$ will progress towards a more severe stage of disease within 5 years. This was associated with a strong increased risk of clinical events, underlining the importance of surveillance.

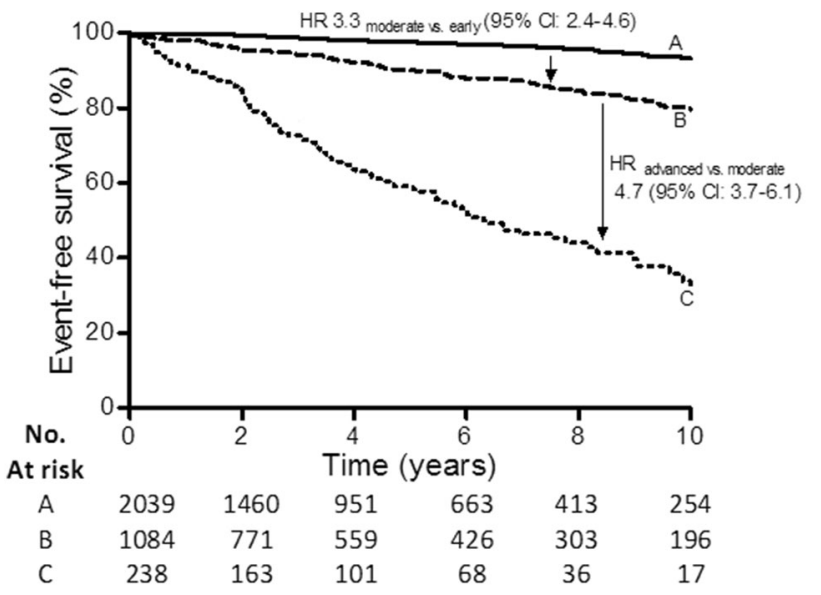

- Patients without transition from biochemical early to moderate or advanced stage - -. Patients that transited from biochemically early to moderate stage

.... Patients that trans ited from biochemically moderate to advanc ed disease 
O-CHOL-03

Epidemiology of primary biliary cholangitis in Pacific-Asia: a meta-analysis

\section{Na Zeng ${ }^{1}$, Jidong Jia ${ }^{1}$, Hong You ${ }^{1}$, Yuanyuan Kong ${ }^{1}$ \\ ${ }^{1}$ Beijing Friendship Hospital, Capital Medical University, China}

Background: The incidence and prevalence rates of primary biliary cholangitis (PBC) showed wide variation in Pacific-Asia. The variation might be in part attributable to heterogeneity in study population, periods, and the methods of case finding or ascertainment. Therefore, our aim was to systematically review prevalence, incidence, hepatocellular carcinoma and fatality of PBC in Pacific-Asia.

Method: Pubmed, Medline and Emabase were searched on November $1 \mathrm{~h}, 2017$. Population-based studies with available data were included if they report incidence, prevalence or epidemiology information of PBC. Study period, number of patients, number of inhabitants, incidence rates, prevalence rates, fatality rates, method of case-finding, method of case-ascertainment and occurrence of HCC were extracted from retrieved articles. The prevalence and incidence of PBC were summarized with a rate (IR) and 95\% confidence intervals. The test of heterogeneity was performed with the Q statistic. Result: The search retrieved 2941 studies among which 18 articles fulfilled the inclusion criteria. The prevalence rates for PBC range from 1.91 to 65.5 per million inhabitants and 8.57 per million inhabitants. When combining 8 studies which met high quality criteria, the average prevalence rates was 52.1 per million (95\% CI: 51.8-52.3 per million). Only 4 articles reported the incidence rates of PBC, which ranged from 8.57 per million inhabitants in South Korea to 54.30 per million inhabitants in Japan. In addition, the HCC and fatality rate of PBC estimated in Asia were 1.4\% (95\% CI: 1.1-1.9\%) and $2.2 \%$ (95\% CI: $1.8-2.8 \%$ ), respectively.

Conclusion: The prevalence and incidence of PBC in Pacific-Asia were not low compared to the western countries. The lacking of population-based data from developing countries may lead to underestimate of the true frequency of PBC.
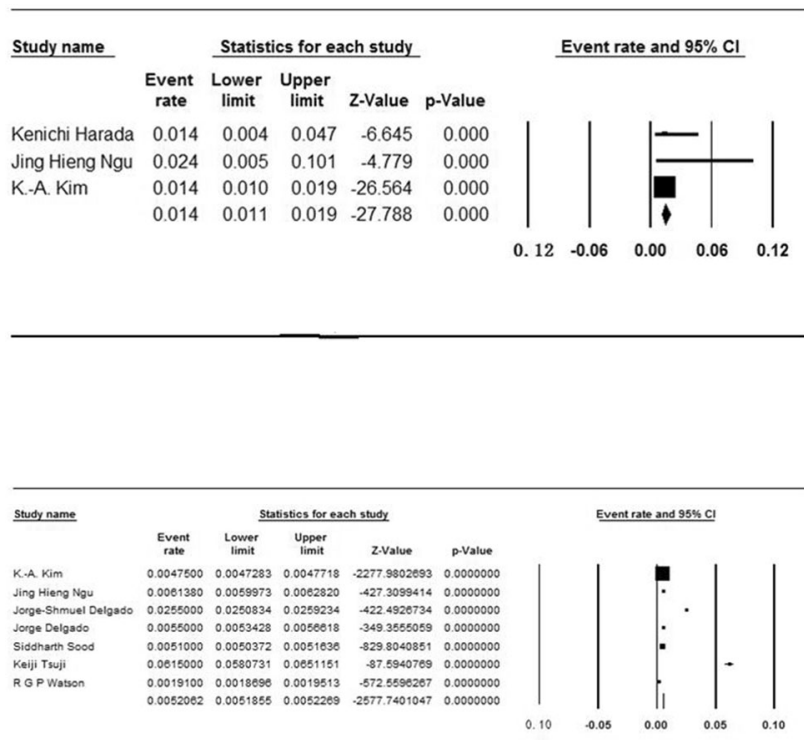

O-CHOL-04

The risk predictive values of UK-PBC and GLOBE scoring system and performance of biochemical response criteria in Chinese patients with primary biliary cholangitis (PBC) on ursodeoxycholic acid

George Lau ${ }^{1}$, Yin Sun ${ }^{2}$, Zhengsheng Zou ${ }^{2}$, Cheng Wang ${ }^{4}$, Jing ${ }_{\text {Chen }}^{4}$, Yudong Wang ${ }^{4}$, Vanessa Wu ${ }^{4}$, George Lau ${ }^{3}$

${ }^{1}$ Humanity and Health Medical Group, Hong Kong; ${ }^{2}$ Beijing 302 Hospital, China, China; ${ }^{3}$ Humanity and Health Medical Center, Hong Kong SAR, China, Hong Kong; ${ }^{4}$ Humanity and Health Research Center, Hong Kong SAR, China, Hong Kong

Background: The biochemical response to ursodeoxycholic acid (UDCA) in primary biliary cholangitis (PBC) is a strong predictor of long-term outcome and thus facilitates the rapid identification of patients needing new therapeutic approaches. Numerous criteria for predicting outcome of treatment have been studied based on biochemical response to UDCA at 1 year. We sought to determine whether biochemical response based on these criteria could identify Chinese patients with PBC at risk of clinical adverse outcome, as defined by liver-related death, liver transplantation, and complications of cirrhosis.

Method: 119 consecutive patients (median age 46, female sex 83.2, median follow-up of 3.8 [range: 1.1-9.1]) all with biopsy-proved PBC treated with UDCA from one of the biggest tertiary hospitals in China (Beijing 302 hospital) were included. Clinical characteristics and liver biochemistry were collected at every 6-month interval. Death, liver transplantation (LT) and complications of cirrhosis were used as clinical adverse outcome. Cox proportional hazards regression analysis was used to identify the risk factors for progression after a year of UDCA treatment. The prognostic ability of UK-PBC score and GLOBE score were evaluated alongside those of the Barcelona, ParisI, Rotterdam, and Paris-II criteria. The Area-Under-the-ReceiverOperating-Curve (AUROC) for survival analysis (c-statistics) was used to determine the performance of each prognostic method.

Result: The survival rates without adverse clinical outcome at 2, 5 and 8 years were $95.8 \%$ and $78.7 \%$ and $61.8 \%$ respectively. Under UDCA therapy, laboratory liver parameters experienced the most prominent improvement at 1 st year (all $\mathrm{p}<0.05$ ). The Paris I and Paris II, but not the Rotterdam and Barcelona definition applied at 1st year after UDCA therapy significantly discriminated the patients in terms of long-term clinical outcome. Paris I was the most powerful predictor among four criteria (HR: 3.6, 95\% CI: 1.5-8.3, AUROC 0.65 ) for non-responders versus responders. According to Paris I criteria 8 -yrs survival was $79.6 \%$ for responders and $43.6 \%$ for nonresponders. The AUROC was 0.78 for GLOBE scoring system (HR: 2.9, 95\% CI: 1.9-4.4). Patients with a GLOBE score $>0.3$ (nonresponders) had a significantly diminished survival compared with those with a score $\leq 0.3$ (responders), and the 3-, 5- and 8-year clinical adverse outcome free survival rates were $95 \%$ vs. $74 \%, 91 \%$ vs. $64 \%$, and $87 \%$ vs. $35 \%$, respectively. The AUROC was 0.739 , 0.738 and 0.738 for 3-, 5-, 8-year UK-PBC risk score.

Conclusion: This analysis confirms the prognostic value of previously proposed response criteria and scoring systems. Paris I was the most powerful predictor among all the criteria while GLOBE score predicted more accurate than UK-PBC score in Chinese cohort. 


\section{O-CHOL-05}

Predictive factors for the effectiveness of primary biliary cholangitis treatment with ursodeoxycholic acid

\section{Peter Jarcuska ${ }^{1}$, Jakub Gazda ${ }^{4}$, Martin Janicko ${ }^{4}$, Dominika} Martinkova $^{3}$, Lucia Mikolajova

${ }^{1}$ University Hospital and University of PJ Safarik, Faculty of Medicine, Slovakia; ${ }^{2}$ Hospital Poprad, Slovakia; ${ }^{3}$ Nemocnica Poprad, Slovakia; ${ }^{4}$ University Hospital and University of PJ Safarik, Faculty of Medicine, Slovakia

Background: Ursodeoxycholic acid (UDCA) is the gold standard in the treatment of primary biliary cholangitis (PBC). Some patients, however, by UDCA therapy progress to liver failure. Aim of the study was to find predictors of the therapeutic response to the UDCA treatment at month 6 and 12 .

Method: 89 patients with PBC (88 female, mean age: $55 \pm 10.1$ years) were enrolled in the retrospective analysis. Therapeutic response to the UDCA treatment was defined as follows: ALP level $<1.67$ ULN and bilirubine level $<2$ ULN at month 6 or 12 of UDCA treatment.

Result: Baseline conjugated bilirubine $(p=0.004), \quad$ AST $(\mathrm{p}=0.004)$, ALT $(\mathrm{p}=0.005)$ and $\operatorname{ALP}(\mathrm{p}=0.001)$ predicted therapeutic response to UDCA treatment at month 6 , while baseline total bilirubine $(\mathrm{p}=0.003)$, conjugated bilirubine $(\mathrm{p}=0.002)$ and ALP $(\mathrm{p}=0.017)$ predicted a therapeutic response to UDCA at month 12 . Therapeutic response to UDCA at month 6 was a strong predictor to achieve a therapeutic response at month 12 of UDCA treatment (OR 12.75, 95\% CI 4.01-40.50; p <0,001). Patients with baseline ALP $\leq 2$ ULN had a significantly higher chance to achieve a therapeutic response at month 6 of UDCA therapy $(p<0.001)$, but not at month 12 of treatment compared to patients with baseline ALP $>2$ ULN. Patients who didńt achieve a therapeutic response to the UDCA at month 6 or 12 had a significantly higher chance to develop hepatic decompensation in the future (at month 6: OR 9.7391, 95\% CI $1.9201-49.3985 ; \mathrm{p}=0.003$; at month 12 : OR $11.5938,95 \% \mathrm{CI}$ 2.1869-61.4641; $\mathrm{p}=0,002)$.

Conclusion: Baseline laboratory parameters have variable statistical power to predict therapeutic response to UDCA therapy at month 6 and 12. Response to UDCA at month 6 is a strong predictor of UDCA response at month 12 . Non-response to UDCA treatment predicts liver decompensation in the future.

\section{O-CHOL-O6}

Self-reported experiences of patients living with primary biliary cholangitis (PBC) and Ursodiol

\section{Kathleen P Ismond $^{1}$, Bishoi Azziz ${ }^{2}$, Gail Wright ${ }^{3}$, Andrew Mason ${ }^{1}$}

${ }^{1}$ University of Alberta, Canada; ${ }^{2}$ Division of Gastroenterology, Canada; ${ }^{3} \mathrm{PBC}$ Society of Canada, Canada

Background: Primary biliary cholangitis (PBC) is a rare, progressive hepatobiliary disease with global prevalence rates of 6.7 to 940 per million-population. In Canada, fewer than 1 in 3000 persons are affected. Treatment for PBC has been limited to ursodiol (or ursodeoxycholic acid, UDCA), a water-soluble bile acid that increases the $\mathrm{pH}$ of bile and lessens the damage caused by hydrophobic bile acids.

Method: In 2017, a semi-synthetic bile acid analogue, obeticholic acid (OCA), was approved for use in Canada for patients intolerant or not responding to UDCA. OCA works as a farnesoid $\mathrm{X}$ receptor agonist by lowering the production of bile acids and decreasing inflammation within the liver. Our aim was to capture the lived experiences and needs of patients with $\mathrm{PBC}$ using an online survey. Anonymous responses were analysed using percentages (binary and Likert-scale) while free-text responses to the open-ended question were manually coded by theme.

Result: The completed survey response rate was $47 \%(\mathrm{n}=119)$. While all participants had experience with UDCA treatment, $7 \%$ were not currently taking the medication. In the prior 12 months, $62 \%$ stated that they required assistance to complete activities of daily living. Specifically, help was required for the following activities: cleaning the home, $58 \%$; grocery shopping, $48 \%$; meal preparation, $46 \%$; and driving a vehicle, $39 \%$. Only $9 \%$ rarely or sometimes required assistance with bathing or dressing. Although on UDCA, $68 \%$ reported significant debilitating side effects of PBC that included: fatigue, $68 \%$; pruritus, $64 \%$; pain or aches, $21 \%$; digestive issues, $18 \%$; or dry eyes/mouth, $5 \%$. Thematic analysis of answers from the open-ended question ("How has PBC affected your life?") indicated that $64 \%$ of respondents adjusted their lifestyles and expectations to accommodate PBC-related symptoms while $30 \%$ reported that their symptom burden negatively affected relationships with family and friends. Nearly one third had progressively worsening symptoms while $42 \%$ were anxious or worried about the future in relation to their health. Financial concerns were expressed by $29 \%$ of respondents which were attributed to either high medication costs or reduced earning potential due to PBC. Nearly one third of patients mentioned the need for more $\mathrm{PBC}$-specific medical treatments other than UDCA and liver transplantation. The PBC Society of Canada provided these results to the Canadian Agency for Drugs \& Technologies in Health (CADTH) in January 2017 as part of the evidence base to determine if the new drug, OCA, should be covered by drug insurers. As of July 25, 2017, CADTH recommended that OCA should only be covered by drug insurers if there was a $60 \%$ reduction in the annual cost of OCA estimated to start at $\$ 36,000$ (CAD) per patient per year. Their decision was based upon evidence that OCA may increase pruritus and overall cost-effectiveness.

Conclusion: Despite the liver-specific health benefits of licensed therapy, patients living with $\mathrm{PBC}$ continue to endure a range of progressive symptoms that are not positively impacted by either UDCA or OCA.

Session: Acute Liver Failure and Acute on chronic Liver failure; Chairpersons; Tatsuo Kanda; S K Sinha; Abby Phillips

$O-A C L F-07$

Presence of Acute kidney injury (AKI) at admission predicts early mortality in patients with acute on chronic liver failure (ACLF): a single centre experience

Chitta Ranjan Khatua ${ }^{1}$, Subhendu Panigrahi ${ }^{1}$, Debakanta Mishra ${ }^{1}$, Sambit Kumar Behera ${ }^{1}$, Suryakanta Parida ${ }^{1}$, Prasant Kumar Parida ${ }^{1}$, Shivaram Prasad Singh ${ }^{1}$

${ }^{1}$ SCB Medical College, India

Background: Acute kidney injury (AKI) occurs commonly in patients with chronic liver disease (CLD) and negatively impacts survival. However, there is scant data on impact of AKI on survival in acute on chronic liver failure (ACLF). We performed a prospective study to evaluate the magnitude of the impact of AKI on the survival in patients with ACLF.

Method: This study was conducted in ACLF patients hospitalised in the Gastroenterology Department, SCB Medical College from 
October 2016 to October 2017. Demographic, clinical, laboratory parameters and outcome were compared between patients with and without AKI.

Result: Consecutive decompensated cirrhosis (DC) patients $(\mathrm{n}=253)$ were screened for ACLF as per The Asian Pacific Association for the Study of the Liver (APASL) criteria. Sixty eight (27\%) of them had ACLF. Out of the 68 ACLF cases, 43 (63\%) had AKI as per International Club of Ascites. Among ACLF patients, those with AKI were younger than those without AKI (44.47 \pm 9.87 vs. $50.14 \pm 10.69 ; \mathrm{p}=0.024)$. Alcohol was the predominant etiology of CLD in ACLF cases (Alcohol vs Hepatitis B vs. Others-68\% vs. $21 \%$ vs. $11 \% ; \mathrm{p}=0.024)$ and it was also significantly associated with AKI $(p=0.001)$. Analysis of the precipitating causes of ACLF with AKI revealed that continued alcohol intake $(76 \%$ vs. $48 \% ; p=0.020)$ was the commonest cause followed by infection $(\mathrm{p}=0.189)$. Patients with ACLF and AKI had higher MELD (unos) (Model for End-stage Liver Disease $) \quad(32.08 \pm 7.7$ vs. $22.66 \pm 3.10 ; \quad \mathrm{p}<0.001)$, $\operatorname{MELD}(\mathrm{Na}+)(33.77 \pm 6.04$ vs. $25.91 \pm 3.21 ; \mathrm{p}<0.001)$ and prolonded hospital stay $(6.53 \pm 2.52$ vs. $4.60 \pm 1.70 ; \mathrm{p}=0.045)$; however, MAP (Mean arterial pressure), BMI (body mass index), total leucocyte count, total bilirubin, serum protein, albumin, CTP (Child-Turcotte-Pugh Classification) score, SAAG (Serum: Ascites Albumin Ratio) were comparable in both groups of ACLF patients. In ACLF patients, kidney was affected in $63 \%$ of cases, liver failure in $48 \%$, coagulation failure in $28 \%$, hepatic encephalopathy in $94 \%$ cases (grade 1 or 2 in $76 \%$ and 3 or 4 in $18 \%$ cases), circulatory failure in $7 \%$ and respiratory failure in $5 \%$. ACLF patients had lower survival both at 28 days (59\% vs. $83 \%$; p < 0.001$)$ and 90 days $(37 \%$ vs. $62 \% ; \mathrm{p}<0.001)$ in comparison to DC patients without ACLF. However, the presence of AKI among ACLF patients further reduced 28 days survival considerably ( $24 \%$ vs. $72 \% ; p=0.041)$, with a trend towards increased late mortality at 90 days though the difference was not statistically significant ( $48 \%$ vs. $30 \% ; \mathrm{p}=0.143$ ).

Conclusion: Over one fourth of DC patients had ACLF and more than half of them had AKI. Although ACLF is a predictor of both reduced early and late survival, a small increase in serum creatinine in ACLF patients should be viewed with caution.

\section{$O-A C L F-08$}

Development of diagnostic criteria and a prognostic score for HBV-related acute-on-chronic liver failure

Jun $\mathrm{Li}^{1}$, Tianzhou $\mathrm{Wu}^{2}$, Jiang $\mathrm{Li}^{2}$, Li Shao ${ }^{2}$, Jiaojiao $\mathrm{Xin}^{2}$, Longyan Jiang ${ }^{2}$, Qian Zhou ${ }^{2}$, Dongyan Shi $^{2}$, Jing Jiang ${ }^{2}$, Suwan Sun $^{2}$, Lingling Yang ${ }^{2}$, Yinyun $\mathbf{L u}^{2}$

${ }^{1}$ The First Affiliated Hospital, Zhejiang University School of Medicine, China; ${ }^{2}$ The First Affiliated Hospital, Zhejiang University School of Medicine, China

Background: The definition of acute-on-chronic liver failure (ACLF) based on cirrhosis, irrespective of etiology, remains controversial. This study aimed to clarify the clinicopathological characteristics of patients with hepatitis B virus-related ACLF (HBV-ACLF) in a prospective study and develop new diagnostic criteria and a prognostic score for such patients.

Method: The clinical data from 1322 hospitalized patients with acute decompensation of cirrhosis or severe liver injure due to chronic hepatitis B (CHB) at 13 liver centers in China were used to develop new diagnostic and prognostic criteria.

Result: Of the patients assessed using Chronic Liver Failure Consortium criteria with the exception of cirrhosis, 391 patients with ACLF were identified: 92 with non-cirrhotic HBV-ACLF, 271 with cirrhotic HBV-ACLF and 28 with ACLF with cirrhosis caused by non-
HBV aetiologies (non-HBV-ACLF). The short-term (28/90 days) mortality of the patients with HBV-ACLF were significantly higher than those of the patients with non-HBV-ACLF. Total bilirubin (TB) $\geq 12 \mathrm{mg} / \mathrm{dL}$ and an international normalized ratio (INR) $\geq 1.5$ was proposed as an additional diagnostic indicator of HBV-ACLF, and $19.3 \%$ of patients with an HBV aetiology were additionally diagnosed with ACLF. The new prognostic score $(0.741 \times \mathrm{INR}+$ $0.523 \times$ HBV-SOFA $+0.026 \times$ age $+0.003 \times \mathrm{TB})$ for short-term mortality was superior to five other scores based on both discovery and external validation studies.

Conclusion: Regardless of the presence of cirrhosis, patients with $\mathrm{CHB}, \mathrm{TB} \geq 12 \mathrm{mg} / \mathrm{dL}$ and $\mathrm{INR} \geq 1.5$ should be diagnosed with ACLF. The new criteria diagnosed nearly $20 \%$ more patients with an HBV aetiology with ACLF, thus increasing their opportunity to receive timely intensive management.

\section{$O-A C L F-09$}

Total serum bile acids in cirrhosis and acute- on- chronic liver failure

Sherin Sarah Thomas ${ }^{1}$, Navneeta Mishra ${ }^{2}$, Preeti Manchanda ${ }^{2}$, Ritu Goyal $^{2}$, Guresh Kumar', Rakhi Maiwall ${ }^{2}$

${ }^{1}$ UGC, India; ${ }^{2}$ ILBS, India

Background: Patients with acute-on-chronic liver failure (ACLF) characteristically have marked systemic inflammation, hyperbilirubinemia and organ dysfunction. There is paucity of data on the association of serum total bile acids (TBAs) with organ dysfunction in patients with cirrhosis and ACLF. We aimed to determine TBAs in patients with cirrhosis and ACLF and to determine diagnostic and prognostic efficacy.

Method: 22 patients with cirrhosis and 15 patients with ACLF were included along with 19 age and sex matched healthy controls in this prospective study.TBA was assessed in all the subjects by enzymatic cycling method using a kit from Diazyme Laboratories (USA).

Result: Serum TBA was significantly raised in ACLF) $\{180.1 \mathrm{mmol} /$ $\mathrm{L}(160.0-250.0)$, median (IQR), $\mathrm{p}<0.001\}$ as compared to cirrhosis \{63.4 (28.9-76.0)\}, and healthy controls $\{4.2(1.0-7.2)\}$ Area Under Receiver Operating Characteristics curve (AUROC) for TBA is 0.98 at a cut off $10.32 \mathrm{mmol} / \mathrm{L}(\mathrm{p}<0.001)$ in controls with a sensitivity of $97.3 \%$ and specificity of $94.7 \%$. In compensated Vs decompensated liver disease AUROC is 0.78 at a cut off $70.0 \mathrm{mmol} / \mathrm{L}(\mathrm{p}<0.001)$ with a sensitivity of $80 \%$ and specificity of $77.8 \%$. AUROC for survivors Vs non-survivors is 0.9 at a cut off $71.0 \mathrm{mmol} / \mathrm{L}$ ( $p<0.001$ ) with a sensitivity of $76.9 \%$ and specificity of $74.4 \%$.

Conclusion: Assessment of TBAs could serve as additional marker for risk stratification in cirrhotic patients with respect to decompensation, ACLF and prediction of death.

\section{$O-A C L F-10$}

Early intervention by antiviral (Tenofovir or Entecavir) therapy in HBV-ACLF patients improves survival rate

\section{Sharker Mohammad Shahadat Hossain ${ }^{1}$, Mamun Al Mahtab ${ }^{2}$, Salimur Rahman ${ }^{2}$ \\ ${ }^{1}$ Kurmitola General Hospital, Bangladesh; ${ }^{2}$ Bangabandhu Sheikh Mujib Medical University, Bangladesh}

Background: Acute on chronic liver failure (ACLF) is an acute hepatic insult manifested as Jaundice and Coagulopathy, complicated within 4 weeks by Clinical Ascites and/or Encephalopathy in a 
patient with previously diagnosed or undiagnosed Chronic Liver Disease/Cirrhosis. It is associated with high 28-day mortality rate ranging from $30 \%$ to $70 \%$. Reactivation of Hepatitis B virus infection and super infection with hepatitis $\mathrm{A}$ or $\mathrm{E}$ are the major causes of ACLF in the Asian region. Liver transplantation is the only definitive therapy though it is not available everywhere and not feasible always. Again MARS therapy (Molecular Adsorbent Recirculating System) didn't reduce mortality significantly. So, antiviral therapy should be started as soon as possible in patients with ACLF due to Hepatitis B irrespective of DNA and ALT status to improve hepatic dysfunction and rescue the patients from mortality.

Aims: This randomized clinical trial was carried out with an aim to see survival among patients with acute on chronic hepatitis B liver failure 03 months after the antiviral (Tenofovir or Entecavir) therapy. Method: In this study a total of 32 acute on chronic Hepatitis B liver failure patients (age $>18$ years with both sexes but male predominant) were included in Hepatology department of Bangabandhu Sheikh Mujib Medical University, Dhaka during January 2013 to December 2015. The patients were randomized into two groups: Tenofovir group $(\mathrm{N}=16)$ and Entecavir group $(\mathrm{N}=16)$ and followed at least for 03 months.

Result: After three months of antiviral therapy, out of 32 patient, total $20(62.5 \%)$ patients were survive. Out of them $13(81.2 \%)$ in tenofovir group and $7(43.7 \%)$ in entecavir group $(p<0.05)$ (Fig 1). Among HBV-ACLF patients started antiviral therapy at different time, early intervention patients have higher survival rate (Fig 2). Out of 15 patients who got antiviral intervention within 7 days of ACLF development or appearance of jaundice and ascites, survival rate and death rate after three month of therapy was $13(86.66 \%)$ and death 02 $(13.33 \%)$, respectively $(p<0.05)$. On the other hand, 10 patients was got antiviral intervention within 8-15 days of ACLF development or appearance of jaundice and ascites. Out of them, survival rate and death rate after three month was $06(60 \%)$ and $04(40 \%)$, respectively $(p<0.05)$. And lastly, Out of 07 patients, who got antiviral intervention within $16-28$ days of ACLF development or appearance of jaundice and ascites, survival rate and death rate after three month was $01(14.28 \%)$ and $06(85.71 \%)$, respectively $(p<0.05)$.

Conclusion: In HBV-ACLF patients, the use of nucleoside and nucleotide analogs has clear survival benefit, which is significantly higher with Tenofovir. Early intervention by antiviral therapy improve survival rates of HBV-ACLF patients and early intervention by tenofovir improves more survival.

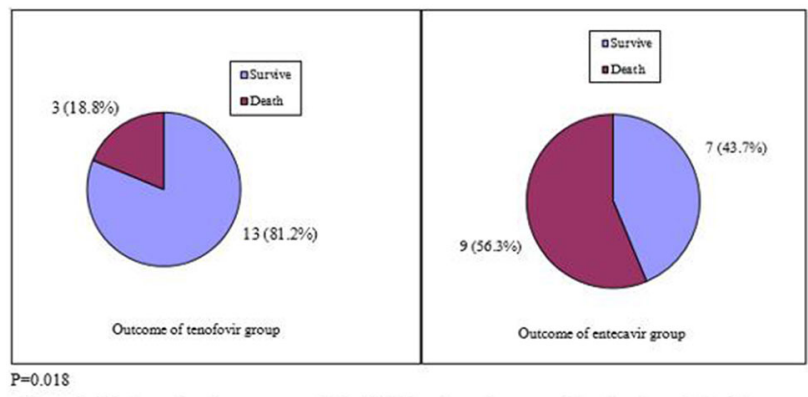

Figure 1: Pie chart showing outcome of the ACLF patients three months after the antiviral therapy.

\begin{tabular}{|c|c|c|c|}
\hline $\begin{array}{l}\text { Intervention by antiviral at different } \\
\text { time }\end{array}$ & No. of patients & $\begin{array}{c}\text { Survival rate } \\
\text { after three month }\end{array}$ & $\begin{array}{c}\text { Death rate } \\
\text { after three month }\end{array}$ \\
\hline $\begin{array}{l}\text { Antiviral intervention with in } 7 \\
\text { days of ACLF development or } \\
\text { appearance of jaundice and ascites. }\end{array}$ & $\begin{array}{c}15 \\
\text { Tenofovir }-07 \\
\text { Entecavir }-08\end{array}$ & $\begin{array}{r}13(86.66 \%) \\
\text { Tenofovir }-07 \\
\text { Entecavir }-06\end{array}$ & $\begin{array}{c}02(13.33 \%) \\
\text { Tenofovir }-00 \\
\text { Entecavir }-02\end{array}$ \\
\hline $\begin{array}{l}\text { Antiviral intervention with in } 8-15 \\
\text { days of ACLF development or } \\
\text { appearance of jaundice and ascites. }\end{array}$ & $\begin{array}{c}10 \\
\text { Tenofovir }-05 \\
\text { Entecavir }-05\end{array}$ & $\begin{array}{c}06(60 \%) \\
\text { Tenofovir }-05 \\
\text { Entecavir }-01\end{array}$ & $\begin{array}{c}04(40 \%) \\
\text { Tenofovir }-00 \\
\text { Entecavir }-04\end{array}$ \\
\hline $\begin{array}{l}\text { Antiviral intervention with in } 16-28 \\
\text { days of ACLF development or } \\
\text { appearance of jaundice and ascites. }\end{array}$ & $\begin{array}{c}07 \\
\text { Tenofovir }-04 \\
\text { Entecavir }-03\end{array}$ & $\begin{array}{l}01(14.28 \%) \\
\text { Tenofovir }-01 \\
\text { Entecavir - } 00\end{array}$ & $\begin{array}{c}06(85.71 \%) \\
\text { Tenofovir }-03 \\
\text { Entecavir }-03\end{array}$ \\
\hline
\end{tabular}

Figure 02: Outcome of the HBV- ACLF patients by antiviral therapy starting at different time

\section{$O-A C L F-11$}

Acute-on-Chronic Liver failure in Wilson's disease-a series of 38 cases from APASL-ACLF research consortium (AARC)

Piyush Gupta $^{1}$, Ashish Goel ${ }^{1}$, Chundamannil Eapen Eapen ${ }^{1}$, Ashok Kumar Choudhury ${ }^{5}$, Soek Siam Tan ${ }^{8}$, Guan Huei Lee ${ }^{11}$, Seng Gee Lim ${ }^{11}$, Harshad Devarbhavi ${ }^{10}$, Yogesh Kumar Chawala $^{7}$, Sunil Taneja ${ }^{7}$, Zhongping Duan ${ }^{3}$, Chen $\mathrm{Yu}^{4}$, Akash Shukla $^{6}$, Padaki N Rao ${ }^{2}$, Zaigham Abbas', Priyanka Jain ${ }^{5}$, Irene Paulson ${ }^{5}$, Shiv Kumar Sarin ${ }^{5}$

${ }^{1}$ christian Medical College, India; ${ }^{10}$ St John Medical College, India;

${ }^{11}$ Yong Loo Lin School of Medicine, Singapore; ${ }^{2}$ Asian Institute of Gastroenterology, India; ${ }^{3}$ Beijing You'an Hospital, Capital Medical University, China; ${ }^{4}$ Capital Medical University Beijing, China;

${ }^{5}$ Institute of Liver and Biliary Sciences, India; ${ }^{6} \mathrm{KEM}$ Hospital and Seth GSMC, India; ${ }^{7}$ PGIMER, India; ${ }^{8}$ Selayang Hospital, Malaysia; ${ }^{9}$ Sindh Institute of Urology and Transplantation, Pakistan

Background: Acute-on-Chronic Liver Failure (ACLF) is a distinct syndrome with high short term mortality due to an acute hepatic insult leading to liver failure and subsequent extra-hepatic organ failure in a patient with chronic liver disease. Wilson's disease flare in a back ground of the chronic disease may result in the syndrome of ACLF, but the data is sparse on this aspect. We analyzed Wilson disease presenting as ACLF from the AARC data base.

Method: 38 cases with Wilson disease, presented with ACLF (APASL definition) were recruited from March 2012 to July 2017. Data was obtained from multiple centres and aggregated on a common platform of AARC. Baseline parameters and further in-hospital course were followed until 90 days for death or liver transplant.

Result: Wilson disease constitutes $1.4 \%$ (38 of 2734) of ACLF with median age of 26 (7-73) years and $24(63 \%)$ males. 14 ACLF cases had defined acute etiological insult (Group-A) and were aged 24 (773) years with 11 males. In rest of the 24 cases the etiology of the acute insult could not be defined (Group-B). They were aged 27 (1157) years with 14 males. All cases had index presentation as ACLF and were diagnosed as having Wilson disease subsequently. The etiology of acute insult was acute viral hepatitis in 7 cases (Hepatitis $\mathrm{E}$ in 3, Hepatitis $\mathrm{A}$ in 3, and Epstein - Barr virus in 1 case), drug induced liver injury in 5 cases ( 3 were on anti-tuberculosis therapy), sepsis and ethanol in 1 case each. 20 cases ( 8 in Group-A) had hepatic encephalopathy (HE) at baseline and 2 developed HE on day 4 (both from Group-A). The duration of jaundice was 0.75 (0.25-4.5) months with all patients having ascites at baseline. Median hospital stay was 24 (range 9-64) and 9 (range 1-36) days in group A and B respectively $(\mathrm{p}<0.001)$. The alkaline phosphatase-to-bilirubin ratio of $<4$ was observed in 9 cases $(24 \%)$ whereas AST-to-ALT ratio was $>2.2$ in 17 cases $(45 \%)$. The mortality with diagnosis of ACLF is higher $(63.9 \%)$ against the overall mortality $(43.8 \%)$ in AARC cohort due to ACLF [HR: $1.83,95 \mathrm{CI} \%, 1.22-2.74, \mathrm{p}=0.003$ ]. The mortality was (8/14) $57 \%$ for Group-A cases which was better than (16/22) $73 \%$ for 
Group-B cases $(\mathrm{p}<0.01)$, while 2 in Group B underwent liver transplant.

Conclusion: This prospective dataset is the largest series of Wilson disease presenting as ACLF. Wilson disease patients with ACLF have a high mortality, which is much higher in absence of an identifiable precipitant for the same. This distinct syndrome needs new and early interventional strategies.

\section{$O-A C L F-12$}

Effect of plasmapheresis on vWF and ADAMTS13 in Acute on Chronic Liver Failure (ACLF)

Mayank Jain $^{1}$, Mouleswaran Sampath ${ }^{1}$, Deepti Sachan ${ }^{1}$, Pushkala Subramanian $^{2}$, Jayanthi Venkataraman ${ }^{1}$, Subhashree Parida ${ }^{1}$, Sipra Bal ${ }^{1}$

${ }^{1}$ Gleneagles Global Health City, India; ${ }^{2}$ Department of Immunology, The TamilnaduDr.M.G.R. Medical University, Chennai, India

Background: Rise inVonWillebrand Factor(vWF) antigen(Ag) levels and fall in ADAMTS13 levels has been studied in various liver disorders. However, there is no data on therapeutic measures to modulate this imbalance and improve clinical outcomes in acute on chronic liver failure (ACLF) patients. Aim of the study was to assess the relation between $\mathrm{vWF}(\mathrm{Ag})$ level and clinical / biochemical parameters in ACLF patients using therapeutic plasma exchange (TPE)

Method: Thirteen patients underwent 21 sessions of TPE at our centre. Their clinical parameters and blood investigations were recorded pre and post TPE. The data was analysed using paired t test. Result: Following TPE, there were statistically significant reductions in serum bilirubin, aminotransferase levels and improvement in coagulopathy. Creatinine value was not significantly altered. There was a significant decline in $\mathrm{vWF} \mathrm{Ag}$ with along with a rise in ADAMTS 13 levels. All patients improved clinically.

Conclusion: Our data provides robust evidence regarding the clearance of VWF antigen and subsequent clinical improvement with TPE in ACLF patients, which needs to be further clarified by multi-centre, large volume studies

Session: Drug Induced Liver Injury. Chairpersons; Naomi Khaing Than Hlaing; S L Broor; Awinash Sinha

\section{O-DILI-01}

MicroRNA-18a down-regulation is involved in rifampicin induced liver injury by enhancing TRADD and BID expression in vitro and in vivo

\section{$\underline{\text { Jun Chen }}^{1}$, Jun Chen ${ }^{1}$, Pengcheng Ou ${ }^{1}$}

${ }^{1}$ The Second Xiangya Hospital, China

Background: Anti-tuberculosis drug induced liver injury (DILI) is a leading cause of drug-induced acute liver failure, nevertheless its molecular mechanism remains unclear. The following study investigates the effect of rifampicin (RFP) on microRNA (miRNAs) and its target genes regulation in hepatic cell in vitro and in vivo.

Method: The expression profiles of miRNAs in RFP treated and untreated human hepatic cell line QSG-7701 were analyzed by microRNA microarray. Gene ontology analysis was applied to analyze the main function of differential miRNAs. The up- and downregulation of miRNAs was examined by qRT-PCR. BID protein expression was detected by western blotting. The binding activity of
miR-18a and TRADD (TNFRSF1A associated via death domain) mRNA 3'-UTR was detected by dual luciferase reporter gene analysis. For in vivo analysis, BALB/c mice were divided into RFP treated and saline treated group. After 7 days treatment, liver were collected and further analyzed using RT-qPCR, western blot and immunohistochemistry method.

Result: 6 up-regulated and 4 down-regulated miRNAs were found in RFP treated cultured cells compared to controls $(\mathrm{p}<0.01)$. After bioinformatics analysis, miR-18a, miR-365a, miR-194, miR-675 and miR-1231 were found to be involved in the process of apoptosis and necrosis of RFP-induced hepatic cells injury; however, only the miR18a was significantly lower in both cells and liver tissue samples compared to controls $(\mathrm{p}<0.05)$. In addition, miR-18a downregulated TRADD expression via 3'-untranslated-region (UTR) pairing; after RFP treatment, BID protein (a pro-apoptotic member of the Bcl-2 family) and TRADD mRNA expression were both increased in hepatic cells/hepatic tissue compared to the control cells/tissue $(\mathrm{p}<0.05)$.

Conclusion: RFP induces liver injury by down-regulating miR-18a and by increasing TRADD and BID expression.

\section{O-DILI-O2}

Glycosyltransferase GLT25D2 acts as a negative regulator of autophagy to promote acetaminophen-induced hepatotoxicity

Feng Ren ${ }^{1}$, Jing Zhang ${ }^{1}$, Xiangying Zhang ${ }^{1}$, Hongbo Shi ${ }^{1}$, Zhongping Duan $^{1}$, Hongshan Wei ${ }^{1}$

${ }^{1}$ Capital Medical University, China

Background: Acetaminophen overdose can cause liver injury by inducing mitochondria damage and subsequent necrosis in hepatocytes, its pathological mechanism still needs to be further explored. The glycosylation of proteins in acetaminophen-induced hepatotoxicity remains enigmatic. Here, the aims of this study is to explore the role of the collagen galactosyltransferase GLT25D2 gene in pathogenesis of acetaminophen-induced hepatotoxicity.

Method: The mice were given a single intraperitoneal (i.p.) injection of acetaminophen. Livers tissues analyzed by histology, reversetranscription polymerase chain reaction, western bolting, immunohistochemistry, and electron microscopy, respectively. Primary hepatocytes were isolated and analyzed. Liver biopsy samples from patients with acetaminophen overdose were analyzed by immunofluorescence staining.

Result: GLT25D2 expression was significantly elevated in the livers of mice exposed to acetaminophen. Knockout of GLT25D2 ameliorated the hepatocellular damage, evidenced by reduced serum alanine aminotransferase levels and well-preserved liver architecture compared with controls. Mechanistic investigations elucidate that GLT25D2 acts as a negative regulator of autophagy, whereby enhancing the susceptibility of hepatocytes to acetaminophen-induced cytotoxicity. Indeed, (1) knockout of GLT25D2 promoted autophagy and enhances autophagy flux in vitro and in vivo; (2) inhibition of autophagy reversed liver protection and hepatocyte protection of GLT25D2 knockout; (3) autophagy induced by GLT25D2 knockout seemed to be selective for damaged mitochondria. More importantly, the expression of GLT25D2 is significantly downregulated in druginduced liver injury patients.

Conclusion: The increased GLT25D2 suppresses autophagy to promote the occurrence and development of acute liver injury and suggest that GLT25D2 can be a potential therapeutic choice for acetaminophen-induced hepatotoxicity. 


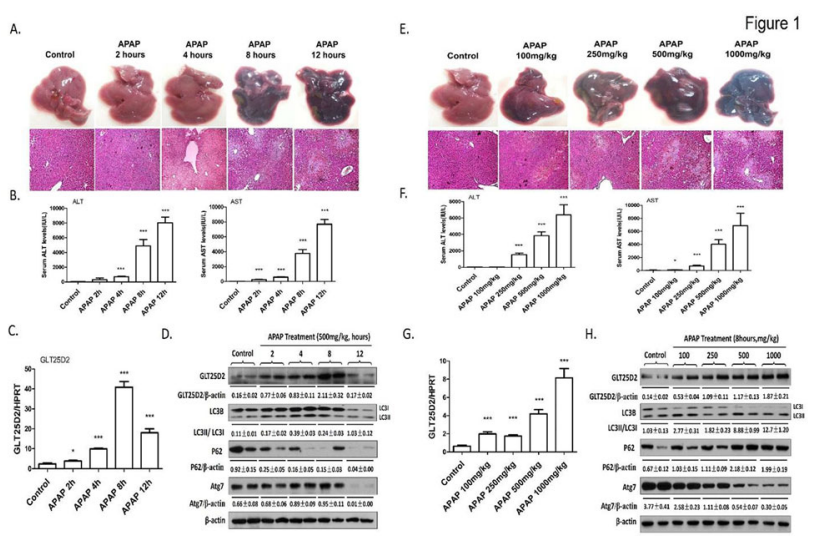

Figure 1 Dynamic profile of GLT25D2 in the progression of APAP-induced hepatotoxicity

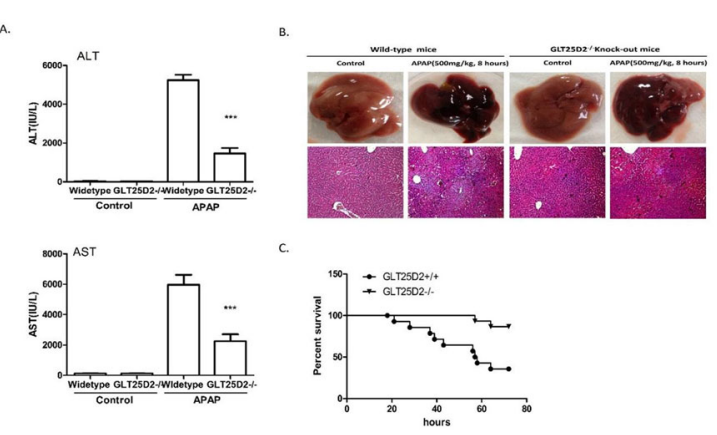

Figure 2 GLT25D2 knockout protects mice from APAP-induced hepatotoxicity

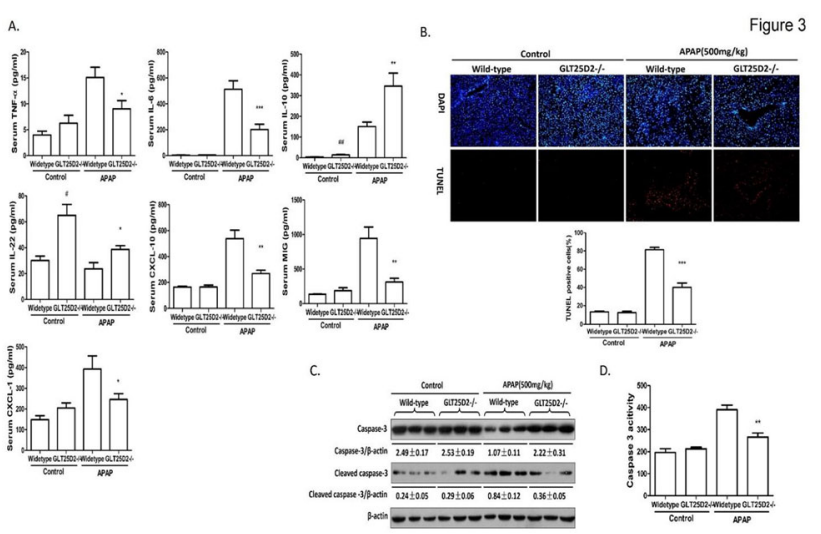

Figure 3 Reduced liver inflammation and hepatocyte death in GLT25D2-/- mice induced by APAP
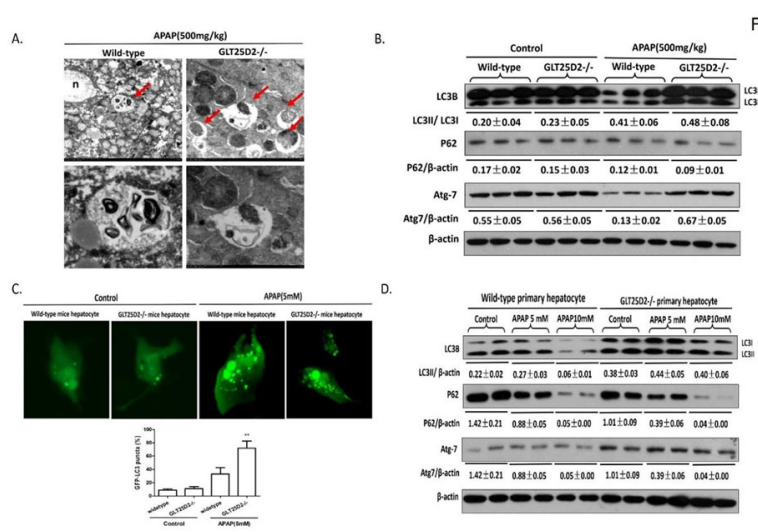

Figure 4 GLT25D2 acts as a negative regulator of autophagy responses to APAP

\section{ica!} $-$

P62 -

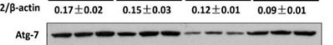

\begin{tabular}{llll} 
& $0.35 \pm 0.02$ & $0.67 \pm 0.05$ \\
\hline
\end{tabular}

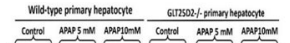

cos

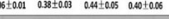

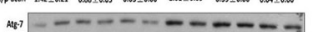

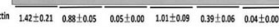

Figure 4
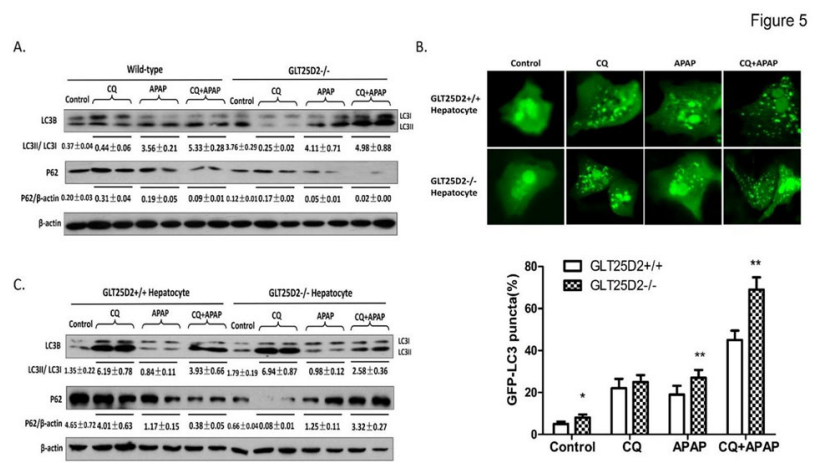

Figure 5 Distinct effects of GLT25D2 on autophagic flux in vivo and in vitro
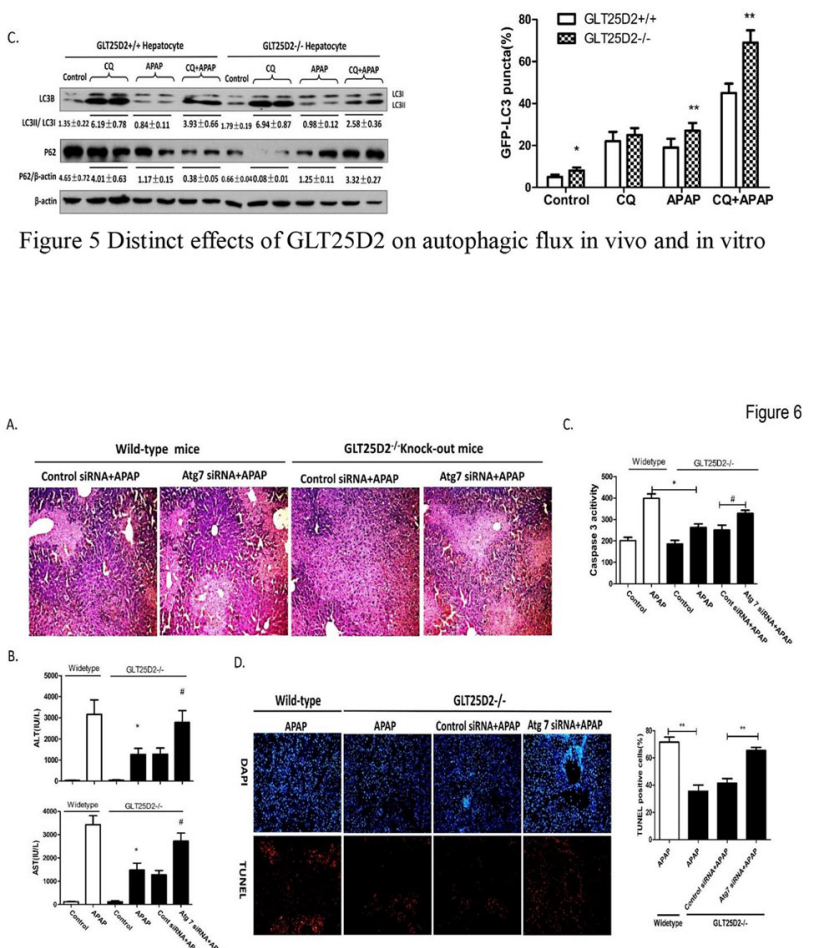

Figure 6 GLT25D2-/- protects mice from APAP-induced hepatotoxicity through Autophagy pathway 


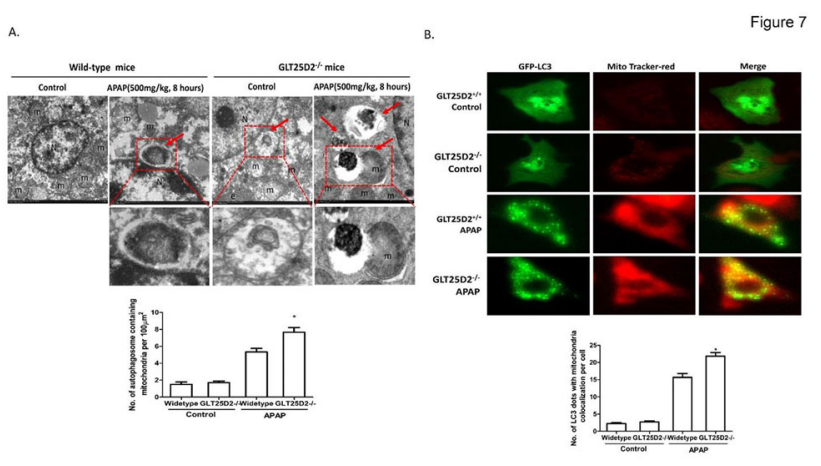

Figure 7 Knockout of GLT25D2 promotes mitophagy

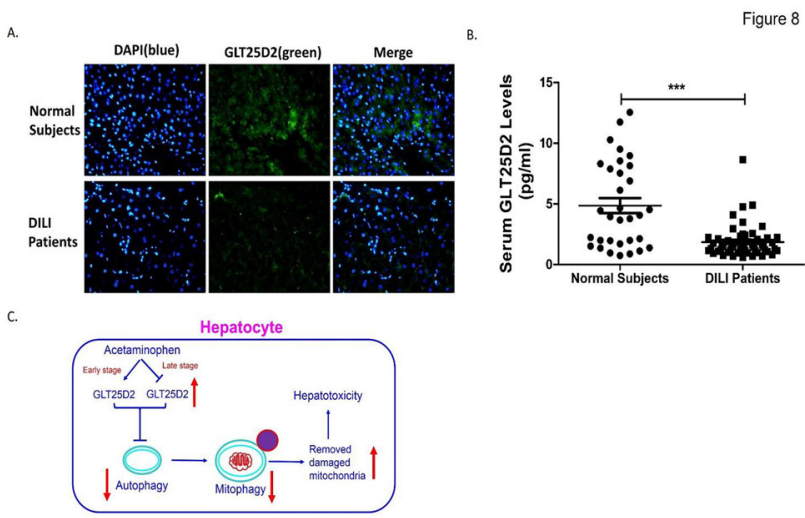

Figure 8 Levels of GLT25D2 in drug-induced liver injury (DILI) patients

\section{O-DILI-03}

Herbal medicines may induce more severe liver injury than western medications

\section{Jun Chen ${ }^{1}$}

${ }^{1}$ The Second Xiangya Hospital, China

Background: The epidemiological and clinical features of herbal medicines (HM) induced liver injury is not very clear in mainland China. From March 2015 to October 2016, China drug induced liver injury (DILI) study group organized a nationwide multicenter cohort retrospective study to intensive investigate the situation of DILI in 308 mainland Chinese hospitals, here, we analyzed the data to clarify the epidemiological and clinical features of HM induced DILI among hospitalized patients in mainland China.

Method: From January 2012 to December 2014, all quantifiable data of hospitalized patients whose diagnosis at discharge was "DILI" were collected, RUCAM score was performed to redefine the diagnosis of DILI. According to the causative agent of DILI, patients were divided into two groups: HM group and western medication (WM) group. The data of demographics, clinical characteristics, suspicious drugs, and outcome assessments of eligible cases were collected. SAS was applied for statistical analyses.

Result: 3684 HM cases and $9005 \mathrm{WM}$ cases were finally enrolled in the study. The mean age of the HM group was $48.2 \pm 14.66$ years old, in contrast, WM group was $44.77 \pm 17.25$ years old $(\mathrm{p}<0.0001)$. Female was predominant in the HM group (male vs female, $40.67 \%$ vs $59.33 \%, \mathrm{p}<0.0001$ ) and an opposite result was found in the WM group. Compare to WM group, duration from drug cessation to symptom disappearance of HM group was longer (20 vs $14, \mathrm{p}<0.0001$ ), serum level of ALT. AST, ALP, TBIL, DBIL was significantly higher, and level of TP, ALB slightly lower in the HM group $(\mathrm{p}<0.0001)$. Meanwhile, incidence of jaundice, itching and digestive symptoms is quite higher in the HM group than WM group $(\mathrm{p}<0.0001)$. Although the general mortality of the two groups had no significant difference $(0.44 \%$ vs $0.29 \%, p=0.2383)$, the liverrelated death accounted for $0.33 \%$ cases in the HM group and $0.12 \%$ cases in the WM group ( $p=0.0387)$.Incidence of acute liver failure and Hy's cases was more frequent in the HM group than WM $\operatorname{group}(0.68 \%$ vs $0.39 \%$. $\mathrm{P}=0.0179 ; 30.32 \%$ vs $8.55 \%$, $\mathrm{p}<0.0001$ ).To patients caused by HM, the mean age of cholestatic pattern tended to be older than hepatocellular or mixed pat$\operatorname{tern}(\mathrm{p}<0.0001)$, moreover, the frequency of chronic DILI in the patients with preexisting liver disease was more higher than patients without preexisting liver disease( $10.46 \%$ vs $6.73 \%, \mathrm{p}=0.0288)$. The median of recovery time of long latency cases ( $>180$ days), days from drug cessation to symptom disappearance, was much longer than short latency cases ( $<7$ days) (days, 18 vs $32, \mathrm{p}<0.0001$ ).

Conclusion: HM induced DILI is more is more prevalent in older people and women. Depending on clinical characteristics and outcomes, the duration from drug cessation to symptom disappearance of HM group was longer than WM group, moreover, HM tends to cause more severe liver injury and liver-related mortality than WM. The independent risk factors for mortality in the HM group were the abnormality of PT, ALB and Cr.

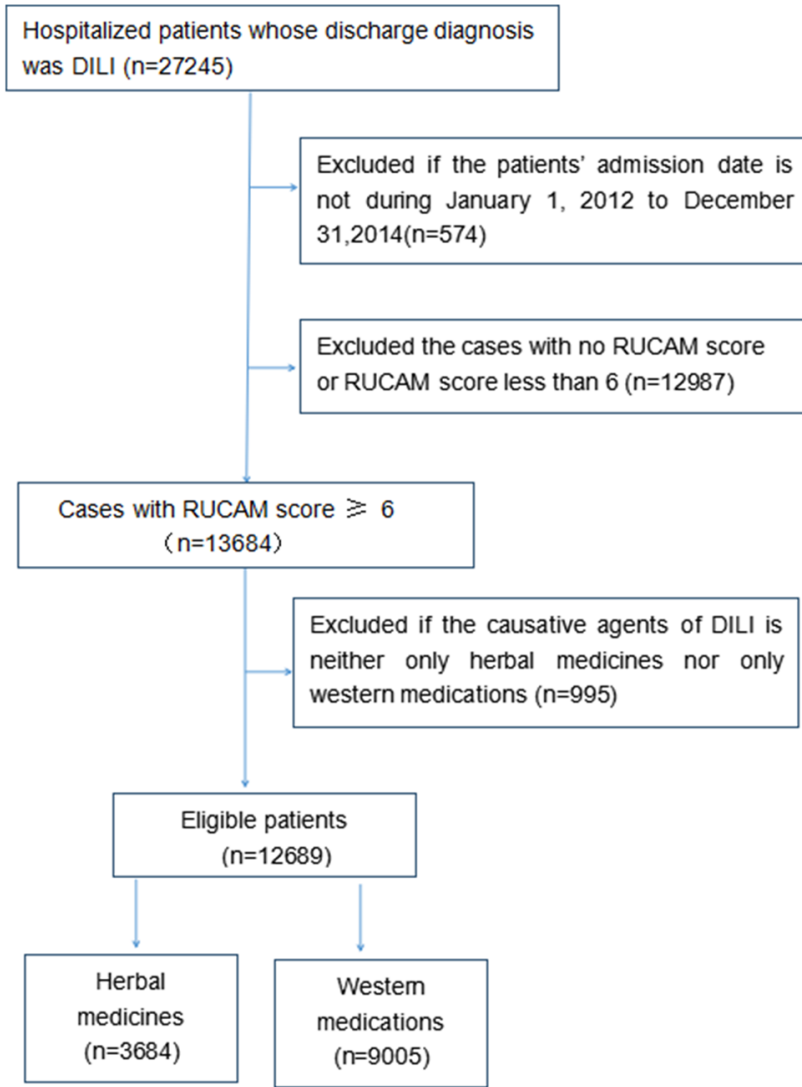

Figure 1. Inclusion and exclusion flow chart 
Table 1. Demographics and clinical characteristics

\begin{tabular}{|c|c|c|c|}
\hline Characteristic & $\begin{array}{c}\mathrm{HM} \\
(\mathrm{N}=3684)\end{array}$ & $\begin{array}{c}\text { WM } \\
(\mathrm{N}=9005)\end{array}$ & $P$ value \\
\hline Age $(y)$, mean $\pm S D$ & $48.24 \pm 14.66$ & $44.77 \pm 17.25$ & $<0.0001$ \\
\hline Female (\%) & 59.33 & 45.04 & $<0.0001$ \\
\hline Alcohol consumption $\mathrm{N}(\%)$ & $268(7.50 \%)$ & $584(6.67 \%)$ & 0.1008 \\
\hline $\begin{array}{l}\text { Days from drug use to } \\
\text { symptom } \\
\text { appearance, Median(Q1- } \\
\text { Q3) }\end{array}$ & $24(8.00-57.00)$ & $23(7.00-57.00)$ & 0.3592 \\
\hline $\begin{array}{l}\text { Days from drug } \\
\text { cessation to symptom } \\
\text { disappearance, } \\
\text { Median(Q1-Q3) }\end{array}$ & $20(11.00-33.00)$ & $14(8.00-25.00)$ & $<0.0001$ \\
\hline \multicolumn{4}{|l|}{ Liver biochemistries } \\
\hline $\operatorname{ALT}(I U / L)$ & $232(93.00-548.00)$ & $86(37.00-207.00)$ & $<0.0001$ \\
\hline \multicolumn{4}{|l|}{ Median(Q1-Q3) } \\
\hline AST(IU/L) & $130(55-320)$ & $52(28-122)$ & $<0.0001$ \\
\hline $\mathrm{ALP}(\mathrm{IU} / \mathrm{L})$ & $130(96.00-181.00)$ & $97(70.40-145.60)$ & $<0.0001$ \\
\hline $\mathrm{TBIL}(\mu \mathrm{mol} / \mathrm{L})$ & $46.5(17.00-141.19)$ & $14.34(9.00-30.00)$ & $<0.0001$ \\
\hline $\mathrm{DBIL}(\mu \mathrm{mol} / \mathrm{L})$ & $27.65(6.97-98.8)$ & $5.2(3.00-14.64)$ & $<0.0001$ \\
\hline $\mathrm{PT}(\mathrm{S})$, mean $\pm \mathrm{SD}$ & $13.40 \pm 4.20$ & $13.49 \pm 4.86$ & 0.9222 \\
\hline INR & $1.03(0.95-1.14)$ & $1.03(0.95-1.15)$ & 0.0668 \\
\hline $\mathrm{TP}(\mathrm{g} / \mathrm{L})$ & $65.08 \pm 8.22$ & $65.69 \pm 8.36$ & $<0.0001$ \\
\hline $\operatorname{ALB}(g / L)$ & $37.42 \pm 5.97$ & $38.21 \pm 6.30$ & $<0.0001$ \\
\hline $\operatorname{Cr}(\mu \mathrm{mol} / \mathrm{L})$ & $60(51.00-72.40)$ & $61.74(50.90-74.00)$ & 0.0445 \\
\hline HBV-DNA positive $n(\%)$ & $80(8.56 \%)$ & $251(15.08 \%)$ & $<0.0001$ \\
\hline \multicolumn{4}{|l|}{ Autoimmune antibodies } \\
\hline ANA positive $\mathrm{n}(\%)$ & $362(13.65 \%)$ & $338(10.37 \%)$ & 0.0001 \\
\hline AMA positive $n(\%)$ & $25(1.08 \%)$ & $19(0.68 \%)$ & 0.1289 \\
\hline AMA-M2 positive $\mathrm{n}(\%)$ & $39(1.57 \%)$ & $42(1.40 \%)$ & 0.6152 \\
\hline ASMA positive $\mathrm{n}(\%)$ & $22(0.99 \%)$ & $18(0.68 \%)$ & 0.2427 \\
\hline anti-LKM positive $n(\%)$ & $4(0.17 \%)$ & $13(0.47 \%)$ & 0.0728 \\
\hline \multicolumn{4}{|l|}{ Pattern of DILI } \\
\hline Hepatocellular(\%) & $1768(51.02 \%)$ & $2458(29.39 \%)$ & $<0.0001$ \\
\hline Cholestatic(\%) & $812(23.43 \%)$ & $3655(43.71 \%)$ & $<0.0001$ \\
\hline Mixed(\%) & $885(25.54 \%)$ & $2249(26.90 \%)$ & 0.1288 \\
\hline
\end{tabular}

Table 3 .Outcomes of DILI patients

\begin{tabular}{cccc}
\hline & $\begin{array}{c}\mathrm{HM} \\
(\mathrm{N}=3684)\end{array}$ & $\begin{array}{c}\mathrm{WM} \\
(\mathrm{N}=9005)\end{array}$ & P value \\
\hline Death cases & $16(0.44 \%)$ & $26(0.29 \%)$ & 0.2383 \\
Liver-related death & $12(0.33 \%)$ & $11(0.12 \%)$ & 0.0387 \\
Acute liver failure & $25(0.68 \%)$ & $35(0.39 \%)$ & 0.0179 \\
Hy's cases & $1117(30.32 \%)$ & $770(8.55 \%)$ & $<0.0001$ \\
Chronic DILI & $257(6.98 \%)$ & $597(6.63 \%)$ & 0.4795 \\
\hline
\end{tabular}

\section{O-DILI-04}

\section{Clinical Features and Outcomes of Hospitalized Patients with Suspected Drug Induced Liver Injury (DILI)}

\author{
Farhana Kayani $^{1}$,Shahab Abid ${ }^{1}$, Adeel Abid ${ }^{1}$, Faryal Subhani ${ }^{1}$ \\ ${ }^{1}$ Aga Khan university Hospital, Pakistan
}

Background: Drug induced liver injury (DILI) is an underestimated cause of liver injury. There is paucity of data from South East Asia. This study aims to provide a clinical spectrum and outcomes of hospitalized patients with DILI.

Method: Patients with discharged diagnosis of DILI were reviewed. The diagnosis of DILI was based upon COIMS/RUCAM score and the exclusion of other hepatic diseases. Liver injury was considered, if there was an increase over 2 fold or normal in alanine aminotransferase (ALT) or conjugated bilirubin or a combined increase in aspartate aminotransferase (AST), alkaline phosphatase (AP), and total bilirubin, with one of them was twofold above normal. The pattern of DILI was classified on basis of $R$ value, ratio between ALT and $\mathrm{AP}$ expressed as hepatocellular; $\mathrm{R}>5$, cholestatic; $\mathrm{R}<2$, and mixed; $\mathrm{R}>2<5$. Severity of DILI was mild (raised ALT and/or AP), moderate (raised ALT and/or AP along with bilirubin level $>2.5 \mathrm{mg} / \mathrm{dl}$ or INR $>1.5$ ) and severe (criteria of moderate +organ failure).In-hospital mortality and prolonged hospital stay ( $>5$ days) were also checked.

Result: 462 cases with the diagnosis of suspected DILI were identified. Mean age was 50 years (range, 20-94 years, $57.6 \%$ were male. DILI was classified as definite or highly probable in $31.1 \%$, probable in $62.5 \%$, and possible in $7.4 \%$. Abdominal pain, vomiting, jaundice \& pruritus was present in $57.1 \%, 57.1 \%, 54.1 \%$, and $42.3 \%$ respectively) and altered mental status in $21.6 \%$. Antituberculous(ATT) drugs was the most common cause $(63.9 \%)$, others were herbal (9.5\%), antiarrhythmic $(8.7 \%)$, statins $(7.3 \%)$, antibiotics $(6.1 \%)$, antifungals $(5.6 \%)$, chemotherapeutic agents $(4.4 \%)$, antidepressants (4.2\%), antimalarials $(2.5 \%) \&$ antiepileptics $(1.7 \%)$.

Pattern of DILI was hepatocellular in $25.1 \%$, cholestatic $56.17 \%$ and mixed $18.72 \%$. Extent of severity of liver injury was mild in $50.6 \%$, moderate in $31.2 \%$ and severe in $18.14 \%$ of cases. In- hospital mortality was $26.5 \%$ and prolonged hospital stay was present in $46.32 \%$. Mortality and prolonged hospital stay was significantly greater in patients with altered mental status $(0.46$ vs. $0.53 \mathrm{p}<0.05)$, male gender ( 0.30 vs. $0.69 \mathrm{p} 0.03)$, DILI by chemotherapeutic agents $(0.7$ vs. $0.2 \mathrm{p}<0.05)$ and antifungals $(0.6$ vs. $0.4 \mathrm{p} 0.02)$.

Conclusion: ATT being the commonest cause and Cholestatic pattern was the most common type of DILI. There is a high mortality (more than quarter) associated with DILI, high index clinical suspicion is required for its diagnosis.

\section{O-DILI-05}

\section{Different methods for judging cholestatic drug-induced liver} injury

\author{
Mindan Xing ${ }^{1}$ \\ ${ }^{1}$ Tianjin Second People's Hospital, Tianjin Institute of Hepatology, \\ China., China
}

Background: The diagnostic criteria established by the Council for International Organizations of Medical Sciences (CIOMS), the diagnostic criteria in the European Association for the Study of the Liver (EASL) Clinical Practice Guidelines: management of cholestasis liver diseases, and liver pathology may be used for judgment of cholestatic 
drug-induced liver injury, but whether the results of these methods are coincident is unclear.

Objective: To explore the coincidence of 3 different sets of diagnostic criteria for cholestatic drug-induced liver injury (DILI).

Method: Three different sets of diagnostic criteria for cholestatic DILI were used separately to classify 133 DILI patients into 2 groups: those with cholestatic DILI and those with non-cholestatic DILI. The 3 sets of criteria were: CIOMS criteria (involving different $\mathrm{R}$ value), EASL criteria, and liver pathology, respectively. Then, the percentages of those with cholestatic DILI were compared to explore the coincidence of the 3 sets of diagnostic criteria.

Result: The differences among the percentages of those with cholestatic DILI diagnosed using 3 different sets of criteria were statistically significant $\left(\alpha=0.05, \chi^{2}=99.97, P<0.001\right)$. In terms of the percentage of those with cholestatic DILI, the rate of concordance between the CIOMS criteria (involving different $\mathrm{R}$ value) and the pathological diagnostic criteria were lower than that of concordance between the EASL criteria and the pathological diagnostic criteria.

Conclusion: Compared with that of cholestatic DILI using the pathological diagnostic criteria, the incidence of cholestatic DILI diagnosed using the CIOMS/EASL criteria based on present biochemical indicators was underestimated.

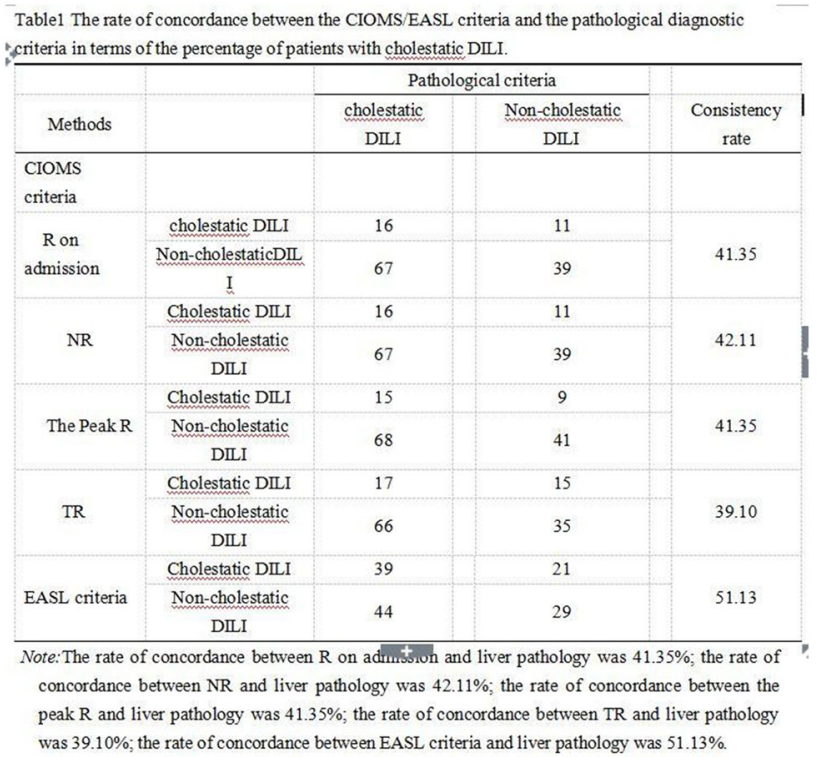

\section{O-DILI-06}

Etiology and clinical features of patients with drug-induced liver injury diagnosed by liver biopsy

George Lau ${ }^{1}$, Dong $\mathrm{Ji}^{2}$, Cheng Wang ${ }^{4}$, Jing Chen ${ }^{4}$, Qing Shao ${ }^{2}$, Bing $\mathrm{Li}^{2}$, Fan $\mathrm{Li}^{2}$, Zhongbin $\mathrm{Li}^{2}$, Yudong Wang ${ }^{4}$, Vanessa $\mathrm{Wu}^{4}$, George $\mathrm{Lau}^{3}$

${ }^{1}$ Humanity and Health Medical Group, Hong Kong; ${ }^{2} 302$ Hospital, Beijing, China., China; ${ }^{3}$ Humanity \& Health Medical Centre, Hong Kong, Hong Kong SAR, China, Hong Kong; ${ }^{4}$ Humanity and Health Research Centre, Hong Kong SAR, China, Hong Kong

Background: Drug-induced liver injury (DILI) is a rare adverse drug reaction and it can lead to jaundice, liver failure, or even death in some patients. Antimicrobials and herbal and dietary supplements are among the most common therapeutic classes to cause DILI in the
Western world. This study aimed to investigate the etiology and clinical features of Chinese patients with DILI.

Method: A total of 194 consecutive DILI inpatients, who underwent liver biopsy in Beijing 302 hospital from January 2015 to December 2015, were enrolled in the study. The etiology, laboratory markers (e.g. ALT, AST, GGT), and the pathological features were analyzed retrospectively. The relationship between liver fibrosis stage and noninvasive measurements, e.g. liver stiffness measured (LSM) by FibroScan ${ }^{\circledR}$ (Echosens, Paris, France), APRI, FIB-4 and portal vein diameter were analyzed by Spearman correlation analysis.

Result: Chinese traditional medicine (TCM) was the most common cause of DILI, which accounted for $46.9 \%$ of patients, followed by acetaminophen-containing regimen (14.4\%), antibiotics $(9.3 \%)$, environmental toxins $(4.6 \%)$, antidepressant $(4.6 \%)$, dietary supplement $(3.1 \%)$, lipid-lowering drugs $(3.1 \%)$, chemotherapeutic agents (3.1\%), and others $(11.3 \%)$. Of 194 DILI patients, hepatocellular type was observed in $78(40.2 \%)$ patients, cholestatic type in $63(32.5 \%)$ patients, and mixed type in $53(27.3 \%)$ patients. Histological findings showed that $70(36.1 \%)$ patients had an acute injury, $124(63.9 \%)$ patients had chronic damages, which composed by G0 (9.8\%), G1 (19.1\%), G2 (21.6\%), G3 (9.8\%), and G4 (3.6\%) inflammation level. LSM, APRI and FIB-4 were positively correlated with liver fibrosis stage, but portal vein diameter had no relationship with liver fibrosis stage.

Conclusion: In Chinese population, almost half of DILI was induced by TCM. Liver stiffness measured by non-invasive biomarkers is correlated with grading from liver biopsy in Chinese patients with DILI.

Session: Hepatitis B Virus Infection- Basic Sciences; Chairperson; Qin Ning; M P Sharma; Vinod Arora

\section{$O-H B V-01$}

Safety and antiviral activity of novel HBV capsid assembly modulator JNJ-56136379 in non-cirrhotic, treatment-naïve patients with chronic hepatitis $B$

Fabien Zoulim ${ }^{1}$, Oliver Lenz ${ }^{6}$, Willem Talloen ${ }^{6}$, Eva Santermans ${ }^{6}$, Christelle Vistuer $^{6}$, Iurie Moscalu ${ }^{8}$, Adrian Streinu Cercel ${ }^{7}$, Stefan Bourgeois', Maria Buti ${ }^{4}$, Javier Crespo ${ }^{3}$, Juan Manuel Pascasio $^{5}$, Lawrence Blatt ${ }^{2}$, John Fry ${ }^{2}$, Jeysen Z Yogaratnam ${ }^{2}$, Joris J Vandenbossche ${ }^{6}$

${ }^{1}$ Hospices Civils de Lyon, and INSERM U1052, Lyon, France, France; ${ }^{2}$ Alios Biopharma (a Janssen Pharmaceutical Company of Johnson \& Johnson), United States; ${ }^{3}$ Hospital Universitario Marques de Valdecilla, Unidad de Ensayos Clinicos, Spai; ${ }^{4}$ Hospital Universitario Vall d'Hebron, Spai; ${ }^{5}$ Hospital Universitario Virgen del Rocio, UGC Enfermedades Digestivas, Spai; ${ }^{6}$ Janssen Pharmaceutica NV, Belgium; ${ }^{7}$ National Institute for Infectious Diseases "Prof. Dr. Matei Bals", Carol Davila University of Medicine and Pharmacy, Romania; ${ }^{8}$ Spitalul Clinic Republican, Moldova; ${ }^{9}$ ZNA Jan Palfijn, Belgium Background: The capsid assembly modulator JNJ-56136379 (JNJ379) is a potent and selective HBV replication inhibitor with a dual mode of action inhibiting early and late steps of the viral life cycle. It was well tolerated in a first-in-human Phase 1a study (NCT02662712). Such an agent may allow patients with chronic hepatitis $\mathrm{B}(\mathrm{CHB})$ to achieve functional cure of infection with a finite treatment regimen.

Method: Treatment-naïve, $\mathrm{HBeAg-positive} \mathrm{or} \mathrm{-negative} \mathrm{CHB}$ patients, with plasma HBV DNA $>2000 \mathrm{IU} / \mathrm{mL}$, METAVIR stage $<$ F3 and ALT/AST $<2.5$ ULN, were enrolled in this ongoing, 
randomized, double-blind, placebo-controlled Phase $1 \mathrm{~b}$ study. While dosing with $150 \mathrm{mg}$ JNJ-379 once daily (QD) is still ongoing, data from dosing with $25 \mathrm{mg}$ QD (after $100 \mathrm{mg}$ loading dose) and $75 \mathrm{mg}$ QD over 28 days are available. Patients were randomized 2:1 to receive JNJ-379 or placebo for each dose.

Result: Across the two dose groups $(n=24)$, patients had a median age of 36 years (range: $21-58$ ) with $88 \%$ males and $75 \%$ Caucasian. Overall, $38 \%$ of patients were $\mathrm{HBeAg}$-positive, and mean $( \pm \mathrm{SD})$ baseline HBV DNA was $5.88( \pm 1.82) \log 10 \mathrm{IU} / \mathrm{mL}$. AEs or laboratory abnormalities $\geq$ Grade 3 were infrequent ( $\leq 2$ patients/dose). Of patients treated with JNJ-379, 56\% (9/16) experienced at least one adverse event (AE) during treatment (5 patients in the $25 \mathrm{mg}$ arm, and 4 patients in the $75 \mathrm{mg}$ arm) compared to $63 \%$ (5/8) in the placebo arm. There were no serious AEs, no discontinuations due to AEs, and no dose-limiting toxicities. After 28 days, mean $( \pm \mathrm{SD})$ reductions in HBV DNA levels from baseline of $2.16( \pm 0.49) \log 10 \mathrm{IU} / \mathrm{mL}$ $(25 \mathrm{mg}$ QD) and $2.89( \pm 0.48) \log 10 \mathrm{IU} / \mathrm{mL}(75 \mathrm{mg}$ QD) were observed. Three patients dosed with $75 \mathrm{mg}$ QD achieved HBV DNA below the level of quantification of the HBV DNA assay but no patients dosed with $25 \mathrm{mg}$ QD reached HBV DNA below quantification. In addition, a decline in HBV RNA levels was observed in both JNJ-379 treatment groups; while reduction in HBV RNA was higher in the $25 \mathrm{mg}$ group than the $75 \mathrm{mg}$ group, more patients in the $75 \mathrm{mg}(\mathrm{n}=6)$ than the $25 \mathrm{mg}$ group $(\mathrm{n}=3)$ achieved HBV RNA below the level of quantification of the HBV RNA assay. No changes of note were observed in $\mathrm{HBsAg}$ or $\mathrm{HBeAg}$.

Conclusion: Preliminary data from this ongoing study in patients with CHB demonstrate that JNJ-379 administered for 28 days is generally safe and well tolerated and has potent antiviral activity against $\mathrm{HBV}$.

\section{$O-H B V-02$}

Safety, tolerability and pharmacokinetics of single ascending doses of JNJ-56136379, a novel HBV capsid assembly modulator, in Japanese adult healthy volunteers

Jeysen Zivan Yogaratnam ${ }^{1}$, Joris J Vandenbossche ${ }^{2}$, Oliver Lenz ${ }^{2}$, Jagadish Gogate $^{3}$, Nathalie Verpoorten ${ }^{2}$, Jeike Biewenga ${ }^{2}$, Jan Snoeys $^{2}$, Lawrence Blatt ${ }^{1}$, John Fry ${ }^{1}$

${ }^{1}$ Alios BioPharma (a Janssen Pharmaceutical Company), United States; ${ }^{2}$ Janssen Pharmaceutica NV, Belgium; ${ }^{3}$ Janssen Research and Development, LLC, United States

Background: JNJ-56136379 (JNJ-379), a capsid assembly modulator, is a potent and selective HBV replication inhibitor in vitro, offering a dual mode of action to disrupt the viral life cycle. JNJ-379 was well tolerated in a first-in-human Phase 1a study (NCT02662712). This study investigated the safety, tolerability and pharmacokinetics (PK) of single ascending doses of JNJ-379 in healthy Japanese volunteers.

Method: In this double-blind, placebo-controlled, Phase 1 study, 32 Japanese adult healthy volunteers were randomized into one of four cohorts (A-D). Within each cohort $(\mathrm{n}=8)$, volunteers were randomized 3:1 to receive either a single oral dose of JNJ-379 $(25,150$, 300 , or $500 \mathrm{mg}$ by cohort, respectively) or placebo in a fasted state. After each dose, safety, tolerability and PK plasma profiles were assessed throughout the 28-day study, and urinary elimination in Cohort $\mathrm{C}$ was assessed for 7 days.

Result: Demographic characteristics were comparable across cohorts, and between volunteers receiving JNJ-379 and placebo. Median age of volunteers was 38.5 years (range: $26-55)$; most were male $(87.5 \%)$. In general, JNJ-379 was well tolerated; of the 24 volunteers receiving JNJ-379, 4 (16.7\%) experienced at least 1 treatment-emergent adverse event (TEAE; 2 in the $150 \mathrm{mg}$ and 2 in the $500 \mathrm{mg}$ groups). The TEAEs reported were hiccups (doubtfully related to JNJ-379), upper respiratory tract infection, nasal congestion, and cough (all unrelated to JNJ-379). All TEAEs were mild and resolved before study end. No dose-response relationship was observed. No Grade 3 or 4 laboratory abnormalities were reported. The most frequently reported $=$ Grade 1 (reported in at least 3 volunteers in any of the treatments groups) laboratory abnormalities were cholesterol increase, fibrinogen decrease, leukocytes decrease, triglyceride increase and total bilirubin increase. No deaths, serious AEs or discontinuations due to AEs were reported. Overall, the increase in JNJ-379 exposure following the single oral dose was proportional over the dose range tested in the 4 cohorts, with dose normalized Cmax of $375,296,308$ and $328 \mathrm{ng} / \mathrm{mL}$ and AUC24 h of 4627, 4298, 4012 and $4382 \mathrm{ng} \mathrm{h} / \mathrm{mL}$ in fasted patients (dose normalized to $25 \mathrm{mg}$ ), respectively. Intersubject variability (\% coefficient of variation) for Cmax ranged from 24.0-42.5\% and for AUC24 h ranged from 13.3-22.1\%. In the 4 cohorts the median tmax ranged from 1.75-3.00 hours across all dose levels (range amongst individuals: 1.00-6.00 hours). Exposures were slightly higher than seen previously in healthy Caucasian volunteers. In Cohort C, $18 \%$ of the administered dose ends up as unchanged drug in urine; mean renal clearance of JNJ-379 $(300 \mathrm{mg})$ was $0.161 \mathrm{~L} / \mathrm{hour}$ and total apparent oral clearance was $0.852 \mathrm{~L} /$ hour; similar to seen previously in healthy Caucasian volunteers.

Conclusion: Results from this study in healthy Japanese volunteers demonstrate that JNJ-379 administered in single ascending doses is generally well tolerated, with a dose-proportional pharmacokinetic profile.

\section{$O-H B V-03$}

Study of Immune response post 3 doses of Hepatitis B vaccine among healthcare workers in a tertiary care public hospital of Mumbai Divyesh Jeram Devaliya
${ }^{1}$, Desma Dsouza
Maitreyee Patwardhan

${ }^{1}$ Non Profit Organization, India; ${ }^{2}$ Lokmanya Tilak Municipal General Hospital \& Medical College, India

Background: Hepatitis B is the most transmissible but vaccine preventable infection. The risk of contracting Hepatitis B to Healthcare workers (HCWs) is four-times greater than that of general adult population as they are in daily contact with blood \& body fluids. In the scenario, where the vaccination status are not well documented and more than half of the injuries goes unreported, knowing the immune response of vaccine becomes imperative.

Method: A retrospective study was conducted in L.T.M.G hospital Mumbai, among vaccinated HCWs, who participated voluntarily with written pre consent in Hepatitis B prevention program. Serum sample was collected from all HCWs to know their Anti Hbs level. Detailed demographic and medical history was taken post their consent. All blood samples were tested by Enzyme Linked Immunosorbent assay (ELISA) for Anti Hbs level so as to know the immune response for different dosage of vaccine.

Result: Among 1676 participants, self-reported status of vaccinated HCWs was, a) H/o 1-Dose 194 HCWs [11\%], b) H/o 2-Dose 518 HCWs [31\%] and c) H/o 3-Dose 964 HCWs [58\%]. Age wise, 50\% were 35 years. Among those found Anti Hbs responsive (Anti Hbs 35 years. Doctors (39\%) and Nurses (36\%) comprised majority of the HCWs receiving 3-doses, while sanitary workers $(18 \%)$ who are at risk were not fully immunized. Among the 58\% who reported receiving 3 doses, 29\% had serum Anti Hbs level below $10 \mathrm{mIU} / \mathrm{ml}$ comprising of Doctors (30\%), Nurses (20\%), Sanitary workers $(23 \%)$ 
and other staff (38\%). Immune response was dose dependent 1-dose $(42 \%)$ and 3 dose $(75 \%)$, while overall $(71 \%)$. The exposure risk to HBV is very high among HCWs hence, a compulsory vaccination program with documented doses should be introduced in health centres \& hospitals. Even among those receiving 3-doses, 29\% HCWs were non-responsive (Anti $\mathrm{Hbs}<10 \mathrm{mIU} / \mathrm{ml}$ ) stressing the need for periodic testing of post vaccination Anti Hbs level among HCWs. The study also found a significant relationship of anti Hbs level with age at which vaccine was taken, education, role of HCWs and highlights the need for early vaccination.

Conclusion: The study stresses on the need for protecting the HCWs from HBV by raising awareness, vaccination, monitoring of Anti Hbs levels and record maintenance.

\section{$O-H B V-04$}

\section{Butyrate reduces Hepatitis B Virus replication by inhibition of sirtulin-1}

\section{Kishor Pant ${ }^{1}$ \\ ${ }^{1}$ South Asian University, India}

Background: The infection of hepatitis B virus is combined with a number of liver disorders is rising significantly. Butyrate has been used for the treatment of a number of body aliments and the metabolic disorders. However, the impact of butyrate on HBV replication still remains unidentified.

Method: We used HepG2, HpG 2215 cells (HBV-expressing hepatocelluar carcinoma cells) and HBV infected patients liver biopsies samples for this study. Immunohistochemistery and western blotting was done using SIRT-1 and Ac-p53 antibodies. The level of HBsAg and HBV was determined by the ELISA and Real Time PCR respectively.

Result: In this present study, the HpG 2215 cells and HBV infected patients tissues samples manifested over-expression of the SIRT-1 and we found that butyrate inhibited HBV induced SIRT1 expression, which in turn inhibited the HBV replication in HBV infected cells. Butyrate suppressed both HBV-DNA replication and the secretion of the hepatitis B surface antigen (HBsAg) in HBV-expressing cells. Inhibition of the SIRT1 by butyrate or SiRNA increased the acetylation of $\mathrm{p} 53$, which subsequently decreased the cell proliferation induced by the HBV-infection.

Conclusion: We have identified that butyrate inhibits HBV replication through inhibition of SIRT-1 expression which further increased the acetylation of p53 and inhibited the HBV-induced cell growth, which depends on SIRT1/Ac-p53 regulatory axis.

\section{$\mathrm{O}-\mathrm{HBV}-05$}

Cytomegalovirus-based vaccine induces robust $\mathbf{T}$ cell responses and exerts an antiviral effect against hepatitis $B$ virus in the hydrodynamic injection mouse model

\section{Hongming Huang ${ }^{1}$, Meike Ruckborn ${ }^{2}$, Dongliang Yang ${ }^{1}$, Mirko Trilling $^{2}$, Jia Liu ${ }^{1}$}

${ }^{1}$ Union Hospital, Tongji Medical College, Huazhong University of Science and Technology, Wuhan, China, China; ${ }^{2}$ University Hospital Essen, University Duisburg-Essen, Germany

Background: Cytomegalovirus (CMV) can elicit a remarkable impact on the immune system of their hosts. Currently, CMV-based vaccines are in the spot-light as they showed superb control and even elimination of simian immunodeficiency infection (SIV) in rhesus macaques. While the potential of CMV vectors for $\mathrm{HBV}$ vaccines has not been studied yet.

Method: BAC mutagenesis was applied to generate recombinant MCMV expressing HBV-derived antigens under the control of the strong eukaryotic EF1 promoter either replacing the gene m157 or $\mathrm{m} 27$. The established HBV hydrodynamic injection (HI) mouse model was used. In a prophylactic regime, mice were vaccinated either with m157-MCMV-HBsAg, m27-MCMV-HBsAg (MCMV replication deficiency strain), the parental MCMV, or PBS mock treatment. Following a booster after 3 weeks, mice were challenged with HBV 6 weeks later. Then HBV viremia and cellular responses were analyzed.

Result: HBsAg expression by MCMV raised specific humoral immunity responses starting 4 weeks post infection. In contrast to the control mice and the MCMV infected mice, m157-MCMV-HBsAg and m27-MCMV-HBsAg vaccinated mice rapidly cleared HBV antigens. The HBsAb response was remarkably higher in MCMVHBs vaccinated mice than the control mice. Interestingly, MCMV vaccinated mice as well as MCVM-HBs vaccinated mice showed increased numbers of total lymphocytes and enhanced effector $\mathrm{T}$ cell responses in the liver and the spleen. Importantly, significant increase of HBsAg specific CD8 $+\mathrm{T}$ cells in liver was only observed in the two MCVM-HBs vaccinated mice groups, but not the MCMV vaccinated mice group. MCMV-based HBsAg vaccination also resulted in enhanced anti-HBsAg and anti-HBcAg specific CD8+ T cell responses in the liver.

Conclusion: Our results demonstrated that HBV-antigen expressing MCMV vectors have significant protective effects and induce robust $\mathrm{T}$ cell responses against $\mathrm{HBV}$, which can be used for designing new therapeutic strategies for CHB treatment.

\section{$O-H B V-06$}

Defective GM-CSF secreting innate response activator B (IRA-B) and humoral immune cells in mother-to-child $\mathrm{HBV}$ vertical transmission

Ashish Kumar Vyas ${ }^{1}$, Sharda Patra ${ }^{2}$, Shiv Kumar Sarin', Nirupma Trehanpati ${ }^{1}$

${ }^{1}$ Institute of liver and Biliary Sciences, India; ${ }^{2}$ Lady Harding Medical College \& Smt. S. K. Hospital, India

Background: Vertical-transmission of Hepatitis-B-virus is the main cause of chronic HBV-infection. B-cell-mediated-responses play an important role in innate and adaptive immunity. GMCSF secreting IRA-B cells play an important role in the pathogenesis of infectious diseases. We performed a comprehensive analysis of HBV-specificB-cells their subsets such as GMCSF-secreting (IRA-B) and regulatory-B-cell-responses in HBV-transmission from mother-to-child(MTC).

Method: 18,461 pregnant females were screened for HBsAg+. 297 were $\mathrm{HBsAg}+(1.63 \%)$ and were followed till delivery and their newborns were tested for HBsAg/HBV DNA in neonate's peripheralblood before HBV vaccination. Immune status of mothers who gave birth to HBsAg/HBV-DNA+, newborns $(\mathrm{Gr}-\mathrm{A}, \mathrm{n}=22)$ and mothers with newborns-ve for $\mathrm{HBV}(\mathrm{Gr}-\mathrm{B}, \mathrm{n}=28)$ was compared. Along with comprehensive subset analysis of B cells. In response to $\mathrm{HBsAg}$ and $\mathrm{HBcAg}$ pooled-peptides stimulation-B-cells, IRA-B and Breg responses (FN- $\gamma$, IL-2, GMCSF, IL-10 and TGF- $\beta$ secretions) were analysed using flow cytometry.

Result: Baseline characteristics of 2-groups were not comparable. Total-B-cells including-mature- $(1.66 \pm 0.20$ vs $2.24 \pm 0.11$, $\mathrm{p}=0.01)$, plasma-B-cells $(4.22 \pm 0.84$ Vs $10.80 \pm 1.75, \mathrm{p}=0.007)$ were decreased in $\mathrm{Gr}$ A. compared to B. Infect early-B cells were 
increased in Gr A than-B $(9.93 \pm 2.33$ Vs $2.20 \pm 0.47, \mathrm{p}=0.03)$. ROC-analysis showed-impact of B-cells in HBV-transmission with highest-specificity-sensitivity. In-response to HBs and HBcAg-peptides, IL-10, TGF- $\beta$ producing-suppressive Bregs (HBs:-IL-10, $36.50 \pm 7.53 \quad$ vs $\quad 8.92 \pm 2.62, \quad \mathrm{p}=0.001) \quad$ (HBs:-TGF- $\beta$, $33.39 \pm 6.91$ vs $13.22 \pm 4.05, \quad \mathrm{p}=0.006$ ), and (HBc:-IL-10, $34.55 \pm 6.04 \quad$ vs $\quad 9.05 \pm 1.54, \quad \mathrm{p}=0.007) \quad(\mathrm{HBc}:-\mathrm{TGF}-\beta$, $30.18 \pm 4.33$ vs $12.38 \pm 3.63, \quad \mathrm{p}=0.005)$ were significantly increased in Gr-A than B. However, HBV-specific GMCSF-secreting IRA-B cells (HBs-9.72 \pm 2.51 vs $22.58 \pm 3.98, \mathrm{p}=0.03$ ), (HBc$12.32 \pm 2.99$ vs $25.97 \pm 3.56, \quad \mathrm{p}=0.03) \quad$ were significantly decreased in Gr A. Other inflammatory-cytokines like IFN- $\gamma$ and IL-2 (HBs: IFN- $\gamma 2.98 \pm 1.02$ vs. $8.31 \pm 1.77, \mathrm{p}=0.01$ ), (HBs: IL-2, $4.44 \pm 0.82$ vs $7.73 \pm 1.64, \mathrm{p}=\mathrm{ns})(\mathrm{HBc}:$ IFN- $\gamma 3.31 \pm 1.43 \mathrm{vs}$ $7.58 \pm 1.75, \mathrm{p}=0.02)$, (HBc: IL-2, $2.54 \pm 0.88$ vs $15.44 \pm 3.94$, $\mathrm{p}=0.005$ ) secreting-B-cells were also decreased-in Gr A vs Gr B. IL-10 and TGF- $\beta$-inhibitory responses by Bregs were increased; however inflammatory responses and IRA-B cells (GMCSF and IFN$\gamma$, IL-2) were-significantly-decreased in mothers transmitting HBV infection to the child.

Conclusion: Effective inflammatory, IRA-B cells-responses and decreased Breg responses have a protective-role in antiviral immunity and help in-prevention of vertical-transmission of HBV.

TABLE I. Baseline Characteristics of the Study Subjects

\begin{tabular}{|c|c|c|c|}
\hline Variables & (Group-A),N=22 & (Group-A), N=28 & $P$-Value \\
\hline Age & $25.0 \pm 2.99$ & $25.65 \pm 3.45$ & 0.50 \\
\hline $\begin{array}{c}\text { HBsAg quant } \\
\text { (IU/ml) }\end{array}$ & $14.93 \times 10^{3}$ & $3.82 \times 10^{3}$ & $<0.0001$ \\
\hline HBV DNA & $6.70 \log 10(0.85-8.04)$ & $3.65 \log 10(0.77-3.71)$ & 0.31 \\
\hline (IU/ml) & & & 0.26 \\
\hline $\begin{array}{c}\text { ALT (IU/ L) } \\
\text { ALB (g/dL) }\end{array}$ & $22.20 \pm 15.04$ & $15.53 \pm 13.57$ & 0.72 \\
\hline $\begin{array}{c}\text { Total bilirubin } \\
\text { (mg/dl) }\end{array}$ & $0.29 \pm 0.07$ & $3.13 \pm 0.85$ & 0.45 \\
\hline
\end{tabular}

Session: Hepatocellular Carcinoma. Chairpersons: Atsushi Tanaka; Ajay jain; Ramesh Kumar

\section{$\mathrm{O}-\mathrm{HCC}-\mathrm{Ol}$}

Androgen receptor drives hepatocellular carcinogenesis by activating enhancer of zeste homolog 2 -mediated Wnt/Bcatenin signaling

\section{Hai Feng ${ }^{1}$, Zhuo Yu ${ }^{4}$, Haibin Song', Jun Feng ${ }^{3}$, Alfred Szelok Cheng $^{5}$}

${ }^{1}$ Northern transformation center, China; ${ }^{2}$ Cancer hospital, Harbin Medical University, China; ${ }^{3}$ School of Pharmacy, Harbin Medical University, China; ${ }^{4}$ Shuguang Hospital Affiliated to Shanghai University of Traditional Chinese Medicine, China; ${ }^{5}$ State Key Laboratory of Digestive Disease, The Chinese University of Hong Kong, China

Background: Epigenetic modification mediated by enhancer of zeste homolog 2 (EZH2) has been recently demonstrated to promote the development of hepatocellular carcinoma (HCC) with male predominance. Androgen receptor (AR) dominates the distinctive feature of gender disparity in driving hepatocarcinogenesis. However, details concerning AR-regulated EZH2 signaling in liver tumorigenicity is yet uninvestigated.

Method: Gene regulation upon AR overexpression or knockdown was assessed by chromatin immunoprecipitation (ChIP-qPCR), luciferase reporter assay, quantitative RT-PCR and western blot. Cellular proliferation, anchorage-independent growth and tumorigenicity were determined by colony formation, soft agar assay and xenograft HCC models. Proteins levels in clinical specimens were statistically analyzed for the correlation of AR-regulated signaling with tumor progression and survival rates.

Result: AR overexpression induced EZH2 expression in LO2 and SK-Hep1 cells. Functional studies demonstrated that AR induced hepatocellular proliferation in vitro and tumorigenesis in vivo, which were counteracted by EZH2 silencing. In mechanism, ChIP-qPCR and luciferase reporter assay revealed that AR bound to EZH2 promoter and stimulated its transcription activity, which promoted EZH2 mRNA transcription and protein expression. Upregulation of EZH2 promoted trimethylation of histone $\mathrm{H} 3$ at lysine 27 (H3K27me3) to cause chromatin remodeling, which silenced tumor suppressors Wnt antagonists including AXIN2, NKD1, PPP2R2B, PRICKLE1 and SFRP5, thus inhibiting Wnt/ $\beta$-catenin signaling. Conversely, knockdown of AR in PLC5 and Huh7 HCC cells reduced transcription activity by decreasing the occupancy of EZH2 promoter, hence suppressing protein expression of EZH2 and $\mathrm{H} 3 \mathrm{~K} 27 \mathrm{me} 3$. Concordant suppression of EZH2 and $\mathrm{H} 3 \mathrm{~K} 27 \mathrm{me} 3$ deregulated Wnt antagonists, which inactivated $\beta$-catenin by forming an inhibitory complex. In a cohort of human HCC patients, concomitant overexpression of AR, EZH2, H3K27me 3 and active $\beta$-catenin was observed in tumors tissues, and correlated with tumor progression and poor prognosis.

Conclusion: These findings uncover a novel mechanism underlying AR-induced hepatocarcinogenesis through EZH2-mediated epigenetic modulation, providing the benefit for the development of HCC therapeutic regime.

\section{$\mathrm{O}-\mathrm{HCC}-\mathrm{O} 2$}

Endoscopic ultrasound guided fine needle aspiration of lymph nodes changes management in a significant number of patients with hepatocellular carcinoma

Narendra Singh Choudhary ${ }^{1}$, Sanjiv Saigal ${ }^{1}$, Rajesh Puri, Haimanti Sarin ${ }^{1}$, Arvinder S Soin ${ }^{1}$

${ }^{1}$ Medanta the Medicity, India

Background: Demonstration of metastatic lymphadenopathy in patients with hepatocellular carcinoma (HCC) rules out option of liver transplantation, or may help in selection or locoregional therapy. Fine needle aspiration (FNA) of enlarged lymph nodes in patients with cirrhosis is difficult by percutaneous methods due to presence of collaterals, presence of coagulopathy and difficult anatomic location. Endoscopic ultrasound (EUS) guided FNA offers advantage of real time monitoring of procedure and proximity to target lymph nodes.

Method: Patients with HCC ( $\mathrm{n}=100)$, who underwent EUS guided lymph node FNA (79 abdominal, 21 mediastinal) over a period of 3 and half years were included. The study was conducted retrospectively at a tertiary care centre at north India. These patients underwent a whole body FDG-18 PET-CT scan and radionuclide bone scan to rule out extra-hepatic disease before FNA if they were candidates for liver transplantation. INR $>1.5$ and platelets $<50000 / \mathrm{cmm}$ were cut-offs for need of fresh frozen plasma or platelet transfusion before EUS FNA. Data is shows as number, percentage and median (25-75 quartiles). 
Result: The study cohort included 100 patients with HCC, 84 were males and 16 were females. All these patients had cirrhosis excep$\mathrm{t}$ one. The age of study cohort was 56 (52-62 years). The severity of liver disease was as following: Child's score 8(6-9), MELD score 10(8-15). A total of 12 patients had fresh frozen plasma and 5 patients had platelet transfusion before EUS FNA procedure. The lymph nodes measured $1.5(1.2-2) \mathrm{cm}$ at long axis and $1(0.7-1.4) \mathrm{cm}$ at short axis. The 22 G FNA needle was used in majority. The cytopathological diagnosis were malignant lymphadenopathy in 26 (24 abdominal, 2 mediastinal), granulomatous change in 8 (5 abdominal, 3 mediastinal) and reactive changes in 59 patients; the cellularity was inadequate for opinion in 7 patients. Two patients had mild self limiting mucosal ooze and one patient had sedation related early encephalopathy that improved with observation.

Conclusion: EUS FNA of lymph nodes in patients with HCC significantly impacts the management in almost one third of patients.

\section{O-HCC-03}

Efficacy and safety of nivolumab in Asian patients with advanced hepatocellular carcinoma (HCC): subanalysis of the CheckMate040 Study

Amy $\mathrm{Ma}^{1}$, Su Pin $\mathrm{Choo}^{8}$, Chiun Hsu', Yoon Koo Kang ${ }^{2}$, Ming Mo $\overline{H o u}^{4}$, Winnie Yeo ${ }^{5}$, Kazushi Numata ${ }^{10}$, Akhil Chopra ${ }^{6}$, Adyb Baakili $^{3}$, Christine Dela $\mathrm{Cruz}^{3}$, Huanyu Zhao ${ }^{3}$, Masatoshi Kudo ${ }^{7}$

${ }^{1}$ Chrysalis Medical Communications, United States; ${ }^{10}$ Yokohama City University Medical Center, Japan; ${ }^{2}$ Asan Medical Center, Korea, Republic of; ${ }^{3}$ Bristol-Myers Squibb, United States; ${ }^{4}$ Chang Gung Memorial Hospital, Taiwan; ${ }^{5}$ Chinese University of Hong Kong, China; ${ }^{6}$ Johns Hopkins Singapore International Medical, Singapore; ${ }^{7}$ Kindai University Faculty of Medicine, Japan; ${ }^{8}$ National Cancer Centre, Singapore; ${ }^{9}$ National Taiwan University Hospital, Taiwan

Background: Many patients with advanced HCC progress on sorafenib therapy. Nivolumab is a fully human anti-programmed death-1 (PD-1) immunoglobulin G4 monoclonal antibody (IgG4 mAb) that demonstrated durable responses and long-term survival in patients with advanced HCC in the CheckMate-040 study (El-Khoueiry AB, Sangro B, et al. Lancet 2017). Here we present survival, durability of response, and safety data in Asian patients with advanced HCC in CheckMate-040.

Method: Patients naive to or previously treated with sorafenib received nivolumab in phase $1 / 2$ dose-escalation (ESC; $0.1-10 \mathrm{mg} /$ $\mathrm{kg}$ ) and expansion (EXP; $3 \mathrm{mg} / \mathrm{kg}$ ) cohorts every 2 weeks regardless of programmed death ligand 1 (PD-L1) status. Primary endpoints were safety and tolerability (in ESC) and objective response rate (ORR) reported by blinded independent central review using RECIST v1.1 (in EXP). Secondary endpoints included duration of response (DOR), disease control rate (DCR), and overall survival.

Result: Asian patients $(\mathrm{N}=107)$ from Hong Kong $(\mathrm{n}=25)$, Japan $(\mathrm{n}=32)$, Korea $(\mathrm{n}=13)$, Singapore $(\mathrm{n}=15)$, and Taiwan $(\mathrm{n}=22)$ were included in the analysis; median follow-up was 19.2 months. Median age was 62 years, and 99\% had Child-Pugh scores of 5-6. Overall, $18 \%$ and $50 \%$ of patients were infected with hepatitis $\mathrm{C}$ or B virus, respectively; $32 \%$ were uninfected. The ORR was $15 \%$ (Table), and the DCR was $48 \%$; the median DOR was 13.8 months; 6 of 16 responses $(38 \%)$ were ongoing. In sorafenib-experienced patients $(\mathrm{n}=85)$, the ORR was $15 \%$, and the DCR was $49 \%$. Median overall survival and overall survival rates in Asian sorafenib-experienced patients $<65$ years and $\geq 65$ years of age were comparable. Overall, treatment-related AEs (TRAEs; any grade) and grade 3-4 TRAEs occurred in $75 \%$ and $11 \%$ of patients, respectively; rates of grade $3-4$ ALT and AST elevations were 1\%-2\%. Additional efficacy and safety data will be presented.
Conclusion: Nivolumab demonstrated durable responses and manageable safety in Asian patients with advanced HCC with or without chronic viral hepatitis.

Table. Nivolumab Efficacy in Asian Patients

\begin{tabular}{|l|c|c|}
\hline \multirow{2}{*}{} & \multicolumn{2}{|c|}{ Asian Patients } \\
\cline { 2 - 3 } & $\begin{array}{c}\text { Sorafenib Experienced } \\
\mathbf{n = 8 5}\end{array}$ & $\begin{array}{c}\text { All Asian Patients } \\
\mathbf{n = 1 0 7}\end{array}$ \\
\hline Objective response, $\mathbf{n}(\%)^{\mathbf{a}}$ & $13(15)$ & $16(15)$ \\
\hline Complete response & $2(2)$ & $2(2)$ \\
\hline Partial response & $11(13)$ & $14(13)$ \\
\hline Stable disease & $29(34)$ & $35(33)$ \\
\hline Progressive disease & $40(47)$ & $51(48)$ \\
\hline Not evaluable & $3(4)$ & $5(5)$ \\
\hline DOR, median (95\% CI), mo $\mathbf{a}^{\mathbf{a}}$ & $9.7(5.55, \mathrm{NE})$ & $13.8(8.31, \mathrm{NE})$ \\
\hline 12-mo OS rate (95\% CI), \% & $60(49.0,69.9)$ & $62(51.6,70.2)$ \\
\hline 18-mo OS rate (95\% CI), \% & $44(32.6,54.1)$ & $44(34.1,53.5)$ \\
\hline
\end{tabular}

RECIST vl.1.

$\mathrm{NE}=$ not estimable.

\section{O-HCC-04}

Combination therapy of transarterial chemoembolization (TACE) and radiofrequency ablation (RFA) for hepatocellular carcinoma (HCC) as compared to TACE and RFA alone

\section{Lalit Garg ${ }^{1}$, Yashwant Patidar', Amar Mukund ${ }^{1}$, Shiv Kumar Sarin $^{1}$ \\ ${ }^{1}$ Institute of Liver and Biliary Sciences, India}

Background: TACE prolongs survival by the arterial injection of drugs and embolizing agents, which subsequently induces ischemic necrosis. The synergistic effect of TACE and RFA has been demonstrated. The decrease in blood flow caused by TACE reduces heat loss, thus permitting larger lesions to be ablated by RFA. Iodized oil particles used in TACE fill the peripheral portal vein around the tumor, thus reducing the portal venous flow.

Method: For patients with HCC combined TACE and RFA will be done. TACE will be done first followed by RFA. It will be done in same sitting. Follow up will be done at 1,3 and 6 months. It is a single center observational study. A retrospective analysis of patients with liver cirrhosis treated with combination therapy for hepatocellular carcinoma at our institute will be done (meeting with inclusion and exclusion criteria).

Result: Majority of patients who underwent combination therapy had 'complete response' (according to modified RECIST criteria) at follow up. They had less tumor recurrence and more disease free survival. Only few patients had 'partial response' at follow up. Combination therapy also leads to improvement in clinical and biochemical parameters.

Conclusion: Combined RFA and TACE appear to be an effective modality and superior to TACE or RFA alone for the treatment of HCC.

\section{O-HCC-05}

The liver autophagy LC3 significantly predicts immediate mortality in patients with hepatocellular carcinoma after surgical resection

Chih Wen Lin ${ }^{1}$, Pohuang Lee ${ }^{1}$, Ginho Lo ${ }^{1}$, Chiachang Hsu', Peimin Hsieh ${ }^{1}$, Kah Wee Koh ${ }^{1}$, Minglung Yu ${ }^{3}$, Yaoli Chen ${ }^{2}$ 
${ }^{1}$ E-DA hospital/I-Shou University, Taiwan; ${ }^{2}$ Changhua Christian Hospital, Taiwan; ${ }^{3}$ Kaohsiung Medical University Hospital, Taiwan

Background: Post-hepatectomy liver failure (PHLF) and motality remains high up to $10 \%$. The identification of predictive factors of IM after surgical resection is of major clinical relevance and may serve as a promising strategy to decrease mortality of HCC patients. Autophagy have an impact on liver regeneration after partial hepatectomy. This study aimed to investigate the role of hepatic autophagy marker(s) in predicting mortality within three months after LR for HCC.

Method: A total of 535 consecutive HCC patients who had underwent the first surgical resection from two hospitals in Taiwan were collected between 2010 and 2014. Clinicopathological data and immediate mortality, defined as all cause-mortality within the first three-month after surgery, were analyzed. The expressions of autophagy proteins (LC3, Beclin-1, and p62) in adjacent non-tumor tissues were scored by using immunohistochemical staining.

Result: About $5 \%$ of the patients had immediate mortality after surgery. The absence of LC3, hypoalbuminemia, high ALT, and major liver surgery were significantly associated with immediate mortality in univariate analyses. Multivariate logistic regression demonstrated that absence of LC3 (HR 40.8, 95\% CI 5.14-325; $p<0.0001$ ) and serum albumin $<3.5 \mathrm{~g} / \mathrm{dl}$ (HR 2.88, CI 1.11-7.52; $p=0.03$ ) were significantly associated with an increase in immediate mortality. The 3-month cumulative incidence of mortality was $12.1 \%$, $13.0 \%, 21.4 \%$ and $0.4 \%$, respectively, among patients with absence of LC3 expression, hypoalbuminemia, both, and neither the absence of LC3 expression nor hypoalbuminemia.

Conclusion: Absence of autophagy marker, LC3 expression, in adjacent non-tumor tissue and hypoalbuminemia were strongly predictive of immediate mortality after resection for HCC.

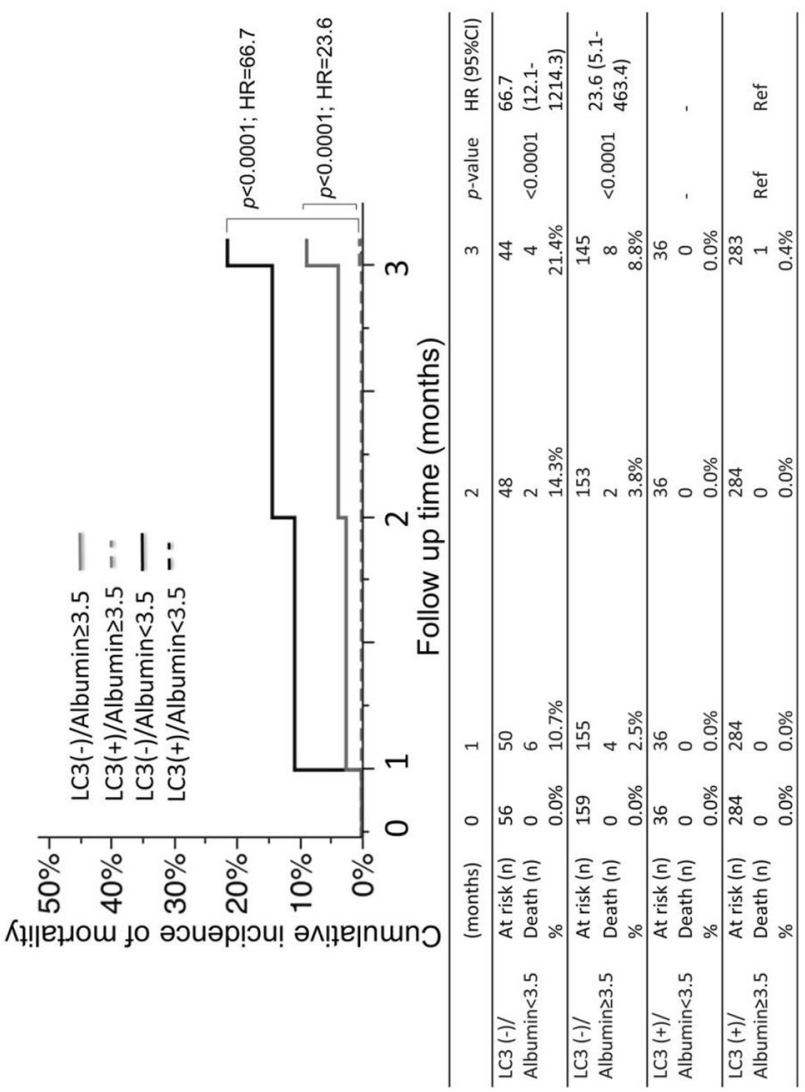

\section{O-HCC-06}

Diabetes mellitus-related hepatocellular carcinoma showed favorable long-term outcomes after hepatectomy

\section{Liang Jing ${ }^{1}$, Yamamoto Masakazu ${ }^{2}$ \\ ${ }^{1}$ Tianjin third central hospital, China; ${ }^{2}$ Tokyo Women's Medical University, Japan}

Background: Many studies have suggested that diabetes mellitus (DM) may play an important role in non-B non-C HCC. Evidence showed that DM was associated with an increased risk for $\mathrm{HCC}(\mathrm{HR}$ 3.64; 94\% CI 2.61-5.07)in Japanese patients. Multiple reports have described the incidence and risk factors of HCC with DM, but no previous studies have assessed the features and surgical outcomes of HCC originating from DM with those of other etiological types of HCC with DM. In this study, we aim to evaluate patients with DMrelated HCC patients who underwent liver resection, with the purpose of comparing the clinicopathological characteristics and surgical outcomes with those of patients with virus-related HCC and alcoholrelated HCC in the background of DM. Furthermore, we aim to classify DM-related HCC according to pathological manifestation (NASH and non-NASH) and compare its characteristics and outcomes.

Method: Consecutive patients who received curative surgery for primary HCC from January 2002 to December 2013 at Tokyo Women's Medical University were recruited. Of 837 HCC patients who underwent liver resection, 189 patients with a history of DM ranging from 2 years to 23 years before the HCC diagnosis were enrolled. We retrospectively studied 189 patients with HCC and DM. Surgical clinicopathological characteristics and outcomes were compared among 75 patients with DM, 84 patients with DM and viral hepatitis, and 30 patients with DM and alcoholic hepatitis.

Result: The mean HbA1c (\%), ICG15 and AFP showed no significant differences among the three groups. Among patients who underwent curative surgery, the overall 5-year survival rate was significantly higher in patients with DM than in patients with DM and viral hepatitis ( $83 \%$ vs $70 \%, p=0.0219)$, whereas there was no significant difference between patients with DM and those with DM and alcoholic hepatitis $(83 \%$ vs $81 \%, \mathrm{p}=0.4)$. The 5 -year recurrence-free survival rate was significantly higher in patients with DM than in patients with $\mathrm{DM}$ and viral hepatitis $(48 \%$ vs $23 \%, \mathrm{p}=0.006)$, whereas there was no significant difference between patients with DM and those with DM and alcoholic hepatitis $(48 \%$ vs $42 \%, \mathrm{p}=0.34)$. Multivariate analysis showed DM to be a significant independent prognostic factor for overall survival and recurrence-free survival. Conclusion: Patients with DM show favorable long-term surgical outcomes among patients with HCC and DM. 
Table 1 Clinicopathological features and outcomes among three groups

\begin{tabular}{llll}
\hline DM & $\begin{array}{l}\text { DM and Viral } \\
\text { hepatitis } \\
(n=84)\end{array}$ & $\begin{array}{l}\text { DMand } \\
\text { alcoholic } \\
\text { hepatitis } \\
(n=30)\end{array}$
\end{tabular}

\begin{tabular}{|c|c|c|c|}
\hline Clinical factors & & & \\
\hline Age (years, mean $\pm s d$ ) & $70.8 \pm 9.1^{\text {v,a }}$ & $66.5 \pm 8.8$ & $66.5 \pm 7.6$ \\
\hline Gender(male/female) & $70 / 5$ & $77 / 7$ & $28 / 2$ \\
\hline $\mathrm{BMI}\left(\mathrm{kg} / \mathrm{m}^{2}\right.$, mean $\left.\pm \mathrm{sd}\right)$ & $24.4 \pm 3.5^{v}$ & $22.5 \pm 3.4$ & $24.8 \pm 3.5$ \\
\hline $\operatorname{ICG15(\% ,~mean\pm sd)~}$ & $13.5 \pm 11.7$ & $14.6 \pm 8.4$ & $15.4 \pm 12.6$ \\
\hline $\mathrm{TBil}(\mathrm{mg} / \mathrm{dl}, \mathrm{mean} \pm \mathrm{sd})$ & $0.6 \pm 0.3$ & $0.7 \pm 0.7$ & $0.7 \pm 0.3$ \\
\hline ALT(iu/l, mean $\pm s d)$ & $37.7 \pm 20.4^{v}$ & $53.8 \pm 36.8$ & $42.6 \pm 28.4$ \\
\hline AST(iu/l, mean $\pm s d)$ & $39.2 \pm 22.4^{v}$ & $55.8 \pm 41.7$ & $38.4 \pm 19.1$ \\
\hline $\mathrm{ALB}(\mathrm{g} / \mathrm{dl}$, mean $\pm \mathrm{sd})$ & $3.9 \pm 0.4^{v}$ & $3.7 \pm 0.5$ & $3.9 \pm 0.4$ \\
\hline ALP(iu/l, mean $\pm s d)$ & $397.0 \pm 319.0$ & $353.5 \pm 189.8$ & $318.5 \pm 310.0$ \\
\hline GGT(iu/l, mean $\pm s d)$ & $145.6 \pm 150.6$ & $123.3 \pm 122.0$ & $166.1 \pm 147.5$ \\
\hline Child-Pugh(A/B) & $70 / 5$ & $74 / 10$ & $29 / 1$ \\
\hline $\operatorname{PLT}\left(10^{4} / u l\right.$, mean $\left.\pm s d\right)$ & $19.2 \pm 7.5^{\mathrm{v}, \mathrm{a}}$ & $13.7 \pm 5.1$ & $14.8 \pm 6.0$ \\
\hline Prothrombin activity (\%, mean \pm sd) & $91.2 \pm 9.4^{v}$ & $87.7 \pm 9.2$ & $87.8 \pm 10.3$ \\
\hline HBA1c(\%, mean $\pm s d)$ & $8.2 \pm 1.7$ & $8.3 \pm 1.6$ & $7.6 \pm 1.4$ \\
\hline $\operatorname{AFP}(\mathrm{ng} / \mathrm{ml}$, median) & 7 & 18.0 & 9.5 \\
\hline PIVKA-II(mAu/ml, median) & 150.0 & 107.5 & 272.5 \\
\hline \multicolumn{4}{|l|}{ Pathological finding } \\
\hline Tumor size $(\mathrm{cm}$, mean $\pm \mathrm{sd})$ & $6.6 \pm 4.1^{\mathrm{va}, \mathrm{a}}$ & $4.2 \pm 2.9$ & $4.8 \pm 2.9$ \\
\hline Vascular invasion (negative/positive) & $56 / 19$ & $59 / 25$ & $22 / 8$ \\
\hline Im(negative/positive) & $60 / 15$ & $59 / 25$ & $24 / 6$ \\
\hline Background liver(LC/non-LC) & $10 / 65^{v, a}$ & $43 / 41$ & $12 / 18$ \\
\hline Macroscopic type(small nodular with & $64 / 9 / 2$ & $68 / 10 / 6$ & $21 / 4 / 5$ \\
\hline \multicolumn{4}{|l|}{ indistinct margin, simple nodular type, } \\
\hline \multicolumn{4}{|l|}{ simple nodular type with extranodular } \\
\hline growth/mutinodular type/mass type) & & & \\
\hline
\end{tabular}

AST, aspartate aminotransferase; ALT, alanine aminotransferase; GGT, gamma-

glutamyltranspeptidase; ALP, alkaline phosphatase; TBil, total bilirubin; PLT, platelet

count;ICG15, indocyanine green retention at 15 min;HBA1c, hemoglobinA1c; AFP, alpha-

fetoprotein; PIVKA-II, protein induced by vitamin K absence or antagonist-II; Im, Intrahepatic

metastasis; LC, liver cirrhosis; v: Comparison with Viral hepatitis group p<0.05.a: Comparison

with Alcohol group, $p<0.05$.
Table 2 Risk factors for Overall Survival in HCC patients with DM

\begin{tabular}{|c|c|c|c|c|c|c|}
\hline \multirow[b]{4}{*}{ Age (years) } & \multicolumn{5}{|c|}{ Overall Survival } & \\
\hline & \multirow{3}{*}{$\begin{array}{l}\text { N 5yearOS\% P } \\
<70\end{array}$} & \multicolumn{2}{|c|}{ univariate analysis } & \multicolumn{3}{|c|}{ multivariate analysis } \\
\hline & & Oddsrat & tio $\mathrm{P}$ & & & \\
\hline & & 90 & $76 \%$ & 0.54 & & \\
\hline & $\geq 70$ & 89 & $80 \%$ & & & \\
\hline \multirow[t]{2}{*}{ Gender } & Male & 154 & $77 \%$ & 0.17 & & \\
\hline & Female & 25 & $84 \%$ & & & \\
\hline BMI & $<23.4$ & 90 & $77 \%$ & 0.57 & & \\
\hline$\left(\mathrm{kg} / \mathrm{m}^{2}\right)$ & $\geq 23.4$ & 89 & $78 \%$ & & & \\
\hline \multirow[t]{3}{*}{ Type } & DM & 72 & $83 \%$ & DM vs Virus 0.02 & 0.484 & $0.04 *$ \\
\hline & Viral hepatitis & 30 & $81 \%$ & DM vs Alcohol & 0.687 & 0.36 \\
\hline & Alcohol & 77 & $70 \%$ & 0.40 & 1 & \\
\hline \multirow[t]{2}{*}{ HBA1c(\%) } & $<8 \%$ & 92 & $77 \%$ & 0.87 & & \\
\hline & $\geq 8 \%$ & 89 & $78 \%$ & & & \\
\hline Childpugh & A & 166 & $78 \%$ & 0.32 & & \\
\hline Class & B & 13 & $66 \%$ & & & \\
\hline ICGR15 & $<10$ & 80 & $85 \%$ & 0.28 & & \\
\hline (\%) & $\geq 10$ & 99 & $71 \%$ & & & \\
\hline PLT & $\geq 15$ & 88 & $74 \%$ & 0.37 & & \\
\hline$\left(* 10^{4} / u l\right)$ & $<15$ & 91 & $79 \%$ & & & \\
\hline AFP & $<11$ & 89 & $82 \%$ & 0.45 & & \\
\hline (ng/ml) & $\geq 11$ & 90 & $72 \%$ & & & \\
\hline PIVKA2 & $<135$ & 90 & $87 \%$ & $0.00 * *$ & 0.748 & 0.43 \\
\hline$(\mathrm{mAu} / \mathrm{ml})$ & $\geq 135$ & 89 & $68 \%$ & & 1 & \\
\hline \multirow[t]{2}{*}{ ASA } & 2 & 93 & $80 \%$ & 0.68 & & \\
\hline & 3 & 86 & $75 \%$ & & & \\
\hline \multirow[t]{2}{*}{ Hepatectomy } & Anatomical & 161 & $79 \%$ & 0.43 & & \\
\hline & Non- Anatomical & 18 & $63 \%$ & & & \\
\hline Blood loss & $<820$ & 92 & $89 \%$ & $0.01 *$ & 0.486 & $0.02 *$ \\
\hline (ml) & $\geq 820$ & 87 & $64 \%$ & & 1 & \\
\hline Operation & $<220$ & 89 & $82 \%$ & 0.35 & & \\
\hline Time(min) & $\geq 220$ & 88 & $74 \%$ & & & \\
\hline Tumor size & $<4$ & 96 & $85 \%$ & $0.03^{*}$ & 0.991 & 0.98 \\
\hline (cm) & $\geq 4$ & 83 & $69 \%$ & & 1 & \\
\hline Macroscopic & SN & 67 & $95 \%$ & $0.00^{* *}$ & 0.72 & 0.37 \\
\hline type & Others & 112 & $63 \%$ & & 1 & \\
\hline \multirow[t]{2}{*}{ Vascular invasion } & Absent & 133 & $85 \%$ & $0.00^{* *}$ & 0.495 & $0.04^{*}$ \\
\hline & Present & 46 & $53 \%$ & & 1 & \\
\hline \multirow[t]{2}{*}{ IM } & Absent & 144 & $85 \%$ & $0.00 * *$ & 0.350 & $0.00 * *$ \\
\hline & Present & 35 & $48 \%$ & & 1 & \\
\hline \multirow[t]{2}{*}{ Cirrhosis } & Absent & 119 & $81 \%$ & 0.10 & 0.55 & $0.02 *$ \\
\hline & Present & 60 & $70 \%$ & & 1 & \\
\hline
\end{tabular}

ASA, American society of anesthesiologist; IM, Intrahepatic metastasis; SN, single nodule 


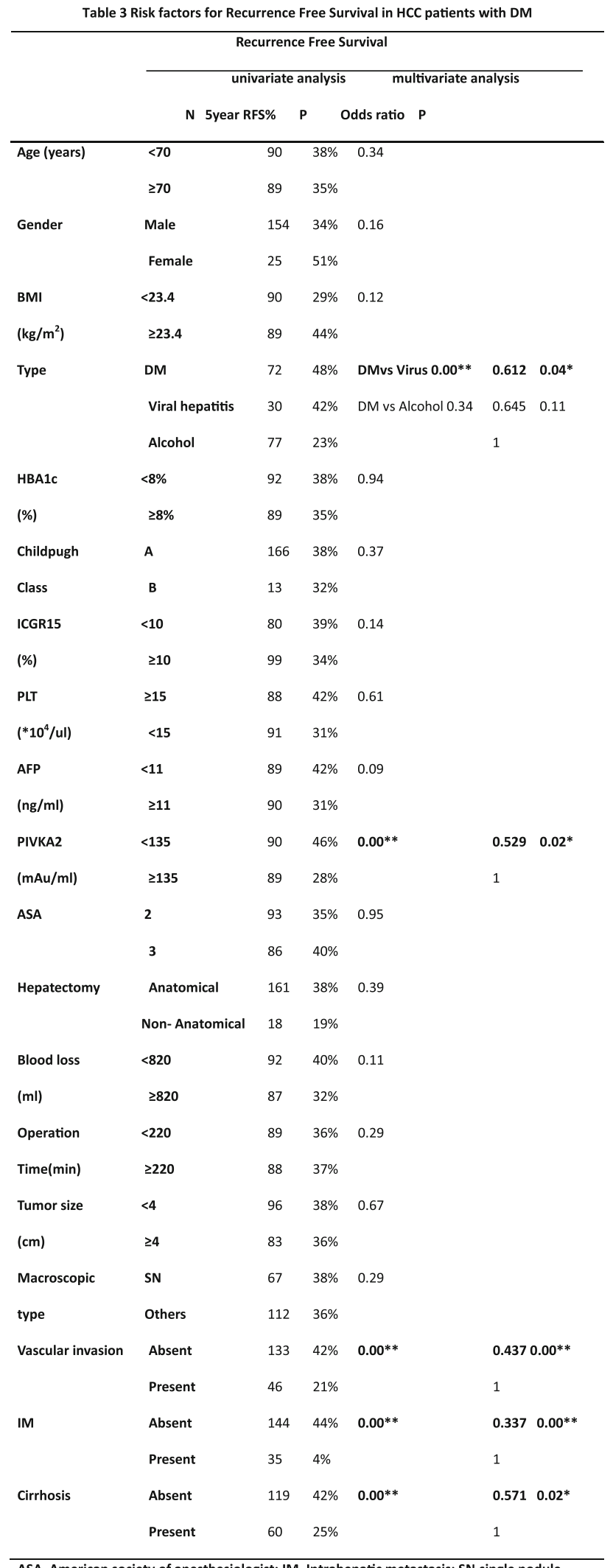

Table 4 Cilinicopathological features and outcomes of DM with NASH and DM without NASH groups

\begin{tabular}{|c|c|c|c|}
\hline & $\begin{array}{l}\text { NASH } \\
(n=37)\end{array}$ & $\begin{array}{l}\text { Non-NASH } \\
(\mathrm{n}=38)\end{array}$ & $\mathbf{P}$ \\
\hline \multicolumn{4}{|l|}{ Clinical factors } \\
\hline Age (years, mean \pm sd) & $71.4 \pm 9.7$ & $70.3 \pm 8.7$ & 0.609 \\
\hline Gender(male/female) & $34 / 3$ & $36 / 2$ & 0.975 \\
\hline $\mathrm{BMI}\left(\mathrm{kg} / \mathrm{m}^{2}\right.$, mean $\left.\pm s \mathrm{~d}\right)$ & $25.6 \pm 3.5$ & $23.2 \pm 3.1$ & 0.002 \\
\hline ICG15(\%, mean $\pm s d)$ & $15.9 \pm 15.5$ & $11.1 \pm 5.4$ & 0.076 \\
\hline TBil(mg/dl, mean $\pm s d)$ & $0.7 \pm 0.3$ & $0.6 \pm 0.3$ & 0.164 \\
\hline ALT(iu/l, mean $\pm s d)$ & $41.8 \pm 22.3$ & $33.6 \pm 17.8$ & 0.081 \\
\hline AST(iu/l, mean $\pm s d)$ & $36.9 \pm 14.8$ & $41.4 \pm 28.0$ & 0.388 \\
\hline $\mathrm{ALB}(\mathrm{g} / \mathrm{dl}$, mean $\pm s d)$ & $4.0 \pm 0.4$ & $3.8 \pm 0.5$ & 0.035 \\
\hline ALP(iu/l, mean $\pm s d)$ & $341.6 \pm 238.0$ & $451.0 \pm 377.4$ & 0.139 \\
\hline GGT(iu/l, mean $\pm s d)$ & $132.9 \pm 134.1$ & $158.0 \pm 166.0$ & 0.474 \\
\hline Child-Pugh(A/B) & $36 / 1$ & $34 / 4$ & 0.371 \\
\hline $\operatorname{PLT}\left(10^{4} / \mathrm{ul}\right.$, mean $\left.\pm \mathrm{sd}\right)$ & $15.9 \pm 5.2$ & $22.4 \pm 8.0$ & 0.000 \\
\hline Prothrombin activity $(\%$, mean $\pm s d)$ & $91.7 \pm 7.7$ & $90.6 \pm 10.9$ & 0.618 \\
\hline HBA1c(\%, mean $\pm s d)$ & $8.6 \pm 1.9$ & $7.8 \pm 1.4$ & 0.031 \\
\hline AFP(ng/ml, median) & 6.0 & 14.0 & 0.056 \\
\hline PIVKA-П(mAu/ml, median) & 76.0 & 1820.0 & 0.02 \\
\hline \multicolumn{4}{|l|}{ Pathological finding } \\
\hline Tumor size $(\mathrm{cm}$, mean $\pm \mathrm{sd})$ & $4.7 \pm 3.2$ & $8.5 \pm 4.2$ & 0.000 \\
\hline Vascular invasion (negative/positive) & $33 / 4$ & $23 / 15$ & 0.015 \\
\hline Im(negative/positive) & $32 / 5$ & $28 / 10$ & 0.273 \\
\hline Background liver(LC/non-LC) & $29 / 8$ & $36 / 2$ & 0.081 \\
\hline Macroscopic type(small nodular with indistinct & $31 / 6 / 0$ & $34 / 3 / 2$ & 0.144 \\
\hline \multicolumn{4}{|l|}{ margin, simple nodular type, simple nodular type } \\
\hline \multicolumn{4}{|l|}{ with extranodular growth/mutinodular type/mass } \\
\hline \multicolumn{4}{|l|}{ type) } \\
\hline \multicolumn{4}{|l|}{ outcomes } \\
\hline Overall survival (month, median) & 68.8 & 40.0 & 0.721 \\
\hline Recurrence free survival (month, median) & 45.1 & 23.5 & 0.467 \\
\hline
\end{tabular}

AST, aspartate aminotransferase; ALT, alanine aminotransferase; GGT, gamma-

glutamyltranspeptidase; ALP, alkaline phosphatase; TBil, total bilirubin; PLT, platelet

count;ICG15, indocyanine green retention at $15 \mathrm{~min}$;HBA1c, hemoglobinA1c; AFP, alpha-

fetoprotein; PIVKA-II, protein induced by vitamin K absence or antagonist-II; Im, Intrahepatic

metastasis; LC, liver cirrhosis

ASA, American society of anesthesiologist; IM, Intrahepatic metastasis; $\mathrm{SN}$,single nodule

${ }^{*} p<0.05 ; * * p<0.01$ 


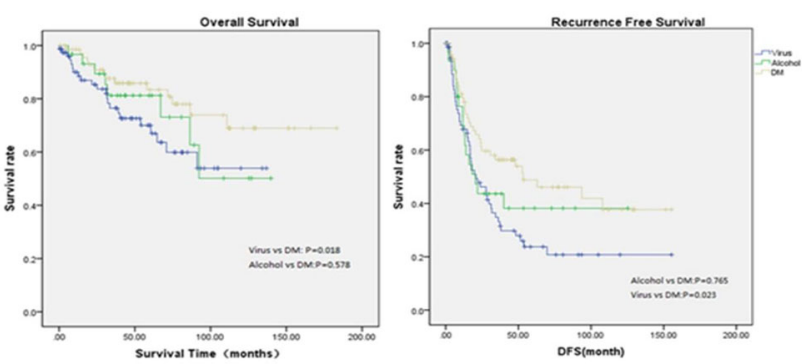

Figure1. Overall Survival and Recurrence-free Survival for DM, Viral hepatitis and Alcohol groups
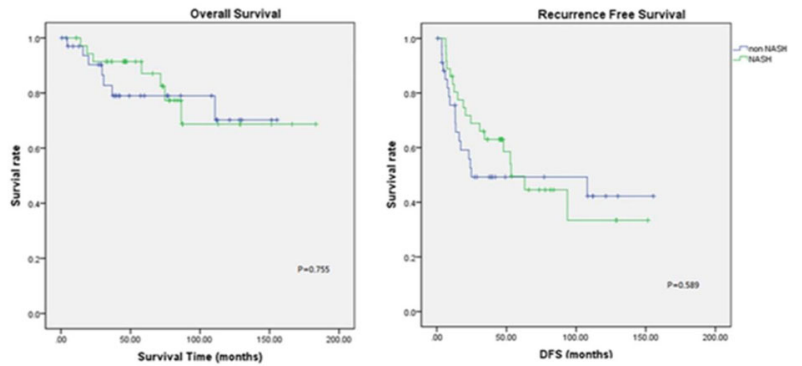

Figure 2 Overall Survival and Recurrence free survival for DM with NASH and DM without NASH groups

\section{Session: Liver Fibrosis. Chairpersons: Sudhamshu K.C; Veena Malhotra; Naveen Gupta}

\section{$O-L F I B-01$}

Transient Elastography monitoring for liver complications of long-term methotrexate use in psoriasis patients

Rabindranath Eswaran ${ }^{1}$ Ashwini R Mahesh ${ }^{1}$, K Premkumar', Kani Shaikh Mohamed $^{1}$, S Ratnakar Kini ${ }^{1}$, U R Dhanalakshmi ${ }^{1}$, A R Venkateswaran ${ }^{1}$, Rajkumar Solomon ${ }^{1}$

${ }^{1}$ Madras Medical College, India

Background: Methotrexate sodium is used as monotherapy in the long-term treatment of severe psoriasis and has long-established safety profile and favorable cost profile. Long term methotrexate is known to cause hepatic fibrosis in psoriasis patients, though the prevalence data varies. The purpose of the study was to evaluate the degree of liver fibrosis by Transient elastography (FibroScan) in psoriasis patients treated with chronic long term methotrexate .

Method: The prospective analytical study was conducted during October 2016 to march 2017 in psoriasis patients receiving long-term methotrexate treatment from the dermatology outpatient department. Clinical and analytical data, duration of treatment, and cumulative dose were obtained. Liver stiffness was evaluated by FibroScan. Measurement for transient elastography (TE), was graded as normal (F0/F1, TE 0-7), mild fibrosis (F1/F2, TE 7.1-9), moderate fibrosis (F2/F3, TE 9.1-12) or severe fibrosis/cirrhosis (F4, TE > 12).

Result: Total 46 psoriasis patients taking methotrexate had fibroscans performed; 1 was excluded due to inadequate imaging (due to obesity) $71.1 \%(\mathrm{n}=32)$ were female and the mean age was 51 years. Most cases were treated with $7.5 \mathrm{mg}$ to $20 \mathrm{mg}$ per week, the mean cumulative dose was $2.25 \mathrm{~g}$ and the mean duration of therapy was
30.6 months. Overall $73.33 \%$ of patients had normal fibroscans (F0/ $\mathrm{F} 1) ; 15.56 \%$ had mild fibrosis (F1/F2), $11.1 \%$ had moderate fibrosis (F2/F3) and no patient had severe fibrosis/cirrhosis (F4). Patients with diabetes, Obesity, significant alcohol use and use of other hepatotoxic agents had significant fibrosis. No significant correlation was observed in fibroscan values with cumulative dose and the duration of drug intake. Also Liver enzymes abnormality did not correlate with fibroscan measurements. Psoriasis patients who develop fibrosis on long term methotrexate often have other risk factors for fatty liver disease. Otherwise fibrosis appears less common and not accurately identified with liver enzymes abnormalities.

Conclusion: Transient elastography could be recommended periodically for monitoring liver toxicity of methotrexate use and liver biopsy could be performed only with high values of TE and/or with chronic liver enzymes abnormalities.

\section{$O-L F I B-02$}

Deposition of elastin as one of features in advanced fibrosis and identification of LOXL1 and FBLN1 as key regulators in elastin deposition

\section{Aiting Yang ${ }^{1}$, Wenshan Zhao', Wei Chen ${ }^{2}$, Huan Wang ${ }^{2}$, Jidong $\mathrm{Jia}^{2}$, Hong You ${ }^{2}$ \\ ${ }^{1}$ Capital Medical University, China; ${ }^{2}$ Beijing Friendship Hospital, Capital Medical University, China}

Background: Liver fibrosis occurs by chronic liver damage and is characterized by excessive production of extracellular matrix (ECM) in the liver. Elastin is especially (over-)expressed at an advanced stage of liver fibrosis. The mechanisms, leading to increased elastin deposition in liver fibrosis are not yet fully understood. We investigated the expression of elastin in CCl4 fibrotic mice and HBV-related liver fibrosis in patients both at an early and advanced stage. Thus, we sought to further reveal the mechanism of elastin deposition in liver fibrosis.

Method: We established $\mathrm{CCl} 4$ induced liver fibrosis/cirrhosis in C57BL/6 mice. We measured the expression of total elastin, secreted elastin (by ELIAS) and insoluble elastin in liver tissue and serum (by photometrical quantification). A chomprensive protein library during the progession/regression of liver fibrosis was established by proteomics analysis. In addition to it, qPCR analysis was performed to validate the protein content on RNA level.

Result: After 8 weeks of $\mathrm{CCl} 4$ liver fibrosis induction in C57BL/6 mice, elastin significantly accumulated in cirrhotic livers compared to fibrotic livers (4 weeks $\mathrm{CCl} 4$ treatment) and healthy control livers (CCl4 vehicle control), as determined on RNA level (by qPCR) or protein level (by western blot). Morphometrical quantification of elastin stained paraffin liver sections supported our previous findings and showed that elastin massively accumulated after 8 weeks of $\mathrm{CCl} 4$ treatment in fibrotic mice. Of note, we discovered that insoluble elastin was significantly increased in cirrhotic livers as compared to fibrotic or health control livers (healthy liver: $207.9 \pm 34.7 \mu \mathrm{g}$, fibrotic liver: $213.5 \pm 26.7 \mu \mathrm{g}$ and cirrhotic liver: $352.0 \pm 57.0 \mu \mathrm{g}$ per $10 \mathrm{mg} ; \mathrm{P}<0.01$ ). Noteworthy, we discovered that accumulation of elastin in murine cirrhotic livers was not only restricted to rodents but could be also found in cirrhotic livers of HBV patients. Here in good accordance to $\mathrm{CCl} 4$ fibrotic mice, elastin significantly accumulated in cirrhotic livers compared fibrotic and healthy controls, respectively. According to proteomics analysis, we identified 4 proteins (ISG15, NRP1, PRDX1, PRDX2), which were gradually upregulated and 13 proteins (APOA2, ORM2, FBLN-1, CCL8, NCAM1, GRN1, APOE, PDCD6, FSTL1, ASGR1, APOA5, LOXL1, LAMA2), which were gradually down-regulated in the serum from 
$\mathrm{CCl} 4$ mice at fibrosis regression ( 8 weeks $\mathrm{CCl} 4$ treatment then stop at 4, 8, 12 weeks) compared to peak fibrosis ( 8 weeks CCl4 treatment). Remarkably, GRN1, LOXL1 and FBLN1 was gradually increased during fibrosis progression, then gradually decreased in the reversal phase as validated by qPCR. Interestingly, both LOXL1 and FBLN1 were involved in the process of elastin assembly.

Conclusion: Elastin is significantly over-expressed especially in $\mathrm{CCl} 4$ cirrhotic livers of mice as well as HBV related cirrhotic livers in patients compared to fibrotic and healthy control livers, respectively. LOXL1 and FBLN1, involved in the process of elastin assembly, are promising therapeutic targets treat liver fibrosis at an advanced stage.

\section{$O-L F I B-03$}

Earlier utilization of transient elastography may yield improved prognostic outcomes in patients with progressive liver disease

Kersitna H Boctor ${ }^{1}$, Magdy Elkhashab ${ }^{1}$, Sirajuddaula Kausar ${ }^{1}$, Marjan Farshadi ${ }^{\text {, Muhammad K Mahmood }}{ }^{1}$, Hamed Dallali ${ }^{1}$, Marzena Magnes', Mark Khan ${ }^{3}$, Jean Palmart ${ }^{2}$, Sunny Loo ${ }^{2}$, Otilia Muraru ${ }^{1}$

${ }^{1}$ Toronto Liver Centre, Canada; ${ }^{2}$ Advisory Physicians Research Services Inc, Canada; ${ }^{3}$ Gilead Sciences Canada, Canada

Background: We aimed to assess the usefulness of transient elastography as a screening tool in the long-term reduction of liver-related mortality. We also assessed whether frequency of testing was a protective factor and finally if fibrosis staging at referral was predictive of long-term development of cirrhosis, hepatocellular carcinoma (HCC), or other primary cancers.

Method: 93 patients who underwent a liver biopsy for diagnostic findings were retrospectively assessed for: disease etiology, demographics, fibrosis staging ((F-score) using biopsy and transient elastography), and development of cirrhosis, and/or HCC.

Result: Of the 93 biopsied patients, 91 underwent TE, all 93 were included in the intention to treat analysis. The mean number of fibroscan tests done per patient was 1.67; however no significant difference was noted in the number of tests in those with $\mathrm{HCC} / \mathrm{Cir}-$ rhosis. Those with HCC / Cirrhosis were significantly more likely to have a higher Fibrosis stage on their first fibroscan test - See Table 1. $75 \%$ of patients with HCC/Cirrhosis had an initial score of $\geq \mathrm{F} 2$ on first visit, while $86 \%$ of those with cirrhosis reported an initial F-score of $\geq \mathrm{F} 2$ on their first visit. $67 \%$ of those with Liver CA reported an initial fibroscan result of F4; $72 \%$ of those with Cirrhosis reported an initial fibroscan result of F4. Interestingly, only 12 patients $(16 \%)$ with $\mathrm{HCC}$ or cirrhosis had an initial F-Score of $<$ F2. $79 \%$ of those without HCC had a F- Score of $<$ F2, while $83 \%$ of those with no cirrhosis reported a F-Score of $<\mathrm{F} 2$.

Steatosis: As can be seen in Table/ Figure 2, steatosis varied by gender and age, where we witnessed clustering of steatosis staging and male gender outweighing the female gender on the extreme ends of the scale regardless of disease etiology.

Serial scans: Among those with multiple fibroscans, $70 \%$ had an F-Score of $<$ F2 on their first and their most recent/last Scan. 5\% (21 patients) had a score that worsened from $<\mathrm{F} 2$ to $\geq \mathrm{F} 2$, while $10 \%$ had an improvement (an initial score of $\geq$ F2 with a recent score of $<$ F2). 1 patient among those with HCC or cirrhosis had a score that worsened, while 5 experienced an improved F-Score that were visible with long-term monitoring via transient elastography.

Conclusion: Although these findings are preliminary, we uncovered that patients with $\mathrm{HCC} /$ cirrhosis were referred too late for intervention to result in best possible prognostic outcome. Limited results show a trend towards patients with initial low fibroscan results $(<\mathrm{F} 2)$ are less likely to have worsening of results in subsequent tests implying earlier diagnosis results in earlier intervention and subsequent optimized management. A larger cohort of patients is currently being assessed to better support these findings and uncover any confounding factors or barriers to care.

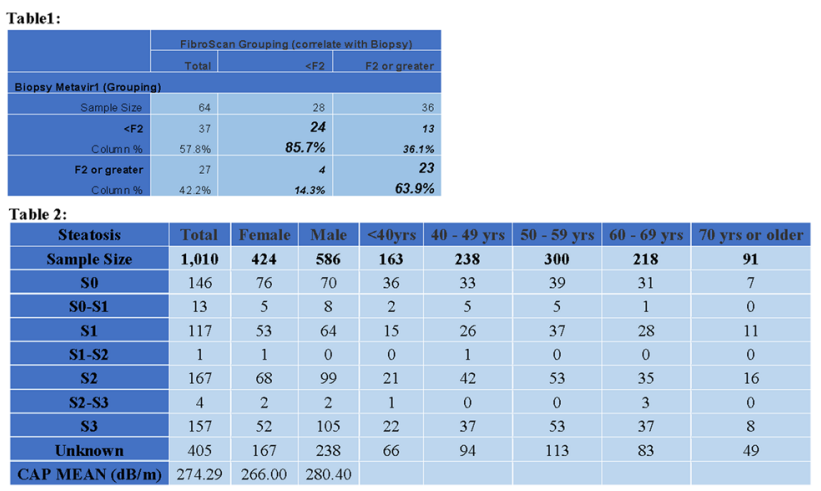

\section{O-LFIB-04}

Changes in hepatic fibrosis in chronic hepatitis $\mathbf{C}$ patients treated with direct-acting antivirals: single centre experience

\section{$\underline{\text { Rajendra Gunjal }}^{1}$ Varun Tadkalkar $^{1}$, B K Bincy ${ }^{1}$, Neehar Shanavas ${ }^{1}, \mathrm{~K}$ V Anoop ${ }^{1}$, Krishnadas Devadas ${ }^{1}$ \\ ${ }^{1}$ Govt Medical College, India}

Background: Treatment with Direct Acting Antiviral Drugs (DAA) results in sustained viral response (SVR) in more than $90 \%$ of treated individuals with subsequent improvement in liver function and liver fibrosis. The aim of our study was to assess changes in liver fibrosis by transient elastography (TE) and fibrosis-4 (FIB-4) score and ASTPlatelet ratio (APRI) in patients with chronic hepatitis $\mathrm{C}(\mathrm{CHC})$ treated with DAA.

Method: Between April 2015 and April 2017, 110 consecutive CHC patients treated with DAAs at our centre were included. TE, FIB4, APRI score were performed before treatment and after 12 weeks of completion of therapy, the time of assessing sustained virological response (SVR12) and after 24 weeks of completion of therapy. Changes in scores were analysed by paired t test.

Result: The median age of the patients was 51 years (Range: 28-70yrs), $46.3 \%$ were males, $57 \%$ had compensated cirrhosis, $79 \%$ were treatment naïve, $92.7 \%$ achieved SVR12. Most common genotype was type $3(62 \%)$ followed by genotype 4 (20\%), genotype 1 (18\%). There was significant improvement in fibrosis scores among all patients as shown in Figure1. No relationshipwas found between significant improvements in liver fibrosis and baseline serum HCVRNA, HCV genotype, DAA treatment modality and ribavirin use. Patients with advanced fibrosis $(\mathrm{F} 3-\mathrm{F} 4, \mathrm{TE}>9.5 \mathrm{KPa})$ had significant improvement in fibrosis scores compared to those without advanced fibrosis(F0-F2, TE < 9.5) as shown in Figure1. Patients who have not achieved SVR12 (non-SVR12) had increment in fibrosis scores as shown in Figure1. Patients who achieved SVR12 have shown improvement in liver enzymes along with fibrosis. For these patients, mean SGOT and SGPT improved from baseline $76.26 \mathrm{IU} / \mathrm{ml}$ to $34.42 \mathrm{IU} / \mathrm{ml}$ and $81.53 \mathrm{IU} / \mathrm{ml}$ to $31.49 \mathrm{IU} / \mathrm{ml}$ respectively at SVR12. Non-SVR12 patients had shown increment in liver enzymes along with fibrosis scores. For these patients mean SGOT and SGPT increased from baseline $61.87 \mathrm{IU} / \mathrm{ml}$ to $92.62 \mathrm{IU} / \mathrm{ml}$ and $65.12 \mathrm{IU} / \mathrm{ml}$ to $101.37 \mathrm{IU} / \mathrm{ml}$ respectively. Among those who have achieved SVR12, 78.4\% completed 24 weeks post treatment. For these patients median TE, FIB4, APRI scores improved from $10.6 \mathrm{KPa}, 1.95,0.52$ at 12 weeks post treatment to $9 \mathrm{KPa}, 1.15,0.32$ at 24 weeks post 
treatment respectively. Change in the trends in fibrosis scores for these patients is shown in the figure 2

Conclusion: Most patients with SVR12 using DAA therapy experience a significant reduction in liver fibrosis as assessed using TE, FIB4 and APRI, however no significant improvement was seen at 24 weeks post completion of therapy.

TABLE-1 SHAXCE IX FIBROSIS SCORES AMONG ALL PT

\begin{tabular}{|l|c|c|c|}
\hline & PRE-RX & POST-RX & P value \\
\hline Median TE(Kp:a) & 18 & 13.4 & $<0.01$ \\
\hline Median FIB4 & 2.85 & 1.90 & $<0.01$ \\
\hline Median APRI & 0.92 & 0.54 & $<0.01$ \\
\hline
\end{tabular}

TABLE-2 CHANGE LX.EIBROSIS SCORES IN F3-F4 VS F0-F2

\begin{tabular}{|l|c|c|c|c|}
\hline & \multicolumn{2}{|c|}{ F3-F4(n=81) } & \multicolumn{2}{c|}{ F0-F2(n=29) } \\
\cline { 2 - 5 } & PRE-RX & POST-RX & PRE-RX & POST-RX \\
\hline Median TE(KP,a) & 23.8 & 17.8 & 6.7 & 6.5 \\
\hline Median F1B4 & 3.97 & 2.44 & 1.17 & 0.97 \\
\hline Median APRI & 1.73 & 0.63 & 0.34 & 0.28 \\
\hline
\end{tabular}

TABLE-3 CHNAGE LXEHBROSIS SCORES IN SVR VS NON-SVR

\begin{tabular}{|l|c|c|c|c|}
\hline \multirow{2}{*}{} & \multicolumn{2}{|c|}{ SVR(n=102) } & \multicolumn{2}{c|}{ NON-SVR(n=8) } \\
\cline { 2 - 5 } & PRE-RX & POST-RX & PRE-RX & POST-RX \\
\hline Median TE(Kpa) & 20 & 14 & 19.7 & 28.8 \\
\hline Median F1B4 & 3.56 & 2.41 & 3.6 & 4.3 \\
\hline Median APRI & 1.59 & 0.64 & 1.35 & 2.07 \\
\hline
\end{tabular}

TE
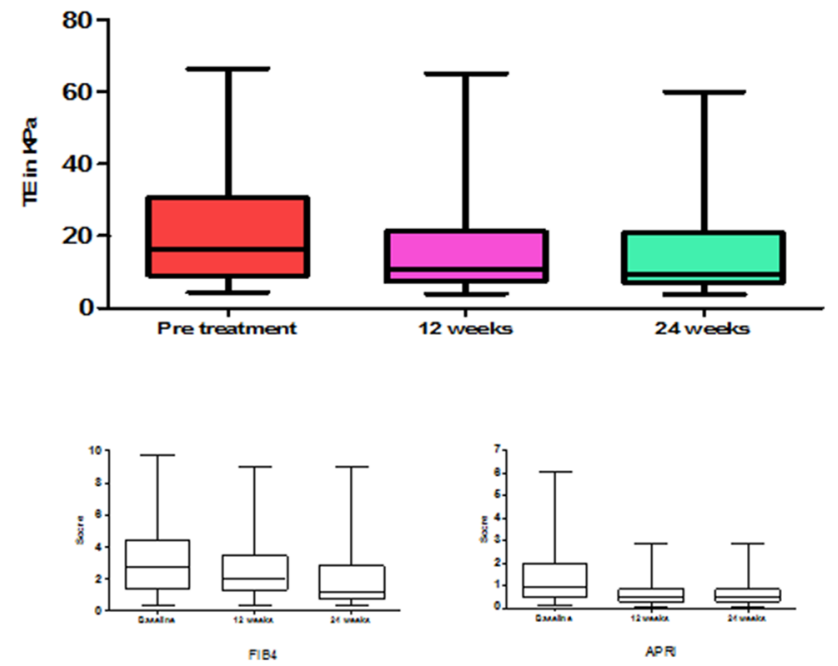

\section{O-LFIB-05}

Assessment of liver fibrosis with FibroScan and FibroTouch in patients with chronic liver disease

\section{Wenping Luo ${ }^{1}$ \\ ${ }^{1}$ Capital Medical University, China}

Background: Assessment of liver fibrosis in patients with chronic liver disease is crucial for therapy and prognosis.In recent years, noninvasive methods for liver fibrosis assessment have been developed.The aim of this study was to research and compare the diagnostic efficacy in liver stiffness of FibroScan (FS) and FibroTouch (FT).

Method: Patients who took percutaneous liver biopsy in Beijing Friendship hospital from 03/2014 to 06/2017 were enrolled. We collected their clinical data and evaluate fibrosis in histology using the
METAVIR scoring system. Using the METAVIR scoring system as the gold standard .Liver stiffnesses were measured by FS and FT within 1 day prior or inferior to liver biopsy. The diagnostic accuracy of FS and FT for fibrosis were assessed by area under the receiver operating characteristic curves (AUROCs). The influencing factors for liver stiffness were explored by multivariate analysis.

Result: 211 patients were enrolled in this study, including viral hepatitis $(\mathrm{n}=48)$,non-alcoholic fatty liver disease $(\mathrm{n}=30)$, primary biliary cirrhosis $(n=27)$,drug induced liver injury $(n=45)$ and other liver diseases $(\mathrm{n}=61)$. (1) FS and FT were correlated with liver fibrosis results, and the correlation coefficient were 0.73 and 0.69 , respectively $(\mathrm{P}<0.01)$. (2) The median of FS and FT in patients with F0, F1, F2, F3, and F4 fibrosis stages were $4.7 \mathrm{kPa}, 6.8 \mathrm{kPa}, 11.8 \mathrm{kPa}, 15.7 \mathrm{k}$ $\mathrm{Pa}, 24.2 \mathrm{kPa}$ and $4.9 \mathrm{kPa}, 7.0 \mathrm{kPa}, 11.3 \mathrm{kPa}, 15.9 \mathrm{kPa}, 24.6 \mathrm{kPa}$ respectively, the difference was significant between $\mathrm{F} 0$ and $\mathrm{F} 1, \mathrm{~F} 1$ and $\mathrm{F} 2, \mathrm{~F} 2$ and $\mathrm{F} 3$ in both FS and FT $(\mathrm{P}<0.05)$. But there was no significant difference between $\mathrm{F} 3$ and $\mathrm{F} 4$ in neither method $(\mathrm{P}=0.942$ vs 0.827$)$.

(3) There was significant difference between F0/F1 and F2, F2 and F3/ F4 for FS and FT when F0, F1 and F3 , F4 were combined as one group separately $(\mathrm{P}=0.002$ vs 0.001$)$. (4) For diagnosing $\mathrm{F} \geq 1, \mathrm{~F} \geq 2$, $\mathrm{F} \geq 3$ and $\mathrm{F}=4$, the AUROC for FS were $0.880,0.835,0.742,0.724$, and the cut-off values were $6.8 \mathrm{kPa}, 9.1 \mathrm{kPa}, 15.3 \mathrm{kPa}, 16.6 \mathrm{kPa}$; the AUROCs for FT were $0.844,0.828,0.734,0.733$ and the cut-off values were $6.5 \mathrm{kPa}, 10.0 \mathrm{kPa}, 16.0 \mathrm{kPa}$ and $18.7 \mathrm{kPa}$, respectively. However, no significant difference of AUROCs existed between FS and FT for diagnosing the same stage of fibrosis $(p>0.05)$. (5) In multivariate analysis, FS and FT were correlated with histological activity grade. Conclusion: Compared with FibroScan, FibroTouch has similar diagnostic efficacy in liver fibrosis.

\begin{tabular}{|c|c|}
\hline & median(range)/n (\%) \\
\hline Age (yr) & $47(35,57)$ \\
\hline Gender (male) & $81(38.6)$ \\
\hline BMI $\left(\mathrm{kg} / \mathrm{m}^{2}\right)$ & $23(20.4,25.3)$ \\
\hline \multicolumn{2}{|l|}{ Etiology } \\
\hline Viral hepatitis & $48(22.7)$ \\
\hline PBC & $27(12.7)$ \\
\hline DILI & $45(21.3)$ \\
\hline NAFLD & $30(14.2)$ \\
\hline Others & $61(28.9)$ \\
\hline \multicolumn{2}{|l|}{ Fibrosis stage } \\
\hline FO & $33(15.6)$ \\
\hline F1 & $86(40.8)$ \\
\hline F2 & $49(23.2)$ \\
\hline F3 & $31(14.7)$ \\
\hline F4 & $12(5.7)$ \\
\hline \multicolumn{2}{|l|}{ Inflammatory słage } \\
\hline GO & $28(13.2)$ \\
\hline G1 & $68(32.2)$ \\
\hline G2 & $81(38.4)$ \\
\hline G3 & $21(10.0)$ \\
\hline G4 & $13(6.2)$ \\
\hline ALT,IU/L & $51(29.8,120.5)$ \\
\hline AST,IU/L & $50(29.3,98.5)$ \\
\hline ALP,IU/L & $106.5(80,181)$ \\
\hline GGT,IU/L & $84(39,244)$ \\
\hline TBIL,umol/L & $15.43(10.92,24.53)$ \\
\hline WBC, $" 10^{\wedge} 9 / \mathrm{L}$ & $4.6(3.7,5.7)$ \\
\hline PLT,'10^9/L & $184.5(132,233)$ \\
\hline$A L B, g / L$ & $39.8(34.9,43.2)$ \\
\hline PTA,\% & $95.7(84.4,106.8)$ \\
\hline $\mathrm{PA}, \mathrm{mg} / \mathrm{L}$ & $167.8(120.6,207.5)$ \\
\hline Fibroscan $(\mathrm{kPa})$ & $9.3(5.9,15.7)$ \\
\hline FibroTouch $(\mathrm{kPa})$ & $9.7(5.7,15.8)$ \\
\hline
\end{tabular}

Qualitative variables are shown in $\mathrm{n}(\%)$ and quantitative variables in median(range). Abbreviations: $\mathrm{BMI}$, body mass index; $\mathrm{PBC}$, primary biliary cinhosis;DILI,drug-induced liver injury; NAFLD,non alcoholic fatty liver disease; $\mathrm{AST}_{r}$ aspartate aminotransferase; ALT, alanine aminotransferase;ALP,alkaline phosphatase;

GGT,gamma-glutamyl transpeptidase;TBIL,total bilrubin;WBC, white blood cell;

PLT, platelet;ALB,albumin;PTA, prothrombin activity;PA, prealbumin; 
Table 2 .Diagnostic Performances of FS and FT for fibrosis.

\begin{tabular}{|c|c|c|c|c|c|c|}
\hline $\begin{array}{l}\text { Fibrosis } \\
\text { stages }\end{array}$ & & Cut-off & $\begin{array}{l}\text { AUC } \\
\text { (95\%Cl) }\end{array}$ & $\mathrm{Se}$ & Sp & $F S$ vs $F T(P)$ \\
\hline \multirow[t]{5}{*}{$F \geqslant 1$} & & & & & & 0.122 \\
\hline & FS & 6.8 & 0.88 & 0.71 & 0.97 & \\
\hline & & & $(0.83-0.92)$ & & & \\
\hline & FT & 6.5 & 0.84 & 0.74 & 0.79 & \\
\hline & & & $(0.78-0.89)$ & & & \\
\hline \multirow[t]{5}{*}{$F \geqslant 2$} & & & & & & 0.202 \\
\hline & FS & 9.1 & 0.84 & 0.80 & 0.75 & \\
\hline & & & $(0.78-0.90)$ & & & \\
\hline & FT & 10.0 & 0.82 & 0.76 & 0.76 & \\
\hline & & & $(0.75-0.88)$ & & & \\
\hline \multirow[t]{5}{*}{$F \geqslant 3$} & & & & & & 0.594 \\
\hline & FS & 15.3 & 0.74 & 0.65 & 0.73 & \\
\hline & & & $(0.63-0.83)$ & & & \\
\hline & FT & 16.0 & 0.73 & 0.60 & 0.75 & \\
\hline & & & $(0.62-0.82)$ & & & \\
\hline \multirow[t]{5}{*}{$F=4$} & & & & & & 0.684 \\
\hline & FS & 16.6 & $0.72(0.55$ & 0.61 & 0.83 & \\
\hline & & & $0.85)$ & & & \\
\hline & FT & 18.7 & 0.73 & 0.83 & 0.64 & \\
\hline & & & $(0.56-0.85)$ & & & \\
\hline
\end{tabular}

Abbreviations: FS,FibroScan; FT,FibroTouch;AUROC, area under the receiver operating characteristics curve; $\mathrm{CI}$, confidence interval.

$P$ value: AUROC of FS versus FT $\rightarrow$ DeLong test
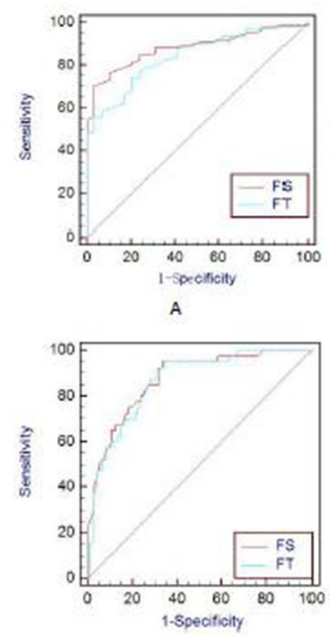

c
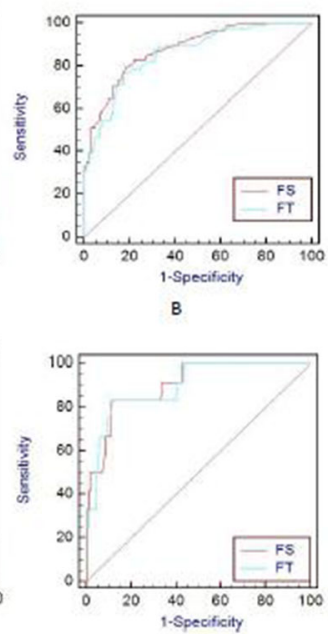

Figure 1. ROC curves for $F S$ and $F T$ for the diagnosis of $F \geqslant 1(A), F \geqslant 2(B), F \geqslant 3(C)$ and $F=4(D)$.

Abbreviations: FS,FibroScan; FT,FibroTouch.

\section{O-LFIB-06}

\section{Hepatocyte telomere shortening and senescence are general markers of human liver cirrhosis}

\section{Ali Abdelrahman Ghweil ${ }^{1}$, Ali Ghweil ${ }^{1}$, Mohamed Monir $\mathrm{Helal}^{3}$, Asheaf Khodery $^{2}$}

${ }^{1} \mathrm{Head}$ of the tropical medicine and gastroenterology, Egypt; ${ }^{2} \mathrm{Clinical}$ pathology, Egypt; ${ }^{3}$ Tropical medicine and gastroentrology, Egypt

Background: Hepatocyte telomere shortening and senescence are general markers of human liver cirrhosis. Telomere shortening limits the number of cell divisions of primary human cells and might affect the regenerative capacity of organ systems during aging and chronic disease. To test whether the telomere hypothesis applies to human cirrhosis, the telomere length was monitored in cirrhosis induced by a broad variety of different etiologies. Telomeres were significantly shorter in cirrhosis compared with noncirrhotic samples independent of the primary etiology and independent of the age of the patients. Quantitative fluorescence in situ hybridization showed that telomere shortening was restricted to hepatocytes whereas lymphocytes and stellate cells in areas of fibrosis had significantly longer telomere reserves. Hepatocyte-specific telomere shortening correlated with senescence-associated $\beta$-galactosidase staining in $84 \%$ of the cirrhosis samples, specifically in hepatocytes, but not in stellate cells or lymphocytes. Hepatocyte telomere shortening and senescence correlated with progression of fibrosis in cirrhosis samples. This study demonstrates for the first time that cell type-specific telomere shortening and senescence are linked to progression of human cirrhosis. These findings give a novel explanation for the pathophysiology of cirrhosis, indicating that fibrotic scarring at the cirrhosis stage is a consequence of hepatocyte telomere shortening and senescence. The data imply that future therapies aiming to restore regenerative capacity during aging and chronic diseases will have to ensure efficient targeting of specific cell types within the affected organs

Method: Hepatocyte telomere shortening and senescence are general markers of human liver cirrhosis. Telomere shortening limits the number of cell divisions of primary human cells and might affect the regenerative capacity of organ systems during aging and chronic disease. To test whether the telomere hypothesis applies to human cirrhosis, the telomere length was monitored in cirrhosis induced by a broad variety of different etiologies. Telomeres were significantly shorter in cirrhosis compared with noncirrhotic samples independent of the primary etiology and independent of the age of the patients. Quantitative fluorescence in situ hybridization showed that telomere shortening was restricted to hepatocytes whereas lymphocytes and stellate cells in areas of fibrosis had significantly longer telomere reserves. Hepatocyte-specific telomere shortening correlated with senescence-associated $\beta$-galactosidase staining in $84 \%$ of the cirrhosis samples, specifically in hepatocytes, but not in stellate cells or lymphocytes. Hepatocyte telomere shortening and senescence correlated with progression of fibrosis in cirrhosis samples. This study demonstrates for the first time that cell type-specific telomere shortening and senescence are linked to progression of human cirrhosis. These findings give a novel explanation for the pathophysiology of cirrhosis, indicating that fibrotic scarring at the cirrhosis stage is a consequence of hepatocyte telomere shortening and senescence. The data imply that future therapies aiming to restore regenerative capacity during aging and chronic diseases will have to ensure efficient targeting of specific cell types within the affected organs

Result: Hepatocyte telomere shortening and senescence are general markers of human liver cirrhosis. Telomere shortening limits the number of cell divisions of primary human cells and might affect the regenerative capacity of organ systems during aging and chronic disease. To test whether the telomere hypothesis applies to human cirrhosis, the telomere length was monitored in cirrhosis induced by a broad variety of different etiologies. Telomeres were significantly shorter in cirrhosis compared with noncirrhotic samples independent of the primary etiology and independent of the age of the patients. Quantitative fluorescence in situ hybridization showed that telomere shortening was restricted to hepatocytes whereas lymphocytes and stellate cells in areas of fibrosis had significantly longer telomere reserves. Hepatocyte-specific telomere shortening correlated with senescence-associated $\beta$-galactosidase staining in $84 \%$ of the cirrhosis samples, specifically in hepatocytes, but not in stellate cells or lymphocytes. Hepatocyte telomere shortening and senescence correlated with progression of fibrosis in cirrhosis samples. This study demonstrates for the first time that cell type-specific telomere shortening and senescence are linked to progression of human cirrhosis. These findings give a novel explanation for the pathophysiology of cirrhosis, 
indicating that fibrotic scarring at the cirrhosis stage is a consequence of hepatocyte telomere shortening and senescence. The data imply that future therapies aiming to restore regenerative capacity during aging and chronic diseases will have to ensure efficient targeting of specific cell types within the affected organs

Conclusion: Hepatocyte telomere shortening and senescence are general markers of human liver cirrhosis. Telomere shortening limits the number of cell divisions of primary human cells and might affect the regenerative capacity of organ systems during aging and chronic disease. To test whether the telomere hypothesis applies to human cirrhosis, the telomere length was monitored in cirrhosis induced by a broad variety of different etiologies. Telomeres were significantly shorter in cirrhosis compared with noncirrhotic samples independent of the primary etiology and independent of the age of the patients. Quantitative fluorescence in situ hybridization showed that telomere shortening was restricted to hepatocytes whereas lymphocytes and stellate cells in areas of fibrosis had significantly longer telomere reserves. Hepatocyte-specific telomere shortening correlated with senescence-associated $\beta$-galactosidase staining in $84 \%$ of the cirrhosis samples, specifically in hepatocytes, but not in stellate cells or lymphocytes. Hepatocyte telomere shortening and senescence correlated with progression of fibrosis in cirrhosis samples. This study demonstrates for the first time that cell type-specific telomere shortening and senescence are linked to progression of human cirrhosis. These findings give a novel explanation for the pathophysiology of cirrhosis, indicating that fibrotic scarring at the cirrhosis stage is a consequence of hepatocyte telomere shortening and senescence. The data imply that future therapies aiming to restore regenerative capacity during aging and chronic diseases will have to ensure efficient targeting of specific cell types within the affected organs

\section{Session: Hepatitis B Virus Infection- Clinical; Chairperson: Cosmas Rinaldi A Lesmana; Mandhir Kumar; Vikash Prakash}

\section{$O-H B V-07$}

Occult hepatitis B virus infection in patients with chronic hepatitis $\mathrm{C}$ and the risk of reactivation during DAA treatment

\section{Sevda Aghayeva}

\section{${ }^{1} \mathrm{MD}$, Azerbaija}

Background: In Azerbaijan, the prevalence of viral hepatitis B and C is $3.2 \%$ and $4.3 \%$. The aim of the study is to examine the prevalence of the occult hepatitis B infection in patients with CHC. Several authors report the evidence of reactivation of hepatitis B in patients with concomitant HBV infection, even in patients with isolated anti$\mathrm{HBc}$ seropositivity. All the patients in our study received treatment with DAA and the impact of $\mathrm{HBV}$ infection and reactivation rate was explored.

Method: The study included 164 chronic hepatitis $\mathrm{C}$ patients with negative HBsAg. Depending on serological activity of anti-HBc-IgG, the patients were divided in 2 groups. Group I included $72(43 \%)$ antiHBc-IgG positive patients (49 men and 23 women, mean age $\pm \mathrm{SD} 47.5 \pm 9.8$ years). Group II included $92(56 \%)$ anti-HBcIgG negative patients ( 57 men and 35 women, mean age \pm SD $43.6 \pm 12.5$ years). All patients received treatment with DAA regimens and evidence of virological/clinical reactivation was monitored. Result: Detectable HBV DNA was found in 18 (25\%) out of 72 anti$\mathrm{HBc}$-positive patients with chronic hepatitis C. None of the patients had pretreatment HBV DNA level more than $2000 \mathrm{IU} / \mathrm{mL}$ (34$223 \mathrm{IU} / \mathrm{mL}$ ) and no NA treatment was initiated. In a group of anti-
HBc-IgG- negative patients, none of them had positive HBV DNA. Anti-HBc-positive patients with chronic hepatitis $\mathrm{C}$ had more evident clinical manifestations and changes in biochemical parameters than anti-HBc-negative patients with chronic hepatitis $\mathrm{C}$ such as higher fibrosis score, higher ALT level etc. All of the patients received treatment with SOF + LED with or without RBV for genotype 1 and SOF + DAK with or without RBV for genotype 2 and 3. Level of HBV DNA along with HCV RNA, ALT, AST were monitored in patients with detectable HBV DNA at week 2, 4, 12 during the treatment and week 12 and 24 after the end of the treatment. None of the patients showed $1 \log$ increase in HBV DNA during and post treatment period. 2 patients relapsed during the study and none had the evidence of HBV reactivation such as ALT/AST increase or increase in HBV DNA. One patient relapsed 12 weeks after the end of treatment and was positive for antiHBcIgG but had negative HBV DNA and the second patients was post-transplant patient with negative antiHBcIgG. The overall SVR rate of HCV treatment was $98.7 \%$. Conclusion: In Azerbaijan occult hepatitis $B$ infection with detectable HBV DNA is seen in $25 \%$ of $\mathrm{HBcIgG}$ positive and in $10.9 \%$ of overall hepatitis $\mathrm{C}$ patients. The treatment with DAA did not provoke any HBV reactivation.

\section{$O-H B V-08$}

Tenofovir alafenamide (TAF) compared with tenofovir disoproxil fumarate (TDF) in patients with chronic HBV: week 96 efficacy and safety results in Chinese patients enrolled in 2 overseas phase 3 studies

Toan Luu Vo ${ }^{1}$, Fung Scott $^{14}$, Jinlin Hou ${ }^{8}$, Calvin Pan ${ }^{11}$, Wan Long Chuang $^{6}$, Ting Tsung Chang', Aric Josun Hui ${ }^{2}$, Chi Yi Chen ${ }^{3}$, Xiao Li Ma ${ }^{4}$, Edward Tam ${ }^{7}$, Tak Yin Tsang ${ }^{12}$, Jenny Yang ${ }^{1}$, John Flaherty $^{1}$, Anuj Gaggar ${ }^{1}$, Shuyuan Mo ${ }^{1}$, Myron Tong ${ }^{5}$, Jia Horng Kao $^{10}$, Henry Lik Yuen Chan ${ }^{13}$

${ }^{1}$ Gilead Sciences, United States; ${ }^{10}$ National Taiwan University Hospital, Taiwan; ${ }^{11}$ NYU Langone Medical Center, United States;

${ }^{12}$ Princess Margaret Hospital, Hong Kong; ${ }^{13}$ The Chinese University of Hong Kong, Hong Kong; ${ }^{14}$ Toronto General Hospital, Canada;

${ }^{2}$ Alice Ho Miu Ling Nethersole Hospital, Hong Kong; ${ }^{3}$ Chiayi Christian Hospital, Taiwan; ${ }^{4}$ Hahnemann University Hospital, United States; ${ }^{5}$ Huntington Memorial Hospital, United States; ${ }^{6}$ Kaohsiung Medical University Hospital, Taiwan; ${ }^{7}$ LAIR Centre, Canada; ${ }^{8}$ Nanfang Hospital, China; ${ }^{9}$ National Cheng Kung University Hospital, Taiwan

Background: TAF, a novel prodrug of tenofovir, has demonstrated efficacy noninferior to that of TDF at Week 48 in patients with chronic HBV (CHB) with significantly reduced bone and renal effects. Here we evaluated the efficacy and safety of TAF vs TDF in the subset of patients of Chinese ethnicity enrolled in two overseas studies.

Method: In 2 Phase 3 studies, HBeAg-negative (Study 108) and HBeAg-positive (Study 110) CHB patients were randomized 2:1 to TAF $25 \mathrm{mg}$ QD or TDF $300 \mathrm{mg}$ QD and treated for 96 weeks. In this analysis, the efficacy (HBV DNA $<29 \mathrm{IU} / \mathrm{mL}$, biochemical and serological responses) and safety, including assessment of bone and renal parameters, were evaluated in the subset of Asian patients enrolled in sites outside of China who self-identified to be of Chinese ethnicity.

Result: Of 1298 patients randomized and treated, 471 (36\%) were of Chinese ethnicity; 156 (TAF 97; TDF 59) and 315 (TAF 207; TDF 108) patients were $\mathrm{HBeAg}$-negative and $\mathrm{HBeAg}$-positive, respectively. For the TAF and TDF groups within each study, baseline characteristics of the study populations were generally similar. Key 
efficacy results at Weeks 48 and 96 are presented in the Table. In both $\mathrm{HBeAg}$-negative and $\mathrm{HBeAg}$-positive patients of Chinese ethnicity, the antiviral efficacy of TAF was similar to that of TDF and results were comparable to those in the overall overseas population. Numerically higher percentages of patients treated with TAF achieved normalization of serum ALT values and anti-HBe seroconversion at Weeks 48 and 96. The safety of TAF and TDF, including changes in renal and bone parameters were similar to results previously reported in the overall overseas population.

Conclusion: In CHB patients of Chinese ethnicity, TAF $25 \mathrm{mg}$ showed similar antiviral efficacy to TDF $300 \mathrm{mg}$, with less change in bone and renal parameters. Results in this subgroup were comparable to those in the overall population.

\begin{tabular}{|c|c|c|c|c|}
\hline \multirow[b]{2}{*}{$\mathbf{n} / \mathbf{N}(\%)$} & \multicolumn{2}{|c|}{$\begin{array}{c}\text { HBeAg-negative Patients } \\
\text { (Study 108) }\end{array}$} & \multicolumn{2}{|c|}{$\begin{array}{c}\text { HBeAg-positive Patients } \\
\text { (Study 110) }\end{array}$} \\
\hline & $\begin{array}{c}\text { TAF } \\
(\mathbf{n}=97)\end{array}$ & $\begin{array}{c}\text { TDF } \\
(\mathbf{n}=59)\end{array}$ & $\begin{array}{c}\text { TAF } \\
(\mathrm{n}=\mathbf{2 0 7})\end{array}$ & $\begin{array}{c}\text { TDF } \\
(\mathrm{n}=108)\end{array}$ \\
\hline \multicolumn{5}{|c|}{ Week 48} \\
\hline HBV DNA $<29 \mathrm{IU} / \mathrm{mL}$ & $90(93 \%)$ & $55(93 \%)$ & $143(69 \%)$ & $76(70 \%)$ \\
\hline ALT normalization & $46 / 94$ & & $100 / 207$ & \\
\hline (AASLD criteria) $^{\mathrm{a}}$ & $(49 \%)$ & $18 / 59(31 \%)$ & $(48 \%)$ & $39 / 108(36 \%)$ \\
\hline HBeAg loss & $\mathrm{NA}^{\mathrm{b}}$ & $\mathrm{NA}$ & $28 / 204(14 \%)$ & $11 / 103(11 \%)$ \\
\hline HBeAg seroconversion & $\mathrm{NA}$ & NA & $23 / 204(11 \%)$ & $8 / 103(8 \%)$ \\
\hline \multicolumn{5}{|c|}{ Week 96} \\
\hline HBV DNA $<29 \mathrm{IU} / \mathrm{mL}$ & $88(91 \%)$ & $57(97 \%)$ & $161(78 \%)$ & $86(80 \%)$ \\
\hline ALT normalization & $46 / 94$ & & $119 / 207$ & \\
\hline$\left(^{A A S L D} \text { criteria }\right)^{\mathrm{a}}$ & $(49 \%)$ & $24 / 59(41 \%)$ & $(57 \%)$ & $46 / 108(43 \%)$ \\
\hline HBeAg loss & $\mathrm{NA}^{\mathrm{b}}$ & NA & $55 / 204(27 \%)$ & $17 / 103(17 \%)$ \\
\hline HBeAg seroconversion & NA & $\mathrm{NA}$ & $47 / 204(23 \%)$ & $13 / 103(13 \%)$ \\
\hline
\end{tabular}

\section{$O-H B V-09$}

Effectiveness of peginterferon alfa-2a therapy in $\mathrm{HBeAg}$-negative Chinese patients with chronic hepatitis $B$ at 1 year posttreatment: a multicenter, prospective observational cohort study

Li Zhang ${ }^{1}$, Xinyue Chen ${ }^{4}$, Qianguo $\mathrm{Mao}^{18}$, Yao Xie ${ }^{2}$, Xiaoguang $\overline{D o u}^{11}$, Qing Xie ${ }^{10}$, Jifang Sheng ${ }^{15}$, Zhiliang Gao ${ }^{17}$, Xiaoling Zhou $^{8}$, Yingxia Liu ${ }^{12}$, Huanwei Zheng ${ }^{14}$, Shuqin $\mathrm{Zhang}^{7}$, Shibo $\mathrm{Li}^{19}$, Fusheng $\mathrm{Zhu}^{5}$, Yuqin $\mathrm{Xu}^{13}$, Mingxiang Zhang ${ }^{16}$, Yaoren $\mathrm{Hu}^{9}$, Xiaoping Chen', Yan Huang ${ }^{1}$, Jidong $\mathrm{Jia}^{3}$

${ }^{1}$ Shanghai Roche Pharmaceuticals Ltd., China; ${ }^{10}$ Rui Jin Hospital, Shanghai Jiao Tong University School of Medicine, China;

${ }^{11}$ Shengjing Hospital of China Medical University, China;

${ }^{12}$ Shenzhen Third People's Hospital, China; ${ }^{13}$ The 211 Hospital of People's Liberation Army, China; ${ }^{14}$ The Fifth Hospital of Shijiazhuang, China; ${ }^{15}$ The First Affiliated Hospital of Zhejing University, China; ${ }^{16}$ The Sixth People's Hospital of Shenyang, China;

${ }^{17}$ The Third Affiliated Hospital, Sun Yat-sen University, China;

${ }^{18}$ Xiamen Hospital of Traditional Chinese Medicine, China;

${ }^{19}$ Zhoushan Hospital of Zhejiang Province, China; ${ }^{2}$ Beijing Ditan Hospital, Capital Medical University, China; ${ }^{3}$ Beijing Friendship Hospital, Capital Medical University, China; ${ }^{4}$ Beijing YouAn Hospital, Capital Medical University, China; ${ }^{5}$ General Hospital of Dagang Oilfield, China; ${ }^{6}$ Guangdong General Hospital, China;

${ }^{7}$ Hepatology Hospital of Jilin Province, China; ${ }^{8}$ Liuzhou Traditional Chinese medical Hospital, China; ${ }^{9}$ Ningbo No. 2 Hospital of Zhejiang Province, China

Background: The incidence of HBeAg-negative chronic hepatitis B (CHB) has increased in China in recent years. However, there are limited data on efficacy and safety of peginterferon (PegIFN) alfa-2a in Chinese $\mathrm{HBeAg}$-negative $\mathrm{CHB}$ patients. The objective of this study is to evaluate the effectiveness and safety of PegIFN alfa-2a in Chinese $\mathrm{HBe} A g$-negative $\mathrm{CHB}$ patients in routine clinical practice.

Method: This was a prospective, multicenter, observational, noninterventional cohort study (NCT01730508) in China. Chinese adult $\mathrm{HBeAg-negative} \mathrm{CHB}$ patients were included who were not co-infected with HIV, HAV, or HCV, not on concomitant telbivudine therapy, and treated with PegIFN alfa-2a (dosing in accordance with approved SmPC in China and as per Chinese standard clinical practice). Patients were followed up for 1 year post-treatment.

Result: A total of 930 patients have been enrolled in this study. 662 patients (81.0\% of male) who met selection criteria were included in the effectiveness analysis. They had a mean age of 37.9 years, 5.61 $\log \mathrm{IU} / \mathrm{mL}$ mean HBV-DNA and $3.083 \log \mathrm{IU} / \mathrm{mL}$ mean baseline HBsAg. The mean ALT ratio was 3.154 times the upper limit of normal (ULN). Of these patients, 476 (71.9\%), 167 (25.2\%) and 71 (10.7\%) patients completed 48 weeks, 72 weeks and 96 weeks of treatment, and $439(66.3 \%)$ patients completed 1 year of post-treatment follow-up. Most patients $(532,80.4 \%)$ received PegIFN alfa-2a as monotherapy, others received NUC add-on treatment during PegIFN alfa-2a therapy $(97,14.7 \%)$ or NUC add-on during follow-up $(33,5.0 \%)$. At 1 year post-treatment, the response rate of HBV-DNA suppression (HBV-DNA $=10 \%$ patients) included White blood cell count decreased $(24.1 \%)$, Platelet count decreased $(22.4 \%)$ and Neutrophil count decreased $(21.4 \%)$, in which it aligns with pervious findings. No drug related deaths were reported.

Conclusion: PegIFN alfa-2a showed good efficacy and was well tolerated in Chinese $\mathrm{HBeAg}$ negative $\mathrm{CHB}$ patients for 1 year post treatment in routine clinical practice.

Table 1. Effectiveness outcomes 1 years post treatment in pre-defined subgroups.

\begin{tabular}{|c|c|c|c|c|}
\hline \multirow[b]{2}{*}{ Subgroups } & \multicolumn{2}{|c|}{ HBV DNA $<2000 \pi / \mathrm{mL}$} & \multicolumn{2}{|c|}{ HBsAg loss } \\
\hline & $\mathrm{n} / \mathrm{N}^{*}(\%)$ & $95 \% \mathrm{CI}$ & $\mathrm{n} / \mathrm{N}^{*}(\%)$ & $95 \% \mathrm{CI}$ \\
\hline PegIFN alfa-2amonotherapy & $185 / 233(79.4)$ & $73.6 \%, 84.4 \%$ & $23 / 216(10.6)$ & $6.9 \%, 15.5 \%$ \\
\hline NA add on during treatment & $46 / 51(90.2)$ & $78.6 \%, 96.7 \%$ & $3 / 42(7.1)$ & $1.5 \%, 19.5 \%$ \\
\hline NA add on during follow-up & $18 / 19(94.7)$ & $74.0 \%, 99.9 \%$ & $0 / 17(0)$ & NA \\
\hline Baseline ALT $\leqslant 2 \mathrm{ULN}$ & $60 / 74(81.1)$ & $70.3 \%, 89.3 \%$ & $9 / 64(15.2)$ & $7.5 \%, 26.1 \%$ \\
\hline Baseline ALT $>2$ ULN and $\leqslant 5 \mathrm{ULN}$ & $85 / 109(78.0)$ & $69.0 \%, 85.4 \%$ & $10 / 106(9.4)$ & $4.6 \%, 16.7 \%$ \\
\hline Baseline ALT $>5 \mathrm{ULN}$ & $40 / 50(80.0)$ & $66.3 \%, 90.0 \%$ & $4 / 46(8.7)$ & $2.4 \%, 20.8 \%$ \\
\hline Age $<35$ years & $67 / 90(74.4 \%)$ & $64.2 \%, 83.1 \%$ & $11 / 84(13.1)$ & $6.7 \%, 22.2 \%$ \\
\hline Age $>35$ years & $118 / 143(82.5)$ & $75.3 \%, 88.4 \%$ & $12 / 132(9.1)$ & $4.8 \%, 15.3 \%$ \\
\hline \multicolumn{5}{|l|}{ PegIFN alfa-2a treatment duration: } \\
\hline 48 weeks ( $252-420$ days) & $96 / 127(75.6)$ & $67.2 \%, 82.8 \%$ & $10 / 117(8.5)$ & $4.2 \%, 15.2 \%$ \\
\hline 72 weeks (421 - 588 days) & $44 / 52(84.6)$ & $71.9 \%, 93.1 \%$ & $5 / 50(10.0)$ & $3.3 \%, 21.8 \%$ \\
\hline 96 weeks (589-756 days) & $17 / 19(89.5)$ & $66.9 \%, 98.7 \%$ & $5 / 18(27.8)$ & $9.7 \%, 53.5 \%$ \\
\hline $\begin{array}{l}\text { HBV DNA decline }>2 \log \text { and ALT } \\
\text { increase at week } 12 \text { compared with } \\
\text { baseline }\end{array}$ & $16 / 25(64.0)$ & $42.5 \%, 82.0 \%$ & $2 / 22(9.1)$ & $1.1 \%, 29.2 \%$ \\
\hline $\begin{array}{l}\text { HBV DNA decline } \leqslant 2 \log \text { and ALT } \\
\text { increase at week } 12 \text { compared with } \\
\text { baseline }\end{array}$ & $131 / 165(79.4)$ & $72.4 \%, 85.3 \%$ & $14 / 149(9.4)$ & $5.2 \%, 15.3 \%$ \\
\hline
\end{tabular}


$O-H B V-10$

Liver histopathology analysis stratified by age and alanine aminotransferase level in patients with chronic hepatitis Bretrospective analysis of $\mathbf{1 0 1 9}$ patients with liver biopsy

Yongpeng Chen ${ }^{1}$, Liwen Huang ${ }^{1}$, Xiaomin $\mathrm{Hu}^{1}$, Jinlin Hou ${ }^{1}$, Xieer Liang', Youfu Zhu'

${ }^{1}$ Nanfang Hospital, Southern Medical University, China

Background: The guidelines for treatment of chronic hepatitis B had been updated recently. To investigate and compare the liver histological features of patients with chronic hepatitis B virus (HBV) infection in different age groups and different serum alanine aminotransferase (ALT) levels in guidelines of EASL, China, and AASLD, we retrospectively analyzed liver biopsies from 1019 treatment naive patients with CHB.

Method: A total of 1019 treatment naive chronic Hepatitis B patients underwent liver biopsies were included. The data of inflammation activity, fibrosis stage, age and ALT were statistically analyzed. Upper limit of normal (ULN) of ALT level were $40 \mathrm{U} / \mathrm{L}$ in tradition, $30 \mathrm{U} / \mathrm{L}$ for males and $19 \mathrm{U} / \mathrm{L}$ for females in AASLD guideline. Categorical variables were compared by chi-squared test or Fisher's exact test as appropriate. Statistical significance was taken as $\mathrm{P}<0.05$.

Result: There is not statistical differences of distribution of inflammation activity and fibrosis stage between patients with age $30-39$ years and age $\geq 40$ years. The percentages of inflammation activity $\geq \mathrm{A} 2, \geq \mathrm{A} 3$ and $\mathrm{A} 4$ in patients with age $<30$ years and $\geq 30$ years were $84.8 \%$ vs $86.3 \%(\mathrm{P}=0.575), 51.85 \%$ vs $59.9 \%$ $(\mathrm{P}=0.009)$ and $12.4 \%$ vs $25 \%(\mathrm{P}<0.001)$, respectively; the percentages of fibrosis stage $\geq F 2, \geq F 3$ and F4 were $68.5 \%$ vs $71.2 \%$ $(\mathrm{P}=0.342), 29.3 \%$ vs $41.3 \%(\mathrm{P}<0.001)$ and $10.4 \%$ vs $22.3 \%$ $(\mathrm{P}<0.001)$ in the same order. With traditional ULN, there is not statistical difference in distribution of fibrosis stage between ALT $<1$ ULN vs $1-2$ ULN and 1-2ULN vs 2-5ULN, and the percentage of significant inflammation $(\geq A 2)$ in patients with ALT $<1$ ULN, 1-2ULN, 2-5ULN and ALT $\geq 5$ ULN was $49.6 \%$, $77.1 \%, 85.7 \%$ and $98.2 \%$, respectively $(\mathrm{P} \leq 0.014)$, the percentage of significant fibrosis $(\geq \mathrm{F} 2)$ in the same order was $48.8 \%, 59.6 \%$, $66.1 \%, 85.3 \%$, respectively $(<1 \mathrm{ULN}$ vs $2-5 \mathrm{ULN}, \quad \mathrm{P}=0.001$; $2-5 \mathrm{ULN}$ vs ALT $\geq 5 \mathrm{ULN}, \mathrm{P}<0.001)$. While using the AASLD ALT ULN, distribution of inflammation activity in ALT $<1$ ULN was not statistically different with that in 1-2ULN, and the distribution of fibrosis score was also not different between ALT $<1$ ULN vs $2-5$ ULN and $1-2 \mathrm{ULN}$ vs $2-5 \mathrm{ULN}$. The percentages of inflammation activity $\geq \mathrm{A} 2$ were $47.3 \%, 64 \%, 81.2 \%$ and $96.3 \%$, respectively $(\mathrm{P} \leq 0.021)$; The percentages of fibrosis stage $\geq \mathrm{F} 2$ were $47.3 \%$, $56.0 \%, 62.3 \%$ and $81.1 \%$, respectively (<1ULN vs $2-5 \mathrm{ULN}$, $\mathrm{P}=0.018 ; 2-5 \mathrm{ULN}$ vs ALT $\geq 5 \mathrm{ULN}, \mathrm{P}<0.001)$. While compared between different guidelines, statistical differences were only found in significant inflammation activity between EASL and China in both of treatment indication and non-treatment indication. Moreover, the percentage of significant fibrosis was approximately $50 \%$ in patients without treatment indication in all guidelines. Among these patients without treatment indication, the proportion of advanced fibrosis was $19.2 \%-26.2 \%$ in age $<30$ years and $24 \%-28.1 \%$ in age $<40$ years; and $6.8 \%-9.5 \%, 13.1 \%-15.6 \%$ of cirrhosis, respectively.

Conclusion: Due to considerable proportion of advanced fibrosis and nearly $10 \%$ of cirrhosis, non-invasive assessment of liver fibrosis and necessary liver biopsy should be recommended for non-treatment indication patients with age $<30$ years.
$O-H B V-11$

Serum HBV RNA level predicts response to peginterferon add-on therapy for hbeag-positive chronic hepatitis B

Bettina Elisabeth Hansen ${ }^{1}$, Margo L A Van Campenhout ${ }^{3}$, Florian Van Bommel $^{7}$, Willem Pieter Elisabeth Brouwer ${ }^{3}$, Qing Xie ${ }^{5}$, Qing Zhang ${ }^{6}$, Fehmi Tabak ${ }^{2}$, Adrian Streinuorcel ${ }^{4}$, Jiyao Wang ${ }^{8}$, Robert J De Knegt ${ }^{3}$, Andre Boonstra ${ }^{3}$, Thomas Berg ${ }^{7}$, Bettina Hansen ${ }^{1}$

${ }^{1}$ Toronto General Hospital, Canada; ${ }^{2}$ Cerrahpasa Medical Faculty, Turkey; ${ }^{3}$ Erasmus MC University Medical Center, Netherlands; ${ }^{4}$ National Institute of Infectious Disease, Romania; ${ }^{5}$ Ruijin Hospital, Jiaotong University, China; ${ }^{6}$ Shanghai Public Health Center, Fu Dan University, China; ${ }^{7}$ University Hospital Leipzig, Germany; ${ }^{8}$ Zhong Shan Hospital, Fu Dan University, China

Background: Hepatitis B RNA (HBV RNA) is a new serum marker that is associated with response to nucleos(t)ide (NA) and peginterferon (PEG-IFN) monotherapy. We aimed to study if HBV RNA can predict response to PEG-IFN add-on therapy.

Method: HBV RNA levels were measured in stored serum samples of $175 \mathrm{HBeAg}$-positive chronic HBV patients who were treated within an international trial (ARES study). All patients started ETV $0.5 \mathrm{mg} /$ day and were after 24 weeks allocated to ETV monotherapy $(\mathrm{n}=90)$ or addition of 24 weeks of PEG-IFN $\alpha 180 \mathrm{mcg} / \mathrm{week}$ $(\mathrm{n}=85)$. All patients were followed until week 72 . HBV RNA was measured at week 0 (baseline), 12, 24 (randomization), and 36 using a RACE-PCR technique (LLQ $800 \mathrm{c} / \mathrm{mL}$ ). We studied the association with $\mathrm{HBeAg}$ loss at week 72 .

Result: The mean age of the study population was 32 (SD 10) years, $125(71 \%)$ patients were male, and HBV genotype distribution was $7 / 19 / 42 / 31 \%$ for genotype A/B/C/D. The mean HBV RNA at baseline was 6.1 (1.1) $\log 10 \mathrm{c} / \mathrm{mL}$. Correlations with serum HBV DNA and qHBsAg were $\mathrm{r}=0.8, \mathrm{p}<0.001$ and $\mathrm{r}=0.6, \mathrm{p}<0.001$. After 24 weeks of ETV, mean HBV RNA had declined to 4.7 (1.5) $\log$ $\mathrm{c} / \mathrm{mL}$, and HBV RNA was < LLQ in $30(17 \%)$ patients. After randomization, mean HBV RNA in the PEG-IFN add-on arm decreased, in contrast to HBV RNA in ETV monotherapy (week 36: -0.97 vs. $+0.30 \log , \mathrm{p}<0.001$ ). At week $72, \mathrm{HBeAg}$ was negative in $27 / 85$ $(32 \%)$ vs. $16 / 90(18 \%, p=0.04)$ patients. In patients allocated to add-on, HBeAg loss occurred in only $12 / 64$ (19\%, Figure) patients with detectable HBV RNA at week 24 vs. in 9/11 (82\%) with HBV RNA < LLQ. Adjusted for therapy allocation, HBV genotype, baseline presence of precore or basal core promoter mutants, and week 24 at randomization, week 24 HBV RNA level was independently associated with HBeAg loss at week 72 (OR 0.4, CI-95\% 0.2$0.6, \mathrm{p}<0.001)$.

Conclusion: PEG-IFN add-on after 24 weeks of ETV pretreatment accelerated HBV RNA decline. HBV RNA level at the end of ETV, before PEG-IFN addition, was associated with subsequent $\mathrm{HBeAg}$ loss. Serum HBV RNA testing may help to select those NA-treated patients who are most likely to benefit from PEG-IFN add-on. 
PEG-IFN add-on - $n=85$

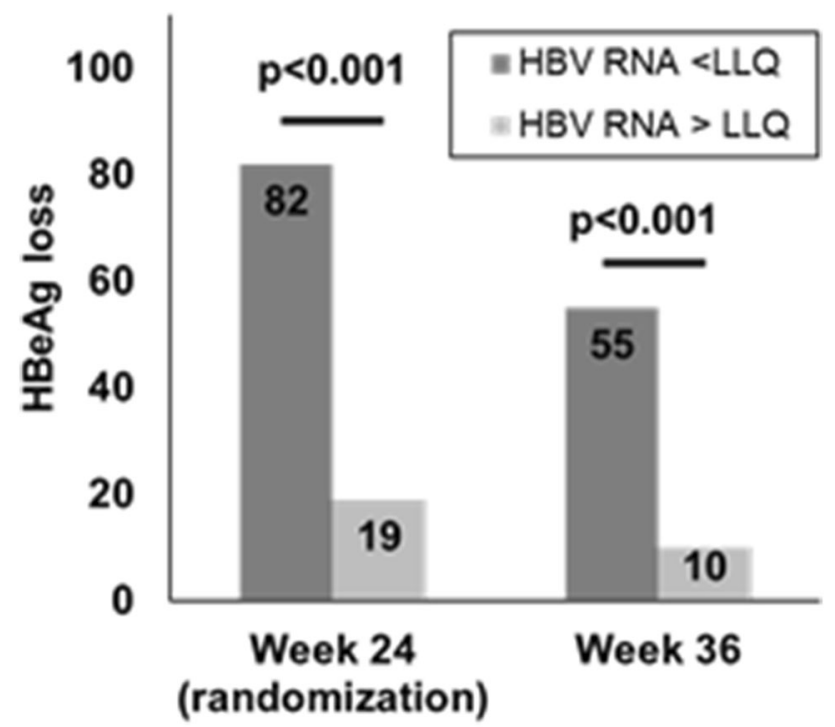

$O-H B V-12$

A phase 3 study comparing tenofovir alafenamide (TAF) to tenofovir disoproxil fumarate (TDF) in patients from China with HBeAg-negative, chronic hepatitis B (CHB): efficacy and safety results at week 48

John F Flaherty ${ }^{1}$, Zhongping Duan ${ }^{5}$, Yu Chen ${ }^{5}$, Fusheng Wang ${ }^{2}$, Qing Xie", Hong Tang ${ }^{13}$, Shanming Wu ${ }^{11}$, Guozhong Gong ${ }^{10}$, Mingxiang Zhang ${ }^{12}$, Feng Lin ${ }^{6}$, Jun Cheng ${ }^{3}$, John Flaherty ${ }^{1}$, Anuj Gaggar $^{1}$, Shuyuan Mo ${ }^{1}$, Andrea Cathcart ${ }^{1}$, G Mani Subramanian ${ }^{1}$, Yan Huang ${ }^{14}$, Jun $\mathrm{Li}^{7}$, Jidong $\mathrm{Jia}^{4}$, Jinlin $\mathrm{Hou}^{8}$

${ }^{1}$ Gilead Sciences, United States; ${ }^{10}$ Second Xiangya Hospital, Central South University, China; ${ }^{11}$ Shanghai Public Health Clinical Center, China; ${ }^{12}$ Sixth People's Hospital of Shenyang, China; ${ }^{13}$ West China Hospital, Sichuan University, China; ${ }^{14}$ Xiangya Hospital, Central South University, China; ${ }^{2}$ Beijing 302 Hospital, China; ${ }^{3}$ Beijing Ditan Hospital, China; ${ }^{4}$ Beijing Friendship Hospital, Capital Medical University, China; ${ }^{5}$ Beijing You An Hospital, Capital Medical University, China; ${ }^{6}$ Hainan General Hospital, China; ${ }^{7}$ Jiangsu Province People's Hospital, China; ${ }^{8}$ Nanfang Hospital, Southern Medical University, China; ${ }^{9}$ Rui Jin Hospital, Shanghai Jiao Tong University, China

Background: TAF, a new prodrug of tenofovir (TFV), has greater plasma stability, enhanced delivery of TFV into hepatocytes, and has lower plasma levels of TFV relative to TDF. In a Phase 3 study (Study 108 ) in non-China patients, TAF has demonstrated efficacy non-inferior to that of TDF at Weeks 48 and 96 with a superior renal and bone safety profile. Here are primary results in a separate cohort of this study enrolled in China.

Method: $154 \mathrm{HBeAg-negative} \mathrm{patients} \mathrm{were} \mathrm{randomized} \mathrm{(2:1)} \mathrm{and}$ received TAF $25 \mathrm{mg}$ QD $(\mathrm{n}=104)$ or TDF $300 \mathrm{mg}$ QD $(\mathrm{n}=50)$. Virologic $(\mathrm{HBV}$ DNA $/=69 \mathrm{IU} / \mathrm{mL}$ ) at Week 48 or $\mathrm{HBV}$ DNA $>/$ $=69 \mathrm{IU} / \mathrm{mL}$ at discontinuation.

Result: Baseline characteristics included: mean age 43 years, $73 \%$ males, $100 \%$ Asian/Chinese, mostly genotypes B (45\%) and C (51\%); mean HBV DNA was $5.5 \log 10 \mathrm{IU} / \mathrm{mL}$ (6 TAF vs 1 TDF patients had HBV DNA $>/=8 \log 10 \mathrm{IU} / \mathrm{mL}$ ), and $40 \%$ were previously treated with nucleos(t)ides. Efficacy and safety results are summarized in the
Table. At Week 48, virologic response rates were $89 \%$ and $98 \%$, in the TAF and TDF groups, respectively, while at Week 64, the response rate with TAF was increased and similar to that of TDF (92\% vs $94 \%$ ). Mean $\log 10$ declines in both HBV DNA and qHBsAg were similar between groups (1 TAF patient experienced HBsAg loss), and a greater proportion of TAF vs TDF patients achieved ALT normalization at Week 48. Overall, adverse events (AE) were similar between groups; 1 patient (TDF group) discontinued treatment for a serious AE of renal impairment. Patients treated with TAF had a small median increase in eGFR-CG compared with a decline in the TDF group at Week 48. A small median percent increase in hip and decrease in spine BMD was seen with TAF relative to larger declines in TDF patients at Week 48. The smaller eGFR-CG and BMD changes were associated with smaller changes in proximal tubular and bone turnover markers in TAF patients. No viral resistance was detected in either group at Week 48.

Conclusion: In HBeAg-negative CHB patients from China, high rates of virologic suppression were achieved with TAF and TDF, with a higher rate of ALT normalization seen with TAF. An improved renal and bone safety profile was observed in TAF patients relative to those receiving TDF.

\begin{tabular}{|c|c|c|}
\hline & $\begin{array}{c}\text { TAF } \\
(N=104)\end{array}$ & $\begin{array}{c}\text { TDF } \\
(\mathrm{N}=50)\end{array}$ \\
\hline \multicolumn{3}{|l|}{ Efficacy Parameters ${ }^{a}$} \\
\hline \multicolumn{3}{|l|}{ HBV DNA $<29 \mathrm{IU} / \mathrm{mL}, \mathrm{n} / \mathrm{N}(\%)$} \\
\hline Week 48 & $93 / 104(89)$ & $49 / 50(98)$ \\
\hline Week 64 & $96 / 104(92)$ & $47 / 50(94)$ \\
\hline HBV DNA, mean $(\mathrm{SD})$ change $\left(\log _{10} \mathrm{IU} / \mathrm{mL}\right)$ & $-4.03(1.68)$ & $-3.89(1.64)$ \\
\hline $\mathrm{qHBSAg}$, mean $(\mathrm{SD})$ change $\left(\log _{10} \mathrm{IU} / \mathrm{mL}\right)$ & $-0.19(0.54)$ & $-0.11(0.44)$ \\
\hline ALT normalization (AASLD criteria), $\mathrm{n} / \mathrm{N}(\%)^{\mathrm{b}}$ & $62 / 95(65)$ & $21 / 44(48)$ \\
\hline \multicolumn{3}{|l|}{ Renal and Bone Parameters } \\
\hline eGFRcG, median (Q1, Q3) change ( $\mathrm{mL} / \mathrm{min})$ & $+0.6(-8.4,+7.2)$ & $-4.8(-11.4,+2.4)$ \\
\hline$>25 \%$ decline in eGFRcG from baseline, $\mathrm{n} / \mathrm{N}(\%)$ & $9 / 102(9)$ & $8 / 50(16)$ \\
\hline Hip $\mathrm{BMD}$, median $(\mathrm{Q} 1, \mathrm{Q} 3) \%$ change & $\begin{array}{c}\mathrm{n}=39 \\
-0.59(-2.19,+0.86)\end{array}$ & $\begin{array}{c}\mathrm{n}=22 \\
-1.38(-2.77,+0.55)\end{array}$ \\
\hline$>3 \%$ decline in hip $\mathrm{BMD}, \mathrm{n} / \mathrm{N}(\%)$ & $2 / 39(5)$ & $5 / 22(23)$ \\
\hline Spine BMD, median (Q1, Q3) \% change & $\begin{array}{c}\mathrm{n}=39 \\
+0.65(-1.37,+3.32)\end{array}$ & $\begin{array}{c}\mathrm{n}=22 \\
-3.82(-4.78,-1.18)\end{array}$ \\
\hline$>3 \%$ decline in spine $\mathrm{BMD}, \mathrm{n} / \mathrm{N}(\%)$ & $3 / 39(8)$ & $12 / 22(55)$ \\
\hline
\end{tabular}

Session: Hepatitis C Virus Infection. Chairperson; Sheikh Mohammad Fazle Akbar; J B Dilawari; Vishal Sharma

\section{$O-H C V-01$}

Direct-acting antiviral therapy restores the phenotype and function of Tfh cells and $B$ cells in hepatitis $C$ virus-infected patients

Arshi Khanam ${ }^{1}$, Shikha Shrivastava ${ }^{1}$, Shyam Kottilil ${ }^{1}$, Eleanor Wilson

${ }^{1}$ Institute of Human Virology, United States

Background: Direct-acting antiviral (DAA) therapies are quite effective in achieving sustained virologic response (SVR) in hepatitis $\mathrm{C}$ virus (HCV) infected patients. However their effects on cellular immunity are not completely defined. Therefore in this study we aimed to investigate the effect of DAA therapy on phenotypic and functional modulation of $\mathrm{T}$ follicular helper ( $\mathrm{Tfh}$ ) cells and $\mathrm{B}$ cells Method: We performed a prospective study of 16 consecutive HCVinfected patients treated with DAA therapy (sofosbuvir and 
ledipasvir) for 12 weeks. Blood samples were collected from these patients at baseline and at the time of SVR (12 weeks following completion of therapy). Peripheral blood mononuclear cells were isolated by density gradient centrifugation. Percent frequencies of Tfh cells, B cells and associated molecules were determined by flow cytometry. PMA/ionomycin stimulation assay was performed to determine the Tfh cells secreted cytokines. Wilcoxon signed-rank test was used to analyze the data.

Result: Frequencies of CD4+ CXCR5+ Tfh cells and CD19+ B cells were comparable in HCV patients pre and post successful DAA treatment. Inducible costimulator (ICOS), required for the generation of Tfh cells and also critical for B cell help, was significantly increased ( $\mathrm{P}$ value) post DAA therapy. However, expression of transcription factor B-cell lymphoma 6 protein (BCL6) and chemokine receptor CXCR3 on Tfh cells remain unaffected post DAA therapy. Importantly, production of IL-27 $(\mathrm{p}<0.01), \quad$ IFN- $\gamma$ $(\mathrm{p}=0.01)$ and TNF- $\alpha(\mathrm{p}=0.03)$ were highly elevated after DAA treatment. Also, higher percentage of $\mathrm{IgD}+\mathrm{CD} 27-$ naive $\mathrm{B}$ cells (pre-therapy vs. post-therapy 53.24 vs. $70.18, \mathrm{p}=0.01$ ) and $\mathrm{IgD}+$ CD27+ non class-switched memory B cells (pre-therapy vs. posttherapy 4.0 vs. $7.0, p=0.01$ ) was seen post DAA treatment. Percent frequency of plasmablasts, precursor cells of short- and long lived immunoglobulin-producing plasma cells, was comparable in HCV patients pre and post treatment. Interestingly the expression of BLIMP-1 transcription factor (pre-therapy vs. post-therapy 29.7 vs. $40.2, p=0.04)$, required for the differentiation of plasmablast into immunoglobulin-producing plasma cells, was significantly elevated post DAA therapy and reflected an increased percentage of plasma cells (pre-therapy vs. post-therapy 52.8 vs. $69.2, \mathrm{p}=0.009$ ).

Conclusion: In this study we conclude that DAA-based therapy restores Tfh cells and $\mathrm{B}$ cells phenotype and function in chronic $\mathrm{HCV}$ infected patients

\section{$\mathrm{O}-\mathrm{HCV}-02$}

Protective role of a novel microRNA in liver tissues against Hepatitis $\mathrm{C}$ infection and its disease progression

\section{Suchandrima Ghosh ${ }^{1}$, Joyeeta Chakraborty ${ }^{2}$, Sayantani} Bhowmick', Amit Ghosh ${ }^{1}$, Susree Roy ${ }^{1}$, Raghunath Chatterjee ${ }^{2}$, Shaleen Agarwal $^{3}$, Subash Gupta ${ }^{3}$, Abhijit Chowdhury ${ }^{1}$, Simanti Datta $^{1}$, Soma Banerjee ${ }^{1}$

${ }^{1}$ Institute of Post Graduate Medical Education \& Research, Kolkata, India; ${ }^{2}$ Indian Statistical Institute, India; ${ }^{3}$ Max Super Speciality Hospital, Saket, India

Background: Hepatitis $\mathrm{C}$ virus (HCV) infected hepatocellular carcinoma (HCC) has an asymptomatic disease progression where an underlying altered gene profile provides us the window of alternative diagnosis and therapeutic approach. Inspite of known regulatory processes, HCV disease progression remains a mystery.

Method: Transcriptome profiling of both small RNA and total RNA was performed with HCC and normal liver tissues on Illumina platform to investigate the deregulated novel miRNAs. Novel miRNAs were predicted by miRDeep2.pl and the corresponding precursor miRNA sequence was predicted by RandFold. mFold was used to predict the stability of the stem loop structure of pre-miRNA and in vitro Dicer knockdown assay to confirm its biogenesis. qRT-PCR and in vitro assays were performed to validate genes and the pathways predicted bioinformatically.

Result: Analysis of small RNA sequencing data of HCC and control sample revealed that about $20 \%$ sequences remained unmapped to the known dataset of miRNAs. Subsequent in depth analysis decoded three novel miRNA like sequences as predicted based on the free energy of stem-loop structure formation, AU content, and Dicer cleavage of the premiRNA. Furthermore, expression analysis in liver tissues of progressive disease stages revealed that only one miRNA, miR-c12 (originated from chromosome 12) was downregulated with the disease stages implicating a protective role of miR-c12 in normal cells, whereas other two miRNAs were upregulated. Target prediction and expression analysis from total RNA sequencing data disclosed that fatty acid catabolism and PPAR signalling axis is totally downregulated in $\mathrm{HCV}$ infected $\mathrm{HCC}$. Interestingly, it has no role in cell proliferation or anchorage independent growth but attenuates $\mathrm{HCV}$ infection by impeding lipid accumulation and by downregulating expression of $\beta$-oxidation genes CPT2 and ACOX1 via CEP350PPAR $\alpha$ axis; hence restoration of miR-c12 could be a novel therapeutics strategy for $\mathrm{HCV}-\mathrm{HCC}$.

Conclusion: Insights into novel miRNAs suggest its importance in understanding the disease progression of $\mathrm{HCV}$ infection. miR-c12 plays a protective role by impeding lipid accumulation inside cell and attenuating $\mathrm{HCV}$ replication and infection.

\section{$\mathrm{O}-\mathrm{HCV}-03$}

Hepatitis $\mathbf{C}$ virus infection among tuberculosis patients in sohag governorate: seroprevalence and associated risk factors

\section{Mohamed Shahat Badawy ${ }^{1}$, Mona Taha ${ }^{2}$, Ahmed Fathy ${ }^{2}$}

${ }^{1}$ South Valley University, Qena Faculty of medicine, Egypt; ${ }^{2}$ Sohag University, Egypt

Background: Tuberculosis (TB) and hepatitis C virus (HCV) infection have emerged as major public health problems in Egypt. Chronic viral infection may increase the risk for development of accelerated lung destruction. Chronic HCV infection is also associated with both direct and indirect effects on pulmonary tissue. No systematic research on HCV/TB co-infection is currently being undertaken.

Method: To determine the prevalence and risk factors for the HCV infection among patients with TB in Sohag. A case-control study was performed to identify risk factors for $\mathrm{HCV}$ infection. Cases were defined as patients with TB who were $\mathrm{HCV}$-seropositive, and controls were defined as patients with TB who were HCV-seronegative

Result: Hundred thirty five tuberculosis patients were fulfilled the inclusion criteria. HCV infection was diagnosed in 21/135 (6.4\%). Goza smokers ( $\mathrm{P}$ value 0.01 Odd's Ratio 3.75, 95\% confidence interval 0.24-0.44), history of operation ( $\mathrm{P}$ value 0.001 ORs 7.67, 95\% CI 0.165-0.263), blood transfusion ( $\mathrm{P}$ value 0.004 ORs $7.2,95 \%$ CI 0.103-0.362), presence of tattoos ( $\mathrm{P}$ value 0.03 ORs $3.4,95 \% \mathrm{CI}$ 0.168-0.338), extra pulmonary tuberculosis ( $\mathrm{P}$ value 0.004 ORs 3.5 , 95\% CI 2.341-3.384), low serum albumin ( $\mathrm{P}$ value 0.002 ORs 0.5 , $95 \%$ CI 0.068-0.317) were the main risk factors associated with HCV infection.

Conclusion: Universal screening for HCV infection in TB patients is highly recommended. There is an urgent need to detect HCV infection in high-risk groups to prevent future $\mathrm{HCV}$ transmission as well as morbidity and mortality associated with TB.

\section{$\mathrm{O}-\mathrm{HCV}-04$}

Mitochondrial function improves after successful clearance of virus by direct acting antivirals (DAA) in chronic HCV infection

$\underline{\text { Alip Ghosh }^{1}}$, Eleanor Wilson ${ }^{1}$, Shyam Kottilil ${ }^{1}$, Bhawna Poonia ${ }^{1}$

${ }^{1}$ University of Maryland, Baltimore, United States 
Background: Mitochondria play critical roles in immune system, particularly in regulating macrophage responses to pathogen infections, tissue damage and inflammation. Recently mitochondrial dysfunction is detected in immune cells in chronic infections including hepatitis B virus (HBV). However, there is scant data regarding alteration of mitochondrial function in immune cells in chronic HCV (CHC) infection.

Method: Here we studied mitochondrial dysfunction in $10 \mathrm{CHC}$ patients and 5 healthy controls (HC). We further investigated the effect of virus cure on change in mitochondrial mass, superoxide level and mitochondrial membrane depolarization in different compartments of immune cells by specific dyes mitotracker-green, MitoSOXred and $\mathrm{JC} 1$, respectively, using flow cytometery on $\mathrm{CHC}$ patients $(\mathrm{n}=5)$ who achieved sustained virological response at 12 week post DAA (NS5A, NS5B, and protease inhibitors) therapy (SVR12). PBMCs were collected pre-treatment and at 12 week post therapy.

Result: Relatively higher mitochondrial mass was observed in monocytes and lymphocytes in CHC patients compared to HC (MFI, monocytes $168.2 \pm 8.03$ vs. $121.8 \pm 5.81, \mathrm{p}<0.005$; lymphocytes $69.2 \pm 6.08$ vs. $48.98 \pm 3.31, \mathrm{p}<0.001)$, which declined significantly at SVR12 (MFI, monocytes 136.2 $\pm 5.56, \quad \mathrm{p}<0.05$; lymphocytes $59.96 \pm 7.87, \mathrm{p}<0.05$ ). Mitochondrial superoxide level was considerably higher in CHC patients compared to healthy controls particularly in CD4 cells and monocytes which was reduced significantly at SVR12 (MFI, CD4 cells $85.46 \pm 14.03$ vs. $64.82 \pm 8.16, \quad \mathrm{p}<0.05 ; \quad$ monocytes $117.6 \pm 53.18 \quad$ vs. $54.02 \pm 11.23, \mathrm{p}<0.05)$ reaching to the level of healthy controls (MFI, CD4 cells $67.54 \pm 8.71$; monocytes $77.07 \pm 10.19$ ). High frequency of depolarized mitochondria in CD4, CD8 T cell and B cells was evident in $\mathrm{CHC}$ patients which was also recovered significantly after SVR following DAA therapy. Interestingly, frequency of $\mathrm{CD} 38$ activation marker was higher in $\mathrm{CHC}$ patients compared to $\mathrm{HC}$ and mitochondrial dysfunction was predominantly observed in CD38 + T cells compared to CD38- T cells.

Conclusion: Chronic HCV infection leads to significant mitochondrial dysfunction in different subpopulation of immune cells and successful clearance of HCV improves mitochondrial functions in immune cells. Thus the improvement in mitochondrial function may play a crucial role for functional recovery of different compartment of immune cells after DAA therapy as indicated in recent studies.

\section{O-HCV-05}

Phenotypes and function of natural killer cells from chronic hepatitis $\mathrm{C}$ patients recovered not at early stage but after the end of sofosbuvir and ledipasvir treatment

Xiaoxiao Wang ${ }^{1}$, Bifen Luo ${ }^{1}$, Hanji Jiang ${ }^{1}$, Xu Cong ${ }^{1}$, Qian Jin¹, Bo Feng $^{1}$, Lai Wei ${ }^{1}$

${ }^{1}$ Peking University People's Hoapital, China

Background: Chronic hepatitis $\mathrm{C}$ virus (HCV) infection remains a major public health problem, highly effective direct-acting antivirals (DAAs) makes chronic hepatitis $\mathrm{C}(\mathrm{CHC})$ a curable disease. Impact of various DAAs on innate immunity was unclear. In this study, we investigated how natural killer (NK) cells were affected in the elimination of $\mathrm{HCV}$ by sofosbuvir/ledipasvir.

Method: 13 naive-treated patients with chronic HCV infection were treated with sofosbuvir/ledipasvir, and peripheral blood was detected at various time points (week 0, week 2, week 4, week 8 and week 12) during therapy and week Pt-12 (Pt, Post of treatment), week Pt-24 after the end of therapy by multicolour flow cytometry, and compared with samples from 13 healthy controls.
Result: All our 13 patients achieved sustained virological response (SVR) by sofosbuvir/ledipasvir therapy. In NK cells subsets, there was a significant decline in CD56bright NK cells frequency at week 8 $(p=0.002)$ and week $12(p=0.003)$, which altered to the level comparable to healthy controls at week Pt-12, but there was no difference in the frequency of CD56dim NK cells. In NK cells phenotypes, compared with NK cells from healthy controls, the baseline expression levels of NKG2A, NKp30, CD94 on NK cells from $\mathrm{CHC}$ patients were higher, and all patients had increased IFNgama and perforin levels. NKG2A, NKp30 and CD94 started to recover at week 12 and reached to the similar level of healthy controls at week Pt-12 or Pt-24. In NK cells function, only IFN-gama started to recover at week 8 and reached to the normalized level at week Pt12; MFI of perforin of NK cells from CHC patients was higher than that from uninfected subjects persistently.

Conclusion: NK cells of naive-treated $\mathrm{CHC}$ patients can be affected by DAAs and phenotypes and function of NK cells recovered not at early stage but after end of sofosbuvir and ledipasvir treatment.
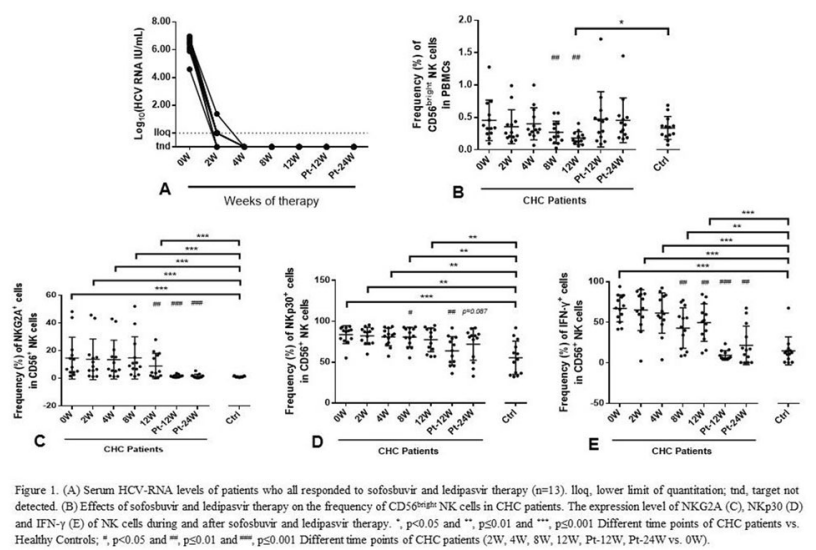

\section{$O-H C V-06$}

Prevalence of HCV infection in Mongolia (more precise estimation)

Yagaanbuyant Borjigon Dahgwahdorj ${ }^{1}$, Baatarsuren Uurtsaikh ${ }^{4}$, Dashtseren Bekhbold $^{2}$, Dashdorj Naranjargal ${ }^{3}$

${ }^{1}$ Mongolian National University of Medical Sciences, Mongolia; 2"Liver center", Mongolia; "3iver" center, Mongolia; "'Onom Foundation" Ulaanbaatar, Mongolia

Background: There is well known, that HCV infection is endemic in Mongolia. So, for elimination of HCV infection, started national campaign for screening of this infection, from June 2017(40-65 age groups). Until 1 October 2017, there was screened 287000 from 741000 (whole population of this age groups).

Purpose of study: To estimate the total number of subjects who is needed anti HCV treatment, in Mongolia.

Method: In all Mongolian family doctors screened 287000 subjects with 40-60 years old, who are before no tested for viral hepatitis. All subjects tested anti-HCV. If anti-HCV tested positive, then done HCV-RNA. For estimation also we used obtained data our previous study in 2012 (J Viral Hepat. V. 24, Issue 9; 2017 pp: 759-767).

Result: In serum of 49386 subjects tested positive for anti-HCV. Positivity of anti-HCV increase by ages (table): 


\begin{tabular}{|l|l|l|l|l|l|l|l|}
\hline $\begin{array}{l}\text { Age } \\
\text { groups } \\
\text { (years) }\end{array}$ & $\begin{array}{l}\text { Screened } \\
\text { population } \\
\text { in } 2017 \\
(\mathrm{n})\end{array}$ & $\begin{array}{l}\text { anti-HCV } \\
\% \\
(+) / 2012\end{array}$ & $\begin{array}{l}\text { anti-HCV } \\
\% \\
(+) / 2017\end{array}$ & $\begin{array}{l}\text { HCV-RNA } \\
\% \\
(+) / 2017\end{array}$ & $\begin{array}{l}\text { Whole } \\
\text { population } \\
(\mathrm{n}) 2016\end{array}$ & $\begin{array}{l}\text { Estimated } \\
\text { anti-HCV } \\
(\mathrm{n})\end{array}$ & $\begin{array}{l}\text { Estimated } \\
\text { with HCV } \\
\text { infection } \\
(\mathrm{n})\end{array}$ \\
\hline $40-44$ & 59910 & 8.5 & 9.93 & 6.65 & $210,897$. & 20942 & 14024 \\
\hline $45-49$ & 59335 & 6.5 & 13.06 & 8.76 & $179,429$. & 23433 & 15718 \\
\hline $50-54$ & 60830 & 16.9 & 16.62 & 11.00 & $157,548$. & 26184 & 17330 \\
\hline $55-59$ & 59882 & 22.4 & 21.04 & 13.75 & $119,171$. & 25073 & 16386 \\
\hline $60-64$ & 46941 & 30.4 & 27.65 & 18.37 & $73,995$. & 20460 & 13593 \\
\hline Tota1 & 286898 & & & & 741040. & 116092 & 77051 \\
\hline
\end{tabular}

Here, a percentage of age related prevalence of anti-HCV is much similar to the data, which obtained from 2012 study. So, we are calculated the age related prevalence by direct standardization on the basis of this two study. Then, we are estimated total subjects with $\mathrm{HCV}$ infection or needed anti viral treatment is no more than 122600 subjects.

Conclusion: Nowadays, in Mongolia living no more 122600 subjects with HCV infection who are needed DAA treatment. So, decision makers and government authorities recommend to take account into this data for planning HCV elimination strategy.

\section{Session: NASH-Clinical; Chairpersons: Chan Wah Kheong; Manisha Bangar; Chandan KN}

\section{$O-N A F L-01$}

Acetyl-CoA carboxylase (ACC) inhibitor GS-0976 leads to significant improvements in MRI-PDFF in a phase 2, randomized, placebo-controlled trial of patients with NASH

Jina Lee ${ }^{1}$, Zeid Kayali ${ }^{5}$, Mazen Noureddin ${ }^{4}$, Peter Ruane ${ }^{8}$, Eric Lawitz $^{9}$, Norman Gitlin ${ }^{2}$, Michael Bennett ${ }^{6}$, Eliza Jing Harting ${ }^{1}$, Bryan J Mccolgan ${ }^{1}$, Macky Natha ${ }^{1}$, Robert P Myers ${ }^{1}$, Mani Subramanian $^{1}$, John G Mchutchison ${ }^{1}$, Michael S Middleton ${ }^{10}$, Claude Sirlin ${ }^{10}$, Michelle $\mathrm{Lai}^{3}$, Michael Charlton ${ }^{11}$, Stephen A Harrison ${ }^{7}$

${ }^{1}$ Gilead Sciences, Inc., United States; ${ }^{10}$ University of California at San Diego, United States; ${ }^{11}$ University of Chicago, United States; ${ }^{2}$ Atlanta Gastroenterology Associates, United States; ${ }^{3}$ Beth Israel Deaconess Medical Center, Harvard Medical School, United States; ${ }^{4}$ Cedars-Sinai Medical Center, United States; ${ }^{5}$ Inland Empire Liver Foundation, United States; ${ }^{6}$ Medical Research Associates Group, United States; ${ }^{7}$ Pinnacle Clinical Research, United States; ${ }^{8}$ Ruane Medical and Liver Health Institute, United States; ${ }^{9}$ Texas Liver Institute, University of Texas Health San Antonio, United States

Background: ACC catalyzes the rate-limiting step in de novo lipogenesis (DNL), which is increased in patients with NASH. Here, we describe the safety and efficacy of GS-0976, a liver-targeted inhibitor of ACC, in a phase 2 trial of patients with NASH.

Method: In this double-blind, placebo-controlled trial, 126 non-cirrhotic subjects with NASH diagnosed noninvasively by MRI proton density fat fraction (PDFF) $\geq 8 \%$ and liver stiffness $\geq 2.5 \mathrm{kPa}$ by MR elastography (MRE), or historical liver biopsy consistent with NASH and stage 1 to 3 fibrosis, were randomized 2:2:1 to receive GS$097620 \mathrm{mg}$, GS-0976 $5 \mathrm{mg}$, or placebo orally QD for 12 weeks (W12). Centrally-read MRI-PDFF and MRE, and FibroScan and serum markers of fibrosis were measured at baseline and W12.

Result: The majority of the subjects were female (65\%) and diabetic (60\%); median MRI-PDFF and MRE-stiffness at baseline were $14.4 \%$ (IQR 11.1-19.0) and $3.43 \mathrm{kPa}$ (2.96-4.20), respectively; $40 \%$ of subjects had MRE $>3.64 \mathrm{kPa}$, consistent with advanced $(\geq \mathrm{F} 3$ ) fibrosis. At W12, GS-0976 $20 \mathrm{mg}$ resulted in a statistically and clinically significant decrease in MRI-PDFF compared with placebo; differences between GS-0976 $5 \mathrm{mg}$ and placebo were not statistically significant (Table). At W12, a $\geq 30 \%$ decline in MRI-PDFF was observed in $48 \%$ of subjects treated with GS-0976 $20 \mathrm{mg}(\mathrm{p}=0.004$ vs. placebo), $23 \%$ treated with GS-0976 $5 \mathrm{mg}$ ( $\mathrm{p}=0.433$ vs. placebo), and $15 \%$ with placebo. Changes in liver stiffness by MRE did not differ between groups. However, subjects treated with GS-0976 $20 \mathrm{mg}$ had significant reductions in serum levels of TIMP-1 (Table); changes in TIMP-1 correlated with changes in PIII-NP $(r=0.47$; $\mathrm{p}=0.001)$ and hyaluronic acid $(\mathrm{r}=0.62 ; \mathrm{p}<0.001)$. Dose-dependent, but not statistically significant reductions in serum ALT, liver stiffness by FibroScan, and PIII-NP were also observed. GS-0976 was generally well-tolerated. At $\mathrm{W} 12$, a median relative change in triglycerides (TG) of $+11 \%,+13 \%$, and $-4 \%$ was observed among subjects treated with GS-0976 $20 \mathrm{mg}$, GS-0976 $5 \mathrm{mg}$, and placebo. Asymptomatic, Grade 3 or 4 TG elevations $(>500 \mathrm{mg} / \mathrm{dL}$ ) were observed in 16 subjects on GS-0976 $20 \mathrm{mg}(\mathrm{n}=7)$ or $5 \mathrm{mg}(\mathrm{n}=9)$. Of these 16 subjects, 4 responded to fibrate or fish oil therapy, and 7 of the remaining 12 subjects resolved without treatment or cessation of GS-0976. The primary factor associated with Grade 3 or 4 TG elevation was a baseline TG level $>250 \mathrm{mg} / \mathrm{dL}(\mathrm{p}<0.001)$.

Conclusion: In this randomized, placebo-controlled study, 12-week therapy with the liver-targeted, oral ACC inhibitor GS-0976 $20 \mathrm{mg}$ QD, led to significant improvements in hepatic steatosis and selected fibrosis markers in patients with NASH.

Table: Relative (\%) Changes in Imaging, ALT, and Serum Fibrosis Markers at W12 *

\begin{tabular}{|c|c|c|c|c|c|}
\hline & \multirow{2}{*}{$\begin{array}{c}\text { GS-0976 } \\
20 \mathrm{mg} \\
(\mathrm{n}=49)\end{array}$} & \multirow{2}{*}{$\begin{array}{c}\text { GS-0976 } \\
5 \mathrm{mg} \\
(n=51)\end{array}$} & \multirow{2}{*}{$\begin{array}{l}\text { Placebo } \\
(n=26)\end{array}$} & \multicolumn{2}{|c|}{ P-values } \\
\hline & & & & $\begin{array}{l}20 \mathrm{mg} \text { vs. } \\
\text { Placebo }\end{array}$ & $\begin{array}{l}5 \mathrm{mg} \text { vs. } \\
\text { Placebo }\end{array}$ \\
\hline MRI-PDFF & -28.9 & -13.0 & -8.4 & 0.002 & 0.142 \\
\hline $\begin{array}{l}\geq 30 \% \text { reduction in MRI- } \\
\mathrm{PDFF}, \%(\mathrm{n} / \mathrm{N})\end{array}$ & $\begin{array}{c}48 \% \\
(22 / 46)\end{array}$ & $\begin{array}{c}23 \% \\
(11 / 47)\end{array}$ & $\begin{array}{c}15 \% \\
(4 / 26)\end{array}$ & 0.004 & 0.433 \\
\hline MRE-stiffness & -5.5 & -9.6 & -12.5 & 0.100 & 0.743 \\
\hline $\begin{array}{l}\geq 15 \% \text { reduction in MRE- } \\
\text { stiffness, } \%(n / N)\end{array}$ & $\begin{array}{c}33 \% \\
(15 / 46)\end{array}$ & $\begin{array}{c}40 \% \\
(19 / 47)\end{array}$ & $\begin{array}{c}35 \% \\
(9 / 26)\end{array}$ & 0.92 & 0.62 \\
\hline Liver stiffness by FibroScan & -11.1 & -8.4 & -3.1 & 0.212 & 0.364 \\
\hline ALT & -20.5 & -9.8 & -6.7 & 0.176 & 0.765 \\
\hline TIMP-1 & -7.9 & -2.9 & -1.5 & 0.022 & 0.301 \\
\hline PIII-NP & -13.9 & -7.0 & -0.5 & 0.107 & 0.605 \\
\hline Hyaluronic acid & -6.9 & -0.7 & -15.3 & 0.386 & 0.576 \\
\hline
\end{tabular}

* Unless indicated, all data are median relative (\%) changes from baseline.

\section{$O-N A F L-02$}

BMS-986036 (pegylated FGF21) in patients with non-alcoholic steatohepatitis: a phase 2 study

Elizabeth Artwick', Yi Luo ${ }^{2}$, Rose Christian², Arun Sanyal', Edgar Charles $^{2}$, Brent Neuschwander Tetri ${ }^{5}$, Rohit Loomba ${ }^{8}$, Stephen Harrison ${ }^{4}$, Manal Abdelmalek ${ }^{3}$, Eric Lawitz ${ }^{6}$, Dina Halegoua Demarzio ${ }^{7}$, Yuping Dong ${ }^{2}$, Stephanie Noviello ${ }^{2}$

${ }^{1}$ Medical Expressions, United States; ${ }^{2}$ Bristol-Myers Squibb, United States; ${ }^{3}$ Duke University, United States; ${ }^{4}$ Pinnacle Clinical Research, United States; ${ }^{5}$ Saint Louis University, United States; ${ }^{6}$ Texas Liver Institute, United States; ${ }^{7}$ Thomas Jefferson University, United States; ${ }^{8}$ University of California "San Diego, United States; ${ }^{9}$ Virginia Commonwealth University, United States 
Background: BMS-986036 is a PEGylated analogue of human fibroblast growth factor 21, a key regulator of metabolism. In preclinical models of non-alcoholic steatohepatitis (NASH), BMS986036 improved steatosis, inflammation, hepatocyte ballooning, and fibrosis. In a Phase 2 study in obese patients with type 2 diabetes, it improved insulin sensitivity, lipids, adiponectin, and fibrosis biomarkers. This Phase 2 study evaluated the safety, tolerability, and efficacy of BMS-986036 in NASH patients.

Method: This was a multicenter, randomized (1:1:1), double-blind, placebo-controlled study in adults with body mass index $=25 \mathrm{~kg} / \mathrm{m}^{2}$, biopsy-confirmed NASH (F1-F3), and hepatic fat fraction $=10 \%$, assessed by magnetic resonance imaging " proton density fat fraction (MRI-PDFF). Randomization was stratified by diabetes status. Patients received subcutaneous injections of BMS-986036 $10 \mathrm{mg}$ daily (QD), BMS-986036 $20 \mathrm{mg}$ weekly (QW), or placebo QD for 16 weeks. The primary efficacy endpoint was absolute change in MRI-PDFF at Week 16; exploratory endpoints included serum pro-C3 (N-terminal type III collagen propeptide, a fibrosis biomarker), ALT, AST, and, in a subset of patients, liver stiffness, assessed by MR elastography (MRE).

Result: 74 patients were treated (median age, 51.5 years; women, $65 \%$; type 2 diabetes, $38 \%$; mean hepatic fat fraction, $19.5 \%$ ). Baseline characteristics, including histology, imaging, and biochemical parameters, were comparable among groups. 68 patients had MRI-PDFF data at both Baseline and Week 16. At Week 16, both BMS-986036 regimens significantly reduced MRI-PDFF versus placebo and improved pro-C3, MRE, adiponectin, ALT and AST (Table). The most frequent AEs in BMS-986036-treated patients were diarrhea ( $17 \%$ vs $8 \%$ [placebo]), nausea ( $15 \%$ vs $8 \%)$, and frequent bowel movements ( $10 \%$ vs $0 \%$ ); most of these were mild, none was severe. Serious AEs occurred in 2 patients; none was considered treatment-related. There were no deaths and no discontinuations due to AEs.

Conclusion: BMS-986036, QD and QW for 16 weeks, compared with placebo, significantly decreased hepatic fat fraction in patients with NASH (F1-F3). BMS-986036 also improved biomarkers of fibrosis (MRE and pro-C3), adiponectin, and markers of hepatic injury (ALT and AST). These results suggest that BMS-986036 has beneficial effects on steatosis, liver injury, and fibrosis in NASH.

\section{$O-N A F L-03$}

\section{Ad libitum mediterranean and low fat diets both significantly reduce hepatic steatosis: a randomized controlled trial}

\section{Catherine Properzi ${ }^{1}$, Therese Sullivan ${ }^{4}$, Jill Sherriff ${ }^{2}$, Leon} Adams $^{6}$, Gary Jeffrey ${ }^{9}$, Helena Ching ${ }^{5}$, Rachel Buckley ${ }^{3}$, George Garas $^{8}$, Gerry Macquillan ${ }^{7}$

${ }^{1}$ Edith Cowan University, Australia; ${ }^{2}$ Curtin Health Innovation Research Institute, Curtin University, Australia; ${ }^{3}$ Massachusetts General Hospital and Harvard Medical School, Boston, MA, United States; ${ }^{4}$ School of Medical and Health Science, Edith Cowan University, Australia; ${ }^{5}$ Sir Charles Gairdner Hospital, Nedlands, Australia, Australia; ${ }^{6}$ The University of Western Australia, Australia; ${ }^{7}$ The University of Western Australia, Nedlands, Australia; ${ }^{8}$ The University of Western Australia, Nedlands, Australia, Australia; ${ }^{9}$ University of Western Australia, Australia

Background: Non-alcoholic fatty liver disease (NAFLD) is associated with increased cardio-metabolic disease. Diet induced weight loss is an important treatment goal in NAFLD, but long-term maintenance is difficult. The optimal diet for either improvement in NAFLD or attenuation of cardio-metabolic risk, regardless of weight loss, is unknown. We examined the effect of two ad libitum isocaloric diets [Mediterranean (MD) or Low Fat (LF)] on hepatic steatosis and cardio-metabolic risk factors.

Method: Subjects with NAFLD were randomized to a 12-week blinded dietary intervention (MD vs LF). Hepatic steatosis was determined via magnetic resonance spectroscopy (MRS). Cardiometabolic risk was assessed using the Framingham Risk Calculator, sphygmocor, and fasting biochemistry. From a total of 56 subjects enrolled, 49 subjects completed the intervention and 48 were included for analysis.

Result: During the intervention, subjects on the MD had significantly higher total fat, and monounsaturated fat but lower carbohydrate and sodium intakes compared to LF subjects $(\mathrm{p}<0.01)$. Hepatic steatosis reduced significantly in both groups $(\mathrm{p}<0.01)$ with similar mean (SD) relative reductions of $25.0 \%( \pm 25.3 \%)$ in $\mathrm{LF}$ and $32.4 \%$ $( \pm 25.5 \%)$ in $\mathrm{MD}(\mathrm{p}=0.32$ between groups). Liver enzymes also improved in both groups. Both groups had minimal weight loss [-1.6 $( \pm 2.1) \mathrm{kg}$ in LF vs $-2.1( \pm 2.5) \mathrm{kg}$ in $\mathrm{MD},(\mathrm{p}=0.52)]$. Significant within-group improvements in cardio-metabolic risk factors including the Framingham risk score, total cholesterol, serum triglyceride, and HbAlc were observed in the MD but not LF $(\mathrm{p}<0.05)$. Adherence was higher for the MD compared to LF ( $88 \%$ vs. $64 \%, p=0.048)$. Conclusion: Ad libitum low fat and Mediterranean diets both significantly improve hepatic steatosis and liver enzymes. Cardiovascular improvements and adherence in the MD indicate it may be the preferred dietary treatment for NAFLD.

\section{$O-N A F L-04$}

Prevalence of non-alcoholic fatty liver disease (NAFLD) in Asia: a systematic review and meta-analysisof 195 Studies and 1,753,168 subjects from 13 countries

Mindie Nguyen ${ }^{1}$, Jie $\mathrm{Li}^{3}$, Biyao Zou ${ }^{1}$, Hideki Fujii ${ }^{2}$, Yee Hui Yeo', Fanpu $\mathrm{Ji}^{4}$, Dong Hyun Lee ${ }^{1}$, Yuemin Feng ${ }^{3}$, Xiaoyu $\mathrm{Xie}^{3}$, Wanhua $\operatorname{Ren}^{3}$, Qiang $\mathrm{Zhu}^{3}$

${ }^{1}$ Stanford University Medical Center, United States; ${ }^{2}$ Osaka City University Graduate School of Medicine, Japa; ${ }^{3}$ Shandong Provincial Hospital Affiliated to Shandong University, China; ${ }^{4}$ the Second Affiliated Hospital of Xi' an Jiaotong University, China

Background: NAFLD is the most prevalent chronic liver disease worldwide and generally correlates with the obesity epidemic. Asia is a large and heterogeneous region with varying socioeconomic levels and obesity prevalence; therefore, our goal was to to estimate the prevalence of NAFLD in Asiathrough a meta-analytic approach and with subanalysis by country and sub-regions within Asia.

Method: PubMed and EMBASE databases were searched from 1989 to 2017 for relevant studies reporting NAFLD prevalence in Asia. Exclusions included studies without data on alcohol consumption or with inclusion of other liver diseases. All studies were reviewed by three independent investigators. We used random-effects models to provide point estimates with $95 \%$ confidence interval (CI) of prevalence. Publication bias was assessed by Egger weighted regression methods.

Result: From the 2700 titles and abstracts reviewed, 195 papers from 13 countries met the inclusion criteria and included 1,753,168 subjects. The overall pooled prevalence for NAFLD in Asia was $31 \%$ (95\% CI: 29-32). Individual country prevalence was shown in Table 1. In countries with more than 3 studies, the lowest prevalence was seen in Japan (24\%, 95\% CI: 21-28) and the highest in Iran (36\%, 95\% CI: 31-41). Notably, pooled prevalence from studies with sample $<1,000$ subjects much higher $(34 \%, 95 \%$ CI:31-38, 45 studies, $\mathrm{n}=23,857)$, compared to pooled prevalence from larger studies ( $\geq 1,000$ subjects) $(30 \%, 95 \%$ CI: $28-31,150$ studies, $\mathrm{n}=172,9311)$. 
By sub-regions within Asia (Table 2), there was significant regional differences $(\mathrm{p}<0.01)$ with the highest NAFLD prevalence seen in West Asia (33\%, 95\% CI: 28-39, 13 studies from Iran, Israel, Saudi Arabia, $\mathrm{n}=32,142)$ and the lowest in Southeast Asia $(24 \%, 95 \% \mathrm{CI}$ : 15-33, 5 studies from Malaysia, Philippine, Singapore, $\mathrm{n}=3457$ ). By country income levels (as defined by World Bank Open Data, data.worldbank.org), NAFLD prevalence was 30\% (95\% CI: 29-32, 89 studies, $\mathrm{n}=1,005,409$ ) for high-income countries (Japan, South Korea, Israel, Saudi Arabia, Singapore, China [Hong Kong, Taiwan]) and different at 31\% (95\% CI: 29-33, 106 studies, $n=747,759)$ for middle-income countries (Iran, Sri Lanka, China [Mainland], Pakistan, Malaysia, Philippine, India, Bangladesh $)(p<0.01)$.The heterogeneity was significant in the overall and each subgroup analysis with the Egger's test p-value of $<0.001$ for all.

Conclusion: Overall NAFLD prevalence in Asia is $31 \%$ similar to Western countries and by country-income levels within Asia but varies by some sub-regions or Asia with highest prevalence in West Asia $(33 \%)$.

Table 1 NAFLD Prevalence in AsiaStratified by Country

\begin{tabular}{|llllll|}
\hline Country & $\begin{array}{l}\text { No. of } \\
\text { Studies }\end{array}$ & $\begin{array}{l}\text { No. of } \\
\text { Patients }\end{array}$ & $\begin{array}{l}\text { Pooled } \\
\text { Prevalence(\%) }\end{array}$ & $\mathbf{9 5 \%}$ CI (\%) & $\mathbf{I}^{\mathbf{2}} \mathbf{( \% )}$ \\
\hline Mainland,China & 76 & 698,603 & 0.31 & $0.29-0.33$ & $99.73 \%$ \\
South Korea & 48 & 848,508 & 0.31 & $0.29-0.33$ & $99.80 \%$ \\
Japan & 19 & 57,208 & 0.24 & $0.21-0.28$ & $99.08 \%$ \\
Taiwan, China & 12 & 91,676 & 0.35 & $0.27-0.43$ & $99.86 \%$ \\
Iran & 10 & 30,291 & 0.36 & $0.31-0.41$ & $98.67 \%$ \\
India & 8 & 3938 & 0.28 & $0.21-0.35$ & $96.50 \%$ \\
Hong Kong, China & 6 & 5978 & 0.34 & $0.24-0.43$ & $98.39 \%$ \\
Sri Lanka & 5 & 8816 & 0.35 & $0.22-0.49$ & $99.44 \%$ \\
Malaysia & 3 & 2167 & 0.24 & $0.10-0.37$ & NA \\
Israel & 2 & 539 & 0.31 & $0.27-0.35$ & NA \\
Pakistan & 2 & 2732 & 0.15 & $0.13-0.16$ & NA \\
Bangladesh & 1 & 110 & 0.44 & $0.34-0.53$ & NA \\
Saudi Arabia & 1 & 1312 & 0.17 & $0.15-0.19$ & NA \\
Philippine & 1 & 1102 & 0.12 & $0.10-0.14$ & NA \\
Singapore & 1 & 188 & 0.40 & $0.33-0.48$ & NA \\
Overall* & 195 & $1,753,168$ & 0.31 & $0.29-0.32$ & $99.72 \%$ \\
\hline *with the Egr's & & & & & \\
\hline
\end{tabular}

Table 2 NAFLD Prevalence in Asia Stratified by sub-regions within Asia

\begin{tabular}{|c|c|c|c|c|c|}
\hline Sub-Regions within Asia & $\begin{array}{l}\text { No. of } \\
\text { Studies }\end{array}$ & \begin{tabular}{|l|} 
No. of \\
Patients
\end{tabular} & \begin{tabular}{|l} 
Pooled \\
Prevalence(\% \\
)
\end{tabular} & \begin{tabular}{|l}
$95 \%$ CI \\
$(\%)$
\end{tabular} & $I^{2}(\%)$ \\
\hline $\begin{array}{l}\text { East Asia } \\
\text { China[Mainland,Taiwan, Hong } \\
\text { Kong], South Korea, Japan }\end{array}$ & 161 & $1,701,973$ & 0.31 & $0.29-0.32$ & $99.75 \%$ \\
\hline $\begin{array}{l}\text { South Asia } \\
\text { India, Sri Lanka, Pakistan, } \\
\text { Bangladesh }\end{array}$ & 16 & 15,596 & 0.30 & $0.22-0.37$ & $99.13 \%$ \\
\hline $\begin{array}{l}\text { West Asia } \\
\text { Iran, Israel, Saudi Arabia }\end{array}$ & 13 & 32,142 & 0.33 & $0.28-0.39$ & $98.92 \%$ \\
\hline $\begin{array}{l}\text { Southeast Asia } \\
\text { Malaysia, Philippine, Singapor }\end{array}$ & 5 & 3,457 & 0.24 & $0.15-0.33$ & $97.65 \%$ \\
\hline
\end{tabular}

O-NAFL-05

Reductions in hepatic proton density fat fraction (PDFF) predict histologic improvement in a multi-center clinical trial of subjects with nonalcoholic steatohepatitis (NASH)

$\underline{\text { Jina Lee }}^{1}$, Eric Lawitz ${ }^{5}$, Saumya Jayakumar ${ }^{7}$, Parvez Mantry ${ }^{6}$, Stephen Caldwell $^{10}$, Anna Mae Diehl ${ }^{3}$, Ren Xü ${ }^{1}$, Ling Han ${ }^{1}$, Yao $\mathbf{L i}^{1}$, Stephen Djedjos ${ }^{1}$, Macky Natha ${ }^{1}$, Robert P Myers', Mani Subramanian $^{1}$, Zachary Goodman ${ }^{4}$, Michael Charlton', Nezam H Afdhal $^{2}$, Claude B Sirlin ${ }^{8}$, Michael S Middleton ${ }^{8}$

${ }^{1}$ Gilead Sciences, Inc., United States; ${ }^{10}$ University of Virginia, United States; ${ }^{2}$ Beth Israel Deaconess Medical Center and Harvard Medical School, United States; ${ }^{3}$ Duke Clinical Research Institute, United States; ${ }^{4}$ Inova Fairfax Hospital, United States; ${ }^{5}$ Texas Liver Institute, University of Texas Health, United States; ${ }^{6}$ The Liver Institute at Methodist Dallas, United States; ${ }^{7}$ University of Calgary, Canada; ${ }^{8}$ University of California San Diego, United States; ${ }^{9}$ University of Chicago, United States

Background: MRI-estimated proton density fat fraction (PDFF) is a noninvasive imaging marker of hepatic steatosis. Although a reduction in PDFF $\geq 30 \%$ has been associated with a histologic response in NASH, this threshold has not been validated. Our objective was to assess the performance of PDFF to predict histologic improvement in a clinical trial of subjects with NASH.

Method: MRI-PDFF was measured at baseline (BL) and Week 24 (W24) in a Phase 2, multi-center trial of 72 subjects with NASH (NAS $\geq 5$ and F2-3 fibrosis) treated with selonsertib $6 \mathrm{mg}$ or $18 \mathrm{mg}$ orally QD alone or in combination with simtuzumab (SIM, $125 \mathrm{mg}$ SQ weekly) or SIM alone for 24 weeks. Liver biopsies at BL and W24 were graded according to the NAFLD Activity Score (NAS) and hepatic fat was quantified by morphometry. The discrimination of MRI-PDFF for NAS response ( $\geq 2$-point reduction) and steatosis improvement ( $\geq 1$-grade reduction) was evaluated using AUROCs and the optimal PDFF thresholds were determined based on the maximal sum of sensitivity and specificity.

Result: Among 65 subjects with evaluable MRI-PDFF and biopsies at BL and W24, a NAS response was observed in $12(18 \%)$ and steatosis improvement in $18(28 \%)$. The AUROC of PDFF to predict NAS response was 0.70 (95\% CI 0.51-0.89) and the optimal threshold was $\mathrm{a} \geq 25 \%$ relative reduction (Table). At this cut-off, MRI-PDFF was $50 \%$ sensitive, $81 \%$ specific, and had positive (PPV) and negative predictive values (NPV) of $38 \%$ and $88 \%$, respectively. Compared with subjects with $<25 \%$ reductions in PDFF, those with $\geq 25 \%$ reductions were more likely to have steatosis improvement $(22 \%$ vs $44 \% ; \mathrm{p}=0.12)$ and larger relative changes in hepatic fat content ($4.2 \%$ vs $-36 \% ; \mathrm{p}=0.0006)$, serum ALT $(-2.9 \%$ vs $-31.8 \%$; $\mathrm{p}=0.0013)$, CK18 M30 (+4.9\% vs $-30.7 \% ; \mathrm{p}=0.0075)$, ELF score $(+0.4 \%$ vs $-2.5 \% ; \mathrm{p}=0.0271)$, and TIMP1 $(+1.7 \%$ vs $-7.8 \%$; $\mathrm{p}=0.084)$. For the prediction of steatosis improvement, the AUROC of PDFF was 0.70 (95\% CI 57-83) and the optimal threshold was a $\geq 0 \%$ relative reduction (sensitivity $89 \%$, specificity $47 \%$, PPV $39 \%$, NPV 92\%).

Conclusion: In this multi-center trial of selonsertib, $\geq 25 \%$ relative reduction in MRI-estimated PDFF was predictive of histologic response. These data support the use of MRI-PDFF as a noninvasive endpoint in clinical trials of interventions for NASH. 
Table: Operating Characteristics of Changes in MRI-PDFF to Predict Histologic Responses *

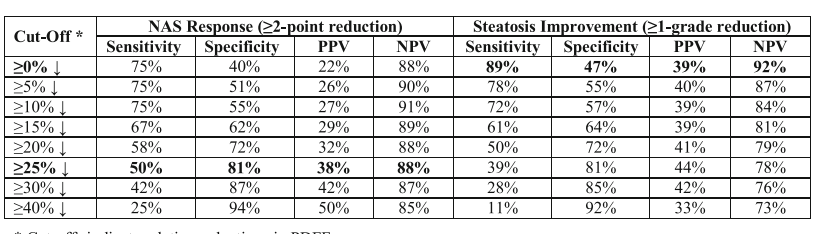

* Cut-offs indicate relative reductions in PDFF.

\section{$O-N A F L-06$}

Survey on knowledge of non alcoholic fatty liver disease (NAFLD) among doctors in Sri Lanka: a multicenter study

\section{Thushara Matthias $^{1}$, Nilesh Fernandopulle ${ }^{3}$, Suranjith L Seneviratne $^{2}$ \\ ${ }^{1}$ National Hospital of Sri Lanka, Sri Lanka; ${ }^{2}$ Institute of Immunity and Transplantation, Royal Free Hospital and university College London, United Kingdom; ${ }^{3}$ Teaching Hospital, Jaffna, Sri Lanka}

Background: In the recent years, Non-alcoholic Fatty Liver Disease (NAFLD) is gaining wide spread interest within the scientific community. Patients with NAFLD are commonly seen by doctors practicing in hospitals on a day to day basis. With the rising prevalence of NAFLD, the objective of this study was to ascertain the knowledge and awareness about NAFLD among non-hepatology/gastroenterology doctors in the state sector hospitals in Sri Lanka Method: A comprehensive anonymous questionnaire (available on request) was given to specialists and specialists doctors in training at three major tertiary care hospital in the country: Colombo, Colombo South and Jaffna.

The first part of the questionnaire consisted of basic demographic data. The second part consisted of risk factors, complications, methods of diagnosis, management options, progression and screening of NAFLD. The responses were open ended and multiple choices were given and they were coded according to predetermined criteria.

Result: The study had 80 grade medical officers/junior doctors and 58 Post graduate trainees/ consultants. A majority $52.9 \%$ had consultations with 12-24 patients. A majority 110 (79.7\%) considered NAFLD to be a major health problem. A large number 97 (70.3\%) thought NAFLD prevalence was $10-40 \%$. The complications of NAFLD: cirrhosis was recognized by a majority and thought about $10-30 \%$ progressed to cirrhosis.Family screening for NAFLD was not recommended by $66(47.8 \%)$. The correct methods of diagnosis for NAFLD was recognized by participants: USS 132 (95.7\%), MRI $34(24.6 \%)$, TE 23 (16.7\%), liver biopsy 88(63.8\%). A smaller number $53(38.4 \%)$ Recognized non invasive methods were available for diagnosis of NAFLD. The correct indications for liver biopsy were identified by $>50 \%$ of the participants: $71(51.4 \%)$ in patients at risk for steatohepatitis and cirrhosis, $70(50.7 \%)$ if other conditions causing steatohepatitis can't be excluded, $47(34.1 \%)$ if other conditions causing steatohepatitis coexist, 26(18.8\%) if metabolic syndrome was present, 66(47.8\%) if high NAFLD fibrosis score, $40(29 \%)$ in children before starting pharmacological therapy. The factors that affect the severity of NAFLD was recognized in the following percentages: older age $48(34.8 \%)$, higher BMI 107 (77.5\%), CRP 45 (32.6\%), High INR35 (25.4\%), Insulin resistance $102(73.9 \%)$ and albumin $54(34.9 \%) .85(61.6 \%)$ refer patients to a gastroenterologist or to a physician. $53(38.4 \%)$ refer patients to a gym, $67(48.6 \%)$ refer to a weight loss clinic and $45(32.6 \%)$ refer to a dietician.Management options: dietary modifications $130(94.2 \%)$ and exercise $123(89.1 \%)$ was noted. A hypocaloric diet $24(17.4 \%)$ was not recommended by many.As for continuous medical education 79 $(57.2 \%)$, did not attend a lecture or read a guideline. The barriers for effective management of NAFLD included: lack of confidence 70 $(50.7 \%)$, time constraints $58(42 \%)$. There was no difference between the two groups in a majority of fields. The postgraduate trainee doctors recognized the availability of non invasive investigations than the non trainee doctors $(\mathrm{P}=0.01)$ and read guidelines on NAFLD $(\mathrm{p}=0.02)$

Conclusion: The gravity of NAFLD and its complications was appreciated but management options were underestimated. More appropriate referrals need to be done and CME activities needs improvement for better management of patients

Session: Hepatitis B Virus Infection-Clinical; Chairperson: Rajnish Monga; $R$ K Himtani; Madhumita P Kumar

\section{$O-H B V-13$}

One year after switching from tenofovir disoproxil fumarate (TDF) to tenofovir alafenamide (TAF): safety results from 2 phase 3 studies in HBeAg-positive and HBeAg-negative patients with chronic hepatitis $B$ (CHB)

Toan Luu Vo ${ }^{1}$, Shuhei Nishiguchi ${ }^{7}$, Hyung Joon $\mathrm{Kim}^{5}$, Edward Gane $^{3}$, Wan Long Chuang ${ }^{8}$, Calvin Pan', Maurizia Brunetto ${ }^{4}$, Aric Josun Hui ${ }^{2}$, Rajiv Mehta ${ }^{10}$, John Flaherty ${ }^{1}$, Vithika Suri ${ }^{6}$, George Wu ${ }^{1}$, Anuj Gaggar ${ }^{1}$, G Mani Subramanian ${ }^{1}$

${ }^{1}$ Gilead Sciences, United States; ${ }^{10}$ Surat Institute of Medical Sciences, India; ${ }^{2}$ Alice Ho Miu Ling Nethersole Hospital, Hong Kong; ${ }^{3}$ Auckland Clinical Studies, New Zealand; ${ }^{4}$ Azienda Ospedaliero-Universitaria Pisana, Italy; ${ }^{5}$ Chung-Ang University Hospital, Korea, Republic of; ${ }^{6}$ Gilead Sciences, India; ${ }^{7}$ Hyogo College of Medicine, Japan; ${ }^{8}$ Kaohsiung Medical University Hospital, Taiwan; ${ }^{9}$ New Discovery LLC, United States

Background: TAF has shown less bone and renal effects with similar efficacy rates compared to TDF in two large multinational Phase 3 studies after 96 weeks (wk) of double-blind (DB) treatment. We evaluated patients who completed 96 wks of DB treatment with TAF or TDF and switched to open label (OL) treatment with TAF, including those with 1 year of data (through Wk 144) to determine changes in bone mineral density (BMD), creatinine clearance (CrCL), and viral suppression.

Method: In 2 identically-designed studies, $1298 \mathrm{CHB}$ patients $(\mathrm{HBeAg}-$ [Study 108; $\mathrm{N}=425$ ] or $\mathrm{HBeAg}+$ [Study $110 ; \mathrm{N}=873])$ were randomized and treated with TAF $25 \mathrm{mg}$ QD or TDF $300 \mathrm{mg}$ QD. At Wk 96, 541 (42\%; TAF 361; TDF 180) patients enrolled in these ongoing 8 year studies had completed DB treatment with TAF or TDF and switched to OL TAF. DXA scans were evaluated every 24 wks as were serial assessments of $\mathrm{CrCL}$ and viral suppression. Analyses included subjects with data at Wk 96 and at Wk 144 for CrCL $(n=401)$, spine $(n=288)$ or hip $(n=287)$ BMD, HBV DNA $(\mathrm{n}=394)$ and ALT normalization by AASLD criteria $(\mathrm{n}=398)$.

Result: CrCL improved significantly in patients who switched from DB TDF to OL TAF at Wk 144 compared to Wk $96(\mathrm{n}=122$, median $(\mathrm{Q} 1, \mathrm{Q} 3)$ change $=+3.6(-4.2,+8.4) \mathrm{ml} / \mathrm{min}, \mathrm{p}<0.001)$; and remained stable in those previously receiving TAF (Figure A). BMD also showed improvements at Wk 144 from Wk 96 among patients switched from DB TDF to OL TAF (hip: $\mathrm{n}=88$, mean (SD) $\%$ change $=+0.94 \%(1.825), \mathrm{p}<0.001 ;$ spine: $\mathrm{n}=88$, mean $(\mathrm{SD})$ $\%$ change $=+1.54 \%(2.680), \mathrm{p}<0.001)$. BMD changes in hip and spine for DB TAF patients entering the OL TAF period were relatively stable (Figure B). Compared to results at Wk 96, high rates of virologic control (HBV DNA $<29 \mathrm{IU} / \mathrm{mL}$ ) were maintained across patients in both treatment groups at Wk 144 (TDF 87\% vs 88\% and 
TAF $91 \%$ vs $89 \%$ ). One year after switching the rate of ALT normalization increased in the TDF group relative to Wk $96(\mathrm{n}=122$; $65 \%$ vs $47 \% ; \mathrm{p}<0.001)$ and did not differ from the TAF group $(65 \%$ vs $66 \% ; \mathrm{p}=0.83$ ) at $\mathrm{Wk} 144$.

Conclusion: Patients who switched from TDF to TAF demonstrated continued improvements in BMD and CrCL over 48 wks of treatment, virologic control was maintained, and rates of ALT normalization increased.
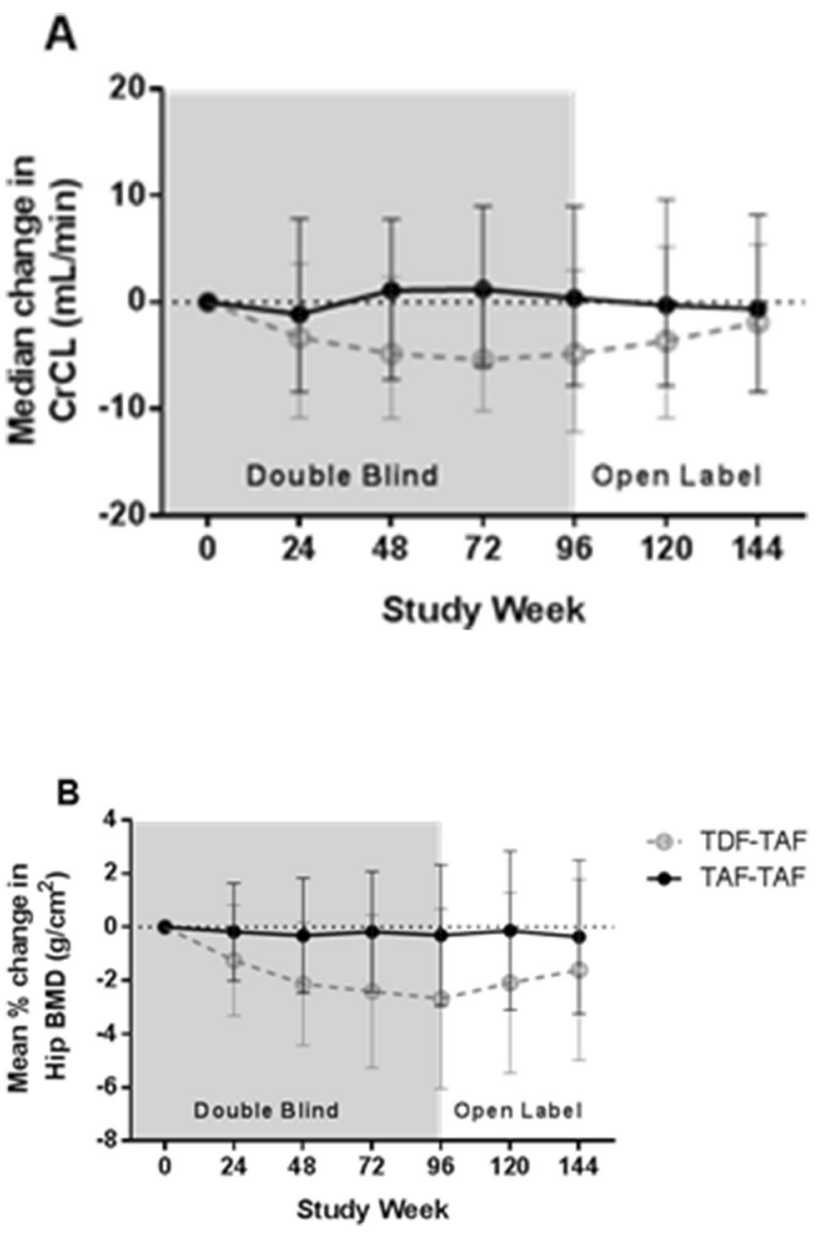

$O-H B V-14$

Racial and gender disparities in hepatitis B vaccination coverage amongst Asian American adults

\section{Mohamed I Elsaid ${ }^{1}$, Navaneeth Narayanan ${ }^{3}$, Jewook $\mathrm{Ha}^{4}$, Vinod Rustgi $^{2}$ \\ ${ }^{1}$ Rutgers, the State University of New Jersey, United States; ${ }^{2}$ Rutgers Robert Wood Johnson Medical School, United States; ${ }^{3}$ Rutgers University, Ernest Mario School of Pharmacy, United States; ${ }^{4} \mathrm{St}$. Joesph's Health Center, United States}

Background: An estimated 257 million individuals are living with hepatitis B Virus (HBV) worldwide. Among those with chronic HBV, $15-40 \%$ of people develop cirrhosis, late stage liver disease or hepatocellular carcinoma. The global burden of $\mathrm{HBV}$ is highest in developing countries, especially those located in Africa, Asia and the Western Pacific. While the aggregate rate of HBV infection has been firmly decreasing in the United States (US), Asian males continue to experience the highest risk of infection. This study aims to investigate the racial and gender disparities in HBV vaccination coverage among Asian American adults through analysis of data from the 2012-2015 National Health Interview Survey (NHIS).

Method: The study sample included 125,399 adults aged 18 to 85 who participated in the 2012-2015 NHIS. The NHIS is an annual health survey that is representative of the non-institutionalized US population. The main outcome of interest was HBV vaccination status. Race/ethnicity was categorized into White-non-Hispanics, Blacknon-Hispanics, Hispanics, Other, Asian-Indian, Chinese, Filipino, and Other-Asian (Korean, Vietnamese, Japanese, and other Asian subgroups). Complex survey methods were applied to all models to provide statistical estimates that are representative of US adults. Multivariable logistic regression models adjusting for age, education, region of residence, survey year, health insurance access, chronic liver disease, influenza vaccination, marital, employment and health status were fit to examine the associations between gender, race/ ethnicity and $\mathrm{HBV}$ vaccinations status.

Result: An estimated 39.66\% (95\% CI; 38.07\%, 41.25\%) of Asian adults living in the US received HBV vaccination. Vaccination prevalence among male Asian adults was lower than their female counterparts $38.05 \%$ (95\% CI; 35.66\%, 40.44\%) versus $41.09 \%$ (95\% CI; $38.96 \%, 43.21 \%$ ). Among Asian adults, the Adjusted Odds Ratio (AOR) of HBV vaccination for females were 1.20 (95\% CI; 1.04 , 1.39) times higher than males. The AORs of HBV vaccination were significantly higher when compared to white 1.21 (95\% CI; 1.03 , 1.41), 1.29 (95\% CI; 1.10, 1.51), respectively for Chinese and Filipino Adults. We observed significant gender disparities in HBV vaccination AOR for Asian-Indian and Chinese adults. In both groups, females had higher AOR of HBV vaccination when compared to males, Asian-Indian 1.42 (95\% CI; 1.04, 1.94) and Chinese 1.39 (95\% CI; $1.07,1.80)$.

Conclusion: Among Asian-Indian and Chinese adult residents of the US, the association between race and $\mathrm{HBV}$ vaccination status differs by gender, with males having lower vaccination rates than females. Health care resources should be directed to these target populations to improve these rates. Asian subgroups should be factored in when analyzing and reporting HBV vaccination statistics for adults.

\section{$O-H B V-15$}

The risk of post-partum hepatitis flares of anti-viral therapy during pregnancy for prevention of $\mathrm{HBV}$ perinatal transmission

\section{Liu Xiao Min ${ }^{1}$}

${ }^{1}$ Bei Jing Di Tan Hospital, China

Background: The paper is to investigate the risk of post-partum hepatitis flares of anti-viral therapy (AVT) during pregnancy for prevention of $\mathrm{HBV}$ perinatal transmission, and to investigate risk factors of post-partum hepatic flares in chronic HBV carriers with high viral load (HBV-DNA $>6 \log \mathrm{IU} / \mathrm{ml}$ ), and to observe the effect of post-partum treatment duration on hepatitis flare rates and severity. Method: This study is a single-center, retrospective study. About 459 chronic HBV infected patients with high viral load and normal liver function before pregnancy and gave birth in Beijing Di Tan Hospital from January 2014 to February 2016 were collected. And divided into Groups (1) Patients were divided into non-antiviral therapy (N-AVT) group and AVT group according to whether received antiviral agents in the third trimester of pregnancy. (2) Patients were divided into two groups with normal or abnormal ALT of post-partum (3) Patients were divided into three groups according to post-partum treatment duration, 0-2 weeks for group 1, 4-6 weeks for group 2 and uncertainty for group 3 
Result: Post-partum liver function of AVT during pregnancy for prevention of HBV perinatal transmission Patients were divided into non-AVT group and AVT group according to whether received antiviral agents in the third trimester of pregnancy. 1.1 Baseline Characteristics Except for hepatitis B viral load, other indexes had no significant difference between the two groups $(P>0.05)$. And through logistic regression found that hepatitis B viral load at baseline didn't related with post-partum liver injury. 1.2 Outcomes (1) ALT, AST in non-AVT group were higher than in AVT group(P 0.05).(2) The incidences of ALT more than ULN, 2 ULN and 5 ULN in nonAVT group were higher than in AVT group(P 0.05). The incidences of AST more than 2 ULN and 5 ULN in non-AVT group were higher than in AVT group (P 0.05) and the abnormal rates of TBIL, DBIL and ALB between the two groups. 2 Risk factors of post-partum hepatitis flare Patients were divided into two groups (normal ALT and abnormal ALT group) according to post-partum ALT level. 2.1 Univariate analysis There were significant differences about age, antiviral treatment during pregnancy, ALT level during pregnancy, baseline ALT level between the two groups. 2.2 Multivariate analysis Age elder than 30 [OR $=1.660,95 \%$ CI $(1.086 \sim 2.538)]$ and ALT level during pregnancy $[\mathrm{OR}=2.469,95 \% \mathrm{CI}(1.349 \sim 4.520)]$ were risk factors. ATV in the third trimester of pregnancy [OR $=0.440$, $95 \%$ CI $(0.281 \sim 0.688)]$ was a protective factor. 3 Comparative analyses about post-partum liver function of patients who stopped antiviral agents at different times after delivery. Patients were divided into three groups (Group 1: patients discontinued antiviral agents at 0-2 weeks after delivery, group 2: patients discontinued antiviral agents at 4-6 weeks after delivery and group 3: patients couldn't remember the exact time when discontinued antiviral agents) according to the time of discontinued AVT. 3.1 Baseline Characteristics All observational indexes had no significant differences between the three groups $(P>0.05)$. 3.2 Outcomes (1) There were no significant differences about the post-partum liver function between the three groups $(\mathrm{P}>0.05)$. (2) There were no significant differences about the incidences of ALT higher than ULN, 2 ULN, 5 ULN and AST higher than ULN, 2 ULN and TBIL abnormal(P.

Conclusion: The risk of developing hepatitis flares after delivery was low in these patients who received antiviral treatment in the third trimester of pregnancy. 2 Eld Age, high ALT level during pregnancy increased the risk of developing liver injury after delivery; antiretroviral treatment in the third trimester of pregnancy reduced the risk of developing hepatitis flares after delivery. 3 Discontinued antiviral agents at 0-2 weeks or 4-6 weeks after delivery did not influence the risk of developing post-partum hepatitis flares.

\section{$O-H B V-16$}

A study of comorbidities, coexisting liver conditions, and cancers in chronic hepatitis $B$ patients in Toronto Liver Centre

Marzena Magnes ${ }^{1}$, Marzena Magnes ${ }^{1}$, Sirajuddaula Kausar', Muhammad Khurram Mahmood ${ }^{1}$, Mark Khan ${ }^{3}$, Jean Palmart ${ }^{2}$, Sunny Loo $^{2}$, Marjan Farshadi ${ }^{1}$, Kerstina Boctor ${ }^{1}$, Magdy Elkhashab $^{1}$

${ }^{1}$ Toronto Liver Centre, Canada; ${ }^{2}$ Advisory Physicians Research Services Inc., Canada; ${ }^{3}$ Gilead Sicences Inc, Canada

Background: Despite immunisation, Hepatitis $\mathrm{B}$ remains to be prevalent, especially in endemic areas. In Canada, it is still common among immigrants from endemic areas. The impact of different comorbidities and coexistent liver conditions on the natural history is of significant importance. The present study is a retrospective analysis of a group of hepatitis B patients seen at an urban centre in Toronto.
Method: We reviewed some of the charts of the patients referred to Toronto Liver Centre from 1995 to 2017. Of the total number of 3610 charts reviewed, 894 chronic Hepatitis B patients were identified. All the data, including demographics, comorbidities, prevalence of cancers, and complications, was extracted and entered into a customized electronic case report form. The data was then analysed by MarketSight ${ }^{\circledR}$.

Result: Of the 894 patients, $56.6 \%$ were males and $43.4 \%$ females. Around $21.9 \%$ were below 40 years of age, $26.3 \% 40-49,28.3 \%$ $50-59,14.4 \% 60-69$, and $9.1 \%$ were 70 or older. Pure chronic hepatitis B was identified in $65.4 \%$ of patients and $34.6 \%$ had one or more coexisting liver conditions. These include non-alcoholic fatty liver in $51.1 \%$, focal liver lesion in $20.1 \%$, chronic hepatitis $\mathrm{C}$ in $8.0 \%$. Comorbidities were present in $45.9 \%$ of all and $36.8 \%$ of pure hepatitis B patients. The most common comorbidities were dyslipidemia and hypertension (Figure 1).The prevalence of cancer was $6.8 \%$ in all and $2.7 \%$ in pure hepatitis B patients. The most common cancer was hepatocellular carcinoma (HCC) $(3.1 \%)$ in all, and breast cancer $(0.7 \%)$ in pure hepatitis B patients (Figure 2). Liver and breast cancers were the most common cancers regardless of any coexisting liver condition. The prevalence of cirrhosis was $6.0 \%$ in all patients and $2.6 \%$ in pure hepatitis B patients. It was higher in patients with other coexisting liver conditions than those with pure disease.

Conclusion: More than one third of the patients had other coexisting liver conditions (NAFLD in over 50\%). Components of metabolic syndrome were the most common comorbidities, even in pure hepatitis B patients.

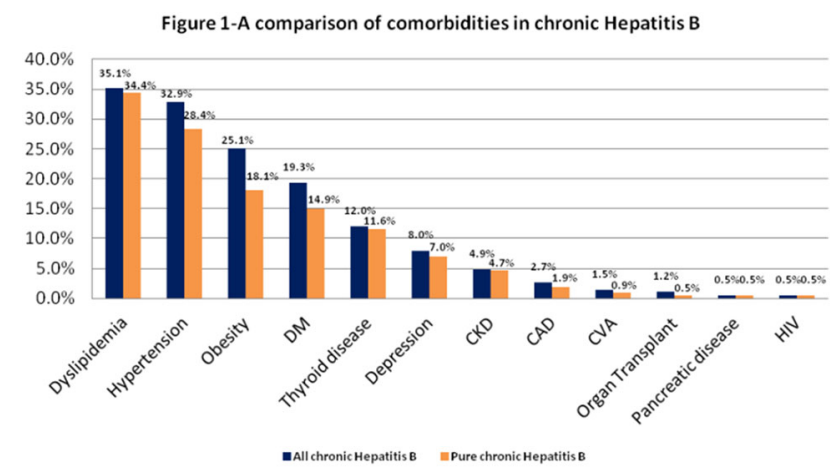

Figure 2-Prevalence of different cancers in chronic hepatitis B

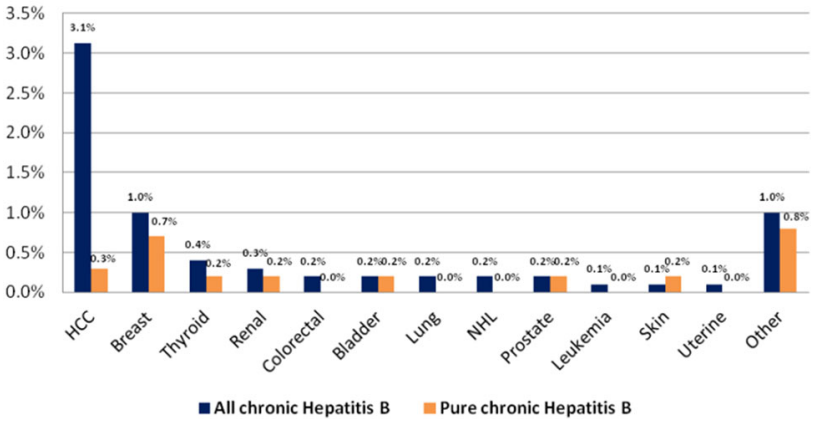




\section{$O-H B V-17$}

Can China achieve 'zero' new HBV-infections $(<0.1 \%$ prevalence in under 5 year olds): disease burden and scenario modelling?

Shevanthi Nayagam ${ }^{1}$, Zheng Hui ${ }^{2}$, Polin Chan $^{3}$, Wang Fuzhen ${ }^{2}$, Miao Ning ${ }^{2}$, Sun Xiaojin ${ }^{2}$, Zundong Yin ${ }^{2}$, Zhang Guomin ${ }^{2}$, Timothy B Hallett ${ }^{1}$

${ }^{1}$ Imperial College, London, United Kingdom; ${ }^{2}$ China CDC, China; ${ }^{3}$ WHO Western Pacific Regional Office, Philippines

Background: In China, the national response to HBV prevention has been strong; with high national coverage of infant vaccination, birth dose vaccination and HBV Immunoglobulin (HBIG). Sequential national serosurveys have already demonstrated a dramatic reduction in chronic HBV prevalence amongst children (0.3\% in 2014). However, in the context of the global elimination targets and the accumulating evidence on the efficacy of reducing vertical transmission further with the use of tenofovir during the last trimester of pregnancy, questions are emerging on the possibility of entirely eliminating the transmission of HBV in China. This study aims to analyse whether current prevention efforts are sufficient to reach a target of $<0.1 \%$ prevalence in under 5 years olds in China, if so by when and to evaluate whether other scale-up strategies could expedite the date of reaching this target?

Method: We developed a simulation model of the HBV epidemic in China, calibrated to HBsAg prevalence, HBeAg prevalence from sequential national serosurveys and HBV-related cancer deaths. We evaluated whether China can reach zero new infections, defined as a target of $0.1 \% \mathrm{HBsAg}$ prevalence in $<5$ year olds. We modelled scenarios to include scaling up HBV vaccination among different target demographics (to include catch-up vaccination and vaccination of healthcare workers) and scale up of prevention of mother to child transmission interventions, to include increasing HBIG coverage and administration of tenofovir to pregnant mothers. We also identified rates of mother-to-child transmission (MTCT) which could be used to evaluate whether China is on course for success.

Result: Maintenance of current high levels of prevention interventions will allow China to reach the target of $<0.1 \% \mathrm{HBsAg}$ prevalence in under 5 year olds by 2029. Drops in coverage will slow down or reverse the decline of new chronic infections. This date can be brought forward to 2024, by further addition of peripartum antiviral therapy for pregnant mothers who are $\mathrm{HBeAg}$ positive, under optimistic assumptions about coverage and effectiveness. Catch-up vaccination among adults and targeting vaccination campaigns among those at risk of exposure do not materially contribute to elimination targets, but do offer individuals benefit and may be cost-effective. Achievement of target by 2025 would be predicted by $<3 \%$ MTCT rate in all $\mathrm{HBs} A g$ positive mothers and $<8.5 \%$ in $\mathrm{HBeAg}+$ mothers. Access to treatment must be increased concomitantly to prevent deaths of the large number of adults already infected.

Conclusion: China is predicted to reach a $<0.1 \% \mathrm{HBsAg}$ prevalence among under 5 year olds by 2029 at current high levels of coverage of prevention interventions. However, the addition of peripartum tenofovir could bring forward the date of achievement of this target by 6 years, if its effectiveness and coverage is high. Further operations research is needed to determine the feasibility and delivery of the widescale expansion of the use of peripartum HBV treatment in China.
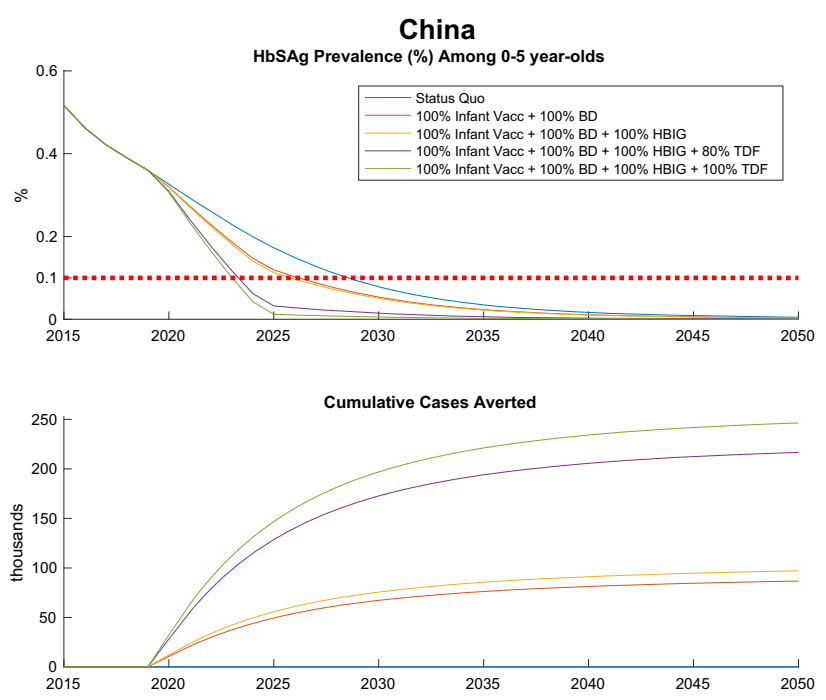

\section{$O-H B V-18$}

Can noninvasive tests predict the regression in hepatic fibrosis? Evaluation of treatment outcomes of hepatitis B patients receiving potent antiviral medication for five years

\section{Serkan Yalaki ${ }^{1}$, Mehmet Suat Yalcin ${ }^{1}$ \\ ${ }^{1}$ Mersin Sehir Hastanesi, Turkey}

Background: Chronic liver disease (CLD) is an important cause of morbidity and mortality worldwide. Hepatitis B virus (HBV) alone causes $10 \%$ of these CLDs. Although complications do not occur in the majority of infected patients, approximately $15-40 \%$ ultimately result in cirrhosis, hepatic failure and hepatocellular carcinoma (HCC). Although hepatic fibrosis may be seen as a regenerative response to liver damage, it is the main factor responsible for the development of cirrhosis. It has been clinically proven that fibrosis and cirrhosis are reversed by the treatment of both viral and nonviral causes underlying inflammation. It is important to be able to demonstrate the presence and severity of fibrosis because the prognosis of CLDs is directly related to the degree of hepatic fibrosis. Biopsy is the gold standard method for evaluating fibrosis in liver diseases. However, liver biopsy is unsuitable for repeated evaluations because it is invasive and can cause major complications, including death. Such limitations have encouraged development of non-invasive methods for assessment of fibrosis. Therefore, several serum biomarkers, combined indices/scores, and imaging techniques for the noninvasive assessment of liver fibrosis have been introduced. The diagnostic value of indirect markers of fibrosis has been demonstrated in numerous studies in chronic hepatitis B patients. In this study, we investigated whether non-invasive tests could predict liver fibrosis regression in naive patients receiving potent antiviral therapy.

Method: This retrospective study was undertaken in Mersin City Hospital. The medical records of patients with a diagnosis of CHB, defined by hepatitis B 's' antigen positivity of more than 6 months duration with a negative anti-HBs antibody, who underwent a liver biopsy between 2008 and 2017 were systematically reviewed and screened for eligibility. Patients with treated potent antiviral therapy were selected from naive patients with liver fibrosis score 2 and above.

Indirect markers of fibrosis: Indirect markers of fibrosis were calculated for each patient based on previously described formulas. (Aspartate aminotransferase/Alanine aminotransferase ratio (AAR), Age/platelet index (API), AST/platelet ratio index (APRI), FIB-4). 
The history of liver biopsies was determined for each patient. This date was accepted as the beginning and the third and fifth year values of the patients were taken. The results of laboratory tests such as platelet (PLT) count and mean platelet volume (MPV) values were noted.

Result: The average age of the patients participating in the study is $48.29+13.79$. Twenty-nine $(30.9 \%)$ of the patients participating in the study were female, and $65(69.1 \%)$ were male. Tenofovir was given to $54(57.4 \%)$ and entecavir to $40(42.6 \%)$ patients. The median values of the initial, third and fifth years of all parameters are measured. There is a statistically significant difference between the 3 measurements in terms of APRI, AAR, FIB-4 score, MPV and PLT distributions in patients receiving tenofovir and entecavir (Friedman $\mathrm{p}<0.05$ ). There was a statistically significant difference between baseline and 3rd year, baseline and 5th year (Wilcoxon $\mathrm{p}<0.016$ Bonferroni correction) in terms of APRI, AAR, MPV and PLT measurements for both drugs according to the post-hoc binary comparison results.

Conclusion: Chronic hepatitis B patients with advanced fibrosis or cirrhosis benefit from antiviral treatment. Five years of ltenofovir and entecavir therapy reduces necroin? Ammatory activity and reverses fibrosis (including cirrhosis) in most patients. As a result, it is known that liver biopsy is the gold standard in the follow-up and treatment of chronic HBV. However, biopsy may not always be possible. In order to predict liver histopathology, various noninvasive tests can be used instead of biopsy.

\section{Session: Hepatitis C Virus Infection; chairpersons-Pham Thi Thu Thuy; Rosmawati Mohammed; Hitender Garg}

\section{$\mathrm{O}-\mathrm{HCV}-07$}

Hepatitis $\mathrm{C}$ in the ED-screening and linkage to care for hepatitis $\mathrm{C}$ infection in the emergency department using point-of-care testing

Joseph Doyle ${ }^{1}$, Jennie Hutton ${ }^{4}$, Rachel Zordan ${ }^{4}$, Anthony Cocco ${ }^{4}$, Sarah Iser $^{4}$, Tracey Weiland ${ }^{5}$, Jessica Howell ${ }^{4}$, Stephanie Fry $^{4}$, ${\text { Kate } \mathrm{New}^{4} \text {, Susanne Glasgow }}^{4}$, Rosemary Sloane ${ }^{4}$, Alisa Pedrana $^{2}$, Bridget Williams ${ }^{2}$, Jennifer Johnson ${ }^{3}$, Sophie Tran ${ }^{5}$, Matthew Jarmin ${ }^{5}$, James Snell ${ }^{5}$, Daniel Phan ${ }^{5}$, Joseph Doyle ${ }^{1}$, Alexander Thompson ${ }^{4}$

${ }^{1}$ Monash University, Australia; ${ }^{2}$ Burnet Institute, Australia; ${ }^{3}$ LaTrobe University, Australia; ${ }^{4}$ St Vincent's Hospital Melbourne, Australia;

${ }^{5}$ University of Melbourne, Australia

Background: Identifying undiagnosed cases of hepatitis $\mathrm{C}$ virus (HCV) infection is central to Australia's effort to eliminate HCV as a public health threat. Emergency department (ED) attendance provides an opportunity for HCV screening which may be particularly useful to reach people facing both significant barriers to accessing health services and high risk of $\mathrm{HCV}$, such as people who inject drugs (PWID). Point-of-care (POC) HCV diagnostics make it possible to provide results at the time of the ED visit, providing an opportunity to fast track $\mathrm{HCV}$ diagnosis and linkage to outpatient treatment. We report on a trial of POC testing for HCV in a metropolitan ED in Melbourne, Australia.

Method: All attendees of the ED over a 92-day study period were assessed for eligibility. Of those eligible, study staff approached patients sequentially to offer study participation. Those who consented then undertook a screening questionnaire to determine the presence of HCV risk factors. Those who identified at least one risk factor for $\mathrm{HCV}$ were then offered screening using the OraQuick HCV antibody test using oral fluid. Those with a reactive result were then offered venepuncture for confirmatory testing and Direct Acting Antiviral (DAA) treatment at the hospital liver clinic.

Result: Over the three months in which the study was conducted there were 3931 presentations to the ED. Of these 1522 patients (39\%) were ineligible. Of the 2409 eligible, 1165 (48\%) participated in the study, $266(11 \%)$ declined, and $978(41 \%)$ were not able to be approached due to time limitations in the emergency room setting. Of the participants, $382(33 \%)$ had one identifiable risk factor and 372 (97\%) underwent the POC HCV antibody test, of whom 50 (13\%) had a positive result. $44(88 \%)$ of these people reported a risk factor of injecting drug use. Of the 72 people who reported a history of injecting drug use $44(61 \%)$ had a reactive POC HCV test result. Of the 50 participants referred to outpatient clinic over a 3-month period, $29(58 \%)$ were HCV PCR positive. Twelve patients (41\%) attended clinic and $8(28 \%)$ commenced DAA treatment. (36\%) of all patients referred to clinic identified as homeless.

Conclusion: Risk factor based screening for $\mathrm{HCV}$ in the metropolitan ED setting identified high risk-factor prevalence, predominantly for PWID. The risk-factor based screening approach using POC tests may be transferable to other settings, to opportunistically reach those at highest risk of $\mathrm{HCV}$.

\section{$\mathrm{O}-\mathrm{HCV}-08$}

High Efficacy and Substantial Improvement of Health-Related Quality of Life (HRQL) in Chinese Patients with Hepatitis C Virus (HCV) Infection Treated with Ledipasvir and Sofosbuvir (LDV/SOF)

Zobair Younossi ${ }^{1}$, Maria Stepanova ${ }^{4}$, Linda Henry ${ }^{4}$, Zhongping $\overline{D u a n}^{3}$, Qing Xie ${ }^{7}$, Jin Lin Hou ${ }^{5}$, Jidong Jia ${ }^{2}$, Wei Lai ${ }^{6}$

${ }^{1}$ Inova Fairfax Hospital, United States; ${ }^{2}$ Beijing Friendship Hospital, China; ${ }^{3}$ Beijing You-An Hospital, United States; ${ }^{4}$ Center for Outcomes Research in Liver Disease, United States; ${ }^{5}$ Nanfang Hospital, China; ${ }^{6}$ Peking University, China; ${ }^{7}$ Shanghai Jiaotong University, China

Background: HCV infection is associated with severe impairment of HRQL. While interferon (IFN) and/or ribavirin (RBV)-containing anti-HCV regimens worsen this impairment, the impact of IFN-free regimens (LDV/SOF) on HRQL of Chinese patients with $\mathrm{HCV}$ has not been reported. Aim: To assess HRQL in Chinese HCV patients treated with LDV/SOF and compare to IFN- and/or RBV-containing regimens.

Method: Chinese HCV patients completed Short Form-36 (SF-36) before, during and after treatment; the HRQL scores were compared between the regimens.

Result: 595 Chinese HCV subjects were enrolled in two phase 3 clinical trials [genotypes (GT) 1, 2, 3 and 6: $62.0 \%, 10.8 \%, 21.2 \%$, $6.0 \%$, respectively; cirrhosis: $15.3 \%$ ]. Patients received IFN + $\mathrm{SOF}+\mathrm{RBV}$ for 12 weeks (GT1 and 6) or SOF + RBV for 12-24 weeks (all GTs) or 12 weeks of LDV/SOF (GT1 only). The SVR-12 rates were $94.6 \%, 94.6 \%$ and $100 \%$, respectively $(\mathrm{p}=0.003)$. Baseline HRQL scores were similar between treatment groups $(p>0.01)$. After 2 weeks of treatment, HRQL scores for patients receiving IFN + SOF + RBV rapidly declined in 6/8 HRQL domains (maximum decline in Bodily pain score: mean -10.3 , $\mathrm{p}<0.001$ ); while HRQL scores for SOF/RBV-treated patients modestly declined in 5/8 domains (maximum decline in Role Physical: mean $-4.1, p=0.0003$ ) and these impairments continued to the end of treatment. In contrast, no significant declines were noted in patients receiving LDV/SOF (all $\mathrm{p}>0.05$ ). Furthermore, patients receiving LDV/SOF experienced improvement of their HRQL as early as 
treatment week 4, and those scores maximized by the end of treatment (Figure). Although post-treatment HRQL scores gradually improved in all IFN-free regimens, the most substantial improvements in HRQL scores were again noted in patients treated with LDV/SOF: subjects who achieved SVR with LDV/SOF had significant improvements in General Health, Vitality, and Mental Health scores with SVR-12 $(\mathrm{p}<0.0001)$.

Conclusion: Unlike anti-HCV regimens that contain IFN and RBV, LDV/SOF in Chinese patients with HCV leads to very high SVR-12 rates and a substantial improvement of HRQL

Figure. Mean Vitality in Chinese patients treated with different regimens.

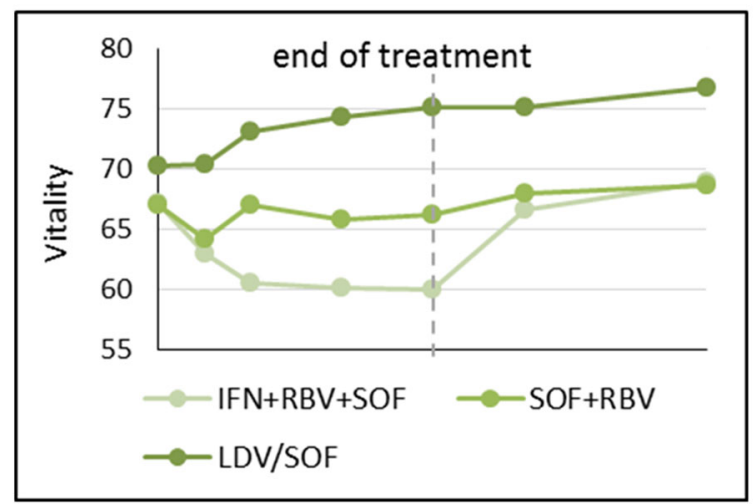

\section{O-HCV-09}

Nosocomal transmission of hepatitis $\mathrm{C}$ viruses $(\mathrm{HCV})$ in a hemodialysis unit: molecular evidence

Elisabeth Lampe ${ }^{1}$, Francisco Campello Do Amaral Mello ${ }^{1}$, Barbara Vieira Do Lago ${ }^{1}$, Vanessa Duarte Da Costa ${ }^{1}$, Giselle Prado Do Nascimento ${ }^{1}$, Paulo Sergio Fonseca De Sousa ${ }^{1}$, Poliana Fernandes Correa $^{1}$, Vincius Mello ${ }^{1}$, Lia Laura Lewisximenez ${ }^{1}$

${ }^{1}$ Fundação Oswaldo Cruz, Brazil

Background: Molecular and epidemiological studies have shown that nosocomial infection is still a relevant form of transmission of hepatitis $\mathrm{C}$ virus (HCV) in patients undergoing hemodialysis (HD). The objective of this study was to evaluate the possible occurrence of HCV transmission in a HD unit located in the state of Rio de Janeiro, who reported seroconversion to anti-HCV in 4 previously negative patients.

Method: A total of 130 patients and 56 employees of the unit were prospectively monitored between December 2016 and March 2017.and HCV-RNA quantification were done using the quantitative Real-Time PCR technique of Abbott platform, with detection limit of $12 \mathrm{IU} / \mathrm{ml}$. For nucleotide sequencing the RT-PCR reaction was used to amplify the NS5B region of the viral genome. The sequences obtained were analyzed phylogenetically in comparison to circulating strains in the country and reference strains

Result: Twenty-three patients $(23 / 130 ; 17.7 \%)$ were positive for HCV-RNA, showing viral loads ranging from $<12$ to $12324.983 \mathrm{IU} /$ $\mathrm{ml}$. All staff were negative $(0 / 53 ; 0 \%)$ for $\mathrm{HCV}-\mathrm{RNA}$. The presence of genotypes 1a $(n=10), 1 b(n-9)$ and $4 a(n=1)$ were detected. Three patients could not be sequenced / genotyped due to low viral load, including one case of recent infection. The phylogenetic analysis of the sequences of this outbreak revealed that four of the nine (44.4\%) samples belonging to genotype $1 \mathrm{~b}$ formed a highly related sequence group, three of them from cases of recent infection and one from a chronic case (index case) demonstrating the existence of a common source of infection. On the other hand, transmission in patients with genotypes 1a and 4a was discarded due to the low similarity found between the sequences (Figure 1).

Conclusion: We identified 18 chronic cases and 4 recent cases of $\mathrm{HCV}$ in the HD unit studied. Phylogenetic analyzes provided evidence of nosocomial transmission among recent cases. These findings demonstrate the importance of molecular analysis in the clarification of $\mathrm{HCV}$ transmission routes and emphasize the need for rigorous adherence to universal biosafety measures in the prevention of $\mathrm{HCV}$ transmission in health units

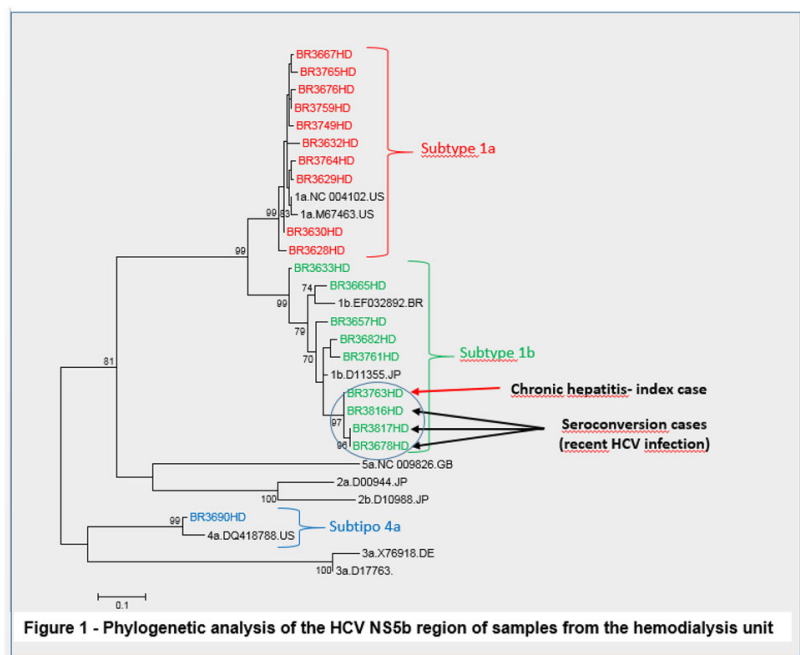

Table 1 Comparison of clinical data between nonalcoholic fatty liver and coronary heart disease group and control group

\section{$O-H C V-10$}

outcomes of sofosbuvir and velpatsavir in chronic hepatitis C G3 patients

\section{Muzzafar Lateef Gill ${ }^{1}$, Ammara Nawaz ${ }^{1}$, Sidra Gill ${ }^{1}$, Arham Baloch $^{1}$, Mehmoona Pervaiz ${ }^{1}$ \\ ${ }^{1}$ Islamabad Gastroenterology Associates, Pakista}

Background: Velpatasvir recently became available in our country. We wanted to study the outcomes of sofosbuvir and Velpatsavir in our chronic hepatitis C G3 patients.

Method: We enrolled 100 consecutive patients with chronic Hepatitis C Genotype 3, who were treatment naïve and without cirrhosis. Exclusion criteria: (1) Patients with platelet count $<125,000$, (2) INR $>1.5$ and (3) FibroScan Value $\geq 12 \mathrm{KPa}$.

Result: $60 \%$ patients were male and $40 \%$ female. Median age 45 years; 96 out of 100 were PCR negative at week 4 (EVR); 2 patients lost follow-up; 94 patients out of 100 were PCR negative at week 12 (ETR); 94 remained HCV PCR negative after 12 weeks of completing the treatment SVR 12; Overall efficacy was $94 \%$.

Conclusion: In our study combination of Sofobuvir and Velpatsavir is very effective and free of side effects in Chronic Hepatitis C G3 patients. Very few patients experienced generalized fatigue and body aches. No other significant side -effects. 


\section{$O-H C V-11$}

Sustained virological response in $\mathrm{HCV}$ patients treated with daclatasvir + sofosbuvir, with or without ribavirin: a large field-practice experience

$\underline{\text { Rodolfo Sacco }}^{1}$, Luigi Elio Adinolfi ${ }^{4}$, Antonio Ascione ${ }^{3}$, Giorgio Barbarini $^{14}$, Angelo Barlattani ${ }^{18}$, Giuseppe Cariti ${ }^{2}$, Raffaele Cozzolongo $^{15}$, Basilio Fimiani ${ }^{10}$, Vincenzo Iovinella ${ }^{5}$, Ruggiero Francavilla $^{12}$, Caterina Furlan ${ }^{17}$, Giovanni Garrucciu ${ }^{19}$, Luca Rinaldi $^{4}$, Massimo Marignani ${ }^{7}$, Paola Begini ${ }^{6}$, Valeria Pace Palitti $^{16}$, Adriano Pellicelli ${ }^{11}$, Gaetano Scifo', Umberto Vespasiani Gentilucci $^{13}$, Giampaolo Bresci ${ }^{1}$, Vincenzo Messina ${ }^{8}$

${ }^{1}$ Pisa University Hospital, Italy; ${ }^{10}$ Umberto Hospital, Italy; ${ }^{11}$ San Camillo-Forlanini Hospital, Italy; ${ }^{12}$ Bisceglie Hospital, Italy; ${ }^{13}$ Campus Biomedico University, Italy; ${ }^{14}$ IRCCS Policlinico San Matteo, Italy; ${ }^{15}$ IRRCSS. De Bellis Hospital, Italy; ${ }^{16}$ Pescara-Penne Hospital, Italy; ${ }^{17}$ Policlinico Umberto 1 , Italy; ${ }^{18}$ Rome Hospital, Italy; ${ }^{19}$ Sassari University Hospital, Italy; ${ }^{2}$ Amedeo di Savoia Hospital Turin, Italy; ${ }^{3}$ Buonconsiglio-Fatebenefratelli Hospital, Italy; ${ }^{4}$ L. Vanvitelli University, Naples, Italy; ${ }^{5}$ San Paolo Hospital, Italy; ${ }^{6}$ Sant ${ }^{\mathrm{TM}}$ Andrea Hospital Rome, Italy; ${ }^{7}$ Sant ${ }^{\mathrm{TM}}$ Andrea Hospital, Italy; ${ }^{8}$ Sant $^{\mathrm{TM}}$ Anna e San Sebastiano Hospital, Italy; ${ }^{9}$ Umberto 1 Hospital, Italy

Background: The combination of daclatasvir (DAC) and sofosbuvir (SOF) in clinical trials achieved sustained virological response at post-treatment week 12 (SVR12) in $>90 \%$ of chronically-infected HCV patients (pts). We evaluated in real-life clinical practice the efficacy of DAC + SOF treatment, with or without ribavirin (RBV), in Genotype 1, 2, 3 and 4 pts with advanced liver disease.

Method: We included 620 consecutive pts fulfilling eligibility criteria of the Italian Drug Agency treated in 19 referral centers of Italy. Fibrosis stage was evaluated by Transient Elastometry (METAVIR F3 $>10 \mathrm{kPa}, \mathrm{F} 4>13 \mathrm{kPa}$ ). Clinical cirrhosis was defined by at least one of the followings: decompensation, oesophageal varices, platelets $<100,000 / \mathrm{mmc}$. Pts received daily SOF + DAC for $12(218)$ or 24 (392) weeks, 248 (40\%) with RBV. The primary efficacy endpoint was SVR12.

Result: Mean age was $60 \pm 9$ years, $63.2 \%$ were males, mean BMI was $24.4 \pm 4.0 \mathrm{~kg} / \mathrm{m} 2,59.5 \%$ were naïve. F4 fibrosis was found in $434(70 \%)$, clinical cirrhosis in $310(50 \%)$. HCV genotypes were: 186 G1 (30\%), 68 G2 (10.9\%), 343 G3 (55.3\%) and 23 G4 (3.7\%). Seven pts $(1.1 \%)$ was co-infected with HBV and $70(11.2 \%)$ with HIV. At baseline, 280/620 (45.1\%) showed HCV RNA $\geq 2 \times 106 \mathrm{IU} / \mathrm{mL}$. Twelve (1.7\%) patients dropped out (3 OLT, 4 HCC, 3 death and 2 lost to follow-up). In pts who completed the study, SVR12 rate was 98\% (596/608): $100 \%$ in G1, $97.5 \%$ in G2, $97.2 \%$ in G3 and $100 \%$ in G4. Virological failures occurred in 8 G2 pts previously treated with $\mathrm{SOF}+\mathrm{RBV}$ and in $4 \mathrm{G} 3$ naïve pts; all had F4 fibrosis (6 clinical cirrhosis) and were treated without RBV. In two (HIV co-infected) G3 pt the viral breakthrough occurred at week 24 of treatment, a classical relapse was observed in the others. On therapy, HCV RNA was undetectable in $30.3 \%$ of the pts at week 2 and in $55.5 \%$ at week 4. At least one adverse events (AE) was reported in $280(46 \%)$ pts; the most frequent one $(30 \%)$ was elevated bilirubin (mean maximum value: $2.2+1.50 \mathrm{mg} / \mathrm{mL}$ ) with grade $3-4$ elevations in $30(4.9 \%)$ and $10(1.6 \%)$, respectively. Anemia was the second AE, reported in $27 \%$ of RBV treated pts and in $18.3 \%$ of the others.

Conclusion: DAC + SOF combination showed an overall $98 \%$ efficacy in an heterogeneous cohort of HCV patients with advanced fibrosis. This study confirms the high efficacy and the pangenotypic action of this combination that also proved well tolerated in clinicalpractice.
$\mathrm{O}-\mathrm{HCV}-12$

The sofosbuvir/velpatasvir single tablet regimen administered for 12 weeks with minimal monitoring in India

Robert H Hyland $^{1}$, Ajay Duseja ${ }^{4}$, Mayank Kabrawala ${ }^{6}$, Robert $\mathbf{H}$ Hyland $^{1}$, Brian Mcnabb ${ }^{1}$, Sophia Lu ${ }^{1}$, Gregory Camus ${ }^{1}$, Luisa M Stamm $^{1}$, Diana M Brainard ${ }^{1}$, Mani Subramanian ${ }^{1}$, Dharmesh Kapoor $^{3}$, . Shalimar ${ }^{2}$, Vivek Saraswat ${ }^{5}$

${ }^{1}$ Gilead Sciences, Inc., United States; ${ }^{2}$ All India Institute of Medical Science, India; ${ }^{3}$ Global Hospital, India; ${ }^{4}$ Post-graduate Institute of Medical Education and Research, India; ${ }^{5}$ Sanjay Gandhi Postgraduate Institute of Medical Sciences, India; ${ }^{6}$ Surat Institute of Digestive Sciences, India

Background: Treatment of chronic hepatitis C virus (HCV) with 12 weeks of sofosbuvir and velpatasvir (SOF/VEL) coformulated as a single tablet regimen has been shown to be safe and effective in Phase 3 studies and real world settings. It is approved in India, Europe, and the USA. The overall safety and high tolerability suggest that SOF/ VEL could be given with minimal safety monitoring, an important consideration for regions with limited medical resources. We tested whether SOF/VEL could be safe and effective when administered in a setting with minimal medical monitoring.

Method: Adult patients with chronic HCV infection of any genotype, including those with prior treatment experience, were eligible to be enrolled. Those with clinical hepatic decompensation, liver transplantation, coinfection with HBV or HIV, or a creatinine clearance Result: One hundred and twenty nine patients were enrolled at 16 sites in India and received at least one dose of the study medication. Most were male $(76,59 \%)$. The median (range) age was 52 (19-75) years and the median (range) BMI was $23(15-40) \mathrm{kg} / \mathrm{m} 2$. The mean (range) HCV RNA was 5.9 (1.9-7.5) $\log 10 \mathrm{IU} / \mathrm{mL}$. Patients were enrolled with HCV genotypes in line with the prevalence in India and included genotype 1 (28, 22\%), $3(89,69 \%)$, and 4 (7, 5\%). Eleven $(9 \%)$ had prior treatment experience. Forty $(31 \%)$ of the patients had cirrhosis. Treatment was well tolerated and there were no discontinuations or interruptions due to AEs; all patients completed therapy. The most frequently reported AEs were headache (4 patients, $3 \%$ ), upper abdominal pain and pyrexia (both 3 patients, $2 \%$ ) which were similar to the most common in the SOF/VEL Phase 3 studies. Grade 3 or 4 laboratory abnormalities were experienced by $12(10 \%)$ patients; the most frequent were decreased haemoglobin (5 patients, $4 \%$ ) and decreased lymphocytes ( 3 patients, $3 \%$ ). Most patients with abnormal laboratory results had had graded abnormalities at screening and/or baseline; none were considered AEs. SVR12 data for all patients will be presented.

Conclusion: SOF/VEL administered without genotype restriction or on-treatment safety assessments was safe and well tolerated. These results suggest that SOF/VEL is a pangenotypic single tablet regimen well suited to use with minimal monitoring in patients in resourcelimited regions. 
Session: NASH-Clinical; Chairpersons- Khin Kyone Kyi; Sanjeev Sachdeva; Sandeep Goyal

O-NAFL-07

The prediction value of liver/spleen CT ratio for the severity of coronary artery stenosis in patients with nonalcoholic fatty liver disease.

\section{$\underline{\text { liang jing }}{ }^{1}$}

${ }^{1}$ Tianjin third central hospital, China

Background: The incidence and mortality of coronary heart disease (CHD) in NAFLD patients were significantly improved.The aim of this study was to investigate the predictive value of computed tomography (CT) ratio of liver/spleen in coronary heart disease (CHD) patients with nonalcoholic fatty liver disease (NAFLD).

Method: 197 patients with nonalcoholic fatty liver disease undergoing coronary angiographywere recruited from 2012 to 2015.All patients were divided into NAFLD with CHD group and NAFLD group according to the diagnosis of coronary angiography. All 158 patients inNAFLDwith CHD groupwere divided into 3 groups according to the CT ratio of liver/spleen:mild, moderate and severe NAFLD group. The severity of coronary artery lesion was compared between these groups.

Result: The Gensini score,number of diseased vessels and the number of the lesions in severe group were higher than mild and moderategroupswith statistically significance $(\mathrm{P}<0.05)$; The alanine transfer enzyme levels in severe group was higher than those in mild group $(\mathrm{P}<0.05)$.The fasting insulin levels in moderate and severe group were higher than mild group $(\mathrm{P}<0.05)$; the insulin resistance index level in severe group is higher than that in mild and moderate$\operatorname{group}(\mathrm{P}<0.05)$.Correlation analysis showed that the liver/spleen $\mathrm{CT}$ ratio were closely associated with Gensini $\operatorname{scores}(\mathrm{r}=-0.848$ $\mathrm{P}<0.01)$

Conclusion: $\mathrm{CT}$ ratio of liver/spleen was associated with the severity of coronary artery disease and it could be used to evaluate the severity of coronary artery disease in CHD patients with NAFLD.

Table 1 Comparison of clinical data between nonalcoholic fatty liver and coronary heart disease group and control group

\begin{tabular}{|c|c|c|c|}
\hline & NAFLD & NAFLD and CHD & tor $x^{2}$ value \\
\hline$n$ & 39 & 158 & \\
\hline Age & $60.03 \pm 6.20$ & $62.56 \pm 8.07^{\circ}$ & 2.15 \\
\hline Gender(male/female) & $26 / 13$ & $108 / 50$ & 0.41 \\
\hline Smoking $(n, \%)$ & $14(35.9)$ & $82(51.9)$ & 3.21 \\
\hline Diabetes $(n, \%)$ & $11(28.2)$ & $49(31.0)$ & 0.12 \\
\hline $\mathrm{TC}(\mathrm{mmol} / \mathrm{L})$ & $4.67 \pm 0.93$ & $5.06 \pm 0.74^{\circ}$ & 2.43 \\
\hline TG (mmol/L) & $1.75 \pm 0.26$ & $1.77 \pm 0.32$ & 0.26 \\
\hline HDL-C (mmo//L) & $1.40 \pm 0.32$ & $1.34 \pm 0.28$ & 1.21 \\
\hline ALT (U/L) & $38.49 \pm 18.83$ & $43.64 \pm 20.53$ & 1.43 \\
\hline $\operatorname{LDL}-\mathrm{C}(\mathrm{mmol} / \mathrm{L})$ & $2.20 \pm 0.60$ & $2.57 \pm 0.42^{*}$ & 3.59 \\
\hline FPG (mmol/L) & $5.16 \pm 0.97$ & $5.51 \pm 0.88^{\circ}$ & 2.16 \\
\hline FINS (mlu/L) & $10.16 \pm 1.71$ & $12.75 \pm 4.33^{* *}$ & 5.87 \\
\hline HOMA-IR & $2.37 \pm 0.75$ & $3.23 \pm 1.53^{*}$ & 5.02 \\
\hline BMI (kg/m2) & $23.78 \pm 1.59$ & $24.75 \pm 2.16^{*}$ & 3.15 \\
\hline Liver/spleen CT ratio & $0.66 \pm 0.10$ & $0.65 \pm 0.18$ & 0.49 \\
\hline Gensini score & $5.33 \pm 3.91$ & $48.29 \pm 13.62^{*}$ & 34.32 \\
\hline
\end{tabular}

Table 2 Comparison of different degrees of non-alcoholic fatty liver combination and coronary heart disease group

\begin{tabular}{|c|c|c|c|c|}
\hline & Mild NAFLD & Moderate NAFLD & Severe NAFLD & \\
\hline$n$ & 58 & 51 & 49 & \\
\hline Age & $61.97 \pm 7.10$ & $62.55 \pm 8.97$ & $63.29 \pm 8.26$ & 0.35 \\
\hline Gender & $40 / 18$ & $36 / 15$ & $32 / 17$ & 0.16 \\
\hline Smoking $(n, \%)$ & $30(51.72)$ & $26(50.98)$ & $26(53.06)$ & 0.04 \\
\hline Diabetes $(n, \%)$ & $17(29.31)$ & $16(31.37)$ & $16(32.65)$ & 0.14 \\
\hline $\mathrm{TC}(\mathrm{mmol} / \mathrm{L})$ & $4.90 \pm 0.65$ & $5.03 \pm 0.77$ & $5.28 \pm 0.78^{\circ}$ & 3.78 \\
\hline $\mathrm{TG}(\mathrm{mmol} / \mathrm{L})$ & $1.75 \pm 0.26$ & $1.78 \pm 0.33$ & $1.77 \pm 0.37$ & 0.13 \\
\hline HDL-C (mmol/L) & $1.37 \pm 0.25$ & $1.38 \pm 0.28$ & $1.27 \pm 0.30$ & 2.37 \\
\hline ALT $(U / L)$ & $40.29 \pm 19.00$ & $46.96 \pm 22.50$ & $44.14 \pm 19.93$ & 1.46 \\
\hline LDL-C (mmol/L) & $2.52 \pm 0.30$ & $2.53 \pm 0.50$ & $2.66 \pm 0.42$ & 1.82 \\
\hline $\mathrm{FPG}(\mathrm{mmo} / \mathrm{L})$ & $5.36 \pm 0.84$ & $5.53 \pm 0.81$ & $5.66 \pm 0.99$ & 1.55 \\
\hline FINS (mlu/L) & $10.93 \pm 2.54$ & $12.27 \pm 3.72^{\circ}$ & $15.39 \pm 5.28^{8 . "}$ & 17.70 \\
\hline HOMA-IR & $2.66 \pm 0.90$ & $3.10 \pm 1.29^{\circ}$ & $4.04 \pm 1.98^{8 . \#}$ & 12.70 \\
\hline Liver/spleen CT ratio & $0.86 \pm 0.07$ & $0.63 \pm 0.05^{\circ}$ & $0.43 \pm 0.04$ & 734.11 \\
\hline BMI (kg/m2) & $24.75 \pm 1.94$ & $24.48 \pm 2.38$ & $25.02 \pm 2.17$ & 0.79 \\
\hline Systolic pressure (mmHg) & $132.43 \pm 19.53$ & $131.38 \pm 20.72$ & $135.73 \pm 19.68$ & 0.65 \\
\hline Diastolic pressure (mmHg) & $76.49 \pm 10.36$ & $77.12 \pm 10.62$ & $77.42 \pm 10.58$ & 0.11 \\
\hline
\end{tabular}

Table 3 The severity of coronary artery lesions among three groups

\begin{tabular}{lccccc}
\hline & \multicolumn{3}{c}{ NAFLD } & F value \\
& Mild & Moderate & Severe & \\
\hline$n$ & 58 & 51 & 49 & \\
GensiniScore & $34.78 \pm 8.44$ & $51.02 \pm 7.70^{*}$ & $61.45 \pm 7.42^{* \#}$ & 115.92 \\
Culprit Arteries & $1.28 \pm 0.55$ & $1.61 \pm 0.57^{*}$ & $2.24 \pm 0.63^{* \#}$ & 36.62 \\
Lesion Number & $1.83 \pm 0.77$ & $2.57 \pm 0.75^{*}$ & $3.36 \pm 0.83^{* \#}$ & 50.78 \\
\hline
\end{tabular}

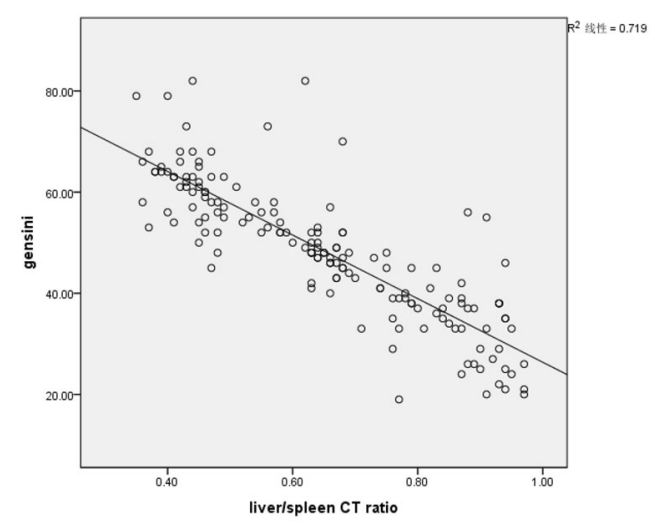

Figure 1 Correlation analysis of liver/spleen CT ratio and Gensini scores . $r=-0.848 \quad P<0.01$ 

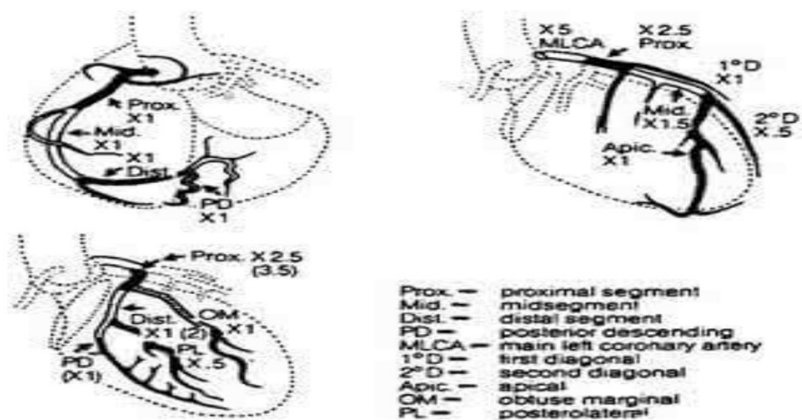

Figure 2 Gensini Score standard

$O-N A F L-08$

Effect of weight reduction on histological activity and fibrosis of lean nonalcoholic steatohepatitis patient

\section{Shahinul Alam $^{1}$, Golam Mustafa ${ }^{1}$, Saiful Islam ${ }^{1}$, Nooruddin} Ahmad $^{1}$

${ }^{1}$ Bangabandhu Sheikh Mujib Medical University, Bangladesh

Background: Weight reduction has evidenced benefit on attenuation of histological activity and fibrosis of nonalcoholic steatohepatitis (NASH), but there is scarcity of data for lean NASH subgroup. We have designed this study to compare effects of weight reduction on histological activity and fibrosis of lean and non lean NASH.

Method: We have included 20 lean and 20 non lean histologically proven NASH patients. BMI

Result: Age, sex, ALT, AST, GGT, HOMAIR, triglyceride, HDL was similar in both group. Non lean had higher incidence of hypertension $(\mathrm{p}=.018)$ and diabetes $(\mathrm{p}=.004)$. Steatosis, ballooning, lobular inflammation, nonalcoholic fatty liver disease activity score (NAS) and fibrosis was similar in 2 groups (Table 1). Weight was reduced in any amount (8/11), 5\% (5/6) and 7\% (2/6) in lean/non lean patients. In lean group weight reduction of any amount was associated with significant reduction of steatosis, ballooning and NAS but not lobular inflammation and fibrosis. Weight reduction of 5\% could significantly reduced steatosis, ballooning and lobular inflammation and NAS but not fibrosis in this group. But in non lean group NAS but not fibrosis could significantly improved with $7 \%$ weight reduction only (Table 2).

Conclusion: Smaller amount of weight reduction had greater benefit of improvement of all the segments of histological activity (NAS) in lean than that of non lean NASH. Fibrosis was not reduced with weight reduction
Taste I: Base lies ctarscterbitio of kesa atd sea kas NASH patias

\begin{tabular}{|c|c|c|c|}
\hline Verchlin & $\operatorname{lem}_{N=15}$ & $\sum_{N=16}$ & P \\
\hline 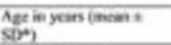 & $34 \mathrm{sa} \pm 8 \mathrm{KS}$ & $3789+5.83$ & 253 \\
\hline somictanks & $E$ & 610 & 50 \\
\hline BMIly & $2326=1.10$ & 27.4423 .59 & $\$ 60$ \\
\hline 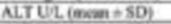 & $46.53+2535$ & $5725+25.48$ & 252 \\
\hline AstuL (men-so) & $3533+15.02$ & $628+259$ & 400 \\
\hline 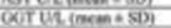 & 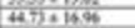 & श्रीभ. $2 \times 10$ & d. \\
\hline LOMA IR Incen is SW & 1500107 & $210=159$ & $5 \pi$ \\
\hline 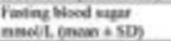 & 4.500 .78 & $5 \times 872150$ & 40 \\
\hline 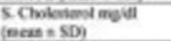 & $195.06 \div-50.01$ & 201.55600 .35 & $\pi m$ \\
\hline 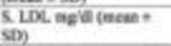 & $11639 \div 41.92$ & $6.64+36.87$ & 375 \\
\hline $\begin{array}{l}\text { shax acta (ancon: } \\
\text { sid) }\end{array}$ & $30203=42 x$ & $3 \times 4=28 \pi$ & 912 \\
\hline 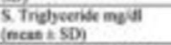 & 22230.161 .141 & 3805.22031 & $2 \%$ \\
\hline 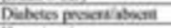 & Gis & 79 & 60 \\
\hline 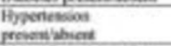 & 174 & 79 & six \\
\hline 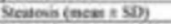 & $20=5$ & $260 \pm 57$ & 786 \\
\hline Ballenning fenen +50 & 1.53 .52 & $144+51$ & ses \\
\hline 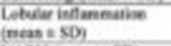 & 1.736 .59 & 1000.88 & $\$ 14$ \\
\hline $\mathrm{NAS}^{*-}(\operatorname{men} \geq \mathrm{SD})$ & $5.27+46$ & $519+.4$ & ans \\
\hline Fitrous $\cos +50)$ & $1.47+.94$ & $1.62+1.03$ & 628 \\
\hline
\end{tabular}

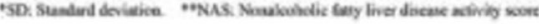

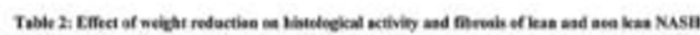

\begin{tabular}{|c|c|c|c|c|c|c|}
\hline & Len & & & Sinten & & \\
\hline Verieter & Broselifer & Aher I yeen & f & Baseline & Ace l yeer & \\
\hline $\begin{array}{l}\text { Wriph in le } \\
\text { (mean + } 50 \text { ) }\end{array}$ & $5 \times 336756$ & $57.47 \div 735$ & 250 & $6 \times 35+409$ & 4.112931 & ass \\
\hline $\begin{array}{l}\text { Whipli } \\
\text { indaction } \\
\text { rovine }\end{array}$ & & 27 & & & 116 & 370 \\
\hline $\begin{array}{l}\text { Stemovis (inesing } \\
\text { isD) }\end{array}$ & 291059 & $137+0 \times 01$ & 301 & 2000044 & $19=6.81$ & 016 \\
\hline $\begin{array}{l}\text { Batiberite } \\
\text { (nemesing }\end{array}$ & 1216002 & 1.2160 .42 & DES & 1556051 & $100 \div 030$ & 016 \\
\hline 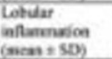 & $1.81 \neq 0.50$ & 1.9220 .48 & 355 & $101=831$ & $136 \geq 0.51$ & .192 \\
\hline 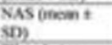 & $5.26 * 0.56$ & $3.59 \div 094$ & 900 & $5.27=0.65$ & $400 \div 69$ & 000 \\
\hline $\begin{array}{l}\text { Fitwovin (mean } \\
\text { isp }\end{array}$ & $1.42 \pm 0.04$ & $0.11 \pm 2.6$ & Sis & $1,35=1.04$ & $D .18=0.60$ & 291 \\
\hline $\begin{array}{l}\text { Weidhit } \\
\text { moduction } 5 \%\end{array}$ & & $5 / 10$ & & & का & sm \\
\hline $\begin{array}{l}\text { Sherbovs inean } \\
\text { (SD) }\end{array}$ & $2.00 \times 0.65$ & $1.36=0 \times 5$ & Dil & $2.17=0.41$ & $107=6.81$ & 9) \\
\hline $\begin{array}{l}\text { Batlowing } \\
\text { (men }+ \text { Sin) }\end{array}$ & $1,45+0.52$ & $1.09+0.30$ & gis. & 1336.052 & $1.00+.000$ & 173 \\
\hline 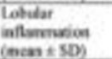 & $1.33 \pm 0.6$ & $1.18 \pm 0.41$ & 566 & 16570.52 & $1.17 \approx 6.41$ & 076 \\
\hline $\begin{array}{l}\text { Nis imanit } \\
\text { sol }\end{array}$ & $3.18 \times 0.41$ & 3.460 .81 & .060 & $5.17 \cdot 0.41$ & $3.85=6.75$ & 010 \\
\hline 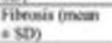 & $1.45 \pm 0.69$ & $1.18+060$ & 062 & T55064 & DJank & 363 \\
\hline 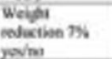 & & 2013 & & & का० & .124 \\
\hline $\begin{array}{l}\text { Siesoven (inen } \\
\text { isD) }\end{array}$ & $2.12 \pm 0.6$ & 1.0250 .44 & an & $2.15=0.41$ & $|n|=6 \times 5$ & 0.76 \\
\hline 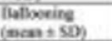 & 13840.52 & 1.1220 .55 & .50 & 133.6052 & $100 \div 600$ & 175 \\
\hline $\begin{array}{l}\text { Lotular } \\
\text { nftormution }\end{array}$ & $1.78 \div 0.46$ & 1.12 .0 .35 & 911 & $1.67+0.52$ & $1.17 * 0.41$ & 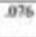 \\
\hline $\begin{array}{l}\text { XAs fincen : } \\
\text { so) }\end{array}$ & $5.25 \div 0.40$ & $3.58 \div 0.64$ & का 1 & $5.17=0.41$ & $3 \times 1 \times=63$ & 060 \\
\hline $\begin{array}{l}\text { ntrowis imean } \\
\text { isd }\end{array}$ & $1.90 \div 0.76$ & 1.25 .671 & .178 & $1.55=824$ & $133=0.8$ & 453 \\
\hline
\end{tabular}




\section{$O-N A F L-09$}

\section{Comparison of diagnostic accuracy of fibroscan and APRI for detecting liver fibrosis in chronic hepatitis B patients combined with non-alcoholic fatty liver disease}

\section{Meijie Shi ${ }^{1}$, Shanshan $\mathrm{Lin}^{2}$, Huanming Xiao ${ }^{1}$, Meijie Shi ${ }^{1}$, Lexin Liu $^{1}$, Xiaoling Chi ${ }^{1}$ \\ ${ }^{1}$ Guangdong Provincial Hospital of Chinese Medicine, China; \\ ${ }^{2}$ GuangzhouUniversity of Chinese Medicine, China}

Background: To comparethe diagnostic accuracy of Fibroscan and APRI for detecting liver fibrosis in Chronic Hepatitis B patients combined with Non-alcoholic Fatty Liver Disease (NAFLD).

Method: In all, $161 \mathrm{CHB}$ patients with NAFLD performed liver biopsy and Fibroscan from May 2014 and July 2017were enrolled in this study. Logistical regression and Area under receiver-operator curve (AUC) were used to determine thediagnostic accuracy of simple tests for liver fibrosis.

Result: Among the 161 patients, 35, 39, 48, 28, 11 patients showed S0?S1?S2?S3?S4, respectively.The summary AUROC values using Fibroscan and APRI for detecting significant fibrosis $(\mathrm{S} \geq 2)$, advanced fibrosis $(S \geq 3)$ and cirrhosis $(S=4)$ were 0.853 vs. 0.758 $(\mathrm{P}<0.05), \quad 0.881 \mathrm{vs}$. $0.742 \quad(\mathrm{P}<0.05)$, and 0.886 vs. 0.717 $(\mathrm{P}>0.05)$. The pooled sensitivity and specificity using Fibroscan for the diagnosis of significant fibrosis, advanced fibrosis and cirrhosis were $83.9 \% \%$ and $74.3 \%, 76.9 \%$ and $89.3 \%, 100 \%$ and $69.3 \%$, respectively. The pooled sensitivity and specificity using APRI for the diagnosis of significant fibrosis, advanced fibrosis and cirrhosis were $73.6 \%$ and $67.6 \%, 71.8 \%$ and $64.8 \%, 72.7 \%$ and66\%, respectively.

Conclusion: Fibroscan is more accurate than APRI in diagnosing liver fibrosis in CHB patients with NAFLD, especially in diagnosing significant fibrosis and advanced fibrosis.

\section{$O-N A F L-10$}

Non-alcoholic hepatic steatosis and liver features among chronic hepatitis $B$ patients

Nazli Begum Ozturk ${ }^{1}$, Enise Yagmur Ozkan ${ }^{1}$, Mertcan Avci ${ }^{1}$, Raim Iliaz $^{1}$, Alp Atasoy ${ }^{1}$, Filiz Akyuz ${ }^{1}$, Kadir Demir ${ }^{1}$, Sabahattin Kaymakoglu $^{1}$, Mine Gulluoglu ${ }^{1}$, Fatih Besisik ${ }^{1}$

${ }^{1}$ Istanbul University, Istanbul Faculty of Medicine, Turkey

Background: Non Alcoholic Steato Hepatitis (NASH)'s incidence is increasing and it is the main cause of chronic liver disease around last decades. Frequency of Chronic Hepatitis $\mathrm{B}(\mathrm{CHB})$ infection is decreasing, however it is still important in low middle income countries. The studies related $\mathrm{CHB}$ infection and co existance of NASH show that patients with $\mathrm{CHB}$ are lower incidence of NASH. Our study's aim is show the hepatic steatosis frequency in CHB.

Method: 279 patients included the study whose HBsAG positive and $\mathrm{HBV}$ DNA $>2000 \mathrm{IU} / \mathrm{ml}$ at least 6 months, from 2013 to 2017 years. To evaluate hepatic steatosis and its percentage, liver biopsy was performed to all patients. ISHAK score was used to evaluate the histologic features. Patients with chronic alcohol consumption were excluded from the study. The patients features were compared both hepatic steatosis and non-found hepatic steatosis groups. SPSS 11 was used for statistical analysis. Statistical significance was defined as $\mathrm{p}<0,05$.

Result: Patients were compared in 2 groups. Hepatic steatosis was found $31.8 \%(n=88)$ in patients with CHB. Male patients were more likely to have hepatic steatosis than female patients (38.8\% vs $21.4 \%$ $\mathrm{p}=0.002)$. Patients with $\mathrm{CHB}$ with hepatic steatosis were older than CHB with non-found hepatic steatosis ( 48 vs $42, p<0.001$ ). In nonfound hepatic steatosis and hepatic steatosis groups mean laboratory values were, AST (42 vs $36, p=0.45$ ), ALT (77 vs $65, \mathrm{p}=0.57$ ), GGT (26 vs $33, p=0.2)$ and ALP (79 vs $74, p=0.39)$. In hepatic steatosis group vs non-found hepatic steatosis group height (173 vs $168 \mathrm{p}=0.003)$, weight $(83$ vs $73 \mathrm{p}<0.001)$, BMI $(27.7$ vs 25.8 $\mathrm{p}=0.004)$ were statistically significant. Although total cholesterol (195 vs $181 \mathrm{p}=0.26$ ), C-Reactive Protein(3 vs 2,4) and fasting plasma glucose(101 vs $96 \mathrm{p}=0.39)$ values were higher, but they were not statically significant. HBV DNA was lower in the non-found hepatic steatosis group however it was statically similar $(27,419$ vs $24,223 \mathrm{p}=0.79)$. HBeAg values were similar in these groups. Histologically activity index $(6.6$ vs $5.5 \mathrm{p}=0,002)$, portal inflammation( 2.3 vs $2.1 \mathrm{p}=0.02$ ), periportal and periseptal hepatitis(2.1 vs $1.7 \mathrm{p}=0.004)$ were higher in hepatic steatosis group. Fibrosis stage was advanced in patients with hepatic steatosis (2.5 vs $2.3 \mathrm{p}=0.04)$.

Conclusion: We found that NASH existence with $\mathrm{CHB}$ especially in older, male patients. We also investigate advanced fibrosis stage in patients with $\mathrm{CHB}$ and NASH unlike the literature. We are planning to widen this study and compare in bigger groups.

\section{$O-N A F L-11$}

Combining hepato-renal ratio (HRR) and shear wave elastography (SWE): the way forward in NAFLD?

Aby Somu ${ }^{1}$ Aby Somu ${ }^{1}$, Maya Peethambaran ${ }^{1}$, M Prashanth ${ }^{1}$, Shibi Mathew ${ }^{1}$, John Mathews ${ }^{1}$, Prakash Zachariass', Mathew Philip $^{1}$

${ }^{1}$ PVS Memorial Hospital, India

Background: The current gold standard for evaluation of NAFLD is liver biopsy, which is invasive. The Hepato-renal ratio (HRR) by ultrasound is a non-invasive simple method that could be used to diagnose significant hepatic steatosis. HRR may be combined with shear wave elastography (SWE) for evaluation of patients with significant steatosis.

Aim: To study the usefulness of combining HRR and SWE in evaluating patients with NAFLD.

Method: The study was conducted in the department of Medical Gastroenterology, PVS memorial hospital, Kochi. Consecutive adult patients with the diagnosis of fatty liver on qualitative ultrasound were selected. The same operator performed all ultrasound examinations. Patients were divided into two groups, HRR $<1.49(<5 \%$ steatosis) and HRR $>1.49$ ( $>5 \%$ steatosis). Liver stiffness measurements (LSM) were recorded using SWE and further stratified according to the METAVIR stage.

Result: Total number of patients were 96.67 patients were males, 29 were females. 34 patients had diabetes and 16 patients were obese and 44 patients were overweight. 22 patients had features of metabolic syndrome. 35 patients had HRR $<1.49$, were as 61 patients had HRR $>1.49$. All patients in the first group with $\mathrm{HRR}<1.49$ and didn't have significant liver stiffness $(<7.1 \mathrm{kPA})$. In the second group with $\mathrm{HRR}>1.49,12$ patients had F2 METAVIR stage, 5 had F3 and 11 patients had F4 stage, rest of the patients were F0-F1. Fibrosis was seen only in the second group of patients with HRR $>1.49$, which was significant statistically ( $\mathrm{p}$ value 0.001 )

Conclusion: (1) Only those patients with significant steatosis as per HRR had fibrosis. (2) The combination of HRR and SWE may help in ideal utilisation of resources.

Limitations: (1) No biopsy correlation. (2) Small sample size. 


\section{$O-N A F L-12$}

\section{Correlation of hepatic steatosis with hepatic fibrosis by fibroscan} in NAFLD patients.

\author{
Varun Mehta $^{1}$ Rishabh Sehgal ${ }^{2}$, Monika Garg ${ }^{2}$, Ramit Mahajan ${ }^{2}$, \\ Ajit Sood $^{2}$ \\ ${ }^{1}$ DMC, India; ${ }^{2}$ B.F.UH.S, India
}

Background: Worldwide NAFLD prevalence ranges from 6- 35\%. The progression of disease is characterized by steatosis, ongoing inflammation and consequent fibrosis. Higher quantity of hepatic fat appears to be an important pathogenic mechanism rather than just an innocent bystander. There is an ongoing debate whether the quantity of hepatic steatosis has correlation with stage of fibrosis. So a study was conducted to find correlation of steatosis and fibrosis in these patients.

Method: All NAFLD patients coming to Dayanand Medical College and Hospital Ludhiana, Punjab for fibroscan examination from 1/1/17 to $30 / 11 / 17$ were analysed for the presence of correlation between hepatic steatosis and hepatic fibrosis using controlled attenuation parameter (CAP) and liver stiffness measurement $(\mathrm{kPa})$.

Result: There were total 446 patients, $312(69.9 \%)$ were males and $134(30.1 \%)$ were females. 9 patients were between age group 0-20 years, 141 were between $20-40$ and 269 were more than $40 \mathrm{yr}$ old. Out of 446 patients 19 (4.2\%) were having steatosis of grade 0 (S0) with mean $\mathrm{kPa}$ of 5.33, $73(16.3 \%)$ were having steatosis of grade 1(S1) with mean $\mathrm{kPa}$ of 5.96, $46(10.3 \%)$ were having steatosis of grade 2 (S2) with mean $\mathrm{kPa}$ of $6.4,308(69 \%)$ were having steatosis of grade 3 (S3) with mean $\mathrm{kPa}$ of 7.63. The difference between these groups was significant $(\mathrm{p}=0.011)$. Overall mean $\mathrm{CAP}(\mathrm{db} / \mathrm{m})$ was $310.5( \pm 61.3)$ and mean $\mathrm{kPa}$ was $7.13( \pm 4.8)$ and there was a significant correlation between the two parameters (Pearson correlation $0.139 ; \mathrm{p}=0.003)$. Also significant correlation was found between $\mathrm{CAP}$ and $\mathrm{kPa}$ in males (Pearson correlation 0.136; $\mathrm{p}=0.016$ ) and with increasing age (Pearson correlation 0. 243; $\mathrm{p}=.004)$.

Conclusion: We conclude that quantification of steatosis by CAP has significant correlation with stage of fibrosis especially in males and with increasing age. The study highlights the need to treat steatosis to prevent fibrosis.

\section{Session: Hepatitis B Virus Infection- Clinical;}

Chairperson; David Handojo Muljono; Manav Wadhawan; Tanmay Vyas

\section{$O-H B V-19$}

\section{Changes of serum CHI3L1 predict changes of liver fibrosis} assessed by Fibroscan or liver biopsies after antiviral treatments for chronic HBV patients

\section{Biaoyang Lin ${ }^{1}$, Yunhua Liu ${ }^{3}$, Weikun $\mathrm{Li}^{3}$, Hongfei Zhang', Yingying $\mathrm{Ma}^{4}$}

${ }^{1}$ Zhejiang Univeristy, United States; ${ }^{2}$ The 302 PLA Hospital, China; ${ }^{3}$ the Third Hospital of Kunming City, China; ${ }^{4}$ Zhejiang Univ, China

Background: $\mathrm{CHI} 3 \mathrm{~L} 1$ is a new marker that have been recently demonstrated to be a excellent non-invasive marker for liver fibrosis caused by various factors including $\mathrm{HBV}, \mathrm{HVC}$, excessive drinking and fatty liver diseases. Secreted by the hepatic stellate cells, CHI3L1 is involved in active liver fibrosis. In this study, we analyzed the utility of changes of serum $\mathrm{CHI} 3 \mathrm{~L} 1$ protein levels in monitoring changes of liver stiffness and antiviral treatment responses of chronic HBV patients.

Method: $201 \mathrm{HBV}$ patients subjected to active treatments at the Third Hospital of Kunming City were enrolled in the study. The treatments include the uses of antiviral nucleoside and nucleotide analogue inhibitors (NAs) or peg2a (pegylated interferon alfa-2a) or the combination of the two. The patients were subjected to Fibroscan. In addition, their serum samples were analyzed for the CHI3L1 concentrations using a CHI3L1 ELISA assay with Chinese FDA approval and CE mark permit. In this analysis, two points recorded as week 0 and week 24 were included.

Result: 201 patients were subjected to the Fibroscan measurement of the liver stiffness at week 0 and week 24 . At the same time, their serum CHI3L1 levels were analyzed at week 0 and week 24. In 201 patients with both the Fibroscan data and the serum CHI3L1 data, we found that, in 95 patients $(95 / 201=47.4 \%)$, the reduction of liver stiffness as measured by Fibroscan is consistent with the reduction of serum CHI3L1 levels. In 189 patients with both the Fibroscan and the APRI measurements, only in 61 patients $(61 / 189=32.3 \%)$ we observed consistency between the reduction of APRI and the reduction by the Fibroscan measurements. Similarly, in 189 patients with both the Fibroscan and FIB4 measurements, only in 62 patients (62/ $189=32.8 \%$ ), consistency between the reduction of FIB4 and the reduction of the Fibroscan measurements were observed. In addition, we found that, in 11 patients $(11 / 201=5.5 \%)$, the liver stiffness measured by Fibroscan increased, and their serum CHI3L1 levels also increased. Together, we observed that, in 106 patients (106/ $201=52.7 \%$ ), the changes of liver stiffness measured by Fibroscan were consistent with the changes of serum CHI3L1 levels. We then analyzed the 95 patients showing consistent reduction of both CHI3L1 and Fibroscan measurement. We found that reduction of APRI or FIB4 were seen only in 54 (56.8\%) and 56 (59\%) of these 95 patients respectively, suggesting that the serum CHI3L1 is far more consistent with Fibroscan than APRI or FIB4. Taking together, these data suggest that serum CHI3L1 is better than APRI and FIB4 in measuring changes of liver stiffness defined by Fibroscan. Finally, for 4 patients, we measured changes of CHI3L1 reductions in serum samples of patients under antiviral treatments and changes of fibrosis stages measured by liver biopsies, we found that the reduction of serum CHI3L1 levels are consistent with reductions of liver fibrosis stages assessed by pathology reports from the four liver biopsies (Table 1).

Conclusion: Changes of serum CHI3L1 levels can be used as an alternative surrogate marker for monitoring changes of liver stiffness and in monitoring treatment responses of $\mathrm{HBV}$ patients subjected to antiviral treatments.

\begin{tabular}{|c|c|c|c|c|c|c|}
\hline $\begin{array}{l}\text { Time } 1 \text {-liver } \\
\text { fibrosis }\end{array}$ & $\begin{array}{l}\text { Time 2 - } \\
\text { liver } \\
\text { fibrosis }\end{array}$ & $\begin{array}{l}\text { Time 1- } \\
\text { Fibro-CHI } \\
\text { test }\end{array}$ & $\begin{array}{l}\text { Time 2- } \\
\text { Fibro-CHI } \\
\text { test }\end{array}$ & $\begin{array}{l}\text { Serum CHI3L1 } \\
\text { reduction }\end{array}$ & $\begin{array}{c}\text { Fibrosis stage } \\
+ \\
\text { inflammation } \\
\text { reduction }\end{array}$ & $\begin{array}{l}\text { The period } \\
\text { between time } 1 \\
\text { and } 2 \text { (months) }\end{array}$ \\
\hline $\mathrm{G} 2, \mathrm{~S} 2-3$ & $\mathrm{G} 1, \mathrm{~S} 2$ & 61.39 & 26.90 & 34.49 & 2 & 14 \\
\hline $\mathrm{G} 3, \mathrm{~S} 3$ & G0-1,S2-3 & 55.35 & 31.40 & 23.95 & 2 & 11 \\
\hline G2,S2-3 & $\mathrm{G} 1, \mathrm{~S} 1$ & 44.38 & 39.70 & 4.68 & 2 & 11 \\
\hline G3,S3-4 & $\mathrm{G} 2, \mathrm{~S} 3$ & 69.02 & 64.00 & 5.02 & 1 & 12 \\
\hline
\end{tabular}




\section{$O-H B V-20$}

\section{Characterization of the hepatitis B virus DNA detected in urine of chronic hepatitis $B$ patients}

Wei Song ${ }^{1}$, Surbhi Jain ${ }^{1}$, Ying Hsiu $\mathrm{Su}^{4}$, Yih Ping $\mathrm{Su}^{4}$, Sierra Mccloud $^{1}$, Ruixia Xue ${ }^{5}$, Taijung Lee ${ }^{4}$, Shu Chuan Lin ${ }^{2}$, Jamin D Steffen $^{3}$, Selena Y Lin ${ }^{6}$

${ }^{1}$ JBS SCIENCE, United States; ${ }^{2}$ Buddhist Tzu Chi General Hospital and Tzu Chi University, Taiwan; ${ }^{3}$ Hepron Molecular Lab, United States; ${ }^{4}$ The Baruch S. Blumberg Institute, United States; ${ }^{5}$ The Second Hospital of Yuncheng, China; ${ }^{6} \mathrm{U}$ Screen Dx, United States

Background: To assess the potential infectivity of urine from patients with chronic hepatitis B virus (HBV) infection (CHB) and correlate urine HBV DNA level with clinical parameters.

Method: Urine from $60 \mathrm{CHB}$ patients with serum viral loads ranging from undetectable to $108 \mathrm{IU} / \mathrm{mL}$ were analyzed for HBV DNA and serum immune markers. HBV DNA was detected from total urine DNA and size-fractionated urine DNA (separated into $\leq 1 \mathrm{~kb}$ and $>1 \mathrm{~kb}$ fractions) by PCR analysis of six regions of the HBV genome.

Result: Twenty-seven of 59 (45.7\%) patients with HBV serum viral load $(\geq 20 \mathrm{IU} / \mathrm{mL})$ contained detectable, fragmented HBV DNA in urine in at least 1 of the 6 PCR assay regions. Only one patient contained HBV DNA detected by all six regions, and was found to have evidence of blood in the urine. Sixteen of 25 urine samples with high viral load $(>105 \mathrm{IU} / \mathrm{mL})$ and 11 of 34 urine samples with low viral load $(<105 \mathrm{IU} / \mathrm{mL})$ contained detectable HBV DNA. Twelve of 27 (44.44\%) patients with detectable HBV DNA in urine were $\mathrm{HBeAg}$ positive, and only 5 of these $\mathrm{HBeAg}$ positive patients were in the group of $33(15.15 \%)$ patients with no detectable HBV DNA in urine. By Fishers' exact test, HBV DNA in urine is significantly associated with high serum viral load $(P=0.0197)$ and $\mathrm{HBeAg}$ $(P=0.0203)$.

Conclusion: We conclude that urine from $\mathrm{CHB}$ patients with healthy kidney function should not contain full-length HBV DNA, and therefore should not be infectious.

\section{$O-H B V-21$}

CHI3L1 performs better than APRI, FIB-4 and GP73 as a noninvasive marker for the staging of liver fibrosis

Biaoyang Lin ${ }^{1}$ Longgen Liu ${ }^{2}$, Tong $\mathrm{Yan}^{2}$, Zhen $\mathrm{Zhu}^{2}$, Zuowei $\mathrm{Bao}^{2}$, Tianmin $\mathrm{Xu}^{2}$, Jing $\mathrm{Xie}^{2}$, Shuqin Zheng ${ }^{2}$, Manman $\mathrm{Cui}^{2}$, Yingying $\mathrm{Ma}^{3}$, Tiangang $\mathrm{Wu}^{3}$, Hong $\mathrm{Li}^{2}$

${ }^{1}$ Zhejiang univeristy, United States; ${ }^{2}$ The third people's hospital of Changzhou, China; ${ }^{3}$ Zhejiang Univ, China

Background: Non-invasive analysis of liver fibrosis is preferable to liver biopsy for indication of antiviral treatments and monitoring treatment responses for chronic HBV (CHB) patients. APRI, FIB-4, CHI3L1 and GP73 have all been proposed for staging of liver fibrosis in CHB patients. We compared the performance of these markers with histological analysis of liver fibrosis stages or inflammation scores from biopsy samples of $\mathrm{CHB}$ patients.

Method: $144 \mathrm{CHB}$ patients were enrolled in the study. Serum samples from these patients were analyzed using ELISA assays for CHI3L1 and GP73, and for routine biochemical analysis for the determination of the aspartate aminotransferase (AST)-to-platelet ratio index (APRI) and the fibrosis index based on four factors (FIB4) scores. In addition, histological readings of fibrotic stages (Metavir scores) and inflammation scores were performed from biopsy samples of these patients. Fibroscan were also analyzed for these patients.

Result: We did correlation analyses of CHI3L1 and GP73 versus histological readings, fibroscan, APRI and FIB4 (Table 1). CHI3L1 showed moderately positive correlations with liver fibrotic stages ( $\mathrm{S}$ stages) $(\mathrm{R} 2=0.304)$, and with Fibroscan $(\mathrm{R} 2=0.334)$, but showed lower positive correlations with FIB-4 $(\mathrm{R} 2=0.193)$ and with inflammation scores $(\mathrm{G}$ scores) $(\mathrm{R} 2=0.165)$ (Table 1$)$. However, we did not observe much correlations between GP73 and liver fibrotic stages or Fibroscan or FIB-4 (Table 1). GP73 showed a very weak correlation with $\mathrm{G}$ scores $(\mathrm{R} 2=0.076)$. We also noted that CHI3L1 showed very little negative correlation with ALT, suggesting that ALT levels are not a major factors affecting the performance of CHI3L1 in assessing liver fibrosis status. Next we did a categorical analysis using Cohen's kappa coefficient, which measures inter-rater agreement for qualitative (categorical) items, for the ability of CHI3L1 and GP73, respectively, to correctly assign the patients into the different fibrotic stages determined by histological analysis of biopsy samples. For a simplified analysis, we only used three categories: S2 or below, S3 and S4. We found that only CHI3L1 has a kappa value of 0.335 , which translated into a fair agreement with the S-stage categories designated by histological stages (Metavir scores). All of the other markers APRI, FIB-3 and GP73 returned kappa values less than or equal to $0.1(0.026,0.1,-0.02$ respectively), suggesting that fibrotic stages determined by CHI3L1 are the only one showing a fair agreement with fibrotic stages determined by biopsies. Considering that histological readings are not perfect with errors as big as $25-30 \%$, and serum analysis assesses the fibrotic status of liver as a whole in contrast to the very limited and local sampling by biopsies, even a perfect marker is unlikely to achieve very high agreement with the fibrotic stages from histological analysis.

Conclusion: CHI3L1 is the best non-invasive marker among the 4 markers that we analyzed (CHI3L1, APRI, FIB-4, GP73) and Fibroscan for staging of liver fibrosis stages determined by histological readings from biopsies.

Table 1. Correlation coefficient analysis of CHI3L1 and GP73 vs. histological readings, fibroscan, APRI and FIB4.

Correlation coefficient

\begin{tabular}{c|c|c|c|c|}
\hline CHI3L1 vs. & & Sampele Size & GP73 vs. & Sampele Size \\
\hline APRI & 0.05695 & 148 & 0.0143 & 146 \\
\hline FIB4 & 0.1929 & 148 & -0.03308 & 146 \\
\hline HA & 0.09253 & 147 & 0.002497 & 145 \\
\hline Fibroscan & 0.3343 & 66 & -0.08012 & 66 \\
\hline S Stages & 0.3041 & 103 & -0.02006 & 102 \\
\hline G scores & 0.1654 & 117 & 0.07573 & 115 \\
\hline & & & & \\
\hline ALT & -0.05377 & 148 & 0.06988 & 146 \\
\hline
\end{tabular}

\section{$O-H B V-22$}

Correlation of Caspase-cleaved cytokeratin18 (CK18-Asp396) fragment M30 with hepatic histological severity (activity) in patients with chronic hepatitis B (CHB)

Kazi Zakir Hossain ${ }^{1}$, Noor Uddin Ahmad ${ }^{2}$, Most Rokshana Begum $^{1}$, Faiz Ahmed Khondoker', Sheikh MD Noor E Alam², Jahangir Alam Sarker ${ }^{1}$, Partho Protik Roy ${ }^{2}$, Mamun Al Mahtab ${ }^{2}$

${ }^{1}$ Shaheed Suhrawardy Medical College Hospital, Bangladesh;

${ }^{2}$ Bangabandhu Sheikh Mujib Medical University, Bangladesh 
Background: Chronic hepatitis B virus (HBV) infection remains a serious public health problem affecting more than 400 million people worldwide. Cytokeratin (CK) 18 is an intermediary filament protein, expressed in hepatocytes, which is proteolytically cleaved during liver damage. M30 epitope of cytokeratin18 (CK18-M30) is involved at different levels in apoptotic pathways. In this study, we aimed to investigate whether serum CK-18 fragment M30 level significantly correlate with the histological severity (activity) in patients with chronic hepatitis B virus infection.

Method: A total of 40 patients with chronic hepatitis B (CHB) were enrolled in this study between January to August 2016. All CHB cases underwent liver biopsy for METAVIR score. Serum CK-18 M30 was measured by ELISA in all patients.

Result: Of the total 40 patients, all were $\mathrm{HBsAg}$ positive, $24(60.0 \%$ ) were $\mathrm{HBeAg}$ negative and $16(40 \%)$ were $\mathrm{HBeAg}$ positive. The mean ALT and AST, HBV DNA levels were $66.4 \pm 28.20 \mathrm{IU} / \mathrm{L}$, $40.2 \pm 20.2 \mathrm{IU} / \mathrm{L}$ and $5.3 \pm 1.7 \mathrm{IU} / \mathrm{ml}$ in logarithmic scale respectively. Among them significant histological activity (A2, A3) were found in $12(30 \%)$ of $\mathrm{HBeAg}$ positive cases And 21 (58.3\%) of $\mathrm{HBeAg}$ negative cases. Mean Serum CK -18 fragment M30 level $128.8 \pm 32.9$ was HBeAg Positive CHB and 123.9 \pm 28.1 in $\mathrm{HBeAg}$ Negative CHB. Results showed that serum concentrations of CK18 were not significantly increased in a stepwise fashion from A0 to A3. A significant correlation was found between ALT, AST level and the METAVIR histological activity scores $(P=0.036$ and 0.016$)$. We found no correlation between the histological activity score and Serum CK-18 fragment M30 level ( $r=-0.073 ; p=0.357)$.

Conclusion: This study indicates there is no correlation between serum CK-18 fragment M30 level and hepatic histologic severity (activity) in patients with chronic hepatitis B virus infection.

\begin{tabular}{|c|c|c|}
\hline Investigation & Range (max, min) & Mean $\pm S D$ \\
\hline Age (in years) & 18,50 & $26.5 \pm 7.7$ \\
\hline Sex (male/female) & - & $31 / 9$ \\
\hline $\mathrm{Hb} \%$ & $11.5,16.2$ & $13.7 \pm 1.3$ \\
\hline TC & 5,12 & $7.4 \pm 1.7$ \\
\hline Neutrophil(\%) & 41,77 & $60.1 \pm 9.4$ \\
\hline Lymphocyte (\%) & 18,46 & $31.2 \pm 7.8$ \\
\hline Monocyte (\%) & 1,8 & $4.5 \pm 1.9$ \\
\hline Eosinophil (\%) & 0,15 & $4.2 \pm 3.0$ \\
\hline Basophil (\%) & 0,2 & $0.10 \pm 0.37$ \\
\hline Platelet count ( $\left.{ }^{*} 10^{9} / \mathrm{L}\right)$ & 100,400 & $234.3 \pm 70.1$ \\
\hline *HBV DNA PCR (IU/ml) & $2.2,8.8$ & $5.3 \pm 1.7$ \\
\hline AST (UL) & 19,118 & $40.2 \pm 20.2$ \\
\hline $\operatorname{ALT}(\mathrm{U} / \mathrm{L})$ & 22,397 & $66.4 \pm 28.2$ \\
\hline Prothrombin Time (sec) & $10.5,15.2$ & $12.4 \pm 0.7$ \\
\hline INR & $0.88,107$ & $1.1 \pm 0.2$ \\
\hline GGT & 12,110 & $30.2 \pm 17.9$ \\
\hline
\end{tabular}

All values are expressed as mean $\pm \mathrm{SD}$ or number (\%)

* HBV DNA PCR values were transformed into logarithmic scale.
Table 2: Distribution of the study patients according to HBeAg status ( $n=40$ )

\begin{tabular}{|c|c|c|c|}
\hline HBeAg status & $\begin{array}{l}\text { All patients } \\
\qquad(n=40)\end{array}$ & $\begin{array}{c}\text { HBeAg Positive } \\
\text { CHB } \\
(n=16)\end{array}$ & $\begin{array}{c}\text { HBeAg Negative CHB } \\
\text { (n=24) }\end{array}$ \\
\hline *Age & $26.5 \pm 7.7$ & $24.9 \pm 9.2$ & $27.6 \pm 6.7$ \\
\hline${ }^{* *}$ Sex (male/female) & $31 / 9$ & $13 / 3$ & $17 / 7$ \\
\hline $\mathrm{Hb} \%$ & $13.7 \pm 1.3$ & $13.7 \pm 1.5$ & $13.7 \pm 1.3$ \\
\hline TC & $7.4 \pm 1.7$ & $7.1 \pm 1.7$ & $7.7 \pm 1.7$ \\
\hline Platelet count ( $\left(10^{9} / \mathrm{L}\right)$ & $234.3 \pm 70.1$ & $218.5 \pm 45.1$ & $244.8 \pm 81.9$ \\
\hline *HBV DNA PCR (IU/ml) & $5.3 \pm 1.7$ & $7.0 \pm 0.9$ & $4.2 \pm 1.0$ \\
\hline AST (UL) & $40.2 \pm 20.2$ & $42.3 \pm 17.8$ & $38.8 \pm 21.9$ \\
\hline $\operatorname{ALT}(U / L)$ & $66.4 \pm 28.2$ & $62.1 \pm 21.5$ & $69.3 \pm 32.0$ \\
\hline Prothrombin Time (sec) & $12.4 \pm 0.7$ & $12.3 \pm 0.7$ & $12.4 \pm 0.8$ \\
\hline INR & $1.1 \pm 0.2$ & $1.1 \pm 0.6$ & $1.2 \pm 0.2$ \\
\hline GGT & $30.2 \pm 17.9$ & $32.6 \pm 23.6$ & $28.6 \pm 13.2$ \\
\hline \multicolumn{4}{|l|}{ Activity score } \\
\hline AO & $0(0.0 \%)$ & $0(0.0 \%)$ & $0(0.0 \%)$ \\
\hline $\mathrm{A} 1$ & $7(17.5 \%)$ & $4(25.0 \%)$ & $3(12.5 \%)$ \\
\hline $\mathrm{A} 2$ & $21(52.5 \%)$ & $8(50.0 \%)$ & $13(54.2 \%)$ \\
\hline A3 & $12(30.0 \%)$ & $4(25.0 \%)$ & $8(33.3 \%)$ \\
\hline
\end{tabular}

All value analysis of *Mean $\pm S D$ and

** number of patients

Table-3: Correlation between factors and activity score of necroinflammation (METAVIR) on spearman correlation test

\begin{tabular}{ccc}
\hline Factors & $\begin{array}{c}\text { Correlation co-efficient(r } \\
\text { value })\end{array}$ & p-value \\
\hline Age & 0.204 & $0.206^{\text {ns }}$ \\
GGT & 0.287 & $0.087^{\text {ns }}$ \\
ALT & 0.333 & $0.036^{\mathrm{s}}$ \\
AST & 0.360 & $0.016^{\mathrm{s}}$ \\
Prothombin time & 0.150 & $0.356^{\mathrm{ns}}$ \\
S.CK-18 m30 & -0.073 & $0.357^{\mathrm{ns}}$ \\
HBV DNA PCR & -0.098 & $0.549^{\text {ns }}$ \\
Platelet count & -0.289 & $0.070^{\text {ns }}$ \\
\hline
\end{tabular}

$O-H B V-23$

Efficacy and safety of combination therapy with interferon and immunomodulators in entecavir-suppressed chronic hepatitis B patients (the Endeavor study)

Jinshang $\mathrm{Hu}^{1}$, Peng Wang ${ }^{1}$, Meifang Han ${ }^{1}$, Yongping Chen ${ }^{10}$, ${ }_{\text {Xinyue Chen }}{ }^{3}$, Qi Xia ${ }^{11}$, Chuanlong Zhu' ${ }^{2}$, Qin Xie ${ }^{8}$, Jiaji Jiang ${ }^{4}$, Lai $\mathrm{Wei}^{7}$, Deming Tan ${ }^{12}$, Xiaoguang Dou', Yanyan $\mathrm{Yu}^{6}$, Jinlin $\mathrm{Hou}^{5}$ 
${ }^{1}$ Tongji Hospital, Tongji Medical College, Huazhong University of Science and Technology, China; ${ }^{10}$ The First Affiliated Hospital of Wenzhou Medical University, China; ${ }^{11}$ The First Affiliated Hospital, School of Medicine, Zhejiang University, China; ${ }^{12}$ Xiangya Hospita, China; ${ }^{2}$ Anhui Provincial Hospital, Anhui Medical University, China; ${ }^{3}$ Beijing You'an Hospital, Capital Medical University, China; ${ }^{4}$ First Affiliated Hospital of Fujian Medical University, China; ${ }^{5}$ Nanfang Hospital, Southern Medical University, China; ${ }^{6}$ Peking University First Hospital, China; ${ }^{7}$ Peking University People's Hospital, China; ${ }^{8}$ Shanghai Ruijin Hospital, Jiaotong University School of Medicine, China; ${ }^{9}$ Shengjing Hospital of China Medical University, China

Background: The ideal endpoint for anti-HBV therapy is the loss of hepatitis B surface antigen (HBsAg). The purpose of the study was to determine the efficacy and safety of sequential combination therapy with interferon (IFN), recombinant human IL-2 (rhIL-2) and therapeutic vaccine in patients treated with long-term entecavir (ETV).The ideal endpoint for anti-HBV therapy is the loss of hepatitis B surface antigen (HBsAg). The purpose of the study was to determine the efficacy and safety of sequential combination therapy with interferon (IFN), recombinant human IL-2 (rhIL-2) and therapeutic vaccine in patients treated with long-term entecavir (ETV).

Method: In this pilot and proof of concept, multicentre and randomized trial, $94 \mathrm{HBeAg}$ positive chronic hepatitis B patients who had received ETV for at least 1 years, with HBV DNA $\leq 1000$ copies/ml and hepatitis B e antigen ( $\mathrm{HBeAg}$ ) loss, were randomly assigned $(1: 1: 1)$ to receive ETV $(0.5 \mathrm{mg} /$ day, oral) for 48 weeks (Group I) or IFN- $\alpha-2 b$ (600 wIU every other day, subcutaneous) for 48 weeks (Group II) or IFN- $\alpha-2 b$ for 48 weeks in combination with rhIL-2 (25 wIU every other day, subcutaneous) for 12 weeks plus vaccine ( $60 \mathrm{ug} / \mathrm{month}$, intramuscular) for 48 weeks (Group III). The primary endpoint was HBsAg loss at week 48 (ClinicalTrials.gov: NCT02360592). Peripheral immune cells were evaluated dynamically.

Result: 94 patients were randomized; 93 received?1 study drug dose. One patient who were $\mathrm{HBeAg}$-positive at baseline was excluded from the modified intention-to-treat population (group I, $\mathrm{n}=27$; group II, $\mathrm{n}=33$; group III, $\mathrm{n}=32$ ). At week $48,3.70 \%$ of subjects in group I, $3.03 \%$ of subjects in group II and $9.38 \%$ of subjects in group III achieved HBsAg loss. Mean HBsAg decline from baseline to week 48 was significantly greater in group III $(0.85 \log 10 \mathrm{IU} / \mathrm{mL})$ and group II $(0.74 \log 10 \mathrm{IU} / \mathrm{mL})$ than in groups I $(0.13 \log 10 \mathrm{IU} / \mathrm{mL}$, respectively, $\mathrm{P}<0.05$ for all comparisons vs group I). Among the patients with $100 \mathrm{IU} / \mathrm{ml}<\mathrm{HBsAg}<1500 \mathrm{IU} / \mathrm{ml}$ at randomisation, $25 \%$ achieved HBsAg loss and mean HBsAg declined $1.65 \log 10 \mathrm{IU} /$ $\mathrm{mL}$ from baseline to week 48 in group III, displaying a significant benefit comparing with group I $(0 \%$ and $0.04 \log 10 \mathrm{IU} / \mathrm{mL}$, respectively), while no significant difference was found between group III and group II (6.67\% and $0.61 \log 10 \mathrm{IU} / \mathrm{mL}$, respectively) (Figure 1). Besides, patients receiving combination treatment showed a significantly higher increase in the CD56bright CD16-NK cells proportions at week 4, patients with good response to combination treatment exhibited a significant decline in Treg proportions from week 12 to week 24 .

Conclusion: For ETV suppressed patients, particularly those with low serum HBsAg level, sequential combination therapy with IFN and immunomodulators may enhance HBsAg loss and led to partial immune restoration.
$O-H B V-24$

Ezetimibe for patients with chronic hepatitis D

$\underline{\text { Zaigham Abbas }}^{1}$, Muhammad Saad ${ }^{1}$, Muhammad Asim ${ }^{1}$, Shoukat $\overline{A l i}^{1}$, Minaam Abbas ${ }^{1}$

${ }^{1}$ Dr. Ziauddin University Hospital, Pakistan

Background: Sodium taurocholate cotransporting polypeptide (NTCP) is the receptor for hepatitis B virus (HBV) and hepatitis D virus (HDV). Ezetimibe, a cholesterol lowering drug, possesses the pharmacophore features to inhibit NTCP. The ability of this drug to impair viral entry using a HDV in vitro infection model based on a NTCP-expressing Huh7 cell line has been documented before. The aim of the study is to evaluate the utility of ezetimibe in patients with chronic HDV infection.

Method: This is a proof of concept phase 2 trial evaluated the efficacy and safety of ezetimibe $10 \mathrm{mg}$ daily in interferon treatment experienced or interferon ineligible patients. Primary therapeutic end point was a decline of HDV RNA $>1 \log$ from baseline at 12 weeks of therapy. Secondary end point was normalization of serum ALT.

Result: The total number of patients was 20, male, 17, age (mean years) $35.9 \pm 9.2$. Clinical cirrhosis was present in 14. Mean HDV RNA level $(\log 10 \mathrm{IU} / \mathrm{ml})$ was $7.5 \pm 1.1$. HBeAg was non-reactive in $18(95 \%)$. HBV DNA was non-detected in $12(63 \%)$. Eight (44\%) patients were on concomitant nucleoside therapy. One patient stopped the treatment at week 4 . According to per protocol analysis $(n=19)$, one $\log$ reduction was seen in $11(58 \%)$, no $\log$ reduction in 5 and $\log$ increase in 3 patients. 10/11 patient with baseline viral load $<6 \log$ responded compared to $1 / 8$ patients with viral $\operatorname{load}>6 \log$ $(p=0.003)$. No effect of concomitant nucleoside analog use or clinical cirrhosis was seen. The drug had a good safety profile.

Conclusion: Treatment of chronic hepatitis D patients with ezetimibe resulted in one log reduction of viral load in 58\% (11/19) of patients who completed 3 months therapy. Further studies are warranted with use in treatment naïve patients, with higher doses of ezetimible or longer duration alone or in combination with other agents.

\section{Session: Hepatitis C Virus Infection. Chairperson; Oidov Baatarkhuu; S V Rana; Amrish Sahney}

\section{$O-H C V-13$}

Tolerable and curable treatment in HIV/HCV co-infected patients by using anti-HCV direct antiviral agents: a real-world observation in China

\section{Da Wei Zhang ${ }^{1}$}

\section{${ }^{1}$ Beijing 302 Hospital, China}

Background: No direct-acting antiviral agents (DAA) is available for treatment of hepatitis $\mathrm{C}$ virus (HCV) patients with thuman immunodeficiency virus-1 (HIV-1) co-infection in China yet, this real-world study aimed to observe the therapeutic safety and efficacy of generic DAAs for HCV/HIV-1 co-infected Chinese patients.

Method: In this real-world study, $176 \mathrm{HIV}-1 / \mathrm{HCV}$ co-infected patients who received anti-HCV DAA treatment together with free of charge ART regimens for HIV infection. Among the 176 patients, 99 patients were treated with sofosbuvir/daclatasvir \pm ribavirin regimen,60 patients with ledipasvir/sofosbuvir \pm ribavirin regimen and 17 patients sofosbuvir/ribavirin \pm Peg-IFN regimen, for 8 or 12 weeks respectively. The primary endpoint of these regimens was 
undetectable HCV RNA at week 12 after the therapy was completed (SVR12). The data of safety, side effects were analyzed.

Result: There were $151 \mathrm{HIV}-1 / \mathrm{HCV}$ co-infected patients who finished the treatment and follow-up check. The date revealed the SVR12 for the patients treated with regimens of sofosbuvir/daclatasvir, sofosbuvir/daclatasvir + ribavirin, sofosbuvir/PegIFN + ribavirin, sofosbuvir/ribavirin, ledipasvir/sofosbuvir, ledipasvir/sofosbuvir + ribavirin for 12 or 24 weeks is $100 \%(75 / 75)$, $100 \%$ (11/11), $100 \%(14 / 14), 95.24 \%(40 / 42)$, and $100 \%(7 / 7)$ respectively. It was found that $\mathrm{HCV} / \mathrm{HIV}-1$ co-infected patients with liver cirrhosis and decompensated cirrhosis also achieved higher SRV12. The frequent adverse events were fatigue, nausea, and muscle pain among the treated patients. Notably, no statistical difference of adverse effects among patients with different baseline CD4 $+\mathrm{T}$ cell counts were found when they received sofosbuvir/daclatasvir or ledipasvir/sofosbuvir regimen. In addition, three or two patients receiving sofosbuvir/daclatasvir or sofosbuvir/daclatasvir + ribavirin regimens respectively discontinued DAA therapy when they considered the possible adverse effects. Two patients relapsed at week 12 since the suspension of ledipasvir/sofosbuvir treatment.

Conclusion: The findings in the real-world observation showed generic sofosbuvir/daclatasvir and ledipasvir/sofosbuvir regimens were better tolerable, less adverse events and high efficient for HIV-1/ $\mathrm{HCV}$ co-infected patients with more than $200 / \mu \mathrm{l}$ of baseline CD4 + T cell counts, compared with the DAA regimens containing Peg-INF and ribavirin. Further study needs to be conducted to confirm the safety and efficacy of DAA treatment in large-cohort HCV/HIV-1 patients.

\section{$\mathrm{O}-\mathrm{HCV}-14$}

Twelve weeks of ledipasvir/sofosbuvir all-oral regimen for patients with chronic hepatitis $C$ genotype 2 infection: integrated analysis of three clinical studies

ChenYu Wang ${ }^{1}$, Chunjen Liu', Edward Gane ${ }^{2}$, Yoshito Itoh ${ }^{5}$, Norifumi Kawada ${ }^{7}$, Yoshiyuki Ueno ${ }^{9}$, Chenyu Wang ${ }^{3}$, Joe Llewellyn $^{3}$, Anu Osinusi ${ }^{3}$, Jenny Svarovskaia ${ }^{3}$, Hongmei $\mathbf{M o}^{3}$, Gerald Crans ${ }^{3}$, Wanlong Chuang ${ }^{4}$, Peijer Chen ${ }^{6}$, Nobuyuki Enomoto $^{8}$

${ }^{1}$ Gilead Sciences, Taiwan; ${ }^{2}$ Auckland Clinical Studies Limited, New Zealand; ${ }^{3}$ Gilead Sciences, United States; ${ }^{4}$ Kaohsiung Medical University Hospital, Taiwan; ${ }^{5}$ Kyoto Prefectural University of Medicine, Japan; ${ }^{6}$ National Taiwan University College of Medicine and Hospital, Taiwan; ${ }^{7}$ Osaka City University, Japan; ${ }^{8}$ University of Yamanashi, Japan; ${ }^{9}$ Yamagata University, Japan

Background: HCV genotype (GT) 2 is the second most common genotype infecting a significant number of patients in several Asian countries including Japan, Taiwan and Korea. Treatment options for chronic HCV GT2 infection remain limited in these countries. The once-daily fixed-dose combination tablet of ledipasvir/sofosbuvir (LDV/SOF) was evaluated for the treatment of GT $2 \mathrm{HCV}$ infection, in patients without cirrhosis or with compensated cirrhosis, in three phase 2 and 3 studies. This integrated analysis describes the safety and efficacy of LDV/SOF for 12 weeks across these 3 clinical studies.

Method: This was a retrospective analysis of subjects treated with LDV/SOF for 12 weeks in the GS-US-337-1655 (Taiwan), GS-US337-1903 (Japan) and GS-US-1468 (New Zealand) studies. Subjects analyzed in this integrated analysis were either mono-infected with HCV GT2 (Japan and New Zealand studies), or co-infected with HCV GT2 and HBV (Taiwan study). The data was pooled and safety and efficacy were analyzed.
Result: Overall 200 subjects were treated and analyzed; $88 \%$ of subjects were Asian, $46 \%$ male, $31 \%$ had prior treatment failure, $15 \%$ were cirrhotic, $25 \%$ were IL28B non-CC, and $34 \%$ were 65 years or older. A total of $22 \%(n=43)$ were co-infected with HBV (HBsAg positive). The overall SVR rate was $97 \%$ (194/200), and was $93 \%$ (27/29) among patients with cirrhosis and 97\% (59/61) in patients who had failed previous therapy. Of the 197 patients with available testing; NS5A resistance-associated substitutions (RASs) were present in $84 \%(165 / 197)$ of patients at baseline. SVR12 rate was $98 \%$ (161/165) in patients with baseline NS5A RASs compared with $100 \%$ (32/32) in patients without NS5A RASs. No new RASs emerged in patients with virologic failure. Treatment with LDV/SOF for 12 weeks was well tolerated. Overall the most common adverse events AEs $(>5 \%)$ were headache and nasopharyngitis. Few subjects experienced serious AEs, 3\% (6/200), none of which were assessed as treatment related. One patient discontinued treatment due to $\mathrm{AE}$.

Conclusion: Treatment with LDV/SOF for 12 weeks is highly effective and well tolerated in patients with GT2 HCV infection, including patients who are treatment experienced and/or have compensated cirrhosis, baseline NS5A RASs and with $\mathrm{HBV} / \mathrm{HCV}$ coinfection.

\section{$O-H C V-15$}

Early occurrence and recurrence of hepatocellular carcinoma in HCV-related cirrhosis treated with direct-acting antivirals

\section{Ali Abdelrahman Ghweil}

${ }^{1} \mathrm{Head}$ of the tropical medicine and gastroenterology, Egypt

Background \& Aims: Hepatocellular carcinoma (HCC) represents a serious complication of $\mathrm{HCV}$-related cirrhosis. New direct-acting antivirals (DAA) cure HCV infection in over $90 \%$ of patients. The aim of this study was to evaluate the early occurrence and recurrence of HCC in cirrhotic patients treated with DAA.

Method: We analysed 180 consecutive cirrhotic patients, without HCC, who were treated with DAA, and followed for 24 weeks. 20 patients had previous.

Result: DAA therapy induced sustained virological response in $94 \%$ of patients. During 24-week follow-up, HCC was detected in 11 patients $(5.5 \%$,) 6 of 20 patients $(30 \%$, with previous $\mathrm{HCC}$ and 5 of 180 patients $(2.78 \%$, without previous HCC. Child-Pugh Class B, more severe liver fibrosis, lower platelet count, and previous HCC were significantly associated with HCC development, at univariate analysis. At multivariate analysis, Child-Pugh class $(\mathrm{p}=0.03$, ) and history of HCC $(p<0.0001$, resulted independently associated with HCC development. Among the 20 patients with previous HCC, younger age and more severe liver fibrosis were significantly associated with $\mathrm{HCC}$ recurrence, both at univariate and at multivariate analysis

Conclusions: In patients with HCV-related cirrhosis, DAA-induced resolution of $\mathrm{HCV}$ infection does not seem to reduce occurrence of $\mathrm{HCC}$, and patients previously treated for $\mathrm{HCC}$ have still a high risk of tumour recurrence, in the short term. For these reasons, all cirrhotic patients should be closely monitored and followed during and after antiviral therapy. 


\section{$\mathrm{O}-\mathrm{HCV}-16$}

A comparison of non-invasive clinical scores to predict advanced liver fibrosis in the ambulatory setting for Hepatitis $\mathrm{C}$ patients with a history of intravenous drug abuse in a multi-ethnic population

\section{Ragesh Babu Thandassery ${ }^{1}$, Jessica Yi Lyn Tan ${ }^{2}$, Prem Harichander Thurairajah $^{2}$, Eng Kiong Teo ${ }^{2}$, John Hsiang ${ }^{2}$ \\ ${ }^{1}$ Hamad Medical Corporation, Qatar; ${ }^{2}$ Changi General Hospital, Singapore}

Background: Hepatitis $\mathrm{C}(\mathrm{HCV})$ remains a major clinical concern among people with history of intravenous drug abuse (IVDA), as most will go on to develop chronic infection leading to liver cirrhosis and hepatocellular carcinoma. Accurate identification of patients with advanced fibrosis is important and utility of non-invasive clinical scores can especially helpful in IVDA patients, who have poorer access to specialist care and high frequency of defaulting medical visits

Method: This is a retrospective study on all HCV patients who were managed in Changi General Hospital, Singapore with history of IVDA, between 2011 to 2016. Data collected include liver stiffness measurement (LSM) by transient elastography. Previously established clinical scores in predicting advanced fibrosis were tabulated and validated by analysing the area under receiver operating characteristic curve (AUROC). Sensitivity, specificity, positive and negative predictive values were examined. Data was analysed by SPSS version 21 with statistical significance defined as $p<0.05$.

Result: A total number of 579 patients were recruited for this study, with $537(92.7 \%)$ male patients. There were $134(23.1 \%)$ Chinese patients, $78(13.5 \%)$ Indian patients and $347(59.9 \%)$ Malay patients. The mean age was 47.5. A majority of patients have HCV genotype 3 (77.7\%). AST-to-platelet ratio index (APRI), modified APRI and Fibrosis-4 (FIB-4) scores were valid in predicting F3 (defined as LSM > 9.5 kPa) (APRI:0.767, (95\% CI: 0.729-0.805); modified APRI:0.779, (0.741-0.816); FIB-4: 0.757, (0.718-0.796)) and F4 (defined as LSM > 12.5 kPa) (APRI:0.770,(0.727-0.814); modified APRI:0.796, (0.754-0.838); FIB-4: 0.789, (0.747-0.830)). Modified APRI was noted to have outperformed APRI in predicting F4 $(\mathrm{p}=0.0023)$. Scores lower than 7.09 were found to have a negative predictive value of $89.5 \%$, scores higher than 19 was found to have a positive predictive value of $77.1 \%$, with a specificity of $96.2 \%$. There was no significant difference between the scores for prediction of F3 stage. Previously established cut-off values for APRI and FIB-4 were found to be inaccurate for the population, especially for F3. For APRI and FIB-4, the established lower cut-off values of 0.5 and 1.45 respectively were noted to poor negative predictive values of $77.8 \%$ and $72.2 \%$ for $\mathrm{F} 3$ fibrosis respectively.

Conclusion: Non-invasive scores are useful for predicting advanced fibrosis in HCV patients with history of IVDA, with modified APRI appearing the better score. However, further studies are required to establish more accurate cut-off values.
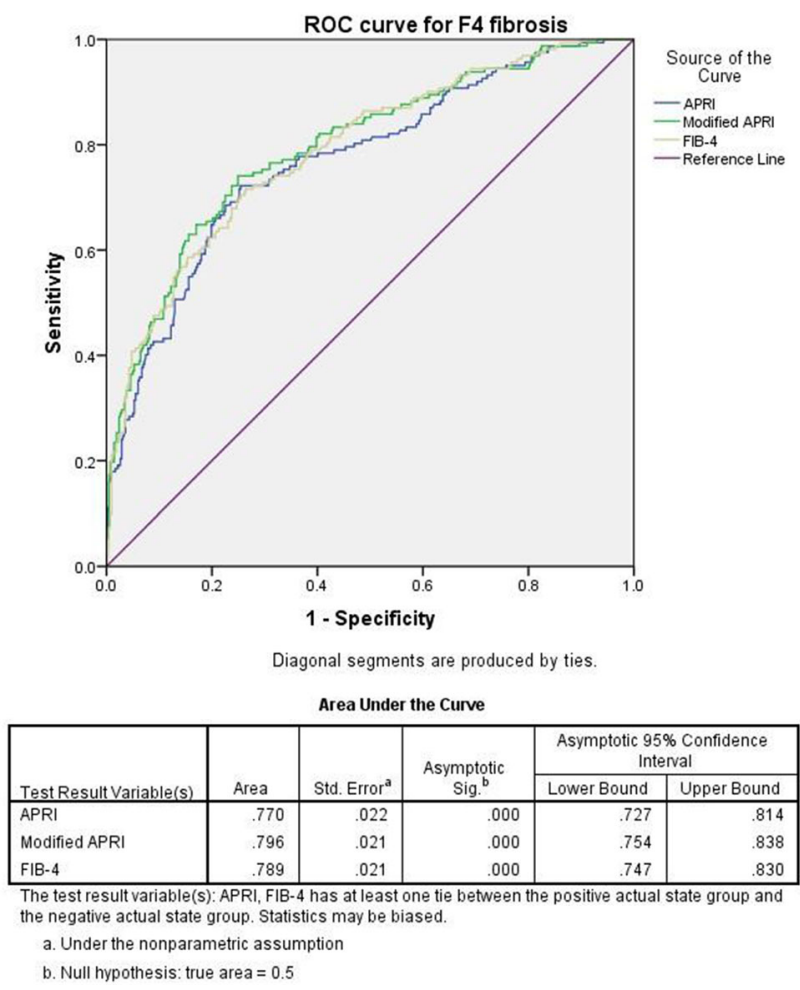

\begin{tabular}{|ll|}
\hline \multicolumn{2}{|c|}{ Comparison of clinical scores for F4 fibrosis } \\
\hline 1) APRI vs FIB-4 & 0.0181 \\
\hline Difference between areas & -0.0104 to 0.0467 \\
\hline $95 \%$ confidence interval & $\mathrm{p}=0.213$ \\
\hline Significance level & 0.0255 \\
\hline 2) APRI vs Modified APRI & 0.00915 to 0.0419 \\
\hline Difference between areas & $\mathrm{p}=0.0023$ \\
\hline 95\% confidence interval & 0.00742 \\
\hline Significance level & -0.0122 to 0.0270 \\
\hline 3) FIB-4 vs Modified APRI & $\mathrm{p}=0.457$ \\
\hline Difference between areas &
\end{tabular}

\section{$O-H C V-17$}

Adverse effects of direct-acting antiviral agents in chronic HCV treated genotype 4 patients; multicenter study

Dina Attia ${ }^{1}$, Dina Attia ${ }^{1}$, Kadry El Saeed ${ }^{2}$, Wafaa Elakel $^{3}$, Tamer El Baz ${ }^{3}$, Ashraf Omar ${ }^{3}$, Ayman Yosri ${ }^{3}$, Manal El Sayed ${ }^{2}$, Maisa El Raziky ${ }^{3}$, Mohamed Abdel Aziz ${ }^{3}$, Mahmoud Anees ${ }^{4}$, Wahid Doss $^{3}$, Yehia El Shazly ${ }^{2}$, Heiner Wedemeyer ${ }^{5}$

${ }^{1}$ Faculty of Medicine, Beni-suef University, Egypt; ${ }^{2}$ Faculty of Medicine, Ain Shams University, Egypt; ${ }^{3}$ Faculty of Medicine, Cairo University, Egypt; ${ }^{4}$ Faculty of Medicine, Tanta University, Egypt; ${ }^{5}$ Hannover Medical School, Germany

Background: Interferon-free regimens are associated with high sustained virological response; however, the associated adverse effects are not yet fully reported. The aim of this study was to evaluate the 
adverse effects associated with the different direct-acting antiviral drugs (DDAs) regimens in the Egyptian patients.

Method: This multicenter retrospective study included all the adverse effects during and after treatment with DAA regimens of 149816 chronic HCV treated Egyptian patients. Patients received SOF/RBV $(\mathrm{n}=21835)$, SOF/SIM $(\mathrm{n}=45188)$, SOF/DCV $(\mathrm{n}=58477)$, SOF/ DCV/RBV $(n=24215)$ and PTV/OBV/r/RBV $(n=101)$. The duration of treatment varied between 12 and 24 weeks. All changes in the treatment regimens, discontinuation, deaths, and serious side effects were reported.

Result: Adverse effects developed in 2475 (1.7\%) [mean age (54 \pm 9 years), male gender (53\%)]. Serious side effects developed in $68 \%, \mathrm{SOF} / \mathrm{RBV}$ was the highest causing regimen $(73 \%$, $\mathrm{p}<0.001)$. Anemia and hyperbilirubinemia were the most common side effects (29.5\% and $24 \%$, respectively), with SOF/RBV (3\% and $1.64 \%$, respectively) had the highest incidence in the treated patients. HCC developed in $1.3 \%$ and death was observed in $3 \%$, with SOF/ RBV was most associated $(76 \%, p=0.243$ and $64 \%, p=0.713$, respectively). Serious side effects $(p=0.045)$ and death cases $(\mathrm{p}=0.025)$ were prominent in liver cirrhosis. Male gender $(\mathrm{p}=0.012)$, lower hemoglobin $(\mathrm{p}<0.001)$, platelets $(\mathrm{p}<0.001)$ and albumin $(\mathrm{p}=0.001)$, higher bilirubin $(\mathrm{p}=0.002)$ and liver cirrhosis $(\mathrm{p}<0.001)$ were factors associated with serious side effects development.

Conclusion: adverse effects associated with DAAs are few, anemia is the most common one. SOF/RBV regimen showed the highest side effects while SOF/DCV was the least one.

\section{$\mathrm{O}-\mathrm{HCV}-18$}

Burden of comorbidities and co-medications in a chronic HCV population compared with a non-HCV population in Japan between 2015 and 2016

\section{Daniel Junichiro Ruzicka ${ }^{1}$, Jumpei Tetsuka ${ }^{1}$, Go Fujimoto ${ }^{1}$, Tatsuya Kanto ${ }^{2}$ \\ ${ }^{1}$ MSD K.K., Japan; ${ }^{2}$ National Center for Global Health and Medicine, Japan}

Background: Direct-acting anti-viral agents have improved the treatment of chronic hepatitis $\mathrm{C}$ virus $(\mathrm{HCV})$ infection, but this treatment is challenging for patients using other comedications because of potential drug-drug interactions. This study aimed to examine the comorbidities and comedications of Japanese chronic $\mathrm{HCV}$ patients in Japan by age group, compared with a non-HCV patient population. This data will inform clinicians around complex profiles of $\mathrm{HCV}$ patients.

Method: This was a retrospective, observational study involving $\mathrm{HCV}$ patients registered in a hospital-based medical claim database. We extracted data for chronic HCV patients aged $\geq 18$ years and age, gender and institution-matched non-HCV patients for the period from January 2015 to November 2016, and then examined chronic comorbidities, long-term co-medications (over 6 months usage), and medications prescribed at least once during the study period.

Result: 128,967 HCV and 515,868 non-HCV patients were included in the analysis (Median age 70 years, $51 \%$ male). More chronic HCV patients than non-HCV patients $(70.5 \%$ vs. $47.1 \%)$ had at least one comorbidity. The proportion of the number of comorbidities increased over age in both HCV/nonHCV patients, and younger HCV patients had higher proportion of comorbidities comparable to 30 years older non-HCV patients proportion, suggesting other contributing factors than an age effect to the development of comorbidities in patients with HCV. For example, in patients with HCV $51.6 \%$ of patients who were 18-44 years old had one or more comorbidities, similar to $51.6 \%$ of non-HCV patients aged 65-74 years (Figure 1). In HCV patients disease of oesophagus, stomach and duodenum was most common (41.7\%/non-HCV: $18.3 \%)$, followed by hypertensive diseases (31.4\%/non-HCV:15.4\%), metabolic disorders (28.2\%/nonHCV:13.9\%), and diabetes mellitus (26.1\%/non-HCV:11.7\%). Comedication usage also increased over age and in regards to chronic/ acute comedication usage, proton pump inhibitors was most frequently used as chronic medication (HCV:14.0\%/31.9\%; nonHCV:6.0\%/17.4\%), followed by calcium antagonists (HCV:12.5\%/ 24.6\%; non-HCV:6.1\%/13.4\%) and angiotensin-II antagonists (HCV:9.0\%/15.2\%; non-HCV: $4.8 \% / 8.6 \%$ ).

Conclusion: To optimize HCV treatment, physicians need to know the exact medication and comorbidity profiles of patients and take appropriate action to manage drug-drug interactions. Further analyses may lead to deeper understanding of the current Japanese HCV patients.

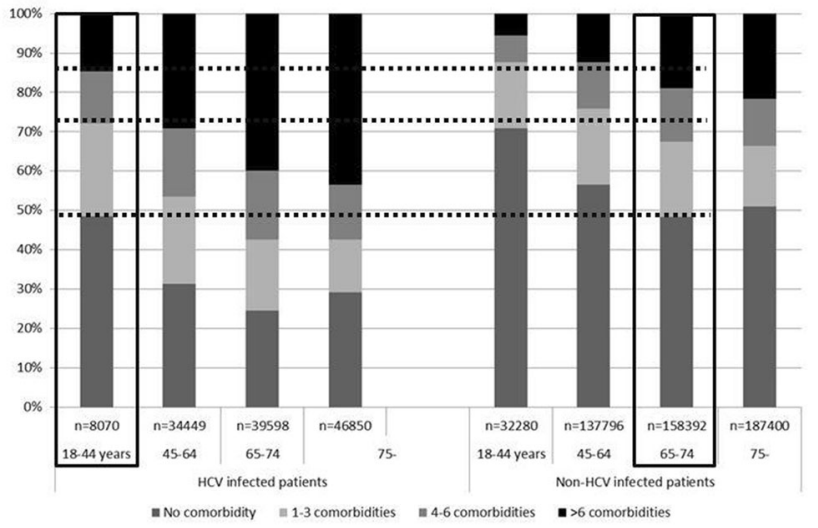

Session: Portal hypertension. Chairperson; Shahab Abid; A S Puri; Tarana Gupta

\section{O-PHT-O1}

The clinical efficacy and safety of simvastatin for reduction of portal hypertension in cirrhotic patients: a systematic review

Radhian Amandito ${ }^{1}$, Raksheeth Agarwal $^{3}$, Oliver Emmanuel Yausep $^{3}$, Matthew Billy ${ }^{3}$, Vito Filbert Jayalie ${ }^{4}$, Rino Alvani Gani ${ }^{2}$

${ }^{1}$ Pondok Indah General Hospital, Jakarta, Indonesia, Indonesia; ${ }^{2}$ Cipto Mangunkusumo General Hospital, Faculty of Medicine, Universitas Indonesia, J1 Pangeran Diponegoro No. 71, Central Jakarta, Jakarta, Indonesia, Indonesia; ${ }^{3}$ Faculty of Medicine, Universitas Indonesia, Jakarta, 10430, Indonesia, Indonesia; ${ }^{4}$ South Tangerang Public Hospital, Tangerang, West Java, Indonesia, Indonesia

Background: Esophageal varies, a complication of portal hypertension in cirrhotic patients, is treated with non-selective beta-blockers (NSBBs) and endoscopic variceal ligation to prevent rebleeding. However, NSBBs are associated with low response rates and systemic adverse effects. Simvastatin is known to induce expression of intrahepatic nitric oxide through the upregulation of Kruppel-Like Factor 2 , decreases portal pressure, and reduces liver fibrosis.

Method: Randomized controlled trials (RCTs) evaluating changes in hepatic venous pressure gradient (HVPG) with use of simvastatin in patients with portal hypertension were retrieved from Pubmed, Cochrane, Scopus, EBSCOhost, ScienceDirect, and ProQuest. The publications were assessed using predetermined selection criteria and 
the Cochrane risk of bias tool before they were included in the review. We selected the HVPG as the primary outcome, with safety and adverse effects of Simvastatin as our secondary outcomes.

Result: Two RCTs of Simvastatin for portal hypertension were included. Both studies have similar baseline characteristics except for the exclusion for child pugh score $>12$ in Abraldes et al. A daily dose of Simvastatin (20 mg with increments up to $40 \mathrm{mg}$ ) could decrease HVPG from baseline to post treatment by $8-15 \%$. The adverse effects were homogenous in control and intervention groups in both studies, therefore they were not caused by the administration of simvastatin. This proves evidence for the clinical efficacy and safety of simvastatin in reducing HVPG in cirrhotic patients with portal hypertension.

Conclusion: Simvastatin effectively reduces HVPG and prevents rebleeding in portal hypertension patients. More evidence is needed to validate this finding in different populations. NSBBs and ligation must still be given alongside simvastatin.

\section{O-PHT-02}

Transjugular intrahepatic portosystemic shunt versus endoscopic therapy in the secondary prophylaxis of variceal re-bleeding in cirrhotic patients: a meta-analysis

\section{Yi Zhou Wang ${ }^{1}$, Chen Xin ${ }^{1}$, Han Mu Lu ${ }^{1}$ \\ ${ }^{1}$ The Affiliated Hospital of Southwest Medical University, China}

Background: The aim of this meta-analysis was to compare the transjugular intrahepatic portosystemic shunt (TIPSS) with endoscopic therapy in the secondary prophylaxis of variceal re-bleeding in cirrhotic patients,to confirm which is more effective and secure.

Method: After the search strategy was established, all relevant studies that compared TIPSS with endoscopic therapy in the secondary prophylaxis of variceal re-bleeding in cirrhotic patients from Medline,PubMed,CNKI were searched and checked by inclusion criteria and exclusion criteria extending up to July 2017, the references which were related to our theme of the articles were also searched and checked. The following items were collected from the selected literetures: first author, year of publication,the number of patients,variceal re-bleeding,mortality,encephalopathy and so on. Then, risk of bias and final result was made out by using the statistical software Revman 5.3

Result: 16 RCTs involving 1163 patients were included. The recurrent total variceal re-bleeding rate $(\mathrm{RR}=0.43,95 \% \mathrm{CI}: 0.36$ to 0.52 , $\mathrm{P}<0.00001)$, the 1 -year $(\mathrm{RR}=0.43,95 \% \quad \mathrm{CI}: \quad 0.27-0.68$, $\mathrm{P}=0.0003$ )and2-year probability of variceal re-bleeding rate ( $\mathrm{RR}=0.51,95 \%$ CI: $0.32-0.79, \mathrm{P}=0.003$ ) was lower in the TIPS group than in the endoscopic group;TIPS and endoscopic group was equal in the respect of the mortality $(\mathrm{RR}=1.04,95 \% \mathrm{CI}$ : 0.86 to $1.27, \mathrm{P}=0.66$ ). In the subgroup analysis of mortality according to the endoscopic type?stent type ?medicine combination?region,there is no difference between the two groups. While the mortality related re-bleeding was lower in the TIPSS group than the endoscopic group $(\mathrm{RR}=0.34,95 \% \mathrm{CI}: 0.18$ to $0.65, \mathrm{P}=0.001)$. The encephalopathy rate was significantly higher in the TIPS group than the endoscopic $\quad$ group $(\mathrm{RR}=1.58, \quad 95 \%$ CI:1.31 to $1.91, \mathrm{P}<0.00001$ ), while in the subgroup analysis according to the stent type,the encephalopathy rate of bare TIPS group was higher than the endoscopic group $(\mathrm{RR}=1.75,95 \% \mathrm{CI}: 1.31$ to 2.34 , $\mathrm{P}=0.0002$ ), and it is equal between covered TIPS group and endoscopic $\operatorname{group}(\mathrm{RR}=0.98,95 \% \mathrm{CI}: 0.6 \sim 1.6, \quad \mathrm{P}=0.94)$.In the respect of the TIPS dysfunction,bare TIPS dysfunction was higher than covered TIPSS. The transfusion units of RBC was less in the TIPS group (WMD $=-1.95,95 \%$ CI:-3.34 to $-0.56, \mathrm{P}=0.006$ ). The time in hospital was shorter in the endoscopic group (WMD $=1.86$, $95 \%$ CI: $1.12 \sim 2.59, \mathrm{P}<0.00001)$.

Conclusion: In terms of variceal re-bleeding rate,TIPSS placement is superior to endoscopic treatment .Furthermore the encephalopathy rate of covered TIPS and survival did not differ to the endoscopic treatment.TIPSS placement is superior to endoscopic treatment for secondary prevention of variceal re-bleeding.

\section{O-PHT-03}

Post-bleeding ascites in children with extrahepatic portal venous obstruction: risk factors and long term outcome

\section{Moinak Sen Sarma ${ }^{1}$, Surender Kumar Yachha ${ }^{3}$, Anshu Srivastava $^{2}$, Ujjal Poddar ${ }^{4}$}

${ }^{1}$ Sanjay Gandhi Postgraduate Institute of Medical Sciences, Lucknow, India; ${ }^{2}$ Additional Professor, India; ${ }^{3}$ Professor and Head, India;

${ }^{4}$ Professor, India

Background: Ascites in a setting of extrahepatic portal venous obstruction (EHPVO) without an underlying chronic liver disease is uncommon. Post-bleeding ascites is an unexplored entity. We aimed to study a) the overall prevalence and etiological profile of ascites and b) risk factors and natural history of post-bleeding ascites in EHPVO patients

Method: All admitted patients of EHPVO with clinically detectable ascites \pm gastrointestinal (GI) bleeding within $<6$ weeks duration were analysed. Post-bleeding ascites (PBA) was defined as appearance of ascites after the index GI bleeding. PBA was compared with the cohort who had GI bleeding without ascites (BWA) admitted during the same period. Concomitant chronic liver diseases were excluded. Laboratory and growth parameters at the index episode were compared with a prior follow-up 3-6 months in non-sick, nonbled state. The difference was termed as fall from baseline.

Result: Of the total 307 analysed EHPVO patients, 26\% $(\mathrm{n}=79)$ presented with ascites. $83 \%(n=66)$ were PBA, mostly variceal bleeding from esophageal varices $(79 \%)$. The rest $(n=13)$ were of other etiologies (figure 1$)$. On comparison of PBA $(n=66)$ vs. BWA $(n=228)$, statistical significance was seen with age of disease onset [6.7 (1-16) vs. $11(0.5-18)$ years; $p=0.03]$, peripheral edema (29 vs. $30 ; \mathrm{p}<0.001$ ), postural symptoms (50 vs. $141 ; \mathrm{p}=0.04)$, height $\mathrm{z}$ scores [-2.3 (-4.3 to 1.5$)$ vs. $-1.7(-3.2$ to 2.0$) ; \mathrm{p}=0.01]$ at admission and weight $\mathrm{z}$ scores [-2.6 (-4.1 to 2.5$)$ vs. $-1.8(-2.2$ to 2.5$) ; \mathrm{p}=0.01]$ at ascites resolution. Statistical significance was also seen between PBA and BWA with index parameters: serum protein [5.5 (3.8-6.9) vs. $6.6(3.6-7.9) \mathrm{mg} / \mathrm{dL} ; \mathrm{p}=0.04]$, albumin $[2.9(1.8-4.3)$ vs. 3.6 $(1.8-5.1) \mathrm{mg} / \mathrm{dL} ; \mathrm{p}=0.02]$, hemoglobin [6.3 (2.9-8.8) vs. $7.2(2.6-$ $11.5) ; \mathrm{p}=0.02]$ and also with fall from baseline: serum protein [1.6 (0.3-2.2) vs. $0.4(0.1-1.4) \mathrm{mg} / \mathrm{dL} ; \mathrm{p}=0.001]$, albumin [0.9 (0.5-1.4) vs. $0.4(0.3-1.1) \mathrm{mg} / \mathrm{dL} ; \mathrm{p}=0.02]$, hemoglobin [4.6 (3.4-6.6) vs. 2.3 $(2.1-3.7) ; \mathrm{p}=0.001]$. Time interval (first bleeding bout to admission) [12 (5-20) vs. 10 (3-20) days; $p=0.7]$, transfusion requirement (57 vs. $180 ; \mathrm{p}=0.2)$ and ongoing endotherapy ( 11 vs. $62, \mathrm{p}=0.8)$ were non-significant between PBA vs. BWA. $32 \%(\mathrm{n}=21)$ had spontaneous ascites resolution (salt restriction) in 3 (2-7) days. 39\% $(\mathrm{n}=26)$ required additional diuretics.29\% $(\mathrm{n}=19)$ had severe ascites requiring large volume paracentesis $[70(53-110) \mathrm{mL} / \mathrm{kg}] .17 \%$ $(\mathrm{n}=11)$ had ascitic fluid infection. Outcome of ascites group $(\mathrm{n}=79)$ is shown in figure 1 .

Conclusion: 26\% EHPVO have ascites after GI bleeding. Early age of disease onset, large volume of GI bleeding and poor nutritional status were risk factors for development of ascites in EHPVO. Overall outcome of EHPVO with ascites is favourable. 


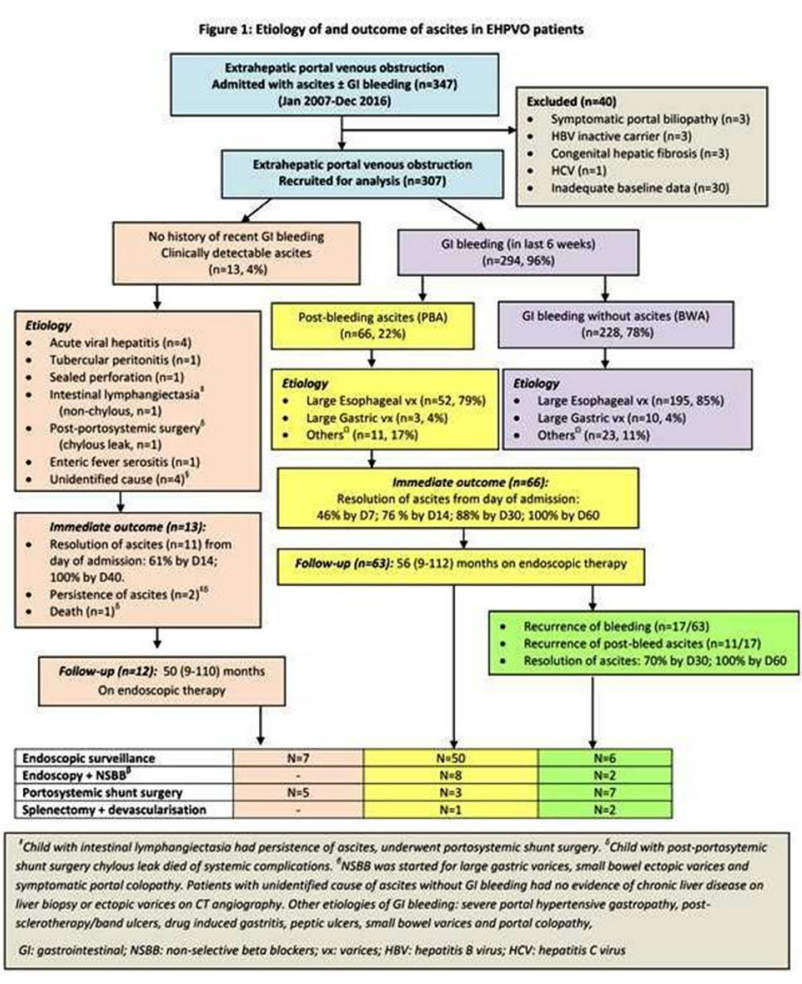

\section{O-PHT-04}

Achieving the World Health Organization (WHO) Global Health Sector Strategies (GHSS) goals for hepatitis C in India: a modelling study

Sarah Robbins ${ }^{1}$, Betty Chiang ${ }^{4}$, Vivek Saraswat ${ }^{7}$, Samir Shah $^{6}$, Gourdas Choudhuri $^{3}$, Anil Anand ${ }^{5}$, Pankaj Puri ${ }^{2}$

${ }^{1}$ Center for Disease Analysis Foundation, United States; ${ }^{2}$ Armed Forces Medical College, India; ${ }^{3}$ Fortis Memorial Research Institute, India; ${ }^{4}$ Gilead Sciences, United States; ${ }^{5} \mathrm{HCV}$ Task Force, Indian

National Association for the Study of Liver, India; ${ }^{6}$ Institute of Liver Diseases, HPB Surgery and Liver Transplant, Global Hospitals, India; ${ }^{7}$ Sanjay Gandhi Post Graduate Institute of Medical Sciences, India

Background: India accounted for $8.5 \%$ of global hepatitis $\mathrm{C}$ virus (HCV) infections, or approximately 6 million individuals in 2015. To address this global health threat, the Ministry of Health in India recently announced plans for the implementation of a national action plan to eliminate hepatitis $\mathrm{C}$ in the country. This study sought to quantify the burden of HCV in India and identify a strategy to achieve the WHO Global Health Sector Strategy Goals (GHSS) for Hepatitis by 2030 .

Method: Using a Markov-progression model, the HCV infected population and future disease progression were forecasted. The impact of a WHO GHSS strategy on projected disease burden was assessed.

Result: Under the current treatment paradigm in India, there were an estimated 6.1 million infections in 2016. This is estimated to decrease by $4 \%$, to 5.9 million infections over the next fifteen years. Liver related mortality is expected to increase by $35 \%$, from 23,000 deaths in 2016 to 32,000 in 2030 . The number of patients with late-stage liver diseases is also expected to increase 15 to $25 \%$. To achieve the GHSS goals of $90 \%$ of the infected population being diagnosed and a $65 \%$ reduction in liver related mortality, treatment must be increased to 500,000 patients annually by 2023 . Similarly, the annual number of newly diagnosed patients will need to be increased to 800,000 by the same year. This will reduce total HCV infections by $90 \%$ and liver related morbidity and mortality by $70-90 \%$ by 2030 . After 2031 , the annual number of treated patients would decline as there become fewer infections overall.

Conclusion: Achieving the GHSS targets for Hepatitis by 2030 are feasible if targeted public health strategies are implemented. The National Action Plan for Elimination will need to include measures combining increased screening and treatment within the next fifteen years to be successful.
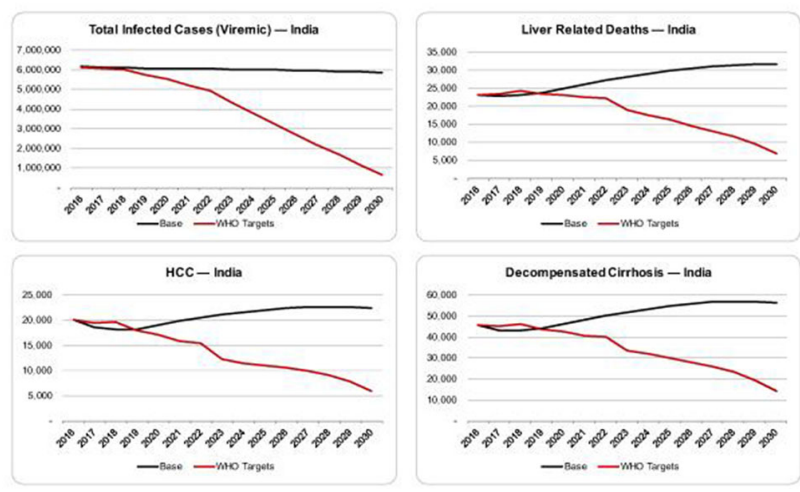

Table 1: Comparison between children undergoing ascitic fluid culture by two methods

\begin{tabular}{|lccc|}
\hline Parameters & $\begin{array}{c}\text { Post-centrifugation } \\
(\mathbf{n}=\mathbf{1 0 0})\end{array}$ & $\begin{array}{c}\text { Routine method } \\
(\mathbf{n}=40)\end{array}$ & P value \\
\hline Median age at presentation $(\mathrm{mo})$ & $84(2.5$ to 216$)$ & $96(3.5$ to 216$)$ & 0.71 \\
Male & $70(70 \%)$ & $27(67.5 \%)$ & 0.77 \\
Ascitic fluid infection & $30(30 \%)$ & $11(27.5 \%)$ & 0.7 \\
SBP & $7(7 \%)$ & $2(5 \%)$ & \\
CNNA & $18(18 \%)$ & $7(17.5 \%)$ & 0.9 \\
MNB & $5(5 \%)$ & $2(5 \%)$ & \\
Positive ascitic fluid culture & $12 / 30(40 \%)$ & $4 / 11(36 \%)$ & 1.0 \\
Other site infections & $29(29 \%)$ & $12(30 \%)$ & 1.0 \\
Antibiotics exposure & $49(49 \%)$ & $18(45 \%)$ & 0.6 \\
CLD & $83(83 \%)$ & $30(75 \%)$ & 0.22 \\
CPT class A/B/C & $0 / 33 / 50$ & $1 / 8 / 21$ & 0.07 \\
\hline
\end{tabular}

CLD: Chronic liver disease, CPT: Child-Pugh-Turcotte, SBP: spontaneous bacterial peritonitis, CNNA: culture negative neutrocytic ascites, MNB: monomicrobial non-neutrocytic bacterascites

\section{O-PHT-05}

Are non-cirrhotic portal fibrosis bleeders different from nonbleeders? A first time analysis in children

Moinak Sen Sarma ${ }^{1}$, Durga Prasad, ${ }^{1}$, Surender Kumar Yachha ${ }^{1}$, Anshu Srivastava $^{1}$, Ujjal Poddar ${ }^{1}$

${ }^{1}$ Sanjay Gandhi Postgraduate Institute of Medical Sciences, Lucknow, India

Background: Non-cirrhotic portal fibrosis (NCPF) is a rare cause of pediatric portal hypertension. Though there is abundant literature in adults, there is paucity of data in children. Endoscopic eradication of varices and conservative management are the mainstay of therapy whereas portosystemic shunt surgery is debatable. We aimed to evaluate and compare the endoscopic and long-term outcomes of biopsy-proven NCPF bleeders and non-bleeders. 
Method: Diagnosis of NCPF was based on all of the following: (1) splenomegaly \pm varices on endoscopy, (2) dilated portal \pm splenic vein (3) preserved liver functions and (4) absence of cirrhosis on liver histology \pm presence of characteristic histology and (5) exclusion of all known chronic liver diseases. The cohort was analysed for their clinical, endoscopic outcome and disease progression. Variceal bleeders and non-bleeders underwent secondary and primary prophylaxis respectively followed by endoscopic surveillance. Betablockers were only indicated for bleeding gastric varices.

Result: 45 NCPF children (21 boys) aged 14 (6-18) years and symptom duration 12 (1-120) months presented as incidental splenomegaly (53\%) and acute variceal bleeding (31\%). Overall $22(49 \%)$ were variceal bleeders. $91 \%$ had hypersplenism with overall splenic size $10(1-17) \mathrm{cm}$ and enlarged liver $(58 \%)$. Percutaneous $(73 \%)$ and transjugular $(27 \%)$ liver biopsy showed characteristic obliterative portal venopathy $(68 \%)$. Initial endoscopic evaluation $(n=45)$ showed esophageal varices $(93 \%)$, gastric varices $(55 \%)$ and portal hypertensive gastropathy (89\%). Table 1 compares primary (nonbleeders, $\mathrm{n}=11$ ) and secondary prophylaxis (bleeders, $\mathrm{n}=22$ ). Modalities used were endoscopic band ligation $(n=30)$ followed by sclerotherapy ( $\mathrm{n}=13$ bleeders) for esophageal varices and glue injection followed by beta-blockers ( $\mathrm{n}=3$ gastric variceal bleeders). Table 2 shows progression of portal hypertension of 12 unbled patients who did not require primary prophylaxis. Overall follow-up duration of 26 (3-120) months, 87\% did not show disease progression. Poor outcome were seen exclusively in six bleeders $(n=3$ death from interval bleeding, $\mathrm{n}=2$ decompensation, $\mathrm{n}=1$ hepatopulmonary syndrome). 4 children $(\mathrm{n}=3$ bleeders $)$ underwent splenectomy with devascularisation (splenic pain and mucocutaneous bleeds due to hypersplenism).

Conclusion: Endoscopic outcome of esophageal varices is similar in bleeders versus non-bleeders. Primary prophylaxis significantly decreases the gastric varices. Though there is progression in portal hypertension in unbled patients, majority of NCPF have preserved liver functions. Small proportion of bleeders have poor outcome and need for surgery.

Table 1: Endoscopic Outcome of patients receiving endotherapy ( $n=33$ )

\begin{tabular}{|c|c|c|c|}
\hline Parameters & $\begin{array}{c}\text { Bleeder }(n=22) \\
\text { Secondary prophylaxis }\end{array}$ & $\begin{array}{l}\text { Non bleeder }(n=11) \\
\text { Primary prophylaxis }\end{array}$ & p-value \\
\hline Required endotherapy & $22(100 \%)$ & $11(100 \%)$ & . \\
\hline Initial grade of esophageal varices (IV/III/11/1/) & $10 / 8 / 4 / 0$ & $7 / 3 / 1 / 0$ & $\cdot$ \\
\hline Total No of endoscopic sessions till $1^{4}$ eradication & $8(2-12)$ & $6(2-9)$ & 0.3 \\
\hline Recurrence of esophageal varices' & $8(36 \%)$ & $4(36 \%)$ & 0.6 \\
\hline $\begin{array}{l}\text { Time interval between } 1^{\text {sh }} \text { eradication and } \\
\text { recurrence }(\mathrm{mo})^{8}\end{array}$ & $8(3-36)$ & $11(6.42)$ & 0.2 \\
\hline Number requiring $2^{2 / 6}$ eradication & $6(27 \%)$ & $2(18 \%)$ & 0.4 \\
\hline Sessions required for $2^{\text {cad }}$ eradication ${ }^{a}$ & $3(2.5)$ & $4(2 \cdot 7)$ & 0.4 \\
\hline $\begin{array}{l}\text { Gastric varices at onset } \\
\left(\text { GOV } 1 / G O V / /(G V)^{5}\right.\end{array}$ & $\begin{array}{l}19(86 \%) \\
(18 / 3 / 3)\end{array}$ & $\begin{array}{l}8(72 \%) \\
(7 / 3 / 1)\end{array}$ & 0.6 \\
\hline $\begin{array}{l}\text { Gastric varices at last endoscopy } \\
(\mathrm{GOV} 1 / \mathrm{GOV} 2 / \mathrm{GGV})^{5}\end{array}$ & $\begin{array}{l}14(63 \%) \\
(12 / 2 / 1)\end{array}$ & $\begin{array}{l}4(36 \%) \\
(4 / 1 / 1)\end{array}$ & 0.001 \\
\hline PHG at onset (mild/severe)" & $\begin{array}{c}22(100 \%) \\
(18 / 4)\end{array}$ & $\begin{array}{c}7(63 \%) \\
(5 / 2)\end{array}$ & 0.003 \\
\hline PHG at last endoscopy (mild/severe)" & $\begin{array}{c}13(59 \%) \\
(9 / 4)\end{array}$ & $\begin{array}{c}4(36 \%) \\
(3 / 1)\end{array}$ & 0.3 \\
\hline \multicolumn{4}{|c|}{ 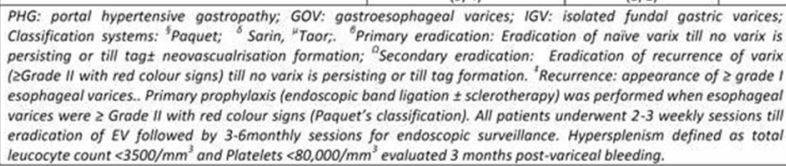 } \\
\hline
\end{tabular}

Table 2: Progression of varices in unbled patients with small varices not on primary prophylaxis $(n=12)$

\begin{tabular}{|l|c|c|}
\hline Parameters & At presentation & Till last follow - up \\
\hline Esophageal varices (Grade IV/III/II/I) & $10(83 \%)$ & $12(100 \%)$ \\
& $(0 / 0 / 5 / 5)$ & $(1 / 3 / 5 / 3)$ \\
\hline Development of large esophageal varices & 0 & $6(50 \%)$ \\
(2 Grade II with red colour signs) & $5(41 \%)$ & $9(75 \%)$ \\
\hline Gastric varices at onset (GOV1/GOV2//GV) & $(4 / 1 / 0)$ & $(6 / 2 / 1)$ \\
\hline & $6(50 \%)$ & $8(66.6 \%)$ \\
\hline PHG (mild/severe) & $(4 / 2)$ & $(5 / 3)$ \\
\hline Classification systems and abbreviations as per table 1 & \multicolumn{2}{|l|}{} \\
\hline
\end{tabular}

\section{O-PHT-06}

Co-existence of portal hypertension and peptic ulcer disease in patients with cirrhosis of liver, irrespective of etiology in a medical college hospital of Bangladesh.

Ahmed Lutful Moben ${ }^{1}$, Mamun Al Mahtab ${ }^{2}$, Md. Noor E Alam', Farjana Majid $^{3}$

${ }^{1}$ MBBS, MD(Hepatology), Bangladesh; ${ }^{2}$ MBBS,

FRCP,MSc,MD(Hepatology), Bangladesh; ${ }^{3}$ MBBS, MPhil(Virology), Bangladesh

Background: Haematemesis in cirrhosis of liver not always from rupture oesophageal varices, rather it may cause by bleeding peptic ulcer disease. This study attempted to evaluate co-existence of portal hypertension and PUD in cirrhotic patients.

Method: Consecutive 96 patients had enrolled who have oesophageal varices at our endoscopy unit during endoscopic evaluations in last 2 months.

Result: Total cirrhotic patients enrolled were $96(\mathrm{M}=61, \mathrm{~F}=35)$, mean age were 51.76 years (22-86 years). HBV was the leading cause of cirrhosis $54.16 \%$, HCV $5.36 \%$, proven NASH were $11.54 \%$ rest were from unknown aetiology. Their average CTP score were 8.6(125), $37.6 \%$ associated with portal hypertensive gastropathy. Grade-3 oesophageal varices found in 52 patients, whereas grade 2 in 25 patients. Surprisingly among this 96 patient 59 (61.45\%) reveals peptic ulcer disease more in the form of gastric ulcer $(n=46)$ then duodenal ulcer $(\mathrm{n}=10)$ and both $(\mathrm{n}=10)$.

Conclusion: Peptic ulcer disease is one of the potential cause of haematemesis in cirrhosis of liver patients, irrespective of cause in Bangladesh. Large multicenter clinical trial needed to comment better.

Session: Hepatitis B Virus Infection; Chairperson: Wasim Jafri; J C Vij; S Sidhu

\section{$O-H B V-25$}

Early intrahepatic TLR4 activation facilitates HBV persistence through strengthening Kupffer cell mediated $T$ cell suppression

\section{Wenqing Zhou ${ }^{1}$, Jinzhuo Luo ${ }^{1}$, Qing Yu ${ }^{1}$, Dongliang Yang', Jia Liu}

${ }^{1}$ Department of Infectious Diseases, Union Hospital, Tongji Medical College, Huazhong University of Science and Technology, China

Background: Chronic hepatitis B virus (HBV) infection is still a major public health problem worldwide, but the mechanism of $\mathrm{HBV}$ persistence remains largely unknown. $\mathrm{HBV}$ is considered as a "stealth" virus since it only triggered a limited innate response during the acute phase of infection, yet it results in acute-resolving infection in most of the infected adult individuals.

Method: In the current study, we investigated the impact of early activation of innate immune response on HBV clearance and the related immune mechanism by using the HBV hydrodynamic injection (HI) mouse model.

Result: 6-8 weeks old male C57BL/6 mice were hydrodynamically injected with TLR4 agonist LPS or HBV replicating plasmid pAAV/ HBV1.2, either separately or in combination. We could show that HI of LPS but not pAAV/HBV1.2 resulted in dramatic elevation of serum proinflammatory cytokine (IL-6, TNF- $\alpha$ and IL-10) levels within 3 hours. Compared to LPS HI alone, HI of LPS and pAAV/ 
HBV1.2 significantly reduced serum IL-6, TNF- $\alpha$ and IL-10 concentrations, suggesting HBV inhibits TLR4 signaling in vivo. Besides, HI of LPS together with pAAV/HBV1.2 led to significant delay of HBV clearance in mice compared with pAAV/HBV1.2 HI alone. Kupffer cells (KCs) were separated from the HI mice and cocultured with $\alpha \mathrm{CD} 3 / \mathrm{CD} 28$ activated CD8 $+\mathrm{T}$ cells. The KCs from HBV plus LPS HI mice showed enhanced ability to suppress IFN- $\gamma$ production by activated $\mathrm{T}$ cells than the $\mathrm{KCs}$ from $\mathrm{HBV} \mathrm{HI}$ alone mice. Strong IL-10 production was detected in these KCs.

Conclusion: Our results suggest that early activation of intrahepatic innate immune responses by TLR 4 agonist strengthens the suppressive properties of KCs and may facilitates the persistence of $\mathrm{HBV}$ infection.

\section{$O-H B V-26$}

\section{Regulation of hepatitis B immunopathology by hypoxia induced regulatory $B$ cells}

\section{Fusheng Wang ${ }^{1}$, Shuang Nan Zhou ${ }^{1}$, Ji Yuan Zhang ${ }^{1}$ \\ ${ }^{1}$ Beijing 302 Hospital, China}

Background: B cells consistently represent abundant cellular components in livers of patients with chronic hepatitis B, but the biological role of $\mathrm{B}$ cells in hepatic inflammation is poor understood in patients with chronic hepatitis B virus infection.

Method: We characterized peripheral and intrahepatic B cells in a cohort of chronic HBV-infected patients at different stages.

Result: We found that B cells were largely accumulated in hypoxic portal area, and these intrahepatic B cells positively correlated with histological activity index and experienced hypoxia as indicated by HIF-1alpha expression. Moreover, we found hypoxia regulated B cells infiltration and detention through relevant chemokine receptors and their ligands. In IA patients, the peripheral expression of CXCR3 on B cell was significantly higher by flow cytometry, and the plasma CXCL10 level was also significantly higher than healthy controls. Meanwhile, CXCL10 was highly expressed in hypoxic livers of IA patients by qPCR. Comparing with fresh ex vivo B cells, hypoxic B cells expressed higher CXCR3 and GLUT1, which were regulated by HIF-1alpha. More importantly, we provided evidence that CXCR3+ GLUT1+ B cells expressed increased IL10, which was the key characteristic of regulatory B cells. Inhibition of HIF-1 activity by adding 2ME2, a HIF-1 inhibitor, reduced the expression of IL10, CXCR3 and GLUT1 on B cells. Compared to healthy people, the proportion of peripheral CXCR3+ GLUT1+ B cell subsets was increased in IA patients and positively correlated with ALT and AST level in CHB patients.

Conclusion: These data demonstrate B cells in response to hypoxic environment take on a regulatory role and contribute to liver immunopathology. In particular, we identify a novel CXCR3+ GLUTI+ B cell subset that exhibits an increased expression of IL10 in CHB patients, and this B cell subset can play an immunomodulatory function. As a hypoxia correlated transcription factor, HIF involves in induction of IL10 and CXCR3+ GLUTI+ Bregs. That indicates that hypoxia plays a significant role in the immunomodulatory function of B cells.
$O-H B V-27$

Hepatitis B virus rtA181T/sW172non-stop mutation may increase resistance fold to adefovir- and entecavir-resistant mutants compared to $\mathrm{rtA181T/sW172stop} \mathrm{mutation}$

Dongping $X u^{1}$, Li Zhao ${ }^{1}$, Xiaodong $\mathrm{Li}^{1}$, Yongqian $\mathrm{Chen}^{1}$, Rongjuan Chen ${ }^{1}$, Jinman Shao', Yi Zhou ${ }^{1}$, Qi Li ${ }^{1}$, Hao Liao ${ }^{1}$, Yangyang Zhao', Lujie Liu ${ }^{1}$, Yan Liu ${ }^{1}$, Dongping $\mathrm{Xu}^{1}$

${ }^{1}$ Beijing 302 Hospital, China

Background: Hepatitis B virus (HBV) rtA181T as a drug-resistance-

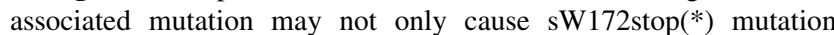
mostly but also non-stop mutation sometimes in overlapping HBV S region. The study aimed to clarify clinical profile and virologic feature of the rtA181T-causative stop and non-stop mutations.

Method: Total of 22,009 patients who visited Beijing 302 Hospital from 2007 to 2016 was enrolled. These patients all received nucleos(t)ide analogues (NAs) treatment and their serum samples were collected for direct and clonal sequence analysis of HBV reverse-transcriptase (RT) and S regions. Phenotypic analysis was performed for representative viral strains in amplicon-transfected HepG2 cells.

Result: The rtA181T mutation was detected in 5.37\% $(1,182 / 22,009)$ of the patients' samples. The rtA181T-causative sW172*, sW172nonstop $(\mathrm{sW} 172 \mathrm{~L} / \mathrm{S})$, and mixed sW172*/non-stop mutations occupied $82.91 \%, 7.70 \%$, and $9.39 \%$, respectively. The detection rate of rtA181T/sW172non-stop mutation had an increasing trend over time, i.e., $4.43 \%$ (27/610) during year 2007-2010, $10.12 \%$ (34/337) during year 2011-2013, and $12.77 \%$ (30/235) during year 2014-2016 of total rtA181T-positive samples $(\mathrm{P}<0.05)$. The patients with $\mathrm{rtA} 181 \mathrm{~T} /$ sW172non-stop mutants had a higher HBV DNA level compared to those with rtA181T/sW172* mutants. $44.33 \%(524 / 1,182) \mathrm{rtA} 181 \mathrm{~T}$ positive samples were detected with signature drug-resistant mutations, including 325 with adefovir-resistant mutation rtA181V/ N236T, 57 with lamivudine-resistant mutation rtM204V/I, 99 with entecavir-resistant mutation rtM204V/I plus rt184/202/250 substitutions, and 43 with multidrug-resistant mutation rtA181V/ $\mathrm{N} 236 \mathrm{~T}+\mathrm{rtM} 204 \mathrm{~V} / \mathrm{I} \pm \mathrm{rt} 184 / 202 / 250$ substitutions. Specifically, the rtA181T/sW172non-stop mutation had a higher ratio of coexistence with adefovir-resistant mutation compared to rtA181T/sW172* mutation $(42.86 \%$ vs. $24.59 \%, \mathrm{P}<0.05)$. Phenotypic analysis showed that rtA181T/sW172S + rtN236T and rtA181T/sW172L + rtN236T mutants exhibited higher HBV DNA production and adefovir resistance fold than that of $\mathrm{rtA} 181 \mathrm{~T} / \mathrm{sW} 172 *+\mathrm{rtN} 236 \mathrm{~T}$ mutant $(98.02 \%$ and $85.5 \%$ vs. $42.1 \%$ in HBV DNA production, and 7.38 -fold and 5.49-fold vs. 3.69-fold in half maximal effective concentration of wild-type strain); rtA181T/sW172L + rtS202G + rtM204V strain exhibited higher HBV DNA production and entecavir resistance fold than that of rtA181T/sW172* $+\mathrm{rtS} 202 \mathrm{G}+\mathrm{rtM} 204 \mathrm{~V}$ strain $(50.98 \%$ vs. $34.49 \%, 524.00$-fold vs. 69.33 -fold).

Conclusion: sW172non-stop mutation ratio in rtA181T-positive samples increased in recent years. Adefovir- and entecavir-resistant mutants with rtA181T/sW172non-stop mutation had higher resistance fold than those with $\mathrm{rtA} 181 \mathrm{~T} / \mathrm{sW} 172 *$ mutation. 


\section{$O-H B V-28$}

EFTUD2 is a novel innate immune regulator inhibiting HBV replication and gene expression through RIG-I mediated pathway

Chuanlong Zhu ${ }^{1}$, Pingping $\mathrm{Hu}^{1}$, Ke Jing ${ }^{1}$, Lianhua Kong ${ }^{1}$, Qian Wang ${ }^{1}$, Shuang $\mathbf{L i}^{1}$, Jun $\mathbf{L i}^{1}$

${ }^{1}$ The First Affiliated Hospital of Nanjing Medical University, China

Background: Recently, RIG-I has been identified dually functions as a sensor in activating innate signaling to counteract viral polymerase in HBV infection (Sato S, et al. Immunity, 2015). We have reported that EFTUD2 exercised antiviral ability via RIG-I signing pathway in HCV JFH1 culture system (Zhu C, et al. J Virol, 2015), but its role in HBV infection remains unclear. In this study, we evaluate whether EFTUD2 can restrict HBV replication through RIG-I mediated pathway in vitro.

Method: We overexpressed EFTUD2 by plasmid transfection, then performed siRNA knockdown of RIG-I in HepG2.2.15 or HBV-infected HepG2-NTCP cells. Selected gene expression were monitored by quantitative PCR and Western-blot. Levels of viral antigen expression and HBV DNA were measured by enzyme-linked immunosorbent assay and quantitative PCR, respectively.

Result: EFTUD2 overexpression inhibited HBV replication from $6,511,626 \pm 1,108,827$ to $3,030,405 \pm 403,128 \mathrm{IU} / \mathrm{ml}(P=0.0069)$ in HepG2.2.15 cells, and meanwhile reduced HBsAg and $\mathrm{HBeAg}$ production by $58.9 \pm 9.1 \% \quad(13.49 \pm 1.27 \quad$ vs $5.54 \pm 1.25$; $P=0.0015)$ and $39.7 \pm 2.0 \%(22.86 \pm 1.83$ vs $13.79 \pm 1.34$; $P=0.0022)$ in culture supernatants, respectively. We further confirmed EFTUD's anti-HBV ability in a novel HBV culture system, namely HepG2-NTCP infected with HBV. Moreover, EFTUD2 overexpression resulted in a increase in RIG-I mature mRNA level in both HepG2.2.15 and HBV-infected HepG2-NTCP, indicating RIG-I mediate EFTUD2's antiviral effect, which could be reversed by specific siRNA against RIG-I.

Conclusion: Our data demonstrate that EFTUD2 restricts HBV replication and gene expression through RIG-I mediated signaling pathways, thereby revealing the participation of EFTUD2 as a novel innate immune regulator and suggesting a potentially targetable antiviral pathway.

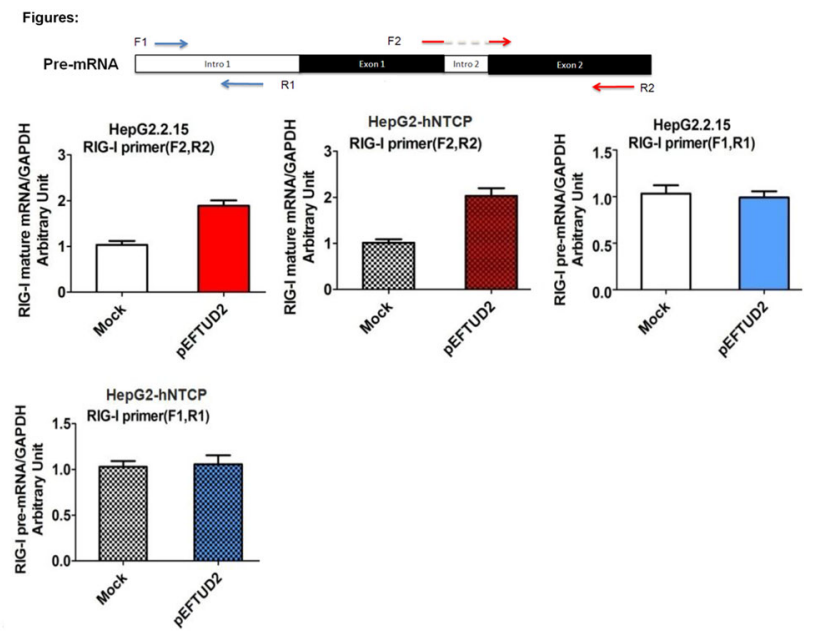

Fig 1: EFTUD2 upregulates RIG-I mature mRNA expression

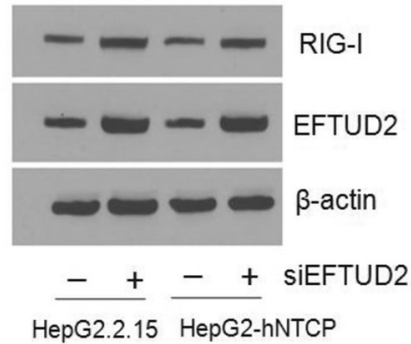

Fig 2: EFTUD2 upregulates RIG-I protein expression

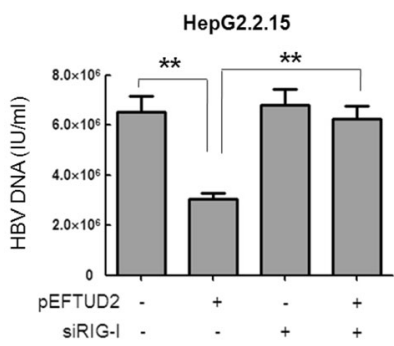

Fig 3: EFTUD2-induced antiviral effect is dependent of RIG-I pathway

\section{$O-H B V-29$}

HBV-specific CD8+ T cells maintain functional exhaustion after antigen reexposure in an acute activation immune environment

Qin Wang ${ }^{1}$, Wen Pan', Yanan Liu', Jinzhuo Luo', Dan Zhu', Yinping Lu', Xuemei Feng ${ }^{1}$, Xuecheng Yang ${ }^{1}$, Ulf Dittmer ${ }^{2}$, Mengji Lu², Dongliang Yang', Jia Liu ${ }^{1}$

${ }^{1}$ Union Hospital, Tongji Medical College, Huazhong University of Science and Technology, China; ${ }^{2}$ University Hospital of Essen, University of Duisburg-Essen, Germany

Background: Chronic hepatitis B virus (HBV) infection is characterized by the presence of functionally exhausted HBV-specific $\mathrm{CD} 8+\mathrm{T}$ cells.

Method: To characterize the possible residual effector ability of these cells, we reexposed CD8 $+\mathrm{T}$ cells from chronically $\mathrm{HBV}$ replicating mice to HBV antigens in an acute activation immune environment. Result: We found that after transfer into naive mice, exhausted $\mathrm{CD} 8+\mathrm{T}$ cells reexpanded in a comparable magnitude as naive $\mathrm{CD} 8+$ $\mathrm{T}$ cells in response to acute HBV infection. The expanded CD8 $+\mathrm{T}$ cells possess similar differentiation phenotype as naive control cells, however, they maintained less activated phenotype, an absence of effector cytokine production and poor antiviral function.

Conclusion: We thus conclude that exhausted CD8+ T cells undergo a stable form of dysfunctional differentiation during chronic HBV infection and switching immune environment alone is not sufficient for the antiviral functional reconstitution of these cells. 


\section{$O-H B V-30$}

\section{Incidence and predictors of significant fibrosis in Incidentally} detected Asymptomatic Hepatitis B positive subjects (IDAHS)

\section{Sridhar Sundaram ${ }^{1}$, Prachi Patil ${ }^{1}$, Shaesta Mehta ${ }^{1}$}

${ }^{1}$ Tata memorial centre, India

Background: Chronic Hepatitis B can present with wide range of manifestations from asymptomatic infection to cirrhosis. Management of Incidentally detected asymptomatic Hepatitis B positive subjects (IDAHS), especially those with normal Alanine transaminase (ALT) has been a topic of debate. We aimed to study the clinical and laboratory profile of IDAHS and patterns of fibrosis as measured by Vibration controlled transient elastography (VCTE).

Method: A retrospective review of IDAHS referred to the Hepatology clinic at Tata Memorial Centre from November 2016 to November 2017 was done. Demographic and laboratory details of the patients were noted. Details of Fibroscan (VCTE) measurements were noted. Fibroscan values of $>8 \mathrm{kPa}$ were classified as significant fibrosis as per the APASL criteria and those with values $>11 \mathrm{kPa}$ were considered as cirrhosis.

Result: 115 patients were reviewed, which included 72 males and 43 females with mean age of 43.9 years. 29 patients (25.2\%) were $\mathrm{HBeAg}$ Reactive. Mean HBV DNA levels were approximately $7 \log$ $\mathrm{IU} / \mathrm{ml}$, median of $1830 \mathrm{IU} / \mathrm{ml}$. HCV ad HIV co-infection was seen in 4 and 2 patients respectively. Diabetes was present in 9 patients $(7.8 \%)$ and hypertension in 12 patients $(10.4 \%)$. ALT was normal in 71 patients $(61.7 \%) .38$ patients $(33 \%)$ had significant fibrosis (> F2 fibrosis), of which 19 had cirrhosis (16.5\%). 8 HBeAg Reactive patients $(27.5 \%)$ and $30 \mathrm{HBeAg}$ Negative $(34.8 \%)$ had significant fibrosis, with no significant difference between these groups. Patients with significant fibrosis were older $(\mathrm{p}<0.05)$ with higher mean ALT $(\mathrm{p}<0.05) .16$ patients with normal ALT had significant fibrosis $(22.5 \%)$. Diabetes and hypertension were significantly correlated with fibrosis.

Conclusion: A large proportion of IDAHS have significant fibrosis and warrant treatment in presence of detectable HBV DNA, despite normal ALT. Age is a predictive factor for increasing fibrosis. Routine non-invasive assessment for fibrosis is warranted and may help guide treatment in IDAHS.

\section{Session: Hepatocellular Carcinoma. Chairperson: \\ Tawesak Tanwandee; Akash Shukla; S P Singh}

\section{O-HCC-07}

\section{An integrated multidisciplinary approach for prediction of fibrosis progression and HCC development in chronic hepatitis $\mathrm{C}$ infected patients utilizing genomics, elastography, imaging and circulating tumor cells}

\section{Emmanuel Thomas $^{1}$, Eugene Schiff ${ }^{1}$, Masato Yoneda ${ }^{1}$, Ashutosh Agarwal $^{1}$, Beatrice Madrazo ${ }^{1}$ \\ ${ }^{1}$ University of MIami School of Medicine, United States}

Background: HCV induced HCC is higher in minority populations in the United States. African Americans (AAs) experience an increased risk of developing and dying from HCC. To serve these communities, it is imperative that we understand the genetic and cellular based mechanisms underpinning the linkages between HCV and HCC. The aims of our study were to assess changes in transient elastography (TE) and fibrosis-4 (FIB-4) score in patients with chronic hepatitis C
(CHC) in a large cohort of patients and in a subcohort who achieved SVR. Patients were also stratified for HCC risk based on genomic and imaging data with inclusion of screening for circulating tumor cells. Method: Our cohort included 2,000 patients and approximately 500 had liver biopsies. Our retrospective prospective study included 60 patients with CHC and a baseline liver biopsy who achieved SVR after treatment with DAA regimens and had a pretreatment TE study and at least one follow up TE measurement at 24 weeks or later post end of treatment response (EOTR). The estimated stage of liver fibrosis based on TE was categorized as F0-F2 $(<9.4 \mathrm{kpa})$, or F3 (9.5-12.4 Kpa), or F4/cirrhotics (TE $>12.5 \mathrm{kpa}$ ).

Result: Approximately 2,000 patients were successfully assessed by fibroscan and comparisons made with clinical parameters of liver disease. The median baseline TE for the 60 patients who achieved SVR was $11.9 \mathrm{Kpa}$ (range 3.8 to 65.2) and at follow up, TE decreased to $7.35 \mathrm{Kpa}$ (range 2.9 to 34.8 ) with a median change in TE of -3.4 $\mathrm{Kpa}$ (range -35.3 to $+1, \mathrm{p}=7.355 \mathrm{e}-11$ ). Follow up median TE done in the cirrhotic population after median time of 39 weeks post EOTR decreased to 11.7 Kpa and FIB4 was 2.3. The median change of TE in cirrhotic patients was $-6.5 \mathrm{kpa}$ (range -35.3 to $+1, \mathrm{p}=1.043 \mathrm{e}-7$ ) and for FIB4 was -1.97 (range -17.47 to -0.33 , $\mathrm{p}=1.49 \mathrm{e}-8$ ). Noncirrhotic patients $(\mathrm{TE}<12.4)$ comprised $55 \%$ of the entire cohort and their median change of TE was $-2.4 \mathrm{Kpa}$ (range -6.4 to 0.7 , $\mathrm{p}=1.539 \mathrm{e}-6$ ) and FIB4 was -0.68 (range -2.8 to $0.41, \mathrm{p}=2.987 \mathrm{e}$ 6). $48 \%$ of the entire cohort down-staged their liver fibrosis as determined by TE. In a multiple logistic regression analysis for factors associated with down-staging in liver fibrosis, we found that patients who were treatment naive were more likely to improve their fibrosis stage (OR 5.73, $\mathrm{p}=0.033$ ). In a subcohort, AAs had higher AST and BMIs and lower albumin levels $(\mathrm{P}<0.05)$.

Conclusion: Liver fibrosis stage, as determined by TE, improved after achieving SVR with DAA treatments in most patients. Although cirrhotic patients had a more significant drop in their median TE when compared to non-cirrhotic patients, they had a lower probability of improving their fibrosis stage and maintained a continued risk to develop HCC. In addition, we identified a group of patients whose TE score did not improve post SVR also representing a cohort of patients whose HCC risk remained. The patients who remain at risk for HCC will be further stratified for increased propensity to develop HCC utilizing genomics, advanced imaging and screening for circulating tumor cells.

\section{O-HCC-08}

Contrast enhanced ultrasound for assessment of therapeutic response following percutaneous ablation in hepatocellular carcinoma

Shashi Bala Paul ${ }^{1}$, Shekhar Singh ${ }^{1}$, Devesh Yadav ${ }^{1}$, Vidyasagar $\bar{R}^{1}$, Ekta Dhamija ${ }^{1}$, Shivanand R Gamanagatti ${ }^{1}$

${ }^{1}$ All India Institute of Medical Sciences, India

Background: Contrast enhanced ultrasound (CEUS) is an advanced ultrasound technique useful for evaluating liver lesions. Due to advantages of less cost, short evaluation time, usability in patients with deranged renal functions, high safety profile, CEUS has carved a special role in specific situations. We illustrate the CEUS findings in hepatocellular carcinoma (HCC) patients following ablation.

Method: HCC patients treated by percutaneous acetic acid (PAI)/ Radiofrequency ablation(RFA), were subjected to CEUS performed on Supersonic aixplorer ultrasound machine using ultrasound contrast (Sonovue, Bracco, Milan, Italy), $2.4 \mathrm{ml}$ per nodule. The enhancement characteristics of liver nodule were evaluated in 3 vascular phasesarterial (AP, at 15-25 seconds), venous (VP) at 45-90 seconds and 
delayed (DP, at 180 seconds) .Interpretation was done on cine recording. Tumor response was based on the mRECIST criteria. CEUS findings were co-related with multiphase computerized Tomography (MPCT)/Magnetic Resonance Imaging (MRI) liver.

Result: Thirty one HCCs in 21 patients, less than $5 \mathrm{~cm}$ size were evaluated by at a mean interval of $2.1+1.8$ months post-ablation. On CEUS, CR was detected in 12(38.7\%) masses.PR was noted in $19(61.3 \%)$ masses and the pattern of residual tumor in these masses was peripheral (11 masses), homogenous (2) and heterogenous (4). CEUS findings corroborated with MPCT/MRI in all cases.

Conclusion: CEUS has the ability to assess post ablation response in focal HCC patients and can be used as an alternative or a complimentary technique to MPCT/MRI in specific situations.

\section{O-HCC-09}

Receiver operating characteristic curve - Independent predictive risk factors for acute hepatic failure after TACE. The area under receiver operating characteristic curve for ICG is 0.972 .
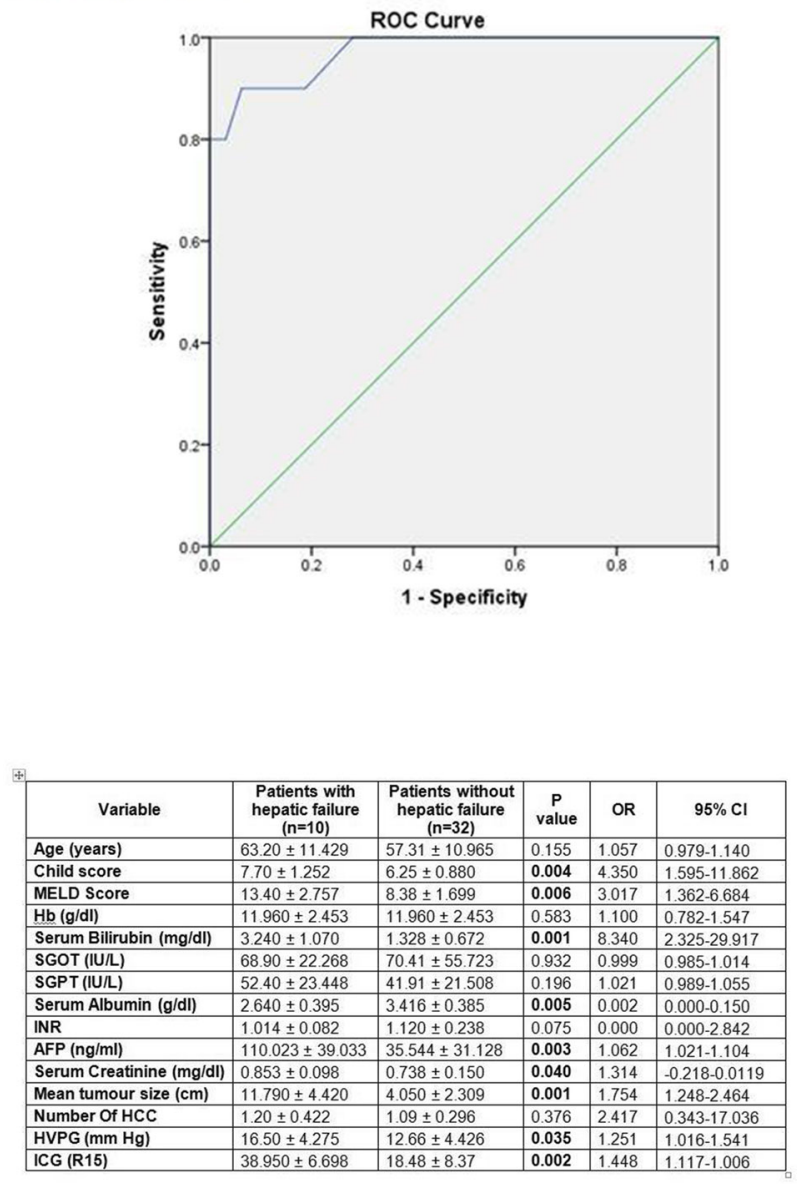

Univariate analysis of risk factors for acute hepatic failure after TACE
O-HCC-10

Outcomes of transarterial therapies in ruptured hepatocellular carcinoma

Deepak Agarwal ${ }^{1}$, Ajit Thapa ${ }^{1}$, Rohit Khandelwal ${ }^{1}$, Saurabh Gupta $^{1}$, Abhay Kapoor ${ }^{1}$, Anubhav Khandelwal ${ }^{1}$, Sanjay Saran Baijal $^{1}$

${ }^{1}$ Medanta-The Medicity, India

Background: Spontaneous rupture of hepatocellular carcinoma is a complication seen in 3\%-15\% of cases with 30-day mortality is $31 \%$ to $38 \%$ and Median survival time is 8.9 to 23 weeks. Present with sudden onset pain abdomen in known or unknown case of HCC. CT Angiography diagnosed hepatic tumor, hemoperitoneum, sub capsular hematoma or active contrast extravasation. So we evaluate the outcome of transarterial embolization in ruptured hepatocellular carcinoma.

Method: Retrospective study of 12 patients (all male aged 31 to 82 years, Mean Age: 59.3 years) with ruptured HCC who underwent transarterial embolization from Nov 2014 to Dec 2017. Ruptured HCC diagnosed on CTA. Seven patients underwent super selective transarterial embolization (TAE, using polyvinyl alcohol with gel foam: $\mathrm{n}=3$, Gel foam: $\mathrm{n}=3$ and PVA: $\mathrm{n}=1$ ) and five patients were treated with supers elective Transarterial chemo-embolization (TACE, c-TACE: $\mathrm{n}=4$; DEB TACE: $\mathrm{n}=1$ ).

Result: From November 2014 to December 2017, 12 male patients with mean age of 59.3 years (Range of 32 to 82 years) presented in intervention radiology department with ruptured HCC. Abdominal pain was presenting symptom in all patients and diagnosis of ruptured HCC was confirmed on computerized tomography angiography (CTA). Distribution of patients in Child Pugh score A, B \& $\mathrm{C}$ was seven, three and two patients respectively. Mean total bilirubin level was $1.95 \mathrm{mg} / \mathrm{dl}$ (Range 0.8 to $3.8 \mathrm{mg} / \mathrm{dl}$ ). Mean size of ruptured HCC was $8.2 \mathrm{~cm}$ (Range $4.4 \mathrm{~cm}$ to $16 \mathrm{~cm}$ ), of which ten seen in right lobe and remaining two tumors involved the left lobe. Two patients with right lobe tumours presented with hemodynamic instability and hypovolaemic shock due to hemoperitoneum and remaining patients presented with contained peri-tumoral bleed. Angiographic demonstration of active extravasation was seen in one patient. Patients undergone transarterial embolization had median serum bilirubin level $1.7 \mathrm{mg} / \mathrm{dl}$ (Range: 1.2 to $38 \mathrm{mg} / \mathrm{dl}$ ) while patients who undergone for TACE had median bilirubin level $1.2 \mathrm{mg} / \mathrm{dl}$ (Range: 0.8 to $3.6 \mathrm{mg} / \mathrm{dl}$ ). Both patients presented with hemoperitoneum were in child $\mathrm{C}$ and died within 30 days. Average hospital stay of the remaining ten patients was 10.6 days and these patients were discharged from hospital in stable condition.

Conclusion: Transarterial embolization is safe and effective method of treatment for ruptured hepatocellular carcinoma.

\begin{tabular}{|c|c|c|c|}
\hline \multicolumn{2}{|l|}{ VARIABLES (N-12) } & VALUE & SURVIVAL ( $>30$ DAYS) \\
\hline \multicolumn{2}{|l|}{ AGE } & 31-82 years & \\
\hline \multicolumn{2}{|c|}{ SEX (MALE/FEMALE) } & Male & \\
\hline \multirow{5}{*}{ EnOLOGY } & \multirow{5}{*}{$\begin{array}{l}\text { HBV } \\
\text { HCV } \\
\text { ALD } \\
\text { NASH } \\
\text { NON-CIRRHOTIC }\end{array}$} & 02 & 02 \\
\hline & & 04 & 03 \\
\hline & & 01 & 01 \\
\hline & & 03 & 02 \\
\hline & & 02 & 02 \\
\hline \multirow{3}{*}{\multicolumn{2}{|c|}{$\begin{array}{r}\text { CHILD SCORE } \\
\text { A } \\
\text { B } \\
\text { C }\end{array}$}} & 07 & 07 \\
\hline & & 03 & 03 \\
\hline & & 02 & 00 \\
\hline \multirow{2}{*}{\multicolumn{2}{|c|}{$\begin{array}{ll}\text { HEMODYNAMIC } & \begin{array}{l}\text { STABLE } \\
\text { UNSTABLE }\end{array} \\
\end{array}$}} & 10 & 10 \\
\hline & & 02 & 00 \\
\hline \multirow[t]{3}{*}{ SIZE } & \multirow{3}{*}{$\begin{array}{l}<5 \mathrm{CM} \\
5-10 \mathrm{~cm} \\
>10 \mathrm{~cm}\end{array}$} & 04 & 03 \\
\hline & & 04 & 04 \\
\hline & & 04 & 03 \\
\hline \multirow[t]{3}{*}{ LOCATION } & \multirow{3}{*}{$\begin{array}{l}\text { LARGE } \\
\text { PERIPHERAL } \\
\text { BULGING }\end{array}$} & 04 & 03 \\
\hline & & 06 & 06 \\
\hline & & 02 & 01 \\
\hline \multirow{2}{*}{\multicolumn{2}{|c|}{ PORTAL VEIN THROMBOSIS }} & 02 & 01 \\
\hline & ABSENT & 10 & 09 \\
\hline
\end{tabular}




\section{$O-H C C-11$}

Survival in patients with hepatocellular carcinoma is related to screening and underlying diagnosis

\section{Ibrahim Hassan ${ }^{1}$, Ed Gane ${ }^{1}$, Debi Prasad ${ }^{1}$, Adam Bartlett ${ }^{1}$, Oonagh Lithgow 1 \\ ${ }^{1}$ New Zealand Liver Transplant unit (NZLTU), Auckland City Hospital, Auckland, New Zealand, New Zealand}

Background: Hepatocellular carcinoma (HCC) is the most rapidly increasing cause of cancer mortality in New Zealand due to endemic hepatitis $\mathrm{B}$ virus (HBV) infection and recent hepatitis $\mathrm{C}$ virus (HCV) and obesity epidemics. The objectives of this audit are to describe the changing landscape of HCC referred to a national HCC service since 1998, in particular, changes in the underlying liver disease, rate of screening uptake and curative treatments and survival.

Method: All newly diagnosed cases of HCC referred to New Zealand Liver Transplant unit (NZLTU) between 1998 and 2016 were included, with follow-up available until June 2017. Data on patient demographics, liver disease aetiology, screening status and HCC stage at diagnosis were collected from chart review. Data were also collected on transplantation, resection and ablation. Kaplan Meier survival estimates were derived from the national mortality database. Recruitment in and adherence with HCC surveillance were recorded. Result: HCC diagnosis rates have increased from 25 cases in 1998 to 260 in 2016, an increase of $20 \%$ per annum. The total of 1683 HCC cases were divided into 3 cohorts (Era 1: Jan 1998 to Dec 2008; Era 2: Jan 2009 to July 2013; Era 3: August 2013 to June 2016), each comprising 561 patients. During the study period, overall survival improved $(\mathrm{p}=0.005)$. The proportion with screen-detected HCC was similar across the 3 cohorts (43\% in Era 1, $42 \%$ in Era 2 and $47 \%$ in Era 3 ) as was the proportion who received curative therapy (35\% in Era 1, 37\% in Era 2 and 41\% in Era 3). 5 and 10-year survival were higher in screen-detected cases $(49 \%$ and $43 \%)$ than in nonscreendetected cases (14\% and 10\%).

Conclusion: The improvement in survival in patients with HCC over the past 20 years likely reflects improved screening uptake and better management of the nonscreen-detected cases. Lower survival in NASH and ALD patients may reflect poor screening uptake and lack of awareness of risk factors in these patients.

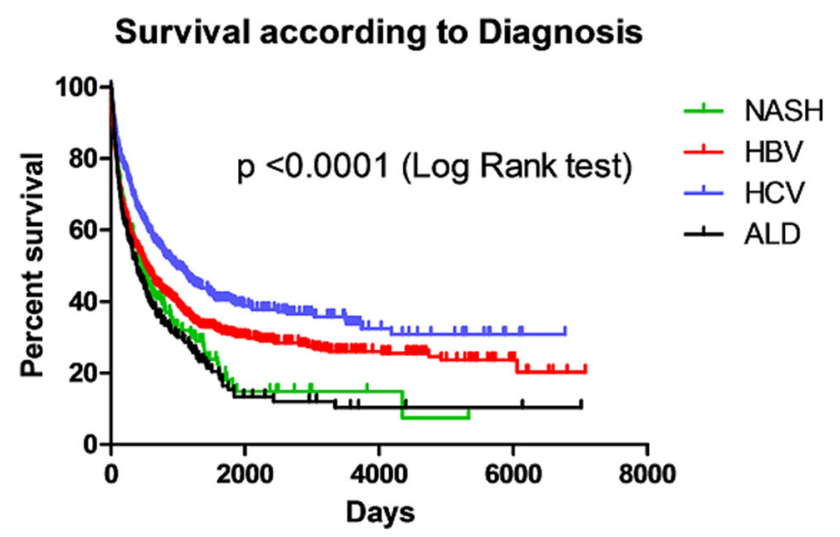

Survival (Total)

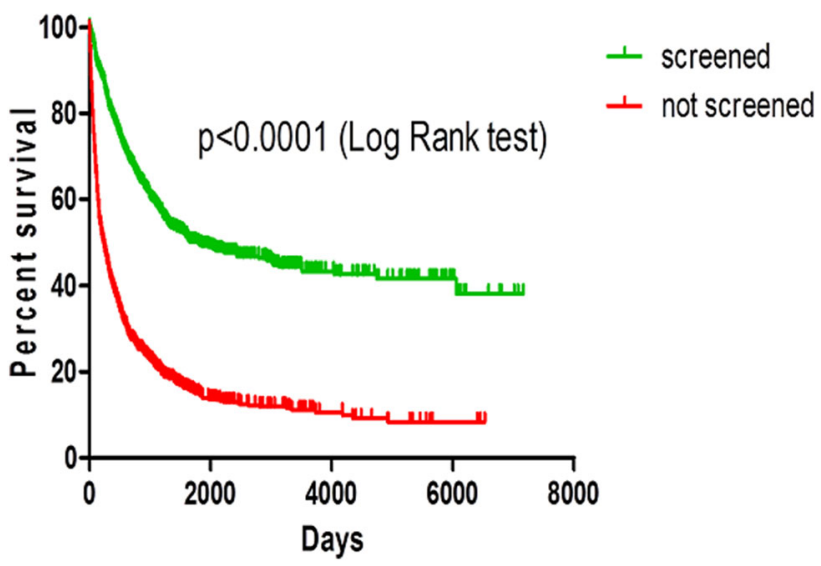

$\mathrm{O}-\mathrm{HCC}-12$

Clinical characteristics, potential etiology, prevalence of cirrhosis and mode of detection for non-B non- $C$ hepatocellular carcinoma in Korea

Jihye Kim ${ }^{1}$, Donghyun Sinn ${ }^{3}$, Wonseok Kang ${ }^{3}$, Geum Youn Gwak $^{3}$, Moon Seok Choi ${ }^{3}$, Yong Han Paik ${ }^{3}$, Joon Hyeok Lee ${ }^{3}$, Kwang Cheol $\mathrm{Koh}^{3}$, Seung Woon Paik ${ }^{3}$, Byung Chul $\mathrm{Yoo}^{2}$

${ }^{1}$ Samsung Medical Center, Sungkyunkwan University School of Medicine, Korea, Republic of; ${ }^{2}$ Konkuk University School of Medicine, Korea, Republic of; ${ }^{3}$ Samsung Medical Center, Korea, Republic of

Background: Regular surveillance of hepatocellular carcinoma (HCC) is recommended in patients with chronic viral hepatitis $(\mathrm{B}, \mathrm{C})$ and/or cirrhosis. Yet, several reports suggest that cirrhosis is not always present in non-B non-C (NBNC) HCC which accounts for one fourth of the HCC in Korea. We aimed to investigate clinical features of NBNC-HCC patients, especially mode of detection and prevalence of cirrhosis.

Method: This single center, retrospective cohort study included consecutive 447 adult treatment-nä̈ve NBNC-HCC patients who diagnosed HCC and received care at Samsung Medical Center in Korea from 2010 to 2013. NBNC was defined as HBsAg negative and anti-HCV antibody negative. Mode of detection was classified as under surveillance, incidental and symptomatic. Cirrhosis was determined by using following criteria: histology if available, radiology, varices by upper endoscopy, fibrosis-4 score.

Result: Heavy alcohol use was most common etiology in NBNC HCC (NBNC-A, alcohol) (267, 59.7\%). Ten patients had other identifiable causes (NBNC-O, other identifiable cause), including autoimmune hepatitis, Budd-Chiary syndrome. Among 170 patients without identifiable causes (NBNC-NA-NO, non-alcohol, no-other identifiable cause), $\mathrm{HBcIgG}$ value was available for 115 patients (67.6\%). HBcIgG were positive for $83.5 \%$ of patients $(96 / 115)$. Presence of metabolic risk factors (diabetes and/or obesity) was observed in $66.7 \%$ of patients $(64 / 96)$ with $\mathrm{HBcIgG}(+)$ and $68.4 \%$ of patients (13/19) with HBcIgG (-). Overall, 148 patients $(33.3 \%)$ had no evidence of cirrhosis, either by histology, radiologic evaluation, upper endoscopy or serum fibrosis marker. Only 114 patients $(25.5 \%)$ were detected by regular surveillance. Among 10 patients with other identifiable cause, $90 \%$ had cirrhosis, $70 \%$ had diabetes or obesity, and $80 \%$ were detected during regular surveillance. Among 267 patients with heavy alcohol use history, $70.4 \%$ had cirrhosis, $65.9 \%$ 
had DM or obesity, and $27.7 \%$ were diagnosed during regular surveillance. Among 170 patients without identifiable cause or heavy alcohol use history (NBNC-NA-NO), 60.0\% had cirrhosis, $67.6 \%$ had $\mathrm{DM}$ or obesity and $18.8 \%$ were diagnosed during regular surveillance. Only $2.9 \%$ were diagnosed under surveillance among NBNC-NA-NO HCC without evidence of cirrhosis.

Conclusion: A significant proportion of NBNC-HCC patients did not show cirrhosis and were not under surveillance at the time of diagnosis, especially for NBNC-NA-NO HCC. Identification of cirrhosis would be inadequate approach to identify NBNC patients who may benefit from HCC surveillance.

Session: Public Health. Chairpersons: Hollinger F. Blaine; Rajoo Singh

\section{$O-P U B H-01$}

Community based Hepatitis B prevention program to improve knowledge, immunization and preventive practices among women of childbearing age in urban slum communities

\section{$\underline{\text { Divyesh Jeram Devaliya }}^{1}$, Shripad Desai ${ }^{1}$, Maitreyee Patwardhan ${ }^{1}$}

${ }^{1}$ Non Profit Organization, India

Background: The HBsAg prevalence among pregnant women in India varies between $0.9 \%$ and $11.2 \%$. Mother-to-child transmission is major mode of transmission in endemic areas where little is known about women knowledge and safe practices. This indicates a considerable need for a community based interventions to protect their offspring. This unique study assess the impact of such program through baseline-midline

Method: Prior implementation of community based hepatitis B prevention program; health workers approached 7560 childbearing women through house visits and with pre consent, baseline data was collected through pretested questionnaire. After 2 years of interventions focused on education, screening, immunization, contact tracing and referrals an external midline evaluation was conducted among sample of 378 women, statistically derived from baseline sample. Data was analyzed to assess the impact of program.

Result: Post interventions, $82 \%$ women were aware about modes of hepatitis B transmission with high accuracy on majority of modes, compared to only $1.8 \%$ in baseline survey. The accuracy was high for majority of different modes of transmission. The correct knowledge on different signs and symptoms was reported by $76 \%$ as against by $1.6 \%$ in baseline. Additionally, $96 \%$ could correctly mention yellowish color of eyes, skin \& urine as a major symptom of Hepatitis B in the midline survey. The baseline record saw only $0.8 \%$ women, ever screened for Hepatitis B, while midline survey recorded $95 \%$ screened for hepatitis B. Women ever vaccinated with at least 1 dose saw an increase from $0.2 \%$ to $94.5 \%$. Program educational and medical interventions encouraged women to come forward for testing and it resulted in doubling the reported cases of hepatitis B from baseline $(0.5 \%)$ to midline $(1.2 \%)$. As a result increased disease awareness helped to encourage testing which helped in mapping the undiagnosed hepatitis B carrier cases. Hepatitis B vaccination to women averted the risk of mother to child transmission among the new born. Overall the program proved to be effective, vital and in right direction towards community based prevention of Hepatitis B Conclusion: The comprehensive program has led to a significant increase in knowledge, practices, vaccination and willingness to test for Hepatitis B among the women and proved to be a replicable program.
O-PUBH-O2

Cost-effectiveness of treatment for Hepatitis $\mathrm{C}$ virus with direct acting antivirals in Phnom Penh, Cambodia

Nyashadzaishe Mafirakureva ${ }^{1}$, Josephine Walker ${ }^{1}$, Momoko Iwamoto $^{2}$, Linda Campbell ${ }^{1}$, Jean Philippe Dousset ${ }^{2}$, Mickael Le Paih $^{2}$, David Maman ${ }^{3}$, Joanna Coast ${ }^{1}$, Anne Loarec ${ }^{3}$, Peter Vickerman $^{1}$

${ }^{1}$ University of Bristol, United Kingdom; ${ }^{2} \mathrm{MA} \odot$ decins Sans

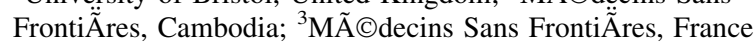

Background: In low and middle-income countries, where $80 \%$ of global Hepatitis $\mathrm{C}$ virus (HCV) infections are found, new and effective HCV treatments (direct acting antivirals, DAA) are not widely available. In 2016, Médecins Sans Frontières (MSF) established the first general population HCV screening and treatment site in Cambodia. We performed cost and cost-effectiveness analyses to compare observed and alternative treatment strategies to a counterfactual of no treatment.

Method: Following standard costing techniques, we identified, quantified and valued the resources used in the screening, diagnosis, and treatment of chronic HCV patients. Patient level data was collected from an on-going prospective, observational cohort study evaluating the 'real-world' effectiveness of the new DAAs in the treatment of $\mathrm{HCV}$ at one centre in Phnom Penh, Cambodia. A Markov model was developed and used to estimate lifetime costs, quality adjusted life years (QALYs) and cost-effectiveness of the intervention using parameters estimated from observed data and literature.

Result: The patients sampled $(n=612)$ comprised $60 \%$ women with a mean age of 57 (range $20-85$ ) years. The liver disease stage composition was pre-cirrhosis $(\mathrm{n}=255,41.7 \%)$, cirrhosis $(\mathrm{n}=328$, 53.6), decompensated cirrhosis (DC, $\mathrm{n}=24,3.9 \%$ ) and hepatocellular carcinoma $(n=5,0.8 \%)$. The average cost of treatment (excluding screening) was $\$ 1151$ (SD $\$ 328$ ), with $\$ 733$ for DAA. Reducing the DAA costs for all patients to the recent negotiated price for MSF in Cambodia of $\$ 120$ reduced the total treatment cost to $\$ 539$ (SD \$84). In the intervention, 63\% of patients were enrolled on treatment, with rates of treatment $>90 \%$ for patients with liver fibrosis stage F3, F4, or DC, and 30\% for fibrosis stages F0-F2. Targeted treatment as implemented (ICER \$4247) was dominated by the strategy in which all individuals were treated at the average rate (ICER \$1306). At a cost-effectiveness threshold of one GDP per capita (\$1270 for Cambodia), these interventions are not cost-effective. With the new DAA cost, targeted treatment has an ICER of \$2317 and equal treatment \$688 (Figure).

Conclusion: The model of care for HCV screening and treatment as implemented by MSF in Cambodia was not cost-effective at a threshold of one GDP per capita, emphasizing the need for lower cost DAA and a simplified model of care to reduce treatment costs. 


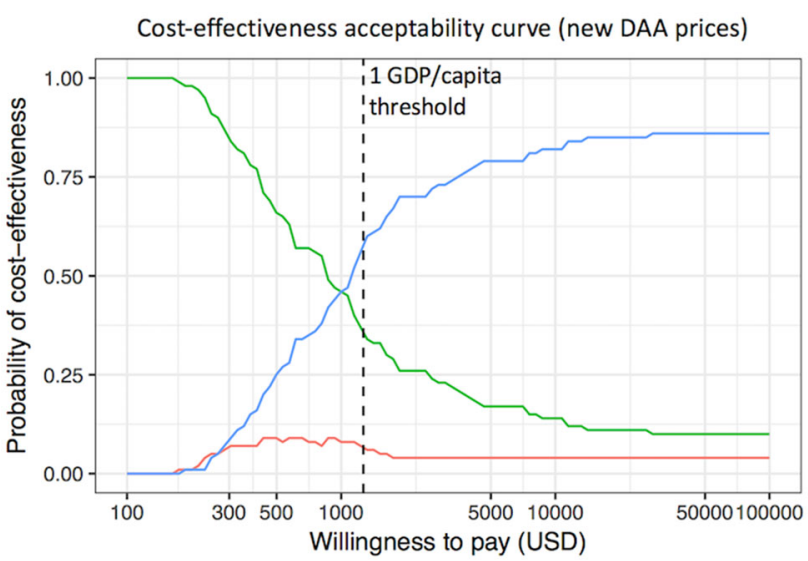

Strategy — Current_treatment - No_treatment - Treat_all

\section{O-PUBH-03}

Meeting the World Health Organization Global Health Sector Strategies for Hepatitis $\mathrm{C}$ in a high burden country, Pakistan

Devin Razavi Shearer ${ }^{1}$, Amir Ghafoor Khan ${ }^{11}$, Betty Chiang ${ }^{10}$, Wasim Jafri $^{2}$, Huma Qureshi $^{14}$, Asad Chaudhry ${ }^{12}$, Zaigham Abbas $^{19}$, Altaf Alam ${ }^{18}$, Altaf Baqir ${ }^{13}$, Javed Iqbal Farooqi ${ }^{16}$, Muhammad Sadik Memon ${ }^{3}$, Arif Nawaz ${ }^{8}$, Amjad Salamat ${ }^{6}$, Masood Siddiq ${ }^{9}$, Arif Mehmood Siddiqui ${ }^{4}$, Ghias Un Nabi Tayyab $^{15}$, Moazzam Ud Din ${ }^{5}$, Aasim Yusuf ${ }^{17}$, Bader Faiyaz Zuberi $^{7}$

${ }^{1}$ The CDA Foundation, United States; ${ }^{10}$ Gilead Sciences, United States; ${ }^{11}$ Khyber Teaching Hospital, Pakistan; ${ }^{12}$ Liver Foundation, Pakistan; ${ }^{13}$ Nishtar Hospital, Pakistan; ${ }^{14}$ Pakistan Medical Research Council, Pakistan; ${ }^{15}$ PGMI, Pakistan; ${ }^{16}$ Post Graduate Medical Institute, Pakistan; ${ }^{17}$ Shaukat Khanum Hospital, Pakistan; ${ }^{18}$ Sheikh Zayed Medical Institute, Pakistan; ${ }^{19}$ Ziauddin University, Pakistan;

${ }^{2}$ Aga Khan University, Pakistan; ${ }^{3}$ AIMS, Pakistan; ${ }^{4}$ Allama Iqbal Medical College, Pakistan; ${ }^{5}$ Bolan Medical College, Pakistan; ${ }^{6} \mathrm{CMH}$, Pakistan; ${ }^{7}$ Dow Medical College, Pakistan; ${ }^{8}$ Fatima Memorial Hospital, Pakistan; ${ }^{9}$ Fauji Foundation Hospital, Pakistan

Background: Pakistan has the largest number of individuals with active hepatitis $\mathrm{C}$ virus (HCV) infection of all countries but China. An estimated $3.7 \%$ of the general population or $7,140,200$ persons were infected with HCV (viremic) in 2016. In high burden countries it is imperative to examine strategies that will not only reduce the disease burden, but the continuing transmission as well on the path towards elimination.

Method: Using a Markov model, the progression of the HCV infected population was quantified and prevention, treatment and screening increases were utilized to meet the World Health Organization Global Health Sector Strategies for Hepatitis. The inputs were gathered through a review of local and international publications, analysis of local data, and discussions with an expert panel.

Result: The elimination of HCV by 2030 is only feasible through aggressive prevention, treatment and screening. An estimated 212,000 new infections occur annually, and while treatment has increased to an estimated 160,700 individuals in 2016, transmission continues to be an issue. Contaminated medical equipment remains a large risk factor, and education campaigns combined with increased blood safety, single use syringes, and the availability of sterile equipment could reduce the new infections by $50 \%$. By increasing screening to keep pace with treatment increases, it is estimated that 450,000 individuals would need to be treated in 2025 and beyond, expanding treatment to the older population and utilizing treatment regimens that result in $90 \%$ of treated individuals having a sustained viral response. This would require 520,000 individuals screened annually starting in 2025.

Conclusion: Meeting the 2030 goals in Pakistan is achievable but it will require wide scale education, implementation of a rigorous blood screening policy, and the widespread availability of single use syringes and sterile equipment, along with screening and treatment. This strategy would avert 116,900 liver related deaths and 76,800 new hepatocellular carcinoma cases in 15 years.

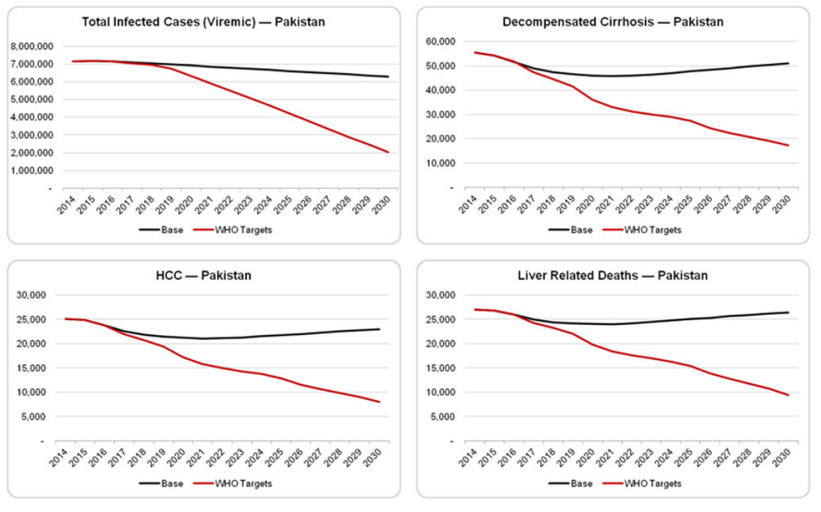

\section{O-PUBH-04}

The Health and Limitation Index (HALex) in Patients with Chronic Liver Disease: A Population Based Study of United States Adults

\section{$\underline{\text { Mohamed I Elsaid }}{ }^{1}$, Jewook $\mathrm{Ha}^{2}$, Tina $\mathrm{John}^{3}$, Vinod Rustgi ${ }^{3}$}

${ }^{1}$ Rutgers, the State University of New Jersey, United States; ${ }^{2}$ New York Medical College/St. Joseph's Health, United States; ${ }^{3}$ Rutgers Robert Wood Johnson Medical School, United States

Background: A significant number of patients with Chronic Liver Disease (CLD) suffer from Multiple Chronic Comorbidities (MCC). Several studies have investigated the relationship between measures of Health Related Quality of Life (HRQoL) among patients with CLD. However, no study to date has examined the effects of the type and total number of MCC on HRQoL among patients with CLD. In addition, no study to date has investigated the association between MCC and disability measures in patients with CLD. The Health and Activity Limitation Index (HALex) is a validated multi-attribute scale that combines patients' activity limitations and perceived health status. Accordingly, we aimed to examine the association between the total number MCC and predictors of health and activity limitations using the HALex in patients with CLD.

Method: The study sample included 1,756 adults with CLD aged 18-85 who participated in the 2014 and 2015 National Health Survey Interview. The outcome of interest was reporting poor HALex score below the 10th percentile for US adults. CLD was limited to adults who reported having CLD in the past 12 months. MCC included selfreported history of 14 chronic conditions: cancer, ulcer, hypertension, diabetes, arthritis, kidney failure, coronary heart disease, angina, myocardial infarction, stroke, other heart conditions, emphysema, COPD, and asthma. We then categorized the number of comorbidities into five groups $(0,1,2,3,4+)$. Multivariable logistic regression models adjusting for age, smoking status, race/ethnicity, gender, education, marital status, body mass index (BMI), health insurance coverage, history of cancer, survey year were used to examine the 
association between MCC and poor health status in patients with CLD.

Result: An estimated $19.65 \%$ of adults with CLD reported having four or more additional MCC. CLD was a significant predictor of poor HALex Adjusted Odds Ratio (AOR) 2.75 (95\% CI; 2.32, 3.26). Increase in the number of MCC was associated with higher odds of poor HALex, poor reported health status and limitations in daily activities in all models (Table). Among adults with CLD, the odds of poor HALex were AOR 9.43 (95\% CI; 5.35, 16.29) higher for those with $4+$ MCC versus adults with just liver disease. In the diseasespecific model for adults with CLD, predictors of poor HALex were having ulcer in the past 12 months, kidney failure, stroke, COPD, asthma hypertension and arthritis. The highest odds of poor HALex were for adults with CLD and history of kidney failure AOR 2.32 (95\% CI; 1.30, 3.82).

Conclusion: Adults with CLD were associated with poor HALex, poor reported health status and limitations in daily activities. The presence of two or more MCC was associated with poor HALex. Adults with CLD and history of kidney failure have the highest odds of limitations to health related quality of life.

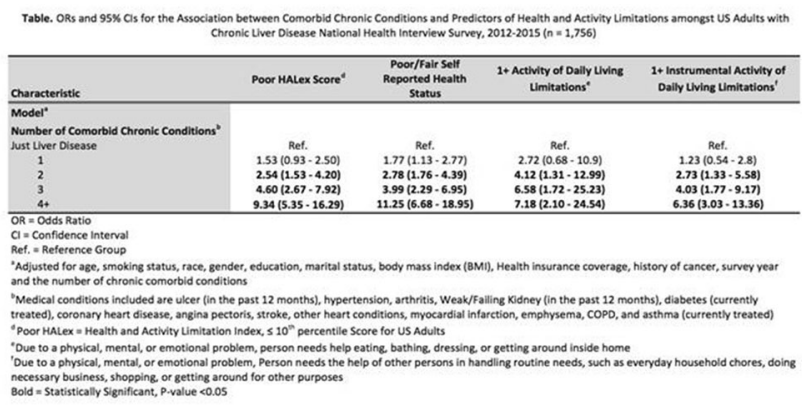

\section{O-PUBH-05}

\section{Achieving the World Health Organization Global Health Sector Strategies for Hepatitis C in Kazakhstan}

\section{Devin Razavi Shearer ${ }^{1}$, Kulpash Kaliaskarova ${ }^{3}$, Aliya} Konysbekova $^{6}$, Yuriy Prokopenko ${ }^{5}$, Roman $\mathrm{Vi}^{2}$, Yesmembetov Yesmembetov $^{4}$

${ }^{1}$ The CDA Foundation, United States; ${ }^{2}$ International HepatoTransplant Group, Astana, Kazakhstan, Kazakhsta; ${ }^{3}$ Ministry of Healthcare and Social Development of the Republic of Kazakhstan, Astana, Kazakhstan, Kazakhsta; ${ }^{4}$ National Scientific Center of Oncology and Transplantology, Astana, Kazakhstan, Kazakhsta; ${ }^{5}$ Republican Coordination Center for Hepatology and Gastroenterology, Astana, Kazakhstan, Kazakhsta; ${ }^{6}$ Republican Diagnostic Center, Astana, Kazakhstan, Kazakhsta

Background: In Kazakhstan, it is estimated that $1.9 \%$ or 331200 individuals are viremically infected with hepatitis $\mathrm{C}$ virus (HCV). With restrictions currently in place on treatment, it is necessary to examine the impact of expanding treatment and what is necessary to meet the World Health Organization Global Health Sector Strategy (GHSS) goals for hepatitis C by 2030.

Method: Using a Markov model, the progression of the HCV infected population was quantified and predicted through 2030. All of the inputs were compiled through a review of local and international publications, analysis of local data, and discussions with an expert panel.

Result: Currently, treatment is restricted to those $\geq$ F3 and while there has been an increase in the use of direct acting anti-virals (DAAs), there still is the usage of older therapies. In order to reach the
WHO GHSS by 2030, treatment will need to be expanded to at least $\geq \mathrm{F} 1$ and at least those aged 74 years old. The sole use of DAAs will also be necessary in order to reduce the number of individuals needing to be retreated. By 2023, treatment would need to increase from 1750 to 22000 individuals treated annually and newly diagnosed would need to increase from 4000 to 23700 . Large increases in the education and prevention of the transmission of $\mathrm{HCV}$ must be implemented by 2022 . Without additional interventions, it is estimated that there will be almost 1500 liver related deaths by 2030 , with large increases seen across all of the later disease stages as compared with today (Figure 1).

Conclusion: Implementing a strategy to attempt to meet the GHSS 2030 goals would avert 7500 liver related deaths and 5000 new hepatocellular carcinoma cases and would set Kazakhstan on a path towards elimination.

Figure 1. Base Case vs WHO Target Scenario 2015-2030, Kazakhstan.

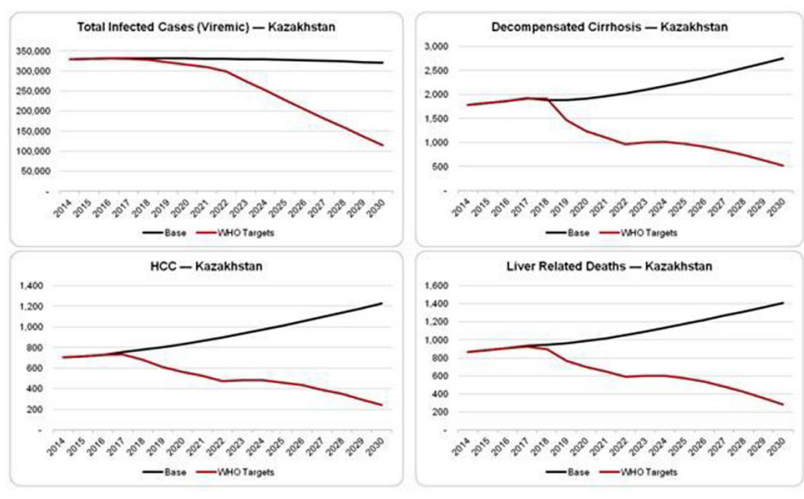

\section{O-PUBH-06}

Modelling the Scale-Up of Screening and Treatment for Hepatitis C Virus Elimination in Pakistan: Quantifying Impact and Efficiency

Aaron G Lim ${ }^{1}$, Josephine G Walker ${ }^{1}$, Huma Qureshi ${ }^{3}$, Hassan Mahmood $^{3}$, Saeed Hamid ${ }^{2}$, Francisco Averhoff ${ }^{4}$, Peter Vickerman $^{1}$

${ }^{1}$ University of Bristol, United Kingdom; ${ }^{2}$ Aga Khan University, Pakista; ${ }^{3}$ Pakistan Health Research Council, Pakista; ${ }^{4}$ U.S. Centers for Disease Control and Prevention, United States

Background: Pakistan has the world's second-largest hepatitis C virus (HCV) burden. With increasing access to highly effective directacting antiviral (DAA) treatment, Pakistan can now aim for the World Health Organization (WHO) HCV elimination targets. However, low levels of diagnosis and referral present challenges to treatment scaleup. We model the impact of screening and treatment interventions in Pakistan to reduce HCV incidence by $90 \%$ and HCV mortality by $65 \%$ by 2030 .

Method: We developed a dynamic HCV transmission model for Pakistan incorporating screening and treatment, and calibrated to national $\mathrm{HCV}$ seroprevalence data from 2007 (4.8\%), surveys in people who inject drugs (PWID, 56-69\%), and HCV prevalence trends in blood-donors. Compared to a counterfactual of no treatment, we determine what screening coverage is needed from 2017 to sustain current treatment levels (150,000 annual treatments). We estimated impact and costs of scaling-up screening, DAA treatment, and prevention interventions directed at achieving WHO HCV-elimination targets. 
Result: Current levels of treatment can be maintained by implementing general population screening with $5 \%$ of individuals screened annually ( 10 million tested/year) and $35 \%$ of diagnosed individuals initiating treatment, resulting in 82 tests per treatment and 5 infections averted per 1000 antibody screenings (IA/1000 Ab). However, incidence and mortality will rise by $6 \%$ and $38 \%$, respectively, by 2030 . Much greater impact is achieved by doubling annual screening to $10 \%$, targeting priority groups with higher prevalence of $\mathrm{HCV}$ infection such as PWID and adults (>20 years), increasing referral rates to $90 \%$, and introducing re-screening every 5 years, with 56 tests per treatment, $10 \mathrm{IA} / 1000 \mathrm{Ab}$, and the WHO mortality target reached by 2041 . However, only with also halving HCV transmission risk in PWID will HCV incidence and mortality approach the WHO targets, now being reached by 2036 . Preliminary costing estimates suggest that screening and treatment scale-up in Pakistan is likely to cost about USD $\$ 2,000$ per cure, with the majority (70\%) of costs coming from screening.

Conclusion: Substantial scale-up of screening and treatment interventions will be required to achieve the WHO HCV elimination targets. This can be optimised if targeted strategies are undertaken, but despite this, costs will be substantial.

\section{Session: Hepatitis B Virus Infection- Clinical ; Chairpersons; Baldev Rana; Zaigam Abbas; Shalimar}

\section{$O-H B V-31$}

Functional remission of HBeAg negative chronic HBV infection after withdrawal of combined therapy with REP 2139 or REP 2165, tenofovir disoproxil fumarate and pegylated interferon a-2a

Andrew Vaillant ${ }^{1}$ Michel Bazinet ${ }^{1}$, Victor Pantea ${ }^{3}$, Gheorghe Placinta $^{3}$, Iurie Moscalu ${ }^{2}$, Valentin Cebotarescu ${ }^{3}$, Lilia Cojuhari ${ }^{3}$, Pavlina Jimbei ${ }^{4}$, Liviu Iarovoi ${ }^{3}$, Valentina Smesnoi ${ }^{4}$, Tatiana Musteata $^{4}$, Alina Jucov ${ }^{3}$, Adalbert Krawczyk ${ }^{5}$

${ }^{1}$ Replicor Inc., Canada; ${ }^{2}$ ARENSIA Exploratory Medicine, Republican Clinical Hospital, Moldova; ${ }^{3}$ Nicolae Testemitanu State University of Medicine and Pharmacy, Moldova; ${ }^{4}$ Toma Ciorba Infectious Clinical Hospital, Moldova; ${ }^{5}$ UniversitÃătsklinikum Essen, Institute for Virology, Germany

Background: The REP 401 protocol (NCT02565719) is a randomized, controlled trial assessing the safety and efficacy of the nucleic acid polymers REP 2139 (lead clinical candidate) or REP 2165 combined with tenofovir disoproxil fumarate (TDF) and pegylated interferon $\alpha$-2a (peg-IFN) in treatment naïve Caucasian patients with chronic $\mathrm{HBeAg}$ negative $\mathrm{HBV}$ infection.

Method: TDF monotherapy ( 24 weeks, $300 \mathrm{mg}$ PO qD) was followed by randomization into experimental and control groups. Experimental patients received 48 weeks of TDF, peg-IFN (180ug SC $\mathrm{qW}$ ) and REP 2139 or REP 2165 (250 mg IV infusion qW). Control patients receive 48 weeks of TDF + peg-IFN but crossover to 48 weeks of experimental therapy in the absence of a $3 \log$ drop in HBsAg after 24 weeks of peg-IFN. Viremia is monitored on the Abbott Architect and Realtime platforms.

Result: Currently, 19/20 control patients have been crossed over and have completed $\geq 24$ weeks of experimental therapy and 19/20 experimental patients have completed treatment and 4-12 weeks of follow-up. Therapy is well tolerated in all except in one crossover patient (REP 2165) who withdrew from therapy due to pegIFN-related depression. Following crossover in the control group, 10/10 patients (REP 2139) and 9/10 patients (REP 2165) have HBsAg reductions $>1 \log$ from baseline. From these 19 responder patients,
14 have achieved $\mathrm{HBsAg}<1 \mathrm{IU} / \mathrm{mL}$ and 11 have achieved $\mathrm{HBsAg} \leq 0.01 \mathrm{IU} / \mathrm{mL}$. In the experimental group, $9 / 10$ patients (REP 2139) and 8/10 patients (REP 2165) had HBsAg reductions $>1 \log$ from baseline. From these 17 responder patients, 14 achieved $\mathrm{HBsAg}<1 \mathrm{IU} / \mathrm{mL}$ and 13 achieved HBsAg $\leq 0.01 \mathrm{IU} / \mathrm{mL}$. HBsA$\mathrm{g}<1 \mathrm{IU} / \mathrm{mL}$ in experimental patients was accompanied by profound increases in anti-HBs (93 to $223,055 \mathrm{mIU} / \mathrm{mL}$ ) in $9 / 14$ patients and strong therapeutic liver flares (ALT/AST $>5 \times$ ULN with normal synthetic liver function) in $12 / 14$ patients. In control patients following crossover to NAP therapy, anti-HBs response is similar (69$68468 \mathrm{mIU} / \mathrm{mL}$ ), however the strength of liver flares with similar HBsAg reductions were markedly attenuated. Functional remission of HBV infection (HBsAg $\leq$ LLOQ, HBV DNA $<$ LLOQ) is persisting 12-24 weeks after removal of therapy in 12 of 13 experimental patients achieving HBsAg $<1 \mathrm{IU} / \mathrm{mL}$ (REP 2139: 8/10, REP 2165: 5/10). Control of HBV infection is stable in the 13th (REP 2139) patient (HBsAg $1.91 \mathrm{IU} / \mathrm{mL}$, HBV DNA $93 \mathrm{IU} / \mathrm{mL}$ ) at 24 weeks follow-up. Serum ALT/AST has normalized in all these patients. Rebound of infection has occurred in all other experimental patients during follow-up, however clinical benefit (reversal of HBV DNA rebound and normal ALT/AST) is apparent in two additional patients. Conclusion: REP 2139-based combination therapy is well tolerated and elicits the establishment of functional control of HBV infection persisting after removal of therapy (functional remission) in $80 \%$ of patients.

\section{$O-H B V-32$}

Greater prevalence of disease severity and associated comorbidities with increasing age: a cross-sectional analysis of chronic hepatitis B patients in Saudi Arabia

Gabriel Wong ${ }^{1}$, Hamdan Alghamdi ${ }^{3}$, Khalid A Alswat ${ }^{5}$, Mohammed A Babatin ${ }^{4}$, Mona Ismail ${ }^{6}$, Waleed Alhamoudi ${ }^{5}$, Abduljaleel Alalwan ${ }^{3}$, Yaser Dahlan ${ }^{3}$, Abdullah Alghamdi ${ }^{4}$, Faleh Alfaleh $^{5}$, Abdulrahman Aljumah ${ }^{3}$, Ibrahim Altraif ${ }^{3}$, Khalid Albeladi $^{3}$, Faisal Batwa ${ }^{3}$, Drifa Belhadi ${ }^{2}$, Victor Genestier ${ }^{2}$, Ayman Abdo ${ }^{5}$

${ }^{1}$ Gilead Sciences, Inc., United States; ${ }^{2}$ Amaris, France; ${ }^{3}$ King Abdulaziz Medical City, Saudi Arabia; ${ }^{4}$ King Fahad Hospital, Saudi Arabia; ${ }^{5}$ King Saud University, Saudi Arabia; ${ }^{6}$ University of Dammam, College of Medicine, Saudi Arabia

Background: Limited evidence is available on the age, prevalence of comorbidities and treatment pattern in patients with chronic hepatitis B (CHB) in Saudi Arabia. Our aim was to compare and characterize CHB patients in 2015 with CHB patients in 2010 and 2012.

Method: We conducted and compared three cross-sectional analyses of CHB patients $\geq 18$ years of age with CHB defined as either positive HBsAg or a documented history of CHB in 2010, 2012 and 2015. Cross-sectional data was accessed from the multicentre Systematic Observatory Liver Disease (SOLID) registry provided by the Liver Disease Research Center of the King Saud University Medical City to retrieve eligible patients.

Result: We identified a total of $765 \mathrm{CHB}$ patients registered in SOLID during different time-points: 274, 256 and 235 in 2010, 2012 and 2015 , respectively. The median age was significantly higher in 2015 (47 years) compared to 2010 and 2012 (41 and 42 years, respectively; $p<0.001)$. The proportion of patients between 2010 and 2015 with hepatocellular carcinoma (HCC; range 1\%-12\%) and cirrhosis (range 5\%-23\%) were consistently higher in 2015 compared to 2010 and 2012 ( $\mathrm{p}<0.0001$; cf. Figure 1). Additionally, more patients had coronary artery disease in 2015 compared with 2010 ( $10 \%$ vs. $4 \%$; $\mathrm{p}=0.006)$; or hyperbilirubinemia ( $18 \%$ vs. $9 \%$; 
$\mathrm{p}=0.006)$; and hepatic steatosis $(32 \%$ vs. $25 \% ; \mathrm{p}=0.075) . \mathrm{A}$ numerical, although non-significant, difference in terms of proportion of patients with diabetes was also seen ( $25 \%$ in 2015 vs. $19 \%$ in 2012; $\mathrm{p}=0.1109 ;$ and $22 \%$ in $2010 ; \mathrm{p}=0.3409)$. The proportion of patients who previously received a treatment for $\mathrm{CHB}$ was also significantly higher in 2015 compared with $2010 / 2012$ (22\% vs. $7 \% / 5 \%$; cf. Figure $2 ; \mathrm{p}<0.0001)$. This difference is mostly driven by the proportion of patients who received lamivudine as a previous treatment (from $3 \%$ in 2010 to $8 \%$ in 2015). Additionally, the rate of patients "currently" receiving a treatment at the year of assessment was also higher in 2015 (56\%) compared to 2010 (18\%) and 2012 $(16 \% ; \mathrm{p}<0.0001)$.

Conclusion: In 2015 relative to 2010/2012, the CHB population in Saudi Arabia was significantly older and more likely to develop comorbidities and/or advanced liver diseases such as cirrhosis and HCC.
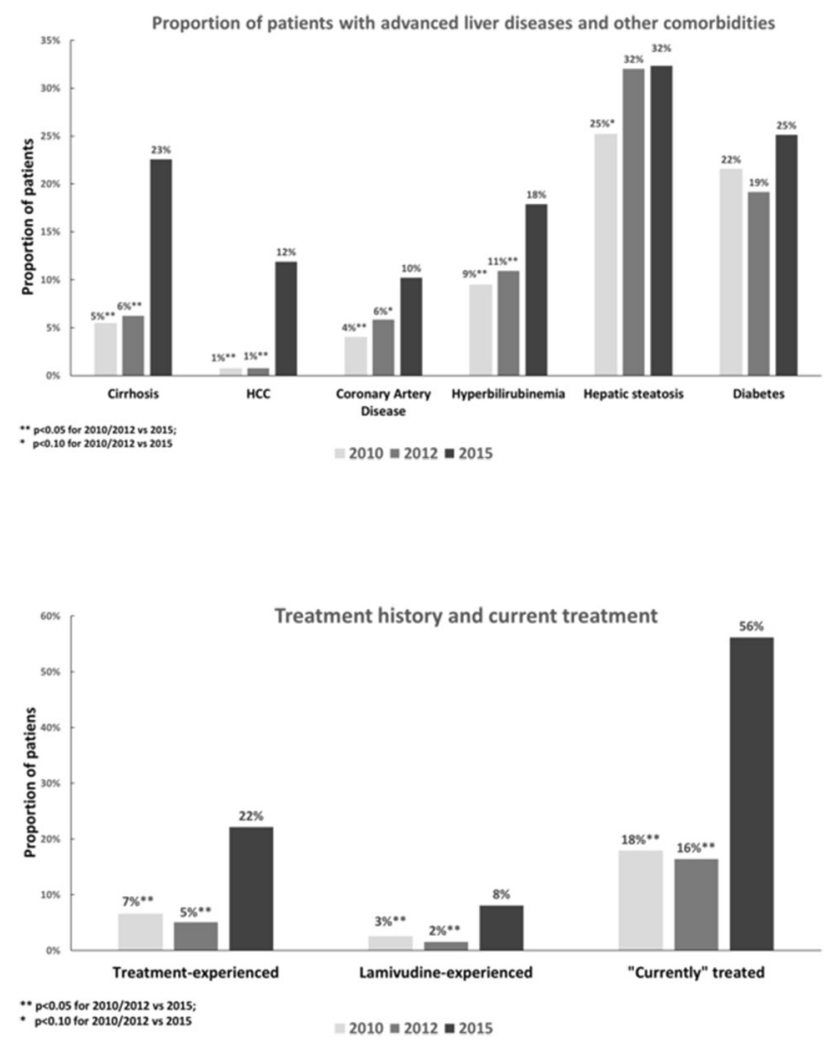

\section{$O-H B V-33$}

HEV decreased HBV replication and aggravated injury of hepatocytes in chronic hepatitis $B$ and hepatitis $E$ virus coinfection

\section{Jinshang $\mathrm{Hu}^{1}$, Meifang Han ${ }^{1}$, Qunqun Jiang ${ }^{1}$, Yongli Wang ${ }^{1}$, Wenyu Wu ${ }^{T}$, Qin Ning ${ }^{1}$}

${ }^{1}$ Tongji Hospital, Tongji Medical College, Huazhong University of Science and Technology, China

Background: Hepatitis B and Hepatitis E virus infections are both endemic in China. Coinfections of HBV and HEV are common and when they occurs, ensues a deranged laboratory liver tests parameters with rapid clinical deterioration. Few available studies on virological influence of Hepatitis B among the HBV/HEV coinfected patients are conflicting. The aim of this study therefore was to assess the impact of HEV infection in CHB patients.

Method: A cross-section study was conducted in the Infectious disease department of Tongji Medical Centre, in Wuhan China. A total of $375 \mathrm{CHB}$ patients were serially recruited and among them 304 were having HBV monoinfection, and 70 were coinfected with HEV and HBV. Among the coinfection with chronic HBV infection and HEV infection, 17 out of patients are grouped into HBV-acute HEV group with HEV IgM positive and 53 of patients are grouped into HBV-exposed HEV group with HEV IgG positive. Clinical, biochemistry, hematological and serological data was obtained from the medical records. HBV DNA was extracted for further genetic analysis. Data was analyzed by STATA program and $P$ value of $<0.05$ was considered to be significant.

Result: The average ages of coinfection groups including HBV-acute HEV group and HBV-exposed HEV group were both higher than that in monoinfection group with $\mathrm{HBV}$ infection (46, 51 versus 31, $\mathrm{P}<0.05$ ). Moreover, a significant elevation of transaminases, bilirubin and INR was observed in the coinfection group. Interestingly, HBV DNA quantification were found to be significantly diminished in HBV and HEV coinfection patients (HBV-acute HEV versus monoinfection, $\mathrm{p}=0.02$; HBV-exposed HEV versus nonoinfection, $\mathrm{p}<0.01)$. Hepatitis $\mathrm{B}$ e antigen $(\mathrm{HBeAg})$ quantification were decreased significantly in $\mathrm{HBeAg}$ positive patients (HBV-acute HEV versus monoinfection, $\mathrm{p}<0.03$; HBV-exposed HEV versus nonoinfection, $\mathrm{p}<0.001)$. Liver cirrhosis, acute on chronic liver failure (ACLF), ascites and hepatic encephalopathy were significantly more common in $\mathrm{HBV} / \mathrm{HEV}$ coinfected patients.

Conclusion: Although HBV replication was diminished during both acute and exposed HEV coinfection groups, HEV infection might aggravated hepatotoxicity and injury of hepatocytes during the coinfection of HBV and HEV coinfection.

\section{$O-H B V-34$}

In a naive chronic hepatitis B patient in a population, lamivudine resistance is a factor affecting the choice of drug with high genetic resistance barrier?

\section{Engin Altinkaya ${ }^{1}$}

${ }^{1}$ Cumhuriyet University Health Application and Research Center, Turkey

Background: In this study, we aimed to determine lamivudine resistance in naive chronic hepatitis B patients in a population and to identify whether this result is predictive for the selection of drugs with a high genetic resistance barrier.

Method: The study comprised 71 chronic hepatitis B patients that have never received treatment for chronic hepatitis B infection before (treatment-naïve). HBV DNA was detected quantitatively by realtime polymerase chain reaction (RT-PCR) method in serum samples collected from the patients. Sequence analysis and reverse hybridization by string method were used to determine Lamivudine motif mutants. Liver biopsy was performed in all patients. Serum samples collected were used to determine $\mathrm{HBeAg}$ and anti-HBeAb levels.

Result: Of the 71 patients participated in the study. Mutation was detected in a total of 8 patients and the prevalence was $11.3 \%(8 / 71)$. The prevalence of mutation was $8.3 \%$ (4/48) in $\mathrm{HBeAg}$-negative patients and it was lower than $\mathrm{HBeAg}$-positive patients, in whom the prevalence was $17 \%(4 / 23)(\mathrm{p}=0.01)$. Comparing the patients with and without mutation in terms of demographic characteristics, ALT levels, HAI and fibrosis scores, no statistically significant difference was determined $(\mathrm{p}=0.29)$. 
Conclusion: As in Turkish society, lamivudine resistance may be seen in naive chronic hepatitis B patients in some societies. We, therefore, recommend taking this into account when choosing drugs with high genetic resistance barrier.

\section{$O-H B V-35$}

\section{Inarigivir: a novel RIG-I agonist for chronic hepatitis B}

Chelsea Macfarlane ${ }^{1}$, Chelsea Macfarlane ${ }^{1}$, Stephen Locarnini ${ }^{10}$, Kathy Jackson ${ }^{10}$, Renae Walsh', Ros Edwards', Rachel Hammond $^{10}$, Carla Coffin ${ }^{7}$, Magdy Elkhashab ${ }^{6}$, Susan Greenbloom $^{5}$, Alnoor Ramji ${ }^{4}$, Henry LY Chan ${ }^{3}$, Won Kim ${ }^{2}$, Nezam Afdhal $^{1}$, Man Fung Yuen ${ }^{8}$, Danny Wong ${ }^{8}$, Radhakrishnan Iyer $^{1}$

${ }^{1}$ Spring Bank Pharmaceuticals, United States; ${ }^{10}$ Victorian Infectious Diseases Reference Laboratory, Australia; ${ }^{2}$ Borame Medical Center, Korea, Democratic People's Republic of; ${ }^{3}$ Chinese University of Hong Kong, Hong Kong; ${ }^{4}$ Pacific Gastroenterology Associates, Canada; ${ }^{5}$ Toronto Digestive Disease Assoc, Inc, Canada; ${ }^{6}$ Toronto Liver Centre, Canada; ${ }^{7}$ University of Calgary, Canada; ${ }^{8}$ University Of Hong Kong, Hong Kong; ${ }^{9}$ Victorian Infectious Disease Reference Laboratory, Australia

Background: Inarigivir (previously SB 9200) is an oral HBV antiviral with both direct acting activity and immune-modulation through activation of the pattern recognition receptor retinoic acidinducible gene 1 (RIG-1). Inarigivir is currently being evaluated in the ACHIEVE trial, double-blind placebo controlled phase II studies of ascending doses of Inarigivir daily monotherapy or placebo (PL) for 12 weeks, followed by a switch to $300 \mathrm{mg}$ Tenofovir (TDF) daily for a further 12 weeks. Here, we report the virological and serological responses of the $1 \mathrm{st}$ cohort inarigivir $25 \mathrm{mg}$ and effects after switching to TDF.

Method: 20 treatment naïve non-cirrhotic HBV patients were randomised 4:1 to $25 \mathrm{mg} \mathrm{SB} 9200$ or placebo. There were M: 12; 1F: 8, mean age 40 yrs, 18 Asian, 16 genotype B/C and 4 A /D with 11 $\mathrm{HBeAg}+\mathrm{ve}$ and $9 \mathrm{HBeAg}-\mathrm{ve}$.

Result: There were no clinical, haematological or biochemical SAEs and no interferon-like side effects. During Inarigivir therapy, 3/7 of the $\mathrm{HBeAg}-\mathrm{ve}$ and $1 / 9$ of the $\mathrm{HBeAg}+\mathrm{ve}$ had greater than 1.0 $\log 10 \mathrm{IU} / \mathrm{ml}$ reduction in HBV DNA (mean $0.6 \log 10, \mathrm{p}=0.01 \mathrm{vs}$ placebo). 3 of $7 \mathrm{HBeAg}-\mathrm{ve}$, and $2 / 9 \mathrm{HBeAg}+\mathrm{ve}$ subjects demonstrated $>0.5 \log 10 \mathrm{IU} / \mathrm{ml}$ decline in serum HBsAg. The serum HBV RNA fell by $>0.5 \log 10$ copies/ml by week 12 in $10 / 16$ patients; seven were $\mathrm{HBeAg}-\mathrm{ve}$, with 5 having $>3.0 \log 10$ copies $/ \mathrm{ml}$ to undetectable whilst 2 had a $>1.0 \log 10$ copies $/ \mathrm{ml}$ decline. In the 3 $\mathrm{HBeAg}+$ ve patients, 1 had a $>1.0 \log 10$ copies $/ \mathrm{ml}$ and 2 had $>0.5$ $\log 10$ copies/ml decline in their serum HBV RNA. Hepatitis B core related antigen ( $\mathrm{HBcrAg}$ ) testing to week 12 revealed a similar antiviral response to the HBV RNA profiles, especially in the $\mathrm{HBeAg}-$ ve group. From 12-24 weeks TDF induced potent suppression of serum HBV DNA of $>3.0 \mathrm{log} \mathrm{IU} / \mathrm{ml}$ decline or to undetectability. In Inarigivir/TDF group 6/16 (38\%) patients achieved a HBsAg response of $>0.5 \mathrm{log} \mathrm{IU} / \mathrm{ml}$ from week $12-24$ which was more enhanced in $\mathrm{HBeAg}+\mathrm{ve}$ patients and 4 of 9 of $\mathrm{HBeAg}+\mathrm{ve}$ also achieved $>0.75 \log$ decline in qHBeAg. Neither HBV RNA or $\mathrm{HBcrAg}$ responses were enhanced following the TDF switch. Patients with $>0.5 \log \mathrm{IU} / \mathrm{ml}$ drop in $\mathrm{HBsAg}$ also demonstrated $\mathrm{HBsAg}$ epitope changes associated with a clearance profile, whilst anti-HBs complexed to HBsAg was observed to develop in $56 \%$ of inarigivir treated patients, possibly reflecting the emergence of an endogenous immune response.
Conclusion: Inarigivir at low dose $25 \mathrm{mg}$ daily, demonstrated safety and significant antiviral effects on HBV replication, presumably at the level of viral RNA packaging, translation and reverse transcription with early suggestion of an immune mediated clearance response more apparent in $\mathrm{HBe} \mathrm{Ag}$-ve patients.

\section{$O-H B V-36$}

Increasing age and comorbidities in 43,316 adult patients with chronic hepatitis B (CHB) from 2011 to 2016 in Japan: results of a real-world analysis

Gabriel Wong ${ }^{1}$, Masayuki Kurosaki ${ }^{3}$, Richard $Z^{2}{ }^{2}$, Steve Sherman $^{2}$, Mindie H Nguyen ${ }^{5}$, Hiroshi Yatsuhashi ${ }^{4}$

${ }^{1}$ Gilead Sciences, Inc., United States; ${ }^{2}$ Creativ Ceutical, United States; ${ }^{3}$ Japanese Red Cross Musahino Hospital, Japan; ${ }^{4}$ Nagasaki Medical Center, Japan; ${ }^{5}$ Stanford University Medical Center, United States

Background: CHB affects approximately $1 \%$ of the general population in Japan with higher prevalence in older people. The aim of this study was to characterize the evolving CHB patient demographics including non-liver comorbidity burden in Japan as well as their changes over the recent 5 years (2011-2016).

Method: We used the Medical Data Vision (MDV) claims database to identify patients $\geq 18$ years with $\geq 1$ inpatient or 2 outpatient ICD-10 codes for CHB (B18.1) and with continuous enrollment for 6 months prior and post index date (first date of $\mathrm{CHB}$ diagnosis) during 1/1/2011-12/31/2016. Patient demographic and comorbidity data was reported from 2011 to 2016 . The proportions of patients with comorbidities for each year was calculated for all CHB patients still observed in the database for that given year.

Result: A total of 43,316 patients met the study inclusion and exclusion criteria during the 2011 to 2016 period. Males made up $57.2 \%$ of the patients in 2011 and decreased to $54.6 \%$ in 2016 $(\mathrm{p}<0.0001)$. The average age of patients was $61.3 \pm 13.1$ years in 2011 and increased to $64.2 \pm 13.2$ years in $2016(\mathrm{p}<0.0001)$. The proportion of the population over 65 increased from $43.1 \%$ in 2011 to $66.0 \%$ in $2016(\mathrm{p}<0.0001)$. Similarly, the proportion of patients over 75 years of age increased from $15.3 \%$ in 2011 to $21.6 \%$ in 2016 $(\mathrm{p}<0.0001)$. The proportion of patients receiving antiviral therapy for HBV was fairly stable, at $32.8 \%$ in 2011 and $31.4 \%$ in 2016 $(p=0.018)$. The Charlson comorbidity index, an overall measure of patient comorbidity (covering 17 conditions), increased from 2.69 to $3.2(\mathrm{p}<0.0001)$ with selected comorbidities summarized in Table 1. In 2016, 21.8\% of CHB patients in Japan had cardiovascular disease (CVD), 36.7\% with hypertension (HTN), $11.8 \%$ with diabetes (DM), $18.2 \%$ with renal impairment (RI), $4.0 \%$ with chronic kidney disease (CKD), 8.6\% with osteoporosis/osteoarthritis/vitamin D deficiency, $4.5 \%$ with fragility (non-traumatic/non-pathological) bone fractures; all of which have increased significantly from 2011 ( $\mathrm{p}<0.001$ to 0.029).

Among CHB patients 65 years or older observed in 2016, almost onethird (29.7\%) had CVD, one-half (47.1\%) with HTN, $14.7 \%$ DM, $5.4 \%$ CKD, $11.4 \%$ metabolic bone disease and $6.2 \%$ non-traumatic/ non-pathological factures. As expected, even more patients 75 or older had comorbidity with over half $(54.1 \%)$ having HTN, onequarter with RI (24.3\%) including $7.2 \%$ with CKD and close to 1 in 10 with fragility bone fracture.

Conclusion: Between 2011 and 2016, the Japanese CHB population has aged $(\sim 2$ in $3 \geq 65)$ and are having more comorbidities, which may affect $\mathrm{CHB}$ management and should be considered in their treatment and monitoring. 


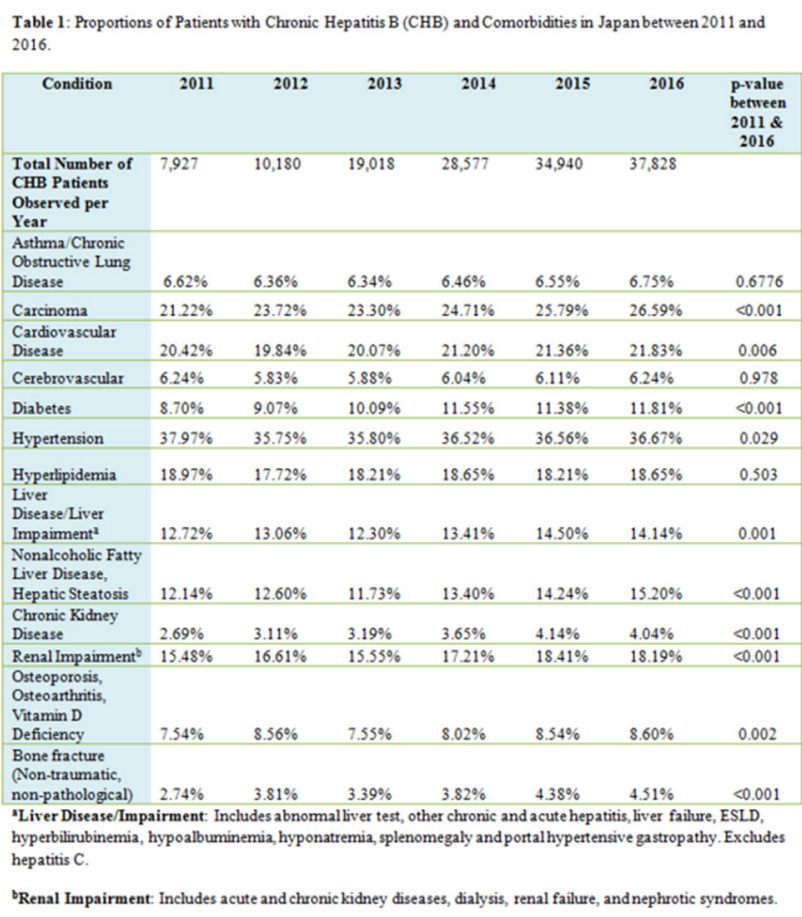

Session: Hepatitis C Virus Infection. Chairperson; A.Kadir Dokmeci; M S Khuroo

\section{$\mathrm{O}-\mathrm{HCV}-19$}

Comparison of event-free survival between DAA and IFN-based therapy, adjusted for disease severity

Bettina Elisabeth Hansen ${ }^{1}$, Lisette A P Krassenburg ${ }^{1}$, Wayel $\mathbf{R}$ Zanjir $^{1}$, Firas Georgie ${ }^{1}$, Orlando Cerrochi ${ }^{1}$, Khalid Munawar ${ }^{1}$, Emily Stotland ${ }^{1}$, Jason Lau ${ }^{1}$, Harry L A Janssen ${ }^{1}$, Jordan J Feld ${ }^{1}$

${ }^{1}$ Toronto General Hospital, Canada

Background: Long-term follow-up studies have shown that sustained virological response (SVR) is associated with improved clinical outcomes with interferon (IFN)-based therapy. However, the causal link of SVR with outcome has been challenged with the argument that SVR may simply be a component of a 'good prognosis' in the natural history of HCV. With markedly improved SVR rates with directacting antivirals (DAAs), the benefit of SVR would be expected to diminish if the association of SVR with outcome is not causal. To evaluate this question, we compared liver-related outcomes in IFNeligible patients treated with DAAs vs. IFN.

Method: Retrospective data were collected for patients treated with IFN-based therapy or DAAs at the Toronto Centre for Liver Disease from Jun/06 to Dec/16. To control for disease severity, patients were categorized as IFN-eligible (IDEAL) based on meeting criteria for the IDEAL trial comparing PegIFNa2a vs PegIFNa2b. Clinical events, defined as decompensation, hepatocellular carcinoma, liver transplantation and all-cause death, were collected from end of treatment to two years.

Result: Of 1110 IDEAL-eligible patients, 58\% received DAAs and $42 \%$ IFN, median (IQR) age 55 (48-61) years and genotype (G) distribution $71 \% / 12 \% / 18 \%$ for $\mathrm{G} 1 / 2 / 3$. Cirrhosis was present in $34 \%$ DAAs vs $14 \%$ IFN $(\mathrm{p}<.001)$. SVR was $97 \%$ with DAAs vs $74 \%$ with IFN ( $\mathrm{p}<.0001)$. The 2 -year cumulative event-free survival with SVR was $98 \%$ for both DAAs and IFN $(\mathrm{p}=.47)$ and $93 \%$ and $88 \%$ for non-SVR $(\mathrm{p}=.68)$. Achieving SVR was associated with improved event-free survival with an adjusted Hazard Ratio (aHR) of $7.69(95 \%$ CI 3-20, p < 0.0001) after controlling for age, sex, genotype, cirrhosis and treatment. After matching IFN non-responders with DAA patients using inverse probability treatment weighting, the overall event rate was $1.1 \%$ in DAA patients compared to $17.9 \%$ in IFN non-responders at 2 years underlining the clinical benefit of higher SVR rates.

Conclusion: In IFN-eligible patients, SVR is more commonly achieved with DAAs and confers a similar clinical benefit as in those treated with IFN. The markedly higher SVR rate but reduced overall event rate with DAAs compared to IFN, despite similar disease severity, unequivocally shows that SVR cannot be due to a 'good prognosis', but rather SVR is a relevant endpoint leading to improved clinical outcomes.

\section{Follow-up (months) 0510152025

Cumulative Survival
1.00
0.95
0.90
0.85
0.80
0.75

Event-free survival in IDEAL-eligible patients
IFN non-SVR-censored
IFN SVR-censored
DAA non-SVR-censored
DAA SVR-censored
IFN non-SVR
IFN SVR
DAA non-SVR

$O-H C V-20$

DAA Hepatitis C treatment in low socioeconomic population at a Federally Qualified Health Center [FQHC]

Imtiaz Alam ${ }^{1}$, Zohha Tariq ${ }^{1}$, Chellyanne Hinds ${ }^{2}$

${ }^{1}$ Austin Hepatitis Center, United States; ${ }^{2}$ Communitycare, United States

Background: CommUnityCare [CUC] Hepatitis C clinic (FQHC) was launched March 2014. CUC serves as a safety net provider to Austin, Texas' high risk uninsured, homeless and refugee population. The clinic offered Hepatitis $\mathrm{C}[\mathrm{HCV}]$ treatment to patients in whom barriers such as cost, unstable living environments and medical comorbidities had previously limited access to HCV therapy.

Method: Single center retrospective cohort study was conducted on HCV patients who received above therapies per AASLD guidelines. HCV RNA levels were measured using a COBAS ${ }^{\circledR}$ Ampliprep/ COBAS $^{\circledR}$ TaqMan $^{\circledR}$ HCV Test with a lower limit of detection of $15 \mathrm{IU} / \mathrm{L}$. Sustained viral response [SVR12] was defined as undetectable HCV RNA at 12 weeks post treatment. The primary endpoint was the proportion of patients who had successfully achieved SVR12. Result: March 2014 - October 2016, 520 patients were treated with the above stated DAAs. Mean age 55 years; $76 \%$ males; $85 \%$ were uninsured; $72 \%$ genotype $1,97 \%$ naive and $8.5 \%$ were cirrhotic. We performed intention to treat analysis (ITT) and per protocol analysis (PPA - patients who completed therapy 12 or 24 week and returned for SVR12). 330 returned for SVR12 results. 326 achieved SVR12 (ITT 63\%), PPA 99\%). LDV/SOF: $\mathrm{n}=408$, ITT (66\%), PPA $(99 \%)$; PrOD: $\mathrm{n}=33$, ITT (81\%), PPA (100\%); SOF/DCV: $\mathrm{n}=47$, ITT (57\%), PPA (100\%); E/G: $\mathrm{n}=10$, ITT $(50 \%)$, PPA (80\%); SOF/ VEL: $\mathrm{n}=22$, ITT $(13 \%)$ PPA $(100 \%)$. Per protocol analysis 4 
patients relapsed [2 LDV/SOF; $1 \mathrm{E} / \mathrm{G}$; and 1 SOF/VEL]. LDV/SOF patients who relapsed were treatment naïve and non-cirrhotic. The E/G patient who relapsed had G1a, NS5a resistance and compensated cirrhosis. The SOF/VEL patient was G3, treatment naïve and cirrhotic.

Conclusion: Treatment with DAAs can allow this complex population to achieve SVR12. Discrepancy between SVR12, ITT and PPA rates are attributed to lack of transportation, relocation of patient and change in patient contact information.

\section{$O-H C V-21$}

Effectiveness of direct anti-viral agents in patients with chronic hepatitis c virus infection and chronic renal diseases. Real life experience

\section{Necati Ormeci ${ }^{1}$, Orhan Sezgin ${ }^{3}$, Ridvan Karaali ${ }^{4}$, Bilgehan} Aygen $^{2}$, Dilara Turan ${ }^{1}$, Serkan Yaras ${ }^{3}$, Lknur Erdem ${ }^{4}$, Orhan Yildiz $^{2}$, Fatih Karakaya ${ }^{1}$, Kenan Ates ${ }^{1}$

${ }^{1}$ Ankara University Medical School, Turkey; ${ }^{2}$ Erciyes University Medical School, Turkey; ${ }^{3}$ Mersin University Medical School, Turkey; ${ }^{4}$ Namik Kemal University Medical School, Turkey

Background: Both hepatitis $\mathrm{C}$ virus infection (HCV) and chronic renal disease (CRD) have co-morbid illnesses which increase morbidity and mortality. Treatment of HCV with Direct Acting Antiviral Agents (DAAs) have revolutionized with the increasing of sustained virological response rates and less advers events since 2016. Aim of the study is to present our real life experience about treatment with PROD regimen (combination of Paritaprevir $150 \mathrm{mg} / \mathrm{day}$, Ritonavir $100 \mathrm{mg} /$ day as a booster, Ombitasvir $25 \mathrm{mg} /$ day and Dasabuvir $250 \mathrm{mg}$ twice/day) in patients with both HCV and CRD.

Method: This is a multicentric, retrospective preliminary cohort study. Seventy three patients with both HCV and CRD (nine stage 2 ,five stage 3 , nine stage 4 and fifty stage 5 ); thirty one male, forty two female, mean age 59.54 (youngest 25,oldest 84) years old, were treated with PROD based regimen. Genotype of the 73 patients were 1a eleven, 1b fifty, 4a twelve respectively. Five patients had compensated liver cirrhosis. Three patients had recurrences for HCV after kidney transplantation. The patients with genotype 1a or compensated liver cirrhosis were treated with PROD and Ribavirin (in a dose of $200 \mathrm{mg}$ in every other day) for 12 weeks. The patients with genotype $1 \mathrm{~b}$ were treated with PROD for 12 weeks. The patients with genotype 4a were treated with Paritaprevir, Ritonavir Ombitasvir and Ribavirin $200 \mathrm{mg}$ every other day.

Result: At the end of the treatment, all patients except one were HCV-RNA negative $(98.6 \%)$. One patient had decompensated after the 4.th day of therapy and she had stop the treatment. She was exitus after two months. Twenty four patients completed the follow up. All of 24 patients had HCV-RNA negative. There were not differences in terms of MELD and CPT score, Plt, serum albumin, INR levels of patients before and after the treatment. Two patients had hyperbilirubinemia temporarily. Nine patients had anemia ( $\mathrm{Hb}$ levels were between 8-10 grams/dl in eight patients, $\mathrm{Hb}$ level was less than 8 gram/dl. Four patients had a diarrhea during the treatment and they resolved after the end of treatment. Two patients had an insomnia, one patient had dyspnea. The dose of Tacrolimus was adjusted during the PROD regimen in three transplanted patients.

Conclusion: PROD regimen is very effective and safe treatment in patients with HCV and CRD which are stage 4 and five. It is necessary to do large clinical trials to support this results.

\section{$O-H C V-22$}

HCV screening can improve substance-use behaviours among patients receiving opioid substitution therapy

Hemant Shah ${ }^{1}$, Hooman Farhang Zangneh ${ }^{1}$, Joseph Eibl' ${ }^{2}$, Graham Gauthier $^{2}$, David Pellegrini ${ }^{2}$, Bettina Hansen ${ }^{1}$, Jordan Feld $^{1}$, David Marsh ${ }^{2}$

${ }^{1}$ University of Toronto, Canada; ${ }^{2}$ Northern Ontario School of Medicine, Canada

Background: In Canada, the province of Ontario has over 50,000 opioid-dependent persons engaged in opioid substitution therapy (OST). Hepatitis C Virus (HCV) infection, with an estimated prevalence of $0.3 \%-0.9 \%$ among all Canadians, is more common in this population. Many experts advocate for testing all OST patients for chronic $\mathrm{HCV}$ infection. To date, the impact of HCV infection diagnosis on the substance use behaviors of OST patients is unknown and we aim to explore that here.

Method: We conducted a retrospective cohort analysis using the electronic health data, urine toxicology and antibody-based HCV infection screening information from a network of 47 addiction treatment clinics in Ontario from 2007 to 2013. We used a logistic regression analysis to determine the impact of $\mathrm{HCV}$ infection testing and diagnosis on substance-use behaviors for patients engaged in OST.

Result: Out of 12386 individuals identified, 8856 patients were screened for HCV infection. 1920 (21.7\%) individuals tested positive for anti-HCV Ab. Patients were followed for a mean of 9.7 months before and 21.2 months after HCV-Ab test. A significant decline in opioids/benzodiazepines consumption was seen after testing for $\mathrm{HCV}-\mathrm{Ab}$. (opioids declined from $35 \%$ to $17 \%, \mathrm{P}<0.001$; benzodiazepines declined from $8.5 \%$ to $6.4 \%, \mathrm{P}<0.001$ ). However, there was no significant decline in overall cocaine use before and after testing for $\mathrm{HCV}-\mathrm{Ab}$ ( $16.7 \%$ before $\mathrm{HCV}-\mathrm{Ab}$ test to $16.5 \%$ after, $\mathrm{P}=0.40$ ). For opioid users, there was no difference between those who tested positive for HCV-Ab versus those tested negative. For benzodiazepines, a significant decrease in use was more often observed among those with positive test result for $\mathrm{HCV}-\mathrm{Ab}$ $(\mathrm{OR}=1.39$, CI95\% 1.24-1.56) after adjustment for age, sex and geographical location. Even though cocaine users did not change their consumption habit overall, patients who tested positive for $\mathrm{HCV}-\mathrm{Ab}$ did decrease their use. $(\mathrm{OR}=1.48$, CI 95\% 1.33-1.66).

Conclusion: We have demonstrated that $\mathrm{HCV}$ infection screening can have a positive impact on substance-use among patients engaged in OST. Expansion and universal screening of OST clients for HCV infection should be encouraged.

\section{$O-H C V-23$}

Liver stiffness predicts relapse after direct acting antiviral therapy against chronic hepatitis $\mathrm{C}$ Virus infection

\section{Mohammad Helal $^{1}$, Ali Ghweil ${ }^{1}$, Mohammad Alsenbesy ${ }^{1}$, Ashraf Khodery $^{2}$ \\ ${ }^{1}$ South Valley University, Egypt; ${ }^{2}$ Sohag University, Egypt}

Background: Over the last decade, several non-invasive methods were proposed for diagnosis of liver fibrosis, including the elastometric measurement of hepatic stiffness, group of clinical and biochemical parameters, and combinations of both methods. It has been suggested that elastography and serum markers are useful techniques for diagnosing severe fibrosis and cirrhosis and for excluding significant fibrosis in hepatitis $\mathrm{C}$ virus patients. 
Method: This is a follow-up study including 100 chronic HCV Egyptian patients treated with Sofosbuvir-based treatment regimen. Transient elastography values were recorded as well as serum fibronectin and APRI were calculated at baseline and SVR12.

Result: There was a significant improvement of platelets counts, ALT and AST levels, which in turn cause significant improvement in APRI scores at SVR12. Liver stiffness measurements were significantly lower at SVR12 $(15.40 \pm 8.96$ vs $8.82 \pm 4.74 \mathrm{kPa}, \mathrm{P}=0.000)$. There was significant decline in serum fibronectin from baseline to SVR $12(524.14 \pm 237.61$ vs $287.48 \pm 137.67, \mathrm{P}=0.000)$.

Conclusion: Compared to pre-treatment values, SVR12 LS scores are significantly reduced which reflects improved liver fibrosis parameters with available DAAs.High LS measurements before treatment can be a predictor of relapse after treatment

\section{$\mathrm{O}-\mathrm{HCV}-24$}

\section{Outcome of hepatitis $\mathrm{C}$ treatment in chronic kidney disease} patients

\section{Anirban Jabbar ${ }^{1}$ \\ ${ }^{1}$ Hospital, India}

Background: Treatment of Hepatitis $\mathrm{C}$ virus infection in patients with chronic kidney disease can be challenging. The availability of direct acting antivirals has dramatically improved HCV cure rates. However there is insufficient data regarding its use in patients with chronic kidney disease (CKD). As sofosbuvir is predominantly excreted via the kidneys, there has been concern about dosing of sofosbuvir in renal disease. The study was carried out to see the response of direct acting antivirals on a cohort of CKD V patients. Method: We retrospectively studied 26 patients of hepatitis $\mathrm{C}$ with $\mathrm{CKD} \mathrm{V}$, of them 22 patients were on hemodialysis. The patients were aged 30-65 years. The patients were either genotype 1 or genotype 3 and all had baseline viral load $>10^{\wedge} 3 \mathrm{iu} / \mathrm{ml}$. The study was carried out at two centers for one year. None of the patient received peg interferon and we excluded patients with Child $\mathrm{C}$ liver disease. Treatment duration was 12 to 24 weeks with direct acting antivirals. All patients received sofosbuvir(400 mg). We recruited 26 patients, 12 of them were genotype 1, 14 of them were genotype 3. Four patients received combination of sofosbuvir $(400 \mathrm{mg})$ and ribavirin (200 mg).(three patients had treatment for 12 weeks and one patient was treated for 24 weeks). Nine patients received sofosbuvir(400 mg) and daclatasvir $(60 \mathrm{mg})$ for $12-24$ weeks. Nine patients received sofosbuvir (400 mg) and ledipasvir (90 mg) for 12 weeks. Rest of the four patients received sofosbuvir $(400 \mathrm{mg})$ and velpatasvir $(100 \mathrm{mg})$ for 12 weeks. (In this group Ribavirin was given to one patient who had previous treatment relapse).

Result: Out of 26 patients treatment failure was seen only in two patients. Rest of 24 patients had undetectable viral load at week 04, week 12 (end of treatment), and 12 weeks after stopping treatment. There was no adverse side effect during treatment with no significant drop in blood count or increased dialysis frequency. We treated two patients who had previous failed treatment. First patient with failed previous treatment who had genotype 3 was given sofosbuvir $(400 \mathrm{mg})$ and daclatasvir $(90 \mathrm{mg})$ for 24 weeks and achieved viral remission, the 2 nd patient which relapsed had genotype 1 and received sofosbuvir $(400 \mathrm{mg})$,velpatasvir(100 $\mathrm{mg})$ and ribavirin $(200 \mathrm{mg}$ ) for 12 weeks and achieved viral remission.

Conclusion: We found sofosbuvir based oral anti HCV regime was highly effective in treating hepatitis $\mathrm{C}$ with chronic kidney disease stage v patients. Sofosbuvir dosage of $400 \mathrm{mg}$ daily was safe to use in CKD patients. The response in patient with previous failed treatment was excellent. Further studies with larger numbers will be useful to confirm our findings.
Session: Miscellaneous. Chairpersons: Prasun Jalal; $K$ Narayansami

\section{$O-M I S C-01$}

Composition of Bile acids in patients with gallstones from north and south India

Mayank Jain ${ }^{1}$, Jayanthi Venkataraman ${ }^{1}$, M Mary Sheeba ${ }^{3}, \mathrm{~K}$ $\overline{\text { Thanigaiarul }}^{2}$, S Sarika ${ }^{2}$, B Mohana ${ }^{2}$, Mohamed Rela ${ }^{1}$, Malay Sharma $^{4}$, R Karvembu ${ }^{3}$, S Narayana Kalkura ${ }^{2}$

${ }^{1}$ Gleneagles Global Health City, India; ${ }^{2}$ Crystal Growth Centre, Anna University, Chennai, India; ${ }^{3}$ Department of Chemistry, National Institute of Technology, India; ${ }^{4}$ JaswantRai Speciality Hospital, Meerut, India, India

Background: We have reported the Chemical and structural analysis of gallstones from the Indian subcontinent and concluded the formation of gallstone is dependent on the regional and dietary factors. High-performance liquid chromatographic (HPLC) method is used for determining and measuring the major bile acids and their conjugates in the human bile. Aim: To determine and measure the various types of bile acids of Cholelithiasis affected patients from South and North India.

Method: A standards set of results of primary, secondary and conjugated bile acids were prepared and concentrations were determined by reversed-phase C 18 HPLC column, running acetate buffer and methanol (30:70). The flow rate was fixed as $1 \mathrm{~mL} / \mathrm{mol}$ and detection was performed at $205 \mathrm{~nm}$. 33 south Indian affected bile acids and 28 north Indian bile acids were taken for the present study

Result: The concentration of the taurolithocholate (TLC) and deoxycholate (DC) was high in the south Indian bile whereas, it is was low in the north Indian samples of bile. The north Indian samples on the other hand had taurochenodeoxycholate (TCDC), taurodeoxycholate (TDC), lithocholic acid (LC) and taurocholate (TCA). On combined analysis of bile acid and their salts, significant difference was noted for chenodeoxycholic acid and its salts and deoxycholic acid and its salts between the two populations (p0.04).

Conclusion: In summary, HPLC is an ideal method for studying individual bile acids in gall bladder bile.There are differences in bile acids and bile salts in north and south Indian subjects.

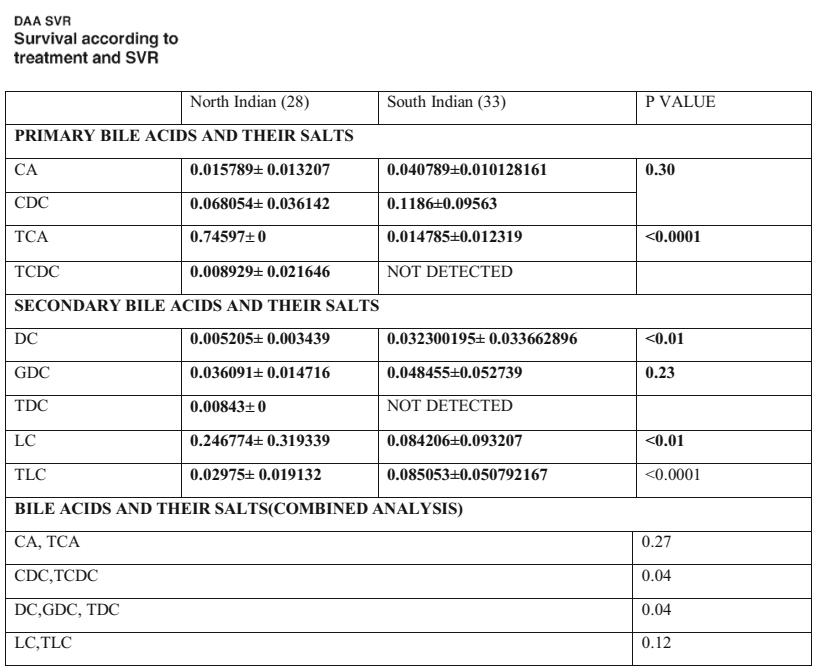




\section{O-MISC-02}

Hepatitis $\mathbf{E}$ virus circulation among immunocompromised and immunosuppressed populations in Brazil

\section{Andreza Salvio Lemos ${ }^{1}$, Amanda De Oliveira Lopes ${ }^{1}$, Noemi} Rovaris Gardinali ${ }^{1}$, Caroline Cordeiro Soares ${ }^{1}$, Rafael Brandao Varella $^{3}$, Fernando Samuel Sion ${ }^{2}$, Luiz Claudio Pereira Ribeiro ${ }^{2}$, Jaqueline Mendes De Oliveira ${ }^{1}$, Marcelo Alves Pinto ${ }^{1}$, Vanessa Salete De Paula ${ }^{1}$

${ }^{1}$ Instituto Oswaldo Cruz, Brazil; ${ }^{2}$ Hospital Universitário Gaffrée \& Guinle, UniRio, Brazil; ${ }^{3}$ Instituto Biomédico, Universidade Federal Fluminense, Brazil

Background: The genotypes 3 and 4 of hepatitis E virus (HEV-3 and HEV-4) have been reported to cause persistent or chronic infections, leading to cirrhosis and death, in immunocompromised and immunosuppressed patients. Once HEV-3 has been the only hepatitis E virus (HEV) genotype reported in Brazil so far, it is important to determinate the real HEV prevalence among special populations, such as kidney transplanted patients and the HIV positive people.

Method: Serum samples from 280 HIV positive patients (2012-2014) and human peripheral blood mononuclear cells (PBMC) from 50 kidney transplanted patients immunosuppressed by tacrolimus (20162017). All nucleic acids from the samples were extracted. The purified products were submitted to a formerly optimized RT-qPCR technique for ORF3 detection, applying a synthetic standard curve and an internal positive control for confirmation of negative results. All RT-qPCR positive samples were also tested for qualitative PCR for ORFs 1 and 2 and for anti-HEV IgM and IgG antibodies detection. Result: No correlation between age, gender or TCD4 + lymphocyte rates of the patients and the presence or absence of HEV-RNA detection was found. From the 280 HIV samples, 10 were positive for HEV coinfection, showing a prevalence of $3.58 \%$ in this population. All viral loads were found between $0.31 \mathrm{IU} / \mathrm{mL}$ to $8.55 \times 107 \mathrm{IU} /$ $\mathrm{mL}$, considered to be low. From the 50 kidney transplanted samples, no one was positive for HEV detection. No samples were positive for anti-HEV IgM or IgG detection and the RNA was detected in only one sample that was amplified, sequenced, and classified as genotype 3 by ORF1 region. All kidney-transplanted samples were negative for serological e molecular tests. The results corroborate the low prevalence found in genotype 3 circulating countries, mainly among special populations.

Conclusion: The HEV infection was rare in HIV infected patients and absent in kidney-transplanted samples. These results contribute to the understanding of the real circulation of HEV in Brazil.

\section{O-MISC-03}

CCR5 + T cells homing to the liver exhibit Inflammatory and pro-fibrogenic signatures in chronic HIV/HCV co-infected patients

\section{Shikha Shrivastava ${ }^{1}$, Haley Ward ${ }^{1}$, Rajiv Kumar Mondal ${ }^{1}$, Manasa Bhatta ${ }^{1}$, Shyam Kottilil ${ }^{1}$ \\ ${ }^{1}$ Institute of Human Virology, United States}

Background: Liver fibrosis is accelerated among patients with HIV/ HCV co-infection, compared to HCV mono-infected patients, although the exact underlying mechanisms are unknown. We hypothesize that $\mathrm{T}$ lymphocytes expressing the HIV co-receptor, CC chemokine receptor 5 (CCR5) preferentially migrate to the inflamed liver contribute to enhanced fibrogenesis Therefore, we aimed (1) to compare the peripheral and intrahepatic CCR5 expression on CD4 and CD8 T-cells in HIV/HCV co-infected patients and chronic HCV mono-infected patients and further functionally characterize the peripheral CCR5 + and CCR5- CD4 and CD8 T-cells. (2) To unravel the functional significance whole transcriptome profiling of sorted CCR5 + and CCR5- T cells was done by RNA sequencing.

Method: Paired PBMCs and liver infiltrating lymphocytes (LILs) from $21 \mathrm{HIV} / \mathrm{HCV}$ co-infected and $14 \mathrm{HCV}$ mono-infected patients were analyzed for CCR5 expression on CD4 and CD8 T-cells by flow cytometry. PBMCs were then sorted for CCR5 + and CCR5- T-cells by BD FACS ARIA with $>98 \%$ purity and stimulated with a pool of HCV peptides and functional characterization was done by flow cytometry. Total RNA was also extracted from the sorted CCR5 + and CCR5- T cells and paired samples were selected for RNA-sequencing on Illumina HiSeq 4000 Sequencing platform. Read counts for each gene were determined using HTseq and differential gene expression were determined by DEseq. Enriched pathways were computed based on the differentially expressed genes, using DAVID and Ingenuity Pathway Analysis. Statistical analysis was done by non-parametric Mann Whitney test to compare HCV mono-infected and $\mathrm{HIV} / \mathrm{HCV}$ co-infected patients and Wilcoxon matched-pair Signed Rank test was used for functional differences in CCR5 + and CCR5- $\mathrm{T}$ cells and for comparing the peripheral blood vs. liver expression.

Result: In the periphery, $\mathrm{CD} 4+\mathrm{CCR} 5+(\mathrm{p}=0.02)$ and $\mathrm{CD} 8+\mathrm{CCR} 5+(\mathrm{p}=0.003) \mathrm{T}$ cells were lower in HIV/HCV coinfected compared to HCV mono-infected patients. While in the liver, $\mathrm{CD} 4+\mathrm{CCR} 5+(\mathrm{p}<0.0001)$ and $\mathrm{CD} 8+\mathrm{CCR} 5+(\mathrm{p}<0.0001)$ were elevated in HIV/HCV co-infected compared to HCV monoinfected patients. We also noted higher percentage of $\mathrm{CD} 4+\mathrm{CCR} 5+(\mathrm{p}<0.0001)$ and CD8 + CCR5 + $(\mathrm{p}<0.0001)$ in the liver of HIV/HCV co-infected patients compared to the peripheral blood. Functional analysis of the CCR5 sorted cells demonstrated increased expression of exhaustion (PD1) $(\mathrm{p}<0.05)$, senescence CD57 ( $\quad$ < $<0.05), \quad$ immune activation markers $\mathrm{CD} 38+\mathrm{HLADR}+(\mathrm{P}<0.05)$, and liver homing chemokine receptor (CXCR3) $(\mathrm{P}<0.05)$. CCR5 $+\mathrm{T}$ cells upon stimulation with HCV peptides secrete more proinflammatory and profibrogenic cytokines and chemokines rather than antiviral cytokines. Furthermore, RNA sequencing analysis revealed that 105 genes were differentially expressed, 68 up- and 37 down-regulated, in CCR5 + T cells compared with CCR5- (FDR $<0.05)$. Dysregulated genes include $C X C R 3, C C R 7, P D E 4 D, I L 2 R B$ involved in liver inflammation, damage and adhesion; IL6ST, KLF10, LGALS3, PLEKHA5, SLC7A5, PRDMI, COLIBA, TNFRSF1B, GZMK CDKN1A associated with liver hyperplasia, fibrosis and hepatocellular carcinoma.

Conclusion: Both functional and transcriptomic analysis of CCR5 $+\mathrm{T}$ cells revealed a pathogeneic role for CCR5 $+\mathrm{T}$ cells in hepatic fibrogenesis. These cells are functionally proinflammatory and fibrotic and preferentially accumulate in liver hastening fibrogenesis, supporting approaches that target CCR5 can be promising for impeding fibrosis (Figure1). 


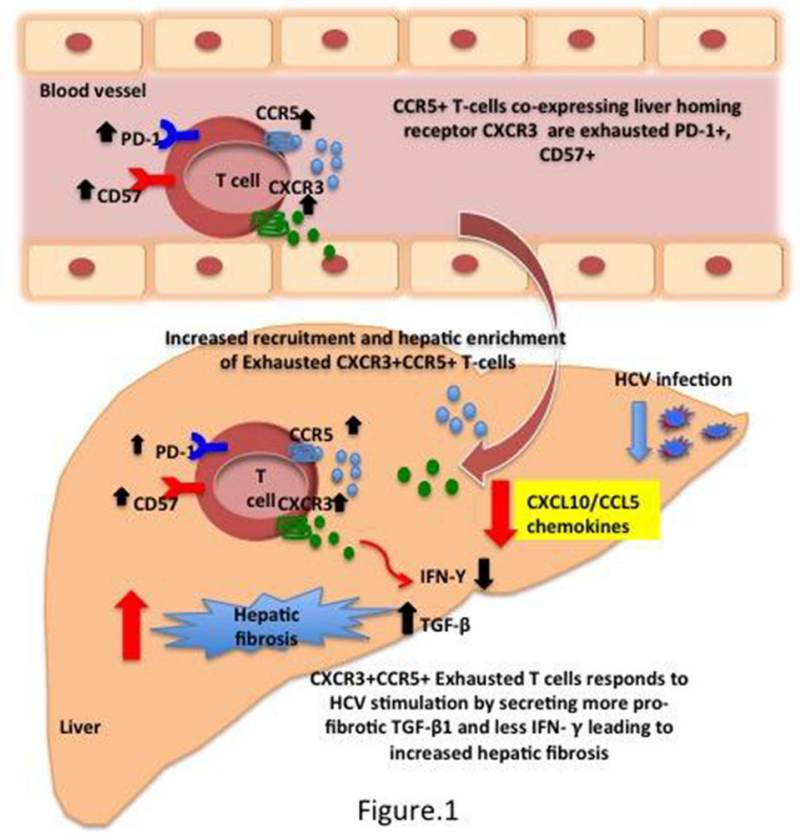

O-MISC-04

Iron mediated regulation of hepcidin: revisiting the dietary iron overload model

\section{Nathan Subramaniam ${ }^{1}$ \\ ${ }^{1}$ Queensland University of Technology, Australia}

Background: Our understanding of the regulation of iron homeostasis has increased steadily in the past few years. Much of this is due to extensive research using animal models of disease and in particular transgenic mice with deletions in genes implicated in systemic or local iron regulation. The effect of these gene deletions on hepatic iron accumulation and expression of hepcidin is usually compared with mice fed an iron-rich diet; a $2 \%$ carbonyl iron diet has been used as a standard.

Method: To understand how iron-enriched diet affects hepatic iron loading and gene expression we performed an analysis of mice fed various concentrations of iron. Male C57B1/6 mice were fed a diet containing either $0.25 \%, 0.5 \%, 1 \%$ or $2 \%$ carbonyl iron for two weeks and compared with mice fed a control diet. The effect of varying concentrations of iron on hepatic iron loading, serum iron levels and the expression of genes involved in iron metabolism was determined. Result: Mice fed diets with increasing levels of iron had significantly lower body weights as compared to those fed a control diet, suggesting a detrimental effect of increasing iron on these mice. Hepatic iron concentration analysis and Perls ${ }^{\mathrm{TM}}$ Prussian blue staining of liver sections indicated that iron levels increased with increasing concentration of iron in the diet. Surprisingly, hepatic gene expression of hepcidin reached a maximum at the $0.25 \%$ carbonyl iron diet level despite increasing hepatic iron accumulation. Similarly transferrin saturation levels also plateaued at this concentration of iron.

Conclusion: Our results indicate that a $0.25 \%$ iron-rich diet for two weeks is sufficient to induce maximal hepatic iron loading without adversely affecting the health of the mice. Importantly these results also demonstrate that in a chronic setting of iron administration, the amount of excess hepatic iron may not influence hepcidin regulation and that expression of hepcidin plateaus at lower hepatic iron levels, providing further insights into the regulation of this important hormone. This data may have implications for previous studies using $2 \%$ carbonyl iron in the diet to examine hepcidin regulation

\section{O-MISC-05}

Outcome of patients with cardiac hepatopathy undergoing heart transplantation preliminary analysis

\section{Natalie LY Ngu ${ }^{1}$, Ammar Majeed ${ }^{1}$, Stuart K Roberts ${ }^{1}$, Peter Bergin $^{1}$, William Kemp ${ }^{1}$ \\ ${ }^{1}$ Alfred Health, Australia}

Background: "Cardiac hepatopathy" describes liver dysfunction in patients with heart failure, which can progress to cirrhosis. There is limited evidence assessing the influence of cardiac hepatopathy on the outcomes of patients after orthotopic heart transplantation (OTHx). The objective of this analysis is to assess baseline characteristics of a population prior to a future analysis with matched non-cirrhotic controls.

Method: A retrospective analysis of patients undergoing OTHx from 2009-2016, at the Alfred Hospital was performed. Cirrhosis was established by histology and/or radiological evidence prior to, or within the 2 months following OTHx. Exclusions were; alternative cause of liver disease, requirement for cardiac assist devices, transplanted prior to 2009 or without documented cirrhosis.

Result: Eleven patients with cirrhosis undergoing OTHx were included. The baseline characteristics are presented in table 1 . The median age was 50 years, and $27 \%$ were females. The median (range) duration of stay in intensive care unit and the total hospital stay after OTHx was 7 (5-11) days and 22 (15-55) days, respectively. Seven patients developed acute kidney injury after OTHx. Other observed complications were sepsis $(n=1)$, bleeding $(n=1)$, re-intervention $(n=1)$ and encephalopathy $(n=1)$. Two participants died at 3 weeks and 6 months after transplant and the remainder were living as of May 2017. Therefore 1 year, 3 year and overall survival at 8 years in this small cohort of patient with cirrhosis was $82 \%$ (Fig 1) 
Table 1: Basic demographics

\begin{tabular}{|c|c|}
\hline Charact eri sti cs & $n=11$ \\
\hline $\begin{array}{l}\text { Age at transplant, yrs } \\
\text { (median, IQR) }\end{array}$ & $\begin{array}{l}50(35- \\
54)\end{array}$ \\
\hline Gender, females n(\%) & $3(27)$ \\
\hline $\begin{array}{l}\text { Indication for } \\
\text { transplantation } n(\%)\end{array}$ & \\
\hline Complex congenital disease & $5(45)$ \\
\hline Cardiomyopathy & $5(45)$ \\
\hline Ischaemic heart disease & $1(10)$ \\
\hline $\begin{array}{l}\text { Modality of cirrhosis } \\
\text { diagnosis } n(\%)\end{array}$ & \\
\hline Histology & $2(18)$ \\
\hline Ultrasound & $9(82)$ \\
\hline Computerised tomography & $5(45)$ \\
\hline $\begin{array}{l}\text { Relevant findings on imaging } \\
n(\%)\end{array}$ & \\
\hline Portal hypertension & $5(45)$ \\
\hline Ascites & $4(36)$ \\
\hline $\begin{array}{l}\text { Laboratory finding (median, } \\
\text { IQR) }\end{array}$ & \\
\hline MELD at Tx & $\begin{array}{l}17(14- \\
28)\end{array}$ \\
\hline Bilirubin $\mu \mathrm{mol} / \mathrm{L}$ & $11(8-24)$ \\
\hline Peak bilirubin $\mu \mathrm{mol} / \mathrm{L}$ & $\begin{array}{l}36(29- \\
64)\end{array}$ \\
\hline $\begin{array}{l}\text { IQR Interquartile range, OTHX } \\
\text { Orthotopic Heart Transplant, MELD } \\
\text { for End - Stage Liver Disease Score }\end{array}$ & Model \\
\hline
\end{tabular}

Figure 1: Kaplan Meier curve of survival after OTHx.

Conclusion: Mortality rate in patients with cardiac hepatopathy after OHTx is low and appears to plateau within the first year after transplant. Further analysis using matched controls will be undertaken in order to assess both improvement in liver function and graft survival following heart transplantation.
O-MISC-06

\section{Deleterious effect of ramadan-fasting in liver transplant recipients}

Moutaz Farouk Derbala ${ }^{1}$, Mohamed Elbadri ${ }^{7}$, Aliaa Mohamed Amer $^{4}$, Saad Alkaabi ${ }^{6}$, Syed Adnan Mohiuddin ${ }^{2}$, Moutaz Farouk Derbala $^{8}$, Yasser Medhat Kamel ${ }^{5}$, Elham Abdel Raouf Abd El Salam Elsayad ${ }^{3}$, Tahany Ahmed Mahgoub ${ }^{9}$

${ }^{1}$ Sr. Consultant Gastroenterology\& Hepatology, Qatar; ${ }^{2}$ Clinical fellow Gastroenterology/Hepatology. Hamad Medical Corporation, Qatar; ${ }^{3}$ Clinical Pharmacist, Qatar; ${ }^{4}$ Consultant Hamad Medical corporation, Qatar; ${ }^{5}$ Consultant, Qatar; ${ }^{6} \mathrm{Head}$ of

Gastroenterology,Hamad Medical Corporation, Qatar; ${ }^{7}$ Specialist Gastroenterology/Hepatolog, Hamad Medical Corporation, Qatar; ${ }^{8}$ Sr. Consultant, Qatar; ${ }^{9}$ Transplant Coordinator, Qatar

Background: Fasting during the lunar month of Ramadan is a ritual followed by Muslims throughout the world. During fasting, Muslims are required to refrain from all intakes of food, water, beverages, smoking from sunrise to sunset for a period of a month.No significant changes, regarding liver function, was reported in healthy subjects or in the chronic hepatitis, non cirrhotic patients. Few clinical trials have addressed that fating to be prohibited in Cirrhotic patients. Liver transplant program in Qatar, was activated in December 2011 and the first liver transplant was performed in on December 6, 2011. Patients with organ transplants represent a special group of patients owing to their special nutritional needs and medication interactions. In a retrospective, observational, controlled study, we studied the effect of fasting Ramadan, in a cohort of 96 liver transplant recipients, on graft function, immunosuppressive level, biochemical and hematological indices. Also, we intend to determine the predictors of abnormal biochemical and hematological indices.

Method: The Hamad liver Transplant Hepatitis Database was utilized, for A retrospective study, including liver transplant patients, who insist to fast Ramadan and are followed in Transplant clinic from August 2008 to January 2016, who were at least 18 years of age. The patients were classified into fasting and non-fasting (Control) groups. The effect of fasting were studied by comparing and intragroupal analysis of the patient data

Result: The study included 96 patients, received a liver transplant in the period of 1986-2014, and followed up in transplant clinic, Hamad Hospital. The patients were mostly men $58(60.4 \%)$, with an average age of $49.71+$ (SD 14.11). No significant differencet, was detecting in any of the biochemical, and hematological indices between patients who fasted and patients who did not.We detected significantly higher albumin $(P<0.0001)$, total proteins $(P<0 \quad .0001)$, cholesterol $(P<.0001)$, creatinine $(P=0.04)$, hemoglobin $(P<0.0001)$, and platelet count $(P<.0001)$, before Ramadan, and after Ramadan compared to during Ramadan. No significant difference, detected in tacrolimus levels $(P=0.96)$ between both groups. Interestingly, fasting, were less likely to have abnormal Tacrolimus levels (OR (95\% CI), 0.2(.05, .85), $P=0.028)$.We detected significantly higher tacrolimus levels $(P=0.02)$, before Ramadan (mean (SE) 4.83 (0.22)), and after Ramadan (mean SE) 4.92 (0.24)), compared to during Ramadan (mean (SE) 3.55 (0.72)). older patients were more likely to develop abnormal cholesterol and triglycerides levels. Only fasting status predicted abnormal total bilirubin (OR 12.82, $P=0.005$ and OR 4.65, $P=0.018$ respectively).

Conclusion: The Recepients with stable graft function, in the absence of cirrhosis, can fast safely and increase Dietary intake of protein is recommended. 


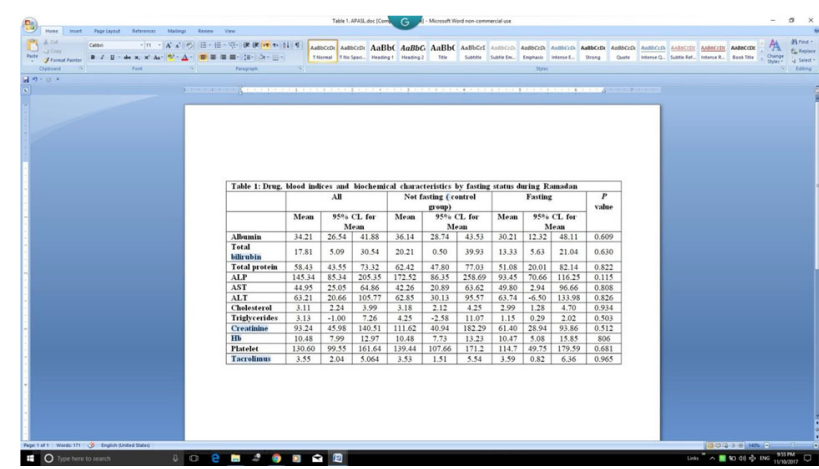

\begin{tabular}{|c|c|c|c|c|c|c|c|c|c|}
\hline & \multicolumn{2}{|c|}{ All } & \multicolumn{2}{|c|}{$\begin{array}{c}\text { Before } \\
\text { Ramadan }\end{array}$} & \multicolumn{2}{|c|}{$\begin{array}{c}\text { During } \\
\text { Ramadan }\end{array}$} & \multicolumn{2}{|c|}{$\begin{array}{c}\text { After } \\
\text { Ramadan }\end{array}$} & \multirow[t]{2}{*}{$P$ value } \\
\hline & Mean & SE & Mean & $\mathrm{SE}$ & Mean & SE & Mean & $\mathrm{SE}$ & \\
\hline Albumin & 39.25 & 0.82 & 40.61 & 0.69 & 34.21 & 3.71 & 39.46 & 0.69 & $<0.0001$ \\
\hline $\begin{array}{l}\text { Total } \\
\text { bilirubin }\end{array}$ & 19.59 & 3.29 & 19.62 & 3.23 & 17.81 & 6.14 & 20.12 & 3.62 & 0.105 \\
\hline Total protein & 70.26 & 1.21 & 71.69 & 0.75 & 58.43 & 7.11 & 72.09 & 0.94 & $<0.0001$ \\
\hline ALP & 136.75 & 13.23 & 124.73 & 12.39 & 145.34 & 28.44 & 145.64 & 15.33 & 0.343 \\
\hline AST & 48.22 & 5.46 & 47.06 & 4.78 & 44.95 & 9.60 & 50.36 & 6.87 & 0.724 \\
\hline ALT & 50.75 & 11.24 & 46.60 & 8.16 & 63.21 & 20.46 & 49.57 & 10.79 & 0.238 \\
\hline Cholesterol & 4.11 & 0.16 & 4.23 & 0.15 & 3.11 & 0.42 & 4.27 & 0.14 & $<0.0001$ \\
\hline Triglycerides & 2.75 & 0.78 & 1.34 & 0.10 & 3.13 & 1.98 & 3.95 & 1.45 & 0.207 \\
\hline Creatinine & 98.54 & 10.25 & 98.33 & 7.34 & 93.24 & 22.79 & 100.30 & 10.65 & 0.036 \\
\hline $\mathbf{H b}$ & 12.65 & 0.27 & 12.97 & 0.21 & 10.48 & 1.20 & 13.02 & 0.25 & $<0.0001$ \\
\hline Platelet & 155.16 & 7.70 & 157.46 & 7.97 & 130.60 & 14.97 & 160.34 & 7.63 & $<0.0001$ \\
\hline Tacrolimus & 4.70 & 0.24 & 4.83 & 0.22 & 3.55 & 0.72 & 4.92 & 0.24 & 0.017 \\
\hline
\end{tabular}

\section{Session: Hepatitis B Virus Infection-Clinical, Chairperson:Shobna Bhatia; Ajit Sood}

\section{$O-H B V-37$}

Is on-treatment change of Model for End-Stage Liver Disease (MELD) score a useful prognostic marker on mortality and hepatic events in chronic hepatitis $B$ patients with cirrhosis? -A cohort study of 1,729 subjects

\section{Cheuk Fung Yip ${ }^{1}$, Grace Lai Hung Wong', Henry Lik Yuen Chan $^{\text {', Yee Kit Tse }}{ }^{1}$, Vincent Wai Sun Wong'}

${ }^{1}$ The Chinese University of Hong Kong, Hong Kong

Background: Antiviral treatments can modify the natural history of chronic viral hepatitis. This is reflected by improving Model for EndStage Liver Disease (MELD) score over time. Whether this improvement correlates with more favorable clinical outcomes remains unclear. This study evaluated the impact of on-treatment change of MELD score in patients with chronic hepatitis B (CHB)related cirrhosis.

Method: CHB patients with cirrhosis who were treated with entecavir (ETV) and/or tenofovir disoproxil fumarate (TDF) for at least 1 year from 2005 to 2016 were identified from a territory-wide cohort in Hong Kong. Patients with MELD scores available at baseline and one year after ETV/TDF were included. The primary and secondary outcomes were all-cause mortality and hepatic events. Patients with cancers including hepatocellular carcinoma previously or during the first year of treatment were excluded.

Result: We identified $1,729 \mathrm{CHB}$ patients $(71.0 \%$ male, mean age $59.8 \pm 11.5$ years) with cirrhosis. The mean MELD score was $11.2 \pm 4.5$ at baseline and $9.9 \pm 3.4$ at one year; $1,059(61.2 \%)$ patients had a decreased MELD score. At a median (interquartile range) follow-up of 4.6 (2.6-6.0) years, 238 (13.8\%) patients died. Among 1,191 patients without prior hepatic events including ascites, spontaneous bacterial peritonitis, variceal hemorrhage, hepatorenal syndrome, hepatic encephalopathy, and liver transplantation based on the International Classification of Diseases, Ninth Revision, Clinical Modification diagnosis codes, 99 (8.3\%) patients developed hepatic events; $709(59.5 \%)$ patients achieved a decreased MELD score. The adjusted hazard ratio (95\% confidence interval) for the decrease of MELD from baseline to Year 1 was $0.87(0.85-0.89 ; P<0.001)$ for all-cause mortality and $0.89(0.84-0.95 ; P<0.001)$ for hepatic events after adjustment of MELD score at baseline. Thus, each unit of improvement in MELD score after one year of antiviral treatment contributed to a $13 \%$ reduction in all-cause mortality and a $11 \%$ reduction in hepatic events in the next 5 years. Among 835 patients who had a MELD score $\geq 10$ at baseline, the 5-year cumulative incidence of all-cause mortality was $23.2 \%$ vs. $42.1 \%$ for patients who achieved more than 1 point decrease in MELD score at Year 1 compared with those who did not. (log-rank test, $P<0.001$ ) (Figure 1). Among 429 patients who had a MELD score $\geq 10$ at baseline and without prior hepatic events, the corresponding 5-year cumulative incidence of hepatic events was $18.3 \%$ vs. $24.3 \%$, respectively. (Gray's test, $P=0.056$ ) (Figure 2).

Conclusion: Improvement in the MELD score after 1 year of antiviral therapy predicts all-cause mortality and hepatic events in cirrhotic CHB patients.

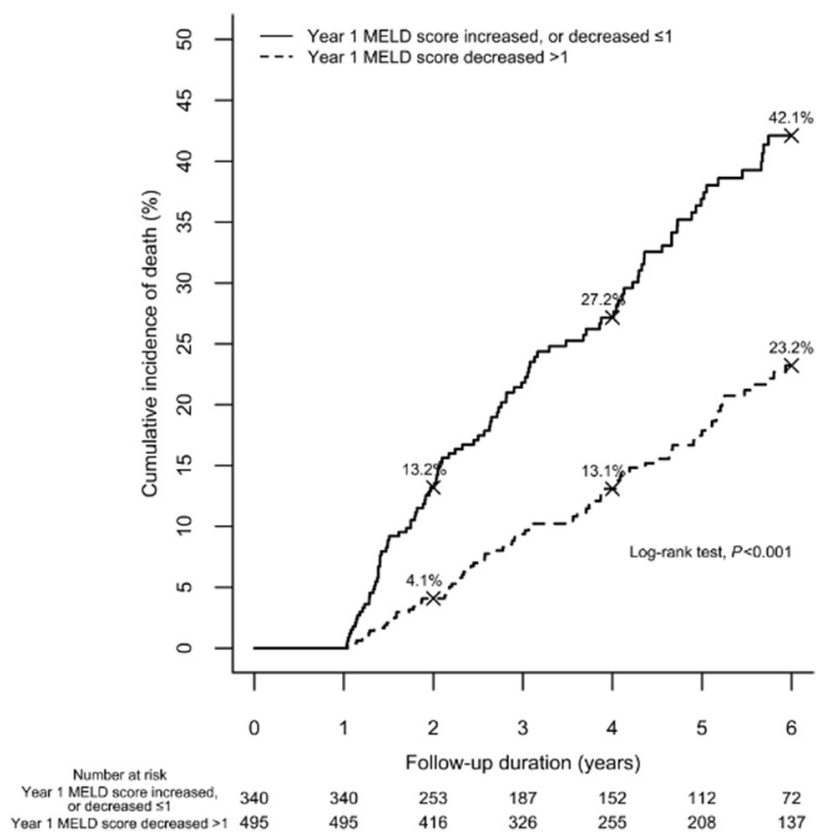




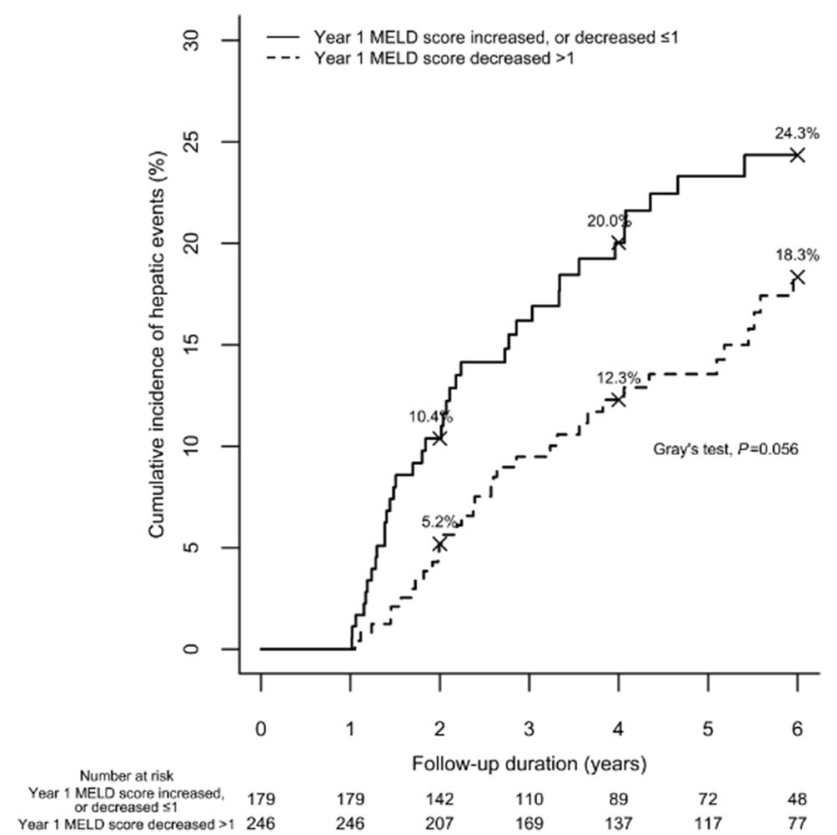

$O-H B V-38$

Is there really any difference between tenofovir monotherapy and tenofovir combination therapy in patients with suboptimal response for entecavir $0.5 \mathrm{mg}$ daily?

\section{Goktug Sirin ${ }^{1}$, Hasan Ylmaz ${ }^{1}$, Ali Erkan Duman', Zeki} Islamoglu $^{1}$, Altay Celebi ${ }^{1}$, Sadettin Hulagu ${ }^{1}$

\section{${ }^{1}$ Kocaeli University Medical School, Turkey}

Background: Although it is one of the most effective antiviral drugs used in the treatment of chronic hepatitis B (CHB), it is not clear how treatment should be continued in patients who do not respond optimally to entecavir (ETV).We aimed to analyze whether there is any difference between two different alternative approaches in terms of reaching the optimal result.

Method: Records of HBV positive patients who treated with oral antiviral agents at Kocaeli University Gastroenterology Department between January 2007 and May 2017 were reviewed. Adult patients with CHB who were partial response to entecavir (EPR) subsequently tenofovir combination treatment (ETV + TDF) or switched to tenofovir (TDF) and their follow-up results examined with respect to demographics and clinical data. Patients who have HIV and/or HCV were excluded from the study. EPR as defined detectable HBV DNA $>60 \mathrm{IU} / \mathrm{mL}$ after? 12 months of ETV. These patients were categorized into two groups as patients who had this lasting positivity longer than 12 months and 24 months despite the treatment. Patients who were treatment non-compliant, could not be followed (drop out) or developed resistance to ETV were excluded. Patients who have been diagnosed with HCC or have undergone therapy, before the rescue therapy beginning, were excluded from the evaluation, too. Total viral suppression ratios (HBV DNA $<60 \mathrm{IU} / \mathrm{mL}$ ) were analyzed using Kaplan-Meier methods and differences between all treatment groups were compared using the log rank test.

Result: 1278 patients with CHB data cards reviewed (613 men and 665 women, with an age range of 19-91 years, and a mean of 61.4 years), then 21 consecutive patients who were subsequently switched to TDF monotherapy and 23 to TDF + ETV combination therapy enrolled to the study. Age, gender and racial differences or HBeAg status ( $71.4 \%$ positive vs. $69.5 \%$ positive, $p=0.26)$ were not found between the groups. The majority of patients had similar baseline ALT. There were slight differences in HBV DNA levels between TDF and TDF + ETV patients, $(7.55 \log 10 \mathrm{IU} / \mathrm{mL}$ vs. 8.92 $\log 10 \mathrm{IU} / \mathrm{mL}, \mathrm{p}=0.01)$ and $(3.45 \log 10 \mathrm{IU} / \mathrm{mL}$ vs. $3.87 \log 10 \mathrm{IU} /$ $\mathrm{mL}, \mathrm{p}=0.05)$ prior to ETV and at the start of rescue therapy, respectively. Viral suppression rates after 6 months of rescue therapy were $78 \%$ for patients on TDF and $87 \%$ for those on TDF + ETV $(\mathrm{p}=0.19)$. After 12 months of rescue therapy, viral suppression rates were nearly identical: $91 \%$ with TDF and $94 \%$ with TDF + ETV $(\mathrm{p}=0.82)$. When Kaplan Meier graphs of viral suppression rates reviewed, log-rank analysis showed that there is not statistically significant difference between the two rescue therapies $(\mathrm{p}=0.69)$. Additionally, multivariate analysis show that HBV DNA prior to ETV and at switch to rescue therapy and time on ETV, TDF + ETV was not an detached predictor for viral suppression compared to TDF alone, also. $(\mathrm{OR}=1.42, \mathrm{p}=0.34)$. HCC developed in 6 patients in the TDF group and in 2 patients in the ETV + TDF group during the study. In patients who were still viremic after 12 months of ETV $0.5 \mathrm{mg}, 40 \%$ were still viremic when they continue to receive treatment after another 12 months. The number of patients who were switched to ETV $1.0 \mathrm{mg}$ daily in both group before starting rescue therapy was 3 and 4, respectively. Complete viral response could not be reached in none of these patients during the time they were treated. Conclusion: TDF monotherapy and TDF + ETV combination therapy seems to have comparable effect in achieving viral suppression in patients without optimal response to ETV. The use of TDF would be more cost-effective than TDF + ETV, if its superiority can be demonstrated by prospective studies in this patient population.

\section{$O-H B V-39$}

Peginterferon is superior to nucleos(t)ide analogues for reduction of chronic hepatitis B-related hepatocellular carcinoma in patients with high-risk score

Peipei Ren ${ }^{1}$, Zhujun $\mathrm{Cao}^{2}$, Ruidong $\mathrm{Mo}^{2}$, Yuhan Liu ${ }^{2}$, Lichang ${ }_{\text {Chen }}{ }^{\text {, Tianhui Zhou }}{ }^{2}$, Ziqiang $\mathrm{Li}^{2}$, Jie Lu ${ }^{2}$, Yunye Liu' ${ }^{2}$, Qing Guo $^{2}$, Rong Chen ${ }^{2}$, Huijuan Zhou ${ }^{2}$, Wei Cai ${ }^{2}$, Hui Wang ${ }^{2}$, Honglian Gui ${ }^{2}$, Qing Xie ${ }^{2}$

${ }^{1}$ Ruijin Hospital Affiliated to Shanghai Jiaotong University School of Medicine, China; ${ }^{2}$ Ruijin Hospital Affiliated to Shanghai Jiaotong University School of Medicine, China

Background: Although a few studies indicated that pegylated interferon (PEG-IFN) was preferable to nucleos(t)ide analogues (NAs) in reducing hepatocellular carcinoma (HCC) incidence, clinical evidence needs to be strengthened. This study was designed to compare the long-term outcomes of PEG-IFN vs NAs therapy in chronic HBV infection (CHB) patients and further evaluated the treatment type effect on the HCC incidence in patients stratified by previously validated HCC risk scores.

Method: Cumulative HCC incidence rates were calculated using the Kaplan-Meier curve and compared with the log-rank test. To reduce the selection bias, propensity score matching (PSM) was utilized. Area under receiver operating characteristic curves (AUROCs) was used for the predictive performance of different HCC risk scores.

Result: 1112 CHB patients (755 naïve case) were enrolled to form the main cohort. During the follow-up (median 5.41 years), we finally observed 31, $21 \mathrm{HCC}$ cases respectively in the entire and naïve cohort. The PEG-IFN group had a lower HCC incidence when compared to the NAs group whether in the entire or naïve cohort $(8.0 \%$ vs $2.3 \%, \mathrm{p}<10$; GAG-HCC $<82) \quad(1.08 \%$ vs $0.00 \%$, $\mathrm{p}=0.3199 ; 0.42 \%$ vs $3.28 \%, \mathrm{p}=0.0848$; respectively). However, the PEG-IFN group had a lower HCC incidence than the NAs group 
in the high-risk score (PAGE-B $=10$; GAG-HCC $=82$ ) patients (3.13\% vs $10.39 \%$, p

Conclusion: Peginterferon is superior to nucleos(t)ide analogues for reduction of chronic hepatitis B-related $\mathrm{HCC}$ in patients with highrisk score (PAGE-B $=10$ or GAG-HCC $=82$ ).

A

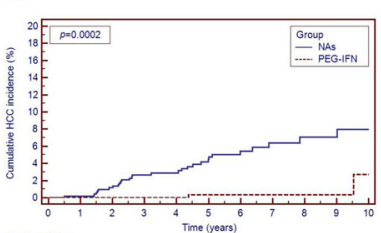

Number at risk
Group: Nas

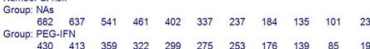

$\mathrm{C}$

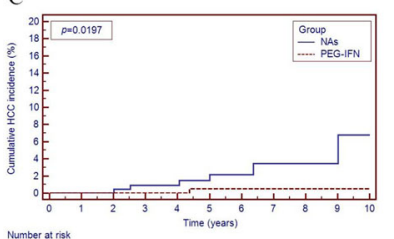

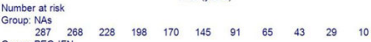

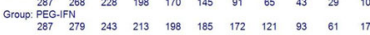

A

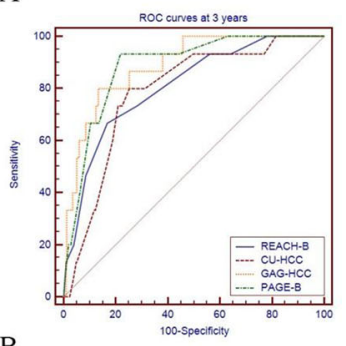

B

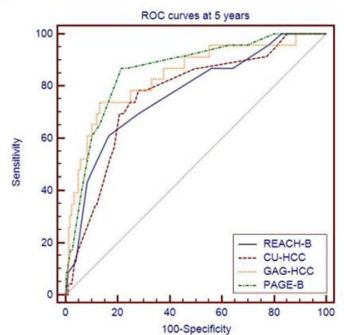

$\mathrm{C}$

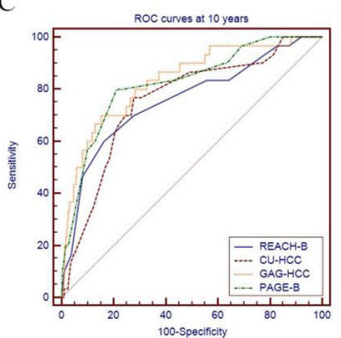

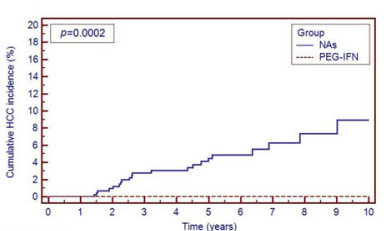

Number at rigk
Group NAs

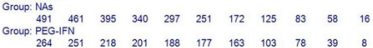
D

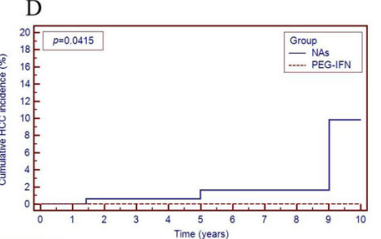

Number at risk
Group: NAs

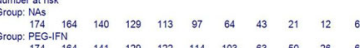

D

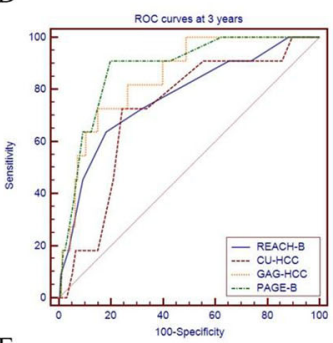

E

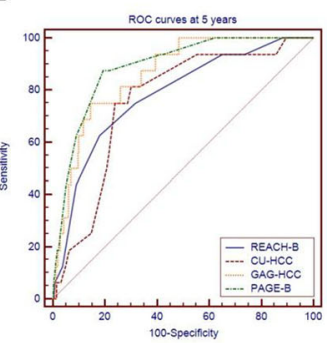

F

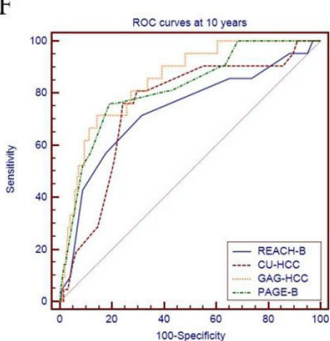

$O-H B V-40$

Prevalence and geographical distribution of anti-Delta antibody in chronic hepatitis $B$ virus carriers in Brazil

Elisabeth Lampe ${ }^{1}$, Barbara Vieira Do Lago Vieira Do Lago', Francisco Campello Do Amaral Mello', Tairine Monteiro De Barros $^{1}$, Vincius Mello ${ }^{1}$, Livia Melo Villar ${ }^{1}$, Lia Laura Lewisximenez 1

${ }^{1}$ Fundação Oswaldo Cruz, Brazil

Background: In Brazil, the Amazon Basin is endemic for hepatitis D virus (HDV) infection; however, studies in other regions of the country are scarce. This study aims to map the seroepidemiological situation of anti-Delta antibodies in chronic hepatitis B carriers in all five Brazilian geographic regions.

Method: Serum samples from $1240 \mathrm{HBsAg}$ positive individuals (55.4\% men; mean age $43.1 \pm 13.4$ years) from 24 of 26 Brazilian states were tested for the presence of anti-Delta antibodies using a commercial immunoassay

Result: Anti-Delta antibodies were detected in 40 samples (3.2\%; $55.5 \%$ males; mean age of $38.1 \pm 13.8$ years). Age less than 20 years was significantly associated with HDV infection $(\mathrm{p}<0.001)$. The median HBV viral load in HDV-negative individuals was $3.7+\mathrm{E} 03$ $\mathrm{UI} / \mathrm{mL}$, while in the HBV/HDV co-infected individuals were slightly lower, $2.5+$ E03 UI/mL. HDV prevalence varied in each Brazilian region and even among the states of the same region, as evidenced in figure 1. The highest prevalence of HDV infection was found in the North $(8.5 \% ; \mathrm{p}<0.001)$, followed by Central West $(2.5 \%)$, Southeast $(1.7 \%)$ and Northeast $(0.8 \%)$. No positive case ware identified in South region. Anti-Delta antibodies were detected in 12 Brazilian states, but more than $60 \%$ of the positive cases were observed in two states, Amazonas and Acre located in the western portion of the Amazon region, where the prevalence were $13.8 \%$ and $24.3 \%$, respectively

Conclusion: The overall HDV prevalence of $3.2 \%$ emphasizes that HDV is far from being a disease in extinction in Brazil. Despite the low anti-Delta prevalence in nonendemic regions, this infection persists in two states (Acre and Amazonas) in the north of the country, indicating that a continuous epidemiological surveillance program should be implemented in all Brazilian regions.

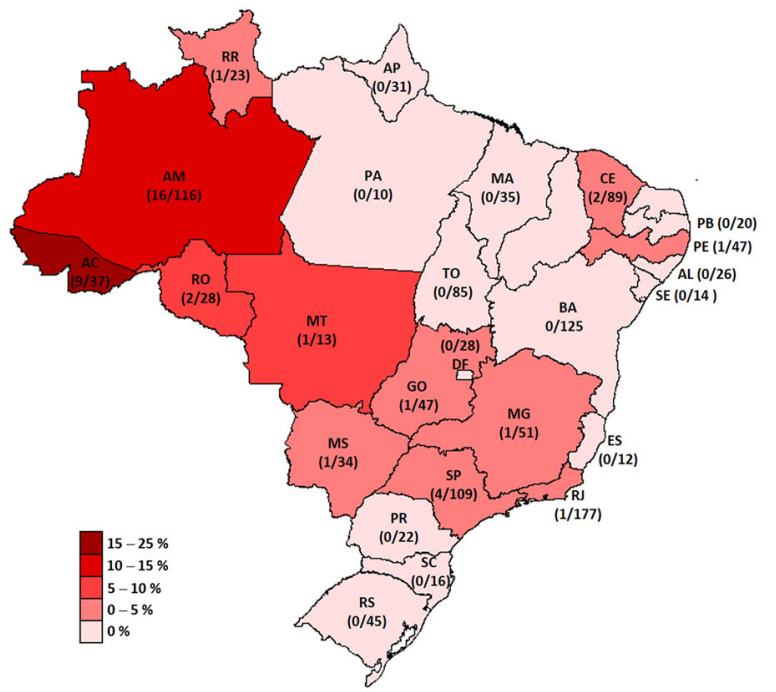

Figure 1. Seroprevalence of anti-Delta-positive samples among chronic hepatitis B carriers according to locality. The number of positive samples and total amount tested in each State are shown in parenthesis. 


\section{$O-H B V-41$}

Screening of significant liver fibrosis in general populations and high risk HBV carriers in China

\author{
Biaoyang Lin ${ }^{1}$, Shengjun $\mathrm{Wu}^{2}$, Xixing $\mathrm{Lin}^{2}$, Tiangang $\mathrm{Wu}^{2}$, \\ Jinlong Chen $^{2}$ \\ ${ }^{1}$ Zhejiang Univeristy, United States; ${ }^{2}$ Zhejiang University, China
}

Background: CHI3L1 is a new marker that have been recently demonstrated to be an excellent non-invasive marker for liver fibrosis caused by various factors including $\mathrm{HBV}, \mathrm{HCV}$, alcoholic and fatty liver diseases. In this study, we screened for significant liver fibrosis (SLF) in general populations and high risk HBV carriers in China in order to estimate prevalence of ALF in general population and in high-risk populations.

Method: Serum samples from 458 healthy individuals (median age 37 years old. $52.7 \%$ female and $47.3 \%$ male)from annual physical check-up population were analyzed for CHI3L1 using the ELISA kit from Hangzhou Proprium Biotech Co. Ltd., which has obtained Chinese FDA approval and CE mark permit. In addition, 217 HBV carriers with detectible HBsAg levels were enrolled and their serum samples analyzed for CHI3L1 levels.

Result: We used the concentration of CHI3L1 > $69.5 \mathrm{ng} / \mathrm{ml}$ (median of biopsy-confirmed S2 liver fibrosis) for significant fibrosis (Huang et al. OMICS, a Journal of Integrative Biology 19(6): 339-345, 2015). 44 of the 458 healthy individuals $(9.61 \%)$ have CHI3L1 levels $>69.5 \mathrm{ng} / \mathrm{ml}$ (Table 1).The prevalence of significant liver fibrosis is $9.61 \%$, which is greater than $6-7 \%$ which was reported for the prevalence rate of liver fibrosis in the adult population without known liver disease in Europe. In Europe, the cause of liver fibrosis is mostly associated with non-alcoholic fatty liver disease, however, in China, they are probably mostly associated with HBV carrier status as the prevalence of HBV carrier is about $8.4 \%$ based on the National Disease Supervision Information Management System of China between 2005 and 2010. (Yan et al. J Clin Transl Hepatol. 2014 Mar; 2(1): 15-22). We next focused on the analysis of significant liver fibrosis for HBV carriers in China. We found that 72 of $217 \mathrm{HBV}$ carriers $(33.18 \%$ ) have CHI3L1 levels $>69.5 \mathrm{ng} / \mathrm{ml}$ (Table 1). High prevalence of liver fibrosis has been reported in other high risk populations. For example, Kwok et al. (Gut 2016; 65: 1359-68) reported a prevalence of $18 \%$ for diabetic populations and Harman et al. (BMJ Open 2015; 5: e007516.) reported 27\% in NFALD and alcoholic liver disease populations.

Conclusion: The prevalence of significant liver fibrosis is $9.61 \%$ in the general populations and as high as $33.2 \%$ in HBV carriers in China. General screening of the general populations, and in particular, in the high risk HBV carriers in China, is warranted to reduce the burden of liver disease in the future.

Table 1. Percentage of individuals with advanced liver fiboris

\begin{tabular}{|cccc|}
\hline Categories & Routine-check-up population & Total & Percentage \\
\hline CHI3L1 $>69.5$ & 44 & 458 & 9.61 \\
\hline & HBV carriers & Total & percentage \\
\hline CHI3L1 > 69.5 & 72 & 217 & 33.18 \\
\hline
\end{tabular}

\section{$O-H B V-42$}

The predictors analysis of early cirrhosis for chronic HBV carriers

\author{
$\underline{\text { Ying Hai Sun }}^{1}$, Yanhui Wang ${ }^{1}$, Ming Wang ${ }^{1}$ \\ ${ }^{1}$ Hepatobiliary Hospital in Jilin Province, China
}

Background: Guidelines for the prevention and treatment of chronic hepatitis B in China for chronic HBV carriers are not recommended, but for chronic HBV carriers aged over 40 years were recommended to do a liver biopsy to identify the liver inflammation and fibrosis level. But limiting the invasive of the liver-biopsy most patients is difficult to accept, this study aimed at explore noninvasive warning systems of early cirrhosis for chronic HBV carriers over 40 years.

Method: 442 chronic HBV carriers over 40 years old (including 40 years) in Hepatobiliary Hospital in Jilin province form August 2012 to August 2015 accepted liver puncture biopsy, 89 cases (liver cirrhosis group) were diagnosed with cirrhosis according to Liver biopsy, 194 cases (control group) were diagnosed with fibrosis below the G2S2 stages (including G2S2). Using statistical software SPSS17.0, statistical analysis on correlation of liver biopsy results with liver function, peripheral blood cell counts, HBV DNA, HBsAg quantitative and Fibroscan.

Result: The cirrhosis group's age, history of hepatitis b, BMI and the proportion of drinkers were $53.3 \pm 8.7$ (years old) $42.7 \pm 7.2$ (years)?29.6 $\pm 3.9 ? 65.17 \%$, the corresponding indicators in control group were $44.6 \pm 3.1 ? 23.6 \pm 5.8 ? 21.8 \pm 3.1 ? 30.41 \%$.

Conclusion: For chronic HBV carriers over 40 years, age, history of hepatitis b infection, obesity, alcohol intake, PLT $<110 \times 109 / 1$, and NEUT $<2.0 \times 109 / 1$ were risk factors for liver cirrhosis. Fibroscan showed good coherence with liver cirrhosis

Session: Hepatitis $C$ Virus Infection. Charpersons: Necati Ormeci; G N Yatoo; Harshad Devarbhavi

\section{$O-H C V-25$}

Patient characteristics and medication burden of chronic hepatitis C (CHC) patients in Japan from a Nationwide Real World Hospital Claims Database

Gabriel Wong ${ }^{1}$, Craig Brooks Rooney ${ }^{3}$, Byrony Langford ${ }^{2}$, Hiroshi Yotsuyanagi ${ }^{4}$

${ }^{1}$ Gilead Sciences, Inc., United States; ${ }^{2}$ Costello Medical Consulting, United Kingdom; ${ }^{3}$ Costello Medical Singapore, Singapore;

${ }^{4}$ University of Tokyo, Japan

Background: Japan has the highest prevalence of Hepatitis $\mathrm{C}$ virus (HCV) infections in the industrialized world with approximately 1.5 million current infections. In addition, Japanese patients with HCV are elderly and thus may possess more comorbidities and higher pill burden. This study describes the pill burden and comorbidities in Japanese HCV patients in the Medical Data Vision (MDV) claims database.

Method: This was a retrospective cohort study of adult ( $\geq 18$ years old) patients with chronic hepatitis C (CHC, ICD-10 code: B18.2) included in the MDV hospital claims database. The database contains information from inpatient and outpatient visits at 287 hospitals in Japan from Apr 2008-June 2016.

Result: Age and gender were derived based on direct reporting from the database, for the most recent $\mathrm{CHC}$ visit. Comorbidity prevalence was assessed using ICD-10 codes, based on a predefined list of comorbidities common to $\mathrm{CHC}$ patients. Patients who had received all oral direct acting antiviral (DAA) therapy were identified through receipt code for a prescription for a DAA. The average number of tablets ('pill burden') taken by DAA-treated patients was calculated for the 90 days prior/post first prescription date of a DAA. The study population included 173,796 patients (mean \pm SD age $69 \pm 14$, $51.7 \%$ male), with a large proportion being over the age of 75 $(40.7 \%)$. Highly prevalent comorbidities included hypertension 
(41.4\%) and type 2 diabetes (18.3\%). Patients within the sample experienced a mean of $3.54 \pm 2.98$ comorbidities, with elderly HCV patients experiencing more comorbidities than younger $\mathrm{HCV}$ patients (age group $18-34$ vs. $75+; 1.17 \pm 1.64$ vs. $4.33 \pm 3.09 ; \mathrm{p}<0.001$ ). The 10 most prevalent comorbidities are displayed in Table 1. Of note, $14.1 \%$ of patients had hepatocellular carcinoma, which rose to $19.6 \%$ in patients $75+$. The prevalence of most comorbidities increased with age. 19,683 patients satisfied criteria for pill burden analysis. Pre- and post-DAA treatment initiation mean pill burden was $4.23 \pm 11.4$ pills/day and $6.72 \pm 12.17$ pills/day, respectively. Pill burden was higher among older patients, with those aged over 75 experiencing mean pill counts of $5.2 \pm 13.27$ and $7.12 \pm 12.05$ preand post-index, respectively. A greater proportion of elderly patients had a pill burden of $>10$ after initiating DAA treatment, compared to those in younger age groups (age group 18-34 vs. $75+; 4.2 \%$ vs. $21.6 \% ; \mathrm{p}<0.001)$.

Conclusion: The majority of Japanese CHC patients are elderly with several comorbidities and high pill burden pre-DAA treatment. Patient pill burden may be an important consideration for HCV regimen selection.

\begin{tabular}{|c|c|c|c|c|c|c|c|}
\hline \multirow{3}{*}{$\begin{array}{l}\text { Comorbidity } \\
\text { ( } \% \text { of patients) }\end{array}$} & \multirow{2}{*}{ All patients } & \multicolumn{6}{|c|}{ Age group (years) } \\
\hline & & $18-34$ & $35-44$ & $45-54$ & $55-64$ & $65-74$ & $75+$ \\
\hline & $n=173,796$ & 4,171 & 7,437 & 14,797 & 28,130 & 48,604 & 70,657 \\
\hline Hypertension & 41.4 & 3.5 & 9.5 & 20.5 & 32.2 & 42.5 & 54.4 \\
\hline $\begin{array}{l}\text { Gastroesophageal } \\
\text { Reflux Disease }\end{array}$ & 30.6 & 10.4 & 16.0 & 22.4 & 28.0 & 31.9 & 35.1 \\
\hline $\begin{array}{l}\text { IBS and } \\
\text { Constipation }\end{array}$ & 29.5 & 11.8 & 13.7 & 17.0 & 21.5 & 28.6 & 38.6 \\
\hline Chronic Gastritis & 26.7 & 12.7 & 16.8 & 21.0 & 24.6 & 28.4 & 29.4 \\
\hline Insomnia & 23.9 & 9.1 & 14.7 & 19.5 & 21.2 & 24.7 & 27.1 \\
\hline $\begin{array}{l}\text { Chronic Pain } \\
\text { Syndromes }\end{array}$ & 23.8 & 12.2 & 16.1 & 19.2 & 22.5 & 24.7 & 26.3 \\
\hline Type 2 Diabetes & 18.3 & 4.4 & 8.6 & 13.5 & 18.5 & 20.8 & 19.3 \\
\hline Dyslipidemia & 17.9 & 6.4 & 11.3 & 14.9 & 18.4 & 19.9 & 18.3 \\
\hline $\begin{array}{l}\text { Hepatocellular } \\
\text { Carcinoma }\end{array}$ & 14.1 & 0.5 & 0.7 & 3.0 & 9.7 & 15.3 & 19.6 \\
\hline $\begin{array}{l}\text { Congestive Heart } \\
\text { Failure }\end{array}$ & 13.5 & 2.9 & 4.4 & 5.7 & 8.5 & 11.5 & 20.2 \\
\hline
\end{tabular}

\section{$O-H C V-26$}

\section{Real World Referral and Treatment Patterns in a Large Community Hospital Hepatitis C Clinic: Immigrant Populations and Genotype 3 in Brampton, Ontario, Canada}

\section{Sergio Marcelo Borgia ${ }^{1}$}

${ }^{1}$ William Osler Health System, Canada

Background: Brampton, Ontario has 600,000 residents, $75 \%$ are foreign born and nearly half the population originates from Punjab, India. Whereas the estimated prevalence of Hepatitis C (HCV) infection in Canada is $1 \%$, Brampton is unique in that $\mathrm{HCV}$ prevalence reflects the residents' country of origin epidemiological burden. Unique immigration patterns allow for HCV epidemiological analyses including treatment outcomes from patterns of imported $\mathrm{HCV}$ infection in a large Canadian city.

Method: Demographic and treatment patterns for 922 consecutively referred HCV infected patients to the Osler Hepatitis Centre (OHC) at Brampton Civic Hospital between 2007-2017 were interrogated. Parameters included descriptors for age, sex, country of origin, genotype (GT), viral load, fibrosis status, co-infection with HIV/HBV and presence of hepatocellular carcinoma. Data were collected prospectively and analyzed for treatment regimen (and access source), treatment outcome, risk factor(s) for $\mathrm{HCV}$ acquisition and lost to follow-up (LTF) rate using a proprietary database (MS Excel ${ }^{\circledR}$ ) housed on a secure hospital server.
Result: A total of 922 patients from 49 countries were referred to the $\mathrm{OHC}$ for HCV infection. Patients born in India or Pakistan accounted for $30 \%$ and $8 \%$. GT distribution mirrored the GT prevalence found in patients' country of origin: Canadian patients GT1 (220/329, 67\%), Indian patients GT3 (127/190, 67\%). Cirrhosis was present in 213/581 (36\%) of patients assessed. Largest risk factors for HCV infection were birth in a country of high HCV endemicity (55\%) and recreational drug use (RDU) at any time (37\%) (Table 1). In patients who completed any treatment, 341/411 (82.9\%) achieved virologic cure at SVR12/24. Virologic failure was documented in 44/411 (10.7\%) of all treated patients: $21 / 44$ (48\%) had cirrhosis, 33/44 (75\%) failed an INF-based regimen; only 12/44 (28\%) failed a DAA regimen. Twenty-six patients died or were LTF after treatment. There were 396 patients with a known treatment regimen (15 patients were treated before referral); the proportion of patients who achieved virologic cure treated with an INF-based regimen was 103/148 (70\%) compared to $223 / 248$ (90\%) for those treated with DAAs. SVR rates by GT and treatment regimen are shown in Figure 2. Overall, 86/770 viremic patients were LTF: 68 after their first visit but prior to treatment and 18 after starting or completing treatment. Only 27 of 770 eligible patients $(3.5 \%)$ declined any form of treatment, surprisingly $8 / 27$ (30\%) declined treatment with a DAA. Thirty-two patients died after referral: 25 prior to treatment, 3 on or post-treatment but before obtaining a SVR result, 2 after achieving SVR12 with DAA treatment and 2 after relapse with INF-RBV. Currently 70 patients are on treatment and 188 untreated patients remain linked to care. Among GT3, a greater proportion of Canadian patients $(24 / 50,48 \%)$ presented with cirrhosis than Indian patients (39/123, 30\%) despite similar mean age. In GT3 patients, RDU was more frequent in Canadian $(49 / 59,83 \%)$ than Indian patients $(4 / 174,2.3 \%)$. Canadian GT3 patients were more likely to be LTF $(9 / 59,15 \%)$ than Indian patients $(3 / 174,1.7 \%)$. Canadian GT3 LTF patients were younger (mean 44y) than Indian patients (66y); all had a history of RDU compared to none in the Indian population. Interestingly, 6 Indian GT3 patients declined treatment whereas no Canadian GT3 patients did.

Conclusion: OHC in Brampton, Canada is enriched for imported cases of GT3 HCV infection from Punjab, India. Indian patients were less likely to present with cirrhosis, reported negligible RDU, and had very low LTF rates. Treatment outcomes, particularly high with DAAs, were similar to previously described real world efficacy data.

Table 1: Patient Characteristics of Osler Hepatitis C Clinic: Brampton, Canada

\begin{tabular}{|l|l|}
\hline Patient Characteristic & $\begin{array}{l}\text { Osler Hepatitis Center HCV Patients } \\
(\text { n=922, viremic=770) }\end{array}$ \\
\hline Age, mean (range) & $50(1-89)$ \\
\hline Male, n (\%) & $534(58 \%)$ \\
\hline Country of Birth: Canada/ India / Pakistan & $435(47 \%) / 273(30 \%) / 76(8 \%)$ \\
\hline Ab+/Undetectable Viral Load & $150(16 \%)$ \\
\hline Still on treatment / SVR12 Pending & $70 / 770(9.0 \%)$ \\
\hline Baseline VL <6.0M IU/mL (GT1:TN/NC) & $251 / 300(83.7 \%)$ \\
\hline Privately Insured Patients & $100 / 879(11.3 \%)$ \\
\hline Patients Diagnosed with HCC & 12 \\
\hline Patients co-infected with HIV / HBV & $17 / 6$ \\
\hline SVR (INF-based Treated Patients) & $103 / 148(70 \%)$ \\
\hline SVR (DAA Treated Patients) & $223 / 248(90 \%)$ \\
\hline Patients treated more than once (not exclusively at & $60(45$ achieved SVR; 15 still on treatment) \\
\hline OHC) & $319,48.3 \%$ \\
\hline GT1 & $56,8.5 \%$ \\
\hline GT2 & $248,37.5 \%$ \\
\hline GT3 & $18,2.7 \%$ \\
\hline GT4 & $2,0.3 \%$ \\
\hline GT6 & $6,09 \%$ \\
\hline GT8 & $2,0.3 \%$ \\
\hline Mixed GT & $12,1.8 \%$ \\
\hline Fo/F1/F2/F3,n (\%) & $85(15 \%) / 123(21 \%) / 114(20 \%) / 47(8 \%)$ \\
\hline Cirrhosis,n (\%) & $213(37 \%)$ \\
\hline & \\
\hline
\end{tabular}




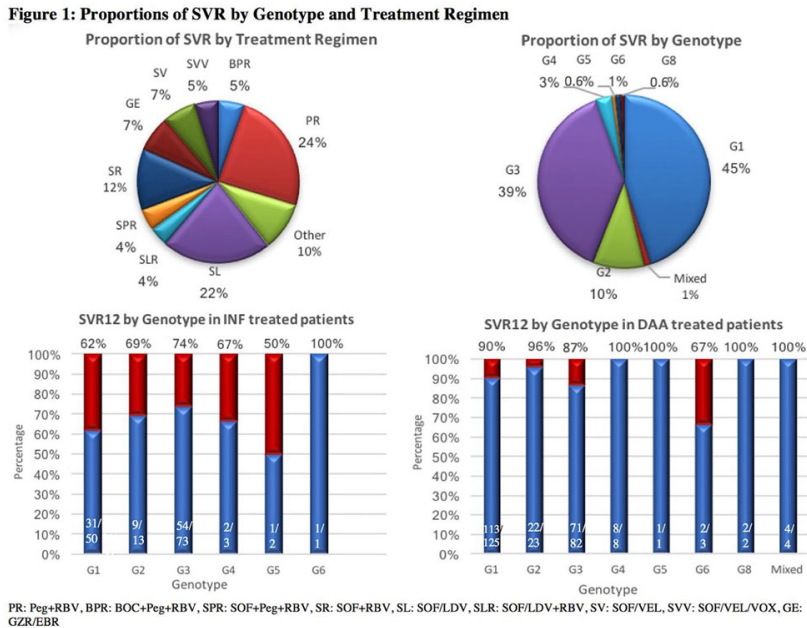

\section{$O-H C V-27$}

Real-world effectiveness and tolerability of direct-acting antiviral (DAA) for chronic hepatitis $\mathrm{C}$ genotype 1: a systematic review and meta-analysis

Mindie Nguyen ${ }^{1}$, Fanpu $\mathrm{Ji}^{4}$, Bin Wei ${ }^{1}$, Yee Hui Yeo ${ }^{1}$, Eiichi Ogawa $^{1}$, Biyao Zou ${ }^{1}$, Christopher D Stave ${ }^{1}$, Zongfang $\mathbf{L i}^{3}$, Ramsey C Cheung ${ }^{1}$, Norihiro Furusyo ${ }^{2}$, Shuangsuo Dang ${ }^{3}$, Mindie H Nguyen ${ }^{1}$

${ }^{1}$ Stanford University Medical Center, United States; ${ }^{2}$ Kyushu University Hospital, Japa; ${ }^{3}$ the Second Affiliated Hospital of Xi' an Jiaotong University, China; ${ }^{4}$ the Second Affiliated Hospital of $\mathrm{Xi}^{\mathrm{TM}}$ an Jiaotong University, China

Background: Asia has the highest HCV disease burden of the world with genotype 1/1b (GT 1/1b) being the most prevalent genotype. Robust real-world evidence of DAA therapy for HCV GT1infected patients in Asia is still lacking. Our goal was to perform a systematic review and meta-analysis to estimate the pooled SVR12 rate of DAA treatment for HCV GT1 in Asia, including subgroup analysis by cirrhosis, prior treatment failure, and pre-treatment resistance associated variants (RAVs).

Method: PubMed and EMBASE databases up to May 31, 2017 were searched for relevant data using a specific search term. Inclusion criteria were real-world study in Asia, GT 1, aged $>18$, and available SVR12 assessed in $\geq 15$ patients in any DAA regimen. Exclusion criteria were clinical trials or analysis patients included in previous clinical trials and meeting abstracts. The pooled SVR rates were computed with a DerSimonian-Laird random-effects model. Subgroup analysis and meta-regression were performed to determine how pre-planned variables affected the pooled estimates.

Result: Of the 8262 citations from PubMed and EMBASE, and 23 additional manually searched articles evaluated, forty-one studies from 8 countries and regions, with a total of 8574 HCV GT1 patients treated with different regimens were included. The pooled SVR12 rates for GT1 were $89.9 \%$ (95\% CI 88.6-91.1, $I 2=55.1 \%$ ) with daclatasvir/asunaprevir (DCV/ASV) and $98.1 \%$ (95\% CI 97.0-99.0, $I 2=41.0 \%$ ) with ledipasvir/sofosbuvir \pm ribavirin (LDV/ $\mathrm{SOF} \pm \mathrm{RBV}$ ). For $\mathrm{SOF} / \mathrm{DCV} \pm \mathrm{RBV}, \mathrm{SOF}+\mathrm{RBV}, 2 \mathrm{D} / 3 \mathrm{D} \pm \mathrm{RBV}$ regimens, the pooled SVR12 rates were $97.4 \%$ (95\% CI 90.9-100, $I 2=56.7 \%)$, $94.1 \%$ (95\% CI $79.1-100, I 2=64.4 \%)$, and $95.9 \%$
(95\% CI 89.5-99.7, I2=0.0\%), respectively. Subgroup analysis [severity of liver disease, prior treatment history, RAVs] for SVR12 in patients treated with DCV/ASV and LDV/SOF were shown in Fig.1. Baseline cirrhosis but not prior treatment history attenuated the effectiveness of both regimens. On meta-regression analysis, cirrhotic patients had $2.81 \%$ (95\% CI -1.85-7.49) and 2.29\% (95\% CI -0.935.60) reduction in SVR12 rate compared to non-cirrhotic patients treated with DCV/ASV and LDV/SOF, respectively. Baseline RAVs severely attenuated SVR12 of DCV/ASV (65.4 vs. $94.3 \%$, $\mathrm{p}<0.001$ ), and only minimally with LDV/SOF (94.5 vs. $99.2 \%$, $\mathrm{p}=0.003)$, giving a $31.6 \%(95 \%$ CI $23.5-39.4)$ and $6.15 \%(95 \%$ CI 1.30-10.60) reduction in SVR12 rates compared to those without RAVs, respectively. Pooled results of adverse events for patients treated with DCV/ASV and LDV/SOF regimen were summarized in Fig.2.

Conclusion: All oral DAA regimens for treatment of HCV GT 1/1b patients in Asia achieved a high cure rates ranged from 89.9\%-98.1\% for different regimens and the side effect profiles were excellent in real-world practice.
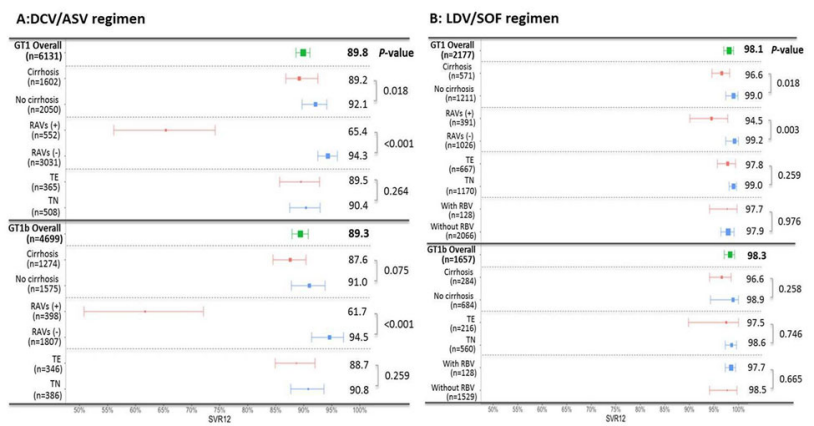

\section{$O-H C V-28$}

Safety and efficacy of elbasvir/grazoprevir (EBR/GZR) in Asian participants with hepatitis C virus (HCV) genotype (GT)1 or 4 infection: an integrated analysis of data from 11 Phase $2 / 3$ trials

Lai Wei ${ }^{1}$, Hiromitsu Kumada ${ }^{9}$, Ponni Perumalswami ${ }^{3}$, Tawesak Tawandee $^{8}$, Wendy Cheng ${ }^{7}$, Jeong $\mathrm{Heo}^{2}$, Pin Nan Cheng ${ }^{6}$, Peggy Hwang ${ }^{4}$, Sheng Mei Mu${ }^{5}$, Xu Min Zhao ${ }^{5}$, Michael Robertson ${ }^{4}$, Barbara Haber ${ }^{4}$, Rohit Talwani ${ }^{4}$

${ }^{1}$ Peking University People ${ }^{\mathrm{TM}} \mathrm{s}$ Hospital, China; ${ }^{2}$ College of Medicine, Pusan National University, Korea, Republic of; ${ }^{3}$ Icahn School of Medicine at Mount Sinai, United States; ${ }^{4}$ Merck \& Co., Inc., United States; ${ }^{5}$ Merck, Sharp \& Dohme, China; ${ }^{6}$ National Cheng Kung University, Taiwan; ${ }^{7}$ Royal Perth Hospital, Australia; ${ }^{8}$ Siriraj Hospital, Thailand; ${ }^{9}$ Toranomon Hospital, Japan

Background: Experience with direct-acting antiviral agents (DAA) for $\mathrm{HCV}$ infection is emerging in the Asia-Pacific region, where $\sim 90$ million people have HCV infection. The combination of EBR/GZR is approved for treatment of HCV GT1 and 4 infection in many countries. We conducted an integrated analysis of the safety and efficacy of EBR/GZR in Asian participants enrolled in phase 2/3 studies.

Method: We reviewed pooled data of all self-identified Asian participants in the EBR/GZR global phase $2 / 3$ program. All participants received EBR $50 \mathrm{mg} / \mathrm{GZR} 100 \mathrm{mg}$ alone for 12 weeks or in combination with ribavirin (RBV) for 16 weeks. The primary endpoint of all studies was sustained virologic response 12 weeks after end of therapy (SVR12; HCV RNA 
Result: A total of 780 Asian participants with HCV GT1 or 4 infection from 15 countries received the combination regimen of EBR/GZR in 11 clinical trials (GT1b, $\mathrm{n}=715$; GT1non-b, $\mathrm{n}=63$; $\mathrm{GT} 4, \mathrm{n}=2)$. Most participants were enrolled from Japan $(\mathrm{n}=366$, $46.9 \%)$, mainland China $(\mathrm{n}=146,18.7 \%)$, Taiwan $(\mathrm{n}=109,14.0 \%)$ and South Korea $(\mathrm{n}=90,11.5 \%)$. Mean age was 55 years (range: $20-80$ years), mean body mass index was $23.4 \mathrm{~kg} / \mathrm{m} 2$ (range: 15.5 $37.4 \mathrm{~kg} / \mathrm{m} 2$ ), and $43.8 \%$ were male. Overall, $12.4 \%$ of participants had cirrhosis, and $20.4 \%$ were treatment experienced. SVR12 was achieved by $96.9 \%(756 / 780 ; 95 \%$ confidence interval [CI]: 95.5 , 98.0) of all Asian participants, including $96.9 \%$ (748/772; $95 \% \mathrm{CI}$ : 95.4, 98.0) who received EBR/GZR for 12 weeks and $100 \%$ (8/8; 95\% CI: 63.1, 100.0) who received EBR/GZR + RBV for 16 weeks. The frequency of safety events among Asian participants was: any adverse event (AE), 58.1\% (453/780); drug-related AEs, 23.6\% (184/ 780 ); serious AEs, $2.6 \%$ (20/780): and discontinuation due to an $\mathrm{AE}$, $0.9 \%$ (7/780). Fifteen participants (1.9\%) had elevated alanine aminotransferase (ALT)/aspartate aminotransferase (AST) levels that met the criteria for an event of clinical interest (ALT/AST $>3$ baseline and $>100 \mathrm{U} / \mathrm{L}$ ), and 3 discontinued treatment. The efficacy and safety profile of EBR/GZR was comparable to that observed among non-Asians.

Conclusion: EBR/GZR was highly effective in Asian participants with primarily GT1b infection, with a safety profile similar to that observed in non-Asians.

\section{$O-H C V-29$}

Safety of treatment of genotype 4 adolescents chronic HCV infected patients with Ledipasvir/Sofosbuvir combination

Dina Attia ${ }^{1}$ Hesham El Khayat ${ }^{6}$, Inas Kamal ${ }^{5}$, Manal El Sayed ${ }^{3}$, Mortada Elshabrawy ${ }^{4}$, Hisham Ayoub ${ }^{3}$, Amal Rizk ${ }^{4}$, Mohsen Maher $^{2}$, Reem Yehia ${ }^{5}$, Yasser Foad ${ }^{5}$, Dina Attia ${ }^{1}$

${ }^{1}$ Faculty of Medicine, Beni-suef University, Egypt; ${ }^{2}$ Egyptian Military Academy, Egypt; ${ }^{3}$ Faculty of Medicine, Ain Shams University, Egypt; ${ }^{4}$ Faculty of Medicine, Cairo University, Egypt; ${ }^{5}$ Faculty of Medicine, Minia University Hospitals, Egypt; ${ }^{6}$ Theodore Bilharz Research Institute, Egypt

Background: The efficacy and safety of Ledipasvir/Sofosbuvir in treatment-naïve adolescent (12-17 years) HCV genotype 1 patients was shown in few clinical trials. The aim of this study was to investigate the effectiveness and safety of Ledipasvir/Sofosbuvir in chronic HCV adolescent patients genotype 4 in the real-world.

Method: This prospective multicenter (6 centers) open label study included 144 adolescent chronic HCV patients with genotype 4 (mean age $14 \pm 2,69 \%$ males). All patients received a combination tablet containing $90 \mathrm{mg}$ Ledipasvir and $400 \mathrm{mg}$ Sofosbuvir once daily for 12 weeks. Laboratory and virological markers were evaluated at baseline, week4, week8 and week12 (EOT), and 12 weeks after end of treatment SVR12.

Result: SVR12 was observed in $142 / 144$ patients (99\%). The relapsers were previous naïve patients $(n=2 / 128,2 \%)$ while the experienced patients showed $100 \%$ SVR12. ALT normalized at W4 with $88 \%$ decrease from baseline $(p<0.001), 89 \%$ decrease at W8 $(\mathrm{p}<0.001)$ and $90 \%$ decrease at EOT $(\mathrm{p}<0.001)$. AST also normalized at W4 with $86 \%$ decrease from baseline $(\mathrm{p}<0.001), 86 \%$ decrease $(\mathrm{p}<0.001)$ and $87 \%$ decrease $(\mathrm{p}<0.001)$ at EOT. No serious side effects were associated. Headache was the most common side effect in all patients (20\%). in the experienced patients, Pruritis $(31 \%, \mathrm{p}=0.007)$, diarrhea $(44 \%, \mathrm{p}<0.001)$ and skin rash $(19 \%$, $\mathrm{p}=0.002)$ were higher than the naïve patients.

Conclusion: Ledipasvir/Sofosbuvir regimen is well tolerated and effective and can be used safely in treating adolescent patients with chronic hepatitis $\mathrm{C}$ genotype 4 .

\section{$\mathrm{O}-\mathrm{HCV}-30$}

\section{Seroconversion of hepatitis $\mathbf{C}$ during dialysis in major cities of Pakistan}

\section{Talal Khurshid ${ }^{1}$}

${ }^{1}$ Holy Family Hospital, Pakistan

Background: Hepatitis C is highly prevalent in Pakistan. Several studies worldwide have shown that patients undergoing hemodialysis are at a risk for developing Hepatitis C. So this study was carried out to determine the proportion of patients undergoing hemodialysis who seroconverted from $\mathrm{HCV}$ negative to $\mathrm{HCV}$ positive status in our hospitals.

Method: This descriptive cross-sectional study was conducted at four tertiary care hospitals of Punjab from January 2016 to March 2016 on patients undergoing hemodialysis currently. With the help of WHO Sample Size Calculator, at confidence level 95\%, absolute precision $5 \%$ and anticipated population proportion $14 \%$, the minimally required sample size was calculated to be 186 patients but we included 190 patients in our study. Sampling technique was stratified random sampling based on hospital and gender. Our inclusion criterion was all those patients who were Hepatitis $\mathrm{C}$ negative (determined by HCV serology, based on the principle of immunochromatography) at the initiation of dialysis and remained negative for the subsequent six months after the initiation of hemodialysis. Our exclusion criteria was all those patients who seroconverted to $\mathrm{HCV}$ positive with six months of initiation of hemodialysis (the period corresponding to the incubation period of hepatitis $\mathrm{C}$ virus.) and those who were dialyzed on emergency basis. The patients' records were thoroughly checked and information regarding their HCV status at initiation of dialysis and HCV status in later serology tests was recorded. Patients who were $\mathrm{HCV}$ negative at baseline but later confirmed to be HCV positive, based on HCV serology were considered seroconverted. All the data was entered and analyzed in Statistical Package of Social Sciences, SPSS (version 22). For All the categorical variables like gender of patient, renal diagnosis, HCV status positive or negative, etc. frequencies along with percentages were calculated. For continuous variables like age of patient, duration since initiation of dialysis (in months) and duration since seroconverted (in months), mode, mean along with standard deviation were calculated.

Result: Out of 190 patients who were HCV negative at the initiation of dialysis, 93 (i.e. $48.9 \%$ ) patients converted to $\mathrm{HCV}$ positive status whereas 97 (i.e. $51.05 \%$ ) patients remained HCV negative throughout the study. The mean time taken for seroconversion was 18.04 months $(\mathrm{SD} \pm 15.43)$ months). The median was 12 months, with an inter quartile range of 14 months.

Conclusion: The proportion of $\mathrm{HCV}$ seroconversion in our hemodialysis units is very high. 
Session: Miscellaneous. Chairpersons: Ravi Ravinuthala; C E Eapen

\section{O-MISC-07}

Assessment of severity of acute liver injury and its outcome in patients with Dengue fever in Lahore and Rawalpindi.

\section{Talal Khurshid $^{1}$}

${ }^{1}$ holy family hospital, Pakistan

Background: To evaluate the impact of Dengue Virus infections on liver by measuring aminotransferase levels of the patients suffering from DF during epidemic in Lahore in 2011 and in Rawalpindi in 2015.

Material and methods: It is a multi-centered retrospective analysis of 1700 patients (1000 from LGH Lahore and 700 from HFH, Rawalpindi in 2011 \& 2015 respectively. Data was analyzed in SPSS 19 with 16 variables on which relevant details were noted.

Degree of liver damage: The degree to which the liver was affected was evaluated in these patients and classified into four groups according to AST and ALT levels (The laboratory reference values of AST \& ALT for males and females were 28 \& 26 IU and 30 \& 29 IU/ L, respectively) at the time of presentation. (1) Group A: Patients with normal AST and ALT levels. (2) Group B: Patients with one of the aminotransferases increased but not $>$ UNL X 3. (3) Group C: Patients with transaminitis of 3-10 X reference values. (4) Group D: Patients with transaminitis $>10 \mathrm{X}$ reference values.

Result: The patients were classified in to classical DF, DHF and DSS $(77.6 \%, 20.6 \%$ and $1.8 \%$ respectively. The degree of rise in aminotransferases indicating liver injury observed in LGH, Lahore was 34.9\% (Grade A), 48.5\% (Grade B), 14.8\% (Grade C) and $1.8 \%$ (Grade D). However, in BBH, Rawalpindi, it was observed as $43.8 \%$ (Grade A), 49.3\% (Grade B), 6.6\% (Grade C) and 0.3\% (Grade D). In classic DF patients, (5\% vs $2.8 \%$ ) were having grade $\mathrm{C} \& \mathrm{D}$ liver damage and (95\% vs $97.2 \%$ ) have no significant liver injury in LGH and HFH respectively. In DHF, aminotransferases were high in $(83.2 \%$ vs $68.6 \%)$ of which $(71.3 \%$ vs $54.8 \%$ patients have Grade C and $(31.8 \%$ vs $17.3 \%)$ have Grade D Liver injury. In patients with DSS, (10\% vs $3.1 \%$ ) have Grade C and $90 \%$ vs $83.2 \%$ ) with Grade D (Highest mortality and long term morbidity) in LGH and HFH respectively.

Conclusion: In Dengue outbreaks in Lahore (2011) and Rawalpindi (2015), majority of patients suffered from DF and a rise in liver enzymes was observed in majority of patients though a significant rise of liver enzymes (Grade D) was observed in patients suffering from DHS and DSS patients only. However, there is significant rise in liver enzymes in 2011 epidemic as compared to 2015 in comparative analysis of two Divisions of Punjab.

\section{O-MISC-08}

Hepatic dysfunction in malaria: clinical spectrum and predictors of mortality

\section{Prerna Goyal ${ }^{1}$, Omesh Goyal ${ }^{2}$, Subhadra Prashar ${ }^{2}$, Sandeep Puri ${ }^{2}$ \\ ${ }^{1}$ RG Stone Hospital, India; ${ }^{2}$ D.M.C. and Hospital, India}

Background: The extent of hepatic dysfunction in malaria varies from mild abnormalities in liver function tests to hepatic failure. Jaundice is a common clinical presentation in severe malaria, seen in approximately $2.5 \%$ patients with Plasmodium falciparum infection but hepatitis is unusual. Recent studies have reported liver involvement in Plasmodium vivax infection also. Patients with hepatic dysfunction in malaria are more prone to develop complications, and may have a poorer outcome. It is important to know about this entity in order to recognize it early, distinguish it from fulminant hepatic failure and offer prompt and specific treatment. We aimed to study the clinical spectrum of hepatic dysfunction and factors predicting outcome in patients with Plasmodium falciparum and Plasmodium vivax malaria admitted to a tertiary care hospital in northern India.

Method: This retrospective study included all hospitalized patients diagnosed to have malaria over 1 year period. Patients with co-infections and/or significant co-morbidities were excluded. Their clinical and biochemical parameters especially the liver function tests, complications and outcome were recorded. Multivariate analysis was performed to find the factors affecting mortality.

Result: A total of 115 patients were included. Out of these, 85(73.9\%) had P. vivax infection and $30(26.1 \%)$ had P. falciparum infection. The mean age was $36.7 \pm 16.1$ years and male:female ratio was $2.7: 1$. History of fever was present in all the cases and clinical jaundice was seen in $34.7 \%$ (40) cases. Other symptoms were chills $(33.1 \%)$, vomiting $(36.5 \%)$ and abdominal pain $(25.2 \%)$. Bleeding episodes were seen in 6 patients ( 3 had hematuria, 3 had hemetemesis/malena). Overall, liver involvement was seen in $37.4 \%$ (43) patients. Predominantly conjugated hyperbilirubinemia was seen in $88.4 \%$ (38) and predominantly unconjugated hyperbilirubinemia in $11.6 \%$ (5) cases. The mean bilirubin, AST and ALT in patients with hepatic dysfunction were $7.7 \pm 7.3 \mathrm{mg} / \mathrm{dL}, 97.1 \pm 103.3 \mathrm{IU} / \mathrm{L}$ and $72.3 \pm 87.8 \mathrm{IU} / \mathrm{L}$ respectively. Significntly greater percentage of falciparum malaria patients had hepatic dysfunction compared to patients of vivax malaria ( $50 \%$ vs. $31.8 \%$; $\mathrm{p}=0.082$ ). Patients with falciparum malaria had significantly higher levels of mean bilirubin, AST, urea and creatinine compared to those with vivax malaria. Patients with hepatic dysfunction had higher rate of complications like renal failure, shock, acute respiratory distress syndrome (ARDS), and mortality. On multivariate analysis- bilirubin $>3 \mathrm{mg} / \mathrm{dl}$, creatitine $>2 \mathrm{mg} / \mathrm{dl}$ and shock were independent predictors of mortality. Conclusion: Hepatic dysfunction was more common and more severe in patients with Plamodium falciparum malaria compared to Plasmodium vivax malaria. Patients with hepatic dysfunction had higher rates of complications and higher mortality.

\section{O-MISC-09}

The analysis of pathogens and drug-resistance in adult patients with liver abscess

\section{Longfeng Jiang ${ }^{1}$, Yao $\mathrm{Li}^{1}$, Yuyun Shao ${ }^{1}$, Ping $\mathrm{Shi}^{1}$, Jun $\mathrm{Li}^{1}$}

${ }^{1}$ The first affiliated hospital with Nanjing Medical University, China

Background: To explore the pathogens and drug-resistance in adult patients with liver abscess(LA).

Method: We included 202 LA patients with pathogens and drug susceptibility test results treated at Jiangsu Province Hospital from March 2009 to February 2017, retrospectively.215 pathogens were identified in the blood or pus cultures of 202 LA patients. Clinical characteristics, pathogens, drug susceptibility test results, prognosis of these patients were collected and analyzed.We applied descriptive analysis to expound the characteristics of pathogens.

Result: (1) Of the 215 pathogens included in adult patients with liver abscess, the most common organism identified was Klebsiella pneumonia (KP) $(67.0 \%)$, but Escherichia coli was less frequently isolated (13.0\%). Of the 27 gram-positive bacteria, Streptococcus and Enterococcus accounted for $5.6 \%$ and $4.2 \%$ respectively. Cultured fungi in the LA patients were all Candida. (2) The drug-resistance rate of KP to piperacillin was more than $50 \%$. The drug-resistance rate of E.coli to ampicillin, piperacillin, aztreonam, cotrimoxazole, levofloxacin, 
cefazolin, and cefuroxime were more than $50 \%$.Besides, the susceptibility rate of E.coli to third and fourth generation cephalosporins,such as cefotaxime,ceftazidime and cefepime were less than $60 \%$. In addition, the drug-resistance rate to $\beta$-lactam was obviously higher than $\beta$-lactam with enzyme inhibitor in KP and E. coli. The susceptibility rate of gram-positive bacteria to penicillin were less than $60 \%$.The susceptibility rate of fungi to amphotericin B was $100 \%$. (3) The mortality of non-Klebsiella pneumoniae liver abscess was higher than Klebsiella pneumoniae liver abscess. The mortality of fungi and E.coli liver abscess was $60.0 \%$ and $17.9 \%$ respectively.

Conclusion: The most common organism identified in LA patients was Klebsiella pneumonia, followed by Escherichia coli. The $\beta$-lactam with tazobactam and carbapenem antibiotic still keep a high antibacterial activity, which could be used as selection of empiric antibacterial therapy in LA patients. The mortality of non-Klebsiella pneumoniae liver abscess was higher, clinical doctors should pay more attention to those.

\section{O-MISC-11}

Knowledge, attitudes and practices on hepatitis $B$ and $C$ at the out-patient Department of Cardinal Santos Medical Center (CSMC) during World Hepatitis Day 2015-2017 Ellaine M. Wei, MD1 Diana A. Payawal, MD1 Department of Internal Medicine, Cardinal Santos Medical Canter, Manila Philippines

\section{Ellaine Martinez Wei $^{1}$}

${ }^{1}$ Cardinal Santos Medical Center, Philippines

Background: World Health Organization hepatitis report 2017 reveals that 325 million people around the world are suffering from hepatitis $\mathrm{B}$ and $\mathrm{C}$ infection. This paper compares mean knowledge, attitude and practice scores (KAPs) of patients in the out-patient department of a Tertiary Level Hospital - CSMC, regarding viral hepatitis B and C to identify their level of awareness and the success of the Hepatitis Day campaign throughout the years 2015 to 2017.

Method: This a cross sectional, questionnaire-based research study. The questionnaire was distributed during the celebration of World Hepatitis Day last July 28, 2017 held at the Out-patient Department of CSMC. The included participants were requested to answer the 73-item questionnaire on the spot, after obtaining their consent and were subsequently collected after completion. The data were subsequently analyzed and comparison with the same questionnaires used in 2015 and 2016 was done.

Result: A total of 50 questionnaires were distributed during the event. A total response rate of $92 \%$ or 46 questionnaires were returned with complete data and answers. Majority of the group (43\%) belongs to $18-40$ years old age group, resides in the city center of Manila (85\%), are predominantly female $(61 \%)$, college graduates $(74 \%)$ and are employed $(59 \%)$ with a monthly income less than $<10,000(65 \%)$. There was a trend of increase in mean scores for knowledge, attitudes and practices towards Hepatitis B and hepatitis C from 2015 to 2017. There were also no significant differences in the mean KAP scores within group comparisons according to age, gender, employment, income bracket and location of residence on. Although there was noted an increasing trend on the mean scores, the number of respondents who passed at $80 \%$ passing rate is low. Only $59 \%$ of the respondents passed on knowledge on viral hepatitis B and C. Only 7\% has positive attitude and $37 \%$ with good practice. The knowledge of public sector regarding viral hepatitis is growing. Information and practices with regard to Hepatitis $\mathrm{B}$ and $\mathrm{C}$, is not affected by socioeconomic factors that may affect availability and dissemination of information on prevention, disease transmission, symptomatology, screening and treatment. This may be attributed to the programs by World Health Organization during World Hepatitis Day in conjunction with the year-round local government programs, especially every January.

Conclusion: The health programs should be continued and intensified to eventually eradicate viral hepatitis and a continuation of this yearly KAP score assessment should be done with a bigger population and possibly a multi-center study design.

\section{O-MISC-12}

Novel remote data collection algorithm as a linkage to care mechanism for patients with hepatitis $B$ virus

Ameer Abutaleb ${ }^{1}$, Pratibha Milton ${ }^{3}$, Simanti Datta ${ }^{7}$, Sk. Mahiuddin Ahammed $^{7}$, Koshy George ${ }^{2}$, Partha Sarathi Mukherjee $^{4}$, Amal Santra ${ }^{4}$, Shyamasundaran Kottilil' ${ }^{6}$, Abhijit Chowdhury ${ }^{5}$

${ }^{1}$ University of Maryland, United States; ${ }^{2}$ Baptist Christian Hospital, India; ${ }^{3}$ Baptist Chritian Hospital, India; ${ }^{4}$ Indian Institute of Liver and Digestive Sciences, India; ${ }^{5}$ Insitute of Post Graduate Medical Education and Research, India; ${ }^{6}$ Institute of Human Virology, United States; ${ }^{7}$ Institute of Post Graduate Medical Education \& Research, India

Background: Populations in remote terrains face logistic burden for linkage to hepatitis B virus (HBV) care despite effective screening tests and vaccines. The aim of our study was to develop a novel software application which can monitor and track a HBV screening and vaccination program at the patient and population levels. We conducted our study in the West Kameng district of Arunachal Pradesh, India.

Method: A web-based application in the nearest city (Bomdila) was used to generate non-identifiable barcodes for laboratory specimens. A field team then traveled to remote villages and engaged patients using an offline tablet application to record patient data, collect, barcode, and link their blood samples. Demographic data, biochemistry, and microbiology samples were synchronized in the Cloud after return to Bomdila. Reports were generated in Bomdila and returned to patients when complete.

Result: Over 8000 patients from the West Kameng district have been approached by our field workers over the past year in groups of batches with each associated field visit (range 198-943 patients). Of them, 7716 consented to HBV screening blood tests. Biochemistry and microbiology reports were generated using our software application, 4025 of which have been delivered to the patients with an explanation of findings. Hepatitis B surface antigen prevalence was $3 \%(\mathrm{n}=235)$. Twenty four patients had alanine aminotransferase (ALT) levels greater than $40 \mathrm{U} / \mathrm{L}$. Five patients had alpha feto-protein (AFP) above the normal range $(>8.6 \mathrm{ng} / \mathrm{ml})$. One hundred and forty patients had very low HBV DNA levels (10,000 copies/ml). Genotypic analysis showed the majority of $\mathrm{HBV}$ infections as $\mathrm{C} / \mathrm{D}$ recombinants, previously characterized in Tibetan populations. Our HBV vaccination program is underway for all eligible patients, with 5,513 patients receiving first and second dose of vaccine, and 2243 completing the entire series to date. Vaccine administration is trackable at the tribe, village, circle, and district levels.

Conclusion: HBV care can be effectively delivered and vaccination campaigns easily tracked in difficult terrains without existing power or internet infrastructure through Cloud-based software algorithms. 
Session: Hepatitis B Virus Infection-Clinical;

Chairpersons: Ian Homer Y Cua; Govind Makharia;

Rajesh Sharma

\section{$O-H B V-43$}

The gamma-glutamyl transpeptidase to platelet ratio: a novel index for predicting significant liver inflammation in chronic hepatitis B

Rui Huang ${ }^{1}$, Jian Wang ${ }^{1}$, Xiaomin Yan ${ }^{1}$, Yong Liu ${ }^{1}$, Yuxin Chen ${ }^{1}$, Zhaoping Zhang ${ }^{1}$, Weimao Ding ${ }^{2}$, Chao $\mathrm{Wu}^{1}$

${ }^{1}$ Nanjing Drum Tower Hospital, The Affiliated Hospital of Nanjing University Medical School, China; ${ }^{2}$ Huai' an No. 4 People's Hospital, China

Background: Evaluating the degree of liver inflammation is important for patients with chronic hepatitis $\mathrm{B}(\mathrm{CHB})$. However, few indexes can predict significant liver inflammation accurately in $\mathrm{CHB}$ patients. We aimed to develop a simple predictive index for significant liver inflammation in $\mathrm{CHB}$ using the routinely clinical parameters.

Method: A total of 384 treatment-naïve CHB patients who had undergone liver biopsy were enrolled in this study. The relation between hematological parameters and liver inflammation were analyzed and a novel predictive index for significant liver inflammation ( $\geq$ G3) based on these parameters was developed. The diagnostic values of this novel index for liver inflammation were compared with other conventional inflammation parameters by the areas under the receiver-operating characteristic curves (AUROCs).

Result: Based on routinely clinical parameters, gamma-glutamyl transpeptidase (GGT) and platelets(PLT) were independent predictors of significant liver inflammation by multivariable analysis in entire patients $(\mathrm{P}<0.001$ and $\mathrm{P}<0.001$, respectively), hepatitis $\mathrm{B}$ e antigen $(\mathrm{HBeAg})$ positive $(\mathrm{P}=0.021$ and $\mathrm{P}=0.019$, respectively $)$ and HBeAg negative patients $(\mathrm{P}=0.009$ and $\mathrm{P}=0.006$, respectively). Accordingly, a novel index, the GGT to PLT ratio (GPR) was developed to amplify the opposing effects of liver inflammation on the GGT and PLT. The AUROCs of GPR in predicting significant liver inflammation were $0.793(95 \%$ CI 0.748 to 0.839$), 0.799$ (95\% CI 0.738 to 0.859 ) and $0.786(95 \%$ CI 0.712 to 0.859$)$ in entire patients, $\mathrm{HBeAg}$ positive and $\mathrm{HBeAg}$ negative $\mathrm{CHB}$ patients, respectively. Further comparisons showed the diagnostic performance of GPR for significant liver inflammation was significantly superior than that of alanine aminotransferase(ALT) (AUROC: 0.691, $\mathrm{P}<0.001$; AUROC: $0.679, \quad \mathrm{P}=0.002$, respectively), aspartate transaminase(AST) (AUROC: 0.716, $\mathrm{P}=0.006$; AUROC: 0.710 , $\mathrm{P}=0.015$, respectively) and GGT (AUROC: $0.768, \mathrm{P}=0.040$; AUROC: $0.764, P=0.020$, respectively) in entire patients and HBeAg positive $\mathrm{CHB}$ patients, but was comparable with AST(AUROC: 0.717, $\mathrm{P}=0.123$ ) and GGT (AUROC: 0.770, $\mathrm{P}=0.437$ ) in HBeAg negative CHB patients.

Conclusion: The GPR index has a better diagnostic value than conventionally predictive parameters to assess liver inflammation in $\mathrm{CHB}$ patients, especially for $\mathrm{HBeAg}$ positive $\mathrm{CHB}$. The application of the novel index may reduce the need for liver biopsy in CHB patients in clinical practice.
$O-H B V-44$

Hepatitis B virus reactivation in patients with previously resolved hepatitis $B$ treated with rituximab-containing chemotherapy for B-cell lymphoma

\section{Erol Cakmak}

${ }^{1}$ Cumhuriyet University, Turkey

Background: In patients with B-cell lymphoma, treatment containing hepatitis B surface antigen ( $\mathrm{HBsAg}$ ) positive rituximab is known to cause an increase in hepatitis B virus (HBV) reactivation. The aim of this study was to determine the frequency of HBV reactivation and potential predictive factors in resolved $\mathrm{HBV}$ patients who have received rituximab-containing chemotherapy for B-Cell lymphoma.

Method: A retrospective examination was made of HBV-related markers in 106 patients before and after receiving rituximab-containing chemotherapy for CD20-positive B-Cell lymphoma.

Result: The 106 patients with CD20-positive B-Cell lymphoma who received rituximab-containing chemotherapy comprised $98 \mathrm{HBsAg}$ negative and $8 \mathrm{HBsAg}$ positive patients. The 98 patients were 64 $(65.7 \%)$ antiHBc negative and $34(34.7 \%)$ antiHBc positive. Of the 34 CD20-positive B-Cell lymphoma patients with resolved HBV infection who received rituximab-containing chemotherapy, $26(76.5 \%)$ were antiHBs-positive and $8(23.5 \%)$ antiHBs-negative. Of the 8 antiHBs-negative patients, HBV reactivation occurred in $4(50 \%)$ and no $\mathrm{HBV}$ reactivation occurred in any of the 26 antiHBs-positive patients. In comparison with the antiHBs-positive patients, the rate of $\mathrm{HBV}$ reactivation in B-cell lymphoma patients with antiHBs-negative and resolved HBV infection was statistically significantly high $(\mathrm{p}<0.001)$.

Conclusion: In conclusion, in patients with resolved HBV infection receiving rituximab-containing chemotherapy for B-cell lymphoma, antiHBs negativity is an extremely significant risk factor for $\mathrm{HBV}$ reactivation

\section{$O-H B V-45$}

Long-term tenofovir monotherapy is effective following treatment failure with multiple nucleos(t)ides in patients with chronic hepatitis B: 96-week results

Pei $\mathrm{Hu}^{1}$ Xieer Liang ${ }^{10}$, Qing $\mathrm{Xie}^{13}$, Jia Shang ${ }^{8}$, Hong Tang ${ }^{14}$, Min $\mathrm{Xu}^{7}$, Qinghua Meng ${ }^{5}$, Jiming Zhang', Pujun Gao ${ }^{2}$, Jifang Sheng ${ }^{3}$, Hao Wang ${ }^{12}$, Jidong Jia ${ }^{4}$, Guiqiang Wang ${ }^{11}$, Cui Xiong ${ }^{6}$, Pei $\mathrm{Hu}^{6}$

${ }^{1}$ GSK, China; ${ }^{10}$ Nanfang Hospital, China; ${ }^{11}$ Peking University First Hospital, China; ${ }^{12}$ Peking University People's Hospital, China;

${ }^{13}$ Ruijin Hospital Affiliated to Jiaotong University, China; ${ }^{14}$ West China Hospital, Sichuan University, China; ${ }^{2} 1$ st Affiliated Hospital of Jilin University, China; ${ }^{3} 1$ st Affiliated Hospital of ZheJiang

University, China; ${ }^{4}$ Beijing Friendship Hospital, Capital University, China; ${ }^{5}$ Beijing YouAn Hospital, Capital University, China;

${ }^{6}$ GlaxoSmithKline, China; ${ }^{7}$ Guangzhou Eighth Municipal People's Hospital, China; ${ }^{8}$ Henan Provincial People's Hospital, China;

${ }^{9}$ Huashan Hospital Affiliated to Fudan University, China

Background: The increasing number of Chronic hepatitis B (CHB) patients who fail multiple antiviral therapy with nucleos(t)ide analogs (NUCs) is a major burden on clinical practice. Tenofovir disoproxil fumarate (TDF), a potent NUC with a high resistance barrier, may be an effective rescue therapy. This study evaluates the efficacy and safety of TDF monotherapy in CHB patients who have failed treatment with multiple NUCs. 
Method: In this Phase IV, multi-center, single-arm, open-label study, 213 CHB patients who had failed multiple NUC treatment were switched to TDF $(300 \mathrm{mg}$ ) for 144 weeks. Treatment failure was defined as HBV DNA level $>200 \mathrm{IU} / \mathrm{mL}$ after therapy with $=2$ NUCs for $=6$ months each and $=12$ months in total, adherence confirmed. Patients who received at least one dose of TDF were included in the analysis. Patients with hepatocellular carcinoma (HCC) before or at screening, prior exposure to TDF within 6 months or decompensated liver disease were excluded. The primary endpoint is the proportion of patients with serum HBV DNA

Result: At baseline, 89.2\% (190/213) of patients were $\mathrm{HBeAg}(+)$ and mean HBV DNA was $4.4 \log 10 \mathrm{IU} / \mathrm{mL}$. Patients had received $=2$ different NUCs, either adefovir (ADV 90.1\%), lamivudine (LAM 62.9\%), entecavir (ETV 55.9\%), or telbivudine (LdT 24.4\%) sequentially or in combination for $>12$ months. A large proportion had single- or multidrug resistance (MDR) mutations (LAM-R 71.8\%, ETV-R 15.0\%, ADV-R 17.8\%, MDR 17.8\%). The proportion of patients achieving a virologic response (HBV DNA

Conclusion: TDF mono rescue therapy was effective and well tolerated in NUC-experienced patients who have failed prior NUCs. Most patients achieved virologic suppression over 96 weeks regardless of mutation pattern and without treatment-emergent resistance occurring. Study funded by GlaxoSmithKline (NCT02195518).

Figure 1 Distribution of HBV DNA Levels Over 96 weeks

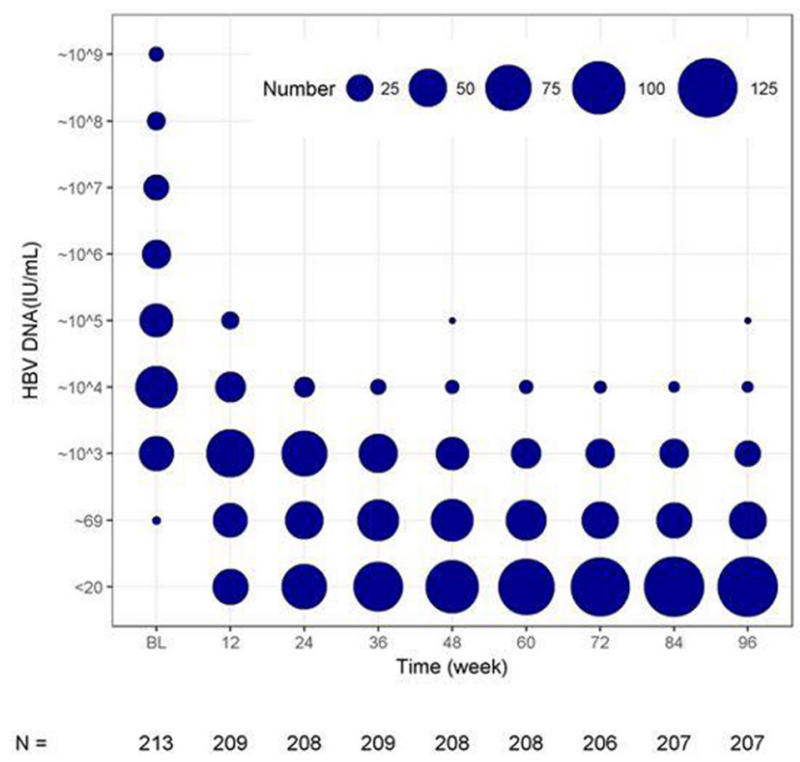

BL: Baseline

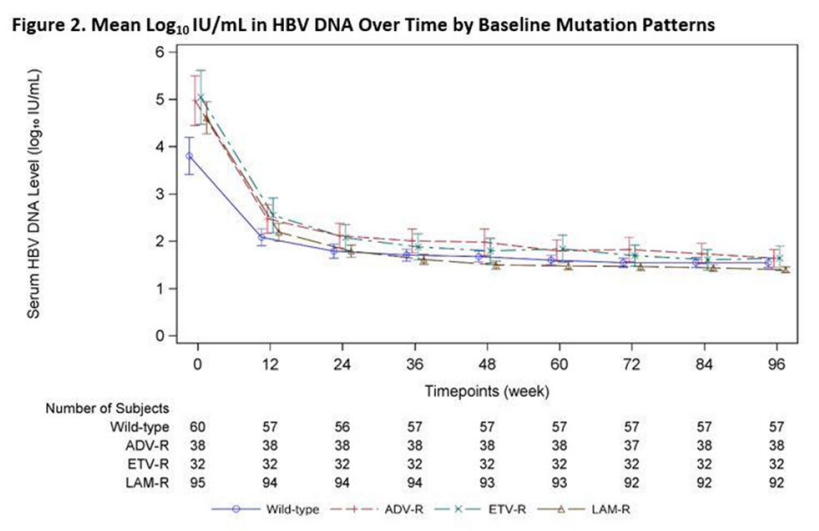

ADV-R: A181T/N(n=27); $N 236 T(n=1) ; A 181 T N+N 236 T(n=10) ; 12 p a t i e n t s$ also had various combinations of resistance mutations to

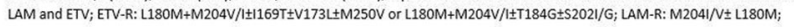

\section{$O-H B V-46$}

No resistance to tenofovir alafenamide (TAF) detected through 48 weeks of treatment in patients with chronic hepatitis B from China

Jinlin Hou ${ }^{1}$, Zhongping Duan ${ }^{2}$, Yu Chen ${ }^{2}$, Neeru Bhardwaj ${ }^{1}$, Andrea Cathcart ${ }^{1}$, Yang Liu ${ }^{1}$, Bandita Parhy ${ }^{1}$, Eric Zhou ${ }^{1}$, Hongmei Mo', John F Flaherty ${ }^{1}$, Anuj Gaggar ${ }^{1}$, Hong Tang ${ }^{4}$, Qing Xie ${ }^{3}$, Qin Ning ${ }^{5}$

${ }^{1}$ Gilead Sciences, United States; ${ }^{2}$ Capital Medical University, China; ${ }^{3}$ Shanghai Jiao Tong University, China; ${ }^{4}$ Sichuan University, China; ${ }^{5}$ Tongji Medical University, China

Background: Presented herein are the integrated Week 48 resistance analyses for a separate cohort of subjects enrolled in 2 Phase 3 studies (GS-US-320-0108 and GS-US-320-0110) in Mainland China which evaluate TAF versus tenofovir disoproxil fumarate (TDF) for the treatment of chronic hepatitis $\mathrm{B}(\mathrm{CHB})$ in $\mathrm{HBeAg}+$ and $\mathrm{HBeAg}-$ treatment-naïve or treatment-experienced adults. In the population of subjects enrolled in these 2 studies $(\mathrm{N}=1298)$ outside of China, TAF was shown to be non-inferior in efficacy to TDF with no resistance observed through 96 weeks of treatment.

Method: Subjects were randomized 2:1 and stratified by HBV DNA level and oral antiviral treatment status to receive TAF $25 \mathrm{mg}$ or TDF $300 \mathrm{mg}$ both given once daily. HBV polymerase (pol)/reverse transcriptase (RT) population sequencing was conducted for subjects with $\geq 24$ weeks of treatment who experienced virologic breakthrough (VB) at Week 48, or at early discontinuation (ED) with HBV DNA $\geq 69 \mathrm{IU} / \mathrm{mL}$. VB was defined as 2 visits with HBV DNA $\geq 69 \mathrm{IU} / \mathrm{mL}$ after achieving $<69 \mathrm{IU} / \mathrm{mL}$, or with a $\geq 1.0-\log 10$ increase from nadir. Sequence changes from Baseline at Week 48/ED are reported herein. Phenotypic analysis was performed for subjects who experienced virologic breakthrough while adherent to study drug, and those with emergent conserved site substitution(s), or polymorphic substitutions when detected in $>1$ patient. Phenotypic analysis of pol/RT substitution(s) was performed in HepG2 cells using site-directed mutagenesis of an HBV genotype (GT) A plasmid. Result: A total of 334 subjects were randomized and treated across both studies (TAF: $\mathrm{n}=227$; TDF: $\mathrm{n}=107$ ); $54 \%$ were $\mathrm{HBeAg}$ positive, $61 \%$ were GT C, and $37 \%$ had prior oral nucleos(t)ide experience. At Week 48, the number of subjects that qualified for HBV sequence analysis was low: 6 of 227 subjects (2.6\%) in the TAF group and 1 of 107 subjects $(0.9 \%)$ in the TDF group. All 6 TAF subjects qualified for sequence analysis due to VB, while in the TDF group, 1 subject qualified for sequence analysis due to ED. Of the 6 subjects in the TAF group, 1 had no change in pol/RT from baseline, 
3 were unable to be sequenced (low HBV DNA level), and 2 had unique polymorphic site substitutions. In the 1 TDF subject, unique polymorphic site substitutions in pol/RT were detected. There were no conserved site substitutions detected in any subject in either group, or polymorphic site substitutions that emerged in $>1$ subject. One subject in the TAF group met the criteria for phenotypic analysis (rtS135Y); no reduction in susceptibility was shown in vitro.

Conclusion: Overall, a small percentage of patients in the TAF and TDF groups qualified for sequence analysis at Week 48. Consistent with non-China results, no substitutions associated with resistance to TAF were detected through 48 weeks of treatment in CHB patients enrolled in China.

\section{$O-H B V-47$}

ALT level \& APRI (Aspartate Platelet Ratio Index) for predicting histological changes of liver in patients with chronic hepatitis $B$

Most. Rokshana Begum ${ }^{1}$, Mamun Al Mahtab ${ }^{2}$, Sheikh

Mohammad Noor E Alam², Nooruddin Ahmad ${ }^{2}$, Faiz Ahmed Khondaker $^{1}$, Kazi Zakir Hossain ${ }^{1}$

${ }^{1}$ SHSMCH, Bangladesh; ${ }^{2}$ BSMMU, Bangladesh

Background: According to World health organization 30\% of world population have serologic evidence of chronic hepatitis $\mathrm{B}(\mathrm{CHB})$. The disease spectrum of chronic $\mathrm{HBV}$ infection ranges from an inactive carrier state to hepatocellular carcinoma. Liver histology is important to determine prognosis and need for treatment. Thus, liver biopsy is the gold standard. But this is invasive and has risk of complications. To optimize the risk non-invasive test is needed.

Method: This was an observational cross-sectional study conducted in the department of hepatology, BSMMU. All patients who met the inclusion criteria were counselled and forty of them were included in the study. Per cutaneous transthoracic liver biopsy was done. All biopsies were fixed with $10 \%$ formalin solution and sent to pathology department, BSMMU. Biopsies was evaluated by METAVIR scoring system using hematoxylin-eosin and Masson's trichrome staining. Then scorings were calculated and compared with histological data. All data were presented as mean \pm SD and analyzed by SPSS.

Result: Among the $40 \mathrm{CHB}$ patients the age range was 18 to 50 years and the mean age was $27.1 \pm 8.5$ years. The highest frequency was found at 21-30 age groups with 29 males and 11 females. The mean HBV DNA PCR (in logarithmic scale) was found to be $5.3 \pm 1.7 .18$ patients were $\mathrm{HBeAg}$ positive and the rest were $\mathrm{HBeAg}$ negative. $\mathrm{HBeAg}$ negative $\mathrm{CHB}$ patients were older, AST and HBV DNA levels were lower. In both group, significant fibrosis $(78.8 \%$ vs $81.8 \%$ ) were found irrespective of $\mathrm{HBeAg}$ status. Among all the patients $7(17.5 \%)$ showed non-significant fibrosis and majority (82.5\%) had significant fibrosis. Among all the subjects 33 (77.5\%) patients had significant histological activity index. For ALT, majority of patients had values in between 35 to 50 , both at significant or nonsignificant fibrosis or Activity scores group. P value for ALT was 0.730 for fibrosis and 0.4475 for histological activity index. The mean APRI in F2-F4 group as well as in A2-A3 group was higher but it was statistically significant only to measure histological activity index with a $\mathrm{p}$ value 0.048 . For measuring fibrosis, the area under the receiver operator characteristic curve (AUROC) for APRI was 0.654 which gives a cut off value of 0.33 with $72.7 \%$ sensitivity and $57.1 \%$ specificity. The result was better to predict histological activity index using same cut off value where AUROC becomes 0.829 with sensitivity $80.6 \%$ and specificity $77.8 \%$. Scatter diagram showed not significant positive correlation between APRI and Metavir scoring for fibrosis but has positive significant correlation between APRI and Metavir Histological Activity Index.
Conclusion: This study indicates that both ALT and APRI are not good predictor for significant fibrosis in patient with chronic Hepatitis B but APRI can predict significant histological activity Index.

Table 01: ALT with liver histology.

\begin{tabular}{|c|c|c|c|c|c|c|c|c|c|}
\hline \multicolumn{10}{|l|}{$\overline{\mathrm{ALT}(\mathrm{U} / \mathrm{L})}$} \\
\hline$<35$ & 6 & 18.2 & 1 & 16.7 & 6 & 19.4 & 1 & 11.1 & \\
\hline $35-50$ & 16 & 48.5 & 3 & 50.0 & 14 & 45.2 & 5 & 55.6 & $0.871^{125}$ \\
\hline$>50$ & 11 & 33.3 & 3 & 50.0 & 11 & 35.4 & 1 & 11.1 & \\
\hline$\overline{\text { Mean } \pm \text { SD }}$ & & $=31.8$ & $\overline{48}$ & \pm 14.4 & & \pm 32.6 & & \pm 13.2 & \\
\hline
\end{tabular}

Table 02: APRI with liver histology.

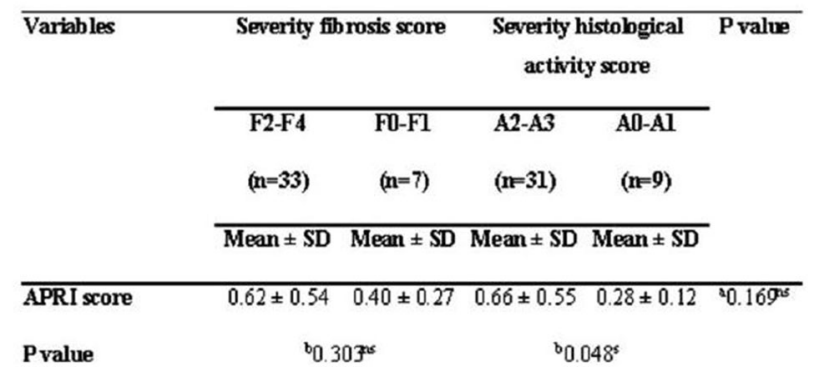

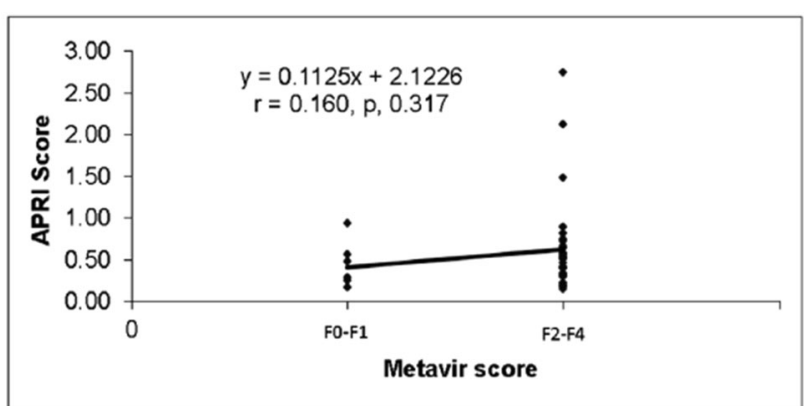

Figure 01: Scatter diagram showing not significant positive correlation $(r=0.160$ $p=0.317$ ) between APRI and Metavir Fibrosis score.

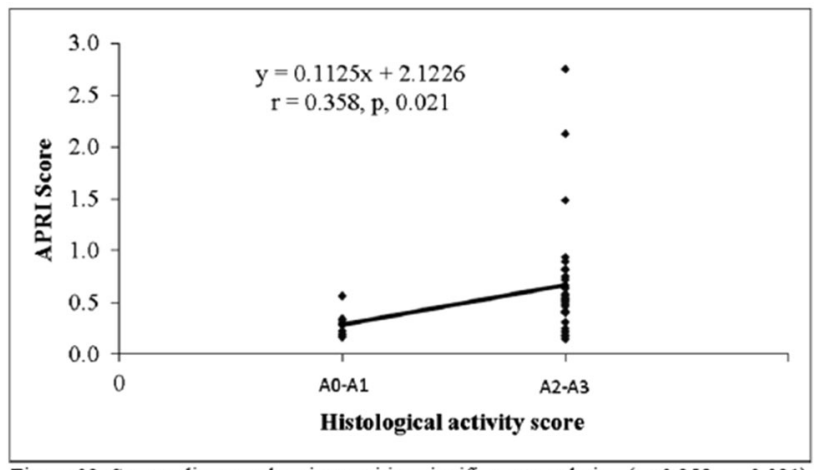

Figure 02: Scatter diagram showing positive significant correlation $(r=0.358 ; p=0.021)$ between APRI and Metavir Histological Activity Index. 
Session: Hepatitis C Virus Infection. Chairpersons: Keyur Patel; V K Dixit; S P Mishra

\section{$O-H C V-31$}

The impact of ombitasvir/paritaprevir/ritonavir with dasabuvir in patients with $\mathrm{HCV}$ associated cryoglobulinemia-experience of a single center

Elena Laura Iliescu ${ }^{1}$, Mihaela Baciuureche ${ }^{1}$, Adriana Mercan ${ }^{1}$, Mihai Dodot $^{1}$, Radu Dumitru ${ }^{1}$, Letitia Toma ${ }^{1}$

${ }^{1}$ Fundeni Clinical Institute, Romania

Background: Chronic HCV infection is associated with a series of extrahepatic manifestations, frequently including mixed cryoglobulinemia, with cutaneous and renal involvement (proteinuria). All oral antiviral therapy has shown excellent results in terms of virologic response and survival of patients with HCV chronic hepatitis or cirrhosis but data on their impact on HCV comorbidities is scarce. We aim to study the effects of all oral antiviral treatment with ombitasvir/paritaprevir/ritonavir and dasabuvir (OMB/PTV/ $r+$ DSV) on clinical and laboratory manifestations of cryoglobulinemia.

Method: This is a prospective observational study including 381 patients with $\mathrm{HCV}$ chronic hepatitis or compensated cirrhosis undergoing treatment with $\mathrm{OMB} / \mathrm{PTV} / \mathrm{r}+\mathrm{DSV}$. At initiation of therapy we determined the serum levels of cryoglobulines, proteinuria and clinically assessed the presence of purpura. All patients were monitored monthly by liver function tests, serum creatinine and blood cell count. In patients with cryoglobulinemia we adittionaly determined proteinuria, serum levels of cryoglobulines, $\mathrm{C}$ reactive protein (CRP), erythrocyte sedimentation rate (ESR) and C3 and C4 fractions of the complement.

Result: There were 28 patients $(7.34 \%)$ with cryoglobulineimia in the study group, with a mean age of $56 \pm 12.7$ years (similar to that of patients without cryoglobulinemia), with a predominance of female gender $(67.8 \%)$. Degrees of liver fibrosis were as follows: $53.5 \%$ with F2, 28.5\% with F3 and $17.8 \%$ with F4. At the initiation of antiviral therapy, 15 patients $(53.5 \%)$ of patients had active purpuric lesions and 25 patients $(89.2 \%)$ had serum cryoglobulins. The mean level of proteinuria was $1.12 \mathrm{~g} / 24$ hours (0.3-2.2 g/24 hours). Immunouppression therapy with Prednisone $5 \mathrm{mg} /$ day was continued in 3 patients. Throughout the duration of antiviral therapy, we noticed a constant decrease in the levels of proteinuria $(p<0.01)$, serum cryoglobulins ( $p<0.01)$, CRP $(p=0.02)$ and ESR $(p=0.03)$, with an increase in $\mathrm{C} 3$ and $\mathrm{C} 4(\mathrm{p}=0.04, \mathrm{p}=0.03$ respectively). Thse changes were evident from week 4 of treatment, regardless of the degree of liver fibrosis. Also, the patients with purpura presented complete clinical remission by the end of the treatment. Immune suppressive therapy was discontinued by week 4 in all three patients. All patients had undetectable HCV-RNA at the end of treatment and 20 patients achieved sustained virologic response, with test results pending in the remaining 8 patients.

Conclusion: We conclude that antiviral treatment with OMB/PTV/ $r+$ DSV is efficient not only in obtaining virologic cure but also in controlling autoimmune manifestations of HVC infection such as cryoglobulinemia, reducing the need for immunosuppression and diminishing inflammation.

\section{$O-H C V-32$}

\section{Outcomes of SOFOSBUVIR and VELPATASVIR versus SOFOSBUVIR and RIBAVIRIN therapy IN HEP C genotype-3 patients}

\author{
Muzzafar Lateef Gill ${ }^{1}$, Ammara Nawaz ${ }^{1}$, Sidra Gill ${ }^{1}$, Mehmoona \\ Pervaiz $^{1}$, Arham Baloch ${ }^{1}$ \\ ${ }^{1}$ Islamabad Gastroenterology Associates, Pakista
}

Background: We are still finding best combination of DAA in HEP C G3 patients. There is a recent availability of SOFSBUVIR and VELPATASVIR in our Country. We decided to study the outcomes of two combination therapies in HEP C G3 patients

Method: We enrolled 100 patients in each arm.

Group A: SOFOSBUVIR and RIBAVIRIN for 24 weeks.

Group B: SOFOSBUVIR and VELPATASVIR for 12 weeks.

Inclusion criteria: All patients with HEP C G3 who are treatment naïve without cirrhosis.

Exclusion criteria: Patients with platelets $<100 \mathrm{k}$; INR $>1.5$; FibroScan $>18 \mathrm{kPa}$; Primary Endpoint; SVR 12 weeks after therapy Result: Both groups were comparable in age, gender and viral load. Group A: SOF and RIBA patients achieved Early Virological Response EVR (4 weeks after treatment) 90/100 patients 90\%. Endof-Treatment ETR 88/100 88\%. 12 weeks after the treatment 86/100 $86 \%$.

Group B: SOF and VEL Patients achieved; Early Virological Response (EVR) 96/100 (96\%); End-of-Treatment (ETR) 96/100 (96\%); Sustained Virological Response (SVR) 96/100 (96\%). Group $\mathrm{B}$ sof +VAL combination was more efficacious than GroupA, $\mathrm{SOF}+\mathrm{RIBA}$ combination. $\mathrm{P}<0.04$.

Conclusion: In our experience SOFOSBUVIR and VELPATASVIR is superior therapy as compared to SOFOSBUVIR and RIBAVIRIN in HEP C G3 patients.

\section{$O-H C V-33$}

Outcomes post treatment with different regimens of new direct acting antiviral agents in Egyptian patients with chronic hepatitis $\mathrm{C}$ and decompensated cirrhosis.

\section{Mohamed Hassan Elnadry ${ }^{1}$ \\ ${ }^{1}$ AL-Azhar Univerity, Egypt}

Background\& aims: In Egypt, approximately $15 \%$ of the population has hepatitis $\mathrm{C}$ infection and genotype 4 infection accounts for more than $90 \%$ of the HCV infections, Available data with DAAs in the treatment of genotype 4 infection suggest SVR12 rates are greater than $95 \%$. The study aimed to evaluate the virological response 12 weeks post treatment (SVR12), change in the Model for End-stage Liver Disease (MELD) score, and adverse clinical events during the study period.

Methods: Prospective study of 451 patients with chronic hepatitis C and decompensated cirrhosis, 396 of them received 12 weeks of alloral DAAs. Treatment was with sofosbuvir plus daclatasvir, or ledipasvir, or Ombitasvir/paritaprevir/ritonavir with or without ribavirin. The enrolled patients were classified into three groups: Group I: Including 162 patients (compensated and decompensated cirrhosis), Group II: Including 234 patients known to have chronic hepatitis C .Group III: Including 55 patients with liver cirrhosis not subjected to DAAs therapy as a control group.

Results: We included 451 subjects with chronic hepatitis $\mathrm{C}$ infection and liver cirrhosis . Patients were $47.8 \%$ male, $84.4 \%$ treatment naive, $54.9 \%$ with cirrhosis, and all of them with HCV genotype 4 . Of the 
study participants 396 patients only were subjected to the treatment, 150 patients in group I, and 53 patient in GII received sofosbuvir + daclatasvir + ribavirin, 183 patients in GII received daclatasvir + sofosbuvir, 7patients in GII received sofosbuvir + ledipasvir, 5 patients received sofosbuvir + ledipasvir + ribavirin (in GI) and 7 patient in GI and 9 patients in GII received Ombitasvir/paritaprevir/ritonavir + ribavirin. After 12 weeks post-treatment, sustained virologic response- 12 were observed in GI \&II regardless of the regimen of therapy, Treated patients in group I had a mean negative change in MELD (_0.722, SD 2.603) representing improvement in liver function, whereas untreated patients in GIII had a minimal mean positive change (0.00, SD 2.92) representing deterioration in liver function $(\mathrm{p}<0$. 001). Improvements have been observed in the Child-score (CPT) in GI versus untreated patent in GIII. Hepatic encephalopathy was evident in $6.1 \%$ in GI post treatment versus $38.1 \%$ in untreated patients (GIII) $\mathrm{p}<0.001$, also ascites were developed in $30.2 \%$ post treatment (GI) versus $65.4 \%$ in untreated patients in GIII $\mathrm{p}<0.001$

Conclusions: This study suggests that newer all-oral regimens of DAAs is effective in the treatment of genotype 4 infection and in patients with decompensated cirrhosis led to prolonged improvement in liver function.

\section{$\mathrm{O}-\mathrm{HCV}-34$}

\section{Reactivation of herpesvirus in patients with hepatitis $\mathrm{C}$ treated} with direct-acting antiviral agents

\section{Ali Abdelrahman Ghweil ${ }^{1}$, Ali Abdelrahman Ghweil ${ }^{1}$, Mohamed Monir Helal ${ }^{1}$ \\ ${ }^{1}$ Head of the tropical medicine and gastroenterology, Egypt}

Background: Reactivation of Herpesvirus in Patients With Hepatitis C Treated With Direct-Acting Antiviral Agents. We performed a case-series analysis of reactivation of herpesvirus in patients with hepatitis $\mathrm{C}$ virus $(\mathrm{HCV})$ infection treated with direct-acting antiviral (DAA) agents. We collected data from 100 patients with $\mathrm{HCV}$ infection treated with DAA. Herpesvirus was reactivated in 10 patients who received DAA therapy (2 patients had cirrhosis and 6 patients are not cirrhotic), a median of 8 weeks after the therapy was initiated. Patients with herpesvirus reactivation were receiving the DAA agents sofosbuvir with ledipasvir (with or without ribavirin. 5 patients with herpesvirus reactivation achieved a sustained virologic response. Immune changes that follow clearance of HCV might lead to reactivation of other viruses, such as herpesvirus. Patients with $\mathrm{HCV}$ infection suspected of having herpesvirus infection should be treated immediately. Some groups also might be screened for herpesvirus infection.

Method: Reactivation of Herpesvirus in Patients With Hepatitis C Treated With Direct-Acting Antiviral Agents. We performed a caseseries analysis of reactivation of herpesvirus in patients with hepatitis $\mathrm{C}$ virus $(\mathrm{HCV})$ infection treated with direct-acting antiviral (DAA) agents. We collected data from 100 patients with $\mathrm{HCV}$ infection treated with DAA. Herpesvirus was reactivated in 10 patients who received DAA therapy (2 patients had cirrhosis and 6 patients are not cirrhotic), a median of 8 weeks after the therapy was initiated. Patients with herpesvirus reactivation were receiving the DAA agents sofosbuvir with ledipasvir (with or without ribavirin. 5 patients with herpesvirus reactivation achieved a sustained virologic response. Immune changes that follow clearance of HCV might lead to reactivation of other viruses, such as herpesvirus. Patients with HCV infection suspected of having herpesvirus infection should be treated immediately. Some groups also might be screened for herpesvirus infection.
Result: Reactivation of Herpesvirus in Patients With Hepatitis C Treated With Direct-Acting Antiviral Agents. We performed a caseseries analysis of reactivation of herpesvirus in patients with hepatitis $\mathrm{C}$ virus (HCV) infection treated with direct-acting antiviral (DAA) agents. We collected data from 100 patients with HCV infection treated with DAA. Herpesvirus was reactivated in 10 patients who received DAA therapy (2 patients had cirrhosis and 6 patients are not cirrhotic), a median of 8 weeks after the therapy was initiated. Patients with herpesvirus reactivation were receiving the DAA agents sofosbuvir with ledipasvir (with or without ribavirin, . 5patients with herpesvirus reactivation achieved a sustained virologic response. Immune changes that follow clearance of $\mathrm{HCV}$ might lead to reactivation of other viruses, such as herpesvirus. Patients with HCV infection suspected of having herpesvirus infection should be treated immediately. Some groups also might be screened for herpesvirus infection.

Conclusion: Reactivation of Herpesvirus in Patients With Hepatitis C Treated With Direct-Acting Antiviral Agents. We performed a caseseries analysis of reactivation of herpesvirus in patients with hepatitis $\mathrm{C}$ virus (HCV) infection treated with direct-acting antiviral (DAA) agents. We collected data from 100 patients with HCV infection treated with DAA. Herpesvirus was reactivated in 10 patients who received DAA therapy (2 patients had cirrhosis and 6patients are not cirrhotic), a median of 8 weeks after the therapy was initiated. Patients with herpesvirus reactivation were receiving the DAA agents sofosbuvir with ledipasvir (with or without ribavirin, . 5patients with herpesvirus reactivation achieved a sustained virologic response. Immune changes that follow clearance of HCV might lead to reactivation of other viruses, such as herpesvirus. Patients with HCV infection suspected of having herpesvirus infection should be treated immediately. Some groups also might be screened for herpesvirus infection.

\section{$O-H C V-35$}

Real world referral and treatment patterns in a Large Community Hospital hepatitis $\mathrm{C}$ clinic: immigrant populations and genotype 3 in Brampton, Ontario, Canada

\section{Sergio Marcelo Borgia ${ }^{1}$}

${ }^{1}$ William Osler Health System, Canada

Background: Brampton, Ontario has 600,000 residents, $75 \%$ are foreign born and nearly half the population originates from Punjab, India. Whereas the estimated prevalence of Hepatitis C (HCV) infection in Canada is $1 \%$, Brampton is unique in that $\mathrm{HCV}$ prevalence reflects the residents' country of origin epidemiological burden. Unique immigration patterns allow for $\mathrm{HCV}$ epidemiological analyses including treatment outcomes from patterns of imported $\mathrm{HCV}$ infection in a large Canadian city.

Method: Demographic and treatment patterns for 922 consecutively referred $\mathrm{HCV}$ infected patients to the Osler Hepatitis Centre (OHC) at Brampton Civic Hospital between 2007-2017 were interrogated. Parameters included descriptors for age, sex, country of origin, genotype (GT), viral load, fibrosis status, co-infection with HIV/HBV and presence of hepatocellular carcinoma. Data were collected prospectively and analyzed for treatment regimen (and access source), treatment outcome, risk factor(s) for $\mathrm{HCV}$ acquisition and lost to follow-up (LTF) rate using a proprietary database (MS Excel ${ }^{\circledR}$ ) housed on a secure hospital server.

Result: A total of 922 patients from 49 countries were referred to the $\mathrm{OHC}$ for HCV infection. Patients born in India or Pakistan accounted for $30 \%$ and $8 \%$. GT distribution mirrored the GT prevalence found in patients' country of origin: Canadian patients GT1 (220/329, 67\%), 
Indian patients GT3 (127/190, 67\%). Cirrhosis was present in 213/581 $(36 \%)$ of patients assessed. Largest risk factors for HCV infection were birth in a country of high HCV endemicity (55\%) and recreational drug use (RDU) at any time (37\%) (Table 1). In patients who completed any treatment, 341/411 (82.9\%) achieved virologic cure at SVR12/24. Virologic failure was documented in 44/411 (10.7\%) of all treated patients: $21 / 44(48 \%)$ had cirrhosis, 33/44 (75\%) failed an INF-based regimen; only $12 / 44(28 \%)$ failed a DAA regimen. Twenty-six patients died or were LTF after treatment. There were 396 patients with a known treatment regimen (15 patients were treated before referral); the proportion of patients who achieved virologic cure treated with an INF-based regimen was 103/148 (70\%) compared to $224 / 249$ (90\%) for those treated with DAAs. SVR rates by GT and treatment regimen are shown in Figure 2. Overall, 86/770 viremic patients were LTF: 68 after their first visit but prior to treatment and 18 after starting or completing treatment. Only 27 of 770 eligible patients $(3.5 \%)$ declined any form of treatment, surprisingly $8 / 27$ (30\%) declined treatment with a DAA. Thirty-two patients died after referral: 25 prior to treatment, 3 on or post-treatment but before obtaining a SVR result, 2 after achieving SVR12 with DAA treatment and 2 after relapse with INF-RBV. Currently 69 patients are on treatment and 188 untreated patients remain linked to care. Among GT3, a greater proportion of Canadian patients $(24 / 50,48 \%)$ presented with cirrhosis than Indian patients $(39 / 123,30 \%)$ despite similar mean age. In GT3 patients, RDU was more frequent in Canadian $(49 / 59,83 \%)$ than Indian patients $(4 / 174,2.3 \%)$. Canadian GT3 patients were more likely to be LTF $(9 / 59,15 \%)$ than Indian patients $(3 / 174,1.7 \%)$. Canadian GT3 LTF patients were younger (mean 44y) than Indian patients (66y); all had a history of RDU compared to none in the Indian population. Interestingly, 6 Indian GT3 patients declined treatment whereas no Canadian GT3 patients did.

Conclusion: OHC in Brampton, Canada is enriched for imported cases of GT3 HCV infection from Punjab, India. Indian patients were less likely to present with cirrhosis, reported negligible RDU, and had very low LTF rates. Treatment outcomes, particularly high with DAAs, were similar to previously described real world efficacy data.

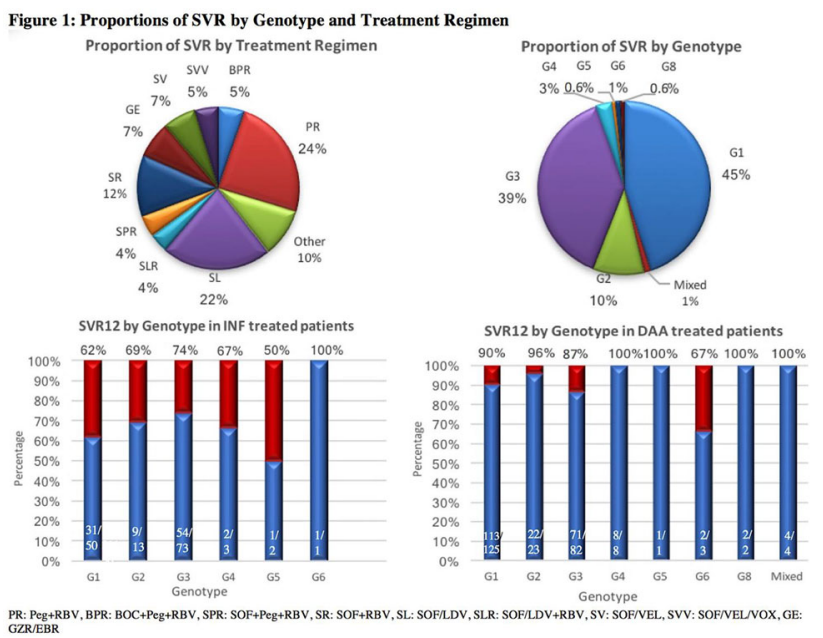

\begin{tabular}{|c|c|}
\hline Patient Characteristic & $\begin{array}{l}\text { Osler Hepatitis Center HCV Patients } \\
(\mathrm{n}=922 \text {, viremic }=770)\end{array}$ \\
\hline Age, mean (range) & $50(1-89)$ \\
\hline Male, $\mathbf{n}(\%)$ & $534(58 \%)$ \\
\hline Country of Birth: Canada/ India / Pakistan & $435(47 \%) / 273(30 \%) / 76(8 \%)$ \\
\hline Ab+/Undetectable Viral Load & $150(16 \%)$ \\
\hline Still on treatment / SVR12 Pending & $69 / 770(9.0 \%)$ \\
\hline Baseline VL $<6.0 \mathrm{M}$ IU/mL (GT1:TN/NC) & $251 / 300(83.7 \%)$ \\
\hline Privately Insured Patients & 100/879 (11.3\%) \\
\hline Patients Diagnosed with $\mathrm{HCC}$ & 12 \\
\hline Patients co-infected with HIV / HBV & $17 / 6$ \\
\hline SVR (INF-based Treated Patients) & $103 / 148(70 \%)$ \\
\hline SVR (DAA Treated Patients) & $224 / 249(90 \%)$ \\
\hline $\begin{array}{l}\text { Patients treated more than once (not exclusively at } \\
\text { OHC) }\end{array}$ & 60 (45 achieved SVR; 15 still on treatment) \\
\hline GT1 & $319,48.3 \%$ \\
\hline GT2 & $56,8.5 \%$ \\
\hline GT3 & $248,37.5 \%$ \\
\hline GT4 & $18,2.7 \%$ \\
\hline GT5 & $2,0.3 \%$ \\
\hline GT6 & $6,0.9 \%$ \\
\hline GT8 & $2,0.3 \%$ \\
\hline Mixed GT & $12,1.8 \%$ \\
\hline F0/F1/F2/F3, n (\%) & $85(15 \%) / 123(21 \%) / 114(20 \%) / 47(8 \%)$ \\
\hline Cirrhosis,n (\%) & $213(37 \%)$ \\
\hline
\end{tabular}

\section{$O-H C V-36$}

Sofosbuvir/velpatasvir is safe and effective in a phase 3 study of genotype 1-3 HCV infected Russian and Swedish patients

Robert H Hyland ${ }^{1}$, Ola Weiland ${ }^{2}$, Konstantin Zhdanov ${ }^{3}$, Brian Mcnabb ${ }^{1}$, Sophia Lu', Evguenia S Svarovskaia ${ }^{1}$, Robert $\mathbf{H}$ Hyland $^{1}$, Diana M Brainard ${ }^{1}$, G Mani Subramanian ${ }^{1}$, Dzhamal Abdurakhmanov ${ }^{4}$

${ }^{1}$ Gilead Sciences, Inc., United States; ${ }^{2}$ Division of Infectious Diseases, Karolinska Institutet at Karolinska University Hospital Huddinge, Swede; ${ }^{3}$ Military Medical Academy, Russian Federatio; ${ }^{4}$ Sechenov First Moscow State University, Russian Federatio

Background: Chronic hepatitis $\mathrm{C}$ virus (HCV) infection is highly burdensome in Russia with an estimated prevalence of 3-4\%, comprised mainly of genotype (GT) 1 (56\%), GT2 (8\%), and GT3 (35\%) $\mathrm{HCV}$ infections, making the need for a pangenotypic HCV regimen critical for this region. Treatment with sofosbuvir (SOF)/velpatasvir (VEL) for 12 weeks results in high sustained virologic response 12 weeks after therapy (SVR12) rates in GT 1-6 HCV infected patients in clinical trials and real-world settings. This study evaluated the efficacy and safety of SOF/VEL for 12 weeks in Russian and Swedish patients with chronic GT 1-6 HCV infection.

Method: Treatment-experienced and treatment-na $\overline{\tilde{A}} v e$ patients with chronic GT 1-6 HCV infection without cirrhosis or with compensated cirrhosis were eligible to enroll in a single-arm, open-label trial to receive a fixed dosed combination of SOF/VEL 400/100 mg daily for 12 weeks. Patients were recruited from 13 sites in Russia and 2 Sites in Sweden. The primary efficacy endpoint was SVR12 using the CAP/ CTM HCV 2.0 assay ( $L L O Q=15 \mathrm{IU} / \mathrm{mL}$ ) and the primary safety endpoint was adverse events (AEs) leading to SOF/VEL discontinuation.

Result: A total of 119 (103 [87\%] Russian and 16 [13\%] Swedish) patients were enrolled and treated. Of these, $50 \%$ were male, $98 \%$ were white, $18 \%$ had compensated cirrhosis, $24 \%$ were treatment experienced. Enrollment by HCV genotype was: GT1, 66\% (GT1a, 8 patients; GT1b 70 patients); GT2, 6\% (7 patients); and GT3, 29\% (34 patients). The mean (range) age and body mass index of enrolled subjects were 44 (18-71) years and $26(18-36) \mathrm{kg} / \mathrm{m}^{2}$, respectively. The overall SVR12 rate for subjects was $99 \%$ (118/119). Of the 118 
patients with SVR12, 110 returned for a posttreatment week 24 visit all of whom also achieved SVR24. The single virologic failure was a female patient without cirrhosis and with HCV GT 3 infection who relapsed between post-treatment weeks 4 and 12. All 21 patients with cirrhosis (4 of whom were treatment experienced), including 10 GT3 HCV-infected patients with cirrhosis (2 of whom were treatmentexperienced) achieved SVR12. Data regarding resistance associated substitutions at baseline (all patients) and relapse $(\mathrm{N}=1)$ will be presented. All subjects completed 12 weeks of SOF/VEL with no discontinuations due to AEs. The most frequent adverse events were headache (19 patients, $16 \%$ ), fatigue ( 8 patients, $7 \%$ ), and asthenia ( 7 patients, $6 \%$ ). No serious AEs were assessed by the investigator as related to study drug and there were no deaths.

Conclusion: Treatment with the single tablet regimen of SOF/VEL for 12 weeks resulted in $99 \%$ SVR12 rates and was well tolerated in a predominantly Russian population of genotype 1-3 HCV-infected patients with and without cirrhosis.

\section{Session: Pediatric Hepatology}

\section{O-PEDS-01}

Proteomic analysis of blood and atretic bile duct reveals candidate biomarkers for biliary atresia

\section{$\underline{\text { Anil Ram }}^{1}$, Yashwant Kumar', Baburam Thapa ${ }^{1}$ \\ ${ }^{1}$ PGIMER, Chandigarh, India}

Background: Biliary atresia (BA) is the most common neonatal cholestatic disorder, which if untreated, has a survival of less than 2 years. Liver transplantation is curative but is hardly available to these patients. Kasai procedure if performed within 2-3 months of life can prolong life but unfortunately, many patients by the time diagnosed are inoperable. Reasons for delayed diagnosis are lack of an efficient serological biomarker, newborn screening program or convenient screening methods and postnatal routine care visit. Discovery of a new serum or urine biomarker of BA in first few weeks of life is therefore highly desirable. With the aim to find out novel antigens, which may be potential candidates for a biomarker, we did proteomic profiling of blood and atretic tissue proteins in infants with BA.

Method: Twenty confirmed cases of BA and 10 controls were recruited, their blood and tissue proteins were extracted and quantified. Both tissue and plasma proteins were separated by onedimension followed by two-dimension gel electrophoresis using IPG strips. Protein spots identified both in patients and control subjects were compared, and differentially expressed spots were subjected to MALDI TOF/TOF. After that peptide mass spectrum, results were analyzed using online Mascot program against SwissProt database. The protein scores greater than 56 were considered significant $(\mathrm{p}<0.05)$.

Result: Out of more than 100 spots revealed by two-dimension gel electrophoresis, 10 spots were found to be differentially expressed on comparison with control subjects. These proteins were identified and characterized as Apo lipoprotein A-I, Apo lipoprotein C-III, Apo lipoprotein A-IV, Apo lipoprotein-V, Haptoglobin, Immunoglobin heavy chain variable, Opalin, Heparan sulphate glucosamine, Beta defensin-1 (in plasma) and B melanoma antigen-5 (in tissue). Of these opalin and Apolipoprotein A-I were downregulated in patients and others were upregulated except Immunoglobin heavy chain variable, which was expressed in controls only.

Conclusion: The study identified differentially expressed proteins in patients of BA. These are the proteins, that are otherwise involved in the inflammatory process and lipid metabolism therefore are likely to play an important role in the disease pathogenesis. Not only that, if studied further, these proteins individually or in combination may prove to be candidates for a biomarker of BA.

\section{O-PEDS-02}

Progressive familial intrahepatic cholestasis: an emerging cause of neonatal cholestasis in young infants

\section{$\underline{\text { Sandeep Yadav }}{ }^{1}$, Lalit Bharadia ${ }^{1}$, Ashwini Gupta ${ }^{1}$}

12 year, India

Background: Progressive Familial Intrahepatic Cholestasis (PFIC) constitutes an important differential in children with cholestatic liver disease. In infants ( $<6$ months old) Cholestatic liver disease comprises of Biliary atresia and Neonatal hepatitis in one third each. PFIC is now known cause of neonatal cholestasis in young infants but there is limited data on this from Rajasthan

Method: This was a prospective study conducted in the Department of Pediatrics, at a tertiary care centre in Rajasthan from January 2017 to December 2017.Clinical and laboratory data, liver histology and outcomes were recorded in a preset performa of infants $<6$ months of age with conjugated hyperbilirubinemia. Etiological diagnosis was made on the basis of biochemistry, histology and genetic test.

Result: There were 21 cases of conjugated hyperbilirubinemia during the study period. The mean age of presentation was 2 months (range 15 days to 6 month) with 13 males and 8 females. In these 19 were term and 2 were preterm. Jaundice was the presenting symptom in all. Stool colour was pale in $57 \%$, clay in $24 \%$ cases and remaining had pigmented stools. 6 infants had history of perinatal insult/sepsis. Liver biopsy was done in 11 infants. Diagnosis was PFIC (I/II) in7, Biliary atresia in 6, GSD in 1, Idiopathic neonatal hepatitis in 4 and sepsis in 3 infants. PFIC was type II in 6 and type I in 1 (based on onset and progression). PFIC patients had progressive pruritus and one of them developed hepatocellular carcinoma and succumbed later on. 3 patients with Biliary Atresia opted for Kasai portoenterostomy, rest three did not opt for Kasai surgery. All patients with PFIC and those with biliary atresia who did not opt for Kasai portoenterostomy were advised for liver transplantation. Idiopathic neonatal hepatitis resolved in 3 out of 4, 4th one is stable on follow up. One preterm with idiopathic neonatal cholestasis also had ileal atresia and cataract, which was operated initially for ileal atresia and then for cataract, Galactosemia and intrauterine infections were ruled out in that baby. Conclusion: PFIC constituted 33\% of cholestasis in young infants and should be suspected in cases of cholestasis with normal GGT. Biliary atresia continue to be common in our series constituting $28 \%$ of cases.

\section{O-PEDS-03}

Tacrolimus and mycophenolate mofetil as second-line therapies for pediatric patients with autoimmune hepatitis

Cumali Efe $^{1}$, Tugrul Purnak ${ }^{1}$, Luigi Muratori ${ }^{5}$, Marten Werner ${ }^{6}$, Paolo Muratori ${ }^{5}$, Daniel Klintman ${ }^{6}$, Aldo J. Montano Loza ${ }^{2}$, Thomas D Schiano ${ }^{8}$, Fin Stolze Larsen ${ }^{3}$, Thomas Berg ${ }^{4}$, Naim Alkhouri $^{8}$, Ersan Ozaslan ${ }^{1}$, Michael A Heneghan ${ }^{7}$, Eric M Yoshida $^{2}$, Staffan Wahlin ${ }^{6}$, Haider Al Taii ${ }^{8}$, Henriette Ytting ${ }^{3}$, Niklas F Muller ${ }^{4}$, Rahima A Bhanji ${ }^{2}$, Hannes Hagstrom ${ }^{6}$

${ }^{1} \mathrm{MD}$, Turkey; ${ }^{2} \mathrm{MD}$, Canada; ${ }^{3} \mathrm{MD}$, Denmark; ${ }^{4} \mathrm{MD}$, Germany; ${ }^{5} \mathrm{MD}$, Italy; ${ }^{6} \mathrm{MD}$, Swede; ${ }^{7} \mathrm{MD}$, United Kingdom; ${ }^{8} \mathrm{MD}$, United States

Background: Predniso(lo)ne, alone or in combination with azathioprine, is the standard therapy for autoimmune hepatitis (AIH). 
However, the this therapy is poorly tolerated or does not control disease activity in up to $20 \%$ of patients. We studied the efficacy and safety of mycophenolate mofetil (MMF) and tacrolimus as secondline therapy in pediatric AIH patients who were intolerant or nonresponders to standard medical therapy.

Method: We performed a retrospective study of data from 13 centers in Europe, USA and Canada. Thirty-eight patients $(<18$ years old $)$ who received second-line therapy (18 MMF and 20 tacrolimus), for a median of 72 months (range, 8-182) were evaluated. Patients were categorized in two groups: Group $1(\mathrm{n}=17)$ were intolerant to corticosteroid or azathioprine and group $2(n=21)$ were non-responders to standard therapy.

Result: Overall complete response rates were similar in patients treated with MMF and tacrolimus $(55.6 \%$ vs $65 \%, \mathrm{p}=.552)$. In group 1, MMF and tacrolimus maintained a biochemical remission in $88.9 \%$ and $87.5 \%$ of patients, respectively $(\mathrm{p}=.929)$. More patients in group 2 given tacrolimus compared to MMF had a complete response, but the difference was not statistically significant $(50.0 \%$ vs. $22.2 \%, p=.195)$. Overall, $71.1 \%(27 / 38)$ of patients responded to MMF and/or tacrolimus therapy. Decompensated cirrhosis/liver transplantation was more commonly seen in MMF and/or tacrolimus non-responders than in responders $(45.5 \%$ vs $7.4 \%, \mathrm{p}=0.006)$. Five patients who received second-line therapy ( $2 \mathrm{MMF}$ and 3 tacrolimus) developed side-effects that led to therapy withdrawal.

Conclusion: This is the largest study shows that long-term therapy with MMF or tacrolimus was generally well tolerated by pediatric patients with AIH. Both MMF and tacrolimus had excellent efficacy in patients intolerant to corticosteroid or azathioprine. Tacrolimus might be better than MMF in patients failing previous therapy.

\section{O-PEDS-04}

Ascitic fluid culture after centrifugation in children with liver disease: Is it better than routine bedside method?

\section{Sumit Kumar Singh ${ }^{1}$, Ujjal Poddar ${ }^{1}$, Richa Mishra ${ }^{1}$, Surender Kumar Yachha $^{1}$, Anshu Srivastava ${ }^{1}$}

${ }^{1}$ Sanjay Gandhi Post-graduate Institute of Medical sciences, India

Background: Occurrence of ascites in cirrhosis is associated with increased mortality. Culture positivity rates (yield) of ascitic fluid infection (AFI) in children are low as compared to adults $(\leq 50 \%$ in children vs. $80 \%$ in adults). Centrifugation of peritoneal fluid dialysate prior to culture in patients with peritoneal dialysis has been shown to increases the yield to $>90 \%$. Our aim was to find whether centrifugation of ascitic fluid prior to culture increases the yield or not?

Method: Children with liver disease-related ascites were enrolled during 2 year study period from May 2015 to April 2017. For the 1st 18 months, ascitic fluid culture was carried out after centrifugation and for next 6 months by routine bedside method. $50 \mathrm{~mL}$ of the ascitic fluid was taken and centrifuged at $3000 \mathrm{rpm}$ for 15 minutes. Sediment was resuspended in $10 \mathrm{~mL}$ of saline and inoculated into BACTEC aerobic and anaerobic bottles. AFI was classified as spontaneous bacterial peritonitis (SBP), culture negative neutrocytic ascites (CNNA) and monomicrobial non-neutrocytic bacterascites (MNB).

Result: One hundred forty children (97 boys; age 84 [2-216] months), were enrolled. Ascitic fluid culture after centrifugation and routine method was done in 100 and 40 children respectively. 29\% $(\mathrm{n}=41)$ had AFI $(\mathrm{SBP}=9, \mathrm{CNNA}=25, \mathrm{MNB}=7)$. Comparison between children undergoing ascitic fluid culture by two methods is shown in table 1 with no difference in ascitic fluid culture yield between the two methods. Gram-negative bacilli constituted $62.5 \%$ of isolated organisms with $80 \%$ of them producing extended spectrum beta-lactamases (ESBLs). More children with CNNA (56\%) had antibiotics exposure as compared to those with positive culture $(31 \%)$, however not significant $(\mathrm{p}=0.12)$. Children with AFI had more frequent complications of cirrhosis and higher mortality compared to no- AFI as shown in Table 2.

Conclusion: Ascitic fluid infection was found in about one-third of children. ESBLs producing gram negative bacilli were the most frequently isolated organism. Ascitic fluid culture after centrifugation did not improve the yield.

Table 1: Comparison between children undergoing ascitic fluid culture by two methods

\begin{tabular}{|lccc|}
\hline Parameters & $\begin{array}{c}\text { Post-centrifugation } \\
(\mathbf{n}=\mathbf{1 0 0})\end{array}$ & $\begin{array}{c}\text { Routine method } \\
(\mathbf{n}=40)\end{array}$ & P value \\
\hline Median age at presentation (mo) & $84(2.5$ to 216$)$ & $96(3.5$ to 216$)$ & 0.71 \\
Male & $70(70 \%)$ & $27(67.5 \%)$ & 0.77 \\
Ascitic fluid infection & $30(30 \%)$ & $11(27.5 \%)$ & 0.7 \\
SBP & $7(7 \%)$ & $2(5 \%)$ & \\
CNNA & $18(18 \%)$ & $7(17.5 \%)$ & 0.9 \\
MNB & $5(5 \%)$ & $2(5 \%)$ & \\
Positive ascitic fluid culture & $12 / 30(40 \%)$ & $4 / 11(36 \%)$ & 1.0 \\
Other site infections & $29(29 \%)$ & $12(30 \%)$ & 1.0 \\
Antibiotics exposure & $49(49 \%)$ & $18(45 \%)$ & 0.6 \\
CLD & $83(83 \%)$ & $30(75 \%)$ & 0.22 \\
CPT class A/B/C & $0 / 33 / 50$ & $1 / 8 / 21$ & 0.07 \\
\hline
\end{tabular}

CLD: Chronic liver disease, CPT: Child-Pugh-Turcotte, SBP: spontaneous bacterial peritonitis, CNNA: culture negative neutrocytic ascites, MNB: monomicrobial non-neutrocytic bacterascites

Table 2: Comparison between ascitic fluid infection (AFI) and and no-AFI groups
\begin{tabular}{|lccc|}
\hline Parameter & AFI (n=41) & No-AFI (n=99) & P value \\
\hline Median age at presentation (mo) & $8 \mathrm{y}(3.5 \mathrm{mo}$ to $18 \mathrm{y})$ & $6.5 \mathrm{y}(2.5 \mathrm{mo}$ to $18 \mathrm{y})$ & 0.16 \\
Male & $30(73.2 \%)$ & $67(67.7 \%)$ & 0.52 \\
Duration of illness (mo) & $5(0.2-132)$ & $3(0.1-75)$ & 0.10 \\
Abdominal pain & $12(29 \%)$ & $4(4 \%)$ & 0.000 \\
Fever & $17(42 \%)$ & $16(16.2 \%)$ & 0.001 \\
Antibiotics exposure & $18(44 \%)$ & $49(49.5 \%)$ & 0.54 \\
Bacteraemia & $6(14.6 \%)$ & $3(3 \%)$ & 0.01 \\
Pneumonia & $6(14.6 \%)$ & $14(14.1 \%)$ & 0.94 \\
Uriary tract infection & $4(10.3 \%)$ & $5(4.1 \%)$ & 0.44 \\
Chronic liver disease & $35(85 \%)$ & $78(79 \%)$ & 0.71 \\
Child-Pugh class C & $24 / 34(70 \%)$ & $37 / 79(47 \%)$ & 0.02 \\
Renal failure & $4(9.8 \%)$ & $6(6.1 \%)$ & 0.47 \\
Hepatic encephalopathy & $19(46.3 \%)$ & $22(22 \%)$ & 0.004 \\
Death (in-hospital) & $11 / 34(32 \%)$ & $11 / 79(14 \%)$ & 0.02 \\
\hline
\end{tabular}

\section{O-PEDS-05}

Pediatric Wilson disease: a study on clinical profile and treatment outcome from a tertiary care center in North India

\section{Jagadeesh Menon $^{1}$, B R Thapa ${ }^{1}$, Sadhna Lal ${ }^{1}$, K P Srikanth ${ }^{1}$, Subhamoy Das $^{1}$, Shruti Sharma ${ }^{1}$, Aditi Kumar ${ }^{1}$ \\ ${ }^{1}$ Post Graduate Institute of Medical Education \& Research,(PGIMER), Chandigarh, India}

Background: Wilson disease is a rare disorder of copper metabolism. Its protean hepatic presentations include acute hepatitis, acute liver failure, acute on chronic liver failure, portal hypertension, asymptomatic hepatomegaly, raised liver enzymes and gall stones. 
Extrahepatic manifestations are also well known. There is a paucity of data on this disease and its outcome. We here present one of the largest cohort of pediatric Wilson disease patients managed at our center.

Method: Clinical profile \& outcome of 107 pediatric Wilson disease cases from year 2006 to 2016 were retrospectively analysed. Diagnosis was based on KF rings, low serum ceruloplasmin \& high 24 hour urine copper as per standard cut offs \pm liver histology. Cases with atleast 12 months follow up post chelation were analysed. Outcome was categorised as (1) Complete improvement: Asymptomatic with normal synthetic function (2) Partial improvement: AST/ ALT elevation and synthetic dysfunction which are resolving (3) Deterioration: Worsening synthetic function or neurological status \& (4) Death.

Result: Of the 107 patients with age ranging from 3 years to 18 years managed at our center, presenting symptoms were jaundice in 60 $(56.07 \%)$, ascites in $49(45.7 \%)$, cola colored urine in $5(4.6 \%)$, encephalopathy in $11(10.2 \%)$, GI bleed in $18(16.8 \%)$ and extra GI bleed (cutaneous, hematuria, epistaxis) in 13 (12.1\%).Neurological presentation was seen in in $23(21.4 \%)$ and psychiatric presentation in $5(4.6 \%)$ patients. Consanguinity was present in $15(14 \%)$ whereas family history of Wilson (hepatic/neurological/hepatic with neuro) was seen in $44(41.1 \%)$ patients. Clinical examination showed hepatomegaly in $88(82.2 \%)$, splenomegaly in $69(64.4 \%)$, ascites in 40 (37.3\%), signs of cirrhosis (including clubbing, spider angioma, skin changes) in $39(36.4 \%)$ and sunflower cataract in $4(3.7 \%$ patients).Gall stones in $2(1.8 \%)$, nephrolithiasis in $3(2.8 \%)$, MPGN and pancreatic involvement in $1(0.9 \%)$ were also seen. Familial Wilson disease was present in $13(12 \%) .4(3.7 \%)$ of the patients' parents (3 male and 1 female) were diagnosed subsequently. Types of presentation was hepatic in $87(81.3 \%)$ or hepatolenticular in $20(18.7 \%)$. The PELD score at admission varied from 0 - 45.8 (mean 11.36).Child Pugh Score was A in 53 (49.5\%), B in $13(12.1 \%)$ and $C$ in $41(38.3 \%)$ patients. KF ring was present in $63(72.4 \%)$ of hepatic Wilson and $18(90 \%)$ of hepatolenticular Wilson patients. Serum Ceruloplamin was low $<20 \mathrm{mg} / \mathrm{dl})$ in $81(93 \%)$ of hepatic and 19 $(95 \%)$ of hepatolenticular wilson.67.2\% of them had value $<$ or $=10 \mathrm{mg} / \mathrm{dl}$.Urine copper was performed in 90 patients with $66(73.3 \%)$ having value $>100 \mu \mathrm{g} / 24$ hours and $11(12.2 \%)$ with value $>40 \mu \mathrm{g} / 24$ hours. Liver biopsy was done in $53(49.5 \%)$ cases contributing to diagnosis. Types of hepatic presentation in were cirrhosis in $45(46.3 \%)$, chronic liver disease in 7 (7.2\%), ACLF in 13 $(13.4 \% \& 53.8 \%$ due to Wilson flare up, ALF in $2(2.06 \%)$, acute hepatitis in $18(18.5 \%)$ and asymptomatic in $13(13.4 \%)$. Patients were started on chelation therapy with combined D Penicillamine and Zinc and was followed up after 1 year.64 (59.8\%) had complete resolution, 26(24.2\%) had partial improvement, 9 (14\%) had deterioration of which 2 patients underwent liver transplant, 4 (3.7\%) were lost to follow up \& 4 (3.7\%) died.

Conclusion: Wilson disease is a common metabolic liver disease in children and it responds well to chelation therapy. Patients who present with ALF/ACLF and those with end stage liver disease may require liver transplantation

\section{O-PEDS-06}

High efficacy of D-penicillamine in Wilson's disease: contribution of noncompliance to the occurrence of D-penicillamine treatment "failure"

Dan Han ${ }^{1}$ Guoliang Li ${ }^{1}$, Taotao Yan', Jingjing Sun ${ }^{1}$, Dan Han', Wanhu Fan ${ }^{1}$

${ }^{1}$ The first affiliated hospital of Xi' an Jiaotong University, China
Background: Wilson's disease (WD), a genetic disorder of copper metabolism due to the mutant ATP7B enzyme, is characterized by reduced excretion, disordered accumulation and abnormal deposits of copper cause the toxic damage to various organs. D-penicillamine(DPCA) is an effective oral chelation agent for WD.

Method: N/A.

Result: A WD patient with clinical manifestations were reversed by D-PCA. However, the patient thought that he was healthy enough to lead to the administration of penicillamine being terminated. The reoccurrence of many manifestations of Wilson's disease were reported but the regular fellow-up were also stopped. In addition, his liver function after drinking alcohol started to deteriorate with the findings of symptoms such as progressive jaundice (yellowing of the skin and eyes), dark urine, fatigue, nausea, vomiting, migraine headaches, lower extremity edema, diarrhea. There was no signs of alleviation of Wilson's disease after taking a few pairs of Chinese herbs. Approximately 4 months after the administration of D-PCA treatment, improvement in jaundice and dysphoria, reduced serum levels of alanine aminotransferase, and elevated ceruloplasmin, as well as stabilization in the radiographic of neurologic and hepatic examination, were observe.

Conclusion: Our case provides major recommendations for improving the compliance of WD patients and it is a lifelong challenge in terms of the management of WD.

Session: Portal Hypertension; Chairpersons: J H Kao; Vaishali Bhardwaj; Brij Sharma

\section{O-PHT-07}

Derivation and validation of a radiomics-based assessment for noninvasive detection of clinically significant portal hypertension in cirrhosis: a prospective multicenter study (CHESS1701)

Yanna Liu ${ }^{1}$, Yanna Liu ${ }^{1}$, Huiguo Ding ${ }^{4}$, Yongping Yang ${ }^{2}$, Guoxin $\mathrm{Li}^{1}$, Hongwu Luo ${ }^{13}$, Weimin $\mathrm{An}^{2}$, Yifei Huang ${ }^{1}$, Jialiang Zou ${ }^{1}$, Xinrui Yuan', Sizhe Chen ${ }^{1}$, Shuoyu Xu' ${ }^{1}$, Yuening Zhang ${ }^{4}$, Changchun Liu ${ }^{2}$, Lei Wang ${ }^{3}$, Ruizhao $\mathrm{Qi}^{2}$, Chuan Liu ${ }^{1}$, Dawei Zhao $^{4}$, Yongli Duan ${ }^{3}$, Xiaojie $\mathrm{Lu}^{8}$, Hongbo $\mathrm{Xu}^{13}$, Zhentai Lu' ${ }^{9}$, Di Dong ${ }^{7}$, Xingshun $\mathrm{Qi}^{5}$, Li Liu ${ }^{1}$, Dengxiang Liu ${ }^{14}$, Xiaoshun $\mathrm{He}^{11}$, Yongxiang $\mathrm{Yi}^{12}$, Yajin Chen ${ }^{10}$, Zaiyi Liu ${ }^{6}$, Jinlin Hou ${ }^{1}$, Jie Tian ${ }^{7}$, Yu Zhang, Fuquan $\mathrm{Liu}^{3}$, Zhiwei $\mathbf{L i}^{2}$, Xiaolong $\mathbf{Q i}^{1}$

${ }^{1}$ Nanfang Hospital, Southern Medical University, China; ${ }^{10}$ Sun YatSen Memorial Hospital, Sun Yat-Sen University, China; ${ }^{11}$ The First Affiliated Hospital of Sun Yat-sen University, China; ${ }^{12}$ The Second Hospital of Nanjing Affiliated to Southeast University, China; ${ }^{13}$ The Third Xiangya Hospital of Central South University, China; ${ }^{14}$ Xingtai People Hospital, China; ${ }^{2} 302$ Hospital of PLA, China; ${ }^{3}$ Beijing Shijitan Hospital, Capital Medical University, China; ${ }^{4}$ Beijing You'an Hospital, Capital Medical University, China; ${ }^{5}$ General Hospital of Shenyang Military Area, China; ${ }^{6}$ Guangdong General Hospital, Guangdong Academy of Medical Sciences, China; ${ }^{7}$ Institute of Automation, Chinese Academy of Sciences, China; ${ }^{8}$ Nanjing Medical University, China; ${ }^{9}$ Southern Medical University, China

Background: Hepatic venous pressure gradient (HVPG) is the gold standard for diagnosing clinically significant portal hypertension $(\mathrm{CSPH})$. However, HVPG measurement is invasive and thus, impractical for routine clinical practice. Noninvasive tests that able to accurately determine CSPH are urgently needed. The study aims to develop and validate a radiomics-based method as a noninvasive surrogate of HVPG for accurate detection of CSPH in cirrhosis. 
Method: We performed a prospective multicenter diagnostic performance trial (CHESS1701, NCT03138915), which involved 370 patients with cirrhosis from 4 high-volume liver centers in China between August 2016 and September 2017. Patients who had a transjugular HVPG measurement, contrast-enhanced abdominal CT within 14 days prior to the catheterization, and complete clinical data were collected.The noninvasive model was developed in a primary cohort that consisted of 222 consecutive patients. The least absolute shrinkage and selection operator regression model was used for data dimension reduction, feature selection, and signature construction from radiomics features extracted from portal venous-phase CT images (Figure 1A and 1B). The diagnostic performance of the radiomics-based signature for $\mathrm{CSPH}$ was prospectively assessed in 148 consecutive eligible patients in 3 external validation cohorts.

Result: The specific signature was identified based on 7 hepatic and 4 splenic radiomics features, which we termed radiomics-based HVPG (rHVPG). The hierarchical clustering revealed clusters of patients with similar expression patterns based on $r$ HVPG (Figure 1C). And the $r$ HVPG-based histogram for the whole population showed that most of the non-CSPH patients had relatively lower score than the CSPH patients (Figure 1D). In the primary cohort, compared to other noninvasive models including three imaging-based and three serumbased models, $r$ HVPG showed the highest diagnostic accuracy for detection of CSPH with a C-index of 0.849 (95\% CI: 0.789-0.911), and the sensitivity, specificity of $78.7 \%$ and $76.9 \%$, respectively (Figure 1E). Application of $r \mathrm{HVPG}$ in 3 external prospective validation cohorts still gave excellent performance with C-index of 0.753 (95\% CI: 0.464-1.000, 0.800 (95\% CI: 0.614-0.986) and 0.917 (95\% CI: 0.772-1.000), respectively (Figure 1F). Besides, intraclass correlation coefficients for inter- and intra-observer agreement were 0.92 0.99 and $0.97-0.99$, respectively.

Conclusion: A novel radiomics signature, termed $r \mathrm{HVPG}$, was developed and prospectively validated as an accurate surrogate of HVPG for noninvasive detection of CSPH in cirrhosis. The tool of $r$ HVPG assessment can facilitate identifying CSPH quickly and easily when invasive transjugular procedure is not available.

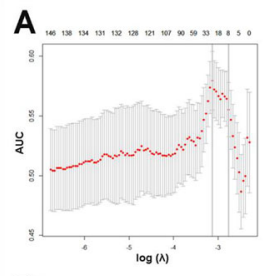

D
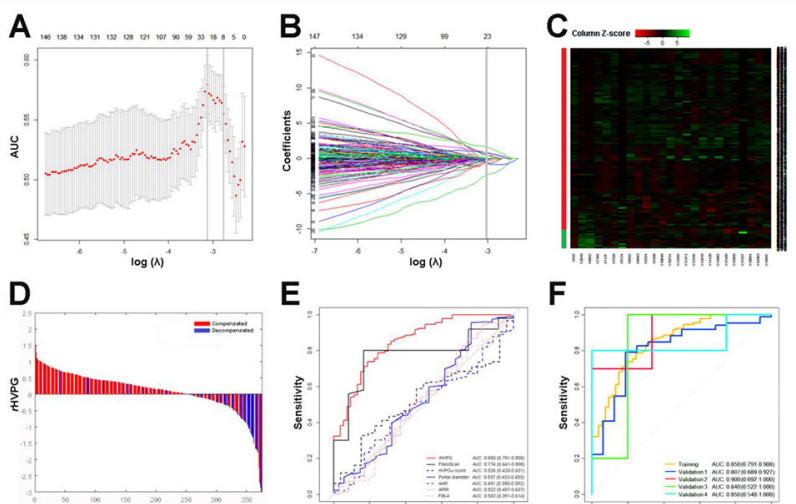

E

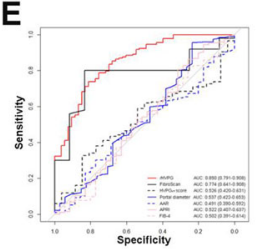

$\mathbf{F}$

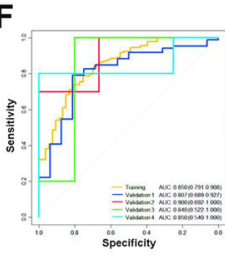

Figure 1. Radiomics features selection, and diagnostic performance of the radiomics siganture for decompensation stage. (A) Tuning parameter $(\lambda)$ selection in the least absolute shrinkage and selection operator (LASSO) model used 10-fold cross-validation via minimum criteria. Dotted vertical lines were drawn both at the optimal (left) and minimum (right) values by using the minimum criteria and the 1 standard error of the minimum criteria. $A \lambda$ value of 0.04358 , with $\log (\lambda)$, -3.1332 was chosen using 10-fold cross-validation. (B) LASSO coefficient profiles of the 20648 features. A coeffient

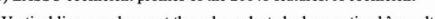
profile plot was prodaced vests in $\log (\lambda)$ sequec. Verical hine was drawn a the value selected where optimal $\lambda$ res in 25 nonzero coefficients using 10-fold cross-validation. (C) Radiomics heat map. Hierarchical clustering of patients $(\mathrm{n}=385)$ on the $\mathrm{y}$ axis and radiomics feature expression on the $\mathrm{x}$ axis, revealed clusters of patients with similar radiomics expression patterns. (D) Radiomics signature for the whole population ( $\mathrm{n}=385$ ) where compensated and decompensated patients were marked with different colors. (E) Receiver operating characteristic curves of noninvasive models for decompensation stage prediction in the training cohort. (F) Diagnostic accuracy of the radiomics signature for decompensation stage prediction in the training cohort. (F) Diagnostic accuracy
decompensation stage in the training cohort and 4 external validation cohorts.

\section{O-PHT-08}

Diagnostic accuracy of radiomics signature for noninvasive prediction of decompensation in patients with cirrhosis: a prospective multicenter study

Yanna Liu ${ }^{1}$, Yanna Liu ${ }^{1}$, Zhenyuan Ning', Huiguo Ding ${ }^{4}$, Yongping Yang', Guoxin $\mathrm{Li}^{1}$, Hongwu Luo's, Weimin An², Yifei Huang ${ }^{1}$, Jialiang Zou ${ }^{1}$, Xinrui Yuan', Sizhe Chen ${ }^{1}$, Shuoyu Xu' ${ }^{1}$, Yuening Zhang ${ }^{4}$, Changchun Liu ${ }^{2}$, Lei Wang ${ }^{3}$, Ruizhao $\mathbf{Q i}^{2}$, Chuan Liu', Dawei Zhao ${ }^{4}$, Yongli Duan ${ }^{3}$, Xiaojie Lu ${ }^{8}$, Hongbo $\mathrm{Xu}^{13}$, Zhentai Lu', Li Liu', Dengxiang Liu' ${ }^{14}$, Xiaoshun $\mathrm{He}^{11}$, Yongxiang $\mathrm{Yi}^{12}$, Yajin Chen ${ }^{10}$, Zaiyi Liu ${ }^{6}$, Jinlin Hou ${ }^{1}$, Jie Tian ${ }^{7}$, Yu Zhang ${ }^{9}$, Fuquan $\mathrm{Liu}^{3}$, Zhiwei $\mathrm{Li}^{2}$, Di Dong ${ }^{7}$, Xingshun $\mathrm{Qi}^{5}$, Xiaolong Qi ${ }^{1}$

${ }^{1}$ Nanfang Hospital, Southern Medical University, China; ${ }^{10}$ Sun YatSen Memorial Hospital, Sun Yat-Sen University, China; ${ }^{11}$ The First Affiliated Hospital of Sun Yat-sen University, China; ${ }^{12}$ The Second Hospital of Nanjing Affiliated to Southeast University, China; ${ }^{13}$ The Third Xiangya Hospital of Central South University, China; ${ }^{14}$ Xingtai People Hospital, China; ${ }^{2} 302$ Hospital of PLA, China; ${ }^{3}$ Beijing Shijitan Hospital, Capital Medical University, China; ${ }^{4}$ Beijing You' an Hospital, Capital Medical University, China; ${ }^{5}$ General Hospital of Shenyang Military Area, China; ${ }^{6}$ Guangdong General Hospital, Guangdong Academy of Medical Sciences, China; ${ }^{7}$ Institute of Automation, Chinese Academy of Sciences, China; ${ }^{8}$ Nanjing Medical University, China; ${ }^{9}$ Southern Medical University, China

Background: In patients with compensated cirrhosis, a hepatic venous pressure gradient (HVPG) $\geq 12 \mathrm{mmHg}$ suggests the decompensation progression and more importantly, highly implies a variceal hemorrhage risk. However, the HVPG measurement is limited by its invasiveness and high cost thus fails to become a routine practice. This study aims to develop and validate a radiomics signature for noninvasive prediction of decompensation in patients with cirrhosis. Method: We enrolled 385 patients with cirrhosis who underwent abdominal contrast-enhanced CT and invasive HVPG measurement in 5 high-volume liver centers in China between August 2016 and October 2017. For the training cohort, 236 patients were retrospectively recruited from one center and 20648 radiomics features were extracted from imaging of each patient. Besides, the least absolute shrinkage and selection operator regression model was used for data dimension reduction, feature selection, and signature construction (Figure 1A, 1B). The performance of the noninvasive radiomics score for decompensation stage prediction was further evaluated in 149 patients who were prospectively recruited from 4 external centers as validation cohorts.

Result: A radiomics score consisted of 25 selected features was developed. According to the hierarchical clustering, patients could be divided into two clusters, that is, compensation and decompensation (suggested by an HVPG $\geq 12 \mathrm{mmHg}$ ) stages (Figure 1C). Besides, the distribution of the radiomics score was generally consistent with that of the HVPG values (Figure 1D). Compared with noninvasive parameters including FibroScan, CT-based portal pressure score, portal diameter, aspartate aminotransferase to alanine aminotransferase ratio, fibrosis index based on 4 factors, and aspartate aminotransferase to platelet count ratio index, the radiomics score showed the highest diagnostic performance for prediction of decompensated cirrhosis (Figure 1E). Application of the radiomics score in 4 external validation cohorts still gave excellent performance with the C-index of 0.807 (95\% CI: 0.689-0.927), 0.900 (95\% CI: $0.692-1.000$ ), and 0.840 (95\% CI: $0.522-1.000), 0.850$ (95\% CI: $0.540-1.000$ ), respectively (Figure $1 \mathrm{~F}$ ).

Conclusion: A noninvasive radiomics signature was developed based on the contrast-enhanced CT imaging and demonstrated excellent prediction performance for decompensation stage in patients with 
cirrhosis. This radiomics signature could be also used to identify individuals at high risk of variceal hemorrhage and avoid unnecessary endoscopy screening.

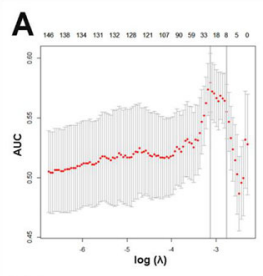

D
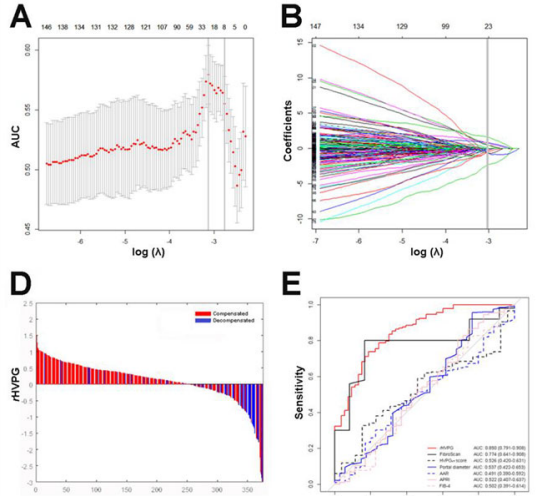

E
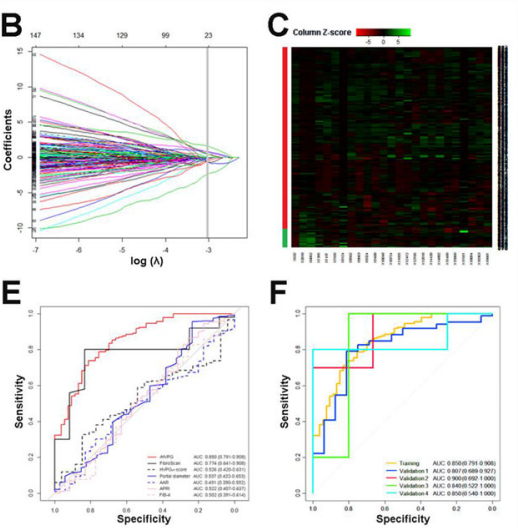

$\mathbf{F}$

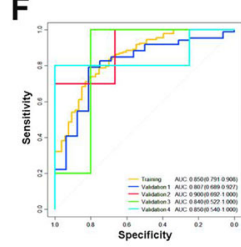

Figure 1. Radiomics features selection, and diagnostic performance of the radiomics siganture for decompensation stage (A) Tuning parameter ( $\lambda$ ) selection in the least absolute shrinkage and selection operator (LASSO) model used 10-fold cross-validation via minimum criteria. Dotted vertical lines were drawn both at the optimal (left) and minimum (right) values by using the minimum criteria and the 1 standard error of the minimum criteria. A $\lambda$ value of 0.04358 , with $\log (\lambda)$, -3.1332 was chosen using 10-fold cross-validation. (B) LASSO coefficient profiles of the 20648 features. A coefficient profile plot was produced versus the $\log (\lambda)$ sequence. Vertical line was drawn at the value selected where optimal $\lambda$ resulted in 25 nonzero coefficients using 10-fold cross-validation. (C) Radiomics heat map. Hierarchical clustering of patients $(\mathrm{n}=385)$ on the $\mathrm{y}$ axis and radiomics feature expression on the $\mathrm{x}$ axis, revealed clusters of patients with similar radiomics expression patterns. (D) Radiomics signature for the whole population ( $\mathrm{n}=385$ ) where compensated and decompensated patients were marked with different colors. (E) Receiver operating characteristic curves of noninvasive models for decompensation stage prediction in the training cohort. (F) Diagnostic accuracy of the radiomics signature for decompensation stage in the training cohort and 4 external validation cohorts.

\section{O-PHT-09}

Indocyanine green retention test (ICG-r15) as a non-invasive predictor of portal hypertension in patients with different severity of cirrhosis

Soren Moller ${ }^{1}$, Else La Cour Sibbesen ${ }^{1}$, Jan Lysgaard Madsen ${ }^{1}$, Flemming Bendtsen ${ }^{1}$

${ }^{1}$ Hvidovre Hospital, Denmark

Background: Patients with cirrhosis and portal hypertension often develop complications relating to hepatic excretory dysfunction. The gold standard measurement of the hepatic excretion is the constant infusion indocyanine green clearance (ICG)-clearance) technique during a liver vein catheterization (LVC). The ICG 15-minutes retention test (ICG-r15) is a less invasive, fast, and inexpensive alternative and the aims of this study were to compare the ICG-r15 test with the gold standard test, to assess relations of ICG-r15 to patient characteristics, and to assess the ICG-r15 level in healthy control subjects.

Method: We included 68 patients with cirrhosis and portal hypertension (Child class A/B/C: 17/37/14) with an average hepatic venous pressure gradient (HVPG) of $15 \mathrm{mmHg}$. All patients underwent a full LVC with determination of ICG-r15. A subset of 38 patients had moreover performed their gold standard ICG-clearance. Sixteen healthy controls subject were included for compiling a reference interval.

Result: The ICG-r15 was increased in the cirrhotic patients with increased liver dysfunction (15/41/58\%) in Child class A/B/C compared with $7 \%$ in the controls (p

Conclusion: The ICG-r15 test can replace the gold standard of ICGclearance determination and may be ideal in pediatric patients. It reflects portal hypertension, the degree of hepatic failure and other aspects of hepatic decompensation and may be useful prior to invasive procedures such as liver transplantation. A more differentiated reference interval needs to be compiled and the predictive value of ICG-r15 should be validated.

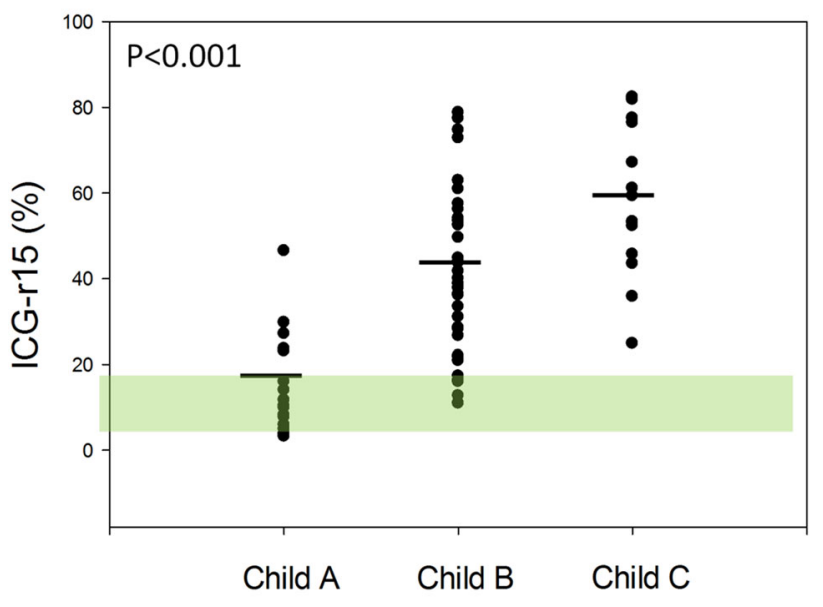

Figure 2

\section{O-PHT-10}

Sepsis in HBV associated decompensated cirrhosis: a comparison between SIRS (Sepsis-1) and qSOFA (Sepsis-3) based criteria

Zhujun Cao ${ }^{1}$, Yuhan Liu', Xiaobo Lu' ${ }^{1}$, Liuying Chen ${ }^{2}$, Shan Yin ${ }^{2}$, ${ }_{\text {Ziqiang Li }}{ }^{1}$, Ruidong Mo ${ }^{1}$, Peipei Ren ${ }^{1}$, Lichang Chen ${ }^{1}$, Jie Lu ${ }^{1}$, Xiaolin Wang ${ }^{1}$, Gangde Zhao ${ }^{1}$, Weiliang Tang ${ }^{1}$, Xiaogang Xiang ${ }^{1}$, Hui Wang ${ }^{1}$, Wei Cai ${ }^{1}$, Hai Li ${ }^{2}$, Qing Xie ${ }^{1}$

${ }^{1}$ Shanghai Ruijin Hospital, China; ${ }^{2}$ Shanghai Renji Hospital, China

Background: The proposed Sepsis-3 criteria based on sequential assessment of quick SOFA (qSOFA) and delta SOFA score has been shown to predict poor outcomes in general population with suspected bacterial infection and was validated in cirrhosis recently (Piano.S, et al. Gut 2017). The current study aimed to compare the Sepsis-1 criteria (based on the combination of SIRS and infection) and Sepsis3 for the ability of risk stratification and prognostic value.

Method: This is a retrospective observational cohort study. Patients with HBV associated cirrhosis were identified from medical records data between 2005 and 2010 at two tertiary teaching hospitals in China. The qSOFA, delta SOFA and SIRS was calculated for each patient at admission and during hospitalization. Pre-admission SOFA was set to 4 based on previous publications (Piano.S, et al. Gut 2017). The primary outcome was 90-day survival. Outcomes were measured using a time-to-event format and were analyzed using competing risk approach with the Fine and Gray method. Liver transplantation was considered as a competing event for death.

Result: A total of 359 decompensated cirrhotic patients with bacterial infection met all study criteria were included from 5102 unique patients with HBV associated cirrhosis. Two hundred and ten (59\%) were confirmed within $48 \mathrm{~h}$ of admission and 149 (41\%) were diagnosed afterwards, i.e., nosocomial infection. The prevalence of positive SIRS ( $\geq 2$ points in four parameters), qSOFA ( $\geq 2$ points in three parameters) and positive delta SOFA (increase of 2 SOFA score) at admission was $29 \%, 15 \%$ and $55 \%$, respectively. Accordingly, Sepsis-1 was diagnosed in $80(22.3 \%)$ patients with $53 \%$ of resolution from BI and $65 \%$ transplant-free mortality; Sepsis-3 was diagnosed in $41(11.4 \%)$ patients with $29 \%$ of resolution from BI and $79 \%$ transplant-free mortality. However, patients with Sepsis-1 had a similar 90-day survival with those without Sepsis-1 (40\% vs 59\%, 
$\mathrm{p}<0.001$ ), though the difference reach significance. In contrast, patients with Sepsis-3 had strikingly lower 90-day survival in comparisons with those without Sepsis-3 (35\% vs 80\%, p <0.001) (Figure 1). In the multivariate analysis, Sepsis-3 (sHR, 2.90 [95\% CI, 1.74-4.83]) rather than Sepsis-1 was an independent predictor for 90-day mortality. In addition, appropriate empirical antibiotics reduce the risk of 90 -day mortality by $56 \%$ (sHR, 0.44 [95\% CI, 0.28-0.69]) independent of the presence of Sepsis-1 or -3 .

Conclusion: In patients with bacterial infection, Sepsis-3 is better than Sepsis-1 in prognosticating cirrhotic patients with bacterial infection. The results may be extended to patients with fungal infection but remains to be confirmed.

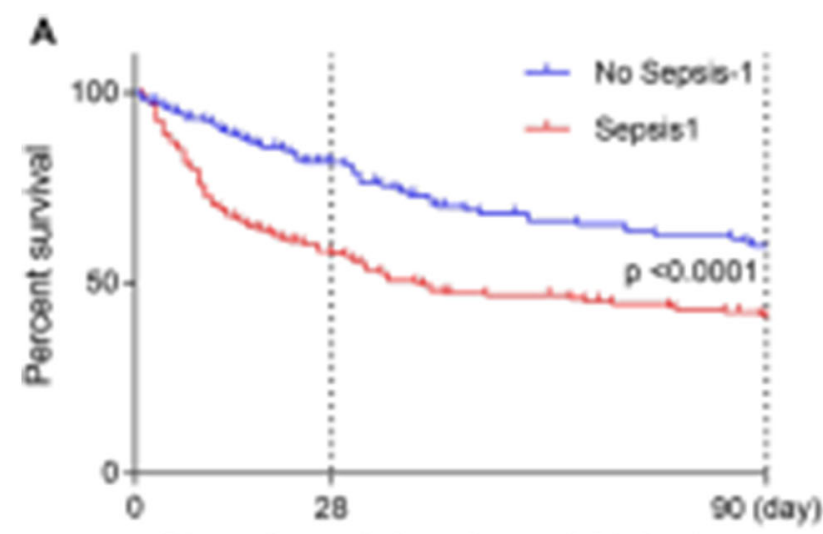

Time elapeed since becterial infection

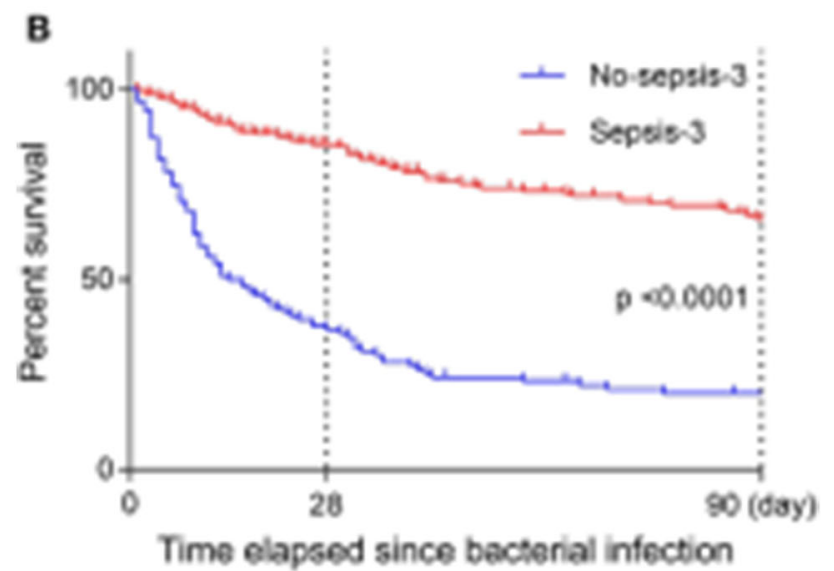

O-PHT-11

Sonoclot signature analysis predicts coagulopathy in cirrhosis

Anupama G Patil ${ }^{1}$ Chhagan Bihari ${ }^{1}$, Hemant D Shewade ${ }^{2}$, Rakhi Maiwall $^{1}$, Neha Nigam ${ }^{1}$, Shiv K Sarin ${ }^{1}$

${ }^{1}$ Institute of Liver \& Biliary Sciences, India; ${ }^{2}$ International Union Against Tuberculosis and Lung Disease (The Union), South-East Asia Office, India

Background: Chronic liver disease (CLD) is characterized by haemostatic changes affecting all aspects of the coagulation system. Predicting the risk of thrombosis or muco-cutaneous bleeding poses a daunting challenge, and conventional assays are known to be poor predictors of thrombotic and muco-cutaneous bleeding risk. Sonoclot signature analysis reflects the status of the entire haemostatic pathway. We investigated whether plasmatic coagulation and platelet function assessment using Sonoclot parameters was suitable for predicting coagulopathy in patients with cirrhosis.

Method: This study involved the analysis of a total of 123 patients admitted to a tertiary care liver hospital from January 2016 to July 2017. This included 72 patients with cirrhosis, 17 with acute-onchronic liver failure and 34 non-cirrhotic controls. Sonoclot variables, namely, activated clotting time (ACT), clot rate (CR), platelet function $(\mathrm{PF})$, time to peak (TP) and peak amplitude (PA) were measured and compared between patients who had a history of thrombotic / coagulopathic bleeding episode and those who did not.

Result: Sonoclot parameters differed significantly between those who had muco-cutaneous bleed and those who did not. Patients who bled $(\mathrm{n}=24)$ had prolonged mean ACT $(245.8 \pm 91.1, \mathrm{p}<0.001)$, depressed CR $(20.3 \pm 12.5, \mathrm{p}=0.006)$, reduced $\mathrm{PF}$ [median $\mathrm{IQR}=1.2(0.1-2.5), \mathrm{p}=0.013]$, longer $\mathrm{TP}(9.0 \pm 2.8, \mathrm{p}=0.036)$ and lesser PA $(58.3 \pm 24.3, \mathrm{p}=0.004)$, compared with non-bleeders. Likewise, patients with thrombosis $(\mathrm{n}=26)$ had significantly lower ACT $(133.6 \pm 53.9, p=0.010)$ and elevated CR (33.0 \pm 17.7 , $\mathrm{p}=0.035$ ). In multivariable model predicting coagulopathic bleeding, ACT, CR and TP were included in the final model. The equation of this model was: [0.01*ACT (seconds) - $0.08 * \mathrm{CR}$ (units/ $\min )+0.09 * \mathrm{TP}$ (minutes) -2.21$]$. Using receiver operating characteristic (ROC) curve analysis of this model, a cut-off value of 0.46 had an optimal specificity of $92.4 \%$ and specificity of $90.9 \%$. For thrombosis, the multivariable analysis revealed that elevated CR was predictive of thrombotic tendency in patients with cirrhosis, the optimal cut-off point being CR $>34$ units/min with sensitivity of 0.81 . Further, the association of Sonoclot parameters with 30-day survival was studied. Patients who died $(\mathrm{n}=26)$ had significantly longer ACT $(\mathrm{p}<0.001)$ and decreased CR $(\mathrm{p}=0.013)$.

Conclusion: In patients with cirrhosis, Sonoclot signature analysis is effective in predicting coagulopathy. A Sonoclot value of $\mathrm{CR}>34$ units/min may predict thrombosis in these patients. We have developed a model that uses ACT, CR and TP to accurately predict coagulopathic bleeding in cirrhotics.

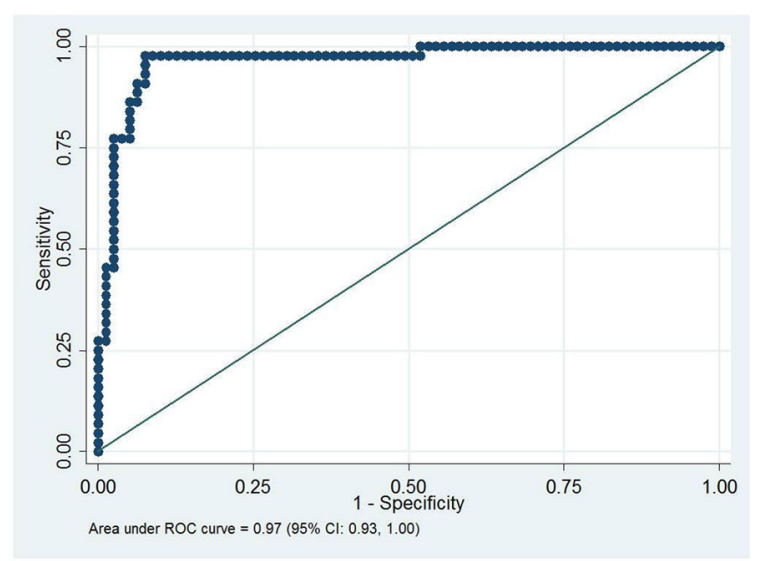

Figure 1: ROC curve of the probit regression model to predict coagulopathic bleeding using Sonoclot parameters (ACT, CR and TP) among patients with cirhosis. The equation of this model was: $\left[0.01^{\star} \mathrm{ACT}\right.$ (seconds) $-0.08^{\star} \mathrm{CR}$ (units/min) $+0.09{ }^{\star} \mathrm{TP}$ (minutes) - 2.21]. $A C T$, activated clotting time; $C R$, clot rate
$\pm S D={ }^{* *}$ Data represented as median (IQR) 


\begin{tabular}{|c|c|c|c|c|c|c|}
\hline \multirow{2}{*}{$\begin{array}{l}\text { Parameter (Mean } \pm \\
\text { SD) }\end{array}$} & \multicolumn{2}{|l|}{ Bleeding } & \multirow{2}{*}{ p-value } & \multicolumn{2}{|l|}{ Thrombosis } & \multirow{2}{*}{ p-value } \\
\hline & $\begin{array}{l}+ \\
(\mathrm{n}=24)\end{array}$ & $(\mathrm{n}=48)$ & & $\begin{array}{l}+ \\
(\mathrm{n}=\mathbf{2 6})\end{array}$ & $(\mathrm{n}=46)$ & \\
\hline ACT, seconds & $245.8 \pm 91.1$ & $174.1 \pm 49.7$ & $<0.001$ & $133.6 \pm 53.9$ & $212.6 \pm 80.9$ & 0.010 \\
\hline $\mathrm{CR}$, unit/min & $20.3 \pm 12.5$ & $30.9+14.9$ & 0.006 & $33.0 \pm 17.7$ & $23.9 \pm 12.2$ & 0.035 \\
\hline $\mathrm{PF} *$ & $1.2(0.1-2.5)$ & $1.5(0.7-3.0)$ & 0.013 & $2.1(0.7-3.2)$ & $0.9(0.3-2.4)$ & 0.068 \\
\hline TP, minutes & $9.0 \pm 2.8$ & $8.3 \pm 3.3$ & 0.036 & $9.0 \pm 2.6$ & $9.62 \pm 4.2$ & 0.226 \\
\hline PA & $58.3 \pm 24.3$ & $75 \pm 23.4$ & 0.004 & $76.7 \pm 22.9$ & $65.1 \pm 25.2$ & 0.072 \\
\hline 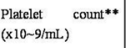 & $50(27-98)$ & $79(51-135)$ & 0.045 & $88(52-135)$ & $57(40-143)$ & 0.189 \\
\hline $\mathrm{INR}^{* *}$ & $2.3(1.7-3.0)$ & $1.6(1.3-3.1)$ & 0.056 & $1.6(1.2-2.1)$ & $1.9(1.5-3.0)$ & 0.084 \\
\hline $\begin{array}{l}\text { Fibrrinogen"* } \\
\text { (mg/dL) }\end{array}$ & $78.1(55.6-118)$ & $155(110-220)$ & $<0.001$ & $200(121-280)$ & $88.8(56.4-148.3)$ & $<0.001$ \\
\hline
\end{tabular}

Table 1: Sonoclot and conventional coagulation parameters in patients with cirrhosis who underwent Sonoclot Signature analysis $(\mathrm{n}=72)$.

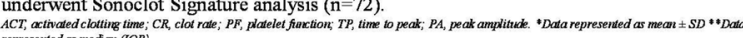

\section{O-PHT-12}

Study of the sleep quality and psychological state of patients with hepatitis B liver cirrhosis

\section{$\underline{\text { Qing } Y e^{1}}$}

${ }^{1}$ Tianjin Medical University, China

Background: Hepatitis B liver cirrhosis is a long-term chronic disease.The patients may suffer from issues such as psychological stress, pain, and economic burden for a long-term. With an altered medical model, the phenomena of chronic physical diseases accompanied by psychological disturbances, such as anxiety and depression, have attracted widespread attention.

Method: We aimed to investigate the sleep quality, mild hepatic encephalopathy, anxiety and depression in patients with hepatitis B cirrhosis by using a variety of scales and questionnaires. A cohort of 341 patients with hepatitis B liver cirrhosis were categorized by Child-Pugh grading (A, B, and C) in this cross-sectional study. Pittsburgh Sleep Quality Index (PSQI), Number Connection Test-A (NCT-A), and Hospital Anxiety and Depression (HAD) evaluated the questionnaires and statistically analyzed the intrinsic correlation.

Result: $213 / 341$ patients presented PSQI index $>5$ points. The PSQI?NCT-A?Anxiety scale (HAD) (a) and depression scale (HAD) (d) of the patients were significantly different. The NCT-A positive patients revealed 214 cases of maybe minimal hepatic encephalopathy (MHE) during a preliminary screening of patients. PSQI score of NCT-A positive patients was significantly elevated as compared to NCT-A negative patients $(\mathrm{P}<0.001)$. HAD (a) and HAD (d) were significantly different between the NCT-A positive and the NCT-A negative groups, $\mathrm{P}=0.002$ and $\mathrm{P}=0.006$, respectively. Univariate Pearson's correlation analysis found that NCT-A and HAD (a) were positively correlated with PSQI, $\mathrm{P}<0.001$ and $\mathrm{P}=0.045$, respectively; however, Child-Pugh score and HAD (d) were not correlated with PSQI, $P=0.061$ and $P=0.059$, respectively.

Conclusion: Patients with hepatitis B liver cirrhosis have a disturbed sleep, which might be an MHE symptom, further causing considerable anxiety.
Session: Hepatitis C Virus Infection. Chairpersons: Pratima Sharma; Uday Ghoshal; Dharmesh Kapoor

$O-H C V-37$

Sustained virological response 12 Weeks after therapy with direct-acting antivirals in select populations with chronic hepatitis $\mathrm{C}$ genotype 1: a meta-analysis of real-world evidence from Asia

Mindie Nguyen ${ }^{1}$, Fanpu $\mathrm{Ji}^{4}$, Bin Wei ${ }^{1}$, Yee Hui Yeo ${ }^{1}$, Biyao Zou ${ }^{1}$, Eiichi Ogawa $^{1}$, Christopher D Stave ${ }^{1}$, Shuangsuo Dang ${ }^{3}$, Ramsey

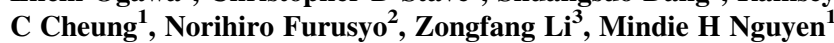

${ }^{1}$ Stanford University Medical Center, United States; ${ }^{2}$ Kyushu University Hospital, Japan; ${ }^{3}$ the Second Affiliated Hospital of Xi' an Jiaotong University, China; ${ }^{4}$ the Second Affiliated Hospital of Xian Jiaotong University, China

Background: Select special populations [patients aged $\geq 75$ years, those with eGFR $<50 \mathrm{~mL} / \mathrm{min} / 1.73 \mathrm{~m}^{2}$ or HCC] have been excluded from registration trials including those conducted in Asia. Our aim was to perform a meta-analysis of real-life data evaluating the effectiveness and safety of DAAs for these historically difficult-totreat HCV genotype 1 (GT 1) select populations in Asia.

Method: We searched PubMed and EMBASE databases using predefined search terms up to May 31, 2017 for published real-world studies reporting SVR12 in at least 15 adult ( $>18$ years) HCV GT 1. The literature was independently screened and data extracted using a case report form by two reviewers. We used random effect modeling to estimate pooled SVR12 rates. Subgroup analysis and meta-regression were performed to determine how pre-planned variables affected the pooled estimates.

Result: In total, $8574 \mathrm{HCV}$ GT1 patients in 41 studies treated with daclatasvir/asunaprevir (DCV/ASV, $\mathrm{n}=6131$ ), ledipasvir/sofosbuvir $(\mathrm{LDV} / \mathrm{SOF}) \pm \mathrm{RBV} \quad(\mathrm{n}=2177), \quad \mathrm{SOF} / \mathrm{DCV} \pm \mathrm{RBV} \quad(\mathrm{n}=133)$, $\mathrm{SOF}+\mathrm{RBV} \quad(\mathrm{n}=61), \quad$ and $\quad$ paritaprevir/ritonavir, ombitasvir \pm dasabuvir $(2 \mathrm{D} / 3 \mathrm{D}) \pm \mathrm{RBV}$ regimens $(\mathrm{n}=72)$ were included. The pooled SVR12 rates were $89.9 \%$ (95\% CI 88.6-91.1, $I 2=55.1 \%)$ for DCV/ASV and $98.1 \%$ (95\% CI 97.0-99.0, $I 2=41.0 \%)$ for $\mathrm{LDV} / \mathrm{SOF} \pm \mathrm{RBV}$. For SOF/DCV $\pm \mathrm{RBV}$, $\mathrm{SOF}+\mathrm{RBV}, 2 \mathrm{D} / 3 \mathrm{D} \pm \mathrm{RBV}$ regimens, the pooled SVR12 rates were $97.4 \%$ (95\% CI $90.9-100, I 2=56.7 \%$ ), $94.1 \%$ (95\% CI $79.1-100$, $I 2=64.4 \%)$, and $95.9 \%(95 \%$ CI $89.5-99.7, I 2=0.0 \%)$, respectively. Subgroup analysis [age, renal function, HCC] for SVR12 in patients treated with DCV/ASV and LDV/SOF were shown in Fig.1.The pooled SVR rate of patients with eGFR $<50 \mathrm{~mL} / \mathrm{min} /$ $1.73 \mathrm{~m}^{2}$ and treated with DCV/ASV was $93.9 \%$ (95\% CI 91.2-96.3), $4.10 \%$ (95\% CI 0.28-7.65) higher than those with eGFR $\geq 50 \mathrm{~mL} /$ $\mathrm{min} / 1.73 \mathrm{~m}^{2}(\mathrm{SVR}=89.8 \%, 95 \%$ CI $88.5-91.0)(P=0.046)$. Especially, the pool SVR rate in DCV/ASV-treated patients with end-stage renal disease (ESRD) was actually $9.38 \%$ (95\% CI 3.84-11.53) higher than those of patients with eGFR $\geq 50 \mathrm{~mL} / \mathrm{min} / 1.73 \mathrm{~m}^{2}(99.2 \%, 95 \%$ CI $94.9-100$ vs. $89.8 \%$, 95\% CI 88.5-91.0, $P=0.009$ ). For DCV/ ASV treatment, patients aged $\geq 80$ years old $(\mathrm{n}=242)$ achieved similar SVR rate as the 1292 patients younger than 80 years old (95.1\%, 95\% CI 88.6-99.1 vs. $92.0 \%, 95 \%$ CI 86.4-96.2, $P=0.480)$. For LDV/SOF regimen, patients with prior history of HCC had a 6.02\% (95\% CI: 1.46-11.19) lower likelihood to achieve SVR compare to those without HCC $(94.1 \%$ vs. $98.7 \%, \mathrm{P}=0.001)$. There were no differences in adverse events and discontinuation in the age and renal dysfunction subgroups treated with DCV/ASV (Fig. 2).

Conclusion: All oral DAA treatment of HCV GT1 resulted in high cure rates and favourable tolerability in select Asian patients in realworld setting including elderly patients and those with ESRD. 


\begin{tabular}{|c|c|c|}
\hline$\overline{G T 1}$ Overall $\mathrm{n}=6131$ & - & 89.8 P-value \\
\hline eGFR $250 \quad n=2234$ & $+\oplus$ & 89.8 \\
\hline eGFR $50 \quad n=489$ & $\mapsto \cdot$ & $93.9^{0.046}$ \\
\hline $\begin{array}{lll}\text { Aged } & 75 & \mathrm{n}=1758\end{array}$ & $\mapsto-1$ & \begin{tabular}{l|l}
89.2 & 0.082
\end{tabular} \\
\hline Aged $275 n=907$ & $+\cdot$ & 92.4 .0 .082 \\
\hline $\mathrm{HCC} \cap \mathrm{n}=234$ & & 88.7 . 0.978 \\
\hline No HCC $n=1064$ & +4 & $88.0^{0.9978}$ \\
\hline GT1b Overall n=4699 & + & 89.3 \\
\hline eGFR $250 n=1687$ & $1+1$ & 89.9 \\
\hline eGFR 550 n=289 & $\mapsto$ & $95.0^{0.039}$ \\
\hline Aged C75 $n=1521$ & $\mapsto$ & $\left.{ }^{89.0}\right]_{0.199}$ \\
\hline Aged $275 n=715$ & +1 & $91.3^{0.199}$ \\
\hline $\mathrm{HCC} \cdot n=200$ & $\longmapsto$ & \\
\hline NoHCC n=978 & $\mapsto \cdot$ & $87.6^{0.496}$ \\
\hline
\end{tabular}

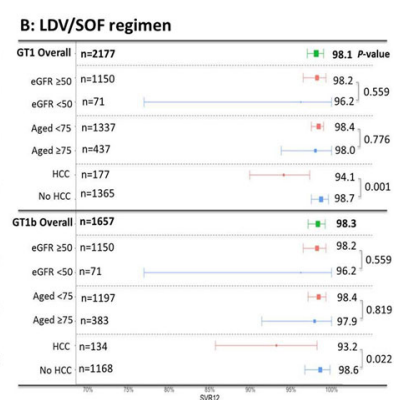

$O-H C V-38$

Treatment of chronic hepatitis $\mathrm{C}$ with ledipasvir/sofosbuvir combination during pregnancy

\section{Ghulam Nabi Yattoo ${ }^{1}$}

${ }^{1}$ SKIMS, India

Background: There are no studies of HCV treatment during pregnancy. Treatment of HCV during pregnancy will reduce vertical transmission. Detection of a chronic infection leads to anxiety among patients and household. Explaining the diseases to patient sometimes leads to treatment by quacks and unauthorized persons. We treated some patients of chronic HCV infection during pregnancy with ledipasvir and sofosbuvir as patients were very anxious and requested the treatment.

Method: Only those pregnant females were enrolled who after explaining the disease and urgency of treatment and psychiatric evaluation were ready for treatment during pregnancy. Treatment was started during second and early third trimester. Only non cirrhotic patients were enrolled.Patients were followed closely clinically an serial investigations and foetal ultrasonography. Patients received ledispavir/sofosbuvir (90/400 mg) for 12 weeks.

Result: We enrolled 15 patients in our study and study is ongoing. Mean age of patients was 27 years with rage of 21 to 36 years. All patients were non cirrhotic with mean fibroscan value of $5.5 \mathrm{kpa}$ and treatment naive. Mean RNA Was 7.2X105 IU/ml. Among enrolled patients genotype 3 constituted $10(67 \%)$, genotype 1 constituted 4 (27\%). All patients achieve RVR as well as SVR12. All patients tolerated treatment well however some patients reported nausea $(40 \%)$, headache $(33 \%)$ and fatigue $(27 \%)$. No serious adverse reaction was reported. All patients had institutional delivery and the babies were reported normal on paediatric examination. Serial ultrasonography antenatal and postnatal were normal. We are following the babies for effec on vertical transmission.

Conclusion: Although study sample is small but it seems that ledipasvir and sofosbuvir is well tolerated and safe in pregnancy. Larger studies are needed.

\section{$O-H C V-39$}

The real-life experience with directly acting antivirals in chronic hepatitis $\mathrm{C}$ treatment: a single center experience from Turkey

Serkan Yaras ${ }^{1}$, Serkan Yaras ${ }^{1}$, Enver Ucbilek ${ }^{1}$, Ibrahim Yilmaz ${ }^{1}$, Osman Ozdogan ${ }^{1}$, Engin Altintas ${ }^{1}$, Orhan Sezgin ${ }^{1}$

${ }^{1}$ Mersin University Faculty of Medicine, Turkey

Background: Directly acting antiviral drugs (DAA) are known to be successful in the treatment of chronic hepatitis $\mathrm{C}(\mathrm{CHC})$. For about
1 year in our country, these drugs are routinely used in the treatment of CHC. We intend to share our experience of our own clinic, Mersin University Faculty of Medicine, Gastroenterology Division.

Method: From July 2016 to August 2017, we started DAA therapy for a total of $230 \mathrm{CHC}$ patients in our clinic.

Result: The mean age of the patients was $63.7 \pm 14.4$ years.Ledipasvir/Sofosbuvir combination treatment started for patients 154/230, and Ombitasvir, Paritaprevir, Dasabuvir, Ritonavir combination (3D) started for 76 patients (table1). The patients generally benefited from the treatment and HCV RNA clearance was achieved during treatment in all but one patient. In the Ledipasvir/Sofosbuvir group, 1 patient was unresponsive to treatment, and 2 patients had recurrence after HCV RNA clearance. No recurrence or no response was observed in the 3D group, so far. Patients generally have tolerated the treatment well. Two patients with decompansated cirrhosis had increase in ascites, resistant to diuretic use. In one of these two patients, denovo ovarian $\mathrm{Ca}$ diagnosed. The increase in bilirubin serum level was observed in 3 patients using 3D, in which the treatment was completed without dose modification. The most common side effects were fatigue and pruritus in both groups. No patient had to discontinue treatment due to side effects.

Conclusion: Our patients almost absolutely got sustained viral response by DAA treatment. We observed that the DAA treatment for patients with chronic hepatitis $\mathrm{C}$ is highly effective and well tolerated.

\begin{tabular}{|c|c|c|}
\hline & $\begin{array}{l}\text { Ledipasvir / Sofosuvir group } \\
(n=154)\end{array}$ & $\begin{array}{l}\text { Ombitasvir, Paritaprevir/ } \\
\text { Dasabuvir/ Ritonavir (3D) } \\
\text { combination group }(n=76)\end{array}$ \\
\hline Male/Female (n/n) & $58 / 96$ & $31 / 45$ \\
\hline Mean age \pm SD & $64.6 \pm 13.2$ & $61.8 \pm 13.9$ \\
\hline Genotype 1a(n) & 3 & 6 \\
\hline Genotype $1 b(n)$ & 122 & 43 \\
\hline Genotype $1(\mathrm{a}-\mathrm{b}$ unknown)(n) & $\frac{122}{22}$ & 26 \\
\hline Genotype 2(n) & 4 & 0 \\
\hline Genotype $3(n)$ & 2 & 0 \\
\hline Genotype 4(n) & 1 & 1 \\
\hline Cirrhotic (n) & 102 & 9 \\
\hline Accompanying Renal Failure(n) & 0 & 25 \\
\hline Naive to treatment (n) & 51 & 56 \\
\hline $\begin{array}{l}\text { PegIFN-Ribavirin treatment in } \\
\text { the past }(n)\end{array}$ & 68 & 18 \\
\hline $\begin{array}{l}\text { Boceprevir treatment in the } \\
\text { past (n) }\end{array}$ & 17 & 2 \\
\hline $\begin{array}{l}\text { Telaprevir treatment in the } \\
\text { past (n) }\end{array}$ & 18 & 0 \\
\hline 12 weeks treatment for $(n)$ & 39 & 76 \\
\hline 24 weeks treatment for $(n)$ & 115 & 0 \\
\hline $\begin{array}{l}\text { Additional Ribavirin treatment } \\
\text { (n) }\end{array}$ & 43 & 9 \\
\hline $\begin{array}{l}\text { End-Treatment Response } \\
\text { Failure }\end{array}$ & 1 & 0 \\
\hline Recurrence (n) & 2 & 0 \\
\hline $\begin{array}{l}\text { End Treatment Viral } \\
\text { Response }(\%)\end{array}$ & 99.4 & 100 \\
\hline Sustained Viral Response(\%) & 99.4 & 100 \\
\hline $\begin{array}{l}\text { Interruption of treatment due } \\
\text { to side effects }(n)\end{array}$ & 0 & 0 \\
\hline
\end{tabular}

\section{$O-H C V-40$}

Bioinformatics Modeling of $\mathrm{HCV}$ proteins with mutations of resistance to antivirals

\section{$\underline{\text { Karina Alejandra Salvatierra }}^{1}$}

${ }^{1}$ Universidad Nacional de Misiones, Argentina

Background: Chronic hepatitis $\mathrm{C}$ virus (HCV) infection remains a worldwide public health problem, resulting in about 10,000 deaths a year. It is estimated that $3 \%$ of the world's population (200 million people in the world) are infected by this virus. Therefore, it is important to analyze the HCV resistance mutations and their possible impact on the treatment with direct-acting antiviral (DAA) to prevent potential therapeutic failures.

Objetive: Model the NS3, NS5A and NS5B proteins with antiviral resistance mutations.

Method: In order to design and develop an online information system, we used different computer applications, technological tools, 
standard languages, systems infrastructure and algorithms. To analyze the resistance mutations in the three-dimensional structure of proteins, we used the PyMol program (http://pymol.sourceforge.net). As a working structure for the protein, we used for NS3 protease the HCV BK isolate genotype $1 \mathrm{~b}$ (code PDB 1CU1), NS5 the structure of the isolate Con1 of HCV genotype 1b code (3FQQ), and for the NS5B polymerase the structure of the isolate Con 1 of $\mathrm{HCV}$ genotype $1 \mathrm{~b}$ (code PDB 3FQL) extracted from the Protein Data Bank (www.ebi.ac.uk/pdbsum).

Result: A software named Biomedical Mutation Analysis (http:// bma.itiud.org/).The algorithm of the online information system developed allows computer analysis of possible changes in nucleotide and amino acid positions associated with resistance mutations to DAAs in NS3, NS5A and NS5B of HCV. Some mutations associated with resistance to the NS3 protease, NS5A and NS5B polymerase were near to the active site, while other mutations were far from the active site. Therefore, mutations at positions near to the active site could affect the binding or contact of the antiviral, whereas those away from the active site could affect the interaction with the antiviral by an indirect mechanism.

Conclusion: We have built an online software tool called Biomedical Mutation Analysis (BMA). Resistance mutations near to the active site, such as in distant areas of the NS3 protease, NS5A and the NS5B polymerase, could interfere in the correct binding or contact of the antivirals, affecting their efficacy and possible therapeutic failure.

\section{$O-H C V-41$}

Comparison of the diagnostic performance of SD Bioline HCV (Standard Diagnostics) rapid test on capillary blood versus venous blood samples

Momoko Iwamoto ${ }^{1}$, Aurelie Calzia ${ }^{1}$, Bun Sreng ${ }^{3}$, Sokchea Yann ${ }^{1}$, Sorphorn Pin $^{1}$, Celine Lastrucci ${ }^{1}$, San Kimchamroeun ${ }^{1}$, Chhit Dimanche $^{4}$, Jean Phillipe Dousset ${ }^{1}$, Mickael Le Paih ${ }^{1}$, Suna Balkan $^{1}$, Tonia Marquardt ${ }^{1}$, Valentina Carnimeo ${ }^{2}$, Anne Loarec ${ }^{2}$, David Maman', Momoko Iwamoto ${ }^{1}$

${ }^{1}$ MÃ@decins Sans Frontires, Cambodia; ${ }^{2}$ Médecins Sans Frontières, France; ${ }^{3}$ Ministry of Health, Cambodia; ${ }^{4}$ Preah Kossamak Hospital, Cambodia

Background: SD Bioline Hepatitis C Virus (HCV) test is a low-cost in vitro immunochromatographic rapid diagnostic assay, which received prequalification from the World Health Organization in Nov 2016 for its use on serum, plasma, and venous whole blood samples, but not for its use on capillary blood. We therefore aimed to compare the performance of SD Bioline HCV test using capillary blood against venous whole blood as the reference test.

Method: Adult patients seeking to be screened for $\mathrm{HCV}$ at Medicines San Frontiers' (MSF) clinic inside Preah Kossamak Hospital (Phnom Penh, Cambodia) were consecutively recruited. Two SD Bioline tests were performed for each patient, with four blinded readers assigned to each patient - two for the capillary test and two for venous whole blood. A third reader was called when the two readers disagreed, and samples with discrepancies between capillary and venous blood test were further tested using ELISA (enzyme-linked immunosorbent assay) test at the Pasteur Institute in Cambodia.

Result: We tested patients with SD Bioline using venous whole blood and capillary blood samples from 421 consenting patients between
October and November, 2017. The mean age of patients was 52.6 (range 19-81), and 36.3\% were male, and 64\% were positive for SD Bioline (as venous blood as the reference test result). Disagreement between two readers occurred in $0.7 \%$ of the tests for both venous blood and capillary tests (Kappa 0.99 for both tests). Discrepancy between capillary and venous sample results occurred in $0.7 \%$ $(n=3)$ of the tests. Two patients with weakly positive venous test and negative result on capillary, and one patient with negative results on venous and weakly positive on capillary test, but all three patients were positive on ELISA. The resulting sensitivity and specificity of tests performed with capillary blood samples were $99.2 \%(95 \%$ confidence interval [CI], 97.3-99.9) and 98.6\% (95\% CI, 95.3-99.8), respectively.

Conclusion: Capillary blood had excellent performance on SD Bioline HCV compared to venous blood. Capillary SD Bioline test could serve as an alternative method for rapid HCV testing in resourcelimited settings or when venipuncture is difficult.

\section{$O-H C V-42$}

Effect of sustained viral response on the regression of fibrosis and portal hypertension in cirrhotic HCV patients treated with Oral antiviral therapy. Punit mehrotra* Pulkit mehrotra** *Lucknow gastroenterology centre **SRMC Chennai

\section{$\underline{\text { Punit Mehrotra }}^{1}$, Pulkit Mehrotra ${ }^{2}$}

${ }^{1}$ lucknow gastroenterology centre, India; ${ }^{2}$ SRMC chennai, India

Background and aims: There is very little Indian data on regression of liver fibrosis (stiffness) and portal hypertension $(\mathrm{PH})$ and their effect on natural history of compensated hepatitis $\mathrm{C}$ virus (HCV)related cirrhosis following sustained virologic response (SVR) by currently available oral antiviral medication. Our aim was to study effect of SVR on child score, variceal status and stiffness score based on fiborscan.

Method: Twenty one patients with compensated HCV genotype 3-related cirrhosis with or without ascites and variceal bleed with Child pugh score $\mathrm{B}$ or $\mathrm{C}$ were given combination of sofosbuvir and daclatasvir without ribavarin for six months. All patients were assessed for Quality of life score, complete liver profile, fibroscan and endoscopy at end of therapy and on follow up at three months and eight months after end of last dose of therapy

Result: All these patients had Fibroscan before the start of therapy (mean score of $24.6 \mathrm{Kpa}$ ) nad endoscopy for vairceal grading based on Sarin's classification, At the end of therapy all the cases achieved SVR. The variceal grading had significant downgrading or disappearance either at the end of therapy or six to eight months of follow up. Fibroscan stiffness score showed significant reduction (24.6 vs $10.4 \mathrm{KpA} ; \mathrm{p}<0.005)$.Quality of life and clinical symptoms were significantly better at end of therapy and on follow up

Conclusion: Complete clinical response, variceal regression and regression of stiffness based on fibroscan following oral antivral therapy in compensated HCV cirrhosis is achievable, and may indicate regression of fibrosis and and decrease in portal pressure The improvement in quality of life following therapy is another milestone in treatment of $\mathrm{HCV}$.. 
Session: Late Breaking Abstracts. Chairpersons: Puneet Puri; Gagan Sood; Ashish Goyal

\section{O-LBRK-02}

Aggressive nutrition therapy significantly improves 28-day survival in critically ill cirrhotic with high nutrition risk as assessed by mNUTRIC score.

Harshita Tripathi ${ }^{1}$, Jaya Benjamin ${ }^{2}$, Puja Bhatia ${ }^{2}$, Varsha Shasthry $^{2}$, Rakhi Maiwall ${ }^{2}$, Lalita Gauri Mitra ${ }^{2}$, Yogendra Kumar Joshi' ${ }^{2}$, Shiv Kumar Sarin ${ }^{2}$, Guresh Kumar ${ }^{2}$

${ }^{1}$ Institute of Liver and Biliary Sciences, India; ${ }^{2}$ Institute of Lver and Biliary Sciences, India

Background: Malnutrition is universal and increases the risk of mortality in critically ill cirrhotics. Nutrition Risk in Critically ill (NUTRIC) score is a validated tool to identify patients at nutrition risk that may benefit the most from aggressive nutrition therapy.

Aim: To assess the impact of aggressive nutritional therapy on survival in critically ill cirrhotic at nutritional risk assessed by the mNUTRIC score.

Methods: A prospective nutritional intervention study in critically ill adult cirrhotics was designed with the collection of baseline and follow-up data during ICU stay. The information pertaining NUTRIC score, clinical, hemodynamic, biochemical, nutritional parameters, use of mechanical ventilation (MV), length of ICU stay (LOS), and development of new-onset infection (NOI) were recorded. Daily nutritional adequacy was calculated as energy received energy required $\times 100$. Logistic regression analysis was used with 28-day mortality as the outcome of interest.

Result: 150 patients [(M-83\%, age-51 $\pm 12.1, \mathrm{BMI}-24 \pm 4.7 \mathrm{~kg} / \mathrm{m}^{2}$; cirrhosis $(74.2 \%)$, acute on chronic liver failure $(25.8 \%)$, median LOS 6 (2-24 days)] were studied. At baseline $116(77 \%)$ had high NUTRIC Score (HNS, $\geq 5$ ) and $34(23 \%)$ low NUTRIC score (LNS, $\leq 4)$. Patients with HNS had significantly higher mortality (69.8\% vs. $44.1 \% ; \mathrm{p}=0.007$;OR $(95 \% \mathrm{CI})$ unadjusted $3.14(1.42$, $6.96 ; \mathrm{p}=0.005)$; adjusted for age, shock, diagnosis, renal failure, comorbidity, $\mathrm{FiO} 2=3.53(1.11,11.2 ; \mathrm{p}=0.032)$, total days on $\mathrm{MV}$ [5(2-24) vs. 3(2-24); $\mathrm{p}=0.02]$, incidence of NOI [41.4\% vs $11.7 \%$; $\mathrm{p}=0.002$; OR $(95 \%$ CI: $7(2,24.5)]$ compared to LNS. Nutritional adequacy was aimed in all patients and achieving it, significantly improved survival, though only in patients with HNS (Fig).

Conclusion: The NUTRIC score in critically ill cirrhotics is a valuable tool in recognizing nutrition risk and predicting mortality. Aggressive nutrition therapy, especially in patients with HNS can significantly improve survival in this cohort.

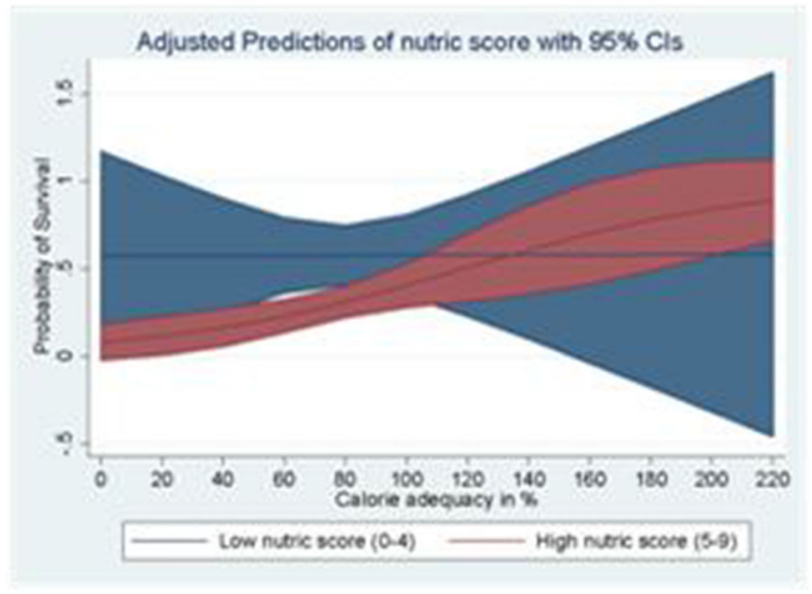

\section{$O-L B R K-05$}

The role of autologous bone marrow derived hepatocyte like cells transplantation in patients with decompensated liver cirrhosis: Assiut University experience

Doaa Abdeltawab ${ }^{1}$, Saad Zaky ${ }^{3}$, Ehab Fawzy Moustafa ${ }^{3}$, Heba Saadeldien $^{5}$, Shaaban Redwan Helal ${ }^{4}$, Hala Gabr ${ }^{4}$, Wael Abou Elkheir $^{2}$

${ }^{1}$ specialist of Tropical Medicine and Gastroenterology, Egypt; ${ }^{2}$ professor of Immunology, Military Medical Academy, Egypt; 3 professor of Tropical medicine and Gastroenterology, Egypt; ${ }^{4}$ professor of Clinical Pathology, Egypt; ${ }^{5}$ professor of Histology and Cell Biology, Egypt

Background: Although liver transplantation is considered gold standard treatment for patients with end stage liver disease, nevertheless shortage of available donors underscores urgent need for alternative therapeutic strategies including stem cell based therapy. In our study we assessed safety and efficacy of hepatocyte like cells differentiated from autologous bone marrow (BM) derived mesenchymal stem cells (MSCs) transplantation in decompensated liver cirrhosis.

Method: 40 patients with $\mathrm{HCV}$ liver cirrhosis were randomly assigned into two groups: group I: included 18 patients who received hepatocyte like cells transplantation via ultrasound guided percutaneous portal vein infusion plus regular liver support, and group II (control): included 22 patients who received regular liver support only. All patients were followed up monthly for 6 months using performance status scale, clinical evaluation, laboratory investigations, abdominal ultrasound, Child-Pugh and model for end-stage liver disease (MELD) calculation.

Result: In hepatocyte like cells-infused patients (GI); statistically significant increase was detected in serum albumin $(P=0.000)$, prothrombin concentration $(\mathrm{P}=0.000)$, and significant decrease in total bilirubin $(\mathrm{P}=0.004)$, international normalization ratio (INR) $(\mathrm{P}=0.003)$ and alanine transaminase (ALT) level $(\mathrm{P}=0.038)$ compared to GII. Regarding clinical data there were no statstical significant differences detected in (frequency of attacks of hematmasis, hepatic encphelopathy or bleeding tendency) between 2 studied groups. There was statstical significant improvemen was deteced in ascites after 3 months of treatment; $55.6 \%$ had no ascites in GI compared to $20.0 \%$ in GII $(\mathrm{P}=0.023)$ but there was no statsticalsginifant difference after 6 months of treament between 2 studied groups. Also the results showed improvement in performance status $(0$ score) was $(44.4 \%)$ in GI in comparison to $(0.0 \%)$ in GII $(\mathrm{P}=0.001)$, Child-Pugh was (Child B; $61.1 \%$ for GI and $15.0 \%$ for GII), (Child C; $38.9 \%$ for GI and $85.0 \%$ for GII). Also an improvment was detected in MELD score; dropping from mean score of (15.3 to 14.6) after 6 months of tretment for GI compared to GII as mean score increased from (15.8 to 18.2) $(\mathrm{P}=0.002)$.

Conclusion: Autologous hepatocyte like cells infusion have a supportive role, short-term beneficial effects on liver synthetic functions and a satisfactory tolerability with no complications in patients with $\mathrm{HCV}$ induced liver cirrhosis. 


\section{$O-L B R K-06$}

Intermittent viral blips reveal Hepatitis B virus polymerase and surface region mutational signatures in patients responding favorably to Tenofovir therapy

Ranjit Chauhan
Sheetalnath Rooge $^{2}$ Avishek Kumar Singh
3

${ }^{1}$ Memorial University of Newfoundland, Canada; ${ }^{2}$ Institute of Liver and Biliary Sciences, India; ${ }^{3}$ Institute of Liver and Biliary Sciences, New Delhi, India, India

Background: Tenofovir is an effective antiviral drug and had shown remarkable efficacy in patients treated over many years with no associated resistant mutations. However, there is scant data on the mutational patterns that emerge during intermittent viral blips in patients favorably responding to the tenofovir disoproxil fumarate (TDF) therapy.

Method: 53 chronic Hepatitis B-infected patients were selected from a previous study who had excellent response to the TDF at the end of the therapy. Using highly sensitive nested polymerase chain reaction $\mathrm{HBV}$ reverse transcriptase region was sequenced, and mutations were analysed for reverse transcriptase overlapping surface region in whom intermittent viral blip/s were detected (> $40 \mathrm{IU}$ ) after achieving initial complete virological response [ICVR (HBV DNA $<20 \mathrm{IU} / \mathrm{ml})$ ] designated as (group A) and initial suboptimal viral suppression [ISOVS (HBV DNA in range 20-40 IU/ml)] designated as (group B). The viral blip load/definition was independent of classical definitions of virological non-response.

Result: The treatment duration length and response were variable and patients achieving complete viral suppression within 36 Weeks were $(\mathrm{n}=5)$, within 2 years $(\mathrm{n}=11)$, in 3.5 year $(\mathrm{n}=18)$ and viral suppression within 5 years were $(\mathrm{n}=19)$. Before the complete viral suppression at the end of the therapy, four types of intermittent viral blips were observed: Initial Blips (IB) detected within 1.5 year of the therapy and even a single blip during this time-period formed this group $[\mathrm{n}=10$, group A $5(50 \%)$; group B $5(50 \%)]$. Early Suppression Late Blips (ESLB) detected from 1.5 year onwards till 4 year of the therapy and even a single blip during this time-period formed this group [n $=9$, group A $8(88.8 \%)$; group B $1(11.1 \%)]$. Two Blips Till 2 Year (TBTTY) having viral blips at two-time points within twoyear period $[\mathrm{n}=16$, group A $11(68.7 \%)$; group B $(31.25 \%)]$. Continues Trace blips $(C T B)$ having at least 3 viral blips $[\mathrm{n}=13$, group A $6(46.1 \%)$ group B $(53.8 \%)$ ] and there were 5 patients in whom no viral blips were detected in entire duration of the TDF therapy and virus remained suppressed throughout. Sequencing analysis during above mentioned all viral blips categories IB, ESLB, TBTTY and CTB revealed $\mathrm{HBV}$ reverse transcriptase and/or surface region mutations in $34(63 \%)$ patients. Of the 34 patients in $17(50 \%)$ of them had reverse transcriptase overlapping surface region mutations. The distribution of mutations in each of these categories was, in [7/10 $(70 \%)]$; in ESLB [7/9 (77.7\%)]; in TBTT [13/16 (81.25\%) and in CTB [7/13 (53.8\%)] patients had HBV reverse transcriptase and/or surface region mutations.

Conclusion: Our data reveal that despite complete viral suppression at the end of the therapy and favorable response, there is still existence of minimal residual viremia during tenofovir antiviral therapy which often goes un-noticed. Mutations detected even during single viral blip may have fitness advantage and should be characterized thoroughly.
Session: Portal Hypertension. Chairpersons; Han-Chieh

Lin; P N Rao, Deepak Lahoti

O-PHT-13

Second-look endoscopy and prolonged use of proton pump inhibitor and octreotide after variceal ligation reduce early bleeding rate in cirrhosis with large gastroesophaegeal varices: A single-blinded, single-centered and randomized controlled trial

Anjiang Wang ${ }^{1}$, Xuelian Zheng ${ }^{1}$, Yue Gong ${ }^{1}$, Jiawei Zhong ${ }^{1}$, Junbo Hong ${ }^{1}$, Jianping $\mathrm{Wu}^{1}$, Haiying Xiao ${ }^{1}$, Jian Wang ${ }^{1}$, Yu You $^{1}$, Bushan Xie ${ }^{1}$, Guihai Guo ${ }^{1}$, Yang $\mathrm{Hu}^{1}$, Xuan $\mathrm{Zhu}^{1}$, Bimin $\mathbf{L i}^{1}$

${ }^{1}$ The first affiliated hospital of Nanchang University, China

Background: The efficacy of routine second-look endoscopy (SLE) and prolonged use of proton pump inhibitor and octreotide (PUPAO) to prevent early bleeding after successful endoscopic variceal ligation (EVL) in cirrhosis with large gastroesophageal varices has not yet been validated. The aim of this study was to determine whether SLE and PUPAO affected clinical outcomes including early bleeding rate and mortality after EVL in cirrhosis.

Method: Consecutive cirrhotic patients received successful EVL for large esophageal varices (EV) or gastroesophageal varices type I (GOV1) (Grade III) for secondary prophylaxis were included. At the $10 \pm 1$ th day after index EVL, patients in SLE group received sedated SLE and additional endoscopic therapy, and were administered PUPAO for additional 3-6 days based on the endoscopic signs during SLE if necessary. Patients in no-SLE group received no SLE. The time to stop proton pump inhibitor and octreotide was at discretion after randomization by physicians in charge.

Result: A total of 438 patients were screened and 252 patients fulfilled the criteria. 126 patients were assigned to the SLE (age: $50.9 \pm 9.7$ yrs, male gender: $68.3 \%$ ) and 126 to no-SLE groups (age: $49.8 \pm 10.7$ yrs, male gender: $69.8 \%$ ), respectively. Two groups were observed closely for 6 weeks. Early bleeding occurred during follow up in 6 patients in SLE group and in 17 ones in no-SLE group (4.8\% v.s. $13.5 \%, P=0.02$ ). There was no significant difference in allcause mortality $(4.0 \%$ in SLE v.s. $6.3 \%$ in no-SLE, $P=0.02)$, but bleeding caused mortality was lower in SLE group (0 v.s. $4.8 \%$, $P=0.03)$. After multivariate logistic analysis, patients with Childpugh classification $\mathrm{B} / \mathrm{C}(O R: 9.99,95 \% C I: 1.12-89.15, P=0.039)$, active bleeding on index EVL (OR: 5.17, 95\% CI: 1.51-17.67, $P=0.009)$, non-selective beta blocker discontinuation during follow up (OR: 5.28, 95\% CI: 1.005-27.73, $P=0.049)$ and patients without SLE after index EVL (OR: 4.45, 95\% CI: 1.05-18.90, $P=0.038$ ) were at a higher risk of bleeding.

Conclusion: Routine sedated SLE after EVL in cirrhotic patients with large EV or GOV is effective in reducing early bleeding rate and its associated mortality.(Clinical trial registration number: ChiCTR-IIR16007963) 


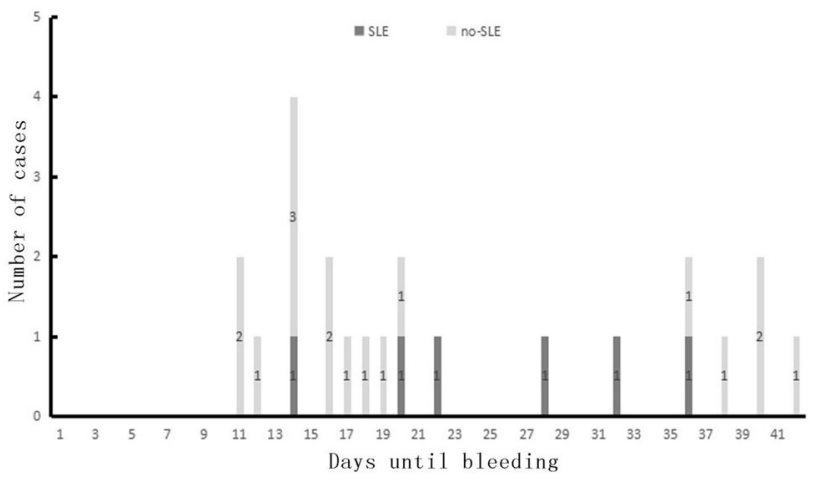

O-PHT-14

A comparison of child-turcotte-pugh classification with six models of end-stage liver disease-based prognostic system for spontaneous bacterial peritionitis in cirrhosis

\section{Pin Cheng Chen ${ }^{1}$}

${ }^{1}$ Linkou Chang Gung Memorial Hospital, Taiwan

Background: For prediction of the prognosis of spontaneous bacterial peritonitis (SBP) in patients with cirrhosis, no direct comparisons have been made among the seven models, the Child-Turcotte-Pugh classification (CTP), the model for end-stage liver disease (MELD), the model for end-stage liver disease with the incorporation of serum sodium (MELD-Na), the integrated model for end-stage liver disease (iMELD) score, and the model for end-stage liver disease to sodium (MESO) index, the modification of the Model for End-Stage Liver Disease (MELD) scoring system (Refit MELD) and the modification of MELD-Na (Refit MELDNa)

Method: Between January 2005 and July 2017, 187 patients who met the criteria for liver cirrhosis with first episode of SBP were enrolled in this retrospective study. Patients' clinical and laboratory data were obtained at diagnosis, and the Child-Turcotte-Pugh (CTP) and 6 MELD-based scores were calculated accordingly. Patients were followed up until August 2017 or until death.

Result: The in-hospital mortality rate was $38 \%$. The cumulative 3 -month and 6-month mortality rates were $51.9 \%$ and $60.1 \%$, respectively. The CTP score and 6 MELD-based model scores could all predict the in-hospital, 3-month, and 6-month mortality. For inhospital mortality, iMELD had the best AUC (0.732), but showed no significant difference in comparison with the CTP score $(\mathrm{p}=0.34)$. For the 3-month mortality, Refit MELD had the best AUC (0.75), which was borderline superior to the CTP score $(p=0.08)$. For the 6-month mortality, Refit MELD still had the best AUC (0.742), and was borderline superior to the CTP score $(\mathrm{p}=0.05)$.

Conclusion: All of the 6 MELD-based models, iMELD, MELD-Na, MESO, MELD, Refit MELD, and Refit MELDNa had no significant better prediction abilities than the CTP classification on in-hospital mortality, 3-month, and 6-month mortality of SBP patients. Only Refit MELD showed borderline superiority to CTP score on 3-month and 6-month mortality of SBP patients.
O-PHT-15

Validation of Baveno criteria to rule out high-risk varices (HRV) in patients with compensated cirrhosis

Varun R Tadkalkar $^{1}$, Rajendra Gunjal ${ }^{1}$, B K Bincy ${ }^{1}$, C J Rathan ${ }^{1}$, H Athul $^{1}$, Krishnadas Devadas ${ }^{1}$

${ }^{1}$ Government medical college thiruvananthapuram, India

Background: Variceal screening is recommended in patients with cirrhosis to identify varices at high risk of bleeding. Non-invasive criteria to rule out high-risk varices (HRV) will avoid unnecessary endoscopies Baveno VI criteria defines patients with compensated cirrhosis in whom endoscopy can be avoided as those with a liver stiffness (LSM) by TE $<20 \mathrm{kPa}$ and platelet count $>150,000 / \mathrm{mm}^{3}$. The aim was to validate Baveno criteria in our cohort from India.

Method: Cross-sectional study evaluating patients with liver stiffness $>10 \mathrm{kPa}$ who had endoscopy within 6 months of TE evaluation at our centre in South India. Patients who had LSM $<20$ and platelet $>150,000 / \mathrm{mm}^{3}$ were considered as satisfying Baveno criteria.

Result: This study included 272 patients who underwent Transient elastography (TE) and upper GI endoscopy from September 2015 to August 2017. 168 (61\%) patients were male while 104 (38\%) patients were female. Most common etiology was HCV (43\%) followed by $\mathrm{HBV}$, NASH and ethanol. Any grades of Varix was present in 98 (36\%) patients and HRV was present in $65(23 \%)$ patients. The mean platelet count among patients who had HRV and the patients who did not have HRV was $101,600 / \mathrm{mm}^{3}$ and $181,884 / \mathrm{mm}^{3}$ respectively. The mean LSM among patients with and without HRV was 29.5 and $18.6 \mathrm{kPa}$ respectively. Out of 192 patients satisfying Baveno criteria $\left(\right.$ LSM $<20 \mathrm{kPa}$ and platelet $\left.>150,000 / \mathrm{mm}^{3}\right), 190$ patients did not have HRV while 2 patients had HRV. Sensitivity, specificity, Positive predictive value (PPV) and Negative predictive value (NPV) was $98 \%, 78 \%, 91 \%$ and $97 \%$ respectively. AUC for Baveno criteria was 0.89. By using Baveno criteria, 190 (70\%) endoscopies could have been circumvented.

Conclusion: The Baveno VI criteria correctly identifies $99 \%$ of patients who can avoid endoscopy. It can be used safely to avoid screening endoscopies in resource constrained settings.

\section{O-PHT-16}

Periscreen strip test-rapid diagnosis of spontaneous bacterial peritonitis

\section{Dinesh Jothimani ${ }^{1}$, Jayanti Venkatraman ${ }^{3}$, Ezhil Arasan ${ }^{1}$, Ponmugil Kalpana ${ }^{2}$, Mohamed Rela ${ }^{3}$ \\ ${ }^{1}$ Consultant, India; ${ }^{2}$ Assistant, India; ${ }^{3}$ Professor, India}

Background: Delay in the diagnosis of Spontaneous bacterial peritonitis (SBP) is associated with significant morbidity and mortality. Conventional diagnosis of SBP is based on the presence of ascitic fluid White Cell Count (WCC) $\geq 500$ cells $/ \mathrm{mm}^{3}$ or Absolute Neutrophil Count $(\mathrm{ANC}) \geq 250 \mathrm{cells} / \mathrm{mm}^{3}$. Periscreen (Serim Research Corp, USA) is a new reagent strip specifically designed to test leukocyte esterase activity, an enzyme released by activated neutrophils.

Aim: We prospectively evaluated the usefulness of Periscreen strip test in the diagnosis of SBP.

Method: All consecutive patients with ascites from June 2016 to March 2017 were evaluated for SBP. Collected samples were analysed for WCC and ANC using standard laboratory techniques. Periscreen strip was dipped in the same sample for 30 seconds and 
observed for 5 minutes. Colour change on the strip was compared with cell count.

Result: 72 patients with ascites underwent both Periscreen and laboratory WCC analysis. Median age was 52 years, M:F, 4:1. Twenty four $(33.3 \%)$ patients were diagnosed with SBP based on WCC $\geq 500$ cells $/ \mathrm{mm}^{3}$. Sensitivity and specificity of Periscreen was $70.8 \%$ and $100 \%$, respectively. However, when ANC $\geq 250$ cells/ $\mathrm{mm}^{3}$ was defined as SBP (20.8\% patients), the sensitivity increased to $100 \%$ and the specificity was $96 \%$. AUROC score for excluding SBP with Periscreen was 0.93 .

Conclusion: Periscreen Strip test is a useful test for rapid diagnosis of SBP with excellent sensitivity and specificity. ANC has a better diagnostic value than WCC. This test can be easily performed at the bedside for early initiation of treatment.

Disclosure: Periscreen Strips were supplied by Serim Research Corp, Indiana, USA

\section{O-PHT-17}

Serum and ascitic amyloid $\mathrm{A}$ as a novel marker in early diagnosis of spontaneous bacterial peritonitis (SBP)

\section{Sherief Abd Elsalam ${ }^{1}$, Rehab Badawi ${ }^{1}$, Shaimaa Soliman ${ }^{2}$}

${ }^{1}$ Tanta University Faculty of medicine, Egypt; ${ }^{2}$ Menofia University, Egypt

Background: Ascitic fluid culture (AF) is the gold standard for the diagnosis of spontaneous bacterial peritonitis (SBP). However, cultures have been negative in about $60 \%$ of patients despite the clinical manifestations suggestive of SBP and the increased ascites neutrophil count. The aim of this study is todetermine the diagnostic value of amyloid A in both serum and ascitic fluid as early biomarkers of diagnosis of spontaneous bacterial peritonitis in relation to other biomarkers of inflammation namely $\mathrm{C}$-reactive protein and neutrophil / lymphocytic ratio.

Method: This case control study was carried on 37 patients with decompensated cirrhosis with ascites (22 of whom had SBP and 15 cases without infections as controls). Ascitic fluid (AF) leukocyte ascitic fluid amyloid A, C-reactive protein (CRP), neutrophil-tolymphocyte ratio (NLR) and serum amyloid A measurements were carried out prior to the use of antibiotics.

Result: There was significant increase of serum amyloid A, CRP and ascitic fluid amyloid ( $\mathrm{p}$ value $<0.001,0.004$ and $<0.001$ ) in patients presented with SBP than those without SBP respectively. The areas under the curve of serum amyloid $\mathrm{A}$ and ascitic fluid amyloid for diagnosis of SBP was1 and0.91 respectively. The cut-off point of serum amyloid A at which SBP can be diagnosed was $9.25 \mathrm{ug} / \mathrm{ml}$ with sensitivity and specifity of $100 \%$ and $100 \%$ respectively. The cut-off point of ascitic fluid amyloid was $2.85 \mathrm{ug} / \mathrm{ml}$ with sensitivity and specifity of $90.09 \%$ and $60 \%$ respectively.

Conclusion: Serum and ascitic fluid amyloid A are good biomarker for early diagnosis of SBP.

\section{O-PHT-18}

Bovine immunoglobulin orally with modified doses of rifaximin for advanced cirrhotics in overt hepatic encephalopathy: a randomized placebo control prospective clinical pilot trial. (BRAIN trial)

\section{Nimy John ${ }^{1}$, Mark Aloysius ${ }^{2}$, Menisa Zaman ${ }^{3}$}

${ }^{1}$ Saint Vincent Hospital, United States; ${ }^{2}$ JJP VAMC Ichann School of Medicine, United States; ${ }^{3}$ Weil Cornell Medicine, United States
Background: Overt Hepatic Encephalopathy (OHE) is integral clinical syndrome in decompensated cirrhosis with significant morbidity. Recurrent hospitalization carries Hospital acquired infections with threatens risk and further decompensation. Imposes hazards: driving skill, domestic affair, social conduct affecting Global QOL score. Rifaximin, lactulose, Metronidazole and are existing therapy of choice. Several others have been postulated: LOPA, Probiotics, Rivastigmine with moderate efficacy. This clinical trial will evaluate added efficacy and shorten course of Rifaximin in clinically OHE

Method: Overt Hepatic Encephalopathy patients recruited in three groups: A single center: dedicated Psychologist, Dedicated lab; single driving instructor, 3 arm study (groups A, B and C), $\mathrm{n}=20$ in each Exclusion criteria: MELD 23 or greater, Uncontrolled DM, SBP, Severe Constipation, HCC, CDAD, Active drug or Alcohol use, On DAAs therapy, PBC, PSC, Schistosoma, AIH, Post-Transplant, HBV, HIV.

Interventions: Group A = Rifaximin $550 \mathrm{mg}$ BID, BCAA $\mathrm{x}$ 3 months; Group $\mathrm{B}=$ Rifaximin $550 \mathrm{mg}$ BID, Bovine IG $\mathrm{x}$ 3 months; Group C = Rifaximin $550 \mathrm{mg}$ BID, Bovine IG, BCAAx 3 months.

Result: Primary End point: Global Encephalopathy score; Group A median 43\%; Group B median 63\%; Group C median 66\%. Secondary End point: Cognitive skill improvement; Group A median $21 \%$; Group B median 33\%; Group C median $31 \%$; impaired Social skill (driving test). Recurrent Hospitalization Time in six months; Group A median 65\%; Group B median 68\%; Group C median $71 \%$.

\begin{tabular}{|c|c|c|c|}
\hline Parameter & $\begin{array}{l}\text { Group A } \\
\text { Rifaximin, } \\
\text { BCCA) x } 3 \\
\text { months }\end{array}$ & $\begin{array}{l}\text { Group B } \\
\text { Rifaximin, } \\
\text { Bovine IG) } x \\
3 \text { months }\end{array}$ & $\begin{array}{l}\text { Group C } \\
\text { Rifaximin, } \\
\text { Bovine IgG, } \\
\text { BCCA) x } 3 \\
\text { months }\end{array}$ \\
\hline Cirrhosis & 6 & 7 & 6 \\
\hline NASH & 4 & 3 & 4 \\
\hline $\mathrm{HCV}$ & 10 & 10 & 10 \\
\hline Gender M:F & $14: 6$ & $16: 4$ & $16: 4$ \\
\hline Age (IQR) & $56-76$ & $56-76$ & $56-76$ \\
\hline Race $(\mathrm{B}: \mathrm{W}: \mathrm{H})$ & $8: 6: 6$ & $10: 6: 4$ & $8: 6: 6$ \\
\hline Mean BMI & 24.6 & 25 & 25.6 \\
\hline Mean MELD & 16 & 14 & 12 \\
\hline Mean Platelets & 78 & 102 & 112 \\
\hline Mean AST & 57 & 48 & 51 \\
\hline Mean $\mathrm{Hb}$ & 10.4 & 10.8 & 10.3 \\
\hline Mean Creatinine & 1. 1 & 0.9 & 1 \\
\hline Mean albumin & 2.9 & 3.1 & 3.3 \\
\hline Mean Sodium & 134 & 136 & 135 \\
\hline Mean WBC & 3.8 & 4.8 & 4 \\
\hline Mean T Bili & 2. 1 & 2 & 1.8 \\
\hline Mean Ammonia & 129 & 122 & 96 \\
\hline Esophageal Varices & $18 / 20$ & $17 / 20$ & $12 / 20$ \\
\hline Ascites & 9 & 10 & 6 \\
\hline $\begin{array}{l}\text { OHE Score (flicker test) } \\
\text { mod: severe }\end{array}$ & $18: 2$ & $17: 3$ & $17: 3$ \\
\hline Number connection test & $130 \mathrm{~s}: 220 \mathrm{~s}$ & 98: 187 & $78: 159$ \\
\hline $\begin{array}{l}\text { Modified dementia score } \\
\text { (mild: mod: severe) }\end{array}$ & $12: 7: 1$ & $17: 3: 0$ & $13: 3: 0$ \\
\hline $\begin{array}{l}\text { Global encephalopathy } \\
\text { score (grade } 2: \text { grade } 3 \text { ) }\end{array}$ & 15/20: $5 / 20$ & 16/20: $4 / 20$ & $16 / 20: 2 / 20$ \\
\hline
\end{tabular}

Conclusion: For morbidity in CLF and ACLF with Cognitive and Psychosocial impairment rifaximin is the standard of care. Oral 
Bovine Immunoglobulin has been used as gut sterilizer and generates Gut specific immune Restitution. This study evaluates adding Oral Bovine immunoglobulin in OHE and concludes that it is not beneficial over standard of care and not cost effective.

\section{Session: Hepatitis C Virus Infection. Chairpersons: Kalyan Ram Bhamidimarri; Priya Abraham,Arun kumar}

\section{$\mathrm{O}-\mathrm{HCV}-43$}

Effectiveness of 12 Week elbasvir/grazoprevir (EBR/GZR) in patients with genotype 1 (GT1) chronic hepatitis $\mathrm{C}$ (HCV) and chronic kidney disease (CKD)

Chizoba Nwankwo ${ }^{1}$, Michael Curry ${ }^{2}$, Bruce Bacon ${ }^{4}$, Steven Flamm $^{3}$, Kris Kowdley ${ }^{5}$, Scott Milligan ${ }^{6}$, Naoky Tsai ${ }^{7}$

${ }^{1}$ Merck \& Co., Inc., United States; ${ }^{2}$ Beth Israel Deaconess Medical Center, United States; ${ }^{3}$ Northwestern Feinberg School of Medicine, United States; ${ }^{4}$ Saint Louis University School of Medicine, United States; ${ }^{5}$ Swedish Medical Center, United States; ${ }^{6}$ Trio Health Analytics, United States; ${ }^{7}$ University of Hawaii, United States

Background: Elbasvir/grazoprevir (EBR/GZR) is recommended for use in the treatment of chronic HCV genotype (GT) 1 and 4 patients including those with renal impairment. The purpose of this study is to describe the real-world effectiveness of 12 week EBR/GZR in patients with GT1 chronic HCV and CKD.

Method: Data were collected from US providers and specialty pharmacies through Trio Health's disease management platform. Patients with CKD and GT1 HCV who initiated 12 week EBR/GZR therapy between Jan 28, 2016 (FDA approval) to Dec 31, 2016 were included in the analyses. CKD was defined as renal impairment of baseline eGFR $<90 \mathrm{ml} / \mathrm{min}$. Effectiveness was defined as attainment of per protocol sustained virological response at week 12 posttreatment (PP SVR12).

Result: 228 patients with GT1 HCV and CKD were treated for 12 weeks with EBR/GZR and ribavirin (RBV) was added for 6 patients. $25 \%(56 / 228)$ of patients had mild stage 2 CKD, $21 \%$ (48/ 228) had moderate stage 3 CKD and $54 \%$ (124/228) had severe renal impairment (CKD stages 4-5). Other characteristics provided in (TABLE). At time of abstract submission, 144 of 228 were evaluable for PP SVR12; 98\% (135/138) of patients treated with EBR/GZR and $100 \%(6 / 6)$ of patients treated with EBR/GZR + RBV achieved PP SVR12.

Conclusion: In the treatment of patients with GT1 chronic HCV and CKD, 12 week EBR/GZR was highly effective with an overall SVR12 (PP) of 98\% (141/144).

\begin{tabular}{|c|c|}
\hline \multicolumn{2}{|c|}{ Baseline Characteristics of EBR/GZR Treated G1 Patients } \\
\hline CHARACTERISTICS - no. (\%) & 12 week EBR/GZR (n=228) \\
\hline +RBV & $6(3 \%)$ \\
\hline Treatment Naïve & $191(84 \%)$ \\
\hline Cirrhosis & $59 / 227(26 \%)$ \\
\hline Baseline Plts <100 K/ml & $27 / 215(13 \%)$ \\
\hline Demographics \\
\hline Age - mean (range) & $62(24-88)$ \\
\hline Male - no. (\%) & $141(62 \%)$ \\
\hline BMI - mean (SD) & $28(7) \mathrm{n}=181$ \\
\hline Virology - no. (\%) & \\
\hline Baseline Viral <800 IU/mL & $86 / 226(38 \%)$ \\
\hline GT1A & $144(63 \%)$ \\
\hline NS5A Resist. Tested & $86 / 144(60 \%)$ \\
\hline NS5A Resist. Possible & $3 / 86(3 \%)$ \\
\hline Comorbids - no. (\%) & \\
\hline Diabetes & $83 / 224(37 \%)$ \\
\hline Hypertension & $158 / 225(70 \%)$ \\
\hline Stage 4-5 CKD & $124 / 228(54 \%)$ \\
\hline
\end{tabular}

\section{$\mathrm{O}-\mathrm{HCV}-44$}

Experience of directly acting antiviral (DAA) for chronic hepatitis $\mathrm{C}$ in local population

\section{Shahid Sarwar ${ }^{1}$}

${ }^{1}$ Services Institute of Medical Sciences, Pakista

Background: As newer antiviral agents are being available in this part of world, it is pertinent to determine treatment outcome with these drugs. This study was planned to determine the outcome of direct acting antiviral treatment of chronic hepatitis $\mathrm{C}$ in local population.

Method: Patients of chronic hepatitis $\mathrm{C}$ with positive HCV RNA and no life threatening co-morbid illness were included in study. After initial diagnostic workup, DAA treatment was started depending on genotype of virus, availability of drugs and patients economic status. Duration of treatment and follow up was as per standard recommendations. Negative HCV RNA 12 week after treatment completion (SVR12) was the primary end-point of study. SPSS ${ }^{\circledR} 20$ was used for analysis.

Result: Total of 360 patients were included. Mean age was 50.06 ( \pm 11.9$)$ with male to female ratio of 1.05 (185/175). Among study population, $201(55.8 \%)$ had evidence of cirrhosis, $44(12.2 \%)$ had history of variceal bleeding, $46(12.81 \%)$ had ascites, $7(1.9 \%)$ had experienced hepatic encephalopathy and $63(17.5 \%)$ were diabetics. Treatment naïve patients were $252(70 \%)$ while $108(30 \%)$ were treatment experienced. Sofosbuvir/Ribavirin (Sof/Riba) was used in $268(74.4 \%)$ patients, $44(12.2 \%)$ received pegylated interferon/sofosbuvir/ribavirin (PegIFN/Sof/Riba), 15(4.2\%) were treated with sofosbuvir/Daclatasvir (Sof/Dacla), 29 (8.1\%) with sofosbuvir/Daclatasvir/Ribavirin (Sof/Dacla/Riba) and 4 patients received Sofosbuvir/Ledipasvir (Sof/Ledi). Treatment was completed in 342 $(95 \%)$ patients, 13 patients failed to complete treatment and 5 were lost to follow up. SVR12 was achieved by $309(90.3 \%)$ patients, 230 (92.7\%) patients in Sof/Riba group, 42 (95.4\%) patients in PegIFN/ Sof/Riba treatment cohort, $13(86.5 \%)$ with Sof/Dacla treatment, 20 $(68.9 \%)$ in Sof/Dacla/Riba group and all 4 patients of Sof/ledi group. SVR12 was significantly better in treatment naïve patients as compared to treatment experienced group ( $\mathrm{p}$ value 0.05 ). Patients with cirrhosis had significantly inferior SVR $1280.5 \%$ as compared to noncirrhotic patients $92.3 \%$ ( $\mathrm{p}$ value $<0.00$ ).

Conclusion: Directly antiviral drugs have excellent outcome in treating patients of chronic hepatitis $\mathrm{C}$, especially in treatment naïve 
and non-cirrhotic patients as compared to treatment experienced and patients with cirrhosis respectively.

\section{$\mathrm{O}-\mathrm{HCV}-46$}

Estimating the net value of treating hepatitis $\mathrm{C}$ virus using a newly available direct-acting antiviral in India

\section{Alexander Jurius Khoury $^{1}$, David Bloom ${ }^{1}$, V Seenu Srinivasan ${ }^{2}$ \\ ${ }^{1}$ Harvard T.H. Chan School of Public Health, United States; ${ }^{2}$ Stanford Graduate School of Business, United States}

Background: Recently developed direct-activing antiviral (DAA) treatments for HCV have been groundbreaking for their high efficacy across disease genotype and lack of severe side effects. However, there is disagreement over whether the benefits of these new treatments justify their cost. This study uses a cost-of-illness (COI) approach to estimate the net value conferred by one of these novel drug combinations, Velpanat, recently licensed for generic manufacture in India.

Method: This study considers COI from lifetime earnings lost due to disability and premature death from HCV infection. Risk of death and disability in future years is calculated using a Markov state-transition model with parameters determined from the literature. The future earnings of sampled patients are predicted using an empirical earnings model with coefficients determined from India Human Development Survey data. Costs to the patient and secondarily-infected individuals are both considered.

Result: Preliminary results suggest that curing individuals diagnosed with chronic HCV in India would preserve 3,745,803 INR in earnings per person. The largest expected economic benefits are conferred to compensated cirrhotic (CC) men because they have a high risk of experiencing disability and premature death if untreated and are anticipated to have relatively high earnings if they remain healthy. For non-cirrhotic (NC) and CC individuals, the expected benefits associated with prevented secondary infections are worth between $1 \%$ and $41 \%$ of the value of benefits conferred to the diagnosed individual (depending on sex and extent of liver damage). Treating decompensated cirrhotic individuals (DC) with DAAs alone offers minimal earnings benefits because these individuals will likely remain disabled and unable to work without liver transplantation. Expected net benefits of treatment are substantial for $\mathrm{NC}$ and $\mathrm{CC}$ patients (ranging from 640,349 INR for NC women to 10,680,848 INR for CC men). The cost of treatment for DC individuals exceeds the expected earnings benefits. These results suggest that treating $10 \%$ of chronic HCV carriers in India could yield as much as 3.19 trillion INR in increased earnings.

Conclusion: For average NC and $\mathrm{CC}$ individuals, the cost of treatment with Velpanat is offset by the benefits of increased future productivity. Increased earnings are not sufficient to offset cost of treatment for DC individuals.

\section{$\mathrm{O}-\mathrm{HCV}-47$}

Implementation of a unique hepatitis $\mathbf{C}$ Care continuum model in international resource-limited settings

Poonam Mathur ${ }^{1}$ Emily Comstock ${ }^{1}$, Ben Emmanuel ${ }^{1}$, Natalia Mercer $^{1}$, David Riedel ${ }^{1}$, Shyam Kottilil ${ }^{1}$

${ }^{1}$ University of Maryland School of Medicine, United States

Background: There has been a rapid evolution of chronic hepatitis $\mathrm{C}$ (HCV) treatment with the introduction of direct-acting antivirals.
Despite this advancement, the World Health Organization (WHO) has identified that only $7 \%$ of those diagnosed with $\mathrm{HCV}$ (1.1 million people) had started treatment in 2015. One barrier to the escalation of treatment, especially in resource-limited settings (RLS), is a lack of trained providers who can treat $\mathrm{HCV}$. Recently, we conducted the NIH ASCEND study in the U.S., which demonstrated that provider task-shifting is a safe and effective method to expand HCV treatment. We sought to translate the ASCEND model internationally to RLS in order to meet the demand for HCV treatment in high-burden areas. Method: ASCEND-model didactic training programs were established in Kigali, Rwanda and Imphal, India for providers who had never treated HCV. Our training sessions focused on HCV diagnosis and management, as outlined by the American Association for the Study of Liver Diseases, Asian Pacific Association for the Study of the Liver, and Rwanda National Guidelines. We evaluated the effectiveness of our training by administering a 10-question, multiplechoice test to each provider prior to the start of the training and immediately after (the same test was used). An independent t-test analysis was used to determine difference in test score pre- and posttraining overall and by country. We also developed a data-capture technique operated by local team members and a sister software application was developed in our India hub station, which uploads and integrates all field-collected data to a cloud-based database for storage.

Result: We analyzed the pre- and post-test results for 11 providers in Rwanda, 18 providers in the U.S. (as part of the ASCEND study), and 29 providers in India. Among the group of Indian providers, 12 providers took the pre-test and 29 providers took the post-test. Overall, the mean difference in score among providers in all 3 countries was an increase of $16 \%$ (from $45 \%$ to $61 \%, \mathrm{p}=0.0002$ ). The mean difference in score among U.S, Rwandan and Indian providers was $21 \%(\mathrm{p}=0.005), 17 \%(\mathrm{p}=0.03)$, and $11 \%(\mathrm{p}=0.15)$, respectively (Figure 1).

Conclusion: The ASCEND model is highly effective in training new providers and can be applied to RLS. Providers' knowledge of HCV diagnosis and management improved after the ASCEND training didactic session, and the same improvement was seen in Rwanda. Although the post-test scores among Indian providers were higher than pre-test scores, review of the post-test answers shows that providers demonstrated difficulty in understanding management of $\mathrm{HCV}$ in HIV and HBV co-infected patients and post-SVR HCC screening. This information highlights that gaps in knowledge remain among providers in India. These topics will need to be addressed thoroughly during subsequent training sessions, so that the $\mathrm{HCV}$ care continuum can be successfully escalated using the ASCEND training model.

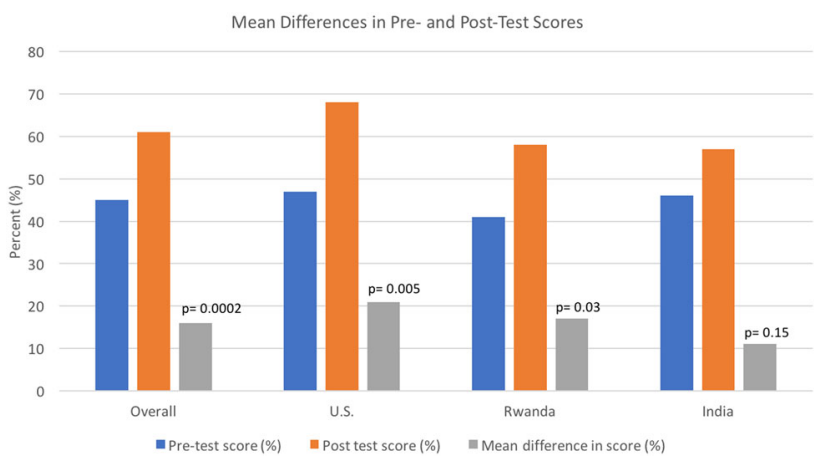




\section{$\mathrm{O}-\mathrm{HCV}-48$}

Improving cascade of hepatitis $\mathrm{C}$ care through linkage to $\mathrm{C}$ care program [LTC]

\section{Imtiaz Alam $^{1}$ Zohha Tariq $^{1}$, Hong Bui ${ }^{1}$, Denise Cook ${ }^{1}$ \\ ${ }^{1}$ Austin Hepatitis Center, United States}

Background: There is an emerging epidemic of people who inject drugs (PWID) and contract the hepatitis $\mathrm{C}$ virus (HCV). At least $75 \%$ of new HCV infections in the United States result from injection drug use. Our aim is to link PWID individuals that are HCV RNA positive to care, and utilize our online patient database management system (PDMS) with our Substance Abuse Treatment Center partners to fulfill the linkage portion.

Method: Longitudinal prospective cohort study with HCV screening at the above centers and utilization of a HIPPA compliant online patient database management system (PDMS) (www.linkagetocare.com). A centrally located Linkage to Care Specialist (LTCS) is notified immediately when an individual's information is entered in the system by the treatment center or self-referred. The LTCS educates the individual and proceeds to link that person to care.

Result: January 2017-August 2017, 428 HCV RNA positive patients referred (18\% self-referred and $82 \%$ referred from 26 facilities in 16 states; 92\% Texas); 69\% patients were uninsured; Median age of patients 39 years (19-77); 59\% males. 395 (91\%) patients were contacted by LTCS; 299 (70\%) HCV patients referred by the LTCS to a medical provider; $15 \%$ patients awaiting lab results while $15 \%$ are on hold because of the facilities does not allow the LTCS to contact the patient until they are discharged. Overall, patients were connected with an LTCS within 2 days after referral. On average, patients were contacted twice before scheduling their first appointment. $123(41 \%)$ patients made it to their first appointment and 23 (19\%) of them initiated HCV treatment; 18 (15\%) patients finished therapy; $97 \%$ patients were seen in office vs. $3 \%$ through telemedicine. Additionally, $54 \%$ of patients were from sober living homes and $46 \%$ from methadone clinics.

Conclusion: Targeting PWID is an effective way of reducing the prevalence of HCV infection. With the LTC program, there is an increase in patient compliance with linkage to care as compared to current care models.

\section{Presidential Poster Clinical}

\section{March 2018}

$P P-C-01$

Comparison between treatment outcome of Sitagliptin plus life style modification versus only life style modification in patients with biopsy proven nonalcoholic steatohepatitis

\section{$\underline{\text { Jhumur Ghosh }}{ }^{1}$, Md. Shahinul Alam², Md. Golam Mustafa ${ }^{2}$ \\ ${ }^{1}$ M H Samorita Medical College and Hospital, Bangladesh; ${ }^{2}$ Bangabandhu Sheikh Mujib Medical University, Bangladesh}

Background: Non-alcoholic fatty liver disease (NAFLD) is currently the most common cause of liver disease worldwide. To date, no therapy provided evidence of significant efficacy, and as a consequence, no approved therapeutic options are available worldwide. Sitagliptin, a Dipeptidyl peptidase-4 (DPP-4) inhibitor stimulates beta oxidation and inhibit hepatic fat accumulation by decreasing hepatic expression of Dipeptidyl peptidase-4 enzyme.
Method: Total 30 biopsy proven NASH patients with or without type 2 diabetes mellitus were included in this study. 20 patients were treated with Sitagliptin plus life style modification (SL) and 10 patients were treated with only life style modification (L) for one year. Nonalcoholic steatohepatitis score improvement $\geq 2$ and fibrosis score improvement $\geq 1$ were defined as histological responder. The comparison between pretreatment and end of treatment histological score were done by paired t-test. Logistic regression analysis was done to find out the best predictor of histological response.

Result: Nonalcoholic steatohepatitis score (NAS) $\geq 2$ improvement occurred in 13 patients out of 20 patients in SL group $(65 \%)$ and 2 patients out of 10 in L group (20\%). Fibrosis score $\geq 1$ improvement occurred in 5 patients $(25 \%)$ in SL group, whereas in L group, 2 patients had this improvement $(20 \%)$.This current Case Control study revealed that Sitagliptin improved NAS score $(P=0.002)$ and steatosis $(\mathrm{P}=0.001)$ in SL group. The difference of NAS score improvement between SL and L group was statistically significant (P $=0.003$ ). Hepatocyte ballooning improvement was borderline significant $(\mathrm{P}=0.055)$ in SL group. There was no improvement in the lobular inflammation and fibrosis score in both SL and L groups. Among 13 patients in SL group 7 patients were diabetic and 6 patients were nondiabetic. There was no significant difference of histological response in diabetic and nondiabetic NASH patients with Sitagliptin. In SL group only 4 patients lost $\geq 7 \%$ body weight. Therefore, significant body weight loss was not associated with significant histological improvement $(\mathrm{P}=0.194)$ in SL group. HOMA-IR improvement was not significant in responders and non-responders $(\mathrm{P}$ $=0.94$ ). This current study showed that Sitagliptin did not improve the insulin resistance (HOMA-IR) in NASH patients. Logistic regression analysis revealed that only treatment with Sitagliptin resulted significant improvement of NAS score, steatosis and borderline improvement of hepatocyte ballooning than that of weight reduction and glycaemic control.

Conclusion: Sitagliptin may improve NAS score, steatosis and hepatocyte ballooning in NASH patients with or without type 2 diabetes mellitus. Further large RCT studies for longer duration are necessary to confirm and expand these findings.

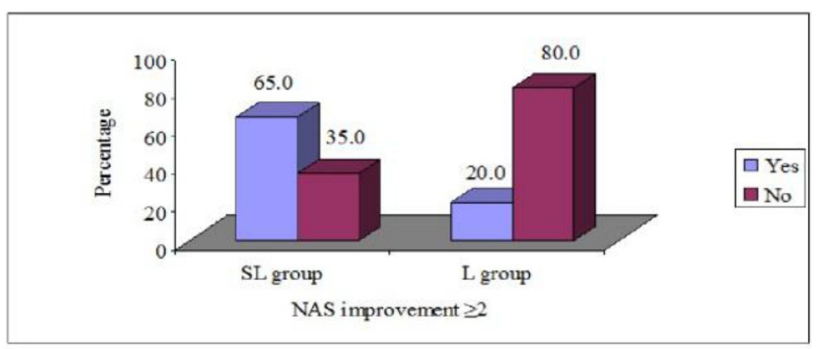

Figure 1: Distribution of NAS score improvement $\geq \mathbf{2}$ in SL and L group

Table 1: Comparison of Histopathological changes of NASH patients

\begin{tabular}{|l|l|l|l|l|l|l|}
\hline Variables & \multicolumn{3}{|c|}{ SL group (n-20) } & \multicolumn{3}{c|}{ L group (n-10) } \\
\hline & $\begin{array}{l}\text { Before } \\
\text { Intervention }\end{array}$ & $\begin{array}{c}\text { After } \\
\text { Intervention }\end{array}$ & P value & $\begin{array}{c}\text { Before } \\
\text { Intervention }\end{array}$ & $\begin{array}{l}\text { After } \\
\text { Intervention }\end{array}$ & P value \\
\hline $\begin{array}{l}\text { NAFLD activity } \\
\text { score }\end{array}$ & $5.7 \pm 0.8$ & $3.9 \pm 1.4$ & 0.002 & $5.4 \pm 0.7$ & $5.0 \pm 1.2$ & 0.269 \\
\hline Steatosis & $2.2 \pm 0.6$ & $1.2 \pm 0.7$ & 0.001 & $2.1 \pm 0.6$ & $1.8 \pm 0.6$ & 0.193 \\
\hline $\begin{array}{l}\text { Lobular } \\
\text { Innammation }\end{array}$ & $1.8 \pm 0.4$ & $1.5 \pm 0.5$ & 0.096 & $1.7 \pm 0.5$ & $1.6 \pm 0.5$ & 0.591 \\
\hline $\begin{array}{l}\text { Hepatocytes } \\
\text { balloonimg }\end{array}$ & $1.7 \pm 0.6$ & $1.4 \pm 0.5$ & 0.055 & $1.6 \pm 0.5$ & $1.6 \pm 0.5$ & 1.00 \\
\hline Fibrosis score & $1.8 \pm 0.7$ & $1.7 \pm 0.9$ & 0.591 & $1.9 \pm 0.9$ & $1.6 \pm 0.8$ & 0.468 \\
\hline
\end{tabular}

$P$ value calculated by paired t-test 


\section{$P P-C-02$}

Changes in liver stiffness during tenofovir disoproxil fumarate therapy in Chinese chronic hepatitis B patients with advanced fibrosis and compensated cirrhosis: Results from a prospective, multi-center, cohort study

Lai Wei ${ }^{1}$, Jia Shang ${ }^{8}$, Qing Xie ${ }^{13}$, Zhansheng Jia ${ }^{16}$, Pujun Gao ${ }^{19}$, Junping Shi ${ }^{17}$, Xinyue Chen ${ }^{3}$, Jiefei Wang ${ }^{14}$, Min $\mathrm{Xu}^{7}$, Liaoyun Zhang $^{4}$, Yingren Zhao ${ }^{18}$, Qing Mao ${ }^{15}$, Maorong Wang ${ }^{12}$, Wei Zhao $^{20}$, Zong Zhang ${ }^{11}$, Jidong Jia ${ }^{2}$, Hong Tang ${ }^{21}$, Jiming Zhang', Xin Zheng ${ }^{10}$, Cui Xiong ${ }^{6}$, Mingfen $\mathrm{Zhu}^{5}$

${ }^{1}$ Peking University People's Hospital, Peking University Hepatology Institute, China; ${ }^{10}$ Institute of Infection and Immunology, Affiliated Union Hospital of Tongji Medical College, HuaZhong University of Science and Technology, China; ${ }^{11}$ Jinan Infectious Disease Hospital, Shandong University, China; ${ }^{12}$ No. 81 Hospital of People's Liberation Army, Nanjing, China; ${ }^{13}$ Ruijin Hospital, Affiliated to Shanghai Jiaotong University, School of Medicine, China; ${ }^{14}$ Shanghai Public Health Clinical Center, China; ${ }^{15}$ Southwest Hospital, Third Military Medical University, China; ${ }^{16}$ Tangdu Hospital, The Fourth Military Medical University,, China; ${ }^{17}$ The Affiliated Hospital of Hangzhou Normal University, Hangzhou, China; ${ }^{18}$ The First Affiliated Hospital of Xi' an Jiaotong University, Xi'an,, China; ${ }^{19}$ The First Hospital of Jilin University, China; ${ }^{2}$ Beijing Friendship Hospital, Capital Medical University, China; ${ }^{20}$ The Second Hospital of Nanjing, China; ${ }^{21}$ West China Hospital, Sichuan University, China; ${ }^{3}$ Beijing YouAn Hospital, Capital Medical University, China; ${ }^{4}$ First Hospital of Shanxi Medical University, China; ${ }^{5}$ GlaxoSmithKline (China) Investment Company Limited, China; ${ }^{6}$ GlaxoSmithKline (China) R\&D Company Limited, China; ${ }^{7}$ Guangzhou Eighth Municipal People's Hospital, China; ${ }^{8}$ Henan Provincial People's Hospital, Zhengzhou, China; ${ }^{9}$ Huashan Hospital Affiliated to Fudan University, China

Background: Tenofovir disoproxil fumarate (TDF) long-term treatment in patients with chronic hepatitis B $(\mathrm{CHB})$ is associated with liver fibrosis regression. This ongoing, prospective, open-label, cohort study (GSK [NCT02224456]) assessed the change in liver stiffness measurement (LSM) during TDF therapy in the subset of Chinese CHB patients; 48-week results are presented here.

Method: Nave patients aged 18-60 years with advanced fibrosis or compensated cirrhosis (diagnosed clinically [LSM plus abnormal ultrasound or platelets

Result: Of total 195 (modified intent-to-treat) patients (75.9\% men, mean age 43.3 years), $37.4 \%$ patients were diagnosed with advanced fibrosis and $36.9 \%$ with compensated cirrhosis by LSM at baseline. Results showed a considerable reduction in mean LSM from $14.4 \mathrm{kPa}$ (baseline) to $11.1 \mathrm{kPa}$ (week 48) (p

Conclusion: Treatment with TDF for 48 weeks significantly decreased LSM value, improved liver fibrosis and was well tolerated in Chinese patients.

Table: Fibrosis stage by LSM during 48 weeks of TDF treatment (mITT)

\begin{tabular}{|l|c|c|c|c|c|}
\hline $\mathbf{n}(\%)$ & $\begin{array}{c}\text { Baseline } \\
\mathrm{n}=187\end{array}$ & $\begin{array}{c}\text { Week 12 } \\
\mathrm{n}=192\end{array}$ & $\begin{array}{c}\text { Week 24 } \\
\mathrm{n}=191\end{array}$ & $\begin{array}{c}\text { Week 36 } \\
\mathrm{n}=190\end{array}$ & $\begin{array}{c}\text { Week 48 } \\
\mathrm{n}=190\end{array}$ \\
\hline Patients with compensated cirrhosis & $69(36.9)$ & $55(28.6)$ & $46(24.1)$ & $40(21.1)$ & $39(20.5)$ \\
Patients with advanced fibrosis & $70(37.4)$ & $53(27.6)$ & $40(20.9)$ & $41(21.6)$ & $43(22.6)$ \\
\hline
\end{tabular}

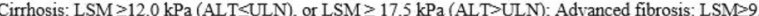
to $<12.0 \mathrm{kPa}(A L T \leq U L N)$ or $L S M \geq 12.4$ to $<17.5 \mathrm{kPa}(A L T>U L N)$.
PP-C-03

Comparison of triceps skinfold thickness and bioelectrical impedance analysis with dual energy $\mathrm{X}$-ray absorptiometery for assessment of fat mass and lean body mass in the patients with cirrhosis

Anoop Saraya ${ }^{1}$, Namrata Singh ${ }^{2}$, Deepak Gunjan ${ }^{2}$, Hem Chander Sati $^{2}$, Vipin Gupta ${ }^{2}$, Kanav Kaushal ${ }^{2}$, Anoop Saraya ${ }^{2}$

${ }^{1}$ AIIMS, India; ${ }^{2}$ AIIMS, N Delhi, India

Background: Nutritional assessment is extremely important issue in cirrhosis and closely related to morbidity and mortality in these patients. Dual energy X-ray absorptiometery (DEXA) is considered as the reference method in assessing fat mass (FM) and lean body mass (LBM). Due to unavailability and high cost, DEXA is used only in research setting. However, Bioelectrical Impedance Analysis (BIA) and Triceps skinfold thickness (TSF-T) can be used in clinical setting. In a developing nation like India, Methods like BIA and TSF-T may prove beneficial and cost effective methods as compare to DEXA. AIM: We aim to evaluate TSF-T and BIA for assessing Fat Mass and Lean body mass using DEXA as gold standard, in patients with cirrhosis.

Method: Cirrhotic patients with all etiologies were included. Disease severity was assessed by Child- Turcotte- Pugh Score (CTP) and catagoried into CTP (A, B and C). FM and LBM was assessed by DEXA and BIA (Tanita-215, leg to leg analyzer). TSF-T was measured using Harpenden\&rsquo;s skinfold calliper, Mid arm muscle circumference $($ MAMC) was calculated by MAMC $=$ MUAC $(\mathrm{cm})$ [3.14*TSF-T $(\mathrm{cm})]$. Analysis of variance, intra-class correlation coefficient(ICC) and Bland and Altman plot analysis were used for comparative analysis among the methods.

Result: 343 patients (273 male, 70 female) were included in this study. DEXA showed significantly higher fat mass in females (14.4 $\forall$ $6.63)$ as compared to males $(12.54 \pm 5.95)$ [P=0.02.] Fat mass decreases; significantly; in patients with CTP B $(11.8 \pm 5.98)$ as compared to CTP A $(13.58 \pm 6.16)$ [p $=0.02]$. Good intra-class correlation coefficients [r(p)] were found between fat mass of DEXA with BIA $0.73(0.0001)$, Lean body mass of DEXA with BIA 0.84(0.0001) and fat mass of DEXA with TSF-T 0.68(0.0001). DEXA showed a relatively good agreement [ICC $(95 \% \mathrm{CI})]$ of fat mass with both BIA [0.83(0.79, 0.86)] and TSF-T[0.80(0.75,0.84)]; and Lean body mass with BIA $[0.91(0.89,0.93)]$ in all patients according to Bland and altman plot analysis(95\% limits of agreement). No correlation was found between DEXA and MAMC for Lean body mass. Conclusion: Simple, cost effective and long established method of Nutritional assessment (BIA and TSF-T) can replace DEXA for FM assessment and BIA can replace DEXA for LBM assessment, which can be easily applied in routine clinical practice. 

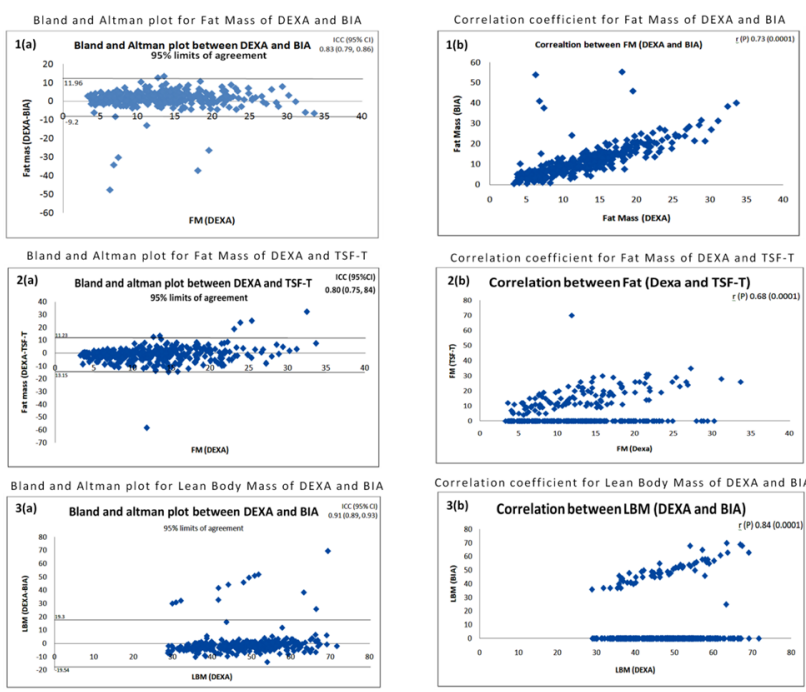

Correlation coellicient for fat mass or Dexa a d Ts.t

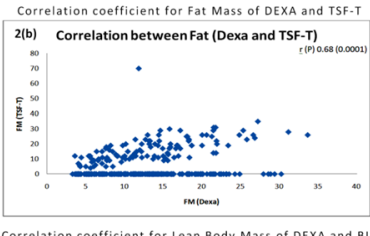

Correlation coefficient for Lean Body Mass of DEXA and B

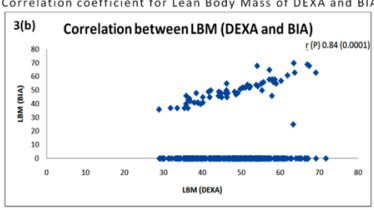

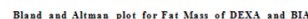
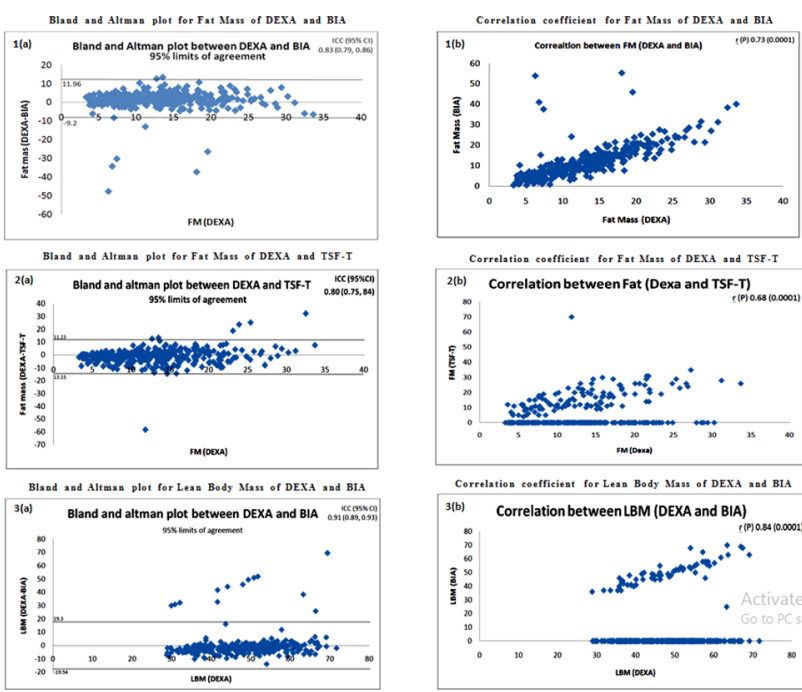

Correlation coefficient for Fat Mass of DEXA and TSF-T
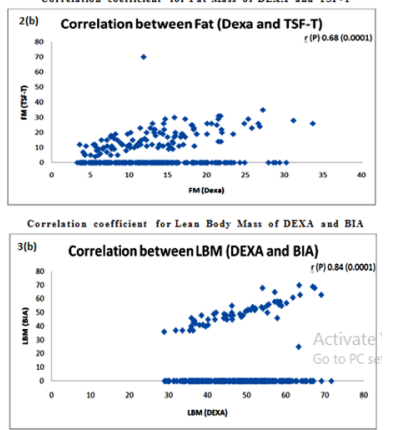

PP-C-04

Porcine (Bepcidin-containing Biological p.o. drug derived from porcine-placenta) can completely replace Phlebotomy in the treatment of Hereditary Hemochromatosis - New Era of Regulating Iron Metabolism

\section{$\underline{\text { Yuki Hamada }}^{1}$}

${ }^{1}$ Hamada Clinic for Gastroenterology, Japan

Background: Recent advance in iron metabolism clarified that most causes of hereditary hemochromatosis (H.H.) are due to partial or total loss of the activity of anti-microbial peptide hormone produced by the liver, hepcidin, which normally regulates iron entry into the circulation. This implies that H.H. genes encode molecules regulating hepcidin synthesis. We have previously shown that the treatment with LaennecÂA ${ }^{\circledR}$ containing hepcidin improves H.H. without repeated phlebotomy for more than 5 years. Here we show that PorcineÂA (Biological p.o. drug derived from porcine-placenta) can also replace the phlebotomy.
Method: 48 years-old male patient that developed type 2 diabetes mellitus had elevated serum ferritin level $(10,191 \mathrm{ng} / \mathrm{ml})$ and a decreased hepcidin-25 level $(0.5-1.6 \mathrm{ng} / \mathrm{ml})$. Liver biopsy revealed remarkable iron deposition and fibrosis. Chromosomal analysis revealed the presence of TfR2 mutations. As the substitute for the repeated phlebotomy, p.o. administration with Porcine ${ }^{\circledR}(9$ tabs $/ d)$ has been prepared for these 6 months consequently after Laennec $\hat{A} \circledast$ treatment.

Result: At the end of the treatment the serum ferritin level was decreased to $600 \mathrm{ng} / \mathrm{ml}$. HbAlc also improved with the same dose of insulin $(8.8 ? 6.8 \%)$. The histological evaluation revealed remarkable improvement of liver fibrosis and iron deposition as has been shown by the treatment with LaennecÂA .

Conclusion: Iron plays important roles in cellular metabolism, but, in excess, it catalyzes the formation of free radicals leading to oxidative stress and cell damage. The placenta-derived Laennec $\hat{A}{ }^{\circledR}$ as well as Porcine $\hat{A}{ }^{\circledR}$ which contains hepcidin actually improved iron overload of H.H. patient without repeated phlebotomy. The results suggest that both Laennec $\hat{A} \circledast$ and Porcine $\hat{A} \circledast$ can take the place of venesection for H.H. and other hepcidin-deficient diseases. Further study is necessary to confirm the results that we obtained.

\section{$P P-C-05$}

Outcomes of major laparoscopic liver resection for hepatocellular carcinoma

\section{Jai Young Cho ${ }^{1}$, Hanisah Guro ${ }^{2}$}

${ }^{1}$ Seoul National University Bundang Hospital, Korea, Republic of; ${ }^{2}$ Amai Pakpak Medical Center, Philippines

Background: To compare the surgical outcomes of major laparoscopic liver resection (LLR) and open liver resection (OLR) for hepatocellular carcinoma (HCC).

Method: We retrospectively reviewed the medical records of 177 patients who underwent major liver resection for HCC between January 2004 and June 2015. We divided the 177 patients into two groups according to the type of procedure: major LLR (LLR group; $n=67$ ) and major OLR (OLR group; $n=110$ ).

Result: Procedures in the LLR group were right hepatectomy (30 patients), right posterior sectionectomy (28), left hepatectomy (11), right anterior sectionectomy (6), extended right hepatectomy (6), and central bisectionectomy (2). Tumor size was greater in the OLR group than in the LLR group $(6.3 \pm 3.8$ vs $4.1 \pm 2.4 \mathrm{~cm} ; P=0.016)$. The mean indocyanine green retention rate at $15 \mathrm{~min}(P=0.698)$ and serum $\alpha$-fetoprotein $(P=0.186)$ were similar in both groups. The mean operation time was longer in the LLR group (416.6 \pm 166.9 vs $332.5 \pm 105.4 \mathrm{~min} ; P=0.002)$. Blood loss $(P=0.319)$, transfusion rate $(P=0.260)$, and $\mathrm{R} 0$ rate $(P=0.255)$ were similar in both groups. Hospital stay was shorter ( $11.3 \pm 8.3$ vs. $18 \pm 21.4$ days; $P=0.007)$ and the complication rate was lower $(20.5 \%$ vs. $38.7 \% ; P=0.005)$ in the LLR group. The 5 -year overall survival $(77.3 \%$ vs $60.2 \% ; P=$ $0.087)$ and disease-free survival $(50.8 \%$ vs $40.1 \% ; P=0.139)$ rates were comparable in both groups.

Conclusion: Major LLR of HCC is feasible and oncologically safe when performed by experienced surgeons. Further refinements of the surgical technique are needed to reduce operation time. 

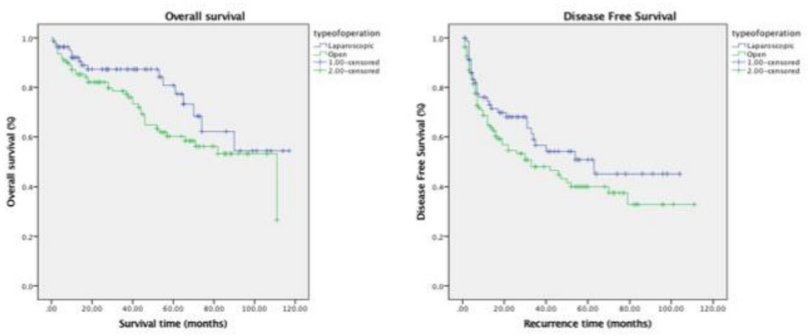

PP-C-O6

The interplay between steatosis and chronic hepatitis B infection on liver fibrosis

Yean Kong Yong ${ }^{1}$ Yi Wen Ting ${ }^{3}$, Sui Weng Wong ${ }^{3}$, Hong Yien Tan $^{4}$, Kok Keng Tee $^{3}$, Rosmawati Mohamed ${ }^{2}$

${ }^{1}$ Xiamen University Malaysia, Malaysia; ${ }^{2}$ University of Malaya Medical Centre, Malaysia; ${ }^{3}$ University of Malaya, Malaysia; ${ }^{4}$ Xiamen University, Malaysia

Background/ Aim: Chronic hepatitis B (CHB) infection and NonAlcoholic Fatty Liver Disease (NAFLD) are the two most prevalent liver diseases worldwide, both capable of driving liver disease progression. To date, the potential interaction between these two liver diseases has not been well defined. The rising prevalence of NAFLD underscore the need for a reliable and accurate non-invasive assessment of liver steatosis, such as the controlled attenuation parameter (CAP). The aim of this study is to investigate the relationship between steatosis, components of the metabolic syndrome and Hepatitis B status with fibrosis stage.

Method: We recruited 522 consecutive $\mathrm{CHB}$ patients with and without antiviral treatments, who had underwent transient elastography in University Malaya Medical Centre (UMMC), Malaysia from 2013 to 2016. 67 patients with significant alcohol consumption, HCV co-infection and other concomitant liver diseases were excluded, the remaining $455 \mathrm{CHB}$ patients were included in this study. Liver steatosis and fibrosis were assessed by CAP and liver stiffness measurement (LSM) respectively. We considered LSM of $7.2 \mathrm{kPa}$ for fibrosis stage $\mathrm{F} \geq 2,9.4 \mathrm{kPa}$ for $\mathrm{F} \geq 3$ and $12.2 \mathrm{kPa}$ for $\mathrm{F} 4$ (cirrhosis) respectively. Mild, moderate and severe steatosis (CAP $=1,2$ and 3, respectively) were defined as $>248 \mathrm{~dB} / \mathrm{m},>268 \mathrm{~dB} / \mathrm{m}$ and $>280 \mathrm{~dB} /$ $\mathrm{m}$ respectively. Data on basic demographics, clinical components of metabolic syndrome, and Hepatitis B status were collected.

Result: $46.6 \%$ of the entire CHB cohort had hepatic steatosis $(\mathrm{n}=$ 212), among which 55 (26\%) had mild steatosis, 31 (15\%) had moderate steatosis and $126(59 \%)$ had severe steatosis. Among treatment naïve patients with steatosis $(\mathrm{n}=181)$, mild steatosis was positively correlated with $\mathrm{HBV}$ VL $(\mathrm{P}=0.0003)$ whilst severe steatosis was inversely correlated with $\mathrm{HBV}$ VL $(\mathrm{P}=0.0014)$. On multivariate analysis, fasting blood glucose $(\mathrm{FBG})$ level $(\mathrm{OR}=29.89$; 95\% CI: 13.54-46.24; $\mathrm{P}<0.0001)$ and serum triglyceride $(\mathrm{TG})(\mathrm{OR}=$ 4.446; 95\% CI: 1.184-7.708; $\mathrm{P}=0.008$ ) were significantly associated with steatosis. Higher FBG level was associated with increased liver fibrosis $(\mathrm{P}<0.001)$. On multivariate analysis, FBG was independently associated with increased liver fibrosis. Every increase of FBG per unit was associated with increase of LSM by $0.52 \mathrm{kPa}(95 \% \mathrm{CI}$ : $0.04-0.995 ; \mathrm{P}=0.034)$. Liver fibrosis and steatosis were positively correlated $(\mathrm{P}<0.0001)$. Age, alanine aminotransferase (ALT), platelet, FBG and CAP score were independently associated with LSM on multivariate analysis, however HBV VL was not associated with liver fibrosis.

Conclusion: Concurrent NAFLD among patients with CHB is frequent. In patients without antiviral treatment, severe hepatic steatosis associated with lower hepatitis B viral load suggests the potential effect of NAFLD on Hepatitis B viral replication. The presence of metabolic syndrome in CHB patients is likely to have an impact on the risk of disease progression to cirrhosis.

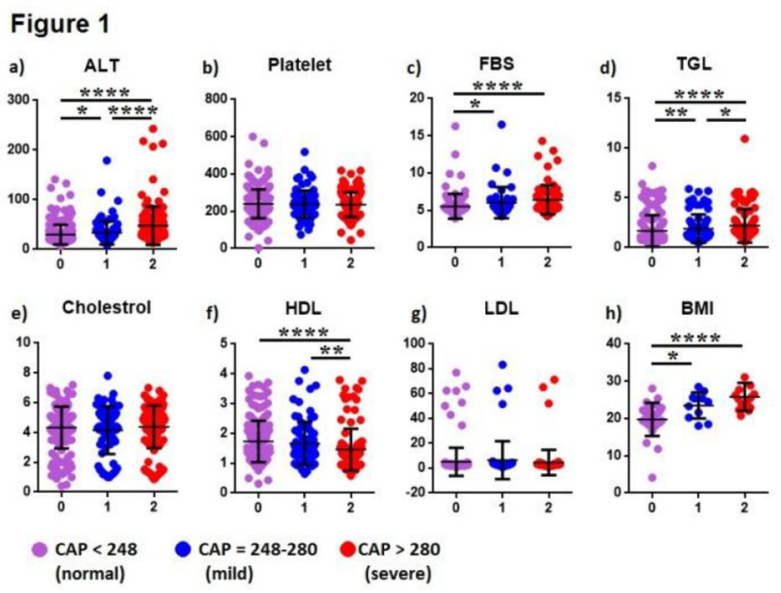

Figure 2
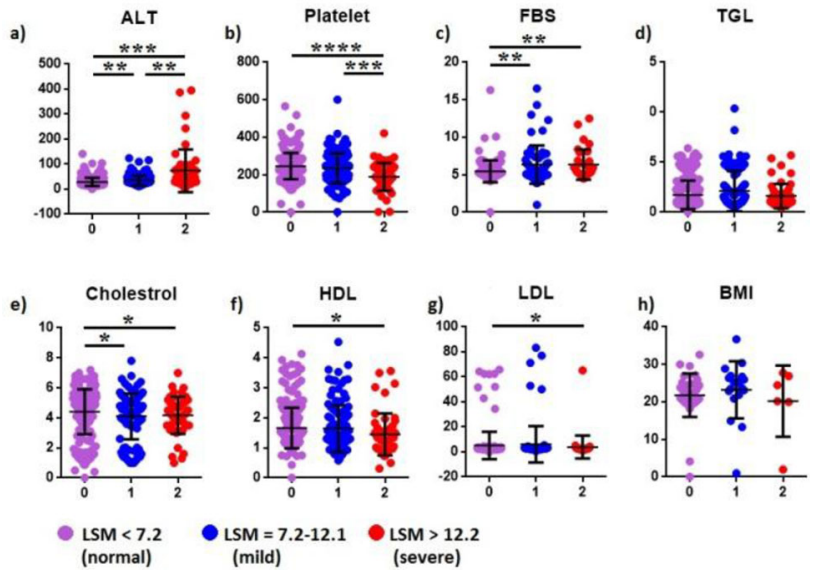

$P P-C-07$

Efficacy Of Fixed Dose Combination Of Sofosbuvir And LEDIPASVIR (SOF/LDV) $\hat{\mathbf{A}} \pm$ Ribavirin In Patients (n=130) infected with HCV genotype 6 (REAL world Myanmar experience)

Soe Thiha Maung ${ }^{1}$, Khin Maung Win ${ }^{2}$, Aung Hlaing Bwa ${ }^{1}$, Khin Aye Wint Han ${ }^{1}$, Si Thu Sein Win ${ }^{1}$, Su Su Htar ${ }^{1}$, Naomi Khaing Than Hlaing, Soe Thu Aung ${ }^{1}$, Moe Pwint Oo ${ }^{1}$, Lei Yin Wine ${ }^{1}$, Wint Wah Ko

${ }^{1}$ Yangon GI and Liver Centre, Myanmar; ${ }^{2}$ Honorary Professor, Myanmar; ${ }^{3}$ Professor and Head of Department, Myanmar

Background: In Myanmar, the prevalence rate of HCV Genotype 6 is estimated to account for $36 \%$ of all HCV infections in Myanmar. Although HCV Genotype 6 is encountered predominately in Southeast Asia, data on optimal treatment strategy is limited.SOF/LDV is the first all-oral DAA approved for HCV Genotype 6. This study is 
aimed to assess the efficacy of SOF/LDV with or without ribavirin, in Myanmar patients infected with HCV genotype 6

Method: A retrospective cohort study of 130 patients infected with chronic HCV genotype 6 who were treated with SOF/LDV+/- ribavirin for 12 or 24 weeks was performed. This single-centre study was conducted in Yangon GI and Liver Centre, Myanmar, from January 2016 to September 2017 as an investigator-initiated study. The rates of SVR12 were stratified by age, sex, presence of cirrhosis, treatment history and HCV viral load at the start of treatment. HCV viral load and HCV Genotype were analysed by various machines namely Roche ${ }^{\circledR}$ COBAS $\AA$ HCV quantitative nucleic acid test 4800 system. Result: In a total of 130 patients, $43 \%$ of patients were male $(n=56)$ and $57 \%$ were female $(n=74)$. The mean age at the time of treatment initiation was 53.1 years (SD 11.69). The majority of patients were treatment-naïve $(87 \%, \mathrm{n}=113)$ and about $52 \%(\mathrm{n}=68)$ of patients had cirrhosis at the time of treatment initiation (FibroScan ${ }^{\circledR}$ Metavir Score F3-F4 is regarded as cirrhosis). The mean baseline viral load prior to start of treatment was 3.3 million $\mathrm{IU} / \mathrm{mL}$ (range 11,000 to 22,322,717). Among all the patients, 7 patients were co-infected with chronic hepatitis B infection and all patients were treatment-naïve and all achieved SVR12. Overall SVR rates among the female and male patients were not significantly different $(77 \%$ vs $76 \%$ ). However, apparently higher SVR rate was noted among the non-cirrhotic patients ( $80 \%$ vs $74 \%$ ) though not statistically significant. The statistically significant higher SVR rate was observed among the treatment-experienced patients compared to treatment-naïve patents (94\% vs $74 \%$ ). When SVR rates were analysed according to the treatment regimens, SVR 12 was achieved in $74 \%$ among the treatment-naïve patients $(\mathrm{n}=113)$. The rates of SVR12 were $71 \%(\mathrm{n}=$ $66 / 93)$ in those receiving SOF/LDV alone and $90 \%(n=18 / 20)$ in those receiving SOF/LDV with ribavirin. For the treatment-experienced patients, the SOF/LDV with ribavirin for 24 weeks regimen was adopted and achieved 94\% SVR rate. There was no significant effect on the SVR 12 by neither BMI nor baseline HCV viral load. The SVR rates were also evaluated by treatment regimens. Among patients treated with SOF/LDV regimen for 12 weeks, $65 \%(\mathrm{n}=47)$ achieved SVR12 and in those with SOF/LDV/RBV for 12 weeks, $82 \%(n=9)$ achieved SVR 12. Among patients who received SOF/LDV for 24 weeks, 91\% $(\mathrm{n}=19)$ achieved SVR12. There were 26 patients who received SOF/LED with ribavirin for 24 weeks and achieved $96 \%$ SVR rate with one relapser. The relapser was 63-year-old Chin ethnic national of Myanmar-Indian border who had treatment-experienced (SOF/LDV 12 weeks) with cirrhosis and baseline HCV viral load 1.7 million IU/mL.

Conclusion: Treatment with SOF/LDV achieved only low SVR rate $(77 \%)$ indicating the unsatisfactory efficacy and genotype 6 is found to be most difficult to treat genotype. But addition of RBV to SOF/ LDV can increase the efficacy slightly.

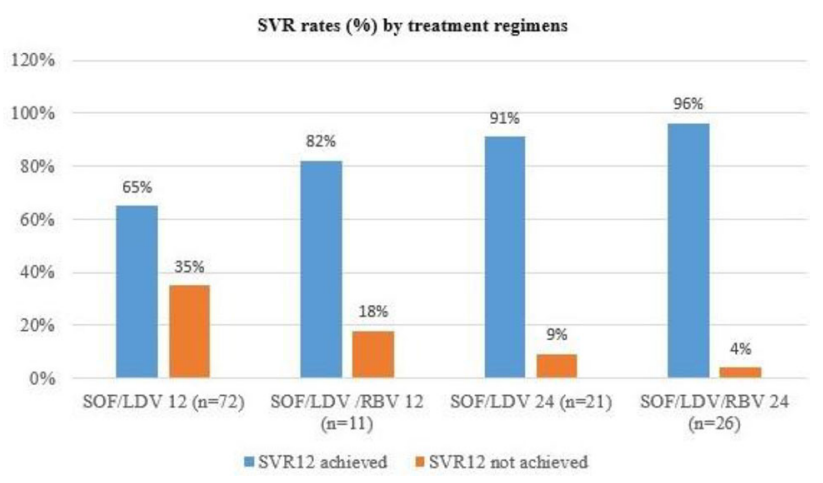

\begin{tabular}{|l|l|c|c|}
\hline \multicolumn{2}{|c|}{} & \multicolumn{2}{|c|}{ SVR12 rates } \\
\cline { 3 - 4 } \multicolumn{2}{|c|}{ Overall } & Percent (\%) & Number (N) \\
\hline \multirow{2}{*}{ Sex } & Male & $77 \%$ & 100 \\
\cline { 2 - 4 } & Female & $76 \%$ & 77 \\
\hline \multirow{2}{*}{ Cirrhosis status } & Cirrhosis & $77 \%$ & 57 \\
\cline { 2 - 4 } & No cirrhosis & $74 \%$ & 50 \\
\hline \multirow{2}{*}{ Treatment History } & Treatment-naive & $80 \%$ & 50 \\
\cline { 2 - 4 } & Treatment-experienced & $74 \%$ & $84 \%$ \\
\hline \multirow{2}{*}{ BMI } & BMI $<25$ & $75 \%$ & 16 \\
\cline { 2 - 4 } & BMI $\geq 25$ & $81 \%$ & 98 \\
\hline
\end{tabular}

\section{PP-C-O8}

parenteral use of prednisolone in severe acute alcoholic hepatitis Olga Ivanovna Tarasova ${ }^{1}$, Pavel Ogurtsov ${ }^{1}$, Nataliya Mazurchik ${ }^{1}$ ${ }^{1}$ RUDN University, Russian Federatio

Background: Patients with severe acute alcoholic hepatitis (AAH) are at a high risk of early death, at a rate of $50 \%$ or greater within 30 days. Patients with severe alcoholic hepatitis may benefit over the short term from specific therapies directed toward reducing liver injury, enhancing hepatic regeneration, and suppressing inflammation. The aim of our study was to find of the efficacy of therapy with prednisolone in patients with severe AAH.

Method: The study included 54 patients with a severe AAH Maddrey index $(\mathrm{MI})>52$. Patients of the control group (36 people) received symptomatic therapy. Patients of the study group (18 patients) additionally received prednisolone in a dose of 60-120 mg iv. Groups of patients were comparable in age, MI, frequency of AAH symptoms (fever, leukocytosis, jaundice and ascites). For the analysis of the efficacy of prednisolone therapy, all patients were divided into 2 subgroups according to the level of creatinine on admission: with normal values ??(up to $110 \mu \mathrm{mol} / \mathrm{l}$ ) and more than $110 \mu \mathrm{mol} / 1$.

Result: The prognosis was evaluated depending on the result of MI (up to 92 or more). Statistical processing of data was carried out with the help of the program "StatGraphics Plus". The differences in the indices in the comparison groups were considered significant at $\mathrm{p}<$ 0.05

Results. At the initially normal level of creatinine in patients receiving prednisolone, hospital mortality was significantly lower: $0 \%$ vs $33 \%(\mathrm{p}<0.05)$. In patients with an initially elevated creatinine level with prednisolone therapy, mortality was significantly higher: $100 \%$ vs $50 \%$ ( $p<0.05)$. With IM $<92$, the mortality of patients receiving prednisolone was less: $17 \%$ vs $30 \%(\mathrm{p}=0.35)$. With $\mathrm{MI}>$ 92 , the lethality in the prednisolone therapy group was higher: $60 \%$ vs $100 \%$ ( $\mathrm{p}=0.11)$.

Conclusion: Parenteral use of $60-120 \mathrm{mg}$ prednisolone improves hospital survival of patients with baseline normal creatinine levels. With an elevated level of creatinine, prednisolone therapy worsens the prognosis and is probably unreasonable.

\section{PP-C-09}

Genotypes distribution of hepatitis $\mathrm{C}$ virus infections in Central Anatolia

Necla Tulek ${ }^{1}$, Sami Kinikli ${ }^{1}$, Kader Dogan ${ }^{1}$, Necla Tulek ${ }^{1}$, Cigdem Ataman Hatipoglu' ${ }^{1}$, Esra Kaya Kilic ${ }^{1}$, Sebnem Erdinc ${ }^{1}$

${ }^{1}$ Ankara Training and Research Hospital, Turkey

Background: Hepatitis C virus (HCV) has different genotypes and sub genotypes throughout the World. Identification of virus genotype are essential to decide the optimal antiviral type and determine the 
duration of treatment. Furthermore, HCV genotyping is also important for epidemiological data. We aimed to investigate the distribution of HCV genotypes among our patients.

Method: This study was carried out retrospectively at Ankara Training and Research Hospital. Our hospital is a tertiary care hospital located at central Anatolia. Patients diagnosed and treated with hepatitis $\mathrm{C}$ infection in the last two years were included into the study. The age, gender, duration of disease and probable transmission way of the patients were recorded. HCV genotype was determined by using RT-PCR.

Result: Forty six males and 42 females, totally 88 patients with mean age of 55, 8 years (18-88 years old) were included into the study. The most common identified genotype was genotype $1(87.5 \%)$ with genotype $1 \mathrm{~b}$ dominance $(75 \%)$, followed by genotype $2(8 \%)$, genotype $3(3,4 \%)$ and genotype $4(1,1 \%)$. The mean age was $56.1,47.7$ and 39 years old for genotype 1, genotype 2 and genotype 3 respectively. It could not be obtained any story related to transmission in 30 of the patients (34\%). We observed a much more genotype 2 infections in the intravenous drug users $(37.5 \%)$ than those of others. Conclusion: Although the most predominant HCV genotype is still genotype $1 \mathrm{~b}$ in our region, we observed genotype 2 and 3 has been increasing especially among the young patients and intravenous drug users.

$P P-C-10$

Endoscopic treatment of patients with bleedings from varicously expanded veins of the esophage in liver cirrhosis.

\section{Javohir Farxodovich Toshnazarov ${ }^{1}$}

${ }^{1}$ Tashkent Medical Academy, Uzbekistan

Background: To improve the results of treatment of patients with bleeding in portal hypertension using endoscopic interventions.

Method: On the basis of the faculty surgery of the Tashkent Medical Academy in the Republican Hospital No. 1 we use various endoscopic methods in the treatment of patients with varicose veins of the esophagus against portal hypertension: endoscopic vein clipping, endoscopic obliteration of veins by varicocid, thrombovar; endogenous veins. Since 2012, 292 patients have received endoscopic treatment.

Result: In 13 patients, vein clipping was performed, incl. in 9 - in combination with sclerotherapy. In this case, after the administration of the sclerosant solution, an endoscopic clipper applied clips to the vein above and below the bleeding zone, which prevents unwanted spread of the sclerosing agent through the vessels, creating the necessary depot at the injection site. After 3-4 days after stopping bleeding, additional sclerotherapy of the remaining veins was performed. Endoscopic sclerotherapy with bleeding from esophageal varices in the background of portal hypertension was used in 28 patients ( 23 men and 5 women) whose mean age was $54.2 \pm 2.4$ years. Endoscopy was performed with endoscopes from Olympus. As a sclerosing agent, $35 \%$ ethanol solution was used, which was directly injected paravasally in an amount of 4 to $10 \mathrm{ml}$. After sclerotherapy, six patients developed chest pains; in 5 patients - ulcers and erosive changes in the esophagus at the injection sites. In 2 patients there was a relapse of bleeding, which required the use of the SengstakenBlakemore probe. The method of applying endoligators is applied in 251 patients with varicose veins of the esophagus to stop bleeding, or for its prevention in terms of preparing patients for port-caval shunting. Of these, in 56 cases, this method was applied in the districts and cities of Tashkent when traveling along the line of sanaviation. Simultaneously, 4-8 endoligatures were applied to the veins. The use of endoliguration in 206 patients at the height of the esophageal bleeding became the final method of stopping it.

Conclusion: Thus, we believe that endolysis of veins is a highly effective method of preventing complications in varicose veins of the esophagus, as well as bleeding from the veins, especially in patients with portal hypertension with a high risk of surgical intervention.

\section{Presidential Poster Basic}

\section{March 2018}

\section{$P P-B-01$}

Decreased ratio of Tregs to Th17 cells exacerbates disease progression of autoimmune hepatitis

\section{Jinshang $\mathrm{Hu}^{1}$, Weiming Yan ${ }^{1}$, Wei Yuan ${ }^{1}$, Peng Wang ${ }^{1}$, Da Huang $^{1}$, Qin Ning ${ }^{1}$ \\ ${ }^{1}$ Tongji Hospital, Tongji Medical College, Huazhong University of Science and Technology, China}

Background: CD4 + CD25 + Foxp3 + regulatory $\mathrm{T}$ cells (Tregs) functions to restrain excessive effector $\mathrm{T}$ cell responses, IL-17-producing Th cells (Th17) is a key player in the pathogenesis of autoimmune disorder. In this study, we aimed to explore the role of Treg/Th17 balance in a murine model of autoimmune hepatitis (AIH) induced by human cytochrome P4502D6 (CYP2D6) and patients with AIH.

Method: The AIH murine model was established by delivering adenovirus vector containing CYP2D6 (Ad-2D6) into the liver of C57BL/6 mice intravenously and intraperitoneally. Treg/Th17 balance in the liver of Ad-2D6 mice including their frequencies and production of functional cytokines were evaluated by multi-colour flow cytometric assay and Enzyme-linked immunosorbent assay (ELISA) respectively. An immunotherapy strategy was performed on Ad-2D6 mice by adoptively transferring with $\mathrm{CD} 4+\mathrm{CD} 25+\mathrm{T}$ cells isolated from healthy mice using MACS microbeads at week 3 and week 4 post infection. The peripheral Treg/Th17 balance was also detected in $18 \mathrm{AIH}$ patients.

Result: Compared with control group, Ad-2D6 mice exhibited progressive liver damage including inflammatory cell infiltration and piecemeal necrosis in the portal area and central vein from 2-4 weeks post infection. A significantly higher serum titers of $\operatorname{IgG}$ (week 2 and week $4, \mathrm{P}<0.05$ ) and detectable autoantibody against CYP2D6 were observed. Interestingly, Ad-2D6 mice showed a significant and sustained decrease in proportion of hepatic Tregs and ratio of Treg/Th17 (week 4 and week $6, \mathrm{P}<0.05$ ), and an obvious increase in hepatic IL17 and IL-22 expression (week 4, $\mathrm{P}<0.05$ ). Adoptive transfer with $\mathrm{CD} 4+\mathrm{CD} 25+\mathrm{T}$ cells resulted in improved hepatic pathological feature, decreased $\operatorname{IgG}$ and autoantibody titers, which might be attributed to the significantly increased Treg/Th17 ratio $(\mathrm{P}<0.05)$ and decreased levels of hepatic IL-17 $(\mathrm{P}<0.05)$ and IL-22. AIH patients with Child-Pugh class $\mathrm{B}$ or $\mathrm{C}$ showed significant lower treg proportions $(\mathrm{P}<0.05)$ and relative lower ratio of Treg/Th17 than those of patients with Child-Pugh class A.

Conclusion: The decreased ratio of Treg/Th17 was involved in the immunopathogenesis of AIH. Adoptive immunotherapy with CD4+CD25+T cells could be in favor of correcting Treg/Th17 imbalance and preventing poor prognosis of AIH. 
$P P-B-02$

Glutamate-cysteine ligase catalytic subunit attenuate hepatitis C virus-related liver fibrosis through suppressing endoplasmic reticulum stress

\section{Nan Yuemin ${ }^{1}$, Lu Yu ${ }^{1}$, Zhao Wen ${ }^{1}$, Zhang Siyu ${ }^{1}$, Liu Lingdi ${ }^{1}$, Kong Lingbo ${ }^{1}$, Du Jinghua ${ }^{1}$ \\ ${ }^{1}$ Third Hospital of Hebei Medical University, China}

Background: Glutamate-cysteine ligase catalytic subunit (GCLC) could regulate the production of glutathione (GSH) and play the antioxidative role in liver, but the role and molecular mechanisms of GCLC in Hepatitis C virus (HCV)-related liver fibrosis remains largely unknown.

Method: Differentiate expressed mRNAs in plasma of the patients with HCV-related liver fibrosis were detected by digital gene expression sequencing method, and further validated by qRT-PCR. The stages of liver fibrosis was determined by H\&E and Masson staining in liver sections, and protein expression of $\alpha$-SMA, Col1A1 and GCLC was detected by IHC. Total RNA and proteins were extracted from liver samples of patients with HCV-related liver fibrosis and actvated HSCs for detecting GCLC, ER stress marker proteins, inflammatory factors and profibrogenesis genes by qRTPCR and Western blot.

Result: GCLC was dramatically decreased in liver tissue of HCVrelated hepatic fibrosis and activated HSCs, meanwhile, ER stress marker proteins including glucose-regulated protein 78 (GRP78), CCAAT/enhancer- binding protein homologous protein (CHOP), and inflammatory factors such as nuclear factor kappa B (NF- $\kappa B)$ and tumor necrosis factor alpha (TNF- $\alpha$ ), and profibrogenesis factors including transforming growth factor beta 1 (TGF $\beta 1)$, tissue inhibitor of metalloproteinase (TIMP) 1 were dramatically increased. Overexpression of GCLC in HSCs could suppress the expression of $\alpha$ SMA and Col1A1 mRNA and protein in LX-2 cells, and increase the GSH level as well as decrease ROS content, concomitant with decreasing expression of GRP78, CHOP, NF- $\kappa \mathrm{B}, \mathrm{TNF}-\alpha, \mathrm{TGF} \beta 1$ and TIMP1.

Conclusion: GCLC was a negative regulatory factor in the progression of HCV-related liver fibrosis, and might function as a novel antifibrosis factor and could be a potential therapeutic target for HCVrelated liver fibrosis.

\section{$P P-B-03$}

Circulating exosomal non-coding RNAs as prognostic biomarkers in human hepatocellular carcinoma

Yu Rim Lee ${ }^{1}$, Soo Young Park ${ }^{1}$, Keun Hur ${ }^{3}$, Gyeonghwa Kim ${ }^{3}$, Se Young Jang ${ }^{1}$, Won Young Tak ${ }^{1}$, Young Oh Kweon ${ }^{1}$, Bina Jeong ${ }^{1}$, Gyoun Eun Kang', Sangkyung Seo', Hye Won Lee ${ }^{2}$, Jung Gil Park $^{4}$

${ }^{1}$ Kyungpook National University Hospital, Korea, Republic of; ${ }^{2}$ Dongsan Medical Center, School of Medicine, Keimyung University, Korea, Republic of; ${ }^{3}$ School of Medicine, Kyungpook National University, Korea, Republic of; ${ }^{4}$ Yeungnam University Medical Center, Korea, Republic of

Background: Exosomal non-coding RNAs (ncRNAs) have unique expression profiles reflecting the characteristics of a tumor, and their role in tumor progression and metastasis is emerging. However, the significance of circulating exosomal ncRNAs in the prognosis of hepatocellular carcinoma (HCC) remains to be elucidated. We therefore determined the prognostic significance of circulating exosomal ncRNAs (miRNA-21 and lncRNA-ATB) for human HCC. Method: This prospective study enrolled 79 HCC patients who attended Kyungpook National University Hospital, Republic of Korea between October 2014 and September 2015. Exosomes were extracted from serum samples using the ExoQuick Exosome Precipitation Solution (System Biosciences). NcRNAs were isolated from exosomes using the miRNeasy serum/plasma micro kit (Qiagen).

Result: During the study period (median 14.0 months), disease progression and mortality were found in 44 and 34 patients, respectively. Exosomal miRNA-21 and lncRNA-ATB were successfully detected in the HCC serum samples. Both circulating exosomal miRNA-21 and IncRNA-ATB were related to TNM stage and other prognostic factors, including the $\mathrm{T}$ stage and portal vein thrombosis, but they were not associated with age, sex, presence of cirrhosis, or etiology. Multivariate analysis using the Cox regression test identified that both higher miRNA-21 and higher lncRNA-ATB were independent predictors of mortality and disease progression, along with larger tumor size and higher $\mathrm{C}$-reactive protein (all $P<0.05)$. The overall survival was significantly lower in patients with higher circulating levels of exosomal miRNA-21 and lncRNA-ATB (log-rank test: both $P<0.05$ ). Conclusion: This study has provided strong evidence that circulating exosomal ncRNAs (miRNA-21 and lncRNA-ATB) are novel prognostic markers and therapeutic targets for HCC.

\section{Figure 1}

A

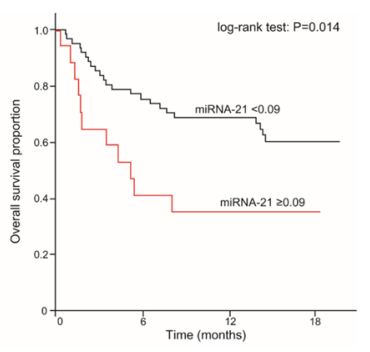

B

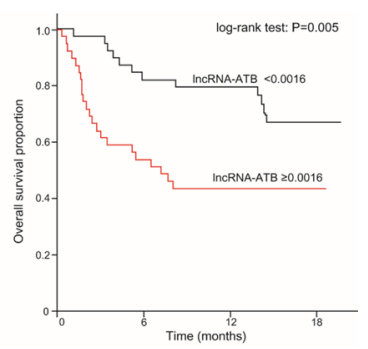

PP-B-04

Hepatitis B virus $\mathbf{X}$ protein maintains hepatic stellate cell activation by regulating peroxisome proliferator-activated receptor gama

\section{Huayu $\mathrm{Xu}^{1}$, Shuang $\mathrm{Li}^{2}$, Donghui Zhou ${ }^{3}$}

${ }^{1}$ The First Affiliated Hospital of Soochow University, China; ${ }^{2}$ The First Affiliated Hospital of Nanjing Medical University, China; ${ }^{3}$ The First Affiliated Hospital of Nanjing Medical University, China

Background: Chronic hepatitis B virus (HBV) infection is a major cause of hepatic fibrosis, and the activation of hepatic stellate cells (HSCs) is the main mechanism of fibrosis. However, the mechanism of hepatic fibrosis induced by HBV is not well elucidated. Hepatitis B virus $\mathrm{X}$ protein $(\mathrm{HBx})$, one of the $\mathrm{HBV}$-related proteins, induces fibrosis in a paracrine way. Peroxisome proliferator-activated receptor $\gamma(\operatorname{PPAR} \gamma)$ inhibits the activation of HSCs and even switches the cell phenotype from activated to quiescent. The aim of this study was to determine the interaction of $\mathrm{HBx}$ and $\operatorname{PPAR} \gamma$ in stellate cell activation.

Method: A stable cell line, LX-2-X, which expressed HBx, was established by infecting LX-2 cells with lentivirus. The Cell Counting Kit-8 (CCK-8) assay was used to detect cell proliferation. The 
expression of PPAR $\gamma$, transforming growth factor- $\beta 1$ (TGF $\beta 1$ ), $\alpha$ smooth muscle actin ( $\alpha$-SMA) and collagen I was measured by quantitative real-time PCR (qRT-PCR), Western blot or ELISA. For the interaction of $\mathrm{HBx}$ and $\operatorname{PPAR} \gamma$, co-immunoprecipitation and luciferase reporter assays were performed.

Result: LX-2-X cells showed increased proliferation compared to control cells, and the PPAR $\gamma$ ligand troglitazone $(0,5,10 \mu \mathrm{mol} / \mathrm{L})$ inhibited LX-2-X cell proliferation in a dose-dependent manner. The expression of TGF- $\beta 1, \alpha$-SMA, and collagen I increased, while PPAR $\gamma$ decreased in LX-2-X cells. HBx bound to PPAR $\gamma$ and suppressed the transcriptional activity of PPAR $\gamma$.

Conclusion: $\mathrm{HBx}$ can maintain stellate cell activation by interacting with PPAR $\gamma$ in vitro.

A

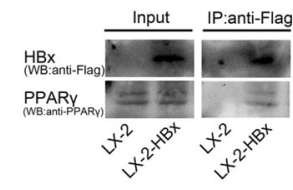

c
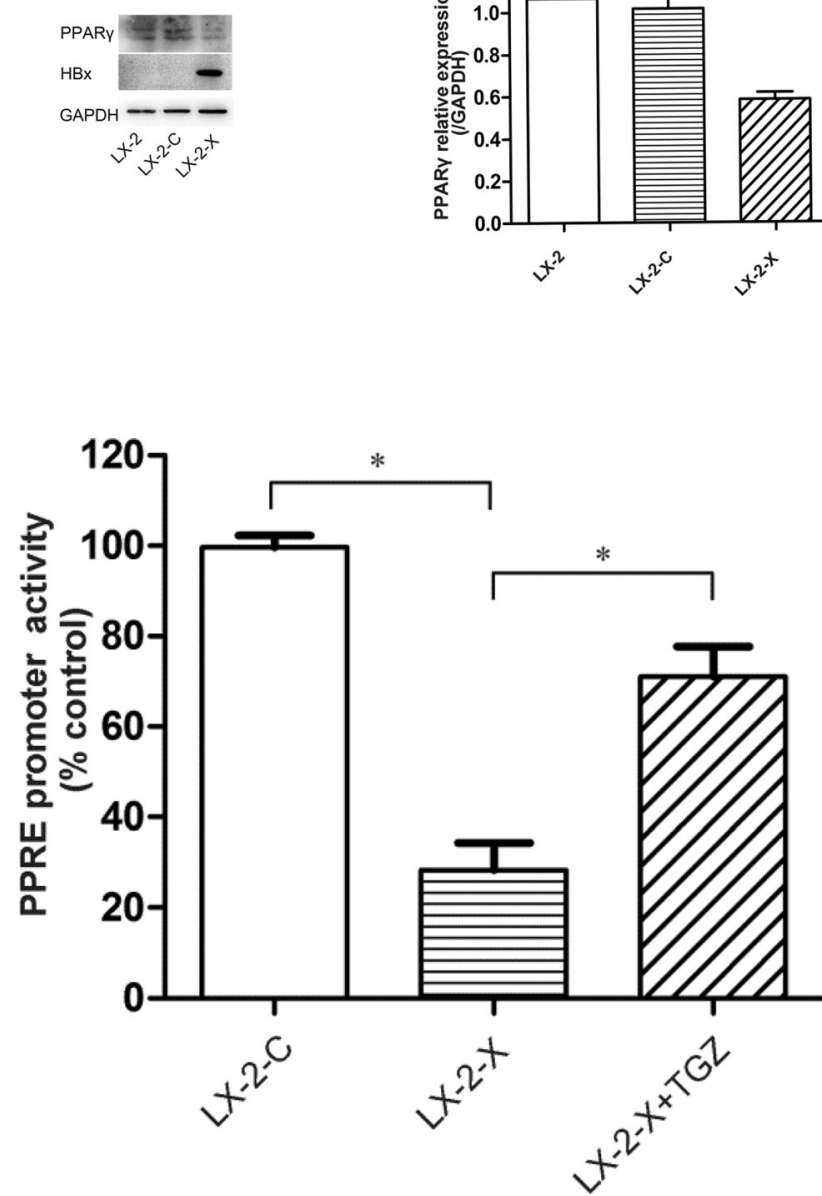

$P P-B-05$

Analysis of resistance-associated substitutions of daclatasvir plus asunaprevir treatment: viral evolution and fitness change after treatment failure

Yechan Jeong ${ }^{1}$, Yechan Jeong ${ }^{1}$, Bora Jin ${ }^{1}$, Hye Won Lee ${ }^{1}$, Hye Jung Park', Do Young Kim ${ }^{1}$, Kwang Hyub Han ${ }^{1}$, Seungtaek Kim $^{1}$, Sang Hoon Ahn ${ }^{1}$

${ }^{1}$ Yonsei University College of Medicine, Korea, Republic of

Background: Daclatasvir plus asunaprevir (DCV+ASV) therapy has a high sustained virologic response (SVR) rate and is the first directacting antiviral (DAA) therapy in Korea for the genotype 1b HCVinfected patients. But, this therapy sometimes failed in patients without baseline NS5A polymorphisms. In this study, we aimed to analyze the change of resistance-associated substitutions (RASs) of NS3 and NS5A after treatment failures and the effect of those RASs on viral fitness.

Method: We continually followed up RASs identified in patients who failed in DCV+ASV therapy. Their sera were collected and viral RNA was isolated. The viral RNA sequences were determined by direct sequencing after RT-PCR . To assess the effect of RASs on viral fitness, they were introduced in the genotype 1 HCV RNA either as single or multiple substitutions and investigated by reporter assay and focus-forming assay after transfection into the Huh7.5 cells. To create quasi-species in vitro, RNAs with or without RAS were cotransfected into cells in various ratio and measured by reporter assay. Result: Typical RASs of NS3 at D168 and those of NS5A at L31 and Y93 were commonly observed after DCV+ASV treatment failures. Actually, those RASs were observed in our samples of patient who failed in DCV+ASV treatment. Interestingly, the RAS at D168 of NS3 reverted to the wild-type amino acid within one year after cessation of DCV+ASV treatment due to treatment failures. However, the RASs of NS5A stably remained over one year after cessation of DCV+ASV treatment. The effect of NS3 RAS (D168V) and NS5A RASs $(\mathrm{L} 31 \mathrm{~V}, \mathrm{Y} 93 \mathrm{H})$ on viral RNA replication was analyzed by mutagenesis into the genotype $1 \mathrm{~b}$ HCV RNA and RNA replication capacity was measured by Gaussia luciferase (GLuc) reporter assay. Among the single RASs, the effect of D168V (NS3 RAS) was more significant than the others for RNA replication and the effect of the triple substitutions combination (D168V + L31V + Y93H) was the most severe. The RAS at Y93 (NS5A RAS) affected both viral RNA replication and virus production when it was tested with infectious genotype 1a HCV RNA. Also, the RNA replication capacity of Y93N substitution increased and wild type decreased when it was cotransfected with wild type and Y93N RNAs into Huh7.5 cells.

Finally, the increase in the RNA replication capacity of Y93N substitutions can be assessed as the effect of trans-complementation of NS5A protein. This our study result suggests that such trans-complementation effect of NS5A protein may help maintain the RASs of NS5A for a long time even after cessation of the DAA treatment.

Conclusion: The RASs of NS3 and NS5A after DCV+ASV treatment failures were analyzed with regard to viral evolution and fitness change. This study results would help understand the fitness change and persistence of RASs after DAA treatment failures.

\section{$P P-B-06$}

Organic anion metabolism disorder when infectious liver failure after hepatectomy

Yusuke Wakasa $^{1}$, Yusuke Wakasa ${ }^{1}$, Norihisa Kimura ${ }^{1}$, Keinosuke Ishido $^{1}$, Daisuke Kudo ${ }^{1}$, Kenichi Hakamada ${ }^{1}$ 
${ }^{1}$ Hirosaki University Graduate School of Medicine, Japan

Background: It has become clear that the impairment of hepatocyte membrane transporters, which are responsible for cell membrane transport of organic anions, such as bilirubin and bile acids, can lead to intrahepatic cholestasis after hepatectomy. This pathophysiologic study aimed to clarify the mechanism of organic anion metabolism abnormality and liver regeneration failure during infectious hepatic failure after hepatectomy from the viewpoint of molecular biology "abnormality of organic anion transporters."

Method: Male Sprague-Dawley rats weighing 180-220 g and aged 6 weeks underwent $70 \%$ hepatectomy with lipopolysaccharide (LPS) administration in the inferior vena cava under general anesthesia. At 3 time-points (24, 72, and 168 hours after hepatectomy) the rats were sacrificed to obtain liver samples by exsanguination. We divided the rats into 4 groups: LPS $+70 \%$ hepatectomy group, sham operation group, LPS alone administration group, and $70 \%$ hepatectomy group. The hepatobiliary enzymes and bilirubin levels were measured from the obtained blood. The RNAs were extracted from the liver samples, and the gene expression profile was analyzed by microarray.

Result: Transaminase, total bilirubin, and bile acid levels were elevated after 24 hours in the LPS $+70 \%$ hepatectomy group compared with the $70 \%$ hepatectomy group. In the microarray analysis, the levels of organic anion transporting polypeptides (Oatps), which are sinusoidal side transporters that extract the bilirubin in the blood, and sodium taurocholate cotransporting polypeptide (Ntcp) that uptakes the bile acids from the blood, and ATP binding cassette protein $\mathrm{C} 2$ (Mrp2) that excrete the bile acids into bile duct, tended to decrease compared with the $70 \%$ hepatic resection group. In the RT-PCR, the similar decrease trend was confirmed.

Conclusion: This study suggested that during liver regeneration in the case of infection after hepatectomy versus normal hepatectomy, the organic anion transporter impairments on the hepatocyte membrane might be enhanced and hyperbilirubinemia would be prolonged.

$P P-B-07$

The Influence Of MTHFR Gene Polymorphism On The Long Term Course In G1 Hepatitis C Patients Who Are Achieved Sustained Viral Response With Treated With Pegylated Interferon Plus Ribavirin.

$\underline{\text { Engin Altintas }}^{1}$, Zuhal Mert Altintas ${ }^{1}$, Ibrahim Yilmaz ${ }^{1}$, Halil Celik $^{1}$, Enver Ucbilek ${ }^{1}$, Serkan Yaras ${ }^{1}$, Osman Ozdogan', Fehmi Ates $^{1}$, Orhan Sezgin ${ }^{1}$

${ }^{1}$ Mersin University Faculty of Medicine, Turkey

Background: A genetic history, such as the MTHFR polymorphism responsible for hyperhomocysteinemia, plays a role in the development of high-grade steatosis and accelerates the progression of liver fibrosis in chronic hepatitis c. The aim of this study was to investigate the long-term effects of MTHFR gene polymorphism in genotype 1 (G1) hepatitis C patients who achieved sustained viral response (SVR) with pegylated interferon plus ribavirin (PIR) therapy.

Method: 58 G1 hepatitis C patients who had SVR with PIR therapy and who had been followed since 2008 were included in the study. MTHFR gene polymorphisms (C677T and A1298C) were tested by PCR-RFLP. Medical records of patients were screened between 2008 and 2017 and cases of cirrhosis and hepatocellular carcinoma (HCC) were recorded. Fibrosis status was assessed by the non-invasive methods (APRI, FIB-4, FORNS index). The difference between the values between 2008 and 2017 was recorded as $\Delta$ APRI, $\Delta$ FIB-4, $\Delta$ FORNS index.

Result: One patient had HCC and 7 patients had cirrhosis. Because there were few patients, cirrhosis and HCC were taken as event group.
For MTHFR C677T, 34 patients had wild type and 24 had mutant alleles. For MTHFR A1298C, 32 patients were wild type and 25 patients had mutant alleles. There was no statistically significant relationship between MTHFR C677T and A1298C and events occurring in the course ( $\mathrm{p}=0.859, \mathrm{p}=0.273$, respectively). There was no interaction for both C677T and A1298C in the change of APRI, FIB-4, FORNS index over years ( $p>0.05$ ). Those of the MTHFR C677T wild type; the mean value of the APRI parameter was decreased from $0.73 \pm 0.49$ to $0.29 \pm 0.09$ and there was a significant difference ( $\mathrm{p}<0.001$ ). Those with MTHFR C677T mutant alleles; the mean value of the APRI parameter was increased from $0.87 \pm 0.68$ to $1.75 \pm 4.80$ and this change was not significant $(\mathrm{p}=0.551)$. The $\Delta$ APRI difference between the two groups was significant $(\mathrm{p}=0.022)$. There was no significant difference in $\Delta$ values for MTHFR A1298C and for other criteria.

Conclusion: Improvement in fibrosis appears to be adversely affected in patients with the MTHFR C677T allele just for APRI score but not for others. It should be evaulated or supported by more works.

\section{PP-B-08}

\section{Decellularization and recellularization of liver scaffold with human hepatic progenitor cells for the development of neo- organ}

Mohd Aejaz Habeeb ${ }^{1}$ Avinash Bardia ${ }^{1}$, Nagarapu Raju ${ }^{1}$, Safwaan Habeeb $^{1}$, Aleem Ahmed Khan ${ }^{1}$, Mohd Aejaz Habeeb ${ }^{1}$

${ }^{1}$ Centre for Liver Research \& Diagnostics, India

Background: Acute as well as chronic liver failures are major fatal problems which lead up to $60-80 \%$ mortality every year. In acute condition, systemic inflammation and accumulation of toxic compounds (ammonia) in liver results in multi-organ failure which leads to hepatic encephalopathy. Pharmacological drugs are not enough capable of removing toxins from liver; whereas in chronic condition, liver transplantation is the only option. However, liver transplantation is limited due to timely unavailability of enough donors, post-transplantation complications and high cost involvement. The present study demonstrates a promising strategy of using natural platform of bioartificial extracorporeal liver support system prepared through decellularization and repopulation of xenogenic liver with human hepatic progenitor cells (hHPCs)

Method: Xenogenic liver was decellularized by perfusion method using retrograde change of detergents and other chemicals. Removal of nuclear components, retention of ECM and vascular integrity of whole decellularized liver was identified. DiD-labeled hHPCs were infused to identify the cells distribution and engraftment efficiency. Repopulation of hHPCs within the decellularized liver scaffold was determined by SEM analysis whereas the functional activity of repopulated cells was determined by ammonia detoxification experiment by urea quantification

Result: The decellularized liver vascular network was able to withstand fluid flow that entered through a central inlet vessel, branched into an extensive capillary bed, and coalesced into a single outlet vessel. Repopulated hHPCs showed homogeneous distribution within the decellularized liver scaffold showing defined vascular tree with multiple branching and residual niches of proliferating cells. Ammonia detoxification showed the functional activity and future applicability of repopulated humanized liver scaffold as extra-corporeal natural organ support system.

Conclusion: This study demonstrate an innovative technology for bioengineering humanized extra-corporeal liver system as better approach for ammonia detoxification as temporary support to the failing liver. 
$P P-B-10$

Correlation of IP-10 gene expression with Serum Alanin transaminase (ALT) levels and Hepatitis B viral load in cirrhosis and hepatocellular carcinoma (HCC) patients.

Umme Shahera $^{1}$, Munira Jahan ${ }^{1}$, Afzalun Nessa ${ }^{1}$, Shahinul Alam $^{1}$, Saifullah Munshi ${ }^{1}$, Shahina Tabassum ${ }^{1}$

${ }^{1}$ BSMMU, Bangladesh

Background: Interferon-gamma induced protein 10 (IP-10), a chemokine is suggested to involve in liver injury during Hepatitis B virus infection (HBV). The increase of IP-10 is a critical step for recruitment of inflammatory cells and hepatopathology. This study was designed to assess the correlation of IP-10 gene expression in liver with HBV-DNA and Serum ALT in patients with cirrhosis and HCC. Method: The study was conducted among 60 patients with chronic HBV infection. The study participants were divided into four groups (15 in each groups), namely HBV positive cirrhosis, HBV negative cirrhosis, HBV positive HCC and HBV negative HCC. Expression of IP-10 gene was observed using real time PCR technique. IP-10 gene expressions in the above mentioned groups were correlated with Serum ALT level and HBV viral load.

Result: IP-10 gene expression in liver was not either correlated with the serum levels of ALT in HBV positive HCC patients nor with HBV negative $\mathrm{HCC}, \mathrm{HBV}$ positive cirrhosis and HBV negative cirrhosis patients. HBV- DNA load also did not correlated with IP-10 gene expression in HBV positive $\mathrm{HCC}$ and $\mathrm{HBV}$ positive cirrhosis patients. Although IP-10 gene was significantly higher in HBV-positive patients with liver cancer than HBV-positive cirrhosis. Similarly, the expression of IP-10 was significantly higher in HBV-positive HCC than HBV-negative HCC patients. However, the expression of IP-10 gene was reduced in HBV-positive cirrhosis in comparison with HBV-negative cirrhosis.

Conclusion: This study shows that there was no significant change with the expression of IP-10 gene and ALT level or viral load, though differential expression of IP-10 gene were observed in cirrhosis and HCC patients.

\section{Poster Presentation}

\section{March 2018}

$H B V-B 1$

Myeloid-derived suppressor cells (MDSC) mediate regulatory $T$ cell induction by TGF- $\tilde{A} \ddot{Y}$ and IL-10 driven pathways in chronically $\mathrm{HBV}$ infected patients with high levels of hepatitis $B$ surface antigen

Simanti Datta ${ }^{1}$, Sourina Pal ${ }^{1}$, Madhuparna Nandi ${ }^{1}$, Debangana $\overline{D e y}^{1}$, Soma Banerjee ${ }^{1}$, Amal Santra ${ }^{1}$, SK Mahiuddin Ahammed ${ }^{1}$, Abhijit Chowdhury

${ }^{1}$ Institute of Post Graduate Medical Education \& Research, Kolkata, India

Background: Patients with chronic HBV infection (CHI) are characterized by increased prevalence of regulatory T-cells (Treg) that inhibit virus-specific T-cell immunity, although mechanisms leading to Treg generation remain ambiguous. MDSC have recently emerged as key mediators of immunosuppression in cancer and other diseases. Here we characterized MDSC in patients representing different phases of $\mathrm{CHI}$ and investigated their role in Treg induction.
Method: Prevalence/subsets/phenotype of MDSC and Treg were studied by flow cytometry in Immune-tolerant (IT), chronic hepatitis $\mathrm{B}(\mathrm{CHB})$ [HBeAg positive/negative (EP/EN)] patients, Inactive carrier (IC) and healthy controls (HC). HBsAg levels in sera of patients were quantified using Abbott Architect i1000sr platform and impact of HBsAg on MDSC generation was tested in vitro. HLA-DRCD33+-MDSC were sorted from study subjects and co-cultured with autologous MDSC-depleted PBMC in presence/absence of transwellinserts or inhibitors and frequencies of Treg-subsets were determined. Result: Significantly greater frequency of HLA-DRCD11b+CD33hi-Monocytic (M)-MDSC was observed in IT and EP/ $\mathrm{EN}-\mathrm{CHB}$ as compared to IC and HC. Further, treatment of PBMC from $\mathrm{HC}$ with exogenous $\mathrm{HBsAg}$ resulted in enhancement of M-MDSC frequency in dose-dependent manner, signifying a pivotal role of HBsAg in the development of M-MDSC. The HBsAg titer was high in serum of IT and CHB patients but markedly low in IC and it showed a significant positive correlation with M-MDSC frequency $(\mathrm{r}=0.606 ; \mathrm{p}=0.006)$. Both IT and EP/EN-CHB displayed elevated percentage of $\mathrm{CD} 4+\mathrm{CD} 25+\mathrm{FOXP} 3+$ Treg and its frequency positively correlated with that of M-MDSC ( $\mathrm{r}=0.609 ; p=0.004)$, implying potential involvement of MDSC in Treg development. The cytokines, TGF- $\beta$ and IL-10, known to favor Treg differentiation were expressed at significantly higher levels by both MDSC-subsets, M-MDSC and granulocytic (G)-MDSC from IT and CHB relative to IC/HC, although M-MDSC exhibited a superior cytokine producing ability than G-MDSC. Co-culture of sorted HLA-DR-CD33+-MDSC with autologous MDSC depleted-PBMC from IT and CHB but not from IC or $\mathrm{HC}$, caused significant increase in $\mathrm{CD} 4+\mathrm{CD} 25+\mathrm{FOXP} 3+-\mathrm{iTreg}$ and CD4+FOXP3-IL-10+-Tr1-cells, whereas CD4+FOXP3-TGF$\beta+$-Th3-cells remained unchanged. This process was dependent on soluble factors/cytokines rather than cell-cell contact as evident from transwell experiments. Moreover, MDSC-mediated iTreg generation was reversed by addition of TGF- $\beta$ RI kinase inhibitor and neutralizing anti-IL-10 while anti-IL-10 treatment alone diminished $\operatorname{Tr} 1$ numbers.

Conclusion: Our results reveal a hitherto unknown role of MDSC in Treg development in IT/CHB via TGF- $\beta /$ IL-10-dependent pathways, suggesting that therapeutic targeting of MDSC or reducing circulating HBsAg level could boost anti-HBV immunity.

\section{$H B V-B 2$}

Pro-fibrogenic mediator Osteopontin is intimately involved in Chronic Hepatitis B and promotes cccDNA production

Sandra Phillips $^{1}$, Jason Coombes ${ }^{1}$, Sameer Mistry ${ }^{1}$, Marietta Simonova $^{2}$, Tanya Hadzhiolova LEBEAU ${ }^{5}$, Slava Pavlova ${ }^{5}$, Krum Katzarov $^{5}$, Patrick TF Kennedy ${ }^{3}$, Upkar S Gill ${ }^{3}$, T A Pereira ${ }^{4}$, F. E Pereira $^{6}$, Roger Williams ${ }^{1}$, Wing Syn ${ }^{1}$, Shilpa Chokshi ${ }^{1}$

${ }^{1}$ Institute of Hepatology, United Kingdom; ${ }^{2} \mathrm{Head}$ of Section, Clinic of Gastroenterology, Bulgaria; ${ }^{3}$ Hepatology, Centre for Immunobiology, Blizard, Institute, Barts and The London School of Medicine and Dentistry, United Kingdom; ${ }^{4}$ Laboratório de Patologia Experimental, Brazil; ${ }^{5}$ Military Medical Academy, Bulgaria; ${ }^{6}$ Núcleo de Doenças Infecciosas, Brazil

Background: We have previously shown that host proteins actively participate in HBV replication (Phillips et al, Gastroenterology 2015). This study aimed to dissect the role of host-protein and pro-fibrogenic mediator Osteopontin (OPN) in chronic HBV infection (CHB) during the clinical phases of $\mathrm{CHB}, \mathrm{HBV}$-driven liver injury and to also evaluate its role in driving covalently closed circular DNA(cccDNA) formation. 
Method: OPN levels were measured by ELISA in sera/plasma (S/P) of healthy controls (HC) and $102 \mathrm{CHB}$ patients during disease phases; $\mathrm{HBeAg}(+) \mathrm{CHB}(\mathrm{CI}$; Immunotolerant), $\mathrm{HBeAg}(+)$ chronic hepatitis $(\mathrm{CH}$; Immune active), $\mathrm{HBeAg}(-) \mathrm{CI}$ (Inactive carrier) and $\mathrm{HBeAg}(-$ )CH. OPN was also quantitated in $\mathrm{CHB}$ patients with different fibrosis stages $(n=81)$ intrahepatically, in $\mathrm{S} / \mathrm{P}$ and in paired $\mathrm{CHB}$ patients undergoing NUC treatment $(n=9)$. Finally, HepaRG cell-line infected with $\mathrm{HBV}$ was cultured over $72 \mathrm{~h}$ in presence/absence of recombinant OPN(recOPN) and neutralizing OPN-specific aptamers. Cells and supernatants were harvested at 24, 48 and 72 hours. HBV replication and antigenemia were assessed by qPCR and cccDNA was quantified using digital droplet PCR.

Result: S/P OPN levels were elevated by 4-fold in CHB ( $\mathrm{p}=0.002)$ and increased with fibrosis stages $(\mathrm{p}=0.006)$. $\mathrm{In} \mathrm{HBeAg}(-) \mathrm{CI}$ and $\mathrm{CH}$, OPN levels were elevated in comparison to $\mathrm{HC}(4.3$-fold; $\mathrm{p}=0.054$; 4.7-fold; $\mathrm{p}=0.005)$. However, $\mathrm{HBeAg}(+) \mathrm{CI}$ exhibited decreased OPN levels compared to all other groups; $\mathrm{HC}$ (5.2-fold; $\mathrm{p}=0.001)$, $\mathrm{HBeAg}(+) \mathrm{CH}$ (13.8-fold; $\mathrm{p}=0.06), \mathrm{HBeAg}(-) \mathrm{CI}(22.6$-fold; $\mathrm{p}<0.001)$ and $\mathrm{HBeAg}(-) \mathrm{CH}$ (24.7-fold; $\mathrm{p}<0.001)$. Interestingly, CHB patients with no fibrosis but with elevated viraemia displayed increased level of $\mathrm{S} / \mathrm{P}$ OPN compared to $\mathrm{HC}(4.5$-fold; $\mathrm{p}=0.0003)$. Furthermore, patients whose viral load became undetectable during antiviral treatment had a significant reduction in S/P OPN (1.5-fold; $p=0.039$ ). In vitro, HepaRG treatment with recOPN significantly enhanced viral replication, antigen production and cccDNA levels (34-fold; $\mathrm{p}=0.02$ ) whereas neutralising OPN aptamers decreased all viral parameters and cccDNA levels $(\mathrm{p}=0.04)$

Conclusion: OPN levels directly correlate with viral replication rates, severity of HBV-driven fibrosis and exhibit differential expression during the different phases of $\mathrm{CHB}$ disease, thus identifying a marker for $\mathrm{CHB}$ driven liver disease. We also demonstrate that OPN is directly involved in HBV replication and can augment cccDNA levels. Finally, we show that neutralisation of OPN can reduce cccDNA and reveal a novel therapeutic strategy for CHB.

\section{$H B V-B 3$}

\section{Targeting NF-kappaB and DNA dependent intrinsic innate} immune pathways to facilitate $\mathrm{HBV}$ infection

\section{Emmanuel Thomas}

${ }^{1}$ University of Miami School of Medicine, United States

Background: More robust in vitro models of HBV infection are needed to facilitate the development of targeted therapeutics. Cell intrinsic innate immunity provides a rapid first line of defense to thwart invading viral pathogens, including HBV, through the production of antiviral cytokines and inflammatory chemokines. However, the presence of many of these signaling pathways in the liver and their role in HBV pathogenesis is unknown. Recent identification of intracellular DNA sensing pathways and involvement in numerous diverse disease processes, including viral pathogenesis and carcinogenesis, suggests a role for these processes in HBV infection that has a DNA genome and RNA intermediates. Inhibition of these pathways would facilitate more robust models of HBV infection.

Method: In order to improve HBV infection, we functionally characterized HBV targeted intrinsic innate immune responses and the role of DNA and RNA sensing pathways in the liver during HBV infection. We utilized in vitro models including unbiased analysis of gene expression. In addition, mRNA and protein expression was measured in HBV stimulated and DNA treated hepatoma cell lines and primary human hepatocytes. Specific RNA and DNA dependent innate and inflammatory pathways, such as RIG-I/MDA5 and IFI16/ cGAS signaling, were studied as well as additional pathways involving the production of chemokines such as CXCL10 and CCL5 that may prevent infection in vivo. siRNA experiments were utilized to functionally determine the signaling pathways that suppress HBV infection.

Result: Blocking signaling pathways involved in the recognition of $\mathrm{HBV}$ and foreign nucleic acids, including MDA5 and NF-KappaB facilitated HBV infection of hepatocytes in vitro. Treatment with antiviral chemokines including interferon alpha stimulates recognition of HBV and foreign nucleic acids in hepatocytes as demonstrated by increased production of CXCL10 and CCL5. The induction of chemokines, including IP-10, was mediated predominantly by MDA5 and NF-KappaB-dependent pathways following HBV stimulation.

Conclusion: HBV stimulated pathways would provide novel targets for suppression to promote viral infection. HBV predominantly activated a rapid inflammatory response whereas HCV stimulates a much stronger Interferon antiviral response. Understanding the mechanism underlying these inflammatory and antiviral responses may provide new strategies to facilitate HBV infection in vitro. In addition, these data offer additional insight into HBV driven pathogenesis.

\section{$H B V-B 4$}

The role and mechanism of MDSCs in promoting HBsAg negative conversion in patients with long-term anti-hepatitis $B$ virus therapy

\section{bingqing wang $^{1}$, Yongfang Jiang ${ }^{1}$}

${ }^{1} 86-18573834272$, China

Background: The clearance of HBsAg has been a challenge in the treatment of chronic hepatitis B. HBsAg conversion rates are still very low in patients with hepatitis B, and individuals have differences. Some studies have shown that HBsAg clearance occurs mainly in viremia rather than $\mathrm{HBsAg}$ itself Mutation, in which the immune factors play an important role in it. At present, we have found that myeloid suppressor cells (MDSCs), as a group of immature cells with immunosuppressive function, inhibit the response of $\mathrm{T}$ cells in patients with chronic hepatitis $\mathrm{B}$, and $\mathrm{HBsAg}$ can promote MDSCs through the ERK/IL-6/STAT3 signaling pathway Amplification, whether MDSCs can also affect the clearance of HBsAg,and HBsAg is related with the mechanism of MDSCs, for our long-term acceptance of entecavir antiviral therapy 8 years after hepatitis B patients (liver function, HBV-DNA, HBsAg, Peripheral blood MDSCs, CD4 ,$+ \mathrm{CD} 8+\mathrm{T}$ cells, $\mathrm{P} 47$ phox, etc) to explore the relevant causes of HBsAg clearance and the role of MDSCs in it.

Method: 160 patients with chronic hepatitis B who needed to receive antiviral therapy were enrolled from May 2008 to July 2016 in 200 patients hospitalized in Xiangya second Affiliated Hospital .They were eligible for anti-Virus drugs (entecavir) treatment for 8 years, after further treatment in the treatment of hepatitis B decompensated cirrhosis (such as ascites, esophageal variceal bleeding, etc.) or even liver failure or combined with viral infections (such as hepatitis A, hepatitis C, hepatitis D, HIV and other infections) And other complications of patients excluded from the experimental group, the final screening of 90 cases of chronic hepatitis B patients into the case group, the same period of 14 healthy people taking the same period as a healthy control group. The liver function, HBV-DNA, HBsAg and HBsAg were measured by intravenous blood samples from healthy subjects and healthy subjects. The percentage of MDSCs was measured by flow cytometry in peripheral blood of 12 healthy subjects. After 8 years of antiviral therapy, the liver function, HBV-DNA, hepatitis B three pairs, HBsAg quantitative detection, flow cytometry to detect peripheral blood MDSCs, CD4 and CD8 cell ratio and Western blot method to determine the content of P47phox. The levels 
of MDSCs, CD4 +, CD8 + T cells were compared between the control group and the surface antigen titer $(0.05 \mathrm{IU} / \mathrm{ml}<\mathrm{HBsAg}$ $<1000 \mathrm{IU} / \mathrm{ml}, 1000 \mathrm{IU} / \mathrm{ml}<\mathrm{HBsAg}<5000 \mathrm{IU} / \mathrm{ml}, 5000 \mathrm{IU} / \mathrm{ml}$ $<$ HBsAg), P47phox and related experimental indicators whether there is no correlation with the titer of HBsAg.

Result: 1 . There was a significant proportion of MDSCs in peripheral blood of healthy controls and chronic hepatitis B patients.The frequency of MDSCs in patients with chronic hepatitis B (1.73\%) was significantly higher than that in normal controls $(0.35 \%)(\mathrm{P}<0.05)$.

2. There was a significant difference in the ratio of $\mathrm{CHB}$ to $\mathrm{HBsAg}(-)$ peripheral blood MDSC after antiviral treatment $(\mathrm{P}=0.0026<0.05)$. There was also a significant difference in the ratio of $\mathrm{CHB}$ to $\mathrm{HBsAg}$ $(+)$ peripheral blood MDSCs before treatment $(\mathrm{P}=0.0001<0.05)$. There was a significant difference in $\mathrm{HBsAg}(-)$ and $\mathrm{HBsAg}(+)$ peripheral blood MDSCs in patients with CHB after antiviral therapy $(\mathrm{P}=0.049<0.05)$. HBsAg $(+)$ patients after antiviral therapy were divided into three groups according to the indicated antigen content: the low content group $(0.05 \mathrm{IU} / \mathrm{ml}<\mathrm{HBsAg}<1000 \mathrm{IU} / \mathrm{ml})$, the middle content group $(1000 \mathrm{IU} / \mathrm{ml}<\mathrm{HBsAg}<5000 \mathrm{IU} / \mathrm{ml})$ and the high content group ( $\mathrm{HBsAg}>5000 \mathrm{IU} / \mathrm{ml}) / \mathrm{Ml}$ ) had no significant difference $(\mathrm{P}=0.061,0.20>0.05)$. $\mathrm{HBsAg}(+)$ patients were divided into HBV-DNA (+) and HBV-DNA (-) groups according to HBVDNA, two group had no significant difference in peripheral blood MDSCs $(\mathrm{P}=0.52>0.05)$.

3. There was a significant difference in CD4 and CD8 frequencies between $\mathrm{HBsAg}(-)$ patients and $\mathrm{HBsAg}(+)$ patients after antiviral therapy $(\mathrm{P}=0.006,0.023,0.05)$.

4. There were P47phox in the peripheral blood of healthy control group and chronic hepatitis B patients, but $\mathrm{HBsAg}(+)$ patients were more than HBsAg (-)patients and healthy subjects $(\mathrm{P}<0.05)$. There was no significant difference in the content of $\mathrm{P} 47$ phox between HBsAg (-)group and $\mathrm{HBsAg}<1000 \mathrm{IU} / \mathrm{ml}$ group, but not significantly different from $\mathrm{HBsAg}>5000 \mathrm{IU} / \mathrm{ml}$ group $(\mathrm{P}<0.05)$. There was also significant difference in $\mathrm{HBsAg}(+)$ DNA $(+)$ group $(\mathrm{HBsAg}$ $<1000 \mathrm{IU} / \mathrm{ml}$ group and $\mathrm{HBsAg}>5000 \mathrm{IU} / \mathrm{ml}$ group $)(\mathrm{P}<0.05)$. Conclusion: 1 . The frequency of MDSCs in peripheral blood of $\mathrm{CHB}$ patients was significantly higher than that of healthy ones, suggesting that tolerance to $\mathrm{T}$ cells in patients with hepatitis $\mathrm{B}$ may be related to the inhibition of $\mathrm{T}$ cell responses with MDSCs.

2. MDSCs were significantly lower in antiviral treatment HBV patients than in healthy subjects and patients before treatment, suggesting that antiviral therapy could help to remove the virus and inhibit the amplification of MDSCs. The frequency of MDSCs in $\mathrm{HBsAg}$ positive patients was significantly higher than that in negative patients, while HBsAg positive patients, CD8 T cells were significantly lower than those of negative patients suggesting that HBsAg in the induction of MDSCs in the augmentation of the role, and the higher the higher the titer induction, and HBsAg positive patients with low titer and high titer peripheral blood MDSCs frequency no significant difference And HBsAg clearance abnormalities.

3. There was no significant correlation between HBV-DNA value and peripheral blood content of MDSCs after antiviral therapy, suggesting that the level of HBV-DNA was not the factor of MDSCs induction.

4. The content of P47phox in peripheral blood of patients with HBsAg positive after antiviral therapy was higher than that of negative and healthy. The high level of HBsAg was higher than that of low content. It is suggested that HBsAg low-content and high-dose MDSCs can still inhibit T cells through ROS pathway. P47phox plays an important role in inducing MDSCs to activate the NADPH oxidase-controlled ROS pathway to play T cell inhibition. The effect of low content group was weaker than that of HBsAg high content group.
$H B V-B 9$

Genetic association between single nucleotide polymorphisms in IL-21 and IL-21R genes and susceptibility to hepatitis B virus chronic infection

SeyedReza Mohebbi ${ }^{1}$, Shahrzad Shoraka ${ }^{1}$, Armin Hosseini Razavi $^{1}$, Afsaneh Sharifian ${ }^{1}$, Hamid Asadzadeh Aghdaei ${ }^{1}$, Seyed Masoud Hosseini' ${ }^{2}$, Mohammad Reza Zali ${ }^{1}$

${ }^{1}$ Shahid Beheshti University of Medical Sciences, Iran, Islamic Republic of; ${ }^{2}$ Shahid Beheshti University, Iran, Islamic Republic of

Background: The clinical outcome of Hepatitis B virus (HBV) infection varies from spontaneous clearance of the virus after infection to a chronic persistent infection that may progress to severe complications such as liver cirrhosis or hepatocellular carcinoma. The mechanisms underlying resolution of $\mathrm{HBV}$ infection or its progression to a chronic disease remain unknown. Many recent studies have suggested host genetic variations such as single nucleotide polymorphisms (SNPs) in cytokines and their receptors genes as influential factors in development of chronic condition or viral clearance. Interleukin-21 (IL-21) is a recently discovered multifunctional cytokine which has crucial roles in accumulation and survival of Ag-specific CD8+ effector T-cells and regulation of B-cell functions. The aim of this study was to analyze genetic association between single nucleotide polymorphisms in IL-21 (rs2055979) and IL-21R (rs3093390) genes and susceptibility to hepatitis B virus chronic infection.

Method: Blood samples were collected from 200 patients with chronic $\mathrm{HBV}$ infection (HBsAg and $\mathrm{HBcAb}$ and $\mathrm{HBV}$ DNA positive), 100 individuals with $\mathrm{HBcAb}$ positive, HBsAg Negative and Anti$\mathrm{HBs} A \mathrm{~b}$ positive results as cleared virus and 200 healthy individuals (HBsAg and $\mathrm{HBcAb}$ negative). Genomic DNA was extracted by standard salting-out method and then for each position, SNP was genotyped by the dedicated PCR-RFLP method.

Result: The genotype frequencies of rs2055979 and rs3093390 did not show any significant difference between chronic HBV patients, controls and clearance group. Genotypes frequencies for rs2055979 were $30.0 \% \mathrm{GG}, 53.5 \% \mathrm{GT}, 16.5 \%$ TT in chronic patients and $24.5 \%$ GG, $54.0 \%$ GT, $21.5 \%$ TT in control group and $23.0 \%$ GG, $54.0 \%$ GT, $23.0 \%$ TT in clearance group (chronic vs control $\mathrm{p}$ value $=0.297$, chronic vs clearance $\mathrm{p}$ value $=0.209$ ). In another SNP (rs3093390), genotypes frequencies were $66.0 \% \mathrm{CC}, 28.5 \% \mathrm{CT}, 5.5 \% \mathrm{TT}$ in chronic and $61.4 \% \mathrm{CC}, 29.7 \% \mathrm{CT}, 9.0 \% \mathrm{TT}$ in control group and $59.0 \% \mathrm{CC}, 31.0 \% \mathrm{CT}, 10.0 \% \mathrm{TT}$ in clearance group (chronic vs control $p$ value $=0.717$, chronic vs clearance $p$ value $=0.275$ ).

Conclusion: No association was detected between the IL-21 (rs2055979) and IL-21R (rs3093390) genetic variants and chronic hepatitis B infection. It seems that these SNPs may not be involved in susceptibility to chronic HBV infection in Iranian population.

\section{$H B V-B 10$}

Hepatitis B virus genotypes A1, $\mathrm{A} 2$ and $\mathrm{E}$ in Cape Verde: unequal distribution through islands and association with human flows

Barbara Vieira Lago ${ }^{1}$, Isabel Iniza Pina Araujo ${ }^{2}$, Natalia Spitz ${ }^{1}$, Caroline Soares $^{1}$, Christian Niel ${ }^{1}$, Barbara Lago ${ }^{1}$, Selma Gomes ${ }^{1}$

${ }^{1}$ Fundação Oswaldo Cruz, Brazil; ${ }^{2}$ Universidade de Cabo Verde, Cape Verde

Background: Hepatitis B virus (HBV) diversity has not been previously studied in Cape Verde. The archipelago was discovered in 1460 by Portuguese explorers, who brought African slaves to colonise the 
islands. In this study, we investigated the HBV molecular characteristics and genetic diversity from 183 HBsAg-positive Cape Verdean individuals

Method: All $183 \mathrm{HBsAg-positive} \mathrm{Samples} \mathrm{were} \mathrm{submitted} \mathrm{to} \mathrm{PCR}$ amplification and to phylogenetic analysis of the pre-S/S region and the full-length genomes

Result: A total of 95 sequences were obteined. Phylogenetic analysis revealed that 54 isolates clustered with HBV/A1 (57\%), 21 with HBV/A2 (22\%), 19 with HBV/E (20\%), and one with HBV/D (1\%). HBV genotypes/subgenotypes were unequally distributed through the islands. In São Vicente, the main northern island, most isolates (84\%) belonged to the African-originated HBV/A1, with the remaining isolates belonging to HBV/A2, which is prevalent in Europe. Interestingly, the HBV/A1 isolates from São Vicente were closely related to Brazilian sequences into the Asian-American clade, which suggests the dissemination of common African ancestors through slave trade. In contrast, in Santiago and nearby southern islands, where a recent influx from different populations circulates, a higher diversity of HBV was observed: HBV/A1 (40\%); HBV/E (32\%); HBV/A2 (28\%); and one HBV/D (1\%). HBV/E is a recent HBV genotype exclusively from Africa that was absent in the era of the slave trade.

Conclusion: Genotypes A1, A2 and E were found and phylogenetically analysed. Cape Verde presented an genotypic unequal distribution through islands. African and European human flows at different times of the history may explain the HBV diversity in Cape Verde. The possible origin and specific characteristics of each HBV genotype circulating in Cape Verde, as well as the molecular signatures were evidenced.

\section{$H B V-B 11$}

Investigation of $\mathrm{HBV}$ multidrug-resistant mutants in a large number of nucleos(t)ide analogs-treated Chinese patients

Dongping $\mathrm{Xu}^{1}$, Yan Liu ${ }^{1}$, Rongjuan $\mathrm{Chen}^{1}$, Xiaodong $\mathrm{Li}^{1}$, Lanlan $\mathrm{Si}^{1}$, Li Zhao ${ }^{\mathrm{I}}$, Jinman Shao ${ }^{1}$, Le $\mathrm{Li}^{1}$, Dongping $\mathrm{Xu}^{1}$

${ }^{1}$ Beijing 302 Hospital, China

Background: Multidrug-resistant (MDR) HBV is defined as those harbor mutations resistant to both nucleoside analogs [lamivudine, telbivudine, and entecavir (ETV)] and nucleotide analog [adefovir $(\mathrm{ADV})]$. The study aimed to investigate the profile and virologic features of MDR mutants in real clinical practice.

Method: Total of 29,639 serum samples were collected from 22,009 patients who received resistant testing (direct sequencing) in Beijing 302 Hospital from 2007 to 2016. The patients all received various sequential/combined therapies of nucleos(t)ide analogs. MDR mutants were verified by clonal sequencing and phenotypic analysis was performed for representative viral clones.

Result: MDR mutants were detected in $0.698 \%(207 / 29,639)$ samples. Among them, 160 samples were detected with MDR without ETV-resistant mutation [MDR-ETVr(-)] and 47 samples with ETVresistant mutation [MDR-ETVr(+)]. Major mutation patterns were rtL180M+A181V+M204V (66.9\%) for MDR-ETVr(-) mutants and $\mathrm{rtL} 180 \mathrm{M}+\mathrm{A} 181 \mathrm{~V}+\mathrm{S} 202 \mathrm{G}+\mathrm{M} 204 \mathrm{~V}$ (40.4\%) for MDR-ETVr(+) mutants. MDR mutants had lower replication capacities than wildtype strain and the activity of MDR-ETVr $(+)$ mutants were relatively lower than MDR-ETVr(-) mutants. TDF or TDF+ETV treatment had higher inhibitory rates in general compared to ETV+ADV for both MDR-ETVr(-) mutants (86.1\%-98.9\% vs. 80.6\%-92.5\%) and MDR$\operatorname{ETVr}(+)$ mutants $(62.9 \%-97.0 \%$ vs. $62.1 \%-79.5 \%)$. Interestingly, TDF+ETV had greater inhibition on M9 with dual ADV-resistant mutations $(88.9 \%$ vs. $76.0 \%)$ and M10 with dual ETV-resistant mutations ( $80.0 \%$ vs. $62.9 \%)$; and the three treatments all had inadequate inhibition on M11 (Table 1).

Conclusion: More primary resistant mutations confer HBV greater resistance but lower replication capacity. TDF is superior to $\mathrm{ADV}+\mathrm{ETV}$ for suppressing MDR-ETVr(+) mutants and TDF+ETV is superior to TDF for suppressing complex MDR-ETVr(+) mutants.

Table 1. Inhibitory effects of ETV+ADV, TDF, and TDF+ETV on MDR HBV mutants in vitro

\begin{tabular}{|c|c|c|c|}
\hline \multirow{2}{*}{ Mutational pattem (clonal sequencing) } & $\mathrm{ETV}+\mathrm{ADV}$ & TDF & TDF+ETV \\
\hline & \multicolumn{3}{|c|}{ Inhibitory rate $(\dot{x} \pm \mathrm{SD}, \%)$} \\
\hline Wild-type & $99.3 \pm 3.2$ & $98.5 \pm 2.4$ & $99.5 \pm 4.0$ \\
\hline $\mathrm{M} 1, \mathrm{rtA} 181 \mathrm{~V}+\mathrm{M} 204 \mathrm{I}$ & $83.8 \pm 3.7$ & $98.9 \pm 1.3$ & $97.1 \pm 3.7$ \\
\hline $\mathrm{M} 2, \mathrm{rtL} 180 \mathrm{M}+\mathrm{M} 204 \mathrm{I}+\mathrm{N} 236 \mathrm{~T}$ & $92.4 \pm 4.2$ & $98.2 \pm 3.1$ & $94.9 \pm 7.7$ \\
\hline $\mathrm{M} 3, \mathrm{rtL} 180 \mathrm{M}+\mathrm{A} 181 \mathrm{~V}+\mathrm{M} 204 \mathrm{~V}$ & $92.5 \pm 4.6$ & $97.6 \pm 5.6$ & $97.5 \pm 4.0$ \\
\hline $\mathrm{M} 4, \mathrm{xtL} 180 \mathrm{M}+\mathrm{A} 181 \mathrm{~V}+\mathrm{M} 204 \mathrm{~V}+\mathrm{N} 236 \mathrm{~T}$ & $92.2 \pm 5.2$ & $96.9 \pm 4.0$ & $96.7 \pm 5.2$ \\
\hline $\mathrm{M} 5, \mathrm{rtL} 1 \mathrm{SOM}+\mathrm{M} 204 \mathrm{~V}+\mathrm{N} 236 \mathrm{~T}$ & $80.6 \pm 1.2$ & $89.2 \pm 2.0$ & $\$ 6.1 \pm 5.0$ \\
\hline $\mathrm{M} 6, \mathrm{rLL} 180 \mathrm{M}+\mathrm{M} 204 \mathrm{~V}+\mathrm{N} 236 \mathrm{~T}+\mathrm{M} 250 \mathrm{~L}$ & $75.2 \pm 5.1$ & $94.3 \pm 3.7$ & $94.8 \pm 5.5$ \\
\hline $\mathrm{M} 7, \mathrm{rL} 180 \mathrm{M}+\mathrm{A} 181 \mathrm{~V}+\mathrm{M} 204 \mathrm{~V}+\mathrm{M} 250 \mathrm{~L}$ & $79.5 \pm 1.7$ & $95.8 \pm 4.2$ & $\$ 8.7 \pm 4.7$ \\
\hline $\mathrm{M} 8, \mathrm{rtL} 180 \mathrm{M}+\mathrm{A} 181 \mathrm{~V}+\mathrm{S} 202 \mathrm{G}+\mathrm{M} 204 \mathrm{~V}$ & $78.0 \pm 1.7$ & $96.4 \pm 1.3$ & $97.0 \pm 2.0$ \\
\hline $\mathrm{M} 9, \mathrm{rL} 180 \mathrm{M}+\mathrm{A} 181 \mathrm{~V}+\mathrm{S} 202 \mathrm{G}+\mathrm{M} 204 \mathrm{~V}+\mathrm{N} 236 \mathrm{~T}$ & $66.9 \pm 2.7$ & $76.0 \pm 1.7$ & $\$ 8.9 \pm 1.8$ \\
\hline $\mathrm{M} 10, \mathrm{rL} 180 \mathrm{M}+\mathrm{A} 181 \mathrm{~V}+\mathrm{T} 184 \mathrm{~A}+\mathrm{S} 202 \mathrm{G}+\mathrm{M} 204 \mathrm{~V}$ & $70.0 \pm 5.5$ & $62.9 \pm 6.0$ & $80.0 \pm 3.5$ \\
\hline $\mathrm{M} 11, \mathrm{rL} 180 \mathrm{M}+\mathrm{A} 181 \mathrm{~V}+\mathrm{T} 184 \mathrm{I}+\mathrm{M} 204 \mathrm{~V}$ & $60.2 \pm 4.5$ & $78.8 \pm 2.5$ & $74.5 \pm 6.8$ \\
\hline $\mathrm{M} 12, \mathrm{rL} 180 \mathrm{M}-\mathrm{S} 202 \mathrm{G}+\mathrm{M} 204 \mathrm{~V}+\mathrm{N} 236 \mathrm{~T}$ & $62.1 \pm 3.2$ & $90.3 \pm 2.8$ & $90.4 \pm 2.6$ \\
\hline $\mathrm{M} 13$, rtL180M+T184A+M204V+N236T & $66.8 \pm 4.8$ & $88.6 \pm 3.8$ & $92.7 \pm 5.0$ \\
\hline
\end{tabular}

\section{$H B V-B 12$}

Lack of association between vitamin D levels and polymorphism of vitamin $D$ receptor (VDR) according virological and biochemical data in hepatitis B virus (HBV) patients

Livia Melo Villar ${ }^{1}$, Leticia Paula Scalioni ${ }^{1}$, Moyra Machado Portilho $^{1}$, Ana Carolina Fonseca Mendonca ${ }^{1}$, Janai Moreira Lima $^{1}$, Ketlyn Araujo Fraga ${ }^{1}$, Lia Laura Lewis Ximenez, Elisabeth Lampe ${ }^{1}$

${ }^{1}$ FIOCRUZ, Brazil

Background: Vitamin D has been identified as an immune-modulator due to its immunomodulatory effects on cells of the immune system. Normal serum vitamin $D$ levels are associated with spontaneous clearance of HBsAg in chronic inactive hepatitis B virus (HBV) patients. Vitamin $D$ deficiency were shown to influence mortality risk in HBV infected individuals. However, few data are available regarding the influence of vitamin $\mathrm{D}$ aspects in HBV infection. The objective of this study is to investigate the association among vitamin D levels and some SNPs of vitamin D receptor according virological and biochemical data of HBV infected individuals.

Method: A total of $81 \mathrm{HBV}$ patients (41 females and mean \pm SD age of $43,88 \pm 15,80$ years) were recruited at Viral Hepatitis Ambulatory (Viral Hepatitis Laboratory, Oswaldo Cruz Institute, FIOCRUZ) during 2012 to 2014 years. All of them had HBsAg and HBV DNA detected in serum and none of them were positive for serum antihepatitis $\mathrm{C}$ virus or anti-human immunodeficiency virus antibody, or exhibited other causes of hepatocellular injury (e.g., any history of alcoholism, autoimmune hepatitis, advanced cirrhosis, or treatment with hepatotoxic drugs) and, concomitant use of drugs known to affect serum vitamin D concentration and intravenous drug use. Serum was tested for ALT, AST, gamma-glutamyltransferase (GGT), phosphate alkaline (PA) and bilirubin using the Automatic Biochemistry analyzer (Labmax Plenno, Labtest, Brazil). HBe Ag and anti-HBe were detected using commercial enzyme immunoassays (Diasorin, Italy). HBV DNA was quantified using Abbott real time HBV. Serum 25-hydroxyvitamin D levels were assessed using automated immunochemiluminometric assay (ICMA) (Liason $25(\mathrm{OH})$ Vitamin D, Diasorin, Varceli, Italy) and those presenting values lower 
than $20 \mathrm{ng} / \mathrm{mL}$ were considered deficient. Genomic DNA was obtained from whole blood samples using QIamp DNA Mini Kit (Qiagen, Valencia, CA). The allelic discrimination analysis for SNPs in $V D R$ genes (Fok, Apa, TaqI) was performed using the TaqMan assays (Applied Biosystems, Foster City, CA, USA) at Rotor-Gene Q 5-plex HRM (Qiagen). SPSS version 20 (IBM Corporation, USA) was used for all statistical analyses. A p-value of less than 0.05 was considered to indicate statistical significant differences between groups

Result: In this study, 18 (22.2\%) HBV patients had vitamin D deficiency (levels <20ng/mL), 12 (14.8\%) had HBeAg, $69(85.2 \%)$ presented anti-HBe and HBV mean viral load was 2.84 x $107 \pm 1.57$ $\mathrm{x} 108$ copies $/ \mathrm{mL}$. Vitamin D deficiency was not associated to biochemical (ALT, AST, PA, GGT and bilirubin) and virological data (HBV viral load, anti-HBe and $\mathrm{HBeAg}$ status). VDR Apa, Fok and TaqI SNPs were not associated to viral load, anti-HBe and HBeAg presence.

Conclusion: Vitamin D deficiency was not frequent in this population and VDR SNPs were not associated to virological data in HBV patients.

\section{$H B V-B 13$}

\section{Expression of miR-122 in hepatitis B virus related liver disease}

\section{Tania Islam Resma ${ }^{1}$, Munira Jahan $^{2}$, Shahina Tabassum ${ }^{2}$}

${ }^{1}$ Dhaka Medical College, Bangladesh; ${ }^{2}$ Bangabandhu Sheikh Mujib Medical University, Bangladesh

Background: MicroRNA 122 (miR-122) is the most abundant miRNA in human liver and is released into blood in huge amount from injured liver tissues. The amount of miRNA may be associated with persistent chronic infection and disease progression. The study was designed to determine the plasma levels of microRNA-122 among chronic hepatitis B (CHB) patients, and to correlate these findings with levels of HBV DNA viral load, alanin aminotransferase (ALT) and alpha fetoprotein (AFP) of the respective groups.

Methods: A total of 70 participants consisting of 20 patients with HBV related chronic hepatitis, 20 patients with HBV related hepatocellular carcinoma (HCC), 20 patients with HBV related cirrhosis, along with 10 healthy controls (HC) were enrolled in the study. Total RNA was extracted from plasma samples followed by cDNA synthesis and finally level of miR-122 was analyzed using real time PCR technique

Results: The level of miR-122 in plasma samples were increased significantly in all patient groups compared to healthy controls. It was $63.28 \pm 18.52$ (mean \pm SEM) fold higher with HCC, $32.8 \pm 7.37$ (mean \pm SEM) fold higher with cirrhosis and $125.84 \pm 24.8$ (mean \pm SEM) fold higher in the CHB group without HCC and cirrhosis than in healthy control group. Plasma miR-122 level correlates positively with HBV DNA viral load in all groups of $\mathrm{CHB}$ patients but has no significant correlation with serum ALT and AFP levels. The receiver-operator characteristic (ROC) curves of plasma levels of miR-122 were generated to determine the specificity and sensitivity of this miRNA in distinguishing patient groups from healthy controls. The plasma miR-122 showed sensitivity and specificity of $95 \%$ and $90 \%, 85 \%$ and $90 \%, 100 \%$ and $90 \%$ respectively when differentiating with $\mathrm{HBV}$ related $\mathrm{HCC}$, with $\mathrm{HBV}$ related cirrhosis and $\mathrm{CHB}$ group without $\mathrm{HCC}$ and cirrhosis from healthy controls. In comparison to AFP levels, the plasma concentration of miR-122 exhibited earlier changes from normal in HBV related HCC patients.
Conclusion: Circulating miR-122 may be considered as a biomarker for prediction of $\mathrm{HBV}$ induced chronic liver diseases and $\mathrm{HBV}$ related $\mathrm{HCC}$.

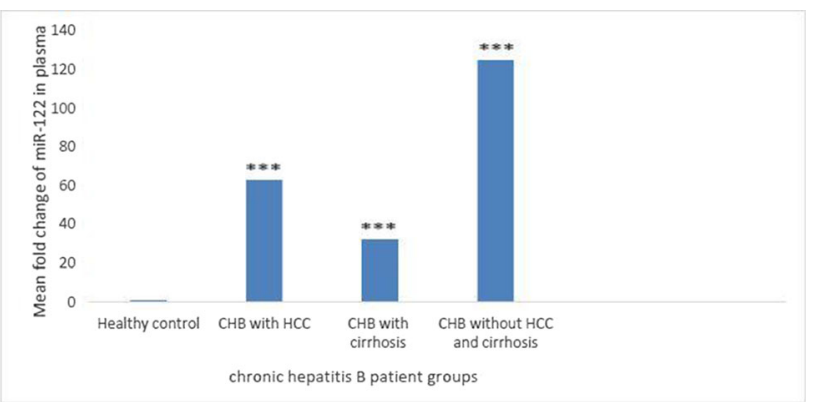

Figure 4.11: Fold changes of miR-122 in plasma among the study groups. Differences were evaluated with a Mann-Whitney $U$ test. ${ }^{* * *} \mathrm{p}<0.001$, compared with the healthy control group.

Table - 4.4: Comparison of increased plasma level of miR-122 and AFP among

CHB patients with $\mathrm{HCC}$ :

\begin{tabular}{|c|c|c|c|}
\hline & $\begin{array}{c}\text { Increased } \\
\text { Plasma levels above } \\
\text { normal in CHB } \\
\text { patients with } \\
\text { HCC } \\
{[\mathrm{n}(\%)]}\end{array}$ & $\begin{array}{c}\text { No increase in } \\
\text { Plasma levels above } \\
\text { normal in CHB patients } \\
\text { with } \\
\text { HCC } \\
{[\mathrm{n}(\%)]}\end{array}$ & Significance \\
\hline $\begin{array}{l}\text { Plasma } \\
\text { miR-122 }\end{array}$ & $19(95 \%)$ & $01(05 \%)$ & \multirow{2}{*}{$\mathrm{P}=0.034^{*}$} \\
\hline AFP & $13(65 \%)$ & $07(35 \%)$ & \\
\hline
\end{tabular}

$$
\begin{aligned}
& \text { Statistical analysis was done by Wilcoxon Signed Ranks test among patients. } \\
& *=\text { Significant } \\
& n=\text { Number of subjects. }
\end{aligned}
$$

\section{$H B V-B 14$}

Enumeration and characterization of HBsAg-specific memory B cells by enzyme linked immunospot assays

Chen Tian ${ }^{1}$, Yong Liu ${ }^{1}$, Yuxin Chen ${ }^{1}$, Yang $\mathrm{Li}^{1}$, Guiyang Wang ${ }^{1}$, Shan Lu' ${ }^{2}$ Chao Wu ${ }^{1}$

${ }^{1}$ Medical School of Nanjing University, China; ${ }^{2}$ The University of Massachusetts, Medical School, United States

Background: Hepatitis B surface antibody (HBsAb) plays a critical role in protecting against infection of hepatitis B virus. As a central component of humoral responses, hepatitis B surface antigen (HBsAg)-specific memory B cells could rapidly secrete $\mathrm{HBsAb}$ in respond to re-exposure to HBsAg. However, HBsAg-specific memory B cell responses in healthy individuals and Chronic HBV (CHB) patients have not been carefully characterized. 
Method: 21 immunized healthy controls (HCs) and $51 \mathrm{HBV}$ patients were enrolled and PBMCs were prepared from $10 \mathrm{~mL}$ fresh heparinized peripheral blood specimens. R848 and recombinant human (rh) IL-2 were added to effectively stimulate B cells. Cells were subsequently incubated for five days and $\mathrm{HBsAg}$-specific memory B cell were detected by Enzyme Linked ImmunoSpot (ELISpot) assay. Result: $17(80.95 \%)$ out of $21 \mathrm{HCs}$ had positive HBsAg-specific memory B cells, despite they had HBV vaccine immunization at least two years ago. HCs with higher than $200 \mathrm{mIU} / \mathrm{mL}$ of $\mathrm{HBsAb}$ had a significantly elevated level of HBsAg-specific B cells compared to those with less than $200 \mathrm{mIU} / \mathrm{mL}$ of $\mathrm{HBsAb}(98.33 \pm 10.89$ vs. $18.4 \pm 6.81$ per $10^{\wedge} 6$ PBMCs $)(\mathrm{p}<0.0001)$. A strong correlation between HBsAb level and the frequency of HBsAg-specific B cells among healthy individuals was founded $(\mathrm{p}<0.0001, \mathrm{r}=0.909) .16$ (31.4\%) out of $51 \mathrm{CHB}$ patients (4 out of 13 IT patients, 8 out of 17 IA patients, and 4 out of 21 IC patients) had positive $\mathrm{HBsAb}$-secreting $\mathrm{B}$ cell responses revealed by B-cell ELISpot assay. CHB patients with relative high level of $\mathrm{HBsAb}(>1 \mathrm{mIU} / \mathrm{mL})$ had significantly elevated level of HBsAg-specific B cells compared to CHB patients with low HBsAb level $(<1 \mathrm{mI} / \mathrm{mL}) \quad(14.58 \pm 6.61$ vs. $4.69 \pm 2.24) \quad(\mathrm{p}<0.05)$. However, we did not find any significant correlation of HBsAb and specific B cells level among CHB patients. The level of HBsAgspecific memory B cells between healthy controls and CHB patients was further compared. As expected, the frequency of HBsAg-specific $\mathrm{IgG}+$ secreting B cells was significantly lower in CHB patients than in immunized healthy individuals $(7.4 \pm 2.5$ vs $52.66 \pm 10.64$, $\mathrm{p}<0.0001$ ). Further, IT patients had an average of $8.75 \pm 6.46$ specific memory B cells, IA patients had an average of $11.15 \pm 4.67$, while IC patients had an average of $3.5 \pm 2.41$ specific B cells among $10^{\wedge} 6$ PBMC, significantly lower compared to the frequency of healthy subjects. Higher frequency of specific B cells (as percentage of total $\mathrm{IgG}+\mathrm{B}$ cells) were observed in IA phase of $\mathrm{CHB}$ patients, compared to IT and IC phase of CHB patients $(0.44 \pm 0.22 \%$ vs. $0.29 \pm 0.19 \%$ and $0.28 \pm 0.24 \%$ ).

Conclusion: Our study recommended that HBsAg-specific B cells demonstrated by B-cell ELISpot assay was a unique immune biomarker distinct from $\mathrm{HBsAb}$, particularly for $\mathrm{CHB}$ patients.

\section{$H B V-B 15$}

Usefulness of oral fluid samples for identification of hepatitis B virus (HBV) genotypes

Moyra Machado Portilho ${ }^{1}$, Ana Carolina Da Fonseca Mendonca', Cristianne De Sousa Bezerra', Barbara Vieira Lago ${ }^{1}$, Leticia Nabuco $^{3}$, Cristiane Villela Nogueira ${ }^{3}$, Claudia Alexandra Pontes Ivantes $^{2}$, Lia Laura Lewis Ximenez ${ }^{1}$, Elisabeth Lampe ${ }^{1}$, Livia Melo Villar ${ }^{1}$

${ }^{1}$ Oswaldo Cruz Institute, Brazil; ${ }^{2}$ Center of Guidance and Orientation (COA), Brazil; ${ }^{3}$ Clementino Fraga Filho University Hospital, Brazil

Background: Hepatitis B virus (HBV) has a partially double-stranded DNA genome and is classified into 10 genotypes (A-J), which have a nucleotide divergence greater than $8 \%$ in the complete genome. In Brazil, genotypes A, D and F are the most frequent. The gold standard method for the determination of HBV genotype is nucleotide sequencing using serum samples but the use of alternative samples, such as oral fluid, may increase access to the molecular diagnosis of hepatitis $\mathrm{B}$, since collection is easier and non-invasive. The objective of this study is to investigate the applicability of oral fluid samples for the identification of HBV genotypes

Method: Samples of blood and oral fluid collected with Salivette (Sarstedt, Germany) were obtained from 64 individuals with chronic hepatitis B. Serum samples were analyzed for hepatitis B serologic markers and quantified by real-time PCR (Abbott Real Time HBV, Abbott Laboratories, USA). All samples were submitted to a qualitative in-house PCR for amplification of the HBV polymerase gene. Products of positive samples were purified using the QIAquick PCR Purification kit (Qiagen, Valencia, USA) and sequenced with forward and reverse primers using a BigDye Terminator 3.1 Cycle Sequencing kit (Thermo Fisher Scientific). The reactions were performed in an automatic ABI PRISM 3100 Genetic Analyzer Sequencer (Thermo Fisher Scientific) and sequences of 847 bp were analyzed by Mega Software v7.0 (Mega Software) and genotypes and subgenotypes determined by phylogenetic tree and using the Geno2pheno HBV website.

Result: Among 64 serum samples, all were $\mathrm{HBsAg}$ and anti-HBc reagents, 49 anti-HBe reagents, $13 \mathrm{HBeAg}$ reagents and with detectable HBV DNA (mean viral load of $3.56 \pm 2.09 \mathrm{log} \mathrm{IU} / \mathrm{mL}$ ). Among serum samples, 43 belonged to genotype A (67.18\%), 16 to genotype $\mathrm{D}(25 \%)$, one to genotype $\mathrm{E}(1.56 \%)$ and four to genotype $\mathrm{F}$ (6.25\%). HBV DNA was detected in 19 oral fluid samples (mean viral load of $3.69 \pm 2.13 \mathrm{log} \mathrm{IU} / \mathrm{mL}$ in paired serum). From these, it was possible to obtain HBV sequence of 10 samples which presented almost $100 \%$ of homology with the paired serum, being classified into the same subgenotype (four pairs of A1, two A2, two D3, one F2 and one E).

Conclusion: In this study, genotype A was the most prevalent, followed by genotypes D and F, such as happens in Brazil. Furthermore, it was identified a genotype E sample, that is not common in Brazil, suggesting the virus migration. Pairs of serum and oral fluid samples were classified into the same subgenotype and most of them are genetically identical, demonstrating that the same viral lineage was identified and suggesting that oral fluid could be used to identify genotypes of HBV such as serum.

\section{$H B V-B 16$}

\section{CD56bright natural killer cell mediated reduction of HBsAg via cytolysis and cccDNA clearance in chronic Hepatitis B patients}

\section{Jinshang $\mathrm{Hu}^{1}$, Xiaoping Zhang ${ }^{1}$, Fang Xiao ${ }^{1}$, Lin $\mathrm{Zhu}^{1}$, Weiming}

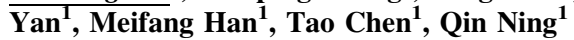

${ }^{1}$ Tongji Hospital, Tongji Medical College, Huazhong University of Science and Technology, China

Background: HBV surface antigen ( $\mathrm{HBsAg}$ ) reduction is well observed in chronic hepatitis $\mathrm{B}(\mathrm{CHB})$ patients treated with pegylate interferon-alpha-2a (Peg-IFN $\alpha$ ). However, the mechanism of HBsAg suppression has not been fully elucidated.

Method: Twenty-seven of 55 Entecavir-treated CHB e antigen positive patients were switched to Peg-IFN $\alpha$ treatment (Group A) whereas 28 patients continued entecavir treatment (Group B). The percentage or absolute number of CD56bright/CD56dim NK cells, expression of receptors and cytokines were evaluated by flow cytometry for 48 weeks and correlated with treatment efficacy. In Vitro, purified NK cells were co-cultured with HepAD38 cells for measurement of $\mathrm{HBsAg}$, apoptosis and covalently closed circular DNA (cccDNA).

Result: In association with a reduction of HBsAg, the percentage and absolute number of CD56bright NK cell was significantly elevated in patients in group A, especially in Virologic Responders (VRs, HBsAg decreased). Furthermore, percentage of NKp30+, NKp46+, TRAIL,+ TNF- $\alpha+$ and IFN $\gamma+$ CD56bright NK cells were significantly expanded in Group A, which were positively correlated with the decline of HBsAg at week 48. In Vitro, peripheral NK cells from Group A induced decline of HBsAg in comparison with NK cells 
from Group B which was significantly inhibited by anti-TRAIL, antiTNF- $\alpha$ and anti-IFN $\gamma$ antibodies. Furthermore, apoptosis of HepAD38 cells and levels of cccDNA, were significantly reduced by TRAIL + and TNF- $\alpha+/$ IFN $\gamma+$ NK cells from Group A respectively. Conclusion: A functional restoration of CD56bright NK cells in Entecavir-treated patients who were switched to Peg-IFN $\alpha$ contributes to HBsAg and cccDNA clearance through TRAIL-induced cytolysis and TNF- $\alpha / \mathrm{IFN} \gamma$-mediated noncytolytic pathways.

\section{$H B V-B 17$}

Development and characterization of oral combination vaccine against hepatitis B \& anthrax

\section{Saurabh Bhargava ${ }^{1}$}

${ }^{1}$ Himalayan University, India

Background: Vaccination has not only become vital but a lot of revolutionary changes are being observable in field of vaccine delivery. Vaccine antigens administered by oral route are often degraded during gastrointestinal transit. Bile salt stabilized vesicles ie bilosomes are found to be effective in preventing antigen degradation and enhance mucosal penetration. The aim of present work was to prepare a combination vaccine system against hepatitis-B (HBsAg) \& anthrax(rPA).

Method: Bilosomes containing HBsAg and rPA were prepared by a lipid cast film method. Antigen loaded bilosomes were characterized in-vitro for their shape, size, percent antigen entrapment and stability. Fluorescence microscopy was carried out to confirm the uptake of bilosomes. The in-vivo study comprised of estimation of IgG response in serum and $\operatorname{sIgA}$ in various body secretions using specific ELISA. Result: Oral immunization induces both mucosal \& systemic immune responses, whereas mucosal responses are not generally observed from systemic immunization. Bilosomes provide needle free, painless approach for immunization, thereby increasing patient compliance and consequently increasing vaccination coverage.

Bilosomes formed were multilamellar and were stable in gastric and intestinal fluids. Fluorescence microscopy suggested that bilosomes were taken up by the gut associated lymphoid tissues. In-vivo data demonstrates that bilosomes produced both systemic as well as mucosal antibody responses upon oral administration at higher dose levels as compared to intramuscular immunization but fail to produce any synergistic effect.

Thus, HBsAg potentiates the production anti-rPA antibody. Also measurable sIgA in mucosal secretions were observed. Thus, the bilosomes are a promising carrier for oral combination vaccines.

Conclusion: This approach can be adapted for human use because mucosal surfaces are initial site of infection $\&$ it is logical to develop vaccination strategies that evoke appropriate localized responses to counteract early events of pathogenesis.
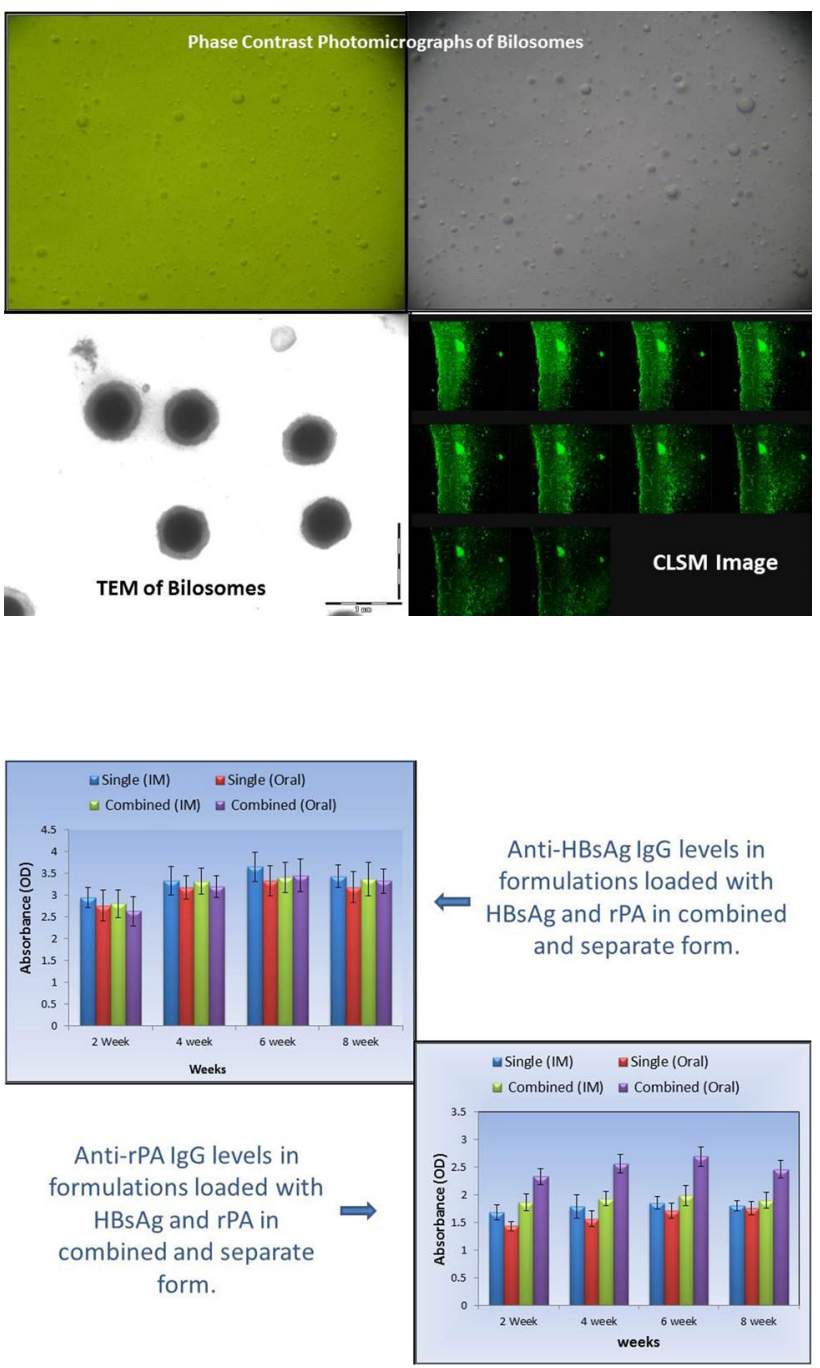

$H B V-B 18$

Whole-genome characteristics of hepatitis B virus using nextgeneration sequencing

\section{Hyun Woong Lee ${ }^{1}$, Jung Il Lee ${ }^{1}$, Kwan Sik Lee ${ }^{1}$}

${ }^{1}$ Yonsei University College of Medicine, Korea, Republic of

Background: Hepatitis B virus (HBV), discovered in 1966, infects more than 350 million people worldwide. $\mathrm{HBV}$ is a leading cause of chronic hepatitis, cirrhosis, and hepatocellular carcinoma, accounting for 1 million deaths annually. Nevertheless, until recently, genetic testing of the hepatitis B virus is currently limited to a small fraction of the entire viral sequence. In this study, we aimed to find all variants of HBV by analyzing the whole genome sequence of it.

Method: Twenty-three newly diagnosed, untreated HBV infected patients participated in this study. DNA extraction and quantification of HBV were conducted as follows: DNA was extracted from $200 \mu \mathrm{l}$ of serum samples of enrolled patients. The amplified products were obtained by PCR. A library of PCR products of the viral genome $(0.2 \mathrm{ng})$ was prepared using Nextera XT DNA Sample Prep Kit (Illumina). The PCR products were sheared into 300- to 1000-bp fragments using the kit, and then analyzed on a MiSeq sequencer (Illumina, USA) for paired-end 150 -bp sequencing. After a quality 
check and data trimming, BWA-MEM v0.6 was applied to map the sequences against the reference HBV genome (DQ683578.1). The sequences whose quality was estimated to be greater than QD30 (quality score normalized by depth 30 ) were selected. GATK v3.7 Mutect 2 was used to call SNPs and indels in the sequences.

Result: The characteristics of the subjects were summarized in tables. We sequenced and analyzed the whole genome of HBV including DNA polymerase, $\mathrm{preS} 1 / \mathrm{preS} 2 / \mathrm{S}$, protein $\mathrm{X}$, and precore/core region. The average depth of coverage spanning 3215 nucleotides was 1359 (min-max; 389-2484). Total 1546 variants (1516 snp and 30 indels) were detected in 23 samples. Among them, 605 variants in polymerase, 360 variants in preS1/preS2/S, 94 variants in $\mathrm{X}, 165$ variants in precore/core region were non-synonymous variants, which make changes in amino acid and protein sequence. The most variable region was g.1950 encoding the core protein, which showed 4 types of variants.

Conclusion: It is expected that the next-generation sequencing technology will provide important clues to the identification of the drug resistance mechanism of HBV because it can read the entire nucleotide sequence of the HBV. And these clues should contribute to the development of effective therapeutic agents to the HBV.

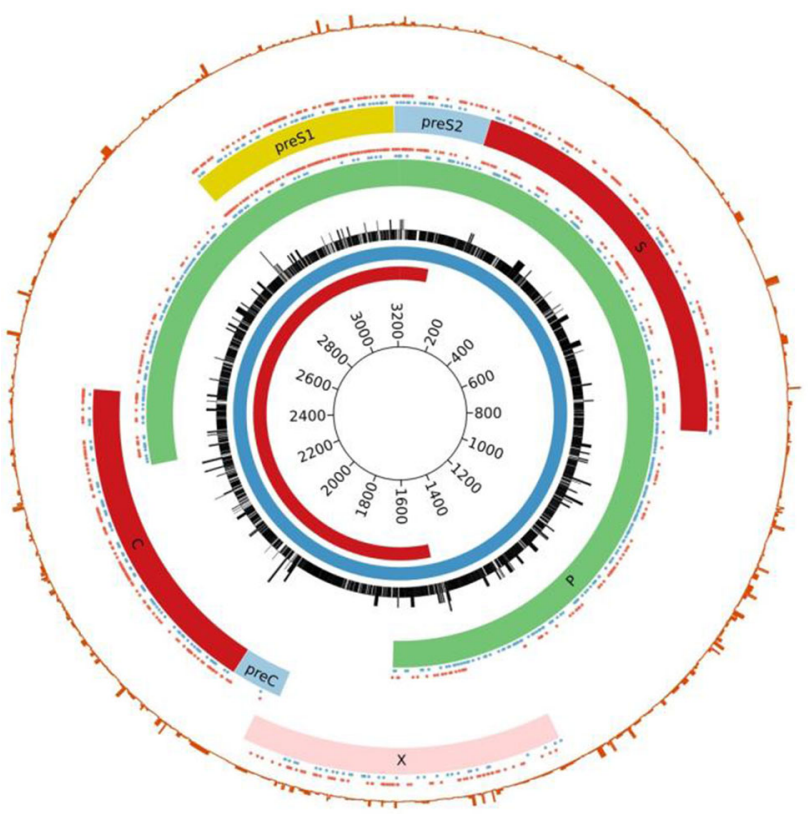

$H B V-B 19$

Characteristics of amino acid substitutions within the "a" determinant region of $\mathrm{HBV}$ surface protein in $\mathrm{CHB}$ patients with coexisting HBsAg and anti-HBs

\section{Wei Hou}

${ }^{1}$ Tianjin Second People's Hospital, China

Background: Chronic hepatitis B (CHB) patients with both hepatitis B surface antigen (HBsAg) and antibodies to HBsAg (anti-HBs) have been described elsewhere. The mutations within the "a" determinant region of HBV Surface (S) gene, which could alter the antigenic conformation and antigenicity of $\mathrm{HBsAg}$, might be one of the possible mechanisms underlying this atypical serological profile. The aim of this study was to analyze the characteristics of amino acid substitutions within "a" determinant region in a large cohort of CHB patients with coexistence of HBsAg and anti-HBs.
Method: In total $8687 \mathrm{CHB}$ patients, of which 505 had coexisting $\mathrm{HBsAg}$ and anti-HBs, were enrolled in this study. Mutations within the "a" determinant region in $131 \mathrm{HBsAg}+/ \mathrm{anti}-\mathrm{HBs}+$ patients and 150 age and gender matched HBsAg+/anti-HBs- patients were determined by Sanger sequencing and the characteristics of amino acid substitutions were analyzed.

Result: The prevalence of coexistence of HBsAg and anti-HBs in the CHB populations was $5.81 \%$. Two HBV genotypes (B and C) were identified in the 281 samples, respectively. Among them, $43(15.3 \%)$ patients were infected with genotype B, 238 (84.7\%) with genotype C. Compared to the control subjects, there were more amino acid substitutions in the HBsAg +/anti-HBs + patients (30.5\% vs. $12.7 \%, \mathrm{P}$ $<0.001)$. In the subgroup analysis stratified by HBV genotypes, more amino acids variability occurred within the "a" determinant region in $\mathrm{HBsAg}+/$ anti-HBs + patients with genotype C $(29.0 \%$ vs. $12.4 \%, \mathrm{P}=$ 0.001),but not with genotype B. When stratified with the loop of "a" determinant region, more amino acid substitutions were observed within the first loop (pooled, $26.7 \%$ vs. $10.0 \%, \mathrm{P}<0.001$; genotype $\mathrm{B}, 35.7 \%$ vs. $6.7 \%, \mathrm{P}=0.05$; genotype $\mathrm{C}, 25.6 \%$ vs. $10.7 \%$, $\mathrm{P}=0.003$ ), but not within the second loop. Amino acid substitutions within "a" determinant were detected at positions s126, s127, s129, s130, s131, s133, s134, s140, s137, s143, s144 and s145. Compared to the control subjects, the most frequent substitution was located at position s126 in HBsAg+/anti-HBs+ patients $(\mathrm{P}=0.002)$, especially in the patients with genotype $\mathrm{C}(\mathrm{P}=0.011)$. In terms of position $\mathrm{s} 126$, the predominant substitution was I126T $(64.0 \%)$ in HBsAg+/antiHBs+ patients, followed by I126s (12.0\%), I126N (8.0\%) and I126V $(8.0 \%)$, respectively. Most of the I126T substitutions (14/16, 87.5\%) belong to single amino acid substitution, only $12.5 \%(2 / 16)$ combined with other substitutions.

Conclusion: The increased amino acid substitutions within the "a" determinant region of HBV S gene might contribute to the coexistence of HBsAg and anti-HBs. These findings extend understanding of the genetic mechanism of the atypical serological profile in $\mathrm{CHB}$ patients.

\section{$H B V-B 20$}

HBcAg-induced up-regulated of 4-1BB ligand on $B$ cells activate CD4 $\mathrm{T}$ cells and regulate cytokines secretion during chronic hepatitis $B$ infection

Yong Liu ${ }^{1}$, Guiyang Wang ${ }^{1}$, Rui Huang ${ }^{1}$, Chen Tian ${ }^{1}$, Yang $\mathbf{L i}^{1}$, Yuxin Chen ${ }^{1}$

${ }^{1}$ Nanjing Drum Tower Hospital, Nanjing University Medical School, China

Background: The dysfunction of $\mathrm{B}$ cells in chronic hepatitis $\mathrm{B}$ (CHB) infection remained to be obscure. Several limited studies have indicated hyperactive $\mathrm{B}$ cell responses. The expression of 4-1BB ligand (4-1BBL) on B cells is associated with cell activation, differentiation or suppressive, primarily through interaction of 4-1BB on $\mathrm{T}$ cells. In this study, we aimed to characterize 4-1BBL on B cells and investigate the role of $4-1 \mathrm{BBL}$ on $\mathrm{B}$ cells among chronic hepatitis $\mathrm{B}$ patients.

Method: 4-1BBL expression on B cells was monitored by flow cytometry in a total of CHB patients and healthy individuals. B cells were stimulated by $\mathrm{sCD} 40 \mathrm{~L}, \mathrm{HBs}$ g or $\mathrm{HBcAg}$, then the level of 4-1BBL on B cells was quantified. The 4-1BBL + B cells and 4-1BBL- B cells were separately sorted by FACS and co-cultured with $\mathrm{T}$ cells, the supernatants of co-culture of $\mathrm{T}$ cells and 4-1BBL $+\mathrm{B}$ cells or 4-1BBL- B cells, respectively, were collected. Cytokines from supernatant and CD69 and 4-1BB expression on T cells were further determined. 
Result: The 4-1BBL expression on B cells was significantly higher in CHB patients than that in healthy subjects. After stimulated with sCD40L or HBcAg, B cells had higher level of 4-1BBL compared with control groups. Meanwhile, 4-1BBL + B cells had higher level of costimulatory molecules, including CD80, CD86, MHC classes I and II than 4-1BBL- B cells. After co-cultured with 4-1BBL+ B cells, the expressions of CD69 and 4-1BB on CD4 T cells were significantly higher than that co-cultured with 4-1BBL- B cells. Cytokines in the culture supernatant including IL-2, IL- 4 and IL-6 were significantly higher in co-culture group of $4-1 \mathrm{BBL}+\mathrm{B}$ cells and $\mathrm{T}$ cells than that in co-culture group of 4-1BBL- B cells and T cells and $\mathrm{T}$ cells alone group. However, the levels of IFN- $\gamma$ and TNF- $\alpha$ in $4-1 \mathrm{BBL}+\mathrm{B}$ cells group were significantly lower than that in $4-1 \mathrm{BBL}-\mathrm{B}$ cells group and $\mathrm{T}$ cells alone group. Thus, hyperactive status of $\mathrm{B}$ cells observed in CHB patients could be partially derived from high level 4-1BBL expression on B cells triggered by HBcAg. Further, 4-1BBL+ B cells could activate CD4 $\mathrm{T}$ cells and affect the cytokines secretion.

Conclusion: 4-1BBL signalling pathway is involved in B cells activation status, and further regulate $B$ cell- $T$ cells interaction by modulating cytokine secretion, which might be critical in B cells dysfunction during $\mathrm{CHB}$ infection.

\section{$H B V-B 21$}

Lipid based nanoparticulate system for effective vaccine delivery

\section{Mani Bhargava ${ }^{1}$, Saurabh Bhargava ${ }^{2}$ \\ ${ }^{1}$ GTB Hospital, India; ${ }^{2}$ Himalayan University, India}

Background: The search for innovative ways of vaccination has intensified recently with declining vaccine coverage and growing public concern about new virulent disease outbreaks. Immunization is a prophylactic approach through which the body is shielded from any incoming pathogenic invasion. The work envisaged here concerns exploring potential of Solid Lipid Nanoparticles (SLN) in efficient protein delivery ( $\mathrm{HBsAg}$ ) through surface modifications, which will in turn; enhance loading efficiency and cellular uptake of SLN using subcutaneous route.

Method: The SLN were prepared by Solvent Injection Method. SLN were optimized for various parameters as lipid, surfactant concentration, stirring time and speed. By considering particle size, polydispersity index (PI) and entrapment efficiency. The characterization parameters included Transmission \& Scanning Electron Microscopy, X-Ray Diffraction Analysis, In-vitro release, Kinetics of uptake by flow cytometer, Evaluation of cell apoptosis, T-cell proliferative response assay, $\mathrm{TH} 1 / \mathrm{TH} 2$ cytokine profile and Internalization studies by spectral bioimaging. The in-vivo study comprised of fluorescence studies and estimation of $\operatorname{IgG}$ response in serum and $\operatorname{sIgA}$ in various body secretions using specific ELISA.

Result: The particulate system is better carrier system for immunization because of less diffusivity and restricted movement. SLNs themselves act as signal for the phagocytic cells. Surface modified SLNs can entrap greater amount of antigen, provide its sustained release and rapidly internalized by the antigen presenting cells. Invitro $\mathrm{T}$ cell proliferation and induction of $\mathrm{TH} 1$ type of immune response clearly marks the potential of this novel carrier system. Fluorescence uptake studies showed better uptake of surface modified SLNs. Higher and more sustained antibody titer obtained with surface modified SLNs suggests their better immunological potential. Thus, subcutaneous immunization could be an efficient alternative approach for vaccination against hepatitis.

Conclusion: The formulations developed in this study can be further explored for the incorporation and delivery of other proteins and peptides and should subsequently be subjected to pilot plant scale-up as well as clinical trial to establish their potential for subcutaneous immunization against hepatitis $\mathrm{B}$.

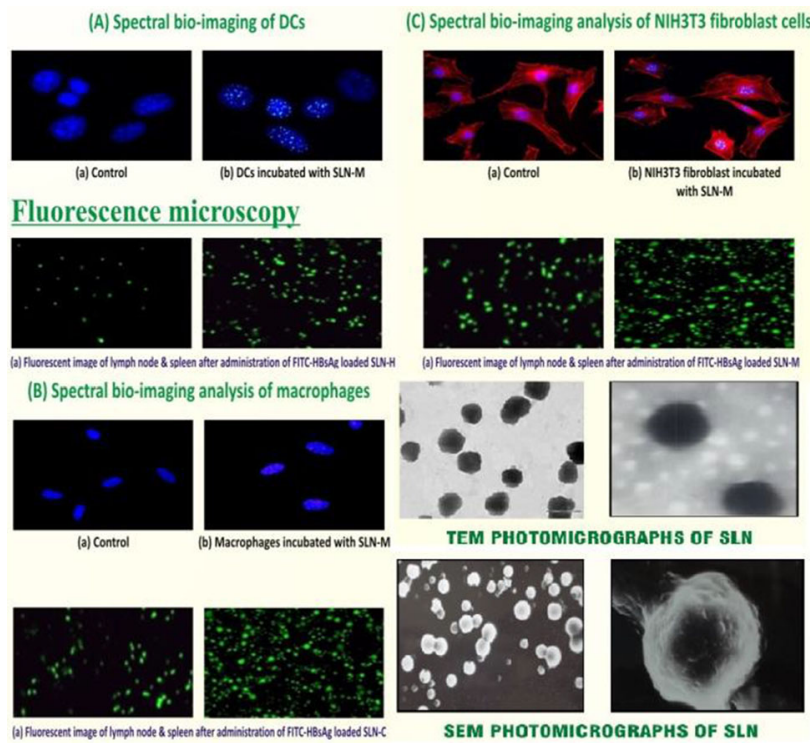

$H B V-B 22$

Development and characterization of surface modified chitosan nanoparticles for selective targeting of lamivudine to hepatocyte

$\underline{\text { Aakanchha Jain }}^{1}$, Mani Bhargava ${ }^{2}$

${ }^{1}$ Bhagyodaya Pharmacy College, India; ${ }^{2}$ GTB Hospital, India

Background: Hepatitis B is an infection of the liver caused by the hepatitis B virus (HBV). It is a major cause of infectious liver disease throughout the world. Viral hepatitis resides primarily in the liver; hence drug targeting with ligand anchored moiety can be an effective strategy in management of this disease. Lamivudine a "nucleoside analogue" is commonly used in treatment of Hepatitis B and effectively inhibit viral replication. However it shows extra-hepatic toxicity.

In the light of above, it was envisaged that the use of receptor-mediated endocytosis may permit the realization of the potential of drug targeting that reduces the side effects. This necessitates developing surface modified chitosan nanoparticles for hepatocyte selective targeting via conjugation of a ligand (glycyrrhizin).

Method: The chitosan nanoparticles were prepared by Low Molecular Weight Chitosan (LMWC) by Ionotropic gelation method and ligand was anchored. The nanoparticles were then characterized invitro for their shape, size, drug entrapment, in-vitro drug release and stability. The in-vivo study comprised of biodistribution studies in various organs and fluorescence microscopy was performed, hematological and histological examinations were done.

Result: Finally it could be concluded that encapsulation of lamivudine in glycyrrhizin coupled LMWC nanoparticles enhances the residence time. Further bioavailability of the drug in liver is increased which could be utilized in reducing the dosing frequency as well as the dose. This could help in the reduction of dose related toxicity associated with this antiviral drug.

Conclusion: Ligand mediated bio-deposition and cellular interaction of LMWC nanoparticles especially at the site would be a focal paradigm for the upcoming research in the field of antiviral drug delivery. 


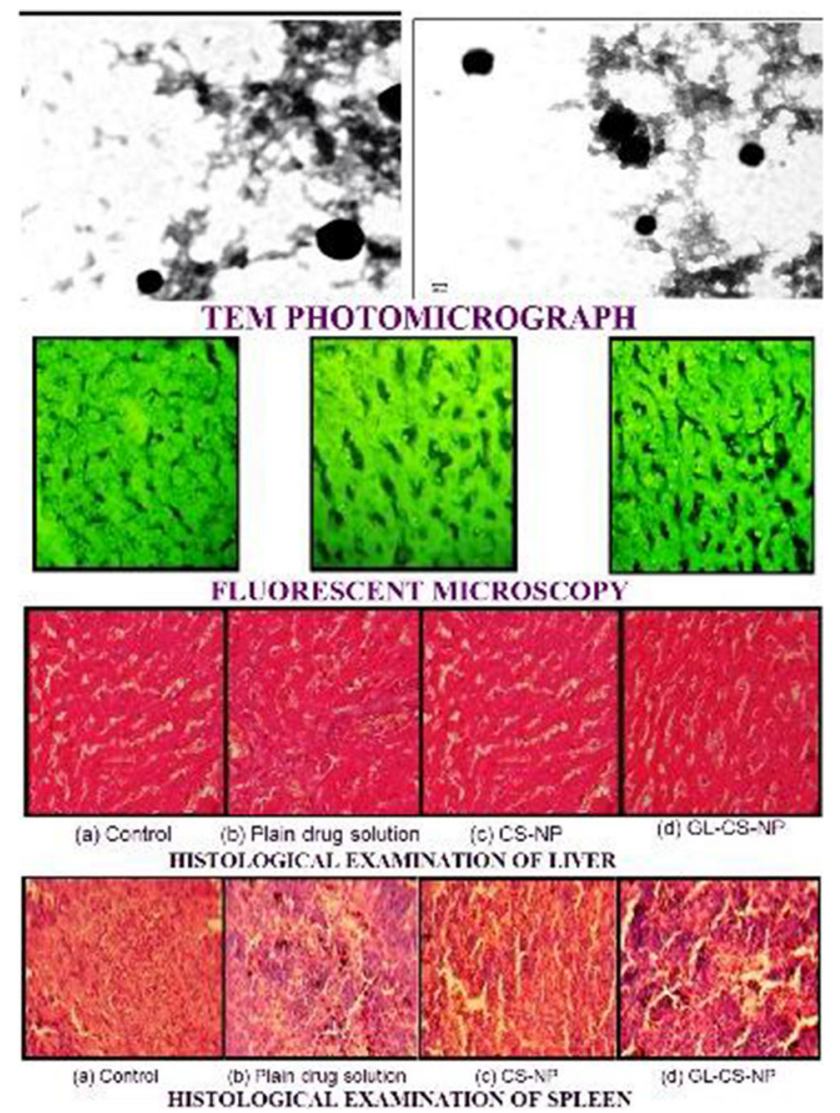

$H B V-B 23$

Development of bipolymer based novel nanoparticles in microsphere system as vaccine adjuvant

\section{Aakanchha Jain ${ }^{1}$}

${ }^{1}$ Bhagyodaya Pharmacy College, India

Background: Novel strategies are required for the achievement of safe and effective immunization beyond the conventional strategies. Frequent booster dosing can be avoided by the development of a mucosal/adjuvant vaccine delivery system, which can safely produce high and long lasting immune responses. Mucosal immunization is an attractive alternative to parenteral as with the appropriate delivery system it is possible to stimulate both humoral and cell-mediated responses.

The research work envisaged promotes the advantages and overcomes the disadvantages of the hydrophilic and hydrophobic polymeric systems, by a combined hydrophilic (gelatin nanoparticles, GN) with a hydrophobic polymeric system (PLGA microspheres). This combination creates a new biodegradable system for HBsAg delivery.

Method: GN \& PLGA microspheres were prepared by double emulsification method and composite system was prepared by phase separation method. Antigen loaded composites were optimized and characterized in-vitro for their shape, size, $\%$ antigen entrapment and stability. Fluorescence microscopy was carried out to confirm the uptake of composites. The in-vivo part of the study comprised of estimation of $\mathrm{IgG}$ response in serum and $\operatorname{sIgA}$ in various body secretions using specific ELISA. The external morphology was studied by Scanning \& Transmission Electron Microscopy.
Result: The in-vitro studies exhibited an initial burst release from gelatin nanoparticles, degradation of antigen from PLGA microspheres \& a continuous release from composite system. This supports the hypothesis to formulate single shot vaccine with such system (to mimic booster dosing). The fluorescence studies showed the selective uptake of composites by NALT.

Conclusion: Humoral response generated by single dose of composites was comparative to marketed formulation that received the booster dose. Further, composite system generated the effective sIgA antibody which was not elicited by the marketed formulation. Thus, it could be concluded from the present study that bipolymer based composite system are capable to provide sufficient protein stability and can be a promising candidate for development of single shot vaccine, not only against Hepatitis but against all those diseases that invade the host by the mucosal surfaces.

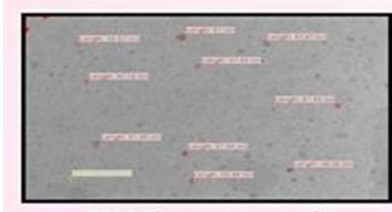

TEM Photomicrograph of Gelatin Nanoparticles

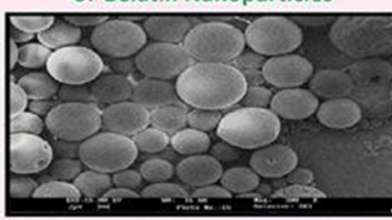

SEM Photomicrographs of

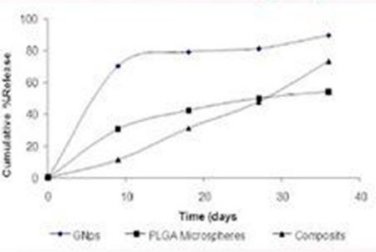

In-vitro Release

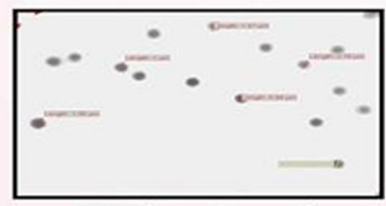

TEM Photomicrograph of PLGA Microspheres
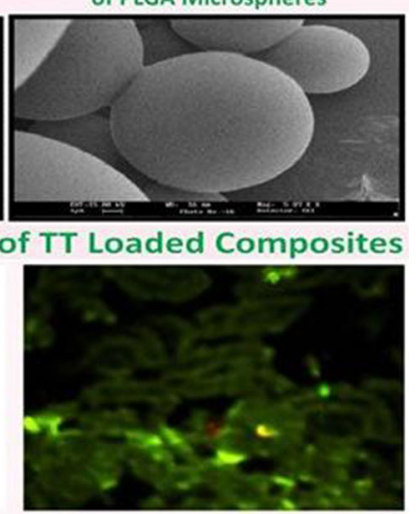

Fluorescence Microscopy of Nasal Mucosa

\section{$H B V-B 24$}

Hunan circulating macrophage inflammatory protein-1beta: a novel prognostic marker of $\mathrm{HCC}$ in chronic hepatitis B patients

Shuaibu Abdullahi Hudu', Mohd Taib Niazlin², Syafinaz Amin

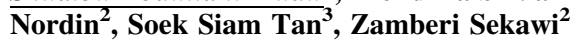

${ }^{1}$ Faculty of Basic Medical Sciences, College of Health Sciences, Usmanu Danfodiyo University Sokoto, Nigeria; ${ }^{2}$ Faculty of Medicine and Health Sciences, Universiti Putra Malaysia, Malaysia; ${ }^{3}$ Selayang Hospital, Lebuh Selayang Batu Cave Selangor, Malaysia, Malaysia

Background: Chronic liver infections are mostly characterised by the expression of pro- and anti-inflammatory cytokines which are the driving force of many inflammatory diseases of the liver, resulting in fibrosis, cirrhosis and subsequent hepatocellular carcinoma (HCC). Therefore, this study aimed to determine a novel prognostic biomarker of HCC in chronic hepatitis B patients

Method: Plasma samples were examined from chronic hepatitis B patients and normal healthy control. Liver function test results were obtained from hepatology department of Selayang hospital. Quantitative hepatitis surface and $e$ antigen was performed using enzyme linked immunosorbent assay according to manufacturer's instruction. 
Hepatitis B viral DNA was extracted from all the plasma samples and quantified using real time PCR. Magnetic beads multiplex Luminex assay, plate was then read immediately using the Luminex analyser. Result: Bivariate correlation reveals that, Macrophage Inflammatory Protein-1 beta (MIP-1beta) in chronic hepatitis B patients is significantly associated elevated liver enzymes such as ALT ( $r=0.314$; $p<0.005$; Figure 1A), and AST ( $\mathrm{r}=0.317 ; p<0.005$; Figure 1B) which are secreted by the hepatocytes in response to liver injury as in viral hepatitis, leading to liver damage. Hepatitis B viral DNA was also found to exhibit positive significant correlation with MIP-1beta $(\mathrm{r}=0.272 ; p<0.05$; Figure 2). Hepatitis $\mathrm{B}$ viral $e$ antigen is an indicator of active viral replication which is detected in the early phase of infection following the appearance of HBsAg. The titres of these antigens rapidly rise during the period of viral replication. MIP-1beta were significantly associated with elevated HBsAg $\left(\chi^{2}=2411.71\right.$; $\mathrm{DF}=2244 ; p<0.005)$ and $\mathrm{HBeAg}\left(\chi^{2}=2302.99 ; \mathrm{DF}=2142 ; p<0.05\right)$.

Conclusion: MIP-1beta can bind carcinomatous tissue and kupffer cells in the liver therefore, it detection in cirrhotic patients might serves as a prognostic factor of HCC.
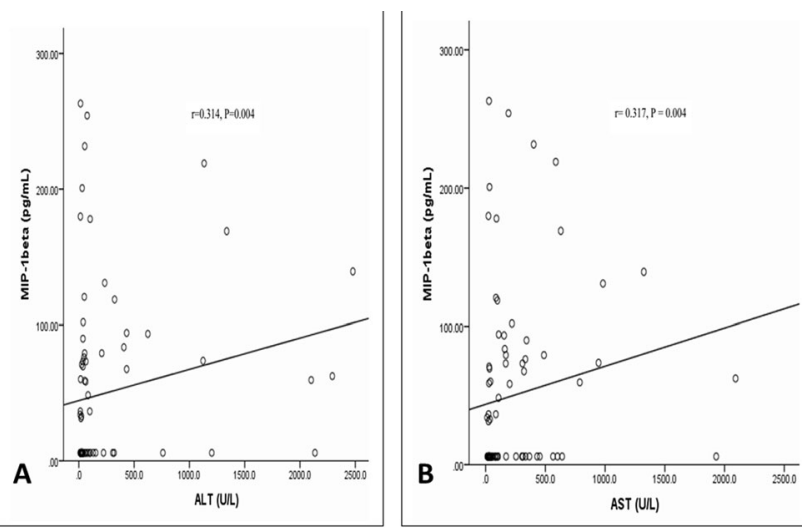

Figure 1. (A): Scattered plot correlation analysis of Macrophage Inflammatory ProteinInflammatory Protein-1 with AST. 1(MIP-1beta) with ALT; (B): Scattered plot correlation analysis of Macrophage

\section{$H B V-B 25$}

RNA sequencing analysis of diversity of hepatitis B virus transcripts in hepatocytes derived from chimeric mice

Shingo Nakamoto ${ }^{1}$, Tatsuo Kanda ${ }^{2}$, Shuang $\mathrm{Wu}^{1}$, Koji

Takahashi $^{1}$, Masato Nakamura ${ }^{1}$, Shin Yasui ${ }^{1}$, Sadahisa

Ogasawara ${ }^{1}$, Eiichiro Suzuki ${ }^{1}$, Yoshihiko Ooka ${ }^{1}$, Akinobu

Tawada ${ }^{1}$, Tetsuhiro Chiba ${ }^{1}$, Makoto Arai ${ }^{1}$, Kazufumi

Kobayashi $^{1}$, Soichiro Kiyono ${ }^{1}$, Hitoshi Maruyama ${ }^{1}$, Naoya Kato ${ }^{1}$, Hiroshi Shirasawa ${ }^{1}$

${ }^{1}$ Chiba University, Japan; ${ }^{2}$ Nihon University, Japan

Background: Viral quasispecies is associated with clinical pathogenesis and levels of immune response. We characterized the diversity of $\mathrm{HBV}$ whole genome in the HBV-infected hepatocyte by analyzing sequences of viral transcripts using in vitro infection system.

Method: Human hepatocytes derived from chimeric mice with humanized liver (PXB cell) were cultured on the cell culture plate and infected with 5 or 25 genome equivalents of HBV (genotype C) per cell (HBV-L or HBV-H, respectively). RNA was collected 5 days and 23 days after infection (D5, D23, respectively). RNA sequencing was carried out on an Illumina Hiseq 1500 sequencer. Reads are mapped to HBV parental sequence. Consensus sequence was determined, and mutation was analyzed by comparing with original sequences. Minor variation frequency was also analyzed.

Result: Thirteen thousand reads were mapped to HBV genome in the D5-L compared to 58 thousand reads in the D5-H. Consensus sequence was identical to the parental sequence under the all conditions studied. Eleven percent of the genome positions had minor variations detected more than $1 \%$ in the $\mathrm{D} 5-\mathrm{L}$ compared to $6 \%$ in the $\mathrm{D} 5-\mathrm{H}$ or $6 \%$ in the D23. In the D5-L, there were 5 positions which have minor variants with the frequency of more than $10 \%$ in the region with low coverage $(<100)$ compared to no positions in the D5$\mathrm{H}$ and 4 positions with low coverage in the $\mathrm{D} 23$.

Conclusion: Our results suggest that HBV genome is less affected by hepatocyte they infect and relatively stable without immune pressure from the immune cells.

$H B V-B 26$

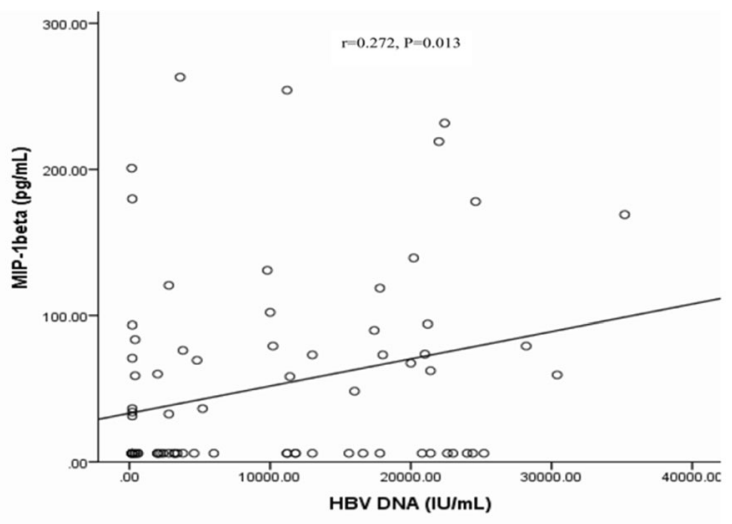

Figure 2: Scattered plot correlation analysis of Macrophage Inflammatory Protein-1 (MIP1beta) with hepatitis B viral DNA loads

\section{The stapled peptides derived hepatitis $B$ virus core protein interfere with viral replication}

\author{
Jinchao Hank ${ }^{1}$, Xiaoxiao Wang ${ }^{1}$, Yuqing $\mathrm{Lu}^{1}$, Xu Cong ${ }^{1}$, Xiaoben \\ Pan $^{1}$, Lai Wei ${ }^{1}$ \\ ${ }^{1}$ Peking University Pepole's Hospital, China
}

Background: Protein-protein interactions (PPI) are involved in all aspects of the viral lifecycle, including genome packaging, reverse transcription, intracellular trafficking and cccDNA epigenetic regulation. Those processes depend on the correct function and conformation of HBV core protein. To develop PPI inhibitors targeting core protein is attractive for HBV therapy. Here we designed a series of stapled peptides based on a-helix domains of core protein, and completed antiviral activity assay.

Method: Helical peptides with hydrocarbon staples were synthesized, uptake of peptide by cell and stabilization were assayed. Antiviral activity was measured by qPCR from HepG2 cells transiently transfected with four genotype expressing vectors $(A \sim D)$. HBsAg and $\mathrm{HBeAg}$ were measured by ELISA in HepDes19 cells. HBV replicative DNA intermediates and viral RNA were detected by southern and 
northern blot analysis. The interaction of stapled peptides with core protein was determined by co-immunoprecipitation.

Result: Those stapled had more highly levels of cell penetration, proteolytic resistance, and target affinity than natural a-helix peptides. Several stapled peptides derived from core protein intra-dimmer interface exhibited potent viral load reduction in vitro. Surprised, Stapled peptides inhibited $\mathrm{HBsAg}$ and $\mathrm{HBeAg}$ expression(EC50 $50 \sim 100 \mathrm{mM}$ ). HBV cccDNA was reduced in HepDeS19 cells treated with stapled peptides.

Conclusion: Hydrocarbon stapled a-helix peptides derived from core protein interfere HBV replication, which could be attractive tools for HBV therapy.

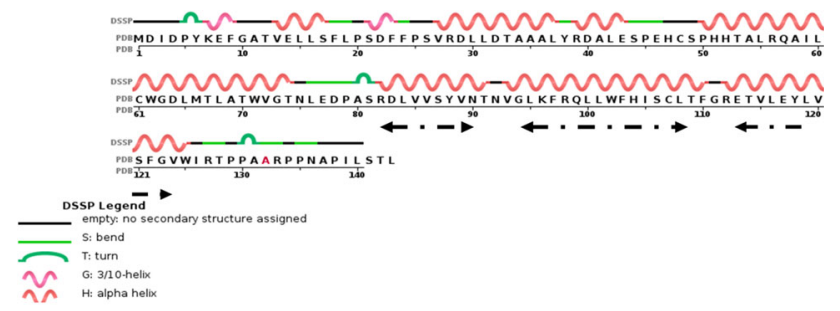

图1 HBV核心蛋白序列和二级结构图, $\leftarrow \cdots$ - 上方为订书肽设计区域

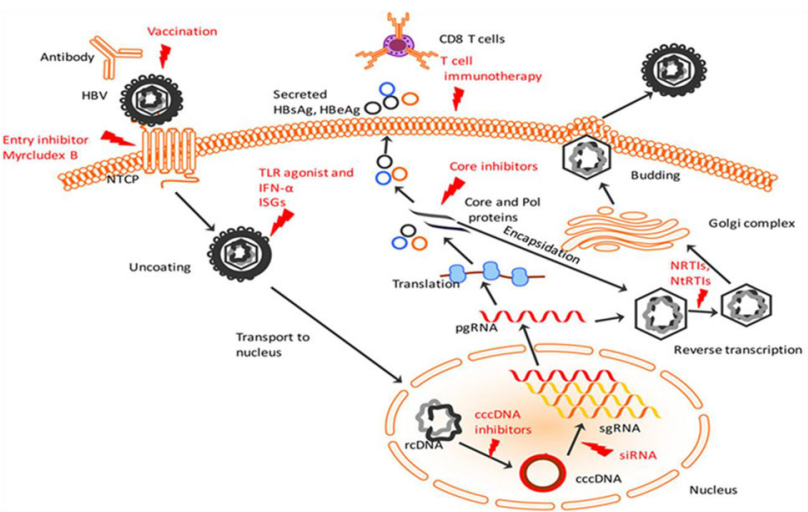

图1：HBV复制周期和抗病毒策略

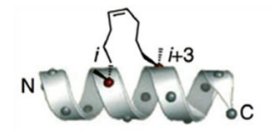

$R_{i, i+3} S(8)$ staple

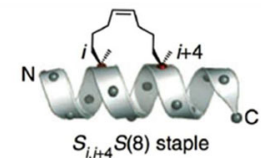

(n)
图2 HBV核心蛋白源性订书肽合成模式图

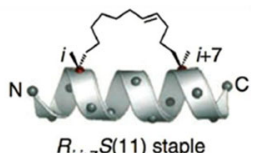

$R_{i, i+7} S(11)$ staple

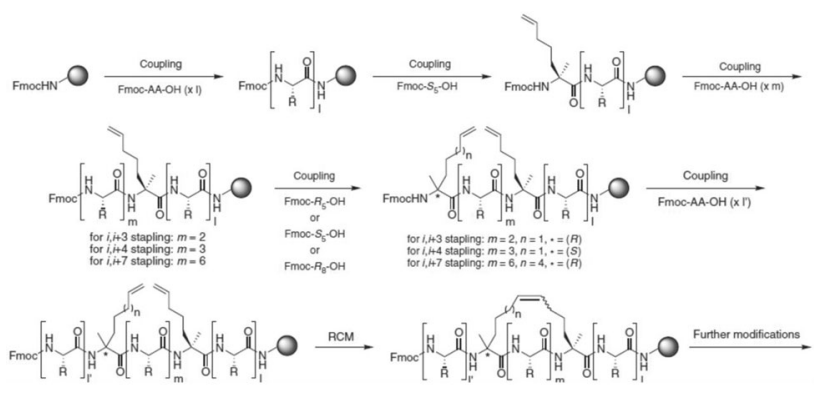

图2 $\alpha$-烯烃侧链的非天然氨基酸和全碳氢链订书肽合成图

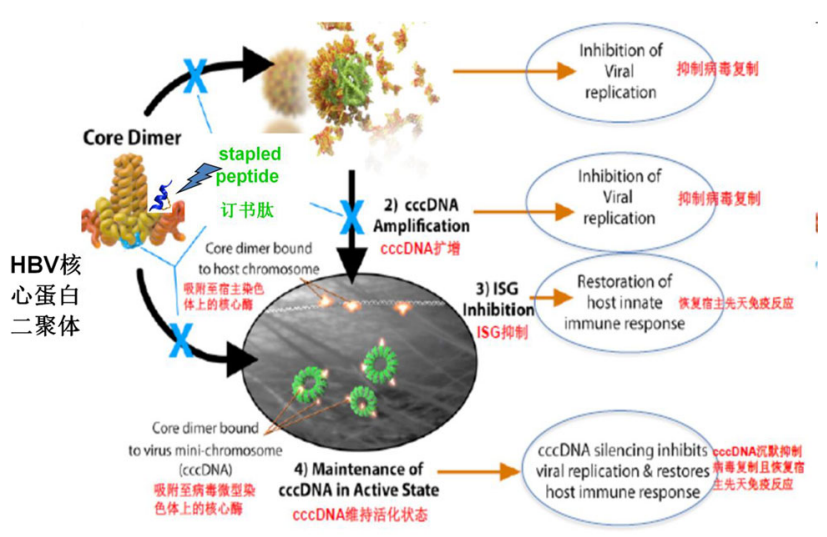

图3 订书肽抗HBV可能机制
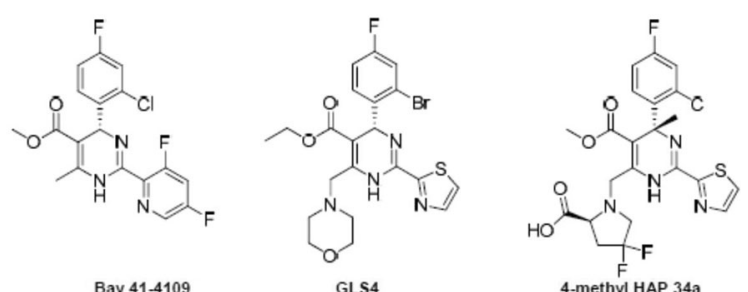

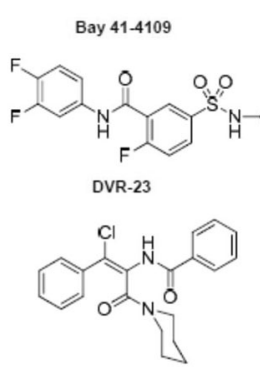

AT-61

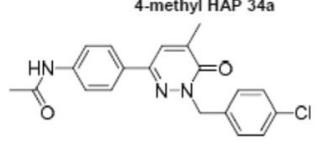

3711

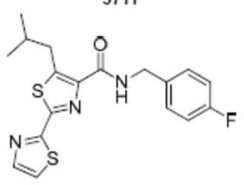

NZ-4

图2 五类小分子HBV核心蛋白别构剂 


\section{$H B V-B 27$}

\section{Surface engineered vesicular carriers for transmucosal} immunization via the nasal route

\section{Sourabh Jain ${ }^{1}$ \\ ${ }^{1}$ Bhagyodaya Tirth Pharmacy College, India}

Background: The critical role of vaccine delivery system in "rational vaccine design" has been widely recognized. Thus research work was envisaged involving development of $\mathrm{IgG}$ antibody immobilized on the surface of hepatitis B surface antigen ( $\mathrm{HBsAg}$ ) antigen-loaded liposomes. which offered increased uptake of nanoliposome through transmucosal surface of nasal route and sustaining release of $\mathrm{HBsAg}$ to evoke relatively high IgA titre in mucosal surface.

Method: Liposomes were prepared by a lipid cast film method \& then $\mathrm{IgG}$ antibody was cross linked on the surface. Coated liposomes were characterized in-vitro for their shape, size, polydispersity index, entrapment efficiency, zeta potential and stability. Fluorescence microscopy was performed to confirm the deposition pattern in respiratory tract. The in-vivo part of the study was conducted to visualize targeting potential, localization pattern, and immunogenicity. In addition, immune response was compared with alum-HBsAg vaccine injected intramuscularly.

Result: Observation of fluorescence images of nasal mucosa, lungs and spleen, revealed that these antibody coated liposome, were significantly taken up by mice respiratory mucosal surface, which made them promising carriers for mucosal vaccination. Considerable immune responses were produced by the developed system that may be due to the induction of MALT as well as contribution of the peripheral airways.

Conclusion: The higher immunity induced by ACL HBsAg may be attributed to its cationic nature, antibody coating and subsequent mucoadhesive property. Thus mucosal immunization with lipid vesicle through nasal administration may be effective in prophylaxis of diseases transmitted through mucosal routes as well as systemic infections. The strategy can be made more appropriate by determination of paracellular transport, nasal mucociliary clearance, mucosal toxicity assessment etc.

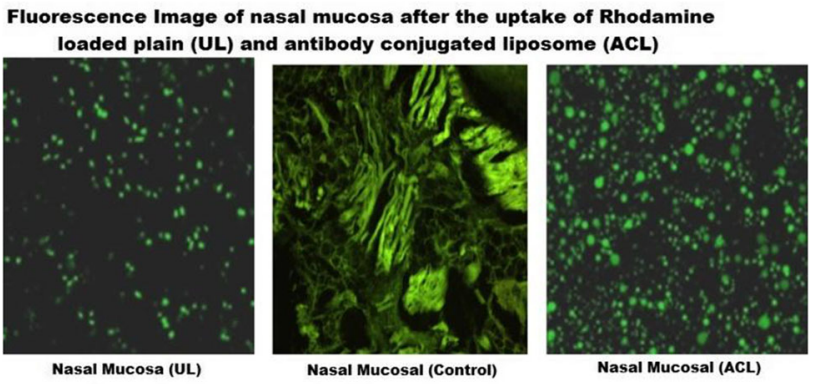

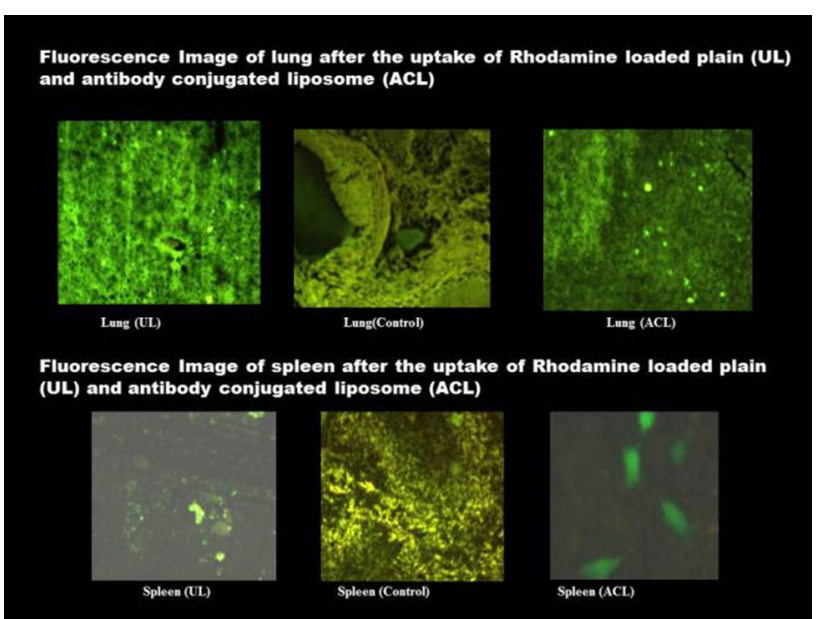

$\mathrm{HBV}-\mathrm{Cl}$

Do chronic hepatitis B patients with normal levels of aminotransferase need treatment?

\section{Huseyin Tarakci ${ }^{1}$}

${ }^{1}$ NO, Turkey

Background: Liver biopsy is the gold standard for assessing liver injury in the patients with chronic viral hepatitis. Treatment of $\mathrm{HBeAg-negative} \mathrm{patients} \mathrm{with} \mathrm{high} \mathrm{HBV-DNA} \mathrm{and} \mathrm{sustained} \mathrm{normal}$ levels of aminotransferase (SNAT) is the subject on which current studies are needed as was reported in all guidelines for the diagnosis and treatment of hepatitis B.

Method: The present study enrolled $\mathrm{HBeAg-negative} \mathrm{chronic} \mathrm{hep-}$ atitis B (CHB) patients with high levels of HBV DNA and SNAT, who had been followed between 2005 and 2017, with regard to the histological examination of liver biopsies of the patients, a histological activity index (HAI) of 6 and higher according to the Ishak Scoring was considered as marked inflammation and a fibrosis score of 2 and higher was considered as marked fibrosis.

Result: A total of $128 \mathrm{CHB}$ patients who met the eligibility criteria were enrolled in the study. Patients were divided into two subgroups according to their thrombocyte count; those with a thrombocyte count within the range of $100000-150000 / \mathrm{ml}$ and those with thrombocyte count higher than 150000/ml. Alanine aminotransferase (ALT) and aspartate aminotransferase (AST) levels were divided into two groups as before liver biopsy and after liver biopsy. Likewise, HBV DNA values were also divided into three groups $(>10000$ copy $/ \mathrm{ml}$, $>100000 \mathrm{copy} / \mathrm{ml}$, and $>1000000 \mathrm{copy} / \mathrm{ml}$ ). Those with an abdominal ultrasonography indicating suspicion of cirrhosis, a thrombocyte count lower than 100000/ml, ALT level higher than 1.25 folds of the normal, another liver disease, documented cirrhosis, and those treated for $\mathrm{CHB}$ were excluded. The patients were compared in terms of gender, age, body mass index, family history of hepatitis B, thrombocyte count, and ALT, AST and HBV DNA values as well as HAI scores and fibrosis scores. Increase in HAI scores were considered significant in the patients with ALT level higher than $40 \mathrm{IU} / \mathrm{l}$ but within a range not exceeding 1.25 fold of the upper limit of the normal and in the patients with HBV DNA level $>1000000$ copy $/ \mathrm{ml}$. Conclusion: HAI might be high in the patients with high HBV DNA level and sustained normal aminotransferase levels with an ALT level close to the upper limit of the normal and close monitoring of such patients is important in terms of need for treatment. 


\section{$\mathrm{HBV}-\mathrm{C} 2$}

Risk prediction model for hepatocellular carcinoma in treatmentnaïve, non-cirrhotic chronic hepatitis $B$ patients without alanine aminotransferase elevation

\section{YoungSuk Lim ${ }^{1}$, Seungbong Han', Giae Kim¹, Jonggi Choi ${ }^{1}$, Gwanghyeon Choi ${ }^{1}$ \\ ${ }^{1}$ Asan Medical Center, University of Ulsan College of Medicine, Korea, Republic of; ${ }^{2}$ Gachon University, Korea, Republic of}

Background: Precise prediction of hepatocellular carcinoma (HCC) risk is crucial in making decisions on antiviral treatment and surveillance in chronic hepatitis B (CHB). This study aimed to develop and validate a HCC risk prediction model for the CHB patients who are not traditionally subject to antiviral treatment due to no significant elevation of alanine aminotransferase (ALT) levels.

Method: The study subjects were recruited from a historical cohort of 5495 treatment-naïve, non-cirrhotic CHB patients with serum ALT levels $<2$ ' upper limit of normal (females, $<19 \mathrm{IU} / \mathrm{mL}$; males, $<30$ $\mathrm{IU} / \mathrm{mL}$ ) at a tertiary referral hospital in Korea. The patients were randomly assigned to either the development cohort or the validation cohort in 4:1 ratio. Cox proportional hazards regression model was used to predict HCC risks.

Result: During the total follow-up of 29,538 person-years, 221 patients $(5.1 \%)$ in the development cohort and $45(4.1 \%)$ in the validation cohort developed HCC. Old age, male gender, and lower platelet counts were found to be independently associated with an increased risk of HCC. Regarding $\mathrm{HBeAg}$ status and HBV DNA levels, HBeAg-positive patients with HBV DNA level $<6 \log 10$ IU/ $\mathrm{mL}$ (hazard ratio, 6.89, 95\% confidence interval [CI], 4.20-11.29) showed the highest risk. Based on these predictors, a 29-point risk model was developed, with HCC risk ranging from $0.01 \%$ to $42.0 \%$ at 3 years, $0.03 \%$ to $73.12 \%$ at 5 years, and $0.08 \%$ to $96.75 \%$ at 10 years. In the validation cohort, the area under receiver operating curves were $0.69(95 \% \mathrm{CI}, 0.55-0.85)$ at 3 years, $0.74(95 \% \mathrm{CI}$, $0.63-0.83)$ at 5 years, and $0.83(95 \% \mathrm{CI}, 0.77-0.88)$ at 10 years.

Conclusion: A HCC risk prediction model was developed and validated based on the non-cirrhotic CHB patients with normal or mildly elevated ALT levels. The model could help assess the HCC risk among patients who are not subject to antiviral treatment which consequently call attention to the necessity of developing a new treatment guideline.

\section{$\mathrm{HBV}-\mathrm{C} 3$}

Economic gains related to hepatocellular carcinoma (HCC) and decompensated cirrhosis (DCC) reduction in Japan is expected from treatment of chronic hepatitis $\mathrm{C}$ (CHC)

Gabriel Wong ${ }^{1}$, Atsushi Tanaka ${ }^{6}$, Tuichiro Eguchi ${ }^{5}$, Linda Henry $^{2}$, Rachel Beckerman ${ }^{3}$, Masashi Mizokami ${ }^{4}$

${ }^{1}$ Gilead Sciences, Inc., United States; ${ }^{2}$ Center for Outcomes research in Liver Diseases, United States; ${ }^{3}$ Maple Health Group LLC, United States; ${ }^{4}$ National Center for Global Health and Medicine, Japan; ${ }^{5}$ Saga University Hospital, Japan; ${ }^{6}$ Teikyo University School of Medicine, Japan

Background: Japanese $\mathrm{CHC}$ patients are infected for a longer period of time, and at risk for HCC, an important complication of $\mathrm{CHC}$. Highly effective Oral Direct Acting Antiviral (DAA) regimens for CHC can lead to high SVR rates which can reduce CHC complications and costs. This study used a decision analytic Markov model to estimate the economic benefit of $\mathrm{HCV}$ cure by reducing $\mathrm{HCC}$ and DCC in Japan.

Method: A hypothetical cohort of 10,000 HCV GT1b Japanese patients with a mean age of 70 was modeled with a hybrid decision tree and Markov state-transition model capturing the natural history of HCV infection over a lifetime horizon.

Result: It was assumed that $15 \%$ of the cohort had cirrhosis and $20 \%$ were treatment-experienced. Treatment options were assumed to be approved all-oral DAAs vs. no treatment (NT). Treatment efficacy was based on randomized controlled trials of DAA regimens. Transition rates and costs were obtained from Japan-specific data. The number of cases of DCC, HCC and quality-adjusted life years (QALYs) were projected for patients treated with an all oral DAA vs. NT. QALYs were monetized using a willingness to pay (WTP) threshold which varied from $¥ 4$ to $¥ 6$ million. The incremental savings associated with treatment were calculated by adding the projected cost of complications avoided to the monetized gains in QALYs.

The model showed that DAA treatment can avoid 1583 cases of HCC and 1162 cases of DCC, saving $¥ 618,076$ and $¥ 251,329$ per treated patient; respectively. If we combine both DCC and HCC as serious complications of HCV-cirrhosis in Japan, treatment leads to avoidance of 2745 cases of complications and the associated savings of $¥ 869,405$ per treated patient. Additionally, DAA treatment can lead to an additional 1.59 QALYs gained per patient treated. The indirect economic gains associated with treatment-related QALY improvements were estimated to be $¥ 6,360,000, ¥ 7,950,000$ and $¥ 9,540,000$ per patient at WTP thresholds of $¥ 4$ million, $¥ 5$ million and $¥ 6$ million. Total economic savings of HCV GT1 treatment with DAAs (vs. NT) was $¥ 7,229,405, ¥ 8,819,405$ and $¥ 10,409,405$ at these different WTP thresholds.

Conclusion: Treatment of HCV GT1b with all Oral DAAs in Japan can lead to significant savings related to avoidance of HCC and DCC.

\section{$\mathrm{HBV}-\mathrm{C} 4$}

Early hepatitis B surface antigen seroclearance after commencement of antiviral treatment in patients with de novo $\mathrm{HBV}$ reactivation

Hae Lim Lee ${ }^{1}$, Hae Lim Lee ${ }^{1}$, Sung Won Lee ${ }^{1}$, Si Hyun Bae ${ }^{2}$, Jong Young Choi ${ }^{2}$, Seung Kew Yoon ${ }^{2}$

${ }^{1}$ Bucheon St. Mary's Hospital, Korea, Republic of; ${ }^{2}$ Seoul St. Mary's Hospital, Korea, Republic of

Background: Hepatitis B virus (HBV) reactivation can occur not only in chronic hepatitis $\mathrm{B}(\mathrm{CHB})$ patients, but even in patients with resolved infection, especially in those who had undergone hematopoietic stem cell transplantation (HSCT) or rituximab treatment. We evaluated the virologic-serologic responses after commencement of antiviral treatment in patients with de novo HBV reactivation.

Method: We reviewed 1,101 consecutive patients treated with rituximab or HSCT who had tested for HBV serum markers at a tertiary center from January 2006 to August 2014. Among them, a total of $341 \mathrm{HBs} A g$-negative/anti-HBc-positive patients who were followed up with HBV markers were included in the study. Clinical outcomes of the patients with de novo HBV reactivation were then compared with $34 \mathrm{CHB}$ patients who started antiviral therapy during rituximab-based therapy or HSCT for hematological diseases.

Result: Forty-three out of the 341 patients (12.6\%) experienced de novo HBV reactivation at median 25.1 months and the cumulative rates were $5 \%, 13 \%$, and $26 \%$ at 1,2 and 4 years, respectively. The median value of HBV DNA at reactivation was $6.2 \mathrm{X} 106 \mathrm{IU} / \mathrm{mL}$. 
Twenty-seven patients $(62.8 \%)$ received antiviral therapy. There was no difference in the complete virologic response rate but significantly higher rate of HBsAg seroclearance was observed in the de novo reactivation group than the CHB group ( $75 \%$ vs. $11.8 \%$, respectively, $\mathrm{p}<0.001)$. Early drop in HBsAg titer and higher ALT levels were associated with HBsAg seroclearance. For the de novo reactivation group, the cumulative rates of $\mathrm{HBsAg}$ seroclearance were $38 \%$ and $60 \%$ at 6 and 12 months, respectively, with a median time to $\mathrm{HBsAg}$ seroclearance of 6.6 months after antiviral initiation. All of the patients with $\mathrm{HBsAg}$ seroclearance except 1 patient without follow-up result also developed anti-HBs antibodies.

Conclusion: De novo HBV reactivation occurs considerably in settings of rituximab-based therapy or HSCT. The early HBsAg seroclearance following antiviral therapy suggests the differential phenotype as well as shorter duration of chronic infection in such patients with de novo HBV reactivation.

\section{$\mathrm{HBV}-\mathrm{C} 5$}

HBeAg quantification to differentiating inactive carrier from HBeAg negative chronic hepatitis B patients

\section{Fehmi Tabak $^{1}$, Sibel Yildiz Kaya ${ }^{1}$, Bilgul Mete ${ }^{1}$, Ilker Inanc Balkan $^{1}$, Nese Saltoglu ${ }^{1}$ \\ ${ }^{1}$ Cerrahpasa School of Medicine, Turkey}

Background: We aimed to determine the contribution of $\mathrm{HBsAg}$ quantification (qHBsAg) to differentiating inactive carrier from active hepatitis in $\mathrm{HBeAg}$ negative chronic $\mathrm{HBV}$ infection patients with HBV DNA $2000-20.000 \mathrm{IU} / \mathrm{ml}$.

Method: We included 100 patients who were treatment naive $\mathrm{HBeAg}$ negative chronic $\mathrm{HBV}$ infection, followed in our department between 2014-2017. We excluded the patients with HDV, HCV and HIV coinfection, cirrhosis and hepatoscellular carcinoma. Patients divided into 3 groups based on HBV DNA level: group 1 (HBV DNA $\leq 2000$ $\mathrm{IU} / \mathrm{ml}$ ), group 2 (HBV DNA 2000-20.000 IU/ml) and group 3 (HBV DNA $>20.000 \mathrm{IU} / \mathrm{ml})$. We collected serum from all patients for qHBsAg analysis. We compared serum qHBsAg levels with biochemical parameters and HBV DNA levels and liver biopsy results. Result: Fifty-eight patients were female and the mean age was 41 years. The median value of qHBsAg of all patients was 3028 (range: 13-41.804) IU/ml. None of the patients whose HBV DNA were $2000-20,000 \mathrm{IU} / \mathrm{ml}$ and had active hepatitis was detected $\mathrm{qHBsAg}$ $\leq 1000 \mathrm{IU} / \mathrm{ml}$. The ratio of $\mathrm{HBsAg}$ quantitation $(\log \mathrm{IU} / \mathrm{ml})$ to $\mathrm{HBV}$ DNA $(\log \mathrm{IU} / \mathrm{ml})$ was noted to be significantly higher in inactive carriers compared with chronic hepatitis $\mathrm{B}(\mathrm{CHB})$ patients. When all patients were examined, there was a positive correlation between qHBsAg and HBV DNA, ALT, HAI score and disease stage. The cut off level of qHBsAg was determined as $4526 \mathrm{IU} / \mathrm{ml}$ to differentiate inactive carrier from $\mathrm{HBeAg}$ negative $\mathrm{CHB}$. With the test specificity of $\% 95$, we found qHBsAg cut off values $1187 \mathrm{IU} / \mathrm{ml}$ and $15848 \mathrm{IU} /$ $\mathrm{ml}$ for the diagnose of inactive carriers and $\mathrm{CHB}$, respectively.

Conclusion: Previous studies have demonstrated that HBV DNA $\leq 2000 \mathrm{IU} / \mathrm{ml}$ with serum $\mathrm{qHBsAg} \leq 1000 \mathrm{IU} / \mathrm{ml}$ are useful to diagnose inactive carrier. Our study observed that this cut-off can be used for diagnosis of inactive carrier in patients with HBV-DNA 2000-20.000 IU/ml as well. In addition, it may be considered to initiate antiviral therapy without advanced tests such as liver biopsy in patients with $\mathrm{HBV}$ DNA $>2000 \mathrm{IU} / \mathrm{ml}$ and $\mathrm{qHBsAg}>15.000 \mathrm{IU} / \mathrm{ml}$.
$H B V-C 6$

Impact of metabolic syndrome on the clinical outcomes of patients with chronic hepatitis $B$ with nucleos(t)ide analogues treatment

\section{Hong Joo Kim ${ }^{1}$ \\ ${ }^{1}$ Sungkyunkwan University Kangbuk Samsung Hospital, Korea, Republic of}

Background: The prevalence of combined metabolic syndrome (MS) and chronic hepatitis $\mathrm{B}(\mathrm{CHB})$ was known to be $0.99 \sim 1.74 \%$ and a known risk factor for increased occurrence of cirrhosis and hepatocellular carcinoma (HCC). However, no data are available for the impact of MS on the clinical outcomes in CHB patients with oral nucleos(t)ide analogues (NUCs) treatment. We aimed to elucidate whether the association of MS and $\mathrm{CHB}$ can impact the clinical outcomes in CHB patients with oral NUCs treatment.

Method: Five hundred eighty-seven CHB patients who received NUCs treatment in our institution from January 2006 to March 2016 were enrolled in the current study. The diagnosis of MS was made by International Diabetes Federation (IDF) consensus definition. Disease progression (PD) was defined as at least one of the followings (newly appeared liver cirrhosis, newly developed HCC, escalation of ChildTurcotte-Pugh (CTP) class, and newly appeared complications of liver cirrhosis, such as ascites, hepatic encephalopathy and/or variceal bleeding).

Result: Overall adverse outcomes (OAO) were defined as the occurrence of at least one of the followings during the study period (emergence of virologic breakthrough and genotypic resistance, new HCC development, PD, and/or death due to liver diseases). Seventy $(11.9 \%)$ out of 587 our enrolled patients were compatible with the definition of MS by IDF consensus definition. There were no significant differences in decrease of $\log 10 \mathrm{HBV}-\mathrm{DNA}$ (mean $\pm \mathrm{SD}$, copies/ml) between CHB patients with MS and without MS 3, 6, 9, $12,24,36,48$, and 60 months after the initiation of NUCs treatment. The occurrence rate of complete response (CR) was also not significantly different between CHB patients with MS and without MS. The normalization rate of alanine aminotransferase (ALT) was significantly higher in $\mathrm{CHB}$ patients without MS compared to those with MS (76.6\% vs. $54.3 \%, \mathrm{P}$

Conclusion: Presence of MS in CHB patients with oral NUCs treatment adversely impacts the clinical outcomes in these patients by increasing cumulative occurrence rates of genotypic resistance, HCC, and PD with resultant poor survival without affecting the virologic responses.

\section{$H B V-C 7$}

Medication nonadherence increases hepatocellular carcinoma, cirrhotic complications and mortality in chronic hepatitis B patients treated with entecavir

Seung Bum Lee ${ }^{1}$, Jung Woo Shin ${ }^{1}$, Seok Won Jung ${ }^{1}$, Byung Uk Lee $^{1}$, Bo Ryung Park ${ }^{1}$, Eun Ji Park ${ }^{1}$, Seung Bum Lee ${ }^{1}$, Jae Ho Park', Byung Gyu Kim', In Du Jeong', Sung Jo Bang', Neung Hwa Park ${ }^{1}$

${ }^{1}$ Ulsan University Hospital, Korea, Republic of

Background: Optimal adherence to nucleos(t)ide analog treatment is necessary to achieve undetectable levels of HBV DNA in patients with chronic hepatitis $\mathrm{B}(\mathrm{CHB})$, and to prevent cirrhotic complications. However, no large long-term follow-up study has investigated the effect of adherence to entecavir (ETV) treatment on specific liver- 
related events (LREs), namely, hepatocellular carcinoma (HCC), cirrhotic complications and mortality.

Method: This was a 10-year longitudinal observational study of treatment-naïve patients with CHB who received ETV treatment. The primary outcome was the cumulative probability of LREs. The cumulative level of adherence to medication was categorized as good $(=90 \%)$ or poor (

Result: Data from 894 treatment-naïve CHB patients who received ETV were analyzed. Overall mean adherence rates were $89.1 \%$. Patients with poor adherence had a higher risk of virologic breakthrough (VBT) (HR, 22.42; 95\% CI, 19.57-52.52; P

Conclusion: Poor adherence to medication was associated with a higher mortality and greater risk of HCC and cirrhotic complications, particularly among patients with liver cirrhosis.

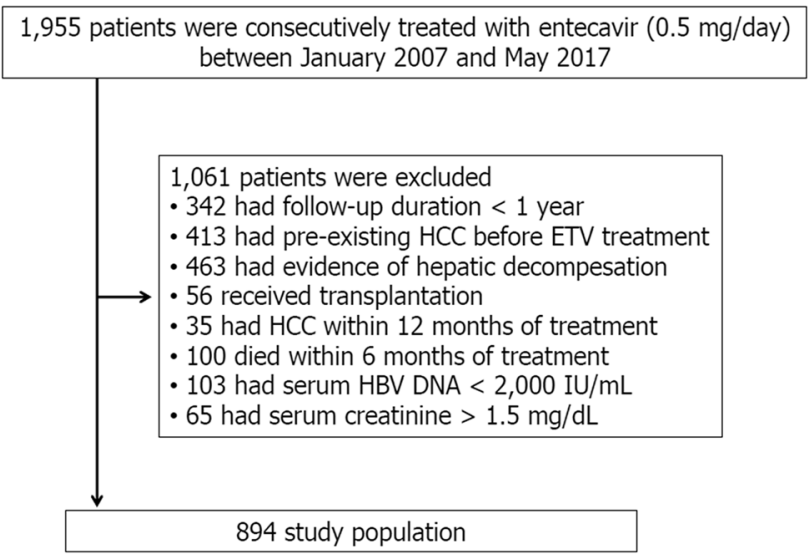

A

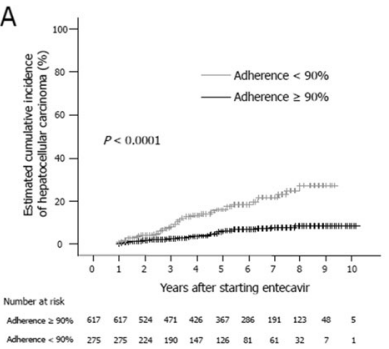

C

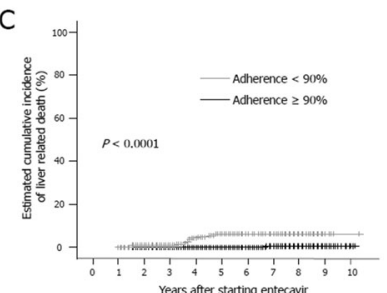

B

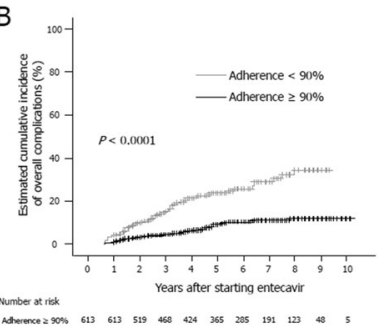

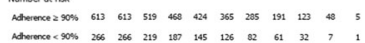

D

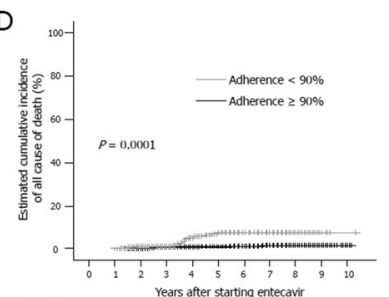

number $x$ mitive

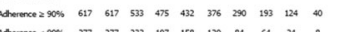

$H B V-C 8$

Mortality, liver transplantation, and hepatic complications in patients with treatment-naïve chronic hepatitis $B$ treated with entecavir vs tenofovir

Seung Bum Lee ${ }^{1}$, Sung Jo Bang1, Jung Woo Shin ${ }^{1}$, Neung Hwa Park $^{1}$, Seung Bum Lee ${ }^{1}$, Hojune Lee ${ }^{1}$, Byung Uk Lee ${ }^{1}$, Jae Ho Park $^{1}$, Byung Gyu Kim ${ }^{1}$, Seok Won Jung ${ }^{1}$, In Du Jeong ${ }^{1}$

${ }^{1}$ Ulsan University Hospital, Korea, Republic of

Background: Few studies have directly compared the long-term clinical outcomes of entecavir (ETV) and tenofovir disoproxil fumarate (TDF). This study aimed to compare the risk of mortality, liver transplantation, and hepatic complications including hepatocellular carcinoma (HCC) and hepatic decompensation between these drugs in treatment-naïve chronic hepatitis B (CHB).

Method: We performed a longitudinal observational analysis of data from 1325 consecutive adult CHB patients with a cumulative adherence of $=80 \%$ to treatment with ETV $(n=721)$ or TDF $(n=604)$ at a tertiary referral hospital in Ulsan, Korea, from January 1, 2007, through April 31, 2017. Among the patients, 708 were analyzed using propensity score matching with a ratio of $1: 1$.

Result: In the follow-up period of up to 5 years, 5 patients $(0.4 \%)$ died, 3 patients $(0.2 \%)$ underwent liver transplantation (LT), and 54 patients $(4.1 \%)$ developed HCC. Hepatic decompensation occurred in $24(1.8 \%)$ patients. ETV therapy did not differ from TDF therapy in terms of the risk of liver-related death or LT (HR 0.96; 95\% CI, 0.234.07; log-rank $\mathrm{P}=0.955)$, HCC (HR, 1.36; 95\% CI, 0.72-2.56; logrank $\mathrm{P}=0.340$ ), and hepatic decompensation (HR, 1.64; 95\% CI, 0.674.00; log-rank $\mathrm{P}=0.276$ ). In the 354 propensity-matched pairs, ETV and TDF were also showed no difference in risks of mortality, LT, and hepatic complications.

Conclusion: In a longitudinal observational study of 1325 patients with CHB, ETV and TDF therapy were effective on the risk of mortality, HCC, LT, and hepatic decompensation.

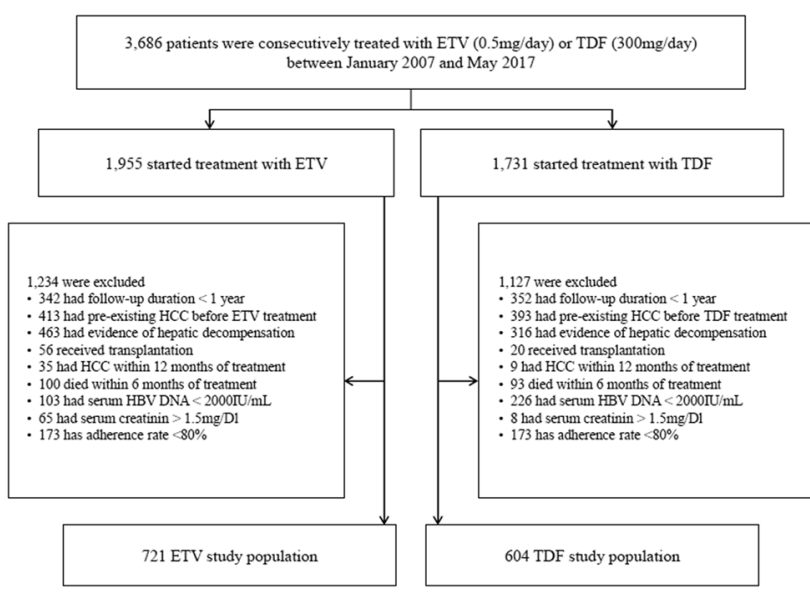

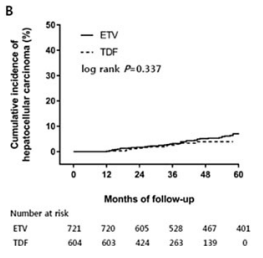

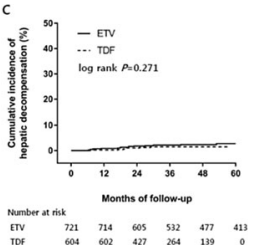




\section{$\mathrm{HBV}-\mathrm{C} 9$}

REST-B: a significant proportion of chronic hepatitis B (CHB) patients on long-term tenofovir disoproxil fumarate (TDF) monotherapy should be considered for changing therapy

Hariklia Kranidioti $^{1}$, Hariklia Kranidioti ${ }^{3}$, John Goulis ${ }^{2}$, Christos Triantos $^{5}$, Anastasia Kourikou ${ }^{3}$, Evangelia Tsentemidou ${ }^{2}$, Christos Tsolias ${ }^{5}$, Konstantinos Thomopoulos ${ }^{5}$, Theodoros Voulgaris ${ }^{4}$, Nikolaos Papadopoulos ${ }^{3}$, Melanie Deutsch ${ }^{3}$, George Papatheodoridis ${ }^{4}$, Spilios Manolakopoulos ${ }^{3}$

${ }^{1}$ Medical School of National and Kapodistrian University of Athens, Greece; ${ }^{2}$ Aristotle University of Thessaloniki, Greece; ${ }^{3}$ Medical School of National \& Kapodistrian University of Athens, Greece; ${ }^{4}$ Medical School of National and Kapodistrian University of Athens, Laiko General Hospital, Athens, Greece; ${ }^{5}$ University Hospital of Patras, Greece

Background: Entecavir, TDF and tenofovir alafenamide (TAF) are the current therapy for CHB. According to the recent EASL guidelines, patients on TDF who are at risk for or have renal/bone toxicity should be considered for a switch to entecavir or TAF, depending upon prior lamivudine (LAM) exposure. We aimed to evaluate the safety profile of long-term TDF therapy and to explore the proportion of patients who may be considered for TDF switch in a sizeable real world cohort of CHB patients

Method: This is a retrospective-prospective study of CHB patients under long-term treatment with TDF. We enrolled 275 CHB patients from four Academic liver units (2 in Athens, 1 in Thessaloniki, 1 Patras). Before TDF initiation, mean age was $56 \pm 14$ years, 168 were males, 15 were $\mathrm{HBeAg}$-positive, 27 had signs of portal hypertension, median HBV DNA was 3.6 (1.6-9.5) $\log 10 \mathrm{IU} / \mathrm{mL}]$. Virological remission was defined by undetectable serum HBV DNA with sensitive PCR assays. Clinical management was based on clinicians' judgment.

Result: We analyzed 212 (15 HBeAg-positive) patients who have been treated for $>24$ months with TDF. 55 (26\%) of our patients had comorbidities (diabetes, hypertension, dyslipidemia) and $47 \%$ had a history of prior LAM therapy. Mean follow-up was $66 \pm 23$ months. Virological and biochemical remission was observed in $96.5 \%$ and $88 \%$ at 24 months and $99 \%$ and $91 \%$ at 48 months, respectively. Age, gender, diabetes, BMI, hypertension, and therapy with statins were not associated with response to therapy. Five patients $(1 \mathrm{HBeAg}$ positive) presented HBsAg clearance after 36 (24-92) months of therapy. Patients $>60$ years old were $47 \%$ at baseline and $59 \%$ at last visit. eGFR $<60 \mathrm{ml} / \mathrm{min}$ was observed in $14 \%$ of the patients at baseline and in $15 \%$ at the last visit. In addition, 28 patients $(10 \%)$ had hypophosphatemia $(<2.5 \mathrm{mg} / \mathrm{dl})$ during the follow-up with $5 / 28$ having eGFR $<60 \mathrm{ml} / \mathrm{min}$. Multivariate analysis revealed that diabetes $(\mathrm{p}=0.004)$ and hypertension $(\mathrm{p}<0.001)$ are associated with the EASL indications for switching TDF to entecavir or TAF.

Conclusion: One fourth of our cohort had comorbidities. 2/3 of our TDF patients could be considered for a switch to entecavir or TAF according to EASL with a significant proportion of them having history of LAM exposure, indicating TAF may be preferred
$\mathrm{HBV}-\mathrm{ClO}$

Durability of HBsAg loss during or after antiviral treatment in a predominantly middle European collective and impact on clinical outcome

Gerlinde Teuber $^{1}$, Martin Sprinzl ${ }^{8}$, Dietrich Hueppe ${ }^{4}$, Renate Heyne $^{6}$, Wolf Peter Hofmann ${ }^{3}$, Markus Cornberg ${ }^{7}$, Arne Schaefer $^{2}$, Joerg Petersen ${ }^{5}$

${ }^{1}$ Praxis PD Dr. med. G. Teuber, Germany; ${ }^{2}$ Diabetes Zentrum Mergentheim, Germany; ${ }^{3}$ Gastroenterologie am Bayerischen Platz, Germany; ${ }^{4}$ Hepatologische Schwerpunktpraxis, Gibraltar; ${ }^{5}$ IFI-institute for interdisciplinary medicine, Germany; ${ }^{6}$ Leberzentrum am Checkpoint, Germany; ${ }^{7}$ Medizinische Hochschule Hannover, Germany; ${ }^{8}$ Medizinische Klinik, Germany

Background: Concerning the durability of HBsAg loss during or after antiviral treatment only a few data are available indicating a high rate of reactivation with recurrence of $\mathrm{HBsAg}$ and/or HBV-DNA of up to $16 \%$ in Asian patients. This may be due to the high percentage of patients with perinatal infection in these countries. There are no data from European patients available concerning the durability of HBsAg loss during or after antiviral treatment and the impact on further clinical outcome.

Method: In this retrospective german multicentre study, 143 patients with chronic hepatitis B (mean age: $43+13.8$ years, 93 males, 50 females) who lost HBsAg during or after antiviral treatment with peginterferon-a and/or nucleos(t)ides between april 2008 and july 2014 were included. Before antiviral treatment, 15 patients had established liver cirrhosis and 2/15 hepatocellular carcinoma, receiving partial liver resection. Primary end points were reactivation with recurrence of HBsAg Further endpoints were clinical progressive liver disease, liver transplantation and death.

Result: During the follow-up period (mean: $3.0+2.1$ years) a recurrence of HBsAg was observed in only $3 / 143$ patients $(2.1 \%)$, none with previous seroconversion to Anti-HBs. The HBV reactivation in these patients was not associated with detectable HBV-DNA levels or a rise in ALT-levels. 2/3 patients had baseline cirrhosis and $1 / 2$ subsequently died due to recurrent multifocal hepatocellular carcinoma. Among the 140 patients with persisting HBsAg loss, two initially cirrhotic patients died and one received liver transplantation all due to hepatocellular carcinoma.

Conclusion: In a predominantly Caucasian patient population $\mathrm{HBsAg}$ loss during and/or after antiviral treatment seems to be durable with low rates of reactivation. Cirrhotic patients, however, have a high risk for developing hepatocellular carcinoma even after HBsAg loss and regular surveillance in these patients seems to be mandatory.

\section{$H B V-C 11$}

No resistance to tenofovir alafenamide detected through 96 weeks of treatment in patients with chronic hepatitis B

Neeru Bhardwaj ${ }^{1}$, Henry Likyuen Chan ${ }^{6}$, Patrick Marcellin ${ }^{5}$, Calvin Q Pan ${ }^{4}$, Andrea Cathcart ${ }^{1}$, Yang Liu ${ }^{1}$, Stephanie Cox ${ }^{1}$, Bandita Parhy' ${ }^{1}$, Eric Zhou ${ }^{1}$, John F Flaherty ${ }^{1}$, Michael D Miller ${ }^{1}$, Anuj Gaggar ${ }^{1}$, Dr Shalimar ${ }^{2}$, Namiki Izumi ${ }^{7}$, Young Suk Lim ${ }^{3}$

${ }^{1}$ Gilead Sciences, United States; ${ }^{2}$ All India Institute of Medical Sciences, India; ${ }^{3}$ Asan Medical Center, Korea, Republic of; ${ }^{4}$ Division of Gastroenterology and Hepatology, United States; ${ }^{5}$ Hôpital Beaujon, France; ${ }^{6}$ Institute of Digestive Disease and State Key Laboratory of Digestive Disease, Hong Kong; ${ }^{7}$ Musashino Red Cross Hospital, Japan 
Background: Presented herein are the post Week 48 through Week 96 resistance analyses for 2 Phase 3 studies (GS-US-320-0108 and GS-US-320-0110) evaluating tenofovir alafenamide (TAF) versus tenofovir disoproxil fumarate (TDF) for the treatment of chronic hepatitis $\mathrm{B}(\mathrm{CHB})$ in $\mathrm{HBeAg}+$ and $\mathrm{HBeAg}$ - treatment-naïve or treatment-experienced adults.

Method: Patients were randomized 2:1 and stratified by HBV DNA and treatment status to receive TAF or TDF. HBV pol/RT population or deep sequencing was conducted for patients with $\geq 24$ weeks of treatment with viremia (HBV DNA $\geq 69 \mathrm{IU} / \mathrm{mL}$ ) at Week 96 or at early discontinuation post Week 48 . Deep sequencing was conducted for patients with HBV DNA $>159 \mathrm{IU} / \mathrm{mL}$ and sequence changes at the consensus sequence level (15\%) are reported. Virologic breakthrough (VB) was defined as HBV DNA $\geq 69 \mathrm{IU} / \mathrm{mL}$ after achieving $<69 \mathrm{IU} /$ $\mathrm{mL}$ or $\mathrm{a} \geq 1.0-\log 10$ increase from nadir. Phenotypic analysis using recombinant $\mathrm{HBV}$ in HepG2 cells was performed for VB patients who were adherent to study drug (plasma drug levels), patients with conserved site substitutions, or for polymorphic substitutions emergent in $>1$ patient.

Result: 1298 patients were randomized and treated (TAF: $n=866$; TDF: $n=432$ ). A similar percentage of patients in the TAF or TDF arms qualified for sequence analysis post Week 48 through Week 96 of treatment (TAF: $10.5 \%$, TDF: $10.9 \%$ ). In the TAF arm, 87 patients qualified at Week 96: 31 had no sequence change from baseline, 15 were unable to sequence (UTS), 32 had polymorphic site substitutions, and 9 had conserved site substitutions. In the TDF arm, 45 patients qualified at Week 96: 26 had no sequence change, 6 were UTS, 11 had polymorphic site substitutions, and 2 had conserved site substitutions. With the exception of the ADV-resistance substitution rtA181T, each detected conserved site substitution was observed in a single patient. Detection of the rtA181T substitution in 2 patients, 1 from each arm, was not associated with increasing plasma HBV DNA levels. At Week 96, a small percentage of patients experienced VB (TAF: $2.4 \%$, TDF: $3.0 \%$ ), and VB was often associated with documented study drug nonadherence (TAF: 22\%, TDF: 46\%). 27 patients qualified for phenotypic analysis post Week 48 through Week 96 (TAF: $n=19$, TDF: $n=8$ ) and no patient isolates tested showed a reduction in susceptibility to TAF or tenofovir, respectively.

Conclusion: Overall, the proportion of patients analyzed and the HBV sequence changes observed were similar between patients in the TAF and TDF arms of these Phase 3 studies. Most substitutions occurred at polymorphic positions and no substitutions associated with resistance to TAF were detected through 96 weeks of treatment.

\section{$\mathrm{HBV}-\mathrm{C} 12$}

Real life state of anti-HBV drug choice in child-bearing age male Hepatitis B patients and its effect on fertility

\section{Chan Xie ${ }^{1}$, Zhaoxia $\mathrm{Hu}^{1}$, Yinong $\mathrm{Ye}^{4}$, Weigang $\mathrm{Wu}^{2}$, Xujing Liang $^{3}$, Zhiliang Gao \\ ${ }^{1}$ The Third Affiliated Hospital of Sun Yat-Sen University, China; ${ }^{2}$ Shenzhen Third People's Hospital, China; ${ }^{3}$ The First Affiliated Hospital of Jinan University, China; ${ }^{4}$ The First People's Hospital of Foshan, China}

Background: Despite limited research on the effect of anti-HBV nucleoside analogs on male fertility, physicians continue to be concerned about safety of nucleoside analogs in pregnancy. Despite limited research on the effect of anti-HBV nucleoside analogs on male fertility, physicians continue to be concerned about safety of nucleoside analogs in pregnancy.

Method: CHB patients in Guangdong province were surveyed using a structured questionnaire. Inclusion criteria: Age between $18-50$ years, continuous evidence of positive HBV marker for $>6$ months. Exclusion criteria: Patients with liver cirrhosis, hepatocellular carcinoma or concurrent diagnosis of other severe vital organ diseases, patients whose wives had HBV infection. Meanwhile, a survey of the knowledge of antiviral nucleoside analogs safety in fertility of male patients and drug selection was conducted among physicians nationwide using WeChat network platform.

Result: A total of $1050 \mathrm{HBV}$ positive male patients were screened. Reasons for not receiving antiviral treatment in 150 patients included "did not meet criteria for antiviral therapy", fertility, and financial condition. A total of 900 participants received antiviral therapy, of which 792 patients had children, and $15.15 \%$ patients took anti-HBV treatment when they were preparing for pregnancy. In the childbearing group, $88.33 \%$ patients received telbivudine, while the non child-bearing group mainly received entecavir (87.20\%). There was no significant difference on birth defect incidence rate between the two groups. A total of 558 physicians nationwide participated in the questionnaire survey. Reasons that influenced drug selection included "patient's illness condition", "fertility demand", "financial condition", and "compliance". Telbivudine was the first choice drug $(32.80 \%, 183 / 558)$, and second was tenofovir $(2.69 \%, 15 / 558) .19 \%$ of physicians suggested to delay antiviral therapy and follow-up closely until childbirth.

Conclusion: Anti-HBV nucleoside analogs had no effect on male fertility. Desire for pregnancy influenced drug selection and timing of administration by physicians. More systematic research on male fertility is needed.

\section{$H B V-C 13$}

The change of biomarkers in peripheral blood during interferon therapy and its response predication value in the chronic hepatitis B

Yiqi Yu ${ }^{1}$, Yiqi Yu ${ }^{2}$, Jing Wang ${ }^{2}$, Chuan Shen ${ }^{4}$, Guojun $\mathrm{Li}^{3}$, Chao Qiu $^{2}$, Wenhong Zhang ${ }^{2}$

${ }^{1}$ Huashan Hospital affiliated to Fudan University, China; ${ }^{2}$ Huashan Hospital, Fudan University, China; ${ }^{3}$ The Second Hospital of Yinzhou of Ningbo, China; ${ }^{4}$ The Third Hospital of Hebei Medical University, China

Background: Underlying the potential mechanism of peginterferon will benefit patients with chronic hepatitis B infection. This study screened the cytokines related with peginterferon, and further evaluated these cytokines and their correlation with clinical data of HBeAg positive patients. Then, RNA-seq technique was used to study early genomics change of peginterferon therapy.

Method: The luminex technique was used to evaluate 27 cytokines of the serum samples of 10 paired $\mathrm{HBeAg}$ positive patients of week 0 , week 4 , week 12 , week 24 , week 48 , including IFN- $\alpha 2$, IL-10, IL12p70, IL-13, IL-17, IL-1 $\beta$, IL-2, IL-3, IL-4, IL-5, IL-6, IL-7, Mip$1 \alpha$, IL-29, IL-33, IL-21, TGF- $\beta 3$, G-CSF, IFN- $\gamma$, IL-8, IP-10, TNF- $\alpha$, CXCL9, IL-16, IL-27, TGF- $\beta 1$, TGF- $\beta 2$. We further chose 10 cytokines of high expression rate among the 27 cytokines which included G-CSF, IFN- $\gamma$, IL-8, IP-10, TNF- $\alpha$, CXCL9, IL-16, IL-27, TGF- $\beta 1$, TGF- $\beta 2$. 6 cytokines of the 10 had a significant different between $10 \mathrm{HBeAg}$ positive patients and 8 healthy controls, which included IP-10, CXCL9, IFN- $\gamma$, IL-16, TGF- $\beta 2$, IL-27 (P.

Result: Twelve weeks therapy of peginterferon lead to higher expression of G-CSF, IFN- $\gamma$, IL- 8 , IP-10, TNF- $\alpha$, IL-16 but lower expression of TGF- $\beta 1$ and TGF- $\beta 2$ in most of patients. The decline of ALT from week 0 to week 12 and the fold change of IP-10 from week 12 to week 0 were correlated though the analyzing the relation between the 8 cytokines and the clinical data during the peginterferon 
therapy( $\mathrm{r}=0.2675, \mathrm{P}=0.0024)$. To exam the early genomics change in the peginterferon therapy, we used RNA-seq to detect HBeAg positive patients with the treatment of peginterferon which includes 4 patients with treatment response and 6 with no response. We found 217 different expressed genes by comparing the response and no response groups. Go analysis showed that the response of peginterferon therapy was related with innate immunity and type 1 interferon signal pathway, etc.

Conclusion: The different expressed biomarkers were found during interferon therapy, which may provide clues for the further understanding of interferon therapy.

\section{$\mathrm{HBV}-\mathrm{C} 14$}

Assessment of treatment response by transient elastography during the tenofovir in nucleos(t)ide-naïve patients with chronic hepatitis B

\section{HoJung Jung ${ }^{1}$}

${ }^{1}$ Kangbuk Samsung Hospital, Korea, Republic of

Background: Several longitudinal studies have demonstrated that elastography could be a useful tool for monitoring of liver fibrosis during the treatment of chronic hepatitis B. However, not enough studies assessing regression of liver fibrosis by elastography are available to date. Therefore, the purpose of this study was to evaluate the liver stiffness before and during the treatment and assess the usefulness of this non-invasive modality.

Method: 127 treatment-naïve patients started treatment with tenofovir. Liver stiffness measurement by a transient elastography was performed at baseline, after $6,12,18$, and 24 months. The difference of results between baseline and each months of elastography were compared respectively by paired t-test methods.

Result: 115, 104, 75 and 54 patients had performed elastography at 6, 12, 18, and 24 months after tenofovir medication. Median liver stiffness values were decreased from $6.79 \mathrm{kPa}(95 \% \mathrm{CI}, 6.03-7.56)$ at baseline to 5.61 (95\% CI, 4.93 - 6.28) 5.44 (95\% CI, 4.75 - 6.12), $5.20(95 \% \mathrm{CI}, 4.36-6.05)$ and $5.15(95 \% \mathrm{CI}, 4.08-6.21) \mathrm{kPa}$ to 6 , $12,18,24$, and 30 months after tenofovir medication. Liver stiffness was significantly decreased at 6 months $(\mathrm{P}=0.007), 12$ months $(\mathrm{P}=$ 0.001) 18 months $(P=0.015)$ compared with that of pretreatment Conclusion: Liver stiffness was significantly improved in chronic hepatitis B patients with tenofovir treatment during 24 months. Considering the liver biopsy is invasive, costly, and associated with possible complications, transient elastography is useful to assess the progression or regression of liver fibrosis safely and serially.

\section{$\mathrm{HBV}-\mathrm{C} 15$}

Projection of health outcomes using tenofovir alafenamide (TAF) for the management of chronic hepatitis B (CHB) in Japan

Gabriel Wong $^{1}$, Ali Tafazzoli ${ }^{2}$, Catherine Saint Laurent Thibault ${ }^{2}$, Masayuki Kurosaki ${ }^{3}$

${ }^{1}$ Gilead Sciences, Inc., United States; ${ }^{2}$ Evidera, United States;

${ }^{3}$ Japanese Red Cross Musahino Hospital, Japan

Background: An estimated 1.2 million people in Japan have CHB. Current Japan Society of Hepatology guidelines recommend TAF, tenofovir disoproxil fumarate (TDF) and entecavir (ETV) as first-line treatment options. The 2017 EASL guidelines recommend that CHB patients with declining renal function/osteoporosis should be considered for switch to ETV or TAF with TAF preferred in lamivudine experienced patients.

Method: We estimated the health outcomes of a Japanese CHB population comparing TAF to ETV or TDF in 1,000 patients over a lifetime. Our model was developed using the Discretely Integrated Condition-Event (DICE) Simulation framework, which conceptualizes the disease and its management in terms of patients' conditions and events impacting these conditions. Model inputs were drawn from published RCTs, peer-reviewed Japanese literature, and real-world database analyses.

Result: Based on results of two pivotal registration trials, the model applied similar hepatitis B virus (HBV) suppression and resistance rates between TAF and TDF, but improved ALT normalization and bone/renal safety. Efficacy and safety data for ETV were obtained from published studies. From published literature, the model assumed that $20 \%$ of TE patients were lamivudine exposed and thus had higher viral resistance rates to ETV. Two treatment modalities were modeled: (1) 1st line treatment-naïve (TN) monotherapy and (2) TN and TE sequential therapy wherein treatments could be switched based on viremia and resistance.

Over a lifetime, TN patients initiated on TAF experienced better liver outcomes in terms of fewer compensated cirrhosis (CC), decompensated cirrhosis (DC), and hepatocellular carcinoma (HCC) events compared to ETV (Table 1). In terms of safety, patients that started on TAF had fewer chronic kidney disease stage 3 (CKDIII, 26 to 30 less per 1000 patients) and similar or fewer bone fracture ( 3 more to 14 less per 1000 patients) events compared to both ETV and TDF. However, it was found that the slight increase in fractures in TAF compared to ETV were due to TAF patients living longer and being able to accrue more fractures. Those who started on TAF similarly experienced higher life years (LY's, +0.1 to 0.2 ) and quality-adjusted life years (QALYs, +0.1 to 0.2 ).

Under sequential therapy, treatment sequences starting on TAF therapy experienced less CC (5 to 27 fewer per 1000 patients) and HCC (9 to 22 fewer per 1000 patients) events and higher LY's $(+0.1$ to 0.2 ) and QALYs (+0.1 to 0.2$)$ compared to ETV and TDF starts. Additionally, patients given TAF as 1st or 2nd line experienced less CKDIII (107 to 155 fewer per 1000 patients) events and fractures (7 to 20 fewer per 1000 patients) compared to other treatment sequences. Conclusion: TAF is projected to have fewer hepatic complications, and renal events when compared to TDF and ETV over a lifetime, driven by its favorable efficacy, safety and resistance profile.

Table 1. Number of Events over Lifetime

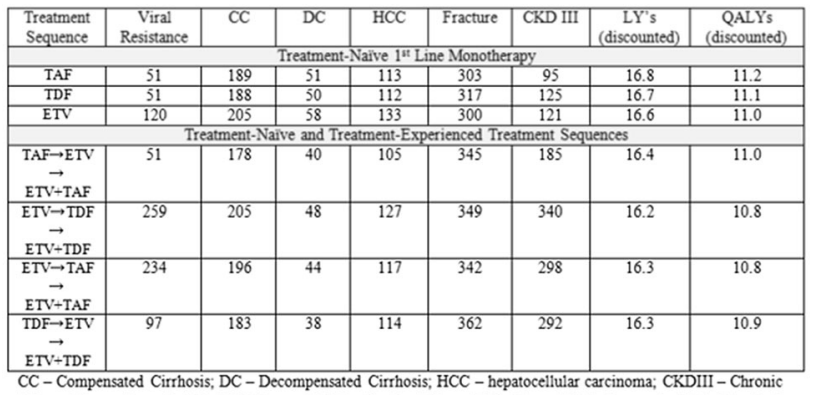

X - Compensated Cirthosis, DC - Decompensated Cirnosis, $\mathrm{CCC}$ - hepatocellular carcinoma, CKDII - Cironic Alafenamide: TDF - Temofovir Disoprovil Fumarte 


\section{$\mathrm{HBV}-\mathrm{Cl6}$}

The 96-week efficacy and safety of telbivudine-based optimization strategy in HBeAg-positive chronic hepatitis B patients with compensated cirrhosis

\section{Hanwei ${ }^{1}{ }^{1}$, Lijun Shen ${ }^{1}$, Bo Jin ${ }^{1}$, Jingjing Han $^{1}$, Xuemei Ma ${ }^{1}$, Feng Wen ${ }^{1}$, Xiaoli Yu ${ }^{1}$, Haiqin Wu ${ }^{1}$ \\ ${ }^{1}$ Beijing 302 Hospital, China}

Background: Efficacy and safety profile of Telbivudine (LdT)-based optimization strategy has been reported in chronic hepatitis B patients. But limited data showed the efficacy of LdT optimized treatment in cirrhosis patients.

Method: This prospective, single arm, open-label study was undertaken in HBeAg-positive, nucleoside-naïve adult HBV-related compensated cirrhosis patients. Patients received LdT for 24 weeks, after which those suboptimal responders with HBV DNA $\geq 300$ copies/ml received LdT plus adefovir(ADV), while the early responders (HBV DNA <300 copies/ml) continued LdT monotherapy. Patients with LdT monotherapy had ADV added on if primary non-response(HBV DNA decline $<1 \log 10 \mathrm{IU} / \mathrm{ml}$ at week 12) or virological breakthrough developed. Efficacy and safety were evaluated during 96 week treatment.

Result: 120 patients screened and 108 were eligible for efficacy analysis. At baseline, the mean HBV DNA level was 5.7 $\pm 1.7 \log 10$ $\mathrm{IU} / \mathrm{ml}$ and $70.4 \%$ patients had abnormal ALT level( $>40 \mathrm{IU} / \mathrm{ml}) .9$ patients received ADV add-on due to primary non-response. In the 99 patients without primary non-response, 70 had undetectable HBV DNA at Week 24 and continued LdT monotherapy, while 29 received ADV add-on. At Week 96, the overall proportion of undetectable HBV DNA was $79.6 \% \quad(86 / 108)$. ALT normalization occurred in $61.8 \%$ (47/76) patients. $19.4 \%(21 / 108) / 11.1 \%(12 / 108)$ patients achieved $\mathrm{HBeAg}$ loss /seroconversion. 25 patients developed virological breakthrough and completed sequencing of HBV polymerase gene, and genotypic resistance confirmed in 18 patients. 1 patient achieved HBsAg loss at week 84 and HBsAg seroconversion at week 96. For early responders, $92.9 \%$ achieved undetectable HBV DNA at week 96 , compared with $58.6 \%$ in suboptimal responders $(\mathrm{P}=0.0001)$, while $\mathrm{HBeAg}$ loss/seroconversion rate were comparable $(24.3 \%$ vs. $10.3 \%, \mathrm{P}>0.05 ; 12.9 \%$ vs. $6.9 \%, \mathrm{P}>0.05)$. LdT was well tolerated in most patients, no myopathy, myositis or rhabdomyolysis occurred. At week 96, eGFR level increased by $3.3 \mathrm{ml} /$ $\mathrm{min} / 1.73 \mathrm{~m}^{2}$ versus baseline (from 99.6 to 102.9 ). For patients with normal eGFR level at baseline (eGFR $\geq 90 \mathrm{ml} / \mathrm{min} / 1.73 \mathrm{~m}^{2}$ ), no obvious change occured at Week $96(\mathrm{P}=0.2684)$. For patients with low baseline eGFR level (eGFR $<90 \mathrm{ml} / \mathrm{min} / 1.73 \mathrm{~m}^{2}$ ), eGFR increased by $9.6 \mathrm{ml} / \mathrm{min} / 1.73 \mathrm{~m}^{2}$, (from $82.6 \pm 5.9$ to $92.2 \pm 10.8$, $\mathrm{P}=0.0007) .50 .0 \%(11 / 22)$ patients with low baseline eGFR had normal eGFR at Week 96.

Conclusion: In HBeAg-positive cirrhosis patients, LdT optimization strategy was effective and well tolerated. Comparing to suboptimal responders, early responder can achieve better virological response. Patients with low baseline eGFR level got obvious eGFR improvement after LdT optimization treatment.

\section{$\mathrm{HBV}-\mathrm{C} 17$}

Fibrosis-4 index predicts cirrhosis risk and liver-related mortality in patients with chronic $\mathrm{HBV}$ infection

TaiChung Tseng ${ }^{1}$, Chunjen Liu ${ }^{1}$, Tunghung $\mathrm{Su}^{1}$, Wanting Yang ${ }^{1}$, Chiling Chen $^{3}$, Hungchih Yang ${ }^{1}$, Peijer Chen ${ }^{3}$, Dingshinn Chen ${ }^{2}$, Jiahorng Kao ${ }^{1}$
${ }^{1}$ National Taiwan University Hospital, Taiwan; ${ }^{2}$ Academia Sinica, Taiwan., Taiwan; ${ }^{3}$ National Taiwan University College of Medicine, Taiwan

Background: Fibrosis-4 index (FIB-4) is a surrogate marker for hepatic fibrosis and serves as an independent risk predictor for hepatitis B virus (HBV)-related hepatocellular carcinoma. However, it is unclear whether a higher FIB-4 index is associated with increased risks of other liver-related adverse outcomes.

Method: A total of 2075 treatment-naïve Taiwanese patients with chronic HBV infection who had no evidence of liver cirrhosis at enrolment were reviewed to explore whether baseline FIB-4 index could stratify the risks of cirrhosis, cirrhosis-related complications, and liver-related mortality.

Results: The average period of follow-up is 15.47 years, and the annual incidence of cirrhosis and liver-related mortality were $0.76 \%$ and $0.19 \%$, respectively. A higher FIB-4 index was associated with a higher incidence of all liver-related adverse outcomes. Patients with FIB-4 $>1.29$, compared to those with FIB- $4<1.29$, were associated with increased risks of cirrhosis, cirrhosis-related complications, and liver-related mortality with the hazard ratio (95\% confidence interval) of 6.19 (4.76-8.05), 6.88, (3.68-12.86), and 7.79, (4.54-13.37), respectively. FIB- 4 index $<1.29$ was able to stratify the risks of liverrelated adverse outcomes even in $\mathrm{HBeAg}$-negative patients with a low risk of disease progression (HBV DNA $<2000 \mathrm{IU} / \mathrm{mL}, \mathrm{HBsAg}<1000$ $\mathrm{IU} / \mathrm{mL}$ and ALT $<40 \mathrm{U} / \mathrm{L})$. Only 1 patient with FIB-4 index $<1.29$ developed cirrhosis but without other events within 15 years of follow-up.

Result: In non-cirrhotic patients with chronic HBV infection, FIB-4 index $<1.29$ is useful for the prediction of the lowest risks of cirrhosis, cirrhosis-related complications, and liver-related mortality. Conclusion: chronic hepatitis B; HBV; HBsAg; FIB-4; APRI; HCC; liver disease; inactive carrier.

\section{$\mathrm{HBV}-\mathrm{C} 18$}

Association between IFN-gamma +874 polymorphisms and hepatitis $\mathrm{C}$ virus infection

Ozlem Kandemir ${ }^{1}$, Ozlem Kandemir ${ }^{2}$, Nurcan Aras ${ }^{3}$, Gulay Borekci $^{4}$, Aysegul Cetinkaya ${ }^{3}$, Irem Bekalp Yilmaz ${ }^{3}$, Guhan Orekici Temel $^{5}$

${ }^{1}$ Mersin University, Turkey; ${ }^{2}$ Mersin University Faculty of Medicine, Department of Clinical Microbiology and Infectious, Turkey; ${ }^{3}$ Mersin University Faculty of Medicine, Department of Medical Biology, Mersin, Turkey., Turkey; ${ }^{4}$ Mersin University School of Health, Mersin, Turkey, Turkey; ${ }^{5}$ Mersin University, Faculty of Medicine, Department of Biostatistics, Turkey

Background: Genetic polymorphisms in cytokines have been shown to affect HCV infection. The aim of the study was to evaluate the association between the gene polymorphisms in interferon gamma (IFN- $\gamma$ ) gene and chronic hepatitis $\mathrm{C}$ virus $(\mathrm{HCV})$ infection among our patients.

Method: IFN- $\gamma+874$ T/A genotypes were determined in 79 chronic HCV patients and 48 healthy controls using Real-Time Polymerase Chain Reaction (RT-PCR) from the DNAs. Genomic DNA was isolated using DNA isolation kit (Roche, Switzerland).

Result: In patients and control groups IFN- $\gamma+874$ TT, TA, AA genotypes were detected $27(31,4 \%), 34(\% 39,5), 25(\% 29,1)$ and 11 $(22,9 \%), 18(\% 37,5), 19(\% 39,6)$ respectively. Although there is no statistical significant was observed, in patient group TT and TA genotype's ratio were higher than control group $(\mathrm{p}=0.396)$. When the distribution of allele frequencies of IFN $-\gamma+874$ T/A polymorphism was evaluated in the patients and control groups, the ratio of $\mathrm{T}$ alleles 
in patient population was higher than control group $(51,8 \% 41,7 \%$ respectively), but it was not statistically significant ( $\mathrm{p}=0.136)$.

Conclusion: As a result IFN- $\gamma+874$ T/A polymorphisms had not a strong association with susceptibility to HCV infection. But studies with larger patient populations can help to demonstration of relationship with polymorphisms in interferon gamma (IFN- $\gamma$ ) gene and chronic hepatitis $\mathrm{C}$.

\section{$H B V-C 19$}

How much success we got with oral antivirals in the treatment of chronic hepatitis $B$ infection

\section{Esragul AKINCI $^{1}$, Esragul Akinci ${ }^{1}$, Ayse But ${ }^{1}$, Hurrem Bodur ${ }^{1}$ \\ ${ }^{1}$ Ankara Numune Education and Research Hospital, Turkey}

Background: Hepatitis B virus (HBV) infection remains a global public health problem in the world. Although we can talk about the cure in hepatitis C infection with more than $90 \%$ sustained virological response with direct acting antivirals, achieving the optimal treatment endpoint in hepatitis B infection is very difficult. In this study, we aimed to overview the success of the oral antiviral treatment in our patients with chronic hepatitis B infection.

Methods: In this study, we collected the data of the patients with chronic hepatitis B infection from the patient-records retrospectively. The patients having oral antiviral agents at least 2 years, taking medications without any interruption and coming to controls regularly were included in the study. The response of the therapy was evaluated with HBV-DNA suppression, ALT normalization, HBe-Ag loss, with or without anti-HBe seroconversion and $\mathrm{HBs}-\mathrm{Ag}$ loss, with or without anti-HBs seroconversion. HBs-Ag loss, with or without anti-HBs seroconversion was defined as an optimal treatment endpoint.

Results: Totally 131 patient-records were obtained between 2002 and 2015 according to the inclusion criteria. Of these patients, 98 were male and mean age was 44 years old (25-76). Mean follow up period was detected as 5.7 years (2-15). Mean HBV-DNA was 4 x 108 copies/ml (104-1010), mean ALT $70 \mathrm{IU} / \mathrm{ml}$ (11-392), mean fibrosis score 2 (1-4) and mean histological activity index 7 (2-13). The number of the patients according to the type of the oral antiviral regiments was as follows: Tenofovir 63, entecavir 58, lamivudine 9 and adefovir 1 patient. Twenty-seven $(20.6 \%)$ patients were $\mathrm{HBe}-\mathrm{Ag}$ positive. Of these patients, in 14 patients $(51.9 \%) \mathrm{HBe}-\mathrm{Ag}$ loss and in 12 patients $(44.4 \%)$ anti-HBe seroconversion were occurred. HBVDNA suppression was achieved in all of the patients $(100 \%)$ by the end of 2 year-treatment. HBs-Ag loss was gained in 4 patients (3\%).and Anti-HBs developed in three of them (2.3\%). In two patients, HBs-Ag loss developed during the antiviral treatment, 6 and 10 years after beginning of the treatment, respectively. In one patient, HBs-Ag loss was occurred 12 years later after stopping 3 year-antiviral treatment and after 2 years from HBs-Ag loss, Anti-HBs developed in this patient. In the other patient, HBs-Ag lost without Anti-HBs seroconversion.

Conclusion: Consequently, follow-ups of the 131 patients for average 5.7 years showed that only a few patients achieved the optimal treatment endpoint. So we can say that optimal treatment of hepatitis $B$ is very difficult with current treatment options. We need more effective drugs.

\section{$H B V-C 20$}

Prognostic value as to 3-month mortality of neutrophil-tolymphocyte ratio, lymphocyte-to-monocyte ratio and NLR and LMR ratio among patients with decompensated liver cirrhosis secondary to hepatitis B

Henedine Gae Dizon Chua ${ }^{1}$, Juliet Gopez Cervantes ${ }^{2}$, Cynthia $\overline{\text { Abad Mapua }}^{2}$, Mark Pierre Sijo Dimamay ${ }^{2}$, Michael Octubre Baclig $^{2}$

${ }^{1}$ St. Luke's Medical Center, Quezon City, Philippines; ${ }^{2}$ St. Luke's Medical Center, Philippines

Background: Philippines is still a hyperendemic area for hepatitis B which is one of the causes liver cirrhosis. It is estimated that 1 million deaths worldwide last 2010 were attributed to liver cirrhosis. A low socioeconomic status is associated with the infection. This paper aims to evaluate the prognostic utility of NLR, LMR, NLR:LMR among cirrhotic patients that are easily available and accessible among average Filipino patients.

Method: This is a retrospective cohort study of 92 adult in-patients from 2009 to August 2017 diagnosed with decompensated liver cirrhosis secondary to hepatitis B who have no malignancy, infection, $\mathrm{CKD}$, autoimmune disease, myocardial infarction, steroid use or other causes of liver cirrhosis. CBC from admission day was recorded. NLR, LMR, NLR:LMR were computed based on the CBC result. Mortality at three months from the admission day was recorded. However, 19 patients were lost to follow up. Descriptive statistics, independent t-test, chi-square test of homogeneity and ROC curve were used to analyze the data of the 72 patients.

Result: Majority of the patients were male, had Child Pugh score B and with mean age of 60 . NLR, LMR and NLR:LMR were not significantly associated with 3-month mortality. However, NLR was seen to be significantly associated with MELD score $(r=0.39$, $\mathrm{p}=0.0007$ ).

Conclusion: Our study failed to show an impact of NLR, LMR and NLR: LMR on short term survival and thus do not represent a robust prognostic factor in patients with hepatitis B related liver cirrhosis. However, NLR has shown correlation with MELD score.

Table 2: Association of NLR, LMR, and NLR-LMR Ratio with Demographic Profile

\begin{tabular}{|c|c|c|c|c|c|c|}
\hline \multicolumn{7}{|c|}{ Association of NLR, LMR, and NLR-LMR with Demographic Proffle $(\mathrm{N}=74)$} \\
\hline & \multicolumn{2}{|c|}{ NLR } & \multicolumn{2}{|c|}{ LMR } & \multicolumn{2}{|c|}{ NLR-LMR Ratio } \\
\hline & $\begin{array}{l}\text { Correlation } \\
\text { Coefficient }\end{array}$ & p-value & $\begin{array}{l}\text { Correlation } \\
\text { Coefficient }\end{array}$ & p-value & $\begin{array}{l}\text { Correlation } \\
\text { Coefficient }\end{array}$ & p-value \\
\hline Age & -0.03 & 0.7984 & -0.07 & 0.5525 & 0.14 & 0.2455 \\
\hline Sex (Fomale) & -0.01 & 0.9326 & 0.01 & 0.9288 & -0.04 & 0.7508 \\
\hline MELD Score & $0.39 \%$ & 0.0007 & -0.03 & 0.7914 & 0.01 & 0.9287 \\
\hline Child Turcotte Pugh Score & 0.14 & 0.246 & -0.12 & 0.298 & -0.19 & 0.099 \\
\hline Mortality (Non-Surviving) : & 0.14 & 0.2379 & 0.09 & 0.4470 & -0.04 & 0.7568 \\
\hline
\end{tabular}




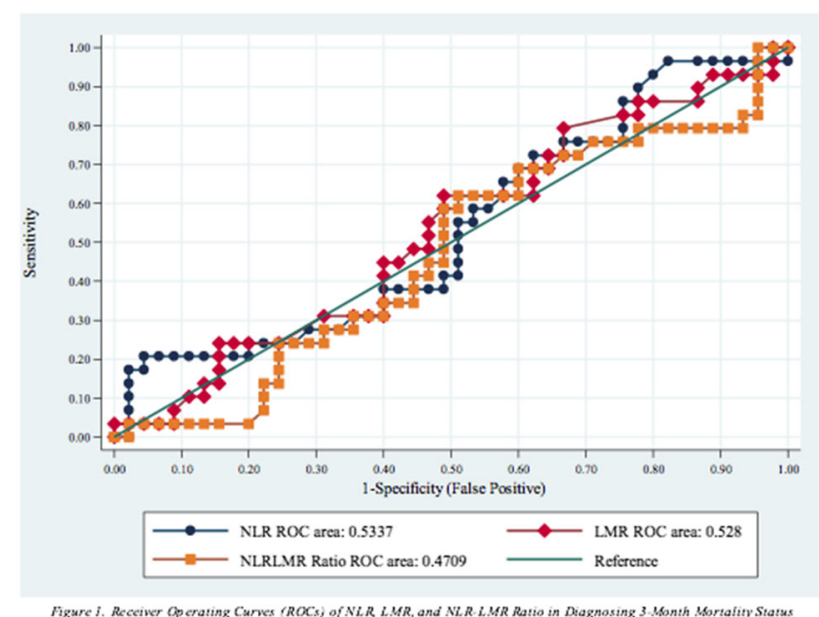

$\mathrm{HBV}-\mathrm{C} 21$

Real-life data of antiviral therapy in chronic hepatitis B patients with chronic kidney disease (Multicenter study)

Seok Hyun Kim $^{1}$, Jong Seok Joo ${ }^{1}$, Hyuk Soo Eun ${ }^{1}$, Eaum Seok Lee $^{1}$, Byung Seok Lee ${ }^{1}$, Myeong Jun Song ${ }^{3}$, Ji Woong Jang ${ }^{5}$, Seok Bae Kim ${ }^{4}$, Il Han Song ${ }^{4}$, Sae Hwan Lee ${ }^{6}$, Hong Soo Kim ${ }^{6}$, Woo Sun Rou ${ }^{1}$, Hee Bok Chae ${ }^{2}$

${ }^{1}$ Chungnam National University Hospital, Korea, Republic of; ${ }^{2}$ Chungbuk National University Hospital, Korea, Republic of; ${ }^{3}$ Daejeon St. Mary's Hospital, Korea, Republic of; ${ }^{4}$ Dankook University College of Medicine, Korea, Republic of; ${ }^{5}$ Eulji University Hospital, Korea, Republic of; ${ }^{6}$ Soonchunhyang University College of Medicine, Korea, Republic of

Background: Approximately $2 \%$ to $15 \%$ of patients with chronic kidney disease (CKD) have chronic hepatitis B. CKD in chronic hepatitis B patients may be associated with hepatitis B virus (HBV) infection or other causes. There is a possibility of renal toxicity of prescribed drugs, and the hepatitis B virus infection itself can aggravate renal dysfunction. At present, there are very limited data to support an analysis of treatment efficacy of antiviral agents in chronic hepatitis B patients, with dosage adjusted according to renal function. The purpose of this study was to investigate the efficacy of adjusteddosage antiviral agents in chronic hepatitis B patients with CKD.

Method: In this retrospective study, we compared records from January 2006 to April 2016 of 27 chronic hepatitis B patients who had an initial creatinine clearance of $50 \mathrm{ml} / \mathrm{min}$ or less (CKD group) and 54 chronic hepatitis B patients who had normal renal function (nonCKD group). Propensity score matching (PSM) was used to identify similar populations in the CKD and non-CKD groups. These patients were aged 18 years or older and had received antivirals for at least 18 months. In the comparison of the CKD group and the non-CKD group, baseline characteristics such as age, gender, serum alanine transaminase (ALT), HBV DNA, presence of liver cirrhosis, HBeAgpositive status, and previous antiviral treatment did not differ significantly between the two groups.

Result: The cumulative virological response (VR) rate of the CKD group was $40.7 \%, 62.9 \%$, and $66.7 \%$ at 12,24 , and 48 months, respectively, while the cumulative VR rate of the non-CKD group was $63.4 \%, 80.4 \%$, and $95.3 \%$ at 12,24 , and 48 months, respectively $(\mathrm{P}=.003)$. The cumulative biochemical response $(\mathrm{BR})$ rate did not differ significantly between the two groups. $(\mathrm{P}=.181)$.

Conclusion: In the current study, the treatment efficacy of the antiviral agent in the non-CKD group was higher than in the CKD group. The results of this study suggest that tenofovir is potent antiviral agent in CKD patients as well as in normal renal function patients, and the therapeutic effect is maintained even in dose reduction.

$H B V-C 22$

The efficacy of de no entecavir monotherapy and telbivudine combined with adefovir were both favourable in compensated cirrhosis patients with $\mathrm{HBV}$ infection

Xiangyong Li ${ }^{1}$, Changhao $\mathrm{Zhu}^{4}$, Jian $\mathrm{Liu}^{3}$, Jie Luo ${ }^{2}$

${ }^{1}$ The third affiliated hospital of Sun Yat-sen University, China; ${ }^{2}$ Shenzhen Luohu Peoples Hospital, China; ${ }^{3}$ The Fifth Affiliated Hospital of Sun Yat-sen University, China; ${ }^{4}$ The First Affiliated Hospital of Shantou University Medical College, China

Background: To evaluate the efficacy and safety of de no telbivudine combined with adefovir and entecavir in compensated cirrhosis patients with HBV infection.

Method: 87 patients with compensated hepatitis B cirrhosis were scrut from June 2011 to June 2016, and then were divided into 2 groups, combination therapy group $(\mathrm{n}=40)$ and monotherapy group $(n=47)$. Regular follow-up took place every 12-24 weeks. Alanine aminotransferase (ALT), total bilirubin, albumin, HBV DNA, and HBsAg levels were compared between the two groups. The primary endpoint was achieving a cumulative probable virologic response. The secondary endpoints were the rate of alanine aminotransferase (ALT) normalization, HBeAg seroconversion in HBeAg-positive patients, and elevated creatinine and creatine kinase.

Result: The baseline parameters and characteristics were similar between the two groups. After a median follow-up of 120 weeks, there was no significant difference in ALT, ALB, TBIL, ALT normalization rate, serum $\mathrm{HBV}$ DNA undetectable rate, serum $\mathrm{HBeAg}$ loss rate, serum $\mathrm{HBe} \mathrm{Ag} / \mathrm{HBe} \mathrm{Ab}$ sero-conversion rate and Child-pugh scores. During the follow-up, 4 patients suffered from virological breakthrough; 1 failed to achieve viral response, the other 3 patients continued NUs therapy, and HBV DNA level fell under the low limit again. Slightly elevated creatinine was observed in 2 patient with de no telbivudine combined with adefovir, whereas creatine kinase did not increase during the follow-up.

Conclusion: The efficacy of de no entecavir monotherapy and telbivudine combined with adefovir were both favourable in compensated cirrhosis patients with HBV infection

\section{$H B V-C 23$}

Lower baseline quantitative HBcAb may help predict response to sequential combination therapy with IFN, rhIL-2 and HBV therapeutic vaccine in entecavir-suppressed CHB patients

\section{Jinshang $\mathrm{Hu}^{1}$, Quan $\mathrm{Yuan}^{2}$, Di Wu ${ }^{1}$, Meifang Han ${ }^{1}$, Guang}

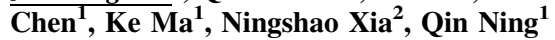

${ }^{1}$ Tongji Hospital, Tongji Medical College, Huazhong University of Science and Technology, China; ${ }^{2}$ National Institute of Diagnostics and Vaccine Development in Infectious Diseases, School of Public Health, China

Background: $\mathrm{HBcAb}$ has been revealed to be closely correlated with hepatic inflammatory activities and treatment response in $\mathrm{HBeAg}$ positive chronic hepatitis $\mathrm{B}(\mathrm{CHB})$ patients recently. The aim of this study was to investigate the relationship of quantitative serum $\mathrm{HBcAb}$ levels and antiviral efficacy in 94CHB patients undergoing a multi- 
center, randomized trial (Endeavor Study, ClinicalTrials.gov: NCT02360592).

Method: $\mathrm{HBeAg}$ positive $\mathrm{CHB}$ patients who had received entecavir (ETV) for $1-5$ years, with HBeAg loss and HBVDNA $\leq 1000$ copies/ $\mathrm{ml}$, were randomized to continue with ETV therapy for 48 weeks (Group I) or switch to conventional interferon (IFN)- $\alpha$-2bfor 48 weeks (Group II) or IFN- $\alpha-2 b$ for 48 weeks in combination with recombinant human interleukin-2 (rhIL-2) for 12 weeks plus HBV therapeutic vaccine for 48 weeks (Group III). Treatment response was defined as HBsAg loss and/or HBeAg seroconversion at week 48 . Serum $\mathrm{HBcAb}$ was quantified in a blinded fashion by using a newly developed double-antigen sandwich immune-assay calibrated by WHO standards.

Result: Compared with the patients continuing with monotherapy (Group I), patients switching to combined therapy (Group II and III) exhibited a significant higher rate of response $(7.41 \%$ vs. $23.88 \%$, $P=0.048$ ). A positive correlation between baseline $\mathrm{HBcAb}$ levels and HBsAg levels was observed in all patients included $(r=0.364$, $P=0.001$ ), while positive correlation between the two variables during treatment only existed among patients undergoing combined therapy ( $r=0.446,0.315,0.315, P=0.000,0.014,0.026$ at week 0 , week 12 , week 48 , respectively). In contrast to monotherapy which had no obvious influence on serum $\mathrm{HBcAb}$ levels, combined therapy resulted in a significant decline in $\mathrm{HBcAb}$ levels (week $12 \mathrm{VS}$. week 0 , $P=0.038$, week 36 VS. week $0, P=0.034$ ). Compared with non-responders within combined therapy group, responders showed significantly lower $\mathrm{HBcAb}$ levels during early phase of treatment $(P=0.017,0.016,0.012$ at week 0 , week 4 , and week 12 , respectively). Furthermore, baseline $\mathrm{HBcAb}$ level appeared to be an ideal predictor for response to combined therapy, including having a larger AUROC curve $(0.714,95 \%$ CI $0.588-0.840, P=0.003)$. We defined $2.695 \log 10$ $\mathrm{IU} / \mathrm{mL}$ as the optimal cut-off value of baseline $\mathrm{HBcAb}$ level to predict response (sensitivity $80.8 \%$, specificity $60.1 \%$, PPV $56.76 \%$, NPV 83.33\%). Patients with baseline $\mathrm{HBcAb} \leq 2.695 \log 10 \mathrm{IU} / \mathrm{mL}$ had significantly more chances to achieve response than those with baseline $\mathrm{HBcAb}>2.695 \log 10 \mathrm{IU} / \mathrm{mL}$ (56.76\% [21/37] VS. $16.67 \%$ [5/30]).

Conclusion: For $\mathrm{CHB}$ patients with $\mathrm{HBeAg}$ loss post ETV treatment, baseline quantitative $\mathrm{HBcAb}$ titer might be a novel non-invasive biomarker for predicting treatment response to sequential combination therapy with IFN, rhIL-2 and HBV therapeutic vaccine.

\section{$H B V-C 24$}

\section{A Study of Etiological and Clinical Profile of Acute Viral Hepatitis at a Tertiary Care Hospital in South India}

\section{Swapnil Babuappa Kankare ${ }^{1}$, Aby Somu ${ }^{1}$, Maya $\mathbf{P}^{1}$, Prashanth $\mathbf{M}^{1}$, Shibi Mathew ${ }^{1}$, John Mathews ${ }^{1}$, Prakash Zacharias ${ }^{1}$, Mathew Philip ${ }^{1}$}

${ }^{1}$ PVS Institute of Digestive Diseases, PVS Memorial Hospital, India

Background: The variability in nature of Acute viral hepatitis (AVH) with regards to its clinical course and complications requires clinical attention from time to time. There is paucity of data from South India with most studies done a decade back. Hence, this study was done to gain the understanding of etiological spectrum, symptomatology, clinical features, pattern of investigations, severity and complications during the course of AVH at a tertiary care hospital in Kerala.

Method: This prospective, descriptive observational study enrolled consecutive OPD/IPD patients above age of 18 years having AVH (diagnosed clinically and serologically) from May 2015 to April 2016. Patients with Chronic Liver disease, clinical or radiological evidence of biliary obstruction/ Drug induced Liver Injury/
Autoimmune hepatitis were excluded. A complete clinical, virological, biochemical and radiological assesement was done at baseline with follow up upto 6 months. Appropriate statistical tests were used for analysis and a $\mathrm{p}$ value of $<0.05$ was considered significant.

Result: Total of 204 patients with AVH fulfilling the inclusion criteria were enrolled in this study. The results as discussed below were observed. 1. AVH more common $(47.1 \%)$ in younger age group (2040 yrs) with male (4.23:1) preponderance. 2. Acute hepatitis B was the most common $(55.8 \%)$ etiology and among it $40-60$ year age group was most commonly (49.1\%) affected. 3. Jaundice (87.7\%) was the most common presenting symptom followed by nausea/vomiting, fever and anorexia. 4. Significant number of patients $(25.4 \%)$ with acute hepatitis B had arthralgia. 5. History of recent travel was the most common risk factors for hepatitis A/E. 6. For Hepatitis B, recent dental extraction (14\%) was most common risk factor. 7. Icterus and splenomegaly were most common clinical findings. 8. Hepatitis B showed slower reduction rate for bilirubin with mild increase (non significant) at 1 week as compared to other etiologies. 9. Acute hepatitis B had significantly high SGPT and SGOT on follow up as compared to other etiologies. 8. Majority of patients achieved normalization of bilirubin and transaminases by end of 8 weeks. 9 . Majority of patients with acute hepatitis B had HBV DNA of $>$ 10,00,000 copies/ml. 10. HBV DNA levels and baseline bilirubin, SGOT, SGPT and INR showed a weak negative correlation. 11. A statistically significant positive correlation was found between $\mathrm{HBV}$ DNA and SGOT, SGPT at 2 weeks. 12. Mean duration for HBsAg loss was $3.54+1.08$ months. 13. Gall bladder wall thickening was the most common sonological finding. 14. Coagulopathy was the most common (18.6\%) complication. 16. Acute liver failure developed in $1.5 \%$ patients and hepatitis A was the most common etiology. 17. Mortality rate observed in the study population was $1 \%$.

Conclusion: This study showed Acute Hepatitis B as the commonest cause of AVH with slower biochemical recovery as compared to other etiologies.

\section{$H B V-C 25$}

A multistep approach for the prevention of hepatitis $B$ infection in a recipient of bone marrow transplantation

\section{Tugrul Purnak ${ }^{1}$, Hayrettin Koklu ${ }^{1}$, Nomingerel Tsveldorj ${ }^{1}$,} Halis Simsek $^{1}$, Yasemin Balaban ${ }^{1}$, Bulent Sivri ${ }^{1}$

${ }^{1}$ Hacettepe University Medical School, Turkey

Background: Allogeneic bone marrow transplantation (ABMT) is a curative treatment option in some hematologic diseases and with newly introduced effective treatments, patients with chronic viral hepatitis may also be evaluated as donors in ABMT. In our country, chronic viral hepatitis B infection is still a public health problem in some regions and immunosuppressive treatment may cause hepatitis $B$ reactivation if prophylactic treatment is not initiated in high risk patients. In the present case, we successfully managed to protect an ABMT recipient from hepatitis $B$ infection since the donor was HbsAg (hepatitis B surface antigen) positive. We aimed to share our experience with the readers.

Method: A 55-year old male patient with myelodysplastic syndrome and romatoid arthritis admitted to the hospital for ABMT. Patient had antibody against HbsAg with vaccination and had no liver disease in history. At the end of donor evaluation by the hematology team, a newly diagnosed hepatitis B infection was diagnosed in the donor and despite all efforts, no other healthy donor could be recruited. The situation was consulted to us before the transplantation. First, we decided to start treatment for donor. Donor's serologic profile was compatible with chronic hepatitis B virus infection (see the table). 
According to government's reimbursement rules for the treatment of chronic hepatitis B infection, liver biopsy was performed (HAI: 5, fibrosis score 2, according to Metavir scoring system) and tenofovir treatment $1 \times 245 \mathrm{mg}$ PO was started. In the beginning of the treatment, liver biochemical tests including ALT, AST, ALP, GGT were all normal. Donor was received tenofovir treatment for a month and Hepatitis B DNA level was returned to negative just before the ABMT. As to recipient, a week before the transplantation, tenofovir treatment was started to ensure the steady state of the blood drug level in the patient in the day of transplantation. Also, Hepatitis B Immunoglobulin at the dose of $800 \mathrm{IU} /$ day was initiated in day 1 and was continued for 7 days. During the treatment, anti-Hbs titers were checked daily and if the dosage was not enough for the protection, additional boluses were ordered in order to maintain the anti-HBs level $>1000 \mathrm{IU} / \mathrm{L}$. In the day 2, recipient's HbsAg became positive but this positivity was seen temporarily and HbsAg returned to negative in day 8. Patient was continued to tenofovir treatment for 12 months and no HBV reactivation was seen in the follow-up. Donor is still receiving tenofovir treatment and follow-up of this patient until now didn't show any complication.

Result: Hepatitis B virus (HBV) reactivation is a major concern among patients who have been receiving immunosuppressive treatment and fortunately clear-cut treatment recommendations have recently emerged for these patients. However, some group of patients may cause to uncertainties for the physicians with respect to management of these patients. In this case, we met several challenges in the management. First, our donor was HbsAg positive and had high viral load in the sera. Secondly, we had limited time to achieve successful management because of the recipient's limited treatment options for his primary hematologic disease and there was no time for delay. We preferred to use tenofovir for the treatment of donor since it is a potent antiviral drug with high genetic barrier to resistance. In literature, there are some case reports using lamivudine for this purpose but if we had chosen lamivudine as a treatment option for our donor, we couldn't have achieved HBV-DNA negativity in the sera rapidly. We also did choose tenofovir as the prophylactic regime for the recipient to prevent $\mathrm{HBV}$ reactivation since $\mathrm{HBV}$ positive blood cells were transferred to the recipient as part of ABMT and HBV infection was unavoidable. The recipient's anti-Hbs titers was above the protective level (see the table) and we didn't consider additional bolus vaccination for enough anti-Hbs titer. However, during the first days of ABMT, hepatitis B immunoglobulin was given to the patients to neutralize HbsAg sourced from donor blood cells.

Conclusion: In conclusion, successful ABMT from $\mathrm{HbsAg}$ positive donor can be performed if necessary measures are taken and tenofovir treatment can be used for this purpose if time is a limit and rapid response is necessary. Recipient's infection with HBV can also successfully prevented with this multistep approach.

\begin{tabular}{|c|c|c|c|}
\hline \multicolumn{2}{|c|}{ Before transplantation } & \multicolumn{2}{|c|}{ Aftec transplantation } \\
\hline Donor's serologic profile & Recipient's serologic profile & Donor's serologic profle & Recipient's serologic profile \\
\hline Hbsag: Positive & Hbsag; negative & HbsAB; Negative & HbsAg; Positive \\
\hline Antillbs: Negative & AntiHbs: & Antillbs: $246 \mathrm{IU} / \mathrm{ml}$ & Antillbs: Negative \\
\hline HbeAs: Nerative & HbeAs: Negative & HbeAs: Nerative & HbeAs: Negative \\
\hline Antillbe; Positive & AntiHbe: Negative & Antillbe; Positive & Antilbbe: Positive \\
\hline AntiHbe IgG: Positi & AntiHbc I8G; Nerative & AntiHbG IgG; Positive & AntiHbG IRG; Positive \\
\hline HBV ONA: 36986 copy/ml & HBV DNA: negative & HBV DNA: Negative & HBV DNA: Negative \\
\hline
\end{tabular}

\section{$H B V-C 26$}

An observational study about long-term renal outcome in chronic hepatitis B patients treated with tenofovir disoproxil fumarate in Korea

Tae Seop Lim ${ }^{1}$, Hye Won Lee ${ }^{1}$, Mi Young Jeon', Beom Kyung Kim ${ }^{1}$, Seung Up Kim ${ }^{1}$, Jun Yong Park ${ }^{1}$, Do Young Kim ${ }^{1}$, Kwanghyub Han', Sang Hoon Ahn' ${ }^{1}$
${ }^{1}$ Yonsei University College of Medicine, Korea, Republic of

Background: Tenofovir disoproxil fumarate (TDF) is known to be an effective and safe antiviral agent for chronic hepatitis B (CHB). However, its long-term effects on renal function have been controversial. This study aimed to analyze the real-world long-term effects of TDF on renal function in Korean patients with CHB.

Method: We analyzed a cohort of consecutive treatment-naïve patients with CHB who were treated with TDF between May 2012 and December 2015 at Severance Hospital, Seoul, Korea, Republic of. Patients receiving TDF $>12$ months with well-preserved liver function were recruited. Patients with a history of hepatocellular carcinoma at the enrollment were excluded. Estimated glomerular filtration rate(eGFR) was calculated with Chronic Kidney Disease Epidemiology Collaboration (CKD-EPI).

Result: A total of 668 patients were analyzed. The mean age was 51.5 years old, and $40.1 \%$ were male. The proportions of hypertension and diabetes mellitus (DM) were $14.5 \%$ and $11.5 \%$, respectively, and that of liver cirrhosis was $20.7 \%$. During the follow-up (median 36.0 months), using linear mixed model, serum creatinine increased from $0.80 \pm 0.57 \mathrm{mg} / \mathrm{dL}$ to $0.92 \pm 0.34 \mathrm{mg} / \mathrm{dL}(\mathrm{p}<0.001)$ and eGFR decreased from $102.2 \pm 15.6 \mathrm{~mL} / \mathrm{min} / 1.73 \mathrm{~m}^{2}$ to $89.9 \pm 20.4 \mathrm{~mL} /$ $\min / 1.73 \mathrm{~m} 2\left(\mathrm{p}<60 \mathrm{~mL} / \mathrm{min} / 1.73 \mathrm{~m}^{2}\right.$, age $>60$ years old, male gender, and hypertension are potential risk factors for a significant renal event (Hazard ratio [HR] 77.967, HR 4.379, HR 3.788, and HR 2.785, respectively). However, DM and liver cirrhosis did not affect the development of a significant renal event.

Conclusion: Under real-world long-term TDF therapy in Korea, overall renal function was decreased moderately and a certain proportion of CHB patients experienced a significant renal event.

\section{$H B V-C 27$}

Attitude and knowledge level of $\mathrm{HBV}$ infected patients' relatives about HBV disease: a cross-sectional survey study

Ayse Kaya Kalem ${ }^{1}$, Ayse Kaya Kalem ${ }^{1}$, Fatma Eser ${ }^{2}$, Imran Hasanoglu ${ }^{4}$, Gul Ruhsar Yilmaz ${ }^{3}$, Rahmet Guner ${ }^{4}$, Mehmet Akin Tasyaran ${ }^{4}$

${ }^{1}$ Ataturk Education and Research Hospital, Turkey; ${ }^{2}$ Diskapi Yildirim Beyazit Education and Research Hospital, Turkey; ${ }^{3}$ Süleyman Demirel University Faculty of Medicine, Turkey; ${ }^{4}$ Yildirim Beyazit University Faculty of Medicine, Ataturk Training and Research Hospital, Turkey

Background: Education and knowledge takes the lead in avoiding dissemination of Hepatitis B virus (HBV). This research aims to examine the level of knowledge, attitude, and manners of HBV infected patients' relatives.

Method: A survey consisting of 28 open ended and multiple-choice questions has been carried out among first degree relatives of HBV infected patients in Atatürk training and research Hospital. Questions were designed to reveal demographic characteristics, knowledge and understanding of the disease, and anxiety. SPSSS v.15 software has been used for the statistical analysis.

Result: 106 relatives have been included in this research with the mean age of $40,6 \pm 11,8.76 .4 \%$ were aware of the effects of HBV to liver, $58.5 \%$ were aware that $\mathrm{HBV}$ is contagious. $84 \%$ knew the concept of inactive HBV carrier. $86.8 \%$ of the participants knew they were at risk themselves. All relatives knew transmission was possible through blood. Vaccination $(89.6 \%)$ and separation of personal belongings carrying the risk of blood contact $(86.8 \%)$ were the most well-known preventive measures. Women were found to have a better understanding of transmission and preventive measures whereas age was found to be inversely related with awareness of these issues. 
$72.6 \%$ of the participants knew that HBV is a treatable disease. Educational attainment was found to be positively associated with general comprehension of surveyed topics.

Conclusion: The knowledge levels of HBV infected patients' relatives have been found to be sufficient despite some shortcomings.

\section{$H B V-C 28$}

Comparison of HBsAg change between HBeAg-negative patients who discontinued entecavir without retreatment or continued entecavir therapy

\section{ChienHung Chen ${ }^{1}$, Shengnan Lu' ${ }^{1}$, Chao Hung Hung', Jing Houng Wang ${ }^{1}$, Tsung Hui $\mathrm{Hu}^{1}$ \\ ${ }^{1}$ Kaohsiung Chang Gung Memorial Hospital, Taiwan}

Background: The aim of this study is to compare the HBsAg change between HBeAg-negative patients who discontinued entecavir without retreatment or continued entecavir therapy.

Method: A total of $126 \mathrm{HBeAg-negative} \mathrm{patients} \mathrm{who} \mathrm{have} \mathrm{stopped}$ entecavir treatment at least 12 months without retreatment (discontinued group) and $84 \mathrm{HBeAg}$-negative patients who had continued entecavir treatment for at least 4 years (continued group) were recruited. All patients in discontinued group fulfilled the stopping criteria set forth by APASL 2012. HBsAg decline was determined from end of treatment in discontinued group and third year of treatment continued group.

Result: Of the 126 patients in discontinued group, 88 patients who achieved sustained virological response (SVR) (HBV DNA $\leq 2000 \mathrm{IU} / \mathrm{mL}$ ) during last 2 years of follow-up and 39 experienced persistent or intermittent virological relapse (HBV DNA>2000 IU/ $\mathrm{mL}$ ) (Group III). Of the 88 patients with SVR, 61 did not experienced virological relapse (Group I) and 26 experienced transient virological or clinical relapse (Group II) during follow-up. The HBsAg levels were lower in Group II compared to Group III at the end of treatment $(P=0.008)$ and 6 months after treatment $(P=0.009)$. There was no significant difference in HBsAg levels at 12 and 24 months after treatment $(P=0.1$ and $P=0.26)$ and in HBsAg loss $(P=0.65)$ between Group I and II. Compared to patients in Group I, patients in Group III had a higher HBsAg levels at the end of treatment, and 6, 12, 24 months after treatment. Compared to patients in Group II, patients in Group III had a higher HBsAg levels at 12 and 24 months after treatment, but not at the end of treatment and 6 months after treatment. Patients in Group I and II had higher levels of HBsAg decline at 12 and 24 months after treatment than those in continued group at fourth and fifth year. There was no significant difference in HBsAg decline between patients in Group III and continued groups.

Conclusion: Patients who discontinued entecavir and achieved SVR had higher levels of HBsAg decline than those who continued entecavir treatment.

\section{$H B V-C 29$}

Cost-effectiveness according to the indication of antiviral therapy for hepatitis B virus for the prevention of hepatocellular carcinoma

\section{Dong Hyun Sinn ${ }^{1}$, Beom Kyung Kim', Sung Eun Kim²,} Ji Hoon Kim ${ }^{3}$, Moon Seok Choi ${ }^{1}$

${ }^{1}$ Samsung Medical Center, Korea, Republic of; ${ }^{2}$ Hallym University Sacred Heart Hospital, Korea, Republic of; ${ }^{3}$ Korea University College of Medicine, Korea, Republic of; ${ }^{4}$ Yonsei University College of Medicine, Korea, Republic of

Background: Antiviral therapy for chronic hepatitis B virus (HBV) infection can reduce the risk of hepatocellular carcinoma (HCC). We evaluated cost-effectiveness of different scenario of antiviral treatment in terms of preventing $\mathrm{HCC}$ using large retrospective cohort of patients with chronic HBV infection.

Method: We used multi-center, retrospective cohort that included a total of 3,624 patients (age $4.8 \pm 11.9$ years, male $=75.3 \%$, cirrhosis $=15.9 \%$ ) who were monitored without antiviral treatment. Using observed 5-years HCC incidence rate, 5-years cumulative incidence rate of $\mathrm{HCC}$ was estimated, assuming that patients who met certain treatment criteria have received antiviral treatment that would have reduced HCC risk with a hazard ratio of 0.4 . The tested scenario were 1) cirrhotic patients with elevated HBV DNA levels $(>2,000 \mathrm{U} / \mathrm{L})$ and chronic hepatitis B (CHB) patients with elevated HBV DNA levels plus alanine aminotransferase (ALT) $>2$ upper limit of normal (ULN) (current); 2) expanding treatment for cirrhotic patients with low viral levels (expansion 1);3) further expanding treatment for CHB patients with elevated HBV DNA levels plus ALT >1 ULN (expansion 2); 4) further expanding treatment for all patients with detectable HBV DNA levels (expansion 3).

Result: During a median 4.6 years of follow-up (range: 0.5-13.0 years), 161 patients were newly diagnosed with HCC. Under current treatment criteria, $66.4 \%$ of $\mathrm{HCC}$ developed outside the treatment recommendation and the number to treat (NNT) to prevent one HCC was 17.9. Percentage of HCC diagnosed outside the treatment recommendation decreased with a expansion of treatment indication $(49.5 \%, 34.6 \%$ and $7.5 \%$ for expansion 1 , expansion 2 and expansion 3 ), but with additional cost for antiviral therapy. The NNT was 16.1, 18.2 and 38.5 for expansion 1,2 and 3, respectively. In this retrospective cohort, net direct cost (additional cost for antiviral therapy direct cost saved for preventing HCC) showed that expanding antiviral therapy indication reduced net direct cost for expansion 1 , while net direct cost increased for expansion 2 and expansion 3 . However, when indirect cost, morbidity and mortality cost of HCC were considered, expanding treatment criteria always showed net cost-benefit.

Conclusion: Expansion of antiviral treatment indication increases additional cost for antiviral therapy, yet, in turn saved cost related to HCC by preventing development of HCC. Net cost-benefit was observed for expansion 1 when direct medical costs was considered. When indirect cost, morbidity and mortality cost from HCC were considered, expansion of antiviral treatment indication always resulted in net cost-benefit.

\section{$H B V-C 30$}

Entecavir and tenofovir disoproxil fumarate in nucleos(t)idenaïve patients with chronic hepatitis B: long-term outcomes and safety in the real-world setting

\section{Il Han Song ${ }^{1}$, Byeong Wook Cho', Suk Bae Kim ${ }^{1}$ \\ ${ }^{1}$ Dankook University College of Medicine, Dankook University Hospital, Korea, Republic of}

Background: Entecavir (ETV) and tenofovir disoproxil fumarate (TDF) are globally named among the potent antivirals for the treatment of chronic hepatitis B virus (HBV) infection. The purpose of the present study is to compare long-term outcomes and safety of ETV and TDF in chronic hepatitis B patients in the real-world setting. Method: We performed a retrospective, observational, and comparative study of 318 nucleos(t)ide-naïve chronic hepatitis B patients who were given ETV $0.5 \mathrm{mg} /$ day $(\mathrm{n}=166)$ or TDF $300 \mathrm{mg} /$ day 
$(\mathrm{n}=152)$ at Dankook University Hospital. We assessed and compared the development of hepatocellular carcinoma (HCC), overall mortality, and renal dysfunction as well as virologic and biochemical responses.

Result:There was no significant differences between the two arms (ETV vs. TDF) in baseline characteristics (age 45 vs. 44 years; accompanying liver cirrhosis $40 \%$ vs. $33 \%$; HBV DNA 6.5 vs. $6.4 \mathrm{log}$ IU/mL; ALT 108 vs. $110 \mathrm{U} / \mathrm{L}$; creatinine 0.79 vs. $0.78 \mathrm{mg} / \mathrm{dL}$; eGFR 101.9 vs. $106.2 \mathrm{~mL} / \mathrm{min})$. Cumulative probabilities of complete virologic response (CVR) and ALT normalization were 90\% vs. 93\% and $98 \%$ vs. $94 \%$, respectively, at 36 months. The cumulative incidence rates of HCC tended to be higher in ETV than TDF arm (1.2\% vs. $0 \%$ at 1 - year; $4.3 \%$ vs. $1.0 \%$ at 2 -years; $6.7 \%$ vs. $1.8 \%$ at 3 -years). Overall mortality in ETV arm were $0 \%$ at 1 -year, $0.7 \%$ at 2 -years, and $1.7 \%$ at 3 -years, while those of TDF were $0 \%, 1.0 \%$, and $3.5 \%$ at each year. Cumulative probabilities of low eGFR (

Conclusion: Long-term clinical outcomes such as the development of HCC, overall mortality, and renal toxicity may be different between ETV and TDF, although both antivirals are comparable in antiviral efficacy of nucleos(t)ide-naive patients with chronic hepatitis B. So, the decision on which antiviral agent to select should be given cautiously consideration based on the risk of individual hepatic and renal dysfunction.

\section{$H B V-C 31$}

\section{Evaluation of treatment response to entecavir}

\section{$\underline{\text { Ufuk Sonmez }}^{1}$, Seher Ayten Coskuner ${ }^{1}$, Sebnem Calik ${ }^{1}$, Selma Tosun ${ }^{1}$ \\ ${ }^{1}$ University of Health Sciences Izmir Bozyaka Education and Research Hospital, Turkey}

Background: Hepatitis B is an important public health problem both in the world and in Turkey. Currently, potent agents such as tenofovir and entecavir are used in the treatment of hepatitis B. Entecavir is a potent guanosine analogue that prevents the proliferation of hepatitis $B$ virus. In addition, the resistance barrier is high and it's a successful agent that suppresses serum HBV DNA levels. We aimed to evaluate the treatment response rates of entecavir using patients.

Method: Forty patients were included in this study who were started using entecavir between 2007-2016. Biochemical response was defined as normal alanin aminotransferase levels, virologic response was defined as negative HBV DNA levels. During the treatment, annual follow-up was performed to detect possible $\mathrm{HBeAg}$ and HBsAg loss.

Result: Forty patients of which $12(30 \%)$ of them were female, and the mean age was 33.9(23-75). A total of 10 patients $(25 \%)$ had a pretreatment $\mathrm{HBeAg}$ positivity. The mean initial ALT level of the patients was $118.6(17-503) \mathrm{IU} / \mathrm{mL}$, while the initial HBV DNA level of $28(70 \%)$ patients was above $20,000 \mathrm{IU} / \mathrm{mL}$. Thirteen of the cases $(32.5 \%)$ were treatment experienced. A total of $6(15 \%)$ patients were pegylated interferon experienced, $2(5 \%)$ patient were adefovir experienced, $4(10 \%)$ were lamivudine experienced, $3(7.5 \%)$ were tenofovir experienced and $1(2.5 \%)$ was telbivudine experienced. Lamivudine experienced patients were taking entecavir treatment from $1 \mathrm{mg} /$ day dose. HBV DNA levels of $9(22.5 \%)$ patients were positive at the end of the first year of treatment. [Mean HBV DNA level was $25.6 \mathrm{IU} / \mathrm{mL}(4,5-115,8)]$. The mean ALT level at the end of the first year of treatment was $24.6(11-66) \mathrm{IU} / \mathrm{mL}$. The median duration of treatment was 6.3 (1-10) years. There was no loss of HBsAg in any patient during the follow-up. Ten HbeAg positive patients of which $3(30 \%)$ of them had antigen loss.(One had in third, and others had in 4th and 6th years of the treatment).
Conclusion: Entecavir- a potent antiviral agent-regresses HBV DNA and ALT levels significantly in a year, just as in our study. No patients had lost HBsAg, but three (30\%) patients had lost HBeAg in our study. In another study $\mathrm{HBeAg}$ loss rate in entecavir using patients was found as $21 \%$ (1).

\section{Reference}

1. Chang TT, Gish RG, Man RD. A comparison of Entecavir and Lamivudine for HBeAg-positive chronic hepatitis B. N Engl J Med 2006;354:1001-1010

\section{$H B V-C 32$}

Evaluation of simple noninvasive scoring models and transient elastography for the prediction of significant fibrosis in biopsyproven chronic hepatitis $B$ patients

$\underline{\text { Jiyuan Zhou }}^{1}$, Guiqiang Wang ${ }^{2}$

${ }^{1}$ Peking University Shenzhen Hospital, Shenzhen PKU-HKUST Medical Center, China; ${ }^{2}$ Peking University First Hospital, China

Background: We aimed to assess the diagnostic value of GPR-APRIFIB-4 and transient elastography (TE, FibroScan $\left.{ }^{\circledR}\right)$ for predicting significant fibrosis in chronic hepatitis B patients who needed biopsies to determine treatment decisions.

Method: From October 2013 to December 2015, 593 treatment-naïve patients who underwent liver biopsy in 24 teaching hospitals located in China were recruited into this study. At the time of liver biopsy, routine laboratory test and TE were performed. Significant fibrosis was defined as ISHAK score $\geq 3$.

Result: Of 511 patients, 290 cases did not meet the criteria for antiviral therapy but these treatment decisions depend on liver biopsy. GPR showed areas under receiver-operating characteristic curve (AUC) of 0.78 (95\% CI 0.73-0.82)for predicting significant fibrosis, which was significantly higher than APRI (AUC $=0.72,95 \%$ CI $0.66-$ $0.77, P=0.019$ ), and had a better trend than FIB-4 (AUC $=0.72,95 \%$ CI $0.67-0.77, P=0.063)$, but significantly lower than TE $(0.86$, $95 \%$ CI $0.82-0.90, \mathrm{P}=0.002$ ) in these 290 patients.

Conclusion: The diagnostic efficiency of GPR for predicting significant fibrosisis superior to Fib-4 and APRI in HBV infected patients. The GPR may be a promising alternative to liver biopsy and TE to guide antiviral decision-making, especially in resource-limited setting.

\section{$H B V-C 33$}

GSRS questionnaire in assessing the quality of life of patients with chronic viral hepatitis $B$

Saidrakhim Nodirovich Lukmonov, Kurbonboy Abdullayevich Madatov', Umid Abdullayevich Allazarov', Muzaffar Uktamovich Ismailov², Oybek Otabekovich Usmanov ${ }^{1}$

${ }^{1}$ Tashkent Medical Academy, Uzbekistan; ${ }^{2}$ Tashkent State Pediatric Institute, Uzbekistan

Background: To study the quality of life (QOL) in patients with chronic viral hepatitis B (CHB) with the help of a specialized questionnaire of gastroenterological patient GSRS.

Method: 112 patients with chronic obstructive pulmonary disease on the basis of faculty surgery of the Tashkent Medical Academy were examined. There were 63 men and 49 women. The average age of patients with CHB was $29 \pm 7.8$ years. 
Result: The level of ALT was increased in all patients studied by more than 3-5 times, an average of $158 \pm 39$ units. In all patients with CHB, HBV DNA was detected, in a quantitative sense, the replication level in $68(60.7 \%)$ patients was more than 2 million copies in $1 \mathrm{ml}$ and $44(39.3 \%)$ were at the level of 200 thousand - 2 million copies in Ml. The assessment of QOL was carried out with the help of the Russian-language GSRS questionnaire, adapted and recommended for use by the Interethnic Center for the Study of QOL. The control group consisted of 86 healthy subjects who were socially comparable with the study group of patients with CHB. Of these, 54 men and 32 women. The mean age in the control group was $36 \pm 8.5$ years. The data obtained in the control group were comparable with the results obtained in the pilot assessment of QOL by the GSRS questionnaire from 2114 healthy residents of the city of Tashkent. Based on the assessment of QOL, the GSRS significantly increased the indices for four of the five scales under study, namely, "abdominal pain" (5.2 \pm $2.5, \mathrm{P}<0.05)$, "dyspeptic syndrome" $(13.4 \pm 3,2, \mathrm{P}<0,05)$, «gastric reflux syndrome» $(8,3 \pm 1,6, \mathrm{P}<0,05)$ and «obstipation syndrome» $(9,6 \pm 2,4, \mathrm{P}<0,05)$, which certainly speaks of pathological changes in the functions of the entire digestive system.

Conclusion: The patients with CHB showed a decrease in the level of QOL, expressed as a deterioration in the performance of the digestive system. Thus, the specialized questionnaire GSRS is sensitive and valid and can be used in the study of QOL in patients with CHB.

\section{$H B V-C 34$}

HBsAg loss in HBeAg positive and HBeAg negative patients with chronic HBV treated with Tenofovir disoproxil: a retrospective case series

\section{Isil Tuzcuoglu', Murvet Sungur', Kamile Kurt ${ }^{1}$}

${ }^{1}$ Merkez Efendi State Hospital, Turkey

Background: Tenofovir disoproxil has been licensed in Turkey since 2008. We retrospectively investigated our patients who have been followed up in our gastroenterology and infectious diseases clinic between August 2008 and August 2017. All the patients were followed up at least 6 months before therapy to ensure that they had chronic hepatitis B. Every patient had liver biopsy procedure to assess the liver pathology.

Method: Of the patients who were started tenofovir disoproxil fumarate treatment 188 patients had enrolled for this retrospective assesment. All the patients have had continuous treatment. Of these patients 39 were $\mathrm{HBeAg}$ positive ( 25 male, 14 female) and 149 HBeAg negative patients (104 males, 45 female) with chronic HBV infection, treatment initiated starting from 2008 till 2017. All the follow-ups for liver biochemistry were done every 3 months and HBV DNA was assessed every 6 months. HBsAg was controlled yearly.

Result: Total of 11 patients (5.8\%) have had HBsAg loss (3 patients of $\mathrm{HBeAg}+$, and 8 patients $\mathrm{HBeAg}-)$ Overall, the mean time to HBsAg loss was 4 years \pm 6.5 months in $\mathrm{HBeAg}(+)$ patients and 4.5 years \pm 4.5 months in $\mathrm{HBe} \mathrm{Ag}(-)$ group. In this case series, $\mathrm{HBsAg}$ loss was observed both in $\mathrm{HBeAg}$ positive patients and in $\mathrm{HBeAg}$ negative patients.

Conclusion: Our results are consistent with the previous reports. Therefore, it may be suggested that treatment with tenofovir could be associated to HBsAg loss in a period of time, in both $\mathrm{HBeAg}$ positive and $\mathrm{HBeAg}$ negative $\mathrm{HBV}$ patients.

\section{$H B V-C 35$}

Prevalence of hepatitis delta virus infection in chronic hepatitis B patients: a seroepidemiological investigation from central Anatoli

Necla Tulek ${ }^{1}$, Necla Tulek ${ }^{1}$, Gunay Tuncer Ertem ${ }^{1}$, Fatma Sebnem Erdinc ${ }^{1}$, Metin Ozsoy ${ }^{1}$, Ebru Aktepe ${ }^{1}$

${ }^{1}$ Ankara Training and Research Hospital, Turkey

Background: Hepatitis delta virus is a RNA virus and hepatitis B virus is required for its replication. Hepatitis delta virus super infection causes more severe disease on hepatitis B infection and more than $80 \%$ of patients develop chronic delta infection. Approximately, $5 \%$ of HBsAg positive people are co-infected with delta virus worldwide and the prevalence rates vary between the countries and geographic regions. We aimed to determine the prevalence of hepatitis delta virus infection among patients chronically infected with hepatitis B virus in our region.

Method: This study was conducted at Department of Infectious Diseases and Clinical Microbiology of Ankara Training and Research Hospital. Our hospital is tertiary care hospital located at Central Anatolia. Patients diagnosed with chronic hepatitis B infection in the last five years were included into the study retrospectively. All the patients were screened by hepatitis delta immunoglobulin by ELISA after the diagnosis of chronic hepatitis B infection and then each 3-6 monthly periods or when the transaminases were elevated. Further tests were performed if required. Initial hepatitis delta test results of the patients were evaluated. Migrants were excluded from the study. Result: At the study period, 825 males (56.4\%), 637 females (43.6\%), totally 1462 patients with a mean age of 42 years old (SD; ?27.5) were diagnosed as chronic hepatitis B infection. Among the patients with chronic hepatitis B infection, anti-hepatitis delta virus antibody were detected as reactive in $8(0.5 \%)$ ( 4 females, 4 males with a mean age of 46 years old) of. One of the patients with hepatitis delta virus infection had cirrhosis at admission.

Conclusion: These findings demonstrated seroprevalence rate is lower than the other regions of the Turkey and hepatitis delta virus infection is not endemic at Central Anatolia.

\section{$H B V-C 36$}

Risk of $\mathrm{HBV}$ reactivation in hematologic and oncologic malignant patients: is screening efficient?

Ayse Kaya Kalem ${ }^{1}$, Rahmet Guner ${ }^{2}$, Ayse Kaya Kalem ${ }^{1}$, Imran Hasanoglu $^{2}$, Turan Buzgan ${ }^{2}$, Bircan Kayaaslan², Zeynep Atalay Altinkaynak ${ }^{2}$, Mehmet Akin Tasyaran²

${ }^{1}$ Ataturk Education and Research Hospital, Turkey; ${ }^{2}$ Yildirim Beyazit University Faculty of Medicine, Ataturk Training and Research Hospital, Turkey

Background: Hepatitis B virus (HBV) serology monitorization is recommended for all patients prior chemotherapy and immunosuppressive therapy due to risk of reactivation. In this study, we aimed to evaluate HBV serology, antiviral usage, and reactivation rates in patients receiving immunosuppressive treatment.

Method: Hematologic and oncologic malignant patients with $\mathrm{HBsAg}$ \pm anti $\mathrm{HBc}$ IgG positivity between 2011-2017 are included in the study. Patients' characteristics are recorded from follow up charts. HBV reactivation was defined as an increase at least $1 \log 10$ in HBV DNA level in patients who previously had a detectable HBV DNA or detectable HBV DNA in patients who previously had undetectable HBV DNA. For anti $\mathrm{HBcIgG}(+)$ patients reactivation was defined as seroreversion in which develops detectable HBsAg, HBV 
DNA and/or biochemical hepatitis. The patients are followed for reactivation up to 6 months after the antiviral prophylaxis completed. Antiviral prophylaxis is initiated 1-2 week prior or concurrent with immunosuppressive therapy and should be maintained until 6 months after immunosuppressive treatment. Patients received rituximab or patients with high basal HBV DNA levels or reactivation patients' antiviral prophylaxis are extended to 12 months after discontinuation of immunosuppressive treatment.

Result: A total of 74 patients were enrolled. Mean age was 64 years and $58 \%$ were male. The most observed malignancies were nonHodgkin lenfoma (28.3\%), chronic lymphocytic leukemia (14.8\%), and multipl myeloma (14.8\%). In terms of HBV reactivation, $83.7 \%$ of the patients were at high-risk, and $14.8 \%$ were at moderate risk. HBV reactivation risk groups, HBV serology profiles, reactivation rates, and antiviral prophylaxis are summarized in Table 1. No death due to HBV reactivation was observed. All patients who developed reactivation $(n=4)$ were in the high-risk group. Only 1 of them received lamivudine prophylaxis for $\mathrm{HBcIgG}(+)$, the others did not. Reactivation occurred in the lamivudine prophylaxis receiving patient 6 months after discontinuation of immunosuppressive treatment. In other cases, reactivation occurred while immunosuppressive treatment was discontinued.

Table 1: Patients' reactivation risk groups, HBV serologies, reactivation rate.

\begin{tabular}{|c|c|c|c|}
\hline & Risk groups & $\begin{array}{l}\text { High } \\
n=62\end{array}$ & $\begin{array}{l}\text { Moderate } \\
\mathrm{n}=11\end{array}$ \\
\hline \multirow{2}{*}{$\begin{array}{l}\text { HBV } \\
\text { serology }\end{array}$} & HBsAg $(+)$ & 7 & 6 \\
\hline & Anti HBcIgG (+) & 53 & 5 \\
\hline \multirow{4}{*}{$\begin{array}{l}\text { Outcome } \\
\text { of } \\
\text { preemptive } \\
\text { group }\end{array}$} & Reactivation & 3 & - \\
\hline & Without reactivation & 12 & 1 \\
\hline & Ongoing follow-up & 3 & 1 \\
\hline & Exitus & - & - \\
\hline \multirow{4}{*}{$\begin{array}{l}\text { Outcome } \\
\text { of } \\
\text { prophylaxis } \\
\text { group }\end{array}$} & Reactivation & 1 & \\
\hline & Without reactivation & 27 & 6 \\
\hline & Ongoing follow-up & 11 & 1 \\
\hline & Exitus & 5 & 2 \\
\hline \multirow{3}{*}{$\begin{array}{l}\text { Antiviral } \\
\text { treatment }\end{array}$} & Lamivudine & 15 & 3 \\
\hline & Entecavir & 28 & 6 \\
\hline & Tenofovir & 2 & - \\
\hline
\end{tabular}

Conclusion: HBV reactivation due to chemotherapy or immunosuppression can lead to life threatening complications like death and liver failure. Therefore, screening HBV serology in these patients remain essential.

\section{$H B V-C 37$}

The effect of entecavir and tenofovir in chronic hepatitis B patients with liver decompensation

YunHao Tai ${ }^{1}$, Yihsiang Huang ${ }^{1}$, Kenghsin Lan ${ }^{1}$, Kueichuan Lee ${ }^{1}$, Yuanlung Cheng', Kebin Chang ${ }^{1}$

${ }^{1}$ Hepato-Gastroenterology, Taiwan

Background: Entecavir (ETV) $0.5 \mathrm{mg}$ and tenofovir disoproxil fumarate (TDF) $300 \mathrm{mg}$ are the recommended first-line therapies for chronic hepatitis B (CHB) given their high efficacy, but it is not thoroughly studied among CHB with liver decompensation patients. Besides, Taiwan national health insurance started to pay ETV 1mg for these patients since 2015/12/01. Therefore, we aim to compare responses to ETV $0.5 \mathrm{mg}$, ETV $1 \mathrm{mg}$ and TDF $300 \mathrm{mg}$ therapy in these treatment groups.

Method: This was a single-center retrospective study including 266 CHB patients with liver decompensation who received ETV $0.5 \mathrm{mg}$, ETV $1 \mathrm{mg}$ or TDF $300 \mathrm{mg}$ from January 2015 through March 2017. The primary endpoint was 30-days mortality. The secondary endpoints were alanine aminotransferase (ALT) normalization and undetectable HBV-DNA rate. We used Chi-Square and linear regression analysis to assess effect on mortality, ALT normalization and complete virologic response (CVR).

Result: Taiwan national health insurance defined $\mathrm{CHB}$ with liver decompensation as (1) total bilirubin (T/B) level $\geq 2.0 \mathrm{mg} / \mathrm{dL}$ or (2) prothrombin time (PT) prolongs $\geq 3$ seconds. Of these 266 patients, 74 received ETV $0.5 \mathrm{mg}, 165$ received ETV $1 \mathrm{mg}$ and 27 received TDF $300 \mathrm{mg}$. Their mean age was 59.54 years, $75.1 \%$ were male. The baseline ALT and HBV DNA level was 544.55 U/L and 16843319.87 IU/ML respectively. 30-days mortality rate was $37.8 \%, 29.1 \%$ and $44.4 \%$ in ETV $0.5 \mathrm{mg}$, ETV $1 \mathrm{mg}$, TDF $300 \mathrm{mg}$ treatment group respectively ( $\mathrm{p}=0.172$ ). $35.1 \%$ of ETV $0.5 \mathrm{mg}$-treatment group, $24.2 \%$ of ETV $1 \mathrm{mg}$-treatment group and $37.0 \%$ of TDF $300 \mathrm{mg}$ treatment group achieved ALT normalization ( $\mathrm{p}=0.134) .51 .4 \%$ of ETV 0.5 mg-treatment group, $58.9 \%$ of ETV 1 mg-treatment group and $54.5 \%$ of TDF $300 \mathrm{mg}$-treatment group achieved CVR ( $\mathrm{p}=0.769)$. Conclusion: In CHB with liver decompensation patients, ETV $1 \mathrm{mg}$ had no statistical significance when compared to ETV $0.5 \mathrm{mg}$ and TDF $300 \mathrm{mg}$ in mortality, CVR and ALT normalization.

\section{$H B V-C 38$}

Usefulness of stopping rule and clinical predictors for relapse after cessation of nucleoside analogues in chronic hepatitis $B$ patients

Do Seon Song ${ }^{1}$, U Im Chang ${ }^{1}$, Jin Mo Yang ${ }^{1}$, Jung Hyun Kwon ${ }^{1}$, Sun Hong Yoo', Jeong Won Jang ${ }^{1}$, Si Hyun Bae ${ }^{1}$, Seung Kew Yoon ${ }^{1}$

${ }^{1}$ The Catholic University of Korea, Korea, Republic of

Background: The stopping rule of international guidelines in chronic hepatitis $\mathrm{B}(\mathrm{CHB})$ patients treated with nucleoside analogues (NA) has not been verified. We assessed the usefulness of stopping rule of Asia-Pacific Association for the Study of Liver (APASL), and investigated predictors of post-treatment relapse, including HBsAg/ $\mathrm{HBeAg}$, clinico-biochemical, and fibrosis parameters.

Method: Patients who stopped NA were included regardless of meeting stopping rule of APASL guideline. Virologic relapse was defined as HBV DNA $>2,000 \mathrm{IU} / \mathrm{mL}$, while clinical relapse was defined as HBV DNA $>2,000 \mathrm{IU} / \mathrm{mL}$ and ALT $>2 \times \mathrm{xULN}$.

Result: A total of $359 \mathrm{CHB}$ patients (172 HBeAg-positive and 187 $\mathrm{HBeAg}$-negative) were recruited for the study: 246 entecavir (ETV)treated and 113 lamivudine (LAM)-treated patients. Patients who met the stopping rule showed lower one-year virologic and clinical relapse rates than those not. There was no difference in virologic and clinical relapse rates between cessation of ETV and LAM. For the entire patients, higher baseline HBV DNA levels and shorter consolidation were significantly associated with clinical relapse. When stopped NA according to APASL guideline, older age (over 30), lower serum albumin and shorter consolidation were significant risk factors for virologic and clinical relapse. Relapse rate was similar between $\mathrm{HBeAg}$-positive and -negative patients (one-year clinical relapse 
rates of $29.8 \%$ and $21.1 \%$, respectively). In HBeAg-positive patients, higher baseline HBV DNA and shorter consolidation were significant predictors for virologic relapse, and Older age (over 30) and lower serum albumin were significant predictors for clinical relapse. However, in $\mathrm{HBeAg}$-negative patients, there were no significant predictors for virologic and clinical relapase. Low HBsAg titers and non-invasive fibrosis markers, such as fibrosis-4 (FIB-4) score and AST to platelet ratio index (APRI) at cessation of NA were not significant predictors for virologic and clinical relapse.

Conclusion: Stopping rule of APASL guideline is generally acceptable and useful as a guide in decision-making when cost and long-term safety of NA is doubted. Younger patients and good synthetic function who met the stopping rule is best considered for NA discontinuation.

\section{$H B V-C 39$}

\section{Acute Viral Hepatitis in Mongolia: A Time Trend Analysis}

Nyamsuren Naranzul ${ }^{1}$, Ts Gerelchimeg ${ }^{2}$, Naranzul Nyamsuren ${ }^{1}$, S Ariunaa ${ }^{1}$, Oidov Baatarkhuu ${ }^{1}$

${ }^{1}$ Mongolian National University of Medical Sciences, Mongolia; ${ }^{2}$ Department of Infectious Diseases, Mongolian National University of Medical Sciences, Mongolia

Background: Mongolia has one of the highest viral hepatitis A, B, C and $\mathrm{D}$ infection incidences worldwide. We sought to investigate changes in the proportion of acute viral hepatitis types in Mongolia over the last decade.

Method: The cohort comprised 546 consecutive patients clinically diagnosed with acute viral hepatitis from January 2012 to December 2014 in National Center for Communicable Diseases, Ulaanbaatar, Mongolia. A time trend analysis investigating the change in proportion of acute hepatitis A virus (HAV), hepatits C virus (HCV), hepatitis B virus (HBV), and hepatitis D virus (HDV) infection among the cohort with respect to a previous published study was undertaken.

Result: Acute hepatitis A, B and C was diagnosed 50.9\%, 26.2\%, and $6.0 \%$ of the cohort. Notably, $16.8 \%$ of the cohort had a dual infection. The etiologies of acute viral hepatitis were varied by age groups. The most common cause of acute viral hepatitis among 2-19 year olds was hepatitis A, HBV and superinfection with HDV among 20-40 year olds, and HCV among 40-49 year olds. Patients with more than one hepatitis virus infection, were significantly older, more likely to be male and had a higher prevalence of all risk factors for disease acquisition. These patients also had more severe liver disease at presentation compared to those with mono-infection.

Conclusion: Acute viral hepatitis is still prevalent in Mongolia. Thus, the need for proper infection control is increasing in Mongolia.

\section{$H B V-C 40$}

Correlation between liver elasticity and natural history of chronic hepatitis B in Dr. Soetomo General Hospital Surabaya

Ummi Maimunah ${ }^{1}$, Kartika Wensdi Renantriandani ${ }^{2}$, Iswan Abbas Nusi ${ }^{1}$, Retno Handajani ${ }^{2}$, Herry Purbayu ${ }^{1}$, Titong Sugihartono ${ }^{1}$, Ulfa Kholili ${ }^{1}$, Budi Widodo ${ }^{1}$, Husin Thamrin ${ }^{1}$, Amie Vidyani ${ }^{1}$, Muhammad Miftahussurur ${ }^{1}$, Priyo Budi Purwono ${ }^{2}$, Poernomo Boedi Setiawan ${ }^{1}$

${ }^{1}$ Dr. Soetomo General Hospital, Indonesia; ${ }^{2}$ Universitas Airlangga, Indonesia

Background: Chronic HBV infection is an important cause of cirrhosis and development of following diseases. HBV infection is a global problem with mortality reaching 1 million lives each year caused by its complications. Precise estimation of liver fibrosis grade is important to estimate the prognosis and treatment of the patients. The aim of this study is to know the correlation between liver elasticity and chronic hepatitis B natural history in Dr. Soetomo General Hospital.

Method: This is a retrospective study of outpatients with chronic hepatitis B in Gastroenterology and Hepatology Center of Dr. Soetomo General Hospital Surabaya. Total sample is 77 patients with purposive sampling method.

Result: Most patients in each group of $\mathrm{HBeAg}$ positive and negative were males (69.4\% and 63.4\%), with average years of age was 39.4 and 52.5 respectively. The average of serum aspartate transaminase (AST) and alanine aminotransferase (ALT) level was 1.4 (x ULN) and 2.0 (x ULN) for HBeAg positive and 1.6 (x ULN) and 2.1 (x ULN) for $\mathrm{HBeAg}$ negative. The average of platelet count and HBV DNA was $227(109 / \mathrm{L})$ and $2.7 \times 105 \mathrm{IU} / \mathrm{mL}$ for $\mathrm{HBeAg}$ positive and $200(109 / \mathrm{L})$ and $2.8 \times 103 \mathrm{IU} / \mathrm{mL}$ for $\mathrm{HBeAg}$ negative. For F2-F4 fibrosis stage, $40 \%$ were found in phase 4 , and for F1 fibrosis stage, $40.4 \%$ were also found in phase 4 . For low APRI score, $39.1 \%$ were found in phase 2, and $69.2 \%$ high APRI score were found in phase 4 . For low FIB-4 score, $42.9 \%$ were found in phase 2, and $75 \%$ high FIB-4 score were found in phase 4. Spearman's correlation test showed that there was not any correlation between liver elasticity and natural history of chronic hepatitis B based on transient elastography $(\mathrm{p}>0.05)$.

Conclusion: There was not any correlation between liver elasticity and natural history of chronic hepatitis B based on transient elastography.

\begin{tabular}{lcc}
\hline \multicolumn{1}{c}{ Characteristics } & $\begin{array}{c}\text { HBeAg-Positive } \\
(\mathbf{n}=\mathbf{3 6})\end{array}$ & $\begin{array}{c}\text { HBeAg-Negative } \\
(\mathbf{n}=\mathbf{4 1})\end{array}$ \\
\hline Sex & & \\
$\quad$ Male/Female & $25 / 11$ & $26 / 15$ \\
Years of age - mean & $39.4 \pm 13.3$ & $52.5 \pm 11.2$ \\
AST (x ULN) & $1.4 \pm 0.9$ & $1.6 \pm 2.0$ \\
ALT (x ULN) & $2.0 \pm 1.6$ & $2.1 \pm 3.2$ \\
Platelet count $\left(10^{9} / \mathrm{L}\right)$ & $227 \pm 76$ & $200 \pm 85$ \\
HBV DNA $(\mathrm{IU} / \mathrm{mL})$ & $2.7 \times 10^{5} \pm 2.5 \times 10^{3}$ & $2.8 \times 10^{3} \pm 3 \times 10^{3}$ \\
\hline
\end{tabular}

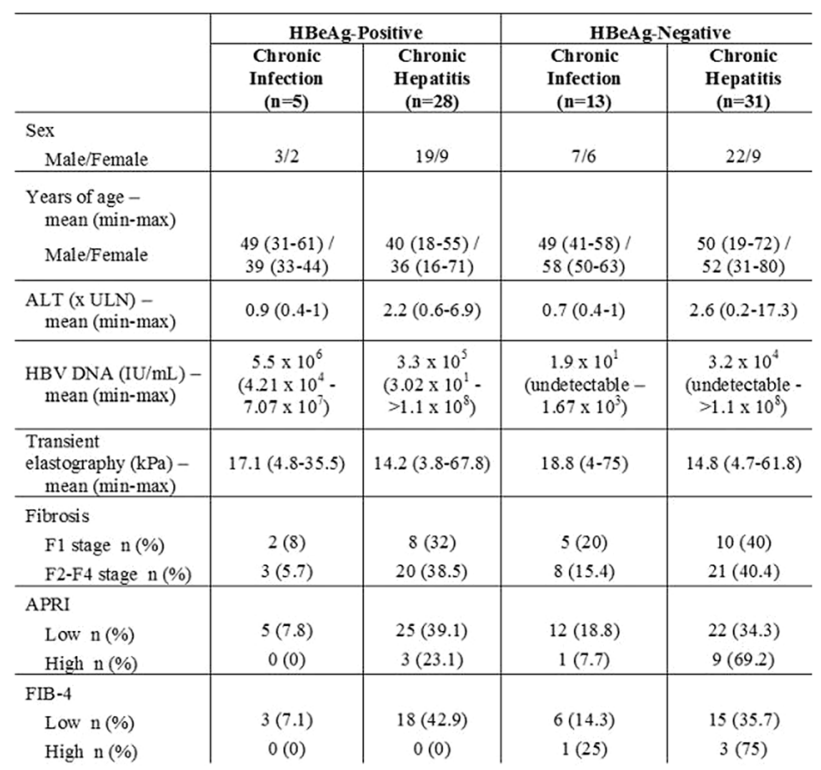




\section{$H B V-C 41$}

A pilot study to explore the use of telbivudine and lamivudine to prevent chemotherapy-related reactivation of hepatitis $B$ infection Lianda Siregar $^{1}{ }^{1}$, Agus Sudiro Waspodo ${ }^{1}$, Siti Nadliroh $^{1}$,

${ }^{1}$ Dharmais National Cancer Center Hospital, Indonesia

Background: Prophylactic drug of choice for preventing hepatitis B virus reactivation induced by cytotoxic chemotherapy is still controversial.

Method: This is an open-label, prospective, interventional, randomized, pilot study to compare telbivudine and lamivudine for preventing chemotherapy-related reactivation of hepatitis B infection. Patients with $\mathrm{HBsAg}$ positive or anti $\mathrm{HBc}$ positive, who were referred by oncologists from May 2014 to April 2017, were randomized to receive either telbivudine or lamivudine. Eligible patients were grouped according to whether they received lamivudine or telbivudine, and were observed during chemotherapy until six months after their last cycles.

Result: A total of fifty-nine patients were enrolled in this study. Median age was 49 years (interquartile range 17,74). Telbivudine (TDF) and lamivudine (LAM) groups consist of 31 patients and 28 patients, respectively. Number of patients with HBsAg positive in the TDF and LAM group was similar, i.e. 25 patients. Rituximab-based treatment was given in nine patients in the TDF group and five patients in the LAM group. Number of patients who complete observation was 11 patients in TDF group and 15 patients in LAM group. Patients, who were loss to follow up, were mostly did not survive due to their late stages of cancer. No reactivation was observed in both groups. One patient in each group experienced virological breakthrough on week 12 after starting antiviral treatment. The HBV DNA of the patient in TDF group became undetectable on three months and six months after chemotherapy, but the patient in LAM group did not complete observation. One patient in LAM group experienced virological breakthrough six months after last chemotherapy cycle.

Conclusion: Telbivudine and lamivudine seems to have same efficacy to prevent reactivation of hepatitis B induced by chemotherapy. Larger number of patients and longer duration of observation are needed to determine the drug of choice.

\section{$H B V-C 42$}

Alteration of serum CHI3L1 levels is associated with the pathological progression of chronic hepatitis $B$

Xiaoning Wang ${ }^{1}$, Ping Liu ${ }^{1}$, Biaoyang Lin ${ }^{2}$, Xinzhu Liu ${ }^{1}$, Xiaoxian Wu ${ }^{1}$, Jiannan Qiu ${ }^{1}$, Zhun Xiao ${ }^{1}$, Li Qi $^{1}$, Jia Liu ${ }^{1}$

${ }^{1}$ Shanghai University of Traditional Chinese Medicine, China;

${ }^{2}$ Zhejiang University, China

Background: It has been confirmed that serum chitinase 3-like $1(\mathrm{CHI}$ L 1) can be used as a noninvasive biomarker of liver fibrosis. However, we still do not know the characteristics of serum CHI3L1 in patients with liver cirrhosis. Here, we explore whether the alteration of serum CHI3L1 levels is associated with pathological progression of liver fibrosis in patients with chronic hepatitis B.

Method: A total of 97 healthy controls, 349 Chronic hepatitis B (CHB) patients diagnosed as liver fibrosis ( $\mathrm{S} 0-\mathrm{S} 4$ stages) by liver biopsies, and $35 \mathrm{CHB}$ decompensated cirrhosis patients diagnosed by clinicians were enrolled in this study. Blood routine tests and liver functions were measured by automatic clinical chemistry analyzers. The levels of serum CHI3L1 were quantified by ELISA.

Result: The serum CHI3L1 levels in patients with CHB (S1-S4) and decompensated cirrhosis were significantly increased, compared with those in the healthy controls. The CHI3L1 levels not only were able to differentiate early stages of liver fibrosis (S0-S2) from late stages of liver fibrosis (S3-S4), but also able to stratify CHB with compensated cirrhosis (S4, Child-Pugh scores $<=6$ ) from those with decompensated cirrhosis (Child-Pugh scores $>=7$ ). The alteration of CHI3L1 levels were associated with the liver function deterioration. We further showed that CHI3L1 is a good marker of liver cirrhosis induced by CHB, with areas under the ROC curves (AUCs) of 0.916 for compensated cirrhosis and 0.983 for decompensated cirrhosis.

Conclusion: Serum CHI3L1 is a potential biomarker for monitoring the pathological progression of chronic hepatitis B. It may also be used to rapidly screen for the patients with decompensated cirrhosis.

\section{$\mathrm{HBV}-\mathrm{C} 43$}

Association between serum cytokeratin-18 neoepitope M30 (CK-18 M30) levels and chronic hepatitis B: a meta-analysis

Changfei Li ${ }^{1}$, Shousheng Liu ${ }^{2}$, Linlin $\mathrm{Lu}^{2}$, Quanjiang Dong ${ }^{2}$, Shiying Xuan', Yongning Xin ${ }^{2}$

${ }^{1}$ Qingdao University, China; ${ }^{2}$ Qingdao Municipal Hospital, China

Background: Cytokeratin-18 Neoepitope M30 (CK-18 M30) has been reported to be associated with chronic HBV infection and the severity of liver injury, but the results of these studies were inconsistent. We sought to investigate to association between serum CK-18 M30 levels and the severity of liver injury in chronic hepatitis B (CHB) patients.

Method: A systematic literature search was performed in PubMed, Embase and Cochrane Library literature databases for relevant studies published in English up to August 2017. Heterogeneity among individual studies were investigated for summarizing all the studies. Standard mean difference (SMD) and 95\% confidence interval (CI) were calculated using random-effects model or fixed-effects model. Finally, sensitivity analysis and publication biases were performed to evaluated the accuracy of this meta-analysis. Statistical analysis were conducted using the Review Manager 5.3 and STATA 12.0 software. Result: Five case-control studies were included in the ultimate analysis, which containing $488 \mathrm{CHB}$ patients, 276 inactive carriers and 193 healthy controls. The major results of the meta-analysis revealed a significant elevated serum CK-18 M30 levels in chronic HBV infected patients which included the CHB patients with severe liver injury and inactive HBV carriers compared to healthy controls $(\mathrm{SMD}=1.13,95 \% \mathrm{CI}: 0.75-1.50, P<0.001 ; \mathrm{SMD}=0.63,95 \% \mathrm{CI}:$ $0.38-0.89, P<0.001$, respectively). Furthermore, the serum CK-18 M30 levels in CHB patients with severe liver injury were significant higher than in inactive carriers (SMD $=1.29,95 \%$ CI: $0.60-1.98, P<$ $0.001)$. Sensitivity and publication biases analysis verified the stability and reliability of our analysis.

Conclusion: The elevated serum CK-18 M30 levels could be regarded as a useful non-invasive biomarker for the diagnosis of chronic $\mathrm{HBV}$ infection, and were associated with the severity of liver injury in chronic Hepatitis B patients. The serum CK-18 M30 levels could reflect the liver inflammation in inactive carriers, which represented the early stage of chronic HBV infection. 


\section{$\mathrm{HBV}-\mathrm{C} 44$}

Evaluation of the long-term efficacy between tenofovir and entecavir in treatment- naïve chronic hepatitis $B$ patients

Gule Cinar ${ }^{1}$, Elif Nur Haliloglu', Ebru Atalay ${ }^{1}$, Ahmet Mert Cavnar ${ }^{1}$, Fazilet Ayan ${ }^{1}$, Elif Mulime Saricaoglu', Tazegul Gul ${ }^{1}$, Utku Can Sarica ${ }^{1}$, Ismail Balik ${ }^{1}$

${ }^{1}$ Ankara University Faculty of Medicine, Turkey

Background: Although tenofovir disoproxil fumarate (TDF) and entecavir hydrate (ETV) have been used widely, few clinical studies have evaluated and compared the long-term efficacy between tenofovir TDF and ETV. We aimed to evaluate the long-term efficacy between them and safety of TDF and ETV in the treatment of naïve chronic hepatitis $\mathrm{B}(\mathrm{CHB})$ patients.

Method: We evaluated retrospectively $166 \mathrm{CHB}$ patients who received first line therapy with $245 \mathrm{mg}$ TDF $(\mathrm{n}=110)$ or $0.5 \mathrm{mg}$ ETV $(\mathrm{n}=56)$.

Result: Two groups showed no difference in baseline characteristics. The change (mean $\pm \mathrm{SE}$ ) in serum hepatitis B virus (HBV)-DNA levels from baseline to week 24 were $5.42 \pm 0.034$ and $5.03 \pm 0.036$ $\log 10 \mathrm{IU} / \mathrm{mL}$ in the TDF and ETV groups, respectively, indicating the non-inferiority of TDF to ETV $(\mathrm{p}<0.01)$. The proportion of subjects with undetectable HBV-DNA increased from 64 to $92 \%$ and 51 to $79 \%$ in the TDF and ETV arms with continuation of the treatment from week 24 to 48, respectively. Although, both groups showed no difference in terms of the serologic, biochemical and virologic (VR) response at week 48, reduction in HBV DNA level were greater in subjects with hepatitis $B$ envelope antigen $(+)(\mathrm{p}=0.03)$ and high alanine aminotransferase levels $(\geq 80 \mathrm{IU} / \mathrm{L})(\mathrm{p}<0.01)$. Prevalence of drug-related adverse events at week 48 was $8 \%$ and $14 \%$ in the TDF and ETV groups.

Conclusion: Although either TDF or ETV, overall, may show a comparable long-term antiviral efficacy in treatment-naïve CHB, TDF has noninferiority to ETV in treatment effectiveness (lowering of serum HBV-DNA level) in CHB patients.

\section{$H B V-C 45$}

\section{The Frequency of Autoantibodies in Patients with Chronic Hepatitis B}

\section{Elif Yorulmaz ${ }^{1}$, Umut Aykanat ${ }^{1}$, Fatma Eksi Polat ${ }^{1}$, Munevver Gul Avsar', Mehmet Oncu', Ayse Banu Esen', Umit Seza Tetikkurt ${ }^{1}$ \\ ${ }^{1}$ Bagcilar Education and Treatment Hospital, Turkey}

Background: In this study, we aimed to evaluate retrospectively the frequency of autoantibodies in newly diagnosed patients with chronic hepatitis $\mathrm{B}(\mathrm{CHB})$.

Method: Between the years January 2010 - August 2015 study, admitted to the outpatient Gastroenterology and Infectious Diseases, CHB diagnosed between ages 17-80 enrolled 122 patients and 117 healthy control group. In both groups ANA, ASMA, AMA and antiLKM positive and negative rates were compared.

Result: None of the patients in the control group patients, the AMA and LKM1 not seen. In $9.8 \%$ of patients in the patient group, $8.5 \%$ of patients in the control group ANA result is positive, there was no statistically significant difference between them. In $5.7 \%$ of patients in the patient group, $0.9 \%$ of patients in the control group tested positive ASMA is very close to that, but there was no statistically significant difference between them. Hepatosteatosis level of the patient group were statistically significantly lower than the control group.

Conclusion: In our study, the low temperature of autoantibody according to all the studies could depend on the views of $\mathrm{CHB}$ patients receiving treatment. In newly diagnosed chronic hepatitis B patients before treatment and after that may arise to look autoantibodies autoimmune phenomena and extrahepatic findings may provide insight terms.

\section{$H B V-C 46$}

HBeAg serology status of chronic hepatitis B patients in Dr. Soetomo General Hospital Surabaya

Kartika Wensdi Renantriandani ${ }^{1}$, Ummi Maimunah ${ }^{2}$, Iswan Abbas Nusi ${ }^{2}$, Retno Handajani ${ }^{1}$, Priyo Budi Purwono', Herry Purbayu ${ }^{2}$, Titong Sugihartono ${ }^{2}$, Ulfa Kholili ${ }^{2}$, Budi Widodo $^{2}$, Husin Thamrin ${ }^{2}$, Amie Vidyani ${ }^{2}$, Muhammad Miftahussurur', Nanda Della Alyda ${ }^{1}$, Poernomo Boedi Setiawan ${ }^{2}$

${ }^{1}$ Universitas Airlangga, Indonesia; ${ }^{2}$ Dr. Soetomo General Hospital, Indonesia

Background: Seropositive $\mathrm{HBeAg}$ indicates active viral replication and is a significant risk factor for cirrhosis and hepatocellular carcinoma. The important end-point therapy is to achieve $\mathrm{HBeAg}$ seroconversion, ALT normalization, undetectable HBV DNA, liver histology improvement, and reduced liver disease severity. The aim of this study is to know the HBeAg serology status of chronic hepatitis B patients in Dr. Soetomo General Hospital.

Method: This is a retrospective study using medical records and sera of patients with chronic hepatitis B which its period was from September 2012 to September 2017 in Gastroenterology and Hepatology Center of Dr. Soetomo General Hospital Surabaya. Total sample was 56 patients which have high level of ALT with purposive sampling method. HBeAg loss tested using Enzyme-linked Immunosorbent Assay (ELISA) method with HBeAg ELISA kit manufactured by Sciencewerke corporate.

Result: Total sample was 56 patients, which $44.6 \%$ were $\mathrm{HBeAg}$ positive. Males patients were $69.6 \%$ with ratio of male and female was $2.3: 1$. The distribution of patients based on age shows that $28.6 \%$ of the patients are $50-59$ years old $(49.4 \pm 13.2)$. Based on the diagnosis, chronic hepatitis $\mathrm{B}$; cirrhosis; and hepatocellular carcinoma, $\mathrm{HBeAg}$ negative patients are always many more than $\mathrm{HBeAg}$ positive. The mean of serum ALT level is 100.54 IU/L (9-882 IU/L). The group of patients who received Telbivudine therapy experienced $\mathrm{HBeAg}$ loss at most, as many as $33.33 \%$ with $1-2$ years of therapy. The group of patients with Lamivudine therapy showed as much as $25 \%$ of patients with $\mathrm{HBeAg}$ loss each treated for $<1$ year, $1-2$ years, and $>2$ years. The group of patients with Tenofovir therapy showed that $40 \%$ of patients had HBeAg loss each treated for $<1$ year and $>2$ years. Total $\mathrm{HBeAg}$ loss treated for $<1$ year, 1-2 years, and $>2$ years were $16 \%, 24 \%$, and $24 \%$ respectively, which total $\mathrm{HBeAg}$ loss of all type and duration of therapy was $64 \%$.

The group of patients who received Telbivudine therapy showed as many as $19.35 \%$ of patients had normal ALT values treated for 1-2 years. The group of patients who received Lamivudine therapy showed as many as $22.22 \%$ of patients had normal ALT values treated for $<1$ year. The group of patients received Tenofovir therapy showed as many as $14.28 \%$ of patients had normal ALT each treated for $<1$ year, 1-2 years, and $>2$ years. Total normal serum ALT level was $42.86 \%$. 
Conclusion: Patients with chronic hepatitis B were mostly 50-59 year-old males. HBeAg negative was many more than $\mathrm{HBeAg}$ positive. Total HBeAg loss of all type and duration of therapy was $64 \%$.

\begin{tabular}{|c|c|c|c|c|c|}
\hline \multirow{2}{*}{ Therapy } & HBeAg positive & \multicolumn{4}{|c|}{ HBeAg loss $-\mathrm{n}(\%)$} \\
\cline { 3 - 6 } & $\mathrm{n}$ & $<1$ year & $1-2$ years & $>2$ years & TOTAL \\
\hline Telbivudine & 12 & $0(0)$ & $4(33.33)$ & $2(16.67)$ & $6(50)$ \\
\hline Lamivudine & 8 & $2(25)$ & $2(25)$ & $2(25)$ & $6(75)$ \\
\hline Tenofovir & 5 & $2(40)$ & $0(0)$ & $2(40)$ & $4(80)$ \\
\hline TOTAL & 25 & $4(16)$ & $6(24)$ & $6(24)$ & $16(64)$ \\
\hline
\end{tabular}

\begin{tabular}{|c|c|c|c|c|c|}
\hline \multirow{2}{*}{ Therapy } & \multirow{2}{*}{$\mathrm{n}$} & \multicolumn{4}{|c|}{ Normal ALT $\mathrm{n}(\%)$} \\
\cline { 3 - 6 } & & $<1$ year & $1-2$ years & $>2$ years & TOTAL \\
\hline Telbivudine & 31 & $5(16.12)$ & $6(19.35)$ & $3(9.67)$ & $14(45.16)$ \\
\hline Lamivudine & 18 & $4(22.22)$ & $2(11.11)$ & $1(5.55)$ & $7(38.89)$ \\
\hline Tenofovir & 7 & $1(14.28)$ & $1(14.28)$ & $1(14.28)$ & $3(42.85)$ \\
\hline TOTAL & 56 & $10(17.86)$ & $9(16.07)$ & $5(8.93)$ & $24(42.86)$ \\
\hline
\end{tabular}

\section{$H B V-C 47$}

Results of treatment with nucleotide analogs in patient which have been infected by hepatitis B virus, for a two-year period

Silva Gjika ${ }^{1}$, Liri Cuko', Adriana Babameto ${ }^{1}$, Stela Taci ${ }^{1}$, Ilir Bibolli' ${ }^{1}$, Irgen Tafaj ${ }^{1}$, Silva Gjika ${ }^{1}$, Jonida Lavdari ${ }^{1}$, Bajram Begaj ${ }^{1}$

\section{${ }^{1}$ QSU NENE TEREZA, Albania}

Background: Chronic hepatopathies from hepatitis B infection remain a major problem all over the world. Numerous long-term studies up to 8 years with nucleotide analogues (TDF) have shown no resistance to it. It can be used in both variants of HBV infection, such as $\mathrm{HBeAg}$ negative and those with $\mathrm{HBeAg}$ positive.

The purpose of this study was to evaluate the results of treatment with Tenofovir disoproxil fumarate (TDF), and its impact on renal problems in patients with chronic viral hepatopathy $\mathrm{B}$.

Method: This is a retrospective study, where the data of the patients are from 2014 to January 2017. The age of the patients is $44.8 \pm 13.3$, 68 males and 32 females. The inclusion criteria in the study were chronic HBV infection, viral HBV DNA (PCR) viral load > $2000 \mathrm{UI} /$ $\mathrm{ml}$, increased transaminases (ALT), quantitative $\mathrm{HbsAg}>1000 \mathrm{UI} /$ $\mathrm{ml}$, and the use of nucleotide analogue (TDF) overlapping per year. The study did not include cases with HCC, or with HCV co-infection. Patients were followed every 3-6 months, with biochemical response (ALT) and virological response (HBV DNA).

Result: There are 54 patients, all negative $\mathrm{HBeAG}$, followed for two years. The biochemical response (ALT $<40 \mathrm{IU} / \mathrm{L}$ ) was in 100 , the virological response (HBV DNA $<20 \mathrm{IU} / \mathrm{ml}$ ) to $87 \%$ in the first year and $100 \%$ in the second year. Patients had no kidney problems during two years of treatment. Quantitative HBsAg did not make any significant changes during this period.

Conclusion: Treatment with Nucleotide Analogs is considered effective, safe and well tolerated in chronic viral hepatopathies B.

\section{$H B V-C 48$}

Randomized study of the relationship between virologic responses to entecavir or other $\mathrm{HBV}$ nucleos(t)ide analogues and long-term clinical outcomes in chinese patients with chronic hepatitis B virus infection

Jinlin $\mathrm{Hou}^{1}$, Wei Zhao ${ }^{10}$, Yuming Wang ${ }^{9}$, Wukui Cao ${ }^{14}$, Guozhong Gong ${ }^{12}$, Mingliang Cheng ${ }^{2}$, Xiaoping Tang ${ }^{6}$,
Deming Tan ${ }^{16}$, Hong Ren ${ }^{11}$, Zhongping Duan ${ }^{4}$, Hong Tang ${ }^{15}$, Zhiliang Gao ${ }^{13}$, Jidong $\mathrm{Jia}^{3}$, Lai Wei ${ }^{7}$, Alexandra Thiry ${ }^{5}$, Elizabeth Cooney ${ }^{5}$, Qing Xie ${ }^{8}$

${ }^{1}$ Nanfang Hospital, Southern Medical University, China; ${ }^{10}$ The 2nd Hospital of Nanjing, China; ${ }^{11}$ The Second Affiliated Hospital of Chongqing Medical University, China; ${ }^{12}$ The Second Xiangya Hospital of Central South University, China; ${ }^{13}$ The Third Affiliated Hospital, Sun Yat-Sen University, China; ${ }^{14}$ Tianjin Second People's Hospital, China; ${ }^{15}$ West China Hospital, Sichuan University, China; ${ }^{16}$ Xiangya Hospital, Central South University, China; ${ }^{2}$ Affiliated Hospital of Guiyang Medical College, China; ${ }^{3}$ Beijing Friendship Hospital, Capital Medical University, China; ${ }^{4}$ Beijing YouAn Hospital, Capital Medical University, China; ${ }^{5}$ Bristol-Myers Squibb, United States; ${ }^{6}$ Guangzhou No.8 People's Hospital, China; ${ }^{7}$ Peking University People's Hospital and Peking University Hepatology Institute, China; ${ }^{8}$ Ruijin Hospital, Shanghai Jiao Tong University School of Medicine, China; ${ }^{9}$ Southwest Hospital, China

Background: In clinical studies of patients with chronic hepatitis B (CHB), entecavir (ETV) showed sustained viral suppression, reduced HBV disease progression and favorable safety. The REALM study assessed clinical outcome events (COEs) during up to 10 years of therapy with ETV or other nucleos(t)ide analogues (nucs) in a large, global, observational study. Results from patients in China are reported.

Method: Patients with $\mathrm{CHB}$ were randomly assigned (1:1) to monotherapy with ETV ( 0.5 or $1.0 \mathrm{mg}$ QD, based on prior treatment status) or an investigator-selected non-ETV nuc, and assessed for HBV DNA response (see Results) and safety (frequencies and relative risks of COEs). Investigator-reported COEs were adjudicated by an independent committee to ensure diagnostic consistency. The relationship between HBV DNA response and time to HBV-related COEs was assessed.

Result:There were 5305 randomized patients in China treated with ETV $(n=2659)$ or a non-ETV nuc $(n=2646)$. At baseline, $81 \%$ were male and the mean age was $36.1 \mathrm{yr} ; 34 \%$ were HBV nuc-experienced, $66 \%$ were $\mathrm{HBeAg}+, 12 \%$ had cirrhosis and the mean serum HBV DNA level was $6.5 \log 10 \mathrm{IU} / \mathrm{mL}$. In the ETV group, mean serum ALT was $114 \mathrm{IU} / \mathrm{mL}$. Patients remained on initial therapy for a mean 99 months (ETV) or 95 months (non-ETV); 3\% of ETV recipients switched to a non-ETV regimen after a mean 95 months; $8 \%$ of nonETV recipients switched to ETV monotherapy or an ETV combination regimen after a mean 55 months. There were no clinically relevant differences between treatment groups in rates of adjudicated COEs (Table). HBV DNA response, defined as confirmed HBV DNA $2 \mathrm{xULN}$ vs $=2 \mathrm{xULN}(83.6 \%$ vs $76.8 \%$ ), and HBeAg-negative vs positive status $(88.1 \%$ vs $76.3 \%)$. Similarly, in the non-ETV group, response rates were higher in patients with HBV DNA levels Conclusion: HBV DNA response was higher with ETV vs non-ETV, and response vs nonresponse was associated with a lower rate of HBV-related COEs in both treatment groups.

Adjudicated COEs by Treatment Group

\begin{tabular}{|c|c|c|}
\hline Adjudicated COEs, n (\%) & $\begin{array}{c}\text { ETV } \\
(\mathrm{N}=2659)\end{array}$ & $\begin{array}{l}\text { Non-ETV } \\
(\mathrm{N}=2646)\end{array}$ \\
\hline Liver-related HBV disease progression $^{3}$ & $95(3.6)$ & $114(4.3)$ \\
\hline Hepatocellular carcinoma (HCC) & $69(2.6)$ & $87(3.3)$ \\
\hline Non-HCC HBV disease progression & $36(1.4)$ & $44(1.7)$ \\
\hline Liver-related death & $3(0.1)$ & $6(0.2)$ \\
\hline Overall malignant neoplasms & $90(3.4)$ & $104(3.9)$ \\
\hline Non-HCC malignant neoplasms & $22(0.8)$ & $18(0.7)$ \\
\hline Deaths & $62(2.3)$ & $74(2.8)$ \\
\hline
\end{tabular}

${ }^{a}$ Composite of non-HCC HBV disease progression, $\mathrm{HCC}$, and liver-related death. 
Time to Adjudicated Liver-related HBV Disease Progression by Treatment Group and HBV DNA Response

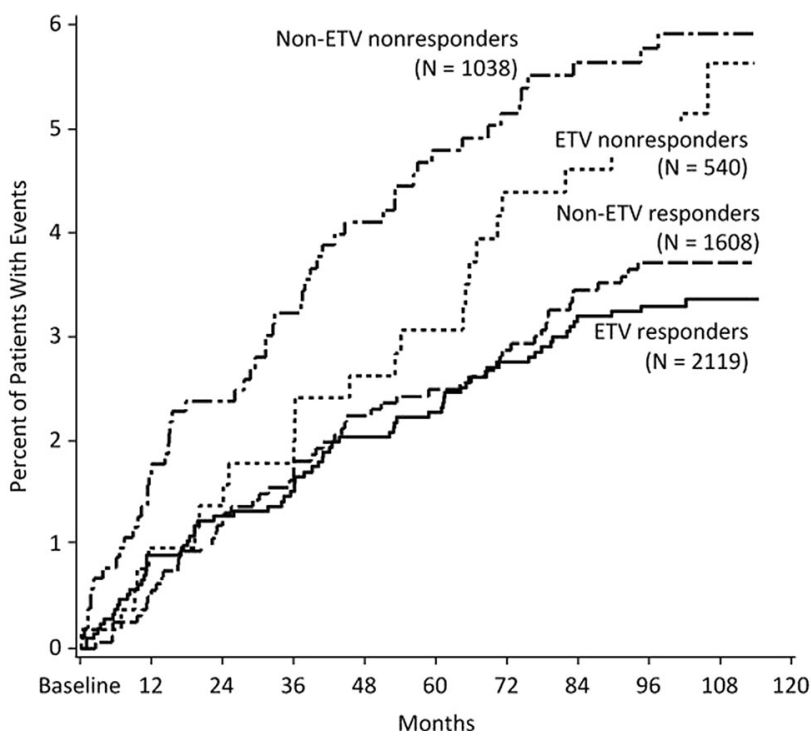

$H B V-C 49$

Analysis of the factors influencing the serum HBsAg negative in chronic hepatitis B patients with NUs treatment

Xiangyong Li $^{1}$, Yufeng Zhang ${ }^{1}$, Zhiwei Zhang ${ }^{1}$, Xinhua $\mathrm{Li}^{1}$, Jiao Gong $^{1}$, Yuankai Wu ${ }^{1}$, Yusheng Jie ${ }^{1}$, Yutian Chong ${ }^{1}$

${ }^{1}$ The Third Affiliated Hospital of Sun Yat-sen University, China

Background: To screen out the factors that may affect spontaneous serum HBsAg negative such as demographic characteristics, baseline characteristics, dynamic changes of the biochemical, virological, serological and imagery indicator during the follow-up period of the 1205 CHB patients with NUs antiviral treatment in the SCHEMA study cohort of our department.

Method: Collect the factors that may affect serum HBsAg negative in CHB patients without antiviral treatment, which include demographic data, baseline data, indicators such as biochemical, virological, serological, liver and spleen imagery and their dynamic variations. Influence factors of serum $\mathrm{HBsAg}$ negative or clearance was performed on COX single factor regression analysis and multivariate regression analysis; annual growth rate of HBsAg negative seroconversion or clearance was performed on Kaplan-Meier life table analysis (Deadline of collecting data is 31st, May, 2015).

Result:A total of 1205 cases of CHB patients were followed up for 10 years. 50 cases of chronic HBV infected persons had negative seroconversion of serum HBsAg, and the annual rate of negative rotation was $1.0 \%$. Univariate analysis showed that the age of more than 50 years $(\mathrm{HR}=1.774, \mathrm{P}=0.047)$, baseline PLT count $[\mathrm{HR}$ value was 0.994, $\mathrm{P}=0.037$ ], baseline $\mathrm{HBeAg}$ negative $(\mathrm{HR}=0.335, \mathrm{P}=0.001)$, spleen length increased $(\mathrm{HR}=1.017, \mathrm{P}=0.013)$, low baseline $\mathrm{HBsAg}$ titer, especially $<2000 \mathrm{IU} / \mathrm{mL}(\mathrm{HR}=0.099, \mathrm{P}<0.001)$, the numbers of change antiviral therapy [HR was $0.028, \mathrm{P}$

Conclusion: For the CHB patients treated with NUCs, Baseline HBsAg levels, the numbers of changing antiviral solution and achieving IVR, EVR,CVR were independent factors of $\mathrm{HBsAg}$ seroconversion. and the patients with baseline HBsAg low levels $(<$ $2000 \mathrm{IU} / \mathrm{mL}$ ) and the numbers of changing antiviral solution less, who achieving IVR, EVR, CVR were more likely to occur serum HBsAg negative.
$\mathrm{HBV}-\mathrm{C} 50$

Analysis of the factors influencing the spontaneous HBsAg serum negative in chronic hepatitis $B$ patients

Xiangyong $L i^{1}$, Xin Shu ${ }^{1}$, Yufeng Zhang ${ }^{1}$, Zhanyi $\mathrm{Li}^{1}$, Zeqian $\overline{W u}^{1}$, Sujuan $\mathrm{Xi}^{1}$, Yutian Chong ${ }^{1}$

${ }^{1}$ The Third Affiliated Hospital of Sun Yat-sen University, China

Background: To screen out the factors that may affect spontaneous serum HBsAg negative such as demographic characteristics, baseline characteristics, dynamic changes of the biochemical, virological, serological and imagery indicator during the follow-up period of the 974 CHB patients without antiviral treatment in the SCHEMA study cohort of our department.

Method: Collect the factors that may affect serum HBsAg negative in CHB patients without antiviral treatment, which include demographic data, baseline data, indicators such as biochemical, virological, serological, liver and spleen imagery and their dynamic variations. Influence factors of serum HBsAg negative or clearance was performed on COX single factor regression analysis and multivariate regression analysis; annual growth rate of HBsAg negative seroconversion or clearance was performed on Kaplan-Meier life table analysis. (Deadline of collecting data is 31st, May, 2015)

Result: In the follow-up period to December 2016, 54 cases of chronic HBV infected persons had spontaneous negative seroconversion of serum HBsAg, and the spontaneous negative conversion rate was about $1.3 \%$ every year. Univariate analysis showed that age, especially over 50 years $(\mathrm{HR}=2.647, \mathrm{P}=0.001)$, $\mathrm{BMI}$, especially more than 28 , namely the special obesity $(\mathrm{HR}=3.401, \mathrm{P}=0.004)$, the baseline HBV DNA level, especially less than E4 IU/mL $(\mathrm{HR}=$ 11.798, $\mathrm{P}=0.001$ ), $\mathrm{HBeAg}$ (baseline state $\mathrm{HR}=0.192, \mathrm{P}=0.001$ ), baseline HBsAg titer, especially $2000 \mathrm{IU} / \mathrm{mL}$ (HR $=0.056, \mathrm{P} 0.001)$, spleen length $(\mathrm{HR}=1.017, \mathrm{P}=0.013)$, right hepatic oblique diameter of [HR was 1.026, $\mathrm{P}=0.007$ ], were the influence factors of serum HBsAg negative conversion. Multivariate analysis showed that baseline HBsAg titer [HR value was 0.071, $\mathrm{P}<0.001$ ] and baseline HBV DNA [HR value was $0.819, \mathrm{P}=0.005$ ] and right hepatic oblique diameter [HR value was $1.031, \mathrm{P}=0.027$ ] were the independent influence factors of serum HBsAg negative conversion.

Conclusion: For the patients without antiviral treatment, Baseline HBV DNA levels and baseline HBsAg levels were independent factors of HBsAg seroconversion. and the patients with baseline $\mathrm{HBV}$ DNA low levels $(<104 \mathrm{IU} / \mathrm{mL})$ and baseline HBsAg low levels $(<$ $2000 \mathrm{IU} / \mathrm{mL}$ ) were more likely to occur serum HBsAg negative.

\section{$H B V-C 51$}

Disease burden of hepatitis B in the Kyrgyz Republic: a focus on prevention

Devin Razavi Shearer ${ }^{1}$, M A Atabekova', Anara Dzhumagulova ${ }^{3}$, G I Jumagulova $^{12}$, K A Nogoibaeva ${ }^{4}$, Omor T Kasymov ${ }^{7}$, Ainara Keshikbaeva ${ }^{6}$, Ainura Kutmanova ${ }^{8}$, Olga Plotnikova ${ }^{11}$, Homie Razavi ${ }^{13}$, A B Satybaldieva ${ }^{10}$, Sultan Suranbaev ${ }^{2}$, Gulmira Syibildaevna Suranbaeva ${ }^{7}$, Vera Toygombaeva ${ }^{5}$, Devin Michael Razavi Shearer ${ }^{13}$

${ }^{1}$ The CDA Foundation, United States; ${ }^{10}$ Republican Blood Center, Kyrgyz Republic; ${ }^{11}$ Republican Center for Immunoprofilaxis, Kyrgyz Republic; ${ }^{12}$ Scientific Production Association Preventive Medicine, Kyrgyz Republic; ${ }^{13}$ The Polaris Observatory, United States; ${ }^{2}$ Department for Disease Prevention and State Sanitary and Epidemiological Surveillance, Kyrgyz Republic; ${ }^{3}$ Kyrgyz State Medical Academy, Kyrgyz Republic; ${ }^{4}$ Kyrgyz State Medical Institute 
of Retraining and Improvement of Qualification, Kyrgyz Republic; ${ }^{5}$ Kyrgyz-Russian Slavic University, B.N. Yeltsin, Kyrgyz Republic; ${ }^{6}$ Mandatory Health Insurance Fund, Kyrgyz Republic; ${ }^{7}$ Ministry of Health of the Kyrgyz Republic, Kyrgyz Republic; ${ }^{8}$ MWMS Head of Department Infectious and Tropical Diseases, Kyrgyz Republic; ${ }^{9} \mathrm{NG}$, Kyrgyz Republic

Background: Central Asian countries have historically had higher levels of hepatitis b virus (HBV) disease burden and the Kyrgyz Republic is no different. Currently, there is a renewed sense of urgency regarding $\mathrm{HBV}$ and the potential to further reduce incidence nationally. The aim of this study is to quantify the HBV disease burden in the Kyrgyz Republic and to examine the impact of prevention measures through modeling.

Method: A literature review was conducted and then expert consensus regarding inputs was built at an in country meeting in September 2017. A dynamic country-level transmission and disease burden model was used to estimate the impact of vaccination, Hepatitis B immune globulin (HBIG), treatment of mothers, aging and disease progression and mortality in the infected population. A scenario was then developed that would meet the Global Health Sector Strategy (GHSS) goal for hepatitis B prevalence among five year olds in $2030, \leq 0.1 \%$, combined with a catch-up vaccination program that would cover $98 \%$ of $20-29$ year olds over a 7 year period starting in 2018.

Result: In 2015, it was estimated that 9\% (4.4-13.2\%) of the adult population was $\mathrm{HBsAg}+$. When expanded to the total population it was estimated that there were 374000 (UI: 245 000-504 000) infected individuals in 2016. It is estimated that at least $25 \%$ of those infected with HBV are also infected with hepatitis delta. Currently the Kyrgyz Republic has high levels of vaccination, $99 \%$ of infants receiving the first dose within 24 hours and $96 \%$ of one year olds having received the complete HBV vaccination schedule. Without intervention, it is estimated that in 2030 the prevalence among five year olds would be $0.2 \%$.

In order to meet the GHSS target of $\leq 0.1 \%$ in 2030 a scenario was developed in which starting in $2018,25 \%$ of infants born to HBsAg+ mothers that received birth dose would also receive HBIG. This was kept constant through 2030. In addition, from 2018-2021, 25\% of mothers with a high viral load $(\geq 20,000 \mathrm{IU} / \mathrm{mL})$ received peripartum anti-viral therapy, increasing to $30 \%$ in 2022 , and $65 \%$ in 2025 and beyond. In addition, a national catch-up vaccination program targeting 20-29 year olds was started in 2018, covering $98 \%$ of this cohort by 2025 .

This scenario resulted in a prevalence of $0.1 \%$ among five year olds in 2030, as well as an estimated 10800 acute and 1800 chronic cases averted (Figure 1).

Conclusion: Currently, there is no cure for HBV or HDV, and the current treatment for HDV is not very effective. Thus, prevention of new cases is imperative in reducing the future disease burden in the Kyrgyz Republic. The catch-up vaccinations strategy targeting the generation born before vaccination will help stop future infections in this age group. This strategy would require the screening of pregnant women, but only $25 \%$ of those found positive would require their infants to receive $\mathrm{HBIG}$, and the antiviral treatment can increase along with the health system capacities from 2018 to 2025 .

\section{$H B V-C 52$}

\section{Droplet digital PCR assay for serum HBV-RNA}

Jing Wang ${ }^{1}$, Wenhong Zhang ${ }^{1}$, Yiqi Y Yu ${ }^{1}$, Zhefeng Meng ${ }^{2}$,
${ }^{1}$ Huashan Hospital, Fudan University, China; ${ }^{2}$ Minhang Branch of Zhongshan Hospital, Fudan University, China

Background: Serum HBV-RNA levels has been considered as an potential approach to diagnosing of hepatitis $\mathrm{B}$ virus infection. However, there was no recognized method able to precisely quantify it with an improved sensitivity and specificity. Recently droplet digital PCR (ddPCR) has been reported to provide an absolute quantification of molecules without use of standard curves. Thus, in this study, we established a way to assess the capacity of ddPCR to detect HBV-RNA in serum.

Method: At least $1 \mathrm{~mL}$ serum of 5 patients with chronic hepatitis B infection were collected with known serum HBV DNA levels. In order to determine the lower detection limit of our assay, we chose serial diluted HBV-DNA standard provided by positive control from HBV nucleic acid quantitative test Kit of Shanghai Kehua, which has been proved by China FDA. The ddPCR reaction consisted of a mix provided by Bio-Rad. HBV-RNA primers which had been validated were used for PCR reaction with an adapted temperature procedure. Each sample was tested in 5 replicates to determine the result. Then, using 106 copies/mL HCV serum as a external control, after serum RNA was extracted and puried with RNase-free DNase and reversely transcribed, products were tested in quintuplicate in the same run by a QX100 ddPCR system with the positive control mentioned above. At last, in order to test the specificity of our assay, precluding the contamination of HBV-DNA, we set up an panel of control conditions and compared with treatment of the extracted nucleic acids with RNAse or omitting reverse transcription.

Result: In summary, we confirmed 3 copies of target molecule was the lower detection limit of our ddPCR system, even considering $75 \%$ recovery rate of HBV-RNA during experiment, thus was approximately 187 copies of HBV-RNA in $1 \mathrm{~mL}$ of serum sample. The assay to test the specificity was also been confirmed that the signals were generated by HBV-RNA but not HBV-DNA (Figure1).

Conclusion: The ddPCR system for testing serum HBV-RNA with absolute quantification and higher sensitivity and specificity might be an interesting alternative to conventional realtime-PCR assay.

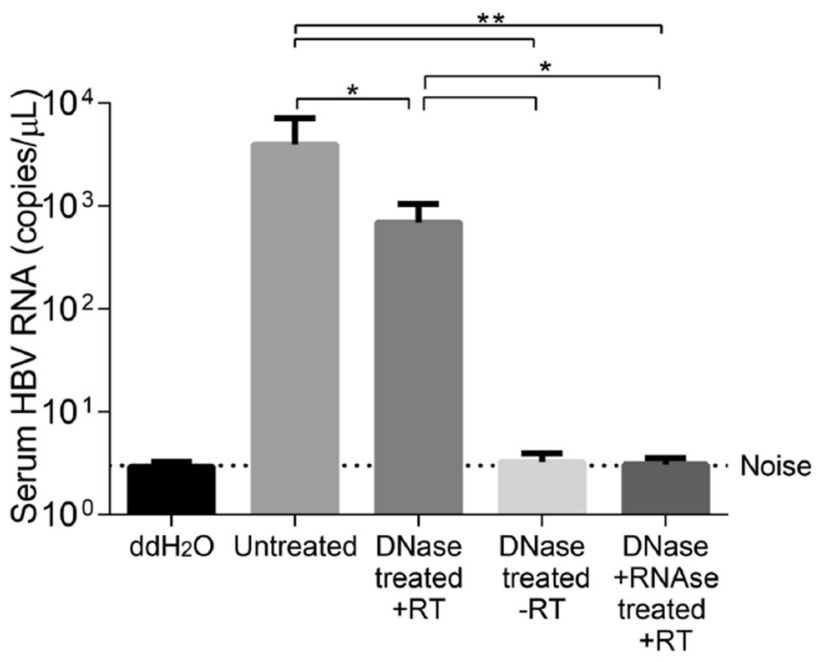

Figure 1. Specificity of ddPCR assay.

RT: Reverse transcription. 


\section{$H B V-C 53$}

Benefits of long-term entecavir therapy in HBeAg-negative patients with chronic hepatitis $B$

\section{Meijie Shi ${ }^{1}$, Xiaoling Chi ${ }^{1}$, Huanming Xiao ${ }^{1}$, Yubao $\mathrm{Xie}^{1}$, Meijie Shi $^{1}$, Gaoshu Cai ${ }^{1}$, Shuduo Wu ${ }^{1}$, Pengtao Zhao ${ }^{1}$, Huijun Chen ${ }^{1}$, Junmin Jiang ${ }^{1}$, Jiezhen Chen ${ }^{1}$, Chaozhen Zhang ${ }^{1}$ \\ ${ }^{1}$ Guangdong Provincial Hospital of Chinese Medicine, China}

Background: Liver stiffness measurement (LSM) has been validated for non-invasive liver fibrosis assessment in pre-treatment chronic hepatitis B. However, data on LSM for fibrosis monitoring during antiviral therapy is still limited today. Thus, the aim of this study was to evaluate the efficacy of Entecavir (ETV) in HBeAg-negative patients with Chronic Hepatitis B (CHB) and the role of LSM for fibrosis monitoring during ETV treatment.

Method: We studied $100 \mathrm{HBeAg-negative} \mathrm{patients} \mathrm{who} \mathrm{were} \mathrm{treated}$ with ETV for more than 48 weeks. The biochemical, virological and serologic parameters as well as the liver stiffness measurement (LSM) were performed at baseline and every 24 weeks during antiviral treatment.

Result: After 48-week ETV treatment, 98\% patients (2/100) achieved alanine aminotransferase (ALT) normalization, $100 \%$ patients achieved HBVDNA seroclearance. After 144-week treatment, all the patients achieved ALT normalization and HBVDNA seroclearance. More importantly, Liver stiffness decreased rapidly in parallel with ALT from $14.5 \mathrm{kPa}$ at baseline to $9.1 \mathrm{kPa}$ at week 48 . None of the patients showed ETV resistance during the antiviral treatment. However, no significant decrease was observed in serum HBsAg after 144-week treatment.

Conclusion: Long-term ETV treatment had good benefits for $\mathrm{HBe} A g$-negative patients with $\mathrm{CHB}$, which could achieved higher ALT normalization and HBVDNA seroclearance rates and good fibrosis improvement. LSM should be assessed for fibrosis monitoring during ETV treatment.

\section{$H B V-C 54$}

Chronic hepatitis B within the Victorian Prisons, Australia - high prevalence of cirrhosis and HBV-HCV and HBV-HDV coinfection

Tim Papaluca ${ }^{1}$, Tim Papaluca ${ }^{1}$, Jessica Howell ${ }^{1}$, Lucy Mcdonald ${ }^{1}$, Ann Craigie $^{T}$, David Iser ${ }^{1}$

${ }^{1}$ St Vincent's Hospital Melbourne, Australia

Background: The prison provides opportunity to engage/treat high risk persons with the viral hepatitis. The Statewide Hepatitis Program (SHP) operates across 14 prisons in Victoria, Australia, and provides management for viral hepatitis to all prisoners $(n=6,200)$. Within the prison, $\mathrm{HCV}$ prevalence is high $(35 \%)$ and $>1000$ prisoners have commenced treatment. The impact of chronic hepatitis B (CHB) however is less defined. We characterized $\mathrm{CHB}$ amongst prisoners referred for assessment for viral hepatitis

Method: All prisoners are offered screening for viral hepatitis on prison entry. Seropositive prisoners are referred for protocol-driven face-to-face assessment by a SHP Clinical Nurse Consultant (CNC) in prison, including clinical assessment, liver stiffness measurement using transient elastography (FibroScan), and arranging relevant blood tests. CNCs are supported by specialist hepatologists (telehealth or face-to-face assessment). We report the results of the first 24 months of the program from October 2015 to October 2017.
Result: There are approximately 6,200 prisoners in Victoria. The SHP has assessed 1571 prisoners referred for viral hepatitis management. The majority of assessments related to HCV totalling 1502 compared with 69 prisoners referred for assessment of CHB.

\begin{tabular}{|l|l|}
\hline \multicolumn{1}{|l|}{ Table 1} & $\mathrm{~N}=1571$ \\
\hline HBsAg + & $\mathrm{N}=69(4.4 \%)$ \\
\hline \hline - HBsAg + / anti-HDV + & $\mathrm{N}=7(10 \%)$ \\
\hline - HBsAg + / anti-HCV + & $\mathrm{N}=32(46 \%)$ \\
\hline - HBsAg + / HCV RNA + & $\mathrm{N}=16(23 \%)$ \\
\hline - HBsAg + / HIV + & 0 \\
\hline Anti-HCV + / HBsAg - & \\
\hline - anti-HBc + / anti-HBs + & $\mathrm{N}=113 / 397(28 \%)^{*}$ \\
\hline - anti-HBc - / anti-HBs + & $\mathrm{N}=318 / 397(72 \%)^{*}$ \\
\hline *estimates from a population of 397 analysed from the HCV Ab + pool \\
\hline
\end{tabular}

Among the HBV infected patients $(n=69)$, mean age was 40 [34-48], the majority were male $(n=62,90 \%)$ and born overseas $(n=39,57 \%)$, particularly from Vietnam $(29 \%, n=20) .55 \%(n=38)$ had a history of injecting drug use, $37 \%$ of these had shared injecting apparatus in prison $(n=14 / 38) .27 \%(n=18)$ were newly diagnosed at current prison entry. Importantly, 77\% ( $\mathrm{n}=53)$ had never previously received specialist HBV care. The majority were in phase III $(n=40,58 \%)$, however 10 (14\%) were phase II and 14 (20\%) were phase IV. 42\% $(n=29)$ received HBV antiviral therapy, initiated by the SHP in $28 / 29$. HBV HCV \& HBV HDV coinfection was common (table 1). 12 received DAA therapy for $\mathrm{HCV}, 2$ experienced a $\mathrm{HCV}$ treatmentassociated increase in HBV viral load, without biochemical or clinical reactivation. $22 \%(\mathrm{n}=15)$ had cirrhosis, more commonly in $\mathrm{HCV}$ coinfected prisoners ( $54 \%$ vs $34 \%, \mathrm{p}<.05)$. 36 prisoners $(53 \%)$ fulfilled criteria for hepatoma screening. The majority $(24,73 \%)$ had received timely screening within $6 / 12$.

Conclusion: $\mathrm{CHB}$ is less common than $\mathrm{HCV}$ in prisoners. The $\mathrm{CHB}$ population was characterized by high rates of HCV and HDV coinfection, as well as high rates of cirrhosis, despite a median age of 40 .

\section{$H B V-C 55$}

Efficacy of tenofovir disoproxil fumarate switch therapy in chronic hepatitis $B$ for suboptimal response to adefovir-based combination therapy

\section{Xiangyong $L^{1}$, Yeqiong $\mathrm{Zhang}^{1}$, Zeqian $\mathrm{Wu}^{1}$, Zhanyi $\mathrm{Li}^{1}$, Zhiwei Zhang $^{1}$, Sujuan Xi ${ }^{1}$, Yutian Chong ${ }^{1}$ \\ ${ }^{1}$ The Third Affiliated Hospital of Sun Yat-sen University, China}

Background: Efficacy and safety analysis of tenofovir disoproxil fumarate (TDF) switch therapy in chronic hepatitis B patients for suboptimal response to adefovir (ADV)-based combination therapy. Method: The efficacy of TDF switch therapy was retrospectively evaluated in 50 chronic hepatitis B patients after failed response to ADV-based combination therapeutics. The primary endpoint was achieving a cumulative probable virologic response. The secondary endpoints were the rate of alanine aminotransferase (ALT) normalization, $\mathrm{HBeAg}$ seroconversion in $\mathrm{HBeAg}$-positive patients, and elevated creatinine and creatine kinase.

Result:48 patients, median age 35 years, were $\mathrm{HBeAg-positive.} \mathrm{17,}$ 14 , and 19 patients were prior treated with lamivudine (LAM) plus ADV, telbivudine (LdT) plus ADV, and entecavir (ETV) plus ADV, respectively. The mean serum HBV DNA level was $4.8 \pm 1.6 \log 10$ $\mathrm{IU} / \mathrm{mL}$ at baseline. 41 patients were treated with TDF alone and 9 with TDF plus ETV. The median follow-up of TDF with or without 
ETV treatment was 102 weeks. The cumulative probability of achieving a virological response at $24,48,96$, and 108 weeks was $52.0 \%, 76.0 \%, 89.8 \%$, and $94.9 \%$, respectively. The cumulative probability of normal ALT at 24, 48, 84, 120, and 132 weeks was $44.0 \%, 53.0 \%, 65.5 \%, 71.3 \%, 77.0 \%$, and $82.8 \%$, respectively. $\mathrm{HBeAg}$ seroconversion occurred in 5 patients. During the follow-up, 6 patients suffered from virological breakthrough; 3 failed to achieve the response. Slightly elevated creatinine was observed in one patient, whereas creatine kinase did not increase.

Conclusion: TDF switch therapy is efficient and safe for chronic hepatitis B patients with suboptimal response to ADV-based combination therapy.

\section{$H B V-C 56$}

Evaluation of predictors to differentiate chronic hepatitis b with reactivation from acute hepatitis $B$

\section{KULDEEP SAHARAWAT ${ }^{1}$, VISHWA MOHAN DAYAL ${ }^{1}$}

${ }^{1} \mathrm{MCI}$, India

Background: Patients of acute hepatitis B (AVHB) and chronic hepatitis $\mathrm{B}$ with reactivation (CHBR) have similar serologic and clinical profile. Differentiation between these patients is difficult specially when they present with jaundice and IgM anti-HBc positive. We aim to study the factors which help in differentiation between AVHB and CHBR.

Method: A total of 75 patients of acute hepatitis diagnosed to be HBsAg positive were enrolled prospectively and divided in to two groups AVHB and CHBR. AVHB group was defined as patients with clinical sign and symptoms of acute hepatitis without prior history of $\mathrm{HBV}$ infection and loss of hepatitis B surface antigen (HBsAg) within 6 months. CHBR group was defined as elevation of serum alanine aminotransferase (ALT) $>10$ times the upper limit of normal, in patients who were IgM- anti $\mathrm{Hbc}$ positive and persistence of $\mathrm{HBsAg}$ at 6 months and/or other features of chronic liver disease. Virological and biochemical profile of the groups were compared to determine the valuable diagnostic factors.

Result: $30.6 \%$ (23) patients were female and $69.4 \%$ (52) were male. The sample/cut-off (S/CO) ratio for IgM anti-HBc was higher in AVHB group and HBV DNA level was higher in CHBR group. The area under receiver operating characteristic curve (AUROC) of S/CO ratio for IgM anti-HBc was 0.960 and taking a cut off value of 6.38 had a sensitivity and specificity of $93.3 \%$ and $86.7 \%$ respectively. The AUROC for HBV DNA level was 0.986 and taking a cut off value of $6.09 \log 10 \mathrm{IU} / \mathrm{ml}$ had a sensitivity and specificity of $93.3 \%$ and $98.3 \%$ respectively.

Conclusion: In patients with acute hepatitis, the value of S/CO ratio for IgM anti-HBc and HBV DNA level, can help in differentiation between AVHB and CHBR.

\section{$H B V-C 57$}

Incidence and predictors of significant fibrosis in incidentally detected asymptomatic hepatitis B positive subjects (IDAHS)

\section{Sridhar Sundaram ${ }^{1}$, Prachi Patil ${ }^{1}$, Shaesta Mehta ${ }^{1}$}

${ }^{1}$ Tata memorial centre, India

Background: Chronic Hepatitis B can present with wide range of manifestations from asymptomatic infection to cirrhosis. Management of Incidentally detected asymptomatic Hepatitis B positive subjects (IDAHS), especially those with normal Alanine transaminase
(ALT) has been a topic of debate. We aimed to study the clinical and laboratory profile of IDAHS and patterns of fibrosis as measured by Vibration controlled transient elastography (VCTE).

Method: A retrospective review of IDAHS referred to the Hepatology clinic at Tata Memorial Centre from November 2016 to November 2017 was done. Demographic and laboratory details of the patients were noted. Details of Fibroscan (VCTE) measurements were noted. Fibroscan values of $>8 \mathrm{kPa}$ were classified as significant fibrosis as per the APASL criteria and those with values $>11 \mathrm{kPa}$ were considered as cirrhosis.

Result: 87 patients were reviewed, which included 49 males and 38 females with mean age of 45.6 years. 22 patients $(25.2 \%)$ were HBeAg Reactive. Mean HBV DNA levels were approximately $7 \log$ $\mathrm{IU} / \mathrm{ml}$, median of $1280 \mathrm{IU} / \mathrm{ml}$. HCV ad HIV co-infection was seen in 3 and 2 patients respectively. Diabetes was present in 8 patients (9\%) and hypertension in 11 patients $(12.6 \%)$. ALT was normal in 53 patients $(60.9 \%) .30$ patients $(34.4 \%)$ had significant fibrosis $(>\mathrm{F} 2$ fibrosis), of which 10 had cirrhosis (11.4\%). $6 \mathrm{HBeAg}$ Reactive patients (27\%) and $24 \mathrm{HBeAg}$ Negative (36\%) had significant fibrosis, with no significant difference between these groups. Patients with significant fibrosis were older $(\mathrm{p}=0.018)$ with higher mean ALT $(\mathrm{p}=0.002) .14$ patients with normal ALT had significant fibrosis $(26.4 \%)$. Diabetes and hypertension were significantly correlated with fibrosis.

Conclusion: A large proportion of IDAHS have significant fibrosis and warrant treatment in presence of detectable HBV DNA, despite normal ALT. Age is a predictive factor for increasing fibrosis. Routine non-invasive assessment for fibrosis is warranted and may help guide treatment in IDAHS.

\section{$H B V-C 58$}

\section{Natural history of serum HBV-RNA in chronic HBV infection}

Jing Wang ${ }^{1}$, Yiqi $\mathrm{Yu}^{1}$, Guojun $\mathrm{Li}^{2}$, Chuan $\mathrm{Shen}^{3}$, Jing $\mathrm{Li}^{1}$, Chao Qiu ${ }^{1}$, Jiming Zhang ${ }^{1}$, Wenhong Zhang ${ }^{1}$

${ }^{1}$ Huashan Hospital, Fudan University, China; ${ }^{2}$ the Second Hospital of Yinzhou of Ningbo, China; ${ }^{3}$ the Third Hospital of Hebei Medical University, China

Background: Virus-like particles encapsulating HBV-RNA represent a serum biomarker for assessing viral replication activity in clinical practice. However, baseline levels of serum HBV-RNA and their associations with viral replicative intermediates and liver disease in phases of chronic hepatitis B remain unknown.

Method: In this cross-sectional study, 102 patients were categorized into immune tolerant (IT), $\mathrm{HBeAg}$-positive immune active (HBeAg+IA), inactive carrier (IC), and $\mathrm{HBeAg-negative} \mathrm{immune} \mathrm{active}$ (HBeAg-IA) phases. HBV-RNA in serum samples and in 66 paired liver biopsies were quantified and correlated with serum ALT levels, histopathological scores, and the levels of other viral replicative intermediates.

Result: Mean levels of serum HBV-RNA differed among phases, with the highest levels among IT $(6.78 \pm 0.83 \log 10$ copies $\mathrm{mL}-1)$ patients, followed by HBeAg+IA $(5.73 \pm 1.16 \log 10$ copies mL-1), HBeAg-IA $4.52 \pm 1.25(\log 10$ copies mL- 1$)$, and IC $(2.96 \pm 0.40$ $\log 10$ copies mL-1) patients (Figure 1). Serum HBV-RNA levels correlated with HBV DNA in all phases, though correlations with other viral replicative intermediates weakened or disappeared when cases were stratified into phases. Distinct compositions of viral products were found among phases: ratio of HBsAg to serum HBVRNA was highest in IC patients, ratio of serum HBV-RNA to intrahepatic HBV-RNA and ratio of intrahepatic HBV-DNA to intrahepatic HBV-RNA was significantly higher in IT patients. 
However, no correlations between severity of histopathology and HBV-RNA levels were observed.

Conclusion: Baseline levels of HBV-RNA and the composition of viral replicative intermediates differ significantly across the natural course of chronic HBV infection. These findings shed light on the nature of viral replication and pathogenesis of disease among different phases of chronic HBV infection.

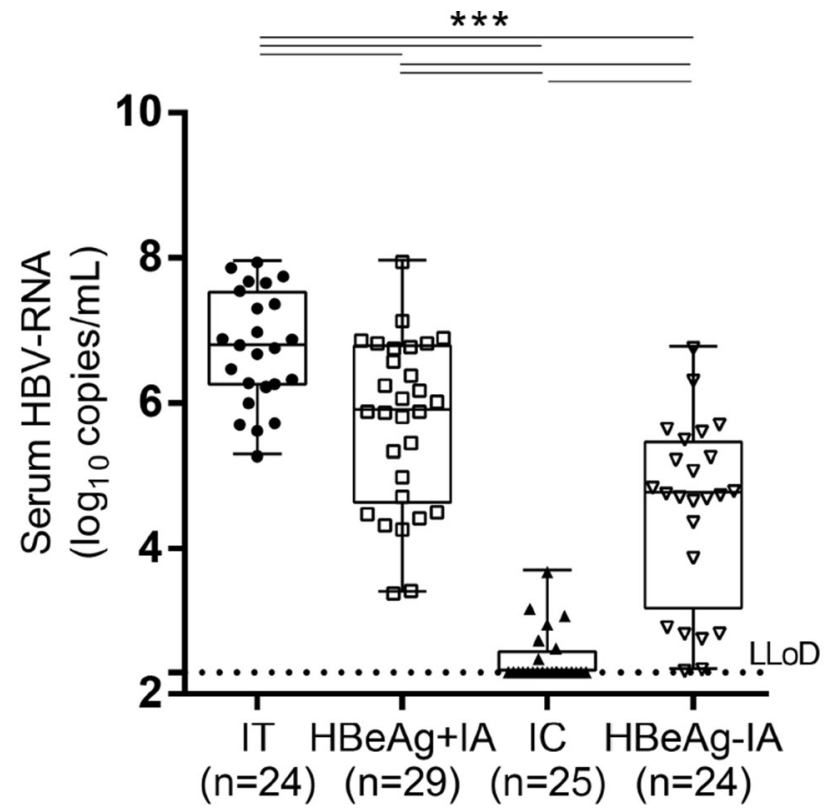

Figure1. Levels of HBV-RNA in serum

\section{$H B V-C 59$}

Diagnosis and monitoring of hepatitis B virus DNA level among the diverse group of Indian population

\section{$\underline{\text { Jagdish Chandra Kandpal }}^{1}$}

${ }^{1}$ Kumaun University Nainital, India

Background: Diagnosis and monitoring scale for infectious diseases among diverse population are the most important aspects of epidemiological studies to control the disease for the destination countries. Data about hepatitis B virus (HBV) DNA level among the Indian population are limited.

Method: A total of 515 suspected cases including 378 (73.39\%) male and $137(26.60 \%)$ female, with ages ranging between 06 and 75 years (mean \pm standard deviation: $35.90 \pm 14.14$ years) participated in this study. From those, blood sample were collected and viral DNAs were extracted from the plasma and analyzed by Real-time PCR system with the combination of artus ${ }^{\circledR}$ HBV RG PCR assay for the specific region of HBV genome.

Result: HBV DNA was detected in a total of 360/515 (69.90\%) plasma samples; $269(74.72 \%)$ male and $91(25.27 \%)$ female. The copy number of HBV DNA ranged from 10.5 to $>2 \times 107 \mathrm{IU} / \mathrm{ml}$ in the plasma samples (median $461 \mathrm{IU} / \mathrm{ml}$ ). On the evaluation of demographic data for the age group and gender, the comparison of viral loads between the age groups and gender indicated no significant correlation $(\mathrm{P}>0.05)$. However, the plasma HBV DNA infection rate among female significantly decreased in the age group of 16-30 years $(\mathrm{P}=0.04)$. There was no significant difference of viral load between the genders in the study population $(\mathrm{P}>0.05)$.
Conclusion: Considering the results of the HBV DNA viral load and evaluation of studied group outcome can help to implement the diagnosis and prevention procedures, as well as to emphasize the risk of HBV transmission in the group of population.

\section{$H B V-C 60$}

Monitoring of liver fibrosis with transient elastography in egyptian patients receiving oral antiviral treatment for hepatitis $B$ related chronic liver disease

Eman Abdelsameea ${ }^{1}$, Omkolsoum Alhaddad ${ }^{3}$, Mohsen Salama ${ }^{3}$, Maha El Sabawy $^{\top}$, Gasser El Azab ${ }^{3}$, Ashraf El Jacky ${ }^{1}$, Ayat Roshdy ${ }^{1}$, Reda Badr ${ }^{2}$

${ }^{1}$ Assistant Professor, Egypt; ${ }^{2}$ Lecturer, Egypt; ${ }^{3}$ Professor, Egypt

Background: Chronic hepatitis B (CHB) often leads to serious health conditions including decompensated liver cirrhosis and hepatocellular carcinoma. Transient elastography (TE) has been suggested to determine prognosis \& possibly to monitor response to treatment in patients with chronic liver disease. In contrast to liver biopsy, TE has the great advantage of being non invasive along with more patient acceptability. Aim: To evaluate changes in degree of hepatic fibrosis through liver stiffness measurement (LSM) using fibroscan in relation to various antiviral therapies in Egyptian patients with hepatitis B related chronic liver disease

Method: This study was prospectively conducted on a total of 217 patients with hepatitis B related chronic liver disease. After eligibility for oral antiviral treatment \& obtaining an informed consent, assessment of liver stiffness\& degree of fibrosis by using TE had been undertaken in two occasions. The first occasion was at the time of initiating treatment versus the time to be enrolled in the study. At least, six months should be elapsed before the 2nd elastography examination.

Result: The mean \pm SD of age was $37.47 \pm 10.15$ years old, $73.7 \%$ $(n=160)$ were males. For all studied patients, the mean \pm SD of the initial and 2nd follow up LSM were 9.59 \pm 7.45 and $7.97 \pm 5.86$ kilopascal $(\mathrm{kPa})$ respectively $(\mathrm{p}<0.001)$. The mean interval between two measures of liver stiffness (LS) was $12.4 \pm 8.07$ months. The mean degree of LS regression was $0.15 \pm 0.37 \mathrm{kPa}$ /month. The value of LSM improved in 177 (81.6\%) patients during follow up period. Patients with advanced fibrosis (F3, F4) demonstrated the highest monthly decline in LS value during antiviral therapy $(0.27 \pm 0.28$ and $0.6 \pm 0.85 \mathrm{kPa} /$ month respectively, $\mathrm{p}<0.001)$. Eighty eight patients $(40.6 \%)$ showed improvement in fibrosis stage by LSM $(\geq 1$ decrease in fibrosis stage), $116(53.5 \%)$ showed stationary stage, while 13 patients $(6 \%)$ showed increase in fibrosis by $\geq 1$ stage. Importantly, LSM significantly decreased in patients who received tenofovir versus those received lamivudine $(\mathrm{p}=0.01$, Table 1$)$. A significant decline in LS values $(\mathrm{P}=0.003)$ was achieved in treatment naive patients $(0.27 \pm$ $0.5 \mathrm{kPa} /$ month $)$ than those who were on treatment $(0.08 \pm 0.27$ $\mathrm{kPa} /$ month)

Conclusion: Treating CHB patients with oral antiviral therapy resulted in significant improvement of liver stiffness in majority of patients 
Table 1: Antiviral medications and change in LS values per month among studied patients $(n=217)$.

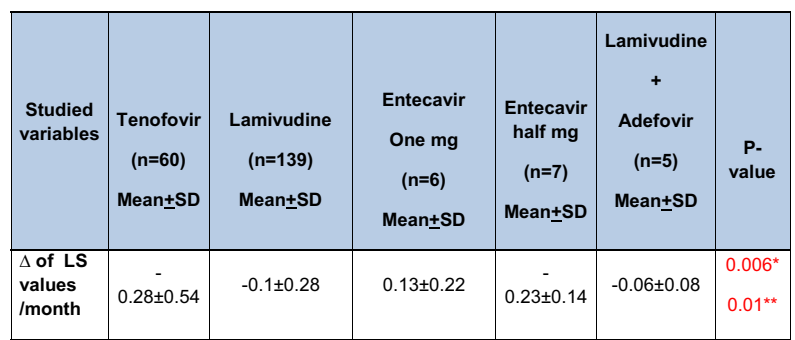

$\mathrm{SD}=$ Standard deviation $* \Delta=$ Delta

*P-value between groups.

** P-value between Tenofovir and Lamivudine

\section{$H B V-C 61$}

Pattern of chronic hepatitis B and treatment modalities in Kerala. South India

\section{Shenoy $K T^{1}$, Leena $\mathrm{K} \mathrm{B}^{2}$ \\ ${ }^{1}$ Sree Gokulam Medical College and Research Foundation, India; ${ }^{2}$ Population Health and Research Institute, India}

Background: Very little data is available from Kerala on the patterns of chronic hepatitis B and the treatment modalities. We evaluated the biochemical, virological and treatment responses in a cohort of chronic HBV as defined by the Asia Pacific Guidelines.

Method: One thousand six hundred and sixty subjects (male 1177 and females 483) were recruited from Medical College, Trivandrum (2005 September to April 2006) and Sree Gokulam Medical College (2006 Mat till 2016 December) and followed up prospectively every 3 to 6 months . AST, ALT. HBeAg were estimated in all the study subjects and HBV DNA quantification and HBsAg quantification was carried out in 878 subjects.

Result: Mean age (SD) was $36.45( \pm 13.25)$. Cirrhosis was noted in the baseline in $242(14.6 \%)$ and $9 \%$ were $\mathrm{HBeAg}$ positive; non cirrhotics $1418(85.4 \%)$ and $11.2 \%$ were $\mathrm{HBeAg}$ positive. ALT was abnormal in $38 \%$. DNA quantification showed high values $(>20,000$ $\mathrm{IU} / \mathrm{mL}$ ) in $22 \%$ and moderate values (2000 to $20,000 \mathrm{IU} / \mathrm{mL}$ ) in $42 \%$ and in the rest, it was $<1500 \mathrm{IU} / \mathrm{ml} / .72$ patients received pegylated interferon for 48 weeks and 12 received sequential therapy with interferon and antiviral. 312 subjects received antivirals for 12 months or more. $6.6 \%$ in the interferon treated lost HBsAg at 48 weeks and none in the oral therapy group .

Conclusion: Treatment with interferon shows promising result and need further long term studies

\section{$H B V-C 62$}

Virological and histological response of peg-interferon in chronic hepatitis B management (preliminary real cases study)

\section{Hery Djagat Purnomo ${ }^{1}$}

${ }^{1}$ Dr. Kariadi Hospital, Indonesia

Background: Chronic hepatitis B (CHB) infection is a serious disorder that can result in cirrhosis, liver failure and hepatocellular carcinoma that remains a major global health problem. Peg-Interferon (Peg-IFN) is recommended as a potential first line treatment for the management of CHB infection but studies on treatment outcomes are still limited. This study was aimed to evaluate virological and histological outcomes and predictor response after a complete therapy of Peg-IFN.

Method: This was an observational study of 50 patients with $\mathrm{CHB}$ receiving Peg-INF at Dr. Kariadi Hospital Semarang. Inclusion criteria was CHB patients who received completed Peg-INF therapy. Exclusion criteria were proven as decompensated cirrhosis and coinfection with hepatitis C. Outcomes were assassed based on virological parameters and fibrosis severity using transient elastography. Thirty one patients were available for follow up for fibrosis value pre and post therapy.

Result: Baseline characteristics: $72 \%$ were males, median age was 38.5 (15-64 years old), 52\% was HbeAg negative, $32 \%$ had elevated ALT (16/50), median baseline of viral load was $106 \mathrm{IU} / \mathrm{cc}$, median fibroscan value was $12.2(7.8-75 \mathrm{kPa})$.

Viral load was decreased up to $2 \log$ in $69,2 \%(18 / 26)$ of HbeAg negative and $75 \%(18 / 24)$ of HbeAg positive patients at twelfth week. Virological response (VR) was achieved in 55\% (10/18) of HbeAg negative and $61,1 \%(11 / 18)$ of $\mathrm{HbeAg}$ positive patients at twelfth week. In HbeAg negative patients, VR were $38,4 \%$ and $73 \%$ at twenty fourth and forty eighth week. In HbeAg positive patients, $54,1 \%$ and $79,1 \%$ achieved a VR at twenty fourth and forty eighth week respectively. Decreasing fibrosis value was observed in $71 \%(22 / 31)$ patients. Among 8 patients with $\mathrm{F} 4(>15 \mathrm{kPa}), 25 \%$ had reached $<\mathrm{F} 2$ $(<7.7 \mathrm{kPa}), 37.5 \%$ had reached $>\mathrm{F} 2(>7.7 \mathrm{kPa})$. Among 20 patients with $>\mathrm{F} 2,39 \%$ had reached $<\mathrm{F} 2$. From multivariate analysis, HbeAg positive and HBV DNA $<\log 6$ were a strong predictor for VR at forty eighth week $(\mathrm{p}<0.005)$ (table 1$)$.

Conclusion: Peg-INF therapy in management of $\mathrm{CHB}$ had good virological and histological response. HbeAg positive and HBV DNA $<\log 6$ were strong predictors for virological response.

\begin{tabular}{|c|c|c|c|c|}
\hline \multicolumn{3}{|c|}{ Bivariate } & \multicolumn{2}{|c|}{ Multivariate } \\
\hline & OR $(95 \% \mathrm{CI})$ & $\mathrm{p}$ & $\mathrm{OR}(95 \% \mathrm{CI})$ & $P$ \\
\hline Age $<35$ yo & $0.271(0.083-0.878)$ & 0.027 & $0.120-1.625$ & 0.219 \\
\hline Sex male & $1.25(0.363-4.306)$ & 0.723 & & $\mathrm{NA}$ \\
\hline HbeAg positif & $0.257(0.079-0.0840)$ & 0.022 & $1.138-17.861$ & 0.018 \\
\hline ABV DNA $<\log$ & $4.41(1.183-16.449)$ & 0.022 & $1.325-23.497$ & 0.019 \\
\hline
\end{tabular}

\section{$H B V-C 63$}

A cure for chronic hepatitis B (CHB)? Exploring the potential clinical and economic consequences of increasing sustained offtherapy hepatitis B virus surface antigen (HBsAg) loss as a costeffective strategy

Mahmoud Hashim ${ }^{1}$, Urbano Sbarigia ${ }^{2}$, Karin Cerri ${ }^{2}$, Bart Heeg ${ }^{1}$

${ }^{1}$ Ingress-health, Netherlands; ${ }^{2}$ Janssen Pharmaceutica NV, Belgium

Background: In patients with CHB infection, the ideal endpoint is sustained 'off-therapy' HBsAg loss, associated with a sustained reduction in HBV DNA and other disease markers, defined as "functional cure". We assessed how HBsAg loss was incorporated in currently published health economic (HE) models of CHB infection. Then we developed and validated a new conceptual model $(\mathrm{CM})$ of CHB natural history, to allow us to capture the clinical and economic value of a functional cure. 
Method: Initially, we conducted a targeted Medline literature review of published models. Data extraction items included: population characteristics ( $\mathrm{HBeAg}$ status, liver damage status), interventions (treatments, therapy line, duration), models (economic evaluation types, models, time interval, health states, discounting, sensitivity analyses) and effectiveness/costs input variables. Following a critical analysis of the literature, a multidisciplinary steering group (experts on HE, epidemiology, and clinical medicine) was convened to develop a CM. The CM identified and described qualitative associations between CHB attributes, progression, and outcomes.

Result: Overall, 53 models from 17 different national payer perspectives were included. Thirty models were for both $\mathrm{HBeAg}$-positive and negative patients and most of these $(85 \%)$ included patients with no cirrhosis at baseline. Most models were cost-utility analyses (77\%). Treatment-specific transition probabilities to morbidity and mortality health states were based on $\mathrm{HBeAg}$ seroconversion, viral response, and viral resistance development. HBsAg loss health state was included in 14 models. Transition probabilities to HBsAg loss health states were not directly treatment dependent. Only in three models (one-way deterministic sensitivity analyses) were outputs mildly sensitive to HBsAg loss rate. Figure shows our developed CM. Health states included HBsAg loss, HBsAg reduction, HBeAg seroconversion, viral load suppression, compensated and decompensated cirrhosis, HCC health states, liver transplant, and death. Compared to previous models, our developed model has additional health states: $\mathrm{HBsAg}$ reduction and four health states for $\mathrm{HCC}$ (early HCC, where patients receive treatment with curative intent; two advanced/metastatic stages, and HCC-related death). Disease progression attributes incorporated into the $\mathrm{CM}$ included $\mathrm{CHB} \mathrm{HBeAg}$ status, genotype, cirrhosis status, co-infection status, and whether the patients' $\mathrm{CHB}$ $\mathrm{HBe} A g$ reactivated.

Conclusion: The consequences of sustained off-therapy HBsAg loss in published models is not well captured, as it is often not directly related to modeled treatments. This could be explained by the fact that only a small $\%$ of patients $(0-10 \%)$ treated with currently available treatments reach sustained HBsAg loss. Our proposed CM can be used as the foundation for assessing the clinical and economic value of novel treatment combination regimens, fully capturing the value of functional cure for CHB patients.

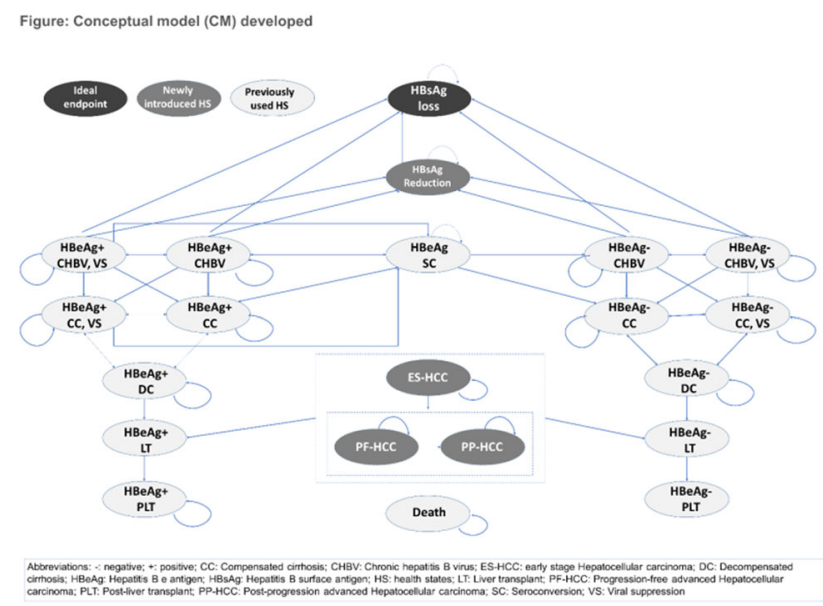

$H B V-C 64$

\section{Assessment of hepatic fibrosis regression by transient elastography in patients with chronic hepatitis B treated with oral antiviral}

\section{Ali Abdelrahman Ghweil}

${ }^{1}$ Head of the tropical medicine and gastroenterology, Egypt

Background: Assessment of Hepatic Fibrosis Regression by Transient Elastography in Patients with Chronic Hepatitis B Treated with Oral Antiviral Agents

Abstract

Transient elastography (TE) has been used as a non-invasive method for liver stiffness measurement (LSM) in patients with chronic liver disease. This study was performed to assess the change of LSM by TE

Method: Assessment of Hepatic Fibrosis Regression by Transient Elastography in Patients with Chronic Hepatitis B Treated with Oral Antiviral Agents

Abstract

Transient elastography (TE) has been used as a non-invasive method for liver stiffness measurement (LSM) in patients with chronic liver disease. This study was performed to assess the change of LSM by TE and to assess its clinical usefulness during long-term oral antiviral therapy in patients with chronic hepatitis $\mathrm{B}(\mathrm{CHB})$. We retrospectively reviewed $80 \mathrm{CHB}$ patients. The mean interval between two LSM was one year Initial and follow-up LSM was $17.15 \pm 12.41$ $\mathrm{kPa}$ and $10.21 \pm 6.36 \mathrm{kPa}$, respectively $(\mathrm{P}<0.001)$. The degree of regression of liver stiffness was. The fibrosis stage classified by LSM value improved in $37(47.5 \%)$ patients during oral antiviral therapy. Of the $30(37.5 \%)$ patients with $\mathrm{LSM} \geq 14.1 \mathrm{kPa}$ (cirrhosis) at $1 \mathrm{st}$ LSM, 7 proved to no longer have cirrhosis ( $\geq 1$ decrease in fibrosis stage) at 2nd LSM. LSM significantly decreased in both baseline high $(>$ upper limit of normal $[\mathrm{ULN}] \times 2)$ and low $(\leq \mathrm{ULN} \times 2)$ alanine aminotransferase groups during antiviral therapy $(\mathrm{P}<0.001 ; \mathrm{P}=$ 0.001 , respectively). Long-term oral antiviral therapy resulted in the improvement of liver stiffness in a substantial portion of patients with CHB. TE may be used a useful clinical tool to assess disease progression in CHB patients.

Result: Assessment of Hepatic Fibrosis Regression by Transient Elastography in Patients with Chronic Hepatitis B Treated with Oral Antiviral Agents

Abstract

Transient elastography (TE) has been used as a non-invasive method for liver stiffness measurement (LSM) in patients with chronic liver disease. This study was performed to assess the change of LSM by TE and to assess its clinical usefulness during long-term oral antiviral therapy in patients with chronic hepatitis $\mathrm{B}(\mathrm{CHB})$. We retrospectively reviewed $80 \mathrm{CHB}$ patients. The mean interval between two LSM was one year Initial and follow-up LSM was 17.15 \pm 12.41 $\mathrm{kPa}$ and $10.21 \pm 6.36 \mathrm{kPa}$, respectively $(\mathrm{P}<0.001)$. The degree of regression of liver stiffness was. The fibrosis stage classified by LSM value improved in $37(47.5 \%)$ patients during oral antiviral therapy. Of the $30(37.5 \%)$ patients with LSM $\geq 14.1 \mathrm{kPa}$ (cirrhosis) at $1 \mathrm{st}$ LSM, 7 proved to no longer have cirrhosis ( $\geq 1$ decrease in fibrosis stage) at 2nd LSM. LSM significantly decreased in both baseline high $(>$ upper limit of normal $[\mathrm{ULN}] \times 2)$ and low $(\leq \mathrm{ULN} \times 2)$ alanine aminotransferase groups during antiviral therapy $(\mathrm{P}<0.001 ; \mathrm{P}=$ 0.001, respectively). Long-term oral antiviral therapy resulted in the improvement of liver stiffness in a substantial portion of patients with CHB. TE may be used a useful clinical tool to assess disease progression in CHB patients.

Conclusion: Assessment of Hepatic Fibrosis Regression by Transient Elastography in Patients with Chronic Hepatitis B Treated with Oral Antiviral Agents 


\section{Abstract}

Transient elastography (TE) has been used as a non-invasive method for liver stiffness measurement (LSM) in patients with chronic liver disease. This study was performed to assess the change of LSM by TE and to assess its clinical usefulness during long-term oral antiviral therapy in patients with chronic hepatitis B (CHB). We retrospectively reviewed $80 \mathrm{CHB}$ patients. The mean interval between two LSM was one year Initial and follow-up LSM was 17.15 \pm 12.41 $\mathrm{kPa}$ and $10.21 \pm 6.36 \mathrm{kPa}$, respectively $(\mathrm{P}<0.001)$. The degree of regression of liver stiffness was . The fibrosis stage classified by LSM value improved in $37(47.5 \%)$ patients during oral antiviral therapy. Of the $30(37.5 \%)$ patients with $\mathrm{LSM} \geq 14.1 \mathrm{kPa}$ (cirrhosis) at $1 \mathrm{st}$ LSM, 7 proved to no longer have cirrhosis ( $\geq 1$ decrease in fibrosis stage) at 2nd LSM. LSM significantly decreased in both baseline high $(>$ upper limit of normal $[\mathrm{ULN}] \times 2)$ and low $(\leq \mathrm{ULN} \times 2)$ alanine aminotransferase groups during antiviral therapy $(\mathrm{P}<0.001 ; \mathrm{P}=$ 0.001 , respectively). Long-term oral antiviral therapy resulted in the improvement of liver stiffness in a substantial portion of patients with CHB. TE may be used a useful clinical tool to assess disease progression in CHB patients.

\section{$H B V-C 65$}

Axonal motor neuropathy, a variant of Guillain Barre syndrome associated with Hepatitis B virus reactivation - a case series

\section{Rahul D Arora ${ }^{1}$}

${ }^{1}$ Tata Memorial Centre, Tata Memorial Hospital, India

Background: Hepatitis B reactivation is one of the common causes of acute on chronic liver failure in east and south east asian countries. Neurological syndromes associated with Hepatitis B virus infection include mononeuritis, auditory neuritis, seizures and Guillain Barre syndrome.

Method: We report rare association of acute axonal polyneuropathy complicating HBV reactivation.

Result: Case one

A 53 year old male presented with history of yellowish discolouration of the eyes and urine since the past 6 days along with progressive asymmetric weakness involving both the legs $(L>R)$. He provided a history of paraesthesias which progressed to limping within 72 hours with involvement of both the arms on the fourth day. Anti $\mathrm{HBe}$ antibody and HBs Ag were reactive. HBV DNA was $2.44 \times 10^{\wedge} 6 \mathrm{IU} /$ $\mathrm{ml}$. Anti HCV and HIV serology were non reactive. Electrophysiological studies were suggestive of inconsistent $F$ waves in both common peroneal and posterior tibial nerves. Decreased $\mathrm{H}$ wave amplitude was noted bilaterally. NCV was suggestive of motor polyneuropathy of axonal type. Power was $4 / 5$ in the left lower limb and $4+/ 5$ in the right lower limb. Reflexes were absent. His neurological symptoms improved with IV Ig, $2 \mathrm{~g} / \mathrm{kg}$ over 5 days.

Case two

A 64 year old male presented with complaints of yellowish discolouration of the eyes and urine since the past one month associated with gradually progressive asymmetric weakness of the legs since the past 4 days which progressed to involve the arms over the course of 24 hours. Power in the right upper limb was 0/5 (shoulder-flexion/ extension) and right lower limb was 1/5 (hip -flexion/extension). Areflexia was present. HBsAg, Anti HBc antibody, Anti HAV antibody were reactive. $\mathrm{HBe} \mathrm{Ag}$ was non reactive. HBV DNA was $2.29 \mathrm{x}$ $10^{\wedge} 9 \mathrm{IU} / \mathrm{ml}$. Prolonged $\mathrm{F}$ wave latency was seen in the bilateral posterior tibial and right common peroneal nerves. Nerve conduction studies were suggestive of axonal motor sensory neuropathy involving both the lower limbs.

Results
Axonal motor polyneuropathy, a variant of Guillain Barre syndrome represents a rare neurological manifestation in the natural history of HBV infection.

Conclusion: The mechanisms underlying these neurological phenomenon, therapeutic role of steroids and antivirals as adjuncts in treatment, efficacy of intravenous immunoglobulins on the resolution of symptoms and effect of treatment on HBV replication is a subject of further research.

\section{$H B V-C 66$}

Continuing besifovir dipivoxil maleate versus switching from tenofovir disoproxil fumarate for treatment of chronic hepatitis B (CHB): 96 weeks results of phase 3 trial

Junghee Won ${ }^{1}$, Won $\mathrm{Kim}^{17}$, Hyung Joon Yim ${ }^{14}$, Jin Mo Yang Jae Young Jang ${ }^{7}$, Yong Oh Kweon ${ }^{15}$, Yong Kyun Cho ${ }^{13}$, Yoon Jun Kim ${ }^{18}$, Gun Young Hong', Dong Joon Kim ${ }^{10}$, Soon Ho Um ${ }^{14}$, Joo Hyun Sohn' ${ }^{11}$, Jin Woo Lee ${ }^{12}$, Sung Jae Park ${ }^{16}$, Byung Seok Lee ${ }^{6}$, Ju Hyun Kim ${ }^{8}$, Hong Soo Kim ${ }^{19}$, Seung Kew Yoon ${ }^{4}$, Moon Young Kim ${ }^{21}$, Kwan Sik Lee ${ }^{20}$, Young Suk Lim², Wan Sik Lee

${ }^{1}$ Medical Writer, Korea, Republic of; ${ }^{10}$ Hallym University College of Medicine, Korea, Republic of; ${ }^{11}$ Hanyang University College of Medicine, Korea, Republic of; ${ }^{12}$ Inha University College of Medicine, Korea, Republic of; ${ }^{13}$ Kangbuk Samsung Hospital, Korea, Republic of; ${ }^{14}$ Korea University College of Medicine, Korea, Republic of; ${ }^{15}$ Kyungpook National University College of Medicine, Korea, Republic of; ${ }^{16}$ Paik Hospital, Inje University, Korea, Republic of; ${ }^{17}$ Seoul Metropolitan Government Boramae Medical Center, Korea, Republic of; ${ }^{18}$ Seoul National University College of Medicine, Korea, Republic of; ${ }^{19}$ Soonchunhyang University College of Medicine, Korea, Republic of; ${ }^{2}$ Asan Medical Center, University of Ulsan College of Medicine, Korea, Republic of; ${ }^{20}$ Yonsei University College of Medicine, Korea, Republic of; ${ }^{21}$ Yonsei University Wonju College of Medicine, Korea, Republic of; ${ }^{3}$ Catholic University Medical College St. Vincent's Hospital, Korea, Republic of; ${ }^{4}$ Catholic University of Korea, Korea, Republic of; ${ }^{5}$ Chonnam University Medical School, Korea, Republic of; ${ }^{6}$ Chungnam National University School of Medicine, Korea, Republic of; ${ }^{7}$ College of Medicine, Soonchunhyang University, Korea, Republic of; ${ }^{8}$ Gacheon University College of Medicine, Korea, Republic of; ${ }^{9}$ Gwangju Christian Hospital, Korea, Republic of

Background: Besifovir dipivoxil maleate (BSV) is an acyclic nucleotide phosphonate with a potent antiviral activity against hepatitis B virus (HBV). In the former phase 3 study, an antiviral efficacy of BSV for forty-eight week was shown to be comparable to tenofovir disoproxil fumarate (TDF) in treatment-naïve CHB patients. We evaluated long-term efficacy and safety of BSV in chronic hepatitis B patients who were positive or negative for hepatitis $B$ e antigen ( $\mathrm{HBeAg}+$ or $\mathrm{HBeAg}-$ ) in the extended follow up of a phase 3 study. Method: After 48 weeks of double-blind comparison of BSV to TDF, eligible patients who had agreed with the extended study continued to participate in the open-label BSV study. The presented data were collected for 96 weeks. We evaluated virological, serological, and biochemical responses for efficacy analysis, and bone mineral density (BMD) and renal outcomes for safety analysis for both BSV group (BSV-BSV) and the group switched from TDF (TDF-BSV). The primary endpoint was the proportion with HBV DNA $<400$ copies/ $\mathrm{mL}$ (response rate).

Result: Among 197 patients who received randomized treatments, $170(87 \%)$ patients entered the open-label phase, and $161(82 \%)$ completed 96 weeks of the study. The response rate of those who have 
taken BSV over 96 weeks is $87.21 \%$ while $85.71 \%$ of patients who switched the treatment from TDF were respondent. HBeAg seroconversion and ALT normalization were shown in $11.54 \%$ and $73.26 \%$ of patients in the BSV-BSV group, respectively. There were no drug resistant mutations to BSV and no adverse events related to bone mineral density or renal function. The safety profile was similar for the two groups.

Conclusion: BSV maintained efficacy in both suppression of HBV DNA and ALT normalization over 96 weeks without any evidence of resistance to BSV. Also, BSV is safe, well tolerated, and effective for those who have switched to BSV from TDF.

\section{$H B V-C 67$}

Demographic, epidemiological, clinical, severity, and treatment response characteristics of hepatitis b Egyptian patients

Maha Elsabaawy ${ }^{1}$, Gasser Alazab ${ }^{1}$, Haitham Sakr ${ }^{1}$, Mohammed Elsherify ${ }^{1}$, Soha Elshinawy ${ }^{1}$, Mahmoud Eltahawy ${ }^{1}$ ${ }^{1}$ phd, Egypt

Background: In the era of HCV eradication by the direct anti-virals, Egypt had to pay attention to hepatitis b virus (HBV) two million. Aim: characterize epidemiological, demographic, clinical, severity and treatment responses of chronic HBV Egyptians.

Method: This observational cross-sectional study was conducted on 183 chronic HBV Egyptians. Demographic, epidemiologic, clinical, laboratory and treatment data were collected from patient registries with the exclusion of all cases with incomplete registries.

Result: HBV positive HBe Ag represented $18.04 \%$ of the whole HBV cohort. They were younger $(31.09 \pm 8.542$ to $38.22 \pm 10.6$ years $)$ $(\mathrm{p}<0.05)$, with higher alanine aminotransferase (ALT) $(84.91 \pm 67.855$ to $53.75 \pm 55.575 \mathrm{U} / \mathrm{L})(\mathrm{p}<0.05)$, basal viral loads $(3.58 \times 108 \pm$ $16.49 \times 108$ to $1.74 \times 106 \pm 10.1 \times 106 \mathrm{IU} / \mathrm{mL})(\mathrm{P}<0.05)$ particularly in chronic active carrier states. unsafe personal hygienic procedures (sharing toothbrushes and shaving razors) were the main infective routes $(73.7 \%)$. coinfection with $\mathrm{HCV}$ was documented in $14.7 \%$, and in $16.3 \%$ with the schistosomal infestation, while HDV coinfection was reported in $8.9 \%$ of the studied cohort. radiologically, $44 \%$ of cases were cirrhotic with splenomegaly in $20.7 \%$, while histologically, $40.2 \%$ were proved to have significant pathology (A2, F2>2). $70.5 \%$ were subjected to Lamidine with unfair response $(16.3 \%)$ occurred mainly in the positive $\mathrm{HBeAg}$ group $(71.6 \%)$. The multivariate analysis had verified positive $\mathrm{HBeAg}$ status and Schistosomiasis to be associated with poor response to oral antiviral therapies $(\mathrm{p}<0.05)$.

Conclusion: More classified Governmental censorship on health care notably private organizations along with viral awareness levitation programs is promptly mandated. Additionally, the reported poor response to oral antivirals in $\mathrm{HBe} \mathrm{Ag}$ positive patients; had assigned interferon as a first line treatment option for this cohort.

\section{$H B V-C 68$}

\section{Diagnosis and monitoring of hepatitis B virus DNA level} among the diverse group of Indian population

\section{Jagdish Chandra Kandpal}

${ }^{1}$ Kumaun University Nainital, India

Background: Diagnosis and monitoring scale for infectious diseases among diverse population are the most important aspects of epidemiological studies to control the disease for the destination countries. Data about hepatitis B virus DNA level among the Indian population are limited.

Method: To the best of HBV diagnosis and monitoring, the present study was conducted to determine the virus DNA level, and the frequency of infection among the age groups and genders in Indian population. A total of 515 suspected cases including $378(73.39 \%)$ male and 137 (26.60\%) female, with ages ranging between 06 and 75 years (mean \pm standard deviation: $35.90 \pm 14.14$ years) participated. From those, blood sample were collected and viral DNAs were extracted from the plasma and analyzed by Real-time PCR system with the combination of artus ${ }^{\circledR}$ HBV RG PCR assay for the specific region of HBV genome.

Result: HBV DNA was detected in a total of 360/515 (69.90\%) plasma samples; $269(74.72 \%)$ male and $91(25.27 \%)$ female. The level for viral load of HBV DNA ranged from 10.5 to $>2 \times 107 \mathrm{IU} / \mathrm{ml}$ in the plasma samples (median $461 \mathrm{IU} / \mathrm{ml}$ ). On the evaluation of demographic data for the age groups and genders, the comparison of viral loads between the age groups and genders indicated no significant correlation $(\mathrm{P}>0.05)$. However, the plasma HBV DNA infection rate among female significantly decreased in the age group of 16-30 years $(\mathrm{P}=0.04)$. There was no significant difference of viral load between the genders in the study population $(\mathrm{P}>0.05)$.

Conclusion: The results of the HBV DNA viral load and evaluation of studied group outcome can help to implement the diagnosis and prevention procedures in such cases, as well as to emphasize the risk of $\mathrm{HBV}$ transmission in the group of population.

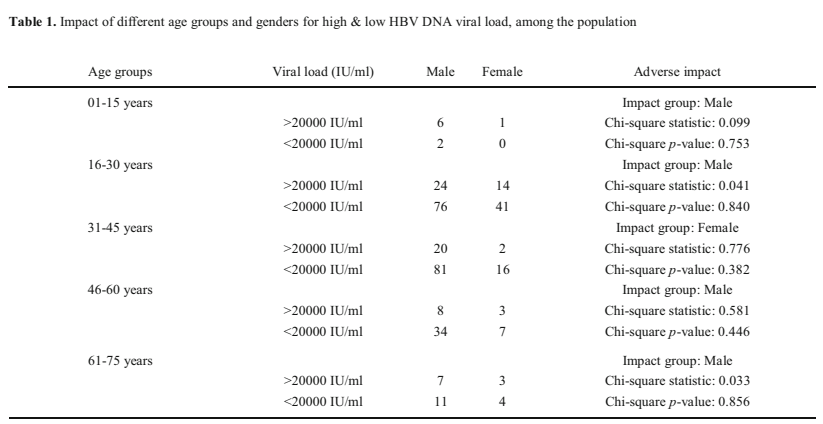




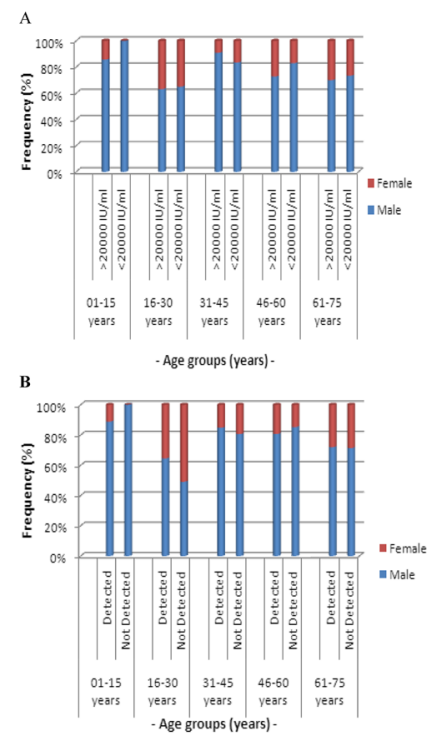

Figure 1. Demographic frequencies of HBV DNA in different age

$H B V-C 69$

Every-two-week ropeginterferon alfa-2b is safe with better efficacy in interferon naïve patients with chronic hepatitis $B$ virus infection: a phase 2, open-label, randomized, active control, dose finding study

Kuanchiao Tseng ${ }^{1}$, Peijer Chen ${ }^{9}$, Chaowei $\mathrm{Hsu}^{7}$, Shengnan $\mathrm{Lu}^{4}$, Minglung $\mathrm{Yu}^{5}$, Chienwei $\mathrm{Su}^{12}$, Weiwen $\mathrm{Su}^{2}$, Rongnan Chien ${ }^{6}$, Chingsheng Hsu ${ }^{11}$, Shihjer $\mathrm{Hsu}^{8}$, Hsuehchou Lai ${ }^{3}$, Kuanchiao Tseng ${ }^{10}$

${ }^{1}$ PharmaEssentia Corp., Taiwan; ${ }^{10}$ PharmaEssentia Corp, Taiwan;

${ }^{11}$ Taipei Tzu Chi General Hospital, Taiwan; ${ }^{12}$ Taipei Veterans General Hospital, Taiwan; ${ }^{2}$ Changhua Christian Hospital, Taiwan; ${ }^{3}$ China Medical University Hospital, Taiwan; ${ }^{4}$ Kaohsiung Chang Gung Memorial Hospital, Taiwan; ${ }^{5}$ Kaohsiung Medical University Hospital, Taiwan; ${ }^{6}$ Keelung Chang Gung Memorial Hospital, Taiwan; ${ }^{7}$ Linkou Chang Gung Memorial Hospital, Taiwan; ${ }^{8}$ National Taiwan University Hospital Yun-Lin Branch, Taiwan; ${ }^{9}$ National Taiwan University Hospital, Taiwan

Background: Ropeginterferon alfa- $2 \mathrm{~b}$ (P1101) is a novel mono-pegylated interferon alfa- $2 b$ with longer duration of action, allowing every-two-week ( $\mathrm{q} 2 \mathrm{w}$ ) injections with high tolerability shown from 2 Phase 2 studies for chronic hepatitis $C$ virus infection genotype 1 and 2 and a pivotal Phase 3 study in treating polycythemia vera. The current study aims to find the optimal dose of P1101 by comparing antiviral activity, safety and tolerability across treatment groups in interferon naïve patients with chronic hepatitis B virus (HBV) infection.

Method: A total of 62 interferon naïve patients with baseline alanine aminotransferase (ALT) between 1.5 -10 times upper limit of normal were recruited. Thirty-one hepatitis $\mathrm{B}$ e antigen ( $\mathrm{HBeAg}$ )-positive subjects with baseline HBV DNA >20,000 IU/ml and $31 \mathrm{HBeAg}-$ negative subjects with baseline HBV DNA $>2,000 \mathrm{IU} / \mathrm{ml}$ were randomized at 1:1:1 ratio to subcutaneous treatment of $\mathrm{q} 2 \mathrm{w}$ P1101 $350 \mu \mathrm{g}$ (Group 1), q2w P1101 $450 \mu \mathrm{g}$ (Group 2), or weekly (q1w) peginterferon alfa-2a $180 \mu \mathrm{g}$ (Group 3, control) respectively. Every patient received 48-week treatment (TW48) and 24-week post-treatment follow-up (FW24). The primary endpoint is HBV DNA $<2,000$ $\mathrm{IU} / \mathrm{ml}$ at FW24. At the time of analysis, all subjects completed 48-week treatment.
Result: Among $\mathrm{HBeAg}$-positive subjects, cumulative $\mathrm{HBeAg}$ seroconversion rate was $27 \%(3 / 11), 36 \%(4 / 11), 11 \%$ (1/9) $(P$ for trend $=0.21$ ) with median time to $\mathrm{HBeAg}$ seroconversion of 24,24 , and 48 weeks ( $P$ for trend=0.28), in Group 1, 2, 3 respectively. At TW48, HBV DNA < 2,000 IU/ml was $27 \%$ (3/11), 36\% (4/11), and $22 \%(2 / 9)$ in Group $1,2,3$ respectively $(P$ for trend $=0.49$ ). Among HBeAg-negative subjects at TW48, undetectable HBV DNA was $70 \%(7 / 10), 73 \%(8 / 11), 50 \%(5 / 10)$ in Group 1, 2, 3 respectively ( $P$ for trend=0.29). Adverse event $(\mathrm{AE})$ profile is similar across groups. Over $90 \%$ of treatment-emergent AEs were Grade 1 or 2. HBeAg-positive and negative subjects pooled together, $10 \%(2 / 21)$, $5 \%(1 / 22), 11 \%(2 / 19)$ discontinued treatment due to AE in Group 1, 2, 3 respectively. The AEs for discontinuation were Grade 3 myocardial infarction (MI) and Grade 4 ALT increased in Group 1, multiple Grade 1 AEs in 1 subject of Group 2, and Grade 3 ALT increased in 2 subjects of Group 3. The subject with MI has risk factors of smoking, and hyperlipidemia. About 14\% (3/21), 14\% (3/ $22), 11 \%(2 / 19)$ reduced dose in Group 1, 2, 3 respectively. All were due to Grade 3 neutropenia or ALT increased.

Conclusion: P1101 is safe and tolerable for chronic HBV infection. P1101 $450 \mu \mathrm{g} \mathrm{q} 2 \mathrm{w}$ has yielded the highest HBeAg seroconversion rate in all groups. Shorter time to $\mathrm{HBeAg}$ seroconversion was observed in both $\mathrm{P} 1101$ groups than in peginterferon alfa-2a group. P1101 $450 \mu \mathrm{g} \mathrm{q} 2 \mathrm{w}$ is selected for a Phase 3 study to confirm efficacy and safety on $\mathrm{HBeAg}$-positive patients.

\section{$H B V-C 70$}

Finite versus indefinite nucleos(t)ide analogue treatment of patients with chronic hepatitis B and HBsAg levels

Xiangyong $\mathrm{Li}^{1}$, Jie $\mathrm{Luo}^{2}$, Xing $\mathrm{Li}^{1}$, Zhiwei Zhang ${ }^{1}$, Yusheng $\mathrm{Jie}^{1}$, Zeqian $\mathrm{Wu}^{1}$, Sujuan $\mathrm{Xi}^{1}$, Xinhua $\mathrm{Li}^{1}$, Jiao Gong ${ }^{1}$, Yuankai $\mathrm{Wu}^{1}$, Zhanyi Li ${ }^{1}$, Yutian Chong ${ }^{1}$

${ }^{1}$ The Third Affiliated Hospital of Sun Yat-sen University, China;

${ }^{2}$ Shenzhen Luohu Peoples Hospital, China

Background: No markers have been established as evidence-based endpoints of finite nucleos(t)ide analogue (NUC) treatment beyond which viral relapse can be ruled out.

Method: This study was aimed to compare finite versus indefinite NUC treatment in patients with chronic hepatitis B (CHB) achieving HBsAg levels

Result: 104 patients achieved HBsAg levels $<20 \mathrm{IU} / \mathrm{mL}$ and 71 could be included in the analysis: 31 received finite NUCs and 40 received indefinite NUCs. In the finite group, 15 patients received short-term (1 year) therapy. Alanine aminotransferase (ALT), total bilirubin, albumin, HBV DNA, and HBsAg levels were compared between the two groups. The baseline parameters and characteristics when $\mathrm{HBsAg}$ levels fell

Conclusion: Discontinuation of NUCs after achieving very low levels of HBsAg could be acceptable in patients whose HBsAg levels fell

\section{$H B V-C 71$}

HBsAg clearance in HBeAg-CHB patients using entecavir with add on or switch to PEG-IFN

Lei Yan ${ }^{1}$, Liwen Chen ${ }^{1}$, Yezhou Ding ${ }^{1}$, Yumin $\mathrm{Xu}^{1}$, Qing Xie ${ }^{1}$, Hui Wang ${ }^{1}$

${ }^{1}$ Rui Jin Hospital Affiliated to Shanghai Jiao Tong University School of Medicine, China 
Background: Chronic hepatitis B (CHB) patients rarely achieve HBsAg loss with nucleoside/nucleotide analogue (NA) therapy. Chronic hepatitis B (CHB) patients rarely achieve HBsAg loss with nucleoside/nucleotide analogue (NA) therapy.

Method: It was evaluated HBsAg loss in HBeAg- patients with entecavir switched to or added on pegylated interferon (PEG-IFN) in a retrospective study. Totally $87 \mathrm{HBeAg}$ - CHB patients treated with entecavir for 24 weeks were studied, 21 switched to PEG-IFN, 13 added on PEG-IFN and 53 continued entecavir therapy (ETV group). Result: HBsAg clearance at week 48 was reported in 2/21 (10\%) patients in switch-to group, $2 / 13(15 \%)$ patients in add-on group; whereas none was in ETV group. HBsAg reduction at week 48 was $1.055 \log \mathrm{IU} / \mathrm{mL}, 0.6452 \log \mathrm{IU} / \mathrm{m}$ or $0.014 \log \mathrm{IU} / \mathrm{mL}$ respectively in switch-to, add-on, or ETV group. The response rate of switch-to, addon and ETV monotherapy was, $44 \%, 38 \%$ or $2 \%$, respectively. It was analyzed that age, BMI, ALT, AST, PLT, liver stiffness, HBsAg titer at baseline, or HBsAg reduction at 24-week, showing that HBsAg titers at baseline and HBsAg reduction at week 24 were associated with HBsAg reduction and clearance. HBsAg reduction was associated with the response rate at week 24 , showing that ROC Curve analyze the response versus HBsAg at week 48, the AUROC was 0.865 . The PPV for response was $70 \%$ and NPV was $100 \%$ with the cut-off value $0.2 \log \mathrm{IU} / \mathrm{ml}$.

Conclusion: In summary, we demonstrated that PEG-IFN enhanced HBsAg loss in $\mathrm{HBeAg}-\mathrm{CHB}$ population. Patients with HBsAg titers at baseline less than $1000 \mathrm{IU} / \mathrm{mL}$ and $\mathrm{HBsAg}$ reduction more than $0.2 \log \mathrm{IU} / \mathrm{mL}$ achieved HBsAg loss with a higher chance.

\begin{tabular}{lcccc}
\multicolumn{4}{l}{ Table 2 characteristic of patients at baseline } \\
\hline GROUP & SWITCH-TO & ADD-ON & ETV & P VALUE \\
\hline Age $(\mathrm{Yr})$ & $34.9(25-50)$ & $43.92(27-60)$ & $45.6(28-65)$ & $<0.001$ \\
Male & $20 / 21$ & $12 / 13$ & $37 / 53$ & 0.02 \\
BMI & 11 vs 10 & 8 vs 5 & 32 vs 21 & NS \\
$<24$ vs $>24$ & $1277( \pm 1776)$ & $1555( \pm 2017)$ & $1898( \pm 1317)$ & NS \\
HBsAg $(\mathrm{IU} / \mathrm{ml})$ & 21 & 13 & 53 & NS \\
HBV DNA undetectable & $32.89( \pm 13.96)$ & $32.77( \pm 12.41)$ & $29.8( \pm 12.98)$ & NS \\
ALT $(I U / m l)$ & $29.05( \pm 14.88)$ & $30.6( \pm 12.71)$ & $26.37( \pm 9.118)$ & NS \\
AST & $45.74( \pm 3.413)$ & $45( \pm 2.404)$ & $43.9( \pm 3.977)$ & NS \\
ALB & $176.6( \pm 49.28)$ & $197.2( \pm 55.24)$ & $154.9( \pm 53.53)$ & NS \\
PLT & 16 vs 5 & 8 vs 5 & 33 vs 20 & NS \\
Liver stiffness $(\mathrm{kPa})$ & & &
\end{tabular}

Continuous data are expressed as the mean $\pm \mathrm{SD}$, age shows with the mean(range). Categorical data shows with number.

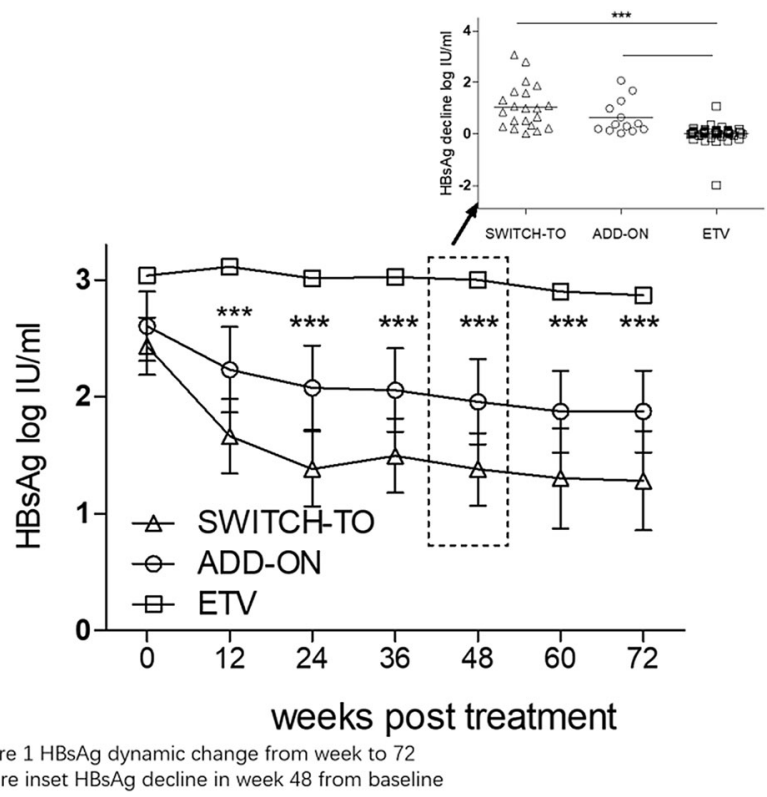

$\mathrm{HBV}-\mathrm{C} 72$

Hepatic events in patients who achieved complete viral suppression with or without hepatitis B surface antigen seroclearance - A territory-wide cohort study of 17,725 subjects

Cheuk Fung Yip ${ }^{1}$, Vincent Wai Sun Wong ${ }^{1}$, Henry Lik Yuen Chan', Yee Kit Tse ${ }^{1}$, Grace Lai Hung Wong ${ }^{1}$

${ }^{1}$ The Chinese University of Hong Kong, Hong Kong

Background: In patients with chronic hepatitis B (CHB) who achieved complete viral suppression in potent antiviral treatment, it is unclear if hepatitis B surface antigen (HBsAg) seroclearance confers additional protection against hepatic events. We compared the risk of hepatic events in treated patients who achieved complete viral suppression with and without HBsAg seroclearance.

Method: All CHB patients who had received first-line nucleos(t)ide analogues (NA), i.e. entecavir and/or tenofovir disoproxil fumarate, for at least 6 months from January 2005 to November 2016 were identified. Complete viral suppression was defined as undetectable serum HBV DNA $(<20 \mathrm{IU} / \mathrm{mL})$ while on NA treatment maintained till the last clinic visit. HBsAg seroclearance was defined as loss of HBsAg detectability for at least once. The primary outcome was a composite of hepatic events.

Result: We identified 17,725 NA-treated CHB patients. At a median (interquartile range [IQR]) follow-up of $4.0(2.2-5.0)$ years, 482 patients developed hepatic events including ascites, spontaneous bacterial peritonitis, variceal bleeding, hepatic encephalopathy, hepatorenal syndrome, hepatocellular carcinoma (HCC) and liver-related death based on the ICD-9-CM diagnosis codes. Complete viral suppression was achieved in 14,845 (83.6\%) patients; $210(1.4 \%)$ patients further had HBsAg seroclearance. Patients with complete viral suppression had a median (IQR) suppression duration of 1.8 (0.9-3.0) years; they were more likely to be male, had negative $\mathrm{HBeAg}$, liver cirrhosis, lower platelet counts, serum total bilirubin and alanine aminotransferase (ALT), and less likely to have detectable hepatitis B virus (HBV) DNA at baseline as compared to the patients without complete viral suppression. Patients with $\mathrm{HBsAg}$ seroclearance were more likely to be male, less likely to have detectable HBV DNA at baseline, had higher platelet counts, serum total bilirubin and ALT. Compared to patients with complete viral suppression, patients without complete viral suppression had a significantly higher risk of hepatic events (adjusted hazard ratio [aHR] $3.00,95 \%$ CI $2.42-3.72 ; P<0.001)$ after adjustment for important covariates; patients with HBsAg seroclearance did not have further risk reduction (aHR 1.16, 0.46-2.94; $P=0.759$ ). Similar findings were observed for HCC, liver-related death, and all-cause death, with aHRs ranging from 2.42 to 5.33 for patients without complete viral suppression (all $P<0.001$ ), and aHRs ranging from 0.59 to 1.49 for patients with HBsAg seroclearance $(P=0.337-0.817)$ (Figures 1-2). Sensitivity analysis on patients who received at least one year of treatment also showed similar results.

Conclusion: Patients who achieved complete viral suppression with NA treatment had similarly low risk of hepatic events, liver-related death, and all-cause death with or without HBsAg seroclearance. 

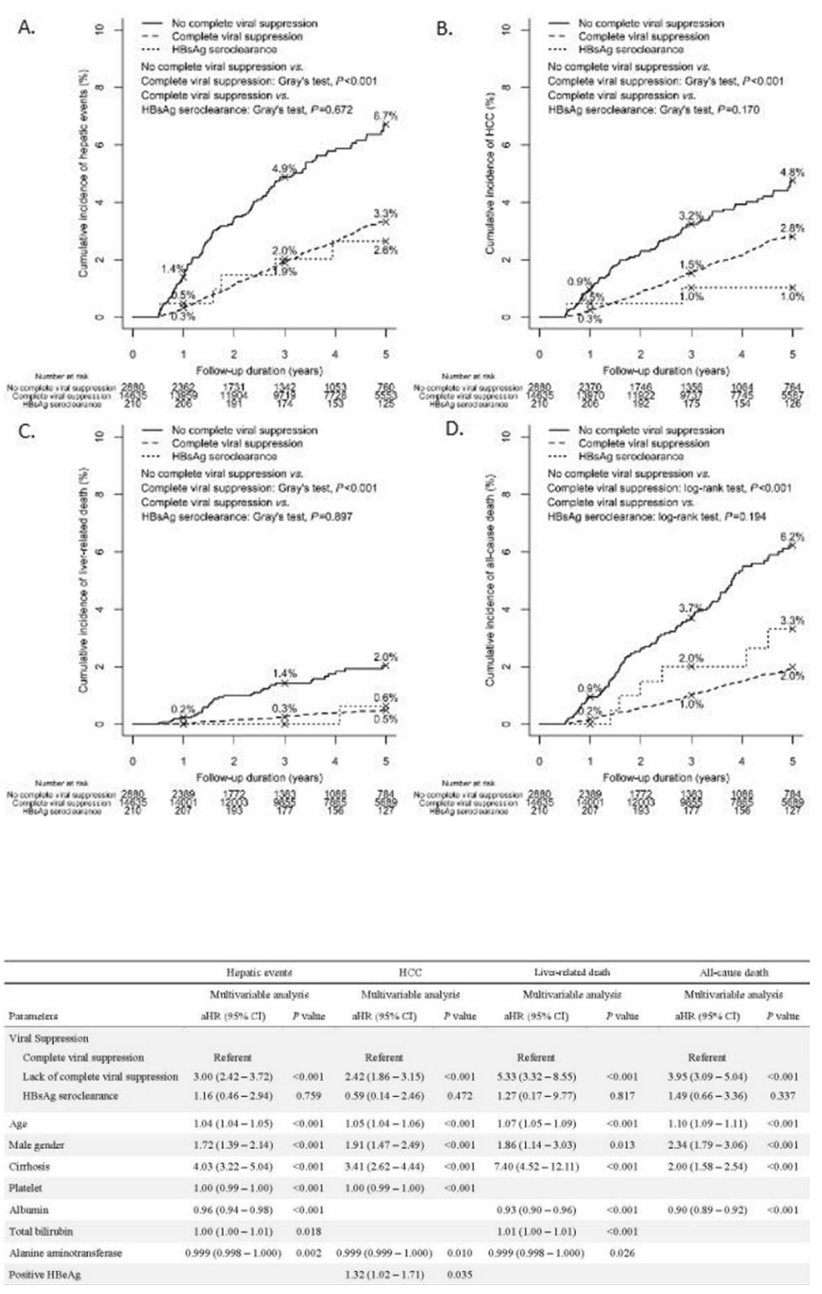

$H B V-C 73$

Hepatitis B virus screening rate at the onset of chemotherapy in patients with hematological maligancy

\section{Sebnem Calik ${ }^{1}$, Sebnem Calik ${ }^{2}$, Seher Ayten Coskuner ${ }^{2}$, Ufuk Sonmez}

${ }^{1}$ Saglik Bilimleri University Izmir Bozyaka Training and Research Hospital, Turkey; ${ }^{2}$ University of Health Science Izmir Bozyaka Training and Research Hospital, Turkey

Background: Patients with hepatitis B virus (HBV) infection are at risk for reactivation after chemotherapy. Effective prophylaxis is available but depends on detection of prior infection. We aimed to evaluate the patients' hepatitis B surface antigen ( $\mathrm{HbsAg}$ ), hepatitis B surface antigen (AntiHBs) and hepatitis B core protein antibody (AntiHBcIgG) screening ratios and the ratio of recommendation of prophylaxis according to the scan results prior to the therapy.

Method: We conducted a retrospective cohort study of patients with newly diagnosed hematological malignancy who received chemotherapy between January 2012 and December 2016 at a training hospital. Data from patient's medical records and the electronic patient data monitoring system were recorded.

Result: Two hundred patients who received chemotherapy were included. One hundred eleven of them $(55.5 \%)$ were acute leukemia, $36(18 \%)$ of them were lymphoma, $24(12 \%)$ of them were multiple myeloma, 18 (9\%) of them were chronic leukemia and $6(3 \%)$ of them were myelodysplastic syndrome. Of 200 patients, $183(91.5 \%)$ underwent Hepatitis B screening. In 153 (76.5\%) patients, HBsAg, antiHBc and antiHBs screening tests were performed, while in 30 (15\%) patients only HBsAg and antiHBS test were performed. Sixty (30\%) patients were anti-HBc and antiHbs positive. The prevalance of only antiHBc positivity was $2.5 \%$. Twelve patient received antiviral prophylaxy and hepatitis B exacerbation developed in one of the patients without antiviral prophylaxis. The prevalence of chronic HBV infection was $2.5 \%$ and all of them received antiviral prophylaxy and hepatitis B exacerbation were not developed.

Conclusion: HBV screening among patients with hematological malignancy is high, but there was a deficiency in the screening test panel. Infectious disease specialists should be in cooperation with other clinicians about hepatitis B screening programs and awareness must raise.

\section{$\mathrm{HBV}-\mathrm{C} 74$}

Hepatitis B: changes in epidemiological and molecular features of Afro-Brazilian communities, Central Brazil

BARBARA VIEIRA LAGO ${ }^{1}$, Livia ORTIZ Lima ${ }^{4}$, Gabriela Alves Cesar ${ }^{3}$, Sabrina Weis ${ }^{3}$, Larissa Bandeira ${ }^{3}$, Grazielli Rocha Rezende ${ }^{3}$, Tayana Ortiz Tanaka ${ }^{3}$, ${\text { Christian } \mathrm{Niel}^{2} \text {, Selma Gomes }}^{2}$, Ana Rita Motta Castro ${ }^{3}$

${ }^{1}$ Fundação Oswaldo Cruz, Brazil; ${ }^{2}$ Fundaçao Oswaldo Cruz, Brazil; ${ }^{3}$ University Federal of Mato Grosso do Sul, Brazil; ${ }^{4}$ University of Mato Grosso do Sul, Brazil

Background: Hepatitis B virus (HBV) is a major cause of liver disease worldwide and persists as an important public health problem in vulnerable populations. Over ten years ago, previous studies conducted in two Afro-Brazilian communities, Furnas dos Dionisios (FD) and São Benedito (SB), detected high prevalence rates of HBV infection $(42.7 \%$ and $16.0 \%)$, susceptible individuals $(55.3 \%$ and $63.0 \%)$ and a low prevalence of anti-HBs alone (2.0\% and $21.0 \%)$. The prevalence of occult HBV infection (OBI) was found to be $18.3 \%$ in FD. These findings resulted in actions for health promotion such as HBV vaccine supply and referral of active hepatitis B cases for clinical care. Reassessing the epidemiological situation of HBV infection nowadays in these communities is essential to qualify the effectiveness of the measures adopted and to design future interventions.

Method: Between October 2015 and July 2016, blood samples were collected from 331 Afro-descendants (207 from FD and 124 from SB) previously interviewed regarding socioeconomic and behavioral characteristics. All serum samples were tested for HBV serological markers by enzyme-linked immunosorbent assay, screened for HBVDNA, sequenced and phylogenetically analyzed.

Result: Considering the two communities together, the overall prevalence of $\mathrm{HBV}$ infection and the chronic carriers remained respectively around $30 \%$ and $5.5 \%$. All subjects presented $\mathrm{HBeAg}-/$ anti-HBe+ status. After more than one decade of the first interventions, the proportion of individuals susceptible to HBV infection fell from $58.9 \%$ to $26.3 \%(\mathrm{p}<0.0001)$, while the vaccinated individuals increased from $10.7 \%$ to $43.5 \%$ ( $p<0.0001$ ). In FD community vaccinated individuals increased from $2 \%$ to $45.9 \%$ (p $<0.0001$ ), while susceptibles to HBV fell from $55.3 \%$ to $18.8 \%$ ( $p<0.0001$ ). In $\mathrm{SB}$, vaccinated individuals increased from $21.0 \%$ to $39.5 \%$ ( $\mathrm{p}<$ 0.001 ) and of susceptibles fell from $63.0 \%$ to $38.7 \%$ ( $p<0.001$ ), Compared to SB, FD has a lower proportion of individuals who are still susceptible to HBV ( $18.8 \%$ vs $38.7 \%$, p < 0.0001$)$, however it has a higher overall prevalence of this infection $(35.3 \%$ vs $21.8 \%$, p < 
0.013). After multivariate analysis, history of family hepatitis and increasing age were factors associated with exposure to $\mathrm{HBV}$. Regarding OBI, nowadays FD has a prevalence of $0.5 \%$ while in SB is $2.4 \%$. HBV viral loads were low, ranging from $2.4 \times 10(1)-7.4 \mathrm{x}$ 10(4) $\mathrm{IU} / \mathrm{ml}$ (mean $6.3 \times 10(3) \mathrm{IU} / \mathrm{ml}$ ). All samples clustered with sequences from the Asian-American clade of the HBV subgenotype A1. Genetic distances $(0.8 \pm 0.3 \%)$ among isolates from the communities were smaller than the intragroup divergence found among A1 Brazilian sequences.

Conclusion: These results demonstrate a significant decrease in the number of individuals susceptible to $\mathrm{HBV}$ infection in FD and SB communities, but also the maintenance of high levels of chronic hepatitis B infection. Epidemiological and molecular studies in Afrodescendant communities are needed for the design of effective strategies to prevent and control HBV vulnerable populations.

\section{$H B V-C 75$}

\section{Hepatitis D infection in Brazil: prevalence and geographical} distribution of anti-Delta antibody

\section{Elisabeth Lampe ${ }^{1}$, Francisco Campello Do Amaral Mello ${ }^{1}$, Tairine Barros $^{\top}$, Vinicius Mello ${ }^{1}$, Livia Melo Villar ${ }^{1}$, Lia Laura Lewis Ximenez ${ }^{1}$, Barbara Vieira Lago ${ }^{1}$ \\ ${ }^{1}$ Fundação Oswaldo Cruz, Brazil}

Background: In Brazil, the Amazon Basin is an endemic area for hepatitis Delta virus (HDV) infection; however, studies in other regions of the country are scarce. This study aims to map the seroepidemiological situation of anti-Delta antibodies in chronic hepatitis B carriers in all five Brazilian geographic regions.

Method: Serum samples from $1240 \mathrm{HBsAg}$ positive individuals from 24 of 26 Brazilian states were tested for the presence of anti-HDV antibodies using the commercial ETI-AB-DELTAK-2 assay.

Result: A total of 1240 samples $(55.4 \%$ men; mean age $43.1 \pm 13.4$ years) were studied. Anti-HDV antibodies were detected in 40 samples $(3.2 \%$; $55.5 \%$ males; mean age of $38.1 \pm 13.8$ years $)$. Age less than 20 years was significantly associated with HDV infection $(\mathrm{p}<0.000)$. The median HBV viral load in HDV-negative individuals was $3.7+\mathrm{E} 03 \mathrm{UI} / \mathrm{mL}$, while in the HBV/HDV co-infected individuals were slightly lower, $2.5+\mathrm{E} 03 \mathrm{UI} / \mathrm{mL}$. The distributions of anti-HDV differed markedly in the diverse regions of the country. The highest prevalence of HDV infection was found in the North region $(8.5 \%$; $\mathrm{p}<0.000)$, followed by Central West $(2.5 \%)$, Southeast $(1.7 \%)$ and Northeast $(0.8 \%)$. No positive case was identified in South region. Anti-HDV antibodies were detected in 12 Brazilian states, but $60 \%$ of the positive cases were observed in two states, Amazonas and Acre located in the western portion of the Amazon region, where the prevalences were $13.8 \%$ and $24,3 \%$, respectively.

Conclusion: The overall HDV prevalence of $3.2 \%$ emphasizes that HDV is far from being a disease in extinction in Brazil. HDV infection was found out in low prevalence in nonendemic areas and remains a major problem in two states (Acre and Amazonas) in the north of the country, indicating that a continuous epidemiological surveillance program should be implemented in all Brazilian regions.

\section{$H B V-C 76$}

Levels of intrahepatic HBV RNA as a potential virological set point for withdrawal of NA therapy

Yiqi Yu ${ }^{1}$, Yiqi $\mathrm{Yu}^{1}$, Jing Wang ${ }^{1}$, Guojun $\mathrm{Li}^{3}$, Chuan Shen ${ }^{4}$, Jun Huang ${ }^{1}$, Xinyu Wang', Yuxian Huang', Xuanyi Wang ${ }^{2}$, Jiming Zhang ${ }^{1}$, Chao Qiu ${ }^{1}$, Wenhong Zhang ${ }^{1}$

${ }^{1}$ Huashan Hospital affiliated to Fudan University, China; ${ }^{2}$ Fudan University, China; ${ }^{3}$ The Second Hospital of Yinzhou of Ningbo, China; ${ }^{4}$ The Third Hospital of Hebei Medical University, China

Background: Viral rebound developed after nucleos(t)ide analogue (NA) withdrawal in most patients who had achieved suppression of serum HBV DNA to an undetectable level. The unresolved question regarding the optimal virological set point for the spontaneous control of HBV replication remained. We evaluated whether such a set point could be accomplished through long-term NA therapy.

Method: We compared the levels of the viral replicative forms of $\mathrm{HBV}$ in serum and liver samples between $\mathrm{HBeAg}$-negative patients who had been successfully treated with entecavir (ETV) for $>1$ year and inactive carriers. Treatment-naive HBeAg-negative chronic patients experiencing active hepatitis were also included for comparison.

Result: Significantly lower levels of serum HBV RNA were observed in ETV-treated HBeAg-negative patients (2.57 $\log 10$ copies $/ \mathrm{mL}$, range $=2.00-3.73 \log 10$ copies $/ \mathrm{mL}$ ) than in treatment-naive patients $(4.74 \log 10$ copies $/ \mathrm{mL}$, range $=2.76-5.75 \log 10$ copies $/ \mathrm{mL} ; P<$ $0.0001)$ ss shown in Fig. 1A. Serum HBV RNA $(<200$ copies $/ \mathrm{mL})$ was undetectable in 6/22 (27.3\%) ETV-treated patients. However, cases with undetectable serum HBV RNA were more frequent (12/16, $75 \%$ ) among inactive carriers (Fig. 1A $P=0.0076$ ). In liver biopsies, intrahepatic HBV RNA was detectable for all patients. The intrahepatic HBV RNA levels of patients treated with ETV $(2.76 \log 10$ copies $/ \mathrm{mL}$, range $=0.30-4.40 \log 10$ copies $/ \mathrm{mL}$ ) were not significantly lower $(P=0.2786)$ than those of treatment-naive patients $(2.06 \log 10$ copies $/ \mathrm{mL}$, range $=1.09-3.10 \log 10 \mathrm{copies} / \mathrm{mL})$, and remained higher than those of inactive carriers $(1.21 \log 10$ copies $/ \mathrm{mL}$, range $=0-1.87$ $\log 10$ copies $/ \mathrm{mL} ; P=0.0019)$ (Fig. 1B). In comparison to treatmentnaive patients, levels of intrahepatic total HBV DNA (Fig. 1C) and covalently closed circular DNA (cccDNA) (Fig. 1D) were significantly decreased in ETV-treated patients and inactive carriers, but no differences were observed between the latter two groups.

Conclusion: These data suggested that although NA therapy induced the remission of liver disease and reduced the size of the cccDNA pool, the levels of intrahepatic HBV RNA could not be reduced to the comparably low levels of inactive carriers. Lower levels of intrahepatic HBV RNA approaching those of inactive carriers, might also be considered a useful virological endpoint for cessation of NA therapy. 

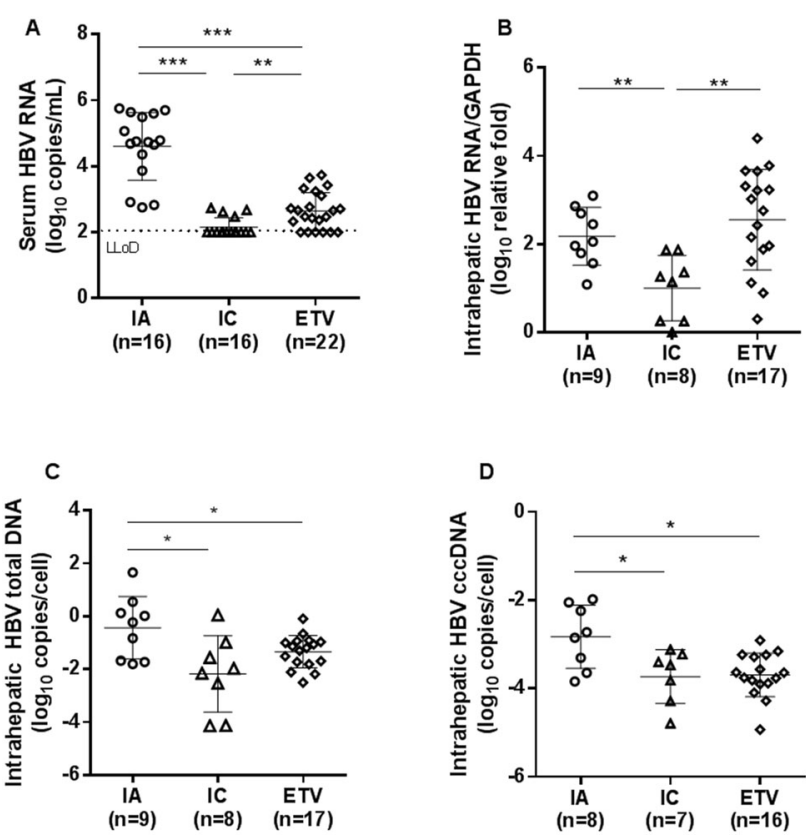

$\mathrm{HBV}-\mathrm{C} 78$

Prospective study of hepatitis B virus infection and its genotypes in Sikkim. Sir Thutop Namgyal Memorial Refferal Hospital Gangtok Sikkim India. Kotwal Raj, Rasielly, Chintamani

\section{Mool Raj Kotwal ${ }^{1}$, Suresh Rasiley ${ }^{3}$, Chintamani Sharma ${ }^{2}$}

${ }^{1}$ Government of Sikkim,SMIMS, India; ${ }^{2}$ Government of Sikkim, STNM Hospital, India; ${ }^{3}$ Government of Sikkim, STNM Hospital, India

Background: Objective: Hepatitis B virus infection causes substantial morbidity and mortality worldwide including India. To generate data as regards the seropravelence of Hepatitis B infection and its genotypes of liver diseases. A total of 593 different types of liver disease cases were collected during 3 years.

Methods: Besides epidemiological data Samples were collected in EDTA or clot activator vials. Serum /plasma were separated by centrifugation method. Hepatitis B surface Antigen, Anti Hepatitis B core Antigen, Hepatitis B core IgM, Anti HCV, Hepatitis A core IgM, Hepatitis $\mathrm{E}$ core $\operatorname{IgM}$ were done using specific kit. HBsAg positive cases were subjected to HBV DNA extraction and further polymerase chain Reaction with specific primers.

Results: A total of $182(30.7 \%)$ acute viral hepatitis cases were collected. The prevalence of Hepatitis B surface antigen among acute viral hepatitis cases was $83.0 \%$ (151/182) with median age of 33 years (SD+-14.9). ranging from 12- 86 years. Males accounted for $66.8 \%$ $(101 / 151)$ of cases while females accounted for the rest $33.2 \%$ (50/ 151). Hepatitis $C$ related cases were $1.1 \%(2 / 182), 1.6 \%$ and hepatitis A virus IgM and hepatitis E IgM were detected in $19.8 \%(36 / 182)$ cases respectively. None of the cases were related to Non A, Non B, and Non C. Among the AVH due to hepatitis B virus infection 47.0\% (71/151) cases were DNA positive. Genotyping results were available for 71 cases. The most common genotype of hepatitis B virus infection detected in AVH cases was genotype D 83.0\% (59/71) and genotype C $9.8 \%$ (7/71) followed by A 7.0\% (5/71).

Conclusion: The prevalence of common type of Hepatitis B among all the cases was found to be $27.2 \%$ (262/963). The most common genotype of Hepatitis B, genotype D $88.9 \%$, genotype C $5.1 \%$ followed by genotype a $5.4 \%$. Surveillance for chronic hepatitis, cirrhosis and Hepatocellular carcinoma would give valuable insights into long-term disease burden in Sikkim, and might help to identify unrecognized modes of transmission and population at risk for infection.

\section{$H B V-C 79$}

Red blood cell distribution width to albumin ratio is a potential index for predicting long-term prognosis for hepatitis B virusrelated liver cirrhosis

Chao $\mathrm{Wu}^{1}$, Jian Wang ${ }^{1}$, Rui Huang', Weimao Ding ${ }^{2}$, Juan $\mathrm{Xia}^{1}$, Xiaomin Yan', Yong Liu', Yuxin Chen ${ }^{1}$

${ }^{1}$ Nanjing Drum Tower Hospital, The Affiliated Hospital of Nanjing University Medical School, China; ${ }^{2}$ Huaan No. 4 People's Hospital, China

Background: It is essential to identify the severity of HBV-related liver diseases and predict long-term prognosis. We aimed to evaluate a novel index, the red blood cell distribution width (RDW)-to-albumin ratio(RAR), for the prediction of liver-related mortality in $\mathrm{HBV}$ related liver cirrhosis.

Method: 223 patients with HBV-related liver cirrhosis, 318 chronic hepatitis $\mathrm{B}(\mathrm{CHB})$ patients and 197 healthy controls(HC) were recruited as a training cohort. 265 patients with cirrhosis, $194 \mathrm{CHB}$ patients and $167 \mathrm{HC}$ from another center were recruited as an external validation cohort. The diagnostic values of RAR for the severity of HBV-related liver cirrhosis were calculated by ROC curves and compared with other noninvasive methods. The prognostic value of baseline RAR were assessed using Kaplan-Meier analysis.

Result: In the training set, median RAR values were significantly higher in patients with HBV-related liver cirrhosis as compared with CHB patients $(\mathrm{P}<0.001)$ and $\mathrm{HC}(\mathrm{P}<0.001)$. RAR was positively correlated with Child-Pugh scores $(\mathrm{r}=0.569, \mathrm{P}<0.001)$ and MELD scores $(\mathrm{r}=0.139, \mathrm{P}=0.038)$. The AUCs of RAR were significantly higher than that of AST-to-platelet (PLT) ratio index (APRI), fibrosis4 index (FIB-4), gamma-glutamyl transpeptidase (GGT)to PLT ratio (GPR), RDW-to-PLT ratio (RPR), AST-to-ALT ratio(AAR) and neutrophil-to-lymphocyte ratio (NLR) in predicting HBV-related liver cirrhosis and decompensated cirrhosis. The performance of RAR in identifying the severity of HBV-related liver cirrhosis was similar in the validation cohort. The mean follow-up duration was 34.9 (IQR, 25.0-49.5) months in cirrhosis patients in the training cohort, during which 21 patients died. The cirrhosis patients were followed for 33.2 (IQR, 16.6-45.9) month in the validation cohort. RAR was an independent predictor of mortality by Cox Regression models(HR 30.6; 95\% CI 2.3-409.6; $\mathrm{P}=0.01$ ). The patients with HBV-related liver cirrhosis were divided into to group by the median $(0.48)$ of RAR value with an incidence rate for liver-related death of 5.9\%, $19.0 \%$ in the training cohort, and $5.6 \%, 26.4 \%$ in the validation cohort, respectively. Kaplan-Meier analysis showed high RAR values was significantly associated with higher cumulative incidence of death throughout follow-up both in the training cohort $(\mathrm{P}=0.005)$ and validation cohort $(\mathrm{P}<0.001)$.

Conclusion: RAR can be used as a novel index for the prediction of severity in HBV-related liver diseases. More importantly, RAR is a useful prognostic index for HBV-related liver cirrhosis. 

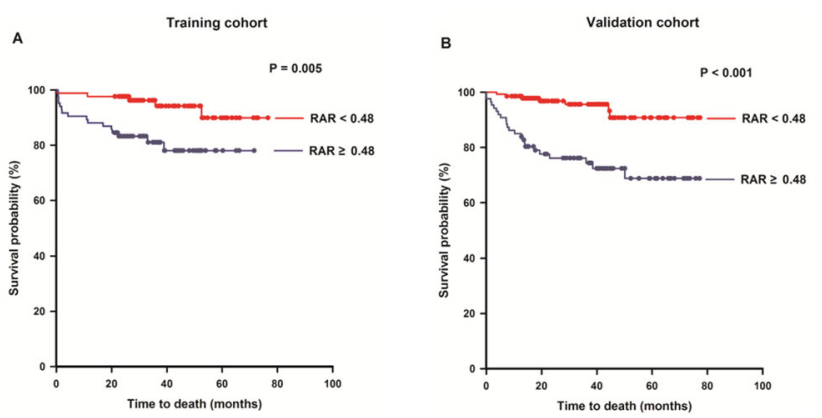

$H B V-C 81$

Risk of hepatitis B reactivation in patients treated with directacting antivirals for hepatitis $\mathrm{C}$

Ali Abdelrahman Ghweil ${ }^{1}$

${ }^{1}$ Head of the tropical medicine and gastroenterology, Egypt

Background: Risk of hepatitis B reactivation in patients treated with direct-acting antivirals for hepatitis c

Abstract The recent introduction of direct-acting antiviral drugs (DAAs) for treatment of the hepatitis $\mathrm{C}$ virus (HCV) has greatly improved the management of $\mathrm{HCV}$ for infected patients. These viral protein inhibitors act rapidly, allowing $\mathrm{HCV}$ clearance and increasing the sustained virological response rates. However, hepatitis B virus (HBV) reactivation has been reported in $\mathrm{HCV} / \mathrm{HBV}$ co-infected patients. Hepatitis $B$ reactivation refers to an abrupt increase in the HBV and is well-documented in patients with previously undetected HBV DNA due to inactive or resolved HBV infection. Reactivation can occur spontaneously, but in most cases, it is triggered by various factors. Reactivation can be transient, without clinical symptoms; however, it usually causes a hepatitis flare. HBV reactivation may occur regardless of $\mathrm{HCV}$ genotype and type of DAA regimen. HBV screening is strongly recommended for co-infected $\mathrm{HCV} / \mathrm{HBV}$ patients before initiation and during DAA therapy regardless of $\mathrm{HBV}$ status, HCV genotype and class of DAAs used. HBV reactivation can be prevented with pretreatment screening and prophylactic treatment when necessary. Additional data are required to evaluate the underlying mechanisms of $\mathrm{HBV}$ reactivation in this setting.

Keywords: Hepatitis B, Hepatitis C, Hepatitis B virus reactivation, Direct-acting antivirals, Pretreatment screening

Method: Risk of hepatitis $\mathrm{B}$ reactivation in patients treated with direct-acting antivirals for hepatitis $\mathrm{C}$

Result: Risk of hepatitis B reactivation in patients treated with directacting antivirals for hepatitis $\mathrm{C}$

Conclusion: Risk of hepatitis B reactivation in patients treated with direct-acting antivirals for hepatitis C.

Result: 206 patients were treated with TDF. 128 patients were excluded for the following reasons. 59 patients had malignancy (including HCC), 36 patients had baseline Phosphorus check days not within 2 months of the first day of TDF administration, 14 patients were co-treated with TDF and other anti-viral agents, 14 patients were not followed up, and 5 patients had other reasons. Consequently, 78 patients were analyzed in this study. Median follow up duration was 350.5 days. Follow up duration is the period from the day of tenofovir initiation to lowest phosphorus level. A total of $50(64.1 \%)$ patients developed hypophosphatemia. There were 28 patients $(35.8 \%)$ with less than $3 \mathrm{mg} / \mathrm{dL}, 16$ patients $(20.5 \%)$ with less than $2.5 \mathrm{mg} / \mathrm{dL}$ and 6 patients $(7.6 \%)$ with less than $2.0 \mathrm{mg} / \mathrm{dL}$. Using univariate analysis, we found that male $(\mathrm{HR}=3.397, \mathrm{P}=0.022)$ and use of diuretics $(\mathrm{HR}=$ $12, \mathrm{P}=0.021)$ and liver cirrhosis $(\mathrm{HR}=3.375, \mathrm{P}=0.041)$ were significantly associated with hypophosphatemia. Using multivariate analysis, we found that male $(\mathrm{HR}=3.836, \mathrm{P}=0.024)$ and liver cirrhosis $(\mathrm{HR}=6.062, \mathrm{P}=0.002)$ was significantly associated with hypophosphatemia. Hypophosphatemia treatment was performed with nuts, dairy, protein intake( 34 patients) or IV phosphorus(3 patient), or recommended stop drinking( 3 patient) and 25 out of 50 patients with hypophosphatemia recovered to normal levels.

Conclusion: When using TDF in HBV patients, hypophosphatemia may be more likely to occur in men or in patients with liver cirrhosis. Therefore, in these patients, phosphorus should be closely monitored when TDF is used and appropriate treatment should be performed in case of hypophosphatemia.

\section{$H B V-C 82$}

A phase 3 study comparing tenofovir alafenamide (TAF) to tenofovir disoproxil fumarate (TDF) in patients from China with HBeAg-positive, chronic hepatitis B: efficacy and safety results at week 48

John F Flaherty ${ }^{1}$, Lunli Zhang ${ }^{5}$, Zhongping Duan ${ }^{4}$, Yu Chen ${ }^{4}$, Shanming $\mathrm{Wu}^{12}$, Fusheng Wang ${ }^{2}$, Qing Xie ${ }^{10}$, Tang Hong ${ }^{15}$, Jun $\mathrm{Li}^{8}$, Yongfeng Yang9, Guozhong Gong ${ }^{11}$, Feng Lin', John Flaherty ${ }^{1}$, Anuj Gaggar ${ }^{1}$, Lanjia Lin ${ }^{1}$, Neeru Bhardwaj', G Mani Subramanian', Chengwei Chen ${ }^{7}$, Yan Huang ${ }^{16}$, Jidong $\mathrm{Jia}^{3}$, Mingxiang Zhang ${ }^{13}$, Qin Ning ${ }^{14}$

${ }^{1}$ Gilead Sciences, United States; ${ }^{10}$ Rui Jin Hospital, Shanghai Jiao Tong University, China; ${ }^{11}$ Second Xiangya Hospital, Central South University, China; ${ }^{12}$ Shanghai Public Health Clinical Center, China; ${ }^{13}$ Sixth People's Hospital of Shenyang, China; ${ }^{14}$ Tongji Hospital Affiliated to Tongji Medical University, China; ${ }^{15}$ West China Hospital, Sichuan University, China; ${ }^{16}$ Xiangya Hospital, Central South University, China; ${ }^{2}$ Beijing 302 Hospital, China; ${ }^{3}$ Beijing Friendship Hospital, Capital Medical University, China; ${ }^{4}$ Beijing You An Hospital, Capital Medical University, China; ${ }^{5}$ First Affiliated Hospital of Nanchang University, China; ${ }^{6}$ Hainan General Hospital, China; ${ }^{7}$ Hospital 85 People's Liberation Army, China; ${ }^{8}$ Jiangsu Province People's Hospital, China; ${ }^{9}$ Nanjing Second Hospital, China 
Background: TAF, a new prodrug of tenofovir (TFV), has greater plasma stability, enhanced delivery of TFV into hepatocytes, and has lower plasma levels of TFV relative to TDF. In a Phase 3 study (Study $110)$ in non-China patients, TAF has demonstrated efficacy non-inferior to that of TDF at Weeks 48 and 96 with a superior renal and bone safety profile. Here are primary results in a separate cohort of this study enrolled in China.

Method: $180 \mathrm{HBeAg-positive} \mathrm{patients} \mathrm{were} \mathrm{randomized} \mathrm{(2:1)} \mathrm{and}$ received TAF $25 \mathrm{mg}$ QD $(\mathrm{n}=123)$ or TDF $300 \mathrm{mg}$ QD $(\mathrm{n}=57)$. Virologic $(\mathrm{HBV}$ DNA $/=69 \mathrm{IU} / \mathrm{mL})$ at Week 48 or HBV DNA $>/=69$ $\mathrm{IU} / \mathrm{mL}$ at discontinuation.

Result: Baseline characteristics included: mean age 34 years, $73 \%$ males, $100 \%$ Asian/Chinese, mostly genotypes B (29\%) and C (70\%); mean HBV DNA was $7.2 \log 10 \mathrm{IU} / \mathrm{mL}(40 \%$ and $37 \%$ of TAF and TDF patients had HBV DNA $>/=8 \log 10 \mathrm{IU} / \mathrm{mL}$ ), and $35 \%$ were previously treated with nucleos(t)ides. Efficacy and safety results are summarized in the Table. Virologic response rates were $61 \%$ and $68 \%$ at Week 48 in the TAF and TDF groups, respectively; at Week 72 an equivalent virologic response rate was seen $(72 \%$ and $72 \%)$. Mean $\log 10$ declines in HBV DNA and qHBsAg were similar between treatment groups and a similar proportion in each group experienced $\mathrm{HBeAg}$ seroconversion at Week 48; no patient experienced HBsAg loss. A greater proportion of TAF vs TDF patients achieved ALT normalization at Week 48. Overall, adverse events (AEs) and serious AEs were similar between groups. Patients treated with TAF showed a small median increase in eGFR-CG compared with a decline in the TDF group at Week 48. Small median percentage increases in hip and spine BMD were seen in TAF patients compared with decreases in TDF patients at Week 48. The smaller changes in eGFR-CG and BMD in TAF patients were associated with smaller changes in proximal tubular and bone turnover markers. No viral resistance was detected in either treatment group.

Conclusion: In HBeAg-positive CHB patients from China, high and similar rates of virologic suppression were achieved with TAF and TDF with a higher rate of ALT normalization seen with TAF. An improved renal and bone safety profile was observed in TAF patients relative to those receiving TDF.

\begin{tabular}{|c|c|c|}
\hline & $\begin{array}{c}\text { TAF } \\
(\mathrm{N}=123)\end{array}$ & $\begin{array}{c}\text { TDF } \\
(\mathrm{N}=57)\end{array}$ \\
\hline \multicolumn{3}{|l|}{ Efficacy Parameters: } \\
\hline \multicolumn{3}{|l|}{ HBV DNA $<29 \mathrm{IU} / \mathrm{mL}, \mathrm{n} / \mathrm{N}(\%)$} \\
\hline Week 48 & $75 / 123(61)$ & $39 / 57(68)$ \\
\hline Week 72 & $88 / 123(72)$ & $41 / 57(72)$ \\
\hline HBV DNA, mean (SD) change $\left(\log _{10} \mathrm{IU} / \mathrm{mL}\right)$ & $-5.35(1.65)$ & $-5.55(1.49)$ \\
\hline HBeAgloss/seroconversion, $\mathrm{n} / \mathrm{N}(\%)$ & $\begin{array}{l}18 / 118(15) \\
13 / 118(11)\end{array}$ & $\begin{array}{c}6 / 57(11) \\
5 / 57(9)\end{array}$ \\
\hline HBsAg, mean (SD) change ( $\left.\log _{10} \mathrm{IU} / \mathrm{mL}\right)$ & $-0.53(0.99)$ & $-0.49(0.74)$ \\
\hline ALT normalization (AASLD criteria), $\mathrm{n} / \mathrm{N}(\%)^{b}$ & $69 / 117(59)$ & $26 / 55(47)$ \\
\hline \multicolumn{3}{|l|}{ Renal and Bone Parameters } \\
\hline eGFR ${ }_{c G}$, median $(\mathrm{Q} 1, \mathrm{Q} 3)$ change $(\mathrm{mL} / \mathrm{min})$ & $+0.6(-5.4,6.6)$ & $-3.0(-15.6,4.2)$ \\
\hline$>25 \%$ decline in eGFR $c$ from baseline, $\mathrm{n} / \mathrm{N}(\%)$ & $3 / 123(2)$ & $9 / 57(16)$ \\
\hline Hip $\mathrm{BMD}$, median (Q1, Q3) \% change & $\begin{array}{c}\mathrm{n}=53 \\
+0.19(-0.82,+1.27)\end{array}$ & $\begin{array}{c}\mathrm{n}=31 \\
-1.43(-2.58,+0.10)\end{array}$ \\
\hline$>3 \%$ decline in hip BMD, $\mathrm{n} / \mathrm{N}(\%)$ & $2 / 53(4)$ & $7 / 31(23)$ \\
\hline Spine BMD, median (Q1, Q3) \% change & $\begin{array}{c}\mathrm{n}=54 \\
+0.71(-1.33,+2.47)\end{array}$ & $\begin{array}{c}\mathrm{n}=31 \\
-1.61(-4.59,+0.49)\end{array}$ \\
\hline$>3 \%$ decline in spine $\mathrm{BMD}, \mathrm{n} / \mathrm{N}(\%)$ & $6 / 54(11)$ & $11 / 31(35)$ \\
\hline
\end{tabular}

\section{$H B V-C 83$}

Results of nucleotide and nucleoside analogues treatment in patients with chronic hepatitis $B$

George Sebastian Gherlan', George Sebastian Gherlan', Adriana Motoc $^{2}$, Augustina Culinescu' ${ }^{2}$, Laurentiu Filip ${ }^{2}$, Stefan Dragos Lazar ${ }^{1}$, Dana Obretin ${ }^{2}$, Aysel Simin Florescu ${ }^{1}$, Emanoil Ceausu ${ }^{1}$, Petre Iacob Calistru ${ }^{1}$

${ }^{1}$ Carol Davila University of Medicine and Pharmacy, Romania; ${ }^{2}$ Dr. Victor Babes Hospital of Infectious Diseases, Romania

Background: We aimed to analyse the groups of indications for treatment in our patients according to local guidelines (that are going to be presented in summary). We tried to identify the factors thatcan predict a rapid normalisation of ALT and non-detectability of HBV DNA divided by medication used.

Method: We included 150 patients (59 women) in this retrospective study. We collected demographic data (age, sex), weight, medication, previous treatments, $\mathrm{HBe}$ status, blood tests results (hematology, chemistry, HBV DNA) and liver fibrosis (appreciated by noninvasive means). We also collected the time to ALT normalisation and to nondetectable HBV DNA. The follow-up period varied for 12 months to 72 months.

Result: Patients ages were between 18-72 years (median 51, stdev 13.52) and median weight was 78 kilograms (stdev 15.072). The mejority of the patients were treated wit Entecavir (133). $60 \%$ of the patients had no previous treatment. Of those previously treated, most were peg-interferon experienced. $11 \%$ were $\mathrm{HBe} \mathrm{Ag}$ positive. Most of the patients were initiated on therapy based on elevated ALT criteria, followed by fibrosis combined with viral load over $2000 \mathrm{IU} / \mathrm{ml}$. $58.6 \%$ of our patients obtained non-detectable HBV DNA in 6 months (faster for patients treated with entecavir). The only identified factors that correlated with faster negativation of HBV DNA were viral load at initiation $(\mathrm{p}=0.03)$ and Negative $\mathrm{HBe} \mathrm{Ag}(\mathrm{p}=0.01)$. ALT normalisation was found in $75 \%$ of the patients at 6 months and the factors that correlated were the same: lower viral load $(p=0.01)$ and $\mathrm{HBe} \mathrm{Ag}(\mathrm{p}=0.01)$. ALT value at the beginning was not a good predictor. Only 5 patients in our group were entecavir resistant $(3.33 \%)$, of which 4 were included in the study with resistance already known at the beginning of monitoring period.

Conclusion: ALT normalisation and negativation of HBV DNA occur in most patients in the first 6 months of the treatment, faster in patients with lower HBV DNA and HBe AG negative.

\section{$H B V-C 84$}

Usefulness of dried blood spot for detection of occult hepatitis B

Livia Melo Villar ${ }^{1}$, Cristiane Sousa Bezerra ${ }^{1}$, Moyra Machado Portilho ${ }^{1}$, Jakeline Ribeiro Barbosa ${ }^{1}$, Jose Napoleao Monte Da Cruz ${ }^{2}$, Cristiane Cunha Frota ${ }^{3}$, Lia Laura Lewis Ximenez ${ }^{1}$, Elisabeth Lampe ${ }^{1}$

${ }^{1}$ FIOCRUZ, Brazil; ${ }^{2}$ Central Public Health Laboratory, Brazil; ${ }^{3}$ Federal University of Ceara, Brazil

Background: Occult HBV can be defined as detection of HBV DNA in liver tissue when HBsAg is not detected in serum or plasma. However, the analysis of liver samples is difficult because of the invasive nature, so the use of serum or dried blood spot samples (DBS) could facilitate access to diagnosis of occult hepatitis B. The objective of this study is to evaluate the usefulness of DBS samples for the detection of HBV DNA in individuals presenting isolated anti$\mathrm{HBc}$ marker in serum. 
Method: Twenty-eight subjects were recruited with isolated anti-HBc and HBV DNA detected in the serum (Abbott RealTime HBV test) which also provided DBS samples. Viral DNA was extracted in serum and DBS using High Pure Viral Nucleic Acid (Roche Diagnostics) where 3 disks of $3 \mathrm{~mm}$ of diameter were used for DBS extraction. In house qualitative PCR for amplification of viral polymerase ( $\mathrm{S} / \mathrm{pol})$ gene were used in serum and DBS samples.

Result: Participants had mean age of $50.79 \pm 14.3$ years and most of them were male $(53.57 \%)$, HBV mean viral load was $528.04 \pm$ $1182.07 \mathrm{IU} / \mathrm{mL}$ in serum $(1.91 \pm 0.87 \mathrm{log} \mathrm{IU} / \mathrm{mL})$, mean values of AST ( $24 \mathrm{U} / \mathrm{L} \pm 27.45)$ and ALT (17.63 U / L \pm 12.83 ) were within the normal range for adults. Nine individuals were anti-HCV or antiHIV reagents $(32.1 \%)$. It was possible to detect HBV DNA by qualitative PCR in 9 serum samples $(9 / 28=32.14 \%)$. These positive serum samples had a mean viral load of $1.53 \log \mathrm{IU} / \mathrm{mL}( \pm 0.72)$, while serum samples without HBV DNA showed a mean viral load of $2.09 \log \mathrm{IU} / \mathrm{mL} \pm 0.90$. Four DBS samples out of nine HBV DNA viremic individuals tested positive by qualitative test $(4 / 28=$ $14.29 \%)$.

Conclusion: HBV DNA was detected in DBS samples from individuals with serum anti-HBc suggesting the presence of occult infection and the usefulness of this sample to increase the access of diagnosis in low resource areas.

\section{$H B V-C 85$}

Intranasal administration of HeberNasvac, a therapeutic vaccine for chronic hepatitis B

\section{Julio Cesar Aguilar ${ }^{1}$}

${ }^{1}$ Center for Genetic Engineering and Biotechnology, Cuba

Background: A novel therapeutic vaccine for chronic hepatitis B (CHB) treatment comprising the recombinant hepatitis B surface ( $\mathrm{HBsAg}$ ) and nucleocapsid ( $\mathrm{HBcAg}$ ) antigens has been developed in the last two decades upto registration. The aim of the present study is to evaluate the long term efficacy results in the first 6 patients treated with this product (HeberNasvac) by the IN route in order to evaluate the effect of the vaccine by IN route alone after five years follow-up. Method: The study was conducted in $6 \mathrm{CHB}$ patients previously treated with a-IFN, 3 of them $\mathrm{HBeAg}$ positive. Patients were immunized with $100 \mathrm{mcg}$ HBsAg and $100 \mathrm{mcg}$ HBcAg (HeberNasvac), via nasal spray in ten administrations, every two weeks. Samples from these patients were preserved for a period of five years after the end of treatment to retrospectively analyze the serological and virological variables. Clinical efficacy was monitored by assessing the levels of HBV DNA, ALT, HBeAg to anti-HBeAg seroconversion as well as by qualitative/ quantitative HBsAg serology during this period of time.

Result: The use of the intranasal route deserves further evaluation as most of the patients with CHB lives in countries where unsafe injections are frequent and, in addition, the nasal route may further potentiate the immune response in the liver. After five year follow-up $\mathrm{HBeAg}$ loss was verified in the three $\mathrm{HBeAg}(+)$ patients, in two cases with seroconversion to anti-HBeAg. A reduction to undetectable viral load was observed in 5 out of 6 patients and in two cases there were HBsAg seroconversion. ALT increases above $2 X$ ULN were only detected in $\mathrm{HBeAg}(+)$ patients and associated to $\mathrm{HBe}$ antigen loss. All patients were negative for cirrhosis or moderate fibrosis ( $<7.8 \mathrm{KPa}$ by Fibroscan assessment) at the end of this period. It was not required any alternative medication due to sustainedly normal LFTs and absence of treatment criteria.

Conclusion: The vaccine was not associated to safety concern 5 years after the end of treatment. Virological, biochemical, serological variables evidenced the efficacy of HeberNasvac in difficult to treat patients when administered by IN route.

\section{$H B V-C 86$}

Longitudinal analysis of drug-resistant hepatitis B virus mutants for 12 entecavir-resistant patients with suboptimal response to subsequent adefovir plus entecavir rescue therapy

Dongping $X u^{1}$, Jinman Shao ${ }^{1}$, Yan Liu', Rongjuan Chen ${ }^{1}$, $\mathrm{Li}_{\text {Zhao }}{ }^{1}$, Yi Zhou ${ }^{1}$, Jialiang Liu ${ }^{1}$, Le Li ${ }^{1}$, Xiaodong $\mathrm{Li}^{1}$, Yangyang Zhao ${ }^{1}$, Zilin Tang ${ }^{1}$, Dongping $\mathrm{Xu}^{1}$

\section{${ }^{1}$ Beijing 302 Hospital, China}

Background: Adefovir dipivoxil plus entecavir (ADV+ETV) is one of recommended rescue therapies for ETV resistance patients with chronic hepatitis B virus (HBV) infection in many Asian countries including China, while the therapeutic efficacy was poor in some cases in clinical practice. The study aimed to clarify the evolution of drug-resistant HBV mutants with clinical responses for such cases.

Method: Twelve ETV resistance patients were enrolled. Resistant mutant dynamics was analyzed by sequencing of HBV reverse-transcriptase region ( $\geq 20$ clones/sample). Phenotypic analysis of drug resistance was determined in amplicon-transfected HepG2 cells.

Result: All patients were detected with dominant ETV-resistant mutants before ADV+ETV therapy and none of them achieved virological response (serum HBV DNA

Conclusion: The poor efficacy of ADV+ETV may lie on relatively weak suppression of ADV to ETV-resistant mutants and emergence of multidrug-resistant mutants. TDF-based therapy is superior to extending ADV+ETV therapy for these patients.

\section{$H B V-C 87$}

Hepatitis D: a multifaceted disease, one center six-month experience

\section{Abdurrahman Sahin ${ }^{1}$, Emrah Ozkan', Salih Kilic', Nurettin Tunc $^{1}$, Ulvi Demirel' ${ }^{1}$, Orhan Kursat Poyrazoglu', Mehmet Yalniz ${ }^{1}$}

${ }^{1}$ Firat University, Turkey

Background: Chronic hepatitis D is the most severe form of viral hepatitis, with the clinical features of accelerated progression to cirrhosis, increased risk of hepatocellular carcinoma and early decompensation in patients with cirrhosis. In this prospective study we aimed to demonstrate the clinical features of HDV carriers.

Method: Consecutive subjects with Anti-HDV seropositivity who attending to gastroenterology clinic of our hospital between April 2017 and October 2017 were recruited to the study. Demographic and clinical features, laboratory data, previous and ongoing treatments and HBV and HDV serology were noted. Patients were divided into four groups according to the baseline predominant virus that were determined based on HBV DNA and HDV RNA results; HDV dominant, HBV dominant, HBV \& HDV dual dominant and dual inactive who couldn't correspond treatment criteria for both viruses. Result: A total of 44 subjects were recruited. The mean age was $53.1 \pm 11.8$ years and, $25(57 \%)$ of them were male. Two of them HBe ag positive and, only one patient had HBs loss in the course of disease. Eleven (25\%) of them had HDV dominant, 15(34\%) had HBV dominant, 15 (34\%) had also dual dominant and 3(7\%) had dual inactive disease. Five $(11 \%)$ patients, who presented with portal hypertension related manifestations, had stable disease course. 
Another 7 (16\%) patients had diagnosed as HCC. Six (14\%) patients have been treated with Peg-IFN, 15 (34\%) patients with only nucleotide analogs (NA) and, 11(25\%) patients with Peg-IFN and NA combination. Among 14 patients treated with Peg-IFN previously, 4 of them $(28 \%)$ were responder to Peg-IFN treatment, 10 were nonresponder. Another 3 patients were on the treatment of Peg-IFN.

Conclusion: The clinical scenario of HDV infection is variable. Our results showed that beside severe course and high rate of HCC development, benign clinical course of the disease including inactive patients, stable patients and even HBs ag loss is also evident.

\section{DILI-1}

Toxicity study of narlaprevir, ritonavir, tenofovir, raltegravir combinations in rats

\section{Elena Shipaeva $^{1}$, Emiliya Krasavina ${ }^{1}$, Anastasiya Dmitrieva ${ }^{1}$, Elena Tarkhova ${ }^{1}$, Elena Shekunova ${ }^{2}$, Marina Makarova ${ }^{2}$, Alexey Kravtchenko ${ }^{3}$, Mikhail Samsonov ${ }^{1}$}

${ }^{1} \mathrm{JSC}$ «R-Pharm», Russian Federation; ${ }^{2}$ Closed Joint-stock company "Research and manufacturing company "HOUM OF PHARMACY", Russian Federation; ${ }^{3}$ Federal Budget Institution of Science "Central Research Institute of Epidemiology", Russian Federation

Background: Narlaprevir (NVR, SCH900518) is potent HCV protease inhibitor. NVR is used with CYP3A4 inhibitor Ritonavir (RTV). $\mathrm{HCV}$ treatment regimens comprising RTV-boosted NVR in patients with HCV and the human immunodeficiency virus (HIV) coinfection will be evaluated. Toxicity of NVR/RTV and antiretroviral drugs coadministration should be investigated in animal study before clinical trials.

Method: Objective of the study was comparative assessment of NVR, RTV, Tenofovir (TDF), Raltegravir (RAL) toxicity (monotherapy vs. combinations) in rats following daily oral 28 days administration with 28 days treatment free period. The 208 male and 208 female outbreed rats were allocated to 13 groups (16 male, 16 female in each) (Table 1). Necropsy was done: on Day 29 “ $50 \%$ animals from each group, on Day 57 “ recovery animals. Following examinations were evaluated: clinical signs, body weights, individual behaviour, clinical pathology, urinalysis, gross pathology, organ weight, hystopathomorphological examination, estimation of local tolerance. Statistical analysis was performed using Shapiro-Wilk W test, Kruskal-Wallis nonparametric test, procedure ANOVA (Tukey test analysis, HSD).

Result:NVR, RTV, TDF, RAL combinations administration as well as monotherapy did not cause any effects on body weights, individual behavior, hematology parameters. A number of findings were observed in all groups. All dose groups changes without significant difference between monotherapy and combinations: -tendency to alanine aminotransferase, aspartate aminotransferase, alkaline phosphatase values increase; -individual deviations of kidneys functions (protein, erythrocytes rise); -hepatocytes fat dystrophy, inflammatory infiltrates in liver parenchyma; -sporadic cases of nephritis; -gastritis, enteritis; -ovaries follicles dystrophy; $-2.4 \%$ male, $6.7 \%$ female deaths. Combinations-related findings: -testicular dystrophy in male rats in all groups doses evaluated, changes frequency was significant higher for combinations in comparison with monotherapy. According to the previous preclinical and clinical studies results, the testicular abnormalities were considered as rodent-specific finding. Animal deaths were not suspected to study drugs (they were assessed as individual reaction to excipients). Phase I clinical trial of drug interaction study with pharmacokinetics of NVR/RTV and antiretroviral drugs in healthy voluntaries is ongoing in Russian Federation. Conclusion: Combination therapy of NVR, RTV, TDF, RAL has shown no significant differences in toxicity effects in comparison with monotherapy of each drug.

\begin{tabular}{|c|c|}
\hline Group description & Dose Level (mg/kg) \\
\hline Control & 0 \\
\hline \multirow{2}{*}{ NVR } & 16 \\
\hline & 80 \\
\hline \multirow{2}{*}{ RTV } & 8 \\
\hline & 40 \\
\hline \multirow{2}{*}{ TDF } & 24 \\
\hline & 120 \\
\hline \multirow{2}{*}{ RAL } & 32 \\
\hline & 160 \\
\hline \multirow{2}{*}{ NVR+RTV+TDF (Combination 1) } & $16+8+24$ \\
\hline & $80+40+120$ \\
\hline \multirow{2}{*}{ NVR+RTV+ RAL (Combination 2) } & $16+8+32$ \\
\hline & $80+40+160$ \\
\hline
\end{tabular}

\section{DILI-2}

In vitro model for hepatotoxicity studies based on a vascularized bioengineered liver platform

\section{$\underline{\text { Qiao } W u^{1}}$}

${ }^{1}$ Capital Medical University, China

Background: Drug-induced liver injury (DILI) is a leading cause of acute liver failure. One of the major obstacles in prediction or evaluation of DILI is the lack of experimental models that recapitulate the stable and physiologically relevant liver functions and reflect the actual levels of drug hepatotoxicity. Under liver physiological conditions, cell-cell and cell-extracellular matrix (ECM) communications play a crucial role in cell differentiation and polarization, thereby influencing liver-specific function. Organotypic liver culture models for hepatotoxicity studies that mimic in vivo hepatic functionality could help facilitate improved strategies for early safety risk assessment during drug development. Interspecies differences in drug sensitivity and mechanistic profiles, low predictive capacity, and limitations of conventional monocultures of human hepatocytes, with high attrition rates remain major challenges.

Method: Using an organ engineering approach and relevant cell lines, we designed a perfusion system that delivers discrete mechanical forces inside an acellular liver extracellular matrix scaffold to study the effects of ECM and cell to cell communications in hepatic metabolic enzyme. We not only have studied the changes of hepatic nuclear receptors, transporters and phase I and phase II enzymes in tissue engineering from the RNA level, but also systematically analyzed the drug enzyme by liquid chromatography-mass spectrometry. In order to further test whether this system can be used as a platform for testing hepatotoxicity, we added diclofenac sodium for repeated perfusion.

Result: We show stable, cell-type specific phenotype/cellular polarity with differentiated functionality in human HepG2 (enhanced metabolizing enzyme activity/albumin synthesis) when in co-culture with human vascular endothelial cells (HUVECs), thus demonstrating biocompatibility and relevance for evaluating drug metabolism and toxicity. We found that $40.4 \mathrm{umol} / \mathrm{L}$ diclofenac sodium $(\mathrm{Cmax}=$ $10.1 \mathrm{umol} / \mathrm{L}$ in human body) perfused in this system can cause hepatotoxicity. Surprisingly, we firstly find this drug cause endothelial cells damaging in the first place. Then we further prove that endothelial cells are more sensitive than hepatocytes to the metabolic poison of diclofenac sodium.

Conclusion: This human co-cultured liver organoid model may represent a sustainable and physiologically relevant alternative cell system to PHHs, complementary to animal testing, for initial hepatotoxicity screening or mechanistic studies of candidate compounds differentially targeting hepatocytes and endothelial cells. 
DILI-3

Yellow phosphorus as rodenticide -predictive factors of liver injury and its outcome analysis in humans consuming rodenticide on suicidal intention: a single centre tertiary care experience from South India

Radhika Venugopal $^{1}$, Radhika Venugopal ${ }^{1}$, Krishnasamy Narayanasamy ${ }^{1}$, Chezhian Annasamy', Senthilkumar $\mathbf{R}^{\mathbf{1}}$, Premkumar $\mathbf{K}^{\mathbf{1}}$, Akilandeswari A $\mathbf{R}^{\mathbf{1}}$, Santhi Selvi $\mathbf{A}^{1}$

${ }^{1}$ Madras Medical College, Rajiv Gandhi Government General Hospital, India

Background: Rodenticide is a common means of suicidal poisoning. Unlike west many rodenticide in India contain hepatotoxic drugs leading on to Acute Liver failure, of which yellow phosphorus is most lethal. In the absence of specific antidote, consumption is often fatal if left untreated. We aimed at finding the socio-demographic characteristics of patients with rodenticide poisoning and to analyse the predictive factors leading to acute liver injury and its outcome.

Method: A total of 101 patients admitted with alleged history of rodenticide paste poisoning (3\% yellow phosphorus) at our department over a period of 6 months were consecutively analysed, probable predictive factors which could influence the outcome were recorded along with other baseline social and demographic characters. Serial charting of liver function tests and other parameters were done.underlying liver diseases were ruled out. All the patients received standard of care management and $\mathrm{N}$ Acetyl Cysteine.Their clinical course and outcomes were recorded.

Result: Of the 101 patients $76 \%$ were from rural areas and $24 \%$ urban. Two thirds $62 \%$ were female and the rest $38 \%$ were males. Age group ranged from 15-65 showing that suicide occured across ages. Most fell between 20-30 yrs. The mode of ingestion was recorded, 34\% had it raw, 30\% with water, $20 \%$ with alcohol and in $17 \%$ it was with food. Most common presenting symptom was vomiting followed by abdominal pain and headache. The Time of manifestation of first symptom was $<6$ hours in $41 \%$ patients, $6-12$ hours in $42 \%$ patients and $>12$ hours in $17 \%$ patients. Time taken to health care facility after ingestion ranged from 1-48 hours. $46 \%$ patients out of 101 sought care within 3 hours, $41 \%$ took medical attention within 3-10 hours and 13\% patients were brought after 10 hours. The International normalized ratio (INR) was $<2$ in 69 patients, $2-3$ in 11 patients and $>3$ INR in 21 patients. MELD score was calculated and 9 had a MELD of $<10,30$ were in 11-20 range, 35 in 21-30 and $>30$ MELD was found in 27 patients. All of those having a MELD of $>40$ died. Spectrum of Liver injury showed Acute Liver Injury in $42(41 \%)$, Acute Liver Failure (ALF) in 21(21\%) patients, Acute Kidney Injury (AKI) in 19\% patients and multiple organ failure was seen in $19 \%$ patients. Two had Myocarditis and one developed Atrial fibrillation. 3 patients developed acute pancreatitis. The overall mortality rate was $19 \%$, with a $22 \%$ mortality among females and $13 \%$ among male. The other 82 patients recovered and were discharged with mean hospital duration of 8.88 days. Psychiatric analysis of most patients revealed para suicide rather than true intention to die \& none had underlying psychosis.

Conclusion: Almost all patients with rodenticide poisoning had liver injury. Lethal dosage and late presentation to medical facility led to development of acute liver failure and mortality. Creating awareness, promoting mental health and suicide prevention is the best option to reduce mortality.
DILI-4

Delayed onset of symptoms in drug-induced liver injury is conditioned by a combination of host factors and drug properties

Camilla Stephens $^{1}$, A Gonzlez Jimnez ${ }^{3}$, I Medina Caliz ${ }^{3}$, M Robles Diaz ${ }^{3}$, A Ortega Alonso ${ }^{3}$, M Chen ${ }^{4}, K_{\text {Mceuen }}^{4}$, A Suzuki $^{2}$, MI Lucena ${ }^{3}$, C Stephens ${ }^{3}$, R J Andrade ${ }^{3}$

${ }^{1}$ Universidad de Málaga, Spain; ${ }^{2}$ Durham VA Medical Center, Duke University, United States; ${ }^{3}$ Instituto de Investigación Biomédica de Málaga (IBIMA), Hospital Universitario Virgen de la Victoria, Universidad de Málaga, CIBERehd, Spain; ${ }^{4}$ National Center for Toxicological Research, US Food and Drug Administration, United States

Background: Most patients with drug-induced liver injury (DILI) manifest clinical symptoms while on therapy, however some develop symptoms several days after drug cessation (delayed onset, DO), which particularly challenges DILI causality assessment. Factors contributing to the delayed onset phenotype are unknown. We aimed to explore potential factors contributing to delayed onset of DILI, by analysing culprit drug properties, host factors and their interactions in a large patient population from the Spanish DILI Registry

Method: 413 DILI cases induced by a single causative drug, which was responsible for at least three cases in the Spanish DILI Registry, were included. Clinical information from the 413 patients, of which $95(23 \%)$ presented DO, and drug properties of 42 causative DILI agents were analysed. A two-tier regression-based model was used to assess host/drug interactions affecting the probability of DO. The findings were validated in an independent cohort of 188 Latin American DILI cases, of which 32 (17\%) presented DO.

Result: Antibacterial and anti-inflammatory drugs accounted for most of the cases presenting with delayed onset. Drug property of $200 \mathrm{mg}$ [OR: 2.9, 95\% CI: 1.2-7.6, $\mathrm{p}=0.0123$ ] and the absence of pre-existing conditions in a patient [OR: $1.9,95 \% \mathrm{CI}: 1.1-3.4, \mathrm{p}=0.0245]$ ) were independently associated with delayed onset. Likewise, drug properties of mitochondrial liability and high Pauling electronegativity were associated with delayed onset, but depending on specific host factors such as age (cut-off 60 years), gender, and pre-existing cardiac diseases. The DO cases in the external cohort were similarly induced by antibacterial and anti-inflammatory drugs. These cases were associated with higher daily dose treatments (mean: 1717 vs $493 \mathrm{mg}$, $\mathrm{p}=$ ?0.001) and hepatic metabolism

Conclusion: This study suggests that delayed onset presentation is conditioned by a combination of host and drug factors. Healthier DILI patients with regards to comorbidities, taking higher daily doses of medications with low hepatic metabolism are at higher risk of manifesting DO. Funding: FondosFEDER (PI15/01440), AEMPS, CIBERehd by ISCIII

\section{DILI-5}

20 years with the Spanish DILI Registry: an analysis of 843 enrolled DILI cases

I Medina ${ }^{1}$, I Medina ${ }^{2}$, C Stephens ${ }^{2}$, M Slim $^{2}$, J Sanabria $^{2}$, M Robles Diaz ${ }^{2}$, A Gonzalez Jimenez ${ }^{2}$, A Ortega Alonso', M Garcia Cortes 2 , B Garcia ${ }^{2}$, R Sanjuan Jimenez ${ }^{2}$, MI Lucena ${ }^{2}$, RJ Andrade ${ }^{2}$

${ }^{1}$ Instituto de Investigación Biomédica de Málaga (IBIMA). Hospital Universitario Virgen de la Victoria. Universidad de Malaga, CIBERehd, Malaga, Spain., Spain; ${ }^{2}$ Instituto de Investigación Biomédica de Málaga (IBIMA). Hospital Universitario Virgen de la Victoria. Universidad de Malaga, CIBERehd, Malaga, Spain, Spain 
Background: Idiosyncratic drug-induced liver injury (DILI) is a rare but potentially serious adverse drug reaction. The ability to enhance the understanding of DILI requires collaborative efforts to obtain sufficient case information that enables well powered studies to be performed. This has led to the development of DILI registries worldwide. The Spanish DILI Registry is a pioneer in this area and has prospectively collected DILI cases since its initiation in 1994. The aim of this study was to assess demographics, clinical characteristics, outcomes and drugs more frequently implicated in hepatotoxicity in Spain.

Method: 843 DILI cases induced by a single or multiple drugs and included in the Spanish DILI Registry from 1994 to 2015 were analysed. Demographics, clinical presentation and outcome was compared between DILI cases stratified by type of liver injury (hepatocellular: HEP, cholestatic: Chol and mixed: Mix).

Result: The study cohort had a similar sex distribution, $48 \%$ females. Age of the entire cohort ranged from 11-90 years with a median of 57 years and a body mass index mean of $25.9 \pm 3.8 \mathrm{~kg} / \mathrm{m}^{2}$. Regarding liver damage type, $57 \%$ presented with Hep, $21 \%$ Chol and $22 \%$ Mix. Age differed significantly between the three groups with Chol and Mix including older patients than Hep (64 vs 61 vs 52 years, $p=<0.001$ ). Time to onset from drug initiation to start of liver reaction varied significantly with Hep cases having a median time of 31 days, Chol 22 days and Mix 21 days, $p<0.001$. Jaundice was a common symptom in all groups ranging from $67 \%$ in Hep to $77 \%$ in Chol. The proportion of severe + fatal/liver transplant cases was highest in Hep cases $(12.8 \%)$ compared to Chol $(7.0 \%)$ and Mix (5.6\%). However, more Chol patients required hospitalization (69\%) than Mix (59\%) and Hep (57\%). The use of 1-2 concomitant drugs was the most common scenario in all three groups, but polypharmacy ( $>4$ drugs) was most frequent among Chol patients (19\%) compared to Mix (14\%) and Hep (10\%). The most commonly implicated causative agents belonged to the therapeutic classes antiinfectives (37\%), central nervous system (14\%), cardiovascular (11\%) and antiinflammatory drugs (9\%). Herbal and dietary supplements contributed to $6 \%$ of the DILI cases. Amoxicillin-clavulanate remains the single agent responsible for the highest number of DILI cases $(21 \%)$. These epidemiological findings are similar to those obtained from the 446 idiosyncratic DILI cases in the first 10 years of the Spanish DILI registry. However, a substantial increase in anabolic androgenic steroid hepatotoxicity was observed in the latter half of the registry's operating time, which has led to regulatory measures.

Conclusion: The Spanish DILI Registry has proved to be valuable for hepatotoxicity in-depth phenotyping, providing consistent figures in clinical characteristics, outcomes and implicated drugs. It also constitutes an important tool for public health promotion in postmarketing drug surveillance.

Funding: AEMPS, Fondos FEDER PI15/01440. CIBERehd-ISCIII

DILI-6

\section{Hepatotoxicity due to Helis weed}

\section{Abdurrahman Sahin ${ }^{1}$, Salih Kilic ${ }^{1}$, Nurettin Tunc ${ }^{1}$, Ulvi Demirel ${ }^{1}$,} Orhan Kursat Poyrazoglu ${ }^{1}$, Mehmet Yalniz ${ }^{1}$

${ }^{1}$ Firat University, Turkey

Background: Hepatotoxicity is liver damage; that is caused by drugs, foods and chemical substances. The degree of liver damage may vary from nonspecific liver enzyme elevation to acute hepatitis, extended cholestasis and chronic hepatitis development due to fulminant hepatic failure. Thousands of years have been benefited from plants that are believed to have preventive effects in order to remain healthy for both therapeutic purposes and staying healthy. However, side effects and toxicities of plants are not fully known.

Method: We presented here 9 cases manifesting with hepatotoxicity after the consumption of helis weed.

Result: Case Series

A total of 9 patients were followed in our clinic due to hepatotoxicity after consumption of helis weed. Patients were from 3 different families. All of the patients had complaints of headache, fatigue, weakness, nausea and vomiting, which started about 24 hours after the consumption of helis. When detailed inquiries were made, it was learned that some people cooked helis weed in oil, consumed some as raw and others consumed as pickles.

In laboratory examinations; there was a rise in AST and ALT values ??in all patients and a slight increase in INR value in 1 patient. None of the ultrasonographic examinations revealed pathologic findings. Patients were followed up with symptomatic treatment in addition to liver-protective diet and IV hydration. It was observed that liver function tests were normalized in all patients. No complication developed during admission in patients.

Demographic findings and laboratory data of the patients are summarized in Table.

Conclusion: In the literature, helis weed is also called Ferula orientalis and ferula rigidula. This plant grows especially in Eastern Anatolia and Southeastern Anatolia; is often used by locals in making herbal cheese. It is consumed in different forms such as pickling, frying and pickling on cheese for daily use. The fact that our patients enter the toxic hepatitis using different forms of helis weed shows that the route of use does not affect the shape of hepatic damage. Hepatocellular toxicity that is caused by plants can be observed in 3 forms as; hepatocellular, cholestatic and mixed type. As a result; Helis weed must be keep in mind as an agent in cases of hepatotoxicity, especially in the eastern and southeastern Anatolia.

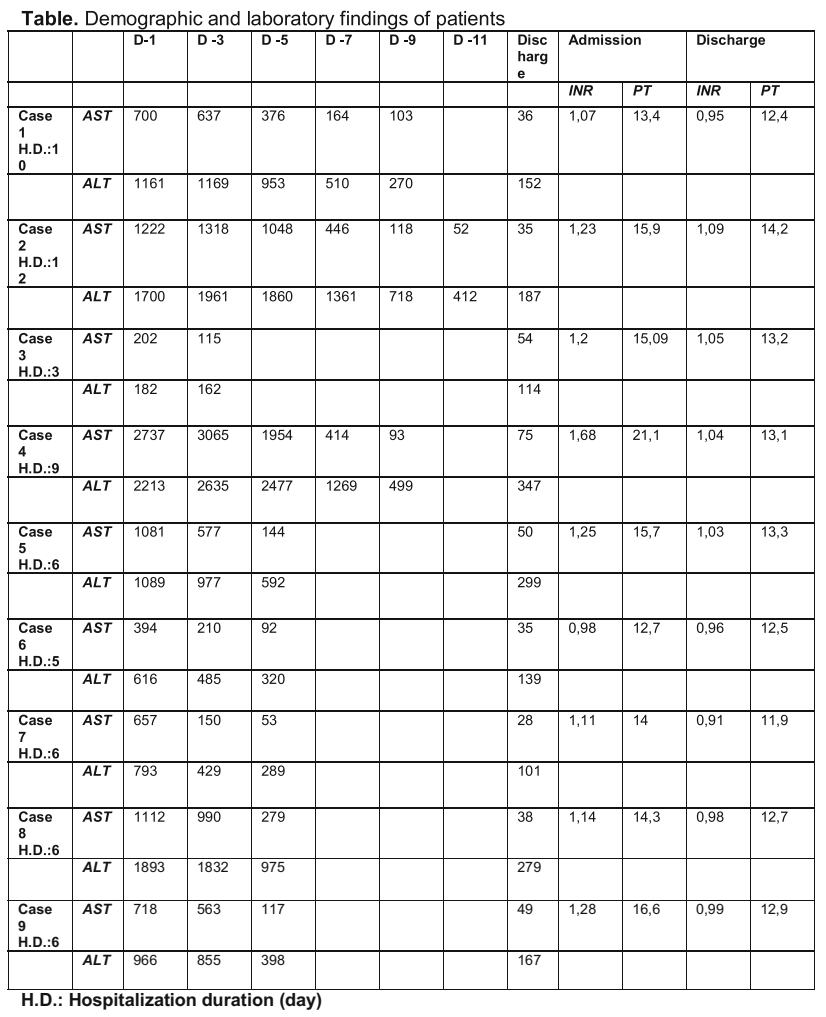


DILI-8

Pyrrolizidine alkaloid-induced hepatic sinusoidal obstruction syndrome: gadoxetic acid-enhanced MR imaging with clinicalimaging correlation

\section{$\underline{\text { Yuhu Song }}{ }^{1}$, Xiaoqian Yang', Tingting Guo ${ }^{1},{\mathrm{Xin} \mathrm{Li}^{1}}^{1}$}

${ }^{1}$ Union Hospital, Tongji Medical College, Huazhong University of Science and Technology, China

Background: To retrospectively evaluate the imaging features of pyrrolizidine alkaloids (PAs)-induced hepatic sinusoidal obstruction syndrome (HSOS) on gadoxetic acid-enhanced MRI, susceptibilityweighted imaging (SWI) and $\mathrm{T} 2 *$ weighted imaging; and then to determine the correlation between image signs and a panel of liver function.

Method: The retrospective study was approved by the institutional review board, and informed consent was waived. 28 PAs-induced HSOS patients were enrolled from Feb, 2013, to Apr, 2017. Medical records of the patients were collected, and MR images were reviewed independently and in consensus by two radiologists.

Result: Abnormal liver function was observed in most of PAs-induced HSOS. Heterogeneity of liver parenchyma in hepatobiliary phase of gadoxetic acid-enhanced MR scans occurred in $100 \%$ of the PAs-induced HSOS patients. Common features of heterogeneous hypointensity were multifocal distribution (mild) in 4 patients (14.29\%), multifocal distribution (severe) in 15 cases $(53.57 \%)$, and diffuse distribution in 9 patients (32.14\%). Hypointense in SWI and T2* imaging was observed in PAs-induced HSOS, and the distribution of hypointense in SWI and T2* imaging was similar to that of portal-venous phase of MRI scan. The severity of heterogeneous hypointensity scored by volume fraction in PAs-induced HSOS was positively correlated with PT and INR.

Conclusion: The patients with PAs-induced HSOS displayed distinct features in gadoxetic acid-enhanced, susceptibility-weighted and $\mathrm{T} 2 *$ weighted imaging; thus, it will provide evidences for the diagnosis of PA-induced HSOS.
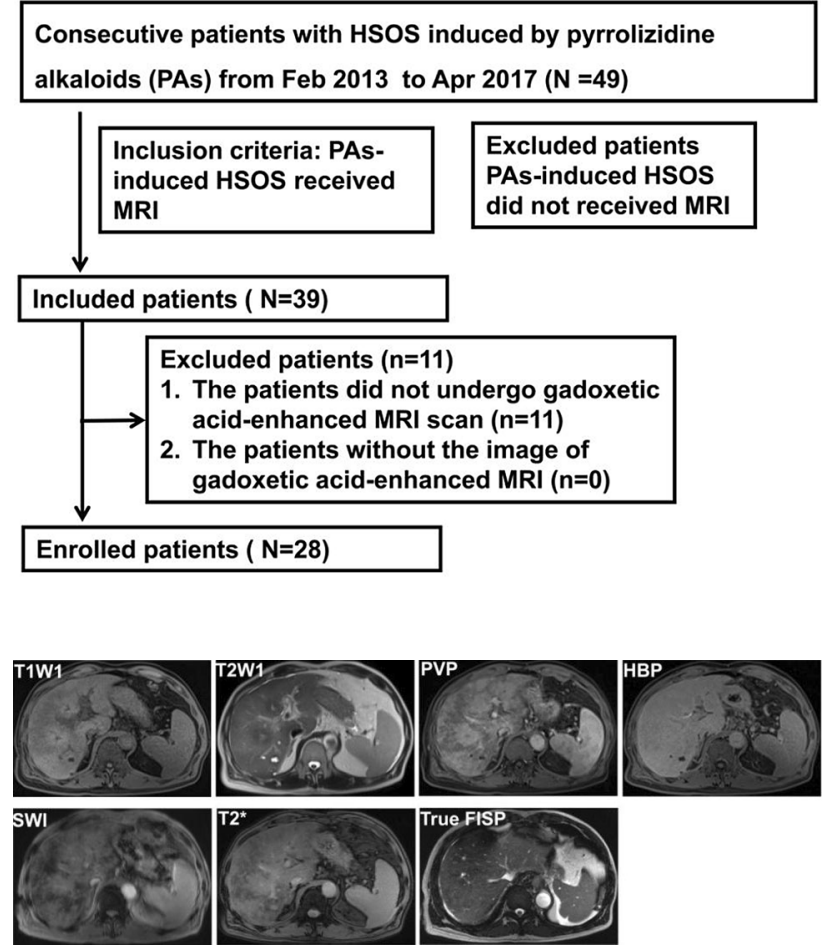

\section{DILI-9}

Halothane Hepatitis is not a thing of past - a series of 20 cases

\author{
Swati Singh $^{1}$, Deepak Deepak ${ }^{2}$ \\ ${ }^{1} \mathrm{MD}$ Anaesthesia and Critical Care, PGIMER Chandigarh, India; \\ ${ }^{2}$ patna medical college, India
}

Background: Halothane was introduced as an anesthetic in the 1950s and was considered a revolutionary agent in the field of anesthesia. Soon after, halothane-induced hepatitis became a concern, leading to the development of less toxic gases that induced a lower incidence of side effects. Two types of halothane-related hepatotoxicity have been described: type 1, or mild hepatitis, is associated with elevated transaminase levels and self-limiting symptoms, and type 2, or severe hepatotoxicity, is associated with acute fatal liver failure and is fatal in most cases. Hepatotoxicity is most likely to be immune-related, based on much evidence.

Method: In our study, we analyze 20 cases of halothane hepatitis notified in the institutional pharmacovigilance between 2012-2016. It concerns 18 women and 2 men, aged between 35 years -65 years.

Result: The injury was cholestatic in the 12 cases and in 8 cases cytolytic. Three cases turned out to be fulmitant resulting in death of the patient. Free radicals that are produced by the metabolism of halothane in the liver can modify cellular proteins and introduce neoantigens to the immune system. Sensitization to these neo-antigens induces a more severe response after multiple exposures; most cases of type 2 hepatitis occur after repeated contact.

Conclusion: New halogenated anesthetics such as enflurane, sevoflurane, and desflurane, are not metabolized in the liver, causing few cases of sensitization. Compared with halothane, these anesthetics are expensive. As a result, replacement of halothane with new halogenated anesthetics requires a precise cost-benefit analysis, especially in developing countries.

\section{DILI-10}

Silymarin for prevention of anti-Tuberculosis drug-induced liver injury: a meta-analysis

Geraldine Claire Floro $^{1}$, Mark Anthony De Lusong1,

${ }^{1}$ University of the Philippines - Philippine General Hospital, Philippines

Background: Drug induced liver injury is considered as one of the most common adverse drug reactions among patients taking antituberculosis drugs. Several hepatoprotectants have been evaluated in preventing anti-tuberculosis drug-induced liver injury (AT-DILI). Most studies are small and limited no drug is proven to significantly prevent AT-DILI. Silymarin, a traditional herbal drug extracted from Silybum marianum, has been used as a hepatoprotectant has been shown to prevent AT-DILI in several animal studies. Recent treatment trials in humans, have conflicting results.

Method: The objective of this study is to evaluate the hepatoprotective effect of Silymarin in preventing drug-induced liver injury in adult patients being treated with anti-tuberculosis drugs.

A comprehensive search of Medline (via Pubmed), the Cochrane Library, Science Direct, Biomed Central and EMBASE was performed for English-language literature about Silymarin and AT-DILI from inception to November 2016. The papers were filtered using predefined inclusion and exclusion criteria. 
Included studies were randomized controlled trials comparing Silymarin to placebo in preventing the development of AT-DILI in adults diagnosed with Tuberculosis who are to receive the standard anti-TB regimen.

Result: Three papers were identified and included in this metaanalysis. The sample comprised a pooled total of 494 patients, of which 244 and 250 patients took Silymarin and placebo, respectively. The difference between Silymarin and placebo groups in incidence of AT-DILI in adult patients with tuberculosis was not statistically significant $(\mathrm{RR}=1.04 ; 95 \% \mathrm{CI}=0.34-3.23 ; \mathrm{p}=0.03)$. Results show significant heterogeneity, which were attributed to differences in ATDILI definitions, frequency and duration of laboratory monitoring, duration of hepatoprotectant treatment, and small sample sizes. The difference in incidence of other adverse events was also not significant between Silymarin and placebo groups $(\mathrm{RR}=1.07$; $95 \% \mathrm{CI}=0.84-$ 1.37; $\mathrm{P}=0.02$ ).

Conclusion: While Silymarin has an acceptable safety profile, available evidence from limited studies suggest that it exhibits no significant hepatoprotective effect against anti-Tuberculosis drug-related liver injury.
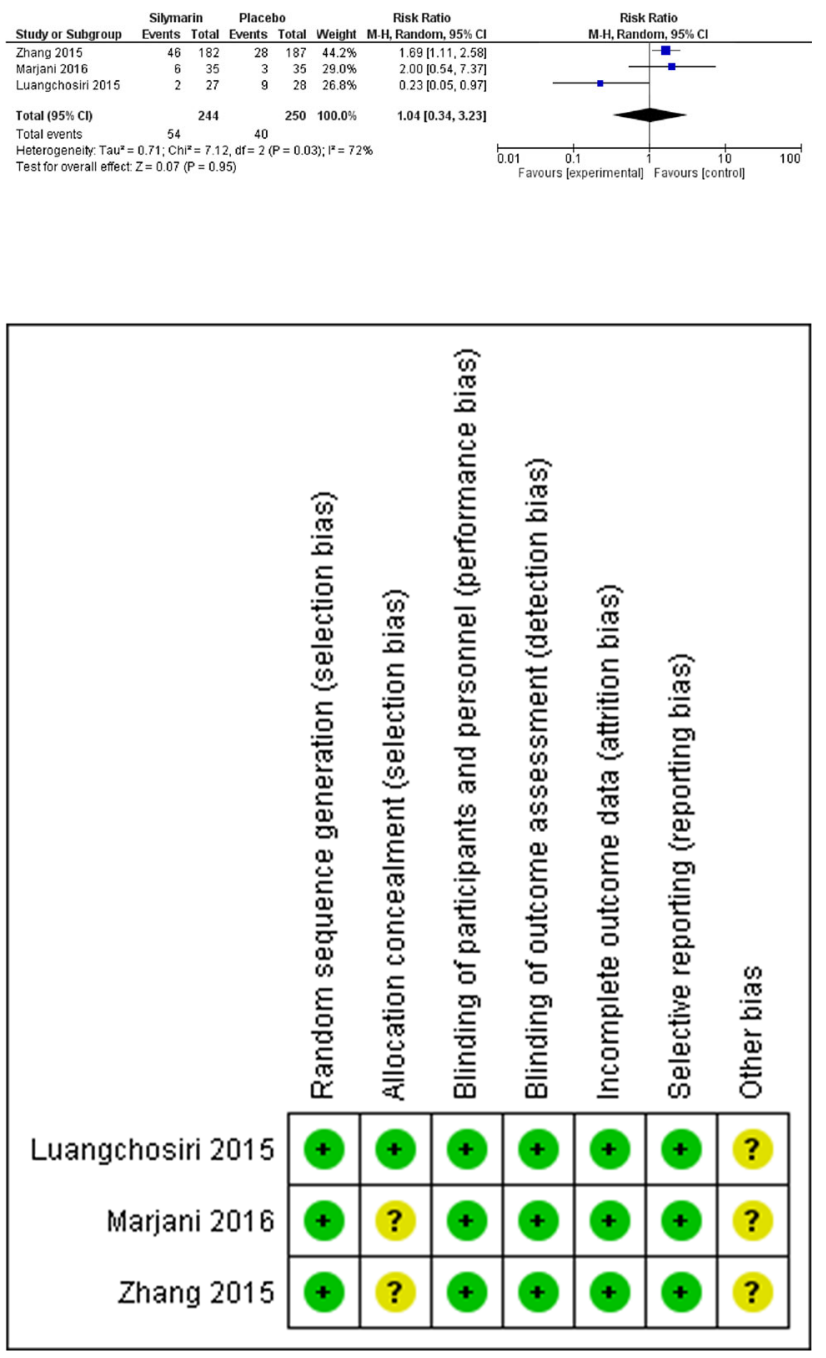

$F I B-1$

Salvianolic acid B inhibits autophagy and activation of hepatic stellate cells through downregulating MAPK signaling pathway

\section{Na Jiang ${ }^{1}$, Jian Ping ${ }^{1}$, Ming Lie $\mathrm{Xu}^{1}$}

${ }^{1}$ Shuguang Hospital Affiliated to Shanghai University of Traditional Chinese Medicine, China

Background: To investigate Salvianolic acid B (SA-B),extracted from a Chinese herb medicine, inhibits autophagy and activation of hepatic stellate cells (HSC) through downregulating MAPK signaling pathway. To investigate Salvianolic acid B (SA-B), extracted from a Chinese herb medicine, inhibits autophagy and activation of hepatic stellate cells (HSC) through downregulating MAPK signaling pathway.

Method: 1. JS-1 or LX-2 (mouse or human HSC cell lines) and primary mouse HSC were incubated with CQ or 3-MA, both are inhibitors of autophagy, for $2 \mathrm{~h}$ and then treated with TGF- $\beta 1$ for $12 \mathrm{~h}$. Rapamycin, an autophagy agonist, was added at last $2 \mathrm{~h}$ before harvest of cells. If using inhibitors of MAPK signaling pathway, the cells were pre-incubated with PD98059, SB203580 or SP600125 for $1 \mathrm{~h}$, respectively. Autophagy flow, and the markers of autophagy and active HSC were detected. 2. JS-1 was incubated with 10-5M SA-B for $24 \mathrm{~h}$, and TGF- $\beta 1$ was added at last $12 \mathrm{~h}$ and rapamycin was added at last $2 \mathrm{~h}$. The inhibitors of MAPK signaling pathway pre-incubated cells for $1 \mathrm{~h}$, respectively. The markers of autophagy and active HSC were detected. 3. Autophagy associated gene ATG5 overexpressed in JS-1 cell lines by lentivirus transfection. The cells were treated with SA-B followed by TGF- $\beta 1$ and the markers of autophagy were detected.

Result: 1. TGF- $\beta 1$ could promote the expression of a-SMA and collagen 1 (Col.1) in HSCs with increasing level of autophagy. CQ or 3-MA could not only significantly depress the autophagy flow and promote the expression of p62 $(P<0.05)$ but also significantly inhibit expression of Col.1 and enhance expression of c-caspase 3 in JS-1 induced by TGF- $\beta 1(P<0.05)$. The effect of rapamycin, however, was on the contrary. The protein lever of p-ERK, p-JNK and p-p38 was significantly increased in JS- 1 induced by TGF- $\beta 1(P<0.05)$. Inhibition of ERK and JNK pathway could decrease the expression of LC3B but inhibitor of p38 could significantly increase it $(P<0.05) .2$. SA-B could significantly decrease expression of autophagy related protein LC3B, ATG5 and ATG7 and both mRNA and protein expression of $\alpha$-SMA and Col.1 in HSC induced by TGF$\beta 1(P<0.05)$.Companied by decreased expression of LC3B, the expression of p-ERK, p-p38, $\alpha$-SMA and Col.1 also significantly reduced in TGF- $\beta 1$-indused LX-2 treated with SA-B $(P<0.05)$. 3.The expression of Atg5, LC3B, $\alpha$-SMA and Col.1 was increase in ATG5 overexpression stable cell lines $(P<0.05)$. But SA-B could reduce these increase expression $(P<0.05)$.

Conclusion: TGF- $\beta 1$ could induce autophagy and activation of HSC via up-regulating MAPK signaling pathway. SA-B could inhibit autophagy and activation of HSC induced by TGF-b1 or overexpression of ATG5 through downregulating MAPK pathway.

\section{$F I B-2$}

The acetylated regulation in IL-13 (TGF-Ã $\ddot{Y}$ )/microRNA-21/ Smad7 signal pathway during liver fibrosis

$\underline{\text { Zuojiong Gong }}{ }^{1}$, Yao Wang ${ }^{1}$, Fangzhou Jiao', Wenbin Zhang ${ }^{1}$, Qian Chen $^{1}$

${ }^{1}$ Renmin Hospital of Wuhan University, China 
Background: Interleukin 13 (IL-13) (transforming growth factor (TGF)- $\beta$ ) / microRNA (miR) -21/smad 7 signal pathway is involved in the activation of hepatic stellate cells (HSCs) and histone deacetylases (HDACs) have been widely played important role during liver fibrosis. This study investigated the acetylated regulation in IL13 (TGF- $\beta$ )/miR-21/smad 7 signal pathway caused by different pathogeny and whether miR-21 could regulate the acetylated process. Method: In vivo experiment, the serum was got for elisa to detect IL13 and TGF- $\beta$. Pathological examination and immunohistochemistry (IHC) were used to analysis the histopathological changes and the expression of connective tissue growth factor (CTGF) in liver. In vitro experiments, the transfection was based on the ability of the GV273miR-21-enhanced green fluorescent protein (EGFP) and GV369miR-21-EGFP lentiviral system to down- or upregulate the miR-21 gene in LX2 cells. Real-time polymerase chain reaction (RT-PCR) was employed to detect the mRNA levels of miR-21, smad7 and CTGF, and western blotting was used to examine the protein levels of CTGF, HDAC2, HDAC6, HDAC8, Histone 3 and Acetyl-Histone 3 (Ac-Histone 3).

Result: Pathological examination showed the liver fibrosis pathological damage in two model groups. The serum IL-13 and TGF- $\beta$ levels, the mRNA levels of miR-21 and CTGF and protein expression levels of CTGF, $\alpha$-SMA, HDAC2, HDAC6 and HDAC8 were increased in the model groups compared with the normal groups $(\mathrm{P}<0.01)$, whereas the Smad7 mRNA level and Ac-Histone 3 protein level was significantly decreased $(\mathrm{P}<0.01)$. After miR-21 was respectively down- or up-regulated by the lentiviral system in LX2, the miR-21/samd7 signal pathway and HDACs (2 and 6) molecule were activated and inhabited. But the HDAC8 and Ac-Histone 3 presented the opposite change with miR-21. IL-13 and TGF- $\beta$ can both furtherly active the miR-21/samd7 signal pathway and HDACs (2, 6 and 8 ) molecule in the transfected LX2 cells, but the Ac-Histone 3 protein level decreased $(\mathrm{P}<0.01)$.

Conclusion: The acetylated regulation played an important role in IL13 (TGF- $\beta$ )/microRNA-21/Smad7 signal pathway during the schistosomiasis and $\mathrm{CCl} 4$ induced liver fibrosis, during which process miR-21 may regulated the HDACs molecules.

\section{FIB-3}

Evaluation of non-invasive bio-markers, AST to platelet ratio index (APRI) and fibrosis-4 (FIB-4) in the assessment of liver fibrosis 1 Ruchit B Patel MD., 2 T.J. Vadivukkarasi MD., DM., 3 P Ganesh MD., DM., 1. Senior resident 2. Asst. Professor 3. Professor and HOD Department of MGE, SRMC, Chennai

\section{Ruchit Patel $^{1}$, P Ganesh ${ }^{3}$, T J Vadivukkarasi ${ }^{2}$}

${ }^{1}$ Senior Resident,, Department of MGE, Sri Ramachandra Medical College and Research Centre, Chennai, India; ${ }^{2}$ Asst. Professor, Department of MGE, Sri Ramachandra Medical College and Research Centre, Chennai, India; ${ }^{3}$ Professor and HOD, Department of MGE, Sri Ramachandra Medical College and Research Centre, Chennai, India

Background: AIMS AND OBJECTIVES OF THE STUDY: To evaluate liver fibrosis using non-invasive bio-markers APRI and FIB4 and to validate them as primary tool against liver elastography for diagnosing liver fibrosis

Method: From November 2015 to November 2017, patients presenting to our liver clinic with chronic liver disease (compensated cirrhosis and chronic hepatitis) of any etiology-alcohol, viral, autoimmune and cryptogenic were included in the study with informed consent. Patients of age $\leq 18$ years and $>75$ years, pregnant women, those with acute hepatitis, acute on chronic liver failure, decompensated chronic liver disease, hepatic space occupying lesions were excluded from the study group. Appropriate baseline lab and radiological parameters were done. AST, ALT was calculated using International Federation of Clinical Chemistry without pyridoxal phosphate (P-5-P) Kinetics. Platelets count were calculated by Coulter electronic counter and by manual count. APRI was calculated using the formula -(AST/upper limit of normal)/Platelet count (109/L) $\mathrm{x} 100$. FIB-4 was calculated using the formula- [Age (years) $\times$ AST (IU/L)]/ [Platelet count $(109 / \mathrm{L}) \times \sqrt{ }$ ALT (IU/L)].?Liver elastography was done using Toshiba APLIO 500 ultrasound and ARFI was also generated. The values of non-invasive indices were compared with the results of liver elastography in order to detect advanced fibrosis (F3 and F4).

Result: Among 203 patients studied, males: $72 \%$, age in years: 46.72 \pm 13.56 , mean $\pm \mathrm{SD}$. Viral etiology noted in $48.3 \%$, alcohol in $5.9 \%$, other etiology noted in $45.8 \%$. With APRI cut-off of 1 the specificity, sensitivity and positive predictive value (PPV) to detect advanced fibrosis was $82 \%, 38 \%$ and $81 \%$ respectively. Using Fib-4 cut off of $>3.25$ the specificity, sensitivity and positive predictive value to detect advanced fibrosis was $88 \%, 24 \%$ and $80 \%$ respectively.

Conclusion: APRI and FIB-4 had specificity, sensitivity and PPV of $82 \%, 38 \%, 81 \%$ and $88 \%, 24 \%, 80 \%$ to diagnose advanced fibrosis in our study

Key words: AST to Platelet Ratio Index, APRI, Fibrosis-4, FIB-4, Liver Elastography, Fibrosis

\section{$F I B-4$}

Evaluation of serum nesfatin level in patients with compensated and decompensated liver cirrhosis

\section{Ahmet Uyanikoglu', Ferzan Aydin', Ahmet Uyanikoglu', Huseyin Taskiran ${ }^{2}$, Fehmi Kas ${ }^{2}$, Necati Yenice ${ }^{2}$ \\ ${ }^{1}$ Harran University, Turkey; ${ }^{2}$ Harran University, Medical Faculty, Turkey}

Background: Nesfatin has been shown to regulates the eating behavior and reduce body weight. Nesfatin is located brain tissue and located also adipose tissue, stomach, pancreatic islets, liver and testis. But their impact is unclear here. This study aims to measure the serum nesfatin level in patients with compensated and decompensated liver cirrhosis, compare the results with healthy individuals, the role of anorexia and cachexia seen in cirrhosis patients and investigate their usability as a marker in the diagnosis and follow-up of patients with cirrhosis.

Method: Gastroenterology clinic inpatient or outpatient follow-up and patients diagnosed with liver cirrhosis based on liver biopsy and/ or biochemical and ultrasonographic findings obtained clinically in laboratory settings were included in the study. Patients were divided into 2 groups as decompensated and compensated according to their clinical stages. The patients who developed esophageal variceal bleeding, acid, hepatic encephalopathy were included in the decompensated cirrhosis group. Detailed medical history, physical examination, laboratory and radiological assessments of the patients included in the study was obtained. One tube of blood was taken from each individuals in the patient and control groups and kept at -85 degrees Celsius. Markers were studied using the Elisa method.

Result: A total of 97 patients with cirrhosis and 51 healthy control patients were included in the study. Of the patients, 43 were compensated $(44.3 \%)$, and 54 were decompensated $(55.7 \%)$. The average age of all patients with cirrhosis was $53.98 \pm 15.67$ (19-89), the average age in the compensated cirrhosis group was $50.74 \pm 15.94$ (19-85), it was $56.56 \pm 15.10(19-89)$ in the decompensated cirrhosis group, and the average age in the control group was $44.75 \pm 11.46$ (18- 
72) yaers. Compensated cirrhosis 29 patients (67.4\%), decompensated cirrhosis 28 patients $(51.8 \%)$ in the control group of 18 patients $(35.2 \%)$ were male. The level of nesfatin was found to be statistically significantly higher in all patients and patients with decompensated cirrhosis $(\mathrm{p}<0.001)$. There was no statistically significant difference between the control group of patients with compensated cirrhosis ( $\mathrm{p}>0.05)$.

Conclusion: The higher level of nesfatin found in all patients with cirrhosis and patients with decompensated cirrhosis suggests that it may have a role in anorexia and cachexia seen in the decompensated cirrhosis period in particular, and can be used as a marker.

\section{$F I B-5$}

Evaluation of serum procollagen C-proteinase enhancer 1 (PCPE1) level as a fibrosis marker in patients with chronic hepatitis $B$

Onur Gokce ${ }^{1}$, Seren Ozenirler ${ }^{1}$, Aysegul Atak Yucel ${ }^{1}$, Nihan Oruklu', Guldal Esendagli Yilmaz', Sevilay Karahan ${ }^{2}$

${ }^{1}$ Gazi University Faculty of Medicine, Turkey; ${ }^{2}$ Hacettepe University Faculty of Medicine, Turkey

Background: Liver biopsy has been considered the gold standard for diagnosing chronic liver diseases and staging fibrosis. Because of the risks of liver biopsy, several noninvasive methods have been introduced for the assessment of liver fibrosis: serum biomarkers and imaging techniques. In this study, we aimed to assess the diagnostic value of serum procollagen C- proteinase enhancer 1 as a non-invasive fibrosis marker in the treatment-naive chronic hepatitis $\mathrm{B}$ patients.

Method: This study included 126 biopsy proven patients with a diagnosis of $\mathrm{CHB}$ virus infection and 50 healthy controls. Fibrosis stage and necroinflammatory activity were assessed in liver biopsy specimens. Fibrosis stage was determined using Ishak's scoring system. Procollagen C-proteinase enhancer 1 level was measured by ELISA assay; and aspartate aminotransferase to platelet ratio index (APRI) and FIB-4 indexes were calculated using the formulas taken from previous publications.

Result: The fibrosis stages identified upon liver biopsy was F0 in 40 patients $(31.7 \%), \mathrm{F} 1-2$ in $64(50.8 \%)$ and F3-6 in 22 (17.5\%). Serum PCPE-1 levels of chronic hepatitis B patients were found to be significantly lower than those of healthy controls $(4.49 \pm 2.74 \mathrm{pg} / \mathrm{ml} \mathrm{vs}$ $42.9 \pm 59.6 \mathrm{pg} / \mathrm{ml}$, respectively, $\mathrm{P}<0.001)$. There was statistically significant negative correlation between serum PCPE-1 level and fibrosis stage $(P=0.011 ; r=-0.226)$. Besides, a statistically significant negative correlation was found between serum PCPE-1 level and necroinflammatory activity $(\mathrm{P}=.030 ; \mathrm{r}=-.194)$. The AUROC of PCPE-1 levels was 0.955 , to differentiate patients with liver fibrosis score of F1-2 from healthy controls was significantly higher $(\mathrm{P}<0.001)$. The AUROC of PCPE-1 levels was 0.615 for predicting fibrosis (F0 vs F1-6). The diagnostic performance of PCPE-1 level in distinguishing moderate and severe fibrosis (Ishak score, $\geq \mathrm{F} 3$ ) was 0,642 ( $\mathrm{P}=0.037$, statistically significant).

Conclusion: In CHB patients, serum PCPE-1 might be used as a noninvasive marker of liver fibrosis. Further animal and human studies are needed to assess the usefulness of this marker.
Table Diagnostic performance of non-invasive indexes in distinguishing different stages of fibrosis (AUROC)

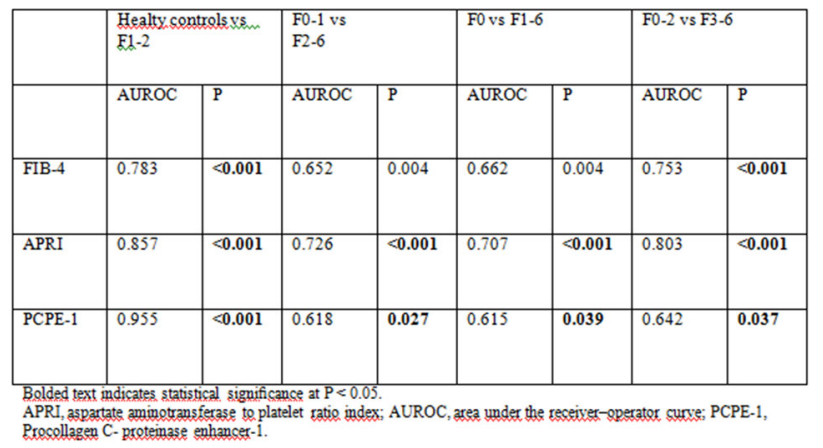

\section{FIB-6}

Increased collagen 1 and fibronectin during liver injury promotes the activation of hepatic progenitor cells

\section{Hamda Siddiqui $^{1}$, Preety Rawal ${ }^{2}$, Mojahidul Islam ${ }^{1}$, Chaggan Bihari $^{1}$, Savneet Kaur ${ }^{1}$ \\ ${ }^{1}$ ILBS, India; ${ }^{2}$ gautam Buddha University, India}

Background: Liver regeneration after partial hepatectomy involves the proliferation of hepatocytes. However, during liver injury, bipotential hepatic progenitor cells (HPC) or oval cells (in rodents) are also known to participate in liver repair and regeneration. Since liver extracellular matrix (ECM) largely regulates HPC behavior, in the current study, we investigated the role of ECM factors in the activation and proliferation of oval cells in a choline-deficient, ethioninesupplemented (CDE) dietary mice model.

Method: Four-week-old mice were fed either normal chow (control) or a CDE diet (at different time points (10 day, 20 day, 10 day withdrawal). Liver biochemical parameters, histology, oval cell markers [evaluated by CK19 and EPCAM immunohistochemistry (IHC)] and expression of important ECM genes were determined in different animal groups. The effect of ECM factors on oval cell proliferation was investigated by MTT assay and flow cytometry analysis in the in vitro studies.

Result: IHC results showed significant expression of oval cells in the periportal regions in mice after 10 and 20 days of diet-induced injury. The oval cell response decreased by about $40-50 \%$ in mice in which diet was withdrawn after 10 days as compared to the mice which were given the diet for 20 days. The ECM gene expression results illustrated maximum expression of collagen 1a and fibronectin on day 20. Further, in vitro studies showed an increase of more than $50 \%$ in the proliferation of hepatic cells and an increase of about $20 \%$ in the expression of hepatic progenitor cell markers on collagen 1 and fibronectin-coated wells as compared to untreated wells.

Conclusion: The study shows that an increase in collagen 1 and fibronectin during liver injury promote the proliferation of HPCs, suggesting a crucial role of ECM modulation in HPC-mediated liver repair. 


\section{FIB-7}

Insulin like growth factor 1 as a marker of severity of liver cirrhosis

\section{Ehab Ahmed Abdelatti ${ }^{1}$, Waleed Mohamed Fathy ${ }^{2}$}

${ }^{1}$ Professor of Hepatology, Egypt; ${ }^{2}$ Professor of Clinical Pathology, Egypt

Background: Insulin-like growth factor 1 (IGF-1) is a soluble protein mainly produced by the liver secondary to the growth hormone stimulation on hepatocytes. IGF-1 plays an essential role in growth, development, and overall cellular regulation in the human body in health as well as in the pathophysiology of various diseases. The aim of the work is to study the relationship between insulin like growth factor 1 and the severity of liver cirrhosis.

Method: The study included 69 patients with liver cirrhosis of various etiologies from Menoufia University Hospitals (Egypt) and 18 healthy volunteers as a control group. They underwent physical examination and laboratory investigations (CBC, liver profile \{ALT, AST, serum albumin, bilirubin, PT \% \}, urea, creatinine, fasting glucose, postprandial glucose and serum IGF-1). Abdominal ultrasonography was done for all patients. Child-Pugh Score, MELD score and AST/Platelet ratio index (APRI score) were calculated for all patients

Result: IGF-1 of cirrhotic patients $(73.1 \pm 42.3 \mathrm{ng} / \mathrm{ml})$ was significantly lower than controls $(243.2 \pm 78.1 \mathrm{ng} / \mathrm{ml})(\mathrm{P}=0.0001)$. IGF-1 was significantly lower in Child class $C(46.11 \pm 6.66 \mathrm{ng} / \mathrm{ml})$ than Child class B $(64.8 \pm 4.4 \mathrm{ng} / \mathrm{ml})$ and A $(149.1 \pm 43.8 \mathrm{ng} / \mathrm{ml})(\mathrm{P}=0.002$ and 0.0001 respectively). Also IGF-1 was significantly lower in Child class $B$ than class A $(\mathrm{P}=0.0001)$. IGF-1 was significantly lower in patients with MELD score $<20(45.62 \pm 6.72 \mathrm{ng} / \mathrm{ml})$ than MELD score $10-19(61.28 \pm 13.85 \mathrm{ng} / \mathrm{ml})$ and MELD score $>10(152.1 \pm 44.30 \mathrm{ng} /$ $\mathrm{ml})(\mathrm{P}=0.0001)$. IGF-1 was significantly lower in patients with APRI $<1.5(46.62 \pm 7.103 \mathrm{ng} / \mathrm{ml})$ than APRI $1-1.5(68.52 \pm 13.99 \mathrm{ng} / \mathrm{ml})$ and APRI $>0.5(155 \pm 45.24 \mathrm{ng} / \mathrm{ml})(\mathrm{P}=0.0001)$.

In cirrhotic patients group, there were an inverse correlation between IGF-1 and APRI score ( $\mathrm{r}=-0.49)$, MELD score $(\mathrm{r}=-0.78)$, Child score $(\mathrm{r}=-0.77)$ and bilirubin $(\mathrm{r}=-0.54)(\mathrm{P}=0.0001$ for all $)$. There were a strong direct correlation between IGF-1 and albumin ( $\mathrm{r}=0.71), \mathrm{PT} \%$ $(\mathrm{r}=0.79)$ and platelet count $(\mathrm{r}=0.58(\mathrm{P}=0.0001$ for all $)$

Conclusion: IGF-1 can be used as an index for evaluating the severity of cirrhosis as it is correlated with variables of hepatic dysfunctions like serum bilirubin, albumin and PT\% and indices of hepatic dysfunctions and fibrosis like Child-Pugh score, MELD score and APRI score.

$F I B-8$

Metalloproteinase inhibitor 1 closely correlates with the severity of liver disease in Egyptian patients Khaled Metwally, Tamer Refaat, Nashwa Shible, Hassan Zaghla, Eman Abdel Sameea, Mona Aref and Fatma Khalaf Hepatology and gastroenterology Unit and Clinical Biochemistry, National Liver Institute

\section{Khaled Elsayed Amer ${ }^{1}$}

${ }^{1}$ Hepatology, Egypt

Background: Liver disease prevalence is high in Egypt and finding a reliable seromarker is needed for diagnosis and prognosis of fibrosis. Tissue inhibitor of metalloproteinase -1 (TIMP-1) previously showed a good relation with fibrosis but few data is available from Egypt.

Method: Forty six patients ( $>18$ years) were recruited from liver clinics at the National Liver Institute, University of Menoufyia from
February to July 2016, where sixteen patients had liver cirrhosis. Evaluation of the degree of fibrosis and liver dysfunction was done by clinical examinations, laboratory tests, and imaging tests (Ultrasound and Fibroscan) with/ without histological examinations. TIMP-1 was determined in plasma samples using the MAC15 TIMP-1 ELISA

Results: Median age was 51 years ( $42-63$ years) and $67 \%$ were men. The primary cause of liver disease was hepatitis C, $89.1 \%$. TIMP-1 values showed significant relation to the degree of fibrosis or cirrhosis assessed by liver biopsy or fibroscan $(\mathrm{p}<0.001)$. Its level increases progressively with the development of encephalopathy and ascites and with the worsening of Child class $(\mathrm{P}=0.025,0.018$ and 0.039 ; respectively).

Conclusion: TIMP-1 increases significantly with liver cirrhosis and its degree of increase correlates with the degree of liver dysfunction.

Table 1: AST, ALT and APRI values tested against TIMP-1 in different patient groups

\begin{tabular}{|l|l|l|l|}
\hline & AST & ALT & APRI \\
\hline All patients $(\mathrm{N}=46)$ & $\mathrm{P}=0.001$ & $\mathrm{P}=0.002$ & $\mathrm{P}=0.002$ \\
\hline Non- cirrhotic $(\mathrm{N}=30)$ & $\mathrm{P}=0.003$ & $\mathrm{P}=0.005$ & $\mathrm{P}<0.001$ \\
\hline Cirrhotic $(\mathrm{N}=16)$ & 0.013 & 0.025 & 0.019 \\
\hline
\end{tabular}

Table 2: Liver dysfunction in relation to TIMP-1

\begin{tabular}{|c|c|c|c|c|}
\hline & & $\begin{array}{l}\text { Patients } \\
\text { number }\end{array}$ & $\begin{array}{l}\text { TIMP-1 } \\
\text { means }\end{array}$ & p values \\
\hline \multirow{3}{*}{$\begin{array}{l}\text { Child - Pugh } \\
\text { score }\end{array}$} & A & 1 & 150 & \multirow{3}{*}{$\mathrm{P}=0.025$} \\
\hline & B & 8 & $229+/-55$ & \\
\hline & $\mathrm{C}$ & 7 & $368+/-120$ & \\
\hline \multirow{2}{*}{$\begin{array}{l}\text { Hepatic } \\
\text { encephalopathy }\end{array}$} & Absent & 9 & $217+/-53$ & \multirow[t]{2}{*}{$P=0.018$} \\
\hline & Present & 7 & $335+/-117$ & \\
\hline \multirow[t]{4}{*}{ Ascites } & Absent & 6 & $219+/-47$ & \multirow{3}{*}{$P=0.039$} \\
\hline & Mild & 7 & $247+/-83$ & \\
\hline & Moderate & 1 & 398 & \\
\hline & Tense & 2 & $400+/-140$ & \\
\hline
\end{tabular}

FIB-9

Yiqi Huoxue recipe protects against carbon tetrachloride-induced liver fibrosis by inhibiting endoplasmic reticulum stress mediated inflammation and apoptosis

Nan Yuemin ${ }^{1}$, Xuemin $\mathrm{Niu}^{2}$, Baoyu Wang ${ }^{2}, \mathrm{Na} \mathrm{Fu}^{2}$, Yang Wang ${ }^{2}$, Yu Lu', Suxian $\mathrm{Zhao}^{2}$, Jinghua $\mathrm{Du}^{2}$, Yuemin $\mathrm{Nan}^{2}$ 
${ }^{1}$ Third Hospital of Hebei Medical University, China; ${ }^{2}$ The Third Hospital of Hebei Medical University, China

Background: Yiqi Huoxue recipe (YQHX), a compound of Chinese herbal medicine, was used to improve liver fibrosis in our current clinical study. YQHX recipe mainly contain the active components are astragalus, salvia miltiorrhiza, amomum, poria cocos, curcuma aromatic. However, the anti-fibrotic mechanisms of YQHX remain unclear. This study aimed to investigate the protective effect and related molecular mechanism of YQHX against carbon tetrachloride (CCl4-) induced liver fibrosis in rats. Yiqi Huoxue recipe (YQHX), a compound of Chinese herbal medicine, was used to improve liver fibrosis in our current clinical study. YQHX recipe mainly contain the active components are astragalus, salvia miltiorrhiza, amomum, poria cocos, curcuma aromatic. However, the anti-fibrotic mechanisms of YQHX remain unclear. This study aimed to investigate the protective effect and related molecular mechanism of YQHX against CCl4-induced liver fibrosis in rats.

Method: Wistar rats were administrated with a mixture of $\mathrm{CCl} 4$ $(30 \%)$ twice a week for 8 weeks by intraperitoneal injection to induce liver fibrosis, YQHX recipe were administered to the rats, respectively. Fuzheng Huayu (FZHY) as positive control. The effects of YQHX and FZHY were assessed by comparing the hepatic histology, levels of ER stress, inflammatory and fibrogenic related genes, and apoptosis genes. The datas were analyzed by one-way analysis of variance (ANOVA), with LSD test analysis for comparison among groups.

Result: Rat administrated with $\mathrm{CCl} 4$ for 8 weeks showed severe hepatic injury including necro-inflammation and fibrosis, pseudo lobular formation. The results showed that YQHX and FZHY significantly decreased the activities of serum ALT/AST and inhibition the expression of pro-fibrotic genes including a-smooth muscle actin $(\alpha$-SMA), transforming growth factor beta1(TGF- $\beta 1$ ), collagen type I (Col1A1) and Col4 in CCl4-induced liver fibrosis. Pretreatment with YQHX and FZHY could attenuate CCl4-induced the ER stress-associated protein GRP78 and CHOP. The expression levels of inflammation cytokines NF- $\kappa \mathrm{B}$, IKK- $\beta$ and TNF- $\alpha$ were decreased after oral of YQHX and FZHX. Additionally, YQHX and FZHY down-regulated BAX, caspase9 and PARP, up-regulated Bcl-2 by suppressed the IRE1 $\alpha$ activation.

Conclusion: Our study demonstrated the protective of YQHX in ameliorating liver fibrosis. The anti-fibrotic mechanism of YQHX might through alleviate ER stress to regulate NF- $\kappa \mathrm{B} / \mathrm{IKK}$ signaling pathway to inhibit inflammation, and regulated $\mathrm{Bcl}-2 / \mathrm{Bax}$ antiapoptosis signaling pathway to prevent liver cell apoptosis.

\section{$F I B-10$}

The impact of inflammation grade of liver histology on the improvement of liver stiffness assessed by transient elastography

\section{Jeong Ju Yoo' ${ }^{1}$, Jeong Ju Yoo', Sang Gyune Kim, Young Seok Kim ${ }^{1}$, Hae Won Yoo ${ }^{1}$ \\ ${ }^{1}$ Soonchunhyang Bucheon Hospital, Korea, Republic of}

Background: Transient elastography (TE; Fibroscan) is now almost indispensable tool to estimate liver fibrosis. Although many clinical factors are known as confounding factors of liver stiffness (LS), there is no knowledge of who will achieve an improvement of liver stiffness if they have a similar liver fibrosis stage. The aim of this study is to see whether baseline hepatic inflammation may affect accurate LS measurement and which factors are associated with improvement of LS in Fibroscan.
Method: This retrospective study included consecutive 678 patients who underwent baseline liver biopsy and sequential LS assessment from 2006 to 2016 at 6 tertiary hospitals in Korea. Liver fibrosis and inflammation were graded on the basis of standard guideline proposed by the Korean Study Group for the Pathology of Digestive Diseases. LS measurement was performed at baseline and 1, 3, 5 years. Improvement of LS was defined as decreased LS value compared with baseline. Logistic regression was used to evaluate factors associated with improvement of LS in Fibroscan.

Result: Mean age of the patients was $47.12 \pm 12.25$ years and $48.5 \%$ were male. Six hundred 2 patients had viral hepatitis (419 HBV; 183 HCV), 76 non-viral hepatitis. Fibrosis stages $0,1,2,3$ and 4 were identified in $13(1.9 \%), 96(14.2 \%), 132(19.5 \%), 186(27.4 \%)$ and $251(37.0 \%)$ patients, inflammation grade $0,1,2$ and 3 were in 28 (4.1\%), $278(14.0 \%), 279(41.2 \%)$, and 93 (13.7\%), respectively. Baseline inflammation grade was correlated with baseline LSM value, and showed linear correlation with $\triangle \mathrm{LSM}$. In addition, as the grade of inflammation increased, the higher percentage of patients showed improvement of LSM. A multivariate analysis showed that higher degree of hepatic inflammation was an independent good predictor for LS improvement (adjusted hazard ratio, 3.33; 95\% confidence interval, 1.20-9.26; $P=0.021$ ) after adjustment for fibrosis stage, platelet count, total bilirubin and alanine aminotransferase level. The association of LSM and hepatic inflammation was more significant in viral hepatitis compared with non-viral etiology.

Conclusion: Baseline hepatic inflammation has significant impact on LS value and improvement of LSM, and should be considered as one of the confounding factors of measuring liver stiffness using Fibroscan.

FIB-11

Factors influencing detection of liver stiffness by transient elastography

\section{Anoop K Varghese ${ }^{1}$, RAJENDRA Gunjal ${ }^{1}$, Biji Benny ${ }^{1}$, Neehar} Shanavas ${ }^{1}$, Prasanth $\mathbf{S}^{1}$

${ }^{1}$ Government medical college Trivandrum, KUHS, India

Background: Transient elastography (TEE), is an useful, noninvasive, quantitative method for evaluating liver fibrosis by measuring the liver stiffness (LSM). When detecting the liver stiffness by TEE, certain parameters may affect the detection results. This study investigated factors affecting detection of liver fibrosis by TEE, in cases where there is a discordance of more than one fibrosis stage between TEE and liver biopsy.

Method: Cross sectional study of patients undergoing liver biopsy because of high TEE with persistent transaminitis/ normal TEE with persistent transaminitis/ high TEE with normal transaminases. Gender, Age, BMI, CBC, RFT, LFT, Etiology, TEE fibrosis score (within 3 months of liver biopsy), Liver biopsy fibrosis score (Metavir). TEE fibrosis score of study population was compared to liver biopsy fibrosis scores, which is considered as gold standard, and patients with discordance of more than one fibrosis stage was analyzed with above mentioned variables which can affect TEE.

Result: 54 patients, 35 male and 19 female were included in the study. Mean age of population was 42.2 years. 32(59\%) had NASH, $14(25.9 \%)$ had Chronic hepatitis B, 6(11.1\%) had Chronic hepatitis $\mathrm{C}$ and 2 (3.7\%) had Autoimmune hepatitis. 27 patients had TEE score discordant with liver biopsy fibrosis score. 2 patients had TEE fibrosis score less than liver biopsy fibrosis score and rest of the patients had a TEE fibrosis score $>1$ liver biopsy fibrosis score.

Correlation analysis: After applying Spearman's correlation, INR had moderate strength of positive correlation (rs) value of 0.421 and $\mathrm{P}$ 
value of 0.029. A weak positive correlation was found between Age (0.208), Total bilirubin (0.397), Direct bilirubin (0.286), ALP (0.302), RBS (0.247), HDL (0.279) and LSM. A weak negative correlation was found between Albumin (-0.313), Urea (-0.208), Creatinine (0.325), Ceruloplasmin (-0.221) and LSM. There was no independent affecting factor of LSM detection after multiple regression analysis. Conclusion: Age, Bilirubin, ALP, Albumin, RFT, RBS, HDL, Ceruloplasmin, INR, affects the LSM detection by TEE and should be taken into account while interpreting TEE scores. There was no correlation of transaminases with TEE score.
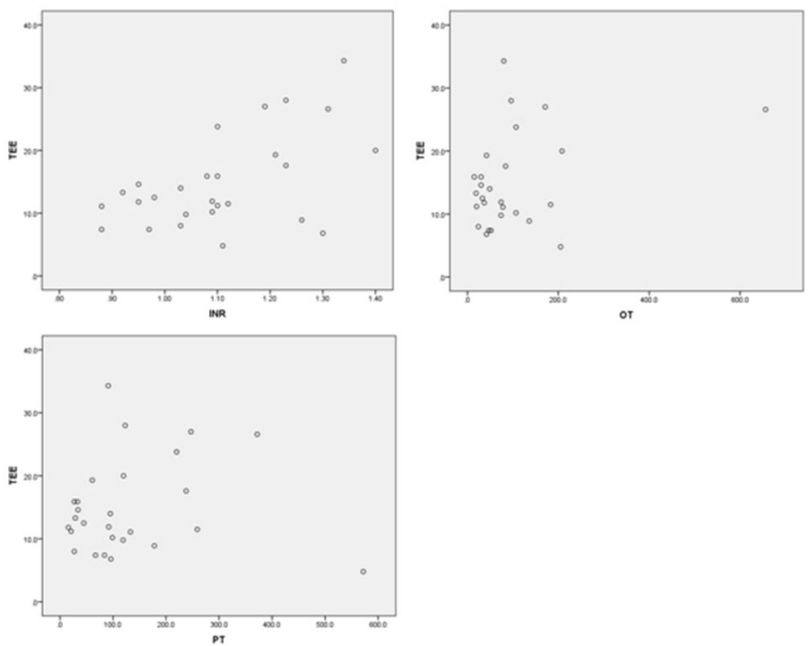

(2D-SWE) which is different from transient elastography has not been sufficiently validated for this purpose. The aim of the study is to evaluate how well non-invasive tools combined with 2D-SWE can predict the presence of esophageal varices and varices needing treatment (VNT).

Method: Between January 2015 and October 2017, 619 patients with compensated advanced chronic liver disease (cACLD) who underwent non-invasive tests (liver stiffness measurement by 2D-SWE, splenic volume by ultrasonography, platelet count) and endoscopy consecutively were enrolled. Among them, 42 patients were excluded for the reasons as follows: 35 for concurrent hepatocellular carcinoma (HCC), 1 for post-transplant state, 6 for extra-hepatic malignancy.

Result: Total 577 patients were eligible for the inclusion of analysis. The mean age was $55.0 \pm 10.7$ years and most common etiology was chronic hepatitis B $(39.7 \%)$. Prevalence of all-size varices and varices needing treatment (VNT) were $22.5 \%$ and $10.7 \%$, respectively. 2DSWE alone has good ability to discriminate varices (AUROCs: 0.868 , $95 \% \mathrm{CI}, 0.849$ to 0.905$)$. Combining platelet count or splenic volume with 2D-SWE has better discriminative ability for varices and AUROCs were 0.879 (95\% CI, 0.838 to 0.896 ) and 0.886 (95\% CI, 0.857 to 0.911 ) respectively. When $2 \mathrm{D}-\mathrm{SWE}$, splenic volume, and platelet count were combined, the patients who carries esophageal varices were best predicted (AUROCs: $0.888,95 \% \mathrm{CI}: 0.859$ to 0.914). In addition, combining $2 \mathrm{D}-\mathrm{SWE}$ and platelet count for predicting the presence of VNT shows the excellent discriminative ability (AUROCs: $0.892,95 \%$ CI, 0.863 to 0.916 )

Conclusion: 2D-SWE combined with platelet count and splenic volume seem to be useful to predict esophageal varices and VNT. This proposal will need to be prospectively validated in the future.

FIB-13

A new noninvasive index for NAFLD.- Sonographic NAFLD index

\begin{tabular}{|c|c|c|c|c|c|c|}
\hline & & & TEE & OT & PT & INR \\
\hline \multirow[t]{12}{*}{ Spearman's rho } & \multirow[t]{3}{*}{ TEE } & Correlation Coefficient & 1.000 & .176 & .112 & $.421^{n}$ \\
\hline & & Sig. (2-tailed) & . & .379 & .577 & .029 \\
\hline & & $\mathrm{N}$ & 27 & 27 & 27 & 27 \\
\hline & \multirow[t]{3}{*}{ OT } & Correlation Coefficient & .176 & 1.000 & $.915^{\prime \prime}$ & $.558^{\circ \prime}$ \\
\hline & & Sig. (2-tailed) & .379 & . & .000 & .002 \\
\hline & & $\mathrm{N}$ & 27 & 27 & 27 & 27 \\
\hline & \multirow[t]{3}{*}{ PT } & Correlation Coefficient & .112 & $.915^{\mathrm{Nx}}$ & 1.000 & $.502^{\mathrm{Nm}}$ \\
\hline & & Sig. (2-tailed) & .577 & .000 & . & .008 \\
\hline & & $\mathrm{N}$ & 27 & 27 & 27 & 27 \\
\hline & \multirow[t]{3}{*}{ INR } & Correlation Coefficient & $.421^{\circ}$ & $.558^{\mathrm{Nn}}$ & $.502^{\mathrm{kn}}$ & 1.000 \\
\hline & & Sig. (2-tailed) & .029 & .002 & .008 & \\
\hline & & $\mathrm{N}$ & 27 & 27 & 27 & 27 \\
\hline
\end{tabular}

**. Correlation is significant at the 0.01 level (2-tailed).

FIB-12

Risk of esophageal varices and non-invasive tools including liver stiffness by two-dimensional shear wave elastography

HAE WON YOO ${ }^{1}$, Young Seok Kim ${ }^{1}$, Sang Gyune Kim ${ }^{1}$, Jeongju Yoo', Jae Woo Park', Gab Jin Cheon'2, Jae Young Jang ${ }^{1}$, Young Don Kim², Soung Won Jeong', Sae Hwan Lee', Baekgyu Jun ${ }^{2}$, Hong Soo Kim ${ }^{1}$, Young Suk Lim ${ }^{2}$, Boo Sung Kim ${ }^{1}$

${ }^{1}$ Soonchunhyang University hospital, Korea, Republic of; ${ }^{2}$ Asan Hospital, Korea, Republic of

Background: According to the Baveno VI criteria, endoscopy for screening varices can be withheld if liver stiffness (LS) measured by transient elastography is less than $20 \mathrm{kPa}$ and platelet count is greater than 150,000 cells $/ \mu \mathrm{L}$. Two-dimensional shear wave elastography

\section{Neeraj Nagaich ${ }^{1}$, Neeraj Nagaich ${ }^{1}$, Radha Sharma ${ }^{3}$,} Mahipal Singh $^{2}$

${ }^{1}$ Fortis Hospital Jaipur, India; ${ }^{2}$ Metro Hospital, India; ${ }^{3}$ ruhs CMS Jaipur, India

Background: NAFLD is an emerging disease in developing countries, which remains silent for years. Noninvasive methods are required for early diagnosis. Sonographic evaluation and grading of steatosis has limitations in dearth of absolute echo amplitude standards and validation of other suggestive criteria. All the available scoring system based on subjective assessment are observer dependent and may be inaccurate . Predictive models and scoring systems may be useful but none have yet been validated There are limited study in which sonographic scoring has been prospectively validated. A quantitative reproducible score which can be applied early would be helpful in early stratification. This study was planned to evaluate accuracy of a new quantitative NAFLD index in predicting hepatic disease.

Method: This prospective and retrospective observational study was conducted in 352 patient with liver steatosis on abdominal imaging and fulfilling eligibility criteria. Details of the patient were recorded along with ultrasonographic finding and liver histology in patients with risk factors. NAFLD index was calculated by summation of individual sonographic score for each variable like liver echogenicity (0-3), visibility of hepatic vessels (0-3), portal vein / biliary radicals, (0-3) gall bladder wall (0-3) and diaphragm (0-3), (total score ranging from 0-15).Cut off value for NAFLD index in predicting disease in relation to histology was calculated after statistical analysis . 
Result: Fatty liver was diagnosed in 352 patients based on sonographic criteria .NAFLD Index showed a positive correlation with histological features of steatosis \& NASH (with a Spearman's coefficient of 0.86). The area under the receiver operating characteristic (ROC) curve for ultrasonographic detection of moderate to severe steatosis was 0.89 . A $p<0.05$ was considered statistically significant. Serum ALT and AST were not associated with histological grade of steatosis and showed no correlation with NAFLD index. NAFLD Index was an independent predictor of NASH $(\mathrm{OR} 2.16 ; \mathrm{P}=0.069)$ and a score $<8$ had a high negative predictive value (91\%) in ruling out the diagnosis of severe NAFLD.

Conclusion: This study demonstrates the utility of this index for noninvasive diagnosis and estimation of hepatic steatosis. NAFLD index score $>8$ was predictive of significant steatosis and compared well with liver histology Application of this index at the outset would be helpful in better selection of patient for liver biopsy and better prognostication of disease for future treatment

\section{FIB-14}

\section{Diagnosis value of MRI IDEAL-IQ and FibroTouch} for the hepatic steatosis

\section{Wenjia Wang ${ }^{1}$, Lixian $\mathrm{Wu}^{1}$}

${ }^{1}$ The first people's Hospital of Foshan, China

Background: To discuss the diagnostic value of FibroTouch for hepatic steatosis with magnetic resonance IDEAL-IQ sequence as the criterion.

Method: Subjects were recruited in the Foshan First People 's Hospital from January 2017 from June 2017. All subjects were collected alcohol history, BMI, hypertension, blood glucose, blood lipids, uric acid, transaminase, and so on. To explore the diagnostic value and the quantitative analysis of liver fat from mild to moderate fatty liver with FibroTouch, choosing MRI IDEAL-IQ sequence as the criterion.

Result: Total of 104 subjects were recruited, the male-to-female ratio was 2.47: 1 , the average age was $41.55 \pm 12.24$, and the fat attenuation parameter was $288.24 \pm 5.07 \mathrm{db} / \mathrm{m}$. The number of patients were diagnosed as fatty liver moderate or severe fatty liver patients severe patients by FibroTouch was 82, 66 and 50 respectively. The detection rates were $78.85 \%, 64.46 \%$ and $48.08 \%$ respectively. The number of patients were diagnosed as fatty liver moderate or severe fatty liver patients severe patients by MRI IDEAL-IQ was 82, 52 and 10 respectively. The detection rate was $78.85 \%, 50.00 \%, 9.62 \%$. Compared with the ROC curve of MRI IDEAL-IQ,the area under the curve of ROC were 0.956,0.956,0.839, All P values were $<0.01$.

Conclusion: FibroTouch has similar diagnostic value in terms of qualitative and quantitative diagnosis of fatty liver with MRI IDEALIQ. FibroTouch is the most economical and reproducible noninvasive method of examination, and is expected to be the preferred method of screening for fatty liver screening and treatment.
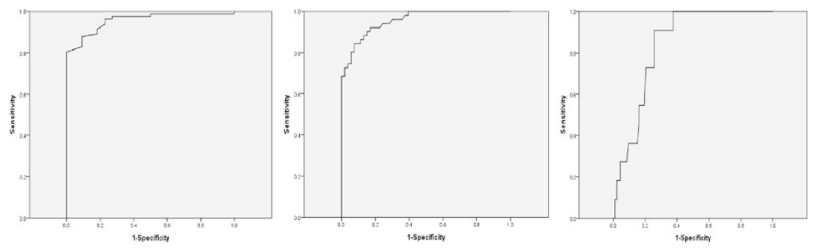

Fig A. The areas under the ROC curve of hepatic steatosis $>5 \%$.

Fig B. The areas under the ROC curve of hepatic steatosis $>10 \%$.

Fig C. The areas under the ROC curve of hepatic steatosis $>25 \%$.

Tab 1.The patient characteristics

\begin{tabular}{ll}
\hline Factors & $\mathrm{M} \pm \mathrm{S}$ \\
\hline Gender(M/F) & $74 / 30$ \\
Age & $41.55 \pm 12.24$ \\
BMI $(\mathrm{kg} / \mathrm{m} 2)$ & $26.10 \pm 4.77$ \\
$\mathrm{ALT}(\mathrm{IU} / \mathrm{L})$ & $59.10 \pm 72.44$ \\
$\mathrm{AST}(\mathrm{IU} / \mathrm{L})$ & $37.67 \pm 42.56$ \\
$\mathrm{GGT}(\mathrm{IU} / \mathrm{L})$ & $67.73 \pm 63.97$ \\
$\mathrm{CR}(\mu \mathrm{mol} / \mathrm{L})$ & $70.43 \pm 14.08$
\end{tabular}

Tag: BMI: Body mass index; ALT: Alanine aminotransferase; AST: Aspartate aminotransferase; GGT:Glutamyl transpeptidase; CR:Creatinine.

FIB-15

Significance of non-invasive markers of liver fibrosis in patients with chronic hepatitis B

Faroque Ahmed $^{1}$, Abul Hayat Manik ${ }^{1}$, Salimur Rahman ${ }^{2}$

${ }^{1}$ Dhaka Medical College Hospital, Bangladesh; ${ }^{2}$ Bangabandhu Sheikh Mujib Medical University, Bangladesh

Background: Liver biopsy, an integral part of management of patients with Chronic Hepatitis B (CHB), is helpful in confirming diagnosis, assessing grade of inflammation and stage of fibrosis and also in guiding treatment strategy. Although liver biopsy is relatively safe, morbidity is significant, so various non-invasive markers have been developed worldwide to asses liver histology

Method: Comparison of works done in Bangabandhu Sheikh Mujib Medical University (BSMMU), Dhaka, Bangladesh, during the period between 2006 and 2010. Evaluation and correlation done between non-invasive markers like AST to Platelet Ratio Index (APRI), AgeSpleen Platelet Ratio Index (ASPRI), Hyaluronic Acid (HA, an unbranched high molecular weight polysaccharide) level, and Transient elastography (Fibroscan) with different stages of hepatic fibrosis by liver biopsy

Result: In cross sectional studies, APRI in 30 patients and ASPRI in 51 patients had no significant relationship with liver fibrosis. HA level was normal (mean $18.3 \mathrm{ng} / \mathrm{ml}$ ) in controls and raised (mean $70.3 \mathrm{ng} /$ $\mathrm{ml}$ ) in $30 \mathrm{CHB}$ patients; Fibroscan was correlated in $60 \mathrm{CHB}$ patients having $\mathrm{F} 1$ fibrosis in $40(66.7 \%), \mathrm{F} 2$ in $3(5 \%), \mathrm{F} 3$ in $13(21.7 \%)$, and F4 in $4(6.6 \%)$ patients. Both HA and Fibroscan shown statistically significant relationship with liver fibrosis. 
Conclusion: In series of studies with CHB patients done in BSMMU, Dhaka, Bangladesh we found no significant correlation between liver fibrosis and some non-invasive markers like APRI, ASPRI etc. whereas, Hyaluronic acid and Fibroscan shown significant correlation. Further studies with larger study population may help in this regard. For evaluating non-invasive marker of liver fibrosis in patients of chronic hepatitis B.

\section{FIB-16}

non-invasive assessment of disease severity by lectinomics in patients with nonalcoholic fatty liver disease (NAFLD)

\author{
Ajay Duseja ${ }^{1}$, Gautam Mandal' ${ }^{2}$, Ajay Duseja ${ }^{4}$, \\ Bishnupada Chatterjee ${ }^{2}$, Suvradeep Mitra ${ }^{3}$, Ashim Dass \\ ${ }^{1}$ Postgraduate Institute of Medical Education and Research, \\ Chandigarh, India; ${ }^{2}$ Maulana Abul Kalam Azad University of \\ Technology, Salt Lake, India; ${ }^{3}$ Post Graduate Institute of Medical \\ Education and Research, Chandigarh, India; ${ }^{4}$ Post Graduate Institute \\ of Medical Education and Research, Chandigarh, India
}

Background: Liver histology still remains the gold standard for assessing the disease severity and differentiating patients with NASH (Non-alcoholic Steatohepatitis) and NAFL (Non-alcoholic fatty liver) (no-NASH) but is an invasive procedure and not free of complications. The aim of the present study was to assess the role of lectinomics in differentiating between NASH and no-NASH in patients with nonalcoholic fatty liver disease (NAFLD).

Method: Thirty eight patients categorised as NASH $(n=10)$ and noNASH $(n=28)$ as per NAFLD activity score $(\mathrm{NAS}>$ or $<5)$ were included in the study. In the glycoprotein analysis of the stored sera, glycosylation patterns in serum proteins of NASH and no-NASH patients were studied using different lectins such as SNA, AAL, DSA, WGA and PNA by ELISA. Protein expression was also studied by SDS-PAGE.

Result: Out of 38 patients with NAFLD (18 males, mean age $38.10 \pm$ 8.8 years) 10 patients $(26.3 \%)$ had histological NASH whereas 28 patients $(73.6 \%)$ had no-NASH.In the glycoprotein analysis of the stored sera, glycosylation patterns in serum proteins of NASH and noNASH patients were studied using different lectins such as sialic acidspecific Sambucus nigra agglutinin (SNA), fucose specific Aleuria aurantia agglutinin (AAL),branching-specific Datura stramonium agglutinin (DSA), GlcNAc specific wheat germ agglutinin (WGA) and $O$-glycan specific peanut agglutinin (PNA) by ELISA. An increase of WGA-binding with serum protein was observed in NASH $(0.215 \pm 0.041, p<0.005)$ patients in comparison to no-NASH patients $(0.193 \pm 0.025)$ indicating high level of GlcNAc. The high binding of serum protein with DSA was observed in patients with NASH $(0.222 \pm 0.039)$, in comparison to patients with no-NASH $(0.199 \pm 0.031)(p<0.005)$ which indicate high glycan branching in serum proteins. There were no differences in sialylation, fucosylation and $O$-glycan between the patients with NASH and no-NASH. On SDS-PAGE analysis one glycoprotein around $75 \mathrm{kDa}$ was expressed higher (1.5 fold) in no-NASH patients than in patients with NASH. Conclusion: Serum glycoprotein(s) is helpful in differentiating between NASH and no-NASH amongst patients with NAFLD.
FIB-17

Efficacy of enhanced liver fibrosis score \& transient elastography for assessment of severity of fibrosis in chronic hepatitis C

\section{Manish Kumar ${ }^{1}$ \\ ${ }^{1}$ GIPMER, India}

Background: Chronic hepatitis $\mathrm{C}$ (CHC) patients, especially with Genotype 3, have advanced fibrosis or cirrhosis. They need a longer duration of therapy and sustained virological response rate is lower. Enhanced liver fibrosis (ELF) score and transient electrography (TE) are noninvasive methods for grading fibrosis, have not been evaluated in Indian CHC patients. Aim to compare ELF and TE for grading the fibrosis and ruling out advance fibrosis or cirrhosis in CHC patients. Method: Consecutive CHC patients attending OPD at GIPMER from Sept. 2016 to May 2017 were included in the study. Patients with decompensated cirrhosis, BMI $\geq 30 \mathrm{~kg} / \mathrm{m}^{2}$ or additional etiology for liver disease excluded. The accuracy of ELF and TE for grading the fibrosis was done by receiver operator characteristic curve analysis with liver biopsy as the gold standard for grading fibrosis. The METAVIR scoring system was used for grading fibrosis in liver biopsy.

Result: Out of 62 patients evaluated, 30 were included (18 Males and 12 females, mean age was 34.7 years). All underwent TE, ELF score calculation and liver biopsy. Mean ELF score and TE score for patients was 9.28 and $9.04 \mathrm{kPa}$ respectively. METAVIR score 4 was taken as severe fibrosis and $2 / 3$ as moderate fibrosis. $10(33 \%)$ patients had moderate and $2(6.6 \%)$ had severe fibrosis. Taking METAVIR as the gold standard, the area under the receiver operator characteristic curve (AUROC) of ELF was 0.620 [CI], with a sensitivity of $33 \%$ and specificity of $67 \%$ for severe fibrosis. Similarly, for TE, AUROC was 0.903 [CI], with sensitivity and specificity $33 \%$ and $100 \%$ respectively at $12.35 \mathrm{kPa}$. Compared to liver biopsy, TE performed better than ELF score for severe and moderate fibrosis with higher AUROC.

Conclusion: TE, as compared to ELF, is a better noninvasive method for ruling out advance fibrosis or cirrhosis is in CHC patients.

\section{FIB-19}

Assessing the severity of liver fibrosis and steatosis in biopsyproven NAFLD patients using MR imaging, transient elastography and serum biomarker

Young Sun Lee ${ }^{1}$, Ji Hoon Kim ${ }^{1}$, In Hee Kim², Chang Hee Lee Lee ${ }^{1}$, Jong Eun Yeon ${ }^{1}$, So Young Kwon ${ }^{3}$, Kwan Soo Byun ${ }^{1}$, Sehwa Kim ${ }^{1}$, Haein Bak ${ }^{1}$

${ }^{1}$ Korea University College of Medicine, Korea, Republic of; ${ }^{2}$ Chonbuk National University Medical School, Korea, Republic of; ${ }^{3}$ Department of Internal Medicine, Korea, Republic of

Background: Because nonalcoholic fatty liver disease (NAFLD) is becoming a leading cause of chronic liver disease, a non-invasive diagnosis of the disease severity is urgently needed. To diagnose liver fibrosis, transient elastography (TE) has an acceptable accuracy in viral related liver disease. The aim of our study is to analyze hepatic fibrosis, steatosis, and inflammation in patients with biopsy-proven NAFLD using MR imaging, TE, and serum biomarkers.

Method: This is a multicenter prospective study of patients with biopsy-proven NAFLD. The patients underwent liver biopsy, MRI and TE 6 months before enrollment. Sera were collected at the time of enrollment. MRI examination included mDIXON, MR spectroscopy (MRS), and MR elastography (MRE). TE measured liver stiffness and 
controlled attenuation parameter (CAP). Twenty serum biomarkers were analyzed with the Luminex Multiplex Assay.

Result: Thirty-five patients with biopsy-proven NAFLD were enrolled from October 2016 to March 2017. Mean age and BMI were 50.6 \pm 0.48 years and $28.50 \pm 4.87 \mathrm{~kg} / \mathrm{m} 2$, respectively. Female patients were dominant $(23,65.7 \%)$, and other co-morbidities were diabetes $(\mathrm{n}=15,42.9 \%)$, hypertension $(\mathrm{n}=12,34.3 \%)$ and dyslipidemia $(\mathrm{n}=11$, $31.4 \%$ ). For the diagnosis of advanced fibrosis (stage 3-4), the AUROC of the MRE tended to be superior $(0.89 ; 95 \%$ CI, 0.72-0.98) compared with the TE $(0.83 ; 95 \%$ CI, 0.66-0.93) $(\mathrm{P}=0.40)$. For the diagnosis of severe steatosis (stage 2-3), CAP (0.7; 95\% CI, 0.52$0.84)$ had a lower AUROC compared with the mDIXON $(0.83 ; 95 \%$ CI, 0.65-0.94; $\mathrm{P}=0.11)$ and $\operatorname{MRS}(0.82 ; 95 \% \mathrm{CI}, 0.63-0.93 ; \mathrm{P}=0.28)$, respectively. In the serum biomarker analysis, increased resistin had a significant association with severe steatosis (stage 2-3) compared to mild steatosis (stage 0-1) $(\mathrm{OR}=1.44 ; 95 \% \mathrm{CI}, 1.01-2.06 ; \mathrm{P}=0.04)$. Increased IFN $-\gamma$ was associated with severe inflammation (stage 2-3) compared to mild inflammation (stage $0-1)(\mathrm{OR}=1.36$; $95 \% \mathrm{CI}, 1.01$ 1.83; $\mathrm{P}=0.04)$. Total PAI-1 showed a tendency for association with the presence of NASH (OR=1.063; 95\% CI, 0.99-1.14; $\mathrm{P}=0.07)$.

Conclusion: Non-invasive modalities using MRI and serum biomarkers could be potential tools for the diagnosis and classification of disease severity in patients with NAFLD.

\section{FIB-20}

\section{Assessment of liver fibrosis by different non invasive methods} in type $2 \mathrm{dm}$ with NAFLD

\author{
Niyanta Karki ${ }^{1}$, Sudhamsu Kc ${ }^{1}$, Jeetendra Kaji Shrestha ${ }^{1}$, \\ Bikash Jaisi $^{1}$ \\ ${ }^{1}$ NASL, Nepal
}

Background: INTRODUCTION: Nonalcoholic fatty liver disease (NAFLD) is quickly becoming one of the most prominent causes of liver disease worldwide and the incidence is increasing even in the developing part of world like ours. NAFLD is the hepatic manifestation of metabolic syndrome, and its prevalence is rising with increased frequency of DM, obesity.

Methods: A hospital based observational study was conducted from October 2015 -April 2016 in the Liver Unit. All patients with known or newly diagnosed DM attending endocrine and liver OPD after assessing the exclusion criteria were enrolled in the study. Fatty liver and its severity was identified by Ultrasonogram. Tests were done to calculate different fibrosis scores(NAFLD Fibrosis, BARD, FIB4) and compared with fibroscan score.

Results: Of 54 cases of which $55.6 \%$ were male and $44.6 \%$ were female. Mean age of the patient was 49.5 and $66.7 \%$ fall in $41-60$ years group. Metabolic syndrome was seen in about $44.4 \%$ of total patients. About $75.9 \%$ had fatty liver- $33.3 \%$ had mild, $31.5 \%$ had moderate and $11.1 \%$ had severe fatty liver in total. Higher the BMI, greater the severity of fatty liver. The fibrosis scores were slightly higher in patients with metabolic syndrome and with duration of DM $>10$ years. Low platelets count was correlated with high NAFLD Fibrosis score and FIB4 score with significant $\mathrm{P}$ values whereas high AST/ALT ratio correlated only with BARD score. In this study, fibroscan did not correlate with different fibrosis scores related to smaller sample size and minimal fibrosis which can be underestimated by fibroscan.

Conclusion: The incidence and severity of fatty liver with higher fibrosis scores correlated with higher BMI, the presence of metabolic syndrome and duration of DM. Different fibroscores like NAFLD fibrosis, BARD, FIB4 scores, platelets count and AST/ALT can be used as a simple tool to assess fibrosis where fibroscan is not available.
FIB-21

Correlation of fibrosis as assessed by Transient Elastography with CIMT in NAFLD patients

\section{Sahil Nohria $^{1}$, Sahil Nohria ${ }^{1}$ \\ ${ }^{1}$ Baba Farid University, India}

Background: Fatty liver is associated with an increase in cardiovascular morbidity and mortality specially in patients with NASH. Liver biopsy is the gold standard for liver fibrosis evaluation, but it is a painful and invasive procedure. Carotid intima-media thickness (IMT) is one of the markers for subclinical atherosclerosis. Liver stiffness (LS) measurement using transient elastography (TE) has emerged as a promising noninvasive tool for assessing the degree of liver fibrosis. Studies done in NAFLD patients have shown good correlation with CIMT when compared to controls

Method: Objectives: To determine the correlation of varying grades of fibrosis with atherosclerosis in 100 patients of NAFLD in Dayanand Medical College and Hospital. Methods: Patients with fatty liver who underwent Transient Elastography for assessment of fibrosis and further agreed to undergo carotid Doppler for CIMT were taken in this study

Results: There was significant correlation found in our study with varying grades of fibrosis and CIMT values. Increased fibrosis as assessed by transient elastography correlated well with increased carotid intima thickness. Also NAFLD patients who were diabetic had higher fibrosis and CIMT values with $\mathrm{p}$ value being $<0.05$

Conclusion: The present study showed that fibrosis as assessed by TE can assess the atherosclerosis risk in patients with NAFLD so that patients can be successfully managed with appropriate lifestyle modification and pharmacological intervention to decrease CVD risk in this group of patients.
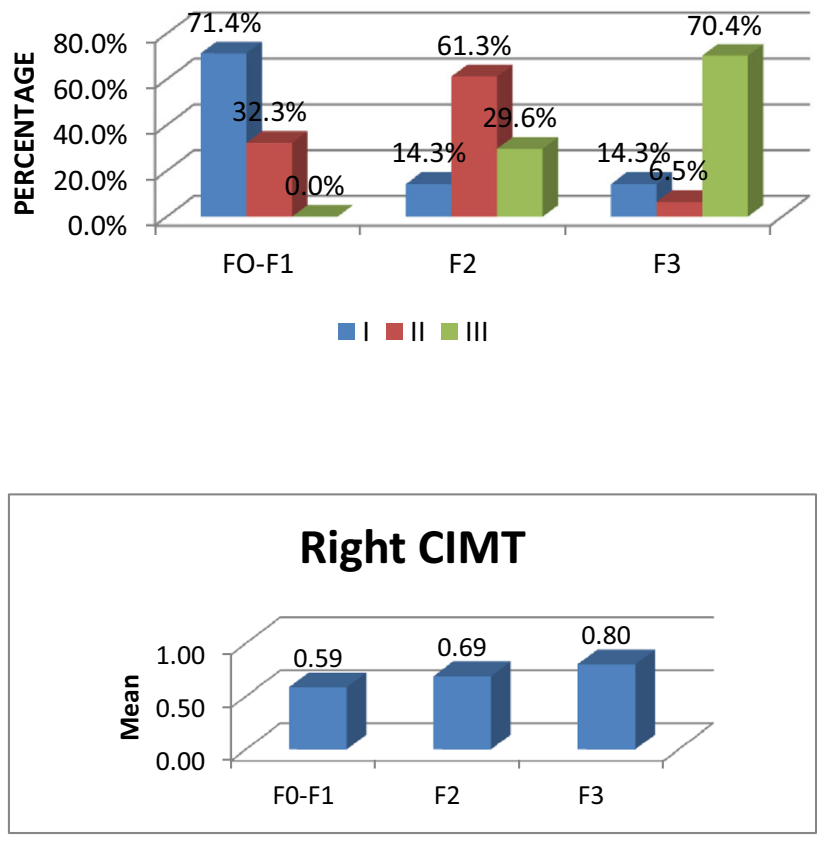
FIB-22

Liver fibrosis assessment in chronic pancreatitis patients having normal bilirubin using transient elastography

Rabindranath Eswaran ${ }^{1}$, Premkumar $\mathbf{K}^{\mathbf{1}}$, Venkateswaran A $\mathbf{R}^{\mathbf{1}}$, Rajkumar Solomon $^{1}$, Ratnakar Kini ${ }^{1}$, Kani Shaikh Mohammed ${ }^{1}$, Chandrashekar Patil ${ }^{1}$, Sriram P B ${ }^{1}$

${ }^{1}$ Madras Medical College, India

Background: Chronic pancreatitis (CP) is an inflammatory disease, in its progressive course, causes significant loss of exocrine and endocrine functions. Common bile duct (CBD) stenosis is a common complication of chronic pancreatitis. The purpose of the study is to analyze the rate of liver fibrosis using Transient elastography (TE) in chronic pancreatitis patients with normal bilirubin, transaminases levels and without CBD stenosis.

Method: All consecutive chronic pancreatitis patients with normal transaminases and bilirubin levels referred to our service were considered and prospectively included in our study between September 2016 to October 2017, after excluding concomitant alcoholic cirrhosis. Clinical and analytical data, duration of disease, duration of exocrine and endocrine insufficiency were obtained. Measurement for transient elastography (TE), was graded as normal (F0/F1, TE 0-7), mild fibrosis (F1/F2, TE 7.1-9), moderate fibrosis (F2/F3, TE 9.1-12) or severe fibrosis/cirrhosis (F4, TE > 12).

Result: Total of $131 \mathrm{CP}$ cases were enrolled, 12 had CBD stenosis and 7 had concomitant alcoholic cirrhosis, were excluded from the study.CBD stenosis was suspected on the basis of radiologic findings. 112 patients enrolled in study group underwent TE, 89 cases were male, $73.21 \%$ had alcoholic etiology and ages ranged from 26 years to 79 years $($ mean $=50.4$ years $) .7 .14 \%$ had moderate fibrosis, $23.21 \%$ had mild fibrosis and no case had values consistent with possible cirrhosis. Alcoholic $\mathrm{CP}$ patients had more significant fibrosis $(\mathrm{p}=$ 0.01 , median $7.9 \mathrm{kPa}$ ). $17.8 \%$ cases had raised alkaline phosphatase up to 1.5 times the normal level. Correlation of rate of fibrosis was found with raised alkaline phosphatase $(p=0.03$, median $7.4 \mathrm{kPa})$, pancreas atrophy $(\mathrm{p}=0.04$, median $7.2 \mathrm{kPa})$ and duration of endocrine insufficiency $(\mathrm{p}=0.01$, median $7.7 \mathrm{kPa}$ ).BMI, pancreatic stone, pancreatic duct stricture, previous endotherapy, duration of $\mathrm{CP}$ disease per se and presence or duration of exocrine insufficiency were not significantly associated.

Conclusion: The study suggests that even $\mathrm{CP}$ patients having normal bilirubin and absent CBD stenosis may have fibrosis of the liver. TE may be useful for surveillance and detection of early liver disease in the group so that management can be planned.

\section{$F I B-23$}

\section{Liver stiffness values using transient elastography in Egyptian} patients with acute viral hepatitis

Mohamed Abdel Samiee ${ }^{1}$, Eman Abdelsameea ${ }^{2}$, Nashwa Shebl ${ }^{2}$, Khalid Gameel $^{2}$, Hanaa Badran ${ }^{2}$, Eman Rowisha ${ }^{2}$

${ }^{1}$ National Liver Institute, Egypt; ${ }^{2}$ National Liver Institute, Menoufia University, Egypt

Background: Transient elastography (TE) is a non-invasive and reproducible tool to assess liver fibrosis/ cirrhosis. However, it remains to be determined if ALT flare interferes with fibrosis assessment.

Aim: To determine the effect of increased serum ALT on liver stiffness measurement in patients with acute viral hepatitis.
Method: Thirty consecutive patients without a previous clinical history of liver disease were prospectively included. Blood samples were collected and TE was done initially and after resolution of hepatitis. Patients with high BMI which could affect fibroscan and patients with cirrhosis were excluded. For determination of the etiology of hepatitis, a detailed physical examination and history taking were performed in all patients.

Result: Patients were $32.87 \pm 10.2$ years old and males were 14 (46.7\%).In all patients, the degree of liver stiffness at the time of the peak increase in aminotransferases exceeded the cutoff values proposed for the prediction of significant fibrosis or cirrhosis. The mean value of LSM at the time of inclusion in the study was $13.91 \pm 6.7 \mathrm{kPa}$ and the mean value of LSM after resolution of hepatitis was $7.7 \pm 3.08$ $\mathrm{kPa}$. Reduction of mean value to $6.21 \pm 1.14 \mathrm{kPa}$ was observed in 10 patients after 1 year of ALT normalization. A progressive significant reduction in liver stiffness values was observed $(\mathrm{P}<0.01)$ in the follow-up period in parallel with the reduction of ALT levels $(\mathrm{P}<0.01)$. Moreover, a statistically significant, positive correlation between ALT and LSM at the onset of acute viral hepatitis was found $(\mathrm{r}=0.38, \mathrm{P}<$ 0.05).The decrease in the mean level of LSM value was statistically significant $(\mathrm{P}<0.01)$. No significant correlation was found between increased total bilirubin level and LSM $(r=0.3$ and $P>0.05)$.

Conclusion: TE has not demonstrated reliable diagnostic accuracy in patients with acute viral hepatitis.

\section{FIB-24}

\section{Noninvasive assessment of liver fibrosis in chronic hepatitis B}

\section{Berna Bozca ${ }^{1}$, Turgut Kacan ${ }^{1}$}

${ }^{1}$ State Hospital, Turkey

Background: Liver biopsy is the best method for evaluating hepatic fibrosis, and recommended for patients with chronic hepatitis B (CHB). But biopsy may cause several complications. In this study, we aim to verify the usefulness of several non-invasive markers of liver fibrosis such as; AAR (Aspartate aminotransferase (AST)/ Alanine aminotransferase (ALT)), APRI (AST/platelet ratio index), API (ageplatelet index), and FIB4 score (fibrosis index based on the four factors).

Method: We retrospectively investigated 323 patients with CHB who performed liver biopsy from 2010 to 2017 . The clinical and laboratory parameters were recorded from each patient at the time of liver biopsy. Samples were reviewed by a single pathologist using Ishak scoring. We further grouped the fibrosis stages as F0-1 (minimal fibrosis) and F2-6 (significant fibrosis). For statistical analysis we used student's t-test, chi-square test, receiver operating characteristics (ROC) analyses with area under the curve (AUC) measurements. $\mathrm{P}<0.05$ considered to be statistically significant.

Result: A total of 323 patients with CHB who had liver biopsy between 2010 to 2017 were included in this study. Mean age was 39,2 +- 11,4 and mean age of illness was 10,3 years. One hundred and sixty nine of the patients $(52,3 \%)$ were female and $93,5 \%$ were AntiHbe positive. Mean HBV-DNA level was 2810782,7 IU/ML. According the pathology results a total of $14(4,3 \%)$ patients were had minimal fibrosis (F0-1) and $309(95,7 \%)$ patients were had is (F2-6). We analyzed the correlations between the values of non-invasive models and fibrosis scores, to evaluate whether non-invasive models are suitable for staging liver fibrosis in CHB patients. We observed that correlations between fibrosis score and respectively for AAR; 49,5\%, AP; $15,5 \%$, FIB4; 9\%, and for APRI; $11,5 \%$. We couldn't observed any strong correlations for each tests. Moderate correlation was observed for AAR test and weak correlations were observed for APRI, FIB4 and AP tests. 
Conclusion: We couldn't observed any strong correlation to determine liver fibrosis with non-invasive markers as well as liver biopsy. It may be due to that our study doesn't include any cirrhosis patients.

FIB-25

Related factors with the correlation between the liver biopsy and transient elastography $\left(\right.$ Fibroscan $\left.^{\circledR}\right)$

Fulya Gunsar ${ }^{1}$, Nilay Danis ${ }^{2}$, Ulus Salih Akarca ${ }^{2}$, Ilker Turan ${ }^{2}$, Zeki Karasu $^{2}$, Galip Ersoz ${ }^{2}$, Funda Yilmaz ${ }^{2}$, Deniz Nart ${ }^{2}$, Fulya Gunsar ${ }^{1}$

${ }^{1}$ Ege University Medical School, Turkey; ${ }^{2}$ Ege University, Turkey

Background: Liver biopsy, used as a gold standard for evaluating liver fibrosis, is invasive and difficult to use because of its low reproducibility. The Fibroscan ${ }^{\circledR}$ (FS) has found widespread application as a validated method for measuring liver fibrosis. However, there is still doubt about this method's compatibility with liver biopsy. In this study we investigated the related factors with the correlation between the liver biopsy and FS

Method: In this study the correlation of stages of the liver fibrosis calculated by FS and detected from synchronous liver biopsies was investigated, after December 2014. It is accepted, as stage 0-2 was mild, 3-4 was moderate, 5-6 were severe fibrosis in liver biopsy according to Ishak score. FS results were considered to be $<6.5 \mathrm{kPa}$ mild, 6.5-12, moderate, $>12-17$ severe, with minor differences according to different diseases. The probable factors, like sex, age, disease etiology, experience with FS, HBV DNA levels, affecting the correlation between FS and biopsy were investigated.

Result: In our hepatology unit a total of 847 patients had liver biopsies in between 2014-2016, of whom 297 patients also had FS. Of the biopsied patients, $52 \%$ were female and $48 \%$ were male(mean age was $48.3 \pm 14.1$ years). In the liver biopsies, mean grade was $6.23 \pm$ 2.70, mean stage: $2.11 \pm 1.63$, and Fibroscan values were $10.4 \pm$ $9.2 \mathrm{kPa}$ (median 7.8). In the biopsies, $60 \%$ of patients had mild fibrosis, $30 \%$ had moderate fibrosis, and $10 \%$ had severe fibrosis. These values were found as $45.1 \%, 30.0 \%$ and $24.9 \%$ in FS measurements. There was a significant correlation between stage of biopsies and FS measurements: $r=0.578, p<0.0001$. According to the diagnosis, these correlations were: HBV: $\mathrm{r}=0.503 ; \mathrm{p}<0.0001$; HCV: $r=0.725 ; p<0.0001 ;$ NAFLD: $r=0.604 ; p=0.001$. Sensitivity of predicting mild fibrosis detected in the liver biopsy by FS was $70.27 \%$ (CI: $58.52 \%-80.34 \%$ ), specificity $85.71 \%$ (95\% CI: $71.46 \%$ $94.57 \%)$, the positive predictive value was $89.66 \%(80.28 \%$ $94.86 \%)$, the negative predictive value was $62.07 \%(53.2-70.35 \%)$ and the AUC was $0.78(0.69-0.85)$. The negative predictive value of severe fibrosis detected in the liver biopsy by FS was $95.6 \%(90,58-$ $97,96 \%)$. In 2014, when there was no agreement between the two methods, the relationship between the two methods was significant ( $\mathrm{p}$ $=0.04)$ in 2015, and a stronger correlation was observed in $2016(\mathrm{p}$ $<0.0001)$. The correlation between these methods was not related with sex or age.

Conclusion: Mild fibrosis in liver biopsy predicted by FS is close to $90 \%$. The absence of severe fibrosis in FS indicates that $96 \%$ of the cases are not severe fibrosis. As the experience with FS increases, the correlation between FS and biopsy is increasing.
FIB-26

The association of hepatic venous pressure gradient with liver stiffness assessed by two-dimensional shear wave elastography and transient elastography in patients with liver cirrhosis

Hae Won Yoo ${ }^{1}$, Young Seok Kim ${ }^{1}$, Sang Gyune Kim ${ }^{1}$, Jae Myeong Lee ${ }^{1}$, Jeongju Yoo', Jae Young Jang ${ }^{1}$, Soung Won Jeong ${ }^{1}$, Sae Hwan Lee ${ }^{1}$, Hong Soo Kim ${ }^{1}$, Boo Sung Kim ${ }^{1}$

${ }^{1}$ Soonchunhyang University hospital, Korea, Republic of Korea

Background: Although the measurement of hepatic venous pressure gradient (HVPG) is the most accurate way to evaluate portal hypertension. Due to its invasiveness, it is necessary to find out the indirect way to identify portal hypertension. The aims of this study are to estimate the correlation between liver stiffness (LS) by two-dimensional shear wave elastography (2D-SWE) and transient elastography (TE, Fibroscan and HVPG, to identify the optimal cut-off value of 2D-SWE for clinically significant portal hypertension (CSPH, HVPG $=10 \mathrm{mmHg}$ ) and severe portal hypertension $(\mathrm{SPH}, \mathrm{HVPG}=12$ $\mathrm{mmHg}$ )

Method: Between January 2015 and October 2017, 111 patients with advanced chronic liver disease who measured HVPG and liver stiffness by 2D-SWE consecutively within 6 months (183 days) were enrolled. 9 patients were excluded for the reasons as follows: 7 for concurrent hepatocellular carcinoma and 1 for post-transplantation state. Among them, 74 patients who received TE simultaneously were involved in the comparison analysis.

Result: Total 103 patients were eligible for the analysis. The mean age was $54.1 \pm 9.7$ years and $72.8 \%$ was male. Mean Child-Pugh score was $7.36 \pm 1.80 .82$ patients $(79.6 \%)$ were proved to have CSPH and 75 patients $(72.8 \%)$ were proved to have SPH. The AUROCs of 2DSWE for predicting CSPH and SPH was 0.724 (95\% CI, 0.627-0.807) and 0.675 (95\% CI, 0.576-0.764) respectively. The optimal cut-off values for determining CSPH and SPH were $12.44 \mathrm{kPa}$ (Sensitivity, $77 \%$; Specificity, $71 \%$ ) and $13.21 \mathrm{kPa}$ (Sensitivity, 57\%; Specificity, $75 \%$ ) respectively. When compared with TE for predicting $\mathrm{CSPH}$, 2D-SWE showed almost comparable performance $[0.757$ (95\% CI, $0.643-0.849$ ) vs. 0.821 (95\% CI, 0.714-0.900), $\mathrm{p}=0.435]$.

Conclusion: 2D-SWE might be as useful as TE to predict CSPH or $\mathrm{SPH}$, it may help to predict portal hypertension indirectly to patients who cannot perform transient elastography.

\section{FIB-27}

Clinical study on the combination of FibroTouch with FIB-4, APRI and GPRI in diagnosis of liver fibrosis

\section{Nan Yuemin ${ }^{1}$}

${ }^{1}$ Third Hospital of Hebei Medical University, China

Background: To explore the value of the combination of FibroTouch, fibrosis index based on the 4 factor (FIB-4), AST-to-ALT ratio index (APRI) and GGT-to-PLT (GPRI) in diagnosis of liver fibrosis in patients with chronic liver disease.

Method: A total of 337 patients were selected and performed liver biopsy from January 2014 to April 2017. The GPRI, APRI, FIB-4 were calculated and liver stiffness was measured by FibroTouch. The diagnostic values of FibroTouch, GPRI, APRI and FIB-4 for liver fibrosis degree were compared by receiver operating characteristic (ROC) curves. The correlation between LSM, APRI, GPRI, FIB-4 and clinical characteristics were analyzed according to Spearman 
analysis. Posterior probability was calculated to explore the accuracy of combining four methods in diagnosis of liver fibrosis.

Result: The patients were divided into significant liver fibrosis group (a liver fibrosis stage of $\geq S 2, n=169$ ), advanced liver fibrosis group (a liver fibrosis stage of $\geq \mathrm{S} 3, \mathrm{n}=86$ ), liver cirrhosis group (a liver fibrosis stage of $=S 4, n=42$ ). The AUROCs of the LSM for predicting significant liver fibrosis, advanced liver fibrosis and liver cirrhosis were $0.826,0.882,0.920$, which were significantly higher than FIB-4 $(0.734,0.711,0.739)$, APRI $(0.662,0.669,0.719)$ and GPRI $(0.621$, $0.674,0.720$ ); LSM was correlated with histopathological fibrosis $(\mathrm{r}=0.564 P=0.000)$ and inflammation grade $(\mathrm{r}=0.651, P=0.000)$. The probability of LSM in diagnosing liver fibrosis of $\mathrm{S} \geq 2, \mathrm{~S} \geq 3$ and $\mathrm{S}=4$ was $72.44 \%, 80.52 \%, 84.21 \%$. When FIB-4, APRI and GPRI were combined with LSM in diagnosing liver fibrosis of $\mathrm{S} \geq 2, \mathrm{~S} \geq 3$ and $\mathrm{S}=4$, the posterior probability was $95.71 \%, 98.6 \%, 99.08 \%$.

Conclusion: A combination of FibroTouch and FIB-4 can improve the accuracy of diagnosing liver fibrosis, and the demand of liver biopsy will decrease significantly when combined with APRI and GPRI further.

\section{$F I B-28$}

Comparison of acoustic radiation force impulse and transient electrography for the diagnosis of fibrosis in patients with nonalcoholic fatty liver disease

\section{Min Wang ${ }^{1}$, Yu Wang ${ }^{1}$, Wenping Luo ${ }^{1}$}

${ }^{1}$ Beijing Friendship Hospital, Capital Medial University, China

Background: Nonalcoholic fatty liver disease (NAFLD) has become a major public health issue. In this study, we performed a cohort study to compare ultrasound-based Acoustic Radiation Force Impulse (ARFI) and Transient Electrography (TE) for the diagnosing fibrosis in patients with biopsy-proven NAFLD.

Method: The patients with biopsy-proven NAFLD were consecutively enrolled in this study from September 2012 to June 2017 in Beijing Friendship Hospital, Capital Medical University. Liver stiffnesses were measured by ARFI and TE within 1 week prior or inferior to liver biopsy. Histological evaluation of fibrosis was regarded as the gold standard. The diagnostic performances of ARFI and TE for fibrosis were evaluated using area under the receiver operating characteristic curves (AUROCs). The influencing factors for liver stiffness of ARFI and TE were explored using multivariate analysis. Result: A total of 61 patients with biopsy-proven NAFLD were enrolled. The mean age and body mass index were 49 years and $25.2 \mathrm{~kg} / \mathrm{m} 2$, respectively. The median liver stiffness measured by ARFI in patients with F0, F1, F2, F3, and F4 fibrosis stages were: 1.0 $\mathrm{m} / \mathrm{s}, 1.4 \mathrm{~m} / \mathrm{s}, 1.5 \mathrm{~m} / \mathrm{s}, 1.9 \mathrm{~m} / \mathrm{s}$ and $2.5 \mathrm{~m} / \mathrm{s}$; Those measured by TE were: $5.0 \mathrm{kPa}, 6.8 \mathrm{kPa}, 9.8 \mathrm{kPa}, 12.0 \mathrm{kPa}$ and $23.1 \mathrm{kPa}$. Liver stiffnesses by ARFI and TE were significantly correlated with liver fibrosis, and the correlation coefficients were 0.801 and 0.798 , respectively $(\mathrm{p}<0.001)$. For diagnosing $\mathrm{F} \geq 1, \mathrm{~F} \geq 2, \mathrm{~F} \geq 3$ and $\mathrm{F}=4$, the AUROC for ARFI were $0.940,0.880,0.885,0.925$, and the cut-off values were $1.16 \mathrm{~m} / \mathrm{s}, 1.50 \mathrm{~m} / \mathrm{s}, 1.86 \mathrm{~m} / \mathrm{s}$ and $1.92 \mathrm{~m} / \mathrm{s}$; the AUROCs for TE were $0.889,0.912,0.909,0.918$ and the cut-off values were 5.2 $\mathrm{kPa}, 7.3 \mathrm{kPa}, 11.9 \mathrm{kPa}, 16.5 \mathrm{kPa}$, respectively. However, no significant difference of AUROCs existed between ARFI and TE for diagnosing the same stage of fibrosis $(p>0.05)$. Multivariate analysis demonstrated that ballooning, triglyceride(TG), platelet (PLT) count were associated with ARFI values.

Conclusion: ARFI has a similar accuracy with TE for diagnosing liver fibrosis in NAFLD patients, and ARFI is a potential and valuable non-invasive technique for liver fibrosis assessment.
Table 1. Clinical Characteristics of Subjects with Biopsy-Proven NAFLD

\begin{tabular}{|c|c|}
\hline Variables & NAFLD $(\mathrm{N}=61)$ \\
\hline \multicolumn{2}{|l|}{ Demographic variables } \\
\hline Sex (Male/female) & $22 / 39$ \\
\hline Age, years & $49(33,57)$ \\
\hline BMI, $\mathrm{kg} / \mathrm{m}^{2}$ & $25.2(23.4,27.4)$ \\
\hline Type 2 diabetes & $19(31.1)$ \\
\hline Hypertension & $20(32.8)$ \\
\hline \multicolumn{2}{|l|}{ Biochemical profile } \\
\hline $\mathrm{ALT}, \mathrm{IU} / \mathrm{L}$ & $78.0(37.5,126.5)$ \\
\hline $\mathrm{AST}, \mathrm{IU} / \mathrm{L}$ & $55.6(31.0,78.5)$ \\
\hline ALP, IU/L & $98.0(70.5,117.0)$ \\
\hline $\mathrm{GGT}, \mathrm{IU} / \mathrm{L}$ & $67.0(40.5,112.5)$ \\
\hline TBIL, umol/L & $11.9(9.8,17.1)$ \\
\hline DBIL, umol/L & $2.6(1.8,3.7)$ \\
\hline ALB, $g / L$ & $42.0(39.5,44.9)$ \\
\hline CHE, KU/I & $8.6(7.2,9.7)$ \\
\hline $\mathrm{CHOL}, \mathrm{mmol} / \mathrm{L}$ & $5.0(4.5,5.5)$ \\
\hline $\mathrm{TG}, \mathrm{mmol} / \mathrm{L}$ & $1.8(1.3,2.4)$ \\
\hline PLT count, $\times 10^{9} / \mathrm{L}$ & $204.0(148.5,236.0)$ \\
\hline PTA, $\%$ & $94.0(83.0,110.5)$ \\
\hline \multicolumn{2}{|l|}{ Imaging } \\
\hline $\mathrm{TE}(\mathrm{kPa})$ & $8.8(6.1,14.2)$ \\
\hline $\operatorname{ARFI}(\mathrm{m} / \mathrm{s})$ & $1.48(1.19,2.07)$ \\
\hline \multicolumn{2}{|l|}{ Liver biopsy } \\
\hline NAFLD activity score & $5.0(3.0,5.5)$ \\
\hline \multicolumn{2}{|l|}{ Fibrosis stage } \\
\hline F0 & $11(18.0)$ \\
\hline $\mathrm{F} 1$ & $16(26.3)$ \\
\hline $\mathrm{F} 2$ & $10(16.4)$ \\
\hline F3 & $11(18.0)$ \\
\hline $\mathrm{F} 4$ & $13(21.3)$ \\
\hline \multicolumn{2}{|l|}{ Steatosis grade } \\
\hline $\mathrm{S} 1(5 \%-33 \%)$ & $39(64.0)$ \\
\hline $\mathrm{S} 2(34 \%-66 \%)$ & $16(26.2)$ \\
\hline $\mathrm{S} 3(>66 \%)$ & $6(9.8)$ \\
\hline \multicolumn{2}{|l|}{ Ballooning } \\
\hline 0 & $8(13.1)$ \\
\hline 1 & $18(29.5)$ \\
\hline 2 & $35(57.4)$ \\
\hline \multicolumn{2}{|l|}{ Inflammation grade } \\
\hline 0 & $4(6.6)$ \\
\hline 1 & $29(47.5)$ \\
\hline 2 & $20(32.8)$ \\
\hline 3 & $8(13.1)$ \\
\hline
\end{tabular}

Unless indicated otherwise, results are expressed as median (quartile) or number(percentage)

Abbreviations: BMI, body mass index; ALT, alanine aminotransferase; AST, aspartate transaminase;ALP, alkaline phosphatase;GGT, gamma-glutamyl transpeptidase;TBIL, total bilirubin; DBIL, direct bilirubin; ALB, albumin; $\mathrm{CHE}$, cholinesterase; $\mathrm{CHOL}$, cholesterol; TG, triglyceride; PLT, platelets; PTA, prothrombin activity; TE, transient electrography; ARFI, acoustic radiation force impulse; NAFLD, nonalcoholic fatty liver disease. 
Table 2 Diagnostic Performances of ARFI and TE (M probe of FibroScan) for fibrosis

\begin{tabular}{|c|c|c|c|c|c|c|c|}
\hline $\begin{array}{l}\text { Fibrosis } \\
\text { Stage }\end{array}$ & Cut-off & A AUROC $(95 \% \mathrm{CI}$ & & Sensitiv & & Specificity & ARFI vs TE $(P)$ \\
\hline $\mathrm{F} \geq 1$ & & & & & & & 0.17 \\
\hline $\mathrm{ARFI}, \mathrm{m} / \mathrm{s}$ & 1.16 & $0.974(0.933,1.000)$ & & 0.940 & & 1.000 & \\
\hline $\mathrm{TE}, \mathrm{kPa}$ & 5.2 & $0.889(0.779,0.999)$ & & 0.96 & & 0.727 & \\
\hline $\mathrm{F} \geq 2$ & & & & & & & 0.49 \\
\hline $\mathrm{ARFI}, \mathrm{m} / \mathrm{s}$ & 1.50 & $0.880(0.793,0.966)$ & & 0.735 & & 0.889 & \\
\hline $\mathrm{TE}, \mathrm{kPa}$ & 7.3 & $0.912(0.839,0.984)$ & 0.971 & & 0.741 & & \\
\hline $\mathrm{F} \geq 3$ & & & & & & 0.50 & \\
\hline $\mathrm{ARFI}, \mathrm{m} / \mathrm{s}$ & 1.86 & $0.885(0.800,0.970)$ & 0.750 & & 0.892 & & \\
\hline $\mathrm{TE}, \mathrm{kPa}$ & 11.9 & $0.909(0.838,0.981)$ & 0.708 & & 0.973 & & \\
\hline F4 & & & & & & 0.90 & \\
\hline ARFI,m/s & 1.92 & $0.925(0.848,1.000)$ & 0.846 & & 0.812 & & \\
\hline $\mathrm{TE}, \mathrm{kPa}$ & 16.5 & $0.918(0.822,1.000)$ & 0.846 & & 0.958 & & \\
\hline
\end{tabular}

Abbreviations: ARFI, acoustic radiation force impulse; TE, transient electrography; AUROC, area under the receiver operating characteristics curve; $\mathrm{CI}$,confidence interval.

$P$ value: AUROC of ARFI versus TE $\rightarrow$ DeLong test

Table 3. The influencing factors of liver stiffness measured by ARFI in Multivariate analysis

\begin{tabular}{lll}
\hline Varibles & beta & $p$ \\
\hline Ballooning & 0.417 & 0.003 \\
TG & -0.214 & 0.044 \\
PLT & -0.288 & 0.020
\end{tabular}

Abbreviations: TG, triglyceride; PLT, platelets.
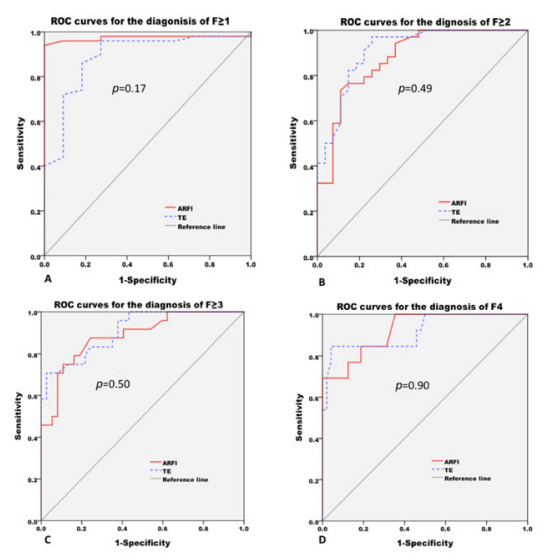

Figure 1. ROC curves for ARFI and TE for the diagnosis of $F \geq 1(A), F \geq 2(B), F \geq 3(C)$ and $F=4(D)$
Liver fibrosis measured by a non-invasive marker (APRI) in a large HIV cohort long-term on antiretroviral treatment in Cambodia

$\underline{\text { An Sokkab }}^{1}$

${ }^{1}$ Sihanouk Hospital Center of HOPE, Cambodia

Background: We investigated frequency, and associated factors, of significant liver fibrosis and cirrhosis in a large cohort of people living with HIV (PLWH) long-term on antiretroviral treatment (ART) in Phnom Penh, Cambodia.

Method: Aspartate-to-platelet ratio index (APRI), hepatitis $\mathrm{C}$ and hepatitis B surface antigen ( $\mathrm{HbsAg}$ ) testing was offered, between 11/2014 and 05/2016, to all adult PLWH followed up in Sihanouk Hospital Center of Hope. We categorized the APRI scores according to standard thresholds for significant fibrosis (APRI $\geq 1.5$ ) and cirrhosis (APRI $\geq 2$ ). Using multivariable logistic regression we identified risk factors for elevated APRI $(\geq 0.5, \geq 1.0)$ including demographic characteristics, CD4 (nadir, current), HIV viral load, comorbidities, past and current alcohol use.

Result: A total of 3046 PLWH were enrolled, median age was 42.3 years, male/female ratio 0.75 . Most $(97.6 \%)$ were on ART (median duration: 6.9 years), with a median CD4 count of 464 cells $/ \mu 1$ and $96.7 \%$ with undetectable HIV viral load $(<50$ copies $/ \mathrm{mL})$. Hundred and seven PLWH (3.5\%) had a chronic HCV infection and 311 (10.2\%) tested positive for HbsAg. The majority $(83.5 \%)$ had an APRI below 0.5. Sixty-four PLWH (2.1\%) had a score indicative of significant fibrosis and $38(1.2 \%)$ of cirrhosis. Risk factors for APRI $\geq 0.5$ were $\mathrm{HCV}$ coinfection (adjusted OR 12.0; CI 7.8-18.4), HbsAg positivity (aOR 1.8; 1.4-2.4), current CD4 below 200 (aOR 1.4; 1.02.1), duration on ART below 12 months (aOR 1.7; 1.2-2.6), current alcohol use $>140 \mathrm{~g} /$ week (aOR $2.4 ; 1.4-4.2$ ), past alcohol use $>140 \mathrm{~g} /$ week (aOR 1.4; 1.0-1.9), female gender (aOR 0.6; 0.4-0.7) and body mass index $>25$ (aOR 1.4; 1.1-1.9). For APRI $>1.0, \mathrm{HCV}$ coinfection (aOR 17.7, 10.7-29.2), HbsAg positivity (aOR 4.1; 2.66.5), CD4 below 200 (aOR 2.2; 1.3-3.8), current alcohol use $>140 \mathrm{~g} /$ week (aOR 2.8; 1.3-6.3), gender (aOR 0.6; 0.4-0.8) and diabetes mellitus (aOR $2.4 ; 1.2-4.6$ ) were significantly associated.

Conclusion: Our results indicate a low prevalence of significant liver fibrosis and cirrhosis in this Cambodian HIV cohort, despite about $15 \%$ being coinfected with hepatitis B or C. Correlation of standard cut-offs for fibrosis markers as APRI, with elastography, should however be further investigated in Asian PLWH long-term on ART to assure no gross underestimation of fibrosis occurred.

FIB-30

The accuracy of liver stiffness measurement, platelet count and platelet count/liver stiffness measurement ratio in identifying the presence of esophageal varices in patients with liver cirrhosis

Mary Ann Uy Bielgo ${ }^{1}$, Juliet Gopez Cervantes ${ }^{1}$, Cynthia Abad Mapua $^{1}$, Mark Pierre Sijo Dimamay ${ }^{1}$, Michael Octubre Baclig ${ }^{1}$

${ }^{1}$ St. Luke's Medical Center, Philippines

Background: Esophageal varices (EV) are major complication of portal hypertension and detected in about $50 \%$ of cirrhotic patients. An upper endoscopy in all cirrhotic would be very expensive. Furthermore, repeated gastroscopies are often poorly accepted by patients. Noninvasive methods are needed to identify clinically significant esophageal varices in patients with cirrhosis. We looked for 
markers of the presence of esophageal varices in patients with cirrhosis.

Method: This is a retrospective cross sectional study done in St. Luke's Medical Center - Quezon City and Global City from 2013 to 2017 involving total of 111 cirrhotic patients who had undergone endoscopy and LSM along with PC. Diagnostic abilities and cut off values were assessed by the area under the receiver operating characteristic curve and multivariate logistic regression.

Result: Liver stiffness measurement (LSM), platelet count (PC) and platelet count/liver stiffness measurement (PC/LSM) ratio had AUC of more than 0.70 . shown as $0.73,0.92$, and 0.91 respectively.

The optimal cut off values of LSM in identifying EV was $\geq 20.66 \mathrm{Kpa}$ with sensitivity of $77.08 \%$ and specificity of $65.08 \%$. The optimal value of $\mathrm{PC}$ was $\leq 150,650 / \mathrm{mm}^{3}$ with $93.75 \%$ and $74.60 \%$ sensitivity and specificity respectively. The PC/LSM ratio had an optimal cut off value of 6.87 with sensitivity of $93.75 \%$ and specificity of $71.43 \%$.

This study demonstrated that using the previous cut off of $\geq 20 \mathrm{kpa}$, has sensitivity of $77.1 \%$ (95\% CI 63.5-86.7) and specificity of $57.1 \%$ (95\% CI 44.9-68.6) with PPV $57.8 \%$ (95\% CI 45.6-69.1) and NPV $76.6 \%$ (95\% CI 62. 8-86.4), with accuracy of $65.8 \%$ (95\% CI 56.5-73.9) and LR (+) 1.8 (95\% CI 1.6-2.0) and LR (-) 0.4 (95\% CI $0.3-0.5)$. Using the previous cut off of $\leq 150,000 / \mathrm{mm}^{3}$ showed 93.8\% (95\% CI 83.2-97.8) and 74.6\% (95\% CI 62.7-83.7) specificity and sensitivity respectively. The PPV was $73.8 \%$ (95\% CI 61.6-83.2) with NPV of $94 \%$ (95\% CI 83.8-97.9). The accuracy was $82.9 \%(95 \%$ CI 74.8-88.8) with LR (+) 3.7 (95\% CI 3.3-4.2) and LR (-) 0.1 (95\% CI .0-0.2).

Conclusion: The LSM, PC and PC/LSM Ratio shown promise as predictive markers of Esophageal Varices. We can used these noninvasive parameters to identify patients who have EV and who may delay or need to undergo surveillance endoscopy.

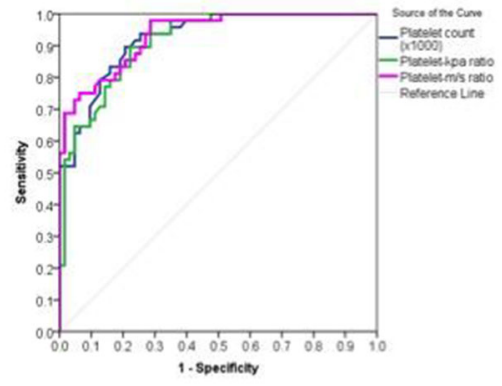

\begin{tabular}{|l|l|l|}
\hline Test & $\begin{array}{l}\text { Area under the } \\
\text { curve }\end{array}$ & value \\
\hline $\begin{array}{l}\text { Platelet count } \\
\text { (x1000) }\end{array}$ & $0.92(0.88-0.82)$ & $<0.001$ \\
\hline Platelet-kpa ratio & $0.91(0.86-0.96)$ & $<0.001$ \\
\hline
\end{tabular}

Figure 4. Area under the curve using Platelet count/Liver stiffness measurement ratio in identifying esophageal yarices

FIB-31

The easy liver fibrosis test (eLIFT) for predicting liver fibrosis in patients with chronic hepatitis B: a retrospective cohort study in China

Jian Wang ${ }^{1}$, Rui Huang ${ }^{1}$, Xiaomin Yan ${ }^{1}$, Juan Xia ${ }^{1}$, Yong Liu ${ }^{1}$, Zhaoping Zhang', Weimao Ding ${ }^{2}$, Chao Wu ${ }^{1}$, Jian Wang ${ }^{1}$

${ }^{1}$ Nanjing Drum Tower Hospital, The Affiliated Hospital of Nanjing University Medical School, China; ${ }^{2}$ Huaian No. 4 Peoples Hospital, China
Background: The easy liver fibrosis test (eLIFT) is a novel index to assess liver fibrosis in chronic liver diseases. We aimed to assess the diagnostic accuracy of eLIFT for live fibrosis in patients with chronic hepatitis $\mathrm{B}(\mathrm{CHB})$ in China, and compare the diagnostic accuracy of eLIFT with other non-invasive fibrosis indexes based on blood parameters.

Method: 294 CHB patients with liver biopsy were enrolled in this study. The diagnostic accuracy of eLIFT for liver fibrosis was evaluated and compared with aspartate aminotransferase-to-platelet ratio index(APRI), fibrosis-4 score(FIB-4), the gamma-glutamyl transpeptidase-to-platelet ratio(GPR) and red cell distribution width-toplatelet ratio(RPR) by the receiver operating characteristic(ROC) curves.

Result: The area under the ROC curves (AUROCs) of eLIFT in predicting significant liver fibrosis, advanced liver fibrosis and liver cirrhosis were $0.687(95 \%$ CI 0.621 to 0.753$), 0.710$ (95\% CI 0.650 to 0.769 ) and 0.750 (95\% CI 0.692 to 0.808 ), respectively. However, according to the Youden index, the optimal cut-off values of eLIFT for predicting significant fibrosis, advanced fibrosis and liver cirrhosis were all 9.5. Furthermore, the diagnostic performance of eLIFT was not significantly different with APRI, FIB-4, GPR and RPR in identifying significant fibrosis, advanced fibrosis and cirrhosis in both $\mathrm{HBeAg}$ positive $\mathrm{CHB}$ and $\mathrm{HBeAg}$ negative $\mathrm{CHB}$.

Conclusion: The eLIFT is a novel index for detecting liver fibrosis with a moderate sensitivity and accuracy. However, it is difficult for eLIFT to distinguishing significant fibrosis, advanced fibrosis and cirrhosis. Further, the diagnostic accuracy of eLIFT was not significantly superior than APRI, FIB-4, GPR and RPR in identifying liver fibrosis in $\mathrm{CHB}$ in China.

FIB-32

Use of vibration controlled transient elastography (fibroscan) to predict presence of oesophageal varices in Indian population

Abhimanrao Manikrao Pawar ${ }^{1}$, N Murugan ${ }^{1}$, M Hariharan ${ }^{1}$, Balasubramaniam Ramakrishnan ${ }^{1}$

${ }^{1}$ Apollo Hospitals, Chennai, India

Background: Screening for Oesophageal Varices in cirrhosis is done by upper gastrointestinal endoscopy. Vibration Controlled Transient Elastography (Fibroscan) measures liver stiffness noninvasively and also correlates with degree of portal hypertension. Liver stiffness $>15$ $\mathrm{kPa}$ is highly suggestive of compensated advanced chronic liver disease (cACLD). Aim is to study the utility of Fibroscan to predict oesophageal varices in Indian patients with cACLD.

Method: A total of 56 Indian patients with liver stiffness $>10 \mathrm{kPa}$ who had undergone upper gastrointestinal endoscopy were studied prospectively. Patients with ascites, Hepatocellular carcinoma, total bilirubin $>5 \mathrm{mg} / \mathrm{dl}$ and AST/ALT $>5$ times upper limit of normal were excluded. Endoscopic varices were graded into no varices, small varices or large varices. All patients underwent $\mathrm{CBC}$, liver function tests, PT-INR, HBsAg, Anti-HCV, serum albumin, renal function tests.

Result: Mean age was 49.8 years. Mean age when there were no varices was 45.9 years, when there were small varices was 50.8 years and when there were large varices was 52.3 years. 42 were males, and 14 were females. Most common etiological factors were probable NASH $(25 ; 44.64 \%)$ followed by HBV $(11 ; 19.64 \%)$,Alcoholic (10; $17.86 \%)$,Others $(8 ; 14.29 \%)$, HCV $(2 ; 3.57 \%)$. Total $18(32.14 \%)$ patients had no varices, $21(37.5 \%)$ patients had small varices and $17(30.35 \%)$ patients had large varices. Mean platelet count when there were no varices was $1,89,833 / \mathrm{mm}^{3}$, when there were small varices was $1,45,285 / \mathrm{mm}^{3}$, and when there were large varices was 
$1,19,412 / \mathrm{mm}^{3}$. Mean liver stiffness for large varices was $35.71 \mathrm{kPa}$, for small varices was $34.86 \mathrm{kPa}$, whereas it was $23.36 \mathrm{kPa}$ when varices were absent. An optimized cutoff of liver stiffness of 17.45 $\mathrm{kPa}$ had $89.5 \%$ sensitivity and $50 \%$ specificity in distinguishing between presence and absence of oesophageal varices with AUROC 0.715 . Whereas, an optimal cutoff of $21.1 \mathrm{kPa}$ had $94.1 \%$ sensitivity and $41 \%$ specificity, positive predictive value of $41 \%$ and negative predictive value of $94.1 \%$ to distinguish between large varices and small or absent varices, AUROC being 0.667.

Conclusion: Fibroscan has good sensitivity but limited specificity in predicting presence of oesophageal varices and also distinguishing large oesophageal varices vs. small or absent oesophageal varices in Indian population.

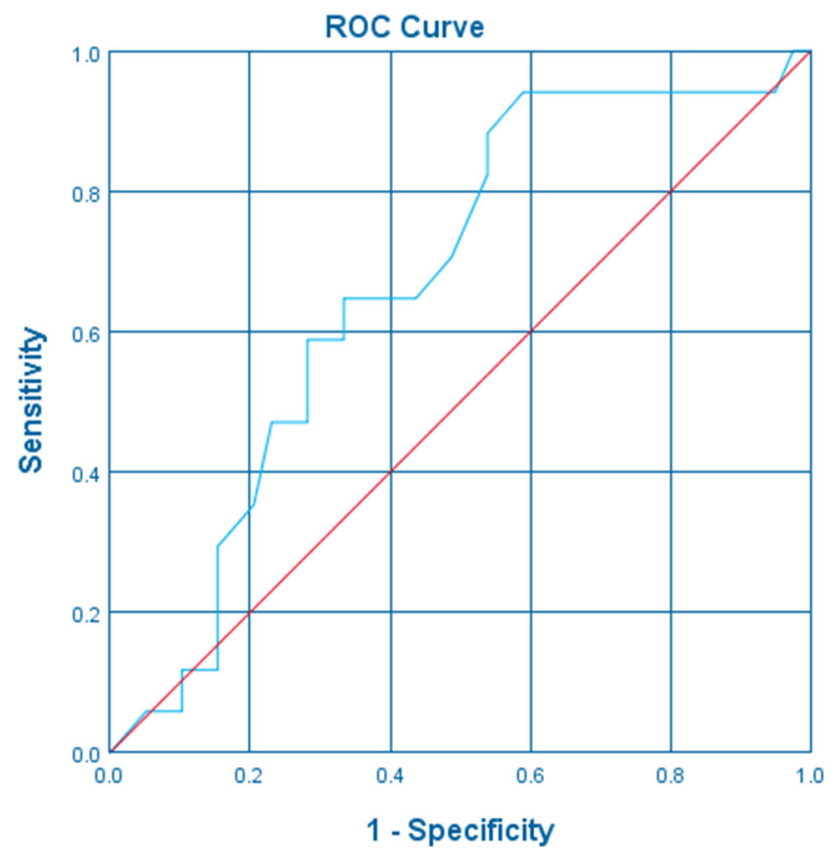

FIB-34

Changes of serum transforming growth factor- $\tilde{\mathrm{A}} \ddot{\mathbf{Y}} 1$ (TGF-Ã $\ddot{Y} 1$ ) in chronic hepatitis $B$ related liver fibrosis

\section{Xiaoqin Dong ${ }^{1}$, Guiqiang Wang ${ }^{1}$, Hong Zhao ${ }^{1}$}

${ }^{1}$ Peking University First Hospital, China

Background: Transforming growth factor-betal (TGF- $\beta 1$ ) is an important cytokine which has multiple profibrogenic but also antiinflammatory and immunosuppressive effects. TGF- $\beta 1$ stimulates the quiescent hepatic stellate cells to myofibroblasts, which is the initiating factor of hepatic fibrosis. However, the changes of TGF- $\beta 1$ in the progress of liver fibrosis are controversial. This article mainly discusses the correlation between serum TGF- $\beta 1$ and liver fibrosis in patients with chronic hepatitis $\mathrm{B}(\mathrm{CHB})$.

Method: A total of 678 Chinese treatment-naïve CHB patients who had undergone percutaneous liver biopsies were enrolled in this study. Ishak histological scores of activity and fibrosis were assessed (HAI04 no/mild inflammatory, HAI5-6 moderate inflammatory, $\mathrm{HAI} \geq 7$ severe inflammatory. F0-1 no/mild fibrosis, F2 moderate fibrosis, F3 significant fibrosis, F4-6 advanced fibrosis or higher). The detailed clinical trial protocol has been registered (NCT01962155 and ChiCTR-DDT-13003724). Serum TGF- $\beta 1$ was quantify by enzyme- linked immunosorbent assay (ELISA). All statistical analyses were performed using SPSS 17.0.

Result: Serum TGF- $\beta 1$ levels decreased in accordance with the progression of liver fibrosis staging, especially in patients with early cirrhosis and cirrhosis $(\mathrm{P}<0.05)$. In CHB patients with ALT $<2 \times$ $\mathrm{ULN}$, the trends in serological changes of TGF- $\beta 1$ were consistent with those in the overall patient (Fig 1,2). There was no significant difference in the serum levels of TGF- $\beta 1$ among CHB patients with different degrees of liver inflammation (Fig 3).

Conclusion: Unlike previous studies, our study showed that serum levels of TGF- $\beta 1$ were significantly reduced in CHB patients with significant fibrosis and cirrhosis.

\section{FIB-35}

Collagen parameters quantitation can have the equivalence to Ishak staging but a higher efficiency in evaluating the hepatitis B-related longitudinal non-advanced liver fibrosis

\section{Yan Wang ${ }^{1}$, Xieer Liang ${ }^{1}$, Jinlian Yang ${ }^{1}$, Jian Sun ${ }^{1}$, Jinlin HOU \\ ${ }^{1}$ Southern Medical University Nanfang Hospital, China}

Background: Evaluation of longitudinal fibrosis is relevant for identification of disease progression and predication of liver-related outcomes for the individualized management of hepatitis B patients. For them, non-advanced fibrosis is a common scenario, however current evaluation tools generally lack sufficient performance in detecting its longitudinal change. We aimed to identify whether using quantitative fibrous collagen parameters ( $\mathrm{q}-\mathrm{FP})$ can be as accurate as but more sensitive than the standard histology for addressing this issue.

Method: A well-established CHB cohort (NCT00962533) with mostly the non-advanced fibrosis were used for this study. 301 subjects with paired liver biopsies were consecutively included. Of them, 139 subjects were used to establish the test and the rests for internal validation. Fibrosis change between baseline and 104 weeks of treatment was blindly assessed with Ishak fibrosis staging and q-FP. Result: $70 \%$ subjects had Ishak F0-2 at baseline. Imaging data were collected yielding a dataset of 69 q-FPs per biopsy specimen, which then composed a single score, q-FS representing the overall fibrosis status. Using q-FP profile, variations of fibrosis response in different clinical settings could be extensively visualized. For diagnosing overall fibrosis endpoint, $67 \%$ (93/139) patients got decrease in their Ishak stages $(\mathrm{p}<0.0001)$, and the decrease extent showed a significant association with baseline Ishak stage (OR 6.7, 95\% CI 4.4-10.5, $\mathrm{p}<0.0001)$; on $\mathrm{q}-\mathrm{FP}$ analysis, $68 \%$ (94/139) patients achieved decrease in their q-FS $(\mathrm{p}<0.0001)$, of which the extent also demonstrated a significant association with baseline q-FS (Spearman $\mathrm{r}-0.7$, 95\% CI -0.7- $-0.6, \mathrm{p}<0.0001)$. However, while q-FP identified the independent association between fibrosis regression and antiviral efficacy (OR 3.0, 95\% CI 1.4-6.5, p=0.005), Ishak failed the detection (OR 0.6, 95\% CI 0.3-1.3, p=0.24). On further analysis of statistical consistency, there showed no difference between their diagnoses of fibrosis endpoint (difference 0.7\%, 95\% CI -9.2-10.6, $\mathrm{p}=1.00$ ). The indices of positive (ppos) and negative (pneg) agreement were 0.8 (95\% CI 0.7-0.8) and 0.5 (95\% CI 0.4-0.7), respectively, for which, the disagreement was identified more likely occurring to the baseline Ishak staging $(\mathrm{p}=0.003)$, mainly involving some pairs with baseline moderate fibrosis, but had not relation to Ishak staging at week 104 $(\mathrm{p}=0.55)$. In contrast, the disagreement had no significant relation to $\mathrm{q}-\mathrm{FP}$ assessment, either at baseline $(\mathrm{p}=0.18)$ or at week $104(\mathrm{p}=0.78)$. Moreover, neither did the disagreement correlate to the biopsy size at baseline $(\mathrm{p}=0.22)$ or at week $104(\mathrm{p}=0.37)$. These findings were verified by reviewing through the paired Ishak staging and q-FP score 
for every subject. Eventually, all the above results were confirmed in the validation subjects.

Conclusion: Compared with standard histology, q-FP could have an equivalent accuracy as well as a higher sensitivity in monitoring the longitudinal non-advanced fibrosis.

FIB-36

Transient elastography with controlled attenuation parameter in the assessment of hepatic steatosis and significant liver fibrosis in patients with scleroderma

Yusuf Yilmaz ${ }^{1}$, Haluk Tarik Kani ${ }^{1}$, Yasemin Sahinkaya ${ }^{1}$, Ilknur Deliktas $^{1}$, Huseyin Hakan Mursaloglu², Munir Aziziy ${ }^{1}$, Guzide Nevsun Inanc ${ }^{1}$, Rafi Haner Direskeneli ${ }^{1}$

${ }^{1}$ Marmara University, School of Medicine, Turkey; ${ }^{2}$ Marmara University Pendik Education and Research Hospital, Turkey

Background: Scleroderma is a fibrotic condition characterized by immunological abnormalities, vascular injury, and increased accumulation of extracellular matrix proteins in the skin. Hepatic involvement in patients with scleroderma is generally considered rare but it is potentially underestimated. We investigated whether measurement of controlled attenuation parameter (CAP), a non-invasive method to quantify steatosis, can assist in screening for chronic liver disease in patients with scleroderma.

Method: We performed transient elastography (TE), to measure liver stiffness, and made CAP measurements in 35 patients with scleroderma (mean age: $52.73 \pm 12.00$ years; 31 females). We also collected information on patients' anthropometric measurements and laboratory data. Based on the published literature, the cutoff value for defining liver steatosis was $\mathrm{CAP} \geq 238 \mathrm{~dB} / \mathrm{m}$, and the cutoff value for defining the presence of significant fibrosis $(\mathrm{F} \geq 2)$ was liver stiffness of $>8.0 \mathrm{kPa}$.

Result: The patients' mean body mass index was $26.76 \pm 5.52 \mathrm{~kg} / \mathrm{m}^{2}$. The levels of aspartate aminotransferase (AST) and alanine aminotransferase (ALT) were $21.03 \pm 5.98 \mathrm{U} / \mathrm{L}$ and $18.03 \pm 6.60 \mathrm{U} / \mathrm{L}$, respectively. The prevalence of steatosis and significant fibrosis in our sample of patients with scleroderma were $48.6 \%(\mathrm{n}=17)$ and $31.4 \%(\mathrm{n}=11)$, respectively.

Conclusion: Hepatic steatosis - determined by CAP measurement and significant liver fibrosis - as assessed by liver stiffness - are common in patients with scleroderma. In this patient group, noninvasive screening for chronic liver injury through TE is clinically warranted.

FIB-37

Validation of simple non-invasive indirect markers of fibrosis in patients with chronic hepatitis $\mathrm{C}$ infection against biopsy as gold standard: a diagnostic validation study

Ajeet Singh Bhadoria ${ }^{1}$, Rakhi Maiwall ${ }^{2}$, Guresh Kumar ${ }^{2}$, Ankur Jindal $^{2}$, Archana Rastogi ${ }^{2}$, Chhagan Bihari ${ }^{2}$, Ankit Bhardwaj ${ }^{2}$, Manoj Kumar Sharma ${ }^{2}$, Shiv Kumar Sarin ${ }^{2}$

${ }^{1}$ All India Institute of Medical Sciences, Rishikesh, India; ${ }^{2}$ Institute of Liver And Biliary Sciences, India

Background: Hepatic fibrosis is an important factor to decide treatment regimen and its duration for management of chronic hepatitis $\mathrm{C}$ (CHC). Liver biopsy is the reference standard for assessing hepatic fibrosis. However, many non-invasive diagnostic approaches are available to detect hepatic fibrosis. We planned to validate simple non-invasive fibrosis biomarkers in $\mathrm{CHC}$ patients against biopsy as the gold standard, for their utilization in public health approaches.

Method: All CHC patients who underwent biopsy at a tertiary care centre in northern India during the period 2010-2015 were included. The non-invasive markers like age-platelet index (AP index), aspartate aminotransferase (AST)-alanine aminotransferase (ALT) ratio (AAR), AST-platelet ratio index (APRI), fibrosis-4 (FIB-4) index, FibroQ, Goteborg University Cirrhosis Index (GUCI), Lok Index and transient elastography (TE) were tested for their diagnostic accuracies in predicting advanced fibrosis (METAVIR score $3 \& 4$ ). Diagnostic characteristics were tested by ROC curve analysis.

Result: In this diagnostic validation study (NCT02658786), a total of $532 \mathrm{CHC}$ (age $43.8 \pm 13.3$ years, $67.1 \%$ male, BMI- $23.5 \pm 2.7 \mathrm{Kg} / \mathrm{m}^{2}$ ) were enrolled. Genotype 3 was the commonest, seen in $378(70.9 \%)$ of the cases, genotype 1 in 133 (24.9\%), genotype 4 in $16(3.01 \%)$ and genotypes 2 and 5 in $5(0.9 \%)$ each. Mean HCV RNA load was $4.54 \pm 2.23 \log 10 \mathrm{IU} / \mathrm{ml}$. Distribution of cases as per METAVIR F-0, 1, 2, 3, 4 was $60(11.3 \%), 134$ (25.2\%), $103(19.4 \%), 79(14.8 \%)$ and $156(29.3 \%)$, respectively. The diagnostic accuracy for advanced fibrosis as per area under ROC curve (with 95\% CI) were 88.5 (84.192.9), 79.2 (73.4-84.9), 77.0 (71.2-82.9), 75.2 (69.0-81.4), 74.2 (67.980.5), 73.5 (67.1-79.8), 71.6 (65.1-78.1), respectively for TE, APRI, AAR, FIB-4, AP index, Fibro Q, GUCI, and Lok Index (Figure 1). Conclusion: Liver stiffness measurement by simple non invasive biomarkers showed impressive diagnostic characteristics for evaluating advanced liver fibrosis in $\mathrm{CHC}$ patients. These scores may further be validated to include in national plan.

Figure 1: Receiver-Operating Characteristic (ROC) curve analysis

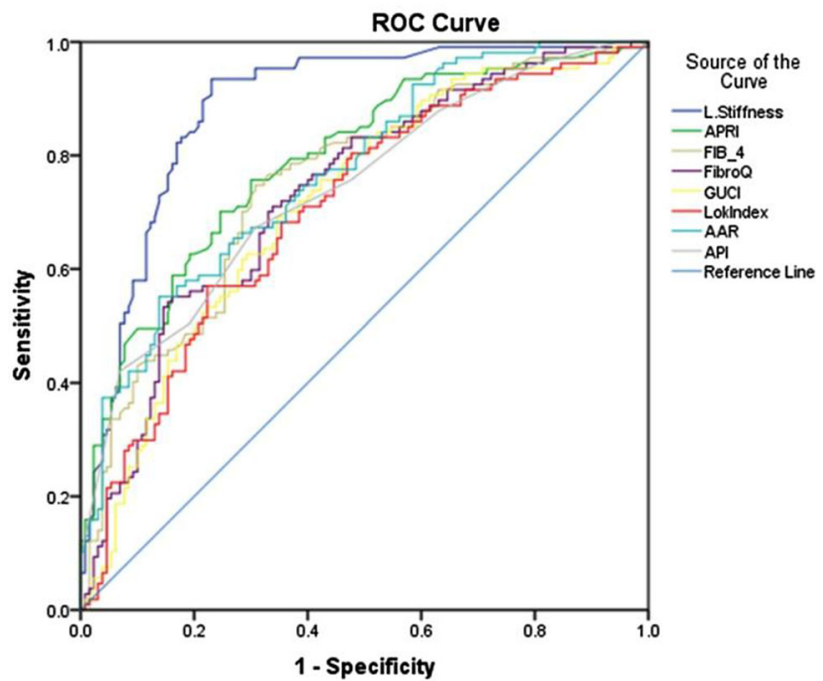

FIB-38

Fatty liver is a potential cause of advanced liver diseases: usefulness of screening for liver fibrosis in the general population

George Lau ${ }^{1}$, Ke $\mathrm{Xu}^{4}$, Jing $\mathrm{Li}^{4}$, Tong Sun ${ }^{4}$, Shengjiang Zhang ${ }^{4}$, Jinhua Shao ${ }^{6}$, Jin Sun ${ }^{6}$, Qiong $\mathrm{He}^{5}$, Jianwen Luo ${ }^{5}$, Cheng Wang ${ }^{3}$, Jing Chen ${ }^{3}$, Yudong Wang, ${ }^{3}$ Vanessa $\mathrm{Wu}^{3}$, George Lau ${ }^{2}$

${ }^{1}$ Humanity and Health Medical Group, Hong Kong; ${ }^{2}$ Humanity and Health Medical Center, Hong Kong SAR, China, Hong Kong;

${ }^{3}$ Humanity and Health Research Center, Hong Kong SAR, China, Hong Kong; ${ }^{4}$ Meinian Onehealth Healthcare (Group) Co., China, 
China; ${ }^{5}$ School of Medicine, Tsinghua University, China, China; ${ }^{6}$ Wuxi Hisky Medical Technologies Co., Ltd, China, China

Background: Non-invasive test of liver fibrosis with transient elastography (TE) has recently been suggested to be a useful tool in stratification of chronic liver disease in the general population. We sought to evaluate the usefulness of TE in screening for liver fibrosis and investigate the prevalence and risk factors associated with liver fibrosis in a large cohort of Chinese general population with normal alanine aminotransferase (ALT $<=40 \mathrm{U} / \mathrm{L}$ ).

Method: In 2016, liver fibrosis and hepatic steatosis in 205,631 consecutive Chinese subjects in 291 cities of 29 provinces in mainland China and Hong Kong SAR were assessed by Fibrotouch ${ }^{\circledR}$ (Wuxi Hisky Medical Technologies Co., Ltd, Beijing China). Each participant had fasting blood sample and anthropometric measurements collected. Validation of FibroTouch to FibroScan ${ }^{\circledR}$ (Echosens, Paris, France) was first done in 578 Chinese patients who visited Humanity and Health $(\mathrm{HnH})$ Medical Centre by Spearman rank correlation analysis, concordance test and receiver operating characteristic (ROC) curve. Multivariable logistic regression using forward stepwise selection procedure was used to identify the risk factors associated with advanced liver fibrosis.

Result: The concordance test showed strong agreement between Fibrotouch and Fibroscan in reliable readings from both TEs (1007 data points, $89 \%) \quad(\mathrm{rho}=0.902$, $\mathrm{z}$-transformed $\mathrm{p}<0.001)$. Using $\mathrm{LSM}=9.5 \mathrm{kPa} \& 9.0 \mathrm{kPa} \& 5.8 \mathrm{kPa}$ as the cutoff for advanced fibrosis from Fibroscan (criteria for HCV \& HBV \& NAFLD), the ROC was 0.986 (95\% CI 0.973 - 0.999), 0.986 (95\% CI, $0.974-0.998)$ and 0.935 (95\% CI $0.920-0.950)$ for readings from Fibrotouch against these criterion.

In subjects aged 18 to 75 years with normal ALT and HBsAg negative, without any missing data $(n=7350)$, the proportion of subjects with clinically relevant fibrosis (LSM 7.3-9.7), advanced fibrosis (LSM 9.7-12.4) and cirrhosis (LSM $>=12.5 \mathrm{kPa}$ ) was $8.8 \%(95 \% \mathrm{CI}$ $8.2-9.5 \%), 1.4 \%$ (95\% CI $1.1-1.6 \%$ ) and $0.7 \%$ (95\% CI $0.5-1.0 \%$ ) respectively. Severe steatosis (UAP $>295$, OR 2.6, 95\% CI 1.6-4.1), higher BMI (OR 1.2, 95\% CI 1.1-1.3), and male sex (OR 2.2, 95\% CI 1.4-3.4) were independently associated with advanced fibrosis. In subjects with advance fibrosis, normal ALT and $\mathrm{HBsAg}$ negative $(n=150), 19.3 \%$ with elevated GGT $(>50 \mathrm{U} / \mathrm{L}), 16.7 \%$ with impaired glucose $(\mathrm{FBG}>=6.1 \mathrm{mmol} / \mathrm{L}), 34.7 \%$ with level of cholesterol $>5.2$ $\mathrm{mmol} / \mathrm{L}$ (ULN), $34.0 \%$ with level of HDL $>1.42$ (ULN), $20.7 \%$ with level of LDL $<2.1$ (LLN) and $45.3 \%$ with level of triglyceride $>1.7$ (ULN). Moreover, 37.3\% had moderate or severe steatosis.

Conclusion: Though with normal ALT and negative HBsAg, male with severe steatosis and higher BMI are still at risk for advanced fibrosis in Chinese general population. Those with negative HBsAg and normal ALT in advanced fibrosis, as high as $40 \%$ had dyslipidemia. Fatty liver is a potential cause of advanced liver diseases in this population.

\section{$H A V-1$}

\section{Japanese miso extract suppresses HAV replication} with the upregulation of glucose-regulated protein 78

Tatsuo Kanda ${ }^{1}$, Nan Nwe $\mathrm{Win}^{2}$, Shingo Nakamoto ${ }^{2}$, Xia Jiang ${ }^{4}$, Mitsuhiko Moriyama ${ }^{1}$, Yutaka Tamura ${ }^{2}$, Akiko Suganami ${ }^{2}$, Hiroshi Shirasawa ${ }^{2}$, Hiroaki Okamoto ${ }^{3}$

${ }^{1}$ Nihon University School of Medicine, Japan; ${ }^{2}$ Chiba University, Graduate School of Medicine, Japan; ${ }^{3}$ Jichi Medical University School of Medicine, Japan; ${ }^{4}$ The First Hospital of Hebei Medical University, China
Background: Hepatitis A virus (HAV) infection is still one of the major cause of acute hepatitis all over the world although the effective vaccine has been developed. We have recently reported that endoplasmic reticulum (ER) stress-associated molecule glucose-regulated protein 78 (GRP78) has antiviral function for HAV. In the present study, we developed human hepatocytes (PXB cells) from a humanized severe combined immunodeficiency albumin promoter/ enhancer driven-urokinase-type plasminogen activator mouse for HAV infection, and examined the effects of Japanese Miso extract on HAV replication.

Method: The human hepatoma cell line Huh7, which is susceptible for HAV infection and PXB cells (Phoenix Bio, Higashi-Hiroshima, Japan) were used. Kurasaigetsuusujiomiso (KU) and Igou-miso (IG) were obtained from Ando Brewery, Kakunodate, Japan. Cells were infected with HAV HA-11-1299 genotype IIIA at 0.1 MOI and treated with $0.5 \%$ Miso extract. HAV RNA and GRP78 were evaluated by real-time RT-PCR and Western blotting at 7 days after HAV infection.

Result: (1) PXB cells supported HAV replication. (2) In PXB cells, KU induced GRP78 (9.4-fold, compared to control; $\mathrm{p}<0.05$ ) and reduce HAV RNA levels $(0.47$-fold, compared to control; $\mathrm{p}<0.05)$. (3) In Huh7 cells, KU and IG, respectively, induced GRP78 (1.2 and 1.2-fold, compared to control; $\mathrm{p}<0.05$ ). (4) HAV RNA were reduced to 0.25 and 0.23 -fold by the treatment with $\mathrm{KU}$ and IG, respectively. Conclusion: Japanese Miso extract suppresses HAV replication along with the enhancement of GRP78 expression. Together, these results show that Japanese Miso extract modulates ER stress pathways for inhibition of HAV replication and can be used as a dietary component for prevention of severe hepatitis A.

\section{HCC-1}

Fgl2 induces a suppressive immunological microenvironment in mice hepatocellular carcinoma model

\section{Jinshang $\mathrm{Hu}^{1}$, Zhongwei Zhang ${ }^{2}$, Jiajia Chen ${ }^{2}$, Xiaoping Luo ${ }^{2}$, Dong $\mathrm{Xi}^{2}$, Qin Ning ${ }^{2}$}

${ }^{1}$ Tongji Hospital, Tongji Medical College, Huazhong University of Science and Technology, China; ${ }^{2}$ Tongji Hospital of Tongji Medical College, Huazhong University of Science and Technology, China

Background: Immunosuppressive molecules are vital to hepatocellular carcinoma (HCC) by evading of immune surveillance. Fgl2, known as a prothrombinase, has been demonstrated as a immunological inhibitor and it has been found overexpression in plasma and tumor tissue in HCC patients in our previous studies. Aim of the study was to elucidate whether and how fgl2 regulate the HCC tumor immunological microenvironment.

Method: $1 \times 107$ Hepa1-6 cells were subcutaneously injected in C57BL/6 mice. Tumor volume were measured and the mice were treated with $50 \mu \mathrm{g}$ fgl2 depletion antibody against FRED every 3 days when the volume is over $100 \mathrm{~mm}^{3}$. After 2 weeks treatment mice were sacrificed. The phenotypes and proportions of immunocytes and cytokines level in tumor and draining lymph node (dLN) were examined .

Result: The tumor volume of fgl2 depletion antibody treatment group was significantly smaller than control group. After 14 days of treatment, the volume of the treatment group $(n=10)$ was $(240.0 \pm 38.86)$ $\mathrm{mm}^{3}$ and that of the control group $(\mathrm{n}=10)$ was $(558.9 \pm 114.6) \mathrm{mm}^{3}$, $P<0.05$. IL-10 and IL-35 level in tumor were greatly lower in treatment group(IL-10 level was $(143.9 \pm 27.54) \mathrm{ng} / \mathrm{ml}$ in treatment group versus $(726.5 \pm 261.8) \mathrm{ng} / \mathrm{ml}$ in control, $P=0.09$ and IL-35 level was $(2.093 \pm 0.5525) \mathrm{ng} / \mathrm{ml}$ in treatment group versus $(4.173 \pm 0.3413) \mathrm{ng} /$ $\mathrm{ml}$ in control, $P<0.05)$. With fgl2 depletion, the numbers of $\mathrm{CD} 8+\mathrm{T}$ 
cells and dendritic cells (DCs) were upregulated(the percent of $\mathrm{CD} 8+\mathrm{T}$ in $\mathrm{CD} 45+$ cells was $(12.25 \pm 2.322) \%$ in treatment group versus $(5.094 \pm 1.239) \%$ in control, $P<0.05$ and that of DCs in CD45+ cells was $(2.840 \pm 0.3960) \%$ in treatment group versus $(1.402 \pm 0.2623) \%$ in control, $P<0.05)$ and M2 was downregulated(the percent of $\mathrm{M} 2$ in macrophages was $(2.025 \pm 1.478) \%$ in treatment group versus $(10.85 \pm 4.256) \%$ in control, $P<0.05)$

Conclusion: fgl2 reduced the numbers of CD8+T cells and DCs which were crucial to anti-tumor immunology. fgl2 upregulated M2 numbers, IL-10 and IL-35 levels, and induced an immunosuppressive state in HCC tumor microenvironment.

\section{$H C C-2$}

Plectranthus amboinicus (Lour.) spreng exerts antitumor activity against p53-competent but not p53-deficient hepatocellular carcinoma both in vivo and in vitro

\section{Chi Tan $\mathrm{Hu}^{1}$}

${ }^{1}$ Division of Gastroenterology, Taiwan

Background: Plectranthus amboinicus (Lour.) Spreng is a perennial herb which belongs to the family Lamiaceae. The plant is distributed throughout tropical countries where it is widely used for treating respiratory tract disorders such as bronchoconstriction, tussis, and expectorant conditions. The objective of this study was to investigate the anticancer activity of the aqueous extract of Plectranthus amboinicus (PA-AEX) in hepatocellular carcinoma (HCC).

Method: High performance liquid chromatography (HPLC) and gas chromatography-mass spectrometry (GC-MS) analyzed the components of PA-AEX. Cell culture systems and a xenograft animal model were used to show the anti-HCC activity of PA-AEX.

Result: HPLC and GC-MS revealed that PA-AEX contained carvacrol, thymol, sabinol, docosenamide, stigmasterol and 1, 2 benzenedicarboxylic acid. The results of a MTT assay demonstrated that PA-AEX induced a strong cytotoxic effect towards p53-competent HepG2 and Huh 7 cells but not in p53-deficeint Hep3B cells. Furthermore, the xenograft model demonstrated that PA-AEX was able to suppress tumor growth as shown in the cellular studies.

Conclusion: This report is the first to demonstrate the anti-HCC activity of fresh PA-AEX and provides support for the application of PA-AEX as a chemopreventive functional food. Most importantly, the results will provide future developments at a preclinical and prospectively a clinical, personalized treatment for HCC.

\begin{tabular}{|c|c|c|}
\hline Cell line & p53 mutant & $\begin{array}{c}\text { Response to } \\
\text { PA-AEX }\end{array}$ \\
\hline U937 & mutant inactive & $\mathrm{x}$ \\
\hline K562 & negative & $x$ \\
\hline Calu-1 & deleted & $x$ \\
\hline $\mathbf{T L}$ & negative & $x$ \\
\hline Jurkat & negative & $x$ \\
\hline HepG2 & Wild type & 0 \\
\hline Huh 7 & Mutant active & 0 \\
\hline Hep3B & Null & $x$ \\
\hline
\end{tabular}

HCC-3

Association of ANG1, ANG2 and FGF genetic polymorphism and their expression analysis in $\mathrm{HBV}$ related hepatocellular carcinoma

\section{$\underline{\text { Dipu bharali }}^{1}$, Suresh Kumar ${ }^{1}$, Premashis Kar $^{2}$}

${ }^{1}$ Maulana Azad Medical College, India; ${ }^{2}$ Max Super speciality Hospital, Vaishali, India

Background: Hepatocellular carcinoma (HCC) is one of the common causes of cancer death in India. Angiogenesis, hypervascularization process in $\mathrm{HCC}$ is closely regulated by angiogenic factors such as Ang1, Ang2 and FGF. The aim is to find out the genetic polymorphism and differential expression of these factors in HCC in Indian population.

Method: Total 75 confirmed diagnosed HBV related HCC cases and 150 controls without HCC were included. Ang1, Ang2 and FGF DNA polymorphism were analyzed by direct sequencing of the amplified PCR products and their differential expression were done using real time PCR.

Result: The Ang1 CC and AC genotype and C, T allele was a risk in chronic liver diseases developing to HCC. Ang2 AA genotype, A allele are individual risk for of HCC in CLD patients of HCC in CLD patients. FGF1 TT, FGF2 CC genotype and Allele T and allele $\mathrm{C}$ are individual risks of HCC in CLD patients. The expression level of Ang1 was lower and Ang2 and FGF mRNA expression level was higher in HCC compared to controls without HCC.

Conclusion: Abnormal vasculature of HCC might be due to Ang1, Ang2 and FGF gene polymorphism and differential expression. High expression of Ang-2, FGF and low expression of Ang-1 in HCC might play key role in carcinogenesis and progression of HCC via angiogenesis by constituting a system that regulates vascular maturity and development of complex vascular networks. The association of polymorphism of angiogenic factors such as Ang1, Ang2 and FGF gene and their expression level might play an important role in the angiogenic and differentiation processes in HCC in CLD and also in initiation and progression of angiogenesis in tumors. Therefore genetic polymorphism of angiogenic factors might be a risk to develop HCC from chronic liver diseases in Indian population.

\section{HCC -4}

Gold nanoparticle tagged anti-tumor drug in targeted killing of chemo-resistant human hepatocellular carcinoma cell line

Mohd Aejaz Habeeb ${ }^{1}$, Sandeep K Vishwakarma ${ }^{1}$, Nagarapu Raju$^{1}$, Safwaan Habeeb ${ }^{1}$, Mohd Aejaz Habeeb ${ }^{1}$

${ }^{1}$ Centre for Liver Research \& Diagnostics, India

Background: Hepatocellular carcinoma (HCC) is the fifth most common solid tumor worldwide and the fourth leading cause of cancer-related death, with an estimated death rate of more than $5,00,000$ per year. Existing treatment including loco-regional or systemic chemotherapy, fail largely due to the chemo résistance properties of cancer cells. High doses of drugs lead to systemic toxicity resulting in a multitude of unwanted adverse reactions due to their lack of availability at tumor site, poor tumor intake of drugs and rapid elimination. In addition most conventional methods for delivering chemotherapeutic agents fail to achieve therapeutic concentration of drugs, despite reaching toxic systemic levels. In present study, we investigated the effect of tagged anti-tumor drugs 
with ultra-small gold nanoparticles (AuNPs) to investigate their effect on HCC and drug resistant HCC cells.

Method: Gold nanoparticles (GNPs) were synthesized by instantaneous reduction of $0.2 \mathrm{mM}$ of $\mathrm{AuCl} 3$ with $6 \mathrm{mM}$ of LiBH4 by adding freshly prepared $\mathrm{AuCl} 3$ with $\mathrm{LiBH} 4$. Sorafenib solution was prepared by taking $285 \mathrm{mg}$ of SF tablet powder dispersed in $40 \mathrm{ml}$ of water by sonicating it for $15 \mathrm{~min}$. Further it was diluted to make a solution of $\sim 0.5 \mathrm{mg} / \mathrm{mL}$ SF as a stock. For preparation of SF-GNP Nanoconjugates (SF-GNP), 2 to 1 volume to volume ratio of GNP and SF stock solution mixed well by sonicating for 5 mins and lef at $4{ }^{\circ} \mathrm{C}$ for $72 \mathrm{hr}$. HepG2 cells were cultured and IC50 values of SF from dose response curve optimized. The IC50 of SF in sensitive HepG2 cells was observed $17.5 \mu \mathrm{g} / \mathrm{mL}$ among different range of concentrations tested. Finally the SF resistant HepG2 cells were found to grow exponentially at $52.48 \mu \mathrm{g} / \mathrm{mL}$ concentration of SF. The cell viability was determined by MTT assay, at each passage of day 3 after SF treatment. The SF treated HepG2 cells passage with viability of approximately $100 \%$ or similar to control group was considered as SF resistant cells which was further utilized to determine the SF-GNP therapeutic efficacy in 3D solid HCC tumor model system.

Result: We observed that nanoformulated drugs (AuNP-QC, AuNP$\mathrm{SF}$ and AuNP-CC) are more effective on normal tumorigenic as well as drug-resistant $\mathrm{HCC}$ cells. Among three nanoformulated drugs (QC, $\mathrm{SF}$ and $\mathrm{CC}$ ), AuNP-tagged SF is more effective in killing of normal tumorigenic as well as drug-resistant HCC cells. In conclusion, this study offers a controlled, safe and more effective drug delivery approach at very less concentration of drugs with pre-determined rates for predefined periods at the target normal and drug-resistant cancer cells.

Conclusion: This particular strategy of using SF-GNP conjugate in treating SF resistant HCC cells may augment the possibility of SF and related drugs to reduce the load of drug resistance with lower dose and reduced adverse events in advanced cancer by evading the drug efflux mechanisms. Further studies will be required to better understand the internalization and subsequent release of SF from SF-GNP nanoconjugates. In addition, the long term therapeutic efficacy of SFGNPs in preclinical model system with detailed mechanism for uptake of SF-GNP nanoconjugates and reversal of drug resistance needs to be identified.

\section{HCC-5}

Hepatitis B-X protein ( $\mathrm{HBx})$ enhances angiogenenic properties of endothelial cells in the progression of hepatocellular carcinoma (HCC)

\section{Preety Rawal ${ }^{1}$, Hamda Siddiqui ${ }^{3}$, Vikrant Nain $^{2}$, Savneet Kaur ${ }^{3}$}

${ }^{1}$ Gautam Buudha University, India; ${ }^{2}$ Gautam Buddha University, India; ${ }^{3}$ Institute of Liver and Biliary Sciences, India

Background: Hepatitis B-X Protein (HBx) encoded in Hepatitis B virus (HBV) genome modulates host cellular responses and plays critical role in hepatocellular carcinoma. In the current study, we investigated how $\mathrm{HBx}$-infected hepatoma cells interact with the neighbouring liver stromal cells (endothelial and stellate cells) by a series of in vitro studies.

Method: Huh7 were cultured and transfected with the mammalian expression vector pGFP-HBx plasmid. Co-culture assays were performed between HBx transfected cells and stromal cells [endothelial cell lines (EC) and hepatic stellate cell lines (SC)]. The expression of angiogenic genes in untransfected and transfected Huh7 co-cultured stromal cells was studied by real time PCR. Subsequently, the role of HBx in tumor invasion was studied by functional assays like migration, proliferation (wound healing), LDL uptake and tube formation in the stromal cells after co-cultures with $\mathrm{HBx}$ transfected hepatoma cells.

Result: A transfection efficiency of $85 \%$ was observed in the $\mathrm{HBx}$ transfected hepatoma cells. Real time PCR data showed that in comparison to EC co-cultures with untransfected cells, there was a substantial upregulation of angiogenic genes, MMP12 and vegfr1, while a downregulation of vegfr2 and MMP14 in the ECs after cocultures with the transfected hepatoma cells. Functional assays revealed that in comparison to stromal cell co-cultures with untransfected cells, there was significantly more migration $(42.85 \%$ in $\mathrm{SC}$ co-cultures and $60 \%$ in EC co-cultures, $\mathrm{P}<0.05$ ), proliferation and wound healing (more than $70 \%$ in EC co-cultures, $\mathrm{P}<0.05$ ), uptake of DiI-LDL (33\% more uptake in EC co-cultures, $\mathrm{P}<0.05)$, tube formation $(31.25 \%$ more networks formed in EC co-cultures, $\mathrm{P}<0.05)$ in co-cultures of ECs and SCs with HBx-transfected cells.

Conclusion: The study indicates that HBV-HBx plays an important role in $\mathrm{HCC}$ invasion by significantly affecting the angiogenic and invasive properties of the neighbouring endothelial cells in the liver.

\section{HCC-6}

Histone lysine methyltransferase G9a is a novel epigenetic target for the treatment of hepatocellular carcinoma

Masayuki Yokoyama ${ }^{1}$, Tetsuhiro Chiba ${ }^{1}$, Zen Yoh $^{2}$, Yuko Kusakabe $^{1}$, Masanori Inoue ${ }^{1}$, Toru Wakamatsu ${ }^{1}$, Tomoko Saito ${ }^{1}$, Sadahisa Ogasawara ${ }^{1}$, Eiichiro Suzuki ${ }^{1}$, Yoshihiko Ooka ${ }^{1}$, Akinobu Tawada ${ }^{1}$, Masayuki Otsuka ${ }^{1}$, Masaru Miyazaki', Osamu Yokosuka ${ }^{1}$, Naoya Kato ${ }^{1}$

${ }^{1}$ Graduate School of Medicine, Chiba University, Japan; ${ }^{2}$ Kobe University Graduate School of Medicine, Japan

Background: Methylation of histone $\mathrm{H} 3$ at lysine 9 (H3K9) and histone $\mathrm{H} 3$ at lysine 27 (H3K27) is involved in transcriptional repression. Histone $\mathrm{H} 3$ lysine 9 dimethylation (H3K9me2) is mainly regulated by the histone lysine methyltransferase G9a. G9a overexpression has been reported in various types of cancers, including hepatocellular carcinoma (HCC). However, both the role of G9a and the significance of $\mathrm{H} 3 \mathrm{~K} 9 \mathrm{me} 2$ in $\mathrm{HCC}$ cells remain unclear.

Method: We performed loss-of-function analyses of G9a using lentivirus-mediated knockdown of G9a in culture. We tried to detect Candidate targets of G9a by chromatin immunoprecipitation followed by sequencing (ChIP-seq) and RNA-sequencing (RNA-seq). Additionally, pharmacological disruption of G9a was conducted in culture and in vivo xenograft models. Finally, G9a expression levels and $\mathrm{H} 3 \mathrm{~K} 9 \mathrm{me} 2$ levels in primary HCC surgical samples were determined by immunohistochemical analyses.

Result: Knockdown of G9a reduced H3K9me2 levels and impaired both HCC cell growth and sphere formation. However, transforming growth factor $\tilde{\mathrm{A}} \ddot{Y} 1$-induced epithelial mesenchymal transition (EMT) was not suppressed by G9a knockdown. Combined analyses of chromatin immunoprecipitation followed by sequencing and RNAsequencing led to successful identification of 96 candidate epigenetic targets of G9a. Pharmacological inhibition of G9a by BIX-01294 resulted in both cell growth inhibition and induction of apoptosis in HCC cells. Intraperitoneal administration of BIX-01294 suppressed the growth of xenograft tumors generated by implantation of HCC cells in non-obese diabetic/severe combined immunodeficient mice. Immunohistochemical analyses revealed high levels of G9a and $\mathrm{H} 3 \mathrm{~K} 9 \mathrm{me} 2$ in $36(66.7 \%)$ and $35(64.8 \%)$ primary HCC tissues, respectively. G9a expression levels were significantly positively correlated with $\mathrm{H} 3 \mathrm{~K} 9 \mathrm{me} 2$ levels in tumor tissues. In contrast, in nontumor tissues, G9a and H3K9me2 were only observed in biliary epithelial cells and periportal hepatocytes. 
Conclusion: G9a inhibition impairs cell growth, but not EMT in HCC cells. Our data indicate that pharmacological interference of G9a might be a novel epigenetic approach for the treatment of HCC.

\section{HCC -7}

Isolation, culture and characterization of patient derived CD34+ liver cancer stem cells

\section{Su Cheol Park ${ }^{1}$, Jae Hoon Jeoung ${ }^{2}$, Yong Jin Jung ${ }^{3}$}

${ }^{1}$ Korea Institute of Radiological and Medical Sciences, Korea Cancer Center Hospital, Korea, Republic of; ${ }^{2}$ Korea Institute of Radiological and Medical Sciences, Korea, Republic of; ${ }^{3}$ Seoul National University College of Medicine, SMG-SNU Boramae Hospital, Korea, Republic of

Background: Radiotherapy and chemotherapy can be used as a salvage therapy for advanced hepatocellular carcinoma (HCC) however the responses of the treatments are insufficient due to the resistance. Liver cancer stem cells can be a possible cause of radioresistance and recurrence of HCC. CD34+ stem cells play an important role during liver development and regeneration. Previously we isolated and cultured CD34+ cells from conventional hepatoma cell line (PLC/PRF/5 cells). In this study we isolated and cultured CD34+ cells from human HCC tissues and evaluated the characteristics of cancer stem cells.

Method: The HCC specimens and data used in this study were provided by the Radiation Tissue Resources Bank of Korea Cancer Center Hospital (2015-1-01-H/C10/1 10). Patient derived CD 34+ cancer stem cells were isolated from human HCC tissues using flow fluorescence-activated cell sorting (FACS). Purified CD34+ cells have been cultured in vitro on mouse embryonic fibroblast feeder cells. Furthermore, by employing transwell culture system, CD34+ cells were cultured in isolated compartment and used for further characterization. Then we explored the expression of CD34 and stem cell markers. Also we compared the expression of stem cell markers between CD34+ cells and CD34- cells.

Result: The expressions of CD34, CD44 and CD133 were $9.8 \%$, $4.8 \%$, and $0.16 \%$ respectively. The HCC cells and fibroblasts were isolated and cultured, however the growth of cells was not maintained. CD34+ cells isolated from human HCC tissues and cultured using transwell were not proliferated in $2 \mathrm{D}$ culture condition. We can observe that Nanog, Oct4, Sox2, CD44 and CD133, the stem cell markers were highly expressed in CD $34+$ cells than CD34- cells.

Conclusion: We could isolate and characterize the CD34+ cancer stem cells from human HCC tissues. Thus CD34+ liver cancer stem cells can be used for the studies of radiation and chemo-resistant HCC. In addition, further study for the optimal culture method with the patient derived cancer stem cells is needed.

\section{$H C C-8$}

LINE-1 demethylation is associated with expression of CD133 and ST18 in hepatocellular carcinoma

Yu Rim Lee ${ }^{1}$, Soo Young Park', Keun Hur ${ }^{3}$, Gyeonghwa Kim ${ }^{3}$, Hye Won Lee ${ }^{2}$, Se Young Jang ${ }^{1}$, Won Young Tak', Young Oh Kweon ${ }^{1}$, Bina Jeong', Gyoun Eun Kang', Sangkyung Seo ${ }^{1}$, Jung Gil Park ${ }^{4}$

${ }^{1}$ Kyungpook National University Hospital, Korea, Republic of; ${ }^{2}$ Dongsan Medical Center, School of Medicine, Keimyung University, Korea, Republic of; ${ }^{3}$ School of Medicine, Kyungpook
National University, Korea, Republic of; ${ }^{4}$ Yeungnam University Medical Center, Korea, Republic of

Background: Long interspersed nuclear element-1 (LINE-1) hypomethylation, representing global DNA methylation level, is associated with prognosis via activation of oncogenic functions of genes. This experiment was performed to evaluate prognostic implication of LINE-1 methylation in patients with hepatocellular carcinoma (HCC) and the possible mechanisms related to oncogene activation.

Method: Ninety-six HCC patients between October 2014 and September 2015 at Kyungpook National University Hospital, Daegu, South Korea were enrolled for this prospective study. Quantitative pyrosequencing was performed to quantify the methylation level of for CpG sites in the LINE-1 promotor. The expression of CD133 and ST18 were measured by immunohistochemistry and their correlation with LINE-1 methylation levels were analyzed.

Result: LINE-1 was significantly hypomethylated in tumor tissues compared with nontumor tissues $(64.0 \pm 11.6 \%$ vs. $75.6 \pm 4.0 \%$, respectively, $p<0.0001)$. In this study population, LINE-1 hypomethylation group in HCC patients was associated with large proportion of female gender, smaller tumor size, and nonexistence of ascites $(p<0.05)$. Contrary to previous reports, LINE-1 hypomethylation was not an independent risk factor for overall survival and disease progression (all $p>0.05$ ). A total of $81(84.4 \%)$ patients had demethylation of LINE-1 $(\Delta \mathrm{MI}<0)$, and $15(15.6 \%)$ patients had hypermethylation of LINE-1 $(\Delta \mathrm{MI} \geq 0)$. HCC with demethylation of LINE-1 $(\Delta \mathrm{MI}<0)$ had higher $\mathrm{CD} 133$ expression than $\mathrm{HCC}$ with hypermethylation of LINE-1 $(\Delta \mathrm{MI} \geq 0)(p=0.011)$. Moreover, when patients divided into two groups based on the mean value of tumor line-1 methylation, ST18 showed borderline significance in distinguishing the LINE-1 hypomethylation group than the other group $(p=0.053)$.

Conclusion: LINE-1 demethylation is associated with expression of CD133 and ST18 in Hepatocellular carcinoma. LINE-1 hypomethylation was not an independent risk factor for overall survival and disease progression in this study, and this is probably due to the various stage of enrolled HCC patients.

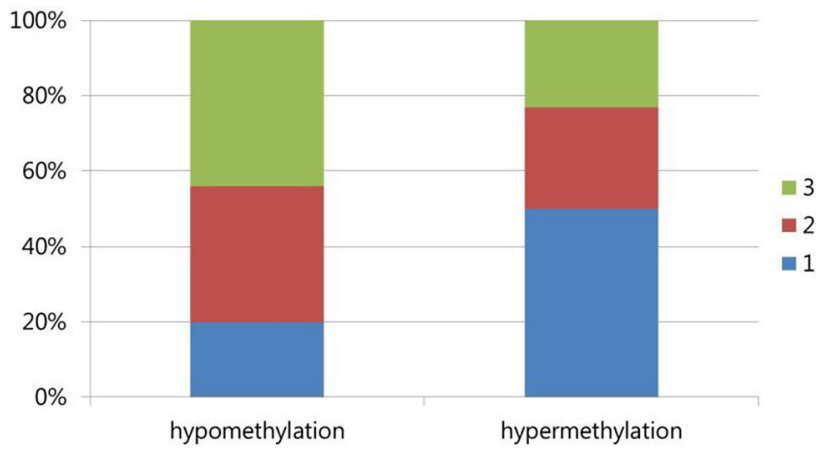

HCC-9

miR-520a is a potential molecular marker of in human hepatocellular carcinoma and functions as a tumor suppressor by targeting IRF2 and E2F7

\section{$\underline{\text { Mingxin Zhang }}^{1}$, Manli Cui ${ }^{1}$, Suna Zhou ${ }^{1}$, Jingjie Wang ${ }^{1}$ \\ ${ }^{1}$ Tangdu Hospital, Fourth Military Medical University, China}

Background: Growing evidence indicates that miR-520a was involved in the complement attack and migration of tumor cells, but nonetheless, the role of miR-520a in human hepatocellular carcinoma 
(HCC) and its exact mechanism is not clear. The present study was to evaluate the biomarker significance and function of miR-520a in HCC and its mechanism.

Method: We used real-time reverse transcriptase-polymerase chain reaction to quantify the expression of miR-520a in $50 \mathrm{HCC}$ tissues and analyzed its relationship with clinicopathological factors and survival. Then, the effects of miR-520a on proliferation, apoptosis, and invasion of HCC cells was detected using MTT, flow cytometric analysis, and transwell invasion assays, respectively. Moreover, we investigated the post-transcriptional regulation of IRF2 and E2F7 expression using immunoblot analysis and luciferase reporter assays. Finally, the effect of inhibiting expression of IRF2 and E2F7 by shRNA on HCC malignant behaviors were also tested.

Result: miR-520a was down-regulated in HCC, and expression of miR-520a was correlated with tumor differentiation, lymph node metastasis, and tumor size. Univariate and multivariate analyses indicated that low miR-520a expression might be a poor prognostic factor. Then, miR-520a inhibited proliferation by inducing apoptosis and repressed invasion in the HCC cell lines SMMC-7721 and Bel7402. Further studies demonstrated that miR-520a post-transcriptionally down-regulated the expression of RF2 and E2F7 in vitro. Moreover, inhibiting of IRF2 and E2F7 got the same effects as upregulating of miR-520a.

Conclusion: miR-520a is a potential molecular marker of HCC and functions as a tumor suppressor by post-transcriptionally regulating IRF2 and E2F7.

\section{HCC-10}

\section{A dangerous relationship between copper and Myc promoting hepatocellular carcinoma}

Clara Balsano ${ }^{1}$, Laura Antonucci ${ }^{1}$, Barbara Barbaro ${ }^{1}$, Barbara Illi $^{2}$, Sergio Nasi ${ }^{2}$, Anna Licata ${ }^{4}$, Luca Miele ${ }^{3}$, Antonio Grieco $^{3}$

${ }^{1}$ University of L'Aquila, Italy; ${ }^{2}$ National Research Council, Italy; ${ }^{3}$ Universit $\tilde{A}$ Cattolica del Sacro Cuore, Italy; ${ }^{4}$ University of Palermo, Italy

Background: Free serum copper correlates with incidence and progression of human cancers, including hepatocellular carcinoma (HCC). Inadequate copper serum concentration is involved in the pathogenesis of Non alcoholic fatty liver disease (NAFLD), which is becoming a major cause of liver damage progression and HCC incidence. Finally, MYC is over-expressed in most of HCCs. We evaluated if serum copper correlates to the progression of NAFLDcirrhosis toward HCC.

Method: We evaluated, in hepatoma cell lines, if exogenous copper had a role in liver tumorigenesis, deepening on the possible interconnection with MYC.

Result: NAFLD-cirrhotic patients were characterized by a statistical significant enhancement of serum copper levels in NAFLD-cirrhotic patients, even more evident in HCC patients. We demonstrated that extracellular copper sensitizes liver cells to proliferate and invade by modulating MYC expression. We highlighted that MYC binds a specific region of the promoter of CTR 1 , a copper transporter whose expression is regulated to avoid excessive intracellular copper entry, regulating its transcription and thus the intracellular copper levels.

Conclusion: This work provides novel insights on the molecular mechanisms by which copper may favor the progression from cirrhosis to cancer. The $\mathrm{Cu} / \mathrm{MYC}$ interplay opens a window to refine HCC diagnosis and design new combined therapies.
HCC-11

Bufalin inhibits HBV-associated hepatocarcinogenesis by blocking HBx-induced AR-CCRK signaling pathway

\section{Zhuo $\mathrm{Yu}^{1}$, Xuehua Sun ${ }^{1}$, Man $\mathrm{Li}^{1}$, Zhenhua Zhou ${ }^{1}$, Lingying Huang ${ }^{1}$, Xin Zhang ${ }^{1}$, Xiaojun Zhu ${ }^{1}$, Hai Feng ${ }^{2}$, Alfred Szelok Cheng ${ }^{3}$, Yueqiu Gao ${ }^{1}$}

${ }^{1}$ Shuguang Hospital affiliated to Shanghai University of Traditional Chinese Medicine, China; ${ }^{2}$ Harbin Medical University, China; ${ }^{3}$ The Chinese University of Hong Kong, Hong Kong

Background: Hepatitis B virus (HBV)-associated hepatocellular carcinoma (HCC) is the main subgroup of malignant hepatoma refractory to treatment especially at advanced stage. Bufalin, a digoxin-like bioactive component secreted by the skin and parotid venom glands of Bufo gargarizans, has been reported to effectively inhibit HBV infection and HCC development. However, seldom is known about the effect of bufalin on HBV-associated HCC progression.

Method: In vitro, cell viability, anchorage-dependent and -independent growths were performed to evaluate the effect of bufalin on $\mathrm{HBx}$-induced hepatocellular proliferation and malignant transformation. PCR array was used to examine the alteration of $\mathrm{HBx}$-induced oncogenic signaling. In vivo xenograft and orthograft mice models, tumor size and incidence were recorded and abnormal proliferation were detected by Ki67 immunostaining. Western blot and RT-qPCR were conducted to examine the expression of $\mathrm{HBx}$-induced oncogenic signaling both in vitro and in vivo samples.

Result: Bufalin showed more efficient effect on inhibiting proliferation and malignant transformation in HBV-associated HCC cells rather than non $\mathrm{HBV}$-associated $\mathrm{HCC}$ cells and immortal hepatocytes both by dose- and time-dependent manner. In mechanism, Bufalin had no effect in HBx which acts as a transcriptional factor during hepatic tumorigenicity, but had a critical influence on $\mathrm{HBx}$-induced AR-CCRK oncogenic signaling. Bufalin greatly decreased the transcript level and protein expression of CCRK through phosphorylated AR impediment, thus subsequently disrupted the activation of $\mathrm{Wnt} / \mathrm{B}$ catenin signaling cascade induced by CCRK. Marked reduction in $\mathrm{HBx}-\mathrm{LO} 2$ cells induced tumor growth and incidence were clearly observed after bufalin treatment in both xenograft and orthograft mice models. Significant suppression of pAR, CCRK and active $\beta$-catenin by bufalin treatment was further verified in tumor tissues.

Conclusion: Bufalin is a potential anti-HCC therapeutic candidate which is more effective in $\mathrm{HBV}$-associated HCC. The underlying mechanism is related to the perturbation of $\mathrm{HBx}$ induced AR-CCRK oncogenic signaling.

\section{HCC-12}

Coexpression of PD-1 and BTLA identifies a novel T-cell exhaustion phenotype in hepatocellular carcinoma patients

\section{Liu Jiayu $^{1}$, Zhao Qiyi ${ }^{1}$, Gao Zhiliang ${ }^{1}$}

${ }^{1}$ Third Affiliated Hospital, Sun Yat-sen University, Guangzhou, PR China, China

Background: Although immunotherapy targeting PD-1/PD-L1 pathway is being applied in clinic, the response outcomes are heterogeneous, suggesting existences of distinctive subsets within PD-1-expressing $\mathrm{T}$ cells that react differently to PD-1/PDL1 blockade. However, markers to demarcate these subsets in human cancers remain unclear. 
Method: CD4+ T cells were purified from tumor and nontumor liver tissues of hepatocellular carcinoma (HCC) patients. Phenotypic and functional characteristics of BTLA (B and T lymphocyte attenuator)+ PD-1+ and BTLA-PD-1+ CD4+ T cells were determined by flow cytometry. Effects of PD-1/PD-L1 and BTLA/HVEM blockade were assessed by ex vivo studies.

Result: We identified a novel phenotype of exhausted CD4+ T cells in advanced human hepatocellular carcinoma. This phenotype was characterized by the coexpression of BTLA and PD-1 on CD4+ T cells from tumor tissue but not nontumor liver tissue. BTLA+PD-1+ tumor-infiltrating $\mathrm{CD} 4+\mathrm{T}$ cells were more severe exhausted than BTLA-PD-1+ and BTLA-PD-1-counterparts, producing less IFN- $\gamma$, TNF- $\alpha$ and IL-2. Importantly, blockade of PD-L1 could restore the IFN- $\gamma / \mathrm{TNF}-\alpha$ production in BTLA + PD- $1+$ tumor CD4+ T cells but partially suppressed the activation of BTLA-PD-1+ CD4+ T cells. Moreover, we provided evidence that interaction of BTLA and its ligand herpesvirus entry mediator (HVEM) also participated in suppressing $\mathrm{CD} 4+\mathrm{T}$ cell function in $\mathrm{HCC}$ tumor.

Conclusion: BTLA could identify distinct function of PD-1 expressing $\mathrm{CD} 4+\mathrm{T}$ cells in human HCC, which might have important implications for future personalized T-cell-base immunotherapy strategies targeting PD-1/PD-L1 pathway.

\section{HCC -13}

Decoction of FuZheng JieDu Xiaoji influences HCC progression through PKB/cyclinD1-P21/ P27 pathway regulation

\section{$\underline{\text { Xue Yang }}{ }^{1}$, Ying Feng ${ }^{1}$, Xianbo Wang ${ }^{1}$ \\ ${ }^{1}$ Beijing Ditan Hospital, China}

Background: The FuZheng JieDu Xiaoji decoction (FZJD) is a traditional Chinese herbal medicine used for the treatment of HCC. We previously found that FZJD combination treatments significantly improved the survival rate of patients with HCC compared to the survival achieved with standard treatments alone, but the results required verification in a larger clinical study. Moreover, the mechanisms underlying the effects of FZJD on HCC tumor progression should be determined.

Method: To evaluate the effectiveness of FJZD, we systematically investigated the major precipitating factors and one-year survival rates of 338 propensity score-matched patients with HCC that were treated with FZJD/standard treatments or standard treatments only. The molecular mechanisms underlying the effects of FZJD on patients with HCC were examined by using biological assays. Highperformance liquid chromatography-mass spectrometry (HPLC-MS) was used to identify the constituents of FZJD extract.

Result: The analysis showed that alkaline phosphates, the neutrophilto-lymphocyte ratio, alpha-fetoprotein levels of $\geq 500 \mathrm{ng} / \mathrm{mL}$, Barcelona Clinic Liver Cancer (BCLC) stage, and the co-administration of FZJD were independent prognostic factors for the survival of patients with HCC. Kaplan-Meier (KM) analysis showed that FZJDtreated patients showed a significantly better prognosis, especially those with BCLC stage-B/C/D. In vitro experiments showed that the addition of medicated serum significantly reduced the proliferation and metastasis of HCC cells through the inhibition of phospho-protein kinase B (PKB) and cyclin D1 expression and increased levels of p21 and p27. The HPLC-MS analysis showed that polyphyllin VI, acteoside, and chlorogenic acid were the main active ingredients of FZJD.

Conclusion: The results suggested that FZJD extends the survival time of patients with $\mathrm{HCC}$ and inhibits cell proliferation through the $\mathrm{PKB} /$ cyclinD1/P21/P27 pathway.
HCC-14

Distinct changes of BTLA and HVEM expressions in circulating CD4+ and CD8+ $\mathrm{T}$ cells in hepatocellular carcinoma patients

\author{
Liu jiayu $^{1}$, Zhao Qiyi ${ }^{1}$, Gao Zhiliang ${ }^{1}$ \\ ${ }^{1}$ Third Affiliated Hospital, Sun Yat-sen University, Guangzhou, PR \\ China, China
}

Background: BTLA/HVEM (B and T lymphocyte attenuator/herpes virus entry mediator) pathways play a critical role in T-cell suppression in tumor. However, its dynamic changes in different $\mathrm{T}$ cell subsets in peripheral blood and their clinical significance are largely unclear in cancer patients.

Method: Peripheral blood lymphocytes were isolated from the blood of HCC patients and healthy donors by Ficoll density gradient centrifugation, and then used for flow cytometry analysis or cell culture. In some experiments, cells were pretreated with a blocking Ab against BTLA or a control IgG. Peripheral blood lymphocytes were stained with fluorochrome-conjugated mAbs for CD3, CD4, CD8, HVEM, IFN- $\gamma$, BTLA or control Ab. CD4+ and CD8 $+\mathrm{T}$ cells were gated based on CD3 and CD4 or CD8 expression, respectively.

Result: In our study, we showed distinct changes of BTLA and HVEM expressions on peripheral blood CD4+ and CD8+ T cells in patients with hepatocellular carcinoma (HCC): BTLA expression were significantly up-regulated on circulating CD4+ but not CD8+ T cells. In sharp contrast, the levels of HVEM expression were significantly down-regulated on circulating CD8+ but not CD4+ T cells. A strong positive correlation between BTLA expression on circulating $\mathrm{CD} 4+\mathrm{T}$ cells and BTLA expression on autologous CD8+ counterparts was observed in healthy donors but absent in HCC patients. More importantly, we found that blockade of BTLA/ HVEM pathway increased IFN- $\gamma$ production in both circulating $\mathrm{CD} 4+$ and $\mathrm{CD} 8+\mathrm{T}$ cells.

Conclusion: Our data suggested that BTLA/HVEM pathway contributes to peripheral T-cell suppression in HCC patients and BTLA/ HVEM may serve attractive targets for HCC immunotherapy.

\section{HCC-15}

Effects of mesenchymal stem cells on solid tumor metastasis in experimental cancer models: a systematic review and metaanalysis

\section{Jinghuan $\mathrm{Li}^{1}$, Jian $\mathrm{Li}^{2}$, Wenshuai $\mathrm{Fan}^{3}$, Zhenggang $\operatorname{Ren}^{3}$}

${ }^{1}$ Zhongshan Hospital, China; ${ }^{2}$ Peking university shenzhen hospital, China; ${ }^{3}$ Zhongshan hospital, Fudan university, China

Background: It has been reported that mesenchymal stem cells (MSCs) are recruited to and become integral parts of the tumor microenvironment. MSCs might have an active role in solid tumor progression, especially cancer metastasis. However, the contribution of MSCs in the process of cancer metastasis is still controversial. Moreover, an analysis of preclinical evidence has so far been lacking. In this review, we performed a meta-analysis on the effects of MSCs administration on cancer metastasis based on published preclinical studies.

Method: The PRISMA guidelines were used to conduct this review. Studies were identified from PubMed, EMBASE, and Cochrane library until March 2017. A total of 42 publications met the inclusion criteria. Outcome data on the incidence and the number of cancer metastasis as well as study characteristics were extracted. Quality of the studies was assessed according to SYRCLE Risk of Bias tool. Random-effects meta-analysis was used to pool estimates. 
Result: Of the 42 studies included, 32 reported that MSCs administration promoted outcome events (numbers or incidences of cancer metastasis), and 39 reported data that suitable for meta-analysis. The median effect size was 1.23 (95\% CI, $0.43 \sim 2.03$ ) for the number of cancer metastasis and $2.04(95 \% \mathrm{CI}, 1.57 \sim 2.65)$ for the incidence of cancer metastasis. Heterogeneity was observed, with the greater impact based on the study length and the metastasis site.

Conclusion: In conclusions, our results suggested that MSCs administration significantly increases the number and the incidence of cancer metastasis. High heterogeneity and poor reported risk of bias limit the quality and validity of these findings.
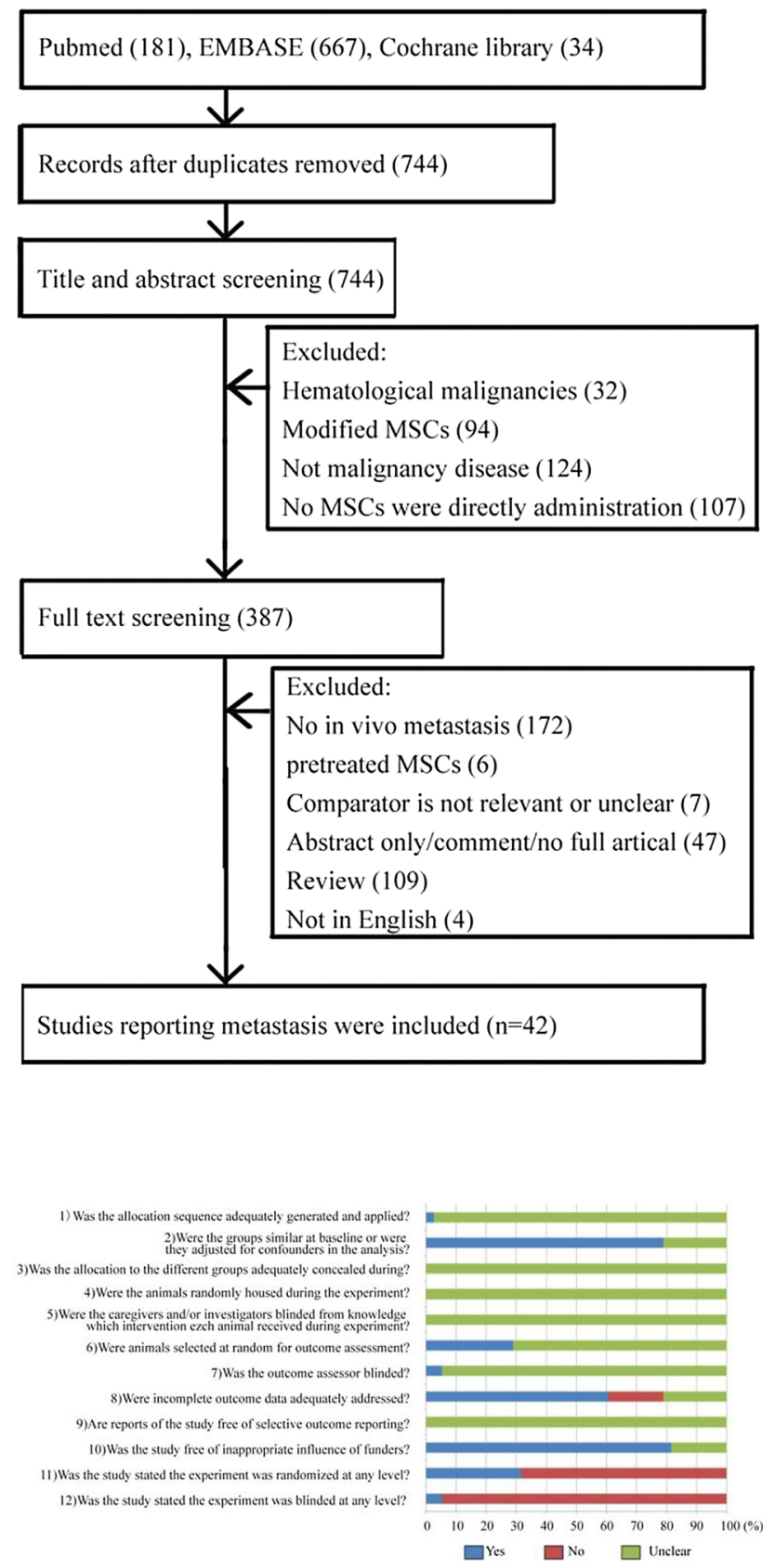

HCC-16

Inhibition of FoxH1 by tumor suppressor HNF4-alpha in hepatooncogenesis

$\underline{\text { DaeGhon Kim }}^{1}$, Jun Zhang ${ }^{1}$, Lan Liu ${ }^{2}$, Mijin Lee ${ }^{1}$, Goungran Yu ${ }^{1}$

${ }^{1}$ Chonbuk National University Medical School, Korea, Republic of;

${ }^{2}$ Yanbian University Hospital, China

Background: HNF4-alpha expression is associated with tumor suppression in hepatocellular carcinoma (HCC), but the molecular mechanisms involved in this signaling pathway remain elusive.

Method: HNF4-alpha expression was investigated by Western blot and immunohistochemistry (IHC) analyses in HCC cell lines and tissues, respectively. Colony forming ability and tumorigenicity were examined in vitro and in a mouse model system through studies of gain or loss of function. Molecular mechanisms of this signaling pathway were explored.

Result: HNF4-alpha expression inhibited the tumorigenicity of SH-J1 cells and its knockdown enhanced tumorigenicity of Alexander (AXL) cells, in vitro and in a xenotransplanted mouse model. Transcription factor array data revealed that FoxH1 expression increased in the nucleus of AXL cells with HNF4 $\alpha$ knockdown. The transcription factor, FoxH1, was shown to be inversely regulated by HNF4-alpha expression in HCC cells. AXL cells stably expressing FoxH1 grew fast in vitro and were more tumorigenic in the animal model system. SK-HEP-1 cells with FoxH1 knockdown grew slowly and were less tumorigenic than control cells. FoxH1 was associated with $\beta$-catenin activation in vitro. Therefore, HNF4-alpha suppressed $\beta$-catenin activation through the inhibition of FoxH1 expression as a tumor suppressor.

Conclusion: HNF4- $\alpha$ suppresses hepato-oncogenesis through inhibition of FoxH1-mediated $\beta$-catenin activation. These downstream signaling molecules may be potential therapeutic targets in HCC.

HCC-17

Internalization of nanoparticles within liver cancer cells: realtime assessment of cellular dynamics based on flow cytometry analysis

Mohd Aejaz Habeeb ${ }^{1}$, Sandeep K Vishwakarma ${ }^{1}$, Avinash Bardia $^{1}$, Nagarapu Raju ${ }^{1}$, Safwaan Habeeb ${ }^{1}$, Mohd Aejaz Habeeb ${ }^{1}$

${ }^{1}$ Centre for Liver Research \& Diagnostics,, India

Background: Since last decades various kinds of nanoparticles have been functionalized to improve their biomedical applications. However, the biological effect of un-modified/non-functionalized bimetallic magnetic nanoparticles remains under investigated. Herein we demonstrate a multifaceted non-functionalized bi-metallic inorganic Gd-SPIO nanoparticle which passes dual high MRI contrast and can kill the cancer cells through several mechanisms. Since last decades various kinds of nanoparticles have been functionalized to improve their biomedical applications. However, the biological effect of un-modified/non-functionalized bi-metallic magnetic nanoparticles remains under investigated. Herein we demonstrate a multifaceted non-functionalized bi-metallic inorganic Gd-SPIO nanoparticle which passes dual high MRI contrast and can kill the cancer cells through several mechanisms.

Method: All in vitro studies were conducted as per the guidelines. Gd-SPIO nanoparticles were synthesized using colloidal hydrolytic synthesis method. SEM images of Gd-SPIO nanoparticles were obtained using a Hitachi S520 Scanning Electron Microscope. The 
biocompatibility of Gd-SPIO nanoparticles with human cells was identified using human liver cells which were incubated with different concentrations of Gd-SPIO nanoparticles ranging from $10 \mu \mathrm{g} / \mathrm{ml}$ to $1 \mathrm{mg} / \mathrm{ml}$ for 72 hours in $\mathrm{CO} 2$ incubator. The dual MRI-contrast property of Gd-SPIO nanoparticles was identified in vitro using 1.5T clinical Magnetome. To establish a real-time quantification for interaction between nanoparticles and cancer cells, human hepatoblastoma cell line (HepG2) was cultured in vitro and exposed to GdSPIO nanoparticles.

Result: The results of the present study demonstrate that Gd-SPIO nanoparticles have potential to induce cancer cell death by production of reactive oxygen species and apoptotic events. Furthermore, GdSPIO nanoparticles also enhance the expression levels of miRNA199a and miRNA-181a-7p which results in decreased levels of cancer markers such as C-met, TGF- $\beta$ and hURP. One very interesting finding of this study reveals side scatter-based real-time analysis of nanoparticles uptake in cancer cells using flow cytometry analysis. Conclusion: In conclusion, this study paves a way for future investigation of un-modified inorganic nanoparticles to purport enhanced therapeutic effect in combination with potential anti-tumor drugs/molecules in cancer cells.

\section{HCC -18}

\section{Long noncoding RNA TXNDC3-1 inhibits hepatocellular carcinoma tumorigenesis via RNA-RNA crosstalk-mediated CDCA3 inhibition}

Yongzhen Liu ${ }^{1}$, Yongzhen Liu ${ }^{1}$, Yongfeng Wang', Guiwen Guan', Jing Zhang ${ }^{1}$, Ting Zhang ${ }^{1}$, Xiangmei Chen ${ }^{1}$, Fengmin Lu$^{1}$

${ }^{1}$ Peking University Health Science Center, China

Background: Hepatocellular carcinoma (HCC) is one of the leading causes of cancer-related death worldwide. However, the underlying mechanism of its oncogenesis remains to be elucidated. Recent evidences have demonstrated that the long non-coding RNAs (lncRNAs) are frequently and aberrantly expressed in HCC and are known to play a role in cancer pathogenesis. The aim of this study was to analyze the expression of a novel lncRNA, TXNDC3-1 in HCC and investigated the biological roles and clinicopathological implications of TXNDC3-1 in hepatocarcinogenesis.

Method: TXNDC3-1 expression was measured in 96 paired HCC tissues and the corresponding para-tumor tissues by real-time PCR. The prognostic predicting value of TXNDC3-1 was evaluated through Kaplan-Meier analysis. The effects of TXNDC3-1 on cell proliferation of HCC cells were studied by overexpression in vitro and in vivo. The mechanism of TXNDC3-1 in regulating CDCA3 was evaluated by bioinformatics analyses, RNA pull down assays, and CRISPR based RNA immunoprecipitation (CRIP). Alterations in CDCA3 expression were identified and verified by RT-PCR and Western blotting.

Result: LncRNA-TXNDC3-1 was significantly down-regulated in HCC tissues compared with their corresponding para-tumor tissues. TXNDC3-1 down-regulation was correlated with shorter overall survival in HCC patients $(\mathrm{p}=0.0014)$. By bioinformatics analyses, we identified CDCA3, a cell cycle related gene, which had a high sequence similarity with TXNDC3-1. RNA pull down and CRIP assays revealed that TXNDC3-1 could bind to the CDCA3 mRNA. The 1422-1465 sequences in CDCA3 mRNA and 106-138 sequences in TXNDC3-1 are essential for this binding. The prediction of RNA secondary structure indicated that this 33-nt fragment could form a RNA hairpin structure in TXNDC3-1 and CDCA3 mRNA respectively, which provide a more stable binding motif for the interaction between TXNDC3-1 and CDCA3 mRNA. Overexpression of
TXNDC3-1 in HCC cell lines inhibited CDCA3 protein expression, leaded to significant G1 arrest and cell proliferation repression. The inhibition effect of TXNDC3-1 on CDCA3 dramatically decreased when the essential binding motif were deleted or mutated. Consistently, the expression of CDCA3 was significantly up-regulated in HCC tissues and higher CDCA3 expression indicated a shorter overall survival in HCC patients.

Conclusion: We identified a novel tumor-suppressive lncRNA, TXNDC3-1, which was significantly down-regulated in HCC and decreased expression of TXNDC3-1 in HCC tissues is linked to poor prognosis. TXNDC3-1 could inhibit CDCA3 expression by directly binding to the 1422-1465 motif of CDCA3 mRNA. This finding suggest that the molecular axis comprising TXNDC3-1 and CDCA3 may play an important role in the hepatocarcinogenesis and provide a potential novel diagnostic and therapeutic target for HCC.

\section{HCC-19}

MiRNA profile as a prognostic biomarker of hepatocellular carcinoma risk after successful HCV treatment

\section{Alisa Petkevich ${ }^{1}$, Alisa Petkevich ${ }^{3}$, Pavel Ogurtsov ${ }^{4}$, $\overline{\text { Elena Kuhareva }}^{4}$, Mikhail Kiselevskiy ${ }^{2}$}

${ }^{1}$ N. N. Blokhin Russian Oncological Research Center, Russian Federation; ${ }^{2} \mathrm{~N}$. N. Blokhin Russian Oncological Research Center, Russian Federation; ${ }^{3}$ N. N. Blokhin Russian Research Oncological Center, Russian Federation; ${ }^{4}$ Peoples' Friendship University of Russia (RUDN)), Russian Federation

Background: Hepatocellular carcinoma $(\mathrm{HCC})$ is a great challenge for global medical society. In 2012 it was the second leading cause of cancer related death and deaths from liver cancer increases at the highest rate. Many factors such as hepatitis $\mathrm{C}$ virus (HCV), elder age, cirrhosis and others are supposed to predict HCC. However, still there is no defenite monitoring scheme for patients from risk group for HCC. In this study we referred to miRNAs as possible prognostic markers for HCC.

Method: Blood (n28), liver tissue (n19), saliva (n12) and cheek swabs (n28) from patients with HCV. qRT-PCR for 7 miRNA and electrophoresis in polyacrylamide gel were performed according to standard protocol. miRNA concentration was determined by NanoDrop. Plant extracts were obtained by standard alcohol distillation and assessed for exosomes presence. MiRNA profile of Huh7 and Hep2G cell lines were determined after incubation with plant extracts.

Result: All miRNAs except for miRNA 126 were detected in all biological samples, miRNA was not detected in saliva. Moreover, there was a strong correlation with miRNA profile in all biological samples from the same patients. MiRNA profile of cell lines was different from data we obtained from patients, not all of 7 chosen miRNAs were determined in cell lines. There was some shift in miRNA profile after incubation with plat extracts.

Conclusion: MiRNA profile identification in biological fluids seems to be not easy for standardization, there are a lot of opened question whether freezing is crucial for results or not, how to normalize obtained data etc. Further research is needed not only for putative miRNAs markers of cancer identification but for creation of complete protocol as well. 
HCC-20

Overexpression of SCAMP3 is an indicator of poor prognosis in hepatocellular carcinoma

\author{
Rongkuan $\mathrm{Li}^{1}$ \\ ${ }^{1}$ The 2nd Hospital of Dalian Medical University, China
}

Background: SCAMP3, an isoform of the secretory carrier membrane proteins (SCAMPs) family, is a membrane-trafficking protein involved in endosome transport. Previous microarray data showed that SCAMP3 mRNA is highly expressed in hepatocellular carcinoma (HCC)

Method: In this study, we investigated the expression and clinical significance of SCAMP3 in 100 pairs of HCC and adjacent normal tissue. SiRNA transfection was performed to silence SCAMP3 expression in HCC cells. The MTS assay and flow cytometry were used to detect the proliferation, cell cycle progression and apoptosis of HCC cells.

Result: Compared with adjacent normal tissues, SCAMP3 expression was dramatically increased in HCC tissues demonstrated by Western blotting $(P<0.05)$. In immunohistochemistry, compared with the adjacent normal tissues, SCAMP3 was detected in $96 \%$ of the HCC samples with a significant increase in intensity and number of stained cells $(P<0.05)$. Also, high SCAMP3 expression was found in $86 \%$ of the HCC samples $(P<0.05)$. The increased SCAMP3 expression was significantly correlated with vascular invasion $(P=0.004)$ and tumor stage $(P=0.001)$. Univariate and multivariate survival analyses showed that the expression of SCAMP3 was an independent prognostic factor of overall survival of HCC patients. Knockdown of SCAMP3 expression led to suppression of cell proliferation and blockage of cell cycle of HCC cells.

Conclusion: In conclusion, our present study suggested that SCAMP3 may serve as a promising prognostic biomarker and molecular target of HCC and further investigation is warranted.

\section{HCC-21}

PCDH20 enhances chemosensitivity in hepatocellular carcinoma by down-regulating MDR1 and NANOG expression via inhibiting the phosphorylation of SMAD3

\section{wu yan qin $^{1}$}

${ }^{1}$ The First Affiliated Hospital, Sun Yat-sen University, China

Background: Chemotherapy is one of the main treatments for HCC, but drug resistance seriously limits the effect. Protocadherin 20 has been reported as a tumor suppressor gene in several cancers. Nevertheless, there is no study with large scale samples evaluating the relationship between $\mathrm{PCDH} 20$ protein expression and prognosis of HCC patients, and its role in mediating HCC chemotherapy.

Method: The stably transfected cell lines expressing PCDH20 was constructed in SMMC7721. Cell viability and IC50 were detected by CCK8. Apoptosis, cell cycles, the population of cells with CD44+CD133+phenotype and the drug efflux function were analyzed by flow cytometry. Related genes were detected by Real-time PCR or Western blot. Subsequently, resistant strain SMMC7721/R was constructed, and then transfected with PCDH20. Related genes and biological characteristics were detected in the same way above. Result: Ectopic expression of PCDH20 resulted in a promotion in the apoptosis induced by Oxaliplatin, and a reduction of the IC50 value of Oxaliplatin and Paclitaxel, the expression of P-SMAD3, MDR1, NANOG, CD44, CD133, the population of cells with CD44+CD133+ phenotype, and the drug efflux function in
SMMC7721cell line. Compared to the parental strain, SMMC7721/R exhibited much higher IC50 value, with lower expression of PCDH20, higher expression of P-SMAD3, MDR1, NANOG, CD44, CD133, larger number of population of cells with CD44+CD133+ phenotype, and stronger drug efflux function in SMMC7721/R. When transfected with PCDH20 plasmid, SMMC7721/R turned up a remarkable reduction of the IC50 value of Oxaliplatin and Paclitaxel, the expression of P-SMAD3, MDR1, NANOG, CD44 and CD133, the population of cells with $\mathrm{CD} 44+\mathrm{CD} 133+$ phenotype, and the drug efflux function, as well as an increase of the late phase rate of apoptosis.

Conclusion: PCDH20 enhances chemosensitivity by suppressing MDR1 and NANOG through inhibiting the phosphorylation of SMAD3 in HCC, via upregulation of apoptosis, downregulation of drug efflux and stemness.

\section{HCC-22}

Primary hepatic resection versus transarterial chemoembolization for hepatocellular carcinoma in intermediate and advanced-stage (BCLC B and C) patients: systematic review and meta-analysis

YoungSun Lee $^{1}$, Myung Han Hyun ${ }^{1}$, Minjin Lee ${ }^{1}$, Ha Seok Lee ${ }^{1}$, Chan Uk Lee $^{1}$, Ji Hoon Kim ${ }^{1}$, Jong Eun Yeon ${ }^{1}$, Kwan Soo Byun ${ }^{1}$, Sehwa Kim¹, Haein Bak ${ }^{1}$

${ }^{1}$ Korea University College of Medicine, Korea, Republic of

Background: To compare primary hepatectomy $(\mathrm{PH})$ and transarterial chemoembolization (TACE) in patients with Barcelona Clinic Liver Cancer intermediate (BCLC B) to advanced (BCLC C) stage hepatocellular carcinoma (HCC).

Method: A systematic review was conducted using PubMed, EMBASE, and Cochrane databases through July 2017. Eligible studies comparing PH with TACE in BCLC B/C HCC patients with survival outcomes were included. A stratified additive model using random effect analysis was conducted. Sensitivity and meta-regression analyses according to the BCLC stage and baseline characteristics were performed.

Result: Twenty-two trials ( 1 randomized controlled trial [RCT], 5 propensity-score matching non-RCTs [NRCT], and 16 NRCTs) including 7985 patients were examined. We found a significant survival benefit for $\mathrm{PH}$ over TACE in BCLC B/C patients (Hazard ratio $[\mathrm{HR}]=0.59,95 \%$ confidence interval $[\mathrm{CI}], 0.52-0.66, P<.00001, I 2$ $=83 \%$ ). According to BCLC stage, both B and C patients showed significantly better overall survival for PH compared to TACE $(\mathrm{HR}=$ $0.53,95 \%$ CI $, 0.45-0.61, P<.00001, I 2=74 \%$; HR $=0.67,95 \%$ CI, $0.59-0.75, P<.00001, I 2=75 \%$, respectively). Five-year survival rates for $\mathrm{PH}$ were significantly higher than for TACE in BCLC B/C, BCLC B, and BCLC C patients $(\mathrm{OR}=3.19,3.38$, and 3.01, respectively; all $P<.00001)$. Survival benefits persisted across subgroup, sensitivity, and meta-regression analyses; inter-study heterogeneity remained constant.

Conclusion: This meta-analysis suggests that surgical resection provides survival benefits in patients with intermediate to advanced HCC. We argue that evidence exists in support of extending PH to carefully selected BCLC B and C HCC patients. 


\section{HCC-23}

Treatment for intermediate-stage hepatocellular carcinoma: current practice and outcome in tertiary hospitals

Irsan Hasan ${ }^{1}$, Imelda M Loho², Rino Alvani Gani', Lianda Siregar ${ }^{2}$, Agus Sudiro Waspodo ${ }^{2}$, Andri Sanityoso Sulaiman', Cosmas Rinaldi A Lesmana', Juferdy Kurniawan ${ }^{1}$, Chyntia Olivia Jasirwan ${ }^{1}$, Kemal Fariz Kalista ${ }^{1}$

${ }^{1}$ Faculty of Medicine, Universitas Indonesia, Indonesia; ${ }^{2}$ Dharmais National Cancer Center Hospital, Indonesia

Background: Intermediate-stage hepatocellular carcinoma (HCC) is a very heterogenous disease, which comprise of patients with single to multiple huge tumors and various stage of liver function, without extrahepatic disease. The first line treatment for this group is transarterial chemoembolization (TACE), however, in clinical practice, not all patients are suitable for TACE. This study aims to evaluate current treatment practice and outcome of patients with intermediate-stage HCC.

Method: We retrospectively analyzed HCC patients database from 2013 to 2016 in Cipto Mangunkusumo Hospital and Dharmais Cancer Hospital. Patients with intermediate stage HCC (BCLC-B) were included in this study. Diagnosis of HCC was made based on AsiaPacific clinical practice guidelines of HCC.

Result: This study included 149 patients with intermediate-stage HCC. Of these, $88(59.1 \%)$ came from Cipto Mangunkusumo Hospital. As a first-line treatment, TACE was done in 52 patients $(34.9 \%)$. Liver resection, as a first-line treatment, was done in 12 patients $(8 \%)$. Three patients underwent liver resection after TACE. Sixty-seven patients $(45 \%)$ were given best supportive care due to disease progression while waiting for treatment. Frequency of TACE varied from one to eleven times. Overall median survival was 617 days (1.7 years). The median survival of patients, who were given any treatment, was longer compared with supportive treatment (2.2 years versus 5.7 months). Among 52 patients who got TACE as first line, the number of partial response, stable disease, and progressive disease, based on RECIST criteria, was six, nine, and thirteen patients, respectively. TACE response could not be determined in 24 patients due to incomplete data.

Conclusion: Either given as a combination or a single treatment, TACE brings better prognosis. However, not all patients have the opportunity to get treatment.

\section{HCC-24}

\section{Genetic polymorphism of IFNL3 rs12979860 influences susceptibility to $\mathrm{HCV}$ related hepatocellular carcinoma in a Chinese population: a case-control study}

\section{Wei $\mathrm{Hou}^{1}$}

${ }^{1}$ Tianjin Second People's Hospital, China

Background: The association between interferon lambda-3(IFNL3, also known as interleukin 28B, IL28B) rs12979860 polymorphism and the development of hepatocellular carcinoma (HCC) has also been examined in a few studies with inconclusive results. IFNL3 rs12979860 polymorphism has been shown a marked differential distribution with regional and ethnic variation. Whether this single nucleotide polymorphism (SNP) influences susceptibility to HCV related HCC in the Chinese population is largely unknown.

Method: In this case-control study, a total of 157 Chinese Han patients with chronic HCV infection were enrolled, including $62 \mathrm{HCV}$ related $\mathrm{HCC}$ patients and 95 chronic hepatitis $\mathrm{C}(\mathrm{CHC})$ patients without HCC, and the genetic polymorphism of IFNL3 rs12979860 was genotyped via a DNA microarray-based assay. The logistical regression analysis was employed to determine the correlation between the genetic polymorphism and risk of HCV-related HCC.

Result: The allele frequency of IFNL3 rs12979860 (C/T) was not statistical different between the HCC and control groups $(\mathrm{P}>0.05)$. Under the genetic model of allele frequency, the $\mathrm{T}$ allele was associated with elevated risk of HCV-related HCC in the Chinese population compared to $\mathrm{C}$ allele after an adjustment for age, gender, BMI, HCV infection duration and HCV genotypes $(\mathrm{P}=0.046)$. In the subgroup analysis stratified by $\mathrm{HCV}$ genotype, subjects with $\mathrm{CHC}$ genotype 1b infection carrying rs12979860 T allele and CT+TT genotype had higher susceptibility to HCC than those with $\mathrm{C}$ allele and CC genotype $(\mathrm{P}=0.020, \mathrm{P}=0.037$, respectively).

Conclusion: IFNL3 rs12979860 polymorphism with T allele could be a factor that increases the risk of $\mathrm{HCV}$ related $\mathrm{HCC}$ in the Chinese population, especially those subjects with $\mathrm{CHC}$ genotype $1 \mathrm{~b}$ infection.

HCC-26

Lipiodol CT: a technique for treating small hepatocellular carcinomas

Shashi Bala Paul ${ }^{1}$, Vidyasagar $\mathbf{R}^{1}$, Ekta Dhamija ${ }^{1}$, Shivanand R Gamanagatti ${ }^{1}$, Shalimar $S^{1}$, Subrat Kumar Acharya ${ }^{1}$

${ }^{1}$ All India Institute of Medical Sciences, India

Background: Lipiodol Computerized Tomography (CT) has been used in the past for preoperative staging of patients of HCC as it has sensitivity in detecting small intrahepatic nodules. Thus Lipiodol CT plays a vital role in visualizing small $\mathrm{HCC}$ which are seen on MRI but not detected on either US or MPCT . At our centre, we are using this technique for treating patients of small HCC which are not seen on ultrasound or MPCT . We illustrate two representative cases managed successfully using this technique.

Method: Diagnosed HCC patients were evaluated for treatment allocation. We encountered five patients with single HCC (less than $2 \mathrm{~cm}$ ), seen only on MRI. These patients had Child's B cirrhosis and were planned for percutaneous ablation. Lipiodol CT was planned and an angiogram through the transfemoral route was initially performed. The catheter was advanced into the hepatic artery and after superselective cannulation of the feeding hepatic artery, 5-10 $\mathrm{ml}$ of lipiodol was injected intraarterially which was taken up by the malignant liver nodule (HCC) and retained within. On post procedure CT, the tumor laden lipiodol was seen as a hyperattenuating nodule which served as a target for subsequent ablative treatment.

Result: Case one was a 51 year female who had a small $1.5 \mathrm{~cm} \mathrm{HCC}$ seen only on MRI. Angiogram depicted the tumor blush following which lipiodol was injected. Post procedure CT depicted lipiodol within the hepatic tumor which was targeted under CT guidance for radiofrequency ablation. Complete response was achieved subsequently.

Case 2 was a 50 year old male who had undergone right extended hepatectomy for HCC and was detected to have a small recurrence(less than $1 \mathrm{~cm}$ ) seen on only on follow up MRI at performed at eight months. Angiogram was done through the transfemoral route and intraarterial lipiodol injection through the hepatic artery delineated multiple small hepatic nodules scattered in the remaining right lobe. This led to change in management from ablation to Tran-arterial chemoembolization due to increase in the stage of the disease. 
Conclusion: Lipiodol CT is a very sensitive tool to detect small HCC nodules and hence can be used for treating those small HCC which are not visualized either on Ultrasound /MPCT

\section{HCC-27}

\section{Microwave ablation for hepatocellular carcinoma: assessment of safety}

Antony Joseph Kolothumveettil ${ }^{1}$, Rajesh Antony ${ }^{1}$, Antony Paul Chettupuzha ${ }^{1}$, Roy J Mukkada ${ }^{1}$, Pradeep George Mathew', Shelley Paul Chireyath', Francis Jose $\mathbf{V}^{\mathbf{1}}$, Abraham Koshy ${ }^{1}$

${ }^{1}$ VPS Lakeshore hospital, India

Background: In hepatocellular carcinoma (HCC), Radiofrequency ablation (RFA) and surgical resection are considered curative for lesions $<5 \mathrm{~cm}$. Few studies have suggested that microwave ablation may be better than RFA. Our aim was to study the safety of microwave ablation for HCC.

Method: Patients with $\mathrm{HCC} \leq 5 \mathrm{~cm}$ and having cirrhosis of liver were included for the period from July to November 2017. The procedure was done under local anaesthesia and Propofol + Midazolam sedation. A power of 50 Watts was applied for 3 to 6 minutes, with the help of an antennae which is either made of ceramic or steel. Ceramic and steel antennae are used for spherical and ovoid type of HCC, respectively. The duration of heat application to the tumour core is based on the chart provided by the device manufacturer. A percutaneous ultrasound (US) guided method is used.

Result: Ten patients with 14 tumours with size ranging from 1 to 5 $\mathrm{cm}$ were enrolled. Safety was assessed and no complications were observed in this group. The possible complications after microwave ablation are significant pain, haemorrhage at probe insertion site, pneumothorax, gall bladder injury and need for blood transfusion due to hematoma in the liver. All the patients were discharged from the hospital within 24 hours and were stable at one month follow up. Conclusion: Ultrasound guided percutaneous microwave ablation appears to be safe

\section{HCC -28}

Active treatment of elderly patients of hepatocellular carcinoma: age need not be a deterrant

\section{Shashi Bala Paul ${ }^{1}$, Shekhar Singh ${ }^{1}$, Neeti Nadda ${ }^{1}$, Vishnubhatla Sreenivas ${ }^{1}$, Shivanand R Gamanagatti ${ }^{1}$ \\ ${ }^{1}$ All India Institute of Medical Sciences, India}

Background: With increase in life expectancy, the proportion of elderly patients with different malignancies is on an increase and their treatment poses a huge challenge. However, in certain malignancies, improved outcomes following treatment have been shown in the elderly. Liver is likely to be less affected by increasing age in comparison to other organs. This study was done to compare the clinical profile, treatment outcome and survival rate of elderly HCC patients with their younger counterparts.

Method: In this retrospective study, case records of treated HCC patients were examined. Patients with complete clinical, demographical, imaging and follow up details were included and divided into two groups- Group 1 - age upto 60 years (younger) and Group 2 age more than 60 years(older). Tumor response and overall survival of both groups were estimated.
Result: Of the 195 treated HCC patients, group 1 were 131 and Group 2 were 64 . On comparing their clinical and demographic profile, no difference was noted in the performance status, etiology of HCC, gender, BCLC staging, mass size and laboratory parameters of the patients of the two groups. Proportion of Child's B patients were more ( $29 \%$ vs $15.6 \%$ ) in Group I compare to Group 2. Additionally, proportion of patients with normal AFP level $<20 \mathrm{ng}$ ) was more in group 2 than those with raised AFP patients $->20 \mathrm{ng} / \mathrm{m}$ (group 1- $31.3 \%$ and $68.7 \%$ vs $43.7 \%$ and $56.3 \%, p=0.09$ ) The tumor response was comparable but the overall survival was better in group 2 (mean + $\mathrm{SD}$, group $124.0+1.74$ months vs $29.7+2.41$ months, $\mathrm{P}=0.06$, difference of 5.7 months). After adjusting for the difference in the proportion of patients with AFP levels less than $20 \mathrm{ng} / \mathrm{ml}$ or more and Childs' status of A/B, the survival of older patients was better following treatment (greater by 4.7 months).

Conclusion: Following treatment, elderly HCC patients have better survival than their younger counterparts. Hence, like the younger HCC patients, active treatment should also be offered to the elderly HCC patients and age need pose as a deterrant. More Studies are needed to validate these results

\section{HCC -29}

\section{Alpha-fetoprotein response after selective internal radiation therapy versus sorafenib in locally advanced HCC in Mongolia (SIRveNIB)}

\section{Nyamsuren Naranzul $^{1}$, R Sanduijav ${ }^{1}$, Oidov Baatarkhuu ${ }^{1}$ \\ ${ }^{1}$ Mongolian National University of Medical Sciences, Mongolia}

Background: Alpha-fetoprotein (AFP) is considered to be an indicator of tumor activity in hepatocellular carcinoma (HCC). We present a novel correlation of AFP response to radiologic response and overall survival (OS) in patients treated with Selective Internal Radiation Therapy (SIRT) and Sorafenib therapies.

Method: Participants from a phase III multicenter randomized trial of SIRT versus Sorafenib in HCC were studied. Thirty five patients with HCC were treated with selective internal radiation therapy or Sorafenib at our institution. Thirty one patients with baseline AFP higher than $20 \mathrm{ng} / \mathrm{ml}$ were studied for analysis. AFP response was defined as more than $50 \%$ decrease from baseline. Twenty six patients with follow-up imaging were studied for the AFP imaging correlation analysis. We studied the relationship between AFP response and treatment outcome in terms of radiologic response and overall survival.

Result: Of 39 patients, 31 patients $(79.4 \%)$ with elevated serum AFP $(>20 \mathrm{ng} / \mathrm{ml})$ and documented radiologic evaluation every 12 weeks. AFP response was seen in $3(17.6 \%)$ of 17 and $6(40 \%)$ of 15 of patients treated with Sorafenib and Selective internal radiation therapy, respectively $(\mathrm{P}=0.16)$. The hazard ratio in AFP nonresponders compared with responders was 1.12 (95\% CI, 0.46-2.69). AFP responders had better survival than nonresponders (15 and 6.95 months, respectively; $\mathrm{P}<.79$ ), and AFP response was strongly associated with survival (hazard ratio, 1.12; 95\% CI, 0.46 to 2.69 ; $\mathrm{P}<$ 0.79). AFP response were frequently observed in patients with radiologically stable disease (SD) and tended to indentify a subgroup of SD patients with better survival.

Conclusion: The data presented support the use of AFP response seen after locoregional therapy as an ancillary method of assessing tumor response and survival, as well as an early objective screening tool for progression by imaging. 


\section{HCC-30}

\section{Artificial ascites in radio frequency ablation for liver cancer}

\section{$\underline{R i n o}$ Alvani Gani $^{1}$, Juferdy Kurniawan ${ }^{1}$, Kemal Fariz Kalista ${ }^{1}$}

${ }^{1}$ Cipto Mangunkusumo National Hospital, Faculty of Medicine Universitas Indonesia, Indonesia

Background: Radio Frequency Ablation (RFA) is one of the treatment modality for liver tumor either as primary tumor as well as secondary malignancy. Occasionally, a good ablation can not be performed due to the tumor location. To assists the ablation in this particular case, some fluid can be deposited inside the abdomen which is called as artificial ascites. The aim of this study is to report and evaluate the practical method to induce artificial ascites in RFA Method: This was a case series study consist of 15 consecutive patients, 35 artificial ascites procedure that had been treated with ultrasound-guided RFA from 2015 to 2017. The method of artificial ascites were using the modification technique for artificial ascites that had been published elsewhere. The modifications were done to suit the conditions and availability of apparatus in Indonesia

Result: Artificial ascites was successfully performed in all 15 patients $(100 \%)$ with total of 40 hepatocellular carcinoma (HCC) in 21 RFA's sessions and tumor size ranges from $10 \mathrm{~mm}$ to $50 \mathrm{~mm}$. Artificial ascites was performed using $5 \%$ dextrose in water $(\mathrm{D} / \mathrm{W})$ solution ranging from $250 \mathrm{ml}$ to $1500 \mathrm{ml}$. No adverse effect occurred during and after the procedure.

Conclusion: Percutaneous RFA using modification artificial ascites technique was more practical, safe, and effective for treating liver cancer

\section{HCC-31}

Clinical comparison of cirrhotic and non-cirrhotic hepatocellular carcinoma in patients with alcoholic liver disease

\section{Young Joo Jin}

${ }^{1}$ Inha University Hospital, Korea, Republic of

Background: We analyzed retrospectively overall survivals (OSs) according to the presence of cirrhosis in hepatocellular carcinoma (HCC) patients with alcoholic liver disease.

Method: The medical records of 186 alcohol-associated HCC patients diagnosed between 2005 and 2015 were retrospectively analyzed. OSs of these patients were comparatively analyzed based on the presence of cirrhosis and Child-Turcotte Pugh (CTP) classification.

Result: Median follow-up duration was 11.8 months (range, 0.1101.6 months). Non-cirrhotic HCC was observed in $37.1 \%$ of the 186 patients. Frequency of CTP class A $(77.1 \%$ vs. $43.8 \%$, p

Conclusion: OS was found to be better for non-cirrhotic than cirrhotic patients with alcohol-related HCC. Survivals of alcohol-related HCC patients without cirrhosis were comparable for CTP class A and B.

\section{HCC-32}

Clinical patterns of non-B non-C, non-alcoholic hepatocellular carcinoma in hepatitis $B$ virus endemic area

Hae Lim Lee ${ }^{1}$, Hae Lim Lee ${ }^{2}$, Jeong Won Jang ${ }^{2}$, Si Hyun Bae', Jong Young Choi ${ }^{2}$, Seung Kew Yoon ${ }^{2}$
${ }^{1}$ Bucheon St. Mary's hospital, Korea, Republic of; ${ }^{2}$ Catholic medical center, Korea, Republic of

Background: Incidence of NAFLD is increasing as a cause of chronic liver disease and past HBV infection has been also considered as major cause of HCC in HBV endemic area. Focused on that, this study was aimed to investigate clinical characteristics and possible etiologies of viral and alcohol-unrelated, NBNC-NA HCC.

Method: We reviewed a total of 1853 consecutive patients with newly diagnosed HCC between 2002 and 2014 at a tertiary center. After excluding rare causes of HCC, $149(8.1 \%)$ of total $1829 \mathrm{HCC}$ patients were classified to NBNC-NA HCC. They were compared to patients with $\mathrm{HBV}$ or $\mathrm{HCV}$ infection or alcohol-related $\mathrm{HCC}$ patients. Result: Proportion of NBNC-NA as a cause of HCC was the third (8\%) following $\mathrm{HBV}(58 \%), \mathrm{HCV}(12 \%)$ and similar with alcohol $(8 \%)$ among the total HCC patients. Baseline liver function in NBNCNA group was relatively better than other groups (APRI and CTP score were significantly lower in NBNC-NA group, $\mathrm{p}<0.001,0.017$, respectively). However in tumor characteristics, lower proportion of early stage and higher of advanced stage were in NBNC-NA group according to mUICC and BCLC system. There was no difference in overall survival between NBNC-NA and the other HCC group. Although proportion of NBNC-NA HCC increased in correlation with age, $2.7 \%$ in up to $40 \mathrm{~s}, 6 \%$ in $50 \mathrm{~s}-60 \mathrm{~s}, 23 \%$ in after $70 \mathrm{~s}$, respectively, and also in recent years in 2012-2014 (12\%) compared to past in 2002-2011 (7\%), proportion of anti-HBc positivity in NBNC-NA group was similar among the different age groups as about $80 \%$. Proportion of diabetes also increased with ages and dyslipidemia as well as diabetes were more prevalent in NBNC-NA than other groups (in both, $\mathrm{p}<0.001$ ).

Conclusion: Past HBV infection and NAFLD-associated metabolic risk factors would have significant importance as etiologies of NBNCNA HCC with increasing prevalence, thus surveillance of those should be helpful to detect NBNC-NA HCC at more earlier stage.

HCC -33

Differences between AFP and PIVKA values as early detection method for hepatocellular carcinoma and cirrhosis

\section{Bogi Pratomo Wibowo}

${ }^{1}$ Medical Faculty Brawijaya University/Saiful Anwar Hospital, Indonesia

Background: Hepatocellular carcinoma (HCC) is the 5th most prevalent cancer in the world, with 500,000 new cases each year. In individuals with normal liver functions, the prothrombin precursor undergoes carboxylation before it is released into the peripheral blood. Carboxylase is dependent on vitamin $\mathrm{K}$ and not found in HCC. This causes abnormal prothrombin to be secreted. To investigate if protein induced by vitamin $\mathrm{K}$ absence or antagonist II (PIVKA-II) can be used as an early detection method to diagnose HCC.

Method: A cross sectional study on in-patients or out-patients at Saiful Anwar Malang Hospital from July 2016 to October 2016.

Result: The p-value ( $>>0.05$ ) obtained using Kolmogorov-Smirnov was 0.166 for diagnosis of HCC and 0.147 for the diagnosis of hepatic cirrhosis. The $\mathrm{p}$-value $(\mathrm{p}>0.05)$ obtained using Shapiro-Wilk was 0.103 for diagnosis of HCC and 0.087 for the diagnosis of cirrhosis. Comparative test using the LSD method showed PIVKA serum levels in HCC as compared to hepatic cirrhosis as significant with a p-value less than $0.05(\mathrm{p}<0.05)$, that is 0.025 . However comparative test using the Tukey HSD method showed that the results obtained were not significant. According to the PIVKA cut off value, the sensitivity and specificity to detect cirrhosis and HCC was as large as $100 \%$. 
According to the AFP cut off value, the sensitivity to detect cirrhosis and $\mathrm{HCC}$ was $93.3 \%$ and the specificity was $76.92 \%$.

Conclusion: Both PIVKA and AFP can be used to detect cirrhosis and HCC. However PIVKA exhibited better sensitivity and specificity in the detection of cirrhosis and HCC.

\section{HCC -34}

Diverse radiological findings of combined hepatocellular carcinoma and cholangiocarcinoma in liver; pictorial essay

\section{Yong Moon Shin ${ }^{1}$}

${ }^{1}$ Asan medical center, Korea, Republic of

Background: Combined Hepatocellular carcinoma-Cholangiocarcinoma is an uncommon primary liver cancer. And its radiological findings are very diverse from typical HCC pattern to typical cholangiocarcinoma feature. Other purpose is to present the spectrum of radiological findings of combined HCC-CCC, and to help the imaging diagnosis of this tumor

Method: There were many pathological proven combined hepatocellular carcinoma and cholangiocarcinoma cases. We evaluated retrospectively reviewed the imaging findings of those tumors. Variable findings of CT and MRI scans of combined HCC-CCC will be shown.

Result: There are very diverse feature of radiological findings of combined HCC-CCC; From synchronous tumor to metachronous tumor. For example, combined HCC-CCC presented typical single HCC pattern mass, or typical single nodular CCC pattern mass, or heterogeneously combined feature of enhancing pattern, or multiple liver mass with atypical radiological features.

Conclusion: Combined HCC-CCC showed veryThe pre-operative diagnosis of combined HCC-CCC is often difficult. However, we can achieve the diagnostic success rate when we are familiar with the diverse radiological findings of this uncommon tumor.

\section{HCC-35}

Etiologic Profile and Risk Factors of Hepatocellular Carcinoma in Patients with Cirrhosis In Armenia 1 H. Ghazinyan, 2 R. Gish, 1 A. Asoyan, 1 A. Mkhitaryan, 1 T. Galstyan, 3 R. Safaryan 1Hepatology, Nork infection hospital, 2 Hepatology, Stanford University Medical Center, 3 Gastroenterology, YSMU

\section{Hasmik Ghazinyan 1, Ara Asoyan', Aregnaz Mkhitaryan', Tsovinar Galstyan $^{1}$, Rusanna Safaryan ${ }^{3}$, Robert Gish ${ }^{2}$}

${ }^{1}$ Nork Clinical Hospital of Infectious Diseases, Armenia; ${ }^{2}$ Stanford University Medical Center, United States; ${ }^{3}$ Yerevan State Medical University, Armenia

Background: Hepatocellular carcinoma (HCC) is the dominant form of primary liver cancer, resulting in at least 500000 death per year. The major risk factors involved in the etiology of HCC include chronic infection with hepatitis $\mathrm{C}$ virus (HCV), hepatitis B virus (HBV), alcoholic liver disease and nonalcoholic steatohepatitis (NASH). The risk factors vary by geographic location and influence the disease course.

Aim: To evaluate the etiologic profile and main risk factors of HCC in cirrhotic patients in Armenia, according to data of one clinical centre.

Method: We studied 652 patients with cirrhosis for 10 years (20062016 years). Data from detailed history, clinical, biochemical, alfafetoprotein's, virological (HBV, HBsAg, HBeAg, Anti-HBeAg,
HBV-DNA), hepatits C virus (HCV, Anti-HCV, HCV-RNA), hepatits D virus (HDV, Anti-HDV and HDV-RNA) and instrumental studies (ultrasound and computed tomography) were analysed.

Result: HCC were diagnosed in $72(11 \%)$ patients from the total 652 with cirrhosis. Mean age of patients with $\mathrm{HCC}$ was $56 \pm 0,51$ (range 27-77). Distribution by sex was men 63 (87\%). Evidence of HCV infection was present in $50(70 \%)$, infection with $\mathrm{HBV}$ alone was detected in $10(14 \%)$ and dual HBV and HDV infection in $4(5,5 \%)$ Alcoholic and cryptogenic (possible nonalcoholic fatty liver disease) cirrhosis with HCC were present in $4(5,5 \%)$ respectively. Mean duration of development of $\mathrm{HCC}$ in cirrhotic patients (after diagnosis of cirrhosis) was 6 years (range 2-10). In 52 out of 72 patients with $\mathrm{HCC}$ the alfa-fetoprotein 's (AFP) as a tumor marker, positivity was $80 \%$, mainly in HBV and HCV infected patients .Mean level of serum AFP was $8300 \pm 6.46 \mathrm{ng} / \mathrm{ml}$ (range 4-12100). According to our data significantly increased risk of HCC was found in cirrhotic patients with high body mass index (BMI) as well as in patients with longer time of cirrhosis . Mean BMI was 28 (range 22-35).In 48 (66\%) out of 72 patients with HCC BMI was 30-35, and in $51(70 \%)$ patients the duration of development of $\mathrm{HCC}$ after diagnosis of cirrhosis was 7-10 years.

Conclusion: $\mathrm{HCV}$ is the main etiologic risk factor for $\mathrm{HCC}$, followed by HBV. Male sex, higher BMI and longer duration of cirrhosis are important predictors of $\mathrm{HCC}$ in cirrhotic patients, indipended ethiology.

HCC -36

Genetic polymorphism of DEPDC5 rs5998152 influences susceptibility to HCV-related hepatocellular carcinoma in a Chinese Han population: a case-control study

\section{Wei Hou ${ }^{1}$}

${ }^{1}$ Tianjin Second People's Hospital, China

Background: Genetic polymorphism in the DEP domain-containing 5 (DEPDC, rs5998152) gene has been demonstrated to be associated with the development of hepatocellular carcinoma (HCC). Whether this single nucleotide polymorphism (SNP) influences susceptibility to HCV-related $\mathrm{HCC}$ in the Chinese Han population is largely unknown.

Method: In this case-control study, a total of 101 Chinese Han patients with chronic HCV infection, including $46 \mathrm{HCV}$-related $\mathrm{HCC}$ patients, and 55 chronic hepatitis $\mathrm{C}$ (CHC) patients without HCC were enrolled, and the genetic polymorphism was genotyped via a matrix-assisted laser desorption/ ionization time of flight mass spectrometry (MALDI-TOF MS) assay. The logistical regression analysis was employed to determine the correlation between the genetic polymorphism and risk of $\mathrm{HCV}$-related HCC.

Result: The distribution of DEPDC rs5998152 alleles and genotypes were not significantly different between $\mathrm{HCC}$ patients and $\mathrm{CHC}$ subjects $(\mathrm{P}>0.05)$. Under the genetic model of allele frequency, $\mathrm{C}$ allele was significantly associated with elevated risk of HCV-related HCC compared to $\mathrm{T}$ allele after an adjustment on age and gender (OR $=2.123,95 \%$ CI: $1.044-4.315, \mathrm{P}=0.038)$. Under the dominant model, the $\mathrm{CC}+\mathrm{CT}$ genotype was associated with increased risk of HCC compared to the TT genotype $(\mathrm{OR}=2.428,95 \% \mathrm{CI}: 1.020-5.784 ; \mathrm{P}=$ 0.045).

Conclusion: DEPDC rs5998152 polymorphism with $\mathrm{C}$ allele was associated with higher susceptibility to HCV-related HCC in the Chinese Han population. Screening of host genetic polymorphisms might be helpful in designing effective and efficient HCC surveillance programs for chronic $\mathrm{HCV}$-infected patients. 


\section{HCC-37}

Pretreatment risk factors, including fibrosis stage, and time to diagnosis of hepatocellular carcinoma (HCC) after eradication of hepatitis $\mathrm{C}$ virus with interferon and ribavirin

Dimitrios - Dimitroulopoulow ${ }^{1}$, Dimitrios Kypreos ${ }^{1}$, Matina Hatzinikolaou $^{1}$, Stavros Stavrinidis ${ }^{1}$, Georgios Sofianidis ${ }^{1}$, Nikolaos Saribyogloy ${ }^{1}$, Klisthenis Tsamakidis ${ }^{1}$

${ }^{1}$ Agios Savvas Hospital, Greece

Background: The aim of the present study was to investigate a possible correlation between HCC development and the liver fibrosis pretreatment stage, age, alcohol consumption, and some laboratory parameters, in patients,successfully treated for chronic $\mathrm{HCV}$ infection.

Method: The records of $357(284 \mathrm{M}, 73 \mathrm{~F})$ consecutive patients that underwent successful treatment for chronic $\mathrm{HCV}$ infection with pegylated interferon $\alpha$ and ribavirin between the years 2004-2014 were reviewed. Age, sex, alcohol consumption, viral genotype and pre-treatment levels of viral load, platelets, bilirubin, $\gamma$-globulins, INR, HbA1c, AFP, CA 19-9 and also fibrosis stage were taken into account in the performed multivariate analysis. Patients were followed routinely every six months with liver ultrasound and AFP levels. The median follow up period after SVR was 62 months (range 11-120).

Result: During the follow-up period 14(11M, 3F, 3.92\%) out of 357 patients developed HCC. The median period from SVR to HCC diagnosis was 35 months (range 21-139). Alcohol consumption (HR 7.3: 95\% CI 3-15.9; $\mathrm{P}<0.0001$ ), age $\geq 55$ years (HR 3.3: 95\% CI 1.56.4: $\mathrm{P}=0.009)$ and advanced $(\mathrm{F} 3 / \mathrm{F} 4)$ fibrosis stage (HR 3.7: $95 \% \mathrm{CI}$ 1.8-7.6; $\mathrm{P}<0.0001$ ) were identified as independent and statistically significant factors for development of HCC after successful eradication of $\mathrm{HCV}$.

Conclusion: Pretreatment advanced fibrosis stage, alcohol consumption and age can consider as significantly important factors correlated with the development of $\mathrm{HCC}$ in non-viremic $\mathrm{HCV}$ patients prior treated with interferon a and ribavirin.

\section{HCC-38}

Risk factors for hepatocellular carcinoma and its mortality rate: a prospective multicenter study

Chyntia Olivia Jasirwan ${ }^{1}$, Kemal Fariz Kalista ${ }^{1}$, Putra Nur Hidayat $^{1}$, Imelda Maria Loho ${ }^{2}$, Gita Aprilicia ${ }^{1}$, Rino Alvani Gani', Irsan Hasan', Andri Sanityoso', Cosmas Rinaldi Lesmana ${ }^{1}$, Juferdy Kurniawan ${ }^{1}$, Saut Horas Nababan ${ }^{1}$

${ }^{1}$ Ciptomangunkusumo National Hospital, Faculty of Medicine Universitas Indonesia, Indonesia; ${ }^{2}$ Dharmais National Cancer Center Hospital, Indonesia

Background: Hepatocellular carcinoma (HCC) is an increasing cause of mortality and remains a major burden despite its considerable preventive measures, screening tools, and treatment modalities. Population-based cancer registry is an essential element to provide a baseline and comprehensive analysis of patient's risk factors. We present a system based multicenter HCC registry at two hospital in Indonesia.

Method: We performed a follow up in HCC patients admitted between 2015 January and 2017 November in Cipto Mangunkusumo National Hospital and Dharmais Hospital Jakarta. Baseline characteristics and their clinical features were collected at the time of inclusion. Primary outcome was patient's death which also the end point of follow up evaluation. We conducted multivariate analysis by using logistic regression. Odds ratio (OR) with $95 \%$ confidence intervals (CIs) were calculated.

Result: There were 302 admissions for patients with HCC in this study with median of age $55 \pm 12.53$ years. Of these, 159 patients were included from Cipto Mangunkusumo National Hospital and 143 patients were from Dharmais Hospital. As much as $75.5 \%$ (228/302) patients were male, with hepatitis B virus (HBV) as the most common etiology found in the patients $(61.3 \% ; 185 / 302)$. Most of patients were referred from other hospital without any complete HCC surveillance data $(92.1 \%$; 278/302). At the time of last follow up, 104 $(34.4 \%)$ patients have died and the rest are still evaluated. Lower odds of mortality were found in male (OR 0.82 ; 95\% CI $0.47-1.42$; $\mathrm{P}=0.485)$ and patients above 50 years old (OR $0.97 ; 95 \%$ CI 0.58 1.61; $\mathrm{P}=0.897)$. Patients with hepatitis $\mathrm{C}$ virus $(\mathrm{HCV})$ infection $(\mathrm{OR}$ 1.47; 95\% CI 0.77-2.82; $\mathrm{P}=0.242$ ) and HBV-HCV coinfection (OR $1.71 ; 95 \%$ CI $0.83-3.53 ; \mathrm{P}=0.148$ ) had higher odds of mortality than patients with HBV infection. Based on Child Pugh (CP) classification, the odds increase progressively in $\mathrm{CP} B$ patients $(\mathrm{OR} 1.24 ; 95 \% \mathrm{CI}$ 0.69-2.22; $\mathrm{P}=0.464)$ and $\mathrm{CP} C(\mathrm{OR} 3.20 ; 95 \%$ CI 1.25-8.19; $\mathrm{P}=0.015)$ compared to $\mathrm{CP}$ A patients. Progressive increase was also found in higher Barcelona Clinic Liver Cancer (BCLC) stadium of HCC. The odds in BCLC B, C, and D patients were 2.12 (95\% CI 0.58-7.72; $\mathrm{P}=0.256), 4.10$ (95\% CI 1.14-14.7; $\mathrm{P}=0.030$ ), and 5.66 (95\% CI 1.2824.9; $\mathrm{P}=0.022$ ) respectively. The presence of cirrhosis increase the odds of mortality (OR $1.13 ; 95 \% \mathrm{CI} 0.70-1.84 ; \mathrm{P}=0.627$ ) compared to those without cirrhosis. On the other hand, routine HCC surveillance reduce mortality (OR 0.36 ; 95\% CI $0.12-1.07 ; \mathrm{P}=0.072$ ) in these patients. Based on treatment modality according to their clinical characteristics, lowest mortality were found in patients who received curative treatment. Higher mortality were found in patients with palliative care (OR 1.55; 95\% CI 0.60-3.98; $\mathrm{P}=0.361)$ and supportive care (OR 2.76; 95\% CI 1.14-6.66; $\mathrm{P}=0.024$ ).

Conclusion: Child Pugh classification, BCLC stadium, and treatment modality might predict mortality in $\mathrm{HCC}$ patients while routine surveillance could act as protective factors. Other parameters need further evaluation.

HCC-39

Role of sorafenib combined transcatheter arterial chemoembolization (TACE) in patients with advanced hepatocellular carcinoma

\section{$\underline{K e b i n}$ Chang $^{1}$, Kueichuan LEE $^{1}$, Yee Chao ${ }^{1}$, Yihsiang Huang ${ }^{1}$}

${ }^{1}$ Taipei Veterans General Hospital, Taiwan

Background: Sorafenib is the standard of care for advanced hepatocellular carcinoma (HCC). START study suggests the safety of the transcatheter arterial chemoembolization (TACE) in combination with sorafenib in BCLC B HCC patients. Whether combination TACE with sorafenib could provide survival benefit in BCLC C HCC patients is unclear. Sorafenib is the standard of care for advanced hepatocellular carcinoma (HCC). START study suggests the safety of the transcatheter arterial chemoembolization (TACE) in combination with sorafenib in BCLC B HCC patients. Whether combination TACE with sorafenib could provide survival benefit in BCLC C HCC patients is unclear.

Method: This retrospective study recruited 311 patients with advanced HCC in Taipei Veterans General Hospital from October 2007 to December 2016. After matching in age, gender, tumor size, vessel involvement, and extrahepatic invasion, a total of 174 patients 
including TACE alone $(\mathrm{n}=58)$, TACE/sorafenib $(\mathrm{n}=58)$, and sorafenib alone $(n=58)$ were enrolled in the analysis. We compared the outcomes of the three groups, using Kaplan-Meier method via SPSS software version 21

Result: The mean age was 64.1 years old in TACE, 63.2 in TACE/sorafenib and 64.5 in sorafenib group with male predominant. The maximal tumor size was $26 \mathrm{~cm}$ in TACE, $21.5 \mathrm{~cm}$ in TACE/sorafenib and $17.3 \mathrm{~cm}$ in sorafenib group $(\mathrm{p}=0.172)$. After a median of 6.6 months of follow-up, TACE and TACE/sorafenib groups were superior to sorafenib group in OS (median OS: 231 days vs 231 days vs 128 days, respectively, $\mathrm{p}=0.003$ ). By stratification of patients with or without main portal vein (MPV) invasion, TACE/sorafenib and TACE had longer OS than sorafenib (median OS: 212 days vs 231 days vs 145 days, respectively, $p=0.008$ ) in patient without MPV invasion. In patient with MPV involvement, there was no significance in OS among the three groups.

Conclusion: Combination TACE with sorafenib can prolong the OS in advanced HCC without MPV involvement.

\section{HCC-40}

Role of surveillance in cirrhosis of liver in improving outcome of hepatocellular carcinoma: a retrospective study from tertiary care centre in South India

Balaji Musunuri $^{1}$, Vishwanath Tantry ${ }^{1}$, Sandeep Gopal ${ }^{1}$, Suresh Shenoy $^{1}$, Anurag Shetty ${ }^{1}$

${ }^{1}$ KMC Hospital, India

Background: Surveillance for hepatocellular carcinoma (HCC) is performed to detect the disease at an early stage. Prognosis depends on tumor stage at diagnosis, with curative therapy available for patients diagnosed at an early stage. In India, HCC presents at an advanced stage due to lack of awareness of surveillance in high-risk group population. Our study done in South Indian Tertiary centre tries to assess the role of surveillance in cirrhosis in improving the outcome in HCC.

Method: Retrospective, single centre observational study done in HCC patients who were admitted to KMC Hospital, Mangalore from Jan 2014 to Oct 2017. Data regarding demographic profile, investigations, therapy received and outcome were collected. HCC was diagnosed based on imaging. Severity of cirrhosis was assessed by CTP and MELD score. Staging of HCC was done using BCLC staging. Patients were divided into two groups. Group A included HCC patients detected by surveillance. Group B included patients presenting with denovo HCC. Groups were compared for outcomes like survival period.

Result: Eighty three patients with HCC were included. Etiology in majority was NASH related in $48 \%$ followed by alcohol in $45 \% .52$ patients were in group A and 31 patients in group B. The mean duration of follow-up in Group A before detection of HCC was $20.32+/-15$ months. In group A, rise in AFP levels and lesions on USG were seen in $49 \%$ of cases, while USG alone picked suspicious lesions in other $51 \%$ of cases. Poor liver function (CTP C) was noted less frequently in Group A than B (22\% vs 35\%). Terminal stage (BCLC D) was less common in Group A compared to Group B (18\% vs $36 \%)$. Portal vein invasion was more common in Group B (58\%) when compared to Group A (36\%). Mean CTP score was significantly higher in Group B (9.2) when compared to Group $\mathrm{A}(8.1)(\mathrm{p}=0.033)$.
Mean MELD scores were higher in Group B (18.4) than in Group A (16.1), though not significant. AFP levels were significantly higher in Group B (12478 ng/ml) when compared to Group A (3049 ng/ $\mathrm{ml})(\mathrm{p}=0.032)$. Early stage HCC i.e., BCLC 0 and A, where curative therapy was possible was seen in $14 \%$ and $10 \%$ of cases in Group A and $B$ respectively. The median survival period was $9+/-1.5$ months in group A while it was $6+/-2.1$ months in Group B ( $\mathrm{p}=0.001)$.

Conclusion: Poor liver function, Terminal stage and vascular invasion were seen less frequently in HCC surveillance group. Overall Survival in HCC surveillance group was marginally better when compared to HCC detected denovo.

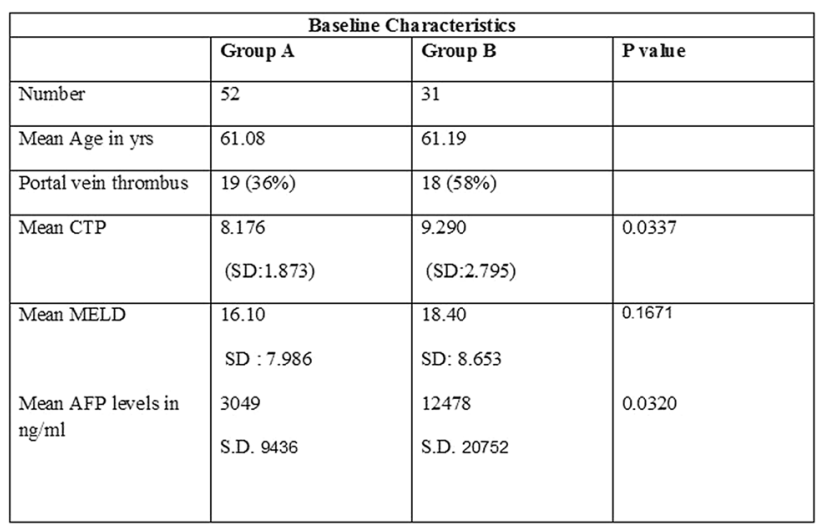

HCC-41

Safe radiofrequency ablation for low platelet count patients with novel platelet count raising drug (Lusutrombopag)

\section{$\underline{\text { Hideo Yoshida }}{ }^{1}$, Hiroyoshi Taniguchi ${ }^{1}$, Akiko Saito ${ }^{1}$, Ryo Nakata ${ }^{1}$}

${ }^{1}$ Japanese Red Cross Medical Center, Japan

Background: Blood transfusion of platelet was the practical way to increase the number of platelet count before invasive hepatic procedure such as radiofrequency ablation (RFA) for liver cancer. Recently, novel drug which raising platelet count by acting on thrombopoietin receptor has become available.

Method: Lusutrombopag was orally administered 3mg daily for seven days for the patients underwent RFA for liver tumor with low platelet count (mainly less than 50 thousands $/ \mu \mathrm{L}$ ). Medication was started 7-19 days before procedure. We collected demographic data, liver function, and platelet count of the patients.

Result: Lusutrombopag was administered for 15 patients. Seven were females and 8 were males. Median age was 70yo (range 54-82). Six patients had hepatitis $\mathrm{C}$ virus, 5 had alcoholic liver disease, 1 had $\mathrm{NASH}$, and 2 had other disease as background liver disease. Median Child Pugh score was 6 (range 5-10). Eight patients were Child A Class, 6 were Child B class, and 1 was Child C Class. Five patients had stage I tumor, 7 had Stage II, and 3 had stage III. Platelet count was elevated from $3.8 \times 104 \pm 1.5 \times 104$ to $7.2 \times 104 \pm 1.8 \times 104$. Eleven patients $(73 \%)$ out of 15 need not platelet blood transfusion by Lusutrombopag administration. No patients had bleeding complication after RFA procedure.

Conclusion: Lusutrombopag administration made the number of patient who need platelet blood transfusion lower at the time of RFA procedure for liver cancer. 


\section{HCC-42}

\section{Tumor infiltrating lymphocytes in hepatocellular carcinoma}

\section{$\underline{\text { Baekhui Kim }}^{1}$, Hyeyoon Chang ${ }^{1}$}

${ }^{1}$ Korea University Guro Hospital, Korea, Republic of

Background: Development of immunotherapeutic agents raised the prognostic and therapeutic importance of tumor infiltrating lymphocytes (TILs) in malignant tumors. In hepatocellular carcinoma (HCC), dense infiltration of TILs is usually not observed. Although small numbers of TILs are observed in HCC, there could be difference in the prognosis of HCC patients according to TILs. We analyzed CD3 and CD8 TILs in HCC tissues and its prognostic implication.

Method: A total of $284 \mathrm{HCC}$ patients who underwent surgical resection in our hospital were enrolled in this study. Tissue microarray was constructed and unstained slides with 4 um thickness were used for stainings. Hematoxylin and Eosin staining, CD3, and CD8 stainings were done. Microscopic images were captured and image analyzer was used for positive cell counting. Median number of CD3 and CD8 positive cells was used for cut-off value in defining high-CD3+ TIL group, low-CD3+ TIL group, high-CD8+ TIL group, and low-CD8+ TIL group.

Result: In the survival analysis of HCC patients, higher tumor stage (p

Conclusion: In many reports of TILs in malignant neoplasm, higher TIL number is usually associated with good prognosis. In this study, CD3+ TIL number did not show difference in patient survival, but, high CD8+ TIL number was associated with poor prognosis. Escaping of host immune mechanism of tumor cells are accomplished in several ways. In HCC, CD8+ TILs may be the target of tumor immuno-regulatory mechanism, either through activation of immunoregulatory molecules or induction of regulatory $\mathrm{T}$ cells.

\section{HCC -43}

Hepatocellular carcinoma following direct acting antivirals for hepatitis $\mathrm{C}$ treatment: a report of case series from tertiary care center in Western Tamilnadu

Prudhvi Krishna Chandolu ${ }^{1}$, Venkatakrishnan $\mathrm{L}^{1}$, Mukundan Swaminathan $^{1}$, Siddharth R Dharamsi' ${ }^{1}$, Vishnu Abhishek ${ }^{1}$, Kartikayan R K $\mathbf{K}^{\mathbf{1}}$, Bibin $\mathbf{P}^{1}$

${ }^{1}$ Dr MGR Medical University, India

Background: Hepatocellular carcinoma (HCC) is one of the most dreadful sequels of hepatitis $\mathrm{C}$ virus (HCV)-related cirrhosis. New direct-acting antivirals (DAA) had successfully created a new era of $\mathrm{HCV}$ elimination. However, their role in moderating the incidence of $\mathrm{HCC}$ in those patients is still questionable. This is the report of case series from tertiary care center in Western Tamilnadu; registering 9 primary HCC cases respective to DAAs therapy after achieving sustained viral response.

Method: All 9 patients were diagnosed as Child A HCV-related cirrhosis. They received management as inpatients in the Clinical Gastroenterology Department. They were diagnosed as having HCV infection during the least 4 years. Pre DAAs treatment evaluation, laboratory, endoscopic as well as tedious professional abdominal imaging [either abdominal ultrasound or computerized tomography (CT) scan] were available for all patients. There was no evidence of HCC pre HCV treatment.

Result: All patients received IFN-free, sofosbuvir-based regimens. Sofosbuvir plus ribavirin was prescribed to $2(22.2 \%)$, sofosbuvir plus ribavirin and daclatasvir were given to 2 patients $(22.2 \%)$. Ledipasvir and Sofosbuvir were given to 5 patients (55.5\%). SVR at week 12 post treatment was achieved in 9 cases $(100 \%)$.

The newer sonography and CT imaging in 9 patients had surprisingly unveiled presence of predominantly small HCC. Most of these new lesions were small; less than $3 \mathrm{~cm}$ in 5 patients $(55.5 \%), 3-5 \mathrm{~cm}$ in 3 cases $(33.3 \%)$, while 1 patient who was diagnosed with a lesion more than $5 \mathrm{~cm}$. The small-sized lesions added to the mean timing for HCC detection $(5.19 \pm 3.48$ months post-treatment). Remaining one patient presented with HCC after SVR 48. Significant biochemical derangements were reported following revelation of HCC. They were significant enough to transfer most of the affected patients from Child class A to Child C cirrhosis. 5 out of 9 patients (55.5\%) underwent TACE. One of the 9 underwent RFA followed by liver transplantation. 3 patients lost for followup.

Conclusion: Risk assessment for HCC should be rigorously undertaken before DAAs, and those at risk should have attentive surveillance during treatment and afterward. It is important to be on continued surveillance after $\mathrm{HCV}$ eradication.

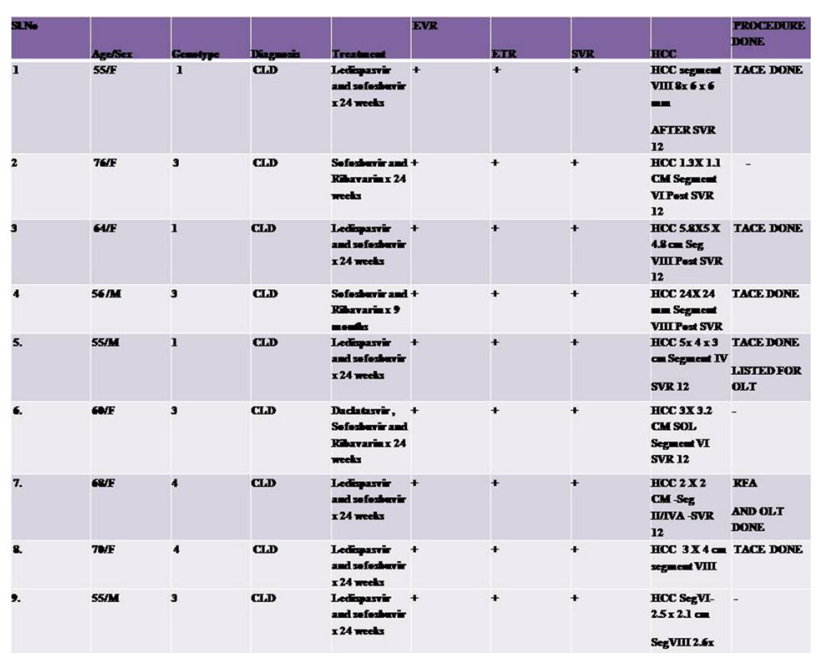

HCC-44

Laparoscopic liver resection of hepatocellular carcinoma located in segments 7 or 8

\section{$\underline{\text { Jai Young Cho }}^{1}$, Soomin Ahn'}

${ }^{1}$ Seoul National University Bundang Hospital, Korea, Republic of

Background: Many centers consider hepatocellular carcinoma (HCC) located in segments 7 or 8 to be unsuitable for laparoscopic liver resection (LLR). We evaluated the safety of LLR of HCC in segments 7 or 8 following the introduction of new laparoscopic techniques.

Method: This retrospective study included 104 patients who underwent LLR $(n=46)$ or open liver resection (OLR) $(n=58)$ for HCC located in segments 7 or 8 between October 2004 and June 2015. The LLR group was subdivided into two subgroups according to whether LLR was performed before (Lap1; $n=29$ ) or after (Lap2; $n=17$ ) the introduction of the Pringle maneuver, intercostal trocars, and semilateral patient positioning.

Result: Non-anatomical resection was more frequent $(63.0 \%$ vs. $29.3 \% ; P<0.001)$ and tumor size was smaller $(2.8$ vs. $4.7 \mathrm{~cm} ; P<$ $0.001)$ in the LLR group than in the OLR group. Blood transfusion $(P=0.526)$, operation time $(P=0.267)$, postoperative complications $(P=0.051)$, and resection margin $(P=0.705)$ were similar in both groups. LLR was associated with less blood loss (550 vs. $700 \mathrm{ml}, P=$ 
0.030 ) and shorter hospital stay ( 8 vs. 10 days; $P=0.001$ ). The 3 -year overall $(90.2 \%$ vs $81.2 \%, P=0.096)$ and disease-free survival $(15.1 \%$ vs. $12.1 \% ; P=0.857)$ rates were similar in both groups. The Lap2 group has less blood loss $(230$ vs. $500 \mathrm{ml} ; P=0.005)$ and shorter hospital stay ( 7 vs 9 days; $P=0.038$ ) compared with the Lap1 group. Conclusion: LLR can be safely performed for HCC located in segments 7 or 8 with recent improvements in surgical techniques and accumulated experience.

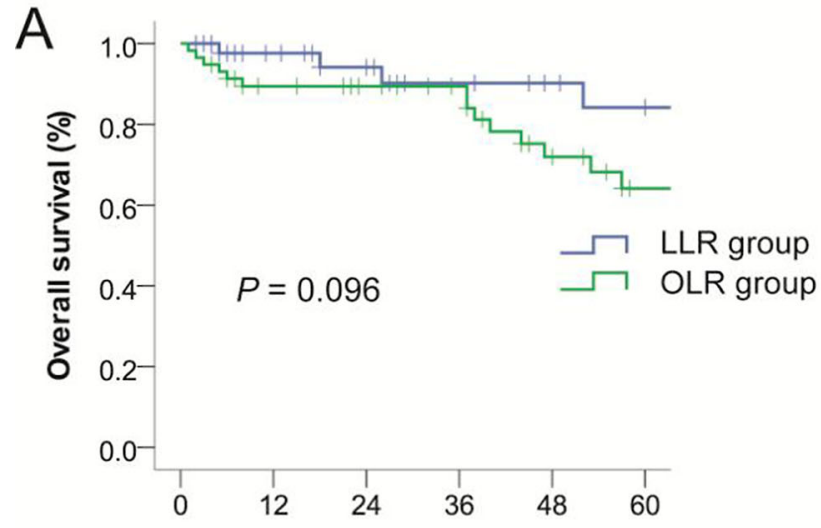

Time after operation (months)

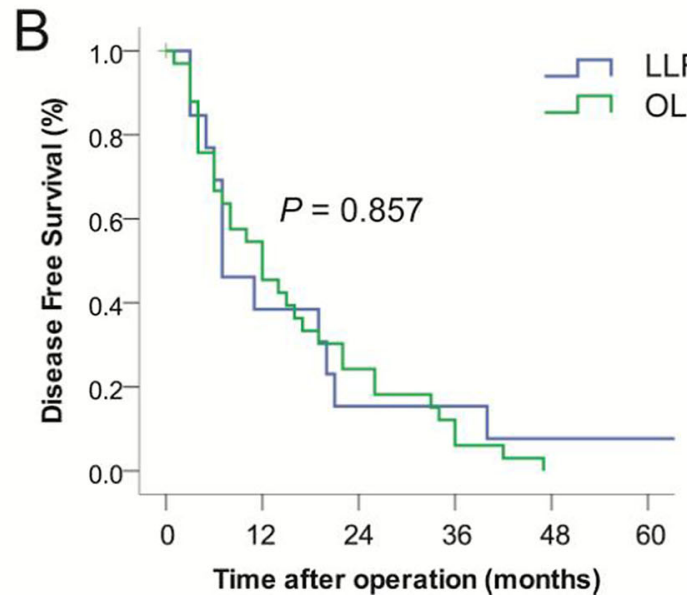

HCC -45

Percutaneous microwave and radiofrequency ablative therapy for hepatocellular carcinoma: a retrospective comparative study

Sandeep V Kumbar ${ }^{1}$, Lijesh Kumar ${ }^{2}$, Prashant Menon ${ }^{2}$, Maya Peethambaran $^{2}$, Aby Somu ${ }^{2}$, Shibi Mathew ${ }^{2}$, John Mathews ${ }^{2}$, Prakash Zacharias ${ }^{2}$, Mathew Philip ${ }^{2}$

${ }^{1}$ PVS Memorial Hospital, India; ${ }^{2}$ PVS Memorial Hospital, India

Background: Ablative therapies have gained acceptance as effective modality for small hepatocellular carcinoma (HCC).There are only a few clinical studies comparing the response to radio frequency ablation (RFA) and microwave ablation (MWA). Aim of the study is to compare the efficacy and complications of ablative therapies.

Methods: Retrospective analysis of 50 cirrhosis patients diagnosed HCC based on CT criteria over period of 15 months. 25 patients (35 nodules) were treated percutaneously with MWA and 25 patients (33 nodules) with RFA. Four weeks outcome of both therapeutic modalities, in terms of morphological tumour response (number, size, location), AFP levels and complications associated with therapy were assessed. Modified RECIST criteria was used for tumour response assessment.

Result: In MWA group, 23 male (92\%) and 2 females (8\%). 13 patients (52\%) in CHILD A group, 8 patients (32\%) in CHILD B, and 4 patients $(16 \%)$ in CHILD C group. Commonest ethology for cirrhosis was ethanol related 16 patients $(64 \%)$. 26 nodules $(74 \%)$ were less than $3 \mathrm{~cm}$ in size and 9 nodules (26\%) of size more than $3 \mathrm{~cm}$. Total 18 patients $(72 \%)$ had single lesion and 7 patients $(28 \%)$ had multiple lesions. Total $21(84 \%)$ out of 25 patients had lesion near to vital structures. $6(24 \%)$ patients had lesion near to inferior vena cava and hepatic veins (IVC/HV). 5 (20\%) patients had lesion near to sub diaphragmatic area. $4(16 \%)$ patients had lesion near to gallbladder. 6 (24\%) patients had lesion near to both IVC/HV and sub diaphragmatic area. In RFA group $22(88 \%)$ were male and $3(12 \%)$ were female patients. There are $17(68 \%)$ patients in CHILD A, 8 patients (32\%) in CHILD B. Commonest ethology being ethanol related 18 (72\%) patients. Out of 33 nodules, 29 nodules (87\%) had size of less than $3 \mathrm{~cm}$ and 4 nodules (13\%) of size more than $3 \mathrm{~cm}$. Total 11 (44\%) out of 25 patients had lesion near to vital structures. $7(28 \%)$ patients had lesion near IVC/HV. 1 (4\%) patient had lesion near gallbladder and $1(4 \%)$ patient had lesion near to both IVC/HV and sub diaphragmatic area. Age group ranges from 40 to 85 years in both the groups. The complete ablative rates were $88 \%$ (26/33) nodules using microwave ablation Vs $88 \%$ (28/35) nodules in RFA (p-0.75) after 4 weeks of assessment. There is no significant differences of ablative rates in tumour size less than or more than $3 \mathrm{~cm}(\mathrm{p}-0.09)$ or in single vs multiple lesions (p-0.9). Similar rates of AFP reduction ( $\mathrm{p}-$ $0.1)$ was noted. No significant difference of variations in Liver function tests $(\mathrm{p}-0.4)$ noted in both groups after 24 hours of ablation. Though we could not compare lesions near vital structures like (IVC/ $\mathrm{HV}$ or sub diaphragmatic or near gall bladder) because of selection bias, ablative response was better with microwave group (76\%). However major complications, mainly pulmonary were associated with microwave ablation $16 \%(4 / 25)(\mathrm{p}-0.001)$. Time required for the procedure was significantly lower in microwave ablation group (58 min vs $148 \mathrm{~min})(\mathrm{p}-0.02)$.

Conclusion: The efficacy of MWA and RFA in HCC are equally good. Microwave ablation is better for lesions near vital structures. Major complications are more with MWA. Time taken for microwave ablation was much less compared to RFA.

HCC -46

Technique and effect of percutaneous transhepatic and free radioactive seed implantation combined with portal vein stent implantation for the treatment of tumor thrombus in portal vein

Fuliang $\mathrm{He}^{1}$, Fuquan Liu ${ }^{1}$, Lei Wang ${ }^{1}$, Yifan $\mathrm{Wu}^{1}$, Zhendong Yue $^{1}$

${ }^{1}$ Beijing Shijitan Hospital, Capital Medical University, China

Background: To analyze the efficacy of percutaneous transhepatic and free radioactive particle implantation combined with portal vein stent implantation in treating the portal vein tumor thrombus.

Method: According to the enter criteria and exclusion criteria, we had elected 57 patients with tumor thrombus in the main portal vein to be treated with stent implantation only(the group A) and 46 patients with tumor thrombus in the main portal vein to be treated with free radioactive particles combined with stent implantation (the group B) from 2010.01 to 2015.01. The two groups were recorded in the aspects of the survival rate, median survival time and stent stenosis rate. 
Result: The cumulative 6-, 12-, and 24-month survival rates for the group A and group B were, respectively, $50.9 \%$ and $73.9 \%, 3.5 \%$ and $39.1 \%, 0 \%$ and $13 \%(\mathrm{P}<0.01)$.

Conclusion: Percutaneous transhepatic and free radioactive particle implantation combined with portal vein stent implantation in treating portal vein tumor thrombus is simple, reliable and safe. It can improve the patient's survival time and the quality of patients' life was significantly better than the quality of patients' life who were treated with only portal vein stent.

\section{HCC-47}

To assess the diagnostic accuracy of HCC-Alpha fetoproteinroutine test (HCC-ART) score based on age, AFP, aspartate aminotransferase (AST)/alanine aminotransferase (ALT) ratio, alkaline phosphatase (ALP), and albumin (Alb) for early detection of hepatocellular carcinoma in cirrhotic patients

Arun Iyer $^{1}$, Krishnadas Devdas ${ }^{1}$, Srijaya Sreesh ${ }^{1}$, Shanid Sathar ${ }^{1}$, $\overline{\text { Sandesh K}}^{1}$, Anirrudha Pratap Singh ${ }^{1}$

${ }^{1}$ Dept of Medical Gastroenterology, Govt Medical College \& Hospital, Thiruvananthapuram, Kerala University of Health Sciences, India

Background: The use of AFP as a screening test for HCC is doubtful on account of its sensitivity of $39-64 \%$, specificity of $76-91 \%$ and low positive predictive value of $9-32 \%$. Similarly, USG is prone to high interobserver variability and poor sensitivity of $35-37 \%$. Therefore AFP in combination with USG is a poor predictive model for detecting HCC. An ideal predictive model for the same must be convenient, inexpensive, widely available, reproducible and minimally or non-invasive.

Method: We conducted a retrospective observational study and the data of all patients who were admitted or evaluated in OPD setting with a diagnosis of HCC; or those with chronic liver disease (CLD) based on standard clinical, biochemical and USG criteria with a clinical suspicion of HCC was analysed. A control group was also analysed, which included patients who had cirrhosis and who were under regular surveillance with biannual USG and AFP monitoring. The HCC-ART score was calculated for all included patients as follows $=(\log$ AFP X AST/ALT ratio X Age X ALP $) / S$. Albumin.

Result: 322 patients were included out of which 203 patients (171 males and 32 females) had documented HCC on triple phase CT and 119 patients ( 81 males and 38 females) were taken as controls. We had taken two cut off values of AFP as $16 \mathrm{ng} / \mathrm{ml}$ and $200 \mathrm{ng} / \mathrm{ml}$ for suspecting $\mathrm{HCC}$ in previously diagnosed cirrhotic patients, which was in accordance to some of the previous studies in India. In our study the best mix of sensitivity and specificity of AFP $(81.3 \%$ and $96.6 \%$, respectively) was seen at a level $9.75 \mathrm{ng} / \mathrm{ml}$. In a large scale multicentre study done in Egypt by Attallah et al in 2014 the HCC-ART score was derived and validated in a cohort of 3559 patients which comprised of an estimation group (1351 patients) and validation group (2208 patients). They had suggested a cut off value as 280 for suspecting HCC cases. We did the analysis in our cohort in accordance with the same cut off value.

As per our cohort analysis for the HCC ART score (with a cut off value of 280 ) the Sensitivity was $86.2 \%$, specificity was 81.5 , positive predictive value was $88.8 \%$ and negative predictive value was $77.6 \%$. Whereas with a cut off level of $16 \mathrm{ng} / \mathrm{ml}$ for serum AFP the sensitivity was $75.4 \%$, specificity was $97.5 \%$ and with a cut off level of $200 \mathrm{ng} /$ $\mathrm{ml}$ the sensitivity dropped down to $75.4 \%$ but with $100 \%$ specificity. As the level of AFP increased, the sensitivity decreased and the specificity showed an increase. At $400 \mathrm{ng} / \mathrm{ml}$ cut-off for all sizes of
HCC the sensitivity was poor $(25.7 \%)$; however, the specificity was perfect $(100 \%)$.

Ours is a setting where the burden of CLD is high. Also, due to higher number of infective aetiologies (hepatitis B and hepatitis C) of CLD, the burden of HCC is also higher. Hence this score can be useful in surveillance programmes for CLD patients. Owing to the complex formula used in the calculation of the score we have also developed a mobile application named "HCC predictor" (link given below) which can be downloaded free from Google play store and hence this app can be used to calculate the score within seconds and hence screen the CLD patients in OPD settings when there is lack of time to evaluate (https://play.google.com/store/apps/details?id=proj.me.scoreapp)

Conclusion: Therefore, this score can be used as a marker in HCC surveillance programme using ultrasonography. In addition, it is highly important to know whether this score will decrease in response to HCC therapy and reductions in tumour burden.

Table 1: Baseline characteristics of cases and controls
\begin{tabular}{|l|c|c|c|c|}
\hline \multirow{2}{*}{ Parameters } & \multicolumn{2}{|c|}{ Cases (N=203) } & \multicolumn{2}{c|}{ Controls (N=119) } \\
\cline { 2 - 5 } & mean & $\begin{array}{c}\text { Standard } \\
\text { deviation }\end{array}$ & mean & $\begin{array}{c}\text { Standard } \\
\text { deviation }\end{array}$ \\
\hline AGE (in years) & 56.87 & 12.10 & 52.66 & 8.61 \\
\hline Child-Turcotte-Pugh score & 8.54 & 2.47 & 8.25 & 2.65 \\
\hline MELD score & 11.17 & 5.12 & 14.23 & 6.39 \\
\hline AST (U/ L) & 153.67 & 156.01 & 65.18 & 38.94 \\
\hline ALT (U/L) & 84.88 & 113.45 & 51.26 & 40.14 \\
\hline ALP (U/L) & 225.44 & 194.03 & 69.92 & 58.75 \\
\hline Serum Albumin (gm/dl) & 2.97 & 0.81 & 30.66 & 42.85 \\
\hline $\begin{array}{l}\text { Serum Alpha Fetoprotein } \\
\text { (ng/ ml) }\end{array}$ & 6166.21 & 28718.51 & 4.81 & 4.14 \\
\hline LOG AFP & 2.29 & 1.19 & 0.60 & 0.26 \\
\hline HCC ART SCORE & 2608.11 & 4033.86 & 147.29 & 174.31 \\
\hline
\end{tabular}

Table 2: Hepatocellular carcinoma staging was conducted as per Barcelona Clinic Liver Cancer group (BCLC). BCLC distribution of our cases of HCC

\begin{tabular}{|l|c|c|}
\hline $\begin{array}{l}\text { BCL } \\
\text { C }\end{array}$ & $\begin{array}{c}\text { FREQUEN } \\
\text { CY }\end{array}$ & $\begin{array}{c}\text { Perce } \\
\text { nt }\end{array}$ \\
\hline 0 & 1 & 0.5 \\
\hline A & 18 & 8.9 \\
\hline B & 35 & 17.3 \\
\hline C & 71 & 35.1 \\
\hline D & 77 & 38.1 \\
\hline Total & 202 & 100 \\
\hline
\end{tabular}

Table 3: Diagnostic performance of AFP

\begin{tabular}{|r|r|r|}
\hline $\begin{array}{r}\text { AFP cut off } \\
\text { level } \\
(\mathrm{ng} / \mathrm{ml})\end{array}$ & Sensitivity & Specificity \\
\hline$>9.75$ & 0.813 & 0.966 \\
\hline$>16$ & .754 & $\mathbf{0 . 9 7 5}$ \\
\hline$>200$ & 0.527 & 100 \\
\hline
\end{tabular}

Table 4: Diagnostic performance of HCC ART score in our cohort.

\begin{tabular}{|c|c|c|c|c|c|}
\hline & \multicolumn{2}{|c|}{ Category } & \multirow[b]{2}{*}{ Total } \\
\hline & & & Cases & Controls & \\
\hline \multirow{4}{*}{$\begin{array}{l}\text { HCC } \\
\text { ART } \\
\text { score }\end{array}$} & \multirow[t]{2}{*}{$>280$} & Number of patients & 175 & 22 & 197 \\
\hline & & $\begin{array}{l}\% \text { with score more } \\
\text { than } 280\end{array}$ & $86.2 \%$ & $18.5 \%$ & $61.2 \%$ \\
\hline & \multirow[t]{2}{*}{$<280$} & Number of patients & 28 & 97 & 125 \\
\hline & & $\begin{array}{l}\% \text { with score less than } \\
280\end{array}$ & $13.8 \%$ & $81.5 \%$ & $38.8 \%$ \\
\hline \multicolumn{2}{|l|}{ Total } & Number of patients & 203 & 119 & 322 \\
\hline
\end{tabular}




\section{HCC -48}

Usefulness of radiofrequency ablation training program for liver tumors

Shuichiro Shiina ${ }^{1}$, Koki Sato ${ }^{1}$, Motonori Shimizu', Hideko Ohama $^{\mathrm{T}}$, Shigeto Ishii ${ }^{1}$, Ryo Kanazawa ${ }^{1}$, Ryo Shimizu ${ }^{1}$, Manabu Hayashi ${ }^{1}$, Takeshi Hatanaka ${ }^{1}$, Nobuhito Taniki ${ }^{1}$, Masashi Takawa ${ }^{1}$, Hiroaki Nagamatsu ${ }^{1}$, Yasuharu Imai ${ }^{1}$

${ }^{1}$ Juntendo University, Japan

Background: RFA, which is now a standard treatment modality, is highly operator-dependent. Its skills and outcomes are different from operator to operator. It would be useful to see a sophisticated RFA procedure at a high volume center to improve their skills. Juntendo is the highest volume center of RFA in Japan.

Method: To disseminate skills and know-hows for RFA and to standardize the procedure, we have held 10 domestic training programs in which 170 Japanese doctors participated. In addition, we have held four international ones in which 49 doctors from abroad attended.

Result: The Programs are designed for intermediate to senior experienced doctors who have experience in RFA as a main operator. The programs are composed of lectures, live demonstrations and case studies. Lecture contents are indications and complications of RFA, RFA devices, US, and others. In live demonstrations, RFA is performed on a wide variety of cases: cases of newly diagnosed cancer not difficult to ablate judging from size and location, a tumor just below the diaphragm requiring artificial ascites, a tumor in the caudate lobe, a tumor adjacent to the heart, a portal vein or hepatic vein, a tumor over 5 centimeters, more than five tumors, hepatic metastasis, etc. We demonstrate the importance to have appropriate patient posture, usefulness of our original dedicated probe for interventional procedures and our RFA dedicated operation table, and ways to perform ablation under Sonazoid guidance, and with multimodality fusion imaging. In case studies, difficult to ablate cases from participants' hospitals are presented and discussed. Many participants remarked on the benefit of being directly trained by noted interventional oncologists in an academic environment. Questionnaire surveys revealed overwhelmingly positive feedback.

Conclusion: Our programs may be useful to provide opportunities to understand basic concepts and learn essential technical tips for successful ablation.

\section{HCC -49}

Computed diffusion weighted imaging of the liver using extrapolation technique in patients who underwent liver transplantation with hepatocellular carcinomas: initial experience and feasibility study

\section{$\underline{\text { Joonil Choi }}^{1}$, Michael Yong Park ${ }^{1}$}

${ }^{1}$ Seoul St.Mary's Hospital, The Catholic Univ. of Korea, Korea, Republic of

Background: Computed diffusion weighted imaging (cDWI) is a recently developed technique in which higher b-value DWIs can be synthesized mathematically from low b-value images without direct image acquisition. In this technique, the apparent diffusion coefficient (ADC) is calculated from low b-value images using extrapolation. The purpose of this study was to evaluate the feasibility and image quality of cDWI of liver in patients with hepatocellular carcinoma (HCC).
Method: Twenty-four patients underwent liver transplantation with HCC were enrolled. Computed DWI was synthesized for b-values of 800 (cDWI800) and $1200 \mathrm{~s} / \mathrm{mm}^{2}$ (cDWI1200) using directly acquired DWI with b-values of 0,50 and $500 \mathrm{~s} / \mathrm{mm}^{2}$. Signal intensity of HCC, background liver and contrast-to-noise ratio (CNR) were evaluated for directly acquired DWI of $800 \mathrm{~s} / \mathrm{mm}^{2}$ (dDWI800), cDWI800, and cDWI1200. Two radiologists evaluated the image quality for contrast between HCC and liver, suppression of background signal and T2 shine-through, and overall image quality.

Result: Quantitative analysis The Friedman test revealed significant differences in the signal intensity of HCC ( $p$

Conclusion: Generating cDWI of higher b-values in HCC patients was feasible. In cDWI of high b-values, contrast between HCC and background liver was very low, even though better suppression of background and less $\mathrm{T} 2$ shine-through effect

Table 1. Comparison of quantitative measurements between diffusion-weighted sequences

\begin{tabular}{|c|c|c|c|c|c|c|c|}
\hline $\begin{array}{l}\text { Quantitative } \\
\text { parameters }\end{array}$ & $\begin{array}{l}\text { dDWIsoo } \\
\text { (A) }\end{array}$ & $\begin{array}{l}\text { cDWI800 } \\
\text { (B) }\end{array}$ & $\begin{array}{l}\mathrm{cDWI}_{1200} \\
\text { (C) }\end{array}$ & $\begin{array}{l}\text { p-values }{ }^{\mathrm{a}} \\
\text { (Friedman) }\end{array}$ & $\begin{array}{l}\text { p-values* } \\
\text { (A vs. B) }\end{array}$ & $\begin{array}{l}\text { p-values* } \\
\text { (A vs. C) }\end{array}$ & $\begin{array}{l}\text { p-values* } \\
\text { (B vs. C) }\end{array}$ \\
\hline $\begin{array}{l}\text { Signal intensity of } \\
\mathrm{HCC}\end{array}$ & 42.2 & 37.5 & 19.4 & $<0.001$ & 0.012 & 0.003 & 0.005 \\
\hline $\begin{array}{l}\text { Signal intensity of } \\
\text { background liver }\end{array}$ & 19.3 & 16.7 & 9.0 & 0.002 & 0.050 & 0.003 & 0.003 \\
\hline Noise & 1.9 & 2.7 & 3.0 & 0.112 & $\mathrm{n} / \mathrm{a}$ & $n / a$ & $n / a$ \\
\hline CNR & 12.4 & 7.5 & 3.4 & 0.038 & 0.003 & 0.016 & 0.041 \\
\hline
\end{tabular}

Table 2. Comparison of qualitative measurements between diffusion-weighted sequences

\begin{tabular}{|c|c|c|c|c|c|c|c|}
\hline Qualitative parameters & $\begin{array}{l}\text { dDWI800 } \\
\text { (A) }\end{array}$ & $\begin{array}{l}\text { cDWIsoo } \\
\text { (B) }\end{array}$ & $\begin{array}{l}\text { cDWI } 1200 \\
\text { (C) }\end{array}$ & $\begin{array}{l}\text { p-values" } \\
\text { (Friedman) }\end{array}$ & $\begin{array}{l}\text {-values* } \\
\text { (A vs. B) }\end{array}$ & $\begin{array}{l}\text { p-values* } \\
\text { (A vs. C) }\end{array}$ & $\begin{array}{l}\text {-values* } \\
\text { (B vs. C) }\end{array}$ \\
\hline $\begin{array}{l}\text { Contrast between HCC } \\
\text { and background liver }\end{array}$ & 3.2 & 3.1 & 2.5 & $<0.001$ & 0.317 & 0.011 & 0.003 \\
\hline $\begin{array}{l}\text { Background liver } \\
\text { suppression }\end{array}$ & 2.9 & 3.4 & 3.6 & 0.002 & 0.025 & 0.011 & 0.180 \\
\hline $\begin{array}{l}\text { Suppression of } \mathrm{T} 2 \\
\text { shine-through effect }\end{array}$ & 2.4 & 2.7 & 3.6 & $<0.001<$ & 0.083 & 0.002 & 0.004 \\
\hline Overall image quality & 3.0 & 3.1 & 2.7 & 0.674 & n/a & n/a & n/a \\
\hline
\end{tabular}

HCC -50

Multi-disciplinary approach to the management of hepatocellular carcinoma: a case report

\section{Kenji Madriaga Sato ${ }^{1}$}

${ }^{1}$ Cardinal Santos Medical Center, Philippines

Background: A 58/F diagnosed case of HCC secondary to NASH initially presenting as right sided abdominal pain. She underwent trisegmentectomy V, VI, VII. She was advised to have close followup. 1 month prior to admission patient she experienced abdominal distention. Progression of symptoms prompted consult for which repeat $\mathrm{CT}$ scan of the whole abdomen was done showing increase in the size of enhancing masses in the liver. Pertinent PE include fluid wave test and bipedal edema

Method: Based on the new imaging findings and laboratory results, Child Pugh Classification was now at Class B, with BCLC Stage C. She was started with Sorafenib. Work up for hypercoagulable state was done revealed, low levels of Protein $\mathrm{C}$ and Protein $\mathrm{S}$ and an increasing trend in FDP levels, hence Enoxaparin was started. Signs 
of decompensation such as ascites and hepatic encephalopathy were managed. She developed bacterial peritonitis and hospital acquired pneumonia hence several antibiotics were given. Persistence of abdominal and low back pain lead to referral to pain medicine.

Result: HCC is the second leading cause of cancer-related deaths worldwide. The rising incidence, genetic heterogeneity, multiple etiologies, and concurrent chronic liver diseases make diagnosis, staging, and selection of treatment options challenging in patients with HCC. best approach to optimize the management of HCC is one that utilizes a core multidisciplinary liver tumor board consisting of hepatologists, pathologists, interventional radiologists, oncologists, hepatobiliary and transplant surgeons, nurses, and general practitioners. The role of the multidisciplinary team is crucial in promptly reconfirming the diagnosis, staging the HCC, and formulating an individualized treatment plan.

The clinical decision making during the evaluation by the multidisciplinary liver tumor board relies heavily on the accuracy of diagnosis and staging of HCC before outlining a treatment plan. A multidisciplinary approach ensures that HCC is accurately staged and that treatment recommendations are evidence-based, patient-centered, and reached by consensus. Specialists with different roles, expertise, and functions are required to manage $\mathrm{HCC}$ due to its complex genetic, pathological, etiological, and oncological features. The contribution of each team member varies and depends on the institution-specific expertise and the stage of $\mathrm{HCC}$ at diagnosis.

Conclusion: MDT plays a role in tailoring the therapy to the specific needs of each patient, taking into account international guidelines, local regulations, in order to increase the clinical benefits of therapy and improve patient's quality of life

\section{HCC -51}

3 year survival prognosis factors of hepatocellular carcinoma treated by percutaneous radiofrequency ablation

\section{$\underline{\text { Hang Viet Dao }}^{1}$, Long Van Dao ${ }^{1}$ \\ ${ }^{1}$ Hanoi Medical University, Vietnam}

Background: Hepatocellular carcinoma (HCC) is a common malignancy worldwide and in Vietnam. Radiofrequency ablation (RFA) is a local therapy to destroy tumor by heat, however, not many studies in our country have been conducted to analyze factors correlating to survival prognosis. Aims: This study was conducted to evaluate overall survival after 1 year, 2 years and 3 years and prognostic factors relating in HCC patients treated by RFA.

Method: This study was an interventional, follow-up study on 130 HCC patients stage Barcelona (BCLC) 0 and A treated RFA from Oct/2011 to June/2017 in Gastroenterology department of Bach Mai hospital with mean follow up time being 38.5 months (SD-13.5 months).

Result: Overall survival time was 52.1 months (CI 95\%: 48.1 - 56.1 months). 40 patients $(30.8 \%)$ died during follow-up time with mean survival time being 26.4 months. The survival rate after 1 year, 2 years and 3 years were $95.3 \%, 85.4 \%$ and $75.4 \%$, respectively. There was no difference of survival rate among different groups of number of tumors, tumor size, types of needles, Child Pugh score, initial AFP level. Cox regression analysis recorded BCLC stage (0 and A) had correlation with survival prognosis $(\mathrm{p}<0.001$ and $\mathrm{r}=0.36)$.

Conclusion: RFA is a curative therapy to improve overall survival; BLCL stage is the only survival prognosis factor for HCC patients treated by RFA.
HCC-52

ANGPT2 polymorphisms and clinical outcome in advanced hepatocellular carcinoma patients receiving sorafenib

Andrea Casadei Gardini ${ }^{1}$

${ }^{1}$ IRST-IRCCS, Italy

Background: Sorafenib (S) represents the standard of care for advanced hepatocellular carcinoma (HCC), even if a large number of patients reports limited efficacy with respect to toxic effects. Biomarkers of S efficacy or resistance have yet to be identified. We analyzed the role of $A N G P T 2$ polymorphisms in relation to clinical outcome in advanced HCC patients receiving S.

Method: Our retrospective study included 130 advanced HCC patients all undergoing S treatment from June 2009 to April 2015. These preliminary data were carried out on one hundred patients and ANGPT2 polymorphisms (rs3739390, rs3739391, rs3739391, rs1961222, rs3020221, rs6559167, rs2916747, rs17063434) were analyzed by direct sequencing in relation to progression-free survival (PFS) and overall survival (OS).

Result: All genotype frequencies followed the Hardy-Weinberg equilibrium. Patients homozygous for ANGPT2 rs55633437 GG genotype showed significantly longer PFS (8.3 vs 2.4 month, $p=$ $0.0022)$ and OS (16.7 vs 6.4 months, $p=0.0024)$ than those with the other genotypes respectively (GT+TT). Median PFS was 3.9 (95\% CI 3.1-5.3), while median OS was 11.8 months (95\% CI 8.7-14.3).

Conclusion: Our preliminary results suggest that $A N G P T 2$ gene may be capable of identifying a subset of HCC patients who are more responsive to $\mathrm{S}$.

\section{HCC -53}

Changing epidemiology and clinical profile of Hepatocellular carcinoma (HCC) in India

Vaneet Jearth $^{1}$, Shaesta Mehta ${ }^{1}$, Prachi Patil ${ }^{1}$, Sridhar Sundaram ${ }^{1}$

${ }^{1}$ Tata Memorial Hospital, Mumbai, India

Background: There is very limited data on epidemiology of HCC in India. The number of patients of HCC with non-viral etiology is increasing as the prevalence of lifestyle-related factors like chronic alcoholism, diabetes and metabolic syndrome change globally. Unlike $\mathrm{HBV} / \mathrm{HCV}$ related HCC (HBHC HCC), little is known about the characteristics of $\mathrm{HCC}$ negative for markers of chronic viral hepatitis labeled as Non-B Non-C(NBNC) HCC .Our aim was to study the change in epidemiology and compare clinical characteristics of patients with NBNC HCC with those having viral etiology for HCC. Method: 155 consecutive patients diagnosed with $\mathrm{HCC}$ with age $>18$ years without history of any prior surgical or interventional treatment for HCC, registered between June 2017 and November 2017 at Tata Memorial Hospital, Mumbai were prospectively enrolled. All patients with negative $\mathrm{HbsAg}$ and Anti HCV ab were evaluated for IgG Anti $\mathrm{Hbc}$ and HBVDNA in serum. Patients who detected negative for viral markers were labeled as NBNC HCC. Patients with presence of only core antibody with non detectable HBVDNA in serum were also grouped under NBNC category in view of doubtful oncological significance

Result: Most of patients were males $(n=139,89.7 \%)$ with mean $( \pm$ SD) age of $56.1 \pm 13.16$.Total number of patients in NBNC HCC group were $66(42.6 \%)$.Risk factors in these patients were diabetes mellitus, metabolic syndrome, alcoholic liver disease, Budd Chiari syndrome and cryptogenic cirrhosis. Overall HCC was found to be 
most commonly associated with HBV in $39.4 \%$ patients $(n=61) .1$ patient in this group had Occult Hepatitis B (IgG Anti Hbc positive with low detectable HBVDNA in blood).HCV alone was found in $11.6 \%(\mathrm{n}=18)$. More than one concomitant risk factor was seen in 6.4 $\%(\mathrm{n}=10)$ cases, out of which association of alcoholic liver disease and Hepatitis $\mathrm{C}$ with $\mathrm{HCC}$ was found in $3.8 \%(\mathrm{n}=6)$. Abdominal pain was the most common presenting symptom in both groups. Cirrhosis was present at presentation in $65.5 \%(n=43)$ of patient with NBNC $\mathrm{HCC}$ and $86.5 \%(\mathrm{n}=77)$ of patients with $\mathrm{HBHC} \mathrm{HCC}(\mathrm{p}$ value $=$ 0.002). Out of patients with cirrhosis CTP A, B and C were $34.2 \%$, $34.2 \%$ and $12.3 \%$ respectively with no significant difference in both the groups. When sub classified as per BCLC staging, BCLC A, B, C and $D$ were $3.2 \%(n=5), 30.3 \%(n=47), 51.6 \%(n=80)$ and $14.8 \%$ $(\mathrm{n}=23)$ respectively with no significant difference with both groups. Major vascular invasion was found to be more common in HBHC HCC $(56.1 \%$ vs $51.5 \%$, p value $=0.55)$. There was no significant difference in incidence of extra hepatic metastasis in both groups $(20.2 \%$ vs $21.2 \%$, p value $=0.81)$.Overall most common sites of metastasis were lungs and lymph nodes in $8.4 \%$ each $(n=13) .6$ patients had obstructive jaundice as per imaging (Intrahepatic Biliary Radicle Dilatation) requiring PTBD. Treatment plan was made as per Liver joint clinic, which is depicted in Table 1 . However post treatment outcomes and survival could not assessed in this short duration and will be analyzed prospectively. Table 2 depicts biochemical parameters, tumor size (largest lesion) and AFP (Alfa fetoprotein)levels in both groups.

Conclusion: Incidence of $\mathrm{HCC}$ is increasing rapidly in India. Although HBV still remains the leading cause of HCC in India, non viral etiologies are on the rise accounting for approximately $40 \%$ (past estimate was 5-15\%). Follow up is needed to assess the differential response to treatment between viral and non viral HCC.

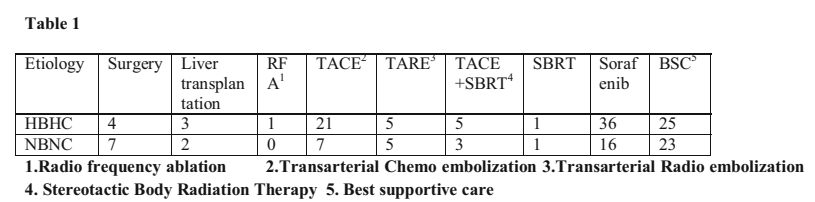

\begin{tabular}{|c|c|c|c|c|c|c|c|c|}
\hline \multirow[t]{2}{*}{$\mathrm{HCC} \diamond$} & \multicolumn{3}{|c|}{ ……....ngFr } & \multirow{2}{*}{ 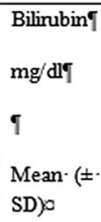 } & \multirow{2}{*}{$\begin{array}{l}\begin{array}{l}\text { Size - of } \\
\text { largest } \\
\text { lesion. } \\
(\mathrm{cm})\end{array} \\
\text { Mean } \quad( \pm \cdot \\
\text { SD)o }\end{array}$} & \multirow{2}{*}{$\begin{array}{l}\text { Albuminf } \\
\text {-g/dlr } \\
\text { Mean } \quad( \pm \cdot \\
\text { SD)a }\end{array}$} & \multirow[t]{2}{*}{ Asciteso } & [o Table.2 \\
\hline & $<100$ & $\begin{array}{l}10- \\
4000\end{array}$ & $>4000$ & & & & & Q \\
\hline HBHCo & 200 & 250 & 440 & $2.2=3.40$ & $8.77=3.50$ & $3.25 \pm 0.64 \mathrm{c}$ & 290 & $\varnothing$ \\
\hline $\mathrm{NBNC}$ & 150 & 170 & 340 & $3.3 \pm 6.00$ & $9.1 \pm 3.90$ & $\begin{array}{l}3.4 \pm 0.51 \text { 于 } \\
0\end{array}$ & 150 & a \\
\hline
\end{tabular}

HCC-54

Efficacy of radiofrequency in patients with hepatocellular carcinoma: a meta-analysis

\section{Andrea Casadei Gardini ${ }^{1}$ \\ ${ }^{1}$ IRST-IRCCS, Italy}

Background: There are no randomized studies in radiofrequency versus best supportive care in the literature, and there are no meta- analysis that have evaluated survival and recurrence free survival factors in radiofrequency patients only.

Method: The purpose of this meta-analysis is to evaluate predictive prognostic factors of survival and recurrence free survival in patients treated with radiofrequency, with the aim of identifying parameters that can help clinicians in the therapeutic choice and to identify stratification factors for future studies in this subset of patients.

Result: Clinical trials that values prognostic factors of radiofrequency ablation were considered. We excluded randomized controlled trials between radiofrequency and surgery. We excluded radiofrequency with microwave and with ethanol. 35 studies published from 2003 to 2017 were analyzed. They included 11.216 HCC patients treated with radiofrequency. For overall survival the analysis of the liver functionality show that child Pugh B versus Child Pugh A(HR=2.32, 95\% $\mathrm{CI}=2.201-2.69 ; \quad P<0.000)$, increase of bilirubin $(\mathrm{HR}=1.03,95 \%$ $\mathrm{CI}=1.01-1.04 ; \quad P<0.0001)$,presence of Portosystemic collaterals(HR $=1.54,95 \% \mathrm{CI}=1.311 .82 ; P<0.0001)$ and ALBI score 1 vs 0 $(\mathrm{HR}=2.69,95 \% \mathrm{CI}=2.10-3.44 ; \mathrm{p}<0.0001)$ was predicted of poor $\mathrm{OS}$. Decrease of Prothrombin activity (HR=0.97, 95\% CI $=0.96-0.99$; $P<0.0001)$ and increase of albumin $(\mathrm{HR}=0.90,95 \% \mathrm{CI}=0.87-0.94$; $P<0.0001)$ was predicted of good OS. The analysis of tumor size show that if considered the cut off of $2 \mathrm{~cm} \quad(\mathrm{HR}=1.41,95 \%$ $\mathrm{CI}=1.23-1.61 ; P<0.0001)$ was predictive of poor OS. If considered the number of nodules the data show that the presence of $>1$ nodules (HR $=1.59,95 \% \mathrm{CI}=1.46-1.74 ; P<0.0001)$ was predictive of poor OS. For alfafetoprotein the data show that only the cutoff of $20 \mathrm{ng} / \mathrm{mL}$ $(>20 \mathrm{ng} / \mathrm{mL}$ vs $<20 \mathrm{ng} / \mathrm{mL} \mathrm{HR}=1.46,95 \% \mathrm{CI}=1.25-1.70 ; P<0.0001)$ was predictive of poor prognosis.

Conclusion: In conclusion, our meta-analysis shows that the maximum benefit of RFA is in child Pugh A, ALBI score 1 , tumor size $<2$ $\mathrm{cm}$ with single nodule and with alpha-fetoprotein less than $20 \mathrm{ng} / \mathrm{mL}$.

HCC-55

Elevated serum urokinase plasminogen activator level predicts poor prognosis in hepatocellular carcinoma after resection

Ming Chao Tsai ${ }^{1}$, Chihche $\operatorname{Lin}^{3}$, Yi Hao Yen ${ }^{3}$, Kuo Chin Chang ${ }^{3}$, Cheng Kun $\mathrm{Wu}^{3}$, Chao Hung Hung ${ }^{1}$, Sheng Nan $\mathrm{Lu}^{2}$, Jing Houng Wang $^{1}$, Chien Hung Chen ${ }^{1}$, Kwong Ming Kee ${ }^{1}$, Ming Tsung Lin ${ }^{1}$, Tsung Hui Hung ${ }^{1}$

${ }^{1}$ Kaohsiung Chang Gung Memorial Hospital and Chang Gung University College of Medicine, Kaohsiung, Taiwan, Taiwan; ${ }^{2}$ Chiayi Chang Gung Memorial Hospital and Chang Gung University College of Medicine, Kaohsiung, Taiwan, Taiwan; ${ }^{3}$ Kaohsiung Chang Gung Memorial Hospital, Taiwan

Background: Urokinase plasminogen activator (uPA) is an extracellular matrix-degrading protease involved in cancer invasion and progression. Although there is good evidence that uPA expression is a clinically relevant biomarker in some solid tumors, its role in hepatocellular carcinoma (HCC) is uncertain. Our aim is to evaluate the prognostic value of serum uPA before surgery in HCC patients receiving curative resection.

Method: Serum uPA levels were determined by enzyme-linked immunosorbent assay in $282 \mathrm{HCC}$ patients who receive complete liver resection at Kaohsiung Chang Gung Memorial Hospital. Overall and recurrence-free survival (RFS) curves were constructed using the Kaplan-Meier method and compared by the lon-rank test. A Cox proportional-hazards regression model was performed to identify independent prognostic factors. Median follow-up time was 52 months.

Result: Patients with higher pretreatment serum uPA had significantly shorter overall and RFS ( $p=0.031$ and $p=0.02$, respectively). 
Multivariate Cox regression analyses indicated that pretreatment high serum uPA was associated with shorter overall and RFS [hazard ratio $(\mathrm{HR})=1.230,95 \%$ confidence interval $(\mathrm{CI}): 1.093-1.385, p=0.001$; $\mathrm{HR}=1.526,95 \%$ CI: 1.044-2.229, $p=0.029$; respectively]. Higher uPA expression is associated with liver cirrhosis $(p=0.045)$ and advanced pathological stage $(p=0.022)$.

Conclusion: We conclude that the uPA is a clinical relevant biomarker in HCC patients receiving curative resection, with higher expression of uPA associated with higher risk disease and poorer prognosis.

\section{HCC-56}

Endocan, a vascular endothelium derived proteoglycan, can be used to detect hepatocellular carcinoma with portal vein thrombosis

\section{Partho Pratik Roy ${ }^{1}$, Salimur Rahman ${ }^{4}$, Mamun Al Mahtab ${ }^{3}$, Ayub} Al Mamun ${ }^{3}$, Noor E Alam ${ }^{2}$

${ }^{1}$ Resident physician, Bangladesh; ${ }^{2}$ Assistant Professor, Bangladesh; ${ }^{3}$ Associate Professor, Bangladesh; ${ }^{4}$ Professor, Bangladesh

Background: Hepatocellular carcinoma is the most common primary liver tumor. Novel biomarkers for the early diagnosis of HCC are required. Endocan is a novel endothelium derived soluble dermatan sulfate proteoglycan. An increase in serum level of endocan reflects endothelial activation and neovascularization which are the prime steps in pathways of neoangiogenesis. The aim of the study is to assess the diagnostic value of serum endocan in detecting hepatocellular carcinoma.

Method: It is a cross sectional observational study which was done in department of Hepatology BSMMU from March 2016 to March 2017. Serum endocan levels were measured in 75 HCC patients who were naïve to treatment, and 35 apparently healthy subjects. ROC curve analysis was done to detect sensitivity and specificity of serum endocan value for differentiating HCC from healthy controls. Association of serum endocan value to clinical, radiological variables were analyzed.

Result: The mean serum endocan levels were $0.33( \pm 0.27) \mathrm{ng} / \mathrm{mL}$ (range, $0.16-1.28 \mathrm{ng} / \mathrm{mL}$ ) in healthy controls and $2.45( \pm 2.41) \mathrm{ng} / \mathrm{ml}$ (range, $0.17-11.10 \mathrm{ng} / \mathrm{mL}$ ) in HCC patients. Levels of endocan were significantly elevated in the serum of patients with HCC as compared to serum from healthy control. Serum endocan can differentiate HCC patients from healthy control with $100 \%$ sensitivity and $76.06 \%$ specificity. Only 18 (24\%) HCC cases have portal vein thrombosis, but $15(83.33 \%)$ of them have high endocan value. Which is statistically significant $(\mathrm{p}=0.01)$ indicating a close link between serum endocan levels and HCC aggressiveness. This findings is similar to the result of the study done by Nault et al. 2013, which has shown that patients with tumor portal thrombosis exhibited higher rates of serum endocan than patients without tumor portal thrombosis.

Conclusion: Portal vein thrombosis is a sign of advanced HCC and its aggressiveness and invasiveness. Serum endocan can be used to detect presence of portal vein thrombosis in advanced, invasive HCC.

Table 1: Association of serum Endocan levels with portal vein thrombosis in HCC patients.
\begin{tabular}{|c|c|c|c|c|}
\hline Trait & Serum Endocan level in HCC (ng/ml) & Total & \multirow{2}{*}{ P value } \\
\cline { 2 - 3 } & $<1.65$ & $\geq 1.65$ & & \\
\hline $\begin{array}{l}\text { Portal vein } \\
\text { thrombosis }\end{array}$ & & & & \\
\hline$\bullet \quad$ Yes & $03(16.67 \%)$ & $15(83.33 \%)$ & $18(24 \%)$ & 0.01 \\
\hline - No & $28(49.13 \%)$ & $29(50.87 \%)$ & $57(76 \%)$ & \\
\hline
\end{tabular}

HCC-57

Evaluation of serum prolactin as a potential tumor marker for detection of hepatocellular carcinoma

Ehab Ahmed Abdelatti ${ }^{1}$, Elsayed Ibrahim Elshayeb ${ }^{1}$, Ashraf Elsayed Abdelghani ${ }^{2}$

${ }^{1}$ Professor of Hepatology, Egypt; ${ }^{2}$ Professor of Oncology, Egypt

Background: Prolactin is a pleiotropic hormone with an established role in the molecular carcinogenesis of breast cancer and other malignancies. Increased circulating prolactin levels and generation of liver cancer cells through PRLR/JAK2 signaling have all been proposed as mechanisms that could contribute to the development of HCC. Aim of this work is to evaluate serum prolactin as a potential tumor marker in detection of hepatocellular carcinoma.

Method: The study included 40 patients with liver cirrhosis (cirrhotic group) and 40 patients with liver cirrhosis and HCC (HCC group) from Menoufia University Hospitals (Egypt) and 20 healthy volunteers as a control group. They underwent physical examination and laboratory investigations (CBC, liver profile \{ALT, AST, serum albumin, bilirubin, PT \% \}, HCV Ab, HBsAg, HCV PCR, AFP and serum prolactin). Abdominal ultrasonography was done for all patients. HCC is further confirmed by triphasic CT and MRI. ChildPugh Score, MELD score and AST/Platelet index were calculated for all patients.

Result: AFP level of HCC group $(458.9 \pm 566.6 \mathrm{ng} / \mathrm{dl})$ was significantly higher than cirrhotic group $(42.1 \pm 16.5 \mathrm{ng} / \mathrm{dl})$ and controls $(6.3 \pm 2.2 \mathrm{ng} / \mathrm{dl})(\mathrm{P}=0.0001)$. AFP level of cirrhotic group is higher than controls $(\mathrm{p}<0.05)$. Prolactin level of HCC group $(67.7 \pm 20.2 \mathrm{ng} /$ dl) was significantly higher than cirrhotic group $(35.9 \pm 12.1 \mathrm{ng} / \mathrm{dl})$ and controls $(15.6 \pm 4.9 \mathrm{ng} / \mathrm{dl})(\mathrm{P}=0.0001)$. Also, Prolactin in cirrhotic group was significantly higher than that of controls $(\mathrm{p}=0.0001)$. Prolactin level was significantly higher in patients with multifocal HCC $(85.2 \pm 15.5 \mathrm{ng} / \mathrm{dl})$ than in patients with single HCC $(62.6 \pm 18.6$ $\mathrm{ng} / \mathrm{dl})(\mathrm{P}=0.002)$. There was a positive correlation between prolactin and APRI index $(\mathrm{r}=+0.36, \quad \mathrm{P}=0.02)$, tumour size $(\mathrm{r}=+0.82$, $\mathrm{P}=0.0001)$, and $\mathrm{AFP}(\mathrm{r}=+0.76, \mathrm{P}=0.0001)$ in $\mathrm{HCC}$ group. Diagnostic validity of serum AFP at cutoff level $51 \mathrm{ng} / \mathrm{dl}$ for prediction of HCC in cirrhotic patients (sensitivity $84 \%$, specificity $65 \%$, positive predictive value $73 \%$, negative predictive value $94 \%$, area under the curve (AUC) (0.9569) and diagnostic accuracy 83\%). Diagnostic validity of serum prolactin at cutoff level $44.5 \mathrm{ng} / \mathrm{dl}$ for prediction of HCC in cirrhotic patients (sensitivity $81 \%$, specificity $78 \%$, positive predictive value $80 \%$, negative predictive value $91 \%$, area under the curve (AUC) (0.9297) and diagnostic accuracy 86\%). Diagnostic validity of combined AFP and prolactin for prediction of HCC in cirrhotic patients (sensitivity $88 \%$, specificity $90 \%$, positive predictive value $90 \%$, negative predictive value $88 \%$ and diagnostic accuracy $89 \%)$.

Conclusion: Serum prolactin is elevated in HCC patients. Therefore it is considered as a promising and potentially complementary biomarker with AFP for detection of HCC. Significant elevation of prolactin levels is very useful for HCC screening. 


\section{HCC -58}

Evaluation of Talin-1 as a tumour marker for hepatocellular carcinoma

\section{Mohamed Nabil Hassan Kamal Mahrous ${ }^{1}$}

${ }^{1}$ Shebin Elkom Fever Hospital, Egypt

Background: Prognosis of patients with Hepatocellular Carcinoma (HCC) depends on tumour stage, with curative therapies available only for patients detected at an early stage; therefore, new and specific markers for HCC are critically needed. Talin-1 is a cytoskeleton protein that plays a role in tumour formation and metastasis in different cancer types. Talin-1 is overexpressed in HCC. The aim was to assess the value of Talin-1 gene expression in Egyptian patients with HCC.

Method: This study included 40 patients with HCC, 30 patients with liver cirrhosis and 30 healthy controls. For all groups, clinical data and image findings were studied; serum alpha-fetoprotein detected by enzyme immunoassay (EIA) kit \& Talin-1 gene expression were detected by using real time polymerase chain reaction (PCR). Tumor characteristics were assessed including size, number and site. Tumor staging was done using Okuda, CLIP, VISUM and Tokyo staging systems.

Result: Talin-1 gene expression was significantly higher in HCC patients compared to cirrhotic patients and controls. The sensitivity and specificity in diagnosis of HCC were $93.3 \%$ and $80 \%$ respectively at cutoff of 9.5 (2-ct) with $85.7 \%$ accuracy. Talin-1 gene expression has no significant correlation with Child score, MELD, uMELD, tumour number, tumour size, AFP, Okuda, CLIP, Tokyo or VISUM staging systems.

Conclusion: Talin-1 gene expression could be a useful diagnostic marker for detection of HCC.

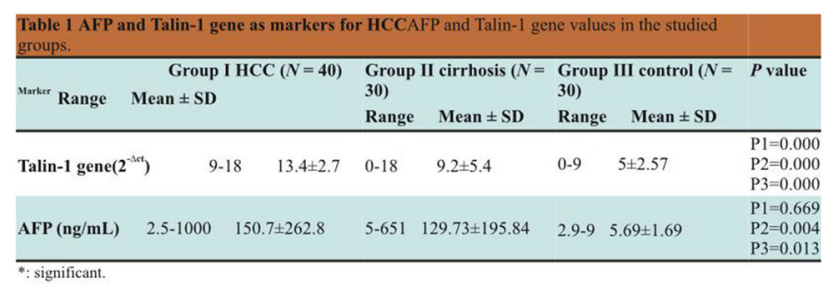

\begin{tabular}{|c|c|c|c|c|c|c|c|c|c|c|}
\hline \multicolumn{11}{|c|}{$\begin{array}{l}\text { Table } 2 \text { AFP and Talin-1 gene as markers for } \\
\text { HCC. }\end{array}$} \\
\hline \multirow[t]{2}{*}{ Test } & \multirow{2}{*}{\multicolumn{2}{|c|}{ Cut off Sensitivity \% }} & & \multicolumn{3}{|c|}{ Specificity } & \multicolumn{3}{|c|}{ NPV } & \multirow[t]{2}{*}{$P$-value } \\
\hline & & & & $\%$ & PPV \% & & $\%$ & AUC $\%$ & & \\
\hline \multicolumn{2}{|c|}{ Talin-1 gene( $\left({ }^{-\Delta c t}\right)$} & 9.5 & 93.3 & & 80 & 100 & & 93.7 & 85.7 & \multirow{2}{*}{$\begin{array}{l}0.000^{*} \\
0.018^{*}\end{array}$} \\
\hline AFP & 13.1 & 60 & & & 69.9 & 100 & & 71.4 & 65.4 & \\
\hline
\end{tabular}

*=significant, $\mathrm{PPV}=$ positive predictive value, $\mathrm{NPV}=$ negative predictive value, $\mathrm{AUC}=$ area under curve:, $2^{-\Delta c t}=$ relative

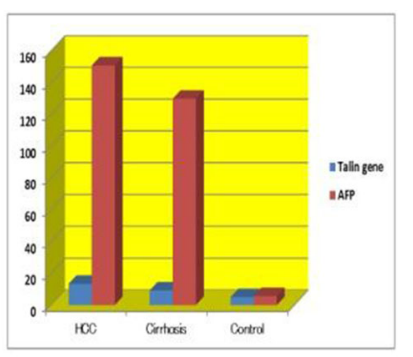

Figure 1: Talin-1 gene \& AFP levels among the studied patients

\section{ROC Curve}

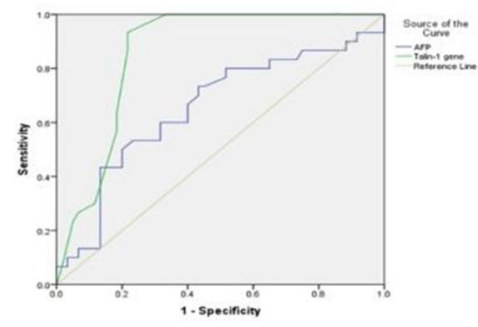

Figure 2: ROC curve analysis for Talin-1 gene and AFP as markers for HCC

HCC -59

Experience of irreversible electroporation for improving survival in locally advanced liver hilum cancer

\section{Kaiwen Huang ${ }^{1}$ \\ ${ }^{1}$ National Taiwan University Hospital, Taiwan}

Background: Irreversible electroporation (IRE) is a non-thermal focal therapy that utilizes high-voltage electric pulses for permanently rupturing the cellular membrane and inducing apoptosis. In this study, we evaluated the safety and efficacy of a protocol based on ultrasound-guided IRE in patients with inoperable liver hilum cancer. Data were collected from 3 countries in Asia.

Method: From 2012 to 2016, we performed IRE under US guidance in 15 patients with stage III liver hilum cancer. These patients were enrolled into a prospective institutional review board approved trial. Result: No IRE-related deaths occurred. A median follow-up of 38 months showed that $8 \%$ of patients sustained complications with a median grade of 1.2 (range $1-3$, no grade 4 or 5 complications were observed). 3 patients ( $20 \%$ ) experienced local recurrence, and $40 \%$ experienced distant progression. Overall survival was 31 months. Conclusion: Our study suggests that IRE is safe and effective in controlling locally advanced liver cancer. We surmise that the addition of IRE to the empirical treatment may provide a survival advantage. 


\section{HCC-60}

HBV and HCV Related HCC-a comparison. Single centre experience from South India

\section{Shahna S Fathima ${ }^{1}$ \\ ${ }^{1}$ Department of Medical GastroEnterology Government Medical College Hospital Thiruvananthapuram, India}

Background: Hepatitis B Virus (HBV) and Hepatitis C Virus (HCV) statistically are the most commonly implicated risk factors in HCC. Clinical series do not usually subset results according the underlying viral risk factors . Aim of this study is to evaluate potentially significant clinical-pathologic differences in HCCs that develop in association with chronic HBV vs. HCV.

Method: Retrospective record based study

All HCC admitted from 2008 to 2017 were included.HBV and HCV co-infection and alcohol intake in cirrhogenic doses were excluded.HCC diagnosis was based on AASLD/EASL imaging criteria.

Statistical analyses

Quantitative variables were summarized as mean with standard deviation. Qualitative variables were summarized as frequencies and proportions Bivariable analysis was conducted with independent sample T test, Chi square test and Mann Whitney test. p value of $<$ 0.05 was considered statistically significant.

Result: 130 patients were having HCC due to either HBV $(n=97,75 \%)$ or $\mathrm{HCV}(n=33,25 \%)$. Of these $106(82 \%)$ were males and $24(18 \%)$ females. Differences between HBV and HCV related HCCs were analysed under the following headings.

Demographics, age distribution, and underlying comorbidities.

Mean age of diagnosis was earlier in HBV related HCC (51) than HCV related HCC $(61)(p=0.00)$. HCC developed more commonly among males in both groups.

Comorbidities like in general as a whole were more common in HCV HCC ( $\mathrm{p}=0.007)$.Specifically diabetes was more in HCV HCC $(\mathrm{p}=0.018)$.

Oncologic variables

HBV HCC were larger at the time of diagnosis $(7.02 \mathrm{~cm})$ $(\mathrm{p}=0.001)$. Also more HBV HCC were out of Milan criteria at the time of presentation $(\mathrm{p}=0.032)$. AFP was found to be significantly elevated in HBV HCC than HCV HCC ( $\mathrm{p}=0.008)$. Similarly synchronous metastasis was not seen in the HCV related tumors $(\mathrm{n}=1,3 \%)$ and occurred in $14 \%(\mathrm{n}=14)$ in HBV related cirrhosis

Cirrhosis and severity

All the de novo HCC $(n=5)$ occurred in HBV related group, with none in HCV HCC .CHILD,MELD and CTP score was also similar between the 2 groups.

Upper GI bleed, hepatic encephalopathy,ascites,SBP, were similar between the groups

Blood investigations

In LFT ALP was significantly elevated in HBV HCC (210.4 \pm 147 $\mathrm{mg} / \mathrm{dL})$ than HCV HCC $(147.4 \pm 78)(\mathrm{p}=0.04)$.SGOT was also more in HBV HCC than HCV HCC.In hemogram platelet count was significantly lesser in HCV related HCC $(1.23 \pm 0.58 \mathrm{~L} / \mathrm{dL})(\mathrm{p}=0.005)$.

Conclusion: HBV cause HCC at a young age. HBV HCC had larger tumours and were out of Mian criteria at time of diagnosis. HCV HCC had higher number of comorbidities especially diabetes. HBV HCC had higher AFP levels.ALP and SGOT. Platelets are less in HCV HCC.

\begin{tabular}{|c|c|c|c|}
\hline Diabetes(present,absent & $25(26), 72(74)$ & $16(49), 17(51)$ & 0.018 \\
\hline $\begin{array}{l}\text { Coexisting } \\
\text { Cirrhosis(present,absent } \\
\text { ) }\end{array}$ & \begin{tabular}{|l|}
$92(95), 5(5)$ \\
\end{tabular} & $33(100), 0(0)$ & NS \\
\hline CHILD(A B C) & $30(31), 3(32) 1,31(32)$ & $11(33), 13(40), 9(27)$ & NS \\
\hline CTP & $7.6 \pm 2.9$ & $8 \pm 2.4$ & NS \\
\hline MELD & $13.86 \pm 6.2$ & $12.83 \pm 5.5$ & NS \\
\hline$B C L C(0 A B C D)$ & $\begin{array}{l}1(1), 11(11), 15(16), 38(39 \\
1,32(33)\end{array}$ & $0(0), 6(18), 5(15), 14(43), 8(24)$ & NS \\
\hline Size $(\mathrm{cm})$ & \begin{tabular}{|l|}
$7.02 \pm 3.9$ \\
\end{tabular} & $4.9 \pm 2.9$ & 0.001 \\
\hline Mets(present,absent) & $14(14), 83(86)$ & $1(3), 32(97)$ & NS \\
\hline $\begin{array}{l}\text { Pattern( Single,Multiple } \\
\text { Bilobar, Diffuse) }\end{array}$ & $\begin{array}{l}48(50), 18(19), 20(21), 11( \\
11)\end{array}$ & $20(61), 7(21), 4(12), 2(6)$ & NS \\
\hline Pvt,BCS,Both,None & \begin{tabular}{|l}
$49(51), 3(3), 6(6), 39(40)$ \\
\end{tabular} & $12(36), 1(3), 1(3), 19(58)$ & NS \\
\hline AFP(ng/ml) & $9764 \pm 36583$ & $2090 \pm 9348$ & NS \\
\hline $\operatorname{AFP}(<200,>200)$ & $35(36), 62(64)$ & $21(64), 12(36)$ & 0.008 \\
\hline $\begin{array}{l}\text { Milan criteria(Satisfied } \\
\text { Not satisfied) }\end{array}$ & $18(18), 78(81)$ & $13(39), 20(61)$ & 0.032 \\
\hline $\mathrm{Hb}(\mathrm{g} / \mathrm{dl})$ & $11.5 \pm 2.4$ & $11.07 \pm 2$ & NS \\
\hline $\mathrm{Tc}(/ \mathrm{microl})$ & $7841 \pm 3438$ & $6789 \pm 4221$ & NS \\
\hline Neutrophil(\%) & $67 \pm 10$ & $63 \pm 11$ & NS \\
\hline Lymphocyte(\%) & $28 \pm 9.8$ & $29 \pm 9.4$ & NS \\
\hline $\begin{array}{l}\text { Platelet count(laks per } \\
\text { microL) }\end{array}$ & $1.7 \pm 0.8$ & $1.2 \pm 0.6$ & 0.000 \\
\hline Bilirubin(mg/dL) & $2.6 \pm 3$ & $2.9 \pm 5.6$ & NS \\
\hline Direct(mg/dL) & $1.2 \pm 2.1$ & $1.6 \pm 4$ & NS \\
\hline$T P(g / d L)$ & $6.9 \pm 2$ & $6.9 \pm 2.1$ & NS \\
\hline Alb(g/dL) & \begin{tabular}{|l|l|}
$3.14 \pm 0.7$ \\
\end{tabular} & $3.12 \pm 0.6$ & NS \\
\hline $\mathrm{SGOT}(\mathrm{IU} / \mathrm{mL})$ & $201 \pm 286.5$ & $118 \pm 148.6$ & 0.035 \\
\hline SGPT(IU/mL) & $129 \pm 222.4$ & $62 \pm 38.6$ & NS \\
\hline ALP(IU/mL) & $219 \pm 170$ & $143 \pm 78$ & 0.014 \\
\hline INR & $1.35 \pm 0.35$ & $1.28 \pm 0.22$ & NS \\
\hline Creatinine $(\mathrm{mg} / \mathrm{dL})$ & \begin{tabular}{|l|}
$1.07 \pm 0.66$ \\
\end{tabular} & $1.01 \pm 0.55$ & NS \\
\hline HE(present,absent) & \begin{tabular}{|l|}
$3(3), 94(97)$ \\
\end{tabular} & $3(9), 30(91)$ & NS \\
\hline Ascites(present,absent) & $56(58), 40(42)$ & $17(51), 16(49)$ & NS \\
\hline SBP(present,absent) & \begin{tabular}{|l|}
$7(7), 90(93)$ \\
\end{tabular} & $3(9), 30(91)$ & NS \\
\hline UGIB(present,absent) & $10(10), 87(90)$ & $7(21), 26(79)$ & NS \\
\hline
\end{tabular}

Qualitative variables expressed as number with percentage in parenthesis

Quantitative variables expressed as mean with standard deviation

\section{HCC-62}

Imaging profile of hepatocellular carcinoma (HCC) in Kerala, South India

\section{Shenoy $K T^{1}$, Shenoy $\mathrm{K} \mathrm{T}^{2}$}

${ }^{1}$ Sree Gokulam Medical College and Research Foundation, India; ${ }^{2}$ Population Health and Research Institute, India

Background: Ultrasound imaging is useful in determining the extent of lesion as well as vascular involvement and use of contrast $\mathrm{C}$ T scan and or MTR imaging can give additional information on the operability.

Method: 242 subjects ( 210 males and 32 females) with HCC were evaluated between May 2006 to June 2017 in the Dept of Gastroenterology and Radiology of Sree Gokulam Medical College and Research Foundation, Trivandrum using Ultrasound scan followed by either contrast CT scan and or MR imaging with 1.5/ $3 \mathrm{~T}$ scanners. Lesions were identified and vascular invasion was assessed.

Result: At U S Scan, 213 (88\%) had coarse echoes in the liver with shrunken lobes and dilated portal vein or splenomegaly to suggest of chronic liver disease. 110 had solitary mass, while 45 had multicentric lesions and the rest had diffuse pattern. $48 \%$ had thrombus in the main portal vein and or branches. Contrast CT was able to identify tumor thrombus in $87 \%$ of those with portal venous occlusion. 10 patients had I V C invasion and $92 \%$ were in BCLC stage C or D.and were inoperable

Conclusion: Majority of HCC were in advanced stage of the disease with either large lesions or vascular invasion and inoperable 
HCC-63

Incidence and risk factors of acute kidney injury after transarterial chemoembolization among patients with hepatocellular carcinoma in a large tertiary hospital: a single-center retrospective cohort study

Jose Guillain Esteban Cataluna ${ }^{1}$, Juliet Gopez Cervantes ${ }^{1}$, Cynthia Mapua $^{1}$, Mark Pierre Dimamay ${ }^{1}$

${ }^{1}$ St. Lukes Medical Center Quezon City, Philippines

Background: Transarterial chemoembolization (TACE) remains as the standard treatment for intermediate-stage hepatocellular carcinoma (HCC). It may result to renal injury leading to significant morbidity, especially in high-risk patients. The incidence, risk factors, and prognosis remain unclear. We aimed to determine the incidence and risk factors of post-TACE acute kidney injury (AKI) in adult patients with HCC in our institution.

Method: This was a retrospective cohort study, which included adult patients with HCC who underwent TACE from 2013 to 2017. We measured the proportion of patients who developed post-TACE AKI, based on the Acute Kidney Injury Network (AKIN) criteria. Multivariate regression analysis was performed using odds ratio (ORs).

Result: A final sample size of 125 patients was analyzed. Fourteen patients (11.20\%) developed post-TACE AKI. Based on univariate logistic regressions, the following variables were significantly associated with post-TACE AKI: alcoholic liver disease (OR 3.94, 95\% CI 1.127-13.149, $\mathrm{p}=0.032$ ), MELD score (OR 1.10, 90\% CI 0.997$1.249 \mathrm{p}=0.057$ ), baseline bilirubin (OR 5.52, 95\% CI 1.719-17.781, $\mathrm{p}=0.004$ ) and sodium levels (OR 0.86, 95\% CI 0.736-1.017, $\mathrm{p}=0.081$ ). Only elevated bilirubin remained significant using multivariate regression (OR 4.49, 95\% CI 1.298-15.542, $\mathrm{p}=0.018$ ).

Conclusion: The incidence of post-TACE AKI in our study is relatively higher than the reported rates. The presence of alcoholic liver disease as the etiology for cirrhosis, MELD score, bilirubin, and sodium levels were found to be independent risk factors for postTACE AKI. Studies with larger sample sizes are recommended and may include long-term follow-up on survival to assess prognosis.

\begin{tabular}{|c|c|c|c|}
\hline Variable & OR & p-value & $95 \% \mathrm{Cl}$ \\
\hline Age (years) & 0.03 & 0.250 & $.0226616 \quad .0870771$ \\
\hline Gender (Male) & 0.55 & 0.350 & $.1564000 \quad 1.930281$ \\
\hline \# of previous TACE & 1.14 & 0.551 & $.7350276 \quad 1.780438$ \\
\hline Volume of Contrast & 0.002 & 0.798 & $-.0148928 \quad .0193746$ \\
\hline Child-Pugh Score A & 0.45 & 0.182 & $.143413 \quad 1.445929$ \\
\hline Child-Pugh Score B & 2.12 & 0.203 & $.6670529 \quad 6.722749$ \\
\hline Co-existing liver cirrhosis & 1.83 & 0.446 & $.3856376 \quad 8.734375$ \\
\hline \multicolumn{4}{|l|}{ Etiology of cirrhosis } \\
\hline Chronic Hepatitis B & 1.06 & 0.913 & $.3341029 \quad 3.409208$ \\
\hline Chronic Hepatitis C & - & - & - \\
\hline Alcoholic liver disease & 3.84 & $0.032^{\circ}$ & $1.126829 \quad 13.14875$ \\
\hline NAFLD & 0.8 & 0.721 & $.2350426 \quad 2.722211$ \\
\hline Diabetes & 1.01 & 0.978 & $.3304215 \quad 3.123278$ \\
\hline Hypertension & 0.72 & 0.615 & $.209865 \quad 2.516651$ \\
\hline $\begin{array}{l}\text { Co-incidence of Diabetes and } \\
\text { Hypertension }\end{array}$ & 0.79 & 0.683 & $.2577803 \quad 2.43128$ \\
\hline MELD Score & 1.10 & $0.057^{* *}$ & $.996596 \quad 1.248795$ \\
\hline Creatinine & -0.31 & 0.713 & -1.983351 .356774 \\
\hline Elevated bilirubin $(>1.2)$ & 5.52 & $0.004^{\circ}$ & $1.719457 \quad 17.78143$ \\
\hline Elevated PT/INR (>1.1) & 2.04 & 0.226 & $.6434224 \quad 6.482555$ \\
\hline $\mathrm{NA}(\mathrm{mmol} / \mathrm{L})$ & 0.86 & $0.081^{* *}$ & $.7362428 \quad 1.017896$ \\
\hline Volume of lipiodol & 0.98 & 0.866 & $.7935354 \quad 1.214971$ \\
\hline $\mathrm{Hgb}(\mathrm{g} / \mathrm{L})$ & 0.86 & 0.248 & $.6787618 \quad 1.105335$ \\
\hline Platelet (log of values) & -0.48 & 0.438 & $\begin{array}{ll}-1.713939 & .7420351\end{array}$ \\
\hline Serum albumin (g/dL) & 1.02 & 0.465 & $.9650392 \quad 1.080975$ \\
\hline BUN (mg/dL) & 1.04 & 0.199 & $.976966 \quad 1.118205$ \\
\hline ALT & 1.00 & 0.734 & $.9911589 \quad 1.012691$ \\
\hline
\end{tabular}

\begin{tabular}{|l|c|c|lll|}
\hline \multicolumn{5}{|l|}{ Table 4. Results after multivariate logistic regression of risk factors against AKI (N=125) } \\
\hline Variable & OR & p-value & $95 \%$ Cl & \\
\hline Alcoholic liver disease & 2.88 & 0.124 & .7485696 & 11.09874 \\
\hline Elevated bilirubin & 4.49 & 0.018 & 1.298019 & 15.54234 \\
\hline Sodium level & 0.94 & 0.554 & .7974429 & 1.129107 \\
\hline MELD score & 0.97 & 0.786 & 0.8086008 & 1.17428 \\
\hline
\end{tabular}

\section{HCC -64}

Initial experiences with sorafenib and regorafenib sequential therapies in Japanese patients with advanced hepatocellular carcinoma

Sadahisa Ogasawara ${ }^{1}$, Tetsuhiro Chiba ${ }^{1}$, Yoshihiko Ooka ${ }^{1}$, Eiichiro Suzuki ${ }^{1}$, Takahiro Maeda', Masayuki Yokoyama ${ }^{1}$, Kazufumi Kobayashi' ${ }^{1}$, Soichiro Kiyono', Masato Nakamura', Tomoko Saito ${ }^{1}$, Shingo Nakamoto ${ }^{1}$, Shin Yasui ${ }^{1}$, Akinobu Tawada ${ }^{1}$, Makoto Arai ${ }^{1}$, Tatsuo Kanda ${ }^{1}$, Hitoshi Maruyama ${ }^{1}$, Osamu Yokosuka ${ }^{1}$, Naoya Kato ${ }^{1}$

${ }^{1}$ Chiba University, Graduate School of Medicine, Japan

Background: In 2016, the RESORCE trial showed that regorafenib significantly improved survival compared to placebo in sorafenibfailed advanced hepatocellular carcinoma (HCC) patients. At the present time, conversion from sorafenib to regorafenib is the first evidence-based treatment strategy of multi-TKIs in patients with advanced HCC. The aim of this study was to assess the safety and efficacy of sorafenib and regorafenib sequential therapy in patients with advanced HCC.

Method: We retrieved medical records of patients with advanced HCC receiving regorafenib in Chiba University Hospital. Radiological responses were evaluated using both RECIST version 1.1 and modified RECIST. CTCAE version 4.0 was used for assessments of adverse events.

Result: Between June 2013 and October 2017, 18 patients were administered regorafenib for advanced HCC. Median age was 68 years old and the majority of them were male (15 patients, $83 \%)$. The most common etiology was hepatitis $\mathrm{C}$ virus (7 patients, 39\%), followed by hepatitis B virus ( 3 patients, $17 \%$ ). According to baseline radiological assessments, 6 and 12 patients were BCLC B and C, respectively. All 18 patients received sorafenib directly before regorafenib and continued sorafenib at more than $400 \mathrm{mg} / \mathrm{day}$ for the last 20 out of the 28 days. In terms of the dose of sorafenib, 15 patients $(83 \%)$ and 3 patients $(17 \%)$ started sorafenib $800 \mathrm{mg} /$ day and 400 $\mathrm{mg} /$ day, respectively. Similarly, 5 patients $(28 \%), 3$ patients $(17 \%)$, and 10 patients $(56 \%)$ received $800 \mathrm{mg} /$ day, $600 \mathrm{mg} /$ day, and 400 $\mathrm{mg} /$ day of sorafenib at the end of their treatments. Median and mean duration of sorafenib treatment were 16.2 months and 8.6 months, respectively (range: 1.4-88.2 months). Regarding regorafenib treatments (excluding 2 patients who observed less than 4 weeks), 14 of 16 patients $(88 \%)$ required dose modifications. The most common adverse events due to dose modifications were hand foot skin reaction ( 8 patients, $50 \%$ ). In terms of the final dose at the time of the end of the observation period, 2 patients (13\%), 4 patients (25\%), 9 patients $(56 \%)$, and 1 patient $(6 \%)$ were $160 \mathrm{mg} / \mathrm{day}, 120 \mathrm{mg} / \mathrm{day}, 80 \mathrm{mg} / \mathrm{day}$, and $40 \mathrm{mg} /$ day, respectively. During the end of the observation period, 2 patients discontinued treatments due to adverse events.

Conclusion: The majority of the patients receiving regorafenib in this cohort continued sorafenib therapy for an extended period of time, similar to the RESORCE trial. The outcomes of regorafenib in HCC patients who have received sorafenib for a short time period are still controversial. 


\section{HCC-65}

Intra-individual comparison of the MRI contrast agents gadoxetic acid versus gadoteric acid in liver MRI of patients with HCC and underlying cirrhosis

\section{Marta Della Seta ${ }^{1}$, Federico Collettini ${ }^{1}$, Timm Denecke ${ }^{1}$, Bernd Hamm $^{1}$, Tobias Penzkofer ${ }^{1}$, Moritz Schmelzle ${ }^{1}$ \\ ${ }^{1}$ Charité Universitätsmedizin Berlin, Germany}

Background: To the best of our knowledge, the ability of liverspecific contrast agents to produce enhancement characteristics analogous to those obtained with extracellular contrast agents during the early perfusion phases was never confirmed. The aim of our prospective study is to intra-individually compare the two contrast media in patients with HCC and underlying cirrhosis.

Method: Twenty patients with HCC diagnosis underwent 2 liver MR examinations at 3T, one with gadoteric acid and one with gadoxetic acid. Quantitative evaluation using regions of interest (ROI) was performed using the Medical Imaging Interaction Toolkit (MITK). Primary endpoint was the evaluation of peak maximum enhancement between both contrast agents in the lesion with reference to normal tissue. Secondary endpoints comprised signal-to-noise and contrastto-noise values.

Result: Twenty patients were enrolled and assessed. One patient was excluded from final analysis due to poor image quality. In the remaining 19 patients, the relative peak enhancement was significantly higher $(p=$ $0,002)$ for gadoteric acid $(160 \pm 89 ; 95 \%$ confidence interval $[\mathrm{CI}]$ : $200,5 ; 120,1)$ than gadoxetic acid $(96 \pm 76 ; 95 \%$ CI: 129,$9 ; 61,2)$. The CNR after administration of the liver specific contrast agent ( $85 \pm 98$; $95 \%$ CI: 129,$2 ; 40,8)$ and the extracellular contrast agents $(185 \pm 208$; $95 \%$ CI: 278,$3 ; 90)$ did not show significant differences $(p=0,035)$. Also the SNR after the administration of gadoxetic acid $(459 \pm 155 ; 95 \% \mathrm{CI}$ : $528,4 ; 388,9)$ and gadoteric acid $(706 \pm 570 ; 95 \%$ CI: $962 ; 449,1)$ wasn't significantly different $(p=0,041)$.

Conclusion: Gadoteric acid shows a significantly higher relative peak enhancement when compared with gadoxetic acid. CNR and SNR after the administration of the two contrast agents did not differ significantly.

\section{HCC-66}

Liver cancer registry in Bangladesh- where we are where to go?

\section{Md Fazal Karim ${ }^{1}$, Salimur Rahman ${ }^{2}$}

${ }^{1}$ Sir Salimullah Medical College Mitford Hospital, Dhaka, Bangladesh, Bangladesh; ${ }^{2}$ Bangabandhu Sheikh Mujib Medical University, Dhaka, Bangladesh, Bangladesh

Background: Hepatocellular carcinoma (HCC) is a major world health problem. It is responsible for approximately $600,000-700,000$ deaths worldwide. It is highly prevalent in the Asia-Pacific region and Africa. Liver cancer ranks third among the cancers in male patient in Bangladesh. Most HCCs develop in patients with underlying chronic liver disease. Chronic viral hepatitis $\mathrm{B}$ and $\mathrm{C}$ are the major causes of liver cirrhosis and HCC. Study shows HBV major cause of HCC in Bangladesh. Liver cancer registry was created in 2015. This is investigator initiated study. It is an online prospective, observational, multicentre register of patients with probable or confirmed primary or secondary liver cancer. We treat patients but do not efficiently keep their records, what are their socioeconomic conditions, precipitating factors, age of presentation and many other issues. Therefore there is always a need for liver cancer registry at national level. Creating a registry on liver cancer will help in identifying the area, groups, etiologic factor, local condition that may be associated with the disease as well as control of this complication of chronic liver disease. Method: Data of patients having either primary or secondary liver cancer attending private chambers were recorded. There were 4 centers situated in capital city Dhaka. The register recorded the patients personal details, laboratory profiles and treatment. The Liver Cancer Registry database was searched till October 31, 2017; the records of liver cancer patients were analyzed.

Result: Four centers in Dhaka data of 329 patients were recorded in the registry. There are 328 males, 81 females. 50-59 age group comprised majority $24.62 \%$. Most are Muslims $96.66 \%$. HBsAg positive $55.87 \%$, Anti HCV positive $6.03 \%$, both positive $1.9 \%$, non B non C $40 \%$. Here most of patients are having primary liver cancer $(90.06 \%)$, followed by $9.94 \%$ secondary cancers.

Conclusion: This is a time demanded step in documentation of liver cancer patients in Bangladesh a country where there are many patients with cancers but data is lost as no documentation at government or private levels. The monitoring of quality of data recorded and frequency of usage will lead to revision and improvement of the registry; to provide the scientific community with an update of disease trend; help in formulation of guidelines from observational evidence of hepatocellular carcinoma in Bangladesh. Therefore setup of multicenters countrywide at national level is needed for more representative picture.

\section{HCC-67}

Long term follow-up for hepatic arterial infusion chemotherapy of 5-fluorouracil and cisplatin after curative resection of hepatocellular carcinoma

Seoung Yoon Rho ${ }^{1}$, Seung Up Kim ${ }^{2}$, Do Young Kim ${ }^{2}$, Sang Hoon $\overline{A h n}^{2}$, Kyung Sik Kim ${ }^{2}$

${ }^{1}$ Yonsei University College of Medicine, Korea, Republic of;

${ }^{2}$ Severance Hospital, Yonsei University College of Medicine, Korea, Republic of

Background: Many recent studies suggest that postoperative hepatic artery-infusion chemotherapy (HAIC) in hepatocellular carcinoma (HCC) can effectively and safely reduce intrahepatic recurrence and improve the overall survival. However, there are not many reports on the long - term period overall survival and disease-free survival rate of HAIC after curative resection of HCC. Thus, in this study, we investigated 10 years follow-up of patients who underwent HAIC after curative resection of HCC.

Method: We retrospectively reviewed medical records in last 10 years follow-up period of prospectively recruited HCC patients who underwent the adjuvant HAIC with 5-FU and cisplatin after curative resection of HCC. Total 128 patients were included and 31 patients underwent HAIC who were highly suspected recurrence in final pathologic report. Every 4 weeks, four cycles of HAIC were given to patients in HAIC group.

Result: Platelet count and alpha-fetoprotein (AFP) levels HAIC group were higher than non-HAIC group in statistically. However MELD score was higher in non-HAIC group. Proportion of above T2 and portal vein, microvascular invasion in HAIC group were statistically different from non-HAIC group. There were no statistically differences between two groups regarding to not only overall survival ( $p$ value $=0.568$ ) but also disease-free survival $(p$ value $=0.975$ ). Even after propensity score matching, there were no statistically significant differences in analysis of overall survival ( $p$ value $=0.740$ ) and disease-free survival (- value $=0.769$ ). In subgroup analysis, especially above T2, HAIC group seems to be more favorable in overall survival, however there was no statistically significance between two groups $(\mathrm{p}$ value $=0.512$ ). 
Conclusion: In this study, we couldn't find any statistical oncologic benefit in long-term overall survival and disease-free survival in statistically. Further study in large and various subgroup of cohort will be needed.

Table 1. Patient characteristics and laboratory findings

\begin{tabular}{lrrr}
\hline \multicolumn{1}{c}{ Variables } & \multicolumn{1}{c}{$\begin{array}{c}\text { HAIC group } \\
\text { (N=31) }\end{array}$} & $\begin{array}{r}\text { Non-HAIC group } \\
(\mathbf{N}=\mathbf{9 7})\end{array}$ & p-value \\
\hline Sex & & & 0.522 \\
$\quad$ Male & $24(77.4 \%)$ & $82(84.5 \%)$ & \\
$\quad$ Female & $7(22.6 \%)$ & $15(15.5 \%)$ & \\
Ages, years & $51.1 \pm 9.4$ & $53.5 \pm 10.0$ & 0.231 \\
Etiology & & & 0.186 \\
$\quad$ Hepatitis B & $26(83.9 \%)$ & $87(89.7 \%)$ & \\
Hepatitis C & $1(3.2 \%)$ & $6(6.2 \%)$ & \\
Others & $4(12.9 \%)$ & $4(4.1 \%)$ & \\
Serum album,g/dL & $4.4 \pm 0.4$ & $4.3 \pm 0.4$ & 0.453 \\
Serum bilirubin, mg/dL & $0.7 \pm 0.3$ & $0.8 \pm 0.4$ & 0.195 \\
Prothrombin INR & $1.0 \pm 0.1$ & $1.1 \pm 0.4$ & 0.033 \\
Serum creatinine, mg/dL & $0.9 \pm 0.1$ & $1.0 \pm 0.2$ & 0.162 \\
Serum sodium, mmol/L & $140.1 \pm 2.3$ & $140.3 \pm 2.7$ & 0.777 \\
Platelet count, 10 $3 / \mu \mathrm{L}$ & $209.1 \pm 47.6$ & $164.2 \pm 61.8$ & 0.000 \\
MELD score & $3.6 \pm 2.4$ & $5.5 \pm 2.8$ & 0.002 \\
MELDNa score & $4.7 \pm 2.7$ & $5.3 \pm 3.3$ & 0.462 \\
ICG R15. \% & $10.6 \pm 5.8$ & $10.4 \pm 6.8$ & 0.872 \\
AFP, ng/mL & $6021.9 \pm 11130.9$ & $760.4 \pm 3244.3$ & 0.014 \\
\hline
\end{tabular}

Table 2. Pathologic characteristics and microscopic findings.

\begin{tabular}{|c|c|c|c|}
\hline Variables & $\begin{array}{l}\text { HAIC group } \\
\quad(\mathrm{N}=31)\end{array}$ & $\begin{array}{c}\text { Non-HAIC group } \\
(\mathrm{N}=97)\end{array}$ & p-value \\
\hline Tumor_size, $\mathrm{cm}$ & $4.7 \pm 2.4$ & $3.5 \pm 2.1$ & 0.008 \\
\hline Number & & & 0.435 \\
\hline One & $28(90.3 \%)$ & $88(90.7 \%)$ & \\
\hline Two & $2(6.5 \%)$ & $7(7.2 \%)$ & \\
\hline Three or more & $1(3.2 \%)$ & $2(2.1 \%)$ & \\
\hline $8^{\text {th }}$ AJCC TNM stage & & & 0.000 \\
\hline T1a & $2(6.5 \%)$ & $21(21.6 \%)$ & \\
\hline $\mathrm{T} 1 \mathrm{~b}$ & $1(3.2 \%)$ & $34(35.1 \%)$ & \\
\hline $\mathrm{T} 2$ & $27(87.1 \%)$ & $40(41.2 \%)$ & \\
\hline $\mathrm{T} 3$ & $1(3.2 \%)$ & $2(2.1 \%)$ & \\
\hline Operation type & & & 0.177 \\
\hline Segmentectomy & $1(3.2 \%)$ & $11(11.3 \%)$ & \\
\hline Bisegmentectomy & $6(19.4 \%)$ & $25(25.8 \%)$ & \\
\hline Trisegmentectomy & $10(32.3 \%)$ & $16(16.5 \%)$ & \\
\hline Hemihepatectomy & $14(45.2 \%)$ & $45(46.4 \%)$ & \\
\hline Differentiation & & & 0.193 \\
\hline Edmonson-steiner Grade I & $0(0.0 \%)$ & $6(6.2 \%)$ & \\
\hline Edmonson-steiner Grade II & $12(38.7 \%)$ & $47(48.5 \%)$ & \\
\hline Edmonson-steiner Grade III & $15(48.4 \%)$ & $40(41.2 \%)$ & \\
\hline $\begin{array}{l}\text { Edmonson-steiner Grade } \\
\text { IV }\end{array}$ & $2(6.5 \%)$ & $1(1.0 \%)$ & \\
\hline Mixed $\mathrm{HCC}$ and $\mathrm{CCC}$ & $2(6.5 \%)$ & $2(2.1 \%)$ & \\
\hline Sarcomatoid HCC & $0(0.0 \%)$ & $1(1.0 \%)$ & \\
\hline Capsular invasion & $20(64.5 \%)$ & $64(66.0 \%)$ & 1.000 \\
\hline Surface invasion & $23(74.2 \%)$ & $52(55.9 \%)$ & 0.112 \\
\hline Portal vein invasion & $11(35.5 \%)$ & $12(12.4 \%)$ & 0.008 \\
\hline Bile duct invasion & $1(3.2 \%)$ & $0(0.0 \%)$ & 0.546 \\
\hline Hepatic vein invasion & $1(3.2 \%)$ & $0(0.0 \%)$ & 0.546 \\
\hline Hepatic artery invasion & $0(0.0 \%)$ & $1(1.0 \%)$ & 1.000 \\
\hline Microvascular invasion & $25(80.6 \%)$ & $38(39.2 \%)$ & 0.000 \\
\hline Intrahepatic metastasis & $2(6.5 \%)$ & $3(3.1 \%)$ & 0.758 \\
\hline
\end{tabular}

Table 3. Clinicopathologic characteristics (after propensity score matching)

\begin{tabular}{|c|c|c|c|}
\hline Variables & $\begin{array}{l}\text { HAIC group } \\
\qquad(\mathrm{N}=\mathbf{3 1})\end{array}$ & $\begin{array}{l}\text { Non-HAIC group } \\
\quad(\mathbf{N}=\mathbf{3 1})\end{array}$ & p-value \\
\hline Sex & & & 0.748 \\
\hline Male & $24(77.4 \%)$ & $26(83.9 \%)$ & \\
\hline Female & $7(22.6 \%)$ & $5(16.1 \%)$ & \\
\hline Ages, years & $51.1 \pm 9.4$ & $51.4 \pm 9.8$ & 0.885 \\
\hline Serum album, $\mathrm{g} / \mathrm{dL}$ & $4.4 \pm 0.4$ & $4.5 \pm 0.4$ & 0.543 \\
\hline Serum bilirubin, $\mathrm{mg} / \mathrm{dL}$ & $0.7 \pm 0.3$ & $0.7 \pm 0.2$ & 0.895 \\
\hline Prothrombin INR & $1.0 \pm 0.1$ & $1.0 \pm 0.1$ & 0.107 \\
\hline Serum creatinine, $\mathrm{mg} / \mathrm{dL}$ & $0.9 \pm 0.1$ & $1.0 \pm 0.3$ & 0.137 \\
\hline MELD score & $3.6 \pm 2.4$ & $5.2 \pm 2.6$ & 0.021 \\
\hline Serum sodium, $\mathrm{mmol} / \mathrm{L}$ & $140.1 \pm 2.3$ & $139.9 \pm 2.5$ & 0.751 \\
\hline MELDNa score & $4.7 \pm 2.7$ & $5.4 \pm 2.8$ & 0.390 \\
\hline Platelet count, $10^{3} / \mu \mathrm{L}$ & $209.1 \pm 47.6$ & $181.6 \pm 64.0$ & 0.060 \\
\hline ICG R15. \% & $10.3 \pm 5.6$ & $10.5 \pm 8.7$ & 0.925 \\
\hline AFP, ng/mL & $6021.9 \pm 11130.9$ & $1932.7 \pm 5608.3$ & 0.075 \\
\hline Etiology & & & 0.400 \\
\hline Hepatitis B & $26(83.9 \%)$ & $29(93.5 \%)$ & \\
\hline Hepatitis C & $1(20.0 \%)$ & $0(0.0 \%)$ & \\
\hline Others & $4(80.0 \%)$ & $2(100.0 \%)$ & \\
\hline Operation type & & & 0.148 \\
\hline Segmentectomy & $1(3.2 \%)$ & $3(9.7 \%)$ & \\
\hline Bisegmentectomy & $6(19.4 \%)$ & $7(22.6 \%)$ & \\
\hline Trisegmentectomy & $10(32.3 \%)$ & $3(9.7 \%)$ & \\
\hline Hemihepatectomy & $14(45.2 \%)$ & $18(58.1 \%)$ & \\
\hline Number & & & 0.429 \\
\hline One & $28(90.3 \%)$ & $25(80.6 \%)$ & \\
\hline Two & $2(6.5 \%)$ & $4(12.9 \%)$ & \\
\hline Three or more & $1(3.2 \%)$ & $2(6.4 \%)$ & \\
\hline Tumor_size, $\mathrm{cm}$ & $4.7 \pm 2.4$ & $4.3 \pm 2.7$ & 0.544 \\
\hline Differentiation & & & 0.277 \\
\hline Edmonson-steiner Grade I & $0(0.0 \%)$ & $1(3.2 \%)$ & \\
\hline Edmonson-steiner Grade II & $12(38.7 \%)$ & $11(35.5 \%)$ & \\
\hline Edmonson-steiner Grade III & $15(48.4 \%)$ & $18(58.1 \%)$ & \\
\hline $\begin{array}{l}\text { Edmonson-steiner Grade } \\
\text { IV }\end{array}$ & $2(6.5 \%)$ & $0(0.0 \%)$ & \\
\hline Mixed HCC and CCC & $2(6.5 \%)$ & $0(0.0 \%)$ & \\
\hline Sarcomatoid HCC & $0(0.0 \%)$ & $1(3.2 \%)$ & \\
\hline $8^{\text {th }}$ AJCC TNM stage & & & 0.484 \\
\hline Tla & $2(6.5 \%)$ & $2(6.5 \%)$ & \\
\hline $\mathrm{T} 1 \mathrm{~b}$ & $1(3.2 \%)$ & $4(12.9 \%)$ & \\
\hline $\mathrm{T} 2$ & $27(87.1 \%)$ & $23(74.2 \%)$ & \\
\hline $\mathrm{T} 3$ & $1(3.2 \%)$ & $2(6.5 \%)$ & \\
\hline
\end{tabular}

Figure 1-1. Comparison of overall survival between HAIC group/non-HAIC group. (pvalue $=0.568$ )

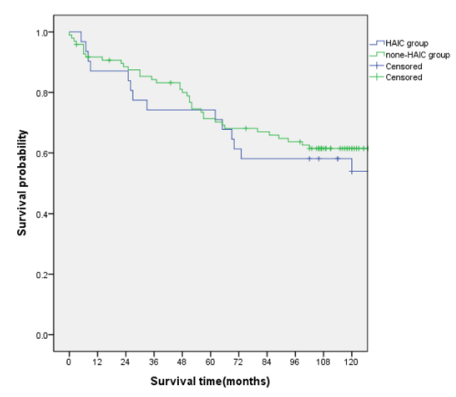


Figure 1-2. Comparison of disease-free survival between HAIC group/non-HAIC group. (pvalue $=0.975$ )

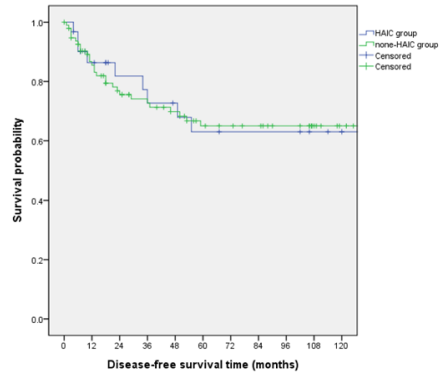

Figure 2-1. Comparison of overall survival between HAIC group/non-HAIC group after propensity score matching $(\mathrm{p}$ value $=0.740)$

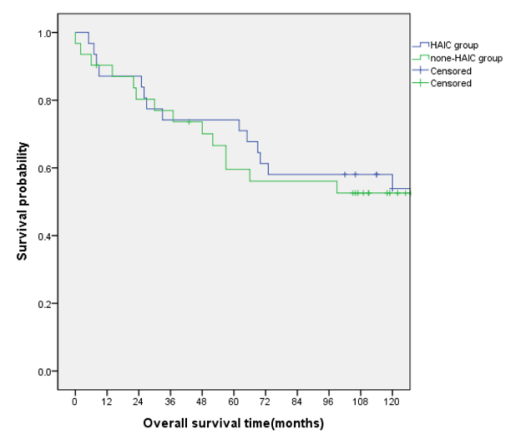

Figure 2-2. Comparison of disease-free survival between HAIC group/non-HAIC group after propensity score matching ( $\mathrm{p}$-value $=0.769$ )

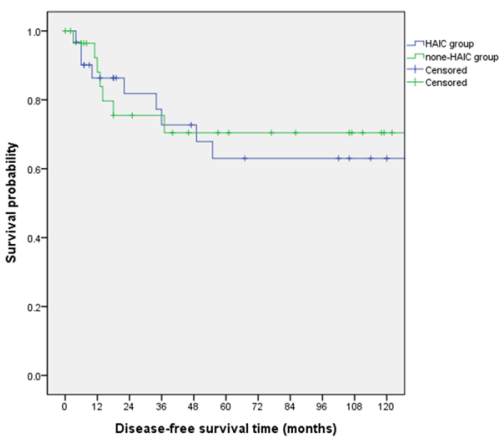

Figure 3-1. Comparison of overall survival between HAIC/non-HAIC group in more than T2 (p-value $=0.621)$

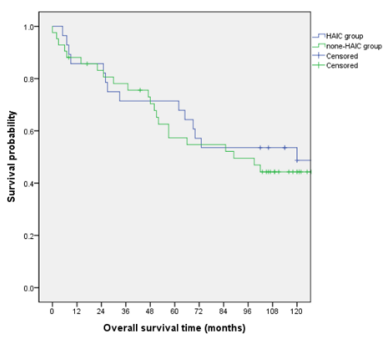

Figure 3-2. Comparison of overall survival between HAIC/non-HAIC group in more than T2 (p-value $=0.512)$

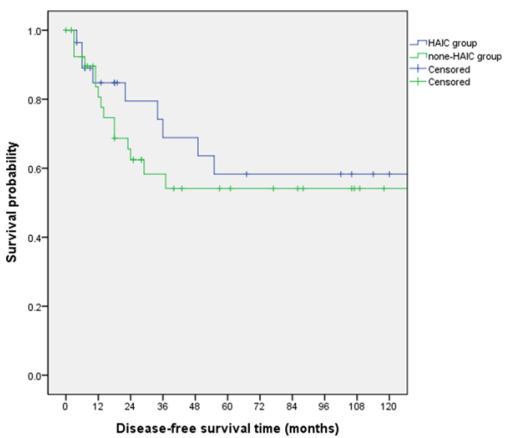

Table 4. Recurrence site and treatment (after propensity score matching)

\begin{tabular}{|c|c|c|c|}
\hline Variables & $\begin{array}{l}\text { HAIC group } \\
\quad(\mathbf{N}=\mathbf{3 1})\end{array}$ & $\begin{array}{c}\text { Non-HAIC group } \\
(\mathbf{N}=\mathbf{3 1})\end{array}$ & p-value \\
\hline First recur & $17(54.8 \%)$ & $17(54.8 \%)$ & 0.595 \\
\hline Recur site - Intrahepatic & $13(76.5 \%)$ & $14(82.4 \%)$ & \\
\hline Extrahepatic & $3(17.6 \%)$ & $3(17.6 \%)$ & \\
\hline Intra/extrahepatic & $1(5.9 \%)$ & $0(0.0 \%)$ & \\
\hline Treatment for first recurrence ${ }^{+}$ & & & 0.720 \\
\hline TACE & 7 & 7 & \\
\hline TACI & 1 & 1 & \\
\hline Systemic chemotherapy & 3 & 3 & \\
\hline RFA & 2 & 3 & \\
\hline Operation & 3 & 1 & \\
\hline Radiation therapy & 1 & 0 & \\
\hline Gamma surgical knife & 1 & 0 & \\
\hline Percutaneous ethanol & 0 & 1 & \\
\hline injection & & & \\
\hline Supportive care & 0 & 1 & \\
\hline $\begin{array}{l}\text { Recent recur (more than } \\
\text { twice) }\end{array}$ & $14(45.2 \%)$ & $15(48.4 \%)$ & 0.824 \\
\hline Recur site - Intrahepatic & $8(57.1 \%)$ & $10(66.7 \%)$ & \\
\hline Extrahepatic & $3(21.4 \%)$ & $3(20.0 \%)$ & \\
\hline Intra/extrahepatic & $3(21.4 \%)$ & $2(13.3 \%)$ & \\
\hline Treatment for first recurrence ${ }^{+}$ & & & 0.302 \\
\hline TACE & 5 & 5 & \\
\hline TACI & 2 & 0 & \\
\hline Systemic chemotherapy & 5 & 5 & \\
\hline RFA & 2 & 0 & \\
\hline Operation & 1 & 0 & \\
\hline Radiation therapy & 2 & 4 & \\
\hline Cryoablation & 0 & 2 & \\
\hline
\end{tabular}

\section{HCC -68}

Long-term sorafenib treatment in a large cohort of HCC patients: a multicenter study

Rodolfo Sacco $^{1}$, Alessandro Granito ${ }^{2}$, Teresa Zolfoino ${ }^{3}$, Carlo Saitta $^{4}$, Luca Marzi ${ }^{5}$, Giampaolo Bresci ${ }^{1}$, Giuseppe Cabibbo ${ }^{6}$

${ }^{1}$ Pisa University Hospital, Italy; ${ }^{2}$ Bologna University, Italy; ${ }^{3}$ Brotzu Hospital, Italy; ${ }^{4}$ Messina University, Italy; ${ }^{5}$ Modena University, Italy; ${ }^{6}$ Palermo university, Italy 
Background: Given the introduction of regorafenib as a second-line therapy for hepatocellular carcinoma (HCC), the therapeutic algorithm for this disease will be further developed. Therefore, it is important to have information on factors predictive of the duration of and response to systemic therapy with sorafenib. In this multicenter, field-practice study, we evaluated outcomes of long-term sorafenib in a cohort of patients with HCC.

Method: HCC patients consecutively enrolled and treated with Sorafenib were identified from 6 Italian referral centers. We considered to analyze those subjects who had received sorafenib treatment for $\geq 12$ months.

Result: Out of a total of 800 patients treated with Sorafenib, 81 (10\%) received long-term treatment with this systemic therapy. Sorafenib was administered in the first-line setting in 19 patients $(23.5 \%)$. Median duration of sorafenib treatment was 22.7 months (range 12.392.6). Adverse events (AEs) of any grade were reported in 74 patients $(91 \%)$; however, only $21(26 \%)$ reported grade 3/4 AEs. Only four patients $(6 \%)$ permanently discontinued sorafenib due to AEs.

Best response to sorafenib was CR in 11 patients (14\%) and PR in 5 (6\%). Median duration of objective response was 18.1 months (4-83.5). Median OS was 34.8 months (95\% CI 29.9-44.3), with 2-, 3and 5 -years survival rates of $76.3 \%, 46.4 \%$ and $25.4 \%$, respectively. The only parameter associated with survival was baseline Child-Pugh class, with median survival of 36.5 months in Child A (95\% CI 29.947.4) and 23.3 months (95\% CI 14.6-41.8) in Child B patients $(\mathrm{P}=0.012)$.

Conclusion: This large 'field-practice' study highlights that sorafenib treatment could result in long-term control of HCC in a relevant proportion of patients. Given also the availability of regorafenib in the second-line setting, an earlier introduction of systemic therapy may be considered for those patients who may be ineligible or non-responders to TACE or to other treatments, when they have preserved liver function.

\section{HCC-69}

\section{Multiple antigen stimulating cellular therapy (MASCT) for hepatocellular carcinoma after curative treatment: a retrospective study}

Yajing $H e^{1}$, Yajing $\mathrm{He}^{1}$, Yabing Guo ${ }^{1}$, Jinzhang Chen ${ }^{1}$, Xiaoyun $\mathrm{Hu}^{1}$, Xiaoshuang $\mathrm{Li}^{2}$, Yanjun Kong ${ }^{2}$, Xiaoyong Zhang ${ }^{1}$, Xiangjun Zhou ${ }^{2}$, Li Liu ${ }^{1}$, Jinlin Hou ${ }^{1}$

${ }^{1}$ Nanfang Hospital, Southern Medical University, China; ${ }^{2} \mathrm{HRYZ}$ Biotech Co., China

Background: The prognosis of hepatocellular carcinoma (HCC) remains poor and available treatment options are limited. This retrospective study evaluated the efficacy of Multiple Antigen Stimulating Cell Therapy (MASCT) as an adjuvant therapy for the treatment of HCC after curative treatment.

Method: Patients who underwent HCC curative treatments were classified into two groups: the MASCT group, in which patients received MASCT treatment after curative treatment $(n=47)$, and the control group, in which patients did not receive any treatment after curative treatment $(n=99)$. Patients who received $\geq 5$ courses of MASCT treatment before recurrence or death $(n=26)$ were further stratified into a subgroup (multiple-course MASCT group) for analysis. The primary endpoint was overall survival (OS). The secondary endpoints were disease-free survival (DFS) and safety.

Result: Kaplan-Meier analysis showed no statistically significant difference in OS between the MASCT group and the control group $(P=0.132)$, nor in DFS $(P=0.310)$ (median: 36.17 vs. 24.27 months). However, when comparing the multiple-course MASCT treated group to the control group, Kaplan-Meier analysis showed a significant difference in OS $(P=0.011)$, but not in DFS $(P=0.104)$ (median: 47.10 vs. 24.27 months). The overall incidences of treatment-related adverse events in the MASCT group and control group were $14.89 \%$ (7/47) and 19.19\% (19/99), respectively. No MASCT treatment-related serious adverse events were reported.

Conclusion: Although the MASCT group was not associated with significantly longer OS or DFS, the multiple-course MASCT group showed significantly improved overall survival after curative treatment, and the treatment procedures were well-tolerated. Multiplecourse MASCT may therefore provide another choice for patients with HCC after curative treatment.

\section{HCC -70}

Prediction model for early intrahepatic recurrence after hepatectomy for patients with hepatocellular carcinoma: An implication for adjuvant treatment

Kelvin $\mathrm{K} . \mathrm{Ng}^{1}$, Chung Mau Lo ${ }^{1}$, Tan To Cheung ${ }^{1}$, Tiffany C Wong ${ }^{1}$, James Y Fung ${ }^{1}$, Ka Wing Ma ${ }^{1}$, Jeff W Dai ${ }^{1}$

${ }^{1}$ The University of Hong Kong, Hong Kong

Background: Efficacy of hepatic resection for hepatocellular carcinoma (HCC) is limited by the high incidence of intrahepatic recurrence, which is related to intrahepatic tumor metastasis in early postoperative period. Accurate prediction of this early tumor recurrence ascertains the role of adjuvant treatment following curative hepatic resection for HCC.This retrospective study aims to develop and test a prediction model for early intrahepatic recurrence.

Method: From 1989 to 2014, 1411 patients with HCC underwent curative hepatic resection. They were divided into development group $(n=706)$ and validation group $(n=705)$. In development group, risk factors for early intrahepatic recurrence $(<18$ months after hepatic resection) were identified by multivariate analysis and a prediction scoring model was developed. The internal validation of this model was assessed in the validation group.

Result: The early tumor recurrent rate was $36.8 \%$ (260 out of 706 patients) in development group. Five clinicopathologic factors were identified as independent risk factors (bilobar disease, multiple tumors $>3$, cirrhosis, microvascular invasion and satellite nodules). A risk scoring model based on the odds ratio of these factors (bilobar disease [2 points], multiple tumors $>3$ [2 points], cirrhosis [2 points], microvascular invasion [ 4 points] and satellite nodules [ 3 points]) was obtained. Patients were stratified into low risk (points $0-4$ ), intermediate risk (points $5-9$ ) and high risk (points $>9$ ) groups with significantly different early tumor recurrence rates $(25.6 \%$ vs. $51.4 \%$ vs. $73.5 \%$ ). The 1-, 3-, and 5-year recurrence-free survival rates of low risk, intermediate risk and high risk groups were $78.9 \%, 59.9 \%$ and $46.8 \%$ vs. $46.8 \%, 29.3 \%$ and $24.4 \%$ vs. $28.6 \%, 9.5 \%$, and $9.5 \%(\mathrm{P}$ $<0.001)$. This risk scoring model was internally validated in the validation group, which had $34.4 \%$ (243 of 705 patients) early tumor recurrent rate. The accuracy of this prediction model as assessed by receiver operating curves showed the area under the curve was $69.7 \%$ with $95 \%$ confidence interval $(65.6 \%-73.9 \%)(\mathrm{P}<0.001)$. The scatter plot between observed and predicted rate of early recurrence using the model showed positive correlation (value 0.925) $(\mathrm{P}=0.000)$ Conclusion: The derived prediction scoring model can accurately predict early intrahepatic recurrence after curative hepatic resection for HCC. This may form the basis of offering adjuvant treatment to high risk patient group after hepatectomy. 


\section{HCC-71}

\section{Predictive factors for GI bleeding in intermediate stage HCC patients receiving TACE treatment}

Po Ting Lin ${ }^{1}$, Wei Teng ${ }^{2}$, Chien Hao Huang ${ }^{2}$, Wen Juei Jeng², Ka Wai Liu ${ }^{3}$, Yi Chung Hsieh², Yi Cheng Chen ${ }^{2}$, Chen Fu Hung ${ }^{3}$, Chen Chun Lin', Chun Yen Lin', Shi Ming Lin', I Shyan Sheen

${ }^{1}$ Linkou Chang Gung Memorial Hospital, Taiwan; ${ }^{2}$ Department of Gastroenterology and Hepatology, Chang Gung Memorial Hospital, Linkou Branch, Taiwan, Taiwan; ${ }^{3}$ Department of Radiology, Chang Gung Memorial Hospital, Linkou Branch, Taiwan, Taiwan

Background: Transarterial chemoembolization (TACE) is the standard of care for intermediate stage HCC patients that majorly composed of cirrhotic patients. Gastrointestinal (GI) bleeding has been a life-threatening issue in cirrhotic patients and may deteriorate liver function and consequently discontinue HCC treatment. This study aims to elucidate the risk factors for GI bleeding in intermediate HCC patients receiving TACE treatment.

Method: From 2014 to 2015, a total of 271 treatment-naïve HCC patients receiving first time TACE treatment in Chang Gung Memorial Hospital, Linkou Medical Center were recruited. PreTACE status including age, gender, etiology of HCC, smoking, alcoholism, prior history of ascites, history of b-blocker used, ChildPugh score, MELD score, and HCC TNM stage are analyzed. All the variables were compared between patients with and without variceal bleeding. Cox regression analysis was applied for the predictive factors for variceal bleeding. Statistics are performed with SPSS V.23 (IBM, USA).

Result: Among the 271 patients, the mean age is $66.6 \pm 11.527$ years old, and $76 \%$ were male. Bleeding episode was documented in 52 patients (19.1\%) with mean time to event as 475 (IQR: 295-737) days. 25 patients $(48 \%)$ experienced variceal bleeding $(\mathrm{EV}, \mathrm{N}=24, \mathrm{GV}$, $\mathrm{N}=2$ ). 8 patients $(15.4 \%)$ had ulcer bleeding while cause of GI bleeding in the other 19 patients $(36.5 \%)$ remained uncertain. The overall mortality rate was higher in patients experienced GI bleeding than those without ( $40.4 \%$ vs. $22,7 \%, \mathrm{P}=0.033)$. By comparison, patients with GI bleeding had older age, higher proportion of alcoholism, smoking history and prior history of ascites, and MELD score higher than 10. Multivariate cox regression analysis revealed prior history of ascites (adjusted HR: $2.48(1.423-4.679), \mathrm{P}=0.002)$ is the only independent predictive factors for GI bleeding in these intermediate stage HCC patients receiving TACE treatment.

Conclusion: GI bleeding in intermediate stage HCC patients receiving TACE treatment had poorer survival. Variceal bleeding accounts for nearly half of the cause. Prior history of ascites preTACE is the independent predictors for GI bleeding.

\section{HCC-72}

Predictive factors that determine response to trans-arterial chemo-embolization in patients with hepatocellular carcinoma

\section{Mahmoud Zaki Abdelsamea Elkadeem ${ }^{1}$}

${ }^{1}$ Egypt - Tanta University, Egypt

Background: Hepatocellular carcinoma is one of the leading causes of cancer deaths worldwide. Transarterial chemoembolization (TACE) is a standard for intermediate stage of liver cancer. It is associated with higher survival than supportive care. However, It may have a risk of mortality and morbidity. Many factors other than radiological response determine TACE outcomes. The aim is to analyze the outcomes after TACE comparing with different radiological responses.

Method: Sixty two patients who underwent transarterial chemoembolization were subjected to clinical examination, laboratory investigations, triphasic abdominal CT with contrast, and evaluation of Child Pugh score, Model for End Stage Liver Disease, and Barcelona Clinic Liver Cancer staging before and one month after therapy. Tumor response according to Modified response evaluation criteria in solid tumors was calculated by one radiologist. Patients were followed up for six months or till death. Statistical analysis including regression and survival analysis were performed.

Result: Study was carried out on 62 Egyptian patients over a two year period between July 2014 and July 2016. These patients had hepatocellular carcinoma and underwent transarterial chemoembolization (TACE). As regard to Modified response criteria in solid tumors: 25 patients $(40.3 \%)$ showed complete response, 15 patients $(24.2 \%)$ showed partial response, 2 patients $(3.2 \%)$ showed stable disease, and 20 patients (32.3\%) showed progressive disease. As regard decompensation after TACE (which was considered as increase Child Pugh score one or more than the score before TACE), 36 patients (58\%) had decompensation, and 26 patients(42\%) did not. As regard 6 month survival, $11(17.7 \%)$ of 62 patients died within 6 months follow up after TACE. The cumulative proportion of surviving was $82 \%$. Significant difference was detected in patients with different radiological responses as regard tumor criteria (size, invasion of portal vein, Barcelona Clinic Liver Cancer stage, and technique of TACE; (superselective and selective)). Size of lesions (two dimensions) $(40.87 \mathrm{~cm} 2)$ is predictive of progressive disease with sensitivity $55 \%$, specificity $83.3 \%$ and positive predictive value $61.1 \%$. High Child Pugh score, left lobe tumors, heterogenous enhanced lesions, serum bilirubin: (cut off value $1.45 \mathrm{mg} / \mathrm{dl}$, sensitivity $38.9 \%$, specificity $88.5 \%$, and positive predictive value $82 \%$ ), AST level: (cut off value $77.5 \mathrm{IU} / \mathrm{L}$, sensitivity $36.1 \%$, specificity $96.2 \%$, and positive predictive; value 93\%), INR (cut off value 1.2 , sensitivity $61.1 \%$, specificity $92.3 \%$, and positive predictive value $92 \%$ ), Model for End Stage Liver Disease (MELD) (cut off value 8.5, sensitivity $80.6 \%$, specificity $73.1 \%$, and positive predictive value $80.6 \%$ ), and Low platelet count (cut off value 80x109/L, sensitivity 33.3\%, specificity $96.2 \%$, and positive predictive value $92.3 \%$ ) were detected to be associated with decompensation after TACE. High serum bilirubin, INR, MELD score, and alphafetoprotein, low platelet count, and progressive tumor response were detected to be negatively affecting 6 month survival.

Conclusion: Radiological response can not be considered alone to determine the outcome after transaterial chemoembolization (TACE). Child-Pugh score, tumor criteria, liver functions, and platelet count also affect the outcome after TACE.

HCC-73

Radiological features and outcomes of mixed hepatocellular and cholangiocarcinoma in patients undergoing surgical resection

ChenTa Chi ${ }^{1}$, Rheunchuan Lee ${ }^{1}$, Mingchih Hou ${ }^{1}$, Fayauh Lee ${ }^{1}$, Yihsiang Huang ${ }^{1}$

${ }^{1}$ Taipei Veterans General Hospital, Taipei, Taiwan., Taiwan

Background: Mixed hepatocellular cholangiocarcinoma (HCC-CCA) is a rare type of primary liver cancer. The radiological features and outcomes of HCC-CCA are poorly understood.

Method: From August 2010 to September 2016, 29 patients with pathology-proven HCC-CCA undergoing surgical resection in Taipei Veterans General Hospital were retrospectively reviewed. Radiological features of contrast-enhanced computed tomography (CT) or 
magnetic resonance imaging (MRI) were reevaluated by a radiologist. Detailed demographic and pathological features, tumor characteristics of the cases were analyzed their association with recurrence-free and overall survivals.

Result: The mean age was 59.2 years old with male predominant $(69.0 \%)$. Of them, $21(72.4 \%)$ were hepatitis B surface antigen (HBsAg)-positive and $4(13.8 \%)$ were antibody to hepatitis $\mathrm{C}$ virus (anti-HCV)-positive. Twenty-three patients $(79.3 \%$ ) had single tumor. The distributions of tumor stage were $5(17.2 \%)$ in T1 stage, 21 $(72.4 \%)$ in $\mathrm{T} 2$ stage, and $3(10.3 \%)$ in T3 stage. Pathological classification included 21 with classic type, 3 with stem cell type, 5 cases had HCC and CCA in different liver tumors. Most of the cases had atypical radiological pattern for HCC, either with early enhancement only $(n=18)$ or lack of both early enhancement and early washout $(\mathrm{n}=1)$. Only 10 patients had typical pattern for HCC. The 1-, 3-, 5 -year disease-free survival rates were 54.2, 41.8, and $34.8 \%$, respectively. The 1-, 3-, 5-year overall survival rates were 80.7, 65.5, and $32.8 \%$, respectively. In multivariate analysis, CLIP score and extra-hepatic metastasis were associated with disease-free survival, whereas solitary tumor and $\mathrm{AFP} \leq 400 \mathrm{ng} / \mathrm{mL}$ were significantly associated with overall survival.

Conclusion: HCC-CCA mostly has atypical radiological feature and high recurrence rate. Single tumor and low AFP level are associated with better survival after surgical resection.

\section{HCC -74}

Randomized trial of preoperative administration of oral pregabalin for postoperative analgesia in patients scheduled for radiofrequency ablation of focal lesions in the liver

\section{Sherief Abd Elsalam ${ }^{1}$, Amr Magdy Abdelfattah ${ }^{2}$ \\ ${ }^{1}$ Tanta University Faculty of medicine, Egypt; ${ }^{2}$ Tanta University, Egypt}

Background: The aim of this study was to evaluate the effect of preoperative pregabalin on postoperative analgesia in patients presented for radiofrequency ablation (RFA) of hepatic focal lesions (HFLs).

Method: This randomized controlled study was carried out on 70 adult patients with hepatocellular carcinoma presented for RFA of HFLs. They were randomized into two groups: Group I: 35 Patients in this group received placebo and Group II: 35 patients in this group received $150 \mathrm{mg}$ of oral pregabalin one hour before the procedure. The primary outcome was the analgesic effect in the form of the severity of postoperative pain and the need of opioid analgesics.

Result: The pain assessed by visual analogue pain scale (VAS Pain) did not show significant difference between the medians of the studied groups immediately postoperative $(p=0.84)$. However, the medians of VAS Pain decreased significantly $(p<0.001)$ in group II compared with group I $(3,2,1,1,1,0$ VS $4,3,3,2,2,2$ respectively) when measured every 4 hours until 24 hours. The number of required doses of rescue analgesia and total required dose of morphine in the first 48 hours postoperatively decreased significantly in group II compared with group I $(p<0.001)$. Side effects as bradycardia, nausea and vomiting and delayed discharge were significantly $(p=0.02,0.01$, 0.01 respectively) less frequent in group II compared with group I (20\% VS $45.7 \%, 17.1 \%$ VS $45.7 \%$ and $11.4 \%$ VS $37.1 \%$ respectively).

Conclusion: Oral pregabalin is safe and effective for postoperative analgesia in the patients scheduled for radiofrequency ablation of focal lesions in liver.

[ClinicalTrials.gov Identifier: NCT03151213]
HCC-75

Relationship between XRCC1 gene polymorphism and hepatocellular carcinoma in Egyptian population

Mohamed AbdelSamiee ${ }^{1}$, Osama Hamam ${ }^{2}$, Mary Naguib ${ }^{3}$, Mohamed Helwa $^{2}$, Mohamed Soliman ${ }^{2}$, Eman Abdelsameea ${ }^{3}$

${ }^{1}$ National Liver Institute, Egypt; ${ }^{2}$ Faculty of Medicine, Menoufia University, Egypt; ${ }^{3}$ National Liver Institute, Menoufia University, Egypt

Background: Several major risk factors for hepatocellular carcinoma (HCC)have been identified, including chronic infection of hepatitis B virus(HBV) and hepatitis $\mathrm{C}$ virus(HCV). Nevertheless, only a fraction of infected patients develops HCC during their lifetime suggesting that genetic factors might modulate HCC development. X-ray repair cross complementing group 1 (XRCC1) participates in the base excision repair pathway of DNA.

Method: Aim: To investigate the association of X-ray repair cross complementing group 1 (XRCC1) gene polymorphism with HCC in Egyptian population.

Methods: This study was assessed on 40 patients with $\mathrm{HCC}$ and 20 cirrhotic patients on top of $\mathrm{HCV}$ and 40 age and gender matched healthy subjects as control group. After collection of relevant clinical data and basic laboratory tests,c. $1517 \mathrm{G}>\mathrm{C}$ SNPof XRCC1 gene polymorphism was performed by (PCR-RFLP) technique.

Result: Our study revealed statistical difference in XRCC1 (CC, GC) genotypes with increased (C) allele frequency in patients with $\mathrm{HCC}$ in comparison with cirrhotic patients as well as control group. In addition, patients with CC, GC genotypes showed significant higher number and larger size of tumor foci and significantly higher Child Pugh grades. The multivariate analysis showed that the presence of c. $1517 \mathrm{G}>\mathrm{C}$ SNPof XRCC1 gene is an independent risk factor for the development of HCC in chronic HCV patients with 3.742 folds increased risk of HCC development.

Conclusion: XRCC1gene polymorphism could be associated with increased risk of HCC development in chronic HCV infected Egyptian patients.

\section{HCC -76}

Risk factors for the development of hepatocellular carcinoma in patients with chronic hepatitis $B$ and $C$ after effective treatment

\section{KuoChin Chang ${ }^{1}$, Yihao Yen ${ }^{1}$, Chengkun $\mathrm{Wu}^{1}$, Mingtsung Lin ${ }^{1}$,} Tsunghui Hu ${ }^{1}$

${ }^{1}$ Kaohsiung Chang Gung Memorial Hospital and Chang Gung University College of Medicine, Kaohsiung, Taiwan, Taiwan

Background: Effective antiviral therapy can reduce the risk of liver cirrhosis (LC)-related hepatocellular carcinoma (HCC) in patients with chronic hepatitis $\mathrm{B}(\mathrm{CHB})$ and $\mathrm{C}(\mathrm{CHC})$ virus infection. Yet, the risk of $\mathrm{HCC}$ development in $\mathrm{HBV}$ and $\mathrm{HCV}$ patients with $\mathrm{LC}$ after effective antiviral therapy treatment is unknown.

Method: From March 2002 to December 2013, 1363 patients with HBV treated with nucleos(t)ide analogues with completely suppressed virus, and patients with $\mathrm{HCV}$ treated with pegylated interferon (peg-IFN)/ribavirin (RBV) combination therapy who achieved SVR were enrolled.

Result: Among the 1363 patients enrolled, 261 developed HCC within a median follow-up of 4.25 years. At the 1 st, $3 \mathrm{rd}$, and 5 th year of follow-up, the cumulative incidences of HCC for all patients were 
$2.7 \%, 8.2 \%$, and $18.3 \%$, respectively. In univariate analysis, patients who developed HCC tended to be of older age ( $p$

Conclusion: Old age, low platelet counts, high AFP levels, and CHB status were independent risk factors for HCC development after effective antiviral therapy in $\mathrm{CHB}$ or $\mathrm{CHC}$ patients with LC.

\section{HCC-77}

rupture of hepatocellular carcinoma: a tale of 20 cases from a tertiary care center in northern India

\section{Srimanta Kumar Sahu ${ }^{1}$, Sunil Taneja ${ }^{2}$, Naveen Kalra ${ }^{2}$, Radha Krishan Dhiman $^{2}$, Yogesh Kumar Chawla ${ }^{2}$, Virendra Singh ${ }^{2}$, Ajay Duseja ${ }^{2}$, Ujjwal Gorsi ${ }^{2}$ \\ ${ }^{1}$ PGIMER and Dr. RML Hospital, New Delhi, India; ${ }^{2}$ PGIMER, Chandigarh, India}

Background: Spontaneous rupture is a potentially life-threatening complication of hepato cellular carcinoma (HCC) and is the third most common cause of death due to $\mathrm{HCC}$ after tumour progression and liver failure. In Asia approximately $10 \%$ of patients with HCC die of rupture each year. In the West however HCC rupture is relatively uncommon, with an incidence of less than $3 \%$. There is no data on the exact incidence of HCC rupture from India.

Method: This was a prospective observational study analysing the clinical and radiological spectrum of consecutive patients presenting with ruptured HCC between Jul 2014 and Jun 2017. HCC was diagnosed as per the AASLD practice guidelines. Rupture of HCC was diagnosed by Triple phase computed tomography (TPCT), with at least two of the following findings a) peripherally located tumour with a contour bulge b) discontinuity of the hepatic surface c) subcapsular hematoma or hemoperitoneum or by demonstration of active extravasation of contrast on $\mathrm{CT}$ and/or angiography.

Result: Twenty patients presented with rupture of HCC with a mean (SD) age $57.5 \pm 10.99$ years, $95 \%$ of them were males (Table 1 ). Cirrhosis was documented in all patients (100\%). The etiology of cirrhosis was most commonly due to infections with chronic hepatitis $\mathrm{C}$ virus (40\%) followed by hepatitis B virus (20\%), alcohol (20\%) and Non alcoholic steato-hepatitis (10\%). In $30 \%$ of patients rupture of HCC was the first clinical presentation of cirrhosis. The most common symptom at presentation was pain abdomen $(85 \%)$ followed by shock in $40 \%$ of patients. The diagnoses of ruptured HCC were ascertained by TPCT in all twenty cases. On TPCT the size of the HCC was $\geq 5 \mathrm{~cm}$ in $80 \%$, HCC was sub-capsular in $75 \%$, capsular breach was seen in $70 \%$, hemoperitoneum was documented in $75 \%$, active extravasation of contrast was seen in $40 \%$ and "Enucleation sign" in 25\% (Table 1). The "Enucleation sign" is an important CT finding in rupture of HCC and was defined as separation of tumour content with intraperitoneal rupture into the perihepatic space which is seen as low attenuating lesion from peripheral enhancing rim on arterial phase imaging (Figure 1). The most common sign on hepatic artery angiography was tumour blush, which was seen in $86 \%$, however active extravasation of contrast was documented in only $36 \%$. Trans-arterial embolization (TAE) was successfully performed in $70 \%$ of patients, $25 \%$ were managed conservatively and only one patient undergone hepatic resection. The one month mortality in patients receiving conservative management, TAE and hepatic resection were $100 \%, 29 \%$ and $0 \%$ respectively. The mean (SD) duration of hospital stay was $7.9 \pm 5.05$ days and the mortality at 3 month was $65 \%$.

Conclusion: Ruptured HCC can be the first manifestation of cirrhosis. TAE achieves hemostasis with a high success rate in the acute phase. Hepatic resection is potentially curative but can be offered to a small subset of patients with ruptured HCC.

\begin{tabular}{|c|c|}
\hline Patients profile & Frequency (\%) \\
\hline Age $($ Mean \pm SD) Years & $57.5 \pm 10.99$ \\
\hline Gender (Male) & $19(95)$ \\
\hline Co-morbidity (Hypertension) & $8(40)$ \\
\hline Cirrhosis & $20(100)$ \\
\hline Cirrhosis due to chronic viral hepatitis & $12(60)$ \\
\hline Rupture as first presentation of cirrhosis & $6(30)$ \\
\hline Decompensated Cirrhosis & $15(75)$ \\
\hline $\begin{array}{r}\text { CTP Score A } \\
\text { B } \\
\text { C }\end{array}$ & $\begin{array}{l}4(20) \\
10(50) \\
6(30)\end{array}$ \\
\hline MELD Score (Mean \pm SD) & $16.2 \pm 8.57$ \\
\hline $\begin{array}{r}\text { BCLC Stage A } \\
\text { B } \\
\text { C } \\
\text { D }\end{array}$ & $\begin{array}{l}1(5) \\
10(50) \\
6(30) \\
3(15)\end{array}$ \\
\hline Multifocal HCC & $11(55)$ \\
\hline $\begin{aligned} & \text { Lobar Distribution of HCC }- \text { Right Lobe } \\
& \text { Left Lobe } \\
& \text { Bilobar }\end{aligned}$ & $\begin{array}{r}15(75) \\
2(10) \\
3(15)\end{array}$ \\
\hline $\begin{array}{ll}\text { Size of HCC } & <5 \mathrm{~cm} \\
& 5.1-10 \mathrm{~cm}\end{array}$ & $\begin{array}{r}4(20) \\
14(70) \\
2(10)\end{array}$ \\
\hline $\begin{array}{cl}\text { Signs on } & \text { TPCT suggesting rupture of HCC } \\
\text { i) } & \text { Subcapsular rocation of HCC } \\
\text { ii) } & \text { Exophytic HCC } \\
\text { iii) } & \text { Hemoperitoneum } \\
\text { iv) } & \text { Breech of liver capsule } \\
\text { v) } & \text { Active contrast extravasation } \\
\text { vi) } & \text { "Enucleation Sign" } \\
\end{array}$ & $\begin{array}{r}15(75) \\
14(70) \\
15(75) \\
14(70) \\
8(40) \\
5(25)\end{array}$ \\
\hline $\begin{array}{l}\text { Signs on Angiography suggesting rupture of } \mathrm{H} \\
\text { i) Abnormal tumour blush } \\
\text { ii) Active extravasation of contrast }\end{array}$ & $\begin{array}{r}12(86) \\
5(36)\end{array}$ \\
\hline $\begin{array}{cc}\text { Serum AFP Levels } & <20 \mathrm{ng} / \mathrm{ml} \\
& 20-200 \mathrm{ng} / \mathrm{ml} \\
& >200 \mathrm{ng} / \mathrm{ml}\end{array}$ & $\begin{array}{l}7(35) \\
5(25) \\
8(40)\end{array}$ \\
\hline Macrovascular Invasion (Present) & $3(15)$ \\
\hline Extrahepatic Spread (Present) & $4(20)$ \\
\hline $\begin{array}{cl}\text { Management } & \begin{array}{l}\text { Trans-arterial Embolization } \\
\text { Conservative } \\
\text { Hepatic Resection }\end{array}\end{array}$ & $\begin{array}{l}14(70) \\
5(25) \\
1(5)\end{array}$ \\
\hline
\end{tabular}

Table 1: Demographic, clinical and radiological profiles of patients with ruptured hepatocellular carcinoma at presentation $(\mathrm{n}=20)$.

Legend (Table 1): Data has been presented as number (percentage) or mean $( \pm$ SD) wherever applicable. CTP (Child Turcotte Pugh); MELD (Model for End Stage Liver Disease); BCLC (Barcelona Clinic Liver Cancer); HCC (Hepatocellular Carcinoma); TPCT (Triple Phase Computed Tomography); AFP (Alfa Feto Protein). *
hepatic artective angiography as part of Trans-arterial embolization (TNE) was done in 14 patients with ruptured HCC.

Figure Legend (Figure 1): The "Enucleation sign" in a case of rupture of hepatocellular carcinoma in segment VII (white open arrow) with separation of tumour content into the perihepatic space seen as hypodense center with peripheral rim enhancement on arterial phase of Triple phase computed tomography (TPCT).

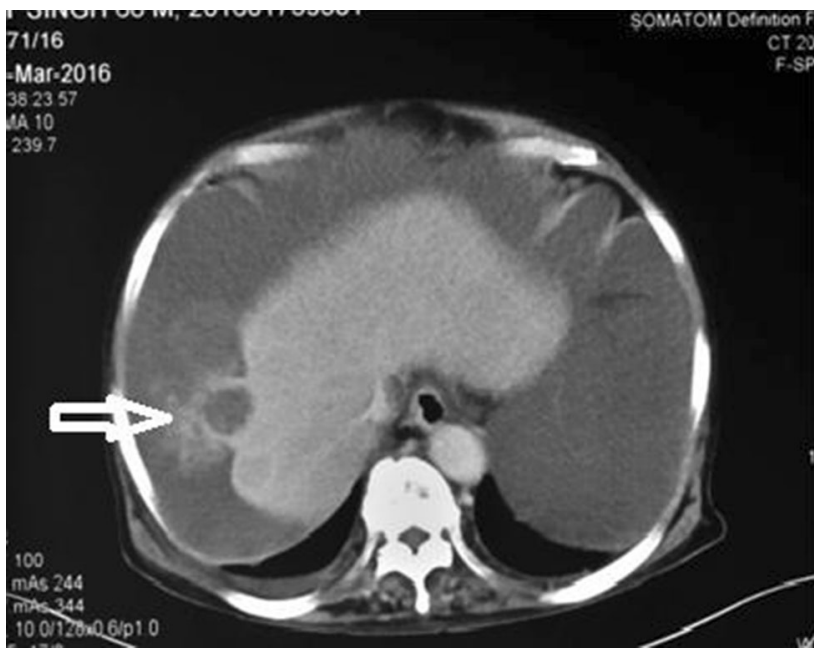


HCC-78

\section{Survival-related factors in hepatocellular carcinoma, a single center experience}

\section{Ulus Salih Akarca1, Nilay Danis², Fulya Gunsar², Ilker Turan², Galip Ersoz $^{2}$, Zeki Karasu', Ulus Salih Akarca ${ }^{2}$ \\ ${ }^{1}$ Ege University, Turkey; ${ }^{2}$ Ege University Medical Faculty, Turkey}

Background: Hepatocellular carcinoma (HCC) is the third most common cause of cancer death. Etiologies and the prognostic factors may differ in various parts of the world. AIM: To determine the basal survival-related factors in $\mathrm{HCC}$ in a single center experience representing the Western part of Turkey.

Method: A retrospective analysis was conducted of $788 \mathrm{HCC}$ patients who were followed up in Ege University Hospital from January 2005 to December 2016. The associations of basal demographic data, blood tests, etiologies of underlying liver disease, tumor diameters and numbers, complications of cirrhosis, treatment modalities, HCC scoring systems with overall survival and transplantation free survival were assessed using Kaplan-Meier or Cox regression analysis. Death records were obtained from Social Security System.

Result: Of 788 patients, 623 was male, 65 female, mean age was $60.5 \pm 10.9$ years. The major etiologes were hepatitis B $(56 \%)$ and hepatitis C $(20,6 \%)$. HCC developed from cirrhotic background in $76.3 \%$ of the patients. Percentages of Child-Pugh A, B and C were $56.4 \%, 28.1 \%$, and $15.5 \%$, respectively. Ascites was present in $32 \%$, hepatic encephalopathy in $6 \%$, esophageal variceal bleeding history in $9 \%$, portal vein involvement in $21.4 \%$, distant metastasis in $4.5 \%$. Thirty-four percent of the patients had diabetes mellitus. $60.3 \%$ were diagnosed under $\mathrm{HCC}$ surveillance. Liver transplantation was performed in 101, resection in 64 , radiofrequency ablation in 98, transarterial chemoembolisation in 266 patients. Mean duration of follow-up was 2.19 years ((\%95 CI: $1,9672-2,4172)$. Mean tumor number was 3.02 (95\% CI: 2.78-3.27) (median 1); mean highest tumor diameter was $5.95 \mathrm{~cm}$ (95\% CI: 5.57-6.29). Barcelona stages from 0 to $\mathrm{D}$ were $11.3,37.4,17.6,18.4,15.2 \%$, respectively. Overall mean survival time was 2.19 year (\%95 CI: 1,731 - 2,481). Transplantation free survival was 1.608 (95\% CI: 1.261-1.956. Among the four most prevalent etiologies, namely hepatitis B, hepatitis C, alcohol and delta hepatitis, HCC derived from alcoholrelated liver disease had the least duration of survival. The following basal parameters were found to be related to survival: Neutrophil count, lymphocyte count, neutrophil/lymphocyte ratio, platelet/lymphocyte ratio, tumor number, tumor size, AST, ALT, alkaline phosphatase, GGT, Na, platelet count, total protein, albumin levels, total bilirubin, AFP, portal hypertension, ascites, hepatic encephalopathy, distant metastasis, portal vein involvement, identification of the tumor under surveillance. Among these parameters, tumor size, AST, GGT, INR, Total bilirubin, portal vein involvement, presence of ascites were independently related to survival in multivariate Cox regression analysis.

Conclusion: Considering that none of the prognostic scoring systems contains biochemical parameters, new scoring systems containing AST, GGT or bilirubin may increase the precision of the prognostic scores.
HCC -79

The outcome of transarterial chemoembolization versus resection for early stage hepatocellular carcinoma in a real world study

\section{Peng $\mathrm{Hu}^{1}$, Xiaoling $\mathrm{Wu}^{1}$, Hu Li ${ }^{1}$, Hong $\operatorname{Ren}^{1}$}

${ }^{1}$ Department of Infectious Diseases, Institute for Viral Hepatitis, The Key Laboratory of Molecular Biology for Infectious Diseases, Chinese Ministry of, China

Background: Although the Barcelona Clinic Liver Cancer system and the China consensus recommend patients with early stage hepatocellular carcinoma (HCC) to be treated with hepatic resection, many patients in real world with early stage $\mathrm{HCC}$ received treatment with transarterial chemoembolization (TACE).In this study, we compared the recurrence rate of resection versus TACE for stage Ia HCC and identified risk factors that influence the recurrence of stage Ia HCC. Method: Total 167 patients with stage Ia HCC were retrospectively analyzed, comprising 111 treated by TACE and 56 by resection. The recurrence rate was compared between the two groups using the Kaplan-Meier method. The independent predictors of recurrence were identified by Cox regression analysis.

Result: According to the analysis of recurrence rate for stage Ia HCC, we found the cumulative recurrence rate in resection group and TACE group were $35.6 \%$ versus $64.0 \%(\mathrm{p}=0.003)$ at 48 weeks. The Cox regression analysis showed poorly differentiated carcinoma ( $\mathrm{HR}=294.2, \mathrm{p}=0.002)$, hepatic cirrhosis $(\mathrm{HR}=10.1, \mathrm{p}=0.038)$, intraoperative bleeding volume $(\mathrm{HR}=1.0, \mathrm{p}=0.022)$, the changes of erythrocyte $(\mathrm{HR}=0.5, \mathrm{p}=0.036)$ and the alphafetoprotein levels $>1210$ $\mathrm{ng} / \mathrm{ml}(\mathrm{HR}=8.8, \mathrm{p}=0.041)$ were independent predictors of higher recurrence rate after treatment with resection. In TACE group, the independent risk factors for recurrence of $\mathrm{HCC}$ were the serum creatinine levels $(\mathrm{HR}=1.1, \mathrm{p}=0.001)$, tumor location more than one lobe (HR=9.1, $\mathrm{p}=0.003)$,esophageal varices $(\mathrm{HR}=2.2, \mathrm{p}=0.026)$, and antiviral therapy for hepatitis B virus after $\operatorname{TACE}(\mathrm{HR}=0.4, \mathrm{p}=0.039)$. Conclusion: Early stage HCC should be treated with resection instead of TACE in order to reduce the recurrence rate.

\section{HCC -80}

Alphafetoprotein (AFP) affects survival after transarterial chemoembolization (TACE) for hepatocellular carcinoma (HCC)

\section{$\underline{\text { Amitabh Suman }^{1}, \text { Alpana Limaye }}{ }^{1}$, Oxana Norkina ${ }^{1}$}

${ }^{1}$ University of Florida, United States

Background: Alphafetoprotein (AFP) has been recently shown to predict survival after liver transplantation for hepatocellular carcinoma (HCC). However, its effect on survival after locoregional therapy has not been appreciated. The aim of the current study is to judge the role of AFP on survival after transarterial chemoembolization

Method: A retrospective analysis of HCC patients treated with only TACE from January 2001 to December 2010 at the VA Medical Center, Gainesville, Florida was undertaken with a view to figure out factors associated with survival. Patients who got any other form of therapy were excluded.

Result: A total of 82 patients were included in the analysis. They were 43-86 years old, 59 (72\%) were white and all were males. All the patients had underlying cirrhosis with Hepatitis C /alcohol /both accounting for a majority $68(82.9 \%)$ of patients. Mean MELD score at the beginning was 9.6 and $65(79.3 \%)$ belonged to Child-Pugh Class A. Mean size of the tumor was $5.6 \mathrm{cms}$ and $11(13.4 \%)$ had multiple tumors. Portal vein thrombosis was reported only in 3 
(3.7\%). They were treated by conventional TACE (37 patients) or drug eluting beads (DEB) (45 patients) and were followed up for a mean of 18.6 months. The peak AFP values ranged from 1.5 to 60,500 (mean 6748, median 319). At the time of analysis 68(82.9\%) had died and $14(17.1 \%)$ were surviving. Univariate analysis using Fisher's exact test and Mann Whitney U test showed peak AFP ( $\mathrm{p}=0.0002)$, Type of TACE ( $\mathrm{p}=0.022)$, MELD score $(\mathrm{p}=0.028)$, CP class $(\mathrm{p}=0.038)$ and response after first TACE $(\mathrm{p}=0.006)$ to be associated with survival. Multivariate analysis using Cox Proportional Hazard Model showed DEB TACE, MELD score, CP class A and peak AFP (Hazard ration $1.15,95 \% \mathrm{CI}=[1.6,1.26], \mathrm{p}=0.0001)$ to be associated with survival

Conclusion: Peak AFP value is associated with survival after TACE for HCC.

\section{HCC -81}

\section{An investigation for the risk factors and prognosis in $\mathrm{HCC}$ patients receiving transarterial chemoembolization with postembolization fever}

Chien Wei Peng ${ }^{1}$, Wei Teng ${ }^{1}$, Wen Juei Jeng ${ }^{1}$, Yi Chung Hsieh ${ }^{1}$, Ka Wai Liu ${ }^{1}$, Wei Ting Chen ${ }^{1}$, Yi Cheng Chen ${ }^{1}$, Chien Hao Huang ${ }^{1}$, Chen Fu Hung ${ }^{1}$, Chun Yen Lin ${ }^{1}$, Chen Chun Lin ${ }^{1}$, Shi Ming Lin ${ }^{1}$, I Shyan Sheen ${ }^{1}$

${ }^{1}$ Chang Gung Memorial Hospital, Linkou Medical Center, Taiwan

Background: The transarterial chemoembolization (TACE) is the standard of care for intermediate stage HCC patients. The post-embolization fever (PEF) is usually observed in 3 days after TACE. Limited data available evaluates the predictors of PEF and its impact on the long-term outcome. Therefore, the aim of this study is to elucidate the predictive factors of PEF and its impact on clinical outcome of HCC patients receiving TACE.

Method: From 2014 to 2015, a total of 271 patients with HCC and receiving first TACE treatment in Chang Gung Memorial Hospital, Linkou Medical Center were recruited. Age, gender, etiologies of HCC (viral status, alcohol), cirrhosis, tumor status, white blood cell counts, aspartate aminotransferase (AST), alanine aminotransferase (ALT), $\alpha$-fetoprotein (AFP), bilirubin level, and prothrombin time were compared between patients with and without PEF. Logistic regression analysis was applied for predictive factors for PEF. Statistics were performed with SPSS V.22 (IBM, USA).

Result: Among the 271 patients, the mean age is $66.6 \pm 11.527$ years old, and $76 \%$ were male. Hepatitis B and C account for $52.5 \%$ and $35.7 \%$ of all the HCC patients respectively. The mean follow-up duration was $20.79 \pm 8.35$ months. The PEF was observed in 103 patients $(38.0 \%)$. In univariate analysis, PEF was associated with higher TNM stage (OR: 2.165, 95\% CI: $1.589-2.950, \mathrm{P}<0.001$ ), infiltrated type HCC (OR: 4.010, 95\% CI: 1.013-15.873, P=0.048), higher platelet count $(\mathrm{OR}=1.003,95 \% \mathrm{CI}: 1.001-1.006, \mathrm{P}=0.017)$ and higher AST level (OR=1.011, 95\% CI: 1.004-1.019, $\mathrm{P}<0.001)$. The larger TNM stage (OR: 1.941, 95\% CI: 1.370-2.749, $\mathrm{P}<0.001)$ and higher AST level (OR: 1.012, 95\% CI: 1.004-1.019, P=0.002) were the independent risk factors in the multivariate analysis. By Kaplan Meir analysis, the cumulative overall survival rate appeared borderline significance between patients with and without PEF (Log rank test, $\mathrm{P}=0.081$ ).

Conclusion: Higher TNM stage and higher AST level were significant risk factors for PEF. Patient with PEF prone to have shorter survival with borderline statistical significance.

\begin{tabular}{|c|c|c|c|c|}
\hline & All patients $(\mathrm{N}=271)$ & without PEF ( $\mathrm{N}=168$ ) & with PEF ( $N=103$ ) & $P$ value \\
\hline Age & $66.6 \pm 11.527$ & $66.41 \pm 11.301$ & $65.49 \pm 11.924$ & 0.523 \\
\hline Gender (Male) & $205(76 \%)$ & $128(76.2 \%)$ & $77(74.8 \%)$ & 0.790 \\
\hline HBV & $135(\mathrm{~N}=257,52.5 \%)$ & $84(\mathrm{~N}=157,53.5 \%)$ & $51(\mathrm{~N}=100,51 \%)$ & 0.695 \\
\hline $\mathrm{HCV}$ & $91(\mathrm{~N}=255,35.7 \%)$ & $57(\mathrm{~N}=157,36.1 \%)$ & $34(\mathrm{~N}=98,34.7 \%)$ & 0.794 \\
\hline Alcohol & $64(23.6 \%)$ & $33(19.6 \%)$ & $31(30 \%)$ & 0.049 \\
\hline DM & $80(29.5 \%)$ & $52(31.0 \%)$ & $28(27.2 \%)$ & 0.509 \\
\hline \multicolumn{4}{|l|}{ TNM stage } & \multirow{5}{*}{$\leq 0.001$} \\
\hline 1 & $84(31.0 \%)$ & $65(38.7 \%)$ & 19 (18.4\%) & \\
\hline 2 & $82(30.2 \%)$ & $58(34.5 \%)$ & $24(23.3 \%)$ & \\
\hline 3 & $98(36.2 \%)$ & $43(25.6 \%)$ & $55(53.4 \%)$ & \\
\hline 4 & $7(2.6 \%)$ & $2(1.2 \%)$ & $5(4.9 \%)$ & \\
\hline \multicolumn{4}{|l|}{ ECOG } & \multirow{3}{*}{0.849} \\
\hline 0 & $251(92.6 \%)$ & $156(92.9 \%)$ & $95(92.2 \%)$ & \\
\hline 1 & $20(7.3 \%)$ & $12(7.1 \%)$ & $8(7.8 \%)$ & \\
\hline Cirrhosis & 252 (93.0\%) & 157 (93.5\%) & $95(92.2 \%)$ & 0.703 \\
\hline $\begin{array}{l}\text { Maximal tumor } \\
\text { size }(\mathrm{cm})\end{array}$ & 4.2 & 2.62 & 4.43 & $\leq 0.001$ \\
\hline Vascular invasion & 44 (16.2\%) & $18(10.7 \%)$ & 26 (25.2\%) & 0.002 \\
\hline Infiltrative & $10(3.7 \%)$ & $3(1.8 \%)$ & $7(6.8 \%)$ & $\underline{0.046}$ \\
\hline WBC & 5100 & 5000 & 5600 & 0.12 \\
\hline Hemoglobin & $12.33 \pm 2.1$ & $12.4 \pm 2.18$ & $12.2 \pm 1.96$ & 0.477 \\
\hline Segment & $59.285 \pm 10.75$ & $59.19 \pm 10.75$ & $59.44 \pm 10.8$ & 0.857 \\
\hline Lymphocyte & $29.1 \pm 9.34$ & $29.16 \pm 9.27$ & $29.04 \pm 9.49$ & 0.922 \\
\hline Platelet & 134 & 127 & 141 & 0.025 \\
\hline INR & 1.2 & 1.2 & 1.2 & 0.279 \\
\hline AFP & 29.1 & 20.9 & 62.6 & 0.045 \\
\hline Alb & 3.73 & 3.75 & 3.65 & 0.65 \\
\hline Bilirubin & 0.8 & 0.8 & 0.8 & 0.586 \\
\hline AST & 54 & 51.5 & 60 & 0.004 \\
\hline ALT & 39 & 36 & 42 & 0.139 \\
\hline AST/ALT & 1.241 & 1.215 & 1.292 & 0.217 \\
\hline Creatinine & 0.75 & 0.74 & 0.785 & 0.413 \\
\hline \multicolumn{5}{|l|}{ TACE type } \\
\hline Conventional TACE & 248 (91.5\%) & $153(91.1 \%)$ & $95(92.2 \%)$ & \multirow[b]{2}{*}{0.739} \\
\hline $\begin{array}{l}\text { Non-conventional } \\
\text { TACE }\end{array}$ & $23(8.5 \%)$ & $15(8.9 \%)$ & $8(7.8 \%)$ & \\
\hline Lipiodol dose & 6 & 5 & 8 & $\leq 0.001$ \\
\hline Adriamycin dose & $\begin{array}{l}23.33 \pm 10.991 \\
\text { (Median:20) }\end{array}$ & $\begin{array}{l}22.04 \pm 9.634 \\
\text { (Median:20) }\end{array}$ & $\begin{array}{l}25.44 \pm 12.667 \\
\text { (Median:20) }\end{array}$ & $\leq 0.001$ \\
\hline
\end{tabular}

Table 2. Predictor for fever

Table 2. Predictor for fever
\begin{tabular}{|l|l|l|l|l|}
\hline Value & Crude OR (95\% Cl) & P value & Adjusted OR $(95 \% \mathrm{Cl})$ & P value \\
\hline Alcohol & $1.761(0.999-3.107)$ & 0.051 & & \\
\hline TNM stage & $2.165(1.589-2.950)$ & $\underline{\leq 0.001}$ & $1.941(1.370-2.749)$ & $\leq 0.001$ \\
\hline Infiltrative HCC & $4.010(1.013-15.873)$ & $\underline{0.048}$ & $2.254(0.534-9.515)$ & 0.269 \\
\hline Platelet & $1.003(1.001-1.006)$ & $\underline{0.017}$ & $1.001(0.998-1.004)$ & 0.459 \\
\hline AFP & $1.000(1.000-1.000)$ & 0.303 & & \\
\hline AST & $1.011(1.004-1.019)$ & $\underline{0.001}$ & $1.012(1.004-1.019)$ & $\underline{0.002}$ \\
\hline
\end{tabular}

Figure 1. Kaplan-Meier curve and Log-rank test

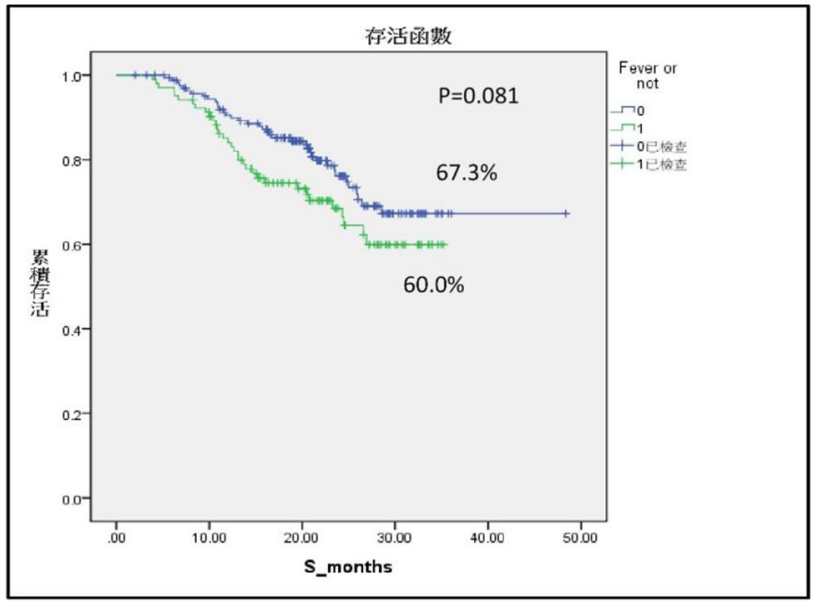




\section{HCC-82}

Evaluation of $\mathrm{CD} 25$ in patients with hepatitis $\mathrm{C}$ positive liver cirrhosis and hepatocellular carcinoma

Ehab Ahmed Abdelatti ${ }^{1}$, Ehab Ebrahim Abdelatti ${ }^{2}$, Waleed Mohamed Fathy ${ }^{3}$, Alsaid Ebrahim Alshaieb ${ }^{1}$

${ }^{1}$ Professor of Hepatology, Egypt; ${ }^{2}$ Faculty of medicine Menoufia University, Egypt; ${ }^{3}$ Professor of Clinical Pathology, Egypt

Background: The immune system employs various defense mechanisms to inhibit cancer proliferation. Soluble CD25 (sCD25) is an immune factor that is part of the immune-suppressive network of cancer proliferation. sCD25 also has novel functional properties with an ability to inhibit, in a dose-related manner, antitumor T-cell responses. The aim of this work is to verify the reliability of CD25 as a marker for diagnosis of hepatocellular carcinoma (HCC)

Method: The study included 30 patients with liver cirrhosis (cirrhotic group) and 30 patients with liver cirrhosis and HCC (HCC group) from Menoufia University Hospitals (Egypt) and 20 healthy volunteers as a control group. They underwent physical examination and laboratory investigations (CBC, liver profile \{ALT, AST, serum albumin, bilirubin, PT \% , HCV Ab, HBsAg, HCV PCR, AFP and serum CD 25). Abdominal ultrasonography was done for all patients. $\mathrm{HCC}$ is further confirmed by triphasic CT. Child-Pugh Score, MELD score and AST/Platelet index were calculated for all patients

Result: AFP level of HCC group $(385.9 \pm 290.8 \mathrm{ng} / \mathrm{dl})$ was significantly higher than cirrhotic group $(28.7 \pm 44.7 \mathrm{ng} / \mathrm{dl})$ and controls $(10.48 \pm 2.2 \mathrm{ng} / \mathrm{dl})(\mathrm{P}=0.0001)$. There was no significant difference between cirrhotic group and controls $(\mathrm{P}=0.08)$. CD25 level of HCC group $(6514.9 \pm 1673.5 \mathrm{ng} / \mathrm{dl})$ was significantly higher than cirrhotic group $(3976 \pm 677.8 \mathrm{ng} / \mathrm{dl})$ and controls $(962.8 \pm 239.6 \mathrm{ng} / \mathrm{dl})$ $(\mathrm{P}=0.0001)$. Also $\mathrm{CD} 25$ in cirrhotic group was significantly higher than that of controls $(\mathrm{p}=0.0001)$. There was a high significant positive correlation between CD25 and AFP ( $\mathrm{r}=0.65$ and $\mathrm{P}=0.0001)$.

Diagnostic validity of serum AFP at cutoff level $200 \mathrm{ng} / \mathrm{dl}$ for prediction of HCC in cirrhotic patients (sensitivity $70 \%$, specificity $80 \%$, positive predictive value $67.7 \%$, negative predictive value $82 \%$ and diagnostic accuracy $76.5 \%$ ).

Diagnostic validity of serum CD 25 at cutoff level $2275 \mathrm{ng} / \mathrm{dl}$ for prediction of HCC in cirrhotic patients (sensitivity $93 \%$, specificity $42 \%$, positive predictive value $48.3 \%$, negative predictive value $91.3 \%$ and diagnostic accuracy 60\%). Diagnostic validity of combined AFP and CD 25 for prediction of HCC in cirrhotic patients (sensitivity $66.6 \%$, specificity $80.4 \%$, positive predictive value $66.6 \%$, negative predictive value $80 \%$ and diagnostic accuracy $75.3 \%$ )

Conclusion: CD25 is more sensitive but less specific than AFP in diagnosis of HCC. Significant elevation of CD25 levels is very useful for HCC screening in patients with liver cirrhosis.

\section{HCC-83}

Prevalence and predictors of macro-vascular invasion in hepatocellular carcinoma at diagnosis

\section{Srimanta Kumar Sahu ${ }^{1}$, Yogesh Kumar Chawla ${ }^{2}$, Naveen Kalra ${ }^{2}$, Radha Krishan Dhiman', Virendra Singh ${ }^{2}$, Ajay Duseja ${ }^{2}$, Sunil Taneja ${ }^{2}$}

${ }^{1}$ PGIMER and Dr. RML Hospital, New Delhi, India; ${ }^{2}$ PGIMER, Chandigarh, India

Background: Tumoural portal vein thrombosis (TPVT) is associated with poor prognosis in hepatocellular carcinoma (HCC). Approximately $10 \%-40 \%$ patients with HCC can have TPVT at the time of diagnosis. Patients with TPVT are more likely to have metastatic disease, fewer therapeutic options, and shortened overall survival compared to those without TPVT. Thrombus involving the main portal vein (MPV) has worse prognosis compared to thrombus involving a branch of the MPV.

Method: Macrovascular invasion (MVI) was assessed on Triple phase computed tomography (TPCT) and considered as tumoral if at least two of the following features were met a) thrombus with arterial hypervascularity b) thrombus causing expansion of the vein c) thrombus in a vein adjacent to or in the same segment as of the tumour and d) direct invasion of the veins. The predictors of MVI were identified on univariate logistic regression analysis and the variables with $\mathrm{p}<0.05$ were subjected to multivariate logistic regression analysis to determine the independent predictors of MVI in HCC.

Result: Among 368 patients with HCC, MVI was seen in $35.9 \%$, most commonly involved the major branches of the MPV (43.9\%), followed by the MPV (17.4\%), MPV plus one or more of its major branch (16.7\%). Extensive MVI involving MPV and its branches with extension to superior mesenteric vein (SMV) and/or splenic vein was seen in $11.4 \%$. Involvements of hepatic vein (s) with thrombosis at $\geq 1$ vein (s) of the spleno-portal axis were seen in 9\%. Isolated involvement of Hepatic vein (s) or SMV was seen in $3 \%$ and $2.3 \%$ of cases respectively (Figure 1).

Patients with MVI were relatively younger (mean age $57.12 \pm 10.8$ vs $60.34 \pm 10.1$, p 0.005), often symptomatic $(97.0 \%$ vs $83.9 \%$, p $<0.001)$, had decompensations $(66.7 \%$ vs $39.4 \%, \mathrm{p}<0.001)$, Child $\mathrm{C}$ cirrhosis $(14.4 \%$ vs $7.6 \%$, p 0.040$)$, multifocal tumours $(65.9 \%$ vs $51.7 \%$, p 0.008 ), larger (size $>5 \mathrm{~cm}$ ) tumours $(75.8 \%$ vs $47.0 \%, \mathrm{p}$ $<0.001)$ and higher $(\geq 200 \mathrm{ng} / \mathrm{ml})$ serum AFP levels $(40.2 \%$ vs $23.1 \%$, p 0.003) compared to those without MVI. More patients in HCC of viral etiology than HCC in alcoholic liver disease had MVI (45.3\% vs $28.9 \%$, p 0.012). At presentation age $\leq 55$ yrs (OR 2.170, 95\% CI 1.3-3.6), being symptomatic (OR 3.194, 95\% CI 1.0-9.8) decompensated cirrhosis (OR 3.2, 95\% CI 1.9-5.3), tumour size $>5$ $\mathrm{cm}$ (OR 3.055, 95\% CI 1.8-5.2) and serum AFP levels $>200 \mathrm{ng} / \mathrm{ml}$ (OR 2.021, 95\% CI 1.2-3.3) were independent predictors of MVI (Table 1).

Conclusion: The present study showed that younger age ( $\leq 55 \mathrm{yrs})$, presence of symptoms and decompensation at diagnosis, tumour size $>5 \mathrm{~cm}$, and serum AFP levels $>200 \mathrm{ng} / \mathrm{ml}$ were independently associated with MVI in HCC. More patients in HCC of viral etiology than HCC in alcoholic liver disease had MVI (45.3\% vs $28.9 \%$, p 0.012).

Figure 1: Distribution of macro-vascular invasions (MVI) in hepatocellular carcinoma (HCC) at diagnoses $(\mathrm{n}=132)$

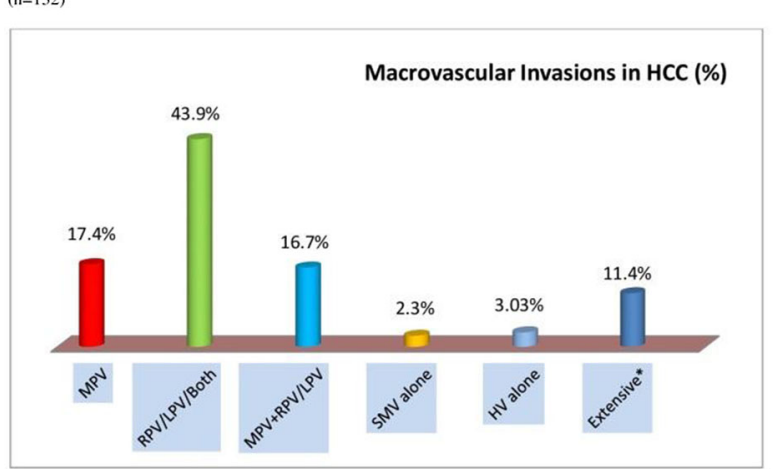

Legend (Figure 1): MPV (Main Portal Vein), RPV (Right branch of MPV), LPV (Left branch of MPV), HV (Hepatic Vein), SMV (Superior Mesenteric Vein). * Extensive, indicates involvement of $\geq 3$ veins in the splenoportal axis. 
Table 1: Independent Predictors of Macrovascular invasion in hepatocellular carcinoma (HCC)

(Multivariate Logistic regression analysis)

\begin{tabular}{|l|l|l|l|}
\hline \multirow{2}{*}{ Variables } & \multicolumn{3}{|c|}{ Multivariate Analysis } \\
\cline { 2 - 4 } & & $\begin{array}{l}\text { 95\% CONFIDENCE } \\
\text { INTERVAL }\end{array}$ & P value \\
\hline Young Age ( 555 years) & 2.170 & $1.321-3.566$ & 0.002 \\
\hline Symptomatic at Presentation & 3.194 & $1.041-9.792$ & 0.042 \\
\hline Decompensation at Presentation & 3.228 & $1.973-5.279$ & $<0.001$ \\
\hline HCC Size $>5 \mathrm{~cm}$ & 3.055 & $1.785-5.227$ & $<0.001$ \\
\hline AFP $>200 \mathrm{ng} / \mathrm{ml}$ & 2.021 & $1.232-3.315$ & 0.005 \\
\hline
\end{tabular}

Legends: AFP (Serum alfa fetoprotein)

\section{HCC-84}

Hepatocellular carcinoma due to chronic liver disease: impact of surveillance on survival

\section{Amey Dilip Sonavane ${ }^{1}$, Pratibha Sonawane', Deepak N Amarapurkar ${ }^{1}$ \\ ${ }^{1}$ Bombay Hospital and Institute of Medical Sciences, India}

Background: Incidence of hepatocellular carcinoma (HCC) in India is increasing. It is still not clear whether surveillance strategies improve survival outcome. In this prospective case control study, we assessed the impact of surveillance versus no-surveillance strategies on HCC staging and survival.

Method: Group A consisted of patients with CLD who were detected with HCC after being on surveillance protocol (6-monthly ultrasound \pm alpha-feto protein $\{\mathrm{AFP}\}$ ) for at least one year. Group B included patients with CLD not subjected to surveillance and diagnosed with $\mathrm{HCC}$ at the time of presentation. Diagnosis of CLD was made on the basis of history, clinical examination, biochemical parameters, imaging, endoscopic findings \pm liver biopsy. Diagnosis of HCC was based on typical imaging features \pm histology. Patients were followed prospectively during the study period.

Result: Out of 734 CLD patients who attended our referral center during the study period (2014-2016), 204 patients were under surveillance protocol. 24 new cases of HCC were diagnosed accounting to an incidence of $1.63 \%$ per year (Hepatitis B $2.36 \%$, Hepatitis C $1.74 \%$, alcohol $1.62 \%$, non-alcoholic steatohepatitis $0.8 \%$, others $1.96 \%$ ) (Group A). 84 patients (Group B) had HCC at initial presentation and were not on any surveillance protocol. Both groups were similar regarding patient demographics (age, gender, comorbidities), etiology of CLD, presence of ascites and esophageal varices, Child-Turcotte-Pugh class, Okuda stage and Model for EndStage Liver Disease (MELD) score. Symptoms, portal vein thrombosis, elevated AFP, mean Cancer of the Liver Italian Program (CLIP) score and metastasis was more common in Group B $(\mathrm{p}<0.05)$. $66.67 \%$ patients in group A were detected in early Barcelona-Clinic Liver Cancer (BCLC) stage $(0, A, B)$ as compared to $9.76 \%$ patients in group B. All patients in group A received treatment $(25 \%$ liver transplant (LT); $58.3 \%$ loco-regional therapy $\{\mathrm{LRT}\})$ compared to $60.5 \%$ patients in group B $(3.7 \%$ LT; $34.6 \%$ LRT) $(\mathrm{p}=0.001)$. Duration of surveillance in group A was 5.06 years/person. Survival was significantly better in group A ( $\mathrm{p}=0.0002$; HR 3.4488); early BCLC stage $(\mathrm{p}<0.0001 ;$ HR 11.28) and those who received treatment $(\mathrm{p}<0.0001)$. On multivariate analysis, detection at an early BCLC stage was independently associated with improved survival.

Conclusion: Surveillance helps to detect HCC at an early BCLC stage, thus amenable to curative treatment resulting in improved survival.
HCC-85

Liver function changes after transarterial chemoembolization (TACE) in US patients (pts) with hepatocellular carcinoma (HCC): The LiverT study

\section{Marc Fellous ${ }^{1}$, Rebecca A Miksad ${ }^{2}$, Fang Xia ${ }^{1}$, Marc Fellous ${ }^{1}$, Fabio Piscaglia ${ }^{3}$ \\ ${ }^{1}$ Bayer HealthCare Pharmaceuticals, United States; ${ }^{2}$ Beth Israel Deaconess Medical Center and Harvard Medical School, United States; ${ }^{3}$ University of Bologna, Italy}

Background: Acute elevations of serum transaminases and bilirubin after TACE are commonly observed in pts with HCC. However, the real-world incidence of chronic liver damage after TACE is unclear. LiverT is a retrospective, observational study to assess the potential chronic deterioration of liver function after a single TACE in pts with HCC in US real-world practice.

Method: Eligible pts with HCC underwent index TACE between Jan 1, 2010 and Mar 31, 2016, and did not undergo repeat/additional HCC treatment in the subsequent 90 days. Pts with at least one laboratory value at baseline, 0-29 days (acute period) and 30-90 days (chronic period) after TACE were identified from Optum's integrated database. Due to a lack of universally accepted liver function deterioration criteria, clinically meaningful changes in laboratory parameters were predefined by experts.

Result: Of 5142 Optum TACE pts, 572 (11\%) were eligible; exclusions were primarily due to lack of HCC diagnostic code and laboratory availability; of eligible pts, $411(72 \%)$ were male and the median age was 62 years. Although lower than acute liver deterioration (0-29 days post TACE), 30-90 day chronic liver deterioration varied by parameter and ranged from $15 \%$ to $31 \%$ (Table). Overall, subgroups analyzed (HCC etiology, diabetes status, anticoagulation use, and presence of portal vein thrombosis) showed similar patterns. Conclusion: We report real-world chronic deterioration of liver function after a single TACE in HCC pts, which may limit subsequent treatment. Further characterization of post-TACE liver dysfunction is important.

\begin{tabular}{|c|c|c|c|c|c|}
\hline \multicolumn{6}{|c|}{ Incidence of liver deterioration post TACE } \\
\hline & & & & & \\
\hline & $\mathrm{n}^{*}$ & $\%$ & $95 \% \mathrm{CI}$ & $\%$ & $95 \% \mathrm{CI}$ \\
\hline Total bilirubin: $50 \%$ increase & 462 & 30 & 26,34 & 23 & 19,27 \\
\hline Albumin: $\geq 0.3 \mathrm{~g} / \mathrm{dL}$ decrease & 442 & 52 & 47,57 & 31 & 27,36 \\
\hline AST: $>25 \%$ increase & 446 & 44 & 39,48 & 30 & 26,35 \\
\hline ALT: $>25 \%$ increase & 441 & 43 & 38,47 & 25 & 21,29 \\
\hline INR: $25 \%$ increase & 251 & 25 & 19,30 & 15 & 10,19 \\
\hline
\end{tabular}

HCC -86

Long term outcome of endoscopic variceal band ligation (EVL) of esophageal varices in Indian patients of chronic liver disease

\section{Gautam Ray}

${ }^{1}$ B.R.Singh (Railway) Hospital, India

Background: Background: EVL is regarded as an effective standard of care for prevention and treatment of variceal bleeding. There is scanty Indian data on long term outcome of EVL for esophageal varices.

Method: Adult patients suffering from chronic liver disease (CLD) who underwent EVL of esophageal varices grade 2 and above between January 2006-December 2015 were prospectively followed for redevelopment of varices, worsening of portal hypertensive gastropathy (PHG), rebleeding and mortality. EVL was done as primary prophylaxis of bleed in 72 and as secondary prophylaxis in the rest. It 
was done as emergency procedure in 12. After first EVL reendoscopy was done 2 weekly till variceal eradication and thereafter repeated 6 monthly. All patients received propranolol after EVL if there were no contraindication

Result: Results: 247 patients (mean age $48.35+16.73$ (range 28-74) years, 175 males) underwent 306 EVL sessions. The etiology of CLD were alcohol 131(53\%), viral hepatitis B \& C 52(21\%), cryptogenic cirrhosis $59(24 \%)$, others $5(4 \%)$. Varices were grade 2 in $27(11 \%), 3$ in $168(68 \%)$ and 4 in $52(21 \%)$ patients and associated with PHG in $189(76.5 \%)$, gastric cardia varices in $8(3.2 \%)$. Child class was A in $86(35 \%), B$ in $114(46 \%)$ and $C$ in $47(19 \%)$. Immediate post EVL bleed occurred in none, rebleeding from esophageal ulcers occurred in $6(2.5 \%)$ within mean $3.8+1.2$ days but there was no immediate mortality from bleed. Mean follow up was 68.3 (28-108) months. Obliteration of varices was achieved in $100 \%$ with $1.3+1.2(1-3)$ endoscopy sessions over a period of $6+2.8$ (3-10) weeks. Varices recurred in $47(19 \%)$ patients within a mean of $35+14.3$ months (range 18-64). New PHG after eradication of varices developed in $44(18 \%)$ and worsening occurred in $94(38 \%)$. No new gastric varices developed. Overall rebleed occurred in $31(12.6 \%)$ and that from recurrent varices was $11(4.3 \%)$. Most of late bleeds were from PHG and esophageal neovascularisation following EVL. There was no difference in rate of redevelopment of varices (16.7\% vs $20 \%)$ and incidence of rebleed $(9.7 \%$ vs $13.7 \%$ ) between patients of primary and secondary prophylaxis. Cumulative rebleeding rate after 1,3,5,7 years were $1.6 \%, 4.8 \%, 7.8 \%$ and $9.4 \%$. Subgroup analysis showed alcohol, larger size of varices, severe PHG and concomitant presence of gastric varices were associated with higher risk of rebleeding but significant association was not found with Child status, thrombocytopenia, coagulopathy. The overall mortality was $164(66.4 \%)$ mostly from progressive CLD and only $18(7.3 \%)$ was due to rebleed. The survival rates at 1,3,5 and 7 years were $95 \%, 63 \%, 44 \%$ and $31 \%$. Multivariate analysis identified alcohol, Child class-C and PHG as significant factors associated with death.

Conclusion: EVL is effective in long term for both primary and secondary prophylaxis of esophageal variceal bleed with low risk of rebleeding and mortality.

\section{$L T-1$}

Does ticagrelor attenuates apoptosis of liver cells induced by abdominal aorta ischemia/ reperfusion?

\section{Goktug Sirin ${ }^{1}$, Melda YARDIMOGLU YILMAZ ${ }^{1}$, Yusufhan Yazir ${ }^{1}$, Orhan Findik ${ }^{2}$}

${ }^{1}$ Kocaeli University Medical School, Turkey; ${ }^{2}$ Derince Education and Research Hospitali, Turkey

Background: Ischemia-reperfusion injury occurs after different surgical treatments and may have an effect in remote organs, causing multiple organ dysfunction syndrome and death.

This study aimed to analyze the effect of ticagrelor pretreatment on the prevention of liver injury induced by abdominal aorta ischemia and reperfusion $(I / R)$ and also to determine the effective dose for achieving this

Method: This study was approved by the Institution of Animal Care and Use Committee at Kocaeli University and complied with the Guide for the Care and Use of Laboratory Animals.

Thirty-five male Sprague-Dawley rats weighing 350-400 $\mathrm{g}$ were randomized into five groups. In group 1, only laparotomy was performed. In all groups except for the sham group (group 1), ischemiareperfusion injury was induced by clamping the aorta with atraumatic vascular clamp infrarenally, for two hours, followed by 4 hours of reperfusion. In group 2-5, animals received with $0.1 \mathrm{ml} / \mathrm{kg}$ saline, ticagrelor at doses of $7.5 \mathrm{mg} / \mathrm{kg}, 15 \mathrm{mg} / \mathrm{kg}$ and $25 \mathrm{mg} / \mathrm{kg}$ orally via gastric gavage, before the ischemic period, respectively. After sacrifice, liver of the animals were extracted for both histopathological (hematoxylin-eosin staining and TdT-mediated dUTP nick-end labeling (TUNEL) method for detection of apoptosis) and biochemical (malondialdehyde assay (MDA), glutathione reductase (GR) and glutathione peroxidase (GPx) assays) analysis. Determination of MDA, GPx and GR levels were performed by enzyme-linked immunosorbent (ELISA) assay. The levels of these oxidant and antioxidant enzymes in liver tissues were measured based on the Biotin double antibody sandwich technology (Bioassay Technology Laboratory, Shanghai, China).

Result: There was a significant difference between the animals that received $7.5 \mathrm{mg} / \mathrm{kg}$ and $25 \mathrm{mg} / \mathrm{kg}$ and $15 \mathrm{mg} / \mathrm{kg}$ and $25 \mathrm{mg} / \mathrm{kg}$ dose of ticagrelor regarding tissue malondialdehyde (MDA), and glutathione reductase levels in liver. Ticagrelor treatment at $25 \mathrm{mg} / \mathrm{kg}$ led to normal liver architecture against $\mathrm{I} / \mathrm{R}$ induced injury. The number of TdT-mediated dUTP nick-end labeling (TUNEL)-positive cells in liver were increased in the sections from saline (I/R) group rats, and decreased following $25 \mathrm{mg} / \mathrm{kg}$ ticagrelor treatment.

Conclusion: Ticagrelor dose-dependently inhibits platelet aggregation, increases cyclooxygenase-2 and also inhibits cellular uptake of adenosine all resulting in attenuation of $\mathrm{I} / \mathrm{R}$ injury. Ticagrelor at 25 $\mathrm{mg} / \mathrm{kg}$ was determined as the dose effective against I/R-induced injury in liver in Sprague-Dawley rats in the present study.

\section{$L T-2$}

Donor to recipient transmission of Factor XII deficiency by orthotropic liver transplantation: case report and review of the literature

Hussien Elsiesy ${ }^{1}$, Waleed Alhamoudi ${ }^{1}$, Hany Elbeshbeshy ${ }^{1}$, Mohamed Shawakat $^{1}$, John Renz ${ }^{2}$, Mohamed Alsebayel ${ }^{1}$

${ }^{1}$ King Faisal Specialist Hospital \& Research Center, Saudi Arabia; ${ }^{2}$ University of Chicago, United States

Background: Transmission of congenital clotting factor deficiencies after liver transplantation is rare. Prior to this report, there was only one case reported of liver donor to recipient transmission of Factor XII deficiencies.

There are also reports of liver donor to recipient transmission of protein $\mathrm{C}$ deficiency with dysfibrinogenemia, protein $\mathrm{S}$, factor VII, Factor VIII and factor XI deficiencies. We report a case of transmission of factor XII deficiency with liver transplantation in a patient primary hyperoxaluria. The patient activated partial thromboplastin time (aPTT) was normal before liver transplantation but he has persistent elevation of the aPTT, but no evidence of bleeding. Knowing the possibilities of transmission of deficiencies of coagulation factors will help in early diagnosis in the transplant recipients.

Method: An 18-year-old male with type 1 primary hyperoxaluria with mild renal impairment with an estimated glomerular filtration rate (eGFR) of 46, received preemptive orthotopic liver transplantation in February 2017. Coagulation tests before liver transplantation showed a normal activated partial thromboplastin time (aPTT) of 29, normal international normalized ratio (INR) of 1 . After successful orthotopic liver transplant, the patient was maintained on Cellcept and low dose tacrolimus. Six month after liver transplantation, the laboratory evaluation before ERCP for biliary stricture revealed a prolonged aPTT at $>150 \mathrm{~s}$ (normal 21-33 s), and review of his laboratory values since transplantation revealed a persistently prolonged aPTT $(>150$ s since liver transplantation). He had not experienced any abnormal bleeding episodes or evidence of recurrent thrombosis. 
Result: revealed normal INR; 0.9, normal Prothrombin Time (PT); 13 seconds, normal Thrombin Time (TT); 19 seconds and high aPTT $>$ 150 seconds (normal 30.5-40.4 seconds) which is corrected on mixing study (indicating no inhibitors). Investigation also revealed factor XII deficiency; level $<0.10 \mathrm{IU} / \mathrm{ml}$ (normal levels $0.60-1.50 \mathrm{IU} / \mathrm{ml}$ ) and mixing study is negative for inhibitors. Results of repeat factor analyses are shown in Table 1; there was evidence of a severe factor XII deficiency. As aPTT was normal, there was no indication to test for Factor XII level.

It was felt that factor XII deficiency, the cause of his prolonged aPTT, was not associated with an increased risk of bleeding and the patient subsequently had endoscopic retrograde cholangio-pancreatography (ERCP) followed by percutaneous transhepatic cholangiography (PTC) both without complications.

The liver donor was an11 year-old female without prior medical or surgical history. She presented with head trauma secondary to gun shot with subsequent rapid brain death. On admission, her aPTT was markedly prolonged at $132 \mathrm{~s}$ with a normal prothrombin time, platelet count, hemoglobin and basic metabolic panel. The etiology of her elevated aPTT was unclear. Her aPTT subsequently dropped to 65 then 48 after FFP. Her outside medical records was not available for review.

Conclusion: Both cases reported were diagnosed after liver transplantation despite having prolonged aPTT in both donors on initial presentation, proper identification before liver transplantation may prevent the transmission of coagulation defect that can adversely affect liver transplant outcome.

\section{$L T-3$}

\section{Successful living donor liver transplantation in a case of mean} pulmonary arterial pressure of $52 \mathrm{mmHg}$

Yang Won Nah ${ }^{1}$, Hyung Woo Park ${ }^{1}$, In Young Yang Huh ${ }^{1}$, Sun Eun Yang Park', Seo Hee Yang Ahn' ${ }^{1}$, Shin Jae Kim ${ }^{1}$, Jin A Kwon ${ }^{1}$

${ }^{1}$ Ulsan University Hospital, Korea, Republic of

Background: Pulmonary hypertension $(\mathrm{PH})$ is defined as an elevated mean pulmonary arterial pressure $(\mathrm{mPAP}) \geq 25 \mathrm{mmHg}$ at rest. The perioperative management can be complicated by hemodynamic instability resulting in severe hypoxemia, acute right heart failure/circulatory collapse and death. Portal hypertension is a curable cause for PH. However, Krowka et al reported that mPAP of 50 $\mathrm{mmHg}$ or greater was associated with $100 \%$ cardiopulmonary mortality in liver transplantation (LT) setting

Method: A 45 years old male patient underwent LDLT for HBV + alcoholic cirrhosis and HCC. MELD score was 28. Echocardiography performed 2 months before the operation showed right ventricular systolic pressure (RVSP) $62 \mathrm{mmHg}$ and maximum tricuspid regurgitant jet velocity (TRVmax) $3.8 \mathrm{~m} / \mathrm{sec}$, suggesting moderate resting PH. During anesthetic induction, pulmonary vascular resistance (PVR) was measured as 246 dynes/sec/cm-5 and mPAP as $52 \mathrm{mmHg}$. One hour later, they were 217 and 40, respectively, with infusion of Isosorbide $1 \mathrm{mcg} / \mathrm{Kg} / \mathrm{min}$. Vital signs were stable and $\mathrm{PaO} 2$ level was $223 \mathrm{mmHg}$. So we decided to proceed with surgery despite of moderate $\mathrm{PH}$.

Result: Graft weighed $550 \mathrm{~g}$ and GRWR was 0.74 . The living donor LT procedure was done with modified right lobe graft. Additionally portal vein endthrombectomy, direct ligation of a large splenorenal shunt and splenic artery ligation was done. The operation took 850 minutes and 24 pints of RBC, 16 pints of FFP, 8 pints of PC and 10 pints of cryoprecipitate. PVR and mPAP were in the range of $217 \sim 149$ and $46 \sim 31$, respectively. Alprostadil $7 \mathrm{mcg} / \mathrm{hr}$ was started just after finishing arterial anastomosis and was infused for 7 days thereafter. Extubation was done 9 hours after the operation and liquid diet was begun 2 days after the operation. The day after LT, RVSP was $96 \mathrm{mmHg}$ and TRVmax $4.9 \mathrm{~m} / \mathrm{sec}$. And 5 days after LT, he complained of dyspnea and Beraprost (prostacyclin pathway agonist) was given orally. Even with mild dyspnea, the patient was discharged on foot 19 days after LT. 6 weeks after LT RVSP was measured as 84 $\mathrm{mmHg}$ and TRVmax $4.0 \mathrm{~m} / \mathrm{sec}$. $\mathrm{mPAP}$ was $63 \mathrm{mmHg}$ on right heart catheterization. On CT scan, there was marked hepatic congestion resulting from pulmonary hypertension. Endothelin receptor antagonist Ambrisentan was given orally. The patient's breathing difficulty almost disappeared soon. Now, 3 months after the operation, he returned to work.

Conclusion: The authors conducted LDLT in a case of severe pulmonary hypertension with mean PAP $50 \mathrm{mmHg}$ successfully by careful intraoperative monitoring and postoperative management. When encountered a case of moderate to severe pulmonary hypertension during LT, initial response to anesthetic management might dictate whether to proceed with LT or not.

\section{$L T-4$}

Dyslipidemia after liver transplantation: single-center experience

\section{Anna Babich ${ }^{1}$}

${ }^{1}$ Scientific Research Institution - Ochapovski Regional Clinical Hospital, Russian Federation

Background: Cardiovascular diseases are among the most frequent causes of mortality after liver transplantation. Post-transplant dyslipidemia is highly prevalent and attention to dyslipidemia is warranted because interventions for dyslipidemia have an impact on reducing cardiac events, morbidity and mortality in transplant population.

Method: The aim of this retrospective clinical study was to estimate the frequency, management of dyslipidemia after successful liver transplantation and the role of immunosuppressive drugs in the pathogenesis of dyslipidemia in liver-transplant recipients. A total of 88 patients (age $47.5 \pm 12.1$ years; 33 women and 55 men) who had undergone successful liver transplantation between 2010 and 2015 at the Scientific Research Institute-Regional Clinical Hospital ?1 and completed 36 months follow-up were studied. The results are presented as means with standard deviations.

Result: Levels of serum lipids (total cholesterol, triglycerides and LDL cholesterol) were studied before liver transplantation and repeated after liver transplantation. At 6,12, and 36 months after liver transplantation, the prevalence of dyslipidemia were $44.3 \%, 54.5 \%$, and $62.5 \%$, respectively. Elevated LDL cholesterol were $13.5 \%$, $24.1 \%$ and $30.2 \%$, respectively. The prevalence of hypertriglyceridemia were $18.4 \%, 26.7 \%, 30.2 \%$, respectively. Hyperglycemia was noticed in $30.1 \%, 44.5 \%, 37.8 \%$ at 6,12 , and 36 months after liver transplantation. Obesity was observed in $28 \%$ of patients at 3 years post-LT. There was significant difference for the development of dyslipidemia, which seemed to be more related to cyclosporine $(65.5 \%)$ than to tacrolimus (34.5\%) Statins were not administrated in $40 \%$ of cases, despite of elevated cholesterol.

Conclusion: The development of dyslipidemia arises after liver transplantation. We suggest that it is vitally important to manage transplant-related dyslipidemia effectively by paying our physicians' attention to administration of statins. Doctors need to motivate patients to lose weight. 
$L T-5$

Low dose HBIG prophylaxis is associated with significant risk of the graft reinfection in Hepatitis B patients undergoing liver transplantation

\section{$\underline{\text { Mahesh Uttamrao Patil }}{ }^{1}$, Sharat Putta ${ }^{2}$, Vishal Vilas Akude ${ }^{1}$ \\ ${ }^{1}$ DND STUDENT, India; ${ }^{2}$ Consultant, India}

Background: The risk of HBV reinfection after liver transplantation without prophylaxis is approximately $80 \%$. The current recommendation for HBV prophylaxis is a combination of a high-dose hepatitis B immune globulin (HBIG) and nucleoside/tide analogue for life. In recent years Liver transplant centers across the country and world are following no HBIG protocol during transplantation in patients with low viral titers.

Method: Comparative study between patient who received standard dose $5000 \mathrm{U}$ during an hepatic phase and 5000-7000 U in first week of post-transplant period with patients low dose (5000 U) over last 5 years period.

Result: HBsAg was measured monthly until 2 consecutive samples were negative and then every 6 months. Anti-HBs antibody titers were measured 6 months after transplantation. HBsAg was also measured if patient is having transaminitis. If a patient was negative for anti-HBs antibodies, he was given a course of HBV vaccination, and his titers were checked 1 month after the completion of his vaccination. A recurrent $\mathrm{HBV}$ infection was defined as the reappearance of HBsAg or HBV DNA after transplantation after initial seroclearance/DNA negativity

Diagnostic Assays HBsAg and anti-HBs antibodies were detected with an enzyme-linked immunosorbent assay technique (VIDAS HBsAg Ultra and VIDAS anti-HBs Total Quick, BioMerieux, France). HBV DNA was determined with a polymerase chain reaction method (COBAS TaqMan assay, Roche Molecular Systems, Branchburg, NJ). Patients were monitored via HBV DNA counts every month until there were 2 successive undetectable values and then every 6 months. HbsAg was tested monthly until negative findings and then every 6 months. The anti-HBs antibody titer was checked 6 months after transplantation, after attempts at vaccination, and subsequently at 6-month interval.

Results

Demographic profile

- Between 2012 to 201715 patients underwent liver transplantation for HBV related CLD.

- All patients have HBV DNA <20 and HBS Ag positive

- All were on standard antiviral therapy including Entecavir in 14 patients and Lamivudine in 1.

- All patients were negative for Hbe AG

- One patient was positive for HDV.

- Decompensated cirrhosis 11

- Hepatocellular carcinoma 5

Type of transplant performed

- DDLT -9

- LDLT - 1

- OLT-4

- Split Liver transplant-1

7 Patients who received modified regimen i.e. $5000 \mathrm{u}$ of HBIG during an hepatic phase and 5000-7000 U during first week 1 patient died due to sepsis and 6 patients are still HBs negative and are on immunosuppressants with regular follow up.

Out of 8 patients who received low dose of HBIG i.e 5000 units in an hepatic phase 3 patients are found to have HBsAg positive on follow up although they are on antivirals.
Conclusion: In patients undergoing transplantation for HBV related Liver Disease Low dose of HBIG prophylaxis is associated with significant risk of reinfection of graft. Optimal dose of HBIG is still needed to be defined in such patients.

\section{$L T-6$}

Pneumocystis pneumonia is associated with poor outcome after living donor liver transplantation

$\underline{\text { Sanjiv Saigal }}^{\mathbf{1}}$, Narendra Choudhary ${ }^{1}$, Neeraj Saraf ${ }^{\mathbf{1}}$, Navin Kumar $^{1}$, Amit Rastogi ${ }^{1}$, Sanjay Goja ${ }^{1}$, Prashant Bhangui ${ }^{1}$, Thiagarajan Srinivasan $^{1}$, Deepak Govil ${ }^{1}$, Arvinder S Soin ${ }^{1}$

${ }^{1}$ Medanta The Medicity, India

Background: Pneumocystis Jirovecii is an opportunistic fungus that can cause pneumonia in immunoscompromised hosts and is associated with significant morbidity and mortality. We describe our experience of Pneumocystis pneumonia (PCP) in recipients with living donor liver transplantation (LDLT).

Method: The study is retrospective analysis of data at a tertiary care center in north India. The immunosuppression protocol consisted of Tacrolimus, Mycophenolate and steroids (for 3 months), PCP prophylaxis is given for 3 months with trimethoprim and sulfamethoxazole at our center. Forty-four patients underwent bronchospic alveolar lavage for pneumonia from 2014 to 2016, 7 of these patients were positive for Pneumocystis Jirovecii by multiplex PCR. Result: The study cohort included 6 males and 1 female. The mean age of study group was $52 \pm 13$ years. The etiologies of liver disease in these patients before LDLT were cryptogenic $(n=3)$, hepatitis B $(n=1)$, hepatitis $(n=1)$, alcoholic $(n=1)$ and Wilson's disease $(n=1)$. One patient had PCP at 5 days after LDLT; PCP occurred after end of prophylaxis at a median period of 18 months (range 6 to 48 months) in rest of patients. All patients were treated with high dose Trimethoprim and Sulfamethoxazole, five (71\%) of patients died despite treatment.

Conclusion: Pneumocystis pneumonia (PCP) is associated with poor outcome after living donor liver transplantation.

\section{$L T-7$}

Efficacy, renal function and safety in living donor liver transplant recipients treated with everolimus plus reduced-exposure tacrolimus compared to standard-exposure tacrolimus: 12-month results from $\mathbf{H 2 3 0 7}$ study

Pragna Bachewal ${ }^{1}$, LB Jeng 4 , SG Lee ${ }^{2}$, WC Lee ${ }^{3}$, KS Suh ${ }^{8}$, T Yoshizumi $^{5}$, M Meier ${ }^{6}$, J Kochuparampil ${ }^{6}$, C Sips ${ }^{6}$, S Kaneko ${ }^{7}$, P Bernhardt ${ }^{6}$, G Levy ${ }^{9}$

${ }^{1}$ Novartis Healthcare Pvt ltd, India; ${ }^{2}$ Asan Medical Center, Korea, Republic of; ${ }^{3}$ Chang Gung Memorial Hospital, Taiwan; ${ }^{4}$ China Medical University Hospital, Taiwan; ${ }^{5}$ Kyushu University Hospital, Japan; ${ }^{6}$ Novartis Pharma AG, Switzerland; ${ }^{7}$ Novartis Pharma KK, Japan; ${ }^{8}$ Seoul National University Hospital, Korea, Republic of; ${ }^{9}$ University of Toronto, Canada

Background: The H2304 study has shown that everolimus with reduced-exposure tacrolimus (EVR+rTAC) vs TAC-control (TAC-C) exhibited significant improvement in renal function along with comparable efficacy and safety post-liver transplantation (LT) in de novo deceased donor LT recipients. Here, we present 12 month (M) results from the $\mathrm{H} 2307$ study (NCT01888432), conducted to 
determine whether EVR+rTAC provides similar beneficial effects post-LT in de novo living donor (LD) LT recipients.

Method: In this 24M, multicenter, open-label, phase III, controlled study, recipients of LDLT were randomized to EVR (trough levels [C0]: $3-8 \mathrm{ng} / \mathrm{mL})+$ rTAC $(\mathrm{C} 0: 3-5 \mathrm{ng} / \mathrm{mL} ; \mathrm{N}=142)$ or TAC-C (C0: $8-12 \mathrm{ng} / \mathrm{mL}$ from Week [W] 5 to $\mathrm{M} 4$ and C0: $6-10 \mathrm{ng} / \mathrm{mL}$ from M4 to M24; $\mathrm{N}=142) \pm$ corticosteroids after a run-in period of $30 \pm 5$ days post-LT. Primary objective was to demonstrate comparable efficacy of EVR+rTAC and TAC-C (composite of treated biopsyproven acute rejection [tBPAR], graft loss, or death) at M12 post-LT. Secondary objective was to at least demonstrate comparable renal function (estimated glomerular filtration rate [eGFR]) in EVR+rTAC and TAC-C arms from randomization (RND) to M12. Other objectives were safety and hepatocellular carcinoma (HCC) recurrence.

Result: Of 284 patients, $92.3 \%(\mathrm{n}=131)$ in EVR+rTAC and $93.7 \%$ $(\mathrm{n}=133)$ in TAC-C arm completed the $12 \mathrm{M}$ study. Demographics and baseline characteristics were balanced between the arms. At M12, incidence of composite efficacy failure was comparable in EVR $+r$ TAC vs TAC-C arms; EVR+rTAC arm was non-inferior (NI) to TAC-C arm $(P<0.001$; Table 1). Moderate or severe episodes of tBPAR occurred only in TAC-C arm. Mean change in eGFR from RND to M12 in EVR+rTAC and TAC-C arms were -7.9 and -12.1 $\mathrm{mL} / \mathrm{min} / 1.73 \mathrm{~m} 2$, respectively. The eGFR was higher in EVR $+\mathrm{rTAC}$ vs TAC-C arms at all visits from W6 to M12; EVR+rTAC arm was NI (NI margin: $-6 \mathrm{~mL} / \mathrm{min} / 1.73 \mathrm{~m} 2 ; P<0.001,90 \% \mathrm{CI}:-0.09$, $8.40)$, but not superior $(P=0.108)$ to TAC-C arm at M12. Using imputation for missing eGFR data, mean change in eGFR was in favor of EVR+rTAC vs TAC-C arm (-8.0 vs $-13.3 \mathrm{~mL} / \mathrm{min} / 1.73 \mathrm{~m} 2)$ with a difference of $5.3 \mathrm{~mL} / \mathrm{min} / 1.73 \mathrm{~m} 2(P=0.046)$. Incidence of adverse events (AE) and serious $\mathrm{AE}$ was comparable between the arms (Table 2). Most frequent AE leading to study drug discontinuation was proteinuria in EVR+rTAC $(2.1 \%)$ and increased blood creatinine level in TAC-C arm (2.8\%). Of 118 patients with HCC, recurrence was seen only in TAC-C arm $(5 / 62,8.1 \%)$. One hepatitis B virus (HBV) recurrence and one $\mathrm{HBV}$ de novo infection occurred in EVR+rTAC and TAC-C arms, respectively.

Conclusion: In LDLT, early introduction of EVR+rTAC at $30 \pm 5$ days post-LT demonstrated comparable efficacy and better renal function to TAC-C at M12. No new safety signal was reported in EVR + rTAC arm. No HCC recurrence was reported in EVR+rTAC arm, whereas 5 patients in TAC-C arm experienced $\mathrm{HCC}$ recurrence. M24 follow-up results will better define these findings.

\begin{tabular}{|c|c|c|c|}
\hline Parameter & $\begin{array}{c}\text { EVR+rTAC } \\
(\mathrm{N}=142)\end{array}$ & $\begin{array}{c}\text { TAC-C } \\
(N=142)\end{array}$ & $\begin{array}{c}\text { Difference, \% } \\
(90 \% \mathrm{CI})\end{array}$ \\
\hline \multicolumn{4}{|l|}{ Efficacy, n (KM \%) } \\
\hline Composite efficacy (tBPAR, graft loss, or death) & $7(5.1)$ & $8(5.8)$ & $-0.7(-5.2,3.7)$ \\
\hline tBPAR & $3(2.2)$ & $5(3.6)$ & $-1.4(-4.7,2.0)$ \\
\hline Borderline & 2 & 1 & \\
\hline Mild & 1 & 1 & \\
\hline Moderate & 0 & 2 & \\
\hline Severe & 0 & 1 & \\
\hline Graft loss & $0(0.0)$ & $0(0.0)$ & - \\
\hline Death & $4(2.9)$ & $3(2.2)$ & $0.7(-2.5,3.8)$ \\
\hline Renal function & $n=130$ & $\mathrm{n}=130$ & \\
\hline $\begin{array}{l}\left.\text { eGFR (MDRD4; } \mathrm{mL} / \mathrm{min} / 1.73 \mathrm{~m}^{2}\right) \text { at Ml2, mean } \\
\text { (SD) }\end{array}$ & $81.2(28.6)$ & $76.5(24.2)$ & - \\
\hline Recurrent $\mathrm{HCC}^{*}, \mathrm{n}(\%)$ & $0(0.0)$ & $5(8.1)$ & - \\
\hline
\end{tabular}

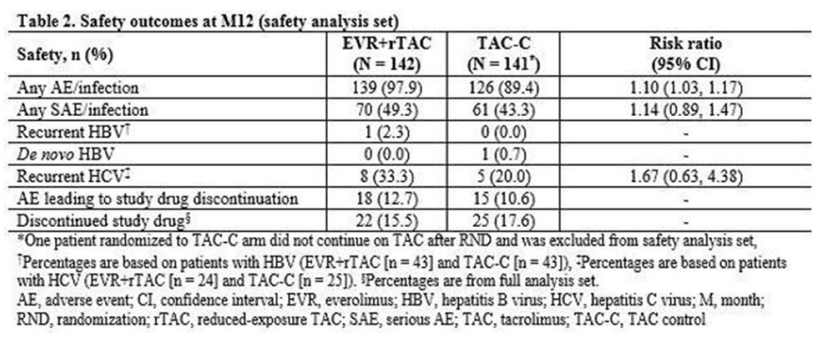

$L T-8$

Liver transplantation for familial amyloid polyneuropathy (FAP - TTR - ValMet30) - A long-term follow up study in a non-transplantation centre

Melina Kavousanaki ${ }^{1}$, Minas Tzagournissakis ${ }^{1}$, John Zaganas ${ }^{1}$, Konstandinos Stylianou ${ }^{1}$, Alexandros Patrianakos ${ }^{1}$

${ }^{1}$ University Hospital of Heraklion, Greece

Background: Familial amyloidosis polyneuropathy (FAP) is a rare, progressive and ultimately life-threatening disease, inherited in the autosomal dominant way and caused by a point mutation on the transthyretin (TTR) gene. Liver transplantation is the only diseasemodifying treatment to date. Our aim is to study the long-term outcomes of patients transplanted for FAP, under a multi-disciplinary team (MDT) care.

Method: We included adult patients that were transplanted for FAP indication and were followed up in a relevant clinic or admitted in our department. All data were kept in a data base prospectively.

Result: Twelve patients (six male), mean age of 43 years and mean follow up post transplant 100 months, were included. Three patients died in this period (one man in a road traffic accident, one woman with end-stage kidney disease/in hemodialysis, due to cerebral hemorrhage, and one woman due to cardiopulmonary syncope/multiple organ insufficiency). All patients had peripheral neuropathy $(25 \%$ severe), a $83 \%$ had autonomic nervous system dysfunction, whereas all men patients had erectile dysfunction. Three patients suffered from significant renal impairment (MDRD4 $<50 \mathrm{ml} / \mathrm{min}$ ), while 6 patients had various genitourinary manifestations. Gastrointestinal involvement typically consisted of diarrhea/constipation interchange, and was present in $75 \%$ of the patients. In a $34 \%$ of the patients there were ocular problems, mainly glaucoma. Maintenance immunosuppression consisted of tacrolimus alone in $42 \%$ of the patients, tacrolimus combined with azathioprine in $33.3 \%$ and tacrolimus combined with mycophenolate in $17 \%$. Only one patient received tacrolimus combined with sirolimus. The severity of several complications related to FAP and worsening quality of life, were found to be associated with waiting in the transplant list more than 12 months.

Conclusion: Patients that transplanted for FAP have a long survival. Prolonged stay in the transplant waiting list is associated with frequency and severity of disease complications. These patients are best managed in the context of MDT care.

\section{$L T-9$}

Living donor liver transplantation using small for size grafts

Abhishek Agrawal ${ }^{1}$, Pulkit Sethi ${ }^{1}$, Sudhindran $\mathrm{S}^{\mathbf{1}}$, $\overline{\text { Ramachandran }}^{1}$, Dinesh $\mathbf{B}^{1}$, Mayank Nautiyal ${ }^{1}$, Unnikrishnan $\mathbf{G}^{\mathbf{1}}$, Binoj $\mathrm{ST}^{\mathbf{1}}$

${ }^{1}$ Amrita Institute of Medical Sciences (AIMS), Kochi, India 
Background: In living donor liver transplantation, graft to recipient weight ratio (GRWR) $>0.8$ is perceived as the critical graft size. This lower limit of GRWR (0.8) has been challenged over the last decade owing to the technical refinements, especially related to inflow and outflow modulation techniques. Our aim was to compare the outcome of small for size grafts $(\mathrm{GRWR}<0.8)$ to normal sized grafts $($ GRWR $>0.8)$ and to determine the risk factors for mortality when small for size grafts were used.

Method: Data of 200 transplant recipients and their donors was analysed over a period of two years. Routine practice of harvesting MHV or reconstructing anterior sectoral veins into neo-MHV was followed during LDLT. Outcomes were compared in terms of mortality, hospital stay, ICU stay and occurrence of various complications such as functional small for size syndrome (F-SFSS), hepatic artery thrombosis (HAT), early allograft dysfunction (EAD), portal vein thrombosis (PVT) and post-operative sepsis. A multivariate analysis was also done to determine the risk factors for mortality in both the groups.

Result: Recipient and donor characteristics, intraoperative variables and demographical data was comparable in both the groups $(G R W R<0.8$ and $G R W R \geq 0.8)$. Postoperative 90 day mortality $(15.5 \%$ vs. $22.85 \%)$, mean ICU stay (10 vs. 10.32 days) and mean hospital stay ( 21.4 vs. 20.76 days) was statistically similar in both the groups. There was no difference in post-operative outcomes like occurrence of SFSS, HAT, PVT, EAD or sepsis between both the groups. Thrombosis of MHV/reconstructed MHV was a risk factor for mortality in grafts with GRWR $<0.8$ but not in those with GRWR $>0.8$.

Conclusion: Graft survival after LDLT using a small for sized right lobe graft (GRWR $<0.8$ ) is as good as with normal grafts. However, patency of anterior sectoral outflow by MHV or reconstructed MHV is crucial to maintain graft function.

\section{$L T-10$}

Recidivism after liver transplantation presenting as delirium tremens in a post liver transplant patient for severe alcoholic hepatitis(AH)

$\underline{\text { Md Nadeem Parvez }}^{1}$

${ }^{1}$ WBMC, MCI, India

Background: CASE REPORT

Method: CASE REPORT

Result: CASE REPORT

Conclusion: CASE REPORT

\section{$L T-11$}

Short-term evaluation of the impact of sofosbuvir-based therapy on hepatic fibrosis in patients with recurrent $\mathrm{HCV}$ infection posttransplant

\footnotetext{
Ahmed Shawkat ${ }^{1}$, Alberto Zanetto ${ }^{3}$, Alberto Ferrarese ${ }^{3}$, Sarah Shalaby $^{3}$, Enrica Franceschet ${ }^{3}$, Ilaria Bortoluzzi ${ }^{3}$, ${\text { Elena } \mathrm{Nadal}^{3} \text {, Martina Gambato }}^{3}$, Giacomo Germani ${ }^{3}$, Marco Senzolo ${ }^{3}$, Enico Gringeri ${ }^{3}$, Giacomo Zanus ${ }^{3}$, Patrizia Boccagni $^{3}$, Abeer Sharafeldin Abdelrehim ${ }^{2}$, Ahlam Mohammed Farghaly ${ }^{2}$, Umberto Cillo ${ }^{3}$, Patrizia Burra ${ }^{3}$ Francesco Paolo Russo ${ }^{3}$

${ }^{1}$ Assiut University Hospital, Padua University Hospital, Egypt;

${ }^{2}$ Assiut University Hospital, Egypt; ${ }^{3}$ Padua University Hospital, Italy
}

Background: The degree of fibrosis determines the stage of liver disease. Liver fibrosis can regress in patients with chronic hepatitis when the cause of liver damage is treated, but most studies assessing benefits of viral eradication were performed in the era of IFN-based therapy and in pre-transplant settings. Aim of the study is to assess noninvasively effect of sofosbuvir-based antiviral therapy on liver fibrosis in patients with post-transplant recurrent HCV infection.

Method: This is a prospective study, in which patients with recurrent $\mathrm{HCV}$ infection post-transplant, treated with sofosbuvir plus ribavirin were enrolled. Clinical, biochemical findings (including APRI and FIB-4 scores) and liver stiffness (LS), measured by Fibroscan at baseline and at 3 months after end of therapy, virological data, type of immunosuppression and response to antiviral therapy were evaluated. Patients were further divided into two groups, according to antiviral response

Result: From August 2014 till January 2016, sixty patients were enrolled, $78.3 \%$ were male, $67.8 \%$ had genotype 1 and $61.7 \%$ received previous $\mathrm{HCV}$ treatment. At baseline, 21 patients $(35 \%)$ had severe fibrosis according to Ishak score. The median time interval from LT was 51 months (5-284), immunosuppressive therapy was tacrolimus based in $78.4 \%$. The median baseline HCV-RNA was 2.341.172 UI/ml (770-52.330.800 UI/ml). SVR was achieved in 43 patients $(71.6 \%)$. There was significant decrease in LS, FIB-4 and APRI scores in patients who achieved SVR in comparison to those who did not achieve SVR (Median change of LS, FIB-4 and APRI in SVR vs. NSVR group was 4.9 vs. 2.2 ( $P=0.000$ vs. 0.074$), 1.06$ vs. $0.6(P=0.000$ vs. 0.177$)$ and 0.97 vs. $0.44(P=0.000$ vs. 0.177$)$ respectively). The percentage of the patients with improved LS, FIB4 and APRI scores in SVR vs. NSVR group was $86 \%$ vs. $58.8 \%$, $79.1 \%$ vs. $70.5 \%$ and $86 \%$ vs. $64.7 \%$ respectively. Multiple linear regression was done for patients with SVR and it was found that the change in FIB-4 and APRI was significantly associated with pretreatment levels of AST, GGT, bilirubin, INR and Child Pugh score, while the change in LS was associated only with high pre-treatment LS values.

Conclusion: Eradication of $\mathrm{HCV}$ with sofosbuvir-based regimen leads to significant regression in Liver fibrosis, assessed by combined non-invasive tests in post-transplant setting as early as 3 months posttherapy.

\section{$L T-12$}

\section{Predictors of post liver transplant HCC recurrence}

\section{Ahmet Gurakar ${ }^{1}$, Bahnam Saberi ${ }^{1}$, Benjamin Philosophe ${ }^{1}$, Natarajan Ravendhran ${ }^{1}$ \\ ${ }^{1}$ Johns Hopkins, United States}

Background: The aim of the current study was to explore the clinical and pathological differences between patients with $\mathrm{HCC}$ recurrence versus no-HCC recurrence following liver transplantation.

Method: We retrospectively evaluated 145 consecutive patients who underwent deceased donor liver transplant for hepatocellular carcinoma at our liver transplant program. We evaluated the clinical and pathological differences between patients who had tumor recurrence compared to patients without recurrence.

Result: Overall 22 (15.2\%) of the recipients had tumor recurrence following liver transplant. The MELD score was similar between the two groups. Overall $40.9 \%$ of patient who had HCC recurrence were outside of Milan and UCSF criteria based on explant pathology. Patients with HCC recurrence had higher serum AFP levels compared to the non-recurrence group both before and after liver transplant, median 41 vs. $11.9 \mathrm{ng} / \mathrm{mL}$ pre-LT $(\mathrm{P}=0.002)$ and 10.9 vs. $3.0 \mathrm{ng} / \mathrm{mL}$ post-LT $(\mathrm{p}<0.001)$, respectively. The patients with HCC recurrence 
had higher rate of poor differentiation $(40.9 \%$ vs. $4.9 \%, \mathrm{P}<0.001)$, and microvascular invasion $(\mathrm{MVI})(72.8 \%$ vs. $18.6 \%, \mathrm{P}<0.001)$ when compared to non-recurrence group. None of the 15 patients who had well differentiated tumors on explant had HCC recurrence following liver transplant. Of the patients with MVI on explant, $41 \%$ developed HCC recurrence compared to $59 \%$ who did not develop recurrence. On the other hand out of 90 patients who did not have MVI on pathology, only 5\% developed HCC recurrence following LT. Total of 15 patients had poor differentiation on explant. Out of these 15 patient, $60 \%$ had tumor recurrence following LT versus $40 \%$ with no recurrence. The overall 6-month, 1-year, 3-year, and 5-year survival for patients in the HCC non-recurrence group was significantly higher than HCC recurrence groups $91.9 \%, 87 \%, 81 \%, 78.3 \%$ compared to $100 \%, 86.4 \%, 30 \%, 6.7 \%$ respectively,(P

Conclusion: The rate of tumor recurrence following liver transplant in our series was similar to reported in the literature. The patients with tumor recurrence have worse tumor biology as shown by higher AFP and higher rate of microvascular invasion, and poor differentiation. There are limited effective treatment options for HCC recurrence following liver transplant and the outcomes are exceedingly poor. Early detection by closer HCC surveillance is important aspect of post-transplant management.

\section{Figure 3}

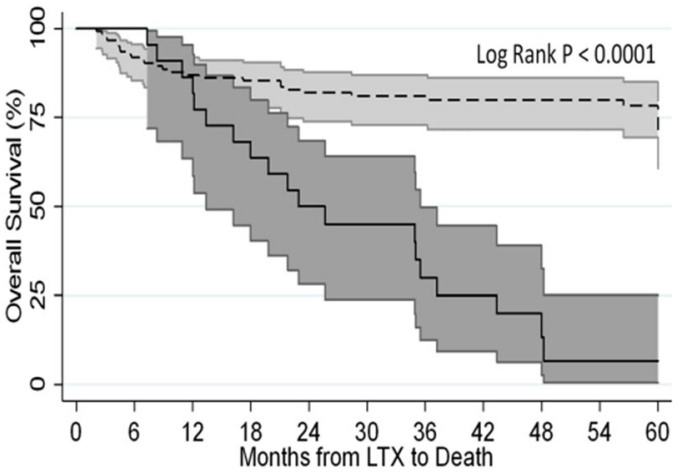

Number at risk

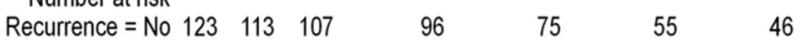

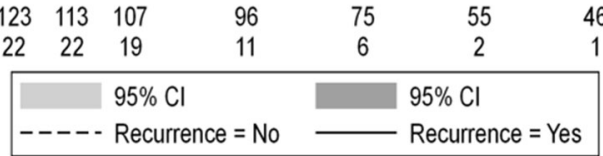

MISC-1

Histone deacetylase 2 inhibitor CAY10683 attenuates TLR4/NF?B signaling pathways in lipopolysaccharide-stimulated microglia

\section{Zuojiong Gong1, Fangzhou Jiao', Yao Wang', Haiyue Zhang', Wenbin Zhang ${ }^{1}$, Luwen Wang ${ }^{1}$}

${ }^{1}$ Renmin Hospital of Wuhan University, China

Background: Neuroinflammation involve in the progression of many central nervous system (CNS) diseases. Several studies have shown that HDAC inhibitors modulated inflammatory responses in LPSstimulated microglia, while, the mechanism remain unclear. The aim of present study was to investigate the effect of HDAC2 inhibitor CAY10683 on inflammatory responses and TLR4/NF- $\kappa B$ signaling pathways in LPS-activated microglia in vitro and in vivo.

Method: The effect of CAY10683 on cell viability of BV2 cells was detected by CCK-8. The expressions of inflammatory cytokines were analyzed by western blotting and RT-PCR. The TLR4 protein expression was measured by western blotting, Immunofluorescence, immunohistochemistry. The MYD88, Phospho-NF- $\kappa \mathrm{B}$ р65, NF- $\kappa \mathrm{B}-$ p65, AH3, H3, and HDAC2 were analyzed by western blotting.

Result: CAY10683 could inhibit expression levels of inflammatory cytokine TNF- $\alpha$ and IL-1 $\beta$ in LPS activated microglia. And it could induce TLR4, MYD88, Phospho-NF- $\mathrm{BB}$ p65, and HDAC2 expressions. Moreover, CAY10683 increased the acetylation of histones H3 in LPS-activated BV2 cells and LPS-induced mouse model.

Conclusion: HDAC2 inhibitor, CAY10683, could suppress inflammatory responses and TLR4/NF- $\kappa B$ signaling pathways by acetylation after LPS stimulation.

\section{MISC-2}

Oleuropein and copper: a novel opportunity against liver disease Clara Balsano $^{1}$, Clara Balsano ${ }^{1}$, Cristiana Porcu ${ }^{1}$, Silvia Sideri ${ }^{1}$

${ }^{1}$ University of L'Aquila, Italy

Background: Incorrect feeding results in overweight or obesity that are associated with liver steatosis. This pathology can progress toward cirrhosis and hepatocarcinoma (HCC). In such processes, transition metals play a key role. Accordingly, altered copper homeostasis has been observed in NAFLD.

We evaluated the effect of Oleuropein (Ole), a phenol able to bind copper, on intracellular copper levels and its role in counteracting liver damage related to high fat diet (HFD).

Method: Real-time PCR, atomic absorption, BioPlex multiplex biometric ELISA-based immunoassays, adipored and western blots were performed for in vitro and in vivo experiments.

Result: In vitro, fatty acids induce intracellular copper modulation. Oleuropein leads to a significant reduction in both the intracellular content of $\mathrm{Cu}$ and lipid accumulation. In a HFD mouse model treated with Ole we highlighted a significant reduction in levels of $\mathrm{Cu}$ in liver tissue, hepatic steatosis and related inflammatory conditions. In particular, the levels of chemokines MCP1 and CXCL1, both correlated with the progression of liver disease, were significantly reduced. The involvement of genes (e.g. tp53, Myc etc.), involved in the control of the entry and efflux of copper in cells, is under investigation.

Conclusion: Our results demonstrate that Ole has inhibitory effects on the progression of liver disease that correlates with its ability to modulate copper.

MISC-3

Study on lipid profile among adults with $\mathrm{HBV}$ and $\mathrm{HCV}$ related chronic liver disease in Bangladesh -report from a tertiary centre

Dr.Harun Or Rashid ${ }^{1}$, Dr.Md.Khalilur Rahman ${ }^{1}$, Dr.Mohammad Mahbubur Rahman Khan', Dr.Mamun Al Mahtab², Dr. Md Abdul Alim ${ }^{1}$

${ }^{1}$ Rajshahi Medical College, Bangladesh; ${ }^{2}$ Bangabandhu Sheikh Mujib Medical University, Bangladesh

Background: Chronic Hepatitis B and Chronic Hepatitis C infections are the major causes of cirrhosis of Liver and Hepatocellular carcinoma in Bangladesh. Development of cirrhosis of liver result in derangement of metabolic functions. This study was conducted to find out the changes of lipid profile among adult with Chronic Viral Hepatitis B and C.

Method: This was a cross sectional descriptive study in Medicine Inpatient Department of Rajshahi Medical College Hospital from 
June 2015 to July 2016. Total Number of Patients were 130. Among them 114 were Chronic Hepatitis B and 16 patients were chronic Hepatitis C. Age of the Patients between 18-65 years. Male 96, female 34 .The Patients were selected by clinical examinations and Laboratory investigations. There were no comorbidities of these patients. Result: Among 130 Patients 116 were child Paugh A and 14 were Paugh. In HBV group Male were 96, Female 18 and in HCV group Male 11, Female 05. Serum Total cholesterol, HDL, LDL and Serum Triglyceride were done in all patients in six monthly interval. The results were reduction of Serum Total Cholesterol and HDL in 45 $(39.47 \%)$ and increased; Serum Triglyceride in $11(09.65 \%)$ patients in HBV group .In HCV group increased Serum Total cholesterol and Serum Triglyceride in $07(43.75 \%)$ and reduction of HDL in 03 $(18.75 \%)$ patients.

Conclusion: Developing countries like Bangladesh are likely to face an enormous burden of chronic liver diseases and prevention and early diagnosis is essential to reduce economic loss as well as health system burden. Dyslipidemia is a frequent finding in chronic liver diseases, which have found associated with severity of the diseases. Moreover, it may acts as a good predictor of chronic liver management. Till now, no needful studies are available in Bangladesh addressing this crucial point. Nevertheless, understanding the pattern of Dyslipidemia of hepatitis B \&amp; C related chronic liver disease in Bangladesh may enrich the clinician and may act as a baseline study for further research.

\section{MISC-4}

Biochemical parameters abnormalities in EBV-associated infectious mononucleosis

\section{Narina Sargsyants ${ }^{1}$ \\ ${ }^{1}$ Armenicum Clinical Center, Armenia}

Background: Acute primary infection with Epstein-Barr virus (EBV) or human herpesvirus 4 can manifested as infectious mononucleosis (IM). The most patients with EBV-associated mononucleosis have abnormal liver function tests, however mechanism of liver damage has not been well defined. The severity of the liver affection probable linked not only to an elevated EBV viremia, but also to a preponderant infection of CD8+ cells infiltrating the liver.

Method: We study biochemical parameters abnormality, common blood count (CBC), epidemiological, clinical peculiarities of IM in 20 patients (75\% male) from 19 to 39 years old (average $24.3 \pm 4.9$ ).

Result: The onset of disease was with febrile temperature in $80 \%$ of patients. In majority of patients diagnosis was done after 7 days from the onset of disease (average $11.0 \pm 4.6$ ), in $90 \%$ only after infectious disease specialist consultation. No cases among family members (household contact). Maculopapular rash we noticed in two cases and were associated with amoxicillin intake. Periorbital edema nasal breath difficulties mentioned in $80 \%$ of patients, hypernasal voice - in $40 \%$. Neck edema around enlarged lymphatic nodes took place only in one patient. Catarrhal nasopharingytis we observed in $40 \%$ of cases, while follicular and lacunar tonsillitis in $60 \%$ of patients with acute IM. Best effect on fever and tonsillitis resolution among antibacterial drugs was associated with azithromycin. Generalized lymphadenopathy reveled in all patients with maximal enlargement of angular-mandibular lymphatic nodes. Splenomegaly enrolled in all patients, hepatomegaly in vast majority.

Lymphocytosis (absolutely and relative) and elevation of ALT and AST we observed in all patients. Laboratory parameters $(\mathrm{m} \pm \mathrm{SD})$ were following: Lymphocytes 5.03 \pm 1.5 ; Lymphocytes-\% 55.4 \pm 9.3 ; Monocytes-\%14.3 3 .1; Leucocytes 9.8 \pm 2.8 ; ESR 14.4 \pm 7.8 ; ALT $229.6 \pm 135.0$; AST $122.1 \pm 64.5$; GGT $177.4 \pm 44.1(\mathrm{~m} \pm \mathrm{SE})$; ALP
$237.4 \pm 162.8$. Jaundice with bilirubin elevation were mentioned only in three patients. Interestingly in majority of patients, we observed abnormality in prothrombin parameters: prolonged prothrombin time, decreasing prothrombin index and increasing of INR. Albumin level was normal in all patients. Biochemical parameters normalized in average in 2-3 weeks without cases of severe hepatitis. Mild thrombocytopenia and late neutropenia (from 2-3 week of disease) noticed in $25 \%$ of patients. The heterophile antibodies agglutination test with horse RBCs was positive in 8 patients, in 12 patients diagnosis was conformed by positive result of VCA IgM, EA-D IgM and VCA IgG detection.

Conclusion: EBV-associated liver damage characterized by the transient, self-limited duration with elevation of liver enzymes accomplish with characteristic clinical signs and diagnostically useful parameters (lymphomonocytosis, atypical lymphocytes etc.) serological tests for antibodies specific for EBV antigens (viral capsid antigen (VCA) IgG, VCA IgM and EBV nuclear antigen (EBNA)-1 $\mathrm{IgG})$ are used to define infection status and for the differential diagnosis of acute IM.

\section{MISC-5}

Cancer-positive margins as a risk factor affecting fatal complications and hepatic insufficiency after extensive hepatobiliary resection for hepatobiliary disease

Norihisa Kimura ${ }^{1}$, Keinosuke Ishido ${ }^{1}$, Daisuke Kudo ${ }^{1}$, $\overline{\text { Shingo Sakuraba }}^{1}$, Takafumi Yachi ${ }^{1}$, Yusuke Wakasa ${ }^{1}$, Kenichi Hakamada ${ }^{1}$

${ }^{1}$ Hirosaki University Graduate School of Medicine, Japan

Background: Major hepatectomy with biliary reconstruction is more complex and difficult to perform and more risky than simple hepatectomy, therefore, this type of hepatectomy can lead to postoperative complications or hepatic insufficiency. The aim of this study was to evaluate the short-term outcomes after this complex surgery for hepatobiliary disease.

Method: Between January 2001 and December 2015, 112 patients underwent major hepatectomy with biliary reconstruction. Patient records were reviewed and factors for postoperative complications and hepatic insufficiency were assessed retrospectively. Fatal complications were defined as grades 4 and 5 according to the ClavienDindo classification system. Hepatic insufficiency was defined as total bilirubin of $>7.0 \mathrm{mg} / \mathrm{dL}$ postoperatively or within $50-50$ criteria (total bilirubin of $>3.0 \mathrm{mg} / \mathrm{dL}$ and prothrombin time

Result: Fatal morbidity and mortality were $7.1 \%(\mathrm{n}=8)$ and $2.7 \%$ $(n=3)$, respectively. Hepatic insufficiency occurred in 23 patients $(20.5 \%)$. Univariate analysis disclosed that cancer-positive margin was the only predicting factor for fetal morbidity $(\mathrm{p}=0.007)$. On the other hand, male $(p=0.036)$, cancer-positive margin $(p=0.001)$, right side hepatectomy $(\mathrm{p}=0.028)$, preoperative jaundice $(\mathrm{p}=0.040)$, operative blood loss $>1500 \mathrm{~mL}$, and perioperative blood transfusion were predicting factors for hepatic insufficiency in univariate analysis and cancer-positive margin (odds ratio $(\mathrm{OR})=4.55, \mathrm{p}=0.001$ ) was the only independent risk factor in multivariate analysis.

Conclusion: Extensive hepatobiliary resection for hepatobiliary malignancies is known to be the most effective treatment for acquisition of negative margins and improved long-term survival. The current study indicated that cancer-positive surgical margins influenced not only poor prognosis but also fetal complications and hepatic insufficiency after extensive hepatobiliary resection. Incomplete surgical resection in addition to excessive surgical stress such as extensive hepatobiliary resection might cause hypercytokinemia and result in postoperative fatal condition. 


\section{MISC-6}

\section{Diagnostic imaging of bile duct adenoma}

Ryoji Tatsumi $^{1}$, Masakatsu Yamaguchi ${ }^{1}$, Mutsuumi Kimura ${ }^{1}$, Tomohiro Arakawa ${ }^{1}$, Tomoaki Nakajima ${ }^{1}$, Yasuaki Kuwata ${ }^{1}$, Itaru Ozeki $^{1}$, Takumi Ohmura ${ }^{1}$, Shuhei Hige ${ }^{1}$, Yasuyoshi Karino ${ }^{1}$

${ }^{1}$ Sapporo Kousei General Hospital, Japan

Background: Bile duct adenoma (BDA) is a rare benign lesion that arises from the epithelium of the intrahepatic bile duct and is often incidentally detected during surgery or by biopsy. The objective of this study was to determine the imaging characteristics of BDA by retrospectively reviewing the images of patients histologically diagnosed with BDA.

Method: Images of 8 patients (9 lesions) histologically diagnosed with BDA after surgical resection were retrospectively reviewed (ultrasound [US] in 3, contrast-enhanced ultrasound [CEUS] in 2, computed tomography $[\mathrm{CT}]$ in 5, EOB-magnetic resonance imaging [MRI] in 6, and CT angiography [AG-CT] in 9 patients). The median age was 56 years, and all were men. The background liver condition was normal liver in 1 , chronic hepatitis in 3 , and cirrhosis in 5 patients, and hepatitis $\mathrm{B}$ and $\mathrm{C}$ infections were present in 2 and 5 patients, respectively. All patients had concurrent malignant tumor and underwent surgery: hepatocellular carcinoma in 6, intrahepatic cholangiocarcinoma in 4 , and cholangiolocellular carcinoma in 1 patient.

Result: The median diameter of BDA was $6 \mathrm{~mm}$. Of the 9 lesions, 8 were located on the surface of the liver, and the shapes of the lesions were semicircular in 5 and circular in 4 . US was performed in 3 lesions and showed ill-defined hypo- to isoechoic nodules. CEUS was performed in 2 lesions and showed enhancement, and defect image was observed in the post vascular phase. CT was performed in 6 lesions and 2 lesions were identified, in which there was enhancement with a prolonged retention. EOB-MRI was performed in 6 lesions and 3 lesions were identified: the lesions showed low signal intensity on T1-weighted imaging and high signal intensity on T2-weighted imaging and diffusion weighted imaging, with enhancement, and low signal intensity in the hepatobiliary phase. AG-CT was performed in all lesions and all lesions were identified. On CT during arterial portography, defect image was observed in all lesions. On CT during hepatic arteriography, enhancement in the first phase and prolonged enhancement in the second phase were observed in 7 lesions. In addition, 2 lesions showed ring-like enhancement in the first phase. Conclusion: The imaging characteristics of BDA are a small semicircular mass located in the liver margin, enhancement with a prolonged retention, and an ill-defined hypo- to isoechoic lesion on US. When these characteristics are present, inclusion of BDA in the differential diagnosis can be useful in making treatment decisions.

\section{MISC-7}

\section{Appearance and clinical characteristics of contrast enhanced ultrasonography in pyogenic liver abscess}

\section{Hong Soo Kim ${ }^{1}$ \\ ${ }^{1}$ Soonchunhyang university, Korea, Republic of}

Background: Contrast enhanced ultrasound(CEUS) have improved the detection rate of liver focal mass in recent years, however there are relatively few clinical information to get from CEUS images in liver abscess. The purpose of this study was to evaluate CEUS findings of liver abscess and clinical characteristic according to CEUS image.
Method: From June 2012 until December 2014, twenty-six patients with pyogenic liver abscess were evaluated with CEUS, conventional US enhanced computed tomography (CT). A realtime, contrastspecific mode of contrast pulse sequencing and a sulphur hexafluoride-filled microbubble contrast agent were used for CEUS. CEUS image in arterial phase and conventional US image were observed and analyzed with clinical characteristic.

Result: Liver abscess images were classified into two groups following to CEUS images. Seventeen patients (65.4\%, Group A) showed rim enhancement with central non-enhancement or septa enhancement in CEUS images. Nine patients (34.6\%, Group B) showed rim non-enhancement with central enhancement or partial non- enhancement in CEUS images. Body temperature $(\mathrm{P}<0.01)$, $\mathrm{C}$-reactive $\operatorname{protein}(\mathrm{p}=0.003)$ and white blood cell $(\mathrm{p}=0.012)$ is higher with Group A. Drainage with antibiotics was used in 15(88.2\%) of 17 patients in group A and 4(44.4\%) of 9 patients in group B. Conventional US images in Group A showed that 6(35.3\%) of 17 patients have irregular margin and iso or hypoechogenecity and $11(64.7 \%)$ of 17 patients have irregular margin and cystic echogenecity. Conventional US images in Group B showed that 8(88.9\%) of 9 patients have regular margin and various echogenecity.

Conclusion: Rim enhancement with central non-enhancement or septa enhancement in CEUS is associated with the time with clinical symptom. CEUS images in liver abscess are useful to decide time to perform intervention compared to conventional US.

\section{MISC-8}

\section{Diagnosis and surgical treatment of patients with Mirizzi} syndrome

\section{Zafar Fayzullo o'g'li Abdullaev ${ }^{1}$}

\section{${ }^{1}$ Tashkent Medical Academy, Uzbekistan}

Background: Among patients, who the cholecystectomy is performed, the Mirizzi syndrome (MS) occurs from $0,06 \%$ to 5,7\%, and it is a difficult problem in all stages of diagnosis and surgical treatment. To make a correct diagnosis before surgical intervention, even in the using of all modern diagnostic methods, may be done less than in $12-32 \%$ cases.

Method: From 1994 to 2016122 patients with MS were operated. The age of patients varied from 20 to 84 years old (on average $50,9 \pm 0,5$ years old). Patients were separated with the help of classification of Csendes A. Taking into account the development of diagnosis system, the choice of tactics and mode of operative treatment all patients were divided into two groups. In control group it was included 69 patients, who were operated from 1994 to 2008. The main group contained 53 patients operated from 2009 to 2016. On a number of clinical and laboratory methods of investigation it was applied modern instrumental investigation methods such as: $\mathrm{x}$-ray examination of gastro-intestinal tract, ultrasound investigation, multi spiral computer tomography, endoscopic examination of stomach and duodenum, endoscopic retrograde pancreatocholangiography, percutaneously-transhepatic cholangiography.

Result: The diagnostic value of USI before operation was in the following: suspicion of I type of MS was noted in $24,3 \%$ of patients, in $37,6 \%$ of patients it was suspected the existent of II-IY types. On USI before operation in $5,4 \%$ of patients it was verified I type and in $18,8 \%$ of patients II-IY type of MS. The using of MRI and MSCT in verification of MS may increase the efficacy of diagnosis particularly in 2 times in the comparison with USI. But, for patients with MS 1 type this indication remains relatively low and composes only $33,3 \%$. This method of diagnosis is more effective in patients with MS 2-4 type because of exact diagnosis in $75,0 \%$ cases. The most detailed 
verification was noted on ERPCHG in MS 1 type (83,3\%). In SM 2-4 type the efficacy of ERPCHG was $81,4 \%$. Uncomplicated post-operative period was noted in $83,0 \%$ of patients of main group, while in patients of control group this indication was only $56,5 \%$. Such difference was noted both in indications of lethality $(1,9 \%$ in main group against $7,2 \%$ in control) and in specific complications $(15,1 \%$ in main group against $39,1 \%$ in control). In pre-established MS 1-2 type it is more effectively the using of laparoscopic interventions, and in cases of MS 3-4 type a priority remains for the choice of open operations (CHEC+draining of choledocha by Ker).

Conclusion: It was noted the significant increasing of topical diagnosis level of MS 1 type in main group (till 42,9\%), and for patients with MS 2-4 type this indication in main group increased till $19,2 \%$ in the comparison with control group. At the same time, stepwise use of all complex of diagnostic monitoring may increase the efficacy of correct diagnosis till $97-99 \%$ in patients with MS 2-4 type.

\section{MISC-9}

Efficacy of nalfurafine hydrochloride in patients with chronic liver disease with refractory pruritus

\section{Koichi Takaguchi ${ }^{1}$, Akemi Tsutsui ${ }^{1}$, Tomonori Senoo ${ }^{1}$, Takuya Nagano $^{1}$, Takacshi Kumada ${ }^{4}$, Tomonori Shimada ${ }^{5}$, Hiraoka Atsushi ${ }^{2}$, Masanori Atsukawa ${ }^{3}$}

${ }^{1}$ Kagawa Prefectural Central Hospital, Japan; ${ }^{2}$ Ehime Prefectural Central Hospital, Japan; ${ }^{3}$ Nippon Medical School Chiba Hokusoh Hospital, Japan; ${ }^{4}$ Ogaki Munisupal Hospital, Japan; ${ }^{5}$ Ootakanomori Hospital, Japan

Background: About more than $50 \%$ patients with chronic liver disease have pruritus or experiencing pruritus, About $5-10 \%$ of them are said to be refractory pruritus and it causes insomnia and markedly decreased QOL. In this study we investigated the pruritus frequency of patients with chronic liver disease and effect of Nalfurafine newly approved pruritus improvement medicine which is a selective $\kappa$ opioid receptor agonist.

Method: 111 patients were admitted to our hospital or other cooperated hospitals after approval of Nalfurafine, their therapeutic effects were judged by the VAS value of pruritus and the severity of Kawashima pruritus score, and the patient background was also examined. There were 2 cases chronic hepatitis B, 11 cases chronic hepatitis C, 3 cases cirrhosis of hepatitis B, 48 cases cirrhosis of hepatitis C, 3 cases of AIH, 9 cases of PBC, 9 cases of alcoholic liver cirrhosis, 6 cases of NASH, 16 cases of HCC and 16 cases unknown. Result: 70 cases of 111 cases were effective and remaining 41 cases were not effective or unknown effect of Nalfurafine.In all patients, the VAS value decreased from $72.96 \mathrm{~mm}$ to $38.95 \mathrm{~mm}$, among the effective examples it improved significantly difference from 71.40 $\mathrm{mm}$ to $35.89 \mathrm{~mm}$, among ineffective or unknown patients it was not improved from $76.14 \mathrm{~mm}$ to $66.25 \mathrm{~mm}$. The pruritus score of Kawashima also improved significantly from 2.92 to 1.55 in the effective patients, but it did not significantly change in patients with not effective or unknown effect from 2.70 to 2.48. Comparing the patients background between effective patients and ineffective patients, there was no difference in gender and age, but T. Bil was high in the ineffective cases $(1.28 \pm 1.23$ and $4.60 \pm 7.02)$, albumin was low tendency $(3.63 \pm 0.72,3.30 \pm 0.92)$ in the ineffective cases. There were 46 cases of continuation of administration and 54 cases of discontinuation of administration, and effective subjects improved pruritus with significant difference. Among 60 patients with discontinuation, 22 cases were stop for improvement of symptoms, 12 cases with insufficiency, 8 cases for side effects, and 16 cases unknown.
Insomnia, wobble, fatigue, kidney failure, etc. were observed in the case of withdrawal of side effects.

Conclusion: Nalfurafine was effective at $64 \%$ after 4 weeks of administration and early treatment effect was recognized, and about $40 \%$ was discontinued of administration. There was no difference in effect between gender, age, and disease causes, but there were many ineffective cases in patients with progressive liver disease.

MISC-

\section{Hyperferritinemia in liver disease- role of genetic testing}

\section{Irshad Ali $^{1}$, Gayathri Gopalakrishnan ${ }^{2}$, Sharat Damodar ${ }^{2}$}

${ }^{1}$ Narayana Health, India; ${ }^{2}$ Senior consultant and Head, Mazumdar Shaw Medical Center, Narayana Health, India

Background: Iron can accumulate in the liver in a variety of conditions, including congenital, systemic iron-loading conditions (hereditary hemochromatosis), conditions associated with systemic macrophage iron accumulation (transfusions, hemolytic conditions, anemia of chronic disease, etc), in some hepatitidies (hepatitis C, alcoholic liver disease, porphyria cutanea tarda), and liver-specific iron accumulation of uncertain pathogenesis in cirrhosis. The pathologist will be faced with the task of determining whether iron accumulation in the liver is significant and, if so, the nature of the disease that lead to the accumulation (ie diagnosis). The tools available to the clinician include (most importantly) clinical history, histologic examination with iron stain, quantitative iron analysis, laboratory iron tests (serum iron and iron-binding capacity, serum ferritin), imaging and germline genetic analysis for mutations in genes known to be associated with primary hemochromatosis (HFE, ferroportin, hepcidin, hemojuvelin, transferrin receptor-2) and secondary hemochromatosis (haematological conditions with iron overload)

Here are two case descriptions with iron overload syndromes where etiological diagnosis were possible only because of availability of genetic testing.

Method: case reports

Result:

Case 1: 52 year old male, Vegetarian, newly detected Diabetic since 3 months with Chronic anemia since december 2015 was diagnosed myelodysplastic syndrome based on Bone marrow study. He has received 4 packed cell transfusions since 2 years. CT abdomen done elsewhere for evaluation of altered liver function was suggestive of chronic liver disease. Hence was referred to our hospital for further workup. On clinical examination, he was pale and had no stigmata of chronic liver disease. Liver was palpable, firm and nodular. Spleen was palpable $8 \mathrm{~cm}$ below costal margin. On investigation he had anemia, decreased MCV and mild transaminitis. Iron profile was suggestive of iron overload ( $\mathrm{Sr}$ Ferritin: $3723.7 \mathrm{ng} / \mathrm{ml}$, Serum Transferrin: $122 \mathrm{mg} / \mathrm{dl}$, Transferrin saturation: 97.44\%). MRI ferri scan showed severe iron loading in the liver (Iron concentration of liver: $34.5 \mathrm{mg} / \mathrm{gm}$ of dry weight and T2 value: $0.9 \mathrm{~ms}$ ). Bone marrow showed Hypercellular marrow with erythroid hyperplasia and subtle dyshematopoiesis consistent with MDS. USG guided liver biopsy was performed which showed Grade 4 iron mainly in hepatocytes and also in kupffer cells, stromal cells and biliary epithelium. There was mild to moderate fibrosis, but no cirrhosis. On genetic analysis he was found to harbor X linked Hemizygous missense variation in exon 9 of ALAS2 gene signifying $X$ linked sideroblastic anemia (a variant of MDS). This syndrome comprises of hypochromic microcytic anemia, marrow ringed sideroblast, variable response to pyridoxine and systemic iron overload secondary to ineffective erythropoiesis. At present he is on oral iron chelator (Tab Deferiprone $2 \mathrm{gms}$ in divided 
doses). Therapeutic phlebotomy was not an option in him as he had anemia.

Case 2: $48 \mathrm{yr}$ old male, married with 2 normal children underwent splenectomy at $16 \mathrm{yrs}$ of age for unknown indication. He was well until 4 yrs ago and then he was diagnosed as $\mathrm{HbE} / \mathrm{B}$ Thalassemia. He requires blood transfusion every 3 months since $4 y r s \quad(\sim 32$ units transfused). He was referred for Altered LFT. He was recently diagnosed as Diabetic on ADM and insulin. Also being evaluated for Erectile dysfunction and loss of libido in Endocrine unit. On clinical examination he had pallor, icterus, gynacomastia and slate grey colour skin. Liver was palpable, $6 \mathrm{~cm}$ below right costal margin; firm and nodular .His clinical ADAM score was suggestive of androgen insufficiency. Serial Ferritin measurements were consistently elevated (2872(2015)-> 3017 -> $2020->3740$ (2017). On investigation he had indirect hyperbilirubinemia. Iron profile was suggestive of iron overload (Sr Ferritin: 2799.8 ng/ml, TSats- 95\%). Hormonal analysis showed hypopituitarism and hypogonadism. MRI ferri scan showed severe iron loading in the liver (Iron concentration of liver: $23.8 \mathrm{mg}$ / gm of dry weight and T2 value: $1.3 \mathrm{~ms}$ ). On genetic analysis he was found to harbor Heterogeneous missense variation in Exon 1 of HBB gene on chromosome 11 signifying Delta/ beta thalassemia. This syndrome comprises of decreased or absent Delta/ beta globin chain, compensatory increase in fetal $\mathrm{Hb} \%$, presents as microcytic and hypochromic anemia and secondary iron overload

Conclusion: Iron overload disorders are common. Inexpensive screening tests as well as confirmatory diagnostic tests are widely available. Increased awareness of the causes and importance of early diagnosis and knowledge of the appropriate use of genetic testing are encouraged. The availability of novel treatments should increase therapeutic options for patients with iron overload disorders.

\section{MISC-11}

Peculiarities of the infection of the extrahepatic bile ducts in mechanical jaundice on the background of viral hepatitis

\section{Saidrakhmon Nodirovich Khoshimov ${ }^{1}$}

${ }^{1}$ Tashkent State Pediatric Institute, Uzbekistan

Background: The aim of our work was to study the effect of viral hepatitis $(\mathrm{VH})$ on the course of benign mechanical jaundice (MJ) and the peculiarities of biliary decompression in this group of patients. The aim of our work was to study the effect of viral hepatitis (VH) on the course of benign mechanical jaundice (MJ) and the peculiarities of biliary decompression in this group of patients.

Method: The results of diagnostics and treatment of 112 patients with MJ of benign genesis at the age of 16 to 81 years were analyzed. They were in the clinic of RKB No. 1 of the Tashkent Medical Academy faculty surgery. Of these, 61 patients with $\mathrm{MJ}$ of benign origin, developed on the background of $\mathrm{VH}$, which formed the main observation group. The control group consisted of 51 patients with $\mathrm{MJ}$ of benign origin without concomitant $\mathrm{VH}$. The presented groups were homogeneous and comparable in age and sex composition.

Result: With MJ, and especially against the background of $\mathrm{VH}$, there was an increased bleeding of the tissues, more pronounced in the zone of cicatricial fusion in the gallbladder, and in some cases refractory to the usual methods of hemostasis. Nevertheless, in both groups in the postoperative period, 1 patient had postoperative bleeding, which required relaparotomy. A number of patients $(10 \%)$ in the main group was performed $\mathrm{CE}$ by Pribram. Also, the nature of the lesion of extrahepatic bile ducts in patients on the background of $\mathrm{CVH}$ differed in the manifestations of fibrinous, purulent cholangitis (statistically more significant with $\mathrm{VH}$ ). The walls of the common bile duct are thick, tense, sclerosed, compacted, which made it even more difficult to manipulate the biliary tract. Fibrosis of the biliary tract was observed in $25 \%$ of cases. The phenomena of cholangitis with $\mathrm{VH}$ were more pronounced, despite the presence of a small "flotating" concrement in choledochus. On the contrary, with $\mathrm{MJ}$ without $\mathrm{VH}$, the common bile duct was clogged with concrements with minimal hyperemia and thickening of the wall. It is likely that with the already existing inflammatory changes in the biliary tract in patients with $\mathrm{CVH}$, by the time of biliary obstruction, the risk of purulent cholangitis increases significantly. The period after biliary decompression, depending on the activity of $\mathrm{CVH}$, was characterized by a significant disturbance of the hepatic-renal function, the development of multi-organ failure.

Conclusion: Thus, the presence of VH in patients with benign breast cancer significantly changes the intraoperative picture of the disease, increases the likelihood of postoperative complications and creates difficulties at the stage of diagnostic search and operational support.

MISC-12

\section{Abnormal transaminase level in brucellosis}

Firdevs Aksoy, ${ }^{1}$, Nurten Nur Aydn ${ }^{1}$, Gurdal Yilmaz ${ }^{1}$, Selcuk Kaya $^{1}$, Suleyman Caner Karahan ${ }^{1}$, Iftihar Koksal ${ }^{1}$

${ }^{1}$ Karadeniz Technical University, Turkey

Background: Brucellosis in humans is a systemic infection in which bacteria localize within mononuclear phagocytes of the reticuloendothelial system. Brucellosis involves the liver in varying ways; transaminase levels are only mildly elevated or can be normal. In our study, We aimed to evaluate whether serum transaminase levels are related to system involvement in patients with brucellosis.

Method: Our study involves retrospective case series of 182 patients admitted to a hospital from 2000 to 2017. The diagnosis of brucellosis was established by isolating Brucella from blood or detecting specific antibodies in serum standard tube agglutination (STA). Admission blood tests included complete blood count, C-reactive protein (CRP), transaminase [aspartate aminotransferase (AST), alanine aminotransferase (ALT)].

Result: We assessed the transaminase level in 182 patients suffering from brucellosis admitted to our department over the last ten years. ALT and AST levels were high in 71(Group A) and normal in 111 (Group B) of 182 patients at the time of admission. The mean age of the patients was $40.9 \pm 14.8$ in Group A and $48.2 \pm 18.0$ in Group B. Transaminase levels were significantly higher in younger patients $(\mathrm{p}=$ 0.005). Grup A'da CRP düzeyleri daha yüksek ( $\mathrm{p}=0.001$ ), platelet düzeyleri $(\mathrm{p}=0.001)$ ve beyaz küre düzeyleri $(\mathrm{p}=0.011)$ daha düsüktü. In Group A, CRP levels were higher $(\mathrm{p}=0.001)$, platelets $(\mathrm{p}=0.001)$ and white blood cell $(\mathrm{WBC})$ levels $(\mathrm{p}=0.011)$ were lower. The incidence of bacteremia was higher in Group A $(p=0.001)$. STA levels were lower in Group A $(p=0.010)$, spondylodiscitis and relapse development were independent of transaminase levels. The demographic and laboratory characteristics of the patients are shown in table 1 .

Conclusion: The results of our study show that when transaminase levels are found to be high, brucellosis can progress with bacteraemia in patients whose epidemiologic history and clinical findings may be consistent with brucellosis. 
MISC-13

Hepatitis A, B, C and D seroprevalence among Turkish HIV/Aids patients

Esra Zerdali ${ }^{1}$, Merve Vanli ${ }^{1}$, Mediha Bozkurt ${ }^{1}$, Inci Yilmaz Nakir $^{1}$, Vahibe Aydin ${ }^{1}$, Melike Nur Kultur ${ }^{1}$, Merve Kilic ${ }^{1}$, Aysegul Inci Sezen ${ }^{1}$, Berna Gurbuz Demirok ${ }^{1}$, Mustafa Yildirim ${ }^{1}$

${ }^{1}$ Saglik bilimleri üniversitesi istanbul haseki egitim ve arastirma hastanesi, Turkey

Background: Liver injury due to hepatitis A (HAV), hepatitis B (HBV) and hepatitis $\mathrm{C}$ virus (HCV) co-infections in adult patients with HIV/AIDS causes significant morbidity and mortality. We aimed to determine the frequency of $\mathrm{HAV}, \mathrm{HBV}$ and $\mathrm{HCV}$ infections among our HIV/AIDS patients.

Method: We retrospectively evaluated results of anti-HAV $\mathrm{IgG}$, $\mathrm{HBsAg}$, anti-HBc IgG, anti $\mathrm{HBs,} \mathrm{anti-HCV}$, HBV-DNA, and HCVRNA at first admission of adult patients with HIV/AIDS who were followed up in our outpatient clinic for at least one year between 2006 and 2016.

Result: Of 706 HIV/AIDS patients, $82(11.61 \%)$ were female and 624 $(88.38 \%)$ were male, mean age was 41 , range $18-87$ years. HAV (Hepatitis A) seropositivity was found in $500(79.7 \%)$ of 627 cases and Anti-HCV was found in 8 of 684 cases. HCV-RNA was also detected in 5 of the anti-HCV positive cases. The serological results in 431 cases that HBV serology was evaluated are presented in the table. HDV infection was detected in 1 of $35 \mathrm{HIV} / \mathrm{HBV}$ coinfection cases. HBV-DNA was not detected in 25 of 77 cases with isolated anti-HBc IgG positivity. One of 146 patients with negative HBV serologic tests had an acute HBV infection before starting an antiretroviral therapy and developed anti-HBs. 123 of 146 patients were vaccinated, but $57(46.3 \%)$ of them did not develop detectable anti-HBs

Conclusion: The rate of HIV/HBV coinfected patients in our study was higher than its rate $(4 \%)$ in the general population of Turkey, which is a moderate endemic region for HBV infection. The low HCV coinfection rates in our study population is similar to the rates in the general population in our country and may be due to low intravenous drug use. In adults, particularly in HIV/AIDS patients, HAV, HBV and HDV infections may lead to fulminant disease and protection is possible via vaccination. $\mathrm{HCV}$ infection is a curable disease and selection of appropriate antiretroviral therapy for HIV-infected patients is important. Thus, serologic evaluation is important in such patients.

\begin{tabular}{|l|l|}
\hline HBVserology & N $(\%)$ \\
\hline HBsAg + , Anti-HBclgG + , Anti-HBs - & $35(\% 8.1)$ \\
\hline HBsAg, Anti-HBclgG + , Anti-HBs + & $77(\% 17.9)$ \\
\hline HBsAg,- Anti-HBclgG,+ Anti-HBs - & $84(\% 19.4)$ \\
\hline HBsAg - , Anti-HBclgG - , Anti-HBs + & $89(\% 20.6)$ \\
\hline HBsAg - , Anti-HBclgG,- Anti-HBs - & $146(\% 33.9)$ \\
\hline
\end{tabular}

MISC-14

Impact of hemodilutional autologous blood transfusion on outcomes after extensive hepatobiliary resection for perihilar cholangiocarcinoma

Norihisa Kimura ${ }^{1}$, Keinosuke Ishido ${ }^{1}$, Daisuke Kudo ${ }^{1}$, Shingo Sakuraba ${ }^{1}$, Takafumi Yachi', Yusuke Wakasa', Kenichi Hakamada

${ }^{1}$ Hirosaki University Graduate School of Medicine, Japan

Background: Extensive hepatobiliary resection for perihilar cholangiocarcinoma (PHCCA) can lead to excessive blood loss that requires perioperative blood transfusion (BT). We often perform hemodilutional autologous blood transfusion (HAT) in patients undergoing such an aggressive surgery. The aim of this study was to assess the impact of HAT on short- and long-term outcomes for PHCCA.

Method: One hundred patients undergoing major hepatectomies with en bloc resection of the caudate lobe and extrahepatic bile duct for PHCCA between 1999 and 2016 were enrolled. In principle, HAT was performed in 57 patients $(57 \%)$ with preoperative hemoglobin $(\mathrm{Hb})>11 \mathrm{~g} / \mathrm{dL}$ and no hypotension or severe cardiac disease. The relationship between HAT and postoperative outcomes was retrospectively assessed.

Result: Disease-specific survival (DSS) rates at 1, 3, and 5 years were $87.5 \%, 43.7 \%$, and $34.5 \%$, respectively, with a median DSS time of 29.8 months. Patients with HAT were younger (P

Conclusion: The current study indicated that HAT can reduce intraoperative blood loss and avoid allogeneic BT. Moreover, HAT could not only increase postoperative complications but also improve survival for PHCCA.

\section{MISC-15}

Prevalence of tuberculosis in patients with liver cirrhosis: a nepalese study

\section{Dilip Sharma ${ }^{1}$}

${ }^{1}$ National Academy of Medical Sciences, Nepal

Background: Studies have shown that patients with liver cirrhosis are more susceptible to tuberculosis (TB) because of immune dysfunction; however there are only limited data. There is no study on prevalence of TB in patients with liver cirrhosis from Nepal. We carried out this study to know the prevalence and clinical characteristics of TB in patients with liver cirrhosis.

Method: In this retrospective study, medical files of 200 consecutive patients admitted with diagnosis of liver cirrhosis in the medical ward of liver unit, Bir Hospital from January 2011 to December 2014 were evaluated for the presence of TB and clinical characteristics of these patients were analyzed.

Result: Eighteen patients were diagnosed to have TB with a prevalence of $9 \%$. The median age of patients with tuberculosis and liver cirrhosis was 53 (35-71) years and $\mathrm{M}$ : $\mathrm{F}$ ratio was $2: 1.67 \%, \mathrm{P}=0.01)$. Median CTP score of patients were 8.5 (5-12) (CTP A: B: C: 3:7:8). Extra pulmonary $\mathrm{TB}$ was more common $(67 \%)$ than pulmonary (33\%). In extra pulmonary TB distribution was as pleural effusion, $\mathrm{n}=4$, abdominal, $\mathrm{n}=4$, intestinal, $\mathrm{n}=2$, peritoneal, $\mathrm{n}=9$, Pott's spine, $\mathrm{n}=2$.

Conclusion: Our study showed that prevalence of tuberculosis in cirrhotic patients is $9 \%$, which is higher than general population. Extra pulmonary TB was more compared to pulmonary TB. Tuberculosis was common in decompensated LC than compensated LC.

\section{MISC-16}

\section{Profile of obstructive jaundice in patients from northern Rajasthan}

\section{Zubin Sharma}

${ }^{1}$ Aditya Nursing Home \& Gastro Centre, India

Background: Biliary Obstruction (EHBO) is one of the most common issue we encounter in patients presenting with jaundice. There have been several studies with different etiologies in different parts our country. Its treatment depends on correction of the underlying 
cause of the disease. The aim of the present study is to identify cause, site of biliary obstruction, difference in history of the disease causing obstructive biliopathy as seen in our centre in patients from Northern Rajasthan.

Method: Patients with obstructive jaundice over a period of 4 years from March 2014- March 2017. Diagnosis was made on the basis of clinical, biochemical and ultrasound, CT scan, MRCP, Side view endoscopy and in few cases Endoscopic ultrasound and USG guided FNA were used for confirmation of etiology. ERCP findings were evaluated and Biopsy details were noted.

Result: Patients with obstructive jaundice over a period of 4 years from March 2014- March 2017. Diagnosis was made on the basis of clinical, biochemical and ultrasound, CT scan, MRCP, Side view endoscopy and in few cases Endoscopic ultrasound and USG guided FNA were used for confirmation of etiology. ERCP findings were evaluated and Biopsy details were noted.

Conclusion: Malignancy was the most common cause of obstructive biliopathy. Carcinoma of Gall bladder was the most commonest followed by Carcinoma of head of pancreas and choledocolithasis were seen in Northern Rajasthan

\section{MISC-17}

Minimally invasive biliary decompression methods in management of obstructive jaundice of malignant etiology

\section{Saidrakhmon Nodirovich Khoshimov ${ }^{1}$}

${ }^{1}$ Tashkent State Pediatric Institute, Uzbekistan

Background: The aim of our study was to explore the possibility of minimally invasive decompression methods of the biliary tract on obstructive jaundice of malignant etiology.

Method: During the period from 2014 to 2016 in the surgical department of the Republican Clinical Hospital ? $1 \mathrm{MH}$ of Uzbekistan were treated 38 patients with obstructive jaundice of malignant etiology. There were male -16 , female - 22. Ages were from 40 to 85 years. The localizations of tumors were following cancer of pancreas head-24, major duodenal papilla carcinoma-5, hepaticocholedochus cancer-5, gall bladder cancer-3, and liver cancer (metastatic) -1 . In addition to the standard diagnostic algorithm of clinical and laboratory studies were included: a routine ultrasound examination, fibrogastroduodenoscopy with papillary checkup, by indications computed tomography, endoscopic retrograde cholangiopancreatography (ERCP), percutaneous transhepatic cholangiography, angiography, laparoscopy, percutaneous puncture- aspiration biopsy under ultrasound guidance.

Result: Step cares were performed to the patients. The first step was the decompression of the biliary tract in the next 6-24 hours after diagnosis and preoperative preparation. To do this, we used the following methods: percutaneous transhepatic biliary drainage (PTBD) under ultrasound guidance - 12 cases, laparoscopic cholecystostomy - 1 case, PTBD under X-ray guidance with previous percutaneous transhepatic cholangiography-16 cases, ERCP with endoscopic papillosphincterotomy (EPST) - 4 cases, transpapillary common bile duct drainage (Endoprosthesis) - 1 case. In 2 cases were performed PTBD and endoscopic retrograde decompression.

Biliary decompression methods primarily depend on the location of the tumor. In the presence of distal obstruction hepaticocholedochus (pancreatic head tumor, the terminal part of the common bile duct, Major duodenal papilla) there were performed PTBD with bougienage of tumors or holangiostomy. In the presence of major duodenal papilla tumor unsprouted to the pancreatic head it is attempting ERCP and EPST with transpapillary drainage of the common bile duct. If unsuccessful outcome of retrograde drainage we performed PTBD.
Choosing between cholecystostomy and PTBD in low block depended on the presence of tumor obstruction of cystic duct, the conditions for implementing the intervention, the degree of biliary hypertension, anatomical structure of the ductal and vascular systems. For tumors of the proximal hepaticocholedochus (cancer of common hepatic duct, gallbladder, metastatic cancer of the porta of hepar) it were carried out PTBD with the stage probing of tumor and stent placement. Percutaneous transhepatic cholangiography previously performed to clarify the extent and degree of localization of the block. PTBD with bougienage of tumor was the final step in the treatment of incurable patients.

Conclusion: Thus, staged treatment of biliary decompression, in the earliest time after diagnosis, in patients with obstructive jaundice is depending on the location of tumor growth. Preferences were given to PTBD as universal method that provides the most adequate decompression of the biliery tract.

\section{MISC-20}

Comparative assessment of quality of life using SF36 (generic specific) and CLD Q HRQOL (disease specific) questionnaire in patients with liver cirrhosis

\section{Mayank Jain $^{1}$, Janani $\mathbf{K}^{1}$, Joy Varghese ${ }^{1}$, Vijaya Srinivasan ${ }^{1}$, Tom Michael ${ }^{1}$ \\ ${ }^{1}$ Gleneagles Global Health City, India}

Background: Health related quality of life (HRQOL) is influenced by the disease state, associated complications and their management. In patients with liver cirrhosis co-morbidity, severity of liver disease and their complications are likely to affect the QOL. Aim of the study was to determine the factors that are likely to influence the domains of HRQOL using SF 36 and CLDQ questionnaires in patients with liver cirrhosis

Method: Methods: Patients with liver cirrhosis were compared with age-gender matched healthy controls for physical and mental components of SF-36 score. Effects of age, co-morbidity namely diabetes, severity of liver disease and complications of liver cirrhosis on HRQOL using self administered or by direct interview SF-36 and CLDQ questionnaire was studied. Patients too ill to complete the questionnaire were excluded. SPSS version 20 was used for Chi Square test, ANOVA, Kruskal Wallis test and stepwise linear regression. A $p$ value of $<0.05$ was considered significant

Result: By SF-36 score, except for body pain, 149 patients had a significantly low individual and composite domain score ( $\mathrm{p}$ value $<0.0001)$ compared to age gender matched controls. Patients below 45 years, majority who belonged to Child Turcotte Pugh (CTP) C with a high MELD and higher rates of complication had low SF - 36 for body pain $(\mathrm{KW} \mathrm{p}<0.005)$ and those above 55 years for physical function $(\mathrm{p}<0.05)$. Both the physical components had a major impact on mental composite score (MCS) $(\mathrm{KW} \mathrm{p}<0.05)$. The overall CLDQ score was also low in patients below 45 years $(\mathrm{p}<0.05)$. Diabetes with or without other co-morbid conditions had no effect on SF-36 or CLD Q scores, while non diabetic co-morbid conditions did on physical domains (physical function, body pain and role physical) and Physical component Score of SF-36 (KW p $<0.01$ to $<0.0001$ ). Severity of liver disease, CTP C had significant effect on all domains and composite scores of both SF-36 and CLD Q (except activity) (p value $<0.05$ to $<0.00001$ ) but not the cirrhosis related complications ( $p$ NS). By linear regression, MELD had a direct and significant association with overall PCS, MCS and CLDQ.

Conclusion: Patients 45 years and below in CTP C, with high MELD and cirrhosis related complications like hepatic encephalopathy and ascites have lower mental component and CLD Q score. 
MISC-21

Effect of Celecoxib on inflammatory mediators in acute hemorrhagic necrotic pancreatitis

\author{
chen xin $^{1}$, Han Mu Lu ${ }^{1}$, Qiong Zhong Wang ${ }^{1}$ \\ ${ }^{1}$ THE Affiliated Hospital of Southwest Medical University, China
}

Background: to investigate the effect of Celecoxib on the levels of inflammatory mediators in acute hemorrhagic necrotic pancreatitis.

Method: Using retrograde cholangiopancreatography injection in rats to make acute hemorrhagic necrotizing pancreatitis (AHNP) model. The experiment were randomly divided into sham operation group (S group), AHNP group and Celecoxib pretreatment group (C+AHNP group).Collecting rats blood in 3,6,12, $24 \mathrm{~h}$. Using the nitro reduction method to observe the level of serum NO at each time point of each group survival, TNF- $\alpha$ was measured by radioimmunoassay, turbidimetric method for quantitative detection of serum endotoxin content.>

Result: the serum NO level of AHNP rats were rising trend in the $3 \mathrm{~h}$ and $6 \mathrm{~h}$ levels, which was significantly lower than $\mathrm{S}$ group. The level of $12 \mathrm{~h}$ was similar to $\mathrm{S}$ group, but at the level of $24 \mathrm{~h}$ was significantly higher than that of $\mathrm{S}$ group. Celecoxib pretreatment significantly increased serum NO level AHNP on 3-12 h, and NO reached the peak level in $12 \mathrm{~h}$ then, the $24 \mathrm{~h}$ dropped significantly, slightly higher than the control group NO level. AHNP rat serum TNF- $\alpha$ and endotoxin levels were significantly increased, Celecoxib pretreatment significantly decreased the levels of TNF- $\alpha$, but has no effect on the level of endotoxin.

Conclusion: Celecoxib can inhibit the transcription and release of TNF-\&alpha; and other inflammatory mediators such as NO, but it has no effect on endotoxin level in acute pancreatitis.

\section{MISC-22}

Expression and clinical significance of interleukin-22 in liver abscess patients with diabetes mellitus

\section{Longfeng Jiang ${ }^{1}$, Yao Liu ${ }^{1}$, Yuyun Shao ${ }^{1}$, Ping Shi ${ }^{1}$, Jun $\mathrm{Li}^{1}$}

${ }^{1}$ The First Affiliated Hospital with Nanjing Medical University, China

Background: To investigate plasma interleukin-22 expression and clinical significance in liver abscess patients with diabetes mellitus. Method: Plasma was obtained from 20 cases of type 2 diabetes mellitus and 20 cases of liver abscess. Plasma from 10 healthy donors was also collected as control. Plasma IL-22 levels were measured by enzyme-linked immunosorbent assay (ELISA)method. Difference between groups was determined bu unpaired t test.

Result: The IL-22 levels of plasma in type 2 diabetes mellitus patients $[(115.2 \pm 17.04) \mathrm{pg} / \mathrm{mL}]$, liver abscess patients $[(116.2 \pm 16.43) \mathrm{pg} / \mathrm{mL}]$ were lower significantly compared with that of healthy controls $[(161.7 \pm 23.22) \mathrm{pg} / \mathrm{mL}](\mathrm{p}<0.01)$. The IL-22 levels of plasma in liver abscess patients with diabetes mellitus [(123.1 \pm 13.96$) \mathrm{pg} / \mathrm{mL}]$ were higher than that of liver abscess patients without diabetes mellitus $[(103.3 \pm 13.00) \mathrm{pg} / \mathrm{mL}]$, The difference between tow groups were statistically significant $(\mathrm{P}<0.01)$.

Conclusion: The IL-22 levels of plasma in type 2 diabetes mellitus patients, liver abscess patients and liver abscess patients with diabetes mellitus were lower significantly compared with that of healthy controls. The IL-22 levels of plasma in liver abscess patients without diabetes mellitus were lower significantly compared to liver abscess patients with diabetes mellitus.
MISC-23

Factors affecting the health related quality of life amongst outpatients with cirrhosis

Gaurav Kapur ${ }^{1}$, Aniruddha Pratap Singh ${ }^{1}$, Sandeep Narayanan, ${\text { Neeraj } V^{1} \text {, Jijo Varghese }}^{1}$, Srijaya Sreesh ${ }^{1}$, Sandesh $K^{1}$, Krishnadas Devadas ${ }^{1}$

${ }^{1}$ Government Medical College Hospital, Trivandrum, Kerala, India

Background: Cirrhosis is usually associated with poor health related quality of life (HRQOL). Treatment strategies for cirrhosis usually consider clinical end points, but do not consider HRQOL. Measuring HRQOL can reflect the actual implications of medical conditions and treatments, more important to the patient. Identifying factors affecting HRQOL may help improve patient outcomes and guide future research. We aimed to evaluate the predictors of HRQOL in this study.

Method: Cross sectional study of outpatient cirrhotics was done. Patients with overt hepatic encephalopathy and Hepatocellular carcinoma were excluded. Malayalam version of Chronic Liver disease questionnaire (CLDQ) was developed and used for measuring HRQOL. Factors affecting HRQOL such as Child Turcotte Pugh (CTP), Model for End stage Liver disease (MELD) Minimal hepatic encephalopathy(MHE), age etc. were studied. MHE was evaluated using Number connectivity tests NCT A and NCT B (adjusted for education levels). Univariate and Multivariate regression was used for analyses.

Result: Total 103 patients with a mean age of 52.2 years, which included $79.6 \%$ males were studied. Alcohol was the major etiology accounting for $49.5 \%$ cases. Others were NASH $24.3 \%$, HBV $15.5 \%$ and HCV $10.6 \%$. Among all patients, $60.2 \%$ were CHILD B, $29.1 \%$ were CHILD A and $10.75 \%$ had CHILD C status. In the studied population median MELD was 12 and CTP was 7. MHE was present in $51.5 \%$ of subjects. CLDQ global was $5.26 \pm 1.06$ for the study subjects and $29.12 \%$ of the patients had poor HRQOL with low global CLDQ $(<5)$. A maximum number of patients had lower scores on emotion component of CLDQ (45.6\%). Of the various factors, female sex, body mass index (BMI), S. albumin, MELD, CTP, presence of MHE and Viral etiology(both Hepatitis B and Hepatitis C) were found to be significant factors in univariate analysis for poor quality of life. Out of 30 patients with poor health rated quality of life, $86.67 \%$ of had MHE. MHE, MELD \& Viral etiology were found significant in multivariate analysis for predicting HRQOL.

Conclusion: MHE appears to be an important factor in determining HRQOL, along with already well established factors like etiology of cirrhosis and severity of disease. So patients with poor health related quality of life should be evaluated for MHE.

\begin{tabular}{|l|r|r|r|}
\hline & N & Mean & Std. Deviation \\
\hline CLDQ & 103 & 5.259 & 1.0563 \\
\hline Abdominal & 103 & 5.434 & 1.6161 \\
\hline Fatigue & 103 & 4.986 & 1.2773 \\
\hline Systemic & 103 & 5.542 & 1.1966 \\
\hline Emotion & 103 & 5.050 & 1.5903 \\
\hline Worry & 103 & 5.383 & 1.1335 \\
\hline Activity & 103 & 5.421 & 1.3382 \\
\hline
\end{tabular}




\section{Multivariate binary logistic regression model}

\begin{tabular}{|l|c|c|c|c|}
\hline & $p$ & OR & \multicolumn{2}{|c|}{$95 \%$ C.I.for OR } \\
\hline & & & Lower & Upper \\
\hline SEX & 0.849 & 1.157 & 0.259 & 5.173 \\
\hline MHE & 0.002 & 12.298 & 2.612 & 57.908 \\
\hline BMI & 0.325 & 1.895 & 0.531 & 6.758 \\
\hline Viraletiology & 0.001 & 10.642 & 2.600 & 43.557 \\
\hline MELD & 0.012 & 0.747 & 0.594 & 0.939 \\
\hline child & 0.360 & 2.750 & 0.315 & 24.022 \\
\hline
\end{tabular}

MISC-24

Immunotherapy in surgery of obstructive jaundice benign etiology

\section{Umarbek Usarov ${ }^{1}$}

${ }^{1}$ Emergency medicine, Uzbekistan

Background: One of the major factors contributing to the obstructive jaundice syndrome is the development of septic complications in these patients at various stages of surgical treatment. According to different authors purulent-septic complications are from 2.9 to $59.4 \%$. In recent years, an important practical meaning takes on studying the role of immunocompetence of the body for the disease and the occurrence of septic complications.

Method: New data on the nature of immunological disorders in patients with obstructive jaundice of benign origin that deepens the existing ideas about the pathogenesis of this disease. Pathogenetically expediency destination data immunocorrecting sick funds. The efficiency of application imunofan in treatment of patients with obstructive jaundice moderate and severe degrees of severity

Result: 1. Patients with obstructive jaundice develops benign genesis induced form of secondary immunodeficiency manifested decrease in the number of immune system cells, their functional impairment, imbalance in the cytokine regulation, inhibition of phagocytic activity of leukocytes. Severity of these disorders depends on the severity of the jaundice. 2. The objective criterion of the depth and effectiveness of endogenous intoxication detoxification therapy is the ECA. Mild jaundice, this indicator decreased by $25.5 \%$ with $37.9 \%$. 3. Predict of the development of inflammatory complications of obstructive jaundice are indicators of pro-inflammatory cytokine interleukin- 6 and the effective concentration of albumin. An increase of serum IL- 6 by $37 \%$ and reducing the concentration of ECA on $42 \%$ of the patients must be referred to the risk of inflammatory complications. 4 . The results of the clinical and immunological studies suggest the need for immunoorientirovannoy therapy in patients with obstructive jaundice moderate and severe degrees of severity.

Conclusion: So, this patients must be treated by special doctors and department.
MISC-25

Impact of nutrition status on performance status and quality of life in end stage liver disease patients

\author{
Neha Bakshi ${ }^{1}$, Kalyani Singh ${ }^{2}$ \\ ${ }^{1} \mathrm{Ph} . \mathrm{D}$. Scholar, India; ${ }^{2}$ Associate Professor, Ph.D., India
}

Background: Malnutrition in End Stage Liver Disease (ESLD) patients has been associated with various prognostic and clinical factors but there is dearth of data on non conventional parameters like Quality of Life (QoL) and Performance Status (PS). Both QoL and PS are new outcome measuring techniques for chronic disease treatments like ESLD. The present exploratory study aimed to analyse the effect of malnutrition on QoL and PS of these patients.

Method: We recruited 54 adult ESLD patients. Since, there is no gold standard for performing nutrition status assessment, the study used various recommended tools for nutrition status assessment of the patients like Subjective Global Assessment (SGA), Mid Upper Arm Circumference, Triceps, Mid Arm Muscle Circumference, and Body Mass Index (BMI) for ascites. QoL and PS were assessed by Short Form-36 Interview and Eastern Cooperative Oncology Group Performance Status scales respectively.

Result: Malnutrition in ESLD patients ranged from $24.1 \%$ to $88.9 \%$ by various nutrition assessment techniques. ESLD patients showed lower levels of QoL and PS. SGA showed malnourished patients were having significantly lower Performance Status grades than normal patients $(\mathrm{p}<0.05)$. The chi-square test with adjusted $\mathrm{z}$-scores showed that moderately malnourished patients were having significantly higher PS Grade-3 (capable of only limited self-care) than normal; also severely malnourished patients had significantly higher PS Grade-4 (completely disabled) than normal and moderately malnourished patients $(\mathrm{p}<0.004)$. Furthermore, malnutrition also showed lower QoL scores. After Bonferroni correction, SGA showed significantly lower emotional wellbeing scores in severely malnourished patients. BMI for ascites showed significantly lower Physical Functioning and Physical Component Summary scores of Quality of Life among severely malnourished patients. Serum albumin levels showed a significant and a positive correlation $(\mathrm{r}=0.326, \mathrm{p}=0.016)$ with emotional wellbeing of the patients.

Attention to maintenance of good nutritional status is often ignored in patients with ESLD with more focus on treating symptoms by medications or other medical procedures. The data revealed higher degree malnutrition is associated with lower QoL scores and PS grade in ESLD patients.

Conclusion: Hence, interventions focusing on improving the nutrition state can help to improve the non conventional parameters like Quality of Life and Performance Status, which can lead to holistic wellbeing of the patient.

\section{MISC-26}

\section{Liver Cirrhosis in women with ciliopathy syndrome}

\section{Syifa Mustika}

${ }^{1}$ Faculty of Medicine Brawijaya University, Indonesia

Background: Ciliopathy syndrome is a congenital abnormality of structure and/or function of cilia, which causes pleiotropic developmental disorder with varied spectrum, including liver cirrhosis. The aim of this case reports is to describe a unique case of liver cirrhosis with possible aetiology of ciliopathy syndrome

Method: A 44 year-old women with chief complain hematemesis had diabetes mellitus, obesity, dyslipidaemia, oligomenorrhoea since 
juvenile, amenorrhoea in the last 4 months, and often got unconscious. Physical examination shows short stature, short fingers (brachydactyly) (so did in her older sister),hyperpigmented maculae in body and four limbs, and hepatosplenomegaly. Laboratory results shows haemoglobin $7.4 \mathrm{~g} / \mathrm{dl}$; Albumin $2.42 \mathrm{~g} / \mathrm{dl}$; urea $84.8 \mathrm{mg} / \mathrm{dl}$;creatinin $2.4 \mathrm{mg} / \mathrm{dl}$;prolactin $138.8 \mathrm{ng} / \mathrm{ml}$, while $\mathrm{HBsAg}$ and anti-HCV were non-reactive. Abdominal ultrasound showed liver cirrhosis; endoscopy showed grade 3 oesophageal varicose; liver biopsies showed hydropic degeneration and fibrosis, and exclude autoimmunehepatitis; and head CT scan showed a chronic lacunar infarction and mega cysterna magna occipital.

Result: Ciliopathy related genes cause multiple abnormalities in the kidneys, brain, eyes, ears, liver, bone, and are also associated with obesity and insulin resistance. Short stature, short fingers, mega cisterna magna, and liver cirrhosis were found in ciliopathy syndrome, among them: Dandy-Walker syndrome, Alstrom syndrome, and Bardet-Biedl syndrome. The last two syndromes are well known for their relationship with insulin resistance and hyperphagic obesity. The gold standard for diagnosis of ciliopathy syndrome is gene mutations tests, like Alms and Bbs. Follow-up of other organ abnormalities, such as renal failure, is very important, so is screening of family members. The prognosis of ciliopathy is poor.

Conclusion: We reported female with hematemesis, short stature, short fingers, hyperpigmented maculae, liver cirrhosis, and mega cisterna magna, which is likely to suffer from ciliopathy syndrome. However, genetic tests hasn't been done yet.

\section{MISC-27}

\section{Recurrent hemobilia from a hepatic artery pseudoaneurysm: a rare complication of Hepatobiliary Tuberculosis}

\section{Geraldine Claire Floro ${ }^{1}$}

${ }^{1}$ University of the Philippines - Philippine General Hospital, Philippines

Background: Hemobilia is a rare cause of upper gastrointestinal bleed, occurring in $<2 \%$ of cases. Hepatic artery pseudoaneurysm from a tuberculous infection is an uncommon cause of hemobilia. We report a rare case of a hepatic artery pseudoaneurysm in a patient with hepatobiliary tuberculosis.

Method: We present a case of a hepatic artery pseudoaneurysm in a patient with hepatobiliary tuberculosis.

Result: A 34-year old male was admitted for recurrent hematemesis and jaundice. He was recently diagnosed with disseminated extrapulmonary tuberculosis of the liver, adrenals and gastrointestinal tract when he presented with new-onset fever, weight loss and right upper quadrant pain. He was on his 2nd month of anti-Koch's treatment when he started having recurrent episodes of massive upper gastrointestinal bleeding associated with generalized jaundice. Drug induced hepatotoxicity was considered and anti-Koch's treatment was put on hold. Repeated upper gastrointestinal endoscopies were undiagnostic. On the 4th endoscopy, blood was seen trickling from the ampulla, and biliary bleeding was then considered. A computed tomography angiography scan was done which revealed a pseudoaneurysm of the left hepatic artery. Embolization of the left hepatic artery was done using three steel coils. Anti-TB medications were resumed and the patient was discharged with no recurrence of jaundice and upper gastrointestinal bleed.

Conclusion: This was a rare case of hemobilia from hepatic artery pseudoaneurysm secondary from HBTB. This case states the importance of maintaining a high index of suspicion in HBTB patients with recurrent obstructive jaundice with concomitant upper GI bleed.
MISC-28

Patients of cirrhosis of liver operated for surgery other than liver transplant: a retrospective analysis

Kishore Kumar Kuduva Subramanian ${ }^{1}$, Manish Tandon ${ }^{1}$, Chandra Kant Pandey ${ }^{1}$

${ }^{1}$ Institute of Liver \& Biliary Sciences, India

Background: AIMS: In patients with cirrhosis of liver operated upon for surgery other than liver transplant

1. Evaluate Mayo clinical online calculator for predicting postoperative mortality risk

2. Correlate observed mortality with MELD \& MELD Na score and with CTP class.

Method: We analysed data of 133 cirrhotic patients who underwent various gastro-intestinal, hepato-pancreatico-biliary \& urological surgeries under anaesthesia care from October 2009 to June 2017 by reviewing the electronic data stored in the hospital information system including the scanned case files. All included patients had cirrhosis documented by histological, clinical or radiological findings consistent with cirrhosis. Patients of Acute liver failure, Acute on chronic liver disease and post liver transplant were excluded.

Result: A total of 133 patients were included in the study. For each patient, ASA status, the CTP class \& MELD score calculated from lab values obtained pre-operatively within 7 days of surgery were noted. Post-operative mortality risk was calculated using the online Mayo calculator \& follow up done for outcome for 1 year.

Males formed $84 \%$ of the included patients. Mean age of the study population was $51 \pm 12$ years. Alcoholic liver disease was closely followed by viral aetiology as cause for the cirrhosis of liver

The Mayo online calculator was good at predicting mortality at day 30, day 90 and at 1 year after surgery. However, the efficacy at predicting the outcome decreased with increase in the duration after surgery (Table 1). With every unit increase in MELD, MELD Na score and CTP class, there is significant rise in the mortality in our study group. Among the aforementioned 3 score, rise in CTP class predicts the mortality to the best at all the time points of 30 day, 90 day and 1 year (Table 2).

Conclusion: Mayo online risk calculator may be used for predicting mortality after non transplant surgery in cirrhotics. There is need for development of locally suited risk score for better prognostication \& utilization of medical resources.

\begin{tabular}{|c|c|c|c|c|}
\hline Days & Area under $\mathrm{ROC}$ & $\begin{array}{l}\text { ROC cul-off value for } \\
\text { prodisted mortality } \\
\text { uaing Mayo risk } \\
\text { calculator }\end{array}$ & Sensizivity & Specificiny \\
\hline 30 days & $0.816^{\circ}$ & $29.5 \%$ & 733 & 955 \\
\hline 90 days & $0.826^{\circ}$ & $41.5 \%$ & 33.1 & 75 \\
\hline 1 year & $0.744^{\circ}$ & $38 \%$ & 67.6 & 656 \\
\hline
\end{tabular}




\begin{tabular}{|l|c|c|}
\hline \multicolumn{1}{|c|}{ Variables } & Mortality rise per increase in variable \\
\hline MELD Score & $16 \%$ \\
& 30 days & $16 \%$ \\
& 90 days & $12 \%$ \\
& 1 year & \\
\hline MELD Na Score & & $13 \%$ \\
& 30 days & $12 \%$ \\
& 90 days & $8 \%$ \\
& 1 year & $86 \%$ \\
\hline CTP Class & 30 days & $56 \%$ \\
& 90 days & $35 \%$ \\
\hline
\end{tabular}

\section{MISC-29}

Retrospective analysis of Liver disease burden and closely tied comorbidities over the years

Kersitna H Boctor $^{1}$, Magdy Elkhashab ${ }^{1}$, Hamed Dallali ${ }^{1}$, Marjan Farshadi ${ }^{\text {, Muhammad Khurram Mahmood }}{ }^{1}$, Sirajuddaula Kausar ${ }^{1}$, Otilia Muraru', Mark Khan ${ }^{3}$, Jean Palmart ${ }^{2}$, Sunny Loo², Marzena Magnes ${ }^{1}$

${ }^{1}$ Toronto Liver Centre, Canada; ${ }^{2}$ Advisory Physicians, Canada; ${ }^{3}$ Gilead Sciences Inc., Canada

Background: With the increasing use of highly effective therapies for hepatitis $\mathrm{C}$ treatment, there has been an underlying shift in the distribution of liver-related morbidity and mortality worldwide. To better understand the burden of liver disease in Toronto, we are in the process of undertaking a comprehensive retrospective analysis of all patients evaluated at the Toronto Liver Centre (TLC) with an approximate data review of more than 10,000 patient charts. We estimate that more than fifty percent of patients evaluated at TLC will have been or are currently diagnosed with NAFLD; with the remaining focus on patients with $\mathrm{HCV}$, and $\mathrm{HBV}$, and to a lesser extent patients with ALD, AIH, PSC, PBC, or other liver conditions. Method: 1531 patients charts at the TLC were reviewed (Dating from Feb 1995 to Feb 2017) and assessed for: reason(s) for referral, demographics, liver disease etiology, fibrosis staging based on imaging (Ultrasound, FibroScan ${ }^{\circledR}, \mathrm{CT}$ ), biopsy, and blood work. We also assessed frequency of follow-up visits, co-morbidities, and therapy utilized. Patient grouping was categorized by primary liver etiology: HBV, HCV, NAFLD/NASH, ALD, AIH, or other. Patients were further sub-categorized by one or $>1$ liver disease from the previously mentioned combination.

Result: Patient Demographics: 1531 patient charts reviewed: 865 males, 666 female; distribution of liver disease was as follows: HBV 353, HCV 391, NAFLD 713, NASH 92, ALD 61, AIH 14, with $34.5 \%$ over 60 years of age. 1021 patients were reported to have only 1 liver disease; 374 to have two, 108 to have three, and 28 to have four or more liver-related diseases from the previously mentioned list. Co-Morbidities (males, female): $66.2 \% \quad(579,434)$ of patients comorbidities, the most significant being 269 T2DM (136,133), 437 dyslipidemia (261,176), 414 hypertension $(225,189), 373$ obesity $(223,150)$, and 125 thyroid Disease $(44,81)$. Cancer (males, females): $110(49,61)$ cases of cancer were identified; $23.6 \%$ HCC $(15,11)$. HCC distribution was as follows: (7 HBV, $12 \mathrm{HCV}, 5$
NAFLD, 5 all other); Non-HCC distribution was as follows (21 HBV, $34 \mathrm{HCV}, 48$ NAFLD, 19 all other).

Conclusion: NAFLD was the largest single disease category, outweighing chronic hepatitis in terms of overall disease burden. There was also substantial differences in the gender distribution of comorbidities with males outnumbering females in terms of hypertension, dyslipidemia and obesity. Additionally, we found an unexpectedly large number of cancer cases (primary HCC and nonliver). We plan to investigate in detail these findings in a larger population set.

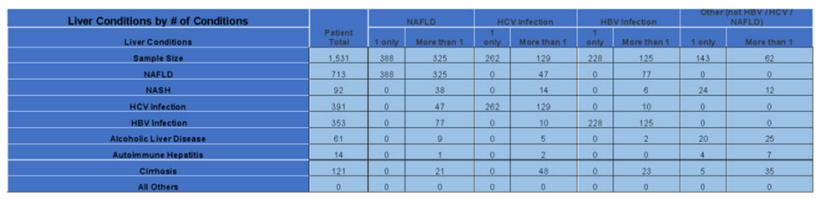

MISC-30

Social and economical impact of chronic liver disease; a least explored aspect

\section{Fakhar Ali Qazi Arisar ${ }^{1}$, Wasim Jafri ${ }^{1}$, Ramlah Nadeem ${ }^{1}$,} Muhammad Kamran ${ }^{1}$

${ }^{1}$ The Aga Khan University Hospital, Pakistan

Background: Chronic liver disease poses a paramount burden not only on the patients but also on their care givers. There has been research on the effects it has on health related quality of life of patients suffering from CLD, however, little has been done on the effects it has on mental, emotional health and economic situation of the entire family unit. This study purposes to look into the financial implications on the family due to chronic liver disease.

Method: This is a cross sectional observational study where patients recruited at GI clinic from January to December 2016 were required to fill out validated questionnaires based on modified McArthur Social Status questionnaire.

Result: A total of 175 CLD patients were enrolled, which include 116 $(66.28 \%)$ males. Mean age of cohort was $49.96 \pm 12.089$ years. Predominant aetiology of CLD was hepatitis C (64\%). Majority of patients were child class B (63\%). Mean MELD score was $11.48 \pm$ 3.556. Diabetes and hypertension were present in $24 \%$ \& $19 \%$ patients respectively. Mean duration of CLD diagnosis before study was $7.81 \pm 6.161$ years. Majority of patients $(90 \%)$ belong to lower or middle class social status with average maximum education of $8.86 \pm$ 5.641 years. Number of dependent family members was $9.1 \pm 7.79$. CLD significantly impacted the patients as $81 \%$ and $66 \%$ of patients blame their disease for worsening of their social and economic situation respectively. $69 \%$ patients had to borrow money for their medical expenses. $59 \%$ of patients were not able to maintain their current standard of life for even a month if their source of income get vanished. Nearly $50 \%$ had to leave or cut short their medicines or miss doctor's appointment due to expenses. $46 \%$ patients had to delay the education of their children. $15 \%$ patients left their houses in search of cheaper place. Moreover male patient appeared to have more severe disease with mean MELD score of $12.19 \pm 3.74$ as compare to females having mean MELD score of $10.24 \pm 2.83$ (pvalue: 0.004 ). Male patients give more importance to work as compare to females $(93 \%$ vs $52 \%$; p-value: $<0.0001)$. Females appear to be dependent on others to bear their expenses ( $77 \%$ vs $24 \%$; p-value: $<0.0001)$. Males had to cut-short their medicines more $(62 \%$ vs $44 \%$; p-value: 0.033 ) or miss surgical procedures (37\% vs $15.7 \%$; p-value: 0.006). 
Conclusion: CLD imposes a tremendous socioeconomic and emotional burden on patients and their caregivers. CLD related expenses impact the family's unit daily functioning and medical adherence. There is an urgent need of improvement in health structure with emphasis on better preventive, diagnostic and therapeutic facilities.

MISC-31

The case for early advanced care planning (ACP) in cirrhosis: a quality improvement project

Kathleen P Ismond ${ }^{1}$, Ashleigh Sprange ${ }^{3}$, Emma Hjartarson ${ }^{4}$, Swati Chavda $^{4}$, Michelle Carbonneau ${ }^{2}$, Amanda Brisebois ${ }^{3}$, Puneeta Tandon ${ }^{4}$

${ }^{1}$ University of Alberta, Canada; ${ }^{2}$ Alberta Health Services, Canada; ${ }^{3}$ Palliative Care Medicine, Canada; ${ }^{4}$ The Cirrhosis Care Clinic, Canada

Background: Advance Care Planning (ACP) is a practitioner-led process for patients to convey their disease-modifying and palliative care preferences. Early integration of ACP in a disease trajectory has improved patients' quality of life and reduced unwanted hospitalizations and use of life-sustaining treatments. Although cirrhosis is a life-limiting chronic disease, few patient (18-26\%) are engaged in ACP discussions.

Method: A common barrier for practitioners is knowing when to initiate ACP in relation to patient readiness and/or liver disease status. Our study objectives were to assess patient preferences for ACP and $\mathrm{ACP}$ completion rates. We conducted a prospective analysis of consecutively consenting patients attending two urban tertiary cirrhosis clinics. Patients received a definition of ACP then completed a survey regarding cirrhosis disease trajectory, experience, preferences regarding ACP discussions, and demographics. Data analyses used descriptive statistics.

Result: The survey response rate was $71.6 \%$ (101/141). The mean age was 61.8 years (SD 9.72 years) and $56.4 \%$ were male. The mean MELD score was $11.5 \pm 5.3$ and the mean Child-Pugh score was 6.2 \pm 1.5 (Class A, 71.3\%; Class B, 26.7\%; Class C, 2.0\%). The etiology of cirrhosis was due to alcoholic hepatitis (39.6\%), non-alcoholic fatty liver disease (28.7\%), viral hepatitis $(15.9 \%)$, or other $(15.9 \%)$. Only $53 \%$ percent of patients felt that cirrhosis would affect their quality of life at some point in the illness in keeping with low selfawareness of health status. In total, $85 \%$ of patients indicated that they wanted ACP discussions performed in a stable outpatient clinic setting instead of during hospital stay. Patients $(97 \%)$ stated it was very important to know the reality about their health condition and prognosis, even if it was bad news. Almost universally, patients expressed that it was important the practitioner carrying out the ACP understood their medical issues, was trusted by them, and did not rush through ACP without being available to answer subsequent questions at another time. The patients placed little emphasis on if the practitioner conducting the ACP had a long-standing relationship with them. Important features were for the ACP discussion from the patients' perspective were honesty, clarity, and empathy. Emphasizing the unpredictable trajectory of cirrhosis, 23 study patients were unexpectedly hospitalized within 8 months from the study's baseline and another 7 died during this time.

Conclusion: Our findings indicate that patients prefer engaging in ACP discussions shortly after being diagnosed with cirrhosis. At this time, they are the healthiest and most able to make informed decisions regarding their future medical care.
MISC-32

A study on an associated dietary factors causing anemia in college female students

\section{Shwetma Mishra ${ }^{1}$, Girija Sankar Shukla ${ }^{2}$ \\ ${ }^{1}$ Central Government, India; ${ }^{2}$ State Government, India}

Background: Anemia, a multifactorial causative problem, has severe impact on the health of human body and stands as a challenge for the society. The severity of anemia depends on the various factors which need at the earliest diagnosis and treatment. Lots of researches on this issue have been carried-out and published but still there are certain points which have to be evaluated at micro level in various walks of society. Diet is one of the most important factor behind causation of anemia. Current cultural scenario among youths and teenagers in India is significantly influenced by western culture.

Method: The Aim of current research under taken by me is an initiative in diagnosis of Anemia among females of age group 17- 30 years studying in the college/any higher academic institutions. A well-structured Questionnaire and Consent form has been designed to collect the necessary information from the respondents in this regard. The Clinical data is taken included blood sampling through Venipuncture and all the test has been performed with Automated Analyzers. The family history has also been collected.

Result: A number of 1070 college going female student's samples from Allahabad and Delhi NCR region was selected for the study. In which 610 females having Anemia and 460 females show no anemia. When going through with their routine analysis it was found that their eating habits lead the main role in causing illness in their body. In this especially fast food/ junk food habits play an important role. The present study result shows that the dietary supplements deficiency affected the college-going female students with the upcoming diseases or disorders in their later life in which fast food eating habits are more common among them. The data shows that the female student having anemia having regular, frequent, rarely fast food habits was $40.28 \%, 33.55 \%$ and $26.17 \%$ respectively. When the detailed study on this was done then it was found that college-going female students who lived in hostels were suffered the most from this disorder symptomatically because of improper dietary food habits. They were eating either in mess or outside and their food was not to be a nutritionally rich diet but it was only fulfilling their hunger and taste and in which fast food habits are more common among them. Skipping food like lunch or dinner was also common in them, which is also a responsible factor for their illness. Lack of nutritional deficiency harms them and cause of so many diseases later on in their life. This study shows that the nutritional deficiency and eating fast food habit was affected college going females with this symptomatic disorder, which later on originates different disorder or disease in their body. No disease comes in a day its creation takes time. Regular eating unhealthy of nutritional deficient diet slowly - slowly becomes in form of some chronic illness. It was also noted this habit also decreases their immune response to fight with different infections/ illnesses.

Conclusion: Thus good nutrition can play a lead role in the well immune response, protection and prevention of several chronic diseases, including obesity, PCOD, Coronary heart disease, certain types of cancers, stroke, and type 2 diabetes. 
MISC-33

Esophageal varices- the most frequent and rising endoscopic finding in patients with upper gi bleed in Pakistani population

\section{Talal Khurshid $^{1}$}

${ }^{1}$ Holy Family Hospital, Pakistan

\section{Background:}

To find out the upper GI endoscopy findings in patients presenting with UGIB in relation to age, gender and etiology.

Method: A retrospective analysis of 3910 patients presented to GI Department from January 2011 to October 2014 for endoscopic evaluation of UGIB. The data was analyzed on SPSS20, descriptive statistics were recorded and results were analyzed as in given table. Result: The pattern of pathologies on EGD is as shown as:-

\begin{tabular}{|l|l|l|l|l|l|}
\hline No & Endoscopic Finding & Number & \%age & $\begin{array}{l}\text { Mean Age } \\
\text { (yrs) }\end{array}$ & $\begin{array}{l}\text { Male: } \\
\text { Female ratio }\end{array}$ \\
\hline 1 & Esophageal varices (EV) & 2998 & $76.6 \%$ & 46 & $1.95: 1$ \\
\hline 2 & Peptic ulcer disease (PUD) & 381 & $9.7 \%$ & 40 & $1: 1$ \\
\hline 3 & Gastritis & 298 & $7.6 \%$ & 39 & $1: 1.5$ \\
\hline 4 & Normal EGD & 165 & $4.2 \%$ & 35 & $1: 1.4$ \\
\hline 5 & Portal Gastropathy (PG) & 22 & $0.5 \%$ & 34 & $1: 1$ \\
\hline 6 & Mallory Weiss & 14 & $0.4 \%$ & 32 & $1: 1.3$ \\
\hline 7 & Gastric Neoplasm & 11 & $0.3 \%$ & 45 & $1.4: 1$ \\
\hline 8 & Fundal Varices (FV) & 10 & $0.3 \%$ & 42 & $1.3: 1$ \\
\hline 9 & Esophageal Neoplasm & 10 & $0.3 \%$ & 49 & $1.8: 1$ \\
\hline
\end{tabular}

Conclusion: The majority of the patients with UGIB presented with haematemesis alone $(\mathrm{n}=3034,77.6 \%)$ followed by combined hematemesis \& malena $(n=540,13.8 \%)$ and malena alone $(n=336$, 8.6\%). EV were the most frequent finding of UGIB ( $\mathrm{n}=2998,76.6 \%)$ followed by PUD $(n=381,9.7 \%) .93 \%$ of the patients with EV were infected by $\mathrm{HCV}$. The bleeding site was accurately identified in $95.8 \%$ of patients and re-bleeding was reported in $5.8 \%$.

Conclusion: EV is the commonest cause of UGIB in our territory manifested mainly by hemetemesis and it is due to high endemic nature of $\mathrm{HCV}$ infection among the male population in Central Punjab as compared to the western world which is mainly PUD. A good number of patients had normal EGD which warrants us to locate for other causes that mimic UGIB

\section{MISC-34}

Balloon assisted enteroscopy in patients with cirrhosis an experience at a tertiary care centre

\section{Pankaj Kumar Shrimal, Mohan Ramchandani ${ }^{1}$, Palle Manohar Reddy , Duvvur Nageshwar Reddy ${ }^{1}$ \\ ${ }^{1}$ Asian Institute of Gastroenterology, India}

Background: Balloon assisted enteroscopy (BAE) has revolutionised the diagnosis and treatment of small bowel diseases. But limited data is available for its role in patients with cirrhosis.

This study is aimed at evaluating the role of BAE in diagnosis and management of small bowel diseases in patients with cirrhosis.

Method: The data of all the patients of cirrhosis who underwent BAE with single balloon enteroscope (SBE) were retrospectively analysed between January 2013 to Aug 2017. The safety, efficacy, diagnostic potential and therapeutic outcomes were analysed.

Result: A total of 50 patients underwent SBE in the given period. Mean age of patients was $56.46 \pm 12.91$ years, 34 were males and 16 were females. Etiology was NASH in 20 patients, alcohol in 8 patients, chronic hepatitis B in 6 patients, cryptogenic in 9 patients and others in 7 patients. According to Child Turcotte Pugh score, 26 were child A, 20 were child B and 4 were child C status. Mean MELD score was $12.29 \pm 4.23$.

Indications for enteroscopy were obscure gastro-intestinal bleeding (OGIB) in 45 patients and pain abdomen suggestive of small bowel obstruction in 5 patients.

In patients with OGIB, most common cause was portal hypertensive enteropathy seen in 30 patients for which 27 patients underwent 36 procedures of argon plasma coagulation, other lesions were: dieulafoy's lesion in 2 patients (underwent hemoclip application), ileal inflammatory bleeding polyp in 1 patient (underwent hemoclip application), 1 patient had jejunal Non-Hodgkins lymphoma for which surgery followed by chemotherapy was given, 1 patient underwent intra-operative enteroscopy which showed caecal ulcer bleeding for which ileo-caecal resection was done and 8 patients had normal enteroscopy. Two patients died due to persistent GI bleed.

In patients with pain abdomen, 1 patient had jejunal neuroendocrine tumor for which surgery was done, 1 patient had Crohn's disease, 1 patient had portal hypertensive enteropathy with no evidence of obstruction and 2 patients had normal enteroscopy, all improved with conservative treatment.

Capsule endoscopy was done in 30 patients, of which 20 patients $(66.66 \%)$ had findings consistent with enteroscopy. Of the remaining 10 patients, visibility was impaired due to presence of blood in 6 patients (20\%) and in 4 patients $(13 \%)$ findings were discordant with enteroscopy.

There were no procedure related mortality or major complications. Conclusion: Balloon assisted enteroscopy is a safe and useful procedure for diagnosis and management of small bowel diseases in patients with cirrhosis.

\section{MISC-35}

Diagnostic accuracy of computed tomography scan, keeping endoscopic ultrasonography as gold standard for detection of pancreatic carcinoma in Pakistani population

\section{Talal Khurshid}

${ }^{1}$ Holy Family Hospital, Pakistan

Background: Endoscopic Ultra sonography (EUS) and Computed Tomography (CT) scan are diagnostic techniques that are considerably important in preoperative diagnosis of pancreatic carcinoma (CA). Even though EUS has been confirmed to be more effective in accurate diagnosis but $\mathrm{CT}$ scan is still used commonly being easily accessible, cost effective and non invasive in most of developing countries. The objective of this study was to determine the diagnostic accuracy of CT scan findings keeping EUS as the Gold Standard procedure.

Methods \& materials: This cross sectional study was conducted at the Liver centre of Holy Family Hospital, where all 75 suspected cases of pancreatic CA. patients who underwent both; Computed Tomography and EUS, each diagnostic procedure performed by same team of radiologists and gastroenterologists respectively, in the year 2014 were included. The diagnosis of each individual patient for carcinoma of pancreas, confirmed through EUS was taken as gold standard. Sensitivity, Specificity, Positive and Negative predictive values along with 95\% confidence intervals (CI) were calculated. Diagnostic accuracy of CT scan compared to EUS was also thereby calculated.

Result: Sensitivity and Specificity of CT scan was found to be as 97.14\% ( $\mathrm{CI}=85.08 \%-99.93 \%)$ and $95 \%(\mathrm{CI}=83.08 \%-99.39 \%)$. The positive predictive value was calculated as $94.44 \%$ ( $\mathrm{CI}=81.74 \%$ - 
99.32\%) while negative predictive value was $97.44 \%$ (CI $=86.52 \%$ 99.94\%). Diagnostic accuracy was $96 \%$.

Conclusion: The diagnostic capability of CT Scan in diagnosis of pancreatic CA is almost nearly effective to EUS

\section{MISC-36}

Dysphagia in Pakistani population; what are the patterns of endoscopic findings in them?

\section{Talal Khurshid}

${ }^{1}$ Holy Family Hospital, Pakistan

Background: To see the pattern of upper GI endoscopy findings in patients presenting with Dysphagia in relation with age, gender and symptoms duration

Material and methods: A retrospective analysis of 738 patients presented at A\&E and GI OPD from January 2011 to October 2014 with history of dysphagia for solid or liquids. These patients were enrolled, an upper GI endoscopy was performed and findings were recorded. The data was analyzed on SPSS 20 and descriptive statistics recorded and results were analyzed as in given table.

Result: The pattern of pathologies on EGD is as shown in Table 1.

\begin{tabular}{|l|l|l|l|l|l|}
\hline No. & Endoscopic Finding & Number & $\%$ Mage & $\begin{array}{l}\text { Mean Age } \\
\text { (yrs) }\end{array}$ & $\begin{array}{l}\text { Male : Female } \\
\text { ratio }\end{array}$ \\
\hline 1 & Esophageal Neoplasm & 180 & $24.4 \%$ & 54 & $1.95: 1$ \\
\hline 2 & $\begin{array}{l}\text { Post Corrosive Esophageal } \\
\text { Stricture }\end{array}$ & 161 & $21.8 \%$ & 28 & $1: 1.46$ \\
\hline 3 & Normal EGD & 158 & $21.4 \%$ & 38 & $1: 1$ \\
\hline 4 & Hiatus Hernia & 111 & $15.1 \%$ & 41 & $1.2: 1$ \\
\hline 5 & Achalasia Cardia & 45 & $6.1 \%$ & 42 & $1: 1$ \\
\hline 6 & Esophageal Candidiasis & 37 & $5.1 \%$ & 42 & $1: 1.5$ \\
\hline 7 & Esophageal Foreign Body & 29 & $3.8 \%$ & 45 & $1: 1.2$ \\
\hline 8 & Tracheo-Esophageal Fistula & 09 & $1.1 \%$ & 29 & $1: 0$ \\
\hline 9 & Esophageal Web & 07 & $1 \%$ & 48 & $1: 1.5$ \\
\hline 10 & Zenker's Diverticulum & 01 & $0.2 \%$ & 43 & $1: 0$ \\
\hline
\end{tabular}

In the patients above the age of $40(n=354,48 \%)$, esophageal neoplasm was the predominant lesion $(\mathrm{n}=92,51 \%)$ followed by Normal EGD $(n=27,15 \%)$. While in patients under the age of 40 years $(\mathrm{n}=384,52 \%)$, the predominant lesion is esophageal stricture $(\mathrm{n}=111$, $29 \%)$ followed by a normal EGD finding which comprises of $(n=107$, $28 \%$ ). In the patients suffering from esophageal neoplasm, the mean duration of onset of symptoms to the diagnosis was 4 months.

Conclusion: Although a good number of patients had a non mechanical etiology of dysphagia but majority of the patients had an underlying mechanical cause of obstruction. Majority of patients presenting with dysphagia and age above 40 had a neoplasia. Majority of patients presenting with dysphagia and age below 40 had post corrosive intake injury of the esophagus.

\section{MISC-37}

Etiological and clinical profile of space occupying lesions of liver in cirrhosis

\section{Shaik Nagoor Basha ${ }^{1}$, Girinadh LRS ${ }^{1}$, Kiran Repana' \\ ${ }^{1}$ Dr NTRUHS, India}

Background: Identifying aetiology of space occupying lesion (SOL) of cirrhotic liver as it is important for proper management of the case and to access the most common clinical presentation in various types of SOLs in cirrhotic liver.
Method: This observational study was done in adult cirrhotic patients $(n=69)$ with SOLs in their liver, which are detected either incidentally or during surveillance in King George hospital from February 2015 to December 2016. Multiphasic CECT was performed as the first imaging modality for evaluation of an ultrasonographically detected lesion in the liver and CEMRI, FNAC were performed only if the features were atypical and equivocal. Carcino-embryonic antigens, CA19-9 are positive and alpha-fetoprotein is negative in cholangiocarcinoma.

Result: The average age of presentation is $50.08 \pm 10.8$ years. HCC was the most common aetiology of SOL which constituted $38(65 \%)$ cases. HCC has its highest incidence in 51-60 years group. The most common symptom being abdominal distention or right upper quadrant pain seen in $27(52 \%)$ of cases. Out of 23 patients who underwent FNAC/biopsy 10 patients diagnosed as having HCC. Most of the lesions above the size of $2 \mathrm{~cm}$ were because of HCC.

Conclusion: $\mathrm{HCC}$ is the most common space occupying lesion in the cirrhotic liver. Benign lesions make up for $34 \%$ of space occupying lesions and are more frequent in lesions with less than $2 \mathrm{~cm}$ size. HBV is the most common aetiology for HCC in our geographical area.

\section{MISC-38}

Primary hepatic leiomyosarcoma a space occupying lesion in the liver: an enigma for diagnosis

Rajdeep Bhagwant More ${ }^{1}$, Ashwini Kumar Setya ${ }^{1}$, Vivek Raj ${ }^{1}$, Suneel Chakravarty ${ }^{1}$, Abhishek Deo ${ }^{1}$

${ }^{1}$ Max Superspeciality Hospital, Saket, New Delhi, India

\section{Background:}

Primary hepatic leiomyosarcoma are rare soft tissue sarcomas with about only about 60 cases reported in the English literature. Due to its rare existence and limited literature, the diagnostic algorithms and standards of care have not been adequately defined. This has led to a delayed diagnosis of such patients. We herein present a 72 year old lady with primary hepatic leiomyosarcoma treated with chemotherapy and attempt to delineate the line of management .

Method: A 72 year old Nigerian lady presented to our department with complaints of abdominal pain and swelling in the epigastrium and right hypochondrium over 3 months prior to presentation with other non specific symptoms such as generalized weakness, loss of appetite and weight loss.

Result: Primary hepatic leiomyosarcomas (PHL) account for 1-2\% of all liver malignancies. Due to the rarity of primary hepatic sarcomas, and PHL in particular, the diagnostic algorithms and standards of care have not been adequately defined. In worldwide literature there are only a few cases series are available. The standard management consensus guidelines for these tumors have not been yet established and most of the management strategies are based on expert opinion. This has led to a delayed diagnosis and subsequently a poor prognosis and survival of such patients.

Conclusion: Primary Hepatic Leiomyosarcoma is a rare primary hepatic malignancy

$\&$ is usually a diagnosis of exclusion. It should be subjected to histopathological evaluation after liver biopsy of the lesion \& further immunohistochemistry tests. 

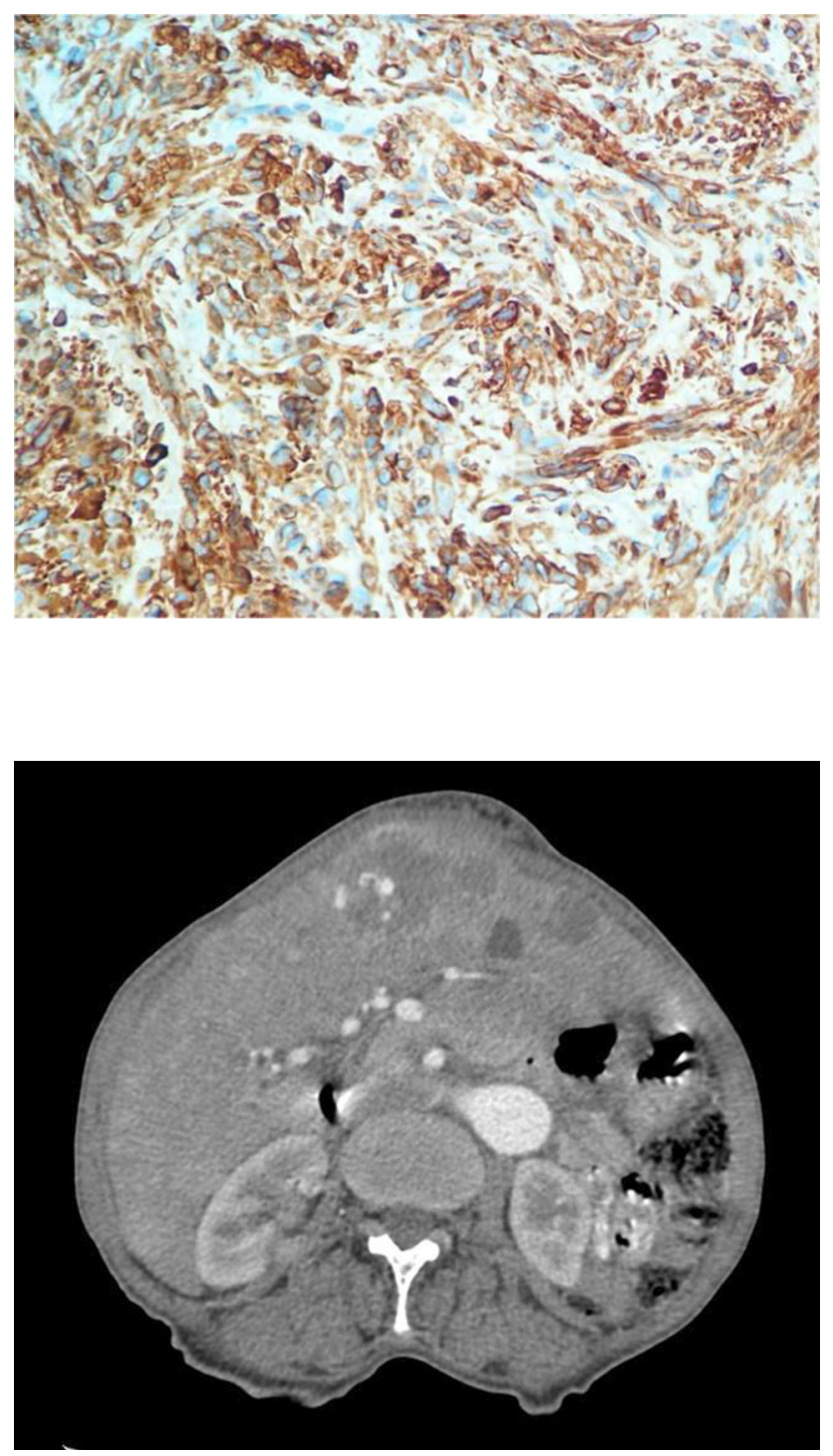

MISC-39

Risk of failure and complications of endoscopic retrograde cholangiopancreatography; comparison of elderly and young Pakistani patients

\section{Talal Khurshid ${ }^{1}$}

${ }^{1}$ Holy Family Hospital, Pakistan

Background: Endoscopic Retrograde Cholangiopancreatography (ERCP) is an effective diagnostic and therapeutic procedure, widely performed in patients, irrespective of age. The objective of the study was to compare the risk of failure and procedural complications in young and elderly patients.

Method: This cohort study was conducted at Holy Family Hospital, where all 362 patients who underwent the therapeutic or diagnostic
ERCP performed, in the year 2014 were included and categorized as 276 young (aged $20-59$ years) and 86 elderly (60 years and above) patients. The procedural and post procedural records of both study groups were followed up prospectively to compare the risk of failure of procedure and the complications during and after procedure. Chi square test was applied at $5 \%$ level of significance and Relative risks (RR) along with $95 \%$ confidence intervals (CI) were also determined through SPSS.

Result: Successful therapeutic intended procedures were observed in $95.08 \%$ of elderly and $97.32 \%$ of young patients. (RR of failure 0.64 , CI 0.19-2.85, $\mathrm{p}$ value 0.47). Similarly Successful diagnostic intended procedures were performed in $88 \%$ of elderly and $91.1 \%$ of young patients. (RR of failure 1.35, CI 0.37-4.84, p value 0.64). At least one or more Procedural and post procedural complications were observed in $9.3 \%$ and $8.3 \%$ of elderly and young patients respectively ( $\mathrm{p}$ value 0.77), where risk of complications was also observed to be the same with relative risk of 1.11 (CI $0.51-2.40$ ).

Conclusion: The success rates, risk of failure and complications of the procedure in elderly was same as that of young, providing evidence that it is an equally safe procedure for elderly too.

KEY WORDS: Cholangiopancreatography, Endoscopic Retrograde, Age groups, risk, complications

\section{March 2018}

MISC-40

A rare polyp of the common bile duct: spindle cell carcinoma

Kailash Marotrao Kolhe ${ }^{1}$, Shamsher Singh Chauhan ${ }^{1}$, Harshad Khairnar ${ }^{1}$, Dhaval Choksi ${ }^{5}$, Akash Shukla ${ }^{4}$, Meghraj Ingle ${ }^{3}$, Vikas Pandey ${ }^{2}$

${ }^{1}$ Resident, India; ${ }^{2}$ Assistant Professor, India; ${ }^{3}$ Associate Professor, India; ${ }^{4}$ Professor and Head, India; ${ }^{5}$ Super Speciality Medical Officer, India

Background: Spindle cell tumour of the bile duct is an uncommon finding with only 2 cases reported in literature so far. We report a case of an elderly female who presented to us with recurrent cholangitis and was diagnosed to have a common bile duct sarcoma.

Method: A fifty years old female presented with recurrent cholangitis since three months. Laboratory evaluation showed cholestatic picture with high WBC counts suggestive of cholangitis. Ultrasound of abdomen showed a dilated common bile duct. Endoscopic ultrasound showed a polypoidal mass in proximal common bile duct with tiny CBD calculi. During ERCP balloon sweeps were taken; globular smooth walled, fleshy mass was seen prolapsing intermittently out of papilla. Biopsy was taken which was suggestive of spindle cell tumour on histopathology and immunohistochemistry. Cholangioscopy was performed for better visualisation of the tumour mass. A contrast enhanced CT of the abdomen did not show metastasis. Surgery for resection was planned but the patient was unwilling. Currently she is asymptomatic and on regular follow up.

Result: The recurrent cholangitis in our patient is due to spindle cell tumour.

Conclusion: Spindle cell tumour of CBD is a rare tumour with only few case reports worldwide. 
MISC-41

Role endobiliary intervention in complex treatment of patients with mechanical jaundice

\section{Elyor Sayfutdinov ${ }^{1}$ \\ ${ }^{1}$ Tashkent Medical Academy, Uzbekistan}

Background: Surgical treatment of patients with mechanical jaundice is an actual problem of abdominal surgery. One of the major factors contributing to the disease is the development of septic complications in these patients at various stages of surgical treatment. In recent years, an important practical meaning takes on studying the role endobiliary intervention in complex treatment of patients with mechanical jaundice.

Method: Period from 2015 to 2016 in surgical department of Republic Clinic Hospital? 1 were examined and treated 24 patients with the syndrome of obstructive jaundice, 13 women (54.2\%) and 11 men $(45.8 \%)$. The patients' age - from 28 to 75 years, average age 51 years. The majority were patients with a tumor of the pancreas head - $13(54.2 \%)$ and Klatskin tumor - $4(16.7 \%)$. The remaining patients were diagnosed: papillary tumor $-4(16.7 \%)$, hepatocellular carcinoma - $3(12.4 \%)$

Result: All patients urgently with a view to the biliary tract decompression percutaneous trans-hepatic drainage of the bile duct was performed. Most patients $15(62.5 \%)$, external-internal drainage performed simultaneously, in two stages $2(8.3 \%)$ patients, $1(4.15 \%)$ were performed only external drainage. Two-stage holding externally-internal drainage was caused by a high degree of hyperbilirubinemia $(120 \mathrm{~mol} / \mathrm{L})$, the presence of purulent cholangitis and marked dilatation of the intrahepatic ducts, which impeded the passage of occluded segment and would lead to an increase in trauma intervention. $4(16.7 \%)$ patients with tumor Klatskin performed bilateral drainage. After resolving jaundice patients conducted further examination, including ultrasound and $\mathrm{CT}$ with bolus strengthening of the abdominal cavity. Histological in some cases performed puncture aspiration biopsy under ultrasound guidance (a tumor of the head of the pancreas, primary liver cancer). With cavernous contraindications performed surgery. Open surgical interventions were performed 2 $(8.3 \%)$ patients; one of them $(4.2 \%)$ - pancreatoduodenal resection was performed Endobiliary stenting 13 (30.9\%) patients, including "Y"-shaped stent was used in $3(23.01 \%)$ cases.

The mortality in the early postoperative period (3-7 days) after the drainage of the bile ducts percutaneous trans-hepatic drainage - 1 $(4.2 \%)$ patients; group of stenting of the bile ducts -. 1 (4.2\%) Cause of death in most cases the phenomenon of severe renal and hepatic failure.

Conclusion: Given the high risk of open surgery in patients with obstructive jaundice, we believe that endobiliary intervention should be considered not only as a stage of preoperative preparation.

\section{NAFL-1}

\section{Adenovirus-mediated over-expression of BMP-9 deteriorates MCD-induced non-alcoholic steatohepatitis in mice}

\footnotetext{
Qi Li ${ }^{1}$, Beibei Liu ${ }^{4}$, Honglei Weng ${ }^{3}$, Katia Breitkopf Heinlein ${ }^{3}$, Steven Dooley ${ }^{3}$, Keshu $\mathrm{Xu}^{4}$, Huiguo Ding ${ }^{2}$

${ }^{1}$ Beijing You'an Hospital Affiliated with Capital Medical University, China; ${ }^{2}$ Beijing You'an Hospital Affiliated with Capital Medical University, Beijing, China; ${ }^{3}$ Medical Faculty Mannheim, Heidelberg University, Germany; ${ }^{4}$ The Union Hospital, Tongji Medical College, Huazhong University of Science and Technology, China
}

Background: Liver inflammation and macrophage infiltration is a critical step for progression of non-alcoholic steatohepatitis (NASH) from non-alcoholic fatty liver. Bone morphogenetic protein-9 (BMP9), a member of the TGF-beta superfamily, is newly found cytokine involved in the regulation of chemokines and lipogenesis. However, the function of BMP-9 in NASH is still unknown. We tested the hypothesis that BMP-9 contributes to steatohepatitis in NASH mice. Method: BMP- 9 over-expressed mice on C57BL/6 background were fed either MCD diet or control diet for 4 weeks. The mouse orbital blood was taken to measure alanine aminotransferase (ALT) and triglyceride (TG) levels. Liver histology was presented by HE staining. Lipid deposition was displayed by Oil red-staining. Protein expression of F4/80, iNOS, CD206 and alpha-SMA was investigated by immunohistology. RNA expression of pro-inflammatory factors and pro-fibrotic genes was investigated by real-time PCR.

Result: BMP-9 was significantly up-regulated in MCD-induced $\mathrm{NASH}$ mice at the 4th week than the normal control mice. Compared with the other 3 groups, the liver in BMP-9 over-expressed mice displayed more ballooning degeneration of liver cells, focal inflammatory cell infiltration and bullous steatosis. Serum ALT levels were significantly increased in mice fed the MCD diet and it was even higher in BMP-9 over-expressed mice fed the MCD diet. Compared with the $\mathrm{CD}$ diet mice, levels of chemokine monocyte chemoattractant protein-1 (MCP-1) mRNA were increased in lives of MCD diet mice. BMP-9 over-expression in the liver further enhanced this rise tendency of MCP-1 mRNA expression, whereas interleukin (IL)-1beta; and IL-6 mRNA expression was reduced by BMP-9 overexpression. For tumor necrotic factor (TNF)-alpha, IL-10 and C-C Motif Chemokine Receptor 2 (CCR2) mRNA expression, it was not significantly affected by either the MCD diet or BMP-9 over-expression. Hepatic macrophage accumulation increased in mice fed with the MCD diet and further increased with BMP-9 over-expression. Liver F4/80 positive macrophage number was markedly higher in BMP-9 over-expressed liver. Interestingly, the number of iNOS positive macrophages was significantly more in the MCD+Ad-BMP9-HA (c) mice than that in the other 3 groups. There was no markedly difference of Sirius red staining among 4 groups. It was in the same case for alpha-SMA staining. Regarding to the pro-fibrogenic genes such as Collagen I, PAI-1 and MMP-9, we did not found any significantly differences in 4 groups. MCD diet promoted CTGF and TGF-beta1 mRNA expression significantly. BMP-9 over-expression intensified the significance of TGF-betal mRNA expression, however, it did not have the same effect on CTGF mRNA expression. Conclusion: These results indicate that BMP-9 exerts an indirect proinflammatory role via promoting the secretion of inflammatory factors and macrophage infiltration in MCD-induced NASH.

\section{$N A F L-2$}

Circulating long non-coding RNA TCONS_00016452 is inversely correlated with liver steatosis in patients with non-alcoholic fatty liver disease

Jung Gil Park ${ }^{1}$, Soo Young Park ${ }^{3}$, Keun Hur ${ }^{3}$, Heon Ju Lee ${ }^{1}$, Won Young Tak ${ }^{3}$, Young Oh Kweon ${ }^{3}$, Se Young Jang ${ }^{3}$, Yu Rim Lee ${ }^{3}$, Gyeonghwa Kim ${ }^{3}$, Jihyun Lee ${ }^{3}$, Eunhye Lee, Hye Won Lee ${ }^{2}$

${ }^{1}$ College of Medicine, Yeungnam University, Korea, Republic of; ${ }^{2}$ Dongsan Medical Center, School of Medicine, Keimyung University, Korea, Republic of; ${ }^{3}$ School of Medicine, Kyungpook National University, Korea, Republic of

Background: Hepatic steatosis is an initial process in patients with non-alcoholic steatohepatitis (NASH), which is progressed to liver 
fibrosis. Long non-coding RNA (IncRNA) TCONS_00016452 is batch genome conversion of lncRNA LeXis, which is related with cholesterol metabolism and hepatic steatosis in mouse.

Method: To evaluate the circulating lncRNA TCONS_00016452 in patients with non-alcoholic fatty liver disease (NAFLD), we analyzed a total of fifty patients who underwent percutaneous liver biopsy to confirm NASH, which is reviewed by one pathologist. Expression of circulating lncRNA TCONS_00016452 in plasma was compared using quantitative real-time polymerase chain reaction according to pathologic results.

Result: Expression of circulating lncRNA TCONS_00016452 was significantly decreased in patients with severe steatosis compared to patients with minimal to moderate steatosis $(13.9 \pm 16.0$ vs $7.4 \pm 5.3, p=0.039$. As the grade of steatosis was advanced, circulating lncRNA TCONS_00016452 was decreased $(r$-value $=-0.29$, $p=0.004)$. However, circulating lncRNA TCONS_00016452 was decreased in patients with significant fibrosis compare to without significant fibrosis $(8.3 \pm 5.1$ vs $17.2 \pm 19.6, p=0.040)$.

Conclusion: Circulating lncRNA TCONS_00016452 is inversely correlated with grade of liver steatosis in patients with NAFLD.

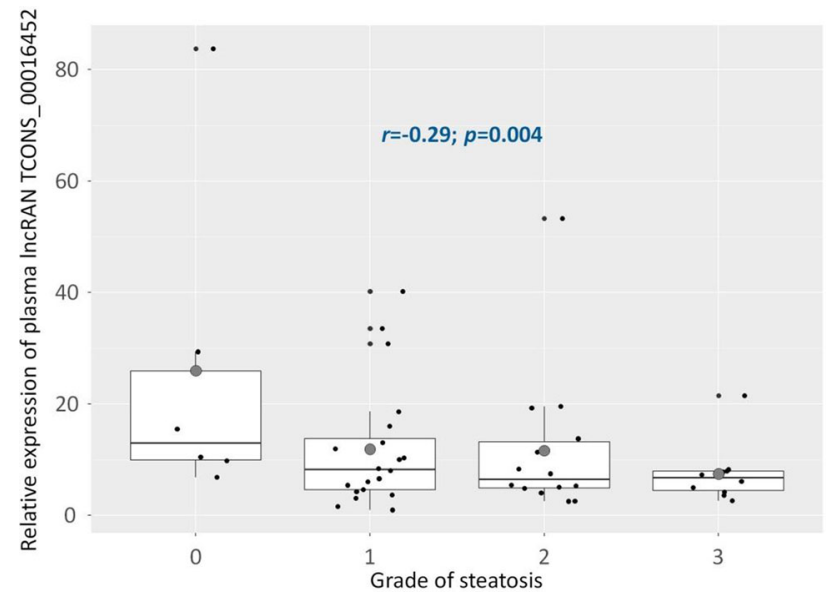

$N A F L-3$

Differential DNA methylation of PPAR-gamma genes in patients with non-alcoholic fatty liver disease

Yu Rim Lee ${ }^{1}$, Soo Young Park ${ }^{1}$, Keun Hur ${ }^{3}$, Eunhye Lee ${ }^{3}$, Se Young Jang', Won Young Tak ${ }^{1}$, Young Oh Kweon ${ }^{1}$, Bina Jeong', Gyouneun Kang', Sangkyung Seo ${ }^{1}$, Jung Gil Park ${ }^{4}$, Hye Won Lee ${ }^{2}$

${ }^{1}$ Kyungpook National University Hospital, Korea, Republic of; ${ }^{2}$ Dongsan Medical Center, School of Medicine, Keimyung University, Korea, Republic of; ${ }^{3}$ School of Medicine, Kyungpook National University, Korea, Republic of; ${ }^{4}$ Yeungnam University Medical Center, Korea, Republic of

Background: NAFLD can lead to the development of liver fibrosis and cirrhosis but only in a minority of patients. Differential liver DNA methylation of peroxisome proliferator-activated receptor gamma (PPAR-gamma) gene promoter has been shown to distinguish patients in terms of fibrosis severity in NAFLD. However, methylation of PPAR-gamma gene promoter in human with sufficient number is still needed. We therefore determined the prognostic significance of DNA methylation of PPAR-gamma gene promoter for patients with NAFLD.
Method: This prospective study enrolled 18 normal and 54 biopsy proven NAFLD patients who attended Kyungpook National University Hospital, Korea, Republic of between March 2015 and October 2016. We extracted genomic DNA from liver tissue obtained by biopsy. Bisulfite modification of genomic DNA was performed and that was confirmed for PPAR-gamma methylation levels with pyrosequencing.

Result: The average of $4 \mathrm{CpG}$ methylation of PPAR-gamma promoter had significantly lower in the NAFLD group (15.03\% DNA methylation) when compared to the normal patients (19.55\%). Quantitative DNA methylation of PPAR-gamma stratified patients into mild (Kleiner 0-2) and severe (Kleiner 3-4) fibrosis (14.21\% vs $17.87 \%$, $\mathrm{p}=0.007)$. Moreover, hypermethylation at the PPAR-gamma promoter of liver were also associated with higher NAFLD fibrosis score, degree of steatosis, and the presence of DM ( $\mathrm{p}=0.012,0.027,0.024$, respectively), but they were not associated with presence of liver inflammation, ballooning, presence of NASH, NAFLD activity score, and other liver function test. Patients with advanced fibrosis exhibited significantly higher NAFLD fibrosis score, FIB-4, and PPAR-gamma DNA methylation (all $\mathrm{p}<0.05$ ) and showed borderline significance with transient elastography $(\mathrm{p}=0.084)$. These markers, including NAFLD fibrosis score, FIB-4, and PPAR-gamma DNA methylation, had an area under the receiver operating curve (AUROC) of 0.855 , $0.778,0.75$, respectively for predicting advanced fibrosis.

Conclusion: DNA methylation level PPAR-gamma may be useful in NAFLD patients for predicting progression of liver fibrosis, which is a very important factor in disease progression that is currently impossible to predict. Together with other markers like NAFLD fibrosis score, FIB-4, transient elastography, it may be possible to generate an algorithm that can predict more precisely which patients are likely to progress on to a severe fibrosis in patients with NAFLD.

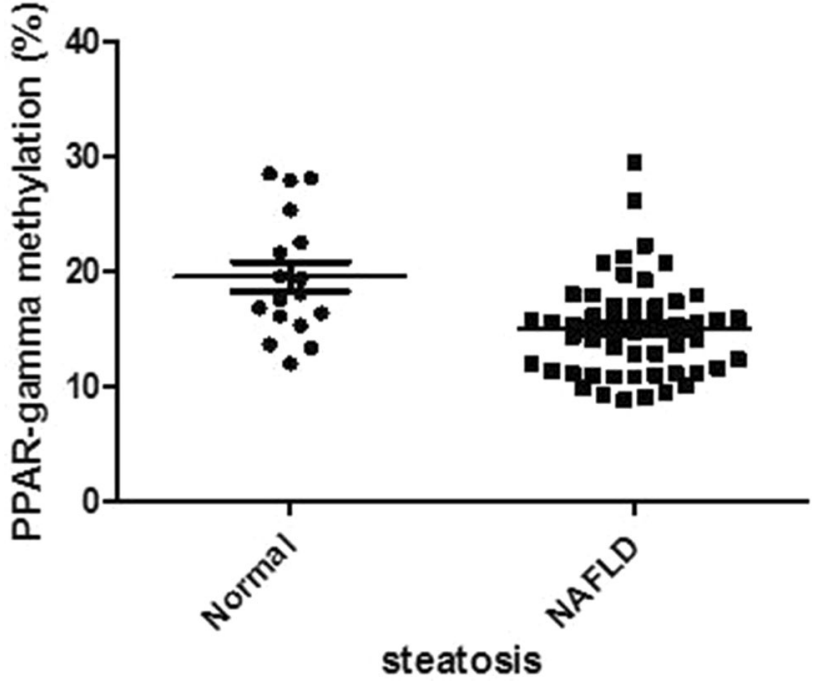




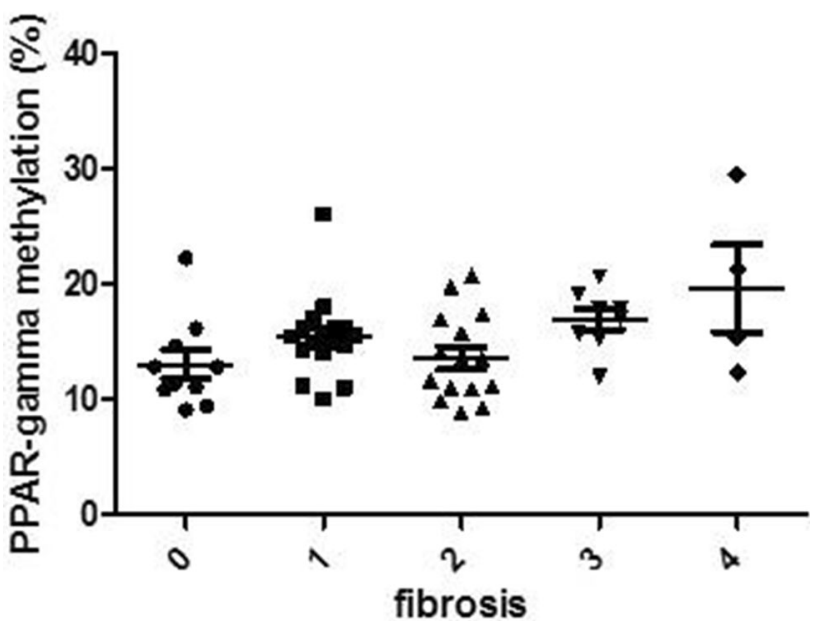

NAFL-4

Liver and circulating long non-coding RNA GAS5 is correlated with liver fibrosis in patients with non-alcoholic fatty liver disease

Jung Gil Park ${ }^{1}$, Soo Young Park ${ }^{3}$, Keun Hur ${ }^{3}$, Heon Ju Lee ${ }^{4}$, Won Young Tak ${ }^{3}$, Young Oh Kweon ${ }^{3}$, Se Young Jang ${ }^{3}$, Yu Rim Lee ${ }^{3}$, Gyeonghwa Kim ${ }^{3}$, Jihyun Lee ${ }^{3}$, Eunhye Lee ${ }^{3}$, Hye Won Lee ${ }^{2}$

${ }^{1}$ College of Medicine, Yeungnam University, Korea, Republic of; ${ }^{2}$ Dongsan Medical Center, School of Medicine, Keimyung University, Korea, Republic of; ${ }^{3}$ School of Medicine, Kyungpook National University, Korea, Republic of; ${ }^{4}$ Yeungnam University, Korea, Republic of

Background: Non-alcoholic steatohepatitis is an important process in progressing liver fibrosis. However, presence of advanced liver fibrosis is more important prognostic factor in patients with nonalcoholic fatty liver disease (NAFLD). Long non-coding RNA (lncRNA) GAS5 is related with inhibition of liver fibrogenesis by competing endogenous RNA.

Method: To evaluate the both liver and circulating lncRNA GAS5 in patients with NAFLD, we analyzed a total of fifty-one patients with NAFLD confirmed by percutaneous liver biopsy, which was reviewed by one pathologist. Expression of lncRNA GAS5 in both liver and plasma was analyzed using quantitative real-time polymerase chain reaction in NAFLD patients according to stage of fibrosis $(\mathrm{F} 0 ; \mathrm{n}=8$, $\mathrm{F} 1 ; \mathrm{n}=21, \mathrm{~F} 2 ; \mathrm{n}=10, \mathrm{~F} 3 ; \mathrm{n}=6, \mathrm{~F} 4 ; \mathrm{n}=6$ ).

Result: Expression of lncRNA GAS5 was significantly increased in patients with advanced fibrosis compared to patients without advanced fibrosis (liver, $4.5 \pm 1.6$ vs $6.3 \pm 2.8, p=0.026$; plasma, $8.7 \pm 4.9,17.1 \pm 6.0, p=0.000)$. As the fibrosis was progressed, expression of lncRNA GAS5 in both liver and plasma was increased except cirrhosis (liver; $r$-value $=0.305 ; p=0.042$, plasma; $r$ value $=0.452 ; p=0.002$ ). However, expression of lncRNA GAS5 in plasma was decreased in patients with cirrhosis compare to advanced fibrosis $(8.8 \pm 5.9$ vs $17.1 \pm 6.0, p=0.036)$.

Conclusion: Both liver and circulating LncRNA GAS5 is correlated with stage of liver fibrosis before cirrhosis occurs.
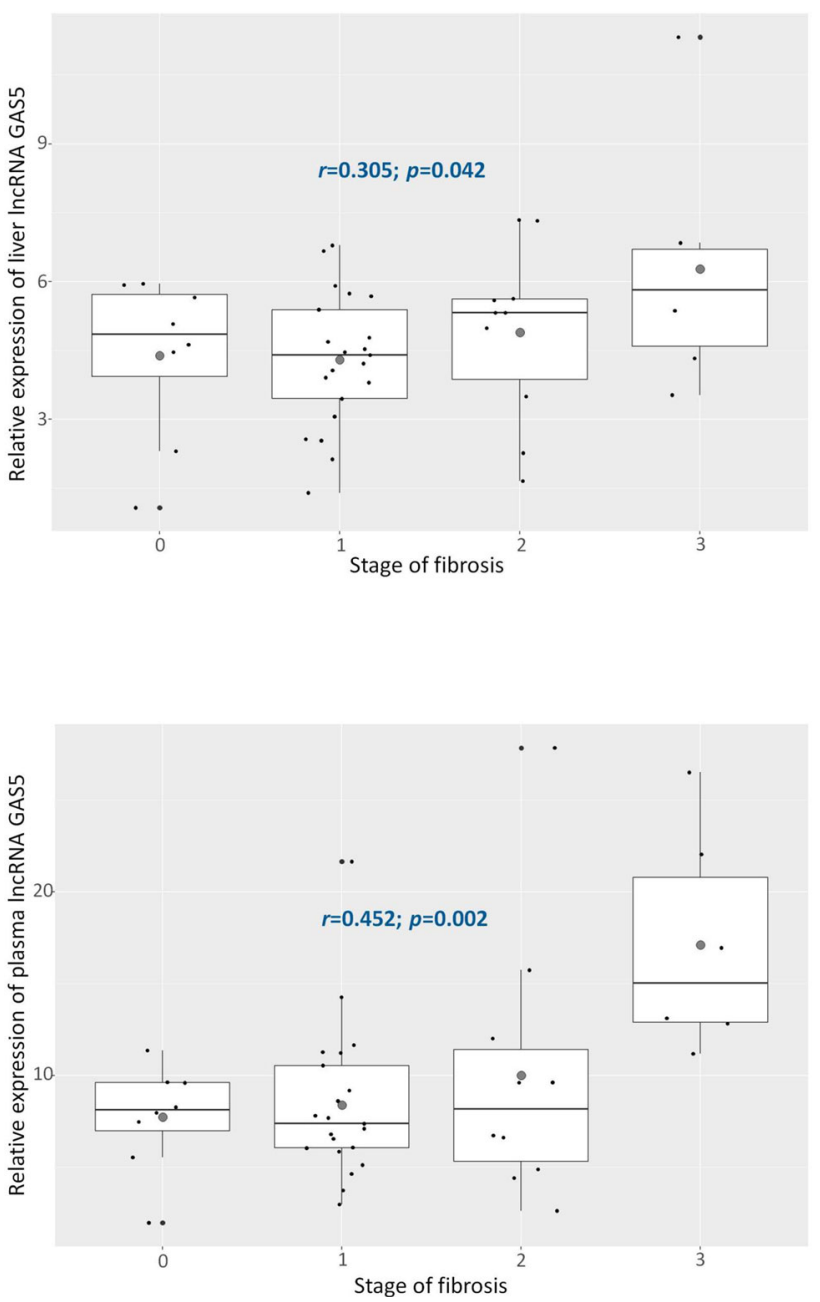

NAFL-5

Urinary Lipocalin 2 and non alcoholic fatty liver disease

Eman Abdelsameea ${ }^{1}$, Maha Elsabawy ${ }^{2}$, Soha El Shinnawy ${ }^{2}$, Nabil Omar ${ }^{2}$, Mary Naguib ${ }^{2}$

${ }^{1}$ Assistant Professor, Egypt; ${ }^{2}$ National Liver Institute, Menoufia University, Egypt

Background: Early diagnosis and appropriate management of Non alcoholic Fatty Liver Disease (NAFLD) might have a great effect on minimizing complications. However, liver biopsy is still the gold standard method for diagnose.

Aim of the work: To evaluate urinary Lipocalin 2 as a possible noninvasive marker of NAFLD diagnosis and progression.

Method: This case control study was conducted on 40 NAFLD patients merely diagnosed by liver biopsy with assessment of steatosis $\&$ inflammation grades by NAFLD scoring system. Forty apparently healthy subjects with well-matched age- and sex were also enlisted. Demographic, clinical, laboratory, ultrasonographic, and biopsy data of all subjects were registered. Urinary Lipocalin 2 was assessed by enzyme-linked immunosorbent assay.

Result: The mean age of NFLD patients was $35.35 \pm 9.53$ years old, with essentially male predominance $77.5 \%(\mathrm{n}=31)$. Urinary Lipocalin 2 was significantly higher in NAFLD patients $(5085.2 \mathrm{pg} / \mathrm{ml})$, than control group $(238.46 \mathrm{pg} / \mathrm{ml})(\mathrm{p}=0.001)$. A cutoff value of 
$855 \mathrm{pg} / \mathrm{ml}$ or more, was determined to define NAFLD with $97.4 \%$ sensitivity $100 \%$ specificity $(\mathrm{P}$-value $=0.001)$, accuracy $98.7 \%$, and AUR 0.974. According to histopathological examination of liver biopsy; NAFLD patients were furtherly subdivided into simple steatosis (31 patients) and nonalcoholic steatohepatitis (NASH) (9 patients). In spite of being high in both subgroups $(5070.9 \mathrm{pg} / \mathrm{ml}$ in simple steatosis and $5148.57 \mathrm{pg} / \mathrm{ml}$ in NASH), urinary Lipocalin 2 failed to distinguish between both subgroups $(\mathrm{p}=0.05)$.

Conclusion: Urinary Lipocalin 2 could be a simple, a non-invasive diagnostic rather than prognostic test of NAFLD.

\section{NAFL-6}

\section{Constructing engineering liver from recellularization of naturally-sourced scaffolds as ex vivo model of NAFLD}

\section{Qiao $\mathrm{Wu}^{1}$}

${ }^{1}$ Capital Medical University, China

Background: Currently, non-alcoholic fatty liver (NAFLD) is considered to be one of the most common liver disease in the world. In spite of Intense research efforts, knowledge about how to identify disease and molecular mechanisms are still limited. It is urgent to develop more accurate model to investigate this disease. This study is to construct a human ex vivo model of non-alcoholic fatty liver disease (NAFLD), utilising HepG2 recellularizing rat decellularized liver scaffolds in a three-dimensional (3D) perfused bioreactor platform.

Method: Using an organ engineering approach and relevant cell lines, we designed a perfusion system that delivers medium with free fat acid to cells resided in acellular liver extracellular matrix scaffold to study the effects of anti-steatosis drug in hepatic function. Oil Red O staining was used to measure fat loading in the hepatocytes and the consumption of free fatty acids (FFA) from culture medium was monitored. Hepatic functions and gene expression profiles were compared for cells cultured in $3 \mathrm{D}$ and $2 \mathrm{D}$ conditions. To determine if fat loading in the system could be modulated perfused with known anti-steatotic compounds.

Result: Human HepG2 demonstrate enhanced metabolizing enzyme activity/albumin synthesis in 3D perfusion ECM system, thus demonstrating biocompatibility and relevance for evaluating drug metabolism and toxicity. HepG2 cultured in fatty medium were found to accumulate more fat than control medium and fat uptake was continuous over a 5-d culture. Fat loading of HepG2 did not cause any hepatotoxicity and significantly increased albumin and urea production. Adipokines were expressed by fatty cells and genes associated with NAFLD and liver disease were upregulated. The utility of the model for drug screening was demonstrated by measuring the effects of known antisteatotic compounds. HepG2, cultured under fatty conditions and treated with metformin, had a reduced cellular fat content compared to untreated controls and consumed less FFA from cell culture medium.

Conclusion: The bioengineered liver as in vitro NAFLD model recapitulates features of clinical NAFLD and is an ideal tool for analysing the efficacy of anti-steatotic compounds.
$N A F L-7$

\section{Elafibranor and obeticholic acid differentially alter NASH and lipid metabolism in diet-induced NASH mouse and hamster models}

Francois Briand $^{1}$, Emmanuel Brousseau ${ }^{1}$, Nourdine Faresse ${ }^{1}$, Maureen Pont $^{\top}$, Clement Costard ${ }^{1}$, Isabelle Urbain ${ }^{1}$, Marjolaine Quinsat ${ }^{1}$, Thierry Sulpice ${ }^{1}$

${ }^{1}$ PHYSIOGENEX, France

Background: Non-alcoholic steato-hepatitis (NASH) mouse models present limitations for evaluating drugs, due to a different lipid/bile acids metabolism as compared with human. To overcome this issue, we developed a Diet-induced NASH (DIN) hamster model with features of human-like lipid/bile acids metabolism. Here we evaluated the benchmarks elafibranor (GFT505) and obeticholic acid (OCA), in both DIN mouse and hamster models.

Method: Effects of GFT505 and OCA both at $15 \mathrm{mg} / \mathrm{kg} / \mathrm{day}$ were evaluated in DIN C57BL6/J mice fed a high fat/cholesterol/fructose rich diet, or DIN hamsters fed a cafeteria diet. Fasting lipids assays, liver histology and NAS scoring were performed at the end of treatment.

Result: In the DIN mouse, OCA and GFT505 significantly reduced total NAS score (both $\mathrm{p}<0.001$ vs. vehicle), including reduced steatosis, hepatocyte ballooning, and fibrosis, while inflammation was only reduced by OCA. Plasma total cholesterol (TC) and LDL-cholesterol levels were reduced by $33 \%$ and $45 \%$ with OCA (both $\mathrm{p}<0.001$ vs. vehicle). GFT505 did not change plasma TC but reduced plasma triglycerides $(-32 \%)$ and strongly increased plasma ketone bodies $(+307 \%)$ levels (both $\mathrm{p}<0.001$ vs. vehicle), in line with an inhibition of diet-induced body weight gain.

In the DIN hamster, both OCA and GFT505 reduced total NAS score, including significant reduction in inflammation and trends towards lower ballooning scores, while no significant change was observed for liver steatosis and fibrosis. Hamsters treated with OCA showed significantly higher plasma cholesteryl ester transfer protein activity by $18 \%$, higher LDL-cholesterol levels by $27 \%$, and lower HDL-cholesterol levels by $20 \%$ vs. vehicle. Trends towards lower LDLreceptor and higher Scavenger Receptor class B type I hepatic protein levels were also observed. GFT505 reduced fasting plasma total cholesterol, triglycerides and free fatty acids levels by 13,10 and $16 \%$ (all $\mathrm{p}<0.05$ vs. control), while fasting plasma ketone bodies levels increased by $35 \%(\mathrm{p}<0.05)$. GFT505 reduced hepatic fatty acids levels by $22 \%$ ( $p<0.05$ vs. control) and induced a $40 \%$ lower liver gene expression of Glucose-6-phosphatase, and 35\% higher gene expression of carnitine palmitoyltransferase $1 \mathrm{~A}$ (both $\mathrm{p}<0.05$ ).

Conclusion: OCA and GFT505 differentially alter lipid metabolism and NASH depending on the DIN model. While both drugs markedly improved lipid profile and NASH in DIN mice, moderate improvements were also seen in DIN hamsters. Importantly, the DIN hamster is the only model showing the same dyslipidemic effect observed in humans treated with OCA. For better translation to human, drug development should not be limited to mouse, but rather extended with models showing a lipid and NASH profile closer to human, such as the DIN hamster. 
$N A F L-8$

Exome-wide association study with hepatic fat content in nonalcoholic fatty liver disease reveals significant association with 5 novel QTLs

Ankita Chatterjee $^{1}$, Kausik Das ${ }^{3}$, Pankaj Singh ${ }^{5}$, Dipankar Mondal ${ }^{5}$, Ranajoy Ghosh ${ }^{7}$, Abhijit Chowdhury ${ }^{8}$, Badal Dey', Analabha Basu' ${ }^{3}$, Partha P. Majumder ${ }^{4}$, Biswanath Bhattacharyya ${ }^{6}$, Priyadarshi Basu ${ }^{2}$

${ }^{1}$ RESEARCH SCHOLAR, India; ${ }^{2}$ ASSISTANT PROFESSOR, India; ${ }^{3}$ ASSOCIATE PROFESSOR, India; ${ }^{4}$ Distinguished Professor, India; ${ }^{5} \mathrm{DM}$, STUDENT, India; ${ }^{6}$ FIELD ASSISTANT, India;

${ }^{7}$ HISTOPATHOLOGIST, India; ${ }^{8} \mathrm{HOD}$, India; ${ }^{9}$ LABORATORY MANAGER, India

Background: Non-alcoholic fatty liver disease (NAFLD) is a global epidemic related to metabolic syndrome and obesity. In contrast to most world populations, lean NAFLD phenotype is prevalent in Indians and generally to South and South-East Asians suggesting a unique phenotypic presentation. In spite of various genetic association studies with NAFLD performed across different populations, studies from Indian populations are rare. In this study we have used an exome-wide approach to identify genetic determinants of hepatic fat content (HFC) in NAFLD.

Method: HFC was measured for 261 individuals using MRS and for a subset of 146 individuals liver biopsy was performed for histopathological evaluation. Relationship of the clinico-pathological features with the HFC was assessed using linear regression. We genotyped the 261 individuals using Illumina-Human Exome Bead chip version 1.2. After data curation, and adjusting for age, gender and other covariates; quantitative trait association analysis was performed with HFC on 34320 SNPs. Associations of the significant QTLs with disease severity were further tested among 146 individuals for whom liver biopsy data was available.

Result: Our study identified positive correlations of ALT, BMI, serum triglyceride and fasting blood glucose with HFC. A total of 9 SNPs were found to be significantly associated (p-value $<0.0001$ ) with the HFC adjusted for age, gender, ALT, BMI, Triglyceride and fasting blood glucose. Of these 9 QTLs, 4 SNPs including rs738409 and rs2281135 (PNPLA3), rs58542926 (TM6SF2) and rs3761472 in SAMM50 were previously reported to be associated with NAFLD. We thus have validated the robust association of SNPs in PNPLA3 and TM6SF2 gene with NAFLD in our population. We also identified 5 novel genetic loci in FAM161A (rs17513722), PNPLIRP3 (rs2116286), EVC (rs16837598), PKHD1 (rs2397060) and NPIPL1 (rs4788084) to be associated with HFC.

Conclusion: Our study also identified 5 novel SNPs associated with HFC and disease severity in NAFLD, among whom 2 SNPs have potentially damaging effect on protein function-rs16837598 (EVC) and rs2116286 (PNPLIRP3).

\section{NAFL-9}

\section{Hepatic Argonaute2 (Ago2): a molecular target of NASH} and obesity

Jashdeep Bhattacharjee ${ }^{1}$, Jashdeep Bhattacharjee ${ }^{1}$, Esam Salem ${ }^{2}$, Elise Bernhard $^{2}$, Vishnupriya J Borra ${ }^{2}$, Kazutoshi Murakami ${ }^{3}$, Rosa Maria Salazar Gonzalez ${ }^{1}$, Mikako Warren', Takahisa Nakamura ${ }^{3}$

${ }^{1}$ Children's Hospital Los Angeles, United States; ${ }^{2}$ Cincinnati Children's Hospital Medical Center, United States; ${ }^{3}$ Cincinnati Children's Hospital Medical Centre, United States
Background: In mammals, there are four Argonaute proteins (Ago), in which Ago2 uniquely possesses an endoribonuclease activity regulating biogenesis of microRNA and RNA silencing. Sleeve gastrectomy (VSG) is known to trigger a cascade of molecular events and induce weight loss and reversal of obesity related liver disease (NASH). We performed VSG on diet-induced obese mice to investigate the role of hepatic Ago2 on NASH improvement post VSG.

Method: 4-6 weeks old male C57BL6/J mice (wildtype, WT) and liver-specific Ago2 knockout mice (Ago2KO) were fed on high fat diet $(60 \mathrm{kcal} \%)$ for 30 weeks. The mice were randomly distributed in two groups (1) Sleeve gastrectomy (VSG) ( $n=4-6)$ and (2) sham operated (SHAM) $(n=4-6)$ for both WT and Ago2KO. Post-surgery the animals were observed for 28 days before sacrifice and subsequent pathological and molecular analysis of collected samples were performed.

Result: As expected post-op weight loss was lower in the SHAM groups compared to the corresponding VSG groups $(-5.36 \pm 1.44 \mathrm{~g}$ (WT SHAM) vs $-14.03 \pm 2.04 \mathrm{~g}$ (WT VSG); $-5.50 \pm 1.70 \mathrm{~g}$ (Ago2KO SHAM) vs $-9.93 \pm 1.55 \mathrm{~g}$ (Ago2KO VSG)). However, the Ago2KO VSG mice lost less weight post-VSG compared to WT mice that underwent VSG $(\mathrm{P}=0.0007)$ (Figure 1). Again as expected blood glucose levels of WT SHAM mice were higher than WT VSG group $(575.50 \pm 36.63 \mathrm{mg} / \mathrm{dl}$ vs. $434.25 \pm 14.23 \mathrm{mg} / \mathrm{dl} ; \mathrm{P}=0.01)$ however no significant difference was observed between the Ago2KO SHAM and Ago2KO VSG groups. The liver histological NAFLD activity score (NAS) was lowest for the WT VSG group but again no difference was observed between the Ago2KO groups (1.00 \pm 0.71 (WT VSG) vs $6.00 \pm 0.5774$ (WT SHAM); $4.75 \pm 0.95$ (Ago2KO SHAM); $4.34 \pm 0.67$ (Ago2KO VSG) (Figure 2). Though not statistically significant a similar trend was seen for plasma ALT. Further, with qPCR analysis we observed that $\alpha$-smooth muscle actin ( $\alpha$-SMA) was decreased in WT VSG compared to WT SHAM, but no such differ-

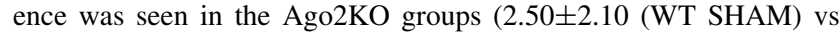
$0.72 \pm 0.07$ (WT VSG); $0.42 \pm 0.09$ (Ago2KO SHAM); $0.46 \pm 0.06$ (Ago2KO VSG)).

Conclusion: Ago2 appears to have a critical role in the outcomes of VSG. Ago2KO mice loose less weight post-VSG and do not have the same improvement in NASH seen in WT mice. Hepatic Ago2 may therefore be a novel therapeutic target for NASH and obesity.

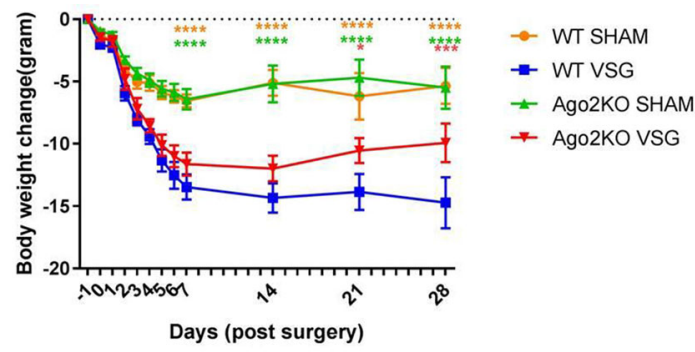

Figure 1.

Graphical representation of post VSG body weight change in VSG and SHAM group of WT and Ago2KO. ( 2 way ANOVA, $* * * * \mathrm{P}<0.0001 ; * * * \mathrm{P}<0.001 ; * \mathrm{p}<0.05$ ) 


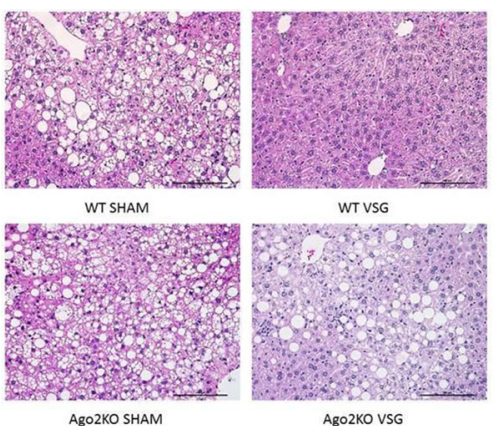

(A)

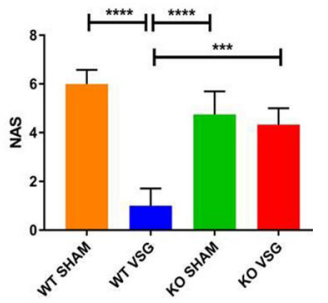

(B)
Figure 2.

(A) Representative histological images of liver (200X magnification) and

(B) NAFLD Activity Score (NAS) (2 way ANOVA, ${ }^{* * * *} \mathrm{P}<0.0001$; ${ }^{* * *} \mathrm{P}<0.001$ )

\section{NAFL-11}

Liver transcriptome study and its relation to clinicopathological features identify insulin resistance as a key player in the early stages of NAFLD among Indians

Priyadarshi Basu ${ }^{1}$, Ankita Chatterjee ${ }^{7}$, Analabha Basu' ${ }^{2}$, Kausik Das ${ }^{2}$, Pankaj Singh ${ }^{3}$, Dipankar Mondal ${ }^{3}$, Badal Dey ${ }^{5}$, Pratap Pandit ${ }^{8}$, Shweta Roychoudhury ${ }^{8}$, Ranajoy Ghosh ${ }^{4}$, Partha P. Majumder ${ }^{6}$, Abhijit Chowdhury ${ }^{6}$

${ }^{1}$ National Institute of Biomedical Genomics, India; ${ }^{2}$ ASSOCIATE PROFESSOR, India; ${ }^{3}$ DM STUDENT, India;

${ }^{4}$ HISTOPATHOLOGIST, India; ${ }^{5}$ LABORATORY MANAGER, India; ${ }^{6}$ PROFESSOR, India; ${ }^{7}$ RESEARCH FELLOW, India; ${ }^{8}$ RESEARCH TECHNICIAN, India

Background: Non-alcoholic fatty liver disease (NAFLD) is a multistage disease with heterogeneous outcomes and is related to obesity and Type II Diabetes. Molecular mechanisms at early stages of the disease are unclear. We aimed to study the risk factors and molecular changes in the early stages of NAFLD spectrum in the Indian context which is often not associated with overall obesity.

Method: In this study, two study groups were chosen - Mild NAFLD (NAFLD Activity Score (NAS) $=0 \& 1$ ) and Advanced NAFLD $(\mathrm{NAS}=4 \& 5)$. Clinico-pathological risk factors were compared between 72 Mild and 51 Advanced NAFLD subjects. Among them, difference in liver tissue transcriptome of 28 Mild and 20 Advanced NAFLD patients was studied using Illumina HT12v4 microarray. Validation of differential gene expression was done on an independent cohort (12 Mild and 11 Advanced NAFLD samples) using RTPCR.

Result: Among the risk factors studied, significant increase of weight $(\mathrm{p}$-value $=0.013), \mathrm{HbA1c}(\mathrm{p}$-value $=0.005)$ and insulin resistance $(I R$, $\mathrm{p}$-value $=0.025$ ) were found in Advanced NAFLD patients. 28 Differentially Expressed Genes (DEGs) involved in fatty acid uptake, ROS production and regulation, cell proliferation, cell adhesion and migration, ER stress and deregulated metabolism, were identified after multiple testing corrections $(q-v a l u e<0.05)$. Significant positive correlations between expressions of DEGs and severe diabetic profile were observed. PI3K-AKT pathway which functions in insulin signaling was identified as the most enriched pathway using pathway enrichment analysis with the DEGs.

Conclusion: Our evidences suggest that increased fat deposition due to increased fatty acid uptake and development of IR may cause deregulation of PI3K-AKT pathway in early stages of NAFLD spectrum. Subsequent changes like cell proliferation and migration along with deregulated metabolism, ROS production and ER stress, may lead to disease progression.

\section{$N A F L-12$}

Pathogenic role of IncRNA-AK012226 in $\mathrm{db} / \mathrm{db}$ mice fatty liver and NCTC1469 nonalcoholic fatty liver cell model

Hui Yang ${ }^{1}$, Xingtian Chen ${ }^{1}$, Yangzhi Xu ${ }^{1}$, Dan Zhao ${ }^{1}$, Ting Chen ${ }^{1}$, Chengxin $\mathrm{Gu}^{1}$, Ganxiang $\mathrm{Yu}^{1}$, Ken Chen ${ }^{2}$, Jie $\mathrm{He}^{3}$, Yun Zhong ${ }^{4}$, Shiming Liu ${ }^{4}$, Yuqiang $\mathrm{Nie}^{3}$

${ }^{1}$ Second Affiliated Hospital of Guangzhou Medical University, China; ${ }^{2}$ Guangdong Pharmaceutical University, China; ${ }^{3}$ Guangzhou First People's Hospital, China; ${ }^{4}$ Guangzhou Institute of Cardiovascular Disease, China

Background: Obesity and the related metabolic abnormalities are increasing in the world. Nonalcoholic fatty liver disease (NAFLD) is a common disorder in obese people and is becoming the leading cause of HCC worldwide. Recently, long non-coding RNA (lncRNA) has been proved to play remarkable roles in numerous biological processes and human diseases, including NAFLD. However, the function of lncRNA in NAFLD pathogenesis remains largely unknown. The aim of this study is to explore the lncRNA expression profile in NAFLD mice and identify novel lncRNAs involved in the pathogenesis of NAFLD.

Method: A microarray analysis (Mouse LncRNA Microarray V2.0, Arraystar) was performed to study the liver lncRNA and mRNA profiles of diabetic $\mathrm{db} / \mathrm{db}$ mice with NAFL as compared to normal C57BLK/S mice. Some interested differentially expressed (DE) IncRNAs was confirmed by quantitative RT-PCR in the mice liver samples and NCTC1469 cells treated with free fatty acid (FFA). Next, co-expression network of AK012226-mRNAs was constructed and Kyoto Encyclopedia of Genes and Genomes (KEGG) pathway analysis and GO (Gene Ontology) analysis were performed. We knocked down the AK012226 expression by siRNA and studied the effect on lipid accumulation in NCTC1469 cells treated with FFA via Nile red staining and flow cytometric analysis.

Result: A total of 3360 lncRNAs (2048 up-regulated and 1312 downregulated) and 2685 mRNAs (1195 up-regulated and 1490 downregulated) were found differentially expressed between NAFL group and control group. Five DE lncRNAs (AK012226, B430212C06Rik, Fabp3-ps1, NR-040532, AK052193) that were selected for qPCR validation in the liver samples were consistent with the microarray results. Besides, the up-regulated lncRNA AK012226 was also significantly increased in the NCTC1469 NAFLD cellular model. Thus, the up-regulated IncRNA AK012226 was chosen for the further study. Subsequently, the co-expression network of AK012226-mRNAs revealed 218 co-expressed mRNAs and bioinformatic analysis of these co-expressed mRNAs indicated that they were enriched in the PPAR signaling pathway. Further, Nile Red Staining and flow cytometry analysis reveal that knockdown of AK012226 by siRNA significantly reduced lipid accumulation in NCTC1469 cells treated with free fatty acid.

Conclusion: The current study identified dysregulated lncRNAs and mRNAs involved in NAFLD, and in particular, we identified a novel lncRNA AK012226 that was associated with lipid accumulation in NAFLD and may provide a potential therapeutic target for NAFLD. 
NAFL-13

PNPLA3, IL-28 and IL-6 single nucleotide polymorphisms and co-occurrence of non alcoholic fatty liver disease in nongenotypes-3 chronic hepatitis $\mathrm{C}$

Ragesh Babu Thandassery ${ }^{1}$, Antony Raphael Joy ${ }^{1}$, Fayaz Ahmad Mir $^{1}$, Madiha Emran Soofi ${ }^{1}$, Hamid Ullah Wani ${ }^{1}$, Saad Al Kaabi ${ }^{1}$

${ }^{1}$ Hamad Medical Corporation, Qatar

Background: The genetic predisposition to non alcoholic fatty liver disease (NAFLD) is not well described in Middle Eastern population. Patients with chronic hepatitis $\mathrm{C}$ (CHC) could have coexisting NAFLD. Non-genotypes-3 CHC is believed to develop hepatic steatosis from non viral factors. In this study we attempt to describe correlation between hepatic steatosis and single nucleotide polymorphisms (SNP) in 3 candidate genes- PNPLA3, IL-6 and IL-28 in nongenotypes-3 $\mathrm{CHC}$ patients.

Method: We analyzed the role of SNPs rs738409 in PNPLA3, rs 12980275 in Il28B and rs2069837 in IL 6 in patients with CHC who have undergone liver biopsy (LB) as part of treatment evaluation between January 2013 and December 2014. DNA extraction was done from LB specimens and SNPs identified and characterized in triplicate. Associations of alleles with the presence and/or severity of hepatic steatosis were evaluated by univariate and multivariate logistic regression analysis, considering all relevant covariates.

Result: Of the total 512 patients, mean age was $44.8 \pm 11.4$ years (86.6\% males), predominantly genotype $4(66.1 \%)$, followed by genotype $1(11.9 \%)$ and genotype $3(10.3 \%)$. Pretreatment LB showed (Scheuer classification) stage-0 fibrosis (F0) in 24.6\%, stage-1 (F1) in $34.6 \%$, stage-2 (F2) in $22.9 \%$, stage- 3 (F3) in $12.4 \%$ and stage -4 (F4) in $5.6 \%$ patients. Of the SNPSs studied rs 738409 of PNPLA3 was independently associated with presence of hepatic steatosis ((odds ratio $=1.7,95 \%$ confidence interval $=1.4-2.3, p=0.002)$. rs 12980275 in Il28B and rs2069837 in IL 6 did not show a correction with presence or severity of hepatic steatosis on univariate analysis. The presence of metabolic syndrome, body mass index, hepatis $\mathrm{C}$ viral genotype and viral load were not significantly related to occurrence and severity of hepatic steatosis. There was no correlation between any of the SNPs studied and fibrosis stage in LB, grade of inflammation in LB or treatment response (pegylated interferon-ribavirin and direct acting antivirals) to $\mathrm{CHC}$.

Conclusion: In a Middle Eastern cohort of patients with chronic nongenotypes-3 hepatitis C virus infection, rs738409 SNP of PNPLA3 was independently associated with presence of hepatic steatosis. However rs12980275 SNP in Il28B and rs2069837 SNP in IL 6 were not associated with presence and/or severity of hepatic steatosis.

\section{NAFL-14}

Normalizing iron metabolism by Laennec ${ }^{\circledR}$ derived from humanplacenta through the action of hepcidin can improve NASH complicating with type 2 diabetes

\section{Yuki Hamada ${ }^{1}$}

\section{${ }^{1}$ HAMADA CLINIC for Gastroenterology, Japan}

Background: The discovery of hepcidin in 2001 and the subsequent rapid understandings on its correlation with many kinds of metabolic disorders have dramatically changed our clinical understandings of human diseases relating with iron metabolism. Both liver and pancreas should be regarded as endocrine organs in the interaction of hepcidin and insulin. In many NASH cases complicating with type 2 diabetes, remarkable declines of serum ferritin and HbAlc were observed after treating with Laennec ${ }^{\circledR}$ (derived from human placenta). Then, we examined whether Laennec ${ }^{\circledR}$ can improve the pathological background of type 2 diabetes through regulating iron metabolism

Method: We divided 68 NASH cases (all cases liver biopsied) into two groups retrospectively. Laennec ${ }^{\circledR}$-treated 38 cases were treated with the infusion of 2 ampules (224 mg) of Laennec ${ }^{\circledR} 1-2$ times/w, in addition to the ordinary liver supporting. Serum ferritin, ALT and $\mathrm{HbA1c}$ were measured, and liver re-biopsy was carried out to evaluate changes of iron deposition in 20 cases.

Result: By infusing Laennec ${ }^{\circledR}$, serum ferritin level declined from $268.4 \pm 308.3 \mathrm{ng} / \mathrm{ml}$ (before medication) to $59.3 \pm 46.1$ (after) (Wilcoxon $\mathrm{P}<0.01$ )

Conclusion: The improvement of type 2 diabetes complicating with NASH by the administration with Laennec ${ }^{\circledR}$ suggests the importance of iron regulation on insulin resistant type 2 diabetes which shows hyperferritinemia.

NAFL-15

Associations between metabolic syndrome and hepatic fat content measured by MRI in NAFLD patients

Yong Kyun Cho ${ }^{1}$, Woo Kyu Jeon ${ }^{1}$, Hong Joo Kim ${ }^{1}$, Dong Il Park ${ }^{1}$, Seung Jae Lee ${ }^{1}$, You Gyung Kim¹, Heon Ju Kwon', Byung Ik Kim ${ }^{1}$

${ }^{1}$ Kangbuk Samsung Hospital, Korea, Republic of

Background: Hepatic fat accumulation have key role in pathogenesis of insulin resistance and metabolic syndrome in patients with NAFLD (Non alcoholic fatty liver disease), but the relationship between hepatic fat content measured by MRI and metabolic syndrome components has not been determined.

Method: A total of 649 middle-aged nondiabetic NAFLD subjects with and without metabolic syndrome [male $\mathrm{n}=539(83.1 \%)$, female $\mathrm{n}=110$ subjects $(16.9 \%)$, median age, 51.8 years; range, 31-79] quantified hepatic fat by using $1.5 \mathrm{~T}$ MRI examination including double-echo chemical shift imaging for calculating fat fraction in the liver. The liver fat fraction was defined as follow: (signal intensity inphase - signal intensity out-of-phase)/(2x signal intensity in-phase). We also examined how hepatic fat content is related to individual component of the metabolic syndrome using multivariate logistics regression analysis.

Result: Hepatic fat content was 3-fold higher in subjects with $(\mathrm{n}=146$, $7.85 \pm 7.5 \%$ mean $\pm \mathrm{SD})$ than without $(\mathrm{n}=494,2.58 \pm 4.8 \% \mathrm{P}<0.001)$ the metabolic syndrome. The increase in hepatic fat content remained significant after adjusting for age, gender. Hepatic fat content was correlated significantly with HOMA-IR $(\mathrm{OR}=2.81,95 \% \mathrm{CI} 2.26-3.48$, $\mathrm{P}<0.001)$, presence of metabolic syndrome $(\mathrm{OR}=5.56$, 95\% CI 3.70 8.34, $\mathrm{P}<0.001)$, number of metabolic syndrome components using WHO clinical criteria $(\mathrm{OR}=2.08,95 \% \mathrm{CI} 1.76-2.45, \mathrm{P}<0.001)$. Among metabolic syndrome components, the best correlates of hepatic fat content was BMI $(\mathrm{OR}=1.46,95 \% \mathrm{CI} 1.34-1.58, \mathrm{P}<0.001)$.

Conclusion: Hepatic fat content was significantly higher in middleaged nondiabetic NAFLD patient with metabolic syndrome. Insulin resistance and presence of metabolic syndrome were key determinants of increased hepatic fat content in NAFLD. 
NAFL-16

Comparison of metabolic risk factors amongst urban and rural patients with NAFLD - a community based study

\section{Ajay Duseja ${ }^{1}$, Ajay Duseja ${ }^{3}$, Anuradha Chakraborti ${ }^{2}$,} P. V. M. Lakshmi ${ }^{2}$

${ }^{1}$ Postgraduate Institute of Medical Education and Research, Chandigarh, India; ${ }^{2}$ Post Graduate Institute of Medical Education and Research, Chandigarh, India; ${ }^{3}$ Post Graduate Institute of Medical Education and Research, Chandigarh, India

Background: There is a limited epidemiological community-based data on the prevalence of nonalcoholic fatty liver disease (NAFLD) and its metabolic risk factors from India. Majority of available data is from hospital based studies on small number of patients. Present study assessed the prevalence of NAFLD in urban and rural communities of north India and measured the metabolic risk factors among them.

Method: In a prospective epidemiological cross-sectional study done over a period of one year, 171 urban and 130 rural subjects were analyzed for the prevalence of NAFLD based on ultrasound. Metabolic syndrome (MS) was assessed by International Diabetic Federation criteria with modified waist for the Asia Pacific region. Patients with $\geq 3$ components out of 5 were defined as having MS. Clinical examination, anthropometric measurements, biochemical assessment and viral markers were done in all subjects.

Result: In urban community, the prevalence of NAFLD was 56\% (95/ 171). Out of 95 patients with NAFLD, 66(69.4\%) had mild steatosis, $18(19.0 \%)$ had moderate steatosis, $11(11.6 \%)$ had severe steatosis. MS was present in $70(73.7 \%)$ patients with NAFLD. 90(94.7\%) NAFLD patients were either overweight [12(12.6\%)] or obese [78(82.1\%)], with mean BMI $29.0 \pm 4.6 \mathrm{~kg} / \mathrm{m}^{2}$. Among NAFLD patients, $94(98.9 \%)$ had central obesity, 32(33.7\%) had dysglycemia, $49(51.6 \%)$ had high blood pressure, $36(37.9 \%)$ had hypertriglyceridemia and 79(83.2\%) had low HDL cholesterol.

In rural community, the prevalence of NAFLD was 33\% (43/130). Out of 43 patients with NAFLD, 32(74.4\%) had mild steatosis, $8(18.6 \%)$ had moderate steatosis, $3(7.0 \%)$ had severe steatosis. MS was present in 24(55.8\%) patients with NAFLD. 34(79.1\%) NAFLD patients were either overweight [7(16.3\%)] or obese [27(62.8\%)], with mean BMI $26.5 \pm 4.9 \mathrm{~kg} / \mathrm{m}^{2}$. Among NAFLD patients, $36(83.7 \%)$ had central obesity, $17(39.5 \%)$ had dysglycemia, $24(55.8 \%)$ had high blood pressure, $18(41.9 \%)$ had hypertriglyceridemia and $31(72.1 \%)$ had low HDL cholesterol.

The prevalence NAFLD was higher in urban in comparison to rural community $(56 \%$ vs $33 \%)(\mathrm{p}=<0.001)$. The urban participants [mean age $47.2 \pm 16.1$ years, males $=56(32.7 \%)$ ] were older than rural participants [mean age 39.6 \pm 15.4 years, males $=43(33.1 \%)$ ] $(\mathrm{p}=<0.001)$. They had significantly higher mean weight $(70.4 \pm 12.3 \mathrm{~kg} \quad$ vs $60.1 \pm 13.8 \mathrm{~kg}, \quad \mathrm{p}=<0.001)$, mean BMI $\left(27.2 \pm 4.5 \mathrm{~kg} / \mathrm{m}^{2}\right.$ vs $\left.23.6 \pm 5.0 \mathrm{~kg} / \mathrm{m}^{2}, \mathrm{p}=<0.001\right)$, central obesity ( $88.9 \%$ vs $60.0 \%, p=<0.001)$, low HDL cholesterol $(81.3 \%$ vs $63.8 \%$, $\mathrm{p}=0.001)$ and $\mathrm{MS}(55.6 \%$ vs $40.0 \%, \mathrm{p}=0.007)$ in comparison to rural participants.

NAFLD was significantly associated with MS in urban [Odds ratio (OR) 5.7, 95\% confidence interval (CI) 2.9-11.1 ( $<<0.001)]$ and rural community (OR 2.7, 95\% CI 1.3-5.6 $(\mathrm{p}=0.010)$ ].

Conclusion: Prevalence of NAFLD is significantly higher in urban population as compared to rural population of north India. Metabolic risk factors are more common in urban as compared to rural patients with NAFLD.
NAFL-17

Epidemiological survey of prevalence of fatty liver disease and its risk factors in urban population of Tibet Autonomous Region China

\section{$\underline{\text { Qingping Wen }}{ }^{1}$, Suolangpuchi Suolangpuchi ${ }^{3}$, Xiyue $\mathbf{L u}^{2}$, Qingping Wen ${ }^{1}$}

${ }^{1}$ TIBET AUTONOMOUS REGION PEOPLE'S HOSPITAL, China; ${ }^{2}$ Tibet Fukang Hospital Physical Examination Center, China; ${ }^{3}$ Tibet Fukang Hospital, China

Background: To investigate the prevalence and risk factors of fatty liver among adult residents in Tibet Autonomous Region. The purpose is to understand the characteristics of the population in the area and to provide evidence for prevention, intervention and management of fatty liver.

Method: A total of 25785 cases with Abdominal ultrasound examination and diagnosis of fatty liver in our physical examination center from January 2017 to July 2017 were included in this study. The age, body mass index, blood pressure, biochemical test results, blood glucose, triglyceride, total cholesterol, low density lipoprotein and high density lipoprotein in patients with fatty liver were compared with those in non-fatty liver group. FibroTouch testing and analysis of the prevalence of fatty liver were performed on some randomized subjects.

Result: The cohort included 18-95 years old, with an average age of (36.62 \pm 11.88$)$ years and a prevalence of fatty liver of $15.40 \%$ $(\mathrm{n}=4007)$, and a prevalence rate of $18.82 \%(3117 / 16566)$ in males, the prevalence of females was $9.65 \%$ (890/9219), the prevalence of males was significantly higher than that of females $(\mathrm{P}<0.01)$. Among 18-70 years of age, the prevalence increases with age, with different age groups $(18-29,0-39,40-49,50-59,60-69)$ respectively $7.77 \%(-$ male: $10.54 \%$, female: $3.05 \%$ ), $16.67 \%$ (male: $21.84 \%$, fe-

male: $7.25 \%$ ), $19.61 \%$ (male: $21.68 \%$, female: $14.93 \%$ ), $25.70 \%$ (male: $26.85 \%$, female: $23.73 \%$ ), 25.96 (male: $30.60 \%$, female: $20.79 \%$ ). Univariate analysis showed that, BMI, blood pressure, blood glucose, triglyceride, total cholesterol and low density lipoprotein fatty liver were significantly higher than those in non-fatty liver $(\mathrm{P}<0.01)$, High-density lipoprotein was significantly lower than non-fatty liver group ( $<<0.01$ ); FibroTouch was detected in 456 randomly selected subjects, the prevalence of fatty liver was $29.17 \%$, of which $45.11 \%$ was mild fatty. The prevalence of fatty liver in B-ultrasound was $23.9 \%$ in this population, of which mild fatty liver accounted for $18.37 \%$.

Conclusion: The prevalence of fatty liver among male and female in the general population in Tibet Autonomous Region is significant at different ages. Age, body mass index, blood pressure, and biochemical results of blood glucose, triglycerides, total cholesterol, low density lipoprotein, high density lipoprotein were significantly associated with the occurrence of fatty liver; FibroTouch can be used as an effective evaluation of fatty liver, the detection rate of patients with mild fatty liver is higher than B ultrasound, the specific reasons need further analysis.

\begin{tabular}{|c|c|c|c|c|c|c|c|}
\hline \multirow{2}{*}{$\begin{array}{l}\text { Age } \\
\text { (years) }\end{array}$} & \multicolumn{2}{|r|}{ Over all } & \multicolumn{2}{|r|}{ Male } & \multicolumn{2}{|r|}{ Femsle } & \multirow[b]{2}{*}{$\mathrm{P}$} \\
\hline & $\mathrm{n}$ & Prevalence $(\%)(n)$ & $\mathrm{n}$ & Prevalence(\%) (n) & $n$ & Prevalence $(\%)(n)$ & \\
\hline 18. & 8558 & $7.76(664)$ & 5378 & $1054(567)$ & 3180 & $3.05(97)$ & 0.000 \\
\hline 30. & 7932 & $16.67(1322)$ & 5119 & $21.84(1118)$ & 2813 & $7.25(204)$ & 0.000 \\
\hline 40. & 5512 & $19.61(1081)$ & 3824 & $21.68(829)$ & 1688 & $1493(252)$ & 0.000 \\
\hline 50 . & 2607 & $25.7(670)$ & 1646 & $26.85(442)$ & 961 & $23.73(228)$ & 0.000 \\
\hline 60. & $\$ 55$ & $25.96(222)$ & 451 & $30.6(138)$ & 404 & $20.79(84)$ & 0.000 \\
\hline 70 - & 321 & $14.95(48)$ & 148 & $15.54\langle 23\rangle$ & 173 & $14.45\langle 25\rangle$ & 0.003 \\
\hline Total & 25785 & $15.4(4007)$ & 16566 & $18.82(3117)$ & 9219 & $9.65(890)$ & \\
\hline
\end{tabular}




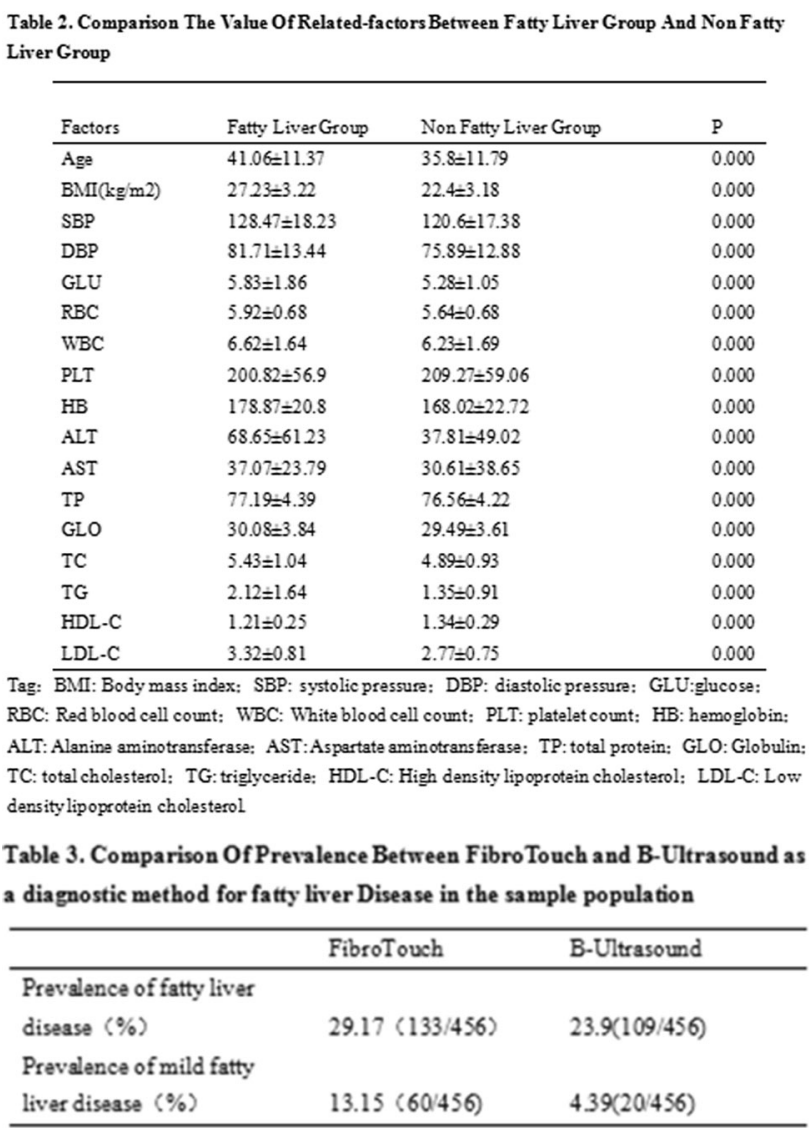

NAFL-18

Liver fibrosis is associated with risk for colorectal adenoma in patients with non-alcoholic fatty liver disease

Min Cheol Kim ${ }^{1}$, Jung Gil Park ${ }^{1}$, Byung Ik Jang ${ }^{1}$, Heo Lee ${ }^{1}$, Min Kyu Kang 1 , Soo Young Park ${ }^{3}$, Won Young Tak ${ }^{3}$, Young Oh Kweon ${ }^{3}$, Se Young Jang ${ }^{3}$, Yu Rim Lee ${ }^{3}$, Keun Hur ${ }^{3}$, Hye Won Lee

${ }^{1}$ College of Medicine, Yeungnam University, Korea, Republic of; ${ }^{2}$ Medical Center, School of Medicine, Keimyung Univ, Korea, Republic of; ${ }^{3}$ School of Medicine, Kyungpook National University, Korea, Republic of

Background: Non-alcoholic fatty liver disease (NAFLD) is associated with risk for the colorectal adenoma, which is related with various metabolic factors. However, there is limited study to evaluate liver fibrosis caused by progression of NAFLD. This study is to evaluate the association between the NAFLD with advanced fibrosis and the risk for colorectal adenoma.

Method: We retrospectively analyzed the adults who underwent ultrasound and first-time colonoscopy on same-day in a health screening program for 8 years. NAFLD was diagnosed using ultrasound, of which fibrosis is evaluated using non-invasive score model. We evaluated adenoma detection rate and presence of fibrosis using complex score models including NAFLD fibrosis score, fibrosis- 4 index, and simple score models including APRI and BARD score in patients with NAFLD. Result: Among a total of 6332 subjects, the subjects without NAFLD, and with NALFD were 3937 (62.18\%), and 2395 (37.82\%), respectively. The subjects with NAFLD had a higher prevalence of colorectal adenoma, advanced adenoma and multiple adenomas as well. In a multivariate analysis adjusting for demographic and metabolic factors, NAFLD was significantly independent risk factor for colorectal adenoma (odd ratio [OR] 1.15; 95\% CI 1.02-1.30), advanced adenoma (OR 1.50; 95\% CI 1.12-2.01), and multiple adenomas (OR 1.32, 95\% CI 1.01-1.73). When NAFLD was further stratified as degree of fibrosis using non-invasive score models, the subjects with NAFLD and advanced fibrosis had significantly higher risk for colorectal adenoma, advanced adenoma, and multiple adenomas compared to those with NAFLD and non-advanced fibrosis.

Conclusion: Non-alcoholic fatty liver disease is independent risk factor for colorectal adenoma. Moreover, NAFLD with advanced fibrosis has higher risk for colorectal adenoma compared to NAFLD without advanced fibrosis.

\section{NAFL-19}

Association between increasing level plasminogen activator inhibitor-1 and severity of non alcoholic fatty liver disease (NAFLD)

\section{Didik Indiarso $^{1}$}

${ }^{1}$ Kariadi Hospital, Indonesia

Background: Plasminogen activator inhibitor-1 (PAI-1) is a prothrombotic factor that increased risk of cardiovascular disease (CVD). It is known that there are a strong link between NAFLD and increased risk of CVD in individuals with or without coexisting metabolic syndrome. We previously demonstrated that PAI-1 levels were closely related to steatohepatitis and as independent factor in addition of metabolic syndrome.

Method: In 40 patient group proven fatty liver based on Ultrasonography (US) and liver biopsy, and another group of 40 patient without fatty liver on US, we were measure liver test, anthropometry, metabolic syndrome, insulin resistance and level of PAI-1. Hepatic histological features were systematically scored. Trend analysis was applied to determine the correlation of plasma PAI-1 levels with above variable and with the histological features in the liver.

Result: We found that plasma PAI-1 levels were significantly increased with severity of NAFLD $(\mathrm{p}=0,032)$.

Conclusion: PAI-1 appears to be tightly related to histologic severity of NAFLD, further more it was independent factor for increasing level PAI-1. PAI-1 may be a mediator of disease progression in NAFLD.

\section{$N A F L-20$}

Gut-liver interactions in patients with nonalcoholic fatty liver disease: role of intestinal flora and toll-like receptors

Eman Abdelsameea ${ }^{1}$, Olfat Hendy ${ }^{2}$, Ayat Roshdy ${ }^{1}$, Mona Aref ${ }^{1}$, Maha Allam ${ }^{1}$, Fatma A Younis ${ }^{1}$

${ }^{1}$ Assistant Professor, Egypt; ${ }^{2}$ Professor, Egypt

Background: Disturbed gut microbiome composition is associated with multiple diseases in humans. Impaired balance between nutritional absorption and energy storage is involved in pathogenesis and progression of non-alcoholic fatty liver disease (NAFLD).

Objectives: To investigate whether gut bacterial microbiome could have a role in inflammatory process leading to NAFLD development and decipher their effect on immune response represented by Toll like receptor-4 levels.

Method: One hundred and five adult participates were enrolled, 70 patients diagnosed with NAFLD, they were further subdivided into 32 cases with pure steatosis (SS), and 38 cases with non alcoholic steatohepatitis (NASH), as well as 35 healthy subjects, all matched 
for age, gender. Body mass index (BMI) was calculated, abdominal ultrasonography and liver biopsy were done. Liver tests, lipid profile, blood sugar, insulin and C-peptide, fasting insulin, and serum levels of Toll like receptor (TLR-4) were measured. Fresh stool samples were homogenized and either used for immediate aerobic and anaerobic cultures of bacteria, or for measurement of bacterial flora levels using quantitative real time PCR of genes encoding 16S rRNA. Result: BMI, Fasting glucose, LDL Cholesterol, HOMA-IR, AST, ALT and GGT were significantly increased in NAFLD group when compared to control group. Serum levels of TLR-4 and levels of bacteroides and lactobacilli were significantly increased in NAFLD groups compared to control group and its level was significantly increased in NASH compared to simple steatosis $(\mathrm{p}<0.001)$. Clostridia, bifidobacteria, and E. coli showed no significant difference among groups. Increased copy numbers of bacteroides as well as lactobacilli were positively correlated with BMI, ALT, AST, fasting glucose, triglycerides and, TLR-4 levels.

Conclusion: Gut microbiota play an important role in NAFLD-related pathophysiology. Further studies are recommended to understand mechanism of such effect and to evaluate possible therapeutic targets for prevention and control of NAFLD.

Table 1: Comparison between NAFLD subgroups and control groups regarding clinical and routine laboratory data of studied groups.

\begin{tabular}{|c|c|c|c|c|c|}
\hline Variables & $\begin{array}{c}\text { Controls } \\
n=30 \\
\text { Mean+SD }\end{array}$ & $\begin{array}{c}\text { Simple steatosis } \\
\quad n=32 \\
\text { Mean+SD }\end{array}$ & $\begin{array}{c}\text { NASH } \\
n=38 \\
\text { Mean+SD }\end{array}$ & $P$-value & $\begin{array}{c}\text { Within groups } \\
p \text {-value* }\end{array}$ \\
\hline HOMA & $2.32+0.68$ & $3.50+1.04$ & $2.58+0.78$ & $<0.001$ & $\begin{array}{l}P 1=<0.001 \\
P 2=0.210 \\
P 3=<0.001\end{array}$ \\
\hline $\begin{array}{l}\text { Triglyceride } \\
\text { (mg/dl) }\end{array}$ & $141.63+11.47$ & $178.09+19.51$ & $167.78+19.97$ & $<0.001$ & $\begin{array}{l}P 1=<0.001 \\
P 2=<0.001 \\
P 3=0.017\end{array}$ \\
\hline Cholesterol (mg/dl) & $190.53+17.34$ & $198.75+19.67$ & $194.63+19.56$ & 0.239 & $\begin{array}{l}P 1=0.091 \\
P 2=0.379 \\
P 3=0.368\end{array}$ \\
\hline HDL (U/L) & $38.96+4.72$ & $30.75+3.31$ & $32.31+3.79$ & $<0.001$ & $\begin{array}{l}P 1=<0.001 \\
P 2=<0.001 \\
P 3=0.103\end{array}$ \\
\hline LDL (U/L) & $113.00+14.08$ & $107.75+10.01$ & $119.00+10.48$ & $<0.001$ & $\begin{array}{l}P 1=0.77 \\
P 2=<0.001 \\
P 3=<0.001\end{array}$ \\
\hline $\begin{array}{l}\text { Fasting glucose } \\
(\mathrm{mg} / \mathrm{dl})\end{array}$ & $84.23+7.42$ & $93.21+8.01$ & $93.89+7.89$ & $<0.001$ & $\begin{array}{l}P 1=<0.001 \\
P 2=<0.001 \\
P 3=0.719\end{array}$ \\
\hline Insulin (mIU/ml) & $6.54+1.16$ & $7.89+0.49$ & $9.41+1.41$ & $<0.001$ & $\begin{array}{l}P 1=<0.001 \\
P 2=<0.001 \\
P 3=<0.001\end{array}$ \\
\hline Albumin (g/dl) & $4.44+0.36$ & $3.91+0.32$ & $3.92+0.26$ & $<0.001$ & $\begin{array}{l}P 1=<0.001 \\
P 2=<0.001 \\
P 3=0.965\end{array}$ \\
\hline AST (U/L) & $26.83+6.29$ & $34.59+5.68$ & $52.63+9.61$ & $<0.001$ & $\begin{array}{l}P 1=<0.001 \\
P 2=<0.001 \\
P 3=<0.001\end{array}$ \\
\hline ALT (U/L) & $26.23+5.43$ & $62.28+6.09$ & $67.39+10.62$ & $<0.001$ & $\begin{array}{l}P 1=<0.001 \\
P 2=<0.001 \\
P 3=<0.001\end{array}$ \\
\hline GGT (U/L) & $43.13+4.74$ & $70.22+7.96$ & $63.37+9.44$ & $<0.001$ & $\begin{array}{l}P 1=<0.001 \\
P 2=<0.001 \\
P 3=<0.001\end{array}$ \\
\hline $\operatorname{ALP}(U / L)$ & $52.43+8.59$ & $65.28+9.64$ & $63.11+7.19$ & $<0.001$ & $\begin{array}{l}P 1=<0.001 \\
P 2=<0.001 \\
P 3=0.286\end{array}$ \\
\hline TLR-4 (ng/ml) & $3.45+0.62$ & $3.75+0.87$ & $6.84+2.03$ & $<0.001$ & $\begin{array}{l}P 1=0.404 \\
P 2=<0.001 \\
P 3=<0.001\end{array}$ \\
\hline
\end{tabular}

$N A F L-21$

Regulation of the motor function of the gallbladder and intestinal transit in non-alcoholic fatty liver disease

$\underline{\text { Javohir Farxodovich Toshnazarov }}^{1}$

${ }^{1}$ Tashkent Medical Academy, Uzbekistan

Background: To detect violations of the motor function of the gallbladder and sigmoid colon at various stages of NAFLD.

Method: There were 44 patients with NAFLD - 24 patients in the stage of steatosis of the liver and 20 patients with steatohepatitis. The slow-wave and spike activity of the gallbladder and sigmoid colon was registered with the help of the hardware-software complex Conan-M with a measurement error of $\pm 5 \%$.

Result: Clinically, the asymptomatic course of NAFLD is noted on the background of a slight increase in liver size and insignificant hyperfermentemia (ALT and AST levels elevated from 1.2 to 1.5 times). Electromyographically, with steatosis of the liver, accompanied mainly by constipation, it was noted that the slow-wave activity of the gallbladder was within the norm, amounting to $8.4 \pm 0.4$ per min and $0.09 \pm 0.001 \mathrm{mV}$ (respectively, frequency and amplitude). At the same time, there was an increased spike activity, the frequency of spikes was $3.4 \pm 0.25(70 \%, p<0.05)$. In patients with steatosis of the liver, a slowed-down slow wave (frequency $4.9 \pm 0.3$ per min (18.3\%, p <0.05), amplitude $0.11 \pm 0.002 \mathrm{mV}$ ) was noted and increased spike activity was $3.8 \pm 0,15(26,6 \%, \mathrm{p}<0,05)$ and $0,03 \pm$ 0,002 ) of the sigmoid colon, which led to the development of constipation. With steatohepatitis, accompanied by the development of diarrhea, there was an increase in the frequency of slow-wave activity of the gallbladder to $10.6 \pm 0.5$ per $\min (32.5 \%, \mathrm{p}<0.05)$ with normal spike activity. There was an increase in slow wave activity of sigmoid colon (frequency $9.5 \pm 0.7$ per minute $-58.3 \%, \mathrm{p}<0.05$ ) and spike activity (frequency - $3.7 \pm 0.2$ - by $48 \%, \mathrm{p}<0.05$ ), which indicated an increase in intestinal transit.

Conclusion: The severity of the NAFLD current determines the degree of impairment of the motor function of the gallbladder and intestinal transit. The increase in the motor function of smooth muscles can be associated with an increase in the level of toxic lithocholic acid and a decrease in the level of acetic acid in the pool of bile acids in patients with NAFLD.

$N A F L-22$

The association between NAFLD and breast cancer: high prevalence and poor prognosis

YoungSun Lee ${ }^{1}$, Ha Seok Lee ${ }^{1}$, Minjin Lee ${ }^{1}$, Chan Uk Lee ${ }^{1}$, Sang Jun Suh $^{1}$, Young Kul Jung ${ }^{1}$, Ji Hoon Kim ${ }^{1}$, Yeon Seok Seo', Hyung Joon Yim ${ }^{1}$, Jong Eun Yeon ${ }^{1}$, Kwan Soo Byun ${ }^{1}$, Sehwa Kim ${ }^{1}$, Haein Bak ${ }^{1}$

${ }^{1}$ Korea University College of Medicine, Korea, Republic of

Background: Breast cancer is most common cancer in women worldwide, and it is a main cause of death in women. The incidence of breast cancer is correlated with metabolic component including diabetes, hypertension, and obesity. Likewise breast cancer, metabolic components are important risk factors for development of NAFLD. In this study, we analyzed the prevalence of NAFLD in patients with breast cancer and the effect of NAFLD on the prognosis of breast cancer.

Method: Patients who had other chronic liver disease including chronic viral hepatitis $\mathrm{B} / \mathrm{C}$, autoimmune hepatitis, and primary biliary 
cholangitis and significant alcohol abuse (more than $140 \mathrm{~g} /$ week in women and $210 \mathrm{~g} /$ week in men) were excluded. Hepatic steatosis was evaluated by nonenhanced computed tomography (CT) scan. We measured values of regions of interest (ROI) for 5 times in liver and spleen, respectively. We diagnosed NAFLD when the average ROI of liver is higher than it of spleen 15 or more. 135 healthy controls who took non-enhanced CT scan were also analyzed.

Result: Total 492 patients with breast cancer who received operation were enrolled from January 2010 to June 2014. Mean age and BMI were $51.6 \pm 11.0$ years and $24.7 \pm 9.9 \mathrm{~kg} / \mathrm{m}^{2}$, respectively. The prevalence of DM and hypertension were $9.5 \%$ and $24.6 \%$, respectively. The prevalence of NAFLD in patients with breast cancer was $43.5 \%(214 / 492)$ and it was significantly higher comparing with healthy control $(29.6 \%, 40 / 135)(\mathrm{P}=0.004)$. Overall survival did not showed significant difference between NAFLD group and nonNAFLD group ( $\mathrm{P}=0.958$ by log-rank test). However, recurrence rate was significantly higher in patients with NAFLD $(10.7 \%, 23 / 214)$ comparing with those without NAFLD $(5.4 \%, 15 / 278)(\mathrm{P}=0.027)$. The disease free survival was significantly higher in in patients with NAFLD comparing with those without NAFLD ( $P=0.033$ by $\log$ rank test). $15.9 \%$ of patients (34/214) experienced ALT elevation above 3 times of upper normal range, whereas only $10.4 \%$ of patients (29/278) showed elevation of ALT above 3 times of upper normal range $(\mathrm{P}=0.049)$.

Conclusion: The prevalence of NAFLD in patients with breast cancer is significantly high compared to healthy control group. Moreover, breast cancer patients with NALFD showed poor prognosis in aspect of recurrence. Therefore, diagnostic evaluation of NAFLD would be important in patients with breast cancer.

\section{$N A F L-23$}

The incidence rate and risk factors of hepatocellular carcinoma in diabetic patients at a specialized clinic for the diagnosis and management of DM

\section{Masataka Seike ${ }^{1}$, Mizuki Endo ${ }^{1}$, Koichi Honda ${ }^{1}$, Junya Oribe ${ }^{1}$, Masanori Tokoro ${ }^{1}$, Masao Iwao ${ }^{1}$, Mie Arakawa ${ }^{1}$, Kazunori Murakami $^{1}$ \\ ${ }^{1}$ Oita University Faculty of Medicine, Japan}

Background: Objectives: Diabetes mellitus (DM) is a risk factor for hepatocellular carcinoma (HCC) and cardiovascular disease. However, the incidence rate of $\mathrm{HCC}$ in patients with DM remains unclear. We tried to determine the incidence of HCC in patients with DM related to prognosis and to elucidate the utility of two non-invasive markers of liver fibrosis: the Fibrosis-4 (FIB4) index (which includes the three variables; age, aspartate aminotransferase [AST], and platelets) and the AST to platelet ratio (APRI) in predicting HCC in patients with DM. Our study protocol was approved by the Institutional Review Board of our institution.

Method: Methods: Study design: A prospective cohort study was performed at a specialized clinic for the diagnosis and management of T2DM in Oita. A total of 670 patients with DM (age, 40-79 years) who underwent abdominal ultrasonography between April 2003 and March 2004 were enrolled. Of these patients, 279 were excluded from analysis because they were positive for the hepatitis B surface antigen, hepatitis $\mathrm{C}$ antibody, they abused alcohol or they had dropped out of our study within the 12 years following. The remaining 391 patients were enrolled. The primary endpoint was the onset of HCC. The evaluation was performed using the Kaplan-Meier method.

Result: Results: Five patients with HCC (median age, 76 years old; four males and one female) were diagnosed during the observation period. The cumulative incidence rates of HCC with DM were $0.5 \%$,
$0.5 \%, 1.1 \%$, and $1.5 \%$ at $5,7,10$, and 12 years, respectively. The annual yearly incidence of HCC with DM was $0.107 \%$. Three patients with HCC had a history of drinking of alcohol. The remaining two patients had unknown etiology and nonalcoholic fatty liver disease. Five patients with HCC showed low platelet levels and high levels of AST and gamma glutamic pyruvic transaminase. Furthermore, the FIB4 index and APRI at the start of the observation time in the patients with HCC group was significantly higher than the FIB4 index of those without HCC. Optimal cutoff level for the FIB4 index and APRI were 2.5 and 0.7 , respectively. The area under the receiver operating characteristic curve values for FIB4 and APRI were 0.911 and 0.893 , respectively.

Conclusion: These results indicate that the annual incidence rate of HCC among diabetic patients at a specialized clinic for the diagnosis and management of DM is $0.107 \%$. As the number of patients with DM has increased in Japan, they should be monitored carefully during follow-up using the FIB4 index and APRI to ensure early diagnosis and treatment of $\mathrm{HCC}$.

\section{$N A F L-24$}

The possibility of laboratory-instrumental diagnostics of nonalcoholic fat liver disease.

\section{Javohir Farxodovich Toshnazarov ${ }^{1}$}

\section{${ }^{1}$ Tashkent Medical Academy, Uzbekistan}

Background: To determine the effectiveness of the diagnosis of NAFLD with laboratory and instrumental researches.

Method: A retrospective analysis of 280 case histories of patients with NAFLD aged between 23 and 64 years old (mean age $48 \pm$ 14.7 years) was produced. Among the patients, women predominated $(68 \%)$. Of the classical risk factors for the development of NAFLD, $175(62.5 \%)$ people suffered from obesity, type 2 diabetes mellitus 55 $(19.6 \%)$, severe hyperlipidemia $38(13.6 \%)$, intestinal diseases 7 $(2.5 \%)$, long-term hepatotoxic drugs - $5(1.8 \%)$ patients. Verification of the diagnosis of NAFLD was carried out according to clinical, biochemical and instrumental studies. According to anamnestic and personal data, alcoholic character of liver damage was excluded. As instrumental methods, all patients underwent ultrasound examination (ultrasound) of the liver. For diagnostic purposes, computed tomography $(\mathrm{CT})$ and magnetic resonance imaging (MRI) were performed using phase contrast.

Result: When analyzing the biochemical parameters of blood serum, in $96 \%$ of patients there was a significant increase in the activity of aminotransferases by 2-4 times. In patients with obesity and atherogenic dyslipidemia, the AST concentration was increased by an average of $1.9(\mathrm{p}<0.05)$, and ALT - in $2.2(\mathrm{p}<0.05)$ times. In patients with a violation of carbohydrate metabolism, the values of AST and ALT were significantly higher, respectively, $2.5(\mathrm{p}<0.05)$ and $3.4(\mathrm{p}$ $<0.05$ ) times. Only a small group of patients had laboratory signs of hepatic-cell insufficiency and cholestasis. Thus, the increase in the level of total bilirubin was in $29(10.3 \%)$ patients, AP and $\gamma$-GTP, respectively, in $16(5.7 \%)$ and $31(11.0 \%)$ patient. Ultrasound of the liver made it possible to detect hepatomegaly in 237 (84.6\%) patients, an increase in organ echogenicity in 257 (91.8\%), vascular inconsistency and distal echo loss in $48(17.1 \%)$ and $12(4.3 \%)$ patients. CT performed by $12(4.3 \%)$ patients allowed an indirect assessment of the degree of steatosis and a decrease in the radiographic density of the liver in patients with NAFLD-MRI using phase contrast, conducted by $5(1.8 \%)$ patients, made it possible to estimate the amount of fat tissue and local accumulation of fat in the liver.

Conclusion: The possibility of verifying the diagnosis of NAFLD in patients with classical risk factors that have deviations in biochemical 
laboratory tests, ultrasound and CT examinations, is quite high. This allows in most cases for a final judgment about the diagnosis not to perform a biopsy-morphological study of the liver.

\section{$N A F L-25$}

Variant in ABCA1 is associated with nonalcoholic fatty liver disease in a Chinese Han Population

\author{
Cong Wang ${ }^{1}$, Songling Liao', Haiyan Yue ${ }^{1}$, Yongning Xin ${ }^{2}$, \\ Shiying Xuan ${ }^{2}$ \\ ${ }^{1}$ Dalian Medical University, China; ${ }^{2}$ Qingdao Municipal Hospital, \\ China
}

Background: Nonalcoholic fatty liver disease (NAFLD) is a burgeoning health problem as the leading cause of chronic liver disease in adults and children. The disease of unknown etiology shows closely related with dyslipidemia. Adenosine triphosphate binding cassette transporter (ABCA1) involved in cholesterol efflux, fatty acid oxidation and inflammation. We investigated possible associations between the ABCA1 variants and NAFLD.

Method: The ABCA1 gene were determined in 265 NAFLD patients and 126 healthy controls using Matrix-Assisted Laser Desorption/ Ionization Time-of-Flight Mass Spectrometry (MALDI-TOF MS). Meanwhile, serum liver function profiles and liver enzymes were estimated using standard clinical laboratory methods.

Result: The genotype and allele frequencies of the ABCA1 rs $1800977 \mathrm{G} / \mathrm{A}$ polymorphisms between the NAFLD group and the healthy control group were significantly different $(\mathrm{P}<0.05)$. No significant differences in genotype and allele frequencies of the ABCA1 rs2066714T/C, rs2066715T/C, rs2230808T/C, rs2515629A/ $\mathrm{G}, \mathrm{rs} 363717 \mathrm{~T} / \mathrm{C}$ and $\mathrm{rs} 4149341 \mathrm{~T} / \mathrm{C}$ was found between the NAFLD and heathy. Compared with the noncarriers in NAFLD, the rs2066714 $\mathrm{C}$ in $\mathrm{ABCA} 1$ had significantly higher levels of low-density lipoprotein (LDL) $(\mathrm{P}=0.045)$ and $\mathrm{rs} 2230808 \mathrm{~T}$ had significantly lower levels of high-density lipoprotein (HDL) $(\mathrm{P}=0.039)$. Nevertheless, differences in other serum between the carriers and the noncarriers were not statistically significant.

Conclusion: We first demonstrated that the ABCA1 gene associated with NAFLD in a Chinese Han population. The rs1800977 polymorphisms may predict the predisposition to NAFLD.

\section{$N A F L-26$}

\section{Vitamin D deficiency increases the risk of metabolic syndrome but does not increase the severity of fatty liver in subjects with nonalcoholic fatty liver disease: a community-based cross- sectional study}

\section{LiWei Chen ${ }^{1}$, Rongnan Chien ${ }^{1}$}

${ }^{1}$ Chang-Gung Memorial Hospital at Keelung, Taiwan

Background: Previous studies have revealed that vitamin D deficiency can significantly increase the risks of insulin resistance and metabolic syndrome. Vitamin D may influence hepatocytes and nonparenchymal hepatic cells (hepatic stellate cells, Kupffer cells) in nonalcoholic fatty liver disease (NAFLD) via metabolic, anti-inflammatory and anti-fibrotic effects. This study aimed to evaluate the association between serum vitamin D levels and NAFLD parameters, including metabolic syndrome risk and hepatic steatosis.

Method: From August 2013 to August 2016, a community-based study was performed in the north-eastern region of Taiwan. All subjects participated in a demographic survey, blood testing and abdominal ultrasonography (US). The vitamin D level was evaluated by quartile divide or used the classification of deficiency $(30 \mathrm{ng} / \mathrm{ml})$. Exclusion criteria were: (1) men who consumed more than $40 \mathrm{~g}$ alcohol per day and women who consumed more than $20 \mathrm{~g}$ alcohol per day. (2) Secondary causes of steatosis (e.g., corticosteroid use, gastric bypass surgery). (3) Seropositivity for hepatitis B surface antigen or anti-hepatitis C virus antibody. (4) People who had received vitamin $\mathrm{D}$ supplementation in the previous 3 months before enrolling in this study.

Result: Subjects were divided into NAFLD group and normal control (subjects number=564 in each group) following abdominal US study and excluding alcohol abuse and matching age and gender. The mean age was 57.1 years in NAFLD group and 57.5 in control group. Subjects with serum vitamin D deficiency $(30 \mathrm{ng} / \mathrm{ml})$ [deficiency vs. sufficiency, adjusted odds ratio $(\mathrm{OR})=1.860(95 \% \mathrm{CI}=1.234-2.804)$, $\mathrm{P}=0.003$; insufficiency vs. sufficiency, adjusted $\mathrm{OR}=1.669(95 \%$ $\mathrm{CI}=1.237-2.251$ ), $\mathrm{P}=0.001$ ]. Subjects in the quartile 1 (the lowest vitamin D) had a higher risk for metabolic syndrome than those in the quartile 4 (the highest vitamin $\mathrm{D})(\mathrm{OR}=2.792,95 \% \mathrm{CI}=1.719-4.538$, P0.05].

Conclusion: Subjects with low vitamin D level increased the risk of developing metabolic syndrome compared to those with high vitamin D levels. The prevalence of hepatic steatosis (as measured by abdominal US) was similar in subjects with NAFLD among the different vitamin D levels.

\section{$N A F L-27$}

\section{An update on NAFLD and NASH in Asia}

\section{ChingSheng Hsu ${ }^{1}$, Jiahorng Kao ${ }^{2}$}

${ }^{1}$ Director, Taiwan; ${ }^{2}$ National Chair Professor, Graduate Institute of Clinical Medicine, National Taiwan University College of Medicine, Taiwan

Background: Non-alcoholic fatty liver disease (NAFLD) has become the most overwhelming liver disease in Asia. Growing data indicate that NAFLD may be a systemic disease, not just confined to liverspecific disorders, but also associated with several extrahepatic manifestations, such as cardiovascular diseases, and malignancy. Moreover, issues related to the impact of NAFLD on the clinical outcomes and management of viral hepatitis remain to be elucidated. Method: We conducted a narrative review and searched for literature from PubMed, Ovid MEDLINE, and the Cochrane Library database till August 2016. Studies relevant to the emerging data of NAFLD in Asia, including the diagnosis, risk factors, the assessment and management of Asian NAFLD patients were examined.

Result: NAFLD is not uncommon in some parts of Asia, affecting $2.04 \%-52 \%$ of adults across the Asian countries. After the control of viral hepatitis in the foreseeable future, NAFLD is anticipated to be the most prevalent and clinically significant liver disease in Asia. Because Asia varies greatly across the region in ethnic groups, cultures, environments, economics, and health systems, there exist substantial regional variations in the prevalence, presentations, underlying mechanisms and the management of NAFLD in Asian countries. Of particular note is that NAFLD, NASH, and its progression to cirrhosis and HCC, indeed exist in Asia. In consideration of its high prevalence, metabolic associations, co-morbidities and complications, prophylactic measures, as well as active management strategies, should be timely planned and implemented. Unfortunately, awareness of this disease remains poor in most Asian countries, and enhanced public education about NAFLD is warranted to improve the understanding, prevention, and management of this disease in this region. Moreover, non-invasive assessment of liver fibrosis is not 
widely available in the daily practice of most Asian countries and the use of liver biopsy is usually deferred. Therefore, the development of an effective and practical measurement to assess the severity of NAFLD is urgently required. Finally, as viral hepatitis remains rampant in Asia, an interaction between NAFLD and viral hepatitis is a concern and deserves additional studies.

Conclusion: The situations of NAFLD are much more complicated in Asia. Collaboration in Asian countries to develop an effective and practical measurement to assess the severity of NAFLD is urgently required in this region.

\section{$N A F L-28$}

\section{Aspartate aminotransferase (AST) is a strong predictor of NAFLD activity score for diagnosing non-alcoholic steatohepatitis (NASH)}

Dulal Chandra Das ${ }^{1}$, Mamun Al Mahtab ${ }^{3}$, Sheikh Mohammad Noor E Alam², Partho Pratik Roy ${ }^{5}$, Salimur Rahman ${ }^{4}$

${ }^{1}$ Medical Officer, Bangladesh; ${ }^{2}$ Assistant Professor, Bangladesh;

${ }^{3}$ Associate Professor, Bangladesh; ${ }^{4}$ Professor, Bangladesh; ${ }^{5}$ Resident Physician, Bangladesh

Background: Nonalcoholic fatty liver disease (NAFLD) is a hepatic manifestation of metabolic syndrome. Non-alcoholic steatohepatitis (NASH) can lead to cirrhosis and hepatocellular carcinoma.

The aim of this study is to determine AST value that will accurately predict NASH.

Method: This was a Observational, Cross sectional study. 50 patients of Non alcoholic fatty liver disease (NAFLD) attending at outpatient and inpatient Department of Hepatology, Bangabandhu Sheikh Mujib Medical University, Dhaka, Bangladesh were included in the study and underwent liver biopsy. Liver biopsies were scored using NASH clinical research network (NASH CRN) system.

Result: 25 were NASH and 25 were non- NASH. Overall, twenty eight $(56 \%)$ had normal AST. AST in NASH group were $55.2 \pm$ $30.1 \mathrm{IU} / \mathrm{L}$ and in Non-NASH group were $33.6 \pm 20 \mathrm{IU} / \mathrm{L}$. In NASH group significantly higher percentage of raised AST had NASH compared with normal AST ( $68 \%$ vs. $32 \%$ ). In Non-NASH group significantly higher percentage of normal AST than raised AST $(80 \%$ vs. $20 \%$ ). Overall $16 \%$ of normal AST had NASH whereas $10 \%$ of elevated AST had non NASH. There was significant difference in the NAFLD activity score for diagnosing NASH between elevated and normal AST ( $P$ 0.004). Higher AST values correlated with higher specificity. By multivariate analysis AST were found to be significant, revealed that AST $>37 \mathrm{U} / \mathrm{L}$ have the best possibility (6 times) of NASH.

Conclusion: AST level has the strong predictive value for diagnosing NASH in NAFLD patients.

\section{$N A F L-29$}

Clinical, biochemical, anthropometric and histologic character of nonalcoholic fatty liver disease without insulin resistance

\section{Md Kamrul Anam}

${ }^{1}$ Railway General Hospital, Bangladesh

Background: Nonalcoholic fatty liver disease (NAFLD) is thought to be a hepatic manifestation of metabolic syndrome or insulin resistance (IR). The aim of the study was to compare the clinical including anthropometric, metabolic, biochemical and histological profile of
NAFLD patients with or without insulin resistance (IR) and to understand the differences between the groups.

Method: Total 851 patients with sonographic evidence fatty liver were included. These patients underwent clinical evaluation, anthropometric measurements, and blood tests. IR was calculated using the homeostatic model assessment (HOMA) and HOMA-IR value above 2 was considered to indicate IR. Liver biopsy had been performed in 285 patients who consented for the procedure and who had metabolic syndrome or raised ALT.

Result: Among 851 NAFLD patients, 561(65.9\%) patients were without IR and $290(34.1 \%)$ patients were having IR. Males were significantly higher in non-IR group $(41.0 \%$ vs $30.7 \%, P=0.046)$. The proportion of patients with diabetes $[39.0 \%$ vs. $19.10 \% ; P=$ $0.000]$ and metabolic syndrome $[78.10 \%$ vs. $58.80 \% ; P=0.014]$ were significantly higher in IR group. Though not significant, IR group had greater number of obese (BMI $>25)$ patients $[74.50 \%$ vs. $63.80 \%$; $P=0.210]$ and Hypertensive patients $(36.60 \%$ vs $23.50 \% ; P=0.085)$. Waist circumference $(95.9 \pm 8.4$ vs. $93.3 \pm 8.4 \mathrm{~cm} P=0.003)$ and BMI $\left(27.9 \pm 4.3\right.$ vs $\left.26.6 \pm 3.5 \mathrm{~kg} / \mathrm{m}^{2} P=0.002\right)$ were also significantly higher in IR group. Biochemical markers including lipid profile, ALT, AST and ALP differences were not statistically significant between the two groups. Among the 275 patients with available histopathology reports, lobular inflammation, ballooning and fibrosis score were having no significant differences between the two groups, only steatosis score was significantly higher in IR group $(2.0 \pm 0.7$ vs $1.8 \pm 0.8 ; P=0.007)$. There was also significantly higher the mean NAFLD Activity Score (NAS) $(4.9 \pm 1.2$ vs. $4.5 \pm$ $1.4 ; P=0.03$ ) and nonalcoholic steatohepatitis (NNFL/NASH) $[28 / 66$ vs. $87 / 94 ; P=0.004]$ in IR group. In multivariate analysis, presence of diabetes mellitus and IR $[P=0.003$ and $P=0.041$, respectively] were significantly associated with NASH.

Conclusion: This study characterizes patients of NAFLD without IR as a distinct entity. IR is not the sole factor for NAFLD in our population, since only one-third of the NAFLD patients had IR. NAFLD in our region is possibly a heterogeneous disorder.

$N A F L-30$

\section{Metabolic syndrome and non-alcoholic fatty liver disease}

Azizbek Abbosovich Ismatov ${ }^{1}$, Saidrakhim Nodirovich Lukmonov ${ }^{1}$

${ }^{1}$ Tashkent Medical Academy, Uzbekistan

Background: Generalization of our long-term observations and studies of metabolic disorders in patients with metabolic syndrome (MS) and non-alcoholic fatty liver disease (NAFLD), as well as the identification of the characteristics of NAFLD in men and women.

Method: 164 patients with NAFLD were examined on the basis of faculty surgery of the Tashkent Medical Academy in the RCH ?1, of which 103 men aged from 29 to 64 years and 61 women aged from 44 to 63 years. All women were examined in the phase of physiological menopause. The 1st group included 124 patients with diagnostic criteria of MS, the 2nd group consisted of 40 patients without MS.

Result: The results of our clinical studies give grounds to distinguish a number of patterns during the NAFLD in both men and women. The developed lipid profile was determined: Triglycerides (TG); The level of total cholesterol (CSt). The state of carbohydrate metabolism was assessed by fasting glucose level. Some of the patients underwent a standard glucose tolerance test. All patients had a mandatory criterion for MS - abdominal obesity. Consequently, the provision is confirmed that the NAFLD is a component of MS. According to our data, heavy hypercholesterolemia (more than $7.8 \mathrm{mmol} / \mathrm{l}$ ) was more often recorded in men. Dyslipidemia in NAFLD is characterized by an increase in TG levels of more than $1.7 \mathrm{mmol} / \mathrm{L}$ and hypoalpha 
cholesterolemia, in which the level of HDL cholesterol is $<1.0 \mathrm{mmol} /$ $\mathrm{L}$ in men and $<1.2 \mathrm{mmol} / \mathrm{L}$ in women. IR is the leading pathogenetic factor in the formation of NAFLD, and its calculated index of the index IR-HOMA-IR allows you to assess the sensitivity to insulin. The HOMA-IR index was also significantly higher. Higher hypertriglyceridemia in combination with IR suggests that the combination of these disorders and their greater severity contribute to the formation of liver steatosis in men much earlier. The cluster of constituent factors of MS is more pronounced in men. The main pathophysiological factors contributing to the formation of NAFLD, namely, IR and dyslipidemia were more significant in men. This may be due to the earlier development of liver steatosis in men and their younger age in comparison with women.

Conclusion: Thus, summing up the results of our studies, it should be noted that NAFLD in most patients is detected against the background of MS. Our observations and studies confirm that male gender is a risk factor for NAFLD and this disease is more often diagnosed in men.

\section{NAFL-31}

\section{Non-alcoholic fatty liver disease in people of different age categories}

\section{Azizbek Abbosovich Ismatov ${ }^{1}$ \\ ${ }^{1}$ Tashkent Medical Academy, Uzbekistan}

Background: Study of the clinical course and metabolic disorders in patients with non-alcoholic fatty liver disease (NAFLD) of different age categories.

Method: 113 patients (63 men and 50 women) were examined on the basis of faculty surgery of the Tashkent Medical Academy. The patients were divided into two groups. The 1st group includes 29 elderly people aged of 60 to 77 years (mean age $67.4 \pm 1.2$ years). The second group consisted of 84 patients of middle age from 45 to 59 years (mean age $53.3 \pm 0.6$ years).

Result: All patients have internal pathology. However, if isolated CHD was recorded with the same frequency in both groups, then arterial hypertension was isolated and was only diagnosed for the first time mainly in patients of the 2 nd group. The comorbid cardiovascular pathology was 2.5 times more frequently observed in patients of the 1st group. It should be noted that gastroesophageal reflux disease in patients of the 1st group in half of the cases associated with a hiatal hernia, and in patients of the 2 nd group there was noted combination with Barrett's esophagus. Secondly, in assessing the state of carbohydrate metabolism the following changes have been identified: Disorders in the metabolism characterized by registering in violation of the standard glucose tolerance test and verification of type 2 diabetes mellitus mainly in patients in group 2 . Fourth. To assess the state of the porphyrin exchange, the most informative is the determination of the excretory profile of the porphyrin metabolism indices, since it allows us to verify a larger spectrum of disturbances both at the early stages that we used. In porphyrin metabolism disorders, it was found that early disturbances (increased excretion of porphyrin precursors, as well as a change in the ratio of porphyrin fractions) were more common in patients in the 2 nd group. On the contrary, the fractional disorders that are formed with the prescription of the disease are predominantly noted in patients of the 1st group. Porphyrin metabolism disorders are a reflection of general pathophysiological processes. This is facilitated by the occurrence of more significant disorders in carbohydrate and lipid metabolism.

Conclusion: Thus, the NAFLD in the patients surveyed by us is predominantly formed at a young age. While, the concept of the formation of the NAFLD at the moment can not be finally substantiated and requires further research.
$N A F L-32$

\section{Non-alcoholic fatty liver disease}

\section{Nodirjon Fazliddinovich Ruzimurodov ${ }^{1}$}

${ }^{1}$ Tashkent Medical Academy, Uzbekistan

Background: To determine the criteria for diagnosing this disease in the early stages of the disease with the first simple symptoms on ultrasound findings.

Method: The work was carried out at the 1st Republican Clinical Hospital under the Ministry of Healthcare of the Republic of Uzbekistan in the Department of Surgery and Therapy, which came in patients diagnosed with fatty hepatosis and steatohepatitis. In the material, 25 patients were collected who applied from April 2017 to August 2017.

Result: 1. Non-alcoholic fatty liver disease was diagnosed in 20 $(92,8 \%)$ of 25 patients with obesity, with fatty hepatosis detected in $19 \%$, non-alcoholic steatohepatitis - in $6 \%$.

2. The clinical picture of fatty hepatosis is characterized by the presence of abdominal obesity, ultrasound signs of liver steatosis, total cholesterol $(5.34 \pm 0.83 \mathrm{mmol} / \mathrm{L})$, low density lipoproteins $(3$, $35 \pm 0.86 \mathrm{mmol} / \mathrm{l})$, triglycerides $(1.92 \pm 0.78 \mathrm{mmol} / \mathrm{l})$, free fatty acids $(1.7 \pm 0.8 \mathrm{mmol} / \mathrm{l})$.

3. Morphological picture of non-alcoholic steatohepatitis is characterized by the presence of large-droplet fatty dystrophy, inflammatory-necrotic changes in hepatocytes (balloon dystrophy, lobular and portal inflammation) and fibrosis of varying severity.

Conclusion: When detecting fatty hepatosis or steatohepatitis on ultrasound, it should be suspected of NAFLD and make further analyzes to provide this disease in severe stages.

$N A F L-33$

Non-alcoholic fatty liver (NAFLD) - a retrospective analysis of patients referred to Toronto Liver Centre from 1974 to 2017

Marzena Magnes ${ }^{1}$, Mark Khan ${ }^{3}$, Muhammad Khurram Mahmood $^{1}$, Hamed Dallali ${ }^{1}$, Sirajuddaula Kausar ${ }^{1}$, Jean Palmart ${ }^{2}$, Sunny Loo ${ }^{2}$, Kerstina Boktor ${ }^{1}$, Marzena Magnes ${ }^{1}$, Magdy Elkhashab ${ }^{1}$

${ }^{1}$ Toronto Liver Centre, Canada; ${ }^{2}$ Advisory Physicians Research Services Inc., Canada; ${ }^{3}$ Gilead Sciences Inc., Canada

Background: Non-alcoholic fatty liver disease (NAFLD) is described as a condition of hepatic steatosis with or without hepatocellular injury. Prevalence of NAFLD is increasing worldwide and in North America. To better understand the burden of liver disease in Ontario, we are conducting a comprehensive retrospective analysis of all patients evaluated at the Toronto Liver Centre (TLC), and we are presenting the preliminary data related to NAFLD in this abstract.

Method: A total of 3610 medical charts at the Toronto Liver Centre were reviewed, dating from Feb. 1994 to Sep. 2017. Data regarding demography, liver disease etiology, liver fibrosis stage based on Transient Elastography (Fibroscan ${ }^{\circledR}$ ), liver biopsy, imaging diagnosis, and blood work, were collected from the charts and entered onto electronic case report forms. Data was analyzed using MarketSight ${ }^{\circledR}$ software.

Result: Of the 3610 patients, $1615(44.7 \%)$ had NAFLD, including $58.5 \%$ males and $41.5 \%$ females. Majority of patients $(30.3 \%)$ were $50-59$ yrs old followed by the patients in their 60's and 40's respectively. The main presentation of fatty liver among NAFLD patients were hepatomegaly $(33.8 \%)$ and elevated liver enzymes (26.9\%); and the major co-morbidities were obesity $(41.0 \%)$, 
dyslipidemia (37.8\%), hypertension $(32.0 \%)$ and diabetes mellitus (21.7\%). $10.6 \%$ of NAFLD patients had some type of thyroid disease, with hypothyroidism being the most common $(70.9 \%)$. Breast cancer was the most common cancer (31.2\%) among 96 (5.9\%) patients with cancer.

Ultrasound was able to detect fatty liver in $84.7 \%$ of patients; and left $15.3 \%$ undiagnosed.

Among 58 patients who had biopsy and Fibroscan ${ }^{\circledR}$ within one year period of time, Metavir score measured by Fibroscan ${ }^{\circledR}$ correlated reasonably with biopsy-derived Metavir score in $77.6 \%$ of cases.

Nearly $18.0 \%$ of the NAFLD patients with Metavir score on biopsy (total of 128) had moderate to advanced fibrosis. Fibroscan ${ }^{\circledR}$ of 1,259 patients showed moderate to advanced fibrosis in $9.3 \%$, and severe steatosis in $35.8 \%$. Cirrhosis was found in 38 patients $(2.3 \%)$. Conclusion: Hypothyroidism and breast cancer were markedly high among NAFLD patients, which concur with earlier findings. Studies suggest hypothyroidism and resultant hypometabolism are associated with NAFLD.

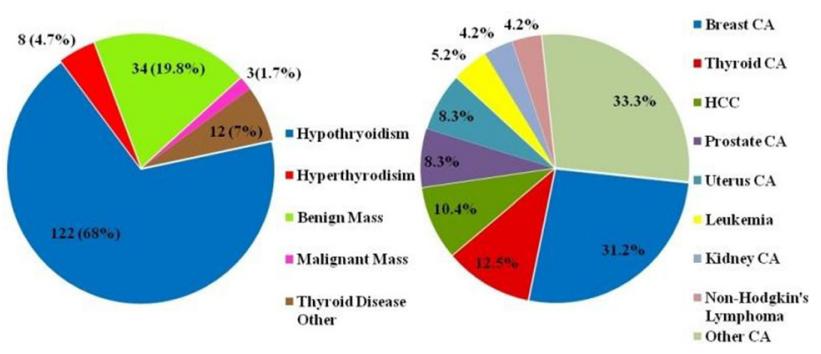

Figure 1 - Prevalence of thyroid diseases and cancers

Table 1 - Distribution of cancers among patients with NAFLD

\begin{tabular}{|c|c|}
\hline Cancer reported & Number $(\%)^{*}$ \\
\hline Breast $\mathrm{CA}$ & $30(31.3 \%)$ \\
\hline Thyroid CA & $12(12.5 \%)$ \\
\hline HCC & $10(10.4 \%)$ \\
\hline Prostate $\mathrm{CA}$ & $8(8.3 \%)$ \\
\hline Uterus CA & $8(8.3 \%)$ \\
\hline Leukemia & $5(5.2 \%)$ \\
\hline Non-Hodgkin's Lymphoma & $4(4.2 \%)$ \\
\hline Kidney CA & $4(4.2 \%)$ \\
\hline Other CA & $32(33.3 \%)$ \\
\hline
\end{tabular}

$N A F L-34$

Nonalcoholic fatty liver disease and liver fibrosis as estimated by ultrasound shear wave elastography in the visitors of the World Hepatitis Day

\section{Shoukat Ali Samejo ${ }^{1}$, Zaigham Abbas ${ }^{1}$, Muhammad Asim ${ }^{1}$ \\ ${ }^{1}$ Dr Ziauddin University Hospital Clifton, Pakistan}

Background: Nonalcoholic fatty liver disease (NAFLD) has become a major concern all over the world. The clinical course of the disease is not always benign and leads to cirrhosis and hepatocellular carcinoma in a significant number of patients. To evaluate the presence of fatty liver and associated fibrosis, we prospectively evaluated our attendees of the World Hepatitis Day Program.

Method: The visitors were evaluated after informed consent for the presence of any liver disease by blood counts, biochemical profile, viral serology and ultrasound. Those positive for hepatitis $\mathrm{B}$ and $\mathrm{C}$ were excluded from analysis. The non-B, non- $\mathrm{C}$ visitors having fatty liver on ultrasound were further evaluated by shear wave elastography performed on Toshiba Aplio 500 platinum version 06. Presence of fibrosis was estimated in $\mathrm{kPa}$ and hepatic steatosis was assessed in a semi-quantitative way on a scale of $0-3$.

Result: Total number of attendees evaluated were 96 . Sixteen visitors (16.7\%) were positive for hepatitis C while $3(3.1 \%)$ for hepatitis B, hence excluded. The rest of the 77 attendees had age range of 14-63 with a mean age of 33.8. Male to female ratio was 1:2. Mean BMI was 23.9 with a range from $16-38.5 \mathrm{~kg} / \mathrm{m}^{2}$. Eighteen $(23.4 \%)$ were hypertensive and $4(5.2 \%)$ had a history of ischemic heart disease. Five $(6.4 \%)$ were found to be diabetic. Thirty four persons $(44.2 \%)$ had a BMI of less than 23 while $43(55.8 \%$ ) had BMI $>23$ (overweight or obese). Steatosis was present in $24(31.2 \%)$ of the non-B, non-C visitors; $14(18.2 \%)$ had stage I steatosis, 9 (11.7\%) stage II and $1(1.3 \%)$ with stage III steatosis. $79.16 \%(19 / 24)$ of the visitors with steatosis had a BMI of $>23$. Four out of five patients with diabetes had steatosis. Significant fibrosis F2 or more was present in $16.6 \%(4 / 24)$ persons with steatosis, while $13(54.16 \%)$ had elevated ALT suggestive of steatohepatitis. There was a significant correlation between liver elasticity and steatosis ( $\mathrm{p}$ value $=0.03$ ).

Conclusion: Presence of NAFLD is common in our population. Some of these patients have steatohepatitis and advanced fibrosis. Evaluation of NAFLD should be included in the screening programs for the liver disease in the general population in addition to the viral serology.

$N A F L-35$

Nutritional status and energy metabolism in patients with nonalcoholic fatty liver disease

Qing $Y e^{1}$

${ }^{1}$ Tianjin Medical University, China

Background: In recent years, with the high incidence of obesity, diabetes and hyperlipidemia, the prevalence of non-alcoholic fatty liver disease has increased significantly. It has become a medical and social problem of universal concern. NAFLD patients are characterized by overnutrition and metabolic disorders, so it is of important clinical significance to assess and monitor the nutritional and metabolic status of NAFLD patients.

Method: 359 cases of NAFLD patients from Department of Gastroenterology Tianjin Third Central Hospital during June 2015 to March 2017 as the research object, divided into moderate and severe fatty liver group and mild fatty liver group, 50 healthy volunteers as control group, analysis of patient and control cells in application of body composition analyzer; application of metabolic cart were measured in energy metabolism, including resting energy expenditure (REE), respiratory quotient (RQ), and the three major nutrients oxidation rate $(\mathrm{CHO} \%, \mathrm{FAT} \%$ and $\mathrm{PRO} \%)$.

Result: Severe fatty liver group compared with mild fatty liver group and control group, the BMI, waist circumference, waist to hip ratio were significantly increased $(\mathrm{P}<0.001)$, the serum ALT, TG, TC, HDL, LDL and FBS were significantly increased $(\mathrm{P}<0.05)$.

Body composition analysis showed that the content of skeletal muscle in the three groups had no statistical difference, $\mathrm{P}=0.067$; in severe 
fatty liver in patients with ICW, ECW, protein, fat content of mild fatty liver group and control group were significantly increased $(\mathrm{P}<0.01)$, but there were no obvious differences between mild fatty liver group and control group; VFA in the three groups were statistically significant, moderate to severe fatty liver group VFA significantly increased.

The result of metabolic vehicle test showed that the respiratory quotient (RQ) of the moderate and severe fatty liver group and the mild fatty liver group was $0.72 \pm 0.08$ and $0.78 \pm 0.06$, respectively, which were lower than that of the control group $(0.80 \pm 0.02)$, $\mathrm{P}=0.004$. The resting energy expenditure (REE) was not significantly different between the moderate and severe fatty liver group $(\mathrm{P}=0.207)$, but it was significantly higher than that of the control group (all $\mathrm{P}<0.001$ ). In the moderate and severe fatty liver group, $\mathrm{CHO} \%, \mathrm{FAT} \%$ and $\mathrm{PRO} \%$ were $(19.49 \pm 9.71) \%,(66.23 \pm 12.54) \%$, $(14.22 \pm 6.11) \%$, and compared with the control group, $\mathrm{CHO} \%$ decreased and FAT\% increased.

Conclusion: There are different levels of nutritional excess and metabolic disorder in patients with NAFLD. The body composition and energy metabolism vehicle can be used to evaluate and monitor the nutritional and metabolic status of NAFLD patients.

\section{$N A F L-36$}

Prevalence and clinico-epidemiology of nonalcoholic fatty liver disease (NAFLD) in patients with metabolic syndrome

Ajay Duseja ${ }^{1}$, Abdul Khaliq ${ }^{4}$, CC Choubal ${ }^{8}$, Dipesh Sonawane ${ }^{2}$, Dyotona Sen Roy ${ }^{2}$, Harshal Gadhikar ${ }^{3}$, Manisha Bangar', Mukesh Agarwal ${ }^{7}$, Rashmi Hegde ${ }^{2}$, Ramesh Roop Rai ${ }^{11}$, Rahul Kothari $^{10}$, Samir Shah ${ }^{5}$, Sanjiv Saigal ${ }^{6}$

${ }^{1}$ Postgraduate Institute of Medical Education and Research, Chandigarh, India; ${ }^{10}$ Shree Hospital, India; ${ }^{11}$ SR Kalla Memorial Gastro \& General Hospital, India; ${ }^{2}$ Abbott India Limited, India; ${ }^{3}$ Deenanath Mangeshkar Hospital and Research Hospital, India; ${ }^{4} \mathrm{FS}$ Endocrine and Diabetic Center, India; ${ }^{5}$ Institute of Liver Disease, HPB Surgery and Transplant, Global Hospital, India; ${ }^{6}$ Medanta Institute of Digestive \& Hepatobiliary Sciences, India; ${ }^{7}$ Only Research, India; ${ }^{8}$ People's College of Medical Sciences \& Research, India; ${ }^{9}$ Princess Esra Hospital, India

Background: Limited information is available on the prevalence and clinico-epidemiology of NAFLD in Indian patients with metabolic syndrome (MetS). The present study assessed the prevalence, clinical and epidemiological factors of NAFLD in patients with MetS.

Method: In this cross-sectional study, 432 adult patients with an established diagnosis of MetS (ATP III criteria with modified waist) were enrolled across 10 centers in India over one year. NAFLD was defined as the presence of fatty liver on ultrasound with exclusion of alcohol and other causes of fatty liver. Non-invasive assessment of hepatic fibrosis was done by NAFLD fibrosis score (NFS).

Result: Amongst 432 patients with MetS, central obesity was the most common component [424(98.14\%)] followed by low HDL-C levels [365(84.5\%)], elevated SBP [346(80.09\%)], high fasting glucose levels [288(66.67\%)] and elevated triglycerides [169 (39.12\%)]. Insulin resistance with HOMA-IR > 1.64 was present in $383(88.6 \%)$ patients.

Two hundred and ninety-eight $(69 \%)$ patients with MetS were diagnosed with NAFLD (mean age $48.6 \pm 10.61$ years, mean BMI $30 \pm$ $4.94 \mathrm{~kg} / \mathrm{m}^{2}$ ) with higher prevalence in females [186 (62.4\%)] in comparison to males [112 (37.6\%)]. Most common risk factors associated with NAFLD were high waist circumference [292 (98\%)] and HOMA-IR [273(91.61\%)] followed by obesity [265(88.92\%)] and high fasting glucose levels [231(77.51\%)].
In total $117(39.26 \%)$ patients had NFS $>0.675$ suggesting presence of advanced fibrosis whereas low probability of advanced fibrosis was present in $38(12.75 \%)$ patients (NFS $<-1.455)$. Risk factors most commonly observed in patients with advanced fibrosis were central obesity [116 (99.14\%)], HOMA IR >1.64 [108 (92.3\%)], low HDL-C levels [102 (87.17\%)], high SBP [94 (80.34\%)] and fasting glucose [84 (71.79\%)].

Conclusion: Patients with MetS have high prevalence of NAFLD. Central obesity and insulin resistance are the most common factors associated with NAFLD in these patients. Large proportion of NAFLD patients has advanced hepatic fibrosis.

$N A F L-37$

A low phase angle can predict advanced fibrosis in patients with non alcoholic steatohepatitis

\section{Varsha Shasthry $^{1}$}

${ }^{1} \mathrm{PhD}$ Scholar, India

Background: Nonalcoholic steatohepatitis (NASH) presents with variable degrees of fibrosis and liver biopsy remains the gold standard for its diagnosis. There is a need of non-invasive reproducible tool to identify patients with an increased risk of fibrosis. Phase angle (PhA) assessed by bioelectrical impedance analysis has been used as a predictor in different clinical conditions.

Aim: To study the association of $\mathrm{PhA}$ and degree of fibrosis in patients with biopsy proven NASH.

Method: Biopsy proven NASH patients evaluated between 2014 to 2016 were studied. Data of biochemical parameters (LFT, lipid profile, HbA1c) and bioelectrical impedance analysis (BIA) measurements (BMI in $\mathrm{kg} / \mathrm{m}^{2}$, Phase angle- $\mathrm{PhA} 0$, Fat percentageF\%, Visceral fat rating, Trunk fat percentage- $\mathrm{TrF} \%$, appendicular skeletal muscle index- ASMI in $\mathrm{kg} / \mathrm{m}^{2}$ ) within three months of the date of liver biopsy were retrieved from the hospital information system. Fibrosis stages F 0,1 and 2 were clubbed together and termed as mild fibrosis (MiF); F 3 and 4 were termed together as advanced fibrosis (AdF).

Result: Total of 150 NASH patients [M-73.3\%, BMI-28.4 \pm 5.1 , age$41.9 \pm 11.9$ years; Lean and normal weight- $11.4 \%$; overweight- $14.1 \%$; obese-50.3\%; morbidly obese-24.2\% were studied. Overall (both males and females) between fibrosis categories, AdF patients were of older age (47.7 vs. 37.8 years; $\mathrm{p}<0.001)$, had lower ALT levels $(59.8$ vs. $78.9 \mathrm{IU} / \mathrm{ml}$; p-0.009), low cholesterol (167 vs. $188 \mathrm{mg} / \mathrm{dl}$; $\mathrm{p}<0.001)$ and a high HbA1c (6.6 vs. 6.1 ; p-0.001) compared to MiF. Body composition-wise, AdF patients had significantly high F\%(33.6 vs. 30.6; p-0.04), high $\mathrm{TrF} \%$ (36.4 vs. 32.6; p-0.04) and a low PhA (5.8 vs. $6.3 ; \mathrm{p}-0.04$ ) compared to $\mathrm{MiF}$ patients. The findings were similar in males but in females, only age, $\mathrm{HbA} 1 \mathrm{c}$ and $\mathrm{PhA}$ were significantly different. Logistic regression analysis revealed $\mathrm{PhA}$ (OR:0.51; 95\%CI:0.22-0.77; p:0.02) as predictor of AdF in NASH even after adjusting for age, gender and BMI. Taking F1 as the reference category, multinomial logistic regression analysis suggested a decrease in the degree of fibrosis by $63 \%$ in F3 and $75 \%$ in F4 with every unit increase in PA (Table:1)

Conclusion: A low $\mathrm{PhA}$ is associated with advanced fibrosis irrespective of age, BMI and gender. Body composition analysis using BIA would be recommended to assess nutritional status and also to predict risk of fibrosis by Phase angle in NASH patients 


\begin{tabular}{|c|c|c|c|c|}
\hline \multicolumn{5}{|c|}{ Table: 1 Multinomial Logistic regression analysis } \\
\hline Fibrosis stage & mean \pm SD & OR & $\mathbf{9 5 \%} \mathbf{C I}$ & p-value \\
\hline 1 & $6.42 \pm 0.89$ & - & - & - \\
\hline 2 & $6.26 \pm 0.69$ & 0.79 & $0.49-1.3$ & 0.36 \\
\hline 3 & $5.89 \pm 0.67$ & 0.37 & $0.18-0.77$ & 0.009 \\
\hline 4 & $5.72 \pm 0.97$ & 0.25 & $0.114-0.57$ & 0.001 \\
\hline
\end{tabular}

NAFL-38

\section{Appropriateness of liver biopsy for nonalcoholic fatty liver disease in obese patients during laparoscopic cholecystectomy for gallstone disease}

\section{Kyung Sik Kim ${ }^{1}$, Sang Hyub Han ${ }^{1}$, Ji Hae Nahm ${ }^{1}$, Do Young Kim $^{1}$, Seung Woo Park ${ }^{1}$, Young Nyun Park ${ }^{1}$ \\ ${ }^{1}$ Severance Hospital, Yonsei University College of Medicine, Korea, Republic of}

Background: Obesity is well known as a risk factor for gallstone disease and is also a risk factor for fatty liver. Before laparoscopic cholecystectomy, it is not easy to detect fatty liver, except for the cases of severe fatty liver by blood chemistry and imaging diagnostic tools. However liver biopsy is particularly useful in patients without definite clinical manifestations. Liver biopsy is invasive and is not recommended except in special cases, but hepatic biopsy is not a relatively risky procedure during laparoscopic cholecystectomy. Therefore, we investigated the clinical characteristics in biopsy proven non-alcoholic fatty liver disease and non-alcoholic steatohepatitis in obese patients during laparoscopic cholecystectomy for gallstone disease.

Method: We retrospectively reviewed medical records in obese patients with gallstone disease who underwent laparoscopic cholecystectomy with liver biopsy. The definition of obesity was based on the Asian obesity criteria. (overweight:23 24.9, obese class I: $>25, \&$ obese class II $>30$ ). Total 74 patients were included, and 30 patients were diagnosed as fatty liver (A) and compared with patients without fatty liver disease (B) using various parameters.

Result: There were 9 male and 21 female in fatty liver group and 18 male and 26 female without fatty liver. In the fatty liver group, 6 overweight, 19 obese class I, \& 5 obese class II, but in the group without fatty liver, 21 overweight, 19 obese class I, \& 4 obese class II. The degree of obesity in the fatty liver group was more severe $(\mathrm{p}=.022)$. Except level of aminotransferase, all biochemical parameters were not different between two groups.

Conclusion: The liver biopsy could be recommended in obese I \& II patients with abnormal levels of aminotransferase.

\section{NAFL-39}

Characteristics of nonalcoholic fatty liver disease in the Pakistani population

\section{Shoukat Ali Samejo ${ }^{1}$, Zaigham Abbas ${ }^{1}$, Muhammad Asim ${ }^{1}$ \\ ${ }^{1}$ Dr Ziauddin University Hospital Clifton, Pakistan}

Background: Nonalcoholic fatty liver disease (NAFLD) has become an important cause of chronic liver disease and cirrhosis all over the world. Not much work has been done to address the characteristics of this disease in our population. The aim of this study is to evaluate our patients of NAFLD for risk factors, demographic features and presence of fibrosis.

Method: In this prospective ongoing study, patients who were found to have fatty liver on ultrasound examination of liver were further evaluated by anthropometric measurements, metabolic profile, insulin resistance, and fibroscan to assess the degree of fibrosis along with controlled attenuation parameter (CAP) for the stage of steatosis. Insulin resistance was calculated by homeostatic model assessment (HOMA-IR).

Result: Total number of patients assessed so far are 40; M:F 4:1, age $47 \pm 12.61$ (range 24 to 78 years). Nine patients (22.5\%) had diabetes, $7(17.5 \%)$ hypertension, 26(65\%) hypercholesterolemia, and 18 (45\%) hypertriglyceridemia. Seven patients $(17.5 \%)$ had BMI $<23 \mathrm{~kg} /$ $\mathrm{m}^{2}, 4(10 \%)$ with BMI $23-25$ and $29(72.5 \%)$ having BMI of $>25$. Ten percent (4/40) had waist circumference of $<80 \mathrm{~cm}$ and $90 \%$ (36/ 40) had a waist circumference of $>80 \mathrm{~cm}$. Twenty five percent $(10 / 40)$ had a HOMA score of $<3,30 \%(12 / 40)$ had a HOMA score of 3 to 5 , $15 \%(6 / 40)$ had a HOMA score of $>5$. Six patients $(15 \%)$ fulfilled the criteria of metabolic syndrome. Nineteen patients $(47.5 \%)$ patients had elevated ALT suggesting steatohepatitis. Ten patients $(25 \%)$ had F0-F1 fibrosis and 12 (30\%) had F2-F4 fibrosis. Four patients (10\%) patients had established cirrhosis, none of them had hepatoma.

Conclusion: In our population, many patients with NAFLD don't have insulin resistance, the predisposing factor in these patients may be dyslipidemia. A significant number of patients have F2-F4 fibrosis. Lean NAFLD is not uncommon.

\section{$N A F L-40$}

Coexistence of liver steatosis with other chronic liver diseases: lessons learnt from histopathological findings

Cosmas Rinaldi Adithya Lesmana ${ }^{1}$, Lidwina Cahyadinata ${ }^{1}$, Agnita Irawaty $^{1}$, Levina Pakasi ${ }^{1}$, Laurentius Adrianto Lesmana ${ }^{1}$

${ }^{1}$ Medistra Hospital, Indonesia

Background: Liver steatosis is a histopathological hallmark of nonalcoholic fatty liver disease (NAFLD), which is a risk factor of nonalcoholic steatohepatitis (NASH) and liver fibrosis.

Method: A cross-sectional study was done on patients who underwent liver biopsy for various indications. Adult patients were enrolled if they showed more than 5\% steatosis on histopathology assessment. NAFLD activity score (NAS) was evaluated and a NAS of 5-8 was regarded as NASH. Fibrosis stage was assessed according to the METAVIR system; F2 or more was considered as significant fibrosis. Result: A total of 28 patients were enrolled; men and women were equally distributed. Patients' mean age was $41.3+11.5$ years old. Obesity (body mass index $>25 \mathrm{~kg} / \mathrm{m}^{2}$ ) was found in $15(53.6 \%$ ) patients. Only $2(7.1 \%)$ patients had diabetes. There were $11(39.3 \%)$ patients with chronic hepatitis B and $5(17.9 \%)$ patients with autoimmune hepatitis (AIH). Fibrosis evaluation showed: no fibrosis in $10(35.7 \%), \mathrm{F} 1$ in $11(39.3 \%), \mathrm{F} 2$ in $4(14.3 \%)$, and $\mathrm{F} 3$ in 3 $(10.7 \%)$. No cirrhosis (F4) was found, but 1 patient with mild steatosis showed evidence of hepatocellular carcinoma. NASH was found in $9(32.1 \%)$ patients. There was no association between NASH and obesity, hepatitis B, or AIH. From 9 patients with NASH, 3 $(33.3 \%)$ showed no fibrosis, $5(55.6 \%)$ had F1, and 1 (11.1\%) had F2. On the other hand, only chronic hepatitis B was strongly associated with significant fibrosis ( $p=0.007$; Fisher's exact test).

Conclusion: Liver steatosis is not exclusive for patients with metabolic disorders. Chronic hepatitis B and autoimmune hepatitis B patients are often co-exist with non-alcoholic fatty liver disease. 
Further study is need to elucidate the role of liver steatosis in other chronic liver diseases and its association with clinical outcome.

\section{$N A F L-41$}

Eight-week modified alternate-day calorie restriction is an effective dietary strategy for non-alcoholic fatty liver disease with moderate steatosis and mild fibrosis

Muhammad Izzad Johari ${ }^{1}$, Muhammad Ilham Abdul Hafidz ${ }^{4}$, Mung Seong Wong ${ }^{3}$, Rona Marie Lawenko ${ }^{2}$, Zheng Feei Ma ${ }^{3}$, Khairiah Mat Yusoff ${ }^{3}$, Khairun Nisah Ibrahim ${ }^{3}$, Juhara Haron ${ }^{3}$, Chandran Nadarajan ${ }^{3}$

${ }^{1}$ MALAYSIAN SOCIETY OF GASTROENTEROLOGY AND HEPATOLOGY, Malaysia; ${ }^{2}$ DE LA SALLE HEALTH SCIENCES INSTITUTE, Philippines; ${ }^{3}$ UNIVERSITI SAINS MALAYSIA, Malaysia; ${ }^{4}$ UNIVERSITI TEKNOLOGI MARA, Malaysia

Background: Easier to comply and with proven efficacy, modified alternate-day calorie restriction (MACR) involves alternate day of $70 \%$ calorie restriction and habitual energy intake. The aim of study was to assess efficacy of 8 -week MACR in reduction of steatosis, fibrosis, and biochemical parameters in non-alcoholic fatty liver disease (NAFLD).

Method: Consecutive participants with NAFLD but without other liver diseases were consented. After 2-week period of stable eating and activity habits, participants begun their 8-week MACR through advice of a dietitian. Besides diary, participants received phone-calls and 2-weekly dietitian appointment to ensure adherence. At baseline and 8th 8 weeks after intervention, BMI, bloods (lipid profiles, glucose, and liver enzymes), and ultrasound (SuperSonic Imagine Aixplorer, France) to assess liver steatosis grading (mild, moderate, severe) and shear-wave elastography (SWE) were measured.

Result: A total of 105 patients were screened, 41 consented but 11 withdrew, and 30 participants (mean age 43.9 years and BMI $31.5 \mathrm{~kg} /$ $\mathrm{m}^{2}$, males $70 \%$, diabetes $53 \%$ ) completed the study. With 8 -week MACR, significant reductions were observed of grading of liver steatosis (40\% reduction in those with moderate steatosis, $\mathrm{P}=0.001)$, $\mathrm{SWE}$ (mean difference 0.9, $\mathrm{P}=0.001$ ), BMI (mean difference $0.6 \mathrm{~kg}$ / $\mathrm{m}^{2}, \mathrm{P}=0.003$ ), glucose (median difference $0.3 \mathrm{mmol} / \mathrm{L} \mathrm{P}=0.01$ ), ALT (median difference $20.5 \mathrm{U} / \mathrm{L}, \mathrm{P}=0.001$ ), and AST (median difference $9 \mathrm{U} / \mathrm{L}, \mathrm{P}=0.002$ ).

Conclusion: Eight-week MACR appears to be an effective dietary strategy for NAFLD especially with moderate steatosis and mild fibrosis.

$N A F L-42$

Serum copper predicts carotid intima-media thickness in obesityrelated nonalcoholic fatty liver disease patients with low prevalence of co-morbidities

\section{Clara Balsano $^{1}$, Giovanni Tarantino ${ }^{2}$, Cristiana Porcu ${ }^{1}$, Mario Arciello ${ }^{1}$, Paola Andreozzi ${ }^{1}$}

${ }^{1}$ University of Aquila, Italy; ${ }^{2}$ Federico II University Medical School of Naples, Italy

Background: The association of occurrence of obesity, nonalcoholic fatty liver disease (NAFLD) and cardiovascular (CV) diseases with altered metals homeostasis is intriguing. Copper in particular was identified as key player in various metabolic derangements. Thus, we investigated the possible association of serum copper with an indicator of early CV risk as the Intima-Media Thickness (IMT) of carotid artery and its predictive value in a selected population of obese patients.

Method: We performed a cross-sectional study recruiting 100 obese patients characterized by a low prevalence of co-morbidities. Ultrasound investigation for hepatic steatosis and IMT evaluation was performed.

Serum samples were collected and then analyzed through atomic absorption spectrometry to evaluate their copper content. Possible correlations between copper bioavailability and biochemical, clinical and anthropometric characteristics of patients were sought.

Result: Age negatively predicted copper serum levels of patients $(\mathrm{p}=.009)$. However, the most interesting finding is the negative prediction of IMT by the copper serum levels $(\mathrm{t}-2.23, \mathrm{P}=0.028$, Least Absolute Deviations Regression). Factor analysis confirmed therefore mentioned inverse correlation and highlighted the strong inverse correlation between smoking and copper serum levels.

Conclusion: Our data show that an altered copper bioavailability predicts early atherosclerosis as main $\mathrm{CV}$ risk in obesity-related NAFLD patients, shedding some light in this pathological scenario.

\section{$N A F L-43$}

Factors predicting significant and advanced fibrosis in patients with non alcohol fatty liver disease

\section{Jayshri Shah ${ }^{1}$}

${ }^{1}$ Ansh Liver Clinic, India

Background: Non Alcohol Fatty Liver Disease (NAFLD) is a common cause of chronic liver disease associated with varied etiological factors. NAFLD ranges from simple fatty liver to steatohepatitis which may lead to cirrhosis. Knowledge of predictors of significant fibrosis, will allow timely intervention to prevent further progression to cirrhosis. The aim of the study was to assess risk factors that influence presence of significant $\&$ advanced fibrosis in asymptomatic patients.

Method: This is a prospective observational study among patients $(\mathrm{N}=629)$ referred to outpatient Liver clinic from March 2014 to April 2017 for evaluation of asymptomatic incidentally detected fatty liver on ultrasound (USG). Of these, 321 patients were diagnosed with NAFLD. Patients with history of alcohol, coexisting hepatitis B, C infection were excluded. BMI, Lipid profile, Diabetes, Hypertension and hypothyroidism were recorded.

Result: NAFLD patients $(\mathrm{N}=321)$ were classified into 3 groups depending on $\mathrm{E}$ score $(\mathrm{kPa})$ on Fibroscan (FS) (502 echosens) at time of referral: Group A mild or no fibrosis $(<7.0<\mathrm{F} 2$, mean 5.5, median 5.6 range 3.0-7.0), Group B significant fibrosis (7.0- 10.2, F2-3, mean 8.3, median 8.15, range 7.1-10.2) Group $\mathrm{C}$ advanced fibrosis $(>10.2$ $\mathrm{F} 4$, mean 23.08, median 15.5, range 10.3-75). M probe was used in $72.3 \%$ cases and XL probe in $27.7 \%$.

BMI $>25$ was the commonest risk factor associated with NAFLD, followed by dyslipidemia, diabetes, hypertension and hypothyroidism. Table I shows the comparison of various variables which can predict the severity of fibrosis detected on FS in patients with NAFLD. The variables were analysed using the Chi-Square test. Higher age and CAP score, abnormal AST or ALT value, presence of metabolic syndrome, BMI $>25$, diabetes, hypertension and dyslipidemia were statistically significant predictors of significant and advanced fibrosis. In stepwise multiple logistic regression analysis using $<F 2$ (no significant fibrosis), $>=F 2$ (significant fibrosis) as dependent variable and age, gender, diabetes, hypertension, dyslipidemia, BMI $>25$ as independent variables, only age $(\mathrm{p}=0.029)$ and diabetes $(\mathrm{p}=0.009)$ were found to be statistically significant predictors of significant fibrosis 
Conclusion: NAFLD can be silently associated with significant \& advanced fibrosis in approximately half of patients, despite normal transaminases in more than two third. Higher age and diabetes significantly increases the risk of fibrosis.

Table: Comparison of variables and severity of Fibrosis

\begin{tabular}{|c|c|c|c|c|c|}
\hline Variable & \begin{tabular}{|l} 
Group A (KE2) \\
$\mathrm{N}=144(44.9 \%$ \\
)
\end{tabular} & \begin{tabular}{|l|} 
Group B( $E 2-3)$ \\
$\mathrm{N}=72(22.4 \%)$
\end{tabular} & $\begin{array}{l}\text { Group C (EA) } \\
N=105(32.7 \% \\
)\end{array}$ & \begin{tabular}{|l|} 
Total \\
$\mathrm{N}=321$
\end{tabular} & $p$ \\
\hline $\begin{array}{l}\text { 1) Gender } \\
\text { Male } \\
\text { Female }\end{array}$ & $\begin{array}{l}82(47.4 \%) \\
62(41.9 \%)\end{array}$ & $\begin{array}{l}42(24.3 \%) \\
30(20.3 \%)\end{array}$ & $\begin{array}{l}49(28.3 \%) \\
56(37.8 \%)\end{array}$ & $\begin{array}{l}173(53.9 \%) \\
148(46 \%)\end{array}$ & 0.19 \\
\hline $\begin{array}{l}\text { 2) Age (years) } \\
\text { Mean } \\
\text { Median } \\
\text { Range }\end{array}$ & \begin{tabular}{|l|}
43.86 \\
41 \\
$14-73$
\end{tabular} & $\begin{array}{l}46.89 \\
48.5 \\
15-72\end{array}$ & $\begin{array}{l}51.59 \\
51 \\
24-82\end{array}$ & $\begin{array}{l}47.07 \\
47 \\
14-82\end{array}$ & $200 \mathrm{E}-05$ \\
\hline $\begin{array}{l}\text { 3) FS CAP score }(\mathrm{dB} / \mathrm{m}) \\
\text { Mean } \\
\text { Median } \\
\text { Range }\end{array}$ & \begin{tabular}{|l|}
289.85 \\
292 \\
$165-400$
\end{tabular} & $\begin{array}{l}315.46 \\
317 \\
192-394\end{array}$ & \begin{tabular}{|l}
311.86 \\
317 \\
$151-400$
\end{tabular} & \begin{tabular}{|l|}
302.79 \\
305 \\
$151-400$
\end{tabular} & 0.00012 \\
\hline $\begin{array}{l}\text { 4) Normal AST ALT } \\
\text { Yes } \\
\text { No }\end{array}$ & \begin{tabular}{|l}
$100(52.6 \%)$ \\
$44(33.6 \%)$
\end{tabular} & $\begin{array}{l}43(22.6 \%) \\
29(22.1 \%)\end{array}$ & $\begin{array}{l}47(24.7 \%) \\
58(44.3 \%)\end{array}$ & $\begin{array}{l}190(59.1 \%) \\
131(40.8 \%)\end{array}$ & 0.00047 \\
\hline $\begin{array}{l}\text { 5)Metabolic } \\
\text { syndrome } \\
\text { Yes } \\
\text { No } \\
\end{array}$ & $\begin{array}{l}37(24.3 \%) \\
107(63.3 \%)\end{array}$ & $\begin{array}{l}37(24.3 \%) \\
35(20.7 \%)\end{array}$ & $\begin{array}{l}78(51.3 \%) \\
27(16 \%)\end{array}$ & $\begin{array}{l}152(47.3 \%) \\
169(52.6 \%)\end{array}$ & $244 E-13$ \\
\hline $\begin{array}{l}\text { 6) BMI }>25 \\
\text { Yes } \\
\text { No } \\
\text { Mean } \\
\text { Median } \\
\text { Range }\end{array}$ & \begin{tabular}{|l}
$104(41.9 \%)$ \\
$40(54.8 \%)$ \\
28.36 \\
27.25 \\
$20-44$
\end{tabular} & $\begin{array}{l}54(21.8 \%) \\
18(24.7 \%) \\
28.33 \\
27.4 \\
21-47.9\end{array}$ & $\begin{array}{l}90(36.3 \%) \\
15(20.5 \%) \\
28.82 \\
28.2 \\
22-43.1\end{array}$ & \begin{tabular}{|l}
$248(77.2 \%)$ \\
$73(22.7 \%)$ \\
28.5 \\
27.8 \\
$20-47.9$
\end{tabular} & 0.03 \\
\hline $\begin{array}{l}\text { 7)DM } \\
\text { Yes } \\
\text { No } \\
\end{array}$ & \begin{tabular}{|l}
$49(32.5 \%)$ \\
$95(55.9 \%)$ \\
\end{tabular} & \begin{tabular}{|l|}
$30(19.9 \%)$ \\
$42(24.7 \%)$ \\
\end{tabular} & $\begin{array}{l}72(47.7 \%) \\
33(19.4 \%) \\
\end{array}$ & \begin{tabular}{|l|}
$151(47 \%)$ \\
$170(52.9 \%)$ \\
\end{tabular} & $2,8 E-07$ \\
\hline $\begin{array}{l}\text { 8)Dxslipidemia } \\
\text { Yes } \\
\text { No }\end{array}$ & $\begin{array}{l}98(43.4 \%) \\
46(48.4 \%)\end{array}$ & $\begin{array}{l}56(24.8 \%) \\
16(16.8 \%)\end{array}$ & $\begin{array}{l}72(31.9 \%) \\
33(34.7 \%)\end{array}$ & $\begin{array}{l}226(70.4 \%) \\
95(29.5 \%)\end{array}$ & 0.29 \\
\hline $\begin{array}{l}\text { 9)Hypertension } \\
\text { Yes } \\
\text { No }\end{array}$ & \begin{tabular}{|l}
$44(37.3 \%)$ \\
$100(49.3 \%)$
\end{tabular} & $\begin{array}{l}25(21.2 \%) \\
47(23.2 \%)\end{array}$ & $\begin{array}{l}49(41.5 \%) \\
56(27.6 \%)\end{array}$ & $\begin{array}{l}118(36.7 \%) \\
203(63.2 \%)\end{array}$ & 0.03 \\
\hline $\begin{array}{l}\text { 10)Hxpothxroidsim } \\
\text { Yes } \\
\text { No }\end{array}$ & \begin{tabular}{|l|}
$21(31.8 \%)$ \\
$123(48.2 \%)$
\end{tabular} & $\begin{array}{l}13(19.7 \%) \\
59(23 \%)\end{array}$ & $\begin{array}{l}32(48.5 \%) \\
73(28.6 \%)\end{array}$ & $\begin{array}{l}66(20.5 \%) \\
255(79.4 \%)\end{array}$ & 0.008 \\
\hline
\end{tabular}

$N A F L-44$

\section{Hepatic biomarkers to improve cardiovascular risk assessment}

\section{Clara Balsano $^{1}$, Cristiana Porcu ${ }^{1}$, Silvia Sideri ${ }^{1}$, Clara Balsano ${ }^{1}$}

${ }^{1}$ University of Aquila, Italy

Background: Non-Alcoholic Fatty Liver Disease (NAFLD) is associated with enhanced cardiovascular risk (CVR). However, the mechanisms and the biomarkers linking liver steatosis and an increased CVR are poorly defined at the molecular level. To identify new potential biomarkers able to predict CVR, we evaluated the expression of 82 adipogenesis-related genes in livers and hearts of a mouse model of liver steatosis.

Method: Sixty C57BL/6J mice were housed individually in a temperature-controlled room, on a 12 hours light-dark cycle. Mice were randomly divided into six different groups (10 animals per group) and fed with one of the following type of diet: High Fat Diet (HFD) or Normal-Fat Diet (NFD). After 6 and 12 months, mice were sacrificed and livers and hearts collected to perform gene expression analysis using the Mouse Adipogenesis RT2 Profiler Qiagen PCR Array System, according to the manufacturer's instructions. Unsupervised clustering, t-test and Analysis of Variance (ANOVA) were performed.
Result: Unsupervised analysis showed a specific tissue and timedependent adipogenesis-related gene regulation. Additionally, a differential pattern of expression between NFD $v s$ HFD mice was observed. Supervised analyses corroborated all these findings. In fact, t-test comparing livers $v s$ hearts of NFD and HFD mice allowed to identify 13 and 34 significantly differentially expressed genes at 6 and 12 months, respectively. In particular, in line with the literature, we remarked the up-regulation of Agt, Cebpa, Fasn and Shp in liver and the overexpression of $\mathrm{Lpl}$, Glut4, Bmp 4, Fgfl, Ppargcla, Ppargclb, Sfrpl and Sfrp5 in heart, confirming a specific tissue regulation of lipid metabolism between these two organs. Furthermore, ANOVA highlighted that at 6 months in livers and at 12 months in hearts, HFD-fed mice displayed the up-regulation of the following set of genes: Adipoq, Dlk1, Dkk1, Foxc2, Jun, Lep, Lipe, Sfrp5 and Ucp1. Q-PCR analyses to validate the expression of these transcripts identified by arrays were performed.

Further microarray analyses on peripheral blood mononuclear cells from healthy donors and NAFLD patients are currently ongoing. Conclusion: Our findings evidenced the existence of a liver-heart axis, indicating that liver is the first organ responding to the insult derived from high fat intake. A panel of genes representing potential predictors of CVR was identified.

$N A F L-45$

Impact of lifestyle modification induced $=7 \%$ weight loss in the management of ultrasound diagnosed non-alcoholic fatty liver disease in type 2 diabetics

\section{Kuhu Roy ${ }^{1}$, Uma Iyer ${ }^{4}$, Rumin Shah ${ }^{3}$, Tushar Vaishnav ${ }^{2}$}

${ }^{1}$ Independent nutrition consultant, India; ${ }^{2}$ Alkapuri -ray and Sonography Clinic, India; ${ }^{3}$ Diabetology Clinic, India; ${ }^{4}$ The M.S. University of Baroda, India

Background: The management of non-alcoholic fatty liver disease (NAFLD) revolves around correcting the underlying metabolic aberrations. There are limited studies with hepatic end point from India that have assessed the impact of lifestyle modification induced weight loss in the management of NAFLD in type 2 diabetics. Therefore, the aim of the research was to assess the impact of lifestyle modification induced $\geq 7 \%$ weight loss in the management of NAFLD in type 2 diabetics.

Method: It was a pre-test post-test experimental arm only study. Thirty type 2 diabetic ultrasound diagnosed NAFLD subjects were enrolled from a diabetic clinic to receive individual counselling on lifestyle modification (dietary, physical activity recommendations and positive behaviours) for a period of four months, in addition to standard care. Data on diet, physical activity and biochemical profile was assessed. Weight loss of $7 \%$ was selected as the outcome measure considering it is beneficial in improving liver profile. Ultrasonography was used as a guide to weight loss.

Result: Majority of the subjects lost $\geq 7 \%$ weight $(60 \%)$ whereas $40 \%$ of the subjects lost $<7 \%$ weight. The age, duration of diabetes and drug regime was similar in subjects who lost $<7 \%$ or $\geq 7 \%$ weight. Subjects with $\geq 7 \%$ weight loss had a significant increase in proportion of protein intake (13\% vs. $11.04 \%, \mathrm{P} 0.0017)$ and high density lipoprotein cholesterol $(48.2 \mathrm{mg} / \mathrm{dl}$ to $53.1 \mathrm{mg} / \mathrm{dl}, \mathrm{P} 0.0007)$ and liver span reduced significantly (179 $\mathrm{mm}$ to $167.3 \mathrm{~mm}, \mathrm{P} 0.004)$. They also ate out less frequently as compared to subjects with $<7 \%$ weight loss (5.5\% vs. $41.6 \%, \mathrm{P} 0.025)$. Subjects with $\geq 7 \%$ weight loss had a significant increase in total MET minutes/week (1063 to 1622 , $P$ 0.012) and became significantly higher from the subjects who lost $<7 \%$ weight ( 1622 vs. 819.3 , P 0.032 ). The prevalence of hypertriglyceridemia became significantly lower in subjects who lost $\geq 7 \%$ 
weight compared to subjects who lost $<7 \%$ weight $(16.7 \%$ vs. $58.3 \%$, $P$ 0.045). Gamma glutamyl transferase reduced significantly in subjects with $\geq 7 \%$ weight loss (28 U/L to $22.4 \mathrm{U} / \mathrm{L}, \mathrm{P} 0.007$ ) and also became significantly lower from subjects who lost $<7 \%$ weight (22.4 U/L vs. $32.3 \mathrm{U} / \mathrm{L}, \mathrm{P}$ 0.033). The prevalence of metabolic syndrome $(77.7 \%$ to $44.4 \%, \mathrm{P} 0.043)$ and NAFLD declined significantly ( $100 \%$ to $61.1 \%$, P 0.007 ) in subjects who lost $\geq 7 \%$ weight.

Conclusion: Weight loss $\geq 7 \%$ achieved through lifestyle modification was effective in the management of ultrasound diagnosed NAFLD in type 2 diabetics and therefore can be used as a therapeutic strategy for NAFLD.

\section{$N A F L-46$}

Lower vitamin D levels are associated with severe fibrosis in patients with non-alcoholic fatty liver disease: a populationbased study

\author{
Su Lin ${ }^{1}$, Yuxiu Liu' ${ }^{3}$, Yueyong $\mathrm{Zhu}^{2}$ \\ ${ }^{1}$ The First Affiliated Hospital of Fujian Medical University, China; \\ ${ }^{2}$ First Affiliated Hospital of Fujian Medical University, China; ${ }^{3}$ Fujian \\ Medical University, China
}

Background: Previous studies have shown that vitamin D (Vit D) deficiency is associated with liver steatosis. However, the relationship between Vit $\mathrm{D}$ and liver fibrosis in non-alcoholic fatty liver disease (NAFLD) remains unclear. This study aims to better outline the relationship between Vit D and liver fibrosis in NAFLD patients.

Method: This cross-sectional study was conducted based on data from the National Health and Nutrition Examination Survey (19881994). Liver steatosis was diagnosed by ultrasonography. Hepatic fibrosis was assessed using two non-invasive models; the AST to platelet ratio index (APRI) score and the NAFLD fibrosis score (NFS). Linear association of chi-square tests and ordinal logistic regression were used to analyze the relationship between Vit D deficiency and liver fibrosis.

Result: Among the 10,450 participants, 4,867 (46.6\%) had Vit D deficiency and $3,763(36.0 \%)$ had Vit D insufficiency. Participants with Vit D deficiency were more likely to be females $(61.0 \%)$, and had a higher body mass index $\left(28.32 \mathrm{~kg} / \mathrm{m}^{2}\right)$. NFS and APRI scores were higher in both the Vit D insufficient and deficient patients compared to those with sufficient Vit D levels. The percentage of significant fibrosis decreased with increasing Vit D levels, irrespective of which non-invasive model was employed. After adjusting for age, sex, body mass index, race, season, renal function, viral hepatitis, diabetes history and hypertension history, ordinal logistic regression revealed that Vit D level was independently associated with the degree of liver fibrosis.

Conclusion: Lower Vit D levels are associated with advanced liver fibrosis in NAFLD.

\section{$N A F L-47$}

\section{Limited implication of high density lipoprotein for diagnosing NASH}

\section{Sheikh Mohammad Noor E Alam ${ }^{1}$, Biplob Kumar Saha ${ }^{4}$, Dulal Chandra Das ${ }^{3}$, Amit Das ${ }^{3}$, Partho Pratik Roy ${ }^{5}$, Mamun Al Mahtab ${ }^{2}$}

${ }^{1}$ Assistant Professor, Bangladesh; ${ }^{2}$ Associate Professor, Bangladesh; ${ }^{3}$ Medical Officer, Bangladesh; ${ }^{4}$ Resident Medical Officer,

Bangladesh; ${ }^{5}$ Resident Physician, Bangladesh
Background: Nonalcoholic fatty liver disease (NAFLD) is a metabolic liver disease encompasses a wide spectrum of conditions ranging from simple steatosis to nonalcoholic steatohepatitis (NASH), fibrosis, and end stage liver disease.

The objective of this study is to evaluate high density lipoprotein (HDL) level for diagnosing NASH.

Method: An observational, cross sectional study was carried out in the Department of Hepatology, Bangabandhu Sheikh Mujib Medical University, Dhaka. 43 patients of Non-alcoholic fatty liver disease (NAFLD) attending at outpatient and inpatient department of Hepatology were selected and underwent for liver biopsy with NAFLD Activity Score (NAS). HDL was also analyzed.

Result: Mean serum HDL level in the study population was 37.1 (9.8) $\mathrm{mg} / \mathrm{dl}$. And $41.8 \% \mathrm{NASH}$ patients had serum HDL level in the metabolic range (male $<40 \mathrm{mg} / \mathrm{dl}$, female $<50 \mathrm{mg} / \mathrm{dl}$ ) according to NCEP ATP III 2001 and International Diabetes Federation 2006 criteria for South Asians. Serum HDL level difference had no significant correlation ( $\mathrm{p}=0.17$, chi-square test) in between NNFL and NASH groups.

Conclusion: Serum high density lipoprotein (HDL) has very limited implication for diagnosing NASH. Biopsy is the only way to estimate steatohepatitis and/or fibrosis.

$N A F L-48$

Non-alcoholic fatty liver disease and carotid artery intima-media thickness: a positive association

Fahmida Shams Azam

${ }^{1}$ Dhaka Medical College, Bangladesh

Background: Prevalence of non-alcoholic fatty liver disease (NAFLD) is rapidly increasing worldwide. Increased carotid intima media thickness (CIMT) is generally accepted as an early indicator of atherosclerosis and has been related to cardiovascular disease including incidence of myocardial infarction and stroke.

Method: This cross sectional study was carried out at the department of Radiology and Imaging of Dhaka Medical College Hospital during the period of July 2016 to June 2017. Study population comprised of the outpatients who underwent abdominal ultrasonogram. Measurement of CIMT was done by high resolution real-time B-mode ultrasonogram at the same time. Body weight, height, blood pressure and available biochemical test reports were recorded along with a brief clinical history.

Result: A total of 101 subjects were included in this study. Of them, on ultrasonographic examination of abdomen $49(48.5 \%)$ had normal liver assigned as group A and 52(51.5\%) patients had fatty liver disease assigned as group B. Mean age of group A and group B patients were $36.8 \pm 12.4$ and $46.15 \pm 10.3$ years, and male:female ratio was 20:29 and 28:24 respectively. Family history of cardiovascular disease, diabetes mellitus and current smoking history were $14.3 \%$ versus $19.2 \%, 24.5 \%$ versus $44.2 \%$ and $14.3 \%$ versus $11.5 \%$ respectively among group A and group B. Frequency of NAFLD was grade I fatty liver $32.6 \%$, grade II fatty liver $38.4 \%$ and grade I II fatty liver was $28.8 \%$. Mean BMI was $24.8 \pm 4.1$ versus $27.9 \pm 3.5$ $(\mathrm{p}=0.000)$ and serum total cholesterol $(\mathrm{mg} / \mathrm{dl})$ was $175.1 \pm 41.3$ versus $207.3 \pm 52.6(\mathrm{P}=0.030)$ among two groups. On the other hand mean systolic blood pressure $(\mathrm{mm} \mathrm{Hg}) 127.5 \pm 16.1$ versus $127.5 \pm 16.1$ $(\mathrm{P}=0.836)$, mean diastolic blood pressure $(\mathrm{mm} \mathrm{Hg}) 81.6 \pm 11.6$ versus $82.4 \pm 8.7(\mathrm{P}=0.7 .8)$, serum triglyceride level $(\mathrm{mg} / \mathrm{dl}) \quad 175.3 \pm 106.1$ versus $213.4 \pm 167.4(\mathrm{P}=0.404)$ were not significant among groups. Mean CIMT $(\mathrm{mm})$ was $0.62 \pm 0.15$ in group A and $0.77 \pm 0.17$ in group $\mathrm{B}(\mathrm{P}=0.000)$ respectively. This result is highly significant. Accuracy of the CIMT in patients with or without NAFLD by ROC curve 
showed the area under curve (AUC) was 0.752 which was fair outcome of the study. CIMT cut-off at $0.680 \mathrm{~mm}$ showed the highest sensitivity $(75 \%)$ and specificity $(63 \%)$ for the presence of fatty liver disease by ultrasonogram.

Conclusion: There is a positive correlation between NAFLD with CIMT. High BMI and raised serum cholesterol has significant role in the development of NAFLD. A large scale study is recommended to find more accurate cut off of CIMT in the relationship with NAFLD.

\section{$N A F L-49$}

Non-alcoholic fatty liver disease in patients with Ulcerative colitis: a prospective Indian data. Mehrotra Punit*, Mehrotra Pulkit** *Lucknow Gastroenterology Centre **SRMC Chennai

\section{Punit Mehrotra $^{1}$, Pulkit Mehrotra ${ }^{2}$}

${ }^{1}$ Lucknow Gastroenterology Centre, India; ${ }^{2}$ SRMC Chennai, India

Background: Ulcerative colitis (UC) is a immune mediated disorder and Non alcoholic fatty liver disease is a spectrum of hepatic disorder from simple steatosis to cirrhosis. Fatty liver based on ultrasound is frequently seen in patients with UC. Recent increase in awareness of NAFLD in UC is evident from several publications. Metabolic risk factors are associated with non-alcoholic fatty liver disease (NAFLD), but they are less frequent in UC. To our knowledge there are no studies of NAFLD in IBD from India based on FIBROSCAN and Controlled attenuation Parameter (CAP score.

Aim: This study evaluates the frequency of NAFLD in patients of UC using Fibroscan and CAP score and data was later compared to control population.

Methods: All UC patients presenting to gastroenterology clinic between June 2016 to June 2017 were enrolled. All cases underwent Fibroscan and biochemical tests to assess liver function. UC patients with NAFLD were compared with those who had no evidence of NAFLD (control).

Result: Among 128 UC patients, 23 (17.96\%) had evidence of NAFLD by Fibroscan, and when compared to control (10/102: $9.80 \%$ ) was significantly higher. On comparison of metabolic risk factors there was no significant difference between control and UC group with NAFLD. There was no association between the age and onset of illness. On analyzing the UC group further based on extent of illness there was no association between extent of disease and prevalence of NAFLD. In patients with UC group with NAFLD CAP score was significantly higher in those with severe disease based on Truelove and Wits classification (323.5 vs $258 \mathrm{p}<0.005)$.

Conclusion: NAFLD occurred in $17.96 \%$ of the UC population. There was no effect of age and onset of illness on presence of NAFLD in UC patients. Metabolic risk factors did not play a significant role in prevalence of NAFLD in UC implying other confounding factor may be the cause of NAFLD. Association of severity of disease with NAFLD may help clinician to decide mode of therapy and choose the appropriate medication.

Keywords: Ulcerative colitis, NAFLD, NASH, fibroscan fatty liver

NAFL-50

Non-alcoholic fatty liver disease in pregnancy and associated fetomaternal outcomes in a tertiary care hospital in Dhaka, Bangladesh

Md. Akmat Ali ${ }^{1}$, Md. Zakiul Hassan ${ }^{2}$
${ }^{1}$ Ad-din Women's Medical College Hospital, Bangladesh;

${ }^{2}$ International Centre for Diarrhoeal Disease Research, Bangladesh, Bangladesh

Background: Non Alcoholic Fatty Liver Disease (NAFLD) is a growing public health concern globally. NAFLD has been linked with adverse pregnancy outcomes. We aimed to describe the clinical and laboratory profile and pregnancy outcomes of patients with fatty liver disease.

Method: We conducted the study from January 2017 to October 2017 at a tertiary care teaching hospital in Dhaka, Bangladesh. We enrolled pregnant women who consented to participate in the study. We collected demographic and clinical information using a pre-tested questionnaire. We investigated for evidence of fatty liver disease by ultrasonographs. Other investigations which included blood glucose level, lipid profile and liver function tests were also performed. Pregnancy outcomes of all enrolled participants were recorded during delivery.

Result: We enrolled 103 patients. Mean age of the patients was 27 years (range 18-39 years). Eighteen patients were enrolled during first trimester, 32 in second and 53 in third trimesters. Of 103, 3 $(2.9 \%)$ patients had known history of fatty liver disease, $11(10.7 \%)$ had hypertension, $11(10.7 \%)$ had Gestational Diabetes Mellitus but none had previous history of Diabetes Mellitus. Among 103 patients, $8(7.8 \%)$ patients had previous pregnancy related complications and $32(31 \%)$ had foetal complications including still birth, abortion, neonatal death and intrauterine death (IUD). Of all patients, $92(89 \%)$ had grade $1,10(9.7 \%)$ had grade 2 and one $(0.9 \%)$ patient had grade 3 fatty liver. Of 103, 35 (32\%) patients had high fasting blood sugar level and $26(24.1 \%)$ patients had positive Oral Glucose Tolerance Test (OGTT). Of all patients, 31\% (34/103) had a high cholesterol level, none had abnormal High Density Lipid (HDL), 5.8\% (6/103) had high Low Density Lipid (LDL) and 78.6\% (81/103) had high Triglyceride (TG) level. Of 103 patients, USG identified 3 patients with abnormal foetal profile. Only 2 patients had adverse pregnancy outcome: one IUD and one abortion.

Conclusion: Non-alcoholic fatty liver disease increases the risk of maternal and foetal complications. Pregnant women should regularly screen for fatty liver disease for early detection and intervention.

\section{$N A F L-51$}

Profile of incidentally detected fatty liver on ultrasonogram in females- ultrasound detected fatty liver innocent?

\section{Paul T. Joyes ${ }^{1}$}

${ }^{1}$ Senior Resident, India

Background: NAFLD is increasing in females due to increased prevalence of obesity in middle aged females belonging to low socioeconomic status. Prevalence in India ranges from $23-46 \%$. PCOD is strongly associated with NAFLD. This study is exclusively done in females as use of alcohol is rare in rural indian women. Aimto delineate clinical and biochemical profile of NAFLD in females. To detect metabolic syndrome and to assess fibrosis in this group. Method: Inclusion: Females diagnosed with fatty liver on ultrasonogram attending MGE OPD from 1st november 2016- 30th november 2017. Exclusion: significant alcohol consumption. overt chronic liver disease and other diseases that contribute to fat accumulation in liver. Study design: descriptive study. Methodology: After history \& clinical examination, patients will undergo CBC, LFT, FLP, HbsAg, anti HCV and fibroscan. IDF criteria used for metabolic syndrome and $\mathrm{Tg} / \mathrm{HDL}$ ratio used for insulin resistance.

Result: This study included incidentally detected fatty liver on ultrasonogram exclusively in female patients who presented to 
medical gastroenterology OPD of our institute. We have 51 patients with incidentally detected fatty liver and they were diagnosed with Non alcoholic fatty liver disease after clinical and biochemical evaluation. Mean age was 45 years. $47 \%$ belonged to $40-50$ years age group. $69 \%$ of patients belonged to lower middle class, $10 \%$ in upper middle class and $21 \%$ upper lower class according to modified Kuppuswamys SES scale. $47 \%$ of females in our study belonged to overweight and $43 \%$ were obese according to BMI. $90 \%$ of females in our study had a waist circumference more than $80 \mathrm{~cm}$ showing that truncal obesity can be a confounding factor to NAFLD. 51 patients in our population were not previously diagnosed to be diabetics and yet $51 \%$ had fasting blood sugars more than $100 \mathrm{mg} / \mathrm{dL}$. Hypertriglyceridemia with low HDL level is common biochemical abnormality in NAFLD. Mean TG level in our study was $154+1-42.46 \%$ had TG level more than $150 \mathrm{mg} / \mathrm{dL} .88 \%$ of females in our study had a HDl level less than $50.15 \%$ of patients had high blood pressure. Elevated liver enzymes were noted in $25 \%$ of patients. In our study we followed the international diabetic federation criteria for metabolic syndrome and $70 \%$ had metabolic syndrome. Tg/HDL ratio more than 3.5 was used as a marker for insulin resistance. $63 \%$ had aTg/HDL ratio more than 3.5 which showed insulin resistance is one of the confounding mechanism for NAFLD. Fibroscan assessment in this 51 showed that $66 \%$ had no evidence of fibrosis (F0), $21 \%$ had F1 and $13 \%$ had F2 fibrosis. No cases were detected to have Poly cystic ovarian disease (PCOD) in our study. Incidentally detected fatty liver could be benign as in this study here was no evidence of fibrosis in majority of patients. Since there is strong association with metabolic syndrome and insulin resistance, early detection and life style modification might help in reversal of the disease.

Conclusion: This study is unique as we selected a non alcoholic group. Truncal obesity, dyslipidemia and fasting hyperglycemia were seen majority. No PCODs in the study group. Overweight and obesity were common among middle aged women.

\section{$N A F L-52$}

Reductions in liver stiffness by magnetic resonance elastography (MRE) predict fibrosis improvement in a multi-center clinical trial of subjects with nonalcoholic steatohepatitis (NASH)

Jina Lee ${ }^{1}$, Eric Lawitz ${ }^{6}$, Reem Ghalib ${ }^{5}$, Magdy Elkhashab ${ }^{7}$, Stephen Caldwell $^{10}$, Manal Abdelmalek ${ }^{2}$, Kris Kowdley ${ }^{4}$, Ren $\mathrm{Xu}^{1}$, Ling $\mathrm{Han}^{1}$, Yao $\mathrm{Li}^{4}$, Stephen Djedjos ${ }^{1}$, Macky Natha', Robert P Myers ${ }^{1}$, Mani Subramanian', Zachary Goodman ${ }^{3}$, Michael Charlton', Claude B Sirlin ${ }^{8}$, Michael Middleton ${ }^{8}$

${ }^{1}$ Gilead Sciences, Inc., United States; ${ }^{10}$ University of Virginia, United States; ${ }^{2}$ Duke University Medical Center, United States; ${ }^{3}$ Inova Fairfax Hospital, United States; ${ }^{4}$ Swedish Medical Center, United States; ${ }^{5}$ Texas Clinical Research Institute, United States; ${ }^{6}$ Texas Liver Institute, University of Texas Health, United States; ${ }^{7}$ Toronto Liver Centre, Canada; ${ }^{8}$ University of California San Diego, United States; ${ }^{9}$ University of Chicago, United States

Background: MRE is a noninvasive imaging technique that estimates liver stiffness. The clinically important difference in MRE-stiffness is unknown. Our objective was to assess the performance of liver stiffness measurement by MRE to predict fibrosis responses.

Method: Centrally-read 2D, $60 \mathrm{~Hz}$ MRE was performed at baseline (BL) and Week 24 (W24) in a Phase 2 multi-center clinical trial of 72 subjects with NASH (NAS $\geq 5$ and F2-3 fibrosis) treated with selonsertib $6 \mathrm{mg}$ or $18 \mathrm{mg}$ orally QD alone or in combination with simtuzumab (SIM, $125 \mathrm{mg}$ SQ weekly) or SIM alone for 24 weeks. Liver biopsies at BL and W24 were staged according to the NASH Clinical Research Network (CRN) classification and hepatic collagen was quantified by morphometry. The discrimination of MRE-stiffness for fibrosis improvement ( $\geq 1$-stage reduction) and fibrosis progression ( $\geq 1$-stage increase) was evaluated using AUROCs and the optimal stiffness thresholds were determined based on the maximal sum of sensitivity and specificity.

Result: Among 54 subjects with evaluable MRE and biopsies at BL and W24 (63\% F3; median BL MRE-stiffness, $3.64 \mathrm{kPa}), 18$ (33\%) had fibrosis improvement and $8(15 \%)$ had fibrosis progression. The AUROC of MRE-stiffness to predict fibrosis improvement was 0.62 (95\% CI $0.46-0.78)$ and the optimal threshold was a $\geq 0 \%$ relative reduction (Table). At this cut-off, MRE was $67 \%$ sensitive, $64 \%$ specific, and had positive (PPV) and negative predictive values (NPV) of $48 \%$ and $79 \%$, respectively. Compared with subjects with no reduction in MRE-stiffness, those with any reduction had greater relative changes in hepatic collagen content $+2.8 \%$ vs $-21.9 \%$, $\mathrm{p}=0.61)$, liver stiffness by FibroScan $(-2.7 \%$ vs $-4.0 \%$; $=0.40)$, ELF $(+0.5 \%$ vs $-1.7 \% ; \mathrm{p}=0.03)$, and TIMP1 $(+5.7 \%$ vs $-9.0 \% ; \mathrm{p}=0.016)$. MRE performed better for predicting fibrosis improvement in subjects with F3 compared with F2 fibrosis at BL (sensitivity/specificity: F3, $69 \% / 86 \%$ vs $\mathrm{F} 2,60 \% / 33 \%$ ). For detecting fibrosis progression, the AUROC of MRE-stiffness was 0.57 (95\% CI 0.36-0.79). At an optimal threshold of $\geq 0 \%$ increase, MRE-stiffness was $63 \%$ sensitive, $46 \%$ specific, and had PPV and NPV of $17 \%$ and $88 \%$, respectively. Conclusion: In this multi-center trial of SEL in patients with F2-3 fibrosis, any reduction in MRE-stiffness was predictive of fibrosis improvement. These data support the use of MRE as a noninvasive endpoint in clinical trials of patients with NASH.

\section{$N A F L-53$}

Significant body weight change and liver or cardiovascular (CVD) complications in Asian patients with histological proven nonalcoholic fatty liver patients (NAFLD); a prospective cohort study

Sombat Treeprasertsuk ${ }^{1}$, Julalak Karuehardsuwan ${ }^{1}$, Roongruedee Chaiteerakij $^{1}$, Panida Piyachaturawat ${ }^{1}$, Kanokwan Sonsiri ${ }^{1}$, Chonlada Phathong ${ }^{1}$, Pisit Tangkijvanich ${ }^{1}$, Piyawat Komolmit ${ }^{1}$

${ }^{1}$ Chulalongkorn University, Thailand

Background: The long-term follow-up of liver or cardiovascular (CVD) outcomes of Asian population with NAFLD is clearly reported. We aimed to compare the outcome of body weight changes, as well as the liver or CVD complications in Asian NAFLD patients with or without morbid obesity.

Method: We prospectively enrolled 222 NAFLD patients with biopsy-proven during the 10-year period between January 2007 and December 2016. The diagnosis of NAFLD was based on liver histopathological exam with NAS score of 1-8, with exclusion of significant alcohol intake, use of medications that can cause hepatic steatosis, as well as viral hepatitis B and C infection. Baseline characteristics, liver enzymes and clinical outcomes were recorded. All patients were followed up of at least 1 years. Body weight change (\%), the liver or CVD complication was recorded including those who developed cirrhosis, hepatocellular carcinoma, stroke or myocardial infarction. Predictors of significant weight change of at least 5\% among patients with NAFLD were identified using Cox Regression analysis. Ethical approval was obtained from IRB, Faculty of Medicine, Chulalongkorn University.

Result: The mean age of NAFLD patients was $42.6 \pm 14.0$ years with male and female of $44.6 \%$ and $55.4 \%$. The prevalence of DM, HT, dyslipidemia and morbid obesity was $40.1 \%, 53.6 \%, 58.1 \%$ and $47.3 \%$, respectively (Table 1 ). NASH patients with histological proven (NAS $>4$ ) were found in $48.6 \%$ whereas significant fibrosis (F24) was observed in $36.9 \%$. With an average follow up duration of 44.2 
\pm 25.2 months, 17 patients $(7.7 \%)$ had experiences of complications including 14 patients who developed liver complication and 3 patients developed CVD complication. At the end of follow-up, 186 NAFLD patients had completed data of body weight change as well as complications. One hundred patients $(53.8 \%)$ achieved significant weight loss of at least $5 \%$. Seventy patients $(37.6 \%)$ underwent bariatric surgery including sleeve gastrectomy $(\mathrm{n}=34)$ and gastric bypass surgery $(n=36)$. All patients who underwent bariatric surgery were diagnosed as morbid obesity. By comparison NAFLD patients with and without morbid obesity, we found that NAFLD patients with morbid obesity had significantly less proportion of patients with advanced fibrosis ( $\mathrm{p}$ 0.001), and less frequent liver complications ( $\mathrm{p}$ 0.008 ) than those without morbid obesity. The outcome of the group with significant weight change $(5 \%)$ was mainly consequence of bariatric surgery (Table 2)

Conclusion: Advanced liver fibrosis and liver complications were found more often in NAFLD patients without morbid obesity than those with morbid obesity. This finding secondary to bariatric surgery in those NAFLD patients with morbid obesity. About $7.7 \%$ of a cohort of biopsy-proven NAFLD patients had experience of liver or CVD complications, during an average duration of follow up of 4 years.

\section{$R E G-1$}

3D Bioprinted liver tissue constructs for patient-specific implants and drug testing

\section{$\underline{\text { Swati Midha }}{ }^{1}$, Neha Kaushik ${ }^{1}$, Amtoj Kaur ${ }^{1}$, Sujata Mohanty ${ }^{1}$ \\ ${ }^{1}$ AIIMS, India}

Background: A discrepancy between the increasing demand for potential liver graft recipients and the number of donors available demands alternative strategies. Conventional tissue engineering strategies have attempted to create artificial liver substitutes which lack structural and compositional compliance with host tissue. Therefore, in order to mimic native liver tissue, 3D bioprinting aims to develop customized grafts for rendering patient-specificity. Beyond grafting, such in vitro 3D substitutes of liver tissue will act as appropriate models for drug testing by pharmaceuticals. Here, we test the feasibility of 3D hydrogel scaffolds by designing novel bioink compositions for liver tissue engineering.

Method: We fabricated hydrogel-based 3D bioprinted constructs using Pluronic F-127 and compared against commercial bioink in terms of rheology, printability, bioink composition and cell survival. Optimization was conducted using different concentrations of ink (10$30 \% \mathrm{w} / \mathrm{v}$ Pluronic F-127) in 1X PBS and complete culture media. Stability of the bioprinted constructs was tested using degradation studies by media immersion for 7 days. Cytocomptability was assessed by encapsulating WRL-68 and HepG2 cells for 7 days in the bioprinted matrix and tested for Live/Dead staining followed by immunofluorescence studies for morphology and matrix deposition.

Result: Using 3D bioprinting, we attempted to prepare functional liver constructs (Figure 1). Using varied bioink concentrations, we demonstrated that $25 \% \mathrm{w} / \mathrm{v}$ of Pluronic F-127 in complete culture media provides optimal printability for 3-5 layered constructs. 3D bioprinted constructs above this critical conc. gelled immediately upon the addition of cells, resulting in non-homogenous cell mixing in the bioink. Degradation studies revealed stable 3D bioprinted constructs only above $20 \%$ w/v concentration of Pluronic F-127 bioink. Morphological analysis of the scaffolds revealed homogenous appearance of the bioink with uniformly distributed cells. Live/dead staining demonstrated $<90 \%$ viable cell population after 1 and 7 days respectively indicating no adverse effects of printing parameters on cell survival. Morphological analysis revealed characteristic cellular morphology retained up to 7 days in culture with subsequent matrix synthesis.

Conclusion: 3D bioprinted Pluronic F-127 constructs provide a functional bioink for subsequent differentiation into liver tissue. Future studies will focus on assessing long-term cell viability and key mechanisms that regulate cellular differentiation towards mature and stable liver tissue.

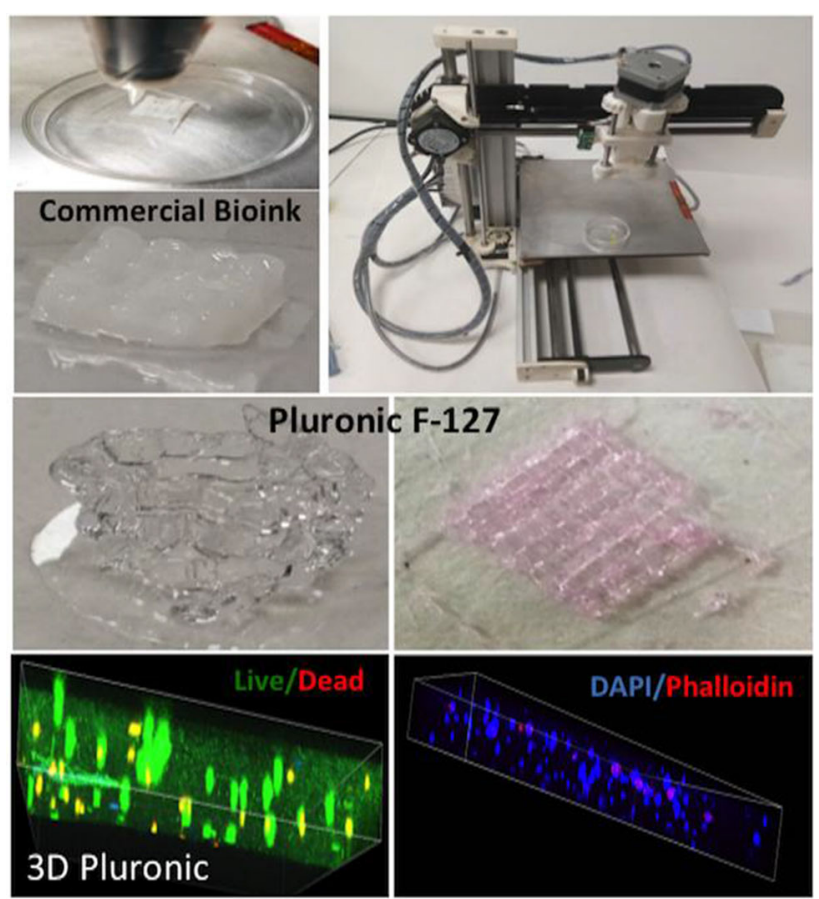

Figure 1: In vitro optimization and characterization of various bioinks (Commercial, Pluronic F-127) in terms of printability, post-printing gelation and cytocompatibility. Confocal micrographs demonstrate that majority of encapsulated WRL-68 cells were live after 7 days (Live/Dead staining) in the 3D bioprinted construct and demonstrated characteristic morphology as observed using phalloidin/DAPI.

\section{$R E G-2$}

Exosome derived from human mesenchymal stem cells alleviate liver injury through proliferation of damaged hepatocytes

\section{Suchi Gupta ${ }^{1}$, Sonali Rawat ${ }^{1}$, Sanjana Vig ${ }^{1}$, Sujata Mohanty ${ }^{1}$ ${ }^{1}$ AIIMS, India}

Background: Liver diseases are one of the leading cause of deaths in India. India ranks 61 with $2.5 \%$ of the total death due to liver failure. In spite of high regenerative potential of liver, at some stages of progression of disease, it loses its regenerative capacity. During this progression, apoptosis of primary hepatocytes occurs followed by activation of quiescent Hepatic Stellate Cells (HSCs). Currently, liver transplantation is the only effective therapy available. Alternatively, use of human Mesenchymal Stem cells (hMSCs) has shown tremendous potential for alleviating liver diseases. Recently, many research groups have shown through in vitro studies that Nano vesicles called exosomes derived from these hMSCs mimics their effect by repairing liver in liver injury. However the mechanism of repair is still unknown.

Method: To study the above hypothesis, we have isolated exosomes from tissue specific hMSCs (Bone marrow and Adipose tissue) and characterized them by flow cytometry. These MSCs were then 
cultured in serum free media for $48 \mathrm{~h}$ to collect exosomes and isolate them by ultracentrifugation. Primary hepatocyte cell line WRL-68 was given oxidative damage and then co-cultured with exosomes for $24 \mathrm{~h}$. Proliferation and cell cycle markers were studied by qRT-PCR. Also, hepatic stellate cells-HSCs (LX-2) were activated and co-cultured with these exosomes. The effect of these exosomes on LX-2 was evaluated by flow cytometry for the expression of activation markers after treatment ( $\alpha$-SMA and vimentin).

Result: It was observed that these hMSCs derived exosomes were able to enhance the proliferation of these damaged hepatocytes as evaluated by upregulation of cell cycle markers (Cyclin D1 and PCNA) and down regulation of apoptotic genes (Bcl-2). Also, these exosomes were able to revert activated hepatic stellate cells to senescence/ quiescent stage as observed by decrease in expression of $\alpha$-SMA and vimentin.

Conclusion: This study showed that hMSCs derived exosomes can alleviate liver fibrosis by enhancing the proliferation of damaged hepatocytes and leading to senescence of HSCs. These findings represent a potentially novel contributing mechanism of liver regeneration and have important implications for new therapeutic approaches to liver disease. Exosomes can in future be used as CELL FREE THERAPY.

\section{$R E G-3$}

Insight into the different forms of graphene in PCL scaffolds: a physiochemical study

Manu Dalela ${ }^{1}$, Manu Dalela ${ }^{2}$, Krishnan Gopal Jain ${ }^{1}$, Debika Debnath $^{4}$, Ankarao Kalluri ${ }^{4}$, Prabir K. Patra ${ }^{4}$, Kaushik Chatterjee $^{3}$, Sujata Mohanty ${ }^{1}$

${ }^{1}$ AIIMS DELHI, India; ${ }^{2}$ All India Institute of Medical Sciences, India; ${ }^{3}$ Indian Institute of Science, Bangalore, India; ${ }^{4}$ University of Bridgeport, United States

Background: The goal of this present study was to find the quantitative relationship between the biomaterials and stem cells. Towards designing, the biocompatible materials for various applications we design poly( $\varepsilon$-caprolactone) composites containing graphene. Poly $(\varepsilon-$ caprolactone) (PCL) is a synthetic biodegradable polyester extensively used in biomedical applications and FDA approved also. Graphene, a single layer carbon crystal, based nanomaterials have recently gained considerable interest for tissue engineering applications including osteogenic, neural and differentiation in other lineages due to their favorable chemical, electrical and mechanical properties. Method: In this study, we have prepared polycaprolactone (PCL) composites using graphene and graphene oxide (GO) by two methods. Firstly, solvent precipitation technique was used to synthesized PCL composites scaffolds using graphene oxide (GO), reduced GO (RGO), and amine-functionalized GO (AGO) at different filler content. Secondly, electrospinning method PCL-graphene nanostructured scaffolds were synthesized using different weight percentage of graphene.

Result: Protein Adsorption, cell culture and in vitro cell adhesion studies showed that presence of amine groups on AGO surface was most effective for promoting stem cells adhesion and spreading.

Conclusion: This work demonstrates the key role of graphene in terms of surface functionalization and increasing the weight percentage as filler in composites scaffolds and nanostructured scaffolds which significantly provide permissive surfaces for protein and cell adhesion which subsequently increases the biological responses. Thus, we envisaged that such a platform can serve as a powerful tool for developing future therapies for any diseases and injuries.

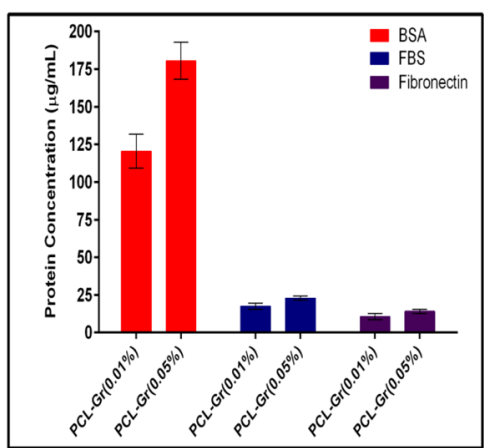

Fig1. Protein adsorption study on the PCL-graphene nanostructured scaffolds.

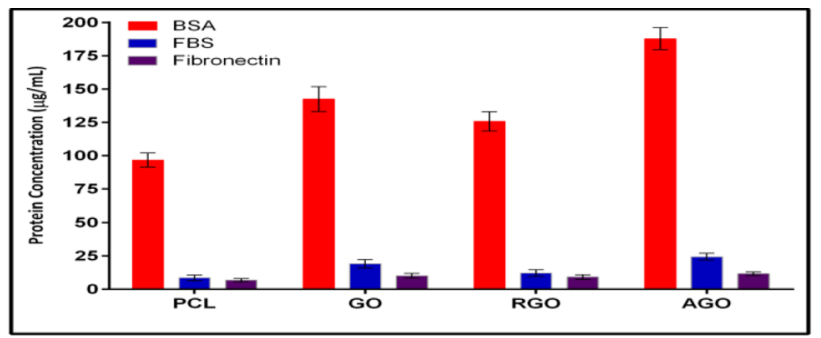

Fig2. Protein adsorption study on the PCL composites scaffolds

\section{REG-6}

Inhibition of apoptosis stimulating protein two of $\mathrm{p53}$ promotes liver regeneration in mice by activation of mammalian target of rapamycin

\section{Hongbo $\mathrm{Shi}^{1}$}

${ }^{1}$ Beijing You'an Hospital, China

Background: Although liver regeneration (LR) has been intensively studied in various ways, the mechanisms of LR are still elusive. ASPP2 was discovered as a binding partner of p53 and plays an important role in regulating cell apoptosis and growth. However, the role of ASPP2 in liver regeneration has not been reported.

Method: ASPP2 expression profile was detected in a mouse model by $70 \%$ partial hepatectomy (PHX). Liver regeneration and hepatocyte proliferation were studied in ASPP2 +/- mouse with PHX. The mammalian target of rapamycin (mTOR) and autophagy pathways were analyzed in ASPP2 +/- mouse with PHX. After rapamycin or 3-MA treatment, proliferation and regeneration were detected in the mouse model with PHX.

Result: ASPP2 expression displayed a significant upregulation at early stage and a downregulation at late stage. Compared to normal mice, Liver regeneration was enhanced in ASPP2+/- mice with $70 \%$ PHX. The mTORC1 pathway was significantly upregulated and autophagic pathway was downregulated in ASPP2+/-mice with 70\% PHX. Inhibition of mTORC1 pathway significantly reduced liver regeneration in ASPP2+/- mice with 70\% PHX. Disruption of autophagic pathway did not further enhance liver regeneration in ASPP2+/- mice with $70 \%$ PHX.

Conclusion: ASPP2 inhibition can promote liver regeneration after hepatectomy mainly through activating mTOR pathway and minorly through suppressing autophagic pathway. 
REG-7

Human umbilical cord derived mesenchymal stem cell infusion improves survival of the patients with $\mathrm{HBV}$ related decompensated liver cirrhosis

\section{Fusheng Wang ${ }^{1}$, Ming Shi ${ }^{1}$}

${ }^{1}$ Beijing 302 Hospital, China

Background: Human Bone marrow derived mesenchymal stem cells (BM-MSC) have been reported to improve liver function in patients with liver cirrhosis or liver failure. This retrospective study aims to investigate whether human umbilical cord derived mesenchymal stem cells (UC-MSC) infusion can improve the survival rate for the patients with the decompensated liver cirrhosis associated with chronic hepatitis B virus (HBV) infection.

Method: One hundred twenty-five patients with HBV-related decompensated liver cirrhosis received conventional medical treatment in combination with three times of UC-MSC infusions at a dose of $1.0 \times 106 / \mathrm{kg}$ body weight at 4-week interval. Other 120 patients with HBV-related decompensated liver cirrhosis received conventional medical treatment as control group. All participants were with follow-up check for 80 months since onset of UC-MSC infusions. The survival status, liver function and adverse events were evaluated throughout the follow-up period.

Result: The average survival time in the UC-MSC treated group was significantly longer than that in control group during the 80-month follow-up period (74.4 months vs 65.2 months, $\mathrm{P}<0.05)$. In particular, the UC-MSC infusions significantly increased serum albumin level and prothrombin activity at 24-week after three times of UCMSC in treated patients as compared with control patients. The HCC developed rates in UC-MSC patients showed no significant deviations as compared with control group throughout the 80-month follow-up check (25.2\% vs $29.9 \%)$. Furthermore, no side-effects were found in the UC-MSC treated patients.

Conclusion: UC-MSC treatment is not only safe, but also improves the survival rate for the patients with decompensated liver cirrhosis, indicating that MSC therapy may serve as a novel therapeutic approach for this disease.

\section{$A C L F-1$}

Simvastatin efficiently prevents the progression of acute-onchronic liver failure in portal hypertensive cirrhotic rats

Jordi Gracia Sancho ${ }^{1}$, Dinesh Mani Tripathi ${ }^{1}$, Marina Vilaseca ${ }^{1}$, Erica Lafoz ${ }^{1}$, Hector Garcia ${ }^{1}$, Gabriela Viegas ${ }^{1}$, Jarbas Rodriguez De Oliveira ${ }^{2}$, Juan Carlos Garcia Pagan ${ }^{1}$, Jaime Bosch $^{1}$

${ }^{1}$ IDIBAPS Research Institute, Spain; ${ }^{2}$ University of Porto Alegre, Brazil

Background: Cirrhosis may be further aggravated by bacterial infections, ultimately leading to ACLF. Although the devastating effects of ACLF in cirrhotic patients are well recognized, its cellular and molecular mechanisms are unknown. We aimed at understanding the effects of acute endotoxemia on hepatic microcirculation, liver cells phenotype, and survival in pre-clinical models of cirrhosis. In addition, the potential protective effect of simvastatin was analyzed. Method: ACLF was precipitated by a single administration of LPS in rats with compensated and decompensated advanced chronic liver disease (due to chronic CCl4, TAA or BDL) pre-treated with simvastatin or vehicle. Changes in hepatic and systemic hemodynamics, hepatic microvascular phenotype and function, overall survival, and underlying cellular mechanisms were determined.

Result: LPS administration aggravated portal hypertension in cirrhotic rats by further deteriorating intrahepatic microvascular dysfunction. Underlying mechanisms included worsening of endothelial dysfunction, exacerbated hepatic inflammation and oxidative stress, HSC activation, and neutrophil recruitment and activation, altogether negatively impacting on animals' survival.

Simvastatin pre-treatment prevented most of ACLF-derived complications and mortality through a variety of sinusoidal-protective, antiinflammatory and antioxidant pathways.

Conclusion: Development of ACLF by LPS administration to cirrhotic rats markedly exacerbates hepatic and systemic inflammatory pathways, aggravating liver microvascular dysfunction and portal hypertension and decreasing survival. Simvastatin has strong protective effects in this pre-clinical scenario, which further supports its use in the treatment of patients with advanced chronic liver disease.

$A C L F-2$

Patients with infection related ACLF (i-ACLF) and hepatic insult related ACLF (h-ACLF) have different clinical and cytokine profile.

Ajay Duseja ${ }^{1}$, Manu Mehta ${ }^{2}$, Ajay Duseja ${ }^{2}$, Sunil Taneja ${ }^{2}$, Vikas Guatam $^{2}$, Radha Krishan Dhiman ${ }^{2}$, Yogesh Kumar Chawla ${ }^{2}$

${ }^{1}$ Postgraduate Institute of Medical Education and Research, Chandigarh, India; ${ }^{2}$ Post Graduate Institute of Medical Education and Research, Chandigarh, India

Background: There is sparse data on the clinical and cytokine profile of patients with infection related acute-on-chronic liver failure (iACLF) and hepatic insult related ACLF (h-ACLF). Aim of the present study was to compare clinical presentation and cytokine profile amongst patients of infection related ACLF (i-ACLF) with patients with ACLF related to hepatic insults (h-ACLF) in a homogenous group of patients with alcohol related cirrhosis.

Method: Consecutive patients with alcohol related cirrhosis presenting as ACLF as per APASL or CANONIC or NACSELD criteria were included prospectively. Based on the acute insults, patients were divided into i-ACLF (infections as precipitating events) and h-ACLF (hepatic insults like alcoholic hepatitis, acute viral hepatitis, DILI, etc. as acute precipitating events). Parameters including hospital stay, prognostic scores, cytokine profile (TNF- alfa, IL-6, LPS) and morality were compared between patients with i-ACLF and h-ACLF. All patients gave an informed consent and study had the approval of the institute's ethics committee.

Result: A total 127 patients of ACLF with alcohol as chronic etiology (mean age 45.6 \pm 9.6 years) were included. Hepatic insults (h-ACLF) were acute precipitants in $77(60.6 \%)$ patients with $67(76.13 \%)$ patients having alcoholic hepatitis, (mean MDF 92 \pm 46.5 ) followed by i-ACLF in $36(28.3 \%$ ), both in $11(8.7 \%$ ) and unknown in $3(2.4 \%)$ patients. Patients with i-ACLF had significantly higher past history of decompensation $(88.8 \%$ vs. $37.6 \%)$ and hospital stay (mean $19.4 \pm 15.2$ vs. $12.0 \pm 9.3$ days). Mean platelet count and haemoglobin was significantly lower in patients with $\mathrm{i}$-ACLF than h-ACLF $\left(88.25 \pm 54.88 \times 10^{3} / \mathrm{mm}^{3}\right.$ vs. $125.5 \pm 76.7 \times 10^{3} / \mathrm{mm}^{3}$ and $8.51 \pm 1.69 \mathrm{~g} / \mathrm{dl}$ vs. $9.59 \pm 2.26 \mathrm{~g} / \mathrm{dl}$ respectively). Hepatic injury-related parameters, such as AST, ALT and serum bilirubin were significantly higher in the h-ACLF than i-ACLF. Liver failure (Day1, 3 , 5) was more common in h-ACLF patients, whereas i-ACLF patients had higher frequency of extrahepatic organ failures (circulation day 1 and respiration day 5). Grades of ACLF, severity scores and in-hospital, 1, 3 and 6 month mortality were similar across all 
patient groups. Independent predictors of mortality were $\mathrm{HE}$ at presentation (OR 4.7) and development of second infection (OR 5.3). LPS levels were significantly higher in patients with i-ACLF and those developing second infection. Grade of ACLF, number of organ failures, SOFA and CLIF-C-OF score positively correlated with TNF$\alpha$ levels $(\mathrm{p}<0.05$ for all). Mean IL-6 level in was significantly higher in non-survivors (in hospital, 1 month and 3 month) than in survivors Conclusion: i-ACLF is different from h-ACLF with higher past history of decompensation, hospital stay, extrahepatic organ failures, LPS level and lower mean platelet count, haemoglobin, AST, ALT and serum bilirubin levels.

ACLF-3

Role of NLRP3 inflammasome on the pathogenesis of HBV related acute-on-chronic liver failure

\section{Yanhong Jia ${ }^{1}$, Chuan Shen ${ }^{1}$, Ziyue Li $^{1}$, Yadong Wang ${ }^{1}$, Wei Wang $^{1}$, Caiyan Zhao ${ }^{1}$ \\ ${ }^{1}$ Third hospital of Hebei Medical University, China}

Background: Hepatitis B viral related Acute-on-chronic liver failure (HBV-ACLF) is a life threatening syndrome featured with damaged hepatic function and multiple organ dysfunction. Immune dysfunction seems to play a crucial role in the development of HBV-ACLF. Monocytes proposed as the key cell population that mediates inflammation. NLRP3 inflammasome is an intracellular multiprotein complex which is the part of innate immunity. We aim to explore the role of NLRP3 inflammasome in the pathogenesis of HBV-ACLF by investigating the levels ofNLRP3 in peripheral monocytes and liver tissues.

Method: Total of 75 subjects were enrolled in our study, including HBV-ACLF patients $(n=30)$, chronic hepatitis B patients (CHB) $(n=30)$ and healthy controls (HC) $(n=15)$. NLRP3 expression on monocytes were appropriately evaluated using flow cytometry, and its relationship with disease was evaluated by MELD score. Liver NLRP3 expression was also analyzed by real-time PCR and immunohistochemistry.

Result: The mRNA levels of NLRP3 and NLRP3 related genes (caspase-1, IL-1 $\beta$ and IL-18) in Peripheral Blood Mononuclear Cell (PBMC) of HBV-ACLF patients $(0.45 \pm 0.21)$ were lower than CHB patients $(0.74 \pm 0.24)$ and healthy controls $(P<0.05)$. The expression of NLRP3 (46.43 \pm 10.86$)$ were lower than CHB group (53.56 \pm 6.56$)$ and HCs group $(60.80 \pm 7.23)$.

Subgroup of monocytes include CD14++CD16- monocytes, CD14+CD16+monocytes and CD16++CD14- monocytes; NLRP3 in CD14+CD16+monocytes of HBV-ACLF patients (51.86 \pm 11.16$)$ had lower expression compared with CHB patients $(60.87 \pm 7.55)$ and HCs (71.48 \pm 8.16 ); so as NLRP3 related genes in CD14+ monocytes. on CD14++CD16- monocytes CD16++CD14- monocytes, groups of data in each group of between comparisons, not obvious difference.

In liver tissue, NLRP3 had higher expression of HBV -ACLF patients (17.2 \pm 4.14 compared with CHB patients (1.8 \pm 0.84$)$ $(P<0.01)$. So as the mRNA levels of NLRP3 and NLRP3 related genes; The expression of NLRP3 were gathered in interlobular portal areas and hepatic lobule, especially in hepatic lobule. NLRP3 were showed mainly in Kupffer cell cytoplasm by co-expression of CD68.

The expression of NLRP3 in CD14+CD16+ monocytes of HBVACLF patients were negatively correlated with the MELD score $(\mathrm{R}=-$ $0.74)(P<0.01)$.
Conclusion: NLRP3 were mainly expressed in CD14+CD16+monocytes and express lower in the peripheral blood of HBV - ACLF, they may be gathered to liver to damage hepatocyte and may be partial mechanism of HBV-ACLF immunosuppression.

$A C L F-4$

Clinical profile and mortality in patients with ACLF according to CLIF-SOFA criteria from a tertiary care centre in Kerala.

Mary George $^{1}$, Benoy Sebastian ${ }^{1}$, Sunil K Mathai ${ }^{1}$, Ashfaq Ahmed $^{1}$, Saji Varghese', Sandheep Janardhanan', Waseem Raja $^{1}$, Sameer Sakker ${ }^{1}$

${ }^{1}$ Travancore Cochin Medical Council, India

Background: In this study clinical profile and mortality in patients with ACLF according to CLIF-SOFA criteria was assessed in patients with ACLF from a tertiary care centre in Kerala.

Method: Study was done as a single centre prospective descriptive study. We had total 198 patients, out of which we had 150 patients who met the inclusion and exclusion criteria. We had 41 patients in CLIF-SOFA group. Study duration was from JUNE 2015-MAY 2016. Patients with chronic liver disease with acute decompensation were included. Previously compensated and decompensated patients were enrolled in the study provided the previous decompensation had improved after treatment. All patients were followed up for 3 months from inclusion into the study or until mortality, whichever was earlier. Result: We got the complete data from 150 patients who met the inclusion and exclusion criteria. Out of this 150 patients ACLF was present according to CLIF-SOFA in 41 (27.3\%) patients.

In CLIF-SOFA, NO ACLF group 28 day mortality was $11 \%$, and in the ACLF group mortality was $36.5 \%$. (p value $=0.001$ ). 28 day survival in CLIF SOFA group was significantly lower in the ACLF group (log-rank test: chi square value 13.67; p value $<0.001$ ). In CLIF-SOFA, NO ACLF group 90 day mortality was $11.9 \%$, while in ACLF group it was $41.4 \%$. (p value $=0.001$ ). Survival in CLIF-SOFA NO-ACLF group is significantly better than ACLF group. (log-rank test, chi-square-16.971: $\mathrm{p}<0.001$. AUROC for 28-d mortality for CLIF-SOFA, APACHE-II, Child-Pugh and MELD scores was 0.757, $0.734,0.732$ and 0.734 respectively. Backward conditional logistic regression was done to compare the utility of these scores. Only APACHE II score was found to be predictor of mortality with an odds ratio of 1.15 . ( $\mathrm{p}$ value $<0.01$ ).

Conclusion: The 28 day mortality in CLIF-SOFA group with ACLF was $36.5 \%$, while in NO ACLF group it was $11 \%$.. Mortality was higher in patients with ACLF according to CLIF-SOFA criteria. When we compared the prognostic scores Child-Pugh score, MELD score, APACHE II, CLIF-SOFA score AUROC was higher for CLIFSOFA score and APACHE II score. The AUROC was .757, .734, .734, .732 for CLIF-SOFA, APACHE II, MELD, Child-Pugh score respectively. On backward conditional logistic regression analysis for the utility of these score for 28 day mortality only APACHE II was found to be significant with an Odds ratio of 1.15. The sample size in our study was relatively small and this might not be representative of all India, we need more well defined studies to characterize the clinical profile and mortality in patients with ACLF. 
ACLF-5

Does transfer factor (TF) given in the golden window of acute-onchronic liver failure (ACLF) influence selected immunological parameters: a case-control study

Lubomir Skladany ${ }^{1}$, Tomas Koller ${ }^{2}$, Kristina Kropacekova ${ }^{1}$, Janka Vnencakova ${ }^{1}$, Ludek Hochmuth ${ }^{1}$, Daniela Jancekova ${ }^{1}$

${ }^{1}$ F.D.ROOSEVELT UNIVERSITY HOSPITAL, Slovakia;

${ }^{2}$ UNIVERSITY HOSPITAL RUZINOV, Slovakia

Background: ACLF is characterized by a distinctive immunopathology which overlaps with on-label indications of TF; this immunomodulator a.o. replenishes lymphoid lineages from CD34+ cells to NK cells, enhances immunity to Stph. aureus, C. albicans, etc. Part of HEGITO7 Registry (RH7, hospitalizations with decomp. [d]ACLD) focused on immune parameters of ACLF pts; here we present first results on influence of $\mathrm{TF}$ on selected immune parameters as compared to SOC.

Method: Case-control study from RH7. Inclusion criteria: Hospital admission with acute decompensation (AD) of ACLD; informed consent. Exclusion criteria: malignancy. Variables: age; gender; ACLD etiology; AD/ACLF according to CANONIC truncated by APASLs' 4-weeks interval from event to hospital admission; TF (s.c.); CRP (mg/ml); IL6 (ng/L); CD3 T-Ly (x10E9/L), CD4, CD8, NK-cells, HLA-DR on T-Ly (CD4, CD8); phagocytosis; oxidative burst; 30-day mortality.

Result: During study interval of 14 months, 87 pts with AD were enrolled; mean age: 52.7 ; 38 females (43\%); age: $19-77$ years; ACLD etiology: alcoholic liver disease (ALD) - 73 patients (pts, [84\%]), viral hepatitis - 5 pts $(2,8 \%)$, autoimmune - 7 (8\%), and NASH - 5 $(5,7 \%)$. Stratification of AD on admission: ACLF-0 (no ACLF, pure AD): 51 pts $(58,6 \%)$, ACLF-1 - 13 pts (14,9\%), ACLF-2 - 15 pts $(17,2 \%)$, ACLF-3 - 8 pts $(9,1 \%)$; cases and controls differed in $\%$ of females $(56,8$ vs. $30,2 \%)$, but not in other baseline characteristics (age, etiology, ACLF, CTPS, MELD) (Table 1). 44 cases were treated with TF, and 43 controls with SOC (without TF). 25 patients (28.7\%) have died within 30 days of inclusion $-25 \%$ receiving TF, and $32,6 \%$ of controls $(\mathrm{p}=0,44)$. In pts with ACLF-0 at baseline, 30 -day mortality was $8.7 \%$ in TF treated pts $v s .14 .8 \%$ in controls on SOC $(\mathrm{p}=0,52)$. Survivors at 30 day had lower baseline values of IL-6 $(27,7$ vs. $133, \mathrm{p}<0,001)$, procalcitonin $(0,27$ vs. $0,9, \mathrm{p}<0.001)$ and CRP (21.7 vs. 51.75; p=0.001), and higher baseline lymphocyte counts: $\mathrm{CD} 3$ ( 0,957 vs. $0,53, \mathrm{p}=0,001), \mathrm{CD} 4$ (0.66 vs. $0.42, \mathrm{p}=0.0032)$ and CD8 lymphocytes $(0,24$ vs. $0,14, \mathrm{p}=0,003)$, NK cells $(0,133$ vs. $0,05, \mathrm{p}=0,001)$, levels of $\mathrm{C} 4$ ( 0,16 vs. $0,12, \mathrm{p}=0,027), \mathrm{C} 3$ ( 0,87 vs. $0,57, \mathrm{p}=0,0025)$ and higher oxidative burst $(34,69$ vs. $12,8, \mathrm{p}<0.001)$.

Survivors at 30 days were evaluated for changes in immunological parameters from baseline. Patients receiving TF $(n=19)$ had significantly increased their absolute counts of HLA DR lymphocytes $(0,15$ vs. $0,24, \mathrm{p}=0,016)$ and HLADR CD8 lymphocytes ( 0,029 vs. 0,33 , $\mathrm{p}=0,0062)$ compared with the group receiving SOC $(\mathrm{n}=11)$ : HLA DR $(0,246$ vs. $0,222, \mathrm{p}=0,52)$ and HLADR CD8 (0,015 vs. $0,048, \mathrm{p}=0,36)$. Both groups had a trend in higher HLADR CD4 counts $(0,02$ vs. $0,036, \mathrm{p}=0,06$, and 0,023 vs $0,038, \mathrm{p}=0,053$ ) (Table 1 .)

Conclusion: AD pts surviving 30 days had significantly higher baseline: lymphocytes, complement, and oxidative burst; TF led to an increase in overall HLA DR expression, particularly on HLADR CD8 cells, that was not observed in patients on SOC.

\begin{tabular}{|c|c|c|c|c|c|}
\hline & \multicolumn{2}{|c|}{$\begin{array}{c}\text { Cases - with TF } \\
(\mathrm{N}=44)\end{array}$} & \multicolumn{2}{|c|}{$\begin{array}{c}\text { SOC Controls - } \\
\text { without TF } \\
(\mathrm{N}=43)\end{array}$} & \multirow[t]{2}{*}{$\mathbf{P}$} \\
\hline & Baseline & Day 30 & Baseline & Day 30 & \\
\hline Age [years] & \multicolumn{2}{|c|}{52.7} & \multicolumn{2}{|c|}{52,7} & ns \\
\hline Females (\%) & \multicolumn{2}{|c|}{56,8} & \multicolumn{2}{|c|}{30,2} & 0,021 \\
\hline $\begin{array}{c}\text { Etiology ACLD (\%) } \\
-\quad \text { ALD } \\
- \text { HBV } \\
- \text { HCV } \\
- \text { PBC/PSC }\end{array}$ & \multicolumn{2}{|c|}{$\begin{array}{c}84,1 \\
2,3 \\
6,8 \\
9,1\end{array}$} & \multicolumn{2}{|c|}{$\begin{array}{c}83,7 \\
2,3 \\
0 \\
7\end{array}$} & $\begin{array}{l}\text { ns } \\
\text { ns } \\
\text { ns } \\
\text { ns }\end{array}$ \\
\hline $\begin{array}{c}\text { AD subxypes (\%) } \\
-\quad \text { ACLF 0 } \\
-\quad \text { ACLF 1 } \\
-\quad \text { ACLF 2 } \\
-\quad \text { ACLF 3 }\end{array}$ & $\begin{array}{l}54,5 \\
13,6 \\
20,5 \\
11,4\end{array}$ & & $\begin{array}{c}62,8 \\
16,3 \\
14 \\
7\end{array}$ & & $\begin{array}{l}\text { ns } \\
\text { ns } \\
\text { ns } \\
\text { ns }\end{array}$ \\
\hline MELD (points) & \multicolumn{2}{|c|}{24,1} & \multicolumn{2}{|c|}{21,9} & ns \\
\hline Child-Pugh & \multicolumn{2}{|c|}{10.7} & \multicolumn{2}{|c|}{10,6} & ns \\
\hline CRP $(\mathrm{mg} / \mathrm{ml})$ & $21,7^{\circ}$ & $12,83^{*}$ & 23,26 & $9,44^{\circ}$ & ${ }^{\circ} 0,06 ;{ }^{* 0} 0.0027$ \\
\hline IL-6 (ng/L) & 37,6 & 31,19 & 13,67 & 14,36 & \\
\hline $\begin{array}{l}\text { CD3+4+HelpT-Ly } \\
\text { (abs count } \times 10^{\circ} / \mathrm{L} \text { ) }\end{array}$ & 0,43 & 0,55 & 0,79 & 0,91 & \\
\hline $\begin{array}{l}\text { CD3+B+Cytotoxic } \\
\text { T-Ly (abs) }\end{array}$ & $0,19^{\circ}$ & $0,2595^{\circ}$ & 0,252 & 0,277 & $P=0,07$ \\
\hline CD16+56+NK (abs) & 0,067 & 0,106 & 0,1575 & 0,1745 & ns \\
\hline C.D19+B-Ly & 0,10 & 0,10 & 0,146 & 0,148 & ns \\
\hline $\begin{array}{l}\text { HLA DR expression } \\
\text { on T-Ly: }\end{array}$ & & & & & \\
\hline $\begin{array}{l}\text { HLA DR } \\
\text { HLA DR CD3 }\end{array}$ & $\begin{array}{c}0,158^{*} \\
0,034\end{array}$ & $\begin{array}{l}0,246^{*} \\
0,033\end{array}$ & $\begin{array}{c}0,246 \\
0,0896\end{array}$ & $\begin{array}{c}0,222 \\
0,0585\end{array}$ & $\begin{array}{l}* p=0,016 \\
" p=0,06\end{array}$ \\
\hline $\begin{array}{l}\text { HLA DR CD4 } \\
\text { HLA DR CD8 }\end{array}$ & $\begin{array}{c}0,02^{\circ} \\
0,029^{\circ}\end{array}$ & $\begin{array}{l}0,036^{*} \\
0,033^{*}\end{array}$ & $\begin{array}{c}0,023^{\prime *} \\
0,015\end{array}$ & $\begin{array}{c}0,038^{* *} \\
0,048\end{array}$ & $\begin{array}{l}* p=0,054 \\
* p=0,0062\end{array}$ \\
\hline Phagocytosis & 92,75 & 93,65 & 94,1 & 93,9 & ns \\
\hline Oxidative burst & 27,95 & 37,8 & 35,4 & 38,0 & ns \\
\hline $\begin{array}{c}\text { Complement } \\
-\quad \text { C3 } \\
-\quad \text { C4 } \\
\end{array}$ & $\begin{array}{l}0,61 \\
0,14\end{array}$ & $\begin{array}{l}0,65 \\
0,15\end{array}$ & $\begin{array}{l}0,96 \\
0,17\end{array}$ & $\begin{array}{l}1,07 \\
0,18\end{array}$ & \\
\hline $\begin{array}{l}\text { 30-d mortality (\%) } \\
\text { ACLF } 0(=A D) \\
\text { ACLF } 1 \\
\text { ACLF } 2 \\
\text { ACLF } 3\end{array}$ & $\begin{array}{c}25 \\
8,7 \\
16,6 \\
33,3 \\
100 \\
\end{array}$ & & $\begin{array}{c}32.56 \\
14,8 \\
60 \\
57,1 \\
75 \\
\end{array}$ & & $\begin{array}{l}0,44 \\
0,51 \\
0,16 \\
0,43 \\
0,24\end{array}$ \\
\hline
\end{tabular}

\section{ACLF-6}

Improved 28-day survival with continuous slow infusion of Furosemide, Albumin with Terlipressin in patients with ACLF and $\mathrm{AKI}$

Alok Kumar ${ }^{1}$, Gaurav Pandey ${ }^{1}$, Prabhat Narayan Mishra', Vivek Anand Saraswat ${ }^{1}$, Samir Mohindra ${ }^{1}$, Krishna VP' ${ }^{1}$, Manjunath Hatti $^{1}$, Shreya P Butala ${ }^{1}$, Arun Singh Bhadauria ${ }^{1}$

${ }^{1}$ SGPGI, India

Background: Pathophysiology of ACLF involves dysbalanced systemic inflammation, worsened hyperdynamic circulation and altered gut permeability leading to multi organ dysfunction including renal dysfunctions. A combination of continuous, slow infusion of furosemide, albumin, with Terlipressin administered according to a response-guided protocol in patients with ACLF and AKI, causing pathophysiological changes may impact survival

Method: 30 patients in Arm 1 with $\mathrm{Sr}$ creatinine > 2, were managed with SAFI(T) and another group of 15 patients $(\mathrm{Sr}$ creatinine $>2)$ in Arm 2, were managed with standard medical treatment. Patients were diagnosed with HRS according to standard guidelines. Arm 1 were given Furosemide infusion at $2 \mathrm{mg} / \mathrm{hr}$, albumin infusion at $2 \mathrm{gm} / \mathrm{hr}$ (20-40 gm/d) and Terlipressin infusion at $4 \mathrm{mg} / 24 \mathrm{hrs}$. If 24-hr Urine $\mathrm{Na}$ persisted below $80 \mathrm{meq} / \mathrm{day}$, response guided increase in terlipressin ( $1 \mathrm{mg} / 12$ hrly) was done ( $\max 8 \mathrm{mg} / 24$ hours).

Result: Both the Arms were comparable in baseline CTP, MELD, CLIF-SOFA scores and $\mathrm{Sr}$ Creatinine values. If 24-hr Urine $\mathrm{Na}$ 
persisted below $80 \mathrm{meq} / \mathrm{day}$, response guided increase in terlipressin ( $1 \mathrm{mg} / 12 \mathrm{hrly}$ ) was done (max $8 \mathrm{mg} / 24$ hours) after correcting anaemia $(\geq 8 \mathrm{~g} / \mathrm{dl})$ and excluding cardiac conduction anomalies with baseline ECG (and repeated 12 hourly) till reduction/normalization of $\mathrm{Sr}$ creatinine. Graded increase of Furosemide infusion was done at a rate of $1 \mathrm{mg} / 12 \mathrm{hrs}$, if $\mathrm{UNa}<80 \mathrm{meq} /$ day, with aggressive potassium supplementation as needed.

Results : Baseline Characteristics : Sr Creatinine $>2$
\begin{tabular}{|l|l|l|}
\hline & Arm $1[$ SAFI(T)] & Arm 2 [SMT] \\
\hline N & 30 & 15 \\
\hline CTP & $11.1 \pm 3.2$ & $11.9 \pm 1.4$ \\
\hline CLIF-SOFA & $11.6 \pm 1.8$ & $11.3 \pm 2.3$ \\
\hline MELD & $32 \pm 7.8$ & $37.1 \pm 8$ \\
\hline Creatinine $(\mathrm{mg} /$ dl) & $2.9 \pm 0.76$ & $3.2 \pm 1.1$ \\
\hline U Na day0 $(\mathrm{ml} /$ day) & $23.8 \pm 18$ & $32.4 \pm 21$ \\
\hline U Na day4 $(\mathrm{ml} /$ day $)$ & $256 \pm 182$ & $\mathbf{7 2} \pm 43$ \\
\hline
\end{tabular}

At 28 days survival in arm 1 was $69.1 \%$ vs $41.4 \%$ arm 2

Conclusion: Survival is better with continuous slow infusion of Furosemide, Albumin with Terlipressin in patients with ACLF and AKI.

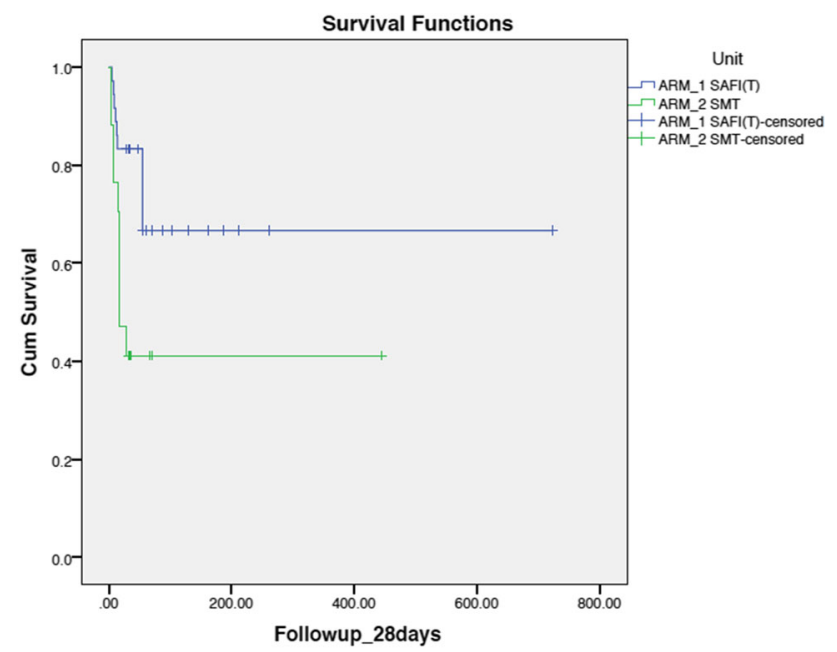

ACLF-7

Slow infusion of furosemide and albumin

with or without terlipressin (SIFA-T) improves deranged systemic hemodynamics and survival in severe acute on chronic liver failure

Prabhat Narain Sharma ${ }^{1}$, Gaurav Pandey ${ }^{1}$, Krishna VP ${ }^{1}$, Alok Kumar $^{1}$, Manjunath Hatti ${ }^{1}$, Arun Singh ${ }^{1}$, Vivek Anand Saraswat ${ }^{1}$, Prabhat Narain Sharma ${ }^{1}$

${ }^{1}$ SGPGI, Lucknow, India

Background: ACLF is characterized by abrupt onset of severe liver dysfunction with extrahepatic organ failure(s) but with potential for reversal once the acute insult resolves. Although deranged systemic hemodynamics underpins extrahepatic organ failure, changes in hemodynamics after recovery or complete mobilization of ascites have not been reported. We studied hemodynamics in ACLF before and after complete mobilization of ascites with slow infusion of furosemide and albumin with/without terlipressin [SIFA (T)] and its impact on determining the clinical outcomes.

Method: 25 consecutive patients with ACLF fulfilling the EASLCLIF criteria and with large ascites admitted between Oct 2016 and
March 2017 were enrolled. Patients with active GI bleed, severe comorbidities, malignancy, BCS, $<18$ yrs, pregnancy were excluded. Besides the standard workup for ACLF, systemic hemodynamic parameters were determined by clinical and echocardiographic examination including cardiac index, systemic vascular resistance index and stroke work. All patients were treated with a response guided protocol with SIFA(T) for mobilization of ascites. Changes in clinical, biochemical, organ failure, hemodynamic parameters measured after resolution of ascites were compared with baseline data.

Result: $11(44 \%)$ patients had ACLF grade 3, while six (24\%) had ACLF grade 2. Five (25\%) patients died during the hospitalization. Systemic hemodynamics could be reassessed after complete mobilization of ascites in 20 patients using $\operatorname{SIFA}(\mathrm{T})$ regimen given for a mean period of $15.1 \pm 4.6$ days. At discharge there was significant improvement in all the parameters assessed $(\mathrm{p}<.0001$ for cardiac index, systemic vascular resistance index) as well as CTP and MELD scores $(\mathrm{p}<.0001$ for both).

Conclusion: Complete mobilization of ascitic and third space fluid in severe ACLF could be successfully achieved in $75 \%$ patients SIFA(T) with minimal adverse events. This was associated with significant improvement in liver function, improvement in organ failure(s), in systemic hemodynamics, and in improved survival. We suggest that improving systemic hemodynamics in ACLF with SIFA(T) therapy may reverse extra-hepatic organ failure and improve survival.

\begin{tabular}{|c|c|}
\hline \multicolumn{2}{|c|}{ Table 1: Baseline characteristics of all patients(n=25) } \\
\hline Variable & Value \\
\hline Age (years) & $44 \pm 9(22-59)$ \\
\hline Sex(M/F) & $22 / 3$ \\
\hline Etiology (alcohol/other) & $17 / 8$ \\
\hline CTP score & $13(11-15)$ \\
\hline MELD score & $32(21-46)$ \\
\hline ACLF Grade 1/2/3 & $8(32 \%) / 6(24 \%) / 11(44 \%)$ \\
\hline Creatinine(mg/dl) & $2.18 \pm 1.33(0.30-5.90)$ \\
\hline Albumin(g/dL) & $2.46 \pm 0.28(2.0-3.10)$ \\
\hline Bilirubin(mg/dt) & $14.04 \pm 6.96(5.1-27.4)$ \\
\hline INR & $2.9 \pm 0.86(1.7-4.8)$ \\
\hline 24 hour UNa (mmol/L) & $34.96 \pm 23.21(5-87)$ \\
\hline BSA(m²) & $1.86 \pm 0.23(1.28-2.52)$ \\
\hline
\end{tabular}

\begin{tabular}{|c|c|c|c|}
\hline Variable & Adminision & Dechlarge & pvadue \\
\hline $\operatorname{mad}$ & $30(21-46)$ & $22(15-31)$ & 80.001 \\
\hline CIP & 13(12-14\} & $10(9-10.25)$ & 40.001 \\
\hline Creatinine(me/dl) & $1.7 \pm 1.27(0.8-5.9)$ & $0.89+0.23(0.6-1.2)$ & 80.001 \\
\hline Albumin (g/dl) & $25+0.28(20-3.1)$ & $33+0.47(25-42)$ & 0.001 \\
\hline Etrubin (mg/dl) & $11.2+5.66(5.4 .27 .1)$ & $7.6 \pm 4.71(25-17.8)$ & 80.001 \\
\hline $\mathbf{N R}$ & $27 \pm 080(1.748)$ & $23+0.6 /(1.5-3.7)$ & 0.001 \\
\hline $24 \mathrm{HH}$ Wa $(\mathrm{mmol} / \mathrm{L})$ & $389+253$ & $3845+202.6$ & 80.001 \\
\hline $\operatorname{Bsa}\left(m^{2}\right)$ & $182+023$ & $1.69+0.19$ & 0.001 \\
\hline Mean arterid pressure (man He) & $81.6+6.52$ & $939+4.98$ & 80.001 \\
\hline Systotic blood pressure (man Hel) & 109+9 & $122 \pm 8$ & 8001 \\
\hline 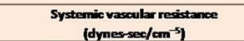 & $668.8+234.13$ & 1299.5+531.45 & 80001 \\
\hline 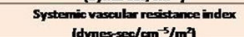 & $12068 \pm 3988$ & 219274907.6 & snot \\
\hline Heart rate (bpm) & $97.2+8.26$ & $81.9+8.88$ & 80.001 \\
\hline Stroke Volume(mL1/beat) & $92.1+25.09$ & $725+24.44$ & 8001 \\
\hline 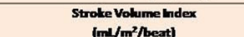 & $509+13.95$ & 43.45+15.4 & 0.001 \\
\hline Cardiac outpurt (l/mmin) & $89+2.62$ & $60 \pm 2.28$ & 8001 \\
\hline Cardiax index $\left(\left[/ \mathrm{m} m / \mathrm{m}^{2}\right)\right.$ & $49+141$ & $3.6 \pm 1.42$ & $\$ 0.001$ \\
\hline Stroke work [e/beat) & $14.0 \pm 3.93$ & $12.4 \pm 4.33$ & 0.013 \\
\hline $\begin{array}{l}\text { Arterid compliance } \\
\text { (m/mmm }\end{array}$ & $229 \pm 0.66$ & 1.770 .63 & $<0001$ \\
\hline
\end{tabular}




\section{$A L F-1$}

The protective mechanism of CAY10683 on intestinal mucosal barrier in acute liver failure through LPS/TLR4/MyD88 pathway

\section{Zuojiong Gong ${ }^{1}$, Yao Wang', Qian Chen ${ }^{1}$, Fangzhou Jiao', Wenbin Zhang 1 \\ ${ }^{1}$ Renmin Hospital of Wuhan University, China}

Background: The purpose of this study is to investigate the protective mechanism of the selective HDAC2 inhibitor CAY10683 on intestinal mucosal barrier in acute liver failure (ALF) through inhibiting LPS/TLR4/MyD88 pathway in vivo and in vitro.

Method: In order to establish ALF-induced intestinal epithelial barrier disruption external and internal models, D-galactosamine/LPS and LPS were respectively used with rats and human colonic epithelial cell line NCM460. The groups were then administrated with CAY10683. Transepithelial electrical resistance (TEER) was measured to detect the permeability of cells. The protein expressions of TLR4 in cells were detected by immunofluorescence. Real-time PCR and western blotting were employed to detect the mRNA and protein levels of TLR4, MyD88, TRIF, TRAF6, zonula occludens (ZO)-1 and occluding. And the intestinal epithelial tissue pathology was detected with hematoxylin-eosin (HE) staining. Immunohistochemistry was used to examine the expression of TLR4 in intestinal epithelial tissues.

Result: After interfering with CAY10683, the mRNA and protein levels of TLR4, MyD88, TRIF and TRAF6 was decreased when compared with model group $(\mathrm{P}<0.05)$, whereas the mRNA and protein levels of $\mathrm{ZO}-1$ and occluding were elevated $(\mathrm{P}<0.05)$. The permeability of intestinal epithelial cells was elevated in CAY10683 interfered groups, when compared with model group $(\mathrm{P}<0.05)$. And the degree of intestinal epithelial tissue pathological damage in CAY10683 group was significantly reduced. Moreover, CAY10683 significantly decreased the protein level of TLR4, and increased ZO-1 and occluding staining in animal tissue $(\mathrm{P}<0.05)$.

Conclusion: The HDAC2 inhibitor CAY10683 could promote the damage of intestinal mucosal barrier in acute liver failure (ALF) through inhibiting LPS/TLR4/MyD88 pathway in vivo and in vitro, which might serve as a new protective agent for ALF associated intestinal mucosal barrier disruption.

\section{$A L F-2$}

\section{ACUTE LIVER FAILURE A looking back.}

\section{Ankur Jain ${ }^{1}$, Rajesh Gopalakrishna ${ }^{1}$, Sudhindran S ${ }^{1}$, Shweta Mallick $^{1}$, Shine Sadasivan ${ }^{1}$, Dr. Harshavardhan Raob ${ }^{1}$ \\ ${ }^{1}$ Amrita Institute of Medical Sciences, India}

Background: Acute liver failure (ALF) although relatively uncommon, tends to affect young individuals and is the leading cause for emergency liver transplantation (LT) the world over. We carried out this study to look at the disease spectrum and to identify the possible predictors of poor outcomes Acute liver failure (ALF) although relatively uncommon, tends to affect young individuals and is the leading cause for emergency liver transplantation (LT) the world over. We carried out this study to look at the disease spectrum and to identify the possible predictors of poor outcomes

Method: We analysed retrospectively data of 69 cases (median age 24 yrs, M:F 2:1) of LT performed for ALF at our institution from April 2007 to April 2017 (out of total of 560 (12\%)).
Result: The aetiology was identified in 52 cases $[\operatorname{HAV}(n=14)$, $\operatorname{HBV}(n=5), \operatorname{HEV}(n=1)$, ATT induced $n=(6)$, Acute Wilson's $(n=3)$, Autoimmune $(\mathrm{n}=5)$ and $\mathrm{Zn}$ Phosphide poisoning $(\mathrm{n}=16)]$ and remaining 17 cases were of indeterminate aetiology. Aetiology differed between adults $(>12$ years, $n=61)$ and children $(n=8)$. Whilst the most common aetiology in adults were indeterminate followed by HAV and Zinc Phosphide poisoning, equal incidence of indeterminate, Acute Wilson's and ATT induced liver failure were seen among children. Upon meeting King's College criteria, 68 had live donor LT(55 Right lobe grafts, 3 left lobe grafts, 4 lefts lateral segment grafts and 5 cases were APOLT) and 1 deceased donor LT. Postoperative morbidity seen were bile leak (23\%), sepsis (17.3\%), ACR (14.4\%), AKI (13\%), graft dysfunction (5\%), HAT (2.8\%) and PVT $(1.4 \%)$. Overall mortality was $36 \%(50 \%$ in the paediatric and $34 \%$ in adults), vast majority of which (92\%) occurred within 30 days of transplant. The major cause of mortality was sepsis with MODS. Risk factors for mortality included age less than 20 years, pretransplant renal dysfunction, Grade 4 encephalopathy and hepatitis A aetiology. Over a median follow up of 8 years, $95 \%$ of perioperative survivors are leading a normal life.

Conclusion: LT for ALF carry high mortality (36\%) in those presenting with renal dysfunction, Grade 4 encephalopathy, HAV aetiology and age less than 20 years. Nevertheless, if they survive the perioperative period, long term survival is excellent.

$A L F-3$

Acute liver failure associated with parvovirus B19 Infection

Arthur Daniel Rocha Alves ${ }^{1}$, Marcelo Alves Pinto ${ }^{3}$, Rita De Cassia Nasser Cubel Garcia ${ }^{2}$, Jessica Vasques Raposo ${ }^{1}$, Vanessa Salete De Paula ${ }^{3}$, Luciane Almeida Amado Leon ${ }^{3}$

${ }^{1} \mathrm{PhD}$ student, Brazil; ${ }^{2}$ Professor, Brazil; ${ }^{3}$ Researcher in public health, Brazil

Background: The Parvovirus B19 (B19V) infection is commonly acute, self-limited and may cause a variety of clinical manifestations. However, the spectrum of B19V-linked diseases has increased in past few years, due to the correlation between B19V-persistent infection in non-erythroid tissues and development of atypical diseases, such as hepatitis. Related to liver, B19V may cause since biochemical changes until acute liver failure (ALF). Therefore, B19V has been proposed to cause ALF in patients based on isolation and characterization of B19V genome in liver tissue. The objective of this study was to determine the role of the B19V as etiological agent of ALF cases.

Method: Archived liver tissue and serum samples obtained from thirty patients with ALF were investigated for B19V infection. For molecular diagnostics, DNA was extracted and qPCR was performed by Taqman methodology using probe and primers for NS1 region. Anti-B19V IgM and IgG antibodies were evaluated by commercial ELISA, in serum samples. The presence of B19V mRNA in the liver tissue was analyzed to evaluate viral replication.

Result: The study population was divided into 3 groups according to etiology and clinical condition: a) virual-induced $\operatorname{ALF}(n=11)$; $b$ ) nonviral induced $\operatorname{ALF}(n=8)$, which include drug $(n=5)$ and autoimmune $(n=3)$ causes; $c)$ undefined etiology $\operatorname{ALF}(n=11)$. Seven patients were B19V-DNA positive in serum and liver through qPCR. Among them, six were IgM anti-B19V negative and IgG anti-B19V positive, and one patient had IgM and IgG anti-B19 undetectable. Among B19VDNA positive patients $(n=7)$, there were 2 co-infected with hepatitis $B$ virus, 1 co-infected with cytomegalovirus, 3 were indeterminate causes and 1 was drug-induced ALF. Four patients infected with B19V progressed to death. Among them, 2 were co-infected with 
HBV and 2 were undefined etiology cases. The B19V mRNA was present in liver from 3 patients with undefined etiology of ALF and in the drug-induced ALF patient, indicating presence of viral replication in these cases.

Conclusion: These laboratory findings suggest a diagnosis of B19Vassociated ALF. Because there is a rare occurrence, B19V is likely under-diagnosed as a cause of acute hepatic injury. Our study highlights the importance of considering this virus in the differential diagnosis of ALF, mostly in patients with haematological abnormalities, and during outbreak periods of erythema infectiosum.

\section{$A L F-4$}

Prognostication in acute liver failure: comparison of the King's College Hospital Criteria with the Sequential Organ Failure Assessment Score (SOFA), Acute Physiology and Chronic Health Evaluation II (APACHE II) and Acute Liver Failure Early Dynamic model (ALFED).

Vandana Saluja ${ }^{1}$, Anamika Sharma ${ }^{2}$, Lalita Gouri Mitra ${ }^{1}$, Prashant Agarwal $^{1}$, Rakhi Maiwall, ${ }^{1}$, Seema Alam ${ }^{1}$, Guresh Kumar $^{1}$

${ }^{1}$ Institute of liver and biliary sciences, India; ${ }^{2}$ AII India Institute of Medical Sciences, India

Background: The need for emergency liver transplantation in acute liver failure (ALF) is based on the Kings College criteria (KCC) score, derived two decades ago. Since then Non transplant survival has improved considerably due to sophistication of intensive care and earlier referral of patients.

The Acute Physiology and Chronic Health Evaluation II score (APACHE II) and SOFA scores have been validated in patients with cirrhosis with acute deterioration.

The Acute Liver Failure Early Dynamic (ALFED) score is a recently derived model for determining outcome in patients with acute liver failure.

The SOFA, APACHE II, and KCC scores have been compared in acetaminophen induced acute liver failure.

Aim: We aimed to compare the KCC score with SOFA, APACHE II and the ALFED score in patients with acute liver failure.

Method: Consecutive patients with ALF who are admitted to the ICU of Institute of liver and Biliary Sciences from January 2014-December 2016 were evaluated. The severity of liver disease was evaluated with the KCC criteria and the ALFED score. APACHE II was used for the classification of illness severity, and SOFA score (at admission and 48 hours) was used for grading organ dysfunction.

Those who underwent an emergency liver transplant, left against medical advice or were shifted from outside hospitals were excluded. Outcome Measures: Primary outcome was prediction of mortality. Result: 166 patients of ALF presented to our Institute from January 2014 to December 2016.

80 patients were managed with conservative medical management. 45 out of 80 patients survived $(56 \%)$ and $35(44 \%)$ patients died.

Total of 80 patients were analysed.

\begin{tabular}{|l|l|l|l|l|l|l|l|}
\hline Score & $\begin{array}{l}\text { AUC(area } \\
\text { under Curve) }\end{array}$ & Cut off & $\begin{array}{l}\text { Sensitivity } \\
(\%)\end{array}$ & $\begin{array}{l}\text { Specificity } \\
(\%)\end{array}$ & $\begin{array}{l}\text { Positive } \\
\text { predictive } \\
\text { value }\end{array}$ & $\begin{array}{l}\text { Negative } \\
\text { predictive } \\
\text { value }\end{array}$ & $\begin{array}{l}\text { Percentage } \\
\text { correctly } \\
\text { classified }\end{array}$ \\
\hline KCC & $\begin{array}{l}0.82(0.72- \\
0.92)\end{array}$ & $>2$ & 77.1 & 82.2 & 77.1 & 82.2 & $80 \%$ \\
\hline $\begin{array}{l}\text { SOFA } \\
\text { admission }\end{array}$ & $\begin{array}{l}0.65(0.531- \\
0.77)\end{array}$ & $>9$ & 62 & 62.2 & 56.4 & 68.3 & $62.5 \%$ \\
\hline $\begin{array}{l}\text { SOFA 48 } \\
\text { hours }\end{array}$ & $\begin{array}{l}0.85(0.76- \\
0.94)\end{array}$ & $>9$ & 88.6 & 73.3 & 72.1 & 89.2 & $80 \%$ \\
\hline $\begin{array}{l}\text { APACHE } \\
\text { II }\end{array}$ & $\begin{array}{l}\text { II } \\
0.88)\end{array}$ & $>15$ & 74.3 & 75.6 & 70.3 & 79.1 & $75 \%$ \\
\hline ALFED & $\begin{array}{l}0.88(0.80- \\
0.95)\end{array}$ & $>4$ & 85.7 & 82.2 & 78.9 & 88.1 & $83.8 \%$ \\
\hline
\end{tabular}

\begin{tabular}{|l|l|l|l|}
\hline Parameter & Odds Ratio (OR) & $\mathbf{9 5 \%}$ CI & P value \\
\hline KCC & 2.79 & $1.77-4.39$ & 0.00 \\
\hline SOFA admission & 1.26 & $1.03-1.53$ & 0.025 \\
\hline SOFA 48 hours & 1.73 & $1.34-2.23$ & 0.00 \\
\hline APACHE II & 1.16 & $1.07-1.26$ & 0.00 \\
\hline ALFED & 6.2 & $2.68-14.68$ & 0.00 \\
\hline
\end{tabular}

Conclusion: The ALFED score performed better than the KCC in our Group of patients. SOFA score at 48 hours proved to be comparable to KCC. Thus, in our study dynamic scores showed a good performance in patients with acute liver failure.

\section{ALF-5}

Acute liver failure: betaherpesvirus as possible etiology

Jessica Vasques Raposo ${ }^{1}$, Arthur Daniel Rocha Alves ${ }^{1}$, Vanessa Salete De Paula ${ }^{2}$, Marcelo Alves Pinto ${ }^{2}$

${ }^{1} \mathrm{PhD}$ student, Brazil; ${ }^{2}$ Researcher, Brazil

Background: The etiology of acute liver failure (ALF) cases is often unknown. Herpesviruses have been associated to cases of ALF such as betaherpesvirus, Human Cytomegalovirus (HCMV), Human Herpes Virus 6 (HHV-6) and Human Herpesvirus 7 (HHV-7). The poor prognosis of evolution for hepatitis in cases of herpes are associated with late diagnosis. This study investigated the betaherpesvirus infection in patients with ALF unknown etiology, using the qPCR multiplex and sequencing.

Method: Liver samples pre-transplant from 14 patients with ALF of unknown etiology were analyzed. These samples were collected in a public hospital of Rio de Janeiro. All the samples were tested in qPCR multiplex to betaherpesvirus. All positive samples were sequenced to confirm herpes infection. Besides that, biochemical analysis and the levels of liver enzymes were evaluated.

Result: The mean age of patients was 29 years old and $85,7 \%$ were female. Seven $(50 \%)$ cases of infection from previously unrecognized viral pathogens were positive to betaherpesvirus; six $(42.8 \%)$ cases of HHV-6, one case $(7.1 \%)$ of HCMV and two cases $(14.2 \%)$ of dual infection HHV-7/ HHV-6 and HHV-7 and HCMV. The HHV-7 was only identified in the presence of other betaherpesvirus. The sequencing information confirmed the presence of betaherpesvirus before transplant. In all positives patients, the values of Alanine transaminase (ALT) and Aspartate transaminase (AST) were higher than $1000 \mathrm{U} / \mathrm{L}$. Alkaline phosphatase (ALP) enzyme presents small elevations in hepatics levels.

Conclusion: The results of this study were relevant to demonstrate the betaherpesvirus as possible etiologic agents in cases of ALF, therefore suggest that ALF patients should be screened for the presence of uncommon viruses as betaherpesvirus. 
AUTO-1

\section{Autoimmune liver disease - clinical and Immunohistochemical profile, An Insight}

\section{Neeraj Nagaich ${ }^{1}$, Neeraj Nagaich ${ }^{1}$, Radha Sharma ${ }^{2}$, Ajay Yadav ${ }^{3}$ \\ ${ }^{1}$ Fortis Hospital Jaipur, India; ${ }^{2}$ RUHS, India; ${ }^{3}$ SMS Medical College, India}

Background: Combination of clinical, laboratory, and pathologic criteria play a pivotal role in solving the puzzle of Autoimmune liver disease spectrum. There is histopathologic overlap among the different autoimmune liver diseases making diagnosis a challenge based on morphologic features alone seen on routine stains. There is dearth of data on immunophenotype of plasma cells in autoimmune liver diseases in relation to clinical phenotype. This prospective and retrospective study was done to study role of IHC in diagnosing autoimmune liver disease and their clinical correlation..

Method: Liver biopsies from 72 patients diagnosed with autoimmune liver diseases during period of 2010-2017 (autoimmune hepatitis; Primary biliary cirrhosis \& Primary sclerosing cholangitis) were selected who fulfilled the eligibility criteria. Count of plasma cells showing unequivocal immunohistochemical expression of either IgM or $\mathrm{IgG}$ was done. Absolute count and the concentration of positive cells for each immunoglobulin subtype were evaluated. Clinical profile of patients in each subgroup was also analysed.

Result: Thirty seven patients had a diagnosis of AIH (, 26 females, 9 males mean age 29 , range $9-53$ ).

Twenty one patients had a diagnosis of PBC ( 19 females, 2 male mean age 46 range 21-67). Associated autoimmune conditions were seen in $8 \%$ of patients. The combined number of $\operatorname{IgM}$ and $\mathrm{IgG}+$ plasma cells in AIH (mean $=9.79$ cells $/ \mathrm{mm}^{2}$ ) was higher than that seen in PBC or PSC (mean $=7.1$ cells $/ \mathrm{mm}^{2} 5.5$ cells $/ \mathrm{mm}^{2}$, respectively, $\mathrm{P}<0.05$ )

An $\mathrm{IgM} / \mathrm{IgG}$ ratio $\geq 1$ predicted the distinction of $\mathrm{PBC}$ from other groups and accurately distinguished $\mathrm{PBC}$ from $\mathrm{AIH}$ in $88.9 \%$ of cases, PBC from either AIH or PSC in $83.8 \%$ of cases, and PBC from all other groups in $85 \%$.

Conclusion: Plasma infiltrate in liver tissue with $\operatorname{IgM} / \operatorname{IgG}$ ratio $\geq 1$ suggests $\mathrm{PBC}$, while an $\mathrm{IgM} / \mathrm{IgG}$ ratio $<1$ decreases the likelihood of PBC. IgG was the predominant immunoglobulin subclass in the patients, except for the PBC group, in which an IgM predominance was seen. IgG predominant infiltrates, are relatively nonspecific and may be seen in a variety of conditions. Predominantly IgM+ plasma cell infiltrate, although not pathognomonic, favour the diagnosis of PBC. The IgM/IgG ratio is particularly useful in distinguishing PBC from AIH.

Characterization of plasma cells in liver biopsies of autoimmune liver disease patients by $\operatorname{IgM}$ and $\operatorname{IgG}$ immunohistochemistry can be a valuable tool in solving the puzzle of AIH.

\section{AUTO-2}

Efficacy and safety of budesonide as a maintenance therapy for patients with autoimmune hepatitis

\section{Fehmi Ates $^{1}$, Mehmet Demir ${ }^{2}$, Engin Altintas ${ }^{1}$, Orhan Sezgin ${ }^{1}$ \\ ${ }^{1}$ Mersin University, Turkey; ${ }^{2}$ Mustafa Kemal University, Turkey}

Background: The initial treatment of choice for autoimmune hepatitis (AIH) is prednisone alone or with azathioprine. A significant number of patients with AIH develop steroid-specific side effects or require doses of steroids that are unacceptable for long-term treatment. We investigated the efficacy and safety of budesonide as an alternative steroid maintenance therapy for patients which were previously treated with prednisolone remission induction therapy.

Method: We performed a retrospective analysis of data from 36 patients (30 female) with non-cirrhotic AIH who were treated initially with prednisolone but then switched to budesonide, conducted at two centers in Turkey from 2009 through November 2017. Patients were evaluated after 6 months, 12 months, 24 months, 36 months, and at the last follow-up evaluation; response to treatment with budesonide was assessed based on normal serum levels of aminotransferases and IgG (biochemical response).

Result: Twenty patients were switched to budesonide therapy because of prednisolone-induced side effects and 16 patients were switched because of prednisolone dependency. Completely, a biochemical response was detected in $58 \%$ of patients after 6 months of budesonide treatment, in $75 \%$ after 12 months, and in $72 \%$ after 24 months. At the last follow-up evaluation (mean time, $60 \mathrm{mo}) 12$ patients $(33 \%)$ were still receiving budesonide treatment. Six patients (17\%) had stopped budesonide therapy because of insufficient response to budesonide or its side effects. Nine patients with osteopenia at the beginning of budesonide treatment were followed up and evaluated by dual-energy X-ray absorptiometry. After a median of 24 months of budesonide treatment, bone mineral density had improved in 5 patients, remained stable in 4 patients.

Conclusion: Oral budesonide, in combination with azathioprine, can effectively maintain remission in patients with non-cirrhotic $\mathrm{AIH}$, with a low rate of steroid-specific side effects. The combination of budesonide with azathioprine may therefore become a new standard of remission maintenance therapy for non-cirrhotic patients with $\mathrm{AIH}$.

\section{AUTO-3}

Liver cirrhosis caused by autoimmune hepatitis and the possible impact of metabolic factors on liver disease progression

Cosmas Rinaldi Adithya Lesmana ${ }^{1}$, Lidwina Cahyadinata ${ }^{1}$, Agnita Irawaty $^{1}$, Levina Pakasi ${ }^{1}$, Laurentius Adrianto Lesmana ${ }^{1}$

${ }^{1}$ Medistra Hospital, Indonesia

Background: Patients with autoimmune hepatitis (AIH) are often presented with cirrhosis due to delayed diagnosis. Early identification is important to prevent further complications. Little is known about the impact of metabolic conditions for liver disease progression in AIH.

Method: A retrospective cohort study was performed on AIH patients between 2011 and 2016 in Medistra Hospital, Jakarta. Demographic and laboratory data were retrieved from the patient's medical record. Diagnosis of AIH was established by positive antinuclear antibodies (ANA) or liver biopsy after excluding viral hepatitis. Cirrhosis was evaluated by histopathology, abdominal ultrasound or clinical symptoms portal hypertension. Association between clinical factor and cirrhosis was tested using Chi-square or Fisher's exact test.

Result: A total of 36 cases were retrieved during the study period; 23 $(63.9 \%)$ of them were women. Patients' mean age was $55.1+$ 16.9 years. Liver cirrhosis was present in $21(58.3 \%)$ patients. There were $13(36.1 \%)$ patients with obesity and $10(27.8 \%)$ with type 2 diabetes. Cirrhosis tended to be associated with obesity $(p=0.089)$ and all patients $(100 \%)$ with diabetes also had cirrhosis $(p=0.002)$. Gender and age were not associated with cirrhosis.

Conclusion: About half of the patients with autoimmune liver disease presented with cirrhosis. Obesity and diabetes may have aggravated the underlying liver fibrosis. Further study is needed to confirm the risk of autoimmune hepatitis and liver cirrhosis among patients with metabolic syndrome. 


\section{AUTO-4}

Clinical characteristics and treatment response of autoimmune hepatitis in Indonesia

Cosmas Rinaldi Adithya Lesmana ${ }^{1}$, Lidwina Cahyadinata ${ }^{1}$, Agnita Irawaty $^{1}$, Levina Pakasi ${ }^{1}$, Laurentius Adrianto Lesmana ${ }^{1}$

${ }^{1}$ Medistra Hospital, Indonesia

Background: Autoimmune hepatitis (AIH) has non-specific symptom and is only diagnosed after excluding other causes of hepatitis. Immunosuppressive treatment is the standard practice; however, predictors of treatment response have not been evaluated widely.

Method: A retrospective cohort study was performed on AIH patients between 2011 and 2016 in Medistra Hospital, Jakarta. Demographic and laboratory data were retrieved from the patient's medical record. Diagnosis of AIH was established by positive antinuclear antibodies (ANA) or liver biopsy after excluding viral hepatitis. Patients were treated with low-dose corticosteroid or azathioprine or both. Normalization of liver enzymes on the last visit was regarded as good treatment response. Duration of treatment response was analyzed using the Kaplan-Meier estimation curve. A log-rank $p$ of $<0.05$ was considered significant.

Result: There are $36 \mathrm{AIH}$ cases retrieved and women were predominated (63.9\%). Cirrhosis was present in $21(58.3 \%)$ patients, whereas jaundice was observed in $17(47.2 \%)$ and ascites in 14 $(38.9 \%)$ patients. Diagnosis of AIH was established using serology only in $19(52.8 \%)$, biopsy only in $2(5.6 \%)$ and both methods in 15 $(41.7 \%)$. There were 34 patients with ANA titer available; $24(66.7 \%)$ had a titer of $1 / 1000$ or more. Only 28 cases with follow-up data at least for 3 months were eligible for further analyses. Good treatment response was observed in $22(78.6 \%)$ patients. Poor treatment response was associated with the presence of cirrhosis $(p=0.024)$, jaundice $(p=0.005)$, and ascites $(p=0.022)$. The median duration of treatment response was significantly shorter patients with cirrhosis (log-rank $p=0.034)$, jaundice (log-rank $p=0.02$ ), ascites (log-rank $p=0.026)$, and diabetes (log-rank $p=0.024)$.

Conclusion: Patients with AIH often diagnosed with cirrhotic liver, jaundice and ascites, which often require emergency management. Treatment response in AIH is poorer in patients with cirrhosis and diabetes. Failure to give immunosuppressive treatment in these patients may be the reason of not having optimal response.

\section{$A L D-1$}

To explore and investigate the synergistic effect of polyherbal formulation against high fat diet and alcohol induced hepatotoxicity in rodents

\section{Onkar Bedi ${ }^{1}$, Puneet Kumar ${ }^{2}$, Vinod Gauttam ${ }^{2}$}

${ }^{1}$ Punjabi University, Patiala, India; ${ }^{2}$ Associate Professor, India

Background: Non alcoholic fatty liver and alcoholic fatty liver disorders includes a wide spectrum of liver conditions ranging from simple steatosis to nonalcoholic steatohepatitis and advanced hepatic fibrosis. The scientific and traditional data revealed that Phyllanthus niruri Linn. (Euphorbiaceae) (PN), Andrographis paniculata Nees. (Acanthaceae) (AP) and Piper longum Linn. (Piperaceae) (PL) are associated with many therapeutic activities like anti-inflammatory, antioxidant and antihyperlipidemic. Similarly, the aim of the present study was to investigate the synergistic and mechanistic approaches of these herbs with respect to hemeoxygenase-1 pathway (HO-1) to produce hepatoprotective effect against alcohol and HFD (high fat diet) induced hepatotoxicity in wistar rats.
Method: The hepatoprotective activity of three herbal extract combinations $P N, A P$ and $P L$ were first studied in in-vitro on HepG-2 cell lines by using ethanol $(100 \mathrm{mM})$ which further analyzed through invivo activity against alcohol $(40 \% ; 2 \mathrm{ml} / 100 \mathrm{~g})$ and HFD induced hepatotoxicity in wistar rats. Various parameters such as oxidative stress, liver enzymes, lipid profile level were assessed and various histopathological changes were also observed in different experimental animal groups. The body weight and urine analysis was done on 7 th, 14th, 21st day.

Result: The in vitro (HepG-2 cell lines) and rodent animal model experimentation with different herbal combinations (AP: PN: PL, $1: 1: 1,2: 2: 1,3: 1: 1)$ showed significant hepatoprotective effect. However, herbal combination with equal ratio showed significant hepatoprotective effect as compared to other dosage regimens. The herbal combination groups showed significant reduction in oxidative stress parameters and improved liver enzymes level.

Conclusion: The present study suggested that the combination of herbal drugs have shown synergistic hepatoprotective effect possibly through antioxidant and HO-1 induction mechanism. The combination of these three herbal drugs may provide basis for their clinical implications in liver disorders.

$A L D-2$

Increased plasma sST2, but not IL-33, is associated with the severity of alcoholic liver disease

Zijian Sun ${ }^{1}$, Miaomiao Gao', Ang Huang ${ }^{2}$, Binxia Chang², Ying $\mathrm{Sun}^{2}$, Ning $\mathbf{L i}^{2}$, Jiyuan Zhang ${ }^{2}$, Zhengsheng Zou ${ }^{1}$

${ }^{1}$ Peking University, China; ${ }^{2}$ Beijing 302 Hospital, China

Background: Interleukin-33 (IL-33) exerts its biological function through binding with its receptor ST2. ST2 protein exists in two uppermost isoforms: a transmembrane form and a soluble form (sST2). sST2 is regarded as a decoy receptor blocking the IL-33 signaling pathway by binding with IL-33. Recently, several experimental and clinical evidence have indicated that IL-33-ST2 axis is associated with various liver diseases. However, the role of IL-33ST2 axis in alcoholic liver disease (ALD) remains unknown.

Method: Blood samples were collected from ALD patients and healthy controls (HCs) and the plasma IL-33 and sST2 were measured by ELISA. The expression levels of IL-33 and ST2 mRNA in liver tissues from HCs and ALD patients were performed through affymetrix human gene expression array and further validated by qPCR. Result: There were no significant differences in plasma levels of IL33 between HCs $(n=20)$ and ALD patients $(n=47)$. In addition, the levels of plasma IL-33 in ALD was not associated with liver damage markers (total bilirubin: TBil; prothrombin time: PT). Furthermore, no differences in the IL-33 and ST2 mRNA expression between liver tissues from ALD patients $(n=5)$ and HCs $(n=5)$ through human gene expression array and qPCR. Interestingly, the concentration of plasma sST2 was markedly elevated in ALD patients (53.8 IQR 34.3-71.7 $\mathrm{pg} / \mathrm{mL}$ ) as compared to HCs (21.6 IQR $15.3-37.7 \mathrm{pg} / \mathrm{mL} ; \mathrm{p}<0.0001)$. Especially in patients with severe alcoholic hepatitis, the levels of plasma sST2 was higher. Further analysis showed that elevated sST2 concentration exhibited strong positive correlation with TBil $(\mathrm{r}=$ $0.65 ; \mathrm{p}<0.0001)$ and PT $(\mathrm{r}=0.71 ; \mathrm{p}<0.0001)$.

Conclusion: Plasma sST2 was markedly elevated in ALD patients and showed a strong positive correlation with TBil and PT. These data suggest the possible role of $\mathrm{sST} 2$ in predicting severe alcoholic hepatitis. 
$A L D-3$

A new prognostic model for severe alcoholic hepatitis- ABCDE INDEX

Neeraj Nagaich $^{1}$, Neeraj Nagaich ${ }^{1}$, Radha Sharma ${ }^{2}$, Subhash Nepalia $^{3}$, Sandeep Nijhawan ${ }^{3}$, Jayant Sharma ${ }^{1}$, Abhinav Sharma ${ }^{1}$

${ }^{1}$ Fortis Hospital Jaipur, India; ${ }^{2}$ RUHS CMS Jaipur, India; ${ }^{3}$ SMS Medical College, India

Background: Alcoholic hepatitis ( $\mathrm{AH})$ is an acute life- and healththreatening disease. However, accurate and representative epidemiological data on its incidence and prognosis are not available. Prediction of survival in patients with Alcoholic hepatitis, has been done with various scoring systems but no studies have stratified patients with Alcoholic hepatitis, as having good, intermediate, or poor prognosis. A prognostic index which can be applied early and more predictive of long term survival would be useful in deciding treatment plans.

Method: 352 patients which diagnosed as severe alcoholic hepatitis based on Maddrey's discriminant factor (DF) and fulfilled eligibility criteria were evaluated in this prospective and retrospective study.

DF has been used in clinical practice for more than 30 years [9]. A DF of 32 is used to stratify a patient's severity of AH, patients with a score of $\geq 32$ having a high short-term mortality The index was calculated at admission in all patients. The patients were followed up for six months to assess the mortality predictive strength of this new index in comparison to DF. Throughout the study patient were given standard of care treatment.

Multivariate analysis identified age, bilirubin level, Delta PT (prothrombin time) (Normal PT value - PT of patient.), creatinine level, grade of encephalopathy as independent predictors of 6- month mortality. The score combining these 5 variables was named the ABCDE Score for Alcoholic Cirrhosis and compared to DF. The prognostic value of two scoring systems was determined by generating a receiver operating (ROC) curve and the area under the curve was calculated for survival at 30 days, 90 days and 6 months. Abstinence from alcohol was significantly $(P<0.05)$ associated with survival at 6 months. The predictive value of this index was better than DF. By stepwise multivariate analysis, this index was identified as independently associated with 6-month mortality. The new ABCDE - INDEX may improve the prognostic accuracy to predict the 6-month outcome.

Result: Out of this cohort of 352 patient the total mortality was $41 \%$ (144).

The respective 1-month and 6-month cumulative mortality in this cohort was $61 \%(87 / 144)$ and $39 \%(57 / 144)$.

$\mathrm{ABCDE}$ index score greater than 7 was associated with increased risk of mortality even after steroid therapy as dictated by DF.

Patients with a high score of this index showed more readmissions and mortality. $\mathrm{ABCDE}$ index higher predictability as compared to DF, AUROC 0.87 (0.73-0.97) vs. $0.63(0.42-0.81)$.

Conclusion: The new ABCDE - INDEX may improve the prognostic accuracy to predict the 6-month outcome.

A score greater than 7 was associated with increased risk of six month mortality even after steroid therapy as indicated by DF score.

$\mathrm{ABCDE}$ index may help in early risk stratification as this group of patient would require more rigorous treatment and follow up to prevent mortality in this cohort.

Table-1 Univariate analysis of variables at randomization associated with survival (significant difference)

\begin{tabular}{|l|l|l|l|}
\hline Variable & Survivors (n=208) & Non-survivors(n=144) & p value \\
\hline Age (years) & $38(22-69)$ & $49.0(33.0-75.0)$ & \\
\hline Bilirubin $(\mathrm{mg} / \mathrm{d}$ ) & $21.5(2.9-52.2)$ & $30.5(5.4-47.9)$ & \\
\hline Creatinine (mg/dl) & $1.0(0.4-6.1)$ & $5.1(1.1-14.9)$ & \\
\hline PT & $68(30-245)$ & $68(39-328)$ & \\
\hline Encephalopathy & $1(1-2)$ & $2(2-4)$ & \\
\hline
\end{tabular}

Table- 2 Comparison of two indices.

\begin{tabular}{|l|l|l|l|}
\hline Index & Survivors (n=208) & Non-survivors(n=144) & p value \\
\hline $\mathrm{ABCDE}$ index. & $5.9(4-8)$ & $9(6-12)$ & 0.02 \\
\hline $\begin{array}{l}\text { Maddrey's discriminant } \\
\text { factor (MDF) (®/SD) }\end{array}$ & $30(24-36)$ & $42(30-75)$ & 0.04 \\
\hline & & & \\
\hline
\end{tabular}

Table-2 Scores assigned for calculating $\mathrm{ABCDE}$ index

\begin{tabular}{|l|c|c|c|}
\hline \multirow{2}{*}{ Parameter } & \multicolumn{3}{|c|}{ score } \\
\cline { 2 - 4 } & $\mathbf{1}$ & $\mathbf{2}$ & $\mathbf{3}$ \\
\hline Age (yr) & $<40$ & $40-50$ & $>50$ \\
\hline Bilirubin (mg/dl) & $<10$ & $10-20$ & $>20$ \\
\hline Creatinine (mmol/L) & $<1.5$ & $1.5-3$ & $>3$ \\
\hline $\begin{array}{l}\text { Delta PT } \\
\text { (Normal PT value - PT of patient.) }\end{array}$ & $0-4.00$ & $4.0-10.0$ & $>10$ \\
\hline Encephalopathy grade (1-4) & $0-1$ & 2 & $\geq 3$ \\
\hline
\end{tabular}

\begin{tabular}{|l|l|l|}
\hline ABCDE score & $\mathbf{3 0}$ day mortality. & $\mathbf{6}$ month mortality. \\
\hline$<7 \%$ & $10 \%$ & $16 \%$ \\
\hline$\geq 7 \%$ & $53 \%$ & $60 \%$ \\
\hline
\end{tabular}

ALD-4

Effects of probiotics (Lactobacillus rhamnosus R0011 and Lactobacillus acidophilus R0052) on modulation of gut microbiota in the treatment of mild alcoholic liver disease

\section{Ki Tae Suk ${ }^{1}$, Dong Joon Kim ${ }^{1}$}

${ }^{1}$ Hallym University College of Medicine, Korea, Republic of

Background: Alcohol cause abnormal bowel overgrowth of gramnegative bacteria and disruption the gut barrier, consequently, promotes the translocation of lipopolysaccharide (LPS). Probiotics might restore bowel flora by reducing gut-derived microbial LPS in patients with alcoholic liver disease (ALD). To evaluate the relationship between alcohol administration and changes on gut microbiota, its involvement in the pathogenesis of alcohol liver disease, and how gut microbiota modulation could be a target for the treatment of ALD. We evaluated the therapeutic effects of probiotics on gut microbiota in patients with mild ALD.

Method: 101 patients with ALD (probiotics: 53 and placebo: 48) and normal patients were prospectively randomized to receive the 7 days of Lactobacillus rhamnosus R0011 and Lactobacillus acidophilus $\mathrm{R} 0052$ at $120 \mathrm{mg} /$ day or placebo. All patients, except normal patients were hospitalized and were not permitted alcohol. Liver function and LPS level were evaluated by enzyme-linked immunosorbent assay (ELISA), and to determine the relative importance of gut bacterial changes for patients were demonstrated using PCR-denaturing gradient gel electrophoresis (PCR-DGGE) with 16S rRNA, and analyzed for the evaluation of microbial species before and after probiotics treatment for 7 days.

Result: In both groups, liver function were significantly improved after 7 days of abstinence and dominant types of bacteria were gram negative at baseline. In ALD patients with probiotics treatment (baseline and after), the mean level of alanine aminotransferase $(64+117$ and $27+24, p=0.049)$, Child-Pugh score $(79+2.5$ and $7.2+2.1, \mathrm{p})$. 
Conclusion: Seven-day of supplementation with Lactobacillus rhamnosus R0011 and Lactobacillus acidophilus R0052 was associated with restoration of bowel flora and probiotics might be effective in the treatment of alcoholic hepatitis by regulation gut-liver axis. And, indicating that a short-term administration of probiotics can change the gut microbiota, leading to the amelioration of liver injury induced by chronic alcohol consumption.

\section{$A L D-5$}

A Lille model for predicting prognosis after corticosteroid treatment of severe alcoholic hepatitis in Japanese patients

\section{Yuji Suzuki ${ }^{1}$, Keisuke Kakisaka', Akiko Suzuki ${ }^{1}$, Yasuhiro Takikawa $^{1}$}

${ }^{1}$ Iwate Medical University School of Medicine, Japan

Background: Corticosteroids are the most widely used agents for the treatment of severe alcoholic hepatitis ( $\mathrm{AH})$. The therapeutic efficacy of corticosteroids is assessed by the Lille model, which has been validated well in western countries; however, the usefulness of the Lille model has not yet been confirmed independently in Japanese patients. The purpose of the present study was to determine whether the Lille model could predict the prognosis of severe AH in Japanese patients.

Method: This was a retrospective cohort study of patients who were admitted with clinically severe AH from April 2011 to December 2017 at Iwate Medical University. Patient records were reviewed, and the findings of the clinical examination were documented. The inclusion criteria were as follows: recent onset of jaundice $(<$ 8 weeks), history of excessive alcohol consumption ( $>60 \mathrm{~g} /$ day), Maddrey's discriminant function (MDF) $>32$. Patients with the MDF $>32$ or a Model for End-stage Liver Disease (MELD) score of $>20$ were treated with corticosteroids unless they had any contraindications.

Result: Twenty-six patients satisfied the inclusion criteria for severe AH. Twenty-two out of the 26 patients were treated with corticosteroids (median age of 43 years, aspartate aminotransferase $144 \mathrm{U} / \mathrm{l}$, bilirubin $19.8 \mathrm{mg} / \mathrm{dl}$, INR 1.91, MDF 76, and MELD score 28). Fourteen of the patients $(64 \%)$ were male. Median daily alcoholic intake at the time of admission was $114.5 \mathrm{~g}$. Three patients (14\%) had non-gastrointestinal hemorrhagic complications at the time of admission. Hepatic encephalopathy occurred in eight patients $(36 \%)$. During corticosteroid treatment, bacterial infections occurred in six patients $(27 \%)$. In addition, six $(27 \%)$ patients received renal replacement therapy for acute kidney injury. One patient received a living donor liver transplantation at 26 days of admission. The transplant-free survival rates in the group were $78 \%$ (17 survivors) and 64\% (14 survivors) at day 28 and six months, respectively. A multivariate regression model identified only the Lille score as an independent predictor of outcome after six months $(95 \% \mathrm{CI}$, $0.08-6.61, p=0.0434)$. When classified as corticosteroid responders or non-responders (with non-responders obtaining a Lille score of $\geq$ 0.45 ), the six-month transplant-free survival rate was $83 \%$ in responders and $40 \%$ in non-responders $(p=0.0339$ by the log-rank test).

Conclusion: The Lille model might be useful for predicting the sixmonth prognosis of Japanese severe AH patients who are treated with corticosteroid therapy. A future larger study will be required to verify the researchers' findings.

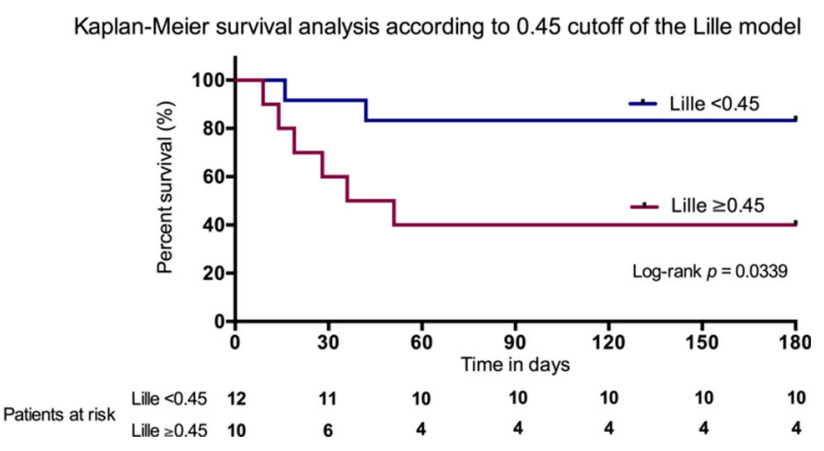

ALD-6

Non invasive markers in alcoholic liver disease

Harit Goverdhan Kothari ${ }^{1}$, Sudhir Jagdishprasad Gupta ${ }^{1}$, Nitin Rangrao Gaikwad $^{1}$, Amol Rajendra Samarth ${ }^{1}$, Tushar Hiralal Sankalecha ${ }^{1}$

${ }^{1}$ Government Medical College \& Super-Speciality Hospital, Nagpur, India

Aim: To assess non-invasive markers for prediction of Esophageal Varices and risk of bleeding in Alcoholic liver disease patients.

Background: In cirrhotic, there is propensity for development of esophageal varices due to portal hypertension. Esophageal varices have tendency to bleed. Parameters like clinical, biochemical and ultrasonographic findings can be utilised for prediction of risk of esophageal varices and variceal bleed. Upper gastrointestinal endoscopy is utilised for screening for esophageal varices.

Method: It's an analytical Cross sectional study done in Department of Gastroenterology, Government Medical College and Super Specialty Hospital, Nagpur. Adult male patients with significant alcohol intake, who had cirrhosis of liver with clinical, biochemical, radiological and endoscopic findings were included in the study. Upper gastrointestinal endoscopy kept as gold standard for assessment of esophageal varices. Patient aged below 18 years, cirrhosis due to other etiology, hepatocellular carcinoma were excluded. Child Turcotte Pugh scores, AST ALT Ratio (AAR), AST Platelet Ratio Index (APRI), FIB-4 and Platelet count-Spleen diameter ratio (PC/SD) were calculated for all patients.

Result: Total 202 male patients were enrolled into study. Mean age was $43.77 \pm 9$ years. Out of them 188(93\%) patients had esophageal varices. According to Child Pugh classification patients were divided in Class A, 10(4.9\%) Class B, 59(29.2\%) and Class C, 133(65.8\%) respectively. 91(48.4\%) patients had variceal bleeding. FIB-4 values in variceal bleeding patients were $6.36 \pm 3.5$ as compared to 4.18 \pm 2.5 in non-bleeding varices $(\mathrm{p}<0.05)$. PC/SD ratio in bleeding varices was $965.35 \pm 310$ as compared to $1396.4 \pm 550$ in nonbleeding varices $(\mathrm{p}<0.05)$. APRI was $2.17 \pm 1.2$ for bleeding varices as compared to $1.26 \pm 0.85$ for non-bleeding varices $(\mathrm{p}>0.05)$. AAR was $2.35 \pm 1.3$ for non-bleeding varices as compared to $2.04 \pm 0.9$ for bleeding varices $(\mathrm{p}>0.05)$.

Conclusion: FIB-4 and PC/SD may be useful non-invasive markers for predicting esophageal varices and risk of variceal bleeding in alcoholic liver disease. 


\section{CASE-1}

\section{Prolonged cholestasis after endoscopic retrograde} cholangiopancreatography: a case report and review of the literature

\section{Cheng Kuan Lin ${ }^{1}$}

${ }^{1}$ Far Eastern Memorial Hospital, Taiwan

Background: Prolonged cholestasis after ERCP is a rare complication. We report a case of worsening cholestasis associated with severe pruritus following ERCP after removal of a common bile duct stone. Method: A 68-year-old man presented with intermittent epigastralgia with tea-color urine for one week. He denied hepatitis history or alcohol consumption. Physical examination revealed icteric sclera and epigastric tenderness. Laboratory data showed WBC: $6100 / \mu \mathrm{L}, \mathrm{AST}$ : $76 \mathrm{IU} / \mathrm{L}$ (normal value).

Result: The exact mechanism for the prolonged post-ERCP cholestasis remains unclear. It might be caused by allergy to the contrast agent or the prophylactic antibiotics. There were eight reported cases in the English literature. In the analysis of these cases and our patient, the age was $53 \pm 11$ year-old, and male predominant (67\%). Three cases received second times of ERCP for a concern of retained stone or inadequate biliary drainage. As an unintentional rechallenge test of contrast material, the jaundice subsequently worsened. The serum total bilirubin was up to $45.2 \mathrm{mg} / \mathrm{dL}$ in one case. The cholestasis was recovered after $54 \pm 22$ th days later. UDCA and cholestyramine were the most used therapies. Five cases had good response by adding oral prednisolone. The overall prognosis was favorable with no long-term consequences.

Conclusion: Prolonged cholestasis can develop after successful ERCP. The clinician should be aware of this uncommon complication of iodinated contrast agents and avoid repeated ERCP to induce the worsening jaundice.

\section{CASE-2}

Gallbladder polyps was the only biliary disease that had its prevalence proportion persistently increased over the past 10 years in in-hospital Chinese patients

\section{Yan WANG ${ }^{1}$, Meihong $\mathrm{Lin}^{3}$, Zhihua $\mathrm{Su}^{3}$, Feng Zhang ${ }^{3}$, Ziming Liu $^{3}$, Jinlian Yang ${ }^{3}$, Zesheng Jiang ${ }^{2}$ \\ ${ }^{1}$ Southern Medical University Nanfang Hospital, China; ${ }^{2}$ Southern Medical University Zhujiang Hospital, China; ${ }^{3}$ Southern Medical University, China}

Background: Biliary disease (BD) is a major public health burden. We aimed to identify the dynamic spectrum of BDs among the inhospital Chinese patients over the past 10 years.

Method: We performed retrospective serial annual surveys at a tertiary medical centre with overall 72,000 in-hospitals per year in Guangzhou China. Patients data were collected from electronic record database with IRB approval. Inclusion criteria were: in-hospitals with primary diagnosis of BD and discharge between 01 JAN 2006 and 31 MAY 2016. We excluded the ones with biliary complications caused by other system diseases, or with diagnosis not coded in ICD-10.

Result: Totally 22,978 subjects were included. Overall median age was 57 years, $57.0 \%$ were males, and the etiologies related to gallstone $62.6 \%$, neoplasms and polyps $16.8 \%$, chronic inflammation and infection $14.6 \%$, bile tract malformation $0.7 \%$, parasitic disease $0.3 \%$, and autoimmune disease $0.2 \%$. The profiles of BDs with top five proportions were mostly the same for every year, i.e. gallbladder stone $(48.4 \%, 95 \% \mathrm{CI} 47 \%-50 \%)$, gallbladder polyps $(12.8 \%, 95 \% \mathrm{CI}$
9.8\%-14.6\%), cholecystitis $(11.5 \%, 95 \%$ CI $10.6 \%-14.1 \%)$, intrahepatic $(9.1 \%, 95 \%$ CI $8.1 \%-9.9 \%)$ and extrahepatic cholelithiasis $(4.1 \%$, 95\% CI $3.3 \%-4.4 \%$ ). The proportion of neoplasms was relatively stable between $4.8 \%-3.8 \%$. Of them, the malignancies had a median of $2.9 \%(1.9 \%-4.8 \%)$, showing a generally decreasing trend $(-0.3 \%$ per year, Skewness $=0.96$, Kurtosis $=1.4, \mathrm{r}=-0.8, \mathrm{p}=0.002$ ) attributed mainly to the change in proportion of gallbladder cancer $(-0.1 \%$ per year, Skewness $=2.2$, Kurtosis $=5.6, r=-0.7, p=0.028$ ). Among all BDs, gallbladder polyps (GBP) was the only one that had its proportion persistently increased from $5.2 \%$ to $17.6 \%$ over the 10 years. For these GBP subjects, median age was 50.0 years, $62.2 \%$ were males, and BMI was $23.5 \mathrm{~kg} / \mathrm{m}^{2}$. $16.6 \%$ were smokers and $8.2 \%$ were alcohol consumers. $14.4 \%$ were T2DM and $36.8 \%$ had hypertension. Regarding concomitant liver disease, $27.6 \%$ had fatty liver, $16.8 \%$ had hepatic cyst, $8.7 \%$ hep B, $0.6 \%$ hep C, and $0.4 \%$ had DILI. $2.2 \%$ had cirrhosis and $7.8 \%$ had liver tumor. Most median levels of laboratory tests were at the normal ranges.

Conclusion: The major spectrum of BDs among local in-hospital patients was relatively stable over the past 10 years. GBP showed an exceptionally increasing trend in proportion and appeared to have more concomitance with fatty liver, T2DM and hypertension than with other non-biliary diseases. It was proposed that increasing incidence of GBP could be due to availability of advanced imaging technology, however the linear trend of increase does not support a main role of this presumption. As there meanwhile showed an opposite trend of change in proportions of biliary malignancies, it seems GBP may not have a promotive impact on their occurrence.

\section{CASE-3}

\section{A case of cholestatic DILI - dress syndrome in a patient on phenytoin}

\section{Raajeev Vijay Hingorani $^{1}$, Murali $\mathrm{A}^{2}$, Palaniappan Singaram ${ }^{3}$}

${ }^{1}$ DNB Resident, India; ${ }^{2}$ H.O.D., India; ${ }^{3}$ Senior Consultant, India

Introduction: Cholestatic pattern of liver injury is common in presentation. Various offending drugs have been noted to cause DILI. However DRESS syndrome is one of the uncommon presentations. Here is a case of DRESS (Drug Rash, Eosinophilia and Systemic Symptoms) in a patient on Phenytoin, which settled immediately after stopping the offending drug, and symptomatic management.

Case Description: 76 year old elderly male Mr. P was treated elsewhere 3 months prior for hypertensive intracerebral hemorrhage with convulsions. On presentation here, he had complaints of significant pruritus, gradual weight loss, anorexia and severe weakness over past 15 days. He did not have fever, altered bowel habits, altered sensorium, GI bleed. On examination, he had high temperature, left axillary $1 \mathrm{~cm}$ lymph node palpable, and also rashes and small plaques over elbow and periorbital region were noted. Rest of general and systemic examination was unremarkable. Investigations revealed GGT 372, ALP142, AST 29, ALT 20, Albumin normal, Platelets normal. WBC was 28000 with eosinophilic predominance $(37 \%)$ suggestive of cholestasis with eosinophilia. HAV, HEV, HbsAg, HCV, ANA, blood cultures all were negative. Excision biopsy of left axillary lymph node showed sinus histiocytosis with reactive changes. DILI was strongly suspected. RUCAM's score was 9 ( $>=7$ is positive). Phenytoin was changed to Levetiracetam as per Neurologists advise. Symptomatic intravenous steroids and cholestyramine were instituted, after which patient settled eosinophilia reduced, general condition was better and patient recovered on followup. 


\section{CASE-4}

A case report: hepatic decompensation during ombitasvir/ ritonavir/paritaprevir and dasabuvir treatment in patients with $\mathrm{HCV}$ and $\mathrm{HBV}$ coinfection. Is it caused by immune exacerbation?

\section{Nese Demirturk ${ }^{1}$, Zerrin ASCI ${ }^{2}$, Hatice Sule Kendir ${ }^{2}$, Emine Turkoglu}

${ }^{1}$ Kocatepe University Medical Faculty, Turkey; ${ }^{2}$ Afyonkarahisar Public Hospital, Turkey

Background: HCV is noncytopathic virus and the capacity to induce chronic liver disease, which is thought to be immune-mediated. Combination with DSV and OBV/r/PTV is one of the direct acting antiviral treatment options for CHC. This treatment usually tolerated well and effectively reduces viral load. Cases of HBV reactivation, during or after DAA therapy have been reported in $\mathrm{HBV} / \mathrm{HCV}$ coinfected patients.

Method: A 64 year-old male patient was admitted to our policlinic for $\mathrm{HBV} / \mathrm{HCV}$ coinfection. HBsAg positivity was determined two years ago. Anti-HCV positivity was determined recently; HCV RNA was found $5001589 \mathrm{IU} / \mathrm{ml}$ in November 2016. He was infected by HCV GT1b. In histopathologic examination of the patient's liver HAI score was $9 / 18$ and his stage of fibrosis was $5 / 6$. Laboratory studies disclosed ALT $64 \mathrm{IU} / \mathrm{ml}$, AST $62 \mathrm{IU} / \mathrm{ml}$, albumin $4 \mathrm{~g} / \mathrm{dl}$, total bilirubin $0.5 \mathrm{mg} / \mathrm{dl}$, PT $1.5 \mathrm{~s}$ and creatinin $1.1 \mathrm{mg} / \mathrm{dl}$.

Result: The patient's serum HBV DNA level was $1564 \mathrm{IU} / \mathrm{ml}$. He was evaluated as compensated cirrhosis caused by $\mathrm{CHC}$. He was treated with $\mathrm{OBV} / \mathrm{r} / \mathrm{PTV}$ and DSV.

After 19 days of starting treatment ascites, pretibial edema and hyperbilirubinaemia developed in the patient. His clinical condition worsened. Laboratory studies disclosed total bilirubin $7.9 \mathrm{mg} / \mathrm{dl}$, prothrombin time $13.5 \mathrm{~s}$ and creatinin $1.9 \mathrm{mg} / \mathrm{dl}$. OBV/PTV/r and DSV treatment was stopped immediately. He underwent hospitalization. Tenofovir treatment was started against the possibility of HBV reactivation. His HBV DNA and HCV RNA serum levels were checked and both found negative.

The patient followed in hospital for two weeks. Several paracenthesis and hemodialysis were applied to the patient. After then the patient's clinical condition improved. Serum bilirubinemia, creatinin and prothrombin time returned normal limits. Ascites has disappeared. So the patient was discharged healthy from the hospital.

After 12 weeks of treatment discontinuation HCV RNA was found negative in the patient so he was considered to have SVR. HBV DNA and HCV RNA are still negative when the patient comes to check in October 2017. It is quite interesting to obtain SVR with such very short duration of treatment.

The issue that needs to be discussed is hepatic decompensation observed in the patient caused by treatment side effect or exacerbation of the patient's immunity? When the clinical condition of the patient worsens, the presence of a negative HBV DNA may suggest an immunological exacerbation.

Conclusion: Hepatic decompensation is rare but severe complication of DAA drug therapy in HCV infected patients with compensated cirrhosis. But, there may be immunological exacerbation by lowering the HCV viral load.

\section{CASE-5}

EBV induced subacute hepatic failure in a patient on antituberculous therapy

\section{Raajeev Vijay Hingorani ${ }^{1}$, Murali $\mathrm{A}^{2}$, Palaniappan Singaram ${ }^{3}$}

${ }^{1}$ DNB Resident, India; ${ }^{2}$ H.O.D., India; ${ }^{3}$ Senior Consultant, India

Introduction: Patients on DILI can have hepatic decompensations including ATT-induced liver failure. But subacute hepatic failure due to viral etiologies other than Hepatitis A, B, C, E are uncommon.

Here is an unusual presentation of EBV-induced subacute hepatic failure in a patient on ATT for Pleural TB.

Case Description: 21 years young female Miss. N presented elsewhere with complaints of moderate grade evening rise of temperature, mild dry cough, anorexia, significant weight loss for one month. No history of dysuria, abdominal pain, watery stools. General and systemic examination suggested pleural effusion, which was detected to be exudative in nature. Based on clinicopathological correlation, she was started empirically on 4-drug ATT. After an initial month of tapering steroids, she was continued on ATT (1 more month of intensive regimen and then continuation regimen), which she tolerated well, weight and appetite improved. After total 4 months of ATT, she developed jaundice, fever. LFT showed transaminitis. Viral profile for Hepatitis A, B, C and E was negative. ATT was withheld. Fever settled, but jaundice and anorexia persisted for further 5 weeks, then she presented to us. Investigations showed coagulopathy (INR 2.4), hyperbilirubinemia (Total bilirubin 4.0) and moderate ascites with features of hepatitis on CT. Ascites was high SAAG and low protein in nature. Also HSV IgM, CMV IgM were negative. Transjugular liver biopsy showed features of hepatitis, no evidence of chronic liver disease. IHC (liver biopsy) was sent for EBV which was positive. Serum EBV EA-IgG, CA-IgG, CA-IgG (with urea) all were positive. Serum EBV CA- IgM was negative, EBNA was negative. She was not in encephalopathy. She improved symptomatically. After LFT and INR gradually settled, 3-drug ATT was restarted. She did not develop relapse of Liver-related clinical issues and she recovered as noted on 1 month followup.

\section{CASE-6}

A bleeding lesion in the stomach: a direct extension of the hepatocellular carcinoma and the challenges faced!

Antony Joseph Kolothumveettil ${ }^{1}$, Antony Paul Chettupuzzha ${ }^{1}$, Roy J. Mukkada ${ }^{1}$, Pradeep G. Mathew ${ }^{1}$, Francis Jose $V^{1}$, Pushpa Mahadevan', Shelley P. Chireyath ${ }^{1}$, Rajesh Antony', Abraham Koshy ${ }^{1}$

${ }^{1}$ VPS Lakeshore Hospital, India

Background: Introduction: Primary Hepatocellular carcinoma (HCC) is the 2nd most common cause of cancer deaths in the world and is the eight most common cause of cancer deaths in the United States. The frequency of Gastro Intestinal (GI) bleeding due to direct invasion by HCC into adjacent organs is around $2.0 \%$, and may be life threatening. Common causes of upper GI hemorrhage in a patient with HCC includes bleeding gastro-esophageal varices and congestive gastropathy, when associated with portal hypertension; and peptic ulcer. Stomach invasion was the most common in a case series involving 11 out of 2237 HCC patients, who presented with Upper GI bleeding and had a median survival of 5 months. Here, we report a case of HCC with direct invasion of the stomach leading to massive GI bleeding.

Method: Case report.

Result: Case description.

An Eighty-five-years old male with no background history of cirrhosis of liver presented with severe pain in the epigastrium and hypotension, in July 2014. He was extensively evaluated to find out the cause of HCC. He was negative for HBV infection. He required hemodynamic stabilisation with multiple transfusion of blood products. A triple phase CT scan abdomen was suggestive of a HCC with intra- 
tumoral bleed, with a $5 \mathrm{~cm} \times 5 \mathrm{~cm}$ exophytic lesion in the segment 2,3 of the liver. He had undergone trans-arterial coil embolization (TAE) of the feeder vessel during the hospitalisation and was clinically stabilized. He also complained of malena and an Esophagogastro-duodenoscopy was suggestive of angioectasias in the fundus of the stomach, which were argon plasma coagulated. He underwent a trans-arterial chemoembolization (TACE) for the HCC in January 2015. He was doing well and was on follow up with his AFP value being in the normal range. A CT scan abdomen was repeated in May 2016 which showed a similar sized lesion and his $\alpha$-feto protein (AFP) value was $6 \mathrm{ng} / \mathrm{ml}$.

He presented to us with massive upper GI hemorrhage in the first week of December 2016, and was stabilised hemodynamically. An Upper GI endoscopy at that time, revealed a tumor protruding into the antrum of stomach (Fig 1) and HCC was proven on histopathology examination. A conventional angiography of the splanchnic vessels revealed a dual blood supply to the tumour, namely from the left gastric artery and the left hepatic artery. Coil embolization of the Left gastric artery and gel foam embolization of the left hepatic artery was done. A cyanoacrylate injection into the ulcerating mass in the stomach in late December 2016, failed to stop the hemorrhage and he continued to have malena and haemoglobin drop. In January 2017, it was decided to perform an endoscopic ultrasound (EUS) guided Sodium Tetradecyl Sulphate (STD) injection into the vessels (Fig 2). Fibroprotein was used for microscopic bleeds from the friable tumour bed to stop oozing, and he was stabilised hemodynamically. He was doing well, but with a palpable epigastric mass and an ECOG score of 2. In April 2017, he finally had a massive upper GI bleed and expired. Conclusion: In a locally invasive hepatocellular carcinoma, treatment can be challenging as in a situation like this. With advancement of treatment options including TAE/TACE, such tumour extension from liver segments closer to the bowel may become more common in the near future. With the aim of prolonging life, the application of simpler methods, which may not have been tried or proven previously and which will cause no harm to the patient can be implemented.

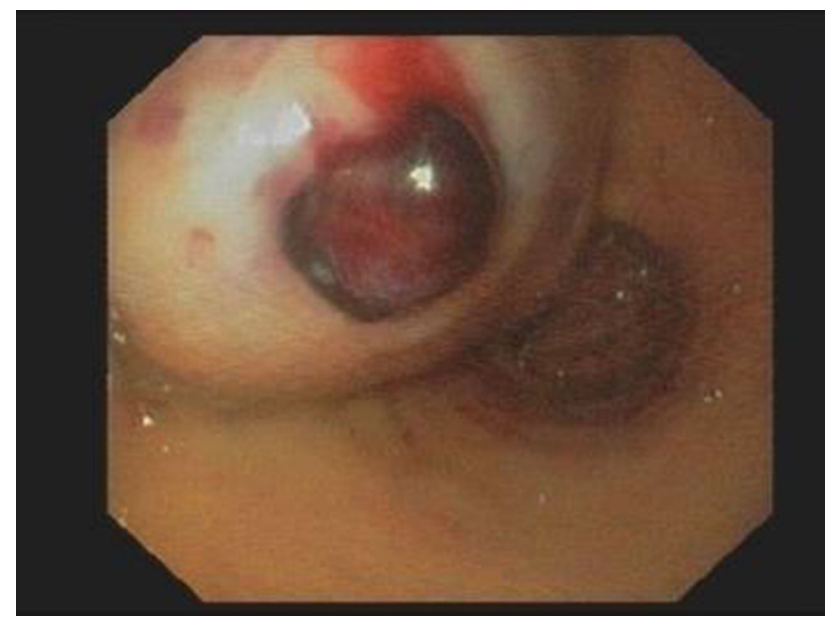

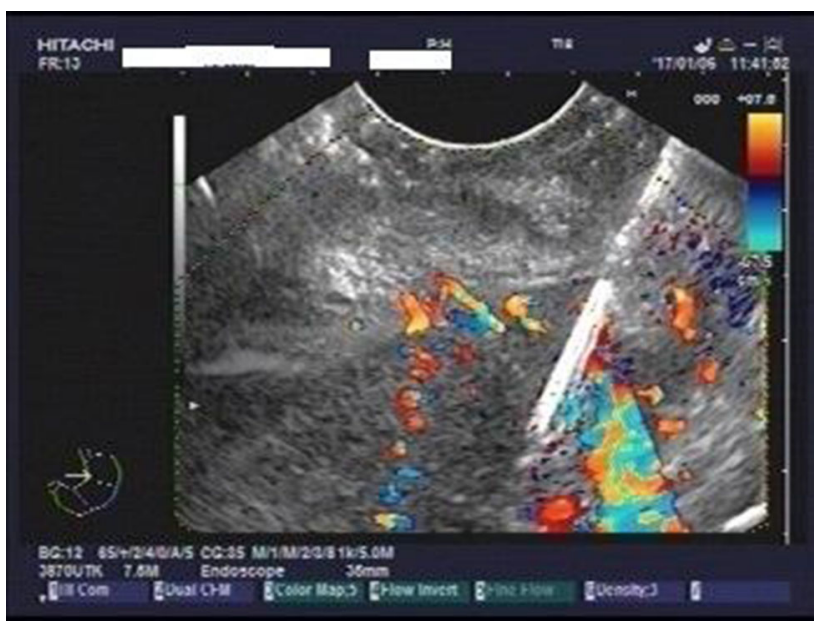

\section{CASE-7}

A rare case of hepatic squamous cell carcinoma diagnosed with endoscopic ultrasound guided fine needle aspiration

\section{Koki Yamada $^{1}$, Kaoru Kikuchi ${ }^{1}$}

${ }^{1}$ Okinawa Chubu Hospital, Japan

Background: Primary hepatic squamous cell carcinoma (SCC) is very rare disease of the liver. Also, this is first case report of hepatic squamous cell carcinoma diagnosed with endoscopic ultrasound guided fine needle aspiration (EUS-FNA).

Method: CASE Description.

A 52-year-old male who had a past medical history of decompensated liver cirrhosis secondary to chronic hepatitis B and alcohol abuse came to our emergency room with right upper quadrant abdominal pain and a $4 \mathrm{~kg}$ weight gain.

Result: Analysis of ascites showed white blood cell count increase and diagnosed as spontaneous bacterial peritonitis (SBP). Simultaneously, a computed tomography (CT) scan of the abdomen with contrast revealed approximately $8 \mathrm{~cm}$ hypodense mass in the left hepatic lobe with mild peripheral enhancement in delayed imaging. Given the ascites and coagulopathy secondary to liver cirrhosis, we provided EUS-FNA ( 22 Gauge, 3 passes) for diagnosis of liver mass.

Histopathologically, squamous cell carcinoma (SCC) was made. Finally, we diagnosed him as primary hepatic SCC. As laryngoscope, EGD and PET CT were all unremarkable except for the liver lesion and lymph nodes. Finally, the patient was diagnosed as Primary Hepatic SCC with systemic lymph node metastasis. As soon as we finished SBP treatment, we started CDDP +5 -FU with radiation therapy. He developed for liver and renal failure with underlining 3 cycles of chemotherapy. In the end, He passed away 134 days after his diagnosis of hepatic SCC.

Conclusion: Clinical course of Primary Hepatic SCC is aggressive and resectability is most important prognostic factor. EUS-FNA of liver mass is reliable, safe, feasible diagnostic modality especially for liver malignancies. 


\section{CASE-8}

\section{Allopurinol administered with azathioprine for autoimmune hepatitis treatment allows do decrease azathioprine dose - case report}

\section{Marek Woynarowski ${ }^{1}$ \\ ${ }^{1}$ Children's Health Memorial Institute, Poland}

Background: Azathioprine therapy should be monitored with 6-thioguanine blood concentration and azathioprine dose should be adjusted to maintain therapeutic values of blood 6-thioguanine concentration. Allopurinol alters azathioprine metabolism allowing to decrease the dose.

Method: This is a case report of patient treated with combination of azathioprine and allopurinol.

Result: Female patient HG with inborn cataract, ASD and recurrent syncopes presented liver dysfunction at the age of 6 years. The initial presentation of liver disease was fever, throat infection, hepatosplenomegaly, hypertransaminazemia (ALT $2100 \mathrm{U} / \mathrm{l}$ ) and hyperbilirubinemia $(3,9 \mathrm{mg} \%)$. Diagnostic work-up showed autoimmune hepatitis with high positivity for LKM (1:81820), increased concentration of $\operatorname{IgG}(2200 \mathrm{mg} / \mathrm{dl})$ and gamma-globulins $(25,7 \mathrm{~g} / \mathrm{l})$. Liver biopsy showed high inflammation and mild cirrhosis (G3/S1 according to Batts and Ludwig scale). Patient received standard prednisone and azathioprine therapy with rapid decrease of liver function tests and reduction of the therapy to maintenance doses of prednisone and azathioprine. Liver biopsy done two years after presentation showed marked improvement but not complete resolution of liver inflammation (G1/S2) thus patient continued maintenance therapy.

Three years after diagnosis liver function tests deteriorated. Patient had mild steroid related symptoms thus azathioprine dose was increased under control of blood 6-thioguanine level. Over the next 6 months the azathioprine dose was increased from $37,5 \mathrm{mg}(1,5 \mathrm{mg} /$ $\mathrm{kg})$ to $150 \mathrm{mg}(5,5 \mathrm{mg} / \mathrm{kg})$ but therapeutic concentration of 6-thioguanine in blood was not achieved and ALT activity remained slightly increased. Due to inadequate therapeutic effect we decided to modify therapy. The steroids was continued, azathioprine dose was lowered to $25 \mathrm{mg} / \mathrm{d}$ and allopurinol $(25 \mathrm{mg} / \mathrm{d})$ was added to increase azathioprine metabolites. This approach increased blood 6-thioguanine levels to therapeutic values however ALT was not normalized and ALT flares requiring increase of steroid doses were noted. Four years after diagnosis liver biopsy showed mild inflammation and fibrosis (G1/S1) and patient continues on deflazacort, low dose of azathioprine $(25 \mathrm{mg} / \mathrm{d})$ and allopurinol $(25 \mathrm{mg} / \mathrm{d})$.

Conclusion: The case presents that allopurinol can alter the azathioprine metabolism allowing for marked decrease of the azathioprine dose but it does not necessarily effect in normalization of ALT

\section{CASE-9}

\section{Autoimmune encephalopathy and autoimmune hepatitis - case} report

\section{Marek Woynarowski ${ }^{1}$}

${ }^{1}$ Children's Health Memorial Institute, Poland

Background: Autoimmune inflammation may have different clinical presentations. Coincidence of autoimmune hepatitis with thyroid disease, IBD diabetes mellitus or coeliac disease is well known. Method: We present the patient with coincidence of autoimmune encephalopathy and autoimmune hepatitis.
Result: Four years old girl was hospitalized due to neurological abnormalities (ataxia and myoclonic tremor of lower and upper extremities). EEG and brain MRI were normal, cerebrospinal fluid examination was normal. Cerebrospinal fluid and serum amino acids including dopamine and serotonin metabolites were normal. Patient was positive for ANA (1:640), ANCA, anti-neuronal (against cerebellum antigens) and anti Ma2/Ta antibodies typical for autoimmune encephalopathy. Patient received immunomodulatory synacthen-depot therapy with initial improvement of neurological symptoms but within the next three years patient experienced five episodes of encephalopathy relapse treated with intravenous methylprednisolone and immunoglobulins.

At the age of 7 years patient had increased aminotransferase activity. Viral liver disease, Wilson disease and alfa-1-antiprotease deficiency were excluded as the causes of liver disease. Patient had slightly increased $\mathrm{IgG}(1570 \mathrm{mg} / \mathrm{dl})$, and gamma globulins $(22,4 \mathrm{~g} / \mathrm{l})$. Patient was positive for ANA and SMA. Liver biopsy showed moderate lymphocytic infiltration (G3/S0 according to Batts and Ludwig classification). Liver problem was diagnosed as autoimmune hepatitis and patient received prednisone $1 \mathrm{mg} / \mathrm{kg}$ tapered down after 4 weeks to maintenance dose and azathioprine $1,5 \mathrm{mg} / \mathrm{kg}$. Liver function improved and within one year of the therapy no relapses of encephalopathy or liver function impairment were noted.

Conclusion: Patient presented in this case report had most probably the same etiology of neurological symptoms and liver inflammation. Both conditions can be successfully treated with standard immunosuppression.

\section{CASE-10}

Danis Ella Stent placement in the management of a lower end esophageal bleeding in a post liver transplant patient: a case report

\section{Chetan Kalal $^{1}$, Akash Shukla ${ }^{1}$, Mihir Vora ${ }^{1}$, Ravi Mohanka ${ }^{1}$, Amit Maydeo}

${ }^{1}$ Global Hospital, Mumbai, India

Background: 44 years old male, a chronic alcoholic since last 20 years was diagnosed with decompensated chronic liver disease one year back. He had frequent hospital admissions during last year in view ascites, haematemesis, encephalopathy and hepato-renal syndrome respectively. He was evaluated for living donor liver transplantation after 6 months of abstinence. His MELD at the time of admission was 22 and Child score of 11. After thorough donor work up he underwent LDLT.

Method: Right lobe graft without MHV was taken. Graft weight was 665 grams with GRWR of 1.2. Post operative course was complicated by high ascitic fluid drainage, sepsis related acute renal failure. Post operatively (day 5) patient had malena and fall in hemoglobin $(1.5 \mathrm{gm} \%)$. He required blood and blood products transfusions and inotropic support in view of hemodynamic instability. Antimicrobials were escalated empirically. Sepsis screen was sent.

Result: Upper GI endoscopy revealed diffuse mucosal oozing from the lower end of esophagus along with small isolated gastric varices. Glue injection was done, however patient continued to bleed despite supportive measures. Repeat UGI scopy was done, which showed continued generalized ooze from lower end of esophagus. Finally endoscopic guided Ella Danis stent was placed with upper end at $25 \mathrm{~cm}$ from GE junction. The patient was stabilized and moved to the intensive care unit. There was no subsequent evidence of upper GI bleed, however he continued to deteriorate gradually due to pulmonary sepsis and eventually succumbed on POD 22. 
In summary, we report the first ever use of Danis Ella stent for diffuse mucosal bleeding from lower esophagus in the setting of post liver transplant. This stent can be used a bridge to stabilize the patient prior to definitive treatment.

Conclusion: Further well executed randomized trials are needed to confirm the safety and efficacy of this approach in setting of post liver transplant.

\section{CASE-11}

Extrahepatic portal venous obstruction with severe celiac artery stenosis as a presentation of celiac disease - an unheard entity

\section{Apurva Shah ${ }^{1}$ \\ ${ }^{1}$ Apollo Hospitals International Limited, Ahmedabad, India}

Background: A newly explored area of celiac disease is hypercoagulability and the resulting thromboembolic phenomena. We report first case to our knowledge of celiac disease presenting as extrahepatic portal venous obstruction and superior mesenteric vein thrombosis with severe celiac artery stenosis, managed successfully with arterial stenting.

Method: A 26 year old female presented with severe persistent upper abdominal pain worse after eating, anorexia, vomiting since last one month and chronic diarrhoea, weight loss, severe growth retardation since childhood. Amenorrhoea and chronic anaemia was significant past history. Physical examination revealed severe growth retardation (BMI- $15.92 \mathrm{~kg} / \mathrm{m}^{2}$ ), pallor, icterus and soft mildly tender abdomen. Investigations revealed iron deficiency anemia, increased bilirubin (3 mg/dl), SGOT (63U/L), CRP, prothrombin time (PT), LDH.

Result: Viral markers (B and C) negative, autoimmune liver profile was negative with normal IgG. Ultrasound revealed mild splenomegaly, mildly altered echo texture of liver, portal and SMV thrombosis and changes of portal biliopathy. Thrombophilia work up showed mildly reduced Protein C, Protein S, antithrombin III. Gastroscopy revealed Grade II esophageal varices, flat duodenal fold. Diagnosis of celiac disease was made based on clinical features, blood parameters, positive serology (anti endomysial antibody), D2 biopsy report of total villus atrophy. CECT abdomen showed 70\% stenosis of celiac artery trunk, SMV and portal vein thrombosis, changes of portal biliopathy with mild ascites. No relief of abdominal pain even after gluten free diet and anticoagulation with intravenous heparin for a week. Abdominal aortography revealed ostial $80 \%$ stenosis of celiac artery, same treated with PTA, abdominal pain subsided within days. She was kept on gluten free diet, antiplatelet and oral anticoagulation on discharge.

Conclusion: She required endoscopic variceal ligation for primary prophylaxis of esophageal varices, excellent improvement seen in form of no abdominal pain, weight gain and normalization of haemoglobin, PT, LFT at one year follow up.

\section{CASE-12}

\section{Multiple malignancies, hepatocellular carcinoma and multiple myeloma: a case report}

Kemal Fariz Kalista ${ }^{1}$, Rino Alvani Gani ${ }^{1}$, Irsan Hasan ${ }^{1}$, Andri Sanityoso $^{1}$, Cosmas Rinaldi Lesmana ${ }^{1}$, Juferdy Kurniawan ${ }^{1}$, Chyntia Olivia Jasirwan ${ }^{1}$, Saut Horas Nababan ${ }^{1}$, Rahmat Cahyanur $^{1}$, Alvina Widhani ${ }^{1}$, Gurmeet Singh ${ }^{1}$, Sahat Matondang ${ }^{1}$, Ening Krisnuhoni ${ }^{1}$, Marini Stephanie ${ }^{1}$
${ }^{1}$ Cipto Mangunkusumo National Hospital, Faculty of Medicine Universitas Indonesia, Indonesia

Background: Hepatocellular carcinoma (HCC) still remain a big burden in Indonesia. Multiple malignancies in patient with $\mathrm{HCC}$ is extremely rare case.

Method: Case Report.

Result: We present 62 year old woman, with HCC, multiple myeloma (MM) and diabetes mellitus type-2. Patient came with abdominal discomfort. We performed imaging study and we found no cirrhotic liver and we found huge mass in liver segment $6 \& 7$ with atypical pattern enhancement. Laboratory findings: hepatitis marker for B \& C were negative, AFP $22 \mathrm{IU} / \mathrm{mL}$. This patient had no cirrhosis but she had low level albumin $(2.5 \mathrm{~g} / \mathrm{dL})$ and high level globulin $(6.2 \mathrm{~g} / \mathrm{dL})$. We elaborate further about hyperglobulinemia so we checked Ig G level, protein electrophoresis and immuno fixation. We found high level Ig G $3390 \mathrm{mg} / \mathrm{dL}$. Protein electrophoresis revealed elevation beta- 2 fraction and gamma globulin with monoclonal pattern. Immuno fixation revealed Ig G monoclonality. BMP was also done and revealed elevated plasmocyte. All of this result lead us to multiple myeloma. Because of no viral hepatitis, low AFP and no typical pattern enhancement in abdominal CT, we did liver nodule biopsy and nodule histology suit with hepatocellular carcinoma.

Conclusion: Multiple malignancies, especially HCC and MM, is very rare case. We report here HCC case with very unusual feature. We have to concern and elaborate further about this unusual feature and we can found unusual and rare diagnosis.

\section{CASE-13}

Spontaneous rupture of hepatocellular carcinoma under ledipasvir-sofosbuvir treatment: a case report

\section{Bulent Colak ${ }^{1}$}

${ }^{1}$ NONE, Turkey

Background: Affecting nearly $130-170$ million persons throughout the world, hepatitis $\mathrm{C}$ virus (HCV) is the leading cause of liver-related mortality and liver transplantation. The combination therapy of ledipasvir/sofosbuvir provides several advantages compared to other regimens, including use of a single-pill regimen, efficacy in patients exposed to protease inhibitors, safety in decompensated cirrhosis, and potential to avoid ribavirin.

Method: Case report.

Result: Case: A 62-years old male patient admitted to the hospital with abdominal distention and pruritus. Laboratory analyses revealed leucocyte $5170 / \mathrm{mm}^{3}$, hemoglobin $12.45 \mathrm{gr} / \mathrm{dL}$, platelet $57000 / \mathrm{mm}^{3}$, total bilirubin $3.35 \mathrm{mg} / \mathrm{dL}$, direct bilirubin $1.6 \mathrm{gr} / \mathrm{dL}$, indirect bilirubin $1.75 \mathrm{mg} / \mathrm{dL}$, alanine aminotransferase (ALT) $102 \mathrm{IU} / \mathrm{L}$, aspartate aminotransferase (AST) $154 \mathrm{IU} / \mathrm{L}$, creatinine $0.78 \mathrm{mg} / \mathrm{dL}$, albumin $2.1 \mathrm{gr} / \mathrm{dL}$, protrombin time $18.4 \mathrm{sec}$, INR 1.55 , alpha-fetoprotein $27.7 \mathrm{ng} / \mathrm{mL}$. Anti-HCV was also positive in his test. HCV RNA level was $77763 \mathrm{IU} / \mathrm{mL}$ and genotype was 1b Ultrasound showed liver parenchyma was coarse granular, $74 \times 58 \mathrm{~mm}$ isoechoic heterogenous solid lesion in right lobe anterior segment which is adjacent to vena cava inferior, spleen is $190 \mathrm{~mm}$ and ascites in all quadrant. Contrast enhanced computed tomography (CT) revealed hepatocellular carcinoma with hepatocellular carcinoma (HCC) wash-out characteristics. Ledipasvir $90 \mathrm{mg}$ and Sofosbuvir $400 \mathrm{mg}$ combination a tablet per day as antiviral treatment was 2 started. Weakness, fatigue and increase in abdominal bloating were marked in 21th day of treatment. Hemoglobin $8.9 \mathrm{gr} / \mathrm{dL}$, platelet $76000 / \mathrm{mm}^{3}$, AST $176 \mathrm{IU} / \mathrm{L}$, ALT $146 \mathrm{IU} / \mathrm{L}$, serum albumin $2 \mathrm{gr} / \mathrm{dL}$, protrombin time $40 \mathrm{sec}$, INR 1.56, creatinine $1.37 \mathrm{mg} / \mathrm{dL}$, total bilirubin $3.56 \mathrm{mg} / \mathrm{dL}$ and HCV RNA was 
negative in laboratory test. There was no bleeding in esophagogastroduodenoscopy. There was hemorrhagic ascites in paracentesis. Hemoglobin 4,1 gr/dl, hemotocrite 14.1 in ascitic fluid analysis. HCC rupture was revealed in contrast enhanced abdominal CT. Despite blood transfusion, hypotension was marked and patient was exitus.

Conclusion: Underlying cause of the rupture of HCC as spontaneous or as a complication of treatment was not understood. Ledipasvir and Sofosbuvir combination should be followed closely for detection of anti viral treatment complication.

\section{CASE-14}

Eosinophilic liver abscess resolved with albendazole treatment: a case report

Cosmas Rinaldi Adithya Lesmana ${ }^{1}$, Agnita Irawaty ${ }^{1}$, Sri Inggriani $^{1}$, Ening Krisnuhoni ${ }^{1}$, Ibrahim Basir ${ }^{1}$, Heru Sundaru ${ }^{1}$, Cosmas Rinaldi Adithya Lesmana ${ }^{1}$, Laurentius Adrianto Lesmana $^{1}$

${ }^{1}$ Medistra Hospital, Indonesia

Background: Eosinophilic liver abscess (ELA) is an eosinophilic infiltration and necrosis of the liver accompanied by peripheral eosinophilia. It is associated with parasitic infestation, allergic disease or hypereosinophilic syndrome involving the liver. However, the etiology cannot be identified in many cases. We report a difficult case of ELA without identified etiology who responded to anthelmintic treatment.

Method: A Case Report.

Result: A 45-year old woman was complaining abdominal pain and prolonged fever for 2 months. She also lost $5 \mathrm{~kg}$ of body weight in the last 3 months. No history of any allergic disease. On physical examination, she was febrile and there is a tenderness at the epigastric and right upper quadrant regions. The initial laboratory test showed marked eosinophilia (33\%) with a slight leukocytosis $(10,830$ per $\mathrm{mm}^{3}$ ) corresponded to a total eosinophil count of $5,429 / \mu \mathrm{L}$. Total IgE level was high $(658.9 \mathrm{kIU} / \mathrm{L})$. Liver enzymes only showed mild elevation of gamma glutamyl transferase (48 U/L) and alkaline phosphatase (193 U/L). Abdominal ultrasound showed multiple solid nodules with cystic parts in the right and left lobes of the liver. Magnetic resonance imaging showed non-specific coalescing multiple cysts in the liver. Diagnostic laparoscopy revealed an abscess with extensive tissue necrosis. Pathology assessment found heavy eosinophilic infiltration with Charcot-Leyden crystal. Immunohistochemistry staining was negative for parasitic, fungal, or tuberculous infection. Toxocara antibody was also negative. The diagnosis was eosinophilic liver abscess suggestive of visceral larva migrants. The patient was treated with albendazole $400 \mathrm{mg}$ daily and corticosteroid. Clinical improvement was shown after 6 months therapy. Eosinophilia was reduced and resolution of the lesions was observed on imaging evaluation.

Conclusion: Eosinophilic liver abscess is rarely found in Indonesia. Clinical manifestation and liver imaging are not specific; peripheral eosinophilia may be the only symptom. Eosinophilic abscess with Charcot-Leyden crystals is important to rule out malignant or metastatic disease. Long-term anthelminthic treatment should be tried and may confirm nematode infestation (visceral larva migrants) if clinical improvement occurs.
CASE-15

A rare case of $\mathbf{4 4}$ years old female with local hepatic tuberculosis

\section{$\underline{\text { Syifa Mustika }}^{1}$}

${ }^{1}$ Faculty of Medicine Brawijaya University, Indonesia

Background: Mycobacterium tuberculosis (TB) infection of the liver, known as hepatic tuberculosis, is a rare extrapulmonary manifestation of an active TB infection, with incidence less than $1 \%$ from all TB infection. Hepatic tuberculosis has become more prevalent, especially in human immunodeficiency virus (HIV) patient. Hepatic tuberculosis is uncommon, lack of specific clinical manifestations and imaging features, so it can easily be misdiagnosed in clinical.

Method: We reported a 44 years old woman with chief complaint right upper quadrant abdominal pain. She was diagnosed with HIV infection 5 months earlier. Physical examination suggest a hepatomegaly and tenderness in right upper quadrant abdominal area. Laboratory result show anemia, leucopenia, limfopenia and low level of CD4. The result of ICT TB, AFB SMS sputum test and Xpert MTB/Rif are negative, and thorax X-ray is normal.

Result: Abdominal ultrasonography findings was a multiple hypoechoic lesion liver segment VI. Patient underwent image-guided fine needle aspiration biopsy with result confirmed hepatic tuberculosis. Patient diagnosed as hepatic tuberculosis and treated with first-line antituberculosis drugs for 9 months with good therapeutic response. Conclusion: We reported a case which clinical features of hepatic tuberculosis are nonspecific. Radiologic imaging and fine needle aspiration biopsy is the best diagnostic tool for hepatic tuberculosis. Anti-TB treatment is giving good results.

\section{CASE-16}

A rare presentation of spontaneous iliopsoas hematoma in a patient with liver cirrhosis

\section{$\underline{\text { Abhimanrao Manikrao Pawar }}{ }^{1}$, N. Murugan ${ }^{1}$}

${ }^{1}$ Apollo Hospitals, Chennai, India

Background: An iliopsoas hematoma can occur either spontaneously or secondary to trauma or bleeding tendency due to hemophilia and anticoagulant therapy. Although liver cirrhosis is commonly associated with coagulopathy, iliopsoas hematoma is very rare, but in view of high mortality we should cautiously diagnose it and treat it.

Method: We herein present a case of a 52 years old diabetic man with alcoholic cirrhosis Child class $\mathrm{C}$, who was admitted with features of hepatic encephalopathy and severe pain in left groin and inability to straighten his left leg since 2 weeks. Clinically he was drowsy, disoriented and had asterixis. His heart rate was 94/min; BP was $110 / 70 \mathrm{~mm} \mathrm{Hg}$, temperature of $98.60 \mathrm{~F}$. He had no tenderness with palpation of the abdomen and groin and bowel sounds were active in all quadrants but the abdomen was much distended. His left thigh was in flexed posture and he was wincing on attempting to straighten his left thigh. There was no history of trauma or antiplatelet or anticoagulant ingestion. He didn't had history of hematemesis or malena. $\mathrm{His} \mathrm{Hb}$ was $9 \mathrm{gm} / \mathrm{dl}$, white cell count of $6800 / \mathrm{mm}^{3}$ with neutrophils $65 \%$, platelet count $1,20,000 / \mathrm{mm}^{3}$, INR 2.2 Initially he was treated with lactulose orally as well as with enema along with Rifaximin $550 \mathrm{mg}$ BD. His encephalopathy got recovered within 2 days. He was evaluated by ultrasound of groin which was normal \& MRI spine done to rule out radicular pain was normal. MRI pelvis however showed presence of left iliopsoas collection? Abscess or hematoma $(4.4 \times 4.9 \times 9 \mathrm{cms} \sim 90 \mathrm{cc})$. 
Result: In view of deranged prothrombin time, he was transfused 4 Fresh frozen plasma followed by a CT guided aspiration of the same was carried out and it turned out to be a hematoma which was formed spontaneously. There were no intra or post procedure complications. Post procedure his pain was improved significantly and he was ambulatory on the 2 nd post procedure day. His upper GI endoscopy revealed large oesophageal varices for which endoscopic variceal ligation were done. He was then discharged in a stable condition.

Conclusion: This case illustrates the rarely suspected cause of leg pain in cirrhosis and emphasizes that physician should have a high degree of suspicion for spontaneous iliopsoas hematoma and its treatment.

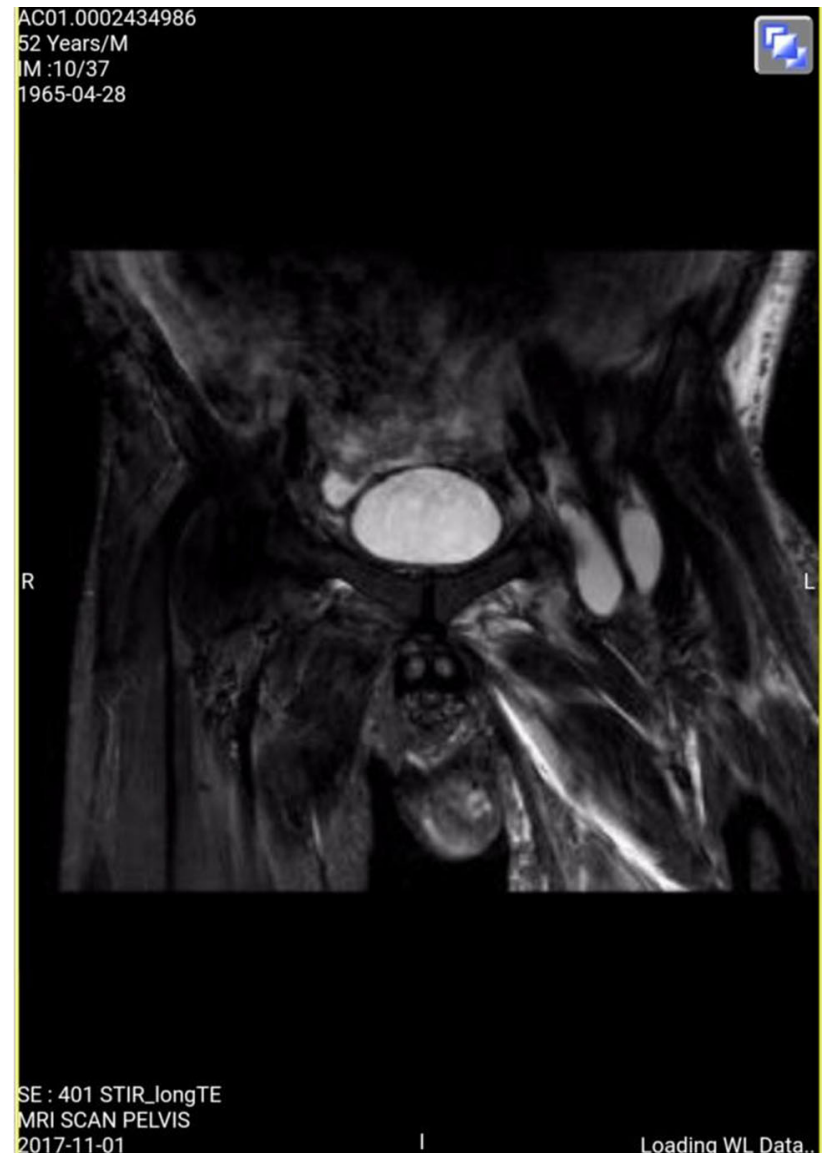

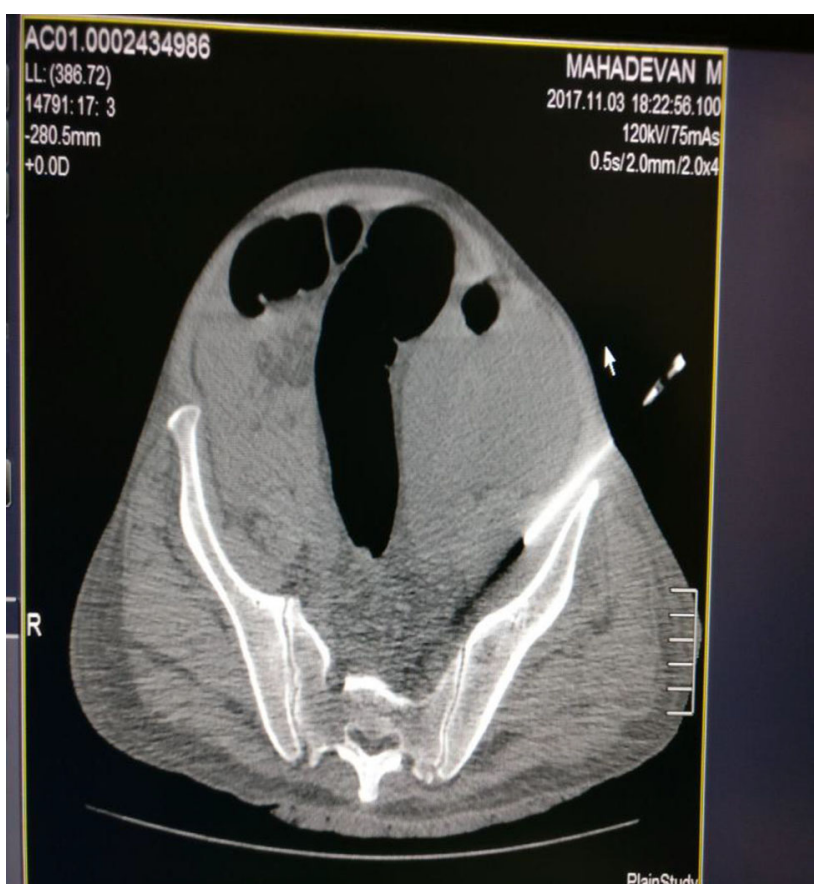

CASE-17

Chronic hepatitis B reactivation subsequent to chronic hepatitis $\mathbf{C}$ treatment: a case report

\section{Habip Gedik}

${ }^{1}$ Ministry of Health Bakirköy Sadi Konuk Training and Research Hospital, Turkey

Background: $\mathrm{CHB}$ and $\mathrm{CHC}$ infections are the important global health problems. Because of their shared modes of transmission, coinfection with HBV and HCV is frequent. Patients who are coinfected have a higher risk of progression to severe liver disease (cirrhosis, decompensated liver disease), increased risk of hepatocellular carcinoma, and unresponsiveness to treatment. In this article, a case with reactivated $\mathrm{CHB}$ subsequent to $\mathrm{CHC}$ treatment is presented.

Method: A 42-year-old woman with HBV and HCV was admitted to infectious diseases clinic with a major complaint of fatigue for three months. There was no sense of the patient's history and family history. There was no smoking, alcohol, and any drug in the history. Her physical examination and vital signs were normal at the admission. The patient had no history of drug hypersensitivity, liver diseases, blood transfusion, or surgery. She refuted to use an over-the-counter and traditional Chinese medicine. Other virological and autoimmunity markers were negative. Finding were normal by USG.

Result: Laboratory tests revealed an alanine transaminase (ALT) of 182 IU/L (Normal range: 0-55 IU/L), aspartate transaminase (AST) of $150 \mathrm{IU} / \mathrm{L}$ (Normal range: 0-34 IU/L), HCV-RNA of $2080195 \mathrm{IU} /$ $\mathrm{mL}, \mathrm{HCV}$ genotype $1 \mathrm{~b}$, Hepatitis B surface antigen (HBs Ag), HBVDNA of $1184 \mathrm{IU} / \mathrm{mL}$, whereas the results of other tests were within the normal range. Pegylated interferon (Peg-IFN) alpha-2a $180 \mathrm{mcg} /$ week and ribavirin (RBV) tablet $1200 \mathrm{mg} /$ day were initiated in April 2015 for $\mathrm{CHC}$ treatment. At the third and sixth months of $\mathrm{CHC}$ treatment, the liver enzymes were slightly elevated and HCV-RNA negative. HCV-RNA remained to be negative in the first year after the treatment of $\mathrm{CHC}$. Patients presented with fatigue once again and elevated liver enzymes to our department after one year of $\mathrm{CHC}$ 
treatment. A liver biopsy was performed according to the health insurance guideline of Turkey in order to receive CHB treatment due to a HBV-DNA of $878215 \mathrm{IU} / \mathrm{ml}$ and elevated liver enzymes. The histopathological examination of liver tissue revealed a mildly active histologic activity (6/18) and a mildly active histological activity (1/6) according to ISHAK scoring. Tenofovir tablet $245 \mathrm{mg} / \mathrm{day}$ was administered for the CHB treatment. The patient is still followed up without any complaint at the ambulatory clinic of infectious diseases. Reactivation of HBV is thought to be a result of an immunological response to infected hepatocytes that replicate $\mathrm{HBV}$ after the restoration of both innate (restoration of NK cell phenotype and function) and adaptive (improvement in $\mathrm{CD} 8+\mathrm{T}$ cell function) immunological mechanisms with interferon treatment against $\mathrm{CHC}$. Spontaneous fluctuations in the HBV-DNA levels are common in patients with $\mathrm{HBs}$ Ag-positive. Maylin and colleagues reported to occur the spontaneous fluctuations $(>0.5 \log 10)$ in $63 \%$ of 87 patients with HBs Ag positive, normal ALT and AST. Papatheodoridis and colleagues reported that almost $50 \%$ of patients with CHB followed during a median period of time of 7.5 months (HbeAg negative) had $>1 \log 10 \mathrm{IU} / \mathrm{mL}$ fluctuations in the HBV-DNA levels. The probable risk of liver failure due to $\mathrm{HBV}$ reactivation, although slight, urges us to screen all patients receiving hepatitis $\mathrm{C}$ treatment for the presence of HBs Ag.

Conclusion: As a result, patients coinfected with $\mathrm{HBV}$ and $\mathrm{HCV}$ should be followed up for the cirrhosis and hepatocellular carcinoma after $\mathrm{CHC}$ treatment in terms of $\mathrm{HBV}$ reactivation, even though they have undetectable viral load for HBV and HCV.

Table: Laboratory findings of the patient

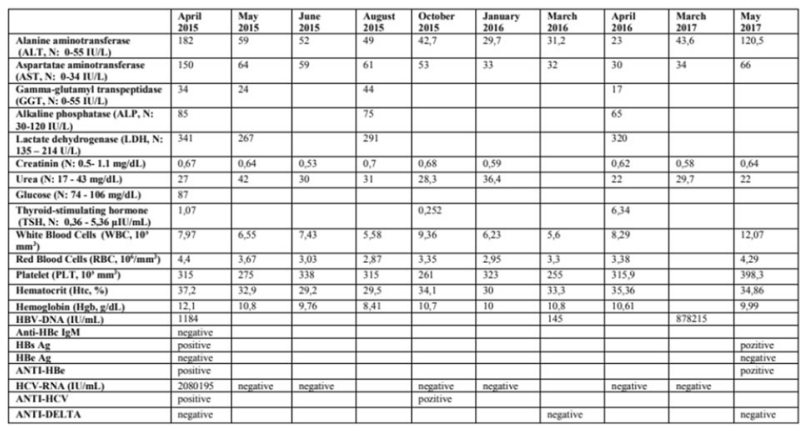

CASE-18

Congenital hypopituitarism: underdiagnosed and missed cause of neonatal cholestasis - a case series

\section{$\underline{\text { Karunesh Kumar }}^{1}$, Akshay Kapoor ${ }^{1}$, Inderpal Singh Kochar ${ }^{1}$ \\ ${ }^{1}$ Apollo Hospital, India}

Background: Congenital panhypopituitarism is a rare and usually missed but important cause of cholestasis because it is treatable with hormone therapy. Many case series have described different common manifestations of panhypopituitarism like jaundice, development delay but data is scarce. Panhypopituitarism presenting as neonatal cholestasis along with associated chronic eczematous dermatitis and alopecia so far has not been reported in literature.

Method: Observational and descriptive case series at our center.

\section{Result:}

Case 1: This child was 5 months old preterm, low birth weight who developed jaundice on Day 3. His jaundice progressed and he developed acholic stools and skin lesions in the second week of life. Alopecia total is was observed along with indurated, erythematous, eczematous, plaques over limbs, face and scalp with excoriation. On examination he had hepatosplenomegaly and ascites. Initial investigations and liver biopsy was inconclusive for etiology. His Cortisol was and TSH was high $9.85 \mathrm{Uu} / \mathrm{ML}$. Hence, MRI brain was done which showed small sized pituitary for age. Subsequently his growth hormone stimulation test showed severe growth hormone deficiency. Based on these investigations diagnosis of hypothyroidism with hypopituitarism was made and started on appropriate treatment.

Case 2: 17 months old male developed jaundice on day 3 of life. In 4th month of age child developed abdominal distension which was progressive. At 1 year of age child started losing hairs over scalp and face along with skin changes in form of dry, rough and thick skin. For these complaints child was evaluated. He also had pallor and icterus. Alopecia total is was observed along with eczematous skin with excoriation. On examination child had hepatomegaly and ascites. His development was delayed in all domains. Initial hormonal evaluation showed normal Cortisol $23.22 \mu \mathrm{g} / \mathrm{dL}$ and high TSH of $30.73 \mathrm{Uu} / \mathrm{ML}$ with negative anti-TPO. Subsequently his growth hormone stimulation test showed severe growth hormone deficiency with IGF-1 $<25 \mathrm{ng} / \mathrm{ml}$ and IGF BP3 $0.6 \mathrm{mcg} / \mathrm{ml}$. Based on these investigations diagnosis of hypothyroidism with hypopituitarism was made and treated.

Case 3: This was a 2 months old male, borderline preterm at 36 weeks and of low birth weight. He remained well till one month of age when he was admitted with lethargy followed by seizures (status) and encephalopathy. Jaundice was also noted at the same time. Child was evaluated to rule out sepsis and common metabolic conditions which can cause seizure and cholestasis. Ultrasound of the abdomen was suggestive of obstructive etiology but biopsy had features of bile duct paucity. HIDA scan was non-excretory. His Cortisol was $0.5 \mu \mathrm{g} /$ $\mathrm{dL}$ and TSH was high (14.23 Uu/ML). Subsequent tests showed low ACTH level with low IGF-1. Based on these investigations diagnosis of hypothyroidism with hypopituitarism was made.

Conclusion: Congenital hypopituitarism may be caused by one of a number of genetic disorders or be part of a developmental defect involving the hypothalamus and/or pituitary gland. Very rarely it can present as cholestatic jaundice in the neonatal period. High index of suspicion is required to diagnose hypopituitarism as a cause of neonatal cholestasis. Early intervention can dramatically change the outcome as it is treatable by adequate hormone supplementation.

\section{CASE-19}

Lymphomatous involvement of the liver in a persistently septic elderly patient: a case report on a therapeutic dilemma

\section{Jose Guillain Esteban Cataluna ${ }^{1}$}

\section{${ }^{1}$ St. Luke Medical Center Quezon City, Philippines}

Background: Hepatic lymphoma is a rare disease condition. In particular, secondary lymphomatous involvement of the liver is associated with disseminated disease and can present as a diagnostic dilemma. In an elderly patient, therapeutic dilemma can also ensue due to multiple comorbidities, sepsis, and advanced age itself.

Method: We report a case of a 72-year old woman who presented with a five-month history of generalized weakness and persistent fever.

\section{Result:}

Management: Abdominal imaging (ultrasound and CT scan) detected a hepatic mass. A diagnosis of diffuse large B-cell lymphoma was made using a percutaneous liver biopsy. Further diagnostic testing with a PET scan showed involvement of abdominal lymph nodes. The patient eventually expired due to multiple organ failure secondary to 
septic shock. Here, we review the treatment options for lymphomatous involvement of the liver in advanced lymphoma.

\section{Conclusion:}

Recommendation: Chemotherapy with rituximab, cyclophosphamide, doxorubicin, vincristine, and prednisone (R-CHOP) remains as the choice of treatment for disseminated disease. While advanced age has been found to be a poor prognostic factor, it is not considered as a contraindication to treatment. This case demonstrates that hepatic lymphoma should be considered in the differential diagnosis of spaceoccupying liver lesions in an elderly non-cirrhotic liver with normal levels of alpha-fetoprotein and carcinoembryogenic antigen and with no pre-existing chronic liver disease or significant findings relating to an infectious process.

\section{CASE-20}

\section{Terlipressin induced neuropathy: a case report}

Vandana Saluja ${ }^{1}$, Lalita Gouri Mitra ${ }^{1}$, Prashant Agarwal ${ }^{1}$, Ashok Choudhury $^{1}$, Satyendra Kumar ${ }^{1}$

${ }^{1}$ Institute of liver and biliary sciences, India

\section{Background:}

Introduction: Terlipressin is used as therapy in patients with hepatorenal syndrome and variceal bleed. It is also known for its vascular complications.

In spite of use with caution, complications like limb ischemia, gangrene, and osteomyelitis have been reported.

\section{Method:}

Introduction: Terlipressin is used as therapy in patients with hepatorenal syndrome and variceal bleed. It is also known for its vascular complications.

In spite of use with caution, complications like limb ischemia, gangrene, and osteomyelitis have been reported.

\section{Result:}

Case Description: A 37-year-old male with acute on chronic liver failure was admitted due jaundice, distension of abdomen. He had swelling of the right upper extremity secondary to thrombophlebitis. He was started on Injection Terlipressin infusion on day 3 for hepatorenal syndrome. 24 hours after starting of infusion, he developed cyanosis of finger tips of both the upper limbs. Terlipressin infusion was discontinued.

Although the cyanosis recovered, he complained of weakness of right upper limb associated with sensory loss.

Nerve conduction studies revealed decreased amplitude and velocity of sensory nerve conduction and absent motor conduction. He developed wrist drop along with sensory loss in the right upper limb. He received an upper limb splint along with regular physiotherapy. However there was a poor recovery of neurological deficit. He left against medical advice 4 days later.

Peripheral cyanosis, gangrene and osteomyelitis have been reported with terlipressin. Our case report is the first to describe nerve injury secondary to use of terlipressin.

Terlipressin is a vasopressin analogue that is converted to lysine vasopressin. Vasopressin has been shown to constrict the vasa nervorum in the peripheral nerves in animal models. Ischemic events secondary to terlipressin therapy are seen in the presence of risk factors like hypovolemia, concomitantly administered pressor drugs. Both symmetric and asymmetric involvement of extremities has been reported.

In our case we believe the anoxia of peripheral nerves due to constriction of vasa nervorum contributed to the neuropathy. Pre existing swelling of the upper limb probably caused a compartment like syndrome and predisposed him to prolonged ischemia of the right upper limb.

Vasodilators like alprostadil, sildenafil and nitrates have been tried. But use in ischemic neuropathy is controversial.

Conclusion: Despite its rarity, ischemic neuropathy is a serious complication due to terlipressin, and special attention should be paid in high risk individuals.

\section{CASE-21}

Upper gastrointestinal bleed- a rare delayed manifestation of hepatic artery pseudo aneurysm post trans jugular liver biopsy: a case report and review of literature

Saurabh Gupta ${ }^{1}$, Anubhav Khandelwal ${ }^{1}$, Azimudin Haja ${ }^{1}$, Sanjiv Saigal $^{1}$, Sanjay Saran Baijal ${ }^{1}$

${ }^{1}$ Medanta-The Medicity, India

Background: A CASE REPORT AND REVIEW OF LITERATURE. Method: A CASE REPORT AND REVIEW OF LITERATURE.

Result: Introduction: Trans jugular liver biopsy (TJLB) is a method to obtain liver tissue for histopathological diagnosis of acute or chronic liver disease, without injuring the liver capsule, in patients with contraindications to the percutaneous liver biopsy. It is generally performed in setting of ascites, coagulopathy, severe fatty liver or peliosis hepatis. The procedure is not free from complications, however major complications are rare. Overall rate of major complications of TJLB in English literature ranges between 0 to $2.8 \%$, with only a few case reports of post procedure hepatic artery injury or pseudo aneurysm. Hepatic artery injury can present as intra parenchymal hematoma, subcapsular hematoma, intraperitoneal bleed/ hemoperitoneum or hemobilia. Only two case reports are published in the PubMed literature where hepatic artery pseudo aneurysm after TJLB has presented as upper gastrointestinal (GI) bleed in form of hemobilia (both within a week of procedure) while similar cases were because of arteriobiliary fistula ( 2 cases) or gall bladder rupture ( 2 cases) or without radiological abnormality in patients with coagulopathies (3 cases).

Case Description: We present a case of post TJLB hepatic artery pseudo aneurysm that presented clinically after 10 days as upper GI bleed and was endoscopically diagnosed as hemobilia. Subsequent CT angiography revealed a segmental hepatic artery pseudo aneurysm with hemobilia and dilated biliary radicals. It was successfully embolized using metallic coils and glue with improvement in the symptomatology of the patient, who however had a prolonged hospital course because of other comorbidities.

Conclusion: A Case report and review of literature.

\section{CASE-22}

A 24-year old female with indeterminate hyperacute liver failure: a case report

\section{Maria Elizabeth Capuno Ching ${ }^{1}$}

${ }^{1}$ St. Luke's Medical Center-Global City, Philippines

Background: ALF refers to sudden massive hepatic necrosis with encephalopathy and impaired synthetic function without pre-existing cirrhosis. A previously healthy 24 -year old female with a history of lacrimal gland tumor on chronic oral prednisone $(40 \mathrm{mg})$ for a year was admitted for acute decreased sensorium, generalized jaundice, tea-colored urine, anorexia and undocumented fever. 
Method: Laboratory findings showed hyperbilirubinemia, transaminitis (ALT 5507, AST 3549), elevated alkaline phosphatase, impaired coagulation hyperammonemia and normal platelets. Extensive work-up including hepatitis panel, paracetamol, methamphetamine, cannabinoids, benzodiazepine, barbiturates, cocaine, opiates, phencyclidine, cytomegalovirus IgM, EBV, HSV1, HSV2, C3, anti-Sm and anti-mitochondrial antibody, LKM1, ceruloplasmin, strepA throat screen test, malarial smear and leptospiral IgM were all unremarkable.

Result: Whole abdominal ultrasound revealed unremarkable liver, biliary tree and pancreas, with splenomegaly $13 \times 14.5 \mathrm{~cm}$. Medical and supportive treatment were promptly provided. Orthotopic liver transplantation (OLT) was contemplated, however, cerebral edema and hemorrhage ensued on Day 5 leading to demise.

Etiology varies widely among toxic, viral, metabolic and vascular insults. Steroids are known to be safe as far as hepatotoxicity is concerned. It is in fact the treatment of choice for autoimmune hepatitis. Our patient has been on chronic prednisone ( $40 \mathrm{mg}$ once daily) for a year as anti-inflammatory adjunct for lacrimal gland tumor There have been some rare reported cases of severe liver injury with repeated steroid administration, in turn leading to poor outcome. Such injury is categorized under drug induced liver injury (DILI), speculated to be an idiosyncratic reaction to steroids and their metabolites, or to compounds used as preservatives. Literature search revealed 15 case reports and two case series of methylprednisolone-induced DILI reported between 2005-2015. Most reported cases showed female predilection, with median time of presentation of DILI of 4 weeks (range: 1-10 weeks), median dose of $3.5 \mathrm{~g}$ methylprednisolone ( $4.3 \mathrm{mg}$ prednisone equivalent).

Management consisting of intensive care should be initiated depending on the etiology and chronicity of ALF. OLT has emerged as the only therapeutic intervention with proven benefit for patients with advanced ALF.

Conclusion: We documented a case of a 24-year old patient with no preexisting liver disease presenting as indeterminate hyper-acute liver failure. Chronic steroids which might have precipitated the said condition, supported by few case reports.

\section{CASE-23}

Tuberculous liver abscesses as the initial presentation of disseminated tuberculosis in an immunocompetent patient: a case report

\section{Mariel Dianne Soloria Velasco ${ }^{1}$, Felix Zano ${ }^{1}$, Antonio Comia ${ }^{1}$ \\ ${ }^{1}$ University of the Philippines - Philippine General Hospital, Philippines}

\section{Background:}

Introduction: A tuberculous liver abscess (TLA) is considered a rare manifestation of extrapulmonary tuberculosis, even as a component of disseminated infection.

\section{Method:}

Case Presentation: We present a case of a 36-year old Filipino male admitted for a three-month history of abdominal pain, fever and weight loss. He did not present with any respiratory symptoms. Physical examination did not reveal jaundice nor palpable lymphadenopathies. The abdomen was flat, with normoactive bowel sounds, soft, with note of a vague epigastric fullness with direct tenderness. Human immunodeficiency virus (HIV) screening was negative. Ultrasound revealed a $9 \mathrm{~cm} \times 9 \mathrm{~cm} \times 9 \mathrm{~cm}$ complex left hepatic lobe mass. Aspiration yielded $350 \mathrm{ml}$ of reddish-brown turbid fluid. Gram stain, acid-fast stains and cultures were all negative, but TB-PCR was positive. CT scan also revealed an incidental finding of an ill-defined heterogeneously enhancing mass with central calcification in the posterobasal segment of the left lower lobe and multiple nodular densities throughout both lungs. Lymphadenopathies were also noted at the paracaval, aortocaval and prediaphragmatic regions. He was managed as a case of disseminated tuberculosis and started on quadruple therapy.

Result:

Discussion: The occurrence of a TLA is considered very unusual, with primary cases reported in literature as having a prevalence of only $0.34 \%$. Its clinical presentation is non-specific.

Conclusion: A TLA should be one of the differentials early on in the work up and management of hepatic abscesses, particularly in highprevalence countries and most especially in the setting of disseminated infection

\section{CASE-24}

A serendipitous rechallenge of an innocuous drug - a case report of Quetiapine hepatotoxicity Dr. Sameer Sakkeer Hussain*, Dr. Benoy Sebastian*** *Junior resident, ** Consultant, Department of Gastroenterology, Medical trust Hospital, Kochi, Kerala

\section{Sameer Sakkeer Hussain ${ }^{1}$}

${ }^{1}$ National board of examination, India

\section{Background: \\ Introduction}

We present a case of Quetiapine induced hepatotoxicity; a drug not well known for its hepatotoxicity. The drug was suspected to be the culprit and stopped but the patient unknowingly restarted the medication which acted as a rechallenge and caused hepatitis with mild coagulopathy. Patient recovered uneventfully after withdrawal of drug.

\section{Method:}

\section{Discussion}

Quetiapine is a dibenzothiazepine, an atypical antipsychotic agent. It is metabolized via the hepatic oxidase system via the cytochrome P4503A4 isoenzyme. Quetiapine is generally considered to be a non hepatotoxic drug. Very limited case reports of Quetiapine induced hepatotoxicity have been described in literature. This case report sheds light to the fact that drugs previously considered safe also are potential causes of DILI. There needs to be a high degree of suspicion for DILI so that the offending drug can be identified and withdrawn causing minimal damage to the patient.

\section{Result:}

\section{Case presentation}

34 year old female attended the OP department after complaining of severe fatigue. She was on treatment for bipolar affective disorder with Sodium divalproate, Lithium, Lorazepam and Quetiapine. Her investigations revealed mild transaminitis (SGOT-130 and SGPT 126) with normal bilirubin, alkaline phosphatase and INR levels. Her CBC, RFT, Serum electrolytes, RBS, TFT and USG Abdomen were within normal limits. The following day her transaminitis worsened with SGOT-431, SGPT-422 and INR-1.3(test -16.7, control-14.0).

IgMAntiHAV, IgMHEV, Hbsag (ELISA), Total anti HbC, HCV (ELISA) were negative. Non hepatotropic viruses like IgM HSV and EBV were negative as well. Autoimmune workup of ANA, AMA, AntiLKM1 and Anti SM were negative.

24 hour urine copper $-17 \mathrm{mcg} / \mathrm{d}(<50)$, Serum ceruloplasmin$26.2 \mathrm{mg} / \mathrm{d}$ (15-60). Her serum lithium levels was $0.45 \mathrm{mEq} / \mathrm{L}(0.8-1.2$ -Therapeutic range) and Ferritin $-113 \mathrm{ng} / \mathrm{ml}$.

Brucella IgM, Lyme IgM, and Weil felix were negative.

Blood and urine cultures showed no growth. Procalcitonin was 1.1( $>2-$ Sepsis $)$ 
A strong suspicion of DILI lead to cessation of anti psychotics in liaison with psychiatrist. Sodium divalproate and lithium were tapered and quetiapine continued along with lorazepam. There was gradual worsening of her LFT's in spite of the drug rearrangements. Finally after much deliberation Quetiapine was also stopped. This led to improvement in her LFT's (SGOT- 82, SGPT-256).

Nine days later she had severe fatigue and a repeat LFT showed the following picture -Total Bilirubin- 1.6, Direct bilirubin -0.6, SGOT-1276, SGPT-1258, ALP-182 and INR of 1.4. She had no features of hepatic encephalopathy or bleeding manifestations. On closer scrutiny she revealed she had restarted Quetiapine 6 days back. Quetiapine was withdrawn and she was restarted on Lithium and Sodium divalproate. She improved with standard supportive care and was discharged. Her LFT's came back to normal over a period of 45 days.

Conclusion: Our case report highlights the fact that the drug list causing DILI is ever expanding. Drug review should be an integral part of clinical history taking. Rechallenge had accidentally happened in our case pinpointing to the exact culprit.

\section{CASE-25}

Gall bladder perforation following selective internal radiation therapy (SIRT) for hepatocellular carcinoma: a case report

\section{Meng Chong $\mathrm{Ng}^{1}{ }^{\text {, Lisa Shim }}{ }^{1}$, Cositha Santhakumar ${ }^{1}$, Christine Chan $^{1}$, Charles Chan ${ }^{1}$, Guillermo Becerill ${ }^{1}$ \\ ${ }^{1}$ Concord RGH, Australia}

Background: Selective Internal Radiation Therapy (SIRT) using SirSpheres ${ }^{\circledR}$ Y-90 resin microspheres is an established option for nonsurgical treatment of primary hepatocellular carcinoma (HCC). Cholecystitis is a recognised complication but not gall bladder perforation. This is a report of a case of gall bladder perforation 10 weeks after transarterial SIRT.

Method: The patient is a 82 year old Chinese man on long term entecavir for chronic hepatitis B with past history of partial gastrectomy for duodenal ulcer and two head and neck operations for nasopharyngeal carcinoma. He underwent abdominal ultrasound examination for HCC surveillance regularly and asymptomatic gall stone had been consistently noted. Suspicion of an HCC was raised by ultrasound in mid-2015 and abdominal CT and MRI confirmed arterial enhancing lesion in segment $7 / 8$ consistent with HCC plus possible satellite lesions.

Result: Trans-arterial chemoembolization (TACE) with DC BeadsTM was performed in August 2015 with good response shown on MRI. Two new lesions were seen on CT in September 2016 and DC BeadsTM was given again via TACE. Residual tumours were treated with ultrasound guided ethanol injection and good response was confirmed on follow up CT. However, a new lesion was seen in segment 5 and he received Sir-Spheres ${ }^{\circledR}$ given transarterially on July 7 2017. Ten weeks later he developed abdominal pain, fever and jaundice. Ultrasound and CT revealed perforation of the gall bladder with gall stone at the neck of the gall bladder. He was treated with antibiotics and the fluid collection around the gall bladder was drained through a percutaneous catheter. He underwent cholecystectomy five weeks later without complication. Histology of the gall bladder showed microspheres throughout the wall and the serosal fat being present both inside and outside of vascular spaces. Inflammation and oedema were noted in the wall associated with necrosis, haemorrhage and mucosal ulceration.

Conclusion: Although gall bladder perforation does occur as a complication of cholecystitis, it is likely that, in this case radiation effect on the gall bladder wall contributed to the perforation. In the presence of gall stone, cholecystectomy prior to SIRT should be considered in addition to measures aimed at reducing the chance of entry of microspheres into the cystic artery.

CASE-26

Albendazole as a rare cause of recurrent severe acute hepatitis in a child

Santosh M Narayankar ${ }^{1}$, Abhishek Anil Mufkalwar ${ }^{1}$, D Viswanath $\overline{\text { Reddy }}^{1}$, Y Rami Reddy ${ }^{1}$, Guduru R Srinivas Rao ${ }^{1}$

${ }^{1}$ Yashoda Hospital, Secunderabad, India

Background: Recurrent acute hepatitis has few causes. Identification of etiology helps not only in management but also in prevention of recurrence. We present a rare case of recurrent acute hepatitis due to albendazole.

Method: A 12 year old girl studying in 6th standard presented to us during her third episode of jaundice. Each episode was preceded by prodrome. Liver function tests were suggestive of acute severe hepatitis. Viral markers (A, B, C, E), Wilsons and autoimmune workup was negative. There was history of Albendazole intake prior to onset of jaundice. No history of any other drug intake was there. She was not willing for admission however she improved with medical management. Six months later she again presented to us with acute severe hepatitis after albendazole intake at school.

\begin{tabular}{|l|l|l|l|l|}
\hline LFT & $27 / 02 / 2017$ & $03 / 05 / 2017$ & $01 / 09 / 2017$ & $26 / 09 / 2017$ \\
\hline Total Bilirubin & 12.97 & 0.78 & 18.23 & 2.25 \\
\hline Direct & 8.9 & 0.0 & 11.93 & 0.00 \\
\hline Indirect & 1.83 & 0.21 & 1.99 & 0.71 \\
\hline AST & 1406 & 35 & 635 & 39 \\
\hline ALT & 1274 & 27 & 638 & 41 \\
\hline ALP & 543 & 318 & 383 & 253 \\
\hline Total Protein & 9.2 & 8 & 9.4 & 8.5 \\
\hline Albumin & 4.0 & 4.2 & 4.1 & 4.4 \\
\hline Globulin & 5.1 & 3.8 & 5.3 & 4.1 \\
\hline A/G Ratio & 0.79 & 1.13 & 0.80 & 1.06 \\
\hline GGT & 33 & 12 & 27 & 12 \\
\hline
\end{tabular}

Result: Albendazole is given as a routine prophylaxis in Indian government schools as per national deworming programme. Childs father clearly observed and reported to us development of jaundice each time after deworming treatment at school. Our case shows clear temporal association. Re exposure to offending agent each time caused severe acute hepatitis. Her RUCAM score was 10. Hence albendazole is considered as the causative agent of hepatic injury in our patient.

Conclusion: Our case shows occurrence of severe acute hepatitis even after single dose of albendazole. It should be considered in differential diagnosis any acute hepatitis, particularly in children who are given routine deworming therapy. 
$H C V-1$

Features of expression of cytochrome-c, pcna, actin in the liver in chronic viral hepatitis " $C$ "

\section{Muborakhkhon Abdug'appor Qizi Rozikova ${ }^{1}$}

${ }^{1}$ Tashkent Medical Academy, Uzbekistan

Background: To study the peculiarities of translocation of cytochrome-C from the cytoplasm to the nucleus of cells, expression of PCNA, as well as the distribution of smooth muscle actin in liver cells in chronic viral hepatitis $\mathrm{C}$ (CVHC).

Method: 11 patients with HCVF were examined (F1-3 - 9 cases, F4 2 cases), treated at the Department of Surgery of the Republican Clinical Hospital No.1. The material was collected by the method of percutaneous puncture liver trepan-biopsy from 3 different zones (VII segment), puncture liver biopsy was fixed in $10 \%$ formalin, conducted through a series of alcohols, and paraffin. Histological sections 3$5 \mu \mathrm{m}$ thick were stained with hematoxylin and eosin, and Mallory in the Slinchenko modification. Immunohistochemical detection of PCNA (1: 200), cytochrome-C (1: 100), W-567 (1: 200) (stellate cell marker), actin (1: 100) (manufactured by Chemicon, USA) was performed on dewaxed histological sections of the liver.

Result: Cytochrome-C localization was observed in the cytoplasm of hepatocyte cells, bile ducts, endothelial cells of sinusoids. In single hepatocytes, translocation of cytochrome-C from the cytoplasm to the nucleus of cells was observed. This indicates that the detected cells are in a state of apoptosis. PCNA is expressed in the nuclei of cells of individual hepatocytes and in the nuclei of bile duct cells. The appearance of PCNA-positive cells indicates activation of reparative liver regeneration. In the study, a pronounced diffuse distribution of non-smooth muscle actin (W-567) and actin in the cytoplasm of hepatocytes, bile duct epitheliocytes, as well as in endothelial cells of sinusoids was found. Actin was observed in the form of granular inclusions in the cytoplasm of hepatocytes in a state of granular dystrophy.

Conclusion: Cytochrome-C localization was observed in the cytoplasm of hepatocyte cells, bile ducts, endothelial cells of sinusoids. In single hepatocytes, translocation of cytochrome-C from the cytoplasm to the nucleus of cells was observed. This indicates that the detected cells are in a state of apoptosis. PCNA is expressed in the nuclei of cells of individual hepatocytes and in the nuclei of bile duct cells. The appearance of PCNA-positive cells indicates activation of reparative liver regeneration. In the study, a pronounced diffuse distribution of non-smooth muscle actin (W-567) and actin in the cytoplasm of hepatocytes, bile duct epitheliocytes, as well as in endothelial cells of sinusoids was found. Actin was observed in the form of granular inclusions in the cytoplasm of hepatocytes in a state of granular dystrophy.

\section{$\mathrm{HCV}-2$}

Effects of resistance-associated variants on NS5B polymerase structure of hepatitis $\mathrm{C}$ virus genotype $\mathbf{1 b}$

\author{
Allan Peres da Silva ${ }^{1}$, Deborah Antunes Nascimento ${ }^{1}$, Ernesto \\ Raul Caffarena' ${ }^{1}$, Elisabeth Lampe ${ }^{1}$ \\ ${ }^{1}$ Fundação Oswaldo Cruz, Brazil
}

Background: Sofosbuvir (SOF) is a direct-acting antiviral that targets the hepatitis C virus (HCV) NS5B polymerase and inhibits viral replication. The resistance-associated variants (RAVs) L159F, C316N, A207T and A218S were detected in subjects who failed SOF treatment. In Brazilian HCV-1b infected subjects, $25 \%$ presents the double L159F-C316N variation. However, a lower rate was detected in HCV-1b North American, European and Asian isolates. The effects of genetic diversity from different geographical regions might elucidate the relationship between sequence variations and NS5B protein structural changes. The aims of this study were to build molecular models for NS5B wide-type and variants sequences and subsequently evaluate their effects and stabilities in aqueous solution by molecular dynamics simulations analysis.

Method: HCV-1b protein structures of NS5B wide-type (EF407464) and variant containing L159F, C316N, A207T and A218S (EF407491) were obtained by comparative modeling based on the PDB 4J04, using the program Modeller v.9.11. Molecular dynamics (MD) simulations were performed with AMBER99SB-ILDN force field employing the GROMACS program package. Long-range nonbonded interactions were treated by particle-mesh Ewald summation. The Berendsen scheme was used to maintain temperature $(300 \mathrm{~K})$ and pressure by weak coupling to an external bath. The program CHIMERA v1.12 was used for analysis.

Result: The time evolution of backbone RMSD values was analyzed to verify the stability of each model. Larger root mean square deviation (RMSD) indicates an increase on protein structural flexibility. The RMSD values of the wild-type protein observed over time showed higher values and fluctuations. After $30 \mathrm{~ns}$ of simulation, both structures presented almost the same values. In addition, it was not observed interactions between residues L159 and S282, and the residue $\mathrm{C} 316$ only formed hydrogen bonds with the catalytic residue D139, and not with the catalytic residues D220 and D318. Also, the variant $\mathrm{C} 316 \mathrm{~N}$ was not able to alter the NS5B active site conformation; the only morphological differentiation between structures involved the residues H374, D375, R380, H475 and S476.

Conclusion: These findings might explain the relative high frequencies of variants found in some HCV-1b populations, since they did not cause alterations on the NS5B active site conformation. It is important to notice that these variants are also observed in some nonresponder patients treated in combined therapies with SOF and were studied here as part of the same structure. Additional studies involving molecular docking will clarify if these variants might contribute to model the non-response to SOF.

\section{$H C V-3$}

Polymorphism of genes involved in the reactions of congenital immunity, while viral hepatitis $\mathrm{C}$ in ethnic groups of Buryats and Mongolians

\section{Nyamsuren Naranzul ${ }^{1}$, Malov $\mathrm{IV}^{3}$, Malov $\mathrm{SI}^{3}$, S Ariunaa ${ }^{4}$,} Ogarkov OB ${ }^{6}$, Symbelova TA ${ }^{6}$, Stepanenko LA $^{6}$, Zherbanova LV $^{6}$, P Nymadawa ${ }^{2}$, Savilov ED

${ }^{1}$ Mongolian National University of Medical Sciences, Mongolia; ${ }^{2}$ Gyals Medical Center, Mongolia; ${ }^{3}$ Irkutsk State Medical University, Russian Federation; ${ }^{4}$ National Center for Communicable Diseases, Mongolia; ${ }^{5}$ Republican Clinical Infectious Diseases Hospital, Russian Federation; ${ }^{6}$ Scientific Centre for Family Health and Human Reproduction Problems, Russian Federation

Background: In Mongolia, there is a phenomenon of absolute dominance of $1 \mathrm{~b}$ virus genotype of $-98.8 \%$ (Baatarkhuu O. et al., 2008). In ethnically close Buryatia, its concentration does not exceed $54.8 \%$ (Malov S.I. et al., 2012), and in neighboring China - 56.8\% (Gower E. et al., 2014). A possible explanation for the existing phenomenon may be the presence of a genetic predisposition of the population to the $1 \mathrm{~b}$ genotype, in which this clone of the virus receives a selective advantage when distributed in Mongolia. The aim of this study was to test the proposed hypothesis by the study of the 
SNP genes IFNL4 (rs368234815), IFNL3 (rs12979860 and rs8099917), CD209 (rs4804803), TLR3 rs3775291 and rs13126816 in cohorts of Mongolian patients with hepatitis $\mathrm{C}$ virus and in the ethnically similar Buryat group, and also in patients with Hepatitis C caused by different virus genotypes. The aim of this study was to test the proposed hypothesis by the study of the SNP genes IFNL4 (rs368234815), IFNL3 (rs12979860 and rs8099917), CD209 (rs4804803), TLR3 rs3775291 and rs13126816 in cohorts of Mongolian patients with hepatitis $\mathrm{C}$ virus and in the ethnically similar Buryat group, and also in patients with Hepatitis $\mathrm{C}$ caused by different virus genotypes.

Method: A total of 400 patients with chronic HCV were examined, including 200 from the Republic of Buryatia and 200 from Mongolia. The compared groups of patients completely matched in clinicallaboratory and sex-age indices.

Result: There were no associations of polymorphic variants of the genes CD209, IFNL3, and ethnicity of patients, as well as genotypes of the virus in the Buryat population. Obviously, the internalization of different genotypes of the virus into the cell is universal, and, at least, does not depend on the polymorphism of the CD209 gene. In contrast, as a result of the work performed, two SNPs in the candidate genes TLR3 (rs3775291) and IFNL4 (rs368234815) were detected, polymorphic variants of which occur with different frequency in patients with 1 and not $1(2 / 3)$ genotypes of the virus. Carriers of G-allele rs3775291 TLR3 are 3.1 times more resistant to infection with $2 / 3$ virus genotypes ( $\mathrm{p}<0.0001$ ), and carriers of $\Delta \mathrm{G}$-allele rs 368234815 IFNL4 - 2.0 times $(\mathrm{p}<0.02)$. Consequently, the higher the proportion of human carriers of these alleles and their haplotypes in a population, the higher the tolerance for the spread of $2 / 3$ genotypes of the virus in it. Under these conditions, the first genotype of the virus will receive genetically determined selective advantages, displacing the 2 nd and the 3rd from circulation.

Conclusion: Further studies at the level of practically healthy people in Mongolia and Buryatia, as well as the inclusion of other polymorphisms in the analysis will help establish the role of congenital immunity genes in the selective selection of individual genotypes of the virus.

\section{$\mathrm{HCV}-4$}

Expansion of intrahepatic lymphocytes expressing PD-1 and bcl-2 in chronic hepatitis $\mathrm{C}$ : correlation with disease progression

Eman Abdelsameea ${ }^{1}$, Nermine Ehsan ${ }^{2}$, Ahmed Elrefaey ${ }^{2}$, Mervat Sultan $^{2}$, Ayat Abdallah ${ }^{2}$, Maha Elsabaawy ${ }^{2}$

${ }^{1}$ Assistant Professor, Egypt; ${ }^{2}$ National Liver Institute, Menoufia University, Egypt

Background: Programmed Death-1 (PD-1) plays an important role in balancing antiviral immunity. Recently, PD-1 has been shown to induce immune exhaustion of $\mathrm{T}$ cells in chronic hepatitis $\mathrm{C}(\mathrm{CHC})$. Immune exhaustion and apoptosis are responsible for viral persistence. Little information is available regarding the correlation between PD-1 and anti-apoptotic proteins such as bcl-2.

The purpose of this study was to investigate the relationship between PD-1 and bcl-2 in liver tissue of patients with $\mathrm{CHC}$ infection and disease progression.

Method: This study was conducted on $91 \mathrm{CHC}$ patients and 20 healthy control subjects. Immunohistochemistry was performed on liver tissue sections using the following antibodies: PD-1, bcl-2, CK7, Ki67 and C-kit.

Result: Immunohistochemical results were correlated with demographic, laboratory and histopathological findings. Transaminases, platelets and prothrombin time showed significant relation with PD-1 and bcl-2. PD-1 and bcl-2 demonstrated significant correlation with necroinflammatory activity and fibrosis $(\mathrm{P}<0.000$ and $<0.001$ respectively). PD-1 but not bcl-2 revealed significant correlation with CK7 and C-kit $(\mathrm{P}<0.009$ and $<0.000$ respectively). PD-1 did not show significant correlation with either steatosis or Ki67. There was a strong significant negative correlation between PD-1 and bcl-2 $(\mathrm{r}=0.723, \mathrm{P}=<0.000)$.

Conclusion: Intrahepatic PD-1 are conversely interrelated to bcl-2 with a pivotal role in disease severity and progression.

\section{$H C V-5$}

Investigation of the relationship between gene polymorphisms of TNF a (-1031) and chronic hepatitis C infection

Ozlem Kandemir ${ }^{1}$, Nurcan Aras ${ }^{3}$, Gulay Borekci ${ }^{4}$, Ozlem Kandemir $^{2}$, Aysegul Cetinkaya ${ }^{3}$, Irem Bekalp Yilmaz ${ }^{3}$, Guhan Orekici Temel

${ }^{1}$ Mersin University, Turkey; ${ }^{2}$ Mersin University Faculty of Medicine, Department of Clinical Microbiology and Infectious, Turkey; ${ }^{3}$ Mersin University Faculty of Medicine, Department of Medical Biology, Mersin, Turkey., Turkey; ${ }^{4}$ Mersin University School of Health, Mersin, Turkey, Turkey; ${ }^{5}$ Mersin University, Faculty of Medicine, Department of Biostatistics, Turkey

Background: In this study we were aimed to determine the relationship between TNF- $\alpha$ (-1031 T/C) gene polymorphism and chronic $\mathrm{HCV}$ infections

Method: A total of 99 patients with chronic hepatitis C and 99 healthy subjects were included in the study. Approximately $2 \mathrm{ml}$ of blood from the subjects were taken into tubes containing EDTA, and genomic DNA was isolated using DNA isolation kit (Roche, Switzerland). After the isolation of genomic DNA from blood samples of the patient and control groups, TNF- $\alpha$ (-1031 T/C, rs1799964) polymorphism was investigated by using the Real-Time Polymerase Chain Reaction (RT-PCR) from the DNAs. The data obtained were analyzed using the SPSS package program.

Result: Among chronic hepatitis C group, TNF $-\alpha(-1031)$ TT, TC, CC genotypes were detected in $6(6,1 \%), 27(27,3 \%)$ and $66(66,7 \%)$ of the patients, respectively, while these numbers were $5(5 \%), 34$ $(34,3 \%)$ and $60(60,6 \%)$ in control group, respectively. When the distribution of allele frequencies of TNF- $\alpha$ (-1031) T/C polymorphism was evaluated in the patients and control groups, frequencies of $\mathrm{T}$ and $\mathrm{C}$ alleles were found to be $39(19,7 \%)$ and $159(80,3 \%)$ in the patient cases and $44(22,2 \%)$ and $154(77,8 \%)$ in the controls. However, there was no statistically significant difference between two groups regarding genotypes and alleles distributions for TNF-alpha $(\mathrm{p}>0,05)$.

Conclusion: Our results showed that TNF-alpha (-1031 T/C) polymorphism was not effective in chronic HCV infection. Further studies on this subject can contribute to the elucidation of the molecular mechanisms of chronic hepatitis $\mathrm{C}$.

\section{HCV-6}

NS5B sequence-based genotype analysis of hepatitis C virus from Bangladesh

Nahida Sultana ${ }^{1}$, Mohammad Enayet Hossain ${ }^{2}$, Mariya Kibtiya Sumiya $^{2}$, Mustafizur Rahman ${ }^{2}$, Munira Jahan ${ }^{1}$, Afzalun Nessa ${ }^{1}$, Saifullah Munshi ${ }^{1}$, Shahina Tabassum ${ }^{1}$

${ }^{1}$ BSMMU, Bangladesh; ${ }^{2}$ ICDDRB, Bangladesh 
Background: Worldwide, more than 3.8 million new cases of hepatitis $\mathrm{C}$ virus (HCV) infection is reported annually. Understanding of the HCV genome and proteins has enabled efforts to improve efficacy and tolerability of HCV treatment. The present study was aimed to identify the circulating genotypes and subtypes of HCV by sequence analysis of the NS5B region among the $\mathrm{HCV}$ infected population from Bangladesh.

Method: The study patients were selected from anti-HCV positive patients who provided blood at the Department of Virology, BSMMU from July 2015 to June 2016 for HCV RNA quantification. After HCV viral load quantification, Out of $150 \mathrm{HCV}$ RNA positive samples, 36 specimens were randomly selected and tested for NS5B gene amplification by One Step RT-PCR. A total of 36 readable partial sequences of the NS5B gene were found, and subsequently used for genotyping of circulating HCV by NCBI Genotyping tool.

Result: The majority of $31(86.1 \%)$ study population were infected with genotype 3 , while only $5(13.9 \%)$ were infected with genotype 1 . Subtype $3 \mathrm{~b}$ was the most prevalent $(42 \%)$ in the study population, followed by subtype $3 \mathrm{a}(39 \%), 1 \mathrm{~b}(11 \%), 3 \mathrm{~g}(5 \%)$ and $1 \mathrm{a}(3 \%)$. No other genotypes and subtypes were detected in this study. Phylogenetic analysis of Bangladeshi HCV sequences revealed that circulating strains clustered closely with strains from Pakistan, China, Thailand, Malaysia, Indonesia, Germany, USA, and Canada. All these countries share migration of people for immigration purpose, working aboard and one or two drug trafficking routes, which indicates that Bangladeshi HCV strains are being circulated through these routes. Conclusion: The study revealed that two genotypes with 5 different $\mathrm{HCV}$ subtypes are presently circulating among HCV infected population of Bangladesh, and this information may aid clinicians towards better management of $\mathrm{HCV}$ patients.

\section{$H C V-7$}

Serum miRNA expression profile reveals a novel prognostic panel for HCV patients

\section{Mohd Aejaz Habeeb ${ }^{1}$, Sandeep K Vishwakarma ${ }^{1}$, Rahamathulla Syed $^{1}$, Safwaan Habeeb ${ }^{1}$, Aleem Ahmed Khan ${ }^{1}$ \\ ${ }^{1}$ Centre for Liver Research \& Diagnostics, India}

Background: Currently the primary HCV diagnosis is based on the $\mathrm{HCV}$ antibody detection followed by the HCV-RNA quantification and $\mathrm{HCV}$ genotyping. These approaches can help to identify the HCV infection along with the liver function test (LFT). However, the subpopulation of $\mathrm{HCV}$-antibody positive cases that are negative for viral copies can't be discriminated with normal individuals which may lead to chronic, cirrhosis or HCC, as well as viral relapse status can't be calculated based on these methods. Hence, there is a need to identify more appropriate prognostic/diagnostic panel for $\mathrm{HCV}$ is highly required. In this present study, the unique prognostic panel of three selected miRNAs (miRNA-122, miRNA-21 and miRNA-181a) in combination with viral load was developed with multi-level approach to give valuable information for the assessment of disease status, therapeutic response and relapse in HCV patients.

Method: The expression of selected circulatory miRNAs was quantified in 25 healthy controls and $184 \mathrm{HCV}$ patients with viral load $\geq 1,00,000$ copies $/ \mathrm{ml}$ ( $\geq 1$ Lakh copies/ml), before, during the treatment and after 6 months follow-up after treatment and further compared with the serum biochemical changes and hematological parameters.

Result: Different categories of naïve HCV patients showed significantly high expression level of miRNA-122 $(>2.0$ fold, $\mathrm{p}<0.001)$ in chronic patients as compared to the healthy subjects whereas miRNA181a showed gradual decrease in expression levels with increasing the chronicity of the disease. In addition, miRNA-21 was significantly higher in HCC patients as compared to the healthy subjects and other category of HCV patients. miRNA-122 was showed significantly down regulated $(\mathrm{p}<0.001)$ expression levels during the each treatment regimen, whereas the expression levels of miRNA-181a got significantly enhanced with increasing the treatment duration $(\mathrm{p}<0.001)$. There was no significant miRNA variation was found after 6 months follow up cases when compared with the treated groups of both the genotypes.

Conclusion: Differential expression of miRNA-122 provides a direct measure for HCV quantification in both the genotypes before and during combination therapy and can be used as a prominent diagnostics as well as prognostic marker to develop better therapeutic strategies.

\section{$H C V-8$}

Effectiveness and safety of direct-acting antiviral therapies in treatment-experienced chronic hepatitis $\mathrm{C}$ infection patients in Turkey

Fehmi Tabak ${ }^{1}$, Tansu Yamazhan ${ }^{5}$, Behice Kurtaran ${ }^{4}$, Saban Esen $^{10}$, Nefise Oztoprak Cuvalci ${ }^{3}$, Yusuf Onlen', Gulsen Yoruk ${ }^{8}$, Ilknur Esen Yildiz ${ }^{11}$, Dilara Inan ${ }^{2}$, Esra Yerlikaya Zerdali ${ }^{7}$, Rahmet Guner ${ }^{14}$, Sener Barut ${ }^{6}$, Hayat Kumbasar Karaosmanoglu $^{12}$, Fehmi Tabak ${ }^{1}$, Hepatitis C Study Group ${ }^{13}$

${ }^{1}$ Istanbul University Medical School, Turkey; ${ }^{10}$ Ondokuz Mayis University Medical School, Turkey; ${ }^{11}$ RTE University Medical School, Turkey; ${ }^{12}$ Sadi Konuk Research and Training Hospital, Turkey; ${ }^{13}$ Turkey, Turkey; ${ }^{14}$ Yildirim Beyazit University Medical School, Turkey; ${ }^{2}$ Akdeniz University Medical School, Turkey; ${ }^{3}$ Antalya Research and Training Hospital, Turkey; ${ }^{4}$ Cukurova University Medical School, Turkey; ${ }^{5}$ Ege University Medical School, Turkey; ${ }^{6}$ Gaziosmanpasa University Medical School, Turkey; ${ }^{7}$ Haseki Research and Training Hospital, Turkey; ${ }^{8}$ Istanbul Research and Training Hospital, Turkey; ${ }^{9}$ Mustafa Kemal University Medical School, Turkey

Background: Turkish Viral Hepatitis Society (VHSD) and Infectious Diseases and Clinical Microbiology Specialty Society (EKMUD) created an online database and collect data of patients with chronic hepatitis $\mathrm{C}(\mathrm{CHC})$ patients using direct-acting antiviral therapies (DAA) in Turkey. This abstract aims to evaluate effectiveness and safety of DAA in treatment-experienced chronic hepatitis $\mathrm{C}$ infection patients in Turkey.

Method: Between April 2017 and October 2017, 36 centres from Turkey recorded 1500 patients to the database. Patients $>18$ years with $\mathrm{CHC}$ under direct-acting antiviral therapies were enrolled in this non-interventional observational study. Efficacy and safety results are only given for the patients with SVR12 data. The study was approved by the ethics committee and registered to clinicaltrials.gov (NCT03145844).

Result: Of the 1500 patients; $48.2 \%$ (723/1500) were treatment experienced. Of those, $57.8 \%$ (418/723) were female and mean age was 59.1 (SD:12.4) years. The most common HCV genotype was GT1 $(92.8 \%, 671 / 723$; of those $81.4 \%$ GT1b, $10.1 \%$ GT1a). $14.5 \%$ (105/723) had cirrhosis whereas $13.3 \%$ (14/105) had decompensated cirrhosis (Child-Pugh B-C). Liver biopsy was performed for $39.7 \%$ (287/723) and the median HAI-Knodell score was 8 (IQR:6-10) whereas $94.1 \%(270 / 287)$ had Ishak fibrosis stage $\geq 1$.

As previous treatment, $89.9 \%(650 / 723)$ had used peginterferon/ribavirin, $4.8 \%$ (35/723) peginterferon/ribavirin plus telaprevir and $4.0 \%$ (29/723) peginterferon/ribavirin plus boceprevir. 
Current treatments were ledipasvir/sofosbuvir \pm ribavirin $(60.2 \%, 435 / 723)$, paritaprevir/ritonavir/ombitasvir \pm dasabuvir \pm ribavirin $(33.5 \%, 242 / 723)$ and sofosbuvir+ribavirin $(5.1 \%, 37 / 723)$. Of the 723 treatment experienced patients; $52.6 \%$ (380/723) had an SVR12 evaluation.

Before DAA and at SVR12; median AST was 40.2 U/L and 21.0 $\mathrm{U} / \mathrm{L}$, median ALT was $49.0 \mathrm{U} / \mathrm{L}$ and $17.0 \mathrm{U} / \mathrm{L}$, median INR was 1.03 and 1.01 , median platelet was 170000 count $/ \mathrm{mL}$ and 179000 count/ $\mathrm{mL}$, respectively. Before DAA median HCV RNA was $1.45 \times 106$ copies/mL and $0.3 \%(1 / 380)$ had HCV viral load $<12 \mathrm{IU} / \mathrm{mL}$. At month 1, treatment end and 12 weeks after treatment end (SVR12); 85.3\% (209/245), 99.5\% (364/366) and 98.2\% (373/380) had HCV viral load $<12 \mathrm{IU} / \mathrm{mL}$, respectively (Figure 1 ).

After DAA had been started; $23.4 \%$ (89/380) patients experienced 175 adverse events, reported adverse events were asthenia $(10.8 \%, 41 /$ $380)$, pruritus $(8.4 \%, 32 / 380)$, insomnia $(5.5 \%, 21 / 380)$, headache $(5.3 \%, 20 / 380)$, nausea $(3.9 \%, 15 / 380)$ and others $(12.1 \%, 46 / 380)$.

Conclusion: DAA are effective and safe treatment for $\mathrm{HCV}$ infection among treatment-experienced patients. The results from a real-life setting in Turkey are similar to those from real-life settings in other countries and previous clinical trials.

*Hep-C Study Group

Kaya S, Ince N, Gunduz A, Namiduru M, Batirel A, Günal Ö, Coskuner SA, Karsen H, Köksal I, Akinci E, Tosun GG, Gül HC, Sirmatel F, Türker K, Duygu F, Sünnetcioglu M, Erben N, Karabay O, Sener A, Senates E, Çelebi G, Akbulut A, Baykam N

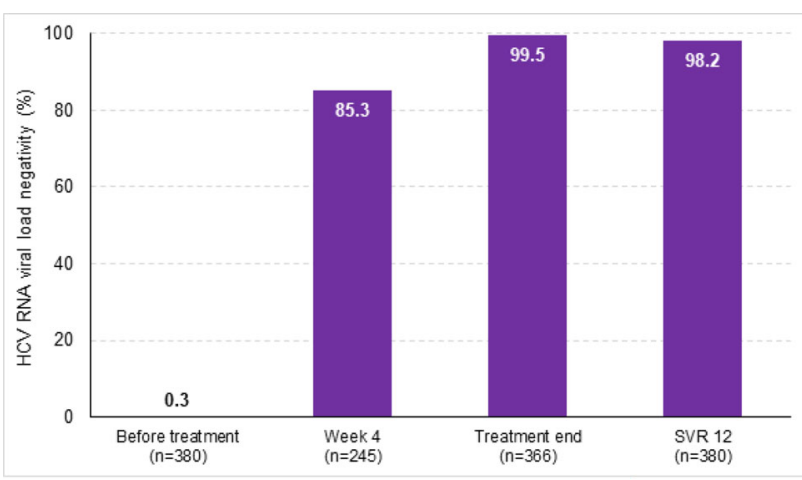

\section{$H C V-9$}

Cholesterol metabolism in the presence of biliary insufficiency in patients with long-term chronic hepatitis $c$

\section{Muborakhkhon Abdug'appor Qizi Rozikova ${ }^{1}$, Saidrakhim Nodirovich Lukmonov \\ ${ }^{1}$ Tashkent Medical Academy, Uzbekistan}

Background: To study the cholesterol metabolism in patients with chronic hepatitis $\mathrm{C}$ with a low replicative level of viral bodies and biliary insufficiency with a duration of disease more than 5 years (CHC).

Method: 28 patients with $\mathrm{CHC}$ were examined, the diagnosis was serologically verified by ELISA test, and the PCR method. The study was carried out on the basis of the Scientific Research Center for the Study of the Liver and Biliary tract. The mean age was $38 \pm 7$. The duration of the disease since the diagnosis of hepatitis was more than 5 years. Diagnosis of biliary insufficiency was carried out by the method of gradual chromatic duodenal sounding. All patients also underwent a study of biochemical blood indices with mandatory examination of liver samples, lipid spectrum and bile acids.
Result: In patients with $\mathrm{CHC}$, violations of the biochemical composition of bile were detected, namely: in the vesicular and hepatic portions of bile, the concentration of cholic acid was significantly decreased and the cholate-cholesterol coefficient was lowered. When calculating the total production rate of the components released into the duodenum one hour after the administration of the stimulus, a significant decrease in the rate of cholic acid, cholate-cholesterol and phospholipid-cholesterol coefficients was also found. When studying lipid metabolism in patients with diagnosed biliary insufficiency, a biochemical blood test revealed an increase in the level of cholesterol, and amounted to $7.3 \pm 2.4 \mathrm{mmol} / 1(\mathrm{p}<0.5)$. The level of blood cholesterol was elevated in 21 patients ( $75 \%$ of cases).

Conclusion: Cholesterol metabolism in long-term $\mathrm{CHC}$ patients is impaired at all stages of its metabolism, and in $75 \%$ hypercholesterolemia is diagnosed.

\section{HCV-10}

Daclatasvir and reduced-dose sofosbuvir: a safe, effective and pangenotypic treatment for hepatitis $C$ in patients with eGFR

Rakesh Aggarwal ${ }^{1}$, Dharmendra Singh Bhadauria ${ }^{1}$, Anupma Kaul $^{1}$, Prashant Verma ${ }^{2}$, Mayank Mehrotra ${ }^{3}$, Amit Gupta ${ }^{1}$, Raj Kumar Sharma ${ }^{1}$, Praveer Rai ${ }^{1}$, Rakesh Aggarwal ${ }^{1}$

${ }^{1}$ Sanjay Gandhi Postgraduate Institute of Medical Sciences (SGPGI), India; ${ }^{2}$ Ram Manohar Lohia Institute of Medical Sciences, India; ${ }^{3}$ Regency Hospital, India

Background: Sofosbuvir is a key agent for HCV treatment. It is not recommended for patients with chronic kidney disease (CKD) and estimated glomerular filtration rate (eGFR) $<30 \mathrm{~mL} / \mathrm{min}$. We report real-life experience of treating a cohort of CKD patients with eGFR $<30 \mathrm{~mL} / \mathrm{min}$ using daclatasvir and half-daily dose of sofosbuvir.

Method: Adults patients who (i) had eGFR $<30 \mathrm{ml} / \mathrm{min}$ and detectable HCV RNA and (ii) were treated with interferon and ribavirin free, DAA based regimens were included. All patients were treated with daily doses of daclatasvir $60 \mathrm{mg}$ and sofosbuvir $200 \mathrm{mg}$. The planned duration of treatment was 12 weeks, except for 24 weeks in those with either clinical evidence of cirrhosis or on immunosuppressive drugs. The end-points of the study were: (i) 12 weeks of follow-up after treatment completion, (ii) treatment discontinuation, or (iii) death or loss to follow-up. Liver cirrhosis was diagnosed if the patient had either gastroesophageal varices at endoscopy or ascites with serum-ascites albumin gradient more than $1.1 \mathrm{~g} / \mathrm{dL}$, with additional supporting clinical and/or biochemical features. Liver stiffness, measured using transient elastography, was not taken into consideration for the diagnosis of cirrhosis. Virological response was assessed by measuring serum HCV RNA concentration after 4 weeks of treatment (rapid virological response $[\mathrm{RVR}]$ ), at the end of the scheduled 12 or 24 weeks of treatment (end-of-treatment response [ETR]) and 12 weeks after stopping treatment (sustained virological response [SVR12]).

Result: Thirty-six (88\%) among 41 included patients (median [range] age: 48 [19-75] years; 25 [61\%] male; genotype 1/3/4 were 17/ 22/2; cirrhosis 5) completed the treatment, two discontinued and three died during treatment. On an intention-to-treat basis, HCV RNA were undetectable at 4 weeks of treatment, treatment completion and after 12 weeks of follow-up in 40/41 (97.6\%), 37/41 (90.2\%) and 37/41 (90.2\%), respectively. None of the patients had a relapse.

Conclusion: Daclatasvir and half-daily dose of sofosbuvir was safe and effective against genotype 1 and $3 \mathrm{HCV}$ infection in patients with eGFR $<30 \mathrm{ml} / \mathrm{min}$. This combination was could be a pangenotypic treatment option for such patients. 
Table 1:Clinical and laboratory characteristics of the patients with HCV infection and renal disease with estimated glomerular filtration rate $<0 \mathrm{~mL} / \mathrm{min}$

\begin{tabular}{|c|c|}
\hline Characteristic & Value \\
\hline \multicolumn{2}{|l|}{ Prior renal transplant } \\
\hline $\begin{array}{l}\text { No } \\
\text { Yes }\end{array}$ & $\begin{aligned} 37 & (90) \\
4 & (10)\end{aligned}$ \\
\hline $\begin{array}{l}\text { Estimated GFR in those not on dialysis }(\mathrm{n}=10) \\
(\mathrm{ml} / \mathrm{min})\end{array}$ & $24(12-28)$ \\
\hline \multicolumn{2}{|l|}{ Cirhosis } \\
\hline $\begin{array}{l}\text { Absent } \\
\text { Present }\end{array}$ & 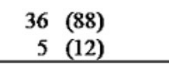 \\
\hline Liver stiffiness $(\mathrm{kPa})$ in those without cirnosis $(\mathrm{n}=31)^{*}$ & $9.8(5.2-34.3)$ \\
\hline \multicolumn{2}{|l|}{ HCV genotype } \\
\hline $\begin{array}{l}1 \\
3 \\
4\end{array}$ & $\begin{aligned} 17 & (42) \\
22 & (54) \\
2 & (5)\end{aligned}$ \\
\hline HCV RNA concentration $\left(\log _{10} \mathrm{IU} / \mathrm{mL}\right)$ & $5.90(4.12-9.90)$ \\
\hline
\end{tabular}

Data are shown as number (\%) or as median (range), as appropriate

* These data were not available for five patients

HCV-11

Declines in HBsAg levels observed during treatment with ledipasvir/sofosbuvir (LDV/SOF) in patients with chronic hepatitis B virus (HBV) and hepatitis $\mathrm{C}$ virus (HCV) infection

ChenYu Wang ${ }^{1}$, Chunjen Liu ${ }^{13}$, Wanlong Chuang ${ }^{10}$, Ishyan Sheen $^{2}$, Horngyuan Wang ${ }^{11}$, Chiyi Chen ${ }^{4}$, Kuochih Tseng ${ }^{7}$, Tingtsung Chang ${ }^{12}$, Benedetta Massetto ${ }^{9}$, Anu Osinusi ${ }^{9}$, Gregory Camus 9, Deyuan Jiang', Fangqiu Zhang', Diana Brainard', John Mchutchison', Tsunghui $\mathrm{Hu}^{2}$, Yaochun $\mathrm{Hsu}^{3}$, Chijen $\mathrm{Chu}^{14}$, Ginho Lo ${ }^{8}$, Jyhjou Chen ${ }^{5}$, Chengyuan Peng ${ }^{6}$, Rongnan Chien ${ }^{2}$, Peijer Chen ${ }^{13}$

${ }^{1}$ Gilead Sciences, Taiwan; ${ }^{10}$ Kaohsiung Medical University Hospital, Taiwan; ${ }^{11}$ Mackay Memorial Hospital, Taiwan; ${ }^{12}$ National Cheng Kung University Hospital, Taiwan; ${ }^{13}$ National Taiwan University College of Medicine and Hospital, Taiwan; ${ }^{14}$ Taipei Veteran Hospital, Taiwan; ${ }^{2}$ Chang Gung Memorial Hospital, Taiwan; ${ }^{3}$ Changhua Christian Hospital, Taiwan; ${ }^{4}$ Chia-Yi Christian Hospital, Taiwan; ${ }^{5}$ ChiMei Hospital, Taiwan; ${ }^{6}$ China Medical University Hospital, Taiwan; ${ }^{7}$ Dalin Tzu Chi Hospital, Buddhist Tzu Chi Medical Foundation, Taiwan; ${ }^{8}$ Eda Hospital, Taiwan; ${ }^{9}$ Gilead Sciences, United States

Background: Clinical HBV reactivation necessitating initiation of HBV treatment occurred in $2 \%(2 / 111)$ of HBsAg positive genotype (GT) 1 or $2 \mathrm{HCV}$ - infected patients treated with LDV/SOF for 12 weeks. In this analysis, we evaluated the kinetics of HBsAg levels in patients with versus without $\mathrm{HBV}$ reactivation, and provide long term safety and efficacy follow up through post treatment Week 48.

Method: This was a retrospective analysis in patients enrolled in the GS-US-337-1655 study. In this study, patients infected with chronic HCV GT 1 or GT2 and HBV (HBsAg+) not currently receiving HBV treatment were treated with LDV/SOF $(90 / 400 \mathrm{mg})$ qd for 12 weeks. HBV DNA and HBsAg levels and other liver tests were monitored at all study visits during treatment and follow up.

Result: At baseline, 74 patients $(67 \%)$ had HBV DNA = the lower limit of quantification (LLOQ, $20 \mathrm{IU} / \mathrm{mL}$ ), the mean (range) of HBsAg titer was $1.84 \log 10 \mathrm{IU} / \mathrm{mL}(-1.31,+3.83 \log 10 \mathrm{IU} / \mathrm{mL})$, and 110 patients $(99 \%)$ were $\mathrm{HBeAg}$ negative. HBsAg declined during treatment followed by return to baseline during the post-treatment follow up: the mean change at Weeks 1,4 and 12 were $-0.14,-0.25$, and $-0.47 \log 10 \mathrm{IU} / \mathrm{mL}$, respectively; the mean change at follow- up week 48 (FU-48) was $-0.10 \log 10 \mathrm{IU} / \mathrm{mL}$. Overall, 50 (45\%) of patients had HBsAg decline $=0.5 \log 10$ at $=1$ time point through PT Week 48: $42(84 \%)$ for the first time during treatment period and 8 (16\%) during the post-treatment follow up Overall, 79 patients $(71 \%)$ experienced HBV reactivation, defined as a change of HBV DNA from less than LLOQ to greater or equal to LLOQ or an increase of = $1 \log 10$ from baseline greater or equal to LLOQ, through FU-48. HBsAg mean change from baseline to Week 12 in patients who had $\mathrm{HBV}$ reactivation $(\mathrm{N}=79)$ and in patients who did not have $\mathrm{HBV}$ reactivation $(\mathrm{N}=32)$ was $-0.49 \log 10 \mathrm{IU} / \mathrm{mL}$ and $-0.43 \log 10 \mathrm{IU} / \mathrm{mL}$, respectively $(\mathrm{p}=0.36)$. A total of 4 patients with $\mathrm{HBV}$ reactivation have initiated HBV therapy through post treatment week 48 , based on local guidelines in Taiwan. All 111 subjects, including 68 GT1 and 43 GT2 patients, achieved SVR48. Concordance between SVR12 and SVR48 was $100 \%$.

Conclusion: A transient HBsAg decline was observed in coinfected patients during treatment with LDV/SOF. A similar decline in $\mathrm{HBsAg}$ levels was observed at Week 12 in patients with and without HBV reactivation. Additional studies are warranted to investigate the mechanism of $\mathrm{HBsAg}$ decline during treatment with direct-acting antivirals in $\mathrm{HBV} / \mathrm{HCV}$ coinfected patients.

\section{$\mathrm{HCV}-12$}

\section{Extrahepatic manifestations of chronic HCV infection}

Saidrakhim Nodirovich Lukmonov ${ }^{1}$, Kurbonboy Abdullayevich

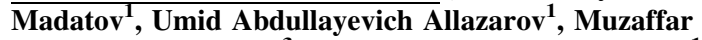
Uktamovich Ismailov ${ }^{3}$, Oybek Otabekovich Usmanov ${ }^{1}$, Asliddin Nurmatovich Sharapov ${ }^{1}$, Akbar Rashidovich Sadullayev ${ }^{2}$, Aziz Abbosovich Ismatov ${ }^{1}$

${ }^{1}$ Tashkent Medical Academy, Uzbekistan; ${ }^{2}$ Samarkand state medical institute, Uzbekistan; ${ }^{3}$ Tashkent State Pediatric Institute, Uzbekistan

Background: The purpose of our study is to study the clinical aspects of chronic HCV infection, paying special attention to extrahepatic manifestations of the disease.

Method: We observed 229 patients with chronic HCV infection in the department of infectious diseases 3 clinics of the Tashkent Medical Academy. Of these, 191 patients had chronic hepatitis, 37 had liver cirrhosis, and 1 had hepatocellular carcinoma. All patients underwent virological examination. HCV infection was confirmed by the determination of total AT-HCV by enzyme immunoassay and the detection of RNA-HCV by polymerase chain reaction.

Result: In 102 patients genotype 1b was registered and in 27 genotype 3a. A complex biochemical examination was performed, during which purposefully evaluated the cytolysis, the exchange of iron and carbohydrates. At a part of patients with late cutaneous porphyria (LCP) studied genetic predisposition. For this purpose, the presence of a polymorphism of C282Y and H63D alleles of the HFE gene was determined. According to the indications for the verification of mixed cryoglobulinemia, a blood test for cryoglobulins was carried out. In a number of patients a study was performed to evaluate liver fibrosis with the biochemical test "Fibromax" and ultrasound study "Fibroscan". In the examination of patients with chronic HCV infection, extrahepatic manifestations were detected in 67 (29.3\%) people. Of these, LCP was diagnosed in 32 , mixed cryoglobulinemia - in 13, autoimmune thyroiditis (AIT) - in 10, diabetes mellitus - in 7, Sjogren's syndrome - in 2, idiopathic fibrosing alveolitis - in 2, chronic recurrent urticaria - in 1 . Special features in the clinical course of these diseases were not observed. Predominantly hepatotropic viral infection was detected in patients under control examinations due to decompensation of the underlying disease. Only in 8 patients with chronic viral hepatitis C AIT was detected during preparation for 
antiviral therapy. HCV infection in patients with LCP was detected, on average, in 10-15 years after its manifestation. Replacement of C282Y and H63D in the HFE gene was found in half of patients with LCP. In patients with anomalous homozygous genotypes C282C and $\mathrm{H} 63 \mathrm{H}$, reliable signs of the syndrome of chronic iron overload were noted. The combination of extrahepatic manifestations was observed in $5(7.5 \%)$ patients. Of these, AIT and diabetes mellitus - in 2, LCP and AIT - in 2 and in 1 patient LCP was combined with AIT and Sjogren's syndrome. In 12 patients with extrahepatic manifestations, including those with co-infections, 2 stages of liver fibrosis were detected with non-invasive evaluation.

Conclusion: Thus, the etiological role of $\mathrm{HCV}$ as a trigger factor for extrahepatic manifestations must be considered in a complex relationship with other risk factors and evaluated as one of the important components of a multifactorial pathogenetic mechanism.

\section{$\mathrm{HCV}-13$}

Impact of direct antiviral agents on health-related quality of life in patients of chronic hepatitis $\mathrm{C}$ with chronic kidney disease

\section{Ajay Duseja ${ }^{1}$, Pramil Tiwari ${ }^{2}$, Ajay Duseja ${ }^{3}$, Raja Ramachandran ${ }^{4}$}

${ }^{1}$ Postgraduate Institute of Medical Education and Research, Chandigarh, India; ${ }^{2}$ NIPER, India; ${ }^{3}$ Post Graduate Institute of Medical Education and Research, Chandigarh, India; ${ }^{4}$ Post Graduate Institute of Medical Education and Research, Chandigarh, India

Background: Patient-reported outcomes (PRO) provide important information related to patients' experience in chronic diseases. Chronic hepatitis $\mathrm{C}$ (CHC) patients with chronic kidney disease (CKD) represent challenging population to treat where the healthrelated quality of life (HRQoL) are affected from both $\mathrm{CHC}$ and CKD. This study assessed effect of directly acting antiviral agents (DAAs) on HRQoL in patients of CHC with CKD.

Method: This prospective study conducted at a public teaching tertiary care hospital included adult patients of CHC with CKD $\left(\right.$ GFR $\left.<60 \mathrm{ml} / \mathrm{min} / 1.73 \mathrm{~m}^{2}\right)$ prescribed with DAAs after ethical approval. HRQoL was assessed using Short Form 12 Health Survey Questionnaire before and at end of treatment (ETR). Physical Composite Score (0-100), Mental Composite Score (0-100), and SF6D utility index (0-1) were calculated where 0 indicated the lowest, 50 or 0.5 normal and 100 or 1 the highest level of health.

Result: A total of 40 patients with positive HCV RNA with or without anti-HCV positive were screened and 30 patients were included in the study [Males $=25$ (83.3\%), mean age $43.3 \pm 12.4$ years]. Cirrhosis was present in $5(17 \%)$ patients and $19(63.3 \%)$ patients were treatment naive. Twenty three $(76.7 \%)$ patients had genotype (GT) 1, 4(13.3\%) had GT3, 2(6.7\%) had GT4, and only one $(3.3 \%)$ had GT2 (Table 1). Patients were divided into two groups including $13(43.3 \%)$ patients with GFR $<30 \mathrm{ml} / \mathrm{min} /$ $1.73 \mathrm{~m}^{2}$ and $17\left(56.7 \%\right.$ ) with GFR $>30 \mathrm{ml} / \mathrm{min} / 1.73 \mathrm{~m}^{2}$. Of 13 patients with GFR $<30 \mathrm{ml} / \mathrm{min} / 1.73 \mathrm{~m}^{2}, 11$ patients were on hemodialysis (HD). All Patients with GFR $<30 \mathrm{ml} / \mathrm{min} / 1.73 \mathrm{~m}^{2}$ were prescribed half dose of sofosbuvir (SOF) $(200 \mathrm{mg}$ ) with daclatasvir (DCV) $(60 \mathrm{mg})$ irrespective of HCV genotype. Patients having GFR $>30 \mathrm{ml} / \mathrm{min} / 1.73 \mathrm{~m}^{2}$ were prescribed DAAs on the basis of their HCV GT [GT $2 \& 3$ prescribed SOF 400mg with DCV 60mg; GT 1\&4 prescribed combination of SOF $400 \mathrm{mg}$ with ledipasvir $90 \mathrm{mg}$ ]. (Table 2) HCV RNA was negative in $30(100 \%)$ patients at ETR. Overall, there was significant improvement in PCS at ETR achieved with DAAs ( $42 \pm 1.97$ vs. $50 \pm 1.34, \mathrm{p}<0.05)$. Similarly, baseline MCS improved after administration of DAAs ( $43.7 \pm 1.36$ vs. $47 \pm 1.43$, $\mathrm{p}<0.05)$ at ETR. SF6D utility index also improved from baseline value $0.62 \pm 0.02$ to $0.73 \pm 0.02(\mathrm{p}<0.05)$. Improvement in PCS (HD, $34.6 \pm 2.28$ vs. $43.1 \pm 1.45, \quad \mathrm{p}<0.05$; non-HD, $45.8 \pm 2.42$ vs. $54.2 \pm 1.96, \mathrm{p}<0.05) \&$ SF6D (HD, $0.56 \pm 0.01$ vs. $0.64 \pm 0.01, \mathrm{p}<0.05$; non-HD, $0.56 \pm 0.02$ vs. $0.66 \pm 0.02, \mathrm{p}<0.05$ ) was seen in all patients irrespective whether they were on HD or not. According to GT, significant improvement in HRQoL after treatment with DAAs was observed in GT1 $(42 \pm 2.1$ vs. $50 \pm 1.45, \mathrm{p}<0.05)$. Cirrhotic patients had improvement in both PCS and MCS (PCS, $39.3 \pm 4.18$ vs. $49.2 \pm 3.83$, $\mathrm{p}<0.05$; MCS, $40.5 \pm 4.63$ vs. $48.2 \pm 3.36, \mathrm{p}<0.05)$ whereas non-cirrhotic patients had improvement only in PCS.(42 \pm 2.23 vs. $50.6 \pm 1.45, \mathrm{p}<0.05)$

Conclusion: Patients with CHC with CKD have impaired HRQoL both in mental and physical aspects. Treatment with DAAs improves HRQoL in these patients.

\begin{tabular}{|c|c|c|c|c|c|c|c|c|c|c|}
\hline Patients & No. & $\begin{array}{l}\text { Enrolment } \\
\text { PCS }\end{array}$ & ETR PCS & p-value & $\begin{array}{l}\text { Enrolment } \\
\text { MCS }\end{array}$ & ETRMCS & p-value & $\begin{array}{l}\text { Enrolment } \\
\text { SF } 60\end{array}$ & ETR SF6D & p.value \\
\hline Global & 30 & $42+1.96$ & $50.4 \div 1.34$ & 0.0001 & $43.8+1.36$ & $47.2+1.44$ & 0.02 & $0.62 \pm 0.02$ & $0.73+0.02$ & 0.0001 \\
\hline Hemodialysis & 10 & $34.6 \pm 2.28$ & $43.1+1.45$ & 0.004 & $40.2+1.58$ & $44.3 \div 1.95$ & 0.067 & $0.56 \pm 0.01$ & $0.64 \pm 0.01$ & 0.001 \\
\hline $\begin{array}{c}\text { GFR }>30 \mathrm{ml} / \mathrm{min} \\
1 / 1.73 \mathrm{~m}^{2}\end{array}$ & 17 & $45.8 \pm 2.42$ & $54.2 \pm 1.96$ & 0.0001 & $47.2 \pm 1.67$ & $49 \div 1.7$ & 0.186 & $0.56 \pm 0.02$ & $0.66 \pm 0.02$ & 0.0001 \\
\hline $\begin{array}{c}\text { Renal } \\
\text { Transplanted }\end{array}$ & 17 & $48.3 \pm 2.15$ & $54.6 \pm 1.19$ & 0.006 & $46.8 \pm 1.77$ & $49 \pm 2.01$ & 0.167 & $0.68 \pm 0.02$ & $0.77 \pm 0.03$ & 0.001 \\
\hline Cirrhotic & 5 & $39.3 \pm 4.18$ & $49.2+3.83$ & 0.0001 & $40.5 \pm 4.63$ & $48.2+3.36$ & 0.038 & $0.61 \pm 0.05$ & $0.76 \pm 0.07$ & 0.026 \\
\hline Non Cirrhotic & 25 & $42 \pm 2.23$ & $50.6 \pm 1.45$ & 0.0001 & $44.7 \pm 1.36$ & $47.1+1.61$ & 0.071 & $0.63 \pm 0.02$ & $0.72 \pm 0.02$ & 0.0001 \\
\hline HCV Genotype1 & 23 & $42 \pm 2.1$ & $50 \pm 1.45$ & 0.0001 & $45 \pm 1.52$ & $49 \pm 1.54$ & 0.004 & $0.63 \pm 0.02$ & $0.73 \pm 0.02$ & 0.0001 \\
\hline GI2 & 1 & 44.91 & 55.1 & NA & 49.02 & 43.1 & NA & $0.62 \pm 12.5$ & $0.75 \pm 0.19$ & 0.253 \\
\hline GT3 & 4 & $39 \div 7.82$ & $51 \pm 5.3$ & 0.062 & $38.3 \pm 4$ & $40.2 \pm 4.22$ & 0.391 & $0.56 \pm 0.04$ & $0.68 \pm 0.04$ & 0.09 \\
\hline GT4 & 2 & $47 \pm 12.51$ & $52.3 \pm 7.89$ & 0.22 & $42.1 \pm 6.07$ & $47 \pm 7.62$ & 0.395 & 0.63 & 0.88 & NA \\
\hline Treatment Naive & 19 & $39+2.49$ & $48 \pm 1.6$ & 0.0001 & $43 * 1.33$ & $46 \pm 1.97$ & 0.053 & $0.6 \pm 0.02$ & $0.7 \pm 0.02$ & 0.0001 \\
\hline Experienced & 11 & $46.1 \pm 2.85$ & $55.3 \pm 1.55$ & 0.013 & $46.1 \pm 2.91$ & $49.5 \pm 1.84$ & 0.122 & $0.66 \pm 0.04$ & $0.77 \pm 0.03$ & 0.01 \\
\hline
\end{tabular}

Table 2: Impact of different combinations of sofosbuvir on HRQoL

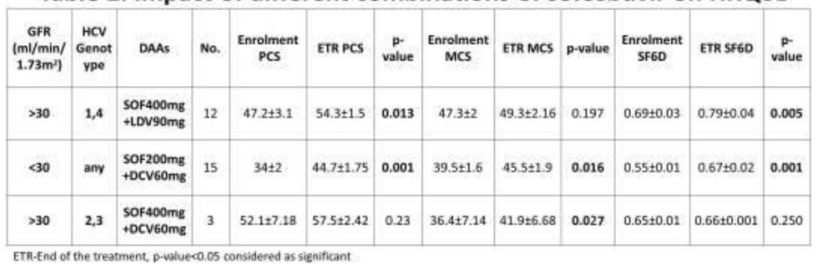

$H C V-14$

Patient-reported outcomes in chronic hepatitis $\mathrm{C}$ patients with or without extrahepatic manifestations (EHMs) in Japan

\section{$\underline{\text { Gabriel Wong }}{ }^{1}$, Umareddy Indira ${ }^{3}$, Dena Jaffe ${ }^{2}$, Namiki Izumi ${ }^{4}$}

${ }^{1}$ Gilead Sciences, Inc., United States; ${ }^{2}$ Kantar Health, Israel; ${ }^{3}$ Kantar Health, Singapore; ${ }^{4}$ Musashino Red Cross Hospital, Japan

Background: Almost two-thirds of patients with Hepatitis C (HCV) infection experience EHMs which have been shown to increase mortality and add to the impairment of Health Related Quality of Life (HRQoL) and costs of management. The objective of this study was 
to estimate the burden of EHMs of HCV in Japan with respect to HRQoL and work productivity.

Method: Data from the Japan 2010-2016 National Health and Wellness Survey (NHWS) $(\mathrm{N}=175,000)$ which is a nationally representative patient-reported survey of adults were used. Respondents who self-reported a doctor diagnosis of at least one of the following conditions in addition to HCV are considered positive for EHMs: Type 2 diabetes, depression, arthritis, fibromyalgia, Sjögren's or kidney disease.

Result: Patients with EHMs ( $n=307)$ were compared to HCV patients without any of the above mentioned conditions $(n=754)$. Groups were compared on HRQoL (assessed via the mental [MCS], physical component summary [PCS] scores, and health state utility score from the SF-36v2), work productivity and activity related impairment (assessed via the WPAI-GH instrument), in a bivariate analysis.

A total of $\mathrm{N}=1,061$ respondents reported a diagnosis of HCV $(49.1 \%$ were female; mean age $=60$ ) of which 307 respondents had any of the following conditions: Type 2 diabetes, depression, arthritis, fibromyalgia, Sjögren's or kidney disease and were classified as "HCV with EHM group" (unadjusted mean age $=56.8$; Charlson comorbidity index score $(\mathrm{CCI})=4$. The rest of the 754 patients classified as control group ("HCV without EHM") were older (unadjusted mean age 61.3) and had a lower CCI score of 1.3.

Compared with controls, respondents with EHMs had significantly lower MCS (41.69 vs. 48.06; p<0.001), PCS (45.08 vs. 49.15; $\mathrm{p}<0.001$ ), and health state utility ( 0.66 vs. $0.74 ; \mathrm{p}<0.001)$. Respondents with EHMs also had significantly greater absenteeism (10.56 vs. 4.67; $\mathrm{p}<0.001$ ), presenteeism (32.9 vs. $18.83 ; \mathrm{p}<0.001$ ), overall work impairment (36.87 vs. $21.32 ; \mathrm{p}<0.001)$, and activity impairment ( 37.75 vs. $24.93 ; \mathrm{p}<0.001)$.

Conclusion: $\mathrm{HCV}$ is a systemic disease and treating $\mathrm{HCV}$ is expected to improve clinical outcomes as well as patient quality of life and productivity resulting from improvement of $\mathrm{HCV}$ related EHMs.

\section{$H C V-15$}

Quality of life of young women of different constitutional groups with $\mathrm{HCV}$ infection.

\section{Saidrakhim Nodirovich Lukmonov ${ }^{1}$, Kurbonboy Abdullayevich Madatov $^{1}$, Umid Abdullayevich Allazarov ${ }^{1}$ \\ ${ }^{1}$ Tashkent Medical Academy, Uzbekistan}

Background: To monitor the quality of life in young women with chronic hepatitis $\mathrm{C}(\mathrm{CHC})$ of various somatotypes.

Method: The study included data from 56 women aged 18 to 39 years, 36 of them were diagnosed with HCV in the replication phase with moderate activity, 20 women were healthy and included in the control group. The study was conducted in the department of infectious diseases 3 - clinic of the Tashkent Medical Academy. The diagnosis of $\mathrm{CHC}$ has been confirmed by the detection in the blood of RNA-HCV, anti-HCV IgM, anti-HCV. The degree of activity of the inflammatory process was determined by increasing ALT value by more than three norms. Quality of life (QoL) was assessed using a general questionnaire MOS SF-36.

Result: MOS SF-36 showed a significant reduction $(\mathrm{P}<0.05)$ of QoL in all scales in patients with $\mathrm{CHC}$ compared to healthy women. Especially sharp changes were observed on the part of the mental component in comparison with physical. Differences in quality of life were unreliable in the group with an indeterminate somatotype, where the indicator "viability" differed little in the study and control groups (65.7 and 70 points, respectively, $\mathrm{p}>0.05$ ). During the time of the study, we were unable to detect a significant difference in the quality of life (except for the RAF scale) in patients with $\mathrm{CHC}$ of various somatotypes.

Conclusion: In patients with chronic hepatitis $\mathrm{C}$, significantly lower quality of life is observed compared with the control group. From the somatotype, changes in mental and physical health indicators in patients with $\mathrm{CHC}$ do not depend significantly.

\section{HCV-16}

\section{Real-world effectiveness and cost per SVR of sofosbuvir/} velpatasvir chronic hepatitis $C$ treatment

\section{Gabriel Wong ${ }^{1}$, Peter Atanasov ${ }^{2}$, Karsten Wursthorn ${ }^{3}$, Albrecht} Stoehr $^{3}$, Jorg Petersen ${ }^{3}$

${ }^{1}$ Gilead Sciences, Inc., United States; ${ }^{2}$ Amaris UK, United Kingdom; ${ }^{3}$ Institut für interdisziplinäre Medizin, Germany

Background: Sofosbuvir/Velpatasvir (SOF/VEL) single tablet regimen (STR) is approved in Europe for the treatment of chronic hepatitis C (CHC) patients with genotypes (GT) 1-6 with the ASTRAL trials showing high sustained virological response (SVR). The aim of the present analysis is to characterize the population receiving SOF/VEL in clinical practice, describe outcomes and estimate the cost per SVR.

Method: This analysis included the first $\mathrm{CHC}$ patients treated with SOF/VEL in a single centre in Germany, and for whom sustained virological response after 12 weeks of follow-up (SVR12) will be available in October 2017. Baseline characteristics, prior treatment history, safety, effectiveness and cost per SVR were investigated. The analysis was performed using descriptive statistics.

Result: 126 patients received a $12 \mathrm{w}$ treatment and were included in the analysis. The mean (standard deviation -SD) age was 49.1 (10.8) years and $72 \%$ were males. Patient's GT distribution was $9 \%, 3 \%$, $9 \%, 75 \%, 1 \%, 1 \%$ and $2 \%$ for GT1a, GT1b, GT2, GT3, GT4, GT5 and GT6, respectively. The METAVIR stage distribution of patients at baseline was $22 \%, 8 \%, 14 \%, 13 \%$ and $43 \%$ for F0, F1, F2, F3 and F4, respectively. 38 patients had RBV added to SOF/VEL (one patient was GT1a-F4, 3 were GT3-F3 and 34 were GT3-F4 with 6 who had decompensated disease) and one patient did not have RBV and had decompensated disease (GT1a-F4). Six patients were HIV co-infected and no patient was HBV co-infected. Overall, $84 \%$ of patients were treatment-naïve. Among the treatment experienced patients $(n=20), 75 \%(n=15)$ had relapses or null responses to prior therapies. At baseline, co-morbidities were reported in $95 \%$ of patients, with arterial hypertension (15\%) and depression (13\%) being most common. The per protocol analysis for patients with available data (50/122) shows that $98 \%(49 / 50)$ achieved SVR12 (100\% in GT3 patients (36/36)). Six adverse events (AEs) occurred; 3 were unrelated to treatment; 2 led to discontinuation and were possibly related to treatment and 1 was moderate and possibly related to treatment. Four patients discontinued the treatment; 2 due to lack of adherence; 1 was lost to follow-up and 1 because of AEs.

Conclusion: Early experience indicates that SOF/VEL is being used across all genotypes with majority use in GT3 population. Observed SVR rates are consistent with rates from pivotal trials. 


\section{$H C V-17$}

The large real-world data in Taiwanese chronic hepatitis $\mathbf{C}$ patients receiving all oral directly acting antivirals

\section{ChungFeng Huang ${ }^{1}$, Minglung $\mathrm{Yu}^{1}$ \\ ${ }^{1}$ Kaohsiung Medical University Hospital, Kaohsiung Medical University, Taiwan}

Background: All-oral directly acting antivirals (DAAs) have emerged as the promising therapeutic regimens for the treatment of hepatitis $\mathrm{C}$ virus (HCV) infection. DAAs were approved in Taiwan since 2016 and was partially reimbursed by National Health Insurance since Feb 2017. To date, large real-world in Taiwanese chronic hepatitis $\mathrm{C}(\mathrm{CHC})$ patients receiving DAAs are limited. We aimed to investigate the real world efficacy and safety profile of all oral DAAs in Taiwan

Method: Consecutive $\mathrm{CHC}$ patients in a tertiary center and two regional hospitals were included in this study. Treatment response was defined as the sustained virological response (unquantifiable hepatitis C RNA) at 12 weeks post end-of-treatment (SVR12). We summarized the characteristics of the patients in this cohort and estimated the on treatment responses and SVR12. The biochemical data were monitored and adverse events were also reported.

Result: A total of 443 patients treated with all oral DAAs between Apr 2016 and June 2017 were enrolled in this real world data analysis. The mean age of the patients was 61.4 years, and $42 \%(n=188)$ of the patients were male. One hundred and eighty-four $(41.5 \%)$ patients had liver cirrhosis and $21(4.7 \%)$ patients were with Child-Pugh B. $82 \%(\mathrm{n}=363)$ of the treated patients were with $\mathrm{HCV}$ genotype 1 (HCV-1) infection, whereas the remaining subjects belonged to HCV2 or mixed HCV-1 and HCV-2 infection. The prescribed regimens were as followed: sofosbuvir/ribavirin $(n=37,8 \%)$, sofosbuvir/ledipasvir + ribavirin $(\mathrm{n}=56,13 \%)$, sofosbuvir/daclatasvir + ribavirin $(\mathrm{n}=42,10 \%)$, paritaprevir/ritonavir/ombitasvir/dasabuvir + ribavirin $(\mathrm{n}=252,57 \%)$ and daclatasvir/asunaprevir $(\mathrm{n}=56,12 \%$; all without NS5A resistance associated substitutions). In the modified intention to treat (mITT) population, the on-treatment response at week 1, week 4, week 8 and end-of-treatment was $25.6 \%, 88.9 \%, 99.2 \%$ and $99.7 \%$ respectively; and the SVR12 rate was $99.1 \%(228 / 230)$. Of the two patients who experienced virological failure, one patient relapsed from 12 weeks of sofosbuvir/ribavirin. The other one relapsed from 24 weeks of daclatasvir/asunaprevir with the occurrence of treatmentemergent L31V and Y93M. The safety profile was satisfactory. Eight $(1.8 \%)$ patients had early discontinuation of the treatment due to side effects or severe adverse events, of which $4(0.9 \%)$ were judged as drug related (drug eruption $[n=1]$, severe vomiting $[n=1]$, myalgia/ weakness $[n=1]$, and dyspnea/palpitation $[n=1]$ ). None received NS3 protease inhibitor-containing regimens experienced liver decompensation.

Conclusion: All oral DAAs were highly effective and safe in Taiwanese $\mathrm{CHC}$ patients in the real world setting.

\section{HCV-18}

Treatment of hepatitis $\mathrm{C}$ virus in kidney transplant recipients with direct acting anti-viral agents

\section{Nagat Bousifi, Lutfia Ben Omer ${ }^{1}$, Abdulfatah Lamin ${ }^{1}$, Dalia Khair $^{1}$, Entisar EInaili ${ }^{1}$, Munir Abudhair ${ }^{1}$ \\ ${ }^{1}$ Central Hospital of Tripoli, Libyan Arab Jamahiriya}

Background: Most $\mathrm{HCV}$-infected kidney transplant recipients are infected while on dialysis, Treatment of Hepatitis $\mathrm{C}$ after kidney transplantation improve post-transplant outcomes. Direct-acting antivirals (DAAs) constitute an effective treatment in kidney transplant recipients with sustained virological response reaches $100 \%$. We aimed to treat kidney transplant patients who are infected with hepatitis $\mathrm{C}$ with available direct-acting antivirals.

Method: With intention to treat we included subsequent hepatitis $\mathrm{C}$ infected kidney transplant recipients that were registered and waiting for availability of DAAs. Epidemiological data were collected, country of kidney transplant, years of dialysis, diagnosis and previous treatment, and status of immunosuppressant drugs. HCV viral load and genotype, abdominal ultrasound to exclude liver cirrhosis. Treatment with DAAs was according to HCV genotype, duration of treatment was from 12 to 24 weeks depending on presence of liver cirrhosis.

Result: A total of 29 patients (17 males and 12 females), mean age 45 years (23 yrs-68 yrs), 16 patients were kidney transplanted in Pakistan, 3 in Egypt, 4 in Jordan, 5 in Libya, and 1 in England. 2 kidney recipients not undergo dialysis. Kidney transplant was in the same year of dialysis in 9 patients, within 1 year in 11 patients, and between 2-3 years in 7 patients. Diagnosis of HCV was before transplant in 17 patients, same year of transplant 6 patients, and after transplant in 6 patients. One patient was treated before transplant, and only one patient treated after transplant but both relapsed. 20 patients were on Cyclosporine, and 9 patients were on Tacrolimus. 22 patients were on prednisolone which was decreased to $5 \mathrm{mg}$ during treatment. $\mathrm{HCV}$ genotypes were; G1 in $11(38 \%), \mathrm{G} 3$ in $6(21 \%)$, and $\mathrm{G} 4$ in $12(41 \%)$ patients. One patient was HBV coinfected and 2 patients were cirrhotic. Treatment was started in 28 patients (Ledipasvir/Sofosbuvir in 23, and Daclatasvir+Sofosbuvir in 5. Week 4 was undetected in 16/16(100\%) patients. End of treatment response was undetected in $25 / 25(100 \%)$, Sustained virological response was undetected in $28 / 28(100 \%)$ patients.

Conclusion: Eradication of HCV infection with DAAs is feasible after kidney transplantation with excellent safety, tolerability in all genotypes of hepatitis $\mathrm{C}$.

\section{HCV-19}

Weight loss and improvement of hepatic fibrosis in Egyptian patients with chronic HCV Khaled Metwally, Eman Abdelsameea, Tamer Refaat, Khaled Gameel and Eman Roweiysha. Liver Unit, National Liver Institute, University of Menoufia

\section{Khaled Elsayed Amer ${ }^{1}$}

${ }^{1}$ Hepatology, Egypt

Background: Liver fibrosis is now considered as a reversible disease, but curing the primary hepatic disease, sometimes, doesn't mean fibrosis improvement. Steatosis could be one of the reasons responsible for this failure. Loss of weight has been shown to improve fibrosis but few data is available from Egypt.

Method: Adult patients ( $>18$ years) were recruited from hepatitis $\mathrm{C}$ out patients clinic, National Liver Institute (NLI), University of Menoufia, from 2013 to 2016.

Result: Thirty CHC patients with moderate and severe steatosis, who were initially assessed by liver biopsy and FibroScan for their fibrosis stage, were followed up by FibroScan after losing $>10 \%$ of their weight. Median age was 44.7 years $(29-59$ years $)$ and $50 \%$ were men. The degree of fibrosis and its values improved with the significant weight loss ( $\mathrm{p}<0.01$ for both).

Conclusion: Weight reduction is associated with improvement of hepatic fibrosis in Egyptian patient with $\mathrm{CHC}$. 
Table 1: Characteristics of patients' liver biopsies

\begin{tabular}{|c|c|c|}
\hline & Frequency & $\%$ \\
\hline \multicolumn{3}{|c|}{ Fibrosis stage } \\
\hline F1 & 11 & 36.7 \\
\hline F2 & 9 & 30 \\
\hline F3 & 8 & 26.6 \\
\hline F4 & 2 & 6.7 \\
\hline \multicolumn{3}{|l|}{ Activity grade } \\
\hline $\mathbf{A 1}$ & 16 & 53.3 \\
\hline A2 & 13 & 43.3 \\
\hline A3 & 1 & 3.4 \\
\hline \multicolumn{3}{|c|}{ Steatosis degree } \\
\hline No & 0 & 0 \\
\hline Mild & 0 & 0 \\
\hline Moderate & 20 & 66.7 \\
\hline Severe & 10 & 33.3 \\
\hline Total & 30 & 100.0 \\
\hline
\end{tabular}

Correlation between FibroScan stages of fibrosis before and after weight loss

\begin{tabular}{|l|c|c|}
\hline \multicolumn{2}{|c|}{} & Fibrosis stage 1 \\
\hline \multirow{2}{*}{ Fibrosis stage 2 } & $R$ & 0.700 \\
\cline { 2 - 3 } & $p$-value & $<0.01$ \\
\hline
\end{tabular}

\section{HCV-20}

Daclatasvir and asunaprevir combination therapy for patients with chronic hepatitis $C$ virus genotype $1 \mathrm{~b}$ infection in real world

\section{Byung Seok Kim ${ }^{1}$, Byoung Kuk Jang ${ }^{3}$, Jung Gil Park ${ }^{5}$, Se Young} Jang $^{4}$, Jeong III Suh ${ }^{2}$

${ }^{1}$ Catholic University of Daegu School of Medicine, Korea, Republic of; ${ }^{2}$ Dongguk University College of Medicine, Korea, Republic of; ${ }^{3}$ Keimyung University School of Medicine, Korea, Republic of; ${ }^{4}$ Kyungpook National University School of Medicine, Korea, Republic of; ${ }^{5}$ Yeungnam University College of Medicine, Korea, Republic of

Background: In previous clinical studies, daclatasvir (DCV) and asunaprevir (ASV) combination therapy showed both high rate of sustained virologic response (SVR) and low rate of serious adverse events. We evaluated the efficacy and safety of DCV and ASV combination therapy for patients with chronic hepatitis $\mathrm{C}$ virus (HCV) genotype $1 \mathrm{~b}$ infection in real world.

Method: A total of 278 patients (184 treatment-naïve patients) with chronic HCV genotype $1 \mathrm{~b}$ infection, who received DCV and ASV combination therapy, were enrolled from five institutes in Daegu and Gyeongsangbuk-do area. We evaluated the rates of rapid virologic response (RVR), end-of-treatment response (ETR), and SVR at 12 weeks after completion of treatment (SVR12). And we also investigated rate of adverse events and predictive factors of SVR12 failure. Result: The mean age was $59.5 \pm 10.6$ years, and 140 patients $(50.2 \%)$ were men. Seventy-seven patients $(27.6 \%)$ had liver cirrhosis. Baseline nonstructural protein 5A (NS5A) sequences were available from 268 patients. Pretreatment NS5A resistance-associated variants (RAVs) were present in $6(2.1 \%)$ patients. The RVR rate was $96.6 \%$ (258/267), and ETR rate was 95.2\% (223/232). Overall SVR12 rate was $91.6 \%$ (197/215). Adverse events occurred in 17 patients (7.9\%). Six patients discontinued treatment due to elevation of liver enzymes $(n=4)$ and severe nausea $(n=2)$. Among them, 4 patients achieved SVR12. Other adverse events included fatigue, headache, diarrhea, dizziness, loss of appetite, skin rash, and mild dyspnea. In a univariate analysis, statistical significant predictive factor of SVR12 failure was not shown.

Conclusion: DCV and ASV combination therapy provided high rates of RVR, ETR, SVR12 in chronic HCV genotype $1 \mathrm{~b}$ infection patients in real world. DCV and ASV combination therapy was well tolerated without serious adverse events.

$H C V-21$

Effectiveness and safety of direct-acting antiviral therapies in chronic hepatitis $\mathrm{C}$ infection patients with diabetes mellitus in Turkey

Fehmi Tabak ${ }^{1}$, Gulsen Yoruk ${ }^{16}$, Nevin Ince ${ }^{7}$, Behice Kurtaran ${ }^{6}$, Mustafa Namiduru $^{10}$, Nefise Oztoprak Cuvalci ${ }^{3}$, Yusuf Onlen ${ }^{19}$, Ilknur Esen Yildiz ${ }^{21}$, Dilara Inan ${ }^{2}$, Esra Yerlikaya Zerdali ${ }^{14}$, Sener Barut $^{11}$, Sibel Kaya ${ }^{1}$, Saban Esen ${ }^{20}$, Ayse Batirel ${ }^{18}$, Seher Ayten Coskuner ${ }^{5}$, Iftihar Koksal ${ }^{17}$, Hanefi Cem Gul ${ }^{13}$, Kamuran Turker ${ }^{4}$, Ebubekir Senates ${ }^{12}$, Ayhan Akbulut ${ }^{9}$, Nurcan Baykam $^{15}$, Tansu Yamazhan ${ }^{8}$, Fehmi Tabak ${ }^{1}$

${ }^{1}$ Istanbul University Medical School, Turkey; ${ }^{10}$ Gaziantep University Medical School, Turkey; ${ }^{11}$ Gaziosmanpasa University Medical School, Turkey; ${ }^{12}$ Goztepe Research and Training Hospital, Turkey; ${ }^{13}$ Gulhane Research and Training Hospital, Turkey; ${ }^{14}$ Haseki

Research and Training Hospital, Turkey; ${ }^{15}$ Hitit University Medical School, Turkey; ${ }^{16}$ Istanbul Research and Training Hospital, Turkey;

${ }^{17}$ Karadeniz Teknik University Medical School, Turkey; ${ }^{18}$ Kartal

Research and Training Hospital, Turkey; ${ }^{19}$ Mustafa Kemal University Medical School, Turkey; ${ }^{2}$ Akdeniz University Medical School, Turkey; ${ }^{20}$ Ondokuz Mayis University Medical School, Turkey; ${ }^{21}$ RTE University Medical School, Turkey; ${ }^{3}$ Antalya Research and Training Hospital, Turkey; ${ }^{4}$ Bagcilar Research and Training Hospital, Turkey; ${ }^{5}$ Bozyaka Research and Training Hospital, Turkey; ${ }^{6}$ Cukurova University Medical School, Turkey; ${ }^{7}$ Duzce University Medical School, Turkey; ${ }^{8}$ Ege University Medical School, Turkey; ${ }^{9}$ Firat University Medical School, Turkey

Background: Turkish Viral Hepatitis Society (VHSD) and Infectious Diseases and Clinical Microbiology Specialty Society (EKMUD) created an online database and collect data of patients with chronic hepatitis $\mathrm{C}(\mathrm{CHC})$ patients using direct-acting antiviral therapies (DAA) in Turkey. This abstract aims to evaluate effectiveness and safety of DAA in chronic hepatitis $\mathrm{C}$ infection patients with diabetes mellitus in Turkey.

Method: Between April 2017 and October 2017, 36 centres from Turkey recorded 1500 patients to the database. Patients $>18$ years with $\mathrm{CHC}$ under direct-acting antiviral therapies were enrolled in this non-interventional observational study. Efficacy and safety results are only given for the patients with SVR12 data. The study was approved by ethics committee and registered to clinicaltrials.gov (NCT03145844). 
Result: Of the 1500 patients; $16.8 \%$ (252/1500) were diabetic. Of those, $64.7 \%(163 / 252)$ were female and mean age was 63.8 (SD:9.8) years. The most common HCV genotype was GT1 $(95.2 \%, 240 / 252$; of those $87.9 \%$ GT1b, $6.7 \%$ GT1a). $17.1 \%$ (43/252) had cirrhosis whereas $11.6 \%(5 / 43)$ had decompensated cirrhosis (Child-Pugh B-C). Liver biopsy was performed for $56.7 \%$ (143/252) and the median HAI-Knodell score was 8 (IQR:6-10) whereas 98.6\% (141/ 143) had Ishak fibrosis stage $\geq 1$.

Of the patients, $56.3 \%(142 / 252)$ were treatment experienced and $90.1 \%(128 / 142)$ had used peginterferon/ribavirin, $4.9 \%(7 / 142)$ peginterferon/ribavirin plus boceprevir and $4.9 \%$ (7/142) peginterferon/ribavirin plus telaprevir. Current treatments were paritaprevir/ ritonavir/ombitasvir \pm dasabuvir \pm ribavirin $\quad(48.8 \%, 123 / 252)$, ledipasvir/sofosbuvir \pm ribavirin $(44.0 \%, 111 / 252)$, sofosbuvir+ribavirin $(3.2 \%, 8 / 252)$.

Of the 252 diabetic patients; $42.1 \%(106 / 252)$ had an SVR12 evaluation. Before DAA and at SVR12; median AST was $41.0 \mathrm{U} / \mathrm{L}$ and 20.3 U/L, median ALT was 49.0 U/L and 17.0 U/L, median INR was 1.04 and 1.00 , median platelet was 171000 count $/ \mathrm{mL}$ and 179000 count $/ \mathrm{mL}$, respectively. Before DAA, median HCV RNA was $1.93 \times 106$ copies $/ \mathrm{mL}$ At month 1 , treatment end and 12 weeks after treatment end (SVR12); 88.9\% (56/63), 100\% (100/100) and 97.2\% (103/106) had HCV viral load $<12 \mathrm{IU} / \mathrm{mL}$, respectively (Figure 1). After DAA had been started; $27.4 \%$ (29/106) patients experienced 48 adverse events, reported adverse events were asthenia $(12.3 \%, 13 /$ $106)$, pruritus $(8.5 \%, 9 / 106)$, headache $(7.5 \%, 8 / 106)$, nausea $(3.8 \%, 4 /$ $106)$, insomnia $(3.8 \%, 4 / 106)$, and others $(9.4 \%, 10 / 106)$.

Conclusion: DAA are effective and safe treatment for $\mathrm{HCV}$ infection among diabetic patients. The results from a real-life setting in Turkey are similar to those from real-life settings in other countries and previous clinical trials.

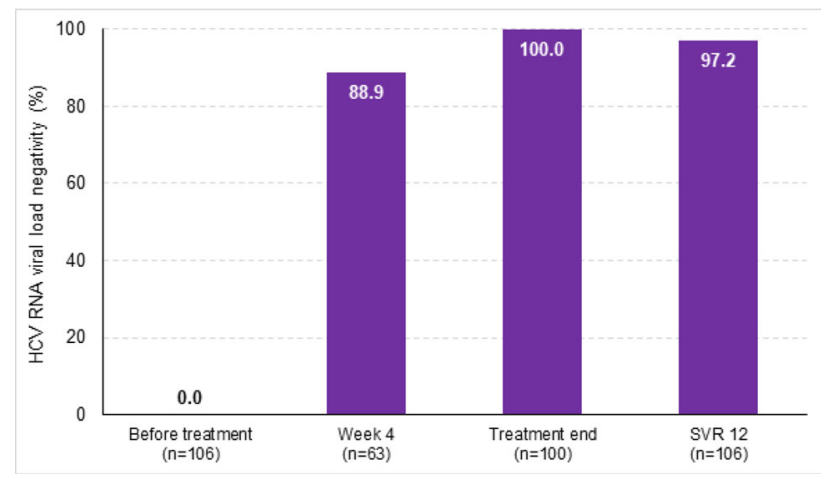

\section{$H C V-22$}

Impact of sustained virological response on indirect serum biomarkers of fibrosis in chronic hepatitis $\mathbf{C}$ patients

\section{Anup S. Nair ${ }^{1}$}

${ }^{1}$ Calicut Govt Medical College, India

Background: Novel direct antiviral agents (DAA) targeting hepatitis $\mathrm{C}$ virus (HCV) have revolutionized the treatment of chronic hepatitis $\mathrm{C}$ infection (CHC). Rates of sustained virological response (SVR) to treatment have drastically improved since introduction of DAA. We examined the changes in the non invasive indirect serum biomarkers of fibrosis scores, FIB -4 and APRI after DAA treatment of CHC.

Method: In an ongoing clinical prospective study, till date 30 patients completed the study. All those patients with chronic hepatitis or compensated cirrhosis of liver received Sofosbuvir $400 \mathrm{mg}$ and Daclatasvir $60 \mathrm{mg}$ for 12 weeks and those with decompensated cirrhosis of liver had it for 24 weeks. FIB -4 and APRI scores were calculated prior to the treatment and after achieving SVR.

Result: 30 patients (22M: $8 \mathrm{~F}$ ) with a mean age of $47 \pm 8$ years were included, of which 13 (43.3\%) had cirrhosis of liver. Of the 13 Cirrhotic patients 9, 3, and 1 were in CTP A, B and C stage respectively. Among the total patients 23(76.7\%), 5(16.7\%), 2(6.7\%) were genotype 3,1 and 4 respectively. Median Viral load was: $499801 \mathrm{IU} / \mathrm{ml}$ (range: 2.4x 103 -7.1x 108). Overall ETR and SVR were 100\% in patients across the genotype. Median FIB -4 and APRI values significantly decreased from 2.54 (IQR $1.65-4.43$ ) and 1.10 (IQR 0.65 2.43 ) to 1.80 (IQR $1.23-2.84, \mathrm{p}<0.001$ ) and 0.43 (IQR $0.3-0.79, \mathrm{p}<$ $0.001)$, respectively

Conclusion: Patients with SVR after DAA therapy showed significant regression of validated fibrosis scores FIB -4 and APRI. Whether this indicates a regression of fibrosis or merely resolution of chronic liver inflammation needs long term follow up.

\section{$H C V-23$}

Oral direct acting antivirals (DAAs) therapy of patients $(n=261)$ with chronic HCV genotype 3 infection - real life Myanmar experience

Si Thu Sein Win ${ }^{1}$, Khin Maung Win ${ }^{2}$, Naomi Khaing Than Hlaing $^{3}$, Aung Hlaing Bwa ${ }^{1}$, Khin Aye Wint Han ${ }^{1}$, Su Su Htar ${ }^{1}$, Soe Thiha Maung ${ }^{1}$, Soe Thu Aung, ${ }^{1}$, Lei Yin Wine ${ }^{1}$, Wint Wah Ko ${ }^{1}$, Moe Pwint Oo ${ }^{1}$

${ }^{1}$ Yangon GI and Liver Center, Myanmar; ${ }^{2}$ Honorary Professor, Myanmar; ${ }^{3}$ Professor, Myanmar

Background: Genotype 3 has emerged as the most difficult-to-cure genotype being associated with lower sustained virologic response at post-treatment week 12 (SVR12) rates, a more accelerated fibrosis progression and the strongest predictor for the occurrence of HCC. Therefore, in the era of DAAs, we retrospectively assessed the treatment outcome and safety of all-oral sofosbuvir (SOF) based regimens for treatment of $\mathrm{HCV}$ genotype 3 infections in a real life setting in Myanmar.

Method: This open-label retrospective cohort study was conducted at the Yangon GI and Liver Center in Yangon, Myanmar between December 2015 and September 2017 as an investigator-initiated study.

The primary end point was SVR12. According to the published international guidelines, choice of DAAs and duration of therapy were determined by degree of fibrosis and \pm previous treatment. However, in Myanmar, it heavily depends on the availability of DAAs and financial status of the patients. Virologic analyses were performed at the baseline, week 4 , week 8 , week 12 , week 24 and SVR 12 of treatment.

Result: Overall, 261 patients with HCV genotype 3 infection received treatment of all-oral DAAs therapy. Patients with mean age of 51 years (range $17-81$ ), 32\% men, 68\% lady, $88.5 \%$ treatment naïve, $11.5 \%$ treatment experience and $67 \%$ with cirrhosis. The mean baseline viral load prior to start treatment was 3.6 million IU/mL (between 8,011 and 176,389,223).

SVR 12 was achieved in 246/261 (94\%) patients. Analysis of the subtypes revealed $3 \mathrm{~b}$ was majority - 176 out of $261(67 \%)$ and genotype subtype 3 a - 60 out of 261 (23\%) with SVR rates of $93 \%$ and $98.3 \%$ respectively. Subtyping was not available in 25 patients (10\%) achieving 96\% SVR12.

A trend towards a poorer response rate was noted in patients with high body mass index (BMI $\geq 25)-89 \%$ (85/96) SVR as compared to $98 \%(161 / 165)$ in BMI $<25$ group. Analysis of SVR12 in patient 
based on baseline characteristics showed no notable difference by gender, age, and HBV or HIV co-infection.

There was no significant difference in achieving SVR12 in treatment naïve and treatment experienced patients - 94\% (217/231) and 97\% (29/30). However, patients with cirrhosis had lower SVR rates than non-cirrhosis - 93\% (163/175) and 96.5\% (83/86) respectively. Similarly, increasing liver stiffness was associated with a decreased chance of achieving SVR.

Furthermore, we stratified the SVR rates by treatment regimens. 108 out of total 261 patients treated with Sofosbuvir and Ribavirin (SOF+RBV) for 24 wks achieved $96 \%$ of SVR rates (104/108). On the other hand, only $93 \%(142 / 153)$ of SVR rates was obtained in 153 patients treated with SOF+DCV (Daclatasvir) \pm RBV for 12 or 24 wks. Moreover, an impressive cure rate revealed $96 \%$ of SVR12 was achieved in patients with cirrhosis under the treatment of SOF+RBV 24 wks $(n=74 / 77)$ and SOF+DCV+RBV 24 wks $(n=25 / 26)$. The most common adverse events (AEs) were fatigue, headache and nausea. No patients discontinued treatment due to AEs.

This study indicated SOF+RBV 24 wks is an effective regimen which can achieve SVR12 rates more than $90 \%$ in both cirrhotic and non-cirrhotic patients. SOF+DCV $12 \mathrm{wks}$ treatment is good only for non-cirrhotic patients. In cirrhotic patients, SOF+DCV treatment should be extended to $24 \mathrm{wks}$ and if tolerable, RBV should be added to achieve better SVR rates.

Conclusion: This retrospective cohort study with real life Myanmar all-oral DAAs experience data on 261 patients will be of great contribution to the scientific data on the treatment of patients with chronic HCV genotype 3 infection.

\begin{tabular}{|c|c|c|c|}
\hline \multicolumn{2}{|l|}{ Characteristics } & Number. (\%) & SVR \% (no.) \\
\hline Mean Age - yr. & \multicolumn{3}{|l|}{$51.6(17-81)$ year } \\
\hline \multirow[t]{2}{*}{ Gender } & Male & $84 / 261(32 \%)$ & $94 \%(79 / 84)$ \\
\hline & Female & $177 / 261(68 \%)$ & $97 \%(167 / 177)$ \\
\hline \multirow[t]{3}{*}{ Genotype Subgroup } & $3 a$ & $60 / 261(23 \%)$ & $98.3 \%(59 / 60)$ \\
\hline & $3 \mathrm{~b}$ & $176 / 261(67 \%)$ & $93 \%(163 / 176)$ \\
\hline & Not available & $25 / 261(10 \%)$ & $96 \%(24 / 25)$ \\
\hline \multirow[t]{2}{*}{ Metavir score for fibrosis } & F3-F4 (Cirrhosis) & $175 / 261(67 \%)$ & $93 \%(163 / 175)$ \\
\hline & F0-F2 (Non cirrhosis) & $86 / 261(33 \%)$ & $96.5 \%(83 / 86)$ \\
\hline \multirow[t]{2}{*}{ Treatment Received } & Naîve & $231 / 261(88.5 \%)$ & $94 \%(217 / 231)$ \\
\hline & Experienced & $30 / 261(11.5 \%)$ & $97 \%(29 / 30)$ \\
\hline \multirow[t]{2}{*}{ Child Pugh Score } & A & $230 / 261(88 \%)$ & $94 \%(216 / 230)$ \\
\hline & B & $31 / 261(12 \%)$ & $97 \%(30 / 31)$ \\
\hline \multirow[t]{2}{*}{ BMI } & $<25$ & $165 / 261(63 \%)$ & $98 \%(161 / 165)$ \\
\hline & $\geq 25$ & $96 / 261(37 \%)$ & $89 \%(85 / 96)$ \\
\hline \multirow[t]{2}{*}{ Co-Infection } & HBV co-infection & $9 / 261(3.4 \%)$ & $100 \%(9 / 9)$ \\
\hline & HIV co-infection & $1 / 261(0.3 \%)$ & $100 \%(1 / 1)$ \\
\hline \begin{tabular}{|l} 
Overall SVR \\
\end{tabular} & & 261 & $94 \%(246 / 261)$ \\
\hline
\end{tabular}

Table1. Characteristics of Patients with HCV Genotype 3 Infection who received Oral DAAs treatment regimens and Predictors of SVR. $(n=261)$

\section{$H C V-24$}

Presence of genotype 3 predicts cirrhosis in chronic hepatitis $\mathbf{C}$ patients

Tarana Gupta $^{1}$, Hari Aggarwal ${ }^{1}$, RAHUL CHAUDA ${ }^{1}$, SUBHASH MEEL $^{1}$, ASWANI BHAVIKATTE ${ }^{1}$, Ajit Lather ${ }^{1}$, Sandeep Goyal $^{2}$, Virendra Singh $^{3}$

${ }^{1}$ PGIMS, Rohtak, India; ${ }^{2}$ Independent Researcher, India; ${ }^{3}$ PGIMER, India

Background: Hepatitis $\mathrm{C}$ has emerged as important cause for cirrhosis and hepatocellular carcinoma. We studied the prevalence of different Genotypes and severity of liver disease in chronic hepatitis $\mathrm{C}$ patients.

Method: We prospectively studied 962 patients with chronic hepatitis C from June 2016 to May 2017. We compared all biochemical parameters, quantitative HCV RNA, HCV genotype, fibroscan, ultrasound, and endoscopic findings in genotype 3 and non-genotype 3 patients. Cirrhosis was diagnosed in patients with liver stiffness measurement $(\mathrm{LSM})$ values $\geq 12.5 \mathrm{Kpa}$.

Result: Out of total 949 patients, there were 546 patients with genotype 3 and 297 patients (genotype 1/4/5; $n=103 / 188 / 6$ ) with nongenotype 3 . The mean HCV RNA values in genotype 3 and nongenotype 3 were 6.9 and 8.7 million copies $/ \mathrm{mL}(\mathrm{P}=0.051)$ respectively. In 106 patients baseline RNA was undetectable. The mean age was not different between genotype 3 and non-genotype 3 (39.6 vs $39.2, \mathrm{P}=0.811)$. The LSM values were available in 618 patients and biochemical parameters were available in 212 patients. Overall, genotype 3 patients had higher LSM (11.3 vs 7.62, $\mathrm{P}=0.000$ ), higher AST (88.4 vs $68.6, \mathrm{P}=0.027)$ and low platelet count (228.4 vs 261 , $\mathrm{P}=0.032$ ) as compared to non-genotype 3 patients. Sub group analysis revealed that non-cirrhotic patients with genotype 3 had higher LSM (6.75 vs $6.11, \mathrm{P}=0.000)$ and higher AST ( 74.4 vs $57, \mathrm{P}=0.01$ ) values as compared to patients with non-genotype 3 . The platelet count was lower in non-cirrhotic genotype 3 as compared to non-genotype 3 (241.1 vs $263.6, \mathrm{P}=0.161$ ) although not statistically significant. Overall, genotype 3 had higher prevalence of cirrhosis than nongenotype $3(115 / 415$ vs 25/245, $\mathrm{P}=0.000)$, respectively. However, once cirrhosis developed decompensation was not different between the two groups ( $32 / 115$ vs $7 / 25, \mathrm{P}=0.986)$.

Conclusion: Chronic liver disease is more advanced in non-cirrhotic chronic hepatitis $\mathrm{C}$ patients with genotype 3 than non-genotype 3 . Genotype 3 patients have higher prevalence of cirrhosis indicated by higher AST, lower platelet count and higher LSM values as compared to non-genotype 3 patients. However, once cirrhosis developed, decompensation was not different between the two groups.

\section{$H C V-25$}

The impact of direct acting antiviral therapy on renal function in patients with chronic hepatitis $\mathrm{C}$ infection: a real-life data

Necla Tulek $^{1}$, Kader Dogan ${ }^{1}$, Sami Kinikli ${ }^{1}$, Necla Tulek ${ }^{1}$, Cigdem Ataman Hatipoglu ${ }^{1}$, Esra Kaya Kilic ${ }^{1}$, Sebnem Erdinc ${ }^{1}$

${ }^{1}$ Ankara Training and Research Hospital, Turkey

Background: Direct-acting antiviral agents have changed the treatment and prognosis of patients with chronic hepatitis $\mathrm{C}$. The choice of a regimen and duration of therapy depends on HCV genotype, presence or absence of compensated cirrhosis, previous therapy, underlying diseases and drug interactions. These drugs are generally regarded as safe and well tolerated, however, there are some concerns 
about using them for patients with hepatic and renal impairment. The aim of this study is to evaluate the effects of direct- acting antiviral treatment on renal function of patients with chronic hepatitis $\mathrm{C}$.

Method: Patients treated with direct-acting antiviral agents for hepatitis $\mathrm{C}$ infection are included inthe study retrospectively. Age, gender, treatment regimen, genotype, underlying diseases, usage of other drugs, blood urea, creatinine, and eGFR values (initial, at the end of the treatment and three months after completing the therapy) were recorded. eGFR values of the patients were compared according to gender, age, presence or absence of comorbidities and other drug usage. None of the patients had chronic renal diseases. The changes in eGFR values were analyzed at the end of the treatment and three months after the therapy. Treatment duration was 12 weeks for paritaprevir, ritonavir, ombitasvir and dasabuvir combination (PrOD), 24 weeks for sofosbuvir-ledipasvir (without ribavirin), 12 weeks for sofosbuvir-ribavirin for genotype 2 and 24 weeks for sofosbuvirribavirin for genotype 3 .

Result: Eighteen female (45\%), 22 male (55\%) totally 40 patients with the mean age of $51.5 \pm 17.5$ years (range $18-81$ years) were included in the study. Duration of the therapies was shown in the Table 1 . The predominant genotype was genotype $1 \mathrm{~b}(90 \%)$, followed by genotype $2(5 \%)$, genotype 1 a $(2.5 \%)$, and genotype $3(2.5 \%)$. Fourteen of the patients $(35 \%)$ had another diseases including hypertension, diabetes mellitus, depression, hypothyroidism; 13 $(32.5 \%)$ was taking antihypertensive drugs, antidepressants etc. Mean values of initial urea, creatinine and eGFR were $29.22 \pm 8.04 \mathrm{mg} / \mathrm{dL}$, $0.77 \pm 0.21 \mathrm{mg} / \mathrm{dL}$ and $92,38 \pm 17,85 \mathrm{ml} / \mathrm{min} / 1.73 \mathrm{~m} 2$, respectively. Initial eGFR rates of the patients were statistically higher in males $(\mathrm{p}=0,04)$, in patients $>60$ years old $(\mathrm{p}$

Conclusion: This study indicated that direct acting antiviral therapies are safe drugs and that they have no negative impact on renal functions for the patients whose functions are initially normal.

Table 1. The effect of direct-acting antivirals on eGFR values of patients with chronic hepatitis $C$ (mean eGFR values before and at the end of the treatment)

\begin{tabular}{|l|l|l|l|}
\hline & $\begin{array}{l}\text { Mean eGFR } \\
\text { Before the treatment }\end{array}$ & $\begin{array}{l}\text { Mean eGFR } \\
\text { At the end of the treatment }\end{array}$ & p value \\
\hline $\begin{array}{l}\text { Ledipasvir-sofobuvir } \\
\text { (24 weeks) } \\
(\mathbf{n}=\mathbf{1 2})\end{array}$ & $85,56 \pm 13,6$ & $96,1 \pm 13,2$ & $\mathbf{0 , 0 1 2}$ \\
\hline $\begin{array}{l}\text { PrOD (12 weeks) } \\
\text { (n=25) }\end{array}$ & $92,45 \pm 17,49$ & $98,02 \pm 17,3$ & $\mathbf{0 , 0 2 2}$ \\
\hline $\begin{array}{l}\text { Sofosbuvir-ribavirin } \\
(\mathbf{1 2} \text { weeks) } \\
(\mathbf{n}=\mathbf{2})\end{array}$ & $118,15 \pm 22,4$ & $131,1 \pm 12,5$ & $\mathbf{0 , 3 1 4}$ \\
\hline
\end{tabular}

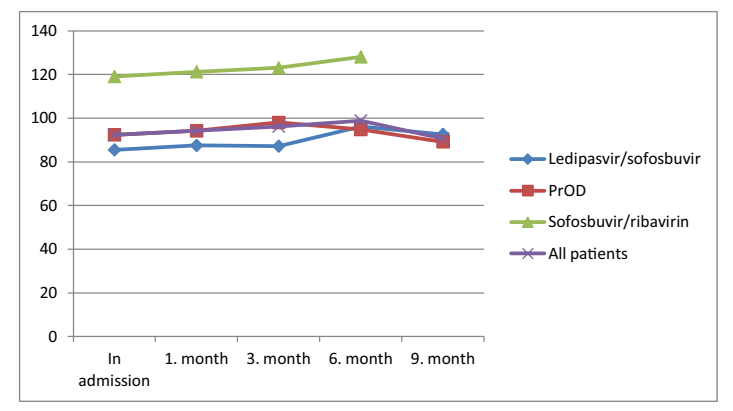

Figure 1. Mean eGFR values of chronic hepatitis $C$ patients according to the regimen received, including the values of three months after therapy
$H C V-26$

\section{Correlation of modified FIB 4 and transient elastography in patients with chronic hepatitis $\mathbf{C}$}

\section{Jose Mathew ${ }^{1}$}

${ }^{1}$ Medical College Trivandrum, India

Background: Hepatitis C virus (HCV) infection is one of the main cause of chronic liver disease worldwide. Several studies have indicated that non-invasive indices AST to Platelet Ratio Index (APRI) and Fibrosis 4(FIB-4) exhibit high reliability for predicting liver fibrosis in Chronic Hepatitis C (CHC). Recently modified FIB-4 has been proposed. Aim of the study is to look whether there is a correlation existing between modified FIB-4 and Transient elastography (TE) in CHC.

Method: All adult patients diagnosed as Chronic Hepatitis C, consulted the Department of Medical Gastroenterology Trivandrum from January 2015 to June 2017 were included in the study. Transient elastography (TE) was done on all those patients. Patient characteristics and laboratory data including AST, ALT, Platelets were collected on the same day of Transient elastography. FIB-4 calculated by the formula (Age x AST) / (Platelets $\mathrm{x}$ (sqr (ALT). mFIB-4 is calculated by the formula $10 \times$ Age (years) $\times$ AST $(\mathrm{U} / \mathrm{L}) /$ Platelet count $(10$ to the power of $9 / \mathrm{L}) \times$ ALT $(\mathrm{U} / \mathrm{L})$.

Result: The study included 106 patients with Chronic Hepatitis C. Baseline characteristics of the study population were as follows: Mean age of the study population was 48.8(ranging from 35 to 57 years). Males were constituting $65 \%$ and females were constituting $35 \%$ of the study population. $64.4 \%$ (66 patients) were infected with Genotype 3, $17.5 \%$ (18 patients) Genotype 1 and 4 each and remaining $1 \%$ with Genotype $2.34 \%$ were (36 patients) were diabetic and $6.6 \%$ (7 patients) were having dyslipidaemia. Correlation of FIB4 with Transient elastography was analysed using a scatter plot. Spearman's correlation coefficient $r$ for FIB-4 with Transient elastography was 0.529 . p value was $<0.01$. Thus a moderate correlation was noted between them. Similarly correlation between mFIB-4 and Transient elastography was also analysed. Spearman's correlation coefficient $r$ was 0.473 with a $p$ value of $<0.01$. Thus, there was a slightly increased correlation was shown by the FIB-4 as compared to the modified FIB-4 in relation with Transient elastography. In subgroup analysis, Genotype 1 had highest correlation with transient elastography followed by genotype 3 and Genotype 4 .

Conclusion: The study showed a moderate positive correlation between FIB-4 and mFIB-4 with TE, and FIB4 had a slightly higher correlation among the two. FIB-4 and mFIB-4 may be used in place of TE for assessing fibrosis in $\mathrm{CHC}$ when TE is not available. 
Scatter diagram for TE with Fib-4

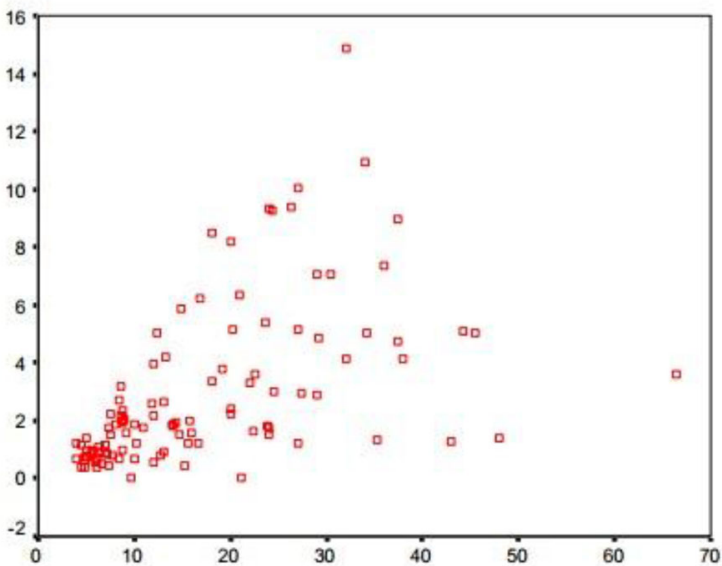

TE

\section{Scatter diagram for TE with $\mathrm{mFIB}$}

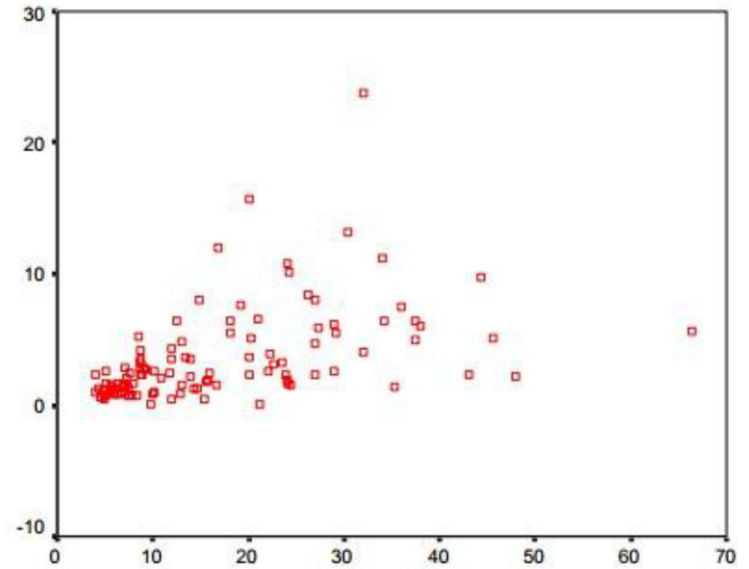

TE

\section{$H C V-27$}

Multiple comorbidities and significant pill burden in hepatitis $\mathrm{C}$ patients in a large US insured population

\section{Gabriel Wong ${ }^{1}$, Janet Lee ${ }^{1}$, Felix $\mathrm{Cao}^{2}$, Josephine Nhu Tran ${ }^{2}$}

${ }^{1}$ Gilead Sciences, Inc., United States; ${ }^{2}$ Optum, United States

Background: Decreasing pill burden and regimen complexity have been associated with improved adherence and clinical outcomes in other diseases and this may extend to hepatitis $\mathrm{C}$ virus (HCV). This study describes the pill burden and comorbidities in patients treated for HCV.

Method: Commercial and Medicare Advantage insurance claims were used to identify patients who filled HCV medications between 11/1/2013 and 7/31/2016 (index date =first fill date of HCV medication). Patients were continuously enrolled in the health plan for 9 months prior and 6 months after index date. Pill burden was calculated as mean \pm SD daily pill count for chronic, oral medications in the 90 days prior to (pre-HCV) and after index date (post-HCV). Result: Descriptive statistics were used to compare patient demographics and pill burden changes by HCV medication. The study population included 9,815 patients (mean \pm SD age 59.1 $\pm 9.7,63 \%$ male, $56 \%$ commercial). Highly prevalent comorbidities included pain $(61 \%)$ and hypertension $(50 \%)$. Pre- and post-HCV pill burden was $5.4 \pm 9.3$ and $7.7 \pm 9.6$, respectively. Pill burden was significantly higher among older patients and in those with more comorbidities $(\mathrm{p}<0.001)$. A greater proportion of Medicare patients had a post-HCV pill burden of $\geq 8$ pills/day than in commercial $(52 \%$ vs. $29 \%$, $\mathrm{p}<0.0001$ ). Post-HCV pill burden was higher among patients treated with more complex regimens, such as ombitasvir/paritaprevir/ritonavir \pm dasabuvir (Figure; $p<0.001$ ). As such, patients with HCV have a substantial pill burden even prior to their initiation of HCV treatment, and more complex HCV regimens further increase the burden by up to $300 \%$.

Conclusion: The majority of Japanese CHC patients are elderly with several comorbidities and high pill burden pre-DAA treatment. Patient pill burden may be an important consideration for $\mathrm{HCV}$ regimen selection.

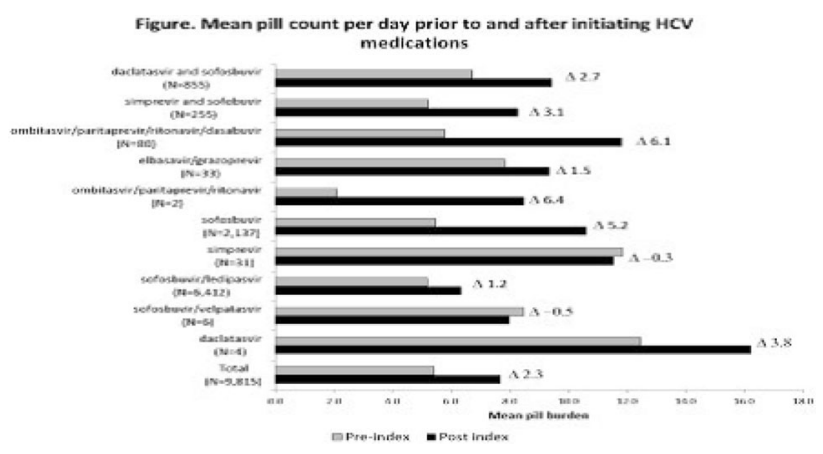

$H C V-28$

Safety and efficacy of paritaprevir/r and ombitasvir combination therapy for $\mathrm{HCV}$ infected patients with Cryoglobulinemia

\author{
Goki Suda $^{1}$, Katsumi Terashita ${ }^{1}$, Koji Ogawa ${ }^{1}$, Kenichi \\ $\overline{\text { Morikawa }}^{1}$, Naoya Sakamoto ${ }^{1}$ \\ ${ }^{1}$ Hokkaido University, Japan
}

Background: Cryoglobulinemia was reported to be observed in nearly $40 \%$ of hepatitis C virus (HCV) infected Japanese patients. So far safety and efficacy of interferon-free direct-acting antiviral agents (DAAs) for such patients has not been well assessed in Japan. In this study, we aimed to evaluate the safety and efficacy of $\mathrm{HCV}$ protease inhibitor (paritaprevir) and NS5A inhibitor (ombitasvir) combination therapy for genotype $1 \mathrm{~b}$ infected patients with cryoglobulinemia.

Method: In this prospective multicenter study, genotype $1 \mathrm{~b} \mathrm{HCV}$ infected Japanese patients, including liver cirrhosis, with an immune disorder, cryoglobulinemia, treated with paritaprevir/ $\mathrm{r}$ and ombitasvir combination therapy between February 2016 and September 2017 were included. We evaluated the sustained virologic response 12-weeks (SVR12), safety, and changes of cryoglobulinemia during and after the treatment.

Result: Eleven patients with cryoglobulinemia were treated with paritaprevir/r and ombitasvir combination therapy. The patients were aged 34-81 years (median, 63 years), and $63.6 \%$ (7/11) were male. One patient had NS5A RAV in L31M and one had NS5A RAV in Y93H at baseline. Of the 11 patients treated, 9 have reached SVR12. SVR12 data for all patients will be presented. Overall 100\% (9/9) of 
the patients achieved SVR12. Of the 9 patients, one patient discontinued the therapy due to hyperkalemia $(\mathrm{K}=7.0 \mathrm{mEq} / \mathrm{l})$ without symptom at 6 weeks after initiation of the therapy. However, after discontinuation of this combination therapy, the laboratory abnormality was restored and this patient achieved SVR12. A total of $89 \%$ (8/9) patients could complete the therapy and experienced no severe AEs, including liver decompensation. In addition, in $89 \%$ (8/9) of the patients, serum cryoglobulin was undetectable at 12 week after the treatment completion.

Conclusion: Paritaprevir/ $\mathrm{r}$ and ombitasvir combination therapy for genotype $1 \mathrm{~b} \mathrm{HCV}$ infected Japanese patients was highly effective and well-tolerated with improvement of cryoglobulinemia.

\section{$H C V-29$}

24 weeks sofosbuvir/velpatasvir/ribavirin treatment in chronic $\mathrm{HCV}$, genotype 3a, cirrhotics failures to sofosbuvir/daclatasvir/ ribavirin combination

Dimitrios - Dimitroulopoulow $^{1}$, Stavros Stavrinidis ${ }^{1}$, Dimitrios Kypreos $^{1}$, Apostolos Malahias ${ }^{1}$, Klisthenis Tsamakidis ${ }^{1}$, Dimitrios Xinopoulos

${ }^{1}$ Agios Savvas Hospital, Greece

Background: To assess the efficacy and safety of Sofosbuvir/Velpatasvir/ribavirin, in treatment-experienced to DAA's, HCV genotype 3 infected cirrhotic patients

Method: $35 \mathrm{HCV}$ patients, cirrhotics (compensated) or with severe fibrosis, previously failures to interferon/ribavirin combination, were treated with DAA's. $11(27,27 \%)$ were infected with the genotype 3 , subtype $\alpha$, of HCV and were treated with the sofosbuvir/daclatasvir/ ribavirin combination for 12 weeks.

Three $(8,57 \%$ of the total, $27,27 \%$ of the genotype 3 infected) of them (males, age 47-58 years) failed to respond to the treatment reported above. All were cirrhotics (Fibroscan 32-75 Kp(a)). For this population, was decided to receive Sofosbuvir/Velpatasvir (400/100 mg, 1x1) plus Ribavirin (1200 mg) for 24 weeks and to be followed for 12 weeks post-treatment. The pre-treatment viral load was between $0,54-$ $0,92 \times 106 \mathrm{IU} / \mathrm{ml}$ and the aminotransferase levels were: ALT 248-99 U/1, AST 179-98 U/l, $\gamma$ GT 54-203 U/l. HCV-RNA was measured at weeks 4,12,24 and 36, general blood count and liver biochemistry were monitored monthly, and measurements of AFP levels and upper abdominal ultrasound were performed every 3 months.

Result: Although, viral load was undetectable at the end of week 4 in all patients, aminotransferase and $\gamma \mathrm{GT}$ levels were abnormal during the first 12 weeks (but with a trend of normalization). At the end of week 16 normalization of liver biochemistry was observed in all patients. At the end of treatment all patients presented normal liver biochemistry and undetectable HCV-RNA. Variceal bleeding was noted in a patient at week 3 of treatment. No other serious side effects or events were observed. 12 weeks after the end of treatment all patients achieved undetectable HCV-RNA.

Conclusion: Sofosbuvir/Velpatasvir/Ribavirin for 24 weeks is an effective treatment option for cirrhotic $\mathrm{HCV}$, genotype 3, patients failures to sofosbuvir/daclatasvir/ribavirin, which achieves rapid HCV-RNA undetectability but no rapid normalization of liver biochemistry
$H C V-30$

Clinical and virological response on indian generic of DAA regimens with/without IFN in $\mathrm{HCV}$-infected patients, real world data

\section{Narina Sargsyants ${ }^{1}$}

${ }^{1}$ Armenicum Clinical Center, Armenia

Background: Real world data about high efficacy and good tolerability of directly acting antiviral agents (DAAs) generics, especially in difficult-to-treat patients could be extremely important for low- and middle-income countries, where health care budgets are not cover treatment expenses and liver transplantation not available.

The aims: Evaluation of adverse events, clinical and virological response on the different sofosbuvir (SOF) contain regimens.

Method: $52 \mathrm{HCV}$-infected patients from 18 to 73 years old (58\% male, $49.4 \pm 13.2$ years old, BMI $26.6 \pm 5.3 \mathrm{~kg} / \mathrm{m}^{2}$, viral load 1466-8897560 IU/ml) treated with DAA-contain regimens with/ without IFN (8/44). Main characteristics of the patients in Table1. IFN-free SOF contain regimens were following: $\mathrm{SOF}+\mathrm{RBV}$ in 5 patients; $\mathrm{SOF}+\mathrm{DCV} \pm \mathrm{RBV}$ in 16 patients, $\mathrm{SOF} / \mathrm{LDV} \pm \mathrm{RBV}$ in 22 patients and SOF+VLP+RBV in 1 patient with cirrhosis, ESRD, genotype $1 b+3$ (treatment was discontinuing due to worsening of eGFR). In 6 naive patients with $1 \mathrm{~b}$ genotype and viral load $<6000000$ $\mathrm{IU} / \mathrm{ml}$ duration of SOF/LDV treatment were shortened to 8 weeks. All patients were checked on 4 week of the treatment and for SVR 12 by Abbott Real-Time PCR. AFP checked in all patients with F 3 and 4 (normal $<8.78 \mathrm{ng} / \mathrm{mL}$ ). Ferritin checked in all patients with metabolic syndrome and advanced fibrosis (normal range 21.81-274.66 ng/mL). Result: Genotypes distribution: 1 and $1 \mathrm{~b}-53.8 \%, 2-9.6 \%, 1 \mathrm{~b}+2-$ $5.8 \%, 3-28.8 \%$ and $1 \mathrm{~b}+3-2 \%$. Prevalence of F4 was $56 \%$ (among them Child-Pugh $\mathrm{C}$ cirrhosis 7 patients and $\mathrm{B}-6$ ), F3-6\%, F2$38 \%$. In patients with compensated liver disease, DAAs well tolerated with non-expressed weakness in few cases and one case of nausea. In 2 experienced patients with decompensated cirrhosis, we observed first episode of severe encephalopathy with normal creatinine/urea level, resolved in 4-5 days in ICU, during which treatment was temporary interrupted, with subsequent SVR. On week $4 \mathrm{HCV}$ were detectable only in 4 patients: 3 males patients with $\mathrm{F} 4-15 \mathrm{IU} / \mathrm{ml}$ (genotype $1 \mathrm{~b}+2, \mathrm{AFP}=132.5$ ), $13 \mathrm{IU} / \mathrm{ml}$ (genotype $1 \mathrm{~b}+2, \mathrm{AFP}=11.8$ ) and $114 \mathrm{IU} / \mathrm{ml}$ (genotype $1 \mathrm{~b}, \mathrm{AFP}=26.1$ ) with subsequent SVR. In one diabetic female with genotype $1 \mathrm{~b}, \mathrm{~F} 3-43 \mathrm{IU} / \mathrm{ml}$ (starting viral load less than $6000000 \mathrm{IU} / \mathrm{ml}$ ) SOF/LDV shortened to 8 weeks, on 12 week after end-of-treatment virus still persist $15 \mathrm{IU} / \mathrm{ml}$ (relapse). 41 patients reached SVR 12 checking point -40 with SVR. AFP in average $14.0 \pm 4.7 \mathrm{ng} / \mathrm{mL}$ (range $2.0-132.0$ ) was elevated in $44 \%$ of patients with F3 or F4. In majority of patients AFP decreased after antiviral therapy, except 3 patients (elevated from 26 to 51.6, 14.3 to 18.9 and 4.2 to 7.6), despite SVR. Ferritin level range 7.3-855.84 $\mathrm{ng} / \mathrm{mL}$. Hyperferritinemia was enrolled in $10 \%$ of checked patients (all with co-morbidities), elevated in majority of patients treated with IFN-contain regimen and normalized in all after the treatment with DAAs

Conclusion: New sofosbuvir contain DAAs genetics are highly effective with good tolerability even in difficult-to-treat populations. Nevertheless, treatment of patients with decompensated cirrhosis required close monitoring by experienced clinicians. 


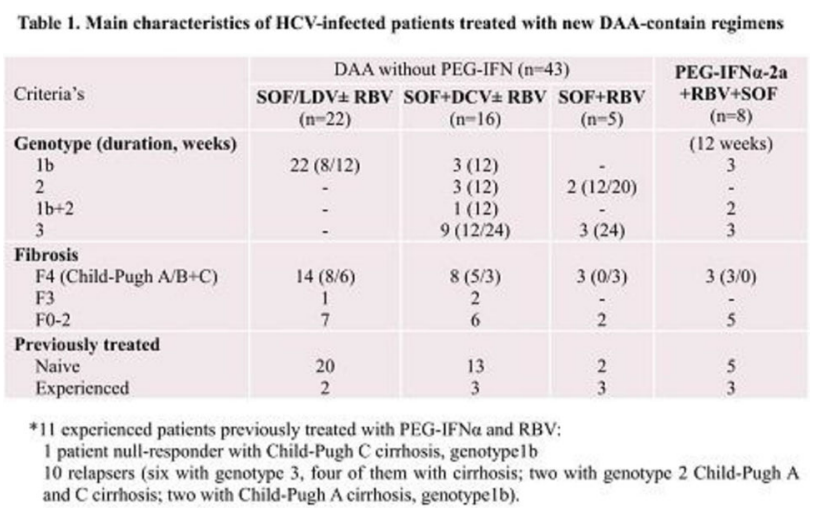

$H C V-31$

Improvement in quality of life in chronic hepatitis $\mathbf{C}$ infected patients after directly acting antiviral therapy

\section{Mridula Sharma $^{1}$, Moni Chaudhary ${ }^{2}$ \\ ${ }^{1}$ Meerut College, India; ${ }^{2}$ Sushila Jaswant Rai Superspeciality Hospital, India}

Background: HCV affects 170 million people worldwide. Extrahepatic manifestations of $\mathrm{HCV}$ like fatigue, anorexia, myalgia, arthralgia result in poor health-related quality of life (HRQOL). The treatment of hepatitis $\mathrm{C}(\mathrm{HCV})$ infections has significantly changed in the past few years due to the introduction of direct-acting antiviral agents (DAAs). The present study was aimed to assess the effect of DAA's on HRQOL in chronic HCV patients.

Method: HRQOL was assessed in 500 control subjects and 492 adult outpatients of HCV in the period from January 2016 to June 2017 by SF-36 values. HRQOL in HCV was done at baseline and at 24 weeks. Exclusion criteria were: co-infection with other viruses; coexistence of other forms of hepatic disease, associated chronic liver disease and extremely low cognitive status precluding reliable participation. The liver function tests, and quantitative measurement of Hepatitis C RNA were done at the beginning of procedure and at 6 months post treatment.

Result: The first table shows baseline pre-treatment values of 492 adult HCV patients with eight health concepts: physical functioning, bodily pain, role limitations due to physical health problems, role limitations due to personal or emotional problems, emotional wellbeing, social functioning energy/fatigue, general health perception and a comparison with age and sex matched controls of 500 normal subjects. Differences were of comparable significance for both men and women (results not shown in the tables). The scores of 36 scales ranged from 0 (lowest) to 100 (highest), with higher scores indicating better HRQOL. Compared with healthy controls, patients with chronic HCV at baseline had significantly lower HRQOL. The first table indicates a direct relationship between the chronic infection and reduction in HRQOL as lower scores were seen in all of the eight scales of the SF-36 $(\mathrm{P}$ value $<.001)$. During the treatment 400 patients achieved SVR at 12 weeks and were followed up till 24 weeks. Repeat HRQOL survey was done at 24 weeks after starting the treatment. 32 patients were lost during follow up and 60 patients did not achieve SVR. The second table shows the effect of successful DAAs and improved HRQOL after viral eradication. Successful therapy with DAAs was associated with improvement in HRQOL and the improvement was related directly to sustained virological response to treatment. The improvement in score was significant in all eight scales of the SF-36 after oral antiviral drugs in cases where sustained viral response was achieved.
Conclusion: Successful oral antiviral therapy provides meaningful improvements in HRQOL in patients with chronic hepatitis C. The effect of successful treatment indicates a direct relationship between the chronic infection and HRQOL.

Table 1

SF-36 physical and mental health parameters comparison (HCV vs normal controls)

\begin{tabular}{|l|l|l|l|}
\hline Parameter & $\begin{array}{l}\text { HCV patients } \\
(\mathbf{m e a n} \pm \mathbf{S D}) \\
\mathbf{n}=\mathbf{4 9 2}\end{array}$ & $\begin{array}{l}\text { Control } \\
(\mathbf{m}=a n \\
\mathbf{n}=\mathbf{5 0 0}\end{array}$ & P value \\
\hline Physical function & $78.2+18.8$ & $90.7+12$ & $<.001$ \\
\hline Role Physical & $68.4+30.2$ & $94.4+23.1$ & $<.001$ \\
\hline Bodily pain & $76.1+18.4$ & $88.8+17.8$ & $<.001$ \\
\hline General Health & $66.2+24.4$ & $84.2+16.6$ & $<.001$ \\
\hline Vitality & $50.1+19.5$ & $64.2+18.2$ & $<.001$ \\
\hline Social function & $74.7+22.1$ & $84.1+18.2$ & $<.001$ \\
\hline Role emotional & $78.9+28.1$ & $86.2+26.6$ & $<.001$ \\
\hline Mental Health & $75.2+17.7$ & $80.5+16.1$ & $<.001$ \\
\hline
\end{tabular}

Table 2

SF-36 physical and mental health parameters comparison post DAAs SVR 12 weeks

\begin{tabular}{|l|l|l|l|}
\hline Parameter & $\begin{array}{l}\text { Pre treatment } \\
(\mathbf{m}=a n \pm \mathbf{S D}) \\
\mathbf{n}=\mathbf{4 0 0}\end{array}$ & $\begin{array}{l}\text { Post treatment } \\
(\mathbf{m}=a n \pm \mathbf{S D}) \\
\mathbf{n}=\mathbf{4 0 0}\end{array}$ & Pvalue \\
\hline Physical function & $76.4+17.6$ & $86.7+14$ & $<0.001$ \\
\hline Role Physical & $69.4+28.2$ & $84.4+20.2$ & $<0.001$ \\
\hline Bodily pain & $74.6+19.4$ & $82.5+14.4$ & $<0.001$ \\
\hline General Health & $67.8+23.6$ & $78.2+18.6$ & $<0.001$ \\
\hline Vitality & $52.2+21.2$ & $60.2+14.4$ & $<0.001$ \\
\hline Social function & $76.8+22.6$ & $82.6+20.8$ & $<0.001$ \\
\hline Role emotional & $77.7+25.4$ & $84+22.4$ & $<0.001$ \\
\hline Mental Health & $75.7+15.7$ & $79.4+19.2$ & $<0.001$ \\
\hline
\end{tabular}

$H C V-32$

\section{Ombistasvir/paritaprevir/ritonavir and dasabuvir treatment} experience in $\mathrm{HCV}$ patients

\section{Mustafa Kemal Celen ${ }^{1}$, Kadim Bayan ${ }^{3}$, Mustafa Kemal Celen ${ }^{1}$,} Recep Tekin ${ }^{1}$, Ozgur Gunal $^{4}$, Tuba Dal ${ }^{5}$, Irem Akdemir ${ }^{2}$

${ }^{1}$ Dicle University Hospital, Turkey; ${ }^{2}$ Batman State Hospital, Turkey; ${ }^{3}$ Private Sultan Hospital, Department of Gastroenterology, Turkey; ${ }^{4}$ Samsun Training \& Educational Hospital, Turkey; ${ }^{5}$ Yildirim Beyazit University Hospital, Turkey

Background: Achieving sustained virologic response (SVR) is critical in HCV patients. In current study, we aimed to investigate the efficacy and safety of DAA (OBV/PTV/r $+\mathrm{DSV} \pm \mathrm{RBV})$ treatment regimen in patients with $\mathrm{HCV}$.

Method: A total of 57 adults with HCV infection who initiated treatment DAA were included in this study. Baseline, 4 weeks and 12 weeks data including clinical characteristics, laboratory results and adverse events (AEs) were recorded.

Result: One patient left treatment at the second week. The majority of patients were female $33(57.9 \%)$ and HCV GT1b $(80.7 \%)$. Of the patients, $43(75.4 \%)$ patients were treatment experienced with pegylated interferon \pm RBV and 14 (25.6\%) patients were naive. Ten (17.5\%) patients had liver cirrhosis, 3 patients renal insufficiency, 7 patients diabetes mellitus, and 6 patients hypertension. Mean baseline ALT and HCV RNA levels was 48.58 $\pm 25.8 \mathrm{U} / \mathrm{L}$ and $2857661 \pm 7231938 \mathrm{IU} / \mathrm{ml}$, respectively. At the end of the week 4 , one patient had $>15 \mathrm{IU} / \mathrm{mL}$ HCVRNA level, HCVRNA was negative in 
remaining patients and mean ALT levels was 34.7 $\pm 17.01 \mathrm{U} / \mathrm{L}$. Three (5.3\%) patient had AEs. At the end of the week 12, HCVRNA was negative in all patients, mean ALT levels was $29.3 \pm 12.2 \mathrm{U} / \mathrm{L}, 8$ (14\%) patients had AEs.

Conclusion: DAA was a safe and effective therapy with $100 \%$ SVR rate and low treatment discontinuation rate $(1.7 \%)$ in patients with HCV GT1, GT1a and GT1b. This was well tolerated and efficient treatment approach in patients with liver cirrhosis, renal insufficiency, diabetes mellitus, and hypertension.

\section{$H C V-33$}

Predictive value of hepcidin in patients with chronic hepatitis $\mathrm{C}$ infection among Egyptians

Eman Abdelsameea ${ }^{1}$, Doaa Ibrahim ${ }^{3}$, Hisham A. Ismail ${ }^{4}$, Mohamed El Assal $^{2}$, Mai Abuzeid ${ }^{5}$

${ }^{1}$ Assistant Professor, Egypt; ${ }^{2}$ Faculty of Medicine, Benha University, Egypt; ${ }^{3}$ Faculty of Science, Menoufia University, Egypt; ${ }^{4}$ GEBR institute, Sadat University, Egypt; ${ }^{5}$ National Liver Institute, Menoufia University, Egypt

Background: Iron is essential for oxygen transport and energy production but it is potentially toxic through generation of oxidative stress. Patients with chronic hepatitis $\mathrm{C}(\mathrm{CHC})$ infection frequently have serum and hepatic iron overload which is a co-factor for disease progression. Human hepcidin, a 25-amino acid peptide produced by hepatocytes, is considered a new mediator of innate immunity and master iron-regulatory hormone.

Method: Aim: To determine clinical relevance of hepcidin in patients with $\mathrm{CHC}$ infection and whether it correlates with markers of hepatic inflammation and iron status in those patients.

Subjects and methods: This study included 80 individual (54 patients, 26 controls) who were matched in age and sex. Serum hepcidin levels and serum iron status were determined. Their levels were correlated to each other and to other laboratory parameters.

Result: Mean serum hepcidin levels were significantly lower in $\mathrm{CHC}$ patients than in controls $(\mathrm{p}=0.001)$. Patients with $\mathrm{CHC}$ had significantly higher mean values of serum iron and ferritin than controls $(\mathrm{p}=$ 0.001 and 0.017 respectively). There was a significant negative correlation between hepcidin levels and ALT, $(\mathrm{r}=-0.454, \mathrm{p}<0.001)$, AST $(r=-0.440 ; p=0.001)$ and serum iron $(r=-0.453, p=0.001)$ while hepcidin levels correlated positively with ferritin $(r=0.105, p=$ 0.017), platelets count $(\mathrm{r}=0.245, \mathrm{p}=0.028)$ and with HCV RNA level $(\mathrm{r}=0.308, \mathrm{p}=0.023)$

Conclusion: Adequate levels of hepcidin may improve severity of liver disease\& may contribute to management of $\mathrm{CHC}$ patients.

\section{$H C V-34$}

Risk of impaired fasting glucose (IFG)/T2DM and atherosclerosis in $\mathrm{F} 3 / \mathrm{F} 4 \mathrm{HCV}$ patients

\section{Peter Jarcuska ${ }^{1}$, Sylvia Drazilova ${ }^{2}$, Martin Janicko ${ }^{3}$, Ivan} Schreter $^{3}$, Pavol Kristian ${ }^{3}$, Ladislav Virag ${ }^{3}$

${ }^{1}$ University Hospital and University of PJ Safarik, Faculty of Medicine, Slovakia; ${ }^{2}$ Hospital Poprad, Slovakia; ${ }^{3}$ University Hospital and University of PJ Safarik, Faculty of Medicine, Slovakia

Background: Aim to the study: To find IFG/T2DM prevalence in HCV patients with advanced fibrosis or cirrhosis. To objective atherogenic risk in $\mathrm{HCV}$ patients with advanced fibrosis or cirrhosis.
Method: We investigated 121 pts (70 women, $59 \%$; mean age $59 \pm 10$ year) before beginning of DAA treatment. Study has retrospective design. 119 patients had genotype 1 (110 pts genotype $1 \mathrm{~b}), 2$ patients had genotype 3.34 patients were naive (28.1\%). 93 pts had stage F4 (76,9\%), 28 pts F3 (23.1\%). 20 pts were treated for T2DM (16.5\%). Prevalence of impaired fasting glucose (IFG) or T2DM and atherogenic risk were evaluated.

Result: Impaired fasting glucose (IFG) or T2DM was found in $60.7 \%$ of F3 pts and in $59.1 \%$ of F4 pts (NS), while $21.4 \%$ of F4 pts and $21.5 \%$ of $\mathrm{F} 3$ pts were diagnosed with T2DM (NS). Triacylglycerols level $>1,7 \mathrm{mmol} / \mathrm{l}$ was observed in $37.2 \%$ of $\mathrm{F} 4$ pts and in $0 \%$ of $\mathrm{F} 3$ pts $(\mathrm{p}=0.012)$. Atherogenic index of plasma in high risk was found in $25.6 \%$ of $\mathrm{F} 4$ pts and in $0 \%$ of $\mathrm{F} 3$ pts $(\mathrm{p}<0.05)$.

Conclusion: F3 and F4 HCV patients have similar, but high risk of IFG or T2DM, bu tF4 patients have significantly higher cardiovascular risk than those with F3 stage of fibrosis.

$H C V-35$

Sustained Virological Response (SVR) at 12 weeks post-treatment (SVR12) is equivalent to SVR at 24 weeks post-treatment (SVR24) in the end point assessment in the treatment of chronic $\mathrm{HCV}$ infection with (Direct-Acting-Antivirals) DAAs - real life experience: Myanmar

Khin Maung Win ${ }^{1}$, Su Su Htar ${ }^{4}$, Soe Thiha Maung ${ }^{4}$, Si Thu Sein Win $^{4}$, Naomi Khaing Than Hlaing ${ }^{3}$, Soe Tint ${ }^{4}$, Urvashi Singh ${ }^{2}$, Aung Hlaing Bwa ${ }^{4}$, Khin Aye Wint $\mathrm{Han}^{4}$

${ }^{1}$ University of Medicine 1, Myanmar; ${ }^{2}$ The Queens University of Belfast, Northern Ireland, UK, United Kingdom; ${ }^{3}$ University of Medicine, Mandalay, Myanmar; ${ }^{4}$ Yangon GI and Liver Centre, Myanmar

Background: SVR is achieved when the viral load is below the threshold of $15 \mathrm{IU} / \mathrm{ml}$ or is undetectable. SVR12 and SVR24 have long been used as therapeutic follow up endpoints in the treatment of chronic HCV. Most organizational guidelines recommend the use of both SVR12 and SVR24 in clinical practice. The aim of this study was to investigate whether there is $100 \%$ concordance between SVR12 and SVR24 in the treatment of HCV by sofosbuvir-based therapy.

Method: This study was conducted upon the 225 patients who received treatment at Yangon GI \& Liver Centre from October 2015 to January 2017. Quantitative HCV RNA measurement was conducted with Roche COBAS AmpliPrep/COBAS TaqMan HCV test and HCV genotyping was done by Roche Cobas 4800 System. Only those who completed treatment monitoring of both SVR 12 and SVR 24 were analyzed. Two main endpoints were used for this study namely SVR12 and SVR24. There 4 main identified genotypes in Myanmar 1,2,3 and 6. Those whose genotype could not be identified were grouped under "indeterminate".

Result: Out of all the patients, the distribution of genotypes was as follows; Genotype-1 49 patients (22\%), Genotype-2 2 patients (1\%), Genotype-3 76 patients (33\%), Genotype-6 93 (42\%) and Genotype indeterminate - 5 patients $(2 \%)$.

In genotype 1 , there were 35 treatment naïve and 14 treatment experienced patients. The 2 patients of genotype 2 were prescribed SOF/PEG/RBV for 12 weeks. Both patients were treatment naïve. In genotype 6 , there were total of 93 patients of which 74 patients were treatment naïve while 14 patients were treatment experienced. In genotype 3, there was a total of 76 patients which were further divided up as 61 treatment naïve and 15 treatment experienced patients.

Six main treatment regimens were given to patients; 
1) Sofosbuvir/Ledipasvir (SOF/LED) \pm Ribavirin (RBV) for $12 / 24$ weeks

2) Sofosbuvir/Ribavirin (SOF/RBV) for 24 weeks

3) Sofosbuvir, Daclatasvir (SOF/DCV) \pm Ribavirin (RBV) for $12 / 24$ weeks

4) Sofosbuvir, Peg-IFN, Ribavirin (SOF/PEG/RBV) for 12 weeks. All 225 patients recruited for the study who achieved SVR12 also achieved SVR24 irrespective of the different treatment regimens and different genotypes. This was also demonstrated by the $100 \%$ positive predictive value for all treatment regimens with varying durations across all genotypes. Thus, it can be inferred that SVR12 and SVR24 are concordant in the case of this data.

In all the patients, cirrhotic or non-cirrhotic, those who achieved SVR12 also achieved SVR24.

The concordance of SVR12 and SVR24 was proven in the case of this study regardless of different Sofosbuvir based regimens, irrespective of prevalent genotypes in Myanmar and in both cirrhotic and non-cirrhotic states. This confirms that SVR12 may be used as a follow up endpoint in clinical practice in all possible resource settings.

Conclusion: It is concluded that SVR12 alone should be used and SVR24 is no longer necessary as the endpoint of the DAA-based HCV therapy. This paper will help modify the future HCV treatment clinical guidelines locally as well as internationally.

\begin{tabular}{|c|c|c|}
\hline GENOTYPE & 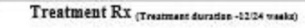 & No. of patients \\
\hline \multirow[t]{5}{*}{1} & SOF/LED 12 & 23 \\
\hline & SOF/LED $/ R^{\prime} V_{12}$ & 1 \\
\hline & SOF/LED/RBV ${ }_{24}$ & 4 \\
\hline & SOF/PEGRBV 12 & 14 \\
\hline & $\mathrm{SOF} / \mathrm{RBV}_{34}$ & 7 \\
\hline 2 & SOF/PEG/RBV 12 & 2 \\
\hline \multirow[t]{5}{*}{3} & SOF $/ D^{\prime} C_{12}$ & 5 \\
\hline & $\mathrm{SOF} / \mathrm{DAC}_{24}$ & 5 \\
\hline & SOF/DACRBV ${ }_{24}$ & 3 \\
\hline & SOF/PEGRBV 12 & 29 \\
\hline & $\mathrm{SOF}_{\mathrm{RBV}} \mathrm{z4}$ & 34 \\
\hline \multirow[t]{6}{*}{6} & SOF/PEG $/ R_{B V} V_{12}$ & 26 \\
\hline & SOF/LED $_{12}$ & 30 \\
\hline & SOF/LED $_{24}$ & 6 \\
\hline & SOF/LED/RBV & 4 \\
\hline & SOF/LED /RBV & 6 \\
\hline & $\mathrm{SOF}_{/ R_{B}}$ & 21 \\
\hline \multirow[t]{3}{*}{ Indeterminable } & SOF/LED 12 & 1 \\
\hline & SOF/LED $_{2 t}$ & 3 \\
\hline & SOF/PEG/RBV 12 & ${ }^{1}$ Active \\
\hline \multicolumn{2}{|c|}{ TOTAL NO OF PATIENTS } & ${ }^{225}$ Go to Se \\
\hline
\end{tabular}

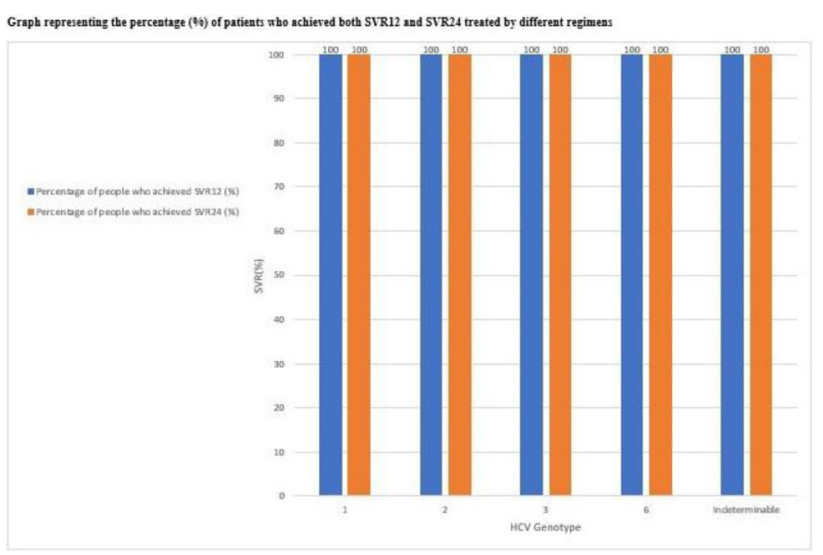

$H C V-36$

Treatment of chronic hepatitis $\mathrm{C}$ genotype $\mathrm{I}$ with ombitasvir/paritaprevir/ritonavir plus dasabuvir

Silva Gjika ${ }^{1}$, Liri Cuko', Adriana Babameto ${ }^{1}$, Stela Taci ${ }^{1}$, Ilir Bibolli $^{1}$, Irgen Tafaj ${ }^{1}$, Silva Gjika ${ }^{1}$, Jonida Lavdari ${ }^{1}$, Bajram Begaj $^{1}$

${ }^{1}$ QSU Nene Tereza, Albania

Background: Direct-acting antiviral (DAAs) have revolutionized the treatment of $\mathrm{HCV}$ with cure rates of 95-100\%. In 2015 European Medicines Agency approved combination of antiviral therapy for chronic hepatitis C 1 genotype with ombitasvir/paritaprevir/ritonavir plus dasabuvir. Concomitant inhibitions of the key viral proteins results in a potent suppressions of HCV replication. This antiviral regimen may be used in patients with severe renal impairment, including hemodialysis patients.

Method: In this study are included 16 experienced patients who were treated for chronic HCV infection 1 genotype with ombitasvir/paritaprevir/ritonavir plus dasabuvir ribavirin between 2016-2017. All patients had previous treatment failurewith interferon. Main laboratory studies were performed in all patients at the baseline and during the treatment. Inclusion criteria were patients with chronic hepatitis $\mathrm{C}$ 1 genotype, including those with cirrhosis Child-Pugh A or B. Are not included patients with confection, hepatocellular carcinoma or decompensate liver disease.

Result: $37.5 \%$ (6) were female and 62\%(10) male. Main age was 44.5 years, history of previous interferon-based treatment failure had $100 \%(16 / 16)$ of patients. Median of baseline viral load was 1000000 $\mathrm{UI} / \mathrm{ml}$. By elastography F1, F2, F3, F4 were $18.7 \%$ (3), 25\%(4),31\% (5),25\% (4) respectively. 13 patients were $1 \mathrm{~b}$ subgenotype and 3 were 1a subgenotype. Among patients with cirrhosis (F4) 2 patients had cirrhosis Child-Pugh A, and 2 Child Pugh B. All patients had negative RNA HCV by PCR at the end of treatment course (SVR 12). 24 weeks after the discontinuation of the treatment only one had the positive RNA HCV, the patient was with cirrhosis Child-Pugh B and needed 24 weeks treatment. SVR 12 were $100 \%$, and SVR 24 were $95 \%$.

Conclusion: The results of the treatment were comparable to the other results pivotal. There were no serious adverse events during the treatment. It was the first experience in our country Albania. The overall observed safety profile of ombitasvir/paritaprevir/ritonavir plus dasabuvir was acceptable. 
$H C V-37$

VIEKIRA PAK (ritonavir-boosted paritavir/ombitasvir and dasabuvir) associated drug-induced interstitial lung disease: case series with systematic review of literature

Yu Jun Wong ${ }^{1}$, Si Yuan Chew ${ }^{1}$, John Hsaing Chen ${ }^{2}$, Prem Harichander Thurairajah ${ }^{2}$, Rahul Kumar ${ }^{2}$, Roshni Sadishiv Gokhle $^{2}$, Imran Mohamed Noor ${ }^{2}$, Yi Lyn Jessica Tan ${ }^{2}$

${ }^{1}$ Singhealth, Singapore; ${ }^{2}$ Changi General Hospital, Singapore

Background: Chronic hepatitis $\mathrm{C}$ virus (HCV) infection is a serious cause of liver-related morbidity and mortality worldwide, affecting more than 170 million patients globally. Oral direct-acting antivirals (DAA) have since replaced conventional interferon and ribavirin as first line HCV therapy due to a better tolerability profile and high efficacy in achieving sustained virological response. VIEKIRA PAK (ritonavir-boosted paritavir/ombitasvir and dasabuvir), a combination of potent DAA, is an effective initial treatment for HCV genotype 1 infection in patients with or without cirrhosis and in patients who may or may not have received interferon-ribavirin therapy before.

Method: We describe two patients who developed severe DILD requiring mechanical ventilation for respiratory failure on day 8 and day 40 of VIEKIRA PAK therapy who recovered after supportive management with systemic corticosteroids and systemically reviewed the literature on VIEKIRA PAK associated drug-induced interstitial lung disease.

Result: Although the incidence of pulmonary toxicity is low in Phase 3 clinical studies, there have been emerging case reports of VIEKIRA PAK associated DILD. While recovery is reported with supportive care in all cases, VIEKIRA PAK associated DILD is often lifethreatening and VIEKIRA PAK may have to be discontinued permanently.

Conclusion: In summary, VIEKIRA PAK associated DILD is a rare yet potentially near-fatal complication that should be recognized early by physicians.

\section{$H C V-38$}

Sofosbuvir and ledipasvir fixed-dose combination without ribavirin in treatment-naive patients with hepatitis $\mathbf{C}$ virus infection in Mongolia

\section{Nyamsuren Naranzul $^{1}$, Oidov Baatarkhuu ${ }^{1}$}

${ }^{1}$ Mongolian National University of Medical Sciences, Mongolia

Background: Mongolia has a large burden of viral hepatitis, especially chronic hepatitis B virus (HBV) and hepatitis C virus (HCV) infections, which are associated with cancer and cirrhosis. Chronic HBV infection is acquired in early childhood in Mongolia, while $\mathrm{HCV}$ and hepatitis delta virus (HDV) transmission is healthcare related. Mongolia also has the highest, and increasing, rate of liver cancer and mortality from liver cancer in the world. Cancer is the second most common cause of death in Mongolia and liver cancer is responsible for $44 \%$ of all cancers. Chronic hepatitis B and C infections are responsible for $95 \%$ of liver cancers in the country. As of December 25, 2015 branded ledipasvir/sofosbuvir (Harvoni), and 4 kinds of generic ledipasvir/sofosbuvir are available in Mongolia. We followed and evaluated treatment outcomes of the patients with HCV infection using Hepcinat-LP (manufactured by Natco pharma).

Method: We started our prospective analysis on December 2016 until June 2017, for 3 months, on 124 patients. All patients were treated with SOF/LDV for 12 weeks and, their treatment was evaluated by quantitative HCV-RNA assays prior and 12 week of treatment.
Sustained Virological Response (SVR) after 12 weeks of treatment was assessed.

Result: 124 patients with an average age of $54 \pm 11$ (range: 29 - 78 years old) were included in the study. In the result, patients were chronic hepatitis C 83\%, liver cirrhosis Child-Pugh A 1.4\%, ChildPugh B $13.4 \%$. According to the study, F0 stage was 49\%, F1 stage $22 \%, \mathrm{~F} 2$ stage $9.5 \%$ and F3 stage $19 \%$. SVR12 rates were $98.77 \%$. AST and ALT levels were significantly decreased and normalized within the first 4 weeks and remained stable until follow-up week 12 of treatment $(\mathrm{P}<0.001$ and $\mathrm{P}<0.001$, respectively). All patients were achieved SVR12 (100\%).

Conclusion: Considering the results of this study and the ease of use (one pill a day) we believe Hepcinat-LP can be the best choice for treating all cases of hepatitis $\mathrm{C}$ in Mongolia.

\section{$H C V-39$}

SVR achievement in Mongolian patients with hepatitis $C$ treated SOF/LED regimen: a multi-center study

Nyamsuren Naranzul, ${ }^{1}$, Oidov Baatarkhuu' ${ }^{1}$, Naranzul Nyamsuren $^{1}$, G Khishigjargal ${ }^{3}$, D Javzmaa ${ }^{7}$, D Oyuntuya ${ }^{6}$, S Nyamaa $^{5}$, T Tumentogtokh ${ }^{2}$, B Saruul $^{4}$

${ }^{1}$ Mongolian National University of Medical Sciences, Mongolia; ${ }^{2}$ Govi-Altai Province Hospital, Mongolia; ${ }^{3}$ Khentii provinces Hospital, Mongolia; ${ }^{4}$ National Center for Communicable Diseases, Mongolia; ${ }^{5}$ Songinokhairkhan District Hospital, Mongolia; ${ }^{6}$ Tuv Provinces Hospital, Mongolia; ${ }^{7}$ Zavkhan provinces Hospital, Mongolia

Background: In Mongolia, previous studies shown HCV prevalence is over $10 \%$ and $97-98 \%$ of people with HCV infection have infected with genotype $1 \mathrm{~b}$. Therefore activities on reducing chronic infection prevalence of hepatitis viruses and preventing complications of hepatitis viral infections have been conducted in the country. One of them is availability of Harvoni treatment for HCV patients since December 2015. To evaluate data on the antiviral efficacy and safety of direct acting antiviral (DAA) treatment with respect to sustained virological response (SVR) 12 weeks after completion of treatment.

Method: We retrospectively analyzed patient monitoring records and patient registration forms for $\mathrm{HCV}$ patients who received Harvoni treatment at NCCD, MNUMS, provinces and districts hospitals. Quantitative methods were applied in that retrospective study. Six hundred and forty-seven patients diagnosed as HCV and treated by Harvoni (ledipasvir/sofosbuvir) were attended the study.

Result: There were totally 647 patients received Harvoni for HCV infection by September 2016. People who received treatment for less than 3 months there $31 \%$ and for longer than 3 months were $8 \%$. Among them $91.9 \%$ have chronic hepatitis and first stage of liver cirrhosis and $8 \%$ have liver cirrhosis and carcinoma. After 1 month of treatment, HCV RNA tests result was negative for $98.8 \%$ of all Harvoni patients and for the rest $1.1 \%$ resulted in decrease of $\mathrm{HCV}$ RNA. After 3 month of trerapy, blood test result showed $100 \%$ recovery on transaminase level. 453/465, 10/465 and 2/465 of them were respectively genotype $1 \mathrm{~b}, 2$ and $1 \mathrm{a}$. APRI score were pretreatment $1.3 \pm 0.58$ and post treatment $0.443 \pm 0.148$. FIB4 score were pre-treatment $3.8 \pm 1.2$ and post treatment $1.65 \pm 0.59$. Occurrences of side effects were mild. $1.2 \%, 5.8 \%$ and $4.6 \%$ of them were respectively with CTP C, CTP B and CTP A scores. $88.2 \%$ of the participants were chronic hepatitis $\mathrm{C}$ and $1.7 \%$ of them were pretreated by interferon.

Conclusion: After treatment by Harvoni tablets, excellent SVR12 results were shown among the study participants' and the favorable side-effect profile were observed for the Mongolian context. 


\section{$\mathrm{HCV}-40$}

The effect of antiviral therapy on serum triglyceride level in chronic hepatitis $\mathrm{C}$

\section{Batbold Batsaikhan}

${ }^{1} \mathrm{PhD}$ candidate, Taiwan

Background: Low lipid profile is associated with hepatitis $\mathrm{C}$ virus (HCV) infection and maybe predicts $\mathrm{HCV}$ treatment response. Chronic HCV infection is the main cause of liver fibrosis and advanced liver fibrosis may alter lipid profiles during HCV treatment. We aimed to evaluate the effect of antiviral therapy on lipid profiles and to investigate the factors related to the changes of lipid profiles in chronic hepatitis $\mathrm{C}$ patients.

Method: Total 863 patients who treated interferon based anti HCV therapy in Kaohsiung Medical University Hospital from 2004 to 2015 included in this study. The lipid profile measured and evaluated in baseline, end of the treatment and after 6 months of the cessation of the anti-HCV treatment.

Result: Sustained virologic response (SVR) was achieved in $81.2 \%$ of all patients. The baseline TG levels in the SVR group $(90.5 \pm 34.3 \mathrm{mg} /$ dL) and non SVR groups $(93.5 \pm 37.3 \mathrm{mg} / \mathrm{dL})$ were similar. The TG levels at 6 months after cessation of the treatment was significantly elevated in SVR group $(102.8 \pm 56.9 \mathrm{mg} / \mathrm{dL}, \mathrm{p}=0.0001)$ but did not elevated in non SVR group $(94.5 \pm 45.6 \mathrm{mg} / \mathrm{dL}, \mathrm{p}=0.708)$ compared with baseline TG levels. After adjusting patients by four indexes for fibrosis (FIB4) in cut-off point 3.25, serum TG levels significantly increased in low FIB4 group $(103.2 \pm 57.9 \mathrm{mg} / \mathrm{dL}, \mathrm{p}=0.0001)$ but not in high FIB4 group $(98.1 \pm 49.6 \mathrm{mg} / \mathrm{dL}, \mathrm{p}=0.095)$ after 6 months end of the treatment. The other lipid profiles such as total cholesterol, high density lipoprotein cholesterol and low density lipoprotein cholesterols were increased in both high and low FIB4 groups. Serum TG level was increased greater in patients who had low FIB4 score and patients who achieved SVR (baseline $89.1 \pm 34.8 \mathrm{mg} / \mathrm{dL} ; 6$ months after treatment $104.3 \pm 59.3 \mathrm{mg} / \mathrm{dL}$, paired $\mathrm{T}$ test $\mathrm{p}=0.0001)$. The change of serum TG level for low FIB4 $(15.1 \pm 53.3 \mathrm{mg} / \mathrm{dL})$ and high FIB4 $(6.7 \pm 47.9 \mathrm{mg} / \mathrm{dL})$ groups were significantly $(\mathrm{p}=0.041)$ different in patients who achieved SVR.

Conclusion: Our study support that SVR is the main determinant of the increase of lipids after Pegylated Interferon and Ribavirin treatment. However mild fibrosis also has an effect in increase of lipids after the treatment.

\section{HCV-41}

Adverse clinical incidents after achieving SVR24 in patients with chronic Hepatitis $C$ and cirrhosis treated with DAA

\section{Jolanta Opoka Kegler ${ }^{1}$, Ewa Marcinkowska ${ }^{1}$, Grazyna Cholewinska $^{1}$ \\ ${ }^{1}$ Hospital for Infectious Diseases, Poland}

Background: Despite the effective treatment of Hepatitis C, advanced liver diseases apart from the SVR were observed in a group of patients. This study presented the incidences of abnormal hepatic markers and HCC after successful treatment and SVR24.

Method: This is an observational study of 140 patients with Hepatitis $C$ at an average age of 62 years, who achieved SVR24 after the 12 -week treatment with 3D. $96 \%$ of them confirmed G1b, two patients had G1a and three G4. All patients had fibrosis at F4 level in the elastography. This study assessed the frequency of adverse incidents, e.g. hypertransaminasis, hyperbilirubinaemia, AFP increased, cirrhosis decompensation, and HCC, which appeared within 18 months since the follow-up from SVR24. The results were evaluated by the $\mathrm{Chi}^{2}$ test.

Result: All of the 140 patients (M-61 K-79) achieved SRV24. During the 18-month follow-up period, 19 patients (13\%) had an adverse clinical incidents, such as persistent hyperbilirubinaemia, elevated transaminases levels, cirrhosis decompensation, or HCC. Among 42 patients with hyperbilirubinemia, 39 (92.8\%) normalized BIL, and ALT/AST normalization were confirmed in 118 (95.1\%) out of 124 with hypertransaminases. Both values were statistically significant ( $\mathrm{p}$ $<0.001$ ). Only two subjects after SVR developed decompensated liver cirrhosis. Prior to treatment, 7 patients were diagnosed with HCC, but in 4 of them after SVR24 occurred progression to multifocal HCC. No tumor progression after treatment was found in 3 patients. In all studied populations $(\mathrm{n}=140), 11$ cases of $\mathrm{HCC}$ were identified, but no significance was found for this analysis $(\mathrm{p}=0.575)$. Four people $(2.8 \%)$ revealed HCC de novo, after obtaining SVR. During the study, 50 subjects $(87.7 \%)$ among those $(n=57)$ who had AFP elevation before treatment, normalized the cancer marker level AFP. All patients without HCC prior to treatment, normalized AFP after SVR $(\mathrm{p}<0.001)$. Patients who developed multifocal changes in liver after SVR achieved, presented more than 10-fold increase in AFP.

Conclusion: Late consequences of chronic liver disease were reported in 19 subjects $(13 \%)$ :

Four individuals $(2,8 \%)$ - developed HCC de novo after SVR24. Patients who achieved SVR should continue the long-term monitoring of the liver function.

\section{$\mathrm{HCV}-42$}

Hepatitis B reactivation among individuals with chronic hepatitis $\mathrm{C}$ treated with pan-oral direct-acting antivirals: a single-center study in Taiwan

Shou Wu Lee ${ }^{1}$, Teng Yu Lee ${ }^{1}$, Sheng Shun Yang ${ }^{1}$, Hong Zen Yeh ${ }^{1}$, Chi Sen Chang ${ }^{1}$

${ }^{1}$ Taichung Veterans General Hospital, Taiwan

Background: Clearance of $\mathrm{HCV}$ has been reported to induce the reactivation of $\mathrm{HBV}$. The aim of this study was to investigate the rate of $\mathrm{HBV}$ reactivation in $\mathrm{HCV}$-infected patients who received treatment with pan-oral direct-acting antivirals (DAAs).

Method: Data from HCV subjects receiving oral DAA therapy were retrospectively collected from October 2015 to May 2017. Patients who were seropositive for $\mathrm{HBsAg}$ or anti-HBc were enrolled. The efficacy of DAAs, including end-of-treatment virologic response (ETVR) and sustained virologic response (SVR) 12, were recorded. $\mathrm{HBV}$ virologic reactivation was defined as a reappearance of $\mathrm{HBsAg}$, or increased HBV DNA by at least one $\log 10 \mathrm{IU} / \mathrm{ml}$. HBV clinical reactivation was defined as virologic reactivation and serum alanine aminotransferase (ALT) over 2-fold of the upper limit of normal.

Result: There were $11(7.2 \%)$ cases and $53(34.6 \%)$ cases in the HBsAg group and the anti-HBc group among all 153 subjects. All individuals achieved ETVR and SVR12. There were no cases with reappearance of HBsAg during DAAs therapy. Among 7 cases in the HBsAg group whose HBV DNA level was determined, HBV virological reactivation was detected in 2 subjects $(28.6 \%)$. Among all 11 subjects in the HBsAg group, there was one $(9.1 \%)$ case with HBV clinical reactivation, which was resolved following treatment with Entecavir. The case with HBV clinical reactivation had a higher baseline HBV DNA viral load $(1,380 \mathrm{IU} / \mathrm{ml})$ compared with that of the other patients $(20-296 \mathrm{IU} / \mathrm{ml})$. 
Conclusion: HBV virological and clinical reactivation occurred in $28.5 \%$ and $9.1 \%$ of subjects with HBsAg seropositivity. No HBV reactivation was observed in the cases with past $\mathrm{HBV}$ infection.

\section{$H C V-43$}

Influence of direct acting antivirals for patients with hepatitis $\mathbf{C}$ on lipid metabolism

\author{
Hidehiro Kamezaki ${ }^{1}$, Miyuki Sensui ${ }^{1}$, Junichi Senoo ${ }^{1}$, Dai \\ Sakamoto \\ ${ }^{1}$ Eastern Chiba Medical Center, Japan
}

Background: With the advent of direct acting antivirals (DAA), it has become possible to eliminate viruses in many patients with hepatitis C. It has also been clarified that hepatic reserve such as the albumin value improves in patients who have achieved sustained viral response (SVR). Along with such improvement in nutritional condition, there have been concerns about influence on lipid metabolism with some patients. In this study, we analyzed patient backgrounds and clinical courses retrospectively and examined the abnormality of lipid metabolism.

Method: Out of the 53 cases in which DAA were introduced at our hospital from October, 2014 to March, 2017, 48 cases (one non responder, two cases of treatment discontinuation, two cases of follow-up discontinuation) for which SVR was confirmed were studied. DCV/ASV was used in 8 cases, SOF/LDV in 16 cases, OBV/PTV/r in 3 cases, SOF/RBV in 20 cases, OBV/PTV/r/RBV in 1 case. There were no patients to whom a BCAA preparation was newly introduced during the course of the treatment. This clinical study has been approved by the ethics committee of our hospital.

Result: The Alb value was $4.17 \pm 0.32 \mathrm{mg} / \mathrm{dL}$ at the start of the treatment, $4.21 \pm 0.29 \mathrm{mg} / \mathrm{dL}(\mathrm{P}=0.354)$ at the end of the treatment, $4.30 \pm 0.30 \mathrm{mg} / \mathrm{dL}(\mathrm{P}=0.002)$ twelve weeks after the end of the treatment, and $4.45 \pm 0.32 \mathrm{mg} / \mathrm{dL}(\mathrm{P})$.

Conclusion: It was clarified by the examination at our hospital too that nutritional condition improves in patients who DAA have been introduced to and have achieved SVR. It was also clarified that LDLChol and non HDL-Chol, a risk factor of arteriosclerosis, also increased.

\section{$\mathrm{HCV}-44$}

\section{Multicentre results of treatment and side effects of new antivirals in hepatitis $\mathbf{C}$}

\section{Aliye Soylu ${ }^{1}$, Aliye Soylu' ${ }^{1}$, Serdal Cakmak ${ }^{1}$, Sule Poturoglu ${ }^{3}$, Kader Irak $^{4}$, Yildiz Okuturlar ${ }^{1}$, Isa Sevindir ${ }^{1}$, Serkan Ipek ${ }^{1}$, Gkturk Ogredici $^{1}$, Ilke Soylu ${ }^{2}$, Asli Cifcibas Ormeci ${ }^{3}$, Erhan Altnorz}

${ }^{1}$ Bakirköy Dr. Sadi Konuk Training and Research Hospital, Turkey; ${ }^{2}$ Bezmi Alem University, Turkey; ${ }^{3}$ Haseki Training and Research Hospital, Turkey; ${ }^{4}$ Kanuni Sultan Süleyman Training and Research Hospital, Turkey

Background: We evaluated the side effects of antiviral therapy in Hepatitis $\mathrm{C}$, the reasons for the failure of the previous treatment, medications used, and the new outcomes in the light of the parameters during the course of treatment.

Method: Patients who were previously been treated at three different centers and had side effects, primary unresponsiveness, relapse-requiring retreatment or receiving treatment for the first time were included in the study. The disease states were classified as chronic hepatitis $\mathrm{C}$ (CHC), compensated cirrhosis (CC) and decompensated cirrhosis (DCC). The medications they have received and the medications they will receive during new treatment, the treatment time, and the side effects have been compared between parameters and the groups.

Result: A total of 136 cases (81F) and mean age $59.96+12.5$ years (24-79) were included. All cases were 60.3\% CHC, 30.9\% CC and $8.8 \%$ DCC. In $23.8 \%$ of patients, treatment was interrupted due to primary missed response and in $17.5 \%$ because of side effects and $58.7 \%$ of patients were relapser. The rate of patients who had treatment for the first time were $51.5 \%$. The percentage of genotype- 1 patients were $86.7 \%$, genotype-24.4\% genotype-3 $7.3 \%$ and genotype- $41.5 \%$. The HCV RNA median level of all cases was 4205708.5 (10291-408371117) IU/ml. There was no difference between the withdrawal rates by recurrence, primer unresponsiveness and side effects $(p=0.219)$ of patients who were treated with Peginterferon alfa- $2 \mathrm{a}$ and $2 \mathrm{~b}$. In DCC group, the most preferred treatment was Ledipasvir+Sofosbuvir (75\%), in CC group Ledipasvir+Sofosbuvir+Ribavirin $\quad(35.7 \%)$ and in $\mathrm{CHC}$ group Ombitasvir+Paritaprevir+Ritonavir+Dasabuvir $(52.4 \%)$. When the patients who had been treated for the first time were evaluated, HCV RNA was negative except 6 of 127 patients. One of these patients had previously received peginterferon alfa- $2 \mathrm{a}$ and other 5 had not received any treatment before. There was no difference for first month treatment response between peginterferon alfa- $2 a$, Peginterferon alfa- $2 b$ and previously untreated groups $(p=0.260)$. Side effects were observed in $15.44 \%$ of cases (itching 10 , weakness 10 , insomnia 3 , hypertension 2, in one case Zone Zoster, gripal complaints, weight loss, nausea). Major side effects were seen in $2.94 \%$ of patients (1 acid, 2 bleeding of varicose veins, 1 hepatic encephalopathy). In $4.4 \%$ of patients there were simultaneous hepatitis B. Hepatitis B had no negative effects or side effects on treatment response. Five patients who had received HCC treatment previously had recurrence and progression, and in 2 cases developed new HCC. At the end of the treatment there was one patient with $\mathrm{HCV} \operatorname{RNA}(+)$. There was no difference in the treatment results of peginterferon alfa-2a, peginterferon alfa- $2 b$ and previously untreated groups $(p=0.109)$.

Conclusion: The treatment results of patients who had previously received treatment (primer unresponsiveness and withdrawal due to side effects and recurrence) did not effect the antiviral treatment response. The most common side effect was itching and nausea. Major side effects were rare. Close follow-up is required for HCC. Hepatitis B coexistence did not affect the treatment response. When the medication choices are evaluated, it is seen that the selection was made according to patient's clinics.

\section{$H C V-45$}

\section{Patient tolerability of Interferon and Interferon free regimen} for hepatitis $\mathbf{C}$ infection

\section{Dexton Johns ${ }^{1}$}

${ }^{1}$ DM Wayanad Institute of Medical Science, India

Background: The treatment goal of Chronic hepatitis C virus (HCV) infection is sustained virological response (SVR) which indicates $\mathrm{HCV}$ eradication. Traditionally pegylated-interferon-alpha (PEGIFN) in combination with ribavirin was used but lately direct-acting antivirals (DAAs) which are specifically designed to target various stages of HCV life cycle.

Objective: To assess the physical and mental health related quality of life (HRQoL) before during and after treatment.

Method: A systematic review and meta-synthesis techniques were adopted to identify, appraise and synthesize the relevant literature 
regarding the experience of of $\mathrm{HCV}$ patients conducted according to the PRISMA guidelines. Several electronic databases such as PubMed, CINAHL, Scopus, PsycINFO and the Cochrane Library databases were searched.

Result: 598 patients from 49 studies were included in the systematic review. All studies showed an increase in the quality of life following treatment. Interferon and ribavirin treatment showed more adverse effect compared to DAAs. HRQOL had a statistically significant correlation with age, sex, educational level, living type, employment status, monthly income level, and comorbidity status. Sofosbuvir and velpatasvir showed better tolerability among the DAAs.

Conclusion: DAAs are better tolerated by the patients and has a significant improvement in the quality of life.

Education, compassion and health care needs to be tailored to improve the overall well being of patients with $\mathrm{HCV}$.

\section{$H C V-46$}

Treatment response and safety of sofosbuvir plus ribavirin for the treatment of $\mathrm{HCV}$ genotype 2 infection

\section{Young Kul Jung ${ }^{1}$, Sang Jun Suh ${ }^{1}$, Hyung Joon Yim ${ }^{1}$, Ji Hoon} $\mathrm{Kim}^{3}$, Jong Eun Yeon ${ }^{3}$, Kwan Soo Byun ${ }^{3}$, Yeon Seok $\mathrm{Seo}^{2}$, Soon Ho $\mathrm{Um}^{2}$

${ }^{1}$ Korea Univ. Ansan Hospital, Korea, Republic of; ${ }^{2}$ Korea Univ. Anam Hospital, Korea, Republic of; ${ }^{3}$ Korea Univ. Guro Hospital, Korea, Republic of

Background: Sofosbuvir (SOF) and ribavirin (RBV) for 12 or 16 weeks is recommended for treatment of patients with HCV genotype (GT) 2 infected patients in KASL guideline based on clinical trials. However, there is a few data in real world setting. So, we investigated effectiveness and safety of this regimen for HCV GT2 infected patients in tertiary hospitals.

Method: Three tertiary center, prospective observational cohort study evaluates clinical practice data (Korea university Anam, Guro, and Ansan) between January 2015 and December 2016. Clinical data were centrally collected from medical records. Selection of treatment regimen and duration was the investigator's choice. The primary efficacy outcome was sustained virological response 12 weeks after therapy (SVR12).

Result: 146 patients were visited and 131 patients were treated SOF plus RBV during 12 weeks $(n=122)$ or 16 weeks $(n=9)$. Overall, EVR, ETR, and SVR 12 by ITT analysis were $90.0 \%, 96.2 \%$ and $87 \%$. In addition, EVR, ETR, and SVR12 by PP analysis $97.5 \%, 99.2 \%$ and $96.3 \%$, respectively. In subgroup analysis, SVR12 in patients with treatment-naïve and treatment-experience were $97.2 \%$ or $94.7 \%$, respectively. SVR12 in patients with and without cirrhosis were $94.4 \%$ and $97.4 \%$, respectively. In patients with high viral load $(>800,000 \mathrm{IU} / \mathrm{mL})$, SVR rate was $95.3 \%$ and there was no differentiation comparing with non-high viral load. In the multivariate analysis, liver cirrhosis, lower serum albumin and RBV dose at baseline were not associated with SVR12. Common adverse events (AEs) included fatigue, anaemia, nausea, headache, insomnia, rash and flu-like symptoms. Discontinuation due to AEs occurred in only 1 patient by severe anemia.

Conclusion: In this clinical practice setting, SOF and RBV was safe and effective for treatment of patients with HCV GT2 infection in Korean.
$H C V-47$

A drug Interaction study to assess the pharmacokinetics of a potent $\mathrm{HCV}$ protease inhibitor narlaprevir and antiretroviral drugs

Emiliya Krasavina $^{1}$, Alexey Kravchenko ${ }^{3}$, Konstantin Zhdanov ${ }^{6}$, Vladimir Chulanov ${ }^{3}$, Vasily Isakov ${ }^{4}$, Svetlana Erofeeva ${ }^{2}$, Elena Shipaeva $^{5}$, Elena Tarkhova ${ }^{5}$, Mikhail Samsonov ${ }^{5}$

${ }^{1}$ Medical Adviser, Russian Federation; ${ }^{2}$ Bessalar Clinic, Russian Federation; ${ }^{3}$ Federal Budget Institution of Science "Central Research Institute of Epidemiology", Russian Federation; ${ }^{4}$ Institute of Nutrition, Russian Federation; ${ }^{5}$ JSC R-Pharm, Russian Federation;

${ }^{6}$ State military educational government-financed institution of higher professional education "Kirov Military Medical Academy", Russian Federation

Background: In Russia hepatitis C diagnosed in $45.2 \%$ of HIV patients during medical check-ups. This study was designed to investigate pharmacokinetic (PK) drug-drug interactions between ritonavir (RTV) boosted HCV protease inhibitor narlaprevir (NVR) and antiretroviral therapy (ART) tenofovir disoproxil fumarate (TDF) and raltegravir (RAL), as a potential treatment of $\mathrm{HCV} / \mathrm{HIV}$ co-infected patients.

Method: This was a phase I, crossover and multiple dose study. The potential for PK drug-drug interactions, safety and tolerability were evaluated when NVR/ RTV and TDF (Part I) and NVR/RTV and RAL (Part II) were administered in healthy adult volunteers. 18 subjects were randomized in each Part, 6 subjects in each sequence (A/B/C, B/C/A, C/A/B), Figure 1. Geometric mean maximum concentration of drug in serum $(\mathrm{Cmax})$ and area under the concentrationtime curve (AUC) for NVR/RTV, TDF or RAL were analyzed.

Result: All subjects completed the study. All subjects in both parts were male. 1 protocol deviation was detected: 1 subject missed 1 dose of RTV in third period Part II Treatment C (NVR/RTV+RAL). No serious adverse events (AEs) were revealed. All AEs were mild by severity and were resolved without any actions. No discontinuations were due to AEs. In Part I co-administration with TDF had no meaningful effect on plasma exposure of NVR/RTV with geometric mean ratios (GMR) close to $100 \%$ (104.20\% for AUC (tau); $101.97 \%$ for Cmax). Co-administration of NVR/RTV increased Cmax of TDF by more than $30 \%$ (GMR $131.08 \%$ ), while had no substantial impact on AUC (tau) and minimal predose concentration (Ctrough) (GMRs were $107.68 \%$ and $101.27 \%$ respectively). Among 5 subjects of Part I with AEs, 2 subjects experienced headache, 1 subject - diarrhea, 1 subject had increased ALT level and 1 subject - LDH level decrease. In Part II co-administration of RAL had no meaningful effect on plasma exposure of NVR/RTV with GMRs close to $100 \%(93.36 \%$ for AUC (tau); $97.78 \%$ for Cmax). Co-administration of NVR/RTV slightly reduced plasma exposure of RAL (GMR of Cmax was $86.21 \%$, for Ctrough - 64.71\%), but had no impact on AUC (tau) (GMR 91.11\%). Reduction in RAL exposure due to co-administration with NVR/RTV observed in this study is not likely to be clinically meaningful and hence no dose adjustment of NVR/RTV or RAL is necessary. In Part II 1 subject experienced 2 AEs - hepatic enzymes increase following combination of Treatment C (NVR/RTV+RAL). Co-administration of NVR/RTV with multiple doses of TDF and RAL in healthy subjects was safe and well tolerated. No significant PK drug-drug interactions were found between NVR/RTV and ART drugs.

Conclusion: This data will be supported the enrollment of HCV/HIV co-infected patients in efficacy and safety phase II study with NVR/ $\mathrm{RTV}+$ Daclatasvir combination 
Figure 1. Study design

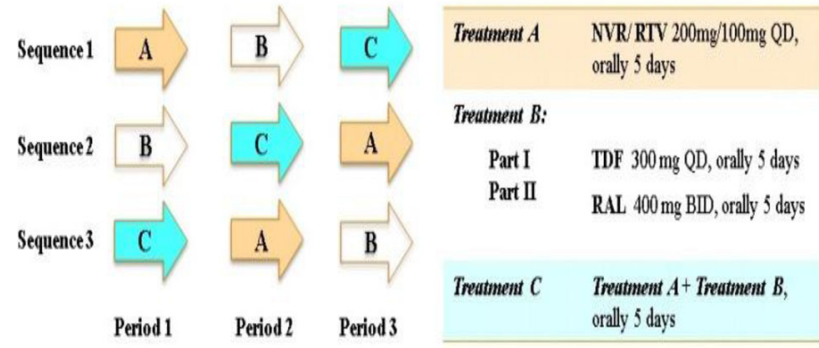

$H C V-48$

\section{Adverse events of HCV treatment using Sofosbuvir/Ledipasvir combination (Real life experience)}

\section{Nyamsuren Naranzul ${ }^{1}$, Oidov Baatarkhuu', Amarsanaa Jazag ${ }^{2}$ \\ ${ }^{1}$ Mongolian National University of Medical Sciences, Mongolia; \\ ${ }^{2}$ Happy Veritas Clinic and Diagnostic Center, Mongolia}

Background: The incident of liver cancer in Mongolia generally caused by HBV and HCV, it is 7 times higher than that world's average. In recent studies, $27 \%$ percent of the population has been diagnose and it is every 1 of 4 people has the virus and most prevalent cause of HCC and causing number one public health issue. Mongolia is one of the first countries that registered Sofosbuvir/ Ledipasvir (SOF/LDV) regimen from developing countries. The HCV treatment program in Mongolia has started on January of 2016.

Method: We followed and evaluated treatment outcomes of the patients with HCV infection using Harvoni (manufactured by Gilead Science). We started our prospective analysis on August until October 2016, for 3 months, on 1230 patients. All patients were treated with SOF/LDV for 12 weeks and, their treatment was evaluated by quantitative HCV-RNA assays prior and W (week) 4 and W12 of treatment. Sustained Virological Response (SVR) after 12 weeks of treatment was assessed. Virus genotype analysis using cDNA microarray, liver enzymes, CBC and drug related adverse events were assessed in every patient. All the tests were conducted at Happy Veritas Laboratories in Ulaanbaatar, Mongolia.

Result: Total of 40 adverse events were observed in 527/1230 patients (43\%). Single adverse events were observed in 358/527 $(68 \%)$, whereas 2 events were observed in $116 / 527(22 \%)$ and 3 or more events were observed 52/527 (10\%) on patients respectively. Age wise 35 or lower aged patients were 43/153 (28\%), age of 36 to $55,295 / 655(45 \%)$ and age of 56 of more, 190/422 (45\%) were adverse events were observed. Our result by gender wise, out of $406 / 781(52 \%)$ on female patients, on male patients, $121 / 449$ (27\%) were observed adverse events.

Conclusion: Treatment of HCV in Mongolia using all-oral dual DAA was divided in 3 phases due to shortness of drugs and logistics arrangements. We were able to include only stage-one patients in this study. We have achieved $95.5 \%$ SVR12W for 3 months treatment with SOF/LDV this time. Despite the identical adverse events were found in other Asian and other regions in the world during treatment, unrecorded adverse were observed such as the facial paralysis, paraproctitis, AFP and facial skin darkening in Mongolia.
$H C V-49$

Antiviral treatment based on Sofosbuvir improve the balance of NK cell receptor of $\mathrm{HCV}$ patients

Nan nan $\mathrm{Hu}^{1}$, Ya Ping $\mathrm{Han}^{2}$, Nan Nan $\mathrm{Hu}^{2}$, Ying Ying $\mathrm{Yan}^{2}$, Nian $\overline{\text { Chen }}^{2}$, Li Dong ${ }^{2}$, Jun $\mathrm{Li}^{2}$

${ }^{1}$ The First Affiliated Hospital with Nanjing Medical University., China; ${ }^{2}$ Department of Infectious Diseases, China

Background: Dynamically monitored the changes of NK cells surface activating receptors and inhibitory receptors of patients with Chronic Hepatitis C (CHC) before and after treatment based on Sofosbuvir and investigated the regulatory effects on NK cell receptor of patients with $\mathrm{CHC}$ by antiviral therapy.

Method: 19 patients with chronic hepatitis $\mathrm{C}$, we treated 5 non cirrhotic patients of chronic hepatitis $\mathrm{C}$ genotype 1 (group A), who started initial treatment, with Sofosbuvir combined Peg-IFN and Ribavirin for 12 weeks; 4 patients of chronic hepatitis C genotype 2 (B group) were treated with Sofosbuvir combined ribavirin for 12 weeks; 10 patients of hepatitis $\mathrm{C}$ cirrhosis genotype 1 or 3 or recurrence after interferon treatment ( $\mathrm{C}$ group) were treated with Sofosbuvir combined ribavirin for 24 weeks.

Result: 1 . Sustained viral response (SVR) rates of 15 cases of hepatitis C patients in group A and group C was $100 \%$, and the rate of SVR in 4 cases of group 2 hepatitis C patients in group B was $75 \% .2$. After the antiviral treatment of Sofosbuvir, NK cells surface activating receptors $\mathrm{NKp} 46$ and $\mathrm{NKp} 30$ were higher in all 3 groups than those before antiviral therapy, whereas the expression level of NK cell surface inhibitory receptor NKG2A decreases dramatically and there was significant difference $(P<0.05)$; meanwhile, the expression level of NKG2DT on lymphocyte surface was significantly higher than that before antiviral therapy, the difference was statistically significant $(P<0.05)$. 3. The expression level of NK cell surface activating receptor NKp46 in group A (Sofosbuvir combined Peg-IFN and Ribavirin) was significantly higher than that of group $\mathrm{B}$ and $\mathrm{C}$ (Sofosbuvir combined Ribavirin) at 12 weeks after antiviral treatment and the difference was statistically significant $(P<$ 0.05). 4. The NK cell surface activation receptors NKp46, NKp30 and inhibitory receptors NKG2A in peripheral blood of one patient, who had not achieved a sustained virologic response in group A after antiviral treatment, were improved at the fourth weeks and twelfth weeks compared with those before antiviral treatment, but there were too few cases for a valid statistical analysis. 5 . The NK cell surface NKp46 and NKp30 of all patients expressed at high level at 12 weeks after the end of Sofosbuvir antiviral therapy.

Conclusion: NK cell surface receptor dysfunction was significantly improved after HCV RNA became negative by Sofosbuvir antiviral therapy, it indicates that hepatitis $\mathrm{C}$ virus itself have an impact on the expression of NK cell related receptors.

$H C V-50$

Change in alpha-feto-protein after direct antiviral agent treatment in chronic hepatitis $C$ and its relation with APRI and FIB-4 scores

Celal Ulasoglu $^{1}$, Yasar Colak ${ }^{1}$, Ilyas Tuncer ${ }^{1}$

${ }^{1}$ Istanbul Medeniyet University Goztepe ERH, Turkey

Background: Chronic hepatitis $\mathrm{C}$ (CHC) as a cause of cirrhosis and hepatocellular carcinoma (HCC) may have AFP elevations in up to $30 \%$ of the patients. We aimed to assess the possible any early change in Alpha-fetoprotein (AFP) levels after the treatment with direct 
acting agents (DAA) and the correlation with biochemical parameters including APRI and FIB-4 scores.

Method: A total of 127 patients with $\mathrm{CHC}$ fulfilling the reimbursement criteria for DAA treatment were included into the study between August 1,2016 and December 31, 2017. All patients had no evidence of HCC prior to treatment. As available treatment options in Turkey either a combination of sofosbuvir $90 \mathrm{mg}+$ ledipasvir $400 \mathrm{mg}$ or ombitasvir $12,5 \mathrm{mg}+$ paritaprevir $75 \mathrm{mg}+$ ritonavir $50 \mathrm{mg}+$ dasabuvir $250 \mathrm{mg}$ for $12-24$ weeks were chosen according to the clinical status and HCV genotype. The biochemical values were recorded and APRI score and FIB-4 scores were also assessed.

Result: The $31 \%$ of cases had abnormal initial AFP levels in according to cut-off level of $10 \mathrm{ng} / \mathrm{ml}$. While this ratio was $12 \%$ when $15 \mathrm{ng} / \mathrm{ml}$ was taken as the cut-off value. Mean initial AFP levels were $8,6 \mathrm{ng} / \mathrm{ml}$ while mean post-treatment AFP level was $5,3 \mathrm{ng} / \mathrm{ml}$ $(\mathrm{p}=0.17)$.

Conclusion: AFP, a glycoprotein with 591 amino acids and a half-life of 5 days decreased in $92.0 \%$ of our cases, while $6,5 \%$ increased and $1,5 \%$ remained the same level at the third month of DAA treatment. In our patients with $\mathrm{CHC}$, treatment with DAA resulted with a $100 \%$ end-of-treatment response and an average change was $29 \%$ and $32 \%$ decrease in AFP levels in females and males, respectively. Median for all cases was $30 \%$. This decline was also significantly correlated with simultaneous decrease in AST, ALT, APRI and FIB-4 scores $(\mathrm{p}<0.001)$, but not with platelet count, drug used or genotype.

References:

1. Sturgeon CM, Duffy MJ, Stenman UH, et al: National Academy of Clinical Biochemistry laboratory medicine practice guidelines for use of tumor markers in testicular, prostate, colorectal, breast, and ovarian cancers. Clin Chem 2008 Dec; 54(12):e11-79

2. Milose JC, Filson CP, Weizer AZ, et al: Role of biochemical markers in testicular cancer: diagnosis, staging, and surveillance. Open Access J Urol 2011 Dec 30;4:1-8

3. Chen CH, Lin ST, Kuo CL, Nien CK. Clinical significance of elevated alpha-fetoprotein (AFP) in chronic hepatitis C without hepatocellular carcinoma. Hepatogastroenterology. 2008 JulAug;55(85):1423-7. PubMed PMID: 18795704.

Table 1: Changes in clinical parameters

\begin{tabular}{|l|r|r|r|r|}
\hline & Min & Max & Mean & \pm SD \\
\hline HCV_RNA IU/ml & 200.000 & $17,700.000$ & $6,214.000$ & 5,6079 \\
\hline AFP & $-29,4 \%$ & $84,8 \%$ & $29,9 \%$ & 19,3 \\
\hline AST & $-236,0 \%$ & $86,5 \%$ & $43,3 \%$ & 44,9 \\
\hline ALT & $-168,8 \%$ & $89,9 \%$ & $49,6 \%$ & 41,1 \\
\hline APRI & $-454,8 \%$ & $96,8 \%$ & $41,2 \%$ & 73,1 \\
\hline FIB4 & $-562,1 \%$ & $95,4 \%$ & $11,7 \%$ & 91,8 \\
\hline
\end{tabular}

Table 2:Comparison of Pre- and post- treatment values

\begin{tabular}{|c|c|c|c|}
\hline & Mean & $\pm S D$ & P VALUE \\
\hline AFP(1) & 8,6 & 4,9 & \multirow{2}{*}{,000 } \\
\hline $\operatorname{AFP}(2)$ & 5,3 & 2,7 & \\
\hline AST1 & 62,0 & 34,0 & \multirow{2}{*}{,011 } \\
\hline AST2 & 27,4 & 11,7 & \\
\hline ALT1 & 66,5 & 51,6 & \multirow{2}{*}{,000 } \\
\hline ALT2 & 24,1 & 11,4 & \\
\hline PLT1 & 161,9 & 66,0 & \multirow{2}{*}{,062 } \\
\hline PLT2 & 173,8 & 64,9 & \\
\hline APRI(1) & 1,5 & 2,2 & \multirow{2}{*}{,001 } \\
\hline APRI(2) & 0,6 & 0,7 & \\
\hline FIB-4(1) & 4,2 & 4,5 & \multirow{2}{*}{,048 } \\
\hline FIB-4(2) & 2,9 & 3,6 & \\
\hline
\end{tabular}

Paired t-test

$H C V-51$

Chronic HCV prevalence based on birth cohort and race/ethnicity in a large HMO population in Southern California

\section{$\underline{\text { Andy Tien }}^{1}$, Amandeep Sahota ${ }^{1}$, Tse Ling Fong ${ }^{2}$ \\ ${ }^{1}$ Kaiser Permanente Los Angeles Medical Center, United States; \\ ${ }^{2}$ USC Keck School of Medicine, United States}

Background: Chronic hepatitis $\mathrm{C}$ virus (HCV) infection is a leading cause of liver disease requiring liver transplant in the United States. With the efficacy of new direct-acting antivirals agents, identifying and treating those with chronic $\mathrm{HCV}$ is recommended. Current guidelines recommend screening the baby boomer population (born between 1945-1965) for hepatitis C. Data from NHANES suggest that there are 2.7 million Americans chronically affected with $\mathrm{HCV}$. However, there is limited data among the prevalence of HCV in Asian Americans. Our study aims to identify the prevalence rates of $\mathrm{HCV}$ among different birth cohorts and race/ethnicities in Southern California.

Method: In a large Health Managed Organization in Southern California with a population size of 3,234,542 members over the age of 18 as of December 2016, we used procedure codes to identify 15,202 patients with at least one detectable HCV RNA or reactive HCV qualitative test since 2004. For data analysis, patients were further divided into birth cohorts and race/ethnicity. The denominator used includes members screened and unscreened for hepatitis $\mathrm{C}$.

Result: The overall chronic HCV prevalence rate is $0.47 \%(15,202 /$ $3,234,542$ ) with the highest prevalence of $1.08 \%$ in the birth cohort born between 1945-1964 $(\mathrm{n}=996,389)$ and the lowest prevalence of $0.04 \%$ in those born between 1985-1998 $(n=815,641)$. Among the 1945-1964 birth cohort, HCV was prevalence in $2.31 \%$ of American Indians ( $\mathrm{n}=2,769), 0.45 \%$ of Asian and Pacific Islanders $(n=116,794)$, $2.09 \%$ of Black $(n=93,379), 0.98 \%$ of Hispanic/Latino $(n=302,367)$, 
$1.14 \%$ of White/Caucasian $(n=434,365), 1.31 \%$ of those identified as multiple race $(n=5954)$, and $0.51 \%$ in those of unknown race $(\mathrm{n}=40,761)$. The overall prevalence is $0.23 \%$ in Asians and Pacific Islanders from all birth cohorts $(\mathrm{n}=374,030)$.

Conclusion: Chronic HCV prevalence trends in a large HMO population in Southern California is consistent with the national data from current studies with the highest prevalence in the American Indian and Black populations. We can further infer from this data that the prevalence of chronic HCV in Asian Americans is $0.23 \%$. Overall, the 1945-1964 birth cohort has the highest prevalence and remains a valuable target for HCV screening.

\section{HCV-52}

Clinical Manifestations of Changes of the Oral Mucous Membrane in Patients with Viral Hepatitis in Armenia For 20152017 YY. Vahe Azatyan1, Lazar Yessayan1, Hasmik Ghazinyan2, Melanya Shmavonyan2 1YSMU after M. Heratsi, 2Norq Clinical Hospital

\section{Hasmik Ghazinyan $^{1}$, Lasar Yessayan ${ }^{2}$, Hasmik Ghazinyan ${ }^{1}$, Melanya Shmavonyan ${ }^{1}$ \\ ${ }^{1}$ Nork Clinical Hospital of Infectious Diseases, Armenia; ${ }^{2}$ Yerevan State Medical University, Armenia}

Background: Background: HBV/HCV infected patients with the acute as well and chronic viral hepatitis, including cirrhosis and hepatocellular carcinoma represent a diverse group with various patterns of viral replication and great variations of immune profiles resulting in different hepatic and extrahepatic manifestations.

It is well- known, that the basis of the pathogenesis of any inflammatory process is the result of combination of two main factors: the effects on the tissue of any irritant and local tissue reaction. The latter depends on the general state of the body, its local and overall immunity. There are many clinical evidences of pathogenic interactions between diseases of internal organs and inflammatory periodontal diseases.

Aim: The aim of this study was to evaluate the changes of the oral mucous membrane in patients with HCV/ HBV infections, admitted Nork clinical infection hospital.

Material and Methods: Our study enrolled 125 patients with HCV and HBV infections, among them HCV-69 (55.2\%) / HBV-56 $(44.8 \%)$. The mean age of patients was 51 ( 25 to $77 \mathrm{yy})$. There were $81(65 \%)$ men.

Bacteriological examination of the tooth-gum fluid from the pathological gingival pockets of patients with $\mathrm{HCV} / \mathrm{HBV}$ infections was performed to determine the microbial landscape, histological study of scrapings of oral mucosa and the carbohydrate metabolism.

Result: The changes of oral mucosa in color - hyperemia and cyanosis have been revealed in all patients. The lagging of the tongue was detected in $22.7 \% \mathrm{HBV}$ and $17.8 \% \mathrm{HCV}$ infected patients. Hemorrhages on mucosa were detected in $42.5 \% \mathrm{HBV}$ and $62.5 \%$ $\mathrm{HCV}$ infected patients, associated with severity of liver disease. Changes in state of gums were manifested by hyperemia $(27.8 \%)$, swelling $(29.3 \%)$, bleeding and soreness $(33.7 \%)$. Suppuration from the tooth-gum pockets $(16.8 \%)$, necrotic areas $(21.1 \%)$ were detected. The conducted bacteriological study of the tooth-gum fluid showed, that most frequently the $\mathrm{HCV} / \mathrm{HBV}$ infections are inoculated with staphylococci $(22.8-25.6 \%)$. The microbial association is represented by Gram-positive and Gram-negative rods, total value made $82.3 \%$ for $\mathrm{HBV}$ and $83.2 \%$ for $\mathrm{HCV}$ infections.

To specify the morphological and functional state of oral mucosa scrapings have been studied the accumulation /clustering/ of glycogen in epitheliocytes and in histological study of the scrapings from the cheek mucosa of the patients with HCV/HBV infections both single accumulations of the epitheliocytes and vast nidi, consisting of sheets. In some observations sheets consisting of basal and spicular cells were revealed. Dystrophia of epitheliocytes with neutrophils and lymphocytes were detected. While staining for glycogen weakly expressed granulicity-decreasing of glycogen amount was revealed in $\mathrm{HBV} / \mathrm{HCV}$ patients.

Conclusion: The changes in oral mucosa in HCV/ HBV infections were characterized by various manifestations associated with severity of liver disease.

The microbial landscape of tooth-gum fluid in HCV/HBV infections was relatively stable, there wasn't any definite pathogen, which was involved in development of changes in the oral mucosa. Histological examination of oral mucosa (cheek area) revealed suppression of carbohydrate metabolism, reduction of resistance and systemic reactivity of body, and disruption of local mucosal response in HCV / $\mathrm{HBV}$ infections.

It has been established that treatment of $\mathrm{HCV} / \mathrm{HBV}$ infections leads to an improvement in oral mucosa state and decrease in the seeding of pathogenic microbes.

\section{$H C V-53$}

\section{Cure in chronic hepatitis c with 4 week treatment}

Esra Zerdali $^{1}$, Aysegul Inci Sezen ${ }^{1}$, Inci Yilmaz Nakir ${ }^{1}$, Merve Vanli $^{1}$, Ozlem Altuntas Aydin², Mustafa Yildirim ${ }^{1}$

${ }^{1}$ Saglik Bilimleri Üniversitesi Istanbul Haseki Egitim Ve Arastirma Hastanesi, Turkey; ${ }^{2}$ Saglik Bilimleri Üniversitesi Istanbul Bakirköy Dr. Sadi Konuk Egitim ve Arastirma Hastanesi, Turkey

Background: $96-100 \%$ cure is possible in the treatment of chronic hepatitis $\mathrm{C}(\mathrm{KHC})$ infection with the use of direct acting antivirals (DAA). DAA have less side effects and shorter time of treatment than previous treatments. New efforts are being made to determine the effectiveness of even shorter treatments. In this study, we present a patient with a permanent virological response with only 4-week DAA use in our polyclinic.

\section{Method:}

Result: A 26-year-old female patient who came from Syria as immigrant to our country was referred to our polyclinic on detection of Anti-HCV (+) in pre-marriage blood tests.

The patient had previous splenectomy and blood transfusions. Her physical examination was normal. In laboratory evaluations, WBC: 10340/uL, Hgb: 13,5 g/dl, PLT: 634000/ uL, AST: 27 U/l, ALT: 47 U/l, ALP: 80 U/l, GGT: 50 U/l, t.bil: 0,5 mg/dl, HCV-RNA: 2057117 $\mathrm{IU} / \mathrm{ml}$, genotype 4 was detected. In liver ultrasound, liver size at the upper limit $(155 \mathrm{~mm})$ was evaluated. Liver biopsy of the patient resulted as HAI: 6 Fibrosis: 0. Then, the patient had to move to another city and attended to another hospital's infectious diseases clinic. There she had received pegile-interferon + ribavirin treatment and she had partial response with this treatment. The case was referred to our policlinic again and had laboratory tests. In the second laboratory evaluation, wbc: $8230 / \mathrm{uL}$, hgb: $17.1 \mathrm{G} / \mathrm{dl}$, plt: $541000 / \mathrm{uL}$, AST: 19U/l, ALT: 38U/l, alp: 80U/l, ggt: 32U/l, t.bil: $1,3 \mathrm{mg} / \mathrm{dl}$,, HCV-RNA: $31880 \mathrm{IU} / \mathrm{mL}$ was detected.

Ledipasvir / sofosbuvir + ribavirin treatment scheduled to last 12 weeks has been started for this non-cirrhotic patient who had experienced previous treatment.

AST: 9U/l, ALT: 15U/l, tbil: 0,6 mg/dl, HCV-RNA: (-) were detected after 4 weeks of drug use by the patient. Unfortunately, her treatment couldn't be continued after 4 weeks because of the changes in the refund policy in our country. 
The patient's HCV-RNA was evaluated as negative after 12 and 24 weeks and one year of the 4-weeks treatment use.

Conclusion: We present this case in the light of studies on the reduction of DAA treatment duration. Although this patient received inadequate duration of treatment of DAA due to current guidelines, her HCV RNA is still negative first year after treatment. Incase of low fibrosis in the histological evaluation of the liver as in this patient, 4 weeks of treatment can be successful. However, long-term follow-up results of these cases need to be evaluated.

\section{$H C V-54$}

Dynamic of clinical and laboratory abnormalities in small cohort of $\mathrm{HCV}$-infected patients treated with sofosbuvir contain regimens

\section{Narina Sargsyants ${ }^{1}$}

${ }^{1}$ Armenicum Clinical Center, Armenia

Background: New highly effective directly acting antiviral agents (DAAs) have been increasingly used for the treatment of chronic hepatitis $\mathrm{C}$ virus (HCV) infections and should be investigated in difficult-to-treat patients, include patients with severe complications, decompensated liver cirrhosis, comorbidities and in elderly patients.

The aims of this report is monitoring of clinical and laboratorial abnormalities before, during and after antiviral treatment with the different sofosbuvir (SOF) contain regimens.

Method: $61 \mathrm{HCV}$-infected patients from 21 to 73 years old $(56 \%$ male, $49.7 \pm 13.0$ years old, BMI $26.0 \pm 4.7 \mathrm{~kg} / \mathrm{m}^{2}$, viral load $118-27485300 \mathrm{IU} / \mathrm{ml}$ ) treated with following regimens: $\mathrm{SOF}+\mathrm{DCV} \pm \mathrm{RBV}$ in 33 patients, $\mathrm{SOF} / \mathrm{LDV} \pm \mathrm{RBV}$ in 22 patients, $\mathrm{SOF}+\mathrm{RBV}$ in 5 patients and $\mathrm{SOF}+\mathrm{VLP}+\mathrm{RBV}$ in 1 patient. 15 patients previously treated with PEG-IFN $\alpha$ and RBV and 1 patient with SOF/LDV: 1 patient null-responder with Child-Pugh C cirrhosis, genotype $1 \mathrm{~b} ; 13$ relapsers (seven with genotype 3 , five of them with cirrhosis; two with genotype 2 Child-Pugh A and C cirrhosis; four with genotype $1 \mathrm{~b}$, two of them with Child-Pugh A cirrhosis) and one patient with re-infection.

Important comorbidities cases: 14 diabetes, 6 autoimmune thyroiditis, 4 renal insufficiency, 3 portal vein thrombosis, 3 positive liver autoimmunity markers (ANA in 3, ASMA in 2), 3 lymphocytosis, 3 elevated RF, 2 HB?-negative chronic HBV-infection (one with HBV DNA $226 \mathrm{IU} / \mathrm{ml}$, next with HBV DNA $<10 \mathrm{IU} / \mathrm{ml}$ previously treated with PEG-IFN $\alpha$ ), 1 idiopathic thrombocytosis (bone marrow evaluation result "primary myelofibrosis"), 1 arrhythmia, 1 ulcerative colitis, 1 progressive keratoconus and retinal degeneration, 2 after low extremity injury.

Result: During the treatment with DAAs, we do not observed reactivation of HBV and negative impacts on other above-mentioned comorbidities. In one patient with cirrhosis, ESRD, genotype $1 b+3$, treated with SOF+VLP and low dose RBV, treatment was discontinuing due to worsening of eGFR. In three other patient with high starting creatinine and low eGFR, renal function remains the same. One patient with decompensated cirrhosis and portal vein thrombosis, during treatment with SOF+DCV+RBV had a constantly high activity of ALT, AST, GGT, AP and developed exacerbation nephrolithiasis with severe pain, conservatively resolved. From three patients with lymphocytosis, in one patient lymphocytes count normalized with decreasing size of enlarged lymph nodes of portal tract. ANA and ASMA become negative in two patients. In three females with increased RF, we enrolled significant reduction. Liver fibrosis decreased in patients with F3 and compensated cirrhosis, but remain on the same degree in patients with metabolic syndrome, obesity and decompensated cirrhosis. Despite SVR, in some patients with decompensated cirrhosis required management of sings of hypersplenism and coagulopathy.

Conclusion: Sofosbuvir contain DAAs regimens characterized with high efficacy and good safety profile in HCV-infected patients with comorbidities. In patients with decompensated cirrhosis even after SVR close monitoring and management of some complications is mandatory.

\section{GENOTYPES}

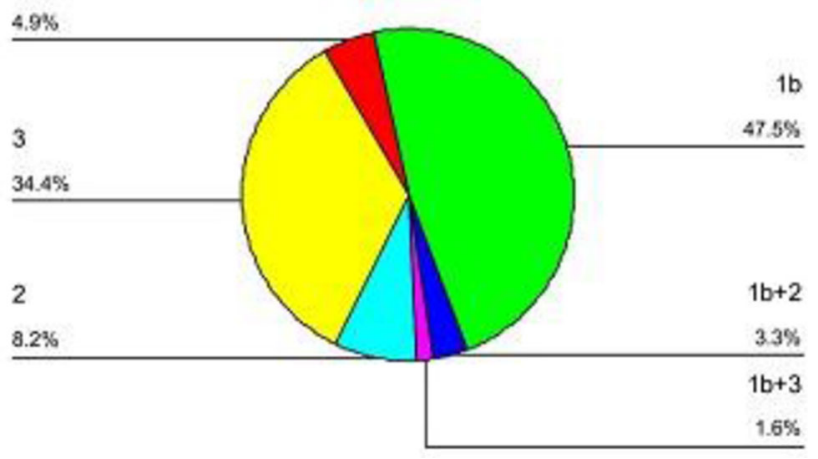

\section{FIBROSIS}

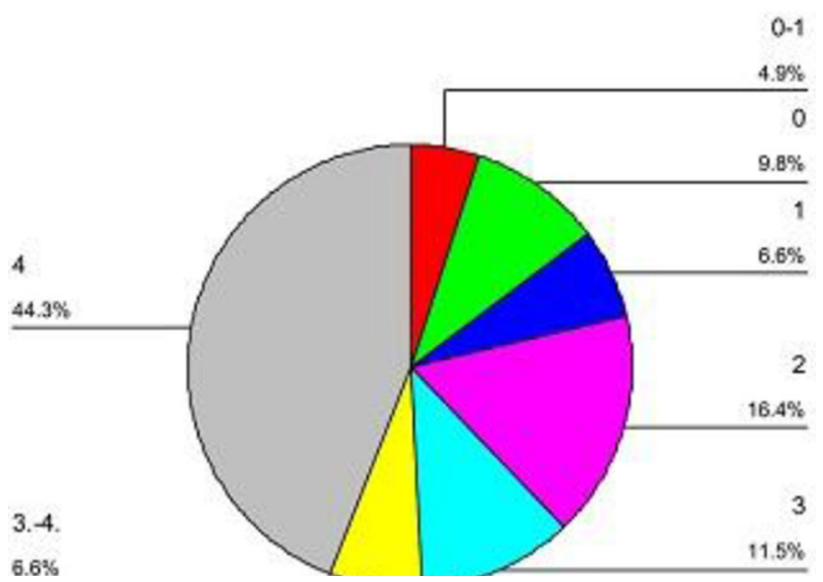

$H C V-55$

Efficacy and safety of sofosbuvir/daclatasvir combination therapy for Taiwanese patients with chronic hepatitis $\mathrm{C}$ virus infection

ChiaYen Dai ${ }^{1}$, Chingi Huang ${ }^{1}$, Minglun Yeh ${ }^{1}$, Chungfeng Huang ${ }^{1}$, Zuyau Lin ${ }^{1}$, Shinncherng Chen ${ }^{1}$, Jeefu Huang', Wanlong Chuang ${ }^{1}$, Minglung Y $\mathbf{u}^{1}$

${ }^{1}$ Kaohsiung Medical University Hospital ad University, Taiwan

Background: The combination therapy with sofosbuvir (SOF) and daclatasvir (DCV) was reported well tolerated and achieved high sustained virological response (SVR) rates in patients with chronic hepatitis $\mathrm{C}$ virus (HCV) infection. Recently, APASL, EASL and AASLD guidelines-recommended for pan-genotype hepatitis $C$ virus 
infection. The studies aimed to survey the efficacy and safety of SOF+DCV dual therapy in Taiwanese patients with HCV genotype 1 and/or 2 infection.

Method: We analyzed clinical data from chronic HCV genotype 1 and/or 2 patients treated with sofosbuvir and daclatasvir (with Ribavirin in 14 patient) at Kaohsiung Medical University Chung-Ho Memorial Hospital. Total 30 patients ( 8 males and 22 females, mean age: $65.6 \pm 10.4$ years) have been treated for 12 weeks and followed up for 12 weeks.

Result: All 30 patients achieve undetectable HCV RNA at 12 weeks after cessation of therapy (SVR12). With sofosbuvir and daclatasvir therapy, $20(66.7 \%)$ patients at week 4, and $29(96.7 \%)$ patients at EOT had undetectable HCV RNA (8 patients at week4, and one patient at EOT with unquantifiable $\mathrm{HCV}$ RNA $<30 \mathrm{IU} / \mathrm{mL}$ but detectable). The serum aminotransaminase levels were improved after the treatment. The mean (range) baseline creatinine, AST, ALT and total bilirubin (T-bil) levels were: $0.85(0.53-2.31) \mathrm{mg} / \mathrm{dL}, 72.0(23-$ 202) IU/L, 67.9 (15-223) IU/L and 1.15 (0.4-3.0) mg/dL. No patient experience acute exacerbation/decompensation at EOF.

Conclusion: We report the real-world experience of sofosbuvir and daclatasvir combination therapy which achieved very high SVR rates and was well tolerated in Taiwanese patients with HCV genotype 1 and/or 2 infection

\section{$H C V-57$}

Efficacy and safety of elbasvir/grazoprevir in elderly chronic hepatitis $\mathrm{C}$ genotype $1 \mathrm{~b}$ patients

\section{Kenichi Kitada ${ }^{1}$, Makoto Kuboki ${ }^{2}$ \\ ${ }^{1}$ Morioka City Hospital, Japan; ${ }^{2}$ Morioka University, Japan}

Background: The emergence of direct-acting antiviral (DAA) agents has dramatically changed the treatment schema for hepatitis $C$ virus (HCV) infection. From sustained virological response (SVR) rates to tolerability, DAA agents have shown outstanding profiles compared with the prior therapy of pegylated interferon with ribavirin. However, the efficacy and safety profiles of DAA therapy in elderly patients are still unclear. We investigated the efficacy and safety of Elbasvir / Grazoprevir which is latest approved in Japan in elderly chronic hepatitis $\mathrm{C}$ genotype $1 \mathrm{~b}$ patients.

Method: We analyzed the consecutive data from the elderly patients (age $>65$ ) who were treated with fixed daily doses of Elbasvir (50 mg) and Grazoprevir (100 mg) for 12 weeks. Baseline resistance-associated variants (RAVs) at $\mathrm{L} 31$ and $\mathrm{Y} 93$ in NS5A were assessed by the PCR-Invader method.

Result: Fifty-eight patients were collected from Dec. 2016 to Mar. 2017. Mean age is 75.9 years old ranging from 65 to 87 years old with 21 males and 37 females. Four patients were treatment naïve, forty nine patients were non responder of PegIFN $\alpha /$ ribavirin and five patients were non responders of PegIFN $\alpha /$ ribavirin/simeprevir. The rate of SVR at 24 weeks is $96.5 \%$ (56/58). Elbasvir/Grazoprevir HCV treatment failure is only two patients who had multiple RAVs with L31 and Y93. There were no patients who have experienced treatment-related serious adverse events and treatment withdrawal.

Conclusion: Elbasvir plus Grazoprevir combination therapy for the elderly hepatitis $\mathrm{C}$ genotype $1 \mathrm{~b}$ patients iswell tolerated and achieved higher SVR.
$H C V-58$

Genotypes distribution of hepatitis $\mathbf{C}$ virus infections in Central Anatolia

Necla Tulek ${ }^{1}$, Sami Kinikli ${ }^{1}$, Kader Dogan ${ }^{1}$, Necla Tulek ${ }^{1}$, Cigdem Ataman Hatipoglu', Esra Kaya Kilic ${ }^{1}$, Fatma Sebnem Erdinc ${ }^{1}$

${ }^{1}$ Ankara Training and Research Hospital, Turkey

Background: Hepatitis $\mathrm{C}$ virus (HCV) has different genotypes and sub genotypes throughout the World. Identification of virus genotype are essential to decide the optimal antiviral type and determine the duration of treatment. Furthermore, HCV genotyping is also important for epidemiological data. We aimed to investigate the distribution of HCV genotypes among our patients.

Method: This study was carried out retrospectively at Ankara Training and Research Hospital. Our hospital is a tertiary care hospital located at central Anatolia. Patients diagnosed and treated with hepatitis $\mathrm{C}$ infection in the last two years were included into the study. The age, gender, duration of disease and probable transmission way of the patients were recorded. HCV genotype was determined by using RT-PCR.

Result: Forty six males and 42 females, totally 88 patients with mean age of 55, 8 years (18-88 years old) were included into the study. The most common identified genotype was genotype $1(87.5 \%)$ with genotype $1 \mathrm{~b}$ dominance $(75 \%)$, followed by genotype $2(8 \%)$, genotype $3(3,4 \%)$ and genotype $4(1,1 \%)$. The mean age was $56.1,47.7$ and 39 years old for genotype 1, genotype 2 and genotype 3 respectively. It could not be obtained any story related to transmission in 30 of the patients $(34 \%)$. We observed a much more genotype 2 infections in the intravenous drug users $(37.5 \%)$ than those of others Conclusion: Although the most predominant HCV genotype is still genotype $1 \mathrm{~b}$ in our region, we observed genotype 2 and 3 has been increasing especially among the young patients and intravenous drug users.

HCV -59

Hepatitis C associated oral lichen planus management using direct-acting antivirals.

Dexton Johns ${ }^{1}$

${ }^{1}$ DM Wayanad institute of medical science, India

Background: Oral Lichen Planus is one of the extraneous manifestation of hepatitis $\mathrm{C}$ infection. Traditionally pegylated-interferonalpha (PEG-IFN) in combination with ribavirin was used but lately direct-acting antivirals (DAAs) which are specifically designed to target various stages of HCV life cycle. The DAAs are well tolerated and with lesser adverse effects

Method: Lichen Planus refractory to conventional steroid treatment was considered as an oral manifestation of $\mathrm{HCV}$ and it was confirmed by anti-HCV by ELISA (third generation) and reverse transcription polymerase chain reaction (RT-PCR) for HCV-RNA. Nine patients with HCV-related OLP received Ledipasvir/sofosbuvir for 12 weeks. Out of nine, five were males with a mean age of 64 . The patient response were assessed before and after treatment.

Result: Sustained virological response was observed in all patients and there was no worsening of lichen planus in any of the treated patients. Clinically refractory lichen planus resolved with DAAs treatment.

Conclusion: We have reported a case series of successful management of interferon free treatment in $\mathrm{HCV}$ associated oral lichen 
planus. Given the strong association, screening for HCV should be considered in patients with oral lichen planus.

\section{HCV-60}

Improvement of liver function parameters and fibrosis scores in hepatitis $\mathrm{C}$ virus infected patients treated with direct-acting antiviral agents

Hae Lim Lee ${ }^{1}$, Sung Won Lee ${ }^{2}$, Chang Wook Kim², Sang Wook Choi $^{2}$, Jin Mo Yang ${ }^{2}$, Jung Hyun Kwon ${ }^{2}$, Myeong Jun Song Song $^{2}$, Jeong Won Jang ${ }^{2}$, Si Hyun Bae ${ }^{2}$, Jong Young Choi Choi ${ }^{2}$, Seung Kew Yoon ${ }^{2}$

${ }^{1}$ Bucheon St. Mary's Hospital, Korea, Republic of; ${ }^{2}$ Catholic Medical Center, Korea, Republic of

Background: The advent of direct-acting antiviral agents (DAA) has enabled high sustained viral response (SVR) rates in hepatitis $C$ virus $(\mathrm{HCV})$ infected patients. We aimed to evaluate longitudinal changes in liver function parameters and fibrosis scores in patients who achieved SVR.

Method: A total of 963 patients who received interferon free DAA combination \pm ribavirin for $\mathrm{HCV}$ infection between December 2015 and February 2017 at the liver unit of the Catholic University of Korea were consecutively recruited. Patients with hepatocellular carcinoma, other liver diseases, or those who received liver transplantation were excluded. Four hundred and thirteen patients who achieved sustained viral response 12 weeks post treatment (SVR12) were analyzed and the parameters of liver function, AST to Platelet Index (APRI) and Fibrosis-4 (FIB-4) scores before treatment was compared to that of SVR12.

Result: Of the 413 patients, 308 (75\%) patients had chronic hepatitis and $105(25 \%)$ patients had liver cirrhosis. In the total patients, aspartate aminotransferase were decreased from 61 and to $28 \mathrm{IU} / \mathrm{L}$ and alanine aminotransferase levels were decreased from 57 to $21 \mathrm{IU} /$ $\mathrm{L}$ between baseline and 4 weeks post treatment $(\mathrm{p}<0.001$, both). In the patients with chronic hepatitis, APRI and FIB-4 scores were all significantly improved; APRI score was $0.93 \pm 1.28$ and $0.51 \pm 0.56$, $\mathrm{p}<0.001$, FIB-4 score was $3.25 \pm 3.79$ and $2.67 \pm 2.21, \mathrm{p}=0.022$, (mean $\pm \mathrm{SD}$ ), at baseline and SVR12, respectively. In the patients with liver cirrhosis, platelet (PLT), albumin, prothrombine time international normalized ratio (PT INR) levels, APRI and FIB-4 scores were all significantly improved; PLT level was $115 \pm 53$ and $166 \pm 82$, $\mathrm{p}<0.001$, albumin level was $3.8 \pm 0.6$ and $4.3 \pm 0.3, \mathrm{p}<0.001$, PT INR level was $1.15 \pm 0.13$ and $1.05 \pm 0.09, \mathrm{p}<0.001$, APRI score was $2.13 \pm 1.59$ and $0.55 \pm 0.42, \mathrm{p}<0.001$, FIB- 4 score was $7.83 \pm 5.56$ and $2.79 \pm 1.73, \quad \mathrm{p}<0.001$, at baseline and SVR12, respectively $($ mean \pm SD)

Conclusion: This study suggested that a significant improvement in liver function parameters and non-invasive fibrosis markers could be achieved in HCV infected patients who achieved SVR.

\section{HCV-61}

Lack of clinically relevant differences in sofosbuvir and ledipasvir exposure across ethnicities

Brian J Kirby $^{1}$, Vinay D Daryani ${ }^{1}$, Amy Meng ${ }^{1}$, Deyuan Jiang ${ }^{1}$, Anu Osinusi ${ }^{1}$, Bryan Mcnabb ${ }^{1}$, KH John Ling ${ }^{1}$, Brian J Kirby ${ }^{1}$

${ }^{1}$ Gilead Sciences, United States

Background: Ledipasvir/sofosbuvir $90 \mathrm{mg} / 400 \mathrm{mg}$ (LDV/SOF) and SOF $400 \mathrm{mg}$ are approved for treatment of $\mathrm{HCV}$ infection in multiple countries worldwide including the U.S. and E.U. In support of global registration, regional Phase 3 studies were conducted in China, Egypt, Japan, Korea, Taiwan, and Vietnam. LDV and SOF are P-gp and BCRP substrates. LDV is primarily excreted in feces unchanged while SOF is metabolized by cathepsin A (CatA) and carboxylesterase 1 (CES1). As differences in activity of these drug metabolizing enzymes and transporters may be present across ethnic groups, ethnic and demographic differences in the exposure of LDV, SOF, and its primary circulating metabolite GS-331007 were explored across the regional Phase 3 studies compared to the predominantly Caucasian U.S./E.U. Phase $2 / 3$ population.

Method: LDV, SOF and GS-331007 exposures were generated for individual subjects with measureable concentrations of each analyte using population PK models. Within each ethnic group (e.g. Chinese, Egyptian, Hispanic, Japanese, Korean, Taiwanese, and Vietnamese) the effect of demographic/patient characteristics (e.g. age, sex, body weight, renal function, and cirrhosis) on LDV, SOF, and GS-331007 exposure was evaluated. Results across ethnic groups were compared to the predominantly Caucasian population.

Result: Figure 1 presents percent geometric mean ratios (\%GMR) and $90 \%$ confidence intervals (CI) for LDV, SOF, and GS-331007 AUCtau across the various ethnic groups compared to the predominantly Caucasian population. Modest changes in the exposure of LDV SOF, or GS-331007 were observed across ethnic groups but were not considered clinically significant based on exposure-response relationships for safety and efficacy established in Phase 3 studies. Similar to findings from the predominantly Caucasian U.S./E.U. Phase $2 / 3$ populations, no clinically significant relationships were identified between demographic/patient characteristics and LDV, SOF, or GS-331007 exposure.

Conclusion: No clinically meaningful differences were observed in the pharmacokinetics of LDV, SOF, and GS-331007 across multiple ethnic groups.

\section{Exposure Changes in Ethnic Groups Compared to Predominantly Caucasian Population}

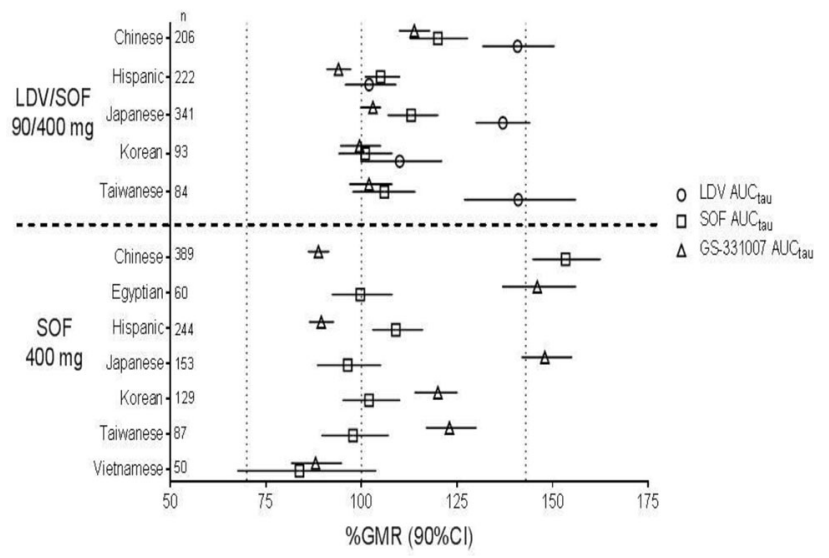

HCV-62

Patients with chronic hepatitis $\mathrm{C}$ receiving sofosbuvir and ribavirin-based treatment, with or without Interferon in Zhejiang, China

\section{$\underline{\text { Xiaoxin } \mathrm{Wu}^{1}}$, Xiaowei $\mathrm{Xu}^{1}$, Hangping $\mathrm{Yao}^{2}$, Lanjuan $\mathrm{Li}^{2}$}

${ }^{1}$ The First Affiliated Hospital, Zhejiang University College of Medicine, China; ${ }^{2}$ The First Affiliated Hospital, School of Medicine, Zhejiang University, China 
Background: Hepatitis C virus (HCV) is one of the important virus as the cause of liver disease in china. The aim of the present study was to explore whether Sofosbuvir and ribavirin -based treatment can cure the patients with chronic hepatitis $\mathrm{C}$ in eastern china.

Method: We examined a cohort of hepatitis $\mathrm{C}$ virus mono-infected patients and nine patients were agreed to participate in our treatment and research. The patients were diagnosed with chronic hepatitis $\mathrm{C}$ with or without cirrosis. Nine patients include 4 female and 5 male were comply with the requirements for selected and willing to participate in this experiment. Sofosbuvir and ribavirin-based treatment with or without interferon was given to patients. Viral loads, serum alanine transaminase (ALT), cytokines and chemokines were recorded while on treatment and after treatment.

Result: After two weeks of Sofosbuvir (SOF) and ribavirin (RBV)based treatment, the viral load of patients were declined to limits of detection. The patients without cirrosis had rapid virological response (RVR) with undetectable viral load at week 4 and sustained virological response (SVR). Eight patients were cured. One patient with cirrosis failed as the virus reappeared during SVR4. The interferoninducible protein-10 (IP-10) was reduced after the treatment.

Conclusion: SOF and RBV-based treatment was effective in treating $\mathrm{HCV}$ patients without cirrosis. When dealing with patients with cirrosis, the common treatment was not enough. The IP-10 has the potential to be the biomarker for the prognosis of $\mathrm{HCV}$.
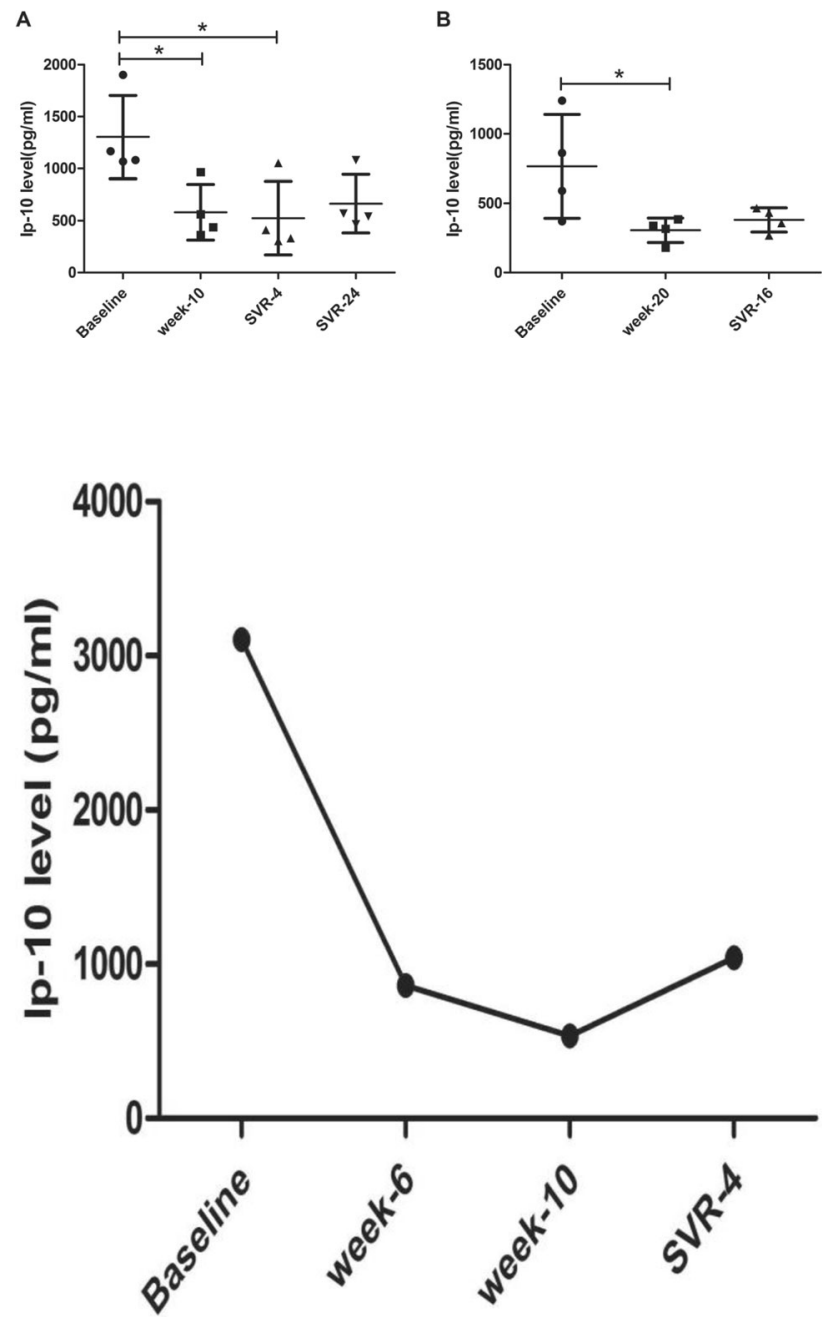

HCV-63

Prevalence of HCV among 40-65 aged population of Arkhangai provinces in Mongolia

Nyamsuren Naranzul ${ }^{1}$, G Buyankhishig ${ }^{2}$, T Ganbyamba ${ }^{2}$, D Munkhbayar $^{2}$, Ts Tserendejid ${ }^{2}$, G Mandakh ${ }^{3}$, R Gandiimaa $^{3}$

${ }^{1}$ Mongolian National University of Medical Sciences, Mongolia; ${ }^{2}$ Central Hospital of Arkhangai province, Mongolia; ${ }^{3} \mathrm{Health}$ Department of Arkhangai Province, Mongolia

Background: Mongolia has a large burden of viral hepatitis, especially chronic hepatitis B virus (HBV) and hepatitis $\mathrm{C}$ virus (HCV) infections, which are associated with cancer and cirrhosis. We aimed to determine $\mathrm{HCV}$ seroprevalence and risk factors for hepatitis $\mathrm{C}$ among 40-65 aged population of Arkhangai province.

Method: The survey is conducted by randomly cross-sectional design of a descriptive study, selected target group of 17600 people, and from 21700 people aged 40-65 in Arkhangai province. We randomly chose 464 participants from 7 soums /from 19 soums of Arkhangai province/, and use questionnaire sheet and HCV Rapid Test for determine risk factor and $\mathrm{HCV}$ infection.

Result: The survey has involved among 464 participants aged 40-65 from 7 soum of Arkhangai province. The average ages of participants were $49.6 \pm 6.86$. 44.6 percent was male participants from total 30.4 percent of participants were $40-44$ year old. 20.3 percent of participants were infected with hepatitis C. From total number of participants who had a HCV infection was, infected from unsafe syringe rate was 83.3 percent, participants live with a person who has hepatitis $\mathrm{C}$ over the past year infection rate was 50 percent, proportion of participants which infected $\mathrm{HCV}$ in non-hospital condition rate was 33.3 percent, participants which infected from HCV infected mother rate was 31.3 percent, and proportion of during dental health care services infection rate was above 25 percent. Dominant risk factors for men are tattoo and changing razors. 14.7 percent of participants haven't any risk factors, 30.8 percent of respondents have one risk factor, and 0.5 percent of respondents have nine risk factors.

Conclusion: The prevalence of anti-HCV was $20.3 \%$ among the participant. Average number of people who had a risk factor was 2.3, and infected average person of study was 2.8 risk factors. The most prevalence risk factor is dental service. Prevalence rate is 75 percent.

$H C V-64$

Real world experience of generic sofosbuvir-based regimens with or without ribavirin in chronic hepatitis $\mathrm{C}$ patients

$\underline{\text { YIJIE HUANG }}^{1}$, Chunghsin Chang ${ }^{1}$, Shengshun Yang ${ }^{1}$

${ }^{1}$ Division of Gastroenterology, Taiwan

Background: We aimed to investigate real-world efficacy and safety of generic sofosbuvir (SOF)-based regimens with or without ribavirin (RBV) in chronic hepatitis $\mathrm{C}(\mathrm{CHC})$ patients.

Method: This is a retrospective single-center study. One hundred and fifteen CHC patients (87 naïve and 28 pegylated interferon-experienced) were enrolled. Hepatic parameter and liver fibrosis score were compared at baseline, during, and 12 weeks after stopping treatment. The primary end point was sustained virologic response at 12 week after end of treatment (SVR12).

Result: Of 115patients treated (mean age 59.2 years, $40 \%$ men, $9.5 \%$ HCC,38.3\% cirrhosis), 45 received sofosbuvir (SOF) plus ledipasvir (5 with RBV), 43 received SOF plus daclatasvir (4 with RBV), 19 received SOF plus velpatasvir (1 with RBV) and 8 received SOF plus RBV. Overall, 99\% patients achieved SVR. One genotype $1 \mathrm{~b}$ patient 
treated by SOF-ledipasvir developed virological relapse. There was a significant improvement of platelet count, AST and ALT levels which resulted in marked reduction of APRI and FIB-4 score from baseline to 12 weeks post-treatment. The most common adverse effect was fatigue or malaise $(11.4 \%)$. No patient experienced serious adverse effect and none discontinued treatment owing to adverse event.

Conclusion: This data demonstrated that generic SOF-based regimens provided a high rate of SVR12and improved hepatic fibrosis score. Generic SOF-base regimens shown to be safe regardless of age and pretreatment fibrosis status. Generic DAAs is effective and could be an alternative choice if brand-named SOF-based regimens are not affordable.
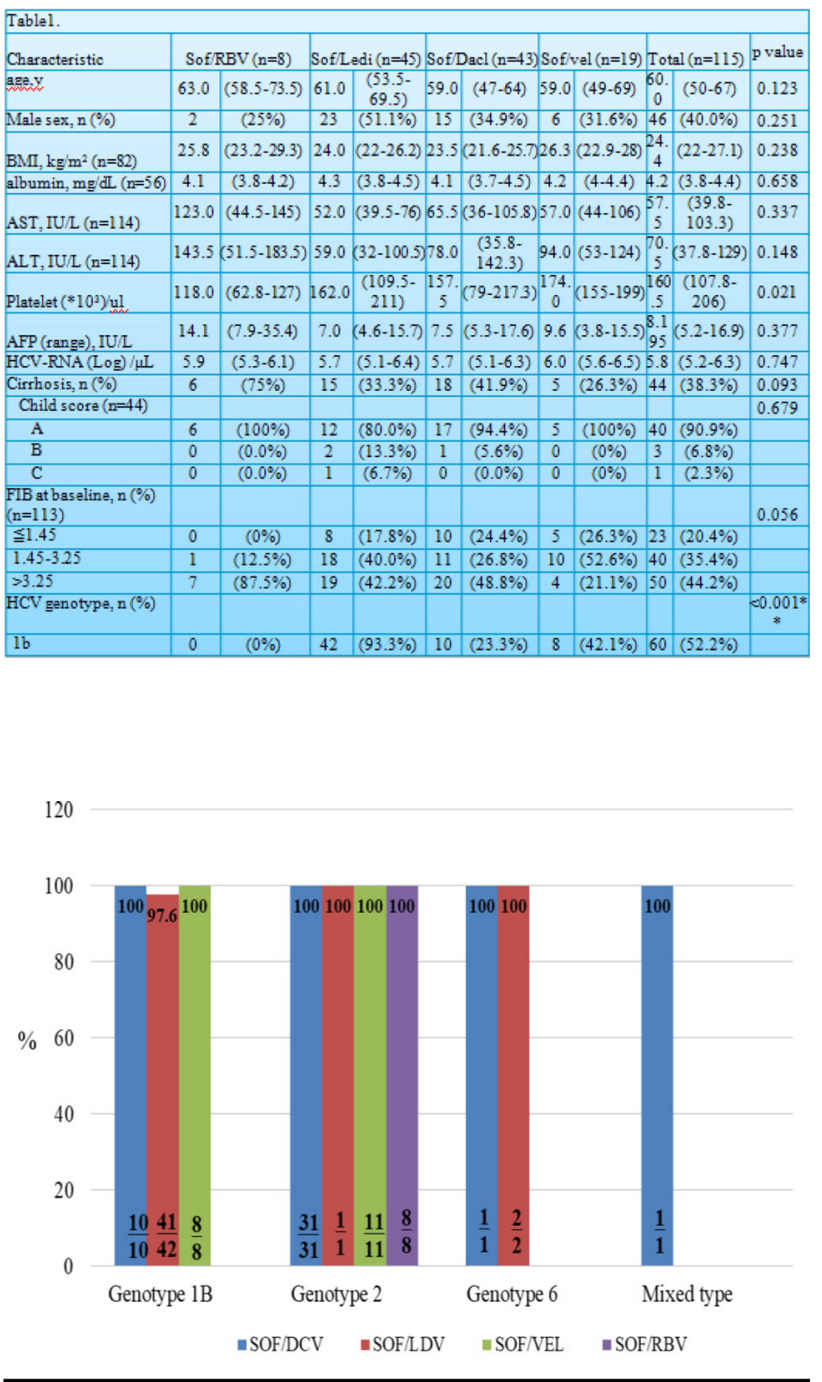

HCV-65

Real-life data on interferon (IFN)-free direct acting antiviral (DAA) therapies for chronic hepatitis C (CHC) in Kocaeli

\section{Sila Akhan ${ }^{1}$, Murat Sayan ${ }^{1}$, Salih Cakiroglu ${ }^{1}$, Ecem Vuslat Gunes $^{1}$ \\ ${ }^{1}$ Kocaeli University Medical Faculty, Turkey}

Background: To evaluate sustained virologic response (SVR) and adverse events (AE) with IFN-free DAA therapies in Kocaeli.
Method: From January 2016 to January 2017, we treated 47 adult patients with chronic hepatitis $\mathrm{C}$ using DAA therapies. This 46 patients were followed up and treated for chronic hepatitis $\mathrm{C}$ in Infectious Diseases policlinic since 2004 in different times. Most patients were infected with HCV genotype $196 \%$ (HCV-1 17 patients (36\%); 1a, 3 patients $(6 \%)$; $1 \mathrm{~b}, 25$ patients $(53 \%)$ followed by HCV-3, 2 patients $(4 \%)$. These percentages were similar to those reported for the country in general. One patient had kidney transplantation and one patient had liver transplantation.

Result: From 47 patient 12 patients (26\%) were treatment naive; 28 patients received sofosbuvir/ledipasvir, 13 patients ombitasvir/paritaprevir/ritonavir (OBV/PTV/r) +dasabuvir, 2 patients sofosbuvir+RBV, 2 patients elbasvir/grazoprevir, 1 patient daclatasvir+asunaprevir and 1 patient sofosbuvir and pegylated interferon. 5 patients (5 sofosbuvir/ledipasvir, 1 OBV/PTV/r+dasabuvir) were on haemodialysis. In sofosbuvir/ledipasvir group including the 4 dialysis patients all have pegylated interferon+RBV experience; mostly twice and 5 of them with telaprevir, 2 of them with boceprevir. Twenty five patients underwent liver biopsy with the results of fibrosis 2 in 2, fibrosis 3 in 13, fibrosis 4 in 6, fibrosis 5 in 4 and two patients have fibroscan results. In all patients were reached the end of the treatment results and HCV RNA were found negative in $100 \%$. The sustained virologic response (SVR) results at 3 months of therapies were found $100 \%$, as well. Anemia was the only side effect and seen only in patients who receiving ribavirin combination (10 patients: 2 patients sofosbuvir+RBV and 8 patients OBV/PTV/r+dasabuvir +RBV) $(22 \%)$. In one patient bilirubin levels increased over $10 \mathrm{mg} / \mathrm{dL}$. After cessation of ribavirin bilirubin turned to normal levels.

Conclusion: Our patients had mostly pegylated interferon experience and for that that reason this new treatment options were very well tolerated. SVR were found $100 \%$ very successful, in our patients.

\section{HCV-66}

Real-world efficacy and safety experience reimbursed HCV direct anti-viral agent for patients with advanced fibrosis in Taiwan

ChiHsien Chang ${ }^{1}$, Chichieh Yang', Chienhua Chen ${ }^{1}$, Jyonghong $\overline{\text { Lee }}^{1}$, Chinsheng $\mathrm{Wu}^{1}$, Yutsai Liu ${ }^{1}$, Kweiming Chen ${ }^{1}$, Yuling Huang ${ }^{1}$, Lihsiang Shin ${ }^{1}$

${ }^{1}$ Show Cheap Memorial Hospital, Taiwan

Background: Only two direct anti-viral regimens, daclatasvir/ asunaprevir (DUAL) \& OMV+PTV/r+DSV (ProD), has been reimbursed by Taiwanese National Health Insurance for F3/F4 population in 2017. Several lethal cases associated with direct anti-viral agent (DAA) occurred. Taiwan is known for high HBV prevalence. HCV/ HBV co-infection is characterized by a more aggressive clinical course and the estimated prevalence in Taiwan of $\mathrm{HCV} \mathrm{Ab}$ among $\operatorname{HBsAg}(+)$ patient is up to $12 \% 1$.

Method: Genotype $1 \mathrm{~b} \mathrm{HCV}$ infection were treated with DUAL therapy from Jan 2017 at Show Chwan Health System in Chung-hua, Taiwan were e Patients with resistance-associated substitutions (RASs) to NS5A inhibitors were excluded. The primary endpoints was SVR12. SVR12 data is collected continuously. In addition, base line $\mathrm{HBsAg}$ positive patients were enroll as $\mathrm{HBV} / \mathrm{HCV}$ co-infection. $\mathrm{HBV}$ viral flare up is defined as HBV DNA increase of one $\log 10$ or undetectable reversed to detectable. HBV biological flare up is defined as ALT 2x increase. This is an interim report.

Result: 42 patients were enrolled. Average age was 62.8 years old. Most patients $(73.8 \%$ ) were female. $69 \%$ of patients was with $\mathrm{F} 3 / \mathrm{F} 4$ status and $41.3 \%$ of them were CP-A6. The baseline mean viral load was $1.64 \times 106.89 \%$ of patients were IFN-experienced. Our patients 
were difficult-to-treat population. There were four patients with concomitant HBV infection. HCV RNA was under LLOQ in 38/42 $(90.5 \%)$ at W4. EOT is still in progress and was achieved by $41 / 42$ (97.6\%) patients. The SVR12 was $18 / 19$ (94.7\%). Two patients didn't achieve RVR were with viral load above average viral load. One patient experienced breakthrough at month 3 EOT \& SVR did not affected by cirrhotic status.

There was no significant safety finding. Only 1 patient $(2.3 \%)$ experienced Grade III (5-10x) ALT elevation and Grade II (1.6-2.5x) bilirubin elevation, respectively. The patient contributed the spike in ALT and bilirubin elevation was because of herbal medications for flu. After discontinuing the herbal medicine, the episode was over.

Totally 5 patient in whom base line $\mathrm{HBsAg}$ was positive. HBV DNA in 3 patients was undetectable and detectable in another two patients: $335 \mathrm{IU} / \mathrm{ml}$ and $70 \mathrm{IU} / \mathrm{ml}$, average $202.5 \mathrm{IU} / \mathrm{ml}$. In this subgroup, HCV RNA was under LLOQ in 5/5 (100\%) at W4 (RVR). EOTVR is still in progress and was achieved by $4 / 4(100 \%)$ patients. The SVR12 was $3 / 3(100 \%)$, The HBV DNA was detectable in W12 in 2 patients: $1258 \mathrm{IU} / \mathrm{ml}$ and $435 \mathrm{IU} / \mathrm{ml}$, both have $1 \mathrm{log}$ increase. In W24 (EOT), one more patient with HBV viral flare up (undetectable to $15 \mathrm{IU} / \mathrm{ml}$ ). Totally $\mathrm{HBV}$ viral flare up is $60 \%$ (3/ 5 ) in our study. There were no biological flare up in this 3 patients. Conclusion: SVR12 and ADR in real world is similar to clinic trial. HBV/HCV co-infection in patients with similar HCV viral response but high rate of $\mathrm{HBV}$ viral flare up were also recorded in our study. Fortunately, no biological flare of HBV was noted.

\section{HCV-67}

Real-world experience of efficacy and safety using paritaprevir/ ritonavir, ombitasvir and dasabuvir for advanced fibrotic genotype 1 chronic hepatitis c patients who failed to previous therapy with pegylated interferon plus ribavirin

\section{Sih $\mathrm{Hsien}^{\mathrm{Wu}} \mathbf{u}^{1}$}

${ }^{1}$ Taipei Veterans General Hospital, Taiwan

Background: Treatment of chronic hepatitis C (CHC) evolved rapidly in recent years due to the invention of interferon-free direct antiviral agents. Based on the data obtained from clinical trials, combination therapy with paritaprevir/ritonavir, ombitasvir and dasabuvir (PrOD) with or without ribavirin (RBV) have been showed to cure over $95 \%$ CHC patients infected by genotype 1 hepatitis $\mathrm{C}$ virus (HCV), regardless cirrhosis or not. However, real-world data regarding the efficacy and safety of PrOD-based therapy in Asian $\mathrm{HCV}$ genotype $1 \mathrm{CHC}$ patients are limited, especially for advanced fibrotic patients who failed previous therapy with pegylated interferon (Peg-IFN) plus RBV. Therefore we conducted this study to address these important issues.

Method: Between January and April 2017, a total of 47 advanced fibrotic ( $>=\mathrm{F} 3$ ) genotype $1 \mathrm{CHC}$ patients who failed to previous therapy with Peg-IFN and received PrOD-based therapy for 12 weeks at Taipei Veterans General Hospital were retrospectively enrolled. Weight-based RBV 800-1200 mg/day was added for all HCV genotype $1 \mathrm{a}$ and $1 \mathrm{~b}$ patients with cirrhosis. Advanced fibrosis was defined by liver stiffness measurement by transient elastography (FibroScan)? $9.5 \mathrm{kPa}$. Patients were excluded for analyses if they had history of hepatic decompensation, mixed infection with $\mathrm{HCV}$ other than genotype 1 and coinfection with hepatitis B virus. Serum quantitative HCV RNA level was determined by real-time PCR (Cobas Taqman HCV Test v2.0, Roche Diagnostics) with lower detection limit of 15 $\mathrm{IU} / \mathrm{mL}$. Sustained virological response (SVR) was defined by undetectable HCV RNA at the end and 12 weeks after the completion of therapy.

Result: The mean age of enrolled population was $63.5+/-9.7$ years, $20(42.6 \%)$ of them was male and $15(31.9 \%)$ diagnosed to have liver cirrhosis. The mean baseline HCV RNA level was $6.28+/-0.84 \log 10$ $\mathrm{IU} / \mathrm{mL}$ and $93.6 \%$ (44/47) were infected by HCV genotype $1 \mathrm{~b}$. Previous treatment history of Peg-IFN was as follows: relapser $68.1 \%$, partial or null responder $27.6 \%$ and early terminated due to intolerance of side effects: $4.3 \%$. After PrOD-based therapy, the rates undetectable $\mathrm{HCV}$ at week 2, 4 and 12 were $59.6 \%, 89.4 \%$ and $100 \%$, respectively. $57.8 \%(11 / 19)$ patients with detectable HCV RNA at week 2 were $<100 \mathrm{IU} / \mathrm{mL}$. Fatigue, pruritus, skin rash, insomnia and headache were most common patient reported adverse effects. Grade $2(1.5 \sim 3.0 \mathrm{X}$ ULN) hyperbilirubinemia were found in $12.8 \%(6 / 47)$ patients during study period and all of them belonged to unconjugated hyperbilirubinemia. No patients had grade 3 or 4 hyperbilirubinemia during study period and all patients finished 12 weeks PrOD-based therapy. After post-treatment follow up, all 47 patients (100\%) achieved SVR.

Conclusion: Our real world data in Taiwan demonstrated PrODbased therapy with or without RBV is well-tolerated and highly effective for genotype $1 \mathrm{CHC}$ patients with advanced fibrosis who failed to previous therapy with Peg-IFN plus RBV.

\section{HCV-68}

Safety, tolerability, and pharmacokinetics of sofosbuvir and velpatasvir fixed-dose combination tablet in healthy Chinese subjects

Kimberly L Garrison ${ }^{1}$, Luisa M Stamm ${ }^{1}$, Gong Shen ${ }^{1}$, Yanhua Ding ${ }^{2}$, Brian Mcnabb ${ }^{1}$, K.H. John Ling ${ }^{1}$, Erik Mogalian', Anita Mathias ${ }^{1}$

${ }^{1}$ Gilead Sciences, United States; ${ }^{2}$ The First Hospital of Jilin University, China

Background: Sofosbuvir/velpatasvir (SOF/VEL) 400/100 $\mathrm{mg}$ is the approved dose in the U.S. and E.U. for the treatment of chronic hepatitis $\mathrm{C}$ virus (HCV) infection. In support of ongoing clinical development in China, a Phase 1 single dose (SD) and multiple dose (MD) study was conducted to evaluate the safety, tolerability and pharmacokinetics (PK) of SOF/VEL 400/100 mg in healthy Chinese subjects.

Method: Single and multiple (7) once-daily doses of SOF/VEL $400 / 100 \mathrm{mg}$ were administered under fasted conditions to healthy Chinese subjects. Exposures of SOF, its predominant circulating inactive metabolite, GS-331007, and VEL were characterized following single and multiple doses. Accumulation ratios and their associated 90\% CIs were calculated for GS-331007 and VEL AUC and $\mathrm{Cmax}$ ([MD]/[SD]). SOF is a pro-drug with a half-life $<0.5$ hours; as such, no accumulation ratio of SOF was calculated. Safety was evaluated throughout the study by assessment of adverse events (AEs) and laboratory abnormalities.

Result: Fourteen ( 7 male and 7 female) Chinese subjects with median (range) age of 29 (19-42) years and mean (standard deviation) body mass index of $21(1.5) \mathrm{kg} / \mathrm{m}^{2}$ were enrolled and completed the study. Exposures of SOF, its metabolite GS-331007, and VEL in Chinese subjects were similar to historical values (Table 1). Consistent with historical values in overseas studies, GS-331007 and VEL showed $18 \%$ to $32 \%$ and $13 \%$ to $43 \%$ accumulation, respectively. No deaths, serious (AEs), severe (Grade 3 or Grade 4) AEs, or discontinuations due to AEs were reported. There were no clinically relevant patterns of AEs or laboratory abnormalities. 
Conclusion: The PK and safety of SOF/VEL 400/100 mg in healthy Chinese subjects was similar to historical data, supporting the 400/100 mg dose of SOF/VEL for use in Chinese patients with HCV infection.

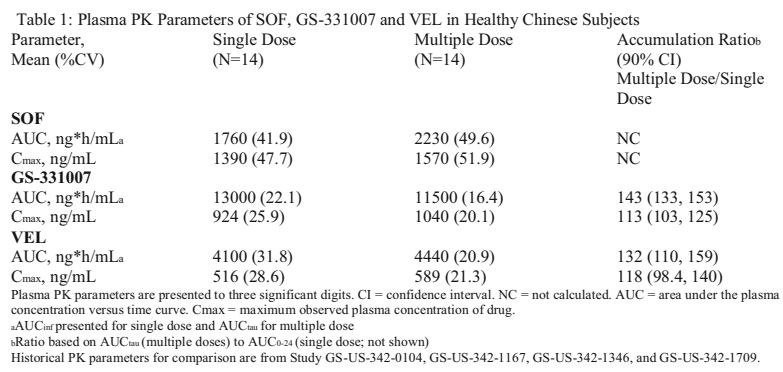

HCV-69

Sustained virological response to dasabuvir and ombitasvir/paritaprevir/ritonavir \pm ribavirin treatment in patients with chronic hepatitis c: real-world data from Turkey

\section{Nese Demirturk ${ }^{1}$, Emine Turkoglu ${ }^{1}$, Petek Sarlak Konya ${ }^{1}$, Havva Tunay ${ }^{1}$ \\ ${ }^{1}$ Kocatepe University Medical Faculty, Turkey}

Background: Today direct acting antiviral drugs are essential for chronic hepatitis $\mathrm{C}(\mathrm{CHC})$ treatment. Combination with dasabuvir and ombitasvir/r/paritaprevir \pm ribavirin is one of the direct acting antiviral treatment options for CHC in Turkey available since June 2016. In this study our purpose is to evaluate the characteristics of patients receiving this therapy and their sustained virological response.

Method: The study was planned retrospectively. The treatment-naive or peginterferon/ribavirin treatment-experience patients treated with dasabuvir ( $250 \mathrm{mg} /$ twice daily) and ombitasvir/ritonavir/paritaprevir (25/150/100 mg once daily) \pm ribavirin for 12 weeks to CHC between June 2016 - July 2017 were included in the study. Primary end point was "end of treatment response" and secondary end-point was "sustained virological response" at 12 week. Side effects were also evaluated in the patients.

Result: DSV and OBV/r/PTV regimen was initiated to 48 patients; 6 of them were also given RBV. 26 patients were female. The mean age of the patients was 58. All subjects were infected with HCV GT1;5 of them were infected GT1a and 43 of them were infected GT1b. 44 patients were naive. 7 of treatment experience patients were not respond to previous treatment, and the remaining patients had relapses. 30 of the patients had elevated pre-treatment ALT; the mean serum ALT level was $59 \mathrm{IU} / \mathrm{ml}$. The pre-treatment HCV RNA was found $3.3 \times 106 \mathrm{IU} / \mathrm{ml}$. There were 12 patients with cirrhosis (all of them Child- Pugh A).

At the end of the study we found that the two patients did not come to the control after the first month of treatment and in a patient the treatment can not be continued with because of sudden loss of consciousness developed on the fifth day of treatment. So the therapy was completed 45 patients. In all patients completed the treatment were obtained "end of treatment response". "Sustained virological response" was obtained in 39 of the 40 patients $(97.5 \%)$ who completed the 12-week follow-up period after the treatment was finished. In one patient developed relapse at the 8 weeks after the cessation of the therapy. This relapsed patient had peg-IFN/ribavirin treatmentexperience and he didn't have cirrhosis and he infected by HCV genotype 1a. So we found that "sustained virological responses" were $100 \%, 100 \%, 96.7 \%$ and $92.8 \%$ with cirrhosis $(10 / 10)$, treatment- naive (26/26), without cirrhosis (29/30) and treatment-experience (13/ 14) patients, respectively.

The treatment regimen was generally tolerated well. Adverse events occured in $17(37.8 \%)$ patients. The treatment was discontinuated a week earlier in a patient because of upper gastrointestinal bleeding. The other mild side effects were fatigue, pruritus, arthralgia, increase in serum bilirubin, headache and cough.

Conclusion: Our real-world data was demonstrated that DSVand $\mathrm{OBV} / \mathrm{r} / \mathrm{PTV} \pm \mathrm{RBV}$ treatment in HCV genotype 1 infected patients is effectiveness and a good safety profile. It is consistent with clinical trials results.

$\mathrm{HCV}-70$

The evaluation of the new treatment regimen given to the hepatitis $\mathrm{C}$ patients

\section{Selcuk Aksoz ${ }^{1}$, Hakan Sezgin Sayiner ${ }^{2}$ \\ ${ }^{1}$ Adiyaman Training and Research Hospital, Turkey; ${ }^{2}$ Adiyaman University, Faculty of Medicine, Turkey}

Background: The Hepatitis $\mathrm{C}$ disease is liver inflammation caused by a virus of RNA genotype called Hepatitis $\mathrm{C}$ virus (HCV). Hepatitis C virus (HCV) is the member of Flaviviridae family and the genom varies highly. $\mathrm{HCV}$ virus has at least six different genotype determined and each genotype includes different subtypes. HCV genotypes distribution vary geographically worldwide. The indication of viral genotype has great importance in the selection of antiviral treatment, following up the treatment duration and response to the treatment. In this research, in Adiyaman city for the last 6 months, the HCV infected patients treatment with ritonavir, ombitasvir, paritaprevir and dasabuvir -which is one of the new treatment regimens- results are being evaluated.

Method: We included 9 Chronic Hepatitis $\mathrm{C}$ (KHC) patients between the ages 20 and 77 in this research. In all the patients, HCV was determined as genotype 1 . Three of the patients were experienced in treatment previously. All the patients were given ritonavir, ombitasvir, paritaprevir 2 tablets once a day and dasabuvir 2 tablets, one at each time, once a day for 3 months and clinical and laboratory followups were done every 15 days.

Result: On the 15th day of treatment, HCV RNA levels were 0 in all patients. During the 3 month treatment, only 1 patient had a significant level of itching complaint that did not require treatment to stop. All the patients completed the 3 month treatment and there was no significant laboratory value change that would require to stop treatment in any of the patients.

Conclusion: New treatment regimens including ritonavir, ombitasvir, paritaprevir and dasabuvir for chronic hepatitis $\mathrm{C}$ patients appear effective and reliable. However, it is important for this research to be supported with a larger group of patients.

\section{$\mathrm{HCV}-71$}

Pegylated IFN-a 2a plus ribavirin combination therapy results in Mongolian patients with hepatitis $\mathbf{C}$ virus genotype $\mathbf{1 b}$

\section{$\underline{\text { Nyamsuren Naranzul }}^{1}$, Oidov Baatarkhuu' ${ }^{1}$, G Uugantsetseg ${ }^{1}$ \\ ${ }^{1}$ Mongolian National University of Medical Sciences, Mongolia}

Background: Mongolia is one of the highest prevalent of hepatitis C virus infection of the world. The standard therapy for patients with genotype 1 chronic hepatitis $\mathrm{C}$ (CHC) is a combination of peg-interferon alfa $-2 \mathrm{a}$ and ribavirin for 48 weeks. However the most 
appropriate duration of treatment remains to be established because of treatment -related side effects and cost. The study objective is compare the efficacies of 24-week and 48-week treatments of peginterferon alfa-2a plus ribavirin in Mongolian patients

Method: A total of 47 patients with genotype $1 \mathrm{CHC}$ was treated between August 2008 and were randomly assigned to treatment and December 2013 and at least one dose of study medication, consisting of $180 \mathrm{mg}$ of peginterferonalfa 2-a once weekly plus daily ribavirin (1000 or $1200 \mathrm{mg}$, depending on body weight). Patients with undetectable HCV RNA at 24 weeks of treatment were allowed to choose either 24 or 48 weeks as the duration of their treatment; 4 patients chose the 24 week treatment regimen and the 43 patients chose the 48-week regimen.

Result: The SYR rate was higher in patients treated for 48 weeks than in those treated for 24 weeks ( $74 \%$ vs $48.8 \%, p=0.0013$ ). In the multivariate analysis, age $<52$ years, platelets $>148000 \mathrm{~mm}^{3}$, and treatment duration for 48 weeks remained significant independent predictors of SVR. Fourteen of the 47 patients who relapsed in the 24-week treatment group received split 24-week therapy, and 3 patients was achieved SVR. The overall SVR rate did not differ significantly between the 24-week treatment group, including those who underwent 24 -week split therapy $48.8 \%$, and the 48 -week treatment group $(48.8 \%$ vs $74 \%)$.

Conclusion: SVR rate was $74 \%$ of the patients in Mongolia. The 24-week therapy following failure is a useful treatment strategy for patients with genotype $1 \mathrm{~b}$ chronic hepatitis $\mathrm{C}$.

\section{HCV-72}

Safety, tolerability and pharmacokinetics of ledipasvir/sofosbuvir fixed-dose combination tablet in healthy Chinese subjects

\section{Rebecca Begley ${ }^{1}$, Yanhua Ding ${ }^{2}$, Brian Mcnabb ${ }^{1}$, Gong Shen ${ }^{1}$, John Ling ${ }^{1}$, Erik Mogalian', Anita Mathias ${ }^{1}$ \\ ${ }^{1}$ Gilead Sciences, Inc, United States; ${ }^{2}$ The First Hospital of Jilin University, Jilin, China, China}

Background: Ledipasvir/sofosbuvir (LDV/SOF) $90 / 400 \mathrm{mg}$ is the approved dose in the U.S. and E.U. for the treatment of chronic hepatitis $\mathrm{C}$ virus (HCV) infection. In support of ongoing clinical development of LDV/SOF in China, a Phase 1 single (SD) and multiple dose (MD) study was conducted to evaluate the safety, tolerability and pharmacokinetics (PK) of LDV/SOF 90/400 $\mathrm{mg}$ in healthy Chinese subjects.

Method: Single and multiple (10) once-daily doses of LDV/SOF 90/400 mg were administered under fasted conditions to healthy Chinese subjects. Exposures of SOF, its predominant circulating inactive metabolite, GS-331007, and LDV were characterized following single and multiple doses. Accumulation ratios (ARs) and their associated 90\% CIs were calculated for GS-331007 and LDV based on a parametric mixed-effects ANOVA model with the natural log-transformed values of AUC and Cmax (MD/SD). SOF is a pro drug with a half-life $<0.5$ hours; as such, no accumulation ratio of SOF was calculated. Safety was evaluated throughout the study by assessment of adverse events (AEs) and laboratory abnormalities.

Result: Fourteen ( 7 male and 7 female) Chinese subjects with median (range) age of 27 (19-42) years and mean (standard deviation) body mass index of $21.7(1.7) \mathrm{kg} / \mathrm{m}^{2}$ were enrolled and completed the study. Exposures of SOF, GS-331007, and LDV in Chinese subjects were similar to historical values in overseas studies (Table 1). Consistent with historical values, GS-331007 and LDV showed 29\%-57\% and $107 \%-177 \%$ accumulation, respectively, upon multiple dosing (Table 1). Overall, LDV/SOF 90/400 mg was well tolerated. No clinically relevant pattern of AEs or laboratory abnormalities were identified during the study and no deaths, serious AEs, severe (Grade 3 or Grade 4) AEs, or discontinuations due to AEs were reported. The only AEs that occurred in $>1$ subject were proteinuria $(\mathrm{N}=3$, all Grade 1, all asymptomatic and transient, and all deemed related to study treatment by investigator) and oropharyngeal pain ( $\mathrm{N}=2$, both Grade 1 and deemed unrelated to study treatment by investigator).

Conclusion: The PK and safety of LDV/SOF 90/400 mg in healthy Chinese subjects was similar to historical data, supporting the 90/400 $\mathrm{mg}$ dose of LDV/SOF for use in Chinese patients with HCV infection.

\begin{tabular}{|c|c|c|c|}
\hline $\begin{array}{l}\text { Parameter, } \\
\text { Mean (\%CV) }\end{array}$ & $\begin{array}{l}\text { Single Dose } \\
\quad(N=14)\end{array}$ & $\begin{array}{l}\text { Multiple Dose } \\
\quad(\mathrm{N}=14)\end{array}$ & $\begin{array}{l}\text { Accumulation Ratio }{ }^{\mathrm{b}}(90 \% \mathrm{Cl}) \\
\text { Multiple Dose/ Single Dose }\end{array}$ \\
\hline \multicolumn{4}{|l|}{ SOF } \\
\hline AUC (ng*h/mL) ${ }^{a}$ & $1750(50.1)$ & $2030(41.7)$ & $\mathrm{NC}$ \\
\hline $\mathrm{C}_{\max }(\mathrm{ng} / \mathrm{mL})$ & 1550 (48.9) & $1530(42.0)$ & $\mathrm{NC}$ \\
\hline \multicolumn{4}{|l|}{ GS-331007 } \\
\hline AUC (ng*h/mL) & $12700(21.8)$ & $12100(15.0)$ & $157(150,165)$ \\
\hline $\mathrm{C}_{\max }(\mathrm{ng} / \mathrm{mL})$ & $836(19.4)$ & $1070(14.3)$ & $129(121,138)$ \\
\hline \multicolumn{4}{|l|}{ LDV } \\
\hline AUC (ng*h/mL) ${ }^{a}$ & $9690(38.2)$ & $10900(35.1)$ & $277(228,337)$ \\
\hline $\mathrm{C}_{\max }(\mathrm{ng} / \mathrm{mL})$ & $322(34.7)$ & $628(31.1)$ & $207(166,259)$ \\
\hline
\end{tabular}

\section{$H C V-73$}

The real-world safety and efficacy of sofosbuvir and ledipasvir for elderly patients

Hideyuki Tamai ${ }^{1}$, Naoki Shingaki ${ }^{1}$, Yoshiyuki $\operatorname{Ida}^{3}$, Ryo Shimizu ${ }^{3}$, Shuya Maeshima ${ }^{3}$, Junpei Okamura ${ }^{2}$, Akira Kawashima ${ }^{2}$, Taisei Nakao ${ }^{2}$, Takeshi Hara ${ }^{1}$

${ }^{1}$ Wakayama Rosai Hospital, Japan; ${ }^{2}$ Naga Municipal Hospital, Japan; ${ }^{3}$ Wakayama Medical University, Japan

Background: In September 2015, the clinical use of sofosbuvir and ledipasvir was approved in Japan for patients infected with genotype 1 hepatitis $\mathrm{C}$ virus. This therapy was well tolerated and achieved a high SVR rate $(100 \%)$ in a Japanese Phase III trial. However, the patients were younger than real-world patients, and the proportion of patients with cirrhosis, or chronic renal disease was small. Furthermore, patients with history of hepatocellular carcinoma (HCC) treatment was excluded. The present study aimed to evaluate the safety and efficacy of sofosbuvir and ledipasvir for elderly patients and to clarify whether extremely high sustained virological response (SVR) rate can be achieved even in a real-world setting.

Method: This was a multicenter prospective cohort study. Exclusion criteria were any of followings: 1) being infected with other genotypes other than genotype 1,2 ) treatment failure of daclatasvir and asunaprevir, 3) estimated glomerular filtration rate (eGFR) $\mathrm{ml} / \mathrm{min} /$ $\left.1.73 \mathrm{~m}^{2}<30,4\right)$ decompensated cirrhosis, 5) any form of cancer. Between September 2015 and February 2017, 424 patients were treated by standard doses of sofosbuvir ( $400 \mathrm{mg} /$ day) and ledipasvir (90 mg/day) for 12 weeks. As 2 patients was lost to follow-up, 422 patients were finally analyzed. The negativity of HCV-RNA at week 12 after the end of therapy was defined as SVR.

Result: Mean age was $68 \pm 12$ years old. There were 219 male and 203 female patients. The SVR rates of overall, patients $<65$ years, patients $\geq 65$ and $<75$ years, and patients $\geq 75$ years were $97 \%$ (411/ 422), 97\% (130/132), 96\% (155/161), and 98\% (126/129), respectively. The SVR rates of ineligible patients to interferon, cirrhotic patients, elderly ( $\geq 65$ years old) and cirrhotic patients, patients with moderate chronic kidney disease $(\mathrm{eGFR}<60$ ), patients with treatment failure of ribavirin and interferon, patients with history of HCC treatment, patients with protease inhibitor failure, and patients with resistance-associated substitutions (RASs) to non-structural protein 5A (NS5A) were 97\% (355/366), 97\% (184/190), 96\% (147/153), $98 \%$ (91/93), $98 \%$ (89/91), 95\% (78/82), 93\% (43/46), and 93\% (37/ 
40), respectively. No significant difference was seen in SVR rate of each factor. Four patients $(1 \%)$ discontinued treatment due to adverse events. Most frequently adverse effect was elevation of serum uric acid level (14\%; 58/422). No significant difference was seen in safety between patients $<75$ years and patient $\geq 75$ years groups.

Conclusion: In a real-world setting, sofosbuvir and ledipasvir was a safe treatment even in patients aged $\geq 75$ years. When patients without pre-existing NS5A RASs and prior protease inhibitor failure would be selected, extremely high SVR rate can be achieved irrespective of age.

\section{HCV-74}

Successful treatment of hepatitis $\mathbf{C}$ virus infection in an advanced aged patient with paritaprevir/ritonavir, ombitasvir + dasabuvir combination: a case report

Necla Tulek ${ }^{1}$, Necla Tulek ${ }^{1}$, Fatma Sebnem Erdinc ${ }^{1}$, Sukran Sevim $^{1}$, Meliha Cagla Sonmezer ${ }^{2}$, Metin Ozsoy ${ }^{1}$, Gunay Tuncer Ertem ${ }^{1}$

${ }^{1}$ Ankara Training and Research Hospital, Turkey; ${ }^{2}$ Hacettepe University, Medical School, Turkey

Background: Hepatitis C treatment with paritaprevir/ritonavir, ombitasvir, dasabuvir Although the direct-acting antivirals have become the cornerstone of hepatitis $\mathrm{C}$ treatment recently, there is limited data to use them in elderly patients. In here, we describe the successful treatment of a patient very advanced age with paritaprevir/ ritonavir, ombitasvir, dasabuvir combination

Method: Newly diagnosed, 86 years old, male patient referred to our clinic for treatment of hepatitis C. He has been on haemodialysis since 2012. Hepatitis C infection has been diagnosed by HCV RNA (70000000 IU/ml) five months ago after two units of blood transfusion. At admission, he had no complaint except fatigue. Laboratory test results showed that haemoglobin was $9,6 \mathrm{~g}$; blood urea $185 \mathrm{mg} / \mathrm{dl}$; creatinine $10.8 \mathrm{mg} / \mathrm{dl}$; eGFR 4; AST $239 \mathrm{U} / \mathrm{L}$ (N: 0-35); AST 239(N:0-35) U/L and anti-HCV negative. He was hospitalized. Acute hepatitis $\mathrm{C}$ could not be differentiated. Blood samples of donor was checked again for hepatitis $\mathrm{C}$ and the result was negative again. $\mathrm{He}$ was followed up and administered supportive treatment. Two weeks after, his ALT, AST levels elevated gradually to $770 \mathrm{U} / \mathrm{L}, 498 \mathrm{U} / \mathrm{L}$ respectively. HCV RNA was $256000000 \mathrm{IU} / \mathrm{mL}$ and genotype was $1 \mathrm{~b}$. We decided to give paritaprevir/ritonavir, ombitasvir, dasabuvir combination for 12 weeks.

Result: After the fourth day of therapy, ALT, AST levels begun to decline. At the second week of treatment, he was discharged with ALT; 299 U/L, AST; 148, and eGFR; 9 values. At the first month of treatment, HCV RNA level was negative. ALT, AST levels returned to normal at two months of treatment. HCV RNA levels were negative at the end of and three months after treatment. No side effect was detected, and treatment was well tolerated.

Conclusion: This case showed that paritaprevir/ritonavir, ombitasvir + dasabuvir combination is safe and effective even in the patients with advanced age.

\section{HCV-75}

Combination of sofosbuvir, pegylated-interferon, and ribavirin for treatment of hepatitis $C$ virus genotype 1 infection in Indonesia

Andri Sanityoso Sulaiman ${ }^{1}$, Rino Alvani Gani ${ }^{1}$, Irsan Hasan', $\overline{\text { Cosmas Rinaldi Lesmana }}^{1}$, Juferdy Kurniawan ${ }^{1}$, Chyntia Olivia

\section{Maurine Jasirwan', Kemal Fariz Kalista ${ }^{1}$, Muhammad Yusuf} Hanif $^{1}$

${ }^{1}$ Cipto Mangunkusumo National Hospital, Faculty of Medicine Universitas Indonesia, Indonesia

Background: The presence of direct-acting antiviral (DAA) has improved the treatment of HCV genotype 1 infection and making it more preferable than Pegylated-interferon (PegIFN) and Ribavirin (RBV) based treatment. However treatment with all DAA combination regimen is limited and expensive in low health care affordability country including Indonesia. The appearance of generic sofosbuvir (SOF) facilitate the utilization of SOF plus PegINF with or with our RBV combination. Therefore, in this study we assessed the efficacy of $\mathrm{SOF}+\mathrm{RBV}$ and $\mathrm{SOF}+\mathrm{RBV}+\mathrm{PegINF}$ combination for treatment of chronic hepatitis $\mathrm{C}$ infections patient with genotype 1 in Indonesia. Method: We performed retrospective study comprising of 128 patients in Cipto Mangunkusumo Hospital with chronic hepatitis C, genotype 1, infection. 36 patients was treated with PegINF+SOF + RBV and 92 patients was treated with SOF+RBV with the duration of therapy was 12 and 24 weeks in both arms. The primary endpoint was sustained virologic response after treatment completion (SVR12).

Result: In the end of treatment, $99.2 \%$ patients achieved undetected HCV RNA in 12 weeks and 24 weeks duration of therapy $(100 \%$ in $\mathrm{PegINF}+\mathrm{SOF}+\mathrm{RBV}$ group and $98.9 \%$ in $\mathrm{SOF}+\mathrm{RBV}$ group). The SVR12 of PegINF+SOF+RBV reach $100 \%$ meanwhile The SVR12 of SOF+RBV reach $88 \%$. No different in SVR12 between cirrhotic and non-cirrhotic patient in $\mathrm{PegINF}+\mathrm{SOF}+\mathrm{RBV}$ group while in SOF+RBV group, the SVR12 was lower in cirrhotic patients $(82.9 \%)$ compared to non-cirrhotic patients $(92.2 \%)$. In multivariate analysis, HIV co-infection is associated with lower SVR12 in SOF+RBV group.

Conclusion: 12 weeks and 24 weeks of PegINF+SOF+RBV and $\mathrm{SOF}+\mathrm{RBV}$ is effective in the treatment of genotype 1 chronic hepatitis $\mathrm{C}$ infection

\section{$\mathrm{HCV}-76$}

Resistance-associated variants in HCV NS5A gene in responders and non-responders Brazilian patients infected with $\mathrm{HCV}$ genotypes 1 and 3 treated with daclatasvir

Elisabeth Lampe $^{1}$, Vanessa Duarte Costa ${ }^{1}$, Carlos Eduardo Brandao Mello ${ }^{2}$, Francisco Campello Do Amaral Mello ${ }^{1}$, Pedro Coroa $^{1}$, Estevao Nunes ${ }^{1}$, Lia Laura Lewis Ximenez ${ }^{1}$, Elisabeth Lampe $^{1}$

${ }^{1}$ Fundação Oswaldo Cruz, Brazil; ${ }^{2}$ Hospital Universitário Gaffree Guinle, Brazil

Background: In Brazil, hepatitis $\mathrm{C}$ virus (HCV) seroprevalence is about $1.3 \%$ and approximately 10,000 cases are notified each year. The most prevalent subtypes are $1 \mathrm{a}$ and $1 \mathrm{~b}$, followed by $3 \mathrm{a}$. Several amino acid changes have been associated with daclatasvir resistance. The aim of this study was to investigate the presence of resistanceassociated variants (RAVs) in HCV NS5A gene in responders and non-responders patients infected with HCV genotypes 1 and 3 treated with daclatasvir.

Method: A total of 119 serum samples (HCV-1a: $n=54$; HCV-1b: $\mathrm{n}=46$; HCV-3: $\mathrm{n}=19$ ) was collected at baseline along with 13 serum samples from non-responders patients (HCV-1a: $n=7$; HCV-1b: $n=1$; HCV-3: n=5) after 12-week of treatment with daclatasvir. Methodology included viral RNA extraction, RT-PCR reactions with specific primers for each genotype and purification followed by nucleotide sequencing reaction. 
Result: Considering HCV-1a responders, RAVs L31M (1/47; 2.1\%) and Q30L $(1 / 47 ; 2.1 \%)$ were identified at baseline. NS5A mutation $\mathrm{L} 31 \mathrm{M}$ has been potentially associated with reduced response rates to daclatasvir in HCV-1a patients, however, here, treatment outcome was not influenced by this particular mutation. Substitutions M28T and Q30R, characteristic of RAVs for NS5A DAAs daclatasvir, ledipasvir and ombitasvir, were identified in HCV sequence from one $(1 / 7 ; 14.2 \%)$ non-responder patient infected with HCV-1a after 12-week treatment with daclatasvir. For HCV-3 responders, amino acid residues at position 31 and 93 were conserved in all sequences analyzed. RAVs at position $62, \mathrm{~S} 62 \mathrm{~T}$ and $\mathrm{S} 62 \mathrm{~L}$, were observed in $4 / 14(28.5 \%)$ and $1 / 14(7.1 \%)$ baseline sequences, respectively. Among five non-responders patients infected with HCV-3, RAVs A30S $(1 / 5 ; 20 \%)$ and A30K $(1 / 5 ; 20 \%)$ were identified in baseline and after therapy, respectively. A30K is highly associated with resistance to daclatasvir.

Conclusion: This study highlights the importance of identifying baseline RAVs for HCV-3 prior treatment, as combined therapy using daclatasvir might not be effective when primary resistance mutations are present in the infecting viral variants.

\section{$\mathrm{HCV}-77$}

Efficacy comparison of sofosbuvir-ribavirin and sofosbuvirdaclatasvir regimen for hepatitis $\mathrm{C}$ treatment in NS5A inhibitor limited source area

\section{Juferdy Kurniawan ${ }^{1}$, Rino Alvani Gani ${ }^{1}$, Irsan Hasan ${ }^{1}$, Andri Sanityoso', Cosmas Rinaldi Lesmana ${ }^{1}$, Chyntia Olivia Jasirwan', Kemal Fariz Kalista ${ }^{1}$}

${ }^{1}$ Ciptomangunkusumo National Hospital, Faculty of Medicine Universitas Indonesia, Indonesia

Background: Sofosbuvir (SOF) and daclatasvir (DCV) regimen is recommended for all genotype whether as first choice therapy or an alternative. However, DCV availability is still inadequate at various places and country. Ribavirin (RBV) is more affordable and has been known as chronic hepatitis $\mathrm{C}$ therapy along with sofosbuvir or interferon. Therefore, we conducted this study to assess the efficacy of $\mathrm{SOF}+\mathrm{RBV}$ and $\mathrm{SOF}+\mathrm{DCV}$ regimen for treatment of chronic hepatitis $\mathrm{C}$ in Indonesia.

Method: We conducted a retrospective cohort study with chronic hepatitis $\mathrm{C}$ who were treated with sofosbuvir. Sofosbuvir-ribavirin data was collected from 2015-2016, while sofosbuvir-daclatasvir was collected from 2016-2017. Baseline characteristics were recorded from medical record unit in Cipto Mangunkusumo Hospital. Response therapy measure at week 12 or 24 (end of treatment) and 12 weeks after therapy-sustained virologic response (SVR12). The primary efficacy measure was the SVR12.

Result: We include 309 patients with median of age $52 \pm 15$ years, $59.7 \%(184 / 309)$ were male, 64.4\% (199/309) had genotype 1 infections, 29.8\% (92/309) with cirrhosis, and 4.9\% (15/309) with HIV co-infection. Out of $54.3 \%$ (168/309) patients were treated with $\mathrm{SOF}+\mathrm{DCV}$ regimen and the rest were given $\mathrm{SOF}+\mathrm{RBV}$ regimen. In the end of treatment, $99.3 \%$ patients both in SOF+RBV group (136/ $137)$ and SOF+DCV group (164/165) have no detectable viral load. The SOF+RBV group has SVR12 rates of $90.8 \%$ (109/120) while SOF+DCV group shows higher SVR12 with $98.2 \%$ (108/110). Based on cirrhotic status, SOF+RBV group achieved SVR12 rates of $84.4 \%$ (38/45) in cirrhotic patients and $94.7 \%$ (71/75) in non cirrhotic patients. The SOF+DCV group achieved SVR12 rates of $100 \%$ (27/ 27 ) in cirrhotic patients and $97.5 \%(81 / 83)$ in non cirrhotic patients. Duration of treatment whether 12 or 24 weeks was determined by patients cirrhotic status and its HCV genotype.
Conclusion: Both regimens show an impressive outcome, with overall SVR12 rates above $90 \%$ irrespective of patients cirrhotic status and HCV genotype. Sofosbuvir-ribavirin regimen still can be used as hepatitis C treatment in NS5A limited source area.

HCV-78

Is there any way to catch untreated $\mathrm{HCV}$ infected patients for therapy?

Bahadr Orkun Ozbay ${ }^{1}$, Hurrem Bodur ${ }^{1}$, Esragul Akinci ${ }^{1}$, Bahadir Orkun Ozbay ${ }^{1}$

${ }^{1}$ Ankara Numune Education and Research Hospital, Turkey

Background: Hepatitis $\mathrm{C}$ patients may remain untreated for years, if the correct orientation isn't done by clinicians. We aimed to identify patients who were tested for anti -HCV for any reason and was found positive but didn't referred to the related clinic.

Anti-HCV positive patients with positive or unknown HCV RNA are scheduled to be called for testing.

Method: Anti HCV results of the patients who admitted to any clinic in our hospital were evaluated between 2010-2017 from electronic records. Then, HCVRNA status of the Anti HCV positive patients were investigated. If the patient with positive HCVRNA wasn't referred for therapy, he/she called for liver biopsy and treatment. In addition, the patients with positive Anti HCV and unknown HCVRNA was also called for testing.

Result: The number of patients with positive Anti HCV was found 501. Of which, 266 patients were treated by infectious diseases or gastroenterology specialist. The number of patients with unknown HCV RNA result was 111, whereas negative was 90 . The number of HCV RNA positive but untreated patients was 16. A total of 127 untreated patients with positive or unknown HCV RNA, were contacted (figure1). Of them, we were able to communicate with 50 patients for HCV RNA testing and 7 patients for liver biopsy.

Conclusion: The high number of patients with positive anti HCV but unknown HCV RNA was evaluated as being remarkable. The patients in this group were called for the examination. When anti HCV was detected positive by any clinicians whatever the cause of testing, it is important to refer these patients for therapy. Otherwise, these patients may remain untreated for years since they don't have significant clinical symptoms. It can also be considered to establish a stimulating system in this direction in hospitals.

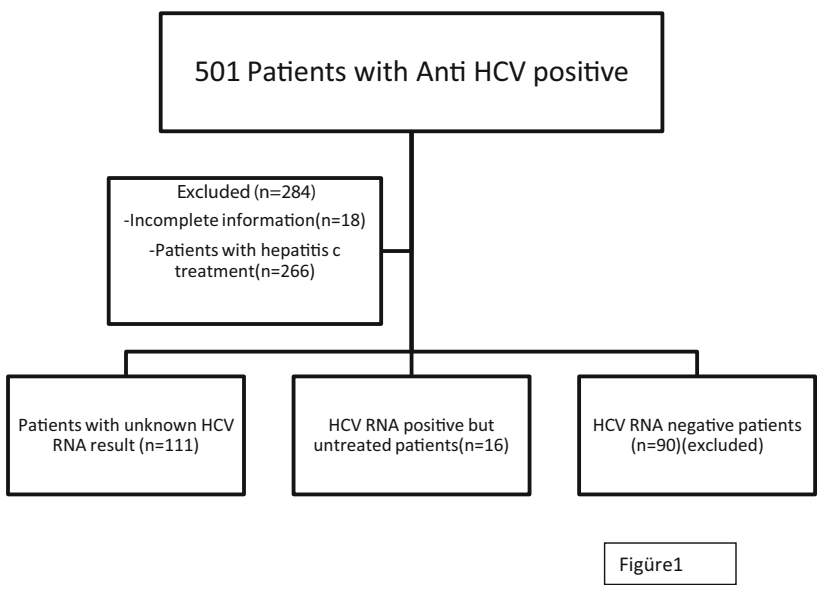




\section{HCV-79}

\section{Some aspects in disease of viral hepatitis $\mathrm{C}$}

\section{Nodirjon Fazliddinovich Ruzimurodov ${ }^{1}$}

${ }^{1}$ Tashkent Medical Academy, Uzbekistan

Background: To study the nature and extent of changes in clinical and laboratory indicators and to determine diagnostic criteria for the different levels of HCV activity at different stages of the disease.

Method: Material for this study was performed by the 1st Republican Clinical Hospital under the Ministry of Healthcare of the Republic of Uzbekistan in the Department of Therapy, which is diagnosed with Viral Hepatitis $\mathrm{C}$ and other co-morbidities. A randomized method was used by 30 patients, $16(53 \%)$ were female, $14(47 \%)$ were male. The average age was $54.3 \pm 0.4$ years.

Result: All patients recommended laboratory-instrumental and morphological examination. With the dynamic observation of patients with $\mathrm{HCV}$ the study of clinical and laboratory indicators and the use of instrumental methods (ultrasound). The need for puncture biopsy (including immunohistochemical examination) in dynamics is shown. It is shown that the timely administration of specific antiviral therapy prevents further progression of inflammatory necrotic processes. Of the total number of patients, 3 of them had a mixed viral hepatitis $\mathrm{C}$ and $\mathrm{B}$ infection, which is characterized by a significant variety of replication activity results, immunohistochemical detection of $\mathrm{B}$ and $\mathrm{C}$ virus antigens, and microscopic data. In these patients there was a deterioration in the analysis and objective status, which is indicative of the different variants of the activity of each virus. The cirrhotic stage is revealed in $2(6.7 \%)$ patients according to the histological data in the absence of its clear clinical and laboratory signs. Using the methods of multifactor analysis, differences in indicators of treatment effectiveness were established. The most informative data were biochemical indicators (ALAT and bilirubin), platelets and Polymerase chain reaction (PCR).

Conclusion: We recommend a quarterly examination of patients with $\mathrm{HCV}$ infection and mixed (hepatitis $\mathrm{C}$ and $\mathrm{B}$ ) with the determination of clinical and biochemical indicators and using instrumental methods. It is recommended quarterly PCR control and a puncture biopsy every 2-3 years.

\section{$\mathrm{HCV}-80$}

\section{Some aspects in the treatment of chronic hepatitis $\mathrm{C}$}

\section{Muzaffarbek O'ktam O'g'li Ismailov ${ }^{1}$}

${ }^{1}$ Tashkent State Pediatric Institute, Uzbekistan

Background: To study the clinical, functional, virological, immunological and morphological features of liver damage in patients with chronic viral hepatitis $\mathrm{C}$.

Method: 20 patients with hepatitis $\mathrm{C}$ virus hepatitis were analyzed in the department of hepatology in the clinical base of the therapy at the Tashkent Medical Academy.

Result: It found out that Chronic hepatitis of the virus C proceeds from different degrees of activity - minimal, moderate and severe, which depends on the level of viral load, genotype of the virus and the involvement of immunopathological mechanisms. The clinical course of this hepatitis is more often symptomatic - one of the leading symptoms is general weakness in combination with dyspepsia. Hepatic manifestations - jaundice, hepato- and splenomegaly, thrombocytopenia and gingival hemorrhage are observed rarely and correlates with high viral replication, ALT activity and pronounced disorders of cellular and humoral immunity. The proposed algorithm for diagnosis, prognosis and antiviral therapy of chronic hepatitis $\mathrm{C}$ allows to identify the nature of the course of the process, but also to clarify the degree of viral replication, the severity of liver damage depending on different genotypes, and the features of the involvement of immune disorders in progression process.

Conclusion: The proposed algorithm for diagnosis, prognosis and antiviral therapy of chronic hepatitis $\mathrm{C}$ allows to identify early clinical signs of the disease. All this will solve the problem of early antiviral therapy.

\section{$H C V-81$}

Effect of vitamin d replacement on sustained virological response in chronic hepatitis $\mathbf{C}$ patients: a propensity score stratification and multilevel analysis

Kriangsak Charoensuk ${ }^{1}$, Orawan Chaiyamahapurk ${ }^{1}$, Woramon Jiamsripong $^{1}$, Wiboon Uthaisaengsuk ${ }^{1}$, Jayanton Patumanond ${ }^{2}$

${ }^{1}$ Buddhachinaraj Hospital School of Medicine, Thailand; ${ }^{2}$ Thammasat University, Thailand

Background: Chronic hepatitis $\mathrm{C}(\mathrm{CHC})$ is one of the main causes of chronic liver disease, cirrhosis and liver related mortality worldwide. Despite recent major therapeutic breakthroughs, Combination of pegylated interferon plus ribavirin still be one of standard of care, SOC in the majority of countries including thailand. The aim of this study was to assess the impact of vitamin D replacement on treatment outcome in $\mathrm{CHC}$ patients.

Method: The retrospective cohort study was conducted in $\mathrm{CHC}$ patients enrolled in hepatitis clinic, Buddhachinaraj Hospital, Phitsanulok Thailand. Since 2010-2015, A total of 1267 patients were included and treated by using a standardized protocol base on National Health Security Office, NHSO policy. There were $795 \mathrm{HCV}$ infected patients treated with SOC alone and 472 patients was received vitamin plus SOC. Baseline characteristics data was collected to case record form. The 25-hydroxyvitamin $\mathrm{D}(25(\mathrm{OH}) \mathrm{D})$ was measured at baseline and at the end of treatment.

Result: A total of $1180 \mathrm{CHC}$ patients were eligible at the end of the study and classified into two group, 744 patients SOC and 436 in SOC plus vitamin D group. At the end of study, only the 25(OH)D levels in vitamin D replacement group were significantly increased from 19.29 to $44.03 \mathrm{ng} / \mathrm{ml},(\mathrm{p}<0.001)$. All CHC patients had 25(OH)D levels below $30 \mathrm{ng} / \mathrm{ml}$ and $51 \%$ of patients had vitamin D deficiency and the remaining had vitamin insufficiency by definition. Multilevel logistic regression with adjusted group stratified propensity score analysis was performed. Significant higher rates os SVR were observed in vitamin D group $(\mathrm{OR}=1.78 ; 95 \% \mathrm{CI}$ : 1.32-2.38). By subgroup genotype 13 and 6, the results of OR were $1.30(0.88-1.92, \mathrm{P}=0.180) 2.87(1.74-$ 4.73, $\mathrm{P}<0.001) 1.77(1.09-2.88, \mathrm{P}=0.021)$ respectively.

Conclusion: Our study demonstrated that vitamin D supplement during treatment with pegylated interferon plus ribavirin therapy in CHC patients resulted in high SVR rate. This important data may had the benefit in $\mathrm{CHC}$ care and implementation. 


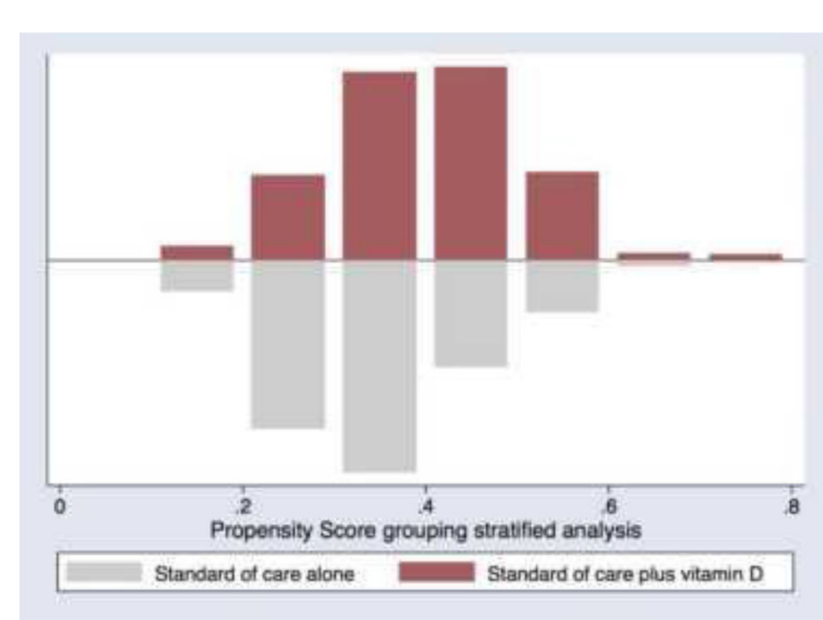

\begin{tabular}{|c|c|c|c|c|}
\hline \multirow[t]{2}{*}{ Genotype } & \multicolumn{2}{|c|}{$\begin{array}{l}\text { Model } 1 \\
\text { Logistic regression analysis }\end{array}$} & \multicolumn{2}{|c|}{$\begin{array}{c}\text { Model } 2 \\
\text { Marginal model } \\
\text { (model based variance) }\end{array}$} \\
\hline & OR $(95 \% \mathrm{Cl})$ & P-value & OR $(95 \%$ Cl) & P-value \\
\hline All & $2.0(1.51-2.67)$ & $<0.001$ & $1.78(1.32-2.38)$ & $<0.001$ \\
\hline Genotype 1 & $1.76(1.46-2.12)$ & $<0.001$ & $1.30(0.88-1.92)$ & 0.180 \\
\hline Genotype 3 & $3.91(3.07-4.99)$ & $<0.001$ & $287(1.74-4.73)$ & $<0.001$ \\
\hline Genotype 6 & $2.95(2.28-3.81)$ & $<0.001$ & $1.77(1.09-2.88)$ & 0.021 \\
\hline
\end{tabular}

\section{$H C V-82$}

Outcomes of sofosbuvir and ribavirin treatment combination in HEP C $G 3$ pediatric patients

\section{Muzzafar Lateef Gill ${ }^{1}$, Ammara Nawaz ${ }^{1}$, Sidra Gill ${ }^{1}$, Arham Baloch $^{1}$, Mehmoona Pervaiz ${ }^{1}$ \\ ${ }^{1}$ Islamabad Gastroenterology Associates, Pakistan}

Background: Children with chronic HEPC have very limited treatment option. We want to evaluate the efficacy of SOFSBUVIR and RIBAVIRIN in HEP C G3 pediatric patients

Method: We consecutively enrolled 50 pediatric patients with chronic HEP C. The dose of SOFOSBUVIR is $400 \mathrm{mg}$ daily and RIBAVIRIN dose is $8 \mathrm{mg}$ per $\mathrm{kg}$ body weight for the duration of 24 weeks.

Primary End-Point

SVR 12 weeks after treatment

Secondary endpoint

Any side effects of treatment

\section{Result:}

50 patients were enrolled

3 failed to follow-up

47 patients completed the treatment

Median age 14 years

FibroScan mean value $6 \mathrm{kPa}$

Platelets count mean value $150 \mathrm{k}$

All were genotype 3

This patients have regular 4 weekly follow-up on outpatient basis All patients are treatment naïve.

EVR (4 weeks after treatment) 44/50 patients were PCR Negative i.e. $88 \%$
ETR 42 out of 50 patients were PCR negative i.e.84\%

SVR (after 12 weeks of therapy i.e. $84 \%$ )

Side effects

$10 \%$ patients have nausea, vomiting and headache.

$7 \%$ have non-specific lethargy and tiredness.

Conclusion: In our experience combination of sofosbuvir and ribavirin is pretty safe and very effective treatment in hep c pediatric population.

$H C V-83$

Prevalence of HCV associated comorbidities in a cohort of $\mathbf{3 6 1 0}$ liver patients in an urban population of Canada

Marzena Magnes ${ }^{1}$, Hamed Dallali ${ }^{1}$, Marjan Farshadi ${ }^{1}$, Mark Khan $^{3}$, Jean Palmart ${ }^{2}$, Sunny Loo ${ }^{2}$, Sirajuddaula Kausar ${ }^{1}$, Marzena Magnes ${ }^{1}$, Kerstina Boctor ${ }^{1}$, Magdy Elkhashab ${ }^{1}$

${ }^{1}$ Toronto Liver Centre, Canada; ${ }^{2}$ Advisory Physicians Research Services Inc, Canada; ${ }^{3}$ Gilead Sciences Inc., Canada

Background: Hepatitis $\mathrm{C}$ virus is a globally occurring pathogen with variation in prevalence. Patients affected can present with a number of other manifestations that may deteriorate its prognosis. A significant number of people are chronically infected with viral hepatitis in Canada. This study aims to determine HCV burden and its associated comorbidities in liver patients presented at urban center in Canada. Method: A total of 3610 charts of patients with primary liver disease that reported to Toronto Liver Centre (TLC) from February 1995 to September 2017 were reviewed and analyzed retrospectively. The demographic, diagnostic and treatment details extracted were transferred to electronic case report forms. MarketSight ${ }^{\circledR}$ software was used to analyze and calculate the rate and frequencies of the various variables.

Result: Our cohort of 3610 patients reported 862 with HCV; 542 with pure $\mathrm{HCV}$ and the rest 320 had one or more liver condition. Over $75 \%$ of these patients seen were in their 5th, 6th and 7th decades of life having a male predominance of $1.4: 1$. Around $64 \%$ patients had some associated comorbid condition; highest being hypertension $(44.5 \%)$ followed by obesity $(27.8 \%)$, dyslipidemia $(25.6 \%)$, diabetes mellitus (24\%), depression (20\%), gastro-esophageal reflux disease $(16.5 \%)$ and thyroid (15.3\%). As Compared to other members of the cohort, HCV had almost thrice the rate of depression and a higher proportion of thyroid, HIV, Crohn's and coronary artery disease associated conditions (Figure 1). It also carried the highest rate of cancer and cirrhosis amongst all. 8.2\% (71) of these HCV patients were also reported to have some associated cancer with number of liver and breast cancers at the top (Figure 2). Cirrhosis rate of $6.6 \%$ was seen in pure $\mathrm{HCV}$ which rose to $13.7 \%$ in patients with an additional hepatic pathology.

Conclusion: HCV had the highest rate of depression, cancer, thyroid, cirrhosis and HIV in all, highlighting the need of effective management of these comorbidities to avoid further burden to the disease. 


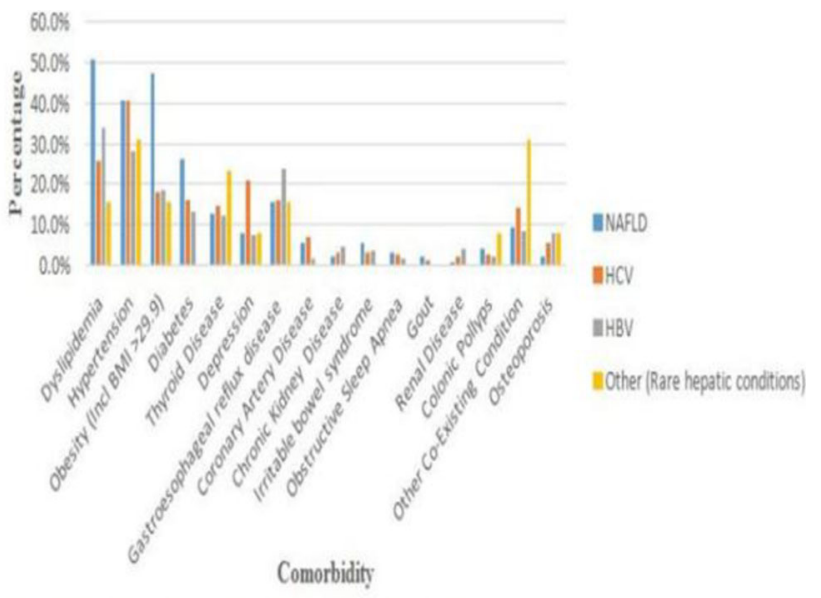

Figure l:Frequenç of Distribution of Comorbididies in Hepatic Pathologetes

\begin{tabular}{|c|c|c|c|c|}
\hline Cancer Type & NAFLD & HCV & HBV & Other (Rare hepatic conditions) \\
\hline Liver (incl HCC) & 10 & 30 & 28 & 0 \\
\hline Colon & 0 & 3 & 2 & 0 \\
\hline Bladder & 2 & 3 & 2 & 0 \\
\hline Breast & 30 & 9 & 9 & 0 \\
\hline Colorectal & 2 & 3 & 0 & 0 \\
\hline Kidney & 4 & 3 & 3 & 0 \\
\hline Leukemia & 5 & 1 & 1 & 0 \\
\hline Lung & 3 & 2 & 2 & 0 \\
\hline Melanoma & 3 & 1 & 1 & 0 \\
\hline Non-Hodgkin's Lymphoma & 4 & 1 & 2 & 0 \\
\hline Ovarian & 1 & 2 & 0 & 0 \\
\hline Pancreatic & 1 & 1 & 0 & 1 \\
\hline Prostate & 8 & 4 & 2 & 0 \\
\hline Thyroid & 12 & 4 & 4 & 0 \\
\hline Uterus & 8 & 4 & 1 & 0 \\
\hline Other & 19 & 11 & 9 & 0 \\
\hline TOTAL & 96 & 71 & 61 & 1 \\
\hline
\end{tabular}

Figure 2: Number of cancer patients in various Liver conditions

\section{$H C V-84$}

Safety and efficacy of ledipasvir/sofosbuvir in a genotype $1 \mathrm{HCV}$ infected Chinese population: results from a phase 3 , clinical trial

Cathy $\mathrm{Lu}^{1}$, Qing $\mathrm{Xie}^{11}$, Jinlin Hou ${ }^{8}$, Hong Tang ${ }^{20}$, Qin Ning ${ }^{19}$, Jun Cheng $^{2}$, Yuemin Nan ${ }^{18}$, Lunli Zhang ${ }^{13}$, Jun Li ${ }^{14}$, Jianning

Jiang $^{12}$, Brian Mcnabb ${ }^{5}$, Fangqiu Zhang ${ }^{5}$, Gregory Camus ${ }^{5}$, Hongmei $\mathrm{Mo}^{5}$, Anu Osinusi ${ }^{5}$, Diana M Brainard ${ }^{5}$, G. Mani Subramanian $^{5}$, Guozhong Gong ${ }^{17}$, Zhuangbo $\mathrm{Mou}^{7}$, Shanming $\mathrm{Wu}^{4}$, Min $\mathrm{Xu}^{6}$, Guiqiang Wang , Peng $\mathrm{Hu}^{16}$, Yanhang Gao ${ }^{15}$, Zhongping Duan ${ }^{3}$, Lai Wei ${ }^{10}$

${ }^{1}$ Gilead Science China, China; ${ }^{10}$ Peking University People's Hospital, China; ${ }^{11}$ Shanghai Jiaotong University Ruijin Hospital, China; ${ }^{12}$ The First Affiliated Hospital of Guangxi Medical University, China; ${ }^{13}$ The First Affiliated Hospital of Nanchang University, China; ${ }^{14}$ The First Affiliated Hospital with Nanjing Medical University, China; ${ }^{15}$ The First Hospital of Jilin University, China; ${ }^{16}$ The Second Affiliated Hospital of Chongqing Medical University, China; ${ }^{17}$ The Second Xiangya Hospital of Central South University, China; ${ }^{18}$ The Third
Hospital of Hebei Medical University, China; ${ }^{19}$ Tongji Hospital of Tongji Medical College, Huanzhong University of Science and Technology, China; ${ }^{2}$ Beijing Ditan Hospital Affiliated to Capital Medical University, China; ${ }^{20}$ West China Hospital, Sichuan University, China; ${ }^{3}$ Beijing You-An Hospital, Capital Medical University, China; ${ }^{4}$ Clinical Center of Shanghai Public Health, China; ${ }^{5}$ Gilead Science Inc., United States; ${ }^{6}$ Guangzhou Eighth People's Hospital, China; ${ }^{7}$ Jinan Infectious Disease Hospital, China; ${ }^{8}$ Nanfang Hospital of Southern Medical University, China; ${ }^{9}$ Peking University First Hospital, China

Background: Chronic hepatitis $\mathrm{C}$ virus (HCV) infection remains a major health threat in China, affecting at least 10 million people, with approximately 58\% having genotype (GT) 1 infection. There is a critical need for simple, all oral, direct-acting anti-viral regimens to treat GT1 HCV in this region. Treatment with ledipasvir (LDV)/sofosbuvir (SOF) results in high sustained virologic response (SVR) rates in GT1 HCV infected patients in clinical trials and real-world settings. This study evaluated the efficacy and safety of LDV/SOF for 12 weeks in Chinese patients with chronic GT1 HCV infection.

Method: Treatment experienced and treatment naïve patients with chronic GT1 HCV infection with no cirrhosis or with compensated cirrhosis were eligible to enroll in a single-arm, open-label trial to receive a fixed dosed combination of LDV/SOF 90/400 mg daily for 12 weeks. Patients were recruited from 18 sites in China. The primary efficacy endpoint was SVR12 weeks after completing treatment (SVR12) using the CAP/CTM HCV 2.0 assay (LLOQ =15 IU/mL). SVR 24 weeks after treatment completion (SVR24) and virologic resistance analysis through deep sequencing ( $>15 \%$ cutoff) are also reported as secondary endpoints. The primary safety endpoint was adverse events (AEs) leading to LDV/SOF discontinuation.

Result: A total of 206 Chinese patients were enrolled and treated. Of these, $50 \%$ were male, $16 \%$ had compensated cirrhosis, $49 \%$ were treatment-experienced, 72\% had IL28B CC genotype, and 100\% had GT1b HCV infection. The mean (range) age and body mass index of enrolled subjects were 47 (21-72) years and $23(14-34) \mathrm{kg} / \mathrm{m}^{2}$, respectively. The overall SVR12 and SVR24 rates were both $100 \%$ (206/206). All 32 patients with cirrhosis (15 of whom were treatmentexperienced), achieved SVR12(24). At baseline, 29 subjects had single $\mathrm{Y} 93 \mathrm{H}$ resistance associated substitutions (RASs), and all 29 achieved SVR12(24). There were no discontinuations due to AEs. There were 120 patients $(58 \%)$ who experienced any treatmentemergent adverse events (AEs), with viral upper respiratory tract infection (18\%), upper respiratory tract infection (14\%), and cough $(6 \%)$ being the only common $(>5 \%)$ AEs. No serious or severe AEs were assessed by the investigator as related to study drug and there were no deaths.

Conclusion: Treatment with the single tablet regimen of LDV/SOF for 12 weeks resulted in $100 \%$ SVR12 and SVR24 and was well tolerated in a Chinese population of treatment experienced and treatment naïve GT1 HCV-infected patients with and without cirrhosis.

Table: SVR12 and SVR24 for GT1 HCV infected Chinese Patients

\begin{tabular}{l|c|c|c}
\hline N (\%) & $\begin{array}{c}\text { Total } \\
\mathbf{N}=\mathbf{2 0 6}\end{array}$ & $\begin{array}{c}\text { Cirrhosis } \\
\mathbf{N}=\mathbf{3 2}\end{array}$ & $\begin{array}{c}\text { No Cirrhosis } \\
\mathbf{N}=\mathbf{1 7 4}\end{array}$ \\
\hline SVR12 & $206 / 206(100)$ & $32 / 32(100)$ & $174 / 174(100)$ \\
\hline SVR24 & $206 / 206(100)$ & $32 / 32(100)$ & $174 / 174(100)$ \\
\hline
\end{tabular}


HCV-85

Sofosbuvir (SOF) and ledipasvir (LDV) combination is associated with high sustained virologic response (SVR) and improvement of health-related quality of life (HRQL) in East Asian patients with hepatitis $\mathrm{C}$ virus (HCV) infection

Zobair Younossi ${ }^{1}$, Maria Stepanova ${ }^{3}$, Linda Henry ${ }^{3}$, Kwanghyub Han $^{10}$, Sang Hoon Ahn ${ }^{10}$, Youngsuk Lim ${ }^{2}$, Wanloung Chuang Jia Horng $\mathrm{Kao}^{7}$, Nguyen Kinh ${ }^{6}$, Ching Lung Lai ${ }^{9}$, Man Fung Yuen', Henry Lik Yuen Chan ${ }^{4}$, Wei Lai ${ }^{8}$

${ }^{1}$ Inova Fairfax Hospital, United States; ${ }^{10}$ Yonsei University College of Medicine, Korea, Democratic People's Republic of; ${ }^{2}$ Asan Medical Center, Korea, Democratic People's Republic of; ${ }^{3}$ Center for Outcomes Research in Liver Disease, United States; ${ }^{4}$ Institute of Digestive Disease, China; ${ }^{5}$ Kaohsiung Medical University Hospital, Taiwan; ${ }^{6}$ National Hospital of Tropical Diseases, Vietnam; ${ }^{7}$ National Taiwan University College of Medicine and Hospital, Taiwan;

${ }^{8}$ Peking University, China; ${ }^{9}$ Queen Mary Hospital, China

Background: While interferon (IFN)-containing regimens for $\mathrm{HCV}$ has a significant negative impact on HRQL, IFN-free regimen and $\mathrm{HCV}$ cure can lead to HRQL improvement. Although HCV infection is highly prevalent in East Asia (EA), these patients have been underrepresented in HRQL studies.

Aim: To assess HRQL in EA HCV patients treated with different anti-HCV SOF-based regimens.

Method: EA HCV patients completed Short Form-36 (SF-36) before, during and after treatment; the HRQL scores were compared between regimens (\#NCT02021643, IFN+SOF+RBV vs. SOF+RBV, $\mathrm{N}=686$, and \#NCT02021656, LDV/SOF, N=384; 2013-2017).

Result: 1070 EA HCV subjects were enrolled in two phase 3 clinical trials [China: $55.6 \%$, S. Korea: $20.7 \%$, Taiwan: $16.1 \%$, Vietnam: 4.7\%, and Hong Kong: $2.9 \%$; Genotype (GT) 2: 26.2\%, GT1: $54.9 \%$, GT3: $11.8 \%$, GT6: $7.2 \%$; cirrhosis: $14.0 \%$ ]. Patients received pegylated interferon, sofosbuvir, and ribavirin (IFN+SOF+RBV) for 12 weeks ( $\mathrm{n}=155$, GT 1 and 6) or SOF+RBV for 12-24 weeks $(n=531$, GT 1, 2, 3 and 6) or IFN-free RBV-free SOF and ledipasvir (LDV) ( $\mathrm{n}=384$, GT1 only). The SVR-12 rates were $95.5 \%, 96.0 \%$, and $99.2 \%$, respectively $(\mathrm{p}=0.008)$. Baseline HRQL scores were similar between treatment groups (all but one $\mathrm{p}>0.05$ ). After 2 weeks of treatment, HRQL scores for the IFN+RBV-containing regimen became significantly lower as compared to the IFN-free regimens (average decline up to -11.2 points, $\mathrm{p}<0.0001$ ). By the end of treatment, IFN-treated group experienced significant declines in most of HRQL scores (up to -13.3 points, $\mathrm{p}<0.02$ for $7 / 8$ HRQL scales). Patients on SOF+RBV had milder HRQL impairments (up to -5.4 points, $\mathrm{p}<0.05$ for $5 / 8$ scales). In contrast, patients receiving IFN- and RBV-free regimen with LDV/SOF had improvement in their HRQL scores (up to +4.3 points by the end of treatment, $p<0.001$ for $3 / 8$ scales) (Figure). Achieving SVR-12 with IFN+RBV+SOF and $\mathrm{SOF}+\mathrm{RBV}$ was associated with improvement in General Health $(\mathrm{GH})$ and Vitality (VT) (up to +2.9 points, $\mathrm{p}<0.05$ ). In contrast, SVR-12 with LDV/SOF was associated with improvement in Physical Functioning, GH, VT, Mental Health, Role Emotional (up to +5.9 points, $\mathrm{p}<0.03$ ). In multivariate analysis, receiving IFN was consistently independently associated with HRQL impairment during treatment $(\beta:-10.4$ to -17.3 points, $\mathrm{p}<0.0001)$.

Conclusion: Treatment of EA HCV patients with IFN is associated with significant HRQL impairment while IFN-free RBV+ regimens are associated with mild impairment. In contrast, treatment with $\mathrm{LDV} / \mathrm{SOF}$ is associated with improvement of HRQL during treatment. Finally, SVR-12 is associated with HRQL improvement, again the most prominently with the LDV/SOF regimen.
Figure- Vitality in EA patients treated with different regimens during and after treatment.

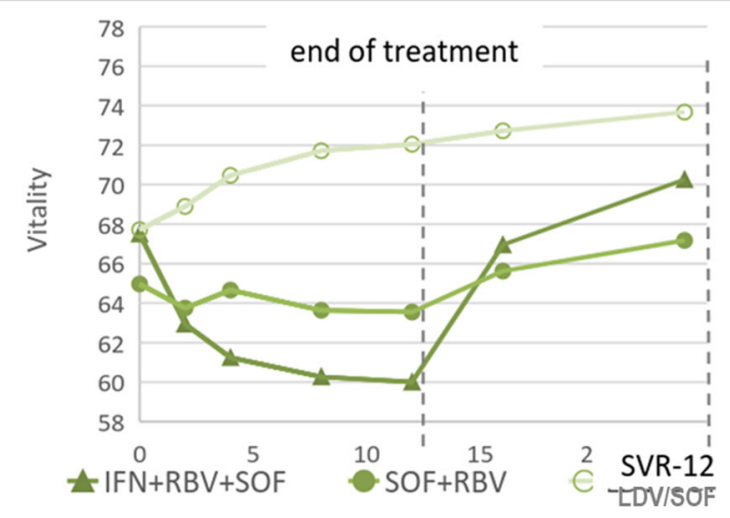

$H C V-86$

SVR12 versus SVR24 as evaluation end-points in chronic HCV Egyptian patients treated with sofosbuvir-based regimens

Maha Elsabaawy ${ }^{1}$, Khaled Gameel ${ }^{1}$, Heba Eldemerdash ${ }^{1}$, Talaat Zakareia $^{1}$, Marwa Fekry ${ }^{1}$, Mary Albert ${ }^{1}$, Mahmoud Eltahawy ${ }^{1}$

${ }^{1}$ phd, Egypt

Background: Hepatitis $\mathrm{C}$ infection represents a major national health problem in Egypt. The recommended reliance on 12 weeks posttreatment sustained virological response (SVR12) instead of SVR24 has been lately challenged.

Aim: Judging concordance between SVR12 and SVR24 among HCV genotype 4 Egyptian patients.

Method: In a prospective study; 91 patients received SOF+Interferon (IFN)+Ribavirin (RV) for 12 weeks, 52 patients received SOF+RV for 24 weeks and 56 patients received SOF+simeprevir (SIM) for 12 weeks. Demographic, and laboratory data, fibrosis stage assessed by transient elastography, treatment regimens, HCV RNA at week 4, at the end of treatment, and $12 \& 24$ weeks post-treatment were reported. Patients who failed to achieve undetectable HCV RNA at the end of therapy were excluded.

Result: Concordance between SVR12 and SVR24 was found to be $96.5 \%$ for the whole study population, with positive predictive value (PPV) $96.4 \%$. Regarding treatment groups it was found to be $95.6 \%$ for SVR24 in SOF+IFN+RV treated patients, $94.2 \%$ in SOF+RV treated patients and $100 \%$ concordance in SOF+SIM treated patients with insignificant values $(\mathrm{p}-=0.2)$. In spite of non-significance, the reported 7 relapsers $(3.5 \%)$ were mainly males ( 5 cases, $\mathrm{p}=0.9)$, naïvely treated ( 5 cases, $\mathrm{p}=0.6$ ), achieved RVR ( 5 cases, $\mathrm{p}>0.005$ ), with advanced fibrosis (F4) by fibroscan $(5$ cases, $\mathrm{p}=0.7)$. Regression analysis failed to detect any predictors of relapse.

Conclusion: In spite of the high grade of concordance between SVR12 and SVR24, the reported rate of relapsers necessitates the backward commitment to SVR24 as a reliable primary end-point of treatment response evaluation. 


\section{$H C V-87$}

A retrospective analysis of the prevalence of various hepatitis $C$ virus $(\mathrm{HCV})$ genotypes with post-treatment sustained viral response (SVR) achieved in a multi-cultural Canadian population

Marzena Magnes ${ }^{1}$, Sirajuddaula Kausar ${ }^{1}$, Marjan Farshadi ${ }^{1}$, Hamed Dallali $^{1}$, Marzena Anna Magnes ${ }^{1}$, Kerstina Boctor ${ }^{1}$, Sara Nasr $^{1}$, Mark Khan ${ }^{3}$, Jean Palmart ${ }^{2}$, Sunny Loo ${ }^{2}$, Magdy Elkhashab $^{1}$

${ }^{1}$ Toronto Liver Centre, Canada; ${ }^{2}$ Advisory Physicians Research Services Inc., Canada; ${ }^{3}$ Gilead Sciences Canada, Inc., Canada

Background: Hepatitis $\mathrm{C}$ virus exhibits high genetic diversity with regional variations in prevalence. The virus genotype and degree of liver damage are used to guide treatment decisions. This study aims to explore the prevalence of various genotypes and sustained viral response achieved in treated patients in an urban Canadian population harbouring immigrants from different ethnic backgrounds of the world.

Method: This retrospective analysis consisted of $862 \mathrm{HCV}$ patients evaluated at Toronto Liver Centre from February 1995 to September 2017. Medical history, management, treatments, and health outcomes data was collected through review of medical records and entered into customised electronic case report forms. This was later analysed using MarketSight ${ }^{\circledR}$ Crosstab software.

Result: A review of 3610 patients' charts of primary liver disease revealed 862 cases of $\mathrm{HCV}$; 542 with pure $\mathrm{HCV}$ and 320 had one or more other liver condition too. All the six major genotypes were reported in our HCV cohort; highest being $1(n=302)$ followed by 3,4 , 2, 6 and 5 in a descending order of sequence. Genotypes 1, 2, 3 and 4 showed a significant male dominance while the rare genotypes 5 and 6 had a slight higher number of females. A significant number of older charts had missing genotyping due to non-availability at that time, some also due to an inadequate viral load in patients' blood (Figure 1). Quiet a convincing number of these patients either lost to follow up $(22.7 \%)$ or were not able to get complete course of treatment $(26.8 \%)$. Four hundred and fifty patients received full course of anti-HCV treatments; high SVR rate was achieved in most of these patients. Patients with genotypes 2a, 2b, 5a and 6 attained 100\% SVR after due course of treatment. It was around $85 \%$ with genotype 3 and comparatively lower in patients with genotypes 1 and 4 . Most of these patients were treated with a combination of peg-interferon and ribavirin which showed a SVR of almost $77 \%$. The achieved SVR was highest in therapies containing some direct-acting antivirals in the regimen (Figure 2).

Conclusion: Our finding depicts diversity in our community. It highlights the therapeutic benefit of pan-genotypic drug therapies. This will also be helpful in developing national treatment strategies.

\begin{tabular}{|c|c|c|c|c|c|c|}
\hline Type / Total & Subtype & Total & $\begin{array}{l}\text { Treated with } \\
\text { No SVR }\end{array}$ & $\begin{array}{c}\text { Treated with } \\
\text { SVR }\end{array}$ & $\begin{array}{l}\text { Never } \\
\text { treated }\end{array}$ & $\begin{array}{l}\text { Lost to } \\
\text { follow up }\end{array}$ \\
\hline \multirow[t]{3}{*}{ Type 1/302 } & Unspecified & 88 & 9 & 35 & 4 & 41 \\
\hline & $1 \mathrm{a}$ & 109 & 20 & 53 & 9 & 29 \\
\hline & $1 \mathrm{~b}$ & 108 & 14 & 63 & 12 & 22 \\
\hline \multirow[t]{4}{*}{ Type 2/57 } & Unspecified & 37 & 2 & 19 & 7 & 9 \\
\hline & $2 a$ & 12 & 0 & 8 & 2 & 2 \\
\hline & $2 \mathrm{~b}$ & 7 & 0 & 6 & 0 & 1 \\
\hline & $2 \mathrm{c}$ & 1 & 0 & 0 & 1 & 0 \\
\hline \multirow[t]{4}{*}{ Type 3/137 } & Unspecified & 50 & 5 & 27 & 5 & 13 \\
\hline & $3 a$ & 84 & 10 & 58 & 3 & 14 \\
\hline & $3 \mathrm{~b}$ & 2 & 0 & 2 & 0 & 0 \\
\hline & Other & 1 & 0 & 1 & 0 & 0 \\
\hline \multirow[t]{6}{*}{ Type 4/118 } & Unspecified & 97 & 27 & 51 & 11 & 14 \\
\hline & $4 a$ & 3 & 1 & 1 & 0 & 1 \\
\hline & $4 \mathrm{c}$ & 11 & 5 & 4 & 2 & 1 \\
\hline & $4 \mathrm{~d}$ & 1 & 0 & 0 & 1 & 0 \\
\hline & $4 \mathrm{e}$ & 1 & 0 & 0 & 1 & 0 \\
\hline & Other & 7 & 2 & 6 & 0 & 0 \\
\hline \multirow[t]{2}{*}{ Type 5/10 } & Unspecified & 5 & 2 & 1 & 1 & 1 \\
\hline & $5 a$ & 4 & 0 & 3 & 0 & 1 \\
\hline Type $6 / 11$ & None & 11 & 0 & 5 & 3 & 3 \\
\hline \multirow{2}{*}{$\begin{array}{c}\text { Unknown / } \\
235\end{array}$} & & & & & & \\
\hline & None & 235 & 2 & 19 & 169 & 45 \\
\hline
\end{tabular}

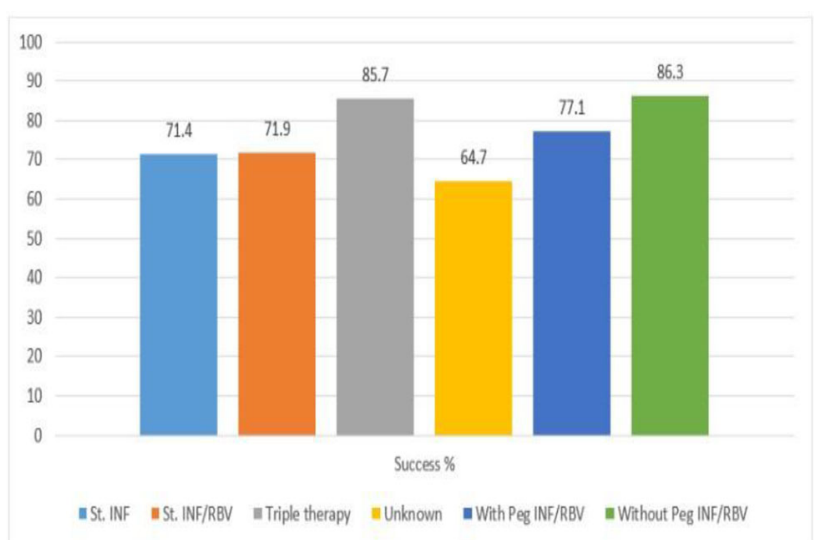

Figure 2: SVR achieved with different $\mathrm{HCV}$ treatment regimens

\section{$H C V-88$}

Exploring the acceptability of community-based, rapid point-ofcare hepatitis $\mathbf{C}$ testing in people who inject drugs - a qualitative study

Bridget Williams ${ }^{1}$, Alisa Pedrana ${ }^{1}$, Joseph Doyle ${ }^{3}$, Jessica Howell ${ }^{1}$, Bridget Williams $^{1}$, Peter Higgs ${ }^{2}$, Margaret Hellard ${ }^{1}$

${ }^{1}$ Burnet Institute, Australia; ${ }^{2}$ Latrobe University, Australia; ${ }^{3}$ The Alfred and Monash University, Australia

Background: To eliminate hepatitis $\mathrm{C}(\mathrm{HCV})$ increasing the number of people who inject drugs (PWID) undergoing HCV treatment is critical. When treated, it has been shown that PWID can achieve similar rates of HCV cure to non-PWID. A known barrier to commencing treatment in this population is the need to attend multiple appointments to be diagnosed. Rapid point-of-care (RPOC) tests provide results within 20 to 120 minutes, allowing for same-day diagnosis, and can be offered opportunistically in non-clinical settings. In this nested qualitative study we explore factors influencing the acceptability of HCV RPOC testing for PWID attending needle syringe program (NSPs)

Method: The Rapid-EC pilot study offered RPOC testing to PWID at three NSPs in Melbourne, Australia. PWID were screened using the OraQuick HCV antibody mouth swab (result in 20 minutes); those who tested positive then underwent venepuncture for an RPOC RNA test: the Xpert HCV Viral Load (result in 105 minutes). Convenience 
sampling was used to select Rapid-EC participants for a semi-structured interview. A hybrid thematic analysis of the interview transcripts was performed, guided by Sekhon's 'Theoretical Framework of Acceptability'.

Result: Nineteen participants were interviewed; all but one participant reported injecting drugs in the preceding month. Three core themes emerged: people and place, method of specimen collection, and rapidity of result return. It was highly acceptable to be offered testing at the NSP by nurses and NSP workers, who were described as competent and non-judgmental. Most participants reported that even if a finger stick RPOC RNA test were an option in the future, they would prefer to undergo venepuncture, as the same sample could be used for pre-treatment workup tests (if required) and bundled HIV and HBV testing. The 20 minutes required to receive the antibody test result was acceptable, whereas the 105 minutes required for the RNA result was largely unacceptable. No participants waited onsite to receive their RNA result, and only five participants received this result on the same day (via telephone).

Conclusion: RPOC diagnostic tests that avoid venepuncture are not necessarily the most attractive to PWID, given that venepuncture is still required for pre-treatment workup and testing for other bloodborne viruses. Currently available RPOC RNA technology was not perceived as rapid and did not allow a diagnosis to be delivered in a single visit.

\section{HCV-89}

Outcomes and predictors of treatment response with sofosbuvir plus daclatasvir with or without ribavirin in Egyptian patients with genotype 4 hepatitis $C$ virus infection

\section{Sherief Abd Elsalam ${ }^{1}$, Osama Ashraf Ahmed², Mohamed Hassan A Fouad $^{2}$ \\ ${ }^{1}$ Tanta University Faculty of medicine, Egypt; ${ }^{2}$ Ainshams University, Egypt}

Background: Treatment of HCV changed dramatically with the introduction of oral direct-acting antiviral (DAA) drugs due to their high antiviral potency and safety profile. Sofosbuvir plus daclatasvir combination therapy was extensively investigated in HCV genotypes 1,2 and 3, while published data regarding its real life application in treatment of genotype 4 is lacking. Therefore, we conducted this study to assess the outcomes and predictors of treatment response with sofosbuvir plus daclatasvir with or without ribavirin in Egyptian patients with genotype 4 hepatitis $C$ virus infection.

Method: This prospective study included 300 Egyptian patients with chronic genotype $4 \mathrm{HCV}$, treated with sofosbuvir plus daclatasvir \pm ribavirin for $12-24$ weeks. Primary outcome was the number of patients who achieved sustained virologic response (SVR12) and secondary outcome was the occurrence of adverse events.

Result: $92.67 \%$ of all patients achieved SVR12. SVR12 rates of $96.55 \%$ and $84.54 \%$ were reported in non-cirrhotic and cirrhotic patients respectively. SVR12 in treatment-naïve and treatment-experienced patients were $94.12 \%$ and $87.01 \%$ respectively. $19.7 \%$ of patients experienced mild adverse events. Older age, cirrhosis and low platelet count were the predictors of treatment non-response.

Conclusion: Based on this multi-center prospective study, sofosbuvir plus daclatasvir \pm ribavirin for $12-24$ weeks appears to have favorable outcomes in treatment of genotype $4 \mathrm{HCV}$ infected Egyptian patients. Older age, cirrhosis especially Child Pugh class B and low platelet count are independent risk factors of treatment nonresponse.
HCV -90

Real world efficacy and tolerability of direct-acting antiviral for treatment of chronic hepatitis $\mathrm{C}$ infection: interim results of a territory-wide study in Hong Kong

YT Hui ${ }^{1}$, Grace Wong ${ }^{4}$, Sienna Liu ${ }^{4}$, Ken Liu ${ }^{4}$, Sharon Cheung ${ }^{4}$, YK Ma ${ }^{13}$, David But ${ }^{11}$, WY Mak ${ }^{1}$, Owen Tsang ${ }^{10}$, KB Lai ${ }^{15}$, CK $\mathrm{Loo}^{5}$, Annie $\mathrm{Ng}^{3}$, MS Lai ${ }^{6}$, CW Chan ${ }^{16}$, Joulen YL Lau ${ }^{8}$, Tina Fan $^{12}$, AJ Hui ${ }^{2}$, CY Lam ${ }^{\text {, WI Cheung }}{ }^{7}$, Jacky Chan ${ }^{10}$, Karen Lam $^{10}$, Lawrence SW Lai ${ }^{9}$, WF Luk ${ }^{10}$, Michael KK $\mathrm{Li}^{14}$, WC Lao $^{8}$, Jodis TW Lam ${ }^{1}$, Steven Tsang ${ }^{12}$, KN Kung ${ }^{15}$, Ronald KN Tong $^{3}$, YK Chan ${ }^{3}$, WH Chow ${ }^{16}$, CS Leung ${ }^{16}$, CT To ${ }^{16}, C_{\text {CW Li }}{ }^{16}$, J Wong $^{16}$, WL Tao ${ }^{16}$, WS Chan ${ }^{16}$, R Chan ${ }^{2}$, K Lee ${ }^{11}$, W Yau ${ }^{14}$, A $\mathrm{Chan}^{16}$, YH Ho ${ }^{5}$, YM Kan ${ }^{5}$, KL Lui ${ }^{14}$, E Shan ${ }^{3}$, James YY Fung ${ }^{11}$, Henry LY Chan ${ }^{4}$, MF Yuen ${ }^{11}$, Vincent WS Wong ${ }^{4}$

${ }^{1}$ Queen Elizabeth Hospital, Hong Kong; ${ }^{10}$ Princess Margaret Hospital, Hong Kong; ${ }^{11}$ Queen Mary Hospital, Hong Kong; ${ }^{12}$ Tseung Kwan O Hospital, Hong Kong; ${ }^{13}$ Tuen Mum Hospital, Hong Kong;

${ }^{14}$ Tuen Mun Hospital, Hong Kong; ${ }^{15}$ United Christian Hospital, Hong Kong; ${ }^{16}$ Yan Chai Hospital, Hong Kong; ${ }^{2}$ Alice Ho Miu Ling

Nethersole Hospital, Hong Kong; ${ }^{3}$ Caritas Medical Centre, Hong Kong; ${ }^{4}$ CUHK, Hong Kong; ${ }^{5}$ Kwong Wah Hospital, Hong Kong; ${ }^{6}$ North District Hospital, Hong Kong; ${ }^{7}$ Our Lady of Maryknoll Hospital, Hong Kong; ${ }^{8}$ Pamela Youde Nethersole Eastern Hospital, Hong Kong; ${ }^{9}$ Pok Oi Hospital, Hong Kong

Background: Direct-acting antivirals (DAA) are highly effective for the treatment of hepatitis $\mathrm{C}$ infection $(\mathrm{HCV})$. These medications were increasingly used in Hong Kong and we would like to report their usage and treatment outcome in our local patients.

Method: Patients with chronic hepatitis $\mathrm{C}$ infection who had received DAA therapy were retrieved from the cohort of a territory-wide study in Hong Kong. The patients' clinical characteristics and treatment outcome were retrospectively reviewed.

Result: From February 2014 to September 2017, a total of 72 DAAtreated patients with sustained virological response (SVR12) data available were included. $88 \%$ of the patients were Chinese, $56.8 \%$ were interferon-experienced and $45.9 \%$ had liver cirrhosis. The genotype distribution were G1b (63.4\%), G1a (12.2\%), G6 (8.5\%), G3 (4.9\%) and G2 (2.8\%). At week 4 of DAA treatment, HCV RNA level became undetectable in $89.6 \%$ of patients. The overall rate of SVR12 was $97.3 \%$ (G1a=90\%, G1b=97.6\%, G2, 3, 6=100\%). The SVR rates of each type of DAA therapy were: ombitasvir/paritaprevir/ ritonavir \& dasabuvir $(\mathrm{N}=40, \mathrm{SVR} 12=100 \%$, fig. 1$)$; sofosbuvir/ ledipasvir (N=14, SVR12=92.9\%, fig. 2); sofosbuvir/pegylated-interferon/ribavirin $(\mathrm{N}=4, \mathrm{SVR} 12=100 \%)$; sofosbuvir/ribavirin $(\mathrm{N}=14$, SVR12=92.9\%). DAA were also highly effective across different special populations (e.g. thalassemia major, chronic kidney diseases, hemophilia, compensated and decompensated cirrhosis) and the treatment were well-tolerated. Two treatment failures were encountered (1) a genotype 1a cirrhotic treatment-experienced HIV coinfected hemophiliac patient who received sofosbuvir/ledipasvir/ribavirin for 12 weeks; (2) a genotype $1 \mathrm{~b}$ treatment-naïve patient who received sofosbuvir/ribavirin for 24 weeks. The reported adverse events include malaise (20\%), skin rash $(6.5 \%)$, nausea $(4.8 \%)$, insomnia $(4.8 \%)$ and grade 3-4 alanine aminotransferase elevation (3.2\%). Premature termination of treatment occurred in one patient only. No serious adverse event was noted.

Conclusion: DAA is a highly effective and well-tolerated treatment for chronic hepatitis $\mathrm{C}$ infection in real-world setting in Hong Kong. 

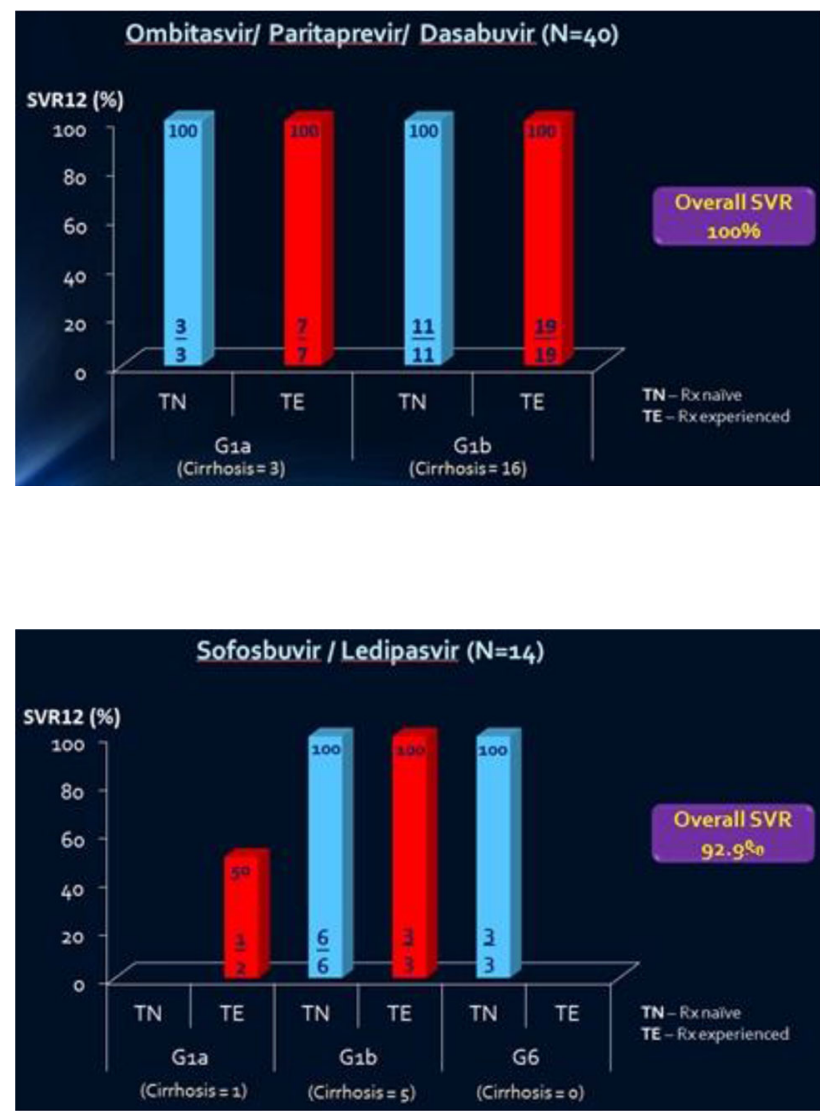

HCV-91

Systematic review with meta-analysis: real-world effectiveness and tolerability of interferon-free direct-acting antiviral regimens for chronic hepatitis $\mathrm{C}$ genotype 1 in Asia

Mindie Nguyen ${ }^{1}$, Bin Wei ${ }^{1}$, Yee Hui Yeo ${ }^{1}$, Eiichi Ogawa ${ }^{1}$, Christopher Stave ${ }^{4}$, Shuangsuo Dang ${ }^{3}$, Zongfang $\mathrm{Li}^{3}$, Norihiro Furusyo $^{2}$, Biyao Zou' ${ }^{1}$, Ramsey Cheung ${ }^{1}$, Mindie Nguyen ${ }^{1}$

${ }^{1}$ Stanford University Medical Center, United States; ${ }^{2}$ Kyushu University Hospital, Japan; ${ }^{3}$ Second Affiliated Hospital of Xi' an Jiaotong University, China; ${ }^{4}$ Stanford University, United States

Background: Direct-acting antiviral (DAA) regimens have shown high efficacy and tolerability for patients with HCV genotype $1 / 1 \mathrm{~b}$ $(\mathrm{GT} 1 / 1 \mathrm{~b})$ in clinical trials. However, robust real-world evidence of DAA treatment for HCV GT1 infected patients in Asia is still lacking. Our goal was to perform a systematic review and meta-analysis the real-world effectiveness of DAA therapy for HCV GT1 infection in Asia.

Method: We included studies that enrolled adult patients with GT1 infection in real-world practice in Asia, using IFN-free DAA regimens, and reported sustained virological response (SVR) after 12 weeks end of treatment by May 31, 2017. Random-effects model was used to estimate pooled SVR12. Subgroup analysis and meta-regression were performed to determine how pre-planned variables affects SVR12.

Result: Of the 8262 citations from PubMed and EMBASE, and 23 additional manually searched articles evaluated, we included 41 studies from 8 countries and regions, comprising of 8574 individuals. The pooled SVR12 rates for GT1 were $89.9 \%$ (95\%CI 88.6-91.1,
$I 2=55.1 \%$ ) with daclatasvir/asunaprevir (DCV/ASV) and $98.1 \%$ (95\%CI 97.0-99.0, I2=41.0\%) with ledipasvir/sofosbuvir \pm ribavirin (LDV/SOF \pm RBV). For SOF/DCV \pm RBV, SOF $+R B V, 2 D / 3 D \pm R B V$ regimens, the pooled SVR12 rates were $97.4 \%$ (95\%CI 90.9-100, $I 2=56.7 \%), 94.1 \%(95 \%$ CI $79.1-100, I 2=64.4 \%)$, and $95.9 \%(95 \% \mathrm{CI}$ $89.5-99.7, I 2=0.0 \%$ ), respectively. Subgroup analysis [age, renal function, cirrhosis, treatment-experience, resistance associated substitutions (RASs) and prior history of HCC)] for SVR12 in patients treated with DCV/ASV and LDV/SOF were shown in Fig.1. Baseline cirrhosis but not prior treatment history and age, attenuated the effectiveness of both regimens. Baseline RASs severely attenuated SVR12 of DCV/ASV (65.4 vs. $94.3 \%, \mathrm{p}<0.001)$, with $31.6 \%$ (95\%CI 23.5-39.4) reduction in SVR rate compared to those without RASs, and only minimally with LDV/SOF ( 94.5 vs. $99.2 \%, \mathrm{p}=0.003)$. The pooled SVR rate of patients with eGFR $<50 \mathrm{~mL} / \mathrm{min} / 1.73 \mathrm{~m} 2$ and treated with DCV/ASV was $93.9 \%$ (95\% CI 91.2-96.3), 4.10\% (95\% CI $0.28-7.65)$ higher than those with eGFR $\geq 50 \mathrm{~mL} / \mathrm{min} / 1.73 \mathrm{~m} 2$ (SVR=89.8\%, 95\% CI 88.5-91.0) ( $\mathrm{P}=0.046)$. Especially, the pool SVR rate in DCV/ASV-treated patients with end-stage renal disease (ESRD) was actually $9.38 \%$ (95\% CI 3.84-11.53) higher than those of patients with eGFR $\geq 50 \mathrm{~mL} / \mathrm{min} / 1.73 \mathrm{~m} 2(99.2 \%, 95 \%$ CI $94.9-100$ vs. $89.8 \%, 95 \%$ CI $88.5-91.0, \mathrm{P}=0.009)$. For $\mathrm{LDV} / \mathrm{SOF}$ regimen, patients with prior history of HCC had a $6.02 \%$ (95\% CI: $1.46-11.19)$ lower likelihood to achieve SVR compare to those without HCC (94.1\% vs. 98.7\%, $\mathrm{P}=0.001$ ). Pooled results of adverse events for patients treated with DCV/ASV and LDV/SOF regimen were summarized in Fig.2.

Conclusion: All oral DAA treatment of HCV GT1 resulted in high cure rates in Asian patients in real-world setting including elderly patients and those with end-stage renal disease.

Fig. 2 The AEs in patients treated with DCV/ASV and LDV/SOF regimens

\begin{tabular}{|c|c|c|c|c|c|c|c|c|}
\hline \multirow{2}{*}{$\begin{array}{l}\text { Regimen } \\
\text { Event }\end{array}$} & \multicolumn{3}{|c|}{ DCV/ASV } & \multicolumn{5}{|c|}{ LOV/SOF $\pm R B V$} \\
\hline & Studies & $\mathrm{N}$ & Rate $(\%)$ & $95 \% \mathrm{Cl}$ & Studies & N & Rate (\%) & $95 \% \mathrm{Cl}$ \\
\hline AnY AEs & 6 & 978 & 28.06 & $13.4-45.52$ & 3 & 874 & 17.04 & $0.67-46.51$ \\
\hline ALT elevation & 9 & 2290 & 16.45 & $8.70-25.96$ & 2 & 102 & 0 & 0.1 .89 \\
\hline SAES & 11 & 2758 & 4.79 & $3.43 \cdot 6.34$ & 5 & 1306 & 1.09 & 0.3 .52 \\
\hline DAA-related & 11 & 2758 & 4.53 & $3.33-5.88$ & 4 & 1252 & 0.56 & $\therefore$ \\
\hline No DAA-related & 11 & 2758 & 0.18 & .* & 4 & 1252 & 0.08 & $\therefore$ \\
\hline Early discontinuation & 18 & 5860 & 7.06 & $5.46-8.83$ & 6 & 1397 & 0.82 & $0.17-1.82$ \\
\hline Death & 18 & 5860 & 0.05 & $\therefore$ & 6 & 1397 & 0.14 & $\therefore$ \\
\hline
\end{tabular}

\section{HCV-92}

Association of type 2 diabetes and hepatitis $\mathrm{C}$ virus infection in Pakistani population: a meta-analysis

\section{Sher Zaman Safi}

${ }^{1}$ COMSATS Institute of Information Technology, Lahore, Pakistan

Background: The relationship between diabetes and Hepatitis $\mathrm{C}$ virus infection (HCV) has been reported in various studies, however the data is inconclusive in a number of ways. In developing countries, including Pakistan, very limited data is available on this subject. We, therefore, conducted this study to evaluate the association of diabetes and HCV infections in Pakistani population.

Method: Studies, evaluating the relationship of type 2 diabetes mellitus and HCV were identified using different key words from various databases including web of science, PubMed, Embase and CINAHL. The primary outcome measure was the association of HCV and type 2 diabetes mellitus in Pakistan. Separate analysis was performed for "HCV infection in diabetic patients" and "diabetes in HCV infected patients". Odds ratios with $95 \%$ CIs were calculated 
through random effects model (dichotomous data). Heterogeneity was calculated using I2 statistics.

Result: From an initial 53 records, a total of 6 observational studies were finally selected for the meta-analysis. Based on random effects model, HCV patients in 3 studies $(n=1,902)$ showed a higher risk of developing diabetes as compared to patients with no HCV (OR 0.01, 95\% CI: 0.00-0.06, I2 = 0\%; RR 0.01, 95\% CI: 0.00-0.07, I $2=0 \%$ ). The other 3 studies $(n=13,710)$ had assessed HCV infections in patients with T2DM and those with no T2DM. Likewise, there was a higher prevalence of $\mathrm{HCV}$ in patients with T2DM (OR 0.7, 95\% CI: $0.17-0.42, \mathrm{I} 2=32 \%$; RR $0.30,95 \%$ CI: $0.20-0.46, \mathrm{I} 2=32 \%$ ) than those with no T2DM.

Conclusion: Our finding show significant association between T2DM and HCV infection. Various prospective studies with sufficient sample sizes are recommended.

\section{HCV-94}

Hepatitis $\mathrm{C}$ virus infection in vulnerable populations: a seroprevalence study of homeless, people who inject drugs and prisoners in London, United Kingdom

Dewi Nur Aisyah ${ }^{1}$, Laura Shallcross ${ }^{1}$, Andrew Hayward ${ }^{1}$, Rob Aldridge $^{1}$, Sara Hemmings ${ }^{1}$, Susan Yates $^{1}$, Gloria Ferenando ${ }^{1}$, Lucia Possas ${ }^{1}$, Elizabeth Garber ${ }^{1}$, John M Watson ${ }^{2}$, Timothy D Mchugh $^{1}$, Marc Lipman ${ }^{1}$, Anna Maria Geretti ${ }^{4}$, Alistair Story ${ }^{3}$

${ }^{1}$ University College London, United Kingdom; ${ }^{2}$ Centre for Public Health Data Science, United Kingdom; ${ }^{3}$ University College London Hospitals, United Kingdom; ${ }^{4}$ University of Liverpool, United Kingdom

Background: People Who Inject Drugs (PWID), homelessness and imprisonment substantially increase the risk of $\mathrm{HCV}$ infection and individuals with these risk behaviours play an important role in disease transmission. Capturing accurate data on disease prevalence within these groups is challenging and this stymies efforts to develop targeted strategies to reduce HCV transmission. The aim of this study was to estimate the prevalence of $\mathrm{HCV}$ in these vulnerable populations.

Method: We conducted a cross-sectional study between May 2011 and June 2013 in London and recruited participants from hostels for the homeless, drug treatment services and a prison alongside with the National Health Services Find and Treat (F\&T) Service. A questionnaire was administered and blood samples were collected to be tested for hepatitis C.

Result: We recruited 491 individuals who were homeless $(40.68 \%)$, 205 drug users (16.98\%), and 511 prisoners (42.34\%). Eight percent of participants (98/1207, 95\% CI: $6.67 \%-9.84 \%)$ had active $\mathrm{HCV}$ infection and $3 \%(38 / 1207,95 \% \mathrm{CI}: 2.27 \%-4.34 \%)$ had a previous HCV infection. This study found that $56.62 \%$ (95\% CI: $47.9 \%-65 \%$, 77/136) of HCV infected participants had a history all three risk factors (homelessness, imprisonment and drug use), 27.3\% (95\% CI: $20.11 \%-35.63 \%)$ had 2 overlapping risk factors, and $15.39 \%(95 \%$ CI: $10.63 \%-23.69 \%$ ) had one risk factor. Multivariate logistic regression identified three factors that were associated with increased risk of $\mathrm{HCV}$ infection: duration of injecting $(<1$ year $\mathrm{OR}=12.62,95 \%$ CI: $6.22-25.57 ; 2-9$ years OR=50.04, 95\% CI: $24.80-100.95$, and $=10$ years $\mathrm{OR}=67.34,95 \%$ CI: $32.29-140.46)$ and age $>50$ yo $(\mathrm{OR}=13.85$, 95\% CI: $1.67-114.85)$.

Conclusion: This study confirmed the high prevalence of HCV among these three groups linked to injecting drug use and age. More than half of HCV infected individuals had the intersecting risk factors of homelessness, prison and drug use, highlighting the vulnerability of these patients. Reducing the burden of HCV among these vulnerable groups is fundamental to lessen HCV transmission.

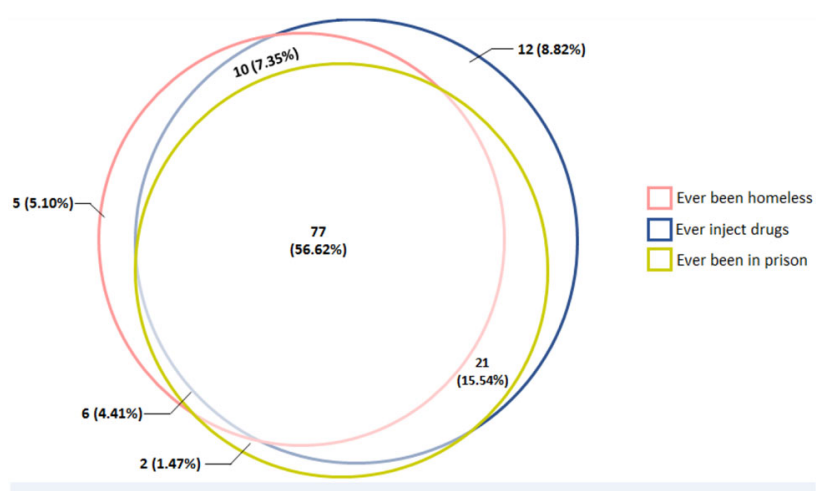

$H C V-95$

Household transmission of hepatitis $\mathrm{C}$ virus infection, Punjab, India

Adam Trickey ${ }^{1}$, Ajit Sood ${ }^{3}$, Shaun Shadaker ${ }^{2}$, Vandana Midha ${ }^{3}$, Vijay Surlikar ${ }^{4}$, William Thompson ${ }^{2}$, Claudia Velozzi ${ }^{2}$, Subodh Kanchi $^{4}$, Peter Vickerman ${ }^{1}$, Margaret T May ${ }^{1}$, Francisco Averhoff $^{2}$

${ }^{1}$ University of Bristol, United Kingdom; ${ }^{2}$ Centers for Disease Control and Prevention, United States; ${ }^{3}$ Dayanand Medical College, India;

${ }^{4}$ MSD India Pvt. Ltd., India

Background: The state of Punjab, India, has a high burden of hepatitis $\mathrm{C}$ virus (HCV) infection and has embarked on an ambitious program to provide treatment to all infected persons. Treatment efforts should be coupled with prevention efforts in order to maximize the effectiveness of $\mathrm{HCV}$ elimination programs. Community and household transmission of $\mathrm{HCV}$ infection is not well understood. We investigate the contribution of transmission at the household level in Punjab, India.

Method: Data from an HCV sero-prevalence survey conducted in Punjab, India, 2013-2014 were analysed. We administered a behavioural questionnaire and linked responses to $\mathrm{HCV}$ antibody test results. We simulated 1000 scenarios with HCV spread randomly throughout the population, and compared these results to actual clustering of HCV observed in the study. Logistic regression adjusted for household size, was used to identify factors associated with multiple HCV infections ( $>1$ household members). Variables associated with multiple HCV infections were used to develop a final model.

Result: There were 5,543 eligible participants from 1,593 households that completed the survey and had a linked HCV test result. The estimated anti-HCV positive $(\mathrm{HCV}+)$ prevalence was $3.6 \%(95 \%$ confidence interval: $3.0 \%, 4.2 \%)$. There were $1,433(90 \%)$ households with no $\mathrm{HCV}+$ individuals, $130(8.2 \%)$ households with just 1 member that tested positive for $\mathrm{HCV}$, and 30 (1.8\%) households had $\geq 2$ members that tested positive for HCV. Of the 1000 simulated scenarios with $\mathrm{HCV}$ infection distributed randomly, the median (interquartile range) number of households that had $\geq 2$ members $\mathrm{HCV}+$ was $15(12,17)$. No scenario had 30 households with $\geq 2$ members $\mathrm{HCV}+(\mathrm{p}<0.0001)$. Variables associated with a household having $\geq 2$ members $\mathrm{HCV}+$ (versus 0 or 1 ) were: the number of members in the household, adjusted odds ratio (aOR) 1.56 per member $(1.34,1.83)$, the household being in the south (versus the north) of the state, aOR $9.71(2.20,42.8)$, and the proportion of members of households that 
have ever smoked opium/bhuki, (aOR for if all members had smoked opium/bhuki) 32.7 (5.8, 184.5).

Conclusion: In Punjab, India, using a large sample of households, we found evidence for clustering of $\mathrm{HCV}$ infection within households. These findings have implications for HCV prevention, screening, and elimination efforts in Punjab.

\section{HCV-96}

Paritaprevir + ritonavir +ombitasvir + dasabuvir \pm ribavirin treatment in $\mathrm{HCV}$ infected chronic hemodialysis patients: a single center experience

Ulus Salih Akarca ${ }^{1}$, Husnu Pullukcu², Tansu Yamazhan², Galip Ersoz $^{2}$, Nalan Gulsen Unal ${ }^{2}$, Fulya Gunsar ${ }^{2}$, Ilker Turan ${ }^{2}$, Zeki Karasu $^{2}$, Ulus Salih Akarca ${ }^{2}$

${ }^{1}$ Ege University, Turkey; ${ }^{2}$ Ege University Medical Faculty, Turkey

Background: The prevalence of HCV in Turkey is 0.5-1.0\%. AntiHCV positivity rate was $6.94 \%$ in chronic hemodialysis patients in Turkey in 2013. HCV infection increases all kind of mortality in hemodialysis patients. In order to decrease the mortality and to eliminate the source of infection, hepatitis $\mathrm{C}$ must be treated. In this study we presented our experiences with Paritaprevir+Ritonavir+Ombitasvir+Dasabuvir (PrOD) regime in patients undergoing hemodialysis.

Method: Since July 2016, 22 patients on chronic hemodialysis have been treated with $\mathrm{PrOD} \pm$ ribavirin. Their demographic data, $\mathrm{HCV}$ genotypes, and HCV RNA and ALT levels at the beginning and week 4, 12, 24 were recorded. Data of adverse effects were collected.

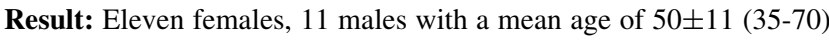
were treated. Two patients had compensated liver cirrhosis, 5 had previous renal transplantation. Basal ALT was $26.8 \pm 12.4 \mathrm{U} / \mathrm{l}, \mathrm{HCV}$ RNA level was $5.2 \pm 0.9 \log \mathrm{IU} / \mathrm{ml}$. Genotype $1 \mathrm{~b}$ was found in $16,1 \mathrm{a}$ in $5,1 \mathrm{a}+1 \mathrm{~b}$ mixed infection in 1 patients. All 1a patients started ribavirin $200 \mathrm{mg} /$ day and dose was adjusted as tolerated. Among 20 tested patients, 17 were HCV RNA negative at week-4, the other 3 patients' HCV RNA levels were 13,63 and $16 \mathrm{IU} / \mathrm{ml}$. Twenty patients who had completed week-12 became HCV RNA negative. All 14 patients reaching posttreatment week 12 had sustained response, 2 reached SVR 8, 4 reached SVR 4. G1a patients tolerated $200 \mathrm{mg} /$ day ribavirin except 1 patient who needed blood transfusion and dose reduction to every other day of $200 \mathrm{mg}$. Two patients complained pruritus, 1 had anemia. One patient died due to heart failure, 1 discontinued the treatment due to pruritus.

Conclusion: In this ongoing study, PrOD \pm ribavirin is highly effective and safe regime in patients undergoing hemodialysis.

\section{HCV-97}

Paritaprevir, ritonavir, ombitasvir, dasabuvir (PrOD) treatment of kidney transplant recipients with hepatitis $\mathrm{C}$ infection

Ulus Salih Akarca ${ }^{1}$, Huseyin Toz $^{2}$, Nalan Gulsen Unal ${ }^{2}$, Mumtaz Yilmaz $^{2}$, Ilker Turan', Fulya Gunsar ${ }^{2}$, Zeki Karasu' ${ }^{2}$, Galip Ersoz $^{2}$, Mehmet Ozkahya ${ }^{2}$, Ulus Salih Akarca ${ }^{2}$

${ }^{1}$ Ege University, Turkey; ${ }^{2}$ Ege University Medical Faculty, Turkey

Background: Renal transplant recipients are difficult patients to treat due to impaired renal function and drug-drug interactions between DAAs and immunosuppressive drugs. Since Turkish Social Security System reimburses only PrOD regime in treatment-naive kidney transplantation (KT) recipients, we treated most of our KT patients with PrOD. The aim was to investigate the efficacy and safety of PrOD in KT patients with HCV infection in a single center real life experience.

Method: KT recipients with a post-transplant follow-up of at least 1 year were included into the study. The patients with genotype $1 \mathrm{~b}$ were treated with PrOD and those with genotype 1a received PrOD+ribavirin. The patients were followed up once a week for the first 2-3 weeks, then biweekly. The blood levels of immunosuppressives were closely followed up and adjusted.

Result: Eighteen patients ( 10 male, 8 female) with the age of $35 \pm 10$ were assessed. 11 received a live donor allograft. Mean duration of dialysis before KT was 7.7 \pm 7.3 (1-23) years. Elapsed time for HCV treatment after KT was $13.1 \pm 9.8(1.1-27.3)$ years. Thirteen patients were under calcineurin inhibitors treatment. Three patients were treatment experienced, the others were naive. Nine patients were infected with G1a, others with G1b. All patients were treated for 12 weeks, except 1 with cirrhosis who was treated for 24 weeks. HCV RNA was undetectable at week 4 in $9(50 \%)$ patients. EOT response was $100 \%$. SVR12 was achieved in all 16 patients completing 12 week post-treatment follow-up. Remaining 2 patients reached SVR4. Cyclosporin of $50 \mathrm{mg}$ was given once a day to once a week in 8 cyclosporin-treated patients. Its blood levels were maintained at $87.9 \pm 39.4 \mathrm{ng} / \mathrm{ml}$. Out of 6 patients taking tacrolimus $(0.5-1.5 \mathrm{mg}) ; 1$ discontinued, 3 patients received it every 10 days, 2 patients received it once a week. With these adjustments $7.7 \pm 0.9 \mathrm{ng} / \mathrm{ml}$ blood drug levels were obtained. Three patients were using AZA + prednisolone, 1 patient was using only prednisolone with no dose adjustment. The most common side effect was anemia mostly seen in patients receiving RBV. Pruritus was observed 1 patient. None of the patients discontinued the treatment. Serum creatinine, urine protein, and eGFR did not changed significantly during treatment period.

Conclusion: In this real life experience, of 18 PrOD treated KT recipients, 16 reached SVR12, 2 SVR4. Tacrolimus or cyclosporine serum levels maintained within the normal range with close monitoring. PrOD regime can be successfully and safely used in KT recipients with $\mathrm{HCV}$ infection with close follow-up.

\section{HCV-98}

Real-world effectiveness and tolerability of sofosbuvir plus ribavirin for chronic hepatitis $\mathrm{C}$ genotype 2 in Asia: a systematic review and meta-analysis of 13 studies and 2208 patients

Mindie Nguyen ${ }^{1}$, Bin Wei ${ }^{1}$, Fanpu $\mathrm{Ji}^{1}$, Yee Hui Yeo ${ }^{1}$, Eiichi $\overline{\text { Ogawa }}^{1}$, Christopher Stave ${ }^{5}$, Shuangsuo Dang ${ }^{4}$, Zongfang $\mathbf{~ L i}^{3}$, Norihiro Furusyo $^{2}$, Ramsey Cheung ${ }^{1}$, Mindie Nguyen ${ }^{1}$

${ }^{1}$ Stanford University Medical Center, United States; ${ }^{2}$ Kyushu University Hospital, Japan; ${ }^{3}$ Second Affiliated Hospital of Xi' an Jiaotong University, China; ${ }^{4}$ Second Affiliated Hospital of Xi' an Jiaotong University, Xi' an, People Republic of China, China; ${ }^{5}$ Stanford University, United States

Background: Sofosbuvir plus ribavirin ( $\mathrm{SOF}+\mathrm{RBV}$ ) for 12 weeks is the standard treatment for chronic hepatitis $\mathrm{C}$ (CHC) genotype 2 (GT2) in most of Asia; however, its real-world effectiveness has only been available in small and/or single-center studies. Our aim was to determine the real-world effectiveness (RWE) and tolerability of 12-week SOF+RBV therapy for CHC GT2 in Asia.

Method: A systematic search in PubMed and Embase were conducted independently two reviewers identified full articles and conference proceedings through June 30, 2017. Eligible studies should include at least $10 \mathrm{CHC}$ GT2 adult patients treated with SOF+RBV for 12 weeks under real-world setting in Asia with the primary outcome of the sustained virological response 12 weeks after 
end of treatment (SVR 12) and/or adverse events (AEs). Meta-analyses and meta-regression were performed in the Freeman-Tukey random-effects model.

Result: A total of 2208 patients from 13 studies were included. The pooled SVR 12 was $95.8 \%$ (95\% CI 94.6\% - 96.9\%) with non-significant heterogeneity $(\mathrm{I} 2=34.4 \%, p=0.11)$. No significant publication bias based on the Egger's test $(p=0.10)$ and Begg's test $(p=0.14)$. A total of $25.9 \%$ patients had RBV dose reduction, but only $0.7 \%$ discontinued therapy prematurely. The most common AEs was Anemia (27.9\%), and serious adverse events (SAEs) occurred in $2.0 \%$ patients. In subgroup analysis, the pooled estimate of SVR 12 for noncirrhotic and cirrhotic subgroups were $97.7 \%$ (95\% CI: $95.9 \%$ $99.1 \%$ ) and $91.3 \%$ (95\% CI: $88.1 \%-94.1 \%)$, respectively $(p<0.0001)$. In the treatment history subgroups, the major prior treatment was interferon $(95.4 \%)$. And, the pooled SVR12 for the treatment-naïve patients and treatment-experienced patients were $97.4 \%$ (95\% CI: $95.2 \%-99.0 \%)$ and $91.8 \%$ (95\% CI: $89.1 \%-94.3 \%),(p=0.0002)$. Meta-regression analyses showed cirrhotic patients have $8.7 \%(95 \%$ CI: $3.8 \%-13.6 \%$ ) lower SVR 12 than non-cirrhotic patients, and treatment-experienced have $7.2 \%$ (95\% CI: $2.5 \%$ - 11.9\%) lower SVR 12 than treatment-naïve patients. Cirrhotic treatment-experienced patients had the lowest SVR 12 at $84.5 \%$. Unlike Peg-IFN era, $\mathrm{SOF}+\mathrm{RBV}$ for 12 weeks treatment showed no significant difference between RBV dose reduction and no reduction groups with $p=0.30$. No significant differences found in pooled SVR 12 among patient subgroups: HCC $(95.0 \%, 95 \%$ CI: $92.0 \%$ - 96.9\%) vs. no-HCC $(89.2 \%, 95 \%$ CI: $77.9 \%$ - 95.1\%) $(p=0.10)$; GT 2a $(95.1 \%, 95 \%$ CI93.3\% - 96.8\%) vs. GT 2b (95.7\%, 95\%CI: 91.9\% - 98.4\%) $(p=0.86)$; age $<65(97.8 \%$, 95\%CI: $94.5 \%-99.8 \%)$ vs. age ${ }^{3} 65$ $(95.1 \%$, 95\%CI: $92.3 \%-97.4 \%)(\mathrm{p}=0.20)$.

Conclusion: In Asia, the pooled RWE of SOF+RBV for 12 weeks treatment was $95.8 \%$ in CHC GT2 patients. Despite frequent occurrence of anemia and RBV dose reduction, it has no impact on SVR 12, premature discontinuation was negligible. However, for the treatment experienced and/or cirrhotic patients, RWE of 12-week therapy with $\mathrm{SOF}+\mathrm{RBV}$ was much lower.

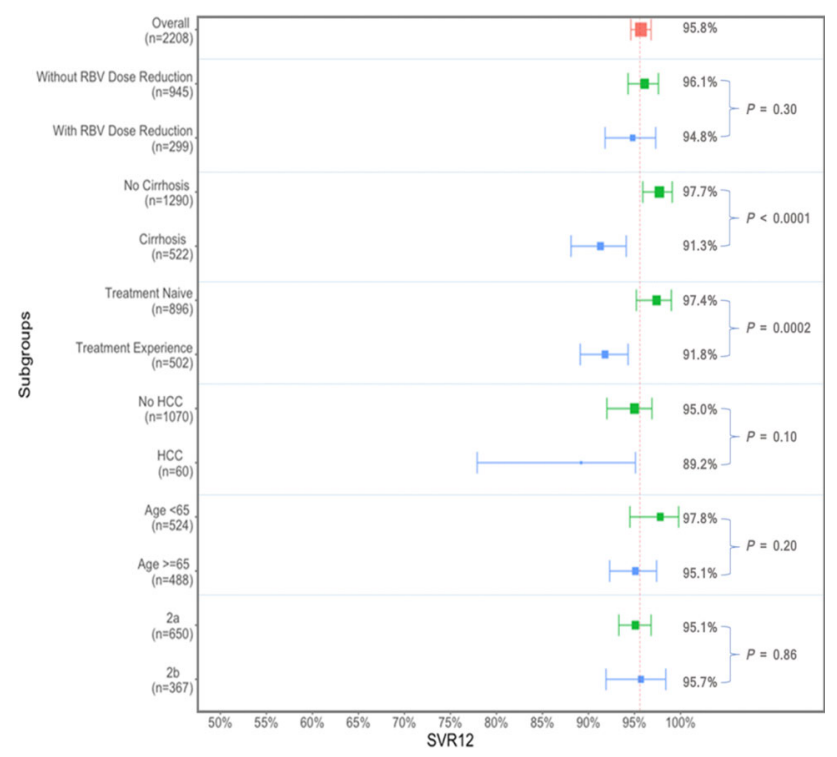

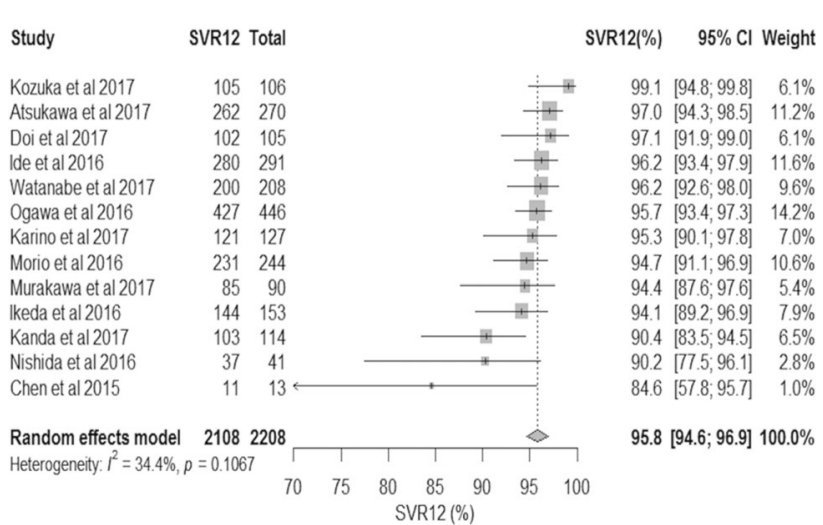

$H C V-99$

Real-world effectiveness of direct-acting antiviral therapies in chronic hepatitis $C$ genotype 3 in Asia: a systemic Review and meta-analysis of 15 studies and 4230 patients

\section{Mindie Nguyen ${ }^{1}$, Bin Wei ${ }^{1}$, Fanpu $\mathrm{Ji}^{1}$, Yee Hui Yeo ${ }^{1}$, Eiichi} $\overline{\text { Ogawa }}^{1}$, Linda Henry ${ }^{1}$, Christopher Stave ${ }^{5}$, Shuangsuo Dang ${ }^{4}$, Zongfang $\mathrm{Li}^{3}$, Norihiro Furusyo ${ }^{2}$, Ramsey Cheung ${ }^{1}$, Mindie Nguyen ${ }^{1}$

${ }^{1}$ Stanford University Medical Center, United States; ${ }^{2}$ Kyushu University Hospital, Japan; ${ }^{3}$ Second Affiliated Hospital of Xi' an Jiaotong University, China; ${ }^{4}$ Second Affiliated Hospital of Xian Jiaotong University, China; ${ }^{5}$ Stanford University, United States

Background: Genotype 3 (GT3) is a common chronic hepatitis C (CHC) genotype in Asia, especially in South Asia (66.7\%). The new direct-acting antivirals (DAAs) treatment regimens have high cure rates but the real-world effective (RWE) results were limited for Asia. This study aims were to perform a systematic review and metaanalysis of the RWE of the DAAs for CHC GT 3 patients in Asia.

Method: A systematic search were performed on Pubmed and Embase for full-article publications and conference proceedings through June 30, 2017. Eligible studies were original studies in Asia under real-world settings with the primary outcome of SVR12 and/or adverse events (AEs) information for CHC GT3 adult patients Two reviewers (BW and FJ) independently identified, screened, extracted, discussed and evaluated eligible studies. A third senior researcher (MN) resolved any discordance. Meta-analyses and meta-regression was performed in random-effects model. The Newcastle-Ottawa Scale (NOS) for cohort studies were conducted to evaluate risk of bias.

Result: A total of 15 studies with 4230 patients yielded a pooled SVR12 of $92.7 \%$ (95\% CI: $88.9 \%-95.8 \%$ ). High heterogeneity was noted among the studies ( $\mathrm{I} 2=93.2 \%, \mathrm{p}<0.0001$ ), which indicated there may exist one or more subgroups caused of the heterogeneity. Publication bias were not observed in either the Begg test $(p=0.80)$ and Egger test $(p=0.14)$. The majority of patients $(n=4069,96 \%)$, were treated with a $\mathrm{SOF}+\mathrm{RBV} \pm \mathrm{Peg}$-IFN therapy: 24 weeks of $\mathrm{SOF}+\mathrm{RBV}$ or 12 weeks of SOF+RBV+Peg-IFN, with a pooled SVR12 rate of $93.0 \%$ (95\%CI: $87.9 \%-96.1 \%, \mathrm{I} 2=89.3 \%)$, and $95.4 \%$ (95\%CI: $90.9 \%-97.7 \%, \mathrm{I} 2=81.9 \%$ ), respectively. There was no statistically significant difference between 24 weeks of SOF+RBV and 12 weeks of SOF+RBV+Peg-IFN for 12 weeks with OR $=1.1$ (I2=47.0\%). However, much higher AEs observed in Peg-IFN group than IFN-free group that myalgia $(49.1 \%$ vs $11.0 \%)$, fatigue $(43.5 \%$ vs $22.9 \%$ ), anemia ( $40.7 \%$ vs $28.1 \%$ ) and insomnia (12.0\% vs $7.8 \%$ ). Moreover, $\mathrm{DCV}+\mathrm{SOF} \pm \mathrm{RBV}$ for 12 or 24 weeks therapy were reported in 149 CHC GT3 patients with an SVR12 of $91.7 \%$ (95\% CI: 
$86.0 \%-95.1 \%$ ). Only 12 patients of total were treated with LDV+SOF for 12 weeks and nine achieved SVR12 (75\%). In subgroup analyses, the pooled estimate of SVR12 for non-cirrhotic and cirrhotic subgroups were $98.9 \%$ (95\%CI: $97.6 \%$ - 99.7\%, I2 =66.1\%) and $88.6 \%$ in $(95 \% \mathrm{CI}: 82.4 \%-93.7 \%$, I2 $=81.4 \%)$, respectively $(p<0.0001)$. Cirrhotic patients had $10.9 \%$ lower SVR12 than noncirrhotic patients, and the status of cirrhosis contributed to $69.5 \%$ of total heterogeneity by R2. No significant SVR12 differences in subgroups: treatment-naïve vs treatment-experienced $(94.6 \%$ vs $94.0 \%$, $\mathrm{p}=0.89$ ); India vs. Pakistan vs Myanmar vs Iran (94.7\% vs $87.6 \%$ vs $97.9 \%$ vs $90.9 \%, \mathrm{p}=0.28)$.

Conclusion: SOF+RBV 24-week therapy showed as high RWE as SOF+RBV+Peg-IFN 12-week therapy with comparatively lower adverse events, but may not optimal for CHC GT3 patients with cirrhosis.
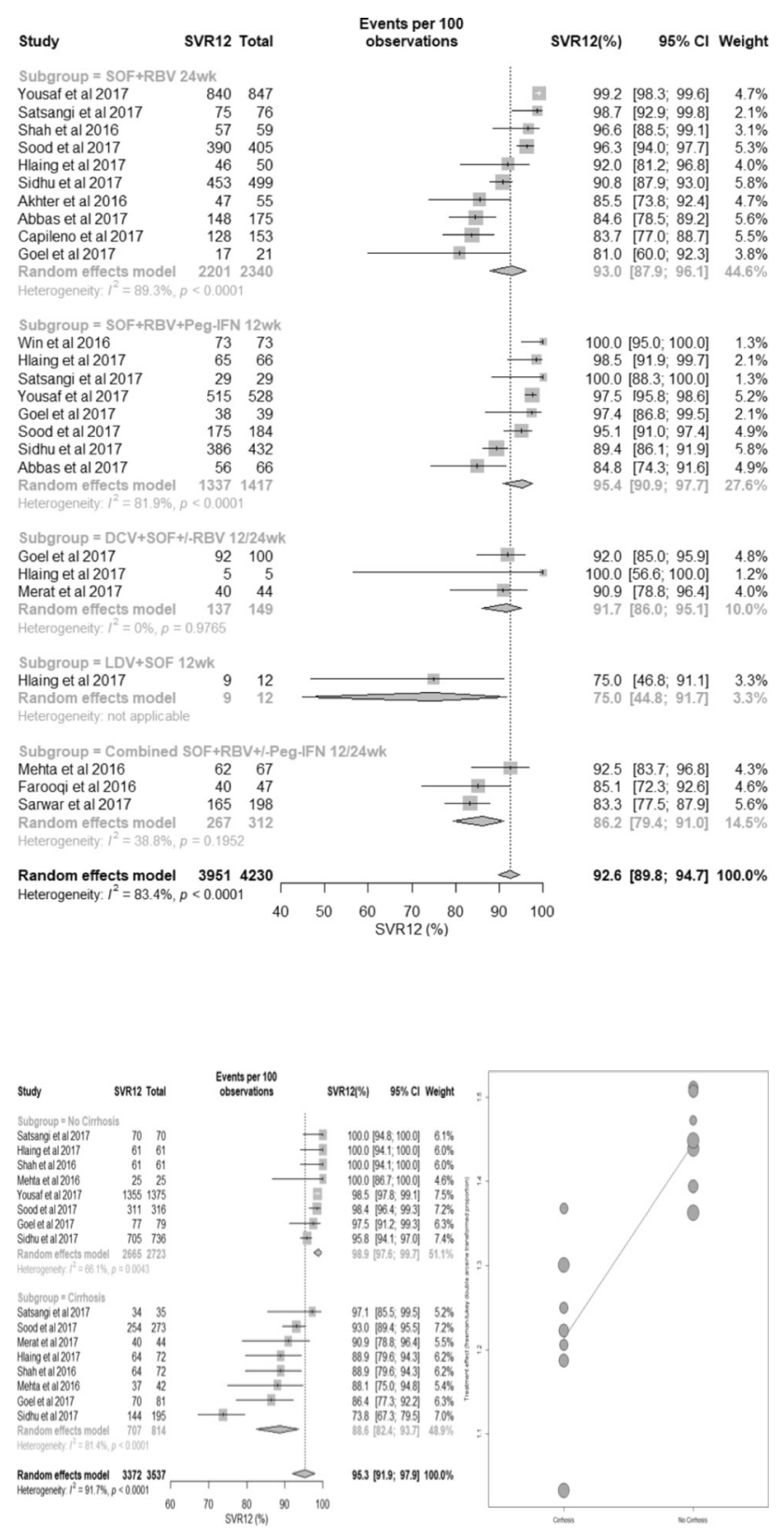

$H C V-100$

Treatment of chronic hepatitis $\mathrm{C}$ genotype 3 with ledipasvir and sofosbuvir

\section{Ghulam Nabi Yattoo}

${ }^{1}$ SKIMS, India

Background: Despite some preliminary data from ELECTRON-2 trial regarding use of ledipasvir and sofosbuvir combination in Chronic Hepatitis C Genotype 3 there are no guidelines recommending this combination in such patients. In Kashmir, India Genotype 3 is the most common genotype of Chronic Hepatis C infection. To increase the compliance and ease of taking DAA in chronic hepatitis $\mathrm{C}$ genotype 3 patients we decided to use single tablet of ledipasvir \& sofosbuvir combination.

Method: It was a Prospective, Open label, Hospital based observational study. All patients of chronic hepatitis C Genotype 3 were randomized into two groups: Non-cirrhotic and Cirrhotic. Both groups were divided further into two groups: Treatment Naïve and Treatment Experienced. Non-cirrhotic patients received ledipasvir//sofosbuvir (90/400 mg) for 12 weeks, however treatment was extended to 24 weeks in Cirrhotics.

Result: We enrolled 104 patients of chronic hepatitis c over a period of 18 months. August 2015 to Females constituted 66 (63.5\%) and males were $38(36.5 \%)$. Average age was 40 years (rage $18-76$ years). $96 \%$ patients were married. Out of 104 patients, $86(82.7 \% \%)$ were genotype $3,15(14.9 \%)$ were genotype 1 and $3(2.9 \%)$ were genotype $4.92(88 \%)$ were non-cirrhotic and $12(11.5 \%)$ were cirrhotic. 95 (95.2\%) were treatment naïve. Among genotype 1 and 4 all patients achieved RVR \& SVR12. Out of 86 genotype 3 patients 78 (90.6\%) were non-cirrhotic and $8(9.3 \%)$ were cirrhotic. Among non-cirrhotic genotype 3 patients $75(96 \%)$ achieved SVR12 while as $6(75 \%)$ among cirrhotics. All patients tolerated the combination well however some patients experienced nausea (26\%), headache (25\%) and fatigue (21\%). No patient had to discontinue therapy due to adverse drug reactions.

Conclusion: Single tablet ledipasvir and sofosbuvir combination is safe and effective in genotype 3 even without ribavirin.

\section{HCV-101}

\section{Analysis of hepatitis $\mathrm{C}$ virus infection in patients under hemodialysis}

\section{Karina Alejandra Salvatierra ${ }^{1}$}

${ }^{1}$ Universidad Nacional de Misiones, Argentina

Background: Hepatitis C virus (HCV) is a major global health problem. Patients under hemodialysis are considered high risk to acquire the Hepatitis $\mathrm{C}$ infection. The present study aimed to determine the prevalence rate of $\mathrm{HCV}$ in hemodialysis patients from the city of Posadas (Argentina).

Method: The study included 172 patients with chronic renal failure under hemodialysis attending hemodialysis centers of the city of Posadas (Argentina). People who participated in the study went daily to a hemodialysis center and participation was voluntary. Blood samples were collected through venipuncture in dry tubes $(9.5 \mathrm{ml})$ with vacuum. After clot retraction, samples were centrifuged at 1,500 $\mathrm{rpm}$ for 5 minutes and stored in $5 \mathrm{~mL}$ aliquots at $-20^{\circ} \mathrm{C}$. Serum samples are tested for antibodies to hepatitis C virus (anti-HCV) using the enzyme-linked immunosorbent assay test (ELISA).

Result: A total of 172 patients were included in the study; 98 are males $(57 \%)$ and 74 females $(43 \%)$, with a mean age of 53.4 years. A total of $9.9 \%(17 / 172)$ of the patients were positive for antiHCV 
reagents. $72.1 \%$ of patients had a hemodialysis treatment time of less than 5 years. A history of having received previous transfusions was observed in $29 \%(5 / 17)$ anti-HCV positive cases.

Conclusion: Hepatitis $\mathrm{C}$ virus (HCV) is a major global health problem. Patients under hemodialysis are considered high risk to acquire the Hepatitis C infection. HCV infection prevalence was $9.9 \%$ in patients with chronic renal failure under hemodialysis from the city of Posadas (Argentina). Despite the biosecurity measures and preventive actions in hemodialysis units, the prevalence of HCV infection among hemodialysis patients is still relatively high when compared with those observed in developed countries.

\section{HCV-102}

Efficacy of NS5A inhibitor-containing regimens and baseline NS5A resistance associated substitutions: a data from China

Lichang Chen ${ }^{1}$, Huijuan Zhou ${ }^{1}$, Ruidong Mo ${ }^{1}$, Peipei Ren ${ }^{1}$, Zhujun Cao', Yuhan Liu $^{1}$, Tianhui Zhou', Qing Guo', Hui Wang $^{1}$, Wei Cai ${ }^{1}$, Qing Xie ${ }^{1}$, Jie Lu ${ }^{1}$

${ }^{1}$ Rui Jin Hospital Affiliated to Shanghai Jiao Tong University School of Medicine, China

Background: NS5A inhibitor-containing regimens for patients with chronic hepatitis $\mathrm{C}$ with variable efficacy dependent on baseline NS5A resistance associated substitutions (RAS) widespread used across the world, nevertheless, a rare data from China is available. We aimed to retrospective evaluate the associations between the sustained virologic responses of 24 weeks after treatment (SVR24) of NS5A inhibitor-based DAA therapy and baseline NS5A RAS in a realworld, observational study.

Method: A total of 35 patients with chronic HCV infection who were intolerant to, or failed to PegIFN plus ribavirin therapy were included. They were treated with different NS5A inhibitor-based DAAs combinations (daclatasvir + asunaprevir / ombitasvir + paritaprevir + ritonavir / ledipasvir + sofosbuvir in treatment of 12 or 24 weeks) according to the access of the drugs and the liver condition. Baseline NS5A RAS was analyzed by Sanger sequencing with quantitative HCV RNA assessed prior to treatment, at treatment week 4, the end of treatment, 12 weeks post-treatment (SVR12) and 24 weeks posttreatment (SVR24) by COBAS TaqMan HCV test 2.0 (LLOQ

Result: HCV genotypes were $100 \%$ G1b and overall, seven (20\%) patients had at least one NS5A RAS at baseline, including L31 and Y93. SVR24s were not achieved by $6 \%$ ( 2 in 35 ). The presence of NS5A RASs at baseline did not increase the risk of initial antiviral treatment failure $(\mathrm{OR}=4.5, \mathrm{p}=0.365)$. However, $\mathrm{SVR} 24$ was affected by different DAAs combinations (p).

\section{$H C V-103$}

Improvement of liver stiffness values measured by transient elastography after treatment of chronic hepatitis $C$ patients with directly acting anti-virals (DAA's)

\section{Muzzafar Lateef Gill', Ammara Nawaz ${ }^{1}$, Sidra Gill ${ }^{1}$, Arham Baloch $^{1}$, Mehmoona Pervaiz ${ }^{1}$ \\ ${ }^{1}$ Islamabad Gastroenterology Associates, Pakistan}

Background: An improvement in liver stiffness (LS) measured by Transient Elastography (TE) have been observed in patients with chronic hep c treated with directly acting antiviral agents (DAA). We wanted to study the effect of DAA on liver stiffness in baseline and 6-months after the treatment with DAA.
Method: With this background we conducted an observational study comparing Liver stiffness at baseline and 24 weeks after sustained virological Response (SVR) HEP C patients, treated with DAA.

Result: Out of 80 patients, $55 \%$ were male and $45 \%$ were female. Median age 45 Years

They were all HEP C genotype 3 Patients. They all have sustained virological response for the last 6 month after treatment.

1. Median TE on Baseline, prior to DAA Treatment was mean 20 (12-30). After the treatment it was

2. Mean 13 (Range 6-18). It was statistically significant showing $30 \%$ decrease in liver stiffness

Conclusion: DAA treatment shows improvement in liver stiffness chronic hep c patients.

\section{HCV-104}

New direct acting antivirals for the treatment of chronic hepatitis C

\section{$\underline{\text { Bengu Tatar }}{ }^{1}$, Bengu Tatar ${ }^{1}$, Nadide Colak ${ }^{1}$, Ayse Ozkan ${ }^{1}$, Melda} Turken

${ }^{1}$ Tepecik Educational and Research Hospital, Turkey

Background: Direct-acting antivirals (DAAs) are currently the treatment of chronic hepatitis $\mathrm{C}$ virus (HCV) infection. These agents produce higher response rates and have fewer toxicities than the interferon-based therapies.

Aim of this study is showing the clinical and laboratory results of our chronic hepatitis $\mathrm{C}(\mathrm{CHC})$ patients.

Method: In our study; CHC outcome patients in ESRD (End Stage Renal Disease) and NRF (normal renal function) group were included, therewithal patients under 18 age, pregnancy or coinfections were excluded. Demographic data, baseline serum HCV RNA levels (PCR, Low limit of detection $15 \mathrm{IU} / \mathrm{mL}$ ), HCV genotype informations, biochemical results, previous treatment history were recorded. According to genotypic and histological examination, $\mathrm{CHC}$ patients were treated with Ledipasvir + Sofosbuvir \pm ribavirin or OmbitasvirParitaprevir-Ritonavir + Dasabuvir \pm ribavirin.

Patients visits were performed on $1,2,4,8$ and 12 weeks during treatment.

Result: Sixty four patients included in study. Mean age of patients was 56.4 years, and $46 \%$ of male. Baseline mean laboratory values of CHC patients are summarized in table 1 and 2. While 3/64 of patients had to stop treatment because of adverse events, this was mainly in people treated with ribavirin. Non of them performed biochemical discharge. Comparison of virologic response rates in ESRD and NRF group had show in Table 3.

Table 1: Baseline mean laboratory values of $\mathrm{CHC}$ patients

\begin{tabular}{|l|l|l|}
\hline Baseline mean values & NRF & ESRD \\
\hline AST & 49,7 & 25,8 \\
\hline ALT & 58,1 & 29,9 \\
\hline GGT & 113,8 & 63,8 \\
\hline LDH & 215,2 & 194,5 \\
\hline Urea & 40,3 & 73,2 \\
\hline creatinin & 0,9 & 6,0 \\
\hline Total bilirubine & 0,8 & 0,7 \\
\hline Albumine & 4,1 & 4,2 \\
\hline Direct bilirubine & 0,2 & 0,2 \\
\hline Alfafetoprotein & 4,9 & 40,9 \\
\hline
\end{tabular}




\begin{tabular}{|l|l|l|}
\hline genotype & NRF & ESRD \\
\hline 3 & $(\mathbf{n : 3 1 )}$ & $(\mathbf{n : 3 3 )}$ \\
\hline 4 & 1 & 0 \\
\hline $1 \mathrm{a}$ & 2 & 1 \\
\hline $1 \mathrm{~b}$ & 6 & 19 \\
\hline $1 \mathrm{~b}$ and 4 & 21 & 13 \\
\hline
\end{tabular}

Table 2: Genotypic distribution of CHC patients

\begin{tabular}{|l|l|l|l|l|l|}
\hline & $\begin{array}{l}\text { HCVRNA } \\
\text { baseline }\end{array}$ & HCV RNA & HCVRNA & $\begin{array}{l}\text { HCVRNA } \\
\text { 4.week }\end{array}$ & $\begin{array}{l}\text { 2. week } \\
\text { week }\end{array}$ \\
\hline Esrd & $1,18 \mathrm{E}+07$ & $4,65 \mathrm{E}+02$ & $2,52 \mathrm{E}+01$ & 0 & 0 \\
\hline (n:33) & & & & & \\
\hline Nrf & $1,56 \mathrm{E}+06$ & $1,87 \mathrm{E}+02$ & $5,60 \mathrm{E}+01$ & $8,10 \mathrm{E}+00$ & 0 \\
\hline (n: 31$)$ & & & & & 0 \\
\hline
\end{tabular}

Table 3: Comparision of virologic response rates of ESRD and NRF group

Conclusion: In conclusion, the rates of virologic response were similar in ESRD and NRF groups.

According our study we think treatment with DAA regimens might be severe cure for $\mathrm{CHC}$, but patients should be followed in terms of long term complications.

\section{HCV-105}

The Rapid-EC Study - a feasibility study of point-of-care testing in community clincs targeted to people who inject drugs in Melbourne, Australia.

Bridget Williams $^{1}$, Alisa Pedrana ${ }^{1}$, Jessica Howell ${ }^{1}$, Joseph Doyle ${ }^{1}$, Alexander Thompson ${ }^{5}$, Frances Bramwell ${ }^{3}$, Dean Membrey ${ }^{3}$, Margaret Mcpherson ${ }^{4}$, Chloe Layton ${ }^{3}$, Bridget Draper ${ }^{1}$, Janine Roney $^{2}$, Ned Latham ${ }^{1}$, Margaret Hellard ${ }^{1}$

${ }^{1}$ Burnet Institute, Australia; ${ }^{2}$ Alfred Health, Australia; ${ }^{3}$ Cohealth, Australia; ${ }^{4}$ North Richmond Community Health, Australia; ${ }^{5} \mathrm{St}$ Vincent's Hospital, Australia

Background: Increasing the number of people who inject drugs (PWID) accessing hepatitis $\mathrm{C}$ virus (HCV) diagnosis and treatment is essential to Australia's success in eliminating HCV. Point-of-care (POC) diagnostics may reduce barriers to HCV care by allowing same-day diagnosis and facilitating testing in locations already frequented by PWID, such as needle and syringe exchange programs (NSPs). The Rapid-EC study was conducted to assess the feasibility and acceptability of POC testing for $\mathrm{HCV}$ in three community clinics with NSPs.

Method: Clients of the NSP service, who were not engaged in HCV care, were invited to participate. Participants underwent OraQuick $\mathrm{HCV}$ antibody mouth swab test (20 minutes to result); those positive were offered venepuncture for the Xpert HCV viral load (105 minutes to result). Participants were offered same-day results delivered on site, via phone or text message, or on return to the service.

Participants were invited for follow-up review and those diagnosed with CHR Clients of the NSP service, who were not engaged in HCV care, were invited to participate. Participants underwent OraQuick $\mathrm{HCV}$ antibody mouth swab test (20 minutes to result); those positive were offered venepuncture for the Xpert HCV viral load (105 minutes to result). Participants were offered same-day results delivered on site, via phone or text message, or on return to the service. Participants were invited for follow-up review and those diagnosed with chronic HCV were assessed for treatment by a nurse and linked to community or specialist prescribers. Analysis of treatment uptake is ongoing. Participants also completed demographic, behavioural and acceptability surveys once HCV were assessed for treatment by a nurse and linked to community or specialist prescribers. Analysis of treatment uptake is ongoing. Participants also completed demographic, behavioural and acceptability surveys.

Result: A total of 174 participants completed POC testing for HCV antibodies, of whom $150(86 \%)$ had a reactive result. Of these, 140 (93\%) underwent a POC HCV RNA test, of which 76 (54\%) had detectable RNA. The median age of participants was 41 years, 118 (67\%) were male, $154(88 \%)$ had injected drugs within six months and $93(53 \%)$ had shared injecting equipment within six months. Of the 67 participants for whom a four-week period had elapsed since $\mathrm{HCV}$ diagnosis, $40(60 \%)$ had been reviewed and linked to care in that time. Only $7(5 \%)$ participants waited on-site to receive their POC RNA result, while $85(61 \%)$ opted to receive their result via phone call or text message. The majority $(85 \%)$ of participants preferred POC compared to standard HCV testing.

Conclusion: Provision of POC testing at community clinics with NSPs was feasible and acceptable to clients at high risk of HCV infection. Point-of-care tests streamlined HCV testing and enhanced linkage to care opportunities, however the ability to provide same-day diagnosis with current tests is limited. The utility of HCV POC diagnostics may be improved by reducing time to result and number of tests to diagnosis.

HCV-106

A comparison of non-invasive clinical scores to predict advanced liver fibrosis in the ambulatory setting for hepatitis $\mathrm{C}$ patients with a history of intravenous drug abuse in a multi-ethnic population

Yue Zhao ${ }^{1}$, Jessica Yi Lyn Tan ${ }^{1}$, Prem Harichander Thurairajah ${ }^{1}$, Eng Kiong Teo ${ }^{1}$, John Hsiang ${ }^{1}$

${ }^{1}$ Changi General Hospital, Singapore

Background: Hepatitis C (HCV) remains a major clinical concern among people with history of intravenous drug abuse (IVDA), as most will go on to develop chronic infection leading to liver cirrhosis and hepatocellular carcinoma. Accurate identification of patients with advanced fibrosis is important and utility of non-invasive clinical scores can especially helpful in IVDA patients, who have poorer access to specialist care and high frequency of defaulting medical visits

Method: This is a retrospective study on all HCV patients who were managed in Changi General Hospital, Singapore with history of IVDA, between 2011 to 2016. Data collected include liver stiffness measurement (LSM) by transient elastography. Previously established clinical scores in predicting advanced fibrosis were tabulated and validated by analysing the area under receiver operating characteristic curve (AUROC). Sensitivity, specificity, positive and negative predictive values were examined. Data was analysed by SPSS version 21 with statistical significance defined as $p<0.05$. 
Result: A total number of 579 patients were recruited for this study, with 537 (92.7\%) male patients. There were 134 (23.1\%) Chinese patients, $78(13.5 \%)$ Indian patients and 347 (59.9\%) Malay patients. The mean age was 47.5. A majority of patients have HCV genotype 3 (77.7\%).

AST-to-platelet ratio index (APRI), modified APRI and Fibrosis-4 (FIB-4) scores were valid in predicting F3 (defined as LSM $>9.5 \mathrm{kPa}$ ) (APRI:0.767, (95\% CI: 0.729-0.805); modified APRI:0.779, (0.7410.816); FIB-4: 0.757, (0.718-0.796)) and F4 (defined as LSM $>12.5 \mathrm{kPa}$ ) (APRI:0.770,(0.727-0.814); modified APRI:0.796, (0.754-0.838); FIB-4: 0.789, (0.747-0.830)).

Modified APRI was noted to have outperformed APRI in predicting F4 ( $\mathrm{p}=0.0023)$. Scores lower than 7.09 were found to have a negative predictive value of $89.5 \%$, scores higher than 19 was found to have a positive predictive value of $77.1 \%$, with a specificity of $96.2 \%$.

There was no significant difference between the scores for prediction of F3 stage. Previously established cut-off values for APRI and FIB-4 were found to be inaccurate for the population, especially for F3. For APRI and FIB-4, the established lower cut-off values of 0.5 and 1.45 respectively were noted to have poor negative predictive values of $77.8 \%$ and $72.2 \%$ for F3 fibrosis respectively.

Conclusion: Non-invasive scores are useful for predicting advanced fibrosis in HCV patients with history of IVDA, with modified APRI appearing the better score. However, further studies are required to establish more accurate cut-off values.

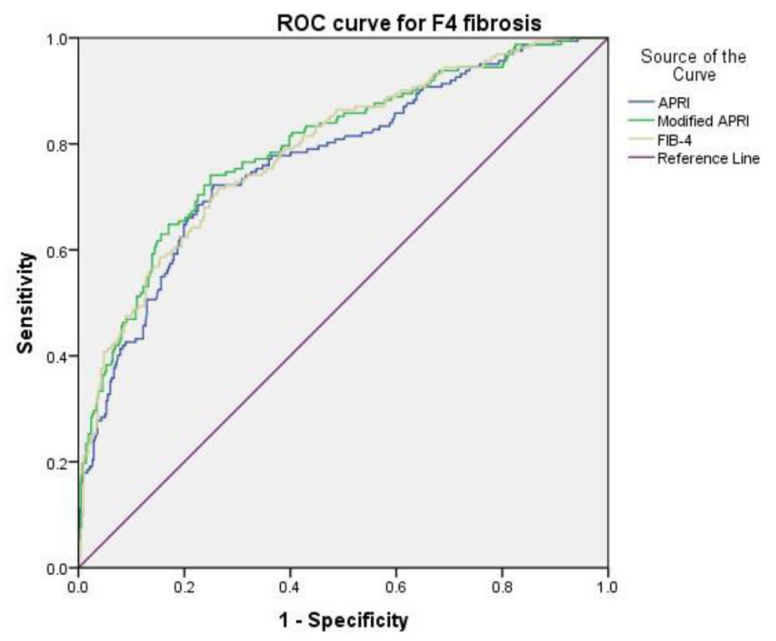

Diagonal segments are produced by ties.

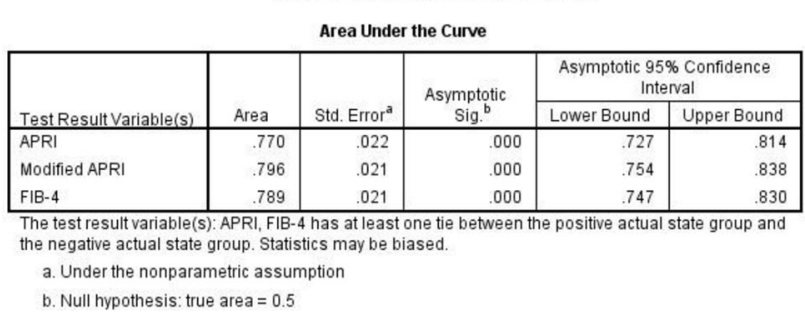

\begin{tabular}{|l|l|}
\hline \multicolumn{2}{|c|}{ Comparison of clinical scores for F4 fibrosis } \\
\hline 1) APRI vs FIB-4 & \\
\hline Difference between areas & 0.0181 \\
\hline $95 \%$ confidence interval & -0.0104 to 0.0467 \\
\hline Significance level & $\mathrm{p}=0.213$ \\
\hline 2) APRI vs Modified APRI & \\
\hline Difference between areas & 0.0255 \\
\hline 95\% confidence interval & 0.00915 to 0.0419 \\
\hline Significance level & $\mathrm{p}=0.0023$ \\
\hline 3) FIB-4 vs Modified APRI & \\
\hline Difference between areas & 0.00742 \\
\hline 95\% confidence interval & -0.0122 to 0.0270 \\
\hline Significance level & $\mathrm{p}=0.457$
\end{tabular}

\section{HCV-107}

A comparison of non-invasive clinical scores to predict advanced liver fibrosis in the ambulatory setting for hepatitis $\mathrm{C}$ patients with a history of intravenous drug abuse in a multi-ethnic population

\section{$\underline{\text { Ragesh Babu Thandassery }}^{1}$}

${ }^{1}$ Hamad Medical Corporation, Qatar

Background: Hepatitis C (HCV) remains a major clinical concern among people with history of intravenous drug abuse (IVDA), as most will go on to develop chronic infection leading to liver cirrhosis and hepatocellular carcinoma. Accurate identification of patients with advanced fibrosis is important and utility of non-invasive clinical scores can especially helpful in IVDA patients, who have poorer access to specialist care and high frequency of defaulting medical visits

Method: This is a retrospective study on all HCV patients who were managed in Changi General Hospital, Singapore with history of IVDA, between 2011 to 2016. Data collected include liver stiffness measurement (LSM) by transient elastography. Previously established clinical scores in predicting advanced fibrosis were tabulated and validated by analysing the area under receiver operating characteristic curve (AUROC). Sensitivity, specificity, positive and negative predictive values were examined. Data was analysed by SPSS version 21 with statistical significance defined as $p<0.05$.

Result: A total number of 579 patients were recruited for this study, with $537(92.7 \%)$ male patients. There were 134 (23.1\%) Chinese patients, 78 (13.5\%) Indian patients and 347 (59.9\%) Malay patients. The mean age was 47.5. A majority of patients have HCV genotype 3 (77.7\%).

Albumin-to-platelet ratio index (APRI), modified APRI and Fibrosis4 (FIB-4) scores were valid in predicting F3 (defined as LSM $>9.5 \mathrm{kPa}) \quad$ (APRI:0.767, (95\% CI: 0.729-0.805); modified APRI:0.779, (0.741-0.816); FIB-4: 0.757, (0.718-0.796)) and F4 (defined as LSM $>12.5 \mathrm{kPa}$ ) (APRI:0.770,(0.727-0.814); modified APRI:0.796, (0.754-0.838); FIB-4: 0.789, (0.747-0.830)).

Modified APRI was noted to have outperformed APRI in predicting F4 ( $\mathrm{p}=0.0023)$. Scores lower than 7.09 were found to have a negative predictive value of $89.5 \%$, scores higher than 19 was found to have a positive predictive value of $77.1 \%$, with a specificity of $96.2 \%$.

There was no significant difference between the scores for prediction of F3 stage. Previously established cut-off values for APRI and FIB-4 were found to be inaccurate for the population, especially for F3. For APRI and FIB-4, the established lower cut-off values of 0.5 and 1.45 respectively were noted to poor negative predictive values of $77.8 \%$ and $72.2 \%$ for $\mathrm{F} 3$ fibrosis respectively. 
Conclusion: Non-invasive scores are useful for predicting advanced fibrosis in HCV patients with history of IVDA, with modified APRI appearing the better score. However, further studies are required to establish more accurate cut-off values.

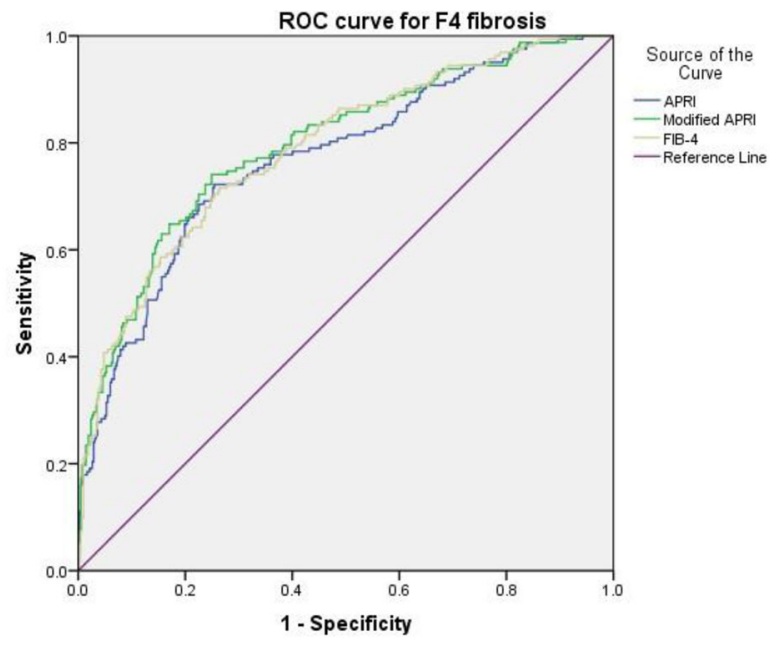

Diagonal segments are produced by ties.

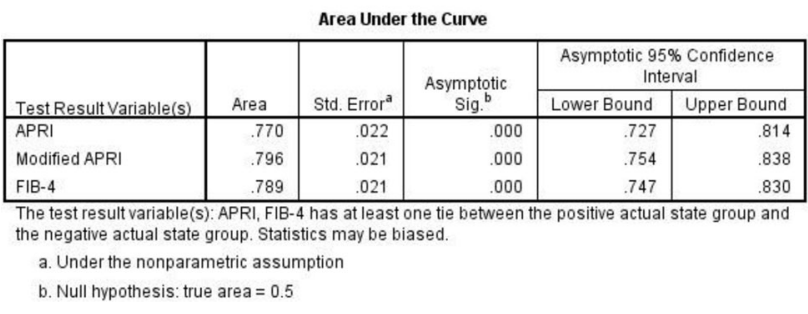

Comparison of clinical scores for F4 fibrosis

1) APRI vs FF-4

Difference between areas

$95 \%$ confidence interval

Significance level

2) APRl vs Modified APR

Difference between areas

$95 \%$ confidence interval

Significancelevel

3) FlB-4 vs ModifiedAPRI

Difference between areas

$95 \%$ confidence interval

Significance level
HCV-109

Analysis of rapid viral relapse factors after DAA treatment

\section{Jun Chen ${ }^{1}$}

${ }^{1}$ The Second Xiangya Hospital, China

Background: The recent rapid development of direct-acting antiviral agents (DAAs) has greatly improved treatment of hepatitis $\mathrm{C}$ virus (HCV) infection. But we observed some rapid viral relapse phenomenon and even liver disease progression with the relapse after DAAs treatment.

Method: To clarify the relapse factors, we reviewed related published literatures from Pubmed, OVID and Web of Science databases including: patients' compliance and relapse, efficacy of generic DAAs, HCV genotype and DAAs choice, resistance associated variants (RAVs), DAA efficacy in patients with decompensated cirrhosis and the incidence of $\mathrm{HCC}$ in patients with cirrhosis treated with DAAs.

Result: For patients with injecting drug use (IDU) history, drug addiction is the most important rapid viral relapse factors (account for $27 \%$ relapse incidence), repeat to IDU was associated with age $<30$ years at treatment and low education level. To the efficacy of generic DAAs, there is no sufficient evidence to prove the efficacy of generic drugs below the original drug, some reports from India assessed the imitation effect was similar with the original drug. On the DAA$\mathrm{s}$ choice for genotype 3 patients, the EASL recommend the use of SOF+VEL+ ribavirin (RBV) for 12 weeks or SOF+VEL24 weeks; if $\mathrm{SOF}+\mathrm{DCV}$ is used, the RBV should be given at the same time, with a course of treatment for 24 weeks. Baseline RAVs has a higher incidence among HCV infections patients, for different DAAs, RAVs affects the efficacy of DAA to varying degrees (range from no affected to greatly affected). At present, there is no sufficient evidence to suggest that DAA can increase the incidence of HCC among patients with decompensated cirrhosis. On the contrary, there are indications that antiviral can significantly improve the prognosis of patients.

Conclusion: Rapid viral relapse factors after DAA treatment may include repeat to IDU, DAAs choice for genotype 3, RAVs and decompensated cirrhosis. For these patients, we need pay more attention about DAAs choice and closely medical observation.

\section{$H C V-110$}

Can we afford not to screen and treat $\mathrm{HCV}$ infection in Canada?

Hemant Shah $^{1}$, William Wong ${ }^{2}$, Alex Haines ${ }^{1}$, Hooman Farhang Zangneh $^{1}$

${ }^{1}$ University of Toronto, Canada; ${ }^{2}$ University of Waterloo, Canada

Background: High hepatitis C (HCV) infection prevalence and the cost of the new direct acting antiviral (DAA) drugs contributed to $61.4 \%$ of overall spending growth in Canada's drug budget in 2016 . Screening for HCV infection followed by DAA treatment in individuals born between 1946 and 1964 is both effective and cost effective; however, the question of affordability remains unresolved. We aim to look into the long term cost and health outcomes of $\mathrm{HCV}$ screening for Ontario up to 2030.

Method: We used a previously validated state-transition model to analyse economic and health impact of two strategies: (1) HCV infection screening followed by DAA treatment in individuals born between 1946 and 1964 and (2) Treatment for HCV infected individuals diagnosed symptomatically born between 1946 and 1964. We used a payer's perspective, in a closed model and discounted costs at 
a $1.5 \%$ annual rate. Outcome metrics were costs, quality-adjusted lifeyears (QALY), hepatocellular carcinoma (HCC) and decompensated cirrhosis (DC) cases detected over a 14-year period.

Result: The "screen \& treat" strategy 1 provided an additional 0.0005 QALYs/person on average after 5 years; given the 3.6 million individuals in the model population in Ontario, it equates to 12,484 QALYs gained. Cost difference between the two strategies increases as time goes on, with strategy 1 initially costing more (\$16.9 million at 5 years) but eventually saving the health service money after 10 years. At 14 years, the difference in the budget impact between the two strategies will be $\$ 911$ million saved by implementing strategy 1 . Sensitivity analysis on DAA cost showed that decreasing this cost to $\$ 16,000$ as opposed to $\$ 55,860$ in strategy 2 , leads to a $\$ 555$ million cost decrease in Ontario, confirming the cost of DAAs as the main driver of cost in this analysis.

Conclusion: The impact of HCV infection screening for Ontario will be on both the budget and the population health. Given the current availability of effective curative treatment options for $\mathrm{HCV}$ and since Canada has agreed to meet WHO targets, additional screening should be implemented.

\section{$H C V-111$}

Changes in body mass index in chronic hepatitis C Egyptian patients treated with direct acting antiviral regimens

Mohamed Alboraie ${ }^{1}$, Mohamed El Kassas ${ }^{4}$, Mohamed Alboraie ${ }^{1}$, Adel Eltahan ${ }^{6}$, Dalia Omran ${ }^{3}$, Mohamed Abdella ${ }^{1}$, Ahmed Fouad $^{2}$, Sameera Ezzat ${ }^{5}$, Inas Moaz ${ }^{5}$, Mohamed Eltabbakh ${ }^{2}$, Mohamed Naguib $\mathrm{Wifi}^{3}$, Mervat Naguib ${ }^{3}$

${ }^{1}$ Al-Azhar University, Egypt; ${ }^{2}$ Ain Shams University, Egypt; ${ }^{3}$ Cairo University, Egypt; ${ }^{4}$ Faculty of Medicine; Helwan University, Egypt; ${ }^{5}$ Menoufia University, Egypt; ${ }^{6} \mathrm{New}$ Cairo Hospital, Egypt

Background: Data regarding changes in the Body mass index after treatment with the new DAA is few. This Cross-sectional study aim to assess the changes in BMI in chronic $\mathrm{HCV}$ patients treated with DAAs in Egypt and to explore other factors influencing this change. Method: Patients with chronic hepatitis $\mathrm{C}$ were recruited. All patients were subjected to full history taking, clinical examination including measuring baseline weight, height and calculating body mass index (BMI) and basic pretreatment laboratory investigations. Viral load was measured at baseline, at end of treatment as well as 12 weeks post treatment completion to assess for sustained virological response. Changes in body weight and BMI were monitored during and after treatment. BMI of $>30$ was taken as cutoff for obese patients.

Result: The studied group included 162 patients with mean age $48.56 \pm 11.498$ years. The percentage of male patients were higher than female patients $(61.1 \% \mathrm{Vs} 38.9 \%)$. Twenty nine percent of our patients were obese. About $12 \%$ of patients were diabetic and $11 \%$ of patients were hypertensive. Only 26 patients were treatment experienced. The most commonly received DAAs regimen was SOF, DCV +/- RBV with percentages $(92 \%)$ and the least used regimens were $\mathrm{PAR} / \mathrm{OMP} / \mathrm{RBV}$ and SOF/SIM respectively. Treatment duration was 12 weeks in $84 \%$ of patients and 24 weeks in $16 \%$ of patients. Regarding the BMI difference between pre and post treatment there was a significant statistical difference $(\mathrm{p}=0.03)$ in the form of increase in BMI post treatment $(28.68 \pm 5.35 \mathrm{Vs} 28.18 \pm 4.55$ pretreatment $)$ as shown in figure 1 . There was no significant difference in BMI $(\mathrm{P}=0.59)$ between ultrasonography normally viewed liver (143 patients) and cirrhotic group (21 patients) $(2.07 \pm 11.43 \mathrm{Vs}$ $0.18 \pm 10.41)$. Also there was no significant difference in BMI between both of treatment naïve and experienced patients $(1.39 \pm 11.99$ and $4.17 \pm 6.11)$ respectively with $\mathrm{P}=0.12$.
Conclusion: Treatment of chronic hepatitis $\mathrm{C}$ with direct acting antivirals can lead to increased body mass index. Further studies are needed to explore if this effect is secondary to treatment with direct acting antivirals or secondary to improvement of liver functions and life style of treated patients.

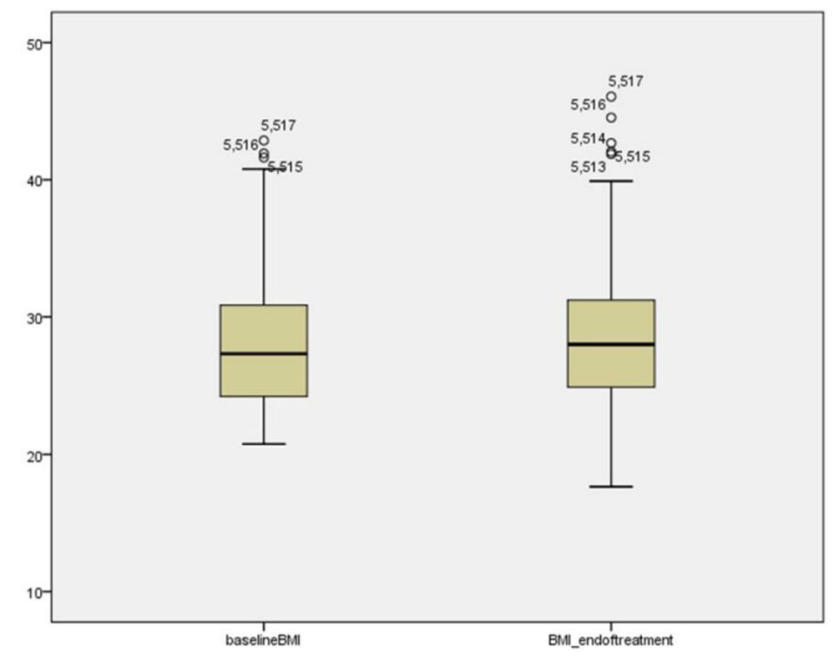

$H C V-112$

Circulating microRNA 17 host gene protein and hepatic expression of PTEN and NF-kB in patients with chronic hepatitis $C$ virus infection: relation to hepatic steatosis, inflammation and fibrosis

$\underline{H o d a ~ E l ~ A g g a n ~}^{1}$, Sabah Mahmoud ${ }^{1}$, Nevine El Deeb ${ }^{1}$, Ehab Hassona $^{1}$, Sally El Demiry ${ }^{1}$

${ }^{1}$ Faculty of Medicine, University of Alexandria, Egypt

Background: The microRNA (miR)-17 92 cluster regulates various biological processes and its host gene encodes for a 70 amino acid polypeptide known as miR-17 host gene $(M I R 17 H G)$ protein. The present study was designed to evaluate circulating $M I R 17 H G$ protein and hepatic expression of phosphatase and tensin homolog (PTEN) and nuclear factor kappa-B (NF-kB) in patients with chronic hepatitis $\mathrm{C}$ virus (HCV) infection in relation to hepatic steatosis, inflammation and fibrosis.

Method: Thirty treatment-naïve patients with chronic HCV infection [18 patients with chronic hepatitis $\mathrm{C}$ (CHC) and 12 patients with cirrhosis] and 15 healthy subjects were included in the study. Quantitative determination of plasma levels of $M I R 17 H G$ protein was performed using sandwich enzyme immunoassay. Core liver biopsies obtained from HCV-infected patients were assessed for METAVIR histological activity grade and fibrosis stage and for grade of steatosis. Immunohistochemical staining of liver specimens was done using anti-human monoclonal antibodies against PTEN and NF-kB.

Result: Plasma MIR17HG protein levels showed a significant increase in patients with chronic $\mathrm{HCV}$ infection compared with healthy subjects $(\mathrm{P}=0.012)$ and in patients with cirrhosis compared with patients with $\mathrm{CHC}(\mathrm{P}<0.001)$. The plasma $M I R 17 H G$ protein levels were inversely correlated with hepatic PTEN expression and positively correlated with serum levels of aminotransferases, METAVIR histological activity grade and fibrosis stage, steatosis grade and hepatic NF-kB expression $(\mathrm{P}<0.05)$. By plotting a receiver-operating characteristic (ROC) curve, the sensitivity and specificity of plasma $M I R 17 H G$ protein levels in discriminating patients with cirrhosis from patients with $\mathrm{CHC}$ were $100 \%$ and $88.9 \%$ 
respectively at a cut-off level of $45.5 \mathrm{pg} / \mathrm{ml}(\mathrm{AUC}=0.995)$ and in discriminating patients with early fibrosis (METAVIR F1 or F2) from patients with advanced fibrosis (METAVIR F3 or F4) were $93.8 \%$ and $92.9 \%$ respectively at a cut-off level of $40.3 \mathrm{pg} / \mathrm{ml}($ AUC $=0.978)$. The hepatic PTEN expression showed inverse correlations with serum gamma glutamyl transpeptidase levels, METAVIR fibrosis stage, steatosis grade and hepatic NF-kB expression $(\mathrm{P}<0.05)$.

Conclusion: Activation of the miR-17 92 cluster may play an important role in the pathogenesis of $\mathrm{HCV}$-related liver injury via PTEN inhibition and NF-kB activation. Circulating $M I R 17 H G$ protein could be a useful biomarker for hepatic fibrosis.

\section{$H C V-113$}

Cost-effectiveness of generic pan-genotypic sofosbuvir/valpatasvir versus sofosbuvir plus daclatasvir/ledipasvir for the treatment of chronic hepatitis $\mathbf{C}$ virus infection in India

\section{Rakesh Aggarwal $^{1}$, Amit Goel ${ }^{1}$, Qiushi Chen ${ }^{2}$, Jagpreet Chhatwal ${ }^{2}$}

${ }^{1}$ Sanjay Gandhi Postgraduate Institute of Medical Sciences (SGPGI), India; ${ }^{2}$ Massachusetts General Hospital, United States

Background: Treatment of HCV infection with low-cost 'generic' direct-acting antivirals (DAAs) available in India and other developing countries needs determination of HCV genotype ('genotypedependent' regimens). Generic velpatasvir, a DAA that obviates the need for genotype determination ('pan-genotype' regimen) recently became available but is costlier. We therefore evaluated the costeffectiveness of 'genotype-dependent' versus 'pan-genotype' DAA treatments in India.

Method: A previously-validated microsimulation model, adapted to Indian population, was used to compare the costs and long-term outcomes of three scenarios: no treatment, and treatment with genotype dependent and pan-genotype regimens. Input parameters were derived from literature. Using a payer's perspective and life-time time horizon, quality-adjusted life years (QALYs), total costs, and incremental cost-effectiveness ratio (ICER) were calculated. Both deterministic and probabilistic sensitivity analyses were also conducted.

Result: At the current price (US\$ 223 for 4 weeks), 'pan-genotype' regimen was cost-saving compared to no treatment. Compared with 'genotype-dependent' regimens, it increased QALYs by 0.07 and increased costs by US\$231, but was deemed cost-effective with an ICER of US\$ 3130 per QALY gained. At the reduced price of US\$ 145 , the 'pan-genotype' regimen will become cost-neutral compared with 'genotype-dependent' regimens (at the current price of US\$100 for 4 weeks); this threshold varied with the cost of 'genotype-dependent' regimens.

Conclusion: At current prices, velpatasvir-based 'pan-genotype' regimen is cost-effective for HCV treatment in India where 'generic' drugs are available. A reduction in the prices of 'pan-genotype' regimen has the potential to make its use cost-saving, while simplifying treatment in community-level programs aimed at $\mathrm{HCV}$ elimination
Fig 1: State-transition model showing the natural history of hepatitis $\mathrm{C}$ virus infection, and used to compare the different treatment strategies.

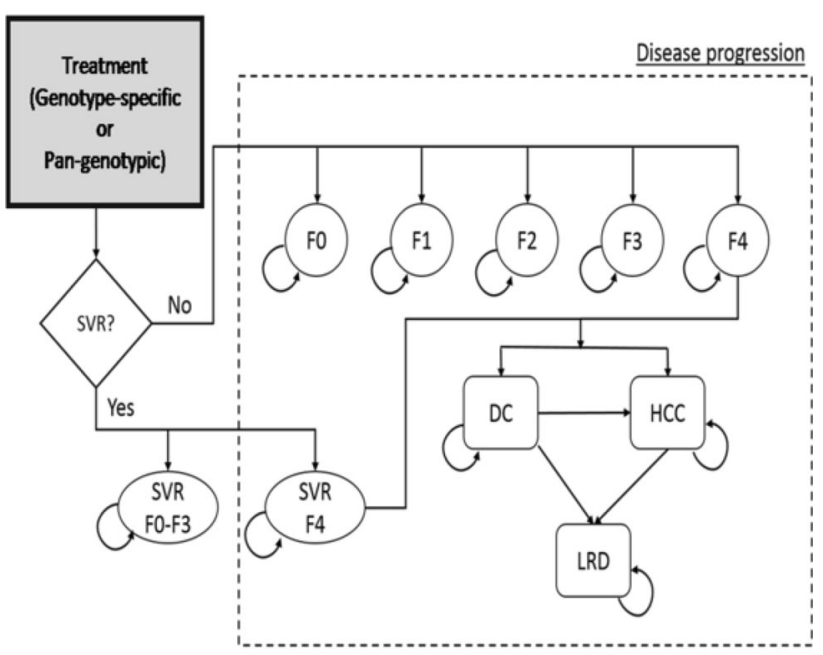

$\mathrm{DC}=$ decompensated cinhosis; $\mathrm{HCC}=$ hepatocellular carcinoma; $\mathrm{HCV}=$ hepatitis $\mathrm{C}$ virus; $L R D=$ liver-related death; $S V R=$ sustained virologic response.

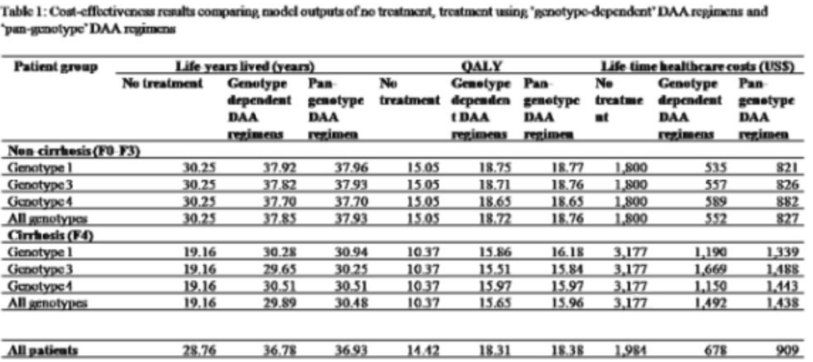

$H C V-114$

Effect of sustained viral response on the regression of fibrosis and portal hypertension in cirrhotic $\mathrm{HCV}$ patients treated with oral antiviral therapy. Punit Mehrotra* Pulkit Mehrotra** *Lucknow Gastroenterology Centre **SRMC Chennai

\section{Punit Mehrotra ${ }^{1}$}

${ }^{1}$ Lucknow Gastroenterology Centre, India

Background and Aims: There is very little Indian data on regression of liver fibrosis (stiffness) and portal hypertension (PH) and their effect on natural history of compensated hepatitis $\mathrm{C}$ virus (HCV)related cirrhosis following sustained virologic response (SVR) by currently available oral antiviral medication. Our aim was to study effect of SVR on child score, variceal status and stiffness score based on fibroscan.

Method: Twenty one patients with compensated HCV genotype 3-related cirrhosis with or without ascites and variceal bleed with Child pugh score $\mathrm{B}$ or $\mathrm{C}$ were given combination of sofosbuvir and daclatasvir without ribavirin for six months. All patients were assessed for Quality of life score, complete liver profile, fibroscan and endoscopy at end of therapy and on follow up at three months and eight months after end of last dose of therapy 
Result: All these patients had Fibroscan before the start of therapy (mean score of $24.6 \mathrm{Kpa}$ ) nad endoscopy for variceal grading based on Sarin's classification, At the end of therapy all the cases achieved SVR. The variceal grading had significant downgrading or disappearance either at the end of therapy or six to eight months of follow up. Fibroscan stiffness score showed significant reduction (24.6 vs $10.4 \mathrm{KpA} ; \mathrm{p}<0.005)$. Quality of life and clinical symptoms were significantly better at end of therapy and on follow up

Conclusion: Complete clinical response, variceal regression and regression of stiffness based on fibroscan following oral antiviral therapy in compensated HCV cirrhosis is achievable, and may indicate regression of fibrosis and decrease in portal pressure The improvement in quality of life following therapy is another milestone in treatment of $\mathrm{HCV}$

\section{$H C V-115$}

Efficacy and safety of sofosbuvir-based regimens for chinese patients with chronic hepatitis C: a 48-week real-world, observational study

\section{Lichang Chen ${ }^{1}$, Jie Lu ${ }^{1}$, Huijuan Zhou ${ }^{1}$, Ruidong Mo ${ }^{1}$, Peipei} Ren $^{1}$, Zhujun Cao', Yuhan Liu', Tianhui Zhou ${ }^{1}$, Qing Guo', Hui Wang $^{1}$, Wei Cai ${ }^{1}$, Qing Xie ${ }^{1}$

${ }^{1}$ Rui Jin Hospital Affiliated to Shanghai Jiao Tong University School of Medicine, China

Background: The prominent efficacy of direct acting antiviral (DAA) treatment with sofosbuvir-containing regimens for patients with chronic hepatitis $\mathrm{C}$ from other countries and regions in the world having been verified, whereas a very limited data of DAA in HCV treatment notably with medium- to long-term follow-up from China is available. We aimed to retrospective evaluate the real-world sustained virologic responses of 48 weeks after treatment (SVR48) of sofosbuvir-based DAA therapy in a real-world, observational study.

Method: A total of 88 patients with chronic HCV infection who were intolerant to, or failed to PegIFN plus ribavirin therapy were included. They were treated with different sofosbuvir-based DAAs combinations (sofosbuvir \pm ledipasvir/daclatasvir/ribavirin in treatment of 12 or 24 weeks) according to the access of the drugs and the liver condition. Quantitative HCV RNA was assessed prior to treatment, the end of treatment, 24 weeks post-treatment (SVR24) and 48 weeks post-treatment (SVR48) by COBAS TaqMan HCV Test 2.0 (LLO$\mathrm{Q}=15 \mathrm{IU})$. Biochemistry tests and severe adverse outcomes (hepatocellular carcinoma, orthotopic liver transplantation or death) as well as safety were recorded.

Result: Baseline characteristics revealed that $61 \%$ were HCV genotype $1 \mathrm{~b}$ infection within $38 \%$ on sofosbuvir+ribavirin and $34 \%$ on sofosbuvir+ledipasvir \pm ribavirin. SVR24s were achieved by $98 \%$ ( 86 in 88) and one patient died by the end of follow-up without any further recurrence or reinfection. No correlation of SVR24 between GT1b and non-GT1b was observed (1b vs. non-1b, p >0.10). Similarly, SVR24 was not affected by baseline viral load and the stage of liver fibrosis (viral load of high vs. low, $\mathrm{p}>0.25$, or cirrhosis vs. noncirrhosis, $\mathrm{p}>0.25$ ). No hepatocellular carcinoma or orthotopic liver transplantation observed but a death, mortality was independent on whether SVR24 was attained but correlated with baseline decompensated state (decompensation vs. compensation, $\mathrm{p}<0.05$ ).

Conclusion: High rates of SVR48 of regimens containing sofosbuvir achieved in Chinese HCV patients who were ineligible for PEG-IFN and RBV combination therapy with safety needing to be confirmed and complemented with further analyses, but in decompensated patients we should try to treat as early as possible to avoid its degeneration.
$H C V-116$

\section{Elimination of hepatitis $\mathrm{C}$ can be possible only with differentiation by decentralization and integration of testing pathways}

\section{Veronica Epah NKIE}

${ }^{1}$ FIND Geneva, United States

Background: HCV is a major cause of chronic liver disease and death and is prevalent in a variety of populations, including Blood Transfusion Recipients (BTR), People Who Inject Drugs (PWIDs), Men who have Sex with Men (MSM), and in mothers and/or their newborn children. The availability of pan-genotypic Direct Acting Antiretrovirals (DAAs) has put $\mathrm{HCV}$ in the global health spotlight and on track towards elimination. As such it is imperative that effective diagnostic testing and treatment options be made available to ensure timely diagnosis and treatment.

Method: Barriers that affect accurate HCV diagnosis in LMICs include the lack of testing at primary care sites, lack of trained personnel to perform complex testing, high costs of testing assays, and a lack of optimal service delivery structure to ensure access to proper patient care and treatment. To address these challenges, the WHO recommends a 3-step algorithm to effective and accurate treatment of HCV: Screening, Diagnosis and Monitoring.

Result: Many LMICs have three levels of patient care: L1, L2, and L3. Patients in rural areas have to travel out to L3 sites, usually located in the urban towns, for testing and treatment. This leads to delay in diagnosis and treatment of Hepatitis C. Successful following of this algorithm will involve differentiation of testing in LMICs. Differentiation in this case implies offering the same testing algorithm in various ways to meet the needs of different populations. This will occur by decentralizing testing to L1 and L2 sites, and integrating $\mathrm{HCV}$ testing into already existing platforms for testing of other infectious diseases.

The table below describes various scenarios for HCV testing decentralization and integration. The first is the current standard of care in many LMICs. The subsequent scenarios depict possible ways by which patient access to testing can be improved depending on available resources. The use of Dried Blood Spot (DBS) testing in these scenarios will facilitate sample collection and transportation, decrease patient travel to L3 sites and allow for diagnosis and monitoring at lower level health facilities.

Hepatitis $\mathrm{C}$ is a prominent and yet very underreported worldwide infection. This is influenced in part by a lack of awareness of individuals about the disease, and a lack of effective testing and monitoring strategies. Decentralization and integration of testing will improve $\mathrm{HCV}$ diagnosis and treatment. However, for effective elimination of the virus, decentralization and integration of treatment must occur as well. Currently most Direct Acting Antiretrovirals (DAAs) for HCV are located in L2 and L3 sites, and sold at very high costs. Making DAAs available at L1 sites will drastically improve efforts for HCV eradication.

Conclusion: Differentiation of testing by differentiation and integration, including the use of DBS testing and availability of DAAs, will greatly improve global efforts to eliminate HCV by the WHO's 2030 target date. 


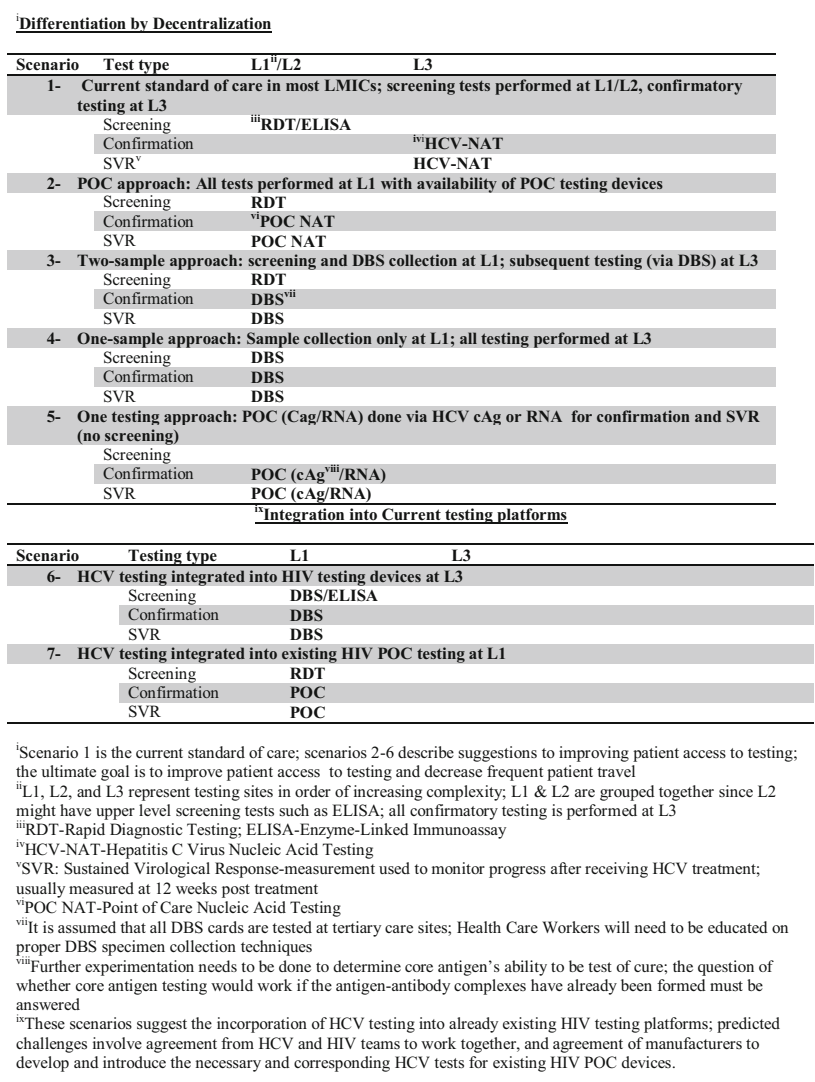

$H C V-117$

$\mathrm{HBV}$ reactivation is rare in chronic hepatitis $\mathrm{C}$ patients with resolved $\mathrm{HBV}$ receiving direct acting antivirals: a systematic review and meta-analysis in cohort studies

George Lau ${ }^{1}$, Guofeng Chen ${ }^{2}$, Jing Chen ${ }^{5}$, Qing Shao', Dong $\mathrm{Ji}^{2}$, Fan $\mathrm{Li}^{2}$, Bing $\mathrm{Li}^{2}$, Tingting $\mathrm{Wu}^{5}$, Yudong Wang ${ }^{4}$, Vanessa $\mathrm{Wu}^{5}$, George $\mathrm{Lau}^{3}$

${ }^{1}$ Humanity and Health Medical Group, Hong Kong; ${ }^{2} 302$ Hospital, Beijing, 100039, China, China; ${ }^{3}$ Humanity \& Health Medical Centre, Hong Kong, Hong Kong SAR, China, Hong Kong; ${ }^{4}$ Humanity and Health Research Centre, Hong Kong SAR, China, China; ${ }^{5}$ Humanity and Health Research Centre, Hong Kong SAR, China, Hong Kong

Background: The increased global awareness of hepatitis $\mathrm{B}$ reactivation $(\mathrm{HBVr})$ in chronic hepatitis $\mathrm{C}(\mathrm{CHC})$ patients coinfected with HBV treated with pan-oral direct acting antivirals (DAAs) had urged the update of AASLD/EASL guidelines as well as add-in the black boxed warning of HBVr into the prescription information of DAAs. We performed a systematic review and meta-analysis to compare the rate of $\mathrm{HBVr}$ in $\mathrm{CHC}$ patients coinfected with chronic HBV infection (hepatitis B surface antigen [HBsAg] positive) and resolved $\mathrm{HBV}$ infection ( $\mathrm{HBsAg}$ negative with positive $\mathrm{HBcAb}$ ).

Method: A systematic search of English literature from database inception to 31 Oct 2017 in Pubmed, Embase, Ovid, and Cochrane databases was conducted in Nov 2017. Only cohort study and manuscript in English were included. A meta-analysis was conducted using a random effects model to assess the HBVr (primary outcome, defined by authors) and hepatitis due to $\mathrm{HBVr}$ (secondary outcome, defined by authors). A meta-regression was conducted to identify sources of between-study heterogeneity in the estimates of the reactivation rate. Publication bias was assessed using the Egger test. Analysis was done by Stata 13 .

Result: Of the 839 CHC patients coinfected with HBV from 9 cohort studies, 188 were with positive $\mathrm{HBsAg}$ and 651 were with resolved $\mathrm{HBV}$. The definition of $\mathrm{HBVr}$ and related hepatitis varied by studies. The pooled incidence rate of $\mathrm{HBVr}$ was significantly higher in $\mathrm{CHC}$ coinfected with current HBV $(23.9 \%, 95 \%$ confidence interval $[\mathrm{CI}]$ 13.4-36.0\%, $\mathrm{p}=0.08$ for between-study heterogeneity) than in $\mathrm{CHC}$ coinfected with resolved HBV $(0.5 \%, 95 \% \mathrm{CI}: 0-1.9 \%, \mathrm{p}=0.06$ for between-study heterogeneity; $\mathrm{p}<0.001$ for between-group heterogeneity). In the meantime, the pooled incidence rate of hepatitis due to $\mathrm{HBV}$ reactivation was significantly higher in $\mathrm{CHC}$ coinfected with current HBV $(3.1 \%, 95 \% \mathrm{CI} 0.1-8.5 \%, \mathrm{p}=0.25$ for between-study heterogeneity) than in $\mathrm{CHC}$ coinfected with resolved $\mathrm{HBV}(0 \%$, $95 \%$ CI $0-0.3 \%, p=0.99$ for between-study heterogeneity; $\mathrm{p}<0.001$ for between-group heterogeneity). No publication bias was presented in the estimates of $\operatorname{HBVr}(\mathrm{p}=0.31)$ and hepatitis due to $\operatorname{HBVr}(\mathrm{p}=0.30)$. Baseline characteristics (e.g. age, sex, genotype, proportion of undetectable HBV DNA) were not associated with $\mathrm{HBVr}$ in both patients with positive HBsAg and resolved HBV (all p>0.05).

Conclusion: Rates of HBV reactivation and hepatitis due to HBV reactivation are rare in $\mathrm{CHC}$ patients with resolved $\mathrm{HBV}$ treated with pan-oral DAAs. No predictor was found for $\mathrm{HBV}$ reactivation in $\mathrm{CHC}$ with resolved HBV receiving pan-oral DAAs.

\section{$H C V-118$}

Improving cascade of hepatitis $\mathrm{C}$ care through linkage to $\mathrm{C}$ care program [LTC] in the PWID population

\section{Imtiaz Alam $^{1}$, Zohha Tariq ${ }^{1}$, Hong Bui Bui ${ }^{1}$ \\ ${ }^{1}$ Austin Hepatitis Center, United States}

Background: There is an emerging epidemic of people who inject drugs (PWID), contract the hepatitis C virus (HCV), and fail to seek treatment. We must work through environments such as methadone clinics and drug rehabilitation centers,. Our aim is to screen the PWID population and increase linkage to care. To achieve this goal, we plan to utilize our online patient database management system (PDMS) with our Substance Abuse Treatment Center partners.

Method: Longitudinal prospective cohort study with HCV screening and utilization of a HIPPA compliant online patient database management system (PDMS) (http://www.linkagetocare.com). A centrally located Linkage to Care Specialist (LTCS) is notified immediately when an individual's information is entered in the system by the treatment center. The LTCS educates the individual and proceeds to link that person to care.

Result: January 2017 - August 2017, 461 PWID population were screened at 6 facilities; 163 (36\%) were HCV Antibody positive; of the 162 RNA tests performed $90(56 \%)$ were HCV RNA positive; $75 \%$ patients were uninsured; Median age 37 years $(79 \% \leq 50$ years); $63 \%$ males. 85 (94\%) have been linked to care by LTCS; 62 (73\%) were awaiting further lab results; while $16(18 \%)$ patients made it to their first appointment; $63 \%$ were seen in office and $38 \%$ were seen through telemedicine; 5 patients initiated therapy; and 7 patients at two methadone clinics have not approved the LTCS to contact the patient. Patients were connected with an LTCS within 2 days after referral. On average, patients were contacted twice before scheduling their first appointment.

Conclusion: High prevalence of $\mathrm{HCV}$ in PWID treatment centers. $79 \%$ of HCV PWID patients age $\leq 50$ years. $94 \%$ of patients have been educated about HCV and have been linked to care. 
$H C V-119$

Multi-target DAA therapy is associated with decline in immunosuppressive $T$ regulatory cells and myeloid derived suppressor cells in HIV/HCV co-infected patients

\section{Shikha Shrivastava ${ }^{1}$, Manasa Bhatta ${ }^{1}$, Arshi Khanam ${ }^{1}$, Shyam Kottilil $^{1}$, Eleanor Wilson ${ }^{1}$ \\ ${ }^{1}$ Institute of Human Virology, United States}

Background: HIV/HCV co-infection is associated with expansion of immunosuppressive myeloid derived suppressor cells (MDSCs) and T regulatory cells (T regs) leading to accelerated fibrogenesis. MDSCs are heterogeneous subset of regulatory Antigen presenting cells (APCs) that inhibits $\mathrm{T}$ cell responses. MDSCs are divided into two categories: monocytic MDSCs (M-MDSCs) and granulocytic MDSCs (G-MDSCs), each of which suppresses immune responses using different mechanisms. MDSCs have been shown to suppress T-cell responses by way of numerous mechanisms including expression of inhibitory cell surface molecules, production of regulatory cytokines, the metabolism of arginine through activation of arginase-1 (Arg-1), production of nitric oxide synthase (i-NOS) and up regulation of (indoleamine 2,3-dioxygenase (IDO) and reactive oxygen species (ROS). The advent of direct acting antivirals to treat $\mathrm{HCV}$ provided us a unique opportunity to explore the impact of different DAAs combinations in normalization of immune responses in order to redefine the better treatment paradigm for co-infected patients. Therefore, we aimed to assess the impact of different combination DAA treatment regimens on the frequencies and functions of immunosuppressive MDSCs and T regs in HIV/HCV co-infected patients.

Method: We analyzed PBMC, at baseline and at the time of sustained viral response $(\mathrm{SVR})$, from subjects (total, $\mathrm{N}=22$ ) treated with three different combination DAA regimens: daclatasvir (DCV) and asunaprevir (ASV) for 24 weeks (CONQUER 2 DAA), DCV/ASV/ beclabuvir (BCV) for 12 weeks (CONQUER 3 DAA), and sofosbuvir (SOF) and ledipasvir (LDV) for 12 weeks (ERADICATE study). Frequencies of Tregs (Foxp3+CD25hiCD4+) and myeloid-derived suppressor cells (M-MDSCs: HLA-DRloCD33+CD11b+ and G-MDSCs: HLA-DRloCD33+CD11b+CD15+CD66b+) were analyzed by multiparametric flow cytometry. Further functional characteristics of MDSCs were analyzed in terms of expression of immunosuppressive mediators or factors through which MDSCs exert their immunosuppressive or regulatory functions such as i-NOS, Arg1 and IDO. Statistical analyses were conducted by Wilcoxon matched-pair Signed Rank test, with $\mathrm{p}$ values $<0.05$ considered to be significant.

Result: Overall, we observed a significant decline in the frequencies of Tregs $(\mathrm{P}=0.0005)$, monocytic-MDSCs $(\mathrm{P}=0.005)$ and granulocyticMDSCs $(\mathrm{P}=0.01)$ following DAA therapy in $\mathrm{HIV} / \mathrm{HCV}$ co-infected patients. Further we also noted significant decline in the expression of molecules that are implicated in immunoregulatory functions of MDSCs such as Arg-1 ( $\mathrm{P}=0.003)$, i-NOS ( $\mathrm{P}=0.005)$ and IDO $(\mathrm{P}=0.009)$.

Conclusion: We demonstrate here in HIV/HCV co-infected patients, sustained virological response is associated with decline in immunosuppressive T-regulatory cells and Myeloid Derived Suppressor cells following DAA therapy.
HCV-120

Outcome of chronic hepatitis $\mathrm{C}$ patients: experience from 10 years of follow up

Imran Hasanoglu ${ }^{1}$, Rahmet Guner ${ }^{1}$, Imran Hasanoglu ${ }^{1}$, Ayse Kaya Kalem $^{1}$, Bircan Kayaaslan ${ }^{1}$, Turan Buzgan ${ }^{1}$, Zeynep Unsal $^{1}$, Mehmet A Tasyaran ${ }^{1}$

${ }^{1}$ Ankara Yildirim Beyazit University School of Medicine, Turkey

Background: Chronic hepatitis $\mathrm{C}$ (CHC) affects many people all over the world and can cause liver failure, cirrhosis, and hepatocellular cancer. In the last decade, rapid evolution of HCV therapeutics brought great hopes with high cure rates. Direct acting agents (DAAs) which have low side-effect profiles, high response rates replaced interferon and ribavirin treatment.

Method: We evaluated our patients with CHC between 2007-2017 retrospectively. Outcomes of the patients are recorded from follow up charts.

Result: A total of 209 patients are included in the study. Fifty-seven percent of the patients were female. Mean age was 56 (range: 23-81) years. Four patients $(2 \%)$ had cirrhosis. Twenty-five percent of the patients had at least 1 co-morbid disease. Mean duration of follow up was 3.5 years. Genotype distribution of the patients were as follows: $90 \%$ genotype 1 (1a: $95 \%$ ), $4 \%$ genotype $4,3 \%$ genotype 2 , and $3 \%$ genotype 3. Among patients with histopathological evaluation, median histology activity index and fibrosis scores were 7 (0-18) and $5(0-$ 5), respectively. Mean HCV RNA level was 4.5x 106 copy/ml. Outcomes of the patients are summarized in Figure 1.

Conclusion: After IFN and ribavirin treatment, which was a challenge for both the clinician and the patients, new DAAs have emerged as rescuers with high response rates, ease of use and low side effect profiles. While many patients have been able to cure with these new therapies, many still remain as candidates.

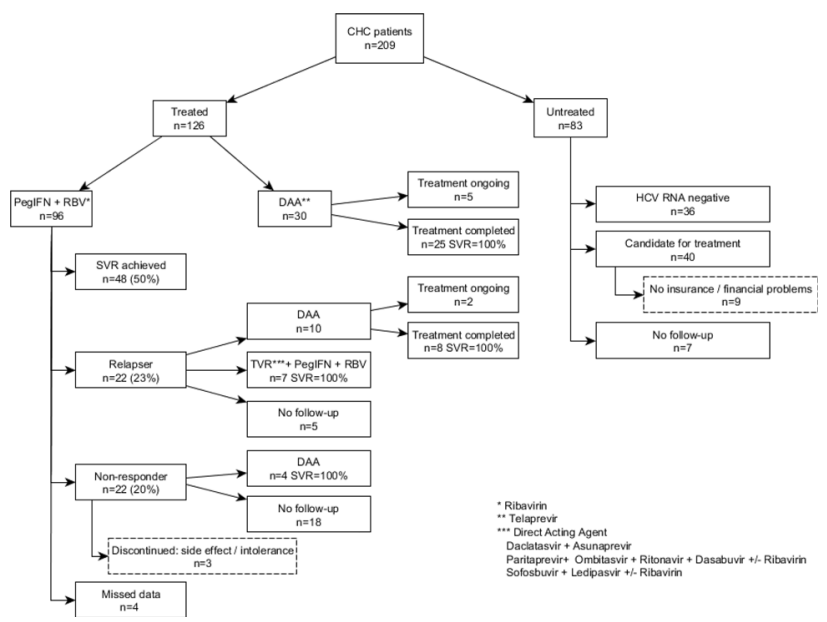

$H C V-121$

Potential drug-drug interactions in chronic hepatitis $\mathrm{C}$ patients with direct-acting antiviral agents

Chun Ming Hong ${ }^{1}$, Chih Hsun Tai ${ }^{1}$, Shu Wen Lin ${ }^{3}$, Jia Horng $\mathrm{KaO}^{2}$

${ }^{1}$ National Taiwan University Hospital, Taiwan; ${ }^{2}$ National Taiwan University, College of Medicine, Taiwan; ${ }^{3}$ National Taiwan

University, Taiwan 
Background: Treatment of chronic hepatitis $\mathrm{C}$ has dramatically evolved since the introduction of direct-acting antivirals (DAAs). DAAs show high sustained virologic response and satisfactory safety profiles. However, several DAAs would interact with common medications, and drug-drug interactions (DDIs) may lead to loss of effectiveness and even an increased risk of toxicity. The aim of our study is to detect the number and severity of potential DDIs in patients with DAAs.

Method: The CHC patients who received DAAs therapy in our hospital from April 1, 2015 to March 31, 2016 were enrolled. Demographic and clinical data including age, sex, HCV genotype, DAAs regimens, treatment duration, concurrent medications, and rate of SVR12 were reviewed from electronic medical records. Potential DDIs were evaluated through different databases of clinical pharmacy including Micromedex, Lexicomp, and Liverpool HEP Interactions. DDIs were classified into 4 categories: no interaction, possible or minor, may adjust dose or monitor, and avoid concurrent use.

Result: A total of 196 patients (82 male, 41.8\%; average age, 65.3 years) who received DAAs therapy were recruited. Among these patients, $99(50.5 \%)$ were cirrhotics and $74(37.8 \%)$ had been treated with interferon and ribavirin. 137 patients $(69.9 \%)$ had HCV genotype 1 infection. The most common DAAs regimen was ledipasvir/sofosbuvir with or without ribavirin (120 patients, $61.2 \%$ ). Only $53(27.0 \%)$ patients had no concurrent medications. Overall, 871 medications were reviewed and 148 potential DDIs were identified in $74(37.8 \%)$ patients. The top three common medications interacting with DAAs were amlodipine, fexofenadine, and magnesium oxide. Among DAA regimens, $36.4 \%$ of patients receiving paritaprevir/ritonavir/ombitasvir/dasabuvir had significant DDIs, followed by daclatasvir/asunaprevir (33.3\%), ledipasvir/sofosbuvir (25.0\%), and sofosbuvir $(0 \%)$. With regard to therapeutic effectiveness, the SVR12 rates were higher than $95 \%$ in general except daclatasvir/asunaprevir $(85.0 \%)$.

Conclusion: Potential DDIs are not uncommon in $\mathrm{CHC}$ patients with DAAs; however, most of them are manageable. Before initiating DAA therapy, DDIs with concurrent medications should be carefully assessed to avoid potential harm.

\section{$H C V-122$}

Real-world efficacy of sofosbuvir and daclatasvir, with and without ribavirin for patients with chronic hepatitis $\mathbf{C}$ in Southeast Asia

Sakkarin Chirapongsathorn $^{1}$, Veerasak Wongpaitoon ${ }^{2}$, Satien Techapaitoon $^{2}$, Patamaporn Phanubol ${ }^{2}$, Charkaphan Osangthamnont $^{2}$, Rujapong Sukhabote ${ }^{2}$, Sinn Anuras ${ }^{2}$

${ }^{1}$ Phramongkutklao Hospital, College of Medicine, Thailand;

${ }^{2}$ Bumrungrad International Hospital, Thailand

Background: Efficacy and well-tolerability of direct antiviral agents are integral to treatment for chronic hepatitis C. However, controversy exists regarding applicability of clinical trials to real world practice, especially for $\mathrm{HCV}$ genotype 6 . We report virologic responses of patients with $\mathrm{HCV}$ infection receiving sofosbuvir (SOF) and daclatasvir (DCV) with or without ribavirin (RBV) therapy for 12 weeks.

Method: Approval was obtained from the Bumrungrad Institutional Review Board. Treatment naïve and treatment experienced (TE) HCV all GT patients, during March 2016 through January 2017 who started SOF/DCV-based therapy were consecutive enrolled by dedicated nurse and analyzed on an intent-to-treat basis.

Result: The efficacy population $(\mathrm{N}=211)$ infected with HCV GT1 (42\%), GT6 (29\%), GT3 (19\%), GT2 (5\%), and GT4 (3\%). All patients started SOF/DCV-based therapy; 135 started SOF/DCV/RBV therapy and 76 started SOF/DCV therapy for 12 weeks. Respective baseline characteristics were median age (57 with IQR 48, 68 years), female $(125,59 \%)$, TE $(136,64 \%)$, cirrhotic $(111,53 \%)$. We report EOT, SVR12 and SVR24 rates of $100 \%, 98 \%$ and $86 \%$ for patients receiving $\mathrm{SOF} / \mathrm{DCV}$ with or without $\mathrm{RBV}$. No any serious adverse side effect was reported.

Conclusion: $\mathrm{DCV}+\mathrm{SOF} \pm \mathrm{RBV}$ achieved high SVR12 in all GT and was well tolerated in this large real-world cohort of $\mathrm{HCV}$ patients in Southeast Asia.

\begin{tabular}{|c|c|}
\hline Characteristics & Overall, $N=211$ \\
\hline Age & $57(48,68)$ \\
\hline Female & $125(59 \%)$ \\
\hline Treatment experienced & $136(64 \%)$ \\
\hline Cirrhotic & $111(53 \%)$ \\
\hline \multicolumn{2}{|l|}{ HCV genotype } \\
\hline - $\mathrm{GT} 1$ & $88(42 \%)$ \\
\hline - $\mathrm{GT} 2$ & $61(29 \%)$ \\
\hline - $\mathrm{GT3}$ & $40(19 \%)$ \\
\hline - others & $22(10 \%)$ \\
\hline SOF/DCV/RBV therapy & $135(64 \%)$ \\
\hline SOF/DCV therapy & $76(36 \%)$ \\
\hline
\end{tabular}

Chirapongsathorn, et al. APASL2018
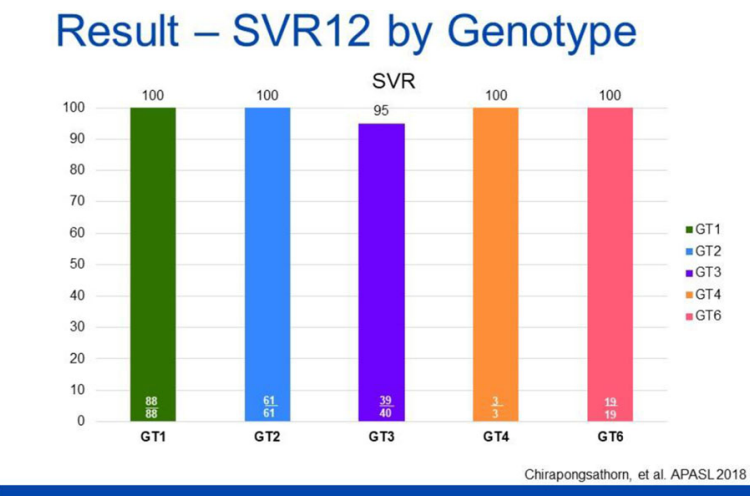

$H C V-123$

Real-world sustained virologic response rates (SVR12) with interferon (IFN)-free direct acting antiviral (DAA) therapy in East Asia-results from REAL-C (real-world effectiveness from the Asia Liver consortium for chronic hepatitis C)

Mindie Nguyen ${ }^{1}$, Mindie H Nguyen ${ }^{1}$, Norihiro Furusyo ${ }^{19}$, Dae Won Jun', Ming Lung Yu'14, Jia Horng Kao ${ }^{22}$, Masaru Enomoto $^{24}$, Eiichi Ogawa ${ }^{19}$, Etsuko Ilio ${ }^{20}$, Chen Hua Liu ${ }^{22}$, Akihiro Tamori ${ }^{24}$, Chia Yen Dai ${ }^{14}$, Sang Bong $\mathrm{Ahn}^{3}$, Koichi Azuma $^{16}$, Wan Long Chuang ${ }^{14}$, Kazufumi Dohmen ${ }^{2}$, Nobuhiko Higashi $^{27}$, Chung Feng Huang ${ }^{14}$, Jae Yoon Jeong ${ }^{8}$, Jang Han Jung $^{6}$, Eiji Kajiwara ${ }^{13}$, Masaki Kato ${ }^{18}$, Akira Kawano ${ }^{15}$, Toshimasa Koyanagi ${ }^{4}$, Seung Ha Park ${ }^{11}$, Takeaki Satoh ${ }^{21}$, Shinji Shimoda ${ }^{18}$, Do Seon Song ${ }^{28}$, Kazuhiro Takahashi ${ }^{7}$, Ming Lun Yeh $^{14}$, Eileen Y Yoon ${ }^{12}$, Jee Fu Huang ${ }^{14}$, Yoshiyuki Ueno ${ }^{30}$, Hwai 
I Yang ${ }^{23}$, Dong Hyun Lee ${ }^{5}$, Grace Wong ${ }^{29}$, Jun Hayashi ${ }^{10}$, Hideyuki Nomura $^{26}$, Makoto Nakamuta ${ }^{17}$, Hiroaki Haga ${ }^{30}$, Mi Jung Jun ${ }^{5}$, Mei Hsuan Lee ${ }^{23}$, Yuichiro Eguchi ${ }^{25}$, Hirokazu Takahashi $^{25}$, Shinji Iwane ${ }^{25}$, Sally Ann Tran', Linda Henry ${ }^{1}$, Yasuhito Tanaka ${ }^{20}$, REAL C Investigators ${ }^{1}$

${ }^{1}$ Stanford University Medical Center, United States; ${ }^{10}$ Haradoi Hospital, Japan; ${ }^{11}$ Inje University Haeundae Paik Hospital, Korea, Republic of; ${ }^{12}$ Inje University Sanggye Paik Hospital, Korea, Republic of; ${ }^{13}$ Kajiwara Clinic, Japan; ${ }^{14}$ Kaohsiung Medical University Hospital, Taiwan; ${ }^{15}$ Kitakyushu Municipal Medical Center, Japan; ${ }^{16}$ Kyushu Central Hospital, Japan; ${ }^{17}$ Kyushu Medical Center, National Hospital Organization, Japan; ${ }^{18}$ Kyushu University Graduate School of Medical Sciences, Japan; ${ }^{19}$ Kyushu University Hospital, Japan; ${ }^{2}$ Chihaya Hospital, Japan; ${ }^{20}$ Nagoya City University Graduate School of Medical Sciences, Japan; ${ }^{21}$ National Hospital Organization Kokura Medical Center, Japan; ${ }^{22}$ National Taiwan University Hospital, Taiwan; ${ }^{23}$ National Yang-Ming University, Taiwan; ${ }^{24}$ Osaka City University Medical School, Japan; ${ }^{25}$ Saga University Hospital, Japan; ${ }^{26}$ Shin-Kokura Hospital, Japan; ${ }^{27}$ Steel Memorial Yawata Hospital, Japan; ${ }^{28}$ The Catholic University of Korea College of Medicine, Korea, Republic of; ${ }^{29}$ The Chinese University of Hong Kong, Hong Kong; ${ }^{3}$ Eulji University Seoul Hospital, Korea, Republic of; ${ }^{30}$ Yamagata University Faculty of Medicine, Japan; ${ }^{4}$ Fukuoka City Hospital, Japan; ${ }^{5}$ Good Gang-An Hospital, Korea, Republic of; ${ }^{6}$ Hallym University Dongtan Sacred Heart Hospital, Korea, Republic of; ${ }^{7}$ Hamanomachi Hospital, Japan; ${ }^{8}$ Hanyang University College of Medicine, Guri Hospital, Korea, Republic of; ${ }^{9}$ Hanyang University Hospital, Korea, Republic of

Background: Since their recent introduction in Asia, IFN-free DAAs have revolutionized treatment of chronic hepatitis $\mathrm{C}$ across all $\mathrm{HCV}$ genotypes. However, experience from large and diverse routine clinical practice is still limited. The aim of this study was to report real-world outcomes from a large multinational cohort of East Asian HCV patients treated with IFN-free DAAs.

Method: Data were obtained using a required case report form from the REAL-C registry of patients who were initiated on IFN-free DAA therapy in routine practice and represented 10 study centers inclusive of 30 clinical sites in Hong Kong, Japan, Korea, and Taiwan. Cirrhosis was determined by liver biopsy, noninvasive tests (elastography/fibroscan, fibrotest), or the presence of clinical, radiologic, endoscopic, laboratory evidence of cirrhosis and/or portal hypertension.

Result: A total of 3702 patients have been registered. Table 1 displays the patient characteristics. The average age was $63.6 \pm 12.8$; $17.7 \%$ had diabetes, $8.7 \%$ had chronic renal impairment, $26 \%$ had cirrhosis (5.1\% decompensated cirrhosis), and $5.4 \%$ had HCC at baseline or prior to DAA treatment initiation. The majority of patients were HCV GT1 (68.7\%), followed by HCV GT2 (30.4\%). Ten different DAA regimens were used, with the majority receiving LDV/ SOF $(43.7 \%)$, followed by SOF+RBV (27.8\%). One-third were treatment experienced ( $24.8 \%$ with prior PEG-IFN+RBV, $4.5 \%$ with prior DAA). SVR12 overall rate was $96.6 \%$. Significant decreases noted in all major liver enzymes at week 12 and 24 post treatment. No increase in creatinine noted across treatments; $3.2 \%$ stopped treatment and $13.4 \%$ had an adverse event with fatigue $(5.6 \%$ in patients treated with RBV vs. $6.4 \%$ in those treated without $R B V, p=0.61$ ) and anemia $(5.6 \%)$ the most reported. Table 2 displays SVR12 rates by cirrhosis and prior treatment status for the most commonly used DAA treatments for GT1 and GT2 patients. SVR12 rates were excellent ranging from $97.1 \%$ (95\%CI: $94.1-98.8 \%$ ) to $99.7 \%$ (95\% CI: $99.0-$ $99.9 \%$ ) for GT1 patients treated with LDV/SOF who did not have cirrhosis regardless of prior treatment history and who were treatment-naive with cirrhosis but lower in the cirrhotic treatmentexperienced group (92.2\%; 95\%CI: 86.7-95.9\%) ( $\mathrm{p}<0.0001)$. Subanalysis results for GT1b were similar, with SVR12 $99.7 \%$ for non- cirrhotic treatment-naive, $99.5 \%$ for non-cirrhotic treatment-experienced, $97.4 \%$ for cirrhotic treatment-naive, and $93.0 \%$ for cirrhotic treatment-experienced, $(\mathrm{p}<0.0001)$. For GT2 patients, SVR12 was excellent for all groups (96.8-98.0\%) except for cirrhotic treatmentexperienced patients $(\mathrm{n}=66)$ who experienced an SVR12 of $87.9 \%$ (95\%CI: $77.5-94.6 \%)(\mathrm{p}=0.002)$.

Conclusion: $\mathrm{HCV}$ cure rates were high overall in the REAL-C cohort-LDV/SOF GT1 98\%; SOF+RBV GT2 96\% except for cirrhotic, treatment-experienced patients especially in GT2, suggesting alternative therapy is needed.

\begin{tabular}{|c|c|}
\hline Baseline Demographics: Total Cohort & $\mathrm{N}=3702$ \\
\hline Male & $1581(43.3)$ \\
\hline Age & $63.6 \pm 12.8$ \\
\hline Body mass index (BMI) & $23.5 \pm 4.1$ \\
\hline \multicolumn{2}{|l|}{ Ethnicity } \\
\hline Chinese, Hong Kong and other & $52(1.4)$ \\
\hline Taiwanese & $450(12.2)$ \\
\hline Korean & $384(10.4)$ \\
\hline Japanese & $2805(75.8)$ \\
\hline Other & $8(0.2)$ \\
\hline Diabetes & $616(17.7)$ \\
\hline Chronic renal insufficiency & $283(8.7)$ \\
\hline Cirrhosis & $957(25.9)$ \\
\hline Decompensated cirrhosis & $53(5.1)$ \\
\hline Hepatocellular carcinoma (HCC) & $195(5.4)$ \\
\hline Other cancer $(n=792)$ & $79(9.6)$ \\
\hline \multicolumn{2}{|l|}{ Direct acting antiviral (DAA) regimen initiated } \\
\hline SOF+RBV (sofosbuvir+ribavirin) & $1028(27.8)$ \\
\hline LDV/SOF (ledipasvir/SOF) & $1617(43.7)$ \\
\hline 3D (paritaprevir/ritonavir, ombitasvir+dasabuvir) & $171(4.6)$ \\
\hline DCV+ASV (daclastavir+asunaprevir) & $706(19.1)$ \\
\hline SOF+DCV & $28(0.8)$ \\
\hline EBR/GZR (elbasvir+grazoprevir) & $27(0.7)$ \\
\hline GLE/PIB (glecaprevir+pibrentasvir) & $20(0.5)$ \\
\hline Other & $104(2.8)$ \\
\hline \multicolumn{2}{|l|}{ HCV genotype (GT) and subtype } \\
\hline GT 1 & $2542(68.7)$ \\
\hline Untyped & $187(5.1)$ \\
\hline 1a & $38(1.0)$ \\
\hline $1 \mathrm{~b}$ & $2317(62.6)$ \\
\hline GT2 & $1122(30.4)$ \\
\hline Untyped & $272(7.4)$ \\
\hline $2 \mathrm{a}$ & $561(15.2)$ \\
\hline $2 \mathrm{~b}$ & $251(6.8)$ \\
\hline $2 \mathrm{a} / 2 \mathrm{~b}$ & $1(0.03)$ \\
\hline $2 \mathrm{a} / 2 \mathrm{c}$ & $37(1.0)$ \\
\hline GT 3 & $8(0.2)$ \\
\hline GT 4 & $3(0.13)$ \\
\hline GT 6 & $22(0.6)$ \\
\hline Multiple GT's & $4(0.1)$ \\
\hline \multicolumn{2}{|l|}{ Prior treatment } \\
\hline IFN (interferon) +/- RBV & $154(4.3)$ \\
\hline PEG-IFN +/- RBV & $886(25.0)$ \\
\hline TVR (telaprevir)+PEG-IFN+RBV & $17(0.5)$ \\
\hline BOC (boceprevir)+PEG-IFN+RBV & $31(0.9)$ \\
\hline SIM (simeprevir)+PEG-IFN+RBV & $79(2.2)$ \\
\hline DCV+ASV & $26(0.7)$ \\
\hline Other DAA & $11(0.3)$ \\
\hline Unknown & $18(0.5)$ \\
\hline
\end{tabular}


Table 2: SVR-12 (95\% Confidence Interval) by Genotype, Treatment Received, Cirrhosis and Treatment Status

\begin{tabular}{|c|c|c|c|c|}
\hline & \multicolumn{2}{|c|}{ Non Cirrhotic } & \multicolumn{2}{|c|}{ Cirrhotic } \\
\hline & $\begin{array}{l}\text { RX Naîve } \\
(\mathrm{N}=1509)\end{array}$ & $\begin{array}{c}\text { RX Experienced } \\
(\mathrm{N}=736)\end{array}$ & $\begin{array}{l}\text { RX Naîve } \\
(\mathrm{N}=448)\end{array}$ & $\begin{array}{c}\text { RX Experienced } \\
(\mathbf{N}=335)\end{array}$ \\
\hline Genotype 1 & $n=950$ & $n=546$ & $n=349$ & $n=269$ \\
\hline LDV/SOF $(\mathrm{n}=1515)$ & $99.7 \%(99.0-99.9 \%)$ & $99.5 \%$ (98.2-99.9\%) & $97.1 \%(94.1-98.8 \%)$ & $92.2 \%$ (86.7-95.9\%) \\
\hline$D C V+A S V(n=599)$ & $92.4 \%(88.3-95.5 \%)$ & $87.7 \%(81.0-92.7 \%)$ & $93.5 \%(87.0-97.3 \%)$ & $90.5 \%(83.7-95.2 \%)$ \\
\hline $\begin{array}{l}\text { Genotype 2 } \\
\text { SOF+RBV (n=914) }\end{array}$ & $\begin{array}{c}n=559 \\
97.0 \%(95.2-98.2 \%)\end{array}$ & $\begin{array}{c}n=190 \\
96.8 \%(93.3-98.8 \%)\end{array}$ & $\begin{array}{c}n=-99 \\
98.0 \%(92.9-99.8 \%)\end{array}$ & $\begin{array}{c}n=66 \\
87.9 \%(77.5-94.6 \%)\end{array}$ \\
\hline
\end{tabular}

\section{$H C V-124$}

Real-world treatment outcome and predictors of response to different direct acting anti-viral regimens in Egyptian patients infected with chronic hepatitis $\mathrm{C}$ : a single center experience

Mohamed Alboraie ${ }^{1}$, Mohamed El Kassas ${ }^{5}$, Mohamed Alboraie ${ }^{1}$, Adel Eltahan $^{8}$, Heba Omar ${ }^{4}$, Sameera Ezzat ${ }^{6}$, Inas Moaz $^{6}$, Mahmoud Abouelkhair ${ }^{4}$, Mohamed Elbadry ${ }^{3}$, Shimaa Afify ${ }^{7}$, Amir Farid ${ }^{2}$, Dalia Omran ${ }^{4}$

${ }^{1}$ Al-Azhar University, Egypt; ${ }^{2}$ Ain Shams University, Egypt; ${ }^{3}$ Aswan University, Egypt; ${ }^{4}$ Cairo University, Egypt; ${ }^{5}$ Faculty of Medicine; Helwan University, Egypt; ${ }^{6}$ Menoufia University, Egypt; ${ }^{7}$ National Hepatology and Tropical Medicine Research Institute, Egypt; ${ }^{8} \mathrm{New}$ Cairo Hospital, Egypt

Background: Direct acting antiviral agents (DAAs) completely replaced interferon based therapies in Egypt. Real-world efficacy data are still emerging in different parts of the world to prove this major step towards effective management of hepatitis $\mathrm{C}$ using these agents. We aimed to report the real-world efficacy of seven regimens including direct acting antiviral in treatment of chronic hepatitis $\mathrm{C}$ patients in Egypt.

Method: Data of chronic hepatitis $\mathrm{C}(\mathrm{CHC})$ patients treated in our center were retrieved. Patients were treated according to the national recommendations for treatment of $\mathrm{CHC}$. Patients were subjected to hematologic panel, liver biochemical profile, and HCV RNA at scheduled visits, end of treatment (EOT) and 12 weeks after discontinuation of treatment. The primary outcome was to determine the efficacy of each regimen by sustained virological response (defined as HCV PCR below LLOQ) at 12 weeks post treatment (SVR12). Secondary outcome was to identify predictors of response to each regimen.

Result: Our study included 5517 CHC patients (51\% females) with mean age of 52 years. The following treatment regimens were used (IFN/SOF/RBV, PAR/OMP/RBV, SOF/DCV, SOF/DCV/RBV, SOF/ LED/RBV, SOF/RBV, SOF/SIM). Out of 5517 patients who started treatment in our center; $216(3.9 \%)$ patients lost follow up after enrollment in the treatment program. In all patients who completed treatment course $(n=5301)$, overall sustained virologic response (SVR12) rate was $95.5 \%$ while per protocol SVR rates were $(91.9 \%$, $98.8 \%, 99.8 \%, 99.2 \%, 100 \%, 82.9 \%$ and 96.4$)$ in the following regimens:(IFN/SOF/RBV, PAR/OMP/RBV, SOF/DCV, SOF/DCV/ RBV, SOF/LED/RBV, SOF/RBV, SOF/SIM) respectively. SVR 12 rates were higher in treatment naive patients $(97.4 \%)$ than in treatment experienced $(95.1 \%)$ and in patients without cirrhosis $(97 \%)$ than in patients with cirrhosis $(88.8 \%)$. Female gender, younger age, lower baseline AST, lower baseline AFP, lower baseline total bilirubin, smaller portal vein diameter, lower baseline FIB-4 score, lower baseline liver stiffness, higher baseline prothrombin concentration and higher baseline albumin were predictors of sustained virologic response 12 weeks after treatment. (Figures $1 \& \mathrm{a} 2$ )

Conclusion: All used regimens are highly effective in treating chronic hepatitis $\mathrm{C}$ in Egypt. SOF/RBV was the least effective therapy and SOF/LDV/RBV was the most effective therapy. Using certain clinical, laboratory and ultrasonographic predictors can improve treatment outcome.
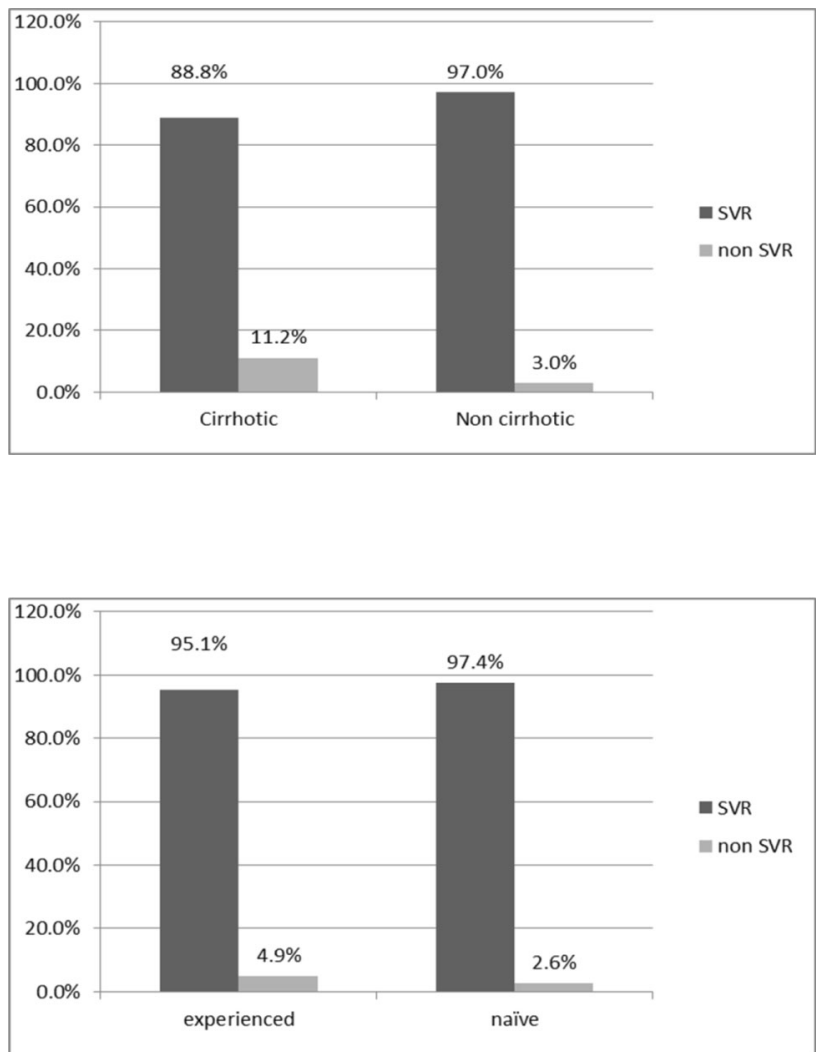

\section{HCV-125}

Real-world use of elbasvir/grazoprevir and outcomes in patients with Chronic Hepatitis C: Retrospective data analyses from the TRIO Network

Chizoba Nwankwo ${ }^{1}$, Bruce Bacon ${ }^{7}$, Michael Curry ${ }^{2}$, Douglas Dieterich $^{4}$, Steven Flamm ${ }^{5}$, Scott Milligan ${ }^{8}$, Naoky Tsai ${ }^{6}$, Zobair Younossi $^{3}$, Nezam Afdhal ${ }^{2}$

${ }^{1}$ Merck \& Co., Inc., United States; ${ }^{2}$ Beth Israel Deaconess Medical Center, United States; ${ }^{3}$ Center for Liver Diseases, Inova Fairfax Hospital, United States; ${ }^{4}$ Icahn School of Medicine at Mount Sinai, United States; ${ }^{5}$ Northwestern University Feinberg School of Medicine, United States; ${ }^{6}$ Queens Medical Center, University of Hawaii, United States; ${ }^{7}$ Saint Louis University School of Medicine, United States; ${ }^{8}$ Trio Health Analytics, United States

Background: Elbasvir/grazoprevir (EBR/GZR) is indicated for genotypes (GT) 1 or 4 chronic HCV. The purpose of this study is to understand the uptake, healthcare utilization and outcomes of chronic HCV patients treated with EBR/GZR in the United States.

Method: Data were collected from providers and specialty pharmacies through Trio Health's Innervation Platform, a cloud-based disease management program. 314 Patients who initiated EBR/GZR from Jan 28, 2016 (EBR/GZR FDA approval date) to Oct 2016 were included in the analyses. For select analyses, data were included from a comparator group of 3303 GT1 patients who initiated non-EBR/ GZR regimens in the same time period.

Result: Patients who received EBR/GZR regimens were treated for GT1a (61\%, 192/314), GT1b (33\%, 103/314), GT1x (1\%, 2/214), 
GT2 $(<1 \%, 1 / 314)$, GT3 $(<1 \%, 1 / 314)$, and GT4 $(5 \%, 15 / 314)$ HCV. 12 week EBR/GZR was the preferred EBR/GZR regimen across all genotypes and received by $90 \%(284 / 314)$ patients. Comparison of GT1 patients who received EBR/GZR (297) to a non-EBR/GZR treated GT1 comparator group (3303) revealed the EBR/GZRtreated population had higher mean serum creatinine (3.0 vs. 1.0, $\mathrm{t}=9.8, \mathrm{p}<0.001)$, prevalence of diabetes $\left(32 \%\right.$ vs. $21 \%, \mathrm{x}^{\wedge} 2=17.6$, $\mathrm{p}<0.001)$, hypertension ( $59 \%$ vs. $\left.48 \%, \mathrm{x}^{\wedge} 2=12.8, \mathrm{p}<0.001\right)$, and stage $4 / 5$ CKD $\left(29 \%\right.$ vs. $\left.2 \%, x^{\wedge} 2=503, p<0.001\right)$ and lower prevalence of stage $2 / 3$ CKD $\left(35 \%\right.$ vs. $\left.58 \%, \mathrm{x}^{\wedge} 2=53.9, \mathrm{p}<0.001\right)$ and HIV coinfection ( $4 \%$ vs. $9 \%, \mathrm{x}^{\wedge} 2=8.9, \mathrm{p}=0.003$ ). (TABLE) Only $16 \mathrm{EBR} /$ GZR-treated patients have completed follow up. Excluding one GT1a patient that discontinued 12 week EBR/GZR in week 7 due to hospitalization unrelated to treatment, the per protocol SVR was $100 \%$ (15/15). Of the 15 patients that achieved SVR, 9/15 were GT1a, 4/15 were GT1b, 1/15 was GT1x and 1/15 was GT3. No viral failures with EBR/GZR were observed to date.

Conclusion: EBR/GZR is highly effective and its use is predominantly in GT1 and as 12-week therapy without RBV, though EBR/ GZR is employed disproportionately in GT1 patients with severe renal impairment or HIV coinfection relative to a non-EBR/GZR treated GT1 population. Full SVR data on EBR/GZR will be presented at the meeting.

\begin{tabular}{|l|c|c|c|}
\hline \multicolumn{1}{|c|}{$\begin{array}{c}\text { GT1 PATIENTS } \\
\text { CHARACTERISTICS }\end{array}$} & $\begin{array}{c}12 \text { week } \\
\text { EBR/GZR } \\
(\mathrm{n}=268)\end{array}$ & $\begin{array}{c}\text { ALL EBR/GZR } \\
\text { REGIMENS } \\
(\mathrm{n}=297)\end{array}$ & $\begin{array}{c}\text { non-EBR/GZR } \\
\text { REGIMENS } \\
(\mathrm{n}=3303)\end{array}$ \\
\hline Age - mean (range) & $59(25-88)$ & $59(25-88)$ & $59(20-92)$ \\
\hline Male - no. (\%) & $173(65 \%)$ & $190(64 \%)$ & $1929(58 \%)$ \\
\hline BMI - mean (range) & $29(17-50)$ & $29(17-50)$ & $29(15-50)$ \\
\hline Baseline ALT - mean (SD) & $56(51) \mathrm{n}=243$ & $58(53) \mathrm{n}=270$ & $70(69) \mathrm{n}=2772$ \\
\hline Baseline AST - mean (SD) & $52(44) \mathrm{n}=236$ & $54(48) \mathrm{n}=262$ & $65(62) \mathrm{n}=2720$ \\
\hline Baseline Hb - mean (SD) & $12.9(2.2) \mathrm{n}=222$ & $12.9(2.2) \mathrm{n}=247$ & $14(1.8) \mathrm{n}=2567$ \\
\hline Baseline Ser. Creatinine-mean (SD) & $3.1(3.2) \mathrm{n}=194$ & $3(3.1) \mathrm{n}=217$ & $1(0.6) \mathrm{n}=2506$ \\
\hline Diabetes - no. (\%) & $82 / 252(33 \%)$ & $90 / 280(32 \%)$ & $623 / 2935(21 \%)$ \\
\hline Hypertension- no. (\%) & $150 / 251(60 \%)$ & $164 / 279(59 \%)$ & $1382 / 2905(48 \%)$ \\
\hline Stage 2/3 CKD-no. (\%) & $88 / 252(35 \%)$ & $99 / 280(35 \%)$ & $1750 / 3011(58 \%)$ \\
\hline Stage 4/5 CKD- no. (\%) & $75 / 252(30 \%)$ & $82 / 280(29 \%)$ & $51 / 3011(2 \%)$ \\
\hline HIV Co-Infection- no. (\%) & $10 / 268(4 \%)$ & $11 / 297(4 \%)$ & $287 / 3299(9 \%)$ \\
\hline HBV Co-Infection- no. (\%) & $2 / 268(1 \%)$ & $2 / 297(1 \%)$ & $28 / 3299(1 \%)$ \\
\hline Fibrosis 3/4 - no. (\%) & $106 / 266(40 \%)$ & $123 / 295(42 \%)$ & $1389 / 3244(43 \%)$ \\
\hline Baseline Viral >6MM IU/ml- no. (\%) & $35 / 247(14 \%)$ & $41 / 273(15 \%)$ & $538 / 2995(18 \%)$ \\
\hline GT1a- no. (\%) & $167(62 \%)$ & $192(65 \%)$ & $2418(73 \%)$ \\
\hline GT1b- no. (\%) & $99(37 \%)$ & $103(35 \%)$ & $746(23 \%)$ \\
\hline GT1x- no. (\%) & $2(1 \%)$ & $2(1 \%)$ & $139(4 \%)$ \\
\hline GT1a NS5A RAV Tested - no. (\%) & $87 / 167(52 \%)$ & $104 / 192(54 \%)$ & tbd \\
\hline Treatment Naïve - no. (\%) & $189 / 226(84 \%)$ & $204 / 251(81 \%)$ & $2386 / 2894(82 \%)$ \\
\hline
\end{tabular}

\section{$H C V-126$}

Restricting the use of direct acting antiviral drugs for chronic hepatitis $\mathbf{C}$ in patients with advance liver disease does not reduce short-term treatment-related cost

Yu Jun Wong ${ }^{1}$, John Hsaing Chen ${ }^{2}$, Yi Lyn Jessica Tan², Rahul Kumar $^{2}$, Eng Kiong Teo ${ }^{2}$, Chong Xuan Deshawn Tan ${ }^{2}$, Prem Harichander Thurairajah ${ }^{2}$

${ }^{1}$ Singhealth, Singapore; ${ }^{2}$ Changi General Hospital, Singapore

Background: The high cost of direct antiviral agent (DAA) remains a barrier to treatment access. While restricting the use of DAA as salvage therapy for advance liver disease and treatment failures alone is an effective rationing strategy in a cost constrained setting, it is unclear if this strategy reduces the immediate short term healthcare burden and cost. We aim to evaluate if DAA, when restricted in its use to treating Pegylated Interferon and Ribavirin (PR) failures and advance liver disease intolerant to PR regime would reduce the immediate healthcare related attendance and cost.
Method: We reviewed $120 \mathrm{HCV}$ patients treated (81 PR and 39 DAA) between 2013 to 2017 in our institution. Among genotype 1 (GT1) HCV, patient either received 48 weeks of PR or 12 weeks of DAA therapy (Viekira Pak or HARVONI). Among genotype 3 (GT3) $\mathrm{HCV}$, patient either received 24 weeks of PR or 12 weeks of DAA therapy (Sofosbuvir (SOF)/ Daclatasvir or SOF + PR regime). The cost for PR and DAA as first line therapy was calculated by including the total cost incurred from inpatient, outpatient and Accident and Emergency (A\&E) visits during the course of treatment. Cost per hospital attendance was calculated by dividing the average cost of hospital visits for inpatients, outpatients and A\&E by the total number of patients and expressed as average cost per patient.

Result: Baseline demographics were summarized in Table 1. Patients treated with DAA had more advance liver disease at baseline as evident by higher mean MELD score ( 9.6 vs $7.8, \mathrm{p}=0.005$ ), more liver related complications such as ascites $(23.1 \%$ vs $2.5 \%, \mathrm{p}=0.001)$ and esophageal varices $(33.3 \%$ vs $13.6 \%, \mathrm{p}=0.009)$. Treatment with DAA was associated with a reduced cost per patient for outpatient clinic visits (S\$1539 vs. S\$2456, P

Conclusion: DAA is highly effective and allowed HCV with advance disease to be treated. Treatment with DAA reduced treatment-related outpatient visit cost as DAA was associated with less side effects and required less frequent monitoring compared to PR regime. However, the average short-term treatment-related cost, particularly from A\&E visit and inpatient admission was not reduced with the use of DAA. This is due to sicker patient being treated with DAA and the complications related to their intrinsic liver disease requiring longer hospital stay. The strategy of restricting the use of DAA for more advance liver disease is not cost saving in the short term. Patients with HCV should be treated at an early stage of disease to reduce the short term cost of hospital related care.

Table 1: Baseline demographic of treated HCV patients

\begin{tabular}{|c|c|c|c|c|}
\hline \multicolumn{2}{|c|}{ Descriptions: } & \multirow{2}{*}{$\frac{N}{51.4(24-67)}$} & \multirow[t]{2}{*}{$\%$} & \multirow{2}{*}{$\frac{\text { Total }}{120}$} \\
\hline Age & (Mean, range) & & & \\
\hline \multirow[t]{2}{*}{ Gender } & Male & 108 & 90.0 & 120 \\
\hline & Female & 12 & 10.0 & \\
\hline \multirow[t]{3}{*}{ Race } & Chinese & 41 & 34.2 & 120 \\
\hline & Malay & 61 & 50.8 & \\
\hline & Indian & 18 & 15.0 & \\
\hline \multirow[t]{2}{*}{ Genotype } & 1 & 25 & 20.8 & 120 \\
\hline & $2 / 3$ & 95 & 79.2 & \\
\hline \multirow[t]{2}{*}{ Baseline Viral load } & $<400,0001 \mathrm{U} / \mathrm{ml}$ & 36 & 30.0 & 120 \\
\hline & $8400,0001 \mathrm{U} / \mathrm{ml}$ & 84 & 70.0 & \\
\hline \multirow[t]{2}{*}{ Cirrhosis } & Yes & 64 & 53.3 & 120 \\
\hline & No & 56 & 46.7 & \\
\hline \multirow[t]{2}{*}{ Ascites: } & res & 11 & 9.2 & 120 \\
\hline & No & 109 & 90.8 & \\
\hline \multirow[t]{2}{*}{ Esophageal varices } & res & 24 & 20.0 & 120 \\
\hline & No & 96 & 80.0 & \\
\hline \multirow[t]{2}{*}{ HE } & Yes & 2 & 17 & 120 \\
\hline & Mo & 118 & 98.3 & \\
\hline MELD score & (Mean, range) & $9[6-20]$ & & \\
\hline \multirow[t]{5}{*}{ Fibrosis seore } & $0=1$ & 18 & 15.0 & 120 \\
\hline & 2 & 10 & 8.3 & \\
\hline & 3 & 18 & 15.0 & \\
\hline & 4 & 64 & 53.3 & \\
\hline & Nat done & 10 & 8.3 & \\
\hline \multirow[t]{2}{*}{ DAA Therapy } & Yes & 39 & 32.5 & 120 \\
\hline & No & 81 & 67.5 & \\
\hline \multirow[t]{3}{*}{ Overal SVR } & Detected & 24 & 20.0 & 120 \\
\hline & Not detected & 96 & 80.0 & \\
\hline & Defaulted/Nat dane & $\mathrm{g}^{*}$ & 8.1 & \\
\hline
\end{tabular}




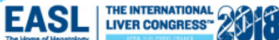

Table 2: Short-term healthcare cost for $\mathrm{HCV}$ patients treated with PR

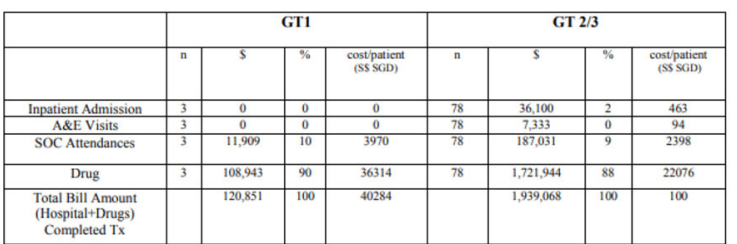

Table 3: Short-term healthcare cost for $\mathrm{HCV}$ patients treated with DAA

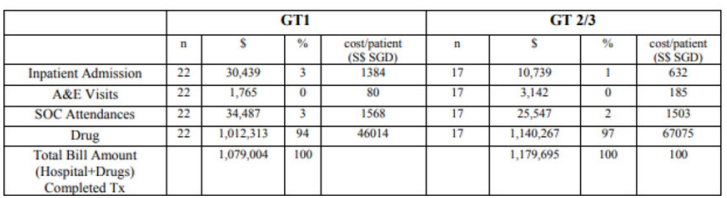

$H C V-127$

Safety and efficacy of sofosbuvir/velpatasvir in a genotype 1-6 HCV infected population from Singapore, Malaysia, Thailand, and Vietnam: results from a phase 3 , clinical trial

ChenYu Wang ${ }^{1}$, Kinh Nguyn Van ${ }^{4}$, Phuong Le ${ }^{5}$, Hoi Poh Tee ${ }^{3}$, Chenyu Wang ${ }^{2}$, Brian Mcnabb ${ }^{2}$, Sofia $\mathrm{Lu}^{2}$, Julia $\mathrm{Lu}^{2}$, Luisa Stamm $^{2}$, Diana Brainard ${ }^{2}$, Mani Subramanian ${ }^{2}$, Chee Kiat Tan ${ }^{7}$, Tawesak Tanwandee ${ }^{8}$, Teerha Piratvisuth ${ }^{6}$

${ }^{1}$ Gilead Sciences, Taiwan; ${ }^{2}$ Gilead Sciences, United States; ${ }^{3}$ Hospital Tengku Ampuan Afzan, Malaysia; ${ }^{4}$ National Hospital for Tropical Diseases, Vietnam; ${ }^{5}$ People's Hospital 115, Vietnam; ${ }^{6}$ Prince of Songkla University, Thailand; ${ }^{7}$ Singapore General Hospital, Singapore; ${ }^{8}$ Siriraj Hospital, Mahidol University, Thailand

Background: The prevalence of hepatitis $\mathrm{C}$ virus (HCV) in Singapore, Malaysia, Thailand, and Vietnam, ranges from 1-3\%. Because of the heterogeneity of genotypes (GTs) in these countries (primarily GT1, 2, 3, and 6), there is a critical need for a pangenotypic, all oral regimen to address the burden of $\mathrm{HCV}$ infection. Treatment with sofosbuvir (SOF)/velpatasvir (VEL) results high sustained virologic response (SVR) rates in GT1-6 HCV infected patients in clinical trials and real-world settings. This study evaluated the efficacy and safety of SOF/VEL for 12 weeks in patients with chronic GT1-6 HCV infection.

Method: Treatment experienced and treatment naïve patients with chronic GT1-6 HCV infection with no cirrhosis or with compensated cirrhosis were eligible to enroll in a single-arm, open-label trial to receive a fixed dosed combination of SOF/VEL 400/100 mg daily for 12 weeks. Patients were recruited from 13 sites in Singapore, Malaysia, Thailand, and Vietnam. The primary efficacy endpoint was SVR 12 weeks after treatment (SVR12) using the CAP/CTM HCV 2.0 assay ( $\mathrm{LLOQ}=15 \mathrm{IU} / \mathrm{mL}$ ). SVR 24 weeks after treatment completion (SVR24) and virologic resistance analysis through deep sequencing ( $>15 \%$ cutoff) are also reported as secondary endpoints. The primary safety endpoint was adverse events (AEs) leading to SOF/VEL discontinuation.

Result: A total of 111 patients were enrolled and treated. Of these, $51 \%$ were male, $14 \%$ had compensated cirrhosis, $18 \%$ were treatment-experienced, 82\% had IL28B CC genotype, 20\% had GT1a, $23 \%$ GT1b, 3\% GT2, 23\% GT3, and 31\% GT6 HCV infection. The mean (range) age and body mass index of enrolled patients were 46 $(23-75)$ years and $24(17-37) \mathrm{kg} / \mathrm{m}^{2}$, respectively. The overall SVR12 and SVR24 rates were both 97\% (108/111), with SVR12 (24) rates according to GT presented in the Table. SVR12 rate was $100 \%$ in the 15 patients with protocol-defined cirrhosis. All 3 patients who did not achieve SVR experienced virologic relapse. All 15 cirrhotic patients, including 8 with GT3 HCV infection achieved SVR12 and 24. No treatment-emergent NS5A resistance associated substitutions (RASs) were identified among the 3 patients with relapse. There were no discontinuations due to AEs. There were 50 patients $(45 \%)$ who experienced any adverse events (AEs), with no AEs occurring in $>10 \%$ of patients. No serious or severe AEs were assessed by the investigator as related to study drug and there were no deaths.

Conclusion: Treatment with the single tablet, pangenotypic regimen of SOF/VEL for 12 weeks was highly effective and well tolerated in a GT 1, 2, 3, and $6 \mathrm{HCV}$ infected population with and without cirrhosis from Singapore, Malaysia, Thailand, and Vietnam.

Table: SVR12 and SVR24 for GT1-6 HCV infected Patients

\begin{tabular}{l|c|c|c|c|c|c|c}
\hline N (\%) & $\begin{array}{c}\text { Total } \\
\text { N=111 }\end{array}$ & $\begin{array}{c}\text { GT1a } \\
\text { N=22 }\end{array}$ & $\begin{array}{c}\text { GT1b } \\
\text { N=25 }\end{array}$ & $\begin{array}{c}\text { GT1 } \\
\text { (total) } \\
\mathbf{N = 4 7}\end{array}$ & $\begin{array}{c}\text { GT2 } \\
\mathbf{N}=\mathbf{3}\end{array}$ & $\begin{array}{c}\text { GT3 } \\
\mathbf{N}=\mathbf{2 5}\end{array}$ & $\begin{array}{c}\text { GT6 } \\
\mathbf{N = 3 6}\end{array}$ \\
\hline SVR12 & $108 / 111(97)$ & $22 / 22$ & $25 / 25$ & $47 / 47$ & $3 / 3(100)$ & $23 / 25(92)$ & $35 / 36(97)$ \\
& & $(100)$ & $(100)$ & $(100)$ & & & \\
\hline SVR24 & $108 / 111(97)$ & $22 / 22$ & $25 / 25$ & $47 / 47$ & $3 / 3(100)$ & $23 / 25(92)$ & $35 / 36(97)$ \\
& & $(100)$ & $(100)$ & $(100)$ & & & \\
\hline
\end{tabular}

$H C V-128$

Scale up of hepatitis $\mathbf{C}$ virus treatment in the era of all-oral directacting antivirals: a multicenter 9-year Greek cohort study

Vasilios Papastergiou ${ }^{1}$, Vasilios Papastergiou ${ }^{4}$, Melanie Deutsch ${ }^{4}$, Maria Mela ${ }^{2}$, Christos Triantos ${ }^{5}$, Hariklia Kranidioti ${ }^{4}$, Christina Chatzievangelinou ${ }^{2}$, Konstantinos Zisimopoulos ${ }^{5}$, Olga Anagnostou $^{3}$, George Papatheodoridis ${ }^{4}$, Spilios Manolakopoulos ${ }^{4}$

${ }^{1}$ Kapodistrian University of Athens, Medical School, Greece; ${ }^{2}$ Evangelismos General Hospital, Greece; ${ }^{3}$ Greek Organization Against Drugs, Greece; ${ }^{4}$ Medical School of the National and Kapodistrian University of Athens, Greece; ${ }^{5}$ University Hospital of Patras, Greece

Background: More than 80.000 people are living with hepatitis C virus $(\mathrm{HCV})$ infection in Greece. All-oral direct-acting antivirals (DAAs) are awaited to increase the HCV treatment uptake, although access to DAAs is prioritized due to their high costs. Recent changes in national DAA reimbursement policies included access to DAAs for uninsured patients (Aug 2016), expansion of prioritization from cirrhosis to F2 fibrosis, and prioritization of patients with hematological/ kidney comorbidities (Jul 2017). In this retrospective, longitudinal, cohort study, we explored the influence of all-oral DAAs and expanded reimbursement policies on HCV treatment uptake.

Method: We analysed consecutive HCV-viraemic adults $(n=832)$ who visited at least once 3 tertiary liver centres ( $n=5$ physicians) over a 9-year period (Jan 2009 -Aug 2017). Kaplan-Meier analyses were used to compare the cumulative incidence of treatment initiation across 4 time periods: peginterferon/ribavirin (PR; Jan 2009-May 2011, $\mathrm{n}=211$ ), first-generation protease inhibitors (PI; Jun 2011-Dec 2013, n=255), early DAA (Jan 2014-Dec 2015, n=233) and late DAA (Jan 2016-Aug 2017, $n=133$ ). Cox regression was used to estimate incidence rate ratios as relative risks (RR) with $95 \%$ confidence intervals (CI).

Result: Overall, 427 (51.3\%) patients started treatment over a median (IQR) follow-up of 25.6 (8.6-73) weeks. The annual HCV treatment uptake remained stable from 2009 to 2013 fluctuating between $24.6 \%$ / year and $31.4 \%$ year, dropped to $19.4 \% /$ year in 2014 , and then raised again peaking to $32.7 \%$ year in 2017 . The ratio of treated with DAAs/ 
treated patients increased from $10.2 \%$ in 2014 to $89.9 \%$ in 2017 . The overall cumulative probability of treatment across all periods was $50 \%, 64.3 \%$ and $66.3 \%$ at 1,3 and 5 years respectively. Previously considered "difficult to treat" populations, including cirrhotics (RR: 2.3; 95\%CI: 1.5-3.6), treatment experienced (RR: 2.1; 95\%CI: $1.3-$ 3.2 ), and genotype 1 -infected (RR: 1.7 ; $95 \% \mathrm{CI}: 1.2-2.3$ ) patients were more likely to initiate therapy after the introduction of 2 nd generation DAAs. By Kaplan-Meier analysis, the HCV treatment uptake was significantly increased in the late DAA period (1-year cumulative treatment initiation: $73.1 \%$ vs $46.1 \%, 52.6 \%$, and $40.6 \%$ for the PR, $\mathrm{PI}$, and early DAA periods respectively; $\mathrm{p}=0.006)$. Non-cirrhotics (RR:2.43; 95\%CI:1.51-3.90), treatment-naïve (RR:2.16; 95\%CI:1.433.27), intravenous drug users (RR:3.05; 95\%CI:1.92-4.82) and nonholders of public insurance (RR:4.87; 95\%CI:2.63-8.70) were more likely to initiate therapy in the late compared with early DAA period, reflecting the expanded prioritization criteria.

Conclusion: Our data show an increase in HCV treatment uptake after the introduction of all-oral DAAs and the expanded DAA prioritization policies. However, as a substantial proportion of patients still remains untreated, a framework of actions for addressing existing barriers is urgently needed to increase the treatment uptake.

\begin{tabular}{|c|c|}
\hline $\begin{array}{l}\text { Age, mean(SD) } \\
\text { Gender, } n(\%)\end{array}$ & $46.5(13.1)$ \\
\hline Male & $591(71)$ \\
\hline Female & $241(29)$ \\
\hline \multicolumn{2}{|l|}{ Origin, $n(\%)$} \\
\hline Born in Greece & $633(76.1)$ \\
\hline Born outside Greece & $199(23.9)$ \\
\hline \multicolumn{2}{|l|}{ Intravenous Drug use, $n(\%)$} \\
\hline Ever injected drugs & $177 / 369[48]$ \\
\hline Active IDU & $53(6.4)$ \\
\hline Treatment drug dependency & $204(24.5)$ \\
\hline $\begin{array}{l}\text { Former IDU history } \\
\text { Unknown }\end{array}$ & $\begin{array}{c}187(22.5) \\
44(5.3)\end{array}$ \\
\hline \multicolumn{2}{|l|}{ Insurance Status, $\mathrm{n}(\%)$} \\
\hline Public Insurance & $465(55.9)$ \\
\hline Unemployment insurance & $198(23.8)$ \\
\hline Uninsured & $97(11.7)$ \\
\hline Unknown & $72(8.7)$ \\
\hline Undergoing fibroscan, $n(\%)$ & $565(77.9)$ \\
\hline Undergoing liver biopsy, $n(\%)$ & $66(7.9)$ \\
\hline \multicolumn{2}{|l|}{ Fibrosis stage ${ }^{\wedge}, \mathrm{n}(\%)$} \\
\hline FO-F1 & $227(27.3)$ \\
\hline F2 & $101(12.1)$ \\
\hline $\mathrm{F} 3$ & $107(12.9)$ \\
\hline F4 & $182(21.9)$ \\
\hline Unknown & $215(25.8)$ \\
\hline HCV-RNA>800.000IU/ml, $n(\%)$ & $437(52.5)$ \\
\hline \multicolumn{2}{|l|}{ Genotype, $n(\%)$} \\
\hline 1 & $293(35.2)$ \\
\hline 2 & $48(5.8)$ \\
\hline 3 & $312(37.5)$ \\
\hline 4 & $151(18.1)$ \\
\hline $5 / 6$ & $2(0.2)$ \\
\hline Unknown & $26(3.1]$ \\
\hline Treatment naïve, $n\left(x_{0}\right)$ & $574(69)$ \\
\hline At least one Comorbidity*, $n(\%)$ & $89(10.7)$ \\
\hline
\end{tabular}

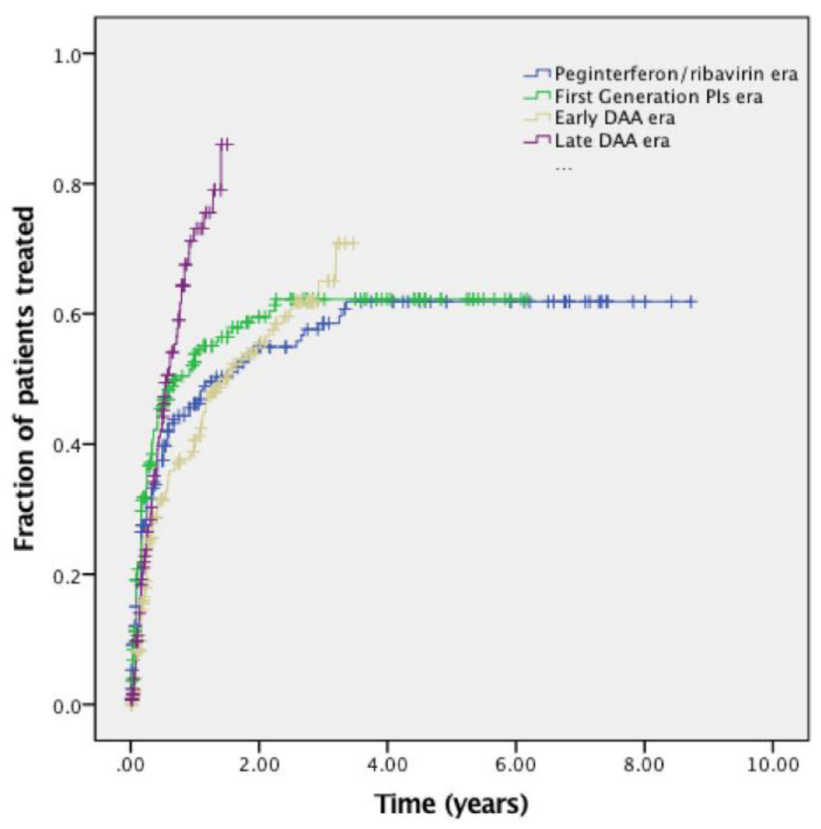

HCV-129

Sofosbuvir and daclatasvir to treat a patient with acute hepatitis $C$ virus genotype 2 monoinfection

\section{Chen ${ }^{1}{ }^{1}$, Jinhua $\mathrm{Hu}^{1}$ \\ Military Hospital, China}

Background: Airect-acting antivirals (DAAs) belongs to first-line treatment for patients with chronic hepatitis $\mathrm{C}$ virus (HCV) infection. However, evidence of its effects on acute hepatitis $\mathrm{C}$ virus infection patients is limited and lack of data for genotype 2 monoinfection patients on the treatment of acute hepatitis $\mathrm{C}$ with DAAs.

Method: A patient with acute hepatitis $C$ virus genotype 2 monoinfection was treated by sofosbuvir and daclatasvir. We conducted a prospective observation for the efficacy of the patient.

Result: A 26 year-old Chinese female was acute infected hepatitis $\mathrm{C}$ virus and HCV RNA had not spontaneous clearance during 12 weeks after infection. The patient received sofosbuvir and daclatasvir combination treatment for 12 weeks. HCV RNA was undetectable at week 4 and anti-HCV antibody were seroconversion at week 12 during treatment of patient. The patient achieved a sustained virological response 36 weeks after the end of treatment.

Conclusion: The patients with acute HCV genotype 2 monoinfection would benefit from antiviral treatment with sofosbuvir and daclatasvir.

Fig. 1. The dynamic trends of HCV RNA viral load and anti-HCV antibody after treatment with SOF and DCV of the patient: HCV RNA viral load was undetectable at weeks 4 and anti-HCV antibody were seroconversion at weeks 12 . The patient achieved a SVR 36 weeks after the end of treatment. 


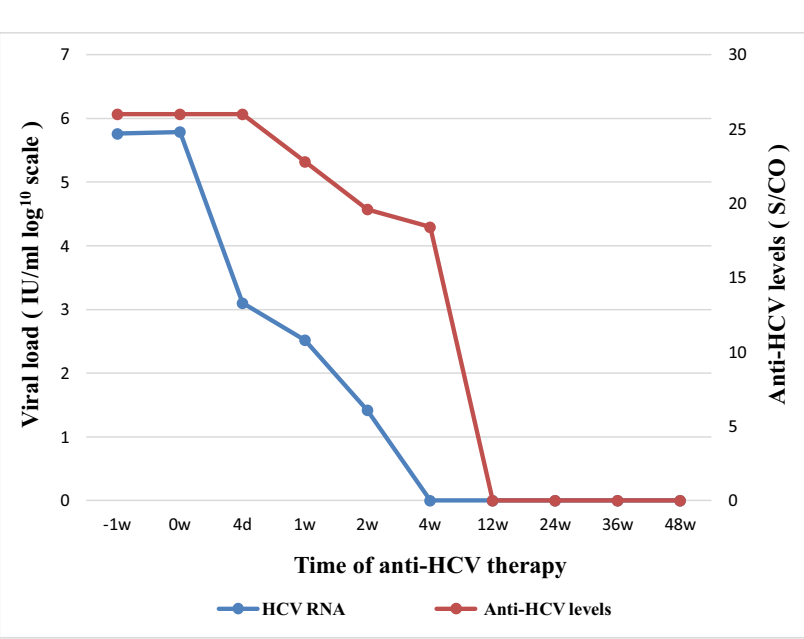

Fig. 2. The dynamic trends of liver function after treatment with SOF and DCV of the patient: liver function gradually improved and returned to normal levels at weeks 4.

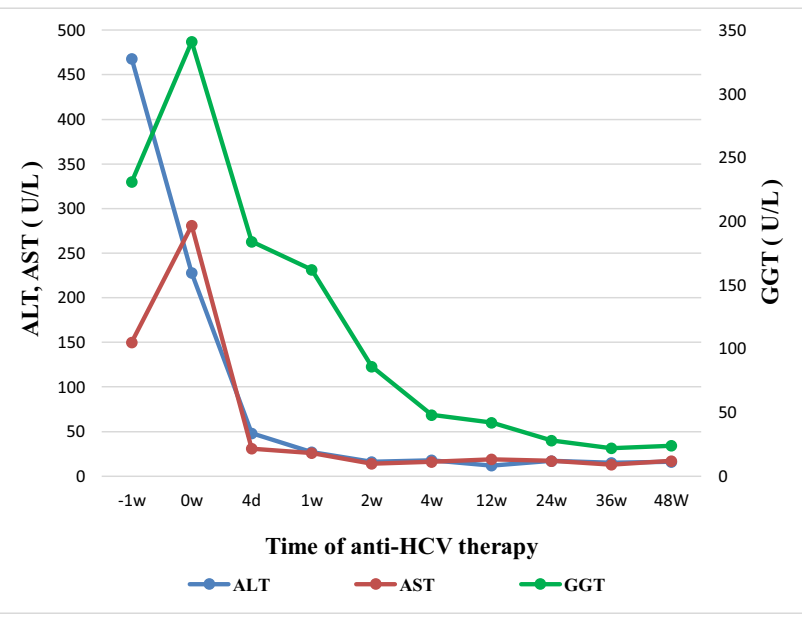

$H C V-130$

Study some of natural killer cell phenotypes in viral hepatitis

Mohammad Helal $^{1}$, Ali Ghweil ${ }^{1}$, Shereen Aziz ${ }^{2}$, Ashraf Khodery ${ }^{2}$

${ }^{1}$ South Valley University, Egypt; ${ }^{2}$ Sohag University, Egypt

Background: Natural killer (NK) cells are an integral part of the innate immune system. They have been suggested to play an important role in both defense against viral hepatitis and the pathogenesis of other liver diseases.

Method: NK cell markers (CD3, CD56, CD16) were studied by flow cytometry from 96 individuals including 76 patients with hepatitis $\mathrm{B}$ and $\mathrm{C}$ as well as 20 healthy subjects as control.

Result: Infection with viral hepatitis was associated with increased frequencies of CD3-\&CD56+NK cells and CD3-\&CD16+ NK cells in the peripheral blood; and severity of hepatitis is correlated with the percentage of NK cells in peripheral blood with no statistically significant difference between HCV and HBV infections.

Conclusion: Alteration of NK cell phenotypes with viral hepatitis depend on disease activity rather than virus-specific factors.
HCV-131

Sustainable and uniform response for treatment of post transplant hepatitis $\mathrm{C}$ with all DAA regimens across all hepatitis $\mathrm{C}$ genotypes

Moutaz Farouk Derbala ${ }^{1}$, Moutaz Farouk Derbala ${ }^{7}$, Syed Mohiuddin $^{4}$, Yara Altahan ${ }^{6}$, Mohammed Elbadri ${ }^{4}$, Yasser Kamel $^{3}$, Prem Chandra ${ }^{2}$, Tahany Mahgoub ${ }^{5}$

${ }^{1}$ Sr. Consultant Gastroenterology\& Hepatology, Qatar; ${ }^{2}$ Consultant Epidemiology, Qatar; ${ }^{3}$ Consultant Gastroenterology, Qatar; ${ }^{4}$ Fellow Gastroenterology \& Hepatology, Qatar; ${ }^{5}$ Liver Transplant Coordinator, Qatar; ${ }^{6}$ Medical Student, Bahrain; ${ }^{7}$ Sr. Consultant Gastroenterology \& Hepatology, Qatar

Background: There is limited data available on the efficacy of Direct Acting Antiviral (DAA's) agents for recurrent Hepatitis $\mathrm{C}$ in liver transplant recipients,, especially genotype 4 . We aim to retrospectively analyse the efficacy and safety of DAA's post liver transplant. Method: 48 liver recipients with recurrent Hepatitis $\mathrm{C}$ were included in a non-Interventional, retrospective, single-centre cohort study conducted in Hamad General Hospital, Qatar. All patients were treated for 12 weeks with DAA's, except for sofosbuvir/ribavirin regimen who were treated for 24 weeks. Chi-square test or Fisher exact and Yates corrected Chi-square test, repeated measure analysis of variance (ANOVA) were used for statistical analysis.

Result: Overall SVR12 was achieved in $95.8 \%$ of the cases. There was no statistical difference observed in SVR12 between different treatment groups $(\mathrm{P}=0.616)$. SVR12 rates in genotype 4, 93.9\%, (95\% CI:80.4 to 98.3), was comparable to other genotypes 1, 2 and 3 $100 \%(\mathrm{P}=0.134)$. SVR12 was found to numerically higher in patients with fibrosis stage (F0-2) (97.1\% vs $92.9 \%$; $\mathrm{P}=0.583)$ and MELD score $<10$ (97\% vs 93.3\%; $\mathrm{P}=0.955$ ). Cirrhotics had lower rates of SVR12 compared to Non-cirrhositic $(\mathrm{P}=0.986)$. SVR12 rate was higher among females compared to males ( $100 \%$ vs $93.3 \%$; $\mathrm{P}=0.386)$, however no significant association was observed with other baseline demographic Mean ALT decreased significantly from Week 0 (mean ALT 59 \pm 10.4 ) to week 24 (mean ALT 25.4 \pm 4.1 ); $\mathrm{P}<0.001$. The most common $\mathrm{AE}$ was raised cholesterol $(6,12.5 \%)$, observed in patients treated with tacrolimus as immunosuppressive. Increased tacrolimus level in those treated with Viekirax.

Conclusion: Treatment of Post transplant hepatitis $\mathrm{C}$ with 12 weeks DAA's is safe and effective across all genotypes with all treatment regimens. Treatment is associated with significant reduction in $\mathrm{t}_{\text {Tables }}$

Association between baseline characteristics with SVR 12W

\begin{tabular}{|c|c|c|c|c|}
\hline & \multicolumn{4}{|c|}{ SVR $12 \mathrm{~W}$} \\
\hline & responder/Total & $\%$ & $95 \% \mathrm{CI}$ & P. value* \\
\hline $\begin{array}{l}\text { Gender } \\
\text { Male } \\
\text { Female }\end{array}$ & $\begin{array}{l}27 / 30 \\
17 / 18\end{array}$ & $\begin{array}{c}90 \\
94.4\end{array}$ & $\begin{array}{c}74.4,96.5 \\
74.2,99\end{array}$ & 0.989 \\
\hline $\begin{array}{c}\text { Age Group } \\
<=55 \text { years } \\
55 \text { to } 65 \text { years } \\
>65 \text { years }\end{array}$ & $\begin{array}{c}19 / 20 \\
18 / 20 \\
7 / 8\end{array}$ & $\begin{array}{l}95 \\
90 \\
87\end{array}$ & $\begin{array}{l}76.4,99.1 \\
69.9,97.2 \\
52.9,97.8\end{array}$ & 0.959 \\
\hline $\begin{array}{c}\text { BMI } \\
<=30 \\
30 \text { to } 35 \\
>35\end{array}$ & $\begin{array}{c}21 / 23 \\
13 / 15 \\
9 / 9\end{array}$ & $\begin{array}{r}91.3 \\
86.7 \\
100\end{array}$ & $\begin{array}{r}73.2,97.6 \\
62.1,96.3 \\
70.1,100\end{array}$ & 0.87 \\
\hline $\begin{array}{c}\text { Cirrhosis } \\
\text { Yes } \\
\text { No }\end{array}$ & $\begin{array}{l}32 / 36 \\
12 / 12\end{array}$ & $\begin{array}{r}88.9 \\
100\end{array}$ & $\begin{array}{r}74.7,95.6 \\
75.8,100\end{array}$ & 0.606 \\
\hline $\begin{array}{l}\mathrm{HCC} \\
\text { Yes } \\
\text { No } \\
\end{array}$ & $\begin{array}{l}12 / 15 \\
32 / 33 \\
\end{array}$ & $\begin{array}{l}80 \\
97\end{array}$ & $\begin{array}{l}54.8,92.9 \\
84.7,99.5\end{array}$ & $0 . .168$ \\
\hline $\begin{array}{c}\text { Transplantation } \\
\text { LRT } \\
\text { OLT }\end{array}$ & $\begin{array}{l}32 / 35 \\
12 / 13\end{array}$ & $\begin{array}{l}91.4 \\
92.3\end{array}$ & $\begin{array}{l}77.6,97 \\
66.7,98.6\end{array}$ & 0.987 \\
\hline
\end{tabular}


Figure 1.Tacrolimus dose over time

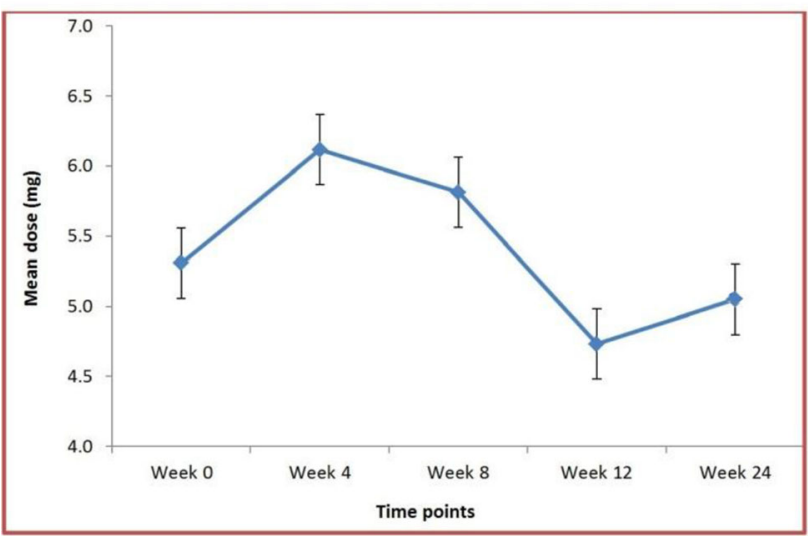

$H C V-132$

The incidence of aggressive de novo or recurrent hepatocellular carcinoma in patients with hepatitis $\mathbf{C}$ virus infection receiving direct-acting antiviral therapy

Hany Ahmed Elbeshbeshy ${ }^{1}$, Sarra Yousif ${ }^{1}$, Faisal Abaalkhail ${ }^{1}$, MOHAMED ALSEBAYEL ${ }^{1}$, Hussein Elsiesy ${ }^{1}$, Waleed Alhamoudi $^{1}$

${ }^{1}$ King Faisal Specialist Hospital \& Research Center, Saudi Arabia

Background: The development of Direct Acting Antivirals (DAA) against Hepatitis C Virus (HCV) and their success in eradicating the virus at very high rates, constituted a breakthrough in the treatment of this disease. This will ultimately improve the long term outcome by reducing the rate of liver decompensation and Hepatocellular Carcinoma (HCC). Recently, new data have emerged showing unexpectedly high rates of de novo HCC or recurrence in patients treated with DAAs. Several reasons have been proposed to explain this increased risk. Rapid reduction of viral loads caused by DAAs leading to rapid reduction of inflammation and increase in serum levels of vascular endothelium growth factor (VEGF) is one rationale. We have noticed increase in HCC cases with aggressive behavior in our cohort of patients treated with DAA's. This data has been previously reported; however, new cases have been identified in the same cohort since the previous report. Therefore, the data, with the new cases included, is presented here. Aim of the study: To evaluate the incidence of de novo HCC or aggressive recurrence in cirrhotic patients or patients transplanted for HCC who received DAAs at our center.

Method: This is a retrospective, observational cross-sectional study that included all patients with HCV who received DAA during the period from January 2014 to December 2016. During the study period, patients were followed regularly with Alfa Feto Protein (AFP) and liver images every 3-6- months. Tumors beyond Milan criteria were considered aggressive.

Result: Out of 258 patients treated at our center, 158 met the criteria. Follow up duration was 1-36 months. All patients received Sofosbuvir-based therapy (predominantly Ledipasvir /Sofosbuvir). 16 $(10.1 \%)$ patients developed either De novo or HCC recurrence post treatment (Figure 1). Out of the 16 patients, 10 were females, 10 had diabetes and 7 were obese (out of 10 overweight). Ten patients had aggressive HCC including 2 post-transplant recurrences (Figure 2). The majority of HCCs $(62.5 \%)$ developed after DAA therapy were De novo tumors (Figure 3). Average time to HCC detection post treatment was 11.2 months (range 4-18 months). 13 patients had elevated AFP (5 patients with AFP $>400 \mu \mathrm{g} / \mathrm{L}$ ). All patients received loco- regional therapy, Sorafenib or both. One patient was for palliative care only and one patient expired during follow up period.

Conclusion: Despite achieving a sustained virological response, HCC occurrence remains a major concern following DAA treatment especially aggressive HCC (62.5\% of cases). Close post-treatment screening and surveillance is required.

Figure 1: Incidence of Hepatocellular Carcinoma after treatment with Direct-Acting Anti-retroviral therapy

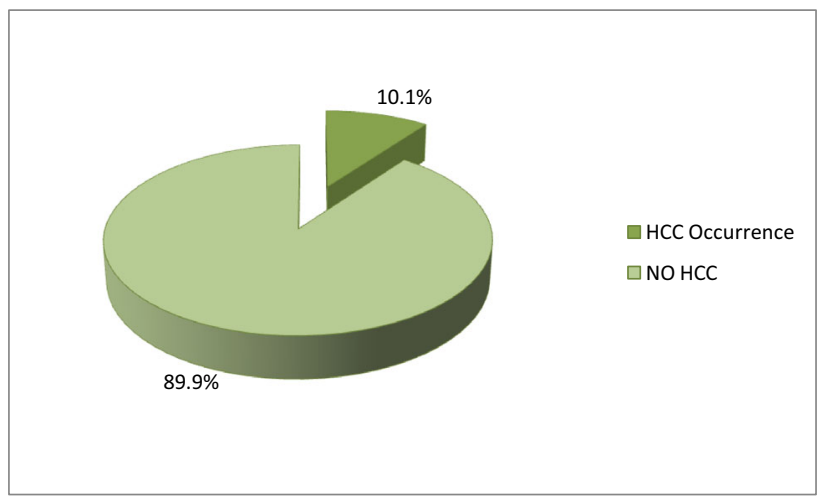

Figure 2: Distribution of Hepatocellular Carcinoma by aggressive behavior

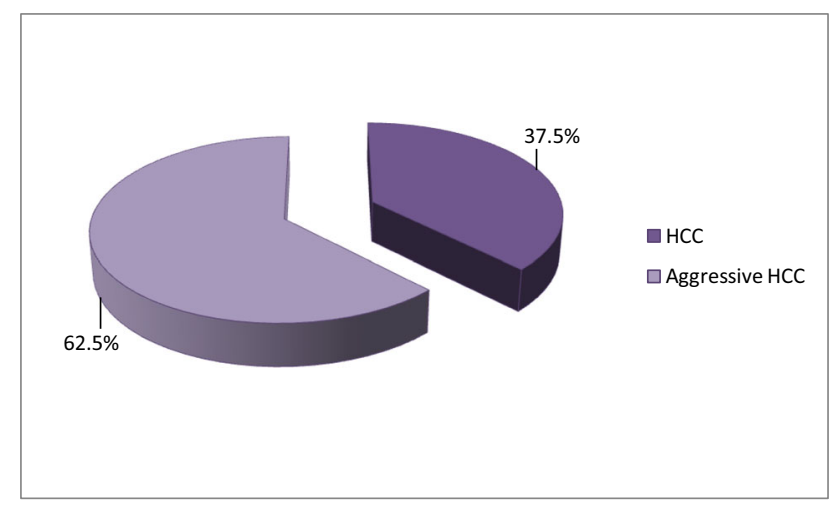

Figure 3: Distribution of Hepatocellular Carcinoma by Recurrence of De novo origin.

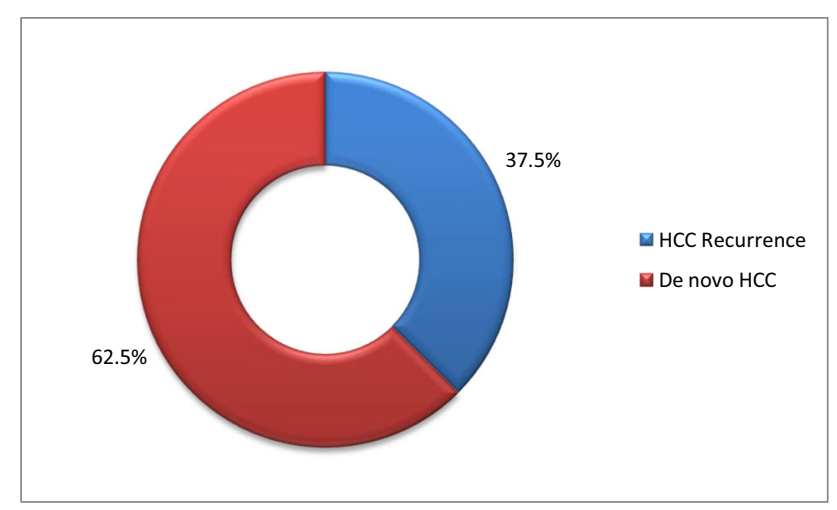




\section{$H C V-133$}

Two years follow up of chronic hepatitis c patients treated with sofosbuvir/daclatasvir: an Egyptian experience

\author{
Karim Elnoemany ${ }^{1}$, Mohamed Badr ${ }^{2}$ \\ ${ }^{1}$ Monofiya Tropical Disease, Egypt; ${ }^{2}$ Menofiya University, Egypt
}

Background: Hepatitis $\mathrm{C}$ virus (HCV) is a leading cause of chronic viral hepatitis affecting 130-150 million people world-wide. The goal of HCV therapy is to achieve sustained virological response (SVR), currently defined as undetectable serum HCV RNA 12 weeks posttreatment. Treatment with the new direct acting antivirals (DAAs) that target the $\mathrm{HCV}$ protease NS3/4A, NS5A and the NS5B polymerase proteins can lead to more than $90 \%$ SVR. The aim is to Study the long-term effect of new DAAs on treatment of HCV for 2 years of treatment and SVR.

Method: Single center cohort of adults with HCV genotype 4. The study was conducted on 1220 patients with chronic hepatitis C (all are of genotype 4) with different stages of fibrosis (F0 - F4). All patients were treated with Sofosbuvir/Daclatasvir (SOF/DAC) with or without ribavirin for 3 months. All patients (100\%) achieved sustained virological response at end of treatment (SVR12) and were enrolled in the follow-up study for 2 years after end of treatment. The patients were assigned in groups according the degree of fibrosis: F0 ( $n=284), F 1$ $(n=299), F 2 \quad(n=250), F 3 \quad(n=195)$ \& F4 $(n=192)$. All patients were followed every three months with history taking, clinical examination, liver function tests, renal function tests, alfa fetoprotein (AFP), complete blood count (CBC), PCR quantitative for $\mathrm{HCV}$, abdominal ultrasound and Fibroscan for 2 years.

Result: The rates of SVR at 12 weeks after end of treatment (SVR 12) were $100 \%$ in all groups. Of 1220 adults, 4 died and 16 lost follow-up. SVR after 24 weeks of end of treatment (SVR 24) were 99.2\%. The percentage was the same every 3 months for 2 years of follow-up. No significance difference in all laboratory and radiological investigations through 2 years. No significant difference in Fibroscan scores through 2 years of follow-up. The adverse effects noted during follow-up were development of hepatocellular carcinoma (HCC) in only 5 patients (all in $\mathrm{F} 4$ group), development of ascites in only 3 patients ( 1 in group F3 and 4 in group F4).

Conclusion: We observed a high rate of SVR 12 with (SOF/DAC). The relapse occurred in SVR 24. Patients who achieved SVR at 24 weeks were the same who achieved SVR after 2 years.

\section{$H C V-134$}

A systematic review of the extrahepatic manifestations of hepatitis $C$ infection in East Asia

Zobair Younossi ${ }^{1}$, Linda Henry ${ }^{3}$, Janus Ong ${ }^{9}$, Atsushi Tanaka ${ }^{8}$, Yuichiro Eguchi ${ }^{7}$, Masashi Mizokami ${ }^{5}$, Youngsuk Lim $^{2}$, Minglung $\mathrm{Yu}^{4}$, Maria Stepanova ${ }^{3}$, Yock Young Dan ${ }^{6}$

${ }^{1}$ Inova Fairfax Hospital, United States; ${ }^{2}$ Asian Medical Center, University of Ulsan College of Medicine, Korea, Democratic People's Republic of; ${ }^{3}$ Center for Outcomes Research in Liver Disease, United States; ${ }^{4}$ Kaohsiung Medical University Hospital, Taiwan; ${ }^{5}$ National Center for Global Health and Medicine, Japan; ${ }^{6}$ National University Hospital, Singapore; ${ }^{7}$ Saga University Hospital, Japan; ${ }^{8}$ Teikyo University School of Medicine, Japan; ${ }^{9}$ University of the Philippines, Philippines

Background: Chronic hepatitis $\mathrm{C}(\mathrm{CHC})$ infection causes a systemic infection with both hepatic and extrahepatic manifestation (EHMs). Although the prevalence of EHMs in the Western countries is well described, the prevalence HCV-related EHMs in the East Asian countries is not well known. To perform a systematic review to quantify the prevalence of selected EHMs among CHC patients in East Asia.

Method: PubMed, Medline, and Japan databases searched (1990-Dec 2016) with "hepatitis c virus" "chronic hepatitis C", "extrahepatic manifestations", and respective EHM's. Data were collected and reviewed by two per PRISMA guidelines excluding review articles. EHMs were by ICD-9 codes or clinically: depression: BDI-2 score $>19$, chronic kidney disease (CKD): eGFR of $<60 \mathrm{~mL} / \mathrm{min} / 1.73$ $\mathrm{m} 2$ per MDRD, diabetes (DM):fasting blood glucose level $>126 \mathrm{mg} /$ dL. Pooled prevalence determined by random effects models.

Result: Initially, 75 articles were identified. After applying the inclusion and exclusion criteria, 23 articles remained (Japan=6; China $=3$, Korea $=4$, Taiwan=9, Asia=1) with a total of 468,656 subjects $(\mathrm{n}=51,160 \mathrm{CHC}$ and $\mathrm{n}=417,496$ non-HCV controls). $\mathrm{HCV}$ patients' age ranged from 44-70 (mean age 55), 50\% were male (range 0\%-67\%), and over $80 \%$ of diagnosis was established through positive anti-body for HCV. CHC subjects had higher risk of lymphoma ( $\mathrm{n}=2$ reports) $4.6 \%$ vs. $2.3 \%, \mathrm{OR}=1.79(1.47-2.19), \mathrm{p}<0.0001$; $\mathrm{DM}$ ( $\mathrm{n}=11$ reports) $16.7 \%$ vs. $9.4 \%, \mathrm{OR}=1.84(1.52-2.21), \mathrm{p}<0.0001$; $\mathrm{SS}$ ( $\mathrm{n}=2$ reports) $9.6 \%$ vs. $3.5 \%, \mathrm{OR}=9.80(1.25-76.56), \mathrm{p}=0.0295 ; \mathrm{LP}$ ( $\mathrm{n}=$ reports) $8.2 \%$ vs. $4.2 \%, \mathrm{OR}=2.45(1.36-4.41), \mathrm{p}=0.0027$; depression $(\mathrm{n}=1$ report) $51.6 \%$ vs. $27.7 \%, \mathrm{OR}=2.77(1.25-6.15), \mathrm{p}=0.0121$; $\mathrm{CKD}(\mathrm{n}=5$ reports) $3.8 \%$ vs. $1.7 \%, \mathrm{OR}=2.00(1.09-3.70), \mathrm{p}=0.026$; RA ( $\mathrm{n}=2$ reports) $0.9 \%$ vs. $0.3 \%, \mathrm{OR}=2.41 \quad(1.54-3.76), \mathrm{p}=0.0001$; CVA ( $\mathrm{n}=2$ reports) $25.3 \%$ vs. $19.3 \%$, hazard ratio $1.38(1.24-1.53)$, $\mathrm{p}<0.05$; IHD ( $\mathrm{n}=1$ report) $25.3 \%$ vs $11.6 \%$, OR=1.76 (1.04-2.96), $\mathrm{p}=0.0 .034$. Additionally, the prevalence of $\mathrm{MC}$ (symptomatic and asymptomatic; $\mathrm{n}=1$ report) in $\mathrm{CHC}$ was $46.9 \%$ in $\mathrm{CHC}$ vs. $1.9 \%$ in general population with a risk ratio of 24.7 (17.2-32.2).

Conclusion: $\mathrm{CHC}$ in East Asia is associated with increased risk for EHMs. This data should inform investigators about the comprehensive impact of $\mathrm{CHC}$ infection with both hepatic and extrahepatic consequences.

\section{$H C V-135$}

Clinically meaningful differences in health-related quality of life and fatigue in patients with hepatitis $\mathrm{C}$ virus $(\mathrm{HCV})$ infection treated with elbasvir/grazoprevir (EBR/GZR) compared to sofosbuvir (SOF) with pegylated interferon and ribavirin (PR)

Chizoba Nwankwo ${ }^{1}$, Bellinda Kingkallimanis ${ }^{5}$, Shelby Corman ${ }^{5}$, Jingjun Qiu $^{3}$, Jean Marie Arduino ${ }^{4}$, Sushma Patel ${ }^{4}$, Jan Sperl ${ }^{2}$

${ }^{1}$ Merck \& Co., Inc., United States; ${ }^{2}$ Institute for Clinical and Experimental Medicine, United States; ${ }^{3}$ Merck \& Co. Inc, United States; ${ }^{4}$ Merck \& Co., Inc, United States; ${ }^{5}$ Pharmerit International, United States

Background: HCV leads to decreased health-related quality of life (HRQoL) and increased fatigue even in the absence of advanced liver disease. HRQoL is further diminished by PR regimens due to poor tolerability. It is important to measure $\mathrm{HRQoL}$ and fatigue in clinical trials, however, the clinical meaningfulness of often reported change scores is not intuitively apparent. The aim of this study is to describe and interpret the HRQoL and fatigue results of C-EDGE $\mathrm{H} 2 \mathrm{H}$ trial comparing EBR/GZR to SOF/PR in patients with HCV infection. Method: C-EDGE H2H was a randomized, open-label, multinational trial in which patients with $\mathrm{HCV}$ genotype 1 or 4 infections received 12 weeks of treatment with EBR/GZR or SOF/PR. The sample consisted of 247 patients with baseline and $\geq 1$ follow-up assessment on the SF-36v2 and FACIT-Fatigue. Changes from baseline to week 4 and 12 on treatment and week 12 after completion of treatment were 
calculated. No minimal clinically important differences (MCID) were available for fatigue or all SF-36v2 health domains except vitality where $>4.2$ has been proposed. Therefore, the difference between treatment means were converted to effect sizes (ES) and compared to a standardized criteria: $<0.2=$ small effect, $0.5=$ moderate effect, and $>0.8=$ large effect.

Result: Baseline mean HRQoL and fatigue scores were similar between treatment groups. During treatment, the ES for the difference between treatment arms on the 8 domains of the SF-36 and its physical and mental component scores (PCS and MCS) ranged from 0.25 (General Health) to 0.58 (Vitality) at week 4 and 0.42 (Bodily Pain) to 0.70 (MCS) at week 12. These differences between treatment arms represent a clinically meaningful impact on HRQoL favoring EBR/GZR, reflecting a moderate ES. After completion of treatment, ES ranged from 0.01 (Bodily Pain) to 0.15 (MCS), reflecting a small effect. Change scores reflected overall improvement in comparison with baseline HRQoL for both groups at follow-up. Difference in mean change from baseline between groups for Vitality exceeded the MCID $>4.2$ during treatment. The same pattern regarding ES was seen for the total FACIT-Fatigue score. The ES was 0.56 at week 4, 0.58 at week 12 , and -0.04 at follow-up.

Conclusion: This study has shown that during treatment, patients randomized to SOF/PR had a clinically meaningful decrease in overall HRQoL and fatigue compared to those in the EBR/GZR group. The largest ES was observed at week 12 for MCS.

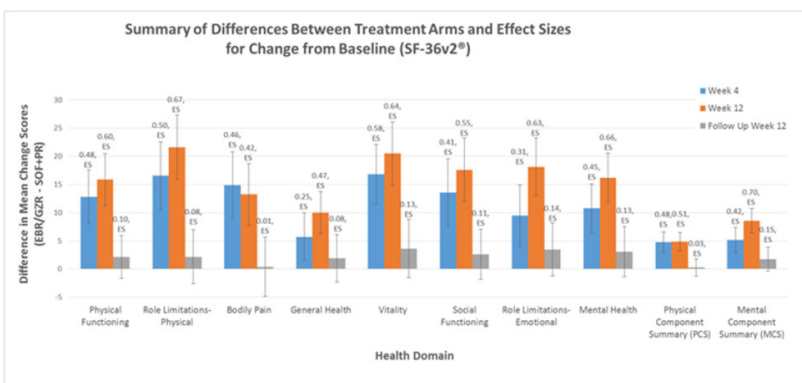

$H C V-136$

Decompensation of hepatitis C patients after direct-acting antiviral treatment

\section{Nyein Mon $\mathrm{Yu}^{1}$, Win Naing ${ }^{1}$}

${ }^{1}$ University of Medicine (I), Yangon, Myanmar, Myanmar

Introduction: With the availability of direct-acting antivirals (DAAs), treatment of a large number of patients becomes possible. Interferon has been contraindicated in decompensated cirrhotic patients and now the guidelines recommend use of DAAs in these patients. But experiences in real world studies are limited. Here we report 2 cases of liver decompensation after DAA treatment.

Case Description: A 61 year old lady with known history of hepatitis $\mathrm{C}$ related liver cirrhosis was started on DAA treatment (Sofosbuvir and Daclatasvir). Her initial viral load was $84,000 \mathrm{IU} / \mathrm{ml}$ and genotype was 3b. Child-Pugh-Turcotte grade was B (9) and MELD score was 13 . The viral load at week 4 was reduced to $17 \mathrm{IU} / \mathrm{ml}$ but she suffered from increasing abdominal distension and intermittent fever followed by jaundice. Her serum bilirubin level increased up to $16 \mathrm{mg} / \mathrm{dL}$, Child's score C (12) and MELD score 23. Ultrasound abdomen showed cirrhosis of liver with massive ascites and oesophago-gastro-duodenoscopy showed Grade IV oesophageal varices with red signs. The DAA treatment was stopped and treated as possible drug-induced liver injury and spontaneous bacterial peritonitis but the patient did not improve with increasing serum bilirubin up to $29 \mathrm{mg} / \mathrm{dL}$ and deteriorating general condition. As deceased donor liver transplantation was not well developed in Myanmar, the patient finally passed away with decompensated cirrhosis.

Result: The second case was about a 49 year old lady with Child (C) Cirrhosis, also of genotype 3 and HCV viral load $21680 \mathrm{IU} / \mathrm{ml}$. Fibroscan showed advanced fibrosis with liver stiffness measuring about $69.2 \mathrm{kPa}$. She was started on Sofosbuvir/Velpatasvir plus ribavirin regimen. The ribavirin dose was $800 \mathrm{mg}$ per day. She also presented with acute abdominal pain after 4 weeks of DAA treatment. HCV RNA was undetectable at week 4 but serum bilirubin increased from $3 \mathrm{mg} / \mathrm{dL}$ to $6.6 \mathrm{mg} / \mathrm{dL}$. She was treated with empirical antibiotics for spontaneous bacterial peritonitis but her serum bilirubin levels increased to $14.4 \mathrm{mg} / \mathrm{dL}, 20 \mathrm{mg} / \mathrm{dL}$, and $33 \mathrm{mg} / \mathrm{dL}$ on Day 47 after DAA. The treatment was stopped finally but the patient's conditions deteriorated further.

Conclusion: Many trials have proved the efficacy of DAAs in decompensated cirrhosis with improved MELD scores. But it is not without risks. Frequent follow-up visits and monitoring are recommended in treating decompensated cirrhotic patients.

\section{$H C V-137$}

Description of the course and outcomes of antiviral treatment of $1000 \mathrm{HCV}$ infected patients Hasmik Ghazinyan, A. Asoyan, A. Muradyan, T. Asatryan, T. Galstyan, V. Sargsyan, N. Sargsyants, N. Stepanyan, A. Mkhitharyan, M. Shmavonyan, V. Asoyan, M. Gemilyan, S. Hakopyan, S. Tarkhanyan, R. Abovyan, N. Romanova

Hasmik Ghazinyan ${ }^{1}$, Ara Asoyan', Anzhela Muradyan', Tigranuhi Asatryan $^{1}$, Violeta Sargsyan ${ }^{2}$, Narina Sargsyants ${ }^{2}$, Naira Stepanyan ${ }^{2}$, Aregnaz Mkhitaryan', Vigen Asoyan ${ }^{5}$, Manik Gemilyan $^{5}$, Sergey Hakobyan ${ }^{5}$, Srbuhi Tarkhanyan ${ }^{5}$, Melanya Shmavonyan $^{5}$, Romela Abovyan ${ }^{3}$, Naira Romanova ${ }^{4}$, Tsovinar Galstyan ${ }^{1}$

${ }^{1}$ Nork Clinical Hospital of Infectious Diseases, Armenia;

${ }^{2}$ Armenicum Medical Center, Armenia; ${ }^{3}$ National Center Diseases control and Prevention, Armenia; ${ }^{4}$ Scientific Centre of Drug and Medical Technology Expertise, Armenia; ${ }^{5}$ Yerevan State Medical University, Armenia

Background: $130-150$ million people globally have Chronic Hepatitis C infection. Approximately 500.000 people die each year from HCV-related liver diseases. Direct-acting antiviral agents (DAA) for HCV have initiated a revolution in the management and control of this important liver disease.

Aim: Our study aimed at describing the course and outcomes of the antiviral treatment with DAA of $1000 \mathrm{HCV}$ infected patients in Armenia.

Method: $1000 \mathrm{HCV}$ infected patients were included in this study. The mean age was 48 (ranging from 17-81) with a male dominance $65 \%$. Pre-treatment evaluation included HCV RNA, HBsAg, Anti-HBcAg, Anti-HIV, genotyping of $\mathrm{HCV}$, staging of fibrosis by elastometry (Fibroscan) as well as biochemical and instrumental investigations. A single regimen was selected for undertaking the treatment - Sovaldi and Daclatasvir for naïve patients with all genotypes. Ribavirin was added to the scheme in treatment-experienced and cirrhotic patients. The duration of treatment was 12 weeks with assessment of efficacy including detection of HCV RNA at 4 weeks of treatment (RVR) and at the end of treatment (EOT).

Result: Out of 1,000 HCV infected patients, 76 (7.6\%) were coinfected with HIV and $3(0.3 \%)$ with HBV; 56 (5.6\%) were patients 
with relapse of HCV infection, 50 (89\%) with previously IFN based treatment. $183(18.3 \%)$ patients were with cirrhosis. Seventy (7\%) patients were PWID. The HCV genotype distribution was as follows: G1-46\%, G2-14\%, G3-37.5\%, G4-1\%, and unknown 1.5\%, and the proportions of fibrosis stages (by Metavir) were: F0-28\%, F1-12\%, F2-17\%, F3-13\%, F4-30\%.

During the treatment, $25(2.5 \%)$ patients were discharged from treatment, 5 of them due to death, 2 of which were due to liver disease progression, 2 due to cardiovascular disease and 1 to hemodialysis complications. Treatment of 8 patients was stopped, 4 of which due to intolerance to drug, 4 due to liver disease decompensation, and 12 patients stopped the treatment arbitrary by themselves. $82(8,2 \%)$ of treated patients had other side effects (SE) that did not cause cessation of treatment. SEs were more in male $(73 \%)$, freguently general impairment in $55 \%$ of them, digestive disorder and neurological impairment in $22 \%$ respectively.

The efficacy of treatment: at 4 weeks, HCV RNA (RVR) was positive in $103(10.3 \%)$ patients. Among 986 patients who completed treatment HCV RNA was positive at EOT in only $38(3.85 \%)$ patients. Among these 38 patients, 30(79\%) were infected with HCV genotype 3, $32(94 \%)$ had cirrhosis and $25(66 \%)$ had relapse of HCV infection.

Conclusion:

- The burden of HCV- related liver disease is increasing in Armenia.

- New treatment with DAA including Sovaldi and Daclatasvir pangenotypic combination is effective and safe $(96 \%$ aviremia at the EOT).

- Genotype 3, fourth stage of fibrosis and relapse of HCV infection were the main risk factors of viremia at the EOT.

- The rates of side effects were comparable to those reported in international studies.

\section{$H C V-138$}

Estimated 5-year acquisition cost of direct acting antiviral (DAA) for the treatment of hepatitis $\mathrm{C}$ in Malaysia in 2018 to 2022

\section{Rosmawati Mohamed ${ }^{1}$, Fatiha Hana Shabaruddin ${ }^{1}$, Maznah} Dahlui $^{1}$, Amirah Azzeri ${ }^{1}$, Scott Alexander Mcdonald ${ }^{2}$

${ }^{1}$ University of Malaya, Malaysia; ${ }^{2}$ Centre of Excellence for Research in AIDS (CERiA), University of Malaya, Kuala Lumpur, Malaysia, Malaysia

Background: Malaysia is yet to adopt direct-acting antivirals (DAA) as standard hepatitis $\mathrm{C}$ treatment. Access to generic DAAs became possible recently through voluntary licensing programs. This analysis aimed to estimate the 5-year DAA drug acquisition costs (2018 to 2022) for the Ministry of Health, based on three possible scenarios for Malaysia. All scenarios assumed DAA with or without ribavirin based on recommendations from the most recent guidelines.

Method: The assumed population is patients within tertiary care, based on projections from a national HCV disease model. Scenario 1 assumed the use of sofosbuvir and velpatasvir at RM6000 (USD 1500 ) for 12 weeks of treatment through private purchasing by individual hospitals. Competitive market pricing through direct government negotiations with voluntary license manufacturers were assumed for: Scenario 2 with the use of sofosbuvir and velpatasvir at RM3000 (USD 750) and Scenario 3 with sofosbuvir and daclatasvir at RM1250 (USD300) respectively for 12 weeks of treatment.

Result: The first-year (2018) costs for Scenarios 1 and 2 with sofosbuvir and velpatasvir are estimated to be RM9.1 (USD 2.2) and RM4.7 (USD 1.2) million respectively, gradually rising to RM21.4
(USD 5.3) and RM10.9 (USD 2.7) million respectively by 2022. The total 5-year DAA acquisition costs for Scenarios 1 and 2 with sofosbuvir and velpatasvir are RM76.5 (USD 18.8) and RM39.1 (USD 9.6) million respectively. The estimated 5-year SVR cure rate for Scenarios 1 and 2 with sofosbuvir and velpatasvir is 97\%. For Scenario 3, the first year cost for sofosbuvir and daclatasvir is estimated to be RM7.8 (USD 1.9) million, rising to RM18.4 (USD 4.5) million in 2022. The total 5-year DAA acquisition costs for sofosbuvir and daclatasvir are RM65.7 (USD 16.2) million with an estimated SVR cure rate of $91 \%$. Treatment with sofosbuvir and velpatasvir (Scenarios 1 and 2) is estimated to achieve higher SVR rate than sofosbuvir and daclatasvir (Scenario 3).

Conclusion: Acquiring sofosbuvir and velpatasvir via direct government negotiations with voluntary license manufacturers (Scenario 2 ) is the most effective and least costly approach to improve access of DAA in Malaysia.

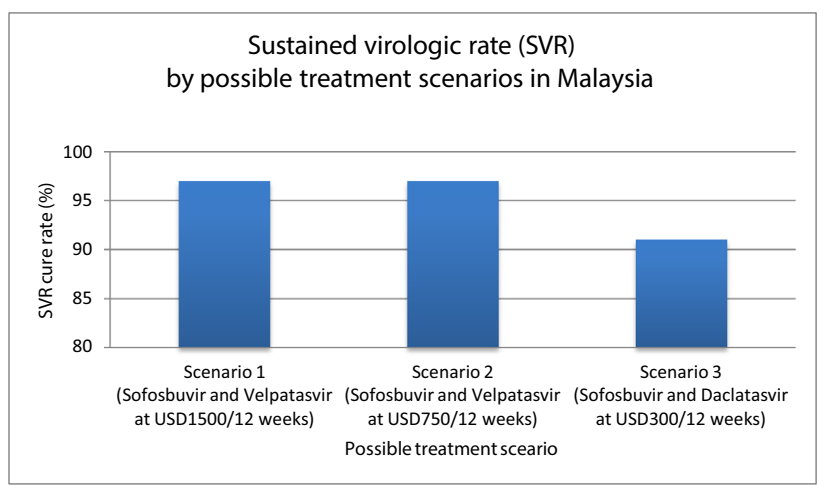

$H C V-139$

Frequency of depression; during pegylated interferon therapy for chronic hepatitis $\mathrm{C}$

\section{Muhammad Adnan}

${ }^{1}$ Isra University, Pakistan

Background: Combine Pegylated interferon therapy and ribavirin is most commonly used treatment in chronic hepatitis $\mathrm{C}$ patients. Along with other complications, different psychiatric disorders are observed during combination therapy including depression. Depression is the major morbidity as it may lead to different destructive ideation including suicide among patients on treatment. We have tried to calculate the frequency of depression along with associated factors in our present study.

Method: Objectives: To nd out the frequency of depression during Pegylated interferon therapy for chronic hepatitis C.

Study Design: Descriptive study. Setting: Liaquat University of Medical \& Health Sciences, Jamshoro/Hyderabad Duration:

The duration of study was six months starting from 01-01-2014 to 31-06-2014.

Methodology: Two hundred and fty two cases of chronic hepatitis $\mathrm{C}$ on the basis of anti HCV (ELISA) and PCR positive were selected in this study.

Result: Results: Out of 252 patients, 111 (44\%) were males and $141(56 \%)$ were females. Most of the patients belonged to 26 to 50 years of age. The duration of treatment was 12 weeks. Two hundred \& eight $(82.5 \%)$ patients had ever married while the remaining $17.5 \%$ were unmarried. Out of 106 (42\%) depressed patients, 39 (37\%) had mild, 31 (29\%) had moderate, 20 (19\%) had moderately severe and 15 $(15 \%)$ had severe depression. 
Conclusion: Conclusion: It was observed that depression is a common during combination therapy with Peg interferon and the ribavirin in chronic $\mathrm{HCV}$ patients.

\section{$H C V-140$}

Hepatitis C therapy; a single dose of ledipasvir / sofosuvir for 6 months? or single dose + ribavirin 3 months? Single center real life data

\section{Ahmet Uyanikoglu ${ }^{1}$, Ahmet Uyanikoglu ${ }^{2}$, Ahmet Konca ${ }^{2}$, Necati Yenice $^{2}$ \\ ${ }^{1}$ Harran University, Turkey; ${ }^{2}$ Harran University, Medical Faculty, Turkey}

Background: The frequency of hepatitis $\mathrm{C}$ virus (HCV) in our country is around $1 \%$. In this study, it was aimed to compare the results of treatment with ledipasvir $90 \mathrm{mg} /$ sofosbuvir $400 \mathrm{mg}$ for 6 months (group 1) and ledipasvir $90 \mathrm{mg} /$ sofosbuvir $400 \mathrm{mg}+$ ribavirin $200 \mathrm{mg}$ (2X3) 3 months (group 2).

Method: The characteristics of patients, treatment side effects and treatment outcomes of patients were evaluated, between October 2016 and March 2017. The patient's monthly policlinic controls were performed. End of the first month, end of the treatment, and 3 month after, end of treatment HCV RNA evaluated. Treatment is continuing in some of the patients and the results of the initial evaluation are presented in this study.

Result: Of the total 35 patients, $18(52 \%)$ were male and mean age was $63.2 \pm 11.9$ (range 22-86) years. Twenty two of the patients (63\%) were cirrhotic. Group 1 had 17 patients (48\%), 9 men $(53 \%)$ and a mean age was $64 \pm 14.3$ (range 22-86), 15 were cirrhotic (88\%). 9 patients were genotype 1,6 genotype $1 \mathrm{~b}, 1$ genotype $1 \mathrm{a}, 1$ genotype 4 . Treatment indications were cirrhosis 10 patients $(59 \%)$, recurrence with/without cirrhosis 5 patients, chronic renal failure (CRF) 1 patient and in post transplantation recurrence 1 patients. 16 of these patients (95\%) achieved rapidly virologic response (RVR), 1 patient was HCV-RNA positive end of first month. End-of-treatment response (EVR) in $8 / 8$ patients, and 12 week after treatment, sustained virological response (SVR12) in 5/5 patient. One patient died of complications of CRF and 1 patient died of advanced hepatocellular cancer (HCC) during the follow-up. Group 2 had 18 patients $(52 \%), 9$ were male $(50 \%)$ and the mean age was $62.3 \pm 9$. (range 35-73). 11 patients were genotype 1,6 genotype $1 \mathrm{~b}, 1$ genotype $1 \mathrm{a}$. Treatment indications in these patients were cirrhosis in 4 patients, recurrence with or without cirrhosis 11 , recurrence after transplantation in 2 patients, and CRF in 1 patient. In group 2, RVR was obtained in all 18 patients, EVR in 16/16 patients and SVR12 in 14/14 patients. In two patient, HCC was detected, second month of treatment and 2 months after the end of treatment. Patient follow-up and treatment were continuing.

Conclusion: Our HCV patients are mostly genotype I, mostly cirrhotic, half of the patients are elderly, two of third treatment experienced. Two the patients were died due to HCC and CRF, and two patients had HCC during and after treatment. In the other patients, the first treatment results were successful in both groups.
HCV-141

Hepatitis $\mathrm{C}$ treatment with ledipasvir and sofosbuvir in a low socioeconomic population at a Federally Qualified Health Center [FQHC]

$\underline{\text { Zohha Tariq Alam }}^{1}$, Hisham Malik ${ }^{1}$, Chellyanne Hinds ${ }^{2}$, Jordan Niquette $^{2}$, Imtiaz Alam ${ }^{1}$

${ }^{1}$ Austin Hepatitis Center, United States; ${ }^{2}$ Community Care, United States

Background: CommUnityCare [CUC] Hepatitis C clinic (FQHC) was launched in March 2014. CUC serves as a safety net provider to Austin, Texas' high risk uninsured, homeless and refugee population. The clinic offered Hepatitis $\mathrm{C}[\mathrm{HCV}]$ treatment to patients in whom barriers such as cost, unstable living environments and medical comorbidities had previously limited access to treatment to therapy. Treatment included direct acting antiviral (DAA), Ledipasvir and Sofosbuvir, for 8,12 or 24 weeks per AASLD guideline recommendations.

Method: Single center retrospective analysis was conducted on HCV patients who received the above therapy. HCV RNA levels were measured using a COBAS ${ }^{8}$ Ampliprep/COBAS ${ }^{\circledR}$ TaqMan ${ }^{\circledR} \mathrm{HCV}$ Test with a lower level of detection $<15$ IU/L. Sustained viral response [SVR12] was defined as undetectable HCV RNA at 12 weeks post treatment. The primary endpoint was the proportion of patients who had undetectable SVR12 weeks post therapy.

Result: May 2015 to August 2017, 513 patients were treated with Ledipasvir and Sofosbuvir. Mean age 53 years; $67 \%$ males; $66 \%$ were uninsured; $98 \%$ had genotype 1 and $2 \%$ were genotypes $2-4 ; 14 \%$ had cirrhosis. We looked at intention to treat analysis (ITT) and per protocol analysis (PPA - patients who completed either 8,12 or 24 weeks of therapy as indicated and returned for SVR12. $n=293$ ). SVR12 was $80 \%$ per ITT vs. $99 \%$ per PPA [ $\mathrm{n}=291] .93 \%$ received 12 weeks treatment vs. $3 \%$ received 24 weeks vs. $3 \%$ received 8 weeks; 2 relapsed. $28 \%$ were lost to follow up [ $\mathrm{n}=144$ ]. 69 patients had undetectable HCV RNA during therapy but never returned for SVR12. 7 patients stopped therapy and 2 patients who died during or shortly after stopping medication had decompensated cirrhosis. 2 African-American patients who relapsed, were genotype 1a, did not have cirrhosis and had completed only 12 weeks of treatment. 2 of the 3 genotype 3 patients achieved SVR 12 and 1 patient stopped therapy. 1 genotype 2 patient achieved SVR12. 2 out of 4 genotype 4 patients achieved SVR12.

Conclusion: Our data demonstrates that treatment with Ledipasvir and Sofosbuvir can allow this vulnerable and complex population to achieve SVR12 [99\% PPA] with rates comparable to published study data. Discrepancy between SVR12 ITT and PPA rates are attributed to the lack of transportation, relocation of patient and change in patient contact information.

$H C V-142$

Eliminating hepatitis C virus (HCV) in Indonesia

Sarah Blach $^{1}$, Irsan Hasan $^{3}$, Naning Nugrahini ${ }^{2}$, Sigit Priohutomo $^{2}$, Eva Yuzwar ${ }^{2}$, Homie Razavi ${ }^{1}$, Sarah Blach ${ }^{1}$, Laurentius A. Lesmana ${ }^{4}$, Ali Sulaiman ${ }^{3}$, Andri Sanityoso Sulaiman $^{3}$, Samsuridjal Djauzi ${ }^{3}$, Rino A Gani ${ }^{3}$

${ }^{1}$ Center for Disease Analysis, United States; ${ }^{2}$ Disease Control \& Environmental Health, Ministry of Health, Jakarta, Indonesia, Indonesia; ${ }^{3}$ Division of Hepatobiliary, Department of Internal Medicine, Faculty of Medicine, University of Indonesia, Dr. Cipto Mangunkusumo Hospital, Jakarta, In, Indonesia; ${ }^{4}$ Division of 
Hepatobiliary, Faculty of Medicine, University of Indonesia, Dr. Cipto Mangunkusumo Hospital, Indonesia

Background: In 2013, the Indonesian National Health Survey (Riskesdas) found an anti-HCV prevalence of $1.0 \%$, with a prevalence among children and adolescents (1-14 years of age) of $0.6 \%$. Despite this high prevalence, only 600 patients were initiated on treatment in 2016. To support strategic planning for HCV elimination by 2030 , treatment and diagnostic targets are needed. This study sought to quantify the burden of $\mathrm{HCV}$ and identify strategies to eliminate $\mathrm{HCV}$ from Indonesia.

Method: The current and future disease progression of the HCV infected population was forecast using an Excel-based Markov model. The impact of intervention strategies, (including prevention, treatment and screening) on the projected disease burden was measured. Result: The average age of the HCV infected population in Indonesia is $40-44$ years. Over the next 13 years, all cause mortality, liver related deaths and treatment will result in a $<5 \%$ reduction in $\mathrm{HCV}$ infections. However, the number of patients with end stage liver diseases and liver related deaths will increase by more than $55 \%$, as the infected population ages (Figure 1).

Achieving the global health sector strategy targets for HCV elimination (90\% reduction in new cases, $90 \%$ diagnosed, $65 \%$ reduction in liver related deaths by 2030) requires a comprehensive approach including increased prevention, diagnosis and treatment, as shown in table 1. In the short term (before 2020) enhancing screening and linkage to care will be especially important, in order to ensure there will be enough patients to treat. After 2020, a test and treat paradigm will be necessary. The resulting impact of these measures on disease burden is shown in figure 1 .

Conclusion: Low baseline treatment in Indonesia is currently prohibitive for HCV disease management and elimination goals. In order to achieve the diagnosis and treatment targets required for elimination, substantial capacity building may be necessary. However, simplified treatment regimens provide a solid first step towards this goal.

\begin{tabular}{|c|c|c|c|c|c|c|}
\hline & \multicolumn{2}{|c|}{ Past efforts } & \multicolumn{4}{|c|}{ Future efforts required for elimination } \\
\hline & 2015 & $\overline{2016}$ & 2017 & 2019 & 2021 & 2023 \\
\hline Treated & 230 & 600 & 15,000 & 60,000 & 80,000 & 95,000 \\
\hline Newly diagnosed & 12,100 & 18,000 & 30,000 & 72,000 & 80,000 & 95,000 \\
\hline Fibrosis stage & $\geq$ F0 & $\geq \mathrm{F} 2$ & $\geq \mathrm{F} 1$ & $\geq \mathrm{F} 0$ & $\geq \mathrm{F} 0$ & $\geq \mathrm{F} 0$ \\
\hline New infections & 24,100 & 19,300 & 14,500 & 10,100 & 5,000 & 2,300 \\
\hline Treated age & $15-64$ & $15-64$ & $15-64$ & $15-69$ & $15-69$ & $15-74$ \\
\hline SVR & $77 \%$ & $87 \%$ & $95 \%$ & $95 \%$ & $95 \%$ & $95 \%$ \\
\hline
\end{tabular}

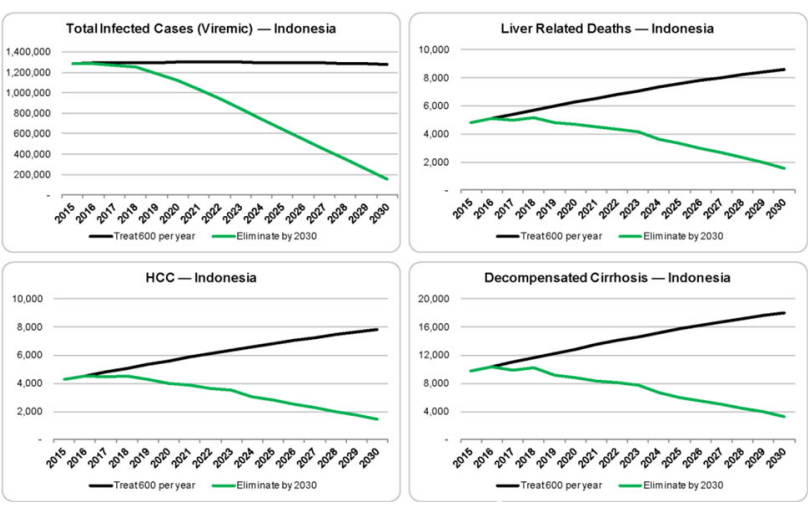

$H C V-143$

Is serum angiotensin-converting enzyme level a useful noninvasive marker for liver fibrosis in patients with chronic hepatitis $\mathbf{C}$ ?

\section{Tark Akar}

${ }^{1}$ Bülent Ecevit University Medical Faculty, Turkey

Background: Chronic hepatitis $\mathrm{C}(\mathrm{CHC})$ is still a critical problem for all over the world. The determination of the stage of liver fibrosis is the main stone of treatment selection and prediction of long-term prognosis. Liver biopsy is still considered as a standard gold method, but, due to unmet needs, new non-invasive markers are required. The aim of this study was to investigate any possible relationship between the serum angiotensin-converting enzyme (ACE) level and the stages of liver fibrosis in patients with CHC.

Method: A total $100 \mathrm{CHC}$ patients and 100 healthy subjects were enrolled in this study. The relationship between the serum ACE level and the stages liver fibrosis were investigated by stratifying the liver fibrosis into three different formats as following; [ $\operatorname{group}(\mathrm{G})-\mathrm{I}$; classic Ishak's Score from F1 to F6; G-II; mild (F1-2), moderate (F3-4) and severe (F5-6); G-III; mild $(<\mathrm{F} 2)$ and advanced $(\mathrm{F}>2)]$. The clinical usability of serum ACE level for both groups was also investigated. Result: The serum median ACE level was higher in the healthy group than in CHC [(42.5 (7-119) vs 36 (7-91) $\mathrm{U} / \mathrm{I}<\mathrm{em}>, \mathrm{p}=0,002</ \mathrm{em}>]$. There was no statistically significant difference among the three different fibrosis groups (G-I, G-II, G-III, $<\mathrm{em}>\mathrm{p}=0.797$, $\mathrm{p}=0.986$, and $\mathrm{p}=0.874</ \mathrm{em}>$ ) and was no correlation between serum ACE level and the stages of liver fibrosis ( $r ; 0.026, p=0.923)$. The usability of serum ACE for evaluated patients with $\mathrm{CHC}$ and healthy subjects were calculated as $47 \%$ and $64 \%$, respectively.

Conclusion: Our study indicated that there was no relationship and correlation between the serum ACE level and the stages of liver fibrosis in patients with CHC. The assessment of serum ACE level using genetically corrected reference values may provide more accurate results than alone.

\section{$H C V-144$}

Rapid development of Hepatocellular Carcinoma after eradication of hepatitis C virus with Directly Acting Antiviral treatment

\section{Fakhar Ali Qazi Arisar ${ }^{1}$}

\section{${ }^{1}$ The Aga Khan University Hospital, Pakistan}

Background: Hepatitis $\mathrm{C}$ is a major risk factor for the development of hepatocellular carcinoma (HCC), arising typically on a background of liver cirrhosis. Treatment of hepatitis $\mathrm{C}$ has been revolutionized by the addition of oral direct-acting antivirals (DAAs) with sustained virological response (SVR) rates above $90 \%$. There is a recent concern under debate about the increased risk of early $\mathrm{HCC}$ recurrence in patients with chronic hepatitis $\mathrm{C}(\mathrm{CHC})$ who were treated with directacting antivirals. Nonetheless, these reports mostly focused on patients who were cirrhotic and were already treated for HCC.

Method: We report four cases of treatment naïve, chronic hepatitis $\mathrm{C}$ patient who were treated with DAAs and rapidly developed infiltrative HCC.

Result: All four cases were treated with sofosbuvir and ribavirin for 6 months, and they develop infiltrative exophytic HCC within a year of completion of therapy, despite achieving SVR. Moreover, in our scenario, one of the patient appeared clinically non-cirrhotic till he developed HCC. 
In all of our patient, there was a rapid development of HCC, supported by recent negative imaging before the actual diagnosis. In two of our patients, at the time of diagnosis, tumor appeared infiltrative on CT scan with portal vein thrombosis making it advance staged. On follow up scan involvement of whole liver parenchyma within 3 months in case 1 also supports the speediness of tumor growth. This also suggests that patients treated for $\mathrm{HCV}$, despite being non-cirrhotic, should be kept in surveillance even after achieving SVR.

Conclusion: In conclusion, there is debatable increased risk of HCC occurrence/early recurrence in patients treated with DAAs. Our cases supports the existing data about the pattern of HCC occurrence after DAA therapy, however, large prospective studies are needed to solve this mystery.
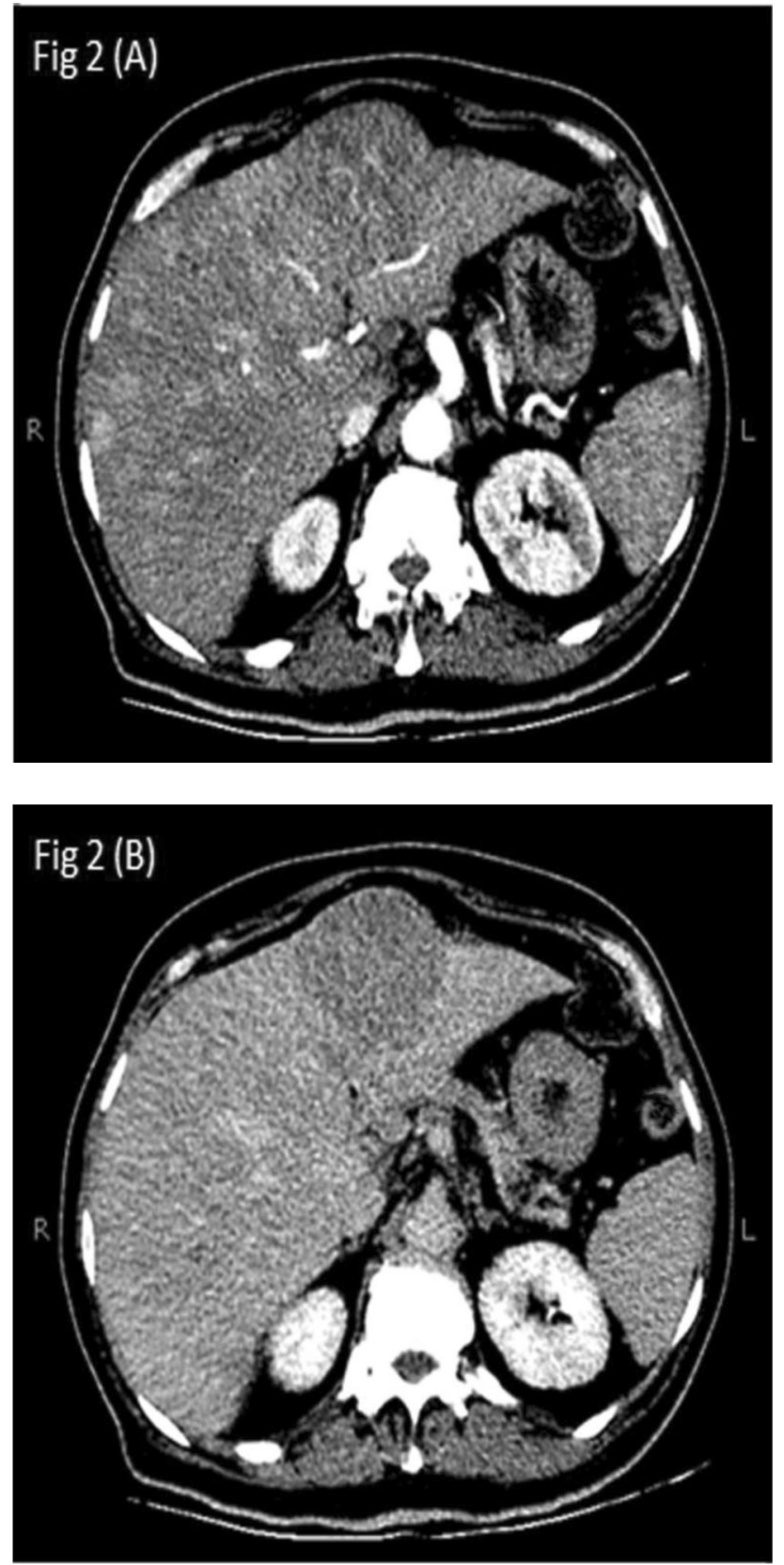

HCV-145

Direct acting antivirals in treatment of $\mathrm{HCV} / \mathrm{HIV}$ co-infected patients: real life experience from Nepal

\section{Sudhamshu KC ${ }^{1}$, Dilip Sharma ${ }^{1}$, Niyanta Karki ${ }^{1}$, Sandip} Khadka $^{1}$, Dipendra Khadka ${ }^{1}$, Kiran Regmi ${ }^{1}$

${ }^{1}$ National Academy of Medical Sciences, Nepal

Background: Direct acting antivirals (DAA) have revolutionized the treatment of chronic hepatitis $\mathrm{C}$ patients. However, the real-life data regarding its use in HIV co-infection from developing country is lacking. We aimed to see the efficacy of DAA in HCV HIV coinfected populations.

Method: In this prospective observational study from Nepal between April 2015 till July 2017, treatment naïve patients undergoing treatment for chronic HCV infection in HIV co-infected individuals with DAA were included in the analysis. Patients on nevirapine were switched to efavirenz or atazanavir. Patients received sofosbuvir/ ledipasvir, sofosbuvir/daclatasvir with or without ribavirine. Dose of daclatasvir and ribavirine was adjusted accordingly. Sustained virological response at week 12 (SVR12), adverse events and treatment compliance were evaluated. Treatment efficacy was compared between cirrhotic and non-cirrhotic patients.

Result: Of 218 patients presenting with anti HCV report $181(83 \%)$ had detectable HCV RNA. 85 (47\%) patients were having ART at presentation. 3 patients could not complete treatment due to gall stone pancreatitis and 82 completed treatment. $29(35 \%)$ were cirrhotic at presentation. $51(62 \%)$ patients were genotype 3, 27 (33\%) were genotype $1,3(4 \%)$ were mixed $1 \mathrm{a} / 3$ and $1(1 \%)$ was 6 . Most of the patients were having undetectable HIV virus. 74 (90\%) had SVR12. Non-cirrhotics had $96 \%$ SVR compared to $79 \%$ in cirrhotics. SVR was in genotype 3 was $88 \%$ while it was $93 \%$ in genotype 1 .

Conclusion: Real life experience showed that the DAAs are equally effective in HCV HIV co-infected patients. In non-cirrhotic patients, the result is comparable to mono infected patients. Genotype 3 coinfected are also difficult to treat patients. Unlike interferon based treatment, DAA treatment is well tolerated in HCV HIV co-infected patients and there was no drop out during treatment.

\section{$H C V-146$}

High sustained virological response rates for patients with hepatitis c genotype 2 treated with sofosbuvir and ribavirin in the real world - an Australian nursing experience

Vincenzo Fragomeli ${ }^{1}$, Saroja Nazareth ${ }^{4}$, Nadine Leembruggen ${ }^{4}$, Anton Colman $^{3}$, Sally Spruce ${ }^{2}$, Susan Mason ${ }^{5}$

${ }^{1}$ Nepean Hospital, Australia; ${ }^{2}$ Mid North Coast Local Health District, Australia; ${ }^{3}$ Royal Adelaide Hospital, Australia; ${ }^{4}$ Royal Perth Hospital, Australia; ${ }^{5}$ Royal Prince Alfred Hospital, Australia

Background: Historically, hepatitis C (HCV) was treated with interferon and ribavirin. Treatment was associated with significant adverse events and suboptimal sustained virological (SVR) rates. The era of direct acting antivirals has dramatically changed the HCV treatment landscape with high SVR rates and minimal side effects. For some patient groups however, ribavirin continues to be an important part of therapy. In Australia, patients with genotype 2 are prescribed ribavirin in combination with sofosbuvir. This study aims assess the safety, tolerability and efficacy of sofosbuvir and ribavirin combination therapy for genotype 2 patients in the real world.

Method: Data was collected from 30 patients with genotype $2 \mathrm{HCV}$ who commenced on sofosbuvir plus ribavirin across four Liver 
Clinics in Australia. Baseline characteristics, treatment outcomes, pathology results, adherence, compliance and adverse events were collected.

Result: Mean age 60 years, $66.7 \%$ male and $96.7 \%$ Caucasian. The majority of patients were naïve to prior $\mathrm{HCV}$ therapy (80\%), $10.0 \%$ prior non-response. 3 patients were cirrhotic (10\%) and 6 had advanced fibrosis (20\%). The overall SVR rate was 96.6\% (29/30). One patient relapsed post cessation of therapy (3.3\%). This patient was a 62 year old Caucasian male with no fibrosis and prior nullresponse to HCV therapy. 24 patients reported adverse events (80\%). In total 57 adverse events were reported, 41(71.9\%) were mild in severity, $15(26.3 \%)$ were moderate. Severe fatigue was recorded by one patient $(3.3 \%)$. Most common adverse events were fatigue $(70 \%)$, headache $(20 \%)$, pruritus $(16.7 \%)$, insomnia $(16.7 \%)$, dyspnoea $(16.7 \%)$ and irritability (16.7\%). One serious adverse event occurred at week 12 of treatment, with a patient hospitalised for pneumonia. Mean haemoglobin at day 1 was $146 \mathrm{~g} / \mathrm{L}$. Haemoglobin decreased during therapy (mean decrease $14 \mathrm{~g} / \mathrm{L}$ ) but returned to baseline levels at week 12 follow-up (mean $148 \mathrm{~g} / \mathrm{L}$ ). One patient experienced haemoglobin below $100 \mathrm{~g} / \mathrm{L}$ (lowest report $97 \mathrm{~g} / \mathrm{L}$ ). Ribavirin dose was reduced and anaemia resolved post treatment. No patients underwent transfusion or erythropoietin therapy. All patients completed 12 weeks of therapy and 12 weeks of follow-up. $90 \%$ of patients attended all scheduled appointments. Three patients (10\%) missed two appointments. Four patients (13.3\%) missed doses of sofosbuvir and/ or ribavirin.

Conclusion: Sofosbuvir and ribavirin combination therapy for genotype $2 \mathrm{HCV}$ was associated with excellent treatment outcomes in the real world setting. The treatment was well-tolerated with most adverse events reported as mild in severity. Laboratory abnormalities were rare with only one patient experiencing anaemia. This small study is an encouraging example of high SVR rates and minimal adverse events in situations where the available therapy continues to include ribavirin.

\section{$H C V-147$}

Successful retreatment of 12 -week combination regimen of grazoprevir and elbasvir for HCV GT1b patient, who failed to be treated by sofosbuvir/ledipasvir due to ventricular tachycardia

Tatsuo Kanda ${ }^{1}$, Mitsuhiko Moriyama ${ }^{1}$, Shingo Nakamoto ${ }^{2}$, Masato Nakamura ${ }^{2}$, Tetsuhiro Chiba ${ }^{2}$, Hitoshi Maruyama ${ }^{2}$, Shunichi Matsuoka ${ }^{1}$, Naoya Kato ${ }^{2}$

${ }^{1}$ Nihon University School of Medicine, Japan; ${ }^{2}$ Chiba University, Graduate School of Medicine, Japan

Background: Sofosbuvir-including regimen elicits cardiovascular events during the treatment. But it is not clear which regimens should be selected to retreat the patients, who experienced cardiovascular events during the sofosbuvir-including regimens. We experienced the successful retreatment of 12-week combination regimen of $\mathrm{HCV}$ NS3/4A inhibitor grazoprevir and NS5A inhibitor elbasvir for 67-year-old Japanese male infected with HCV GT1b, who failed to be treated by NS5B inhibitor sofosbuvir/NS5A inhibitor ledipasvir.

Method: N/A.

Result:
Case: $\mathrm{He}$ is interferon-naïve and had diabetes mellitus and apical hypertrophic cardiomyopathy as comorbidities. His liver stiffness was $7.0 \mathrm{kPa}$ by transient elastography (FibroScan) and platelet count was $19,6000 / \mu \mathrm{L}$, indicating that he had no cirrhosis. His renal function was almost normal (estimated GFR, $50 \mathrm{ml} / \mathrm{min}$ ). When he was 66-year-old, ventricular tachycardia ran at 2 days after the commencement of treatment with sofosbuvir/ledipasvir and this combination treatment was avoided. After this event, his HCV RNA was still positive, and he had no cardiac events without any drugs for cardiovascular diseases, although beta-blocker was transiently used. He was retreated with 12-week combination regimen of grazoprevir and elbasvir without any cardiac events and SVR4 was achieved at present. It has been reported that sofosbuvir caused cardiac events during this treatment. Together, 12-week combination regimen of grazoprevir and elbasvir were safe and effective for the retreatment of HCV GT1-patients who failed to be treated by sofosbuvir/ledipasvir due to ventricular tachycardia.

Conclusion: In conclusion, 12-week combination regimen of grazoprevir and elbasvir should be selected as one of the treatment options for HCV GT-1b patients with cardiovascular diseases.

\section{$H C V-148$}

Successful retreatment with grazoprevir plus elbasvir for cirrhotic patients with hepatitis $C$ virus genotype $1 \mathrm{~b}$, who discontinued the prior DAA treatment

Tatsuo Kanda ${ }^{1}$, Mitsuhiko Moriyama ${ }^{1}$, Masato Nakamura ${ }^{2}$, Shin Yasui $^{2}$, Shingo Nakamoto ${ }^{2}$, Tetsuhiro Chiba ${ }^{2}$, Hitoshi Maruyama $^{2}$, Naoya Kato ${ }^{2}$

${ }^{1}$ Nihon University School of Medicine, Japan; ${ }^{2}$ Chiba University, Graduate School of Medicine, Japan

Background: Combination of DAA treatment results in higher SVR rates, with less serious adverse events in $\mathrm{HCV}$-infected patients. But several patients who discontinued the prior DAA treatment due to adverse events have been observed. In this study, we also experienced the successful retreatment with 12-week combination regimen of HCV NS3/4A inhibitor grazoprevir and HCV NS5A inhibitor elbasvir for three cases infected with HCV GT1b, who discontinued the prior DAA treatment.

Method: Case series.

Result: We present a case series of three patients with HCV GT1b and discontinuation of the prior DAA treatment, who have been successfully retreated with grazoprevir and elbasvir. One case discontinued ombitasvir-paritaprevir-ritonavir at day 12 for renal dysfunction; one case discontinued sofosbuvir-ledipasvir at day 3 for ventricular tachycardia; and the other case discontinued sofosbuvirledipasvir at week 2 for creatine kinase elevation. They commenced to be retreated with grazoprevir and elbasvir at 16 months, 9 months and 13 months, respectively, after stopping the first DAA treatment and completed 12 week-regimens. HCV NS5A resistance-associated variants (RAVs) L31 and Y 93 were wild type, Y93H 33\% and Y93H $>93 \%$, respectively. All patients achieved SVR12.

Conclusion: Retreatment with grazoprevir and elbasvir is effective for HCV GT1b patients who discontinued the prior DAA combination due to adverse events within 4 weeks. 


\section{CHOL-1}

\section{Validation of the GLOBE Score and UK-PBC Risk Score in Japanese patients}

\section{Osamu Yoshida ${ }^{1}$, Masanori Abe ${ }^{1}$, Atsushi Yukimoto ${ }^{1}$, Yusuke Imai $^{1}$, Takao Watanabe ${ }^{1}$, Yohei Koizumi ${ }^{1}$, Masashi Hirooka ${ }^{1}$, Teru Kumagi ${ }^{1}$, Yoichi Hiasa ${ }^{1}$ \\ ${ }^{1}$ Ehime University, Japan}

Background: Primary biliary cholangitis is a progressive liver disease and UDCA is the only available pharmacotherapy for PBC. Two predictive models for PBC long term outcome, the GLOBE Score and the UK-PBC Risk Score, have recently been proposed. These predictive models are derived using data from PBC patients in either North America or Europe. This study therefore aimed to validate the utility of the GLOBE Score and UK-PBC Risk Score in Japanese patients with PBC.

Method: Data from 282 Japanese PBC patients were retrospectively analyzed in this study. Patients with overlapping with autoimmune hepatitis, high ALT ( $>5$ times upper limit of normal) and less than 2 years of follow-up were excluded. The endpoints were defined as liver transplantation or liver-related death due to liver failure, hepatocellular carcinoma and varices bleeding. GLOBE Score and UK-PBC Risk Score were evaluated with the enrolled patients.

Result: A total of 172 patients were enrolled. Nineteen patients $(11.0 \%)$ reached the endpoint during follow-up (mean, 99.5 \pm 61.7 months; range, 16-295 months). ROC analysis revealed that the AUROC for GLOBE score was 0.719 ( 5 years: $p=0.0018$; 10 years: $\mathrm{p}<0.001$; 15 years: $\mathrm{p}=0.0018)$ and the AUROC for UK-PBC Risk Score was 0.748 ( 5 years: $p=0.0593,10$ years: $p=0.0101,15$ years: $\mathrm{p}=0.0027$ ). GLOBE score $>0.30$ (original threshold value) provided $68.4 \%$ sensitivity, $58.8 \%$ specificity, $59.8 \%$ accuracy) whereas GLOBE score $>0.65$ provided higher sensitivity $(63.2 \%)$, higher specificity $(81.0 \%)$ and higher accuracy $(86.0 \%)$.

Conclusion: Both GLOBE Score and UK-BC Risk Score are useful for predicting the prognosis of Japanese PBC patients, but optimization of threshold values may be necessary for the GLOBE Score.

\section{CHOL-2}

Validation of various prognostic models in primary biliary cholangitis in Korean patients

\section{Jeong Ju Yoo ${ }^{1}$, Hae Won Yoo', Jeong Ju Yoo', Eun Ju Cho ${ }^{2}$ \\ ${ }^{1}$ Soonchunhyang Bucheon Hospital, Korea, Republic of; ${ }^{2}$ Seoul National University Hospital, Korea, Republic of}

Background: For primary biliary cholangitis (PBC), various prognostic models have shown good performance in predicting outcomes in Western countries, but have not been validated in Asian population. This study aimed to compare the prognostic performance of suggested models in an independent cohort of PBC patients.

Method: This retrospective study included consecutive 289 patients diagnosed as PBC from 2006 to 2016 by liver biopsy in a tertiary hospital, Korea. Decompensated PBC was defined as presence of ascites, variceal hemorrhage, or jaundice. Event-free survival (EFS) was defined as time until development of decompensation, liver transplantation or death during follow-up. Time-dependent ROC curve analysis was performed to compare the performance of each model.
Result: Mean age of the patients was $57.3 \pm 11.9$ years and $87.9 \%$ were female. Fifteen patients $(5.2 \%)$ had decompensated PBC at initial diagnosis. During a median follow-up of 76.5 months (range, 4-212), 35 patients $(12.1 \%)$ died, and $8(2.8 \%)$ underwent liver transplantation. For the prediction of transplant or liver-related death, PARIS-II, PARIS-I and UK-PBC model showed moderate performance (area under the curves (AUCs), 0.74, 0.74 and 0.72, respectively), whereas GLOBE score and Barcelona model showed poor performance (AUCs, 0.6 and 0.57 , respectively). When considering patients with compensated $\mathrm{PBC}$, similar trends were observed. In addition, PARIS-II, PARIS-I and UK-PBC model showed moderate performance in the prediction of decompensation (AUCs, 0.71, 0.74 and 0.73, respectively), and the performance of GLOBE score and Barcelona model were poor (AUCs, 0.63 and 0.6, respectively). None of the models showed reliable performance in the prediction of EFS (all AUCs $<0.7$ ).

Conclusion: Although most of the published prognostic models for PBC show moderate prediction, their prediction accuracies are relatively low in Korea. So it is necessary to develop new prediction model in PBC defined to Korean patients.

\section{CHOL-4}

Effect of intestinal probiotics combined with UDCA on long-term prognosis of primary biliary cholangitis

\section{$\underline{\text { Ming Wang }}{ }^{1}$, Yanhui Wang', Haiying Sun ${ }^{1}$ \\ ${ }^{1}$ Hepatobiliary Hospital in Jilin Province, China}

Background: Primary biliary cholangitis (PBC) is designed to slow the progression of disease and reduce symptoms and complications, the purpose of this study is to investigate the effect of intestinal probiotics combined with UDCA and UDCA only on the long-term prognosis of primary biliary cholangitis.

Method: 18 cases of PBC were from January 2010 to January 2012 in our hospital, 5 cases were child pugh grade A, 3 cases were grade B, 10 cases were grade $C$, UDCA were daily used, at the same time using intestinal probiotics. 16 patients with $\mathrm{PBC}$ in the same period were as control group, 3 cases were child pugh graded A, 3 cases were grade B, 10 cases were grade C, and UDCA were daily used. No statistics difference was shown with sex, age, course of cirrhosis and other drugs between two groups. Observation index was symptom alleviation, progression, complication and mortality rate in the last 5 years.

Result:

1. Progress of the past 5 years in patients with child pugh grade A and grade B:In the treatment group, 5 patients with child pugh grade $\mathrm{A}$ progressed to grade $\mathrm{B}, 1$ cases progressed to grade $\mathrm{C}, 3$ patients with child pugh grade $\mathrm{B}$ were stable, the overall progression rate was $2 / 8(25 \%)$. In the control group, 2 cases of child pugh grade A progressed to grade B, 1 of 3 patients with grade $\mathrm{B}$ progressed to grade $\mathrm{C}$, the overall progression rate was $3 / 6(50 \%)$. There was significant difference between the two groups $(\mathrm{P}<0.05)$.

2. The symptom alleviation situation was shown in table 1 
table 1 The symptom alleviation situation

\begin{tabular}{|c|c|c|c|c|c|}
\hline symptom & & cases & alleviation & $\begin{array}{l}\text { alleviation } \\
\text { rate }\end{array}$ & $\begin{array}{l}\mathrm{P} \\
\text { value }\end{array}$ \\
\hline \multirow[t]{2}{*}{ Itching } & $\begin{array}{l}\text { treatment } \\
\text { group }\end{array}$ & 15 & 9 & $60 \%$ & \\
\hline & $\begin{array}{l}\text { control } \\
\text { group }\end{array}$ & 15 & 3 & $20 \%$ & $<0.05$ \\
\hline \multirow[t]{2}{*}{$\begin{array}{l}\text { xerosis } \\
\text { cutis }\end{array}$} & $\begin{array}{l}\text { treatment } \\
\text { group }\end{array}$ & 18 & 0 & 0 & \\
\hline & $\begin{array}{l}\text { control } \\
\text { group }\end{array}$ & 16 & 0 & 0 & $>0.05$ \\
\hline \multirow[t]{2}{*}{ fatigue } & $\begin{array}{l}\text { treatment } \\
\text { group }\end{array}$ & 18 & 10 & $55.56 \%$ & \\
\hline & $\begin{array}{l}\text { control } \\
\text { group }\end{array}$ & 16 & 5 & $31.25 \%$ & $<0.05$ \\
\hline \multirow[t]{2}{*}{$\begin{array}{l}\text { Sleep } \\
\text { disorder }\end{array}$} & $\begin{array}{l}\text { treatment } \\
\text { group }\end{array}$ & 10 & 3 & $30 \%$ & \\
\hline & $\begin{array}{l}\text { control } \\
\text { group }\end{array}$ & 12 & 3 & $25 \%$ & $>0.05$ \\
\hline
\end{tabular}

3. Complications occurrence in recent 5 years was shown in table 2

table 2 complications occurrance in recent 5 years

\begin{tabular}{|c|c|c|c|c|}
\hline complications & & cases & occurrance & $P$ value \\
\hline \multirow[t]{2}{*}{ infection } & $\begin{array}{l}\text { treatment } \\
\text { group }(\mathrm{N}=18)\end{array}$ & 3 & $16.67 \%$ & \\
\hline & $\begin{array}{l}\text { control } \\
\text { group }(\mathrm{N}=16)\end{array}$ & 9 & $56.25 \%$ & $<0.05$ \\
\hline \multirow[t]{2}{*}{$\begin{array}{l}\text { hepatic } \\
\text { encephalopathy }\end{array}$} & $\begin{array}{l}\text { treatment } \\
\text { group }(\mathrm{N}=18)\end{array}$ & 2 & $11.11 \%$ & \\
\hline & $\begin{array}{l}\text { control } \\
\text { group }(\mathrm{N}=16)\end{array}$ & 8 & $50.00 \%$ & $<0.05$ \\
\hline \multirow[t]{2}{*}{$\begin{array}{l}\text { gastrointestinal } \\
\text { hemorrhage }\end{array}$} & $\begin{array}{l}\text { treatment } \\
\text { group }(\mathrm{N}=18)\end{array}$ & 4 & $22.22 \%$ & \\
\hline & $\begin{array}{l}\text { control } \\
\text { group }(\mathrm{N}=16)\end{array}$ & 4 & $25.00 \%$ & $>0.05$ \\
\hline \multirow[t]{2}{*}{$\begin{array}{l}\text { Hepatic } \\
\text { carcinoma }\end{array}$} & $\begin{array}{l}\text { treatment } \\
\text { group }(\mathrm{N}=18)\end{array}$ & 1 & $5.56 \%$ & \\
\hline & $\begin{array}{l}\text { control } \\
\text { group }(\mathrm{N}=16)\end{array}$ & 0 & $0 \%$ & \\
\hline \multirow[t]{2}{*}{$\begin{array}{l}\text { Hepatorenal } \\
\text { syndrome }\end{array}$} & $\begin{array}{l}\text { treatment } \\
\text { group }(\mathrm{N}=18)\end{array}$ & 0 & $0 \%$ & \\
\hline & $\begin{array}{l}\text { control } \\
\text { group }(\mathrm{N}=16)\end{array}$ & 1 & $6.25 \%$ & \\
\hline \multirow[t]{2}{*}{$\begin{array}{l}\text { hepatic } \\
\text { hydrothorax }\end{array}$} & $\begin{array}{l}\text { treatment } \\
\text { group }(\mathrm{N}=18)\end{array}$ & 2 & $11.11 \%$ & \\
\hline & $\begin{array}{l}\text { control } \\
\text { group }(\mathrm{N}=16)\end{array}$ & 2 & $12.50 \%$ & $>0.05$ \\
\hline
\end{tabular}

4. 5 years of mortality, mortality rate in the treatment group was $22.22 \%$. Mortality rate in the control group was $31.25 \%$, no significant differences were shown between the two groups $\left(\chi^{2}=0.0147, \mathrm{P}>0.05\right)$.
Conclusion: Intestinal probiotics with UDCA can effectively retard progress of $\mathrm{PBC}$ and relieve itching and fatigue. Incidence of infection and hepatic encephalopathy with intestinal probiotics and UDCA decreased obviously compared with UDCA.

HEV-1

\section{Hepatitis $\mathrm{E}$ virus viremia is infrequent among healthy blood} donors in India

Rakesh Aggarwal ${ }^{1}$, Harshita Katiyar ${ }^{1}$, Vishwajeet Yadav', Atul Sonker $^{1}$, Rajendra Kumar Chaudhary ${ }^{1}$, Amit Goel ${ }^{1}$, Rakesh Aggarwal $^{1}$

${ }^{1}$ Sanjay Gandhi Postgraduate Institute of Medical Sciences (SGPGI), India

Background: Hepatitis E virus (HEV) is transmitted primarily through consumption of contaminated water and food. Recently, HEV viremia in healthy blood donors and transfusion-related transmission of HEV have been reported, leading to calls to screen donated blood for HEV. However, these data are from regions where genotype 3 HEV in predominant. In India, human infections are caused only by genotype $1 \mathrm{HEV}$, with very different epidemiological features. The frequency of subclinical HEV viremia in the Indian population is unknown.

Method: Serum specimens from blood units donated at our blood bank during the year 2016, were prospectively collected and stored at $-80^{\circ} \mathrm{C}$, for HEV RNA and anti-HEV antibody testing. Data on markers of other transmission-transmitted viral infections (HBsAg, anti-HCV and anti-HIV), done as part of routine blood-bank screening, were recorded. Minipools of three specimens each were prepared, and subjected to RNA extraction (QIAamp Viral RNA minikit, Qiagen, Valencia, CA, USA), followed by a sensitive one-step reverse transcription and real-time polymerase chain reaction assay for HEV RNA capable of detecting all HEV genotypes. For all HEV RNA positive specimens, quantitation of HEV viremia and HEV genotyping were planned. A randomly-selected subset of sera were tested for IgG anti-HEV antibodies using a commercial immunoassay (Wantai HEV IgG assay; Beijing Wantai, Beijing, China).

Result: Sera were collected from 1799 blood donors (June-July 2016: 900; November-December 2016: 899). Among 1799 donors (median age: 30 years [range 18-63 years]), 1746 [97.0\%] were men. HBsAg, anti-HCV and anti-HIV antibodies tested positive in $17(0.95 \%), 16$ $(0.90 \%)$ and $3(0.17 \%)$ of these donors, respectively. None of the donors tested positive for HEV RNA. Of the 633 randomly-selected donors $(35.2 \%$ of the entire cohort analyzed; median age 30 [range 18-63] years, 613 [96.8\%] male) tested for serum IgG anti-HEV, 383 $(60.5 \%)$ tested positive. The seropositivity rates increased with increasing age, being 70/136 (52\%), 177/299 (59\%), 100/154 (65\%), $30 / 34(88 \%)$ and $6 / 10(60 \%)$ in the $18-24,25-34,35-44,45-54$ and ${ }^{3} 55$ years age groups, respectively.

Conclusion: HEV viremia was infrequent in our cohort of healthy blood donors from northern India with a high anti-HEV antibody prevalence. This suggests that asymptomatic HEV viremia may be less frequent in areas with genotype $1 \mathrm{HEV}$ predominance than in those with predominance of genotype $3 \mathrm{HEV}$. Though more studies on the issue are warranted, our data suggest that screening of donated blood for HEV RNA may not be required in regions with predominance of genotype $1 \mathrm{HEV}$. 


\section{$H E V-2$}

Efficacy and safety of ribavirin for treatment of acute hepatitis $\mathbf{E}$ infection - a retrospective analysis in a tertiary hospital in Singapore

Rangarajan Purushothaman $^{1}$, Thinesh Lee Krishnamoorthy ${ }^{1}$, Ng Tong Yong ${ }^{1}$, Chow Wan Cheng ${ }^{1}$, Rajneesh Kumar ${ }^{1}$

${ }^{1}$ Singapore General Hospital, Singapore

Background: Ribavirin is used in the treatment of chronic and acute Hepatitis E in immune- compromised patients but data about the efficacy and safety in Singapore population is not clear.

Method: Primary outcome was to study a) Effectiveness of Ribavirin in treatment of acute Hepatitis E infection (HEV) in immunocompromised patients - defined as sustained virological response (SVR) by absence of HEV PCR at 12 weeks of treatment.

b) Mean time taken for normalization of Alanine transaminase values.

Secondary outcome to assess the development of anaemia with use of ribavirin - defined as decrease in hemoglobin by more than $3 \mathrm{gm} /$ $\mathrm{dL}$ attributed to ribavirin use.

Hepatitis E infection is defined as clinical Hepatitis with raised ALT and positive Serology or PCR.

We analysed of data of patients with Hepatitis E infection treated with Ribavirin over past 5 years using electronic medical records

Result: 15 immunocompromised patients with acute Hepatitis E were treated with Ribavirin. Mean age of $60+/-13.7$ years with $60 \%$ males. They were given 10-12 weeks of Ribavirin dosed at 400-600 mg per day.

Mean ALT, AST, Bilirubin and standard error of mean (SE) at start of treatment were $310 \mathrm{U} / \mathrm{L} \pm 77 \mathrm{U} / \mathrm{L}, 321 \mathrm{U} / \mathrm{L} \pm 106 \mathrm{U} / \mathrm{L}$ and $49.5 \mathrm{mg} /$ $\mathrm{dL} \pm 17 \mathrm{mg} / \mathrm{dL}$ respectively. SVR was seen in $87.5 \%$ of patients. Mean time taken for normalisation of ALT was 24 days after initiation of ribavirin with a standard deviation of 13 days.

$3 / 15$ of patients had anaemia with none being symptomatic or requiring transfusion. No adverse events were seen related to Ribavirin use and it was fairly tolerated. None of the patients had de compensation of liver disease with ribavirin use.

Conclusion: Ribavirin is effective in treatment of acute Hepatitis E in immunocompromised patients and is well tolerated, effective and reduces morbidity.

\section{$H E V-3$}

High anti-HEV prevalence was not related to high viremia among individuals from Western Argentina.

Livia Melo Villar ${ }^{1}$, Vanessa Salete De Paula ${ }^{1}$, Andreza Salvio Lemos $^{1}$, Elisangela Ferreira Da Silva ${ }^{1}$, Juliana Custodio Miguel ${ }^{1}$, Hector Cuello ${ }^{2}$, Ivana Lo Castro ${ }^{2}$, Carlos Espul ${ }^{2}$

${ }^{1}$ FIOCRUZ, Brazil; ${ }^{2}$ Central Hospital, Argentina

Background: Hepatitis E virus (HEV) is an etiologic agent of acute hepatitis with a worldwide distribution. Few data are available on the prevalence of HEV infection in Latin America, especially in Argentina. Objective: The objective of this study is to evaluate the prevalence of anti-HEV and HEV RNA in individuals living in the province of Mendoza, Argentina (Western Argentina).

Method: A total of 345 individuals were recruited in the city of Mendoza in 2014 and divided into 3 recruitment sites (hospital setting, rural community and urban population). All participants had more than 18 years old and gave written informed consent to participate in this study. All individuals answered a sociodemographic and risk behavior questionnaire after signing an informed consent form. The project was approved by local ethics committee. Anti-HEV IgG marker was tested in serum samples using commercial HEV IgG immunoenzyme assay, Mikrogen. Reactive anti-HEV samples were submitted to qualitative RT-nested PCR for amplification of ORF1 region and real-time PCR for quantitative detection.

Result: In the study population, mean age was $40 \pm 14.3$ years and the majority were female. Prevalence of anti-HEV IgG was $6.08 \%$ $(21 / 345)$. In this group of 21 individuals, the majority were female $(81 \%)$ and aging more than 50 years old $(57 \%)$. Of these 21 individuals, 11 had information regarding sewage and recreational water use, such as swimming pools, beaches and rivers, being $54.5 \%$ having no sewage system and $36.3 \%$ frequented recreational waters in the last month. No serum sample was HEV RNA reactive by qualitative and quantitative methods.

Conclusion: A high prevalence of anti-HEV antibodies was observed in the study population compared to studies conducted in other Latin America countries, like Brazil, which may be related to characteristics of the population studied, such as pork consumption and lack of sewage network in this population. However, HEV RNA circulation in the studied samples was not observed, demonstrating low viremia in this study.

\section{$H E V-4$}

Prevalence of acute hepatitis $\mathbf{E}$ infection in community: a cross sectional cohort study in New York

\section{Nimy John ${ }^{1}$, Mark Aloysius ${ }^{2}$, Menisa Zaman ${ }^{3}$}

${ }^{1}$ Saint Vincent Hospital, United States; ${ }^{2}$ JJP VAMC Ichan School of Medicine, United States; ${ }^{3}$ Weill Cornell Medicine, United States

Background:

Objective: The prevalence of Acute Hepatitis E has not been explored systematically in US. Sporadic Zoonotic transfer has been reported and recently looked at in post-transplant cohorts. Exotic tourism with exposure to contaminated water sources, Homosexual (MSM) Stool transplant recipients and acquisition of Uncooked products of Hog, Deer meat can transfer Hepatitis E. Distinct Genotype has been established with potential clinical implications. This clinical pilot observational study explores Prevalence of acute Hepatitis E in NY.

Method: Three hundred $(n=300)$ participated prospectively from large reservoir for five years with mild to moderate clinical symptoms: Fatigue (79\%), Nausea (48\%), Itching (20\%), Diarrhea (12\%), Jaundice (40\%), Loss of appetite (93\%), Dysgeusia (81\%) All the participants had Pre-and post HEV IgM and IgG tested. Other Pre-and post-labs included CBC with differential, LFTs, Acute hepatitis profile, HbsAg, HbsAb, HCV, HSV, HIV, EBV, Post infection stool HEV IgM and IgG. 300 participants were divided into 3 groups:

1.Group A $(\mathrm{n}=205) \mathrm{h} / \mathrm{o}$ exotic travel (within 60 days of return) India 42(North and East India), Bangladesh 23(central, east and west), Pakistan 15(east, south and central), Dominican Republic 12(south), Haiti 33(central), Mexico 33(Eastern central), Middle Asia 22(Uzbekistan, Turkmenistan, Tajikistan), West Africa 5(Ivory Coast, Senegal), North Africa 14(Libya, Syria, Egypt), Turkey 6(Central)

2.Group B $(\mathrm{n}=45)$ Homosexual

3.Group $C(\mathrm{n}=43)$ Exotic meat consumers 
Result:

\begin{tabular}{|c|c|c|c|}
\hline & Group A & Group B & Group C \\
\hline Male & 155 & 30 & 33 \\
\hline Female & 50 & 15 & 10 \\
\hline Stool transplant & & 3 & \\
\hline Age group & 18 to 67 & $26-78$ & $27-76$ \\
\hline Mean BMI & 25.7 & $27.30 \%$ & $38 \%$ \\
\hline \multicolumn{4}{|l|}{ HEV genotype } \\
\hline 1. Genotype A & $43 \%$ & $0 \%$ & $0 \%$ \\
\hline 1. Genotype B & $23 \%$ & $19 \%$ & $3 \%$ \\
\hline 1. Genotype C & $34 \%$ & $78 \%$ & $42 \%$ \\
\hline \multicolumn{4}{|l|}{ Race } \\
\hline White & & Male 11, Female 3 & Male 21 , female 1 \\
\hline Black & & male 7, female 1 & Male 10 , female 6 \\
\hline Hispanic & & male 12, female 2 & male 2 , female 3 \\
\hline Transgender & & 8 & \\
\hline \multicolumn{4}{|l|}{ LFTs(mean) } \\
\hline ALT & 46 & 112 & 36 \\
\hline AST & 52 & 156 & 37 \\
\hline ALP & 176 & 209 & 123 \\
\hline Albumin & 3.6 & 3.8 & 4.7 \\
\hline TBili & 3.5 & 9.0 & 1.2 \\
\hline Direct bilirubin & 1.2 & 4 & 0.4 \\
\hline \multicolumn{4}{|l|}{ CBC mean } \\
\hline WBC & 6.2 & 8.2 & 6.6 \\
\hline $\mathrm{Hb}$ & 11.2 & 12.7 & 13.2 \\
\hline Plt count & $322 \mathrm{k}$ & $387 \mathrm{k}$ & $298 k$ \\
\hline US Fatty liver & $9 \%$ & 13 & 21 \\
\hline Stool HEV IgM & $100 \%$ & $100 \%$ & $100 \%$ \\
\hline $\begin{array}{l}\text { Co infection with HIV on } \\
\text { ART }\end{array}$ & 0 & 16 & 0 \\
\hline $\mathrm{HCV}$ coinfection & 3 & 2 & 0 \\
\hline HBV coinfection & 7 & 1 & 0 \\
\hline \multicolumn{4}{|l|}{ Alcohol intake mean } \\
\hline 1. 60 grams & 0 & 0 & 23 \\
\hline 1. 30 grams & 56 & 6 & 13 \\
\hline 1. 20 grams & 123 & 5 & 0 \\
\hline 1. No alcohol intake & 26 & 34 & 7 \\
\hline \multicolumn{4}{|l|}{ Fibrosure } \\
\hline F0 & 197 & 12 & 32 \\
\hline $\mathrm{F} 1$ & 5 & 14 & 7 \\
\hline $\mathrm{F} 2$ & 0 & 6 & 4 \\
\hline F3 & 1 & 2 & 0 \\
\hline IVDU & 0 & 4 (former IVDU) & 0 \\
\hline Pruritis score & 5 & 1 & 2 \\
\hline CDAD recurrent & 0 & 8 & 0 \\
\hline HSV & 0 & 8 & 12 \\
\hline \multicolumn{4}{|l|}{ Food accusation } \\
\hline 1. Wild boar & 0 & 0 & 22 \\
\hline 1. Deer & 0 & 0 & 13 \\
\hline 1. Guinea pig & 0 & 0 & 8 \\
\hline Marijuana use & 0 & 0 & 20 \\
\hline
\end{tabular}

Conclusion: Acute Hepatitis E is an existing clinical entity in evaluating ALD in US, in certain demographic mosaic of exotic travelers, exotic meat eaters and homosexual individuals. It can also be transmitted from stool bank to stool transplant receipt; study encourages to evaluate all stool banks in US and in MSM cohort.
HEV-5

Risk factors of hepatitis $\mathbf{E}$ caused liver failure: a retrospective case-control study

$\underline{\text { Jingmin Zhao }}^{1}$, Yijin Wang ${ }^{1}$

${ }^{1}$ Beijing 302 hospital, China

Background: Hepatitis E virus (HEV) is one of the most common causes of non-chronic liver failure (NCLF) in developing countries. However, the risk factors for HEV patients developing liver failure have not been comprehensively investigated. The aim of this study was to understand the risk factors of HEV complicating with ALF.

Method: 62 patients diagnosed with HEV who developed to NCLF (acute/subacute/acute-on-chronic liver failure) and 210 non-NCLF HEV infected patients as control were enrolled from November 2011 through September 2017. Clinical index and epidemiological records were analyzed. Differences were tested using Student's t test for continuous measures, and Chi-square or Fisher's exact test for categorical measures. The results based on two-sided tests and $\mathrm{P}<0.05$ was defined as statistically significant.

Result: Median age of HEV infected patients with or without NCLF were $54.55 \pm 9.33$ years vs $52.87 \pm 13.58$ years $(P=0.360)$, respectively. Development of NCLF upon HEV infection were more common in male $(98.39 \%$ vs $79.52 \% ; P<0.001)$. Compared with solely HEV infected patients, HEV complicating NCLF presented a lower level of $\gamma$-glutamyltransferase (GGT) (123.36 $\pm 105.97 \mathrm{U} / \mathrm{L}$ vs $236.91 \pm 299.93 \mathrm{U} / \mathrm{L} ; P<0.001)$, and prealbumin (PA) $(50.93 \pm$ $34.41 \mathrm{mg} / \mathrm{L}$ vs $99.04 \pm 64.38 \mathrm{mg} / \mathrm{L} ; P<0.001)$, choline esterase $(3450.25 \pm 1471.04 \mathrm{U} / \mathrm{L}$ vs $4925.83 \pm 1958.22 \mathrm{U} / \mathrm{L} ; P<0.001)$, leucine aminopeptidase $(87.17 \pm 25.95 \mathrm{U} / \mathrm{L}$ vs $104.15 \pm 47.50 \mathrm{U} / \mathrm{L}$; $P<0.001)$, triglyceride $(1.79 \pm 0.72 \mathrm{mmol} / \mathrm{L}$ vs $2.36 \pm 1.16 \mathrm{mmol} / \mathrm{L}$; $P<0.001)$, low density lipoprotein cholesterol $(1.92 \pm 0.59 \mathrm{mmol} / \mathrm{L}$ vs $2.80 \pm 1.13 \mathrm{mmol} / \mathrm{L} ; P<0.001)$ and $\alpha 1$-globulin $(3.56 \pm 0.94$ vs $4.88 \pm 1.01, P<0.001)$. While a higher value of aspartate aminotransferase (AST) $(679.70 \pm 1018.39 \mathrm{U} / \mathrm{L}$ vs $394.07 \pm 475.03 \mathrm{U} / \mathrm{L}$; $P=0.038)$, lactate dehydrogenase $(317.23 \pm 220.59 \mathrm{U} / \mathrm{L}$ vs $252.89 \pm$ 115.27 U/L; $P=0.040)$ and alpha-fetoprotein $(114.96 \pm 201.01 \mathrm{ng} / \mathrm{ml}$ vs $54.43 \pm 111.32 \mathrm{ng} / \mathrm{ml} ; P=0.029)$ were significantly associated with NCLF development in HEV infected patients. Furthermore, a multivariate logistic regression model was constructed to identify independent risk factors of NCLF due to HEV. HEV infected patients with underlying chronic liver disease (CLD) (odds ratio, 2.640; 95\%CI, 1.254, 5.559), including alcoholic liver disease, cirrhosis, hepatitis B virus (HBV) and autoimmune liver disease, high level of AST (odds ratio, 1.002; 95\%CI, 1.001,1.003), lower level of GGT (odds ratio, 0.994; 95\%CI, 0.990,0.998), low level of PA (odds ratio, $0.985 ; 95 \% \mathrm{CI}, 0.973,0.997$ ) were more likely to associate with NCLF. Furthermore, of the $62 \mathrm{HEV}$ related NCLF patients, there is no difference between the improvement rate of NCLF in patients with or without underlying CLD (63.63\% vs $66.67 \%, P=1.000)$.

Conclusion: HEV infected patients with male gender, underlying CLD, higher level of AST, lower level of GGT and lower level of PA are more likely to develop non-chronic liver failure. 


\section{$H E V-6$}

The role of hepatitis $E$ in acute non-traumatic neuropathy in China: a prospective case-control study

\section{Jingmin Zhao ${ }^{1}$, Yijin Wang \\ ${ }^{1}$ Beijing 302 Hospital, China}

Background: Neurological complications have been increasingly reported in hepatitis $\mathrm{E}$ virus (HEV) infected patients in western countries and mainly due to genotype $3 \mathrm{HEV}$. Whether other genotypes of HEV are also related to neurological injury is largely unknown. The aim of this study is to determine the frequency of hepatitis $\mathrm{E}$ in patients with acute non-traumatic neurological disorder in China, where genotype $4 \mathrm{HEV}$ is prevalent.

Method: From September through November 2017, 765 patients consecutively diagnosed with acute non-traumatic neurological injury, including posterior circulation ischemia $(n=210)$, cerebral infarction $(n=171)$, stroke $(n=133)$, neurodegenerative diseases $(n=$ $108)$, central nervous system infections $(n=31)$, and others $(n=112)$ were enrolled, matched with 1171 healthy controls. Serum samples were collected for testing anti-HEV immunoglobulin (Ig) M and IgG by ELISA. Subjects with detectable anti-HEV IgM were also tested for HEV RNA. All patients' demographic and clinical data are documented. Differences were tested using Student's t test for continuous measures, and Chi-square or Fisher's exact test for categorical measures. The results based on two-sided tests and $P<0.05$ was defined as statistically significant.

Result: Anti-HEV IgM were positive in only 1 encephalitis $(0.13 \%)$ of the neurological injured patients, and $8(0.68 \%)$ in healthy controls. The 1 patient and 4 of the 8 controls with positive anti-HEV IgM were also positive for anti-HEV IgG. Overall, 290 of the 764 patients (37.96\%) and 409 of the 1163 controls $(35.2 \%)$ without anti-HEV IgM were detectable for anti-HEV IgG. Further study in our patients' cohort revealed that anti-HEV IgG positivity was more common in male $(181 / 290,62.41 \%$ vs $109 / 290,37.59 \%, P=0.006)$ and older patients $(67.17 \pm 12.65$ vs $64.03 \pm 13.69, P=0.002)$. Of note, stratification analysis showed that central nervous system infections were less likely to be in patients with detectable anti-HEV IgG, compared with of ant-HEV IgG negative patients, although not statistically significant $(2.41 \%, 7 / 290$ vs $4.85 \%, 23 / 474, P=0.124)$.

Conclusion: Non-traumatic neurological injury is rarely associated with current/recent HEV infection in China. Past exposure to HEV seems a protective factor for central nervous system infections.

\section{$H E V-6$}

Clinical features and outcomes of hepatitis $\mathbf{E}$ in patients with underlying chronic liver disease: a respective case-control study

\section{Jingmin Zhao ${ }^{1}$, Yijin Wang ${ }^{1}$}

${ }^{1}$ Beijing 302 Hospital, China

Background: Although hepatitis E virus (HEV) infection generally causes acute and self-limiting disease, fulminant hepatitis are frequently reported in HEV patients with underlying chronic liver disease (CLD). However, the differences of clinical features upon HEV infection in individuals with or without existing chronic liver disease haven't been comprehensively understood. This study aims to investigate the clinical symptoms, manifestations and outcomes of HEV infection in presence or absence of CLD.

Method: From January 2015 to October 2017, 124 patients diagnosed with solely HEV infection and 103 patients diagnosed with HEV infection combining pre-existing CLD, including chronic hepatitis B, $\mathrm{C}$, alcoholic liver disease, moderate/severe fatty liver and cirrhosis by presence of anti-HEV immunoglobulin (Ig) M, were enrolled. Epidemiological and clinical features were collected. Chi-squared or Fisher's exact tests were performed to compare proportions. The results based on two-sided tests and $P<0.05$ was defined as statistically significant.

Result: Analysis of total 227 patients with positive anti-HEV IgM showed an overall high median age (54 years) and overwhelming proportion of males $(189 / 227,83 \%)$. Compared with patients of solely HEV infection, HEV infected patients with pre-existing CLD were more commonly to present abdominal pain/distension (35.92\% vs. $20.97 \%, P=0.017)$, but less likely to present nausea/vomiting (63.11\% vs. $78.23 \%, P=0.013)$. Higher level of procalcitonin $(0.91$ $\pm 0.92 \mu \mathrm{g} / \mathrm{L}$ vs $0.61 \pm 0.52 \mu \mathrm{g} / \mathrm{L} ; P=0.047)$ and alkaline phosphatase (ALP) $(205.54 \pm 97.78 \mathrm{U} / \mathrm{L}$ vs $176.20 \pm 63.41 \mathrm{U} / \mathrm{L} ; P=$ 0.005) were demonstrated to associate with solely HEV infection than CLD combined HEV infection. While the presence of anti-HEV IgM in CLD patients had higher proportion of seroperitoneum $(37.86 \%$ vs. $20.97 \%, \mathrm{p}=0.005)$, pleural effusion $(17.48 \%$ vs. $4.84 \%, P=0.002)$ and peritonitis $(14.56 \%$ vs. $4.84 \%, P=0.02)$. In addition, HEV patients with pre-existing CLD were more likely to develop liver failure $(22.33 \%$ vs. $12.1 \%, P=0.049)$ and shock (3.88\% vs. $0.81 \%$, $P=0.263$ ).

Conclusion: HEV infection in absence of pre-existing CLD presented typical clinical symptoms of acute hepatitis. However, HEV infection with underlying CLD showed more clinical manifestations and severe outcomes than HEV infection alone.

\section{PHT-1}

Randomized control trial of rifaximin and norfloxacin in primary and secondary prophylaxis of spontaneous bacterial peritonitis (SBP) in cirrhotic patients

\section{Dibyalochan Praharaj ${ }^{1}$}

${ }^{1}$ PGIMER, India

Background: Spontaneous bacterial peritonitis (SBP) is a life threatening infection in patients with cirrhosis. Patients with advanced cirrhosis with or with out prior history of SBP have a high risk of developing SBP for which they need either primary or secondary prophylaxis respectively. Norfloxacin has been used for SBP prophylaxis since decades which has led to increase in incidence of SBP on prophylaxis and SBP related to gram positive organisms. Thus alternative oral antibiotic must be used to prevent this life threatening complication in cirrhotic patients. Rifaximin is an oral antibiotic effective against both gram positive and gram negative organisms and is used to prevent and treat hepatic encephalopathy in cirrhotic patients.

Method: 117 patients with decompensated cirrhotic patients with ascites were enrolled into study. 59 patients had past history of SBP and were randomly assigned to receive norfloxacin (400 $\mathrm{mg} \mathrm{OD})$ or rifaximin $(550 \mathrm{mg} \mathrm{BD})$ as secondary SBP prophylaxis. Rest 58 patients with advanced cirrhosis as defined by CTP score of 9 or more, hyperbilirubinemia, hyponatremia but with no history of SBP and were randomly assigned to receive norfloxacin or rifaximin at same dosage. All the patients were followed up for 6 months and were evaluated for presence of SBP at end of 3 months and 6 months or when symptomatic

Result: Thirteen of 33 (39\%) patients receiving norfloxacin for secondary prophylaxis and 6 of 30(20\%) patients receiving norfloxacin for primary prophylaxis developed SBP compared to 2 of $26(7 \%)$ patients receiving rifaximin for secondary prophylaxis and $4 / 28(14 \%)$ 
patients receiving rifaximin for primary prophylaxis. Patients receiving norfloxacin had higher rate of development of SBP compared to rifaximin in both primary $(20 \%$ vs $14 \%$; $\mathrm{P}=0.731)$ and secondary $(39 \%$ vs $7 \% ; \mathrm{P}=0.007)$ arm.

Conclusion: Rifaximin was more effective than norfloxacin in secondary prophylaxis of SBP

\section{PHT-2}

Urine cystatin $\mathrm{C}$ is a strong predictor for short-term mortality and therapeutic response to Terlipressin in cirrhotic patients with serum creatinine greater than $1.5 \mathrm{mg} / \mathrm{dl}$

\section{Jae Woo Park ${ }^{1}$, Jeong Ju Yoo', Young Seok Kim', Sang Gyune Kim ${ }^{1}$ \\ ${ }^{1}$ Department of Gastroenterology and Hepatology, Soonchunhyang University School of Medicine, Korea, Republic of}

Background: Acute kidney injury (AKI) in liver cirrhosis is sometimes accompanied by tubular injury which can lead to poor outcome. Cystatin C is completely reabsorbed in kidney unless renal tubules are damaged. We aimed to find the predictive factors for therapeutic response of terlipressin and short-term mortality in cirrhotic patients with AKI.

Method: During Jan. 2011 to Dec. 2016, out of 225 patients with serum creatinine $(\mathrm{Cr})$ greater than $1.5 \mathrm{mg} / \mathrm{dl}, 145$ met the definition of $\mathrm{AKI}$ and urine sample was collected for the measurement of urine cystatin C (UCC). Therapeutic response was defined as the decrease of $30 \%$ from baseline $\mathrm{Cr}$ within 72 hours. Three-month mortality after developing AKI was analyzed as well. According to the median value of UCC $(3.5 \mathrm{mg} / \mathrm{dL})$ we divided into 3 groups; those whose UCC value was negative (UCC-neg), lower than $3.5 \mathrm{mg} / \mathrm{dL}$ (UCC-low) and $3.5 \mathrm{mg} / \mathrm{dL}$ or more (UCC-high).

Result: Eighty-two (56.6\%) patients died within following 3 months. When UCC-neg was used as a reference, the survival rate of the UCChigh was significantly lower (adjusted hazard ratio, 2.87; 95\% confidence interval, 1.72-4.8; $\mathrm{p}<0.001$ ), but that of UCC-low was not [aHR, $1.17(0.67-2.05)]$. Among 52 patients who were treated with terlipressin, twenty-nine $(55.8 \%)$ achieved therapeutic response. The response rate decreased with increasing UCC as follows; [UCC-neg, 16/18 (88.9\%); UCC-low, 6/10 (60.0\%); UCC-high, 7/24 (29.2\%)]. After adjusted with other variables, UCC-high [OR, 0.05 (0.01-0.23)] and UCC-low [OR, $0.18(0.02-1.3)]$ showed lower response rate compared to UCC-neg.

Conclusion: Urine cystatin $\mathrm{C}$ was a strong predictive marker for therapeutic response of terlipressin and short-term survival in cirrhotic patients who developed AKI. It could be a promising prognostic marker in cirrhotic patients with AKI.

\section{PHT-3}

Hepatic ascites treatment with novel diuretics - vasopressin V2 receptor antagonist (Tolvaptan)

\author{
Rintaro Fukuda ${ }^{1}$, Hdeo Yoshida ${ }^{1}$, Hiroyoshi Taniguchi ${ }^{1}$, Akiko \\ Saito $^{1}$, Ryo Nakata \\ ${ }^{1}$ Japanese Red Cross Medical Center, Japan
}

Background: Medical treatment of hepatic ascites has been based on furosemide and spironolactone for long time. Recently, novel diuretics of Tolvaptan become available in Japan.

Method: Tolvaptan was administered for 57 patients with refractory hepatic ascites between September 2013 and December 2016. Patients received $3.75 \mathrm{mg} /$ day or $7.5 \mathrm{mg} /$ day of Tolvaptan orally. Demographic data, body weight, daily urine volume, serum creatinine, serum sodium concentration, and liver numbers were collected.

Result: In 57 patients, 39 were male and 18 were female. Age of patients ranged between 44 and 91 (median 66 yo). Etiology of liver disease was as follows: HCV 25, Alcoholic liver disease 16, Others 16. Child Pugh score ranged between 8 and 13 (median 10). Twentyseven were Child B and 30 were Child C. Urine volume increased $476 \pm 776 \mathrm{ml}, 588 \pm 767 \mathrm{ml}$, and $93 \pm 900 \mathrm{ml}$ on day 1,7 , and 14 respectively. Thirty-three percent of patients had body weight loss more than $1.5 \mathrm{~kg}$ in 1 week. No severe adverse effect including acute elevation of serum sodium level was seen. Twelve patients stopped taking Tolvaptan because of no effect (two patients), general malaise, encephalopathy, pneumonia, dehydration, and of recovery from ascites (one patient).

Conclusion: Tolvaptan was safely administered for the treatment of hepatic ascites. This novel diuretic provide alternative option for the treatment of end stage liver disease.

\section{PHT-4}

Child Turcotte Pugh vs MELD vs renal resistance index score for assessment of prognosis in liver cirrhosis

\section{Muppa Indrakeela Girish ${ }^{1}$}

${ }^{1}$ King George Hospital, India

Background: CTP and MELD scores have been widely used for the assessment of prognosis in cirrhosis, with some limitations. The resistive index (RI) can be used to assess vascular resistance by Doppler ultrasonography. Cirrhotic patients with elevated intra renal RIs tend to develop HRS, leading to poor prognosis. The study is designed whether intra renal resistive index can be used as a prognostic parameter in cirrhotic patients and compare this with the existing prognostic scores.

Method: Prospective single center study. Cirrhotic Patients $>18 \mathrm{yrs}$ were included. HRS, UGI Bleed, DM, HTN, Decreased kidney size on U/S abdomen were excluded. Patients were followed for 6 months. To discriminate the predictive value of the parameters, a receiver operating characteristic (ROC) curve was established by standard procedures.

Result: Out of 92 patients 56 alive, 26 deceased, 10 lost to follow up. Male/Female - 62/20 Mean Age- 41.13+/-9.24. On comparing the baseline characteristics of both the deceased and alive group, intra renal Resistive index is the only parameter that is statistically different. In ROC analysis CTP score has an AUROC of 0.749, MELD score has 0.836 and RRI has 0.858 . When compared with both CTP and MELD score, renal Resistive index performed better. Kaplan Meir survival analysis showed a significant difference in survival between the groups with Intra renal resistive index $(\mathrm{RI}) \leq 0.71$ (alive) and $\mathrm{RI}>0.71$ (deceased).

Conclusion: When compared with MELD score and CTP score, intra renal resistive index is better in predicting short term survival among cirrhotic patients. 


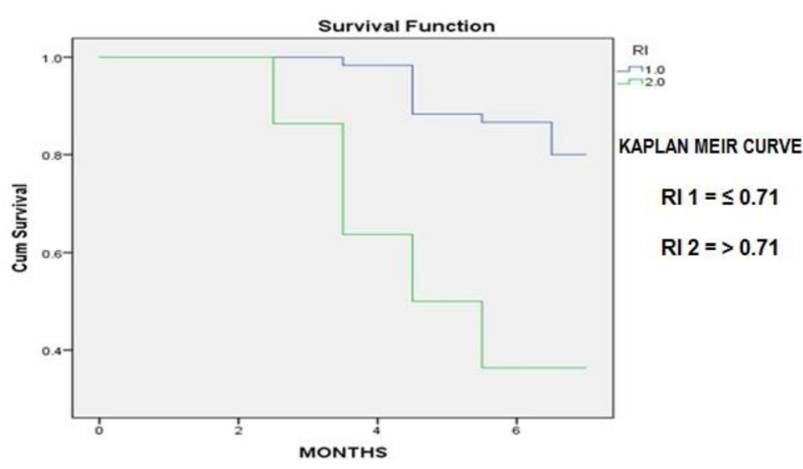

ROC Curve

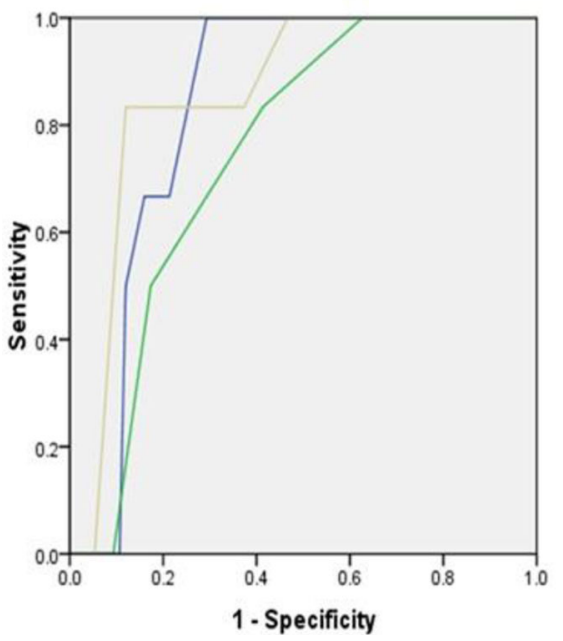

Source of the Curve —MELD ${ }_{\mathrm{RI}}^{\mathrm{CTP}}$

PHT-5

Contrast-enhanced ultrasonography with intraperitoneal injection of Sonazoid for the functional assessment of peritoneovenous shunting

Soichiro Kiyono ${ }^{1}$, Hitoshi Maruyama ${ }^{1}$, Kazufumi Kobayashi ${ }^{1}$, Masato Nakamura $^{1}$, Shingo Nakamoto ${ }^{1}$, Shin Yasui ${ }^{1}$, Sadahisa Ogasawara $^{1}$, Eiichiro Suzuki ${ }^{1}$, Yoshihiko Ooka ${ }^{1}$, Tetsuhiro Chiba $^{1}$, Naoya Kato ${ }^{1}$

${ }^{1}$ Chiba University Graduate School of Medicine, Japan

Background: Peritoneo-venous shunting (PVS) is widely accepted as a useful treatment option in patients with intractable ascites. Proper assessment for PVS function is required because shunt dysfunction is a common problem. The aim of the study was to examine the effect and the safety of contrast-enhanced ultrasound with the transcutaneous intraperitoneal injection of microbubble contrast agent into the ascites to evaluate the function of PVS.

Method: The subject of the study was consecutive 7 patients with chronic liver disease (male 9, female 7; age $44-77$ years) who had dysfunction of PVS placed for the treatment of intractable ascites, and 3 cirrhosis patients (male 2, female 1; age $61-74$ years) showing intractable ascites without a creation of PVS as controls. A shunt dysfunction was defined by the recurrence of ascites diagnosed by ultrasound.
An intraperitoneal injection of contrast agent (Sonazoid, $0.5 \mathrm{~mL}$ ) into the ascites was performed using $23 \mathrm{G}$ needle under local anesthesia (1 $\%$ lidocaine). The enhancement in the systemic circulation was assessed by the observation of heart or the major artery (abdominal aorta or femoral artery) up to $15 \mathrm{~min}$ for the positive/negative migration of microbubble via PVS.

Result: No migration was detected in the systemic circulation in 4 patients without PVS, suggesting no natural migration of microbubble from ascites to the systemic circulation.

The intraperitoneal injection of Sonazoid showed negative migration of microbubble into the systemic circulation in $4 / 7$ patients, just like the control 4 patients, suggesting the complete occlusion of the PVS. Two of them underwent replacement of the PVS, however repeated paracentesis was applied for the other two patients following the removal of the PVS because of a presence of severe venous thrombosis.

Positive migration of microbubble into the systemic circulation was observed in $3 / 7$ patients approximately 1 minute after the agent injection. The result indicated the reason for shunt dysfunction in the 3 patients was not a complete occlusion but a kink of the tube. The ascites in these 3 patients was controlled by occasional paracentesis ( 0.5 to 2 times per month) without replacement of PVS. There was no adverse event during and after the intraperitoneal injection of Sonazoid.

Conclusion: Contrast-enhanced ultrasound after the intraperitoneal injection of microbubble agent into the ascites is effective for the functional assessment of PVS.

\section{PHT-6}

Treatment efficacy of endoscopic variceal obturation for gastric variceal bleeding according to the type of varices

Yeon Seok Seo ${ }^{1}$, Han Ah Lee ${ }^{1}$, Tae Hyung Kim ${ }^{1}$, Young Sun Lee', Sang Jun Suh 1 , Hyun Gil Goh', Young Kul Jung', Ji Hoon Kim¹, Hyung Gin An ${ }^{1}$, Hyung Joon Yim ${ }^{1}$, Jong Eun Yeon ${ }^{1}$, Kwan Soo Byun $^{1}$, Soon HO Um ${ }^{1}$

${ }^{1}$ Korea university, Korea, Republic of

Background: This study was performed to the treatment efficacy of EVO in patients with GV bleeding according to the type of varices. Method: All patients who treated with EVO for GV bleeding were included in this study. Patients with previous history of endoscopic treatment for $\mathrm{GV}$ bleeding $(\mathrm{n}=4)$ and those combined with portal vein invasion by $\mathrm{HCC}(\mathrm{n}=9)$ or other malignancy $(\mathrm{n}=7)$ were excluded. In addition, patients with IGV2 were also excluded because the number was too small for analysis $(\mathrm{n}=2)$.

Result: Age was $59.4 \pm 12.4$ years and 72 patients $(79.1 \%)$ were men. Alcoholic liver disease (47 patients, 51.6\%) was most common cause of liver disease, followed by chronic hepatitis B (24 patients, $26.4 \%)$. HCC was combined in 28 patients $(30.8 \%)$. Type of varices was GOV1, GOV2, and IGV1 in 33 (36.3\%), 31 (34.1\%), and 27 $(29.7 \%)$ patients, respectively. Hemostasis was successful in 88 patients $(96.7 \%)$. Hemostasis success rates according to the type of varices were $100 \%, 96.8 \%$, and $92.6 \%$ in GOV1, GOV2, and IGV1, respectively, which were comparable among three groups $(\mathrm{P}=0.279)$. Hemostasis was successful in 88 patients $(96.7 \%)$. GV was obliterated in 81 patients $(89.0 \%)$. Number of EVO session was comparable among three groups $(1.5 \pm 0.7,1.3 \pm 0.4$, and $1.7 \pm 0.7$ in patients with GOV1, GOV2, and IGV1, respectively), while amount of histoacryl was significantly smaller in patients with GOV1 $(1.4 \pm 0.7$ $\mathrm{ml})$ compared to those with GOV2 $(2.6 \pm 1.3 \mathrm{ml}, \mathrm{P}=0.042)$ and IGV1 $(2.7 \pm 1.4 \mathrm{ml}, \mathrm{P}=0.041)$. Among 81 patients with $\mathrm{GV}$ obliteration, $\mathrm{GV}$ recurrence rate at 24 months was $43.9 \%$. GV recurrence was 
significantly lower in patients with GOV1 (18.7\%) than those with GOV2 (65.0\%; P=0.005) and those with IGV1 (47.0\%; P=0.046). VB recurrence rate at 24 months was $21.3 \%$. VB recurrence rate was significantly lower in patients with GOV1 $(12.1 \%)$ than those with GOV2 (40.5\%; $\mathrm{P}=0.021)$, but it was comparable with patients with IGV1 (11.6\%; P-0.929).

Conclusion: Size of varices and amount of histoacryl for GV obliteration were significantly lower in patients with GOV1 than those with GOV2 or IGV1. GV recurrence, GV rebleeding were significantly lower in patients with GOV1 than those with GOV2.

\section{PHT-7}

"Predictors of non-recovery in cirrhosis with acute kidney injury"

Mithra Prasad ${ }^{1}$, Mohan Prasad VG², Madhura Prasad², KVKSN Murthy $^{2}$, Narayanasamy $\mathbf{K}^{\mathbf{1}}$

${ }^{1}$ Madras Medical College, India; ${ }^{2}$ VGM Gastro Centre, India

\section{Background:}

- To determine the predictors of non- recovery in patients admitted with cirrhosis and acute kidney injury over a period of one year (2016) in two tertiary care hepatology and gastroenterology specialised centres

- To determine the usefulness of MELD, MELD Na and Child Turcott Pugh (CTP) scoring in prognosticating cirrhotics with acute kidney injury.

Method: All cirrhotics with AKI as per ICA-AKI criteria admitted over a period of one year (2016) were included

Patients with AKI not responding to albumin were started on midodrine and octreotide $(\mathrm{M} / \mathrm{O})$ with albumin for five days. In partial responders, the regime was continued up to two weeks. Non responders were given Terlipressin, further hemodialysed or referred for transplant

Variables were analysed by Mean, SD, Chi square, Pearson's correlation coefficient, Kruskal wallis test, To arrive at a predictor model, binary logistic regression was used. Analysis was by SPSS version 16.0

Result: Of 572 cirrhotics, 53 developed hepatorenal syndrome (HRS). The incidence was $9.26 \%$.

The mean age was 51.33 ( $\mathrm{SD} \pm 10.32$ ) years. Renal dysfunction is more commonly found among the middle aged cirrhotics. MELD was significantly higher in the age group from 36-45

There were $51(96 \%)$ male and two (4\%) female patients. The major etiology of cirrhosis was alcohol $(\mathrm{N}=27,50.94 \%)$.

35 patients $(66 \%)$ had type 1 HRS and 18 patients (34\%) had type 2 HRS.

The mean values were Bilirubin $8.52 \mathrm{mg} / \mathrm{dl}$, Creatinine $1.98 \mathrm{mg} /$ dl, PTINR 1.72, SGOT 77.23 IU/ml, SGPT 40.15 IU/l, Albumin 2.78 $\mathrm{g} / \mathrm{dl}$, Sodium $134.08 \mathrm{mg} / \mathrm{dl}$.

$75.47 \%$ of patients belonged to stage 1 of AKI, $18.86 \%$ to stage 2 and $5.66 \%$ to stage 3

$29 / 44$ cases, $54.71 \%$ recovered completely The average days to recovery was 6.34 days. $24 / 44$ cases, $45.28 \%$ patients continued to worsen. They were switched to treatment with terlipressin and albumin and showed no improvement so were subsequently hemodialysed. 5/44, 9.43\% died and the remaining were referred for transplantation.

M/O with albumin infusion was used and $54.71 \%$ (29/53 cases) recovered completely. There were no adverse effects.
There was no relation between age, gender, cause of cirrhosis, stages of AKI, type of HRS, Hepatic encephalopathy, SBP, variceal bleed, hepatocellular carcinoma and outcome

High values of bilirubin, PT and INR were significantly associated with non-recovery by unpaired t test.

High MELD, MELD Na, Child Pugh score were associated with worse outcome.

Low EGFR was associated with worse outcome.

The mean of MELD for the non-recovery group was higher than the mean of MELD for the recovery group (Graph 1)

MELD score was higher in age 36-45 years

Mean estimate of survival was more for type $2 \mathrm{HRS}$ as compared to type $1 \mathrm{HRS}$ for patients with bilirubin $>5.3 \mathrm{mg} / \mathrm{dl}$. In patients older than 48 years, type 2 HRS had better survival

A positive correlation with non-recovery was found with bilirubin, PT INR, MELD, MELD Na, CTP score. Using forward selection regression, MELD and creatinine were found significant for the model and were used to build a predictor model

Output $Z=3.227+(1.012 \times$ creatinine $)-(0.233 \times$ MELD $)$

When the output is greater than 0.5 , it is categorized as recovery.

The prediction accuracy is $72.7 \%$ (Table 1)

Conclusion: HRS commonly was seen in fifth decade males High bilirubin, INR, MELD, CTP and low EGFR are associated with non-recovery

MELD and MELD Na are comparable in HRS.

Type 1 HRS with bilirubin $>5.3 \mathrm{mg} / \mathrm{dl}$ and age $>48$ years had a poor survival curve.

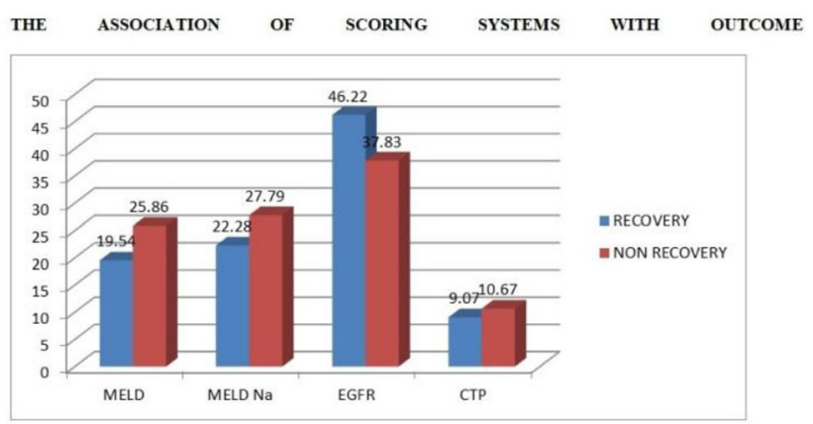

Accuracy of the proposed model in predicting response to treatment with vasoconstrictor and albumin

\begin{tabular}{|l|l|l|l|l|}
\hline \multicolumn{2}{|l|}{} & $\begin{array}{l}\text { Not Recovered } \\
\text { (Actual) }\end{array}$ & $\begin{array}{l}\text { Recovered } \\
\text { (Actual) }\end{array}$ & \\
\hline & Not Recovered (Predicted) & 18 & 9 & 69.6 \\
\cline { 2 - 5 } & Recovered (Predicted) & 7 & 19 & 76.2 \\
\hline \multicolumn{2}{|l|}{ Overall Percentage } & & & 72.7 \\
\hline
\end{tabular}

PHT-8

Bacteriological profile and antibiotic susceptibility of infections in cirrhosis

Biji Benny ${ }^{1}$, Neehar M Shanavas', Anoop K V', Sandeep N', Singh Aniruddha ${ }^{1}$, Shanid $A^{1}$, Krishnadas Devadas ${ }^{1}$

${ }^{1}$ Government Medical College, India

Background: Bacterial infections are common and accounts for about 30\%-50\% mortality in patients with cirrhosis. Aim of the study 
was to identify the bacteriological profile responsible for common infections in cirrhosis and to determine the susceptibility pattern of isolates.

Method: Retrospective cross-sectional study done in 210 cirrhotic patients, admitted in the department of gastroenterology, government medical college, Thiruvananthapuram, from October 2016 to September 2017. They were analyzed for the type of infections, culture yield, type of bacteria and its antibiotic susceptibility and whether it was health care associated (defined as infections diagnosed within 48 hour of hospital admission in patients with any prior 90-day healthcare contact) or community acquired.

Result: Common infections are ascitic fluid infections (AFI) (37.1\%), followed by urinary tract infections (UTI-18.1\%), cellulitis (16.2\%), AFI with UTI (11.9\%), AFI with cellulitis $(9.5 \%)$ and respiratory tract infections (RTI)(7.5\%). Health care associated infections in AFI, UTI, cellulitis, AFI with UTI, AFI with cellulitis and RTI are $61.5 \%, 44.7 \%, 67.6 \%, 52 \%, 45 \%, 40 \%$ respectively. Culture yield is lowest in AFI (11.5\%) and highest in UTI. Most common organism causing AFI is E-Coli in a community acquired infections and in health care associated AFI was klebsiella. In all other types of infections klebsiella was the commonest organism. Sensitivity to third generation cephalosporin in E-coli, Klebsiella and pseudomonas were $57 \%, 46 \%$ and $50 \%$ respectively in community acquired infections and $25 \%, 24 \%$ and $0 \%$ respectively in health care infections. Sensitivity to piperacillin-tazobactam in E-coli, Klebsiella and pseudomonas are $71 \%, 77 \%$ and $100 \%$ respectively in community acquired infections and $50 \%, 41 \%$ and $33 \%$ respectively in health care infections. Sensitivity to carbapenems in E-coli, Klebsiella and pseudomonas were $100 \%, 92 \%$ and $100 \%$ respectively in community acquired infections and $93 \%, 88 \%$ and $66 \%$ respectively in health care infections. Sensitivity to quinolones in E-coli, Klebsiella and pseudomonas were $57 \%, 62 \%$ and $100 \%$ respectively in community acquired infections and $25 \%, 41 \%$ and $0 \%$ respectively in health care infections. Sensitivity to aminoglycosides in E-coli, Klebsiella and pseudomonas were $100 \%, 100 \%$ and $100 \%$ respectively in community acquired infections and $100 \%, 94 \%$ and $100 \%$ respectively in health care infections. Enterococcus and staphylococcus were isolated in health care infections and streptococcus in community acquired infections only. All strains of enterococcus, staphylococcus and streptococcus were sensitive to cloxacillin, vancomycin and linezolid.

Conclusion: Most common organisms were E-coli and Klebsiella. Most of the strains were 3rd generation cephalosporin resistant and aminoglycosides followed by carbapenems had the highest sensitivity.

Table 1:-Percentage of type of infections and common organisms

\begin{tabular}{|l|l|l|l|l|}
\hline TYPE & $\begin{array}{l}\text { TOTAL } \\
(\%)\end{array}$ & $\begin{array}{l}\text { HOSPITAL } \\
\text { CARE } \\
\text { ASSOCLATED } \\
(\%)\end{array}$ & $\begin{array}{l}\text { CULTURE } \\
\text { POSITIVIT } \\
\text { Y (\%) }\end{array}$ & MOST COMMON ORGANISM \\
\hline AFI & $78(37.1)$ & $48(61.5)$ & $9(11.5)$ & E.Coli.Klebsiella.Enteroceccus \\
\hline UTI & $38(18.1)$ & $17(44.7)$ & $16(42.1)$ & Klebsiella.E.Coli \\
\hline CELLULITIS & $34(16.2)$ & $23(67.6)$ & $12(35.3)$ & Klebsiella, Pseudomonas.Streptecocus \\
\hline AFI+ UTI & $25(11.9)$ & $13(52)$ & $8(32)$ & KlebsiellaPneumenia.5.Coli \\
\hline $\begin{array}{l}\text { AFI+ } \\
\text { CELLULITIS }\end{array}$ & $20(9.5)$ & $9(45)$ & $5(25)$ & Klebsiella, Rseudomonas.Streptececus \\
\hline RTI & $15(7.5)$ & $6(40)$ & $5(33)$ & Klebsiella, Staphylococus.Strepte \\
\hline
\end{tabular}

Table 2:- Antibiotic susceptibility of isolates

\begin{tabular}{|c|c|c|c|c|c|c|c|c|c|}
\hline ORCANISM & & $\begin{array}{l}\text { 3RD } \\
\text { GENERAT } \\
\text { ON } \\
\text { CEPHALO } \\
\text { SPORIN }\end{array}$ & $\begin{array}{l}\text { PIPERACI } \\
\text { LLIN } \\
\text { TAZOBBA } \\
\text { CTAMI }\end{array}$ & $\begin{array}{l}\text { CARBAPE } \\
\text { NENIS }\end{array}$ & $\begin{array}{l}\text { QUTNOLO } \\
\text { NES }\end{array}$ & $\begin{array}{l}\text { AManocL } \\
\text { UCOSIDES }\end{array}$ & $\begin{array}{l}\text { CLOXACI } \\
\text { LIN }\end{array}$ & $\begin{array}{l}\text { YANCOM } \\
\text { YCIN }\end{array}$ & LINEZOLID \\
\hline \multirow[t]{2}{*}{ ECOLI } & H & 25 & 50 & 93 & 25 & 100 & & & \\
\hline & c & 57 & $\pi$ & 100 & 57 & 100 & & & \\
\hline \multirow[t]{2}{*}{ KLEBSTELLA } & H & 24 & 41 & $8 s$ & 41 & 94 & & & \\
\hline & c & 46 & 77 & 92 & 62 & 100 & & & \\
\hline \multirow[t]{2}{*}{ PSEUDOMONAS } & H & 0 & 33 & 66 & 0 & 100 & & & \\
\hline & c & 50 & 100 & 100 & 50 & 100 & & & \\
\hline ENIEROCOCCUS & H & & & & & & 100 & 100 & 100 \\
\hline STREPTOCOCCUS & c & & & & & & 100 & 100 & 100 \\
\hline STAPHYLOCOCCUS & H & & & & & & 100 & 100 & 100 \\
\hline
\end{tabular}

\section{PHT-9}

Clinical and bacteriological profile of cellulitis in end stage liver disease

\section{Mayank Jain $^{1}$, Subhashree Parida ${ }^{2}$, Dinesh Jothimoni ${ }^{2}$, Jayanthi} Venkataraman ${ }^{2}$

${ }^{1}$ Gleneagles Global Health City, India; ${ }^{2}$ Gleneagles Global Health City, Chennai 600100, India, India

Background: Patients with liver cirrhosis are highly susceptible to bacterial infections. The risk of cellulitis and bacteriological profile in cirrhotic patients including antibiotic resistance pattern is not well established. Earlier study from our centre has reported that $69 \%$ of bacterial isolates in cirrhotics are multidrug resistant.

Aim: To determine clinical and bacteriological profile of skin infection presenting as cellulitis in patients of liver cirrhosis.

Method: In this prospective study, consecutive cirrhotic patients hospitalized between February 2017 and September 2017 with clinically diagnosed cellulitis were enrolled. Data retrieved included demography, aetiology, co-morbidity, cirrhosis related complications, site of cellulitis, baseline investigations, wound and blood culture reports with antibiotic sensitivity. As per the protocol, all patients are empirically started on piperacillin - tazobactam until results of culture reports and are then subsequently modified.

Result: Three hundred thirty eight patients were admitted with end stage liver disease during the study period. Of these, twenty five had cellulitis and formed the study cohort. The mean age was $52.8+10.4$ years. Majority were men $(88.0 \%)$. The median MELD score was $18.8+10.4$. Non alcoholic steatohepatitis and alcohol contributed to $40 \%$ of cirrhosis followed by cryptogenic (16\%) and hepatitis B (4\%). 9 patients $(36 \%)$ had diabetes mellitus. There was only one past smoker. Other complications during admission included refractory ascites and renal dysfunction in $36 \%$ respectively, SBP in $12 \%$, hepatic encephalopathy and gastrointestinal bleed in $8 \%$ respectively. Three of the 25 patients had history of cellulites in the past. Majority of the patients had unilateral $(68 \%)$ or bilateral pedal edema $(20 \%)$. Two patients had unilateral upper limb involvement (8\%) and scrotal oedema in $4(4 \%)$. The skin was erythematous and inflamed with warmth and tenderness on touch. Fifteen patients $(60 \%)$ had superficial ulcerated lesions and serous fluid ooze from the superficial lesion; one patient had bullous lesion. Culture positivity was noted in thirteen cases (52\%; 3- blood, 11 - discharge from the affected skin). Majority of the cultures showed monomicrobial growth $(9,70 \%)$ and the remaining were polymicrobial. E. coli was the commonest isolate (6/13), followed by Klebsiella (4), Pseudomonas (2) and Staphylococcus aureus (2). Eight isolates $(61 \%)$ had multidrug resistant organisms (ESBL-6, Carbapenemase producing klebsiella-2). Average duration of treatment 10 days ( +4.1 days). Twenty two patients were discharged with healing of the wound; 2 left against medical advice and one died due to multiorgan failure. 
Conclusion: Cellulitis was noted in $7.4 \%$ of hospitalised cirrhotics. Culture positivity was $52 \%$ with predominant monomicrobial infection. E. coli and Klebsiella were the common isolates and $61 \%$ are multidrug resistant.

\section{PHT-10}

\section{Hematological indices in portal hypertension: cirrhosis} versus NCPHT

\section{$\underline{\text { Abdurrahman Sahin }}^{1}$, Hakan Artas ${ }^{1}$, Mehmet Yalniz ${ }^{1}$ \\ ${ }^{1}$ Firat University, Turkey}

Background: We aimed in this study to compare the alterations of hematological indices (HI) in portal hypertension (PHT) related with cirrhosis and non-cirrhotic portal hypertension (NCPHT). We aimed in this study to compare the alterations of hematological indices (HI) in portal hypertension (PHT) related with cirrhosis and non-cirrhotic portal hypertension (NCPHT).

Method: Portal vein Doppler US reports between January 2010 and January 2017 were reviewed and the cases of PHT related with cirrhosis or NCPHT were recruited to this retrospective study. Demographic, clinical, endoscopic features and laboratory data (complete blood count including Neutrophil to lymphocyte ratio (NLR) and platelet to lymphocyte ratio (PLR) at the time of the radiological assessment) were collected from hospital records. Cytopenia was defined as the presence of anemia and/or leukopenia and/or thrombocytopenia.

Result: A total of 239 patients with cirrhosis (91 CTP A class; 88 CTP B class; 61 CTP C class) and 89 patients who had NCPHT were recruited. Demographic, clinical and laboratory features were given in Table. The etiological factors for cirrhosis were HBV (23.8\%), HDV $(8.3 \%)$, HCV $(6.7 \%)$, NASH $(13 \%)$, alcoholic cirrhosis $(6.3 \%)$, autoimmune hepatitis $(4.6 \%)$ and primary biliary cholangitis $(3.8 \%)$. Seventy subjects $(29.3 \%)$ were classified as cryptogenic cirrhosis. The abnormalities in $\mathrm{HI}$ were higher in patients with cirrhosis than NCPHT group (Table 2). Though NLR did not differ between groups $(2.71 \pm 0.14$ for cirrhosis, $2.47 \pm 0.19$ for NCPHT, $\mathrm{p}=0.125)$, PLR was higher in patients with NCPHT then in patients with cirrhosis $(134.8 \pm 8.2$ vs $93.5 \pm 3.9, \mathrm{p}<0.001)$. The comparison of $\mathrm{HI}$ among cirrhotic groups was given in Table 3 . The NLR was the only parameter to differentiate each CTP group from two others. The NLR value has gradually increased from CTP-A to CTP-C, and was significantly correlated with CTP score $(\mathrm{r} 2=0.370, \mathrm{p}<0.001)$ and MELD score $(\mathrm{r} 2=0.371, \mathrm{p}<0.001)$. The AUROC of NLR was $0.692(95 \% \mathrm{CI}$, 0.624-0.759; $\mathrm{p}<0.001)$ differentiating compensated cirrhotic patients (CTP-A) from decompensated patients (CTP-B and CTP-C) at a cut off value of 2.33 with $55 \%$ sensitivity and $74 \%$ specificity.

Conclusion: Hypersplenism is more prominent in cirrhosis compared to NCPHT. The NLR is correlated with the severity of cirrhosis and, this simple test reveals the course of cirrhosis.

\section{PHT-11}

\section{Proton pump inhibitor use in liver cirrhosis}

\author{
Marianne Anastasia De Roza ${ }^{1}$, Marianne Anastasia De Roza ${ }^{1}$, Lim \\ Kai $^{1}$, John Chen Hsiang ${ }^{1}$ \\ ${ }^{1}$ Singhealth, Singapore
}

Background: There are growing concerns about long term use of proton pump inhibitors (PPIs) and associated adverse effects. Patients with liver cirrhosis are more susceptible to infections and PPI usage may promote this. There is also evidence that suggests increased mortality with PPI use in liver cirrhosis. Despite these concerns, PPIs are still widely prescribed in liver cirrhosis patients for long durations. The aim of this study is to determine PPI use in liver cirrhosis.

Method: Data was extracted from an electronic database of patients records at a tertiary 1000-bed hospital in Singapore from January 2012 to June 2017. Patients with ICD10 codes for liver cirrhosis of all aetiologies were identified and those with PPI prescriptions were included into the study. We used a cumulative defined daily dose (cDDD) as recommended by the World Health Organization to measure the prescribed amount of PPI. We then identified those with cDDD $>28$ (i.e. $>1$-month use) which other studies suggest to significantly cause adverse outcomes.

Result: A total of 1446 patients with liver cirrhosis were prescribed PPI and included into the study. 970 (67.1\%) were male. $888(61.4 \%)$ were Chinese, 278 (19.2\%) were Malay and 135 (9.3\%) were Indian. The mean age was 64.8 years (SD 8.7). $1243(86.0 \%)$ of these patients were prescribed PPI with a cDDD $>28$.

Conclusion: Despite growing concerns about PPI use in liver cirrhosis, $86 \%$ of a large cohort of liver cirrhosis patients were prescribed PPI for more than a month (cDDD $>28)$. The adequacy and impact of such prescriptions can be further studied.

\section{PHT-12}

\section{Hyperferritinemia in patients with chronic liver diseases}

\section{Narina Sargsyants ${ }^{1}$}

${ }^{1}$ Armenicum Clinical Center, Armenia

Background: Hyperferritinemia is found in a large spectrum of conditions include increased ferritin synthesis (acquired/genetic conditions with or without iron overload) or increased release of ferritin from damaged cells (hepatocytes cytolysis in acute or chronic liver diseases). Three main mutations in HFE gene associated with risk of hereditary hemochromatosis development: $\mathrm{C} 282 \mathrm{Y}(845 \mathrm{G}>\mathrm{A})$, H63D $(187 \mathrm{C}>\mathrm{G})$ and S65C $(193 \mathrm{~A}>\mathrm{T})$. The vast majority $(85-92 \%)$ of European patients characterized with homozygous for the $\mathrm{C} 282 \mathrm{Y}$ variant in the HFE gene.

The aims of this report is description of hyperferritinemia in patients with chronic hepatitis, including monitoring during antiviral treatment with the different sofosbuvir (SOF) contain regimens.

Method: Ferritin (FERR) level in blood (normal range 21.81-274.66 $\mathrm{ng} / \mathrm{mL}$ ) have been checked in 57 patients with chronic hepatitis: from 21 to $73(52.4 \pm 11.9)$ years old, $58 \%$ male, BMI $27.8 \pm 5.1 \mathrm{~kg} / \mathrm{m}^{2}$. Among them vast majority were $\mathrm{HCV}$-infected patients ( $49 \%$ with $\mathrm{F} 4$ and $17 \%$ with $\mathrm{F} 3$, viral load $118-27485300 \mathrm{IU} / \mathrm{ml}$ ) treated with following regimens: $S O F+D C V \pm R B V$ in 18 patients, $S O F / L D V \pm R B V$ in 13 patients, $\mathrm{SOF}+\mathrm{RBV}$ in 5 patients, $\mathrm{SOF}+\mathrm{PEG}-\mathrm{IFN} \alpha+\mathrm{RBV}$ in 5 patient and PEG-IFN $\alpha+$ RBV in 11 patient.

First time in Armenia patients with high ferritinemia were screened for Hereditary Hemochromatosis HFE by gene analysis with implication of Real-time PCR based single nucleotide polymorphisms (SNPs) genotyping assay (set up in 2015).

Result: Among 57 patients hyperferritinemia noticed in 13 males $(23 \%)$ with mean age $53.1 \pm 11.7$ years old, FERR level $868.85 \pm 503.41 \mathrm{ng} / \mathrm{mL}$. GGT $177.7 \pm 142.39 \mathrm{U} / \mathrm{ml}$. 9 of them were HCV-infected with F4 or F3 and 4 patients with NAFLD without HCV-infection. All patients with hyperferritinemia have co-morbidities: 9 metabolic syndrome, 8 diabetes, 6 alcohol abuse. In 5 patients with FERR more than $500 \mathrm{ng} / \mathrm{mL}$ (minimal - 517.78, maximal - 1946.8) we checked mutation in HFE and revealed $187 \mathrm{C}>\mathrm{G}$ (rs1799945) heterozygous in three and homozygous in one patient. In all patients with chronic hepatitis $\mathrm{C}$ who gain SVR, level of FERR 
normalized, except one patient with primarily level of FERR was 1174.15, then during treatment with PEG-IFN+RBV increased up to 3627.56, and despite SVR still remain elevated 642.03, also was checked on Hereditary Hemochromatosis with heterozygous in gene 187C $>\mathrm{G}$ (rs1799945). Elevation of FERR during antiviral treatment of chronic hepatitis $\mathrm{C}$ mainly observed in patients with advanced fibrosis, during PEG-IFN+RBV contain regimens (only in 5 patients with IFN-free DAAs regimens) and with hyperferritinemia at the beginning. In NAFLD patients level ferritinemia correlate with GGT level.

Conclusion: In HCV-infected patients hyperferritinemia associated with advanced fibrosis or cirrhosis and co-morbidities. Among investigated patients with high ferritinemia C282Y homozygous HFE genotype typical for hereditary hemochromatosis, was not found. Presence of H63D heterozygous HFE genotype reinforce necessity of further investigations among patients with signs of iron overload in Armenia.

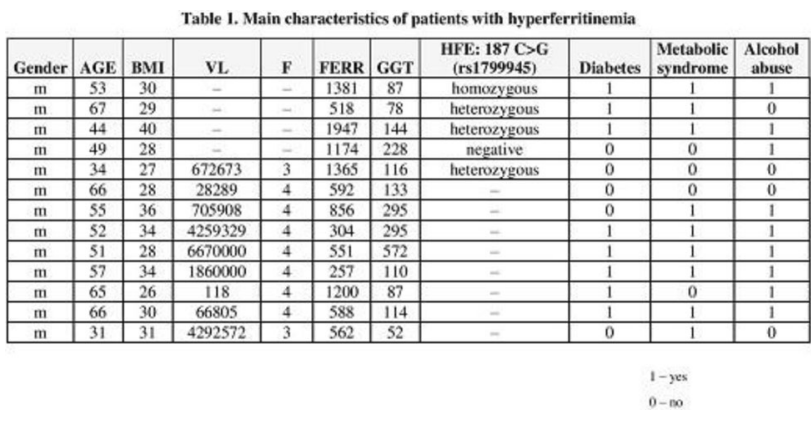

PHT-13

A study for rapid bedside diagnosis of spontaneous bacterial peritonitis using leukocyte esterase test

\section{Mamata Sanjay Lotlikar ${ }^{1}$}

${ }^{1} \mathrm{DM}$, India

Background: Spontaneous Bacterial Peritonitis (SBP) is a potentially life threatening complication of liver cirrhosis with ascites. The prevalence of SBP in hospitalized patients with liver cirrhosis and ascites is up to $30 \%$. The in hospital mortality is also very high (30$50 \%$ ). SBP requires urgent diagnosis and treatment that is usually based on total and differential Leukocyte count of ascitic fluid (AF). Method: We conducted this study by doing bedside leukocyte esterase reagent strip (LERS) test on ascitic fluid and compared with cell count done by counting chamber method.

Result: Out of 75 cases $17(22.6 \%)$ were diagnosed to have SBP by counter chamber method as compared to 13 cases $(17 \%)$ by reagent strip method with cut off +++3 on colorimetric scale. The sensitivity, specificity, positive predictive value, negative predictive value and accuracy of reagent strip were $76.4 \%, 98.2 \%, 76 \%, 93.5 \%, 89 \%$ respectively compared to counting chamber method.

Conclusion: LERS test is useful bedside test for rapid diagnosis of SBP. Even though less sensitive it is highly specific for diagnosing SBP.
PHT-14

An external validation of the Baveno VI criteria and a new proposal for improving the identification of low risk cirrhotic patients who can avoid screening endoscopy for varices

Jiannis Vlachogiannakos ${ }^{1}$, Dimitrios Karagiannakis ${ }^{1}$, Theodoros Voulgaris $^{1}$, Spiros Siakavellas ${ }^{1}$, Despina Kalogera ${ }^{1}$, Theodoros Aggelopoulos $^{1}$, George Papatheodoridis ${ }^{1}$

${ }^{1}$ National and Kapodistrian University of Athens, Medical School, Greece

Background: Varices represent a serious complication of cirrhosis and early endoscopic diagnosis is mandatory to start prophylactic treatment and prevent bleeding. However, it has been suggested that patients with PLTs $>150000$ and liver stiffness (LS) 110000 and LS Method: We retrospectively collected liver elastography, ultrasonographic and laboratory data of patients with chronic liver disease attended the outpatient clinic from 2015 to 2017. Those with LS = $12 \mathrm{kPa}$ proceeded to upper GI endoscopy (UGE) within 1 year after elastography. As high risk varices (HRV) were defined esophageal varices $=5 \mathrm{~mm}$, those with red spots, and any gastric varices. Splenomegaly was defined by diameter $>12 \mathrm{~cm}$. Exclusion criteria were decompensated disease, porto-splenic vein thrombosis, and non-cirrhotic portal hypertension.

Result: We included 108 patients (65 male, 43 female) with chronic liver disease. Mean age was $58 \pm 12$ years. The aetiology of cirrhosis was chronic viral hepatitis in $59(54.6 \%)$, alcoholic liver disease in 13 (12\%), NAFLD in $21(19.4 \%)$ or other in $15(13.9 \%)$. Child-Pugh score A patients represented the vast majority $(103 / 108,95.4 \%)$ whereas $5(4.6 \%)$ were CTP-B. Varices irrespective of size and bleeding risk were documented in $42(38.9 \%)$, whereas HRV were present in $25(23.1 \%)$. PLTs $20 \mathrm{kPa}$ was observed in $56(51.9 \%)$ and $>25 \mathrm{kPa}$ in $35(32.4 \%)$. In our cohort, BAVENO VI criteria could predict the absence of HRV with sensitivity $100 \%$ and NPV $100 \%$. $12 / 59(20.3 \%)$ could avoid UGE. No patient with HRV full filled the BAVENO VI criteria for non-screening endoscopy. Adopting the expanded criteria, 52 (48\%) of patients supposed not to undergo screening UGE. Among them, 10 (9.26\%) had HRV. Sensitivity was $80.8 \%$ and NPV was only $60 \%$. When PLTs replaced by spleen diameter

Conclusion: In our cohort of patients, BAVENO VI criteria reliably distinguished those who could avoid endoscopy although only $20 \%$ of the whole population would benefit. The expanded criteria couldn't be validated, showing high rate of misclassification among patients with HRV. We have also shown that the combination of LS and spleen diameter represent an accurate and easy to perform combination that could accurately recognize a substantial proportion of patients who could safely avoid screening UGE.

\section{PHT-15}

\section{Cardiac dysfunction in portal hypertension: a single centre experience}

Sambit Kumar Behera ${ }^{1}$, Sambit Kumar Behera ${ }^{1}$, Kaibalya Ranjan $\overline{\text { Dash }}^{1}$, Suryakanta Parida ${ }^{1}$, Chitta Ranjan Khatua ${ }^{1}$, Debakanta Mishra $^{1}$, Subhendu Panigrahi ${ }^{1}$

${ }^{1}$ SCB Medical College, India

Background: Cardiac functional abnormalities are known to occur in cirrhosis of liver. However the relative impact of portal hypertension and hepatic dysfunction is not known. We studied patients of cirrhotic 
and noncirrhotic portal hypertension to evaluate the contribution of portal hypertension and/or cirrhosis in causing cardiac abnormalities. Method: A cross sectional observational study was undertaken. Consecutive patients with portal hypertension (cirrhotic and noncirrhotic) were enrolled after excluding known cardiac illness. Noninvasive cardiac assessment was performed using electrocardiogram (ECG) and transthoracic echocardiography (ECHO). Statistical analysis was performed using SPSS software. $\mathrm{P}$ value $<0.05$ was considered significant.

Result: 78 patients of portal hypertension were studied. 12 had extra hepatic portal vein obstruction (EHPVO), 2 had Budd-Chiari syndrome and 64 had cirrhosis of liver due to various aetiologies. All the patients of EHPVO and Budd-Chiari syndrome had normal cardiac function. In patients of cirrhosis of liver, diastolic dysfunction was present in 42 patients (65.62\%). QT prolongation was seen in ECG of 16 patients with cirrhosis $(25 \%)$. There was significant correlation found between diastolic dysfunction and ascites $(\mathrm{P}=0.034)$. There was no significant correlation between MELD Score and diastolic dysfunction $(\mathrm{P}=0.317)$. Cardiac dysfunction was equally prevalent among patients of cirrhosis due to alcohol and other etiologies. ( $\mathrm{P}$ value $=0.92$ ).

Conclusion: Patients with portal hypertension due to extrahepatic causes have normal cardiac function. Two third of patients with cirrhosis of liver have diastolic dysfunction while one fourth had QT prolongation. Diastolic dysfunction in cirrhotics where no other known cardiac risk factors are present may be attributed to cirrhotic cardiomyopathy.

\section{PHT-16}

Changes of esophageal varices after DAAs treatment for compensated cirrhotic patients with $\mathrm{HCV}$

Tomoaki Nakajima $^{1}$, Ryoji Tatsumi ${ }^{1}$, Masakatsu Yamaguchi ${ }^{1}$, Mutsuumi Kimura ${ }^{1}$, Tomohiro Arakawa ${ }^{1}$, Yasuaki Kuwata ${ }^{1}$, Itaru Ozeki ${ }^{1}$, Takumi Ohmura ${ }^{1}$, Shuhei Hige ${ }^{1}$, Yoshiyasu Karino $^{1}$, Joji Toyota ${ }^{1}$

${ }^{1}$ Sapporo-Kosei General Hospital, Japan

Background: Influence of sustained virologic response (SVR) on change of esophageal varices has not been studied previously. We evaluated the influence of SVR on esophageal varices after DAAs treatment in cirrhotic patients.

Method: 48 compensated cirrhotic patients with esophageal varices who accomplished 24-weeks or 12-weeks DAAs treatment were enrolled. We evaluated the change of esophageal varices form by upper endoscopy before and after DAAs treatment retrospectively. 41 $\mathrm{HCV}$ genotype 1 patients were assigned to daclatasvir/asunaprevir for 24 weeks or sofosbuvir/ledipasvir for 12 weeks or Ombitasvir/Paritaprevir/ritonavir for 12 weeks, and 7 genotype 2 patients were assigned to sofosbuvir/RBV for 12 weeks.

Result: SVR24 rate was $81.3 \%$. Esophageal varices were improved in 3 patients, unchaged in 29 patients, and progressing in 16 patients. The rate of past history of esophageal varices therapy was significantly higher in progressing group $(87.5 \%)$ than improved or unchanged group $(40.6 \%)(\mathrm{P}=0.002)$. On the other hand, the rate of SVR was same in both group $(81.3 \% / 81.3 \%)$.

Conclusion: After DAAs treatment for cirrhotic patients, we should monitor esophageal varices, especially in patients with past history of esophageal varices, with and without SVR.
PHT-17

Cirrhosis - alternative approaches to modern medicine.

\section{Muzaffarbek O'ktam O'g'li Ismailov ${ }^{1}$, Saidrakhim Nodirovich Lukmonov $^{2}$ \\ ${ }^{1}$ Tashkent State Pediatric Institute, Uzbekistan; ${ }^{2}$ Tashkent Medical Academy, Uzbekistan}

Background: To find out the etiological structure of liver cirrhosis for improving its diagnosis and prevention of complications.

Method: 57 patients were referred to the Republican Clinical Hospital No. 1 in the Department of Surgery and Therapy from May 2017 to September 2017. All patients were diagnosed with cirrhosis of the liver with different etiologies. During the study, the etiological structure of liver cirrhosis with various etiologies was studied.

Result: The study revealed that the main etiological factors of liver cirrhosis according to the departments are hepatitis viruses in 16 patients $(28 \%)$, alcohol 15 patients $(27 \%)$ and their combination (15\%), less often - autoimmune and cholestatic diseases (14\%). Among patients with liver cirrhosis infected with hepatitis viruses, the hepatitis $\mathrm{C}$ virus was most frequently detected ( 9 patients - 55\%), less often hepatitis B virus (5 patients $32 \%$ ) and mixed infection with two or three viruses ( 2 patients $13 \%)$. In $20(35 \%)$ patients with liver cirrhosis, extrahepatic manifestations were detected, primarily cutaneous vasculitis (in $25 \%$ of cases), renal damage (in $16 \%$ of cases) and peripheral nervous system in the form of multiple mononeuritis and polyneuropathy (in 18\% of cases). Most often, extrahepatic lesions are noted in cirrhosis of the virus viral etiology (in the outcome of HCV infection), especially in patients with mixed cryoglobulinemia.

Conclusion: To establish the etiology of liver cirrhosis, close attention should be paid to the "etiologic ornament" - combinations of symptoms characteristic of the viral, alcoholic and autoimmune and cholestatic of the etiology of liver cirrhosis.

\section{PHT-18}

Early rebleeding after endoscopic variceal ligation in cirrhotic patients in a Tertiary Care Hospital

Ashish $T^{1}$, Premaletha $\mathbf{N}^{1}$, Sunilkumar $\mathbf{K}^{1}$, Prijith Abraham Thomas ${ }^{1}$, Deepak Thomas ${ }^{1}$, Unnikrishnan $\mathrm{S}^{1}$

${ }^{1}$ Govt. Medical College, Kottayam, India

Background: Endoscopic variceal ligation (EVL) is currently the definitive treatment of choice for active variceal hemorrhage. EVL is also recommended as primary prophylaxis of variceal bleeding. However, early re bleeding after EVL (within 6 weeks) may occur and may be fatal at times. Our aim was to find out the proportion of cirrhotic patients developing early rebleeding after endoscopic variceal ligation (EVL) and to assess the clinical and endoscopic factors associated.

Method: 100 consecutive patients with cirrhosis of all etiologies undergoing endoscopic variceal ligation (EVL) were followed up for 6 weeks and assessed clinically and endoscopically every 2 weeks for development of early re bleeding. Clinical and endoscopic factors predisposing to early rebleeding were studied. Data was entered in Microsoft Excel and analyzed using IBM SPSS v16.0

Result: 12 patients developed early rebleeding post EVL. Among them 4 patients developed the complication within first 2 weeks, 3 patients developed massive bleeding requiring multiple blood transfusions and 2 patients died during hospital stay. History of previous variceal bleeding, low platelet count, high prothrombin time and 
number of bands applied were found to be independent predictors of early re bleeding in multivariate logistic regression analysis.

Conclusion: Early rebleeding after EVL is a rare but life threatening complication. Close follow up of patients and early recognition and hospitalization might prevent mortality and improve prognosis.

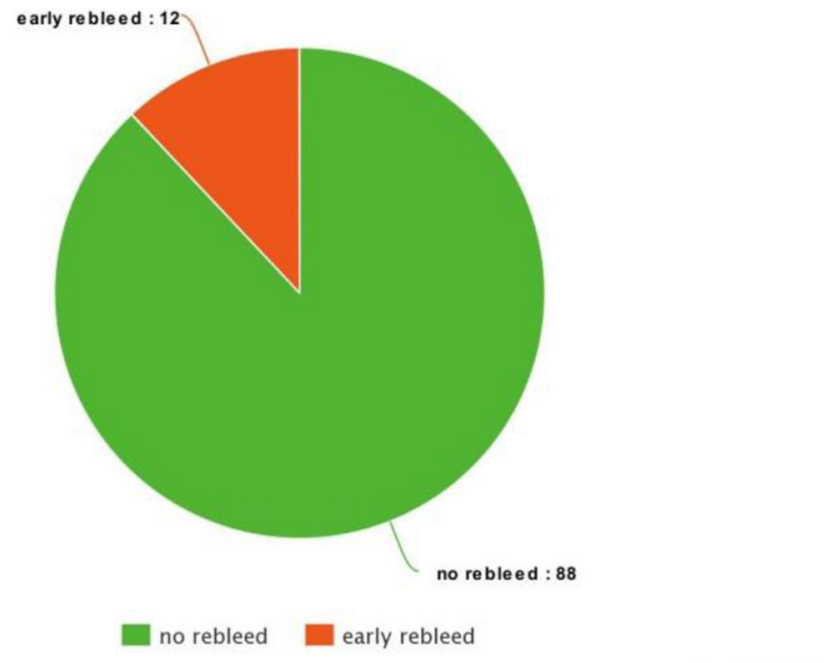

PHT-19

EUS-guided therapy in the management of gastric fundal and ectopic varices - a single center experience

Shibi Mathew ${ }^{1}$, Prakash Zacharias', Prashanth Menon ${ }^{1}$, Maya Peethambaran $^{1}$, Aby Somu ${ }^{1}$, John Mathews ${ }^{1}$, Mathew Philip ${ }^{1}$

${ }^{1}$ PVS Institute of Digestive Diseases, Kochi, India

Background: Gastric fundal varices and ectopic varices can cause fatal variceal hemorrhage. There are reports of EUS guided variceal obliterative therapy in the management of these varices. Aim of this study is to analyze the safety and outcome of EUS guided therapy of gastric fundal and ectopic varices in our group of patients.

Method: Prospective analysis of patients with upper GI bleed due to gastric fundal and ectopic varices who were managed in our center with EUS guided therapy. Study period was 36 months.

Result: Thirty-one patients who underwent EUS guided variceal therapy were analyzed. Mean age was 51.93 yrs. Male: Female ratio was 28:3. All patients except one pregnant lady with extrahepatic portal vein obstruction had cirrhosis as the cause of portal hypertension. Eighteen patients $(60 \%)$ had ethanol as the cause of cirrhosis, while the rest had cryptogenic cirrhosis. Thirteen patients were in Child A status, 14 in Child B and 3 in Child C status. The various types of varices included GOV 1(2), GOV2(22), IGV1(3) and Duodenal (4). Most patients had large varices (74.2\%). The clinical presentations included acute variceal hemorrhage in 17 patients; a recent variceal bleed (within 2 weeks) in 7 ; recurrent uncontrolled bleed in 2 patients and history of remote bleed in 1 patient. Four patients underwent prophylactic EUS guided therapy for the varices. Fifteen patients gave a previous history of glue injection to the same site of varices. A combined therapy of EUS guided intra-variceal glue injection and coil insertion was done in 16 patients, while coil insertion only was done in 3 patients and in the remaining patients only glue injection was done. On repeat EUS evaluation, only 7 patients $(22.58 \%)$ had incomplete obliteration of the varices which needed a repeat EUS guided intervention. In the initial follow up period of 2 weeks, only 3 patients had recurrence of bleed. There were no procedure related complications or clinically evident systemic embolization. Thirty-day mortality was nil. Twenty-four patients $(77.42 \%)$ were under follow up, with a median follow up duration of 210 days. Only two patients had recurrence of bleed during this period.

Conclusion: EUS guided cyanoacrylate glue and coil insertion is a highly effective treatment modality for managing our group of patients with gastric fundal and duodenal varices.

\section{PHT-20}

\section{In-hospital mortality rate and its predictive factors of liver cirrhosis}

Chyntia Olivia Jasirwan ${ }^{1}$, Rino Alvani Gani ${ }^{1}$, Irsan Hasan ${ }^{1}$, Andri Sanityoso $^{1}$, Cosmas Rinaldi Lesmana ${ }^{1}$, Juferdy Kurniawan ${ }^{1}$, Saut Horas Nababan ${ }^{1}$, Kemal Fariz Kalista ${ }^{1}$, Putra Nur Hidayat ${ }^{1}$, Gita Aprilicia $^{1}$

${ }^{1}$ Ciptomangunkusumo National Hospital, Faculty of Medicine Universitas Indonesia, Indonesia

Background: Recent studies estimate that mortality from liver cirrhosis will account for substantial disease burden each year, especially in developing countries. Better understanding of risk factors associated with mortality from patients with liver cirrhosis is needed to improve preventive measures and survival rate. Therefore, we conducted a study at Cipto Mangunkusumo National Hospital Jakarta as tertiary hospital which represent the study population in Indonesia.

Method: Cirrhotic patients admitted between 2017 January and 2017 November in Cipto Mangunkusumo National Hospital Jakarta were prospectively reviewed. Baseline characteristics and their clinical features were collected at the time of inclusion. We conducted multivariate analysis by using logistic regression. Odds ratio (OR) with 95\% confidence intervals (CIs) were calculated.

Result: There were 205 admissions for patients with cirrhosis in this study with median of age $54 \pm 23$ years. As much as $16.1 \%(33 / 205)$ patients had hepatocellular carcinoma (HCC) and 36.1\% (74/205) had portal hypertension. Hepatitis B virus infection was the most frequent etiology found in the patients $(41 \% ; 84 / 205)$. Overall in-hospital mortality was $36.6 \%$ (75/205). Higher odds of mortality were found in male patients (OR, 3.20; 95\% CI, 1.10-9.31; $\mathrm{P}=0.033)$ and patients above 50 years old (OR, $4.31 ; 95 \% \mathrm{CI}, 1.85-10.02 ; \mathrm{P}=0.001)$. Compared to patients with Child Pugh $(\mathrm{CP})$ classification of $\mathrm{A}$, the odds for mortality increased progressively in patients with CP B (OR, 3.71; 95\% CI, 1.25-10.97; $\mathrm{P}=0.018)$ and patients with $\mathrm{CP} C \mathrm{C}(\mathrm{OR}, 5.96 ; 95 \%$ $\mathrm{CI}, 1.59-22.32 ; \mathrm{P}=0.008)$. Higher odds of mortality were also found in the presence of portal hypertension (OR 4.57; 95\% CI, 1.90-10.98; P, $0.001)$, HCC (OR, 4.98; 95\% CI, 1.81-13.70; $\mathrm{P}=0.002)$, hematemesis (OR, 3.17; 95\% CI, 1.25-8.03; $\mathrm{P}=0.015)$, melena $(\mathrm{OR}, 3.34 ; 95 \% \mathrm{CI}$, $0.76-14.65 ; \mathrm{P}=0.110)$, jaundice (OR, $3.42 ; 95 \% \mathrm{CI}, 1.34-8.75$; $\mathrm{P}=0.010$ ), and encephalopathy (OR, 1.77; 95\% CI, 0.62-5.02; $\mathrm{P}=0.286$ ) compared to patients without these features.

Conclusion: The presence of portal hypertension, HCC, and patient's age might be effective for predicting the in-hospital mortality of liver cirrhosis. Other parameters which show insignificant higher odds of mortality should be considered. 
PHT-21

Increased prevalence of asymptomatic peptic ulcers diagnosed during screening endoscopy in patients with cirrhosis

\section{Jiannis Vlachogiannakos ${ }^{1}$, Theodoros Voulgaris ${ }^{1}$, Dimitrios Karagiannakis $^{1}$, Despina Kalogera ${ }^{1}$, Theodoros Aggelopoulos ${ }^{1}$, Elissavet Chloupi ${ }^{1}$, George Papatheodoridis ${ }^{1}$ \\ ${ }^{1}$ National and Kapodistrian University of Athens, Medical School, Greece}

Background: It has been previously reported that patients with liver cirrhosis have increased prevalence of peptic ulcers compared to general population, attributed to impaired mucosal defense mechanisms. However, the small size and the heterogeneity of the studies made the interpretation of the results difficult and thus the relationship between cirrhosis and peptic ulcer remains uncertain. We investigated the prevalence of peptic ulcers in patients with advanced chronic liver disease and their potential association with the underlying etiology, the severity of liver disease as well as the other manifestations of portal hypertension.

Method: All cirrhotic patients who attended the liver clinic of a Greek tertiary hospital during a 6-month period (March 2017 to September 2017) were invited to participate in the study. We excluded patients who were receiving acid suppressing drugs. Screening endoscopy was performed using a push enteroscope and we recorded the presence of gastric or duodenal ulcer, presence and characteristics of gastroesophageal varices, development and severity of portal hypertensive gastropathy (PHG) as well as of portal hypertensive enteropathy (PHE). Cirrhosis was diagnosed based on elastography, liver biopsy, or a combination of clinical, biochemical and imaging data.

Result: We enrolled in the study 86 consecutive cirrhotic patients (M/ F: 52/34) with a mean (SD) age of $60 \pm 14$ years. The cause of liver cirrhosis was chronic hepatitis in 31 (36\%), alcohol in $24(28 \%)$ or other in $31(36 \%)$. Fifty one patients $(59 \%)$ were Child-Pugh stage A, $31(36 \%)$ CTP B and 4 (5\%) CTP C. Peptic ulcer was found in 17 $(19,8 \%)$ patients. Of them, $13(15,2 \%)$ had gastric ulcer while 4 $(4,6 \%)$ a duodenal ulcer. All were asymptomatic. None had been receiving NSAIDs. Helicobacter pylori was not diagnosed in any patient (rapid urease test, histology). Varices of any grade were diagnosed in $52(61 \%)$ patients and $36(42 \%)$ of them needed treatment. PHG was present in $71(83 \%)$ patients (severe in 35\%) and PHE in $15(17.5 \%)$. The presence of ulcer, irrespectively of the origin, was unrelated to the etiology or the severity of the liver disease (CTP and MELD score). Moreover, no statistically significant correlation was found between the presence of peptic ulcer and the other endoscopic manifestations of portal hypertension (gastroesophageal varices and/ or PHG and/or PHE).

Conclusion: According to our results, patients with liver cirrhosis have an increased prevalence of peptic ulcers although they are asymptomatic and have no known risk factors of ulcerogenicity. Peptic ulcer disease was not associated with either the severity of liver disease or the other manifestations of portal hypertension. Further studies are needed to investigate potential pathogenetic mechanisms that associate chronic liver disease with increased frequency of peptic ulcers.
PHT-22

Infrared color liquid crystalline thermography in diagnostics of portal hypertension syndrome in patients with cirrosis of the liver

\section{Saidrakhmon Nodirovich Khoshimov ${ }^{1}$}

${ }^{1}$ Tashkent State Pediatric Institute, Uzbekistan

Background: Clinical evaluation of the possibilities of using infrared color liquid crystal thermography and remote thermal imaging in the diagnosis of portal hypertension $(\mathrm{PH})$ in patients with liver cirrosis. Method: Complex clinical-laboratory, ultrasound, endoscopic and thermographic study of 30 patients with LC complicated by PH at the age of 43 to 70 years (mean age was $63.1 \pm 7.2$ years). Thermography was performed using an infrared camera ThermaAM P 65 (FLIR Systems, Sweden). In addition, thermography was performed in 20 healthy volunteers.

Result: When thermography of healthy people (group 1) recorded skin areas with different color spectra (from white to blue). However, the precise localization of each part of the spectrum of color, characteristic of the pathological process, was not observed. In all patients with a class A (group 2) LC, the white spectrum of color predominantly occupied the right lateral and peripump region of the anterior abdominal wall. The minimum body temperature is $35^{\circ} \mathrm{C}$, the maximum temperature is $36.7^{\circ} \mathrm{C}$. In patients with B-class $\mathrm{C}$ (group 3), white and red color spectra were distributed predominantly in the epigastric and mesogastric areas of the anterior abdominal wall. In addition, the vessels of the anterior abdominal wall were moderately contoured. The minimum body temperature is $32.1{ }^{\circ} \mathrm{C}$, the maximum temperature is $36.4{ }^{\circ} \mathrm{C}$. In patients with $\mathrm{C}$ class $\mathrm{C}$ (group 4), white and red color spectra were also distributed in the epigastric and mesogastric areas of the anterior abdominal wall. At the same time, the considerably widened vessels of the anterior abdominal wall were clearly contoured in the form of pronounced vascular trunks. The minimum body temperature is $35.2{ }^{\circ} \mathrm{C}$, the maximum temperature is $38.2^{\circ} \mathrm{C}$. Of the 30 examined, $19(63.3 \%)$ patients with thermographic signs of high pressure in the portal system and varicose veins of the esophagus of grade II-III were operated. 12 patients underwent surgery of M. D. Patsior, 7 - portocaval shunting, including mesentericocaval anastomosis - in 2, dorsal splenorenal anastomosis in 3, splenorenal H-type anastomosis with a synthetic vascular insert in 2 .

Conclusion: Thermography with a thermal imager ThermaCAM P65 provides objective information on the degree of blood supply to the anterior abdominal wall in patients with $\mathrm{LC}$ complicated by $\mathrm{PH}$ and allows non-invasive monitoring of the patient's condition in the postoperative period.

\section{PHT-23}

Noninvasive indices for prediction of high-risk esophageal varices in compensated advanced chronic liver disease

Rathan Cyriac Joseph ${ }^{1}$, Sandeep Narayanan ${ }^{1}$, Aniruddha Singh ${ }^{1}$, Neeraj Vinayakumar ${ }^{1}$, Jose Mathew ${ }^{1}$, Shanid $A^{1}$, Krishnadas Devadas $^{1}$

${ }^{1}$ Govt. Medical College, Thiruvananthapuram, India

Background: Universal screening endoscopy benefits only a minority of patients with Compensated Advanced Chronic Liver Disease (cACLD). Noninvasive indices are needed to identify cACLD patients at risk of having significant varices. The Aim of the study was to evaluate the performance of Liver stiffness (LS), Liver stiffness- 
Spleen diameter to Platelet ratio score (LSPS), APRI, FIB-4 index and Platelet-Spleen ratio for prediction of High risk varices in cACLD.

Method: A sample of 100 patients with cACLD, defined by Liver stiffness $>10 \mathrm{kPa}$ by Transient elastography, were included. Those who had history of decompensation were excluded. All patients underwent Ultrasound Abdomen \& OGDscopy. High risk varices were defined as large varices or small varices with Red colour signs. The noninvasive indices were assessed according to established formulae. Diagnostic performance was assessed by ROC curve in terms of Sensitivity, Specificity, Positive predictive value (PPV), Negative predictive value (NPV) and Area under curve (AUC).

Result: Majority of the patients had viral-related cACLD with Hepatitis B being the most common etiology in 33\% of cases followed by Hepatitis C(30\%). Prevalence of Esophageal varices was $30 \%$. Prevalence of High risk-varices was $13 \%$. LSPS-based model had the best discriminative ability in predicting High risk-varices (AUC-0.87) followed by Platelet-spleen ratio (AUC-0.81); Liver Stiffness (AUC-0.77); FIB4(AUC-0.68); AST/ALT ratio (AUC-0.58) and APRI (AUC-0.55). The optimal LSPS cutoff value of 2.3 provided a Sensitivity of $76 \%$, Specificity of $87 \%$, PPV of $48 \%$, NPV of $96 \%$. The optimal cutoff value of Platelet-spleen ratio was 916 with a Sensitivity of $54 \%$, Specificity of $93 \%$, PPV of $53 \%$, NPV of $93 \%$. Both LSPS \& Platelet-spleen ratio had good Negative predictive value for High risk varices. Below the optimal cutoff of LSPS $<2.3$, Endoscopy could be safely avoided with less than 5\% risk of missing High-risk varices

Conclusion: Both LSPS and Platelet-spleen ratio was superior to the other indices in identifying cACLD patients with low probability of having High risk Esophageal varices so that unnecessary screening endoscopies can be avoided or delayed.

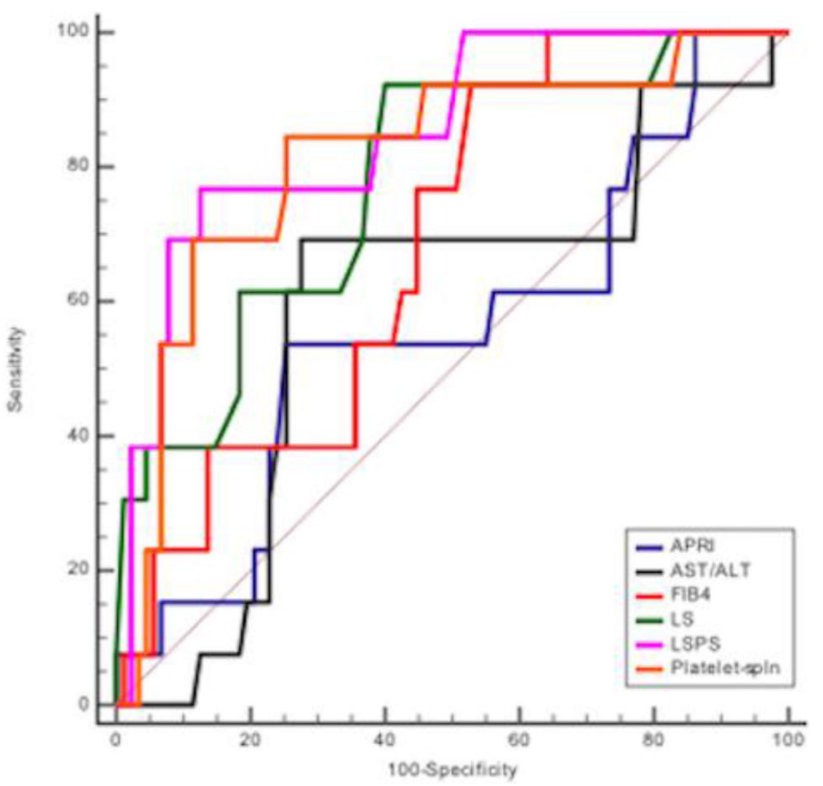

\begin{tabular}{|c|c|c|c|c|c|c|}
\hline Indices & AUC & Cutoff & Sensitivity & Specificity & PPV & NPV \\
\hline LSPS & 0.87 & $\mathbf{2 . 3}$ & $\mathbf{7 6}$ & $\mathbf{8 7}$ & $\mathbf{4 8}$ & 96 \\
\hline PSR & 0.81 & 916 & $\mathbf{5 4}$ & 93 & 53 & 93 \\
\hline LS & 0.77 & $\mathbf{2 0}$ & $\mathbf{8 4}$ & $\mathbf{6 0}$ & $\mathbf{2 2}$ & 93 \\
\hline FIB4 & 0.68 & $\mathbf{2 . 4}$ & $\mathbf{7 6}$ & 55 & 20 & 94 \\
\hline APRI & 0.55 & 1.04 & $\mathbf{3 9}$ & 77 & 20 & 89 \\
\hline AST/ALT & 0.58 & 1.22 & $\mathbf{6 1}$ & $\mathbf{7 4}$ & $\mathbf{2 6}$ & $\mathbf{9 2}$ \\
\hline
\end{tabular}

PHT-24

Patient response to endoscopic therapy for gastroesophageal varices based on endoscopic ultrasound (EUS) findings

\section{Yujen Tseng ${ }^{1}$}

${ }^{1}$ Zhongshan Hospital Fudan University, China

Background: Gastroesophageal variceal hemorrhage is a common complication secondary to portal hypertension. Endoscopic therapy is the current recommended treatment modality for preventing gastroesophageal variceal rebleed. This study is aimed at identifying outstanding characteristics between good and poor responders to endoscopic therapy based on endoscopic ultrasound (EUS) findings to better guide individualized therapy.

Method: A prospective cohort study was conducted, wherein 170 patients who previously received secondary prophylactic treatment for gastroesophageal varices were enrolled and classified into two groups based on their response to repeat endoscopic therapy sessions. Prior to consolidation therapy, all patients received EUS examination to observe for any extraluminal phenomenon. All available records of follow-up endoscopic examinations were retrieved to validate study results.

Result: Of the included subjects, 106 patients were considered poor responders, 64 were considered good responders. Prior to hospital admission or subsequent endoscopic findings. The presence of paragastric veins, gastric perforating veins, and esophageal perforating veins reached a statistical significance of $p<0.001$. While, age, hemoglobin, and prothrombin time (s) had a $p$-value of $0.018,0.024$, and 0.050 respectively.

A binary logistic regression was conducted to evaluate the significant variables from the univariate analysis. The presence of para-gastric vein has the highest odds ratio (OR) of 5.374 (95\% CI 1.013-28.475), followed by the presence of gastric perforating vein, with an OR of 2.908 (95\% CI 1.174-7.201).

An ROC curve was constructed based on the predictive probability of the binary logistic regression. Age, hemoglobin, para-gastric vein, gastric perforating vein yielded a AUROC of 0.758 (95\% CI $0.685-$ 0.830 ), while that of EUS phenomenon alone is 0.698 (95\% CI 0.616 0.779 ), and para-gastric vein alone is 0.641 (95\% CI $0.556-0.726$ ) (Figure 3).

A retrospective review of the hospital database was conducted in attempt identify available follow-up endoscopy examination of all study subjects. Among the poor responders $(n=106), 61(57.5 \%)$ patients received a follow-up endoscopy examination, with an average time to follow-up of $5.92 \pm 2.45$ month. $32(52.5 \%)$ patients had complete variceal obliteration, while $29(47.5 \%)$ had variceal recurrence, requiring further intervention, evident by F3 varix or red wale mark. The results of the follow-up endoscopy findings were closely correlated to the presence of para-gastric vein with a Pearson's correlation of $0.380, p$-value 0.002 .

Conclusion: Presence of para-gastric vein is a characteristic of poor response to endoscopic therapy for treating gastroesophageal varices. Early identification of this subgroup necessitates a change in course of treatment. 


\section{PHT-25}

Prediction of esophageal varices in liver cirrhosis by transient elastography and aspartate aminotransferase-to-platelet ratio index (APRI)

\section{Golam Azam ${ }^{1}$, Debashis K Sarker ${ }^{1}$, Majharul Haque ${ }^{1}$, M Anisur Rahman \\ ${ }^{1}$ BIRDEM General Hospital, Bangladesh}

Background: Cirrhosis is a chronic liver disease that can be caused by almost all progressive liver injuries, such as viral, autoimmune, hereditary, metabolic and toxin mediated liver diseases. Esophageal varix $(E V)$ is a frequent complication of cirrhosis. Although the survival rate of patients with bleeding cirrhosis has improved because of the progress in variceal hemorrhage management, the hospital mortality rate is still around $14.5 \%$ cases. Early detection of EV in all patients with liver cirrhosis is required in order to reduce the mortality.

Method: This observational study was done at department of Gastrointestinal, Hepatobiliary \& Pancreatic Disorders (GHPD), BIRDEM General Hospital, Dhaka, during the period of August 2015 to October 2016. A total of 65 patients with cirrhosis of liver were included. Complete blood count, liver function test, endoscopy of upper GIT, ultrasonography, transient elastogram were done for all patients. Statistical analysis was done with SPSS version 22 .

Result: The study included 65 cirrhotics, among them $66.2 \%$ were male. The mean age was 53.8 years. For predicting EVs, transient elastography at a cutoff value of $18 \mathrm{kpa}$ demonstrated a sensitivity was $88.7 \%$ (95\% CI=82.3-92.7), specificity $75.0 \%$ (95\% CI=46.992.6), PPV 94.0\% (95\% CI=87.2-98.2), NPV 60.0\% (95\% CI=37.5 74.0), AUC was 0.769. In APRI for prediction of EVs at cutoff value 1.00 , sensitivity was $63.3 \%$ (95\% CI=55.6-65.4), specificity $83.3 .0 \%$ (95\% CI=53.7-97.0), PPV 94.3\% (95\% CI=84.1-99.0), NPV 33.3\% (95\% CI=21.5-38.8) and AUC was 0.779 .

Conclusion: There was a significant positive correlation found between transient elastography with EVs in cirrhotic patients. Liver stiffness value at $18 \mathrm{Kpa}$ can predict EVs in cirrhotic patients. On contrary, APRI had a less NPV that proved there is no satisfactory cutoff value for APRI to be used as a predictor of EVs.

PHT-26

Prospective evaluation of role of serum ferritin and CLIF - SOFA score in predicting 30 day outcome in cirrhotic patients requiring intensive care

$\underline{\text { Rohit N Maidur }}{ }^{1}$, Sukanya Bhrugu ${ }^{1}$, Ajit Kumar' ${ }^{1}$, Nayana Joshi ${ }^{1}$

${ }^{1}$ Nizam's Institute of Medical Sciences, India

Background: The short-term prognosis of acutely ill patients with cirrhosis is influenced by the degree of hepatic and extra hepatic organ dysfunction. Whenever the patients with cirrhosis are critically ill the question of futility and/or utility of placing them in ICU arise. Various scoring systems have been evaluated to predict the outcome in such patients. The present study is intended to find the parameters that influence outcome in cirrhotics requiring critical care

Method: This was single-centre, prospective, observational study. Patients more than 18 years of age with liver cirrhosis of any aetiology requiring intensive care were enrolled after excluding subjects according to exclusion criteria. History, physical examination, blood tests, and imaging studies were done. Prognostic scores were calculated on the day of admission and at day 7. Appearance of new events, complications, length of ICU and hospital stay were documented.
Follow up was done at day 30 in person or by telephonically for those who had left the hospital

Result: Total of 96 subjects were enrolled, out of which 12 were lost to follow up. Of the remaining 84 subjects, 55 of them expired within 30 days of admission. Total 77 subjects were male and 7 females. Alcohol was the commonest etiology seen in $76.1 \%(n=64)$ of cirrhosis of our subjects followed by both alcohol and viral hepatitis $(n=9)$, hepatitis B $8.3 \%(n=7)$ and hepatitis C infection $(n=4)$. Higher mean age, presence of ascites at presentation, history of malena, jaundice, oliguria, hepatic encephalopathy and low MAP at admission requiring inotrope support were more common among those who didn't survive beyond 30 days. SBP was commonest infection followed by pulmonary infections, UTI, cellulitis, acute viral hepatitis $\mathrm{A} / \mathrm{E}$ in both groups. However on multivariate analysis older age, presence of malena, oliguria, presence of infective foci, hepatic encephalopathy were significantly associated with mortality.

Low hemoglobin levels, low platelet count, high leucocyte count, raised creatinine, high blood urea were seen in those who did not survive beyond 30 days. Higher lactate and lower $\mathrm{pH}$ levels were noted in non survivors. In our study mean serum ferritin levels were significantly higher $(\mathrm{p}<0.001)$ in non survivors. This study revealed mean serum ferritin levels were significantly higher $(\mathrm{p}<0.001)$ in non survivors (922.95 \pm 319.85 ) as compared to survivors (368.17士 113.87). On multivariate analysis of lab parameters low platelet count, high lactate levels, low $\mathrm{pH}$, high creatinine, high bilirubin and high serum ferritin were predictors of mortality.

In the present study at admission general ICU scores SOFA (0.808), APACHE II (0.855) fared better in predicting outcome than liver specific scores in critically ill liver cirrhotics. Lower ROC was observed for liver specific scores as with CTP (0.727), MELD (0.79) and MELD Na (0.77) as compared to general ICU scores on the day of admission. On comparison of these scores calculated on day of admission with those recalculated scores on day 7 we found higher ROC values on day of admission (CTP 0.72 vs 0.66; MELD 0.79 vs 0.70; MELD-Na 0.77 vs 0.68 ; APACHE II 0.855 vs 0.84 ; SOFA 0.80 vs 0.74 ; CLIF SOFA 0.86 vs 0.83 ). Admission day scores predicted outcome better with higher ROC

Conclusion: Serum ferritin, CLIF SOFA, APACHE II and SOFA scores along with oliguria, malena, hepatic encephalopathy, creatinine, MAP, lactate, $\mathrm{pH}$, platelet count on day of admission predicted 30 day mortality better than CTP and MELD scores

\section{PHT-27}

The impact of kidney dysfunction on mortality in cirrhotic patients with acute deterioration

Soung Won Jeong ${ }^{1}$, Tae Yeob Kim ${ }^{4}$, Eileen L. Yoon ${ }^{3}$, Do Seon Song $^{9}$, Hee Yeon Kim ${ }^{10}$, Chang Wook Kim ${ }^{10}$, Young Kul Jung ${ }^{5}$, Dong Hyun Sinn ${ }^{6}$, Sang Gyune Kim ${ }^{8}$, Jae Young Jang ${ }^{8}$, Won $\mathrm{Kim}^{7}$, Hwi Young Kim ${ }^{2}$, Moon Young Kim ${ }^{11}$, Eunhee Choi ${ }^{11}$

${ }^{1}$ Soonchunhyang University Seoul Hospital, Korea, Republic of; ${ }^{10}$ The Catholic University, Korea, Republic of; ${ }^{11}$ Wonju College of Medicine, Yonsei University, Korea, Republic of; ${ }^{2}$ Ewha Womans University School of Medicine, Korea, Republic of; ${ }^{3}$ Inje University, Korea, Republic of; ${ }^{4}$ Institute of Medical Science, Hanyang University, Korea, Republic of; ${ }^{5}$ Korea University, Korea, Republic of; ${ }^{6}$ Samsung Medical center, Korea, Republic of; ${ }^{7}$ Seoul National University College of Medicine, Korea, Republic of;

${ }^{8}$ Soonchunhyang University, Korea, Republic of; ${ }^{9}$ The Catholic University of Korea, Korea, Republic of 
Background: This study aimed to investigate short-term mortality and associated factors in cirrhotic patients with kidney dysfunction and acute deterioration.

Method: Of the 1204 cirrhotic patients experienced acute deterioration, 1094 patients (male 815, mean age 55.8 years) without kidney failure (defined as serum creatinine level of $2 \mathrm{mg} / \mathrm{dL}$ or above) were retrospective consecutively collected during 2013. Kidney dysfunction was defined by serum creatinine levels from 1.2 to $1.9 \mathrm{mg} / \mathrm{dL}$ at admission based on retrospective KACLiF [Korean Acute-on-Chronic Liver Failure (ACLF)] study. Baseline characteristics and short-term mortality according to the presence of kidney dysfunction were analyzed. Independent risk factors for 90-day mortality in patients with kidney dysfunction were obtained by multiple logistic regression.

Result: The prevalence of kidney dysfunction was 19.2\% (210/1094). The 28-day and 90-day mortality were higher in patients with kidney dysfunction than in those without kidney dysfunction ( $12.4 \%$ vs. $4.4 \%$, $\mathrm{P}<0.001 ; 19.0 \%$ vs. $8.5 \%, \mathrm{P}<0.001$, respectively). Eighty percent $(32 / 40)$ of non-survivor was associated with the presence of ACLF and 18 out of 32 patients $(56.2 \%)$ were ACLF development after admission. In multiple logistic regression, low albumin level $(0.1 \mathrm{mg} / \mathrm{dL}$ increase, OR $0.90,95 \% \mathrm{CI} 0.81-0.99, \mathrm{P}=0.033$ ) and the presence of ACLF (at admission, HR 11.4, 95\% CI 3.8-33.6, P < 0.001; after admission, HR 30.8, 95\% CI 9.9-95.8, $\mathrm{P}<0.001$ ) had independent factors of 90-day mortality. Especially, ACLF experience of grade 2 or above had higher mortality than grade 1 ACLF $(\mathrm{P}<0.001)$.

Conclusion: In cirrhotic patients without kidney failure, kidney dysfunction with acute deterioration had a substantial mortality. To improve the mortality, it is necessary to endeavor to recognize the early renal dysfunction and to prevent additional organ failure and the progression of ACLF.

PHT-28

Urinary neutrophil gelatinase associated lipocalin, as a diagnostic marker of acute kidney injury in cirrhotic patients'

\section{Md Rofiqul Islam ${ }^{1}$, Md Rofiqul Islam ${ }^{1}$, Md Saiful Islam²}

${ }^{1}$ Medical Officer, Bangladesh; ${ }^{2}$ Bangabandhu Sheikh Mujib Medical University, Bangladesh

Background: Acute Kidney injury (AKI) leads the liver cirrhosis patients to the doom of multiorgan failure. The AKI diagnosis in the early possible period in the hospitalized cirrhotic patients can save many lives. But it is difficult to detect AKI early without conventional biochemical tool, serum creatinine. Abrupt increase of the level of serum creatinine by at least $0.3 \mathrm{mg} / \mathrm{dL}$ may be termed as acute kidney injury (AKI). It may occur in about $20 \%$ of hospitalized patients in decompensating liver cirrhosis (Garcia-Tsao $G$ et al. 2008). The commonest causes of AKI in cirrhotic patients are pre-renal azotemia, hepatorenal syndrome and acute tubular necrosis. The aim of this study was to assess of an increase uNGAL level predicts the development of AKI in cirrhotic patients, to determine the accuracy of uNGAL as a diagnostic marker of AKI in cirrhotic patients and to find out the cut off value of UNGAL level to diagnose AKI in cirrhotic patients

Method: This cross sectional study was carried out at Department of Hepatology, Bangabandhu Sheikh Mujib Medical University, Dhaka. A total 70 Patients of decompensated cirrhosis and decompensated cirrhosis with AKI prone conditions admitted into Department of Hepatology in BSMMU were included for the study. They were evaluated by proper history and clinical examination. Initial investigations were done to meet up inclusion and exclusion criteria including liver function test [serum bilirubin (total), serum albumin, prothrombin time (PT)], renal function tests (serum creatinine), ascitic fluid analysis (cytology, total protein, albumin, SAAG), abdominal ultrasonography, CXR-PA view, ECG, echocardiography. Decompensated cirrhosis were diagnosed with a combination of physical, biochemical, radiological and endoscopic findings. The patients were chosen according to purposive sampling. Serum creatinine ( $\mathrm{sCr}$ ) levels 03 months before the admission was collected wherever available and used as baseline $\mathrm{sCr}$. In patients without a previous $\mathrm{sCr}$ value, the $\mathrm{sCr}$ on admission was used as baseline. Where the baseline $\mathrm{sCr}$ was normal then the patients were included for the study. Patients were then monitored with $\mathrm{sCr}$ at 24 hours and 48 hours. The presence of AKI was diagnosed when the patients were fulfilled the criteria proposed by the International club of ascites for cirrhotic patient. Urine sample for NGAL was collected within 24 hours after admission.

Result: The mean age of the respondents was $43.49 \pm 12.46$ years and $46.09 \pm 14.90$ years in Group A and Group B respectively. Twenty six $(74.3 \%)$ were male and $9(25.7 \%)$ were female in Group A. Twenty seven $(77.1 \%)$ were male and $8(22.9 \%)$ were female in group B. Male patients were predominant in both groups. Out of 70 cirrhosis patients, HBV was found $45(64.3 \%)$ cases, cryptogenic $11(15.7 \%)$, NASH 7(10.0\%), HCV 5(7.1\%) and Wilson's disease 2(2.9\%). Out of 35 acute kidney injury (AKI) patients, the contributing conditions of AKI were hepatic encephalopathy (HE) $8(22.9 \%)$, acute on chronic liver failure (ACLF) 7(20.0\%), variceal haemorrhage 6(17.1\%), spontaneous bacterial peritonitis (SBP) 6(17.1\%), pneumonia $2(5.7 \%)$. Regarding laboratory parameters platelet count, prothrombin time, INR, serum albumin were statistically difference between two groups. $\mathrm{Hb} \%$, TC, ESR, AST, ALT, serum bilirubin, serum ALP were not statistically significant between two groups. Mean serum creatinine were $1.02 \pm 0.24$ in Group A and $2.27 \pm 1.01$ in Group B. Comparison of mean uNGAL between Patients of decompensated cirrhosis with AKI and without AKI, uNGAL was significantly higher in AKI group. ROC- AUC of 0.984 (95 \% confidence interval [CI]: $0.962-1.000, \mathrm{p}<0.001)$. The optimal cutoff value was $\geq 50 \mathrm{ng} / \mathrm{mL}$ providing $91.4 \%$ sensitivity, $94.3 \%$ specificity, $92.8 \%$ accuracy, 94.1\% positive predictive value (PPV), $91.7 \%$ negative predictive value (NPV), respectively.

Conclusion: uNGAL is a valid marker for the early detection of AKI in cirrhotic patients with AKI-prone conditions.

Table-5: Comparison of laboratory findings between two groups $(n=70)$

\begin{tabular}{lccc}
\hline Laboratory findings & $\begin{array}{c}\text { Group A } \\
(\mathbf{n}=\mathbf{3 5}) \\
\text { Mean } \pm \text { SD }\end{array}$ & $\begin{array}{c}\text { Group B } \\
(\mathbf{n}=\mathbf{3 5}) \\
\text { Mean } \pm \text { SD }\end{array}$ & P value \\
\hline $\mathrm{Hb} \%(\mathrm{~g} / \mathrm{dl})$ & $9.9 \pm 1.7$ & $9.3 \pm 1.7$ & 0.124 \\
$\mathrm{TC}\left(-\mathrm{-x} 10^{9} / \mathrm{L}\right)$ & $7.1 \pm 2.7$ & $8.7 \pm 4.7$ & 0.082 \\
ESR & $46.3 \pm 31.1$ & $40.7 \pm 26.6$ & 0.421 \\
Platelet count $\left(--10^{9} / \mathrm{L}\right)$ & $143.1 \pm 56.7$ & $111.7 \pm 44.8$ & $0.012^{\mathrm{s}}$ \\
AST (U/L) & $105.4 \pm 75.1$ & $103.7 \pm 80.4$ & 0.927 \\
ALT (U/L) & $62.6 \pm 32.2$ & $67.9 \pm 51.6$ & 0.609 \\
Prothrombin Time: Pt (sec) & $20.7 \pm 5.6$ & $24.9 \pm 8.1$ & $0.015^{\mathrm{s}}$ \\
INR & $1.8 \pm 0.6$ & $2.1 \pm 0.7$ & $0.038^{\mathrm{s}}$ \\
Serum Albumin (g/d1) & $2.4 \pm 0.4$ & $2.1 \pm 0.3$ & $<0.001^{\mathrm{s}}$ \\
Serum Bilirubin $(\mathrm{mg} / \mathrm{dl})$ & $5.8 \pm 8.5$ & $7.0 \pm 7.5$ & 0.517 \\
Serum ALP (U/L) & $166.7 \pm 105.5$ & $155.9 \pm 78.6$ & 0.630 \\
\hline
\end{tabular}

Data were expressed as mean $=\mathrm{SD}$

$P$ value reached from unpaired t-test.

Group A : Patients of decompensated cirrhosis without AKI

Group B : Patients of decompensated cirrhosis with AKI 


\begin{tabular}{|cccc|}
\multicolumn{9}{c|}{ Area Under the Curve } \\
\begin{tabular}{|ccccc|}
\hline Test Result Variable(s): uNGAL & & \\
\hline \multirow{2}{*}{ AUC } & Std. Error & P value & \multicolumn{2}{c|}{ Asymptotic 95\% Confidence } \\
\cline { 3 - 5 } & & & \multicolumn{2}{c|}{ Interval } \\
\cline { 3 - 5 } & & & Lower Bound & Upper Bound \\
\hline 0.984 & 0.011 & $<0.0001$ & 062 & 1.000 \\
\hline
\end{tabular}
\end{tabular}

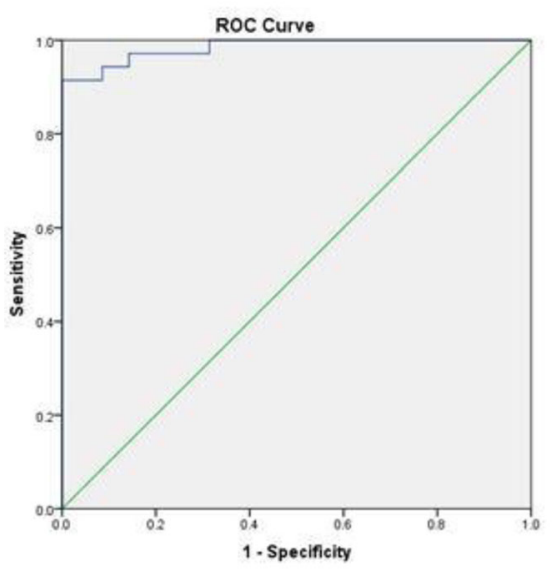

Fig. 1: ROC curve

PHT-29

Prevalence, etiological factors, survival rates, lifetime and causes of death of patients with liver cirrhosis in Tajikistan

\author{
Sayfullo Avezov ${ }^{1}$, Rohatillo Odinaev ${ }^{1}$, Dilorom Ishankulova ${ }^{1}$, \\ Behruz Ganiev \\ ${ }^{1}$ Institute of Gastroenterology, Tajikistan
}

Background: Survival rates, lifetimes and principal causes of patients death with liver cirrhosis (LC) are reflected in numerous studies. However, nowadays there are some indicators, which have not been studied yet in patients with LC in Tajikistan. Aims: To study the prevalence of LC, survival rates, lifetimes and principal causes of patients death, and evaluation of prognostic value of complications of LC in Tajikistan.

Method: There were diagnosed 1374 patients of the LC. Survival was assessed according to the Kaplan-Meier method. The mortality risk of cirrhosis complications was analyzed by a time-dependent Cox regression model.

Result: The main etiological factors of development of LC were: hepatitis B virus (49\%), hepatitis C virus (36\%) and alcohol (4\%). The incidence of viral LC reach 2,32 per 10000 of adult populations. Alcohol-induced LC (ALC) and primary biliary cirrhosis (PBC) are over 10 and 5 times less frequent than in Russia. Lifetime and 3-year survival rate of patients depend on a phase of cirrhotic process compensation. The highest 3 -years survival rate of patients from the producing moment of this diagnosis was $79 \%$ at Child-Pugh grade A vs. $28 \%$ at grade C. The cause of death of $89 \%$ of patients has been directly related to complications of cirrhosis. The main reasons of patients death were: hepatic encephalopathy $(46,7 \%)$, bleeding serve $(18,3 \%)$, hepatorenal syndrome $(12,5 \%)$, spontaneous bacterial peritonitis $(9,2 \%)$ and portal vein thrombosis $(2,5 \%)$. The prognosis of survival is most unfavorable at hepatic encephalopathy in comparison with other complications of the LC. Presence more than one complication increases probability of death of patients more than 2,5 times. The higher relative risk of death has patients with grade B and $\mathrm{C}$ with comparison to grade $\mathrm{A}$.

Conclusion: The main etiological factors of LC are HBV and HCV in Tajikistan. In our country the incidence of viral LC does not differ from that in Russia. ALC and PBC are a few in Tajikistan. Survival rate of patients depend on Child-Pugh grade.

PHT-30

Pronounced coronary arteriosclerosis in cirrhosis. Impact on cardiac function and survival?

Soren Moller ${ }^{1}$, Karen Vagner Danielsen ${ }^{1}$, Signe Wiese ${ }^{1}$, Jens Dalgaard Hove ${ }^{1}$, Flemming Bendtsen ${ }^{1}$

${ }^{1}$ Hvidovre Hospital, Denmark

Background: The relation between excessive alcohol consumption and coronary arteriosclerosis has remained controversial. The etiology of cirrhosis has been considered a substantial risk factor for development of arteriosclerosis. The coronary artery calcium-score derived from coronary CT-angiography is a robust marker of coronary arteriosclerosis. In this study we assessed the burden of coronary arteriosclerosis in cirrhotic patients of various etiologies and the association to cardiac dysfunction and survival.

Method: Times New Roman

Result: In men with cirrhosis median coronary artery calcium-score was 328 vs 9 in age and race-adjusted healthy references and in women 136 vs 0 (p

Conclusion: Coronary arteriosclerosis was extensive in patients with cirrhosis and in particular in those with alcoholic etiology, but coronary arteriosclerosis had only limited influence on cardiac function and survival. Interestingly, no other well-known risk factors than age seems to interfere with coronary arteriosclerosis in cirrhotic patients.

PHT-31

Role of transjugular intrahepatic portosystemic shunt in the management of hepatopulmonary syndrome

\section{Wang Zhou yi ${ }^{1}$, Xin Chen ${ }^{2}$}

${ }^{1}$ The Affiliated Hospital of Southwest Medical University, China; ${ }^{2}$ The Affiliated Hospital of Southwest Medical University, China

Background:

Objective: To evaluate the safety and efficacy of transjugular intrahepatic portosystemic Shunt (TIPS) procedure in patients with Hepatopulmonary Syndrome (HPS).

Method: We prospectively enrolled patients with Hepatopulmonary Syndrome receiving TIPS for portal hypertension caused by cirrhosis from January 2015 to December 2016 in our Hospital, to evaluate the role of TIPS in the management of HPS.

Result: The study consisted of 14 patients, The median oxygen saturation $(\mathrm{SaO} 2)$ of HPS group was $96.32 \% \pm 1.56 \%$, partial pressure of arterial oxygen $(\mathrm{PaO} 2) 82.35 \pm 9.75 \mathrm{mmHg}$, alveolar-arterial oxygen gradient (PA-aO2) $36.22 \pm 6.38 \mathrm{mmHg}$. 1 patients had hepatic encephalopathy 1 month later and 1 patients had hepatic encephalopathy 2 month later. No patient had stent dysfunction and no rebleeding were found after 3 month. statistically significant difference was detected for $\mathrm{SaO} 2, \mathrm{PA}-\mathrm{aO} 2$ between before TIPS procedure and that after 3 days, no significant difference for $\mathrm{PaO} 2$. 
Significant difference was detected for $\mathrm{SaO} 2, \mathrm{PaO} 2, \mathrm{PA}-\mathrm{aO} 2$ between before TIPS procedure and that after 1 month. No Significant difference was detected for $\mathrm{SaO} 2, \mathrm{PaO} 2, \mathrm{PA}-\mathrm{aO} 2$ between before TIPS procedure and that after 3 month.

Conclusion: TIPS was safe in patients with HPS regardless of severity with no deleterious effects on oxygenation. However, It can't improve hypoxemia in early stage after TIPS procedure. Further prospective studies are warranted.

\section{PHT-32}

\section{Serum versus ascitic procalcitonin as a marker for diagnosis} of SBP

\section{Mohamed Hamdy Badr \\ ${ }^{1}$ Consultant, Egypt}

Background: Patients with liver cirrhosis are at high risk of developing bacterial infection, SBP is the most serious of them, Guidelines recommend culture positivity and $\mathrm{PMN}>250$ cells $/ \mathrm{cm}$ for SBP diagnosis, but due to low bacterial density, half of patients have -ve culture. Although paracentesis is generally a safe procedure, it still have some serious complications. Recently some biomarkers have been proposed for detection of SBP and Procalcitonin is one of them. Method: We studied 45 Cirrhotic ascitic patients and we divided them into 2 groups: 1 . SBP (CASE group $=30$ patients), 2 . no SBP (Control group $=15$ patients). SBP diagnosis was based on $\mathrm{PMN}>250$ in ascitic sample at admission. Other infections were rolled out by doing CXR, Urine and Stool analysis, cRP, CBC and ENT consultation. All patients and control were subjected to thorough history taking, complete clinical examination and Lab Investigations. We measured both serum and ascitic Procalcitonin (PCL) for all patients and controls at admission.

Result: Ascitic PCL in SBP group was $1.909 \pm 0.5 \mathrm{ng} / \mathrm{ml}$ and in control group $0.480 \pm 0.346 \mathrm{ng} / \mathrm{ml}$ (P value NS), Serum PCL in SBP group was $1.846 \pm 1.684 \mathrm{ng} / \mathrm{ml}$ and in control group $0.659 \pm 0.610 \mathrm{ng} /$ $\mathrm{ml}$ (P value HS), Serum PCL at cutoff level 0.382 shows sensitivity $67 \%$ and specificity $46 \%$ with AUC 67.8 (P value 0.045 ), Ascitic PCL at cutoff level 0.320 shows sensitivity $67 \%$ and specificity $40 \%$ with AUC 58.9 (Pvalue NS).

Conclusion: Serum PCL is better than Ascitic PCL for diagnosis of SBP, at the same cutoff level serum PCL is better than Ascitic PCL for early detection of SBP although they have low sensitivity and specificity.

\section{March 2018}

\section{PHT-33}

The impact of sepsis and non-communicable diseases on prognostic models to predict the outcome of hospitalized chronic liver disease patients

\section{Fakhar Ali Qazi Arisar ${ }^{1}$, Shahab Abid ${ }^{1}$, Safia Awan ${ }^{1}$}

${ }^{1}$ The Aga Khan University Hospital, Pakistan

Background: Non-communicable diseases (NCDs) such as diabetes, hypertension, chronic kidney disease (CKD) and ischemic heart disease are important associations which contribute in determining the outcome of patients with liver cirrhosis. The aim of this study is to evaluate the impact of NCDs and sepsis on the outcome of decompensated chronic liver disease (CLD) patients. Non-communicable diseases (NCDs) such as diabetes, hypertension, chronic kidney disease (CKD) and ischemic heart disease are important associations which contribute in determining the outcome of patients with liver cirrhosis. The aim of this study is to evaluate the impact of NCDs and sepsis on the outcome of decompensated chronic liver disease (CLD) patients.

Method: In this cross-sectional study we analyzed CLD patients for NCDs and factors responsible for mortality, prolonged hospital stay ( $>5$ days) and early readmission (within 7 days).

Result: A total of 399 patients were reviewed, $258(64.6 \%)$ were male and mean age was $54.3 \pm 11.7$ years. Overall mortality was $13 \%(\mathrm{n}=52)$. Prolonged hospital stay and readmission were present in $18 \%(\mathrm{n}=72)$ and $7 \%(\mathrm{n}=28)$ of patients respectively. NCDs were present in $189(47.4 \%)$ patients. Acute kidney injury (AKI), sepsis and non-ST elevation MI (NSTEMI) were present in 165 (41\%), $70(17.5 \%)$, and $7(1.75 \%)$ of patients respectively. On multivariate analysis AKI, NSTEMI, sepsis, and coagulopathy were found statistically significant predictors of mortality. While CKD, low albumin and high MELD-Na were found statistically significant as predictors of morbidity.

Addition of sepsis in conventional MELD score predicts mortality even better than MELD-Na (AUROC: 0.735 vs 0.686 ; $p<0.001$ ). Among NCDs, CKD was found to increase morbidity independently. Conclusion: Addition of sepsis improve the predictability of MELD score as prognostic marker for mortality in patient with CLD. Presence of CKD increases the morbidity of patients with CLD.

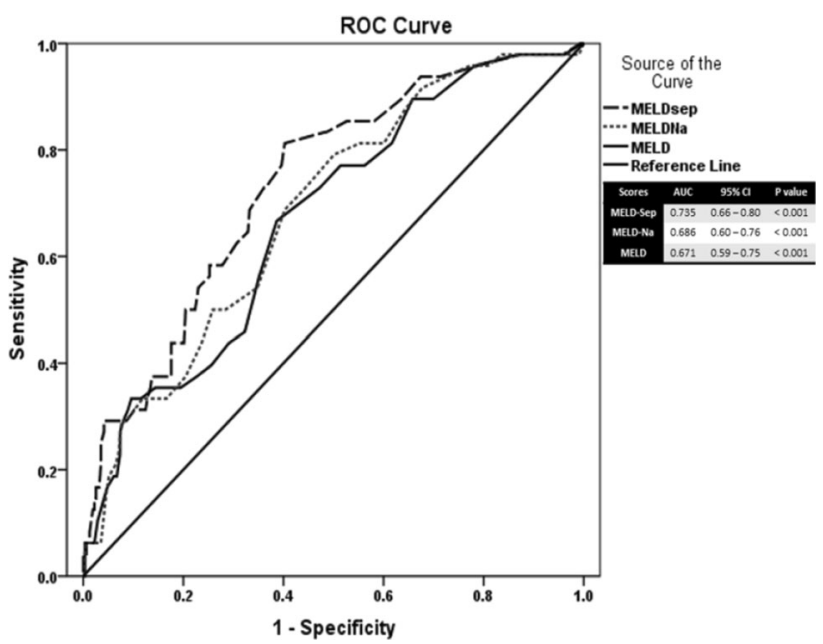

PHT-34

Evaluation of portal hypertension by Doppler ultrasound in cirrhotic patients before and after simvastatin

\author{
Sherief Abd Elsalam ${ }^{1}$, Nadia Elwan', Raafat Salah ${ }^{1}$, Manal \\ Hamesa $^{1}$, Ebtsam Shady ${ }^{1}$ \\ ${ }^{1}$ Tanta University Faculty of medicine, Egypt
}

Background \& Aims: Portal hypertension is the most common complication of cirrhosis. $\beta$-adrenergic blockers \pm organic nitrates are currently used as hypotensive agents. Statins, such as simvastatin seem to be safe for chronic liver diseases and exert multiple pleiotropic effects. The aim of this study was to evaluate portal hypertension by Doppler ultrasound in cirrhotic patients before and after receiving simvastatin.

Methods: This randomized controlled clinical study was conducted on 40 cirrhotic patients who were randomized into two groups Group 
(I): 20 patients were given simvastatin $20 \mathrm{mg} /$ day for 2 weeks (increased to $40 \mathrm{mg} /$ day at day 15 for another 2 weeks); Group (II): 20 patients who did not receive simvastatin as a control group. All patients were subjected to clinical examination, routine laboratory investigations, abdominal and Doppler ultrasound basal and 30 days after, including Portal vein diameter (PVD), Portal vein velocity (PVV), Portal vein blood flow (PVBF), Portal vein flow direction, Hepatic artery resistance index (HARI), Hepatic artery pulsatility index (HAPI), Splenic artery resistance index (SARI), Portal hypertension index (PHI), Liver vascular index (LVI), Modified liver vascular index (MLVI).

Results: There was a highly significant decrease in HARI after 30 days of receiving simvastatin in group I from $0.785 \pm 0.088$ to $0.717 \pm 0.086(\mathrm{P}<0.001)$, There was a significant decrease in PHI in group I after 30 days of receiving simvastatin from $(3.915 \pm 0.973$ $\mathrm{sec} / \mathrm{m})$ to $(3.605 \pm 1.168 \mathrm{sec} / \mathrm{m}),(\mathrm{P}=0.024)$. There was a significant increase in MLVI in group I after 30 days of receiving simvastatin from $11.540 \pm 3.266$ to $13.305 \pm 3.222 \mathrm{~cm} / \mathrm{sec}$ - by $15.3 \%$ from baseline $(\mathrm{P}=0.009)$ No significant side effects detected .

Conclusion: Simvastatin is safe and effective portal hypertensive lowering agent.

[ClinicalTrials.gov Identifier: NCT02994485]

Keywords: Simvastatin; Portal hypertension; Cirrhosis; Doppler; Ultrasound.

\section{PHT-35}

Balloon-occluded retrograde transvenous obliteration (BRTO) a safe and efficacious alternative to transjugular intrahepatic shunts (TIPS) in the management of gastric varices

Taufique Ahmed ${ }^{1}$, Raymond Liang ${ }^{2}$, Suresh B Babu', Chern Hao Chong $^{3}$, Ikram Hussian ${ }^{3}$, Benjamin CH Yip ${ }^{1}$

${ }^{1}$ Khoo Teck Puat Hospital, Singapore; ${ }^{2}$ Tan Tock Seng Hospital, Singapore; ${ }^{3}$ Woodlands Health Campus, Singapore

Background: Balloon-occluded retrograde transvenous obliteration (BRTO) is an endovascular technique used as a therapeutic adjunct or alternative to transjugular intrahepatic shunts (TIPS) in the management of gastric varices

Our aims were to review the patients who underwent BRTO in our institution with a view to assess the technical outcomes, patient outcomes and factors which influences these.

Method: We retrospectively collected data on all patients who underwent BRTO at Khoo Teck Puat Hospital, Singapore from May 2015 to November 2017. A search was done to identify all patients who had BRTO in the time period and a review of their notes was carried out.

Result: We identified 10 patients who underwent BRTO. For 8 of these patients the indication was secondary prophylaxis for bleeding gastric varices. The other two patients underwent BRTO for primary prophylaxis for large gastric varices, both patient had HCC and underwent transarterial chemo-embolization at the same time.

Our local practice is to favour BRTO over TIPS if the ejection fraction is less than $60 \%$, which was the case in three patients. BRTO is also preferred in patients who have a history of recurrent encephalopathy, which was the case in two patients. One of the patients with recurrent encephalopathy had a MELD score of 18 .

The procedure was a technical success in all patients with intraoperative balloon rupture prolonging one case.

The mean length of follow up was 475.5 days (39-934 days). The patient with 39 days of follow up had her BRTO in October 2017. Two patients died from progression of hepatocellular carcinoma at 91 days and 366 days post BRTO. Only one patient had an episode of re- bleeding at 404 days post BRTO, which resulted in death. For those that we have the data, MELD score and Child-Pugh score remained stable over time post BRTO.

Conclusion: In summary BRTO is a safe and efficacious alternative to TIPS for treatment of gastric varices. $50 \%$ of our patients had relative contraindications for TIPIS. No patients had rebleeding within a year post procedure. $88.9 \%$ patients survived at least a year post BRTO.

\section{PHT-36}

Combined radiologic-blood parameters and blood derived noninvasive fibrosis scores in predicting cirrhosis, development of esophageal varices and decompensation

Ragesh Babu Thandassery ${ }^{1}$, Syed Adnan Mohiuddin ${ }^{1}$, Muneera Al Mohannadi ${ }^{1}$, Madiha Emran Soofi ${ }^{1}$, Hamid Ullah Wani ${ }^{1}$, Saad Al Kaabi $^{1}$

${ }^{1}$ Hamad Medical Corporation, Qatar

Background: In patients with chronic hepatitis C (CHC), we aimed to compare combined radiologic-blood parameters (CRBP) and blood derived non-invasive fibrosis scores (NIF) for predicting pre treatment cirrhosis, development of esophageal varices (EV) and liver decompensation post antiviral treatment (AVT).

Method: 1605 patients (Jan 2002 to June 2015) with CHC underwent liver biopsy (LB) and received AVT with pegylated interferon and ribavirin. Three CRBPs (platelet count-bisplenic diameter index [PSI], platelet count-bisplenic diameter-portal vein index [PSPVI], platelet count-bisplenic diameter-portal vein-caudate lobe length index [PSCLI]) and nineteen blood derived NIFs were calculated from routine blood tests and abdominal ultrasound done prior to starting AVT. AUROCs were calculated for each of these parameters for predicting cirrhosis on pre treatment LB and development of EV and decompensation on follow up after AVT.

Result: Mean age was $41.9 \pm 9.7$ years ( $85 \%$ males), predominantly genotypes $4(65 \%)$ and $1(11 \%)$.

Pretreatment LB (Scheuer criteria) showed stage-0 fibrosis in $1.9 \%$, stage- 1 in $32.9 \%$, stage- 2 in $39.5 \%$, stage-3 in $19 \%$, and stage- 4 (cirrhosis) in $6.6 \%$ of the patients. After AVT, there were 1,089 $(67.8 \%)$ responders, $482(30 \%)$ non-responders and $34(2.1 \%)$ relapsers. After median follow-up of 6580.5 patient-years post AVT; 39 (2.4\%) developed EV (2 patients had both esophageal and gastric varices and one had only gastric varices) and $52(3.2 \%)$ had decompensation (bleed-9, ascites-39, jaundice-22, hepatic encephalopathy-7, hepatorenal syndrome-4).

CRBPs had higher accuracy for prediction of cirrhosis and EV, while NIFs had higher accuracy for predicting decompensation (Table 1). The highest AUROCs were seen with PSI for predicting cirrhosis $(A U R O C=0.882)$ and EV $($ AUROC $=0.885)$ and with FIB-4 score for decompensation $($ AUROC $=0.854)$. At a cut of 1200 PSI had sensitivity of $87.2 \%$ and specificity of $75 \%$ for predicting cirrhosis and at a cut off of 1100 PSI had sensitivity of $89.4 \%$ and specificity of 85.7 for predicting EV. At a cut off of 2.5, FIB-4 score had a sensitivity of $82.5 \%$ and specificity of $80.1 \%$ in predicting decompensation.

Conclusion: CRBPs predict cirrhosis and development of esophageal varices with high accuracy. Some of the blood derived NIFs have high accuracy in predicting decompensation post antiviral treatment. Application of these simple scores may help in non-invasive screening of patients at high risk for development of esophageal varices and decompensation after antiviral treatment. 


\begin{tabular}{|c|c|c|c|}
\hline NIFs/CRBPs & \begin{tabular}{|l|} 
Predictive \\
Accuracy \\
for pre treatment cirrhosis \\
$\#$
\end{tabular} & \begin{tabular}{|l|} 
Predictive accuracy \\
for post treatment \\
esophageal varices \#
\end{tabular} & $\begin{array}{l}\text { Predictive accuracy } \\
\text { for post treatment } \\
\text { decompensation \# }\end{array}$ \\
\hline APRI & $0.842(0.800-0.884), 0.021$ & $\begin{array}{l}0.769(0.607- \\
0.932), 0.083\end{array}$ & $0.538(0.063-.783), 0.120$ \\
\hline Fibrosis- 4 & $0.827(0.781-0.874), 0.024$ & $\begin{array}{l}0.841(0.716- \\
0.886), 0.018\end{array}$ & $0.854(0.738-0.956), 0.041$ \\
\hline Lok score & $0.800(0.754-0.846), 0.024$ & $\begin{array}{l}0.820(0.775- \\
0.876), 0.023\end{array}$ & $0.803(.858-0.993), 0.127$ \\
\hline $\mathrm{GUCl}$ & $0.847(0.805-0.889), 0.021$ & \begin{tabular}{|l|}
$0.817(0.667-$ \\
$0.967), 0.077$
\end{tabular} & $0.604(0.062-0.775), 0.144$ \\
\hline Fibro alpha & $0.636(0.568-0.704), 0.035$ & \begin{tabular}{|l|}
$0.622(0.371-$ \\
$0.874), 0.128$
\end{tabular} & $0.644(0.362-0.999), 0.146$ \\
\hline Pohl score & $0.586(0.524-0.649), 0.032$ & $\begin{array}{l}0.588(0.485- \\
0.691), 0.052\end{array}$ & $0.619(0.392-0.836), 0.126$ \\
\hline $\begin{array}{l}\text { Globulin albumin } \\
\text { ratio }\end{array}$ & $0.762(0.708-0.816), 0.027$ & $\begin{array}{l}0.507(0.407- \\
0.607), 0.051\end{array}$ & $0.452(0.225-0.661), 0.110$ \\
\hline Doha score & $0.838(0.797-0.880), 0.021$ & $\begin{array}{l}0.796(0.733- \\
0.858), 0.032\end{array}$ & $0.685(0.586-0.842), 0.044$ \\
\hline PSI & $0.882(0.700-0.963), 0.042$ & $\begin{array}{l}0.885(0.794- \\
0.977), 0.047\end{array}$ & $0.596(0.319-0.874), 0.142$ \\
\hline PSPVI & $0.872(0.709-0.936), 0.033$ & $\begin{array}{l}0.845(0.703- \\
0.983), 0.071\end{array}$ & $0.551(0.300-0.803), 0.128$ \\
\hline PSPVCLI & $0.850(0.737-0.922), 0.047$ & \begin{tabular}{|l|}
$0.852(0.738-0.962)$, \\
0.057
\end{tabular} & $0.495(0.248-0.743), 0.126$ \\
\hline King score & $0.851(0.811-0.892), 0.021$ & \begin{tabular}{|l|}
$0.817(0.667-$ \\
$0.967), 0.077$
\end{tabular} & $0.641(0.016-0.898), 0.150$ \\
\hline Fibrosis index & $0.820(0.771-0.870), 0.021$ & $\begin{array}{l}0.819(0.752- \\
0.869), 0.032\end{array}$ & $0.767(0.529-0.873), 0.079$ \\
\hline $\begin{array}{l}\text { Fibrosis cirrhosis } \\
\text { index }\end{array}$ & $0 . .794(0.745-0.843), 0.025$ & \begin{tabular}{|l|}
$0.801(0.658-$ \\
$0.945), 0.073$
\end{tabular} & $0.646(0.603-0.998), 0.143$ \\
\hline $\begin{array}{l}\text { Mean platelet } \\
\text { volume }\end{array}$ & $0.689(0.563-0.816), 0.064$ & \begin{tabular}{|l|}
$0.461(0.289-$ \\
$0.633), 0.088$
\end{tabular} & $\begin{array}{l}0.648(0.478-0.756) \\
0.067\end{array}$ \\
\hline RPR & $0.485(0.426-0.545), 0.030$ & $\begin{array}{l}0.829(0.816-0.943) \\
0.032\end{array}$ & $0.839(0.742-0.959), 0.060$ \\
\hline $\begin{array}{l}\text { Globulin platelet } \\
\text { index }\end{array}$ & $0.817(0.793-0.881), 0.021$ & $\begin{array}{l}0.500(0.243-0.757) \\
0.131\end{array}$ & $\begin{array}{l}0.500(0.297-0.800), \\
0.146\end{array}$ \\
\hline FibroQ score & $0.785(0.734-0.836), 0.026$ & $\begin{array}{l}0.838(0.740- \\
0.896), 0.014\end{array}$ & $0.781(0.630-0.850), 0.076$ \\
\hline CDS & $0.793(0.747-0.839), 0.023$ & $\begin{array}{l}0.766(0.883- \\
0.849), 0.042\end{array}$ & $\begin{array}{l}0.804(0.610-0.988), \\
0.089\end{array}$ \\
\hline $\begin{array}{l}\text { Age platelet } \\
\text { index }\end{array}$ & $0.819(0.789-0.858), 0.020$ & $\begin{array}{l}0.757(0.686-0.827) \\
0.036\end{array}$ & $0.816(0.663-0.948), 0.078$ \\
\hline AST-ALT ratio & $0.605(0.545-0.665), 0.031$ & \begin{tabular}{|l|}
$0.565(0.356-$ \\
$0.774), 0.107$
\end{tabular} & $0.671(0.405-0.999), 0.137$ \\
\hline
\end{tabular}

\# AUROC, 95\% Confidence interval, standard error,

$\mathrm{CDS}=$ Cirrhosis discriminant score, $\mathrm{RPR}=$ Red cell distribution width to platelet count ratio

\section{PHT-37}

Community acquired spontaneous bacterial peritonitis in cirrhotic patients and molecular concordance with bacteremia

\section{Bipanchi Mahanta ${ }^{1}$, Anup Kumar Das ${ }^{1}$, Achinta Kumar Borthakur $^{1}$, Amrit Kumar Borah ${ }^{1}$, Tarjina Begum ${ }^{1}$, Prajna Anirvan ${ }^{2}$}

${ }^{1}$ Assam Medical College, Dibrugarh, India, India; ${ }^{2}$ Assam Medical College, India

Background: Spontaneous bacterial peritonitis (SBP) is a major cause of mortality in liver cirrhosis. Multi drug resistant bacteria further complicate the management of cirrhosis. Therefore establishing the bacterial etiology and their sensitivity pattern in different geographical areas help in early initiation of empirical treatment. The present study aimed at isolation and sensitivity pattern of bacteria causing SBP including molecular concordance with bacteremia.

Method: All consecutive clinically suspected SBP cases attending Medicine OPD in Assam Medical College and Hospital and hospitalised from July 2017 to October 2017 were included in the study prospectively. Ascitic fluid and blood were collected from all the cases prior to initiation of antibiotics. All isolates were subjected for bacterial culture and antibiotic sensitivity following the Clinical and Laboratory Standards Institute guidelines. Organisms with similar growths in blood and ascitic fluid were studied for molecular similarity of their 16s rRNA by Polymerase Chain Reaction.

Result: Out of 39 clinically suspected cases of SBP, bacterial growth was seen in 19 (48.7\%) ascitic fluid cultures. Staphylococcus aureus $(21.05 \%)$ was the most common isolate followed by Acinetobacter baumannii (15.7\%), Citrobacter species (15.7\%) and Escherichia coli $(10.5 \%)$. Majority of the isolates showed multidrug resistance including the Quinolones (57.9\%) and the Third generation Cephalosporins $(83.3 \%$ in the Gram negative organisms). Only 4 cases $(10.2 \%)$ showed growth in blood cultures after seven days of repeated subculture. Of these, 2 cases $(5.1 \%)$ showed similar growth in blood and ascitic fluid. These two isolates were tested for strain similarity by $16 \mathrm{~s}$ rRNA gene sequencing by Polymerase Chain Reaction and was found to be similar.

Conclusion: Community acquired SBP is a major complication in cirrhotics and Staphylococcus aureus is the most common cause in Upper Assam. Larger molecular studies to trace the source of peritonitis will help physicians to prevent and treat SBP.

\section{PHT-38}

Determinants of post paracentesis acute kidney injury in patients with cirrhosis of liver

\section{Mayank Jain ${ }^{1}$, Jayanthi Venkataraman ${ }^{1}$, Tom Michael ${ }^{1}$, Joy Varghese $^{1}$ \\ ${ }^{1}$ Gleneagles Global Health City, India}

Background: Despite adequate colloidal replacement, a subgroup of patients undergoing paracemtesis develop AKI and the predictors responsible for this injury are yet unknown.

Aim of the study- To determine the prevalence and predictors of paracentesis induced AKI in patients with liver cirrhosis with normal baseline renal parameters

Method: A prospective, observational analytical study undertaken at Gleneagles Global Health City, Chennai between April 2015 to April 2017. All patients undergoing large volume paracentesis were enrolled as per inclusion and exclusion criteria. As per AKIN criteria for acute kidney injury, cohort was divided into AKI and non AKI group based on their reports after 48 hours of paracentesis. All variable were analysed with these two group with both univariate and multivariate regression to determine significant predictors of AKI post paracentesis.

Result: 182 patients underwent 859 therapetuic paracentesis. 94 paracentesis resulted in AKI $(10.9 \%)$. The median number of paracentesis was 10 (range 1-25) and the median volume of fluid drained per paracentesis was $6 \mathrm{~L}$ (1-20 L). Hypotension occurred on 30 (30/ $859 ; 3.5 \%)$ and respiratory distress on $7(7 / 859 ; 0.8 \%)$ occasions. By univariate analysis, aetiology of cirrhosis, diabetes, hypertension and coronary artery disease were significantly associated with development of AKI. Patients who developed post paracentesis AKI were significantly younger, with higher MELD scores and greater volume 
of fluid drained. By univariate logistic regression, for each litre of fluid drained during paracentesis, the risk of AKI increased by 1.24 times. By multivariate logistic regression, the significant predictors of AKI were volume of fluid drained and MELD score

Conclusion: The prevalence of post paracentesis AKI is $10.9 \%$. The volume of abdominal paracentesis and MELD score are definitive predictors of paracentesis induced AKI

\section{PHT-39}

Frequency of minimal hepatic encephalopathy in cirrhotic patients with normal neurological examination

\section{Talal Khurshid ${ }^{1}$}

${ }^{1}$ Holy family hospital, Pakistan

Background: Hepatic encephalopathy is a chronic neuropsychiatric syndrome which is secondary to cirrhosis of liver and carries prognostic implications. Objectives: to detect the frequency of minimal hepatic encephalopathy in patients with cirrhosis who have normal neurological examination presenting in tertiary care setting. Study Design: cross-sectional survey

Method: a total of 150 cases were included in this study. Bispectral index of the patients was calculated by the researcher by applying bispectral index monitor pad on the forehead and graded from 0 to 100 .

Results: 22 patients $(14.7 \%)$ were $20-40$ years old, 98 patients $(65.3 \%)$ were $41-60$ years of age while 30 patients $(20.0 \%)$ were between 61-70 years of age, mean age of the patients was observed $51.25 \pm 11.32$. out of 150 patients, 90 patients $(60.0 \%)$ were male and remaining 60 patients $(40.0 \%)$ were female, minimal hepatic encephalopathy was noted in 91 patients $(60.7 \%)$.

Conclusion: It is concluded that MHE is highly prevalent among patients with cirrhosis. MHE patients exhibit a specific cognitive impairment that negatively impacts their driving capability and work performance and importantly is not evident to the patients themselves. Although testing for MHE and subsequent therapy is not standard of care at this time, it is important to consider this in cirrhotics in order to improve their ability to live their life to the fullest.

\section{PHT -40}

Predictors of mortality in patients with liver cirrhosis -a follow up study

\section{Mayank Jain ${ }^{1}$, Jayanthi Venkataraman ${ }^{1}$, Vijaya Srinivasan ${ }^{1}$ \\ ${ }^{1}$ Gleneagles Global Health City, India}

Background: Background-Patients with liver cirrhosis are predisposed to complications which cause progression of liver disease with resultant mortality.

Aim: To identify individual or combination of cirrhosis related complications (CRC) that predict mortality in patients with liver cirrhosis

Method: Patients with a confirmed diagnosis of liver cirrhosis were included for the study. Baseline information and frequency of variceal bleed, hepatic encephalopathy, ascites, spontaneous bacterial infection, deranged renal function and infection during the follow up period was noted. Complications that predicted survival or mortality were taken as the primary end point of the study. Independent sample t-test, chi square test and Mann Whitney U test were used. Univariate and logistic regression analysis was done for significant variables.
Result: There were 227 survivors and 87 non survivors. Majority of non survivors belonged to CTP $\mathrm{B}$ or $\mathrm{C}$ with a high MELD $(19.8 \pm 5.8$ versus $17.6 \pm 5.3 ; \mathrm{p}$ value 0.002$)$. Complications like variceal bleed $(17.2 \%$ versus $4 \%$ ), spontaneous bacterial peritonitis $(11.5 \%$ versus $4.4 \%)$, hepatic encephalopathy (34.5\% versus $15.4 \%)$ and deranged renal function ( $29.9 \%$ versus $11.9 \%)$ were significantly common among non survivors in the first admission. Renal dysfunction remained a significant complication in second and third admission. The mean survival for patients with no complication was statistically significant compared to those with at least one complication.

Conclusion: CRC were significantly higher in non survivors. Renal dysfunction was the major recurrent complication leading to subsequent admissions. Survival benefit was significant in liver cirrhosis with no complication.

\section{PHT-41}

Risk factors of portal vein thrombosis in patients with liver cirrhosis: the first case-control study in Russia

Khava Kodzoeva ${ }^{1}$, Daria Strelkova ${ }^{1}$, Kseniya Ulyanova ${ }^{1}$, Sofia $\overline{R o g a c h e v a}^{1}$, Alina Volkova ${ }^{1}$, Artem Dekhanov', Mariya Nadinskaya ${ }^{1}$, Vladimir Ivashkin ${ }^{1}$

${ }^{1}$ Sechenov University, Russian Federation

Background: Data of studies concerning the association between clinical and biochemical characteristics of cirrhotic patients and occurrence of portal vein thrombosis (PVT) are contradictory. No such studies have been previously conducted in Russia. The aim of this study was to evaluate risk factors of PVT in cirrhotic patients. Method: We realized a case-control study including 931 patients admitted to our clinic from January 2010 to May 2017. Cases were 91 cirrhotic patients with the diagnosis of PVT based on multislice computed tomography; Controls were 343 cirrhotic patients without PVT matched with cases for age and sex by stratified randomization. The predictive model was designed using the multivariate logistic regression.

Result: The patients of both groups were different in haemoglobin, white blood cells (WBCs), platelets, albumin, total bilirubin, alanine aminotransferase (ALT), international normalized ratio (INR) levels, the diameter of the portal vein, spleen volume, Child-Pugh class, MELD score and in presence of hepatocellular carcinoma (HCC) Table. There were no significant differences between the PVT group and non-PVT group in etiology of cirrhosis, fibrinogen levels. Multivariate logistic regression model included four variables: ChildPugh classes B and C ( $p<0,01$; OR 2.6; 95\% CI: 1.5-4.5), large spleen volume ( $<<0,01$; OR 1.02; 95\% CI: 1.01-1.03), low level of ALT (p < 0,01; OR 0.98; 95\% CI: 0.97-0.99); and presence of HCC ( $\mathrm{p}<0,01$; OR $7.9 ; 95 \%$ CI: 2.6-23.6). After stratifying patients by Child-Pugh all of factors remained stable in the multivariable predictive model.

Conclusion: The occurrence of PVT associates with more advanced liver cirrhosis according to Child-Pugh staging as well as with presence of hepatocellular carcinoma, value of splenomegaly and low level of ALT regardless of Child-Pugh class. 


\begin{tabular}{|c|c|c|c|}
\hline & $\begin{array}{c}\text { Cases } \\
\text { Patients with PVT } \\
(n=91)\end{array}$ & $\begin{array}{c}\text { Controls } \\
\text { Patients without PVT } \\
\quad(n=343)\end{array}$ & $p$-value \\
\hline \multicolumn{4}{|l|}{ Etiology of cirrhosis, n (\%) } \\
\hline Hepatitis C or B & $44(49 \%)$ & $141(41 \%)$ & n.s. \\
\hline Alcoholic liver disease & $24(26 \%)$ & $75(22 \%)$ & n.s. \\
\hline Other & $23(25 \%)$ & $127(37 \%)$ & n.s. \\
\hline$H b, g l$ & $120[99 ; 134]$ & $128[114 ; 140]$ & $<0,01$ \\
\hline WBCs, $\times 10^{9} / \mathrm{L}$ & $4,3[3,2 ; 6,4]$ & $4,9[3,8 ; 6,5]$ & 0,043 \\
\hline PLT, $x 10^{9} / \mathrm{L}$ & $91[64 ; 141]$ & $115[73 ; 171]$ & 0,029 \\
\hline Albumin, gl & $30,4 \pm 6,6$ & $33,3 \pm 7,1$ & 0,001 \\
\hline TBIL, mg/dl & $2,2[1,4 ; 3,6]$ & $1,8[1,0 ; 3,0]$ & 0,01 \\
\hline ALT,UL & $36[25 ; 58]$ & $76[44 ; 131]$ & $<0,01$ \\
\hline INR & $1,1[1,1 ; 1,3]$ & $1,2[1,1 ; 1,4]$ & $<0,01$ \\
\hline Fibrinogen, gl & $2,6[2,0 ; 3,5]$ & $2,8[2,2 ; 3,7]$ & 0,279 \\
\hline Portal vein diameter, mm & $13[11 ; 15]$ & $12[11 ; 13]$ & 0,001 \\
\hline Spleen volume, $\mathrm{cm}^{2}$ & $78[63 ; 105]$ & $60[46 ; 82]$ & $<0,01$ \\
\hline MELD score & $13[10 ; 16]$ & $11[8 ; 14]$ & $<0,01$ \\
\hline $\mathrm{HCC}$ & $21(23 \%)$ & $16(5 \%)$ & $<0,01$ \\
\hline \multicolumn{4}{|l|}{ Child-Pugh class, $\mathrm{n}(\%)$} \\
\hline A & $9(10 \%)$ & $160(47 \%)$ & $<0,01$ \\
\hline B & $55(60 \%)$ & $115(34 \%)$ & $<0,01$ \\
\hline C & $27(30 \%)$ & $67(19 \%)$ & $<0,01$ \\
\hline \multicolumn{4}{|c|}{$\begin{array}{l}\text { Quantitative values are expressed in terms of mean } \pm \text { SD for a symmetrical distribution and median (IQR) fo } \\
\text { unsymmetrical distributions and categorical variables are shown as frequencies (percentage). Hb, Haemoglobin } \\
\text { WBCS, white blood cells; PLT, platelet count; TBIL, total bilirubin; ALT, alanine aminotransferase; INF } \\
\text { international normalized ratio; MELD, Model For End-Stage Liver Disease; HCC, hepatocellular carcinoma, n. } \\
\text { non-significant }\end{array}$} \\
\hline
\end{tabular}

PHT -42

The effect of carvedilol vs propranolol in patients with cirrhosis related gastroesophageal varices for secondary prophylaxis: a randomized controlled trial

\section{Yichao Wei ${ }^{1}$}

${ }^{1}$ Zhongshan Hospital, Fudan University, Shanghai, China, China

Background: The role of Carvedilol in the prevention of recurrent variceal hemorrhage is still unclear due to lack of head-to-head controlled trials. This study aimed to compare the effect of Carvedilol to Propranolol in preventing recurrence and rebleeing of varices after endoscopic eradication.

Method: Consecutive patients in Zhongshan Hospital, Shanghai were enrolled. Among them, those who already took nonselective betablockers(NSBB) entered into Control group1, and the others were randomized into either Carvedilol or Propranolol group. After randomization, patients took the corresponding drug for 6 months, and dosage was individually titrated. The main outcome was need of endoscopic retreatment after 6 months. Randomization was revealed by randomized table. Neither researchers nor patients were blinded, but doctors who conducted endoscopic examination were blinded.

Result: During March 1st and August 31st 2015, we included 37 patients with previous history of variceal bleeding and achieving variceal eradication after endoscopic treatment in this study. Among them, 12 entered into Control group1; 12 were randomized into Propranolol group and 13 were randomized into Carvedilol group (figure 1). The 3 groups were different in gender and cause of cirrhosis composition. The mean dose for Propranolol was $17.73 \mathrm{mg} \pm 9.32 \mathrm{mg}$, and that for Carvedilol was $10 \mathrm{mg}$. During study, 4 and 5 people entered into Control group 2 from Carvedilol and Propranolol group respectively, because of cease of drug use, either because of intolerance of NSBB or reluctance to follow the protocol halfway. The combination of NSBB after variceal eradication can lower patients' 6-month endoscopic retreatment rate compared with Control group2 (Carvedilol group + Propranolol group + Control group1 Vs Control group2, 10/22 Vs 5/5, $\mathrm{P}=0.047)$. Carvedilol and Propranolol were comparable in preventing recurrence and rebleeding of $\mathrm{EGV}(4 / 8 \mathrm{Vs} 7 / 11 \%, \mathrm{P}=0.658$; 0/12 Vs 1/11, P = 0.100). Compared with Propranolol, Carvedilol didn't cause a greater impact on mean arterial pressure, nor did it result in more adverse events.

Conclusion: NSBB has an effect for decreasing the need of endoscopic retreatment after variceal eradication, but the difference between Carvedilol and Propranolol is not significant.

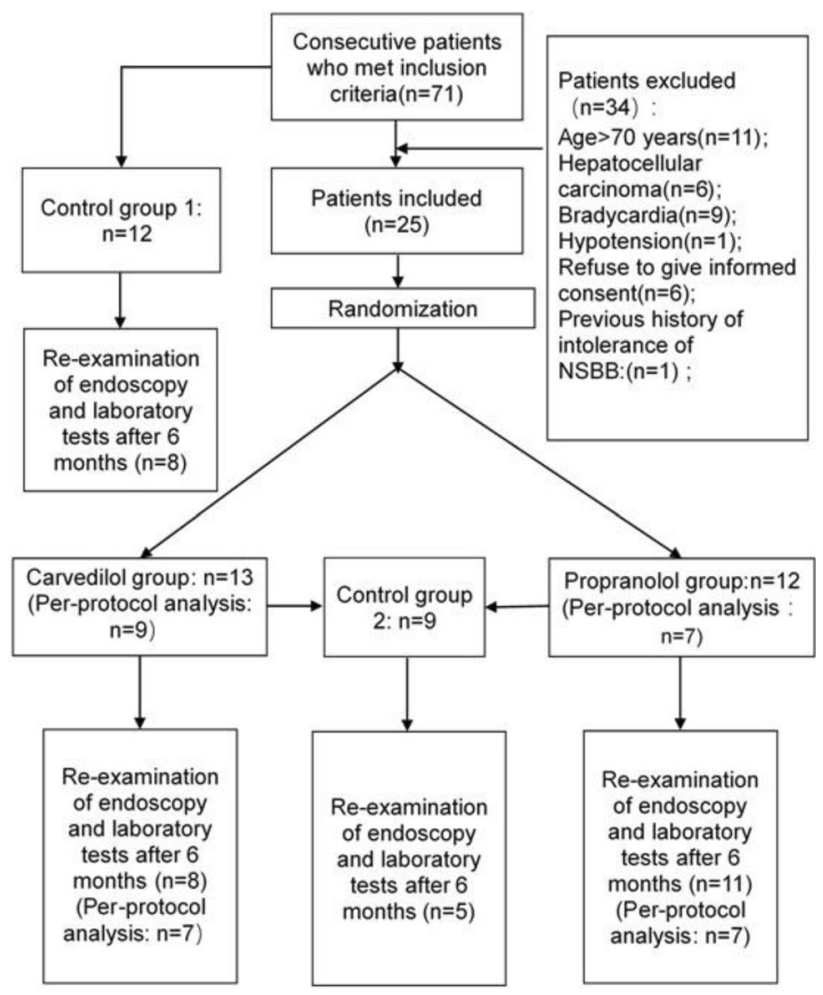

PHT-43

Predictors of spontaneous bacterial peritonitis in Egyptian patients with cirrhotic ascites Khaled Metwally, Medhat Assem, Tamer Reraat and Mohamed Yousery. Liver Unit, National Liver Institute, University of Menoufyia

\section{Khaled Elsayed Amer ${ }^{1}$, Medhat Assem Mahrous ${ }^{1}$}

${ }^{1}$ Hepatology, Egypt

Background: Spontaneous bacterial peritonitis (SBP) is a serious complication of liver cirrhosis and finding a prognostic model to predict it is needed. Objective: to test the ability of different laboratory tests and the new Wehmeyer's SBP scoring system to predict it. Methods: Three hundred patients admitted at the National Liver Institute, University of Menoufyia, Egypt (2015-2016) with liver cirrhosis and ascites were included in our study. SBP was diagnosed if ascetic neutrophils count $\geq 250 / \mu \mathrm{L}$ with no sign of secondary peritonitis. 
Results: Median age 56 (29 -81 years), 60\% men and primary cause of liver disease was hepatitis C, $91.7 \%$. By univariate analysis: age, total bilirubin, AST, creatinine, international normalized ratio, MELD score, total leucocytic count, platelet count and C-reactive protein (CRP) were significant. By multivariate analysis independent predictors were age, platelet count and CRP ( $\mathrm{p}=0.004,0.013$ and $<$ 0.001 , respectively). CRP at a cutoff point $\geq 13.5 \mathrm{mg} / \mathrm{L}$ could predict SBP (sensitivity $86.4 \%$ and specificity $66.0 \%$ ). Wehmeyer's SBP scoring system was able to predict it $(\mathrm{p}<0.001)$, only $4 \%$ of patients with 0 score developed SBP (CRP cutoff is $30 \mathrm{mg} / \mathrm{L}$ ), while $92.8 \%$ with score of 3 or 4 developed it. By using our CRP cutoff value of $13.5 \mathrm{mg} / \mathrm{L}$, no patient with 0 score developed SBP.

Conclusion: age, CRP and platelet count are independent predictors for SBP and a scoring system including them could easily predict it. SBP diagnosis could be excluded in patients with zero score, using CRP cutoff value of $13.5 \mathrm{mg} / \mathrm{L}$.

\section{PHT-44}

\section{Spectrum of acute kidney injury in patients with chronic liver} disease

\section{Amit Soni $^{1}$, Nirmaljeet Singh Malhi ${ }^{1}$ \\ ${ }^{1}$ SPS Hospital, India}

Background: Acute kidney injury is commonly seen in patients with chronic liver disease [CLD]. The aim of this work was to study the spectrum of Acute Kidney Injury (AKI) in patients with CLD and outcome.

Method: We conducted a hospital based study of CLD patients who were admitted between June 2016 and December 2016 in Gastroenterology department at a tertiary care hospital. The patients with AKI were identified. Details of these patients were noted from the data record.

Result: 500 CLD patients were included in this study and Acute kidney injury was present in $18.8 \%(n=94)$ patients. The spectrum of Acute kidney injury was Prerenal failure [dehydration, overuse of diuretics etc.] $42.5 \%(\mathrm{n}=40)$, Hepatorenal syndrome $29.7 \%(\mathrm{n}=$ 28), Sepsis induced AKI 21.27\% $(\mathrm{n}=20)$ and others [Drug induced, Glomerulonephritis] $6.3 \%(\mathrm{n}=6)$. The incidence of renal diseases was $6.3 \%$ in class A, $40.4 \%$ in class B, and $53.1 \%$ in class C cirrhosis. Hemodialysis was needed in $19.14 \%$. 500 CLD patients were included in this study and Acute kidney injury was present in $18.8 \%(n=$ 94) patients. The spectrum of Acute kidney injury was Prerenal failure [dehydration, overuse of diuretics etc.] $42.5 \% \quad(n=40)$, Hepatorenal syndrome $29.7 \%(n=28)$, Sepsis induced AKI $21.27 \%$ $(\mathrm{n}=20)$ and others [Drug induced, Glomerulonephritis] $6.3 \%$ $(n=6)$. The incidence of renal diseases was $6.3 \%$ in class A, $40.4 \%$ in class $\mathrm{B}$, and $53.1 \%$ in class $\mathrm{C}$ cirrhosis. Hemodialysis was needed in $19.14 \%$.

Conclusion: Acute kidney injury was seen in a significant proportion (18.8\%) of CLD patients. Prerenal was the commonest cause of AKI. The incidence of AKI was maximum in patients with child $\mathrm{C}$ status. Majority of the patients had a reversible cause and did not require hemodialysis. Judicious use of diuretics, use of renal safe drugs and early management of infections can prevent a major chunk of AKI among CLD patients.
PHT-45

A prospective study evaluating factors associated with renal dysfunction in cirrhosis and the impact on clinical outcome

Rishabh Prakash Jain ${ }^{1}$, Ajit Kumar ${ }^{1}$, Sukanya $\mathbf{B}^{1}$, Raghavendra $\mathbf{Y}^{1}$, Nayana Joshi ${ }^{1}$, Gaurav $T^{1}$, Vineet $C^{1}$, Ravichandra $T^{1}$

${ }^{1}$ Nizams Institute of Medical Sciences, India

Background: Introduction: Cirrhotic patients with renal dysfunctions have high mortality rates. This study investigated the prevalence, cause and clinical outcome in patients with cirrhosis and renal dysfunction.

Methods: This was a prospective observational study of consecutive patients admitted with cirrhosis at a tertiary centre. Renal dysfunction was classified according to revised recommendations by International Club of Ascites, causes and outcome were analysed.

Results: Total 356 patients were admitted during the study period from 1st January 2016 to 31st December 2016. Renal dysfunction was present in $112(31.4 \%)$ patients. Presentation with abdominal distension, altered sensorium, diuretic use and oliguria at admission were present in patients with renal dysfunction $(\mathrm{p}<0.05)$.Similarly infective foci, ascites, diuretic use and hepatic encephalopathy were associated with higher renal dysfunction $(\mathrm{p}<0.05)$. Urinary tract infections (UTI) were most common infection in renal dysfunction $(29.5 \%)$ as well as without renal dysfunction $(11.1 \%)$ followed by spontaneous bacterial peritonitis. Renal dysfunction with infection $(49.1 \%)$ was most common cause followed by hypovolemia $(28.7 \%)$, hepatorenal syndrome (HRS) $(18.7 \%)$ and parenchymal renal diseases (3.5\%).Mean CTP and MELD scores were higher in patients with renal dysfunction. The mean days of hospital stay $(9.07 \pm 3.54)$ and mean ICU days stay $(4.75 \pm 3.4)$ were significantly higher in patients with cirrhosis with renal dysfunction. The in-hospital mortality was $13.48 \%$, which was significantly higher in patients with cirrhosis with renal dysfunction (29.5\%) than as compared to patients without renal dysfunction $(6.1 \%)$ [p $<0.001]$.

\section{Conclusion:}

Conclusion: Renal failure has a high prevalence in cirrhotics and is associated with high mortality and morbidity. Infections are most common cause of renal failure.

PHT-46

Bacterial infection in decompensated cirrhosis: Data from HBV infected patients in China-Regional retrospective study

Zhujun Cao ${ }^{1}$, Yuhan Liu', Xiaobo Lu', Liuying Chen ${ }^{2}$, Shan Yin ${ }^{2}$, ${ }_{\text {Ziqiang Li }}{ }^{1}$, Ruidong Mo', Peipei Ren ${ }^{1}$, Lichang Chen ${ }^{1}$, Jie Lu' ${ }^{1}$, Xiaolin Wang ${ }^{1}$, Gangde Zhao ${ }^{1}$, Weiliang Tang ${ }^{1}$, Xiaogang Xiang ${ }^{1}$, Hui Wang ${ }^{1}$, Wei Cai ${ }^{1}$, Hai $\mathrm{Li}^{2}$, Qing Xie ${ }^{1}$

${ }^{1}$ Shanghai Ruijin Hospital, China; ${ }^{2}$ Shanghai Renji Hospital, China

Background: Bacterial infection (BI) complicates cirrhosis and frequently precipitates acute-on-chronic liver failure (ACLF), as is proved in cohort from European or North America. Data from China, where HBV associated end-stage liver disease dominates the"killer" list, is currently scarce but also important. This study analyzed the characteristics, incidence of $\mathrm{BI}$ in HBV associated decompensated cirrhosis and assessed its impact on the survival.

Method: Data from two tertiary teaching Hospital at Eastern-China between 2005-2010 were analyzed retrospectively. A total of 5102 HBV-related cirrhotic patients' medical records were identified using ICD code and gone through chart review by two independent investigators. A total of 3920 patients were excluded per protocol and 
finally, 1282 patients with decompensations were included for analysis.

Result: Overall, 399 episodes of bacterial infection occurred in 359 $(28 \%)$ patients with significantly $(\mathrm{p}<0.0001)$ higher incidence in ACLF (52\%) than in those without (17\%). Pneumonia (11\%) was the most common type of proven bacterial infection followed by spontaneous bacterial peritonitis (7\%) and urinary tract infection (3\%). There was relatively a balanced proportion between CA $(29 \%)$ and HCA $(31 \%)$ with higher proportion of HA $(40 \%)$ in non-ACLF patients. In contrast, the proportion of CA $(16 \%)$ was rather low with balanced HCA (42\%) and HA (42\%) in ACLF patients. Twenty-six (7.2\%) patients suffered second infection (17 in ACLF vs 9 in DC). Among the 71 patients (20\%) with culture positive infections, 52 experienced monomicrobial infections while 19 patients experienced polymicrobial infections. The proportion of Gram-negative bacteria $(58 \%)$ was higher than that of Gram-positive bacteria (42\%) but without statistical difference. Multi-drug resistant bacteria were common as observed in 50\% of isolation (42 in non-ACLF vs $58 \%$ in ACLF). Patients with BI had more intense systemic inflammation (blood leucocyte counts), higher prevalence of SIRS, positive qSOFA than non-BI patients. BI complicated disease course as indicated by a significantly higher cumulative incidence of renal dysfunction, liver failure, coagulation failure, cerebral failure, circulatory and/or respiratory system failure during the first 28 day of stay in patients with BI than those without BI at admission. Accordingly, cumulative incidence of ACLF in patients with BI was significantly higher $(23 \%$ vs $9 \%$ by 28 day of stay, $\mathrm{p}<0.001$ ) and 90 -d transplant-free survival ( $44 \%$ vs $71 \%$; p < 0.001) was significantly lower than those without BI. The presence of BI (sHR, 1.6, 95\% CI: 1.2-2.2) or ACLF (sHR, 3.1, 95\% CI: 2.3-4.1) at admission were identified as the two strongest independent predictors of 90-d mortality under multivariate analysis using competing risk approach. In patients with $\mathrm{BI}$, the presence of ACLF, sepsis (Sepsis 3.0 criteria) and inappropriateness of empirical antibiotics were the three independent predictors of 90-d mortality.

Conclusion: Bacterial infection complicated disease course of HBV associated decompensated cirrhosis as is evident in alcoholics. The development of BI impaired 90-day survival independent of the presence of ACLF. Reduced mortality observed in BI patients with appropriate empirical antibiotics further emphasized the importance of prophylactic strategies.

\section{PHT-47}

\section{Bacterial infections in cirrhosis-clinical profile and factors affecting outcome}

\section{Omesh Goyal ${ }^{1}$, Prerna Goyal ${ }^{2}$, Deepinder Kaur ${ }^{1}$, Rajoo Singh Chhina $^{1}$ \\ ${ }^{1}$ D.M.C. and Hospital, India; ${ }^{2}$ R.G. Stone and Superspeciality Hospital, India}

Background: Cirrhosis is an immunocompromised state that predisposes patients to spontaneous bacterial infections, hospitalacquired infections, and a variety of infections from uncommon pathogens. Once infection develops, the excessive response of proinflammatory cytokines on a pre-existing hemodynamic derangement in cirrhosis further facilitate the development of severe complications such as septic shock, multiple organ failure, and death. Early recognition and treatment of infections in cirrhosis is warranted to decrease the morbidity and mortality. This study aimed to analyze the clinical profile and factors affecting outcome of bacterial infections in cirrhotics.

Method: Medical records of all patients with cirrhosis admitted to the Gastroenterolgy Unit of a tertiary care hospital in northern India from January 2013 to December 2016 were analysed. Clinical history, examination and lab investigations were noted. Child-Turcotte Pugh scores (CTP) and Model for end stage liver disease (MELD) scores were calculated. Multiple logistic regression analysis was performed to find the risk factors for development of bacterial infections in cirrhotics, and for factors affecting mortality in cirrhotics with bacterial infection. Various possible risk factors studied were- age, gender, etiology of cirrhosis, serum bilirubin, serum albumin, serum creatinine, prothrombin time index (PTI), CTP score and MELD score.

Result: A total of 716 hospitalized patients with cirrhosis were included. The mean age of patients was $54 \pm 9.3$ years, and the male: female ratio was 5.7:1. Most common etiology of cirrhosis was alcohol $(49.2 \% ; \mathrm{n}=352)$, followed by hepatits $\mathrm{C}$ virus $(29.4 \% ; 211)$, NAFLD $(13.6 \% ; 98)$ and hepatits B virus $(3.9 \%$; 28$)$. Bacterial infections were diagnosed in $24.8 \%(178 / 716)$ of these patients. The various sites of bacterial infection were- spontaneous bacterial peritonitis (SBP) $(43.8 \%$; 314), skin and soft tissue infections (SSTI) $(33.7 \%$; 242), urinary tract infection (UTI) $(26.1 \%$; 187), lower respiratory tract infection $(12.4 \%$; 89$)$, bacteremia $(11.8 \% ; 85)$, and meningitis $(0.7 \%$; 5). Two hundred and six $(28.7 \%)$ patients had multiple infections. Blood/local site cultures were positive in 116 out of $178(65.2 \%)$ patients. The predominant type of organisms isolated were gram negative bacilli (GNB) $(96 / 116 ; 82.7 \%)$, and Gram positive cocci (GPC) (20/116; 17.3\%). On multivariate analysis, high CTP score and high MELD score were found to be independent predictors of developing bacterial infection in cirrhotics $(p=0.001)$. Overall mortality rate was $22.4 \%(\mathrm{n}=161)$. On multivariate analysis, high serum creatinine and high MELD score were found to be independent predictors of mortality.

Conclusion: Bacterial infection occur commonly in cirrhotics, especially in those with high CTP and MELD scores. Most common sites were SBP and SSTI, and the most common organism were GNB. High serum creatinine and high MELD score were independent predictors of mortality.

\section{PHT -48}

Bacterial infections; independent predictors of 30-day mortality in acute decompensated patients with and without ACLF.

Salisa Wejnaruemarn ${ }^{1}$, Jarongkorn Sirimongkolkasem ${ }^{1}$, Tongluk Teerasarntipan $^{1}$, Roongruedee Chaiteerakij ${ }^{1}$, Chonlada Phathong $^{1}$, Piyawat Komolmit ${ }^{1}$, Pisit Tangkijvanich ${ }^{2}$, Sombat Treeprasertsuk $^{1}$

${ }^{1}$ Department of Medicine, Division of Gastroenterology, Faculty of Medicine, Chulalongkorn University, and Thai Red Cross, Bangkok 10330, Thailand, Thailand; ${ }^{2}$ Department of Biochemistry, Faculty of Medicine, Chulalongkorn University, Bangkok 10330, Thailand, Thailand

Background: Bacterial infections are common triggers of acute-on chronic liver failure (ACLF) and showed poorer outcome than noninfection triggered ACLF. In addition, the prevalence of bacterial infections in Asian patients with ACLF is high. Thus, we aim to identify the prevalence and characteristic of bacterial infections as well as their impact on survival of hospitalized cirrhotic patients with acute decompensation (AD).

Method: We prospectively enrolled Thai hospitalized cirrhotic patients at the King Chulalongkorn Memorial Hospital during 5-year study period (Feb 1, 2012 to January 31, 2017). The CLIF-SOFA score, clinical history, laboratory profiles and details of bacterial infections were collected and logistic regression analyses were used to identify predictors of 30-day mortality 
Result: There were 146 cirrhotic patients with $\mathrm{AD}(\mathrm{M}: \mathrm{F}=1.75: 1$, mean MELD $22.7 \pm 8.3$ ) and 81 patients $(55.5 \%)$ were diagnosed with ACLF. Proportion of patients with ACLF grade 0, 1, 2 and 3 were $44.5 \%, 17.8 \%, 23.3 \%$ and $14.4 \%$ respectively whereas 56 patients $(38.3 \%)$ showed CLIF-OF score $>10$. A total of 71 patients (48.6\%) presented bacterial infections and subtype of community acquired (CA), health care associated (HCA) and nosocomial infection were $35.6 \%, 10.3 \%$ and $2.7 \%$ respectively. Spontaneous bacterial peritonitis (SBP; $13.7 \%)$, primary bacteremia $(11.6 \%)$, urinary tract infection (5.5\%), hepatobiliary infection $(4.1 \%)$ and pneumonia $(4.1 \%)$ were the top 5 common sources of infection. The overall 30 -day mortality rate was $21.2 \%$ which was not significant different between those $\mathrm{AD}$ patients with and without bacterial infection (table 1). In addition, patients with ACLF had significantly higher mortality rate than those without ACLF $(30.9 \%$ vs $9.2 \%, \mathrm{p}=0.001)$. The predictors of 30-day mortality was the presence of ACLF $(\mathrm{OR}=$ 4.9 ; $95 \%$ CI 1.8-13.1) and bacterial infection $(\mathrm{OR}=1.6$; $95 \%$ CI 0.7 3.8).

Conclusion: The prevalence of bacterial infection in hospitalized cirrhotic Asian patients with AD was high, up to $48.6 \%$, with 30 -day mortality rate of $21.2 \%$. The predictors of 30 -day mortality were the presence of ACLF and bacterial infection.

Table 1. Characteristic data of 146 cirrhotic patients with acute decompensation (AD)

\begin{tabular}{|l|l|l|l|}
\hline $\begin{array}{l}\text { Variables } \\
\text { Mean +/- SD; number (\%) }\end{array}$ & $\begin{array}{c}\text { With bacterial } \\
\text { infection (n=71) }\end{array}$ & $\begin{array}{l}\text { Without bacterial } \\
\text { infection (n=75) }\end{array}$ & P value \\
\hline Age at enrolled (year) & $57.5+/-13.0$ & $56.1+/-14.7$ & 0.52 \\
$\%$ male & $62 \%$ & $65 \%$ & 0.67 \\
Hb (g/dL) & $9.8+/-2.4$ & $9.7+/-2.3$ & $0.004^{*}$ \\
$\%$ PMN & $75.4+/-13.5$ & $73.1+/-10.8$ & 0.27 \\
BUN (mg/dL) & $30.4+/-24.8$ & $36.3+/-27.1$ & 0.17 \\
Cr (mg/dL) & $1.8+/-1.4$ & $2.0+/ / 1.9$ & 0.39 \\
Albumin (g/dL) & $2.4+/-0.6$ & $2.6+/-0.6$ & $0.049^{*}$ \\
HCO3 (mEq/L) & $16.5+/-5.0$ & $19.2+/-4.9$ & $0.001^{*}$ \\
MELD score on admission & $24.0+/-8.9$ & $21.5+/-7.7$ & 0.07 \\
CLIF-OF score & $9.6+/-2.7$ & $8.6+/-2.1$ & $0.013^{*}$ \\
$\%$ Death at 30-day & $30 \%$ & $20 \%$ & 0.18 \\
\hline
\end{tabular}

PHT-49

Correlation between spleen size, platelet count and MELD Score in cirrhosis.

Manoj Yadav ${ }^{1}$, Jose Mathew ${ }^{1}$, Neeraj Vinayakumar', Aniruddha Pratap Singh $^{1}$, Varun Tadkalkar ${ }^{1}$, Srijaya $S^{1}$

${ }^{1}$ Kerala Institute of Health Sciences (KUHS), India

Background: Thrombocytopenia (Platelets $<1.5$ lakhs) and splenomegaly are frequent findings in cirrhosis. Model for End-stage Liver Disease (MELD) is a chronic liver disease severity scoring system. In this study, primary aim was to evaluate the relation between spleen size, platelet count and MELD score in cirrhosis with a secondary aim to evaluate effect of etiology of cirrhosis on thrombocytopenia.

This study was important as there is no similar study available from India.

Method: In this retrospective study, medical records of 112 patients with cirrhosis admitted in medical gastroenterology department of Government Medical College, Trivandrum were included. Statistical analysis was done using scatter plot and spearman's rank correlation coefficient (SPSS 21).
Result: Out of 112 patients included $75(67 \%)$ were males and 37 $(33 \%)$ were females.

\begin{tabular}{|l|l|l|l}
\hline & $\begin{array}{l}\text { Platelet count } \\
\text { (lakhs/cumm) }\end{array}$ & MELD & Spleen size (cm) \\
\hline $\mathrm{n}$ & 112 & 112 & 112 \\
\hline Mini. - Max. & $0.54-2.8$ & $7-24$ & $8.5-19.5$ \\
\hline Mean (SD) & $1.328(0.427)$ & $15.08(4.202)$ & $11.41(1.786)$ \\
\hline Median & 1.32 & 16 & 11 \\
\hline
\end{tabular}

To examine the relationship between spleen size, platelet count and MELD score, a scatter plot was generated for visual inspection. There was no apparent relationship between these measures (Fig. 1).

Correlation analysis by Spearman method showed non significant negative correlation between platelet count and spleen size $(\mathrm{P}=0.77$, coefficient $=-0.028$ )

We also found a decreased platelet count was significantly more common among patients with cirrhosis of alcoholic etiology $(83.78 \%)$ than in other etiologies of cirrhosis $(52 \%)(\mathrm{P}=0.012)$ (Fig. 2).

Conclusion:

1. There is no correlation between spleen size, platelet count and MELD score in cirrhosis.

2. Thrombocytopenia is more common in cirrhosis caused by alcohol as compared to other etiologies.
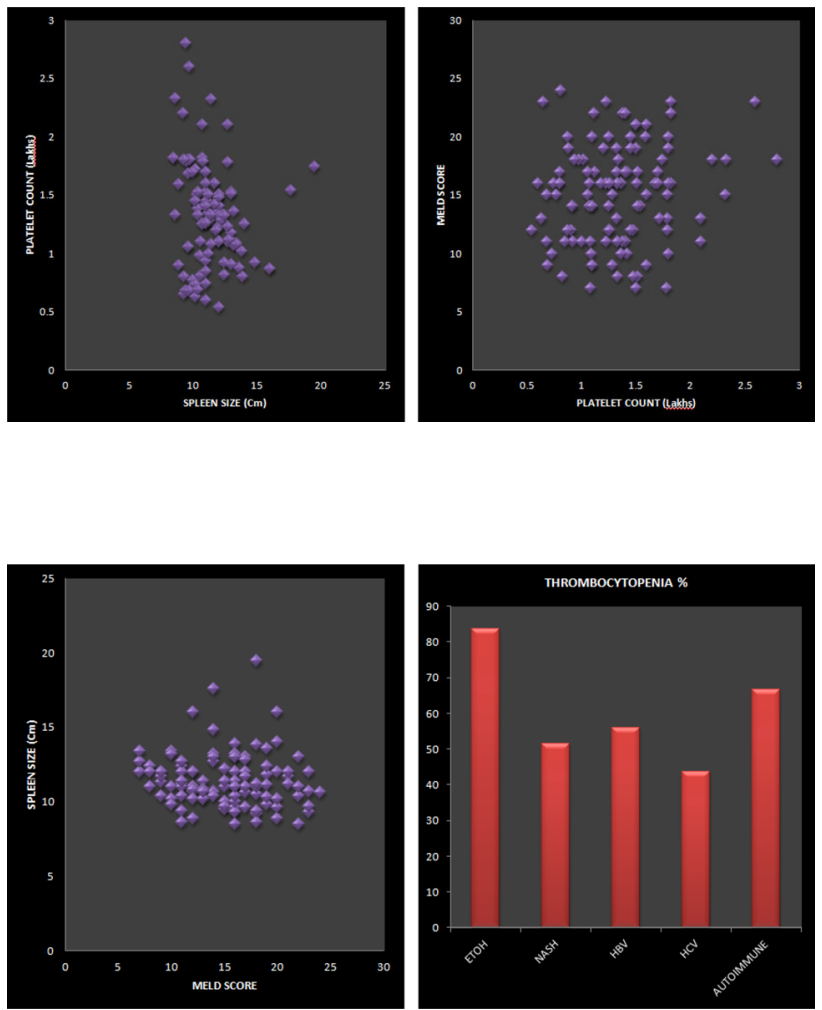

PHT-50

Diagnostic value of ascitic cholesterol in differential diagnosis of ascites

Yuhu Song ${ }^{1}$, Li Du ${ }^{1}$, Shenghua Zhu ${ }^{1}$, Jin Ye ${ }^{1}$, Keshu Xu ${ }^{1}$, Dong $\mathrm{Xu}^{2}$, Jin $\mathrm{Li}^{3}$ 
${ }^{1}$ Union Hospital, Tongji Medical College, Huazhong University of Science and Technology, China; ${ }^{2}$ Tongji Hospital, Tongji Medical College, Huazhong University of Science and Technology, China; ${ }^{3}$ Zhongnan Hospital of Wuhan University, China

Background: Diagnostic value of ascitic cholesterol in differential diagnosis of ascites is controversial, so the aim of our study is to investigate the performance of ascitic cholesterol in discriminating the cause of ascites.

Method: Consecutive patients with new onset ascites were prospectively enrolled. The pertinent data of the patients were collected from a total of 714 patients with all forms of ascites.

Result: In the training cohort, ascitic cholesterol was a quiet effective method of distinguishing non-portal hypertension $(\mathrm{PH})$ from portal hypertension; at the cut-off value of $1.11 \mathrm{mmol} / \mathrm{L}$, area under curve of ROC curve was 0.958 . In whole cohort, it yielded sensitivity of $92 \%$, specificity of $93 \%$, NPV of $93 \%$, and PPV of $92 \%$, accuracy of $92 \%$. The sensitivity of ascitic cholesterol was superior to serum-ascites albumin gradient (SAAG) in identifying non-PH-related ascites. Ascitic cholesterol plus SAAG yielded $96 \%$ sensitivity, combined ascitic cholesterol and SAAG yielded $99 \%$ specificity. In the misdiagnosed patients based on the classification of SAAG, diagnostic accuracy of ascitic cholesterol is $78 \%$. In mixed ascites, ascitic cholesterol had excellent performance in identifying peritoneal lesions, such as metastasis of cancer and peritoneal involvement of benign ascites.

Conclusion: Ascitic cholesterol is an excellent parameter in the differentiation of non-PH from $\mathrm{PH}$, thus this simple and cheap parameter should be determined in the patients with new onset ascites in clinical practice

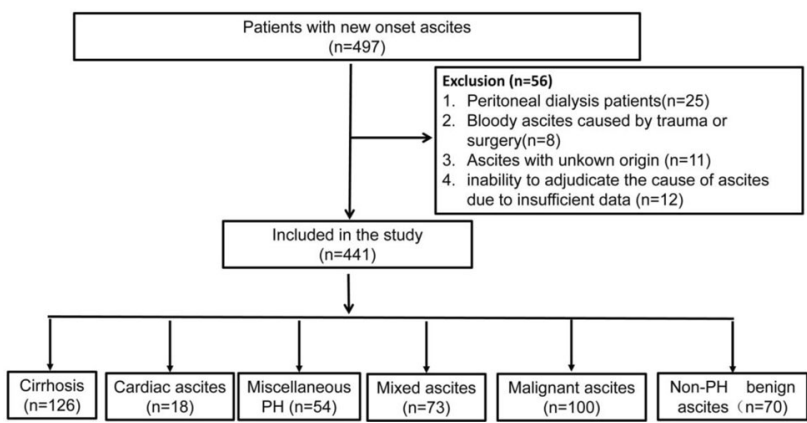

A

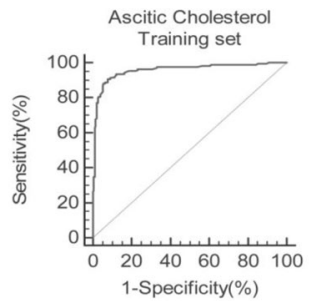

C

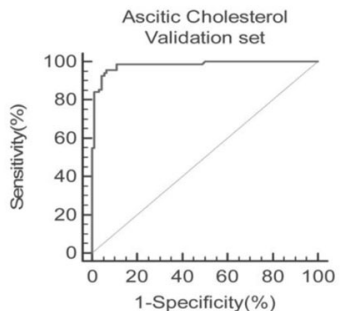

B
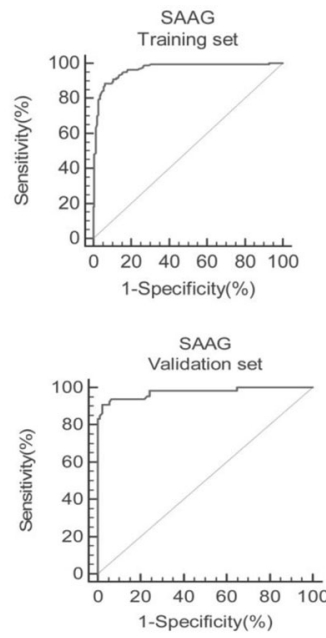

PHT-51

Endocoiling with/without cyanoacrylate injection versus cyanoacrylate injection alone in post cyanoacrylate gastric rebleed - a retrospective study

\section{Abraham Koshy $^{1}$}

${ }^{1}$ VPS Lakeshore Hospital, India

Background: N-butyl cyanoacrylate injection is recommended in bleeding/recently bled gastric varices. However, cyanoacrylate injection is associated with re-bleed in $25-50 \%$ of patients. Endocoiling is an emerging treatment modality for bleeding gastric varices. The aim was to compare EUS guided coil application combined with cyanoacrylate injection to cyanoacrylate injection alone in postcyanoacrylate gastric re-bleed.

Method: A retrospective analysis of a prospectively maintained database was performed. Thirty seven patients who re-bled after cyanoacrylate injection who had endocoiling of varices were included. Fifty one comparison patients had only cyanoacrylate injection. Endocoiling was done under endosonographic guidance. A single coil was placed in 10, two coils in each of 16 patients, three coils in 6 patients, four coils in 3 patients, five coils in one patient and six coil in one patient. In addition, cyanoacrylate glue injection was given in 21 patients. Nine patients had repeat endocoiling 1 month later. Rebleed and mortality were assessed.

Result: Endocoiling: Seven patients $(7 / 37,19 \%)$ re-bled during follow up of 9-365 days. Four out of 37 (11\%) died. One patient died 9 days after the procedure due to acute respiratory distress syndrome, one died one month after the procedure, one died 4 months after the procedure due to a re-bleed and one patient died 5 months after the procedure due to spontaneous bacterial peritonitis.

Glue only: 26/51 patients (51\%) re-bled during follow up of up to 45 to 365 days. Endocoiling resulted in significantly less re-bleed than glue-only by $\log$ rank test of Kaplan Meir plot $(\mathrm{z}=5.4$, $\mathrm{p}<0.001)$.Two out of 51(4\%) died 59 and 186 days after the procedure.

Conclusion: Endocoiling with/without cyanoacrylate injection for obliteration of gastric varices is effective for post cyanoacrylate gastric variceal re-bleed.

Figure 1. Kaplan Meir plot comparing re-bleod in glue imjection group with endocoil group (log-rank test: $z=5.4, p<0.0011$

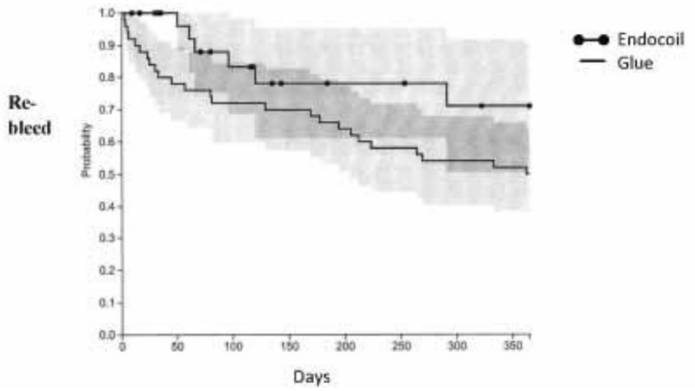

PHT-52

Fungal peritonitis: a neglected entity in critically ill patients with liver cirrhosis

Pratibha Kale ${ }^{1}$, Vikas Khillan ${ }^{2}$, Shiv Kumar Sarin ${ }^{2}$

${ }^{1}$ Institute of Liver and Biliary Sciences, India; ${ }^{2}$ Institute of Liver and Biilary Sciences, India 
Background: Cirrhosis is associated with impaired metabolic function and alters the host defence mechanisms and predisposes to a plethora of infections. Spontaneous peritonitis, especially spontaneous fungal peritonitis (SFP), is an important and potentially fatal complication in cirrhosis. We evaluated risk factors, microbiological findings, and outcome of patients with SFP compared to spontaneous bacterial peritonitis (SBP) in critically ill patients.

Method: The study is a retrospective analysis of 12672 ascitic fluid cultures received from January 2012 to December 2017 to the department of microbiology of tertiary care hepatobiliary centre. Bacterial and fungal identification and antimicrobial susceptibility system were done by automated Vitek 2 system. Laboratory parameters like liver function tests, blood counts, ascitic fluid analyses were compared between bacterial and fungal peritonitis groups.

Result: Of the 12672 ascitic fluid samples 1121 (8.84\%) were culture positive. Amongst these $69(6.11 \%)$ had fungal and $833(74 \%)$ had bacterial peritonitis. CHILD Pugh classification was mainly CHILD C and MELD Score was in patients with SFP $(34 ; 18-40 ; \mathrm{p}<0.001)$, SBP $(32 ; 12-40 \mathrm{p}<0.002)$. There was elevated total bilirubin (mean 7.1 vs $4.6 \mathrm{mg} / \mathrm{dl}$ ) and alanine transaminase (mean 60.07vs $46.29 \mathrm{IU} /$ L) with significantly higher ascitic fluid glucose (123.6 vs $74.68 \mathrm{mg} /$ $\mathrm{dl}, \mathrm{p} 0.05)$ and ascitic fluid protein (2.39 vs $1.15 \mathrm{~g} / \mathrm{dl}, \mathrm{p} 0.003)$ in SFP group. Mortality rate was significantly higher in patients with SFP (90\%, p < 0.001), followed by SBP $(75 \% ; \mathrm{p}<0.001)$. Candida pelliculosa 21 (30.43\%), C. albicans 20 (28.9\%), C. tropicalis 11 (15.9\%), Cryptococcus neoformans $6(8.69 \%)$ were common species isolated.

Conclusion: SFP is common complication of cirrhosis associated with high mortality. Factors predicting SFP are CHILD C liver cirrhosis, elevated MELD score, high ascitic fluid glucose and protein which may guide initiation of antifungal therapy.

\section{PHT-53}

\section{Increased myocardial extracellular volume define patients with more advanced cirrhosis and at higher risk of mortality: results from a cardiac MRI and echo study}

\section{Soren Moller ${ }^{1}$, Signe Wiese ${ }^{1}$, Silje Mo ${ }^{1}$, Jens Dalgaard Hove ${ }^{1}$, Rajeshwar Mookerjee ${ }^{2}$, Flemming Bendtsen ${ }^{1}$ \\ ${ }^{1}$ Hvidovre Hospital, Denmark; ${ }^{2}$ Royal Free Hospital, United Kingdom}

Background: Cirrhotic cardiomyopathy is an established complication in cirrhosis. Cardiac dysfunction has been linked to disease severity, development of hepatorenal syndrome, and increased mortality. However, the pathogenesis and precise definition of cirrhotic cardiomyopathy remain a matter of debate. It has been speculated that structural changes in the myocardium are involved. Cardiac MRI (CMR) with T1-mapping and quantification of extracellular volume (ECV) is a new non-invasive method to assess diffuse myocardial fibrosis in conditions such as amyloidosis. This technique has, to our knowledge, never been applied in cirrhosis. Our aim was therefore to perform a detailed characterization of cardiac structure and function using CMR and echocardiography in cirrhotic patients to determine the relationship between myocardial fibrosis and cardiac function, liver disease severity, and adverse events.

Method: 63 cirrhotic patients and 12 healthy controls were included. All patients underwent contrast-enhanced CMR (including ECV determination), echocardiography with Tissue Doppler Imaging (TDI), and clinical and biochemical assessments. Follow-up with registration of death and liver transplantation was ascertained. In the controls, contrast-enhanced CMR alone was performed.
Result: ECV was higher in the patients compared with healthy controls $(31.1 \pm 6$ vs. $27.4 \pm 3 \%, p=0.04)$. Moreover, ECV increased across Child Pugh A/B/C classes (26.9 $\pm 4 / 31.5 \pm 5 /$ $34.4 \pm 6 \%, \mathrm{p}=0.02)$. During a median follow-up of 22 months (131)16 patients had either died (12) or been transplanted (4) and the ECV was significantly higher than in patients without events $(33.4 \pm 5$ vs. $30.5 \pm 6 \%, p=0.043)$. ECV correlated with MELD $(\mathrm{r}=0.31, \mathrm{p}=0.028)$, cardiac index $(\mathrm{r}=0.35, \mathrm{p}=0.015), \mathrm{CRP}$ $(\mathrm{r}=0.47, \mathrm{p}=0.001)$, proANP $(\mathrm{r}=0.4, \mathrm{p})$.

Conclusion: Cirrhotic patients have an increased ECV compared to healthy controls. ECV is related to disease severity with the highest values in the patients with the most advanced disease and in those who die or are transplanted during follow-up. We suggest that these changes reflect diffuse myocardial fibrosis and are of pathophysiological relevance, defining cirrhotic patients who may develop cirrhotic cardiomyopathy and increased mortality. Further studies on the potential of ECV in the definition of cirrhotic cardiomyopathy are warranted.

\section{PHT-54}

\section{Outcome in patient of portal hypertension with surgical intervention}

Sonny Bherwani ${ }^{1}$, Kaushal Vyas ${ }^{1}$, Sushil Narang ${ }^{1}$, Nilesh Pandav ${ }^{1}$

${ }^{1} \mathrm{NHL}$ MMC, India

Background: To investigate the outcome of Esophagogastric devascularisation surgery with spleenectomy (EGDS) or proximal splenorenal shunt (PSRS) using clinical parameters, portal vein diameter and variceal bleeding in patients of portal hypertension (PHT). We evaluated the safety of procedure, problem encountered, solution for them and analyze the outcome with available literature to reduce the complication and mortality in patients.

Method: Complete blood count, liver function test, Upper gastrointestinal endoscopy and Ultrasonography abdomen with Doppler were done for enrolled patients. Diagnosis of PHT was based on clinical, biochemical, radiological support and confirmed by liver biopsy. The study included 70 patients with variceal bleed due to PHT, 32 patients underwent EGDS and 38 patients were offered PSRS. Patients of Cirrhosis, EHPVO and NCPF with unfavourable anatomy underwent EGDS others underwent PSRS. Endoscopic evaluation of gastroesophageal varices was done before and later at 6 months of surgery. Result: The study enrolled 70 patients out of which, 32 patients underwent EGDS and 38 patient underwent PSRS. Mean age was 30.44 year in EGDS group and 17.36 year in PSRS group. $64(91 \%)$ patients had hematemesis with malena while $6(9 \%)$ patient had only malena at presentation. $35(55 \%)$ patients presented with first episode of UGI bleed, 26 (40\%) presented with second episode while $3(5 \%)$ had history of multiple episodes of UGI bleed in the past.

Endoscopic evaluation of gastroesophageal varices after 6 month of surgery revealed that PSRS had better results compared to EGDS in term of gastroesophageal variceal status after surgery which is considered as one of the important predictive factors in variceal bleeding and also rebleeding. Also the gastroesophageal varices were compared using the following parameters a) disappearance of varices, b) decrease in size of varices, c) remain same or d) increase in size during follow up endoscopic evaluation. Disappearance of varices were observed more frequently in PSRS group than EGDS group ( $21 \%$ vs. 00$), p<0.01$. Though there was no increase in size in both the group, variceal size remained same more in EGDS group when compared to PSRS group ( $12 \%$ vs. 00$), \mathrm{p}<0.01$. Decrease in variceal size was observed in $88 \%$ cases of EGDS group and $79 \%$ cases of PSRS group (table below). 


\begin{tabular}{|l|l|l|}
\hline SURGERY TYPE & EGDS & PSRS \\
\hline Varices disappear & $00(0 \%)$ & $08(21 \%)$ \\
\hline Varices decrease & $28(88 \%)$ & $30(79 \%)$ \\
\hline Varices remain same & $04(12 \%)$ & $00(0 \%)$ \\
\hline Varices increase & 00 & 00 \\
\hline Total & 32 & 38 \\
\hline
\end{tabular}

USG abdomen revealed evidence of cirrhosis of liver in 9 out of 25 cirrhotics while there was altered echo pattern in the remaining patients. Portal vein diameter was measured by Doppler USG in patient undergone EGDS and PSRS procedure, preoperatively and after surgery at 6 months. Mean Portal vein diameter after EGDS in preoperative and postoperative after 6 month was 13.81 and $12.95(\mathrm{p}$ $=0.076) \mathrm{mm}$ respectively. Mean Portal vein diameter after PSRS in preoperative and postoperative after 6 month was 12.25 and $10.5 \mathrm{~mm}$ $(\mathrm{p}<0.05)$ respectively. Shunt was patent in all patient after 6 months confirmed by USG Doppler. Post operative ascites was noticed more in cirrhotic patients who underwent EGDS.

Conclusion: In developing countries there is limitation to access to tertiary facilities and endoscopic procedures. Thus surgical intervention either PSRS or EGDS is safe and prevents mortality due to rebleeding with acceptable morbidity.

\section{PHT-56}

Patient-reported outcomes in viral hepatitis without advanced fibrosis: chronic hepatitis B vs. hepatitis $\mathrm{C}$ infection

Zobair Younossi $^{1}$, Maria Stepanova ${ }^{3}$, Issah Younossi ${ }^{3}$, Fatema Nader $^{3}$, Naoky Tsai ${ }^{5}$, Mindie H Nguyen ${ }^{6}$, Ed Gane ${ }^{2}$, Kosh Agarwal $^{4}$, Harry LA Janssen ${ }^{7}$

${ }^{1}$ Inova Fairfax Hospital, United States; ${ }^{2}$ Auckland Clinical Studies, New Zealand; ${ }^{3}$ Center for Outcomes Research in Liver Disease, United States; ${ }^{4}$ Institute of Liver Studies, United Kingdom; ${ }^{5}$ John A. Burns School of Medicine University of Hawaii, United States; ${ }^{6}$ Stanford University, United States; ${ }^{7}$ Toronto Centre for LIver DIsease, Canada

Background: Chronic infections with hepatitis B and C are associated with adverse clinical outcomes and patient-reported outcomes (PROs) such as health-related quality of life (QoL). The aim is to compare PRO scores in patients with chronic HBV and HCV without advanced liver disease enrolled in recent clinical trials before and after suppression/clearance of their infection.

Method: Subjects with HCV and HBV infection prior to initiation of antiviral treatment and after viral suppression/eradication with antiviral regimens (HCV: sofosbuvir-based regimens, HBV: TDF+GS-9620) completed three PRO questionnaires: SF-36, CLDQ, and WPAI:SHP. For this analysis, only patients with fibrosis stage $\leq 2$ were included. The PRO scores were compared between patients with HBV and HCV before treatment initiation and at the end of posttreatment follow-up (in patients with undetectable viremia).

Result: We included 132 patients with HBV and $132 \mathrm{HCV}$-infected controls (matched by age, gender, race, region of the world, history of type 2 diabetes, anxiety, depression, fatigue, insomnia and stage of fibrosis $\leq 2$ ). Both group were initially viremic and were treated for viral suppression (HBV below $20 \mathrm{IU} / \mathrm{mL}$ ) and viral eradication (HCV SVR) with IFN-free regimens. Baseline PRO scores were significantly higher in patients with HBV in the domains of Physical Functioning, Role Physical, Bodily Pain, Social Functioning, Role Emotional, and Physical Component Summary scores of SF-36, SF-6D utility score, Emotional and Fatigue domains of CLDQ, and Presenteeism and total Work Productivity Impairment of WPAI: SHP, in comparison to patients with HCV by $5.8 \%$ to $13.2 \%$ of a PRO score range ( $<<0.05$ ).
At the end of follow-up (48 weeks of treatment for HBV and SVR-24 for HCV patients), only Physical Functioning and Role Physical scores remained higher in HBV by $6.7 \%-9.9 \%$, while other PRO scores became similar between HBV and HCV groups ( $p>0.05$ ) (Figure).

Conclusion: Prior to treatment, PRO scores are lower in patients with $\mathrm{HCV}$ in comparison to $\mathrm{HBV}$-infected subjects. After successful treatment of both HBV and HCV, most PRO differences between $\mathrm{HCV}$ - and HBV-infected patients disappear confirming the positive impact of viral treatment on PROs.

Figure- Patient Reported Outcomes for Patients with HBV and HCV Before and After Treatment

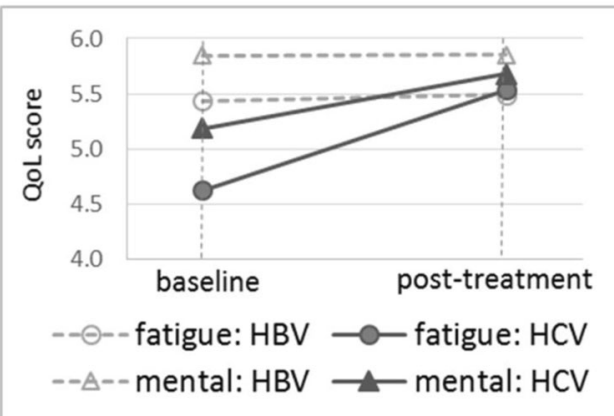

PHT-57

Post-paracentesis circulatory dysfunction with repeated large volume paracentesis in chronic liver disease: prospective longitudinal study in children

Moinak Sen Sarma ${ }^{1}$, Moinak Sen ${ }^{2}$, Surender Kumar Yachha ${ }^{1}$, Vijayalakshmi Bhatia ${ }^{1}$, Anshu Srivastava ${ }^{1}$

${ }^{1}$ Sanjay Gandhi Postgraduate Institute of Medical Sciences, Lucknow, India; ${ }^{2}$ Sanjay Gandhi Postgraduate Institute of Medical Sciences, India

Background: Ascites is a common complication of cirrhosis and is associated with poor prognosis. Post-paracentesis circulatory dysfunction (PPCD) is overall seen in one-third of cirrhotic children who undergo single-time large volume paracentesis (SLVP). It is not known whether PPCD occurs with repeated LVP (RLVP). Our study aimed to analyse the safety, complications and effect of albumin infusion during RLVP on PPCD in children with severe ascites due to chronic liver disease

Method: Enrolled children with severe ascites underwent single time or repeated LVP with albumin infusion (figure 1). Maximal D6 value and delta (difference of baseline and D6) plasma renin activity (PRA) of RLVP were compared with SLVP. Worsening PPCD was defined as $\geq 1.5$ times increase of delta PRA between the sessions in the RLVP group. "At-risk" group was defined as any cirrhotic child with $\geq 2$ worsening PPCD episodes. Their outcome at 3 months and maximal follow-up were noted. Poor outcome was defined as failure of definitive therapy, need for liver transplantation or mortality.

Result: Table 1 shows baseline characteristics of 37 children (SLVP, $\mathrm{n}=17$; RLVP, $\mathrm{n}=20$ ). $92 \%$ had high PRA at the onset. The overall incidence of PPCD was 43\% (SLVP: $12 \%$, RLVP: $70 \%$; $p=0.003$ ). Baseline pediatric end-stage liver disease (PELD) score $>27$ was associated with PPCD (sensitivity: $90 \%$; specificity: $50 \%, \mathrm{p}=0.01$ ) in RLVP group. Worsening PPCD $(n=28 ; 54 \%)$ and at-risk children $(\mathrm{n}=9 ; 45 \%)$ were identified in 52 sessions of RLVP. In the RLVP group, PPCD occurred if cumulative ascitic fluid extraction volume was $>585 \mathrm{~mL} / \mathrm{kg}$ (sensitivity: $90 \%$; specificity: $50 \%, \mathrm{p}=0.01$ ), cumulative albumin infusion $<4.2 \mathrm{~g} / \mathrm{kg}$ (sensitivity: $93 \%$; specificity: $55 \%, \mathrm{p}=0.03$ ) and frequency of sessions were $>2.3 /$ week (sensitivity: $91 \%$; specificity: $57 \%, \mathrm{p}=0.02$ ). In those who developed PPCD, lowest volume of ascites extracted in SLVP and RLVP groups were 117 and $471 \mathrm{~mL} / \mathrm{kg}$ respectively. RLVP patients $(80 \%)$ were 
susceptible to asymptomatic, persistent hyponatremia (baseline vs. day 6 from first LVP, $131 \pm 4$ vs. $124 \pm 4 \mathrm{mEq} / \mathrm{L} ; \mathrm{p}=0.001$ ), hemodynamic changes (25\%), renal impairment (35\%), recurrence of ascites $(60 \%)$ and hospital readmission $(70 \%)$. At risk patients had worsening of PELD scores (baseline: $25 \pm 8$ vs. follow-up $31 \pm 6$; $\mathrm{p}=0.01)$ at 3 months. Poor outcome at maximal follow-up $(18 \pm 7.4$ months) was seen in $35 \%(\mathrm{n}=1, \mathrm{SLVP} ; \mathrm{n}=12$, RLVP) especially in the at-risk group $(\mathrm{n}=8 ; 89 \%)$.

Conclusion: Risk factors of PPCD with repeated LVP are baseline PELD $>27$, cumulative extracted ascites $>585 \mathrm{~mL} / \mathrm{kg}$, albumin infusion $<4.2 \mathrm{~g} / \mathrm{kg}$ and frequency of sessions $>2.3 /$ week. One-third have poor outcome, more in the at-risk group.

Figure 1: Work-up protocol

Study design of single time and repeated large volume paracentesis

$$
\checkmark
$$

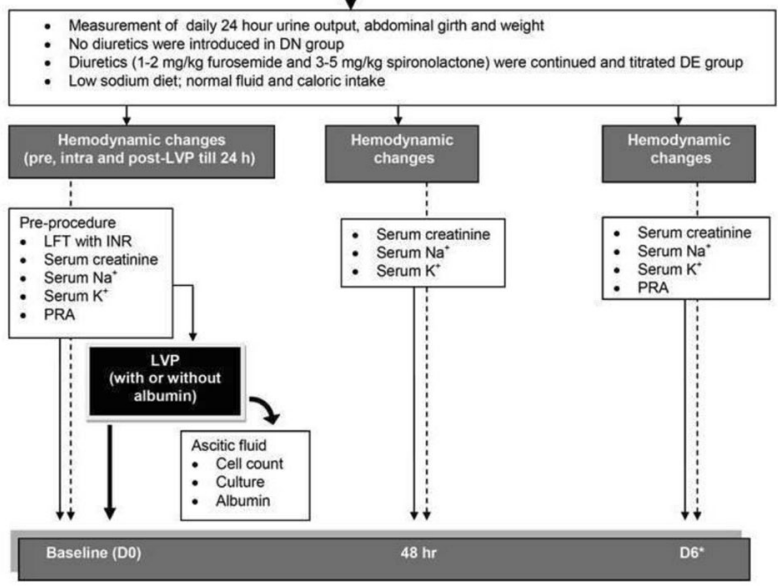

DN: diuretic naive, DE: diuretic exposed, LVP: large volume paracentesis, LFT: liver function test, INR: international normalized ratio, Na: sodium, K: potassium, PRA: plasma renin assay
In RLVP, D6* values were assessed from last performed LVP if $>1$ LVP were performed between D0 and D6 Table 1: Comparison of single time and repeated large volume paracentesis groups

\begin{tabular}{|c|c|c|c|}
\hline & $\begin{array}{l}\text { Single time LVP } \\
\text { group (n=17) }\end{array}$ & $\begin{array}{l}\text { Repeated LVP } \\
\text { group }(n=20)\end{array}$ & $p$ value \\
\hline Age $(y)$, boys $(n)$ & $9.8 \pm 4.4 .13$ & $8.3 \pm 5.1,15$ & $0.77,0.81$ \\
\hline \multicolumn{4}{|l|}{ Etiology of liver disease $(n)$} \\
\hline - Budd-Chiari syndrome & 3 & 7 & \\
\hline - Wilson disease & 5 & 5 & \\
\hline - Autoimmune liver disease & 1 & 4 & \\
\hline - Chronic hepatitis B & 2 & 1 & \\
\hline - Biliary atresia & 1 & 0 & \\
\hline - Alagille syndrome & 1 & 0 & \\
\hline - Cryptogenic & 4 & 3 & \\
\hline Duration of ascites (months) & $3.6 \pm 5.3$ & $7.1 \pm 11.0$ & 0.20 \\
\hline Weight Z-scores & $-1.69 \pm 2.21$ & $-2.51 \pm 1.84$ & 0.26 \\
\hline Height Z-scores & $-0.98 \pm 1.75$ & $-1.39 \pm 1.32$ & 0.46 \\
\hline Child-Pugh score (A/B/C) & $0 / 3 / 14$ & $0 / 4 / 17$ & 0.71 \\
\hline PELD score & $22 \pm 6$ & $28 \pm 10$ & 0.05 \\
\hline Volume of ascites extracted $(\mathrm{mL} / \mathrm{kg})^{q}$ & $143 \pm 77$ & $506 \pm 105$ & 0.001 \\
\hline Ascitic fluid infection (n) & 4 & 7 & 0.47 \\
\hline Baseline PRA ( $\mathrm{ng} / \mathrm{mL} / \mathrm{h}$ ) & $27.5 \pm 18.4$ & $29.1 \pm 20.7$ & 0.65 \\
\hline Maximum value of PRA $(\mathrm{ng} / \mathrm{mL} / \mathrm{h})$ & $28.3 \pm 21.2$ & $73.1 \pm 19.8$ & 0.01 \\
\hline Folds elevation from baseline" & $1.1 \pm 0.5$ & $6.4 \pm 5.3$ & 0.02 \\
\hline Number of subjects with PPCD (n) & 2 & 14 & 0.003 \\
\hline
\end{tabular}

Note: values expressed as mean \pm SD; LVP: large volume paracentesis PELD: pediatric endstage liver disease, PRA: plasma renin activity, PPCD: post-paracentesis circulatory dysfunction. ${ }^{t}$ Volume of ascites extracted $(\mathrm{mL} / \mathrm{kg})=$ Total volume $(\mathrm{mL}) \div$ Dry weight $(\mathrm{kg})$; dry weight was calculated after completion of LVP. 'Folds elevated $=$ Day 6 PRA (SLVP) or maximum noted PRA after 6 of any $L V P(R L V P)+$ Baseline PRA value. High PRA defined as PRA $>2$ SD of nomal. PPCD: PRA rise of $>50 \%$ of baseline value on day 6 of paracentesis to a level 2 standard deviation (SD) above age-appropriate mean pediatric cut-off. Normal mean PRA (+ 2SD) are variable for different age groups, 0.5-1 y: 6.27 (14.49); 1.4 y: 4.47 (10.71); 510 y: $2.33(4.79) ; 11-17 y: 2.07(6.11) \mathrm{ng} / \mathrm{mL} / \mathrm{h}$
PHT-58

Role of argon plasma coagulation (APC) in treatment of severe portal hypertensive gastropathy in patients with liver cirrhosis

\section{Mohamed Hassan Elnadry ${ }^{1}$}

${ }^{1}$ AL-Azhar Univerity, Egypt

Background: PHG mainly causes chronic blood loss and anemia in patients with cirrhosis, and can also cause acute hemorrhage. The bleeding risk associated with mild PHG is approximately $3.5-31 \%$, and the risk increases to $38-62 \%$ in severe PHG. APC is a non contact thermal method of hemeostasis. The study aimed to evaluate the efficacy and safety of APC as a tool of management of patients with severe portal hypertensive gastropathy due to liver cirrhosis.

Method: A prospective study was conducted from the period between January 2017 to July 2017 on 30 persons who fulfilling the designed inclusion criteria. Cirrhotic patients were prospectively included in this study if they presented with overt or occult bleeding related to portal hypertension. Success was defined as; control of bleeding, or significant $\mathrm{Hb}$ increase from pretreatment level, All patients were subjected to full history taking, through clinical examination, routine laboratory investigations, Occult blood in stool, abdominal ultrasound, and upper GIT endoscopy.

Results: All patients had severe portal hypertensive gastropathy, 19 patients $(63.3 \%)$ had their lesions in the body and fundus, 5 patients $(16.7 \%)$ had their lesions in the body, fundus and antrum, 4 patients (13.3\%) had their lesions in the body and 2 patients (6.7\%) had their lesions in the fundus only. APC was done for 30 patients with severe PHG and followed in serial sessions (3-4 weeks interval) up to 3 sessions and duration of follow up was 6 months, 22 patients $(73.3 \%)$ of severe PHG changed into mild form of PHG.

Conclusion: APC is effective and safe in the treatment of cirrhosisrelated sever portal hypertensive gastropathy and our study suggests that APC should be considered as the treatment of choice in sever portal hypertensive gastropathy.

\section{PHT-59}

Role of NGAL in early detection and prediction of mortality in hepato renal syndrome

\section{Mrunmaya Panda ${ }^{1}$}

${ }^{1}$ ABMH Pune, India

Background: Neutrophil gelatinase-associated lipocalin (NGAL) is a novel biomarker of acute kidney injury (AKI) of several etiologies and is increased in both serum and urine 1-3 days before the increase in serum creatinine $(8,9)$. Biomarker NGAL has been found to be low in pre-renal and HRS as compared to acute tubular necrosis (ATN) $(10,11)$. The prognosis of these patients is grim and possible early detection and treatment may improve the outcome.

Method: A total 100 patients with known chronic liver disease were recruited from June 2014 - December 2015, as an prospective case control study from OPD, IPD and ICU of Aditya Birla Memorial Hospital, Pune. Those with normal renal function were controls and those satisfying the criteria of HRS were cases. These patients were evaluated for severity of liver disease, baseline blood NGAL and followed for 3 months for survival.

Blood NGAL levels were compared between stable CLD and those with HRS. Blood NGAL was compared with other markers of severity of liver disease to find a correlation. Various factors including blood NGAL were evaluated as a predictor of mortality in CLD patients. The study was analysed as intention to treat analysis. 
Result: The mean age was not different between cases and controls $(51.4+11.59$ and $50.76+12.79$ years). Mean NGAL was higher in cases as compared to controls (465.86 vs. $161.74 \mathrm{ng} / \mathrm{ml}$, p 0.00 ). There was significant correlation between NGAL and MELD score in cases only and no correlation between NGAL and CPC in either group. Follow up of 3 months showed, mortality of $38 \%$ in cases and $34 \%$ in controls. NGAL was the only predictor of mortality in overall patients of chronic liver disease. The cut-off level of NGAL to be significant was $>250$ as a predictor of mortality.

Conclusion: HRS is associated with higher levels of NGAL. Blood NGAL may be an early marker of hepato-renal syndrome in patients of chronic liver disease and is predictable of mortality beyond levels of 250 .

\section{PHT-60}

Study of ascitic fluid in children with chronic liver disease in different variants of peritonitis

\section{Kamal Hossen}

${ }^{1}$ BSMMU, Bangladesh

Background: Chronic liver disease (CLD) is not uncommon in Bangladesh. Ascites is common feature of CLD patients. Ascites is a culture media for bacterial infection. Spontaneous bacterial peritonitis is a frequent complication of ascites in children with chronic liver disease. The rapid and effective diagnosis of peritonitis will reduced mortality.

Method: It was a cross sectional observational study, conducted at BSMMU, Dhaka, without interrupting standard care practice. The study was done from January 2016 to July 2017. During this period consecutive children CLD with ascites were included in this study. Samples were collected purposively who fulfilled inclusion criteria. The details history, physical examination findings and investigation reports were recorded in a predesigned standard data sheet. Ascitic fluid study specially physical appearance, cytology, total protein, LDH, Gram stain \& culture were done in all case.

Result: A total of 30 children were selected according to selection criteria. After ascitic fluid study, all patients were divided into two groups: Group I included five patients $(16.67 \%)$ with culture negative neutrocytic ascites (CNNA) in which the neutrophil count $\geq 250 / \mathrm{mm}^{3}$ and culture was negative indicate infected group. Group II, twenty five $(83.33 \%)$ patients in which the neutrophil count $<250 / \mathrm{mm}^{3}$ and negative culture indicate non infected group. None of our patients had spontaneous bacterial peritonitis (SBP) or bacterascites. Presence of fever, history of abdominal. pain and tenderness significantly higher in CNNA group $(\mathrm{p}<0.05)$. Ascitic fluid LDH or ascitic fluid total protein was not significant difference between two groups but ascitic serum LDH ratio $\geq 0.5$ was significantly higher in CNNA group. Conclusion: Culture negative neutrocytic ascites (CNNA) was the only variety of ascitic fluid infection in this study. Culture of ascitic fluid is not always diagnostic of infection. Ascitic serum LDH ratio $\geq$ 0.5 add to the diagnostic accuracy.

\section{PHT-61}

The 10-year chronic liver disease spectrum evolution in China: experience from the largest tertiary special hospital with $\mathbf{2 1 3 8 2}$ liver biopsy cases

George Lau ${ }^{1}$, Guofeng Chen ${ }^{2}$, Yiming $\mathrm{Fu}^{2}$, Cheng Wang ${ }^{4}$, Jing $\overline{C h e n}^{4}$, Qing Shao ${ }^{2}$, Bing $\mathrm{Li}^{2}$, Fan $\mathrm{Li}^{2}$, Zhongbin $\mathrm{Li}^{2}$, Vanessa $\mathrm{Wu}^{4}$, Yudong Wang ${ }^{4}$, George $\mathrm{Lau}^{3}$
${ }^{1}$ Humanity and Health Medical Group, Hong Kong; ${ }^{2}$ Beijing 302 Hospital, China; ${ }^{3}$ Humanity \& Health Medical Centre, Hong Kong, Hong Kong SAR, China, Hong Kong; ${ }^{4}$ Humanity and Health Research Centre, Hong Kong SAR, China, Hong Kong

Background: Beijing 302 hospital is the largest tertiary special hospital for liver diseases, which receives patients from all over the China. The patients visited our hospital is representative and we aimed to assess the pattern and evolution of Chinese people liver disease spectrum using data from our patients to provide the evidence for developing population-based effective strategy of chronic liver disease management.

Method: The clinical data of executive patients who underwent liver biopsy by any reasons from 2007 to 2016 were retrospectively collected, and the disease spectrum and biochemical parameters of each patient were recorded, such as sex, age, outcome, etc. Liver biopsy were guided by ultrasound, and HE, Masson and reticular fiber staining were performed routinely in liver tissues, immunohistochemical staining and special staining, such as Congo red and PAS, were optional selected if necessary.

Result: In total, 21382 liver biopsy cases were enrolled and analyzed. The top 5 etiology were chronic hepatitis B (CHB) $(44.1 \%)$, drug induced liver injury (DILI) (14.1\%), chronic hepatitis C (CHC) (9.2\%), cryptogenic liver disease (CLD) 6.9\%, and autoimmune liver diseases (AILD) (5.2\%). The rate of CHB patients decreased from $58.6 \%$ to $32.9 \%$, DILI increased from $3.7 \%$ to $13.7 \%$, CHC increased from $7 \%$ to $22.4 \%$, and then decreased to $2.2 \%$, CLD increased from $4.8 \%$ to $13.3 \%$, and AILD increased from $6.3 \%$ to $13.3 \%$. Besides, non-alcoholic steatohepatitis (NASH) increased from $4.1 \%$ to $11.4 \%$. Conclusion: Chronic liver disease spectrum in China has been changed, as the living standard of Chinese people rising recent decades. The incidences of DILI, CLD, NASH and AILD increased rapidly, while $\mathrm{CHC}$ decreased significantly, implying the DAAs therapy has achieved a great success.

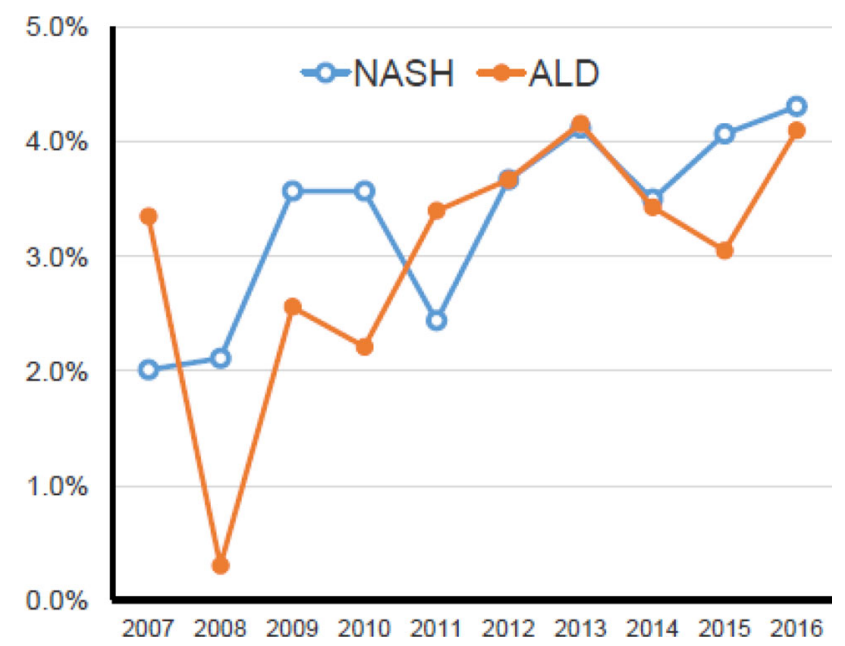




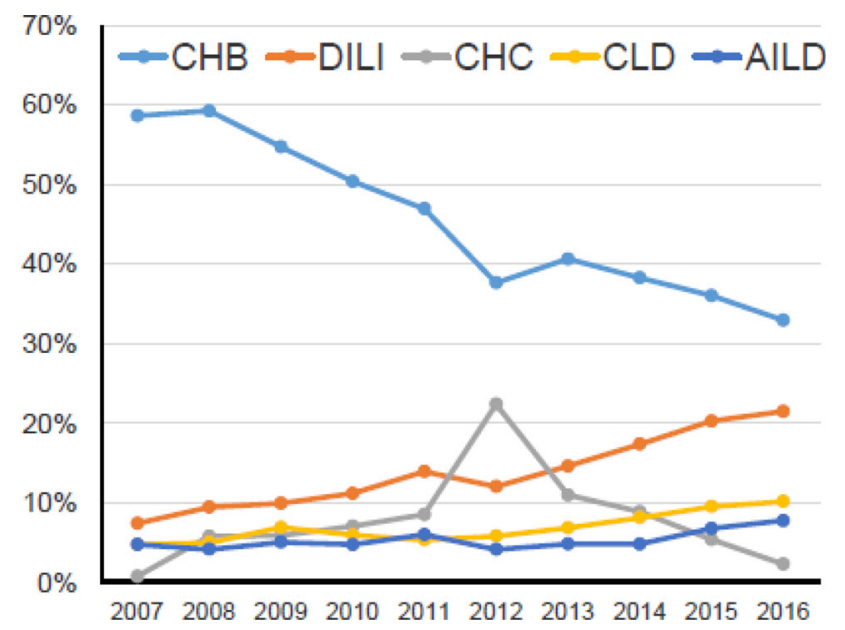

PHT-62

Urinary NGAL - A Novel Diagnostic and Prognostic Biomarker for Differential Diagnosis in Acute Kidney Injury in Cirrhosis

\section{Suhas Udgirkar ${ }^{1}$, Sanjay Chandnani ${ }^{1}$, Sunil Pawar ${ }^{1}$, Nikhil} Sonthalia $^{1}$, Shubham Jain ${ }^{1}$, Ravindra Surude ${ }^{1}$, Qais Contractor ${ }^{1}$, Pravin Rathi ${ }^{1}$

${ }^{1}$ T.N.M.c and B.Y.L Nair Hospital Mumbai, India

Background: Acute kidney injury (AKI) is prone to increase mortality in patients with cirrhosis by four fold. Due to higher volume of distribution and low muscle mass patient may have falsely low values of Sr.creatinine in cirrhosis. This study aimed to determine usefulness of urinary neutrophil gelatinase associated lipocalcin (uNGAL) for differential diagnosis of acute kidney injury in cirrhosis and for predicting transplant free mortality at 30 days.

Method: We prospectively enrolled 94 patients of cirrhosis at a tertiary care institute from western India. Urinary NGAL was measured by CMIA (Chemoluminescence Microparticle Immunoassay) method upon hospital admission (within 24 hrs or after development of AKI) in all patients. FeNa (fractional excretion of sodium) was calculated from urine and serum creatinine and urinary sodium values. Patients with urinary tract infection and anuria were excluded. Patients were followed at 30 days.

Result: 10 (9\%) patients had normal kidney function, 9 (11.37\%) stable chronic kidney disease, 32(29.50\%) prerenal azotemia, 33 (36.37\%) HRS and 10 (13.64\%) had intrinsic acute kidney injury (iAKI). Patients with HRS had uNGAL levels of (median 465.00 $[430.00,599.00])$ intermediate between prerenal azotemia $(65.50$ [47.75, 189.68] and iAKI $1217.50[1128.00,1375.00](\mathrm{p}-<0.001)$. FeNa values did not differentiate between prerenal and HRS group. Patients with SBP (n-13) $608 \mathrm{ng} / \mathrm{ml}$ and and hepatic encephalopathy $(\mathrm{n}-22)(552 \mathrm{ng} / \mathrm{ml})$ had higher mean uNGAL values than patients without SBP (354.09 ng/ml) (p- 0.1031) and hepatic encephalopathy $(343.5 \mathrm{ng} / \mathrm{ml}$ p-0.07849) respectively which not statistically significant. uNGAL level $>299 \mathrm{ng} / \mathrm{ml}$ and $>1102 \mathrm{ng} / \mathrm{ml}$ had highest AUROC in differentiating prerenal from HRS and HRs from iAKI respectively. $18(27 \%)$ patients died at 30 days. uNGAL levels were significantly higher in patients who died at 30 days (717.17 Vs 312.4 $\mathrm{ng} / \mathrm{ml})(\mathrm{p}-0.00338)$. uNGAL + MELD had higher sensitivity and specificity in predicting mortality than uNGAL and MELD in multivariate analysis (AUROC-0.818) with sensitivity and specificity of $72.22 \%$ and $82.89 \%$.
Conclusion: uNGAL level serves as an early marker in differentiating Prerenal, HRS and intrinsic AKI in cirrhotics from each other. MELD + uNGAL give better model in predicting mortality at 30 days.

PHT-63

The prevalence and microbiological profile of spontaneous bacterial peritonitis in cirrhosis of the liver with ascites

\section{$\underline{\text { Swati Singh }}{ }^{1}$ Rakesh Tandon ${ }^{2}$, Vineet Kumar Gupta ${ }^{2}$ \\ ${ }^{1}$ MD Anaesthesia and Critical Care, PGIMER Chandigarh, India; ${ }^{2}$ PSRI HOSPITAL, India}

Background: The microbiological profile of spontaneous bacterial peritonitis (SBP) in Indian patients with cirrhosis of the liver (CL) with ascites is limited. To study the prevalence of SBP and its microbiological profile in CL patients with ascites.

Method: One hundred consecutive patients with $\mathrm{CL}$ and ascites underwent diagnostic paracentesis. SBP was diagnosed when ascitic fluid culture was positive and polymorphonuclear leukocytes (PMN) were $>250 / \mathrm{mm}^{3}$. Two variants were: culture negative neutrocytic ascites (CNNA) when ascitic fluid PMN count was $>250$ cells $/ \mathrm{mm}^{3}$ but culture was negative, and monomicrobial nonneutrocytic bacterascites (MNB) when a single organism was grown but PMN count was

Result: Of hundred cirrhotic patients with ascites, SBP was found in $36 \%$. Among them, $22 \%$ were classical SBP, $72 \%$ were CNNA and $6 \%$ were MNB. E. coli was the commonest organism isolated; all strains of it were resistant to third generation cephalosporins (cefotaxim, ceftrixone, cefoperazone) whereas $78 \%$ were resistant to quinolones (levofloxacin, ciprofloxacin, norfloxacin). All E. coli isolates were sensitive to imipenem, but only $67 \%$ were sensitive to a combination of third generation cephalosporin and beta lactamase inhibitor.

Conclusion: SBP is common in patients with CL with ascites and is mostly caused by E coli. A high percentage of E coli are resistant to cephalosporins and quinolones, but sensitive to imipenum or a combination of 3rd generation cephalosporin and beta lactamase inhibitor.

\section{PED-1}

ratio of aspartate aminotransferase to alanine aminotransferase and alkaline phosphatase to total bilirubin in Wilsonian acute liver failure in children

\section{Afsana Yasmin ${ }^{1}$ MD Rukunuzzaman ${ }^{2}$, ASM Bazlul Karim ${ }^{3}$, $\overline{\text { Rubaiyat Alam }}^{1}$, Kamal Hossen ${ }^{1}$ \\ ${ }^{1}$ Resident,MD, Bangladesh; ${ }^{2}$ Associate Professor, Bangladesh; ${ }^{3}$ PROFESSOR, Bangladesh}

Background: Acute liver failure (ALF) due to Wilson disease (WD) is invariably fatal. Therefore, rapid diagnosis of WD is needed to start immediate management. The lack of sensitive and specific criteria for the rapid diagnosis of Wilsonian acute liver failure (WALF) has made this disease quite difficult to identify. The aim of study to determine the role of AST/ALT ratio and ALP/total bilirubin ratio for diagnosis of Wilsonian acute liver failure.

Method: It is a cross-sectional observational study. Sample was collected purposively who has fulfilled the inclusion criteria of ALF. Serum alanine aminotransferase, aspartate aminotransferase, alkaline phosphatase and total bilirubin were done in same blood sample. At the end of study, we have seen sensitivity and specificity of AST/ALT ratio and ALP/bilirubin ratio in diagnosis of WALF and compared 
clinical, hematological and biochemical parameters of children with acute liver failure due to Wilson disease with other causes of liver failure.

Result: Among total fifty-three consecutive patients of acute liver failure thirty-six are Wilsonian acute liver failure patients and nonWilsonian acute liver failure patients are seventeen. Among nonWilsonian liver failure patients, thirteen Hepatitis A, one hepatitis B, one hepatitis $\mathrm{E}$, one herpes simplex and one indeterminate hepatitis. Among the thirty-six WALF patients, thirteen had consanguinity $(36 \%)$ whereas in non WALF group only $5 \%,(\mathrm{p}=0.018)$. Female are commonly affected in WALF $(63 \%)$ compared with non WALF $(25 \%), p=0.008$. There is no significant difference in mean age of both groups. $78 \%$ patients are between five to ten years in WALF. $\mathrm{K}-\mathrm{F}$ ring is present in $73 \%$ of WALF group and none of non WALF group $(\mathrm{p}=0.001)$. Hemoglobin $(8.4 \pm 2.1$ vs $10.4 \pm 1.8$, p $<0.002)$, ALT $(117 \pm 187$ vs $429 \pm 353,<0.003)$, alkaline phosphatase $(159 \pm 131$ vs $245 \pm 95,<0.020)$, albumin $20.97 \pm 3.74$ vs $25 \pm 7.75, \mathrm{p}=0.021)$, ceruloplasmin $(11.6 \pm 7.1$ vs $21.5 \pm 14.33$, $<0.006)$ and INR $(2.9 \pm 0.7$ vs $3.59 \pm 1.6,<0.05)$ are lower in children with WALF compared to non WALF group. AST/ALT ratio is higher in children with WALF $(2.80 \pm 1.50)$ compared with non WALF $(1.68 \pm 0.84), \mathrm{p}<0.000$. There is no difference in Total bilirubin $(11.5 \pm 10.4 \mathrm{mg} / \mathrm{dl}$ vs $16.5 \pm 9.8 \mathrm{mg} / \mathrm{dl}, \mathrm{p}=0.09)$, AST $(248 \pm 325 \mathrm{IU} / \mathrm{L}$ vs $356 \pm 375 \mathrm{IU} / \mathrm{L}, \mathrm{P}=0.292)$ and $\mathrm{ALP} / \mathrm{TB}$ ( $35.64 \pm 48.30$ vs $37.64 \pm 72.66, \mathrm{P}=0.906)$ ratio in both groups. The sensitivity, specificity, positive predictive value, negative predictive value and diagnostic accuracy of AST to ALT ratio are 67\%, $94 \%, 96 \%, 57 \%$ and $75 \%$ respectively. The sensitivity, specificity, positive predictive value, negative predictive value and diagnostic accuracy of alkaline phosphatase to total bilirubin ratio are $28 \%$, $100 \%, 100 \%, 40 \%$ and $51 \%$ respectively.

Conclusion: From the presented study it may be concluded that AST/ ALT ratio may be an important screening test for early diagnosis of Wilsonian acute liver failure. ALP/Bilirubin ratio is highly specific though sensitivity is very low.

\section{PED-2}

Evalution of mean platelet volume and neutrophil-to-lymphocyte ratio in children with Chronic Hepatitis B patients

\section{$\underline{\text { Recep Tekin }}^{1}$, Fesih Aktar ${ }^{1}$, Muhammet Kosker ${ }^{2}$, Serhat Samanci $^{2}$ \\ ${ }^{1}$ Dicle University, Turkey; ${ }^{2}$ Diyarbakir Children'sHospital, Turkey}

Background: Indirect inflammatory markers such as mean platelet volume (MPV), neutrophil to lymphocyte ratio (NLR) and platelet to lymphocyte ratio (PLR) have been identified as markers of inflammation. The present study aimed to investigate the MPV, NLR and PLR in pediatric chronic hepatitis B patients.

Method: The total study population comprised of 94 pediatric patients with chronic hepatitis B and 88 healthy volunteers as the control group. Routine laboratory methods were used to measure white blood cell count (WBC), platelet count (PLT), neutrophil count, lymphocyte count, MPV, PDW, and NLR in all subjects of both the patient and control groups. A diagnosis of chronic HBV infection state is based on HBsAg positivity longer than six months.

Result: Age and sex distribution did not different among the groups. MPV ( $p=0.011)$ and NLR $(p=0.001)$ was significantly larger in the chronic hepatitis $\mathrm{B}$ patients group than in the control group. Lymphocyte count $(\mathrm{p}<0.001)$ and PLT $(\mathrm{p}<0.001)$ values were significantly lower in the study group. There were no significant differences in WBC and PLR between the study group and the control group. Negative correlation was found between PLR and MPV $(R 2=$
0.512, $\mathrm{p}=0.044)$ in study group. Positive correlation was found between PLR and NLR $(R 2=0.512, \mathrm{p}<0.001)$ in study group.

Conclusion: MPV and NLR levels may be considered as a marker of inflammation. We thought, future research is needed to investigate this relationship with longitudinal data to establish the association between these variables in pediatric chronic hepatitis B patients.

\section{PED-3}

Evalution of mean platelet volume and neutrophil-to-lymphocyte ratio in children with Chronic Hepatitis B patients

\section{$\underline{\text { Recep Tekin }}{ }^{1}$, Muhammet Kosker ${ }^{2}$, Fesih Aktar ${ }^{1}$, Serhat Samanci $^{2}$ \\ ${ }^{1}$ Dicle University, Turkey; ${ }^{2}$ Diyarbakir Children'sHospital, Turkey}

Background: Indirect inflammatory markers such as mean platelet volume (MPV), neutrophil to lymphocyte ratio (NLR) and platelet to lymphocyte ratio (PLR) have been identified as markers of inflammation. The present study aimed to investigate the MPV, NLR and PLR in pediatric inactive chronic hepatitis B patients.

Method: The total study population comprised of 94 pediatric patients with chronic hepatitis B and 88 healthy volunteers as the control group. Routine laboratory methods were used to measure white blood cell count (WBC), platelet count (PLT), neutrophil count, lymphocyte count, MPV, PDW, and NLR in all subjects of both the patient and control groups. A diagnosis of chronic HBV infection state is based on HBsAg positivity longer than six months.

Result: Age and sex distribution did not differ among the groups. MPV $(p=0.011)$ and NLR $(p=0.001)$ was significantly larger in the chronic hepatitis $\mathrm{B}$ patients group than in the control group. Lymphocyte count $(\mathrm{p}<0.001)$ and PLT $(\mathrm{p}<0.001)$ values were significantly lower in the study group. There were no significant differences in WBC and PLR between the study group and the control group. Negative correlation was found between PLR and MPV $(R 2=$ $0.512, \mathrm{p}=0.044)$ in study group. Positive correlation was found between PLR and NLR $(R 2=0.512, \mathrm{p}<0.001)$ in study group.

Conclusion: MPV and NLR levels may be considered as a marker of inflammation in pediatric inactive chronic hepatitis B patients. We thought, future research is needed to investigate this relationship with longitudinal data to establish the association between these variables in pediatric chronic hepatitis B patients.

\section{PED-4}

Correction of the state of the apoptotic-regenerative system of hepatocyte growth factor cytokines and tumor necrosis factor alpha (hgf, tnf-a) in children with chronic cholecystocholangitis

\section{Saidrakhim Nodirovich Lukmonov ${ }^{1}$, Kurbonboy Abdullayevich Madatov $^{1}$, Umid Abdullayevich Allazarov ${ }^{1}$ \\ ${ }^{1}$ Tashkent Medical Academy, Uzbekista}

Background: To investigate the effectiveness of the use of ascorutin and thiotriazoline in a complex therapy regimen in patients with impaired functional state of the liver in children with chronic cholecystocholangitis.

Method: The work was carried out on the basis of the endocrinological clinic of the Tashkent Medical Academy. To achieve the goal and solve the tasks, a detailed comprehensive examination of 78 children with chronic cholecystocholangitis, at the age of 7-18 years was carried out. 40 children received standard therapy, and 38 
children received standard therapy with thiotriazoline and ascorutin. The control group included 30 children without pathology.

Result: In patients with children who received thiotriazolin and ascorutin in a complex standard therapy, there was a positive tendency to reduce the level of HGF. The concentration of HGF in the serum of this group of children was higher and was $1115.77 \pm 320.27$ $\mathrm{pmol} / \mathrm{L}$ before treatment and $753.27 \pm 47.85 \mathrm{pmol} / \mathrm{L}$ after treatment, respectively $(\mathrm{p}<0.05)$. In comparison with the group of healthy children, this indicator was $458.57 \pm 59.73 \mathrm{pmol} / \mathrm{l}$ and was three times higher. In the study of the index of TNF-a in children of this group, the index was before treatment $740.63 \pm 179.30 \mathrm{pmol} / \mathrm{ml}$, and after completion of the course of therapy with thiotriazoline and ascorutin $357,99 \pm 92,02$ pmoles/ml $(\mathrm{p}<0,05)$, with the figures tend to converge tentatively to the corresponding level in healthy $(\mathrm{pN}>$ 0.05). The use of standard therapy also led to a decrease in the TNF- $\alpha$ value to a level of $457.77 \pm 81.89 \mathrm{pmol} / \mathrm{L}$ compared to the same indicator for treatment $(628.39 \pm 100.67, \mathrm{p}>0.05)$. However, the indices obtained as a result of such treatment still exceeded the similar group of comparisons determined in children $(\mathrm{pN}>0.01)$. The positive dynamics of TNF-a stabilization as a result of standard therapy was significant $(p<0.05)$, however, the tendency of its level decrease was noted somewhat more slowly. The use of a course of standard therapy with thiotriazoline and ascorutin in children with chronic cholecystocholangitis led to an increase in the level of the apoptotic regenerative reactivity index of hepatocytes. The stabilization of serum hepatocyte growth factors and tumor necrosis factor- alpha was noted, indicated by therapy. The dynamics of the index of the apoptotic regenerative reactivity of the hepatocyte was only tendentious $(p>0.05)$ and it was not possible to obtain reliable normalization of the levels of indices in the children of the comparison group.

Conclusion: Application in the treatment of schemes with ascorutin and thiotriazolin leads to a possible stabilization of the level of HGF, which, in our opinion, is due to cytoprotective and capillary stabilizing effect on endotheliocytes of sinusoids and hepatocytes as a whole.

\section{PED-5}

Age- And Sex-specific reference intervals of liver chemistries (ALT, AST, AKP, GGT) in Chinese children and its effect on natural history of chronic hepatitis B

\section{Peng $\mathrm{Hu}^{1}$, PENG $\mathrm{HU}^{2}$, Yunan Chang ${ }^{2}$, Hu $\mathrm{Li}^{2}$, Hong Ren ${ }^{2}$}

${ }^{1}$ Department of Infectious Diseases, Institute for Viral Hepatitis, The Key Laboratory of Molecular Biology for Infectious Diseases, Chinese Ministry of, China; ${ }^{2}$ Department of Infectious Diseases, Key Laboratory of Molecular Biology for Infectious Diseases (Ministry of Education), Institute for Viral Hepatitis, China

Background: Increasing evidences indicated that most pediatric laboratory analytes are performed in the context of age- and sexdependent dynamics. In China, however, age- and sex-specific reference intervals (RIs) for ALT, AST, AKP and GGT are not available. In this study, we aimed to establish the age- and sex-related RIs of ALT, AST, AKP and GGT in China and applied the new proposal ALT RI to identify the chronic hepatitis B virus infection.

Method: Children (1-14 years) were collected from healthcare unit of Children's Hospital of Chongqing medical university from April 2014 to November 2017. All children were assessed according to pre-defined exclusion criteria including illness, history of hepatobiliary surgery or hepatotoxic medication, risk factors of metabolic disease or inherited liver disease, underweight, overweight and obese. Age- and sex-specific RIs were determined by using percentile(3rd-97th) method. CHB children were diagnosed by persistently positive HBsAg (> 6 months).

Result: We provided the age- and sex-specific RIs of ALT, AST, AKP and GGT based on 4,232 Chinese healthy children (boys = 2,415; girls $=1,817$ ). ALT percentiles were stabilized over age and the lower limit of normal (LLN) were 11.3-14.7 U/L, 12.1-16.1 U/L in boys and girls respectively, the upper limit of normal (ULN) were 40.4-44.5 U/L, 38.9-45.4 U/L in boys and girls respectively. AST showed substantial age-specific decreased. LLN of AST were 26.6 U/L-15.7 U/L, 24.8-14.1 U/L in boys and girls respectively, ULN were 53.3-34.9 U/L, 50.8-34 U/L in boys and girls respectively. AKP showed complex pattern of change with age, a decline in the first 3 or 4 years was followed by a rise with sex-specific onset, peak and subsequent decline. LLN of AKP were 101.5-131.2 U/L, 46.6-139.9 $\mathrm{U} / \mathrm{L}$ in boys and girls respectively, ULN were 278.1-437.6 U/L, 234.2-351.9 U/L in boys and girls respectively. Based on the newly proposed cut-points of ALT, approximately $16.1 \%$ boys and $19 \%$ girls would be classified as chronic infection phase using the current ALT threshold of children's hospitals, but they were in chronic hepatitis phase.

Conclusion: Based on large healthy population, we established the gender- and age-specific RIs of ALT, AST levels for Chinese children. Meanwhile, newly proposed cutoff of ALT will benefit us to understand the natural history of CHB in children.

\section{PED-6}

Use of stool colour and hepatobiliary scintigraphy in the differentiation of biliary atresia from idiopathic neonatal hepatitis

\section{Shashi Bhushan Thakur ${ }^{1}$}

${ }^{1}$ BSMMU, Bangladesh

Background: Stool color observation and Hepatobiliary scintigraphy are two simple examination and test to diagnose biliary atresia (BA). So these are convenient and reliable methods to differentiate BA from other causes of neonatal cholestasis specially idiopathic neonatal hepatitis (INH).

Method: This cross-sectional observational study was conducted at BSMMU, Dhaka, Bangladesh, from January, 2016 to June, 2017 on purposively sampled infants who developed jaundice before three months of age, had direct bilirubin of $>20 \%$ of total with pale stool and dark urine. Detailed history was taken, thorough physical examination was done, stool color was observed for 3 days and were matched with BC infant stool color card, investigations such as LFT, USG, HIDA scan, and liver biopsy were done. Liver biopsy was taken as a gold standard for diagnosis.

Result: Seventy-two cases were included in the study and their mean age at admission was $87.3 \pm 37.2$ days. Median of serum gamma glutamyl transpeptidase (GGT) in BA was 570.0 U/L (range 45 - 4560 $\mathrm{U} / \mathrm{L}$ ) and in INH it was $138.0 \mathrm{U} / \mathrm{L}$ (range 40.0 - $1117.0 \mathrm{U} / \mathrm{L}$ ) and the difference between them was statistically significant. ( $p-0.000)$. Hepatobiliary scintigraphy was significantly positive $(n=46,86.8 \%)$ among BA than INH $(\mathrm{n}=7,13.2 \%)$ subjects $(\mathrm{p}<0.008)$. The sensitivity, specificity, positive predictive value, negative predictive value and accuracy of hepatobiliary scintigraphy were $86.8 \%, 42.1 \%$, $80.7 \%, 53.3 \%, 75 \%$ respectively. Stool color was persistently pale in $44(83.0 \%)$ cases of BA than $4(21 \%)$ of INH cases which was significant ( $p$ 0.000). The sensitivity, specificity, positive predictive value, negative predictive value and accuracy of stool color were $83.0 \%, 78.9 \%, 91.7 \%, 62.5 \%, 81.9 \%$ respectively. The sensitivity, specificity, positive predictive value, negative predictive value and accuracy of stool color and hepatobiliary scintigraphy when 
combined together came out to be $79.2 \%, 89.5 \%, 95.5 \%, 60.7 \%$, $81.9 \%$ respectively.

Conclusion: This study result showed that biliary atresia and idiopathic neonatal hepatitis can be differentiated by stool color and hepatobiliary scintigraphy.

PED-7

AARC-ACLF model is the best prognostic model in pediatric acute-on-chronic liver failure

\author{
Bikrant Bihari Lal ${ }^{1}$, Vikrant Sood ${ }^{1}$, Rajeev Khanna ${ }^{1}$, Seema \\ Alam ${ }^{1}$ \\ ${ }^{1}$ Institute of Liver and Biliary Sciences, New Delhi, India
}

Background: There are no validated prognostic models for pediatric ACLF. Our objective was to evaluate the applicability of existing prognostic models to pediatric ACLF. We also aimed to evaluate whether pediatric modification of these scores is superior to existing score.

Method: ACLF was defined as per APASL definition. Children dying or requiring LT were included under poor outcome and those surviving beyond 90 days were included in good outcome. PELD, CTP, CLIF-SOFA and AARC-ACLF scores were calculated. Worst parameter in the first 48 hours of admission was used for calculating the score. A modified pediatric CLIF-SOFA score was also calculated by replacing absolute creatinine with grades of AKI and by using age based hypotensive cutoffs. Pediatric modification of AARC-ACLF scores were based on AKI grades rather than absolute creatinine values. The scores were assessed for their ability to discriminate survivors and non-survivors.

Result: A total of 85 children with ACLF were included. The most common underlying CLDs were wilson's disease $(39,45.9 \%)$ and AIH $(30,35.3 \%)$. The most common acute precipitating event was viral hepatitis $(30,35.3 \%)$. Thirty-two children died or required liver transplant and 52 survived with native liver. When the various prognostic models were applied, AARC-ACLF score had the best AUROC for identifying children likely to have poor outcome [Figure 1]. At a cutoff score of more than 10, AARC-ACLF score predicted poor outcome with $90.6 \%$ sensitivity and $90.4 \%$ specificity with AUROC of 0.955 . CLIF-SOFA score $>10$ predicted poor outcome with $81.2 \%$ sensitivity and $90.4 \%$ specificity with AUROC of 0.924. PELD and CTP scores had poor discriminatory value with AUROC of 0.728 and 0.74 respectively. Pediatric modifications of AARC-ACLF scores and CLIF-SOFA scores did not improve their prognostic value. Survival with native liver was $100 \%, 86.7 \%$ and $15.2 \%$ in children with AARC-ACLF grades 1, 2 and 3 respectively [Figure 2].

Conclusion: AARC-ACLF score is the best prognostic score in pediatric ACLF. Children with AARC-ACLF grade 3 have $85 \%$ mortality and warrant an urgent liver transplant.
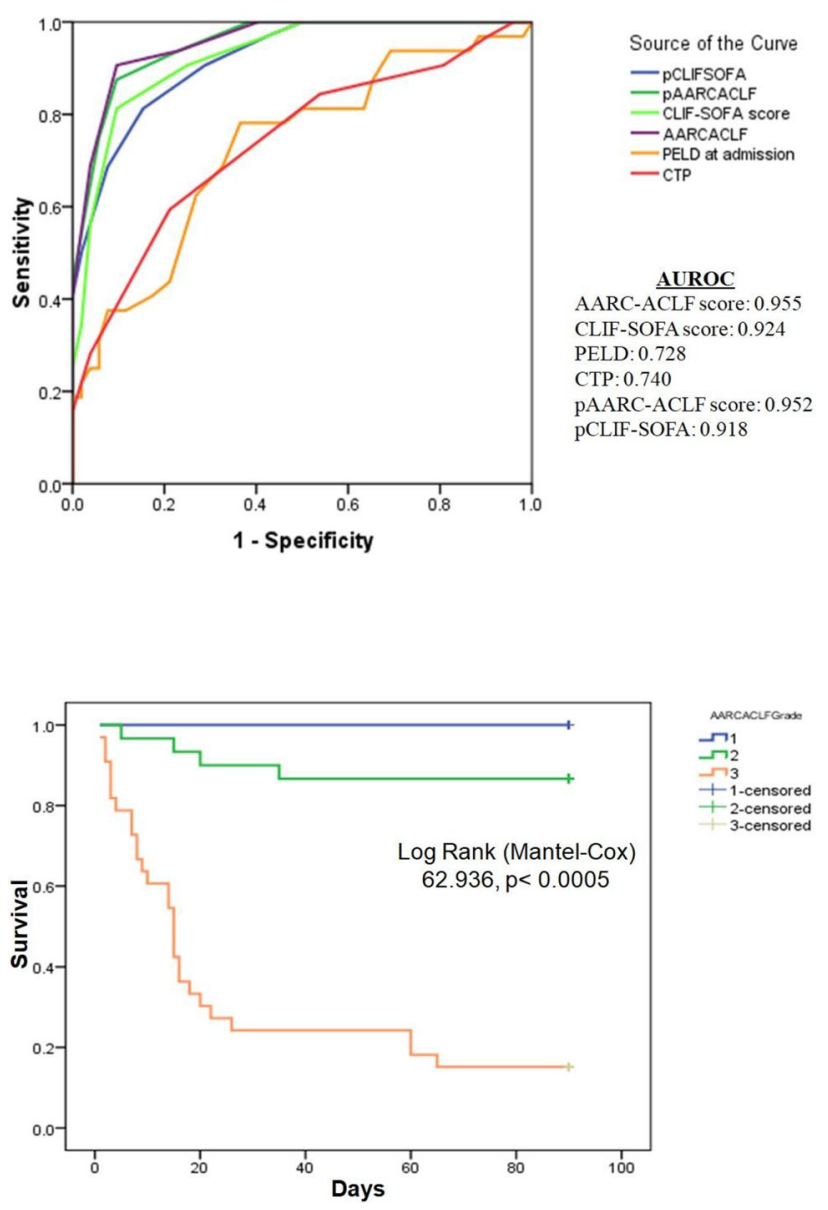

PED-9

Clinical spectrum of congenital hepatic fibrosis (CHF) and progression in children: experience from a tertiary care centre

Subhamoy Das ${ }^{1}$, Suvradeep Mitra ${ }^{2}$, Shrikanth $\mathrm{KP}^{4}$, Ashim Das ${ }^{3}$, Babu Ram Thapa ${ }^{5}$

${ }^{1} \mathrm{DM}$ Student, India; ${ }^{2} \mathrm{DM}$ Student, PGIMER, India; ${ }^{3}$ PGIMER, India; ${ }^{4}$ PGIMER, Chandigarh, India; ${ }^{5}$ Professor \& Head, India

Background: Congenital hepatic fibrosis (CHF) is a rare cause of chronic liver disease in children. We retrospectively analyzed the data of the patients seen over past 10 years to see the clinical spectrum of the disease as well as the progression.

Method: The data of all the cases diagnosed as CHF (with other causes of CLD being excluded) were retrieved from the hospital files. Their demographic features and clinical profiles were studied. Other causes of chronic liver diseases were excluded. They were found to be subjected to upper GI endoscopy, and abdominal ultrasound findings were recorded in almost all the patients. USG guided liver biopsies of these patients were reported by a single histopathologist, experienced in pediatric hepatoopathology.

Result: One hundred and three $(\mathbf{N}=\mathbf{1 0 3})$ cases of CHF were managed over last ten years from the year 2006 to 2016. Age of presentation of children with CHF was between 3 months to 15 years, (mean 38 months) with 49 (49\%) being infants. Male: female ratio was 2:1 (70 males: 33 females). Presentation was with cholestatic jaundice in $55(53 \%)$, abdominal distension (with or without 
organomegaly) in $53(51 \%)$ and UGIE bleeding in $16(15 \%)$ of the patients. An infant with cholangitic type of CHF had bilateral polydactyly in hands and the association is described in several syndromes with CHF (Figure 1). It was seen that majority of the patients in infant group presented with cholestatic jaundice (78\%). had pigmented stools in the neonatal period and 14 of $39(35 \%)$ had pale stools during the initial presentation.

Lliver was enlarged in all with firm to hard consistency and the left lobe was distinctly palpable in $95 \%$ of patients (not documented in 5 patients). Splenomegaly was present in $81(78 \%)$ of the patients. Most of the children had pallor, with mean hemoglobin being $8.44 \mathrm{~g} /$ dL. Ascites was present in 20 (19\%), and ultrasonography revealed alteration in the liver echotexture in $78 \%$ (80 patients) and 17 of 98 $(17 \%$, no data for 5 patients) patients had kidney involvement. Upper GI Endoscopy showed varices in 52 patients $(50 \%)$ with 16 of 52 $(30 \%)$ requiring sclerotherapy or endoscopic variceal ligation (EVL) for eradication of these varices. Eight patients $(8 \%)$ had familial association. Six patients (12\% of 49) in the infant group with episodes of recurrent cholangitis developed decompensated liver disease during follow up. Two patients developed hepatopulmonary syndrome and one patient was transplanted. One patient died of massive GI bleed.

Conclusion: CHF presents as cholestasis, portal hypertension and mixed. Cirrhosis described in 3rd decade but infantile cholangitic type had early cirrhosis. Sporadic was more common and firm hepatosplenomegaly with histology is diagnostic.

Table 8: CHF patients with kidney involvement $(\mathrm{N}=17)$

\begin{tabular}{|c|c|c|c|}
\hline & Investigation & $\begin{array}{l}\text { Number (\%) } \\
\text { Total (N=17) }\end{array}$ & $\begin{array}{l}\text { Family History of } \\
\text { Kidney disease }\end{array}$ \\
\hline Bilateral polycystic kidney & USG & $9(52)$ & 1 \\
\hline $\begin{array}{l}\text { Bilateral medullary sponge } \\
\text { kidney }\end{array}$ & USG and MRI & $2(12)$ & No \\
\hline $\begin{array}{ll}\text { Bilateral medullary } \\
\text { nephrocalcinosis }\end{array}$ & USG & $2(12)$ & No \\
\hline \begin{tabular}{|lr|}
\multicolumn{3}{|c|}{ Bilateral cortical calcification } \\
with renal parenchymal \\
disease
\end{tabular} & USG & $1(6)$ & No \\
\hline Horse shoe kidney & USG & $1(6)$ & No \\
\hline $\begin{array}{l}\text { Bilateral gross hypertrophic } \\
\text { kidney }\end{array}$ & USG & $2(12)$ & No \\
\hline
\end{tabular}

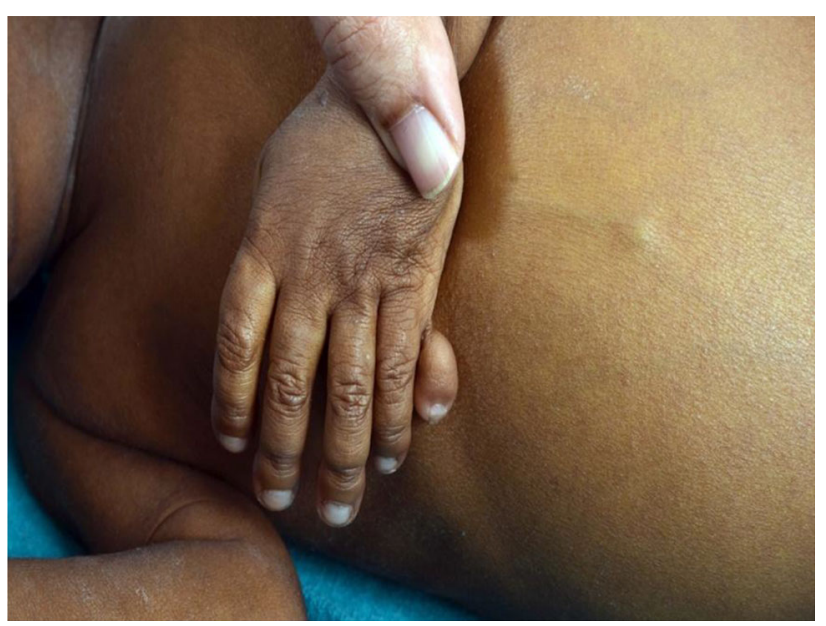

PED-10

Colonic changes in children with extrahepatic portal vein obstruction: prevalence, characteristics and predictors

Nagendra Kumar ${ }^{1}$, Anshu Srivastava ${ }^{1}$, Niraj Kumari ${ }^{1}$, Somit $\overline{M i t t a l}^{1}$, Surender Yachha ${ }^{1}, Z_{\text {Zafar Neyaz }}{ }^{1}$, Ujjal Poddar ${ }^{1}$

${ }^{1}$ Sanjay Gandhi Post Graduate Institute of Medical Sciences, India

Background: Colonic changes in portal hypertension are well described in adults with cirrhosis, but literature is scarce in children and extrahepatic portal venous obstruction (EHPVO). We studied children with EHPVO for a) prevalence and nature of colonic mucosal changes b) relation of colonic changes with disease duration, extent of splenoportal axis thrombosis, portal hypertensive gastropathy $(\mathrm{PHG})$ and $\mathrm{c}$ ) correlation between histological and endoscopic changes.

Method: Children $<18$ years of age with EHPVO (as per APASL criteria) were prospectively enrolled between April 2015 to March 2017. The demography, clinical presentation, laboratory parameters and imaging (extent of porto-venous axis thrombosis) were analysed. Full colonoscopy with biopsy from all segments was taken. Colonic findings were classified as varices (anorectal or colonic) and portal hypertensive colopathy (PHC: colitis like [hyperemia, ulcers] or vascular lesions [cherry red spot, spider angioma, vascular ectasia]). Morphometric analysis of colonic biopsies performed.

Result: 54 EHPVO children [41boys, median age 12 (4-18) years] were evaluated. Hematochezia was present in 12 (22\%). Rectal varices were seen in $51(94 \%)$, colonic varices in $2(3.7 \%)$ and PHC in $40(75 \%)$ cases. In patients with PHC, both vascular and colitis like lesions were present in $48 \%(\mathrm{n}=19)$, followed by only colitis like $(42 \%, \mathrm{n}=17)$ and only vascular $(10 \%, \mathrm{n}=4)$ lesions. In cases with PHC, 52.5\% ( $\mathrm{n}=21 / 40)$ cases had pan-colonic involvement with predominant left side changes $(71 \%, 15 / 21)$. Children with colopathy had higher prevalence of PHG (90\% vs 57\%; p $=0.01$ ), higher number of endotherapy sessions [6 (1-20) vs $1.5(0-8) ; p=0.03]$ and lower prevalence of large esophageal varices $(12.5 \%$ vs $43 \%$; $\mathrm{p}=$ $0.02)$ than those without colopathy. Clinico-laboratory profile and extent of portal venous thrombosis was similar in patients with and without PHC. Similarly, there was no significant difference in the colonic changes between patients with or without hematochezia. We did not find difference in colonoscopic findings in relation to anatomic variation in extent of portal venous axis thrombosis. Reversal of the colonic changes including punched out ulcers was seen in 2 cases after proximal spleno-renal shunt. EHPVO children had higher number of capillaries per crypt as compared to controls. None of the 
histologic morphometric changes had correlation with endoscopic changes.

Conclusion: Colonic changes (varices/PHC) are present in $>90 \%$, but $20 \%$ are symptomatic. PHC is pan-colonic with predominant left sided in 50\%. Punched out ulcer is a feature of PHC. PHG and eradication of esophageal varices predicts presence of PHC.
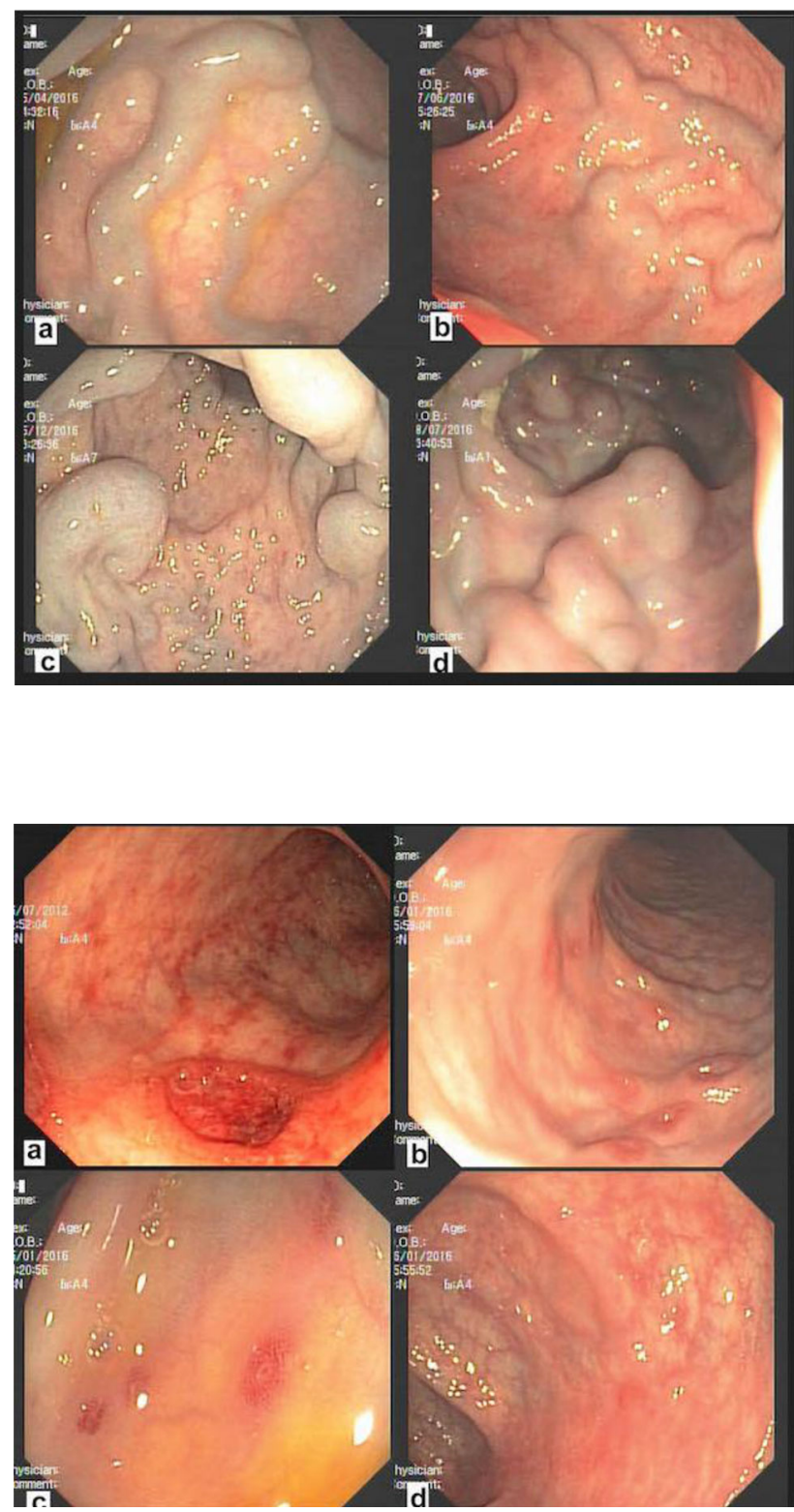

PED-11

Fanconi bickel syndrome: case series of 3 children Aruna S1, Anil V2, Lewin M1, Savita K1, Saurav J1, Vibhavari1, Indumathi CK1, Lewin S1, Department of Paediatrics1, Department of Paediatric Nephrology2, St.Johns Medical College Hospital, Bengaluru

\section{Aruna Sethuraman}

${ }^{1}$ St.Johns Medical College Hospital, India
Background: Fanconi-Bickel syndrome (FBS), also known as glycogen storage disease type XI (GSD XI)1, is a rare autosomal recessive disorder of carbohydrate metabolism. It is caused by mutations in the gene SLC2A2, which encodes for the facilitative glucose transporter GLUT22. The diagnosis of this disorder is often delayed, mainly being treated for proximal renal tubular dysfunction. We describe 3 children who presented at different ages, their followup and management

Method: This was a retrospective, crosssectional descriptive study, case records of consecutive children diagnosed with Fanconi Bickel syndrome were reviewed during July 2014 to September 2017 at St.Johns Medical College hospital. Diagnosis was based on clinical presentation of proximal renal tubular dysfunction, hepatomegaly, hypoglycaemia, supported by laboratory evaluation, liver biopsy findings and one child were confirmed with genetic testing. Family history, anthropometry was also noted. Follow up case records were also noted till the present date of September 2017.

Result: Clinical profile of three children diagnosed to have Fanconi Bickel syndrome were noted. The ages were 3 yrs, 6 months and 3 yrs respectively, with one female and 2 male children. Their age of complaints started at $2 \mathrm{y} 6 \mathrm{~m}$, from birth and $1 \mathrm{y} 6 \mathrm{~m}$ respectively and age of diagnosis were at $3 \mathrm{yrs}, 2 \mathrm{yr} 8 \mathrm{~m}, 3 \mathrm{yrs}$ respectively. Common presenting complaints were polyuria, polydipsia, short stature, genuvarum, abdominal distension, gross motor delay. One child had gum bleeds, epistaxis, fracture of long bone and the younger child at presentation had cataract with hypoglycemic seizures. All were born out of 3rd degree consanguinity with no other significant family history. On followup all three children had h/o recurrent wheeze and developed bronchopneumonia and developed diarrhea on and off. Anthropometry revealed short stature in all with features of rickets and hepatomegaly in all. Lab investigations were s/o Fanconi syndrome with normal liver and renal functions. The younger child had recurrent hypoglycemic seizures and two non hypoglycemic seizures whereas the other two children had asymptomatic hypoglycaemia with elevated triglycerides. Two children liver biopsy was done which was suggestive of glycogen storage disorder and the younger child at presentation was diagnosed with a genetic test.

All children were started treatment for proximal renal tubular dysfunction in the form of phosphate, bicarbonate, calcium and potassium supplementation. Two children are on active Vitamin D3 and cholecalciferol supplementation. Two children were immediately started on corn starch supplementation. The younger child in view of cataract, age of presentation and urine galactose positive was initially treated as galactosemia with withdrawal of breast feeding and lactose free formula.

Follow up till now is for $3 y r s, 2 y r s$ and $1 \mathrm{y} 8 \mathrm{~m}$ duration respectively. The younger child with early presentation was initially followed up like galactosemia, he continued to have hypoglycemic seizure on followup which subsided on starting cornstarch and regular feeds. All three children growth improved after supplementation, compliance issues being a major challenge.

Conclusion: Hypophosphatemic rickets with hepatomegaly one should consider diagnosis of Fanconi Bickel syndrome. Even on treatment it is a challenge to manage proximal RTA. These children also are prone for recurrent infections. 
PED-12

Histological pointers to progressive familial intrahepatic cholestasis: a five year spectrum analysis at a North Indian tertiary referral centre

\section{Kavita Gaur ${ }^{1}$ Puja Sakhuja', Kaushik Majumdar ${ }^{1}$, Geeta Chauhan $^{1}$, Seema Kapoor ${ }^{3}$, Mitali Mukherji ${ }^{2}$, Mohammed Faruq $^{2}$}

${ }^{1}$ GB Pant Institute of Postgraduate Medical Education and Research,New Delhi, India; ${ }^{2}$ Institute of Genomics and Integrative biology, India; ${ }^{3}$ Maulana Azad Medical College, India

Background: Diagnosing Progressive familial intrahepatic cholestasis (PFIC) is a challenge. Clinical manifestations of PFIC overlap with most neonatal cholestasis disorders. Though genetic analysis is considered gold standard, cost issues hinder mutational analysis. The histopathological spectrum of Indian cases of suspected PFIC has seldom been studied. The present study was carried out define the definitive histopathological clues to facilitate a prompt diagnosis.

Method: Cases of clinically/pathologically suspected PFIC were retrieved from the e-database at the Department of Pathology, GIPMER, New Delhi over five years (2013-2017).Clinical presentation and the presence/absence and distribution of cholestasis, hepatocyte ballooning, rosetting, portal tract changes,fibrosis (Ishak's score) and relevant histological findings were documented. Immunohistochemistry for canalicular multidrug resistance protein (MDR3) was performed on all cases. Histology was correlated with clinical and or biochemical data wherever available.

Result: Out of a total of 87 cases of metabolic liver disease analyzed in the same time period, PFIC comprised $(28 / 87=32.1 \%)$ cases. Mean patient age was 2.186 years with a male sex predilection $(2: 1)$. Clinical suspicion was noted in 18/28 (64.2\%) cases. Histology confirmed/suggested PFIC in 24/28 (85.7\%) cases. The most common presentation was jaundice (89.2\%). Mean alkaline phosphatase and gamma glutamyl transferase levels (where available) were $438.94 \pm$ $1.2 \mathrm{IU} / \mathrm{L}$ and $28.33 \pm 0.9$ respectively. The predominant histological picture in our series was of bland canalicular cholestasis (75\%) vis a vis giant cell hepatitis (17.8\%)/exclusive ductular pathology in $7.1 \%$ cases. Loss of MDR3 immunoexpression was not seen in our cohort suggesting a relative lack of PFIC3.Lack of lobular inflammation was another significant pointer $(89.2 \%)$. Follow up data was available in $46.4 \%$ cases.

Conclusion: Traditionally considered as an "orphan" disease, PFIC comprised a significant proportion of pediatric metabolic liver disease at our centre.Bland canalicular cholestasis was the most definitive histological pointer to PFIC. A diligent histopathological examination may be the most important key in unlocking pediatric PFIC in routine practice.

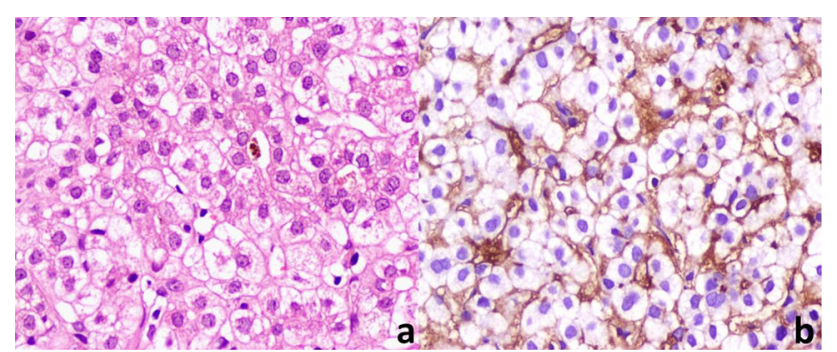

PED-13

Infants with congenital hepatic fibrosis: are they clinically different from those with EHBA?

\section{Sadhna Bhasin Lal ${ }^{1}$, Sadhna Bhasin $\mathrm{Lal}^{2}$, Subhamoy Das², Jagadeesh Menon $^{2}$, Ashim Das ${ }^{2}$, Babu Ram Thapa ${ }^{2}$ \\ ${ }^{1}$ Post Graduate Institute of Medical Education and Research, Chandigarh, India; ${ }^{2}$ PGIMER, Chandigarh, India}

Background: Congenital Hepatic Fibrosis (CHF) is an important cause of Cholestasis Of Infancy, presenting mainly in its Cholangitic Form. The clinical presentation at this age needs to be specifically profiled as it may be often confused with the more common problem of Biliary Atresia for which early Kasai's procedure is required, though not for CHF.

Method: This is a retrospective study. All children lesser than one year of age and diagnosed as CHF and EHBA on liver histology/ preoperatively were included in this study. Their demographic, clinical and biochemical details at first presentation were noted and compared.

Result: Fifty infants with histologically diagnosed CHF were identified, as against sixty-six children with EHBA, however 37 children presented to Pediatric Gastroenterology post Kasai procedure. The median age at presentation of infants with $\mathrm{CHF}$ was $5.67+2.71$ months and EHBA was $2.66+1.47$ months $(\mathrm{p}=0.022)$. A male to female ratio of 2.33:1 was noted, with average age of onset of symptoms was $43.56+74.9$ days in CHF group. In EHBA group, male to female ratio was 1.9:1, along with average age of onset of symptoms being $15.76+17$ days $(p=0.003$ w.r.t. CHF). Jaundice was the presenting complaint in $92 \%$ infants in CHF group and $97 \%$ in EHBA. Persistent clay colored stools seen in only $48 \%$ in $\mathrm{CHF}$ group and $89.6 \%$ in EHBA ( $p=0.00)$. In CHF and EHBA group moderate and massive hepatomegaly was comparable (76\% and $69 \%$ respectively, $\mathrm{p}=0.49$ ), however moderate to massive splenomegaly was found significantly more in $\mathrm{CHF}$ when compared to EHBA group (48\% and $10 \%$ respectively, $\mathrm{p}=0.001)$.

In EHBA group, surgery (Kasai procedure) was done in 55 children. In 11 children, surgery was not done due to advanced cirrhosis, and in 6 children it is not known. Average age of surgery was $73.98+18.63$ days. Outcome was known in $42 \%$ of the infants with CHF, out of which $66.7 \%$ decompensated and $33.3 \%$ were compensated \& $100 \%$ children who later decompensated had esophageal varices Vs. $43.3 \%$ in compensated group at presentation.

Mean Alanine Transaminase (ALT) levels and Aspartate Transaminase (AST) levels were $178.4+155.3 \mathrm{IU} / \mathrm{L}$ and $324.3+$ $297.8 \mathrm{IU} / \mathrm{L}$ in CHF group; and $181.55+122.5 \mathrm{IU} / \mathrm{L}$ and $217.59+$ $122.63 \mathrm{IU} / \mathrm{L}$ respectively in EHBA group (p value: 0.459 and 0.018 for ALT and AST respectively). Serum Bilirubin levels was $11.3+$ $7.8 \mathrm{mg} / \mathrm{dl}$ in CHF group, as against $9.94+3.3 \mathrm{mg} / \mathrm{dl}(\mathrm{p}=0.00)$ in EHBA group. Serum Alkaline phosphatase (ALP) levels were 603.2 $+345.9 \mathrm{IU} / \mathrm{L}$ and $718.97+389.13 \mathrm{IU} / \mathrm{L}$ respectively $(\mathrm{p}=0.974)$ in CHF and EHBA groups.

Conclusion: Infants with CHF present significantly later, are more likely to have intermittent clay colored stools \& moderate/massive splenomegaly, higher AST \& higher bilirubin w.r.t. to infants with EHBA. They also frequently have esophageal varices, which may be taken as an early indicator of decompensation in these children. 
PED-14

Infants with portal hypertension: Long-term endoscopic outcome of primary and secondary variceal prophylaxis in biliary atresia and extrahepatic portal venous obstruction

\section{Moinak Sen Sarma ${ }^{1}$, Surender Kumar Yachha ${ }^{3}$, Anshu Srivastava $^{2}$, Ujjal Poddar ${ }^{4}$ \\ ${ }^{1}$ Sanjay Gandhi Postgraduate Institute of Medical Sciences, Lucknow, India; ${ }^{2}$ Additional Professor, India; ${ }^{3}$ Professor and Head, India; ${ }^{4}$ Professor, India}

Background: Infants with portal hypertension are a high risk subset due to life-threatening variceal bleeding. There is paucity of data regarding the same. We aimed to evaluate the role of primary and secondary prophylaxis and the endoscopic outcome of infants with varices appearing before $1 \mathrm{y}$ of age.

Method: Of the all the infants undergoing endoscopy, the study recruited 2 groups who were not on pre-existing beta-blocker therapy: a) extrahepatic portal venous obstruction (EHPVO) and b) biliary atresia (BA) with successful portoenterostomy. Bleeders underwent secondary prophylaxis (endoscopic sclerotherapy). Those with small esophageal varices (EV) at screening were followed up. Primary prophylaxis (endoscopic band ligation \pm sclerotherapy) was performed when EV were $\geq$ Grade II with red colour signs (Paquet's classification). All patients underwent 2-3 weekly sessions till eradication of EV followed by 3-6monthly sessions for endoscopic surveillance. Beta-blockers were introduced on follow up for recurrence of varices, interval bleeding or large gastric varices

Result: Figure 1 shows the study flow and compares the differences in the endoscopic outcomes of the two groups. Secondary prophylaxis was overall required in 35/45 (78\%). 22\% (BA, $n=9 ;$ EHPVO, $n=1)$ with initial small varices ( $\leq$ grade II) required primary prophylaxis at 15 (12-84) months. Duration of follow-up was 84 (12-120) months. Hypersplenism, large EV, age at endoscopic intervention, total number of endoscopic sessions and recurrence of EV were significantly higher in EHPVO as compared to BA (fig. 1). $6 \%$ had sclerotherapy complications (bleeding ulcers and stricture). 22\% EHPVO required secondary eradication of EV. 16\% BA showed persistence of EV till last follow-up. Both groups had similar outcomes with gastric varices and portal hypertensive gastropathy (fig. 1). Indicators of recurrence of EV were persistence $(63 \%)$ of gastroesophageal varices despite primary eradication of $\mathrm{EV}$ or recurrence $(27 \%)$ of gastroesophageal varices 6 (3-9) months prior to recurrence. $12 \%$ developed isolated gastric varices, one of whom required glue injection. All severe portal hypertensive gastropathy (n $=6$ ) developing prior primary eradication became mild with recurrence of EV or gastric varices. Overall interval bleeding from varices during primary eradication occurred in 34\% within 11 (7-16) days from last endoscopic session. Beta-blockers were given in $67 \%$ (EHPVO, $\mathrm{n}=26$; BA, $\mathrm{n}=4$ ) for $22(19-36)$ months. Non-favourable vascular anatomy for portosystemic shunting was observed (100\% for Meso-Rex bypass; $81 \%$ for non-selective shunts) in EHPVO at onset and follow-up. 38\% BA were referred for liver transplantation.

Conclusion: Majority of EHPVO infants have high degree of portal hypertension as they are early onset bleeders with high recurrence of varices despite eradication. Polytherapy (regular endoscopic surveillance and beta-blocker therapy) is the only option in those with nonshuntable anatomy. In comparison, children with BA have slow progression and better outcome of portal hypertension.

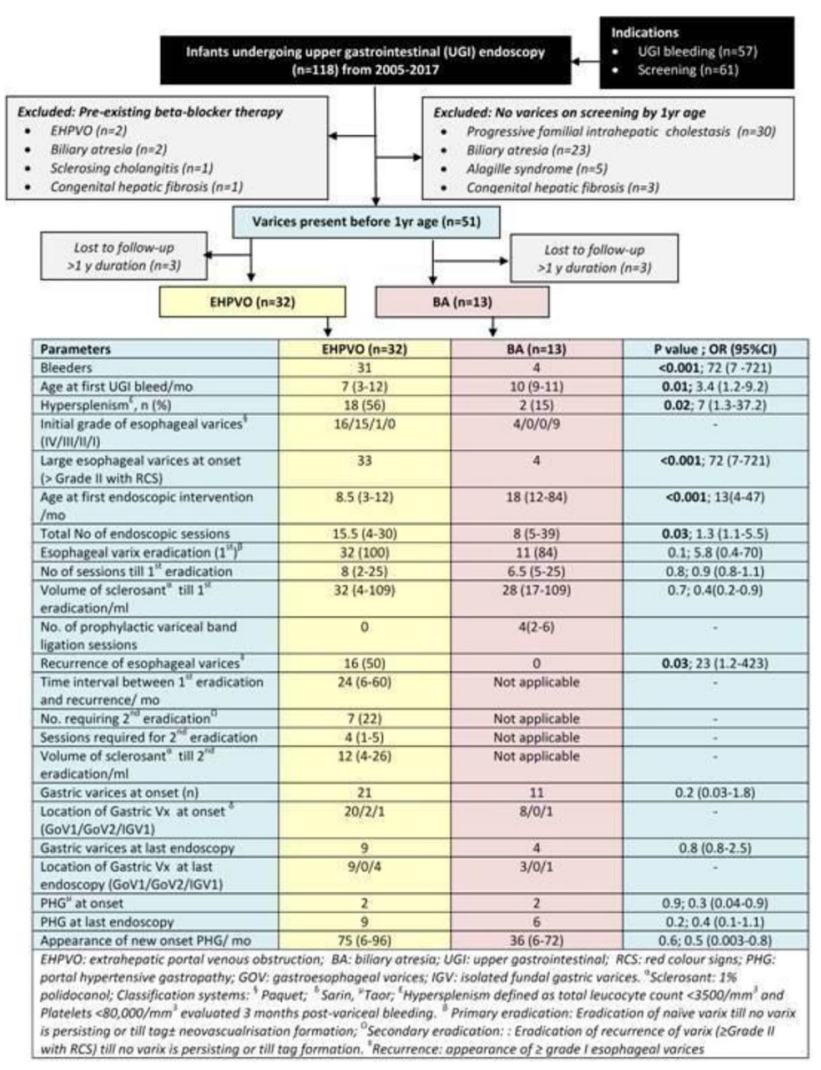

PED-15

Interventional radiological managements of portal vein stenosis in pediatric population after living donor liver transplantationS

Saurabh Gupta $^{1}$, Anubhav Khandelwal ${ }^{1}$, Abhay Kapoor ${ }^{1}$, Deepak Agarwal $^{1}$, Rohit Khandelwal ${ }^{1}$, Sanjay Saran Baijal ${ }^{1}$

\section{${ }^{1}$ MEDANTA- THE MEDICITY, India}

Background: Portal vein (PV) stenosis is uncommon complication after liver transplant with greater incidence in the pediatric recipients (7-27\%), seen after 6 months of surgery and at the surgical anastomosis due to scarring or fibrosis with neo intimal hyperplasia. Interventional radiology (IR) plays an important role in diagnosis with catheter portography being gold standard investigation and procedures like angioplasty with or without stenting for management of same.

Method: Observational study was conducted between December 2011-December 2017 on pediatric recipients with clinical suspicion of PV stenosis. Doppler ultrasonography and computed tomography were done for confirmation and positive cases underwent angioplasty with or without stenting. Peripheral PV puncture were taken under ultrasound guidance with stenosis demonstrated on venogram followed by balloon dilatation. Stenting was performed when elastic recoil of $50 \%$ or more was found after dilating twice. Puncture tracts were embolized with metallic coils $(n=3)$ and coil with glue $(n=1)$. Result: Over a period of 6 years, $5 / 87(5.7 \%)$ pediatric recipients with mean age of $43.9 \pm 65.7$ months at transplant were diagnosed with portal vein stenosis. Average interval between the transplant and first clinical presentation of portal vein stenosis was $8.6 \pm 2.3$ months. Treatments used were angioplasty $(n=3)$, angioplasty and stenting ( $n$ $=1$ ) and 1 patient with spontaneous extensive collateralization was kept on follow up. Post procedural pressure gradients in all patients 
were less than $5 \mathrm{~mm} \mathrm{Hg}$. On follow up, the patient with stenting had patent $\mathrm{PV}$ at 35 months. One patient did not have any clinical presentation to suggest recurrence after one month of procedure and is still on follow up. Two patients experienced recurrent stenosis with average interval of $1.5 \pm 0.5$ months after the first intervention and underwent repeat angioplasty. One of these had patent PV after 5 months and other had recurrent stenosis after 4 months and underwent a third angioplasty and is on follow up. There was no major complication.

Conclusion: Angioplasty with or without stenting is a safe and effective for management of PV stenosis in post liver transplant pediatric recipients.

\section{PED-16}

Low level of humoral immunity against $\mathrm{HBV}$ vaccine in Mongolian children and teenagers

\section{Yagaanbuyant Borjigon Dahgwahdorj ${ }^{1}$, Sereenen Khishigtogtoh ${ }^{2}$,} Murdorj Altankhuu ${ }^{2}$

${ }^{1}$ Mongolian National University of Medical Sciences, Mongolia;

2"Liver" center, Mongolia

Background: There is well known that Mongolia is endemic on viral hepatitis infection. Nationwide mandatory vaccination against HBV infection for every newborn was introduced since 1991 in Mongolia. It was key measure for prevention of acute viral hepatitis at this time. The aim of this study was to investigate the levels of vaccine induced antibody (HBsAb) in different age groups, who born after introduction mandatory HBV immunization program in Mongolia.

Materials and methods: In 2017, we have observed randomly selected 317 subjects $0-20$ years, living in Ulaanbaatar, Mongolia. In serum of all subjects tested for HBsAg and quantity HBsAb by CLIA diagnostic kit (Sysmex). Subjects with positive for HBsAg, rejected or not included in this study.

Results: In $31.9 \%$ of all subjects detected HBsAb, more than $10 \mathrm{IU} / \mathrm{L}$. The concentration of $\mathrm{HBsAb}$ was with a large difference more depending on age, decreasing tendency (table).

\begin{tabular}{|l|l|l|l|l|l|l|l|}
\hline $\begin{array}{l}\text { Age } \\
\text { groups } \\
\text { (years) }\end{array}$ & $\mathrm{n}$ & $\begin{array}{l}>10 \\
\text { IU/L } \\
(\%)\end{array}$ & $\begin{array}{l}<10 \\
\text { IU/L } \\
(\%)\end{array}$ & $\begin{array}{l}10-50 \\
\text { IU/L } \\
(\%)\end{array}$ & $\begin{array}{l}50-100 \\
\text { IU/L (\%) }\end{array}$ & $\begin{array}{l}100-250 \\
\text { IU/L (\%) }\end{array}$ & $\begin{array}{l}>250 \\
\text { IU/L (\%) }\end{array}$ \\
\hline $0-3$ & 38 & 71.1 & 28.9 & 34.2 & 15.7 & 7.8 & 13.1 \\
\hline $4-6$ & 67 & 38.9 & 61.1 & 22.3 & 10.4 & 4.4 & 1.4 \\
\hline $7-10$ & 104 & 24.1 & 75.9 & 18.2 & 2.8 & 1.9 & 0.96 \\
\hline $11-14$ & 60 & 23.4 & 76.6 & 16.6 & 5.0 & 0 & 1.6 \\
\hline $15-20$ & 48 & 18.8 & 81.2 & 10.4 & 2.0 & 0 & 6.25 \\
\hline Total & 317 & $31.9 \%$ & 68.1 & 19.5 & 6.3 & 2.5 & 3.4 \\
\hline
\end{tabular}

Conclusion:

Discussion and conclusion: Although not detected the ethology, after only 2 years of introduction this immunization a morbidity and mortality from acute viral hepatitis there was declined more 2 and 5 fold, respectively. In 2016, in whole country acute HBV infection registered 1.2 case in 10000 . So, a clinical efficacy of HBV vaccination is enough as in international level. But, humoral immunity against recombinant HBV vaccine in Mongolian children and teenagers developing significantly lacked than that international publication data.
PED-17

Total intrahepatic lipid content assessed by magnetic resonance proton spectroscopy in obese children with nonalcoholic fatty liver disease

Dariusz Marek Lebensztejn $^{1}$, Anna Bobruschociej ${ }^{1}$, Irena Bialokozkalinowska ${ }^{2}$, Eugeniusz Tarasow ${ }^{1}$

${ }^{1}$ Medical University of Bialystok, Poland; ${ }^{2}$ Lomza State University of Applied Sciences, Poland

Background: Ectopic hepatic lipid accumulation is closely related to the development of insulin resistance, which is regarded as one of the most significant risk factors of non-alcoholic fatty liver disease (NAFLD). Magnetic resonance proton spectroscopy (1H-MRS) is considered to be a reliable non-invasive diagnostic method in evaluating hepatic lipid content. Therefore, the aim of the study was to evaluate the total intrahepatic lipid content (TILC) in obese children with NAFLD.

Method: The prospective study included 100 obese children (aged 8-17 years, median 12 years) admitted to our Department to diagnose initially suspected liver disease. Patients with viral hepatitis (HCV, HBV, CMV), autoimmune (AIH), toxic and metabolic (Wilson's disease, alfa-1-antitrypsin deficiency) liver diseases were excluded. NAFLD was diagnosed in children with liver steatosis in ultrasound as well as elevated ALT serum activity. The degree of liver steatosis (graded according to Saverymuttu scale) were assessed in ultrasound. Advanced steatosis was defined as a score $>1$. TILC was assessed by $1 \mathrm{H}-\mathrm{MRS}$ in all children in relative units in comparison to unsuppressed water signal. Voxele's size $3 \times 3 \times 3 \mathrm{~cm}\left(27 \mathrm{~cm}^{3}\right)$ was localized in the right liver lobe in the manner that it would not comprise the vessels and bile ducts.

Result:NAFLD was diagnosed in 38 children. Significant positive correlation was found between TILC and ALT $(r=0.45)$, AST $(\mathrm{r}=0.46) \quad$ GGT $\quad(\mathrm{r}=0.39)$, triglycerides $(\mathrm{r}=0.3)$, HOMA-IR $(\mathrm{r}=0.2)$ and steatosis grade in ultrasound $(\mathrm{r}=0.65)$. TILC was significantly higher $(\mathrm{p}<0.001)$.

Conclusion:The higher TILC in NAFLD children assessed in $1 \mathrm{H}-$ MRS and its correlation with biochemical markers of liver injury, triglycerides concentration and insulin resistance suggest intensive ectopic hepatic lipid accumulation in examined group of children.

\section{PED-18}

Efficacy of ribavirin free directly acting antiviral regimens for hepatitis $\mathbf{C}$ infection in thalassemics: real life data

Akash Shukla ${ }^{1}$, Ruchir Patel $^{2}$, Meghraj Ingle ${ }^{2}$, Prashant Dhore ${ }^{3}$, Prabha Sawant $^{2}$, Akash Shukla ${ }^{1}$

${ }^{1}$ LTMMC LTMGH, India; ${ }^{2}$ LTMMC \& LTMGH, India; ${ }^{3}$ Seth GSMC \& KEMH, India

Background: Treatment of hepatitis C (HCV) infection in patients with thalassemia is challenging due to anemia, risk of hemolysis with ribavirin (RBV), concomitant iron overload and early onset of cirrhosis. There is paucity of data with RBV free regimen in thalassemics, especially in genotype (GT) 3 and adolescents. Here, we present real life data of outcomes of RBV free directly acting antiviral drugs (DAA) in a predominantly adolescent population of thalassemics.

Method: Prospective study involving thalassemia patients $(>12$ years) with detectable HCV RNA. Cirrhosis was difined by fibroscan values above $13 \mathrm{Kpa}$. GT1 patients with or without cirrhosis were given Sofosbuvir- Ledipasvir (treatment naïve for 12 weeks, 
treatment experienced for 24 weeks). GT 3 patients, naïve or treatment experienced were given Sofosbuvir-Daclatasvir for 12 weeks (without cirrhosis 12 weeks, with cirrhosis 24 weeks). Primary endpoint was sustained viral response (SVR 12). Secondary end-points were increase in blood transfusion requirements and adverse events. Result: 43 patients (adolescents -27 ; adults -16 ) with thalassemia major (34 patients) or thalassemia intermedia (9 patients) and having detectable HCV RNA levels were studied. GT 1 was the predominant GT with 29 patients and GT 3 was found in 14 patients. Seven patients (5- GT 1 and 2- GT 3) had cirrhosis. The median fibroscan values were $10.2 \mathrm{Kpa}(4.2-33.1 \mathrm{Kpa})$. Seven patients were previously treated with interferon and ribavirin containing regimens (3- genotype 1 and 4- genotype 3). All patients with cirrhosis were treatment naive. The mean haemoglobin value at baseline was $9.6 \mathrm{~g} / \mathrm{dl}$. Rapid virological response (RVR) was achieved in $41 / 43$ patients. Patients who did not achieve RVR were both of genotype 1, treatment naïve and without cirrhosis. One of these achieved SVR. SVR was achieved in 39 out of 40 patients who followed up. 3 patients were lost to follow up post treatment completion (1- genotype 3 treatment naïve without cirrhosis; 2- genotype $1\{1$ treatment naïve with cirrhosis and 1 treatment naïve without cirrhosis\}). Hence SVR was seen in $97.5 \%$ patients (39/40) on a per protocol basis and 90.7\% (39/43) on an intention to treat analysis. None of the patients required blood transfusion above their usual regimen while on treatment. Side effects in the form of fatigue ( 5 patients) and dry cough ( 3 patients) were seen. No severe adverse events were noted. There was no treatment discontinuation due to adverse events.

\begin{tabular}{|c|c|c|c|c|}
\hline & Regimen given & $\begin{array}{l}\text { Duration } \\
\text { (weeks) }\end{array}$ & RVR achieved & SVR achieved \\
\hline $\begin{array}{l}\text { Treatment naïve cirrhotics } \\
\text { genotype } 1(n=5)\end{array}$ & $\begin{array}{l}\text { Sofosbuvir+ } \\
\text { Ledipasvir }\end{array}$ & 24 & $5 / 5(100 \%)$ & $5 / 5(100 \%)$ \\
\hline $\begin{array}{l}\text { Treatment naïve non- } \\
\text { cirrhotics genotype } 1(n=21)\end{array}$ & $\begin{array}{l}\text { Sofosbuvir+ } \\
\text { Ledipasvir }\end{array}$ & 12 & $19 / 21(90.5 \%)$ & $18 / 19(94.75 \%)$ \\
\hline $\begin{array}{l}\text { Treatment experienced non- } \\
\text { cirrhoticsgenotype } 1(\mathrm{n}=3)\end{array}$ & $\begin{array}{l}\text { Sofosbuvir+ } \\
\text { Ledipasvir }\end{array}$ & 12 & $3 / 3(100 \%)$ & $3 / 3(100 \%)$ \\
\hline $\begin{array}{l}\text { Treatment naïve cirrhotics } \\
\text { genotype } 3(n=2)\end{array}$ & $\begin{array}{l}\text { Sofosbuvir+ } \\
\text { Daclatasvir }\end{array}$ & 24 & $2 / 2(100 \%)$ & $2 / 2(100 \%)$ \\
\hline $\begin{array}{l}\text { Treatment naïve non- } \\
\text { cirrhotics genotype } 3(n=8)\end{array}$ & $\begin{array}{l}\text { Sofosbuvir+ } \\
\text { Daclatasvir }\end{array}$ & 12 & $8 / 8(100 \%)$ & $7 / 7(100 \%)$ \\
\hline $\begin{array}{l}\text { Treatment experienced non- } \\
\text { cirrhotics genotype } 3(n=4)\end{array}$ & $\begin{array}{l}\text { Sofosbuvir+ } \\
\text { Daclatasvir }\end{array}$ & 12 & $4 / 4(100 \%)$ & $4 / 4(100 \%)$ \\
\hline Total & & & $41 / 43(95.35 \%)$ & $39 / 40(97.5 \%)$ \\
\hline
\end{tabular}

Conclusion: RBV free DAA regimens in usual doses are safe and efficacious in adolescent and adult patients with thalassemia. These do not cause any increase in need for blood transfusion during the therapy.

\section{PUBH-1}

Hepatitis $\mathrm{C}$ infection in the eastern mediterranean region: the current burden of disease and a road map for achieving the WHO global health sector strategy goals

Jonathan Schmelzer ${ }^{1}$, Sarah Blach ${ }^{1}$, Samantha Brandon ${ }^{1}$, Chris Estes $^{1}$, Ivane Gamkrelidze ${ }^{1}$, Helen Nde ${ }^{1}$, Devin Razavi Shearer ${ }^{1}$, Sarah Robbins ${ }^{1}$, Homie Razavi ${ }^{1}$

${ }^{1}$ CDA Foundation, United States

Background: The 69th World Health Assembly passed a resolution to eliminate viral hepatitis by 2030 . Epidemiological assessment and predictive modeling are needed to develop strategies to achieve this goal. This study quantifies the current hepatitis $\mathrm{C}$ disease burden in the Eastern Mediterranean Region (EMRO) and proposes a strategy for achieving the World Health Organization (WHO) Global Health Sector Strategy (GHSS) goals for Hepatitis by 2030.

Method: HCV disease burden models were developed for 20 countries and aggregated into a regional model which took into consideration new infections, disease progression, mortality and cured to estimate historic and future burden of disease. Regional averages were applied to country populations when country-specific data were not available. Intervention scenarios were developed within the regional model and projected outcomes related to the size of the $\mathrm{HCV}$-infected population by disease stage were assessed from 2016 to 2030.

Result: In 2016, there were an estimated 14.7 million viremic infections in the region, $17 \%$ of which had been previously diagnosed (770,000 newly diagnosed) and 770,000 of which had initiated treatment that year. Maintaining the current treatment and diagnostic paradigm would result in an estimated $30 \%$ reduction in total HCV infections and $3 \%$ to $9 \%$ increase in liver-related morbidity and mortality by 2030 .

To achieve the GHSS goals of diagnosing $90 \%$ of total infections and reducing liver-related mortality by $65 \%$ by 2030 , the region would need to treat an estimated 760,000 patients in 2017 , gradually dropping to 710,000 in 2025 , while gradually increasing the number diagnosed annually to 880,000 by 2025 . Additional prevention efforts will need to be coordinated across the region in order to achieve the GHSS goal of a 90\% reduction in new infections by 2030 .

Conclusion: Achieving the GHSS goals is feasible with a slight increase in the number of annually diagnosed cases. Prevention efforts, easing age restrictions and the expansion of access to highly curative treatment will be necessary components.

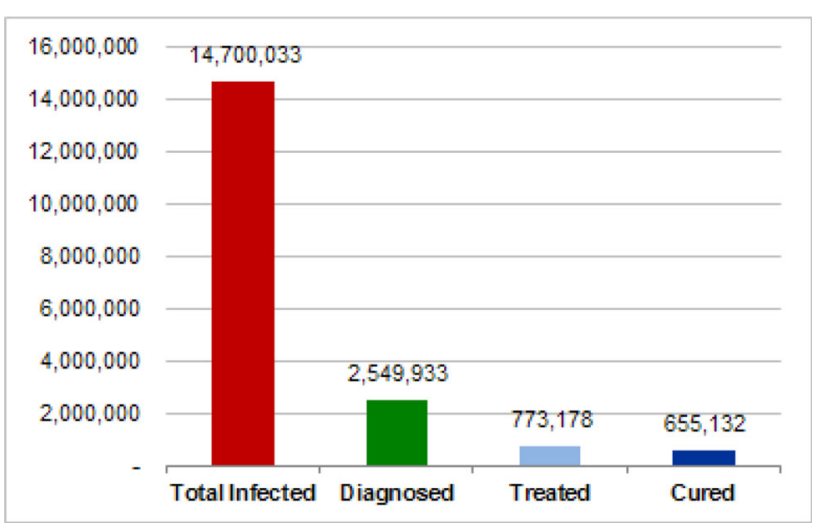

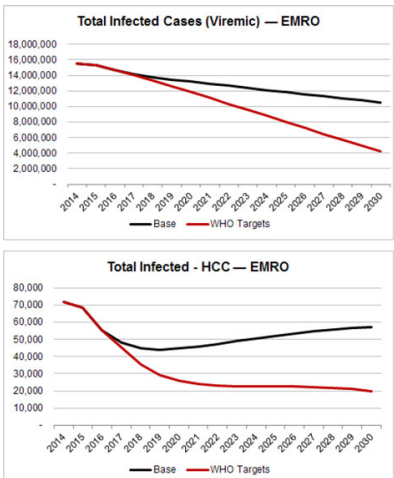

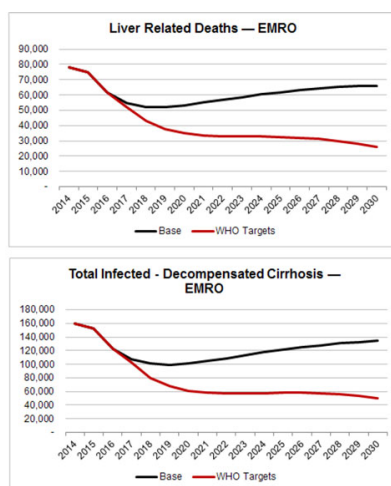




\section{PUBH-3}

Prevalence of hepatitis $B$ and hepatitis $C$ virus infections among nurses in a tertiary hospital in Mongolia

\section{Nyamsuren Naranzul ${ }^{1}$, S Ariunaa ${ }^{1}$, Oidov Baatarkhuu ${ }^{1}$ \\ ${ }^{1}$ Mongolian National University of Medical Sciences, Mongolia}

Background: M.Colombo, W.Lange studies showed that $30-40 \%$ of people became chronic after suffering from Hepatitis $\mathrm{B}$ and $\mathrm{C}$ virus, about $50 \%$ of chronic cases transformed into primary liver cancer. There are few studies in our country were conducted on hepatitis among healthcare professionals, particular nursing personnel. The study was conducted to identify of hepatitis B and C virus among nurses and make recommendations to prevent and control of Hepatitis $B$ virus and Hepatitis $C$ virus.

Method: We carried out cross-sectional study among selected nurses, to determine surface antigen of hepatitis B virus and antibodies to hepatitis $\mathrm{C}$ virus. For identification of these antibodies and antigen, and validation of results Elisa tests from CTK Biotech Company (USA) and simplifying diagnostics were used.

Result: There were 598 nurses from the First Central Hospital, the Second Central Hospital, the Third Central Hospital, Hospital of Ministry of Justice and Internal Affairs, and the National Center of Maternal and Child Health who participated in the study. From 5 hospitals 598 nurses surveyed and revealed the hepatitis B virus surface antigen positive $18.9 \%$, hepatitis $\mathrm{C}$ virus antibodies in $23.1 \%$, $\mathrm{B}$ and $\mathrm{C}$ viruses detected by $1.2 \%$ combined. There is an urgent need to provide knowledge to medical personnel regarding standards during procedures, concerning hepatitis infections, monitoring and improve technology used during procedures.

Conclusion: The study identified that 43.2 percent of nurses surveyed on hepatitis $\mathrm{B}$ and $\mathrm{C}$ viruses were detected; it shows a high prevalence among the nurses.

\section{PUBH-4}

Disease burden of chronic hepatitis $\mathrm{C}$ virus (HCV) infection in mongolia: potential impact of attaining world health organization (WHO) 2030 goals

Chris Estes ${ }^{1}$, Samantha M Brandon ${ }^{1}$, Betty Chiang ${ }^{2}$, Tsendsuren S Oyunsuren $^{4}$, Pagbajabyn Nymadawa ${ }^{3}$, Homie Razavi ${ }^{1}$

${ }^{1}$ Center for Disease Analysis, United States; ${ }^{2}$ Gilead Sciences, United States; ${ }^{3}$ Mongolian Academy of Medical Sciences, Mongolia;

${ }^{4}$ Mongolian Academy of Sciences, Mongolia

Background: Chronic HCV infection is a major cause of advanced liver disease, including cirrhosis and hepatocellular carcinoma (HCC) in Mongolia. Disease progression modeling was used to quantify the future health burden under two scenarios.

Method: HCV infection and related sequelae were tracked between through 2030. Baseline assumptions were extracted from the literature, using Mongolian data where available. One scenario was developed based on continuation of the status quo (base scenario), where annual treated patients gradually decline as the diagnosed population is depleted. Another scenario was developed to reduce future $\mathrm{HCV}$ infection to reach WHO goals for disease burden reduction by 2030 (aggressive scenario), where annual newly diagnosed and treated increase to a maximum of 10,000 and 15,000 annually, respectively, while new infections decline by $90 \%$ in the coming decade.
Result: In 2016, there were an estimated 188,100 viremic HCV infections, 56,800 prevalent advanced stage ( $\geq F 3$ or HCC) cases and 1280 liver-related deaths. Under the Base scenario, total infections are expected to decline by $25 \%(141,300)$ by 2030 , largely due to mortality as the infected population ages.

Under the aggressive scenario, viremic cases in 2030 decline by $>80 \%$ as compared to the base scenario to 24,100 cases. Likewise, prevalent cases of advanced liver disease decline by $>75 \%(10,050$ cases in 2030 as compared to 42,770 cases under base scenario) while liver deaths in 2030 decline by $>60 \%$ (330 deaths as compared to 920 deaths under base scenario).

Conclusion: Under the status quo, HCV prevalence in Mongolia will decrease by 2030 , but cases of advanced liver disease and liver-related deaths will continue at high levels. Increasing diagnosis along with treatment can lead to significant reduction in total infections, mortality, and morbidity.

\section{PUBH-5}

Clinical - epidemiological characteristics of the HCV infected people who inject drugs (PWID). A Greek prospective descriptive analysis

Hariklia Kranidioti $^{1}$, Spilios Manolakopoulos ${ }^{1}$, Christina Chatzievagelinou $^{2}$, Adonis Protopapas ${ }^{4}$, Margarita

Papatheodoridi $^{5}$, Konstantinos Zisimopoulos ${ }^{7}$, Eftychia Evangelidou', Pinelopi Antonakaki ${ }^{1}$, John Vlachogiannakos ${ }^{5}$, Christos Triantos $^{7}$, Ioannis Elefsiniotis ${ }^{6}$, John Goulis ${ }^{4}$, Maria Mela $^{2}$, Olga Anagnostou ${ }^{1}$, Christos Tsoulas ${ }^{3}$, Melanie Deutsch ${ }^{1}$, George Papatheodoridis ${ }^{5}$

${ }^{1}$ Medical School of National and Kapodistrian University of Athens, Greece; ${ }^{2}$ Evangelismos - Ophthalmiateion Athinon - Polycliniki Hospitals, Greece; ${ }^{3}$ Gilead, Greece, Greece; ${ }^{4}$ Medical School of ?ristotle University of Thessaloniki, Greece; ${ }^{5}$ Medical School of National and Kapodistrian University of Athens, Laiko General Hospital, Athens, Greece; ${ }^{6}$ National and Kapodistrian University of Athens, Greece; ${ }^{7}$ University Hospital of Patras, Greece

Background: HCV infection is very common among people who have a history of drug use, while the drug use is the main route of the HCV spread. In Greece, it is estimated that 17,000 current/recent PWID have HCV viremia. The aim of our study was to explore the clinical and epidemiological characteristics of a large cohort of untreated HCV infected PWID.

Method: This is a descriptive analysis of prospectively collected data among PWID with Chronic Hepatitis C. Since 1st of June 2017 until now we have selected data from six outpatient tertiary liver clinics (4 in Athens, 1 in Thessaloniki and 1 In Patras) in order to register epidemiological and clinical characteristics of infected PWID.

Result: We have included 800 PWID with chronic hepatitis C $(78.5 \%$ males, mean age $42 \pm 10$ years) who have not received antiviral treatment with all oral Direct Acting Antivirals (DAAs) until 1st of June 2017. One third (35\%) of the PWID were treatment experienced with pegIFN $\alpha /$ Ribavirin without achieving SVR. Almost one third $(30 \%)$ of the patients had comorbidities (diabetes mellitus, hypertension, psychological disorders etc.). According to the social history, $70 \%$ were active smokers and $27 \%$ alcohol users, $67 \%$ were unemployed, $34 \%$ had education $>12$ years and $29 \%$ were married. The majority of the patients $(65 \%)$ were attending addiction programs; $57 \%$ of them were under opiate substitution therapy with methadone and $36 \%$ with buprenorphine. Of the PWID, 36\% reported sporadic or systemic drug use. Co-infection with HIV and HBV was present in $1.4 \%$ and $2.9 \%$, respectively. The genotype distribution was $20.5,4.6$, $3.3,61$ and $10 \%$ for genotype $1 \mathrm{a}, 1 \mathrm{~b}, 2,3$ and 4 , respectively, without 
significant differences among the participating centres. Mean serum HCV RNA levels were $5.3 \times 106 \pm 3.3 \times 107 \mathrm{IU} / \mathrm{mL}$ and $59 \%$ of the patients with genotype 1a had HCV RNA levels $>800.000 \mathrm{IU} / \mathrm{mL}$. The mean liver stiffness was $9 \pm 7 \mathrm{kPa}(2.8-44 \mathrm{kPa})$, whereas $21 \%$ of the patients had cirrhosis (7\% decompensated) with two of them having $\mathrm{HCC}$ as well. Almost half of the patients (51\%) had F0-F1 stage of liver disease defined as stiffness $\leq 7 \mathrm{kPa}$.

Conclusion: Our real life data confirm that genotype 3 is predominant in Greek HCV PWID. One third of such patients have evidence of comorbidities and approximately one fifth cirrhosis. It is noteworthy that half of the patients have early disease (F0-F1 fibrosis) and thus high probability of remaining without access to DAA if any priority criteria are adopted.

\section{PUBH-6}

Gilead sciences' support of global efforts toward increasing screening, access, and linkage to care for chronic hepatitis B patients

Toan Luu Vo ${ }^{1}$, Anand Reddi ${ }^{1}$, Colleen Hurley ${ }^{1}$, John Flaherty ${ }^{1}$, Joonwoo Bahn ${ }^{2}$, Katherine Macbride ${ }^{1}$, Kelsey Grossman ${ }^{1}$, Lorna Davis Robinson ${ }^{1}$, Manika Lall ${ }^{1}$, Marjoleine Op Den Brouw ${ }^{3}$, Scott Williams ${ }^{1}$, Betty Chiang ${ }^{1}$

${ }^{1}$ Gilead Sciences, United States; ${ }^{2}$ Gilead Sciences, Korea, Republic of; ${ }^{3}$ Gilead Sciences, United Kingdom

Background: Gilead Sciences, Inc. supports the efforts of government agencies, professional and community-based organizations, payors, and healthcare providers (HCPs) who are committed to increasing screening and linkage to care (SLTC) and access for CHB patients around the world.

Method: Multiple departments within Gilead including Access Operations and Emerging Markets (AOEM), Clinical Research, Medical Affairs, Government Affairs and Policy, Public Affairs, and the Independent Medical Education Department (IMED) support programs related to SLTC and access to treatments for chronic hepatitis B patients.

Result: The FOCUS program partners with health systems, governments, and harm reduction orgs to build scalable, sustainable, and innovative SLTC paradigms. Since 2016, 15 FOCUS partners from the US have screened $>63,000 \mathrm{HBV}$ pts $(1.2 \mathrm{~K}$ diagnosed and $72 \%$ linked to care).

Through investigator-sponsored research (ISR) and external collaborations, Gilead is supporting 5 active and 1 planned SLTC studies. Gilead's AOEM team has 6 active HBV ISR studies providing access to 7 countries that address unmet needs in resourcelimited settings such as Latin Am., Africa and Asia, as well as publicprivate partnerships demonstrating Gilead access.

Gilead's AOEM team collaborates with regional partners to introduce high-quality, branded HBV drug in low- and middle-income countries, and generic drug manufacturers to produce highquality, low-cost generic versions of TDF for developing countries. Gilead was the first pharmaceutical company to join the medicine distribution alliance in 2011 with the Medicine Patent Pool, which in turn sub-licenses drugs to generic drug companies.

The Government Affairs and Policy team supports global access to Gilead therapies by leveraging evidence, amplifying best practices, and engaging policy makers, influencers, and advocates to drive political leadership and evidence-based policy development. Working closely with think tanks, academic institutions, and multilateral organizations, this team supports initiatives to advance and strengthen health systems and access to HBV SLTC.

Corporate Grants support the efforts of community-based orgs and public health entities to educate their constituents about HBV and also address barriers to care, reduction of disparities, and the importance of testing and treatment. Gilead Public Affairs works closely with community and advocacy orgs to raise awareness of HBV. In 2016, Gilead created a documentary profiling 2 families impacted by HBV with the goal of raising awareness, decreasing stigma, and encouraging SLTC, as appropriate.

The Gilead IMED supports programs that expand the knowledge and skills of HCPs to manage HBV. In 2017 alone, > 35,000 HCPs worldwide participated in HBV SLTC education programs supported by Gilead.

Conclusion: Gilead Sciences is firmly committed to supporting strategies toward HBV awareness, screening and linkage to care through partnerships with governments, professional societies, community-based organizations, and HCPs.

\section{PUBH-7}

High prevalence of chronic hepatitis c seropositivity among unrecognized high risk populations on the south northern of Thailand; a prospective, pilot study

Kriangsak Charoensuk ${ }^{1}$, Sarawut Suwanpatoomlerd ${ }^{2}$, Gingkan Hanlumyuang ${ }^{2}$, Kulpreeya Ekkaewnumchai ${ }^{4}$, Phutcharaporn Deeyangwai $^{3}$, Somrat Sritrakool ${ }^{3}$, Woramon Jiamsripong ${ }^{1}$, Wiboon Uthaisaengsuk $^{1}$, Orawan Chaiyamahapurk ${ }^{1}$

${ }^{1}$ Buddhachinaraj Hospital School of Medicine, Thailand; ${ }^{2}$ Lomkao Hospital, Thailand; ${ }^{3}$ Lomsak Hospital, Thailand; ${ }^{4}$ Nongphai Hospital, Thailand

Background: Chronic hepatitis $\mathrm{C}(\mathrm{CHC})$ is one of the major cause of liver related mortality. Previous study, the prevalence in general Thai population is lower than $2 \%$ across age group and areas. A large number of $\mathrm{CHC}$ treatment in national registrations were observed especially the south northern area of Thailand. The aim of study was to determine the prevalence of $\mathrm{CHC}$ in this area of thailand and also to determine the possible risk factors associated with infection in this population.

Method: A Prospective, multicenter, pilot study was conducted among emergency (ER) and out patients department (OPD) attendees, 2 sites in Phitsanulok (Buddhachinaraj referal hospital) and 3 sites in Petchabun province (Lomkao, Lomsak and Nongphi, the rural hospital), by using a standardized protocol.Patients, who agreed to participate, $\geq 18$ yearsold, completed a questionnaire with 1 or more risk factors for chronic hepatitis $\mathrm{C}$ identification and were tested for $\mathrm{HCV}$ antibodies.

Result: A total of 955 patients were included and screened.The median age was 53 years (IQR 37-69 years) and 52.5\% were male. Of these, 535 patients $(56.1 \%)$ had more than one risk factors. Overall prevalence of $\mathrm{HCV}$ antibody positive was $8.6 \%$. The highest prevalence was observed among patients in Lomsak hospital 16.7\% (30/ 180), followed by Lomkao Hospital $12.8 \%$ (30/235), Nongphi hospital $5.0 \%$ (9/180), Buddhachinaraj referal hospital 3.8\% (9/240). Among this $\mathrm{CHC}$ patients were male $(81.6 \%)$. A significantly higher prevalence of patients in Pethabun province than patients in Phitsanulok province was identified $(11.6 \%$ vs $3.6 \%, \mathrm{p}<0.001)$. The $\mathrm{CHC}$ associated risk factors was analysed and shown in Fig. 1. There was no significant increase in prevalence among baby boomers group $(\mathrm{p}=0.42)$. History of tattoos and piercing $(32.7 \%)$, Intravenous drug use; IVDU (24.5\%) and alternative medicine (22.4\%) were the major possible causes of $\mathrm{CHC}$ infection in this area.

Conclusion: This study shows a significantly high prevalence of $\mathrm{CHC}$ seropositivity. Tattoos, piercing, IVDU are the major causes of CHC infected patients. The targeted screening guideline or policy for identified at-risk groups are recommended. 


\section{PUBH-8}

How well are we aware of the immune status of hepatitis B and hepatitis $\mathrm{A}$ in chronic hepatitis $\mathrm{C}$ patients? a multicenter retrospective study in Turkey

Nevin Ince $^{1}$, Ayhan Balkan', Mustafa Uguz ${ }^{14}$, Nefise Oztoprak Cuvalci $^{3}$, Ilknur Esen Yildiz ${ }^{13}$, Tugba Yanik Yalcin ${ }^{15}$, Kenan Ugurlu $^{2}$, Neslihan Celik ${ }^{12}$, Gul Durmus ${ }^{6}$, Gulsen Iskender ${ }^{8}$, Seher Ayten Coskuner ${ }^{5}$, Mustafa Dogan ${ }^{7}$, Pinar Ergen ${ }^{11}$, Ramazan Gozukucuk $^{10}$, Muhammed Bekcibasi ${ }^{4}$

${ }^{1}$ Infectious disease, Turkey; ${ }^{10}$ Hisar Hospital, Department of Infectious Diseases and Clinical Microbiology, Turkey; ${ }^{11}$ Istanbul Medeniyet University, Goztepe Training and Research Hospital, Turkey; ${ }^{12}$ Regional Training and Research Hospital, Department of Infectious Diseases and Clinical Microbiology, Erzurum, Turkey;

${ }^{13}$ Rize University School of Medicine, Turkey; ${ }^{14}$ Silifke State Hospital, Turkey; ${ }^{15}$ Sivas Numune Training and Research Hospital, Turkey; ${ }^{2} 25$ Aralik State Hospital, Turkey; ${ }^{3}$ Antalya Training and Research Hospital, Turkey; ${ }^{4}$ Bismil State Hospital, Department of Infectious Diseases and ClinicalMicrobiology, Turkey; ${ }^{5}$ Bozyaka Training and Research Hospital, Department of Infectious Diseases and Clinical Microbiology, Turkey; ${ }^{6}$ Bursa Y $\tilde{A}^{1 / 4 k s e k}$ Ihtisas Training and Research Hospital, Turkey; ${ }^{7}$ Corlu State Hospital, Department of Infectious Diseases and Clinical Microbiology, Turkey; ${ }^{8}$ Dr A.Y. Ankara Oncology Training and Research Hospital, Turkey; ${ }^{9}$ Gaziantep University School of Medicine, Turkey

Background: Hepatitis A, B and C are among the major public health problems in our country and in the world. Recent advances in antiviral therapy with direct-acting antivirals have led to significant improvements in sustained virologic response. National vaccination programs for hepatitis B (HBV) and hepatitis A (HAV) were implemented in the child age group in our country in 1996 and 2012, respectively. However, HAV and HBV immunity rates are thought to be low in adult chronic hepatitis $\mathrm{C}$ patients past the vaccination period. The aim of this study is to highlight the seroprevalence status of HAV and HBV in chronic hepatitis $\mathrm{C}$ patients on whom direct antiviral treatment is planned and to emphasize vaccination of seronegative ones.

Method: A national, multicenter study of HCV infected patients was conducted from July 2016 to October 2017. Serological tests of HBsAg, antiHBs, and antiHAV IgG were evaluated by ELISA, and the files of HCV infected patients at the age of 18 or over who applied to 15 hospitals in 13 different cities of our country were screened.

Result: Eight hundred twenty-eight patients from 15 centers were included in the study. Of those patients, $362(43,8 \%)$ were males while $466(56,2 \%)$ were females, and mean age was found to be 57,85 $\pm 14,75$ years (18-86 years). As a result of the examinations, HBV/ $\mathrm{HCV}$ coinfection was found to be $2,4 \%$ (n:20) while antiHBs positivity was $46,9 \%$ (n: 389 ). Of the 610 patients evaluated in terms of antiHAVIgG serology, $88,85 \%$ (n: 542 ) of them were found to be antiHAVIgG positive while $11,14 \%$ (n: 68) of them were found to be antiHAVIgG negative. Approximately half of $\mathrm{HCV}$ positive patients (47.8\%, n: 396$)$ were found to be antiHBs negative. In addition, a significant proportion of the patients $(26,32 \%, \mathrm{n}: 218)$ were not examined in terms of Hepatitis A serology. The proportion of patients not examined in terms of HBsAg and antiHBs was found to be $1.9 \%$ (n: 16$)$ and $1.8 \%$ (n: 15), respectively.

Conclusion: Screening of other vaccine-preventable hepatitis agents such as HAV and HBV should not be neglected because of the possibility of fulminant hepatitis in the presence of existing viral hepatitis in the case of a liver disease like chronic hepatitis C. Our study shows that significant amounts of seronegativity can be found in patients with $\mathrm{HCV}$, and that these tests may be overlooked at times. Completion of hepatitis A and B vaccination schemes in seronegative individuals after screening will be helpful in preventing complications that may develop.

\section{PUBH-9}

The current and future disease burden of hepatitis B in the general population and among five year olds in the Eastern Mediterranean Region

Jonathan Schmelzer ${ }^{1}$, Chris Estes ${ }^{1}$, Devin Razavi Shearer ${ }^{1}$, Sarah Blach $^{1}$, Samantha Brandon ${ }^{1}$, Ivane Gamkrelidze ${ }^{1}$, Helen Nde ${ }^{1}$, Sarah Robbins ${ }^{1}$, Homie Razavi ${ }^{1}$

${ }^{1}$ CDA Foundation, United States

Background: Accurate national estimates of chronic hepatitis B (CHB) are needed to devise national and regional strategies. Previous studies have either provided prevalence estimates based on a literature review or have depended on regional models. This study quantifies the prevalence of CHB in the Eastern Mediterranean Region among the general population and five year olds from 2016 through 2030 using historical prevalence estimates combined with country level modeling.

Method: A literature review was conducted for hepatitis B surface antigen (HBsAg) prevalence among the general population (by age) and e-antigen prevalence among women of child bearing age. A dynamic country-level transmission and disease burden model was used to estimate the impact of vaccination, Hepatitis B immune globulin, treatment of mothers, aging and disease progression and mortality in the infected population in each country. Results were then aggregated to the region and regional averages were applied to populations of countries without available data.

Result: HBsAg prevalence data was available and models were developed for 18 countries representing $92 \%$ of the region's population. In 2016, the regional CHB prevalence was estimated at $2.2 \%$ (CI: $1.9-2.9 \%$ ) corresponding to 14.9 million (CI: $12.9-19.5$ million) infections after the impact of perinatal prophylaxes was taken into consideration. There were an estimate 1.2 million total cases of cirrhosis, 111,000 cases of decompensated cirrhosis and 112,000 cases of hepatocellular carcinoma (HCC). Among five year olds, the regional prevalence was estimated to be $0.5 \%$ (CI: $0.4-0.7 \%$ ) corresponding to 76,100 infections (CI: 67,300-110,000).

By 2030, total CHB infections in the EMRO region is projected to decline by $9 \%$ in the general population and $6 \%$ among five year olds. However, total cirrhosis, decompensated cirrhosis and HCC are expected to increase by $8 \%, 4 \%$ and $22 \%$ respectively.

Conclusion: This utilization of disease progression modeling; provides a more accurate epidemiological assessment on the current state and future projection of chronic hepatitis B in the EMRO region than is otherwise available.

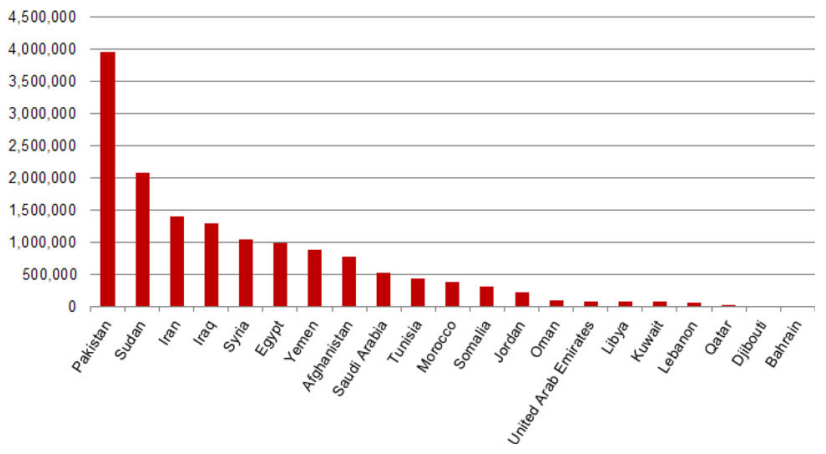




\section{PUBH-10}

The cost-effectiveness analysis of magnetic resonance imaging and ultrasound for hepatocellular carcinoma screening

\section{Su Lin ${ }^{1}$, Qiping Chen ${ }^{2}$, Yueyong Zhu'}

${ }^{1}$ The first affiliated hospital of Fujian Medical University, China; ${ }^{2}$ First Affiliated Hospital of Fujian Medical University, China

Background: Biannual ultrasound (US) is recommended for hepatocellular carcinoma (HCC) screening in patients with cirrhosis. However, the sensitivity of US is suboptimal, especially in patients with cirrhosis. Magnetic resonance imaging (MRI) is characterized with high sensitivity but on the other hand with higher price. The purpose of this study was to evaluate the cost-effectiveness of liverspecific contrast MRI and US in HCC surveillance in cirrhotic patients at high risk of HCC.

Method: A decision-analytic model was established to simulate cirrhotic patients undergoing MRI or US twice a year to screen HCC. The cost-effectiveness ratios of different screening strategies were calculated using TreeAge Pro 2011 software. Costs only included screening and diagnosis costs. The clinical outcome was evaluated using years of life gained (YLG).

Result: The average costs for HCC screening were RMB 2050.2 in MRI group and RMB 262.6 in US group for each person, and the effectiveness were 0.11 YLG and 0.02 YLG, respectively. The costeffectiveness ratios of MRI and US group were close when the prevalence of HCC was $17.8 \%$, with RMB 11445 per gained life year. The sensitivity analysis showed that the cost-effectiveness ratio increased with the cost and decreased with the prevalence of $\mathrm{HCC}$ (Figure 1).

Conclusion: The cost-effectiveness of HCC surveillance is closely related to the prevalence of $\mathrm{HCC}$ and the price of screening mothed. MRI is a cost-effective way to screen for HCC when the risk of is greater than $17.8 \%$
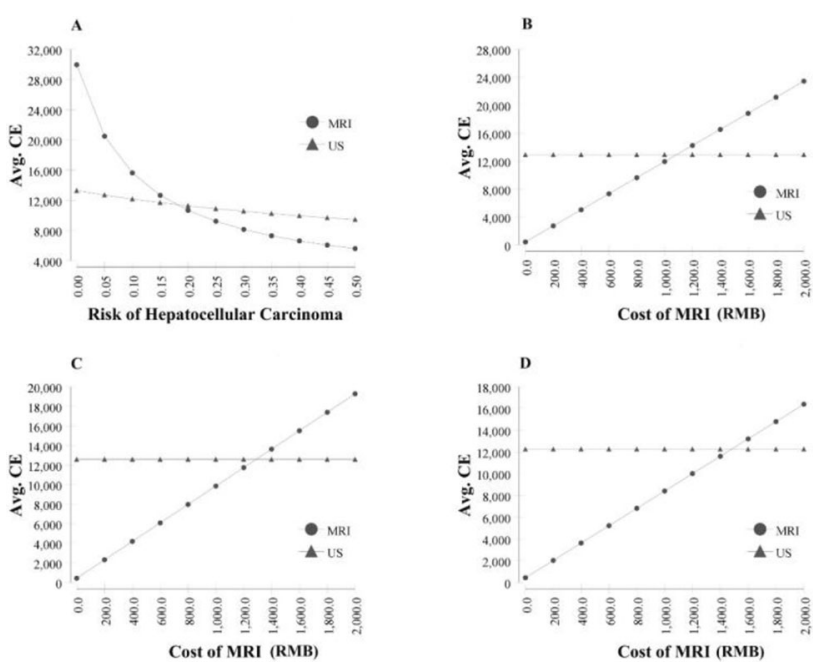

Figure 1 Sensitivity analysis of One-way sensitivity analysis of key model parameters and their effect on average cost-effectiveness.
PUBH-11

Using A transmission dynamic model of hepatitis $\mathbf{C}$ infection to determine the cost-eectiveness of 3-week triple combination of DAAs treatment in China

George Lau ${ }^{1}$, Cheng Wang ${ }^{5}$, Jing Chen ${ }^{5}$, Qing Shao ${ }^{2}$, Dong $\mathrm{Ji}^{2}$, Fan $\mathrm{Li}^{2}$, Bing $\mathrm{Li}^{2}$, Tingting $\mathrm{Wu}^{4}$, Yudong Wang ${ }^{4}$, Vanessa $\mathrm{Wu}^{4}$, George Lau $^{3}$

${ }^{1}$ Humanity and Health Medical Group, Hong Kong; ${ }^{2} 302$ Hospital, China; ${ }^{3}$ Humanity \& Health Medical Centre, Hong Kong; ${ }^{4}$ Humanity and Health Research Centre, Hong Kong; ${ }^{5}$ Humanity and Health Research Centre, Hong Kong SAR, China, Hong Kong

Background: Current guidelines recommend 8-24 weeks of directacting antivirals (DAAs) treatment in chronic hepatitis $\mathrm{C}$ (CHC). The exceptionally high cost is onerous and has adversely affected treatment access and drug compliance. Recent clinical trials showed triple or quadruple combination of DAAs could shorten the duration to 3-6 weeks with $95-100 \%$ sustained virologic response (SVR) rates in selected patients. We aimed to evaluate the cost-effectiveness of 3-week triple DAAs treatment in Chinese HCV patients based on a transimission dynamic model.

Method: We used a previously developed and validated dynamic hepatitis $\mathrm{C}$ virus transimission model in China. This mathematical model using a system of ordinary differential equations simulated the HCV transimission dynamics, which divided the population into 6 classes and included both actue and chronic stage of HCV. We simulated the prevalence of $\mathrm{HCV}$ and medical and treatment costs associated with the disease under two hypothetical scenarios: treatment with standard 12-week sofosbuvir (SOF) + pegIFN + ribavirin (Neutrino, SVR 90\% across all genotype and fibrosis stage) and treatment with 3-week of SOF+ daclatasvir (DCV)+ asunaprevir (ASV) (SVR 67\% across all genotype and fibrosis stage). These two regimens were chosen because they were just licensed in China and most likely will be used in the real-world practice in the future. By estimating the incremental cost effectiveness from a healthcare provider's perspective, we examined the cost-effectiveness of introducing a strategy of 3-week triple DAAs treatment into China's National Program.

Result: Our dynamic model showed that even with a lower SVR rate of 3-week triple DAAs treatment than that of 12-week Neutrino, the substantially decreased cost (US\$2965 vs. US\$10130) increased the treatment rate, i.e. improved the accessibility for patients at the chronic stage. The increased treatment rate reduced the basic reproduction number (R0, the average number of new infections generated by a single infectious individual) from $\mathrm{R} 0=1.4289$ when Neutrino is used to $\mathrm{R} 0=0.7659$ when 3 -week triple DAAs treatment is considered. Based on the model, the predicted number of HCV infection (acute and chronic) will decrease by $14.1 \%$ in 2030 if 3-week triple DAAs treatment is introduced, and US $\$ 1.1$ billion government money will be saved annually.

Conclusion: Introduction of 3-week triple DAAs treatment to China is very cost saving from government perspective, and would likely be instrumental in decreasing morbidity and mortality caused by HCV.

\section{PUBH-12}

Achieving the World Hepatitis Organization Global Health Sector Strategies Goals for Hepatitis in the WHO Western Pacific Region (WPRO): A Modelling Study

Samantha Brandon ${ }^{1}$, Chris Estes ${ }^{1}$, Helen Nde ${ }^{1}$, Sarah Robbins ${ }^{1}$, Jonathan Schmelzer $^{1}$, Homie Razavi ${ }^{1}$ 
${ }^{1}$ CDA Foundation, United States

Background: The Western Pacific Region (WPRO) region accounts for $20 \%$ of the burden of hepatitis C (HCV) globally, but data to guide management strategies are lacking. Progression to decompensated cirrhosis (DC), hepatocellular carcinoma (HCC), and liver related death (LRD) makes the virus a main driver of morbidity in the region. This study sought to quantify the burden of HCV in WPRO and identify a strategy to achieve the Global Health Sector Strategy Goals (GHSS) for Viral Hepatitis Elimination by 2030.

Method: Excel-based disease progression models, built with published data and/or expert consensus were used to assess $2016-2030$ trends in HCV infection and morbidity in 16 WHO WPRO countries and Taiwan (Australia, Cambodia, China, Fiji, Hong Kong, Japan, Laos, Malaysia, Mongolia, New Zealand, Papua New Guinea, Philippines, Samoa, Singapore, South Korea, Vietnam).

Result: In 2016, there were an estimated 13.7 million viremic HCV infections, $21 \%$ have been diagnosed. Under today's treatment paradigm $(300,000$ treated annually), this is expected to decrease by $30 \%$, to 10 million, by 2030 . Liver related mortality is expected to increase 20\%, from 102,000 deaths in 2016 to 124,000 in 2030. The number of patients with end-stage liver disease is also expected to increase $20 \%$.

In order to achieve GHSS goals of diagnosing $90 \%$ of the infected population and reducing liver related mortality by $65 \%$, treatment in the region must be increased to 900,000 patients annually by 2030 . Similarly, the annual number of newly diagnosed patients will need to increase to 750,000 by 2019 (from 350,000 in 2016). These actions will reduce total HCV infections by $90 \%$ and liver related morbidity and mortality by $70 \%$. After 2031 , the number of infections will decline and the number of treated patients can be scaled back accordingly.

Conclusion: Under the status quo, HCV prevalence in WPRO countries will decrease by 2030 , but cases of advanced liver disease and liver-related deaths will continue at a high level. Increasing diagnosis and treatment can lead to significant reductions in total infections, mortality, and morbidity.

\section{PUBH-13}

Management of viral hepatitis: medical education outcomes of physicians in Rural India

\section{Akash Shukla $^{1}$, Rahul Bargaje ${ }^{2}$, Dennis Israelski ${ }^{3}$ \\ ${ }^{1}$ LTMMC LTMGH, India; ${ }^{2}$ Gilead Sciences, India; ${ }^{3}$ Gilead Sciences, United States}

Background: Hepatitis B and Hepatitis C infections are major public health concerns and the role of primary care providers (PCP) is crucial, especially in resource limited settings. There is little data about the knowledge, awareness and management of Hepatitis B and Hepatitis $\mathrm{C}$ and the barriers faced by primary care providers, especially in the rural areas.

Method: A validated questionnaire based survey comprising 25 questions and 3 case study questions. was conducted among the primary care providers in the Bhuj, district of Gujarat (India) to assess the gaps in knowledge of current practices, and potential solutions to improve access to care and treatments of Hepatitis B and Hepatitis C infection in rural India. Following an educational program, the questions related to knowledge were repeated.

Result: Hundred and forty PCP $(70 \%$ men, $53 \%<30$ years) participated. 47\% PCP believed that the largest barrier to screening HBV infection was asymptomatic disease and this percentage was much higher $(\sim 72 \%)$ for $\mathrm{HCV}$ infection. The main barriers to treatment were cost (medications and diagnostic tests) and patients' fear of side effects with long term therapy. Need to improve initial screening and diagnosis was felt by $79 \%$ and $86 \%$ PCP. Only $31 \%$ felt a need for on universal screening policies. About $75 \%$ of primary care providers felt that better primary care education can help in filling the knowledge gaps. The knowledge improved in HBV and HCV after the program $(p=0.00051,0.023$ respectively, Figure 1))

Figure 1 Pre and Post educational program knowledge of PCP

Conclusion: There were gaps in the knowledge of primary care providers regarding diagnosis, screening, treatment availability and management of patients with viral Hepatitis (VH). Educational program targeting these deficits resulted in improvement in the awareness of PCP.

\section{PUBH-14}

Quantifying the impact of achieving the World Health Organization (WHO) Global Health Sector Strategy (GHSS) goals for hepatitis $\mathrm{C}$ in the South East Asian (SEARO) region

Sarah Robbins ${ }^{1}$, Sarah Robbins ${ }^{1}$, Sarah Blach ${ }^{1}$, Chris Estes ${ }^{1}$, Ivane Gamkrelidze ${ }^{1}$, Samantha Brandon ${ }^{1}$, Helen $\mathrm{Nde}^{1}$, Devin Razavishearer $^{1}$, Jonathan Schmelzer ${ }^{1}$, Homie Razavi ${ }^{1}$

${ }^{1}$ Center for Disease Analysis Foundation, United States

Background: In 2015, an estimated 10 million people were living with Hepatitis $\mathrm{C}$ virus (HCV) in the SEARO region and it was responsible for approximately 408,000 deaths, the second highest number of deaths of any WHO region. An understanding of disease burden is necessary to create evidence-based public health strategies for elimination of $\mathrm{HCV}$. We forecasted the disease burden of HCV in the SEARO region and developed a strategy to achieve the WHO GHSS goals for Hepatitis by 2030.

Method: 9 SEARO-country specific models were built and regional averages were applied to country populations when country-specific data was not available. Country estimates were then aggregated into a regional disease burden model. This disease progression model was used to quantify the size of the HCV-infected population by $\mathrm{HCV}$ sequelae from 2016 through 2030.

Result: In 2016, there were an estimated 10.3 million viremic infections in the SEARO region and $60 \%$ of all infections were found in those born between 1954 and 1984. Relative to the current standard of care over the next fifteen years, the total $\mathrm{HCV}$-infected population in the SEARO region is expected to decline minimally, by only $1 \%$ by 2030. Liver related morbidity and mortality is forecasted to increase $60-70 \%$ by the same year.

To achieve the GHSS goals, a significant increase in total number of patients screened and linked to care is necessary. The number of individuals diagnosed annually would need to increase to 800,000 by 2021 and the number of patients treated annually to 775,000 patients by 2025 . Under this scenario, viremic infections are forecast to decline by $85 \%$ from 2016 to 2030; while decompensated cirrhosis cases, hepatocellular carcinoma cases, and liver related deaths will decline by $65 \%-70 \%$ by the same year.

Conclusion: Viremic infections are expected to decrease minimally in the region over the next fifteen years. Targeted screening strategies coupled with increased access to direct acting anti-viral therapy are needed to achieve the GHSS goals. 

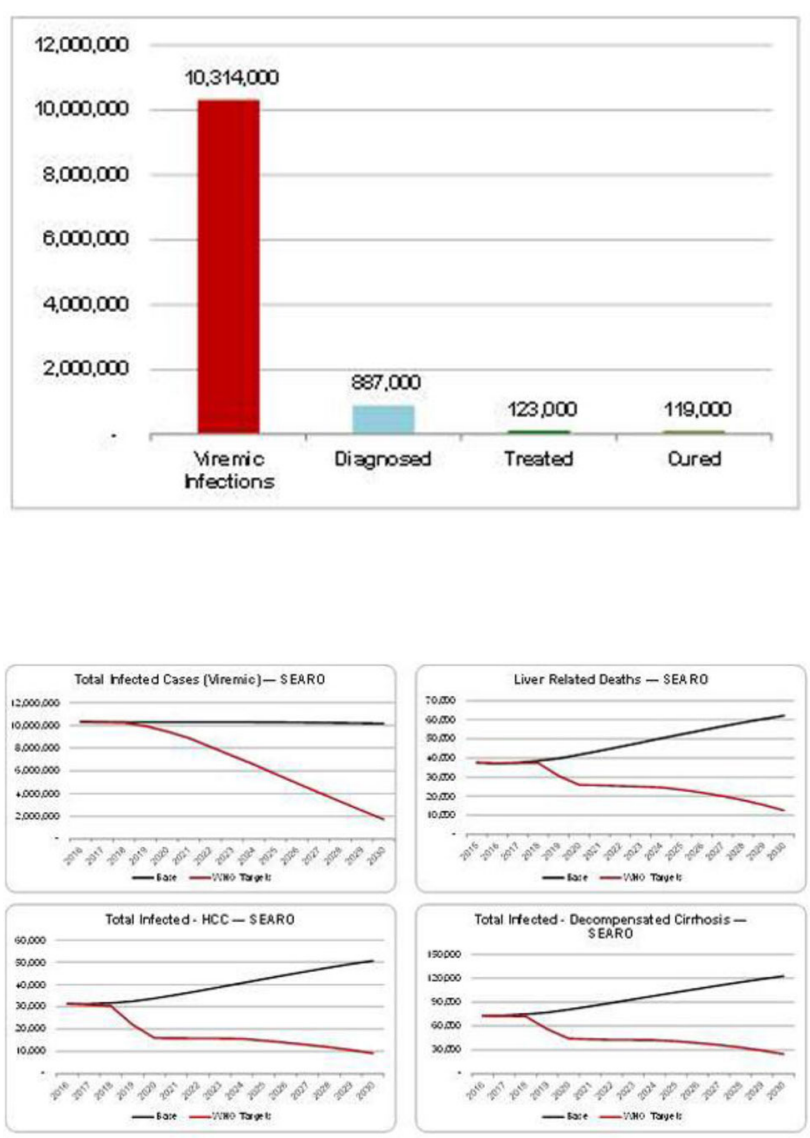

PUBH-15

Study of Hepatitis B positive childbearing women in slum communities of Mumbai

\section{Divyesh Jeram Devaliya $^{1}$, Shripad Desai ${ }^{1}$, Maitreyee Patwardhan ${ }^{1}$}

${ }^{1}$ Non Profit Organization, India

Background: Hepatitis B is a serious global health problem, responsible for 1.4 million deaths every year. India falls in the intermediate endemicity zone (prevalence of $2-7 \%$ ) with a projected disease burden of about 50 million. Hepatitis B is a silent disease major morbidity and mortality. This paper includes findings from Hepatitis Prevention program implemented in slum communities of Mumbai.

Method: The study is a part of community based hepatitis B prevention program focused on childbearing women. The participation was voluntary and with pre consent. The study analyzed the findings found among positive women during the baseline to midline. It also stress the importance of family contact tracing. The baseline was prior the program implementation among known positive cases, while mid line was conducted by external evaluator post 2 years of program implementation among known and new cases.

Result: The program has found 39 positive childbearing women in the community. Out of which 30 were newly diagnosed as a part of voluntary screening intervention while 9 were known cases found during baseline. The knowledge on signs and symptoms of hepatitis B among the positive cases increased from baseline (40\%) to midline $(92 \%)$. Similarly knowledge on modes of transmission saw a record increase from baseline $(30 \%)$ to midline $(100 \%)$. The practice of family contact tracing and immunization saw a change of 59\% during midline, as none of positive women found during baseline had ever screened or vaccinated their family members. Through family contact tracing of 39 positive cases, there were 7 such cases, were atleast one family positive case was detected, while in families of rest 32 women, no such positive cases were identified. Among those 7 positive cases, 6 women had one positive case in their family while one had 2 positive case in family. So total 8 new positive case were identified besides 39 positive women. The contact tracing has shown that in some cases father, mother, sister or brother are Hepatitis B positive, not limiting to spouses and children. Denial to treatment and nonacceptance of test results was seen as a challenge for contact tracing. Avoiding such practices increases the risk of transmitting infection and developing cirrhosis or liver cancer. Low awareness along with high risk practices, increases the risk of acquiring infection among community and especially within family members. Among 39 there were 3 Hepatitis B positive pregnant women who were followed up to avert mother to child transmission among their new born.

Conclusion: Mass awareness among communities and strategies to reduce stigma can prove effective in reducing the incidences. The study highlights the significance of family contact tracing of positive patient.

\section{PUBH-16}

The disease burden of hepatitis B in Republic of Uzbekistan: strategies providing hope for the future

Devin Razavi Shearer ${ }^{1}$, Jamshidbek Ashurov ${ }^{3}$, Intizor Egamova ${ }^{3}$, Aziza Khikmatullaeva ${ }^{3}$, Adkhamjon Mamatkulov ${ }^{3}$, Maya Makhmudova $^{2}$, Homie Razavi ${ }^{4}$, Devin Razavi Shearer ${ }^{4}$

${ }^{1}$ The CDA Foundation, United States; ${ }^{2}$ Republican Blood Center, Uzbekista; ${ }^{3}$ Research Institute of Virology, Uzbekista; ${ }^{4}$ The Polaris Observatory, United States

Background: In July of 2017 in the Republic of Uzbekistan, the Cabinet of Ministers made a Decree to provide additional measures to prevent the spread of infectious diseases. This expansive decree specifically addresses the need to stepwise increase the diagnosis of the hepatitis B virus (HBV). This study aims to quantify the current and future disease burden of HBV in the Republic of Uzbekistan, while exploring two scenarios that can help mitigate the costly later stages of the disease.

Method: A literature review was conducted and then expert consensus regarding inputs was built at an in country meeting in September 2017. The PRoGReSs model, a dynamic country-level transmission and disease burden model was used to estimate the impact of perinatal prophylaxes, treatment, aging and disease progression and mortality in the infected population.

A scenario was then developed with stepwise increases in screening and treatment in a manner that is compatible with the current health care system resources. A second scenario was created to meet the Global Health Sector Strategy for Viral Hepatitis of $90 \%$ of the infected population being diagnosed and $80 \%$ of those diagnosed and eligible receiving treatment.

Result: In 2015, it was estimated that $8.1 \%$ of the population was $\mathrm{HBsAg}+$. This correlates to an estimated 2.5 million (UI: 1.3-3.7) infected individuals in 2016. Due to high levels of vaccination and an aging infected population, the prevalence in the future is expected to decline. However, in the absence of additional interventions, HBVrelated morbidity and mortality are expected to increase (Figure 1). Under the first scenario, "Inc Tx", it was estimate that the number of individuals annually diagnosed could stepwise increase to 80,000 individuals by 2025 and beyond, and that treatment could be stepwise expanded to almost 140,000 individuals by 2025 and beyond. This 
scenario would cause decreases morbidity and mortality, averting 7,000 new cases of hepatocellular carcinoma (HCC), and saving 7,400 lives.

In the second scenario, "WHO 2030", treatment and diagnosis were increased to meet the 2030 targets for diagnosis and treatment. This required diagnosing almost three times as many individuals annually by 2025 and treating almost seven times more individuals as compared to the "Inc Tx" scenario. This scenario would result in averting almost 15,000 new cases of HCC and save almost 14,000 lives but would require large investments not only for the treatment and diagnosis, but in the infrastructure to support such an aggressive program as well

Conclusion: The Decree of the Cabinet Ministers came at a pivotal time and has the foresight to put measures in place to mitigate the disease burden of HBV in the Republic of Uzbekistan. With the current capabilities of the health care system, a large impact can be made and 7,400 lives can be saved. The much more aggressive scenario provides evidence of what could be possible but not currently feasible.
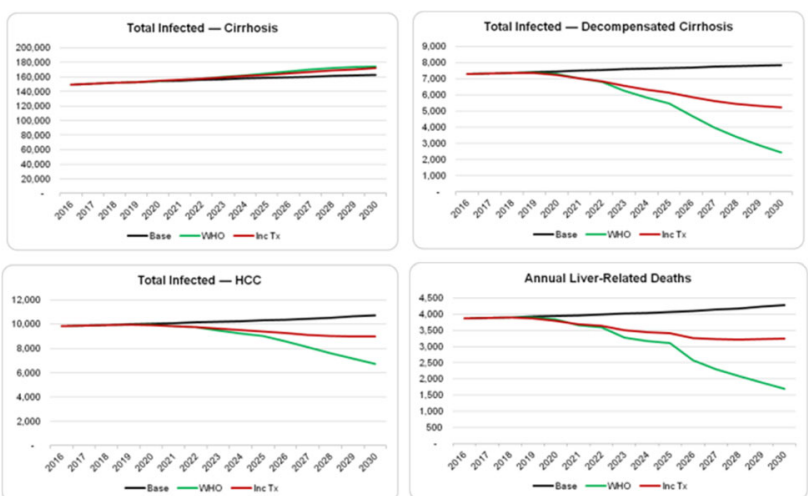

VASC-1

Retrospective cohort study of bleeding complications in patients with budd chiari syndrome on anticoagulation

\section{Anand Sharma $^{1}$, Uday George Zachariah ${ }^{1}$, Shyamkumar N Keshava $^{1}$, Vinu Moses ${ }^{1}$, Jeyaseelan L ${ }^{1}$, Ashish Goel ${ }^{1}$, Chundamannil E Eapen ${ }^{1}$ \\ ${ }^{1}$ Christian Medical College Vellore, India}

Background: Anticoagulation is recommended in patients with Budd Chiari syndrome (BCS), but may cause bleeding complications.

Method: Patients diagnosed with BCS; who had bleeding complications on oral anticoagulants(OAC) from January 2007 to May 2017 were studied in a retrospective cohort study. Baseline data and data on severity of disease, intervention performed, follow up, bleeding episodes, anticoagulation and treatment efficacy were extracted. HASBLED score which is used to predict risk of bleed in patients with atrial fibrillation OAC; was also calculated. We also compared the predictors of bleed in patients with variceal bleed with non variceal bleed in this cohort of patients.

Result: Of 579 patients with BCS, 57 patients [Male 31; Age 33\&plusmn; 11 years, baseline MELD $13 \pm 5$, HAS-BLED[acronym stands for uncontrolled hypertension, abnormal liver or kidney function, history of stroke, bleeding history, labile INR, age $>65$ years, drugs(concomitant antiplatelet or alcohol use)] at the time of bleed $2 \pm 0.69]$ experienced bleeding on OAC. At presentation $8(14 \%)$ patients had refractory ascites and $13(22.8 \%)$ patients had prior history of variceal bleed. All the patients received oral anticoagulation with acenocoumarol with a target INR of 2-3. On Kaplan Meier plot, the median time from start of OAC to first bleeding episode was 59 months (95\% C.I-32 to 86 months). 19(33.9\%) patients had variceal bleed and $38(66.1 \%)$ from other sites.[dental 8(14.03\%), ENT 6(10.5\%), menstrual $4(7.01 \%)$, rectal $6(10.5 \%)$, intramuscular hematomas $5(8.7 \%)$,lungs $2(3.5 \%)$, non variceal upper gastrointestinal 3(5.2\%), CNS(1.75\%)].Major bleed(requiring hospitalization or transfusion or if intracranial, retroperitoneal or fatal) occurred in $27(47.37 \%)$ patients. INR was labile $(<60 \%$ time in therapeutic range) in $5(8.8 \%)$ patients and supratherapeutic during the bleed in $9(15.8 \%)$ patients. Irreparable hepatic veins were more in variceal bleeders [15(78.9\%) vs. 14(36.8\%), p value 0.004$]$ than non variceal bleeders, but type of intervention, presence of refractory ascites at presentation, labile INR, HAS-BLED score, portal vein thrombosis and IVC thrombosis did not differ between the two groups. The baseline pressure gradient across the obstruction and the reduction in pressure after intervention were similar in the two groups. There was no bleeding related mortality.

Conclusion: Of 579 patients with BCS, 57(10\%) patients had a bleed. HAS-BLED score in these patients at the time of bleed was $2 \pm 0.69$. Major bleeding complications in Budd Chiari Syndrome are uncommon and well tolerated with close monitoring of INR.

\begin{tabular}{|c|c|}
\hline \multicolumn{2}{|l|}{ Baseline data of the cohort of patients } \\
\hline Age & $33 \pm 11$ years \\
\hline Sex(No of Males) & 31 \\
\hline BaselineMELD score & $13 \pm 5$ \\
\hline Baseline Child Pugh score & $7 \pm 1.71$ \\
\hline BaselineRotterdam prognostic index & $1.91 \pm 0.59$ \\
\hline Baseline Clichy prognostic index & $4.74 \pm 0.82$ \\
\hline BaselineHAS-BLED score & $2 \pm 0.69$ \\
\hline $\begin{array}{l}\text { Baseline Variceal status (\%) } \\
\text { No Varices } \\
\text { Large esophageal varices } \\
\text { Small esophageal varices } \\
\text { Isolated gastric varices }\end{array}$ & $\begin{array}{l}9(17 \%) \\
16(30.2 \%) \\
12(22.6 \%) \\
4(7.5 \%)\end{array}$ \\
\hline No of patients with Variceal bleed (\%) & $19(33.9 \%)$ \\
\hline No of patients with IVC Obstrnction(\%) & $12(21.1 \%)$ \\
\hline No of patients with Portal Vein thrombosis (\%) & $4(7 \%)$ \\
\hline No of patients with Irreparable hepatic veins (\%) & $30(52.6 \%)$ \\
\hline Typeof interventiondone & \\
\hline No intervention & $13(22.8 \%)$ \\
\hline Angioplasty & $25(43.9 \%)$ \\
\hline Shunt & $19(33.3 \%)$ \\
\hline
\end{tabular}




\begin{tabular}{|l|l|l|l|}
\hline \multicolumn{4}{|l|}{ Factors studied as predictors of variceal bleed, compared to non variceal bleed } \\
\hline Factors & Variceal bleed, n=19(\%) & Non Variceal bleed, n=38(\%) & v value \\
\hline $\begin{array}{c}\text { Typeofintervention } \\
\text { No intervention } \\
\text { Angioplasty } \\
\text { Shunt }\end{array}$ & $\begin{array}{l}4(21.1 \%) \\
6(31.6 \%) \\
9(47.4 \%)\end{array}$ & $\begin{array}{l}9(24.3 \%) \\
19(51.4 \%) \\
9(24.3 \%)\end{array}$ & 0.19 \\
\hline $\begin{array}{l}\text { Refractory ascitesat } \\
\text { presentation }\end{array}$ & $4(21.1 \%)$ & $4(10.8 \%)$ & 0.30 \\
\hline LabileINR & $1(5.3 \%)$ & $4(10.8 \%)$ & 0.49 \\
\hline Portal vein thrombosis & $1(5.3 \%)$ & $3(8.1 \%)$ & 0.70 \\
\hline IVCobstruction & $4(21.1 \%)$ & $8(21.6 \%)$ & 0.96 \\
\hline Irreparableveins & $15(78.9 \%)$ & $14(36.8 \%)$ & 0.004 \\
\hline $\begin{array}{l}\text { HAS-BLED score } \\
\text { (mediantSD) }\end{array}$ & $2 \pm 0.83$ & $1.5 \pm 0.6$ & 0.16 \\
\hline $\begin{array}{l}\text { BaselinePressuregradient } \\
\text { in mm Hg(mediantSD) }\end{array}$ & $25 \pm 8.56$ & $18 \pm 10.26$ & 0.23 \\
\hline $\begin{array}{l}\text { Drop in gradient in } \\
\text { mmHg(mediantSD) }\end{array}$ & $14 \pm 6.04$ & $9 \pm 8.97$ & 0.25 \\
\hline
\end{tabular}

Conclusion: We detected PCC in $24 \%$ of patients with portal cavernoma; it was significantly more prevalent in non-cirrhotic and nonmalignant PVT. Female sex, greater spleen length, and gallbladder varices were significant predictors of PCC.

\begin{tabular}{|c|c|c|c|}
\hline & $\begin{array}{c}\text { Group 1: } \\
\text { patients with PCC }\end{array}$ & $\begin{array}{c}\text { Group 2: } \\
\text { patients without PCC }\end{array}$ & p-valuc \\
\hline Number of patients (n) & 21 & 65 & \\
\hline Male & 6 & 38 & 0.024 \\
\hline Female & 15 & 27 & 0.024 \\
\hline \multicolumn{4}{|l|}{ Cause of PVT: } \\
\hline $\begin{array}{l}\text { liver cirrhosis / malignant hepatobiliary } \\
\text { tumors }\end{array}$ & 1 & 20 & 0.018 \\
\hline non-cirrhotic and non-malignant PVT & 20 & 45 & 0.018 \\
\hline Age at diagnosis of PVT, years Me [Q25-75] & $38.0[20.3 ; 49.5]$ & $44.0[27.8 ; 56.6]$ & 0.188 \\
\hline Bleeding esophageal / gastric varices, $\mathbf{n}(\%)$ & 8 of $18(44 \%)$ & 20 of $55(36 \%)$ & 0.596 \\
\hline Hemoglobin, g/ Me [Q25-75] & $112[105 ; 127]$ & $114[105 ; 133]$ & 0.720 \\
\hline Platelets, " $10 \% 1 \mathrm{Me}[Q 25-75]$ & $288[110 ; 376]$ & $162[110 ; 259]$ & 0.367 \\
\hline INR Me [Q25-75] & $1.16[1.09 ; 1.37]$ & $1.18[1.09 ; 1.32]$ & 0.804 \\
\hline Fibrinogen, g/Me [Q25-75] & $2.95[2.43 ; 3.58]$ & $2,63[2.24 ; 3.3]$ & 0.470 \\
\hline Increased ALP and/or GGT, $n$ (\%) & 5 of $17(29 \%)$ & 16 of $37(43 \%)$ & 0.383 \\
\hline ALP, *ULN Me [Q25-75] & $2[1.6 ; 2.2]$ & $1.2[1.1 ; 1.3]$ & 0.003 \\
\hline GGT, "ULN Me [Q25-75] & $1.9[1.3 ; 3.7]$ & $2.3[1.2 ; 4.2]$ & 0.599 \\
\hline Gallbladder varices, $\mathrm{n}(\%)$ & $8(38 \%)$ & $9(14 \%)$ & 0.025 \\
\hline Spleen length, $\mathrm{cm} \mathrm{M} \pm \mathrm{SD}$ & $18.6 \pm 3.1$ & $15.7 \pm 3.6$ & 0.017 \\
\hline
\end{tabular}

\section{VASC-1}

Risk factors for portal cavernoma cholangiopathy: the first study in Russia

Daria Aleksandrovna Strelkova ${ }^{1}$, Khava Bagaudinovna Kodzoeva $^{1}$, Kseniya Aleksandrovna Ulyanova ${ }^{1}$, Sofia Igorevna Rogacheva $^{1}$, Alina Sergeevna Volkova ${ }^{1}$, Artem Sergeevich Dekhanov $^{1}$, Maria Jurevna Nadinskaya ${ }^{1}$, Vladimir Trofimovich Ivashkin $^{1}$

${ }^{1}$ Sechenov University, Russian Federatio

Background: Portal cavernoma cholangiopathy (PCC) is defined as biliary abnormalities due to portal cavernoma in patients with portal vein thrombosis (PVT). A limited number of studies evaluating predictors of PCC were carried out and there were no such studies in Russia. The aim of the study is to derive a predictive model of the development of PCC.

Method: The study included 86 patients (44 males, 42 females; median age of 42 years $(\mathrm{Q} 25-75[27 ; 56]))$ with the diagnosis of portal cavernoma on multislice computed tomography (MSCT) and/or Doppler ultrasound (US). PCC was defined as biliary dilatation with exclusion of other causes on MSCT and/or US. The patients were classified as Group 1: with PCC $(\mathrm{n}=21)$ and Group 2: without PCC $(\mathrm{n}=65)$. Demographic, clinical, laboratory, and instrumental data were analyzed. Multivariable logistic regression was applied to identify risk factors for the presence of PCC.

Result: There was no difference between the groups in age at diagnosis of PVT, incidences of variceal bleeding or levels of hemoglobin, platelets, INR, and fibrinogen. PCC was significantly more common in female patients, and in patients with non-cirrhotic and non-malignant PVT, greater spleen length, and gallbladder varices. The increase in alkaline phosphatase (ALP) and gammaglutamyltransferase (GGT) occurred with the same frequency in both groups, but the elevated ALP levels were significantly higher in patients with PCC (Table). We observed symptoms (itching, cholangitis and hemobilia) only in two patients with PCC. According to multivariate logistic regression analysis, female sex ( $\mathrm{p}=0.03$; OR $6.0 ; 95 \%$ CI: $1.1-32.4)$, greater spleen length ( $\mathrm{p}=0.03$; OR $1.3 ; 95 \%$ CI: $1-1.5)$, and gallbladder varices $(\mathrm{p}=0.04$; OR 5.1 ; $95 \%$ CI: $1.1-$ 23.6) could be identified as predictive factors of the occurrence of PCC. The area under the ROC curve for the spleen length was 0.74 $(0.60-0.89, ?=0.006)$ with a cut-off of $16.2 \mathrm{~cm}$ (sensitivity $85.7 \%$, specificity $62.2 \%$ ) - Figure.

Figure. ROC curve for spleen length

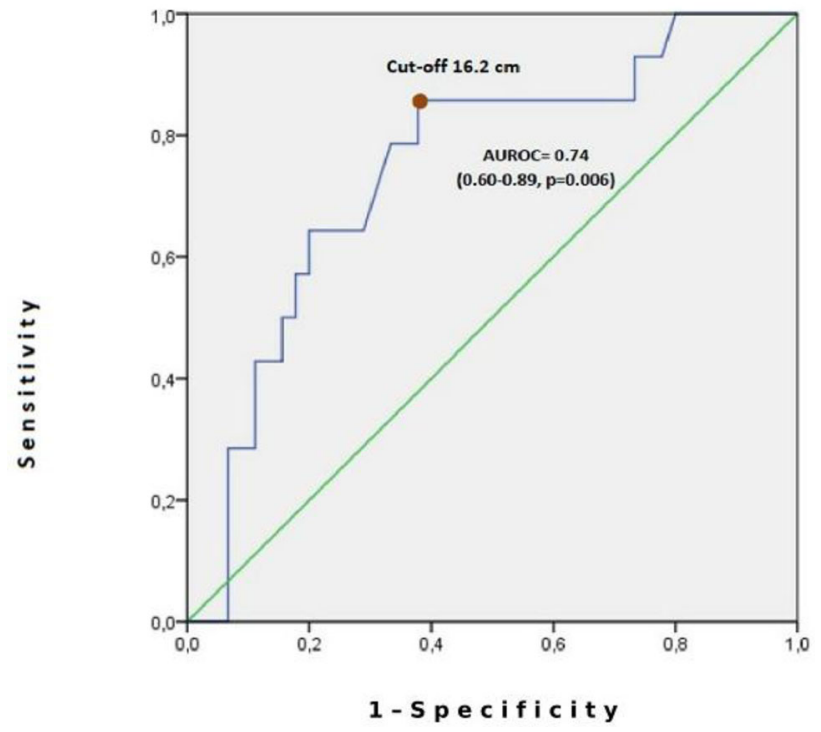

VASC-1

Role of endovascular interventions in BuddChiari syndrome

Chandan J Das ${ }^{1}$, M Soneja ${ }^{1}$

${ }^{1}$ AIIMS, India

Background: Percutaneous endovascular interventions are increasingly being used in management of Budd-Chiari syndrome (BCS). In this retrospective, single centre study, we evaluated the role of endovascular interventions in management of BuddChiari syndrome Method: This study retrospectively analyzed 30 consecutive patients of BCS who underwent endovascular intervention between 2014-17. Preprocedural Doppler and CT venography were performed in all patients. Two patients had underwent 3D CEMRA for planning of treatment. Technical/clinical success, technical difficulties, and complications of endovascular procedures were analysed. Role of Child-Turcotte-Pugh (CTP) in predicting mortality, clinical success, and need for re-interventions was also assessed 
Result: Thirty patients with BCS were treated with various endovascular interventions. Technical success were achieved in 27 patients $(90 \%)$. Following interventions were performed: IVC angioplasty $(\mathrm{n}=7)$, RHV angioplasty and stenting $(\mathrm{n}=5)$, IVC angioplasty with RHV stenting ( $\mathrm{n}=3)$, RHV angioplasty $(\mathrm{n}=3)$, IVC angioplasty \& thrombus aspiration $(\mathrm{n}=2)$, MHV angioplasty $(\mathrm{n}=1)$, LHV angioplasty $(n=1)$, MHV stenting $(n=1)$, IVC stenting $(n=1)$, IVC filter placement $(\mathrm{n}=1)$, intravenous catheter thrombolysis $(\mathrm{n}=$ $1)$, and direct intrahepatic porto-systemic shunt (DIPS) $(\mathrm{n}=1)$. Polytetrafluoroethylene (PTFE)-covered stents were used in one patients to perform DIPS and a $20 \mathrm{~mm} \times 6 \mathrm{~cm}$ stent was used in IVC to keep the lumen patent following angioplasty. Balloon mounted stents $(37 \mathrm{~mm} \times 10 \mathrm{~mm})$ were used in hepatic veins. Follow-up was performed by Doppler ultrasonography. Mean follow-up time was 11.4 months (range: 11-17months). In three patients (10\%), procedure could not be performed due to tight IVC stricture. The 1-, 2-year stent patency rates were $91.91 \%, 88.08 \%$, respectively. Re-interventions were required in 1 (3\%). Overall mortality was 1 (3\%). CTP prognostic score could not predict mortality, clinical success, and need for re-interventions.

Conclusion: Endovascular interventions are very useful in the management of BCS.

\section{NUTR-1}

\section{Sarcopenia in liver cirrhosis can be predicted with hand grip stregnth}

\section{Amanda Trixie Hardigaloeh ${ }^{1}$, Johana Titus ${ }^{1}$, Cleopas Martin Rumede $^{\mathrm{I}}$ \\ ${ }^{1}$ Cipto Mangunkusumo Nasional Hospital, Faculty of Medicine Universitas Indonesia, Indonesia}

Background: Malnutrition is independent factor related to morbidity, mortality and high cost of treatment in liver cirrhosis. Hand grip strength (HGS) is one of the method use for malnutrition detection and prognosis evaluation. The correlation of HGS with liver function (Child Pugh or CP score) and muscle mass is controversial. These important evaluation is not yet available in Indonesia.

Method: This is a cross-sectional study in liver cirrhosis patients at Hepatobiliary clinic of Cipto Mangunkusumo Hospital from February to June 2015. Nutritional status was assessed by HGS. Muscle mass was obtained from bioimpedance. Data were analyzed using Spearman correlation test.

Result: There were 115 patients liver cirrhosis at Hepatobiliary clinic of Cito Mangunkusumo Hospital, 112 patients who fit the inclusion criteria, consisted of 79 men and 33 women with mean age 5, $15 \pm$ 10.55 years, median CP score 6 (5-13) with median HGS 26 (11-50) $\mathrm{KgF}$, mean muscle mass $44,43 \pm 8,12 \mathrm{~kg}$. The median intake of energy $1334,82(604,75$ - 3023,7) kkal, median protein 45,87 (19$114,5)$ gram. Prevalence of malnutrition according HGS was $33 \%$. Hand grip strength is not correlated with CP score $(p 0.046, \mathrm{r}=-0.19)$ however it is correlated with muscle mass $(p<0.001, \mathrm{r}=0.70)$

Conclusion: There are 33\% malnutrition cases based on HGS in out patient liver cirrhosis. There is no correlation between hand grip strength with Child Pigh score however HGS is correlated with muscle mass in liver cirrhosis
NUTR-2

Predictors of unplanned hospitalization or death \& utility of rapid screening with clinical frailty scale in outpatients with cirrhosis

Aniruddha Pratap Haripal Singh ${ }^{1}$, Sandesh $\mathbf{K}^{\mathbf{1}}$, Gaurav Kapur ${ }^{1}$, Neeraj Vinaykumar', Varun Tadkalkar', Manoj Yadav ${ }^{1}$

${ }^{1}$ Government Medical College, India

Background: Hospitalizations in patients are the major cause of morbidity and healthcare expenditure in patients with cirrhosis. Predictive factors determining which outpatients with cirrhosis are at highest risk for unplanned admissions are lacking. We aimed to study factors predicting unplanned hospitalization or death at 3 months \& study utility of Clinical Frailty Scale (CFS) in outpatients with cirrhosis.

Method: This was a Prospective observational study of consecutive outpatients with cirrhosis in a single tertiary care centre in Kerala. Patients with active malignancy, ESRD \& any condition requiring immediate admission were excluded. Frailty was defined as CFS $>4$. Unplanned hospitalization was defined as any admission to acute care hospital that was not planned. Data on socioeconomic status, MELD, CHILD \& Charlson comorbidity index were recorded. The outcome was defined as unplanned hospitalization or/and death at 1 and 3 months. Variables were analyzed by Binary logistic regression.

Result: Overall 124 patients included, 52(41.9\%) were frail, Etiology was predominantly alcohol 63/124 (51\%) followed by HBV 30/124 (24\%) then NASH 12/124 (16.7\%) related cirrhosis. Mean MELD was 20 and CTP score was 10.6 in frail patients.CFS mean value was 5.2 with CRP mean of $3.2 \mathrm{mg} / \mathrm{dl}$ in frail outpatients. 26/52 (50\%) of frail patients as compared to $2 / 72(2.8 \%)$ non-frail patients had unplanned hospitalization at 1 month whereas $44 / 52(84.6 \%)$ as compared to $11 / 72(15.3 \%)$ had unplanned hospitalization at 3 months. $9 / 52(17.3 \%)$ frail patients died in comparison to only $1 / 72(1.46 \%)$ in the non-Frail group at 3 months. On Univariate regression analysis variables found to have $p$ values $<0.05$ were considered for Multivariate Cox regression analysis. Multivariate Binary logistic regression (Backward LR) patients taking B- blocker more than 20 mg with $\mathrm{P}<0.07$, OR 7.16 (CI 1.73-29.6), Frail patients with CFS $>$ $4 \mathrm{P}<0.01$, OR: 5.38 (CI: 1.3-21.6), Presence of AKI with $\mathrm{P}<0.006$, OR: 17.24 (CI: 2.2-133.7), CRP $>2 \mathrm{mg} / \mathrm{dl}$ with $\mathrm{P}<0.001$, OR: 11.23 (CI: 2.6-47) were found to independently predict unplanned admission at 1 month and unplanned admission/death at 3 months. We also observed that CRP with a cut-off of $2 \mathrm{mg} / \mathrm{dl}$ with Sensitivity of $77.5 \%$ and specificity of $92.42 \%$ with AUC of 0.88 predicted unplanned hospitalization/death at 3 months.

Conclusion: Clinical frailty scale greater than 4 as rapid 1-minute screen predicts unplanned hospitalization and death at $1 \& 3$ months and can be utilized in outpatients with cirrhosis for stratifying patients at risk of adverse outcomes. 
Baseline Characteristics stratified by Frailty ( CFS $>4$ )

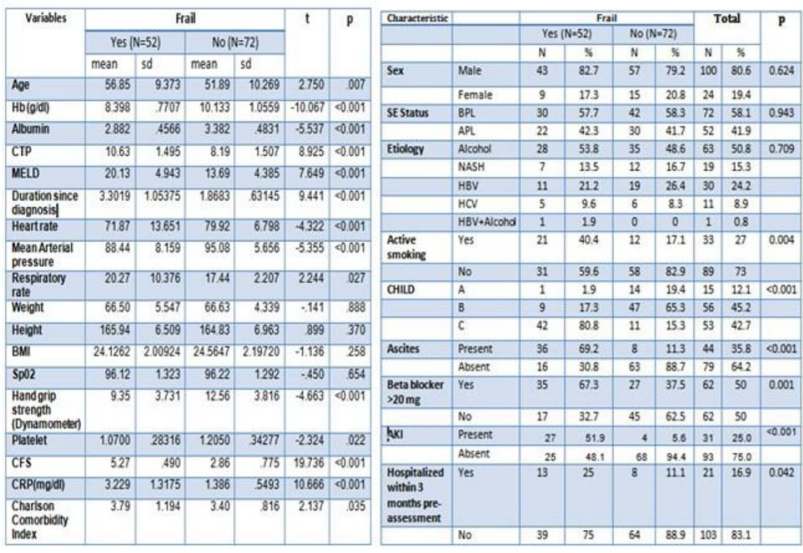

\begin{tabular}{|c|c|c|c|c|c|c|c|c|}
\hline \multirow[t]{3}{*}{ Variable } & & \multicolumn{4}{|c|}{ Frail } & \multirow{2}{*}{\multicolumn{2}{|c|}{ Total }} & \multirow[t]{3}{*}{$P$} \\
\hline & & \multicolumn{2}{|c|}{ Yes $(N=52)$} & \multicolumn{2}{|c|}{ No $(\mathrm{N}=72)$} & & & \\
\hline & & $\mathrm{N}$ & $\%$ & $\mathrm{~N}$ & $\%$ & $\mathrm{~N}$ & $\%$ & \\
\hline \multirow[t]{2}{*}{$\begin{array}{l}\text { Admission } \\
\text { at1 month }\end{array}$} & Yes & 26 & 500 & 2 & 28 & 28 & 22.6 & $<0.00$ \\
\hline & No & 26 & 500 & 70 & 972 & 96 & 774 & \\
\hline \multirow[t]{2}{*}{$\begin{array}{l}\text { Admission } \\
\text { at3month }\end{array}$} & Yes & 44 & 846 & 11 & 3 & 55 & 4 & $<0.001$ \\
\hline & No & 8 & 15.4 & 61 & 84.7 & 69 & 556 & \\
\hline \multirow[t]{2}{*}{ Death } & Yes & 9 & 173 & 1 & 1.4 & 10 & 8.1 & .001 \\
\hline & No & 43 & 827 & 71 & 986 & 114 & 919 & \\
\hline
\end{tabular}

\begin{tabular}{|c|c|c|c|}
\hline \multicolumn{4}{|c|}{$\begin{array}{l}\text { Multivariate Logistic Regression (Bachward IR) for } \\
\text { prediction of hospitalization/death at } 3 \text { months. }\end{array}$} \\
\hline Variables & p.ralue & OR & a \\
\hline $\begin{array}{l}\text { B-Blockers(20 } \\
\text { mg) }\end{array}$ & 0.07 & 7.16 & $173-29.67$ \\
\hline CPS $>4$ & 0.01 & 5.38 & $13-21.6$ \\
\hline AKI & 0.006 & 1724 & $22 \cdot 133.7$ \\
\hline CRP & 0.001 & 11.23 & 2.647 \\
\hline \multicolumn{3}{|c|}{$\begin{array}{l}\text { Hosmer lemeshow goodness off Fittest, } X \text {. }(P \text { - } \\
\text { value) }\end{array}$} & $9.32(0.15)$ \\
\hline
\end{tabular}

PATH-1

A retrospective analysis of a multiethnic cancer cohort with various hepatic pathologies evaluated in an urban-based hepatology clinic

Marzena Magnes ${ }^{1}$, Marzena Magnes ${ }^{1}$, Marjan Farshadi', Muhammad Khurram Mahmood ${ }^{1}$, Hamed Dallali ${ }^{1}$, Kerstina Boctor $^{1}$, Mark Khan ${ }^{3}$, Jean Palmart ${ }^{2}$, Sunny Loo ${ }^{2}$, Magdy Elkhashab $^{1}$

${ }^{1}$ Toronto Liver Centre, Canada; ${ }^{2}$ Advisory Physicians Research Services Inc., Canada; ${ }^{3}$ Gilead Sciences Canada, Inc., Canada

Background: With the prevalence of liver diseases on the rise, it has become increasingly important to understand the risks associated with such conditions. One of the largest health and financial burden, an individual or community can face, is cancer. It is also the leading cause of mortality in Canada. This study aims to assess and describe the cancer population among primary liver diseases in a multiethnic Canadian population at the Toronto Liver Centre within an urban setting.

Method: A total of 3610 patient medical charts from the Toronto Liver Centre were retrospectively analyzed arbitrarily, so far. Medical chart date ranged from February, 1995 to September, 2017. All charts reviewed had a diagnosis of a hepatic pathology. Information regarding patient demographics, liver disease etiology, cancer diagnoses and various co-morbidities present, were collected and entered into a standardized electronic case report form. Data was organized and analyzed using MarketSight.

Result: Of the 3610 patient charts reviewed with a liver disease, 247 $(6.8 \%)$ patients were found to have a form of cancer, $121(49.0 \%)$ of which were males and $126(51.0 \%)$ were females. The prevalence of cancer in age groups under 40, 50-59, 60-69 and over 70 years of age, were $2.0 \%, 6.5 \%, 21.0 \%, 26.3 \%$ and $44.1 \%$ respectively. Linear regression analysis determined a $10.4 \%$ increase in the prevalence of cancer per decade of age $(\mathrm{r} 2=0.979)$. Liver, breast, and thyroid cancer were the highest reported cancers within the cohort (refer to Figure 1). The dominant co-morbidities were hypertension, dyslipidemia, diabetes and obesity (refer to Figure 2). These co-morbidities are also the major factors associated with metabolic syndrome. The prevalence of cancer in non-alcoholic fatty liver disease (NAFLD), hepatitis B (HBV) and hepatitis C (HCV) patients in the cohort was $5.9 \%, 6.8 \%$ and $8.2 \%$ respectively. Liver cancer was the most prevalent cancer found in viral hepatitis patients, encompassing $45.9 \%$ and $42.5 \%$ of $\mathrm{HBV}$ and $\mathrm{HCV}$ patients with cancer respectively. Breast cancer was the most dominant form of cancer in patients with NAFLD. The proportion of breast cancer patients that had NAFLD as their associated liver disease was $58 \%$.

Conclusion: The study results show a strong association between breast cancer and NAFLD, which aligns with recent studies demonstrating a similar relationship. Further research is required to determine the exact nature of this correlation.

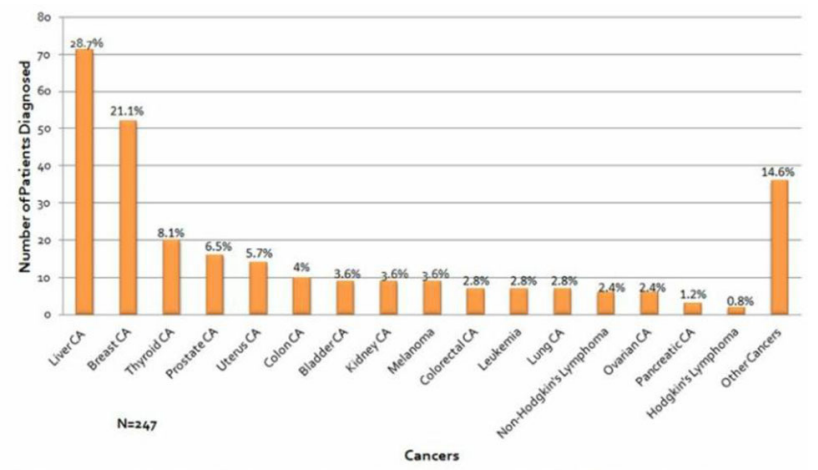

Figure 1: Different types of Cancers reported by Cancer patients with Liver Disease

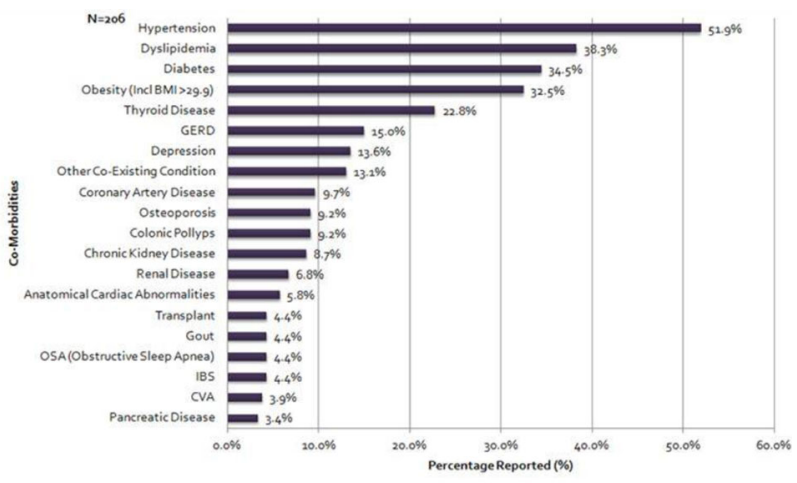

Figure 2: Co-Morbidities reported in Cancer Patients with Liver Disease

\section{PATH-2}

A retrospective analysis comparing gastrointestinal conditions in a multi-ethnic cohort with various hepatic pathologies evaluated in an urban-based hepatology clinic

Marzena Magnes ${ }^{1}$, Jean Palmart ${ }^{2}$, Sunny Loo ${ }^{2}$, Magdy Elkhashab $^{1}$, Hamed Dallali ${ }^{1}$, Muhammad Khurram Mahmood ${ }^{1}$, 
Marjan Farshadi ${ }^{1}$, Marzena Anna Magnes ${ }^{1}$, Sara Nasr ${ }^{1}$, Kerstina Boctor $^{1}$, Mark Khan ${ }^{3}$

${ }^{1}$ Toronto Liver Centre, Canada; ${ }^{2}$ Advisory Physicians Research Services Inc., Canada; ${ }^{3}$ Gilead Sciences Canada, Inc., Canada

Background: There exist an established understanding of the close physiological interaction between the liver and the gastrointestinal (GI) system. However, with both digestive and hepatic diseases being a significant source of morbidity, mortality, and health-care costs in Canada, this study aims to observe the association between GI disorders and various liver pathologies in patients within a multiethnic Canadian population at the Toronto Liver Centre.

Method: A total of 3610 patient medical charts from the Toronto Liver Centre were retrospectively analyzed arbitrarily. Medical chart date ranged from February, 1995 to September, 2017. All charts reviewed had a diagnosis of a hepatic pathology. Information regarding patient demographics, liver disease etiology, and GI disease diagnoses, were collected and entered into a standardized electronic case report form. Data was organized and analyzed using MarketSight ${ }^{\circledR}$.

Result: Of the 3610 patient charts reviewed, a total of $639(17.7 \%)$ patients were found to have GI disorders of interest: irritable bowel syndrome (IBS), inflammatory bowel disease (IBD), gastroesophageal reflux disease (GERD), and colon polyps.

There were 117 (3.2\%) liver disease patients that reported IBS, 66 $(56.4 \%)$ of which were female and $51(43.6 \%)$ were male. IBD was reported by $35(1.0 \%)$ patients, $11(31.4 \%)$ were females and 24 $(68.6 \%)$ were male. GERD was diagnosed in $407(11.3 \%)$ liver disease patients, $195(47.9 \%)$ of which were females and $212(52.1 \%)$ were males. Colon polyps were found in $92(2.5 \%)$ patients, 39 $(42.4 \%)$ female and $53(57.6 \%)$ males. Overall, the prevalence of IBS, GERD and colon polyps tended to increase in older patients; this was seen most drastically in colon polyps. Linear regression analysis demonstrated an $8.91 \%(\mathrm{r} 2=0.856)$ increase of colon polyps prevalence per decade of age in liver disease patients. IBD tended to decrease in prevalence in older patients.

GI conditions analyzed specifically in non-alcoholic fatty liver disease (NAFLD), viral hepatitis B (HBV) and viral hepatitis C (HCV) patients, displayed that the GI diseases of interest were highly prevalent in NAFLD patients compared to HBV or HCV patients (Figure 1).

In our cohort of liver disease patients, 71 patients were diagnosed with hepatocellular carcinoma (HCC). The number of these patients that reported IBS, IBD, GERD or colon polyps was $2(2.8 \%), 0(0 \%)$, $5(7.0 \%)$ and $3(4.2 \%)$ respectively.

Conclusion: Our results demonstrated a high level of association between patients with NAFLD and the GI conditions focused within this study. Further studies will be required to determine the exact nature of this association.

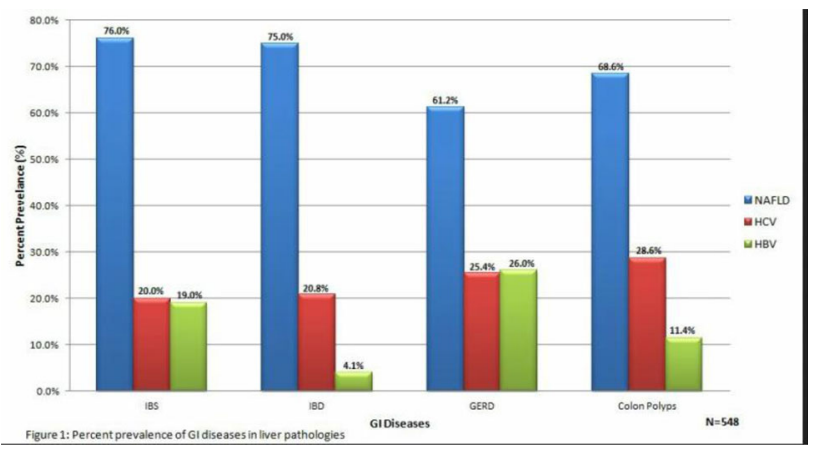

\section{LATEB-1}

Fatty acid oxidation defect masquerading as glycogen storage disease: report of three cases

\section{Ashwini Gupta ${ }^{1}$, Lalit Bharadia ${ }^{1}$, Vivek Jain ${ }^{1}$, Sandeep Kumar ${ }^{1}$}

\section{${ }^{1}$ Santokba Durlabh JI Memorial Hospital, India}

Background: Glycogen storage disease (GSD) has heterogeneous clinical presentation of varying severity ranging from asymptomatic hepatomegaly at one end and severe hypoglycemia, hepatic cirrhosis and progressive muscle weakness at other end.

Fatty acid oxidation defect (FAOD) also has a varied presentation, either neonatal onset (hyper ammonemia, transient hypoglycemia, cardiomyopathy and sudden death) or late onset with rhabdomyolysis and muscle cramps.

In a case of hepatomegaly and hypoglycemia in young children, FAOD is rarely thought as a differential while GSD is more often suspected.

We reports 3 cases which were initially misdiagnosed as GSD but on reevaluation were confirmed to have FAOD (two of these also had a genetic confirmation of fatty acid oxidation defect)

Method: During study period of 2 yr (Jan. 17- Dec. 18) children with suspected GSD based on massive hepatomegaly in second half of infancy, seizures and hypoglycemia were subjected to hemogram, liver function test, fasting blood sugar, prothrombin time, serum uric acid, serum Triglyceride level and liver biopsy. Children with GSD who didn't respond to conventional treatment in the form of uncooked corn starch were further evaluated by TMS with Exome sequencing.

Result: Out of 10 children with suspected GSD 6 were diagnosed to have GSD based on characteristic liver biopsy and suggestive lab reports. Of these, 2 children were eventually diagnosed as FAOD (Patient 1 and 2 in table No. 1). Patient no 3 sibling with similar presentation had succumbed while on treatment for presumptive GSD based on liver biopsy. Hence we straightaway got Exome sequencing which confirmed FAOD suggesting his sibling also must have had FAOD. 
Table No 1

\begin{tabular}{|c|c|c|c|c|c|c|c|c|c|}
\hline $\begin{array}{l}\text { S. } \\
\text { N } \\
\text { o. }\end{array}$ & $\begin{array}{l}\text { Age at } \\
\text { Presentat } \\
\text { ion }\end{array}$ & $\begin{array}{l}\mathrm{Se} \\
\mathrm{x}\end{array}$ & $\begin{array}{l}\text { Consangui } \\
\text { nity \& } \\
\text { Family } \\
\text { History }\end{array}$ & $\begin{array}{l}\text { Symptom } \\
\text { s at } \\
\text { Presentati } \\
\text { on }\end{array}$ & $\begin{array}{l}\text { Initial } \\
\text { Worku } \\
\text { p }\end{array}$ & $\begin{array}{l}\text { Initial } \\
\text { Diagno } \\
\text { sis }\end{array}$ & Course & $\begin{array}{l}\text { Subsequ } \\
\text { ent } \\
\text { Workup }\end{array}$ & $\begin{array}{l}\text { Final } \\
\text { Diagnosis }\end{array}$ \\
\hline \multirow[t]{2}{*}{1} & 9 months & $\mathrm{F}$ & $\begin{array}{l}\text { Consanguin } \\
\text { eous } \\
\text { marriage }\end{array}$ & $\begin{array}{l}\text { Doll's } \\
\text { faces } \\
\text { seizure? }\end{array}$ & $\begin{array}{l}\text { Liver } \\
\text { biopsy }\end{array}$ & GSD & $\begin{array}{l}\text { Not } \\
\text { improved } \\
\text { by }\end{array}$ & $\begin{array}{l}\text { TMS } \\
\text { CPT1 }\end{array}$ & FAD \\
\hline & & & & $\begin{array}{l}\text { Recurrent } \\
\text { resp. } \\
\text { illness, } \\
\text { hepatomeg } \\
\text { aly, no } \\
\text { splenomeg } \\
\text { aly }\end{array}$ & & & $\begin{array}{l}\text { conventi } \\
\text { onal } \\
\text { treatment }\end{array}$ & Def. & \\
\hline 2 & 9 months & $\mathrm{F}$ & $\begin{array}{l}\text { Non } \\
\text { Consanguin } \\
\text { eous } \\
\text { marriage, } \\
\text { two siblings } \\
\text { death within } \\
\text { 1st week of } \\
\text { life }\end{array}$ & $\begin{array}{l}\text { Jaundice } \\
\text {, hepatic } \\
\text { steatosis, } \\
\text { pigmented } \\
\text { stool, } \\
\text { anemia }\end{array}$ & $\begin{array}{l}\text { Liver } \\
\text { biopsy }\end{array}$ & GSD & $\begin{array}{l}\text { Not } \\
\text { improved } \\
\text { by } \\
\text { conventi } \\
\text { onal } \\
\text { treatment }\end{array}$ & \begin{tabular}{|l} 
TMS \\
and \\
Exome \\
sequenci \\
ng \\
showed \\
Carnitine \\
- acyl \\
carnitine \\
transloca \\
se def.
\end{tabular} & FAD \\
\hline 3 & 1 year & $\mathrm{M}$ & $\begin{array}{l}\text { Non } \\
\text { Consanguin } \\
\text { eous } \\
\text { marriage, } \\
\text { sibling } \\
\text { death, } \\
\text { hepatomega } \\
\text { ly fatty } \\
\text { liver. Liver } \\
\text { biopsy - } \\
\text { probable } \\
\text { GSD, } \\
\text { Wolman's } \\
\text { screen } \\
\text { negative }\end{array}$ & $\begin{array}{l}\text { Irritability, } \\
\text { hepatomeg } \\
\text { aly, no } \\
\text { splenomeg } \\
\text { aly }\end{array}$ & $\begin{array}{l}\text { TMS } \\
\text { suggesti } \\
\text { ve of } \\
\text { CPT1 } \\
\text { Deficie } \\
\text { ncy }\end{array}$ & FAD & $\begin{array}{l}\text { Had } \\
\text { Progressi } \\
\text { ve } \\
\text { deteriorat } \\
\text { ion and } \\
\text { succumb } \\
\text { ed }\end{array}$ & $\begin{array}{l}\text { Exome } \\
\text { sequenci } \\
\text { ng } \\
\text { confirme } \\
\text { d CPT1 } \\
\text { def. }\end{array}$ & $\begin{array}{l}\text { Retrospecti } \\
\text { vely, we } \\
\text { presume } \\
\text { that } \\
\text { previous } \\
\text { sibling also } \\
\text { had FAOD }\end{array}$ \\
\hline
\end{tabular}

*CPT1-Carnitine Palmitoyl Transferase Deficiency Type 1

Conclusion: Children with clinical diagnosis of GSD should have TMS with or without Exome sequencing as liver biopsy may not distinguish them from FAOD. TMS is an easily available test to rule out FAOD.

\section{LATEB-2}

Autoimmune liver diseases: dominance, overlap or variantsa large cohort clinicopathologic study

\section{Archana Rastogi ${ }^{1}$, Chhagan Bihari ${ }^{1}$, Mitali Singhal ${ }^{1}$, Shasthry $\mathrm{SM}^{2}$, Shiv Kumar Sarin ${ }^{2}$ \\ ${ }^{1} \mathrm{MD}, \mathrm{DNB}$, India; ${ }^{2} \mathrm{MD}, \mathrm{DM}$, India}

Background: Autoimmune hepatitis (AIH), Primary biliary cholangitis (PBC), Primary sclerosing cholangitis (PSC) are the main phenotypes of autoimmune liver diseases (AILD). Overlap designations lack well-validated diagnostic criteria; recent emphasis on variant phenotypes mandates careful correlation of histology with biochemical, serological and radiologic work-up. Liver histology considered the strongest diagnostic means, present study aimed to analyze autoimmune liver diseases for dominance, true overlaps and variants.

Method: Retrospective study of 415 of 478 patients with histological diagnosis of AILD (2010-2017), constituted the study group. Standard diagnostic criteria for AILDs and overlap syndrome with Histological activity index (HAI) score were applied. Biopsies were relooked in cases with biochemical and immunoserological overlaps or discordance.
Result: AILD (415 cases) were AIH in $250(60.2 \%)$, PBC in 67 (16.1\%), PSC in $38(9.2 \%)$, and overlap of AIH-PBC in $60(14.5 \%)$ cases. 20/250 (8\%) of AIH showed biochemical \&/or Immunoserologic overlap with PBC. 27/67 (40.3\%) of PBC and 14/38 (36.8\%) of PSC showed such overlap with AIH. 19/60 (31.7\%) of overlap cases had laboratory favouring AIH or PBC. Relook of biopsies revealed $\mathrm{HAI}>6$ in $100 \%$ (AIH, overlap) and in none (PBC, PSC). Histological diagnosis of dominant phenotype or overlap remained unaltered in 243/250 (97.2\%) AIH, (100\%) PSC, 64/67 (95.5\%) PBC, 47/60 (78.3\%) overlap syndrome.

Image1: Photomicrographs of AIH (A,D), PBC (B), PSC (C), AIHPBC overlap (E), AIH-PSC overlap (F). (HE, 200x)

Image 2: Table showing demographic \& laboratory parameters of AILD \& various phenotypes

Image3: Flowchart depicting spectrum of AILD with overlaps \& dominance based on histologic, biochemical, immunoserologic \& radiological correlation

Conclusion: AIH followed by $\mathrm{PBC}$ and overlap comprised majority of AILD. Biochemical \& immunoserologic overlap or discordance was least in $\mathrm{AIH}$ and maximum in PBC. Histology along with HAI score is pivotal in recognising dominant, variant and true overlaps.

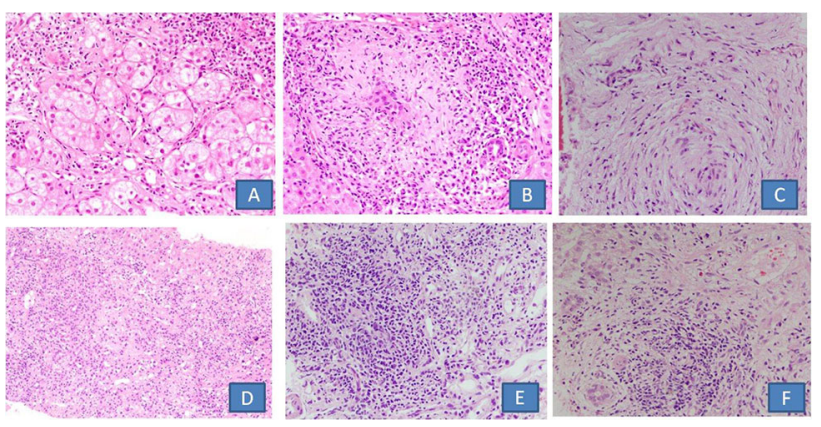

\begin{tabular}{|c|c|c|c|c|c|c|c|c|}
\hline & Age & M.F & $\begin{array}{l}\text { Bil(T) } \\
\text { mg/dl }\end{array}$ & $\begin{array}{l}\text { ALT } \\
(\mathrm{IU} / \mathrm{L})\end{array}$ & $\begin{array}{l}\text { AST } \\
(\mathrm{IU} / \mathrm{L})\end{array}$ & $\begin{array}{l}\text { ALP } \\
(\mathrm{IU} / \mathrm{L})\end{array}$ & $\begin{array}{l}\text { GGT } \\
\text { (IU/L) }\end{array}$ & $\begin{array}{l}\mathrm{IgG} \\
(\mathrm{g} / \mathrm{L})\end{array}$ \\
\hline \begin{tabular}{|l|} 
Total AlLD \\
(415)
\end{tabular} & $42.8 \pm 17.4$ & 1:1.7 & $\begin{array}{l}2.6 \\
(0.2-50)\end{array}$ & \begin{tabular}{|l}
79 \\
$(10-4740)$
\end{tabular} & \begin{tabular}{|l}
113 \\
$(12-2980)$
\end{tabular} & $\begin{array}{l}154 \\
(31-1290)\end{array}$ & \begin{tabular}{|l}
61 \\
$(10-1552)$
\end{tabular} & $22.46+8.10$ \\
\hline $\begin{array}{l}\text { AIH } \\
\text { (250) }\end{array}$ & $42.2+19.4$ & $1: 1.5$ & \begin{tabular}{|l}
2.7 \\
$(0.7-43.9)$
\end{tabular} & $\begin{array}{l}84.5 \\
(10-4740)\end{array}$ & $\begin{array}{l}123 \\
(12-2980)\end{array}$ & $\begin{array}{l}127 \\
(43-710)\end{array}$ & \begin{tabular}{|l}
49.5 \\
$(10-1463)$
\end{tabular} & $23.02+7.58$ \\
\hline $\begin{array}{l}\text { PBC } \\
\text { (67) }\end{array}$ & $48.9 \pm 9.6$ & 1:5.7 & $\begin{array}{l}1.8 \\
(02-26.7)\end{array}$ & $\begin{array}{l}74 \\
(14-820)\end{array}$ & $\begin{array}{l}97 \\
(22-1021)\end{array}$ & $\begin{array}{l}201 \\
(31-912)\end{array}$ & $\begin{array}{l}98 \\
(13-591)\end{array}$ & $20.45 \pm 7.76$ \\
\hline \begin{tabular}{|l} 
PSC \\
(38)
\end{tabular} & $30.2+12.5$ & 3.2:1 & $\begin{array}{l}4.0 \\
(0.5-50)\end{array}$ & $\begin{array}{l}86.5 \\
(18-599)\end{array}$ & $\begin{array}{l}114.5 \\
(44-549)\end{array}$ & $\begin{array}{l}285 \\
(108-1290)\end{array}$ & $\begin{array}{l}140 \\
(77-1552)\end{array}$ & $21.66 \pm 9.41$ \\
\hline \begin{tabular}{|l|} 
Overlap \\
$(60)$
\end{tabular} & $46.9 \pm 12.6$ & $1: 3.3$ & $\begin{array}{l}3.4 \\
(0.4-27.6)\end{array}$ & $\begin{array}{l}76 \\
(12-999)\end{array}$ & $\begin{array}{l}112.5 \\
(23-655)\end{array}$ & $\begin{array}{l}185 \\
(75-842)\end{array}$ & $\begin{array}{l}72.5 \\
(55-539)\end{array}$ & $22.99 \pm 9.23$ \\
\hline
\end{tabular}




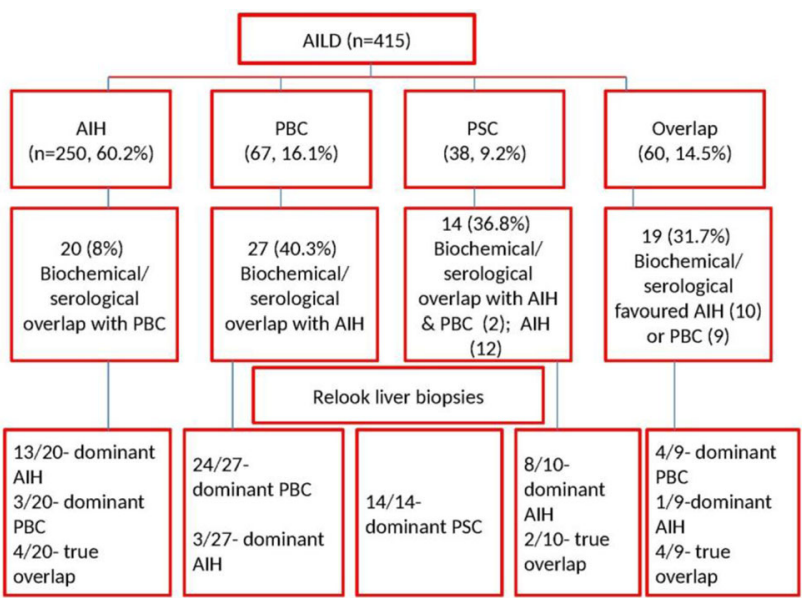

\section{LATEB-3}

Predictive factors associated with hepatitis c antiviral therapy response

\section{$\underline{\text { Ahmed Fottoh }}^{1}$, Ahmed Fottoh ${ }^{2}$, Ali Ghweil ${ }^{2}$ \\ ${ }^{1}$ Qena, Egypt; ${ }^{2}$ South Valley University, Egypt}

Background: Egypt represents the highest prevalence world-wide of $\mathrm{HCV}$ infection. According to EDHS, 15\% among the survey responders had antibodies to $\mathrm{HCV}$.

Method: The study included 200 patients randomized into two groups, the A group given dual therapy (Sofosbuvir and Daclatasvir, $\mathrm{n}$ $=130$ ) and the B group given triple therapy (Sofosbuvir, Daclatasvir and Ribavirin, $\mathrm{n}=70$ ) both for 12 weeks. The end point was the percentage of patients with HCV RNA $<15 \mathrm{IU} / \mathrm{mL} 12$ weeks after stopping therapy (SVR12).

Result: In group A, patients achieving SVR was 95.4\% $(\mathrm{n}=124)$, HCV treatment naïve patients had a higher rate of SVR12 $(97.9 \% \mathrm{n}=$ $95)$ than experienced patients $(87.9 \% \mathrm{n}=29)$ and patients with cirrhosis at baseline had no virological response at all $(0 \%, \mathrm{n}=3)$ compared to those with normal liver $(100 \%, \mathrm{n}=59)$ or abnormal (evidence of fibrosis) $(95.6 \%, \mathrm{n}=65)$. In group $\mathrm{B}$, percentage of patients who achieve SVR12 was $88.6 \%(\mathrm{n}=62)$, HCV treatment naïve patients achieve SVR12 $(90.9 \%, \mathrm{n}=40)$ higher than HCV treatment experienced $(84.6 \%, \mathrm{n}=22)$ and cirrhotic patients showed a good response $(78.4 \%, \mathrm{n}=29)$. The most common AEs were headache, fatigue and insomnia.

Conclusion: According to this study, most of the predictive factors to previous regimens have lost their value and almost have no role in predicting response of treatment. We also recommend Sofosbuvir plus Daclatasvir as a treatment for treatment-naïve and treatmentexperienced HCV patients. With adding Ribavirin to Sofosbuvir and Daclatasvir, triple therapy is a suitable option for patients with evidence of fibrosis or cirrhotic patients.
Table 1: Baseline Demographic Characteristics \& laboratory of two groups of patients

\begin{tabular}{|c|c|c|c|}
\hline \multirow{2}{*}{\multicolumn{2}{|c|}{ Parameter }} & \multicolumn{2}{|l|}{ Mean \pm SD } \\
\hline & & $\begin{array}{l}\text { Group A } \\
\mathrm{N}=130\end{array}$ & $\begin{array}{ll}\text { Group } \\
\mathrm{N}=70\end{array}$ \\
\hline \multicolumn{2}{|l|}{ Age } & $46.22 \pm 11.56$ & $46.96 \pm 11.50$ \\
\hline \multicolumn{2}{|l|}{ BMI } & $29.01 \pm 6.71$ & $27.45 \pm 5.06$ \\
\hline \multicolumn{2}{|l|}{ ALT } & $46.83 \pm 34.65$ & $43.30 \pm 20.61$ \\
\hline \multicolumn{2}{|l|}{ AST } & $43.27 \pm 23.55$ & $46.15 \pm 19.67$ \\
\hline \multicolumn{2}{|c|}{ Serum Albumin } & $4.04 \pm 0.42$ & $4.01 \pm 0.56$ \\
\hline \multicolumn{2}{|c|}{ Total Bilirubin } & $0.74 \pm 0.25$ & $0.88 \pm 0.33$ \\
\hline \multicolumn{2}{|c|}{$\begin{array}{l}\text { Serum } \\
\text { Creatinine }\end{array}$} & $0.97 \pm 0.82$ & $0.93 \pm 0.19$ \\
\hline \multicolumn{2}{|c|}{ WBC } & $6.47 \pm 2.01$ & $6.01 \pm 2.50$ \\
\hline \multicolumn{2}{|c|}{ Hemoglobin } & $14.07 \pm 1.16$ & $13.62 \pm 1.60$ \\
\hline \multicolumn{2}{|c|}{ Platelets } & $230.18 \pm 60.71$ & $\begin{array}{l}200.21 \pm 68.8 \\
6\end{array}$ \\
\hline \multicolumn{2}{|c|}{ HCV RNA PCR } & $\begin{array}{l}1295520.24 \pm \\
1919294.13\end{array}$ & $\begin{array}{l}1492960.71 \pm \\
2996920.55\end{array}$ \\
\hline \multicolumn{2}{|l|}{ FIB-4 } & $1.39 \pm 0.62$ & $2.19 \pm 1.72$ \\
\hline \multicolumn{2}{|c|}{ Blood Glucose } & $92.58 \pm 15.71$ & $96.47 \pm 17.62$ \\
\hline \multicolumn{4}{|c|}{ Number } \\
\hline \multirow[t]{2}{*}{ Sex } & M & 82 & 50 \\
\hline & $\mathrm{F}$ & 48 & 20 \\
\hline \multirow{2}{*}{$\begin{array}{l}\text { Treat- } \\
\text { ment } \\
\text { status }\end{array}$} & Naïve & 97 & 44 \\
\hline & $\begin{array}{l}\text { Experi } \\
\text { enced }\end{array}$ & 33 & 26 \\
\hline \multirow{3}{*}{$\begin{array}{l}\text { Liver } \\
\text { Status }\end{array}$} & Normal & 59 & 19 \\
\hline & $\begin{array}{l}\text { Abn- } \\
\text { ormal* }\end{array}$ & 68 & 14 \\
\hline & Cirrhosis & 3 & 37 \\
\hline
\end{tabular}

Table 2: Antiviral response of 2 groups

\begin{tabular}{|c|c|c|c|}
\hline \multirow[t]{2}{*}{ Parameter } & & \multicolumn{2}{|c|}{ Number(Percent) } \\
\hline & & $\begin{array}{l}\text { Group A } \\
(\mathrm{DCV}+\mathrm{SOF})\end{array}$ & $\begin{array}{l}\text { Group B } \\
(\mathrm{DCV}+\mathrm{SOF}+ \\
\mathrm{RBV})\end{array}$ \\
\hline \multicolumn{4}{|c|}{ Treatment response } \\
\hline \multirow[t]{2}{*}{ EOT* } & Yes & $126(96.9 \%)$ & $63(90 \%)$ \\
\hline & No & $4(3.1 \%)$ & $7(10 \%)$ \\
\hline \multirow[t]{2}{*}{ SVR } & Yes & $124(95.4 \%)$ & $62(88.6 \%)$ \\
\hline & No & $6(4.6 \%)$ & $8(11.4 \%)$ \\
\hline \multicolumn{4}{|c|}{ Treatment status } \\
\hline \multirow[t]{2}{*}{ Naïve } & Yes & $95(97.9 \%)$ & $40(90.9 \%)$ \\
\hline & No & $2(2.1 \%)$ & $4(9.1 \%)$ \\
\hline \multirow[t]{2}{*}{ Experienced } & Yes & $29(87.9 \%)$ & $22(84.6 \%)$ \\
\hline & No & $4(12.1 \%)$ & $4(15.4 \%)$ \\
\hline \multicolumn{4}{|c|}{ Liver condition } \\
\hline \multirow[t]{2}{*}{ Cirrhosis } & Yes & $0(0 \%)$ & $29(78.4 \%)$ \\
\hline & No & $3(100 \%)$ & $8(21.6 \%)$ \\
\hline \multirow[t]{2}{*}{ Normal } & Yes & $59(100 \%)$ & $19(100 \%)$ \\
\hline & No & $0(0 \%)$ & $0(0 \%)$ \\
\hline \multirow[t]{2}{*}{ Abnormal** } & Yes & $65(95.6 \%)$ & $14(100 \%)$ \\
\hline & No & $3(4.4 \%)$ & $0(0 \%)$ \\
\hline
\end{tabular}

* End of treatment

**Evidence of fibrosis 
Table 3: Complications (Safety and Tolerability)

\begin{tabular}{|l|l|}
\hline Parameter, n (\%) & $\begin{array}{l}\text { All Patients } \\
(\mathrm{N}=200)\end{array}$ \\
\hline Death & $0(0)$ \\
\hline AEs leading to discontinuation & $0(0)$ \\
\hline Serious AEs & $0(0)$ \\
\hline Patients with any AEs & $54(27 \%)$ \\
\hline Headache & $44(22 \%)$ \\
\hline Fatigue & $40(20 \%)$ \\
\hline Nausea & $27(13.5 \%)$ \\
\hline Diarrhea & $18(9 \%)$ \\
\hline Insomnia & $12(6 \%)$ \\
\hline Abdominal pain & $10(5 \%)$ \\
\hline Arthralgia & $9(4.5 \%)$ \\
\hline Jaundice & $13(7 \%)$ \\
\hline Anemia & $34(17 \%)$ \\
\hline Thrombocytopenia & $37(18.5 \%)$ \\
\hline Leucopenia & $20(9.5 \%)$ \\
\hline Pancytopenia & $17(8.5 \%)$ \\
\hline Total bilirubin $>2.5$ ULN* & $0(0)$ \\
\hline ALT $>5$ ULN & $0(0)$ \\
\hline AST $>5$ ULN & $0(0)$ \\
\hline RBV dose reductions or stoppage & $7(3.5 \%)$ \\
\hline Pneumonia & $0(0)$ \\
\hline Congestive cardiomyopathy & $0(0)$ \\
\hline Arteriosclerosis & $0(0)$ \\
\hline Basal cell carcinoma & $0(0)$ \\
\hline & \\
\hline
\end{tabular}

*ULN: upper limit of normal

\section{LATEB-4}

Supine chest compression: a rescue therapy in severe ARDS a case series

\author{
Shilpa Tiwari ${ }^{1}$, Geeta Rajput ${ }^{1}$, Lalita Gouri Mitra ${ }^{1}$, Vandana \\ Saluja $^{1}$ \\ ${ }^{1}$ Institute of Liver and Biliary sciences, India
}

Background: Achieving adequate oxygenation is a major challenge in severe ARDS. In liver failure patients with severe ARDS prone ventilation cannot be done due to coagulopathy, severe ascites, high vasopressor supports, cerebral edema and multiple invasive catheters in situ.

Method: In this case series of 5 patients we studied improvement in oxygenation by performing supine chest compression ventilation. By applying $2 \mathrm{KG}$ weight bilaterally over chest in supine position, mechanical ventilation was done. Improvement was assessed by Pao2/Fio2 ratio in sequential blood gas analysis every 6 hourly for next 24 hours.

Result: Improved oxygenation (Pao2/Fio2) was observed in all patients. Respiratory mechanics work to improve oxygenation almost as same as the mechanism proposed for prone position without any major adverse events and serious complications.

\section{Conclusion:}

- Supine chest compression ventilation proved to be a rescue therapy in this group of patients where prone ventilation technique cannot be performed.

- We suggest larger randomised study to determine efficacy and also to find out the optimum weight required to compress the chest.

\section{LATEB-5}

Ayurveda treatment outcome for the correction of thrombocytopenia in alcoholic decompensate cirrhosis: case series

\section{Ashok Kumar Panda ${ }^{1}$ \\ ${ }^{1}$ CARIHD, Bhubaneswar, India}

Background: Cirrhosis of liver is the end stage of every chronic liver disease. Most of the patient attend to our OPD in decompensate stage. Decompensate cirrhosis is defined by the presence of ascites, varicose bleeding, encephalopathy and/or jaundice. Thrombocytopenia (platelet count $<150 \times 109 / \mathrm{L})$ is a frequent complication of decompensate cirrhosis and is considered an indicator of advanced disease. The prevalence is $64 \%-84 \%$ of patients with cirrhosis or fibrosis in decompensate cirrhosis. The low platelet count is due partly to the effects of portal hypertension and hypersplenism, decreased thrombopoietin production, alcohol and virus-induced bone marrow suppression. Thrombopoietin is predominantly produced by the liver and is reduced when liver cell mass is severely damaged. This leads to reduced thrombopoiesis in the bone marrow and consequently to thrombocytopenia in the peripheral blood of patients with advanced-stage liver disease. It is being an indicator of advanced disease and poor prognosis. Platelet transfusion is the option in emergency conditions. Currently, only eltrombopag is approved for usage among patients with thrombocytopenia and chronic hepatitis $\mathrm{C}$ virus infection in order to initiate and maintain interferon-based antiviral treatment. Nevertheless, the optimal management of hematologic abnormalities among patients with chronic liver disease, and its risk for bleeding complications, is still a matter of discussion. The risk of bleeding complications can cause postponement of necessary procedures and therapy. Thromocytopenia is profoundly found in the routine check up of $\mathrm{CBC}$ in decompensate cirrhosis patients as patient opt ayurveda as last resort. Ayurveda treatment plan and procedure were on study to improve survival in decompensated cirrhosis.

There are more than three hundred herbo-mineral preparations in Indian system of medicine for the treatment of jaundice and chronic liver diseases. More than 50\% people of our country relay on Ayurveda and herbal medicine for liver diseases. Undoubtly Ayurveda herbs and products having defined biochemical active component can protect liver from oxidative stress, promote virus elimination, block fibrogenesis, anti inflammatory, immune-modulating, liver regenerating and inhibit tumour growth in vitro and in vivo studies. Phyto extracts of Carica papaya and Tinospora cordifolia (Thrombobliss) enhance platelet in patients with infectious like Dengue and other infections associated with thrombocytopenia. Therefore this attempt was taken to observe the effect of Carica papaya extract along with hepato protective herbs in alcoholic decompensate cirrhosis.

Method:

Aims and Objective: The aim of the study to assess the safety and efficacy of cariden(Phyto extracts of Carica papaya and Tinospora cordifolia) on thrombocytopenia of three pre diagnosed compensated cirrhosis patients. 
Cases presentation and history: Three male patients of alcoholic compensated cirrhosis attended our OPD for the treatment after advised for liver transplant. All patients have the history of thrombocytopenia since six months. All three have jaundice, ascites and one have hepatic encephalopathy .

Treatment design and outcome measure: The treatment protocol was initial digestive, hepato protective and immune-modulator. Ayurveda procedure like- Purgation along with $500 \mathrm{ml}$ milk daily for six months. All patients MELD score and Child -pugh score are assessed before and after treatment. Among three two patient have grade 2 thrombocytopenia and one patient has grade 3 thrombocytopenia. Cap of Cariden (Phyto extracts of Carica papaya 1100mg, Tinospora cordifolia and other $300 \mathrm{mg}$ ) is administrated in dose of one capsule twice daily after food. The base line platelet is compared with every 15 days for three months. The safety of the drug assessed by renal and liver function test

\section{Result:}

Treatment outcome and discussion: Cariden (Phyto extracts of Carica papaya $1100 \mathrm{mg}$ and Tinospora cordifolia $300 \mathrm{mg}$ ) has enhance the platelet count within 15 days and it can be normalise the platelet within 90 days of therapy in all three cases. It is safe as CBC, electrolytes, renal and liver function test of three patients no significant change with base line. Thrombocytopenia occurs frequently in alcoholics through a direct effect on the bone marrow. Alcohol reduces platelet life span and leads to ineffective megakaryopoiesis. This medicine may increased platelet production and maturation in alcoholic compensated cirrhosis patients. Therefore it is also good indicators of good prognosis and improving survival of these treated cases.

Conclusion: Cap of Cariden (Phyto extracts of Carica papaya $1100 \mathrm{mg}$, and Tinospora cordifolia $300 \mathrm{mg}$ ) is safe and it increased platelet production and maturation in three alcoholic compensated cirrhosis patients. Further pilot study is suggested.

\section{LATEB-6}

Circulating endothelial progenitor cells (EPC) present an inflammatory phenotype and function in patients with alcoholic Liver cirrhosis

Rashi Sehgal ${ }^{1}$, Savneet Kaur ${ }^{1}$, Shastry SM${ }^{1}$, Helen Mcguire ${ }^{3}$, Barbara Fazekas, Geoffrey Mccaughan ${ }^{2}$, Shiv K Sarin ${ }^{1}$, Nirupma Trehanpati ${ }^{1}$, Devanshi Seth ${ }^{4}$

${ }^{1}$ Institute of liver and biliary sciences, India; ${ }^{2}$ centenary institute australia, Australia; ${ }^{3}$ Centenary Institute of Cancer Medicine and Cell Biology, Australia; ${ }^{4}$ Royal Prince Alfred Hospital, Australia; ${ }^{5}$ The University of Sydndey, Australia

Background: Endothelial progenitor cells (EPCs) have been implicated in liver injury and repair. However, the phenotype and potential of these heterogenous EPCs remain elusive. In particular, their involvement in the pathogenesis of alcoholic liver cirrhosis (ALC) remains unclear. The current study extensively characterized the phenotype and functions of EPCs to understand their role in ALC pathogenesis.

Method: Patients with ALC $(n=45)$ and healthy controls $(\mathrm{HC}, \mathrm{n}=$ 25) were recruited in the study. Circulating EPCs were identified as CD34+CD133+CD31+ cells by flow cytometry. Circulating EPCs from an independent cohort of HC $(n=5)$ and ALC patients $(n=4)$, matched for age and gender, were comprehensively phenotyped by mass cytometer time of flight (CyTOF). Ex vivo cultures of circulating EPCs from a subset of patients and controls were also tested for their functions, including colony formation, LDL uptake and cytokine secretion (ELISA).
Result: Three distinct populations of circulating EPCs (CD34+CD133+CD31+) could be classified based on their CD45 expression, negative (CD45-), intermediate (CD45int) and high (CD45hi). CD45int and CD45hi EPCs significantly increased in ALC patients compared to controls $(\mathrm{p}<0.05)$. CyTOF data showed that CD45hi EPCs were distinct from CD45- and CD45int EPCs, with higher expression of $\mathrm{T}$ cell and myeloid markers, including $\mathrm{CD} 3$, CD4, HLA-DR, and chemokine receptors, CCR2, CCR5, CCR7 and CX3CR1. Similar to circulating EPCs, percentage of CD45hi CD34+CD31+ EPCs in ex vivo cultures, significantly increased in patients compared to HC ( $p=0.024)$. Cultured EPCs from patients also showed increased LDL uptake and release of TNF-alpha, RANTES, FGF-2 and VEGF.

Conclusion: We report the first extensive characterization of circulating human EPCs with distinct EPC subtypes in HC and ALC patients. Increase in CD45hi EPC subtype with enhanced functions, inflammatory cytokines and angiogenic mediators in patients suggesting an inflammatory role of these cells in ALC.

\section{LATEB-7}

Lower rates of baseline NS5b, NS5a resistance associated substitutions (RASs) in hepatitis $\mathrm{C}$ virus (HCV) infected chronic kidney disease patients than $\mathrm{HCV}$ infected patients with only liver disease: results of a pilot study from India

\section{Ekta Gupta $^{1}$, Manish Choudhary ${ }^{1}$, Suman Lata Nayak ${ }^{1}$, Manoj} Kumar Sharma $^{1}$, Shiv Kumar Sarin ${ }^{1}$

${ }^{1}$ Institute of liver and Biliary sciences, India

Background: Treatment of chronic hepatitis $\mathrm{C}$ virus (HCV) infection with direct-acting antiviral agents (DAA) is associated with high rates of sustained virologic response (SVR). Factors associated with treatment failure include advanced stages of liver fibrosis, response to previous antiviral therapy and viral factors such as baseline viral load and existence of resistance associated substitutions (RASs). Baseline resistance testing is currently not routinely considered or recommended. The role of these substitutions should be studied for treatment decisional support.

Method: In this study $\mathrm{HCV}$ isolates from 88 patients at baseline (before initiation of DAA treatment) were sequenced at the drug acting sites of NS5a and NS5b region by Sanger sequencing. Comparison were made between the isolates obtained from HCV infected patients with only liver disease (group $1, \mathrm{n}=60$ ) and HCV infected patients with coexisting chronic kidney disease (group 2, $\mathrm{n}=28$ ).

Result: The predominant genotype seen in group 1 was Genotype $3: 41(68.3 \%), 3 \mathrm{a}: 32 ; 3 \mathrm{~b}: 5$, while the predominant genotype seen in group 2 was Genotype 1: $17(60 \%)$ and all were subtype $1 \mathrm{a}$. HCV viral load was similar in both the groups 5.7 (R: 4.2-6.8) $\log 10 \mathrm{IU} / \mathrm{ml}$ in group 1 while in group 2 it was $6.07(\mathrm{R}: 3.9-7.7) \log 10 \mathrm{IU} / \mathrm{ml}(\mathrm{p}$ value: 0.25$)$. Overall the prevalence of RASs at baseline was seen in $22.7 \%$ of the study cohort. The prevalence was higher in group 1: $30 \%$ than in group 2: 7.1\%; p value $<0.001$.In group $1,11(18.3 \%)$ patients had NS5a RASs: in 7 patients Q30K, in $1 \mathrm{Y} 93 \mathrm{H}$, in $3 \mathrm{~L} 31 \mathrm{M} /$ L. In 7(11.6\%) NS5b RASs were seen: in 2 patients L159F, in 4 $\mathrm{C} 316 \mathrm{~N} / \mathrm{H}$ and in $1 \mathrm{~V} 321 \mathrm{I}$. In group 2 none of the patients showed RASs in the NS5b region of the virus while in NS5a region it was seen in $2(7.1 \%)$ patients as Q30K.

Conclusion: Prevalence of RAS at baseline in HCV infected patients with liver disease were much higher than HCV infected patients with CKD. Host immune status might influence the occurrence of these RAS in the virus and thereby controlling the response to the treatment. 


\section{LATEB-8}

Spectrum of histopathological changes in patients with noncirrhotic intrahepatic portal hypertension

\author{
Archana Rastogi ${ }^{1}$, Abhijit Chougule ${ }^{3}$, Rakhi Maiwall ${ }^{2}$, Chhagan \\ Bihari $^{3}$, Vikrant Sood ${ }^{2}$, Shiv Kumar Sarin ${ }^{2}$ \\ ${ }^{1} \mathrm{MD}$, DNB, India; ${ }^{2} \mathrm{MD}$, DM, India; ${ }^{3} \mathrm{MD}$, India
}

Background: Non-cirrhotic portal fibrosis (NCPF) is a clinical disorder characterized by features of portal hypertension in the absence of significant fibrosis. It is one of the commonest causes of portal hypertension in India. This study aimed to analyze detailed histomorphological spectrum of NCPF.

Method: Retrospective analysis of cases with clinical diagnosis of NCPF and compatible histopathology available, received between 2011 and 2016, were studied. Laboratory parameters were recorded in each case: haemoglobin $(\mathrm{Hb})$, white blood cell count, platelet count, prothrombin time, international normalized ratio and liver function tests. The serology/molecular analysis for hepatotrophic viruses, liver-specific autoantibody profile or other autoantibodies and results of thrombophilia workup were noted wherever available. Liver stiffness was measured in kilopascals $(\mathrm{kPa})$. The values of HVPG measurement were noted from the clinical files wherever available. The study material consisted of serial step sections of liver biopsy stained with haematoxylin \& eosin (H\&E), Masson's trichrome and orcein stains. In cases where nodularity was appreciated on $\mathrm{H} \& \mathrm{E}$ sections, reticulin silver stain was performed to confirm/exclude changes of NRH. A meticulous review of the following earlier reported histopathological features7 was done: number of portal tracts, number of hypoplastic portal tracts, approximation of portal tracts, portal tract fibrosis, presence or absence of portal vein branches, obliterative sclerosis of portal vein branches, thrombosis of small intrahepatic portal veins, arterialization of portal vein branches, portal angiomatosis, paraportal shunting vessels, nodular regeneration (NRH-like change), megasinusoids, perisinusoidal fibrosis, abnormal parenchymal veins and incomplete septal fibrosis. Other features such as portal inflammation, changes in the bile duct/hepatic artery, extramedullary hematopoiesis, hepatocytic steatosis, siderosis, cholestasis if any were also noted.

The quantitative data were expressed as mean or median values and the qualitative data were enumerated as frequencies and percentages. The comparisons of LS and HVPG with portal/periportal fibrosis and perisinusoidal fibrosis were made.

Result: There were 67 specimens from 66 patients which included 43 $(65.2 \%)$ male and $23(34.8 \%)$ female patients with a mean age of 31 years (range: 7-61 years). The liver function tests showed only a mild derangement. The average length of biopsy was $1.4 \mathrm{~cm}$ (median: $1.2 \mathrm{~cm}$, range: $0.8-3.4 \mathrm{~cm}$ ) and the mean number of portal tracts per biopsy was 11.1 (median: 10, range: 5-30). Most cases showed a combination of histological features; the mean number of histological features noted per biopsy was 7.4 (median: 7, range: 3-12). Obliterative portal venopathy was seen $47.8 \%$ cases. Portal angiomatosis $(61.2 \%)$, paraportal shunt vessels $(61.2 \%)$, portal vein dilatation $(74.6 \%)$, hypoplastic portal tracts $(56.7 \%)$, megasinusoids $(64.1 \%)$ and abnormally dilated central veins $(64.1 \%)$ were other prevalent features. Portal/periportal fibrosis and perisinusoidal fibrosis was seen in $77.6 \%$ and $61.2 \%$ cases; none showed bridging fibrosis or cirrhosis. The median hepatic venous pressure gradient (HVPG) and liver stiffness (LS) values were $8 \mathrm{~mm}$ of $\mathrm{Hg}$ (range: $5-20 \mathrm{~mm}$ of $\mathrm{Hg}$ ) and 9.2 $\mathrm{kPa}$ (range: $4.4-26.3 \mathrm{kPa}$ ). There was no correlation of HVPG or LS with either portal/periportal fibrosis or perisinusoidal fibrosis.

Conclusion: Due to relatively non-specific and non-pathognomonic nature, a combination of different histological features in the absence of significant fibrosis and appropriate clinicoradiological background is needed for diagnosing NCPF. HVPG or LS did not show any correlation with either portal/periportal fibrosis or perisinusoidal fibrosis.

\section{LATEB-9}

Late Liver allograft dysfunction: Histopathological spectrum with analysis of rejection histology

Archana Rastogi ${ }^{1}$, Chhagan Bihari ${ }^{3}$, Sowmya Uthaiah ${ }^{3}$, Ashok Choudhary $^{2}$, Senthil Kumar ${ }^{4}$, Viniyendra Pamecha ${ }^{4}$, Shiv K Sarin $^{2}$

${ }^{1} \mathrm{MD}$, DNB, India; ${ }^{2} \mathrm{MD}$, DM, India; ${ }^{3} \mathrm{MD}$, India; ${ }^{4} \mathrm{MS}$, India

Background: Spectrum of pathologic diagnosis for late graft dysfunction of liver transplantation is broad and overlapping. Increasing need to understand the causes necessitates the study of histological spectrum along with rejection histology.

Method: 198 clinically indicated liver allograft biopsies from 94 recipients, of 410 transplants (mainly LDLT) between 2010 - May 2017, were selected as the study group. Histological diagnosis with single and multiple pathologies were noted. Rejection biopsies were categorized into inflammatory (1), biliary (2) or mixed (3). Inflammation was subcategorized as predominant portal (A), central (B) or mixed (C). Categories 1A, 1B, 1C, 2, 3A, 3B, 3C were correlated with LFTs.

Result: $94 / 410$ (22.9\%) of the recipients had late liver graft dysfunction. Single pathology was noted in $182 / 198$ biopsies $(91.9 \%)$ and combined pathologies in $16 / 198(8.1 \%) .48$ recipients had repeat biopsies- $2(n=24), 3(n=7), 4(n=9), 5(n=4), 6(n=5), 7(n=2)$. Most common cause of late graft dysfunction was rejection- late acute rejection (73), chronic rejection (20). Other pathologies included: biliary (13), recurrent disease (10), vascular (12), steatohepatitis (7), sepsis (6), chronic hepatitis (4), cholestasis (5). Graft biopsies were inadequate (3) and no specific pathology (21). Combined pathologies included rejection with recurrence, biliary, sepsis or steatohepatitis. 93 biopsies of rejection scored 1A (26), 1B (3), 1C (24), 2 (2), 3A (15), 3B (6) and 3C (17). With treatment, improvement in LFT was noted in $1 \mathrm{~A}-17 / 26(65.4 \%), 1 \mathrm{~B}$-none, 1C- 7/24 (29.2\%), 2- none, 3A- 6/15 (40\%), 3B-none, 3C- 5/17 (29.4\%).

Conclusion: Late allograft dysfunction occurred in $22.9 \%$ recipients, rejection being the commonest and combined pathologies observed in $8 \%$. Amongst rejection biopsies, portal based inflammation was the most frequent, followed by mixed portal-perivenular inflammation. Biliary changes and perivenular inflammation showed lesser improvement in LFTs, thus poorer prognosis.

\section{LATEB-11}

\section{Differential diagnosis between intra hepatic metastasis or multicentric carcinogenesis by mutational analysis in hepatocellular carcinoma}

\section{Yoshihiko Ooka $^{1}$, Yosuke Hirotsu ${ }^{3}$, Hitoshi Moshizuki ${ }^{2}$, Kenji Amemiya $^{3}$, Masao Omata ${ }^{3}$ \\ ${ }^{1}$ Chiba university hospital, Japan; ${ }^{2}$ Yamanashi Central Hospital, Japan; ${ }^{3}$ Yamanashi Prefectural Central Hospital, Japan}

Background: The accurate differential diagnosis of Intrahepatic metastasis and multicentric carcinogenesis is often difficult by radiographic and/or pathological findings and/or tumor location. The gene mutations analysis of HCC tissues by using next-generation sequencing may distinguish IM from MC in multifocal HCCs. 
Method: We investigated two patients with multifocal HCCs who received resection and examined the gene mutations of their resected specimen by using next-generation sequencer.

Result: Patient 1 had three nodules (two HCCs and one dysplastic nodule), Patient 2 had two HCCs. The radiological or histopathological findings were difficult to distinguish IM from MC accurately in both two patients. Genomic profiles of three nodules in patient 1 were very different. There was no common conserved mutation between three nodules. Two nodules in patient 2 had a common conserved mutation (TERT promoter 228) with high allele frequency and also had different mutations with low minor allele frequency. The nodules that had different pathological feature in same tumor had a common conserved mutation and some different mutations.

Conclusion: Gene mutation analysis using the next-generation sequencer clarified the dissimilarity between tumors and it also revealed tumor heterogeneity. It brings the differentiation between IM and $\mathrm{MC}$ is one of the application.

\section{LATEB-12}

Post-bleeding ascites in children with extrahepatic portal venous obstruction: risk factors and long term outcome

\section{Moinak Sen Sarma ${ }^{1}$, Anshu Srivastava ${ }^{1}$, Surender Kumar Yachha $^{1}$, Ujjjal Poddar \\ ${ }^{1}$ Sanjay Gandhi Postgraduate Institute of Medical Sciences, Lucknow, India}

Background: Ascites in a setting of extrahepatic portal venous obstruction (EHPVO) without an underlying chronic liver disease is uncommon. Post-bleeding ascites is an unexplored entity. We aimed to study a) the overall prevalence and etiological profile of ascites and b) risk factors and natural history of post-bleeding ascites in EHPVO patients.

Method: All admitted patients of EHPVO with clinically detectable ascites \pm gastrointestinal (GI) bleeding within $<6$ weeks duration were analysed. Post-bleeding ascites (PBA) was defined as appearance of ascites after the index GI bleeding. PBA was compared with the cohort who had GI bleeding without ascites (BWA) admitted during the same period. Concomitant chronic liver diseases were excluded. Laboratory and growth parameters at the index episode were compared with a prior follow-up 3-6months in non-sick, nonbled state. The difference was termed as fall from baseline.

Result: Of the total 307 analysed EHPVO patients, 26\% ( $\mathrm{n}=79)$ presented with ascites. 83\% $(n=66)$ were PBA, mostly variceal bleeding from esophageal varices $(79 \%)$. The rest $(n=13)$ were of other etiologies (figure 1). On comparison of PBA ( $n=66$ ) vs. BWA (n =228), statistical significance was seen with age of disease onset [6.7 (1-16) vs. $11(0.5-18)$ years; $\mathrm{p}=0.03$ ], peripheral edema (29 vs. $30 ; \mathrm{p}$ $<0.001$ ), postural symptoms (50 vs. $141 ; \mathrm{p}=0.04$ ), height $\mathrm{z}$ scores [-2.3 (-4.3 to 1.5$)$ vs. $-1.7(-3.2$ to 2.0$) ; \mathrm{p}=0.01]$ at admission and weight $\mathrm{z}$ scores [-2.6 (-4.1 to 2.5$)$ vs. $-1.8(-2.2$ to 2.5$) ; \mathrm{p}=0.01]$ at ascites resolution. Statistical significance was also seen between PBA and BWA with index parameters: serum protein [5.5 (3.8-6.9) vs. 6.6 (3.6-7.9) $\mathrm{mg} / \mathrm{dL} ; \mathrm{p}=0.04]$, albumin $[2.9(1.8-4.3)$ vs. $3.6(1.8-5.1)$ $\mathrm{mg} / \mathrm{dL} ; \mathrm{p}=0.02]$, hemoglobin $[6.3(2.9-8.8)$ vs. $7.2(2.6-11.5) ; \mathrm{p}=$ $0.02]$ and also with fall from baseline: serum protein $[1.6(0.3-2.2)$ vs. $0.4(0.1-1.4) \mathrm{mg} / \mathrm{dL} ; \mathrm{p}=0.001]$, albumin $[0.9(0.5-1.4)$ vs. $0.4(0.3-$ 1.1) $\mathrm{mg} / \mathrm{dL} ; \mathrm{p}=0.02]$, hemoglobin [4.6 (3.4-6.6) vs. $2.3(2.1-3.7) ; \mathrm{p}=$ $0.001]$. Time interval (first bleeding bout to admission) [12 (5-20) vs. $10(3-20)$ days; $p=0.7$ ], transfusion requirement (57 vs. $180 ; p=0.2$ ) and ongoing endotherapy (11 vs. $62, \mathrm{p}=0.8)$ were non-significant between PBA vs. BWA. 32\% $(\mathrm{n}=21)$ had spontaneous ascites resolution (salt restriction) in $3(2-7)$ days. $39 \%(n=26)$ required additional diuretics.29\% $(\mathrm{n}=19)$ had severe ascites requiring large volume paracentesis [70 $(53-110) \mathrm{mL} / \mathrm{kg}] .17 \%(\mathrm{n}=11)$ had ascitic fluid infection. Outcome of ascites group $(n=79)$ is shown in figure 1 .

Conclusion: 26\% EHPVO have ascites after GI bleeding. Early age of disease onset, large volume of GI bleeding and poor nutritional status were risk factors for development of ascites in EHPVO. Overall outcome of EHPVO with ascites is favourable.

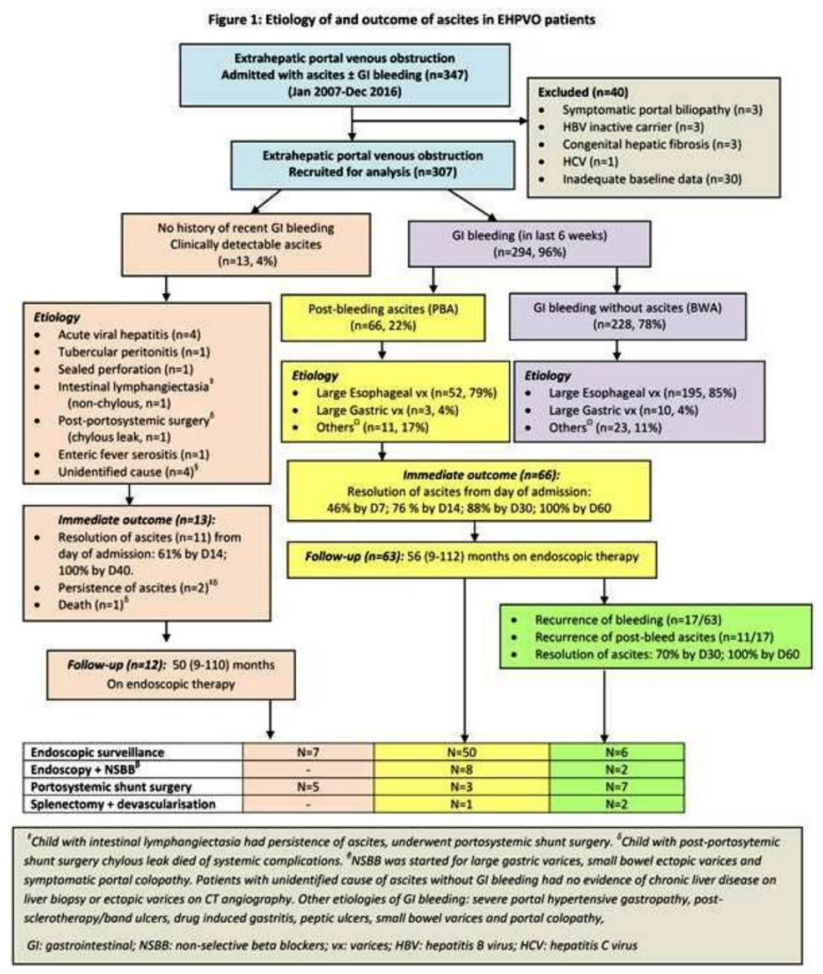

\section{LATEB-13}

Post-paracentesis circulatory dysfunction with repeated large volume paracentesis in chronic liver disease: prospective longitudinal study in children

\section{Moinak Sen Sarma ${ }^{1}$, Surender Kumar Yachha ${ }^{1}$, Anshu Srivastava $^{1}$, Vijayalakshmi Bhatia ${ }^{1}$}

${ }^{1}$ Sanjay Gandhi Postgraduate Institute of Medical Sciences, Lucknow, India

Background: Ascites is a common complication of cirrhosis and is associated with poor prognosis. Post-paracentesis circulatory dysfunction (PPCD) is overall seen in one-third of cirrhotic children who undergo single-time large volume paracentesis (SLVP). It is not known whether PPCD occurs with repeated LVP (RLVP). Our study aimed to analyse the safety, complications and effect of albumin infusion during RLVP on PPCD in children with severe ascites due to chronic liver disease.

Method: Enrolled children with severe ascites underwent single time or repeated LVP with albumin infusion (figure 1). Maximal D6 value and delta (difference of baseline and D6) plasma renin activity (PRA) of RLVP were compared with SLVP. Worsening PPCD was defined as $\geq 1.5$ times increase of delta PRA between the sessions in the RLVP group. "At-risk" group was defined as any cirrhotic child with $\geq 2$ worsening PPCD episodes. Their outcome at 3 months and 
maximal follow-up were noted. Poor outcome was defined as failure of definitive therapy, need for liver transplantation or mortality.

Result: Table 1 shows baseline characteristics of 37 children (SLVP, $\mathrm{n}=17$; RLVP, $\mathrm{n}=20$ ). $92 \%$ had high PRA at the onset. The overall incidence of PPCD was 43\% (SLVP: $12 \%$, RLVP: 70\%; $\mathrm{p}=0.003$ ). Baseline pediatric end-stage liver disease (PELD) score $>27$ was associated with PPCD (sensitivity: $90 \%$; specificity: $50 \%, \mathrm{p}=0.01$ ) in RLVP group. Worsening PPCD $(\mathrm{n}=28 ; 54 \%)$ and at-risk children ( $=9 ; 45 \%$ ) were identified in 52 sessions of RLVP. In the RLVP group, PPCD occurred if cumulative ascitic fluid extraction volume was $>585 \mathrm{~mL} / \mathrm{kg}$ (sensitivity: $90 \%$; specificity: $50 \%, \mathrm{p}=0.01$ ), cumulative albumin infusion $<4.2 \mathrm{~g} / \mathrm{kg}$ (sensitivity: $93 \%$; specificity: $55 \%, \mathrm{p}=0.03$ ) and frequency of sessions were $>2.3 /$ week (sensitivity: $91 \%$; specificity: $57 \%, \mathrm{p}=0.02$ ). In those who developed PPCD, lowest volume of ascites extracted in SLVP and RLVP groups were 117 and $471 \mathrm{~mL} / \mathrm{kg}$ respectively. RLVP patients $(80 \%)$ were susceptible to asymptomatic, persistent hyponatremia (baseline vs. day 6 from first LVP, $131 \pm 4$ vs. $124 \pm 4 \mathrm{mEq} / \mathrm{L} ; \mathrm{p}=0.001$ ), hemodynamic changes $(25 \%)$, renal impairment $(35 \%)$, recurrence of ascites $(60 \%)$ and hospital readmission $(70 \%)$. At risk patients had worsening of PELD scores (baseline: $25 \pm 8$ vs. follow-up $31 \pm 6$; $\mathrm{p}=0.01)$ at 3 months. Poor outcome at maximal follow-up $(18 \pm 7.4$ months) was seen in $35 \%$ ( $n=1$, SLVP; $n=12$, RLVP) especially in the at-risk group $(\mathrm{n}=8 ; 89 \%)$.

Conclusion: Risk factors of PPCD with repeated LVP are baseline PELD $>27$, cumulative extracted ascites $>585 \mathrm{~mL} / \mathrm{kg}$, albumin infusion $<4.2 \mathrm{~g} / \mathrm{kg}$ and frequency of sessions $>2.3 /$ week. One third have poor outcome, more in the at-risk group

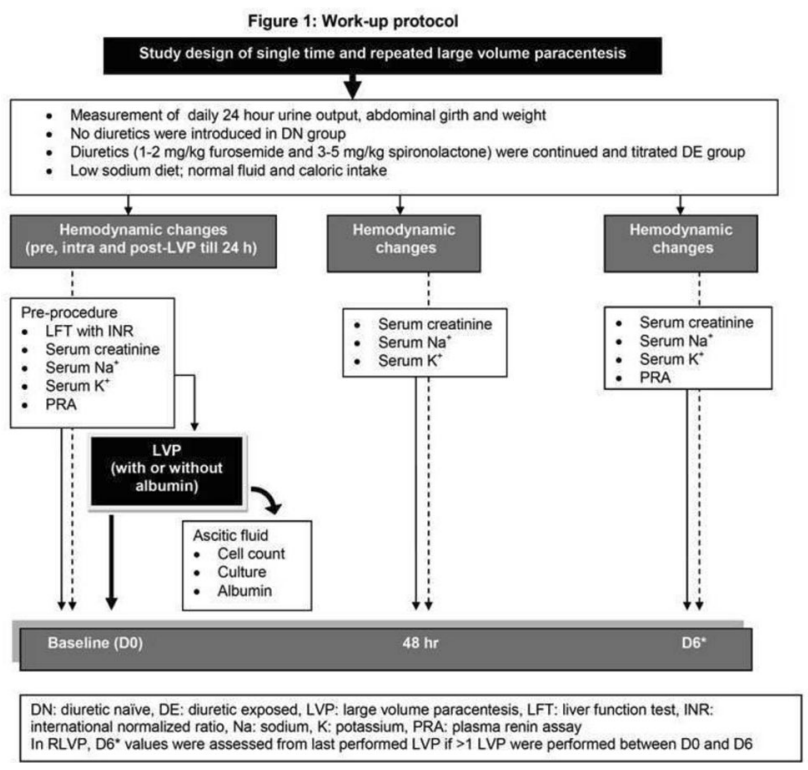

\begin{tabular}{|c|c|c|c|}
\hline & $\begin{array}{c}\text { Single time LVP } \\
\text { group }(n=17)\end{array}$ & $\begin{array}{c}\text { Repeated LVP } \\
\text { group }(n=20)\end{array}$ & p value \\
\hline Age $(y)$, boys (n) & $9.8 \pm 4.4,13$ & $8.3 \pm 5.1,15$ & $0.77,0.81$ \\
\hline \multicolumn{4}{|l|}{ Etiology of liver disease $(n)$} \\
\hline - Budd-Chiari syndrome & 3 & 7 & \\
\hline - Wilson disease & 5 & 5 & \\
\hline - Autoimmune liver disease & 1 & 4 & \\
\hline - Chronic hepatitis B & 2 & 1 & \\
\hline - Biliary atresia & 1 & 0 & \\
\hline - Alagille syndrome & 1 & 0 & \\
\hline - Cryptogenic & 4 & 3 & \\
\hline \begin{tabular}{|l} 
Duration of ascites (months) \\
\end{tabular} & $3.6 \pm 5.3$ & $7.1 \pm 11.0$ & 0.20 \\
\hline Weight Z-scores & $-1.69 \pm 2.21$ & $-2.51 \pm 1.84$ & 0.26 \\
\hline \begin{tabular}{|l} 
Height Z-scores \\
\end{tabular} & $-0.98 \pm 1.75$ & $-1.39 \pm 1.32$ & 0.46 \\
\hline Child-Pugh score (A/B/C) & $0 / 3 / 14$ & $0 / 4 / 17$ & 0.71 \\
\hline PELD score & $22 \pm 6$ & $28 \pm 10$ & 0.05 \\
\hline Volume of ascites extracted $(\mathrm{mL} / \mathrm{kg})^{2}$ & $143 \pm 77$ & $506 \pm 105$ & 0.001 \\
\hline Ascitic fluid infection ( $n$ ) & 4 & 7 & 0.47 \\
\hline Baseline PRA ( $\mathrm{ng} / \mathrm{mL} / \mathrm{h})$ & $27.5 \pm 18.4$ & $29.1 \pm 20.7$ & 0.65 \\
\hline Maximum value of PRA $(\mathrm{ng} / \mathrm{mL} / \mathrm{h})$ & $28.3 \pm 21.2$ & $73.1 \pm 19.8$ & 0.01 \\
\hline Folds elevation from baseline* & $1.1 \pm 0.5$ & $6.4 \pm 5.3$ & 0.02 \\
\hline Number of subjects with PPCD (n) & 2 & 14 & 0.003 \\
\hline \multicolumn{4}{|c|}{ 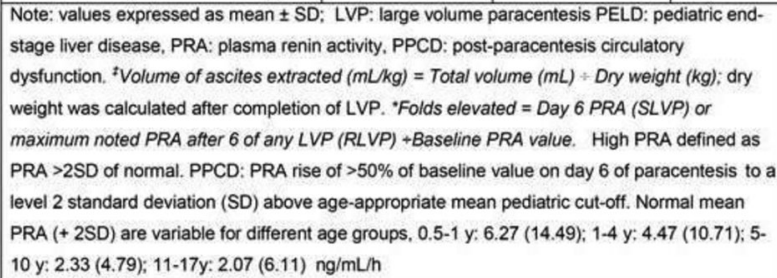 } \\
\hline
\end{tabular}

\section{LATEB-14}

Are non-cirrhotic portal fibrosis bleeders different from nonbleeders? A first time analysis in children

\section{Moinak Sen Sarma ${ }^{1}$, Durga Prasad ${ }^{2}$, Moinak Sen Sarma ${ }^{2}$, Surender Kumar Yachha ${ }^{2}$, Anshu Srivastava ${ }^{2}$, Ujjal Poddar ${ }^{2}$ \\ ${ }^{1}$ Sanjay Gandhi Postgraduate Institute of Medical Sciences, Lucknow, India; ${ }^{2}$ Sanjay Gandhi Postgraduate Institute of Medical Sciences, India}

Background: Non-cirrhotic portal fibrosis (NCPF) is a rare cause of pediatric portal hypertension. Though there is abundant literature in adults, there is paucity of data in children. Endoscopic eradication of varices and conservative management are the mainstay of therapy whereas portosystemic shunt surgery is debatable. We aimed to evaluate and compare the endoscopic and long-term outcomes of biopsy-proven NCPF bleeders and non-bleeders.

Method: Diagnosis of NCPF was based on all of the following: 1) splenomegaly \pm varices on endoscopy, 2) dilated portal \pm splenic vein 3 ) preserved liver functions and 4) absence of cirrhosis on liver histology \pm presence of characteristic histology and 5) exclusion of all known chronic liver diseases. The cohort was analysed for their clinical, endoscopic outcome and disease progression. Variceal bleeders and non-bleeders underwent secondary and primary prophylaxis respectively followed by endoscopic surveillance. Betablockers were only indicated for bleeding gastric varices

Result: 45 NCPF children (21 boys) aged 14 (6-18) years and symptom duration 12 (1-120) months presented as incidental splenomegaly (53\%) and acute variceal bleeding (31\%). Overall $22(49 \%)$ were variceal bleeders. $91 \%$ had hypersplenism with overall splenic size $10(1-17) \mathrm{cm}$ and enlarged liver (58\%). Percutaneous (73\%) and transjugular $(27 \%)$ liver biopsy showed characteristic obliterative portal venopathy $(68 \%)$. Initial endoscopic evaluation $(n=45)$ 
showed esophageal varices (93\%), gastric varices $(55 \%)$ and portal hypertensive gastropathy (89\%). Table 1 compares primary (nonbleeders, $\mathrm{n}=11$ ) and secondary prophylaxis (bleeders, $\mathrm{n}=22$ ). Modalities used were endoscopic band ligation $(n=30)$ followed by sclerotherapy ( $\mathrm{n}=13$ bleeders) for esophageal varices and glue injection followed by beta-blockers ( $\mathrm{n}=3$ gastric variceal bleeders). Table 2 shows progression of portal hypertension of 12 unbled patients who did not require primary prophylaxis. Overall follow-up duration of 26 (3-120) months, $87 \%$ did not show disease progression. Poor outcome were seen exclusively in six bleeders $(n=3$ death from interval bleeding, $\mathrm{n}=2$ decompensation, $\mathrm{n}=1$ hepatopulmonary syndrome). 4 children ( $\mathrm{n}=3$ bleeders) underwent splenectomy with devascularisation (splenic pain and mucocutaneous bleeds due to hypersplenism).

Conclusion: Endoscopic outcome of esophageal varices is similar in bleeders versus non-bleeders. Primary prophylaxis significantly decreases the gastric varices. Though there is progression in portal hypertension in unbled patients, majority of NCPF have preserved liver functions. Small proportion of bleeders have poor outcome and need for surgery.

Table 1: Endoscopic Outcome of patients receiving endotherapy ( $n=33$ )

\begin{tabular}{|c|c|c|c|}
\hline Parameters & $\begin{array}{c}\text { Bleeder }(n=22) \\
\text { Secondary prophylaxis }\end{array}$ & $\begin{array}{l}\text { Non bleeder }(n=11) \\
\text { Primary prophylaxis }\end{array}$ & p-value \\
\hline Required endotherapy & $22(100 \%)$ & $11(100 \%)$ & - \\
\hline Initial grade of esophageal varices (IV/I11/11/1) & $10 / 8 / 4 / 0$ & $7 / 3 / 1 / 0$ & 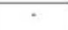 \\
\hline Total No of endoscopic sessions till $1^{11}$ eradication" & $8(2-12)$ & $6(2-9)$ & 0.3 \\
\hline Recurrence of esophageal varices ${ }^{T}$ & $8(36 \%)$ & $4(36 \%)$ & 0.6 \\
\hline $\begin{array}{l}\text { Time interval between } 1^{\text {th }} \text { eradication and } \\
\text { recurrence }(\mathrm{mos})^{\mathrm{b}}\end{array}$ & $8(3-36)$ & $11(6-42)$ & 0.2 \\
\hline Number requiring $2^{\text {no }}$ eradication & $6(27 \%)$ & 2 (18\%) & 0.4 \\
\hline Sessions required for $2^{\text {nd }}$ eradication ${ }^{\circ}$ & $3(2-5)$ & $4(2-7)$ & 0.4 \\
\hline $\begin{array}{l}\text { Gastric varices at onset } \\
(\mathrm{GOV} 1 / \mathrm{GOV} 2 / 1 \mathrm{GV})^{8}\end{array}$ & $\begin{array}{l}19(8) \\
(18)\end{array}$ & $\begin{array}{l}8(7236) \\
(7 / 3 / 1)\end{array}$ & 0.6 \\
\hline $\begin{array}{l}\text { Gastric varices at last endoscopy } \\
\text { (GOV1/GOV2/1GV) }\end{array}$ & $\begin{array}{l}14(63 \%) \\
(12 / 2 / 1)\end{array}$ & $\begin{array}{l}4(36 \%) \\
(4 / 1 / 1)\end{array}$ & 0.001 \\
\hline PHG at onset (mild/severe)" & $\begin{array}{c}22(100 \%) \\
(18 / 4)\end{array}$ & $\begin{array}{c}7(63 \% 6) \\
(5 / 2)\end{array}$ & 0.003 \\
\hline PHG at last endoscopy (mild/severe)" & $\begin{array}{c}13(59 \%) \\
(9 / 4)\end{array}$ & $\begin{array}{c}4(36 \%) \\
(3 / 1)\end{array}$ & 0.3 \\
\hline \multicolumn{4}{|c|}{ 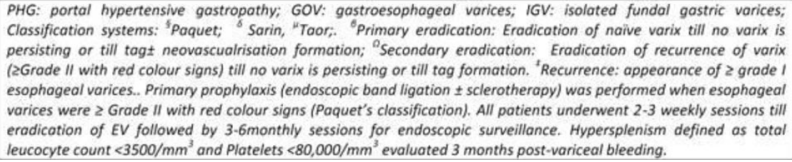 } \\
\hline
\end{tabular}

Table 2: Progression of varices in unbled patients with small varices not on primary prophylaxis ( $n=12)$

\begin{tabular}{|l|c|c|}
\hline Parameters & At presentation & Till last follow - up \\
\hline Esophageal varices (Grade IV/III/II/I) & $10(839)$ & $12(100 \%)$ \\
& $(0 / 0 / 5 / 5)$ & $(1 / 3 / 5 / 3)$ \\
\hline Development of large esophageal varices & 0 & $6(50 \%)$ \\
( $\mathrm{G}$ Grade II with red colour signs) & $5(41 \%)$ & $9(75 \%)$ \\
\hline Gastric varices at onset (GOV1/GOV2//GV) & $(4 / 1 / 0)$ & $(6 / 2 / 1)$ \\
\hline & $6(50 \%)$ & $8(66.6 \%)$ \\
\hline PHG (mild/severe) & $(4 / 2)$ & $(5 / 3)$ \\
\hline Classification systems and abbreviations as per table 1
\end{tabular}

LATEB-15

Pediatric Hepatic Wilson Disease: Effect of chelation therapy and outcome in a large cohort

Moinak Sen Sarma ${ }^{1}$, Mridul Chandra Das ${ }^{2}$, Moinak Sen Sarma ${ }^{2}$, Surender Kumar Yachha ${ }^{2}$, Anshu Srivastava ${ }^{2}$, Ujjal Poddar ${ }^{2}$

${ }^{1}$ Sanjay Gandhi Postgraduate Institute of Medical Sciences, Lucknow, India; ${ }^{2}$ Sanjay Gandhi Postgraduate Institute of Medical Sciences, India
Background: As there is paucity of exclusive literature on pediatric hepatic Wilson disease (WD), this study was aimed to evaluate clinical presentation and outcome of children with hepatic WD.

Method: WD children satisfying $\geq 2$ of 3 criteria (serum ceruloplasmin $<20 \mathrm{mg} / \mathrm{dL}$, positive Kayser-Fleischer ring, 24 hour urine copper $>40 \mu \mathrm{g}) \pm$ liver histology managed in our department from Jan 2007 to June 2016 were analyzed. Patients with $\geq 9$ months of adequate follow-up were evaluated for response to chelation therapy. They were analyzed as following categories: a) complete improvement (asymptomatic + normalization of transaminases, serum albumin and coagulation); b) partial improvement (asymptomatic + near normalization of transaminases + serum albumin or coagulation) c) progression (deterioration of synthetic functions, decompensation or death); d) drug toxicity. To determine the prognostic factors of outcome, the follow-up cohort was divided into 2 groups a) Good outcome ( $>9$ months follow up with complete or partial improvement) and b) Poor outcome defined by worsening liver synthetic functions, need for liver transplantation, lost to follow-up within 9 months of starting therapy or death.

Result: 111 WD children aged 108 (36-180) months and overall PELD score 16 (-11 to 60). Liver histology was consistent with WD in $68 \%(\mathrm{n}=27 / 40)$. Figure 1 shows the presentation and outcome.Chelation was started in $84 \%(n=94) .14 \%(n=16)$ patients died in hospital due to advanced disease. 65with follow up of 43 (9144) months were analyzed for outcome. 92\% ( $n=60)$ were on D-penicillamine monotherapy and $6 \%(\mathrm{n}=4)$ with additional severe neurological presentation were on D-penicillamine and zinc. Among those on D-penicillamine monotherapy, favourable outcome was seen in $58 \%(\mathrm{n}=35) .13 \%(\mathrm{n}=8)$ had drug toxicity and $12 \%(\mathrm{n}=7)$ required addition of zinc due to disease progression. $6 \%(\mathrm{n}=4)$ were started on trientine. Overall favourable outcome was observed in $70 \%$ $(n=46)$. Table 1 shows comparison of good and porr outcome patients. On logistic regression analysis, of all the variable PELD score and Nazar score were best predictors of outcome $(p<0.001)$. Conclusion: In this large pediatric experience of hepatic WD we have shown expanded clinical forms with various manifestations. Majority were manageable with D-penicillamine monotherapy. Addition or change of chelation is required in select situations of drug toxicity and neurological presentation.

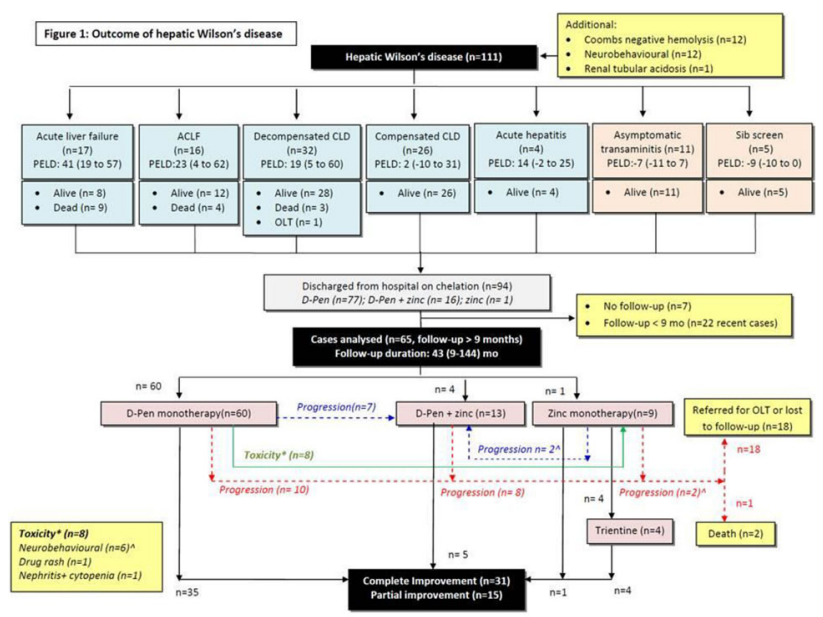




\begin{tabular}{|c|c|c|c|}
\hline \multicolumn{4}{|c|}{ Table 1: Comparison of good and bad outcome patients of Wilson disease } \\
\hline Parameters & Good outcome (n=47) & Poorentcome $(n=60)$ & Prvalue \\
\hline Age of diagnosis (month) & $108(36-174)$ & $108(53-180)$ & 0.23 \\
\hline Age of onset (month) & $96(36-168)$ & $106(53-176)$ & 0.15 \\
\hline Bilirubin (mg/dl) & $1.8(0.32-53.2)$ & $3.4(0.29-54.6)$ & 0.003 \\
\hline INR & $1.6(0.9-5)$ & $3.1(1-15)$ & 0.000 \\
\hline Albumin (gm/dl) & $2.8(1.4-5)$ & $2.5(1.1-4.3)$ & 0.018 \\
\hline PELDscore & $8(-11-48)$ & $23.5(-10-62)$ & $<0.001$ \\
\hline $\operatorname{ALP}(<150$ IU/dl) & $354(31-1297)$ & $158(9-1286)$ & 0.002 \\
\hline Serum Na(meq/L) & $139(128-146)$ & $136(117-150)$ & 0.038 \\
\hline Creatinine (mg/dl) & $0.6(0.2-1.1)$ & $0.6(0.2-1.8)$ & 0.174 \\
\hline Hemoglobin (gm/dl) & $11.3(6-14.9)$ & $9.3(4.4-12.8)$ & 0.000 \\
\hline WBC connt $\left(\times 10^{3}\right)$ & $7.8(2.8-17.9)$ & $7.2(2.6-30.4)$ & 0.688 \\
\hline Platelet connt $\left(\times 10^{3}\right)$ & $149.5(50-490)$ & $104.5(30-375)$ & 0.001 \\
\hline Durationof follow up (month) & $47(9-144)$ & $2.25(0-123)$ & $<0.001$ \\
\hline New Wilson Index & $7(1-17)$ & $11(1-20)$ & 0.007 \\
\hline Nazar's score & $4(0-12)$ & $5.5(0-12)$ & 0.001 \\
\hline Hemolysis (number) & 7 & 16 & $0.16^{\wedge}$ \\
\hline Renal involvement (number) & 4 & 7 & $0.42^{n}$ \\
\hline Neurological involvement (number) & 4 & 7 & $0.42^{n}$ \\
\hline Encephalopathy & 4 & 16 & $0.01 \wedge$ \\
\hline
\end{tabular}

\section{LATEB-16}

Congenital hepatic fibrosis in children: experience from a tertiary care center

Moinak Sen Sarma ${ }^{1}$, Aathira Ravindranath ${ }^{2}$, Moinak Sen Sarma ${ }^{2}$, Surender Kumar Yachha ${ }^{2}$, Anshu Srivastava ${ }^{2}$, Ujjal Poddar ${ }^{2}$

${ }^{1}$ Sanjay Gandhi Postgraduate Institute of Medical Sciences, Lucknow, India; ${ }^{2}$ Sanjay Gandhi Postgraduate Institute of Medical Sciences, India

Background: Congenital Hepatic Fibrosis (CHF) is an uncommon cause of portal hypertension in children. It is a developmental anomaly of the hepatobiliary system which is associated with extrahepatic organ involvement. Our objective is to study the various modes of presentation and evaluate their outcome.

Method: Details of patients with CHF (liver histology proven) \pm Caroli syndrome (based on imaging and portal hypertension without liver biopsy) were retrieved from electronic records from Jan 2002 to Dec 2017. Their clinical, laboratory, endoscopic profiles and outcomes were evaluated. Varices were graded as per Paquet (esophageal), Sarin (gastric location) and Hashizume (gastric size) grading system. Those with follow-up $<6$ months were excluded from outcome analysis. Poor outcome was defined progression of portal hypertension, liver disease, need for portosystemic shunt surgery or liver transplantation

Result: Forty-two cases of CHF $(n=27) \pm$ Caroli syndrome $(n=15)$ constituted $2 \%$ of all portal hypertension. They presented with overall age of 78 (IQR 24-125) months and symptom duration of 12 (IQR $2-24)$ months. Extended family history was contributory in $5 / 42$ (12\%). 13/27 (48\%) liver biopsies had features of ductal plate malformation. All cases of Caroli syndrome were diagnosed by MRI ( $\mathrm{n}=$ 13) and ultrasonography $(n=2)$. Their predominant modes of presentation and outcomes in each group are shown in table 1. Extrahepatic manifestations $(\mathrm{n}=12,29 \%)$ were medullary sponge kidney $(\mathrm{n}=3)$, medullary nephrocalcinosis $(\mathrm{n}=3)$, Autosomal Recessive Polycystic Kidney Disease (ARPKD, $n=3$ ), renal tubular acidosis $(n=1)$, renal calculi $(n=1)$, Joubert syndrome $(n=1)$ and polyarteritis nodosa $(n=1)$. Associated hepatobiliary disease were extra hepatic portal vein obstruction $(n=4)$, bile duct stricture $(n=1)$ and type 1 choledochal cyst $(\mathrm{n}=1)$. The liver outcome was assessed in 29/42 (70\%) over 28 (IQR 10-60) months follow up. 20/29 (69\%) had no progression of portal hypertension with stable liver disease. $9 / 29(31 \%)$ showed progression in disease $[\geq 1$ of the following: variceal bleed/recurrence $(n=5)$, symptomatic hypersplenism $(n=3)$, decompensation $(n=3)$, portal colopathy $(n=1)]$. 4/29 $(14 \%)$ had recurrent cholangitis. Two needed Surgery intervention [portosystemic shunt surgery $(\mathrm{n}=1)$; hepaticojejunostomy + splenectomy $(\mathrm{n}=$ $1)$ ] and referred for liver transplantation $(n=4)$. Comparison of poor outcome between CHF $(n=6)$ and Caroli syndrome $(n=3)$ was not significant $(\mathrm{p}=0.8$ ) 2/3 ARPKD developed stage III chronic kidney disease.

Conclusion: $71 \%$ of CHF present as incidentally detected organomegaly and portal hypertension. $31 \%$ have progression in disease. Liver outcome of CHF is similar to Caroli syndrome.

\begin{tabular}{|c|c|c|c|c|}
\hline & $\begin{array}{l}\text { Hematemesis (n=11) } \\
26.1 \%\end{array}$ & $\begin{array}{l}\text { Incidentally detected } \\
\text { organomegaly }(n=19) \\
45.2 \%\end{array}$ & \begin{tabular}{c|}
$\begin{array}{c}\text { Neonatal cholestasis } \\
(n=7) 16.6 \%\end{array}$ \\
\end{tabular} & $\begin{array}{l}\text { Cholangitis ( } n=5) \\
11.9 \%\end{array}$ \\
\hline $\begin{array}{l}\text { Age at presentation (mo) } \\
\text { median (IQR) }\end{array}$ & 84 (IQR 60-144) & 60 (IQR 30. 84) & 6.5 (IQR $1.5-20)$ & 90 (IQR 24-132) \\
\hline Varices at presentation & 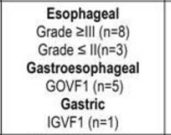 & $\begin{array}{l}\text { Esophageal } \\
\text { Grade } 2 \| 1(n=7) \\
\text { Grade } \leq \|(n=12) \\
\text { Gastroesophagageal } \\
\text { GOVF1 }(n=2)\end{array}$ & $\begin{array}{c}\text { Esophageal } \\
\text { Grade } \geq \| 11(n=1) \\
\text { Grade } \leq \|(n=4) \\
\text { Not evaluated }(n=2) \\
\text { Gastroesophageal } \\
\text { GOVF1 (n=1) }\end{array}$ & $\begin{array}{c}\text { Esophageal } \\
\text { Grade } \geq \| 1 \mid(n=3) \\
\text { Grade } \leq \mid 1(n=2) \\
\text { Gastroessophageal } \\
\text { GOVF1 }(n=3)\end{array}$ \\
\hline $\begin{array}{c}\text { Total no of endoscopic } \\
\text { sessions }\end{array}$ & $\begin{array}{c}E V L+E S T(n=51) \\
\text { Glue }(n=2)\end{array}$ & EVL+EST (n=17) & EST $(n=1)$ & $E V L+E S T(n=12)$ \\
\hline Endoscopic Outcome & $\begin{array}{c}\text { Esophageal } \\
\text { Grade } 1(n=8) \\
\text { Eradicated }(n=3) \\
\text { Gastroesophgeal } \\
\text { GOVIF1 ( } n=5 \text { ) persisting } \\
\text { Gastric } \\
\text { IGVF1 }(n=1) \text { persisting }\end{array}$ & $\begin{array}{l}\text { Esophageal } \\
\text { Grade } 1(n=3) \\
\text { Eradicated }(n=0) \\
\text { Gastroesophgeal } \\
\text { GOV1F1 (n=2) }\end{array}$ & $\begin{array}{c}\text { Esophageal } \\
\text { Grade } \leq \| 1(n=3) \\
\text { No gastric varices }\end{array}$ & $\begin{array}{c}\text { Esophageal } \\
\text { Grade } 1(n=3) \\
\text { Eradicated }(n=0) \\
\text { Gastroesoophgeal } \\
\text { GOV1F1(n=3) }\end{array}$ \\
\hline $\begin{array}{l}\text { Variceal Rebleeding or } \\
\text { recurrence }\end{array}$ & 1 (non-compliance) & 1 (recurrence) & . & - \\
\hline Follow-up (mo) & 12 (IQR 6-53) & 11.5 (IQR 6.5-63.5) & 3 (IQR 2.5-80) & 10 (IQR 8-10) \\
\hline Long term outcome & $\begin{array}{c}\text { Stable liver disease }(n=5) \\
\text { Symptomatic hyypersplenism } \\
\text { (n=2) } \\
\text { Decompensation }(n=1) \\
\text { Portal colopothy y }(n=1) \\
\text { LTF }(n=2)\end{array}$ & $\begin{array}{c}\text { Stable liver disease }(n=9) \\
\text { Decompensation }(n=2) \\
\text { Cholangitis }(n=1) \\
\text { Variceal Bleed }(n=4) \\
\text { LTF }(n=8)\end{array}$ & $\begin{array}{c}\text { Stable liver disease }(n=3) \\
\operatorname{LTF}(n=4)\end{array}$ & $\begin{array}{l}\text { Stable liver disease (n=3) } \\
\text { Symptomatich chypersplenism } \\
\quad(n=1) \\
\text { Recurrent cholangitis }(n=3) \\
\text { LTF }(n=1)\end{array}$ \\
\hline rotherapy; F1: H & astroesophageal varic & $\begin{array}{l}\text { isolated } \\
\text { TF: lost to }\end{array}$ & & ligation; EST: Endoscopic \\
\hline
\end{tabular}

\section{LATEB-17}

Increased collagen 1 and fibronectin during liver injury promotes the activation of hepatic progenitor cells

\section{Hamda Siddiqui $^{1}$, Preety Rawal ${ }^{2}$, Mojahidul Islam ${ }^{1}$, Chaggan Bihari $^{1}$}

${ }^{1}$ ILBS, India; ${ }^{2}$ Gautam Buddha University, India

Background: Liver regeneration after partial hepatectomy involves the proliferation of hepatocytes. However, during liver injury, bipotential hepatic progenitor cells (HPC) or oval cells (in rodents) are also known to participate in liver repair and regeneration. Since liver extracellular matrix (ECM) largely regulates HPC behavior, in the current study, we investigated the role of ECM factors in the activation and proliferation of oval cells in a choline-deficient, ethioninesupplemented (CDE) dietary mice model.

Method: Four-week-old mice were fed either normal chow (control) or a CDE diet (at different time points (10 day, 20 day, 10 day withdrawal). Liver biochemical parameters, histology, oval cell markers [evaluated by CK19 and EPCAM immunohistochemistry (IHC)] and expression of important ECM genes were determined in different animal groups. The effect of ECM factors on oval cell proliferation was investigated by MTT assay and flow cytometry analysis in the in vitro studies.

Result: IHC results showed significant expression of oval cells in the periportal regions in mice after 10 and 20 days of diet-induced injury. The oval cell response decreased by about $40-50 \%$ in mice in which diet was withdrawn after 10 days as compared to the mice which were given the diet for 20 days. The ECM gene expression results illustrated maximum expression of collagen 1a and fibronectin on day 20. 
Further, in vitro studies showed an increase of more than $50 \%$ in the proliferation of hepatic cells and an increase of about $20 \%$ in the expression of hepatic progenitor cell markers on collagen 1 and fibronectin-coated wells as compared to untreated wells.

Conclusion: The study shows that an increase in collagen 1 and fibronectin during liver injury promote the proliferation of HPCs, suggesting a crucial role of ECM modulation in HPC-mediated liver repair

\section{LATEB-18}

Outcome and predictors of mortality in patients with antitubercular drugs induced liver injury: a single center experience

\section{Sunil Kumar $N^{1}$, Thazhath Mavali Ramachandran ${ }^{1}$ \\ ${ }^{1}$ Department of Gastroenterology, Medical college, Kozhikode, India}

Background: Anti-tubercular (ATT) drug-induced liver injury (DILI) is a leading cause of DILI and drug-induced acute liver failure (ALF) in India. Unlike other drugs, re-challenge of primary ATT drug becomes essential for effective management of tuberculosis and hence, knowledge on clinical course and prognosis in ATT DILI is important. We undertook this study to determine clinical characteristics, outcome and predictors of mortality in patients with ATT DILI. Method: This is a prospective observational study conducted in department of Gastroenterology, Medical college, Calicut, India from January 2014 till july 2017. All patients with DILI secondary to ATT drugs were enrolled in the study after exclusion of other causes. Causality assessment was done by RUCAM (Roussel Uclaf Causality Assessment Method) scoring. Patients were followed up for 6 months from the time of diagnosis or until normalization of liver tests.Clinical characteristics, outcome and predictors of mortality in these patients were studied.

Result: There were a total of 133 cases of DILI over a period of 4 years. There were a total of 50 cases with ATT DILI during this period. Mean age of patients was 46.1 years and males constituted $54 \%$. Most common symptoms were Anorexia, vomiting and jaundice. On RUCAM causality assessment, $6.6 \%$ had possible, $44.5 \%$ had probable, $48.9 \%$ had highly probable chance of drug injury $71.1 \%, 17.8 \%$ and $11.1 \%$ had hepatocellular, mixed and cholestatic type of liver injury respectively. Among ATT induced DILI, 10 patients died constituting $20 \%$ mortality. The mean duration of drug exposure after symptom onset was significantly higher among non survivors compared to survivors $(9.9 \pm 3.1$ vs $4.8 \pm 2.6$ days, $\mathrm{p}<$ $0.001)$.On multivariate analysis, MELD score, mean creatinine, mean INR, bilirubin and drug exposure after symptom onset were predictors of mortality in ATT induced DILI patients.

On regression analysis, mean MELD, bilirubin and INR in combination showed a model for predicting mortality in these patients. The Cut-off for these variables and its sensitivity/specificity are shown below. MELD score of $>28$, mean INR of $>2.17$ and bilirubin $>$ 12.45 showed a sensitivity of more than $90 \%$ for mortality in these patients. DILI:

Regression model for predicting mortality in patients with ATT

\begin{tabular}{|l|l|l|l|}
\hline Variables & Cut-off & Sensitivity(\%) & Specificty(\%) \\
\hline MELD & 28 & 90 & 97.1 \\
\hline Mean INR & 2.17 & 100 & 94.3 \\
\hline $\begin{array}{l}\text { Mean } \\
\text { Bilirubin(mg/dl) }\end{array}$ & 12.45 & 90 & 77.1 \\
\hline
\end{tabular}

Conclusion: ATT DILI results in significant morbidity and mortality and has important clinical implications. Clinical and hematological parameters like MELD, INR, bilirubin and creatinine helps in predicting severity of liver injury.

Table 1: Comparison of baseline characteristics between patients having DILI related to ATT and non ATT drugs

\begin{tabular}{|c|c|c|c|}
\hline $\begin{array}{l}\text { Drug } \\
\mathrm{n}=133\end{array}$ & $\begin{array}{l}\text { ATT } \\
\mathrm{n}=50\end{array}$ & $\begin{array}{l}\text { Non ATT } \\
\mathrm{n}=83\end{array}$ & Significance \\
\hline Age(in years) & $46.1+/-13.4$ & $45.6+/-17.7$ & 0.868 \\
\hline Gender(Male/Female) & $27 / 23$ & $42 / 41$ & 0.704 \\
\hline BMI $\left(\mathrm{kg} / \mathrm{m}^{2}\right)$ & $20.3+1-3.4$ & $21.7+1-4.0$ & 0.043 \\
\hline MELD & $17.3+/-10.8$ & $12.6+l-6.5$ & 0.002 \\
\hline DRESS & 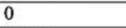 & $15.7 \%$ & 0.003 \\
\hline R factor* & $34 / 9 \pi$ & $48 / 21 / 14$ & 0.491 \\
\hline $\begin{array}{l}\text { Mean duration of drug } \\
\text { exposure(in days) }\end{array}$ & $75.1+1-63.3$ & $43.3+1-61.6$ & 0.006 \\
\hline Ascites & $14 \%$ & $12 \%$ & 0.740 \\
\hline Hepatomegaly & $16 \%$ & $15.7 \%$ & 0.950 \\
\hline $\mathrm{Hb}(\mathrm{gm} / \mathrm{dl})$ & $11.7+/-1.7$ & $12.5+/-1.7$ & 0.012 \\
\hline WBC count $\left(10^{3} / \mathrm{dl}\right)$ & $9385.7+/-4538$ & $9134.5+1-3121$ & 0.706 \\
\hline Platelet(105/dl) & $2.3+/-1$ & $2.6+1-0.8$ & 0.106 \\
\hline Mean INR & $1.89+/-1.2$ & $1.33+l-1.2$ & 0.014 \\
\hline Peak INR & $2.2+1-2.2$ & $1.4+/-1.3$ & 0.013 \\
\hline Mean Bilirubin(mg/dl) & $7.3+1-6.8$ & $5.9+1-6.4$ & 0.225 \\
\hline Peak Bilirubin(mg/dl) & $10.2+/-9.8$ & $7.5+1-8.3$ & 0.089 \\
\hline Protein(gm/dl) & $6.6+1-0.7$ & $6.7+1-0.6$ & 0.466 \\
\hline Albumin(gm/dl) & $3.3+1-0.6$ & $3.6+1-0.5$ & 0.013 \\
\hline Mean ALT(IU/t) & $512.9+/-522$ & $286.7+l-374$ & 0.004 \\
\hline Mean ALP(IU/t) & $178.6+l-110$ & $189.9+l-130$ & 0.608 \\
\hline $50 \%$ reduction in ALT(in days) & $14.9+/-11$ & $16.0+l-13.8$ & 0.669 \\
\hline Normalisation of liver tests & $31.4+1-22.2$ & $31.4+1-22.9$ & 0.988 \\
\hline Recovery & $68 \%$ & $81.9 \%$ & 0.137 \\
\hline
\end{tabular}

*R factor: Hepatocellular/Mixed/Cholestatic.

Abbreviation: BMI: Body mass index; DRESS: Drug reaction with eosinophilia and systemic symptoms; Hb: hemoglobin; WBC: White blood cell; INR: International normalized ratio; ALT: Alanine transaminase; ALP: Alkaline phosphatase.

\begin{tabular}{|c|c|c|c|}
\hline & $\begin{array}{l}\text { Survivors } \\
\mathrm{n}=40\end{array}$ & $\begin{array}{l}\text { Non survivors } \\
\mathrm{n}=10\end{array}$ & Significance \\
\hline Age & $43.8+1-13.6$ & $51.2+1-11.1$ & 0.126 \\
\hline Sex(Male/Female) & 19/21 & $8 / 2$ & 0.078 \\
\hline BMI & $19.9+1-2.9$ & $20.3+1-3$ & 0.744 \\
\hline MELD & $13.2+1-7.4$ & $31.9+/-10.3$ & $<0.00$ \\
\hline Symptoms: Anorexia & $37.1 \%$ & $30 \%$ & 0.677 \\
\hline Symptoms: Jaundice & $17.1 \%$ & $30 \%$ & 0.370 \\
\hline $\begin{array}{l}\text { Symptoms: Pain } \\
\text { abdomen }\end{array}$ & $2.9 \%$ & $10 \%$ & 0.334 \\
\hline $\begin{array}{l}\text { Drug exposure after } \\
\text { symptoms(in days) }\end{array}$ & $4.8+1-2.6$ & $9.9+/-3.1$ & $<0.00$ \\
\hline $\mathrm{Hb}(\mathrm{gm} / \mathrm{dl})$ & $11.9+/-1.5$ & $11.5+1-1.9$ & 0.449 \\
\hline WBC $\operatorname{count}\left(10^{3} / \mathrm{dl}\right)$ & $8637+1-3389$ & $12348+/-7317$ & 0.026 \\
\hline Platelet(105/d1) & $2.6+l-1$ & $1.6+/-0.4$ & 0.004 \\
\hline Creatinine(mg/dl) & $0.8+/-0.1$ & $1.7+/-1.1$ & $<0.00$ \\
\hline Mean INR & $1.4+1-0.7$ & $3.7+/-1.3$ & $<0.00$ \\
\hline Total protein(gm/dl) & $6.7+1-0.7$ & $6.2+1-0.4$ & 0.098 \\
\hline Albumin(gm/dl) & $3.4+1-0.6$ & $3+1-0.5$ & 0.079 \\
\hline Mean Bilirubin(mg/dl) & $4.6+1-4.8$ & $16.4+1-5.5$ & $<0.00$ \\
\hline Mean ALT(IU/t) & $412.4+1-356$ & $789.5+1-782$ & 0.037 \\
\hline Mean ALP(IU/t) & $173.0+/-111$ & $183.7+1-82$ & 0.781 \\
\hline
\end{tabular}

\section{LATEB-19}

Early renal dysfunction in cirrhotic children by renal resistive and pulsatility indices: prospective controlled study to assess effect of paracentesis and albumin therapy

Moinak Sen Sarma ${ }^{1}$, Moinak Sen Sarma ${ }^{1}$, Durga Prasad ${ }^{1}$, Surender Kumar Yachha ${ }^{1}$, Raghunandan Prasad ${ }^{1}$, Anshu Srivastava $^{1}$, Ujjal Poddar ${ }^{1}$

${ }^{1}$ Sanjay Gandhi Postgraduate Institute of Medical Sciences, Lucknow, India 
Background: There are no reliable tools to detect early renal dysfunction (RD) in cirrhotic children. Azotemia is influenced by sarcopenia in liver diseases. Renal resistive index (RI) and pulsatility index (PI), features of renal vasoconstriction are non-invasive methods to detect early RD before azotemia sets in. We aimed to detect early RD in cirrhotic children by renal doppler ultrasonography (DU) and observe the effect of paracentesis and albumin infusion on RI and PI

Method: Healthy controls and consecutive cirrhotics with normal creatinine/glomerular filtration rate were prospectively enrolled. Decompensated patients with tense ascites underwent large volume paracentesis (LVP) with or without albumin infusion and diuretics were initiated at $48 \mathrm{~h}$. RI and PI derived from bilateral renal artery velocity (upper, mid and lower poles) on DU by a single blinded sonologist were measured at baseline, 48h, D7, D30 and D90 of LVP. $\mathrm{RD}$ was defined as RI $>0.7$ or PI $>1.35$ from the average of all poles. Groups compared were: healthy controls vs. compensated cirrhotics, compensated cirrhotics vs. decompensated cirrhotics and decompensated albumin infusion group (AG) vs. non-albumin group (NAG). All cases were followed up for 3 months for complications and mortality

Result: Comparison of healthy controls vs. compensated cirrhotics: RI: $0.53(0.46-0.63)$ vs. $0.55(0.48-0.64) ; \mathrm{p}=0.23$ and PI: $1.06(0.73-$ $1.23)$ vs. $1.11(0.75-1.41) ; \mathrm{p}=0.16$. Table 1 shows the comparison of compensated vs. decompensated cirrhotics. Paracentesis overall improves the RI in cirrhotics. Sustained effect of RI and PI was seen in AG till D30 as compared to NAG group till D7 of LVP. At followup, 20\% decompensated cirrhotics developed renal failure and $23 \%$ died. Renal failure patients $(n=6)$ compared to non-renal failure $(\mathrm{n}=$ 24) had higher RI $[0.77(0.62-0.83)$ vs. $0.67(0.46-0.75), \mathrm{p}<0.001]$ and PI [1.24 (0.95-1.54 vs. $1.11(0.87-141) ; \mathrm{p}<0.001]$. None of the compensated patients developed renal failure in 3 months. Table 2 compares the profile of dead vs. alive patients in decompensated group.

Conclusion: Early renal dysfunction is present in $50 \%$ of non-azotemic decompensated cirrhotics detected by renal resistive and pulsatility indices. Large volume paracentesis and albumin infusion have short term benefit in early renal dysfunction. A significant proportion of decompensated cirrhotics develop renal failure and mortality in follow-up.

\begin{tabular}{|c|c|c|c|c|c|}
\hline & $\begin{array}{c}\text { Compensated } \\
\text { cirrhotic group; } \\
\text { CC (n=30) }\end{array}$ & $\begin{array}{c}\text { Overall } \\
\text { decompensated } \\
\text { cirrhotic group; } \\
\text { DC ( } \mathrm{n}=30 \text { ) }\end{array}$ & \begin{tabular}{c|} 
Decompensated \\
Albumin group: \\
AG $(\mathrm{n}=16)$
\end{tabular} & $\begin{array}{l}\text { Decompensated } \\
\text { Non-albumin } \\
\text { group: } \\
\text { NAG (n=14) }\end{array}$ & $\begin{array}{c}\text { P value } \\
\text { "(CC vs. DC) } \\
\text { "(AG vs. NAG) }\end{array}$ \\
\hline Age (years) & $12(7-18)$ & $10(6-18)$ & $9(6-17)$ & $11(7.18)$ & $0.56^{\circ}, 0.78^{\prime}$ \\
\hline $\begin{array}{l}\text { Etiology } \\
\text { Wilson disease } \\
\text {. Autoimmune liver disease } \\
\text { Budd-chiari syndrome }\end{array}$ & $\begin{array}{l}16 \\
10 \\
4\end{array}$ & $\begin{array}{l}13 \\
6 \\
11\end{array}$ & $\begin{array}{l}6 \\
4 \\
6\end{array}$ & $\begin{array}{l}7 \\
2 \\
5\end{array}$ & - \\
\hline PELD score & $17(9.24)$ & $37.5(14-58)$ & $35(14.55 \cdot 54)$ & $37.5(25-57)$ & $<0.001^{*}, 0.500^{\prime}$ \\
\hline 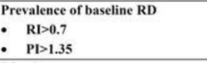 & $\begin{array}{l}0 \\
0\end{array}$ & $\begin{array}{l}14(46 \%) \\
15(50 \%)\end{array}$ & $\begin{array}{l}8(50 \%) \\
8(50 \%)\end{array}$ & $\begin{array}{l}6(37 \%) \\
7(50 \%)\end{array}$ & $\begin{array}{l}<0.001^{*}, 0.12^{*} \\
<0.001^{*}, 0.45^{*}\end{array}$ \\
\hline $\begin{array}{l}\text { RI values } \\
: \quad \text { Baseline } \\
\\
0 . \quad 48 \text { h after LVP } \\
07 \text { after LVP } \\
\text { D30 after LVP } \\
\text { D90 after LVP }\end{array}$ & $\begin{array}{c}0.55(0.48-0.64) \\
\vdots \\
\vdots \\
-\end{array}$ & $\begin{array}{l}0.72(0.45-0.84) \\
0.67(0.47-0.81) \\
0.63(0.49-0.74) \\
0.61(0.47-0.71) \\
0.60(0.46-0.70)\end{array}$ & $\begin{array}{c}0.69(0.45-0.79) \\
0.65(0.48-0.75) \\
0.59(0.49-0.71) \\
0.56(0.46-0.68) \\
0.55(0.48-0.66) \\
(\mathrm{n}=12) \\
\end{array}$ & $\begin{array}{c}0.68(0.49-0.83) \\
0.64(0.51-0.78) \\
0.62(0.56-0.73) \\
0.60(0.52-0.66) \\
0.58(0.53-0.62) \\
(\mathrm{n}=11) \\
\end{array}$ & $\begin{array}{c}<0.001^{*}, 0.440^{\circ} \\
0.540^{*} \\
0.43^{*} \\
0.76^{*} \\
0.45^{*}\end{array}$ \\
\hline $\begin{array}{l}\text { PI values } \\
: \quad \text { Baseline } \\
: \quad 48 \mathrm{~h} \text { after LVP } \\
\text { D7 affer LVP } \\
\text { D30 after LVP } \\
\text { D90 after LVP }\end{array}$ & $\begin{array}{c}1.11(0.75-1.41) \\
\vdots \\
\vdots\end{array}$ & $\begin{array}{l}1.24(0.92-1.54) \\
1.16(0.93-1.39) \\
1.09(0.91-1.33) \\
1.04(0.86-1.31) \\
1.03(0.87-1.35)\end{array}$ & $\begin{array}{l}1.22(0.95-1.49) \\
1.11(0.91-1.37) \\
1.07(0.90-1.35) \\
1.02(0.89-1.37) \\
1.04(0.91-1.31) \\
(\mathrm{n}=12)\end{array}$ & $\begin{array}{l}1.21(0.90-1.49) \\
1.16(0.86-1.51) \\
1.14(0.91-1.47) \\
1.11(0.85-1.46) \\
1.13(0.87-1.35) \\
(\mathrm{n}=11) \\
\end{array}$ & $\begin{array}{l}<0.001^{*}, 0.21^{*} \\
0.43^{*} \\
0.23^{\prime \prime} \\
0.73^{*} \\
0.34^{*}\end{array}$ \\
\hline $\begin{array}{l}\text { Development of renal failure } \\
\text { (S.creatinine }>1.5 \mathrm{mg} / \mathrm{dL} \text { ) in } 3 \mathrm{mo}\end{array}$ & 0 & $6(20 \%)$ & $3(18 \%)$ & $3(21 \%)$ & $<0.001 * 0.55$ \\
\hline Mortality in 3 months & 0 & $7(23 \%)$ & $4(25 \%)$ & $3(21 \%)$ & $<0.001,0.38$ \\
\hline $\begin{array}{l}\text { In AG intergroup comparisons: base } \\
\text { In NAG intergroup comparisons: ba } \\
\text { In AG intergroup comparisons, base } \\
\text { In NAG intergroup comparisons. } \\
\text { PELD: pediarric end-stage liver dise }\end{array}$ & 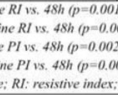 & $\begin{array}{l}\text { ay } 7(p=0.0 \\
\text { day } 7(p=0 . \\
a y>(p=0.00 \\
\text { day } 7(p=0 . \\
\text { pulsatility i }\end{array}$ & $\begin{array}{l}\text { day } 30(n=0.006 \\
D 30(p=0.23) \\
D 30(p=0.007) \\
D 30(p=0.33) \\
R D: \text { : renal dysti }\end{array}$ & $\begin{array}{l}\text { ay } 90(n=0.11) \\
\text { day } 90(n=0.22) \\
D 90(p=0.88) \\
990(p=0.67)\end{array}$ & 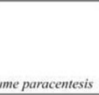 \\
\hline
\end{tabular}

\begin{tabular}{|c|c|c|c|}
\hline \multicolumn{4}{|c|}{ Table 2: Comparison of dead vs. alive patients in decompensated group } \\
\hline Parameters & Dead n=7 & Alive, $\mathrm{n}=\mathbf{2 3}$ & $\mathbf{p}$-value \\
\hline Baseline PELD score & $51(43-54)$ & $34(13-53)$ & 0.005 \\
\hline Baseline S. Na & $133(122-143)$ & $129(124-138)$ & 0.349 \\
\hline RI (baseline) & $0.76(0.73-0.83)$ & $0.67(0.46-0.74)$ & $<0.001$ \\
\hline PI baseline & $1.26(0.91-1.58)$ & $1.01(0.81-143)$ & $<0.001$ \\
\hline GFR at last follow-up & $63(36-101)$ & $112(94-158)$ & $<0.001$ \\
\hline Creatinine at last follow up & $0.94(0.5-2.1)$ & $0.70(0.4-0.9)$ & 0.298 \\
\hline $\begin{array}{c}\text { Renal failure at last follow up } \\
\text { (creatinine }>1.5)\end{array}$ & $4(57 \%)$ & $2(8 \%)$ & $<0.001$ \\
\hline
\end{tabular}

\section{LATEB-20}

Severe hepatopulmonary syndrome in children has a dreaded outcome: challenges faced and strategies adopted to improve survival $>95 \%$

Neelam Mohan ${ }^{1}$, Neelam Mohan ${ }^{1}$, Veena Raghunathan ${ }^{1}$, Maninder Singh Dhaliwal ${ }^{1}$, Sakshi Karkra ${ }^{1}$, Arvinder Singh Soin ${ }^{1}$

${ }^{1}$ Medanta The Medicity Hospital, India

Background: Hepatopulmonary syndrome(HPS) is a dreaded complication of end-stage-liver disease(ESLD).Pre-operative pO2 < $50 \mathrm{mmHg}$ and $>20 \%$ shunting on Technetium99mTc-albumin-aggregated (MAA)scan are considered as strong mortality predictors, making the decision of liver transplantation(LT) difficult1.Data on outcomes of HPS in pediatric-LT is limited.

Aim: To study spectrum of HPS in pediatric-ESLD \& evaluate the complications and role of newer strategies to improve outcome/survival Post LT.

Method: Retrospective-analysis of pediatric-LT patients between 2010-2016. HPS was identified pre-operatively by positive contrastenhanced echocardiogram (CE-ECHO) \& elevated alveolar-arterial oxygen gradient $(\geq 15 \mathrm{~mm} \mathrm{Hg})$. In CE-ECHO, appearance of few leftatrial bubbles was labelled as 'some' pulmonary-arteriovenous malformation; dense opacification was labelled'significant'. Postoperative course of HPS patients was studied.

Results: 22 of 159 children who underwent-LT had HPS. 12 had significant, 10 had some pulmonary-arteriovenous shunting. By oxygenation criteria, 11 had 'mild' $(\mathrm{pO} 2>80 \mathrm{mmHg})$, 5-had 'moderateto-severe' $(50 \mathrm{mmHg}>\mathrm{pO} 2<80 \mathrm{mmHg}$ ) and 6 had 'very severe'HPS(pO2 < 50 mmHg)2.Patients with 'very-severe'HPS underwent MAAscan;shunt fraction ranged from 30-73\%, this dint correlate with severity of hypoxemia or predict post-operative course.4/6 in 'very severe'HPS-group had pO2 $<45 \mathrm{mmHg}$ which seemed to predict higher morbidity and mortality. Of the 4,1 died on 7 th post-operative day due to massive intracranial hemorrhage (ICH). Remaining 3patients had prolonged refractory hypoxemia requiring inhaled nitric oxide(iNO) for $>2$ weeks (Range: 19-42 days, mean: 27.3 days). Their mean-duration of post-operative ventilation was 31 days, mean-ICU-stay-33 days and mean-hospital-stay-47days. This was comparatively much-higher than the remaining HPS-patients (mean:duration of ventilation-3.85 days, ICU stay-8.2 days, hospital stay-23 days). Incidence of vascular thrombosis was high.4/22 of HPS-patients developed hepatic-artery thrombosis; as against 6/137non-HPS patients. Portal-vein thrombosis occurred in 1patient. Other complications were ICH $(\mathrm{n}=2$; both had pre-op pO2 $<45$ 
mmHg), seizures $(n=5)$, ARDS $(n=2)$ and hypertension $(n=4)$. Overall mortality in HPS-group was $1 / 22(4.54 \%)$.

Conclusion: LT is only definitive cure for HPS. LT in severe HPS is challenging, with high risk of vascular thrombosis, prolonged ventilation and ICH. In our experience meticulous care and use of iNO post-LT improved oxygenation in very severe HPS, with good survival of $>95 \%$. $\mathrm{pO} 2<45 \mathrm{mmHg}$, rather than MAAscan predicted a difficult post-operative course.

\section{LATEB-21}

Do nor and recipient CMV status in east - are infants here at higher risk for CMV infection post liver transplant; does preemptive/hybrid gancyclovir therapy help?

\section{Neelam Mohan ${ }^{1}$, Sakshi Karkra', Deepak Goyal ${ }^{1}$, Maninder Singh Dhaliwal $^{1}$, Veena Raghunathan ${ }^{1}$, Neelam Mohan ${ }^{1}$}

${ }^{1}$ Medanta The Medicity Hospital, India

Background: Cytomegalovirus (CMV) is the most common viral infection post liver transplant.

Aim: To analyze the donor and recipient serology pretransplant and study the rate of CMV infection/disease in infants versus older children and to assess if hybrid therapy/preemptive therapy posttransplant reduced the infection/disease rate in pediatric liver transplantation (LT)

Method: Retrospectively collected data of pediatric LDLT was analysed from 2010 to March 2017.CMV-serology was done in all recipients $(\mathrm{R})$ and donors $(\mathrm{D})$. CMV polymerase chain reaction (PCR) $>500$ copies per $/ \mathrm{ml}$ was considered significant. It was carried out at 2, 4, 6, 12 \& 24weeks post LT. CMV infection status on the basis of PCR was ascertained upto 6 months post LT. A comparative analysis of CMV infection/disease incidence in the recipients of $<1 \mathrm{yr}$ and $>$ 1 -yr was done. Hybrid therapy was given (prophylaxis with $5 \mathrm{mg} / \mathrm{Kg}$ of Gancyclovir once a day for 3 weeks followed by preemptive prophylaxis) in infants \& high risk cases. In case of infection: IV Ganciclovir twice a day till 2 consecutive samples at a gap of 2 weeks are negative/oral therapy was considered once viral load was $<$ 10,000 copies $/ \mathrm{ml}$.

Result: There were 160 pediatric LR (Living related)LT with 2 categories of 143 patient in $\mathrm{D}+($ Donor)R+(Recipient) and 15 patient in $\mathrm{D}+\mathrm{R}-$. In $\mathrm{D}+\mathrm{R}+$ and $\mathrm{D}+\mathrm{R}$ - the mean age and weight 73.7 months, $21.1 \mathrm{~kg}$ and 40 months and $11.7 \mathrm{~kg}$ respectively. In $\mathrm{D}+\mathrm{R}+$ there were 35 infant and 34\% developed CMV infection. There were no statistical difference in CMV infection in those who received prophylaxis vs those who didn't but the mean CMV copies/ml were 4737copies/ $\mathrm{ml}$ and 40181 respectively. 24/108 (22\%) D+R+ patient $>1$ year of age had CMV infection $36 \%$ of infants who got hybrid prophylaxis developed CMV disease while $100 \%$ of CMV infection in infants with preemptive prophylaxis progressed to CMV disease. There was statistically significant difference between $<6$ months \& $>6$ month age $(\mathrm{p}<0.005)$ with former getting more infections, however only $28 \%$ of infections converted to disease in hybrid therapy group.

In $\mathrm{D}+\mathrm{R}$ - there were 4 infants and the numbers are too small for statistical analysis within the group. The mean CMV Copies/ml was 37000 in infants and 12065 in others. However, overall CMV infection rate was $46 \%$ as compared to $25 \%$ in $\mathrm{D}+\mathrm{R}+$ which is statistically significant.

Conclusion: $90 \%$ of the pediatric LT patients were in $\mathrm{D}+\mathrm{R}+$ category. $\mathrm{D}+\mathrm{R}-$ had almost double $\mathrm{CMV}$ infection rate than $\mathrm{D}+\mathrm{R}+$ with infants having CMV infection 1.5 times as often, compared to $>1$ year. There was significantly more infections in $<6$ months age in comparison to $>6$ month age $(\mathrm{p}<0.005)$. Prophylaxis for 3 weeks did not reduce the incidence of CMV infection post LT though the viral load was significantly low. Infants especially $<6$ months may be considered as high risk.

\section{LATEB-22}

Chemokines acts as key moderator in Hepatitis $\mathrm{A}$ and $\mathrm{E}$ infection

Rashi Sehgal ${ }^{1}$, Nirupma Trehanpati ${ }^{1}$, Ashish Kumar Vyas ${ }^{1}$, Meenu Bajpai ${ }^{1}$

${ }^{1}$ Institute of Liver and Biliary Sciences, India

Background: Hepatitis A virus (HAV) and hepatitis E virus (HEV) infection usually result in a self-limiting acute disease which affects liver. Liver injury in HEV and HAV infection is mediated by various immune cells through secretion of chemokines,cytokines that attract and regulate $\mathrm{T}$-cell mobilization to the site of inflammation.However,the role of immune cells as well as chemokines during HAV pathogenesis is not yet documented.

Method: In order to investigate the role of chemokines and cytokines in acute viral hepatitis-A (AVH-A) and acute viral hepatitis-E(AVHE)infection, we have recruited patients from AVH-A $(n=15)$, AVH-E $(\mathrm{n}=20)$ and healthy controls $(\mathrm{HC}, \mathrm{n}=12)$.Expression of various CXCRs and CCRs were analysed using flow cytometry.Cytokine secretion(IFN- $\gamma$, TNF- $\alpha$,IL-6,IL-12 and IL-2) by CD3+T-cells after PMA+Ionomycin stimulation was analysed to assess the pro-inflammatory responses among both groups.

Result: Total CD3+T-cells was significantly decreased in AVH-A compared to the AVH-E and HC ( $35 \pm 7$ vs. $118 \pm 13$ vs. $129 \pm 22$, p $=0.003)$ while $\mathrm{CD} 3+\mathrm{CD} 8+\mathrm{T}$ cells $(25 \pm 8$ vs. $32 \pm 9, \mathrm{p}=0.05)$ showed also decreased trend in AVH-A compared to AVH-E but this difference was not significant.However, IFN- $\gamma(16.5 \pm 5$ vs. $5.5 \pm 2$ vs. $6 \pm 3), \mathrm{TNF}-\alpha(16.4 \pm 3$ vs. $12 \pm 3$ vs. $0.7 \pm 0.3)$ and IL- $2(2 \pm 0.3$ vs. $0.5 \pm 0.3$ vs. $1.5 \pm 0.3)$ secretion by T-cells were increased in AVH-A compared to AVH patients and HC.Although,we did not observed significant difference in liver injury markers in both AVH-A and AVH-E group.However,chemokines receptors and chemokines were significantly decreased in AVH-A compared to AVH-E. Interestingly,expression of CXCR3 and CXCR5 on CD3+T cells was also significantly decreased in AVH-A compared to AVH-E in follow-ups at $1 \& 6$ months.

Conclusion: Patients with AVH-A infection showed decreased chemokine receptors and chemokines profile, which is essential for migration of immune cells. This immune impairment might have important consequences on host's immune defence and liver injury in HAV patients.

\section{LATEB-23}

\section{Audit of Upper Gastrointestinal Endoscopy in children}

Bhushan Arun Miraje ${ }^{1}$, Prachi Arun Karanik', Bijal Ashok Mistry $^{1}$, Alka Raja Jadhav', Vibhor Arun Borkar ${ }^{1}$, Anil Arun Tambe $^{1}$

${ }^{1}$ LTMMC \& LTMGH, Sion, Mumbai, India

Background: Upper GI endoscopy has now become a standard diagnostic and therapeutic procedure for many gastrointestinal problems in children. As there is lack of data related to endoscopy and histopathology in children, we carried out this audit of upper GI endoscopy in our setup.

Method: This is a retrospective and prospective observational study carried out at the Division of Pediatric Gastroenterology, Hepatology and Nutrition, Department of Pediatrics, LTMMC \& GH, Sion, 
Mumbai during a one year period from July 2016 to June 2017. All children less than 12 years of age who underwent upper gastrointestinal endoscopy for initial diagnosis were recruited for the present study.

Result: A total of 134 children $<12$ years were included in the study.The mean age was 7.04 years (Figure 3), median was 8 years (range: 4 months to 12 years). There were 85 boys $(63.43 \%$ ) and 49 girls $(36.57 \%)$, the ratio of boys to girls was $1.73: 1$. The most common clinical presentation was hematemesis [43 (33.58\%)] followed by chronic abdominal pain [21(15.67\%)]. In 58/134 (43.28\%) patients, the endoscopy revealed a normal finding. Out of the 43 patients presenting with hematemesis, 17 had extra hepatic portal venous obstruction, 2 were operated biliary atresia (Kasai procedure) and 1 had chronic liver disease. There were 3 other patients with chronic liver disease and 2 operated biliary atresia (Kasai) without hematemesis. Out of 3 patients with hepatosplenomegaly, 1 had portal hypertension. Esophageal varices was the most common pathological finding seen on endoscopy [39(29.10\%)]. A total of 52 patients underwent endoscopy followed by histopathology.In patients with only positive endoscopic findings, the correlation coefficient $(\mathrm{R})$ between endoscopic and histopathology findings was found to be 0.70456(two-tailed p-value 0.00336), indicating a significant positive association. However, there was a poor correlation between the two diagnostic methods (endoscopy and histopathology) in patients with normal endoscopy findings. Out of 26 duodenal biopsy $61.54 \%$ were mild duodenitis.

Conclusion: Esophageal varices was the most common endoscopic finding at our center. In spite of normal endoscopy findings, the histopathology was found to be abnormal in many patients. This suggests a need for doing frequent biopsies from upper GI even in presence of normal endoscopic findings. Mild duodenitis is most common histopathology found even in macroscopically normal duodenum mucosa.

\section{LATEB-24}

Concurrent extrahepatic autoimmune disorders: unexplored dimension of autoimmune liver disease in the children

Vikas Jain $^{1}$, Surender Kumar Yachha ${ }^{2}$, Eesh Bhatia ${ }^{2}$, Moinak Sen Sarma $^{2}$, Anshu Srivastava ${ }^{2}$

${ }^{1}$ Super Speciality Paediatric Hospital \& Post Graduate Teaching Institute, Noid, Uttar Pradesh, India; ${ }^{2}$ Sanjay Gandhi Postgraduate Institute of Medical Sciences, Lucknow, India, India

Background: Prospective and comprehensive data available for concurrent extrahepatic autoimmune disorders (CEAIDs) in autoimmune liver disease (AILD) children is not available. We conducted a study to evaluate CEAIDs in AILD and their effect on outcome of AILD.

Method: AILD in children were diagnosed on the basis of simplified diagnostic criteria and CEAIDs in AILD were labelled as per standard diagnostic criteria. Clinico-pathological profile, treatment response and outcome were compared between AILD with CEAIDs (Group A) and AILD without CEAIDs (Group B).

Result: Among all 62 children with children, CEAIDs were found in $42 \%(n=26)$ [vitiligo $(42 \%)$, celiac disease $(C D)(15 \%)$, potential CD $(15 \%)$, autoimmune hemolytic anemia (AIHA) (15\%)]. CEAIDs were asymptomatic in $75 \%$. Single CEAID was diagnosed in $81 \%(21 / 26)$ and multiple in $19 \%(5 / 26)$. Significantly less biochemical remission (BR) $(46.1 \%$ vs. $74.2 \%, \mathrm{p}=0.03)$, more treatment failure $(23 \%$ vs. $3.2 \%, \mathrm{p}=0.04)$ and higher mortality $(15.3 \%$ vs. $3.2 \%, \mathrm{p}=0.04)$ were seen in Group A as compared to Group B. On multivariate analysis (n $=57)$ less BR in vitiligo $(p=0.04)$; more treatment failure in AIHA ( $p$
$=0.004)$ and vitiligo $(\mathrm{p}=0.04)$; high mortality in AIHA $(\mathrm{p}=0.02)$ subgroups were reported. Outcome of AILD is good in children with coexisting CD treatment. Diabetes mellitus in our AILD cases $(n=8)$ were steroid induced rather than autoimmunity (absence of antibody against tyrosine phosphatase (IA2) and glutamic acid decarboxylase (GAD) and elevated C-peptide)

Conclusion: All AILD children should be screened for CEAIDs as majority are asymptomatic. AILD outcome was favorable in CD but poor in vitiligo and AIHA. We suggest CEAIDs incorporation in pediatric AILD scoring system.

\section{LATEB-25}

Viral load by reverse transcriptase polymerase chain reaction as a marker of severity in children with acute hepatitis $A$

\section{Shrish Bhatnagar ${ }^{1}$, Ayub Anasari ${ }^{1}$, Tasleem Raza ${ }^{1}$}

${ }^{1}$ Eras Lucknow Medical College, India

Background: To study the association between severity and viral load in children suffering from acute Viral A Hepatitis

Method: 50 patients of Acute Viral A Hepatitis were enrolled into the study. They were divided into acute hepatitis (Group I), severe acute hepatitis (Group II) and fulminant hepatitis (Group III), based on PT time and Hepatic encephalopthy. Viral load was assessed by Real Time PCR using quantitative assay

Result: Majority of the patients belonged to acute hepatitis. HAV viral load of Group III $(3.94+0.61 \mathrm{log}$ copies/ml $)$ and Group III $(3.54+0.82 \mathrm{log}$ copies $/ \mathrm{ml})$ was found to be statistically significantly higher than that of Group I $(1.85+0.51 \log$ copies $/ \mathrm{ml})$. Correlation between HAV viral load and Prothrombin time was found to be strong and statistically significant.

Conclusion: Children suffering from Acute Viral A Hepatitis with high viral load have a poor prognosis.

\section{LATEB-26}

Prognostic molecular biomarkers in gall bladder carcinoma in Indian population

Rupal Tripathi ${ }^{1}$, Dinesh Chandra Doval ${ }^{2}$, Saud Azam ${ }^{2}$, Ullas Batra $^{2}$, Anurag Mehta ${ }^{2}$

${ }^{1}$ Institut d'Investigacions Biomèdiques August Pi i Sunyer (IDIBAPS), Hematopathology Unit, Hospital Clinic, CIBERONC, Spain; ${ }^{2}$ Rajiv Gandhi Cancer Institute \& Research Centre, India

Background: Gallbladder carcinoma (GBC) is the most common cancer of the biliary tract with dismal prognosis and poor survival. Its enigmatic aetiology involving altered gene expression is a key phenomenon in this cancer and is usually fatal due to late clinical presentation and lack of effective treatment modalities. Till today, there is scanty information related to the molecular pathogenesis of the disease. The present study was conducted to assess the role of a range of molecular biomarkers including growth factor receptors [epidermal growth factor receptor (EGFR) and vascular endothelial growth factor (VEGF)], cell cycle regulatory molecule (cyclin D1) and marker of cell proliferative activity (Ki-67).

Method: A total of sixty consecutive patients with cholecystectomy/ biopsies from gall bladder bed were included in the study. Archived formalin fixed paraffin embedded tissue blocks were obtained and immunohistochemistry was performed. Clinical information, tumor profile and follow-up was recorded to study the possible correlations. 
Result: Around $70 \%$ of the tumors were moderately differentiated and tubular morphology was observed in $50 \%$ cases. Seventy percent of the patients presented with advanced stages of the disease. Overexpression of EGFR, VEGF and cyclin D1 was observed as 75, 40 and $64 \%$ cases, respectively. The younger patients (age $<40$ years) showed higher expression levels of these biomarkers. The growth factors were strongly expressed in the early stages of the disease and were associated with an absence of nodal metastasis. Ki-67 was significantly higher in the younger patients (p-value $<0.03)$ and poorly differentiated tumors ( $\mathrm{p}$-value $<0.03$ ). Advanced disease and poorly differentiated tumors showed a significantly poor median survival $(\mathrm{P}<0.05)$.

Conclusion: Growth factors (EGFR and VEGF) may be utilized as prognostic molecular biomarkers as GBC may possibly arise from the defects in these cell signaling molecules which might activate the transcription factors and cell cycle regulatory proteins may also be of considerable importance. Further large scale studies are required to validate the role of these biomarkers in the causation and progression of these lethal cancers.

\section{LATEB-27}

\section{SEPTO-OPTIC dysplasia: an unusual cause of neonatal cholestasis}

\section{PRITA MOHANTY' ${ }^{1}$ THOMAS ROSSI ${ }^{2}$}

${ }^{1}$ UCSF Benioff Children's Hospital Oakland, United States;

${ }^{2}$ University of Rochester Medical Center, United States

Background: Septo-optic dysplasia is defined by a variable combination of absence of the septum pellucidum, optic nerve hypoplasia, and hypopituitarism. Severe prolonged cholestatic jaundice is believed to be secondary to hypopituitarism. Although evidence of a specific hormonal deficiency or combination of endocrine deficiencies is unknown, jaundice and liver function do appear to improve after hormone replacement. The present case provides a reminder concerning an association between Septo-optic dysplasia and neonatal cholestasis.

Method: A 4 week old full term girl was evaluated for failure to thrive, prolonged jaundice and hypoglycemia. The neonatal course was complicated by adrenal insufficiency, septic shock and hypoxic respiratory failure. On physical examination, her length and weight were at the 3 rd percentile. She had scleral icterus and nystagmus. There was no hepatosplenomegaly. Labs revealed elevated transaminases, cholestasis and hypoglycemia. Free T4, insulin-like growth factor and IGF binding protein 3 were low. Ophthalmologic examination showed bilateral optic nerve hypoplasia. On Magnetic Resonance Imaging, there was absence of the septum pellucidum and hypoplastic appearance of the optic nerves and chiasm, which is consistent with Septo-optic dysplasia. Because results of free T4 was consistently low, the patient was started on Levothyroxine. Based on the history of recurrent hypoglycemia, persistently elevated transaminases and growth delay, a glucagon test was performed and was found to be compatible with growth hormone deficiency. The patient was started on growth hormone replacement. Repeat liver function tests showed resolution of cholestasis.

Result: The association of liver dysfunction with hypopituitarism was first suggested in 1956. It has been suggested that growth hormone and cortisol deficiencies may be responsible for the development of hyperbilirubinemia since these hormones seem to modulate bile acid synthesis and bile flow. Several studies in animals support the lack of growth hormone as the responsible factor for hepatic dysfunction, and suggest that certain hepatic enzymes are growth hormone dependent. The pattern of liver dysfunction secondary to hormone deficiencies is variable and goes from predominantly indirect hyperbilirubinemia, indirect hyperbilirubinemia that turns into a cholestasis, cholestasis alone, or cholestasis with elevated hepatic enzymes.

Conclusion:The diagnosis of Septo-optic dysplasia must be considered in infants who present for evaluation of cholestasis, particularly if there is associated hypoglycemia and nystagmus. Liver function seems to normalize following hormone replacement.

\section{LATEB-28}

Noninvasive diagnosis of cerebral edema in patients of acute liver failure

Harsh Vardhan Tevethia $^{1}$, Ashok Choudhury ${ }^{2}$, Rakhi Maiwall ${ }^{2}$, Lalita Mitra', Vandana Saluja ${ }^{1}$, Prashant Singh ${ }^{1}$, Aditya Trigatia $^{1}$, Guresh Kumar ${ }^{1}$, Shalini Thapar ${ }^{1}$, Shiv Kumar Sarin ${ }^{1}$

${ }^{1}$ Institute of liver and biliary ciences, New Delhi, India; ${ }^{2}$ ilbs, India

Background: Cerebral edema (CE) is the most common and most fatal complication leading to high mortality in Acute Liver Failure (ALF). The astrocyte swelling positively correlates to blood ammonia level and is considered to be the cause for CE. We studied the correlation of various noninvasive diagnostic modalities i.e. CT scan, Optic Nerve Sheath Diameter (ONSD) and Arterial Ammonia in predicting the cerebral edema.

Method: Consecutive patients of ALF admitted to ICU from January 2016 to December 2017, with age 18-70 yrs. were recruited. All patients undergone noncontrast CT scan of brain at admission followed by monitoring ammonia, ONSD, grade of HE every six hourly. All patients with Grade II-IV were on ventilator and received standard treatment for raised intracranial pressure (ICP). Cerebral edema on CT scan was considered confirmatory and was divided into mild, moderate and diffuse. The data was prospectively collected and analyzed.

Result: Forty ALF patients, predominantly females (55\%) with median age $29+3.2$ years were included. The common etiology was HEV (42.5\%). The jaundice to encephalopathy duration was $6.33 \pm 4.2$ days, presentation with HE in $(100 \%)$ with a baseline bilirubin of $17.33+6.8 \mathrm{mg} / \mathrm{dl}$ and INR of $4.2+2.7$. The Grade III-IV HE was noted in $71.2 \%$ patients. Cerebral edema on CT was found in $73.2 \%$ patients. The cerebral edema was mild, moderate and diffuse in $12 \%, 31 \%, 57 \%$ patients respectively. The Grades of Encephalopathy did not correlate to presence or severity of cerebral edema. Arterial Ammonia correlated with $\mathrm{HE}$ and a value of $>$ $241 \mathrm{mcg} / \mathrm{dl}$ correlated with the CT findings of cerebral edema $(95 \% \mathrm{CI}$ (2.9-73.7), $\mathrm{p} \leq 0.001]$ as was the ONSD value of $>5 \mathrm{~mm}(95 \% \mathrm{CI}$ (2.9-73.7), $\mathrm{p} \leq 0.001]$. ONSD had a sensitivity of $92 \%$ and specificity of $93 \%$ in diagnosing cerebral edema. Grade III-IV HE, ANH3 > 241, ONSD $>5$ predicted the cerebral edema with sensitivity specificity (AUROC curve). On univariate analysis Ammonia $(\mathrm{p}<0.005$ ), heomoglobin and bilirubin were significant variables, but on multivariate analysis, ammonia independently predicted well, the cerebral edema as seen in CT scan of brain.

Conclusion: Arterial Ammonia and ONSD are good and simple bedside parameters in predicting cerebral edema in ALF patients and could guide the treatment plan for raised ICP as well. 


\section{LATEB-29}

Transient elastography and controlled attenuation parameter assessment of liver disease in children and young adults with Cystic fibrosis: a 3 year longitudinal study

Prita Mohanty $^{1}$, Paul D Mitchell ${ }^{2}$, Shanna Wiggins ${ }^{2}$, Denis Nguyen $^{2}$, Sarah Harney ${ }^{2}$, Maureen M Jonas ${ }^{2}$, Christine K Lee ${ }^{2}$

${ }^{1}$ UCSF Benioff Children's Hospital Oakland, United States; ${ }^{2}$ Boston Childrens Hospital, United States

Background: Liver disease is the third leading cause of death in cystic fibrosis (CF) patients. Hepatic steatosis is a common, early sign of CF-related liver disease (CFLD). Controlled attenuation parameter (CAP), obtained during transient elastography (TE), can detect and quantify steatosis.

Method: This was a longitudinal cohort study of CF patients seen for routine outpatient care at Boston Children's Hospital. CAP and LSM were obtained at enrollment (January-October 2013) and annually up to 3 years. CFLD was defined per published criteria as: No CFLD, CFLD without portal hypertension (PHTN) and CFLD with PHTN. CFLD without PHTN criteria:recent ALT $>1.3 x$ ULN, on ursodiol, or abnormal liver echogenicity on imaging. CFLD with PHTN criteria:splenomegaly, esophageal varices on endoscopy, platelet count

Result: A total of 249 patients [53\% male; mean age $14 \pm 7$ y;7(3\%) Conclusion: In this 3 year study, CAP is able to detect changes in CFLD when there is no detectable change in liver fibrosis.

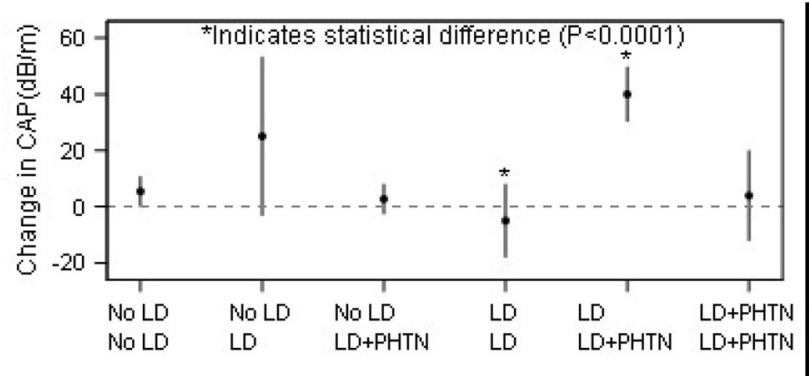

Change in $\mathrm{CAP}(\mathrm{dB} / \mathrm{m})$ across adjacent patient encounters

\section{LATEB-31}

\section{Hepatobiliary dysfunctions in HIV positive children}

\section{Bikrant Bihari Lal ${ }^{1}$, Praveen Kumar ${ }^{2}$, Jagdish Chandra ${ }^{2}$, Anju

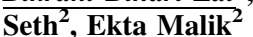 \\ ${ }^{1}$ Institute of Liver and Biliary Sciences, New Delhi, India; ${ }^{2}$ Lady Harding Medical College, India}

Background: Hepatobiliary dysfunctions are seen both as an integral part of HIV infection spectrum and as ART induced toxicity. The range of involvement is highly variable with asymptomatic liver enzyme derangements to life threatening hepatic decompensation. Though multiple studies in adults have described the etiology of hepatobiliary dysfunctions in HIV, such studies are lacking in pediatric population. Objective: To study the hepatobiliary dysfunctions in $\mathrm{HIV}$ positive children and to determine prevalence of $\mathrm{HBV} / \mathrm{HCV}$ coinfection with HIV in children.

Method: Cross-sectional observational study in Pediatric center of excellence ( $\mathrm{pCoE}$ ) in Kalawati Saran Children's Hospital. 173 HIV positive children were enrolled between November 2015-March 2017, aged 6 months to 18 years and divided into three groups: 36 ART naïve (group A), 43 on ART for 4-6 weeks (group B) and 94 on ART $>6$ weeks (group C). All were subjected to history and examination with focus on hepatobiliary system, complete liver function test, serum amylase and lipase, coagulogram, viral markers (HBsAg and Anti-HCV) and abdominal ultrasound. Refusal of consent and known surgical and metabolic hepatobiliary dysfunction were exclusion criteria.

Result: Overall 43\% (76/173) children had hepatobiliary dysfunction in the form of transaminitis. The average AST (70 IU/L) and ALT (52 IU/L) of HIV positive children was higher than normal cutoffs (AST or ALT $<40 \mathrm{IU} / \mathrm{L}$ ). Proportion of children with hepatobiliary dysfunctions in Group A, Group B and Group C were 50, 48 and 39.36\% respectively. Most of these dysfunctions were Grade 1 as per NACO guidelines. ART induced hepatotoxicity accounted for $48.7 \%$ of the overall dysfunctions. Hepatitis B/C coinfection seroprevalence was determined as $12.14 \%$.

Conclusion: Current ART regimens are largely safe and associated with mild hepatotoxicity not reaching the level of clinical significance but a strict vigil is indispensable in avoiding life threatening complications. Future studies with larger sample size and longitudinal model are recommended.

\section{LATEB-32}

Weekly regimen of vitamin D supplementation is more efficacious than stoss regimen for treatment of vitamin $D$ deficiency in children with non-cholestatic chronic liver diseases

\author{
Bikrant Bihari Lal ${ }^{1}$, Seema Alam', Rajeev Khanna', Dinesh \\ Rawat $^{1}$ \\ ${ }^{1}$ Institute of Liver and Biliary Sciences, New Delhi, India
}

Background: There are no evidence based recommendations on the ideal dose and regimen for supplementation of vitamin D in children with CLD. This study aimed to compare the safety and efficacy of weekly and stoss regimens for treatment of vitamin D deficiency in these children.

Method: Children between the ages of 1 to 18 years with CLD and vitamin D deficiency $(25-\mathrm{OH}$ Vitamin D3 $<30 \mathrm{ng} / \mathrm{ml})$ were included. They were randomized to receive either stoss regimen $(6,00,000 \mathrm{IU}$ on day 1$)$ or weekly $(60,000$ IU weekly) regimen of vitamin D. The vitamin D levels at 3 and 6 months were compared in the 2 groups. Result: A total of 210 suspected cases of CLD were assessed for eligibility. Of a total of 67 children satisfying the inclusion criteria, 33 and 34 were randomized to receive stoss and weekly regimen respectively. Final analysis included 28 children in each group. Clinical rickets was seen in $25.4 \%$ of children with hypovitaminosis D. The rise in levels of vitamin D at 3 months was higher with weekly regimen $(34.3 \pm 30.7 \mathrm{ng} / \mathrm{ml})$ as compared to stoss regimen $(17.2 \pm$ $11.5 \mathrm{ng} / \mathrm{ml})(\mathrm{p}=0.009)$. Rise at 6 months as compared to baseline was significantly higher with weekly regimen $(30.7 \pm 24 \mathrm{ng} / \mathrm{ml})$ as compared to stoss regimen $(11 \pm 8.4 \mathrm{ng} / \mathrm{ml})(\mathrm{p}<0.001)$. Normal levels of vitamin D at 6 months was achieved in 24/28 (85.7\%) of those receiving weekly regimen and $9 / 28(32.1 \%)$ of those receiving stoss regimen $(\mathrm{p}<0.001)$. With stoss therapy, vitamin $\mathrm{D}$ increased at 3 months as compared to baseline but thereafter dropped significantly at 6 months $(p=0.008)$. Compliance was similar in both groups. Both the regimens of vitamin $\mathrm{D}$ supplementation were safe.

Conclusion: Weekly regimen of vitamin D supplementation is more effective than stoss regimen for treatment of hypovitaminosis D in children with CLD. Both regimens are safe in these children. The rise in vitamin $\mathrm{D}$ is more sustained with weekly regimen. 


\section{LATEB-33}

Prevalence of diabetes mellitus in newly detected hepatitis $\mathbf{C}$ positive patients

\section{Nisar Ahmad Shah ${ }^{1}$, Nisar Ahmad Shah ${ }^{1}$, Showkat Ahmad Kadla $^{1}$, Roopakshi Pathania ${ }^{1}$, Bilal Ahmad Khan ${ }^{1}$, Asif Iqbal Shah $^{1}$, Shabir Ahmad Sheikh ${ }^{1}$, Mohamad Amin Dar ${ }^{1}$ \\ ${ }^{1}$ GMC Srinagar, India}

Background and Objective: Currently there are 170 to 200 million patients of hepatitis $\mathrm{C}$ all over the world, majority of them belong to Asian sub-continent. A study from Jammu and Kashmir in which around forty thousand replacement blood donors have were screened for Hepatitis $\mathrm{C}$ has revealed a prevalence of $0.169 \%$. In addition to CLD, HCV also causes some extra hepatic manifestations in almost $35 \%$ of infected patients. The extra hepatic manifestations result in spectrum of conditions and are supposed to be autoimmune in nature. The prevalence of type $2 \mathrm{DM}$, which is postulated to be an extra hepatic manifestation of $\mathrm{HCV}$ infection among $\mathrm{HCV}$ infected people, has been reported to be more than the general population. There is a compelling reason to believe this, because studies have shown an increased prevalence of anti $\mathrm{HCV}$ antibodies in type $2 \mathrm{DM}$ patients than in general population. Objectives: Our aims and objectives were (i) To find out the prevalence of Type $2 \mathrm{DM}$ in HCV positive patients, and (ii) To find out which HCV genotype is associated more with abnormal blood sugars and type $2 \mathrm{DM}$ in $\mathrm{HCV}$ positive patients

Material \& Methods: The current study was a hospital based case control study, conducted in the Department of Gastroenterology in collaboration with Department of Medicine, Government Medical College Srinagar. All incidentally detected Hepatitis C patients who consented were enrolled and were investigated as per the study design. A total of 70 cases were included in the study and equally matched 70 healthy volunteers who were HCV negative by ELISA and non-diabetic were taken as Controls. They were matched equally with respect to age, sex and demography. After obtaining the written informed consent, a thorough clinical examination and all the baseline investigation were done. Ultrasonography focusing on features of Portal Hypertension and cirrhosis was performed on all cases. Upper GI Endoscopy for varices and Portal Hypertensive Gastropathy and Quantitative HCV RNA estimation using Cobas Ampliprep and Taqman 48 (Roche) were done. Controls underwent GTT, HbA1c, serum insulin levels, HOMA-IR, however LFT, KFT and Lipid profile were done only in those who voluntered (23 patients). Analysis was done using Open Epi 3.01 (www.openepi.com). A p value of $<0.05$ was considered statistically significant.

Results: Among 70 cases, the mean age was $45.97 \pm 11.982$ years with $47.14 \%$ being males and $52.85 \%$ being females. As far as demographic profile is considered, cases and controls were comparable. In case of Hepatitis C patients, $67.14 \%$ patients were infected with Genotype 3 and $32.85 \%$ with Genotype 1 with $44.28 \%$ patients having HCV RNA levels more than $6,00,000 \mathrm{IU} / \mathrm{ml}$. Percentage of patients and controls was compared on the basis of Fasting Blood sugars in three different ranges of $\leq 100 \mathrm{mg} \%, 101-125 \mathrm{mg} \%$ and $\geq$ $126 \mathrm{mg} \%$ and same was found to be $50 \%, 28.57 \%$ and $21.45 \%$ respectvely in case of patients while in case of controls the respective percentage was $25.71 \%, 58.57 \%$ and $15.71 \%$. When compared on the basis of 2 hour GTT at three different levels of $<140 \mathrm{mg} \%, 140-199$ $\mathrm{mg} \%$ and $\geq 200 \mathrm{mg} \%$, the percentage among infected patients was $51.45 \%, 30 \%$ and $18.6 \%$ respectively while as in case of controls, the same was $68.57 \%, 23 \%$ and $8.57 \%$ respectively. Other parameters on the basis of which cases and controls were compared include $\mathrm{HbA1C}$, serum insulin levels, HOMA-IR, BMI, LFT and lipids. In case of Hepatitis C patients, HbA1C of $<5.6$ was seen in $68.57 \%$ while as in controls $\mathrm{HbA} 1 \mathrm{C}$ of same value was observed in $82.86 \%$. Estimation of serum insulin in case of patients and controls revealed that $85.71 \%$ of cases had it in the range of 2-25 mIU/L and the percentage of controls showing serum insulin level in the same range was $89.99 \%$. Compared on the basis of BMI and lipids there was no statistically significant difference between cases and controls however HOMA-IR of $<2.5$ in cases was seen in only $45.71 \%$ infected patients while as $65.71 \%$ of controls showed HOMA-IR of $<2.5$ and the difference was statistically significant with the $p$ value of 0.0063 . When FBS among two genotypes was compared, it was observed that, $15.71 \%$ of patients with Genotype 3 had FBS of more than $126 \mathrm{mg} / \mathrm{dl}$ while as amongst patients with Genotype 1, only $5.71 \%$ of patients had fasting blood sugar of more than $126 \mathrm{mg} / \mathrm{dl}$. They were also compared on the basis of 2 hour GTT that revealed a level of $\geq 200 \mathrm{mg} \%$ in $5.70 \%$ of those infected with genotype 1 against $12.90 \%$ of genotype 3

Conclusion: Hepatitis $\mathrm{C}$ causes 2.1 fold increase in the prevalence of Type 2 Diabetes in patients without evidence of chronicity, cirrhosis and Portal Hypertension with increased predisposition to Genotype 3 over Genotype 1.

\section{LATEB-34}

Safety and efficacy of bolus versus continuous infusion of terlipressin in acute variceal bleed in patients with cirrhosis " $A$ Randomized trial

Shakti Prasad Choudhury ${ }^{1}$, Ankur Jindal', Ashok Choudhury', Rakhi Maiwall $^{1}$, Shiv Kumar Sarin ${ }^{1}$

${ }^{1}$ Institute of Liver and Biliary Sciences, India

Background: Continuous infusion of terlipressin has been found to be better tolerated and equally effective at lower doses than intravenous boluses in type 1 hepatorenal syndrome (HRS) and septic shock. However, the dose and modality of terlipressin administration in preventing recurrent variceal bleed has not evaluated. We investigated the efficacy, hemodynamics and adverse effects of continuous infusion versus bolus dose of terlipressin in acute esophageal variceal bleed (AVB)

Method: Fifty consecutive cirrhotic patients with AVB were randomised to receive either bolus(BOL)- $2 \mathrm{mg}$ QID or continuous infusion(CI)- $4 \mathrm{mg} / 24$ hour of terlipressin for 72hours after endotherapy. Hepatic venous pressure gradient (HVPG) was measured at baseline, at 12 and 24 hours. Responder was defined as HVPG $<20 \mathrm{mmHg}$ or $>10 \%$ decline from baseline (rHVPG). In Nonresponders (HVPG $>20 \mathrm{mmHg}$ or $<10 \%$ decline from baseline), the terlipressin dose was increased by $2 \mathrm{mg}$ /day at 12 and 24 hours in CI arm. Primary objective was to study the efficacy ofbolus versus continuous infusion of terlipressin in HVPG reduction at 24 hours \& secondary was to study the incidence and resolution of post bleed acute kidney injury \& to compare rebleed and 42 days survival.

Result: Fifty patients ( 25 in each), in BOL (92\% males,mean age 47.7 $\pm 10.7 \mathrm{yr})$ and $\mathrm{Cl}(80 \%$ males, $48.6 \pm 10.6 \mathrm{yr})$ had comparable baseline parameters, severity scores and baseline HVPG. The therapy led to a significant reduction in HVPG at 12 hours (p-.024) and maintained at 24 hours $(\mathrm{p}=0.032)$. HVPG reduced significantly at 12 hours in both CI and BOL arms, [CI: $20.69 \pm 3.66$ to $18.9 \pm 4.09 \mathrm{~mm}$ $\mathrm{Hg}, \mathrm{p}<0.001)$, BOL: $(20.85 \pm 3.96$ reduced to $18.43 \pm 4.35, \mathrm{p}<$ $.001]$ and was sustained at 24 hours- $(20.69 \pm 3.66$ to $17.99 \pm 4.42$ and $20.85 \pm 3.96$ to $16.88 \pm 4.42, \mathrm{p}=0.03)$. The HVPG reduction was greater in $\mathrm{Cl}$ as compared to $\mathrm{BOL}(\mathrm{p}=0.01)$. The reduction in HVPG of $\leq 20 \%$ was higher in CI arm [56.3\% vs $27.2 \%$; $p=0.04)$. The median daily effective dose requirement of terlipressin was lower in the CI [4 mg (range $4-8$ ) vs. $8 \mathrm{mg}, \mathrm{p}<0.001$ ]. With continuous infusion, adverse events were much lower than bolus group $(20.3 \%$ versus $37.5 \%, p=0.04)$. There was no difference in the rebleed rate ( $p$ $=0.71)$ and 42 days survival rate $(\mathrm{p}=0.38)$. 
Conclusion: Use of terlipressin as continuous intravenous infusion, is associated with greater reduction in HVPG at 24 hours. At a lower dose, this is equally effective and is associated with less adverse effects than bolus injections when used for acute variceal bleed. Continuous infusion is recommended for cirrhotics with AVB.

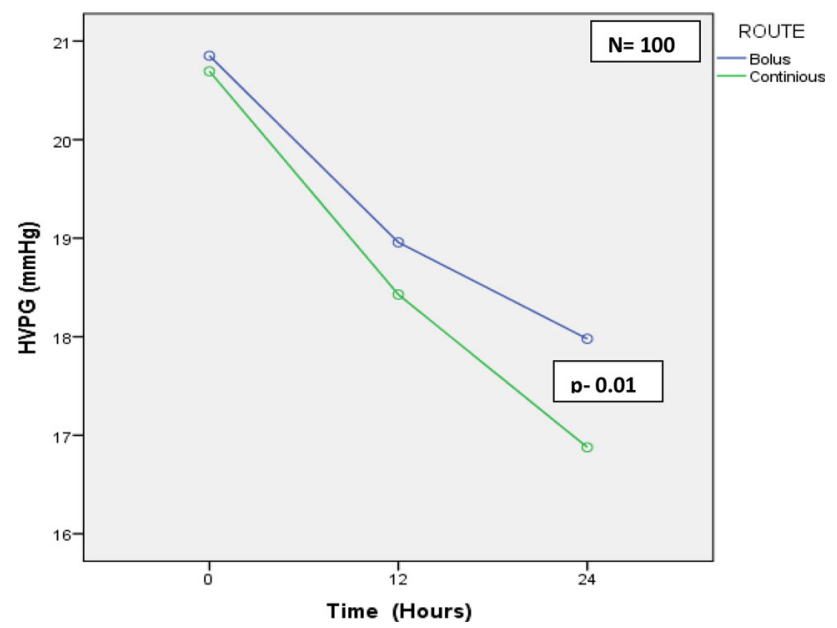

LATEB-35

Endogenous heparinoids are associated with coagulation failure during sepsis in severe alcoholic hepatitis

Madhumita Premkumar', Priyanka Saxena ${ }^{2}$, Roshni Mirza², Priyanka Jain $^{2}$, Guresh Kumar ${ }^{2}$, Puja Bhatia ${ }^{2}$, Sukriti Sukriti ${ }^{2}$, Chhagan Bihari' ${ }^{2}$, Ashok Choudhury ${ }^{2}$, Shiv Sarin ${ }^{2}$

${ }^{1}$ PGIMER, India; ${ }^{2}$ ILBS, India

Background: Rebalanced coagulation in liver disease is set to a lower level, and despite an increased prothrombin time (PT), bleeding may only be caused when superadded factors like infection supervenes. The later could be due to generation of endogenous heparinoids produced due to inflammation associated with sepsis. The coagulation dysfunction in alcoholic hepatitis is not well studied.

Method: Consecutive patients with severe alcoholic hepatitis (SAH), defined as Discriminant function $(\mathrm{DF})>32 ;[\mathrm{DF}=4.6 \times$ (patient's PT (seconds) - control's PT)+serum bilirubin level (in mg/dl)], who had no sepsis at presentation, were assessed at day 0,3 , and 7 for development of systemic inflammatory response syndrome (SIRS)/ sepsis, changes in coagulation parameters, disease progression and bleeding events. (See Figure 1)
Result: A global coagulation assay called SONOCLOT ${ }^{\mathrm{TM}}$ (global and heparinized) to measure the HLE of endogenous heparinoids, and specific assays i.e. factor VIII, vonWillebrand factor (vWF), protein C (PrC) and antithrombin III (AT), tissue plasminogen activator, tissue factor and plasminogen activator inhibitor were measured at the mentioned time points. These were compared with conventional parameters like platelet count, activated partial thromboplastin(APTT), and INR. Consecutive patients with SAH were screened, and 78 (44.3 \pm 11.7 years; $97 \%$ male $)$ with no evidence of sepsis were recruited. At day 0, elevated endogenous heparinoids were present in $20(71.4 \%)$ and $7(25 \%)$ patients with SIRS. Predictors of mortality at day 0 were Factor $8[\mathrm{OR}, 4$; 95\% CI 1.0- 1.45; $(\mathrm{p}=$ $0.03)$ ], and at day 3, low PrC [OR 0.9; 95\% CI 0.89-0.98 (p=0.007)], low AT [OR 0.8; 95\% CI 0.8-0.94 (p = 0.008)] and high INR [OR 6.3; 95\% CI 1.4-27.9 $(\mathrm{p}=0.015)$ ] (See Table 1$)$. Bleeding events were seen in $55 \%$ [skin-39\%, gastrointestinal-18.4\%, and multiple sites$39 \%$ ]. The effects of sepsis on 28day mortality (OR 7.8; 95\% CI, 6.73 to $8.36 ; \mathrm{P}=0.002$ ) and bleed events [OR, 9.01; 95\% CI 6.74 to 9.39 ; ( $\mathrm{p}=0.004)$ ] were significant. A deranged SONOCLOT ${ }^{\mathrm{TM}}$ was a predictor of bleeding (OR 3.5; $\mathrm{p}=0.05)$ and mortality $(\mathrm{OR}=2.9 ; 95 \%$ CI 2.1-6.9 ( $\mathrm{p}=0.056)$. The presence of endogenous heparinoids showed high mortality risk [OR $4.5 ; 95 \%$ CI 2.8 to $5.6 ; \mathrm{p}=0.02$ )] Conclusion: Endogenous heparinoids contribute to coagulation abnormalities in patients with SAH. Presence of endogenous heparinoids in SAH is associated with an increased tendency to bleed, risk of sepsis and mortality.

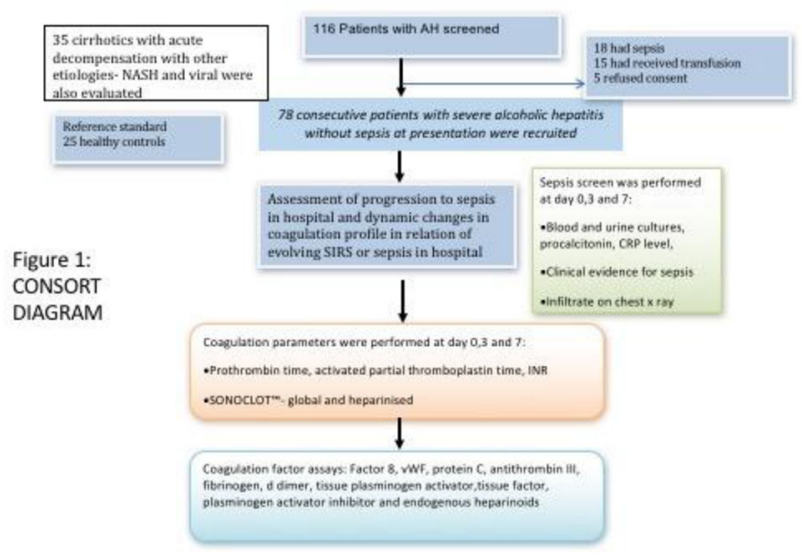


Table 1: Coagulation factor profile of patients in alcoholic hepatitis, and change in relation to evolution of sepsis or SIRS. $\mathrm{N}=78$.

\begin{tabular}{|c|c|c|c|c|c|c|c|c|}
\hline Parameter & 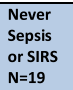 & \begin{tabular}{|l|} 
Only SIRS \\
$\mathrm{N}=8$
\end{tabular} & \begin{tabular}{|l|}
$P$ \\
value
\end{tabular} & \begin{tabular}{|l|} 
SIRS $\rightarrow$ \\
Sepsis \\
N=25
\end{tabular} & \begin{tabular}{|l|}
$P$ \\
value
\end{tabular} & \begin{tabular}{|l} 
De novo sepsis \\
$\mathrm{N}=26$
\end{tabular} & \begin{tabular}{|l|}
$P$ \\
value
\end{tabular} & \begin{tabular}{|l} 
Total \\
subjects \\
$\mathrm{N}=78$
\end{tabular} \\
\hline & (1) & (2) & 1 vs 2 & (3) & \begin{tabular}{|l|}
1 vs \\
3 \\
\end{tabular} & (4) & 1 vs 4 & \\
\hline & \begin{tabular}{|l|} 
Median \\
(Range)
\end{tabular} & & & \begin{tabular}{|l|}
$\begin{array}{l}\text { Median } \\
\text { (Range) }\end{array}$ \\
\end{tabular} & & Median (Range) & & \begin{tabular}{|l|}
$\begin{array}{l}\text { Median } \\
\text { (Range) }\end{array}$ \\
\end{tabular} \\
\hline \multicolumn{9}{|c|}{ 1. Factor $8(\%)$} \\
\hline Day 0 & \begin{tabular}{|l|}
$187(69-$ \\
342 \\
\end{tabular} & \begin{tabular}{|l|}
$187.2(92-$ \\
$328)$
\end{tabular} & 0.618 & \begin{tabular}{|l|}
$178.1(33-$ \\
$182)$ \\
\end{tabular} & 0.809 & 195.5(130-468) & 0.983 & \begin{tabular}{|l|}
$176(30-$ \\
$263)$
\end{tabular} \\
\hline Day 3 & \begin{tabular}{|l|}
$184(76-$ \\
$385)$
\end{tabular} & \begin{tabular}{|l|}
159.5 \\
$(122-349)$ \\
\end{tabular} & 0.905 & \begin{tabular}{|l|}
$189(54-$ \\
$449)$ \\
\end{tabular} & 0.890 & $178.9(56-389)$ & 0.956 & \begin{tabular}{|l}
$179(56-$ \\
$389)$
\end{tabular} \\
\hline Day 7 & $\begin{array}{l}201( \\
117- \\
398) \\
\end{array}$ & \begin{tabular}{|l|}
$156.5(89-$ \\
$292)$
\end{tabular} & 0.116 & \begin{tabular}{|l|}
$245(47-$ \\
$387)$
\end{tabular} & 0.280 & $196(44-544)$ & 0.165 & \begin{tabular}{|l|}
$174.2(44-$ \\
$524)$
\end{tabular} \\
\hline \multicolumn{9}{|l|}{$\begin{array}{l}\text { 2. Protein C } \\
\text { (\%) }\end{array}$} \\
\hline Day 0 & \begin{tabular}{|l|}
$34(9-$ \\
$222)$ \\
\end{tabular} & \begin{tabular}{|l|}
$27.6(10-$ \\
$162)$ \\
\end{tabular} & 0.911 & $22(8-56)$ & 0.282 & $27(10-116)$ & 0.294 & \begin{tabular}{|l|}
$33.5(4-$ \\
$222)$ \\
\end{tabular} \\
\hline Day 3 & $\begin{array}{l}26(8.9- \\
112)\end{array}$ & $\begin{array}{l}30(18.7- \\
56)\end{array}$ & 0.683 & $20(8-56)$ & 0.055 & $22(11.2-122.3)$ & 0.823 & \begin{tabular}{|l|}
$24.6(8.9-$ \\
$122)$ \\
\end{tabular} \\
\hline Day 7 & \begin{tabular}{|l|}
27.8 \\
$(11-$ \\
$125.4)$ \\
\end{tabular} & \begin{tabular}{|l|}
$156.5(89-$ \\
$292)$ \\
\end{tabular} & 0.727 & \begin{tabular}{|l|}
$22.5(9.8-$ \\
$78.4)$
\end{tabular} & 0.069 & $21(11-126)$ & 0.720 & $\begin{array}{l}28(11- \\
126.4)\end{array}$ \\
\hline \multicolumn{9}{|l|}{$3 \mathrm{VWF}(\%)$} \\
\hline Day 0 & $\begin{array}{l}211 \\
(106- \\
311) \\
\end{array}$ & $\begin{array}{l}124(106- \\
219)\end{array}$ & 0.045 & \begin{tabular}{|l|}
$212(54.4-$ \\
$344)$
\end{tabular} & 0.744 & $150(23-423)$ & 0.400 & \begin{tabular}{|l}
$188(23-$ \\
$423)$
\end{tabular} \\
\hline Day 3 & \begin{tabular}{|l|}
134.5 \\
$(87-344)$ \\
\end{tabular} & \begin{tabular}{|l|}
$134(89-$ \\
$322)$ \\
\end{tabular} & 0.870 & \begin{tabular}{|l|}
$145(81-$ \\
$343)$ \\
\end{tabular} & 0.590 & $213(76-454)$ & 0.031 & \begin{tabular}{|l|}
$167(76-$ \\
$554)$ \\
\end{tabular} \\
\hline Day 7 & \begin{tabular}{|l|}
$144(84-$ \\
$345)$ \\
\end{tabular} & \begin{tabular}{|l|}
166.5 \\
$(121-245)$ \\
\end{tabular} & 0.893 & \begin{tabular}{|l|}
$156(68-$ \\
$238)$ \\
\end{tabular} & 0.612 & $189(22.7-546)$ & 0.545 & \begin{tabular}{|l|}
$146(22-$ \\
$516)$ \\
\end{tabular} \\
\hline \multicolumn{9}{|l|}{$\begin{array}{l}4 \\
\text { Antithrombin } \\
3(\%)\end{array}$} \\
\hline Day 0 & \begin{tabular}{|l|}
34 \\
$(13.4-$ \\
$54)$ \\
\end{tabular} & \begin{tabular}{|l|}
$33.5(11-$ \\
$162)$
\end{tabular} & 0.611 & \begin{tabular}{|l|}
$29(14-$ \\
$47.8)$
\end{tabular} & 0.062 & 33.6 (8-78) & 0.663 & \begin{tabular}{|l|}
$34(8-78$ \\
1
\end{tabular} \\
\hline Day 3 & $\begin{array}{l}36.5 \\
(11.2- \\
144) \\
\end{array}$ & \begin{tabular}{|l|}
$29(11-$ \\
$78.9)$
\end{tabular} & 0.266 & \begin{tabular}{|l|}
$21(12.6-$ \\
$134)$
\end{tabular} & 0.051 & $30.8(11-122.3)$ & 0.639 & \begin{tabular}{|l|}
$34(11-$ \\
$144)$
\end{tabular} \\
\hline Day 7 & $\begin{array}{l}32 \\
(15.6- \\
123) \\
\end{array}$ & \begin{tabular}{|l|}
$22(14.5-$ \\
$112)$
\end{tabular} & 0.872 & $23(32-156)$ & 0.449 & $23.5(13-145)$ & 0.257 & \begin{tabular}{|l}
$34(13.2-$ \\
$145)$
\end{tabular} \\
\hline \multicolumn{9}{|l|}{$\begin{array}{l}\text { 5. Tissue } \\
\text { plasminogen } \\
\text { activator } \\
(\mathrm{ng} / \mathrm{ml}) \\
\end{array}$} \\
\hline Day 0 & $\begin{array}{l}9.64 \\
(3.5- \\
14.0) \\
\end{array}$ & \begin{tabular}{|l|}
$10.7(2.8-$ \\
$25)$
\end{tabular} & 0.739 & \begin{tabular}{|l|}
$12.3(2.1-$ \\
$33.5)$
\end{tabular} & 0.344 & $125.5(21.0-372.7)$ & 0.354 & \begin{tabular}{|l|}
$10.6(3.5-$ \\
$46)$
\end{tabular} \\
\hline Day 3 & \begin{tabular}{|l|}
8.39 \\
$(3.7-$ \\
$24.3)$ \\
\end{tabular} & \begin{tabular}{|l|}
$12.3(2.5-$ \\
$20.8)$
\end{tabular} & 0.279 & \begin{tabular}{|l|}
$9.8(1.5-$ \\
$32.3)$
\end{tabular} & 0.353 & 113.1(26.4- 323.9) & 0.128 & \begin{tabular}{|l|}
$9.8(3.7-$ \\
$32.3)$
\end{tabular} \\
\hline Day 7 & $\begin{array}{l}7.17 \\
(2.64- \\
28.0) \\
\end{array}$ & \begin{tabular}{|l|}
$7.8(1.1-$ \\
$15.8)$
\end{tabular} & 0.411 & \begin{tabular}{|l|}
$7.8(1.3-$ \\
$17.5)$
\end{tabular} & 0.666 & \begin{tabular}{|l}
$126.8(36.1-$ \\
$230.79)$
\end{tabular} & 0.703 & \begin{tabular}{|l|}
$7.893 .6-$ \\
$28.0)$
\end{tabular} \\
\hline \multicolumn{9}{|l|}{$\begin{array}{l}6 \\
\text { Plasminogen } \\
\text { activator } \\
\text { inhibitor } \\
\text { (pg/ml) } \\
\end{array}$} \\
\hline Day 0 & \begin{tabular}{|l|}
$2.6(0.7-$ \\
$12.7)$ \\
\end{tabular} & \begin{tabular}{|l|}
$7.2(1.1-$ \\
$11.8)$ \\
\end{tabular} & 0.090 & \begin{tabular}{|l|}
$1.8(0.8-$ \\
$15.4)$ \\
\end{tabular} & 0.606 & $3.9(1.2-16)$ & 0.237 & \begin{tabular}{|l|}
$2.6(0.7-$ \\
$16)$ \\
\end{tabular} \\
\hline Day 3 & \begin{tabular}{|l|}
$2.5(1.5$ \\
$-5.9)$ \\
\end{tabular} & \begin{tabular}{|l|}
$5.5(1.5-$ \\
$15.4)$ \\
\end{tabular} & 0.283 & \begin{tabular}{|l|}
$3.9(1.2-$ \\
$15.1)$ \\
\end{tabular} & 0.131 & $5.6(1.2-20.7)$ & 0.040 & \begin{tabular}{|l|}
$3.0(1.2-$ \\
$20.7)$ \\
\end{tabular} \\
\hline Day 7 & \begin{tabular}{|l|}
$1.9(1.6-$ \\
$4.9)$ \\
\end{tabular} & \begin{tabular}{|l|}
$3.9(1.2-$ \\
$6.2)$ \\
\end{tabular} & 0.660 & \begin{tabular}{|l|}
$4.9(1.2-$ \\
$18.9)$ \\
\end{tabular} & 0.123 & $4.9(1.1-15.9)$ & 0.059 & \begin{tabular}{|l|}
$4.0(1.1-$ \\
$18.9)$ \\
\end{tabular} \\
\hline \multicolumn{9}{|l|}{$\begin{array}{l}7 \text { Tissue } \\
\text { Factor (ng/ml) }\end{array}$} \\
\hline Day 0 & $\begin{array}{l}29.0 \\
(17.2- \\
132.6) \\
\end{array}$ & \begin{tabular}{|l|}
25.1 (9.8- \\
$135.6)$ \\
\end{tabular} & 0.429 & \begin{tabular}{|l}
$41.8(11-$ \\
$478.5)$
\end{tabular} & 0.282 & $33.8(18.5-159)$ & 0.673 & \begin{tabular}{|l|}
$38.3(9.8-$ \\
$478)$
\end{tabular} \\
\hline Day 3 & $\begin{array}{l}39.8 \\
(20.2- \\
194.6) \\
\end{array}$ & \begin{tabular}{|l|}
$29.8(18.2-$ \\
$125.6)$
\end{tabular} & 0.787 & \begin{tabular}{|l}
$45.2(9-$ \\
$228)$
\end{tabular} & 0.952 & $34.1(20.2-360)$ & 0.866 & \begin{tabular}{|l}
$42.5(9-$ \\
$360)$
\end{tabular} \\
\hline Day 7 & \begin{tabular}{|l|}
37.1 \\
$(17.4-$ \\
$102.9)$ \\
\end{tabular} & \begin{tabular}{|l|}
$35.6(18.1-$ \\
$171.8)$
\end{tabular} & 0.172 & \begin{tabular}{|l|}
$55.6(15.5-$ \\
$236.2)$
\end{tabular} & 0.362 & 48.1 (37.1-179.5) & 0.295 & \begin{tabular}{|l|}
$48.0(15.5-$ \\
$236.2)$
\end{tabular} \\
\hline
\end{tabular}

\section{LATEB-36}

Mathematical model gives insights into the development of occult hepatitis $B$ virus infection and its reactivation

\section{Ranjit Chauhan $^{1}$, Ashish Goyal ${ }^{2}$}

${ }^{1}$ Memorial University of Newfoundland, Canada; ${ }^{2}$ Los Alamos National Laboratory, Los Alamos, United States
Background: Out of several phases of HBV infection, the least understood phase is occult hepatitis virus infection (OBI). The paucity of data due to non-availability of biological tissues and secondly due to the prerequisite of ultra-sensitive assays for the detection of OBI prompted us to utilize mathematical modeling in determining mechanisms that lead to OBI and characteristics of HBV infection during OBI.

Method: We proposed a mathematical model $(M)$ and separately considered two phenomenon as reasons for episomal maintenance and accumulation of covalently closed circular DNA (cccDNA) in HBV infected individuals: (i) $M 1$ - Recirculation of the relaxed circular DNA and or double-stranded linear DNA (RC-DNA and or dsLDNA) from cytoplasm to nucleus of the infected hepatocytes, and (ii) $M 2$ - reinfection of the infected hepatocytes with virions. We further incorporated the dynamics of integrated hepatitis B virus (iHBV) and investigated its potential role in the development of OBI as well as its reactivation.

Result: The results showed that the main transmission mechanism in OBI is cell-to-cell transmission (CCT) and not the cell-free virus transmission (CFT). Although in most cases viral suppression of at least $99 \%$ (from its peak production values) was main determinant to the development of OBI, we also found that the inhibition of the production of HBsAg (and not overall viral suppression per se) could also explain the development of OBI. Our analysis also revealed that OBI seropositive cases are more likely to progress into a cirrhosis stage than OBI seronegative cases. The integrated DNA was mostly found to be silent (or non-productive for HBsAg) during OBI and for any reason, if it becomes productive can cause HBV reactivation.

Conclusion: Viral suppression is neither essential nor sufficient to explain the development of OBI. This is the first study that incorporates the dynamics of integrated hepatitis B virus and showed that HBV transmits via cell-cell mechanism in OBI. Lastly, we conclude that the production status of HBsAg from integrated DNA is the key factor in not only the development of OBI but also its reactivation.

\section{LATEB-37}

Identification of miRNAs associated with dendritic cell dysfunction during acute and chronic hepatitis $B$ virus infection

Avishek Kumar Singh ${ }^{1}$, Sheetalnath Babasaheb Rooge ${ }^{1}$, Aditi Varshney $^{1}$, Madavan Vasudevan ${ }^{2}$, Manoj Kumar ${ }^{4}$, Robert Geffers $^{3}$, Vijay Kumar', Shiv Kumar Sarin ${ }^{1}$

${ }^{1}$ ILBS, India; ${ }^{2}$ Bionivid Technology Private Limited, India; ${ }^{3}$ Helmholtz Centre for Infection Research, Germany; ${ }^{4}$ Institute of Liver and Biliary Sciences, India

Background \& aims: Dendritic cells (DCs) are central player in an immune response by taking up pathogen products and their presentation to $\mathrm{T}$ cells resulting in activation of these $\mathrm{T}$ cells. The uptake or expression of hepatitis B virus (HBV) proteins in/by DC is considered to impair their immune-stimulatory function and contribute to viral persistence. Therefore, understanding the expression of miRNA and their gene networks in DCs could provide accurate reasons of their dysfunction in hepatitis B patients.

Methods: We investigated differentially expressed miRNAs by microarray in DCs from different stages of HBV infection and liver disease [immune active (IA; $\mathrm{n}=20)$; low replicative $(\mathrm{LR} ; \mathrm{n}=20$ ); HBeAg negative $(n=20)$; acute viral hepatitis $(\mathrm{AVH}, \mathrm{n}=20)$ and healthy controls $(n=20)]$. The miRNA expression levels were determined by unsupervised hierarchical clustering and principal component analyses.

Results: Analysis of miRNA-mRNA regulatory networks clearly identified 19 miRNAs and 12 target gene interactions with 
interdependent regulation of MHC-I and MHC-II pathways and functioning of CD4 and CD8 T cells and NK cells during disease progression. We observed specific down-regulation of miR-2278, miR-615-3p and miR-3681-3p in immune active group versus health control resulting in elevated expression of seven target genes, viz., CANX, CALR, HSPA1B, HSPA8, HSP90AB1, HLA-A and HLA-B. In low replicative versus immune active group, there was specific upregulation of four miRNAs, viz, miR-7-1-1-3p, miR-192-5p and miR195-5p and miR-32-5p and down-regulation of two viz., miR-152-3p and miR-3613-3p resulting in down-regulation of above target genes except for $C A L R$. In HBeAg negative versus low replicative interpretation, two miRNAs each were either up-regulated (miR-342-3p and $m i R-940)$ or down regulated (miR-152-3p and miR-503-3p) impacting the expression of their cognate genes. Interestingly, in the AVH versus immune active group, all six miRNAs, viz., miR-34a-5p, miR-130b-3p, miR-221-3p, miR-320a, miR-324-5p and miR-484 were up-regulated resulting in down-regulation of multiple genes involved in antigen processing and presentation, such as, $H L A-A$, HLA-B, HLA-C, HSP-70, HSP90, CANX and CALR.

Conclusions: Selective regulation of pathways/molecules by miRNAs in DCs of hepatitis B patients appear to impact primary immunodeficiency, antigen processing and presentation and thus, provide a fresh understanding of the immune regulation in HBVassociated liver diseases. The data may be also useful in developing new therapeutic strategies.

\section{LATEB-39}

\section{Neonatal cholestasis with acholic stools; an unusual presentation} of CMV hepatitis

\section{Sanjeev Kumar Verma ${ }^{1}$, Pranshi Agarwal ${ }^{1}$, Rashmi Kumar ${ }^{1}$, Chanchal Rana ${ }^{2}$, Narendra Krishnani ${ }^{3}$}

${ }^{1}$ King George's Medical University, India; ${ }^{2}$ King George Medical University, India; ${ }^{3}$ Sanjay Gandhi Post Graduate Institute Of Medical Sciences, India

Background: Congenital CMV infection occurring in $0.2-2.5 \%$ of all live births, is infrequent, poorly recognised and treatable cause of neonatal cholestasis. We report a case of neonatal cholestasis with congenital cytomegalovirus (CMV) infection, which presented with prolonged jaundice and acholic stools as the first clinical sign and showed dramatic improvement with valganciclovir.

Method: CASE REPORT: A 60 days old female infant was admitted to our emergency department with the chief complaint of progressively increasing jaundice and pale stools since birth. She was full term normal vaginal delivery with a birth weight of $2.2 \mathrm{~kg}$. His prenatal and perinatal history was uneventful.

At the time of presentation, baby was icteric, was not sick looking taking orally well and was passing clay coloured (acholic) stools. Physical examination showed icteric skin and sclera, hepatomegaly ( $3.5 \mathrm{~cm}$ below the right costal margin, firm, left lobe $4.5 \mathrm{~cm}$ ), and splenomegaly $(1.5 \mathrm{~cm}$ below the left costal margin). Naked eye examination of stool colour confirmed persistent acholic stools for 3 days. The blood biochemistry results at the time of admission, were as follows: total bilirubin $12.28 \mathrm{mg} / \mathrm{dL}$ (direct $7.13 \mathrm{mg} / \mathrm{dL}$ ); alanine aminotransferase $245.3 \mathrm{U} / \mathrm{L}$, aspartate aminotransferase $261.3 \mathrm{U} / \mathrm{L}$, alkaline phosphatase was $1001.1 \mathrm{IU} / \mathrm{L}$; and gamma-glutamyl transpeptidase $440 \mathrm{IU} / \mathrm{L}$. Pre feed and post feed abdominal ultrasonography showed no gall bladder contractility with irregular lumen and non-visualisation of common bile duct lead us for possibility of biliary atresia. But her liver biopsy histopathology examination findings were consistent neonatal hepatitis and no features of bile duct obliteration, proliferation or edema suggestive of biliry atresia. Sepsis screen was negative; serology revealed positive IgM antibodies against CMV and CMV- DNA PCR in liver tissue confirmed, CMV related neonatal hepatitis.

Result: The patient was started on valganciclovir. Significant improvement, in form of change of stool colour (acholic to yellow stools) after 3 weeks of treatment and gradual improvement and normalization of LFTs was seen in our patient after completion of 12 weeks of valganciclovir treatment. Now child is has no organomegaly, growing well, and have normal liver function tests.

\begin{tabular}{|c|c|c|c|c|}
\hline \multicolumn{5}{|c|}{ INVESTIGATIONS } \\
\hline $\begin{array}{l}\text { LIVER } \\
\text { FUNCTION } \\
\text { TESTS }\end{array}$ & $30 / 05 / 2016$ & $\begin{array}{l}\text { 9/06/2016 } \\
\text { (Valganciclovir } \\
\text { started) }\end{array}$ & $\begin{array}{l}\mathbf{0 2 / 0 9 / 2 0 1 6} \\
\text { (Valganciclovir } \\
\text { completed) }\end{array}$ & $04 / 10 / 2016$ \\
\hline $\begin{array}{l}\text { Total/ } \\
\text { Direct } \\
\text { Bilirubin } \\
\\
(\mathrm{mg} / \mathrm{dl})\end{array}$ & $12.28 / 7.13$ & $8.39 / 6.07$ & $0.39 / 0.19$ & $0.6 / 0.2$ \\
\hline $\begin{array}{l}\text { SGPT } \\
(\mathrm{U} / \mathrm{L})\end{array}$ & 245.3 & 273.7 & 71.1 & 32 \\
\hline $\begin{array}{l}\text { SGOT } \\
(\mathrm{U} / \mathrm{L})\end{array}$ & 261.3 & 255.8 & 48.7 & 28 \\
\hline $\begin{array}{l}\text { S. ALP } \\
\text { (U/L) }\end{array}$ & 1001.1 & 1027.3 & 558.5 & 527 \\
\hline S.Protein/ & $6.19 / 3.85$ & - & $1^{-}$ & $5.95 / 3.9$ \\
\hline $\begin{array}{l}\text { S.Albumin } \\
(\mathrm{gm} / \mathrm{dl})\end{array}$ & & & & \\
\hline PT/INR & $13.7 \mathrm{sec} / 1.0$ & & & \\
\hline
\end{tabular}

Conclusion: CMV related neonatal hepatitis presenting as acholic stools is rare. Congenital TORCH infection workup is unnecessary in cases of neonatal cholesatasis with obstructive biliary features. This case emphasizes the importance of liver biopsy in all cases of neonatal cholestasis with or without acholic stools.

\section{LATEB-44}

Histo-pathological data review of granulomatous hepatitis a retrospective study

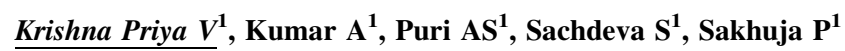
${ }^{1}$ GB Pant Hospital, India

Background: Granulomas in liver are found in $2-15 \%$ of liver biopsies. Etiology of granulomas vary geographically. HCV, sarcoidosis, are the common causes in the west whereas tuberculosis is the commonest cause in Asia.There are only few studies addressing this issue in India.AIMS AND OBJECTIVES- To analyse the clinical,demographic biochemical and histopathological data in cases of granulomas in liver.

Method: This is a retrospective data review of histopathological records from 2011-2015 done at GIPMER,New Delhi. 35 cases with liver biopsy with granulomas in liver were studied.

Result: PUO was the indication in $28(80 \%), 2(5.7 \%)$ were diagnosed as sarcoidosis previously,3 (8.5\%) had HCV positivity, 1 (2.8\%) had for hepatitis B positivity.There were 17 females and 18 males.Mean age was $25.83 \pm 2$ years. $6(17 \%)$ had jaundice, $2(5.7 \%)$ had evidence of extraintestinal Koch's. 31 (88\%) patients had evidence of tuberculosis. 4 (11\%) had AFB positive. 
Conclusion: Tuberculosis is the most common cause found.AFB was positive in only 4 which can be improved with nuclear methods.

\section{LATEB-45}

Nutritional assessment in chronic liver disease: clinical, anthropometric, biochemical and hematological parameters

Thinakar Mani Balusamy ${ }^{1}$ Thinakar Mani ${ }^{1}$, Premkumar $\mathbf{K}^{1}$, Bharat Narasimhan $^{2}$, Malar Vizhi ${ }^{1}$, Venkateswaran $\mathbf{A R}^{\mathbf{1}}$, Mohammed Ali ${ }^{1}$, Pugazhendhi Thangavelu ${ }^{1}$, Sibi Thooran ${ }^{1}$, Radha Krishnan ${ }^{1}$, Mohammed Noufal ${ }^{1}$, Ratnakar Kini ${ }^{1}$, Kani Sheikh $^{1}$, Sri Ram ${ }^{1}$, Rajkumar Solomon ${ }^{1}$

${ }^{1}$ Dr. MGR Medical University, Chennai, India; ${ }^{2}$ Manipal University, KMC Mangalore, Karnataka, India

Background: Aims: Malnutrition is common in patients with chronic liver disease, More than $60 \%$ of compensated cirrhosis and $90 \%$ of decompensated cirrhotic patients found to have various degree of malnutrition. One of modalities in the management of cirrhotic patient is adequate nutritional supplements, which eventually reduce the morbidity and mortality in such patients. It is necessary to investigate the interference of the pathophysiological changes of liver in the methods of diagnosis of the nutritional status in cirrhotic patients.

Objective: To evaluate the frequency of malnutrition among patients with cirrhosis, using different assessment tool for analysing the nutritional state.

Method: Nutritional state was determined clinically by mid-arm muscle circumference (MAMC); mid-arm circumference (MAC); body mass index (BMI); percentage of ideal body weight (BW); triceps skinfold thickness(TST) and subjective global assessment (SGA); By laboratory evaluation of total lymphocyte count (TLC) and serum albumin (ALB). Stage of disease assessed by Child-Turcotte- Pugh (CTP) and Model for End-Stage Liver Disease (MELD) Result: One Hundred patients has been evaluated, $80 \%$ were male and mean age were $40.0 \pm 10$ years. The predominant etiology was alcohol $70 . \%$ of the cases. According to the classification of ChildPugh, $19 \%$ were A, $53 \%$ were B and $28 \%$ were $\mathrm{C}$ and according to MELD < 9 in 9\%, 10-19 in 35\%, 20-29 in 43\%, 30-39 in 11\%, and > 40 in $2 \%$. Variable percentage of prevalence of malnutrition were diagnosed by Different assessment tool, which include $51.5 \%$ (MAMC) $57.8 \%$ (MAC) 16\% (BMI), 20.9\% (BW) 90.6\% (TST) and $55 \%$ with Subjective global assessment scale. The levels of TLC and ALB were compatible with malnutrition diagnosis in $65.2 \%$ and $40 \%$ of the patients, respectively. The frequency of diagnosis of malnutrition increased according to the severity of $\mathrm{HC}$ and it also increased in patients with alcoholic etiology. A greater depletion of muscular tissue in male was demonstrated.

Conclusion: The prevalence of malnutrition in cirrhotic patients varies according to the methods employed for evaluation. The prevalence of malnutrition is greater in advanced cirrhotic patients, particularly alcoholic with higher CTP and MELD. They conclusion from above study is best way to asses malnutrition in cirrhotic patients is Triceps skin fold thickness.

\section{LATEB-46}

Orphan nuclear receptor NR4A1: a novel key regulator of Hepatic stellate cell activation

Dinesh Mani Tripathi
Garcia Caldero $^{1}$, Jaime Boscha Vilaseca
${ }^{1}$, Erica Lafoz $^{1}$, Hector Carlos Garcia Pagan ${ }^{1}$

${ }^{1}$ Hospital Clínic, IDIBAPS, CIBEREHD, Spain

Background: Hepatic stellate cell (HSC) activation mediates its transition from quiescent to highly proliferative \& extracellular matrix producing phenotype, promoting fibrosis. Although several fibrogenic pathways and mediators exist and have been deeply investigated, orphan nuclear receptors and their role in fibrosis have been recently recognized and proposed. Orphan nuclear receptors represent a family of transcription factors responsible for the regulation of intracellular pathways in many diseases, such as cancer, metabolic and proliferative diseases. This study aimed to evaluate and understand the role of the orphan nuclear receptor NR4A1 in HSC activation and liver fibrosis

Method: NR4A1 expression was assessed in healthy and cirrhotic liver tissues from human subjects with different aetiologies and in healthy and cirrhotics rats (thioacetamide (TAA) and carbon tetrachloride (CCl4)). In addition, HSC were isolated from healthy rats, activated in vitro up to 3 passages (p0, p2, p3) and treated with the NR4A1 agonist, cytosporane B (CsnB; $10 \mu \mathrm{g} / \mathrm{ml})$ or vehicle (Veh; DMSO) for $24 \mathrm{~h}$. HSC activation markers ( $\alpha$ SMA, PDGFR $\beta$ and col $1 \alpha 1$ ) and NR4A1 expression were analysed by qPCR, total NR4A1 and phosphorylated/inactivated (pNR4A1) protein expression was analysed by Western Blot.

Result: In cirrhotic livers, we observed significantly increased NR4A1 protein expression compared to healthy, in human (+ 200-350\%, different aetiologies) and in rats (TAA: $+20 \%$; CCl4: + $49 \%$ ). However, such increment was mainly due to a marked increase in the expression of the inactivated (phosphorylated) NR4A1 (pNR4A1, TAA: $+11 \%$; CCl4: $+57 \%$ ), suggesting a pathological inactivation of this transcription factor.

In vitro data demonstrated significant induction of NR4A1 during HSC activation. Indeed, NR4A1 mRNA expression was significantly increased from $\mathrm{p} 0$ to $\mathrm{p} 2$ ( $>8$ fold), and further up-regulated in $\mathrm{p} 3$ ( $>$ 30 fold), in comparison to p0 HSC. Interestingly, increased NR4A1 positively correlated with HSC activation markers ( $\alpha$ SMA, Col1a1 and PDGFBR). Pharmacological activation of NR4A1 by CsnB was capable to prevent HSC de-differentiation as demonstrated by significant down-regulation of the HSC activation markers $\alpha$ SMA (-93\% and $-97 \%)$, PDGFR $\beta(-84 \%$ and $-86 \%)$ and Colla1 $(-49 \%$ and $-75 \%)$ in comparison to vehicle-treated cells in $\mathrm{p} 2$ and $\mathrm{p} 3$, respectively. Conclusion: Our study demonstrates for the first time NR4A1 as a novel modulator of HSC phenotype. Interestingly, inactive form of this transcription factor is highly expressed in cirrhotic livers, thus we herein propose modulation of NR4A1 activity as a promising avenue to treat chronic liver disease. 


\section{LATEB-48}

Higher baseline MAP protects against organ failure in patients of Acute on Chronic Liver Failure (ACLF)

Debi Prasad ${ }^{1}$, Ashok Kumar Choudhury Choudhury ${ }^{23}$, Mamun Al Mahtab Al Mahtab ${ }^{6}$, A.Kadir Dokmeci ${ }^{4}$, Md. Fazal Karim ${ }^{32}$, Diana Alcantarapayawal ${ }^{11}$, Manfung Yuen ${ }^{31}$, V.G. Mohan ${ }^{36}$, George K. Lau ${ }^{20}$, Osamu Yokosuka ${ }^{12}$, Ananta Shrestha Prasad ${ }^{16}$, Priyanka Jain ${ }^{22}$, Irene Paulson ${ }^{23}$, Shiv Kumar Sarin' ${ }^{22}$, Salimur Rahman Rahman', Harshad Devarbhavi Devarbhavi ${ }^{33}$, Zhongping Duan', Chen $\mathrm{Yu}^{8}$, Q Ning ${ }^{34}$, C E Eapen ${ }^{15}$, Ashish Goel Goel $^{14}$, Y K Chawla ${ }^{30}$, Sunil Taneja Taneja ${ }^{29}$, R K Dhiman ${ }^{29}$, Ajay Duseja ${ }^{29}$, Saeed Sadiq Hamid ${ }^{3}$, Amna Subhan Butt ${ }^{2}$, Wasim Jafri $^{2}$, Soeksiam Tan ${ }^{19}$, Dong Joon Kim ${ }^{18}$, Ji Dong Jia ${ }^{7}$, Deepak Narayan Amarapurkar ${ }^{10}$, Hasmik Ghazinian ${ }^{28}$, Jinhua $\mathbf{H u}^{27}$, Guan Huei Lee ${ }^{37}$, Seng Gee Lim ${ }^{37}$, Laurentius A. Lesmana ${ }^{26}$, Cosmas Rinaldi Lesmana ${ }^{25}$, Akash Shukla ${ }^{24}$, Sombat Treeprasertsuk $^{13}$, Samir Shah ${ }^{17}$, Chetan Kalal ${ }^{17}$, Zaigham Abbas $^{38}$, Jose D Sollano ${ }^{35}$, Gian Carpio ${ }^{35}$, Manoj K Sahu ${ }^{21}$, P. N. $\operatorname{Rao}^{5}$

${ }^{1}$ University of Auckland, New Zealand; ${ }^{10}$ Bombay Hospital \& Medical Research Center, Mumbai, India, India; ${ }^{11}$ Cardinal Santos Medical Center 10 Wilson St. Greenhills West San Juan City,, Philippines, Philippines; ${ }^{12}$ Chiba University, Japa; ${ }^{13}$ Chulalongkorn University, Thailand; ${ }^{14} \mathrm{CMC}$ VELLORE, India; ${ }^{15} \mathrm{CMC}$, Vellore, India, India; ${ }^{16}$ Foundation Nepal Sitapaila Height, , Nepal; ${ }^{17}$ Global Hospital, Mumbai, India, India; ${ }^{18}$ Hallym University College of Medicine, South Korea, Korea, Republic of; ${ }^{19}$ Hospital Selayang, Bata Caves, Selangor, Malaysia; ${ }^{2}$ Aga Khan University Hospital, Karachi, India; ${ }^{20}$ Humanity and Health Medical Group, China, China; ${ }^{21}$ IMS \&SUM hospital, Odisa, India; ${ }^{22}$ Institute of Liver and Biliary Sciences Vasant Kunj, India; ${ }^{23}$ Institute of Liver and Biliary Sciences, Vasant Kunj, Delhi, India, India; ${ }^{24}$ KEM Hospital and Seth GSMC, India; ${ }^{25}$ Medistra Hospital, Jakarta, Indonesia, Indonesia; ${ }^{26}$ Medistra Hospital, Indonesia, Indonesia; ${ }^{27}$ Millitary Hospital BeijingChina, China; ${ }^{28}$ Nork Clinical Hospital of Infectious Disease Armenia, Armenia; ${ }^{29}$ PGIMER, Chandigarh India, India; ${ }^{3}$ Aga Khan University Hospital, Pakista; ${ }^{30}$ PGIMER, Chandigarh, India; ${ }^{31}$ Queen Mary Hospital, China, China; ${ }^{32}$ Sir Salimullah Medical College, Mitford Hospital Bangladesh, Bangladesh; ${ }^{33}$ St John Medical College, Bangalore, India, India; ${ }^{34}$ Tongji Hospital, Tongji Medical College, China; ${ }^{35}$ University of Santo Tomas, Manila, Philippines, Philippines; ${ }^{36}$ VGM Hospital Coimbatore, India, India; ${ }^{37}$ Yong Loo Lin School of Medicine, National University of Singapore, Singapore; ${ }^{38}$ Ziauddin University Hospital, Karachi, Pakista; ${ }^{4}$ Ankara University School of Medicine, Turkey; ${ }^{5}$ Asian Institute of Gastroenterology Hyderabad, India; ${ }^{6}$ Bangabandhu Sheikh Mujib Medical university, Dhaka, Bangladesh, Bangladesh; ${ }^{7}$ Beijing Friendship Hospital, Capital University, China, China; ${ }^{8}$ Beijing You'an Hospital/Translational Hepatology Institute Capital Medical University, China; ${ }^{9}$ Beijing You'an Hospital/Translational Hepatology Institute Capital Medical University, India

Background: Acute on chronic liver failure (ACLF) is a frequently encountered syndrome associated with multi organ failure and high short term mortality. Shock on presentation, defined as MAP (mean arterial pressure) $<60 \mathrm{~mm} \mathrm{Hg}$, is a predictor of mortality in ACLF. Little is known however about optimum MAP in ACLF without shock. We aim to compare survival data, complications and organ failure rate between patients with MAP 65-70 vs MAP $>70$.

Method: Patients with ACLF (APASL definition) were recruited from 52 centres across Asia Pacific. Data was prospectively collected in this observational study cohort from October 2012 to September 2017. Total 1436 patients were grouped into low MAP (65-70 mm $\mathrm{Hg})$ and high MAP $(>70 \mathrm{mmHg})$ and were analyzed with respect to organ failure, complications and 90 days surival. Patient with shock,acute variceal bleed or baseline renal failure were excluded. The primary end point was 90day survival and/or liver transplantation. Secondary endpoint was incidence of complications like AVB (Acute Variceal Bleeding), AKI (Acute kidney Injury), Sepsis and number of extrahepatic organ failures.

Result: Total of 1436 patients with $84 \%$ male and Mean age of $44 \mathrm{yrs}$ were anaalyzed. Baseline characteristics like Age, sex, MELD, MELD Na and AARC score were similar between the groups. Majority of patients have ALD (51.9\%), followed by viral hepatitis (24.4\%), NASH (6.3\%) and cryptogenic (8\%) as aetiology of chronic liver disease. Incidence of AVB was statistically significant for higher MAP group (72.3\% vs $43.8 \% \mathrm{p}=0.023)$. Number of Organ failure $>$ 2 was significant for lower MAP group vs higher MAP group $(8.5 \%$ vs $2.4 \%$ with $\mathrm{p}=0.005$ ). No significant difference observed for sepsis and AKI incidence between the groups in the thirty day follow up period. Rate of liver transplant was not statistically significant between both groups. No difference in overall Survival on day 30 or day 90 between two groups. However time to event was shorter for lower MAP group (median survival 123 days vs 212 days). Interestingly cox proportional hazard ratio showed base line AARC score and age as significant predictors of 30 day and 90 day mortality. Other scores like MELD, MELD Na were not statistically significant predictor in our analysis

Conclusion: The baseline MAP above 70 is associated with fewer number of organ failures. Likely Higher MAP equals preserved PVR (peripheral vascular resistance) which leads to improved outcome in terms of organ failure irrespective of disease specific score like MELD Na, MELD and AARC scores at baseline.

\section{LATEB-49}

New insights about genetic determinants in Non cirrhotic portal hypertension (NCIPH)-A targeted NGS analysis

Rekha Athiyarath ${ }^{1}$, Rekha Athiyarath ${ }^{1}$, Aaron Chapla ${ }^{1}$, Nihal Thomas ${ }^{1}$, Balasubramanian $\mathrm{Ka}^{1}$, Elwyn Elias ${ }^{2}$, Eapen $\mathrm{Ce}^{1}$, Ashish Goel $^{1}$

${ }^{1}$ Christian medical college, India; ${ }^{2}$ University Hospital, United Kingdom

Background: NCIPH describes portal hypertension of unknown etiology with no clinical and histological signs of cirrhosis. NCIPH, a chronic thrombotic microangioapthy of portal vein branches, is relatively common in developing countries. Recent studies reported the possible genetic basis of familial NCIPH by identifying mutations in $K C N N 3, D G U O K$ and FOPV genes. In our pilot study we have observed ADAMTS13-VWF imbalance in patients with NCIPH. Therefore we aimed to understand the role of genetic factors in determining/modifying the pathogenesis of NCIPH.

Method: In liver biopsy proven NCIPH patients, we performed next generation targeted sequencing of eleven genes (ADAMTS13, VWF, KCNN3, DGUOK, MBL2, CD46, CFH, CFB, MTHFR, CFI, C3). The frequency of any variants identified were compared with that in public databases (1000 Genome, ExAc).

Result: Twenty-two patients with confirmed NCIPH were included in this study. Total of 397 variants were identified in one or more patients, of which $29 \%$ were in coding region and $40 \%$ were missense variants. We identified 3 novel and 16 rare variants (MAF $<0.01)$ in our study cohort. We have also identified VWF variants (rs1063856, rs216321, rs1800380, rs1063857) were strongly associated with high plasma VWF level in previous studies. The frequency of these variants were found to be higher in our cohort than reported in public databases. We also found variants in complement and ADAMTS13 
genes which are known to be associated with thrombotic microangiopathies

A homozygous rare variant in CD46 c.38C $>\mathrm{T}$ (p.S13F) was also identified in one patient (reported in association with HELLP syndrome).

Another significant finding was the identification of two likely pathogenic variants in MBL2 gene. The patient (31 yr old male having psoriasis and a history of sibling death due to jaundice at 7 years of age, presented with hypersplenism) had a heterozygous variation c.579_580 insAT, p.Q194fs*6 in MBL2 gene resulting in a 200 aminoacids truncated protein and another heterozygous missense variant c.584 T>G; p.F195C in the same gene. Both of these novel variations were predicted to be likey pathogenic. Further,eight out of 22 patients $(36 \%)$ had other reported MBL2 variants (p.R52C, p.G57E, p.G54D) which has been shown to be associated/predispose to various infections. The details of other rare variants are presented in table 1 .

Conclusion: This is the first NGS based comprehensive genetic analysis to delineate the role of genetic factors pertaining to microangiopathy in patients with NCIPH. The present data highlights a possible link of MBL2 gene in NCIPH and the deficiency of which has shown to predispose to infections and could be an underlying mechanism in portal hypertension in NCIPH. Identification of functionally important/VUS genetic variants implies the probable synergistic role of these interacting variants influencing the phenotype. This data provides some insights into the genetic basis of NCIPH.

\begin{tabular}{|c|c|c|c|c|c|}
\hline Gene & NT change & AA change & 1000 Genome & ExAc & Mutation taster \\
\hline C3 & c. $3342 C>G$ & SYN & 0.001 & 0.0001 & Disease causing \\
\hline VWF & $c .1027 G>A$ & V343M & NIL & 0.0006 & Disease causing \\
\hline VWF & c. $7731 \mathrm{G}>\mathrm{A}$ & SYN & NIL & 0.001 & Disease causing \\
\hline VWF & $c .5783 \mathrm{C}>\mathrm{G}$ & P1928R & 0.001 & 0.002 & Polymorphism \\
\hline VWF & $c .7604 \mathrm{G}>\mathrm{A}$ & R2535Q & 0.001 & 0.0006 & Disease causing \\
\hline $\mathrm{CFH}$ & c. $193 \mathrm{G}>\mathrm{A}$ & V65I & NIL & 0.0003 & Polymorphism \\
\hline $\mathrm{CFH}$ & $c .2821 \mathrm{G}>\mathrm{T}$ & V941F & 0.003 & 0.003 & Polymorphism \\
\hline KCNN3 & $c .1389 \mathrm{G}>\mathrm{A}$ & SYN & 0.003 & 0.002 & Disease causing \\
\hline $\mathrm{CFI}$ & $c .1322 \mathrm{G}>\mathrm{A}$ & K441R & 0.003 & 0.003 & Polymorphism \\
\hline ADAMTS13 & $\mathrm{c} .3829 \mathrm{C>T}$ & R1277W & $\mathrm{NIL}$ & 0.0001 & Polymorphism \\
\hline $\mathrm{CFI}$ & $c .1315 \mathrm{G}>\mathrm{A}$ & G439R & 0.001 & 0.001 & Polymorphism \\
\hline DGUOK & c. $757 \mathrm{~A}>\mathrm{G}$ & N253D & NIL & 0.0001 & Polymorphism \\
\hline VWF & $c .7083 \mathrm{C}>\mathrm{G}$ & SYN & 0.001 & 0.002 & Disease causing \\
\hline CD46 & $c .38 \mathrm{C}>\mathrm{T}$ & S13F & 0.005 & 0.003 & Polymorphism \\
\hline $\mathrm{CFH}$ & $c .2867 C>T$ & T956M & 0.004 & 0.002 & Polymorphism \\
\hline $\mathrm{CFH}$ & $c .3019 G>T$ & V1007L & 0.002 & 0.001 & Polymorphism \\
\hline
\end{tabular}

\section{LATEB-50}

Granulomatous liver disease- a case series

\section{Tarana Gupta ${ }^{1}$, Hari K Aggarwal ${ }^{1}$, Pulkit Chabra ${ }^{1}$, Subhash Meel $^{1}$ \\ ${ }^{1}$ PGIMS, ROHTAK, India}

Background: Sarcoidosis is a systemic granulomatous disease characterized by the formation of noncaseatingepithelioid granulomas. Lung is the most common organ involved in sarcoidosis. Liver is incidentally involved in about $12 \%$ of sarcoid patients. Liver involvement as the initial manifestation is an uncommon finding in the absence of respiratory symptoms. The most common presentation is asymptomatic elevation of alkaline phosphatase. The uncommon presentations may be portal hypertension which is either non cirrhotic or cirrhotic in later stages.

Method: Case Report

Result: Here we present a case series of three patients who presented with liver disease as the first presentation of sarcoidosis. First patient was a diabetic presenting with complaint of weight loss since 6 months. After extensive evaluation, diagnosis of hepatic tuberculosis was made and given antitubercular therapy for 5 months. However patient did not respond and was referred to us. Reevaluation revealed noncaseating granulomas in periportal areas in liver biopsy with involvement of liver, lungs and vocal cords. Another patient presented with cirrhosis of liver and significant weight loss since 4 months. However intrahepatic cholestasis and hypercalcemia raised the suspicion of sarcoidosis. Third patient was asymptomatically diagnosed to have cirrhosis of liver. In this young patient, etiological evaluation pointed towards possibility of infiltrative liver disease. All patients were suspected as cirrhosis or tuberculosis, but careful history and clinical examination alongwith extensive histopathological evaluation led to the diagnosis of sarcoidosis and proper institution of steroids resulting in clinical improvement of all the patients.

Conclusion: Infiltrative liver disease is an important diagnosis in a patient presenting with features of chronic liver disease. The proper etiological evaluation leading to targeted therapy improves the long term outcome in these patients and also prevents the advanced fibrosis and development of portal hypertension related complications.

\section{LATEB-51}

Significant increase in intestinal permeability in patients with decompensated cirrhosis after endoscopic variceal ligation

Manjunath Durgappa ${ }^{1}$, Gaurav Pande ${ }^{1}$, Alok Kumar', Krishna V $\overline{\mathbf{P}}^{1}$, Shreya Butala ${ }^{1}, \mathbf{V}$ A Saraswat ${ }^{1}$

${ }^{1}$ SGPGI, India

Background: Increased intestinal permeability (IP), and bacterial translocation (BT) are well known in liver cirrhosis (LC). Endoscopic variceal ligation leads to increase in portal pressure acutely due to obliteration of collaterals. The effect of rapid increase in portal pressure on intestinal permeability after EVL is unknown.

Aim: To compare IP in patients with Extrahepatic portal venous occlusion(EHPVO) and decompensated liver cirrhosis (D-CLD) before and after EVL.

Method: Lactulose/mannitol ratio (LMR) in the urine using 1H-NMR spectroscopy an simple, efficient, validated way to assess intestinal permeability was used in 14 patients each with decompensated cirrhosis and EHPVO before EVL and within 12 hours after EVL and 7 healthy controls. Urinary LMR measurement:Urine specimen was obtained after overnight fast.5 g D-mannitol and $10 \mathrm{~g}(15 \mathrm{~mL})$ lactulose was administered orally. Urine was collected at half hourly interval for two hours, followed by, at hourly interval for next two hours.All baseline lab tests (blood, urine, ascetic fluid) were done.

Result: 30 patients were recruited for the study with 7 controls. Baseline characteristics of D-CLD patients were age $44.7 \pm 9.7$ years, CTP-7 \pm 1.4 ; MELD $-14.5 \pm 2.7$. EHPVO patients had a significantly lower age of $28 \pm 4.7 \mathrm{yrs}(\mathrm{p}=0.04)$. The urinary LMR excretion was higher in D-CLD patients (untreated) at baseline compared to EHPVO. In normal controls, median value was $0.11 \mathrm{mmol}$, range 0.02 to 0.3 , In cirrhotics median was $0.24 \mathrm{mmol}$, (range 0.14 to 1.03 ) which increased to $0.44 \mathrm{mmol}$ (0.30 to 1.44). In EHPVO median was $0.12 \mathrm{mmol}$ (range 0.026 to 0.34 ) (at baseline which increased to 0.14 mmol (range 0.03 to0.42) ( $\mathrm{p}=0.7$ )

Conclusion: Rapid increase in portal pressure in cirrhosis patients leads to increase in intestinal permeability. 


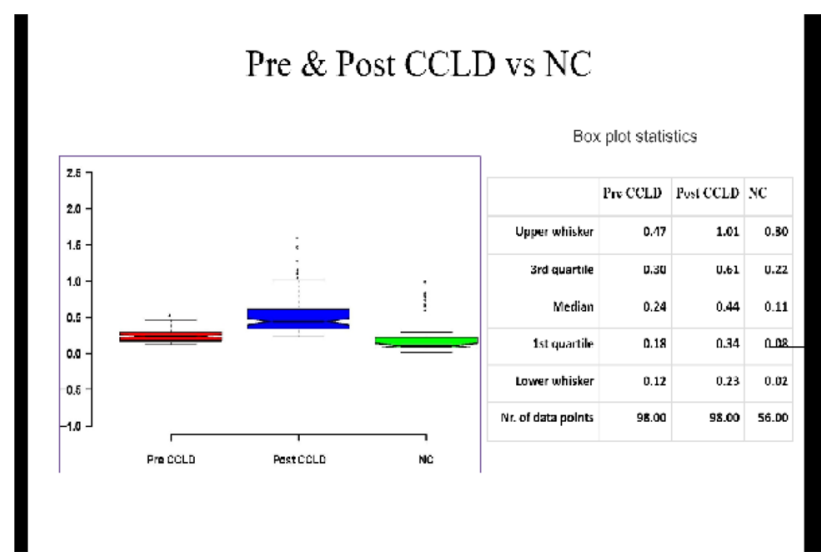

LATEB-52

Langerhans cell histiocytosis with sclerosing cholangitis in children: an entity for recognition

\section{Nagendra Kumar ${ }^{1}$, Anshu Srivastava', Surender Kumar Yachha' ${ }^{1}$ Sanjay Gandhi Post Graduate Institute of Medical Sciences, India}

Background: Langerhans cell histiocytosis $(\mathrm{LCH})$ is an important yet uncommon cause of sclerosing cholangitis (SC) in children. We analyzed the clinical presentation and outcome of patients with $\mathrm{SC}$ and $\mathrm{LCH}$.

Method: Clinical features, investigations and outcome were noted. Diagnosis of SC was based on typical cholangiographic findings with/ without suggestive histology. Children were classified into definite LCH (histology with CD1a positivity) and probable LCH (characteristic multi-systemic involvement $>1$ extra hepatic site).

Result: Overall 15 children (12 boys, age at onset of symptoms- 1.7 [0.2- 7.4] y and age at diagnosis 2.5 [0.9-9] y) with sclerosing cholangitis was analyzed. 7 had definite and 8 had probable $\mathrm{LCH}$. Jaundice (93\%) was the most common symptom followed by pruritus $(67 \%)$, ear discharge $(54 \%)$, polyuria (54\%), seborrhea (60\%), fever $(47 \%)$, weight loss $(47 \%)$, skin rash $(27 \%)$ and tooth fall $(12 \%)$. Hepatomegaly, lymphadenopathy and splenomegaly were present in $100 \%, 73 \%$ and $60 \%$ respectively. Overall, bone was involved in 10 (66\%), skin in $12(53 \%)$, diabetes insipidus in $8(53 \%)$ and lungs in 7 $(40 \%)$ cases. $6(40 \%)$ patients developed multi systemic involvement in follow-up over 18 (6-45) months. Cholangiography showed intrahepatic changes in 11, extrahepatic in 1 and both in 3 cases. Liver biopsy $(\mathrm{n}=10)$ showed changes of SC in all. Biopsy from bone lytic lesion (5/5), lymph nodes $(2 / 2)$, and skin (2/4) was confirmatory, but none of the bone marrow (15/15) and liver biopsies (10/15) showed classical histology. Only 8 children received chemotherapy, of which 3 improved, 3 died during chemotherapy and 2 did not show a response.

Conclusion: $\mathrm{LCH}$ with $\mathrm{SC}$ is a progressive disease with poor outcome. Biopsy from bone lytic lesion, lymph node and skin are useful. High index of suspicion of $\mathrm{LCH}$ is required in SC patients, as $40 \%$ cases do not have extra-hepatic features at presentation

\section{LATEB-54}

Management of walled off pancreatic necrosis: Skin or Scope

Ankush Rambhau Pawar ${ }^{1}$

${ }^{1}$ G B PANT Hospital (GIPMER), India

Background and Aims: Walled of pancreatic necrosis (WON) is a local complication in late phase of severe acute pancreatitis. With advent of minimally invasive therapies over last few decades like percutaneous and endoscopic drainage, paradigm shift has occurred in management of WON. Surgery which was the mainstay in the past is considered now as a salvage therapy. There is a scarcity of data comparing endoscopic guided drainage versus percutaneous drainage in WON.

Method: We evaluated all patients with WON from September 2015 to October 2016. All patients were treated as per our institutional protocol. Percutaneous drainage and endoscopic ultrasound guided metallic stent placement were used as minimally invasive therapies for drainage whenever required. Surgery used as a salvage therapy. We compared data of patients who underwent PCD versus those who underwent endoscopic ultrasound guided metallic stent placement. We assessed demographical profile, etiology, length of hospital stay, mortality and complications in both groups.

Result: Total 219 patients of acute pancreatitis admitted during study period, of these $80(37 \%)$ patients had symptomatic WON. Out of total symptomatic WON 35 (44\%) patients managed conservatively while $45(56 \%)$ patients required intervention. Per-cutaneous drainage (PCD), EUS guided SEMS and combination of both was done in 25 (56\%), $17(38 \%)$ and $3(6 \%)$ patients respectively. We compared 25 patients from PCD group with 17 patients from SEMS group. Those treated with combination therapy were excluded from analysis. Demographic profile, etiology and lab parameters were comparable between both the groups at baseline ( $\mathrm{p}=\mathrm{NS}$ ). Length of hospital stay was significantly less in EUS guided drainage group (mean: $9 \pm 7.5$ days, range 1-28days) as compared to percutaneous drainage group (mean: $27 \pm 21$ days, range2-88 days, p-Value <0.05). $4(16 \%)$ patients in PCD group developed cystocutaneous fistula as an adverse event while $1(6 \%)$ patient had stent migration in endoscopic group. Though trends of adverse events were higher in percutaneous group they did not reach to a significant value $(16 \%$ vs $6 \%, \mathrm{p}-0.3) 3$ patients died in PCD group and 1 in SEMS group (p-NS)

Conclusion: EUS guided SEMS in management of WON is safe and effective modality. EUS guided SEMS has shorter length of hospital stay and fewer complications as compared to percutaneous drainage.

\section{LATEB-55}

Mitochondrial transfer ability and rescue potential of human mesenchymal stem cells vary according to their tissue of origin and may be linked to their intrinsic mitochondrial respiration

Rituparna Chaudhuri $^{1}$ Swati Paliwal $^{1}$, Sonali Rawat ${ }^{1}$, Anurag Agrawal $^{2}$, Sujata Mohanty ${ }^{1}$

${ }^{1}$ All India Institute of Medical Sciences, India; ${ }^{2}$ CSIR-IGIB, India

Background: Mesenchymal stem cell (MSC) therapy has shown promise for cardio-metabolic conditions and other diseases associated with inflammation, tissue damage in many experimental models and also in a few human studies. Notably, the beneficial effects of MSC, apart from being related to engraftment and trans-differentiation, are increasingly being recognized to be due to modulatory effects through donation of healthy mitochondria to stressed/diseased neighbouring cells. While hMSCs for stem cell therapy are typically isolated from 
different tissue sources, such as bone marrow (BM), adipose (AD), dental pulp (DP) and wharton's jelly (WJ), comparative analyses of the mitochondrial donation abilities and thus, rescue potentials of hMSCs from these different tissue sources has not been reported. In this study, we carried out systematic comparisons of hMSCs from $\mathrm{BM}, \mathrm{AD}, \mathrm{DP}$ and $\mathrm{WJ}$ for their mitochondrial transfer capacity and ability to reduce reactive oxygen species (ROS).

Method: hMSCs from $\mathrm{BM}, \mathrm{AD}, \mathrm{DP}$ and $\mathrm{WJ}$ were labeled with Mitotracker Green and antimycin-treated U87-MG cell line and rat cardiomyocytes were labeled with Cell Trace Violet. The labeled cells were subsequently co-cultured for a period of 24 hours to facilitate transfer of mitochondria from hMSCs to oxidatively stressed recipient cells. Double positive cells were quantified using flow cytometer. Comparison of percentage of cells which have taken up Mitotracker Green-labeled mitochondria from hMSCs and the mean fluorescence intensity were used to assess the differential mitochondrial transfer abilities amongst tissue-specific donor MSCs. Mitochondrial ROS levels in recipient cells were assessed by staining with MitoSOX dye to evaluate reduction in ROS, post co-culture with various donor hMSCs. ATP levels of donor hMSCs were checked with colorimetry-based ATP assay kit.

Result: We found that hMSCs derived from AD and BM display higher mitochondrial transfer to stressed recipient cells compared to hMSCs from DP and WJ. Interestingly, the intrinsic ATP levels in AD and $\mathrm{BM}$ were observed to be significantly lower compared to those in DP and WJ. This inverse correlation of mitochondrial transfer to ATP levels suggests that hMSCs with less robust mitochondrial respiration, i.e., $\mathrm{AD}$ and $\mathrm{BM}$, may need to transfer higher amounts of mitochondria to recipient cells to rescue stress, as compared to donor hMSCs with more bioenergetically active mitochondria, i.e., DP and WJ. $P$ values $<0.001$.

Conclusion: This study suggests that hMSC donors vary in their rescue potential, depending on their tissue of origin. This finding provides an important insight into determining the suitable MSC type for specific clinical purposes.

\section{LATEB-56}

Discordance of non-alcoholic fatty liver disease (NAFLD) fibrosis score with fibroscan among asymptomatic patients diagnosed with NAFLD in a wellness clinic

\section{Nicodemus Lim Ong ${ }^{1}$, Diana Payawal ${ }^{1}$ \\ ${ }^{1}$ Cardinal Santos Medical Center, Philippines}

Background: Nonalcoholic fatty liver disease (NAFLD) often remains stable and asymptomatic for a number of years; however, in a subset of patients, particularly those with more advanced fibrosis are at higher risk for progression to decompensated cirrhosis, portal hypertension, hepatocellular carcinoma, or death; these patients would warrant close follow up for surveillance. A recent study in our institution last 2017 showed a prevalence of $39.8 \%$ with all of the patients scoring low risk to develop advanced fibrosis based on the NAFLD Fibrosis Score. This study aims to compare the results of NAFLD Fibrosis Score and Fibroscan in screening patients diagnosed with NAFLD seen at a hepatology clinic.

Method: All patients diagnosed with NAFLD who subsequently underwent Fibroscan at a tertiary medical center were included in the study. NAFLD was diagnosed based on history and ultrasonographic findings, excluded were patients with secondary causes of fatty liver. NAFLD Fibrosis Score was computed for all of the patients and stage of fibrosis using Fibroscan was noted and tabulated in a spreadsheet. Descriptive statistics were computed.
Result: Thirty-two patients were included in this study, with a mean age of $56.3( \pm 12.6$, ranging from $24-77$ years old), majority were females $(62.5 \%), 50 \%$ were hypertensive and $56.3 \%$ were diabetic. Using Fibroscan, 17 patients (53\%) had minimal fibrosis (F0-1), 9 (28\%) had borderline fibrosis (F2-3), 2 (6\%) had advanced fibrosis and $4(12.5 \%)$ had cirrhosis (F4). However, based NAFLD Fibrosis score, all the patients were at low risk to develop advanced fibrosis (mean -26.32 \pm 3.21 ). NAFLD Fibrosis Score was not able to screen $6(18.8 \%)$ patients with advanced fibrosis who were found to have significant fibrosis based on Fibroscan. Most patients diagnosed with advanced fibrosis were Hypertensive and Diabetic.

Conclusion: A significant number of patients with advanced fibrosis were undiagnosed using the NAFLD Fibrosis Score in our study. We recommend further studies with bigger sample sizes; we are also recommending further validation studies of NAFLD Fibrosis Score and Fibroscan paired with liver biopsy in the Filipino population for screening patients at high risk for developing advanced fibrosis.

\begin{tabular}{|c|c|c|c|c|}
\hline \multicolumn{2}{|c|}{ Table 1 Metabolic Parameters (expressed in mean) } \\
\hline Fibrosis Stage (Total $\mathrm{n}=19)$ & $\mathrm{F0}-1(\mathrm{n}=8)$ & $\mathrm{F} 2-3(\mathrm{n}=6)$ & $\mathrm{F3}(\mathrm{n}=2)$ & $\mathrm{F} 4(\mathrm{n}=3)$ \\
\hline & & & & \\
\hline Age & 55.25 & 60.3 & 55.5 & 63 \\
\hline Controlled Attenuation Parameter (CAP) & 296.5 & 328 & 333.5 & 316.67 \\
\hline BMI & 27.693 & 28.775 & 27.52 & 30.52 \\
\hline Platelet Count & 270.6 & 265.3 & 294 & 115.3 \\
\hline Total Cholesterol & 199.41 & 188.88 & 170.72 & 166.92 \\
\hline Triglycerides & 109.4 & 116.98 & 141.56 & 90.45 \\
\hline HDL & 62.1 & 53.31 & 44.84 & 59.72 \\
\hline LDL & 115.67 & 108.7 & 97.68 & 90.86 \\
\hline VLDL & 20.45 & 28.4 & 28.2 & 17.84 \\
\hline AST & 24.6 & 33.42 & 26.32 & 43.59 \\
\hline ALT & 29.98 & 42.56 & 37.76 & 55 \\
\hline Albumin & 45.01 & 44.8 & 40.7 & 37.5 \\
\hline NAFLD Fibrosis Score & -21.4072 & -22.8477 & -16.3288 & -20.9588 \\
\hline
\end{tabular}

\section{LATEB-57}

Handgrip strength in child c cirrhosis patients and it's association with mortality and complications

\section{Veerendra Rajendra Koujalagi ${ }^{1}$, Thazhath Mavali} Ramachandran ${ }^{1}$

\section{${ }^{1}$ Calicut Medical College, India}

Background: Sarcopenia is common in patients with liver disease. Measurement of Hand grip strength using hand grip dynamometer helps to assess sarcopenia objectively. This study aimed to look in to the handgrip strength and its association with prognosis and mortality in patients with child $\mathrm{C}$ cirrhosis.

Method: This is an ongoing prospective observational study. Hand grip strength of Child $\mathrm{C}$ cirrhosis patients was measured using a hand grip dynamometer and were followed up for a period of 6 months to asses mortality and complications

Result: A total of 58 child $\mathrm{C}$ patients were studied .26 of the 58 patients survived and 32 did not survive. The mean handgrip strength among survivors was 27.6 (SD $5.5 \mathrm{~K}$ ) $\mathrm{Kg}$ were as among non survivors it was $14.7 \mathrm{Kg}$ (SD 5). There was a significant association between handgrip strength and survival ( $p$ value $<0.001$ ). The patients with complications like upper GI bleed, AKI, Infection and SBP had mean hand grip strength lower than those without complications but it was not significant.

Conclusion: Sarcopenia is an important predictor of mortality in patients with child c cirrhosis. Hand grip dynamometer is an easy bedside tool to assess the sarcopenia of these patients and is strongly associated with mortality. 


\section{LATEB-58}

Type 2 diabetis mellitus is associated with of severe liver fibrosis and liver steatosis using a combination of non-invasive diagnostic parameter of liver stiffness measurement and controlled attenuation parameter

\section{$\underline{\text { Yonica Marie Tumada Dy }}{ }^{1}$, Jirran Cabatingan ${ }^{1}$}

${ }^{1}$ FUMC, Philippines

Background: Non-Alcoholic Fatty Liver Disease (NAFLD) is frequent and progresses to fibrosis among patients with type 2 diabetes mellitus. It is important to determine the presence of fibrosis and steatosis among Type II diabetic patients using non-invasive parameter, thus helping us identify further risk factors associated with significant liver steatosis and fibrosis among diabetic patients.

Method: All diabetic patients, who underwent FibroScan for assessment of liver fibrosis by liver stiffness measurement (LSM), and steatosis by Controlled Attenuation Parameter (CAP) were included in the study. Demographics and NAFLD Fibrosis score were correlated using chi $\mathrm{T}$ square and ANOVA.

Result: 31 patients were screened with a mean age $50 \pm 11$ years, with BMI $29.5 \pm 6 \mathrm{~kg} / \mathrm{m}$. About $80 \%$ had controlled diabetes $(\mathrm{HbA1C} 5.0 \pm \%)$. Severe fibrosis $(\mathrm{LSM}>8.7 \mathrm{kPa})$ was evidenced in $84 \%$ of patients, whose main risk factors includes age above 50 , increase BMI, higher waist circumference $(\mathrm{p}<0.0001)$ and dyslipidemia.. These factors also correlates with higher CAP(severe steatosis): $330 \pm \mathrm{dB} / \mathrm{m}(\mathrm{p}<0.005)$. There was direct correlation of higher LSM with higher CAP. Higher baseline CAP was related to bigger waist circumference and high triglycerides level $(\mathrm{p}<0.0001)$. However, normal HbA1c, an indication of Diabetes Control, was not significantly correlated with neither CAP nor LSM.

Conclusion: Patients with Type II Diabetes Mellitus have a higher steatosis and severe LSM. The main risk factors includes age above 50, increase BMI, higher waist circumference and dyslipidemia However normal HbA1c, was not significantly correlated with CAP nor LSM.

\section{LATEB-59}

Changes of serum CHI3L1 predict changes of liver fibrosis assessed by Fibroscan or liver biopsies after antiviral treatments for chronic HBV patients

\section{Biaoyang Lin ${ }^{1}$, Yunhua Liu ${ }^{3}$, Weikun $\mathrm{Li}^{3}$, Hongfei Zhang ${ }^{2}$, Yingying $\mathrm{Ma}^{4}$ \\ ${ }^{1}$ Zhejiang univeristy, United States; ${ }^{2}$ The 302 PLA hospital, China; ${ }^{3}$ the Third Hospital of Kunming City, China; ${ }^{4}$ Zhejiang Univ, China}

Background: $\mathrm{CHI} 3 \mathrm{~L} 1$ is a new marker that have been recently demonstrated to be a excellent non-invasive marker for liver fibrosis caused by various factors including HBV, HVC, excessive drinking and fatty liver diseases. Secreted by the hepatic stellate cells,

CHI3L1 is involved in active liver fibrosis. In this study, we analyzed the utility of changes of serum CHI3L1 protein levels in monitoring changes of liver stiffness and antiviral treatment responses of chronic HBV patients.

Method: $201 \mathrm{HBV}$ patients subjected to active treatments at the Third Hospital of Kunming City were enrolled in the study. The treatments include the uses of antiviral nucleoside and nucleotide analogue inhibitors (NAs) or peg2a (pegylated interferon alfa-2a) or the combination of the two. The patients were subjected to Fibroscan. In addition, their serum samples were analyzed for the CHI3L1 concentrations using a CHI3L1 ELISA assay with Chinese FDA approval and CE mark permit. In this analysis, two points recorded as week 0 and week 24 were included.

Result: 201 patients were subjected to the Fibroscan measurement of the liver stiffness at week 0 and week 24 . At the same time, their serum CHI3L1 levels were analyzed at week 0 and week 24. In 201 patients with both the Fibroscan data and the serum CHI3L1 data, we found that, in 95 patients $(95 / 201=47.4 \%)$, the reduction of liver stiffness as measured by Fibroscan is consistent with the reduction of serum CHI3L1 levels. In 189 patients with both the Fibroscan and the APRI measurements, only in 61 patients $(61 / 189=32.3 \%)$ we observed consistency between the reduction of APRI and the reduction by the Fibroscan measurements. Similarly, in 189 patients with both the Fibroscan and FIB4 measurements, only in 62 patients (62/ $189=32.8 \%$ ), consistency between the reduction of FIB4 and the reduction of the Fibroscan measurements were observed. In addition, we found that, in 11 patients $(11 / 201=5.5 \%)$, the liver stiffness measured by Fibroscan increased, and their serum CHI3L1 levels also increased. Together, we observed that, in 106 patients $(106 / 201=$ $52.7 \%$ ), the changes of liver stiffness measured by Fibroscan were consistent with the changes of serum CHI3L1 levels.

We then analyzed the 95 patients showing consistent reduction of both CHI3L1 and Fibroscan measurement. We found that reduction of APRI or FIB4 were seen only in $54(56.8 \%)$ and $56(59 \%)$ of these 95 patients respectively, suggesting that the serum CHI3L1 is far more consistent with Fibroscan than APRI or FIB4. Taking together, these data suggest that serum CHI3L1 is better than APRI and FIB4 in measuring changes of liver stiffness defined by Fibroscan.

Finally, for 4 patients, we measured changes of CHI3L1 reductions in serum samples of patients under antiviral treatments and changes of fibrosis stages measured by liver biopsies, we found that the reduction of serum $\mathrm{CHI} 3 \mathrm{~L} 1$ levels are consistent with reductions of liver fibrosis stages assessed by pathology reports from the four liver biopsies (Table 1).

Conclusion: The serum CHI3L1 is better than APRI and FIB4 in measuring changes of liver stiffness defined by Fibroscan. Reduction of serum CHI3L1 levels is also consistent with reductions of liver fibrosis stages or inflammation scores assessed by liver biopsies. Changes of serum CHI3L1 levels can be used as an alternative surrogate marker for monitoring changes of liver stiffness and in monitoring treatment responses of HBV patients subjected to antiviral treatments.

\begin{tabular}{|ccccccc|}
\hline \multicolumn{7}{|l|}{$\begin{array}{l}\text { Table 1. CHI3L1 reductions in serum samples of patients under antiviral treatments are consistent } \\
\text { with reductions of liver fibrosis stages assessed by pathology reports from four liver biopsies. }\end{array}$} \\
\hline $\begin{array}{c}\text { Time 1 - } \\
\text { liver } \\
\text { fibrosis }\end{array}$ & $\begin{array}{c}\text { Time 2 } \\
\text { liver } \\
\text { fibrosis }\end{array}$ & $\begin{array}{c}\text { Time 1- } \\
\text { Fibro- } \\
\text { CHI test }\end{array}$ & $\begin{array}{c}\text { Time 2- } \\
\text { Fibro- } \\
\text { CHI test }\end{array}$ & $\begin{array}{c}\text { Serum } \\
\text { CHI3L1 } \\
\text { reduction }\end{array}$ & $\begin{array}{c}\text { Fibrosis } \\
\text { stage }+ \\
\text { inflammation } \\
\text { reduction }\end{array}$ & $\begin{array}{c}\text { The period } \\
\text { between } \\
\text { time } 1 \text { and 2 } \\
\text { (months) }\end{array}$ \\
\hline G2,S2-3 & G1,S2 & 61.39 & 26.90 & 34.49 & 2 & 14 \\
G3,S3 & G0-1,S2-3 & 55.35 & 31.40 & 23.95 & 2 & 11 \\
G2,S2-3 & G1,S1 & 44.38 & 39.70 & 4.68 & 2 & 11 \\
G3,S3-4 & G2,S3 & 69.02 & 64.00 & 5.02 & 1 & 12 \\
\hline
\end{tabular}

\section{LATEB-60}

\section{Correlation of APRI Index with Metavir Index in children} with biliary atresia

\section{Himali Abhimanyu Meshram $^{1}$, Ira Shah ${ }^{1}$, Nikita Magdum ${ }^{1}$}

${ }^{1}$ Bai Jerbai Wadia Hospital for children, India

Background: In patients with biliary atresia (BA), liver fibrosis and cirrhosis commonly occur. Although liver biopsy is the gold standard to evaluate liver fibrosis, it is invasive and may result in life- 
threatening complications. The aspartate aminotransferase-to-platelet ratio index (APRI) is a safe and simple method to assess liver fibrosis in patients with chronic liver diseases.

Method: Patients with newly diagnosed BA who underwent the Kasai procedure between March 2006 and May 2009 were analyzed. Several laboratory tests including APRI were performed. Liver wedge biopsy specimens were obtained during the surgical procedure, and histopathologic analyses were performed using the Metavir classification.

Result: Thirty-five patients (12 boys, median age of 1.9 months) were enrolled. Metavir scores were F1 in 0, F2 in 11, F3 in 11, and F4 in 13 patients. The areas under the receiver operating characteristics curves for F3 and F4 were 0.92 and 0.91 , respectively. Distinct optimal cutoff values of APRI for F3 and F4 were obtained (1.01 and 1.41, respectively). With an APRI of 1.57 , the sensitivity and specificity to detect fibrosis was $48 \%$ and $52.17 \%$ respectively. With an APRI of 1.75 , the sensitivity and specificity to detect cirrhosis was $50 \%$ and $57.14 \%$ respectively.

Conclusion: APRI is not an effective tool to measure fibrosis or cirrhosis in patients with biliary atresia in Indian children.

Table 1: Liver function tests and APRI index.

\begin{tabular}{|l|l|}
\hline Parameters & Biliary atresia \\
\hline Total bilirubin $(\mathrm{mg} / \mathrm{dl})$ & $11.09 \pm 4.34$ \\
\hline Direct bilirubin & $6.22 \pm 2.41$ \\
\hline SGOT (IU/L) & $241.7 \pm 140.18$ \\
\hline SGPT & $146.31 \pm 85.24$ \\
\hline Alkaline phosphatase & $870.35 \pm 742.31$ \\
\hline Albumin & $3.77 \pm 0.55$ \\
\hline GGTP & $569.03 \pm 883.89$ \\
\hline Platelet count & $337708.33 \pm 150978.79$ \\
\hline APRI & $2.24 \pm 1.83$ \\
\hline
\end{tabular}

\section{LATEB-62}

Safety and feasibility of new digital suction power control device (SMARTVAC) for intra-abdominal drainage after Hepatic resection

\section{$\underline{K i l ~ H w a n ~ K i m}^{1}$, Jai Young Cho ${ }^{1}$, Pyung Bok Lee ${ }^{1}$, Eun Joo Choi ${ }^{1}$}

${ }^{1}$ Seoul National University Bundang Hospital, Korea, Republic of

Background: In general, an intra-abdominal closed-suction drain has been inserted for early detection of hemorrhage or bile leakage and avoidance of fluid collection after hepatic resection. The conventional drains are included Silicon ball drain (Jackson-Pratt drain), PVC type drain (HemoVAC $\AA$ ). The objective of our study is to evaluate the feasibility of new digital suction power control device (SMARTVAC () by comparing it with traditional closed-suction drainage.

Method: For one month from November 2017, ten patients were prospectively randomized to insert either Externalized closed-suction drainage (Jackson-Pratt group, $\mathrm{n}=5$ ) or Digital suction power control device (SMARTVAC $\AA$ group, $\mathrm{n}=5$ ) after elective hepatic resection. The postoperative outcomes associated with drains of the 2 group of patients were compared. Numerical Rating Scale (NRS) was used to compare the drain site pain of each group.

Result: The amount of drainage from post-operative 1 hour to postoperative 3 days did not differ between the two groups. There was no bleeding, infection at drain site, obstruction and spillage in all patients. One patient in Jackson-Pratt group underwent additional percutaneous drain due to fluid collection around hepatic cutting surface. There were no significant differences between the two groups in terms of postoperative days of drain removal $(5.0 \mathrm{Vs} 3.6, \mathrm{P}=$ $0.310)$, and hospital stay (6.8 Vs $6.6, \mathrm{P}=0.841)$.

Patient-controlled analgesia (PCA) was initiated immediately after surgery in all patients, and there was no significant differences between the two group, NRS (2.6 Vs 3.6), additional injection of opioids ( $1 \mathrm{Vs} 3, \mathrm{P}=0.262$ ), postoperative days of PCA removal $(2.8$ Vs $2.4, \mathrm{P}=0.310)$.

Conclusion: Although the large volume research is necessary, new digital suction power control device is feasible as post-operative abdominal drainage.

\section{LATEB-63}

Clinical Profile of patients with auto-immune hepatitis: a tertiary referral centre experience

\section{Bimal Kumar Sahu ${ }^{1}$}

${ }^{1}$ GB pant Hospital, New Delhi, India

Background and Aims: Autoimmune hepatitis is considered to be rare in Asia-Pacific region. This study was planned to evaluate the clinical, laboratory profile of Patients with auto-immune hepatitis,in a tertiary referral center.

Methods: Retrospectively maintained data of patients attending the liver clinic OPD between 2014 - 2017 was analyzed. AIH was defined by Simplified scoring system.All patients underwent liver biopsy

Result: Total 1300 patients attended liver clinic OPD during the study period.Of these $20(1.5 \%)$ patients were diagnosed to have autoimmune hepatitis. Of 20 patients with $\mathrm{AIH}, 14$ (70\%) were female and $6(30 \%)$ were male. Median age at presentation was $22.5 \mathrm{yrs}$ (range $=4$ to $65 \mathrm{yrs}$ ). 11 patients satisfied the criteria for definite AIH as compared to 7 patients for probable AIH. 2 patients were autoantibody negative. 16 patients are diagnosed as Type I AIH (ANA and/or SMA +), 2 patients as Type II AIH (Anti-LKM +), AIH + PSC overlap in 1 patient.

Clinical features at presentation was jaundice in $11(55 \%)$ patients, ascites- 9(45\%), upper GI bleed -1 (5\%), Asymptomatic-3 (15\%).

At presentation $9(45 \%)$ patients had Cirrhosis. 5 (25\%) patients presented with features suggestive of Acute hepatitis, 2 (10\%) Chronic hepatitis, $1(5 \%)-$ ACLF.

Treatment: 8 patients $(40 \%)$ treated with steroids, 8 patients $(40 \%)$ with steroids + Azathioprine, 1 patient $(5 \%)$ with steroid + Azathioprin + MMF, 3 patients with none.

Conclusion: In our study for the spectrum of AIH at a tertiary care center we found that most of the patients had Type I AIH. The most common presentation of AIH in our study was de-compensation of chronic liver disease. 


\section{LATEB-64}

Effectiveness of ombitasvir/paritaprevir/ritonavir \pm dasabuvir \pm ribavirin in hemodialysis patients with chronic $\mathrm{HCV}$

\author{
Orhan Yildiz $^{1}$, Bilgehan Aygen ${ }^{1}$, Selma Gokahmetoglu ${ }^{1}$, Sinem \\ Baltaci $^{1}$, Necati Mumcu ${ }^{1}$, Esma Eren ${ }^{1}$ \\ ${ }^{1}$ Medical School of Erciyes University, Turkey
}

Background: HCV infection is highly prevalent among patients with end-stage renal disease (ESRD, hemodialysis dependence) and is associated with poor prognosis. Interferon-based therapies have been a standard antiviral therapy of the hemodialysis patients infected with hepatitis $\mathrm{C}$ virus. However, the efficacy of interferons have been unsatisfactory for these patients. Although oral direct-acting antiviral agents have recently become available. Ombitasvir/paritaprevir/ritonavir (OMV/PTVr) \pm dasabuvir (DSV) \pm ribavirin (RBV) as an option in treatment-naive and-experienced patients with chronic HCV GT1 and GT4 infection. Paritaprevir is an NS3-4A protease inhibitor which is metabolised primarily by CYP3A and is given with a low dose of the CYP3A inhibitor ritonavir as a pharmacokinetic enhancer. Ombitasvir is an NS5A inhibitor given in a fixed-dose combination with paritaprevir/ritonavir and dasabuvir, a hepatitis $\mathrm{C}$ virus nonnucleoside NS5B polymerase inhibitor.

In this observational study of efficacy and safety, on hemodialysis (HD) patients with HCV genotype $1 \mathrm{~b}$ and 4 infections treated with $\mathrm{OMV} / \mathrm{PTV} \mathrm{P} \pm \mathrm{DSV} \pm \mathrm{RBV}$ combination therapy.

Method: The study included genotype $1 \mathrm{~b} \mathrm{HCV}$-infected patients with ESRD who received OMV/PTVr/DSV, and genotype $4 \mathrm{HCV}$-infected patients with ESRD who received OMV/PTVr/RBV between May 2016 and May 2017. The treatment with or without RBV was administered for 12 weeks. The sustained virological response (SVR) three months after admission to treatment and safety during the therapy were evaluated.

Result: A total of 25 patients were included in this study. The median age was $62.5 \pm 11.2$ years old, and $44 \%(11 / 25)$ were males. Of the 25 patients, $15(60 \%)$ were infected with genotypes $1 \mathrm{~b}$ and $10(40 \%)$ were infected with genotypes 4 . All of the 25 patients achieved SVR12 (100\%). One patient died from gastrointestinal bleeding during the fourth week of the treatment (unrelated to the treatment). Her HCV RNA before starting treatment was $2.600 .000 \mathrm{IU} / \mathrm{mL}$ and was undetectable at the end of the four weeks. Adverse events were moderate, and no patient had to discontinue treatment. The most common adverse drug events associated with OMV/PTVr/DSV and RBV were nausea, asthenia, anemia pruritus, insomnia and diarrhea. Anemia was more marked in patients receiving ribavirin. No patients required transfusions.

Conclusion: OMV/PTVr/DSV with or without RBV for 12 weeks had moderate adverse events and was effective in hemodialysis patients infected with $\mathrm{HCV}$ genotype $1 \mathrm{~b}$ and 4.

\section{LATEB-65}

Biliary atresia - outcome and the predictive factors - experience from a tertiary care liver centre

\section{Ruchika Kumar $^{1}$, Bikrant Bihari Lal ${ }^{1}$, Vikrant Sood ${ }^{1}$, Rajeev Khanna' $^{1}$, Seema Alam ${ }^{1}$ \\ ${ }^{1}$ Institute of Liver and Biliary Sciences, India}

Background: Biliary atresia, a progressive obliterative cholangiopathy has a fatal outcome, if left untreated and Kasai Portoenterostomy (KPE) is the primary treatment, with sequential liver transplantation in cases in which this is unsuccessful. Several factors influence the outcome of KPE. The aim was to study the outcome of children with biliary atresia and factors affecting it.

Method: Children diagnosed with biliary atresia from January 2010 to May 2016 with age less than one year were included. Predictive factors influencing the outcome and the outcome of KPE, evaluated by the clearance of jaundice ( $\mathrm{S}$. bilirubin $<2 \mathrm{mg} / \mathrm{dL}$ ) in 6 months, and the survival with native liver was analysed.

Result: Of total 108, 66 underwent Kasai Portoenterostomy (KPE) and 42 could not be operated due to late presentation or advanced liver disease and were offered primary liver transplant. Out of the 64 children with complete follow up, $24(39 \%)$ patients cleared their jaundice by 6 months of age and 39 out of 64, (60.9\%) patients survived with native liver (SNL) at one year of age.

On univariate analysis lower age at surgery, higher use of steroids, lower fibroscan value at baseline Pre KPE, higher albumin at 3 months post KPE and lower incidence of cholangitis were significant for clearance of jaundice. And higher albumin and lower bilirubin at 3 months were significant predictors for survival with native liver at 1 year $(\mathrm{p}<0.05)$. On multivariate analysis a lower bilirubin at 3 months post KPE was found to be an independent predictor of successful KPE and survival with native liver at one year. (OR $0.331,95 \%$ CI 0.158 $0.69 \mathrm{p}=0.003$ ). A Cut off bilirubin more than $4.37 \mathrm{mg} / \mathrm{dL}$ at 3 months post KPE was associated with an unsuccessful outcome. Patients with bilirubin more than $6.6 \mathrm{mg} / \mathrm{dL}$ at 3 months post KPE, were unlikely to survive at one year of age.

Conclusion: Bilirubin at 3 months Post KPE is the single most important predictor for success as well as survival with native liver at 1 year. A cut off bilirubin above 6.6 at 3 months post KPE, can be used for listing patients for liver transplant.

\section{LATEB-67}

Mesenchymal stem cell dependent modulation of induced oxidative damaged hepatocytes

\section{$\underline{\text { Sonali Rawat }}^{\mathbf{1}}$ Suchi Gupta $^{1}$, Sanjana Vig ${ }^{1}$, Sujata Mohanty ${ }^{1}$ \\ ${ }^{1}$ AIIMS, New Delhi-29, India}

Background: Abnormalities in the innate and adaptive immune responses and unbalanced immune cell infiltration can disrupt the immune-privileged state of the liver, resulting in inflammation-associated damage of hepatocytes. Last decades' preclinical and clinical trials have led to application of stem cells as a novel therapeutic agent for the treatment of degenerated and immune mediated diseases. Mesenchymal stem cells (MSCs) are a heterogeneous subset of stromal stem cells with immunomodulatory characteristics. Recent studies have shown that MSCs-based therapies may result in reduced liver inflammation, and subsequently improve regeneration of hepatocytes, which could be a promising strategy for patients with immune- mediated liver injuries.

Method: In order to elucidate the therapeutic potential of MSCs and their immunomodulatory molecules in modulation of liver inflammation and fibrosis, primary hepatocyte cell line (WRL-68) was given oxidative stress using $\mathrm{H} 2 \mathrm{O} 2$ to upregulate the ROS level. These damaged cells were co-cultured with tissue specific MSCs and their conditioned media for $24 \mathrm{~h}$.Then, ROS level was evaluated by flow cytometry to confirm the rescue potential of MSCs. In addition, the immunomodulatory molecules, IDO and PGE-2, were evaluated in co-cultured Conditioned Media by ELISA assay.

Result: The current finding is in accordance with MSC-dependent modulation of morphology and function of injured liver cells and compare effects of MSCs versus MSC-generated conditioned medium (MSC-CM). In $\mathrm{H} 2 \mathrm{O} 2$ induced liver injury, WRL-68 were successfully treated with hMSCs which resulted in attenuated hepatocyte 
damage as determined by decreased ROS levels. ELISA assay demonstrated relatively higher level of IDO and PGE-2 in the cocultured condition.

Conclusion: The above evidence showed that MSCs had immunosuppressive and reparative capacities, in rescuing hepatocytes from induced oxidative damage. MSCs are considered to act through multiple mechanisms to coordinate a dynamic, integrated response to liver inflammation and fibrosis, which prevents the progressive distortion of hepatic architecture. Therefore, our subsequent studies will be targeted towards unravelling the key signalling mechanisms in MSCs that are which hold tremendous potential as to reduce the need for liver transplantation.

\section{LATEB-69}

Global transcriptome profiling in the liver biopsies of hepatitis B virus infected patients suggests specific gene signatures of chronic liver diseases

\section{Avishek Kumar Singh ${ }^{1}$, Shiv Kumar Sarin ${ }^{1}$, Sheetalnath Babasaheb Rooge ${ }^{1}$, Aditi Varshney ${ }^{1}$, Madavan Vasudevan ${ }^{2}$, Manoj Kumar ${ }^{4}$, Robert Geffers ${ }^{3}$, Vijay Kumar ${ }^{1}$ \\ ${ }^{1}$ ILBS, India; ${ }^{2}$ Bionivid Technology Private Limited, India; ${ }^{3}$ Helmholtz Centre for Infection Research, Germany; ${ }^{4}$ Institute of Liver and Biliary Sciences, India}

Background: Hepatocyte intrusion by HBVresults in a complex deregulation of host cellular genetic processes to create a favourable environment for viral replication, cellular injury, disease onset and its progression.

Aim: The aim of the present study was to elucidate the molecular mechanisms and processes in the liver of hepatitis B patients involved in HBV replication, liver injury and liver fibrosis.

Methods: We investigated differentially expressed genes by human whole genome gene expression microarray from Agilent Technologies Inc (Santa Clara, USA) in the liver biopsy samples from different stages of HBV infection and liver disease [immune tolerant (IT; $\mathrm{n}=$ $6)$; acute viral hepatitis (AVH; $n=7)$; no fibrosis $(n=13)$; early $(\mathrm{F} 1+$ F2) $(n=17)$ or late fibrosis $(\mathrm{F} 3+\mathrm{F} 4)(\mathrm{n}=14)$ and healthy controls $(\mathrm{n}$ =7)]. Differentially expressed transcripts between patient groups were identified by applying a fold-change threshold of absolute foldchange greater than or equal to 2 . Unsupervised hierarchical clustering of differentially expressed genes between patient groups were done using Pearson Uncentered algorithm with Average linkage rule to identify gene clusters whose expression levels are significantly reproduced across the replicates and different across the patient groups. Differentially expressed genes were filtered based on their $\mathrm{p}$ Value and subjected to gene ontology and pathway enrichment analyses using DAVID tool and BridgeIsland Software and key nodes and edges were identified in each interpretation group. The gene regulatory network was visualized by CytoScape V 2.8.2.

Results: PCA and unsupervised hierarchical clustering analyses clearly discriminated the patient groups based on their whole genome gene expression profiles by ways of distance matrix and co-variance. Healthy and Acute Viral Hepatitis groups clustered wide apart in their total expression profile as compared to Immune Tolerant, Early Fibrosis and Advanced Fibrosis groups. Table-1 shows the number of differentially expressed genes among different patient groups. Analysis of gene regulatory networks identified gene interactions with four distinct nodes each representing a stage-specific gene regulation during disease progression. Major biological events and gene families targeted by differentially expressed genes included Glycosylation, cell cycle, apoptosis, iron metabolism, Innate and adaptive immune response and signal transduction.
Conclusion: Transcriptomic signatures clearly differentiated acute hepatitis B and different stages of chronic hepatitis B infection. Gene regulatory network exhibited a stage specific gene regulation during disease progression and provided fresh insights in the understanding of HBV-associated liver diseases. Further, this data may be helpful in developing early stage disease diagnostics and targeted therapeutics.

\begin{tabular}{|ccc|}
\hline Comparison & Up Regulated & Down Regulated \\
\hline Immune Tolerant Vs Healthy Control & 652 & 673 \\
Acute Viral Hepatitis (AVH) Vs Immune Tolerant & 1559 & 993 \\
Early Fibrosis Vs No Fibrosis & 14 & 17 \\
Advanced Fibrosis Vs Early Fibrosis & 480 & 752 \\
\hline
\end{tabular}

\section{LATEB-70}

Study of local and prothrombotic risk factors in children with Extrahepatic portal vein obstructiol Sheetal Ganju, Rajeev Khanna, Vikrant Sood, Dinesh Rawat, Seema Alam Department of Pediatric Hepatology, Institute of Liver and Biliary Sciences, New Delhi

\section{$\underline{\text { Sheetal Ganju }}^{1}$}

${ }^{1}$ Institute of liver and biliary sciences, India

Background: Extrahepatic portal vein obstruction (EHPVO) comprises $54 \%$ and $11 \%$ of all causes of portal hypertension in children from India and Western world, respectively. Although in $45-72 \%$ of children etiology remains obscure, there is non-uniformity of available literature on this aspect.

Method: Retrospective study of etiological profile of 41 children with EHPVO presenting between January' 2010 and March' 2017. Diagnosis of EHPVO was based on the Baveno 6 consensus i.e. presence of cavernomatous transformation of portal vein in the absence of liver disease or neoplasia.

Result: There were 30 (73\%) males. Median age of presentation was 72 months (4-204 months), 83\% were younger than 10 years of age. Variceal bleed (61\%) and lump abdomen (15\%) were the commonest presentations. Stunting and wasting were seen in $11(26.8 \%)$ patients each. Five children had congenital anomalies. Risk factors were identified in $29(70.7 \%)$ children, 52 prothrombotic risk factors in 25 children, 45 were congenital or genetic, and 7 were acquired. Additionally, 13 local risk factors were present in 12 children - 11 of them being perinatal. Concurrence of more than 1 risk factor was seen in 19 children. There was no association between presence of risk factors (local or prothrombotic) and age at presentation, growth centiles, presence of large varices, variceal bleed and number of variceal bleeding episodes. Also, the number of risk factors did not correlate significantly with age of presentation, number of variceal bleeding episodes or number of endoscopic sessions required to eradicate varices. Protein-S deficiency was the commonest prothrombotic state seen in $17(65.4 \%)$ of 26 children tested. None of the patient had mutation in JAK-2, prothrombin or factor-V leiden, antiphospholipid antibody or abnormal flow cytometry suggestive of paroxysmal nocturnal hemoglobinuria.

Conclusion: Comprehensive evaluation for prothrombotic states helps in identifying children with these disorders and need to be studied in a larger cohort. 


\section{LATEB-71}

Autologus haemopoitic stem cell therapy in decompensated cirrhosis of liver: single centre experience from bangladesh

Mamun Al Mahtab ${ }^{1}$, Md. Abdur Rahim², Sheikh Mohammad Noor E Alam ${ }^{1}$, Md. Ashraful Alam ${ }^{4}$, Faiz Ahmed Khondaker ${ }^{5}$, Ahmed Lutful Moben ${ }^{3}$, Sheikh Anisul Al Haque ${ }^{1}$, Ashraful Hoque $^{1}$, A. G. M. Hasan Al Habib ${ }^{1}$, Nuzhat Al Choudhury ${ }^{1}$, Harisul Hoque ${ }^{1}$, Md. Ashadul Islam ${ }^{1}$, Masuda Begum ${ }^{1}$

${ }^{1}$ Bangabandhu Sheikh Mujib Medical University, Bangladesh; ${ }^{2}$ Abdul Malek Ukil Medical College, Bangladesh; ${ }^{3}$ Kurmitola General Hospital, Bangladesh; ${ }^{4} \mathrm{M}$ Abdur Rahim Medical College, Bangladesh; ${ }^{5}$ Shaheed Suhrawardy Medical College, Bangladesh

Background: Stem cells may aid in hepatocyte mobilization and reversal of decompensated cirrhosis. Aim of this study is to assess safety and primary efficacy of autologous haemopoitic stem cell therapy in decompensated cirrhotics for it's future standardization.

Method: 38 patients have been included so far. All received injection G-CSF (30 IU) s/c daily for 5-6 days along with standard medical therapy. CD34-positive cells were isolated from peripheral blood using cell sorter. Subsequently these were transfused either through hepatic artery or through femoral, internal jugular or cubital veins. Patients are followed up to 120 days. 25 were males and 10 females, aged 27-77 years. 16 had cryptogenic, $14 \mathrm{HBV}, 4 \mathrm{NASH}, 3$ alcoholic and $1 \mathrm{HCV}$ cirrhosis.

Result: 25 patients completed follow up at 30 days, 17 at 90 days and 8 at 120 days. Mean serum bilirubin improved from $2.9 \mathrm{mg} / \mathrm{dl}$ at baseline to $2.5 \mathrm{mg} / \mathrm{dl}, 1.8 \mathrm{mg} / \mathrm{dl}$ and $1 \mathrm{mg} / \mathrm{dl}$ at 30,90 and 120 days respectively. Mean serum albumin rose from $2.8 \mathrm{gm} / \mathrm{L}$ to $2.8 \mathrm{gm} / \mathrm{L}$, $3.0 \mathrm{gm} / \mathrm{L}$ and $3.0 \mathrm{gm} / \mathrm{L}$ at and 30,90 and 120 days. Mean prothombin time showed improvement from $21.1 \mathrm{sec}$ to $19.3 \mathrm{sec}, 18.3 \mathrm{sec}$ and $16.6 \mathrm{sec}$ at 30, 90 and 120 days. While marked and moderate ascites was present in 17 patients, only 5, 1 and 1 patients had similar ascites at and 30, 90 and 120 days. 7/38 patients died at 120 days follow up while 2 were lost on follow up. Causes of death were hepatic failure in 4 , variceal bleeding in 2 patients and HRS in 1. No significant rise in AFP and no hepatic SOL was detected in any.

Conclusion: Autologus haemopoitic stem cell transfusion appears to be safe in patients with decompensated cirrhosis. Patients appear to benefit moderately with this approach.

\section{LATEB-72}

Iron oxide nanoparticles for Labelling and in vivo Tracking of Mesenchymal Stem Cell

Krishan Gopal Jain ${ }^{1}$, Manu Dalela1, Faiyaz Anwar', Sonali Rawat $^{1}$, Neha Kaushik ${ }^{1}$, T Vasanthakumar ${ }^{2}$, Amit Kumar Dinda $^{1}$, Sujata Mohanty

${ }^{1}$ AIIMS, India; ${ }^{2}$ SRM University, India

Background: Transplantation of mesenchymal stem cells (MSCs) has shown promise for the treatment of several degenerative diseases. However, ensuring that MSCs reach their intended targets will be necessary to maximize the ef?cacy of these treatments. This has motivated the development of noninvasive imaging techniques to monitor migration \& homing of cells to target tissues. Iron oxide nanoparticles (IONPs) have been used to label and visualize various cell types with magnetic resonance imaging (MRI). Monitoring magnetically labelled MSCs with MRI has furthered enhanced our understanding of cellular migration and the pathophysiology of diseases in experimental models. These studies shall pave the way for guiding clinical trials using cell-based therapies.
Method: In this work, we carried out a thorough study on the effect of the IONPs in MSCs. We developed super-paramagnetic iron oxide nanoparticles by the decomposition of iron oleate complex. Hydrophilic IONPs were developed by ligand exchange with citric acid with an aim to obtain biocompatible nanoparticle with highly stability and potential imaging properties, which in turn can be used for labeling of various cell types for noninvasive in vivo tracking. MSCs were isolated and expanded from human bone marrow, and were labeled with IONP suspension when cell reached $70 \%-80 \%$ confluency. We analyzed the efficiency of IONPs to label MSCs during short- or longterm exposure ( 4 or $24 \mathrm{~h}$ ). Furthermore, we investigated the retention of IONPs in the cells for up to 5th passage and analyzed the influence of IONPs labeling, on the biological properties (viability, stem cell marker expression and differentiation) of MSCs.

Result: Cell Immunophenotyping, viability and differentiation studies showed that labeling of MSCs with IONPs does not affect their properties i.e. stem cell marker expression, viability and multilineage differentiation potential. Quantification of IONPs in MSCs at each passage showed that nanoparticles stably labeled the cells upto 5th passage.

Conclusion: These findings demonstrate that these magnetic IONP can be efficiently used for labeling stem cells without any cytotoxicity and any change in stemness i.e. expression of stem cell markers, expansion and differentiation potential of stem cell. Our results demonstrate the high potential of long-term IONPs labeling of MSCs for preclinical and clinical applications.

\section{LATEB-73}

Trans-arterial chemoembolization for secondary liver cancer: single centre experience from Bangladesh

Mamun Al Mahtab ${ }^{1}$, Md. Abdur Rahim ${ }^{2}$, Sheikh Mohammad Noor E Alam ${ }^{1}$, Md. Ashraful Alam ${ }^{4}$, Faiz Ahmad Khondaker ${ }^{5}$, Ahmed Lutful Moben ${ }^{3}$

${ }^{1}$ Bangabandhu Sheikh Mujib Medical University, Bangladesh; ${ }^{2}$ Abdul Malek Ukil Medical College, Bangladesh; ${ }^{3}$ Kurmitola General Hospital, Bangladesh; ${ }^{4} \mathrm{M}$ Abdur Rahim Medical College, Bangladesh; ${ }^{5}$ Shaheed Suhrawardy Medical College, Bangladesh

Background: Trans-arterial chemoembolization (TACE) is an accepted modality of treatment for primary liver cancers. The aim of this study to evaluate the scope and efficacy of TACE in patients with secondary liver cancers with primaries from varied sources.

Method: 8 patients have been included in the study. Of them 2 had metastasis from carcinoma head of pancreas, 2 from breast cancer, 1 each from cancers of gall bladder, colon, prostrate and 1 from unknown primary. Injection epirubicin was used as the chemotherapeutic agent in all patients. All received single session of TACE. In addition to standard of care.

Result: At the time of data analysis all 8 patients were surviving. Of them 1 patient is surviving for more than 9 months, 3 for more than 5 months, 1 for more than 3 months and 3 for over 1 month.

Conclusion: TACE seems to be a safe and effective modality for patients with secondary liver cancers with hepatic arterial blood supply. However larger studies are needed to reach a definite conclusion. 


\section{LATEB-74}

Efficacy and safety of ERCP in managing pediatric biliopancreatic disorders: perspective of adult gastreoenterology from tertiary referral center with expertise in ERCP

\author{
Amol S Dahale ${ }^{1}$, Amarender Singh Puri ${ }^{1}$, Ajay Kumar ${ }^{1}$, Sanjeev \\ Sachdeva $^{1}$, Siddharth Srivastava ${ }^{1}$ \\ ${ }^{1}$ Gipmer, New Delhi, India
}

Background: Pediatric ERCP is technically difficult and challenging. Pediatric ERCP procedures are commonly done by adult gastroenterologists not formally trained in pediatric gastroenterology. Studies are scarce from south Asia on the efficacy and safety of ERCP in pediatric patients done by adult gastroenterologists. We aimed to evaluate the efficacy and safety of ERCP in children with pancreatobiliray disorders done by adult gastroenterologist.

Method: We retrospectively analyzed ERCP data base for patients age $\leq 15$ years whounderwent ERCP at G B Pant Institute of Postgraduate Education and Research (GIPMER) between January 2011 to June 2015. The data were analysed for the indication of the procedure, cannulation success rate, procedure success rate, therapeutic interventions, type of anesthesia used and the procedure related complications.

Result: Data of 126 paediatric patients were analyzed. The mean age of the cohort was11.5 \pm 3 years and $67(53.2 \%)$ were male. A total of 170 ERCP procedures were done in the study period with complete data available for $164(96.4 \%)$ procedures. The most common indications for ERCP were CBD stone [ $\mathrm{n}=50,(30 \%)]$, chronic calcific pancreatitis $[\mathrm{n}=38,(23.0 \%)]$, main pancreatic duct injury with leak $[\mathrm{n}=21,(13 \%)]$ and bile leak $[\mathrm{n}=12,(7.0 \%)]$. The overall cannulation success rate was $89 \%(146 / 164)$; procedural success rate was $86 \%$ (141/164) respectively. The complication rate was 5\% (8/164): mild pancreatitis (2), retroperitoneal perforation (2), post prodecural bleed (2) and hypoxia (2). None of the complications resulted in a fatal outcome.

Conclusion: ERCP procedures in children performed by expert endoscopists who have no formal training in pediatric gastroenterology are safe and efficacious.

\section{LATEB-75}

Evaluation of outcome of pre-operative portal vein embolization using glue mixed with lipiodol in hepatobiliary malignancies: a single tertiary centre experience

\section{$\underline{\text { Aniket Mondal }}^{1}$, Senthil Kumar' ${ }^{1}$, Amar Mukund ${ }^{1}$}

${ }^{1}$ Institute of Liver and Biliary Sciences, India

Background: To evaluate safety and outcome of preoperative portal vein embolization using glue mixed with lipoidol in terms of change in future liver remnant volume (FLR), resectablity rate and complications in hepatobiliary malignancy patients.

Method: Between 2011 to 2017, PVE was performed by a percutaneous approach in 24 patients with hepatic malignancy. Data were retrospectively reviewed. Embolization of selected portal vein segments performed was performed using glue mixed with lipiodol (1:4) through percutaneous approach. All patients underwent volumetric computedtomography (CT) assessment before and at 3-5 weeks after PVE and TLV (total liver volume), FLR (future liver remnant) volume \& FLR/TLV ratio were calculated. Complications and respectability rate were also recorded.

Result: Subsegmental, segmental, and sectorial embolization was successfully performed in all patients. None of the patients developed liver insufficiency or fever after embolization. Volumetric CT analysis showed statistically significant increase of FLR volume after PVE $(\mathrm{p}<0.05)$. No major complication was seen after PVE. Resectability rate was also high after PVE.

Conclusion: Preoperative PVE with glue mixed with lipiodol is a safe and effective method for increasing future liver remanant volume before hepatectomy in patients with hepatobiliary malignancy.

\section{LATEB-76}

Correlation of baseline portal pressure (HVPG) and Indocyanine green clearance (ICG) test with post TACE acute hepatic failure

\section{$\underline{\text { Rohit Khisti }}{ }^{1}$, Yashwant Patidar ${ }^{1}$, Lalit Garg ${ }^{1}$, Shivkumar Sarin ${ }^{1}$}

${ }^{1}$ Institute of Liver and Biliary Sciences, India

Background: With advances in imaging technologies, Hepatocellular carcinoma (HCC) is being increasingly diagnosed at earlier stages; hence many treatment options like liver transplantation, resection, and percutaneous ablation are available. Liver transplant is the best curative option for HCC; however it has limitations like availability of donors. Resection is possible in only up to $10-20 \%$ of cases due to associated co-morbidities and advanced cirrhosis. About $80 \%$ of patients with HCC have liver cirrhosis. HCC has many options for interventional management; some of them are complicated by development of portal hypertension (PHT).

The prognosis of cirrhotic patients with $\mathrm{HCC}$ is influenced by presence of PTH and portal pressure measured by HVPG is known to influence the treatment. Patients with HVPG higher than $10 \mathrm{~mm} \mathrm{Hg}$ are not suitable for hepatic resection due to the increased risk of postoperative clinical decompensation. The increase of portal blood flow after TACE can explain the elevation of variceal pressure described in some patients. The correlation between portal hemodynamics and the risk of variceal hemorrhage in portal hypertension is well described.

Dynamic tests to evaluate liver function measure the clearance of tracer substances and provide considerably more information than static tests. Indocyanine green (ICG) is a synthetic dye that is eliminated by the liver without extrahepatic metabolism and excretion, and its blood clearance has been applied to determine the operative risk before hepatectomy, as well as to evaluate donor liver function in transplantation. ICG clearance test has also been found to be useful for assessment of prognosis of patients of cirrhosis.

The aim of present study is to evaluate hepatic venous pressure gradient (HVPG) and Indocyanine green (ICG) clearance test as predictive factors for the development of acute hepatic failure in patients with liver cirrhosis undergoing TACE (Trans-arterial chemoembolization) for hepatocellular carcinoma (HCC)

\section{Method:}

Study population: Between January 2016 to April 2017, a total of 242 patients with liver cirrhosis and hepatocellular carcinoma who underwent management with TACE at our institute were identified in our hospital electronic database. Of these patients 42 patients evaluated with routine liver function tests, ICG retention test, portal pressure measurement (HVPG) were included in our study. The etiology of underlying liver cirrhosis was chronic viral hepatitis (B and C) in 17 patients $(40.5 \%)$, chronic ethanol ingestion in 15 patients $(35.7 \%)$, non-alcoholic steato-hepatitis (NASH) in 6 patients $(14.3 \%)$ while the etiology was cryptogenic in 4 patients $(9.5 \%)$. Treatment decision in these patients was made according to the BCLC staging algorithm. The diagnosis of HCC was based on either pathological confirmation or on the basis of typical imaging findings with elevated serum alpha-fetoprotein (AFP) levels. 
Excluded patients were: patients younger than 18 years and those older than 75 years, poor performance status, Child-Pugh score of more than 9, refractory ascites or hepatic encephalopathy, portal thrombosis, cardiomyopathy, and extra hepatic disease, pregnant females and those with history of allergy to dyes or iodides (ICG injection)

The study was revised and approved by the local ethical committee and institutional review board (IRB). Patient consent was not required for this retrospective study.

Data collection: Baseline demographic information, laboratory measurements, radiological tumor assessment and clinical information were recorded. The tumor assessments included the number and tumour size. According to the Common Terminology Criteria for Adverse Events V.3.0 (CTACE), it is recommended to assess TACErelated adverse events 4 weeks after the procedure. In this study, we recorded occurrence of acute liver failure in patients within 1 month after TACE. Acute hepatic failure was defined as elevation of serum bilirubin three times the baseline or elevation of SGOT/SGPT five times the baseline values occurring within 1 month of TACE.

Statistical analysis: The statistical results are presented as the mean \pm standard deviation or percentages. Continuous variables were compared parametrically using Student's t-test or non-parametrically using the Mann-Whitney U-test.

Univariate and multivariate logistic regression analysis was performed to determine the variables associated with the development of acute hepatic failure after TACE. A two-sided $\mathrm{P}$ value $<0.05$ was considered statistically significant. Statistical analyses were conducted using the SPSS software.

Result: A total of 42 patients with liver cirrhosis and hepatocellular carcinoma were included in this study which underwent TACE according to BCLC staging. Mean age of patients was 58 years with predominantly patients being male $(83.3 \%)$. The most common etiology for liver cirrhosis in the study was chronic viral hepatitis $(40.5 \%)$ while other being ethanol related chronic liver disease $(35.7 \%)$, non-alcoholic steato-hepatitis (14.3\%). The etiology was not known in $9.5 \%$ patients (cryptogenic). Mean Child-Pugh score was 6.60 and mean MELD score was 9.57 in our study population. Mean portal pressure as determined by HVPG was $13.57 \mathrm{~mm} \mathrm{Hg}$ while ICG retention at 15 minutes was 21.57 .

Univariate analysis: Univariate analysis was performed to compare the two groups (patients with and without hepatic failure) for the different pre-procedure variables that might predict acute hepatic failure after TACE

There were 10 patients in the hepatic failure group (group 1) and 32 cases in group without hepatic failure (group 2). Mean Child score was higher in group $1(7.7 \pm 1.252)$ as compared to group $2(6.25 \pm$ 0.880 ) which was found to statistically significant. We found that mean MELD score was significantly higher in group 1 (13.40 \pm $2.757)$ as compared to group $2(8.38 \pm 1.699)$. In group 1 , mean serum albumin levels were significantly lower $(2.640 \pm 0.395)$ than in group 2 (3.416 \pm 0.386$)$. Similarly, as shown in Table 2, mean tumor size, serum bilirubin, serum creatinine and alfa-fetoprotein levels in group 1 were found to be significantly higher than in group 2. Mean ICG retention in group of patients developing acute hepatic failure was found to be higher $(38.95 \pm 6.698)$ than in group of patients which did not develop acute hepatic failure after TACE $(18.48 \pm 8.7)$ and this was statistically significant. Portal pressure as determined by HVPG in group 1 was significantly higher than in group 2 (16.50 \pm $4.275 \mathrm{Vs} 12.66 \pm 7.975)$

There was no significant difference $(\mathrm{p}>0.05)$ between group 1 and group 2 as regards to age, mean hemoglobin level, serum bilirubin, SGOT, SGPT, INR and number of tumours.

Multi-variate analysis: From the above univariate analysis Child score, MELD score, serum bilirubin, serum albumin, alpha-fetoprotein, serum creatinine, mean tumor size and ICG retention were statistically significant factors predictive of acute hepatic failure.
Of these, MELD score, serum albumin, HVPG and ICG were analyzed by stepwise binary logistic regression multivariate analysis. Other variables were not included in multivariate analysis as they reduced the statistical significance of other significant variables due to indirect interdependence. On multivariate analysis, ICG (p value $=0.002$ at $95 \% \mathrm{CI}$ ) was the only statistically significant independent factor predictive of acute hepatic failure after TACE with ORs of 1.339

Receiver operating characteristic curve for independent predictive risk factors for development of acute hepatic failure after TACE revealed the area under receiver operating characteristic curve for ICG to be 0.972 .

Conclusion: Pre-treatment evaluation of routine liver function is of fundamental importance prior to TACE. Patients with higher Child Pugh score, MELD sore, serum bilirubin, serum creatinine, alfa-fetoprotein, larger tumours and lower serum albumin at baseline are likely to have higher chance of acute hepatic failure after TACE

Our study shows higher rate of post TACE acute hepatic failure in patients with- higher ICG retention at 15 minutes and those with higher baseline portal pressure while ICG retention was found to be an independent risk factor for predicting post TACE acute hepatic failure.

\begin{tabular}{|c|c|c|c|c|c|}
\hline Variable & $\begin{array}{c}\text { Patients with } \\
\text { hepatic failure } \\
(n=10)\end{array}$ & $\begin{array}{l}\text { Patients without } \\
\text { hepatic failure } \\
(\mathrm{n}=32)\end{array}$ & $\begin{array}{c}P \\
\text { value }\end{array}$ & OR & $\mathbf{9 5} \% \mathrm{Cl}$ \\
\hline Age (years) & $63.20 \pm 11.429$ & $57.31 \pm 10.965$ & 0.155 & 1.057 & $0.979-1.140$ \\
\hline Child score & $7.70 \pm 1.252$ & $6.25 \pm 0.880$ & 0.004 & 4.350 & $1.595-11.862$ \\
\hline MELD Score & $13.40 \pm 2.757$ & $8.38 \pm 1.699$ & 0.006 & 3.017 & $1.362-6.684$ \\
\hline $\mathrm{Hb}(\mathrm{g} / \mathrm{dl})$ & $11.960 \pm 2.453$ & $11.960 \pm 2.453$ & 0.583 & 1.100 & $0.782-1.547$ \\
\hline Serum Bilirubin (mg/dl) & $3.240 \pm 1.070$ & $1.328 \pm 0.672$ & 0.001 & 8.340 & $2.325-29.917$ \\
\hline SGOT (IU/L) & $68.90 \pm 22.268$ & $70.41 \pm 55.723$ & 0.932 & 0.999 & $0.985-1.014$ \\
\hline SGPT (IU/L) & $52.40 \pm 23.448$ & $41.91 \pm 21.508$ & 0.196 & 1.021 & $0.989-1.055$ \\
\hline Serum Albumin (g/dl) & $2.640 \pm 0.395$ & $3.416 \pm 0.385$ & 0.005 & 0.002 & $0.000-0.150$ \\
\hline INR & $1.014 \pm 0.082$ & $1.120 \pm 0.238$ & 0.075 & 0.000 & $0.000-2.842$ \\
\hline AFP (ng/ml) & $110.023 \pm 39.033$ & $35.544 \pm 31.128$ & 0.003 & 1.062 & $1.021-1.104$ \\
\hline Serum Creatinine (mg/dl) & $0.853 \pm 0.098$ & $0.738 \pm 0.150$ & 0.040 & 1.314 & $-0.218-0.0119$ \\
\hline Mean tumour size $(\mathrm{cm})$ & $11.790 \pm 4.420$ & $4.050 \pm 2.309$ & 0.001 & 1.754 & $1.248-2.464$ \\
\hline Number Of HCC & $1.20 \pm 0.422$ & $1.09 \pm 0.296$ & 0.376 & 2.417 & $0.343-17.036$ \\
\hline HVPG $(\mathrm{mm} \mathrm{Hg})$ & $16.50 \pm 4.275$ & $12.66 \pm 4.426$ & 0.035 & 1.251 & $1.016-1.541$ \\
\hline ICG (R15) & $38.950 \pm 6.698$ & $18.48 \pm 8.37$ & 0.002 & 1.448 & $1.117-1.006$ \\
\hline
\end{tabular}

Univariate analysis of risk factors for acute hepatic failure after TACE

Receiver operating characteristic curve - Independent predictive risk factors for acute hepatic failure after TACE. The area under receiver operating characteristic curve for ICG is 0.972 .

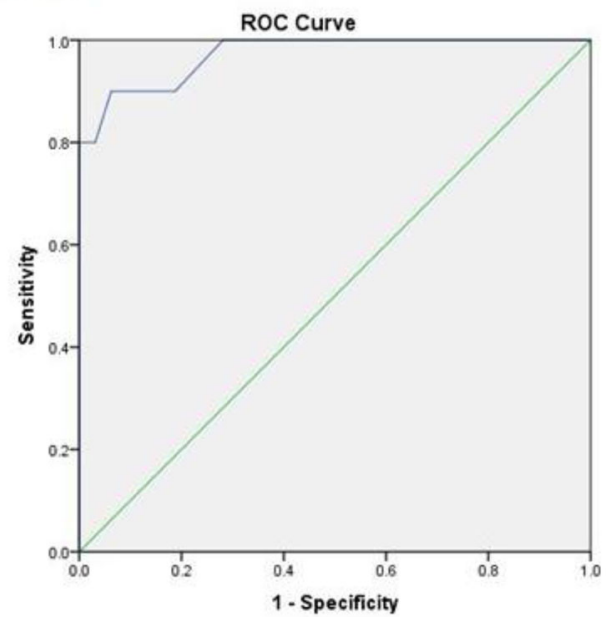




\section{LATEB-77}

\section{Primary sclerosing cholangitis in young- a case report}

\section{Hardik Rameshchandra Rughwani ${ }^{1}$ Hardik Rameshchandra Rughwani $^{1}$, Revathy MS ${ }^{1}$, Chitra $\mathbf{S}^{\mathbf{1}}$, Manimaran $\mathbf{M}^{\mathbf{1}}$ \\ ${ }^{1}$ DR MGR University, India}

\section{Background:}

PSC in young- a case report

Introduction:- Primary Sclerosing Cholangitis (PSC) is an uncommon chronic progressive disease of biliary tract characterized by inflammation and fibrosis of the intrahepatic and extrahepatic biliary ductal systems, leading eventually to biliary cirrhosis. PSC is a pathologic process that occurs in the absence of choledocholithiasis or a history of bile duct surgery. PSC is associated with IBD (predominatly UC in $70 \%$ of adult patients and $50-80 \%$ of children with this disorder). Langerhans cell histiocytosis, immunodeficiency, biliary atresia need to be excluded. Presentation is usually as obstructive jaundice. Diagnosis is established by cholangiography using ERCP and/or MRCP. Treatment of PSC in children is unsatisfactory. Main treatment modality is liver tranplantation. PSC recurs after liver transplantation in about $10 \%$ patients.

Method: Case Report

Result:

Case Discription:- A 5 year old female child presented with generalized pruritus since 3 months, mild upper abdominal pain, progressive obstructive jaundice, clay coloured stools with low grade fever since 1 month. There was no history of abdominal lump, loss of weight, abdominal distension, bloody diarrhoea or altered bowel habits. Antenatal, natal, postnatal periods were uncomplicated. There was no significant history of inflammatory bowel disease or any liver disease. On examination she was deeply jaundiced with moderate hepatomegaly and absence of KF ring. Her reports showed elevated direct bilirubin, presence of bilirubin in urine,with raised serum alkaline phosphatase and GGTP. Viral markers, ANA and ANCA were negative. USG abdomen showed ill defined hypoechoic lesion at Hepatic duct confluence with Bilateral IHBR dilatation. Few subcentimetric lymph nodes seen near hilum. CT abdomen showed $3 * 2$ $\mathrm{cm}$ irregular enhancing mass near hilum involving confluence, causing proximal dilatation. MRCP was done in view of obstruction which showed $2.2 * 1.2 \mathrm{~cm} \mathrm{~T} 2$ hypointense lesion at hilum causing Bilateral IHBRD, possibly hilar cholangiocarcinoma. EUS was done was plan of biopsy of the hilar mass which showed $1.2 \mathrm{~cm}$ hypoechoic lymphnode at hilum, FNA of which showed nonspecific inflammation. So in view of progressive obstructive jaundice patient was planned exploratory laparotomy.

Intraoperative findings showed Liver - surface nodular and irregular, GB - contracted and small, GB opened no bile was found, Prominent cystic lymph node and another solid mass in the perihilar region, Cystic duct,common bile duct,common hepatic duct,right and left heptic duct was sclerosed and narrowed, Dissection upto porta hepatis done and bile duct was transected at that level. So intra operative diagnosis of Sclerosing Cholangitis with sclerosis of CBD,CHD,RHD,LHD was made and Cholecystectomy Roux -en Y PORTO ENTEROSTOMY with liver biopsy was done.

On HPE report of liver biopsy showed effacement of architecture, micro and macrovesicular steatosis, nodular formation, cholestasis, necrosed hepatocytes foci, periductal fibrosis, portal bile duct proliferation with sparse inflammatory infiltrate, bridging fibrosis, Kupffer cell hyperplasia. Findings S/O primary sclerosing cholangitis with early biliary cirrhosis.

Patient was discharged after 10 days and is on follow up with ursodeoxycholic acid and Vitamin A, D, E, K supplements. Patient has marked improvement in pruritus with reduced icterus on 1 month follow up. Patient has been worked for liver transplantation and planned colonoscopy of follow up to rule out IBD.

\begin{tabular}{|l|l|l|l|}
\hline REPORTS & On admission & 10 th day postop & 1 month postop \\
\hline T. billirubin & 22.5 & 16.5 & 13.5 \\
\hline D. billirubin & 16.5 & 10.4 & 8.2 \\
\hline AST/ALT & $248 / 148$ & $110 / 94$ & $78 / 68$ \\
\hline SAP & 814 & 680 & 586 \\
\hline GGTP & 545 & 510 & 286 \\
\hline S. proteins & 7.5 & 7.5 & 7.8 \\
\hline S. albumin & 3.7 & 4.0 & 4.2 \\
\hline PT INR & 1.13 & 1.3 & 1.4 \\
\hline
\end{tabular}

Conclusion: Final treatment of choice for PSC is liver transplantation
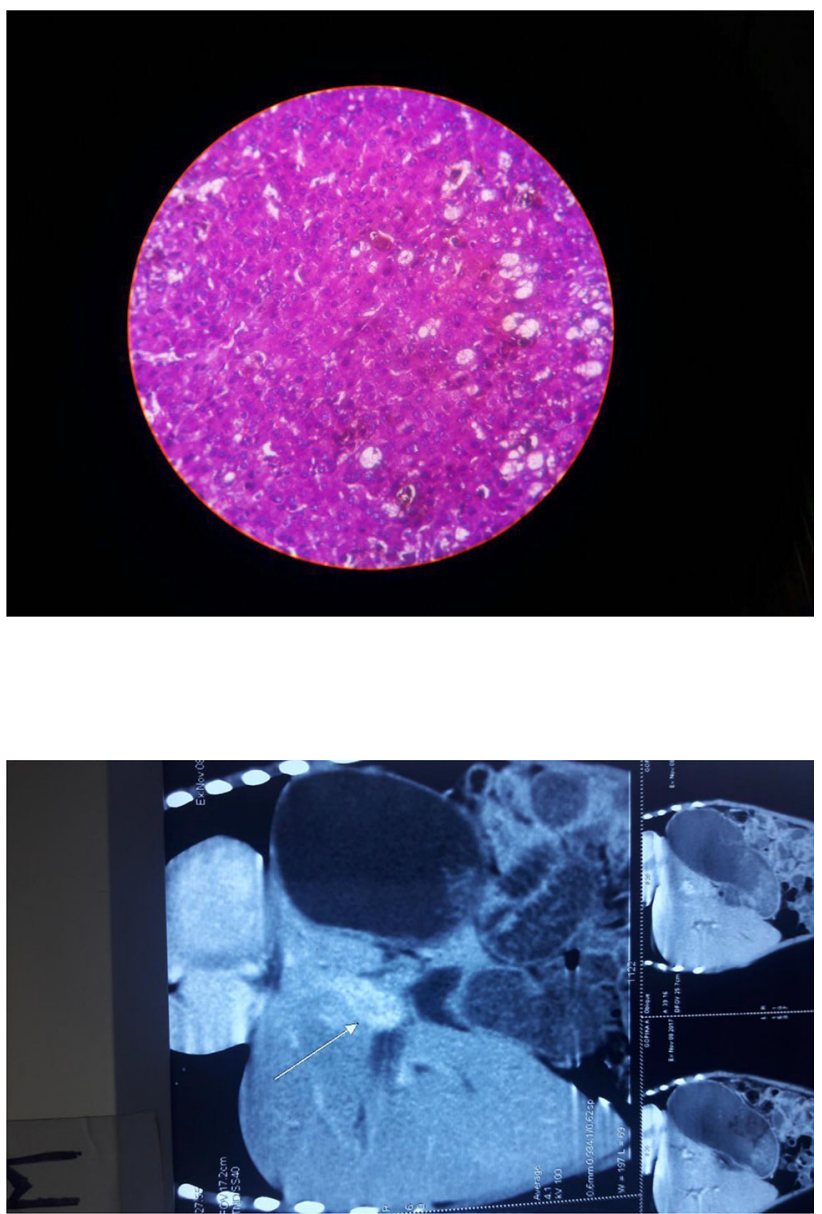

\section{LATEB-78}

Survey of Changes In pH and Electrolytes during Therapeutic Plasma Exchange done on Patients of Liver Disease

\section{Sweta Nayak $^{1}$, Meenu Bajpai ${ }^{2}$, Rakhi Maiwall ${ }^{4}$, Archisman Mohapatra $^{3}$}

${ }^{1}$ Institute of Liver and Biliary Sciences, India; ${ }^{2}$ Additional professor, Institute of Liver and Biliary Sciences, India; ${ }^{3}$ Assistant Professor, Institute of Liver and Biliary Sciences, India; ${ }^{4}$ Associate Professor, Institute of Liver and Biliary Sciences, India

Background: Therapeutic plasma exchange (TPE) is known to disturb the $\mathrm{pH}$ and electrolyte status. We aim to analyze the variation in 
$\mathrm{pH}$, ionized calcium, sodium, potassium and bicarbonate of liver disease patients undergoing TPE.

Method: Patients with liver disease undergoing TPE during July 2016 to August 2017 were analyzed. The pediatric age group patients ( $\leq$ 16), patients whose TPE could not be completed due to adverse effects of TPE and patients with missing data were excluded from the study. Data on patient demographics, details of the TPE procedure, values in the blood gas analysis report and adverse effects of TPE (if any) were collected. The percentage change [Percentage change $=$ 100* (Post levels -Pre levels)/Pre levels] of parameters like $\mathrm{pH}$, ionized calcium, sodium, potassium, bicarbonate, blood pressure and heart rate were computed and analyzed. Correlation (Spearman's rho) between potential predictor covariates for change in parameters was computed.

Result: Of the 107 procedures, 46 (43\%) were done on the MCS plus (Hemonetics Coprporation) and rest $61(57 \%)$ were done on the Spectra Optia (Terumo BCT). The percentage change in ionized calcium, sodium and potassium due to the procedure was found to be statistically significant $(p=0.000)$. The systolic $(p=0.010)$ and diastolic (0.001) blood pressure also changed significantly with the procedure. The percentage change in the $\mathrm{pH}(\mathrm{p}=0.001)$, ionized calcium $(\mathrm{p}=0.002)$ and potassium $(\mathrm{p}=0.004)$ in male patients was found to be significantly different from the female patients. The predictors for the change in ionized calcium were found to be preprocedure ionized calcium $(\mathrm{p}<0.001)$, age of patient $(\mathrm{p}<0.001)$ and the pre-procedure $\mathrm{pH}(\mathrm{p}=0.002)$. The predictors for the change in sodium were found to be pre-procedure sodium, potassium and $\mathrm{pH}(\mathrm{p}$ $<0.001$ each). Similarly, predictors for change in potassium and bicarbonate were their corresponding pre-procedure levels with $\mathrm{p}=$ 0.009 and $\mathrm{p}<0.001$ respectively. Procedure related complications occurred during 30 procedures of which 13 complications $(12.15 \%)$ were categorized as features of hypocalcemia. No association was found between hypocalcemic manifestation and pre-procedure calcium, change in calcium, age or gender of the patient.

Conclusion: The TPE procedure brings about remarkable changes in ionized calcium, sodium, potassium and bicarbonate ions. The decrease in ionized calcium during the procedure is predicted by preprocedure ionized calcium levels, $\mathrm{pH}$ and age of patient.

\section{LATEB-79}

The largest Indian case series of tyrosinemia type 1 - Its various clinical presentations and treatment outcomes

Vaibhav Sameer Shah ${ }^{1}$, Shashank Shrotriya ${ }^{2}$, Ashish Bavdekar ${ }^{2}$

${ }^{1}$ KEM Hospital, Pune, India; ${ }^{2}$ KEM Hospital, India

Background: Tyrosinemia type 1 is an autosomal recessive disease caused due to deficiency of Fumarylacetoacetate hydrolase (FAH) enzyme, and can affect the liver, kidneys and peripheral nerves. The advent of NTBC [2-(2-nitro-4-trifluoromethylbenzoyl)-1, 3 cyclohexanedione]and liver transplantation has revolutionised its outcome. We present a case series of 8 children diagnosed with tyrosinemia, with an aim of describing its varied presentations, treatment options and outcomes.

Method: Observational data of all tyrosinemia patients admitted to KEM Hospital, Pune, between March 1997 and August 2017 was obtained retrospectively and assessed.

Result: While 6 patients presented in infancy, 2 patients presented after 5 years of age. The earliest presentation was at $2 \frac{1}{2}$ months of age, while the oldest was at 9 years of age. Out of the 6 patients presenting in infancy, 2 had neonatal cholestasis while the rest had evidence of chronic liver disease. Among the older children, one had severe rickets with chronic liver disease on presentation, while the other had hepatocellular carcinoma mimicking portal venous obstruction. Renal tubular acidosis was found in 4 patients at the time of presentation, out of which 2 patients had frank rickets. Diagnosis of all patients, except one, were confirmed by the finding of an elevated urinary and blood succinylacetone. In the exceptional case, urinary succinylacetone was normal and diagnosis was confirmed by demonstrating decreased FAH enzyme activity on liver tissue. 3 children were started on dietary therapy and NTBC immediately after diagnosis, out of which, 2 children, in whom medical treatment was initiated before 1 year of age, responded dramatically, with resolution of clinical and bichemical abnormalities, and are currently asymptomatic after 20 years and 13 years of medical treatment respectively, whereas one child in whom medical treatment was started at 6 years of age, developed hepatocellular carcinoma after 2 years of treatment and expired as its consequence. 2 children successfully underwent living donor liver transplantation. The child who presented as hepatocellular carcinoma, underwent chemo- and radiotherapy at an oncology institute, but died within a month of starting treatment. Relatives of the remaining 2 children, refused for treatment citing financial constraints, and were lost to follow up.

Conclusion: Tyrosinemia is a treatable cause of metabolic liver disease. Keeping a high suspiscion for early diagnosis of this disease and institution of early treatment are the two most important factors determining a successful outcome.
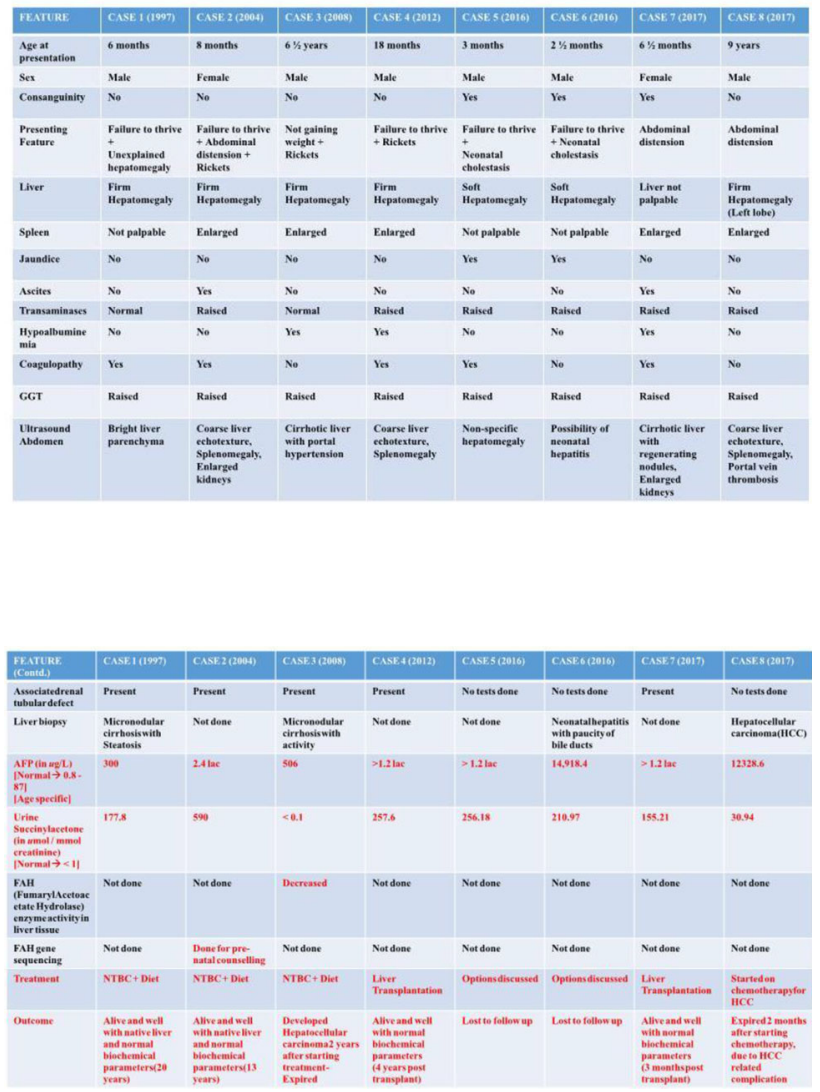


\section{LATEB-80}

Activation of liver $X$ receptors a and by $22(R)$ hydroxycholesterol leads to generation of dopaminergic neuronal cells from human Mesenchymal Stem Cells

\section{Manisha Singh ${ }^{1}$, Sonali Rawat ${ }^{1}$, Lohiny Balendran ${ }^{2}$, Amit Kumar Dinda $^{2}$, Pardeep Kumar Vaishnav ${ }^{2}$, Sujata Mohanty ${ }^{2}$}

${ }^{1}$ All India Institute of Medical Science, India; ${ }^{2}$ All India Institute of Medical Sciences, India

Background: Cholesterol is a type of lipid, which is biosynthesized in all types of animal cells and is an important part of the cell membrane. Human brain consists of as high as $\sim 25 \%$ of the lipids. The lipidome profile of the central nervous system (CNS) is associated with neuronal activity, cognitive behaviour and various neurological disorders. Oxysterols are the oxidized derivatives of cholesterols which show their effect in various functioning through $1 x r-\alpha$ and $1 x r-\beta$. Lxr (liver X receptors) are the nuclear receptors which function through their oxysterol ligands and control various activities like cell division, ventral midbrain neurogenesis and dopaminergic (DAergic) neurogenesis. Hence, in the current study, we aim to investigate the neurogenic effect of 22 (R)- hydroxycholesterol (22-HC), on human MSCs (hMSCs) obtained from bone marrow (BM, adipose tissue (AD) and dental pulp (DP).

Method: The effect of 22-HC on hMSCs was evaluated at morphological, morphometric, ultrastructural, mRNA, protein and functionality levels by qRT-PCR, scanning electron microscopy, transmission electron microscopy, immunoflorescence, western blotting and calcium ion imaging. The effect was evaluated on the basis of detailed morphological and morphometric analysis, expression of genes and proteins associated with maturation of neurons (NF, MAP2, TUJ1, TH), channel ion proteins (Kv4.2 \& SCN5A), chemical functionality (DAT), synapse forming tendency of neurons (Synaptophysin) and transcription factors (Ngn2 \& Pitx3) which play important role in dopaminergic neuron survival and maintenance, efficiency of generation of dopaminergic neurons and functional assessment. The percentage of generation of non- DA cells (Ach, TPH2, S100 and GFAP positive cells) was also evaluated to confirm the selective neurogenic potential of 22-HC.

Result: Post analysis, it was observed that 22-HC yields morphologically more distinct and higher percentage of functional DA neurons and differential effect on various tissue- specific primary human MSCs. There was significant increase in the expression of genes and proteins associated with Daergic neuronal cells in hMSCs. DP-MSCs yield the highest percentage of functional dopaminergic neuronal cells (Figure 1 and 2).

Conclusion: In conclusion, our study provides comprehensive and robust evidences to state the role $22-\mathrm{HC}$ in generating functional DAergic neuronal cells from hMSCs of varied origins. Our study provides the first evidence that oxysterols $(22-\mathrm{HC})$ causes DAergic neurogenesis and leads to their morphological, genetic and functional changes. Our report has also paved the path to investigate the disparate effect of 22-HC on the three types of hMSCs taken under the study. AD-MSCs have shown off beat expression of LXR $\alpha$ as compared to that of LXR $\beta$. These pathways need to be investigated further (by blocking assays) to have an insight of the mode and mechanism of action of 22-HC for midbrain DAergic neurogenesis.

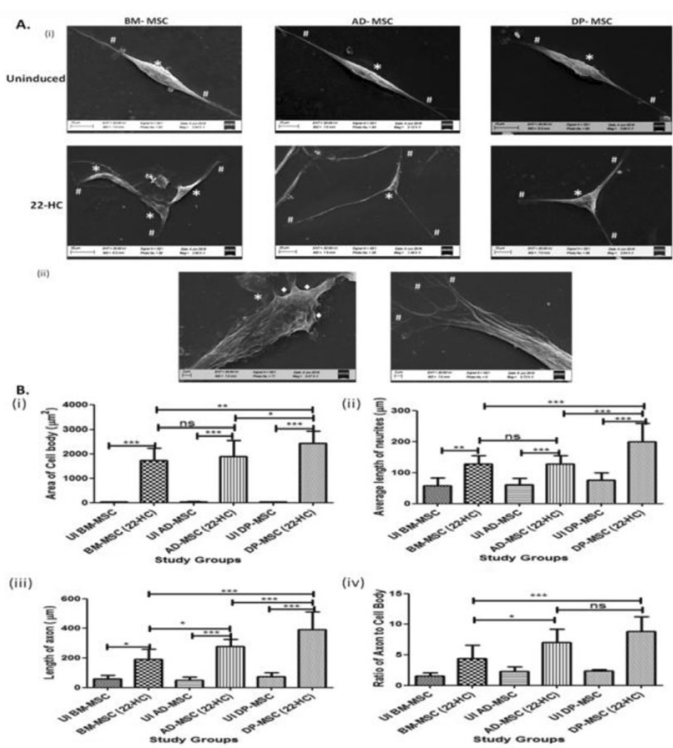

Figure 1: A. Morphological characterization of differentiated \& undifferentiated hMSCs; B. Morphometric characterization of differentiated \& undifferentiated hMSCs
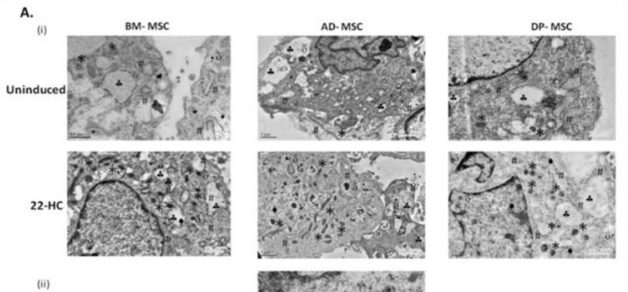

(i)
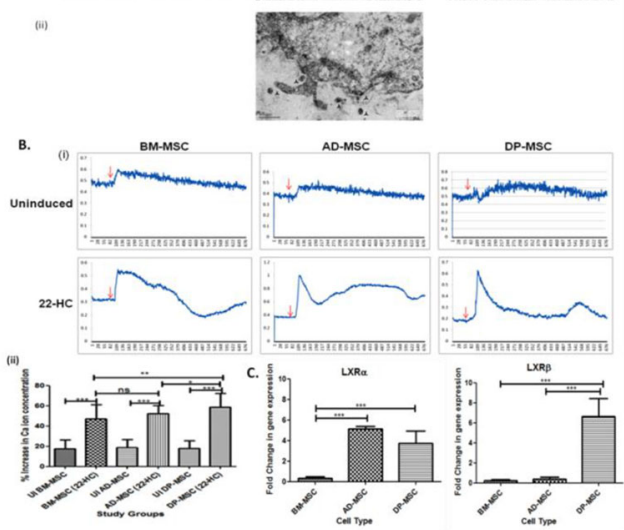

Figure 1: A. Ultrastructural characterization of differentiated \& undifferentiated hMSCs, B. Functional characterization of differentiated \& undifferentiated hMSCs 


\section{LATEB-81}

\section{An unusual complication of Liver Biopsy}

\section{Sandeep Narayanan ${ }^{1}$}

${ }^{1}$ Government Medical College Trivandrum, India

Background: Liver biopsy is a commonly done procedure. We present a rare complication of liver biopsy.

Method: A 61 year old female presented to us with 3 month history of jaundice and pruritus. On evaluation, she had a bilirubin of 21 with direct fraction of 21.5,SGOT/SGPT of 313/700 and ALP of 768.U1trasound and MRCP showed no IHBRD.Viral,metabolic and autoimmune markers were negative.

On taking the history again, it was noted that she had taken CAMS (Kashayam - Auyvedic medicine) for about a month prior to the onset of illness for knee pain. A provisional diagnosis of DILI was made. For confirmation, a liver biopsy was done. The procedure was uneventful. The biopsy reports were consistent with the diagnosis of DILI (Figure 1).

She presented to us again after 1 month with right hypochondrial pain. An ultrasonogram was done which showed a hypoechoic lesion in the liver $7.6 \mathrm{~cm} * 5.6 \mathrm{~cm}$ with doppler signals inside with ying yang sign suggestive of pseudoaneurysm. A CT angiogram confirmed the diagnosis.

Result: Selective celiac angiograms showed a pseudoaneurysm filling from the inferior segment branch of the right hepatic artery. The aneurysm was occluded by trapping technique with two gdc (3m$\mathrm{mx} 8 \mathrm{cms}$ ). Thereafter $1 \mathrm{ml}$ of $25 \%$ NBCA glue was injected into the artery resulting in its occlusion. Post procedure ultrasound doppler showed no doppler signals inside. Her pain disappeared.LFT is normal now.

Conclusion: Pseudoaneurysm is a rare complication of liver biopsy.

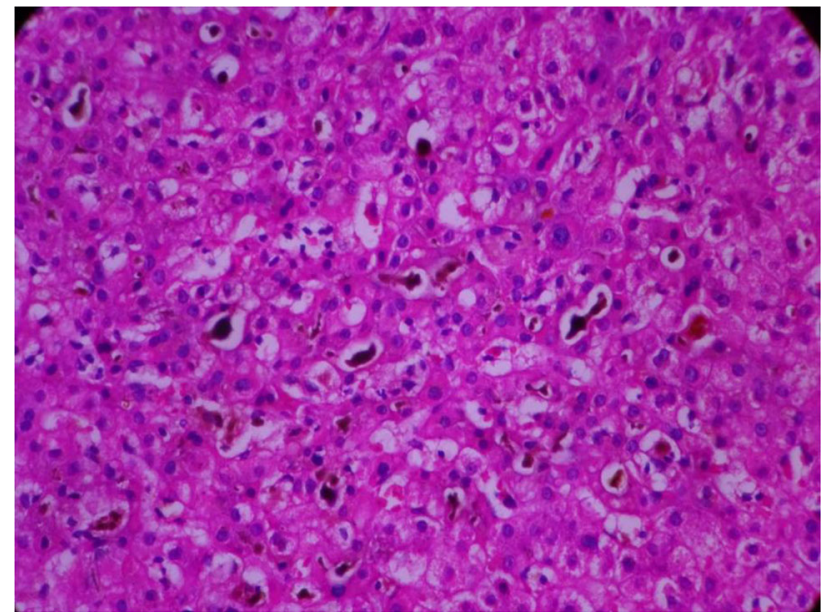

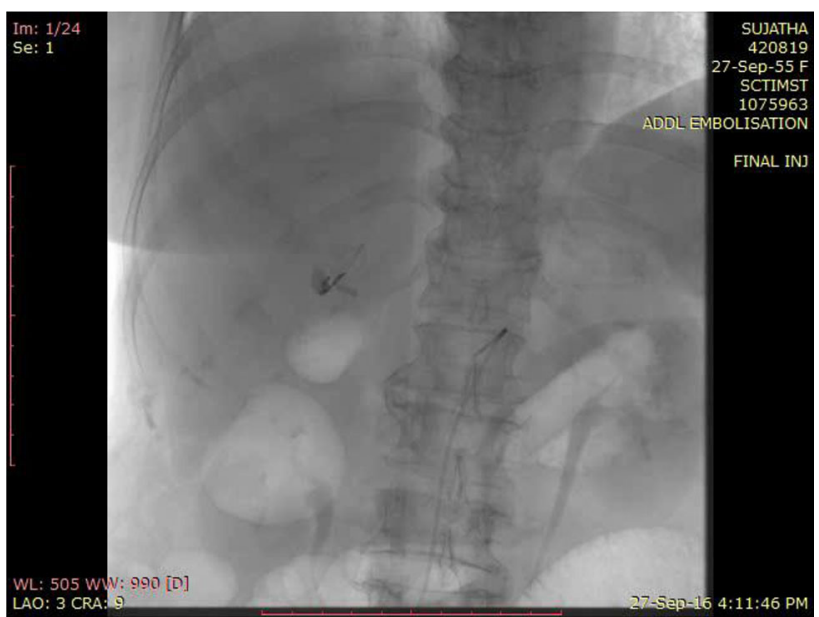

LATEB-82

A study of caregiver burden in Alcoholic liver disease and Nonalcoholic fatty Liver Disease

\section{Murali Krishnan $M^{1}$, Thazhath Mavali Ramachandran ${ }^{1}$}

\section{${ }^{1}$ Calicut Medical College, India}

Background: Cirrhosis of liver, like any other chronic disease increases the socioeconomic and emotional burden on the caregiver. Adherence to therapy and follow-up depends up on the caregiver's physical and mental status. But the problems of caregivers are under recognized. In this background we decided to study the caregiver burden of two groups of cirrhotic patients -Alcoholic liver disease (ALD) and Nonalcoholic Fatty Liver Disease (NAFLD).

Method: This was a descriptive cross-sectional study conducted at a tertiary care centre in Kerala. The study cohort included 50 patients with advanced cirrhosis [Child-Turcotte-Pugh (CHILD) B and C] and their caregivers. The cohort was grouped in to two: ALD and NAFLD. Only the primary caregiver that is, the immediate family member who spent the most time with the patient was included. Caregiver burden was assessed by the Zarit Caregiver Burden Interview (ZBI) scale. Socio demographic variables and clinical variables including age at onset of the illness, duration of the illness, reason for admission, model for end-stage liver Disease (MELD) score, CHILD status were compared.

Result: Baseline characteristics of ALD $(n=25)$ and NAFLD ( $\mathrm{n}=$ 25) patients were comparable. Mean age of the caregivers in ALD was $39 \pm 7.6 y e a r s .21$ females (84\%), spouses 18 (72\%). In NAFLD group mean age of the caregivers was $44 \pm 9.4$ years. 20 females (80\%), spouses $15(60 \%)$. We observed a significantly higher caregiver burden in ALD compared to NAFLD (45.0 \pm 9.7 vs $25.95 \pm 14$ $\mathrm{t}=5.64, p=0.00)$ and in ALD group a significant higher burden was observed when the care giver was not the spouse $(t=3.97, p=0.001)$ Conclusion: Primary caregivers of patients with ALD have significantly higher caregiver burden compared to NAFLD. Among caregivers of ALD patients, burden is significantly higher if the care giver is not the spouse. 


\section{LATEB-83}

Pattern and profile of infections in hospitalized patients with cirrhosis and its role in short term mortality

\author{
Sunu Sara Kurien", Sunu Sara Kurien ${ }^{1}$, Thazhath Mavali \\ Ramachandran $^{1}$ \\ ${ }^{1}$ Calicut Medical College, India
}

Background: Infections are one of the most common causes of admission in cirrhotic patients. This study aimed to assess the clinical profile and short term mortality caused by infections in hospitalized patients with cirrhosis.

Method: This is a prospective study were hospitalized cirrhotics were studied for presence or absence of infections. They were analyzed for both site and type of infection; community-acquired (CA), health care-associated (HCA) or hospital acquired (HA). Both in hospital mortality and mortality at 3 months were studied.

Result: Of the 90 patients included in the study, 51 had infection $(56.6 \%)$ whereas $39(43.4 \%)$ did not. Both groups were comparable in terms of age, sex and etiology of disease. CHILD C status (p-0.038) and MELD status $(\mathrm{p}=0.01)$ were seen to be associated with infection. In those who had infection, the most common was SBP $(70.5 \%)$ followed by cellulitis (19.65) and urinary tract infection (17.6\%), with 8 having more than 1 type of infection. There were 26 cases which were health care associated infections (50.9\%), 16 which were hospital acquired $(31.3 \%)$ and 9 community acquired cases. Cultures were positive in $17(33.3 \%)$ with gram negative infections being more common than gram positive $(52.9 \%$ vs $47.1 \%)$. Those with infection had a significantly greater duration of hospital stay as compared to those without. $(10.6 \pm 6.7 \mathrm{~d}$ vs $6 \pm 3.1 \mathrm{~d}: \mathrm{p}=0.00)$ In hospital mortality was greater in those with infection though not statistically significant. ( 7 vs $1 \mathrm{p}=0.065$ ). 3 month mortality was significantly higher in those with infection. ( 24 vs $4 \mathrm{p}=0.00$ )

Conclusion: Infections are an important determinant of mortality in cirrhotics. Most infections in cirrhotics are either hospital acquired or health care associated. Hence cirrhotic patients should be monitored closely for infections.

\section{LATEB-84}

Inflammatory pseudotumors of the liver: importance of a multimodal approach

Neha Nigam $^{1}$, Chhagan Bihari Sharma ${ }^{2}$, Shikha Singh Rajani ${ }^{2}$, Archana Rastogi ${ }^{2}$

${ }^{1}$ King George Medical University, Lucknow, India; ${ }^{2}$ I.L.B.S., India

Background: Inflammatory pseudotumor (IPT) is a benign mass forming lesion and often mimics malignancy. Clinical diagnosis is difficult with nonspecific symptoms and absent serological evidence of inflammation. Surgical intervention is often performed fortuitously under an over diagnosis of malignancy. If an accurate preoperative diagnosis is made, an unnecessary hepatectomy can be avoided. Study was done to explore various clinicopathological aspects of IPT in liver.

Method: A retrospective analysis of all cases of IPT from June 2009 to December 2016 was done. Seventeen pathologically diagnosed cases of IPT were taken from a total 12075 hepatobiliary specimens, received during Jan 2010 to Jan 2017. Their clinical presentations, biochemical investigations including tumor markers and imaging studies (USG, CT, MRI) were noted. Cytopathology, histopathology and immunohistochemistry findings were reviewed. Treatment details and follow up of the cases were also noted.
Result: A total of 17 patients, diagnosed as inflammatory pseudotumor were included. The age of the patients ranged from 21 to 62 years. Common presenting features were intermittent fever (11), upper abdominal pain (8) and weight loss (5). Radiological diagnosis varied from neoplastic (13) to infectious etiologies (4) with hepatocellular carcinoma being the most common diferential (7/17). Laboratory investigations revealed leucocytosis in (8) patients; hyperbilirubinemia (9), raised transaminases (6), and raised serum alkaline phosphatase (12). Core biopsy of the tumor revealed increased fibrosis along with infiltrating inflammatory cells comprising of plasma cells, eosinophils, neutrophils, foamy histiocytes and occasional giant cells. Eleven cases managed conservatively and showed regression or complete recovery and 6 underwent surgical resection. None of these had any recurrence in on median follow up of 22 months.

Conclusion: Needle biopsy and fine needle aspiration should be routinely practiced in undiagnosed hepatic masses to elute the diagnosis dilemma and to avoid surgical intervention.

\section{LATEB-85}

A novel model using protein $\mathrm{C}$ to predict short-term mortality in cirrhosis

\section{Anupama Patil ${ }^{1}$, Chhagan Bihari ${ }^{1}$, Monika G Thakur ${ }^{1}$, Shiv K $\operatorname{Sarin}^{1}$}

${ }^{1}$ Institute of Liver \& Biliary Sciences, India

Background: End-stage liver disease is characterized by decreased levels of both procoagulant and anticoagulant factors. The validity of the conventional prothrombin-time and related coagulation tests for assessing risk of coagulopathy is questionable, as these tests do not truly represent the balance of coagulation as it occurs in vivo. Also, INR is widely used as a prognostic index to calculate the patient's Child-Pugh (CP) and Model for End-Stage Liver Disease (MELD) scores, which are used to assess disease severity. Further, the coagulation system disturbances (such as acquired protein $\mathrm{C}$ deficiency) have been linked to liver fibrosis progression, plausibly due to formation of micro-thrombi within the hepatic microcirculation. This study was undertaken to assess the relationship between the natural anticoagulants, protein $\mathrm{C}$ (PC), protein S (PS), and anti-thrombin III (AT) with MELD score and hepatic decompensation.

Method: A total of 830 patients comprising of 598 with cirrhosis and 232 non-cirrhotic cases were included and followed for at least 3 months. The functional levels of PC, PS and AT in the plasma were analyzed. MELD scores were used to assess the severity of cirrhosis. A multivariable predictive model was derived using Poisson regression analysis of independent predictors of 3-month mortality.

Result: Cirrhotic patients had lower levels of PC ( $p<0.001)$, PS $(<$ $0.001)$ and AT $(\mathrm{p}<0.001)$ compared with patients having non-cirrhotic liver disease. Also, patients with advanced fibrosis (F3-4) had lower PC levels $($ median $=23.8 \%$, IQR: 16.3-33.0) compared with patients with early fibrosis $(\mathrm{F} 1-2)($ median $=74.9 \%, \mathrm{IQR}=59.7$ 92.5). Low functional PC levels correlated with markers of liver dysfunction and inflammation: INR $(\mathrm{r}=-0.72, \mathrm{p}<0.001)$, serum bilirubin $(\mathrm{r}=-0.620, \mathrm{p}<0.001)$, albumin $(\mathrm{r}=0.539, \mathrm{p}<0.001)$, creatinine $(\mathrm{r}=-0.417, \mathrm{p}<0.001)$, ferritin $(\mathrm{r}=-0.68, \mathrm{p}=0.035)$, procalcitonin $(r=-0.79, p=0.01)$ and raised ESR $(r=0.56, p<$ 0.001). Further, plasma levels of functional PC (but not INR or MELD score) were independent predictors of hepatic encephalopathy $(p=0.002)$ and ascites $(p=0.048)$. However, PS and AT failed to correlate with any of these markers. Patients who died $(n=182)$ at the end of three months had significantly lower functional PC levels $($ median $=23.8 \%$, IQR: 16.3-33.0) compared with those who 
remained alive $($ median $=74.9 \%, \mathrm{IQR}=59.7-92.5) ; \mathrm{p}<0.001$. Using multivariable analyses, low PC level $(\mathrm{p}<0.001)$ and high MELD score $(p=0.038)$ were found to be independent predictors of mortality. A model was derived using these two predictors. This score had better discrimination (area under the receiver operating characteristic curve $=0.84$ ) than a model using MELD alone (area under the receiver operating characteristic curve $=0.62$ ) to predict 3 -month mortality.

Conclusion: In patients with liver cirrhosis, low functional protein $\mathrm{C}$ level correlates with fibrosis, liver dysfunction, inflammation, and sepsis and is an independent predictor of 3-month mortality. This score using functional PC levels and MELD score improves risk prediction of 3-month mortality over MELD alone.

\section{LATEB-86}

Idiosyncratic, immunoallergic severe drug-induced liver injury with skin injury and life threatening thrombocytopenia: challenges in diagnosis and treatment

\section{Tarun Sebastian Joseph ${ }^{1}$, Harshad Devarbhavi ${ }^{1}$}

${ }^{1}$ St. Johns Medical College, India

Background: Twenty-three year old male who was diagnosed to have multibacillary leprosy received dapsone $100 \mathrm{mg}$ daily for 22 days, rifampicin 600 one dose and clofazimine $300 \mathrm{mg}$ one dose. $\mathrm{He}$ developed fever, jaundice and skin rashes after 22 days of treatment. $\mathrm{He}$ was febrile with a temperature of $103 \mathrm{~F}$ with tachycardia and tachypnoea. His physical examination was remarkable for scleral icterus with cervical lymphadenopathy and hepatomegaly. His dermatological examination revealed maculopapular rashes with skin blisters all over the body sparing the mucosal regions.

Method: His initial labs reveal anemia with eosinophilia and thrombocytopenia. His Liver function test was s/o cholestatic and hepatocellular pattern of liver injury with an elevated INR of 3.6.His viral markers were negative so was antinuclear antibodies. He was subjected to a skin biopsy which revealed psoriasiform acantosis with foci of basal cell damage and necrotic keratinocytes in the epidermis and superficial dermis showed perivascular and interface lymphocytic infiltrates suggestive of drug reaction. He was thus considered to have DRESS syndrome secondary to Dapsone

Result: The Dapsone was stopped immediately and he was admitted to intensive care ward. Vitals were monitored. Inj Vitamin $\mathrm{K}$ was given and $\mathrm{N}$ - acetyl infusion was started. A multidisciplinary team consisting of hepatologists, dermatologists, hematologists, intensivists, transfusion specialists was involved with the care of patient. Parentral methylprednisolone $750 \mathrm{mg}$ IV infusion over 1 hour was started erficial dermis showed perivascular and interface lymphocytic infiltrates suggestive of drug reaction. He was thus considered to have DRESS syndrome secondary to Dapsone

Conclusion: On day three he developed epistaxsis and oozing of blood from the skin rashes. Further evaluation showed aPlatelet count of 5000/mm3, INR- 1.11, Hb - $4.60 \mathrm{gm}, \mathrm{WBC}-3600$ Serum LDH 2686, Reticulocyte count $-3.45 \%$, G6-PD level was normal. His Coombs testwas positive and Methemoglobin level was $1.69 \% \mathrm{He}$ received multiple platelets ( 18 units of platelets and once single donor platelet concentrate) and packed cell transfusions. Methyl prednisolone was continued. Nutrition and other supportive care were continued. He was switched to oral steroids after 5 days of parenteral steroids. Despite steroids and multiple platelet transfusions low platelet level persisted $(<5000-20000 / \mathrm{mm} 3)$ for 14 days. Blood culture showed Enterococcus sensitive to Vancomycin and was treated accordingly. Gradually he became afebrile, skin rashes showed healing with hyperpigmentation. Liver functions started improving (see table) and patient completely recovered after which he was reinitiated on rifampicin and clofazamine only without recurrence of liver or skin injury.

\begin{tabular}{|c|c|c|c|c|c|c|c|c|}
\hline & $27 / 2 / 1$ & $3 / 3 / 17$ & $6 / 3 / 17$ & $10 / 3 / 17$ & $15 / 3 / 17$ & $20 / 3 / 17$ & $7 / 6 / 17$ & $5 / 7 / 17$ \\
\hline & 7 & & & & & & & \\
\hline $\begin{array}{l}\text { Total } \\
\text { protein } \\
(\mathrm{g} / \mathrm{dl}) \\
\end{array}$ & 6.37 & 5.42 & 6.05 & 5.61 & 5.81 & 6.46 & 6.92 & 6.97 \\
\hline $\begin{array}{l}\text { Albumin } \\
(\mathrm{g} / \mathrm{dl})\end{array}$ & 1.5 & 1.4 & 1.8 & 2.0 & 2.3 & 2.8 & 3.7 & 4.0 \\
\hline $\begin{array}{l}\text { Total } \\
\text { Bilirubi } \\
\mathrm{n} \\
(\mathrm{mg} / \mathrm{dl})\end{array}$ & 13.45 & 16.43 & 9.34 & 7.18 & 6.67 & 4.33 & 0.3 & 0.7 \\
\hline $\begin{array}{l}\text { Bilirubi } \\
\mathrm{n} \text { Direct } \\
(\mathrm{mg} / \mathrm{dl})\end{array}$ & 10.89 & 12.83 & 7.17 & 6.12 & 5.16 & 3.41 & 0.1 & 0.2 \\
\hline $\begin{array}{l}\text { AST } \\
(\mathrm{U} / \mathrm{L})\end{array}$ & 215 & 345 & 182 & 149 & 116 & 82 & 23 & 23 \\
\hline $\begin{array}{l}\text { ALT } \\
(\mathrm{U} / \mathrm{L})\end{array}$ & 171 & 346 & 330 & 385 & 321 & 217 & 107 & 105 \\
\hline $\begin{array}{l}\text { ALP ( } \\
\mathrm{U} / \mathrm{L}) \\
\end{array}$ & 443 & 557 & 483 & 536 & 504 & 525 & 72 & 77 \\
\hline $\begin{array}{l}\text { GGT } \\
(\mathrm{U} / \mathrm{L})\end{array}$ & 186 & 241 & 293 & 772 & 876 & 736 & 270 & 266 \\
\hline
\end{tabular}

\section{LATEB-87}

A sudden and rapid appearance of a very large liver cell tumour in a young healthy adult Balaji G, Deepak Ganga, Tarun Joseph, Harshad Devarbhavi. Department of Gastroenterology, St. John's Medical College Hospital, Bangalore, Karnataka, India

\section{Balaji Gurappa ${ }^{1}$, Harshad Devarbhavi', Mallikarjuna PatiL ${ }^{2}$ ${ }^{1}$ RGUHS, India; ${ }^{2}$ RGHUS, India}

Background: Introduction: Undifferentiated embryonal sarcoma of liver is a rare and aggressive tumour occurring exclusively in paediatric age groups. Management of such tumours requires multimodal treatment like surgery, chemotherapy and radiation. Presentation in adults is very rare,thus we present such case who presented to our hospital.

Method: A 35 year healthy young male exercising and preparing for a body building competition presented with 2-3 week history of dull aching upper pain abdominal pain of gradually increasing severity, minimal weight loss and fullness in right hypochondrium. Examination revealed hard, tender hepatomegaly with no other positive findings. Investigations that included complete blood counts, liver function and renal function tests were normal. ELISA tests for $\mathrm{HBsAg}$ and $\mathrm{HCV}$ antibodies were negative. AFP was $2 \mathrm{ng} / \mathrm{dl}$. Ultrasonography of abdomen showed a $15 \mathrm{~cm}$ mixed echogenic mass with cystic spaces in the left lobe of the liver. Contrast enhanced CT demonstrated a large heterogenous complex mass measuring $18.5 \mathrm{X} 15.4 \mathrm{X}$ $12.5 \mathrm{~cm}$ arising from and nearly replacing the left lobe. The mass was predominantly cystic with peripherally enhancing solid component that showed heterogenous post contrast enhancement. Hyperdense contents suggestive of hemorrhage were also noted. A diagnosis of embryonal undifferentiated sarcoma was made. In view of increasing severity, intolerable pain and rapid swelling he underwent left hepatectomy with resection of the tumor (see picture). In addition there was focal infiltration of falciform ligament, multiple peritoneal nodules and nodules in the under surface of diaphragm. He had an uneventful post operative recovery and was discharged on 8th post operative day. Histopathology of liver specimen showed undifferentiated embryonal sarcoma of liver with immunohistochemistry showing positivity for CD10, VIMENTIN, P53, and focal positivity for CK and BCL-2. Currently he is receiving chemotherapy and doing 
well. UESL is a very rare tumour that needs urgent and aggressive surgical resection with postoperative chemotherapy. Thus far less than 60-80 cases have been reported worldwide.

Result: A diagnosis of embryonal undifferentiated sarcoma was made. In view of increasing severity, intolerable pain and rapid swelling he underwent left hepatectomy with resection of the tumor (see picture). In addition there was focal infiltration of falciform ligament, multiple peritoneal nodules and nodules in the under surface of diaphragm. He had an uneventful post operative recovery and was discharged on 8th post operative day. Histopathology of liver specimen showed undifferentiated embryonal sarcoma of liver with immunohistochemistry showing positivity for CD10, VIMENTIN, P53, and focal positivity for CK and BCL-2. Currently he is receiving chemotherapy and doing well. UESL is a very rare tumour that needs urgent and aggressive surgical resection with postoperative chemotherapy. Thus far less than 60-80 cases have been reported worldwide.

Conclusion: UESL is a very rare liver tumor to be suspected in a setting of a normal young adult or a child with rapid appearance of a large liver tumor with near normal liver biochemical test and normal AFP. Resection is mainstay of treatment.

\section{LATEB-88}

Fractionated mesenchymal stromal cell derived secretome enriched with exosomes potentiates therapeutic effect in liver injury

\section{Apeksha Damania $^{1}$, Deepika Jaiman ${ }^{2}$, Arun Kumar Teotia ${ }^{2}$, Ashok Kumar ${ }^{2}$ \\ ${ }^{1}$ Aalto University, Finland; ${ }^{2}$ Indian Institute of Technology Kanpur, India}

Background: Over the years, mesenchymal stromal cells (MSCs) have gained widespread acceptability for use in regenerative medicine, primarily due to their enhanced expansion capacity and diverse differentiation potential. In liver injury, also, mesenchymal stem cells have been shown to work either by transdifferentiating into hepatocyte-like cells or by secreting biological factors that inhibit cell death and/or prevent progress to cirrhotic condition. Recently, there has been a paradigm shift from differentiation of MSCs to their paracrine effects at the injury site. Several reports elucidate the role of trophic factors secreted by MSCs towards the repair of injured tissues. We hypothesize that fractionating the MSC secretome will enrich extracellular vesicles known as exosomes, containing soluble bioactive molecules that play a critical role in its therapeutic potential for liver failure.

Method: Rat bone marrow MSCs were isolated and cultured to $70-80 \%$ confluency. The conditioned media collected was filtered, concentrated and ultra-centrifuged to generate fractionated secretome. This fractionated secretome was characterized for presence of exosomes and recovery from liver injury was assessed in liver injury models both in vitro and in vivo.

Result: Better cell recovery and reduced cytotoxicity in in vitro liver injury models using acetaminophen and hydrogen peroxide were observed in presence of fractionated secretome as opposed to unfractionated secretome. Further, the cells exhibited reduced oxidative stress in the presence of fractionated secretome, suggesting a probable anti-oxidative effect. In vivo liver failure models further validated these results. Improved liver regeneration $(0.819 \pm 0.035)$ was observed in presence of fractionated secretome as compared to unfractionated secretome $(0.718 \pm 0.042)$.
Conclusion: Our work provides a proof-of-concept that fractionating the MSC secretome enriches selective bioactive molecules critical in the repair and recovery of injured liver tissue.

\section{LATEB-89}

Cryogel-based bioartificial liver support and bioengineered liver organ for enhanced liver regeneration

\section{Apeksha Damania ${ }^{1}$, Anupam Kumar ${ }^{3}$, Hiroyuki Ijima ${ }^{4}$,} Masamichi Kamihira $^{4}$, Shiv Kumar Sarin ${ }^{3}$, Ashok Kumar ${ }^{2}$

${ }^{1}$ Aalto University, Finland; ${ }^{2}$ Indian Institute of Technology Kanpur, India; ${ }^{3}$ Institute of Liver and Biliary Sciences, India; ${ }^{4}$ Kyushu University, Japa

Background: Liver transplantation is deemed the only existing successful treatment for liver failure. However, the unavailability of adequate donor organs to meet the existing demand has resulted in major organ shortage crises. A major thrust of the work presented here aims at exploring treatment regimens that focus on bridging patients to liver transplantation and improving the liver failure conditions by enhancing the regeneration rate of the failing liver. Our work aims at combining basic tissue engineering ideas with clinical translational biology to develop potential cost-effective strategies to enhance liver regeneration in patients with liver failure. Extracorporeal liver support systems attempt to bridge patients with liver failure until a suitable donor organ is obtained or until the patient's own liver recuperates to resume normal function. One of the major upshots of our work is the development of an integrated hybrid cryogel based bioreactor that can alleviate liver failure conditions. Furthermore, recent progress in the use of decellularized organ scaffolds as regenerative matrices holds great promise in addressing the issue of donor organ shortage. In our work, we develop a cryogel matrix integrated with decellularized liver matrix as a potential hepatocyte carrier for liver tissue engineering applications.

Method: A bioreactor was designed integrating a detoxifying component composed of activated carbon cloth, with a plasma separation component and a cryogel-based hepatic cell-seeded bioreactor in one compact multi-chambered unit. The integrated hybrid cryogel-based bioreactor was evaluated extracorporeally on rodent models of liver failure. Furthermore, offline clinical evaluation of the bioreactor was performed using liver failure patient plasma. In addition, liver decellularized matrix was incorporated within the cryogel matrices as a coating material. These coated matrices were evaluated as implantable tissue constructs as well as bioartificial liver supports both in vitro and in vivo using appropriate liver failure models.

Result: Extracorporeal evaluation of the integrated bioreactor showed $20-40 \%$ improvement in liver function. Offline clinical evaluation of the bioreactor showed its efficacy in improving liver failure conditions and indicated its potential reusability clinically, with utmost two repeated uses before disposal.

Implantation of the coated cryogel scaffold within the lobes of a rodent liver failure model showed a high amount of cellular infiltration indicating integration of the matrix with the neighboring tissue. Improved liver functions in vitro and a $20-60 \%$ improvement in liver function in vivo suggest the potential application of the coated matrix for the generation of a bioengineered organ as an alternative to liver transplantation.

Conclusion: Our work presented here is an amalgamation of various strategies devised to focus on the yet unmet medical need of improving liver regeneration. 


\section{LATEB-90}

Natural history of patients with compensated cirrhosis and a hepatic venous pressure gradient $>20 \mathrm{mmHg}$ : a prospective longitudinal cohort study

Ankit Bhardwaj ${ }^{1}$, Ankur Jindal ${ }^{1}$, V Rajan ${ }^{1}$, Vinod Arora ${ }^{1}$, Manoj Kumar Sharma $^{1}$, Lovkesh Anand ${ }^{1}$, Shiv Kumar Sarin ${ }^{1}$

${ }^{1}$ ILBS, India

Background: HVPG $>10 \mathrm{~mm} \mathrm{Hg}$ predicts clinical decompensation (CD) in compensated cirrhotics. A proportion of cirrhotics have high HVPG $(>20 \mathrm{~mm} \mathrm{Hg}$ ) despite no $\mathrm{CD}$. The natural history, pattern of $\mathrm{CD}$ (ascites, hepatic encephalopathy, variceal bleed) and complications (HCC, mortality) in this group are largely unknown.

Method: Consecutive compensated cirrhosis patients with HVPG $\geq 6$ $\mathrm{mm} \mathrm{Hg}(\mathrm{n} \mathrm{=} 747)$ were followed-up every 3-6 months for any decompensation. Patients were classified based on HVPG $(<12$ $\mathrm{mmHg}$ (low HVPG group), 12-20 mmHg (Intermediate HVPG group) and $>20 \mathrm{mmHg}$ (High HVPG group). We analyzed only the low and high HVPG groups to identify predictors of new CD.

Result: 295(39.4\%) patients developed CD at mean follow-up of 1.9 \pm 0.3 years. In comparison to low HVPG group, first CD in high HVPG group developed early $(1.4 \pm 0.3$ years vs $2 \pm 0.3$ years, $p=$ 0.02 ), more often (ascites- $27.1 \%$ vs $13.2 \%$, variceal bleed- $31.9 \%$ vs $7.9 \%$, AKI- $31 \%$ vs $12.8 \%$, HCC- $12.4 \%$ vs. $3.9 \%$; all $\mathrm{P}<0.05$ ) with higher mortality $(15.9 \%$ vs $1.9 \% ; \mathrm{p}<0.05)$. There was no significant correlation between baseline HVPG level and grade of esophageal varices $(p=0.457)$. All patients in high HVPG group received carvedilol (maximum dose- $25 \mathrm{mg} / \mathrm{d}$, divided) and a repeat HVPG measured after a mean duration of $1.8 \pm 0.4$ years showed suboptimal HVPG response ( $\geq 20 \%$ reduction in HVPG or $<12 \mathrm{mmHg}$ ) in $26.6 \%$ patients (mean HVPG reduction, $3.3 \pm 1.3 \mathrm{~mm} \mathrm{Hg}$ ). Etiology of cirrhosis and grade of varices were not significantly associated with CD or HVPG response. On multivariate analysis, baseline HVPG > $20 \mathrm{~mm} \mathrm{Hg}$ (hazard ratio [HR], 5.09; 95\% confidence interval [CI], 2.909-8.926, $\mathrm{p}=0.001)$ and high MELD score (HR, 1.125; 95\% CI, 1.066- 1.187, $\mathrm{p}=0.001$ ) were independent predictors of $\mathrm{CD}$.

Conclusion: An HVPG of $>20 \mathrm{~mm} \mathrm{Hg}$ independently predicts early and more frequent $\mathrm{CD}$ in compensated cirrhotics. Only a quarter of these patients respond to carvedilol and hence mandates us for careful HVPG monitoring and low threshold for additional drugs or interventions.

\section{LATEB-91}

A study on outcome of hepatitis c patients in a community with predominant genotype 3 with standard of care treatment before and after advent of direct acting antivirals in Kashmir valley a retrospective cum prospective study

Nisar Ahmad Shah ${ }^{1}$, Showkat Ahmad Kadla ${ }^{1}$, Nisar Ahmad Shah $^{1}$, Bilal Ahmad Khan ${ }^{1}$, Asif Iqbal Shah ${ }^{1}$, Shabir Ahmad Sheikh $^{1}$, Rupakshi Pathania ${ }^{1}$, Mohamad Amin Dar ${ }^{1}$

${ }^{1}$ GMC Srinagar, India

Background and objective: Hepatitis $\mathrm{C}$ virus (HCV) was first identified in 1989 and was the major cause of non-A, non-B viral hepatitis. HCV is a globally prevalent pathogen and is a major cause of healthcare burden in India. As per current estimates, more than 165 million people around the world are infected with $\mathrm{HCV}$, of whom 350,000 die each year. The treatment of hepatitis c has evolved by leaps and bounds in last two decades to almost a perfection at present
The aim of our study was to study and compare the response to different regimen during and after the Interferon era.

Materials and Methods: The current study was a hospital based prospective cum retrospective study. For retrospective data, 200 Hepatitis ' $C$ ' patients (Genotype 3) from 2011 to 2014 were analyzed with respect to patients' demography, Quantitative Hepatitis ' $C$ ' RNA before treatment, during treatment and after treatment. For prospective study, 290 patients with Hepatitis C genotype 3 were studied for two years (June 2015 to June 2017). They were enrolled both from IPD and OPD. These patients were detected HCV positive during blood donation, preoperative evaluation, evaluation of liver function tests etc.

Results: For retrospective group,SVR24 was achieved in 90.96\%(171/188) patients on Peg-IFN alpha 2a plus Ribavirin (weight based) combination for a period of 24 weeks.For prospective group, SVR12 was achieved in $94.57 \%$ (157/166) patients on Sofosbuvir plus Ribavirin combination for a period of 12 weeks and 98\% (98/ 100) patients on Sofosbuvir plus Daclatasvir combination for a period of 12 weeks among non-cirrhotics while as among cirrhotics SVR24 was achieved in $83.33 \%$ (20/24) patients on Sofosbuvir plus Ribvirin plus Daclatasvir combination for a period of 24 weeks.

Conclusion: Overall in retrospective group an SVR24 was $90.96 \%$ and in prospective group SVR12 was achieved in $94.57 \%$ on Sofosbuvir plus Ribavirin combination for 12 weeks, $98 \%$ on Sofosbuvir plus Daclatasvir for 12 weeks and in Cirrhotic group, $83.33 \%$ achieved an SVR12 on Sofosbuvir plus Ribvirin plus Daclatasvir combination for 24 weeks.

\section{LATEB-92}

miR-494-3p and miR-422a can be used as non-invasive markers for predicting Hepatitis $\mathbf{C}$ Virus recurrence in patients after liver transplantation

Tanvi Agrawal $^{1}$, Mojahidul Islam ${ }^{1}$, Manish Chandra Choudhary ${ }^{1}$, Senthil Kumar $^{1}$, Gayatri Ramakrishna ${ }^{1}$, Nirupma Trehanpati ${ }^{1}$, Shiv Kumar Sarin ${ }^{1}$

${ }^{1}$ Institute of Liver and Biliary Sciences, India

Background: Outcome of patients undergoing liver transplantation for HCV-related cirrhosis is usually reduced because of the recurrence of hepatitis $\mathrm{C}$ virus (HCV) infections especially in patients who are HCV RNA+ve prior to liver transplant. MicroRNAs (miRNAs) regulate host immune response and thus disease progression. The aim of the study was thus to identify novel miRNA signatures in plasma and liver biopsies which can predict HCV disease recurrence pretransplant.

Method: Total Twenty-two liver transplant patients were enrolled. Liver tissue and plasma were collected from HCV RNA positive $(\mathrm{N}=$ 6) or negative patients $(\mathrm{N}=16)$ at the time of liver transplantation (LT). Post-liver transplant plasma was collected at six months for HCV RNA positive patients. RNA was extracted from biopsy and plasma and subjected to expression panel of 793 miRNAs using Nanostring nCounter (Nanostring Technology). nSolver software was used for analysis of miRNA expression and other bioinformatic tools (TargetScan, MIRDB) were used to predict the targets of identified miRNA.

Result: All HCV RNA positive patients had HCV infection recurrence post-transplant. None of the HCV negative patient had HCV recurrence post- transplant. Three HCV recurrence patients again developed fibrosis (Score-1). Twenty-four miRNAs showed significant differential expression in liver tissue obtained from HCV RNA positive and negative patients, suggesting modulation of these miRNA due to active viral replication. Nine miRNAs (miR-422a, 
miR-4516, miR-4454, 494-3p, miR-1283, 148a-3p, 199a-3p, 223-3p and 26a-5p) were differentially expressed in pre-transplant plasma of patients who developed fibrosis after LT compared to those who does not. Of these 9 miRNA, miR-494-3p and miR-422a were significantly upregulated in plasma as well as the liver tissue.

Conclusion: miRNAs may directly and indirectly regulate $\mathrm{HCV}$ infection contributing to disease recurrence post LT. miR-494-3p and miR-422a can be used as non-invasive markers to identify patients who will develop disease recurrence after LT.

\section{LATEB-93}

Improvement in depression in chronic $\mathrm{HCV}$ patients post DAA therapy

\author{
Mridula Sharma $^{1}$, Moni Chaudhary ${ }^{2}$ \\ ${ }^{1}$ Meerut College, India; ${ }^{2}$ Sushila Jaswant Rai superspeciality \\ Hospital, India
}

Background: Depression in patients with chronic hepatitis $\mathrm{C}$ virus (HCV) infection influence their health-related quality of life. Patient self-assessment instruments are useful in screening, tracking, and documenting the course of depressive symptoms with minimal clinician time. Role of DAA's in improvement in depression in chronic $\mathrm{HCV}$ is not known till date. This study aimed to analyse the prevalence of depression in chronic HCV patients and the role of DAA's in its improvement.

Method: 898 consecutive patients from January 2016 to June 2017 attending the Hepatology clinic in a tertiary care centre were screened for chronic HCV infection. 492 patients suffering from chronic HCV were further screened for depression using patient health questionnaire (PHQ-9) at baseline and at 24 weeks post end of DAA therapy. Exclusion criteria were: co-infection with other viruses; coexistence of other forms of hepatic disease, associated chronic liver disease, associated cardiopulmonary disease, pregnancy, lactation, extremely low cognitive status precluding reliable participation and refusal to participate. The liver function tests, and quantitative measurement of HCV RNA were done at the beginning of the study and at 12 weeks post treatment.

Result: 492 patients who were found to be reactive for HCV were started on DAA and followed till 24 weeks post treatment. Mean age of patients suffering from HCV was $43.3+8$ and $74.2 \%$ of patients were male. $400(81.3 \%)$ patients achieved SVR at 12 weeks post therapy. Of the remaining patients 32 were lost to follow up and 60 patients did not achieve SVR at 12 weeks. Depression severity (minimum score 0 and maximum score 27) was measured by PHQ-9 questionnaire and graded as follows (0-4 none, 5-9 mild, 10-14 moderate, 15-19 moderately severe, 20-27 severe) with patients having score $>5$ considered to be suffering with depression. 338/492 $(68.7 \%)$ were found to be suffering from depression at baseline with mean PHQ-9 score of $15.7+6.2$ (SD) (moderately severe grade of depression). Of the 338 patients who had depression 282 achieved SVR and 160/282 (56.7\%) patients had improvement in PHQ-9 score on follow up with mean PHQ-9 of $8.9+3.6$ (mild grade) suggesting $43.3 \%$ improvement in PHQ-9 score from baseline. Of the 56 patients who did not achieve SVR 8/56 (14.2\%) had improvement in PHQ-9 score with mean PHQ-9 of $12.8+4.6$ (SD) suggesting $18.5 \%$ improvement in PHQ-9. When compared the patients who achieved SVR had a significant more improvement in depression (PHQ-9 $43.3 \%$ vs $18.5 \%)$ [ $p<0.001]$ as compared with those who did not achieve SVR.

Conclusion: Successful oral antiviral therapy provides significant improvements in depression (as shown by PHQ-9 questionnaire) in patients with chronic hepatitis C. $56.7 \%$ of $\mathrm{HCV}$ patients who achieve
SVR 12 have improvement in depression post therapy. The effect of successful treatment indicates a direct relationship between the chronic infection and depression.

Table 1

SF-36 physical and mental health parameters comparison (HCV vs normal controls)

\begin{tabular}{|l|l|l|c|}
\hline Parameter & $\begin{array}{l}\text { HCV patients } \\
(\text { mean } \pm \text { SD }) \\
\mathbf{n = 4 9 2}\end{array}$ & $\begin{array}{l}\text { Control } \\
(\mathbf{m e a n} \pm \mathbf{S D}) \\
\mathbf{n = 5 0 0}\end{array}$ & P value \\
\hline Physical function & $78.2+18.8$ & $90.7+12$ & $<.001$ \\
\hline Role Physical & $68.4+30.2$ & $94.4+23.1$ & $<.001$ \\
\hline Bodily pain & $76.1+18.4$ & $88.8+17.8$ & $<.001$ \\
\hline General Health & $66.2+24.4$ & $84.2+16.6$ & $<.001$ \\
\hline Vitality & $50.1+19.5$ & $64.2+18.2$ & $<.001$ \\
\hline Social function & $74.7+22.1$ & $84.1+18.2$ & $<.001$ \\
\hline Role emotional & $78.9+28.1$ & $86.2+26.6$ & $<.001$ \\
\hline Mental Health & $75.2+17.7$ & $80.5+16.1$ & $<.001$ \\
\hline
\end{tabular}

Table 2

SF-36 physical and mental health parameters comparison post DAAs SVR 12 weeks

\begin{tabular}{|l|l|l|l|}
\hline Parameter & $\begin{array}{c}\text { Pre treatment } \\
(\mathbf{m e a n} \pm \mathbf{S D}) \\
\mathbf{n}=\mathbf{4 0 0}\end{array}$ & $\begin{array}{l}\text { Post treatment } \\
(\mathbf{m e a n} \pm \mathbf{S D}) \\
\mathbf{n}=\mathbf{4 0 0}\end{array}$ & P value \\
\hline Physical function & $76.4+17.6$ & $86.7+14$ & $<0.001$ \\
\hline Role Physical & $69.4+28.2$ & $84.4+20.2$ & $<0.001$ \\
\hline Bodily pain & $74.6+19.4$ & $82.5+14.4$ & $<0.001$ \\
\hline General Health & $67.8+23.6$ & $78.2+18.6$ & $<0.001$ \\
\hline Vitality & $52.2+21.2$ & $60.2+14.4$ & $<0.001$ \\
\hline Social function & $76.8+22.6$ & $82.6+20.8$ & $<0.001$ \\
\hline Role emotional & $77.7+25.4$ & $84+22.4$ & $<0.001$ \\
\hline Mental Health & $75.7+15.7$ & $79.4+19.2$ & $<0.001$ \\
\hline
\end{tabular}

\section{LATEB-95}

Correlation of factors among patients with minimal and advanced fibrosis using fibroscan and controlled attenuation parameter among HBeAg negative patients

Orlando II Villanueva Parinas ${ }^{1}$, Diana Alcantara Payawal, Roberto Lopez ${ }^{1}$, Edwin Alfonso ${ }^{1}$, Marilyn Te ${ }^{1}$

${ }^{1}$ Fatima University Medical Center, Philippines

Background: The aim of this study was to identify factors associated with mild and advanced hepatic fibrosis Using Transient Elastography (FibroScan) and controlled attenuation parameters among $\mathrm{HBeAg}$ negative Patients.

Method: This cross sectional with Chronic HBeAg negative patients were enrolled in the study to evaluate the presence of liver stiffness measurement using Fibroscan. Demographic factors and laboratory and imaging studies were correlated.

Result: A total of 33 patients were enrolled in the study to evaluate the association of liver stiffness measurement using Fibroscan with laboratory and imaging studies among $\mathrm{CHBeAg}$ patients. Patients with mild fibrosis (F0-F1) were grouped into Group I and those with higher fibrosis (F2-F4) wwere grouped into Group II. The following were the findings of the study: (1) age, gender and BMI were not associated with liver stiffness among two groups. (2) There was no difference in the rate of advanced fibrosis between normal and elevated SGOT and SGPT. (3) there is no significant difference between two groups relative to the Ultrasound findings and quantification of steatosis using Controlled Attenuation Parameter (CAP). 
Conclusion: Our results demonstrate that reliance on SGOT, SGPT and liver imaging studies may not accurately assess the extent of hepatic injury. It is therefore recommended that patients with Hepatitis B Virus (HBV) infection should undergo FibroScan.

\section{LATEB-97}

Genomic alterations in coding and non-cording regions of neoplastic and non-neoplastic tissues revealed stepwise mechanism of hepatocarcinogenesis

\section{Yosuke Hirotsu ${ }^{1}$, Hitoshi Mochizuki ${ }^{1}$, Shuntaro Obi ${ }^{2}$, Masao Omata $^{1}$ \\ ${ }^{1}$ Yamanashi Central Hospital, Japa; ${ }^{2}$ Teikyo University Medical Center, Japa}

Background: Hepatocellular carcinoma is a multi-factor disease induced by hepatitis virus infection, metabolic syndrome, alcohol intakes and chemical carcinogens. These conditions cause the chronic hepatitis, liver damage, fibrosis and cirrhosis, and eventually the development of carcinoma. Cellular and histological changes are supposed to be a first steps for hepatocellular carcinoma and the fibrotic liver background is considered as pre-carcinogenic lesion. However, the genetic alterations in non-cancerous cells and the origin of tumor cell are still under investigating.

Method: We performed whole exome sequencing and targeted sequencing of 72 significantly mutated genes assosiated with HCC using paired 56 neoplastic and 27 non-neoplastic tissues from 26 patients with HCC to understand the stepwise mechanism of hepatic carcinogenesis.

Result: Most somatic mutations in neoplastic tissues were high variant allele fraction. We identified recurrently mutated genes which have a role in telomerase, TP53/cell cycle, WNT/b-catenin, chromatin remodeling and epigenetic modifiers pathways. We also found mutations in non-cording region of $A L B$ gene. However, somatic mutations in non-neoplastic tissues and normal liver were relatively low variant allele fraction. Although almost somatic mutations between neoplastic nand non-neoplastic tissues were distinct, mutations in TERT promoter were recurrently mutated even in nontumorous sites. Pyclone analysis based on baysiane model inferred tumor cell clone with TERT promoter mutation was originated from subpopulation of non-neoplastic tissues.

Conclusion: These results suggested the normal liver cells under the chronic stresses have high chances of occurrence to genetic alterations, and eventually driver mutation was pivotal in developing cancer. These results get insight into the stepwise carcinogenic mechanism in hepatocellular carcinoma and origin of tumor cell.

\section{LATEB-99}

Hepatitis C associated hepatocellular carcinoma (HCV-HCC) and response to interferon (IFN)-free direct acting antiviral (DAA) treatment in patients from east Asia: a real-C study with propensity score matching (PSM)

Mindie Nguyen $^{1}$, Mindie H Nguyen ${ }^{1}$, Norihiro Furusyo ${ }^{19}$, Dae Won Jun", Ming Lung Yu ${ }^{14}$, Masaru Enomoto ${ }^{24}$, Jia Horng $\mathrm{Kao}^{22}$, Eiichi Ogawa ${ }^{19}$, Etsuko Ilio ${ }^{20}$, Chen Hua Liu ${ }^{22}$, Hwai I Yang $^{23}$, Akihiro Tamori ${ }^{24}$, Chia Yen Dai ${ }^{14}$, Jee Fu Huang ${ }^{14}$, Yoshiyuki Ueno $^{30}$, Dong Hyun Lee ${ }^{5}$, Grace Wong ${ }^{29}$, Jun Hayashi $^{10}$, Hideyuki Nomura ${ }^{26}$, Makoto Nakamuta ${ }^{17}$, Hiroaki Haga $^{30}$, Mi Jung Jun ${ }^{5}$, Mei Hsuan Lee ${ }^{23}$, Yuichiro Eguchi ${ }^{25}$, Hirokazu Takahashi ${ }^{25}$, Shinji Iwane ${ }^{25}$, Sally Ann Tran', Linda
Henry $^{1}$, Yasuhito Tanaka ${ }^{20}$, REAL C Investigators ${ }^{1}$, Sang Bong $\mathrm{Ahn}^{3}$, Koichi Azuma ${ }^{16}$, Wan Long Chuang ${ }^{14}$, Kazufumi Dohmen $^{2}$, Nobuhiko Higashi ${ }^{27}$, Chung Feng Huang ${ }^{14}$, Jae Yoon Jeong $^{8}$, Jang Han Jung ${ }^{6}$, Eiji Kajiwara ${ }^{13}$, Masaki Kato ${ }^{18}$, Akira Kawano $^{15}$, Toshimasa Koyanagi ${ }^{4}$, Seung Ha Park ${ }^{11}$, Takeaki Satoh $^{21}$, Shinji Shimoda ${ }^{18}$, Do Seon Song ${ }^{28}$, Kazuhiro Takahashi ${ }^{7}$, Ming Lun Yeh ${ }^{14}$, Eileen L. Yoon ${ }^{12}$

${ }^{1}$ Stanford University Medical Center, United States; ${ }^{10}$ Haradoi Hospital, Japa; ${ }^{11}$ Inje University Haeundae Paik Hospital, Korea, Republic of; ${ }^{12}$ Inje University Sanggye Paik Hospital, Korea, Republic of; ${ }^{13}$ Kajiwara Clinic, Japa; ${ }^{14}$ Kaohsiung Medical University Hospital, Taiwan; ${ }^{15}$ Kitakyushu Municipal Medical Center, Japa; ${ }^{16}$ Kyushu Central Hospital, Japa; ${ }^{17}$ Kyushu Medical Center, National Hospital Organization, Japa; ${ }^{18}$ Kyushu University Graduate School of Medical Sciences, Japa; ${ }^{19}$ Kyushu University Hospital, Japa; ${ }^{2}$ Chihaya Hospital, Japa; ${ }^{20}$ Nagoya City University Graduate School of Medical Sciences, Japa; ${ }^{21}$ National Hospital Organization Kokura Medical Center, Japa; ${ }^{22}$ National Taiwan University Hospital, Taiwan; ${ }^{23}$ National Yang-Ming University, Taiwan; ${ }^{24}$ Osaka City University Medical School, Japa; ${ }^{25}$ Saga University Hospital, Japa; ${ }^{26}$ Shin-Kokura Hospital, Japa; ${ }^{27}$ Steel Memorial Yawata Hospital, Japa; ${ }^{28}$ The Catholic University of Korea College of Medicine, Korea, Republic of; ${ }^{29}$ The Chinese University of Hong Kong, Hong Kong; ${ }^{3}$ Eulji University Seoul Hospital, Korea, Republic of; ${ }^{30}$ Yamagata University Faculty of Medicine, Japa; ${ }^{4}$ Fukuoka City Hospital, Japa; ${ }^{5}$ Good Gang-An Hospital, Korea, Republic of; ${ }^{6}$ Hallym University Dongtan Sacred Heart Hospital, Korea, Republic of; ${ }^{7}$ Hamanomachi Hospital, Japa; ${ }^{8}$ Hanyang University College of Medicine, Guri Hospital, Korea, Republic of; ${ }^{9}$ Hanyang University Hospital, Korea, Republic of

Background: Globally, East Asian HCV patients have higher rates of HCV-HCC. Reports suggest HCV-HCC patients do not respond as well to the IFN-free DAAs, but background risks and confounders for treatment failures may not have been adequately controlled. The study aim compared SVR12 of DAAs in East Asian patients with HCV-HCC to those without HCC.

Method: Data were from 10 study centers comprising of 30 clinical sites in Hong Kong, Japan, Korea, and Taiwan representing the RealWorld Evidence from the Asia Liver Consortium for Chronic Hepatitis C (REAL-C)- a registry of patients treated with IFN-free DAAs in routine practice $(\mathrm{n}=3702) .1: 1$ PSM matching on cirrhosis, prior treatment, baseline platelet, age, sex, baseline HCV RNA, treatment regimen, baseline ALT, HCV genotype, and BMI was used to balance the groups at baseline.

Result: In our cohort, there were 195 patients with HCC at baseline or prior to initiation of DAA and 3507 patients who did not have HCC at baseline. Prior to PSM, HCC patients were significantly older with mean age of 70 vs. 63 for non-HCC, more likely male (53 vs. $43 \%$ ), more likely to have renal insufficiency (18 vs. $8 \%$ ), cirrhosis (60.3 vs. $23.4 \%$ ), and decompensation (9.3 vs. 3.6\%) (all $p<0.004$ ). After PSM, there were 342 patients for analysis $(\mathrm{n}=171 \mathrm{HCV}-\mathrm{HCC}$ and $\mathrm{N}=171 \mathrm{HCV}$ non-HCC). Table 1 displays the characteristics of the PSM patients and showed no significant differences in major demographic and clinical characteristics between the two study groups. Mean age was close to 70 for both groups, mean BMI was 23 , the majority had cirrhosis, about half were male, and $40 \%$ were treatment-experienced. GT1b was the predominant $(>60 \%)$ virus strain for both groups. The majority $(>50 \%)$ of both groups were treated with LDV/SOF; eight (three HCC, five non-HCC) stopped treatment before completion while $>10 \%$ had an adverse reaction with the most frequently reported as anemia $(>5 \%)$ and fatigue $(>3 \%)$. There were seven deaths: five in the HCC group with four of these related to liver disease and two non-liver related deaths in the non-HCC group. Overall, SVR12 rate was $>96 \%$ for both groups with no significant differences. (Table 2) 
Conclusion: This PSM study compared treatment for HCV patients with/without HCC, finding no difference in treatment tolerability, completion and cure. DAA treatment should also be considered for HCV-HCC patients.

\begin{tabular}{|c|c|c|c|}
\hline Characteristic & $\begin{array}{c}\mathrm{HCC} \\
(\mathrm{n}=171)\end{array}$ & $\begin{array}{l}\text { No HCC } \\
(\mathrm{n}=171)\end{array}$ & $P$-value \\
\hline Male & $89(52.1)$ & $81(47.4)$ & 0.39 \\
\hline Age & $69.5 \pm 9.4$ & $68.9 \pm 10.0$ & 0.60 \\
\hline Body mass index (BMI) & $23.3 \pm 3.2$ & $23.1 \pm 3.8$ & 0.65 \\
\hline Hypertension & $38(53.5)$ & $17(40.5)$ & 0.18 \\
\hline Hyperlipidemia & $6(4.7)$ & $7(4.9)$ & 0.96 \\
\hline Coronary artery disease & $1(1.5)$ & $2(5.1)$ & 0.28 \\
\hline Chronic renal insufficiency & $20(14.8)$ & $14(9.3)$ & 0.15 \\
\hline $\begin{array}{l}\text { Other cancer } \\
\text { Prostate } \\
\text { Colon } \\
\text { Gastric } \\
\text { Other }\end{array}$ & $\begin{array}{l}1(1.9) \\
1(1.9) \\
0(0.0) \\
2(3.9)\end{array}$ & $\begin{array}{l}1(3.5) \\
1(3.5) \\
1(3.5) \\
1(3.5)\end{array}$ & 0.69 \\
\hline $\begin{array}{l}\text { Cirrhosis } \\
\text { Decompensation }\end{array}$ & $\begin{array}{c}106(62.0) \\
8(8.5)\end{array}$ & $\begin{array}{c}103(60.2) \\
7(6.9)\end{array}$ & 0.67 \\
\hline $\begin{array}{l}\text { Hepatitis C virus (HCV) genotype and subtype } \\
\text { Genotype } 1 \\
\text { Untyped } \\
\text { 1a } \\
\text { 1b } \\
\text { Genotype } 2 \\
\text { Untyped } \\
\text { 2a } \\
2 b \\
\text { 2a/2c } \\
\text { Genotype } 6\end{array}$ & $\begin{array}{c}127(74.2) \\
14(8.2) \\
3(1.8) \\
110(64.3) \\
42(24.6) \\
13(7.6) \\
15(8.9) \\
11(6.4) \\
3(1.8) \\
2(1.2)\end{array}$ & $\begin{array}{l}123(71.9) \\
3(1.8) \\
1(0.6) \\
119(69.6) \\
46(26.9) \\
9(5.3) \\
26(15.2) \\
11(6.4) \\
1(0.6) \\
\mathbf{2 ( 1 . 2 )}\end{array}$ & 0.078 \\
\hline $\begin{array}{l}\text { Prior treatment } \\
\text { Prior direct acting antiviral (DAA) } \\
\text { Prior non-DAA }\end{array}$ & $\begin{array}{l}72(42.1) \\
5(2.9) \\
67(39.2) \\
\end{array}$ & $\begin{array}{l}70(40.9) \\
10(5.9) \\
60(35.1)\end{array}$ & 0.36 \\
\hline log10 HCV RNA, mean (SD) & $5.6 \pm 1.3$ & $5.5 \pm 1.3$ & 0.55 \\
\hline Platelets, median (range) & $115(79-161)$ & $116(84-161)$ & 0.58 \\
\hline Total bilirubin, mean (SD) & $1.1 \pm 0.9$ & $1.0 \pm 0.7$ & 0.20 \\
\hline ALT, median (range) & $46(33-65)$ & $47(29-75)$ & 0.55 \\
\hline $\begin{array}{l}\text { DAA treatment initiated } \\
\text { SOF+RBV (sofosbuvir+ribavirin) } \\
\text { LDV/SOF (ledipasvir/SOF) } \\
\text { 3D (paritaprevir/ritonavir, ombitasvir + dasabuvir) } \\
\text { 2D (paritaprevir/ritonavir, ombitasvir) } \\
\text { DCV+ASV (daclatasvir+asunaprevir) } \\
\text { SOF+DCV } \\
\text { EBR/GZR (elbasvir+grazoprevir) } \\
\text { Other }\end{array}$ & $\begin{array}{l}33(19.3) \\
94(55.0) \\
12(7.0) \\
1(0.6) \\
19(11.1) \\
3(1.8) \\
1(0.6) \\
8(4.7)\end{array}$ & $\begin{array}{c}45(26.3) \\
87(50.9) \\
13(7.6) \\
0(0.0) \\
21(12.3) \\
1(0.6) \\
1(0.6) \\
3(1.8)\end{array}$ & 0.53 \\
\hline
\end{tabular}

Table 2: Tolerability and Outcomes for PSM Patients with and without HCC

\begin{tabular}{|c|c|c|c|}
\hline & HCC & No $\mathrm{HCC}$ & $P$-value \\
\hline $\begin{array}{ll}\text { Death } & \\
& \begin{array}{l}\text { Non-liver related } \\
\text { Liver-related }\end{array}\end{array}$ & $\begin{array}{c}\mathbf{5} \\
1(0.6) \\
4(2.3)\end{array}$ & $\begin{array}{c}\mathbf{2} \\
2(1.2) \\
0(0.0)\end{array}$ & 0.11 \\
\hline Adverse events & $21(12.9)$ & $17(10.1)$ & 0.43 \\
\hline Headache & $0(0.0)$ & $2(3.3)$ & 0.14 \\
\hline Fatigue & $3(4.6)$ & $2(3.3)$ & 0.71 \\
\hline Insomnia & $3(4.6)$ & $1(1.6)$ & 0.35 \\
\hline Nausea & $0(0.0)$ & $1(1.6)$ & 0.30 \\
\hline Arthralgia & $1(1.5)$ & $0(0.0)$ & 0.33 \\
\hline Myalgia & $0(0.0)$ & $1(1.6)$ & 0.30 \\
\hline Rash & $3(2.5)$ & $2(1.5)$ & 0.57 \\
\hline Pruritus & $2(3.0)$ & $0(0.0)$ & 0.17 \\
\hline Anemia & $7(6.0)$ & $7(5.2)$ & 0.78 \\
\hline Dyspnea & $1(1.5)$ & $0(0.0)$ & 0.33 \\
\hline $\begin{array}{l}\text { Study discontinuation } \\
\text { Non-compliance } \\
\text { Psychological side effects } \\
\text { Other } \\
\end{array}$ & $\begin{array}{c}3 \\
1(0.8) \\
0(0.0) \\
2(1.5) \\
\end{array}$ & $\begin{array}{c}\mathbf{5} \\
1(0.7) \\
1(0.7) \\
3(2.0) \\
\end{array}$ & 0.59 \\
\hline SVR-12 rates & $\begin{array}{c}96.8 \\
95 \% \text { CI: } 93-99\end{array}$ & $\begin{array}{c}96.3 \\
95 \% \text { CI: } 92-99\end{array}$ & 0.83 \\
\hline
\end{tabular}

LATEB-100

Dose, duration and type of alcohol consumption in alcoholic liver disease in north western region of India: a tertiary care centre study

\section{Atul Janrao Gawande ${ }^{1}$, Sudhir Maharshi ${ }^{1}$, Shashank Wanjari ${ }^{1}$,} Sandeep Nijhawan

${ }^{1}$ SMS Medical College and Hospital, Jaipur, India

Background: Alcoholic liver disease (ALD) and its complications are one of the major causes of morbidity and mortality. Predisposing factors for ALD includes amount, type, duration of alcohol consumption, patient's genetic predisposition, race, sex and other comorbid conditions. There is paucity of data in literature in Indian population studying effects of dose, duration and type of alcohol consumption on ALD. Aim was to study the dose, duration and type of alcohol consumption in ALD and effect of these parameters on severity and outcome.

Method: Prospective study was conducted at Department of Gastroenterology, SMS Medical College, Jaipur between December 2015 to December 2016. All patients with alcoholic liver disease were enrolled. Data relating to dose, duration, type, pattern of alcohol intake along with biochemical, ultrasonographic and clinical parameters were analyzed

Result: One hundred ten patients (age $43.5 \pm 9.9$, all male) were studied. Mean alcohol consumption was $130.4 \pm 105.4 \mathrm{gm} /$ day. Sixty-three $(57.3 \%)$ patients consumed $<120 \mathrm{gm}, 25(22.7 \%)$ between $120-239 \mathrm{gm}$, while $22(20 \%)$ patients consumed $\geq 240 \mathrm{gm}$ of alcohol per day. Mean duration of alcohol consumption was $15.89 \pm 6.57$ years. Sixty $(54.55 \%)$ patients consumed country made liquor, 26 (23.64\%) whisky, $23(20.91 \%)$ mixed or variable type while 3 $(2.73 \%)$ patients consumed wine. Occurrence of hepatic encephalopathy (HE) significantly associated with dose $(p<0.001)$ and type $(\mathrm{p}<0.001)$ of alcohol. MELD Na score significantly correlated $(r=0.48, p<0.001)$ with dose of alcohol

\begin{tabular}{|l|l|l|l|l|l|}
\hline & N & Minimum & Maximum & Mean & $\begin{array}{l}\text { Std. } \\
\text { Deviation }\end{array}$ \\
\hline Age & 110 & 22 & 65 & 43.57 & 9.954 \\
\hline DSM V & 110 & 1 & 3 & 2.70 & 0.57 \\
\hline CAGE & 110 & 0 & 4 & 2.86 & 1.40 \\
\hline $\begin{array}{l}\text { MELD-Na } \\
\text { score }\end{array}$ & 110 & 7 & 43 & 23.37 & 7.91 \\
\hline CTP score & 110 & 6 & 14 & 10.30 & 1.75 \\
\hline Albumin & 110 & 1 & 4 & 2.41 & 0.40 \\
\hline INR & 110 & 1.0 & 6.4 & 2.01 & 0.90 \\
\hline Hb & 110 & 1.4 & 14.1 & 8.01 & 2.25 \\
\hline LC & 110 & 4.1 & 4878.0 & 1457.99 & 908.52 \\
\hline Platelet count & 110 & .1 & 106.0 & 1.90 & 10.04 \\
\hline Bilirubin & 110 & .5 & 30.0 & 5.32 & 4.92 \\
\hline SGOT & 110 & 3 & 810 & 111.00 & 113.65 \\
\hline SGPT & 110 & 14 & 1190 & 65.57 & 151.92 \\
\hline ALP & 110 & 11.0 & 321.0 & 99.75 & 61.90 \\
\hline TLC & 110 & 2.4 & 60.0 & 10.42 & 7.40 \\
\hline $\begin{array}{l}\text { Dose of } \\
\text { alcohol } \\
\text { (gm/day) }\end{array}$ & 110 & 20 & 530 & 130.47 & 105.373 \\
\hline $\begin{array}{l}\text { Duration of } \\
\text { alcohol } \\
\text { intake (years) }\end{array}$ & 110 & 3 & 32 & 15.89 & 6.568 \\
\hline & & & & & \\
\hline
\end{tabular}

DSM V: Diagnostic and Statistical Manual of Mental Disorder V, MELD-Na: Model for End Stage Liver Disease sodium, CTP: Child-Turcotte-Pugh, LC: Lymphocyte count, TLC: Total leukocyte count. 
Conclusion: Mean alcohol consumption is $130 \mathrm{gm} /$ day for a mean duration of 16 years in patient with ALD in north western region of India. Country made liquor is most common type of alcohol consumed. MELD Na score significantly correlated and occurrence of HE significantly associated with dose of alcohol.

\section{LATEB-102}

Transient elastography (fibroscan) assessment in patients with hepatic and neurological Wilson's disease

\section{Tarun Sebastian Joseph ${ }^{1}$, Harshad Devarbhavi ${ }^{1}$}

${ }^{1}$ St. Johns Medical College, India

Background: Non-invasive assessment of hepatic fibrosis with ultrasound based transient elastography (TE) (or fibroscan) has been validated in a number of chronic liver disease. However, studies involving Wilson disease (WD) patients are rare. Further studies comparing liver stiffness by TE in hepatic and neurological Wilson are lacking. Our aim was to assess liver stiffness by fibroscan in patients with hepatic and neurological Wilson's disease.

Method: Twenty-five patients with well characterized WD underwent TE by FibroscanR, Echosens 402, Paris, France, software - B21). TE was performed in a right intercostal space in a resting respiratory position. Diagnosis of WD was made as per Leipzig criteria. All patients were on follow up and stable on treatment with D-penicillamine \pm zinc. The Fibroscan values were distinguished between hepatic and neurologic Wilson with regard to treatment $\&$ duration of illness. Continuous variables between groups were compared by unpaired $t$ test. $P$ value $<0.05$ was considered significant.

Result: Twenty-five patients (13 with hepatic and 10 with neurologic and 2 with both) underwent TE 3-4 hours after breakfast. Seventeen were males. The mean age of the patients was 20 years and the mean duration of illness was 7.2 years. The demographic and laboratory characteristics between hepatic and neurologic WD are shown in Table. There was no statistical significance between the two in all variables except fibroscan scores.The fibroScan values in patients with neurological WD was $12.94 \pm 5.03$ (Range 7-22) $\mathrm{kPa}$ and in those with hepatic WD was 26.7.6 \pm 16.06 (Range $12.2-75) \mathrm{kKa}(\mathrm{p}$ value $=$ 0.003 ). Scores above $8.4 \mathrm{kPa}$ are considered severe in WD.

Conclusion: Patients with WD have advanced hepatic fibrosis on TE. Hepatic WD patients have significantly higher scores suggestive of cirrhosis while patients with neurologic WD have scores suggestive of severe fibrosis.

\begin{tabular}{|c|c|c|}
\hline Variable & Hepatic & Neurological \\
\hline Age (y) & $20(12-38)$ & $24(10-45)$ \\
\hline $\begin{array}{l}\text { Duration of } \\
\text { illness (y) }\end{array}$ & $6.75(2-17)$ years & $13.2(1-30)$ years \\
\hline Gender (M:F) & $9: 5$ & $8: 3$ \\
\hline $\begin{array}{l}\text { Fibroscan } \\
\text { value (kPa }\end{array}$ & $\begin{array}{l}26.7 \pm 16.1(12.2-75) \\
\mathrm{kpa}\end{array}$ & $12.9 \pm 5.03(7.5-22) \mathrm{kpa}$ \\
\hline $\begin{array}{l}\text { Hemoglobin } \\
\text { (g/dl) }\end{array}$ & $11.8(7.3-14.9)$ & $11(3.8-14.2)$ \\
\hline WBC $10^{3} / \mathrm{dl}$ & $4.9(1.5-8.7)$ & $4.8(1.1-11.6)$ \\
\hline $\begin{array}{l}\text { Platelet } \\
\text { count } 10^{5} / \mathrm{dl}\end{array}$ & $1.3(0.2-3.2)$ & $1.8(0.2-4.1)$ \\
\hline $\begin{array}{l}\text { T. bilirubin } \\
\text { (mg/dl) }\end{array}$ & $1.2(0.5-3) \mathrm{mg} / \mathrm{dl}$ & $0.6(0.2-1.2) \mathrm{mg} / \mathrm{dl}$ \\
\hline AST IU/L & $62.2(21-155) \mathrm{IU} / \mathrm{L}$ & $23.7(12-41) \mathrm{IU} / \mathrm{L}$ \\
\hline ALT IU/L & $59.5(11-187) \mathrm{IU} / \mathrm{L}$ & $24.7(14-38)$ IU/L \\
\hline
\end{tabular}

\section{LATEB-103}

H1N1 influenza presenting as anicteric hepatitis in children a case series

Vaibhav Sameer Shah ${ }^{1}$, Manoj Dungrani ${ }^{3}$, Manohar Choudhary ${ }^{4}$, Mandeep Chaddha ${ }^{4}$, Ashish Bavdekar ${ }^{2}$

${ }^{1}$ KEM Hospital, Pune, India; ${ }^{2}$ KEM Hospital, India; ${ }^{3}$ KEM Hospital, India; ${ }^{4}$ National Institute of Virology, India

Background: Children are particularly affected by the H1N1 pandemic, as evidenced by the age distribution of patients in many settings worldwide. While respiratory and neurologic complications of H1N1 influenza in children have been well described in literature over the years, hepatic complications have seldom been reported. Here we describe a case series of 5 patients having H1N1 infection and presenting with anicteric hepatitis.

Method: Five patients, ranging from 1-7 years of age, presented with a short prodrome of fever, decreased oral intake and cough. Laboratory investigations were suggestive of anicteric hepatitis. Common causes of anicteric hepatitis were ruled out as shown in table 2 . Surprisingly, RT-PCR assay of nasopharyngeal swab confirmed H1N1 infection. Oral Oseltamivir was started along with supportive management. While 3 patients improved and were subsequently discharged, 2 patients succumbed to the illness as requirement for urgent liver transplant could not be met due to financial reasons. 
Result: The common symptoms of H1N1 influenza in children include fever, cough, sore throat, myalgia, vomiting and diarrhea. Complications are more common in children with underlying cardiopulmonary disease and neuromuscular diseases affecting the accessory muscles of breathing. A nationwide German study found pneumonia and ARDS as the most common complications of pediatric H1N1 infection, followed by encephalopathy and myocarditis. These findings were consistent with other large scale pediatric studies conducted in the Americas, Europe and Australia. None of these studies found any hepatic complications of H1N1 infection in the pediatric age group. Alhammadi et al. reported a 22 month old child with H1N1 infection, presenting as anicteric hepatitis, who subsequently improved with supportive care. This presentation was very similar to that observed in our case series. El-Shabrawi et al. published a case of acute myocarditis and fulminant hepatic failure in an infant with pandemic H1N1 in a 10 month old child. There have been isolated case reports of $\mathrm{H} 1 \mathrm{~N} 1$ having hepatic involvement in adults. However, ours is the first case series of H1N1 infection with hepatic involvement in children reported from India or abroad.

Conclusion: Newer strains of H1N1 virus seem to have an affinity for the liver in the pediatric age group. Further studies are required to highlight this novel presentation of $\mathrm{H} 1 \mathrm{~N} 1$ infection in pediatric age group.

\begin{tabular}{|l|l|l|l|l|l|}
\hline Lab Investigations & Case 1 & Case 2 & Case 3 & Case 4 & Case 5 \\
\hline Hb & 11 & 12.6 & 11.6 & 11 & 10.3 \\
\hline TC & 2000 & 4410 & 3600 & 6560 & 5200 \\
\hline APC & 2.95 lac & 32000 & 1.2 lac & 1.35 lac & 71000 \\
\hline SGPT & 2235 & 3104 & 2579 & 2897 & 3151 \\
\hline ALP & 446 & 134 & 486 & 376 & 232 \\
\hline S. Bilirubin & T-0.2 & T-0.2 & T-0.6 & T-0.70 & T-1.2 \\
& & & & & D-0.4 \\
\hline S. Proteins & T-5.3 & T-5.4 & T-5.6 & T-4.3 & T-5.2 \\
& A-3.4 & A-3.1 & A-3.3 & A-2.6 & A-3.2 \\
& G-1.9 & G-2.3 & G-2.3 & G-1.7 & G-2 \\
\hline GGT & 101 & 37 & 56 & 57 & 100 \\
\hline PT / INR & $38 / 1.39$ & $46.3 / 4.18$ & $64 / 5.79$ & $44.6 / 3.78$ & $38.5 / 3.38$ \\
\hline Urea & 40 & 59 & 35 & 16 & 19 \\
\hline Creatinine & 0.86 & 1.43 & 0.45 & 0.31 & 0.53 \\
\hline Ammonia & Not done & Not done & Not done & Not done & 338 \\
\hline Lactate & Not done & Not done & Not done & Not done & 2.8 \\
\hline
\end{tabular}

\begin{tabular}{|c|c|c|c|c|c|}
\hline Special Investigations & Case 1 & Case 2 & Case 3 & Case 4 & Case 5 \\
\hline Hepatitis A IgM & Negative & Negative & Negative & Negative & Negative \\
\hline Hepatitis E IgM & Negative & Negative & Negative & Negative & Negative \\
\hline HBsAg & Negative & Negative & Negative & Negative & Negative \\
\hline Widal test & Negative & Negative & Negative & Not done & Not done \\
\hline Blood culture & No growth & No growth & No growth & Not done & No growth \\
\hline Dengue IgM & Negative & Negative & Negative & Negative & Negative \\
\hline Dengue NS1Ag & Negative & Negative & Negative & Negative & Negative \\
\hline Leptospira IgM & Negative & Negative & Negative & Negative & Negative \\
\hline Rickettsial Antibody IgM & Negative & Negative & Negative & Not done & Not done \\
\hline $\begin{array}{l}\text { Nasopharyngeal swab } \\
\text { for H1N1 (tested by RT- } \\
\text { PCR at NIV, Pune) }\end{array}$ & Positive & Positive & Positive & Positive & Positive \\
\hline TMS // GC/MS & Not done & Not done & Not done & Not done & Normal \\
\hline Treatment & $\begin{array}{c}\text { Oseltamivir } \\
+ \\
\text { Supportive }\end{array}$ & $\begin{array}{c}\text { Oseltamivir } \\
+ \\
\text { Supportive }\end{array}$ & $\begin{array}{c}\text { Oseltamivir } \\
+ \\
\text { Supportive }\end{array}$ & $\begin{array}{c}\text { Oseltamivir } \\
+ \\
\text { Supportive }\end{array}$ & $\begin{array}{c}\text { Oseltamivir } \\
+ \\
\text { Supportive }\end{array}$ \\
\hline Outcome & Recovered & Died & Died & Recovered & Recovered \\
\hline
\end{tabular}

LATEB-104

Effect of low dose steroid regimen during immediate post transplant period in pediatric liver transplant recipients

\author{
Joseph J Valamparampil ${ }^{1}$, Naresh P Shanmugam \\ ${ }^{1}$ Gleneagles Global Health City, India
}

Background: Steroids are the mainstay of immunosuppression in the immediate post-transplant period in liver transplantation (LT) and are associated with significant morbidity. This study was conducted to assess the effects of low dose steroids at immunosuppression induction and initial maintenance in first 3 months after LT in children.

Method: Based on the adult patient protocol in LT, the paediatric protocol was changed to restrict the maximum dose of steroid in the immediate post-transplantation period to 16 milligrams of methylprednisolone irrespective of the weight. In this retrospective study, details of children undergoing LT were collected on the basis of steroid dose used at induction of immunosuppression. Enrolled candidates matched for age and gender with more than 8 kilograms $(\mathrm{kg})$ of weight were divided into either the 'high dose steroid' or 'reduced dose steroid' groups. The first group $(\mathrm{n}=29)$ received steroids at 2 milligrams (mg) per $\mathrm{kg}$ of methylprednisolone (MP) and with a maximum dose of $40 \mathrm{mg}$ while second group $(\mathrm{n}=26)$ received a standard dose of $16 \mathrm{mg}$ of MP at induction. The steroids were tapered to a maintenance dose of $1 \mathrm{mg}$ prednisolone in both the groups. All children received tacrolimus at an initial dose of $0.10-0.15 \mathrm{mg} / \mathrm{kg}$ and were adjusted to maintain serum levels of 10-12 nanogram/milliliter. None of the children received basiliximab. Bivariable analysis was performed using Chi-square and Mann-Whitney U tests after normality assessment with Kolmogrov-Smirnov test.

Result: Both groups were comparable with respect to socio-demographic and etiological exposure variables. There was no statistically significant difference in the occurrence of acute rejection or need for third immunosuppressive drug ( $\mathrm{p}$ value $>0.05$ ) in both groups during first three months. The mean day of normalization of AST, ALT levels and attainment of target levels of tacrolimus were not different between two groups. Also, no significant difference was noted between the treatment groups in terms of occurrence of electrolyte imbalances, number of infective episodes, patient and graft survival. Conclusion: Low dose steroids in induction and initial maintenance in pediatric LT recipients was not associated with increased incidence of acute rejection or need for additional immunosuppression in the first 3 months post-LT. Large studies are needed to confirm the efficacy of low dose steroids in LT.

\section{LATEB-105}

Preserving the native liver for future; auxiliary partial orthotopic liver transplantation (APOLT) for monogenic metabolic liver disease (MLD)

\section{Joseph J Valamparampil ${ }^{1}$, Naresh P Shanmugam ${ }^{1}$}

${ }^{1}$ Gleneagles Global Health City, India

Background: APOLT is a technique by which donor liver graft is placed alongside the native liver following resection of part of the native liver replacing it with a segmental graft. In metabolic liver disease it has the advantage of preserving the native liver and there is the potential for gene therapy in the future with possible withdrawal of immunosuppression. The aim of the study was to determine the efficacy APOLT in correcting the underlying defect and improving quality of life in children with monogenic MLD. 
Method: 12 APOLT procedures were performed in patients with MLD. Median age at transplantation was 32.5 months. All transplants were performed as left auxiliary liver transplants. 5 patients with propionic acidemia (PA), 3 with citrullinemia type 1 (CIT1), and 4 patients with Criggler Najjar syndrome type 1 (CN1) underwent APOLT. Children with PA and CIT1 had a median of 8 and 4 episodes of decompensation per year respectively. Patients with CN1 were receiving an average 16-18 hours of phototherapy per day. Patients with PA and CIT1 had a mean social developmental quotient (DQ) of 49 (<3 standard deviation) as assessed by Vineland Social Maturity Score prior to liver transplantation.

Result: No metabolic decompensation occurred in patients with PA and 2 patients CIT1 in the intra-operatively or in post-transplant period on normal diet over a median follow-up period of 22 months. One child with citrullinemia had graft atrophy with metabolic decompensation. A correctional segmental portal vein embolisation of native liver was performed to cause atrophy of native liver and hypertrophy of graft. This rapidly corrected the metabolic abnormality. All children with $\mathrm{CN} 1$ have normal bilirubin levels without phototherapy on followup. We have $100 \%$ graft and patient survival at mean 22 months of follow up. Progressive improvement in development was seen in children within six months of therapy with a median DQ of 90.

\begin{tabular}{|l|l|l|l|l|l|l|}
\hline No & Disease & $\begin{array}{l}\text { Age at } \\
\text { transplant } \\
\text { (months) }\end{array}$ & $\begin{array}{l}\text { Decompensation } \\
\text { before } \\
\text { transplantation }\end{array}$ & $\begin{array}{l}\text { Decompensation } \\
\text { after } \\
\text { transplantation }\end{array}$ & $\begin{array}{l}\text { Diet restriction } \\
\text { after } \\
\text { transplantation }\end{array}$ & $\begin{array}{l}\text { Follow up } \\
\text { (months) }\end{array}$ \\
\hline 1 & PA & 55 & $8-10 /$ year & None & None & 37 \\
\hline 2 & PA & 21 & $4-6 /$ year & None & None & 19 \\
\hline 3 & PA & 8 & 0 & None & None & 24 \\
\hline 4 & PA & 33 & $4-5 /$ year & None & None & 7 \\
\hline 5 & PA & 38 & $3-4 /$ year & None & None & 28 \\
\hline 6 & CIT-1 & 23 & $3 /$ year & 1 & None & 29 \\
\hline 7 & CIT-1 & 39 & $1 /$ year & None & None & 8 \\
\hline 8 & CIT-1 & 30 & $2-3 /$ year & None & None & 7 \\
\hline
\end{tabular}

Conclusion: This study shows that APOLT corrects the metabolic defect and preserves adequate hepatocyte mass to correct the underlying metabolic abnormality with good quality of life in selected monogenic MLD

\section{LATEB-106}

Transient elastography in non-cirrhotic portal fibrosis: a comparative study with child a cirrhosis in a tertiary care centre

\section{Mohammed Noufal B ${ }^{1}$, Venkateswaran A $\mathbf{R}^{1}$, Rajkumar Solomon $^{1}$, Kani Shaikh $\mathbf{M}^{1}$, Malarvizhi $\mathbf{M}^{1}$, Ratnakar Kini ${ }^{1}$ \\ ${ }^{1}$ THE Tamilnadu DR MGR Medical University, India}

Background: Non-cirrhotic portal hypertension (NCPH) is one of the important causes of variceal bleed in tropical countries. In chronic liver disease a very useful non-invasive method for the diagnosis of fibrosis is Liver stiffness (LS) measurement by transient elastography (TE). There are only limited studies evaluating TE in patients with Non-cirrhotic portal fibrosis (NCPF). Aim of this study was to evaluate role of TE in NCPF.

Method: This is a case control study of retrospectively collected data from 25 patients of NCPF from September 2015 to August 2017. All patients had liver biopsy, TE, computed tomography of abdomen. Twenty-five age and gender matched healthy subjects and fifty age matched patients with cirrhosis with Child's A were taken as controls. Result: The mean age of the patients was 26.5 (15-40) years, with female predominance $($ Female $>$ Male $=19: 6)$. Of the 25 patients 21 had variceal bleed requiring endoscopic band ligation and 3 required glue therapy for fundal varices. The platelet count ( 87 vs $115 \times 10$
$3 / \mathrm{ml}$ ) was lower and total leucocyte count (2.5 vs $5.9 \times 103 /$ cumm P $<0.05$ ), was significantly lower in patients with NCPF compared to patients with cirrhosis.TE (Fibroscan) was significantly low in patients with NCPF compared to cirrhotic patients (8.2 vs $47.4 \mathrm{kPa}, \mathrm{P}$ $<0.005)$ but high compared to healthy controls (8.2 vs $4.7 \mathrm{kPa}, \mathrm{P}<$ 0.005).

Conclusion: Transient elastography (Fibroscan) is significantly low in patients with NCPF compared to patients with cirrhosis. It is a very useful non-invasive technique to differentiate between Child's A cirrhosis and non-cirrhotic portal fibrosis.

\section{LATEB-107}

Acute-on-chronic liver failure (ACLF): a tertiary care center experience

Atul Janrao Gawande ${ }^{1}$, Gaurav Gupta ${ }^{1}$, Bhawar Dhandhu', Shashank Wanjari $^{1}$, Vasudha Goel ${ }^{1}$, Vinay Rathore ${ }^{2}$, Sandeep Nijhawan ${ }^{1}$

${ }^{1}$ SMS Medical College and Hospital, Jaipur, India; ${ }^{2}$ SMS Medical College and Hospital, Jaipur, India

Background: Acute on chronic liver failure (ACLF) is an acute event associated with high mortality. There are chances of regional differences for epidemiology of ACLF. The present study is planned to assess etiologies, course, and outcome in ACLF patients in north western region of India

Method: Two hundred and eight (182 males and 26 females) patient of ACLF fulfilling modified APASL Consensus criteria 2009 admitted to the gastroenterology department of SMS medical college and hospital, Jaipur between october 2015 and January 2017 were included and prospectively evaluated for 3 months, or till death. We evaluate etiology of underlying chronic disease and acute event for decompensation in ACLF

Result: Most common etiology of chronic liver disease (CLD) was alcohol $(72.1 \%)$. Viral, cryptogenic, autoimmune, nonalcoholic steatohepatitis, Wilsons disease as cause of CLD was present in 32 (15.4\%), $12(5.8 \%), 9(4.3 \%), 3(1.4 \%), 2$ (1\%) respectively. Alcohol, sepsis, bleeding, reactivation of hepatitis B, Hepatitis E, antitubercular drugs, and autoimmune hepatitis, as cause of acute event was present in $100(48.08 \%), 34(16.35 \%), 19(9.1 \%), 17(8.2 \%), 15$ $(7.2 \%), 11(5.3 \%), 13(5.25 \%), 2(0.96 \%)$ of patients respectively. In $8(3.85 \%)$ patients the precipitating event was not known. 3 month mortality in this study was $37.5 \%$. Higher MELD score and high CTP score was significantly associated with mortality $(\mathrm{P}<0.001)$. There was no significant difference regarding acute precipitating events between survivors and non survivors.

\begin{tabular}{|l|l|l|}
\hline & Mean & Standard Deviation \\
\hline Age (years) & 43.57 & 10.85 \\
\hline Hb $(\mathrm{g} / \mathrm{dl})$ & 8.91 & 2.31 \\
\hline TLC $($ per $\mathrm{mm} 3)$ & 10.18 & 5.75 \\
\hline Platlet $($ per $\mathrm{mm} 3)$ & 71581.47 & 54978.49 \\
\hline INR & 2.37 & 0.84 \\
\hline Creatinin $(\mathrm{mg} / \mathrm{dl})$ & 1.286 & 0.721 \\
\hline Bilirubin $(\mathrm{mg} / \mathrm{dl})$ & 10.52 & 5.63 \\
\hline SGOT & 164 & 111.85 \\
\hline SGPT & 103.41 & 110.05 \\
\hline Albumin $(\mathrm{g} / \mathrm{dl})$ & 2.48 & 0.613 \\
\hline SAP & 122.36 & 91.17 \\
\hline MELD & 25.56 & 5.95 \\
\hline CTP & 12.60 & 1.73 \\
\hline
\end{tabular}




\begin{tabular}{|l|l|l|l|}
\hline & Survivors $(\mathrm{n}=126)$ & $\begin{array}{l}\text { Non-survivors } \\
(\mathrm{n}=78)\end{array}$ & P value \\
\hline Age (years) & $44.96 \pm 11.08$ & $41.71 \pm 9.93$ & 0.035 \\
\hline CTP Score & $11.63 \pm 1.42$ & $14 \pm 1.39$ & $<0.001$ \\
\hline AST & $164.93 \pm 112.26$ & $163.01 \pm 103.51$ & 0.903 \\
\hline ALT & $103.82 \pm 110.68$ & $103.03 \pm 108.70$ & 0.960 \\
\hline SAP & $126.14 \pm 99.01$ & $117.77 \pm 79.32$ & 0.528 \\
\hline MELD & $23.57 \pm 3.04$ & $31.37 \pm 6.38$ & $<0.001$ \\
\hline INR & $2.06 \pm 0.32$ & $2.83 \pm 1.12$ & $<0.001$ \\
\hline Bilurubin(mg/dl) & $9.38 \pm 4.52$ & $12.59 \pm 6.68$ & $<0.001$ \\
\hline Albumin(g/dl) & $2.56 \pm 0.57$ & $2.35 \pm 0.64$ & 0.016 \\
\hline Hb(g/dl) & $9.26 \pm 2.24$ & $8.51 \pm 2.29$ & 0.022 \\
\hline TLC(Ï103/mm3) & $9.83 \pm 5.4$ & $10.82 \pm 6.34$ & 0.235 \\
\hline Platlet(İ103/mm3) & $69.326 \pm 56.893$ & $75.280 \pm 51.845$ & 0.461 \\
\hline Creatinin(mg/dl) & $1.04 \pm 0.41$ & $1.67 \pm 0.91$ & $<0.001$ \\
\hline HE & $71 / 126$ & $70 / 78$ & $<0.001$ \\
\hline
\end{tabular}

Ascites mobilization in ACLF patients with organ failures is difficult and is associated with frequent side effects.

Method: Patients hospitalised at tertiary centres in India between December 2012 - July 2016 with ascites and ACLF $\geq$ Grade I (CLIFSOFA) were included after excluding patients with age $>$ 65year, malignancy, severe cardiopulmonary disease, chronic kidney disease on dialysis. Consecutive patients were distributed to Arm I and ii in approximate $2: 1$ ratio

Patients in Arm I received SIFA (T) [furosemide $2 \mathrm{mg} / \mathrm{h}$, albumin 2 $\mathrm{gm} / \mathrm{h} ; 20-40 \mathrm{~g} / \mathrm{d}$ ] in addition to standard medical therapy (SMT) for ACLF complications. Furosemide was stepped up by $2 \mathrm{mg} / \mathrm{h}$ every 12 hours if $\mathrm{UNa}+<85 \mathrm{mmol} / \mathrm{d}$ to a maximum of $6 \mathrm{mg} / \mathrm{hr}$.

After $48 \mathrm{hrs}$, if $\mathrm{UNa}+<85 \mathrm{mmol} / \mathrm{d}$, Terlipressin was started at 4 $\mathrm{mg} / 24 \mathrm{hrs}$ (maximum $8 \mathrm{mg} / 24 \mathrm{hr}$ ).

Aggressive potassium supplementation (oral/iv) was done.

Treatment continued till complete ascites mobilisation.

Therapeutic ascitic or pleural tap was only done if the patient was severely dyspnoeic.

Central venous line was placed in oliguric patients.

Arm II- received SMT as per EASL guidelines.

Patients were shifted to oral diuretics at discharge titrating dose to maintain $\mathrm{UNa}>80 \mathrm{mmol} / 24$ hours

Twenty-eight-day mortality, creatinine, urine output, UNa, CTP, MELD were compared between the groups.

Result: 230 patients (143 in Arm I, 87 in Arm II) Aetiology of cirrhosis and acute insult didn't differ qArm I- 87/143 (60.8\%) needed the addition of terlipressin. Patients needing terlipressin had higher creatinine $(1.7 \pm 1.0$ vs $0.9 \pm 0.6)$, lower mean arterial pressure (MAP) (78.0 \pm 7.8 vs $86.4 \pm 7.0)$ and higher MELD score $(31.0 \pm 9.0$ vs $23.4 \pm 5.8)(\mathrm{P}<0.05)$. 28-day survival for ACLF- $1 / 2 / 3$ in the centre I vs II was $87 \% / 90 / 86.7 \%$ vs $70 \% / 66 / 52 \%(\mathrm{P}<0.05)$ three patients in arm I developed persistent diarrhoea, and one required stoppage of treatment worsening of hepatic encephalopathy occurred in 11 patients in arm one vs 23 patients in arm II $(\mathrm{P}<0.05)$. Thirtyseven $(25.8 \%)$ and $39(44.8 \%)$ patients developed secondary infections in arm I and II respectively

Conclusion: qIn this study, SIFA (T) therapy could safely mobilise large ascites in patients with severe liver dysfunction. It may be inferred that

1. It may be rewarding to use established therapy for AKI-HRS (albumin-terlipressin) differently (continuous infusion, with diuretic injection) in patients with ACLF

2. Kidneys are responsive to diuretic therapy and, if side effects are warded off, effective diuresis can be achieved.

Despite lack of improvement in liver function, 28-d survival benefit conferred by SIFA ( $\mathrm{T}$ ) could be due to improved systemic hemodynamics resulting improvement in function of other extrahepatic organs.

Improvement in extra-hepatic organ failure(s) with $\operatorname{SIFA(T)~may~}$ allow the opportunity for LT in an ACLF patient with OF or, rarely, chance for spontaneous recovery form ACLF

Slow infusion of furosemide and albumin with or without terlipressin in acute on chronic liver failure patients with organ failure(s) safely mobilizes ascites and improves 28-day survival

\section{LATEB-109}

\section{Abhimanyu Nehra}

${ }^{1}$ Senior Resident, India

Background: Acute on chronic liver failure (ACLF) patients with organ failures have high short-term mortality.

ACLF is associated with a hyperdynamic circulatory state characterized by an increase in cardiac output and a decrease in peripheral and splanchnic vascular resistance leading to activation of the renin-angiotensin-aldosterone pathway and sodium retention manifesting as ascites.

\section{Renal involvement in children with wilson's disease}

Vaibhav Sameer Shah ${ }^{1}$, Anunaya Katiyar ${ }^{2}$, Namrata Bhagat ${ }^{2}$, Jyoti Sharma $^{2}$, Ashish Bavdekar ${ }^{2}$

${ }^{1}$ KEM Hospital, Pune, India; ${ }^{2}$ KEM Hospital, India

Background: Although renal involvement in Wilson's disease (WD) is rare, it is of significant importance due to the associated morbidity. Initially thought to be uncommon, recent data points to a more frequent occurrence of renal disease in children having Wilson's disease. 
However, very few studies have been done to elaborate this finding. The objective of our study is to describe the prevalence of associated renal disorders in children with WD.

Method: It is a prospective observational study including patients aged 1- 18 years, diagnosed before as WD, visiting the Liver clinic or admitted in the Pediatric ward of KEM Hospital, Pune. Patients with pre-existing renal disease were excluded. Demographic details, laboratory data and sonographic findings were recorded. Standard definitions were used for defining proteinuria, microscopic hematuria and hypercalciuria. Data was analysed using Mann Whitney U test and $\mathrm{z}$ score was calculated to know the significance of difference between the group with and without renal abnormalities.

Result: Out of the 70 patients enrolled in our study, 4 children were excluded as they had pre-existing renal disease. Out of the 66 patients finally evaluated, 24 were girls and 42 were boys. Renal involvement usually occurred early in the course of illness and seemed to occur more frequently in children having WD at an earlier age. 60\% of WD patients with renal manifestations had a positive Kayser-Fleischer (KF) ring. RTA was seen in $11(16.6 \%)$, hematuria in $8(12 \%)$, proteinuria (non-nephrotic) in $6(9 \%)$, calculi in $5(7.5 \%)$ and isolated hypercalciuria in $4(6 \%)$ patients. There was no significant difference between WD patients having renal involvement and those without it, with respect to hepatic, neurological or combined hepatic and neurological presentation of WD. All the children were receiving Penicillamine, except two who were on Zinc maintenance therapy due to Penicillamine allergy. Despite extensive literature search, we found only two pediatric studies worldwide, which highlighted the renal aspect of WD. The most commonly associated renal abnormalities reported in those studies were hematuria, proteinuria and renal tubular acidosis with or without hypercalciuria.

Conclusion: Ours is the only study from India shedding light on the unexplored renal aspect of WD in children. Further studies are required to formulate guidelines for screening of children with WD for renal abnormalities.

\begin{tabular}{|c|c|c|c|}
\hline Parameters & No renal involvement & Renal involvement & P value \\
\hline Total number (N) & $36(54.5 \%)$ & $30(45.4 \%)$ & \\
\hline Boys: girls ratio & $2: 1(23: 13)$ & $1.7: 1(18: 12)$ & \\
\hline Median age at screening & $168(144-204)$ months & $132(105-147)$ months & \\
\hline $\begin{array}{c}\text { Median time since diagnosis } \\
\text { at the time of sereening }\end{array}$ & 62.8 months & 30.9 months & $<\mathbf{0 . 0 5}$ \\
\hline $\begin{array}{c}\text { KF ring positive } \\
\text { "Hepatic', GI symptoms at } \\
\text { Presentation }\end{array}$ & $23(63.88 \%)$ & $20(6 \% \%)$ & \\
\hline $\begin{array}{c}\text { Neurological presentation } \\
\text { Hepatic and Neuro } \\
\text { presentation }\end{array}$ & $20(34.8 \%)$ & $21(31.8 \%)$ & $\mathbf{0 . 8 4}$ \\
\hline
\end{tabular}

Table 2: A comparision of renal involvement in WD with studies from other countries

\begin{tabular}{|l|l|l|l|}
\hline \multicolumn{1}{|c|}{ Parameter } & \multicolumn{1}{|c|}{ Zhuang et al } & \multicolumn{1}{c|}{ Soo Ha et al } & \multicolumn{1}{c|}{ Our Study } \\
\hline Proteinuria & $14 \%$ & $6 \%$ & $9 \%$ \\
\hline $\begin{array}{l}\text { Proteinuria with } \\
\text { Hematuria }\end{array}$ & $5.8 \%$ & & 12 \\
\hline Hematuria & $16.5 \%$ & $26 \%$ & $6 \%$ \\
\hline Hypercalciuria & & $29 \%$ & \\
\hline
\end{tabular}

LATEB-110

Microvesicles from hepatitis $B$ patients function as replication bodies and can transmit $\mathrm{HBV}$ infection

Sukriti Sukriti ${ }^{1}$, Manish Chandra Choudhary ${ }^{2}$, Jaswinder Singh Maras $^{1}$, Sachin Sharma ${ }^{1}$, Sukanta Das ${ }^{1}$, Swati Thangariyal ${ }^{1}$, Mojahidul Islam ${ }^{1}$, Nirupama Trehanpati ${ }^{1}$, Shvetank Sharma ${ }^{1}$, Vijay Kumar', Ekta Gupta ${ }^{1}$, Shiv Kumar Sarin ${ }^{1}$

${ }^{1}$ Institute of Liver and Biliary Sciences, India; ${ }^{2}$ Institute of Liver and Bilary Scinces, India

Background: Evidence suggests that microvesicles (MVs) can transfer genetic material between the cells. However, their role in hepatitis B virus (HBV) infection remains unclear. We investigated whether MVs serve as reservoirs of HBV infection, carry HBV DNA and transmit infection.

Method: MVs were isolated from chronic hepatitis B (CHB) patients, Group1: q HBsAg+ve with undetectable HBV DNA post-antiviral therapy $[\mathrm{n}=9$ ]; Group 2: CHB with detectable HBV DNA > 103 [n = $12]$ and compared with healthy controls $(\mathrm{HC})(\mathrm{n}=20)$. The hepatocyte origin of MVs was confirmed by ASGPR+ and by highly expressed liver specific mir122 levels in MVs. The MV mediated HBV transmission and replication was assessed in Huh7, HepG2 and primary human hepatocytes cell lines.

Result: In Gr1, HBV DNA was detected in MVs in 5 of $9(55 \%)$ patients and in $\mathrm{Gr} 2$, in all patients. Levels of HBV DNA were lower in MVs than in plasma \{median: $2.2 \times 103 \mathrm{IU} / \mathrm{ml}$ versus $6.3 \mathrm{X} 106$ $\mathrm{IU} / \mathrm{ml} ; \mathrm{p}=0.042\}$. The probability of detecting HBV DNA in MVs was higher than plasma in $\mathrm{Gr} 1$ with a relative risk ratio 2.25 (95\% of CI 1.08 to $4.67 ; p=0.02$ ) and odds ratio $28.1(95 \%$ of $\mathrm{CI}-1.27$ to 619.9; $\mathrm{p}=0.03$ ). MVs detected in CHB patients were of hepatocyte origin. MVs from Gr2 naïve (Huh7, HepG2) and high HBV entry receptor, NTCP expressing $(\mathrm{PHH})$ cell lines. HBV replication was also documented in these cell lines.

Conclusion: Hepatic MVs in CHB patients can act as reservoirs of HBV infection. The virus persists in MVs even after clearance of HBV DNA from plasma. These MVs are capable of mediating replication in NTCP-independent manner. This novel data may help in developing new therapeutic strategies for treating low replicative hepatitis B infection. 


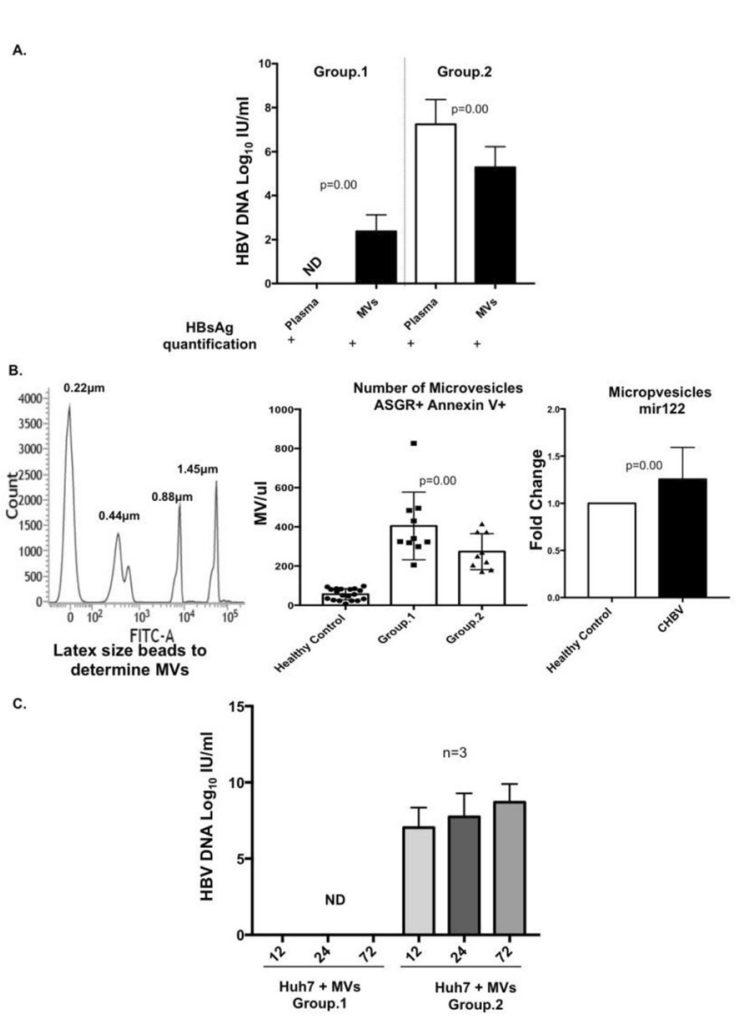

LATEB-111

Catch up growth after liver transplantation in children with biliary atresia

Joseph J Valamparampil $^{1}$

${ }^{1}$ Gleneagles Global Health City, India

Background: To evaluate the growth, nutritional intake in infants with biliary atresia (BA) at the time of liver transplantation (LT) and to determine the rate of catch-up growth in the first year after LT.

Method: 53 children who underwent transplantation between 2013-2016 were followed-up for one year. We analyzed patient demographics, growth, nutritional intake pre-LT and at the time of discharge. Weight-for-age and length-for-age data were used to develop z-scores for each patient using WHOAnthro and WHOAnthroPlus software based on new World Health Organization (WHO) growth charts. Measurements were recorded pre-transplant, 6 months and 1 year after LT. Growth retardation was defined as z-score z-score $<-2$, and catch-up growth as the achievement of $z$-score $\geq 0$. Statistical analysis of age-adjusted z-scores was performed using Chisquare, Friedman and Wilcoxon Sign Rank test after normality assessment with Kolmogrov-Smirnov test.

Result: The median age at LT was 11 months. Mean ad libitum protein and calorie intake in the immediate pre-LT period was $69 \%$ and $74 \%$ of recommended daily allowance $(p<0.05)$ inspite of multiple nutritional interventions. There was statistically significant negative correlation between pretransplant protein intake, energy intake, pretransplant weight $\mathrm{z}$-score and pediatric end stage liver disease score.

Pre-LT albumin, hemoglobin and weight z-score had statistically significant negative correlation with duration of hospital stay. Protein and calorie intake showed statistically significant improvement at the time of discharge without any further nutritional interventions $(p<$
0.05). Significant catch-up in weight and height was seen at 6 months (median z-scores -1.17 and -1.33) and one year (median z-scores 0.53 and -0.38$)$ after LT $(\mathrm{p}<0.05)$.

Conclusion: Children with BA had significant lag in growth despite aggressive nutritional support. LT improves the nutritional intake and nutritional failure should be a strong indication for early LT. Significant catch-up growth was observed after 1 year without any additional nutritional interventions.

\section{LATEB-112}

A randomized study to compare the outcome of carbepenem versus carbepenem plus GM-CSF in Difficult to treat (DTT) Spontaneous Bacterial Peritonitis

\section{Vikash Prakash \\ ${ }^{1} \mathrm{MD}, \mathrm{DM}$, India}

Background-Cirrhotics with SBP have high mortality despite aggressive antibiotic therapy. They show reduced monocyte HLA expression.GM-CSF improves monocytic HLA-DR and decreases gut bacterial translocation.

Methods-131 consecutive cirrhotics with DTT-SBP[defined$\mathrm{SBP}>48 \mathrm{~h}$ of hospitalization or non-response to 3rd Generation Cephalosporin] were randomized to Carbapenem plus $\operatorname{GMCSF}(1.5$ $\mathrm{mcg} / \mathrm{Kg}$ daily IV till resolution or $5 \mathrm{~d}$ ) (Group $\mathrm{A}=65$ )or Carbapenem(1g IV t.i.d x 5d) (Group B =66) in addition to albumin.We evaluated SBP Response rate ( $>75 \%$ decrease in absolute neutrophil count; ANC at $48 \mathrm{~h}$ )and resolution [ANC $<250$ cells/mm3).

Results-Patients with Group A had higher SBP response [Group A: $39 \%$ vs Group B: $21 \%$; p < 0.003] and resolution rates[53\% vs $27 \%$; $<0.03]$. In hospital survival was more in Group A as compared to Group B $(78 \%$ vs $71 \%$; p < 0.33). One patient required discontinuation of GM-CSF due to raising blood white cell count on day 2 but achieved response at $48 \mathrm{~h}$ and resolution by day 4 . On multivariate analysis, presence of pneumonia at enrolment [Hazard ratio; HR-2.99 (95\% CI: 1.29-7.5)], total leucocyte count [HR-1.04(95\% CI: 1.003-1.09)], treatment group [HR-3.01 (95\% CI:1.3-6.9)] predicted mortality.

Conclusion- Addition of GM-CSF to Carbepenemhas greater response rates in DTT-SBP and may improve clinical outcomes.

\section{LATEB-113}

Timely introduction of a combination of low dose terlipressin and noradrenaline as vasopressors is superior to high dose noradrenaline alone in patients of cirrhosis with septic $\operatorname{shock}(\mathrm{NCT02468063)}$

\section{Vikash Prakash}

${ }^{1} \mathrm{MD}, \mathrm{DM}$, India

Background and Aims: Sepsis in cirrhosis leads to rapid deterioration in haemodynamics in an already vasodilated system, with significant down regulation of vasoconstrictive receptors. While timely institution of vasopressors could restore microcirculation, the choice and dosage of vasoconstrictor(s) is not clear. We compared early introduction of a combination of terlipressin and noradrenaline versus noradrenaline alone as vasopressors in patients of cirrhosis with septic shock.

Methods: Cirrhotics with septic shock admitted from April 2015 to January 2017 were screenedand randomized in an open label manner to receive either a combination (Gr. A) of terlipressin ( $2 \mathrm{mg} / 24$ hours 
fixed dose infusion) plus Noradrenaline $(3.75 \mathrm{mcg} / \mathrm{min}$ to $30 \mathrm{mcg} /$ min, $\mathrm{n}=91$ ) or noradrenaline alone $(\mathrm{Gr}$. B, $7.5 \mathrm{mcg} / \mathrm{min}$ to $60 \mathrm{mcg} /$ min, $n=93$ ) to achieve a target Mean Arterial Pressure (MAP) of $>$ $65 \mathrm{mmHg}$ and were followed till death or $48 \mathrm{hrs}$.

Results: 184 cirrhotics with septic shock were randomized to Gr A (N =91) or $\mathrm{B}(\mathrm{N}=93)$, having comparable baseline demographic, clinical and laboratory parameters, MELD (28 vs 30, p = 0.17) and SOFA scores $(12$ vs $13, p=0.33)$. Fortysix $(25 \%)$ patients had 3 organ failures at start of study. At 6 hours, target MAP was achieved more often in Gr A than B (97.8\% vs. 86\%, p = 0.005). Patients in $\mathrm{Gr}$ A had lower incidence of fall in MAP once target MAP was achieved $(1.5 \pm 3.3)$ than $\mathrm{B}(2.7 \pm 4.7)(\mathrm{p}<0.006)$. The frequency $(2$ vs 3 vs $\geq$ 4) of fall in MAP in Gr A (19 vs 5 vs 7) was lower than GrB (26 vs 7 vs 8$)(\mathrm{p}<0.006)$. Adverse effects occured more frequently in Gr. A (18\%) than B (4\%). Most common adverse effect in Gr A was peripheral cyanosis while arrhythmia in Gr B. Overall survival at day 30 was $50 \%$, higher in Gr A than B $(59 \%$ vs, $39 \%, \mathrm{p}=0.01)$. On Multivariate analysis, combination treatment and presence of pneumonia significantly predicted mortality.

Conclusions: Early institution of a combination of low dose terlipressin with noradrenaline achieves greater hemodynamic stability with fewer fluctuations in MAP and a reduced mortality than noradrenaline alone in cirrhotics with septic shock.

\section{LATEB-114}

Rat poisong -acute liver failure to liver transplant - a road map

\author{
Gurudath $G S^{1}$ Mohammed Juned Khan ${ }^{2}$, Anand Vijay ${ }^{4}$, \\ ${\text { Palanivelu } \mathbf{C}^{3}}^{3}$ \\ ${ }^{1}$ GEM Hospital and research centre, India; ${ }^{2}$ Consultant, India; \\ ${ }^{3}$ Director and Head, India; ${ }^{4}$ Liver Transplant Surgeon, India
}

Background: Elemental phosphorus exists in two forms-red and yellow. Red phosphorus is nonvolatile, insoluble, and unabsorbable, and therefore nontoxic when ingested. Yellow phosphorus, on the other hand, is a severe local and systemic toxic affecting multi organs like gastrointestinal tract, liver, cardiovascular, and kidneys. Yellow phosphorus is used as rodenticides and in fireworks. The most readily available source of yellow phosphorus today is rodenticides. Rodenticides are available as powders or pastes contains 2 to $5 \%$ of yellow phosphorus (1). Here we describe a case of rat poisoning containing yellow phosphorus poisoning that led to fulminant hepatic failure and eventually, the patient recovered with liver transplantation

Method:-

Result: -

Conclusion: In conclusion, rat poisoning containing zinc phosphide causes fulminant hepatic failure after 48-72 hrs of consumption. These patients require close observation for development of features of acute liver failure. Liver transplantation is the ideal treatment if MELD 36 or combination of hepatic encephalopathy and INR.

\section{LATEB-115}

Comparison of post mortem liver biopsy findings in ACLF and decompensated liver disease

\author{
Arivarasan Kulandaivelu ${ }^{1}$, Ajay Kumar ${ }^{1}$, Sanjeev Sachdeva ${ }^{1}$, Puja \\ Sakhuja' $^{1}$, Amarender Singh Puri ${ }^{1}$ \\ ${ }^{1}$ GB Pant Hospital, India
}

Background: The Acute on chronic liver failure (ACLF) and decompensated liver disease (DCLD) cannot be differentiated clinically always. The definite role of histopathology in differentiating ACLF and DCLD is also not clear till date. We aim to compare the histological findings of post mortem percutaneous liver biopsy among patients with ACLF and DCLD through a retrospective study.

Method: Percutaneous post mortem liver biopsies (PMLB) of patients with clinically diagnosed ACLF and DCLD were retrospectively analysed. The PMLB was done using $16 \mathrm{G}$ Bard biopsy gun after due consent from patient's attendants. Criteria for ACLF was defined by APASL criteria (2014). Cirrhotic patients with decompensation in absence of acute insult constituted DCLD group. This study was conducted at GIPMER, New Delhi.

Result: A total of 30 patients were analyzed, 15 each in ACLF and DCLD group. Bile duct proliferation(ACLF $\mathrm{n}=12$, DCLD $\mathrm{n}=5$ ), bile duct injury(ACLF $\mathrm{n}=7$, DCLD $\mathrm{n}=2$ ), hepatocyte necrosis(ACLF $\mathrm{n}=$ 6 , DCLD $\mathrm{n}=1$ ), hepatic parenchyma collapse (ACLF $\mathrm{n}=7$, DCLD $\mathrm{n}$ $=0$ ), eosinophilic degeneration of hepatocytes(ACLF $n=4$, DCLD $n$ $=0$ ), ductular transformation(ACLF $\mathrm{n}=10$, DCLD $\mathrm{n}=0$ ), cholesta$\operatorname{sis}(\operatorname{ACLF} n=13$, DCLD $n=9)$, ballooning $(\operatorname{ACLF} n=4$, DCLD $n=1)$ and rossetting (ACLF $\mathrm{n}=3$, DCLD $\mathrm{n}=0$ ) were the common parameters compared. Statistical significant difference was observed in presence of bile duct proliferation, ductular transformation and hepatic parenchyma collapse between these groups $(\mathrm{p} \leq 0.05)$. Chronic inflammatory cell infiltrate was seen in DCLD group whereas in ACLF group, mixed inflammatory infiltrate was observed.

Conclusion: ACLF is clinically and pathologically distinct from DCLD.Hence in cases where there is diagnostic dilemna attempting the liver biopsy may be useful.

\section{LATEB-116}

Serum leptin serve as inflammatory activity markers and can predict steroid responsiveness in autoimmune hepatitis

\section{Chhagan Bihari ${ }^{1}$, Deepika Lal ${ }^{1}$, Monika Thakur ${ }^{1}$, Archana Rastogi $^{1}$, Nirupma Trehanpati ${ }^{1}$, Shiv K Sarin ${ }^{1}$ \\ ${ }^{1}$ Institute of Liver and Biliary Sciences, India}

Background: Adipokines, especially leptin is involved in a wide spectrum of pro-inflammatory functions. Leptin inhibits T regulatory cell (Tregs) proliferation/functions and increases the Th17 cell proliferation and functions, in in vitro models. The study was done to see the leptin levels on the pro-pathogenic process in $\mathrm{AIH}$

Method: We prospectively analyzed leptin levels in treatment naïve autoimmune hepatitis (AIH, $\mathrm{n}=48)$ patients and compared with primary biliary cirrhosis $(\mathrm{PBC}, \mathrm{n}=16)$, chronic hepatitis $\mathrm{B}(\mathrm{CH}, \mathrm{n}=$ $16)$ and healthy controls $(\mathrm{HC}, \mathrm{n}=25)$. AIH patients were treated with steroids and $>50 \%$ reduction in AST/ALT at week 4 was considered as a response, decrease but $>2$ times ULN as partial and $>2$ times ULN of upper normal limits, as non-response.We checked relation of circulatory leptin over liver tests, disease activity, Tregs and TH17 in the liver biopsies. Tregs IHC specific antibodies - CD25 and Fox P3+ and Th17 were done to look for the helper $\mathrm{T}$ cells in the disease activity in liver biopsies of all above 4 groups and counted by Image$\mathrm{J}$.

Result: Serum leptin level was significantly higher in AIH in comparison to $\mathrm{PBC}$, chronic hepatitis and healthy controls \{AIH: 335 (106.2-580)), PBC: 126 (52-381.2), Chronic hepatitis: 67 (3.7-133.5) and healthy controls: $66(40-157.5) \mathrm{pg} / \mathrm{ml}$ with $\mathrm{p}=0.001\}$. In AIH cases; serum leptin levels correlated with hepatic activity index (HAI) $(\mathrm{r}=\mathrm{R}=0.896, \mathrm{p}<0.001)$; serum transaminases (AST: $\mathrm{R}=0.615, \mathrm{p}<$ $0.001, \mathrm{R}=0.551, \mathrm{p}<0.001)$. It inversely related with presence of Tregs in liver biopsies $(\mathrm{p}=-0.711, \mathrm{p}<0.001)$ and positively with Th17 cells $(r=0.650, p<0.001)$. Tregs negatively correlated with severity of disease activity $(r=-0.684, p<0.001)$ and positively with 
Th17 $(\mathrm{r}=0.650, \mathrm{p}=0.001)$. Of 48 AIH cases 18 were negative for leptin $(<200 \mathrm{ng} / \mathrm{ml})$ and 30 were positive for leptin. Leptin positive cases had higher $\operatorname{IgG}(20.5 \pm 6.3$ vs $15.5 \pm 5.9, \mathrm{p}=0.05)$ and more ANA seroposositivity ( 15 vs $4, p=0.047$ ), while no difference noted in absolute neutrophil and eosinophil count. Leptin positivity was found to be associated with steroid responsiveness at 3 months after therapy \{leptin negative- response/partial response/no response: 16/2/ 0 vs leptin positive: $11 / 11 / 8 ; p=0.002$ with a likelihood ratio of 15.4 (9.7-54.76)\}.

Conclusion: Serum leptin serves as a prognostic biomarker for the severity of autoimmune hepatitis and reduced Treg activity. Leptin positivity in hepatic tissue correlates with steroid responsiveness and could help select appropriate patients.

\section{LATEB-117}

Hepatorenal tyrosinemia: a clinical study of 65 cases from a single center in egypt

\section{Hanaa Mostafa El Karaksy ${ }^{1}$, Hala Abdullatif ${ }^{1}$, Engy Mogahed ${ }^{1}$, Carolyne Ghobrial $^{1}$ \\ ${ }^{1}$ Cairo University, Egypt}

Background: Hepatorenal tyrosinemia is a rare autosomal recessive inborn error of metabolism. It mainly affects the liver, kidneys and nervous system. Liver involvement includes liver failure and a tendency to malignant transformation. Treatment with NTBC results in clinical improvement. In resource limited countries, the availability of treatment is questionable and liver transplantation is still an alternative. Aim: We aim to analyze the clinical, laboratory and imaging data of a cohort of children with hepatorenal tyrosinemia presenting to a single tertiary referral center in Egypt, in addition to discussion of chances for therapy.

Method: This study was carried out in the Pediatric Hepatology Unit, Cairo University Pediatric Hospital from 2008-2017. The study protocol was approved by the institutional review board. Data were collected from patients' files.

Result: Over the last 12 years, since the diagnosis of hepatorenal tyrosinemia became available in our center, 65 cases were diagnosed with the disease. The mean age at 1 st symptom was 7.6 months, with a range of 1-30 months; $38(58.5 \%)$ were females. The parents of 53 cases $(81.5 \%)$ were consanguineous; 22 cases $(33.8 \%)$ had a previously affected sib. The commonest presenting symptom was abdominal distention noted by parents in 33 cases (50.7\%). Six cases underwent testing for hepatorenal tyrosinemia after birth because of previously affected sib. Coagulopathy was the presenting feature in 6 cases. Only 2 presented because of jaundice and one case had porphyria-like presentation and was diagnosed (and her deceased brother as Guillian Barre syndrome). Rachitic manifestations were evident in 28 cases $(43 \%)$. Laboratory diagnosis was achieved by a positive test for succinyl acetone in dry blood spot. Ultrasound examination revealed focal hepatic lesions in 26 cases, heterogenous liver in 24 , bright liver in 6 , ascites in 6 and echogenic kidneys which were increased in size in 35 cases. Total serum bilirubin was normal in 22 cases, it ranged between $0.12 \mathrm{mg} / \mathrm{dl}$ to $6.5 \mathrm{mg} / \mathrm{dl}$; ALT was normal in 43 cases $(66 \%)$. Alpha fetoprotein was tested in 58 cases and ranged between $1960 \mathrm{ng} / \mathrm{ml}$ to $339995 \mathrm{ng} / \mathrm{ml}$. Twenty cases were privileged to receive treatment with NTBC and 6 underwent living related liver transplantation, 4 of whom are still surviving 10 years after transplantation with traces of succinyl acetone in urine and/or blood.

Conclusion: In conclusion, hepatorenal tyrosinemia commonly presents with abdominal distention and organomegaly and rarely presents with jaundice. Focal hepatic lesions are common and are associated with highly elevated alpha fetoprotein. Renal involvement manifests as rickets. Treatment with NTBC but often unavailable in resource limited countries.

\section{LATEB-118}

\section{Utility of post mortem liver biopsy (PMLB)}

Arivarasan kulandaivelu $^{1}$, Ajay Kumar ${ }^{1}$, Sanjeev Sachdeva ${ }^{1}$, Puja Sakhuja $^{1}$, Amarender Singh Puri ${ }^{1}$

${ }^{1}$ GB Pant hospital, India

Background: Post mortem liver biopsy (PMLB) has been acknowledged as a useful source of information, capable of stumping the diagnostic skills of one of the most astute physicians. We aimed to audit the histology results of last 50 PMLB performed at our institute. Method: We retrospectively evaluated the clinical records of last 50 PMLB performed at our institute. PMLB was conducted using 16G Bard biopsy gun after due consent from patient's attendants.

Result: Mean age of patients was 39.8 years (range 3-72 years). Males constituted $60 \%$ of the total cases. Ante-mortem clinical diagnosis included decompensated cirrhosis in $48 \%$, Acute on chronic liver failure in $30 \%$ and others $22 \%$ (including acute liver failure, acute febrile illness, suspected granulomatous hepatitis and others). Alcohol was the commonest cause of underlying cirrhosis followed by chronic hepatitis B. In $37(74 \%)$ patients PMLB correlated with ante mortem clinical diagnosis. In $13(26 \%)$ of patients the PMLB did not correlate with ante mortem clinical diagnosis. Two very unusual post mortem diagnosis included rhabdomyosarcoma and granulomatous tuberculosis(on background of Alcoholic cirrhosis)

Conclusion: PMLB is a useful informative tool in both diagnosed and undiagnosed liver disease.

\section{LATEB-119}

Suboptimal level of bone forming cells and increased osteoclast activity in advanced cirrhosis is associated with hepatic osteodystrophy

\section{Chhagan Bihari ${ }^{1}$, Deepika Lal $^{2}$, Dhananjay Mathur ${ }^{1}$, Monika Thakur $^{1}$, Archana Rastogi ${ }^{1}$, Anupam Kumar ${ }^{1}$, Lovkesh Anand ${ }^{1}$, Shiv K Sarin ${ }^{1}$ \\ ${ }^{1}$ Institute of Liver and Biliary Sciences, India; ${ }^{2}$ ILBS, India}

Background: Bone loss is commonly noted in advanced cirrhosis but exact mechanisms are not known. The repair and regeneration of bone occur through the bone forming cells which are derived from bone marrow mesenchymal cells (BM-MSC). We recently reported the loss of BM-MSCs in cirrhosis. In the present study, we aimed to assess the profile and functionality of bone-forming cells and their clinical correlates in cirrhotic patients.

Method: Bone marrow of cirrhotics $(n=61)$, non-cirrhotics $(n=50)$, were studied for bone forming cells. In cirrhotic patients, these were compared with Dual-energy X-ray absorptiometry (DEXA). BM mesenchymal cells were analyzed for fibroblast colony forming units (CFU-F) and their osteogenic (Fibronectin, IGEBP3, Col1, RUNEX2 and alkaline phosphatase) or adipogenic (adiponectin, ALBP, and PPAR- $\gamma$ ) potentials. Bone building proteins were analyzed in BM and peripheral blood.

Result: The CFU-F were lower in cirrhotics $(p=0.0023)$ than controls. Cirrhotic BM- MSCs showed $>2$-fold decrease in osteogenic markers. Cirrhotic patients also showed fewer osteoblasts $(\mathrm{p}<0.001)$, osteocytes $(\mathrm{p}=0.05)$, chondroblasts $(\mathrm{p}<0.001)$, osteocalcin + area $(\mathrm{p}$ $<0.001)$, nestin+ MSCs $(\mathrm{p}=0.001)$ and CD169+ macrophages $(\mathrm{p}<$ 
0.001) [Figure-1]; more so in Child's $\mathrm{C}$ than $\mathrm{A}$ ( $\mathrm{p}<0.001$ each) [Table-1]. DEXA score correlated with osteoblasts $(\mathrm{r}=0.667, \mathrm{p}<$ $0.001)$, osteocytes $(\mathrm{r}=0.670, \mathrm{p}<0.001)$, chondroblasts $(\mathrm{r}=0.340, \mathrm{p}$ $=0.007)$, MSCs $(\mathrm{r}=0.622, \mathrm{p}<0.001)$, osteocalcin + area $(0.419, \mathrm{p}=$ $0.01)$ and CD169+macrophages $(0.525, \mathrm{p}=0.009)$. Low nestin+ MSCs predicted $(\mathrm{p}<0.001)$ bone loss. One unit change in DEXA value was equivalent of 5 unit change in nestin+ MSCs. A $>2$-folds decrease in anti-osteoclastic and increase in pro-osteoclastic factors was observed in Child's $C$ over A. Osteocalcin ( $p=0.008)$, osteonectin $(\mathrm{p}<0.001)$ and BMP-2 $(\mathrm{p}=0.001)$ were decreased, whereas bone resolving factors FGF23 $(\mathrm{p}=0.015)$ and DPP4 $(\mathrm{P}<0.001)$ were increased in $\mathrm{BM}$ and peripheral blood with increasing severity of cirrhosis.

Conclusion: Osteoprogenitor cells are lower in cirrhotics, more so in advanced disease. The loss of osteoprogenitors can contribute to ineffective bone repair and occurrence of osteoporosis in cirrhosis

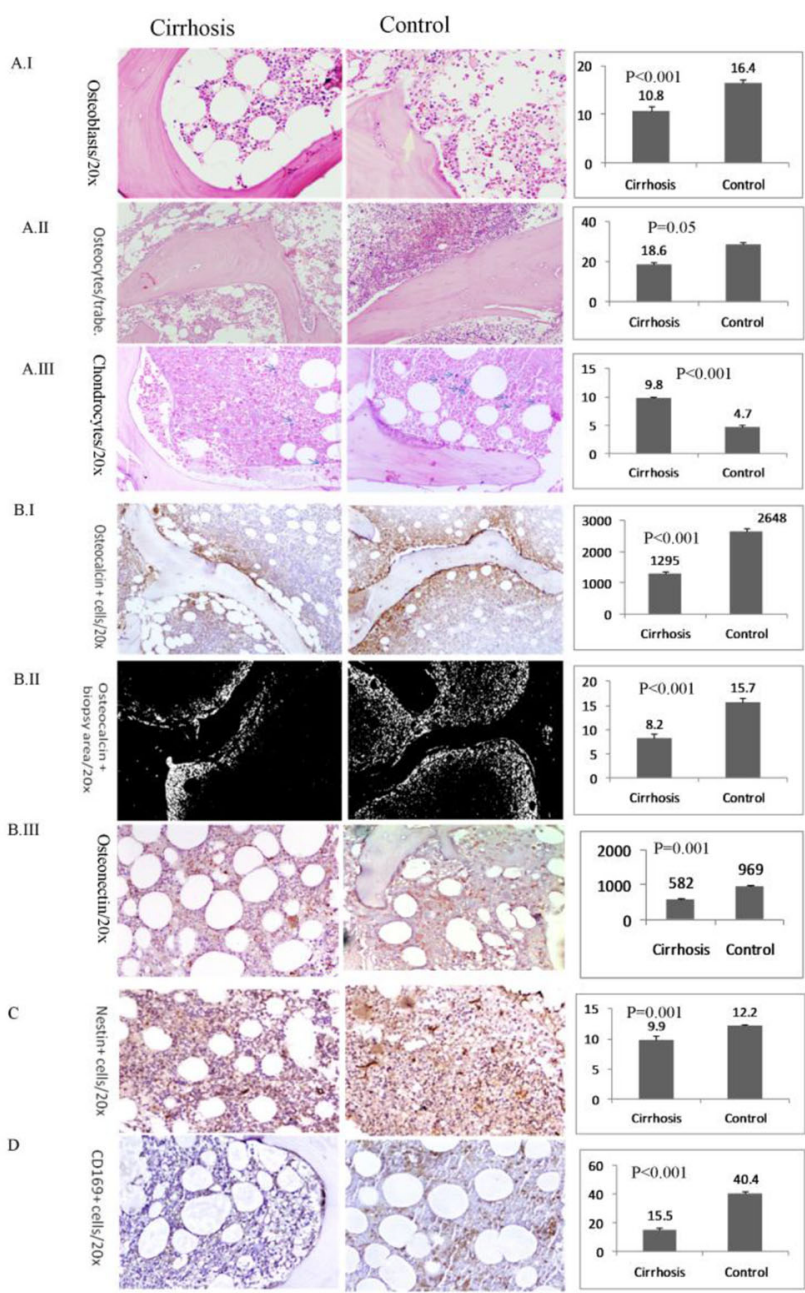

\begin{tabular}{|c|c|c|c|c|c|c|}
\hline Bone forming cells & $\begin{array}{l}\text { Child's A } \\
\text { (10) }\end{array}$ & Child'B (29) & Child's C (22) & $\begin{array}{l}\text { P } \\
\text { value } \\
\text { Child' } \\
\text { s A vs } \\
\text { Child' } \\
\text { s B }\end{array}$ & $\begin{array}{l}\text { P } \\
\text { value } \\
\text { Child' } \\
\text { s A vs } \\
\text { Child' } \\
\text { s C }\end{array}$ & $\begin{array}{l}\mathbf{p} \\
\text { value } \\
\text { Child' } \\
\text { s B vs } \\
\text { Child' } \\
\text { s C }\end{array}$ \\
\hline $\begin{array}{l}\text { Osteoblasts/peritrabecu } \\
\text { lar area }\end{array}$ & $16.57 \pm 3.55$ & $12.13 \pm 4.70$ & $7.27 \pm 5.23$ & 0.093 & $\begin{array}{r}<0.00 \\
1\end{array}$ & 0.002 \\
\hline Osteocytes/trabeculae & $\begin{array}{l}27.57 \pm 10.2 \\
9\end{array}$ & $19.97 \pm 5.27$ & $14.05 \pm 6.65$ & 0.021 & $\begin{array}{r}<0.00 \\
1\end{array}$ & 0.005 \\
\hline Chondrocytes/20x & $8.62 \pm 1.06$ & $5.42 \pm 1.52$ & $2.31 \pm 1.4$ & $\begin{array}{c}<0.00 \\
1\end{array}$ & $\begin{array}{l}<0.00 \\
1\end{array}$ & $\begin{array}{l}<0.00 \\
1\end{array}$ \\
\hline Osteocalcin+cells/20X & $\begin{array}{l}1334 \pm 305.8 \\
3\end{array}$ & $\begin{array}{l}1493.93 \pm 727 . \\
16\end{array}$ & $\begin{array}{l}1001.90 \pm 497 . \\
52\end{array}$ & 1 & 0.582 & 0.016 \\
\hline $\begin{array}{l}\text { Osteocalcin + BM } \\
\text { biopsy area, \% }\end{array}$ & $9.45 \pm 5.41$ & $5.62 \pm 4.20$ & $3.70 \pm 2.87$ & 0.05 & 0.003 & 0.264 \\
\hline Osteonectin+ cells/20x & $1035 \pm 193$ & $654 \pm 162$ & $315 \pm 124$ & $\begin{array}{c}<0.00 \\
1\end{array}$ & $\begin{array}{l}<0.00 \\
1\end{array}$ & $\begin{array}{l}<0.00 \\
1\end{array}$ \\
\hline Nestin+ cells/20x & $15.57 \pm 0.97$ & $11.00 \pm 3.33$ & $6.59 \pm 3.44$ & 0.004 & $\begin{array}{r}<0.00 \\
1\end{array}$ & $\begin{array}{r}<0.00 \\
1\end{array}$ \\
\hline CD169+ cells/20x & $24.00 \pm 6.54$ & $17.25 \pm 6.10$ & $10.09 \pm 4.75$ & 0.013 & $\begin{array}{r}<0.00 \\
1\end{array}$ & $\begin{array}{r}<0.00 \\
1\end{array}$ \\
\hline
\end{tabular}

\section{LATEB-120}

Bone marrow mesenchymal stem cells (Bm-Mscs) from decompensated cirrhotics are not fit for therapeutic uses

Dhananjay Kumar ${ }^{1}$, Dhananjay Kumar', Deepanshu Maheshwari $^{1}$, Nidhi Nautiyal ${ }^{1}$, Smriti Subham ${ }^{1}$, Ashish Vayas ${ }^{1}$, Anupma Prasar ${ }^{1}$, Lovkesh Anand $D^{3}$, Rekha Kumari ${ }^{1}$, Sujata Mohanti $^{2}$, Chhagan Bihari ${ }^{3}$, ANUPAM KUMAR ${ }^{1}$, Shiv Sarin ${ }^{3}$

${ }^{1}$ Institute of Liver and Biliary Sciences, India; ${ }^{2}$ AIIMS, India; ${ }^{3}$ ILBS, India

Background: Autologous bone-marrow-mesenchymal-stemcells(BM-MSCs) are often used for hepatic regeneration in patients with advanced cirrhosis. Use of autologous BM-MSCs in chronic liver disease (CLD) has shown varied clinical response ranging from negligible to mild improvement in CTP scores. In the current study, we aim to investigate the effect of chronic liver damage on tissue repair and regenerative function of BM-MSCs in DecompensatedLiver-Disease (DLD).

Method: MSCs were isolated from BM of DLD patients $(n=10$, DBM-MSC) and there tissue repair and regenerative function were compared with respect to age-matched healthy BM-MSCs $(n=8$, HBM-MSCs). MSC cellular characteristic was confirmed as per the criteria of International Society of Cellular Therapy (ISCT). In-vitro functional efficacy of cells was accessed by studying their potential to suppress the proliferation of cytotoxic t-cells, macrophage polarization, induction of angiogenesis and secretome profile (by cytokine array). The in vivo therapeutic potential was analyzed in the animal model of acetaminophen-induced ALF. Global-transcript profiles of cells were done by next-generation-sequencing (NGS). Metabolic profiles of bone marrow plasma were done by NMR. Bioenergetics of cells were done by analyzing the oxygen-consumption-rates (OCRs) and Extracellular-Acidification-Rate(ECAR) using the Seahorse technology. Stamens property of cells was analyzed by CFU-F assay and senescence property by the SA- $\beta$ Gal assay.

Result: Similar to HBM-MSCs, DBM-MSCs fulfilled the minimal criteria for mesenchymal stromal cells; plastic-adherence, spindleshaped-morphology, inducible-osteo/adipogenesis and specific surface expression patterns. In compression to HMSCs, DMSCs showed 
significant defect in immunomodulatory functions (Figure $1 \mathrm{~A} \& \mathrm{~B}$ ), Angiogenic support (Figure 1C) and paracrine support (Figure 1D). While the infusion of HMSC significantly $(p=0.0012)$ improve the 48-hour survival after APAP induced liver injury DMSC failed to improve the survival (figure 1E), suggesting the loss of therapeutic potential of DMSCs. NGS analysis showed impeachment of genes associated to insulin-resistance in DMSC. DMSCs were significantly $(p=0.0022)$ defective in glucose-uptake (figure $2 \mathrm{~B})$ and are Bioenergetics quiescent (figure 2A). NMR analysis showed an increased level of glucose in DLD BM-plasma in comparison to non-cirrhotic control. This might be responsible for insulin-resistance and loss of function in DMSCs. Further analysis showed the loss of self-renewal capacity (Figure 2C), replicative senescence (figure 2D\&E) in DMSCs.

Conclusion: Bone marrow mesenchymal stem cells in decompensated cirrhosis patients are defective in their regenerative functions due to premature aging and senescence and are not good candidates for MSCs transplantation.
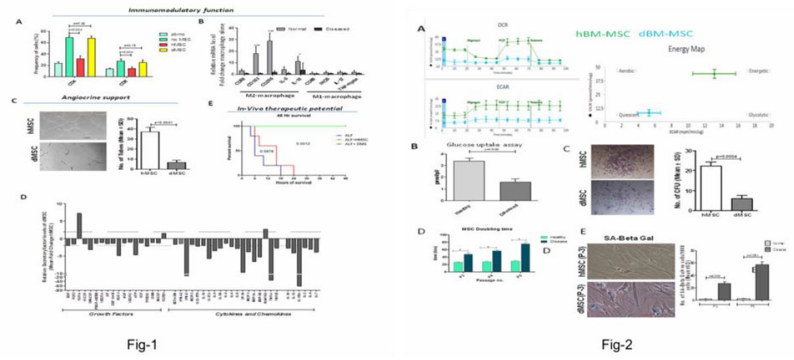

LATEB-122

To determine the utility of thromboelastography (teg) in comparison to conventional coagulation test in cirrhotic patients undergoing central venous cannulation - a tertiary care centre experience

\section{Shivadatta Padhi ${ }^{1}$, Vandana Tyagi ${ }^{2}$, Gaurav Gupta ${ }^{2}$, Ajay Sharma $^{2}$, Sandeep Nijhawan ${ }^{2}$ \\ ${ }^{1}$ SMS Medical College Jaipur, India; ${ }^{2}$ SMS Medical College and Hospital Jaipur, India}

Background And Aims: Hemostasis in patients with chronic liver disease exists in a state of rebalance, in which defects in prohemostatic drivers are compensated for by commensurate changes in antihemostatic drivers. Conventional coagulation tests (CCTs) in cirrhotic patients assess only procoagulant factors and poor predictors of bleeding risk. However CCTs are routinely used prior to invasive procedures and attempts are made to correct these abnormalities before corrective procedures. These practices are not backed by evidences even may be harmful to patients. Thromboelastography (TEG) is a promising tool in evaluating the coagulation status of a cirrhotic patient prior to intervention and hence TEG guided transfusion can avoid unnecessary blood product transfusion to the patients.

Methods: This preliminary prospective study included 33 cirrhotic patients undergoing central line placement in the speciality medical ICU of SMS Medical College and Hospital, jaipur. Conventional coagulation tests (CCTs), thromboplastin generation time (TGT) and Thromboelastography (TEG) were done in all patients and they were observed for postprocedural bleeding. None of the patients received prophylactic transfusion before the procedure

Results: Of the 33 cirrhotic patients (mean age of $45 \mathrm{yrs}, 90.9 \%$ males)with $90.9 \%$ and $9.1 \%$ patients in child class, B, C. All patients underwent central line placement. Bleeding time, clotting time and TGT were normal in all patients. None of the 33 patients developed clinically significant bleeding.Comparison of TEG paramaters ( $R$ time and MA)with INR and platelet counts showed stastically significant lower percentage of abnormal values of $R$ time and MA value compared to INR and platelet count in patients with no post procedural bleed.

Conclusion: Abnormal CCTS in patients with cirrhosis do not predict bleeding risk. Instead of CCTs guided assessment, TEG guided cogulopathy assessment in cirrhotics provides an unique oppurtnunity for rationale transfusion practices in cirrhotic patients prior to invasive procedures like central line placement.

\section{LATEB-122}

To determine the utility of thromboelastograpHY (TEG) in comparison to conventional coagulation test in cirrhotic patients undergoing central venous cannulation - a tertiary care centre experience

\section{Shivadatta Padhi ${ }^{1}$, Vandana Tyagi ${ }^{2}$, Gaurav Gupta ${ }^{2}$, Ajay Sharma $^{2}$, Sandeep Nijhawan ${ }^{2}$ \\ ${ }^{1}$ SMS Medical College Jaipur, India; ${ }^{2}$ SMS Medical College And Hospital Jaipur, India}

Background And Aims: Hemostasis in patients with chronic liver disease exists in a state of rebalance, in which defects in prohemostatic drivers are compensated for by commensurate changes in antihemostatic drivers. Conventional coagulation tests (CCTs) in cirrhotic patients assess only procoagulant factors and poor predictors of bleeding risk. However CCTs are routinely used prior to invasive procedures and attempts are made to correct these abnormalities before corrective procedures. These practices are not backed by evidences even may be harmful to patients. Thromboelastography (TEG) is a promising tool in evaluating the coagulation status of a cirrhotic patient prior to intervention and hence TEG guided transfusion can avoid unnecessary blood product transfusion to the patients.

Methods: This preliminary prospective study included 33 cirrhotic patients undergoing central line placement in the speciality medical ICU of SMS Medical College and Hospital, jaipur. Conventional coagulation tests(CCTs), thromboplastin generation time (TGT) and Thromboelastography (TEG) were done in all patients and they were observed for postprocedural bleeding. None of the patients received prophylactic transfusion before the procedure

Results: of the 33 cirrhotic patients (mean age of $45 \mathrm{yrs}, 90.9 \%$ males)with $90.9 \%$ and $9.1 \%$ patients in child class,B,C. All patients underwent central line placement. Bleeding time, clotting time and TGT were normal in all patients. None of the 33 patients developed clinically significant bleeding.Comparison of TEG paramaters ( $R$ time and MA)with INR and platelet counts showed stastically significant lower percentage of abnormal values of $\mathrm{R}$ time and MA value compared to INR and platelet count in patients with no post procedural bleed.

Conclusion: Abnormal CCTS in patients with cirrhosis do not predict bleeding risk. Instead of CCTs guided assessment,TEG guided cogulopathy assessment in cirrhotics provides an unique oppurtnunity for rationale transfusion practices in cirrhotic patients prior to invasive procedures like central line placement. 


\section{LATEB-124}

A prospective study of prevalence and patterns of infections in cirrhosis patients admitted in intensive care unit

\author{
Niharika Chandra ${ }^{1}$, Padaki Nagaraj Rao ${ }^{1}$, Rajesh Gupta ${ }^{1}$, D \\ Nageshwar Reddy 1 \\ ${ }^{1}$ Asian Institute of Gastroenterology, India
}

Background: Infections are the most important complications leading to increased risk of acute on chronic liver failure, acute kidney injury, multi-organ dysfunction and mortality. Infections commonly seen are spontaneous bacterial peritonitis, cellulitis, pneumonia, urinary tract infection. Aim of our study is to find prevalence and patterns of infection in all cirrhotics and Child Pugh, MELD, INR, albumin.

Method: 41 patients were included from April to December 2017. Pregnancy, malignancy and chronic diseases excluded. Demography,cirrhosis etiology, ascites, hepatic encephalopathy or gastrointestinal bleed, Child-Pugh and MELD. Hemogram, liver function tests, serum procalcitonin, PT, INR, serum ammonia, renal function, urine examination, urine and blood culture, ascitic fluid analysis, culture, USG abdomen, chest $\mathrm{X}$ ray was collected. Prevalence of infection and pattern of infections and correlations with Child Pugh, MELD, renal failure, albumin, INR were assessed.

Result: 41 patients out of 100 screened were studied, $85 \%$ were males and $15 \%$ females. Median age was 50 yrs. $68.3 \%$ were CTP -C, while $29.3 \%$ CTP- B and 2.4\% CTP- A. Median MELD score was 22 and median duration of Cirrhosis was 6 months. Ethanol was the most common with $58.5 \%$ patients having alcohol induced cirrhosis. 19 of 41 patients had evidence of infection at the time of admission which constituted $46.3 \%$ patient studied. $68.4 \%$ of patients with MELD > 20, 21.1\% with MELD 10-20 and 10.5\% with MELD had infection and it was statistically significant, P value- 0.026 . 78.9\% of CTP C, $21.1 \%$ of CTP B and none of CTP A had infection, however this was not statistically significant (p-0.322). Out of these $5(26.3 \%)$ had Pneumonia, $8(42.1 \%)$ had urinary tract infection, $4(21 \%)$ had bacteremia, $3(15.7 \%)$ had cellulitis and $2(10.5 \%)$ had spontaneous bacterial peritonitis (SBP). Negative correlation was found between serum albumin and rate of infection, however it was not statistically significant, P- 0.067. There was no statistical significance found between patients with higher versus lower INR, patients with INR- $<$ $1.5,21.1 \%$ had infection, $15.8 \%$ with INR between 1.5-2.0 and 62.2 with INR $>2.0, \mathrm{P}$ value was 0.329 . No statistically significant correlation was found between serum procalcitonin and rate of infection, $\mathrm{P}$ value was 0.707 and none was found between acute renal failure and infection, P- 0.678 .

Conclusion: $46 \%$ had infection, pneumonia being most common. Significant correlation found with MELD. No correlation found with renal failure, CTP, INR and procalcitonin. Negative correlation was with Albumin.

\section{LATEB-127}

\section{Association between serum 25-hydroxyvitamin D deficiency} and hepatic encephalopathy in chronic liver disease

Thinakar Mani Balusamy ${ }^{1}$, Thinakar Mani', Malar Vizhi', Ratnakar Kini $^{1}$, Bharat Narasimhan ${ }^{2}$, Venkateswaran A $\mathbf{R}^{1}$, Rajkumar Solomon ${ }^{1}$, Kani Sheikh ${ }^{1}$, Premkumar $K^{1}$, Mohammed Ali $^{1}$, Pugazhendhi Thangavelu ${ }^{1}$, Sibi Thooran ${ }^{1}$, Radha Krishnan ${ }^{1}$, Mohammed Noufal ${ }^{1}$, Sri Ram ${ }^{1}$, Tejaswini Dande ${ }^{1}$, Darshan $\mathbf{C}^{1}$

${ }^{1}$ Dr. MGR Medical University, Chennai, India; ${ }^{2}$ Manipal University, KMC Mangalore, Karnataka, India
Background: Aims: To study the association between 25-hydroxyvitamin D (25-OHD) deficiency and hepatic encephalopathy (HE) in patients with chronic liver disease (CLD) at tertiary referral center Method: A retrospective analysis of the results of 250 patients with chronic liver disease who were admitted between 2015 and 2017 was undertaken. Severity of CLD, Hepatic encephalopathy, nutritional status and 25-OHD were analysed in all the patients. Patients who admitted with acute, fulminant or subacute liver disease, with a primary diagnosis of Hepatocellular carcinoma, who did not have a 25-OHD measurement and patient not willing for the study were excluded from the study.

Result: Two fifty patients were included in this analysis. The mean age of all patients with chronic liver diseases was $40 \pm 10$ years. Moderate to severe 25-OHD deficiency was identified in 160 patients of whom 65 had grade 2-3 HE. Mild 25-OHD deficiency was not associated with statistically significant HE. There was a significant correlation between the severity of 25-OHD deficiency and the severity of liver disease and disease severity and the presence of HE.Importantly, individuals with 25-OHD deficiency were more likely to have a diagnosis of overt $\mathrm{HE}$ (OHE) at a significantly Higher Child-Turcotte-Pugh(CTP) and lower model for end stage liver disease (MELD) score than individuals without OHE .

Conclusion: 25-OHD deficiency was observed in the majority of patients with Chronic liver diseases and for the first time was found to be significantly worse in patients with Overt Hepatic encephalopathy.

\section{LATEB-129}

Effect of culture technique on the work up in cirrhotic patients with spontaneous bacterial peritonitis

Usama Ahmed Arafa Morsy ${ }^{1}$, Mahmoud Kamal El Samman', Ali Mahmoud Ahmed Kassem $^{1}$, Leila Mohamed Yousef ${ }^{1}$

\section{${ }^{1}$ Sohag Faculty of Medicine, Egypt}

Background: Spontaneous bacterial peritonitis (SBP) is a serious complication in liver cirrhosis and is associated with significant mortality. Multidrug resistance is an evolving problem in management of SPB. Therefore, early diagnosis and proper selection of antimicrobial therapy are warranted.

Aim of this work was to assess the accuracy of conventional ascetic fluid culture compared to blood culture in diagnosis of SPB and selection of antimicrobial therapy.

Method: One hundred consecutive cirrhotic patients, 54 males and 64 females, with a mean age of $62.02 \pm 10.32$ years, who had moderate to severe ascites and were admitted to the internal medicine department, Sohag university hospital during the period from October 2016 to April 2017 were included. Diagnostic aspiration of ascetic fluid was made for each patient. The aspirated samples underwent chemical and cytological analysis as well as inoculation on conventional cultures and on blood cultures. Positive growths were tested for antibiotic sensitivity.

Result: Forty-seven patients (47\%) among the 100 cirrhotic patients had SBP as indicated by ascetic fluid neutrophilic count $>250$ cell/ $\mathrm{cmm}$ and clinically. Positive growths were detected in 11 patients of them $(23.4 \%)$ by using conventional cultures and in $32(68.1 \%)$ patients by using blood cultures ( 21 by blood culture alone in addition to the 11 sets diagnosed by conventional cultures). By using blood culture as the gold standard, the sensitivity, specificity, positive predictive value and negative predictive value of conventional cultures were $34.38 \%, 100 \%, 100 \%$ and $41.67 \%$ respectively. All isolated bacterial growths (32 cases) were sensitive to meropenem antibiotic whereas resistance to cefotaxime was detected in 20 cases out of the 
32 positive growths $(62.5 \%)$. Other tested drugs showed variable degrees of sensitivity.

Conclusion: Blood culture of ascetic fluid had higher sensitivity and specificity for diagnosis of SBP than conventional culturing techniques. Multidrug resistance (including cefotaxime) was frequent in our patients with ascites and SBP.

\section{LATEB-134}

Prevalence of $\mathrm{HBV}$ infection and age group difference it in Mongolia

Yagaanbuyant Borjigon Dahgwahdorj ${ }^{1}$, Dashdorj Naranbaatar ${ }^{2}$, Baatarsuren Uurtsaikh $^{3}$, Dashdorj Naranjargal ${ }^{4}$, Genden Zulkhuu $^{4}$, Murdorj Altankhuu ${ }^{4}$, Dashtseren Behkbold ${ }^{1}$

${ }^{1}$ Mongolian National University of Medical Sciences, Mongolia;

2"Onom foundation", Mongolia; ${ }^{3}$ Ildengun Hoshuu Co., LTD, Mongolia; ${ }^{4}$ Livercenter, Mongolia

Background: It is best known that viral hepatitis infection is endemic in Mongolia. In 2016 launched big national program on liver diseases. Within this scope, started a national campaign for screening of chronic viral hepatitis infection (in 201740 - 65 age groups and in 2018 remained). Until 1 October 2017, there was screened 287000 subjects (whole population of this age groups is 741000).

Aim was to establish the prevalence of HBV infection in 40 -60 year old ages groups in Mongolia.

Methods and subjects: General practitioners at the primary health care centers screened 287000 people with 40-65 years old, who have not been tested for viral hepatitis. From all participants venous blood vessel, $6 \mathrm{ml}$ blood was taken and were screened by using HBsAg rapid tests. There was estimated total number of subjects by direct standardization method on the basis of results.

Result: Totally, 26193 participants were positive for HBsAg. But, the prevalence rate continually decreased by age. (table).

\begin{tabular}{|c|c|c|c|c|c|}
\hline $\begin{array}{l}\text { Age } \\
\text { groups } \\
\text { (years) }\end{array}$ & $\begin{array}{l}\text { Screened } \\
\text { population } \\
\text { in } 2017 \\
\text { (n) }\end{array}$ & $\begin{array}{l}\text { HBsAg } \\
(+) \\
/ 2017 \\
(n)\end{array}$ & $\begin{array}{l}\text { HBsAg } \\
\%(+) \\
/ 2017\end{array}$ & $\begin{array}{l}\text { Whole } \\
\text { population } \\
\text { (n) } 2016\end{array}$ & $\begin{array}{l}\text { Estimated } \\
\text { with } \\
\text { HBV } \\
\text { infection } \\
\text { (n) }\end{array}$ \\
\hline $40-44$ & 60033 & 6035 & 10.05 & $210,897$. & 21195 \\
\hline $45-49$ & 59412 & 5903 & 9.94 & $179,429$. & 17835 \\
\hline $50-54$ & 60886 & 5750 & 9.44 & $157,548$. & 14873 \\
\hline $55-59$ & 59948 & 5157 & 8.60 & 119,171 & 10249 \\
\hline $60-64$ & 46970 & 3348 & 7.13 & 73,995 . & 5276 \\
\hline Total & 287249 & 26193 & 9.12 & 741040. & 69428 \\
\hline
\end{tabular}

Then, we are estimated total subjects with HBV infection are calculated around 70000 subjects, in this age groups.

Conclusion: The prevalence of HBV infection in studied groups is around $10 \%$. Deference of age group bring two hypothesis: may by HBV carrier situation seriously effected to the age rate or seroconversion of HBsAg non stop continuing in carriers.
LATEB-135

First Report of deletion mutation in ALAS2 associated with decompensated liver cirrhosis in a Chinese Han woman

Xiaoqin Dong ${ }^{1}$, Jun $\mathrm{Li}^{1}$, Hui Liu ${ }^{2}$, Yongwei Wang ${ }^{3}$, He Wang ${ }^{1}$, Na ${ }_{\text {Huo }}{ }^{1}$, Yan Wang ${ }^{1}$, Hong Zhao ${ }^{1}$

${ }^{1}$ Peking University First Hospital, China; ${ }^{2}$ Beijing Youan Hospital, Capital Medical University, China; ${ }^{3}$ Chinese Academy of Sciences Key Laboratory for Biomedical Effects of Nanomaterials and Nanosafety, National Center for Nanoscience and Technology, China

Background: Porphyrias are a group of inherited metabolic disorders resulting from some specific mutations in one of eight genes which encode enzymes of the heme biosynthetic pathway. Seven genes mutations induce partial deficiency of enzymes of heme biosynthesis, and a gain of function mutation in the ALAS2 gene leading to excess production of protoporphyrin (PP) causes a new porphyria, X-linked protoporphyria (XLP). XLP is one form of protoporphyria, characterized by cutaneous photosensitivity and accumulation of freeprotoporphyrin and zinc- protoporphyrin in erythrocytes. Decompensated liver cirrhosis caused by XLP has been rarely reported worldwide.

Method: The non-invasive examinations and liver biopsy were done for determining the cause of liver cirrhosis. The plasma fluorescence emission spectrum analysis was implemented to forerun site index of gene mutations. Then sequencing the exon coding region and splice sites of the related genes.

Result: Liver biopsy specimen showed a slew of dense,dark brown, 'Maltese cross'deposits with various sizes accumulated in portal tracts, hepatocytes, and sinusoids, which indicated protoporphyria. The plasma fluorescence emission spectra showed the peak value was at $634 \mathrm{~nm}$. The screenings tests of the exon coding region and splice sites identified c.1706-1709 del.AGTG mutation of ALAS2 gene, which had been proved a causal link between X-linked Protoporphyria (XLP).

Conclusion: To our knowledge, it's the first case report of XLP, with pathology confirmed sedimentation of protoporphyria, stage 4 biliary fibrosis of the liver and DNA sequencing identified gain-of-function mutation of $A L S A 2$ in a Chinese Han woman. In adult patients with decompensated liver cirrhosis accompanying hepatomegaly should detect concentration of protoporphyrin IX free and/or Zn protoporphyrin IX in erythrocytes and plasma fluorescence emission assay. Gene sequencing analysis will confirm the diagnosis.

\section{LATEB-136}

The distribution of acute viral hepatitis in adult population: a prospective multi-center study in Turkey

Selma Tosun ${ }^{1}$, Ayse Albayrak ${ }^{2}$, Gul Durmus ${ }^{3}$, Irem Cetin ${ }^{1}$, Ayse Serra Ozel $^{4}$, Necla Tulek ${ }^{5}$, Ayten Kadanali ${ }^{4}$, Serpil Erol ${ }^{6}$, Arzu Altuncekic Yildirim ${ }^{7}$, Nurgul Ceran ${ }^{6}$, Ozgur Dagli ${ }^{3}$

1 University of Health Sciences Izmir Bozyaka Education and Research Hospital Department of Infectious Disease and Clinical Microbiology - Izmir, 2 Ataturk University The Faculty of Medicine Department of Infectious Disease and Clinical MicrobiologyErzurum, 3 University of Health Sciences Bursa Education and Research Hospital Department of Infectious Disease and Clinical Microbiology- Bursa, 4 University of Health Sciences Umraniye Education and Research Hospital Department of Infectious Disease and Clinical Microbiology- Istanbul, 5 University of Health Sciences Ankara Education and Research Hospital Department of Infectious Disease and Clinical Microbiology - Ankara, 6 University of Health 
Sciences Haydarpasa Education and Research Hospital Department of Infectious Disease and Clinical Microbiology - Istanbul, 7 Ordu University The Faculty of Medicine Department of Infectious Disease and Clinical Microbiology- Ordu TURKEY

Purpose: In the recent years, apparent changes have been reported in the epidemiology registries of both hepatitis A virus (HAV) and hepatitis B virus (HBV) worldwide and also our country.The present study aims to evaluate the etiology of acute viral hepatitis, clinical course, and prognosis in an adult Turkish population.

Method: This multi-center study was carried out prospectively in 8 hospitals from 6 cities between 2016 and 2017. Data including sociodemographic characteristics of the patients, clinical course and treatment, and laboratory findings were prospectively analyzed.

Findings: Totally 75 patient included in the study, the majority were male 54/75(72\%). The median age was 27 (20-69) years, and most were in the age group of 21 to 35 years and secondly 36-45 years. The most common type were HBV (54 patients 73\%) and HAV (19 patients 26\%). One (1\%) etiologic agent was CMV, and another one was EBV. The most common ways of transmission for HBV were contact with contaminated blood and body fluids and presence of a chronic hepatitis B patient in family; for HAV, consumption of suspicious food or beverages. About half of patients, we couldn't find any source for contamination with infection. There is no seasonal difference. The most frequently reported symptoms were fatigue $(88 \%)$ and nausea (77\%); the most common clinical signs were scleral icterus and acholic stool. Complications were cholestazis, encephalopathy and fulminant hepatitis. Most of the patients with acute HBV developed immunity, four patient were still HBsAg positive. A patient coinfected with HIV infection died due to fulminan hepatitis. All of the acute HAV infected patients are recovered.

Conclusions: Universal HBV vaccination started by year 1998 and for HAV by year 2012 in our country. But adult HAV and HBV vaccination are not at the desired level. Therefore still considerable percentage of population remained unvaccinated, has susceptible to $\mathrm{HAV}$ and HBV. We conclude that larger vaccination programs covering these age groups should be carried out.

Table 1. Distribution of acute HAV and HBV infection cases according to the age groups

\begin{tabular}{|l|c|c|c|}
\hline Age Groups & $\begin{array}{c}\text { Acute HAV } \\
\text { infection }\end{array}$ & $\begin{array}{c}\text { Acute HBV } \\
\text { infection }\end{array}$ & TOTAL \\
\hline $16-20$ Years & 3 & 1 & 4 \\
\hline $21-25$ Years & 6 & 5 & 11 \\
\hline 26-30 Years & 3 & 12 & 15 \\
\hline $31-35$ Years & 6 & 10 & 16 \\
\hline $36-40$ Years & 1 & 5 & 6 \\
\hline $41-45$ Years & 0 & 10 & 10 \\
\hline 46-50 Years & 0 & 6 & 6 \\
\hline 51-55 Years & 0 & 2 & 2 \\
\hline F6-67 Years & 0 & 1 & 1 \\
\hline $68+$ Years & 0 & 2 & 2 \\
\hline TOTAL & & 54 & 73 \\
\hline
\end{tabular}

LATEB-137

Awareness of Vaccinations and Vaccination Status of UnderProtected Diseases of Healthcare Workers

\section{Emine Güner ${ }^{1}$, Selma Tosun ${ }^{2}$}

${ }^{1}$ Hazro District Entegre State Hospital, Family Medicine, Diyarbakır,

${ }^{2}$ Health Sciences University, Bozyaka Training and Research

Hospital, Infectious Diseases and Clinical Microbiology, Izmir, Turkey

Entry: Vaccination is the most effective, safest and least costly approach to protecting human health and preventing infectious diseases. It is the right of every individual to be immunized against diseases which are effective and reliable vaccination. Today, health professionals face a lot of professional risks. For this reason, ensuring the immunity of health workers in vaccine-preventable diseases is an important issue to be addressed.

Materials and Methods: This study is a descriptive study. The research was carried out between January 2016 and June 2016 at University of Health Sciences Izmir Bozyaka Training and Research Hospital. The researcher's universe is composed of 491 health workers who are reached randomly. The data were collected using face-to-face survey method and hospital information system. A questionnaire consisting of 30 questions was prepared to investigate the knowledge, attitudes and behaviors of diseases and vaccines that can be protected by the health workers. 491 health workers were interviewed face to face. The results of the tests were obtained either by themselves or from the hospital automation system. The data were evaluated using the SPSS (Statistical Package for Social Sciences 18 version) program.

Findings: $75.8 \%$ (n: 372 ) of the health workers participating in the survey were female, $24.2 \%$ (n: 119) were male and the mean age was $30.5( \pm 11.06) .99(\% 20,2)$ physicians, $166(33,8 \%)$ nurses, 141 $(28,7 \%)$ trainees, $26(5,3 \%)$ cleaning personnel and 59 persons $(12 \%$ $0)$. The ratio of health workers who do not know the ways of transmission of HAV infection was $17.9 \%$, and $83.3 \%$ of the participants knew the transmission routes of HBV correctly. When HAV vaccination was asked, 117 people $(24 \%)$ were vaccinated. When assessed for the HBV vaccination story, $70.8 \%$ of the participants were vaccinated. It has been determined that tetanus inoculation is the most commonly performed vaccine after $\mathrm{HBV}$ vaccination and it has been observed that tetanus inoculation has been performed for essentially any reason, such as injury or pregnancy, occupation.

Result: Health workers have a high level of knowledge about immunization and immunization. The vaccine that the health care workers have the most and adapt to the hepatitis B vaccine but testing for hepatitis A and the vaccination is not too high. It has been determined that health workers do not have enough knowledge about some vaccines recommended by international guidelines.

\section{LATEB-138}

\section{Anti-HAV IgG seropositivity in medical high school students}

\author{
Selma Tosun ${ }^{1}$, Ali Ilgın Olut ${ }^{1}$, Selda Sayın ${ }^{2}$, Hulya Bayık ${ }^{3}$ \\ ${ }^{1}$ University of Health Sciences Bozyaka Education and Research \\ Hospital Department of Infectious Disease and Clinical \\ Microbiology, 2 University of Health Sciences Bozyaka Education \\ and Research Hospital, 3 University of Health Sciences Bozyaka \\ Education and Research Hospital Department of Pediatrics, Izmir \\ Turkey
}


Aim: Hepatitis A virus (HAV) infection is the most common cause of acute viral hepatitis worldwide but differences exist in endemicity between geographic areas, which is closely related with hygenic and sanitary conditions and other indicators of the level of the development. Turkey is accepted in middle endemicity area according to the WHO. Medical high school students are accepted as high risk group of HAV infection therefore -considering the changes of HAV epidemiology worldwide- it is important to know the serological status of this group and to apply HAV vaccination to susceptible population. In this study we aimed to evaluate HAV seropositivity as well as vaccination history of medical high school students.

Method: Students aged between 16-22 whom were at their internship in hospital clinics were included in to the study. After informed consents and ethical commity aprovals were taken, all students filled a questionnaire about sociodemographic features and vaccination history of HAV infection. Serum anti-HAV IgG were measured by an enzyme linked immunoassay. All seronegative students vaccinated for HAV as a part of the aim of our study .

Findings: A total of 535 student, 528 girls and 87 boys were included in to the study. Serological results revealed that only $21 \%$ of students were anti-HAV IgG seropositive thus $79 \%$ were still susceptible to infection. Among 112 anti-HAV IgG seropositive students, only five notifed that they were vaccinated with HAV vaccine. All data are shown in the table 1.

Conclusion: WHO recommends overall HAV vaccination to low and intermediate endemicity areas worldwide. As being at middle endemic area, Turkey started routine childhood HAV vaccination by the year of 2013. In our study, $79 \%$ of medical vocational high school students whom birth years were 1994-2000, were found to be seronegative for HAV. This finding suggests that still a high percentage of adolescant population remains susceptible to infection thus they must be screened and if seronegative sholud recieve HAV vaccination.

Table 1. HAV seropositivity according to the years of birth of medical vocational high school students

\begin{tabular}{|l|l|l|l|}
\hline $\begin{array}{l}\text { Year } \\
\text { birth }\end{array}$ & Positive & Negative & Total \\
\hline 1994 & $9(28 \%)$ & $23(72 \%)$ & 32 \\
\hline 1995 & $12(23 \%)$ & $41(77 \%)$ & 53 \\
\hline 1996 & $3(11 \%)$ & $24(89 \%)$ & 27 \\
\hline 1997 & $8(26 \%)$ & $23(74 \%)$ & 31 \\
\hline 1998 & $9(11 \%)$ & $\mathbf{7 3 ( 8 9 \% )}$ & 82 \\
\hline 1999 & $56(27 \%)$ & $\mathbf{1 4 9 ( 7 3 \% )}$ & 205 \\
\hline 2000 & $15(14 \%)$ & $\mathbf{9 0 ( 8 6 \% )}$ & 105 \\
\hline Total & $112(21 \%)$ & $\mathbf{4 2 3 ( 7 9 \% )}$ & 535 \\
\hline
\end{tabular}

\section{LATEB-139}

HBsAg positivity of pregnant women in Turkey (changing epidemiology)

Selma Tosun ${ }^{1}$, Ilknur Esen Yildiz ${ }^{2}$, Handan Alay ${ }^{3}$, Haydar Kutlu $^{4}$, Aysenur Evrenos ${ }^{5}$, Semra Sen ${ }^{6}$, Fulya Bayindir Bilman ${ }^{7}$, Recep Kesli ${ }^{8}$, Sule Eren Cakar ${ }^{9}$, Ayten KAdanali ${ }^{9}$, Sibel Baktir Altuntas $^{10}$, Bulent Altuntas ${ }^{11}$, Kenan Ugurlu' ${ }^{12}$, Ali Ilgin Olut ${ }^{1}$

University of Health Sciences Bozyaka Education and Research Hospital Department of Infectious Disease and Clinical Microbiology - Izmir, 2 RTE University Department of Infectious Diseases and Clinical Microbiolgy - Rize, 3 Nene Hatun Hospital Department of Infectious Diseases and Clinical Microbiolgy - Erzurum, 4 Mus State Hospital Department of Microbiology - Mus, 5 Merkez Efendi State Hospital Department of Gynecology and Obstetrics - Manisa, 6 Celal Bayar University The Faculty of Medicine DEpartment of Pediatrics - Manisa, 7 Menemen State Hospital Department of Infectious Diseases and Clinical Microbiolgy - Izmir, 8 Kocatepe University The Faculty of Medicine Department of Microbiology-
Afyonkarahisar, 9 University of Health Sciences Umraniye Education and Research Hospital Department of Infectious Disease and Clinical Microbiology- Istanbu, 10 Esenyurt Family Medicine Center Istanbul, 11 University of Health Sciences Sisli Hamdiye Etfal Education and Research Hospital Department of Family Practioner Istanbul, 12 Twentyfive December State Hospital Department of Infectious Disease and Clinical Microbiology- Gaziantep TURKEY

Aim: According to WHO global hepatitis report (2017), about 257 million people are living with HBV infection. Assuming that women of reproductive age constitute $25.3 \%$ of the world's population, an estimated 65 million women at childbearing age can potentially transmit HBV to their babies. The widespread use of hepatitis B vaccine in infants has considerably reduced the incidence of new chronic HBV infections under 5 years of age from $4.7 \%$ at the prevaccine era to $1.3 \%$ by 2015 . Turkey started universal HBV vaccination of all infants by 1998 . Here we report the results of HBsAg seropositivity of pregnant women from seven different geographichal areas of Turkey for the last eight years.

Method: This multi-center study was carried out in 12 centers from different geographic regions and cities of Turkey. After ethical comittee permission and approval of Turkish Ministry of Health the data were collected fromthe centers and entered to the private surveillance data system and analysed from 2010 to 2017.

Results: From January 2010 to August 2017, totally 35.797 pregnant women data, aged between 17-49 from seven different regions of Turkey were analysed.

The mean seropositivity for the last eight years is found to be $1,6 \%$. The HBsAg seroprevalance rates according to years are given at the Table 1 .

Table 1. HBsAg seropositivity of pregnant women (years 2010-2017)

\begin{tabular}{|c|c|c|c|}
\hline Year & HBsAg POSITIVE & HBsAg NEGATIVE & TOTAL \\
\hline 2010 & $24(1.3 \%)$ & $1821(98.7 \%)$ & 1845 \\
\hline 2011 & $39(1.7 \%)$ & $2197(98.3 \%)$ & 2236 \\
\hline 2012 & $68(1.8 \%)$ & $3709(98.2 \%)$ & 3777 \\
\hline 2013 & $95(1.7 \%)$ & $5359(98.3 \%)$ & 5454 \\
\hline 2014 & $108(1.6 \%)$ & $6776(98.4 \%)$ & 6884 \\
\hline 2015 & $93(1.6 \%)$ & $5616(98.4 \%)$ & 5709 \\
\hline 2016 & $99(1.5 \%)$ & $6364(98.5 \%)$ & 6463 \\
\hline 2017 & $40(1.2 \%)$ & $3389(98.8)$ & 3429 \\
\hline TOTAL & $566(1.6 \%)$ & $35.231(98.4 \%)$ & 35.797 \\
\hline
\end{tabular}

Conclusion: According to the WHO report, Turkey is middle endemic country for HBV. Our study show that HBsAg positivity in pregnant woman is very low. We suggested that HBV epidemiology is changing in our country recently years and Turkey will be better placed at low endemic area for hepatitis B virus infection. Integrating hepatitis $\mathrm{B}$ vaccination with screening -particularly among pregnant women- is essential for hepatitis B elimination. Also HBsAg carrier pregnant women's babies must be immunized with HBV vaccine+HBIG after delivery. 


\section{LATEB-140}

Evaluation of anti-HBs titers and HBsAg status of medical high school students that recieved primary immunization at birth and effects of a booster hepatitis $B$ vaccine administration

\section{Selma Tosun ${ }^{1}$, Selda Sayın ${ }^{2}$ Ali Ilgın Olut ${ }^{1}$, Hulya Bayık ${ }^{3}$}

University of Health Sciences Bozyaka Education and Research Hospital Department of Infectious Disease and Clinical Microbiology, 2 University of Health Sciences Bozyaka Education and Research Hospital Department of Pediatrics, 3 University of Health Sciences Bozyaka Education and Research Hospital, Izmir Turkey

Purpose: Hepatitis B Virus (HBV) immunization at birth has been recommended by World Health Organization since 1991 which successfully applied in many countries and started after the year of 1998 in Turkey. Also Turkish Ministry of Health made a catch-up vaccination programme at years between 2005-2008 to all primary school children born before year 2000, regardless of their previous immunization history. In this study we aimed to evaluate HBV protection status of medical high school students on their internship at hospitals whom all received primary immunization at birth. We also investigated the effect of a booster $\mathrm{HBV}$ vaccination at later ages on $\mathrm{HBsAg}$ status and anti-HBs titers after the primary HBV immunization in infancy by comparing two groups, who had received and born at year 2000 so had not received any booster doses.

Method: Students birth years between 1994-2000 that were in the second, third and the last years of their internship at hospital clinics at years 2014-2017 were included in the study. After informed consents and ethical comity approvals were taken, all students filled a questionnaire about sociodemographic features, knowledge and awareness about HBV infection. Serum anti-HBs and anti-HBc IgG levels was measured by EIA method. If only anti-HBc IgG positive also $\mathrm{HBsAg}$ was measured by the same method.

Findings: Of 490 students, 85 were males and 405 were females. When anti-HBs status were analyzed, in students born before 2000 and had received booster vaccination, $7 \%$ had anti-HBs levels below the protection level $(<10 \mathrm{IU} / \mathrm{mL})$ whereas $59 \%$ of students born at year 2000 and hadn't received booster vaccination had antibody levels below sero-protection. Two female students (birth years are 1997 and 1996) were found to be HBsAg positive and mothers of these two students were also HBsAg carriers. The results are given in table 1 and 2. To students with anti-HBS titers below $10 \mathrm{IU} / \mathrm{ml}$, a rapel dose of $\mathrm{HBV}$ vaccine was administered. All had a protective anti-HBs level when tested one month after the booster dose.

Conclusion: Universal HBV vaccination is the most effective and cheap application for the prevention of HBV infection. The antiHBs titres waning by years but generally protection is going on.

It's now obvious that anti-HBs titers gained by $\mathrm{HB}$ vaccination decline by age from many studies and clinical practices and one dose of booster vaccine given to previous responders with anti-HBs titers below protection level or absent elicits strong amnestic response in more than $90 \%$ of vaccines suggesting the presence of immune memory. Our findings show the waning of antibody titers by years so we prefer application of a booster administration in early adolescents with low or negative anti-HBs titers because of an increased risk of HBV infection is anticipated when adolescents enter into young adulthood through becoming sexually active as HBV infection still remains in middle endemic area in our country.
Table 1. Anti-HBs levels according to the years of birth in adolescents (detailed)

\begin{tabular}{|c|c|c|c|c|}
\hline $\begin{array}{l}\text { Year of } \\
\text { birth }\end{array}$ & $\begin{array}{l}<9 \\
\mathrm{IU} / \mathrm{mL}^{*}\end{array}$ & $\begin{array}{l}10-49 \\
\mathrm{IU} / \mathrm{mL}\end{array}$ & $\begin{array}{l}>100 \mathrm{IU} / \\
\mathrm{mL}\end{array}$ & Total \\
\hline 1994 & 3 & 0 & 30 & 33 \\
\hline 1995 & 3 & 2 & 46 & 51 \\
\hline 1996 & 0 & 0 & 23 & 23 \\
\hline 1997 & 1 & 1 & 21 & 23 \\
\hline 1998 & 1 & 0 & 67 & 68 \\
\hline 1999 & 21 & 22 & 146 & 189 \\
\hline 2000 & 61 & 23 & 19 & 103 \\
\hline Total & 90 (\%48) & $48(\% 10)$ & $352(\% 72)$ & 490 \\
\hline
\end{tabular}

Table 1. Anti-HBs levels according to the years of birth in adolescents

\begin{tabular}{|c|c|c|c|c|}
\hline $\begin{array}{l}\text { Year of } \\
\text { birth }\end{array}$ & $\begin{array}{c}<9 \\
\mathrm{IU} / \mathrm{mL}\end{array}$ & $\begin{array}{c}10-49 \\
\mathrm{IU} / \mathrm{mL}\end{array}$ & $\begin{array}{c}>100 \mathrm{IU} / \\
\mathrm{mL}\end{array}$ & Total \\
\hline $\begin{array}{c}\text { Before } \\
1999\end{array}$ & $29(\% 7) *$ & $25(\% 6)$ & $333(\% 87)$ & 387 \\
\hline 2000 & $61(\% 59)$ & $23(\% 22)$ & $19(\% 19)$ & 103 \\
\hline Total & $90(\% 48)$ & $48(\% 10)$ & $352(\% 72)$ & 490 \\
\hline
\end{tabular}

*Two girl students are HBsAg positive

LATEB-140

CA-01-020: Validation and comparison of AARC score in predicting mortality among patients with ACLF due to hepatitis $\mathbf{E}$ in Asia pacific Region

Amna Subhan Butt ${ }^{1}$, Saeed Hamid ${ }^{1}$, Ashok K Choudhury ${ }^{2}$, Wasim Jafri ${ }^{1}$, Y K Chawla ${ }^{3}$, Sunil Taneja ${ }^{3}$, Z Abbas ${ }^{4}$, Akash Shukla ${ }^{5}$, Mamun Al Mahtab ${ }^{6}$, Deepak N Amarapurkar ${ }^{7}$, Md Fazal Karim $^{8}$, C E Eapen', Asish Goel ${ }^{9}$, Hasmik Ghaziniyan ${ }^{10}$, P N Rao ${ }^{11}$, Manoj K Sahu ${ }^{12}$, Samir Shah ${ }^{13}$, Chetan R Kalal ${ }^{13}$, Harshad Devarbhavi ${ }^{14}$, Z Duan ${ }^{15}$, Chen Yu ${ }^{15}$, S S Tan ${ }^{16}$, Diana A Payawal $^{17}$, O Yokosuka ${ }^{18}$, Priyanka Jain ${ }^{2}$, Irene Paulson ${ }^{2}$, S K Sarin $^{2}$, AARC APASL Working Party.

1. Aga Khan University Hospital, Karachi, Pakistan, 2. Institute of Liver and Biliary Sciences, New Delhi, India, 3. PGIMER, Chandigarh, India, 4. Ziauddin University Hospital, Karachi, Pakistan, 5. KEM Hospital and Seth GSMC, Mumbai, India, 6. Bangabandhu Sheikh Mujib Medical university, Dhaka,Bangladesh, 7. Bombay Hospital \& Medical Research Centre, Mumbai, India, 8. Sir Salimullah Medical College, Mitford Hospital, Bangladesh, 9. Christian Medical College, Vellore, India, 10. Nork Clinical Hospital of Infectious Disease, Armenia, 11. Asian Institute of Gastroenterology, Hyderabad, India, 12. IMS \&SUM hospital, Odisa, India, 13. Global Hospital, Mumbai, India, 14. St John Medical College, Bangalore, India, 15. Beijing You'an Hospital, Translational Hepatology Institute, Capital Medical University, 16.

Departmentment of Hepatology, Hospital Selayang, Bata Caves, Selangor, Malaysia, 17. Cardinal Santos Medical Center, West San Juan City, Metro Manila, Philippines, 18. Chiba University, Japan

Background:Acute-on-chronic liver failure (ACLF) is a distinct entity, associated with high morbidity and mortality. Hepatitis E (HEV) is one of the leading causes of ACLF in Asia. Inclusion of smaller number of HEV related ACLF patients, differences in criteria to define ACLF and absence of validation studies questions the application of existing prognostic models for HEV related ACLF. The 
AARC-ACLF score has been developed using largest data base of 26 Asian countries and found superior to the existing prediction models. It can reliably predict the need for interventions, such as liver transplant, within the first week. However, AARC score has not been validated for HEV related ACLF. Hence, we aim to validate and compare the AARC score in predicting 30, 90 days mortality among patients with ACLF due to hepatitis E.

Methods:APASL-ACLF research consortium (AARC), consisting of 26 tertiary centers across Asia-Pacific regions, maintains an online database for patients diagnosed to have ACLF according to APASL criteria. All patients who had ACLF with acute hepatitis E were reviewed for the current study. The AARC score was validated and compared it with existing prognostic models AUROC.

Result:Out of 2897 patients with ACLF 230 (7.9\%) had acute deterioration due to HEV. Mean age was $48.29 \pm 13.50$ years and $83.9 \%$ were male. The most common cause of chronic liver disease was alcohol (26.5\%) followed by cryptogenic cirrhosis $(25.7 \%)$ and NASH $(25.7 \%)$. Overall $62.2 \%$ survived \& liver transplantation was done in $4.3 \%$ cases. Higher proportion of HE, AKI, organ failure, higher level of creatinine, bilirubin, INR, CTP, MELD, MELD-Na, SOFA, CILF-SOFA, AARC scores were observed among non-survivors as compared to survivors (Table 1). While validating AARC score to predict mortality in HEV related ACLF, The HosmerLemeshow test showed good degree of fit. When we compared AARC score with various prognostic models, AARC score, MELD were found equivalent (AUROC 0.72) but superior to CTP, SOFA, CLIF SOFA, and APACHEE II predicting 30 days mortality (Figure 1). Similar trend was observed while predicting 90 days mortality.

Conclusion:The AARC score has been found equivalent to MELD score but superior to MELD-NA, CTP, SOFA, CLIF-SOFA and APACHEE predicting 30 and 90 days mortality in patients with HEV related ACLF in Asia pacific region. However, considering AUC 0.72 still there is a room to develop a prognostic model with higher accuracy for HEV-ACLF patients. Using such prognostic scores will help to stratify high risk patients on admission and to assess need of liver transplant.

Table 1: Comparison of survivors and non-survivors with ACLF due to HEV

\begin{tabular}{|c|c|c|c|}
\hline & Survivors( $(\mathrm{n}=143)$ & Non-Survivors(n=87) & $p$ value \\
\hline Age (Years) & \begin{tabular}{|l|l|}
$48.5 \pm 13.2$ \\
\end{tabular} & $47.8 \pm 14.0$ & 0.69 \\
\hline $\begin{array}{l}\text { Gender: Male } \\
\text { Female }\end{array}$ & $\begin{array}{l}118(82.5) \\
25(17.5)\end{array}$ & $75(86.2)$ & 0.46 \\
\hline $\begin{array}{c}\text { Female } \\
\text { Ascites: Yes }\end{array}$ & \begin{tabular}{|l}
$25(17.5)$ \\
$115(81.6)$
\end{tabular} & $\begin{array}{l}12(13.8) \\
69(81.2)\end{array}$ & 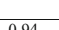 \\
\hline $\begin{array}{r}\text { Ascites: Yes } \\
\text { No }\end{array}$ & $26(18.4)$ & $\begin{array}{l}69(81.2) \\
16(18.8)\end{array}$ & 0.94 \\
\hline HEathy: Yes & $44(31.2)$ & $50(59.5)$ & $<0.001$ \\
\hline & $97(68.8)$ & $34(40.5)$ & \\
\hline Sepsis: Yes & $19(15.4)$ & $16(23.2)$ & 0.18 \\
\hline & $104(84.6)$ & $53(76.8)$ & \\
\hline Variceal bleeding: Yes & $18(66.7)$ & $13(72.2)$ & 0.69 \\
\hline $\begin{array}{ll} & \text { No } \\
\text { AKI: Yes } & \end{array}$ & \begin{tabular}{|l|}
$9(33.3)$ \\
$18(12.9)$ \\
\end{tabular} & \begin{tabular}{|l}
$5(27.8)$ \\
$23(27.1)$
\end{tabular} & 0.007 \\
\hline & $122(87.1)$ & $62(72.9)$ & \\
\hline Organ Failure: 0 & $12(8.4)$ & $5(5.7)$ & 0.01 \\
\hline & $116(81.1)$ & $60(69)$ & \\
\hline 3-5 & $15(10.5)$ & $22(25.3)$ & \\
\hline HR/minutes & $86.1 \pm 13.5$ & $88.3 \pm 16.3$ & 0.29 \\
\hline MAP & \begin{tabular}{|l|l|}
$84.5 \pm 10.0$ \\
\end{tabular} & $84.3 \pm 13.5$ & 0.93 \\
\hline $\mathrm{RR} /$ minutes & $20.7 \pm 2.9$ & $20.4 \pm 3.5$ & 0.46 \\
\hline $\mathrm{Hb}(\mathrm{gm} / \mathrm{dl})$ & $11.2 \pm 2.1$ & $10.9 \pm 2.3$ & 0.30 \\
\hline WBC & $11.8 \pm 6.5$ & $13.7 \pm 11.0$ & \begin{tabular}{|l|l|}
0.13 \\
\end{tabular} \\
\hline Platelets & $174.0 \pm 81.5$ & $162.5 \pm 120.4$ & \\
\hline $\mathrm{Na}(\mathrm{meq} / \mathrm{dl})$ & $131.3 \pm 6.1$ & $130.2 \pm 7.7$ & 0.29 \\
\hline $\mathrm{K}(\mathrm{meq} / \mathrm{dl})$ & $4.0 \pm 0.85$ & $3.9 \pm 0.92$ & 0.79 \\
\hline $\mathrm{Cr}(\mathrm{mg} / \mathrm{dl})\{$ median, IQR $)$ & $0.90[0.61-1.34]$ & $1.2[0.7-2.2]$ & 0.005 \\
\hline Total bilirubin (mg/dl) & $21.7 \pm 9.7$ & $26.4 \pm 9.6$ & $<0.001$ \\
\hline Albumin & $2.3 \pm 0.68$ & $2.36 \pm 0.57$ & 0.82 \\
\hline ALT (IU/ml) \{median, IQR) & $83[55-174]$ & $99[58.2-256.7]$ & 0.18 \\
\hline Alkaline phosphate (IU/ml) \{median, IQR) & $161.3 \pm 134.8$ & $135.8 \pm 113.2$ & 0.03 \\
\hline GGT (IU/ml) \{median, IQR) & $57[39.5-92]$ & $51[32.5-68.2]$ & 0.053 \\
\hline INR & $2.1 \pm 1.1$ & $2.6 \pm 1.5$ & $<0.001$ \\
\hline $\mathrm{PH}$ & $7.1 \pm 0.32$ & $7.15 \pm 0.36$ & 0.55 \\
\hline $\mathrm{PCO} 2$ & $32.3 \pm 7.9$ & $31.5 \pm 8.4$ & \begin{tabular}{|l|}
0.56 \\
\end{tabular} \\
\hline CTP & $11.3 \pm 1.4$ & $12.0 \pm 1.4$ & \begin{tabular}{|l|l|}
0.001 \\
\end{tabular} \\
\hline MELD & $26.5 \pm 6.6$ & $31.0 \pm 6.1$ & $<0.001$ \\
\hline MELD-Na & $29.4 \pm 5.7$ & $33.2 \pm 5.0$ & $<0.001$ \\
\hline SOFA & $8.1 \pm 2.5$ & $10.1 \pm 3.1$ & \begin{tabular}{|l|l|}
0.003 \\
\end{tabular} \\
\hline APACHEE II & $14.5 \pm 5.6$ & $18.4 \pm 7.1$ & \begin{tabular}{|l|l|}
0.01 \\
\end{tabular} \\
\hline CLIF-SOFA & $11.0 \pm 2.6$ & $12.2 \pm 2.5$ & \begin{tabular}{|l|}
0.02 \\
\end{tabular} \\
\hline GAHSBL & $9.3 \pm 1.3$ & $9.8 \pm 1.3$ & 0.10 \\
\hline AARC score & $9.0 \pm 1.8$ & $10.8 \pm 2.0$ & $<0.001$ \\
\hline
\end{tabular}

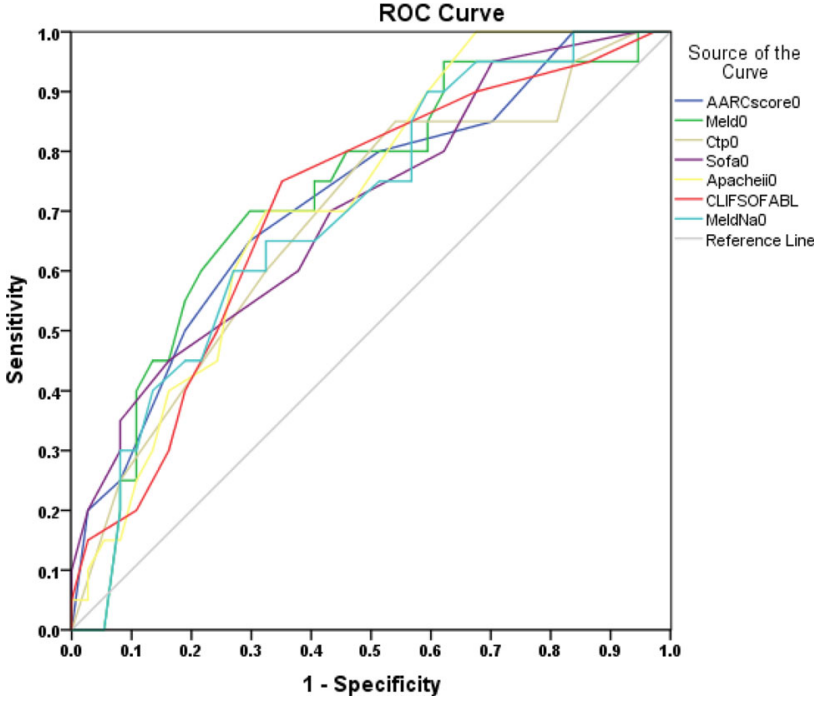

\begin{tabular}{|l|l|l|l|l|l|l|}
\hline & AUROC[95\% CI $]$ & cut & sensitivity & specificity & PPV+ & PPV- \\
\hline AARC score & $0.72[0.57-0.85]$ & 10.5 & 70.3 & 78.1 & 52.0 & 88.6 \\
\hline MELD & $0.72[0.58-0.86]$ & 31.9 & 61.5 & 83.1 & 53.3 & 87.3 \\
\hline CTP & $0.68[0.53-0.83]$ & 12.5 & 46.1 & 77.7 & 40.6 & 81.3 \\
\hline SOFA & $0.70[0.56-0.84]$ & 9.5 & 62.9 & 65.5 & 45.9 & 79.1 \\
\hline CLIF SOFA & $0.70[0.56-0.84]$ & 11.5 & 57.1 & 70 & 52.6 & 73.6 \\
\hline APACHEE II & $0.71[0.58-0.85]$ & 15.5 & 73.9 & 68.8 & 54.8 & 83.7 \\
\hline MELD Na & $0.69[0.56-0.83]$ & 34 & 55.7 & 81.8 & 50 & 85.06 \\
\hline
\end{tabular}

Figure 1: Comparison of various prognostic models for predicting 30 days mortality in patients with ACLF triggered by acute hepatitis E infection

\section{LATEB-142}

Ledipasvir $90 \mathrm{mg} / \mathrm{sof}$ osbuvir $400 \mathrm{mg}$ for 12 weeks for the treatment of CHC genotype 4 adolescents

\section{Shiha $G^{1,2}$, Soliman $R^{1,3}$, ElBasiony $M^{1,2}$, Mikhail $N_{N H}{ }^{1,4}$}

${ }^{1}$ Egyptian Liver Research Institute and Hospital (ELRIAH), Sherbin, El-Mansoura, Egypt, ${ }^{2}$ Hepatology and Gastroenterology unit, Internal Medicine Department, Faculty of Medicine, Mansoura University, Egypt, ${ }^{3}$ Tropical Medicine Department, Faculty of Medicine, Port Said University, Egypt, ${ }^{4}$ Biostatistics and Cancer Epidemiology Department, South Egypt Cancer Institute, Assiut University, Egypt

Introduction: A considerable number of children have chronic HCV infection and are at risk for complications. Approximately 11 out of 115 million of the infected persons in the world are younger than 15 years. Newly developed DAAs provide powerful tool in treatment of chronic HCV patients with good safety profiles.Food and Drug Administration (FDA) in April 2017 approved supplemental applications of sofosbuvir and ledipasvir in treatment of $\mathrm{HCV}$ in children ages 12 to 17 years.

Blood transfusion, some surgical interventions (circumcision) are the main risk factors of $\mathrm{HCV}$ infection in young in the developing country.

Our aim is to evaluate the safety and efficacy of sofosbuvir and ledipasvir in treatment of $\mathrm{HCV}$ infected children (12-17 years) either Naïve or experienced to INF therapy, with or without history of blood disease (leukemia's, lymphomas, thalassemia's) 
ClinicalTrials.gov Identifier: NCT03343444

Patient and Methods: Sixty-eight patients fulfilled inclusion criteria treated in the Egyptian Liver Research Institute and Hospital (ELRIAH), Mansoura, Egypt. 53 of them reached week 12 post treatment. Twelve were Treatment experienced. Fifteen patients had history of blood diseases: Acute lymphocytic leukemia $(n=4)$, Non Hodjikn lymphoma $(\mathrm{n}=4)$, B Thalassemia $(\mathrm{n}=2)$, Heamophilia A ( $=3)$, G6PD $(n=2)$. All patients received treatment in the form of Ledipasvir $90 \mathrm{mg} /$ sofosbuvir $400 \mathrm{mg}$ for 12 weeks.

Results: Sustained viral response after 12 weeks of end of treatment (SVR12) was achieved by $100 \%$. No treatment-related serious adverse events or death were reported

Conclusion: Ledipasvir/sofosbuvir for 12 weeks was highly effective and safe in treatment of CHC genotype 4 children

\section{LATEB-143}

Efficacy and safety of direct acting antivirals in treatment of chronic hepatitis $\mathrm{C}$ renal transplant recipients

Hanzada Mohammed Elmaghrabi(1), Ahmed Yahia Elmowafy (1), Ahmed Farouk Donia(1), Ayman Fathi Refaie(1), Mabrouk Ibrahim Ismail (5), Yasser Abdel-Monem Elhendy (2), Reham Soliman (2,4), Mohammed Elbasiony (2,3), Gamal Shiha $(2,3)$, Mohammed Bakr (1)

${ }^{1}$ Urology and Nephrology Center, Mansoura University, ${ }^{2}$ Egyptian Liver Research Institute and Hospital (ELRIAH), Sherbin, El-

Mansoura, Egypt, ${ }^{3}$ Hepatology and Gastroenterology unit, Internal Medicine Department, Faculty of Medicine, Mansoura University, Egypt, ${ }^{4}$ Tropical Medicine Department, Faculty of Medicine, Port Said University, Egypt, ${ }^{5:}$ Faculty of medicine, Zagazig University, Egypt.

Introduction: Consideration for drug-drug interactions is extremely important in the post-transplantation period in treatment of $\mathrm{HCV}$ infection, particularly with regard to immunosuppressant medications, such as cyclosporine or tacrolimus. The aim of this work is to achieve sustained viral response in hepatitis $\mathrm{C}$ kidney transplant recipients using new direct antiviral agents and preserve the graft function.

Patients and methods: 55 chronic hepatitis $\mathrm{C}$ kidney transplant recipients in Mansoura Urology and Nephrology Center that received kidney in the period between March 1976 and June 2016 were evaluated at the Egyptian Liver Research Institute and Hospital (ELRIAH). Patients with hepatic decompensation, co-infection with hepatitis B virus, human immunodeficiency virus (HIV) or autoimmune hepatitis were excluded. Patients were classified according to renal function into 2 groups: Group A $(n=51)$ with $\mathrm{Crcl}$ more than 30 $\mathrm{ml} / \mathrm{min}$ and they received Sofosbuvir+Daclatasevir. Group B $(\mathrm{n}=4)$ with $\mathrm{Crcl}$ less than $30 \mathrm{ml} / \mathrm{min}$ and they received Ritonavir/Ombitasevir/Paritaprevir. Both groups received treatment for 6 months duration. RBV was added to treatment experienced patients.

Results: Out of the 51 group A patients, 4 patients showed rise of serum creatinine and needed graft biopsy. The 4 group B patients showed significant changes and variability in immunosuppressive drug level and dose modification was done but all of them successfully completed 6 months course of DAAs with SVR 12 rate of 54/55 $(98.1 \%)$. Side effects reported were dizziness, bony aches, fatigue, GIT Upsets and anemia.

Conclusion: SVR could be achieved successfully in kidney transplant recipients using direct antiviral agents.
LATEB-144

Hepatitis B surface antigen quantitation is a useful predictor of treatment response in $\mathrm{HBeAg}$ negative patients treated by nucleos(t)ide analogues

Mohamed Elbasiony1.2, Ayman Eldesoky1.2, Seham Seif 1.2, Neven Farouk1, Reham Soliman 2, Gamal Shiha1.2.

1 Hepatology and Gastroenterology unit, Internal Medicine Department, Faculty of Medicine, Mansoura University, Egypt, 2 Egyptian Liver Hospital (ELH) Mansoura-Egypt.

Background and aim: Surface antigen loss of hepatitis B virus (HBsAg) is a primary aim of therapy in the management of chronic hepatitis B, HBsAg levels may be a surrogate marker of infected hepatocytes, and the only quantitative assay for intrahepatic viral load in treated patients with negative viremia, a rapid HBsAg decline during nucleos(t)ide therapy may suggest patients who will achieve HBsAg seroclearance. Currently, no consensus on the clinical utility of surface antigen monitoring for predicting patient responses to nucleos(t)ide therapy, we aimed to evaluate the possible value of HBsAg quantitation for prediction of treatment response to oral antiviral therapy.

Methods: Treatment with oral antiviral therapy for 130 chronic HBV patients started in outpatient clinic of Egyptian liver Hospital from March 2011 to March 2015. Eighty-five patients were on Lamivudine, 27 patients were on Entecavir, 11 patients were on Tenofovir and 7 patients were on Lamivudine/adifovir. Patients were checked every 6 months after starting treatment for 3 years by HBV DNA, HBsAg level,liver function testes, CBC, fibroscan and abdominal US.

Results: Twelve out of 130 patients partially responded to the therapy, 9 cases were break through, 103 cases were responders and 6 cases achieved HBsAg loss, the baseline HBsAg level has a high predictive value for HBsAg loss vs other patients (AUC $=0.870, \mathrm{P}<$ 0.01 ) at cutoff value $\leq 1927.50 \mathrm{IU} / \mathrm{mL}$ with a sensitivity of $83.3 \%$, a specificity of $73.4 \%$, a PPV of $83.15 \%$, and an NPV of $98.9 \%$. While cut off value of $\leq 128.50$ after 6 months of treatment has a sensitivity of $83.3 \%$, a specificity of $98.4 \%$, a PPV of $71.4 \%$, and an NPV of $99.2 \%$.

Conclusion: Significant $\mathrm{HBsAg}$ decline to $128.5 \mathrm{IU} / \mathrm{ml}$ after 6 monthes of treatment is a useful predictor for patients that achieved HBsAg loss.

\section{LATEB-145}

Ombitasvir, paritaprevir, and ritonavir for treatment of chronic hepatitis $\mathbf{C}$ hemodialysis dependent patients

Ahmed Yahia Elmowafy (1), Hanzada Mohamed Elmaghrabi (1),Mohamed Ahmed Zahab ${ }^{(1)}$, Khaled Farouk Eldahshan ${ }^{(1)}$, Reham Soliman ${ }^{(2.4)}$, Mohamed Elbasiony ${ }^{(2,3)}$ Mohamed Elsayed Mashaly ${ }^{(1)}$, Yasser Elsayed Matter ${ }^{(1)}$ Salwa Mahmoud Elwasif ${ }^{(1)}$,Ahmed Farouk Donia ${ }^{(1)}$, Gamal Mohamed Shiha ${ }^{(2,3)}$, Mohamed Adel Bakr ${ }^{(1)}$

${ }^{1}$ Urology and Nephrology Center, Mansoura University, ${ }^{2}$ Egyptian Liver Research Institute and Hospital (ELRIAH), Sherbin, ElMansoura, Egypt, ${ }^{3}$ Hepatology and Gastroenterology unit, Internal Medicine Department, Faculty of Medicine, Mansoura University, Egypt, ${ }^{4}$ Tropical Medicine Department, Faculty of Medicine, Port Said University, Egypt

Introduction: Among hemodialysis patients, HCV prevalence and transmission is high and frequent, and may be associated with poor prognosis when compared to patients who are not infected. Interferon and ribavirin were poorly tolerated when used to treat $\mathrm{HCV}$ positive 
hemodialysis patients. Direct acting antivirals is now the corner-stone for treating chronic $\mathrm{HCV}$ infection, achieving high SVR rates with fewer side effects and dropout rates than interferon based therapy. One of the available regimens which are metabolized by the liver with minimal renal clearance is Ombitasvir/Paritaorevir/Ritonavir regimen. Our aim is to study the efficacy and safety of this drug regimen among chronic hepatitis $\mathrm{C}$ hemodialysis dependent patients.

Patients and Methods: This is a prospective study which was held in Urology and Nephrology Center, Mansoura University, Egypt. 96 hemodialysis dependent patients were screened for HCV anti-bodies, 38 patients $(39.5 \%)$ were proved to be chronic hepatitis C. Four patients were excluded due to advanced liver cirrhosis, HCC and metastatic breast cancer. Thirty-four patients received Ombitasvir, paritaprevir, and ritonavir for 3 months to treat $\mathrm{HCV}$. All patients were evaluated and received treatment at Egyptian Liver Research Institute and Hospital (ELRIAH).

Results: Mean age was $43.26 \pm 11.91$ years and the majority of the patients are males (67.6\%). About $94 \%$ of the patients showed nonsignificant fibrosis. SVR $24(97 \%)$ was achieved successfully with significant improvement of liver enzymes.No serious treatment-related adverse events were noticed.

Conclusion: Ombitasvir, paritaprevir, and ritonavir regimen is safe and effective in treatment of chronic hepatitis $\mathrm{C}$ hemodialysis dependent patients.

\section{LATEB-146}

Impaired phospholipid methylation results in decreased lipid droplet lipolysis: Role in alcoholic steatosis

\section{Kusum K. Kharbanda ${ }^{\text {a,b }}$, \\ ${ }^{a}$ Departments of Internal Medicine, Biochemistry \& Molecular Biology, University of Nebraska Medical Center; and ${ }^{\mathrm{b}}$ Veterans Affairs Nebraska-Western Iowa Health Care System, Omaha, Nebraska, USA}

Introduction: The earliest manifestation of alcohol consumption is steatosis, which is characterized by the accumulation of lipids as cytoplasmic lipid droplets (LDs) in hepatocytes. Previous studies from our laboratory have shown that alcohol impairs the activity of phosphatidylethanolamine methyltransferase (PEMT) that generates phosphatidylcholine (PC) which is preferentially used in the assembly of very-low density lipoproteins (VLDL). Recent data suggests that VLDL assembly is also regulated by the availability of triglycerides (TGs) stored in LDs via lipolysis and re-esterification. Furthermore, the composition of the phospholipid monolayer is critical in maintaining the structural and functional properties of LDs. In particular, $\mathrm{PC}$, a major class of phospholipid in the LD monolayer, has been shown to is shown to be critically important for stabilizing LDs and preventing their coalescence. The purpose of this study was to determine how ethanol affects the dynamics of hepatic LDs that promotes their accumulation.

Methods: Male Wistar rats (180-200 g) were pair-fed the Lieber DeCarli control and ethanol diet for 4-5 weeks. At the end of the feeding regimen, VLDL production rates were measured and the livers removed and processed for assessing triacylglycerol hydrolase (TGH) activity and LD properties (size \& number). LDs were also purified for lipid composition analysis. Additionally, isolated hepatocytes from control and ethanol-fed rats were exposed overnight to oleic acid-BSA complex containing $\left[{ }^{3} \mathrm{H}\right]$-oleic acid as a tracer to label cellular TGs. To examine cellular lipolysis, the efflux of free $\left[{ }^{3} \mathrm{H}\right]-$ oleic acid was tracked over $4 \mathrm{~h}$ in the presence of an inhibitor that prevents re-esterification of fatty acids.
Results: Qualitative lipidomic analysis revealed significant increases in TGs, cholesterol and monounsaturation of fatty acyl chains concomitant with significant decreases in PC in LDs from the ethanol-fed rats compared to controls $(\mathrm{p}<0.05)$. These findings were confirmed by quantitative analysis of liver LD monolayer PC and PE by thin layer chromatography. These ethanol-induced changes in $\mathrm{PC}$ resulted in supersized LDs which were four times larger as compared to controls $(\mathrm{p}<0.05)$. Further, a 30-40\% decrease in cellular lipolysis ( $\mathrm{p}$ $<0.05)$ and $\mathrm{a} \sim 30 \%$ impairment in VLDL secretion $(\mathrm{p}<0.05)$ was observed in the ethanol-fed rats. However, the content of the main lipase implicated in the hydrolysis of TG stores of LDs for VLDL secretion was unchanged.

Conclusions: Taken together, our data indicates that defective PEMT-mediated methylation alters the phospholipid composition of hepatic LDs that may lead to reduced cellular lipolysis, impaired VLDL secretion, thus contributing to the development of alcoholic steatosis. Treatment with betaine that normalizes PEMT-mediated catalysis could be a safe and effective therapeutic for preventing alcoholic steatosis.

\section{LATEB-147}

\section{A proteomic approach to identify dysregulated platelet} hemostasis in severe alcoholic hepatitis

Background: Severe alcoholic hepatitis (SAH) encompasses a progressive inflammatory liver injury which often leads to early mortality. Platelets are effectors of hemostasis which regulate host inflammatory and immune responses. However, the role of platelet in SAH pathogenesis is obscure. Thus the aim of this study was to investigate the phenotype and effector functions of platelets in SAH. Methods: Phenotypic measurement of platelets was performed in 20 SAH patients as compared to 20 healthy controls (HC). Platelet activation and intracellular calcium were assessed using flow-cytometry. Platelet to platelet adhesion/aggregation was studied by $\mathrm{Gp} 2 \mathrm{~b} / 3 \mathrm{a}$ activation. The effector function of the platelets was studied in $20 \mathrm{SAH}$ patients as compared to 20 healthy controls using advance labeling (iTRAQ) and high-resolution mass spectrometry which was validated in a separate set of SAH patients using real-time PCR.

Results: Platelets of SAH patients documented higher expression of PAC-1 (activated Gp2b/3a receptor marker) as compared to healthy controls $(p<0.05)$. Intracellular calcium levels were also significantly high in SAH platelets $(\mathrm{p}<0.01)$. Platelet to platelet adhesion/ aggregation or $\mathrm{Gp} 2 \mathrm{~b} / 3 \mathrm{a}$ activation was significantly high in $\mathrm{SAH}$ platelets as compared to healthy $(\mathrm{p}<0.05)$. Functional assessment of platelets using iTRAQ labeling and high-resolution mass spectrometry resulted in quantitation of 1236 proteins. Of them, a total of 202 proteins were up-regulated and 321 proteins were down-regulated in SAH vs. HC $(\mathrm{FC}>1.5)$. Further analysis resulted in the identification of 41 platelet specific proteins which were significantly up-regulated and linked to platelet activation (SERPINA1), negative regulation of complement activation/immune system (C1QB) and lipid transportation (APOA5, APOE) in SAH patients. Of the down-regulated 321 proteins a total of 120 platelets specific proteins were linked to alpha granules, dense granules (CSF-1, VWF, TSP-1, ALDH4A1, ACADSB, SSBP1), vesicular transport (FLOT2, HP, TMED7, DYNC1H1, SNAP23, RAB21, VAMP8), positive regulator of blood coagulation (F2, F5), anti-inflammatory and platelets haemostasis (ANXA5, FN1, SELP, PLG, MIF, PSME1, P2RX1,CFH). These results clearly suggest that platelet of SAH patients are activated primarily due to dysregulated calcium signaling. We also observed an impaired intracellular granular content and vesicular transport system in the platelets of SAH patients. On validation, we observed similar gene expression associated with platelets activation (SERPINA1), 
alpha granules \& dense granules (CSF-1, VWF, TSP-1) vesicular transport (TMED7) and anti-inflammatory and platelets hemostasis (CFH, SELP, FN1) in SAH patients as compared to healthy control (p $<0.05)$.

Conclusion: Our study provides the first in-depth characterization of the platelet proteome in SAH patients and indicates severely dysregulated granulation process and vesicular transport system.

\section{LATEB-156}

\section{Anti-fibrotic effect of branched-chain amino acids on activated Hepatic stellate cells}

Hae Lim Lee ${ }^{1,2}$, Jung Hoon $\mathrm{Cha}^{2}$, Seawon Hwang ${ }^{1,2}$, Jang Jeong Won $^{1,2}$, Si Hyun Bae ${ }^{1,2}$, Jong Young Choi ${ }^{1,2}$, Seung Kew Yoon ${ }^{1,2}$

\section{${ }^{1}$ Department of Internal Medicine, College of Medicine, The Catholic University of Korea, Seoul, Republic of Korea, ${ }^{2}$ The Catholic University Liver Research Center, the Catholic University of Korea, Seoul, Republic of Korea}

Introduction: Previous studies suggested that branched-chain amino acids (BCAA) can suppress the incidence of hepatocellular carcinoma and ameliorate hepatic fibrosis progression, but the detailed mechanisms are not clear. This study aimed to evaluate anti-fibrotic effect of BCAA on human hepatic stellate cell line, LX-2 cells.
Methods: LX-2 cells were exposed to varying doses of BCAA for different durations $(0,5,30,50$ and $70 \mathrm{mM}$ for 24 or 48 hours) or to its each components (leucine, isoleucine, and valine) to identify the proliferation of LX-2 cells using proliferation assay. The anti-fibrotic effects of BCAA or of its each components at titrated doses were evaluated using reverse transcription-polymerase chain reaction and Western blotting on LX- 2 cells activated by TGF- $\beta(2.5 \mathrm{ng} / \mathrm{ml})$ or not. Results: LX-2 cell proliferation was inhibited by BCAA or by each one of its component in a dose dependent manner. BCAA treatment decreased the expression of PCNA on LX- 2 cells activated by TGF- $\beta$ or not. Analysis with each component of BCAA application revealed decreased expression of PCNA by valine and leucine, but not by isoleucine. Fibrosis markers including ColI $\alpha 1$ and $\alpha$-SMA were decreased to a greater extent by 48 hours of BCAA treatment than 24 hours. While valine and leucine decreased the expression of ColI $\alpha 1$ and $\alpha$-SMA, isoleucine did not. The expression of N-cadherin, a mesenchymal marker, was repressed by BCAA, valine or leucine, but not by isoleucine administration.

Conclusion: This study identified the anti-fibrotic effect of BCAA on hepatic stellate cell line, LX-2 cells in particular. The results would suggest possible application of oral BCAA supplement in deterring the progression of liver cirrhosis. 